\title{
34th Annual Meeting of the European Association for the Study of Diabetes
}

\author{
Barcelona, Spain, September 1998
}

\section{Abstracts}

\section{Index of Oral Presentations}

OP 1 Indices of Glucose-Intolerance ............... A 3

OP 2 Therapeutic Aspects of Diabetic Nephropathy ........... A 4

OP 3 Experimental and Clinical Islet Transplantation ......... A 6

OP 4 Diabetes and Pregnancy-Clinical Aspects .......... A 7

OP 5 T-Cells in Type 1 Diabetes............ A 9

OP 6 Defect Insulin Signalling Yields Insulin Resistance . . . . . . A 10

OP 7 Antigens and Antibodies in Type 1 Diabetes ......... A 12

OP 8 Ion Channel Activity in $\beta$-Cells .......... A 13

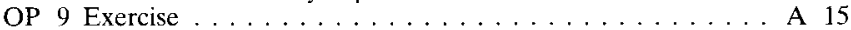

OP 10 Lipids and Late Complications . . . . . . . . . . . . A 16

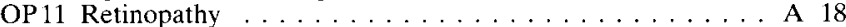

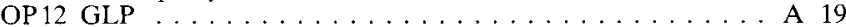

OP 13 Epidemiology of Type 1 Diabetes .............. A 21

OP 14 Islet Metabolism and Insulin Release ............. A 22

OP 15 Cellular Mechanisms of Vascular Dysfunction ......... A 24

OP 16 Lipids and Insulin Resistance ................ A 25

OP 17 Glycation.......... A 27

OP 18 Immunology and Diabetic Pregnancy . . . . . . . . . . . A A 28

OP 19 Cardiovascular Disease, Risk Factors, Prediction and

A 30

OP 20 Genetics of Type 2 Diabetes $\ldots . .$. A 32

OP21 Oxygen Radicals Cause Insulin Resistance . . . . . . . . A 34

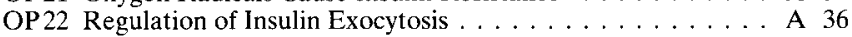

OP23 Pathophysiology of Diabetic Nephropathy .......... A 38

OP 24 Education, Outcome, Health Care Costs ... . . . . . . . . . A A 40

OP 25 Myocardial Infarction in Diabetes .............. . A 42

OP 26 Early Type 2 Diabetes

OP 27 Altered Intra Uterine Development and Insulin ........ A 44

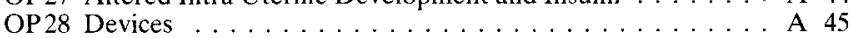

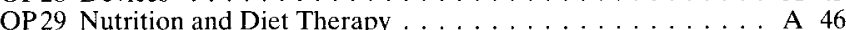

OP 30 Transcriptional Control in $\beta$-Cells ............. A 47

OP31 New Forms of Insulin Therapy ................ . . . . 48

OP32 Neuropathy ...................... A 50

OP33 Insulin Signal Transduction ................ A 52

OP34 Nitric Oxide Vascular Reactivity . . . . . . . . . . . . . A 54

OP 35 Susceptibility and Resistance to $\beta$-Cell Damage ......... . A 56

OP 36 Diabetic Embryopathy-Clinical and Experimental Advances A 58

OP 37 New Therapies for Type 2 Diabetes . . . . . . . . . . . . . A 60

OP 38 Animal Models of Type 1 Diabetes .............. A 62

OP 39 Leptin in Obesity and Pregnancy ................ A 64

OP 40 Development and Regeneration of $\beta$-Cells . . . . . . . A A 66

OP 41 Hypoglycaemia ....................... A 68

OP 42 Epidemiology of Type 2 Diabetes ............ A 70

OP43 Genetics of Type 1 Diabetes ................ A 72

OP 44 Risk Factors of the Diabetic Foot ............ . A 73

OP45 Quality of Life ............ A 74

OP46 PKC-Activation and Vascular Function ............ A 75

OP 47 Patterns of Insulin Secretion from $\beta$-Cells . . . . . . . . . A 76

OP 48 Alterations in Glucose Metabolism . . . . . . . . . . . . . A 77

\section{Index of Poster Presentations}

PS 1 Genetics of Type 1 Diabetes .............. A 78

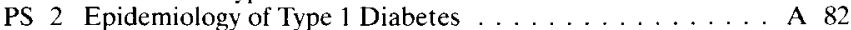

PS 3 Prediction and Prevention of Type 1 Diabetes . . . . . . . . A 86

PS 4 Environmental Factors ......... A 91

PS 5 Clinical Immunology ..................... A 92

PS 6 Experimental Immunology . . . . . . . . . . . . . . . . . A 100

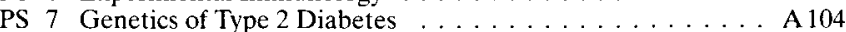

PS 8 Epidemiology of Type 2 Diabetes .............. A 115

PS 9 Prediction and Prevention of Type 2 Diabetes ........ A A 125

PS $10 \beta$-Cell Development, Replication and Insulin Gene

Expression
PS11 Signal Transduction in $\beta$-Cells ............... A 133

PS 12 Ion Channels and Exocytosis ................ A 138

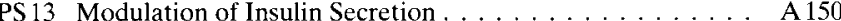

PS 14 Cytokines and $\beta$-Cell Degeneration ............. A 154

PS 15 Islet and Pancreas Transplantation ............. A 163

PS 16 Amylin ........ A 166

PS 17 LADA ............................... A 170

PS 18 Insulin Action: Signal Transduction and Insulin Resistance A 171

PS 19 Insulin Action: Cardiovascular Effects . . . . . . . . . . . . A 174

PS 20 Hormonal Action (Other) . . . . . . . . . . . . . A 176

PS 21 Hormone Receptors . . . . . . . . . . . . . . . . . . . A 179

PS 22 Glucose Transport ......................... A 181

PS 23 Gastro-Entero-Pancreatic Factors .............. A 182

PS 24 Insulin Resistance: Tissue and Cellular Level . . . . . . . . A 185

PS 25 Insulin Resistance: Intracellular . . . . . . . . . . . . A 188

PS 26 Insulin Resistance: Cardiovascular .......... A 191

PS 27 Insulin Resistance: Whole Body . . . . . . . . . . . . . . . A 194

PS 28 Insulin Sensitivity: Methods ... . . . . . . . . . . . . . . A 199

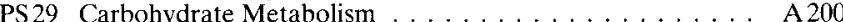

PS 30 Carbohydrate Metabolism: Hepatic Glucose Production . . A 202

PS 31 Protein Metabolism . . . . . . . . . . . . . . . . . . . A 204

PS 32 Lipids $1 \ldots \ldots \ldots \ldots \ldots$ A 205

PS33 Lipoproteins ......................... A 207

PS 34 Free Fatty Acids $\ldots \ldots \ldots \ldots \ldots \ldots$ A209

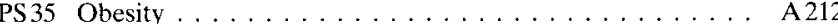

PS 36 Treatment of Obesity ..................... A214

PS 37 Animal Models of Obesity ................ A 215

PS 38 Obesity and TNFa $\ldots \ldots$ A217

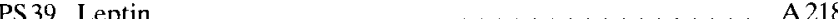

PS 40 Clinical Diabetes . . . . . . . . . . . . . . . A 222

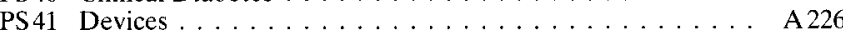

PS 42 Pharmacological Agents ................. A230

PS 43 Hypoglycaemia ....................... A 240

PS 44 Insulin Therapy I . . . . . . . . . . . . . . . . . . . . A 242

PS 45 Insulin Therapy II ................... A 247

PS 46 Diabetes Education - Health Care Delivery ......... A 250

PS 47 Psychology ..................... A255

PS 48 Clinical Pregnancy ..................... A 256

PS49 Experimental Pregnancy . . . . . . . . . . . . . . A 261

PS50 Exercise ......................... . . . . . 263

PS51 Nutrition and Diet Therapy ............... A 265

PS52 Gastrointestinal Autonomic Neuropathy ............ A 267

PS53 Neuropathy ...................... A 270

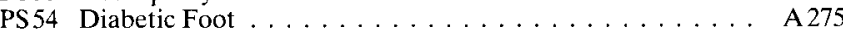

PS55 Retinopathy .............. A280

PS 56 Treatment of Diabetic Nephropathy ... . . . . . . . . . . A 284

PS57 Clinical Nephropathy ........... A285

PS 58 Genetics of Diabetic Nephropathy ............... A 293

PS59 Pathogenesis of Diabetic Nephropathy . . . . . . . . . . A A 297

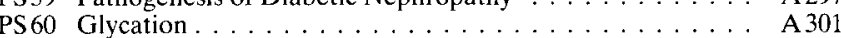

PS61 Autonomic Neuropathy .................. A 303

PS62 Endothelium and Vasomotion . . . . . . . . . . . . . . A 309

PS63 Endothelium in Vitro Studies $\ldots \ldots \ldots \ldots \ldots \ldots \ldots \ldots$ A 316

PS 64 Markers of Endothelial Damage ................. A318

PS65 Adhesion Molecules and Vascular Complications . . . . . . A A 320

PS 66 Oxydative Stress and Complications ............. . A 321

PS67 Bone Density ..... A325

PS68 Pathogenic Mechanisms of Complications . . . . . . . . . A 326

PS 69 Platelets, Coagulation, Rheology .............. . . A 328

PS 70 Atherosclerosis .......... A333

PS 71 Homocysteine and Cardiovascular Disease .......... A336

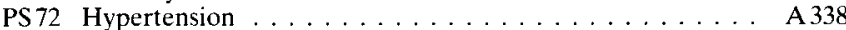

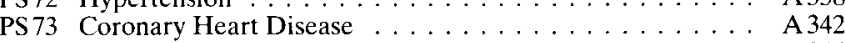

PS 74 Lipids II ... . . . . . . A 346

PS 75 Cardiovascular Risk Factors and Mortality . . . . . . . . . . A A 350 


\section{Indices of Glucose - Intolerance}

\section{1}

IMPACT OF THE APPLICATION OF NEW AMERICAN DIABETES ASSOCIATION DIAGNOSTIC CRITERIA. FEATURES OF THE IMPAIRED FASTING GLUCOSE CATEGORY.

I Conget, A Costa, E Aguilera, M Fernández, F Saval*, R Gomis. Endocrinology and Diabetes Unit. Hospital Clinic i Universitari, *Servei Medic de la Caixa, Barcelona, Spain

American Diabetes Association 1997 (ADA) diagnostic criteria recommends the use of fasting glucose $(126 \mathrm{mg} / \mathrm{dl})$ to diagnose diabetes (DM) and defines the impaired fasting glucose (IFG) category $(110-126 \mathrm{mg} / \mathrm{dl}$ ). Aim. (i) To compare the transcendence of the application of $1997 \mathrm{ADA}$ and the 1985 WHO criteria to diagnose DM. (ii) To analyse clinical characteristics of subjects from a mediterranean area with IFG. Subjects and methods. A sample of 616 subjects, aged 25-65 years, all employees of a bank, were studied. Their previous oral glucose tolerance was unknown. Body mass index (BMI), blood pressure (BP), lipid profile and the response to an oral glucose tolerance test (OGTT) were recorded. According to WHO criteria subjects were classified depending on the $2 \mathrm{~h}$-glucose (G-2h) in; normal glucose tolerance, impaired glucose tolerance (IGT) and DM. Based on the basal glucose (G-0') we divided twice the sample in two groups at the cut point of; G-0 $0^{\prime} \geq 140 \mathrm{mg} / \mathrm{dl}$ and $\mathrm{G}-0^{\prime} \geq 126 \mathrm{mg} / \mathrm{dl}$ (WHO- 85 and $A D A-97$ ). IFG subjects were compared with those subjects with G-0 $<110 \mathrm{mg} / \mathrm{dl}$. Results. $81 \%$ of the sample were men and $35 \%$ of the sample had a BMI $>27 \mathrm{~kg} / \mathrm{m}^{2}$. According to $\mathrm{G}-2 \mathrm{~h}$, we found a $8.2 \%$ of IGT and a $3.2 \%$ of DM. Only $25 \%$ of the subjects with DM, based on OGTT, had a $\mathrm{G}^{-} 0^{\prime} \geq 140 \mathrm{mg} / \mathrm{dl}$ and a $56 \%$ of DM subjects, displayed a $\mathrm{G}-0^{\prime} \geq 126$ $\mathrm{mg} / \mathrm{dl}$. IFG subjects had higher proportions of abnormal glucose tolerance $(1 \overline{4} .8 \%$ DM, 33.3\% IGT) than in the G-0 $<110 \mathrm{mg} / \mathrm{dI}$ group $(0.9 \% \mathrm{DM}, 7 \%$ IGT), $\mathrm{p}<0.01$. In addition, they were more obese (BMI, $\mathrm{kg} / \mathrm{m}^{2} ; 29.0 \pm 4.2$ vs. $25.7 \pm 3.4, \mathrm{p}<0.01$ ) and high blood pressure (BP $\geq 130 / 85 \mathrm{mmHg}$ ) was more frequently detected (SBP; $132.3 \pm 13.6$ vs. $122.9 \pm 13.5$ and DBP; $85.4 \pm 9.4$ vs. $76.6 \pm 9.6, \mathfrak{p}<0.01$ ) Conclusions. The application of ADA criteria in our mediterranean area diminishes the number of people with undiagnosed diabetes compared to WHO criteria. IFG includes subjects with a high rate of IGT, DM and other features of insulinresistance syndrome.

\section{2}

RETROSPECTIVE ANALYSIS OF ORAL GLUCOSE TOLERANCE TESTS APPLYING THE NEW A.D.A. DIAGNOSIS CRITERIA

R. Falip, M. Linares, MP. Navarro, R. Alfayate, M. Mauri and AM. Picó.

Laboratory of Hormones and Department of Endocrinology. Hospital General Universitario de Alicante. Alicante. Spain.

The current diagnostic criteria for Diabetes Mellitus were introduced by the United States National Diabetes Data Group in 1979 and adopted by the World Health Organisation in 1980, with revisions in 1985 and 1994.The Expert Committee on the Diagnosis and Classification of Diabetes Mellitus of the American Diabetes Association, instituted in 1995, reviewed these criteria in July 1997 and recommended a modification in the cutpoint of fasting plasma glucose (FPG) for the diagnosis of diabetes, they proposed to reduce it from 7.8 to $7.0 \mathrm{mmol} /$. They suggest that this change would allow a reduction in the number of oral glucose tolerance tests (OGTT) to perform, reduce the complications derived from it and reduce the economic cost, preserving the diagnostic efficacy.

AIM: To analyse retrospectively the results of OGTTs done in our hospital in order to diagnose diabetes applying the new diagnostic criteria.

SUBJECTS AND METHODS: 531 OGTTs were included. We determined fasting glycemia (after no caloric intake for at least 8 hours) and glycemia 2 hours after an oral glucose load (2hPG) containing $75 \mathrm{gr}$ anhydrous glucose (Glucomédics). Plasma glucose was measured with a hexoquinase method, using an Hitachi analyser (BoehringuerMannheim).

RESULTS: 10 of the subjects included had an FPG level between 7.0 and $7.8 \mathrm{mmol} /$. When we performed OGTT, 7 of them (70\%) were confirmed as diabetic (glycemia $2 \mathrm{~h}$ $\mathrm{PG} \geq 11.1 \mathrm{mmol} / \mathrm{l})$ and the rest $(30 \%)$ were cases of impaired glucose tolerance (IGT) (glycemia $2 \mathrm{hPG}>7.8$ and $<11.1 \mathrm{mmol} /$ ). 521 subjects had $\mathrm{FPG}<7.0 \mathrm{mmol} /$, after OGTT we found 45 diabetics ( $9 \%$ ), 129 IGT patients (25\%) and 347 impaired fasting glucose (IFG) (glycemia $2 \mathrm{hPG}<7.8 \mathrm{mmol} /$ ).

CONCLUSION: All the subjects who presented PPG $_{2} 7.0 \mathrm{mmol} / \mathrm{h}$ had an abnormal OGTT, this patients will benefit from early treatment independently of their diagnosis (DM or IGT). We agree that in this group OGTT could have been avoided, nevertheless the proportional number of tests avoided with this criteria is low. The new cutpoint may be useful in our population to evaluate disturbances in carbohydrate metabolism. This change may offer: rapidity in diagnosis allowing early prevention and treatment, avoid some OGTT and its inherent side effects and improve cost-effectivity of the test.

\section{3}

IGT OR IFG FOR PREDICTING NIDDM. WHO IS RIGHT, W.H.O. OR A.D.A?

JE Shaw, MP de Courten, AM Hodge, D McCcarty, H Gareeboo, P Chitson, KGMM Alberti, PZ Zimmet on behalf of the Mauritius NCD Study Group, Melbourne, Australia.

With the American Diabetes Association's introduction of new fasting plasma glucose (FPG) cut-offs, and impaired fasting glucose (IFG, FPG 6.1-7.0 mmol/l) as a new category of intermediate glucose metabolism, the oral glucose tolerance test could become redundant. We explored the consequences of this for the prediction of NIDDM. In 1987 , fasting and $2 \mathrm{~h}$ plasma glucose ( $2 \mathrm{PG}$ ) were measured on a random sample of the population of Mauritius. This was repeated 5 years later in 3238 of these subjects who were not diabetic in 1987. At baseline, 609 subjects were classified as having impaired glucose tolerance (IGT) (2PG 7.8-11.1mmol/1). Using FPG alone, 328 subjects were classified as IFG. During the 5 year follow up period, 297/3238 subjects progressed to diabetes (FPG $\geq 7.0 \mathrm{mmol} / 1$ or

$2 \mathrm{PG} \geq 11.1 \mathrm{mmol} / 1)$. For IGT, the sensitivity, specificity and positive predictive value (PPV) for future NIDDM were $50 \%, 84 \%$ and $24 \%$ respectively, and for IFG were $26 \%, 94 \%, 29 \%$. The sensitivity in males was IFG-24\%, IGT-37\%, in females IFG- $26 \%$, IGT-66\%. Specificity and PPV did not differ between the sexes. $26 \%$ of progressors were identified from abnormal FPG values alone (IFG), and a further $35 \%$ could be found by considering IGT as well. The baseline $2 \mathrm{~h}$ glucose in IFG subjects was $\geq 11.1 \mathrm{mmol} / 1$ in $19 \%, 7.8$ $11.1 \mathrm{mmol} / \mathrm{h}$ in $36 \%$ and $<7.8 \mathrm{mmol} / 1$ in $45 \%$. The FPG in IGT subjects was $<6.1 \mathrm{mmol} / 1$ in $81 \%$. These data show that within this population, the $2 \mathrm{~h}$ plasma glucose is a much more sensitive predictor of diabetes, especially in women. The omission of the $2 \mathrm{~h}$ measurement could have serious consequences both for the detection and prediction of NIDDM.

\section{4}

CLINICAL SIGNIFICANCE OF THE NEW DIAGNOSTIC CATEGORY OF IMPAIRED FASTING GLUCOSE: A PROSPECTIVE ANALYSIS O. Vaccaro, G. Ruffa, A.A. Rivellese and G. Riccardi. Department of Clinical \& Experimental Medicine. Federico II University, Naples, Italy The ADA has proposed new diagnostic criteria for diabetes based on fasting plasma glucose (FPG). Diabetes has been redefined as FPG $\geq 126 \mathrm{mg} / \mathrm{dl}$, and FPG 106-125 mg/dl has been identified as a new high risk category alternative to the previous IGT class. The study compares on a population basis the conditions of IFG and IGT-respectively identified by ADA and WHO-in terms of prevalence and prognosis. 1233 telephone company employees aged $40-59$ years were studied by OGTT, $52 \%$ were re-examined 11.5 years later. With the new criteria prevalence of diabetes-known + unknown-increased from $6.6 \%$ to $9.2 \%$ (i.e. by $40 \%$ ). The prevalence of IFG was almost twice as that of IGT $(9.1 \%$ vs $5.3 \%, p<0.001)$. The cardiovascular risk factor profile, as judged by the prevalence of at least one condition from among obesity, hypertrigliceridemia $(>200 \mathrm{mg} / \mathrm{dl})$ and hypertension ( $\mathrm{BP} \geq 160 / 95 \mathrm{mmHg}$ or treatment), was similar in both IFG and IGT ( $55 \%$ vs $60 \%$ respectively). The cumulative incidence of diabetes in 11.5 years was slightly, but not significantly higher in the IGT group as compared to the group with IFG $(36.5 \%$ vs $27.3 \%$, ns). However with ADA criteria substantial reclassification of people with IGT has occurred: $11 \%$ meet criteria for diabetes, $42 \%$ for IFG and $47 \%$ for normoglycemia. This latter group, although classified as normoglycemic by ADA, has a cumulative incidence of diabetes of $29 \%$-- i.e. similar to those with IFC $(27.3 \%)$ and significantly higher than the group with normoglycemia $(7.7 \%$, $\mathrm{p}<0.001$ ). In conclusion with the new criteria, the prevalence of diabetes and abnormalities of glucose metabolism is substantially higher than with the use of the existing WHO criteria; furthermore a substantial proportion of people at high risk of diabetes-and therefore susceptible of primary prevention-is not identified. 
SHOULD FASTING PLASMA GLUCOSE LEVELS BE LOWERED FURTHER IN THE DIAGNOSIS OF DIABETES?

V.Mohan, R. Deepa, M.Rema, L.D. Rajasekaran M.V.Diabetes Specialities Centre and Madras Diabetes Research Foundation, 35, Conran Smith Road, Chennai 600086 , India

The recent ADA Expert Committee Report on Classification and Diagnosis of Diabetes suggests a lowering of fasting plasma glucose (FPG) levels from $7.8 \mathrm{mmol} / \mathrm{L}(140 \mathrm{mg} / \mathrm{dl})$ to $7.0 \mathrm{mmol} / \mathrm{L}(126 \mathrm{mg} / \mathrm{dl})$. The new cut off value was proposed so that it would be comparable to the 2 hour post glucose (2Hr PG) level of $11.1 \mathrm{mmol} / \mathrm{L}(200 \mathrm{mg} / \mathrm{dl})$. Few studies however have directly tried to correlate the FPG and $2 \mathrm{Hr}$ PG values during an oral glucose tolerance test (OGTT) We took up a retrospective study based on 5936 GTT's done at our centre and found that an FPG level of $6.4-6.7$ $\mathrm{mmol} / \mathrm{L}(116 \mathrm{mg} / \mathrm{dl}$ to $120 \mathrm{mg} / \mathrm{dl})$ corresponds to a $2 \mathrm{Hr} P G$ of $11.1 \mathrm{mmol} / \mathrm{L}(200 \mathrm{mg} / \mathrm{d} \mathrm{f})$. Using different logistic regression models correlation between the $F P G$ and $2 \mathrm{Hr} P G$ values were done. The regression equation obtained using log-log model which produced the best fit was $\log (\log \mathrm{FPG} \mathrm{mg} / \mathrm{dl})=$ $1.4522+0.00054815(2 \mathrm{Hr} \mathrm{PG} \mathrm{mg} / \mathrm{dl})$. Using this model $2 \mathrm{Hr}$ $P G$ value of $11.1 \mathrm{mmol} / \mathrm{L}(200 \mathrm{mg} / \mathrm{d} /))$ corresponds to an FPG value of $6.5 \mathrm{mmol} / \mathrm{L}(118 \mathrm{mg} / \mathrm{dl})$. Our data suggests that an FPG value of $6.5 \mathrm{mmol} / \mathrm{L}$ (118 $\mathrm{mg} / \mathrm{dl}$ ) corresponds better to a $2 \mathrm{Hr}$ value of $11.1 \mathrm{mmol} / \mathrm{L}(200 \mathrm{mg} / \mathrm{dl})$ than the $7.0 \mathrm{mmol} / \mathrm{L}(126 \mathrm{mg} / \mathrm{dl})$ proposed by the ADA expert committee report
FASTING BLOOD GLUCOSE IS NOT THE BASELINE IN TYPE 2 DIABETES: RELEVANCE FOR THERAPY

F.Tassone, F.Cavalot, M.C.Ponziani, E.Mularoni, P.Massucco, S.Burzacca, P.Perna, A.Giori, G. Anfossi, and M.Trovati. Diabetes Unit, University of Turin, San Luigi Gonzaga Hospital, Orbassano (Turin), ltaly.

Near-normoglycaemia should be reached in type 2 diabetes, blood glucose (BG) control being correlated with vascular complications. UKPDS identified fasting BG (FBG) as the only parameter to decide and check drug therapy, in the assumption that it is the baseline on which post-prandial peaks are superimposed. To verify whether FBG is indeed the baseline, we examined 866 type 2 diabetic patients on diet alone $(\mathrm{M} / \mathrm{F} 491 / 375 ; 60.6 \pm 0.37 \mathrm{yrs} ; 4.9 \pm 0.21$ yrs from diagnosis; BMI $28.5 \pm 0.2$ ), by evaluating: 1)a BG profile carried out with a reflectance meter after overnight fast (h 08.00), 2 hrs after breakfast (h 10.30), 2 (h 14.00) and 4 (h 16.00) hrs after lunch; 2)the corresponding HbAlc (HPLC) Results (mtsem): HbAlc: $6.67 \pm 0.04 \%$; BG h 08.00; $7.15 \pm 0.05$, h $10.30: 6.74 \pm 0.07$; h 14.00: $7.22 \pm 0.07 ;$ h 16.00 : $6.05 \pm 0.06 \mathrm{mmol} / \mathrm{AG}$ at 08.00 is higher than at $10.30(\mathrm{p}=0.0001)$ and at $16.00(p=0.0001)$. Profiles were subdivided according to FBG ranges $(<5.6,5.6-6.7,6.7-7.8,7.8-8.9,8.9-10.0,>10.0 \mathrm{mmol} / \mathrm{l})$ : FBG was higher than $B G$ at $h 16.00$ in profiles with $F B G>5.6 \mathrm{mmol} / \mathrm{l}$ $(p=0.0001)$, delta values being correlated with $F B G(r=0.484$, $\mathrm{p}=0.0001$ ) and progressively increasing. from $0.48 \pm 0.10$ to $2.93 \pm 0.35$ $\mathrm{mmol} / \mathrm{h}$ as FBG ranges increased (ANOVA, $\mathrm{p}=0.0001$ ). In profiles with FBG $>6.7 \mathrm{mmol} / \mathrm{l}, \mathrm{FBG}$ was higher than average daily $\mathrm{BG}$; in profiles with $\mathrm{FBG}>7.8 \mathrm{mmol} / \mathrm{l}, \mathrm{FBG}$ was the highest $\mathrm{BG}$ measured. Thus, since FBG is not the baseline in type 2 diabetes, probably owing to the "dawn phenomenon", before prescribing drugs we should consider afternoon BG, to obtain fasting normoglycaemia without inducing afternoon hypoglycaemia. Therefore, the FBG-based therapeutical approach needs to be revised.

\section{OP 2}

\section{Therapeutic Aspects of Diabetic Nephropathy}

\section{7}

THE STENO TYPE-2 STUDY : INTENSIVE MULTIFACTORIAL INTERVENTION DELAYS PROGRESSION IN DIABETIC MICRO- AND MACROANGIOPATHY IN MICROALBUMINURIC TYPE 2 DIABETIC PATIENTS

P. Gæde, P. Vedel, H.-H. Parving and O. Pedersen, Steno Diabetes Center, Copenhagen, Denmark.

Aim and methods: To asses the effect of intensified multifactorial intervention on diabetic complications over a 4 yr period we performed an open, parallel, randomized intervention trial with 160 type 2 diabetic patients with persistent microalbuminuria randomized to a standard group $(n=80)$ continuing conventional treatment or an intensively treated group undergoing behaviour modification (diet, exercise, smoking habits) and aggressive, stepwise pharmacological treatment focusing on glycaemia (metformin, sulphonylureas, insulin). hypertension (ACE-inhibitors, diuretics, calcium-antagonists, beta-blockers), dyslipidemia (statins, fibrates) and secondary cardiovascular disease prevention with aspirin. Results: A separation of 1,4\% in $\mathrm{HbA}_{1 \mathrm{t}}$ (mean(SE)) $(7,6(0,1)$ vs. $9,0(0,1) \%, p<0,001$ (ANCOVA)) was obtained. The decline in systolic blood pressure ( 9 (2) vs. $4(2) \mathrm{mm} \mathrm{Hg}, p=0,01)$, fasting s-cholesterol $(0,7(0,1)$ vs. $0,2\{0,1)$ mmolli, $p<0,00005)$, fasting s-triglycerides $(0,5(0,2)$ vs. $0,4(0,4)$ $\mathrm{mmol} / \mathrm{h}, 0<0,005)$ and albumin excretion rate (median (range) $(23(-1091-231)$ vs. $0(-1038 ; 162), p<0,005)$ were all significantly greater in the intensively treated group. The progression to overt diabetic nephropathy ( 7 patients vs. 18 $p=0,01$ (multiple logistic regression)), the progression in retinopathy (19 vs. $33, p=0,04)$ and progression in autonomic neuropathy ( 8 vs. $22, p=0,01$ ) was lower in the intensive group compared to the standard group. The number of patients with peripheral vascular disease events was significantly smaller in the intensive group ( 13 vs. $29, p=0,02$ ). The combined incidence of fatal and non-fatal macrovascular events was smaller in the intensively treated group ( 25 vs. $42, p=0$ 03). Conclusion: Intensive multifactorial intervention over a 4 yr period delays the progression in micro- and macrovascular diabetic complications in type 2 diabetic patients with persistent microalbuminuria
8

WHEN SHOULD ACE INHIBITORS BE USED IN IDDM PATIENTS? A COMBINED ANALYSIS OF CLINICAL TRIALS

The ACE Inhibitors in Diabetic Nephropathy Trialist Group. Eurodiab, London, UK Several trials have demonstrated a beneficial effect of ACE inhibitors on renal function in microalbuminuric patients, but the threshold at which to treat remains unclear, and the impact of factors, such as diabetes duration, glycaemic control and blood pressure, is unknown. These questions were addressed by a meta-analysis, which included randomised clinical trials of ACE inhibitors in mictoalbuminuric IDDM patients with at least one year of follow-up. Raw data were obtained to ensure consistency of outcomes. 8 trials (7 European, 1 US) were included, consisting of 240 patients on ACE inhibitor, 234 on placebo. A summary measure of change in albumin excretion rate (AER) was derived for each individual in each study, and this was combined using regression and meta analysis techniques to produce study and combined treatment effects of differences in AER. Overall, ACE inhibitors reduced progression from microalbuminuria to macroalbuminuria by $79 \%$ (odds ratio $0.31,95 \%$ CI $0.19,0.51$ ). Regression to normoalbuminuria occurred more often on ACE inhibitors, (OR 2.64, 95\% CI 1.74,3.99). The \% difference in AER between ACE inhibitor and placebo was calculated; this decreased by length of follow-up. Thus at 1 year, AER was $82 \%$ lower in treatment compared to placebo, at 4 years, this was $36 \%$. Subsequent analyses were performed for 2 years of follow-up, as this had maximum power. Individual trial results varied from a treatment benefit of $30 \%$ to $75 \%$. Overall, at 2 years, AER was $58 \%(95 \%$ CI $40 \%, 70 \%)$ lower on treatment compared to placebo. The treatment effect was $26 \%$ at a baseline AER of $20 \mathrm{\mu g} / \mathrm{min}, 57 \%$ at $50 \mu \mathrm{g} / \mathrm{min}, 71 \%$ at $100 \mathrm{\mu g} / \mathrm{min}, 77 \%$ at $150 \mu \mathrm{g} / \mathrm{min}$, and $81 \%$ at $200 \mu \mathrm{g} / \mathrm{min}(\mathrm{p}=0.01)$. It also varied non-significantly by diabetes duration; $46 \%$ in $<15$ years, $56 \%$ in $15-20$ years, and $67 \%$ in $>20$ years $(p=0.5)$. There were no clear differences in effect by baseline glycaemic control, blood pressure, age and sex. We conclude that the apparent treatment benefit of ACE inhibitors on IDDM nephropathy varies considerably with follow-up. Beneficial effects are observed down to the lowest levels of microalbuminuria; there is little evidence of a threshold effect. There is an indication that the impact of ACE inhibitors may be greatest in patients with longer duration of diabetes, but the treatment effect does not appear to be determined by other factors associated with nephropathy. 
Early ACE-i intervention in microalbuminuria: 24h BP, renal function, and exercise changes.

E. Ebbehøj, P.L. Poulsen, R. Nosadini*, P. Fioretto*, G. Crepaldi*, and C.E. Mogensen, Medical Department $M$, Aarhus, and *Padoa, Italy.

Background: Substantial pathophysiological changes have taken place already in the microalbuminuric stage: BP is elevated with an attenuated circadian rhythm, and vagal function, sympathovagal interaction and kidney ultrastructure are abnormal. Thus, early intervention in microalbuminuria has acquired increasing interest. Design: In 2 randomised placebo controlled double blind studies the effect of 2 years treatment with either lisinopril $(20 \mathrm{mg})$ or placebo was evaluated in normotensive, microalbuminuric IDDM patients. 60 patients with UAE between $20-70 \mu \mathrm{g} / \mathrm{min}$ were analyzed. In the subgroup of patients examined in Aarhus ( $\mathrm{n}=22)$ we performed $24 \mathrm{~h}$ ambulatory blood pressure measurements (AMBP), renal function tests (constant infusion technique) and determinations of exercise induced albuminuria (bicycle ergometer, $70 \%$ of estimated maximal $\mathrm{VO}_{2}$ ). Results: Baseline UAE was almost identical in the two groups (placebo: $36.3 \mu \mathrm{g} / \mathrm{min} \times / \div 1.4$, lisinopril: $35.5 \mu \mathrm{g} / \mathrm{min} \times / \div 1.5$ (geometric mean $\times / \div$ tolerance factor)), whereas development in UAE over the two years was significantly different ( $\mathrm{p}$ 0.02 ) in the two groups with final UAE in the placebo group of $58.8 \mu \mathrm{g} / \mathrm{min} \times / \div 3.2$ and $29.8 \mu \mathrm{g} / \mathrm{min} \times / \div 2.5$ in the lisinopril group. In the lisinopril group 22 patients $(69 \%)$ reversed to normoalbuminuria compared to 6 patients $(21 \%)$ in the placebo group $(\mathbf{p}<0.01)$. AMBP showed small increases in $24 \mathrm{~h}$ systolic and diastolic AMBP $(1.6 \pm 6.2$ and $0.7 \pm 4.9 \mathrm{mmHg}$ over 2 years) in the placebo group, as opposed to significant reductions in the lisinopril group $(-6.0 \pm 8.2$ and $-4.1 \pm 6.4 \mathrm{mmHg})$, ( $\mathrm{p}<0.02$ and $<0.05$ ) Clinic BP measurements did not show significant differences. There were no differences in GFR or RPF in the two groups, but development in UAE and development in filtration fraction (FF) was positively correlated in the intervention group ( $r=0.9$ $p<0.01$ ), i.e. the patients who showed the greatest fall in UAE also were the ones with the greatest fall in FF suggesting fall in filtration pressure. Exercise testing showed a numerical reduction in exercise induced albuminuria in the lisinopril group compared to the placebo group. Conclusions: ACE-i treatment in patients with low-grade microalbuminuria reduces $24 \mathrm{~h}$ AMBP without attenuating diurnal blood pressure variation, reduces $U A E$ significantly, with changes in UAE being strongly associated with changes in FF. Furthermore, ACE-i reverses micro- to normoalbuminuria in a significant fraction of patients compared to placebo.
BOSENTAN NORMALIZES BLOOD PRESSURE, BUT IS NOT RENOPROTECTIVE IN THE DIABETIC REN-2 RAT.

J.L. Wilkinson-Berka, D.J. Kelly, M.E. Cooper* and S.L. Skinner. Departments of Physiology \& Medicine*, The University of Melbourne, Parkville, Australia, 3052

The aim was to determine if the endothelin receptor antagonist, bosentan, prevents the development of severe diabetic nephropathy in the hypertensive transgenic Ren-2 rat (TGR). The TGR displays enhanced tissue renin, and develops diabetic renal failure with similar pathophysiological changes as humans. The initial rise in GFR and albuminuria advancing to severe glomerulosclerosis and hyperkalaemia, which is prevented by ACE inhibition. These findings suggest that tissue Ang II acts as a pathogenic growth factor. As some of the actions of Ang II are mediated by endothelin, the effect of the $\mathrm{ET}_{\mathrm{A}}$ and $\mathrm{ET}_{\mathrm{B}}$ antagonist, bosentan $(\mathrm{B})$ on the kidney lesion was examined. Six week old female TGR were given either $0.1 \mathrm{M}$ citrate buffer (non-diabetic) or streptozotocin $(55 \mathrm{mg} / \mathrm{kg}$, plasma glucose $>18 \mathrm{mmol} / /)$ and gavaged with B $(100 \mathrm{mg} / \mathrm{kg} /$ day $)$ for 12 weeks. Systolic blood pressure in TGR+B was reduced to normotension (nondiabetic, $139 \pm 4 \mathrm{mmHg}$; diabetic, $129 \pm 5$ ) compared with untreated diabetic and non-diabetic TGR $(224 \pm 10, p<0.05)$. Body weight of diabetic TGR+B $(249 \pm 14 \mathrm{~g})$ was less than non-diabetic TGR+B $(330 \pm 10 \mathrm{~g}, p<0.05)$ but similar to untreated diabetic TGR $(223 \pm 8 \mathrm{~g})$. Bosentan did not improve diabetic renal pathology. A $53 \%$ decline in TGR was attenuated but not corrected by $B$ (decline $35 \% \quad p<0.05$ ). Renal renin content of non-diabetic TGR+B $(1.23 \pm 0.36 G \mathrm{G} / \mathrm{kidney})$ and diabetic TGR $(1.42 \pm 0.26)$ was similar to untreated diabetic TGR $(2.1 \pm 0.78)$, but all were elevated compared to untreated non-diabetic TGR $(0.16 \pm 0.03, p<0.05)$. Despite the normotension produced by bosentan, diabetic nephropathy in TGR was not prevented, indicating that neither endothelin nor the hypertension of the TGR are the predominant factors in the development of renal failure in this model. A direct involvement of tissue Ang II through paracrine effects is consistent with these findings.

\section{1}

LOSARTAN MODIFIES GLOMERULAR HYPERFILTRATION AND INSULIN SENSITIVITY IN TYPE 1 DIABETES.

S. Nielsen, K.Y. Hove, J. Dollerup, J.S. Christiansen, O. Schmitz, and C.E. Mogensen. Medical Department M, Aarhus Kommunehospital, Aarhus and Merck Research Laboratories, Copenhagen, Denmark

The effect of the angiotensin II receptor antagonist, losartan on renal hemodynamics and insulin mediated glucose disposal was examined in normotensive, normoalbuminuric Type 1 diabetic patients using a doubleblind, placebo controlled, cross-over design. Diurnal blood pressure, GFR ( ${ }^{125}$ I-iothalamate), RPF ( $\left({ }^{131}\right.$ I-hippuran), UAE were measured and a hyperinsulinaemic, euglycaemic clamp with indirect calorimetry was performed in 9 patients (age $30 \pm 7$ years $\left(m e a n \pm \mathrm{SD}\right.$ ), $\mathrm{HbA}_{1 \mathrm{c}} 8.1 \pm 1.1 \%$ ) following 6 weeks losartan $50 \mathrm{mg} /$ day and 6 weeks placebo. Diurnal blood pressure was significantly reduced after losartan compared with placebo $(122 / 70 \pm 11 / 8$ vs $130 / 76 \pm 12 / 6 \mathrm{mmHg}, \mathrm{p}<0.05)$. A significant decline in GFR $(133 \pm 23 \mathrm{vs} 140 \pm 22 \mathrm{ml} / \mathrm{min},<0.05)$ and filtration fraction (GFR/RPF) $(24.6 \pm 3.5$ vs $26.2 \pm 3.6 \%, \mathrm{p}<0.05$ ) was observed during losartan vs placebo. RPF and UAE did not change. Isotopically determined glucose disposal rates were similar after losartan and placebo in the basal $(2.61 \pm 0.53$ vs $2.98 \pm 0.93 \mathrm{mg} / \mathrm{kg} / \mathrm{min})$ and insulin stimulated states $(6.84 \pm 2.52$ vs $6.97 \pm 3.11$ $\mathrm{mg} / \mathrm{kg} / \mathrm{min}$ ). However, glucose oxidation rate increased significantly after losartan vs placebo in the basal state $(1.72 \pm 0.34$ vs $1.33 \pm 0.18, \mathrm{mg} / \mathrm{kg} / \mathrm{min}$, $\mathrm{p}<0.01)$ and during insulin stimulation $(2.89 \pm 0.75 \mathrm{vs} 2.40 \pm 0.62 \mathrm{mg} / \mathrm{kg} / \mathrm{min}$, $\mathrm{p}<0.03$ ). Basal and insulin stimulated non-oxidative glucose disposal tended to decrease, however not significantly, after losartan. Endogenous glucose production and lipid oxidation were unchanged after treatment and similarly suppressed during hyperinsulinaemia. Glycaemic control, total cholesterol, HDL-cholesterol and triglycerides were stable during losartan and placebo. In conclusion, losartan reduces blood pressure, glomerular hyperfiltration and fittration fraction and improves basal and insulin stimulated glucose oxidation in normotensive, normoalbuminuric Type 1 diabetic patients.

\section{2}

EFFECT OF INTENSIFIED ANTIHYPERTENSIVE TREATMENT ON MORTALITY IN DIABETIC NEPHROPATHY

P. T. Sawicki. U. Didjurgett, I. Mühlhauser, C. Schmidtke, A. Trocha,

R. Bender and M. Berger. Heinrich-Heine University. Düsseldorf, Germany We studied the long-term effect of intensification of antihypertensive treatment on mortality and the need of dialysis in overt diabetic nephropathy. A secpucntial simple of 91 hypertensive Type 1 diabetic patients with overt nephropathy was followed prospectively for 10 years 45 patients were allocated to an intensified antihypertensive therapy group (IT) and 46 patients received routine antihypertensive care (RC) Intensified antihypertensive therapy included self monitoring of blood pressure and self-management of antihypertensive medication aiming at permanent normalisation of blood pressure, i.e. $<140 / 90 \mathrm{mmHg}$ before taking medication (J Hypertens 1995;13:933-8). At baseline, IT and RC patients were comparable with regard to age ( $36 \pm 9$ vs. $37 \pm 11$ years) (mean $\pm S D$ ), diabetes duration ( $24 \pm 3$ vs. $21 \pm 8$ years), hypertension duration ( $3 \pm 3$ vs. $5 \pm 7$ years $)$, cigarette pack years $(11 \pm 15$ vs. $8 \pm 14)$, creatinine clearance $\left(1.3 \pm 0.46\right.$ vs. $\left.1.3 \pm 0.54 \mathrm{mul} \mathrm{s}^{-1} 1.73 \mathrm{~m}^{-2}\right)$, proteinuria $(2.4 \pm 3.3$ vs. $2.5 \pm 2.7 \mathrm{~g}$ $\left.24 \mathrm{~h}^{-1}\right)$ systolic $(15 \pm \pm 19$ vs. $143 \pm 22 \mathrm{mmHg})$ and diastolic $(92 \pm 12$ vs. $87 \pm 11$ mmHg) blood pressurc, HbAlc $(8.2+2.1$ is. $8.5 \pm 1.6 \%)$, total cholesterol $(6.6+1.9$ vs. $7.2+1.9 \mathrm{mM})$ and $\mathrm{HDL}$-cholesterol $(1.43+1.36$ vs. $1.35 \pm 0.39$ $\mathrm{mM}$ ). Systolic/diastolic blood pressure values decreased in the IT group $(-4+24 /-6 \pm 13 \mathrm{mmHg})$ and increased in the $\mathrm{RC}$ group $(+15 \pm 28 /+0.3 \pm 15 \mathrm{~mm}$ $\mathrm{Hg}, \mathrm{p}=0.007$. After $\mathrm{l}(0$ years follow-up, $32 \%$ of all patients required dialysis treatment (IT: $24 \%, \mathrm{RC}: 39 \%$, tog rank $p=0.0367$, life table analysis) and $33 \%$ dicd (IT: $18 \%$, RC: $61 \%, \log$ rank $p=0.0040$ ). The main causes of death were cardiovascular. In the multiple Cox regression analysis including baseline parameters, only lower ase $(p=0,046)$ and intensification of blood pressure control $(j=0.0067)$ were independently associated with improved survival. In conclusion, despite antihypertensive therapy mortality is still high in pittents with overt diabctic nephropathy. Intensification of blood pressure treatment improves survival and preserves renal function in these patients. 


\section{OP 3}

\section{Experimental and Clinical Islet Transplantation}

IN VITRO XENORECOGNITION OF ADULT PIG PANCREATIC ISLET CELLS BY $\mathrm{CD}_{4}+$ AND CD + LYMPHOCYTES FROM TYPE 1 DIABETIC OR HEALTHY SUBJECTS

S. Lalain, L. Chaillous, E. Gouin, P. Sai. Immuno-Endocrinology, University/INRA ENVN, Atianpole, BP 40706, 44307 Nantes cedex 03, France.

In vitro studies were conducted in 45 Type 1 diabetic and 20 healthy subjects in order to investigate the intensity and mechanisms involved in cell-mediated rejection of adult pig islets. Human peripheral mononuclear cells (HPMC) responded to pig islet cells (PIC) by strong proliferations ( $\mathrm{p}<0.0001$ ). The intensity of proliferation was variable among the subjects since the stimulation index ranged from 2 to 215 . The intensity of proliferation was not different in diabetic and healthy subjects. The response to PIC was stronger $(p<0.001)$ than that to pig splenocytes. CD4 T cells were mainly involved in the response to PIC since the proliferation of HPMC was markedly decreased $(p<0.03$ ) when CD4+ were blocked with MoAbs, whereas the blocking of $\mathrm{CD}_{8}{ }^{+}$cells or NK cells gave less marked effects. The response of HPMC to PIC was significantly decreased ( $p<0.01$ ) by $87 \pm 7 \%$, but not abolished, after removal of antigen presenting cells (APC) using magnetic microspheres, indicating that indirect and direct pathways coexisted for the recognition, even though the dominant pathway was indirect. Consistently, purified $\mathrm{CD} 4^{+}$ceils alone did not proliferate in response to PIC but recovered their proliferative ability when mixed with APC (indirect pathway), whereas purified $\mathrm{CD}^{+}$cells alone proliferated (in the presence of $\mathrm{IL}-2$ ) in response to PIC (direct pathway). MHC class II -restricted mechanisms were involved in the proliferation since it was blocked by anti-DR MoAbs. The involvement of Th 2 cells during proliferation in response to PIC appeared to be much greater than that of Th 1 cells since IL10 increased 47-fold ( $p<0.01$ ) while IFN $\gamma$ increased only 1.5 -fold. No statistical differences could be detected between diabetic and control subjects with regards to the lymphocyte subsets and mechanisms of recognition involved, or to the IFNy/IL10 production in response to PIC. These results provide the first detailed information on human cell-mediated xenoreaction to adult pig islets. This discordant situation involves a dominant CD4 class II-restricted Th2 response with indirect pathway for the recognition, but also a less intense CD8 T-cell response resulting from direct recognition. This reaction is strong and constitues a serious obstacle, which could be variable among subjects. The immunogenetics of Type 1 diabetes do not seem to influence the intensity and mechanisms of proliferation in response to PIC.

\section{5}

EFFECTS OF INTERLEUKIN-1B AND TUMOUR NECROSIS FACTOR- $\alpha$ ON MICROENCAPSULATED RAT PANCREATIC ISLETS.

A.King. A. Andersson and S. Sandler. Dept. of Medical Cell Biology, Uppsala University, Uppsala, Sweden.

Microencapsulation of islets of Langerhans should protect against immune rejection of the islets and also possible recurrence of disease. In both of these situations, it is likely that inflammatory cytokines are present in the vicinity of the microencapsulated islets. The aims of this study were to assess the viability of rat islets after microencapsulation using an electrostatic field, and evaluate the effects of the cytokines interleukin-1B (IL-1B) and tumour necrosis factor- $\alpha$ (TNF- $\alpha$ ) on such encapsulated islets in comparison with non-encapsulated free islets. We exposed islets to a high concentration of IL-1B (25 U/ml for $48 \mathrm{~h})$ and measured glucose induced insulin release. At $16.7 \mathrm{mM}$ glucose, both free and microencapsulated islets insulin release rates were significantly decreased when exposed to IL-1B, (from $46 \pm 7$ to $11 \pm 2 \mathrm{ng} / 10$ islets $/ \mathrm{h}$ in free islets, $\mathrm{p}<0.001 ; 35 \pm 5$ to $11 \pm 2 \mathrm{ng} / \mathrm{islets} / \mathrm{h}$ in encapsulated islets; $p<0.001)$. The decrease in insulin release from encapsulated islets compared with control islets was significant $(p<0.05)$. Interestingly, levels of nitrite formed by microencapsulated islets in response to IL- $1 B$ were significantly lower than in free islets $(6.3 \pm 0.9$ and $9.5 \pm 1.4 \mathrm{pmol}$ nitrite $/ 100$ islets $/ \mathrm{h}$. respectively; $p<0.01$ ). We then measured glucose oxidation rates of free and microencapsulated islets exposed to $2.5 \mathrm{U} / \mathrm{ml} \mathrm{IL-} 1 \mathrm{~B}$ for $48 \mathrm{~h}$. At this lower concentration of IL-1B, a significantly decreased rate of glucose oxidation was observed in both free and encapsulated islets, which were detrimentally affected to the same extent (from $188 \pm 30$ to $87 \pm 24 \mathrm{pmol} / 10$ islets $/ 90 \mathrm{~min}$ in free islets; $p<0.001$, and from $148 \pm 21$ to $81 \pm 31 \mathrm{pmol} / 10$ islets/90min in microencapsulated islets; $\mathrm{p}<0.001)$. When TNF- $\alpha$ was added $(1000 \mathrm{U} / \mathrm{ml})$ in combination with $2.5 \mathrm{U} / \mathrm{ml} \mathrm{IL}$ $1 B$, glucose oxidation rates of the islets were decreased further $(54 \pm 19$ and $55 \pm 26$ $\mathrm{pmol} / 10$ islets $/ 90 \mathrm{~min}$; in free and microencapsulated islets respectively). TNF- $\alpha$ alone had no effects on free or microencapsulated islets on the glucose oxidation rate. These data indicate that the alginate/PLL/alginate capsule does not confer protection against the effects of IL- $1 B$ on $\beta$-cell function, even when the IL-1B dose was reduced considerably. These data also indicate that TNF- $\alpha$ penetrates the capsule and in combination with IL- $1 B$ has a detrimental effect on the islets. In conclusion, if soluble cytokines are directly involved in islet damage during rejection, then alginate/PLL/alginate capsules will confer little protection.

\section{4}

FUNCTIONAL MONITORING OF ANTIGEN-PRESENTING CELLS IN ISOLATED HUMAN, PORCINE, AND RODENT ISLETS

H. Jahr, D. Brandhorst, H. Brandhorst, M. Brendel, and R. G. Bretzel. 3rd Medical Department, University of Giessen, Germany

Pre-transplant reduction of islet immunogenicity by depletion from antigenpresenting cells (APCs) may reduce the levels of immunosuppression in clinical islet transplantation and enhance the success rate of tolerance-inducing protocols. Unfortunately, APC-depleting protocols effective for rodent islets are much less efficient for islets from large species. The most stringent test to prove the absence of APCs in cell preparations is the failure to co-stimulate lectin-incubated T-lymphocytes. We adapted this test to monitor the presence of APCs in intact islets. Purified human T-cells $\left(5 \times 10^{4} /\right.$ well) were incubated $\left(4\right.$ days, $\left.37^{\circ} \mathrm{C}\right)$ with $3 \mu \mathrm{g} / \mathrm{ml}$ phytohemagglutinin (PHA) and 15 islets/well. In the absence of islets, T-cell proliferation ( ${ }^{3} \mathrm{H}$-thymidine incorporation) was $<250 \mathrm{cpm}$. At the test conditions used, T-cell response to islets without $\mathrm{PHA}$ were negligible $(<300 \mathrm{cpm})$, too. However, in the combined presence of PHA and islets from mice, rats, pigs, or humans, ${ }^{3} \mathrm{H}$ thymidine incorporation into T-cells was $5,378 \pm 609,13,780 \pm 912,35,611 \pm$ 7,822 , and $18,124 \pm 3,864 \mathrm{cpm}$, respectively ( $\mathrm{n}=5 \mathrm{each}$ ). Low temperature culture (14 days at $22^{\circ} \mathrm{C}$ ) abolished the co-stimulatory capacity of mouse islets, reduced that of rat islets by $92 \pm 4 \%$, but had only a marginal influence on porcine or human islets. These differences may be caused by different susceptibilities of APCs to inhibition by finally radical-mediated mechanisms, including low temperature culture. Four-fold higher concentrations of superoxide or hydrogen peroxide were found to be necessary for inactivation of human peripheral blood APCs compared to rat blood or rat lymph node APCs in the lymphocyte transformation test. In summary, we conclude that the capacity of isolated islets to co-stimulate the proliferation of lectin-incubated $T$-cells may be a reliable and sensitive marker to predict the capacity for "direct" allogeneic immunoactivation vivo. The higher resistance of human APCs to radical-mediated inactivation may at least partly be responsible for the difficulties to transfer APC-depleting protocols from rodent to human islets.

\section{6}

INFLUENCE OF LONG-TERM PANCREATIC ISLET GRAFT FUNCTION ON DEVELOPMENT OF LATE DIABETIC COMPLICATIONS

T.T. Vărkonyi, Cs. Lengyel, Zs. Fülöp ${ }^{\mathrm{a}}$, P. Kempler ${ }^{c}, G$. F. Farkas ${ }^{\mathrm{b}}$ and J. Lonovics. 1st Dept. of Medicine, a Dept. of Ophthalmology, Dept. of Surgery,

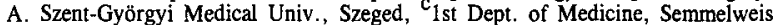
Medical Univ., Budapest, Hungary

Introduction: Pancreatic islet transplantation has been reported to have a beneficial effect on the carbohydrate metabolism soon after the operation in patients with insulin-dependent diabetes mellitus (IDDM). Experience relating to the long-term influence of islet grafting on the development of secondary diabetic complications is so far very limited. The aim of this study was to assess the fate of the neuropathy and the retinopathy in patients with a long history of functioning isle transplants. Patients, methods: 11 IDDM patients who had undergone pancreatic islet transplantation were studied (duration of islet graft function: $9.5 \pm 0.2$ years, age: $41.8 \pm 2.4$ years, duration of DM: $24.9 \pm 2.4$ years, BMI: $26.3 \pm 1.3$ mean $\pm \mathrm{SE}) .10$ non-transplanted IDDM patients with comparable parameters were involved as controls (age: $45.8 \pm 3.0$ years, duration of DM: $22.0 \pm 2.9$ years BMI: $24.7 \pm 1.5$ ). Five cardiovascular tests were performed and a score was calculated to express the severity of autonomic neuropathy (AN). Sensory nerve function was studied with a Neurometer (Neurotron Inc., Baltimore), using constant sine wave transcutaneous nerve stimulation to determine current perception threshold on the peroneal and the median nerves. Retinopathy status was checked by ophthalmography and fluorescence angiography. Results: In patients after islet transplantation the AN score was lower than in the contro group $(3.9 \pm 0.7$ vs $6.0 \pm 0.6 ; \mathrm{p}<0.05)$. The values of beat-to-beat variation were higher in the transplanted group $(13.3 \pm 2.1$ vs $6.5 \pm 1.5 ; \mathrm{p}<0.05)$. Transplanted patients had markedly lower perception thresholds at three frequencies on both limbs in comparison with the non-operated group (median nerve: $3.20+0.22$ vs $3.44 \pm 0.48,1.84 \pm 0.84$ vs $3.18 \pm 1.10,0.71 \pm 0.18$ vs $1.74 \pm 0.93$; peroneal nerve: $3.44 \pm 0.48,1.84 \pm 0.84$ vs $3.18 \pm 1.10,0.71 \pm 0.18$ vs $1.74 \pm 0.93$; peroneal nerve:
$5.37 \pm 0.95$ vs $6.53 \pm 1.10,3.24 \pm 1.10$ vs $5.01 \pm 1.37,1.77 \pm 0.83$ vs $3.96 \pm 1.33$ $\mathrm{mA}$ ). In the follow-up period, the retinopathy improved in 6 of the transplanted patients and remained unchanged in 5 subjects. Conclusions: These results indicate a reduced progression of autonomic and sensory neuropathy a decade after pancreatic islet transplantation. Additionally, the early stage of retinopathy improved in the presence of grafted islets. The data suggest that long-term nearnormoglycaemia associated with a continuous endogenous supply of islet peptides may postpone the development of late complications in IDDM. 
THE IMPACT OF PANCREAS AND KIDNEY TRANSPLANTATION ON LATE DIABETIC COMPLICATIONS AND QUALITY OF LIFE

F. Saudek, M. Adamec, R. Kožnarová, T. Sosna, H. Vondrová, T. Jedináková and P. Bouček. Institute for Clinical and Experimental Medicine, Prague, Czech Republic

The aim of pancreas transplantation is to improve the quality of life and ameliorate the microvascular complications of diabetes. Since 1983, 106 combined pancreas and kidney transplants were performed in uremic NIDDM patients at our center with current 1-year patient and pancreas graft survival rates of 90 and $76 \%$, respectively. The course of diabetic retinopathy, polyneuropathy and quality of life were studied in 3 groups of subjects followed for at least 1 year: recipients with full function of both grafts (PKTx1; $n=30$ ), recipients with pancreatic graft failure (PKTx2; $n=10$ ) and in IDDM recipients of isolated kidney graft $(K T x ; n=18)$. In group PKTxl, the grade of diabetic retinopathy improved, remained stabilized or worsened in 18,60 and $22 \%$ of recipients, respectively. In groups PKTx2 and KTx the findings did not change or worsened in all subjects. Progression of cataract was common in all 3 groups. Clinical neurologic assessment improved, remained stabile or worsened in 96,4 and $0 \%$ of patients in group PKTx 1 and in 65,28 and $7 \%$ of patients in joined groups PKTx2 and KTx $(p<0,05)$. Compared to pre-transplant status, sural nerve velocity increased in $50 \%$ of PKTx1 recipients $(\mathrm{p}<0,05)$. Subjective health improvement in groups PTx 1, PTx2 and KTx was reported by 93,87 and $90 \%$ of subjects, respectively $(p>0,05)$. Superior overall quality of life and more personal free time were found in group PTx1 than in group PTx2 ( $>0,05)$. Overall quality of life in groups PTxl and KTx did not differ significantly $(p>0,05)$. We conclude, that following PKT subjective and objective signs of diabetic neuropathy improve in most recipients. Diabetic retinopathy remains unchanged in most subjects. However, significant improvement, rare in other treatment modalities, may be demonstrated in individual cases. Overall quality of life improves significantly despite no difference between pancreas and kidney and isolated kidney recipients. This may be explained by more frequent pretransplant dialysis treatment in the latter group.
EFFECT OF PANCREAS-KIDNEY TRANSPLANTATION ON MORTALITY IN TYPE 1 DIABETIC PATIENTS WITH END-STAGE RENAL FAILURE YFC Smets, RGJ Westendorp, JW van der Pijl, J Ringers, JW de Fijter and HHPJ Lemkes. Leiden University Medical Centre, Leiden, The Netherlands

Long-term prognosis of patients with type 1 diabetes mellitus and end-stage renal failure appears to be superior after kidney transplantation $(\mathrm{Tx})$ compared with dialysis. Controversy still exists about the additional benefit of a simultaneously transplanted pancreatic graft. In the Netherlands, there is a unique opportunity to perform a population-based follow-up study free from selection on health, because of a) the strict dialysis/transplantation centreallocation; b) the central data collection by the RENINE registry; c) a regional difference in the degree of pancreas-kidney Tx performed (governmentally regulated). Using this regional difference, we set out to study the effect of simultaneous pancreas-kidney $\mathrm{Tx}$ versus kidney $\mathrm{Tx}$ on mortality in type 1 diabetic patients. Between 1985 and 1996, 427 type 1 diabetic patients (agelimit 18-52 y) started on renal replacement therapy and were allocated by RENINE to Leiden area (LB, $n=85$ ) or Netherlands $(N L, n=330$ ) on the basis of their place of residence. 12 recipients of a living-related donor kidney graft were excluded from the current analysis. The two areas were similar with respect to age and sex. Patient survival was higher in LB compared with NL $(\mathrm{RR}=0.5 ; \mathrm{Cl} 950.4-0.8$ ). Survival of 377 patients on dialysis was equal between the two areas. 214 patients were transplanted (kidney-pancreas Tx: LB 73\%; NL 37\%). More pre-emptive Tx were performed in LB ( LB 36\% vs. NL 11\%) and the mean duration of dialysis was shorter in LB (17 vs. 25 months). The mortality risk in LB was 0.4 fold lower (CI95 0.2-0.8) than in NL. This contrast became apparent $>3$ years post $\mathrm{Tx}$ and was independent of duration of dialysis or early transplanted-related deaths. Censored kidney graft survival did not differ between the two areas ( $\log$-rank $p=0.23$ ). Conclusively, we found a $50 \%$ reduction in mortality of type 1 diabetic patients on renal replacement therapy in LB, related to the higher degree of pancreas-kidney transplantation performed.

\section{OP 4 \\ Diabetes and Pregnancy - Clinical Aspects}

19

TRANSIENT INCREASE OF INSULIN REQUIREMENTS IN EARLY
PREGNANCY OF WOMEN WITH TYPE I DIABETES MELLITUS

PREGNANCY OF WOMEN WITH TYPE 1 DIABETES MELLITUS

A. Garcia-Patterson, M. Albareda and R. Corcoy. Servei d'Endocrinologia. Hospital de Sant Pau, Barcelona

It is well-known that in women with Type $1 \mathrm{DM}$, insulin requirements (IR) increase in the second half of pregnancy, whereas information in the first half is more scant and points to a decrease. The occasional observation of women with Type $1 \mathrm{DM}$ requiring transient increases of insulin dose in early pregnancy prompted this study, with the aim of assessing IR in the first part of pregnancy. Thirty-six women with Type $1 \mathrm{DM}$ receiving prepregnancy care and with tight metabolic control before pregnancy ( $\mathrm{HbAlc}<$ mean +3 SD of control population) were studied. Weekly IR were expressed as IU/Kg/day and weekly mean blood glucose (MBG) as the weighted mean of pre and postprandial self-monitoring data. IR and MBG during pregnancy were expressed as its rate to prepregnancy values (mean IR and MBG in the 4 weeks immediately antedating pregnancy). IR did not change in the first 6 weeks of pregnancy $(-0.48 \pm 5.2 \%$, ns), increased at $7-12$ wks $(+5.5+13.1 \%$, $\mathrm{p}<0.001)$ and decreased at $13-18$ wks $(-2.3 \pm 15.6, p<0.001)$. MBG showed a similar pattem, which took place earlier in pregnancy $(-0.6+7.7 \%$ at $1-4$ weeks, ns; $+5.4 \pm 7.4 \%$ at $5-8$ weeks, $p<0.01 ;-5.1 \pm 11.9$ at $9-12$ weeks, $\mathrm{p}<0.001$ ). Fifty-six percent of women showed this characteristic pattern of increase in IR at 7-12 weeks and decrease at 13-18 wks. Five women suffered a severe hypoglycemia, four events taking place at 13-16 weeks. The coincidence of IR increase with the time point where BHCG peak takes place suggests a link to the physiological increase of thyroid hormones. In this line, we analysed the presence of this patterm of IR in women with IDDM either without thyroid disease or with treated hypothyroidism, the frequency being borderline lower in the last group $(18 / 30,60 \%$ vs $2 / 6,33.3 \%, p=0.08)$. We conclude that in pregnant women with Type $1 \mathrm{DM}, \mathrm{IR}$ increase at 7-12 weeks of gestation before decreasing at 13-18 weeks. The transient increase may have a relationship with the physiologic increase in thyroid hormones.

\section{0}

REDUCED INSULIN REQUIREMENTS IN EARLY PREGNANCY IN IDDM

V.J. Aldridge", L. Yaxley , M. Durkan ${ }^{a}$, M.B. Kelly $y^{b}$ R.C. Temple "Bertram Diabetes Centre and bepartment of Obstetrics, Norfolk and Norwich Health Care NHS Trust, Norwich, UK

Increased insulin sensitivity in early pregnancy is recognised but the frequency with which it leads to a reduction in insulin requirements in the first trimester of pregnancy in IDDM is poorly documented. We have retrospectively analysed changing insulin requirements in early pregnancy in IDDM patients presenting at the combined diabetic antenatal clinic between 1990 and 1994. 132 patients presented of which 85 patients $(64 \%)$ were seen at 8 weeks or earlier. In this group there were 8 miscarriages, one ectopic pregnancy and one termination. Data is presented on the remaining 75 patients comparing insulin doses at booking and at 12 weeks. Twenty-nine patients (39\%) required a reduction of insulin dose of greater than $10 \%$. The mean fall in insulin requirement in these patients was $23 \%$ (range $10-48 \%$ ). Fifteen $(52 \%)$ of these 29 patients had a fall in insulin requirement of greater than $20 \%$. Both HbA1c and fructosamine fell significantly between booking and 12 weeks (HbA1c $6.2 \pm 1.5 \%-v-5.4 \pm 1.0 \% p<0.001$, fructosamine $333 \pm$ $67 \mu \mathrm{mol} / \mathrm{L}-\mathrm{v}-282 \pm 38 \mu \mathrm{mol} / \mathrm{L}, \mathrm{p}<0.001$ ). Patients experiencing a fall in insulin dosage had significantly better control at booking (HbA1c 5.71 \pm 0.94 $-v-6.60 \pm 1.76, p<0.01)$. Of the total 132 patients $32(24 \%)$ had at least one severe bypoglycaemic episode (defined as needing external assistance). 24 (75\%) of these patients suffered severe hypoglycaemia in the first 12 weeks only. This study confirms that falling insulin requirement frequently occurs in the first trimester of IDDM pregnancies especially in those well controlled at booking. It is important to advise on this during pre-pregnancy counselling to minimise the risk of severe hypoglycaemia. 
HEMOSTASIS IN PREGNANTS WITH TYPE 1 DIABETES MELLITUS IN PERINATAL PERIOD.

A.Bronisz, D.Rosć, M.Kotschy and A.Graczykowska-Koczorowska,

The L.Rydygier Medical University in Bygoszcz, Poland

The big fluctuations of some hemostatic parameters observed during the pregnanc are connected with the prevention of bleeding in perinatal period. The aim of study was the evaluation of some hemostasis parameters during a perinatal period in pregnants with type 1 diabetes mellitus (DMP). We examined blood plasma of 31 DMP (mean aged 29,1 $\pm 6,2)$ with a good metabolic control $\left(\mathrm{HbA}_{1 \mathrm{c}} 5,3 \pm 1,6 \%\right)$. The blood was taken beteen 36-38 weeks of pregnancy (the III-rd trimester - T3), two hours after delivery of placenta (AD) and after puerperium (AP). The following hemostasi parameters were estimated: platelet count (PLT), activity of antythrombin III (AT III) and plasminogen activator inhibitor type 1 (PAI-1) as well as concentration of tissue plasminogen activator antigen (tPA:Ag), fibrinogen (F) and fibrinogen/fibrin degradation products (FDP). The above parameters in DMP are shown in the table $(\mathrm{M} \pm \mathrm{SD})$. They were compared to those of the I-st trimester of pregnancy $(\mathrm{C})$. The AT III didn't differ significantly, PLT were significantly decreased $(p<0,05)$ in T3 and FDP werc significantly increased AP $(p<0,05)$ in comparison to data from $C$ The $\mathrm{PAA}: \mathrm{Ag}$ and $\mathrm{PAI}-1$ were significantly higher in $\mathrm{T} 3$ in comparison to $\mathrm{C}$ ( $p<0,001$ for two parameters) and $\mathrm{PA}: \mathrm{Ag}$ was still significantly higher $\mathrm{AD}(\mathrm{p}<0,01)$. We also compared values of these parameters in 3 periods.

\begin{tabular}{l|l|l|l|l|l|l}
\hline Period & PLT (G/l) & AT III (\%) & tPA:Ag(ng/mi) & PAI-1(I/ml) & F (g/l) & FDP( $\mu \mathrm{g} / \mathrm{ml})$ \\
\hline
\end{tabular}

\begin{tabular}{l|l|l|l|l|l|l}
\hline $\mathrm{T} 3$ & $172 \pm 43,2$ & $127 \pm 38,6$ & $14,9 \pm 11,5$ & $15,9 \pm 11,4$ & $3,5 \pm 1,0$ & $5,4 \pm 5,7$ \\
\hline $\mathrm{AD}$ & $158 \pm 38,6$ & $115 \pm 33,7$ & $13,5 \pm 11,4$ & $6,1 \pm 9,4 \bullet$ & $2,8 \pm 1,2 \bullet$ & $11,7 \pm 6,5 \bullet$ \\
\hline $\mathrm{AP}$ & $183 \pm 34,1$ & $127 \pm 38,6$ & $8,6 \pm 8,2 \bullet$ & $7,6 \pm 8,7 \bullet$ & $2,7 \pm 0,8 \bullet$ & $13,5 \pm 8,0 \bullet$ \\
\hline \multicolumn{1}{c}{$\mathrm{p}<0,05<\mathrm{p}<0,01$} & $\mathrm{p}<0,001$
\end{tabular}

The significant PAI- 1 activity and F-concentration decrease were observed $A D$ and AP in comparison to T3. We also observed the tPA:Ag decrease AD and AP in comparison to $\mathrm{T} 3$, but the difference between the end of $\mathrm{T} 3$ and puerperium was significant. The statisticaly significant FDP increase was observed AD and AP in comparison to T3. Conclusion: Decrease of the PAI-1 activity and F-concentration with FDP increase during delivery and puerperium indicate to perinatal activation of fibrinolysis in pregnants with type I diabetes (DMP).

\section{3}

METABOLIC ABNORMALITIES IN WOMEN WITH PRIOR GDM

M.Carvalheiro', I.Fagulha', A.Fagulha', L.Gomes', S.Paiva', E.Marta', E.Sobral', F.Leitão ${ }^{3}$, M.L.Pinto', M.M.A.Ruas' and T.Buchanan'. 'Dept of Endocrinology, '2Obstetric Clinic and ${ }^{3}$ Clinical Pathology, University Hospital or Coimbra, Portugal, "University of Southem Califomia, Las Angeles, USA

Our aim was to determine, insulin sensitivity $\left(\mathcal{S}_{j}\right)$, glucose effectiveness $\left(\mathrm{S}_{G}\right)$, glucose tolerance $\left(K_{G}\right)$ and insulin secretion in 40 post-gestational diabetic women (P-GDM) and 18 post-pregnant healthy control women (P-PGC) with similar age 30.1 $\pm 4.7 ; 28.8 \pm 3.3$ in yrs, BMI 25.8 $\pm 4.1 ; 26.8 \pm 6.1$ and WHR $0.84 \pm 0.08 ; 0.84 \pm 0.07$, respectively, in early post-partum period, during an insulin modfied frequently-sampled intravenous glucose tolerance test (FSIVGT: $300 \mathrm{mg}$ glucoselkg body weight, followed in 20 min by 25 -min infusion of insulin $6 \mathrm{mU} / \mathrm{kg} / \mathrm{min}$ and blood sampled 14 times for $240 \mathrm{~min})$. $\mathrm{S}_{1}\left(\times 10^{-} \mathrm{min}-\mathrm{per} \mu \mathrm{U} / \mathrm{ml}\right)$ and $\mathrm{S}_{G}$ $\left(m^{-1}\right)^{-1}$ were estimated by Bergman's minimal model. $K_{G}\left(m^{-1}{ }^{-1} \times 100\right)$ was assessed between 8-19 mins after glucose injection. First phase of insulin secretion was expressed as the area under the insulin curve between $2-8 \mathrm{~min}(\mu \mathrm{U} / \mathrm{m} / \mathrm{xmin})$. "Disposition Index" (DI) was calculated ( $\mathrm{S} \times$ first phase $\left.\times 10^{-1}\right)$ to adjust $\mathrm{B}$-cell function for insulin sensitivity. Fasting glucose (mg/d) and insulin $(\mu \mathrm{U} / \mathrm{m} \mathrm{m})$ and insulin peak, were considered. All the P-GDM had normal glucose tolerance at $75 \mathrm{~g}-0 G \pi$ at 6 weoks post-partum. $S$, was slightly decreased in $P-G D M$ vs P-PGC ( $\sigma=N S)$. $S_{G}$ was significantly docreased in P-GDM vs P-PGC and $K_{G}$ was lower in P-GDM vs P-PGC $(\rho=N S)$. First phase insulin secretion and insulin peak were significantly lower in P-GDM vs P-PGC. Fasting glucose and insulin were higher in P-GDM vs P-PGC ( $\rho=$ NS).

\begin{tabular}{lccc} 
& P-PGC & P-GDM & $p$ \\
\hline $\mathrm{S}_{\mathrm{I}}$ (mean $\pm \mathrm{SD}$ ) & $5.8 \pm 2.3$ & $4.6 \pm 2.7$ & $\mathrm{NS}$ \\
$\mathrm{S}_{\mathrm{G}}$ (mean $\pm \mathrm{SD}$ ) & $0.025 \pm 0.013$ & $0.019 \pm 0.01$ & $<0.02$ \\
$\mathrm{~K}_{\mathrm{G}}$ (mean $\pm \mathrm{SE}$ ) & $2.69 \pm 0.51$ & $1.67 \pm 0.25$ & $\mathrm{NS}$ \\
First Phase (mean $\pm \mathrm{SD}$ ) & $395 \pm 200$ & $277 \pm 159$ & $<0.02$ \\
"Disposition Index" (mean $\pm \mathrm{SD})$ & $2.268 \pm 1.747$ & $1.087 \pm 0.635$ & $<0.01$ \\
Fasting glucose (mean $\pm \mathrm{SD})$ & $82.4 \pm 7.5$ & $85.1 \pm 8.6$ & $\mathrm{NS}$ \\
Fasting insulin (mean $\pm \mathrm{SD})$ & $7.0 \pm 2.7$ & $7.8 \pm 4.0$ & $\mathrm{NS}$ \\
Insulin peak (mean $\pm \mathrm{SD}$ ) & $84.3 \pm 44.2$ & $60.3 \pm 36.3$ & $<0.01$ \\
\hline
\end{tabular}

Thus, we found significant reductions in glucose effectiveness $\left(\mathrm{S}_{\mathrm{G}}\right.$ and insulin secretion in early post-partum in women with normal glucose tolerance and recent GDM when compared to post-pregnant healthy control women. The defect in B-cell function was particularly prominent in view of slightly lower insulin sensitivity in women with prior GDM and may be the major defect in GDM.
THE INFLUENCE OF PREGNANCY ON RENAL FUNCTION LOSS IN INSULIN DEPENDENT DIABETIC PATIENTS WITH DIABETIC NEPHROPATHY

K. Rossing*, P. Jacobsen*, E. Hommel ${ }^{*}$ E. Mathiesen, A. Svenningsen*, P. Rossing ${ }^{*}, \mathrm{H}-\mathrm{H}$. Parving. Steno Diabetes Center Gentofte, Denmark.

We evaluated the long-term impact of pregnancy on renal function and survival in all female IDDM patients ( $n=94$ ) developing diabetic nephropathy between 1970 and 1989 at the Steno Diabetes Center. The observational follow-up study lasted 13 years (range 3-23) from onset of diabetic nephropathy until death or 1996. 25 women became pregnant in average 5 years (range (1-17)) after onset of diabetic nephropathy (Group A). The remaining 69 served as controls (Group B). At onset of diabetic nephropathy the two groups were comparable with regard to demographic data, and s-creatinine was identical: mean (SD) 79 (23) $\mu \mathrm{mol} / \mathrm{l}$ in both groups. At onset of pregnancy all but 2 women had s-creatinine $<100 \mu$ mol/l. All patients received aggressive anthypertensive treatment with on average 2 drugs. During follow-up there was no difference between the two groups in the loss of kidney function as determined by linear regression on reciprocal serum creatinine values (mean (SE)) $0.40(0.10)$ vs. $0.41(0.07) \mathrm{l} / \mathrm{mmol} /$ year (Group A vs. Group B). Furthermore there was no difference in the slope of $1 / \mathrm{s}$-creatinine before and after pregnancy. A doubling of baseline creatinine (to at least 175 $\mu$ moll/) was seen in $7 / 25(28 \%)$ vs $19 / 69(28 \%)$ in group A vs B respectively (Survival analysis Logrank test NS). In 1996 seven (28\%) of the pregnant women had died and four (16\%) had reached ESRD compared to $18(26 \%)$ and 11 $(16 \%)$ of the controls respectively (NS). During follow-up Group A and B had comparable values of $\mathrm{BP}$ (mean (SD) $138(16) / 83$ (9) vs 139 (14)/86 (6) (mm $\mathrm{Hg}$ ), hemoglobin $A_{10} 9.3(1.8)$ vs $9.5(1.2) \%$, and albuminuria (geometric mean (antilog SE)) 603 (1.4) vs 741 (1.1) $\mathrm{mg} / 24 \mathrm{~h}$ ) (NS). In conclusion pregnancy has no adverse long-term impact on renal function and survival in IDDM patients with well preserved kidney function suffering from diabetic nephropathy

\section{4}

PRIOR GESTATIONAL DIABETES: EARLY MANIFESTATION AND/OR PREDICTOR OF A METABOLIC CARDIOVASCULAR SYNDROME. Zs. Kerényi, Á Gy. Tabák, Zs. Bosnyák, E. Madarász, K. Tóth, É. Baranyi, Gy. Csákány and Gy. Tamás, National Ccntre for Diabetes Care, Haynal Univ., Budapest, Hungary

Data are accumulating on the connection between gestational diabetes (GDM) and a metabolic cardiovascular syndrome. To study it former GDM mothers $(\mathrm{n}=119$; mean age: $38.9+6.4$ [SD]yrs; time elapsed since diagnosing GDM: $7.3 \pm 2.5 \mathrm{yrs}$; BMI: $26.3 \pm 5.6 \mathrm{~kg} / \mathrm{m} 2 ; 88$ of them first insulinized [GDM-I], 31 on diet [GDM-D] during gestation) were investigated. Altogether $72(60 \%) \mathrm{GDM}$ (GDM-I vs GDM-D: $P<0.01$ ) could be reclassified with glucose intolerance ( $\mathrm{GF}=$ diab mell. + IGT). Twenty eight pregestational NIDDM first insulinized during gestation (preNIDDM; age: $40.2 \pm 6.5$ yrs; follow-up: $6.8 \pm 2.4 \mathrm{yrs}$; duration $10.8 \pm 3.6 y \mathrm{rs}$; BMI: $29.8 \pm 5.4 \mathrm{~kg} / \mathrm{m} 2[\mathrm{P}<0.05$ vs GDM]) from the same cohor served as controls. Cardiovascular risk factors (blood pressure, body mass index [BMI], - during gestation and at follow up - waist/hip [W/H] ratio, microalbuminuria [MA], lipoprotein lipids at follow up only) were measured Hypertension during gestation (chronic or RR repeatedly $\geq 140 / 90 \mathrm{mmHg}$ ) was found in $35 \mathrm{GDM}$ (29\%; pregnancy induced hypertension [PIH]: 25 [21\%]) and in 10 preNIDDM (36\%, PIH: 9 [32\%]). At follow up hypertension (treated or $\geq$ $160 / 95)$ could be proved in $22(18 \%$; GI: $15 / 2268 \%)$ prior GDM and in $7(25 \%)$ preNIDDM patients, $5(20 \%)$ and $9(100 \%)$ from PLH cases. Correlation between mean systolic $(r=0.38 ; P<0.005)$, diastolic $(r=0.29 ; P<0.05)$ and maximum blood pressure $(=0.37 ; \mathrm{P}<0.005)$ during gestation and $\mathrm{W} / \mathrm{H}$ at follow up were found in prior GDM-I, not however in GDM-D and preNIDDM. Prior GDM with hypertension at follow up had higher W/H compared to nonhypertensive women $(0.86 \pm 0.06$ vs $0.80 \pm 0.06 ; \mathrm{P}<0.005)$. Differences in BMI at follow up between prior GDM women with/without hypertension during gestation $(P<0.05)$ and at follow up $(P<0.0001)$, resp., - not in preNIDDM were observed. No differences in lipoprotein lipids and in MA $(15-28 \%)$ according to hypertension could be proven. GDM seems to be early manifestation of insulin resistance syndrome. Clustering of different risk factors in prior GDM-I with hypertension at follow up (obesity [BMI:30.5+6.2], pathological W/H $[0.86 \pm 0.07]$, MA [28\%], GI[68\%]) proves that prior GDM and insulinization during pregnancy are not only predictors of GI, but also risk indicators of a complex cardiovascular metabolic syndrome to be prevented. 
OP 5

\section{T-Cells in Type 1 Diabetes}

\section{5}

ANALYSIS OF THE T CELLS ISOLATED FROM A DIABETIC PANCREAS

M. Martí. M. Costa, C. Roura-Mir, D. Botello, R. Pujol-Borrell and D. Jaraquemada Unitat d'Immunologia, Hospital Germans Trias i Pujol, Universitat Autònoma de Barcelona. 08193 Bellaterra, Spain.

Type 1 diabetes is an autoimmune disease characterized by the specific destruction of $\beta$ cells by autorreactive $T$ cells. Few studies have been performed using human pancreatic affected tissue and the specific phenotype of $T$ cells involved in the autoimmune attack is still unknown. We have studied the lymphoid cells isolated from the infiltrate of the pancreas of a diabetic donor who died 9 days after the onset of the disease (PB 100$)$. Lymphocytes were isolated from the pancreatic tissue after digestion with collagenase and collection of the supernatant of the islet gradients. The bulk cell culture showed a mixture of CD4 and CD8 T cells and some macrophages. T cell lines and clones were generated by limiting dilution with two different protocols, always using the autologous EBV B-cell line as APC, either with anti-CD3 Ab or incubated with crude islet extract from healthy pancreas donors. In both protocols the majority of $\mathrm{T}$ cell lines obtained were $\mathrm{CD}^{+}$, although some $\mathrm{CD} 8^{+} \mathrm{T}$ cell lines were also isolated. Some rare phenotypes also appeared with very low frequency, such as a double positive $\left(\mathrm{CD}^{+} / \mathrm{CD}^{+}\right)$cell lines (PB 100.32) and a double negative (CD4/CD8 ${ }^{-} \mathrm{CD}^{+}$cell line (PB100.9-24.12). Most cell lines were non-proliferative to islet extracts and only one $\mathrm{CDB}^{+}$cell was found capable of cytotoxicity of islet extract-pulsed autologous EBV cells. When we looked up the TCR expressed by these T cell lines using RT-PCR. the lines showed some oligoclonality, and the most frequent $\mathrm{V} \beta$ segments expressed by these cells were: $V \beta 11, V \beta 13.1, V \beta 7, V \beta 14$. This was found for cells isolated using both protocols confirming that the method used for the generation of $\mathrm{T}$ cell lines did not select a particular set of TCR expressing cells. The $V \alpha$ studies are underway. Expression of a panel of cytokines was analysed by RT-PCR. The main phenotype observed for antigen-isolated $\mathrm{T}$ cells was Thl-like, since all showed a high expression of IFN- $\gamma$ and only some also expressed IL-4. All cell lines isolated with anti-CD3antibodies showed a heterogeneous Th0 pattern, since ail expressed large amounts of IL4 and IFN- $\gamma$. IL-2 and IL-10 were secreted by most of the cells. The pattern of cyrokine expression by these $\mathrm{T}$ cell lines after full stimulation with PMA and ionomicine is now being analysed by flow cytometry. No clear speciticity has been assigned to any of the cell fines. Only one CD8 T cell clone (PBI00.3i) isolated with antigen could be grown and it appears to have some GAD-specific cytotoxicity. In summary. T cells isolated trom the lymphoid infiltrate of a diabetic pancreas show a tendency towards a $T h l$ phenotype and to the restricted use of some $V \beta$ fragments.

\section{7}

\section{ISLET T CELL AUTO-ANTIGENS TARGETED EARLY OR LATE IN PRE-DIABETES \\ H.-M. Dosch\#, R. Cheung\#, M. Pietropaolo+ and D. J. Becker ${ }^{+}$, TORONTO, ONT\# \& PITTSBURGH, PA ${ }^{+}$}

In order to distinguish the course of non-pathogenic and progressive pre-diabetic autoimmunity, we analyzed autoreactive $T$ cells from 132 new onset IDD patients, $10.1 \pm 4.2(1-18)$ yr old, and 268 first degree relatives (FDR) of whom 50 were deemed high IDD risk $\left(\mathrm{ICA}^{+} \&\right.$ high risk DQ). Follow-up samples were obtained in 108 IDD patients at $0.5 \& 1$ yr. 'Early' diabetes test antigens included GAD65, ICA69/BSA, hsp65. 'Late' target antigens included Proinsulin \& IA-2. The presence of autoreactivity to early antigens was prerequisite for the development of $T$ cells targeting late antigens (hence the name). Various combinations of early responses were present at slowly rising rates in low vs. high risk relatives vs. new onset cases, and they remained stable over the first year post diagnosis. Responses to late antigens were present in $87 \%$ of high risk relatives and $83 \%$ of patients, usually $(>60 \%)$ associated with autoreactivities to multiple early \& late autoantigens. Detection of $\mathbf{T}$ cell proliferative responses to early antigens required exogenous $\mathrm{IL} 2$, possibly due to a failure of zap 70 kinase recruitment. Responses to late antigens were competent, without need for exogenous IL2. We propose that early, autoreactive $T$ cell pools tend to undergo anergy upon antigen contact, but they fail to die. Indeed, preliminary data show that patient $\mathrm{T}$ cells have abnormally high resistance to apoptosis. This implies a model where i.) early autoreactivities characterize clinically indolent autoimmunity, and where ii.) progression to aggressive disease is characterized by recruitment of new T cell pools that target Proinsulin and/or IA-2. These latter cells are fully competent and in the islet they might provide IL2 to bypass anergy and support effector functions of early $T$ cell pools thus explaining the aggressive nature of late pre-diabetes.

\section{6}

IA-2 AND INSULIN REACTIVE ISLET INFILTRATING T-LYMPHOCYTES IN HUMAN AUTOIMMUNE DIABETES.

S.Dionisi, V.Viglietta, P.Marchetti, E.Anastasi, C.Tiberti, P.Gottlieb, U.Di Mario and F.Dotta. Univ. of Rome "La Sapienza" and Univ. of Pisa, italy; Barbara Davis Center, Denver, CO- USA. In type 1 diabetes, the target molecules of the T-cell response remain largely uncharacterized. In the NOD mouse, T-cell reactivity has been elucidated in part starting from islet-infiltrating lymphocytes, while data in man are scarce and come from peripheral blood lymphocytes. In type 1 diabetes, a specific T-cell response was shown against the ICA512 fragment of the IA-2 islet tyrosine phosphatase; in addition, we have recently observed a similar response agains the whole IA-2 molecule in newly diagnosed type 1 diabetic patients. We had access to freshly isolated human pancreatic isiets and islet-infiltrating lymphocytes obtained from a 14 year old female organ donor involved in a car accident soon after disease-onset (HLA-DR3 positive; ICA, anti-GAD and anti-1A2 positive). After islet isolation by intraductal collagenase distention, isletinfiltrating lymphocytes have been cultured in RPMI1640 containing rhlL-2 (5Ul/m) and $10 \%$ fresh human serum, and pulsed weekly with freshly isolated human islets $\left(300\right.$ islets $/ 5 \times 10^{\circ}$ lymphocytes) in presence of APCs $(106 / \mathrm{m} \mid)$. Furthermore, since the $6^{\text {th }}$ week of culture, a subline was alternately stimulated weekly with PHA $(1 \mu \mathrm{g} / \mathrm{ml})$ and islets. When enough T-cells were obtained, a T-cell proliferation assay was performed in triplicate and repeated after further 10 stimulation cycles. T-lymphocyles $(20,000 /$ well) were incubated for $72 \mathrm{~h}$ in presence or absence of: insulin, insulin peptide B9-23 or rh|A-2 at a concentration of $10 \mu \mathrm{g} / \mathrm{ml}$ or human or bovine islets $(300$ islets $/ \mathrm{ml})$, with HLA-matched APCs $(50,000 /$ well) and $10 \%$ fresh human serum. After $72 \mathrm{~h}$, ${ }^{3} \mathrm{H}$-thymidine was added for 16 hours. PHA $(10 \mu \mathrm{g} / \mathrm{ml})$ was used as positive control. In addition, T-cells were characterized by fiow cytometry. Finally, the production of IFN $\gamma$ and IL-4 was determined by a ELISA during the proliferation assay. A specific T-cell proliferation (Stimulation Index $\geq 3$ ) against miA-2 (mean S.I. $=6.0$ ) and bovine islets (mean S.I.=12.5) but not towards insulin and insulin peptide B9-23 was observed in the T-cell line cultured with islets and PHA, which also showed a weak reactivity against human islets (mean S.I. $=3.0$ ). The T-cell line grown in presence of human islets, showed reactivity against insulin (mean S.I.=3.4) and human isiets (mean $S . I,=3.4$ ), but not against other antigens tested. By flow cytometry, this line was mainly CD4+(90\%) and HLA-DR+ $(90 \%)$. High levels of IL-4 and IFN $\gamma$ were produced by both lines when stimulated with PHA. In conclusion, in human autoimmune diabetes, a response against $\mid A-2$ can be observed not only in peripheral $T$-cells, but also in islet-infiltrating iymphocyles, suggesting its potential pathogenic importance. In addition to IA-2, the isletderived T-cell lines recognize insulin, are mainly $\mathrm{CO} 4+$ and show both Th1 and Th2 phenotypes.
28

\section{EARLY PROINSULIN T-CELL-EPITOPES IN TYPE-1-DIABETES \\ I. Durinovic-Belló and A-G. Ziegler \\ Diabetes Research Institute, Munich, Germany}

Since insulin and its precursor proinsulin are expressed by pancreatic B-cells exclusively, the hypothesis that epitopes of both molecules may target autoimmune response to $B$ cells, is habitually discussed. We have shown recently that in young pre-diabetic individuals (autoantibody positive; $A D+)$ and IDDM patients cellular reactivity to insulin is higher than in older subjects, whereas cellular reactivity to other autoantigens is equally distributed. Here we analyzed simultaneous memory $T$ cell response to proinsulin and its 15 overlapping peptides $(p)$ at initiation of autoimmunity and at diabetes onset. In addition multiple follow-up samples from one subject were tested and intra-molecular spreading investigated. Proliferation of CD4/CD45RO memory $T$ cells, isolated from peripheral blood lymphocytes of 20 HLA-DRB1*0401/DQB1*0302 positive individuals $13 \mathrm{Ab}+$ relatives and IDDM patients at onset $(12,8 \pm 9$ years) and 7 autoantibodie negative relatives ( $A b$-; $5,7 . \pm 2$ years) - was analyzed. $40 \%(8 / 20)$ of $A b+$ relatives, IDDM patients and $A b-$ relatives exhibited elevated T-cell responses to peptide 11 situated in the central part of proinsulin molecule ( $p 11$; aa18 of the c-peptide to aa1 of the $\alpha$-chain). Significantly increased proliferative response of $A b+$ relatives and IDDM patients compared to Ab- relatives, was observed for insulin $\alpha$-chain $(p<0.005)$, proinsulin peptides 15 and 13 (end of $c$-peptid, aa28 plus entire $\alpha$-chain; $p<0.005$ and $p<0.05$, respectively), and 5 (aa20 of the $\beta$-chain to aa4 of c-peptid; $p<0.05$ ). Multiple follow-up of one individual KM (2 years before IDDM onset to 2 years after), revealed similar pattern of epitope recognition; first was recognized central part of the proinsulin molecule (aa8 to aa24 of the c-peptide), followed by $\alpha$-chain. At IDDM onset additional spreading to peptide 5 and insulin $B$-chain was observed (aa11 of the B-chain to aa4 of c-peptide). Our results reveal that memory $T$ cell response to proinsulin molecule is an dynamic process characterized at the beginning by the recognition of its central part (c-peptide). By pre-diabetic individuals and IDDM patients this is followed by increased cellutar reactivity to insulin $\alpha$-chain and B-chain. 
IMMUNOMODULATION OF INSULIN-SPECIFIC AUTOREACTIVE T-CELLS BY SULFATIDE VARIABLY EXPRESSED IN BETA CELLS K. Buschard, N. Schloot, A. Kaas, T. Bock, T. Horn, P. Fredman, and B.O. Roep. Bartholin Instituttet, Kommunehospitalet, Copenhagen, Denmark; Dept. of Pathology, Herlev Hospital, Herlev, Denmark; Dept. of Neurochemestry, University of Göteborg, Göteborg, Sweden; and Dept. of Immunohematology and Blood Bank, University Hospital, Leiden, The Netherlands.

Sulfatide and insulin are present in the secretory granules and at the surface of beta cells in islets of Langerhans. Insulin autoantibodies and T-cell reactivity against insulin exist in development of insulin-dependent diabetes during which active beta cells have shown to be more vulnerable than passive. In this study we detected median $25 \%$ lower amounts of sulfatide per insulin secretory granule in active, stressed beta cells compared to passive, resting beta cells $(p=0.003)$ using a specific sulfatide monoclonal antibody and electron microscopic evaluation $(n=192$ ultramicrographs). The presence of sulfatide in vitro at doses of 43-8.3 $\mu \mathrm{M}$ resulted in dramatically reduced insulinspecific proliferation $(622 \pm 449$, control value $18001 \pm 2845$, $p=0.0004$ ) of an autoreactive T-cell clone, isolated from an IDDM patient. No inhibition was found using the precursor of sulfatide, galactosylceramide, or GM1. Sulfatide did not reduce aspecific proliferation (induced by PMA) or specific proliferation induced by insulin B-chain (B11-27) peptide epitope. This implies that sulfatide affects processing of the insulin molecule. The findings of the study are suggestive of a (patholphysiological role of sulfatide, variably expressed in beta cells, by modulating the antigenicity of insulin.
THE FaS/APO-1 SURFACE ANTIGEN EXPRESSION AND IL-1 3 SECRETION IN PRECLINICAL AND DIFFERENT CLINICAL STAGES OF TYPE 1 DIABETES

D. Aydemir, M. Araslı, G. Deniz. G.Yıllar, S. Bilgiç, E.Aktaş, F.Salman, Y. Yılmaz. İ.Salman and M.T.Yıimaz. Institute for Experimental Medicine, Department of Immunology and Istanbul Faculty of Medicine, Division of Diabetes, Istanbul University, TURKEY

Beta cell destruction in diabetes could be caused by apoptosis that may be induced by the activation of the Fas (Apo-1/CD95) antigen pathway. The cytokine, interleukin-1 $\beta$ (IL-1 $\beta$ ) has also been implicated to play an important role in the autoimmune $\beta$-cell dantage of type 1 diabetes. The ain of this study was to compare the changes in CD95 expression and IL- $1 \beta$ secretion among four different clinical stages of type 1 diabetes; preclinical stage (first degree of relatives of type 1 diabetics with $I C A \geq 20 J D F u$ and the first phase $(1+3 \mathrm{~min})$ insulin secretion lower than 3 , percentile in the IVGTT test; $<56 \mathrm{ml} . \mathrm{U} / \mathrm{ml}$.) (G1); early clinical stage (duration of diabetes, $<3$ months) (G-2); clinical stage (6-12 months) (G-3); longterm clinical stage (1-5 years) (G-4) and in nondiabetic healthy controls (G-C). The percentage of CD3, CD4, CD8 and CD95 expression was analysed in peripheral blood mononuclear cells by using three colour flow cytometry and serum IL-1 $\beta$ concentration was measured by ELISA. Although the total $\mathrm{T}$ lymphocyles were increased significantly in the G-1 compared to the G-C $(74.8 \pm 5.8 \& 67.7 \pm 5.5$, respectively; $p<0.05)$, there was no significant expression of $\mathrm{CD}^{+}$and $\mathrm{CD}^{+}$T lymphocytes. $\mathrm{CD} 95$ expression was enhanced in G-1 and G-2 compared to G-C $(9.9 \pm 3.7,10.3 \pm 3.1$ $\& 5.8 \pm 2.8$, respectively; $p<0.05$ ), howcver there was no significant increase in apoptotic activity of G-3 and G-4. As to the serum [L-1/3 levels, they were significantly higher in G-1 than in G-C ( $<<0.05$ ). In conclusion, our results show that the increase of $\mathrm{LL}-1 \beta$ secretion and Fas/Apo-1 expression in preclinical stage and their reduction in clinical stage of type 1 diabetes might be two important mechanisms in the pathogenesis of beta cell destruction and death.

\section{OP 6}

\section{Defect Insulin Signalling Yields Insulin Resistance}

\section{1}

$\begin{array}{lll}\text { PROTEIN } & \text { KINASE } & \text { C MEDIATES } \\ \text { INDUCED } & \text { INSULIN } & \text { RESISTANCE } \\ \text { THROUGH SCEMIA }\end{array}$ INDUCED INSULIN RESISTAN
PHOSPHORYLATION OF IRS-1.

A.K. Busch*, I. Castan ${ }^{+}$, E. Degerman ${ }^{+}$, H. Tornqvist ${ }^{\S}$ and L. Mosthaf*. *Department of Molecular Signaling, Hagedorn Research Institute and ${ }^{5}$ Novo Nordisk, Gentofte, Denmark, 'Lund University, Sweden.

Patients with non-insulin-dependent diabetes mellitus show decreased insulin receptor (IR) tyrosine kinase activity as well as decreased tyrosine phosphorylation of IRS-1. In cells overexpressing the two proteins, hyperglycemia induces a decreased tyrosine phosphorylation of both, the IR and IRS-1. Inhibitors of the serine/threonine kinase PKC (protein kinase C) prevent the inhibitory effect of hyperglycemia on these proximal events in insulin signaling, suggesting that PKC mediates the effect through covalent modification of IR and/or IRS-1. In order to detect specific changes in the phosphorylation pattern in response to hyperglycemia we metabolically labeled A293 cells overexpressing the IR and IRS-1 with ${ }^{33} \mathrm{P}_{\text {ind }}$ and performed tryptic digestion of IRS- 1 followed by $2 \mathrm{D}$ phosphopeptide mapping. The nature of the phosphorylation of specific IRS-1 peptides was determined by phosphoamino acid analysis. The 2D phosphopeptide maps showed increased serine phosphorylation of several peptides in response to exposure to hyperglycemia (25mM 2-deoxy-glucose for 30 min) prior to insulin stimulation $\left(10^{-7} \mathrm{M}\right.$ for 5 minutes). This serine phosphorylation was blocked by pretreatment with the PKC inhibitor Gö-6976. We are currently using MALDI technology to determine the identity of these residues. The data suggest that hyperglycemia induces activation of PKC, which phosphorylates IRS-1 on serine residues thereby inhibiting its tyrosine phosphorylation and downstream insulin signaling. Furthermore, the possibility of feedback inhibition from IRS1 to the IR has been suggested. Whether PKC-mediated serine phosphorylation of IRS-1 contributes to insulin resistance in the diabetic state needs to be elucidated.

\section{2}

THE INSULIN RECEPTOR IS DEGRADED BY SPECIFIC PROTEIN KINASE C ISOFORMS

L. Mosthaf, A.K. Busch*, L.F. Juhl*, L. Nielsen, G.S. Olsen, Y. Ikeda and X. Seedorf, Department of Molecular Signaling, Hagedorn Research Institute, Niels Steensens Vej 6, DK-2820 Gentofte, Denmark.

*both authors contributed equally to this work

Protein-kinase C (PKC) has been shown to effect insulin-induced signal transduction and has been suggested to play an important role in insulin resistance. Here we report that specific PKC isoforms have a profound effect on insulin receptor degradation. Coexpression of the insulin receptor with wild-type and in particular constitutively activated PKC $\alpha, \beta 1, \beta 2, \varepsilon, \theta$ and $\eta$ leads to reduced amounts of insulin receptors, while the corresponding kinase inactive isoforms have either no, or only minor effect. Expression of constitutively active PKC $\alpha$ and $\theta$ fully eliminates receptor expression, suggesting that these two PKC isoforms are most potent in regulating insulin receptor degradation. TPA-induced activation of wild-type PKC $\varepsilon$ and $\theta$ induces insulin receptor degradation similar to the constitutively activated isoforms indicating that these isoforms induce receptor degradation also upon activation. To identify the receptor domain which mediates PKC-induced degradation we generated insulin receptors that either lack the juxta-membrane region, the kinase domain, the $\mathrm{C}$-terminal part, the juxta-membrane region and kinase domain, the kinase domain and C-terminal part, or the entire cytosolic part of the $b$ subunit. While all deletion mutants were degradaded by activated PKC $\theta, P K C \alpha-$ mediated receptor degradation required the presence of the insulin receptor tyrosine kinase domain. In conclusion, our data provide substantial evidence for an essential role of specific PKC isoforms in insulin receptor degradation and may provide a novel mechanism of insulin resistance caused by PKC activating agents such as hyperglycemia and TNF $\alpha$. 
33

INSULIN-STIMULATED AKT KINASE ACTIVITY IS REDUCED IN SKELETAL MUSCLE FROM INSULIN RESISTANT NIDDM SUBJECTS

A. Krook, M. Bjömholm, R. A. Roth, J. R. Zierath, and H. WallbergHenriksson, Stockholm, Sweden, and Stanford, USA

The serine/threonine kinase Akt (PKB/Rac), a downstream target of the lipid kinase phosphatidyl inositol (PI3K) 3-kinase, has been implicated to play a role in the insulin signalling pathway to glucose transport. We examined the effect of insulin on PI3 kinase and Akt kinase activity in skeletal muscle from six NIDDM patients and six healthy subjects. Whole body insulin sensitivity, assessed by the euglycemic hyperinsulinemic clamp, was significantly lower in NIDDM subjects $(\mathrm{P}<0.001)$, and this was accompanied by impaired in vitro insulin-stimulated glucose transport in skeletal muscle. Insulin stimulated PI3 kinase tended to be reduced in NIDDM subjects compared to healthy individuals, however, this did not reach statistical significance. In both groups, insulin induced a significant increase in Akt kinase activity, however, the response to maximal insulin $(60 \mathrm{nM})$ was markedly reduced in skeletal muscle from NIDDM subjects ( $66 \%$ of control levels, $p<0.01$ ). Furthermore, impaired Akt kinase activity was not accompanied by decreased protein expression of Akt. Instead, a trend towards increased Akt expression was noted in skeletal muscle from NIDDM subjects $(p<0.1)$. Thus we provide the first evidence that insulinstimulated Akt kinase activity is significantly reduced in skelctal muscle from insulin resistant NIDDM subjects as compared to age and BMI matched healthy controls. Furthermore, the parallel defects in insulinstimulated Akt kinase activity and glucose transport in diabetic skeletal muscle suggest that reduced Akt kinase activity may play a role in the development of insulin resistance in NIDDM.

\section{4}

ROLE OF CELL-PERMEABLE CERAMIDES IN REGULATING GLUCOSE TRANSPORT AND LIPOGENESIS BY INSULIN IN 3T3-L1 ADIPOCYTES

J.Mei, C.N. Wang, L. O'Brien and D.N.Brindley ${ }^{2}$ Dept of Biochemistry and Lipids \& Lipoprotein Research Group, University of Alberta, Edmonton, Canada

TNF- $\alpha$ activates sphingomyelinase through the p55 receptors and produces ceramide. The aim of this study was to investigate the role of the proposed second messenger, ceramide, in producing insulin resistance in 3T3-L1 adipocytes. 3T3L1 adipocytes were cultured in DMEM with $10 \%$ fetal bovine serum and differentiated. Glucose uptake and lipogenesis were measured by the addition of $\left[{ }^{3} \mathrm{H}\right] 2$-deoxyglucose or $\left[{ }^{14} \mathrm{C}\right]-(\mathrm{U})$ glucose, respectively. PI3-kinase and MAP kinase were assayed after immunoprecipitation and $\mathrm{pp} 70 \mathrm{~S} 6 \mathrm{~K}$ by immunoblotting. Incubation of differentiated 3T3-L1 adipocytes with $\mathrm{C}_{2}$-ceramide and TNF- $\mathrm{C}$ increased basal 2-deoxyglucose transport, but decreased the stimulation of glucose transport by insulin. $\mathrm{C}_{2}$-ceramides and TNF- $\alpha$ also decreased the ability of insulin to stimulate glucose incorporation into the fatty acids and the glycerol moieties of triacylglycerol. By contrast, both $\mathrm{C}_{2}$-ceramides and TNF- $\alpha$ alone increased the basal glucose incorporation into the fatty acid and glycerol moieties of triacylglycerol. Treatment of cells with Ly294022, rapamycin and PD98059 (inhibitors for activation of PI 3-kinase, pp70S6K and MAP kinase) respectively for $12 \mathrm{~h}$ blocked the ceramide-induced increase of GLUT1 and thus increased basal glucose transport. These inhibitors also blocked the ceramide-induced increase in basal glucose incorporation into the fatty acid but not the glycerol moieties of triacylglycerol. With ceramide there is a preferential diversion of glucose into glycerol. $\mathrm{C}_{2}$-ceramide increased PI 3-kinase activity associated with IRS-1 in the presence or absence of insulin. Ceramide caused the activation of pp 70S6K and MAP kinase (ERK1 and ERK2) in the absence of insulin, but had no significantly effect on insulin-stimulated activation of pp70S6K and MAP kinase. $C_{2}$-ceramide also blocked the insulin-stimulated increase in acetyl-CoA carboxylase which could explain the decrease in fatty acid synthesis by insulin stimulation after treatment cells with ceramide. It is concluded that cell-permeable ceramides can mimic some effects of TNF- $\alpha$ and these effects are likely mediated by the activation of PI 3-kinase and pp70S6K as well as MAP kinase. Our work provides further understanding for the mechanism of development of insulin resistance in adipocytes.

\section{5}

TUMOR NECROSIS FACTOR ALPHA AND OLEIC ACID ALTER CALCIUM SIGNAL TRANSDUCTION IN FIBROBLASTS FROM TYPE 1 DIABETICS N.R. Husni and B.E. Corkey; Diabetes and Metabolism Unit Boston University School of Medicine; Boston, Massachusetts; USA

This work examined the effects of tumor necrosis factor alpha (TNF- $x$ ) treatment, and the diabetic environment (elevated glucose and fatty acid), on bradykinin-induced $\mathrm{Ca}^{2+}$ mobilization in dermal fibroblasts from type 1 diabetic patients and matched controls. Fibroblasts were exposed to TNF- $\alpha(10 \mathrm{ng} / \mathrm{ml})$ for up to 48 hours. Cells in suspension were then loaded with fura- 2 acetoxymethyl ester, and bradykinin-induced $\mathrm{Ca}^{2+}$ mobilization was measured using fluorescence spectrophotometry. Basal intracellular $\mathrm{Ca}^{2+}$ levels were significantly lower in diabetic fibroblasts than controls $(\mathbf{P}<0.05)$, and $T N F-\alpha$ treatment caused a significant increase in basal $\mathrm{Ca}^{2+}$ in diabetic but not control cells $(P<005)$. Beginning with 1 hour of TNF- $\alpha$ treatment, increases in $\mathrm{Ca}^{2+}$ mobilization in response to bradykinin ( $1 \mathrm{nM}$ to $1 \mu \mathrm{M}$ ) were observed in cells from both controls and diabetics. With 24 hours of treatment, TNF- $\alpha$-induced increments in peak bradykinin response were three-fold greater in diabetics than in controls $(P<0.001)$. Similar results were seen with interleukin -1 beta treatment. $\mathrm{Ca}^{2+}$ transients induced by thapsigargin, an inhibitor of the endoplasmic reticulum $\mathrm{Ca}^{2+}-\mathrm{ATPase}$, were also greater in TNF- $\alpha$ treated fibroblasts than in untreated cells, with an apparently greater increase in cells from diabetic donors. These data indicate that TNF- $\alpha$ caused an increase in intracellular $\mathrm{Ca}^{2+}$ stores, which affected the magnitude of agonist-induced $\mathrm{Ca}^{2+}$ responses. Exposing fibroblasts to a combination of $11 \mathrm{mM}$ glucose and $2 \mathrm{mM}$ oleic acid for 48 hours caused increases in both the peak bradykinin response and the TNF- $\alpha$-induced increment in peak response, which were significantly greater in diabetics than controls $(P<0.001) ; 11 \mathrm{mM}$ glucose alone was without effect. That these phenomena were exhibited to a higher degree in cells from type 1 diabetics than in control cells indicates that fibroblasts from diabetic patients have a heightened sensitivity to TNF- $\alpha$ and oleic acid.

\section{6}

MOLECULAR ANALYSIS OF p85 $\alpha$ PHOSPHOINOSITIDE 3-KINASE IN SEVERE INSULIN RESISTANCE

KCR Baynes ${ }^{\mathrm{a}}$, JP Whitehead ${ }^{\mathrm{a}}$, R Stein ${ }^{\mathrm{b}}$, G Panoyotou ${ }^{\mathrm{b}}$,

T Hansen ', PR Shepherd ' ${ }^{\circ}$ and $S O^{\prime}$ Rahilly ${ }^{\text {a }}$

aDepartments of Clinical Biochemistry and Medicine, University of Cambridge, UK; 'Ludwig Institute of Cancer Research, London, UK;

'Steno Diabetes Center, Gentofte, Denmark and 'Department of

Biochemistry, University College London, UK.

Whilst some $10 \%$ of subjects with Type A insulin resistance syndrome have insulin receptor mutations, the rest remain unexplained at a molecular level. The $p 85 \alpha$ subunit of phosphoinositide 3 -kinase $(P \mid 3 K)$ was examined by SSCP in 21 subjects with features of the Type A syndrome of severe insulin resistance. One subject was heterozygous for a novel amino acid change Arg $409 \mathrm{Gln}$ lying in the p85 $\alpha \mathrm{N}$-terminal SH2 domain. Within the family the mutation appeared to cosegregate with fasting hyperinsulinaemia - median fasting plasma insulin in those with the mutation $(n=4)$ was $218 \mathrm{pmol}^{-1}$ and in wild type members $(n=2)$ was $69.5 \mathrm{pmol}^{-1}$ (reference range $<60 \mathrm{pmol}^{-1}$ ). RFLP analysis of 136 Danish type II diabetics and 135 normal controls found no other individuals with this mutation. The phosphopeptide binding characteristics of wild type and Arg $409 \mathrm{Gln} p 85 \alpha$ GST-fusion proteins were analysed by a sensitive BlAcore competition binding assay. Both wild type and Arg 409 Gin fusion proteins had similar affinity constants for a peptide containing two YMXM motifs ( $\left(\mathrm{C}_{50}\right.$ WT 2.2 , variant $1.8 \mathrm{nmol}^{-1}$ ). Transient transfections in ceils co-expressing $p 85 \alpha$ and $p 110 \alpha$ subunits of P|3K suggest that this mutation does not affect basal PI3K activity. This is the first reported mutation in p85 $\alpha$ in a subject with severe insulin resistance, although it lies within an $\mathrm{SH} 2$ domain it does not appear to affect binding to phosphotyrosine residues nor basal Pl3K activity. 


\section{Antigens and Antibodies in Type 1 Diabetes}

ACTIVE IMMUNE REGULATION FOULOWING ORAL CTB-INSULIN PREVENTS DIABETES IN NOD MOUSE

C. Thivolet, C. Ploix, INSERM U 449 Lyon France

Feeding target antigen is an attractive strategy for preventing organ-specific autoimmune diseases. We have previously demonstrated that CTB, the nontoxic moiety of the cholera toxin (CT) enhanced the tolerogen properties of orally administrated insulin in NOD mice, a model of spontaneous IDDM. Feeding a single dose of microgram amounts of CTBinsulin conjugate prevents islet infiltration by diabetogenic $T$ celis and protects animals against spontaneous and transferred autoimmune diabetes. In order to investigate the mucosal mechanisms of tolerance induction mice were fed with 1 or $10 \mu \mathrm{g}$ of $\mathrm{CT}$ a potent Th2 inducer together with $2 \mu \mathrm{g}$ of CTB-insulin prior to the cotransier with diabetogenic $T$ cells. Recipients of T cells from animals fed either with CTB alone, or CTB-insulin with $1 \mu \mathrm{g}$ or $10 \mu \mathrm{g}$ of CT became diabetic in 34 days $(4 / 5,5 / 5$ and $4 / 5$ respectively) in contrast to mice reconstituted with diabetogenic and CTB-insulin fed donor $T$ cells $(1 / 5, p<0,05)$. Cytokine profiles produced after in vitro stimulation were analysed $24 \mathrm{~h}$ and 7 days after feeding. Mesenteric lymph nodes (MLN) from mice fed with CTB had a threefold increase in IF Ny production between $24 \mathrm{~h}$ and 7 days after oral treatment. This increase was prevented following CTB-insulin but persisted after coadministration of CTB-insulin and CT. In contrast IL-4 production was specifically increased in MLN of mice fed with CTB-insulin. These experiments may elucidate how CTB-insulin can stimulate both local and systemic immunity which may have important consequences for our understanding of oral tolerance in autoimmunity.

\section{8}

EVIDENCE FOR IMMUNOGLOBULIN EPITOPE SPREADING IN GAD65 DURING THE PREDIABETIC PERIOD OF TYPE 1 DIABETES

P. Söhnlein, M. Müller, K. Syren, H.K. Akerblom ${ }^{\#}$, M. Knip ${ }^{\S}$, W. Richter: Department of Internal Medicine 1, University of UIm, Germany; ${ }^{5}$ Dept. of Pediatrics, Medical School University of Tampere, Finland; "Childrens Hospital, University of Helsinki, Finland.

Autoreactive islet cell antibodies directed to glutamate decarboxylase (GAD65-A) are established markers for prediction and diagnosis of insulindependent diabetes mellitus (IDDM). In this study we analysed the complexity and dynamics of the epitope-specific GAD65-A response in GAD65-A individuals during the prediabetic period and at onset of IDDM. Ten human monoclonal islet cell antibodies (MICA) derived from patients at onset of IDDM were used as tools in a immunohistochemical blocking test to probe GAD65-A ${ }^{+}$sera for their epitope recognition of GAD65 in islet cells. The MICA were purified, labelled with digoxigenin and their binding to islets was assessed on cryostat sections of human pancreas, which had been preincubated with GAD65-A ${ }^{+}$sera. The 10 MICA defined six distinct epitopes localised in three independent epitope clusters (EP1 - EP3) of GAD65. EP1 was situated in the middle region of GAD65 within amino acids 245 - 440; EP2 and EP3 were both localised further C-terminal from this region (aa 441-585). At onset of IDDM $56 \% \quad(n=44)$ of the GAD-A ${ }^{+}$sera recognised one or more MICA epitopes, with EP1 being more frequently $(50 \%)$ bound compared to EP2 $(36 \%)$ and EP3 $(39 \%)$. GAD65-A prediabetics $(n=21) 10-96$ months before onset of IDDM and 20 GAD-A healthy individuals at increased risk of IDDM showed a significantly decreased frequency of GAD65-A directed to EP-2. Follow-up sera from 10 prediabetics suggested that EP1 is an early, immunodominant epitope region and revealed epitope spreading from the middle region of GAD65 to the C-terminal epitope clusters. Highly sensitive epitope-specific GAD65-A tests may be useful to distinguish eariy and late stages of $B$ cell destruction before the onset of IDDM.

Supported by the IHFSP $361-95$ and the Ri/707 grant of the DFG.

\section{9}

THREE HUMAN GAD65-SPECIFIC IDDM-RELATED ANTIBODIES ISOLATED FROM AN ANTIBODY PHAGE DISPLAY LIBRARY

W. Richter, P. Soshnlein and. K. M. Jury. Department of Internal Medicine I, University of UIm, Germany

Human monoclonal lgG antibodies (hmabs) are essential tools for the characterisation of the humoral autoimmune response in insulin dependent diabetes meilitus (IDDM). Only few hmabs are available so far from patients at clinical onset of IDDM and all of them are directed to glutamate decarboxylase (GAD65). The phage display technology is a powerful tool for isolation of IDDM-related recombinant human antibody fragments $(\mathrm{Fab})$ specific for a variety of islet cell antigens. The aim of our approach was 1.) to generate a large combinatorial Fab phage display library characteristic for the humoral immune response at onset of IDDM and 2.) to isolate from this library new human islet cell autoantibody fragments directed to any relevant autoantigen in IDDM. The applied techniques for phage display of Fab and expression of soluble Fab molecules in the pComb3HSS system were established using the two human monoclonal GAD65 antibodies MICA 2 and 4 obtained by conventional methods. The antibody phage display library was generated from $10^{8}$ peripheral blood lymphocytes pooled from two individuals with high ICA titers at onset of IDDM. We demonstrated that the chosen phage display system is suitable for enrichment and production of naturally occurring IDDM-related Fabs by isolation of one known (MICA 6) and two new human monoclonal antibodies binding to GAD65 in an ELISA and a RIA. All new antibodies are specific for GAD65 and directed to the immunodominant middle region of GAD65 from amino acid 245 400. The same library will now be screened for high affinity Fabs to other autoantigens relevant for IDDM.

Supported by grants of DFG Ri 707/1-2 and HFSP 361-95 to W.R.

\section{0}

EARLY EXPRESSION AND HIGH PREVALENCE OF ISLET AUTOANTIBODIES FOR DR3/4 HETEROZYGOUS OFFSPRING OF PARENTS WITH TYPE 1 DIABETES: THE BABYDIAB STUDY. M. Schenker ${ }^{1}$, M. Hummel ${ }^{1}$, K. Ferber ${ }^{1}$, E. Keller ${ }^{1}$, E. D. Albert ${ }^{1}$, H.-U. Janka $^{2}$, C. Kastendiek ${ }^{2}$, M. Sorger ${ }^{3}$, F. Louwen ${ }^{4}$, G. S. Eisenbarth, A.-G. Ziegler'; ${ }^{1}$ Munich, ${ }^{2}$ Bremen, ${ }^{3}$ Bonn, "Münster, Germany; Denver, USA.

Encouraging strategies of immunotherapy in animal models of type 1 diabetes have been proposed aiming to intervene early in life to prevent the initiation of islet autoimmunity. Trials of primary intervention in humans could eventually be designed when populations at risk for the development of islet autoimmunity can be genetically defined and risk estimates of the frequency of islet autoantibodies in infancy are available. We therefore determined HLA genotype frequencies in $\mathbf{2 9 6}$ offspring of parents with type 1 diabetes who were followed from birth for at least two years (median follow-up 2.2 years) and who were characterized for the expression of insulin, GAD65, IA-2 and islet cell autoantibodies at birth, 9 months, 2, and 5 years of age. We found that the high risk HLA genotype DRB1*03/04(DQB1*0302) was present in $6.8 \%$ of offspring of parents with type 1 diabetes $(5.9 \%$ of mothers, $9.0 \%$ of fathers and $14.3 \%$ of both parents with type 1 diabetes). The probability to develop persistent or multiple autoantibodies by the age of 2 years was $23.1 \%$ $(95 \% \mathrm{Cl} 6.8-39.2)$ for offspring carrying the DRB1*03/04(DQB1*0302) genotype compared to $4.4 \%(95 \% \mathrm{Cl} 2.4-6.3)$ for offspring without this genotype (odds ratio $5.3, p<0.01$ ). These data show that islet autoantibodies are remarkably frequent for DR3/4 heterozygous offspring and such antibodies appear early in life. This information should aid in the eventual design of trials aiming to prevent islet autoimmunity. 
41

COULD EPITOPE MASKING EXPLAIN WHY PROINSULIN AUTOANTIBODIES ARE LESS CLOSELY ASSOCIATED WITH IDDM THAN INSULIN AUTOANTIBODIES?

AJK Williams, PJ Bingley, RE Chance and Gale EAM, University of Bristol, UK and Eli Lilly, Indianapolis, USA.

It has been suggested that proinsulin and insulin autoantibodies are equally potent markers of Type 1 diabetes. We have investigated this using a new radiobinding assay, adapted for measuring insulin (IAA) and proinsulin (PAA) autoantibodies. Antibodies were measured in sera from 1028 schoolchildren (Median age 11.4, range $9 \cdot 13.7$ years) and 182 newly-diagnosed IDDM patients from the same area (Median age 10.2, range $0.7-20.7$ years). The assay involved two stages: All sera were screened for binding of ${ }^{125} \mathrm{I}$ - labelled insulin or proinsulin. Subsequently all those sera with raised levels ( $>0.4$ units) were re-assayed for competitive displacement with excess unlabelled antigen. The proportion of children with increased insulin and proinsulin binding was similar for schoolchildren (58 vs 53$)$ and cases (140 vs 133), respectively. After competitive displacement, using an antibody threshold that gave a sensitivity of $70 \%(125 / 179)$ in the cases, more than twice as many schoolchildren had PAA $(44 / 1028)$ as IAA $(19 / 1028)(p<0.003) .66 \%$ of cases, but only $9 / 1028$ schoolchildren, had both IAA and PAA above these thresholds. 39 of the 44 schoolchildren with PAA showed competitive displacement with insulin. This suggests that there may be an epitope that is available on ${ }^{125}$ I. labelled proinsulin and native insulin but not on ${ }^{125}$ | - labelled insulin. Paradoxically, blocking of this epitope may reduce the prevalence of insulin autoantibodies in the healthy population without altering disease sensitivity, and may explain why insulin autoantibodies appear to be more specific markers of IDDM than proinsulin autoantibodies. Such differences in epitope specificity of insulin and other autoantibodies may be exploited to enhance our ability to discriminate between health and disease.

\section{OP 8 \\ Ion Channel Activity in $\beta$-Cells}

\section{3}

DYNAMIC IMAGING OF SUB-PLASMA MEMBRANE [ATP] IN $\beta$ CELLS WITH A SNAP25.FIREFLY LUCIFERASE CHIMAERA G.A. Rutter, H.J. Kennedy, and A.E. Pouli. Department of Biochemistry, University Medical School, Bristol BS8 1TD, U.K.,

Increases in free ATP concentration immediately beneath the plasma membrane may couple elevations in blood glucose to $\beta$-cell insulin release, through the closure of ATP-sensitive $\mathrm{K}^{+}$channels. In order to image these changes dynamically in single living cells we have constructed and expressed in primary islet $\beta$ - and derived MIN6 cells cDNA encoding a recombinant chimaera between the palmitoylated t-SNARE, SNAP25, and firefly luciferase. Examined by confocal immunocytochemistry, the SNAP25.luciferase chimaera was targeted efficiently to the plasma membrane. Imaged in single living cells (image capture at $2 \mathrm{~s}$ intervals) with a cooled, intensified charge-coupled device camera, resting [ATP] was similar $(2-5 \mathrm{mM})$, both in the subplasmalemmal region, and in the cytosol (measured with non-targeted luciferase). [ATP] in the subplasmalemmal domain was increased by $21.0 \pm 3.0 \%$ (mean \pm S.E.M.; half-time-to peak, $95 \pm 10 \mathrm{~s} ; n=3$ cells) by elevations in glucose in the physiological range $(3-16 \mathrm{mM})$. Influx of $\mathrm{Ca}^{2+}$ via the activation of $\mathrm{L}$ type $\mathrm{Ca}^{2+}$ channels with $56 \mathrm{mM} \mathrm{K}^{+}$increased [ATP] in the subplasmalemmal region of cells maintained at $3 \mathrm{mM}$ glucose by $20.8 \pm 2.7 \%$ (half-time-to peak, $13.0 \pm 1.0 \mathrm{~s}, n=103$ cells). This effect was entirely blocked by incubation in the absence of extracellular $\mathrm{Ca}^{2+}$-ions. These data indicate that elevated intracellular $\left[\mathrm{Ca}^{2+}\right]$ may stimulate glucose metabolism by activation of intramitochondrial dehydrogenases, providing a feed-forward loop to enhance ATP synthesis. In addition, in cells maximally stimulated with $30 \mathrm{mM}$ glucose and $56 \mathrm{mM} \mathrm{K}^{+}$, baseline oscillations in [ATP] were occasionally apparent, indicative of metabolic changes independent of intracellular $\left[\mathrm{Ca}^{2+}\right]$. This technology will allow changes in free [ATP] to be monitored in living ß-cells in a variety of normal and pathological states.
EVIDENCE FOR THE EXPRESSION IN HUMANS OF ISLET AUTOANTIGENS BY A SUBSET OF THYMIC EPITHELIAL CELLS R. Pujol-Borrell, X. Ferrer-Francesch, O. Dominguez, M. Juan, M. Foz-Sala and M. Sospedra . Immunology Unit. Hospital Universitari Germans Trias i Pujol. 08916 Badalona. Spain.

In type I diabetes mellitus there is a loss of tolerance to several pancreatic islets cell antigens, including insulin, glutamic acid decarboxilase (GAD) and IA2. Our previous results showed that during chilhood, mRNAs for several autoantigens are expressed in the thymus a low but significative levels. These transcription levels of tissue specific antigens detected in human thymus, suggests that thymus could play a role in maintaining tolerance to peripheral antigens. Here we report the initial characterization of four thymic subpopulations, one of them expressing preferentially 'peripheral' self-antigens. Thymic cell fractionation reveals a subpopulation of thymic cells expressing tissue-restricted transcrits of insulin, GAD67, TPO and another peripheral antigens to be present in a fraction enriched for cytokeratin positive cells, that hyperexpressed TAP-1 and as other markers, $\mathrm{HLA}-\mathrm{DR} \mathrm{ht}^{\mathrm{t}}$ and $\mathrm{HLA}-\mathrm{G}$, indicating that they probably are a subset of medullary epithelial cells. Conversely another subpopulation, which is highly enriched in bone marrow-derived antigen-presenting cells, macrophages and dendritic cells, do not express preferentially these selfantigens. Thus, expression of tissue-restricted genes such as insulin in a subset of thymic epithelial cells may serve to limit development of potentially autoreactive $T$ cells in autoimmune diseases.

\section{4}

INTERACTION OF MgATP WITH THE SULPHONYLUREA RECEPTOR SUBUNIT ACTIVATES ATP SENSITIVE K-CHANNELS

F.M. Gribble, S.J. Tucker and F.M. Ashcroft

University Laboratory of Physiology, Parks Road, Oxford, OX1 3PT, UK

The $\beta$-cell ATP-sensitive $\mathrm{K}^{+}\left(\mathrm{K}_{\mathrm{ATP}}\right)$ channel, whose activity regulates insulin secretion, comprises 4 pore-forming Kir6.2 subunits and 4 regulatory sulphonylurea receptors (SUR1). Regulation of $\mathrm{K}_{\mathrm{ATP}}$ channels by glucose involves the interaction of adenine nucleotides at both inhibitory and activatory sites: ATP and ADP inhibit the Kir6.2 subunit directly, whereas activation by MgADP involves the nucleotide binding domains (NBDs) of SUR1. The aim of this study was to investigate whether MgATP also interacts with SUR1. Cloned $K_{\text {ATP }}$ channels were studied electrophysiologically in inside-out membrane patches from mRNA-injected Xenopus oocytes. Kir6.2/SUR1 currents were inhibited by ATP with a $K_{i}$ of $28 \pm 4$ $\mu \mathrm{M}(\mathrm{n}=15)$ in $1.4 \mathrm{mM} \mathrm{Mg}$, but were more sensitive in the absence of $\mathrm{Mg}\left(K_{i}=5.8 \pm\right.$ $1.0 \mu \mathrm{M}, \mathrm{n}=7, \mathrm{p}=0.0007$ ) or when mutations were made in the Walker $\mathrm{B}$ motifs of either NBD of SUR1 (D853N, $K_{i}=13 \pm 0.2 \mu \mathrm{M} ;$ D1505N, $K_{i}=16 \pm 3 \mu \mathrm{M} ; \mathrm{p}=0.05$ ). Truncated Kir6.2 (Kir6.2AC36) currents, expressed without SUR, showed no change in ATP sensitivity on $\mathrm{Mg}$ removal $\left(K_{i}=115 \pm 6 \mu \mathrm{M}\right.$ in $1.4 \mathrm{mM} \mathrm{Mg}, \mathrm{n}=11$; $K_{i}=145 \pm 13 \mu \mathrm{M}$ in $0 \mathrm{mM} \mathrm{Mg}, \mathrm{n}=5$ ). These results suggest that the reduced ATPsensitivity of Kir6.2/SUR1 currents in Mg-containing solutions requires the NBDs of SUR1. Since the strong inhibitory effect of ATP usually dominates the response to ATP, we used an ATP-insensitive mutant of Kir6.2 (R50G, $K_{i}-4 \mathrm{mM}$ ) to investigate whether the interaction of MgATP with SUR1 stimulates channel activity. Kir6.2R50G/SUR1 currents were activated reversibly by MgATP (138 \pm $5 \%$ by $100 \mu \mathrm{M} ; 150 \pm 10 \%$ by $1 \mathrm{mM}$ ). This activation was abolished by mutations in the Walker A motifs of SUR1 (K719A/K1384M). We conclude that MgATP, like MgADP, both activates $K_{A T P}$ channels by interaction with the NBDs of SUR1, and inhibits the currents through a direct effect on Kir6.2. Since the free concentration of MgATP in $\beta$-cells is believed to be much greater than that of MgADP, the stimulatory effect of MgATP on $\mathrm{K}_{\mathrm{ATP}}$ channels may be important in vivo. 
45

IMPAIRED FUNCTION OF HUMAN B-CELLS DUE TO A NOVEL SUR1 GENE MUTATION.

C Ämmälä', R Ashfield ${ }^{2}$, JC Chapman ${ }^{3}$, T Otonkoski ${ }^{4}$, P Thomas $^{5}$ and MJ Dunne ${ }^{3}$; 'Dept. Medical Biophysics, Göteborg University, Sweden, ${ }^{2}$ Physiology Laboratory, University of Oxford, UK, ${ }^{3}$ Dept Biomedical Science, University of Sheffield, ${ }^{4}$ Transplantation Laboratory, University of Helsinki, Finland, 'Dept Pediatrics, University of Michigan, Ann Arbor, USA.

Regulated insulin secretion is of fundamental importance for the maintenance of blood glucose levels, and disruption of B-cell stimulus-response coupling may have dire consequences for glucose homeostasis leading to such diverse diseases as diabetes and persistent hyperinsulinaemic hypoglycaemia of infancy (PHH). Severe, inherited forms of PHHI, which are associated with sustained insulin secretion despite profound hypoglycaemia, have been linked to mutations in the SURI gene coding for the sulphonylurea receptor. SUR1 together with the inwardly rectifying $\mathrm{K}^{+}$channel subunit $\mathrm{Kir} 6.2$ forms the $\beta$-cell $\mathrm{K}_{\mathrm{ATP}}$ channel complex. Here, we have used electrophysiological techniques to investigate the functional consequences of a novel SURI mutation found in a majority of Finnish PHHI patients. In this population a $T$ to A point mutation in exon 4 results in a valine to aspartic acid change at residue 187 (V187D) of the SUR1 gene. Cell-attached patch recordings of human $B$-cells isolated from a Finnish patient following pancreatectomy showed no spontaneous $\mathrm{K}_{\mathrm{ATP}}$ channel activity $(\mathrm{n}=17)$ and no activation of channel activity by diazoxide $(\mathrm{n}=12)$ or somatostatin $(\mathrm{n}=11)$. In excised patches there was a dramatic reduction in channel amplitude $(1.4 \pm 0.4 \mathrm{pA}, \mathrm{n}=12)$ compared to control $B$-cells $(30.3+2.4 \mathrm{pA}, \mathrm{n}=143)$. When SUR1 mRNA engineered to carry the V187D mutation (SUR1-V187D) was co-injected with Kir6.2 mRNA into Xenopus oocytes, no expression of channel activity could be detected either by whole-cell current recordings of metabolically poisoned oocytes $(n=5)$ or in isolated giant patches excised into an ATP-free solution $(n=4)$. This study demonstrates the loss of functional $K_{A T P}$ channels caused by a novel point mutation located in a putative transmembrane region of SUR1. This defect leads to severely impaired insulin release in the Finnish population of PHHI patients.

\section{7}

PHYSICAL INTERACTION BETWEEN SECRETORY GRANULES AND L-TYPE CALCIUM CHANNELS IS REQUIRED FOR FAST EXOCYTOSIS IN B-CELLS

E.Renström, D. Atlas', S. Barg and P.Rorsman, Dept. of Physiology and Neuroscience, Sölvegatan 19, S-223 62 Lund, Sweden and 'Dept. of Biological Chemistry, The Hebrew University of Jerusalem, Jerusalem 91904, Israel.

Exocytosis of insulin-containing secretory granules is elicited by influx of $\mathrm{Ca}^{2+}$ through voltage-sensitive L-type $\mathrm{Ca}^{2+}$-channels in the plasma membrane. Single-channel recordings of $\mathrm{Ca}^{2+}$-channel activity and immunocytochemistry, using an antibody directed against the $\alpha_{1 \mathrm{c}}$-subunit of $\mathrm{L}$-type $\mathrm{Ca}^{2+}$-channels, indicate that the $\mathrm{Ca}^{2+}$ channels are unevenly distributed in the B-cell membrane and that they co-localise with the secretory granules. The molecular/cellular mechanisms behind this organisation and the functional significance (if any) remain unestablished. Recently it was demonstrated that the exocytosis-regulating proteins like syntaxin, SNAP-25 and synaptotagmin interact with a site on the intracellular loop connecting domains II and III of the $\alpha$-subunit of $\mathrm{N}$ - and L-type $\mathrm{Ca}^{2+}$ channels. Peptides containing this synaptic protein interaction-, or synprint-site of the $\mathrm{N}$-type channels, have been demonstrated to inhibit neurotransmission. Here, we have investigated whether such peptides can also interfere with exocytosis in mouse pancreatic B-cells. This was investigated using the standard whole-cell configuration of the patch-clamp technique and capacitance measurements to monitor exocytosis. A $20 \mathrm{kD} \mathrm{L}$-loop peptide, containing the synprintsite of the L-type $\mathrm{Ca}^{2+}$ channel, was obtained by expression in the protease-deficient $E$. coli strain BL21pLysS. Infusion of the L-Joop into the B-cells (by inclusion in the pipette solution that dialyses the cell interior, thus competitively inhibiting endogenous protein-protein interactions), virtually abolished exocytosis evoked by a $500 \mathrm{~ms}$ voltage-clamp depolarisation from $-70 \mathrm{mV}$ to zero. The exocytotic response amounted to $5 \pm 2 \mathrm{fF}$ and $105 \pm 22 \mathrm{fF}$ in the presence and absence of the L-loop, respectively ( $\mathrm{n}=16$ in both groups; $P<0.001$ ). By contrast, LC 753-779, a control peptide with only marginal affinity to the exocytotic proteins, had only minor effects on exocytosis which remained $83 \pm 34 \mathrm{fF}$ ( $n=6 ; n$ s vs. control). It was ascertained that the L-loop neither affected the voltage dependence nor the amplitude of the $\mathrm{Ca}^{2+}$ current. We conclude that physical contact between the secretory granules and the Ltype $\mathrm{Ca}^{2+}$ channels, via the synprint-site, is instrumental for fast depolarization-evoked exocytosis.

\section{6}

MECHANISM OF KATP CHANNEL INHIBITION BY ATP

F.M. Ashcroft, F.M. Gribble, T. Haug, P. Proks, F. Reimann, T.J. Ryder, S. Trapp and S.J. Tucker. University Lab of Physiology, Parks Road, Oxford OX1 3PT, UK ATP-sensitive $\mathrm{K}^{+}\left(\mathrm{K}_{\mathrm{ATP}}\right)$ channels play an important role in regulating insulin release in response to glucose and to sulphonylurea drugs. They are composed of four Kir6.2 and four sulphonylurea receptor (SUR1) subunits. The inhibitory effects of ATP are mediated via Kir6.2, whereas the potentiatory effects of MgADP and diazoxide, and the inhibitory action of tolbutamide are conferred by SUR1. We used a truncated isoform of Kir6.2 (Kir6.2 $\Delta \mathrm{C}$ ), that expresses ATP-sensitive K-channels in the absence of SUR1, to explore the mechanism of nucleotide inhibition. Kir6.2 $\Delta \mathrm{C}$ mRNA was injected into Xenopus oocytes and expressed currents were measured in giant inside-out patches. Kir6.2 was highly selective for ATP: $1 \mathrm{mM}$ of ITP, GTP, CTP and UTP had little effect, while $1 \mathrm{mM}$ ATP caused $~ 90 \%$ block. $K_{i}$ values were $115 \pm 6 \mu \mathrm{M}(n=11)$ for ATP, $260 \pm 22 \mu \mathrm{M}(n=5)$ for ADP and $9.2 \pm$ $0.5 \mathrm{mM}(\mathrm{n}=6)$ for AMP. Thus both the adenine moiety and the $\beta$-phosphate contribute to the specificity for ATP. As Kir6.2AC is highly selective for ATP, we used it as a biosensor to monitor the submembrane ATP concentration. Using the increase in conductance on patch excision, and the measured ATP dose-response curve, we calculated submembrane [ATP] in intact Xenopus oocytes to be $5.8 \pm 0.3$ $\mathrm{mM}(\mathrm{n}=9)$, in the absence of extracellular glucose. This result indicates that submembrane [ATP] is close to that of the bulk cytoplasm. Mutation of six different residues in Kir6.2 significantly reduced ATP-inhibition $\left(K_{i}\right.$ values $>$ $1 \mathrm{mM}$ ). These residues are located in two distinct regions of Kir6.2: the N-terminus preceding, and the $\mathrm{C}$-terminus immediately following, the transmembrane domains. Some mutations (in the C-terminus) also markedly increased the channel open probability: this may explain the decrease in apparent ATP-sensitivity. Other mutations (in both $\mathrm{N}$ - and $\mathrm{C}$-termini) did not affect the single-channel kinetics, and so may reduce ATP-inhibition by interfering with ATP-binding and/or the link between ATP-binding and pore closure. Our results therefore suggest that both the Nand C-terminus of Kir6.2 are involved in $\mathrm{K}_{\mathrm{ATP}}$ channel inhibition by ATP.

\section{8}

THE GENERATION OF GLUCOSE-RESPONSIVE HUMAN INSULN SECRETING CELLS BY TRANSFECTING PHHI-DERIVED ISLET CELLS WITH PDXI, KIR6.2 AND SUR-1.

W.M. Macfarlane*, A.W. Hart*, H.M. Docherty*, R.F.L. James**, A. AynsleyGreen\#, J.C. Chapmant, R.M. Shepherd*, M.N. Hashmi+, K.E. Cosgrove*, M.J. Dunne ${ }_{*}^{+}$, and K. Docherty*. *Department Of Molecular and Cell Biology, University Of Aberdeen, ${ }^{* *}$ Department of Surgery, University of Leicester, \#Institute of Child Health, London, and $\ddagger$ Department of Biomedical Sciences, University of Sheffield, U.K

Persistent hyperinsulinaemic hypoglycaemia of infancy (PHH), is a rare disorder characterised by uncontrolled secretion of insulin. Treatment often involves pancreatectomy. Defects in glucose-sensitive insulin secretion (GSIS) in PHHderived islet cells have been linked to mutations in both subunits of the B-cell $\mathrm{K}_{\mathrm{AT}}$ channel; the sulshonylurea receptor (SUR1), and the $\mathrm{K}^{+}$channel pore Kir6.2. PHHIderived islet cells also exhibit impaired expression of the homeodomain transcription factor PDXI. In order to correct these defects, islet cells (NES 2Y) from a patient with PHHI were stably transfected with CDNAs encoding PDX1, SUR1 and Kir6.2 One resultant clonal line, Nisk9, displayed GSIS within the physiological range. Electrophysiological studies with patch-clamp techniques revealed that these modified cells expressed fully operational $K_{\text {ATP }}$ channeis with an inwardly-rectifying biophysical profile, and sensitivity to intracellular nucleotides (ATP, and ADP) and pharmacological agents (diazoxide, and tolbutamide). The NISK 9 B-cells, unlike the NES $2 \mathrm{Y}$ cells, also expressed operational voltage-dependent $\mathrm{Ca}^{2+}$ channels. These channels were causally linked to depolarisation-dependent rises in cytosolic $\mathrm{Ca}^{2+}$ and insulin secretion when NISK 9 B-cells were exposed to either glucose, tolbutamide and high external concentrations of $\mathrm{KCl}$. These results demonstrate that the defects in PHHI-derived islet cells can be corrected in vitro, for possible autologous transplantation into the patient, and that NISK 9 B-cells are a novel glucose-sensitive B-cell line. 
OP 9

\section{Exercise}

\section{9}

EXERCISE LEADS TO IMPROVED INSULIN ACTION THROUGH OVER EXPRESSION OF KEY PROTEINS IN GLUCOSE METABOLISM.

J.R. Zierath', N. Hjeltnes ${ }^{2}$, D. Galuska', M. Björnholm ${ }^{1}$, A-K. Aksnes ${ }^{2}$, A. Lannem $^{2}$, and H. Wallberg-Henriksson'. 'Dept of Clinical Physiology, Karolinska Hospital, Stockholm, Sweden, "Sunnaas Hospital, Olso, Norway.

Complete spinal cord lesion leads to profound metabolic abnormalities and striking changes in muscle morphology. We assessed the effects of electrically stimulated leg cycling (ESLC) on whole body insulin sensitivity, skeletal muscle glucose metabolism, muscle fiber morphology, enzyme activity and protein expression of key genes involved in glucose uptake and metabolism in five tetraplegic subjects with complete $\mathrm{C}_{5}-\mathrm{C}_{7}$ lesions. ESLC ( 7 sessions/wk for $8 \mathrm{wk}$ ) increased whole body insulin stimulated glucose uptake $(33 \pm 13 \%)$ concomitant with a 2.1 -fold increase in insulin-stimulated 3-O-methylglucose transport in isolated vastus lateralis muscle $\left(1.42 \pm 0.26\right.$ vs. $3.00 \pm 0.46 \mu \mathrm{mol} \mathrm{x} \mathrm{ml}^{-1} \mathrm{x} \mathrm{h}^{-1}$ for pre vs. post training). ESLC lead to a marked increased in protein expression of GLUT4 $(378 \pm 85 \%)$, glycogen synthase $(526 \pm 146 \%)$ and hexokinase II $(204 \pm 47 \%)$ in skeletal muscle, whereas phosphofructokinase $(282 \pm 97 \%)$ was not altered. Hexokinase II activity was increased by $25 \%(\mathrm{P}<0.05)$, whereas enzyme activity of phosphofructokinase, glycogen synthase, and citrate synthase were not changed with training. ESLC training also lead to a $68 \%$ increase in muscle glycogen content $(80.8 \pm 26.7$ vs.

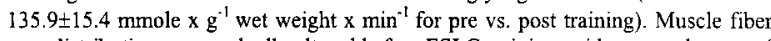
type distribution was markedly altered before ESLC training, with a near absence of type I muscle fibers, a predominant distribution of type IIb fibers $(64 \pm 8 \%)$ and marked general atrophy of all muscle fibers. Muscle fiber type composition was significantly altered following ECSL training. In conclusion, muscle contraction improves insulin action on whole body and cellular glucose uptake in tetraplegic subjects through a major increase in protein expression of key genes involved in the regulation of glucose metabolism. Furthermore, improved insulin action on glucose metabolism preceded changes in muscle fiber type distribution. Thus, physical training is an effective therapy to combat insulin resistance in tetraplegic subjects.

\section{1}

EFFECTS OF EXERCISE AND INSULIN ON MUSCLE GLUCOSE UPTAKE IN ATHLETES AND NORMAL SUBJECTS

Kirsti Larmola, Hannele Yki-Järvinen, Teemu Takala, Vesa Oikonen, Jukka Kemppainen, Hanna Laine, Pauliina Peltoniemi, Ulla Ruotsalainen, Juhani Knuuti and Pirjo Nuutila, Departments of Medicine, University of Turku and Helsinki, and Turku PET Centre, Finland.

Recent in vitro studies have demonstrated that insulin and exercise stimulate glucose uptake via distinct signaling pathways. We determined whether enhanced insulin sensitivity of glucose uptake in athletes also increases the ability of acute exercise to stimulate glucose uptake. For this purpose, muscle glucose uptake $\left.\left({ }^{18} \mathrm{~F}\right]-\mathrm{FDG}\right)$, blood flow $\left({ }^{15} \mathrm{O}-\mathrm{H}_{2} \mathrm{O}\right)$ and oxygen consumption $\left(\left[^{15} \mathrm{O}\right]-\mathrm{O}_{2}\right.$, $)$ were measured with PET in 9 athletes $\left(\mathrm{VO}_{2 \max } 60 \pm 2 \mathrm{ml} / \mathrm{kg} \cdot \mathrm{min}\right)$ and 10 untrained subjects $\left(\mathrm{VO}_{2 \max } 38 \pm 1\right.$ $\mathrm{ml} / \mathrm{kg} \cdot \mathrm{min}, \mathrm{p}<0.001$ ) during euglycemic hyperinsulinemic conditions (serum insulin $70 \mathrm{mU} / \mathrm{L}$, for $60 \mathrm{~min}$ ) and isometric exercise in one leg (rectus femoris muscle, $15 \%$ of maximal power). Muscle oxygen consumption was" 15 -fold higher in the exercising as compared to the resting contralateral muscle and comparable in both groups during exercise ( $36 \pm 6$ vs $32 \pm 5 \mathrm{~mL} / \mathrm{kg}$ muscle min, athletes vs. controls, NS). Insulin increased muscle glucose uptake more in the athletes $(77 \pm 13$ $\mu \mathrm{mol} / \mathrm{kg}$ muscle $\mathrm{min})$ than in the untrained subjects $(43 \pm 5, p<0.05)$, while the increment induced by exercise was comparable in both groups (178 \pm 34 vs $149 \pm 15 \mu \mathrm{mol} / \mathrm{kg}$ muscle min, NS). Muscle blood flow rates in resting muscles ( $38 \pm 7$ vs $27 \pm 3 \mathrm{~mL} / \mathrm{kg}$ muscle $\mathrm{min}$, athletes vs controls, NS), and the increment induceed by exercise ( $231 \pm 3$ vs $221 \pm 3 \mathrm{~mL} / \mathrm{kg}$ muscle min, NS) were not different between the groups. These data provide direct in vivo evidence for differential regulation of glucose uptake by insulin and exercise and demonstrate that physical training increases insulin but not exercise stimulated glucose uptake.

\section{0}

COMPARISON OF INSULIN ACTION ON HEART AND SKELETAL MUSCLE GLUCOSE UPTAKE IN WEIGHT LIFTERS AND RUNNERS

T.O. Takala, P. Nuutila, J.Knuuti, M. Luotolahti and H. Vki-Järvinen. Turku and Helsinki, Finland

We have previously demonstrated that aerobic training (running) is associated with enhanced insulin sensitivity in skeletal muscles, an increase in heart size and and a decrease in insulin stimulated glucose uptake per heart mass. In the present study, we determined how resistance training (weight lifting) influences these parameters. We used $\left[{ }^{18} \mathrm{~F}\right] F D G$ and positron emission tomography combined with the euglycemic hyperinsulinemic clamp technique (insulin infusion rate 1 $\mathrm{mU} / \mathrm{kg} \cdot \mathrm{min}$ ) to quantitate myocardial, skeletal muscle (femoral region) and whole body glucose uptake in weight lifters $(n=8)$, runners $(n=8)$ and in age-matched sedentary males (controls). $\mathrm{VO}_{2}$ max was higher in the runners $(71 \pm 7 \mathrm{ml} / \mathrm{kg} \cdot \mathrm{min}, \mathrm{p}<0.001)$ than in weight lifters $142 \pm 6$ $\mathrm{ml} / \mathrm{kg} \cdot \mathrm{min})$ or controls $(42 \pm 5 \mathrm{ml} / \mathrm{kg} \cdot \mathrm{min})$. Skeletal muscle glucose uptake was enhanced in the runners $(125 \pm 45 \mu \mathrm{mol} / \mathrm{kg} \cdot \mathrm{min}, \mathrm{p}<0.01)$ but unaltered in the weight lifters $(59 \pm 33)$ compared to the controls $(63 \pm 22)$. The rate of glucose uptake per gram of myocardium was similarly decreased in runners $(544 \pm 141 \mu \mathrm{mol} / \mathrm{kg} \cdot \mathrm{min})$ and weight lifters $(651 \pm 128)$ compared with the controls $(1041 \pm 234, p<0.01)$. Both the runners $\left(122 \pm 12 \mathrm{grams} / \mathrm{m}^{2}\right)$ and the weight lifters $(108 \pm 15)$ had a higher left ventricular mass than the controls $(80 \pm 16, p<0.01)$. Consequently, total left ventricular glucose uptake, expressed per body surface area was similar in all groups $168 \pm 16,70 \pm 16$ and $82 \pm 19 \mu \mathrm{mol} / \mathrm{min} \cdot \mathrm{m}^{2}$, respectively). In summary, i) aerobic but not resistance training enhances skeletal muscle insulin sensitivity, ii) both aerobic and resistance training increase myocardial mass and reduce myocardial glucose uptake per muscle mass under resting conditions. These data demonstrate that aerobic and resistance induce similar changes in heart glucose uptake but have distinct effects on skeletal muscle insulin sensitivity.
52

TYPE 2 DIABETIC PATIENTS HAVE TO EXERCISE EVERY DAY. JL.Ardilouze, J.Ménard, D. Panarotto, D.Tessier and P.Maheux. Diabetes Research Group, CHU Sherbrooke, Canada.

Exercise is considered a comerstone in the treatment of diabetes. Scientific studies in this field are quite rare; poorly controlled patients are usually enrolled so statistically significant differences are easily pointed out. The aims of our randomized study were to: 1) evaluate the effects of a moderately intense physical activity program on well controlled $\left(\mathrm{HDA}_{1} \mathrm{C}<130 \%\right.$ of upper limit of normal) non-insulin treated type 2 diabetic patients, and, 2) assess the metabolic evolution during the 3 days following the last exercise session. Twenty five subjects (experimental group $(E) n=12$, control $(C) n=13$ ) were enrolled in a 10-wk aerobic exercise program (3 X60 min/wk, $69.5 \%$ maximal heart rate, with medical supervision). The two groups were similar in terms of age $(\mathrm{E}: 54.1 \pm 6.1 / \mathrm{C}: 54.3 \pm 6.5)$, $\mathrm{BMI}(31.4 \pm 5.1 / 32.4 \pm 5.2), \mathrm{HbA}_{1} \mathrm{C}(7.7 \pm 1.6 /$ $6.9 \pm 1.0 \%)$, fasting glycemia $(8.6 \pm 2.977 .1 \pm 1.9 \mathrm{mM})$, and $\mathrm{Vo}_{2} \max (25.0 \pm 6.3 /$ 23.8 $\left.\pm 6.4 \mathrm{ml} \cdot \mathrm{kg}^{-1} \cdot \mathrm{min}^{-1}\right)$. Lipids profile was optimal: cholesterol $(4.94 \pm 0.76 /$ $5.01 \pm 0.78 \mathrm{mM}), \mathrm{HDL}(1.06 \pm 0.34 / 1.02 \pm 0.34 \mathrm{mM})$, LDL $(2.90 \pm 0.63 / 3.14 \pm 0.79$ $\mathrm{mM}$ ), TG $(2.30 \pm 1.55 / 2.36 \pm 1.66 \mathrm{mM})$. At the end of the program, there was a statistically significant difference for $\mathrm{DVO}_{2} \max \left(\mathrm{E}:+4.1, \mathrm{C}:+0.2 \cdot \mathrm{ml}^{\prime} \mathrm{kg}^{-1} \cdot \mathrm{min}^{-1}\right.$; $p<0.05)$ and $\square L D L(E:+0.19, C:-0.06 \mathrm{mM} ; 0<0.05)$ between the two groups. Moreover, the metabolic evolution 3 days after the last exercise session (D1, $D 2, D 3)$ showed three significant differences: an increase in LDL (D1=3.161 $0.73 / D 2=3.30 \pm 0.77, p<0.005$ ), an increase in fasting insulinemia $(D 1=118.9 \pm$ $91.0 / D 3=324.7 \pm 609.3, p<0.002$ ) as well as an increase in the insulin/glucose ratio (D2 $=15.8 \pm 9.1 / D 3=45.0 \pm 47, p<0.003$ ). In these patients, moderately intense physical exercise had no effect on glycemic control but the interruption of exercise worsened LDL concentrations and insulin sensitivity within 36 hours. These data suggest that well-controlled non-insulin treated type 2 diabetic patients should exercise every day. 


\section{A COMPARISON OF GLUCOSE AND INSULIN EXCURSIONS AFTER INSULIN LISPRO OR HUMULIN R FOLLOWED BY MODERATE EXERCISE}

M. Robinson-Pleadwell', L. Morrical' ${ }^{1}$ S. Hillis' ${ }^{1}$, T. Strack ${ }^{2}$, and G. Bailey' ${ }^{1}$, The Bailey Clinic, Red Deer, Alberta ${ }^{1}$, and Eli Lilly Canada ${ }^{2}$

This randomized open-label crossover trial studied 20 patients with type 1 diabetes with a low fasting C-peptide, mean age 38 years and an average screening HbAlc of 0.087 (normal <0.061). During a 4-8 week lead-in period, diabetes was controlled using insulin lispro ac meals and Humulin ultralente bid and the optimal dose of insulin lispro required prior to a standardized test meal followed by moderate exercise ( $90 \mathrm{kcal}$ semi-quantitated on a bicycle ergometer) was determined for each subject. Glucose and insulin excursions were then determined during and after a moderate energy expenditure $(90 \mathrm{kcal}$ quantitated using a breathby-breath measurement of $\mathrm{O}_{2}$ uptake at $80 \%$ of anaerobic threshold) beginning 90 minutes after a test meal, which was preceded by the optimized dose of insulin lispro or Humulin R. Glucose levels were significantly lower with insulin lispro compared to Humulin $R$ at all time points post exercise ( 30 min-10.35 vs 15.31 , p<.01, 60 min-9.92 vs $14.83, p<.01,90$ min-9.84 vs $14.47, p<.01,120$ min-9.41 vs $13.02, p<.01,150$ min-8.60 vs $11.80, p=.01$ ). Insulin levels $90-120$ minutes post exercise were consistently higher with Humulin R compared to insulin lispro and this correlated with a greater rate of decline of the blood glucose during the postexercise period with Humulin $R$ compared to insulin lispro. Because the patients on Humulin R started at a higher glucose level just prior to exercise, mild hypoglycemia occurred infrequently post exercise with both insulin lispro and Humulin R (6 insulin lispro, 1 Humulin R). No moderate or severe hypoglycemic events occurred during a total of 4.74 patients years of intensive insulin therapy using insulin lispro ac meals and ultralente bid despite a reduction of HbAlc from $0.087 \pm .019$ to $0.070 \pm .011$ over an average study period of 78 days per patient. In conclusion, a regimen of insulin lispro ac meals and ultralente bid significantly improved blood glucose and insulin excursions after moderate exercise and significantly reduced the HbAlc without inducing moderate or severe hypoglycemia.
Glucose homeostasis during a post-prandial exercise in intensively-treated type 1 diabetics (Db1) subjects treated with the basal-bolus insulin regimen (Ultralente-Lispro (LP)).

R. Rabasa-Lhoret, F. Ducros, J. Bourque, and J.-L. Chiasson. Research Center, CHUM, Campus Hôtel-Dieu, Montreal, Canada, $\mathrm{H} 2 \mathrm{~W} 1 \mathrm{~T} 8$

The study was designed to compare 3 different pre-meal doses of LP insulin $(100 \%, 50 \%$ and $25 \%)$ on glucose homeostasis during a postprandial exercise. Well controlled Db1 subjects $(n=6)$ were submitted to a 60 -min exercise at $50 \% \mathrm{VO}_{2}$ max $90 \mathrm{~min}$ after a mixed meal $(600$ $\mathrm{kcal}, 75 \mathrm{~g} \mathrm{CHO}$ ). After exercising with the full pre-meal dose (LP100\%), the subjects were then randomised in a cross-over design to LP $50 \%$ and LP $25 \%$ as the pre-meal dose. Post-prandial glycaemic rose by $0.6 \pm 2.3 \mathrm{mmo} / \mathrm{L}$ after LP $100 \%, 1.5 \pm 0.6$ after LP $50 \%$, and $4.0 \pm 0.5$ after LP $25 \%(p<0.04)$ above basal levels. During exercise, glycaemic levels dropped by $-4.4 \pm 0.4 \mathrm{mmol} / \mathrm{L}$ after LP $100 \%,-4.2 \pm 0.6$ after LP $50 \%$, and $-3.4 \pm 0.5$ after LP $25 \%$, resulting in glycaemic levels at the end of exercise of $-3.8 \pm 2.6,-2.7 \pm$ 0.6 , and $+0.6 \pm 0.6$ respectively in relation to basal levels. During the first hour post-exercise, glycaemic levels increased to $-2.9 \pm$ $1.5 \mathrm{mmol} / \mathrm{L}$ after LP $100 \%,-1.3 \pm 0.9 \mathrm{mmol} / \mathrm{L}$ after $\mathrm{LP} 50 \%$ and $+3.1 \pm 0.9 \mathrm{mmol} / \mathrm{L}$ after LP $25 \%(p<0.04)$ in relation to basal levels. The hypoglycaemic episodes per-exercise were 2 with LP $100 \%, 2$ with LP $50 \%$ and 1 with LP $25 \%$. These data indicate that in $\mathrm{Db} 1$, for a 60 -minute exercise at $50 \% \mathrm{VO}_{2} \max 90 \mathrm{~min}$ after meal: 1) pre-meal LP at $100 \%$ and $50 \%$ are associated with increase hypoglycaemic risk per-exercise; 2) pre-meal LP at $25 \%$ reduces the risk of hypoglycaemia at the cost of a slightly elevated post-prandial plasma glucose. It is suggested that intensively treated $\mathrm{Db} 1$ subjects doing moderate post-prandial exercise for an hour should reduce pre-meal LP to $25 \%$ of their usual doses.

\section{OP 10}

\section{Lipids and Late Complications}

\section{5}

APOLIPOPROTEIN E GENOTYPE IS A DETERMINANT OF LOW DENSITY LIPOPROTEIN CHOLESTEROL AND OF ITS RESPONSE TO A LOW CHOLESTEROL DIET IN IDDM PATIENTS WITH ELEVATED URINARY ALBUMIN EXCRETION

Eddy E. Blaauwwiekel, MD', Berta J. Beusekamp², Wim J. Sluiter, $\mathrm{PhD}$ '

Klaas Hoogenberg MD', Robin P.F. Dullaart MD PhD

Departments of Endocrinology' and Dietetics ${ }^{2}$, University Hospital Groningen, The Netherlands.

Introd uction: Lipoprotein abnormalities are likely to contribute to the increased cardiovascular risk of IDDM patients with microalbuminuria. The apolipoprotein (apo) E genotype is a determant of serum total and low density lipoprotein (LDL) cholesterol.

Methods: We compared lipoprotein levels before and after a I year low cholesterol diet ( $200 \mathrm{mg} /$ day) in 36 IDDM patients with albuminuria between 10 and 200 $\mu \mathrm{g} / \mathrm{min}$. Apo $\mathrm{E}$ genotype was characterized by polymerase chain reaction and restriction isotyping.

Results: In the apo E4 group (at least one $\mathrm{E} 4$ allele, 11 patients) baseline serum total cholesterol and LDL cholesterol as well as apo B levels were higher than in the apo E3 group (without an $\epsilon 4$ allele and with at least one $\epsilon 3$ allele, 25 patients); $6.88 \pm 0.97$ vs $5.86 \pm 1.16 \mathrm{mmol} / 1,4.83 \pm 0.98$ vs $3.9 \pm 1.02 \mathrm{mmol} / \mathrm{l}$ and $1.03 \pm 0.22$ vs $0.86 \pm 0.24 \mathrm{~g} / \mathrm{l}(\mathrm{P}<0.05$ for all). Cholesterol and linoleic acid intake were similar in both groups. At follow-up, cholesterol intake had similarly decreased in both groups, whereas linoleic acid intake did not change. In the apo E4 group, serum total and LDL cholesterol and apo $B$ decreased to $5.98 \pm 1.27 \mathrm{mmol} / 1,4.16 \pm 1.26$ $\mathrm{mmol} / \mathrm{l}$ and $0.86 \pm 0.18 \mathrm{~g} / \mathrm{l}$ at follow-up $(\mathrm{P}<0.01$ for all). These levels were not different from those in the apo $\mathrm{E} 3$ group, and the changes in these parameters were greater $(\mathrm{P}<0.02$ to 0.01$)$ than the unaltered levels in the apo E3 group. Multiple greater $(\mathrm{P}<0.02$ to 0.01$)$ than the unaltered levels in the apo E3 group. Multiple
logistic regresion analysis disclosed that baseline LDL cholesterol was positively logistic regresion analysis disclosed that baseline LDL cholesterol was positively
correlated to cholesterol intake in the apo E4 group only ( $\mathrm{P}=0.004)$, whereas LDL. cholesterol at baseline $(\mathrm{P}=0.006)$ and follow-up $(\mathrm{P}=0.037)$ was negatively related to linoleic acid intake in the whole study population. In the apo $\mathrm{E} 4$ group, changes in the LDL cholesterol were related to changes in cholesterol intake $(\mathrm{P}=0.005)$. Conclusion: The apo E4 allele is associated with atherogenic lipoprotein abnormalities in IDDM patients with minor elevations in albuminuria when they use their habitual diet. Apo E4 carrying IDDM patients respond better to low use their habitual diet. Apo E4 carrying IDDM patients respond better to low
cholesterol diet, possibly as a consequence of an apo E4-related enhanced cholesterol absorption efficacy.

\section{6}

RELATIONSHIP BETWEEN LIPOPROTEIN(a) PHENOTYPES AND ALBUMIN EXCRETION RATE IN DIABETIC PATIENTS

C.Hernández, R.Sinó, L.García-Pascual' ${ }^{1}$, R.Burgos, J.Mesa and P.Chacón ${ }^{2}$ Diabetes Unit and 'Biochemistry Dept. Hospital General Vall d'Hebron.' Endocrinology Dept. Hospital Mutua de Terrassa. Barcelona. Spain. The possible association between $\mathrm{Lp}(\mathrm{a})$ and albumin excretion rate (AER) is a topic that generate conflicting views. The aim of the study was to evaluate the relationship between serum $L p(a)$ concentrations and its phenotypes with AER. For this purpose 191 consecutive diabetic patients (69 IDDM and 122 NIDDM) were included. Lp(a) was determined by ELISA and its phenotypes by SDS-PAGE followed by immunoblotting. Lp(a) phenotypes were grouped by size in small $(\mathrm{F}, \mathrm{B}, \mathrm{S} 1, \mathrm{~S} 2)$, big $(\mathrm{S} 3, \mathrm{S4})$ and null. AER was assessed by RIA and expressed as the mean of three urine samples on 24 hour collections. Statistics: ANOVA, linear, multiple and logistic regression analyses. $L p(a)$ and $A E R$ were $\log$ transformed prior to statistical analysis in view the non-normal distribution. Diabetic patiens with AER > 20 $\mu \mathrm{g} / \mathrm{min}$ (group $1 ; \mathrm{n}=54$ ) presented higher $\mathrm{Lp}(\mathrm{a})$ concentrations than patients with AER $<20 \mu \mathrm{g} / \mathrm{min}$ (group $2 ; \mathrm{n}=137$ ): median $19 \mathrm{mg} / \mathrm{dl}$ vs $5 \mathrm{mg} / \mathrm{dl} ; \mathrm{p}<0.0001$. The diferences remainded at significative level when type of diabetes was considered. A linear correlation between $\mathrm{Lp}(\mathrm{a})$ concentration and AER was observed (IDDM: $r=0.32 ; p=0.01$. NIDDM: $r=0.25 ; p<0.05$ ). In addition, AER was idependently correlated with $\mathrm{Lp}(\mathrm{a})$ concentrations in multiple regression analysis $(\mathrm{p}<0.01)$. On the other hand, the overall frequency distribution of $L p(a)$ phenotypes differed significantly between the two groups: small $58.8 \%$, big $47.2 \%$ and null $0 \%$ (group 1) vs small $38.4 \%$, big $44 \%$ and null $17.6 \%$ (group 2); $<<0.05$. Furthermore, AER $(\mu \mathrm{g} / \mathrm{min})$ was different among $\mathrm{L} \mathrm{p}(\mathrm{a})$ phenotypes: small $55 \pm 122$ (median 4.9 ), big $58 \pm 123$ (median 5.7) and null $3 \pm 2$ (median 2.3); $p=0.01$. None of the patients with null phenotype showed an AER $>10 \mu \mathrm{g} / \mathrm{min}$. Finally, $\mathrm{Lp}$ (a) serum concentration was independently associated to the presence of diabetic nephropathy (AER > $20 \mu \mathrm{g} / \mathrm{min}$ ) in the logistic regression analysis. We conclude that in diabetic patients serum $L p(a)$ concentration is associated with AER. Thus, the elevated cardiovascular risk observed in diabetic patients with high AER might be associated to Lp(a) concentration. Furthermore, patients with null $\mathrm{Lp}$ (a) phenotype could be considered as a group at low risk for the develovment of diabetic nephropathy. 
57

PHOSPHOLIPID TRANSFER PROTEIN (PLTP) MASS CONCENTRATION IN NIDDM.

C.Desrumaux •, L.Lagrost • G.Vaillant*, J.M.Petit*, S.Rudoni*, J.M.Brun*, Ph.Gambert •and B.Vergès*^, * Service Endocrinologie-Diabétologie, • INSERM U498; CHU Dijon, France.

Phospholipid Transfer Protein (PLTP) is supposed to play an important role in lipid metabolism. Indeed, PLTP facilitates the transfer of phospholipids between lipoproteins and is an important determinant of the size distribution of HDI particles. So far, PLTP mass has never been measured in diabetic patients. The aim of the present study was to measure plasma PLTP levels in NIDDM patients. Fifty NIDDM patients ( 23 men, 27 women) and 30 normolipidemic controls were studied. PLTP mass was measured using a competitive enzyme-linked immunosorbent assay (ELISA) with a polyclonal antibody. Mean plasma PLTP mass concentration was significantly higher in NIDDM patients than in controls $(6.76 \pm 1.93$ vs. $3.95 \pm 1.04 \mathrm{mg} / 1 ; \mathrm{p}<0.0001)$. As shown by multiple regression analysis, the difference for PLTP mass concentration between NIDDM subjects and controls was not due to age nor to plasma lipid levels but only due to the diabetic state (or to hyperglycemia). Among NIDDM patients, PLTP levels correlated positively with fasting glycemia $(r=0.34, p=0.02)$ or glycated hemoglobin $(r=0.38, p=0.009)$, but not with plasma lipid values (total cholesterol, triglycerides, $\mathrm{HDL}$-cholesterol). In a multiple linear regression analysis, only fasting glycemia $(t=2.16 ; p=0.03)$ or glycated hemoglobin $(t=2.38 ; p=0.02)$ were significantly associated with plasma PLTP mass concentration. In conclusion: 1) PLTP mass concentration is significantly increased in NIDDM patients. 2) Since the increase of PLTP levels relates only to hyperglycemia, this suggests that regulation of plasma PLTP levels might be independent on plasma lipid levels but would rather relate to glucose metabolism 3 ) Further investigations are needed to highlight the possible consequences of increased plasma PLTP levels in NIDDM and the link between glucose and regulation of PLTP mass.

59

ELECTRONEGATIVE LDL PROPORTION IS RELATED TO NONENZYMATIC GLYCATION IN IDDM BUT NOT IN NIDDM.

几L Sánchcz-Quesada ${ }^{1}$, A. Payés ${ }^{1}, M$. Rigla ${ }^{2}$, A. Caixd̀s ${ }^{2}$, J. Ordóñez-Llanos ${ }^{1}$, A Pérez ${ }^{2}$. Serveis de Bioquímica ${ }^{1}$ i Endocrinologia ${ }^{2}$. Hospital de la Santa Creu i Sant Pau. Barcelona. SPAIN.

Non-enzymatic glycosylation is a process able to modify the biological characteristics of low density lipoprotein (LDL), including an enhancement on the electronegativity of this lipoprotein. We previousiy reported that electronegative LDL (LDL(-)), a potentially atherogenic LDL subform, is abnormally elevated in diabetic patients, and is associated with parameters of glycemic control. Although the exact origin of the negative charge is still unknown, LDL(-) has been described as a citotoxic particle. In order to study the relation of electronegativity and nonenzymatic glycosylation in LDL we have analyzed the effect of glycemic optimization after insulin therapy (IT) upon both LDL(*) and glycated LDL (gLDL) in 10 IDDM and 10 NIDDM patients. Ten normoglycemic subjects were studied as a control group. Chromatographic techniques were used to evaluate LDL(-) (anion exchange), gLDL (phenyl boronate-affinity) and glycated hemoglobin ( $\mathrm{HbAlc}$ ) (anion-exchange). Wilcoxon $t$ and Mann-Whitney $U$ tests were used to evaluate statistical differences after IT (Wilcoxon) and vs controls (Mann-Whitney). Results are shown in the Table, and are expressed as mean $\pm \mathrm{SD}$

\begin{tabular}{cccccc} 
& \multicolumn{2}{c}{ IDDM } & \multicolumn{2}{c}{ NIDDM } \\
$\%$ & Before IT & After IT & Before IT & After IT & Controls \\
\hline HbAIc & $9.7 \pm 2.7^{\dagger}$ & $5.8 \pm 1.3^{\dagger *}$ & $9.5 \pm 1.7^{\dagger}$ & $5.9 \pm 1.0^{+*}$ & $4.6 \pm 0.6$ \\
gLDL & $2.8 \pm 0.6^{\dagger}$ & $1.9 \pm 0.6^{\dagger *}$ & $2.2 \pm 1.7^{\dagger}$ & $1.6 \pm 0.6^{+*}$ & $0.7 \pm 0.2$ \\
LDL $(-)$ & $20.7 \pm 6.1^{+}$ & $15.4 \pm 3.4^{\dagger *}$ & $16.5 \pm 3.9^{\dagger}$ & $16.8 \pm 3.5^{\dagger}$ & $9.2 \pm 2.4$ \\
\hline${ }^{\dagger}<0.05$ vs controls &
\end{tabular}

In conclusion, these data suggest that glycemic control, and therefore, nonenzymatic glycosylation is related to the enhanced LDL(-) proportion found in IDDM patients. However, other processes different than glycation are likely responsible for increased LDL(-) in NIDDM subjects, as LDL(-) proportion is unaffected by glycemic control.

\section{8}

GLYCATION INCREASES ELECTRONEGATIVE LDL SUBFRACTION AND ACCELERATES LDL OXIDATION IN TYPE II DIABETIC PATIENTS.

Moro E., Zambon C., Cazzolato G., Pianetti S., Pais M., Bittolo Bon G.

${ }{ }^{\text {nd }}$ Department of Internal Medicine and Metabolic Diseases, Regional Hospital Venice - Italy

Glycation and oxidation of low density lipoproteins are closely related and potentially accelerate each other, but the relationship between these two processes is not clear. The production of a more electronegative charged LDL is the common denominator of both glycation and oxidation of this lipoprotein. Recently, a more electronegatively charged LDL (LDL) has been identified in human plasma. This LDL subfraction contains oxidative modifications similar to those of in vitro modified LDL such as increased negative charge, : increased contents of conjugated dienes and MDA and dicrease content of vitamin $\mathrm{E}$. In this study we evaluated, if $L D L$ glycation is associated with an increase of $\mathrm{LDL}^{\circ}$ plasma concentration and with a decrease of the LDL resistance to in vitro oxidation. Twenty-four type 2 diabetics and 12 healthy control subjects were selected. Apo B glycation was evaluated using monoclonal antibodies against glycated apo B epitopes. As index of in vivo LDL oxidation we measured the percentage contribution to total LDL of LDL' by ion-exchange HPLC, and the concentration of MDA in plasma and isolated LDL by fluorimetric method. In vitro susceptibility to oxidation of LDL was evaluated following the kinetic of conjugated diene formation and measuring the lag-phase time in presence of $\mathrm{Cu}^{++}$ions. The percentage concentrations of $\operatorname{LDL}^{\prime}(3.88 \pm 1.49 \%$ vs $2.34 \pm 1.03 \% ; p<0.01)$ and of glycated Apo B $(3.33+2.54 \%$ vs $1.24 \pm 71 \%)$ were significantly increased in diabetic patients ( $p<0.01$ for both), while no difference was found in LDL and plasma MDA concentrations. The LDL plasma concentration is significantly correlated with glycated apo B $(r=0.68, p<0.001)$. LDL isolated from type 2 diabetic patients had a significant decrease $(p<0.001)$ of the resistance to oxidative modification as indicated by the shorter lag-phase time $(91+12.6$ vs $120 \pm 24.5$ minutes). The decrease of the lag-phase time is inversely related with glycated apo $B(r=-0.65, p<0.001)$ and $L^{-}$concentrations $(r=-0.69$ $\mathrm{p}<0.001$ ). In conclusion glycation of apo $\mathrm{B}$, in type 2 diabetic patients, is associated with a significative increase of $\mathrm{LDL}^{-}$which might explain the higher susceptibility of these lipoproteins to in vitro oxidation.

\section{0}

HIGH FREE FATTY ACIDS IN THE OFFSPRING ARE ASSOCLATED WITH A PARENTAL HISTORY OF CARDIOVASCULAR DISEASE. M. Carlsson, Y. Wessman and L.C. Groop. Dept. of Endocrinology. Malmö University Hospital, Lund University, Sweden.

NIDDM is associated with an increased risk of coronary heart disease and stroke. Fasting free fatty acids (f-FFA) are elevated in obesity and in NIDDM and predict deterioration of glucose tolerance and development of NIDDM. To examine the relationship between $\mathrm{f}$-FFA concentrations and cardiovascular disease (CVD), we measured f-FFA concentrations in 483 unrelated Swedish NIDDM ( $n=140$, men $=80$, females $=60$ ) and nondiabetic $(N D, n=343$, men=150, females $=193$ ) subjects. Information on parental history of myocardial infarction (MI), stroke and diabetes was obtained by questionnaire. In a subset of 220 subjects indirect calorimetry was performed and basal metabolic rate (BMR) measured. Results: Females had higher f-FFA levels than males (f-FFA $=810 \pm 300 \mu \mathrm{mol} / \mathrm{l}$ vs. $\left.670 \pm 270 \mu \mathrm{mol} / 1 ; \mathrm{p}<1 \times 10^{-6}\right)$ and NIDDM patients had higher f-FFA levels than controls $(810+290 \mu \mathrm{mol} / \mathrm{l}$ vs. $720 \pm 310 \mu \mathrm{mol} / 1 ; p=0.002)$. Data were analysed after dividing the age-adjusted FFA concentrations into quartiles. NIDDM patients, ND controls, men and women were separately considered. There were no differences in fB-glucose, fs-insulin, blood pressure, BMI, fatmass, WHR or BMR between quartiles of $\mathrm{f}-\mathrm{FFA}$ within the groups. ND men from the highest FFA quartile had higher s-cholesterols $(5.8 \pm 1.2 \mathrm{mmol} / 1$ vs. $5.2 \pm 1.2 \mathrm{mmol} / 1 ; \mathrm{p}=0.03)$ and $\mathrm{s}$ triglycerides $(2.1 \pm 1.5 \mathrm{mmol} / \mathrm{l}$ vs. $1.4 \pm 0.7 \mathrm{mmol} / \mathrm{l} ; \mathrm{p}=0.02)$ vs. ND men from the lowest FFA quartile. ND men and females from the highest FFA quartile reported more often a history of MI (men: $41 \%$ vs. $13 \%$; $p=0.007$; females $46 \%$ vs. $25 \%$; $p=0.03$ ) and stroke (men: $30 \%$ vs. $13 \% ; p=0.08$; females: $42 \%$ vs. $14 \% ; p=0.003$ ) in the parents vs. subjects belonging to the lowest FFA quartile. This relationship was not seen in the NIDDM patients. We conclude that high FFA levels in the offspring are associated with a parental history of CVD. It remains to be shown whether elevated FFA levels also predict CVD in the offspring. 


\section{OP 11}

\section{Retinopathy}

\section{1}

VITREOUS LEVELS OF IGF-1 AND IGFBP1/IGFBP3 N PROLIFERATIVE DIABETIC RETINOPATHY: A CASE-CONTROL STUDY

R. Burgos, L. Audi' ' C. Mateo'2, M. García' ' C. Hernández, A. Lecube, J. Mesa, A. Carrascosa ${ }^{1}$ and R. Simó. Diabetes Unit, 'Pediatric Research Unit and ${ }^{2}$ Ophthalmology Dept. Hospital General Vall d'Hebron. Barcelona. Spain. Insulin-like growth factor-1 (IGF-1) has been implicated in the pathogenesis of diabetic retinopathy, and elevated intravitreous IGF-1 levels as well as IGFBP1/IGFBP3 have been reported in diabetic patients with retinal neovascularization. The source of vitreal IGF-1, IGFBP1 and IGFBP3 are presumably ischaemic retina, but increased levels derived from serum diffusion cannot be excluded. Previously, we have demonstrated that intravitreal proteins are elevated in diabetic retinopathy. Therefore, vitreal IGF-1 and IGFBPs should be corrected by proteins in order to avoid the influence of unspecific increase of protein concentration found in diabetic patients. The aim of the study was to determine vitreal levels of IGF-1, IGFBP1 and IGFBP3 in diabetic patients with retinopathy and to investigate whether serum levels could contribute to its intravitreal concentrations. For this purpose we compared 21 diabetic patients with proliferative retinopathy (group A) and 13 nondiabetic patients (group B) in whom a vitrectomy was performed. Both groups were equipared by age, serum IGF-1, IGFBP1 and IGFBP3 levels. IGF-1 and IGFBP3 were measured by RIA and IGFBP1 was measured by immunocnzymometric assay. Vitreal levels of IGF-1 were elevated in group A (median $1.35 \mathrm{ng} / \mathrm{ml}$, range 0.3-8.7) in comparison with group B (median $0.25 \mathrm{ng} / \mathrm{ml}$, range $0-1.38$ ), $\mathrm{p}<0.0001$. After adjusting by vitreal proteins [ratio IGF-1 $(\mathrm{ng} / \mathrm{ml}) /$ protein $(\mathrm{mg} / \mathrm{ml})]$, the difference remainded significant $(p<0.005)$. Vitreal levels of IGFBP1 and IGFBP3 were also elevated in diabetic patients (IGFBP1 group A: median $1.6 \mathrm{ng} / \mathrm{ml}$, range 0.6- 20.7; IGFBP group B: median $0.4 \mathrm{ng} / \mathrm{ml}$, range $0.3-1.9 ; p=0.0001$; IGFBP3 group A: median $102.6 \mathrm{ng} / \mathrm{ml}$, range $53.9-350.8$, IGFBP3 group B: median $29.0 \mathrm{ng} / \mathrm{ml}$, range 3.2 $87.8 ; \mathrm{p}<0.0001)$ However, when the ratio IGFBP/protein was considered the differences were not significant. We concluded that elevated vitreous levels of IGF1 found in diabetic patients are origined by intraocular synthesis rather than serum diffusion. By contrast, unspecific increase of intravitreal proteins could contribute to elevated vitreous levels of IGFBP1 and IGFBP3 found in diabetic patients.

\section{2}

INCREASE OF IGF-1/2 AND IGF-BP3 RESULTS FROM BLOOD RETINA BARRIER BREAKDOWN IN PROLIFERATIVE DIABETIC RETINOPATHY

J. Spranger', J. Bühnen', W.F. Blum ${ }^{2}$, R. Meyer-Schwickerath ${ }^{3}$, H. Schatz ${ }^{1}$ and A. Pfeiffer ${ }^{1}$ Medizinische Klinik Bergmannsheil and ${ }^{3}$ Augenklinik Knappschaftskrkh. Langendreer, Ruhr-Universität Bochum; ${ }^{2}$ Abteilung für Endokrinologie, Kinderklinik, Universităt Gießen

Recently the important function of the IGF system in hypoxic eye disease such as proliferative diabetic retinopathy (PDR) has been demonstrated by showing that retinal neovascularisation was significantly inhibited in transgenic mice with blunted growth hormone function despite regular induction of VEGF by hypoxia. Since normal serum levels of IGFs are already 20 -fold above those in human vitreous even moderate leakage through the blood retina barrier would primarily determine intraocular IGF levels. Therefore we quantified serum and vitreous levels of IGF I / II and IGF-BP 3 and the leakyness of the blood retina barrier by measuring serum and vitreous levels of albumin. Methods: A control group without retinal angiogenesis and patients with PDR were compared. Levels of IGF I/II, IGF-BP3 and albumin (66kDa) were determined by immunological methods. Results: Vitreous levels of albumin were nearly doubled in patients with PDR $(290.4 \pm 47.6 \mathrm{mg} / \mathrm{dl} ; \mathrm{n}=20 ; \mathrm{p}=0.0167)$ compared to controls $(181.3 \pm 61.4 \mathrm{mg} / \mathrm{dl} ; \mathrm{n}=16)$, whereas serum levels did not differ significantly $(4726 \pm 263.5 \mathrm{mg} / \mathrm{dl}$ vs. $4392.5 \pm 808.6 \mathrm{mg} / \mathrm{dl})$. This was comparable to an increase of IGF-I/II and IGF-BP3 in vitreous from PDR patients (IGF-I: $1.1 \pm 0.13 \mu \mathrm{g} / \mathrm{ml} ; \mathrm{p}=0.005$. IGF-II: $33.2 \pm 2.7 \mu \mathrm{g} / \mathrm{ml} ; \mathrm{p}=0.0014$. IGF-BP3: $73.1 \pm 10.9 ; \mathrm{p}=0.0009 ; \mathrm{n}=25$ ) compared to controls (IGF-I: $0.7 \pm 0.1 \mu \mathrm{g} / \mathrm{ml}$. IGF-II: $21.3 \pm 4.2 \mu \mathrm{g} / \mathrm{ml}$. IGF-BP3: $31.3 \pm 4.9 \mu \mathrm{g} / \mathrm{ml}$; $n=19$ ). Senum levels of all investigated peptides did not differ significantly among the groups. Conclusions: This is the first study demonstrating that influx of IGF-I, IGF-II and IGF-BP3 in PDR quantitatively parallels influx of the liver derived serum protein albumin suggesting that leakage of the blood retina barrier and serum levels primarily determine intravitreal IGF levels rather than local synthesis. Novel powerful growth hormone antagonists may therefore provide a promissing approach to prevention of PDR upon improved metabolic control.

\section{3}

IGFBP-4/IGFBP-5 RATIO REGLLATES THE IGF-I EFFECTS ON HUMAN RETINAL ENDOTHELIAL CELLS (HREC)

S. Giannini, B. Cresci, A. Ciucci, L. Pala, C. Manuelli, A. Franchini* and C.M.Rotella. Endocrinology Unit, Section of Metabolic Diseases and Diabetes and Ophthalmology Clinic*, University of Florence, Italy.

Several evidences raised the suspicion that other agents in addition to hyperglycaemia were involved in diabetic retinopathy. Some growth factors, including the IGF-I and its IGFBPs, have been demonstrated to play a role in microangiopathy. Since the IGFBPs control endothelial cell mitosis regulating the IGF bioavailability, the aim of our study has been to check the effect that the growth factors implicated in diabetic retinopathy could have on HREC IGFBP levels and growth. Methods: HREC have been characterized by LDL uptake and factor VIII positivity; $48 \mathrm{~h}$ serum-free culture medium has been loaded on SDS-PAGE in order to check the presence of IGFBPs, which have been first identified by Ligand blot and then characterized by Immunoblot study. Thymidine incorporation has been used to estimate cell growth and Northern blot studies have been applied to determine the expression of IGFBP mRNAs. Our results showed that HREC produce different IGFBPs of $43,34,30$ and $24 \mathrm{kDa}$, respectively characterized as BP-3, BP-2, BP-5 and BP-4, and a fainter proteolitic band of 21-18 kDa. Northern blot studies revealed the presence of mRNA for all the expressed IGFBPs. After $24 \mathrm{~h}$ serum free culture, HREC demonstrated to respond in terms of growth to $10 \mathrm{nM}$ IGF-I and bFGF, which had an additive effect when added together; 10nM PDGF has been capable, although at a less extent, to stimulate HREC mitosis. Conversely, 10nM insulin and $1 \mathrm{nM}$ hGH failed to be mitogenic. When all these growth factors have been tested to control their ability on IGFBPs regulation, we observed that only IGF-I (alone or together with bFGF) dranatically reduced (until the disappearance) the presence of BP-4 and, in the meantime, increased the levels of BP5 , which showed a variation from a single to a doublet $\mathbf{O}$-glycosilated band. These IGF-I induced effects did not modify the expression of BP-4 and BP-5 mRNAs, suggesting that other levels of regulation should be involved. Moreover, the addition of an IGFBP-5 antibody (1:100) significantly reduced the HREC growth. Densitometric quantification of IGFBP bands seems to suggest that IGF-I starts to be a significant mitogenic agent $(0.1 \mathrm{nM})$ when the BP-4/BP- 5 ratio becomes less than 1 . In conclusion, IGF-I could modulate HREC growth altering the BP4/BP5 ratio decreasing the already reported inhibitory action of BP-4 and increasing BP-5, which we demonstrated to have a stimulatory action on IGF-I effects.

\section{4}

LEVELS OF SOLUBLE TGF- $\beta$ RECEPTOR-CD105 AND VASCULAR ENDOTHELIAL GROWTH FACTOR IN THE PLASMA AND VITREOUS OF DIABETIC PATIENTS WITH PROLIFERATIVE RETINOPATHY W. Aziz, R.Malik, L.Cheng, J.Dong, J.A. Olson, A.J.M. Boulton, S. Kumar, J.V. Forrester, D. McLeod. Department of Medicine, Manchester Royal Infirmary, Departments of Ophthalmology, Manchester Royal Eye Hospital and Aberdeen Royal Infirmary, The Medical School, University of Manchester, UK. Proliferative diabetic retinopathy is characterised by angiogenesis which may be regulated by the TGF- $\beta$ receptor (CD105) and/or vascular endothelial growth factor (VEGF). We have assessed the levels (ng/ml-Median (Q1-Q3)) of CD 05 and VEGF simultaneously in the plasma of diabetic patients with background retinopathy $(n=11)(\mathrm{HbAlc}-9.7$ (8.3-10.7), duration of diabetes-21(15-24)) and both plasma and vitreous of diabetic patients with proliferative retinopathy (PR) undergoing vitrectomy ( $\mathrm{n}=11$, HbAlc- 8.4 (7.6-9.5), diabetes duration - 18.5 (12.3-30.8)) and control subjects (C) $(\mathrm{n}=23)$. Plasma CD105 levels were significantly elevated in diabetic patients with background retinopathy $(4.3(4.1-4.5), p<0.0007$. However, there was no significant difference in $\mathrm{CD} 105$ levels between the plasma (PR- 1.4 $(0.05-10.3)$ v $\mathrm{C}-0.4(0.1-6.0), \mathrm{p}=0.71)$ and vitreous $(\mathrm{PR}-0.023(0.02-0.029)$ v $\mathrm{C}-$ $0.029(0.02-0.033), \mathrm{p}=0.14)$ of diabetic patients and control subjects. CD105 levels were significantly greater in the plasma compared to the vitreous of both $\mathrm{C}-0.4$ $(0.09-6.0)$ y $0.029(0.023-0.033)$, $p<0.0001$ and PR- $1.39(0.05-10.3)$ v $0.023(0.02-$ 0.029 ), p<0.006. Plasma VEGF levels did not differ significantly between C- 2.4 (0.6-22.6) and diabetic patients with PR-3.3 (0.38-16.6). However, levels of VEGF were significantly increased in the vitreous of patients with PR- 7.2 (1.9-15.6) compared to $\mathrm{C}-1.8(1.1-3.8), \mathrm{p}<0.01$. There was no significant difference in the levels of VEGF between the plasma and vitreous of either control subjects $2.4(0.6-22.6) \mathrm{v}$ $1.8(1.1-3.8), p=0.44$ or patients with PR- $3.2(0.38-16.6)$ v $7.2(1.9-15.6), p=0.43$. There was a significant inverse correlation between VEGF and CD105 levels in the vitreous of control subjects $(\mathrm{r}=-0.39, \mathrm{p}<0.02)$ and diabetic patients with $P R-(r=-0.51$, $p<0.001$ ). CDI05 levels are elevated in early stages of diabetic retinopathy but fall with the development of proliferative retinopathy. Whereas VEGF levels are elevated in proliferative diabetic retinopathy and an inverse relationship exists between the levels of these substances in the vitreous. CD105 may play a role at an earlier stage of diabetic retinopathy and VEGF may serve to maintain this process in end stage retinopathy. 
65

RETINAL NEOVASCULARIZATION IN THE RODENT IS INFLUENCED BY LOCAL RENIN-ANGIOTENSIN

C.J. Moravski, D.J. Kelly, M.E.Cooper *, S.L. Skinner and J.L. Wilkinson-Berka Depts. of Physiology \& Medicine*, The University of Melbourne, Australia, 3052. This study aimed to determine if an enhanced renin-angiotensin system is associated with increased blood vessel growth in the eye. Currently there exists no rodent model that mimics the pathology of proliferative diabetic retinopathy (PDR). However, like PDR, retinopathy of prematurity (ROP) has hypoxia as a stimulus for neovascularization thus acting as a suitable model for the study of the disease. In rats the retinal circulation is not established at birth resulting in relative tissue hypoxia. This environment stimulates local growth factors and ultimately leads to normal angiogenesis. When newborn rats are housed in high $\mathrm{O}_{2}$ angiogenesis is inhibited and subsequent exposure to room air causes explosive neovascularization, haemorrhage and scarring (ROP). ROP was induced in newborn rats by exposure to $80 \% \mathrm{O}_{2}$ for 11 days followed by 7 days in room air ROP shams were exposed to room air for 18 days from birth. A separate group of ROP and ROP shams received the ACE inhibitor, lisinopril ( $10 \mathrm{mg} / \mathrm{kg} / \mathrm{day}$ ip) from day 11 to 18. Eyes were also examined in two further groups; newborn rats exposed to 11 days of $80 \% \mathrm{O}_{2}$ or 11 days room air. Transgenic Ren-2 rats (TGR) which overexpress tissue renin and Sprague Dawley rats (SDR) were studied. In paraffin sections of ROP retina, vessels protruded into the vitreous and haemorrhages were seen, features not present in ROP shams. In the 11 day $\mathrm{O}_{2}$ group, few vessels were observed compared to shams. Stereology was performed by producing an index of blood vessel profiles (BVP) per histological section of inner retina. The number of BVP increased in ROP compared to ROP shams in both SDR $(25.7 \pm 1.5$ vs $18.7 \pm 1.2, \mathrm{p}<0.05)$ and TGR $(31.6 \pm 0.9$ vs $18.5 \pm 1.3$, $\mathrm{p}<0.05)$, with more BVP in TGR than SDR $(\mathrm{p}<0.05)$; the lisinopril data is currently being analysed. Retinal renin content increased in ROP compared with sham (SDR $12.0 \mathrm{x} / \div 3.1 \mu \mathrm{GU} /$ eye vs $9.5 \mathrm{x} / \div 3.7, \mathrm{p}<0.05$; TGR $85.0 \mathrm{x} / \div 3.7$ vs $48.8 \mathrm{x} / \div 2.3$ $p<0.05$ ). Lisinopril increased eye renin in ROP shams (TGR $785.8 \mathrm{x} / \div 2.5 \mu \mathrm{GU} / \mathrm{eye}$ ) and rose further with ROP $(928.7 \times / \div 2.32)$. These findings are consistent with physiologically regulated retinal renin-angiotensin system, which is sensitive to oxygen tension and participates in neovascularization.
66

RELATION BETWEen ADVANCED GLYCATION END PRODUCTS AND VASCULAR ENDOTHELIAL GROWTH FACTOR RECEPTOR IN DIABETIC EYES

Y. Kanazawa ${ }^{1}$, A. Kakehashi ${ }^{1}$, S. Inoda ${ }^{1}$, M. Kuroki ${ }^{1}$, M. Kaku', Y. Shimizu', S. Makino', M. Kawakami ${ }^{1}$, R. Nagai ${ }^{2}$, and S. Horiuchi ${ }^{2} ;{ }^{1}$ Omiya Medical Center, Jichi Medical School, Omiya; ${ }^{2}$ Kumamoto University Medical School, Kumamoto, Japan

We examined immunohistochemically the localization of advanced glycation end products (AGEs) and flt-1, a vascular endothelial growth factor receptor (VEGFR), to determine their relation to neovascularization in human diabetic eyes. Ocular fluid, a small iris specimen, and proliferative tissue were obtained from 32 patients ( 21 with proliferative diabetic retinopathy [PDR], 11 without diabetes) during intraocular surgery. VEGF levels were assayed by ELISA. Flt-l protein and AGEs were immunostained in the specimens. VEGF levels were higher in patients with diabetes (mean $837 \mathrm{pg} / \mathrm{ml}$ ) than in patients without diabetes (mean $76 \mathrm{pg} / \mathrm{ml})(p=0.004)$. VEGF levels in the aqueous humor were significantly correlated with those in the vitreous $(\mathrm{r}=0.606$; $p=0.001)$. Flt- 1 staining in 19 eyes with PDR was: none $(n=2,10.5 \%)$, moderate $(n=8,42.1 \%)$, and strong $(n=9,47.4 \%)$; flt- 1 staining in nondiabetic disorders wasnone $(n=4,40.0 \%)$, moderate $(n=3,30.0 \%)$, and strong ( $n=3,30.0 \%)$. AGEs were distributed diffusely in extracellular substances; VEGFR was localized in the neovascular endothelium in the proliferative tissue. The expression of flt-1, a VEGFR in the iris, together with the highly elevated VEGF levels in the aqueous humor in patients with diabetes, strongly supports the previous VEGF hyp othesis of angiogenesis in diabetic iris. The coexistence of AGEs and VEGFR in the proliferative tissue in the neovascular endothelium suggests that AGEs may play an important role in neovascularization in diabetic retinopathy.

\section{OP 12 GLP}

67

WITHDRAWN

\section{8}

A POSSIBLE MEDIATOR IN THE GLYCOGENIC EFFECT OF GLP-1 IN HUMAN SKELETAL MUSCLE

M.A. Luque, L. Márquez, I. Valverde and M.L. Villanueva-Peñacarrillo. Fundación Jiménez Díaz. Madrid, Spain.

GLP-1 (7-36)amide (GLP-1) activates glycogen synthesis in rat and human skeletal muscle pieces and human cultured cells, effect which is not mediated by adenylate cyclase. The possible implication of an inositolphosphoglycan (IPG) on the GLP-1 insulinomimetic effects in $\mathrm{BC}_{3} \mathrm{H}-1$ myocytes, HepG 2 cells, rat adipocytes and hepatocytes, has been documented. In this work, we studied the effect of GLP-1, and insulin, on glycogen synthase a activity -as glucosyl incorporation into glycogen from UDP-[U- $\left.{ }^{14} \mathrm{C}\right]-\mathrm{D}$-glucose-, and the kinetics of the glycosylphosphatidylinositols (GPIs), precursors of IPGs, in cultures stablished from satellite cells of human muscle (vastus lateralis) -from subjects without alterations of carbohydrate metabolism undergoing surgery-, grown in SKGM and fused in alpha-MEM. In myotubes (6 subjects), GLP-1 increased $(p<0.001)$ the control (absence of peptide) glycogen synthase $a$ activity $\left(0.028 \pm 3.3 \times 10^{3} \mathrm{U} / \mathrm{g}\right.$, $\mathrm{n}=57)$ at $10^{-10} \mathrm{M}(149 \pm 10 \%$ of control, $\mathrm{n}=24)$ and at $10^{-9} \mathrm{M}(154 \pm 9 \%$, $\mathrm{n}=30)$, with an apparent higher potency than that of insulin $\left(10^{-10} \mathrm{M}\right.$ : $\left.128 \pm 8 \%, n=21, p<0.01 ; 10^{-9} \mathrm{M}: 122 \pm 5 \%, n=26, p<0.001\right) .10^{-9} \mathrm{M}$ GLP-1, in cells prelabelled with myo- $I^{3} \mathrm{H}$ ]inositol, induced a fairly rapid decrease of GPl levels, followed by a recovery of the control value $10.5 \mathrm{~min}: 80 \pm 15 \%$ of control, $n=3$ subjects; $1 \mathrm{~min}: 59 \pm 8 \%$, $n=3, p<0.05 ; 2 \min : 107 \pm 25 \%, n=3 ; 10 \min : 123 \pm 13 \%, n=3)$; the same pattern was detected with $10^{-9} \mathrm{M}$ insulin $10.5 \mathrm{~min}: 85 \pm 20 \%$, $n=3 ; 1 \min : 43 \pm 9 \%, n=3, p<0.05 ; 2 \min : 87 \pm 8 \%, n=3 ; 10 \mathrm{~min}:$ $109 \pm 11 \%, n=3$ ). In conclusion, an IPG could mediate the insulinomimetic effects of GLP-1 in human skeletal muscle, as it seems to occur in other extrapancreatic tissues. 
69

\section{DIFFERENT MODE OF ACTION BY GLP-1 GIVEN BEFORE OR AFTER A} MEAL.

M.K.Gutniak, J. Svartberg, P.M. Hellstrom, S.W. Sanders, B. Ahrén, J.J. Holst and N. Adner. Multidisciplinary Pain Center Kronan, Karolinska Institute, Dept. of Medicine, Stockholm, Sweden, TheraTech Inc. Salt Lake City, USA, Dept. of Medicine, Lund Univ. Hospital, Malmő, Dept. of Medical Physiology, PANUM Institute, Copenhagen, Denmark and Dept. of Medicine, Södersjukhuset, Stockholm, Sweden.

The study was designed to determine the prandial effects of a 3-hour GLP-1 [Glucagon-like peptide 1 (7-36)amide] infusion on serum glucose, insulin, glucagon and gastric emptying in eight NIDDM patients in order to establish the optimal timing for GLP-1 administration during the meal. Different time lags between the start of the meal and administration of the peptide were studied. The study was a placebocontrolled comparison with random assignment to treatment sequence. Insulin infusion was given in order to normalize the glycaemia prior to the experiments. Stepwise infusion of GLP-1 (17 nmol) was started at the onset of a standard meal (550 Kcal)(A), $30 \mathrm{~min}$ (B) and $60 \mathrm{~min}$ after the meal (C). Gastric emptying was measured with paracetamol absorption technique. GLP-1 infusion increased the plasma levels from $20.9 \pm 2.8$ to $70.2 \pm 7.7$ (A), $75.5 \pm 7.8$ (B), and $94.2 \pm 15.4$ (C) $\mathrm{pmol} / \mathrm{L}(\mathrm{p}<0.001)$. The peptide significantly lowers the postprandial glycaemia in all experiments [AUC $2767 \pm 165$ control, $2340 \pm 136(\mathrm{p}<0.002)$ (A), $2262 \pm 90(\mathrm{p}<0.003)$ (B), 2048 \pm 133 $(p<0.03)(C) \mathrm{mmol} / \mathrm{L} / 300 \mathrm{~min}$. The insulin levels were unchanged but the insulinogenic indices increased. The postprandial glucagon levels were lowered only in (A) and (B) $(\mathrm{p}<0.03)$. Gastric emptying (T50) was significantly retarded only in (A) $(p<0.01)$, no effect was observed when GLP-1 was given 30 and $60 \mathrm{~min}$ after the meal. Results of this study suggest that GLP-1 exerts its effect during the meal by retarding gastric emptying in the early phase of digestion. The insulinotropic action is predominant during the later phase. As a potential therapeutic agent GLP-1 may be selectively targeted to act on gastric emptying in treatment of obesity, or insulin release in diabetes, depending on the time of administration in relation to meal.

\section{1}

COEXPRESSION OF GLUCAGON-LIKE PEPTIDE-1 RECEPTOR, VASOPRESSIN AND OXYTOCIN MRNAS IN NEURONS OF RAT SUPRAOPTIC AND PARAVENTRICULAR NUCLE

J.A.Zueco, A.I.Esquifino, J.A.Chowen, E.Alvarez, P.O.Castrillón and E. Blázquez. Instituto Cajal, CSIC and Departamento Bioquímica, Facultad Medicina, Universidad Complutense, Madrid, Spain

This study was designed to gain better insight into the relationships between glucagon-like peptide-1(7-36)amide (GLP-1) and both vasopressin (AVP) and oxytocin (OX). In situ hybridization histochemistry showed colocalization of the mRNAs for GLP-1 receptor, AVP and OX in neurons of the supraoptic (SON) and paraventricular (PVN) hypothalamic nuclei. In the SON, about $80 \%$ and in the PVN, $50 \%$ of neurons expressing AVP or OX also contained detectable levels of GLP-1 receptor mRNA. To determine whether GLP-1 alters AVP and/or OX release, a double in vivo and in vitro experimental study was designed. In vivo, intravenous administration into the jugular vein of 1 $\mu \mathrm{g}$ of GLP-1 significantly decreased plasma vasopressin and oxytocin concentrations. In vitro incubation of the neurohypophysis with GLP-1 did not modify the release of AVP, but it produced a minor increase in the secretion of oxytocin. The coexpression of GLP-1 receptor mRNA with AVP and/or OX mRNAs in SON and PVN provides further support to the already reported central effect of GLP-1 for stimulating AVP release, and other actions induced by this peptide, i.e. behavioral effects. However, our results indicate that, in vivo, the peripheral administration of GLP-1 significantly decreases the circulating levels of AVP and OX, while in vitro incubation of neurohypophysis with the peptide does not modify AVP secretion but slightly increases OX release. These findings therefore suggest a dual secretory response of $A V P$ and $O X$ to the effects of GLP-1 (7-36)amide, which most likely is related to the route of peptide administration or to the amount of GLP-1 administered.
70

INDIRECT EVIDENCE OF DOWN REGULATION OF THE GLP-1 RECEPTOR CAUSED BY LONG TERM EXPOSURE TO GLP-1. B.Brock, S.Gregersen and K.Hermansen. Dept. of Endocrinology and Metabolism, Aarhus Amtssygehus, Aarhus University Hospital, DK-8000 Aarhus C, Denmark.

The glucagon-like petide-1, GLP-1, has a well documented glucose potentiating effect on insulin secretion from beta- cell lines. Previously, we have shown that long term exposure of the beta cell line INS-1 to high glucose down regulates the glucose mediated insulin release and the content of insulin II mRNA. The aim of the present study was to elucidate a) whether GLP. 1 can counteract this glucose mediated down regulation and, b) if the effect of GLP-1 is transient or persistent during long term exposure of the peptide it self. Studies were performed on the insulin secreting beta cell lines INS-1 and betaTC-3 cultured at $16.7 \mathrm{mM}$ glucose with or without addition of $10^{-10} \mathrm{M} / 10^{-8} \mathrm{M}$ GLP-1 for up to 3 days. BetaTC-3 cells were used for competitive binding assays, and insulin II mRNA was determined in INS-I cells by northern blotting while insulin release was examined from both cell lines by RIA Students unpaired t-lest was used to test the degree of significance. After 3 days exposure to $10^{8} \mathrm{M}$ GLP-1, the maximal binding of GLP-1 was reduced to $50 \%$ compared to the binding after 3 hours exposure $(\mathrm{p}<0.001)$. In INS-1 cells, the maximal insulin output was found in cells exposed for 1 hour to $10^{10} \mathrm{M} \mathrm{GLP}-1$, an output declining to $66 \%$ after 3 hours $(p<0.05)$ and to $56 \%$ after 3 days $(p<0.001)$. At $10^{-8} \mathrm{M}$ GLP-1, the maximal insulin secretion was found after 1 hour declining to $69 \%$ after 3 days $(\mathrm{p}<0.01)$. In betaTC-3 cells the insulin release was decreased to $65 \%$ after exposure to $10^{-10} \mathrm{M}$ GLP-1 compared to the release after 3 hours $(p<0.01$ ). Interestingly, both concentrations of GLP-] counteracted the down regulation of insulin release in INS-1 cells exposed to high glucose. In contrast, addition of GLP-1 did not alter the amount of insulin II mRNA in INS-I cells cultured in high glucose neither after 3 hours nor 3 days. In conclusion:Long term exposure to GLP-1 counteracted the glucose induced decrease in insulin release in INS-1 cells, in a manner independent of the insulin II mRNA level. In comparison to the acute action of GLP-1 mediated beta cell effect. long term exposure caused a pronounced decrease in GLP-1 mediated insulin release as well as in the GLP-I binding pointing to a down regulation of the GLP-1 receptor

\section{2}

GLUCAGON-LIKE PEPTIDE-1 (9-36)AMIDE DOES NOT DESENSITISE CONSCIOUS PIGS TO THE ANTI-HYPERGLYCAEMIC ACTION OF GLP-1 CF Deacon, *U Ribel, *B Roed, *HB Jensen Holm, JJ Holst and *RD Carr. Department of Medical Physiology, Panum Institute, University of Copenhagen, and *Diabetes Pharmacology, Novo Nordisk A/S, Bagsværd, Denmark.

Glucagon-like peptide-1 (7-36)amide (GLP-1) is rapidly degraded in vivo by dipeptidyl peptidase IV to form an N-terminally truncated metabolite, des His Ala GLP-1, which is an antagonist in vitro. Desensitisation to GLP-1 in vitro has been reported, and it could be postulated that the antagonistic metabolite may be responsible. This study aimed to see whether prior exposure to the metabolite, at levels similar to those formed during infusion of therapeutic amounts of GLP-1, was sufficient to antagonise the subsequent blood glucose-lowering effect of GLP-1, using conscious, non-fasted pigs given glucose. Animals ( $\mathrm{n}=5-7)$ received $40 \mathrm{~min}$ infusions of GLP-1 $(3.3 \mathrm{pmol} / \mathrm{kg} / \mathrm{min}$ ) or saline, and i.v. glucose $(0.2 \mathrm{~g} / \mathrm{kg})$ given during $\min 21-30$. In a second study, animals $(n=3-4)$ received initial $120 \mathrm{~min}$ infusions of saline or GLP-1 (9-36)amide (2.2 pmol $/ \mathrm{kg} / \mathrm{min}$, designed to mimic metabolite plasma levels achieved during the GLP-1 infusion). After $120 \mathrm{~min}$, these infusions were stopped and followed by infusions of GLP-1 and glucose as before. Blood glucose levels were lower $(P<0.03)$ during the $30 \mathrm{~min}$ period after the glucose load in the GLP-1 group, reaching a nadir by $20 \mathrm{~min}(2.24 \pm 0.25$ vs $3.60 \pm 0.22$ mmol/l, GLP-1 vs saline; $P=0.0018$ ). This was accompanied by a potentiation of the insulin response (incremental area under the curve (AUC) for min 20-70; $12575 \pm 2700$ vs $3733 \pm 856$ pmol $* 50$ min, GLP-1 vs saline: $P=0.0049$ ). During GLP-1 (9-36)amide infusion, plateau levels $(209 \pm 18$ pmol/li) were similar to concentrations of GLP-1 $(9$ 36)amide arising from in vivo degradation of GLP-1 (7-36)amide subsequently infused $(293 \pm 37 \mathrm{pmol} / 1 ; P=0.0873)$. Intact GLP-1 $(7-36)$ amide levels were similar in both groups (132 \pm 21 vs $140 \pm 30$ pmol//, GLP-1 (9-36)amide group vs control; $P=0.8298$ ). There was no effect of the GLP-1 (9-36)amide infusion on subsequent responses to GLP1 (7-36)amide for either glucose or insulin (glucose nadir, $1.82 \pm 0.41$ vs $1.94 \pm 0.37$ $\mathrm{mmol} / \mathrm{l}, P=0.843 \mathrm{l}$; insulin AUC, $7484 \pm 819$ vs $6992 \pm 1443 \mathrm{pmol} * 50 \mathrm{~min}, P=0.7635$; GLP-1 (9-36)amide group vs control). These results suggest that, in vivo, the 2-fold higher levels of GLP-1 (9-36)amide relative to intact GLP-1 obtained during exogenous infusion does not cause desensitisation to the anti-hyperglycaemic effect of GLP-1. 
OP 13

\section{Epidemiology of Type 1 Diabetes}

\section{3}

\author{
INCREASING INCIDENCE OF CHILDHOOD IDDM WORLDWIDE - \\ THE META-ANALYSIS OF THE INCIDENCE TREND DATA \\ S.Vänänen, P.Onkamo, J.Tuomilehto and M.Karvonen. National Public \\ Health Institute, Helsinki, Finland
}

The incidence of childhood IDDM has been studied in many populations for several years and individual reports on secular trends in incidence have been reported. Many of such reports have suggested that the incidence is increasing. The aim of this study was 1 ) to find out whether the incidence of IDDM is increasing globally or restricted to a selected populations only; 2) to estimate the magnitude of the change in incidence. Studies which had reported the yearly incidence for a 8-year period or longer and had at least 5 cases of IDDM registered per year were included in this meta-analysis. A total of 33 studies in 24 countries carried out during 1960 to 1996 with the study period ranging from 8 to 32 years (mean 15 years) fulfilled the inclusion criteria. Modified techniques of meta-analysis were applied to the data. The trends in IDDM incidence were estimated from logarithms of incidence by fitting linear regression. The coefficient in the regression model is the average increase in incidence (\%/year). Results from the pooled data from all 33 populations the overall increase in incidence was $2.9 \% / y e a r$ (95\%CI $2.5: 3.4 ; \mathrm{p}=0001)$. A statistically significant increase was found in $21 / 33$ individual populations. In only one population the trend was not positive $(-0.2,95 \% \mathrm{CI}-2.5 ; 2.2)$ and the largest increase in incidence was $9.5 \% / y e a r$. There was a negative correlation between the increase and the average level of incidence $(r=-.48 ; p=.005)$ but nevertheless the incidence increased significantly in all high incidence populations by $1 \%-3 \% / y e a r$. There is no doubt that the incidence of IDDM is increasing worldwide. Our model predicted that the incidence by 2010 would be still the highest $(50 / 100000)$ in Finland but over 30/100 000 also in many other populations.

\section{5}

CLUSTERING OF TYPE 1 DIABETES MELLITUS IN NORWAY

G. Joner', O. Søvik ${ }^{2}$ and T. Riise ${ }^{3}$, Aker Diabetes Research Centre, Aker University Hospital ${ }^{1}$, Oslo, Dept. of Pediatrics ${ }^{2}$ and Dept. of Public Health ${ }^{3}$, University of Bergen, Norway.

The epidemiology of childhood diabetes in Norway is characterized by a high incidence rate and a north to south gradient with high rates in the most southern counties. The reason for this marked geographical variation is unknown. A prospective and nationwide incidence survey was performed during the years 1989-1995, based on reports from all pediatric hospital departments on incident cases below 15 years of age. In each case, date of birth, date of diagnosis and place of residence at onset have been recorded, and the data were linked to the National Birth Defect Register to obtain place of residence by time of birth. The patient's address was also defined by a map coordinate system(GIS). Clustering in time and space was analysed by the method of Knox. The study revealed 1064 new cases of type 1 diabetes mellitus below 15 years. The overall yearly incidence rate was $23,1 / 10^{5}$ in males, 18,7 in females and 21,3 in both sexes. With one month between dates of birth as cut-off for distance in time and same municipality of residence at onset as definition of closeness in space, the observed number of close pairs was significantly higher than expected $(p<0.02)$. With twelve months between dates of diagnosis as cut off for distance in time and same municipality of residence at onset as definition of closeness in space, the observed number of close pairs was significantly higher than expected $(p<0.05)$. The data also demonstrated that a significanty higher number of cases than expected were born less than 6 months apart and with place of recidence less than $5 \mathrm{~km}$ apart $(p<0.005)$. In conclusion, a general space-time clustering of newly diagnosed cases of Type 1 diabetes have been found, indicating a role of infectious agents in the etiology of the disease.

\section{4}

RECENT TRENDS IN THE INCIDENCE OF TYPE 1 DLABETES IN EUROPEAN CHILDREN

\section{The EURODIAB TIGER Study Group}

In 1989 the EURODIAB TIGER (formerly the EURODIAB ACE) research network established prospective, geographically-defined registers of new cases of type 1 diabetes in children aged under 15 years in a number of European centres (Lancet 1992; 339: 905-909). Multiple sources of ascertainment were used to validate the level of ascertainment in each centre using the capturerecapture method. Other centres whose registries fulfilled the same quality criteria, including many from Eastern Europe, have since joined the group which now comprises 43 centres representing most European countries. Over 16,000 cases were registered in the six-year period 1989-94, and the overall estimate of ascertainment was $97.7 \%$. The standardised average annual incidence rate during the period ranged from 3.2 cases per 100,000 per annum in Macedonia to 40.2 cases per 100,000 per annum in Finland. To analyse trends in incidence during the period, results from centres within each country were pooled to give 31 centre groupings. Poisson regression analysis revealed that there was a significant linear trend $(\mathrm{P}<0.05)$ in nine countries, all but one showing an increase in incidence rate. Pooled over all centres, the annual rate of increase in incidence was $3.6 \%(95 \% \mathrm{Cl} 2.6 \%$ to $4.6 \%)$. However there was evidence of heterogeneity in the trend both between age-groups and between centres. The annual rates of increase in three age-groups pooled over centres were $6.5 \%(95 \% \mathrm{Cl} 4.2 \%$ to $8.8 \%$ ) for $0-4$ year olds, $3.2 \%(95 \% \mathrm{Cl} 1.6 \%$ to $4.9 \%$ ) for $5-9$ year olds and $2.6 \%(95 \% \mathrm{Cl} 1.2$ to $4.0 \%)$ for $10-14$ year olds. A cluster of central European countries showed rates which were increasing rapidly, some by as much as $10 \%$ per annum. The results confirm the extremely large range of incidence rates within Europe and show that incidence rates are increasing more rapidly in some countries than in others. The rapid rate of increase in children aged under 5 years is of particular concern.

\section{6}

EFFECT OF SARDINIAN HERITAGE ON RISK AND AGE AT ONSET OF TYPE 1 DIABETES: A CASE-CONTROL STUDY . G. Bruno, F. Merletti', F. Faggiano', A. De Salvia, N. Grosso, R. Arcari, L. Marsilio $^{2}$, D. Valenti ${ }^{2}$ and G. Pagano. Dept. of Internal Medicine; Dept. of Biomedical Sciences and Human Oncology'; Dept. of Hygiene and Community Medicine $e^{2}$, University of Turin, Italy

Children of Sardinian heritage are at high risk of type 1 diabetes, whereas no data are available in young adults. Age at onset of type 1 diabetes could be associated with different relative weight of genetic susceptibility and environmental determinants in the pathogenesis of the disease. We tested this hypothesis and that of an etiological role of social class in subjects with Sardinian heritage 0-29 yrs of age living in the city of Turin, an highly industrialized area in Northern Italy; 202 cases with onset of type 1 diabetes in the age-group 0-29 yrs in period 1984-91 and 1010 controls randomly selected from residents of the city of Turin, matched by sex and year of birth to cases, were included in this study. Name and place of birth of parents were ascertained by postal inquiry and linkage with city population and census files. Social class was based on the highest educational level of either one or the two parents abstracted from 1991 and 1981 census files. Differential effects of Sardinian heritage and social class on risk of type 1 diabetes in the age groups 0-14 yrs and 15-29 yrs were found. In children with both or one Sardinian parents ORs were $2.06(95 \% \mathrm{CI} \quad 0.85-5.00)$ or $3.18(0.80-12.61)$; in young adults respective ORs were $0.72(0.16-3.19)$ or $1.67(0.45-6.21)$. In children with low social class OR was $1.16(0.68-1.97)$, in young adults $0.66(0.41-1.05)$. Unconditional logistic models confirmed these results. In conclusion, this study showed greater effect of Sardinian heritage on risk of type 1 diabetes in children than in young aduits and a protective effect of low social class in young adults, consistently with the hypothesis of heterogeneity of type 1 diabetes by age at onset, with prevailing genetic effect in childhood and environmental determinants in adulthood. 
77

DECREASED PREVALENCE OF ATOPY IN DIABETIC CHILDREN The EURODIAB ACE Substudy 2 Study Group

Insulin-dependent diabetes mellitus results from a progressive autoimmune destruction of insulin-producing beta-celt of pancreatic islets. In addition to the genetic predisposition, environmental factors contribute to its pathogenesis and these can be either damaging or protective.

Eight European centres (representing a wide range of childhood diabetes incidence) with access to population-based IDDM registries (over $90 \%$ degree of ascertainment) participated in a case-control study focusing on early exposures and risk factors for Type 1 diabetes. Altogether data from 1028 cases amd 2768 controls corresponding to $85.4 \%$ of eligible cases and $76.2 \%$ of controls were analysed. Information (questionnaire of interview) inthis study was collected on atopic diseases (atopic eczema, allergic rhino-conjunctivitis and asthma.)

Allergic rhino-conjunctivitis and asthma were associated with a significantly $(p=0.04$ and $p<0.001$, respectively) decreased risk for Type 1 diabetes without any indication of heterogeneity between the centres. The Mantel-Haenzel combined odds atios and $95 \%$ confidence limits were $0.76(0.58,0.99)$ and $0.64(0.51,0.82)$, respectively. The $\mathrm{MH}$ combined odds ratio for atopic eczema was not decreased (1.03 $0.84,1.26)$. The protective effect was not significantly different in the age groups (0-4 $\mathrm{yr}, 5-9 \mathrm{yr}$ and 10-14 yr) for asthma, but for minoconjuctivitis it was significant only in age group 10-14 years. Adjustment for possible confounders (low birth weight, short duration of breas feeding, older maternal age, lack of preschool Inursery/ care and vilamin D supplementation) in the logistic regression analysis did not significantly alter the protective effect of asthma, but decreased the protective effect of thino-conjunctivitis $(0.85 ; 0.6,1.20)$

These findings indicate that atopy may be protective against the development of IDDM and are consistent with the immunological concept of $T h_{1}$ (IDDM) and $T h_{2}$ (atopy) diseases being mutually exclusive.

\section{8}

VITAMIN D SUPPLEMENT AND RISK FOR TYPE 1 DIABETES IN CHILDHOOD

The EURODIAB ACE Substudy 2 Study Group

The initiation of the immunopathogenetic process that may lead to insulindependent diabetes in childhood probably occurs early in life. Experimental studies in vitro have shown that vitamin $D$ is immunosuppresive and studies in experimental models of autoimmunity including one for autoimmune diabetes have shown vitamin D to be protective. In a European collaborative study (EURODIAB ACE Substudy 2) seven centres with access to population-based and validated case registers of insulin-dependent diabetes patients participated in a case-control study focusing on early exposures and risk for type 1 diabetes. Altogether data from 820 cases and 2335 controls corresponding to $85 \%$ of eligible cases and $76 \%$ of eligible controls were analysed. Questions focused on perinatal events and early eating habits including vitamin D supplementation.

The frequency of vitamin D supplementation in different countries varied from 47 to $97 \%$ among controls. Vitamin D supplementation was associated with a decreased risk for type 1 diabetes without indication of heterogeneity. The Mantel-Haenszel combined odds ratio was 0.67 (95\% confidence limits: $0.53 ; 0.85)$. The protective effect did not differ significantly between three age groups in five year intervals. Adjustment for the possible confounders: a low birth weight, a short duration of breast feeding, older maternal age and study centre in logistic regression analysis did not affect the significant protective effect of vitamin $D$.

Conclusion: This large multicentre trial covering many different European settings consistently showed a significant protective effect of vitamin D supplementation in infancy. The findings indicate that activated vitamin D might contribute to immune modulation and thereby protect or arrest an ongoing autoimmune process initiated in susceptible individuals by early environmental exposures.

\section{OP 14}

\section{Islet Metabolism and Insulin Release}

\section{9}

\section{CLONING AND CHARACTERIZATION OF AN ISLET-SPECIFIC} GLUCOSE-6-PHOSPHATASE

J.C. Hutton, S. Steegers, B. Bergman, T. Zahn and S. Arden. Barbara Davis Center for Childhood Diabetes, Denver, Colorado, USA; Dept. of Clinical Biochemistry, Addenbrooke's Hospital, Cambridge, UK

The recognition of glucose as an insulin secretagogue depends upon its metabolism in the B-cell and the initial steps of glucose metabolism are intimately involved in the recognition of the sugar as a secretagogue. A pancreatic B-cell specific isoform of glucose-6-phosphatase was cloned using a subtractive cDNA expression cloning procedure from mouse insulinoma tissue. The 1901 bp cDNA encoded a 355 aa protein (Mr 40684 ) glycoprotein related in both primary sequence $(48 \%$ overall identity) and overall membrane topology to the previously-described mouse liver isoform. Like the liver isoform its $\mathrm{COOH}$ terminus has an endoplasmic reticulum membrane protein retention motif. Northern blot and RT-PCR analysis indicated that the protein was more highly-

expressed in $\beta$-cell than $\alpha$-cell lines but was not found in other mouse tissues including liver, kidney or brain nor in tissue culture cell lines including those of hepatic (HepG2), renal (COS 1) or neuroendocrine origin (AtT20, PC12). The presence of a unique isoform was consistent with enzymatic studies suggesting that isolated rat islets and mouse insulinoma cell lines possess glucose 6 phosphatase activity with distinct inhibition profile, catalytic properties and immunological reactivity. The co-existence of glucokinase and G6Pase in B-cells could function as a glucose substrate shuttle in vivo and play a key role in the sensing of glucose as a secretagogue and regulating glycolytic flux. The identification of this novel isoform will facilitate further investigations of its transcriptional regulation both in the context of its tissue-specific expression and its involvement in changes in glucose responsiveness of the B-cell of physiological and pathological consequence.

\section{0}

A PIVOTAL ROLE OF NADH SHUTTLES IN GLUCOSEINDUCED INSULIN SECRETION FROM $\beta$ CELLS

K. Eto, Y. Tsubamoto, Y. Terauchi, Y. Yazaki and T. Kadowaki, Tokyo, Japan

In order to determine the role of NADH shuttles comprised of glycerol phosphate (GP) and malate-aspartate (MA) shuttles, which transfer cytosolic NADH into mitochondria for ATP production, in glucose-induced insulin secretion from pancreatic $\beta$ cells, we have generated mice which lack mitochondrial glycerol-3 phosphate dehydrogenase (mGPDH), a rate-limiting enzyme of GP shuttle. Glucose-induced insulin secretion from mGPDH deficient islets with aminooxyacetate, an inhibitor of MA shuttle, added either before or during glucose stimulation was almost completely abrogated. The mGPDH deficient islets showed normal insulin secretory response to glucose. AOA alone only slightly decreased the secretion in wildtype islets. In mGPDH deficient islets, insulin secretory response to glyceraldehyde was similarly affected, whereas response to glibenclamide was essentially normal. Under conditions where both shuttles were halted, glucose utilization in glycolysis and pyruvate transport into mitochondria were not affected, whereas the turnover of TCA cycle was decreased by $58 \%$. Glucose-induced production of $\mathrm{NAD}(\mathrm{P}) \mathrm{H}$, mitochondrial inner membrane hyperpolarization, $\mathrm{Ca}^{2+}$ influx into mitochondria and increase in ATP content were also severely attenuated. This study provides the first direct evidence that NADH shuttles are essential for coupling glycolysis with mitochondrial energy production to trigger and maintain glucoseinduced insulin secretion and that the activity of TCA cycle is partly regulated by NADH shuttles. It also solves so-called pyruvate paradox and thus revises the classical model for metabolic signals in glucose-induced insulin secretion. 
81

\section{SYNCHRONOUS OSCILLATIONS IN OXYGEN TENSION AND} INSULIN RELEASE OF INDIVIDUAL MOUSE ISLETS

H. Ortsäter, P. Liss, P.E. Lund, K. Åkerman and P. Bergsten Departments of Medical Cell Biology and Physiology, Uppsala university, Uppsala, Sweden.

Insulin release from the isolated islet is pulsatile, which is decisive for the appearance of plasma insulin oscillations. Changes in the $\beta$-cell metabolism have been proposed to be important for the generation of the insulin pulses. However, no simultaneous measurements of metabolism and insulin release have been performed on single islets to verify this. We have now measured oxygen tension $\left(\mathrm{pO}_{2}\right)$ and observed oscillations in $\mathrm{pO}_{2}$ corresponding to regular variations in insulin release from individual perifused isolated mouse islets. In the presence of $3 \mathrm{mM}$ glucose average $\mathrm{pO}_{2}$ was $105 \pm 3 \mathrm{~mm} \mathrm{Hg}$ and oscillatory $(0.3 \pm 0.1$ oscillations/min), measured with a modified Clark-type electrode inserted into the islets. Corresponding insulin measurements obtained by analyzing the perifusate with a sensitive ELISA showed oscillations with a similar frequency and a secretory rate of $8 \pm 3$ mmol $^{*} \mathrm{~g}^{-1}{ }^{*} \mathrm{~s}^{-1}$. When the glucose concentration was increased to $11 \mathrm{mM}, \mathrm{pO}_{2}$ decreased to $70 \pm 3$ $\mathrm{mm} \mathrm{Hg}$ within $5 \mathrm{~min}$ with maintained frequency of the oscillations. The corresponding insulin secretory rate rose 10 -fold by increase of the insulin pulse amplitude leaving the frequency unaffected. In contrast, no change in the average $\mathrm{pO}_{2}$ or freqency of $\mathrm{pO}_{2}$ oscillations was observed when the non-metabolizable secretagogue tolbutamide $(1 \mathrm{mM})$ was added to the perifusion medium despite an approximate $30 \%$ increase of the amplitude of the insulin oscillations. When $8 \mathrm{mM}$ non-metabolizable 3-oxymethyl glucose was added to islets perifused in the presence of 3 $\mathrm{mM}$ glucose, neither $\mathrm{pO}_{2}$ nor insulin release were changed. Variations in $\mathrm{pO}_{2}$ and insulin release were synchronous under all conditions tested supporting a role of metabolic oscillations in the generation of pulsatile insulin release.

\section{3}

The Role of the PKC isoform Epsilon in the Augmentation of Insulin Release by Glucose and Palmitate. Susanne G. Straub, Shaohua Tang and Geoffrey W.G. Sharp. Department of Pharmacology, Cornell University, Ithaca, NY, USA Glucose stimulates insulin secretion by activation of KATP channeldependent and KATP channel-independent signaling pathways. It has been suggested that the KATP channel-independent pathway involves a glucose-induced build up of malonyl CoA, inhibition of carnitine palmitoyl transferase I (CPT I), decreased fatty acid oxidation and an increase in cytosolic long chain acyl CoA derivatives. The latter could augment insulin release directly or by the generation of other signaling molecules, such as diacyl glycerol, which activate certain PKC isoforms. The augmentation of insulin release by palmitate, similarly, could be caused by increased long chain acyl CoA derivatives and activation of PKC. Therefore, we studied the translocation of several $\mathrm{PKC}$ isoforms that are known to be present in $\beta$ cells $(\alpha, \beta I I, \delta, \varepsilon, \zeta$, $\eta, \lambda$ and $\mu$ ) in response to glucose and palmitate. We also looked at the effects of etomoxir which inhibits CPT I as does malonyl CoA. HIT cells, which have been used extensively for the study of augmentation pathways, were incubated in the presence of $0.2 \mathrm{mM}$ glucose alone, and with $10 \mu \mathrm{M}$ free palmitate and $1 \mu \mathrm{M}$ etomoxir, and $20 \mathrm{mM}$ glucose alone and in combination with palmitate and etomoxir. At the end of the incubations, the cells were homogenized and cytosol and membrane fractions prepared. The membranes were solubilized and cytosolic and membrane proteins separated by SDS-PAGE. Western blotting and ECL with isoform specific antibodies were used to detect the PKC isoforms and their locations in the cytosol and membrane fractions. The PKC isoforms $\alpha, \varepsilon$ and $\mu$ translocated from the cytosol to the membrane fraction after treatment with $20 \mathrm{mM}$ glucose. PKC $\varepsilon$ translocated to the membrane in response to $10 \mu \mathrm{M}$ palmitate. Both palmitate and etomoxir magnified the glucose-induced translocation of $\mathrm{PKC} \varepsilon$. These data strongly suggest that PKC $\varepsilon$ is a critical signaling molecule in the augmentation of insulin release by glucose and palmitate.

\section{2}

IMPAIRED PYRUVATE METABOLISM AND GLUCOSE OXIDATION IN FATTY ACID CULTURED ISLETS

J. L. Leahy and Y. Q. Liu, University of Vermont, Burlington, USA

Long-term culture of islets with long chain fatty acids (FA) impairs oxidative glucose metabolism and glucose-induced insulin secretion. This finding is of interest because animal models of type 2 diabetes have similar B-cell dysfunction and a raised serum/islet triglyceride level. We investigated rat islets that were cultured with $0.25 \mathrm{mM}$ oleate for 4 days. Protein and DNA contents were equal in the oleatecultured and control islets. Mitochondrial glycerol-3-phosphate dehydrogenase $V_{\max }$ was unchanged in the oleate islets, suggesting there was no change in the glycerol phosphate shuttle. In contrast, the pyruvate content of the oleate islets was increased by $60 \%(p<0.01)$ Investigation of this finding showed a $35 \%$ lowered active pyruvate dehydrogenase $V_{\max }(p<0.015)$. In contrast, pyruvate carboxylase $\mathrm{V}_{\max }$ was unchanged by the oleate culture, suggesting that pyruvate is primarily metabolized through this pathway in the oleate islets. The oxaloacetate that is produced by this reaction enters the citric acid cycle by way of citrate synthase. However, citrate synthase $V_{\max }$ and the citrate content were lowered $50 \%$ in the oleate islets (both $\mathrm{p}<0.01$ ). Alternatively, oxaloacetete can cycle back to pyruvate by way of malate dehydrogenase and malic enzyme, the pyruvate-malate shuttle. Malate dehydrogenase $V_{\max }$ was unchanged in the FA islets, and malic enzyme $V_{\max }$ was minimally reduced. Also, oxaloacetate and malate contents were unchanged. We propose that the altered mitochondrial oxidation of glucose and impaired insulin secretion with FA is secondary to a reduced delivery of substrate into the citric acid cycle because of impaired pyruvate dehydrogenase and citrate synthase activities. As such, there is increased flux through the pyruvate-malate shuttle, thereby creating a futile cycle in terms of pyruvate metabolism.

\section{4}

A NEW GENETIC RAT MODEL OF SEVERE INSULIN DEFICIENCY WITHOUT INSULITIS

V. Esser, K.L. Wyne, S.A. Comerford, R.E. Hammer and J.D. McGarry University of Texas Southwestern Medical Center at Dallas, Dallas, Texas

Carnitine palmitoyltransferase I (CPT I), which exists in at least two forms, the liver $(\mathrm{L})$ and muscle $(\mathrm{M})$ variants, plays a pivotal role in the regulation of fatty acid metabolism by virtue of its potent inhibitability by malonyl-CoA. The malonyl-CoA-CPT I interaction causes elevation of the cytosolic acyl-CoA level which, in turn, has been implicated as a key stimulatory element in insulin secretion. To examine the effect of chronic lowering of $\beta$-cell CPT I activity, a transgenic rat line carrying an anti-sense cDNA for L-CPT I (the isoform expressed in the rat $\beta$-cell) under the control of the rat insulin 1 promoter was generated. Surprisingly, the animals hemizygous for the transgene did not exhibit hyperinsulinemia; on the contrary, they developed insulin deficient diabetes (with no evidence of insulitis) by 5-8 weeks of age, with non-fasting plasma glucose levels of $\sim 400 \mathrm{mg} / \mathrm{dl}$ and a relative insulin:glucose ratio of 0.07 (control values: $\sim 116 \mathrm{mg} / \mathrm{dl}$ and 1.0 , respectively). Tissue survey by Northern blot analysis of a 14 day old transgenic animal confirmed that the anti-sense mRNA is expressed only in the pancreas. At this stage, the islets appear grossly normal histologically and their CPT I activity is comparable to that of control animals. However, at 18-21 days, islet volume begins to decrease, with concomitant loss of both anti-sense and sense CPT I mRNA. A 10-15 fold increase in the number of apoptotic cells within the islet is observed using the TUNEL assay. Furthermore, immunocytochemical analysis demonstrated a decrease of $\beta$-cell mass, a finding supported by the low insulin but normal glucagon content in total pancreas of 8 week old transgenic animals. This is the first transgenic rat model of diabetes to be reported. Its further charcterization is currently under way. 


\section{Cellular Mechanisms of Vascular Dysfunction}

QUALITATIVE DIFFERENCES IN STIMULUS DEPENDENT GENE AND PROTEIN EXPRESSION IN APOPTOSIS OF ENDOTHELIAL CELLS

S.M.Baumgartner-Parzer, M. Artwohl, T. Stulnig, T. Hólzenbein, W. Waldhäusi. Dept. of Internal Medicine III; Division of Clin. Endocrinology \& Metabolism, Wăhringer Gürtel 18-20, A-1090 Vienna, Austria.

Endothelial apoptosis was suggested to be a phenomenon involved in diabetes associated vascular complications. This study was designed to describe the characteristics of apoptosis in cultured vascular endothelia cells as to provide a basis for antiapoptotic strategies. Apoptosis and associated protein and gene expression were evaluated after incubation of HUVECs (human umbilical vein endothelial cells) with high $(30 \mathrm{mM})$ ambient glucose or TNF- $\alpha(330-6600 \mathrm{U} / \mathrm{ml})$ or levamisole $(1-2 \mathrm{mM})$, used as an adjuvans in colon carcinoma therapy. $30 \mathrm{mM}$ glucose, TNF- $\alpha$ and levamisole increased apoptosis up to $120-300 \%(p<0.02)$ of control (set as $100 \%$ ) dependent on the respective stimulus. The tumor suppressor gene p53 and the inhibitor of cyclin dependent kinases p21 were differentially modulated by the three agents used, whereas clusterin expression, originally desribed as a marker of apoptosis, was reduced in all models of apoptosis $(-30 \% ; p<0.02)$. Expression of members of the bcl-2 family of proteins ( $b \mathrm{cl}-$ 2, bak, bcl-xs) was not affected by high ambient glucose, whereas bak was upregulated $(+30 \%)$ by TNF- $\alpha \quad(n=6, p<0.05)$ and levamisol $(+50 \%, n=6$, $p<0.05)$, whereas bcl-2 levels were diminished $(-30 \%, n=6, p<0.05)$ by levamisole only. The hyperphosphorylated form of the Retinoblastoma protein, responsible for cell cycle progression, was markedly affected $(-70 \%)$ by levamisole and TNF- $\alpha(n=6, p<0.02)$, being in line with a growth arrest induced by both agents. Antioxidants as $\mathrm{N}$-acetylcystein and glutathione were able to reduce $(-50 \%, n=5, p<0.05)$ basal and levamisole induced endothelial apoptosis, suggesting possible antiapoptotic intervention by antioxidative strategies. In summary, our data show qualitative differences in the characteristics of stimulus dependent apoptosis in vascular endothelial ceils. These findings are of major interest for the development of antiapoptotic as well as antineoangiogenetic strategies, both of which are of relevance for the prevention of late diabetic vascular complications.

\section{7}

GLUCOSE ACTIVATION OF NFKB IN VASCULAR SMOOTH MUSCLE CELLS. CBT McMullen, KKM Yue, P Anderson, PC Sharpe and ER Trimble. Belfast.

A model has been proposed for the involvement of reactive oxygen species (ROS) in the activation of NFKB by diverse stimuli in different cell types. Evidence however has evolved to question how universal is this model. Therefore the aims of this study were to investigate if elevated glucose levels caused NFKB activation in porcine aortic VSMCs and if the signal transduction pathway for glucose activation of NFxB in these cells involved ROS. VSMCs were cultured in either $5 \mathrm{mmol} / \mathrm{L}$ (NG) or $25 \mathrm{mmol} / \mathrm{L}(\mathrm{HG})$ glucose for 10 days and glucose-induced oxidative stress was demonstrated by a significant decrease in glutathione (GSH) $(-55 \% n=15$ $\mathrm{p}<0.001)$ and an increase in malondialdehyde (MDA) $(+29 \% \mathrm{n}=15 \mathrm{p}, 0.01)$ levels Nuclear extracts were prepared and NFאB binding activity, determined by electrophoretic mobility shift assay, was increased in HG $(+36 \% n=17 p=0.0055)$ The co-incubation of glucose with varying concentrations of $\mathrm{N}$-acetyl cysteinc (NAC) $(0.5,1.0$ and $2.0 \mathrm{mmol} / \mathrm{L})$ did not inhibit glucose activation of NFKB $(\mathrm{p}=0.73,0.98$ and 0.47 respectively). NAC $1.0 \mathrm{mmol} / \mathrm{L}$ was shown to have repleted intracellular GSH levels and incubation of VSMCs with NG plus $10 \mu \mathrm{mol} / \mathrm{L}$ Buthionine sulfoximine (BSO), known to cause GSH depletion, did not cause $N F K B$ activation. These results suggest that GSH levels are not involved in this mechanism of NFKB activation. In addition, the water soluble vitamin $\mathrm{E}$ analogue Trolox $(200 \mu \mathrm{mol} / \mathrm{L})$, which retumed the MDA levels of $\mathrm{HG}$ to that of $\mathrm{NG}$ controls without affecting GSH levels, had no effect on glucose activated NFKB $(+49.8 \%$ $n=5 p=0.97$ ) suggesting that the NFKB activation was not directly associated with peroxidative damage in VSMCs. In summary, we have found activation of NFKB in VSMCs grown under in vitro conditions which mimic those of diabetes but found no evidence for the involvement of oxidative stress in this particular pathway for NFKB activation. The VSMC is another cell type in which the ROS model for $N F K B$ activation may well be restricted to certain stimuli and the presence of such a system is not required for the activation of $\mathrm{NFKB}$ by glucose in these cells

\section{6}

NF-kB activation by high glucose is involved in induction of apoptosis in human umbilical vein endothelial cells (HUVEC). P. Rösen, XL. Du, K. Färber, Diabetes Research Institute, Düsseldorf, Germany

$\mathrm{NF}-\mathrm{\kappa B}$ is a pluripotent transcription factor and has been suggested to play a role in induction of apoptosis by hyperglycaemia. To investigate the underlying mechanisms, we determined the production of ROI by staining the cells with $2^{1} 7$ '-dichlorofluorescein $\left(\mathrm{H}_{2} \mathrm{DCF}\right)$, the activity of NF- $\mathrm{kB}$ by electrophoretic mobile shift assay (EMSA) and in situ using the DNA binding sequence of NF- $\mathrm{KB}$. Induction of apoptosis was identified by the by DNA fragmentation assay. Production of superoxide anions was increased by glucose dose-dependently from $17.5 \pm 3 \mu \mathrm{M}$ (5 mM glucose) to $43 \pm 6 \mu \mathrm{M}$ at $30 \mathrm{mM}$ glucose. NF- $\kappa \mathrm{B}$ was dose-dependently activated by glucose reaching a maximum after for 4 hrs at $30 \mathrm{mM}$ glucose. Similar results were obtained by EMSA. Both, the formation of ROI as well as the activation of NF-KB were prevented by antioxidants ( $\alpha$-tocopherol $10 \mu \mathrm{g} / \mathrm{ml}, \alpha$-thioctic acid $1 \mu \mathrm{M}$, SOD mimetic $100 \mu \mathrm{M}$ ). Interestingly, L-nitro-arginine (L-NNA $100 \mu \mathrm{M}$ ), an inhibitor of NO-synthase, prevented the production of ROI and activation of NF-kB, too. Incubation of HUVEC with high glucose up to $72 \mathrm{hrs}$ induced apoptosis in about $40 \%$ of the cells, which was prevented by the same treatments which inhibited the formation of ROI and the activation of NF-KB (antioxidants and L-NNA). We conclude from these data that the formation and the release of ROI and the activation of NF- $\mathrm{KB}$ are early steps in the signal cascade triggering the induction of apoptosis.
88

THE ROLE OF GLUCOSE IN REDOX STATUS ALTERATIONS IN VASCULAR SMOOTH MUSCLE CELLS

Catherwood M.A., Yue K.K.M. ${ }^{a}$, McMaster D. ${ }^{b}$, Trimble E.R. ${ }^{a}$

${ }^{a}$ Departments of Clinical Biochemistry and ${ }^{b}$ Medicine, Queen's University of Belfast, U.K

We have previously shown that glucose induces oxidative stress in vascular smooth muscle cells (VSMC). The aim of this project was to define specific changes in redox status and the time course of these events. Porcine VSMC (passage 2-5) were cultured in either $5 \mathrm{mmol} \mathrm{l}^{-1}$ (normal glucose, NG) or 25 mmol. $1^{-1}$ glucose (high glucose, HG) for up to 10 days (IOD). Glutathione (GSH) was reduced by $34 \%$ at D2 and $41 \%$ at D10 (2.35 \pm 0.06 Vs $3.5 \pm$ $0.24 \mathrm{nmol} . \mathrm{mg}$ protein ${ }^{-1}, \mathrm{p}<0.005$, HG Vs NG), however increases in $\mathrm{NADH} / \mathrm{NAD}^{+}$(spectrophotometric cycling assay) occurred only after D8 $(0.6 \pm 0.08$ Vs $0.34 \pm 0.08, p<0.005$, HG Vs NG). Although increases in lipid peroxidation (malondialdehyde; HPLC with fluorometric detection) were found only after D8 ( $1.21 \pm 0.05 \mathrm{Vs} 0.8 \pm 0.03 \mathrm{nmol}$. mg protein ${ }^{-1}$ $\mathrm{p}<0.05$, HG Vs NG), membrane changes in fatty acids (decreased C18:1) appeared as early as D4. No evidence of DNA fragmentation (comet assay) was present up to D10. Increases also occurred in $\mathrm{mRNA}$ for $\mathrm{Cu} / \mathrm{Zn}$ superoxide dismutase (SOD), MnSOD (quantitative PCR, $182 \pm 8.6 \%$, $\mathrm{p}<0.005$ and $41 \pm 4.5 \%, \mathrm{p}<0.05$, respectively) and thioredoxin reductase (ribonuclease protection assay, approximately $70 \%$ ) (measured only at D10); there were no significant changes in glutathione peroxidase and catalase mRNA levels. Repletion of GSH by N-acetyl cysteine reverses these changes. It is clear that the early reduction of GSH plays a key role in the cellular events associated with glucose-induced oxidative stress in these cells. 
89

HYPERGLYCEMIA AND VASCULAR SMOOTH MUSCLE CELL SIGNALING

G. Grunberger, P.R. Srinivas and S. Verma. Wayne State University, Detroit, MI, USA

An important early event in the development of atherosclerosis is the proliferation and migration of vascular smooth muscle cells (VSMC) into the neointima of the vessel wall. We now show that hyperglycemia promotes VSMC proliferation by driving specific cellular biochemical pathways. In rat aortic VSMC exposed to chronic hyperglycemia, proliferation is induced through (a) activation of focal adhesion kinase (FAK), and (b) an upregulation of Bd2 expression. This results in the generation of mitogenic anti-apoptotic signals, both of which promote proliferation. VSMC, cultured from rat aorta, incubated in hyperglycemic medium ( $25 \mathrm{mM}$ glucose) for more than $72 \mathrm{~h}$, demonstrate a 2 fold increase in FAK activation. The activation of FAK results in the formation of a dimeric complex with pp60 c-src kinase. Immunoblot analysis shows no induction of FAK expression but rather an increased specific activity. Activated FAK could thus initiate mitogenic signals in the VSMC. Under similar conditions, we detect increased $\mathrm{Bc} / 2$ protein in the mitochondrial membrane fractions of VSMC in high glucose. We also find that there is a parallel increase in p72raf kinase in the membrane fraction. Bcl2 prevents cells from going into apoptosis and in concert with raf confers cells with resistance towards apoptosis. Thus, increased expression of Bcl2 in VSMC under hyperglycemic conditions results in generation of anti-apoptotic signals, promoting proliferation. These data suggest that hyperglycemia drives biochemical pathways that promote VSMC proliferation. This glucose-induced functional change in VSMC could be an important feature in the development of atherosclerosis.

\section{0}

HIGH GLUCOSE-INDUCED FIBRONECTIN OVEREXPRESSION PERICYTES IS MODULATED BY ANTISENSE OLIGONUCLEOTIDES S. ROY and T. ROTH. Schepens Eye Research Institute, Harvard Medical School, Boston, MA, USA

Increased synthesis of fibronectin (FN) is associated with the development of vascular basement membrane thickening, a characteristic lesion of diabetic microangiopathy, and may affect the function of vascular cells. Pericytes located abluminally on the retinal capillaries are known to synthesize significant amounts of fibronectin. Since high glucose upregulates FN synthesis in pericytes, and reduces pericyte proliferation, we investigated whether antisense phosphorothioate oligonucleotides (PS oligos) directed against FN transcript reduce $\mathrm{FN}$ synthesis and modulate proliferation of bovine retinal pericytes cultured in high (30mM) glucose. Cells grown in high giucose for 12 days exhibited increased FN mRNA and protein levels (determined by Reverse Transcription-Polymerase Chain Reaction and western blot analysis) to $212 \pm 46 \%$ and $162 \pm 23 \%$ of control, respectively, and decreased cell count compared to control cells grown in $5 \mathrm{mM}$ glucose (73\% of control, $\mathrm{p}=0.026)$. When cells grown in high glucose were transfected with 0.4 uM FN-antisense PS oligos for $48 \mathrm{~h}$, the FN MRNA and protein level was reduced to near normal level $(95.6 \pm 3 \%$, and $106 \pm 6 \%$ of control, respectively). The cell number in $5 \pm 1$ days

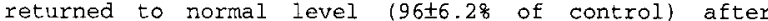
transfection. This indicates the effectiveness of antisense oligonucleotides in reducing excess synthesis of fibronectin by pericytes under high glucose conditions. The antisense strategy may find a wider applicability in reducing synthesis of basement membrane components, and facilitate the understanding of interactions between altered matrix synthesis and cellular function.

\section{OP 16}

\section{Lipids and Insulin Resistance}

\section{1}

PLASMA PHOSPHOLIPID TRANSFER PROTEIN ACTIVITY IS RELATED TO INSULIN RESISTANCE: IMPAIRED ACUTE LOWERING BY INSULIN IN OBESE NIDDM PATIENTS

S.C. Riemens ', A. van Tol', W.J. Sluiter', and R.P.F. Dullaart'. ' Department of Endocrinology, University Hospital Groningen and ${ }^{2}$ Department of Biochemistry, Cardiovascular Research Institute (COEUR), Erasmus University Rotterdam, the Netherlands.

Human plasma contains two lipid transfer proteins, cholesteryl ester transfer protein (CETP) and phospholipid transfer protein (PLTP) that have important functions in high density lipoprotein (HDL) metabolism. We determined the association of plasma activity levels of CETP and PLTP (measured with exogenous substrate assays) with insulin resistance, plasma triglycerides and free fatty acids (FFA), and assessed the lipid transfer protein response to insulin during a 6-7 $\mathrm{h}$ hyperinsulinaemic euglycaemic clamp in non-obese and obese healthy subjects and NIDDM patients ( $\mathrm{n}=\mathbf{8}$ per group). Plasma PLTP activity was positively associated with obesity and NIDDM, insulin resistance, plasma triglycerides and FFA ( $p=0.02$ to $<0.01$ ). In the non-obese healthy subjects insulin decreased plasma triglycerides $(p<0.001)$ and increased the HDL cholesteryl ester/triglycerides (CE/TG) ratio $(p<0.001)$ and these changes were larger than those during saline infusion $(\mathrm{p}<0.01)$. Plasma PLTP activity fell by $14 \%$ at the end of the clamp ( $\mathrm{p}<0.01$ compared to saline infusion). In contrast, plasma CETP activity did not change during hyperinsulinaemia. The decreases in plasma FFA, triglycerides and PLTP activity and the rise in HDL CE/TG were smaller in the obese NIDDM patients compared to non-obese control subjects ( $\mathrm{p}<0.01$ for all), with intermediate changes in the other groups. In the whole study population, baseline HDL CE/TG was negatively correlated with triglycerides $(p<0.001)$ and PLTP activity $(p<0.01)$, but not with CETP activity. Likewise, the rise in HDL CE/TG during the clamp was related to the fall in triglycerides $(p<0.001)$ and in PLTP activity $(\mathrm{p}<0.02)$. It is concluded that plasma activity levels of PLTP, but not of CETP, are regulated by insulin in an acute setting. Insulin resistance is associated with an increase in plasma PLTP activity in combination with altered FFA and triglyceride metabolism. High plasma triglycerides and PLTP activity levels may have coordinate effects on HDL metabolism.

\section{2}

FATTY ACID OXIDATION IN MAN IS REGULATED BY MALONYL CoA LEVELS IN MUSCLE P. Båvenholm*, J. Pigon*, A. Saha*, N.Ruderman**, and S. Efendic*. Division of Medicine Karolinska Hospital and Institute *, and Diabetes and Metabolism Unit, Boston University Hospital**

Malonyl CoA is an inhibitor of carnitine palmitoyl transferase 1 (CPT1), the enzyme that regulates the transfer of long chain fatty acyl $C O A$ (LCFA CoA) acid oxidation in the mitochondria where it is oxidized. To evaluate, whether malonyl CoA plays a major role in regulating fatty acid oxidation in human muscle we measured its concentration in the vastus lateralis, biopsied during an euglycemic hyperinsulinemic clamp. Studies were performed in 11 healthy Swedish men (age $47+6.7$, BMI $26.2+2.0$ ) with normal glucose tolerance. Plasma insulin was clamped at $725+9 \overline{8} \mathrm{mM}$ and glucose was infused at a rate of $8.1 \pm 2.7 \mathrm{mg} / \mathrm{kg} / \mathrm{min}$ to maintain normoglycemia $(5.1 \mathrm{mM})$. After 5 hours of insulin infusion, malonyl CoA levels were increased $(0.21$ vs. $0.25 \mathrm{nmol} / \mathrm{g}, \mathrm{p}<0.001)$ and fatty acid oxidation in the body was decreased $(0.85$ vs. $0.38 \mathrm{mg} / \mathrm{kg} / \mathrm{min}, \mathrm{p}<0.001)$. The increase in malonyl $\mathrm{CoA}$ was associated with increases in the concentrations of citrate (108 vs. $145 \mathrm{nmol} / \mathrm{g} ; \mathrm{p}=0.006)$ an allosteric activator of acetyl CoA carboxylase and of malate (78 vs. $147 \mathrm{nmol} / \mathrm{g}$ $\mathrm{p}<0.05)$ an antiporter for citrate efflux from the mitochondria. The increase in malonyl $\mathrm{COA}$ also correlated with the rate of glucose utilization during hyperinsulinemia $(r=0.55, \mathrm{p}<0.05)$. The results suggest that one mechanism by which muscle restrains fatty acid oxidation when plasma insulin levels are raised is by increasing the concentration of malonyl CoA. They also suggest that such increases in malonyl $\mathrm{CoA}$ are related to increases in the concentration of citrate. Finally, as cytosolic citrate is also an inhibitor of phosphofructokinase, the rise in its concentration when glucose utilization is increased suggests that glucose can also autoregulate its own metabolism. 
93

THE ROLE OF FREE FATTY ACIDS FOR POSTABSORPTIVE ENDOGENOUS GLUCOSE PRODUCTION AND GLUCONEOGENESIS IN MAN

H. Stingl, B.R. Landau*, C. Fümsinn, P. Nowotny, W. Waldhäusl, G.I. Shulman**, and M. Roden; Vienna, Austria: ${ }^{*}$ Cleveland, OH; ${ }^{* *}$ New Haven, CT, USA

To examine direct effects of free fatty acids (FFA) on hepatic glucose metabolism 6 young healthy subjects $\left(22.8 \pm 1.1 \mathrm{~kg} / \mathrm{m}^{2}\right)$ were studied twice. once in the presence of step-wise increased plasma FFA concentrations (LIP; $0-3 \mathrm{~h}: 0.8 \pm 0.1,3-6 \mathrm{~h}: 1.8 \pm 0.2$. 6-9 h: $2.8 \pm 0.3 \mathrm{mM}$ ) by iv triglyceride-heparin infusion and once under control conditions (CON) employing a glycerol infusion to achieve comparable plasma glycero levels ( $9 \mathrm{~h}$; LIP: $0.46 \pm 0.06$. CON: $0.48 \pm 0.04 \mathrm{mM}$ ). In addition, 3 subjects were studied under identical FFA elevation during pancreatic clamps (somatostatin: 0.1 $\mu \mathrm{g} / \mathrm{kg} \mathrm{min}$. insulin: $0.07 \mathrm{mU} / \mathrm{kg}^{\prime} \mathrm{min}$. glucagon: $0.65 \mathrm{ng} / \mathrm{kg}^{\prime} \mathrm{min}$ ). Endogenous glucose production ( $\mathrm{EGP}$ ) and gluconeogenesis (GNG) were estimated employing boluscontinuous D- $\left[6,6-{ }^{2} \mathrm{H}_{2}\right]$-glucose infusion and oral ${ }^{2} \mathrm{H}_{2} \mathrm{O}$ administration $(5 \mathrm{~g} / \mathrm{kg}$ body $\mathrm{H}_{2} \mathrm{O}$ ). The ratio of ${ }^{2} \mathrm{H}$ bound to $\mathrm{C} 2$ and $\mathrm{C} 5$ of glucose (measured by GC-mass spect) gives the fractional contribution of GNG to EGP. Lipid infusion resulted in a small rise of plasma insulin in the first study (basal: $5.8 \pm 0.7 ; 9 \mathrm{~h}: 7.3 \pm 0.9 \mathrm{mU} / \mathrm{l} ; \mathrm{p}=0.01$ ) which was paralleled by a $-10 \%$-decrease in plasma glucose $(\mathrm{p}=0.005)$, while plasma glucose and insulin remained unchanged during CON. After $9 h$, rates of EGP were similar at zero time (LIP: $9.3 \pm 0.5, \mathrm{CON}: 9.0 \pm 0.8 \mu \mathrm{mol} / \mathrm{kg} \mathrm{min}$ ) and declined to $8.4 \pm 0.5$ ( $p<0.01$ vs basal) and $8.2 \pm 0.7 \mu \mathrm{mol} / \mathrm{kg} \min$ ( $p<0.005$ vs basal), respectively. GNG contributed to EGP by $46 \pm 4 \%$ (LIP) and by $52 \pm 3 \%$ (CON) at zero time, and rose to $65 \pm 8 \%(\mathrm{p}<0.05$ vs basal) and $78 \pm 7 \%(\mathrm{p}<0.01$ vs basal) without any difference between both studies. During the pancreatic clamp, plasma insulin and glucagon concentrations were not different from baseline $(9 \mathrm{~h}$; insulin: LIP: $6.0 \pm 0.7$, CON: $6.4 \pm 1.0 \mathrm{mU} /$; glucagon: $80 \pm 20.105 \pm 43 \mathrm{ng} / \mathrm{l}$ ). In contrast to the first study, plasma glucose rose by $50 \%$ during LIP $(135 \pm 16 \mathrm{mg} / \mathrm{dl})$, but decreased by $24 \%$ during CON within $9 \mathrm{~h}(69 \pm 8 \mathrm{mg} / \mathrm{dl}$; $\mathrm{p}<0.05$ vs LIP). Conclusion: Suppression of insulin secretion unmasks the FFA-induced increase of plasma glucose concentration, which is abolished by small increases of insulin secretion under physiological conditions.

\section{5}

Insulin Sensitivity is Related to Skeletal Muscle Lipid Content in Healthy Non-Diabetic Subjects

S JACOB, J MACHANN, A VOLK, K RETT, F SCHICK, W RENN, C CLAUSSEN, O LUTZ, H-U HARING; Univ. Of TUEBINGEN, GERMANY

In the pathogenesis of insulin resistance (IR) the cross talk between adipose tissue (AT) and skeletal muscle (SM) is very important. Non-esterified fatty acids (NEFA) are candidates for regulators of insulin sensitivity (IS) of SM. The role of an increase of lipolysis in AT and an elevation of plasma NEFA in the pathogenesis of IR, however, is controversial.

The aim of the present study was to see whether intramuscular lipid (IML) stores are increased in IR and whether these are related to IS. Therefore, we used the hyperinsulinemic-euglycemic glucose clamp to quantify is [indicated by the metabolic clearance rate for glucose (MCR)] in combination with magnetic resonance (MR)

The relative muscular lipid content $\left(\mathrm{RMLC}_{i}=\right.$ in reference to bone marrow) in two different muscles ( $M$. Tibialis Anterior=TA and M.Soleus $=S O L$ ) were assessed by MR-imaging and the intramyocellular lipid concentration $(=\mid M C L)$ in TA was quantified by MR-proton-spectroscopy. The thirteen healthy, non-diabetic subjects $(5 \mathrm{~m} / 8 \mathrm{f})$, with a mean age of 32 years (range $18-43)$, mean BM? $24,6 \mathrm{~kg} / \mathrm{m} 2(18,9-$ 36,3 ) represented a wide range of IS (MCR $\left.8,0 \mathrm{ml} / \mathrm{kg}^{*} \mathrm{~min} ; 4,1-16,5\right)$. Lipid content was higher in SOL (mean: 2,74 relative\%; range: $1,2-5,3$ ) when compared to TA (1,0 relative\%: $0.4-2,1)$ also $I M C L$ in TA $(1,85$ arbitrary units; $0,5-4,24)$ varied considerably between the subjects. While IMCL showed no significant association with IS the RMLC -both in SOL and in TA- were negatively correlated to MCR (TA: $r=-0.72, p<0.01$; SOL: $r=-0.61, p<0.05$ ).

This study supports the notion, that intramuscular lipid stores play a role in the pathogenesis of IR. As the association between MCR and intramuscular lipids was found in two different muscle types, one can speculate that this observation can be generalized to the skeletal muscle tissue.

Supported by a grant of fortüne, \#428, to SJ and FS

\section{4}

SOLUBLE TNF- $\alpha$ RECEPTOR 1 AND 2 LEVELS CIRCULATE IN PROPORTION TO TOTAL AND LDL-CHOLESTEROL LEVELS C. Gutiérrez", M. Broch^, W. Ricart, , J. Vendrell", R. Casamitjanat, C. Richart* and JM Femández-Real. University Hospital of Girona and Tarragona*. +Hospital Clínic of Barcelona. SPAIN

In the last years, it has been demonstrated that tumor necrosis alpha (TNF- $\alpha$ ) has important effects on whole-body lipid metabolism and insulin resistance. Plasma triglycerides have been found to correlate with plasma TNF- $\alpha$ in patients with ischemic heart disease. The purpose of this study was to explore whether activation of the TNF- $\alpha$. system, as measured by the levels of circulating soluble forms of the TNF receptors 1 and 2 (sTNFR1, sTNFR2) and TNF- $\alpha$ itself, is associated with lipid abnormalities in healthy subjects. Thirty-six subjects [(19 males, mean age $36.2 \pm 1.9$; BMI $28.8 \pm 1.2$, range 22.2-35.7), and 17 females, age $34.9 \pm 1.4$; BMI $28.1 \pm 0.8$, range 19-37.9)] were studied. Plasma sTNFR2 levels, but not sTNFR1, correlated with BMI $(r=0.50, p=0.002)$ and age $(r=0.45, p=0.007)$. In the total population, plasma STNFR 1 correlated with total $(r=0.43, p=0.01)$ and LDL-cholesterol $(\mathrm{g}=0.52, \mathrm{p}=0.002)$ levels, but not with total or $\mathrm{HDL}_{2}-\mathrm{HDL}_{3}$ subfractions of HDL cholesterol, total plasma triglycerides, VLDL-cholesterol or VLDL-triglycerides (all $r<0.11, \mathrm{p}=\mathrm{NS}$ ). Plasma sTNFR2 also correlated with total $(r=0.44, p=0.009)$ and LDL-cholesterol $(r=0.57, p<0.0001)$ levels, and also with $\mathrm{HDL}_{2}$ cholesterol $(r=-0.37, \mathrm{p}=0.029)$. A Multiple Linear Regression in a stepwise manner was constructed to predict total cholesterol levels, with BMI, sex, age, sTNFR1 or sTNFR2 as independent variables. Both sTNFR1 and STNFR2 were significantly associated with total cholesterol $(p=0.031$ and 0.009 ), contributing to $26 \%$ and $19 \%$, respectively, of the variance in total cholesterol levels. In another models in which total cholesterol was substituted for LDL-cholesterol, sTNFR1 or sTNFR2 $(\mathrm{p}=0.0084$ and 0.0005$)$ were significantly associated with LDL-cholesterol, contributing to $39 \%$ and $32 \%$ of the variance in LDL-cholesterol levels, respectively. In summary, plasma levels of STNFR1 and STNFR2 circulate in proportion to total and LDL-cholesterol in healthy subjects.

\section{6}

GLYCEROL IN HUMAN SKELETAL MUSCLE IS HIGH EVEN UNDER HYPERINSULINEMIC CONDITIONS

M Sjöstrand, S Gudbjörnsdottir, L Strindberg, J Wahren*, P Lönnroth. *Dep. of Clinical Physiology, Karolinska Hospital and Lundberg Laboratory for Diabetes Research, Sahlgrenska University Hospital, Sweden.

Hydrolysis of triacylglycerol from muscle cells, intra-muscle adipose tissue and/or plasma may contribute to interstitial skeletal muscle glycerol. To evaluate the glycerol metabolism and the influence of insulin we measured skeletal muscle interstitial glycerol using the forearm model, arterial and deep venous catheterization and intramuscular microdialysis during euglycemic hyperinsulinemic conditions in ten healthy lean young males (group 1). In another group of nine healthy and lean young subjects (group 2), measurements were done in the medial quadriceps muscle and arterilized plasma using the same study protocol. Plasma insulin and glucose concentrations during steady state were $573 \pm 58 \mathrm{mU} / \mathrm{L}$ and $6.1 \pm 0.2 \mathrm{mmol} / \mathrm{L}$, respectively in group 1 and $297 \pm 10 \mathrm{mU} / \mathrm{L}$ and $5.8 \pm 0.7 \mathrm{mmol} / \mathrm{L}$ in group 2 .

Results: Forearm arterial and venous glycerol were $16 \pm 1$ and $18 \pm 1$ $\mu \mathrm{mol} / \mathrm{L}$, respectivily $(p<0.05$ ). Interstitial glycerol concentration in the brachial and medial quadriceps muscle were $33 \pm 3$ and $64 \pm 21$ $\mu \mathrm{mol} / \mathrm{L}$, respectively. The difference between arterial plasma glycerol and interstitial tissue glycerol were $17 \pm 3 \mu \mathrm{mol} / \mathrm{L}(\mathrm{p}<0.05)$ and $41 \pm$ $20 \mu \mathrm{mol} / \mathrm{L}(\mathrm{p}<0.05)$, in the arm and the leg, respectively.

Conclusions: During hyperinsulinemic conditions forearm glycerol release is nearly blunted, whereas the muscle interstitial glycerol concentration is 2-3 times higher than in plasma. The presence of the markedly higher interstitial glycerol levels suggest an in-situ noninsulin regulated hydrolysis /utilization of triglycerides. 
OP 17

\section{Glycation}

97

\section{ACCELERATED DIABETIC GLOMERULOPATHY IN GALECTIN-3 / AGE-RECEPTOR-3 KNOCKOUT MICE} G.Pugliese', F.Pricci', G.Leto', G.Romeo', L.Amadio', S.Catalano', D.Hsu², P.Barsotti ${ }^{1}$, E.Albanese ${ }^{1}$, S.Cordone ${ }^{1}$, L.Frigeri ${ }^{3}$, F. - T. Liu ${ }^{2}$ and U.DiMario' "University of Rome "La Sapienza", Italy; "La Jolla Institute for Allergy and Immunology and ${ }^{3}$ the Scripps Institute, UCSD, CA, USA

Advanced glycation end-products (AGE) have been implicated in the pathogenesis of diabetic glomerulopathy through their binding to cell surface receptors, which mediate both AGE uptake/degradation and AGE-induced cell activation. Galectin-3 (Gai-3), an adhesion molecule of the lectin family, has been recently identified as the AGE-receptor-3. This study was aimed at assessing whether Gal-3 deficiency is capable of interfering with the development of glomerulopathy in experimental diabetes. Male C57BL/6 Gal- 3 knockout $(\mathrm{KO})$ mice, obtained by gene ablation, and wild type $(\mathrm{WT})$ mice were rendered diabetic (D) by i.p. injection of streptozotocin $(180 \mathrm{mg} / \mathrm{kg})$ and killed 4 months later together with age-matched nondiabetic (ND) $K O$ and WT controls. In $D$ animals, metabolic derangement (blood glucose levels: KO-D $23.8 \pm 1.2 \mathrm{mmol} / \mathrm{L}$ and $\mathrm{WT}-\mathrm{D} 23.2 \pm 2.4$ vs. KO-ND $5.5 \pm 0.2$ and $W T-N D 5.7 \pm 0.4$, $p<0.001$ ) and growth impairment (final body weight: KO-D $21.7 \pm 2.5 \mathrm{~g}$ and WTD $21.0 \pm 2.2$ vs. KO-ND $36.1 \pm 3.6$ and WT-ND $32.1 \pm 3.0, p<0.001$ ) were similar in $\mathrm{KO}$ and $\mathrm{WT}$ mice. Conversely, renal functional and structural changes were significantly more pronounced in KO-D than in WT-D mice $(p<0.05-0.001)$. Urinary protein/creatinine ratio was $6.3 \pm 1.4$ in $\mathrm{KO}-\mathrm{D}$ vs. $2.3 \pm 0.8$ in KO-ND $(p<0.001)$ and $3.7 \pm 1.0$ in WT-D vs. $2.0 \pm 0.6$ in WT-ND $(p<0.05)$ and mesangial fractional area was $7.6 \pm 1.2 \%$ in KO-D vs. $5.5 \pm 1.0$ in KO-ND $(p<0.01)$ and $5.2 \pm 0.6$ in WT-D vs. $4.1 \pm 0.4$ in WT-ND $(p<0.05)$. Both extracellular matrix and cell components appeared to be responsible for the more marked mesangial enlargement abserved in KO-D vs. WT-D mice. Kidney weight and mean glomerular area were also increased in $D$ Vs. ND, with no significant difference between KO-D and WT-D. These experiments show that Gal-3 deficiency is associated with accelerated diabetic glomerulopathy, possibly related to a reduced removal of irreversibly glycated molecules and/or to the lack of other Gal-3 actions may be implicated in these changes.

\section{9}

POSSIBLE SIGNIFICANCE OF NON-CARBOXYMETHYLLYSINE ADVANCED GLYCATION END-PRODUCTS IN SERUM OF TYPE 2 DIABETIC PATIENTS.

Z. Makita, M. Takeuchi, Y. Kamada, and T. Koike, Dept. of Internal Med. II, Hokkaido Univ. School of Med., Sapporo, and Dept. of Biochem, Faculty of Pharmaceutical Science, Hokuriku Univ., Kanazawa.

The advanced glycation end-products (AGE) plays an important role in the pathogenesis of diabetic complications. Recent studies demonstrated that $\mathrm{N}^{\varepsilon}$-(carboxymethyl)-L-lysine (CML) is a major epitope for the majority of currently available AGE antibodies. However, recent findings clearly demonstrated that the major source of CML was lipid peroxidation, not glycation. Distinction of CML from non-CML AGE is a prerequisite for understanding the role of AGE toxicity in diabetic complications. We prepared polyclonal antibodies by immunizing rabbits with AGE-rabbit albumin and we then separated the antiserum into antibodies that recognized CML and non-CML by using affinity chromatography on columns coupled with AGE-BSA and CML-BSA. In addition, the relationship between circulating CML or non-CML AGE levels in sixty type 2 diabetic patients and clinical parameters such as $\mathrm{HbAlc}$, mean fasting plasma glucose (FPG) levels before 1-month or before 2-months was then investigated. These CML and non-CML AGE antibodies were used for detection of the size distribution of AGE in serum from type 2 diabetic patients on hemodialysis by Sephadex G-15 chromatography. CML and non-CML AGE were detected as two peaks with an apparent molecular weight of 1.15 and $0.85 \mathrm{kDa}$, respectively. Serum non-CML AGE levels significantly correlated with mean fasting blood glucose levels before 2 -months $(r=0.498, p<0.0001)$ or before 1 month $(r=0.446, p=0.0002)$ and HbA1c $(r=0.375, p=0.0019)$, but the CML levels did not correlate with these clinical parameters. We propose that both CML and non-CML AGE are present in blood circulation, and non-CML AGE rather than CML should be evaluated when attempting to elucidate AGE-related toxicity.

\section{8}

INDUCTION OF GLOMERULAR / MESANGIAL GALECTIN-3 AGE-RECEPTOR-3 EXPRESSION BY THE DIABETIC MILIEU G. Leto', F. Pricci', G. Romeo', S. Catalano', L. Amadio', O.Diaz-Horta', P. Sale', R Gradini', L. Lenti', P. Barsotti' , L. Frigeri², U. Di Mario' and G. Pugliese ${ }^{b}$

l"La Sapienza" University, Rome, Italy; ${ }^{2}$ The Scripps Institute, La Jolla, CA, USA Nonenzymatic glycation has been involved in the pathogenesis of diabetic glomerulopathy, via advanced glycation end-product ( $\mathrm{AGE}$ ) formation and binding to cell receptors, Galectin-3 (Gal-3), an adhesion molecule of the lectin family, has been recently identified as an AGE-binding protein and is now referred as AGE-receptor 3 (AGE-R3). This study was aimed at evaluating the modulation of glomerular/ mesangial expression of Gal-3/AGE-R3 by the diabetic milieu, both in vitro and in vivo. In the in vitro studies, rat mesangial cells (RMC) were (a) cultured for 1-4 week in normal glucose ( $5.5 \mathrm{mM}, \mathrm{NG})$, high glucose $(30 \mathrm{mM}, \mathrm{HG})$, or iso-osmolar mannitol (M); or (b) grown for 4 days on dishes coated with native BSA (BSA), glycated BSA with AGE formation (BSA-AGE), or glycated BSA in which AGE formation was prevented by aminoguanidine (BSA-AM). In the in vivo studies, male SpragueDawley rats were injected either with streptozotocin or vehicle and killed 1 and 2 months later. No Gal-3 was demonstrable in RMC cultured in NG (although it became evident after several passages in culture), whereas cells grown on BSA showed a peak at flowcytometry, corresponding to a diffuse (cytoplasmic) staining at immuno fluorescence. Prolonged exposure (3-4 weeks) of RMC to HG, but not to M, as well as growing cells on BSA-AGE and, to a Jesser extent, BSA-AM, induced or significantly increased Gal-3 protein and mRNA levels, with unchanged medium secretion. RMC under these conditions showed a unique patchy distribution of Gal-3 fluorescence, in addition to the diffuse pattern, with confocal microscopy indicating both a cytoplasmic and surface localization of granules compatible with Gal-3 receptor function. Gal-3 protein and mRNA were not demonstrable in glomeruli from nondiabetic rats, but became detectable in diabetic rats at 2 months. These results indicate that Gal-3/AGE-R3 is not expressed in the mesangium under basal conditions, but it is induced by prolonged exposure to hyperglycemia, both in vivo and in vitro AGEs also induce/up-regulate the expression of their own receptors, thus suggesting that the effect of hyperglycemia may be due to a time-dependent AGE formation.

\section{0}

NOVEL POLYMORPHISMS IN THE CODING REGION OF THE RECEPTOR FOR ADVANCED GLYCATION END PRODUCTS (RAGE) GENE B.I.Hudson, M.H.Stickland and P.J.Grant. Unit of Molecular Vascular Medicine, Level G, Martin Wing. Leeds General Infirmary. Leeds. UK

Advanced glycation end products (AGEs) have been implicated in the pathogenesis of diabetic vascular complications and their effects may be mediated via the Receptor for AGE (RAGE). Evidence indicates a genetic element in the development of these complications and we have therefore screened the coding region of RAGE for allclic variants in 40 Type 2 random diabetes patients and 40 normal volunteers by PCR-SSCP. 9 polvmorphisms were confirmed by sequencing. of which 4 were functional amino acid substitutions: Gly82Ser Thr 187Pro. Gly329Arg and Arg389Gln. To evaluate the ethnic prevalence of the common Gly82Ser polymorphism. 195 Caucasian, 156 Asian and 210 Pima Indians were screened. To investigate the prevalence in diabetics and in relation to cardiovascular disease, 258 Type 2 diabetes patients and 280 Iscaemic Hear Disease (IHD) patients were also screened There was no difference in prevalence of Gly82Ser in Caucasian and Asian subjects ( $87 \% \mathrm{GG}, 12 \% \mathrm{GS}$ and $1 \% \mathrm{SS}$ ) There was a highly significantly lower prevalence of Glv82Ser in the Pima Indian population ( $99 \% \mathrm{GG}, 1 \% \mathrm{GS}) . \chi^{2}$ of $\mathrm{p}<0.00001$. There was no difference in genotype frequencies between Caucasian controls and either Typc 2 diabctics ( $92 \%$ GG and $8 \%$ GS) or IHD patients ( $87 \%$ GG and $13 \%$ GS), $\chi^{2}$ of $p>0.05$. There was also no association found between genotype and macrovascular discasc in the diabetic or IHD patients. In conclusion. the RAGE genc contains common polymorphisms that occur with similar frequencies in Asian and Caucasian populations, but are less common in Pina subjects. The functional nature of this polymorphism is currently being investigated by site-directed mutagencsis and receptor binding studies. Further work is renuired to investigate these polymorphisns for their role in microvascular complications 
A NOVEL INHIBITOR OF ADVANCED GLYCATION END. PRODUCT FORMATION INHIBITS IN VIVO MESENTERIC VASCULAR HYPERTROPHY IN EXPERIMENTAL DIABETES

T. Soulis, S. Sastra, V. Thallas, †SB. Mortensen, †JT. Clausen, †OJ. Bjerrum, †E. Boel and ME Cooper. Department of Medicine, University of Melbourne, Austin and Repatriation Medical Centre, Australia, †Health Care Discovery, Novo Nordisk, Denmark.

Previous studies in our laboratory have demonstrated that the vascular changes in diabetes include hypertrophy of the mesenteric vasculature. The products of the process of advanced glycation known as advanced glycation end products (AGEs) contribute to the development of diabetic complications. We have previously shown that aminoguanidine (AG), an inhibitor of AGEs is able to retard vascular bypertropby associated with diabetes. However, AG is also an inhibitor of various NO synthases and therefore one cannot exclude that the effects of AG may involve inhibition of NO dependant pathways. The present study sought to examine the role of the process of advanced glycation in the development of diabetes vascular disease. The effects of $\mathrm{AC}$ as well as a novel inhibitor of glycation, 2,3-diaminophenazine (NN), which does not inhibit iNOS, were evaluated in a three week model of diabetic vascular disease. Screptozolocin-induced diabetic Sprague-Dawley rats were randomised to receive AG ( $\mathrm{lg} / \mathrm{L}$ in drinking water/day) [n=10], NN (20mg/kg/body weigh/day in drinking water) [n=21] or no treatment, [n=10] and followed for 3 weeks. When compared with control rats $[\mathbf{n}=10]$, diabetes was associated with an increase in mesenteric vascular weight. ${ }^{*} \mathrm{P}<0.01$ vs all groups. Treatment of diabecic rats with AG or NN resulted in the

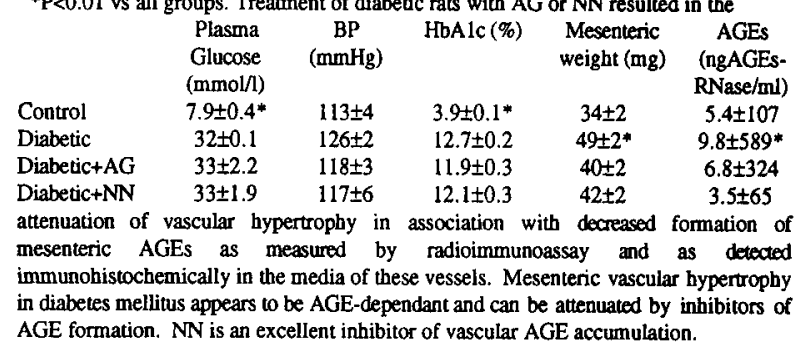

\section{OP 18}

\section{Immunology and Diabetic Pregnancy}

\section{3}

IL-4 AND IFN- $\gamma$ SECRETION BY CORD BLOOD MONONUCLEAR CELLS IN NEWBORNS WITH HLA-DOB1 RISK ALLELES FOR IDDM

P. Klemetti, J.Ilonen, H.K. Ákerblom, O. Vaarala. Hospital for Children and Adolescents, University of Helsinki, Helsinki. Turku Immunology Center and the Department of Virology, University of Turku, Turku, Department of Biochemistry, National Public Health Institute, Helsinki, Finland

To study the functional subtypes of T-lymphocytes in individuals with enhanced genetic risk for IDDM, we collected cord blood samples from newborns with a firs degree relative with IDDM. HLA DQB1 typing was performed by a technique developed for screening of IDDM susceptibility based on the presence of HLA DQB1 alleles associated with a significant risk for the disease (HLA $\mathrm{DQB1} * 0302, * 02)$. Cord blood mononuclear cells $(\mathrm{CBMCs})$ were cultured at $3 \times$ $10^{6}$ cells $(2 \mathrm{ml})$ per well with PHA. Supernatants were collected after $20 \mathrm{~h}$ incubation for IL-4 and after $72 \mathrm{~h}$ incubation for IFN- $\gamma$ measurements by ELISA Median IL-4 levels secreted by PHA-stimulated CBMCs from newborns with increased genetic risk for IDDM (carrying $\mathrm{HLA} \mathrm{DQB1*0302} \mathrm{and/or} \mathrm{*02} \mathrm{alleles:}$ $\mathrm{n}=34$ ) were higher than in the newborns without increased genetic risk for IDDM $(\mathrm{n}=13)(7.0 \mathrm{pg} / \mathrm{ml}$ and $3.5 \mathrm{pg} / \mathrm{ml}$ respectively; $\mathrm{p}=0.01$; Mann-Whitney $\mathrm{U}$ test) Median IFN- $\gamma$ levels did not differ significantly between the groups $[4856 \mathrm{pg} / \mathrm{m}]$ $(n=50)$ vs. $4272 \mathrm{pg} / \mathrm{ml}(\mathrm{n}=21)$, respectively; $\mathrm{p}=0.16$, Mann-Whitney U test]. When newborns were divided to subgroups according to their HLA DQB1 alleles, only CBMCs from the newborns with HLA DQB ${ }^{*} 02,0302$ or HLA DQB $1 * 02 x$ genotype ( $x$ denotes alleles other than $* 0302$ or *02) secreted enhanced levels of IL-4 when compared to the CBMCs from newborns carrying none of the risk alleles for IDDM ( $\mathrm{p}=0.001$ and $\mathrm{p}=0.04)$. On the other hand, CBMCs from the newborns with HLA DQB $1 * 0302, x$ genotype had a tendency towards enhanced IFN- $\gamma$ secretion when compared to the CBMCs from newborns carrying none of the risk alleles for IDDM, medians being 9780 and $4272 \mathrm{pg} / \mathrm{ml}$, respectively ( $\mathrm{p}=0.07$, Mann-Whitney $\mathrm{U}$ test). We conclude that HLA risk allele associated deviations in cytokine production are present from birth on and may affect the development of immune responsiveness in individuals with genetic risk for IDDM.
LEFT VENTRICULAR DIASTOLIC FUNCTION IS ASSOCIATED WITH SERUM LEVELS OF ADVANCED GLYCATION END PRODUCTS IN IDDM PATIENTS

T.J. Berg', O. Snorgaard ${ }^{3}$, P. Hildebrandt ${ }^{3}$, J. Faber ${ }^{3}$, P.A. Torjesen ${ }^{2}$, and K.F. Hanssen, Aker Diabetes Research Centre' and Hormone Laboratory', Aker University Hospital, Oslo, Norway and Frederiksberg Hospital ${ }^{2}$, Copenhagen, Denmark.

Impairment of left ventricular function is common in IDDM patients even without coronary artery disease. This is possibly caused by a reduction in collagen degradation. Advanced glycation end products (AGEs) cross-link tissue collagen and are found within myocardial fibres. To examine whether there is an association between AGEs and left ventricular function we used M-mode and Doppler echocardiography to assess left ventricular diastolic and systolic function in 52 consecutive IDDM patients age $40 \pm 13$ (mean $\pm \mathrm{SD}$ ) years, diabetes duration $17 \pm 13$ years and $\mathrm{HbA}_{1 \mathrm{c}} 8.3 \pm 1.1 \%$. Serum levels of AGEs were measured by a newly developed, competitive immunoassay using polyclonal antibodies made from rabbit immunised with AGE-RNase. A significant correlation was found between the serum levels of AGEs and isovolumetric relaxation time (IVRT), $\mathrm{r}=0.46(\mathrm{p}<0.001$ ), and left ventricular diameter during diastole (LVD), $r=0.37$ ( $p<0.008$ ). Systolic parameters did not correlate with serum levels of AGEs. A stepwise regression analysis with IVRT and LVD as dependent variables showed that $28 \%$ of the IVRT variation could be explained by variation in serum levels of AGEs, $(\mathrm{F}=14.0, \mathrm{p}<0.0005)$ and diabetes duration $(\mathrm{F}=4.6, \mathrm{p}<0.04)$, $R^{2}=0.28$, whereas $\mathrm{HbAlc}$, AER and diastolic blood pressure were of no importance. In conclusion, AGEs may play a role in the increase of heart stiffness seen in patients with type 1 diabetes.

\section{4}

GESTATIONAL AND NON-INSULIN-DEPENDENT DIABETIC PREgNANCIES SHARE A SAME PREVALENCE OF AUTOIMMUNe AND GENETIC DISORDERS

G. Cardellini, E. Sciullo, P. Torresi, C. Tiberti, A. Napoli, A. Buongiomo*, $U$. Di Mario** and F. Fallucca

Cattedra di Diabetologia, "Cattedra di Endocrinologia, Ist. II Clinica Medica, Università "La Sapienza", Roma, "Istituto Superiore Sanità

Epidemiologic and pathophysiologic studies suggest that gestational (GDM) and non-insulin-dependent (NIDDM) diabetes mellitus share many metabolic similarities. The aim of the present study was to investigate autoimmune (Glutamic acid decarboxylase: GADA) and genetic mutations of both the insulin receptor substrate-1 (IRS ${ }_{1}$ ) and $\beta_{3}$-adrenergic receptor in GDM and NIDDM diabetic pregnancies. GADA were measured in 64 control (C), 86 GDM and 83 NIDDM pregnants; in addition, the genetic investigation was done in $87 \mathrm{C}, 77 \mathrm{GDM}$ and 33 NIDDM women. GADA were positive (index $\geq 0.035$ ) in $0 \%$ of $C, 7 \%$ of GDM and $4.8 \%$ of NIDDM, being significant the difference between the GDM and $\mathrm{C}$, whereas GADA of GDM and NIDDM overlapped. Genetic abnormalities of IRS ${ }_{1}$ and $\beta_{3^{-}}$ adrenergic receptor were similar in GDM and NIDDM, being significantly greater in GDM and GDM+NIDDM than in controls for IRS 1 (10.4\% and $10 \%$ vs $2.3 \%$ ). The age and BMI were overlapped GDM and NIDDM, being greater than in controls. Moreover family history for diabetes was very high in the two diabetic conditions $(84 \%$ and $82.7 \%$ as total, $69 \%$ and $68 \%$ as first relatives), being significantly higher than in controls $(26 \%)$. Moreover among the diabetic women metabolic control was poorer and associated with higher $C$-peptide plasma values when $I R S_{1}$ and $\beta_{3}$-adrenergic mutations were observed.

These results suggest that GDM and NIDDM pregnant women, in addition to metabolic finding, share common autoimmune disorders and similar genetic abnormalities which may play a role in the impairment of carbohydrate metabolism. 
THE PREDICTIVE VALUE OF HLA MARKERS FOR THE DEVELOPMENT OF ISLET AUTOANTIBODIES AND IDDM POSTPARTUM IN GDM-WOMEN K. M. Ferber ${ }^{1}, 2$, E. Keller ${ }^{2}$, E.D. Albert ${ }^{2}$ and A.-G. Ziegler ${ }^{1}$ ('Institute of Diabetes Research, Munich, 2Laboratory for Immunogenetics, Munich, Germany)

The aim of the study is to determine the allele frequencies of HLA-DRB1, DQA1, and -DQB1 in Caucasian gestational diabetic women (GDM-women) and to evaluate the predicitive value of HLA markers for the development of IDDM postpartum. Since 1989, $184 \mathrm{GDM}$-women ( 119 white class $\mathrm{A}, 62$ white class $B$ ) were recruited into the study and followed from delivery up to 7 years postpartum (median follow-up time 2.5 years, range $0-7.7$ years). As controls for the HLA allele frequencies we used a panel of 254 unrelated subjects from Germany. The HLA analysis was performed by sequence-specific oligonucleotide typing. Autoantibodies to GAD, IA-2ic and ICA were determined at delivery. During follow-up, 24 GDM-women developed IDDM. In the total group of GDM-women no particular HLA allele were increased or decreased compared to the control population. However, in GDM-women who developed IDDM postpartum there was a significant increase of HLA $D R B 1^{*} 03\left(p_{c}=0.005\right)$. Furthermore, DRB1*03 was significantly associated with the presence of islet antibodies $\left(p_{\mathrm{c}}=0.01\right)$. The risk of GDM-women to develop IDDM within 2 years postpartum is significantly $\left(\rho_{c}=0.0025\right)$ increased in women who are positive for DR3 or DR4 [22\% (12-31)] compared to women who are neither DR3 or DR4 positive $[7 \%(2-12)]$. The combination of the HLA and antibody data revealed that women who are negative for both markers have only a small risk for disease postpartum [3\% (0.7)] compared to women who are positive for antibodies and for DR3 or DR4 $[44 \%(25-64)]$. By combining the antibody measurement and the determination of DR3 or DR4 $92 \%$ of women who developed IDDM postpartum were identified. These results suggest that HLA typing in combination with antibody testing may be a useful strategy to identify women with GDM who develop IDDM within a short time period after deliveny.

\section{7}

PREVALENCE AND TITER OF ISLET CELL ANTIBODIES INCREASE AFTER DELIVERY IN WOMEN WITH GESTATIONAL DIABETES

R. Corcoy, S. Piquer, J. Morales, M. Albareda, A. García-Patterson, M. Puig and A. de Leiva. Servei d'Endocrinologia, Hospital de Sant Pau, Barcelona

Autoimmune diseases characteristically worsen after delivery. The aim of this study was to assess if the same holds true with islet cell antibodies (ICA) as markers of autoimmunity against the $\beta$ cell. ICA were measured in 603 women with gestational diabetes mellitus (GDM) both after diagnosis (Third Workshop Criteria) and at 2-6 months after delivery. ICA were measured by indirect immunofluorescence in a 0 group human pancreas using a prolonged incubation. Statistical analysis: McNemar's test, chi-square test, ANOVA. ICA were present in $10.3 \%$ of women during pregnancy and in $15.3 \%$ after delivery: in $80.6 \%$ of women ICA were - and in $6.1 \%$ were + both during and after pregnancy, in $4.1 \%$ were + during and after whereas in $9.1 \%$ were - during and + after, $p<0.05$. In the 37 women with ICA + both during and after pregnancy, ICA titers did not change in $43.2 \%$, decreased in $16.2 \%$ and increased in $40.5 \%, p<0.05$. The four subgroups of women according to the presence/absence of ICA during and after pregnancy did not differ in clinical characteristics (age, body mass index, family history of DM, previous abnormality of blood glucose, gestational age and oral glucose tolerance test at GDM diagnosis, need of insulin therapy and glucose tolerance after delivery). We conclude that in women with GDM the prevalence and titer of ICA increase after delivery.
Prevalence and predictive value of GAD and IA2 antibodies in a group of women with gestational diabetes mellitus related to ICA.

M. Albareda, R. Corcoy, S. Piquer, I. Vinyets, J. Morales, D. Mauricio, A. GarciaPatterson, E. Bonifacio*, M. Puig-Domingo, J. Adelantado** and A de Leiva. Departments of Endocrinology and **Obstetrics. Hospital de Sant Pau, Barcelona. *Istituto Scientifico San Raffaele, Milan.

Presence of autoantibodies (AA) to pancreatic $\beta$ cell antigens in women with gestational diabetes mellitus (GDM) increases the risk of diabetes (DM). We have investigated the prevalence (P) of ICA (indirect immunofluorescence. prolonged incubation). glutamic acid decarboxylase (GADA) and tyrosine phosphatase (IA2A) antibodies (combined radioligand binding assay) in a group of 1113 women with GDM consecutively recruited. The assocation of AA with glucose tolerance abnormalities (GTA) (standard OGTT) was also investigated in all women $(n=634)$ followed for one year after delivery. Twenty percent of these women developed GTA (17 DM. 107 impaired glucose tolerance/impaired fasting glucose). Prevalence (P) of AA and positive predictive value (PPV) for GTA are shown in the table. In addition, we compared the frequency of association of GADA/IA2A in GDM women with a group of first degree relatives of diabetic subjects (FRD) (see also the table)

\begin{tabular}{|l|c|c|c|c|c|c|}
\hline & ICA & GADA & IA2A & 1AA & 2AA & 3AA \\
\hline P & $8.2 \%$ & $2 \%$ & $0.6 \%$ & $9.9 \%$ & $1.3 \%$ & $0.3 \%$ \\
PPV & $24 \%$ & $25 \%$ & $43 \%$ & $19 \%$ & $25 \%$ & $100 \%$ \\
\hline CDM & GADA & IA2A & FDR & GADA & IA2A \\
\hline ICA + & $7 \%$ & $2.2 \%$ & ICA $+(n=30)$ & $20 \%$ & $\mathbf{1 7} \%$ \\
ICA - & $1.5 \%$ & $0.5 \%$ & ICA $-(n=160)$ & $7 \%$ & $0 \%$ \\
\hline
\end{tabular}

In conclusion, in GDM ICA have a higher $P$ and similar PPV vs GADA remaining as the choice for screening purposes. Association of all AA achieved maximal PPV. In GDM. P of GADA and IA2A were markedly reduced when compared to FDR
108

\section{A STUDY ON T-CELL RECEPTOR $\gamma-\delta$ IN GESTATIONAL DIABETES MELLITUS}

A. Lapolla*, M. Sanzari§̧, C. Betterleş, M. Masin, F. Florianiş, F. Bellio, F. Capovilla, M. Plebanis, D. Fedele

Institute of Internal Medicine- Chair of Metabolic Diseases- Padova University (Italy). §DPT of Laboratory Medicine, Padova (Italy)

Two types of T-cell-recepter (TCR) have been described $\alpha-\beta$ and $\gamma-\delta$ formed by genes rearrangement. The function of $\mathrm{CD} 3 \gamma \delta$ is not yet completely clarified, few studies have shown a significant increase of this marker in different autoimmune diseases and in the first phase of type 1 diabetes development. Gestational Diabetes Mellitus (GDM) is an heterogeneous disease in which the pathogenesis is not completely clarified. In the present study we wished to verify if TCRY6 could be involved in this disease. We evaluated TCR $\gamma$ values in 29 GDM patients (mean age $( \pm \mathrm{SD}) 33.8+3.4 \mathrm{yrs})$ and 21 normal pregnant women matched for age. At the moment of the study (28-36 gestational week) GDM patients showed a good metabolic control (mean fasting plasma glucose $88 \pm 17 \mathrm{mg} / \mathrm{dl}$; mean $\mathrm{HbA}_{\mathrm{lc}} 5.4 \pm 0.5 \%$ ). The lymphocyte subpopulations (CD3-CD3 $\left.\gamma \beta, \mathrm{CD} 3 \gamma 6\right)$ (flow cytometry), islet cell antibodies (ICA) and glutamic acid decarboxylase antibodies (GAD) (RIA method) were evaluated in all patients. The percentage of TCR $\gamma \delta$ was significantly higher in GDM women compared to control group $(5.1 \pm 2.8$ vs. $36 . \pm 1.4 ; p<0.05)$. No significant abnormality of the other lymphocyte subpopulations were found. All subjects were negative for ICA, two GDM patients were positive for GAD. Further studies of follow-up of these patients are imperative to verify if $\gamma \delta$ receptor could be a useful marker for diabetes development 


\section{OP 19}

\section{Cardiovascular Disease, Risk Factors, Prediction and Genetics}

\section{9}

QT INTERVAL PREDICTS MORTALITY IN TYPE I DIABETIC PATIENTS P Rossing, L Breum, A Major-Pedersen, A Sato, $H$ Winding A Pielersen, J Kastrup and H-H Parving, Steno Diabetes Center, DK 2820 Gentofte, Cardiological Lab, Rigshospitalet, Copenhagen, Denmark.

Our aim was to evaluate prolonged QT interval and QT dispersion as predictors of all cause and cardiac mortality in addition to well established risk factors in type I diabetic patients. We identified all subjects with a resting baseline ECG (6-12 leads) ( $\mathrm{n}=697,360 \mathrm{M}$ ) from a cohort of all adult type I diabetic patients, duration of diabetes $>5$ years, attending our clinic in $1984(n=939)$. The patients were followed in a prospective observational study for ten years. At baseline 431 had normoalbuminuria ( $<30 \mathrm{mg} / 24 \mathrm{~h}) 138$ had microalbuminuria (30-299mg/24h) and 128 had macroalbuminuria ( $2300 \mathrm{mg} / 24 \mathrm{~h}$ ) of whom $66(15 \%), 35(25 \%)$ and $61(48 \%)$ died during follow up respectively $(25(6 \%), 14(10 \%), 17(13 \%)$ due to cardiac disease). The QT length (to end of T-wave or nadir between T and U-wave) was measured and corrected for heart rate (QTc). Maximal QTc length (QTc max) and QTC dispersion (maximal -minimal QTc length, adjusted for number of leads) were determined. QTc max was 445(2.0)ms (mean (SE)) for survivors and 461 (4.5) in patients who died ( $p<0.001$ ). Fifty percent had prolonged QTc max (>440ms) $29 \%$ of patients with prolonged QTC max had died compared to $19 \%$ of patients with normal QTC max ( $p=0.001$ ). In a Cox proportional hazards model including baseline values of putative risk factors independent predictors of death were QTC max $(p<0.01)$, age $(p<0.001)$, presence of hypertension $(p=0.001)$ sex $(p<0.001)$, urinary albumin excretion ( $p<0.001)$ smoking $(p=0.04)$ log s-creatinine $(p<0.001)$, height $(p<0.001)$, social class $(p=0.04)$, whereas QTc dispersion, and HbAic were not included. QTC max was also an independent predictor of mortality in the subgroups with normoalbuminuria and with macroalbuminuria. QTc max was not a risk factor for cardiac mortality in the total group, but in the subgroup with macroalbuminuria it was an independent risk factor logether with age hypertension and smoking. In conclusion QTc prolongation, but not increased QTc dispersion, is a marker of increased mortality in type I diabetic palients.

\section{1}

SYSTOLIC BLOOD PRESSURE IS RELATED TO THINNESS AT BIRTH ONLY IN GENETICALLY HYPERTENSION PRONE INDIVIDUALS

$O$. Melander, I. Mattiasson, L. Groop and U.L. Hulthén; Dept of Endocrinology and Medicine, Lund University, Malmö, Sweden

Intrauterine growth retardation, as manifested by low birth weight and low ponderal index (thinness at birth) in relation to the gestational age, are associated with cardiovascular disease, syndrome X, NIDDM and high blood pressure. The aim of this study was to elucidate whether the association between intrauterine growth retardation and higher blood pressure in adulthood is influenced by heredity for hypertension. To address this question we collected anthropometrical data and gestational age from the time of birth from 30 normotensive men with heredity for hypertension (HTREL) and from 27 normotensive men with no heredity for hypertension (CONT) who had been examined in 1990 and 1995. The heredity for hypertension in HTREL consisted of documented, treated primary hypertension in both parents, in one parent and one sibling or in one parent and one grandparent on the maternal or paternal side. In 1990, HTREL were 37.5 \pm 5.9 years old and had BMI $24.9 \pm 2.6 \mathrm{~kg} / \mathrm{m}^{2}$, systolic blood pressure (SBP) $121 \pm 12 \mathrm{mmHg}$ and diastolic blood pressure (DBP) $75.6 \pm 8.6 \mathrm{mmHg}$ and CONT were $36.4 \pm 7.1$ years old and had BMI $24.9 \pm 3.5 \mathrm{~kg} / \mathrm{m}^{2}$, SBP $116 \pm 11 \mathrm{mmHg}$ and DBP $74.5 \pm 6.7 \mathrm{mmHg}$. In $1995 \mathrm{HTREL}$ had BMI $26.1 \pm 3.0 \mathrm{~kg} / \mathrm{m}^{2}$, SBP $127 \pm 13 \mathrm{mmHg}$ and DBP $85.0 \pm 9.5 \mathrm{mmHg}$ and CONT had BMI $25.9 \pm 4.0 \mathrm{~kg} / \mathrm{m}^{2}$, SBP $122 \pm 9.1 \mathrm{mmHg}$ and DBP $77.7 \pm 8.7 \mathrm{mmHg}$. In these parameters HTREL and CONT differed significantly only in DBP $1995(\mathrm{p}=0.009)$ However, HTREL had significantly lower birth weight $(3313 \pm 444 \mathrm{~g}$ vs $3685 \pm 490 \mathrm{~g}$, $\mathrm{p}=0.004)$ and ponderal index $\left(25.8 \pm 2.5 \mathrm{~kg} / \mathrm{m}^{3}\right.$ vs $\left.27.1 \pm 2.2 \mathrm{~kg} / \mathrm{m}^{3}, \mathrm{p}=0.04\right)$ after adjustment for gestational age when compared to CONT. Furthermore, SBP correlated negatively with ponderal index in HTREL in 1990 as well as 1995 ( $r=-$ $0.47 ; p=0.02$ and $r=-0.49 ; p=0.009$, respectively). In contrast, no such correlation existed in CONT neither 1990 ( $r=0.16, \mathrm{NS})$ nor $1995(\mathrm{r}=0.18$, NS). Adjustment of SBP for age did not change these correlations. In conclusion, these findings suggest that the association between intrauterine growth retardation and higher blood pressure in adulthood is limited to individuals with a positive family history of hypertension. The thrifty phenotype may thus serve as a phenotypic marker for a thrifty genotype influencing intrauterine growth.

\section{0}

PROLONGED QTC TIME AND INCREASED HEART RATE AS POWERFUL RISK FACTORS FOR MORTALITY IN NIDDM.

B. Linnemann, H.U. Janka, Zentraikrankenhaus Bremen-Nord Bremen, Germany

The aim of this prospective, community-based study was to analyse predictors for total and cardiovascular mortality in middle-aged, previously hospitalized NIDDM patients over a five-year period. At baseline, diabetic patients with severely consuming diseases (malignancy, liver cirrhosis, chronic renal failure) were excluded. A total of 475 NIDDM subjects (age 55-74 yr) were followed for a median duration of $5.2 \mathrm{yr}, 39 \%$ had signs of coronary artery disease at baseline. At the follow-up examination 85 patients $(20.1 \%)$ had died, $57(67 \%)$ of whom had died from a cardiovascular cause of death. In multiple logistic regression analysis of the traditional risk factors for the endpoint cardiovascular death, only age and low HDL cholesterol were significant $(p=0.018)$, but not total cholesterol, triglycerides, $\mathrm{HbA} 1 \mathrm{c}$, smoking, blood pressure, and form of antidiabetic treatment. On the other hand, age- and sex-adjusted resting ECG criteria as QTc time $(p=0.004)$ and heart rate $(p=0.009)$ were powerful predictors for total as well as cardiovascular mortality. The odds ratio (OR) for cardiovascular death in the upper quartiles of QTc time $(>440 \mathrm{~ms})$ was 4.2 and of increased heart rate $(>90 / \mathrm{min}) 2.9$ in comparison to the lowest quartiles ( $<406 \mathrm{msec} ;<70 / \mathrm{min}$ ). The relative risk of QTc time $>440 \mathrm{~ms}$ at a heart rate of $\geq 75 / \mathrm{min}$ was associated with an OR of 7.0. A significant correlation of QTc time with diabetic neuropathy was not present.

These data suggest that in the last five years of life middle-aged NIDDM patients do not differ in regard to most of the cardiovascular risk factors, but show significant changes of simply detectable ECG variables

\section{2}

ASSOCIATION OF A COMMON POLYMORPHISM IN THE ASUBUNIT GENE OF FXIII WITH MYOCARDIAL INFARCTION AND STROKE. INTERACTIONS WITH PAI-1 LEVELS, PAI-1 4G/5G GENOTYPE AND INSULIN RESISTANCE.

P.J. Grant, H.P. Kohler M.W. Mansfield and M.H. Stickland. Unit of Molecular Vascular Medicine, School of Medicine, University of Leeds, Leeds, UK.

To investigate the relationship between a common $G \rightarrow T$ point mutation in exon 2 of the a-subunit gene of FXIII (FXIIIVal34Leu) and myocardial infarction (MI), genotype frequencies were determined in a case-control study in 398 Caucasian patients with ischaemic heart disease, characterised for atheroma and a history of MI, and 196 healthy controls and 612 cases of acute stroke and 436 healthy controls. Acute stroke was defined by WHO criteria and CT scan. Venous blood was taken for cholesterol and triglycerides, plasminogen activator inhibitor-1 (PAl-1) and PAl-1 4G/5G polymorphisms. We used single stranded conformation polymorphism to determine FXIII genotype and allele specific PCR for PAl-1 genotype. The prevalence of the Val34Leu mutation was lower in patients with $M I$ than in those without MI $(32 \% \vee 50 \%, p=0.0009)$ than in controls $(32 \% v 48 \%, p=0.005)$. In those patients possessing the mutation PAl-1 levels were significantly higher

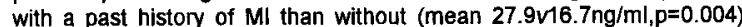
also PAl-1 4 G/5G genotype was commoner in this group (Ml $46 \%$, no $\mathrm{Ml}$ $24 \%, p=0.05$ ). There was a dose-response increase in history of MI in subjects with FXIIIVal34Leu by tertiles of insulin resistance. In stroke patients, the mutation was more frequent in subjects with primary intracerebral haemorrhage $(54.8 \%)$ than in stroke controls $(41.7 \%, p=0.05)$. These results indicate that FXIIIVal34Leu) is protective against $\mathrm{Ml}$ and suggest a mechanism whereby elevated levels of PAI-1 may contribute to vascular risk through co-existent insulin resistance. This mutation may also be associated with primary intracerebral haemorrhage. 
PARAOXONASE 192-GLN-ARG GENE POLYMORPHISM AND CORONARY ARTERY DISEASE IN TYPE 2 DIABETES.

M. Pfohl, M. Koch, J. Füllhase, M. D. Enderle, K. R. Karsch, and H. U. Hăring, Medizinische Universtătsklinik IV, Tubingen, Germany

Paraoxonase is an HDL-associated enzyme implicated in the pathogenesis of atherosclerosis by protecting lipoproteins against peroxidation. Its bialletic gene polymorphism at codon 192 (A for glutamine, B for arginine) is discussed to be associated with coronary artery disease (CAD). To evaluate the role of this paraoxonase gene polymorphism for $C A D$ in type 2 diabetes, we determined the paraoxomase genotype in 288 type 2 diabetic patients (170 with angiographically documented $\mathrm{CAD}$, and 118 without $\mathrm{CAD}$ ). The paraoxonase $192 \mathrm{~A} / \mathrm{B}$ genotype was assessed using polymerase chain reaction followed by Alw I digestion. The frequency of the A allele 0.656 in the $\mathrm{CAD}$ patients and 0.746 in the controls $\left(x^{2}=5.36, p=0.022\right)$. Compared with the AA genotypes, the age-adjusted odds ratio for CAD wos $2.42(95 \%-\mathrm{Cl} 1.01-6.58, \mathrm{p}=0.05)$ in subjects homozygous for the $\mathrm{B}$ allere, and $1.78(95 \%-\mathrm{Cl} 1.08-2.96, \mathrm{p}=0.02)$ in those carrying at least one $\mathrm{B}$ allete indicating an additive effect of the $B$ allele. In the multivariate analysis, this association was even stronger after correction for the possible confounders age, gender, smoking history, and hypertension. There was no association between the PON genotype and history of myocardial infarction $\left(x^{2}=0.89\right.$, $p=0.64$ ), nor with the extent of $C A D$ as judged by the number of vessets diseased $\left(x^{2}=6.48, p=0.37\right)$. Our data indicate that the $192 \mathrm{Arg} / \mathrm{Can}$ polymorphism of the human paraoxonase gene is an independent risk factor for $\mathrm{CAD}$, but not myocardial infarction in type 2 diabetic patients. This could possibly be explained by a reduced ability of the paraoxonase $B$ isoform to protect lipoproteins against peroxidation.
PARAOXONASE-2 GENE G148 VARIANT IS ASSOCIATED WITH ELEVATED PARAOXONASE LEVELS BUT NOT WITH CORONARY HEART DISEASE IN TYPE 2 DIABETES

J. Ruiz ${ }^{1,4}$, E. Castillo', E. Temler', R.W. James ${ }^{2}$, M.C. BlatterGarin $^{2}$, P. Passa ${ }^{3}$, P. Froguel ${ }^{4}$ and R.C. Gaillard'. "Division d'Endocrinologie, CHUV, Lausanne, Suisse; ${ }^{2}$ Division d'Endocrinolgie, HCUG, Genève, Suisse: ${ }^{3}$ Service de Diabétologie, Saint-Louis Hospital, Paris, France : ${ }^{4}$ CNRS EP10, Institut Pasteur de Lille, Lille, France.

The Paraoxonase-1 (PON1) gene has been identified as a genetic risk factor for coronary heart disease (CHD) in type 2 diabetic patients. More recently the PON2 G148 variant was associated with elevated fasting plasma glucose levels in type 2 diabetic patients. Therefore, we investigated the role of PON2 G148 variant on paraoxonase enzyme levels and CHD risk in type 2 diabetic patients. The study was performed in 431 patients (mean age : 60.1 $10.4 \mathrm{yrs}$, male/female $60 / 40 \%$ mean diabetes duration $14.0+8.8$ yrs, $C H D+n=141$ ). The PON2 G/A148 polymorphism was assessed by allele specific PCR for the $G$ and $A$ variants. PON2 $G 148$ was associated with higher levels of PON enzyme (arbritrary unit) (GG: $90.1 \pm 21.2, G A: 91.8 \pm 24.8$, and $A A \cdot 770+23.7, p<0.0001)$. The genotype frequency of PON2 G/A 148 did not differ between type 2 diabetic patients with and without $C H D$ (CHD+: GG : $7.1 \%, G A: 30.5 \%, A A: 62.4 \%$ and $C H D-: G G: 4.5 \%$, GA: $37.9 \%$, AA: $57.6 \%$ ). In multivariate analysis PON2 G/A148 polymorphism accounted for $10 \%$ of the variance of enzyme level. A statistical model was designed including PON2 G/A148, PON1 ML54 polymorphism, total cholesterol and triglycerides, that could overall explain $32 \%$ of PON enzyme level.

These results suggest that the PON2 gene may partially influence circulating levels of paraoxonase, but do not represent an independent risk factor for CHD in our population of type 2 diabetic patients.

\section{5}

PLATELETS: AN IMPORTANT SOURCE OF CIRCULATING VASCULAR ENDOTHELIAL GROWTH FACTOR.

D. Burt, S. Thomas, G Chusney, G Gruden, L. Gnudi, G.C. Viburti Department of Diabetes. Endocrinology and Metabolic Medicine, UMDS, Guy's Hospitai, London, UK.

Serum Vascular Endothelial Growth Factor (VEGF) levels correlate with HbAlc in Type 1 diabetes and are higher after myocardial infarction, conditions associated with abnormal platelet activation. In vitro VEGF is induced by high glucose and is implicated in microvascular complications. AIMS To determine whether 1) platelets are a source of VEGF, 2) Ex vivo VEGF release, during clotting, affects serum VEGF levels. Circulating VEGF was determined by ELISA (range $5-1600 \mathrm{pg} / \mathrm{ml}$ intraassay CV $7.5 \%$ ) in 5 healthy subjects and in purified platelet suspensions. VEGF was measured in paralle! in serum (S), platelet poor plasma (heparin 70U/ml [PH], EDTA [PE] citrate $3.8 \%[P C]$ ) and platelet ich plasma (PRP). VEGF was detectable in platelet lysates. The addition of thrombin $(T 0.5 \mathrm{u} / \mathrm{ml})$ to a purified platelet suspension (PP) increased VEGF levels [PP $50.8(31.4-66.5), P P+T 96(86.5-208.3) \mathrm{pg} / \mathrm{ml}$, median (range), $p=0.02$ ] Serum VEGF levels were higher than plasma levels is $337(249.5$ 525), PH 189(113-284) PC 86(58-127), PE 69(53-103.5, $S$ vs $P$ $p=0.04$ : $P H$ vS $P C$ and $P E p<0.05$, and were similar to those of thrombin stimulated PRP (PRP 0.68. PRP + T 1.3 fold increase vs [S]). Ex vivo platelet activation leads to higher serum VEGF levels. suggesting that plasma is the appropriate sample to detemine circulating VEGF. Platelet derived VEGF is likely to be important in vessel damage and repair.

\section{6}

TEMPORAL DISSOCLATION OF INSULIN'S CENTRAL AND PERIPHERAL VASCULAR, NEURAL AND METABOLIC EFFECTS R. Bergholm, J. Westerbacka, S. Vehkavaara, I. Wilkinson, J. Cockcroft and H. Yki-Järvinen. U.K. and Finland

Physiological concentrations of insulin diminish wave reflection in the aorta, which is due to either an increase in large vessel compliance or vasodilatation of large arteries. In this study we determined how this novel action of insulin relates to its effects on peripheral blood flow (plethysmography), sympathetic activation (spectral power analysis of heart rate variation) and glucose extraction, in 16 normal subjects (age $24 \pm 1$ years, body mass index $22 \pm 1$ $\mathrm{kg} / \mathrm{m}^{2}$ ) during physiologic (insulin $-60 \mathrm{mU} / \mathrm{l}$ for $120 \mathrm{~min}$ ) normoglycemic hyperinsulinemia. Central aortic pressure waves were synthetized from those recorded in the periphery using applanation tonometry and a reverse transfer function every 30 minutes for determination of the augmentation index (AgI, the pressure difference between the first and second systolic peaks expressed as a percentage of pulse pressure). Insulin decreased both augmentation and $\mathrm{AgI}$ from $-2.4 \pm 3.2$ to $-7.3 \pm 3.3 \%(\mathrm{p}<0.01)$ already from $30 \mathrm{~min}$ onwards, while forearm blood flow was not increased until $120 \mathrm{~min}(3.1 \pm 0.2$ vs $2.5 \pm 0.2 \mathrm{ml} / \mathrm{dl} \cdot \mathrm{min}, 120$ vs $0 \mathrm{~min}, \mathrm{p}<0.05$ ). An increase in normalized LF, a measure of sympathetic activation was observed from 30 min onwards $(36 \pm 6$ vs $29 \pm 4, \mathrm{p}<0.01$ ). Glucose extraction increased 5 -fold within $30 \mathrm{~min}$ $(p<0.001)$ and plateaued thereafter. Although forearm blood flow increased clearly later than the AgI, insulin stimulated $(r=-0.63, p<0.02)$ but not basa flow $(r=-0.004)$, or glucose extraction $(r=-0.01)$ were significantly correlated with $\mathrm{Agl}$. These data demonstrate that physiological exposure to insulin markedly affects large vessel function, either via increasing compliance or flow. This effect clearly precedes insulin induced peripheral vasodilatation. Despite their temporal dissociation, effects of insulin on large vessel function and peripheral flow are closely interrelated, and distinct from insulin's effects on glucose extraction. Resistance to insulin's vascular effects could provide a mechanism linking hyperinsulinemia and atherosclerosis. 
OP 20

\section{Genetics of Type 2 Diabetes}

\section{7}

TRANSCRIPTIONAL REGULATION OF HUMAN GLUT2 GENE BY HEPATOCYTE NUCLEAR FACTOR-1ALPHA

Y. Someya, J. Fujita, A. Kubota, Y. Yamada and Y. Seino. Department of Metabolism and Clinical Nutrition, Kyoto University Faculty of Medicine, Kyoto 606-8507, Japan

MODY is a case of hereditary diabetes. Several mutations of HNF-1alpha gene are found in MODY3 pedigrees, but the precise mechanisms that these mutations evoke diabetes are not known. Here, we describe the possibility that mutations of HNF-1alpha cause the depletion of GLUT2, which is known as a glucose sensor of pancreatic beta cells. The 5'-flanking sequences of human GLUT2 gene was cloned. Then the fragment was digested with restriction enzymes to obtain 5'- or 3'-deletion mutants. Subsequently, these fragments were subcloned into luciferase expression plasmid. On the other hand, human HNF-1alpha CDNA was cloned by PCR technique, then deletion mutants from $C$-terminus were made and subcloned into CMV-driven expression plasmid. These luciferase reporter plasmids and HNF-1alpha expression plasmids were transfected to HIT-T15 cells and transcriptional activity was measured. In deletion analysis, pGT2-1291+308Luc (assignation according to J. Takeda et al, Diabetes, 42, 773-777) showed 6-fold increase in transcriptional activity with HNF-1alpha co-expression, and with 5'deletion, pGT2+217+308-Luc showed minimal basal promoter activity but its response to HNF-1alpha was much stronger than pGT2-1291+308Luc. These response to HNF-1alpha was diminished by deletion of sequences between +217 and +308 . In C-terminal deletion study of HNF-1alpha, loss of serine-rich domain decreased the transcriptional inducibility on GLUT2 gene. Mutant with further N. terminal deletion lacking POU domain showed no inducibility on GLUT2 transcriptional activity. Neither mutant construction showed dominant negative effect. In summary, human GLUT2 gene is closely regulated by HNF-1 alpha via sequences downstream of transcription start site, and deletional mutation of HNFlalpha decreases its transcriptional activity and GLUT2 gene transcription.

\section{9}

PED/PEA-15 GENE CONTROLS GLUCOSE TRANSPORT AND IS OVEREXPRESSED IN TYPE 2 DIABETES MELLITUS.

G. Condorelli, G. Vigliotta, A. Cafieri, A. Trencia, and F. Beguinot. Dipartimento di Biologia e Patologia Cellulare e Molecolare and CEOS - CNR, Federico II University of Naples Medical School, Via S. Pansini, 5, Naples 80131, Italy.

Type 2 diabetes mellitus is determined by both environmental and genetic factors. We have used differential display to identify genes whose expression is altered in type 2 diabetes thus contributing to its pathogenesis. One mRNA is overexpressed in fibroblasts from 12 type 2 diabetics compared to 13 non-diabetic individuals $(P<0.01$ by $t$-test analysis), as well as in skeletal muscle $(\mathrm{P}<0.05)$ and adipose tissues $(\mathrm{P}<0.01)$, two major sites of insulin-resistance in type 2 diabetes. The levels of the protein encoded by this mRNA are also 2 -fold elevated in type 2 diabetic skeletal muscle $(\mathrm{P}<0.01)$; thus, we named it $\mathrm{PED}$ for Phosphoprotein Enriched in Diabetes. PED cloning shows that it encodes a $15 \mathrm{KDa}$ phosphoprotein identical to the PKC substrate PEA-15. PED gene maps on human chromosome 1q21-22. Transfection of PED/PEA-15 in differentiating L6 skeletal muscle cells increases the content of Glutl transporters on the plasma membrane by 4 -fold. However, insulin-stimulated 2-deoxy-Dglucose uptake and cell-surface recruitment of Glut4, the major insulin-sensitive glucose transporter, were reduced by $>80 \%$. These effects of PED overexpression are reverted by blocking PKC activity with Staurosporine. Overexpression of PED/PEA-15 gene may contribute to insulin-resistance in glucose uptake in type 2 diabetes.

\section{8}

NOVEL SUSCEPTIBILITY GENE FOR NIDDM IS LOCALISED TO HUMAN CBROMOSOME 120

LIE. Shaw 1 P.K Lovelock ${ }^{1.2}$ D. Duffy ${ }^{3}$. J. Cardinal ${ }^{1}$, J.R. Berkholz ${ }^{1}$ J.B. Kesting ${ }^{l}$,and B. Wainwright ${ }^{2}$ Dept of Diabetes \& Endocrinology, Princess Alexandra Hospital ${ }^{1}$, Centre for Molecular and Cellular Biology, University of Queensland ${ }^{2}$ and Queensland Institute of Medical Research ${ }^{3}$, Brisbane, Australia.

Non-insulin dependent diabetes mellitus (NIDDM) has a substantial genetic component, but to date the nature of this predisposition is largely unknown. Three loci conferring diabetes susceptibility have been defined in differeat pedigrees with Maturity-Onset Diabetes of the Young (MODY), however mutations of these genes do not appear to be major contributors to the more usual forms of late-onset NIDDM. The major pathophysiological abnormality in MODY patients is impaired insulin secretion, whereas both impaired Beta-cell function and insulin resistance are the hallmarks of NIDDM. Pedigree studies are considered difficult in NIDDM because (i) the disorder has late ageat-onset, (ii) there is often bilineal inheritance, (iii) the mode of inheritance is uncertain and (iv) heterogeneity may occur within and between pedigrees. This paper describes a large pedigree of Pacific Islander descent in which a defined phenotype (hyperglycaemia associated with insulin resistance) appears to be inherited in a dominant fashion. The objective of the study is to identify novel mutations which result in diabetes susceptibility. The pedigree includes 16 living diabetic descendants in 3 generations Ten of the subjects had previously diagnosed NIDDM: four treated by diet, five with oral hypoglycaemic therapy and one subject on nocturnal long-acting insulin and oral hypoglycaemic therapy. We performed linkage analysis with the microsatelite markers D12S86, D12S321, D12S807 and D12S342 near the MODY3 (HNF-1 alpha) gene on chromosome $12 \mathrm{q}$ and found significant evidence for linkage (multipoint LOD score + 3.62 at theta $=0.03$ centromeric to marker D12S86). Sequencing of the 10 exons and promoter of HNF-1 lalpha did not identify any causative mutations. The age at diagnosis of diabetes linked with the MODY3 markers was $50( \pm 6)$ years (mean \pm SD) and the BMI of the affected family members was $29 \pm 6 \mathrm{~kg} / \mathrm{m}^{2}$. Pathophysiologically, the affected subjects are insulin insensitive: HOMA \%S $35 \%(16 \%-42 \%)$ (median and interquartile range) and have heterogeneous Beta-cell function: HOMA $\% \mathrm{~B} 92 \%(47 \%$ $140 \%$ ). These phenotypic features are different from those described for patients with mutations in HNF-1 alpha. Our results suggest that the region of chromosome $12 q$ close to the HNF-1alpha locus harbours a novel susceptibility gene or genes for NIDDM.

\section{0}

A P442A MUTATION IN THE MUSCLE GLYCOGEN SYNTHASE GENE RESULTS IN MARKEDLY DECREASED GLYCOGEN SYNTHESIS CAPACITY M. Orho' ${ }^{1}$ H. Shimomura ${ }^{2}$, T. Sanke ${ }^{2}$, K. Nanjo ${ }^{2}$ and L. C. Groop ${ }^{1}$. The Department of Endocrinology, Wallenberg Laboratory, Malmö University Hospital, University of Lund, Malmö, Sweden ${ }^{1}$ and The First Department of Medicine, Wakayama University of Medical Science, Wakayama, Japan ${ }^{2}$

Association between the muscle glycogen synthase gene (GYSI) and NIDDM has been reported in several populations and four aminoacid variants $Q 71 \mathrm{H}, \mathrm{M} 416 \mathrm{~V}$, P442A and G464S, have been identified in the gene. By expression and association studies we have earlier shown that the M416V and G464S variants do not significantly affect the glycogen synthase (GS) activity, nor is the relatively common M416V associated with NIDDM or insulin resistance in Finland. The aim of this study was to estimate the functional importance of the $\mathrm{Q71H}$ and P442A mutations in GYSI. The P442A has earlier been identified in one Japanese patient (age 77 years, BMI $21.9 \mathrm{~kg} / \mathrm{m}^{2}, \mathrm{HbA}_{1 \mathrm{C}} 6.3 \%$, age at onset 58 years) treated with tolbutamide. The mutated cDNAs (GS-71H, GS-442A) were created by PCR, expressed tranciently in COS7 cells and the GS activity was determined from homogenized cell pellets at 0.3 and $7.1 \mathrm{mM}$ UDPG with 0.1 and $10.0 \mathrm{mM}$ G6P. Dose-response curves for UDPG and G6P activation were obtained at $0,0.1,0.3,0.6,1.2$ and $7.1 \mathrm{mM}$ UDPG with $0.1 \mathrm{mM}$ G6P and with $0,0.03,0.1,0.5,0.2$ and $10.0 \mathrm{mM} \mathrm{G6P}$ at 0.3 $\mathrm{mM}$ UDPG. The P442A resulted in significantly decreased activation by both UDPG $\left(\mathrm{K}_{\mathrm{m}} 2.13 \pm 0.45\right.$ vs. $\left.1.31 \pm 0.21 \mathrm{mM}, \mathrm{p}<0.05\right)$ and $\mathrm{G} 6 \mathrm{P}(\mathrm{K}, 1.83 \pm 0.28$ vs. $0.82 \pm 0.13$ $\mathrm{mM}, \mathrm{p}<0.05$ ) when compared to the wild type. GS activity of the GS-442A at high UDPG and low G6P concentrations resulted in $61 \%$ decreased activity $(19 \pm 11$ vs. $49 \pm 5 \mathrm{nmol} / \mathrm{min}$.mg protein, $\mathrm{p}<0.01$ ) and the GS activity at low UDPG and low G6P concentrations was decreased by $91 \%(0.9 \pm 1.5$ vs. $9.5 \pm 2.3 \mathrm{nmol} / \mathrm{min} \cdot \mathrm{mg}$ protein, $\mathrm{p}<0.01$ ) when compared to the wild type. The total GS activity of the GS-442A was decreased by $24 \%(207 \pm 14$ vs. $272 \pm 36 \mathrm{nmol} /$ min.mg protein, $\mathrm{p}<0.05$ ). The GS-71H did not differ significantly from the wild type GS. In conclusion, the P442A mutation resulted in a markedly decreased capability to synthesize glycogen and represents the first naturally occurring mutation in the GYSI gene with functional consequences. Whether this impairment in skeletal muscle glycogen synthesis is sufficient to cause the rnild diabetes in the mutation carrier remains to be shown. 


\section{1}

STUDIES OF THE POTENTIAL INFLUENCE OF AN AMINO ACID VARIANT IN IRS-2 ON INSULIN SECRETION IN TWO SCANDINAVIAN POPULATIONS K. Almind', D. Bernal' ${ }^{2}$, S. Urhammer', T. Hansen ${ }^{1}$, L. Berglund ${ }^{3}$, R. Reneland ${ }^{3}, H$. Lithell $^{3}$, M. White ${ }^{2}$ and O. Pedersen ${ }^{1}$. 'Steno Diabetes Center, Gentofte, Denmark, ${ }^{2}$ Joslin Diabetes Center, Harvard Medical School, Boston, MA, USA and ${ }^{3}$ Department of Geriatrics, Uppsala University, Sweden.

The family of insulin receptor substrates (RS1-4) are cytoplasmic proteins that undergo tyrosine phosphorylation in response to insulin, IGF-1 and various cytokines. Recently it was shown that disruption of IRS-2 causes type 2 diabetes in mice due to impairment in both peripheral insulin signaling and pancreatic $\beta$-cell function. The mice have reduced $\beta$-cell mass which prevents an adequate insulin secretion to compensate for the insulin resistance. The study indicates that IRS-2 has a unique role in regulation of $\beta$-cell neogenesis, proliferation and survival which makes it a potential candidate gene in the pathogenesis of type 2 diabetes. We performed a mutational analysis of the gene encoding human IRS-2 to determine whether variations in the protein are associated with type 2 diabetes or impaired insulin secretion among Caucasians. By using the SSCP (single strand conformation polymorphism) technique we identified a glycine to aspartic acid polymorphism at codon 1057. In an association study of 240 unrelated Danish Caucasian type 2 diabetic patients and 230 matched glucose tolerant control subjects. the allelic frequency of the Gly $1057 \mathrm{Asp}$ polymorphism was $33.8 \%$ in type 2 diabetic patients and $33.9 \%$ in control subjects $(\mathrm{p}=0.981)$. Interestingly, the glucose tolerant subjects homozygous for the polymorphism ( $n=31$ ) had on average a $25 \%$ decrease in fasting serum insulin levels $(\mathrm{p}=0.022)$ before an OGTT and a $28 \%(\mathrm{p}=0.006)$ and $34 \%$ $(\mathrm{p}=0.005)$ decrease in insulin levels at 30 and $60 \mathrm{~min}$, respectively, during an OGTT compared with wildtype carriers $(\mathrm{n}=107)$. The insulin levels remained significantly decreased after adjusting for gender and glucose levels in a multivariate analysis. The amino acid polymorphism was found with similar frequency in a cohort of 640 Swedish males. However, in this population the amino acid variant did not seem to have any impact on insulin secretion. In conclusion: this study suggests that a frequent glycine to aspartic acid polymorphism in human IRS-2 is not associated with type 2 diabetes but it may have a subtle impact on $\beta$-cell function. However, this effect appears only to occur in some populations where other unknown genetic or environmental factors with impact on insulin secretion are operative.

\section{2}

REDUCED BETA-CELL FUNCTION AND BLOOD PRESSURE IN CARRIERS OF INTRON 16-3t VARIANT OF THE SULFONYLUREA RECEPTOR GENE. L.M.'t Hart, J.B. Ruige, J.M. Dekker, G. Nijpels, J.A. Maassen and R.J. Heine. Leiden University, Dept. of Molecular Cellbiology, Wassenaarseweg 72, $2333 \mathrm{AL}$, Leiden and Free University, EMGO Institute, Amsterdam, The Netherlands. Previously we showed an association between the intron $16(-3 c \rightarrow t)$ variant in the sulfonylurea receptor (SUR) gene and NIDDM in The Netherlands. We now examined whether this variant associates with altered $\beta$-cell function and other diabetes-related clinical parameters in a cohort of 94 subjects with impaired glucose tolerance (IGT). Subjects, aged 45 to 74 yrs., with a mean $2 \mathrm{hr}$ plasma glucose value, following two OGTT's, between 8.6 and $11.1 \mathrm{mmol} / \mathrm{l}$ were examined by the hyperglycemic clamp technique. When the various genotypes were compared no significant difference in age, BMI, gender, $\mathrm{HbAlc}$ and fasting glucose (FBG) and insulin levels were detected. We do observe, however, a lower first phase insulin response (incremental area under the curve, $0-10 \mathrm{~min}$.) in $-3 \mathrm{c} / \mathrm{t}$ genotypes compared to the $-3 \mathrm{c} / \mathrm{c}$ (wildtype) genotypes. Also a significant increase in the fasting proinsulin to insulin ratio was observed in this group, which remained significant after correction for age, gender, FBG and BMI in a multiple regression analysis (see table). Remarkably, also a lower systolic and diastolic blood pressure and prevalence of hypertension was found in heterozygous carriers (adjusted for age, BMI, wh ratio and gender). Subjects using anti-hypertensive medication were excluded for these analyses.

\begin{tabular}{|c|c|c|c|c|}
\hline $\begin{array}{l}\text { Intron } 16 \\
\text { Genotype(n) }\end{array}$ & $\begin{array}{l}\text { Ist Phase Insulin } \\
\text { Resp. (mU//min) }\end{array}$ & $\begin{array}{l}\text { Proinsulin to } \\
\text { Insulin Ratio }\end{array}$ & $\begin{array}{l}\text { Diastolic } \\
\mathrm{BP}(\mathrm{mm} \mathrm{Hg})\end{array}$ & $\begin{array}{l}\text { Systolic } \\
\text { BP }(m m ~ H g)\end{array}$ \\
\hline$-3 c / c(31)$ & $13.6(4.9-18.9)$ & $0.072(0.053-0.092)$ & $95 \pm 6$ & $148 \pm 12$ \\
\hline$-3 c / t(43)$ & $8.9(2.7-15.0)$ & $0.089(0.063-0.122)^{\mathrm{a}}$ & $88 \pm 10^{6}$ & $138 \pm 16^{2}$ \\
\hline$-3 t / t \quad(20)$ & $10.9(6.3-21.0)$ & $0.064(0.057-0.112)$ & $88 \pm 10^{b}$ & $141 \pm 16$ \\
\hline
\end{tabular}

Data are expressed as means (SD) or medians (interquartile range).

From our data we conclude that the intron $16.3 t$ variant (in heterozygous form) is associated with reduced blood pressure and altered $\beta$-cell characteristics in subjects with IGT. These altered $\beta$-cell characteristics may also explain the previously observed association between SUR gene variants and type II diabetes mellitus in various populations. The association with reduced blood pressure suggests this variant also affects other tissues. This is supported by the expression pattern of the SUR protein.
123

A MICROSATELLITE VARIANT IN THE ALDOSE REDUCTASE GENE IS ASSOCIATED WITH ELEVATED RISK OF NEPHROPATHY IN TYPE 2 DIABETES

R. Kotter, R. Schnepf, St. Becker, M. Nauck*, K. Badenhoop", R. Petzold ${ }^{+}$, H. Schatz and A. Pfeiffer, Medizinische Universitătsklinik Bergmannsheil, D-44789 Bochum, * Medizinische Universitatskklinik Knappschafts-Krankenhaus, D-44892 Bochum, "Universitatsklinikum Frankfurt, D-60590 Frankfurt, 'Herz- und Diabeteszentrum NRW, University of Bochum, D-32545 Bad Oeynhausen

The aldose reductase gene has been implied in the pathogenesis of diabetic complications. Recently, a microsatellite consisting of an $\mathrm{AC}_{2}$ repeat $2 \mathrm{kB}$ upstream of the transcriptional start site of aldose reductase has been described. One allele, Z-2, was linked to an elevated risk of nephropathy in type I diabetes in England and to early retinopathy in Hongkong. If this marker indeed indicates elevated risk of complications this should also expected for type 2 diabetes patients and should be helpful to identify diabetic individuals at risk. This should be particularly interesting since reproduction of association of risk markers has been relatively infrequent until present. Methods: $30 \times 40 \mathrm{~cm}$ sequencing gels were used to identify microsatellite variants after performing PCR in the presence of $\left[\alpha^{32} \mathrm{P}\right] C T P$ and subsequent detection by autoradiography. 122 NIDDM-patients with established nephropathy as documented by elevated albumin excretion and 127 patients without nephropathy were analysed. Allele frequencies were compared by $\chi^{2}$-test. Results: The marker information content was 0.73 and Hardy Einberg equilibrium was maintained in 140 controls. The most frequent allele in the German population was the $\mathrm{AC}_{24}$ repeat similar to studies in other populations. The Z-2 repeat was significantly associated with nephropathy in the NIDDM population $\left(p=0.025, \chi^{2}\right.$-test). There was no significant association with retinopathy or neuropathy in this population. Conclusion: The aldose reductase gene polymorphism Z-2 is associated with increased occurrence of nephropathy also in type 2 diabetes which suggests a role of the enzyme in the pathogenesis of nephropathy similar to type 1 diabetic patients. Since nephropathy in type 2 diabetic patients is relatively frequently associated with non-diabetic causes the association may not be as high as reported in type I diabetes. However, it appears highly remarkable that the same microsatellite variant is associated with complications in a Chinese and in 2 European populations indicating that this association with aldose reductase or another nearby gene must have been occured before separation of these populations.
124

WITHDRAWN 


\section{OP 21}

\section{Oxygen Radicals Cause Insulin Resistance}

125

PUTATIVE ROLE FOR OXIDATIVE STRESS IN ADIPOCYTE AND SKELETAL MUSCLE INSULIN RESISTANCE.

A. Rudich, A. Tirosh, M. Khamaisi, D. Pessler, R. Potashnik, and N. Bashan Clinical Biochemistry, Ben-Gurion University, Beer-Sheva, Israel.

The association between oxidative stress parameters and metabolic control in diabetic patients is as yet not sufficient to prove cause and effect relationship. To address this question, 3T3-L1 adipocytes and L6 myotubes were exposed to enzymatic reactive oxygen species producing systems, which generated micro molar $\mathrm{H} 2 \mathrm{O} 2$ concentration for up to 24 hours. In both cell lines oxidative stress regulated glucose transport activity and the expression of the glucose transporters GLUT1 $(420 \pm 30 \%)$ and GLUT4 $(40 \pm 4 \%)$. Transcriptional activation of the GLUT1 genc was mediated by enhancer 1 , potentially through an AP-1 binding site. In L6 myotubes but not in 3T3-L1 adipocytes, increased GLUT1 expression could be prevented by rapamycin ( $\mathrm{S} 6$ kinase inhibitor), suggesting cell line specific pathways in the cellular response to oxidation. In 3T3-L1 adipocytes oxidation induced gene suppression of GLUT4 was combined with a selective impairment in insulin stimulated GLUT4 (but not GLUT1) translocation from LDM to the PM. Insulin stimulated tyrosine phophorylation of the insulin receptor and of IRS1, as well as the ability of insulin to activate PI3 kinase were not reduced by oxidation in total cell lysates. However, a marked defect in the ability of insulin to activate Pl3 kinase in the LDM compartment was observed (16-fold in control Vs. 1.3-fold in oxidized cells). These data indicate that oxidation interferes with insulin-mediated compartment-specific PI3 kinase activation. To assess the potential of lipoic acid to protect against diabetes and oxidation associated reduction in GLUT4 expression streptozotocin diabetic rats as well as 3T3-L1 adjpocytes were treated with lipoic acid. In both isolated soleus and 3T3-L1, lipoic acid completely prevented GLUT4 protein reduction, associated with improved insulin stimulated glucose transport activity (normalization in soleus, $80 \pm 7 \%$ protection in $3 \mathrm{~T} 3-\mathrm{Ll}$ adipocytes). In conclusion, these studies support the notion that oxidative stress may play a causative role in peripheral insulin resistance, and may be prevented by antioxidants.

\section{7}

COMPARTMENT SPECIFIC ACTIVATION OF PI 3-KINASE AND PROTEIN KINASE B BY INSULIN IS IMPAIRED BY OXIDATION.

A. Tirosh, A. Rudich, R. Potashnik, and N. Bashan. Clinical Biochemistry, BenGurion University, Beer-Sheva, Israel.

Protein kinase B (PKB, Akt) is a widely expressed $60 \mathrm{kDa}$ serine/threonine kinase which functions downstream of PI 3-kinase. Recent studies indicate that PKB has an important role in mediating some of the acute metabolic effects of insulin including GLUT4 translocation and glycogen synthase kinase-3 inhibition. Previously we have demonstrated that exposure of 3T3-L1 adipocytes to prolonged micromolar concentrations of $\mathrm{H} 2 \mathrm{O} 2$ resulted in impaired insulin stimulated glucose transport and glycogenesis. The current study was designed in order to investigate whether oxidation interferes with insulin induced PKB activation. Exposure of 3T3 $\mathrm{L} 1$ adipocytes to $100 \mathrm{nM}$ insulin resulted in dramatic (50 -fold) elevation in PKB phosphorylation in total cell lysate, with $50 \%$ activation at 2.5 minutes. Insulin induced PKB activation could be also detected in both the plasma membrane and the low density microsomal (LDM) fractions. In oxidized cells, insulin stimulated PKB phosphorylation in cell lysates was dramatically decreased in both total cell lysates and in the various cellular fractions, with no reduction in total cellular PKB content. Since insulin induced PKB activation was found to be downstream of PI 3kinase, we further assessed insulin stimulated PI 3-kinase activation. IRS-1 associated PI 3-kinase activity in total cell lysates was intact following oxidation Insulin induced a 19 fold increase in the amount of the p85 regulatory subunit of PI 3-kinase in the LDM which was associated with $\sim 12$ fold increase in its kinase activity. However, following oxidation a $50 \%$ reduction in the ability of insulin to recruit $\mathrm{p} 85$ to the LDM was observed, and was associated with no significan elevation in PI 3-kinase activity. In conclusion, oxidative stress alters insulin signaling by interfering with compartment specific activation of both PI 3-kinase and PKB. To the best of our knowledge, this is a novel putative cellular mechanism for impaired response to the acute metabolic effects of insulin.

\section{6}

Cellular Stress Impairs Insulin Signaling

H. Kanety, R. Hemi, K. Paz ${ }^{1}$, and A. Karasik . Endocrinology Institute, Sheba Med. Ctr, Tel-Hashomer and ${ }^{1}$ Dep. of Molecular Cell Biology, Weizmann Inst. of Science, Rehovot, Israel.

Previously we have shown that activation of cellular stress pathways by the proinflammatory cytokine tumor necrosis factor (TNF) results in cellular insulin resistance. In the present study we have examined the effect of additional cellular stressors, the protein synthesis inhibitors cycloheximide (CHX) and anisomycin (AN), on insulin signaling. Treatment of rat hepatoma $\mathrm{FAO}$ cells with $\mathrm{CHX}$ and $\mathrm{AN}$ led to a time and dose dependent decrease in insulin-induced tyrosine phosphorylation of insulin receptor substrates, IRS-1 and IRS-2. In addition, it inhibited their downstream association with GRB-2 and phosphatidylinotisol 3-kinase (PI-3 kinase) and impaired the activation of the IRS-associated PI-3 kinase. Similar to TNF, incubation of FAO cells with $\mathrm{CHX}$ and $\mathrm{AN}$ led to a marked decrease in the electrophoretic mobility of IRS-1 and -2 and to a significant reduction in their ability to interact with the juxtamembrane domain of the insulin receptor (IR). Incubation of cell extracts with alkaline phosphatase reversed the inhibitory effects of $\mathrm{CHX}$ and $\mathrm{AN}$.

This work proposes a common pathway by which multiple cellular stressors impair insulin signaling. Protein Ser/Thr kinases activated by stress stimuli enhance Ser/Thr phosphorylation of IRS-1 and IRS2 that impedes their interaction with the $I R$. The impaired interactions of Ser/Thr phosphorylated IRS- 1 and -2 with the IR reduce their Tyr phosphorylation and eliminate their ability to recruit downstream effector molecules resulting in severe impairment of insulin signal transduction. This impairment may be the cellular mechanism underlying insulin resistance in numerous clinical states. The nature of the Ser/Thr kinases responsible for phosphorylating the IRS proteins will be discussed.

\section{8}

INVOLVEMENT OF AP-1 AND C/EBP $\alpha$ IN REGULATING GLUT1 AND GLUT4 EXPRESSION FOLLOWING OXIDATIVE STRESS

D. Pessler, A. Rudich and N. Bashan. Clinical Biochemistry, Ben-Gurion University, Beer-Sheva, Israel.

Oxidative stress has been shown to regulate the expression of various genes by activating transcription factors including AP-1 and NFKB. Recently, we observed that prolonged exposure of 3T3-L1 adipocytes to micromolar $\mathrm{H} 2 \mathrm{O} 2$ concentrations, caused increased GLUT1 and reduced GLUT4 gene expression. The aim of the present study was to evaluate the potential role of several transcription factors in mediating these responses. DNA binding capacity of NFKB and AP-1 assessed by gel mobility shift assay revealed increased activity in nuclear extracts of 3T3-L1 adjpocytes exposed to glucose oxidase. The increased AP-1 binding capacity was associated with a transient activation of c-Jun NH2 terminal kinase (JNK) (at 30 min), a persistent activation of ERK $1 / 2$ (beginning at $2 \mathrm{~h}$ ), and a 2 -fold increase in $m R N A$ leveis of $c$-Fos and c-Jun. A putative role for AP- 1 in increasing GLUT transcription rate was further suggested by the ability of an AP-1 binding sequence from the GLUT1 enhancer to compete with binding capacity to the AP- 1 consensus sequence. The steady state GLUT4 mRNA level was reduced by $40 \pm 4 \%$ in cells exposed to prolonged oxidation. mRNA level of the transcription factor C/EBP $\alpha$ known to be involved in GLUT 4 gene regulation, was reduced in a time-dependen manner, reaching $70 \%$ reduction after 24 hours. This paralleled the time-course reduction in GLUT4 mRNA content, suggesting a possible role in GLUT4 down regulation following oxidative stress. In conclusion, oxidative stress may alter gene expression of glucose transporters by altering expression and function of AP-1 and C/EBP $\alpha$. 
MICROMOLAR CONCENTRATIONS OF $\mathrm{H}_{2} \mathrm{O}_{2}$ INHIBIT INSULIN SIGNALLING

GS Olsen, LL Hansen and L Mosthaf, Department of Molecular Signaling, Hagedom Research Institute, Niels Steensens Vej 6, DK-2820 Gentofte, Denmark.

Non-insulin-dependent diabetes mellitus (NIDDM) is characterised by abnormalities in insulin secretion and insulin resistance in target tissues. Hyperglycemia was found to induce insulin resistance at the level of the insulin receptor (IR). How glucose can mediate this effect is not completely clear. Since hyperglycemia leads to production of hydrogenperoxide and since there is evidence that oxidative stress is increased in patients with diabetes we decided to investigate the effects of physiological concentrations of $\mathrm{H}_{2} \mathrm{O}_{2}$ on insulin signalling. We report here that micromolar concentrations of $\mathrm{H}_{2} \mathrm{O}_{2}$ dramatically inhibit insulin induced insulin receptor tyrosine phosphorylation (i.e. $5 \mathrm{~min} .500 \mu \mathrm{M}$ $\mathrm{H}_{2} \mathrm{O}_{2}$ prior to insulin stimulation reduces tyrosine phosphorylation to $8 \%$ of the insulin stimulated sample) in NIH3T3 cells overexpressing the human insulin receptor. This effect of $\mathrm{H}_{2} \mathrm{O}_{2}$ can be efficiently blocked by preincubation of the cells with Na-orthovanadate, a selective PTPase inhibitor. Similar, the antioxidant $\mathrm{MnCl}_{2}$ prevents this inhibitory effect. Micromolar concentrations of $\mathrm{H}_{2} \mathrm{O}_{2}$ also inhibited IRS-1 phosphorylation, as well as insulin downstream signalling such as PI-3 kinase activation (inhibited to $57 \%$ ), glucose transport (2DG uptake inhibited to $33 \%$ ) and activation of the classical MAPK pathway (50\%). To investigate whether $\mathrm{H}_{2} \mathrm{O}_{2}$ is involved in hyperglycemia induced insulin resistance we preincubated the cells with the $\mathrm{H}_{2} \mathrm{O}_{2}$ scavenger Catalase prior to incubation with $25 \mathrm{mM}$ glucose $(30 \mathrm{~min}$.) or $500 \mathrm{uM}$ $\mathrm{H}_{2} \mathrm{O}_{2}(5 \mathrm{~min}$.). Whereas this treatment totally abolished the inhibitory effect of $\mathrm{H}_{2} \mathrm{O}_{2}$ on insulin-induced tyrosine phosphorylation of the receptor, it had no effect on the inhibition of insulin signalling by hyperglycemia. In conclusion, these results demonstrate that $\mathrm{H}_{2} \mathrm{O}_{2}$ in low concentrations is a potent inhibitor of insulin signalling, however it is not mediating the inhibitory effect of hyperglycemia.
L-ARGININE INCREASES RATES OF INSULIN-MEDIATED GLUCOSE UPTAKE AND GLYCOGEN SYNTHESIS IN SKELETAL MUSCLE IN VITRO

J. Jensen, B. Leighton and M.E. Young. University of Oxford, U.K.

Nitric oxide synthase (NOS) is found within skeletal muscle cells. NOS catalyses the conversion of $\mathrm{L}$-arginine $(\mathrm{L}-\mathrm{Arg})$ to $\mathrm{L}$-citrulline, with the concomitant generation of nitric oxide (NO). NO binds to the haem group of soluble guanylate cyclase, stimulating the generation of cGMP. Recent studies have suggested that the $\mathrm{NO} / \mathrm{cGMP}$ signalling cascade plays an important role in the regulation of skeletal muscle glucose utilisation, and that this cascade is impaired in insulin resistant skeletal muscle. L-Arg, when added to culture media, enhances insulin-mediated glycogen synthesis in 3T3-L1 adipocytes. In addition, L-Arg infusion significantly increases wholc body glucose disposal and insulin-mediated glucose uptake in healthy subjects. As skeletal muscle is the major site of insulin-mediated glucose disposal, the aim of the present study was to investigate whether L-Arg affected insulin-mediated glucose utilisation by skeletal muscle. Incubation of isolated rat soleus muscle preparations in the presence of $\mathrm{L}-\mathrm{Arg}(2 \mathrm{mM})$ significantly increased insulin-mediated $(100 \mu \mathrm{U} / \mathrm{ml}$ ) rates of net lactate release (control, $7.15 \pm 0.43$, versus L-Arg, $8.05 \pm$ $0.22 \mu \mathrm{mol} / \mathrm{h} / \mathrm{g}$ wet wt.; $\mathrm{P}<0.05$ ), ${ }^{14} \mathrm{C}$-labelled lactate release (control, $4.90 \pm 0.50$, versus $\mathrm{L}$-Arg, $6.57 \pm 0.42 \mu \mathrm{mol} / \mathrm{h} / \mathrm{g}$ wet wt; $\mathrm{P}<0.05$ ) and glycogen synthesis (control, $2.84 \pm 0.25$, versus L-Arg, $4.17 \pm 0.09 \mu \mathrm{mol} / \mathrm{h} / \mathrm{g}$ wet wt.; P<0.01). The rate of ${ }^{14} \mathrm{C}$ labelled lactate release is a good indication of the rate of glucose transport. $\mathrm{N}^{\mathrm{G}}-\mathrm{Nitro}$ L-arginine-methyl ester (L-NAME) is a competitive inhibitor of NOS. . L-NAME $(2 \mathrm{mM})$ significantly inhibited L-Arg $(2 \mathrm{mM})$ stimulated rates of insulin-mediated $(100 \mu \mathrm{U} / \mathrm{ml})$ net lactate release ( $\mathrm{L}$-Arg, $8.05 \pm 0.22$, versus $\mathrm{L}$-Arg plus L-NAME, 6.79 $\pm 0.35 \mu \mathrm{mol} / \mathrm{h} / \mathrm{g}$ wet $\mathrm{wt}$.; $\mathrm{P}<0.01)$ and ${ }^{14} \mathrm{C}$-labelled lactate release (L-Arg, $6.57 \pm 0.42$, versus $\mathrm{L}$-Arg plus $\mathrm{L}$-NAME, $4.49 \pm 0.39 \mu \mathrm{mol} / \mathrm{h} / \mathrm{g}$ wet wt.; $\mathrm{P}<0.01$ ), but did not significantly inhibit L-Arg $(2 \mathrm{mM})$ stimulated rates of insulin-mediated $(100 \mu \mathrm{U} / \mathrm{ml})$ glycogen synthesis ( $\mathrm{L}-\mathrm{Arg}, 4.17 \pm 0.09$, versus L-Arg plus L-NAME, $3.86 \pm 0.29$ $\mu \mathrm{mol} / \mathrm{h} / \mathrm{g}$ wet wt.). In conclusion, L-Arg enhances skeletal muscle insulin-mediated glucose utilisation, possibly via improved signalling through the NO/CGMP cascade.

\section{1}

CELLULAR PROTECTIVE MECHANISM AGAINST HYPERGLYCEMIA IN VASCULAR CELLS: ROLE OF REACTIVE OXYGEN SPECIES $S$. Sasson, N. Kaiser and R. Reich. Depts. of Pharmacology and Endocrinology \& Metabolism, Hebrew University-Hadassah Medical Center, Jerusalem, Israel. Hyperglycemia promotes the initiation and progression of atherosclerosis in diabetic patients by modifying vascular cells function. We identified a cellular protective mechanism against the deleterious effects of chronic hyperglycemia in vascular endothelial (VEC) and smooth muscle cells (VSMC). It involves a decrease of $50-70 \%$ in the rate of glucose transport relative to cells grown at $5 \mathrm{mM}$ glucose, associated with a reduction in the total cellular content and plasma membrane abundance of GLUT-1, the typical glucose transporter in these cells. Recently, we have found that 12-lipoxygenase and its product 12-HETE downregulate the rate of hexose transport and GLUT-1 expression in these cells under hyperglycemic conditions. Since glucose-induced formation of reactive oxygen species (ROS) is increased in cells exposed to high glucose levels, we have studied the role of ROS in this autoregulatory process. VEC and VSMC, grown for 5 generations at either 5 or $20 \mathrm{mM}$ glucose, were exposed for $24 \mathrm{hrs}$ to the antioxidants $\mathrm{N}$ acetyl cysteine $(20 \mathrm{mM})$ and lipoic acid $(200 \mu \mathrm{M})$. Both types of cells responded differentially to this treatment: VSMC maintained at $20 \mathrm{mM}$ glucose upregulated the rate of hexose transport nearly 3-folds (half maximal and maximal effects were obtained within 3-6 and $9 \mathrm{hrs}$, respectively), associated with increased expression and plasma membrane abundance of GLUT-1. VEC maintained at $20 \mathrm{mM}$ glucose exhibited only a minor response to antioxidants, increasing the rate of glucose transport by $20-30 \%$, with no apparent alteration in the total GLUT-1 content or its subcellular distribution. Both VSMC and VEC grown at $5 \mathrm{mM}$ glucose did not respond to antioxidant treatment. These results suggest that vascular cells autoregulate their glucose transport system via ROS-dependent and ROSindependent (i.e., via lipoxygenase) pathways. VSMC utilize both mechanisms to downregulate glucose transport under hyperglycemic conditions. VEC use primarily the ROS-independent pathway probably due to the presence of an efficient endogenous antioxidant scavenging activity.

\section{2}

INSULIN-RESISTANCE IN NON-DIABETIC RELATIVES OF TYPE 1 DIABETIC PATIENTS.

E. Matteucci, V. Cinapri and O. Giampietro. Clin. Med. II, Pisa, Italy Oxygen free radicals have been suggested to be involved into the pathogenesis of type 1 diabetes mellitus and its complications. We investigated the antioxidant status of 18 type 1 diabetics (age $36 \pm 11$ years, disease duration $16 \pm 8$ years, HBAlc $8.1 \pm 1.5 \%, 13$ normo- and 5 micro-albuminuric), 18 non-diabetic siblings of diabetic probands ( $32 \pm 8$ yr, 10 siblings of normo- and 8 of micro-albuminurics) and 18 matched healthy controls ( $32 \pm 6 \mathrm{yr}$ ) by measuring circulating levels of transition metals (iron and copper), plasma and cellular antioxidants (serum albumin, glucose, uric acid, bilirubin, transferrin, ferritin, ceruloplasmin, erythrocyte GSH) and circulating indicators of radical-induced damage to lipids (TBARS, thiobarbituric-acid-reactive substances) and proteins (AOPP, advanced oxidation protein products). Type 1 diabetics had higher glycemia and $\mathrm{HbAlc}$ than controls $(\mathrm{p}<0.001)$, lower serum albumin $(\mathrm{p}<0.05)$ and uric acid $(\mathrm{p}<0.01)$. Their siblings had borderline fasting glycemia $(\mathrm{p}<0.05)$ in spite of normal $\mathrm{HbAlc}$, higher serum transferrin $(p<0.01)$ and AOPP $(p<0.001)$. Serum glucose and transferrin were similarly increased in siblings of normo- and micro-albuminuric probands, while AOPP and ceruloplasmin were elevated only in the latter group who had also higher systolic blood pressure $(\mathrm{p}<0.05)$, serum total cholesterol $(\mathrm{p}<0.01)$ and triglycerides $(\mathrm{p}<0.05)$. Thus, not only IDDM probands had lower plasma antioxidants (albumin, uric acid), but also their non-diabetic siblings showed common abnormalities (borderline glycemia and increased serum transferrin) as well as distinctive ones (higher serum cenuloplasmin, AOPP, lipids and systolic pressure, characterizing siblings of normo-albuminurics). Transferrin (and insulin) has a growth-promoting effect and has been related to glucose/insulin metabolism, the expression of transferrin receptors may be influenced by insulinemia. We suggest the presence of a latent insulin-resistant state in non-diabetic siblings of type 1 diabetic people, whose detectable signs are higher serum fasting glucose and transferrin, and that appears more severe in siblings of micro-albuminuric probands. 


\section{Regulation of Insulin Exocytosis}

\section{3}

VISUALISING INSULIN SECRETORY GRANULE DYNAMICS WITH A PHOGRIN.GREEN FLUORESCENT PROTEIN CHIMARA.

A E. Pouli, E Emmanouilidou, C Zhao, C Wasmeier†, J C. Hutton $\dagger$ and G A. Rutter. Biochemistry, University Medical School, Bristol BS8 1TD, U.K., †Barbara Davis Center for Childhood Diabetes, University of Colorado Health Service Center, Denver, CO 80262, U.S.A.

To image single secretory granule movement in living islet $\beta$-cells we have constructed and expressed cDNA encoding a fusion of the densecore secretory granule membrane glycoprotein, phogrin (phosphatase on the granule of insulinoma cells) and enhanced green fluorescent protein $\left({ }^{65} \mathrm{~T}, \mathrm{~V}^{163} \mathrm{~A}\right.$ mutations; EGFP). The chimaera was localized exclusively to dense-core secretory granules (diameter $200-1000 \mathrm{~nm}$ ), identified by coimmunolocalization with anti-(pro-)insulin antibodies. Using laserscanning confocal microscopy and digital analysis of time-lapse images, we have used this chimaera to monitor the effects of secretagogues on the dynamics of secretory granules in single living cells. In unstimulated INS$1 \beta$-cells maintained at $3 \mathrm{mM}$ glucose, granule movement was confined to oscillatory movement (dithering) with period of oscillation $5-10 \mathrm{~s}$ and mean displacement $<1 \mu \mathrm{m}$. Elevated glucose concentrations $(30 \mathrm{mM})$ stimulated insulin release (measured by radio-immunoassay) 1.9-fold and provoked a large (4.6-fold) increase in the movement of granules. In particular, long $(5-10 \mu \mathrm{m})$ saltatory excursions of granules were observed in the presence of high glucose concentrations but were never observed in cells maintained at low glucose. Suggesting a role for increases in intracellular $\left[\mathrm{Ca}^{2+}\right]$, this effect could be mimicked in part by depolarization of the plasma membrane with $\mathrm{K}^{+}$. These results illustrate the potential use of phogrin.EGFP chimeras to study the regulation by glucose and other secretagogues of: (1) secretory granule dynamics; (2) granule / cytoskeletal interactions, and the role of motor proteins in granule movement; (3) the trafficking of a granule-specific transmembrane protein during a cycle of exocytosis and endocytosis.

\section{5}

A $R_{\text {ATP-INDEPENDENT ACTION OF GLUCOSE MAY BE GRANULE }}$ TRANSLOCATION BY INTRACELIUULAR $\mathrm{Ca}^{2+}$ MOBILIZATION . I.Niki, T.Niwa, T.Fukasawa and B.Eidaka Department of Pharmacology, Nagoya Univergity School of Medicine, Nagoya, Japan

Intracellular movement of beta-granules is a requisite stage for release of insulin. We have developed a method to estimate the movement by phase-contrast microscopy and video-imaging. The movement is controlled by insulin secretagogues including glucose. In this study, we investigated the mechanism involved in the control of the movement by glucose using a glucose-responsive beta-cell line, MIN6. The movement was strongly suppressed by intracellular $\mathrm{Ca}^{2+}$-chelator, BAPTA. $\mathrm{Ca}^{2+}$ channel blockers such as nifedipine and nitrendipine weakly inhibited the movement by glucose, while neither high $\mathrm{K}^{+}$nor glibenclamide mimicked the effect of glucose. Inhibition of $\mathrm{Ca}^{2+}$ mobilization by dantrolene or thapsigargin potently suppressed the motile event. Glucose-induced granule traffic was affected by $\mathrm{W}-7$ and ML-9, selective inhibitors of calmodulin and myosin light chain (MLC) kinase, respectively. Selective inhibitors of phopholipase $c$, but not of phospholipase $A_{2}$, caused a potent inhibition of basal and glucoseinduced granule movement. These findings lead us to an idea that control of the movement by glucose results from phosphorylation of MLC, and is more dependent on $\mathrm{IP}_{3}$-induced $\mathrm{Ca}^{2+}$ mobilization rather than $\mathrm{Ca}^{2+}$ influx through voltage-dependent $\mathrm{Ca}^{2+}$ channels, and the pathway may explain one of the potentiating effects of glucose on insulin release independent of the activity of $\mathrm{K}_{\mathrm{ATP}}$.
134

SECRETAGOGUES MODULATE THE $\mathrm{Ca}^{2 *}$ CONCENTRATION IN THE ENDOPLASMIC RETICULUM OF INSULIN SECRETING CELLS MONITORED WITH AEQUORIN.

E. Sebö, E.D. Kennedy, P. Maechler, T. Pozzan and C.B. Wollheim.

Div. de Biochimie Clinique, University of Geneva, Switzerland.

Cellular $\mathrm{Ca}^{2+}$ homeostasis is critically dependent on $\mathrm{Ca}^{2+}$ uptake and release by the endoplasmic reticulum (ER). It has been postulated that glucose-6-phosphate (G6P) stimulates $\mathrm{Ca}^{2+}$ sequestration by the ER in the B-cell, while others have proposed that glucose causes $\mathrm{Ca}^{2+}$ mobilization from the ER. To address these issues, we have directly monitored changes in the free $\mathrm{Ca}^{2+}$ concentration in the lumen of the ER $\left(\left[\mathrm{Ca}^{2+}\right]_{\mathrm{ER}}\right)$. To this end, we established a stable INS-1 rat insulinoma cell line expressing the $\mathrm{Ca}^{2+}$ photoprotein aequorin in the ER by the incorporation of the immunoglobulin Igr2b heavy chain gene upstream of the aequorin cDNA, for ER-targetting. At physiological extracellular $\left[\mathrm{Ca}^{2+}\right]$, the steady state $\left[\mathrm{Ca}^{2+}\right]_{E R}$ was in the range $300-400 \mu \mathrm{mol} / \mathrm{L}$. This concentration was proportional to the extracellular $\left[\mathrm{Ca}^{2+}\right]$ Cyclopiazonic acid, an inhibitor of the ER $\mathrm{Ca}^{2+}$-ATPase rapidly emptied $\left[\mathrm{Ca}^{2+}\right]_{E R}$ reaching $\sim 10 \mu \mathrm{mol} / \mathrm{L}$. [ $\left.\mathrm{Ca}^{2+}\right]_{E R}$ was also lowered by the inositol $(1,4,5)$ trisphosphate ( $\mathrm{Hns}_{3}$ ) generating receptor agonists carbacho and ATP. An increase of the glucose concentration from 2.8 to $10 \mathrm{mmol} / \mathrm{L}$ evoked a small rise in $\left[\mathrm{Ca}^{2+}\right]_{\mathrm{ER}}$, whereas $20 \mathrm{mmol} / \mathrm{L} \mathrm{KCl}$ induced a more marked elevation. The effects of glucose and $\mathrm{KCl}$ were proportional to their enhancement of cytosolic $\left[\mathrm{Ca}^{2+}\right]$. In $\alpha$-toxin permeabilized cells, there was a direct positive correlation between perfused cytosolic $\mathrm{Ca}^{2+}$ and ATP and $\left[\mathrm{Ca}^{2+}\right]_{E R}$, whereas InsP $\mathrm{P}_{3}$ lowered $\left[\mathrm{Ca}^{2+}\right]_{\mathrm{ER}}$ and $1 \mathrm{mmol} / \mathrm{L} \mathrm{G} 6 \mathrm{P}$ had no effect. In conclusion, direct measurements in insulin-secreting cells demonstrated that glucose and $\mathrm{KCl}$ raise whereas $\mathrm{Ins}_{3}$ generating agonists lower $\left[\mathrm{Ca}^{2+}\right]_{\mathrm{P}}$. The cells expressing aequorin in the ER should prove useful for further studies into the role of the organelle in normal and impaired $B$-cell function.

\section{6}

CALBINDIN-D 28K CONTROLS $\left[\mathrm{Ca}^{2+}\right]_{\mathrm{i}}$ AND INSULIN RELEASE IN ISLETS FROM KNOCKOUT MICE AND IN BHC-13 CELLS. T. Schermerhorn, M. Noda, K. Sooy*, S. Christakos*, and G.W.G. Sharp. Dept. of Pharmacology, Cornell University, Ithaca, NY, USA *Dept. of Biochemistry and Molecular Biology, UMDNJ-NJ Medical School, Newark, NJ, USA.

The role of calbindin- $\mathrm{D}_{28 \mathrm{~K}}$, a $28 \mathrm{kD}$, vitamin D-dependent calcium binding protein, in potassium-stimulated increases in $\left[\mathrm{Ca}^{2+}\right]_{\mathrm{i}}$ and insulin release was investigated using pancreatic islets from calbindin- $\mathrm{D}_{28 \mathrm{~K}}$ nullmutant mice (knockouts; KO) and $\beta \mathrm{HC}-13$ cells overexpressing calbindin- $\mathrm{D}_{28 \mathrm{~K}}(\mathrm{CaBP}+)$. Measurement of $\left[\mathrm{Ca}^{2+}\right]_{\mathrm{i}}$ in single islets was performed using indo- 1 microfluorimetry; $\left[\mathrm{Ca}^{2+}\right]_{i}$ in $\beta \mathrm{HC}-13$ cells was measured using fura 2 as the calcium indicator. When stimulated with 45 $\mathrm{mM} \mathrm{KCl}$, the peak $\left[\mathrm{Ca}^{2+}\right]_{i}$ was greater in Ko islets than in wild-type (WT) islets (1260 nM vs $670 \mathrm{nM} ; p<0.05)$. In insulin secretion experiments under perifusion conditions, $\mathrm{KCl}$-stimulated insulin release from KO was enhanced over WT. In islets, $45 \mathrm{mM} \mathrm{K}^{+}$caused an initial rapid peak phase in insulin secretion that was followed by a sustained phase, during which secretion declined over time but remained elevated above basal values as long as $\mathrm{K}^{+}$was present. The magnitude of peak insulin release did not differ between groups but insulin release from Ko islets declined less rapidly over the sustained phase than insulin release from WT islets (time from peak insulin value to its quartile value: $45 \mathrm{~min}$ vs. $29 \mathrm{~min}$, respectively; $p<0.05$ ). The magnitude of $\mathrm{K}^{+}$-stimulated insulin release from CaBPt cells was blunted when compared with $\beta \mathrm{HC}-13$ cells expressing only the vector (VECT) or with BHC-9 cells, which have a large secretory response to $\mathrm{K}^{+}$. At concentrations of $\mathrm{K}^{+}$greater than 20 $\mathrm{mM}$, insulin release from CaBP+ cells was only $5-20 \%$ of that from VECT cells. Basal $\left[\mathrm{Ca}^{2+}\right]$ was slightly lower $(180 \mathrm{nM}$ vs $215 \mathrm{nM})$ and the $\mathrm{K}^{+}$stimulated peak $\left[\mathrm{Ca}^{2+}\right]_{i}$ was markedly inhibited (225 nM vs $820 \mathrm{nM}$ ) in CaBP+ cells when compared to VECT cells. We conclude that calbindin$\mathrm{D}_{28 \mathrm{~K}}$ is acting as a cytosolic buffer for calcium transients in mouse pancreatic $\beta$ cells and controls the rate of insulin secretion via regulation of $\left[\mathrm{Ca}^{2+}\right]_{\mathrm{i}}$. 
137

INTRACELLULARLY APPLIED ION CHANNEL MODULATORS INTERFERE WTTH CALCIUM INDUCED EXOCYTOSIS IN MOUSE PANCREATIC B-CELLS

Sebastian Barg, Erik Renström, and Patrik Rorsman. Department of Physiology and Neuroscience, University of Lund. Sölvegatan 19, S-22362 Lund, Sweden.

It has been postulated that increased osmotic or hydrostatic pressure inside exocytotic granules may facilitate membrane fusion and secretion and that regulation of ion channels in the granular membranes may participate in this process. Pharmacological interference with ion fluxes mediated by these channels would therefore be expected to influence the rate of secretion. We tested this hypothesis in mouse pancreatic B-cells by exposing the cell interior to various ion channel modulators. The regulators were applied by inclusion in the pipette solution which dialyses the cell interior during standard whole-cell recordings. Exocytosis was elicited by infusion of $2 \mu \mathrm{M} \mathrm{Ca}^{2+}{ }_{\text {free }}(9$

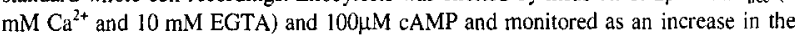
whole cell capacitance. The cell was voltage-clamped at $-70 \mathrm{mV}$ throughout 10 preclude stimulation of secretion by $\mathrm{Ca}^{2+}$-influx through voltage-gated $\mathrm{Ca}^{2+}$-channels. The chloride channel blockers DIDS and niflumic acid, but not NPPB and $9 \mathrm{AC}$ (all at $0.1-0.2 \mathrm{mM}$ ), decreased the rate of exocytosis by approx. $40-70 \%$. Diazoxide $(0.1 \mathrm{mM}$; an activator of ATP-regulated $\mathrm{K}^{+}$-channels; $\mathrm{K}_{\mathrm{ATP}}$-channel) exerted a similar inhibitory action. In addition, ADP ( $5 \mathrm{mM}$; a physiological regulator of the $\mathrm{K}_{\mathrm{ATP}}$-channel) almosi abolished $\mathrm{Ca}^{2+}$-induced exocytosis when applied in the presence of ATP $(3 \mathrm{mM})$. The effects of both diazoxide and ADP were fully antagonised by the sulfonylurea

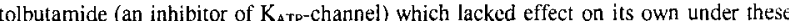
experimental conditions. Our results are consistent with a model in which intragranular accumulation of chloride and potassium leads to the uptake of water into the granule. The resulting increase in osmotic/hydrostatic pressure may provide the energy required for membrane fusion thus facilitating exocytosis. This mechanism is potentially exploitable in the development of novel antidiabetic compounds.

\section{8}

DEPOLARIZATION-EVOKED Ca $a^{2+}$ SIGNALLING IN PANCREATIC $\beta$-CELLS IS AMPLIFIED BY CALCIUM-INDUCED CALCIUM RELEASE

M. S. Islam and P.-O. Berggren. The Rolf Luft Center for Diabetes Research, Department of Molecular Medicine, Karolinska Institute, Karolinska Hospital, 171 76 Stockholm.

The existence of $\mathrm{Ca}^{2+}$-induced $\mathrm{Ca}^{2+}$ release (CICR) in $\beta$-cells has been extensively debated. In this study, using $\mathrm{Sr}^{2+}$ as a $\mathrm{Ca}^{2+}$ surrogate and exploiting the differences in fluorescence properties of $\mathrm{Ca}^{2+}-$ and $\mathrm{Sr}^{2+}$-bound fluo-3, we demonstrate that depolarisation-induced increase in $\left[\mathrm{Ca}^{2+}\right]_{1}$ can be dissected into two components i.e the trigger $\mathrm{Ca}^{2+}$ and the released $\mathrm{Ca}^{2+}$. When extracellular $\mathrm{Ca}^{2+}$ was replaced by $\mathrm{Sr}^{2+}$, depolarisation of fluo-3-loaded cells resulted in clear biphasic increase of fluorescence. The early and low fluorescence was due to strontium entry and subsequent spike-like large increases in fluorescence were due to $\mathrm{Ca}^{2+}$ release from intracellular stores. These spikes were abolished by thapsigargin treatment and increased by ryanodine. In fura- 2 loaded cells, we calculated the integral of increment in $\left[\mathrm{Ca}^{2+}\right]_{\mathrm{i}}$ over time $\left(\int \Delta\left[\mathrm{Ca}^{2+}\right]_{i} \mathrm{~d} t\right)$ as an estimate of amount of $\mathrm{Ca}^{2+}$ presented to the cytoplasm during depolarisation in control cells and in cells whose intracellular $\mathrm{Ca}^{2+}$ stores were depleted by thapsigargine. The time integral of $\left[\mathrm{Ca}^{2+}\right]_{i}$ increment during $300 \mathrm{~s}$ of stimulation by $\mathrm{KCl}$ was significantly higher in the control cells compared to that in the $\mathrm{Ca}^{2+}$ pool-depleted cells. Furthermore, the steady-state $\left[\mathrm{Ca}^{2+}\right]_{i}$ measured at $300 \mathrm{~s}$ of depolarization was significantly lower in thapsigargin-treated cells compared to the control cells. When cells were exposed to $11 \mathrm{mM}$ glucose and $5 \mu \mathrm{M}$ forskolin and membrane potential was clamped at a depolarized level by $\mathrm{KCl}$ and diazoxide, there was generation of large $\mathrm{Ca}^{2+}$ spikes which were blocked by thapsigargin and enhanced by ryanodine. These results demonstrate that following depolarization, there occurs two forms of $\mathrm{Ca}^{2+}$ increase in $\beta$-cells. One form is predominantly due to $\mathrm{Ca}^{2+}$ entry through the plasma membrane $\mathrm{Ca}^{2+}$ channels and the other is due to CICR Furthermore, the latter mechanism appears to involve a ryanodine-receptor like channel.
139

EXTRACELLULAR ATP INHIBITS EXOCYTOSIS OF INSULIN IN MOUSE PANCREATIC B-CELLS

C.R. Poulsen, K. Bokvist and J. Gromada. Dept. Islet Cell Physiology, Novo Nordisk A/S, Symbion, Fruebjergvej 3, DK-2100 Copenhagen, Denmark.

The actions of extracellular ATP have attracted increasing interest as multiple classes of ATP receptors and numerous responsive tissues have been described. Here we have used the patch-clamp technique to investigate the effects of extracellular ATP on membrane potential, ion conductances and exocytosis in single mouse pancreatic B-cells. The standard whole-cell configuration was used throughout this study, except for membrane potential measurements which were perforated patch experiments. In the presence of a sub-stimulatory glucose concentration, ATP $(0.1 \mathrm{mM})$ produced a prompt but minute $(<10 \mathrm{mV})$ depolarization of the membrane potential. Glucose-induced electrical activity $(20 \mathrm{mM}$ ) was slightly enhanced in the presence of ATP. These effects of ATP on electrical activity were associated with a transient $(<1 \mathrm{~min})$ reduction $(32 \%)$ of the ATP-sensitive whole-cell $\mathrm{K}^{+}$current. Surprisingly, ATP inhibited cytoplasmic cAMP production by $29 \%$ in intact islets exposed to forskolin for $2 \mathrm{~min}$. High resolution capacitance measurements of exocytosis were carried out to further explore the effects of ATP on insulin secretion. In experiments where increases in cell capacitance were elicited by 500 ms voltage-clamp depolarisations from -70 to $0 \mathrm{mV}$, ATP inhibited exocytosis by $58 \%$ from $71 \pm 15 \mathrm{fF}$ to $31 \pm 9 \mathrm{fF}$. This decrease was not associated with a reduction in the whole-cell $\mathrm{Ca}^{2+}$-current. When exocytosis was triggered by intracellular dialysis with a $\mathrm{Ca}^{2+}$-EGTA buffer with a free $\mathrm{Ca}^{2+}$ concentration of 2 $\mu \mathrm{M}$ and supplemented with $0.1 \mathrm{mM} \mathrm{cAMP}$, a short application $(30 \mathrm{~s})$ of ATP induced a transient arrest of exocytosis. These data suggest that extracellular ATP has multiple and opposing effects on insulin secretion: 1) ATP slightly stimulates electrical activity by partial and transient closure of ATP-sensitive $\mathrm{K}^{+}$-channels. 2) ATP lowers cAMP production and 3) strongly inhibits exocytosis of secretory granules. These effects of ATP might be important for both para- and autocrine regulation of insulin secretion since ATP is stored and co-secreted with insulin and neurotransmitters important for B-cell function.

\section{0}

CONTRIBUTION OF Na/Ca EXCHANGE TO $\mathrm{Ca}^{2+}$ OUTFLOW AND ENTRY IN THE RAT PANCREATIC $\beta$ CELL.

A. Herchuelz ${ }^{\mathrm{a}}$, , C. Lebeau ${ }^{\mathrm{a}}$, J. Albuquerque ${ }^{\mathrm{b}}$ and F. Van Eylen ${ }^{\mathrm{a}}$. Laboratory of Pharmacology ${ }^{a}$ and Laboratory of Applied Genetics ${ }^{b}$, Université Libre de Bruxelles, School of Medicine, Bât. GE, 808 route de Lennik, B-1070 Brussels ${ }^{2}$ and Faculty of Science, 24 rue de l'industrie, B-1400 Nivelles ${ }^{b}$, Belgium.

To characterize the role played by $\mathrm{Na} / \mathrm{Ca}$ exchange in the pancreatic $\beta$ cell, phosphorothioated antisense oligonucleotides (AS-oligos) were used to knockdown the exchanger in rat pancreatic $\beta$ cells. $\mathrm{Na} / \mathrm{Ca}$ exchange activity was evaluated by measuring cytosolic free $\mathrm{Ca}^{2+}$ concentration $\left(\left[\mathrm{Ca}^{2+}\right]_{\mathrm{i}}\right)$ in single cells using fura-2. Exposure of $\beta$ cells to $500 \mathrm{nM}$ of the AS-oligos for 24 hours inhibited Na/Ca exchange activity by about $77 \%$. In contrast, control oligos (scrambled and mismatched) did not affect $\mathrm{Na} / \mathrm{Ca}$ exchange activity. In AS-oligostreated cells, the increase in $\left[\mathrm{Ca}^{2+}\right]_{\mathrm{i}}$ induced by membrane depolarization ( $\mathrm{K}^{+}$or the hypoglycemic sulfonylurea, tolbutamide) was reduced by $28 \%$ and $40 \%$, respectively. Likewise, the rate of $\left[\mathrm{Ca}^{2+}\right]_{i}$ decrease after $\mathrm{K}^{+}$or tolbutamide removal was reduced by $72 \%$ and $40 \%$, respectively. AS-oligos treatment also abolished the nifedipine resistant increase in $\left[\mathrm{Ca}^{2+}\right]_{i}$ induced by $\mathrm{K}^{+}$and profoundly altered the oscillatory or sustained increases in $\left[\mathrm{Ca}^{2+}\right]_{\mathrm{i}}$ induced by $11.1 \mathrm{mM}$ glucose. The present study shows that AS-oligos may specifically inhibit $\mathrm{Na} / \mathrm{Ca}$ exchange in rat pancreatic $\beta$ cells. In the latter cells, $\mathrm{Na} / \mathrm{Ca}$ exchange appears to mediate $\mathrm{Ca}^{2+}$ entry in response to membrane depolarization and to be responsible for up to $70 \%$ of $\mathrm{Ca}^{2+}$ removal from the cytoplasm upon membrane repolarization. 


\section{Pathophysiology of Diabetic Nephropathy}

\section{1}

SKIN FIBROBLAST NHE- 1 ACTIVITY IN KIDNEY DONORS AND DEVELOPMENT OF DIABETIC GLOMERULOPATHY. JD Walker', LL $\mathrm{Ng}^{2}, \mathrm{GC}$ Viberti', J Basgen', P Jung', K Pinkham', C Dawes', MW Steffes ${ }^{4}$ and SM Mauer'. Departments of Paediatric Nephology', Laboratory Medicine and Pathology ${ }^{4}$, University of Minnesota, Clinical Pharmacology, University of Leicester, ${ }^{2}$, Unit for Metabolic Medicine, Guy’s Hospital, London?

To further explore the cellular mechanisms underlying the development of diabetic glomerulopathy we determined whether skin fibroblast sodiumhydrogen antiport activity (NHE-1) in kidney donors was associated with the development of diabetic glomerulopathy in renal recipients. 14 patients with Type 1 diabetes who had received a living related renal graft which had functioned for a mean (range) of $12.9(8.3-20.2)$ years and their kidney donors were studied. The cells of 1 donor failed to grow. At $5 \mathrm{mM}$ glucose culture there was no correlation between $\mathrm{pH}$ or NHE-I and glomerular structural parameters while at $20 \mathrm{mM}$ glucose culture $\mathrm{pH}$ was correlated with the rate of change of the volume fraction of the mesangium $(r=0.49, p<0.08)$ and NHE- 1 efflux rate was correlated with the rate of change in GBM thickness $(\mathrm{r}=0.50, \mathrm{p}<0.08)$. Renal transplant recipients were divided into 2 groups by the rate of increase in GBM thickness $(<1.25 \mathrm{~nm} / \mathrm{mo}$. (Slow (S) $\mathrm{n}=8$ ) and $>1.25 \mathrm{~nm} / \mathrm{mo}$. (Fast $(\mathrm{F}) \mathrm{n}=5$ )). At $20 \mathrm{mM}$ glucose the NHE-1 efflux rate was higher in the $\mathrm{F}$ compared to the $\mathrm{S}$ group $(35.4 \pm 5.1$ vs. $22.7 \pm 2.9$ mmol//min, respectively, $p<0.04$ ). Age and duration of diabetes at biopsy was similar between $\mathrm{S}$ and $\mathbf{F}$ groups however duration of function at bjopsy was less in the $F$ compared to the $S$ group $(123 \pm 28$ vs. $180 \pm 58$ mo., $p$ $<0.05$ respectively). HbAlc, systolic and diastolic blood pressure levels were no different between groups. In the presence of hyperglycaemia the NHE-1 efflux rate in skin fibroblasts of kidney donors is associated with the rate of thickening of the GBM in this small sample suggesting that factors within the transplanted kidney may influence the rate development of diabetic glomerulopathy

\section{3}

GLUCOSE-INDUCED TGF- $\beta 1$ IN SKIN FIBROBLASTS FROM TYPE 1 DIABETIC PATIENTS WITH DIABETIC NEPHROPATHY

S. Thomas, D. Burt, J. Vanuystel, G. Gruden, L Gnudi, G.C. Vibertl Department of Diabetes, Endocrinology and Metabolic Medicine, UMDS, Guy's Hospital, London, UK.

Hyperglycaemia is the principal risk factor for the development of diabetic glomenulosclerosis, but there are differences in individual susceptibility. TGF- $\beta 1$ a pro-sclerotic growth factor is over-expressed in diabetic nephropathy. We studied TGF- $\beta 1$ production by skin fibroblasts exposed to either normal or high glucose. Cells were obtained from patients with Type 1 diabetes with (1) albuminuria (DNType 1 diabetes $>10$ years $n=18$ ) and $(2)$ normoalbuminuric $(D-$ Type 1 diabetes $>15$ years $n=13$ ) and from age matched non-diabetic controls (NC $n=14$ ). Fibroblasts, cultured in $5 \mathrm{mM}$ glucose (NG), were seeded in either $25 \mathrm{mM}(H G)$ or NG (iso-osmotic with mannitol) for periods from 48 hours to 7 days. TGF- $\beta 1$ mRNA expression was determined by competitive RT-PCR and the supernatant protein level by a two-site immunoassay. HG induced TGF- $\beta 1$ MRNA and protein secretion by 48 hours to a similar degree in all groups (mRNA: 5 fold increase all groups; protein: DN: $1.25 ; \mathrm{D}: 1.5 ; \mathrm{C}: 1.38$ ). Exposure to HG for 7 days resulted in a sustained increase in TGF- $\beta 1$ protein in $D N$. The increase in $D$ was not significant and and did not differ from $C$ (fold increase over control DN: 1.63; D: $1.3 ; C: 1.35$ ANOVA $P$ $<0.05$ ). The addition of LY379196 (Lilly), a specific PKC- $\beta$ inhibitor for the full sevn days at $30 \mathrm{nM}(\mathrm{LY} 30)$ and $60 \mathrm{nM}$ (LY60), prevented glucose induced TGF- $\beta 1$ production in DN with no effect on cell viability ffold increase over control $H G: 1.2 \mathrm{HG}+L Y 30: 1.04 \mathrm{HG}+\mathrm{LY} 60$ : 1. 07]. In patients with Type 1 diabetes, sustained glucose induced TGF- $\beta 1$ production is a feature peculiar to those who develop diabetic nephropathy. This phenomenon seems at least in part to be mediated by PKC- $\beta$.

\section{2}

PROTEIN KINASE C (PKC) BII ISOFORM ACTIVITY IN PERIPHERAL IMMUNE CELLS OF TYPE 2 DIABETIC PATIENTS WITH NEPHROPATHY. S.B.Solerte, A.Saller ${ }^{1}$,A.Pascale ${ }^{2}$,F.Battaini ${ }^{3}$,P.Fioretto ${ }^{1}$,M.Fioravanti ,S.Govoni ${ }^{4}$ E Ferrari G Crepaldi ${ }^{1}$ and R Nosadini 1,5 Department of Internal Medicine ,University of Pavia, Padova ${ }^{1}$, Sassari ${ }^{5}$, Department of Pharmacology, University of Pavia $^{4}$, Milano ${ }^{2}$, Roma Tor Vergata ${ }^{3}$, Italy.

Increased PKC $\beta 11$ isoform activity and levels have been recently linked to the pathogenesis of endotelial dysfunctions and angiopathy in experimental and human diabetes. Within this context, the activity of PKC $\beta$ II isoform was evaluated in basal conditions and after functional activation with phorbol myristate (PMA $160 \mathrm{nM}$ for 15 $\mathrm{min}$ ) in peripheral natural killer (NK) cells of 19 Type 2 diabetic patients and 13 agematched healthy subjects. Diabetic patients were divided as normoalbuminuric ( 7 patients, AER $<20 \mu \mathrm{g} / \mathrm{min}$, mean $\left.\mathrm{GFR}=84 \mathrm{ml} / \mathrm{min} / 1.7 \mathrm{~m}^{2}\right)$ and nephropathic $(12$ patients, AER within $24-806 \mu \mathrm{g} / \mathrm{min}$, mean GFR $\left.=119 \mathrm{ml} / \mathrm{min} / 1.7 \mathrm{~m}^{2}\right)$ and were also classified by means of renal biopsy. NK cells were separated by Ficoll-Hypaque gradient centrifugation at final density of $60 \times 10^{6}$ cells (in $3 \mathrm{~mL}$ PBS). PKC $\beta$ II was measured in cytosol and particulate fractions of NK immune effectors by Western blot analysis. PKC 3 II levels were similar in non nephropathic (mean \pm SD optical density $=1901 \pm 182)$ and nephropathic diabetic patients $(1911 \pm 243)$, as well as in healthy subjects $(1870 \pm 304)$. No changes of PKC $\alpha$ were also reported in these groups (mean values of 957,93l and 1137 respectively). No correlations were found between PKC activity, AER, GFR and glycated hemoglobin in patients with and without nephropathy. The PKC levels of particulate fraction were found to be reduced independently of the degree of albuminuria and of renal hemodynamic variations. No changes of cytosolic PKC $\beta I$ activity during exposure to PMA $(160 \mathrm{nM})$ were finally demonstrated in Type 2 diabetic patients with and without nephropathy $(-43 \%$ and $40 \%$ from baseline respectively), in comparison with healthy subjects $(-41 \%)$. In summary, the functional activity and levels of PKC BII isoform were within the physiological range in diabetic patients with glomerular microvascular alterations. Although PKC activity of NK immune cells cannot reflect the enzymatic activity of glomerular microvasculature, the relevance of a physiologic pattern of PKC $\beta I$ in these cells would seem to leave out the involvement of PKC-system in the pathogenesis of nephropathy in Type 2 diabetes.

\section{4}

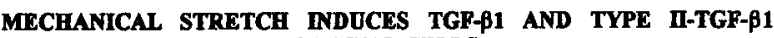
RECEPTOR IN HUMAN MESANGIAL CELLS.

G. Gruden S. Thomas, D. Burt, S. Sacks', L. Gmudi, G.C. Viberti

Department of Diabetes, Endocrinology and Metabolic Medicine, 'Department of Nephrology and Transplantation, UMDS, Guy's Hospital, London, UK. Diabetic and other progressive glomerulopathies are characterised by excess in mesangial matrix deposition. The haemodynamic insult, secondary to glomerular capillary hypertension, has been implicated in the pathogenesis of this alteration. In vitro TGF- $\beta 1$, a pro-sclerotic cytokine, enhances mesangial cell matrix production via an autocrine mechanism, and in vivo, in experimental diabetes, TGF- $\beta$ land/or the TGF- $\beta 1$ type II receptor overexpression parallels mesangial matrix accumulation. We studied the effect of mechanical stretch, which mimics in vitro, the haemodynamic insult on the expression of TGF- $\beta$ land the TGF- $\beta 1$ type II receptor in human mesangial cells. Serum and insulin-deprived mesangial cells were exposed to mechanical stretch (10\% elongation) for 6,12 and 24 hours. Control cells were seeded in non-deformable but otherwise identical plates in parallel. Total RNA was extracted and TGF- $\beta 1$ gene expression, quantitated by competitive RT-PCR Total TGF- $\beta 1$ protein level was determined by ElISA (range: $16-1000 \mathrm{pg} / \mathrm{ml}$, intraassay CV: $1.6 \%$, interassay CV: $7.6 \%$ ) and the TGF- $\beta 1$ type II receptor by western blotting on total protein extracts using a specific rabbit anti-TGF- $\beta 1-R I$ antibody TGF- $11 \mathrm{mRNA}$ and protein levels were significantly greater in stretched cells as compared to control cells (mRNA 12 hrs: 1.8; protein 12-24 hrs: 1.7 and 1.8 fold increase over control $p<0.05$ for all). A parallel $60 \%$ increase in the TGF- $\beta 1$ type II receptor was seen by 24 hours. Stretch induces TGF- $\beta 1$ and upregulates its type II receptor in mesangial cells. This could represent a mechanism by which a mechanical insult leads to increased mesangial matrix deposition in diabetic and other glomerulopathies. 
145

TRANSFORMING GROWTH FACTOR- $\beta 1$ IN IDDM PATIENTS WITH AND WITHOUT DIABETIC NEPHROPATHY

E. Korpinen ${ }^{1.3}$, P.-H. Groop ${ }^{2}$ J. Fagerudd ${ }^{2}$, A.-M. Teppo ${ }^{2}$,H. K. Åkerblom ${ }^{1}$ and $\mathrm{O}$ Vaarala ${ }^{3}$. Hospital for Children and Adolescents ${ }^{1}$, and Department of Medicine, Division of Nephrology ${ }^{2}$, University of Helsinki, and Department of Biochemistry ${ }^{3}$, National Public Health Institute, Helsinki, Finland

Transforming growth factor- $\beta$ (TGF- $\beta$ ) is considered a key mediator in the development of diabetic nephropathy. Our aim was to study TGF- $\beta 1$ protein secretion and mRNA expression by peripheral blood mononuclear cells (PBMC) from IDDM patients with and without diabetic nephropathy. We recruited 52 IDDM patients of whom 28 were classified as having normoalbuminuria, 11 microalbuminuria, 13 overt diabetic nephropathy (DNP), and 13 healthy subjects. One patient from the normoalbuminuric group, 9 patients from the microalbuminuric group, and 11 patients from the DNP group were on ACEinhibitors. Consequently, 2 microalbuminuric patients had regressed to normoalbuminuria, and 3 DNP patients to microalbuminuria. PBMC were cultured for 48 hours in a cell culture medium containing $11 \mathrm{mmol} / \mathrm{g}$ glucose. TGF- $\beta 1$ secretion by PBMC was measured by EIA, and TGF- $\beta 1$ mRNA expression by semiquantitative RT-PCR. After $48 \mathrm{~h}$ of culture, supernatant TGF- $\beta 1$ levels were higher in IDDM patients than in healthy subjects $(4.6 \pm 3.9 \mathrm{vs} .2 .4 \pm 2.7 \mathrm{ng} / \mathrm{ml}$ (mean $\pm S D$ ), $P=0.02$. Supernatant TGF- $\beta$ l levels were $4.5 \pm 3.1 \mathrm{ng} / \mathrm{ml}$ for current normoalbuminuric, $2.4 \pm 2.2 \mathrm{ng} / \mathrm{ml}$ for microalbuminuric, and $7.5 \pm 5.8 \mathrm{ng} / \mathrm{ml}$ for DNP patients, $P=0.02$. TGF- $\beta \perp$ mRNA expression after $24 \mathrm{~h}$ of culture was $3(0-4)$ (median (range) of log titres) for IDDM patients, and $2(0-4)$ for controls. $P=0.16)$. The TGF- $\beta 1$ secretion or mRNA expression did not correlate with AER or $\mathrm{HbA} 1 \mathrm{c}$. We conclude that the TGF- $\beta 1$ secretion by PBMC from IDDM patients is enhanced, and that IDDM patients with persistent macroalbuminuria are high secretors of TGF- $\beta 1$ when compared to other IDDM patients. The results indicate that increased TGF- $\beta 1$ secretion by PBMC may play a role also in orher diabetic complications besides renal disease.

\section{6}

INCREASED ALBUMINURIC RESPONSE TO INFUSION OF ATRIAL NATRIURETIC PEPTIDE IN NORMOALBUMINURIC TYPE 1 DIABETES. J.A. Lutterman, G. Vervoort, J.F.M. Wetzcls, J.H.M. Berden and P. Smits. Depts. of Interial Medicine and Nephrology, University Hospital Nijmegen, The Netherlands. The Atrial Natriuretic Peptide (ANP) is a natriuretic and vasodilating hormone that increases glomerular pressure by dilating the afferent and constricting the efferent glomenilar arteriole. In diabetes increased plasma levels of ANP have been found, fostering the suggestion that ANP contributes to the glomerular hyperfiltration seen in diabetes. Furthermore, ANF increases albuminuria in microalbuminuric diabetes patients. We hypothesised that infusion of ANP might also increase albuminuria in normoalbuminuric diabetes. We have studied 54 normoalbuminuric patients with type 1 diabetes (DP) and 40 healthy controls (C). Measurements were performed before (baseline) and during infusion of ANP $(0.01 \mu \mathrm{g} / \mathrm{kg} / \mathrm{min})$. GFR and ERPF were measured by inulin and $\mathrm{PAH}$ clearances. Filtration fraction (FF) was calculated by GFR/ERPF. Urinary albumin excretion (AER, $\mu \mathrm{g} / \mathrm{min}$ ), plasma ANP, plasma $c-G M P$ (systemic second messenger of ANP) and urinary c-GMP (measure of renal production) were measured by ELISA. Statistical analysis was performed by ANOVA and t-test or Mann-Whitncy, Values are given as means \pm SE. At baseline there were no differences in blood pressure. GFR and ERPF were increased in DP (GFR $12 \mathrm{~J} \pm 2$ vs $105 \pm 2$ in $\mathrm{C}$ and ERPF $568 \pm 15$ vs $523 \pm 14$ in $\mathrm{C}, \mathrm{p}<0.01$ ). FF was not significantly different $(21.7 \pm 0.4 \%$ in $D P$ vs $20.6 \pm 0.5$ in $C, p=0.07)$. AER was increased in DP $(7.4 \pm 0.6 \mathrm{vs} 4.9 \pm 0.3 \mu \mathrm{g} / \mathrm{min}$ in $\mathrm{C}, \mathrm{p}<0.05)$. Plasma ANP was slightly decreased in DP $(7.6 \pm 0.4$ ys $9.4 \pm 0.7 \mathrm{nmol} / \mathrm{l}, \mathrm{p}=0.06)$. Plasma c-GMP differed not significantly $(3.0 \pm 0.3 \mathrm{in} \mathrm{DP}$ vs $2.6 \pm 0.2 \mathrm{mmol} / \mathrm{l})$. The urinary excretion of $\mathrm{c}-\mathrm{GMP}$ was slightly increased in DP ( $4.0 \pm 0.2$ vs $3.3 \pm 0.3 \mathrm{nmol} / \mathrm{min}, \mathrm{p}=0.05$ ). During infusion of ANP, FF increased to $24.1 \pm 0.5 \%$ in DP and $23.2 \pm 0.6$ in C. The increase of AER was significantly greater in DP (AER $18.2 \pm 1.6 \mu \mathrm{g} / \mathrm{min}(173 \%)$ and $9.5 \pm 1.4$ in C $(96 \%)$, $p<0.01$ ). There were no differences in plasma ANP or c-GMP in bolh groups. In DP. a more prominent increase in absolute and fractional urinary c-GMP excretion was observed (c-GMP increased to $24.5 \pm 0.9$ in DP and to $16.7 \pm 1.4 \mathrm{nmol} / \mathrm{min}$ in $\mathrm{C}$, $p<0.01$ and fractional excretion increased from $122 \pm 6 \%$ to $174 \pm 10$ in DP and from $123 \pm 9$ to $123 \pm 10$ in $\mathrm{C}, \mathrm{p}<0.01$ ). We concluded that the albuminuric response to ANP infusion was augmented in nomoalbuminuric type 1 diabetes. Urinary excretion of c-GMP was also increased suggesting an exaggerated renal response to ANP in DP.

\section{7}

Atrial natriuretic peptide induces microalbuminuria in insulin dependent diabetic with normal urinary albumin excretion rates.

K. Mckenna ${ }^{2}$ and $\mathrm{CJ}$. Thompson̈ ${ }^{2}$. 1. Victorìa Infirmary, Glasgow, U.K. 2. Beaumont Bospital, Dublin, Ireland.

Elevated plasma concentrations of atrial natriuretic peptide (ANP) are associated with microalbuminuria in insulin dependent diabetes (IDDM). We have previously demonetrated that intravenous infusions of ANP increase the utinary albuinin excretion rate (UAER) in IDDM patients with taidroalbuminuria. This study aimed to examine if ANP could induce microalbuminuria in IDDM patients with normal UAF. We present the results of a double blind, randomised, placebo controlled study. Eight normoalbuminuric (< $30 \mathrm{mg} \mathrm{day}^{-1}$ ) IDDM patients were studied on thrae occasions. Subjects were euglycaemic clamped, and subsequently water loaded orally !20 $\mathrm{mls}_{\mathrm{kg}}^{-1} \mathrm{plus}$ urinary losses at 15 minute intervals to steady state diuresis. When ateady state was established a 30 minite intravenous infusion of placebo, $0.025 \mathrm{ug} \mathrm{kg}^{-1} \mathrm{~min}^{-7}$ ANP or

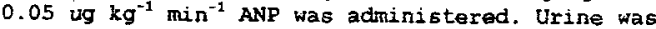
collected at 15 minute intervals for 90 minutes for assay of albumin:creatinine ratio (ACR). Results were analysed by ANOVA. ACR was unaltered by placebo infusion, but increased compared to placebo with both low dose ANP 12.3 \pm 2.3 to $14.6 \pm 13.6 \mathrm{mg}$ rmol ${ }^{-1}, p=0.021$, and high doge ANP $\left(2.1+2.4\right.$ to $28.4 \pm 31 \mathrm{mg}$ manol $\left.\mathrm{I}^{-1} \mathrm{p}=0.01\right)$.

Intravenous infusion of ANP to reproduce

pathophysiological plasma concentrations induces

microalbuminuria in IDDM patients with normal UAER.

\section{8}

ANP INCREASES ALBUMINURIA IN TYPE 1 DLABETES: EVIDENCE FOR BLOCKADE OF TUBULAR PROTEIN REABSORPTION

J.F.M. Wetzels, E.M.G. Jacobs, G. Vervoort, A.J.W. Branten and P. Smits. Depts of Internal Medicine and Nephrology, University Hospital Nijmegen, The Netherlands. The Atrial Natriuretic Peptide (ANP) is a natriuretic and vasodilating hormone. The most striking renal hemodynamic effect of ANP is the increase in glomenular filtration fraction (FF) due to preglomerular vasodilation and postglomenular arteriolar vasoconstriction. In diabetes increased plasma levels of ANP have been reported and it has been suggested that ANP contributes to the glomerular hyperfiltration seen in patients with diabetes mellitus. Infusion of ANP increases the urinary excretion of albumin in patients with diabetes. Although the increased albuminuria has been atributed to a rise in glomerular pressure, alterations in tubular protein handling might be involved. We have studied the effects of ANP in 9 micro-albuminuric patients with type 1 diabetes. After baseline measurements, ANP was infused during 60 minutes at a rate of $0.01 \mu \mathrm{g} / \mathrm{kg} / \mathrm{min}$ after a bolus of 0.05 $\mu \mathrm{g} / \mathrm{kg}$. GFR and ERPF were measured by inulin and PAH clearances respectively. Filiration fraction (FF) was calculated by the quotient of GFR and ERPF. Blood pressure was measured by Dinamap. Urinary albumin, $\beta_{2}$-microglobulin and $\alpha$-light chain concentrations were measured by an ELISA. Proximal and distal sodium reabsorption were calculated by the lithium clearance method. Statistical analysis was performed by ANOVA. Values are given as means \pm SE. GFR increased from $121 \pm 9$ at bascline to $133 \pm 8 \mathrm{ml} / \mathrm{min}(p<0.05$ ) after infusion of ANP whereas ERPF decreased from $564 \pm 5010507 \pm 34 \mathrm{ml} / \mathrm{min}(\mathrm{p}<0.05)$. As a consequence, FF increased from $0.22 \pm 0.01$ to $0.26 \pm 0.01$ ( $p<0.01$ ). ANP attenuated both proximal and distal tubular sodium reabsorption as assessed by the lithium clearance technique. Urinary excretion of albumin increased from $87.6 \pm 21.0$ to $291.4 \pm 67.9 \mu \mathrm{g} / \mathrm{min}(p<0.01)$. The urinary excretion of $\beta_{2}$-microgiobuin increased from $0.28 \pm 0.21$ to $51.9 \pm 10.5 \mu \mathrm{g} / \mathrm{min}$ $(p<0.01)$ and $\alpha$-light chains from $4.7 \pm 1.5$ to $44.9 \pm 4.3 \mu \mathrm{g} / \mathrm{min}$ ( $\mathrm{p}<0.01$ ). During infusion of ANP no significant changes in blood pressure were seen. ANP increases urinary excretion of albumin in micro-albuminuric patients with type I diabetes. Furthermore. ANP attenuates proxinal reabsorption of the low molecular weight proteins $\beta_{2}$-microglobulin and $\alpha$-light chain. Therefore, the increase in albuminuria after infusion of ANP might not simply reflect changes in glomerular pressure but could be explained by inhibition of proximal tubular albumin reabsorption. 
OP 24

\section{Education, Outcome, Health Care Costs}

\section{9}

STAGED DIABETES MANAGEMENT: AN EFFECTIVE TOOL FOR MANAGEMENT OF TYPE 2 DIABETES BY DIABETES EDUCATORS AND PRIMARY CARE PHYSICIANS. L. Blonde ${ }^{1}, R$. Guthrie', M. Tan $^{2}$, J. Parkes ${ }^{2}$, T. O'Brien ${ }^{2}$ and B. Ginsberg ${ }^{2}$. 'The Ochsner Clinic, New Orleans, LA, USA, ${ }^{2}$ Becton Dickinson and Co., Franklin Lakes, NJ, USA.

Staged Diabetes Management (SDM) is a disease state program developed by the International Diabetes Center (IDC). It contains a comprehensive set of practice guidelines and algorithms to guide primary carc physicians (PCPs) and other health care professionals in providing better and more consistent care to their patients with diabetes. SDM was evaluated over a 12-month period at The Ochsner Clinic. In the SDM group, diabetes educators managed 93 patients under the supervision of endocrinologists while PCPs managed diabetic complications and provided non-diabetes care. In the usual care group, Ochsner PCPs managed 73 patients in accordance with usual practice. The primary endpoint was hemoglobin Alc (HbAlc). After 12 months, 77\% of the SDM group experienced an improvement in $\mathrm{HbAlc}$ levels versus $53 \%$ of the usual care group. The mean reduction in HbAlc units was $1.13 \%$ in the SDM group versus $0.47 \%$ in the control group. The table below shows the percentage of patients achieving 4 different levels of reduction in $\mathrm{HbAIc}$ units by treatment group.

\begin{tabular}{|l|l|l|l|l|}
\hline & \multicolumn{4}{|c|}{ Percent Reduction in HbAlc Units } \\
\hline Group & $>0.5 \%$ & $>1.0 \%$ & $>1.5 \%$ & $>2.0 \%$ \\
\hline SDM (n=93) & $66 \%$ & $48 \%$ & $35 \%$ & $24 \%$ \\
\hline Usual Care $(\mathrm{n}=73)$ & $40 \%$ & $26 \%$ & $18 \%$ & $16 \%$ \\
\hline
\end{tabular}

Seventy-percent ( $70 \%$ ) of the SDM group achieved $\mathrm{HbAlc}$ levels below $8 \%$ after 12 months versus $53 \%$ of the usual care group. We conclude that SDM is an effective tool for management of patients with Type 2 diabetes by diabetes educators and PCPs. The significant reduction in HbAlc levels should lead to a reduced incidence of diabetes complications. Reduced complications can in turn assist providers in delivering more appropriate, less costly diabetes care.

\section{1}

BLOOD GLUCOSE SELF-MONITORING IN INSULIN TREATED TYPE 2 DIABETES R. Schiel, U.A. Müller, J. Rauchfuß, H. Sprott and R. Müller, University of Jena Medical School, Department of Internal Medicine II, Jena, Germany

Up to the present there is controvery about blood glucose self-monitoring in type 2 diabetes. In 842 insulin-treated type 2 diabetic patients (age 60,1 $\pm 10,9$, diabetes duration since diagnosis $12,6 \pm 7,6$ years, BMI $28,6 \pm 5,1 \mathrm{~kg} / \mathrm{m}^{2}$, HbA1C $9,34 \pm 1,98 \%$ [HPLC, Diamat $\Theta$, normal range 4,4-5,9\%]) a cross-sectional study was conducted to assess blood-glucose self-monitoring and interactions with quality of diabetes care Among the patients studied there were $90 \%$ of all insulin-treated type 2 diabetic patients aged 16 to 60 years and living in a large city (100424 inhabitants) diabetic patients aged 16 to 60 years and living in a large city (100424 inhabitants) 91 patients were studied, treated at district hospitals. There was a negative correlation $(r=-0,16, p<0,001)$ between the frequency of blood glucose selftests/week and $\mathrm{HbA} 1 \mathrm{c}$. Performing multivariate analysis the most importan parameters associated with HbAic (R-squared $=0,09$ ) were: The frequency of blood glucose self-tests/week $(c=-0,006, p<0,001)$, the insulindose $/ k g$ body wt $(c=0,003, p<0,001)$ and participation in a 5-day structured teaching and treatment programme for patients with conventional insulin therapy according to Berger et al. $(5-T \mathrm{P}, c=0,078, p<0,001)$. Other factors investigated in the model (age, diabetes duration, number of insulin injections/day, sex) showed no associations. Performing a sub-group analysis in patients older than 60 years $(n=396)$ important parameters associated with $\mathrm{HbA1C}$ ( $R$-squared $=0,16$ ) were the participation in a 5TTP $(c=0,004, p<0,001)$ and the frequency of blood-glucose self-tests/week ( $c=-$ $0,006, p<0,001)$ too. In an other sub-group analysis patients $(n=249)$ were studied who have not participated in a 5-TTP. In this sub-group there were no correlation and no association between the frequency of blood-glucose self-monitoring and HbA1c. Then, an intervention was started: 33 of the 249 patients participated in a 5-TTP. At the time of re-examination 1 year after participating in the 5-TTP. HbA1c decreased from $9.5 \pm 1.9 \%$ to $8.3 \pm 1.6 \% \quad(p=0.036)$ and there was a strong association between the frequency of blood glucose self-tests/week and HbA1c $(c=-0,02, p=0,003, R$-squared $=0,24)$. Daily blood glucose self-monitoring is not only important to prevent asymptomatic hypoglycaemia, but also to improve quality of diabetes care and to achieve glycaemic goals. Participation in a 5-TTP and regularly blood glucase self-monitoring is mandatory for all insulin-treated patients with type 2 diabetes mellitus.

\section{0}

SELF-ADJUSTMENT OF BEDTIME INSULIN (SABI):

A KEY TO SUCCESSFUL INSULIN THERAPY IN NIDDM

L. Pekkonen, L. Hyvărinen, R. Hărkönen, M. Riihelă and M. Heikkilä

Espoo, Kotka, Lappeenranta, Rovaniemi FINLAND

We reasoned, based on analysis of our previous multicenter study, and on meta-analyses of insulin treatment trials that use of insufficient insulin doses is due to adjustement of insulin doses exclusively at outpatients visits. In a new Finnish multicenter study we randomized 96 patients with NIDDM (age $58 \pm 1$ years, $\mathrm{HbA}_{1 \mathrm{c}} 9.9 \pm 0.2 \%$, BMI $29 \pm 0.5 \mathrm{~kg} / \mathrm{m}^{2}$ ), for treatment with various bedtime NPH regimens for 12 months. The patients were given oral and written instructions of how to adjust the bedtime NPH dose. The patients were instructed to increase the insulin dose every 3 days by $4 \mathrm{IU} / \mathrm{day}$ if the fasting plasma glucose concentration exceeded $8 \mathrm{mmol} / \mathrm{h}$ and by $2 \mathrm{IU} /$ day if the fasting plasma glucose exceeded $6 \mathrm{mmol} /$. The glycemic target was to lower the fasting plasma glucose concentration below $6 \mathrm{mmol} /$. This was predicted to lower $\mathrm{Hb} \mathrm{A}_{10}$ to less than $7.5 \%$. The dose of bedtime insulin required to lower fasting glucose from 10.5 $\pm 2.1,11.2 \pm 2.3,10.0 \pm 2.3$ and $12.1 \pm 3.1 \mathrm{mmol} / \mathrm{l}$ in groups treated with bedtime NPH and glibenclamide, metformin, both or another injection of NPH in the morning to $6.4 \pm 0.3,6.2 \pm 0.2,6.4 \pm 0.3$ and $6.7 \pm 0.3 \mathrm{mmol} / \mathrm{l}$ ( $<0.001$ for 12 vs 0 months in each group) varied over 20 -fold from 8-168 IU/day. $\mathrm{HbA}_{1 \mathrm{c}}$ decreased to $7.2 \pm 0.2$, $7.7 \pm 0.3,7.8 \pm 0.2$ and $7.9 \pm 0.3 \%(p<0.001)$

No severe hypoglycemias occured, although the frequency of biochemical hypoglycemias significantly increased at fasting glucose concentrations below $6 \mathrm{mmol} / \mathrm{l}$.

In summary: Glycemic targets can be reached safely by instructing the patients to selfadjust their insulin dose. We attribute the better results of this as compared to our previous multicenter study to self-adjustement of insulin dose. These data emphasize the need to not only instruct the patients to perform home-glucose monitoring but also to change treatment according to glucose concentrations.

\section{2}

DIABETIC EMERGENCIES: SOME ERRORS AND COMPLICATIONS OF MANAGEMENT ON PRIMARY CARE LEVEL

R.M.Parhimovich, Moscow Regional Research Clinical Institute, Moscow, Russia. The analysis of 260 cases (age 15-66 years, 128- IDDM and 132- NIDDM) of diabetic emergencies management during first hours or days in ICU of primary care medicine has revealed typical errors and complications and enable to suggest some recommendations PERIOPERATIVE PERIOD in diabetic patients: 1. Insufficient supply of carbohydrate led to increased catabolism and ketoacidosis. Not less than $120-200 \mathrm{~g} / 24 \mathrm{~h}$ infusion of glucose with insulin (potassium and vitamins $\mathrm{B}$, etc.) is necessary in parenteral nutrition. DIABETIC KETOACIDOSIS (DKA): 1.Too fast decision on laparotomy (laparoscopy) in case of DKA abdominal syndrome withou waiting for disappearing the "peritoneal signs" due to the proper DKA therapy during the first hours. . 2.Infusion of colloid instead of crystalloid fluids at the beginning of rehydratation. Colloids is to be used to prevent disequilibrium syndrome or persisting hypotension in spite of sufficient infusion of crystalloids. 3. Overenthusiastic infusion of hypotonic fluids was the main reason for brain oedema. We insist on restriction on the use of hypotonic fluids (instead of $5 \%$ glucose) only in cases of hypernatriemia $>155-160 \mathrm{mmol} / \mathrm{l}$ connected with hyperglycemia $>17 \mathrm{mmol} /$. 4 .Insufficient potassium replacement. 5 . The use of diuretics and cardiac glycosides before fluid and potassium were replaced. 6 . Lowering glycemia by insulin without simultaneous sufficient rehydratation. 7.Delayed or restricted glucose supply after glycemia was lowered $<16 \mathrm{mmol} / 1$ or in cases of "euglycemic DKA". 8. Hypertonic (4\%) bicarbonate infusion $(150-200 \mathrm{ml})$ in $\mathrm{pH}>7.1$. 10. Insufficient awareness for tendency to the recurrence of DKA and development of mixed acidosis during DKA treatment.

HYPOGLYCEMIA: A. Delay with the diagnosis and proper management in some cases with focal stroke-like signs in patients on sullfonylureas. B. Insufficient duration of glucose infusion (or repeated carbohydrate intake) in hypoglycemia due to sulfonylureas (in one case glucose infusion had to be lasted $90 \mathrm{~h}$ ). C. Delay with carbohydrate administration in hypoglycemia in IDDM (as a result - in two young patients hypoglycemia transformed into severe DKA before they were admitted to ICU).EXACERBATION OF RENAL INSUFFICIENCY: 1.Insufficient carbohydrate supply (glucose-insulin infusion enable to stop severe vomiting and acidosis). 2.Insufficient management of urinary tract infection, which often is the main reason for the exacerbation of renal isufficiency. 
153

HOW DOES THE GENERAL PRACTITIONER APPROACH THE CLUSTERING OF CARDIOVASCULAR RISK FACTORS IN TYPE 2 DIABETES?

J.H. Dekker', L.V. Franse', G.D. Valk', R.J. Heine,', and J.Th.M. van Eijk .' EMGOInstitute, ${ }^{2}$ Department of Internal Medicine, Vrije Universiteit Amsterdam

The joint presence of insulin resistance related cardiovascular disease risk factors as hypertension, dyslipidemia and hyperglyceamia is often seen in type 2 disbetic patients. We investigated the prevalence of, and clinical approach by the general practitioner to these cardiovascular risk factors in a type 2 diabetic-population, to assess to which extent the knowledge about the insulin resistance syndrome has been translated in clinical practice. Data of 558 type 2 diabetic patients (aged 32-92), collected in 1995/1996 in 20 general practices in the Netherlands, were crosssectionally analysed. All patients were examined for cardiovascular risk factors as hypertension, dyslipidaemia (high serum cholesterol, high triglyceride, low HDL), hyperglycaemia, obesity and smoking. In our analysis of clustering, patients could 'score' from 0 (good/acceptable levels of all studied riskfactors) to 7 (poor levels of al studied risk factors). Poor glycaemic control ( $\mathrm{HbAl}>7,6)$ was found in 190 patients $(34 \%)$; a low HDL-level $(<0,9 \mathrm{mmol} / \mathrm{l})$ in $24 \%$; a high triglyceride level $(>2,2$ mmol/l) in $33 \%$; and a high serum cholesterol $(26,4 \mathrm{mmol} / \mathrm{l})$ in $28 \%$ of the patients A Body Mass Index greater than $27 \mathrm{~kg} / \mathrm{m}^{2}$ was found in 357 patients $(64 \%)$ hypertension ( $>160 / 95 \mathrm{~mm} \mathrm{Hg}$ ) was seen in 167 patients $(30 \%)$ and $18 \%$ of the patients smoked. A clustering of 3 or more (!) risk factors for cardiovascular diseases was observed in $35 \%$ of this population. Glycaemic control was poor in one third of the patients. A lipid lowering drug was prescribed in only $15 \%$ of the patients with at least one lipid disorder ( $n=223$ ). Of the 167 hypertensive diabetics only $67 \%$ were regularly checked for their bloodpressure and received treatment. It can thus be concluded that the different modifiable risk factors need a more agressive approach to reduce the cardiovascular disease burden of diabetes.

\section{5}

\section{INSULIN AND METFORMIN USE ARE ASSOCIATED WTTH HIGHER} TOTAL MEDICAL COSTS NN TYPE 2 DIABETES

A.W. Bakst, J.B. Brown, G.A. Nichols, and H.S. Glauber; SmithKline Beecham Pharmaceuticals, Collegeville PA; Kaiser Permanente Northwest, Parland OR. We calculated the total annual 1995 medical care costs of persons with Type 2 diabetes (T2DM) (in constant 1993 dollars) and compared these costs across differen antidiabetic therapy groups. Study subjects included ail 12,200 members of Kaiser Permanente Northwest Region Diabetes Registry who had diagnosed T2DM and 12 full months of health plan eligibility in 1995. As shown in Table 1, T2DMs taking insulin, alone or in combination with an SU, had higher costs of every kind, and much higher total costs than other T2DMs. Persons managed on metformin had higher pharmaceutical costs but their other costs were similar to persons managed on SUs and those receiving no antidiabetic medication.

\section{Table 1: 1995 Total Medical Care Costs/Person by Treatment Regimen (S1993)}

\begin{tabular}{rlllll} 
& \multicolumn{1}{|c}{ SU } & $\begin{array}{c}\text { No } \\
\text { Antidiabetic } \\
\text { Drugs }\end{array}$ & $\begin{array}{c}\text { Metformin +/ } \\
\text { other antidiabetic } \\
\text { drugs }\end{array}$ & $\begin{array}{c}\text { Insulin } \\
\text { All Drugs }\end{array}$ & $\begin{array}{c}\text { SU } \\
+ \\
\text { Insulin }\end{array}$ \\
\cline { 2 - 6 } Outpatient & $\$ 1,317$ & $\$ 1,302$ & $\$ 1,304$ & $\$ 1,452$ & $\$ 1,473$ \\
Inpatient & $\$ 1,284$ & $\$ 1,416$ & $\$ 1,633$ & $\$ 1,773$ & $\$ 2,041$ \\
Referral & $\$ 413$ & $\$ 760$ & $\$ 282$ & $\$ 2,728$ & $\$ 3,411$ \\
Imaging & $\$ \$ 328$ & $\$ 328$ & $\$ 345$ & $\$ 935$ & $\$ 784$ \\
TOTAL: & $\$ 4184$ & $\$ 4,398$ & $\$ 4846$ & $\$ 476$ & $\$ 693$ \\
\hline 7364 & $\$ 8402$
\end{tabular}

Progression of therapy from oral agents to insulin is an accepted algorithm for treatment of Type 2 diabetic patients. Total medical care costs were highest for patients receiving insulin as monotherapy or in combination with SU. Abandonment or supplementation of SU therapy may provide an indicator of the progression of T2DM and increasing overall medical care costs. Therapies which have potential to delay or prevent the progression of T2DM to insulin or combination oral therapy may yield healthcare system cost savings
CLINICAL AND ECONOMIC OUTCOMES OF CARE ASSOCIATED WITH REDUCED LEVELS OF HEMOGLOBIN AIC IN PATIENTS WITH TYPE 2 DLABETES. T. O'Brien', N. Ray', D. Ganoczy ${ }^{2}$ and B. Ginsberg ${ }^{2}$ 'Becton Dickinson and Co., Franklin Lakes, NJ, USA. 'Medtap International, Bethesda, MD, USA.

The American Diabeles Association reported that direcl medical and indirect cxpenditures attributable to diabetes in the U.S. were estimated to be $\$ 98$ billion in 1997. Concurrently, acute metabolic and chronic complications were estimated to account for $45 \%$ of the direct medical expenditures. Recent research also indicates that improved glycemic control decreases the risk of diabetes complications and decreases related medical expenditures. Our study examines the effect of different lifetime lcvels of hemoglobin Alc ( $\mathrm{HbAlc}$ ) on the lifetime incidence of these complications and their associated costs. We constructed a series of what if scenarios to cvaluate the effect of a reduction of lifetime $\mathrm{HbAlc}$ levels to $6 \% .8 \%$, $10 \%$ and $12 \%$. The analyses were based on a probabilistic model which was dcycloped by NiH and uses Monte Carlo techniques. The model incorporates risk gradients derived from the DCCT and simulates the life history of Type 2 patients. The results below indicatc that lifetime reductions in $\mathrm{HbAlc}$ decrease both the incidence of complications and related medical expenditures.

\begin{tabular}{|l|c|c|c|c|}
\hline & \multicolumn{5}{|c|}{ HbAlc Level } \\
\hline Type of Complication & $6 \%$ & $8 \%$ & $10 \%$ & $12 \%$ \\
\hline Background retinopathy & $22.3 \%$ & $49.8 \%$ & $95.9 \%$ & $99.6 \%$ \\
\hline Blindness & 9.8 & 18.2 & 53.2 & 59.4 \\
\hline ESRD & 1.4 & 8.7 & 29.0 & 40.2 \\
\hline Neuropathies & 9.1 & 20.8 & 47.2 & 74.4 \\
\hline Total Mcdical Costs & $\$ 85,697$ & $\$ 91.146$ & $\$ 120,903$ & $\$ 132,253$ \\
\hline
\end{tabular}

We conclude that reductions in $\mathrm{HbAlc}$ levels can lead to decreased incidence of complications and lower medical expenditures for Type 2 patients. We anticipate that advances in diabetes treatment, including new monitoring technologies, may lead to improved health status via reductions in $\mathrm{HbAlc}$

\section{6}

The UK Diabetes Information Analysis and Benchmarking Service (UKDIABS) Roxburgh M.A., Vaughan N.J.A.,BDA, London, UK

UKDIABS was established at the British Diabetic Association (BDA) in 1996 to provide UK annual national benchmarking analyses of diabetes population based databases. The project is the culmination of work done through the last decade in the development of locality-based diabetes registers and standardisation of diabetes data collation. An important aspect has been the close collaboration with suppliers of diabetes IT systems and clinicians to secure a reliable standardised data extract for ageregation on a central server at the BDA. Important links have been created with the other bodies including the Central Health Outcomes Unit, CASPE Research, St Mary's Hospital Dept. of Epidemiology, Royal Holloway College Dept of Psychology, DiabCare and the EASD study group DOIT.

About $2 / 3$ of UK districts have some form of population based register project in place, and are now capable of interfacing with UKDIABS. Practical experience has repeatedly shown that the development of such systems takes about two years to reach useful maturity with reasonably complete ascertainment of population based data. For 1995 benchmarking there were about 7 sites in the UK with useable data, about 30 for 1996, and about 40 for 1997. On current trends it is anticipated that this is likely to increase to about 60 districts for 1998 and 80-90 districts for 1999. Benchmarking for 1996 includes data from 19 districts, (approximately 50,000 patient records) and has concentrated on data relevant to monitoring St. Vincent Targets. Initial benchmark data is demonstrated and includes: diabetes-sex prevalence, process and outcome measures for BMI, Systolic BP, Eye and foot examination (including amputation/blindness), incidence of MI, HbAlc process measures, Smoking and Alcohol usage. Wide variations within districts for these measures has been noted, which include variations in amputations ranging from $0.7 \%$ to $6.2 \%$ (ave $2.4 \%$ ), incidence of MI from $0.6 \%$ to $6.9 \%$ (ave 2.3\%) and blindness prevalence ranging from $0 \%$ to $2.4 \%$ (ave $0.34 \%$ ). Results are being fed back to sites for consideration and local use for quality improvement. The project has also initiated a phase involving data validation, from a technical and medical standpoint with appropriate epidemiological analysis including mapping. This is being further facilitated by the creation and active involvement of a User Group, comprising the many clinicians and register managers from the participating districts. The longer-term objective is the refinement of these relatively crude provisional population based measurements to provide a tool for measurement of regional and national propress to achieving the St Vincent objectives in the UK. 


\section{OP 25}

\section{Myocardial Infarction in Diabetes}

\section{7}

DIABETES IS AN INDEPENDENT RISK FACTOR OF SUDDEN DEATH IN PATIENTS FOLLOWING ACUTE MYOCARDIAL INFARCTION. S.Z. Abildstrøm, C. Rask-Madsen, B. Brendorp, C. Torp-Pedersen, L. Køber and P. Hildebrandt ${ }^{2}$, on behalf of the TRACE Study Group. Departments of Cardiology affiliated the University of Copenhagen at Gentofte Hospital, 'Rigshospitalet and ${ }^{2}$ Frederiksberg Hospital, Copenhagen, Denmark. Purpose: Sudden death defined as death within one hour of onset of symptoms is presumably arrhythmic and accounts for approximately $50 \%$ of all cardiovascular deaths after acute myocardial infarction (AMI). The aim of the present study was to assess factors predicting sudden versus non-sudden death in post-infarction patients with left ventricular systolic dysfunction.

Methods: We analysed data from 1749 consecutive patients randomised in the Trandolapril Cardiac Evaluation (TRACE) study. All had left ventricular systolic dysfunction with wall motion index (WMI) $\leq 1.2$ measured by echocardiography 2-6 days after AMI. 237 patients had diabetes. Follow up was 3 years, and all deaths were classified according to cause and mode of death. Multivariate analysis was performed to assess the risk of sudden and non-sudden death associated with the following variables: ventricular fibrillation (VF), sex, age, clinical congestive heart failure (CHF), WM diabetes, history of angina, hypertension, obesity, thrombolysis and trandolapril treatment. Results: Diabetes independently predicted sudden death (risk ratio 1.48 (95\% confidence interval 1.06-2.07)) but not non-sudden death (risk ratio $1.06(0.83-1.37)$ ). History of angina and thrombolytic treatment independently predicted non-sudden death (risk ratios $1.50(1.23-1.83)$ and $0.60(0.48-0.75)$ respectively) but not sudden death. Age, WMI and $\mathrm{CHF}$ predicted both sudden and non-sudden death. Male gender and VF predicted only sudden death. Hypertension, obesity and trandolapril treatment had no predictive value. Conclusion: Diabetes is an independent risk factor of only sudden death in patients with impaired left ventricular systolic function following AMI.

\section{8}

MYOCARDIAL INFARCTION IN DIABETIC PATIENTS. RELATION OF SURVIVAL AND INFARCT SIZE TO GLIBENCLAMIDE THERAPY M. Nauck, P. Sarfert, A. Klamann, V. Launhardt, G. Schulte, and W. H. Schmiegel, Med. Univ.-Klinik, Knappschafts-KH, Bochum, Germany Interactions of sulfonylureas with vascular and cardiac ATP-dependent $\mathbf{K}^{+}-$ channels are well established. The clinical impact of using sulphonylureas (glibenclamide) in patients suffering myocardial infarction, however, has not been evaluated. Therefore, patients admitted with acute myocardial infarction over a 6.5 year period were evaluated retrospectively. 357 non-diabetic patients ( $239 \mathrm{~m} / 118 \mathrm{f} ; 66 \pm 14 \mathrm{y}$.) were compared to 245 Type 2 diabetic patients, who were grouped according to treatment: 76 taking sulphonylurea drugs (glibenclamide $7.4 \pm 3.4 \mathrm{mg} / \mathrm{d} ; 37 \mathrm{~m} / 39 \mathrm{f} ; 72 \pm 10 \mathrm{y}$;; $\mathrm{HbA}_{\mathrm{ic}} 8.4 \pm 1.9$ $\%$ ), and 89 not taking sulphonylurea drugs (i.e. treated by diet alone, metformin, and/or insulin; $54 \mathrm{~m} / 35 \mathrm{f} ; 73 \pm 11 \mathrm{y}$.; $\mathrm{HbA}_{\mathrm{sc}} 7.7 \pm 1.3 \%$ ) and $80 \mathrm{pa}-$ tients with newly diagnosed diabetes $\left(45 \mathrm{~m} / 35 \mathrm{f} ; 72 \pm 12 \mathrm{y}\right.$., $\mathrm{HbA}_{\mathrm{lc}} 7.2 \pm 1.2$ $\%$ ). Increments in $\mathrm{CK}$ and $\mathrm{CK}_{\mathrm{MB}}$ activity were recorded, and the vital status was followed until death or hospital demission. Survival analysis and contingency table analyses $\left(\chi^{2}\right.$-test) were used. Of 357 non-diabetic patients, 72 died (in-hospital mortality $20.2 \%$ ). Of 89 Type 2 diabetic patients not taking glibenclamide, 29 died ( $33.0 \%$ ). Of 76 Type 2 diabetic patients on glibenclamide, 25 died $(32.9 \%$ ). Of 80 newly diagnosed Type 2 diabetic patients 20 died $(25.0 \%)$. Survival analysis indicated a significant difference between groups by $\log$-rank test $(p=0.03)$. In-hospital mortality was significantly higher in known Type 2 diabetic versus in non-diabetic patients ( $<<0.05$ ), whereas no difference existed among known Type 2 diabetic patients receiving or not receiving glibenclamide. The increments in $\mathrm{CK}$ and $\mathrm{CK}_{\mathrm{MB}}$ activity during the initial period tended to be higher in non-diabetic patients $(\mathrm{p}<$ 0.01). In conclusion, in-hospital mortality in Type 2 diabetic patients is higher than in non-diabetic patients suffering acute myocardial infarction, regardless of whether or not they had been treated with sulfonylureas (glibenclamide)

\section{9}

EFFECT OF INTENSE INSULIN TREATMENT ON HYPERGLYCAEMIA IN DIABETIC PATIENTS WITH ACUTE MYOCARDLAL INFARCTION.

K. Malmberg, A. Norhammar and L. Rydén. Department of Cardiology, Karolinska Hospital, Stockholm, Sweden.

Diabetic patients with acute myocardial infarction (AMI) have a dismal prognosis. This may depend on a poor metabolic control. Several studies have shown that admission blood glucose (ABG) is an independent predictor for mortality following an AMI both among patients with and without diabetes mellitus. Knowledge of the effect of lowering the ABG is, however, lacking.

Methods: The effect of intensive insulin treatment in 620 diabetic patients with an AMI was tested in DIGAMI, a prospective randomised study. Long-term mortality (mean 3.4 years; range 1.6-5.6) decreased from $44 \%$ in the control group to $33 \%$ in the insulin group $(\mathrm{p}=0.011)$. The present study reports on treatment effects in different ABG levels.

Results: Mean ABG was $15.6 \pm 4.1 \mathrm{mmol} / \mathrm{l}$ and did not differ between the two groups. In the complete patient cohort both $\mathrm{ABG}$ and glycated haemoglobin was independent predictors for long-term mortality. The figure shows mortality (\%) by $A B G$ tertiles within the two treatment groups.

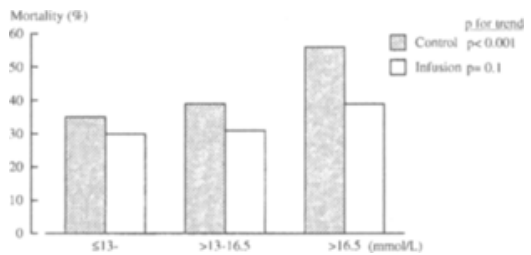

Conclusion: Insulin-glucose infusion followed by intense sc insulin treatment in diabetics with AMI attenuates the harmful effect of elevated $A B G$ on mortality. This effect seems to be most pronounced in patients with the highest ABG levels.

\section{0}

MARKED EFFECT OF ANGIOTENSIN CONVERTING ENZYME INHIBITION IN DIABETIC PATIENTS WITH ACUTE MYOCARDIAL INFARCTION. I. Gustafsson, C. Torp-Pedersen ${ }^{1}$, L. Køber ${ }^{1}$, F. Gustafsson, O. Snorgaard and P. Hildebrandt, on behalf of the TRACE Study Group. Dept. of Cardiology and Endocrinology, Frederiksberg University Hospital; ${ }^{1}$ Dept. of Cardiology, Gentofte University Hospital; Copenhagen, Denmark.

Patients with diabetes mellitus have a high mortality following an acute myocardial infarction (MI), probably due to more pronounced left ventricular (LV) dysfunction and higher risk of reinfarction. We evaluated the effect of angiotensin converting enzyme (ACE) inhibition in diabetic patients with MI and LV dysfunction participating in the Trandolapril Cardiac Evaluation (TRACE) study. The TRACE study was a randomized, double-blind, placebo-controlled trial of trandolapril (1-4 mg daily) in 1749 patients with $\mathrm{MI}$ and LV ejection fraction $\leq 35 \%$. Treatment was started 3-7 days after the MI and the mean follow-up time was 26 months. A history of diabetes was found in $237(14 \%)$ patients. Diabetic patients were slightly older, more often females and had a higher frequency of arterial hypertension, previous $\mathrm{MI}$ and heart failure at randomization. Among the diabetic patients $126(53 \%)$ died during follow-up vs $547(36 \%)$ among the non-diabetic patients. Treatment with trandolapril $\mathrm{vs}$ placebo resulted in a reduced relative risk $(R R)$ of death from any cause in the diabetic group of $0.64(0.45-0.91, p=0.01)$ and in the non-diabetic group of $0.82(0.69-$ $0.97, \mathrm{p}=0.02$ ). In the diabetic group trandolapril reduced the predefined secondary endpoints reinfarction $[R R=0.47(0.24-0.94), p=0.03]$ and progression to severe heart failure $[R R=0.48(0.26-0.90), p=0.02]$ in a multivariate analysis. No significant reduction of these secondary endpoints was found in the non-diabetic group. The interaction between benefit from treatment with trandolapril and diabetes was significant with regard to progression to severe heart failure $(p=0.04)$. We conclude that ACE inhibition after myocardial infarction complicated with left ventricular dysfunction may be of particular importance in patients with diabetes mellitus by saving lives and substantially reducing the occurrence of reinfarction and progression to severe heart failure. 
OP 26

\section{Early Type 2 Diabetes}

\section{1}

THE FASTING SPLIT PROINSULIN CONCENTRATION PREDICTS TYPE 2 DIABETES. N.J.Wareham', C.D.Byme ${ }^{2}$, D.E.M.Williams', D.R.R.Williams ${ }^{3}$, N.E.Day ${ }^{1}$ and C.N.Hales ${ }^{2}$. Departments of Community Medicine $^{1}$ and Clinical Biochemistry ${ }^{2}$. University of Cambridge, Cambridge, UK and Nuffield Institue for Health, Leeds.

Cross-sectional studies have shown that the concentration of all proinsulinlike molecules (PLM) is disproportionately increased in individuals with prevalent Type 2 diabetes. In longitudinal studies, the concentration of total PLM predicts the development of diabetes. It has been suggested that prediction would be enhanced by specific measurement of the different molecules in the proinsulin processing pathway. 32,33 split proinsulin is the predominant form of proinsulin accounting for the hyperproinsulinaemia of diabetes, and therefore, this study was established to examine the liypothesis that the concentration of this molecule would predict diabetes. A populationbased cohort of 1071 caucasian subjects who had a non-diabetic oral glucose tolerance test in 1990-92 were traced and invited to attend for a repeat test 4.5 years later. 937 volunteers attended for rescreening and 26 people were found to have developed diabetes by WHO criteria (crude incidence $6.3 \mathrm{per}$ 1000 person years of follow up). In univariate analyses comparing top with bottom quartile, the risk of progression to diabetes was strongly related to the fasting glucose concentration (relative risk $17.6(2.4-130.4)$ ) and fasting 32,33 split proinsulin (RR $16.4(2.2-121.9)$, but less strongly to fasting insulin (RR 4.41 (1.5-12.9)) or intact proinsulin (RR 5.2 (1.5-17.3)). In multivariate analyses, optimal prediction of diabetes was shown in a model including the fasting glucose, fasting 32,33 split proinsulin and presence of a family history of diabetes. We conclude that the fasting 32,33 split proinsulin concentration predicts the development of diabetes in this cohort study, and that this prediction was better than that observed for either the insulin or intact proinsulin concentrations.

\section{2}

HYPERPROINSULINEMIA AND GLUCOSE TOLERANCE IN THE OFFSPRING OF PATIENTS WITH DIFFERENT PHENOTYPES OF NIDDM

Authors: L. Niskanen ${ }^{1}$, I. Vauhkonen', L. Mykkänen ${ }^{1,2}$, S. Haffner ${ }^{2}$, M. Uusitupa ${ }^{1}$ and M. Laakso'; Departments of Medicine', Kuopio University Hospital, Kuopio, Finland; Department of Medicine/Division of Clinical Epidemiology ${ }^{2}$, The University of Texas, San Antonio, Texas

No studies are available that have examined whether hyperproinsulinemia or increased proinsulin-to-specific (PIsIR) insulin ratio are early markers of the deterioration of glucose tolerance in the offspring of insulin-deficient and insulinresistant probands with NIDDM. Therefore, we measured plasma specific insulin, plasma proinsulin, and plasma C-peptide levels during 2 hour oral glucose tolerance test (OGTT) and during the hyperglycemic clamp in 20 offspring of NIDDM patients with deficient insulin secretion phenotype, 18 offspring of patients with insulin resistant phenotype, and 14 healthy control subjects without a family history of diabetes. The frequency of impaired glucose tolerance was $45 \%$ in the IS-group and $50 \%$ in the IR-group. The IS-group had lower specific insulin-glucose response at $30 \mathrm{~min}$ in the OGTT than the other groups, but they had normal insulin sensitivity measured by the hyperinsulinemic euglycemic clamp. The IR-group had normal specific insulin-glucose response, but they had impaired insulin sensitivity. Fasting plasma proinsulin and plasma proinsulin levels at 30 in the OGTT were comparable among the study groups. The IR-group had lower fasting plasma PIsIR (10.3 \pm 1.1 $\%)$ than the control group $(15.4 \pm 1.4 \% ; P<0.01)$ and the IS-group $(15.0 \pm 2.0 \%$; $P<0.05)$. The IS-group had higher plasma PIsIR at $30 \mathrm{~min}$ in the OGTT $(8.2 \pm 0.7$ $\%)$ than the control group $(6.4 \pm 0.7 \% ; P=0.06)$ and the IR-group $(5.7 \pm 0.5 \% ; P$ $<0.0 \mathrm{l}$ ). However, there were no significant differences in proinsulin-to-C-peptide ratio at fasting stage or at $30 \mathrm{~min}$ in the OGTT among the study groups. Furthermore, there were no significant differences in plasma proinsulin levels and in plasma PIsIR among the study groups during the hyperglycemic clamp. We conclude that impaired processing of proinsulin in $\beta$-cells does not play a fundamental role in the deterioration in glucose tolerance in either groups of offspring of NIDDM patients with deficient insulin secretion or insulin resistant phenotype. The differences found in PIsIR could be due to different clearance rates of proinsulin and specific insulin among the study groups.

\section{3}

NITRIC OXIDE LEVELS ARE ELEVATED IN FIRST DEGREE RELATIVES OF PATIENTS WITH NIDDM.

P.M. Piatti, L.D. Monti, G. Valsecchi, S. Costa, E.P. Sandoli, S. Berni-Canani and A.E. Pontiroli. Istituto Scientifico H. San Raffaele, Milano, Italy.

Recent data indicate that nitric oxide levels through vasodilatation, can affect peripheral glucose metabolism. Since little is known about endothelial function in sisters and brothers, i.e. first degree relatives (FDR) of NIDDM patients, we evaluated nitric oxide levels in FDR of NIDDM patients, to evaluate whether endothelial dysfunction may precede abnormalities of glucose tolerance in these subjects. Three hundred fourty three subjects from 100 families were recruited in the frame of the SPIDER study (an acronyme meaning a study for prevention of diabetes in Lombardia Region). The families were choosen to have at least one NIDDM patient, his/her brothers/sisters and spouses of FDR subjects or NIDDM patients, without family history of NIDDM, as controls. In FDR subjects and in spouses a standard OGTT (75 g p.o.) was performed. Results from 80 NIDDM subjects, 77 FDR subjects (27 with impaired glucose tolerance, IGT; and 50 with normal glucose tolerance, NGT) and 31 spouses are reported. All had normal systolic and diastolic blood pressure and normal myocardial and kidney function. Subjects of the four groups were comparable for age, cigarette smoking and daily physical activity. Nitric oxide levels were evaluated through plasma measurement of NO2/NO3. A moderate degree of overweight, hyperinsulinemia, insulin resistance (HOMA) and hypertriglyceridemia was found in NIDDM and in IGT-FDR subjects. No differences were found between NGT-FDR and controls (fasting true insulin levels: $7.6 \pm 0.9$ vs $6.3 \pm 0.9 \mu \mathrm{U} / \mathrm{ml}$; HOMA: $1.76 \pm 0.15$ vs $1.61 \pm 0.15$; $2 \mathrm{hr}$ OGTT increment insulin levels $22.9 \pm 2.25$ vs $18.2 \pm 2.2 \mu \mathrm{U} / \mathrm{ml}$ ). $\mathrm{NO} 2 / \mathrm{NO} 3$ levels were significantly higher in NIDDM $(29.0 \pm 1.2 \mu \mathrm{M})$ in IGT (33.2 \pm 1.2$)$ and in NGT-FDR (27.8 \pm 1.2$)$ subjects than in control subjects (18.9 \pm 0.9 ; $\mathrm{p}<0.01$ vs NIDDM and FDR), with no differences among NIDDM and FDR subjects. These data suggest that an increase in nitric oxide generation or an alteration in its degradation could precede glucose intolerance and hyperinsulinemia in FDR subjects, and might be one of the primary events in NIDDM.

\section{4}

$24 \mathrm{H}$ INSULIN AND SUBSTRATE PROFLLES: EARLY METABOLIC ABERRATIONS IN OFFSPRING OF TYPE II DIABETIC FAMLIES. B.Nybolm ${ }^{1}$, M.Walker ${ }^{2}$, C.H. Gravholt', P.A. Shearing ${ }^{2}$, K.G.M.M. Alberti ${ }^{2}$ and O.Schmitz'. 'Dept. of Medicine M, Aarhus Kommunehospital, Aarhus, Denmark and ${ }^{2}$ Dept. of Medicine, Framlington Place, Newcastle-upon-Tyne, UK.

An impaired insulin-stimulated glucose uptake (ISGU) is the most common metabolic feature in first-degree relatives (R) of type II (non-insulin dependent) diabetic patients, and is a major risk factor for development of type II diabetes mellitus. To explore aspects of metabolism during daily living in these potentially prediabetic subjects, we determined concentrations of insulin, C-peptide, glucose, and various nutrients during a standardised physiologic $24 \mathrm{~h}$ period (proinsulin was measured following breakfast) in $26 \mathrm{R}$ and 17 anthropometrically matched controls; followed by a hyperinsulinemic (insulin infusion rate: $1.0 \mathrm{mU} / \mathrm{kg} / \mathrm{min}$ ) euglycemic clamp. All had a normal OGTT, but ISGU was reduced in $R(7.76 \pm 0.50$ vs $\$ 1.14 \pm 0.53$ $\mathrm{mg} / \mathrm{kg} / \mathrm{min}, \mathrm{p}<0.001)$. During the profile day, serum insulin was markedly $(70 \%)$ increased in $R(8.00-23.00$ h AUC: $129,406 \pm 12,257$ vs $76,629 \pm 5,083$ pmol/1/. 15 h, $\mathrm{p}<0.01$ ), but not overnight. The same pattern was observed with respect to plasma glucose (day-time AUC: $4,177 \pm 62$ vs $3,974 \pm 40 \mathrm{mmol} / 1 \cdot 15 \mathrm{~h}, \mathrm{p}<0.05$ ), whereas serum $C$-peptide remained elevated in $R$ throughout the $24 \mathrm{~h}$ period ( $p<0.001)$. Fasting serum proinsulin $(13.5 \pm 3.6$ vs $6.1 \pm 0.8 \mathrm{pmol} / 1, p<0.05)$ and proinsulin:insulin $(42 \pm 11$ vs $15 \pm 2 \%, p<0.01)$, as well as serum proinsulin and proinsulin:insulin following breakfast $(p($ both $)<0.05)$ were all significantly increased in R. There were no differences in blood lactate, 3-beta-hydroxy-butyrate, glycerol or serum free fatty acids between the two groups, despite markedly increased circulating insulin concentrations in $R$. In conclusion a considerable metabolic derangement are present in healthy, insulin resistant first-degree relatives of type 2 diabetic patients during conditions that simulates daily living. Moreover, the disproportionately increased level of proinsulin in $R$, signifies that early beta-cell dysfunction also appears to be present in these young potentially prediabetic persons. 


\section{OP 27}

\section{Altered Intra Uterine Development and Insulin}

165

EFFECT OF INTRA-UTERINE GROWTH RETARDATION (IUGR) ON GLUCOSE TOLERANCE AND INSULIN SENSITIVITY AT 25 YR. OF AGE D Jaquet, A Gaboriau, J Léger, D Collin, P Czernichow, C Lévy-Marchal INSERM U 457 - Paris, France

We have previously shown that subjects born full term ( $\geq 36$ wk.) with IUGR (birth weight $\leq 3^{\text {rd }}$ percentile) have normal glucose tolerance but larger insulin and proinsulin excursions under OGTT at age $20 \mathrm{yr}$. than normal birthweight -pairs. At $25 \mathrm{yr}$ of age., subjects from the same cohort born with IUGR and controls born with a normal weight underwent OGGT for follow-up of glucose tolerance, a euglycemic hyperinsulinemic (40 $\mathrm{mU} / \mathrm{min} / \mathrm{m}^{2}$ ) clamp for insulin sensitivity measurement by peripheral glucose uptake (M) and IVGTT $(0.3 \mathrm{~g}$ glucose $/ \mathrm{kg})$ for measurement of first phase insulin release (FPIR). Preliminary results on 15 IUGR and 10 CTRL are given as $m \pm 1 S D$. Non-parametric tests were used for comparisons between the 2 groups.

\section{birth weight $(\mathrm{g})$}

BMI $\left(\mathrm{kg} / \mathrm{m}^{2}\right)$

Systolic BP $(\mathrm{mmHg})$

Glu T0 $(\mathrm{mg} / \mathrm{dl})$

Glu T120 (mg/dl)

[nsul T0 $(\mu \mathrm{U} / \mathrm{ml})$

Insul T120 $(\mu \mathrm{U} / \mathrm{ml})$

Triglycerides $(\mathrm{mmol} / \mathrm{l})$

HDL-Chol. ( $\mathrm{mmol} / \mathrm{l})$

$\mathrm{M}(\mathrm{mg} / \mathrm{min} / \mathrm{kg}$ fat-free-mass $)$

FPIR $(\mu \mathrm{U} / \mathrm{ml})$

$\begin{array}{ccr}\text { IUGR } & \text { CTRL } & p \\ 2390 \pm 280 & 3505 \pm 140 & <.001 \\ 24.7 \pm 5.2 & 23 \pm 1.9 & \text { ns } \\ 118.17 \pm 9.5 & 119.2 \pm 9.2 & \text { ns } \\ 91 \pm 7.4 & 85 \pm 3.8 & .01 \\ 106 \pm 33 & 84.4+14 & .06 \\ 7.6 \pm 4.1 & 4.4 \pm 2.6 & .04 \\ 43.4 \pm 40.3 & 21.2 \pm 8.3 & .09 \\ 1.16 \pm 0.78 & 0.66 \pm 0.23 & .02 \\ 1.5 \pm 0.42 & 1.49 \pm 0.21 & \text { ns } \\ 5.84+2.23 & 8.2 \pm 1.68 & .02 \\ 73.8 \pm 53.4 & 66.4 \pm 25.5 & \text { ns }\end{array}$

Comparison with the OGGT performed $3 \mathrm{yr}$. earlier showed a significant post-load increase in IUGR vs CTRL $(\Delta$ Glu $120=0.14 \pm .26$ vs $-0.14 \pm .22$ $\mathrm{mg} / \mathrm{dl} ; \mathrm{p}=.02$ ). From these data we conclude that 1 ) IUGR is associated with insulin resistance and hypertriglyceridemia, suggestive of early development of syndrome $\mathbf{X}$ in young and non-obese adults with normal glucose tolerance 2) glucose tolerance seems to worsen faster in IUGR than in CTRL at $25 \mathrm{yr}$. of age.

\section{7}

BIRTH WEIGHT AND SERUM INSULIN CONCENTRATIONS IN NONDIABETIC PIMA INDIAN CHILDREN

D. Dabelea, R.L. Hanson, P.H. Bennett, J. Roumain, G. Imperatore, D.J. Pettitt, and W.C. Knowler, NIDDK, National Institutes of Health, Phoenix, Arizona.

Low birth weight may be associated with features of the insulin resistance syndrome. In Pimas, the relation of type 2 diabetes prevalence to birth weight is U-shaped. We have examined the association between birth weight and serum insulin concentrations in $\mathbf{4 8 5 3}$ non-diabetic Pima Indian children. Glucose and insulin levels were measured during a $75 \mathrm{~g}$ oral glucose tolerance test. Multiple linear regression analyses were performed in age groups 5-9, 10-14, and 15-19 years. There was a significant association between birth weight and childhood weight, height, and relative weight ( $<<0.0001$ for each, in each age group) Neither fasting nor post-load insulin levels were correlated with birth weight After adjustment for relative weight, both fasting and post-load insulin levels were negatively correlated with birth weight (fasting insulin: $p=0.04$ at 5-9 years, $p=0.24$ at $10-14$ years, and $p<0.001$ at $15-19$ years; 2 -hour insulin: $p=0.03$ at $5-9$ years, $p=0.01$ at $10-14$ years, and $p<0.0001$ at $15-19$ years). Thus children of lower birth weight appeared more insulin resistant relative to their weight Relative weight had a greater impact on insulin levels than did birth weight. $A$ difference in birth weight of 1 standard deviation $(541 \mathrm{~g})$ was associated with $0.9 \%(95 \%$ confidence interval $0.5-1.3 \%, p=0.03)$ lower fasting insulin, and $2.8 \%$ $(2.2-3.4 \%, \mathrm{p}<0.0001)$ lower 2 -hour insulin. By contrast, a difference in relative weight of 1 SD $(31 \%)$ corresponded to a $16.3 \%(15.9-16.7 \%, p<0.0001)$ higher fasting insulin and a $16.4 \%(15.8-16.9 \%, \mathrm{p}<0.0001)$ higher 2 -hour insulin. The relationship between birth weight and 2-hour glucase concentrations was, however, U-shaped for the 10-14 and 15-19 year age groups, independent of current relative weight. We conclude that low birth weight is a determinant of insulin levels, but only when controlled for childhood size. Both low and high birth weight predict diabetes in Pima children and adults, but the association of birth weight with insulin, adjusted for relative weight, is linear. Thus different mechanisms seem to underlie the association between low and high birth weigh and subsequent type 2 diabetes in Pima Indian children.

\section{6}

SELECTIVE RESISTANCE TO INSULIN IN ADIPOCYTES FROM EARLY GROWTH RETARDED RATS.

S.E. Ozanne, C.L. Wang, M.W. Dorling and C.J. Petry, Department of Clinical Biochemistry, University of Cambridge, Addenbrooke's Hospital, Cambridge, CB2 2QR, U.K.

Many studies have shown a relationship between early growth retardation and non-insulin-dependent diabetes mellitus. Studies in rats have shown that the offspring of rats fed a low protein (LP) diet during pregnancy and lactation have a significantly worse glucose tolerance in late adult life compared to controls. In contrast, in young adult life LP offspring have a better glucose tolerance which is associated with increased insulin-stimulated glucose uptake into skeletal muscle. The aim of the present study was to compare the ability of insulin to stimulate glucose uptake and inhibit lipolysis in adipocytes isolated from LP offspring. LP adipocytes $(n=6)$ had an increased basal ( $55 \pm 5$ compared to $33 \pm 2$ amol glucose/min $/ \mathrm{cell}$, $p$ $<0.001)$ and insulin-stimulated $(96 \pm 8$ compared to $79+8$ amol glucose/min/cell, $\mathrm{p}<0.05$ ) glucose uptake compared to controls. There was no significant difference in basal rates of lipolysis in control and LP adipocytes $(2.5 \pm 0.4$ and $3.0 \pm 0.7$ pmol glycerol released $/ \mathrm{h} / \mathrm{cell}$ for control and LP respectively). Isoproterenol stimulated $(\mathrm{p}<0.001)$ lipolysis in both groups (to $4.0 \pm 0.4$ and 6.8 $\pm 0.06 \mathrm{pmol} / \mathrm{h} / \mathrm{cell}$ in control and low protein, respectively), however had a significantly $(\mathrm{p}<0.001)$ greater effect on LP adipocytes. Insulin reduced $(\mathrm{p}<0.001)$ lipolytic rates in controls to basal levels $(2.2 \pm 0.7 \mathrm{pmol}$ glycerol released $/ \mathrm{h} / \mathrm{cell})$ but had a markedly reduced effect in LP adipocytes ( $5.4 \pm 0.4$ pmol glycerol released/h/cell). These results suggest that early growth retardation causes selective resistance to different metabolic actions of insulin.

\section{8}

GLUCOSE TOLERANCE AND INSULIN RESPONSE IN OFFSPRINGS OF AFRICAN NIDDM PARENTS

J.C. Mbanya', L.N. Pani', J. Ngogang', L. Ashworth' and K.G.M.M. Alberti ${ }^{3}$

Dept. of 'Internal Medicine \& ${ }^{2}$ Biochemistry, University of Yaoundé I, Cameroon and ${ }^{3}$ Wellcome Laboratories, University of Newcastle upon Tyne, UK

Otfsprings of NIDDM parents are predisposed to developing diabetes and early metabolic abnormalities of diabetes may be detectable in these subjects. We studied 69 offsprings (cases) aged 20 years and above of 26 African parents with at least one parent having NIDDM and 62 age and body mass index matched offsprings (controls) of 25 non diabetic African parents with a negative family history of diabetes. Diabetes and impaired glucose tolerance (IGT) was defined by the WHO 1985 criteria following a $75 \mathrm{~g}$ oral glucose tolerance test $(2 \mathrm{hr} 11.1 \mathrm{mmol} / \mathrm{l})$ and first phase insulin secretion was calculated by the ratio of the 0 to 30 minute incremental values of insulin to that of glucose. Fasting blood glucose was different between the cases and the controls $(4.7 \pm 0.6$ vs $3.9 \pm 0.8 \mathrm{mmolli} ; p=0.0001)$, however fasting insulin levels were similar in both groups. There was a four.fold and a nine.fold increase in the prevalence of diabetes and IGT in the cases compared to the background population $14.4 \mathrm{vs} 1.0 \%$ and 18.8 vs $2.0 \%$ for diabetes and IGT respectively). Total glucose intolerance (diabetes and IGT) was greater in the cases than in the controls (23.2 vs $6.4 \% ; p=0.02)$. The prevalence of IGT increased in the cases with increasing number of givcose intolerant parents $(9.8,37.5$ and $50 \%$ respectively with one parent diabetic, one parent IGT and the other diabetic and both parents diabetic compared to $6.5 \%$ in the controls). Both the area under the glucose and insulin curves were different between the two groups (glucose curve, $516 \pm 79$ vs $435 \pm 89 ; \quad p<0.001$; insulin curve $2329 \pm 1264$ vs $3234 \pm 1781 ; 0<0.02$ in cases and controls respectively). The first phase insulin secretion was different in both normal and glucose intolerant cases and controls inormal, 37.9 vs 48.9 and IGT 10.3 vs $17.3 ; F=4.1, p<0.04$ respectively in cases and controls). We conclude that both early glucose and $\beta$-cell abnormalities are frequent in offsprings of African NIDDM parents. 
OP 28

\section{Devices}

169

A THREE-MONTH, MULTICENTER CLINICAL TRIAL OF THERAPY WITH INHALED HUMAN INSULIN IN TYPE 1 DIABETES MELLITUS J. S. Skyler, R.A . Gelfand, and I.A. Kourides for the Inhaled Insulin Phase II Study Group. Miami FL, Groton CT, and San Carlos CA, USA. A new dry powder insulin (I) formulation and aerosol delivery system allow reproducible dosing of rapid-acting I by inhalation. Therapeutic quantities of $I$ are delivered to the systemic circulation with 1-2 inhalations per dose. To compare the efficacy and safety of an inhaled I (INH) with a conventional injection regimen (SC), 70 patients with type 1 diabetes from 10 study sites were randomly assigned, after a l-mo run-in, to $\mathrm{INH}$ or $\mathrm{SC}(\mathrm{n}=35 \mathrm{each})$ treatment for $3 \mathrm{mo}$. INH patients received pre-meal INH plus bedtime injected Ultralente I; SC patients continued their pre-study I (2-3 injections daily). QD home monitoring was reviewed weekly for all patients and I adjusted to target pre-meal glucose of $100-160 \mathrm{mg} / \mathrm{dl}$. Glycemic control, evaluated by $\mathrm{HbA}_{\mathrm{fc}}$, was the same in the two groups at baseline ( $\mathrm{INH}=8.5 \pm 1.1 \%$ and $\mathrm{SC}=8.5$ $\pm 1.1 \%$ ) and at $3 \mathrm{mo}(\mathbb{N H H}=7.9 \pm 1.0 \%$ and $\mathrm{SC}=7.7 \pm 0.9 \%)$ (mean $\pm \mathrm{SD}, \mathrm{p} \mathrm{NS}$ ). Weight gain in the 3 -mo study was $0.1 \pm 2.4 \mathrm{~kg}$ for INH and $0.6 \pm 3.0 \mathrm{~kg}$ for SC (p NS). Hypoglycemia occurred with similar incidence and severity in both groups ( 8 severe episodes in $\mathrm{NH}$ vs 10 in SC; 5.13 mild-moderate episodes per subject-month in INH vs 5.23 in SC). Results of a patient satisfaction questionnaire significantly favored $\mathrm{INH}$, and $80 \%$ of patients treated with $\mathrm{CNH}$ chose to continue in a $1-\mathrm{yr}$ extension of INH therapy. Pulmonary function tests (spirometry, lung volumes, and diffusion capacity) were unchanged after 3 mo of $\mathrm{NH}$ therapy. In conclusion, I treatment with INH was well tolerated with similar hypoglycemia to SC and no weight gain, well liked by patients, and as efficacious as conventional SC I in managing type 1 diabetes mellitus.

\section{1}

NON-INVASIVE BLOOD GLUCOSE MONITORING BY MEANS OF NEAR INFRARED SPECTROSCOPY: LONG-TERM PREDICTION RESULTS

A. Sămann', U. A. Müller' ${ }^{1}$, Ch. Fischbacher', K.U. Jagemann ${ }^{2}$, K. Danzer' ${ }^{2}$ J. Schüler and L. Papenkordt, 'Dept. for Internal Medicine II, Medical School, ${ }^{2}$ Institute of Inorganic and Analytical Chemistry, Friedrich-Schiller-University, Jena, Germany

Modern strategies in the treatment of Diabetes mellitus demand large numbers of blood glucose tests. NIR-spectroscopy is considered to be a method for non-invasive blood glucose testing. The present investigation evaluated the long-term prediction quality of individual (ICM) and overall calibration models (OCM). Spectra of 10 patients were detected over a period of 182 days. Unselected individual, i.e. model includes all detected spectra, and selected overall calibration model, i.e. model with pre-selected spectra, were created at baseline. Spectra pre-selection procedures based on spectra similarities (Pearson's correlation coefficient (r)) and multi-variate distances (leverage value (h)). The calibration models were used to predicted later detected spectra. A clinical, i.e. error grid analysis (EGA [\%]) and a statistical method, i.e. root mean standard error of prediction (RMSP [mmol/l]) were applied to evaluate the predictions of blood glucose. The long-term prediction results for the ICMs ranged from almost sufficient ( $\mathrm{rmsp}=3.1 \mathrm{mmol} / \mathrm{l}$ : EGA: $98 \%$ in the clinical acceptable zone $a+b$, prediction period: 84 days) to insufficient ( $\mathrm{rmsp}=19.7 \mathrm{mmol} / \mathrm{l}$; EGA: $20 \%$ in $a+b$, prediction period: 113 days). The results for the OCMs varied considerably, depending on the selection criteria, e.g. selection criteria $h<0.055$ and $\mathrm{r}>0.9995$ left 120 spectra out of 1327 for prediction; RMSP $=2.8 \mathrm{mmol} / 1$, EGA 98.7 $\%$ in $\mathbf{a}+\mathbf{b}$, prediction period: 144 days. In conclusion, long-term prediction of blood glucose by means of NIR-spectroscopy is feasible. Individual calibration models yielded better results than overall calibration models. Considerable differences in quality of long-term predictions and huge losses of spectra underline the need for further research work.
170

CAUSES FOR THE HIGH ANTIGENICITY OF HOECHST $21 \mathrm{PH}$ INSULIN WHEN DELIVERED INTRAPERITONEALLY BY IMPLANTABLE PUMPS.

E. Delatte*, J. Steibel**, Sapin R**, V Karsten*, A. Belcourt*, M. Pinget* and $\mathrm{N}$. Jeandidier* ${ }^{*} \mathrm{CeeD},{ }^{* *}$ Institut de Physique Biologique, Hôpitaux Universitaires, Strasbourg, France.

Intraperitoneal (ip) insulin delivery with implantable pumps, significantly increases anti insulin antibody (AIA) levels in type 1 diabetic patients. Causes for this high antigenicity are still unknown. Insulin aggregates were shown to induce specific AIA formation and were observed in the device. The aim of the study was to compare the levels of AIA induced by ip injections of Hoechst $21 \mathrm{PH}$ insulin sampled either directly from a vial or from a patient implantable pump reservoir. Two insulins were tested, a less stable one, HOE21PH, which formed aggregates in the device reservoir during clinical use and a new more stable one, HOE21PHv3, for which clinical studies are still under investigation. 3 groups of 5 Wistar rats were injected ip with $200 \mu \mathrm{l}$ of insulin sampled either from a vial (group 1),or from a pump containing either the HOE21PHy3 (group 2) or the HOE21PH (group 3). 5 rats were injected with an antigenic mixture of silicone and HOE $21 \mathrm{PHv} 3$ as control (group 4). Immunizations were performed every 10 days. Antibody levels against human insulin (RIA Pasteur; $\mathrm{N}<2.5 \%$ ) were assessed before immunization and 3 weeks after. Data were transformed into $L n$ and tested with a «Mann Whitney» test. Results showed that AIA mean levels (\%) \pm SEM of $0.0 \pm 0.0$ prior to injection, increased significantly $(\mathrm{p}<0.01)$ to $1.6 \pm 0.1$ (group 1 ) $4.5 \pm 2.9$ (group 2), 9.8 \pm 3.5 (group 3) and 21.7 \pm 9.3 (group 4). AIA levels were higher in groups 2 and $3(\mathrm{p}<0.05)$ as compared to group 1 . Immunizations and AIA dosages are on going with the same protocol in groups 1,2 and 3 with now 10 more rats in each group. Aggregates or other modifications due to the insulin stay in the device could be responsible for the antigenic response observed and perhaps in part for the amplitude observed HOE21PHv3 tending to induce lower AIA levels than HOE21PH.

\section{2}

CALIBRATION-FREE CONTINUOUS ON-LINE TISSUE GLUCOSE MONITORING: THE COMPARATIVE MICRODIALYSIS TECHNIQUE U. Hoss, R. Gessler, B. Kalatz, M.I. Salgado, F. Sternberg and R. Fussgänger. Institute of Diabetes-Technology at the University of Ulm, Ulm, Germany

By the combination of microdialysis and enzymatic amperometric glucose sensing it is possible to measure tissue glucose concentration in men continuously. However, there are problems concerning calibration and response time of the glucose sensor. The comparative microdialysis system is a calibration-free method for quasi-continuous glucose monitoring with short response time. A microdialysis probe is implanted in the subcutaneous tissue and perfused with glucose containing phosphate buffered saline. The flow rate has to be controlled so that a low flow period $(0.4 \mu \mathrm{l} / \mathrm{min})$ is followed by a high flow period $(5.0 \mu \mathrm{l} / \mathrm{min})$. During the low flow rate the dialysate equilibrates to the surrounding tissue, whereas during the high flow rate the original glucose concentration is only slightly changed $(<5 \%)$ and the equilibrated segment of the dialysate is rapidly transported to the downstream located glucose sensing unit. The quotient of the sensor signals belonging to the equilibrated segment of the dialysate and of the dialysate with unchanged perfusate glucose concentration can be used to calculate the absolute tissue glucose concentration. In vitro testing showed that the 'comparative microdialysis' method is linear over a glucose range of $0.22 \mathrm{mmol} /$. The response time is 9 minutes. Continuous glucose monitoring in 6 type 1 diabetic patients over $14 \mathrm{~h}$ has been performed with capillary blood glucose reference measurements every 30 minutes. $97 \%$ of the 'comparative microdialysis' values showed deviations to blood glucose less than $25 \%$. No calibration between the patients was necessary and no signal drift could be observed. The 'comparative microdialysis' technique solves most of the problems normally related with microdialysis systems used for continuous glucose monitoring. Response time is reduced to an acceptable value, sensor drift is eliminated and no calibration is necessary. This method offers a reliable on-line continuous glucose monitor for diabetic patients, including alarm for hypo- and hyperglycemia. 


\section{OP 29}

\section{Nutrition and Diet Therapy}

\section{3}

CHANGES IN SERUM LIPID FATTY ACID COMPOSITION IN RELATION TO GLUCOSE METABOLISM DURING TWO FAT-MODIFIED DIETS

A.M. Louheranta, E.S. Sarkkinen, H.M. Vidgren, U.S. Schwab and M.I.J. Uusitupa, Department of Clinical Nutrition, University of Kuopio, P.O. Box 1627, 70211 Kuopio, Finland

The effects of a high-fat, monounsaturated-fat enriched (Mono) diet and a reducedfat, polyunsaturated-fat enriched (Poly) diet on lipid and glucose metabolism were compared in 31 subjects (18 males, 13 females) with impaired glucose tolerance. After 3 weeks on a run-in diet (37;18:11:5, indicating energy percentages from total fat; saturated:monounsaturated:polyunsaturated fatty acids in the actual diets) subjects were randomized into a Mono diet $(40 ; 11: 19: 8)$ or a Poly diet $(34 ; 11: 10: 10)$ for 8 weeks. In the Mono group fasting plasma glucose (mean + SD) was lower after the test diet than after the run-in period $(6.4 \pm 1.3$ vs. $6.0 \pm 0.8 \mathrm{mmol} / \mathrm{l}$, $\mathrm{p}=0.008)$ but remained unchanged in the Poly group $(6.2 \pm 0.6$ vs. $6.1 \pm 0.7$ $\mathrm{mmol} / \mathrm{l})$. At the end of the study, glucose effectiveness $\left(\mathrm{S}_{\mathrm{C}}\right)$ in the frequently sampled intravenous glucose tolerance test was higher in the Mono group than in the Poly group (1.64 \pm 0.49 vs. $1.11 \pm 0.54 \mathrm{~min}^{-1} \times 10^{2}, \mathrm{p}=0.013$, respectively); in insulin sensitivity there was no difference. For the present study the diet groups were combined and the associations between the changes in the fatty acid composition of serum cholesteryl esters (CE), triglycerides and phospholipids (PL) and fasting plasma glucose and $\mathrm{S}_{\mathrm{S}}$ were examined. An increase in the proportions of oleic acid and $\alpha$-linolenic acid in PL, was associated with a decrease in the fasting plasma glucose concentration $(r=-0.53, p=0.002$ and $r=0.47, p=0.009$, respectively). Similar, but weaker associations were found between the fasting plasma glucose and the proportions of oleic acid $(r=-0.33, p=0.07)$ and $\alpha$-linolenic acid $(r=-0.35, p=0.05)$ in $C E$. An increase in $S_{G}$ was associated with an increase in the proportion of oleic acid $(r=0.55, p=0.004)$ and with a decrease in the proportion of arachidonic acid $(r=-0.40, p=0.04)$ in PL. In conclusion, the Mono diet resulted in beneficial changes in fasting plasma glucose and $\mathrm{S}_{\mathrm{G}}$ and these changes were associated with changes in the proportions of oleic, $\alpha$-linolenic and arachidonic acid in serum lipid fractions.

\section{5}

HIGH FIBRE DIET IMPROVES LONG TERM GLUCOSE CONTROL IN INSULIN DEPENDENT DIABETIC PATIENTS

M.Parillo *, R.Giacco ${ }^{\circ}$,M.R. Pirto , A.M.Rivieccio , A.Giacco, A.A.Rivellese and G.Riccardi. *Caserta General Hospital, ${ }^{\circ}$ I.S.A. CNR - Avellino; Dep. of Clinical and Experimental Medicine - "Federico II" University, Medical School, Naples - Italy Several short term studies have demonstrated that a high fiber diet improves glucose and lipid metabolism in diabetic patients. However ADA dietary recommendations have questioned the beneficial effects of dietary fibre on glucose control in diabetio patients, since they have never been demonstrated in long-term studies. Therefore, the aim of our study was to evaluate the long term (six months) compliance and metabolic effects of a high fibre diet in IDDM patients. A randomized study with parallel groups was undertaken in 63 IDDM patients of both genders. After 4 weeks of an isoenergetic low fibre diet (CHO 58\%, Protein 17\%, Lipid 25\%, Fibre $9 \mathrm{~g} / 1000 \mathrm{kcal} / \mathrm{d}$ ) patients were randomized to either high fibre diet (HF) (CHO 58\%, Protein 18\%, Fat $24 \%$, Fibre $26 \mathrm{~g} / 1000 \mathrm{kcal} / \mathrm{d}$ ) or a low fibre diet (LF) (CHO $58 \%$, Protein $17 \%$, Fat $25 \%$, Fibre $9 \mathrm{~g} / 1000 \mathrm{kcal} / \mathrm{d}$ ) to be followed for 6 months. Of 31 and 32 participants treated with $\mathrm{HF}$ and LF diet, 28 and 27 concluded the study, respectively. Mean age was $32 \pm 12$ and $26 \pm 7$ yrs, BMI $24 \pm 0.6$ and $24 \pm 0.5 \mathrm{~kg} / \mathrm{m}^{2}$, duration of diabetes $11 \pm 7$ and $10 \pm 5$ yrs, $\mathrm{HbAlc} 8.9 \pm 1.4$ and $8.7 \pm 1.4 \%(\mathrm{M} \pm \mathrm{SD})$. Dietary compliance was, in general, satisfactory: beside dietary fibre $(42 \pm 8 \mathrm{~g} / \mathrm{d}$ and $15 \pm 3 \mathrm{~g} / \mathrm{d}$, respectively), diet composition was similar in the two groups (seven- day-food-records). Compliance to the diet was not satisfactory in 7 patients in the $\mathrm{HF}$ group (fibre $<30 \mathrm{~g} / \mathrm{d}$ ) and in 4 patients in the LF group (fibre $>20 \mathrm{~g} / \mathrm{d}$ ). After 6 months of treatment the HF diet, compared with the $L F$, reduced significantly $2 \mathrm{~h}$ postprandial plasma glucose $(195 \pm 92$ vs $255 \pm 91 \mathrm{mg} / \mathrm{dL} ; \mathrm{p}<0.04)$ and $\mathrm{HbAlc}(8.6 \pm 1.0$ vs $9.1 \pm 1.3 \%$; $\mathrm{p}<0.03)$ without changes in insulin dose. No effect on plasma lipids was observed. The number of hypoglycemic events per patient was significantly lower in the $\mathrm{HF}$ than in $\mathrm{LF}$ diet $(4.4 \pm 3.7$ vs $8.8 \pm 7.5 ; p<0.01)$. This study definitely demonstrates that, also in the long term, compliance to the HF diet is satisfactory and that HF diet improves plasma glucose control in IDDM patients and reduces the frequency of hypoglycemia.

\section{4}

NEONATAL FEEDING OF DIABETOGENIC DIET IS PROTECTIVE IN BB RATS: ROLE OF THE GUT TH1/TH2 CYTOKINE BALANCE

S. B. Flohé, P. Rowsell*, P. Jee*, C. Goebel, H. Kolb and F.W. Scott*

Diabetes Research Institute at the University of Düsseldorf, Düsseldorf, Germany, *Nutrition Research Division, Health Canada, Ottawa, Canada

Diet is a major factor in the initiation and progression of pancreatic islet inflammation in diabetes prone BB rats. In this animal model, the feeding of standard cereal based diet from weaning at $23 \mathrm{~d}$ causes destructive insulitis and diabetes while feeding hypoallergenic diets containing hydrolysed proteins does not. Surprisingly, oral feeding of $\mathrm{mg}$ amounts of cereal based diet (NIH-07) suspended in a hypoallergenic, casein hydrolysate formula (Pregestimil 1 , PG) between age 4 to 7 days, significantly inhibited diabetes expression while administration of $P G$ alone in the control group was not protective (31\% versus $56 \%$ of rats diabetic at $130 \mathrm{~d}$ of age, $p=0.02$ ). We hypothesised that oral feeding of the cereal based diet in infancy induced a protective immune response in the gut. We therefore analysed the expression of cytokine genes in the gut $24 \mathrm{~h}$ after feeding with PG alone or PG + NIH-07. Cytokine mRNA levels were determined by semiquantitative RT-PCR. When compared with the PG alone, feeding of pups with $P G+$ cereal based diet caused a significant shift of the Th1/Th2 cytokine balance towards Th2. IFN $\gamma$ gene expression was decreased $(p<0.01)$ while levels of IL-10 and TGF $\beta$ mRNA were similar in both groups. As a consequence, the ratios of $\mathrm{Th} 1 / \mathrm{Th} 2$ cytokines, IFN $\gamma / \mathrm{IL}-10$ or IFN $\gamma / \mathrm{TGF}$ were significantly decreased $(p<0.01)$. We conclude that feeding of otherwise diabetogenic dietary agents during the first week of life, as a minor additive during normal suckling, can induce a protective Th2-dependent oral tolerance response. This may-render the gut immune system resistant to diabetes promoting diets introduced at weaning.

\section{6}

EFFECT OF PROTEIN RESTRICTION ON ALBUMINURIA AND GLOMERULAR FILTRATION RATE IN NIDDM; A RANDOMISED TRIAL L.T.J. Pijls', H. de Vries', A.J.M. Donker ${ }^{2}$ and J.Th.M. van Eijk ${ }^{1}$ 'EMGO-Institute, ${ }^{2}$ Dept. of Internal Medicine, Vrije Universiteit Amsterdam

Protein restriction delays progression of renal disorders in non-diabetic and IDDM patients; does it so in NIDDM without nephropathy? We conducted a randomised trial in 90 NIDDM patients without macroalbuminuria but at risk of progressive albuminuria (i.e. microalbuminuria or at least detectable albuminuria, or diabetes duration $\geq 5 \mathrm{y}$ ). During 24 months the experimental group $(E, n=43)$ received guidance on protein restriction; controls $(C, n=47)$ received usual guidance. Outcomes were albuminuria (mg. $24 \mathrm{~h}^{-1}$, duplicate) and cimetidineinfluenced creatinine clearance as estimate of glomenlar filtration rate (GFR). After 6 months protein intake had become only 0.08 g. $\mathrm{kg}^{-1}(7 \%)$ lower in E than in $\mathrm{C}$; yet, albuminuria had decreased in $\mathrm{E}$ by 12 and increased in $\mathrm{C}$ by 16 $\%(\mathrm{p}=0.02)$. The effect did not differ between normoalbuminuric and microalbuminuric patients. Dose-response analysis over the first 6 months showed that $0.10 \mathrm{~g} . \mathrm{kg}^{-1}$ change in protein intake -animal protein in particularrelated to $9 \%$ change in albuminuria $(\mathrm{p}-0.007)$. Beyond 6 months, protein intake decreased in $\mathrm{C}$; at 24 months it had become $1.07 \pm 0.22$ in $\mathrm{C}$ and $1.10_{ \pm} 0.21 \mathrm{~g} . \mathrm{kg}^{-1}$ in $\mathrm{E}$. We subsequently assessed effects on the longer term, i.e between 6 and 24 months. The initial effect on albuminuria in favour of $E$ was not sustained. GFR, however, decreased more slowly in $\mathrm{E}$ than in C: $3.3 \pm 10$ and $7.6+11 \mathrm{ml} \cdot \mathrm{min}^{-1} .1 .73 \mathrm{~m}^{-2} . \mathrm{y}^{-1}$ respectively $(\mathrm{p}=0.06)$. Surprisingly, not serum, but urinary creatinine developed differently; the latter decreased more in $\mathbf{C}$ than in E. Between protein intake during follow-up and change rate of GFR no doseresponse relationship was observed. We conclude that protein restriction during 24 months in NIDDM patients is hardly feasible, and has no consistent beneficial effect on albuminuria and GPR 
OP 30

\section{Transcriptional Control in $\beta$-Cells}

\section{7}

GLUCOSE STIMULATES TRANSLOCATION OF THE HOMEODOMAIN TRANSCRIPTION FACTOR PDX1 FROM THE CYTOPLASM TO THE NUCLEUS IN PANCREATIC $\beta$ CELLS

C.M. McKinnon, W. M. Macfarlane, Z. M. Felton-Edkins, H. Cragg, R.F.L. James* and K. Docherty. Department of Molecular and Cell Biology, University of Aberdeen, U.K., and *Department of Surgery, University of Leicester, U.K.

Glucose stimulates insulin gene transcription in pancreatic $\beta$ cells through activation of the homeodomain transcription factor PDX1 (Pancreatic/Duodenal homeobox-1) via a stress-activated pathway involving stress-activated protein kinase 2 (SAPK2, also termed $\mathrm{RK} / \mathrm{p} 38$ ). In the present study we show by Western blotting, immunocytochemistry, and electrophoretic mobility shift assay, that in human islets of Langerhans incubated in low glucose ( $3 \mathrm{mM})$ PDX1 exists as an inactive $31 \mathrm{kDa}$ protein localised exclusively in the cytoplasm. Transfer of the islets to high $(16 \mathrm{mM})$ glucose results in rapid (within 10 minutes) conversion of PDX1 to an active $46 \mathrm{kDa}$ form that was present predominantly in the nucleus. This effect of glucose could be mimicked by chemical stress (sodium arsenite), or by over-expression of SAPK2 in the $\beta$-cell line MIN6. The effects of glucose were inhibited by the SAPK2 inhibitor SB 203580, and by wortmannin and LY 294002, which inhibit PI3-Kinase, although the effects of stress (arsenite) were inhibited only by SB 203580. These results demonstrate that glucose regulates the insulin gene promoter through activation and nuclear translocation of PDX1 via the SAPK2 pathway.

\section{8}

GLUCOSE-DEPENDENT TRANSLOCATION OF INSULIN

PROMOTER FACTOR-1 IN MIN6 $\beta$-CELLS.

I. Rafiq and G.A. Rutter. Department of Biochemistry, University Medical School, Bristol BS8 1TD, U.K.

Elevated glucose concentrations stimulate insulin gene transcription in part via the activation of insulin promoter factor-1 (IPF-1, also called PDX-1, STF-1 and IUF-1). Mutations in the IPF-1 gene are linked to an hereditary form of diabetes mellitus (MODY4). In order to determine whether changes in the subcellular distribution of IPF-1 are involved in the activation of the insulin gene by glucose, we have constructed chimaeric cDNAs encoding: (1) IPF-1 plus a c-myc epitope tag and (2) IPF-1 plus enhanced green fluorescent protein (IPF-1.EGFP). When MIN6 $\beta$-cells expressing IPF-1.c-myc were maintained at $3 \mathrm{mM}$ glucose and then analysed by confocal immunocytochemistry, the chimaera was localised largely to the nuclear membrane and nucleoplasm (ratio of nucleoplasmic : nuclear membrane fluorescence $=0.32 \pm 0.089, n=3$ cells). This ratio was increased to $1.22 \pm 0.29(n=4$ cells $)$ after incubation for 90 min. at $30 \mathrm{mM}$ glucose. IPF-1 translocation to the nucleoplasm was associated with a 2.5 fold increase in the activity of a $-260--60$ fragment of the human insulin promoter, assayed in single living MIN6 $\beta$-cells using a firefly luciferase reporter construct. In contrast to myc-tagged-IPF-1, IPF-1.EGFP was localised largely to the nucleoplasm of living MIN6 $\beta$-cells, and was unaffected by incubation at 3 or $30 \mathrm{mM}$ glucose. These data suggest that IPF-1 may be localized to the nuclear membrane of unstimulated cells by an interaction with a specific docking molecule. This interaction, which would be expected to suppress the transactivational potential of IPF-1, can be disrupted by extension of the C-terminus of IPF-1 with EGFP. Elevated glucose concentrations may therefore cause redistribution of IPF-1 to the nucleoplasm by disrupting such an interaction with the nuclear membrane.

\section{9}

SUPPRESSION OF $\mathrm{CA}^{2+} /$ CALMODULIN KINASE II $\delta_{2}$ (CaMkin $\delta_{2}$ ) IN INS- 1 CELLS BY A RETROVIRAL ANTISENSE VECTOR SUPPRESSES INSULIN BIOSYNTHESIS

M. Osterhoff, C. Meier, M. Mơhlig, F. Bikashagi, H. Schatz and A. Pfeiffer, Medizinische Universitătsklinik Bergmannsheil, D-44789 Bochum, Germany

CaMkin II $\delta_{2}$ is highly expressed in INS-1 insulinoma cells at levels similar to those found in rat islets. The importance of the enzyme for insulin secretion has not been clearly established. Methods: Full length CaM kinase II $\delta_{2} \mathrm{cDNA}$ was cloned to yield antisense mRNA into the p50 vector with MPSV LTR, introduced into GPE+86 or PA317 producer cells and supernatants were used for infection of INS-1 cells. After selection with neomycin single clones were isolated and expanded. Immune blots used a polyclonal rabbit antiserum against the association domain of CaMkin II $\delta$, generated against the bacterially expressed protein. Insulin was extracted with the acid ethanol technique and measured by RIA. Results: Of 13 clones generated by insertion of antisense CaMkin II $\delta_{2} 7$ had a completely altered morphology with an egg-like shape and without any fibre outgrowth. The cells grew singly and did not form colonies. Six clones had the normal appearance of INS-1 cells with extensive neurite like fibre outgrowth and formation of confluent cells colonies with extensive intercellular contacts. Immune blots showed normal expression of CaMkin II $\delta_{2}$ in the 6 clones with normal appearance while the enzyme was undetectable by immune blot in the 7 clones with rounded morphology. Determination of insulin levels showed quantities of $1-2 \mu \mathrm{g} / 10^{6}$ cells in normal INS-1 cells and the clones with normal morphology while the 7 clones with rounded morphology had undetectable insulin levels. Analysis of cell growth showed normal growth rates in the cells with CaM kinase II $\delta_{2}$ suppression and an enhanced growth rate in 2 of the 7 clones. Expression of the empty vector or of CaM kinase II $\delta_{2}$ sense clones did not cause rounded morphology or inhibition of insulin biosynthesis. Conclusion: $\mathrm{CaM}$ kin $11 \delta_{2}$ appears to be required for insulin biosynthesis as absence of its expression was associated with absence of insulin in cell extracts. The altered morphology indicates a role for CaMkin II $\delta_{2}$ in cytoskeletal organisation.

\section{0}

AUTOREGULATION OF INSULIN GENE TRANSCRIPTIONAL REPRESSOR C/EBP $\beta$ IN PANCREATIC $\beta$-CELLS

J. Seufert and J. F. Habener, Laboratory of Molecular Endocrinology,

Massachusetts General Hospital, Harvard Medical School, Boston, USA

Supraphysiological glucose levels have been reported to downregulate insulin gene expression in pancreatic $\beta$-cells (glucotoxicity). Previously we have defined the basic leucine zipper transcription factor $C / E B P \beta$ as a repressor of insulin gene promotor activity, which is induced by high glucose levels in vitro and during the development of diabetes in vivo. Here we show that $\mathrm{C} / \mathrm{EBP} \beta$ upregulates its own expression in pancreatic $\beta$-cells by specific binding to defined elements within the C/EBP $\beta$ promoter. 2500 base pairs of the rat $C / E B P \beta$ promoter have been obtained and the transactivational activity was tested in the pancreatic $\beta$-cell line INS- 1 by transient transfection and luciferase reporter gene assays. C/EBP $\beta$-binding sites within the promoter have been mapped by site directed mutagenesis, electrophoretic mobility shift assay (EMSA) and DNase-I footprint analysis. $90 \%$ of the basal promoter activity in INS-1 cells maps to a proximal region of -420 base pairs. In cotransfection experiments C/EBPB transactivates it's own promoter by binding to a proximal CAMP response element (CRE) as a heterodimer with the transcription factor C/ATF (ATF-4). C/ATF is expressed in several pancreatic islet cell lines and in human islets. C/ATF and C/EBP $\beta$ display transactivational synergism on the C/EBP $\beta$ promoter in INS-1 cells, whereas transactivation by phosphorylated cAMP response element binding protein (CREB), which also binds to this CRE, is less pronounced. In gel shift experiments C/EBP $\beta$-C/ATF heterodimers bind with higher affinity to the $\mathrm{CRE}$ in the proximal C/EBP $\beta$ promoter than CREB-homodimers. Finally, in Cos-7 cells transfection of C/ATF leads to upregulation of the endogenous $\mathrm{C} / \mathrm{EBP} \beta$ gene. In summary we provide evidence for a positive autoregulatory loop by which $\mathrm{C} / \mathrm{EBP} \beta$ can upregulate its own promoter activity in pancreatic $\beta$-cells in synergism with C/ATF (ATF-4) and thus enhance its glucose mediated induction, leading to an uncontrolled overexpression and consequently to diminished insulin gene transcription in diabetes mellitus. 
OP 31

\section{New Forms of Insulin Therapy}

\section{1}

\section{LISPRO IN INTENSIVE MANAGMENT OF IDDM: ONE YEAR EXPERIENCE.}

C. Lalli, M. Ciofetta, P. Del Sindaco, P. Brunetti and G. B. Bolli*, DIMISEM Perugia, Italy

In previous studies Lispro insulin has improved 1- and 2-h postmeal blood glucose (BG), but it has been difficult to prove an improvement in \% $\mathrm{HbAlc}$ vs human regular insulin (Hum-R). To establish whether Lispro can be successfully implemented as meal-time insulin in programmes of intensive insulin therapy (IIT) to maintain long-term nearnormoglycemia, $56 \mathrm{IDDM}$ patients were randomized to treatment with either Lispro ( $\mathrm{N}=28)$ or Hum- $\mathrm{R}(\mathrm{N}=28)$ as meal insulin for 1 year (open design, parallel groups). Lispro was injected at mealtime, Hum-R 10-40 min prior to meals (bedtime NPH continued on both occasions). However, with Lispro, NPH was added to Lispro at breakfast (70/30), at lunch $(60 / 40)$ and at supper (80/20) (mixture Lispro/NPH) to optimize pre-meal and bedtime BG. Total daily insulin units were no different in the two treatments, but with Lispro $27 \%$ less short-acting insulin at meals and $29 \%$ more NPH vs Hum-R was needed $(\mathrm{p}<0.05)$. The bedtime $\mathrm{NPH}$ dose was no different. With Lispro, mean daily BG was lower than with Hum-R $(8.2 \pm 0.1$ vs $8.9 \pm 0.1 \mathrm{mmol} / \mathrm{p}, \mathrm{p}<0.05)$; HbAlc was lower $(6.36 \pm 0.09$ vs 6.72 $\pm 0.12 \%$, mean value over 1 year, $p<0.002$ ), and also the frequency of hypoglycemia (hypo, BG $<4.0 \mathrm{mmo} / \mathrm{l}$ ) was lower $(3.9 \pm 0.6$ vs $6.7 \pm 0.9$ episodes/patient-month, $\mathrm{p}<0.05$ ). After 1 year, counterregulatory and symptom responses to experimental, stepped hypo were greater with Lispro vs Hum- $R(p<0.05)$. It is concluded that, if basal insulin is optimally replaced, mealtime injection of Lispro improves the 24-h BG and \% $\mathrm{HbAlc}$ as compared to Hum-R. The improvement can be maintained long-term. Importantly, IIT with Lispro results in less frequent hypo, better awareness and counterregulation to, hypo.

\section{2}

PRE- VERSUS POSTPRANDIAL INSULIN LISPRO: A COMPARATIVE LONG-TERM CROSSOVER TRIAL IN 30 TYPE 1 DIABETIC PATIENTS G. Schernthaner ${ }^{1}$, W. Wein ${ }^{2}$, N. Shnawa ${ }^{2}$, D. Schweighofer ${ }^{2}$ and M. Birkett ${ }^{3}$ Department of Medicine I, Rudolfstiftung Hospital, Vienna, Austria'. Eli Lilly, Austria ${ }^{2}$; Eli Lilly \& Company, Lilly Research Center, Windlesham, UK$^{3}$.

In a previous study in type I diabetics we demonstrated that insulin lispro administered shortly after the start of a low-caloric standard meal displays a glucodynamic control at least as good as regular insulin injected 40,20 or 0 minutes prior to the meal. The current study was designed to investigate the impact of routine postprandial injection of insulin lispro compared to the standard preprandial administration on efficacy and safety using a corssover design where each period lasted for 3 month. 30 type I diabetic patients (between 19-55 years, HbAIc $\leq 8$, informed consent), were randomized to one of the two sequence groups (pre-/postprandial or post/preprandial). In addition all patients performed a time-action profile with a highcaloric standard meal at the end of each period-to compare the glucodynamic control of pre- versus postprandial insulin lispro in this setting. 20 patients were already on intensified treatment with insulin lispro, 10 patients were changed from regular to lispro and enrolled after a stabilisation period. Primary efficacy parameter for the long-term trial was $\mathrm{HbAlc}$, secondary parameters were 8-point blood glucose profiles once monthly, serum cholesterol and lipid levels and fructosamine values, from baseline to last visit. Safety data comprised the number hypoglycemic episodes $(\leq 50$ $\mathrm{mg} / \mathrm{dl}$ ) and adverse events as collected by patient diary. The overall satisfaction of the patients with their treatment was measured by quality-of-life instruments. Results for HbAlc and hypoglycemic events (mean \pm SD): HbAlc baseline value for both groups was $7.21 \pm 0.57$. The change baseline to end-point was:

\begin{tabular}{|l|l|l|l|}
\hline HbAlc & Postprandial $0.09( \pm 0.45)$ & Preprandial $-0.09( \pm 0.45)$ & $\mathrm{p}=0.071$ \\
\hline
\end{tabular} \begin{tabular}{|l|l|l|l|}
\hline Hypo events & Postprandial 31.8 ( \pm 27.1$)$ & Preprandial 32.4 $( \pm 30.6)$ & $p=0.725$ \\
\hline
\end{tabular} No carryover effects were detected, neither for the HbAlc analysis nor for hypoglycemic episodes. In conclusion, this long-term cross-over trial showed that an intensified insulin regimen with insulin lispro injected after the meal is not associated with a significant deterioration in glycemic control nor an increase in the frequency of hypoglycemic events, compared with the standard administration of insulin lispro before the meal.

\section{3}

INSULIN LISPRO IN THE THERAPY OF SULFONYLUREA FAILURE M.E. Trautmann, M.E. Johnson, J.H. Anderson, E.J. Bastyr, L. Vignati, and the IOCE study group, Lilly Research Laboratories, Indianapolis, IN, USA The optimal insulin-containing regimen for patients with Type 2 diabetes at the time of oral agent failure remains speculative. We examined the impact of adding either insulin lispro (LP) before each meal or bedtime NPH insulin to sulfonylurea (SU) versus treatment with mealtime $\mathrm{LP}$ and $\mathrm{NPH}(\mathrm{LP}+\mathrm{NPH})$ at the time of SU failure. 423 patients with Type 2 diabetes (mean age: 60.2+9.6 y., duration of diabetes $10.1+6.6$ y., BMI $29.3+5.5 \mathrm{~kg} / \mathrm{m}^{2}$, HbAlc $9.8+1.7 \%$, means $+\mathrm{SD}$ ) were randomized to one of three treatment arms: 1 . SU (glibenclamide/glyburide) at maximum dose plus preprandial LP, 2. maximum SU plus bedtime NPH or 3. LP plus bedtime NPH without SU. They were evaluated at baseline and two months after the initiation of insulin therapy for treatment success (fasting (FBG)/preprandial glucose $\leq 7.8$ and $2 \mathrm{~h}$ postprandial glucose (PPG) $\leq 10 \mathrm{mmol} / 1$ on $>50 \%$ of measurements on 3 days). In addition to overall rate of occurrence hypoglycemia was analyzed by time of day.

\begin{tabular}{|l|c|c|c|l|}
\hline & LP+SU & NPH+SU & LP+NPH & \multicolumn{1}{|c|}{ Significance } \\
\hline Success (\%) & 24.1 & 23.6 & 28.5 & n.s. \\
\hline FBG (mmol/1) & $9.55 \pm 2.53$ & $\mathbf{8 . 0 5 \pm 2 . 7 0 ^ { * }}$ & $9.09 \pm 2.60$ & ${ }^{*} \mathrm{p}<.05$ \\
\hline PPG (mmol/1) & $9.11 \pm 2.30$ & $11.79 \pm 3.22^{*}$ & $9.12 \pm 2.56$ & ${ }^{*} \mathrm{p}<.05$ \\
\hline HbAlc (\%) & $8.36 \pm 1.32^{*}$ & $\mathbf{8 . 7 4 \pm 1 . 5 2 ^ { * }}$ & $8.54 \pm 1.42^{*}$ & ${ }^{*} \mathrm{p}<.05$ vs. baseline \\
\hline Hypo/pat/mo. & $0.98 \pm 1.74$ & $0.75 \pm 1.5$ & $1.17 \pm 2.4$ & n.s. \\
\hline Midnight-6am & $0.00 \pm .00^{*}$ & $0.13 \pm .47$ & $0.10 \pm .37$ & ${ }^{*} \mathrm{p}<.05$ \\
\hline 6 am - Noon & $0.22 \pm .77$ & $0.34 \pm .89$ & $0.45 \pm 1.60$ & n.s. \\
\hline Noon - 6 pm & $0.48 \pm 1.0$ & $0.20 \pm .70^{*}$ & $0.41 \pm .91$ & ${ }^{*} \mathrm{p}<.05$ \\
\hline 6pm-midnight & $0.27 \pm .74$ & $0.08 \pm .35$ & $0.20 \pm .69$ & n.s. \\
\hline
\end{tabular}

Insulin therapy significantly reduced $\mathrm{HbAlc}$ from baseline with low risk of hypoglycemia in all groups. Similar numbers of patients in each group met the success criteria within two months. Postprandial glucose was improved significantly with LP therapy in combination with either NPH or SU. LP+SU produced significantly less nocturnal hypoglycemia than any NPH-containing regimen. Addition of LP to SU offers a treatment alternative which significantly lowers $\mathrm{HbAlc}$ and postprandial glucose and minimizes noctumal hypoglycemia.

\section{4}

INSULIN LISPRO: SAFE AND EFECTIVE TREATMENT OPTION FOR GESTATIONAL DIABETES.

S.Ilic, L.Jovanovic, D. Pettitt, M. Gutierrez and E.J.Bastyr II. Sansum Medical Research Institute, 2219 Bath Street, Santa Barbara, CA and Indianapolis, IN.

Postprandial hyperglycemia is strongly associated with increased fetal and neonatal morbidity, and the main goal of management of the gestational diabetes should be the achievement of normoglycemia. Therefore we designed our study to compare regular insulin with lispro insulin for the effectivnes of the treatment of woman with gestational diabetes mellitus (GDM).

We followed 35 woman with GDM who failed to achive good glucose control with diet alone. Study was open labeled, and patients were randomized to receive either regular or lispro insulin at the dose of 0.179 U/kg body mass. Patients kept an eight- point daily diary and were evaluated for $\mathrm{HbAlc}$ and antibody testing bi-weekly. All data were analysed using descriptive statistics, and a 2-sample t-test assuming equal variance. Results are shown in the table:

\begin{tabular}{|l|l|l|l|l|}
\hline & $\begin{array}{l}\mathrm{HbA}_{1 \mathrm{o}} \text { at } \\
\text { enrollment }\end{array}$ & $\begin{array}{l}\mathrm{HbA}_{10} \\
6 \mathrm{w} \text { after }\end{array}$ & $\begin{array}{l}\text { Change in } \\
\mathrm{HbAlc}\end{array}$ & Antibody testing \\
\hline LISPRO & $5.49 \pm 0.38$ & $5.12+0.50$ & $0.29(5.66 \%)^{* *}$ & $1.26 \pm 0.69$ \\
\hline REGULAR & $5.35+0.36$ & $5.31+0.57$ & $0.05(2.67 \%)$ & $1.96 \pm 0.31$ \\
\hline p value & $\mathrm{NS}$ & $<0.05$ & $<0.05$ & $<0.05$ \\
\hline ** p=0008
\end{tabular}

** $\mathrm{p}=.0008$

As shown above, woman with GDM on lispro therapy achieved good glucose control defined by significantly lower levels of HbAlc while regular insulin did not. Based on all glucose records the lispro group had fewer hypoglycemic events with overall better glucose control. At the same time the antibody testing showed that insulin lispro is less immunogenic, although levels in both groups were within reference range. According to data shown above we may conclude that lispro insulin should be considered the optimal treatment in patients with GDM. 
185

SIGNIFICANTLY IMPROVED POSTPRANDIAL GLYCAEMIC CONTROL WITH THE NOVEL RAPID-ACTING INSULIN ASPART

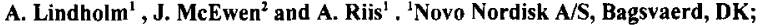
'Drug Development (Scotland) Ltd, Ninewells Hospital and Medical School, Dundee, UK

Introduction: Insulin aspart (Asp ${ }^{828}$, previously $X 14$ )is a novel rapid-acting insulin analogue developed to resemble unmodified human insulin in all biological aspects, but with an earlier onset of action and a shorter duration of effect. Design: The present study was a double-blind, double-dummy injection, crossover trial to compare the postprandial serum glucose control of insulin aspart versus that of human insulin in 24 subjects with Type 1 diabetes. In this meal test study insulin aspart was injected s.c. immediately prior to a standard meal, whereas human insulin was injected s.c. 30 minutes before the meal or immediately before the meal. Results: The postprandial serum glucose excursion over 4 hours was significantly smaller with insulin aspart, being $68 \%$ of that of human insulin injected 30 minutes before the meal $(p<0.0001)$ and $84 \%$ of that of human insulin injected immediately before the meal $(p<0.02)$. Regarding pharmacokinetics insulin aspart was absorbed faster with time to maximum concentration approximately twice as fast as human insulin ( $39 \pm 17$ minutes versus $85 \pm 62$ minutes, $p<0.01)$ and maximum concentration approximately twice as high $(493 \pm 257 \mathrm{pmol} / \mathrm{l}$ versus $239 \pm 131 \mathrm{pmol} / 1, \mathrm{p}<0.0001$ ). Insulin aspart was well tolerated. Conclusion: Insulin aspart improved postprandial glucose control compared to human insulin, and it remains to be elucidated whether this may apply to overall glycaemic control in a treatment situation.
186

NOCTURNAL GLUCOSE REGULATION WTH NPH OR SEMILENTE@ INSULIN ON IDDM WITH AND WITHOUT SEVERE EARLY MORNING HYPERGLYCAEMIA.

J. Wörle, C. Neumann, R. Renner, R. Landgraf" C Strasburger", K-D Hepp, Diabeteszentrum III. Med. Klinik München-Bogenhausen and Medizinische Klinik, Klinikum Innenstadt, Ludwig Maximilians-Universität München', Germany

NPH-ineffectiveness is defined as a blood glucose rise of more than $50 \mathrm{mg} / \mathrm{dl}$ between 2 and 6 a.m. which cannot sufficiently be compensated by increasing the NPH (neutral protamine Hagedorn) insulin -dose combined with a higher risk of nocturnal hypoglycaemic episodes. In the present study (randomised, double blind cross over fashion, statistical analysis with ANOVA) we investigated the influence of a noctumal Semilente(B) (amorphous zinc insulin) versus NPH insulin therapy on blood glucose, insulin levels and counterregulatory hormones. Blood samples were taken every 30 min by a heparinized catheter system for continuous collection. We compared 14 patients with NPHineffectiveness (NPHI) with 10 patients with constant nocturnal glucose levels $\pm 20 \mathrm{mg} / \mathrm{dl}$ (control). The NPHI showed a blood glucose increase between 2 and 6 a.m. from $142 \pm 12,6$ to $213,9 \pm 14,5$, the controls from $138,7 \pm 12,1$ to $144,5 \pm 12,8 \mathrm{mg} / \mathrm{dl}(\mathrm{p}=0,001)$. Obtaining a comparative bedtime insulin dose (NPH $9,2 \pm 0,5$, control $9,3 \pm 0,5$ ) the amount of regular insulin for dinner was significantly higher in the NPHI $(p=0,04)$. The daily insulin dose in the NPHI was $41,2 \pm 2,9$, control $33,7 \pm 2,4$ I.U. During Semilente(8) therapy 8 out of 10 patients in the controls had to receive $10 \mathrm{~g}$ carbohycrates at 4 a.m. because of blood glucose levels lower than $50 \mathrm{mg} / \mathrm{d}$, in the NPHI 4 out of $14(p=0,014)$.

From 1 a.m. onwards senum insulin levels under Semilente $($ were significantly higher compared to NPHinsulin in both groups $(p<0,001)$. The NPHI had significantly higher insulin levels $(0<0,01)$ in both nights, under NPH they showed a continuous decrease of insulin concentration from $22,14 \pm 3,6$ to $5,5 \pm 0,6 \mathrm{\mu U} / \mathrm{ml}$. The controls showed constant insulin levels during treatment with NPH: 10 p.m. $9,2 \pm 1,7,2$ a.m. $9,1 \pm 1,2,6$ a.m. $6,7 \pm 1,8$ a.m. $5,1 \pm 0,7$ $\mathrm{HU} / \mathrm{ml}(p<0,001)$. There were no significant differences for glucagan, cortisol, catecholamines, growth hormone, IGF-1, IGFBP-3 and insulin antibodies.

Summary: Injected subcutaneously at 11 p.m. Semilente (B) shows its maximum effect on blood glucose between 4 and 6 a.m. Patients without NPH ineffectiveness bear an avoidable risk for hypoglycaemic episodes. Patients with NPH ineffectiveness need higher daily insulin doses. Differences in growth hormone levels do not explain the insulin resistance.
187

EFFICACY AND SAFETY OF HOE 901 IN PATIENTS WITH TYPE 1 DIABETES : A FOUR-WEEK RANDOMISED, NPH INSULIN-CONTROLLED TRIAL. T. R. Pieber ${ }^{1}$, I. Eugene-Jolchine ${ }^{2}$ and E. Derobert ${ }^{2}$ for the European Study Group of HOE 901 in Type 1 Diabetes, 'Dep. Internal Medicine, University Graz, Austria and ${ }^{2} \mathrm{HMR}$, Paris, France

HOE 901 is a biosynthetic insulin analogue $\left(21^{\mathrm{A}}-\mathrm{Gly}-30^{\mathrm{B}} \mathrm{a}-\mathrm{L}-\mathrm{Arg}-30^{\mathrm{B}} \mathrm{b}-\mathrm{L}-\mathrm{Arg}\right.$ human insulin) with a prolonged action compared to NPH insulin. The aim of this first multinational, randomised, parallel-group trial was to compare two HOE 901 formulations with NPH in type 1 patients previously treated with a basal-bolus regimen. The two formulations differ only in zinc content, 30 or $80 \mu \mathrm{g} / \mathrm{ml}$. The study could be blinded only for the 2 formulations because HOE 901 (clear solution) can be distinguished from NPH. In addition to pre-meal injections of regular insulin, patients received either HOE 901 at bedtime or NPH once daily at bedtime or twice daily (morning and bedtime) according to the patients' prestudy regimen. The primary endpoint was fasting plasma glucose (FPG) after 4 weeks. A total of 42 centres in 9 European countries treated 333 patients (mean age 36 years, mean (SD) FPG : 12.0 (4.6) mmol/l, HbA I c : $7.96(1.15) \%$ ). FPG was significantly lower on HOE 901 (final adjusted mean value $10.11 \mathrm{mmol} / \mathrm{l}$ vs $12.00 \mathrm{mmol} / \mathrm{l}$ $p=0.0001$, ITT analysis $)$ as was fasting self-monitored blood glucose $(7.13 \mathrm{mmol} / \mathrm{l}$ vs $7.92 \mathrm{mmol} / \mathrm{l}, \mathrm{p}=0.0020$ ). $\mathrm{HbAlc}$ also showed a significant reduction on $\mathrm{HOE}$ $901(-0.14 \%)$ versus NPH ( $\mathrm{p}=0.0299)$. The overall frequency of hypoglycaemia did not differ but the frequency of nocturnal hypoglycaemia was significantly $(p=0.0037)$ lower on HOE $901(36 \%$ vs $55 \%)$. However, when compared to NPH once or twice daily, this effect on nocturnal hypoglycaemia was significant only vs NPH once daily. Both HOE 901 formulations yielded similar results for efficacy and hypoglycaemia. The pattem of adverse events and injection site reactions was similar to NPH. In conclusion, this study indicates that HOE 901 achieves better control of fasting glucose levels and $\mathrm{HbAlc}$ over four weeks and there is a possiblc safety improvement in terms of nocturnal hypoglycaemia. Both HOE 901 formulations appeared to be equally good candidates for use in Phase III.

\section{8}

TIME-ACTION PROFILE OF THE SOLUBLE, FATTY ACID ACYLATED LONG-ACTING INSULIN ANALOGUE NN304

K. Sinha, C. Weyer, M. Loftager", S. Hirschberger"', T. Heise, L. Heinemann; Dep. of Metabolic Dis. A. Nutrition, Heinrich-Heine-University Düsseldorf, Germany; Novo Nordisk, "Denmark, "Germany

Attachment of a free fatty acid to the insulin molecule resulted in the novel long-acting insulin analogue NN304. We studied the pharmacokinetic and pharmacodynamic properties of subcutaneously injected NN304 in comparison to NPH-insulin during euglycaemic glucose clamps in 11 healthy volunteers. On three study days $\mathrm{NN} 304$ was injected in three different doses $(0.15,0.3,0.6 \mathrm{U} / \mathrm{kg}$ body weight $)$, while NPH-insulin $(0.3 \mathrm{U} / \mathrm{kg})$ was injected in identical dose on two separate study days. Subcutaneous injection of NN304 resulted in a linear and proportional increase in total NN304 concentrations $\left(\mathrm{AUC}_{0-1440}: 0.15 \mathrm{U} / \mathrm{kg}: 344 \pm 43,0.3 \mathrm{U} / \mathrm{kg}: 666 \pm 82,0.6 \mathrm{U} / \mathrm{kg}\right.$ $\left.1295 \pm 210 \mathrm{nmol} / \mathrm{l}^{*} 1440 \mathrm{~min} ; \mathrm{p}<0.001\right)$. Maximal concentrations $(609 \pm 140$, $1046 \pm 283,2033 \pm 460 \mathrm{pmol} / \mathrm{l} ; \mathrm{p}<0.001$ ) were registered after $4-6 \mathrm{~h}$. The metabolic response induced by s.c. injection of NN304 did not show the pronounced peak seen with NPH-insulin: GIR $_{\max } 0.15 \mathrm{U} / \mathrm{kg}$ : $2.3 \pm 1.1,0.3$ $\mathrm{U} / \mathrm{kg}: 3.2 \pm 1.1,0.6 \mathrm{U} / \mathrm{kg} ; 3.3 \pm 1.4$ vs. $4.4 \pm 1.8 \mathrm{mg} / \mathrm{kg} / \mathrm{min}(0.3 \mathrm{U} / \mathrm{kg} \mathrm{NPH}-$ insulin; mean of both study days; NPH-insulin vs. $0.15 \mathrm{U} / \mathrm{kg} \mathrm{NN304} \mathrm{p}<0.05$ ). The three different doses of NN304 induced a significantly different glucose consumption in the first $720 \mathrm{~min}$ after injection $\left(\mathrm{AUC}_{0-720} 1.05 \pm 0.62\right.$, $1.89 \pm 0.80,1.73 \pm 0.82 \mathrm{~g} / \mathrm{kg}^{*} 720 \mathrm{~min} ; \mathrm{p}<0.05$ for $0.15 \mathrm{U} / \mathrm{kg}$ ), but not over the whole study period $\left(\mathrm{AUC}_{0-1440} 1.79 \pm 1.12,3.08 \pm 1.34,2.81 \pm 1.40 \mathrm{~g} / \mathrm{kg}^{*} 1440\right.$ $\mathrm{min})$. The area under the GIR profile registered with $\mathrm{NPH}$-insulin $(4.07 \pm 2.20$ $\mathrm{mg} / \mathrm{kg}^{*} 1440 \mathrm{~min}$ ) was higher than that of NN304 in the lowest dose ( $\mathrm{p}<0.05$ ). This first study with the soluble long-acting insulin analogue NN304 in humans showed, that subcutaneous injection of different doses resulted in an increase in total NN304 concentration in a linear dose-response relationship. NN304 induced a more even metabolic effect than NPH-insulin, however, no clear-cut dose-response relationship could be demonstrated. 
OP 32

\section{Neuropathy}

ACTIVATION OF POTASSIUM CHANNELS CORRECTS NERVE DYSFUNCTION IN DIABETIC RATS

TC Hohman ${ }^{1}$, MD Basso', K-D Lai', D Banas ${ }^{1}$, R. Graceffa', T. Argentieri' ${ }^{1}$, J Butera $^{1}$ NE Cameron ${ }^{2}$ and MA Cotter. ${ }^{2}$ Wyeth-Ayerst, Princeton, NJ, USA and ${ }^{2}$ Biomedical Sciences, Aberdeen University, Scotland, UK

Some vasodilators have been shown to correct nerve conduction velocity (NCV) and endoncurial blood flow (EBF) deficits in diabetic rats. Whether vasa nervorum has ATP-sensitive $K$ channels (K-channels) that mediate vasodilation, or whether activation of $\mathbf{K}$ channels could modulate peripheral nerve function is not known. The aim of this study was to examine the effects of two weeks of treatment with the $\mathrm{K}$ channel opener, WAY $135201(3.0 \mathrm{mg} / \mathrm{kg} / \mathrm{day})$, on sciatic motor NCV, EBF, ouabain-sensitive $\mathrm{Na}^{+}-\mathrm{K}^{+}$ATPase activity (ATPase) and reduced glutathione levels (RG) after 6 weeks of untreated streptozotocin-diabetes in rats. $\mathrm{EBF}, 49 \pm 13 \%( \pm 1 \mathrm{SEM})$ reduced $(\mathrm{p}<0.001)$ by diabetes, was restored to the upper quartile of the nondiabetic range by WAY $135201(\mathrm{p}<0.001)$. When compensated for the lower blood pressures in untreated and treated diabetic groups $(17 \pm 14 \%$ and $24 \pm 14 \%$ diminished, respectively; $<<0.01)$, there was a $39 \pm 13 \%$ vascular conductance deficit, $(p<0.001)$ with diabetes whereas conductance was $73 \pm 12 \%$ supernormal $(p<0.01)$ with WAY 135201 treatment. $\mathrm{NCV}$, reduced from $64.3 \pm 10.6 \mathrm{~m} / \mathrm{s}$ to $51.2 \pm 10.6 \mathrm{~m} / \mathrm{s}$ by diabetes $(\mathrm{p}<0.001)$, was corrected by WAY $135201(63.8 \pm 10.6 \mathrm{~m} / \mathrm{s} ; \mathrm{p}<0.001)$. Diabetes diminished ATPase from $16.1 \pm 10.7$ to $8.7 \pm 11.0 \mathrm{mM} \mathrm{ADP} / \mathrm{h} / \mathrm{mg} \mathrm{prt}$. (p<0.001) and WAY 135201 corrected this deficit $(15.4 \pm 10.9 \mathrm{mM} \mathrm{ADP} / \mathrm{h} / \mathrm{mg}$ prt.; $\mathrm{p}<0.001)$. Diabetes also caused a $28.5 \%$ decrease in nerve RG levels $(p<0.01)$ that were unaffected by WAY 135201 treatment. Similar effects on nerve function and biochemistry were observed with a second $K$ channel opener, celikalim. While these studies demonstrate the marked beneficial effects of $\mathrm{K}$ channel activators in experimental diabetic neuropathy, the data also caution that the use of $\mathrm{K}^{+}\left(\mathrm{K}^{+} \mathrm{ATP}\right)$ channel antagonists in glycemic control could theoretically have adverse neurological effects.

\section{0}

Down-Regulation of Sciatic Nerve $\mathrm{Na}^{+}$-Taurine $\mathrm{Co}$-Transporter in Streptozotocin-Diabetic Rats-a potential mediator of glucose-toxicity. R.Pop-Busui, R.Towns, D.Larkin and M.Stevens, Univ.of Michigan, Ann Arbor,MI, USA.

Increased oxidative stress and alterations in glucose-sensitive signal transduction pathways have been invoked in the pathogenesis of experimental diabetic neuropathy (EDN). Activation of the polyol pathway in diabetes and nerve sorbitol accumulation exacerbates oxidative stress and results in depletion of the antioxidant, calcium modulator, neurotrophic agent and neurotransmitter taurine, We have previously shown that prevention of nerve taurine depletion in streptozotocindiabetic (STZ-D) rats corrects neuro-vascular dysfunction, prevents deficits in $\mathrm{Na}, \mathrm{K}$ ATPase activity and attenuates motor and sensory NCV slowing. In STZ-D rats, nerve taurine depletion may result from down-regulation of the energy dependent $\mathrm{Na}^{+}$ -taurine co-transporter (TT). The aim of this study was therefore to assess the effects of diabetes on TT peptide abundance in the sciatic nerve in STZ-D rats. To assess changes in TT protein levels, polyclonal antibodies were generated by immunizing chickens with synthetic peptides corresponding to antigenic $\mathrm{N}$-and $\mathrm{C}$-terminal sequences of the TT. Sciatic nerve proteins, from $4 \mathrm{wk}$ STZ-D and control (C) rats, were isolated and separated by electrophoresis on a $7.5 \%$ SDS-polyacrylamide gel and transferred to a PVDF membrane. A $70-\mathrm{kD}$ protein (the expected TT molecular weight) was detected by Western blot analysis when probed with both the $\mathrm{N}$ - and $\mathrm{C}$ terminal TT antibodies. No band was detected when preimmune chicken IgY was used to probe the blots. The specificity of the antibodies was confirmed by preincubation with the corresponding peptides (which effectively competed) and irrelevant peptides (which had no effect). The abundance of the $70 \mathrm{kD}$ protein in STZ-D vs $C$ rats was compared using a phosphorimager. In STZ-D rats, sciatic nerve TT peptide abundance was decreased 4.5 fold compared with $C$ rats. In conclusion, depletion of sciatic nerve taurine may result in NCV slowing in EDN from down-regulation of the TT. Further studies are required to elucidate the potential mechanisms leading to TT protein depletion.

\section{1}

ALDOSE REDUCTASE (AR) INHIBITION INDUCES AR AND TAURINE TRANSPORTER GENE TRANSCRIPTION IN GLUCOSE-EXPOSED RETINAL PIGMENT EPITHELIAL (RPE) CELLS. M. Stevens and D. Larkin, Univ. of Michigan, Ann Arbor, MI, USA

Glucose-induced depletion of intracellular taurine has been implicated in the pathogenesis of diabetic complications in the rat and is prevented by AR inhibitors (ARI's). Excess intracellular sorbitol may decrease inward Na-taurine cotransport. ARI's improve nerve function and structure in diabetic neuropathy, but in man, poten ARI's are required. The effect of high glucose exposure and the ARI sorbinil $(10 \mu \mathrm{M})$ on AR and human taurine transporter (hTT) gene transcription were studied in high AR-expressing RPE 91 cells. Low AR-expressing RPE 45 and 47 cells or high ARexpressing RPE 91 cells were exposed to 5 or $20 \mathrm{mM}$ glucose \pm ARI for $48 \mathrm{hr}$. AR and hTT mRNA levels were quantitated by filter hybridization with partial human AR and hTT CDNA probes and normalized to $B$-actin. AR and hTT gene transcription were measured in RNA from radiolabeled nuclei and hybridized with filters containing donatured single-stranded DNA targets for human AR, hTT, pBluescript and human genomic DNA (for normalization). Data are expressed relative to RPE 45 in $5 \mathrm{mM}$ glucose and confirmed in an independent study.

\begin{tabular}{l|ccccc} 
RPE Cell Line \# & 45 & 47 & 91 & 91 & 91 \\
Glucose (mM) \pm ARI & 5 & 5 & 5 & 20 & $20+$ ARI \\
\hline AR Transcription & 1 & $1.85 \times$ & $4.13 \times$ & $1.63 \times$ & $10.33 \times$ \\
hTT Transcription & 1 & $0.9 \times$ & $0.1 \times$ & $0.1 \times$ & $4.8 \times$
\end{tabular}
AR and $h T T$ mRNA abundance paralleled transcription. Sorbitol was raised and taurine decreased in RPE 91 cells in 5 and $20 \mathrm{mM}$ glucose and both were normalized by ARI. AR transcription paralleled AR mRNA and activity, implicating increased AR gene transcription as the basis of AR over-expression in RPE 91 cells. Conversely, hTT transcription and activity (not shown) were suppressed in RPE 91 cells even in $5 \mathrm{mM}$ glucose. Exposure to $20 \mathrm{mM}$ glucose suppressed AR gene transcription 3-fold in RPE 91 cells, consistent with osmotic effects of excess sorbitol but did not furthe suppress hTT gene transcription. Inhibition of sorbitol accumulation by ARI in 20 $\mathrm{mM}$ glucose induced AR and hTT gene transcription more than 6-fold and 48-fold, respectively to levels in excess of $5 \mathrm{mM}$ glucose. Thus excess sorbitol suppresses hTT gene transcription in high AR expressing cells. Sorbitol pathway blockade by ARI's may produce a compensatory induction of AR gene transcription in high AR expressing human cells, which may require higher doses or more potent ARI's.
192

THE PRIMARY PATHOLOGY AND PATHOGENESIS OF HUMAN DIABETIC NEUROPATHY.

R.A.Malik, D.Walker, L.H.C. Santos, L.Chimelli, A.A.Barriera and A.J.M. Boulton, Department of Medicine, Manchester Royal Infirmary, Department of Neurology and Neuropathology, Curitiba, Brazil.

The primary pathology and pathogenesis of human diabetic neuropathy are unclear. We have studied 28 children with Type I diabetes aged $13.0 \pm 2.6 \mathrm{yr}$., duration of diabetes $8.5 \pm 3.0$ yr. compared to 28 age matched control subjects. Sural nerve biopsy was performed in 8 patients who fulfilled the minimal criteria for neuropathy based on a significant reduction in peroneal nerve motor conduction velocity $\left(\mathrm{ms}^{-1}\right)(47.1 \pm 6.1$ v $54.1 \pm 5.2, \mathrm{p}<0.007)$ and amplitude $(\mu v)(6.2 \pm 2.3 \vee 9.2 \pm 3.4, p<0.03)$, and sural nerve conduction velocity $\left(\mathrm{ms}^{-1}\right)(35.1 \pm 2.2 \mathrm{v} 45.2 \pm 3.6, \mathrm{p}<0.0001)$ and amplitude $(\mu \mathrm{v})(19.1 \pm$ 11.9 v $31.9+15.6, p<0.04)$. All morphometric data were compared with 5 age matched control subjects. Myelinated fibre density (no $\mathrm{mm}^{-2}$ ) did not differ $(p=0.14)$ between diabetic patients $(9438 \pm 1673)$ and control subjects $(8200 \pm$ 1532). However, teased fibre analysis revealed paranodal abnormalities (16.4 \pm 4.1 v $3.5 \pm 1.0, p<0.01)$ and segmental demyelination $(19.9 \pm 10.2 \times 1.5 \pm$ $0.3, \mathrm{p}<0.01)$ without axonal degeneration $(3.0 \pm 1.8 \times 1.4 \pm 1.2, \mathrm{p}=0.6)$. Myelinated fibre and axonal area did not differ between diabetic patients and control subjects. Diabetic patients demonstrated a reduction in endoneurial capillary luminal area $(12.6 \pm 3.2 \vee 24.9 \pm 4.7, p<0.09)$ and an increase in the endothelial cell profile number $(4.6 \pm 0.2 \times 3.9 \pm 0.2, p<0.03)$ and basement membrane area $(92.7 \pm 9.7 \times 41.1 \pm 5.2, p<0.004)$ without any change in the endothelial cell area $(39.0+3.4 \vee 37.6+7.5 p=0.94)$, pericyte nuclear no. $(0.61 \pm 0.1 \vee 0.6 \pm 0.03, \mathrm{p}=0.82)$ or endothelial/pericyte nuclear ratio $(2.5 \pm$ 0.4 v $2.2 \pm 0.2, p=1.0$ ). The primary pathology of human diabetic neuropathy is paranodal and segmental demyelination without axonal atrophy or axonal degeneration and fibre loss. The early presence of endoneurial capillary abnormalities provides strong support for the role of microangiopathy in the pathogenesis of human diabetic neuropathy. 
193

NEUROPATHY ASSESSMENT BY THE MICHIGAN NEUROPATHY SCREENING INSTRUMENT (MNSI) IN THE POST-DCCT FOLLOW-UP EDIC COHORT D. Greene, C. Martin, E. Feldman, M. Stevens, and P. Cleary for the EDIC Study Group, University of Michigan, Ann Arbor, MI, USA

Clinical research in diabetic polyneuropathy (DPN) often employs complex and difficult end-points. By the end of the Diabetes Control and Complications Trial (DCCT), intensive (Int) glycemic control reduced by $60 \%$ the risk of developing DPN, defined by a neurologist's exam and abnormal electrophysiologic or autonomic tests. In the Epidemilogy of Diabetes Intervention and Complications (EDIC), the follow-up of the DCCT cohort, DPN was monitored by the nurse coordinator or diabetologist with an annual 10-min MNSI. This included a brief physical exam (inspection for foot deformity, dry skin, infection, fissure, ulceration; present-reduced-or-absent ankle reflex; vibration perception $[128 \mathrm{~Hz}$ tuning fork $]$ and pressure sensation $[10 \mathrm{~g}$ filament]). A 15 -item questionaire was self-administered. DPN in EDIC was defined by $\geq 2$ abnormalities on MNSI exam (MNSIE+), or $\geq 7$ questions positive (MNSIQ+). In the first 4-yr of EDIC, mean HbAlc in Int and conventional (Con) treatment ( $\mathrm{Rx}$ ) groups converged $(7.4$ vs $9.1 \%$ at $\mathrm{DCCT}$ end, 8.2 vs $8.3 \%$ at EDIC yr 4). MNSI results were stratified by DCCT DPN status ( + ), DCCT Rx, and by $1^{\circ}$ and $2^{\circ}$ DCCT cohorts (defined by the presence/absence of retinopathy, and diabetes duration $\leq 5$ or $>5 \leq 15 \mathrm{yr}$ at entry). Based on MNSIE+, DCCT subjects originally assigned to Int $\mathrm{Rx}$ continued to have a lower prevalence of DPN than Con Rx sujbects $(20.2 \% \rightarrow 24.6 \%$ for Int vs. $33.6 \% \rightarrow 39.4 \%$ for Con $\mathrm{Rx}$ over the 4 yr of EDIC follow-up in the $2^{\circ}$ cohort, $\mathrm{p}<0.006$ ). MNSIE+ was also less common in Int $\mathrm{Rx}$ subjects for the $1^{\circ}$ cohort, and in subjects who had no neuropathy based on the DCCT classification in both cohorts. We conclude that the MNSIE can serve as a measure of DPN and that Int Rx for $6.5 \mathrm{yr}$ in the DCCT continues to have a beneficial effect on DPN for as long as $4 \mathrm{yr}$ after conclusion of the study.

\section{5}

ERECTILE DYSFUNCTION IN PATIENTS WITH DIABETES: META-ANALYSIS OF 9 SILDENAFIL (VIAGRA ${ }^{\text {TM }}$ ) STUDIES D.E. Price, Morrison Hospital, Swansea, West Glamorgan, UK. Erectile dysfunction (ED) is a common problem in men with diabetes mellitus (DM). Sildenafil, a selective inhibitor of phosphodiesterase type 5 , is an effective and well-tolerated oral treatment for broadspectrum ED. This analysis summarizes the efficacy and safety of sildenafil in men with ED and DM. A total of 633 males (mean age $57 \mathrm{yr}$ ) with ED and DM (21\% type I; $79 \%$ type II) received sildenafil $(\mathrm{N}=388)$ or placebo $(\mathrm{N}=245)$ for 6-26 weeks in 9 double-blind, placebocontrolled studies. The usual dose of sildenafil was $25-100 \mathrm{mg}$. Efficacy was assessed using Questions 3 (Q3: frequency of penetration) and 4(Q4: frequency of maintained erection after penetration) of the International Index of Erectile Function (IIEF) and a global efficacy question (GEQ: Did the treatment improve your erections?). Responses to Q3 and Q4 were graded from 0 to 5, with higher scores indicating better function. Efficacy results are shown in the table below. The majority of adverse events (AEs) were mild or moderate, with headache. dyspepsia, and flushing the most commonly reported all-causality AEs. Only 1 patient on sildenafil discontinued due to a treatment-related AE.

\begin{tabular}{lcccc}
\hline Variable & \multicolumn{2}{c}{ Baseline } & \multicolumn{2}{c}{ End of treatment } \\
& Placebo & Sildenafil & Placebo & Sildenafil \\
\hline Q3 & 1.55 & 1.58 & 1.85 & $2.86^{*}$ \\
Q4 & 1.34 & 1.36 & 1.54 & $2.66^{*}$ \\
\hline GEQ (\% yes) & - & - & $15 \%$ & $59 \%^{*}$ \\
\hline
\end{tabular}

*P value sildenafil vs placebo $<0.0001$

These results indicate that oral sildenafil is an effective and welltolerated treatment for ED in men with DM.
194

AUTONOMIC NEUROPATHY AND THE CARDIOVASCULAR RISK: THE EURODIAB IDDM COMPLICATIONS STUDY

P Kempler $^{1}$, S Tesfaye ${ }^{2}$, N Chaturvedi ${ }^{3}$, LK Stevens ${ }^{3}$, JD Ward ${ }^{2}$, JH Fuller ${ }^{3}$ and the EURODIAB IDDM Study Group. ${ }^{1}$ I.Dept. of Medicine, Semmelweis University, H-1083 Korányi S u 2/a Budapest, Hungary, ${ }^{2}$ Royal Hallamshire Hospital, Sheffield, UK, ${ }^{3}$ Dept. of Epidemiology and Public Health,University College London, UK

Autonomic neuropathy is associated with poor prognosis. However, prevalence data are conflicting and potential risk factors has not been definitely identified up to now. The EURODIAB IDDM Complications Study involved the examination of patients from 31 centres in 16 European counties. Data of 3007 patients were available for the present evaluation. Symptoms and two tests of autonomic function (response of heart rate $/ 30 / 15$ ratio/ and blood pressure from lying to standing) were assessed. The prevalence of autonomic neuropathy was $47 \%$ with no sex but some geographical differences. Significant correlations were observed between the presence of abnormal $30 / 15$ ratio and age $(p<0,01)$, duration of diabetes $(\mathrm{p}<0,0001)$, quality of metabolic control ( $\mathrm{p}<0,0001)$, the presence of retinopathy ( $<0,0001)$, micro- and macroalbuminuria ( $\mathrm{c}<0,0001)$, cardiovascular disease ( $<0,0001)$, severe hypoglycaemia $(\mathrm{p} \ll 0,05)$ and severe ketoacidosis $(\mathrm{p}<0,0001)$. Cardiovascular risk factors such as smoking $(p<0,0001)$, HDL-cholesterol $(p<0,01)$, total cholesterol/HDL cholesterol ratio ( $p<0,001)$, fasting triglyceride $(\mathrm{p} 0,001)$ and body weight $(\mathrm{p}<0,001)$ were also related to abnormal $30 / 15$ ratio. Data were adjusted for age, duration of diabetes and HbAlc. Conclusions: Autonomic neuropathy is one of the most frequent complications in Type 1 diabetes. The study has identified previously known and new potential risk factors which may be important for the development of risk reductions strategies.

\section{6}

DEFECTIVE CORPUS CAVERNOSUM FUNCTION IN DIABETIC RATS: EFFECTS OF ANTIOXIDANTS

M.A. Cotter, A. Keegan and N.E. Cameron. Biomedical Sciences, Aberdeen University, Scotland UK.

Diabetes is associated with impotence in animal models and patients. An invitro rat corpus cavernosum preparation was used to examine the effects of streptozotocin-induced diabetes and treatment with the oxygen free radical scavenger lipoic acid (LA; $300 \mathrm{mg} \mathrm{kg}^{-1}$ day-1 $^{-1}$ ) or the transition metal chelator, trientine, (TRI; $20 \mathrm{mg} \mathrm{kg}^{-1}$ day $^{-1}$ ). In phenylephrine-precontracted corpus cavernosum, relaxation to acetylcholine (ACh) was unaffected by the cyclooxygenase inhibitor, flurbiprofen, or nerve blockade with tetrodotoxin but was markedly attenuated $(>74 \%)$ by the nitric oxide (NO) synthase inhibitor, $\mathrm{N}^{G}$-nitro-L-arginine. This suggests a major contribution by NOmediated endothelial mechanisms. Two months of diabetes caused a $37.1 \pm 4.9 \%$ ( \pm SEM; $p<0.001)$ deficit in maximum relaxation to $\mathrm{ACh}$, which was largely prevented by $\mathrm{LA}(85.1 \pm 19.8 \% ; \mathrm{p}<0.001)$ and partially prevented by TRI $(51.8 \pm 20.2 \%$; $p<0.05)$ treatment. In contrast, maximum endotheliumindependent relaxation to the NO donor, sodium nitroprusside, was unaffected by diabetes or treatment. For phenylephrine-precontracted corpus cavernosum in the presence of atropine $(1 \mu \mathrm{M})$ and guanethidine $(10 \mu \mathrm{M})$, electrical stimulation of autonomic nerves caused NO-mediated relaxation that was abolished by nitro-L-arginine. Nondiabetic rat corpus cavernosum had a maximum neurogenic relaxation of $41.0 \pm 2.3 \%$, which was reduced to $24.3 \pm 2.2 \%(p<0.001)$ by diabetes. Relaxation was in the nondiabetic range for LA $(41.7 \pm 3.1 \%)$, and TRI gave partial protection $(31.9 \pm 2.3 \%$; $\mathrm{p}<0.05)$. Thus, experimental diabetes impairs NO-mediated endothelial and neurogenic relaxation. Together, these NO systems promote vasodilation, therefore, similar defects in patients could contribute to erectile dysfunction. Prevention by antioxidant treatment strategies in diabetic rats suggests potential therapeutic approaches to diabetic impotence. 


\section{Insulin Signal Transduction}

\section{7}

CHARACTERIZATION OF FUNCTIONAL DOMAINS IN THE INSULIN RECEPTOR BY CHIMERIC RECEPTORS

Darryl Telting", Rolf L.L. Smeets, Peter H.G.M. Willems, Gerard C.M. van der Zon"), Antonie Maassen" "Dept. of Molecular Cell Biology, Leiden University, Wassenaarseweg 72, 2333 AL Leiden. The Netherlands

The receptors for insulin (IR) and the epidermal growth factor (EGFR) are members of the tyrosine kinase (TK) receptor family. Although the cytosolic TK domains of the receptors are highly homologous, the receptors have a difference in substrate specificity and response. The IR induces mitogenic and pronounced metabolic responses (i.e. glucose transport, glycogen synthesis, antilipolytic action). In contrast, the EGFR induces predominantly mitogenic responses. The mechanism which gives rise to the receptor responses is largely unknown. To determine the molecular basis of this receptor-specific signaling we have chosen the following approach; regions of the EGFR are exchanged with the IR counterparts (e.g. the juxtamembrane domain, the TK domain and the C-terminal part). These chimeric receptors are expressed in CHO cells, which lack endogenous EGFR. After EGF stimulation the receptor specificity is examined by measurement of insulin-specific responses (i.e. IRS-1 phosphorylation, glucose uptake, glycogen synthesis) or EGF specific responses (i.e. PLC $\gamma_{-1}$ activation, induction cytosolic $\mathrm{Ca}^{2+}$ ). We found that a chimeric receptor in which the IR cytoplasmic domain replaces the complete cytoplasmic EGFR domain induces insulin-like metabolic responses after EGF stimulation. Exchanging both the juxtamembrane and TK domain of the EGFR with their IR counterparts also gives rise to a receptor with IR specific responses (IRS-1 phosphorylation) combined with EGFR specific responses (phosporylation of PLCY-1 and induction of cytosolic $\mathrm{Ca}^{2+}$ ). We conclude that the IR catalytic domain is able to induce EGF-like responses whenever the appropriate substrate (i.e. EGFR c-terminus) is present. Furthermore, the results support a model in which EGFR specificity is created by the juxtamembrane and c-terminal parts of these receptors.

\section{8}

DIFFERENTIAL METABOLIC SIGNALLING IN ADIPOCYTES BY INSULIN RECEPTOR AND IGF-I RECEPTOR TYROSINE KINASES. B. Urs $\varnothing$, H. Kalloo-Hosein, A. Hayward, S. O'Rahilly and K. Siddle Univ. Cambridge, Dept. Clinical Biochemistry, Cambridge CB2 2QR, UK

The insulin and IGF-I receptors mediate diverse effects on cell growth differentiation and metabolism. The extent of similarities and differences in signalling by these receptors remains unclear. To analyse receptorspecific signalling without interference from endogenous receptors we constructed chimeras containing the extracellular domain of the neurotrophin receptor TrkC fused to the intracellular domain of the insulin receptor (TIR) or IGF-I receptor (TIGR), and expressed these in murine 3T3-Ll cells. Matched clones of fibroblasts were differentiated into adipocytes, and the TIR and TIGR chimeras displayed similar NT-3stimulated autophosphorylation (maximum 20-fold, EC50 0.1 nM). Stimulation of PI 3-kinase activity and association with IRS-1 was also similar in TIR and TIGR cells, but stimulation of MAP kinase was approx 2-fold greater in TIGR than in TIR cells. Glucose uptake was differentially stimulated by NT-3 (maximum 8.5 -fold in TIR, 4 -fold in TIGR, compared to 6.5 -fold by insulin). Glycogen synthesis was stimulated to the same level but with different sensitivity in TIR and TIGR cells (EC50 $\sim 0.2 \mathrm{ng} / \mathrm{ml}$ in TIR, $-1 \mathrm{ng} / \mathrm{ml}$ in TIGR). The mRNA for the Fos related antigen 1 (Fra-1) was induced by both receptors. Mutation of chimeras at sites implicated in binding of IRS-1 (Y960F TIR or Y950F TIGR) did not affect receptor autophosphorylation, but abrogated stimulation of IRS-1 phosphorylation, PI 3-kinase activity and glucose transport. We conclude that insulin receptors signal to glucose transport and to glycogen synthesis with a higher efficiency than IGF receptors and that this efficiency relies on IRS- I-dependent factors additional to PI 3-kinase.

\section{9}

CLONING OF NEW MEMBERS OF SIGNALING INOSITOL 5PHOSPHATASES FAMILY.

T. Nishiyama, C.R. Kahn. Joslin Diabetes Center, Boston, MA

Inositol 5-phosphatase (IP 5-Pases) are enzymes which hydrolyze the 5'phosphate from phosphatidylinositol 3,4,5-triphosphate and some inositol polyphosphates. One IP 5-Pase ( $\left.\mathrm{p} 150^{\text {stw }}\right)$ has been shown to induce apoptosis in mammalian cell, and inhibit insulin induced Xenopus oocytes germinal vesicle breakdown suggest its link to tyrosine kinase cell signaling. In this work, we have cloned two alternative splice forms of a new member of the IP 5-Pase family from mouse brain and have named these inositol polyphosphate phosphatase 1 and 2 (IPP-1 and IPP-2). In comparison sequence data between two molecules, both molecules have identical inositol 5-phosphatase domain, including the two conserved 14 amino acids (a.a.) sequence at $\mathrm{N}$ - terminal of 5 phosphatase domain. There is also a second in frame ORF of 195 nucleotides that encodes unique 65 a.a. of IPP-1 C-terminal sequence after the stop codon of IPP-2 cDNA, suggesting the possible mechanisms of alternative splicing of these molecules. On Northern blot analysis, IPP-1 mRNA is expressed at high levels in brain, testis, and kidney. Westem blots using a specific antibody against IPP-1 indicated molecular mass of $60 \mathrm{kDa}$ and an ubiquitous expression in mouse tissues. IPP-1 is expressed in muscle cell lines ( $\mathrm{C} 2 \mathrm{C} 12$, L6), and fully differentiated 3T3-L1 adipocytes, but not in 3T3-L1 preadipocytes nor other insulin insensitive cell lines such as NIH-3T3, CHO, and 32D. In summary, we have cloned a new IP 5-Pase which exhibit a unique pattern of alternative splicing and is specifically expressed in insulin sensitive cell lines such as muscle cell lines. These data suggest that these molecules may play some roles in insulin sensitive tissues, and may regulate metabolic effect by changing $\mathrm{PI}-(3,4,5) \mathrm{P}$, contents in these cells.

\section{0}

Functional Analysis of Class 1a Phopshoinositol 3'-Kinase Isoforms C.A.Beeton, P.Das, and P.Shepherd, Biochem. Dept., University College London, UK

Evidence has accumulated indicating insulin stimulated recruitment and activation of PI3K is neccessary and sufficient for activating signalling pathways leading to downstream responses regulated by insulin. Skeletal muscle is a major insulin target and human muscle studies in our laboratory indicate that signalling in this tissue is complicated bythe presence of various catalytic and adapter subunits of PI3K. These includep $85 \alpha$, and its splice variants p55 $\alpha$ and p50 $\alpha$ which lack the $\mathrm{SH} 3$ and $\mathrm{BH}$ domains, $p 85 \beta$, and $p 55 \gamma$, as well as $p 110 \alpha$ and $p 10 \beta$. The functional significance of these isoforms is unknown and our aim was to undertake transfection studies in HEK293 cells to dissect the role of the different domains and isoforms of PI3K. Briefly, epitope tagged constructs of p85 $\alpha, p 85 \Delta S H 3, p 85 \Delta B H$ were transfected individually, or cotransfected with the catalytic subunit of PI3K. When expressed alone all forms of the adapter subunit were poorly recruited to signalling complexes following insulin stimulation, but this recruitment was greatly increased by cotransfection with $\mathrm{p} 110$. This implies that the catalytic subunit has a role in localisation of PI3K following cell stimulation. The catalytic activity of p85/p110 was not affected by deletion of the SH3 or BH domains, but deletion of the BH domain prevented the stimulation of PI3K activity by the small GTP binding protein Rac. This suggests that adapter isoforms lacking these domains will be regulated differently to the full length isoforms. We find that in resting cells both the lipid and protein kinase activities of pl 10//p85a are $80 \%$ lower than $\mathrm{p} 110 \alpha / \mathrm{p} 85 \alpha$. The protein kinase of p110 targets Ser608 of p85a which in turn reduces lipid kinase activity of $\mathrm{p} 110$. Therefore the current data suggests that either p1 $10 \beta$ has lower intrinsic kinase activity and does not cause autoinhibitory phosphorylation of $\mathrm{p} 85$, or that $\mathrm{p} 110 \beta$ is a highly active protein kinase that maximally phosphorylates $p 85$ even under basal conditions. Studies are currently underway to distinguish between these possibilities. In conclusion, we have defined clear differences in the signalling potential of different isoforms of PI3K adapter and catalytic subunits. Therefore the signalling potential of a cell will be greatly influenced by the relative levels of expression of each of these isoforms. 
PI 3-kinase in normal and typeII diabetic muscle.

B. T. Nave, *H. Wallberg-Henriksson, *J. Zierrath and P. R. Shepherd. Dept. of Biochemistry, University College London, UK and *Dept. of Clinical Physiology, Karolinska Hospital, Stockholm, Sweden.

Activation of phosphatidylinositol 3-kinase (PI3K) by insulin is a key step in the activation of glucose transport in insulin responsive tissues, which is impaired in type II diabetes. In human skeletal muscle, the major site of insulin-stimulated glucose disposal, we find two isoforms of the catalytic subunit (p110 $\alpha$ and $\mathrm{p} 110 \beta$ ), three adapter subunit gene products ( $p 85 \alpha, p 85 \beta$ and $p 55 y$ ) and two splice variants of p85 $\alpha$ (AS53 and p50 2 ). We have previously reported (Shepherd et al., JBC 272, pp.19000) that the levels of adapters recruited to phosphotyrosine complexes after insulin stimulation vary, suggesting a mechanism by which downstream response could be differentially regulated via the PI 3-kinase system. The aim of this study was to characterise the association of adapter and catalytic isoforms of PI3K in normal muscle. Samples from the vastus lateralis muscle were homogenised, equalised for protein content and either immunoprecipitated or whole lysates analysed by SDS-page and western blotting. All adapter antibodies precipitated PI3K activity from normal skeletal muscle, but to different extents ( $\mathrm{p} 50 \alpha \gg \mathrm{p} 85 \alpha>\mathrm{p} 85 \beta=\mathrm{p} 55 \gamma$ ). In contrast, most $\mathrm{p} 110 \beta$ protein was associated with $p 85 \alpha>>p 50 \alpha>p 55 \gamma>p 85 \beta$, while $\mathrm{pl} 10 \alpha$ protein was associated with $\mathrm{p} 55 \gamma \gg>85 \alpha=\mathrm{p} 85 \beta>>\mathrm{p} 55 \alpha, \mathrm{p} 50 \alpha$. This suggests that PI3K associated with $\mathrm{p} 50 \alpha$ has a higher intrinsic catalytic activity and that the ratio of $\mathrm{p} 110 \alpha$ and $\mathrm{p} 110 \mathrm{p}$ associated with individual adapters varies between isoforms. To investigate how PI3K may be altered in diabetic states, vastus lateralis samples were taken from 9 normal (fasting insulin, fasting blood glucose) and 8 diabetic age- and weight matched males. Protein levels of adapter and catalytic subunits were measured and found to be unchanged for p1 $10 \beta$, but slightly higher in diabetics compared to controls for all other adapter and catalytic isoforms tested, although this increase was only significant for $\mathrm{p85 \beta}(180 \% \pm 38 \%$ over basal, $\mathrm{p}<0.05)$. We conclude that down regulation of PI3K proteins is unlikely to be the cause of insulin resistance in muscle of lean type II diabetics.
203

ADENOVIRUS-MEDIATED EXPRESSION OF THE WILDTYPE AND THE ASP905TYR VARLANT OF THE GLYCOGEN-ASSOCIATED REGULATORY SUBUNIT OF PROTEIN PHOPHATASE-1 IN L6 MYOTUBES

S.K. Rasmussen', L. Hansen', E.U. Frevert ${ }^{2}$, P.T.W. Cohen ${ }^{3}$, B.B. Kahn ${ }^{2}$ and O.Pedersen ${ }^{1}$. 'Steno Diabetes Center and Hagedorn Research Institute, Gentofte, DK. ${ }^{2}$ Beth Israel Deaconess Med. Ctr. and Harvard Medical School, Boston, USA. ${ }^{3}$ University of Dundee, Dundee, UK.

The glycogen-associated regulatory subunit of protein phosphatase-1 (PPP1R3) is considered to play an important role in skeletal muscle glycogen turn-over. We previously identified an Asp90STyr polymorphism of the PPPIR3 gene which among healthy subjects was associated with decreased insulin stimulated nonoxidative glucose metabolism (glycogen synthesis). The aim of the present study was to evaluate the effects of overexpression of wildtype (PPP1R3-WT-HA) and mutant (PPPIR3-905-HA) PPP1R3 on basal and insulin stimulated glucose uptake and glycogen synthesis in L6 myotubes. We used adenovirus-mediated gene transfer to express the genes at high level in L6 myotubes. Compared to $\beta$-gal transduced and non-transduced myotubes, overexpression of PPP1R3-WT-HA or PPP1R3-905-HA resulted in an approximately 3 fold increase in both basal (- insulin) and insulin stimulated (100 nM insulin) glycogen synthesis measured as D-[U-14Clglucose incorporation into glycogen. Glycogen synthesis (cpm per well (mean $\pm \mathrm{SD}$ of two experiments in triplicates) in basal and insulin stimulated state was, respectively; no virus $(202 \pm 78$ and $355 \pm 96)$, PPPIR3-WT-HA $(530 \pm 117$ and $1151 \pm 120)$, PPPIR3-905-HA $(607 \pm 83$ and $1296 \pm 116)$, and $\beta$-gal $(274 \pm 48$ and $480 \pm 94)$ ) However, the increased glycogen synthesis due to overexpression of PPP1R3 did not affect either basal or insulin stimulated 2-deoxy-D-glucose uptake when compared to $\beta$-gal transduced cells. Preliminary results show similar effects in CHO-IR cells. In conclusion: overexpression of PPP1R3 in L6 myotubes results in increased glycogen synthesis but does not affect glucose uptake. Importantly, comparison of results obtained from L6 myotubes transduced with PPP1R3-WT-HA and PPP1R3-905-HA showed no significant difference. Therefore the Asp905Tyr polymorphism alone is unlikcly to account for decreased non-oxidative glucose metabolism observed in the previously reported human study.
C-JUN AND ATF-2 ARE PHOSPHORYLATED TO MEDIATE C-JUN GENE EXPRESSION IN RESPONSE TO NNSULIN STIMULATION

D.M. Ouwens, K. Kooistra, D.S. Gomes de Mesquita, G.C.M. van der Zon, J.A.

Maassen, and H. van Dam. Dept. Molecular Cell Biology, Sections Signal

Transduction and Molecular Carcinogenesis, Leiden University Medical Center Leiden, The Netherlands

Insulin stimulates the expression of c-jun in A14 cells. The decisive element in the transcriptional response of the c-jun promoter is the AP1-binding site jun2. To study whether insulin-induced c-jun proceeds via the jun2-element, A14 cells were transiently transfected with a $c$-jun promoter construct carrying five jun2 sequences in front of a luciferase reporter gene. Insulin $(10 \mathrm{nM})$ stimulated the induction of the 5xjun2-luciferase construct approximately $\sim 10$-fold. The jun2-element in the c-jun promoter is constantly occupied by a heterodimer formed by the transcription factors cJun and ATF-2. These pre-bound transcription factors are activated in response to extracellular signals by phosphorylation. Insulin stimulated the phosphorylation of ATF-2 and c-Jun, as determined by Western blot analysis of total cell lysates usine phospho-specific antibodies recognising phosphorylated ATF-2 and c-Jun. To characterise the signaling pathways contributing to c-Jun and ATF-2 phosphorylation and jun2-mediated gene expression, the effects of dominant-negative Ras, of the pharmacological MEK-inhibitor PD98059, and of the p38-inhibitor SB203580 were analysed. The induction of the $5 \times j$ un 2 -luciferase construct by insulin was inhibited by Ras(Asn-17), but not by PD98059 or SB203580. The insulin-induced phosphorylation of the transcription factors was also unaffected by PD98059 and SB203580, indicating that this process does not involve ERK or $\mathrm{p} 38$. We have considered a contribution of JNK for c-jun and ATF-2 phosphorylation. Insulin had no effect on the activation of JNK as determined by Western blot analysis of total cell lysates with antbodies rcognizing the phosphorylated active form of JNK. We conclude from these results that (i) insulin stimulates c-jun expression in a Ras-dependent fashion, (ii) insulin stimulates the phosphorylation of c-Jun and ATF-2, (iii) insulin-induced c-Jun and ATF-2 phosphorylation involves a novel Ras-dependent kinase, that is not identical to ERK, p38, or JNK.

\section{4}

TRANSLOCATION OF LIVER PHOSPHATIDYLINOSITOL-3 KINASE ON MICROSOMES AND GLUCOSE-6 PHOSPHATASE INHIBITION. G. Mithieux, N. Daniele, F. Rajas, B. Payrastre*, G. Mauco*, C.Zitoun, INSERM U.449 and 326*, Lyon, Toulouse*, France

We have studied the molecular mechanism of inhibition of liver microsomal glucose-6 phosphatase (Glc6Pase) during the postprandial period in rats. By using a rapid procedure of isolation of microsomes, we have shown that Glc6Pase activity was decreased after refeeding rats previously unfed for $48 \mathrm{~h}$ : $65 \pm 2$ vs $96.5 \pm 3$ (1 mM glucose-6 phosphate (Glc6P)) and $225 \pm 6$ vs $306 \pm 9$ (20 mM Glc6P) nmol/min/mg prot., means $\pm S . E . M ., n=12, p<0.001$. The amount of Glc6Pase protein (western blot) was not lower in microsomes from refed as compared to fasted rats. Since it has been previously reported that 3phosphoinositides are potent inhibitors of Glc6Pase in vitro, so we tested the hypothesis that phosphatidylinositol-3 kinase (PI3K) be involved in the inhibition. The amount of immunoreactive $\mathrm{p} 85$ (the regulatory subunit of $\mathrm{PI} 3 \mathrm{~K}$ ) and the PI3K catalytic activity were higher by a factor 2.6 and 2.4 , respectively $(p<0.01)$ in microsomes from refed as compared to fasted rats. This resulted from a translocation process since the total amount of p85 was the same in the whole liver homogenates from refed and fasted rats. The amount of immunoreactive insulin receptor substrate 1 (IRS1) was also higher by a factor 2.6 in microsomes from refed rats $(p<0.01)$. Microsome-bound IRS1 was only detected in $\mathrm{p} 85$ immunoprecipitates. Endoplasmic reticulum (ER) was separated from plasma membranes by fractionation on sucrose gradient. We quantified by western blot that 60 to $70 \%$ of microsome-bound p85 was present in ER. These results strongly suggest that an insulin-triggered mechanism of translocation of PI $3 \mathrm{~K}$ onto ER occurs in the liver of rats during the postprandial period. This process, via the lipid products of PI3K, may account for the inhibition of Glc6Pase and participate to the inhibition of hepatic glucose production occuring in this situation. 
OP 34

\section{Nitric Oxide Vascular Reactivity}

\section{5}

ELEVATED SKELETAL MUSCLE BLOOD FLOW IN TYPE 1 DIABETES: ROLE OF NITRIC OXIDE AND SYMPATHETIC TONE G.Vervoort, J.F.M.Wetzels, J.A.Lutterman, J.H.M.Berden and P.Smits. Depts of Medicine and Nephrology, University Hospital Nijmegen, The Netherlands. Capillary hyperperfusion precedes and contributes to the occurrence of diabetic microangiopathy. Baseline vascular tone is regulated by the balance of vasodilating and vasoconstricting factors of which nitric oxide (NO, an endothelium dependent vasodilator) and norepinephrine (a potent vasoconstrictor) are of primary importance. To investigate the role of these factors in hyperperfusion, we have measured forearm blood flow (FBF) in 50 patients with normoalbuminuric type 1 diabetes (DP) and 50 healthy control subjects $(C)$ under baseline conditions and during intra-brachial infusion of $\mathrm{L}$. NMMA, an endothelium-dependent vasoconstrictor, and acetylcholine $(\mathrm{ACh})$ an endothelium-dependent vasodilator. Furthermore, we determined arterial plasma norepinephrine (NE) concentration at baseline, and alpha-adrenergic receptor sensitivity by measuring FBF response to intra-arterially infused NE We found that basal FBF was increased in DP (FBF $2.9 \pm 0.2$ vs $2.0 \pm 0.1$ $\mathrm{ml} / \mathrm{min} / \mathrm{dl}$ in $\mathrm{C}, \mathrm{p}<0.01$ ). L-NMMA caused a similar vasoconstriction in both groups $(28.5 \pm 1.7 \%$ in DP vs $31.2 \pm 2.2 \%$ in $\mathrm{C}, \mathrm{NS})$. Maximum blood flow during infusion of $\mathrm{ACh}$ was not different (FBF $23.3 \pm 1.9$ in DP vs $20.1 \pm 1.6$ $\mathrm{ml} / \mathrm{min} / \mathrm{dl}$ in C). Arterial plasma $\mathrm{NE}$ concentrations were significantly decreased in DP $(0.57 \pm 0.03$ vs $0.81 \pm 0.05 \mathrm{nmol} / \mathrm{l}$ in $\mathrm{C}, \mathrm{p}<0.01)$. The vasoconstrictive effect of NE was increased in DP (slope log dose-response curve $31.3 \pm 1.5$ vs $24.3 \pm 1.8$ in $C, p<0.01$ ). We conclude that basal forearm blood flow is increased in non-complicated type I diabetes mellitus. We have found no evidence for a disturbance of basal or stimulated NO production Arterial plasma NE concentrations are decreased in non-complicated IDDM This may explain the vasodilatation at baseline as well as the increased vascular response to intra-arterially infused NE.

\section{7}

EFFECTS OF THE PROTEIN KINASE C 3 INHIBITOR, LY333531, ON NERVE AND VASCULAR FUNCTION IN DIABETIC RATS.

N.E. Cameron', A. Jack', D.K. Ways ${ }^{2}$ and M.A. Cotter'. 'Biomedical Sciences, Aberdeen University, Scotland, UK and ${ }^{2}$ Lilly Research Laboratories, Indianapolis, IN, USA.

Protein kinase C (PKC) activation by elevated diacylglycerol occurs in several vascular tissues in experimental diabetes and is linked to early retinal and renal complications, which are attenuated by a specific $\mathrm{PKC} \beta$ antagonist, LY333531 (Eli Lilly, Indianapolis, USA). Diabetic neuropathy has a vascular component, therefore, one aim was to test the effects of LY333531 on nerve conduction velocity (NCV) and blood flow deficits. A second aim was to characterize the diabetic vascular deficit and the role of PKC $\beta$ in resistance vessel responses, using the mesenteric vascular bed. After 6 weeks of untreated streptozotocin-diabetes, 2 weeks LY333531 treatment dose-dependently corrected $19.7 \pm 0.8 \%$ ( \pm SEM; $p<0.001)$ sciatic motor and $13.9 \pm 0.7 \% \quad(\mathrm{p}<0.001)$ saphenous sensory $\mathrm{NCV}$ deficits. LY333531 $(10 \mathrm{mg} / \mathrm{kg})$ completely corrected sensory NCV $(p<0.001)$, improved motor NCV by $91.1 \pm 3.3 \% \quad(\mathrm{p}<0.001)$ and totally corrected $(p<0.001)$ a $50.4 \pm 3.2 \%(p<0.001)$ deficit in sciatic endoneurial blood flow. Acetylcholine $(\mathrm{ACh})$ induced relaxation of phenylephrine-precontracted mesenteric vasculature was diminished by 8 weeks of diabetes (control, maximum relaxation $97.8 \pm 0.9 \%,-\log \mathrm{EC}_{50} 7.75 \pm 0.14$; diabetes, $66.2 \pm 4.8 \%$, $7.04 \pm 0.07 ; \mathrm{p}<0.001)$. This was partially prevented by LY333531 $(10 \mathrm{mg} / \mathrm{kg}$; $86.4 \pm 3.2 \%, 7.51 \pm 0.07 ; \mathrm{p}<0.01) . \mathrm{N}^{\mathrm{G}}$-nitro-L-arginine blocked NO-mediated relaxation, unmasking that due to endothelium-derived hyperpolarising factor. ACh maximum relaxation was reduced by diabetes $(14.8 \pm 3.6 \%$ vs $73.8 \pm 4.0 \% ; p<0.001)$ and $L Y 333531$ was protective $(45.7 \pm 7.2 \%, p<0.01)$. Thus, PKC $\beta$ is an important mediator of neurovascular defects in diabetic rats. LY333531's beneficial effect on perfusion of nerve and other tissues depends partly on restoration of multiple endothelial relaxation mechanisms.

\section{6}

ENDOTHELIAL DYSFUNCTION IN ADULT OFFSPRING OF DIABETIC RATS IS COMPROMISED EVEN FURTHER BY A DIET HIGH $N$ SATURATED FAT K. Holemans, R.T. Gerber', L. Poston' and F.A. Van Assche. Depts Ob/ Gyn, Katholieke Universiteit Leuven, Belgium and ' $\mathrm{St}$ Thomas's Hospital, UK. Adult offspring of severely diabetic pregnant rats show vascular dysfunction. Since diets high in saturated fat are involved in the pathogenesis of cardiovascular disease, we investigated the implications of feeding a high fat diet for vascular function in offspring of control Wistar rats and in offspring of streptozotocin-diabetic rats. Offspring of both control rats (C) and of diabetic rats (OD) were fed a normal diet or a diet high in saturated fats (CHF and ODHF, respectively), (16\% lard $4 \%$ corn oil; w/w normal chow) from weaning onward. Body weight was recorded weekly from 7 days of age. Small mesenteric arteries from 100-120 day old offspring were mounted on a myograph. After preconstricting the arteries with $5 \mu \mathrm{M}$ noradrenaline relaxation to acetylcholine (ACh) was performed with and without inhibitors of NO release ( $\mathrm{N}$-nitro L-arginine methylester and oxadiazole quinoxalin) and of prostanoids (indomethacin). On day $98 \mathrm{CHF}$ and ODHF were heavier than $\mathrm{C}$ $(p<0.01)$ and $O D(p<0.01)$, respectively. Their growth patterns showed that $\mathrm{CHF}$ and ODHF started to gain more weight from respectively postnatal days 91 and 63 onward, despite a lower $(\mathrm{CHF}, \mathrm{p}<0.001)$ or normal $(\mathrm{ODHF})$ food intake. In the control group the HF diet reduced $A C h$ induced relaxation $\left[\mathrm{pEC} \mathrm{C}_{50}\right.$ (CHF): $6.61 \pm 0.12$ vs (C): $7.22 \pm 0.09, n=10 ; p<0.0011$. In OD, ACh induced relaxation was impaired compared with $\mathrm{C}\left[\mathrm{pEC}_{50}\right.$ : $(\mathrm{OD}): 6.47 \pm 0.07, \mathrm{n}=9$ vs $(\mathrm{C}): 7.22 \pm 0.09, \mathrm{n}=10 ; \mathrm{p}<$ $0.001]$ and $O D H F$ rats showed a further abnormality, a reduction in maximal relaxation [ODHF: $80.70 \pm 3.96, n=9$ vs OD: $91.19 \pm 2.19, n=9 ; p<0.05$ ]. The impaired endothelium dependent relaxation in $\mathrm{CHF}$ vs $\mathrm{C}$ occurred through inhibition of NO induced relaxation, whereas the difference in relaxation in ODHF vs OD may be attributable to reduced EDHF. In conclusion, the high saturated fat diet (1) reduces endothelium-dependent relaxation in control rats, and (2) may exacerbate vascular dysfunction in the offspring of diabetic rats.

\section{8}

METABOLIC CONTROL DETERMINES THE ENDOTHELIAL FUNCTION IN IDDM PATIENTS

A. Avogaro, L. Calo, F. Piarulli, M. Miola, S. deKreutzenberg, A. Maran, R. Mingardi, A. Tiengo and S. Del Prato. University of Padova, Vicenza Hospital, Italy

In insulin-dependent diabetic (IDDM) patients, loss of metabolic control is associated with increased blood flow. However, a role of endothelium. derived nitric oxide (NO) production has never been assessed. 13 IDDM patients were studied under condition of mild ketosis, in two different occasions. In Study 1, after basal determination, a rapid amelioration of the metabolic picture was obtained by insulin infusion. In Study 2, 7 of the 13 IDDM underwent the same experimental procedure with the exception that fasting plasma glucose was maintained constant. Endothelial function was assessed by measuring forearm blood flow (FBF) during intra-arterial infusion of $\mathrm{N}^{\mathrm{G}}$-monomethyl-L-arginine (L-NMMA), sodium nitroprusside (SNP), and phenylephrine (PE). 13 controls (C) were also studied in baseline conditions and during a hyperinsulinemic.

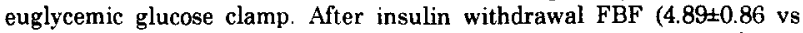
$3.65 \pm 0.59)$ was higher in IDDM than in $C(p<0.05)$. L-NMMA infusion resulted in greater reduction of FBF in ketotic IDDM $(-36 \pm 12 \%$ of the ratio infused:control arm) than in $C(-18 \pm 8 \% ; p<0.01)$ while no difference was observed in the PE and SNP dose-response relationship. After restoration of metabolic control, L-NMMA-mediated FBF decrements ($11 \pm 5$ vs $11 \pm 4 \%$ ) were similar during euglycemia- and hyperglycemichyperinsulinemia. However, these parameters were significantly lower than in $\mathrm{C}$ during baseline and hyperinsulinemic-euglycemia. We conclude that the loss of metabolic control is associated with an inappropriately high NO forearm release. IDDM patients have blunted NO-mediated vasodilatory response to insulin. Normalization of metabolic control does not completely reverse to normal the endothelium function. Hyperglycemia per se, does not seem to acutely affect the endothelial function. 
THE IMPAIRMENT IN ENDOTHELIUM-DEPENDENT

VASODILATION INDUCED BY ACUTE HYPERGLYCAEMIA CAN BE REVERSED BY HYPERINSULINEMLA

A.Fugmann, L.Lind, J.Millgård, C.Berne and H.Lithell. Departments of

Internal Medicine and Geriatrics University Hospital of Uppsala, Sweden

Hyperglycaemia is a well-known cardiovascular risk factor and endothelial dysfunction has been associated with diabetes. In order to assess the effect of acute hyperglycaemia on endothelial function alone, and in combination with hyperinsulinemia, nine healthy subjects was studied with a $2 \mathrm{~h}$ hyperglycemic clamp (12-13 mmol/1) combined with a somatostatin and low dose insulin infusion to achieve euinsulinemia. In three of the subjects this clamp was followed by a hyperglycaemic, hyperinsulinemic clamp for another $2 \mathrm{~h}$. Blood flow was assessed by forearm venous occlusion plethysmography. Endothelium-dependent vasodilation (EDV) was assessed with local infusion of Metacholine ( 2 and $4 \mu \mathrm{g} / \mathrm{min}$ ) and endothelium independent vasodilation (EIDV) by sodium nitroprusside ( 5 and 10 $\mu \mathrm{g} / \mathrm{min}$ ). The euinsulinemic hyperglycaemia increased plasma glucose to $12.4 \pm 0.5 \mathrm{mmol} / \mathrm{l}$, with plasma insulin at fasting level $(10.1 \pm 1.6 \mathrm{mU} / \mathrm{l})$. EDV was significantly impaired by hyperglycaemia. During the euinsulinemic hyperglycaemic period, infusion with Metacholine, $4 \mu \mathrm{g} / \mathrm{min}$ resulted in a forearm blood flow of $19.2 \pm 6.0$ compared with $22.3 \pm 5.2 \mathrm{ml} / \mathrm{min} \cdot 100 \mathrm{ml}$ tissue at baseline $(\mathrm{p}<0.05)$. This impairment was totally reversed by the addition of hyperinsulinemia $(72.0 \pm 2.0 \mathrm{mU} / \mathrm{l})$ to the hyperglycaemic state. Forearm blood flow then increased to $25.9 \pm 10.4 \mathrm{ml} / \mathrm{min} \cdot 100 \mathrm{ml}$ tissue $(\mathrm{p}<0.01)$. EIDV was not significantly changed during the different metabolic states.

In conclusion acute euinsulinemic hyperglycaemia impairs endotheliumdependent vasodilation. This impairment can be reversed by concomitant hyperinsulinemia.
INSULIN STIMULATES A $\mathrm{Ca}^{2+-D E P E N D E N T ~ N I T R I C ~}$ OXIDE SYNTHASE IN HUMAN VASCULAR SMOOTH MUSCLE CELLS.

M.Trovati, L.Mattiello, C.Costamagna*, P.Massucco, G.Anfossi, F.Cavalot, A.Bosia*, and D.Ghigo*. Diabetes Unit, Dept. of Clinical and Biological Sciences, San Luigi Hospital, Orbassano (Turin) Italy and *Dept. of Genetics, Biology and Medical Chemistry, University of Turin, Italy.

Insulin -induced vasodilation is attributed to the endothelial production of nitric oxide (NO), which stimulates cyclic guanosinemonophosphate synthesis in the underlining vascular smooth muscle cells (VSMC). In vitro studies on cultured human VSMC (hVSMC), however, demonstrated that insulin is also able to directly increase cGMP in these cells with a NO-mediated effect, suggesting that insulin stimulates NO synthase (NOS) not only in endothelial cells, but also in hVSMC. hVSMC express an inducible, $\mathrm{Ca}^{2+-i n d e p e n d e n t ~ N O S, ~}$ activated by cytokines and responsible for septic shock. To evaluate whether hVSMC present also a $\mathrm{Ca}^{2+}$ dependent NOS, susceptible to rapid insulin-induced activation in the absence of cytokines, we verified in cultured hVSMC whether ionomycin, a $\mathrm{Ca}^{2+-i o n o p h o r e, ~}$ stimulates NO synthesis, evaluated via $\mathrm{L}-{ }^{-3} \mathrm{H}$-citrulline production from $\mathrm{L}-{ }^{3} \mathrm{H}-a r g i n i n e$, and whether insulin increases in a short time NO and cGMP. Results (mtsem): i) ionomycin $1 \mu \mathrm{mol} / 1$ increases in $5 \mathrm{~min}$ NO synthesis (from $0.07 \pm 0.003$ to $0.6 \pm 0.06 \mathrm{pmol} \mathrm{L}-3 \mathrm{H}$-citrulline $/ \mathrm{min} / \mathrm{mg}$ protein, $\mathrm{p}=0.0001, \mathrm{n}=4$ ); ii)insulin $2 \mathrm{nmol} / \mathrm{l}$ increases in $5 \mathrm{~min}$ both NO (from $0.07 \pm 0.003$ to $0.31 \pm 0.031 \mathrm{pmol} \mathrm{L-3} \mathrm{H}$-citrulline $/ \mathrm{min} / \mathrm{mg}$ protein, $\mathrm{p}=0.0001, \mathrm{n}=4$ ) and cGMP (from $0.7 \pm 0.01$ to $1.5 \pm 0.05$ pmol $/ 10^{6}$ cells, $\mathrm{p}=0.000, \mathrm{n}=6$ ). Thus, hVSMC present a $\mathrm{Ca}^{2+-}$ dependent NOS and insulin increases in a few minutes in these cells NO and, via NO, cGMP. Thus, insulin-induced vasodilation, probably largely dependent on NO production by endothelial cells, can be at least in part attributable to a direct insulin effect on hVSMC.

\section{1}

MICRO- AND MACRO-VASCULAR REACTIVITY IS IMPAIRED IN SUBJECTS AT RISK FOR TYPE 2 DIABETES

AE Caballero, R Saouaf, S Arora, SC Lim, P Smakowski, FW LoGerfo, ES Horton, A Veves. Harvard Medical School, Boston, MA, USA

We have tested the micro- and macro-vascular endothelium dependent and independent vasodilatation in 4 groups: 30 healthy normoglycemic subjects with no history of type $2 \mathrm{DM}$ in a first degree relative [C group, $14 \mathrm{M} / 16 \mathrm{~F}$, age $48 \pm 9$ (mean $\pm \mathrm{sd}$ ) years], 37 healthy normoglycemic subjects with parental history of Type $2 D M(R, 18 M / 19 F$, age $48 \pm 10), 30$ subjects with impaired glucose tolerance (IGT, $13 \mathrm{M} / 17 \mathrm{~F}$, age $51 \pm 10$ ) and 42 type $2 \mathrm{DM}$ patients without vascular complications $(D, 21 \mathrm{M} / 21 \mathrm{~F}$, age 53 \pm 10 , DM duration $4 \pm 4$ years). BMI was lower in C $(26.44 \pm 3.7)$ and $R$ $(28.22 \pm 4.7)$ groups compared to IGT $(32.60 \pm 7.8)$ and $D(32.28 \pm 6.3)(p$ $<0.001)$. Fasting insulin levels were lower in $C(7.1 \pm 8.5 \mu \mathrm{U} / \mathrm{ml})$ and $R(7.8$ $\pm 5.7)$ compared to $1 G T(13.7 \pm 10.2)$ and $D(16.7 \pm 14.9)$ groups $(p$ $<0.001$ ). Fasting glucose, HDA1C, Triglycerides $(\mathrm{Tg}), \mathrm{HDL}$ and total cholesterol (TC) were similar in C and R. IGT and D groups had higher $T C$, LDL and $T g$ than $C$ and $R(p<0.001)$. We employed laser Doppler imaging scanner to measure vasodilatation in the forearm skin in response to iontophoresis of $1 \%$ acetylcholine (Ach, endotheliumdependent) and $1 \%$ sodium nitroprusside (NaNP endotheliumindependent) and ultrasound for the brachial artery diameter changes related to reactive hyperemia after 5 minutes of its occlusion. A stepwise reduction was observed in response to $A$ ch (C $125 \% \pm 66$ increase over baseline, R $99 \pm 48$, IGT $95 \pm 53, \mathrm{D} 73 \pm 44, \mathrm{p}<0.01$ ) and NaNP (C $122 \%$ $\pm 45, R 85 \pm 46$, IGT $82 \pm 49 D 65 \pm 31, p<0.001)$. Similar results were observed in the macrocirculation (C $13.5 \% \pm 6.2$ increase over baseline R $10.4 \pm 6.6$. IGT $9.5 \pm 4.4, D 8.4 \pm 5.0 \mathrm{p}=0.008$ ). Females had a higher response compared to males $(\rho<0.05)$ but a similar stepwise reduction was found in both sexes; e.g. the brachial antery hyperemic response in females was: C $16.4 \% \pm 5.6, R 12 \pm 6.6$, IGT $10.2 \pm 4.6$, D $10.2 \pm 5.2$ (p< 0.05 ) and in males: $C 10.7 \% \pm 5.7, R 8.4 \pm 6.2,1 G T 8.2 \pm 3.8$ and $D 6.2 \pm$ 3.7. Similar results were found in the responses to Ach and NANP. We conclude that vascular reactivity is impaired in the prediabetes stage and that females have a higher vascular reactivity compared to males.

\section{2}

THE INFLUENCE OF GENDER, MENOPAUSAL STATUS AND HORMONAL REPLACEMENT THERAPY ON MICROVASCULAR REACTIVITY IN HEALTHY AND TYPE 2 DIABETIC INDIVIDUALS. S.C. Lim*, S. Arora, A.E. Caballero, P Smakowski, ES Horton, FW LoGerfo and A Veves. Harvard Medical School, MA, USA

Healthy pre-menopausal women are protected from cardiovascular diseases compare to men of similar age; however, women with diabetes are denied of the cardio-proctection associated with estrogens. We have examined the microvascular reactivity in healthy pre-menopausal women $[H$ group, $n=21$, age $39 \pm 8$ years (mean \pm SD)], post-menopausal not receiving hormone replacement treatment (HRT) (HP, $n=9,58 \pm 5$ ), postmenopausal on HRT (HRT, $n=11,53 \pm 4$ ), type 2 diabetic pre-menopausal women (D, $n=8$, age $43 \pm 7$ ), diabetic post-menopausal not on HRT (DP, $n=9,61 \pm 6$ ), diabetic post-menopausal on HRT (DRT, $n=8,54 \pm 5$ ), healthy men (HM, $n=14$, age $51 \pm 10$ ) and type 2 diabetic men ( $D M, n=21$, $55 \pm 10)$. Total cholesterol was lower in $H$ group $(168 \pm 22 \mathrm{mg} / \mathrm{dL})$ compared to D $(222 \pm 65), D P(227 \pm 45)$, DRT $(211 \pm 29)$, HM $(199 \pm 44)$ and DM (202 \pm 33$)$ groups $(\mathrm{p}<0.05)$. LDL cholesterol was lower in $\mathrm{H}$ group $(107 \pm 18 \mathrm{mg} / \mathrm{dl})$ compared to DP $(135 \pm 43), \mathrm{HM}(133 \pm 36)$ and $\mathrm{DM}(130$ $\pm 31)$ groups $(p<0.05)$. We employed laser Doppler imaging scanner to measure vasodilatation in the forearm skin in response to iontophoresis of $1 \%$ acetylcholine (Ach, endothelium-dependent) and 1\% sodium nitroprusside (NaNP, endothelium-independent). The endothelium dependent vasodilation was significantly higher in $\mathrm{H}$ group $(180 \pm 61)(\%$ increase over baseline) compared to HP $(93 \pm 44)$ while it was partially restored in HRT (142 \pm 56 ). It was also reduced in all diabetes groups: D $(92 \pm 52)$, DR $(69 \pm 26)$, DRT $(97 \pm 66)$. A lower response was found in HM $(92 \pm 41)$ and DM $(59+33)(0<0.001)$. Similar pattern of responses were likewise demonstrated in the endothelium independent vasodilation (H $118 \pm 57$, HP $74 \pm 27$, HRT $100 \pm 32$, O $79 \pm 25$, DP $70 \pm 31$, DRT $93 \pm$ 40. HM $92 \pm 40, D M 49 \pm 20 ; p<0.05$ ). We conclude that male gender, post-menopausal status and type 2 diabetes are associated with impaired microvascular reactivity and that HRT may ameliorate this dysfunction in both healthy and diabetic post-menopausal women. 


\section{OP 35}

\section{Susceptibility and Resistance to $\beta$-Cell Damage}

213

DUCTAL CELLS ARE A SOURCE OF NITRIC OXIDE

D. Pavlovic, M.C. Chen, L. Bouwens, D.L. Eizirik and D. Pipeleers. Diabetes Research Center, Vrije Universiteit Brussel, Brussels, Belgium.

The radical nitric oxide (NO) may be a mediator of $\beta$-cell damage in type 1 diabetes. Exposure of human pancreatic islets to cytokines generates NO, but it remains to be identified which cell type(s) are responsible for this effect. Since ductal cells represent 30 to 45 percent of the total cell population in isolated human islet preparations, we investigated whether these cells are a source of NO generation following cytokine exposure. Culture of isolated human ductal cells with interleukin-1 $($ (IL-1 $\beta$ ) plus interferon- $\gamma$ (IFN- $\gamma$ ) induced mRNA expression for the inducible form of NO synthase (iNOS), and increased NO production 12-fold $(p<0.0001, n=5)$ as judged by medium nitrite accumulation. This cytokine-induced nitrite production did not occur in an arginine-free medium or in the presence of methylarginine $(0.5 \mathrm{mmol} / \mathrm{L})$, which is known to inhibit iNOS activity. Immunocytochemical staining indicated that 10 to 20 percent of the cytokine-treated human ductal cells express the iNOS protein. Comparison of the magnitudes of iNOS mRNA expression and nitrite production by ductal and islet cell preparations suggested that $50 \%$ or more of total islet NO production might originate from ductal cells. It is concluded that associated pancreatic ductal cells are a potential source of NO production in the vicinity of human pancreatic $\beta$-cells.

\section{5}

MATURATION FROM A PAE $\beta$-CELL TO A $\beta$-CELL PHENOTYPE IS ASSOCIATED WITH IL-1 13 INDUCED NO DEPENDENT CYTOTOXICITY

K. Nielsen, P. Serup, O.D. Madsen, T. Mandrup Poulsen, J. Nerup and A. E. Karlsen. Steno Diabetes Center and Hagedorn Research Institute, DK-2820 Gentofte, Denmark. Interleukin-1 $(\mathrm{IL-1 \beta})$ induces the expression of the inducible nitric oxide synthase (NOS) and nitric oxide (NO) production in the islets of Langerhans, and is specifically toxic to the $\beta$-cells. We have demonstrated in the MSL-cell system of $\beta$-cells maturation, that IL-1 $\beta$ is highly cytotoxic to the $\beta$-cells, whereas the pre $\beta$-cells are less sensitive. AlM: To analyze the cytotoxic effect of IL-1 $\beta$ induced NO production in the MSL $\beta$-cells and pre $\beta$-cells in the presence or absence of the $L$-arginine analog $N^{0}$-monomethyl-L-arginine (NMMA), an inhibitor of the NO production. METHODS: The MSL-cells display a pre- $\beta$ or $\beta$-cell-type dependent on culture conditions. The two MSL phenotypes were exposed to different concentrations of $I L-1 \beta$ with or without $0.5 \mathrm{mM}$ NMMA for 3 days and the resulting toxicity was determined by the mitochondria activity (MTT-assay). NO production was measured as accumulated nitrite. iNOS mRNA expression was analyzed by quantitative PCR and normalized against TATA-binding protein (TBP) transcripts used as internal standard. RESULTS: A dose-dependent and significant decrease in MTT-level in response to IL-1 $\beta$ was detected both in the pre $\beta$ - and the $\beta$-cells. However the decrease was significantly

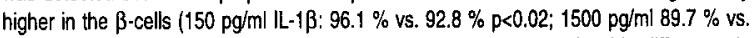
$65.2 \% p<0.001 ; 3000 \mathrm{pg} / \mathrm{ml} 87.4 \%$ vs. $42.5 \% p<0.001)$. Despite this difference in sensitivity to IL-1 $\beta$ we found an equal amount of iNOS mRNA expression and NO production in both cell-types after exposure to $12-1 \beta$. Addition of $0.5 \mathrm{mM}$ NMMA inhibited the IL-1 $\beta$ induced NO production in both phenotypes and no significant decrease in the $M T$-level was detected in both phenotypes under these conditions. CONCLUSION: The $\beta$-cells are more sensitive to IL-1 $\beta$ induced NO production than the pre $\beta$-cells, despite equal amount of NO production in both cells-types. Inhibition of NO production with NMMA protected both celis-types against the toxic effects of IL-1B. It may be speculated that $\beta$ cells after maturation from the endocrine stem-cell may loose part of their ability to scavenge free radicals, like NO. We believe that IL-1 $\beta$ induces a race between protective and deleterious mechanisms and in the $\beta$-cells the deleterious prevail.

\section{4}

THE EFFECTS OF MAP KINASE INHIBITORS ON CYTOKINE-INDUCED NO PRODUCTION, INSULIN SECRETION AND CELL DEATH IN RIN CELLS.

A. M. Ejrnzs, N. Aa. Andersen and T. Mandnup-Poulsen. Steno Diabetes Center, Gentofte, Denmark.

IL-1 induced NO production depends on signalling via the mitogen activated protein (MAP) kinases $\mathrm{p} 38$ and ERK1/2 in rat islets. and inhibition of $\mathrm{p} 38$ reduces IL-1 induced inhibition of insulin secretion. The aim of this study was to investigate the effects of $\mathbf{p} 38$ and ERK $1 / 2$ inhibitors on NO production, insulin secretion and cell death in rat insulinoma (RIN) cells exposed to IL-1 or $\mathrm{L}$. $1+\mathrm{IFN} \gamma+\mathrm{TNF} \alpha$. The hypothesis was that inhibitors of $\mathrm{p} 38$ and ERK $1 / 2$ abolish cytokine-induced NO production, inhibition of insulin secretion and cell death by preventing MAPK signalling dependent iNOS expression. RIN-5AH-T2B cells $(50.000$ cells $/ 200 \mu \mathrm{l})$ were seeded in RPMI $+10 \% \mathrm{FCS}$, precultured for 24 hours, and then incubated with $10 \mu \mathrm{M}$ VK (p38 inhibitor) and $100 \mu \mathrm{M}$ PD (inhibitor of MEK which is upstream of ERKI/2) for one hour prior to cytokine exposure. Cells were then incubated with $0,60,600$ or $1200 \mathrm{U} / \mathrm{ml}$ rhIL-l or a combination of 60 $\mathrm{U} / \mathrm{ml}$ rhll-1. $185 \mathrm{U} / \mathrm{ml} \mathrm{rhTNF} \alpha$ and $0,14 \mathrm{U} / \mathrm{ml} \mathrm{rrIFN} \gamma$ ("mix") for 24 hours. Accumulated insulin secretion was measured by ELISA and NO production by the Griess reaction. Cell death was measured by the propidium iodide assay. IL-l and "mix" treatment resulted in inhibition of insulin secretion to about $50 \%$ of control. The inhibitors alone had no effect on insulin secretion and did not prevent cytokine-induced inhibition of insulin release. NO synthesis increased between 5 and 10 fold by IL-1 and "mix" treatment. The inhibitors reduced IL-1 and "mix" induced NO production by $50 \%$ and $25 \%$ of control respectively. IL-1 and "mix" treatment increased cell death by ten fold, but the inhibitors augmented rather than prevented cell death $(\mathrm{P}<0,05)$. We conclude that inhibition of $\mathrm{p} 38$ and ERK1/2 reduces cytokine-induced NO production but does not prevent cytokine-induced inhibition of insulin secretion and cell death. We suggest that cytokine-induced RIN cell toxicity is independent of NO and signalling via MAPK

\section{6}

CYTOKINE INDUCED NITRIC OXIDE AND INTERLEUKIN-1 CONVERTING ENZYME MAY REPRESENT DIFFERENT PATHWAYS OF CYTOKINE INDUCED B-CELL DESTRUCTION. A.E. Karlsen', D. Pavlovic ${ }^{2}$, K. Nielsen', J. Jensen ${ }^{1}$ H.U. Andersen', F. Pociof', T. MandrupPoulsen', D. Eizirik ${ }^{2}$, J. Nerup'. 'Steno Diabetes Center and Hagedorn Research Institute, Gentofte, Denmark and ${ }^{2} V$ rije Universiteit, Brussel, Belgium.

In rat islets and beta-cell lines cytokine induced nitric oxide (NO) production plays an important role in short term (1-2 days) cytokine induced $\beta$-cell dysfunction and damage. In particular interleukin- $1 \beta$ has been shown to be toxic through NO induction. In contrast, human islets are more resistant to the toxic effect of cytokines and apoptosis is only detectable after $6-9$ days cytokine exposure, primarily induced by a combination of IFN $\gamma$, TNF $\alpha$ and IL-1 $\beta$, with little effect of IL-1 $1 \beta$ alone. Interestingly, cytokine-induced human beta-cell death is indenpendent of NO production. The aim of this study was therefore to analyse whether the cysteine protease, IL-1 converting enzyme (ICE), a caspase considered of key relevance for the apoptotic pathway in different cells and tissues might be induced by cytokines in human islets. Methods: Isolated human islets (from 5 different donors), rat islets and rat insulinoma cells were cultured 24 or 72 hrs with different concentrations and combinations of IL-1 $\beta, T N F \alpha$ and IFN $\gamma$. RNA was isolated and multiplex PCR analysis (26 or 27 cycles) were performed for ICE, iNOS and different house-keeping control genes (here cyclophilin) for normalisation after quantification on a Phospholmager. Visualisation of apoptotic RIN cells was based on the terminal deoxynucleotidyl transferase end-labeling (TUNEL) procedure. Results: ICE mRNA expression as percent of cyclophilin $+/$ SEM, $n=5$ and $p$ values vs. control (ns= not significant)

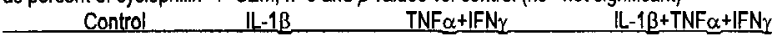

$24 \mathrm{hrs}: 6.2 \pm 2.0 \quad 2.4 \pm 0.9(\mathrm{~ns}) \quad 50.8 \pm 7.8(p<0.001) \quad 20.5 \pm 2.9(\alpha<0.004)$ 72hrs: $3.2 \pm 1.2 \quad 2.1 \pm 0.3$ (ns) $\quad 34.4 \pm 7.0(\not<0.003) \quad 18.4 \pm 3.0(\times 0.002)$ The conditions of induced ICE mRNA expression correlates well with the previously observed NO-independent apoptosis in human islets following 6-9 days cytokine exposure. Analysis of rat islets and RIN cells also showed cytokine induced ICE mRNA expression, in the RIN cells demonstrated to be independent of NO production. Conclusion. These data suggest that different mechanisms may be activated by cytokines to induce $\beta$-cell damage, and that ICE expression may be critically involved in the NO-independent apoptosis induced in human islets by long term exposure to cytokines. 
CYTOKINE MEDIATED NITRIC OXIDE PRODUCTION AND FAS EXPRESSION ACT SYNERGISTICALLY ON $\beta$-CELL DAMAGE

S. Frigerio, G.A. Hollănder and U. Zumsteg. University Children's Hospital and Dept. of Research, CH-4005 Basel, Switzerland.

Inflammatory cytokines and toxic free radicals have been demonstrated to play a major role early in the pathogenesis of IDDM. However, intracellular signalling pathways of beta cell death are poorly understood. Our investigations focus on cytokine induced molecular mechanisms leading to beta cell damage. Isolated OF1 mouse islets and an established beta cell line (NIT-1) exposed to a combination of proinfalmmatory cytokines (IL-1 $\beta$, IFN $\gamma$ and TNF $\alpha$ ) were assayed for mechanisms leading to impaired insulin secretion. After cytokine exposure, a profound decrease in insulin secretion was observed in both islets $(58 \pm 3 \%)$ and NIT-1 cells $(30 \pm 5 \%)$. This effect was mediated by the production of nitric oxide (NO) in the beta cell itself as marked induction of inducible NO synthase (iNOS) was found by RT-PCR in both islets and NIT-1 cells. Furthermore, islets from iNOS deficient mice were resistant to the cytokine induced inhibition of insulin secretion. However, both wild type and iNOS deficient islets showed a significant increase in programmed cell death $(30 \pm 2 \%)$ upon exposure to cytokines. This result suggests an NO-independent signalling pathway leading to apoptosis. Phenotyping of wild type and iNOS deficient islets and NIT-1 cells exposed to cytokines revealed also the upregulation of Fas (CD95/APO-1). Importantly, crosslinking of this surface molecule by soluble Fas-ligand (FasL) resulted in enhanced apoptosis ( $45 \pm 7 \%$ ). In conclusion these data provide support to the contention that proinflammatory cytokines induce beta cell damage via the production of NO radicals and the induction of apoptosis. The latter is also mediated by Fas expression and may be triggered in situ by activated FasL bearing $\mathrm{T}$ cells.

\section{9}

INTERLEUKIN-18 MODIFIES THE PHENOTYPE OF B CELLS MAKING THEM MORE RESISTANT TO CYTOTOXIC ASSAULTS Zhidong Ling, Mark Van De Casteele, Décio L. Eizirik and Daniel Pipeleers Diabetes Research Center, Faculty of Medicine, Vrije Universiteit Brussel. Laarbeeklaan 103, B-1090 Brussels, Beigium

In vitro studies on rat $B$ cells have indicated that these cells can actively participate in their own destructive process, either by modulating their sensitivity to toxic agents, or by activating their repair and/or defence mechanisms. The cytokine interleukin-1/3 (IL-1) is cytotoxic to isolated rat islets but not in cultures of single purified rat $B$ cells. The present study demonstrates that an initial exposure to $\mathrm{IL}-1 \beta$ renders rat $B$ cells more resistant to different cytotoxic agents. Exposure to $\mathrm{IL}-1 \mathrm{~B}(0.1 \mathrm{ng} / \mathrm{ml})$ for $96 \mathrm{~h}$ alters the morphologic phenotype of at least 40 percent rat $\beta$ cells, changing their spherical appearance into spreading over the culture dish. This alteration is associated with an increased cellular expression of heat shock protein 70 (HSP70), heme oxygenase (HO) and manganese superoxide dismutase (MnSOD) ( $\mathrm{P}<0.05 \mathrm{vs}$. control). When tested after $20 \mathrm{~h}$ culture, the IL-I treated B cells exhibited a 70 and 60 percent reduction in cell death ( $\mathrm{p}<0.05$ vs. control) following exposure to, respectively, alloxan $(2 \mathrm{mM})$ and streptozotocin ( $2 \mathrm{mM})$. This protective effect was also observed in an arginine-free medium, indicating its independence of NOproduction. Pretreatment with IL-1 also protected rat $B$ cells against the NO donor GEA $(50 \mu \mathrm{M})$-induced $B$ cell death, but this effect required the presence of arginine. Protein analysis indicates that arginine is needed for the IL-1-induced HO expression but not for MnSOD and HSP70-expression. It is concluded that interleukin-1 $B$ is not necessarily cytotoxic for $B$ cells, but can, instead, make them more resistant to cytotoxic agents. This cytokine-induced protection of $B$ cells is associated with changes in cellular morphology and protein expression.
FAS ENGAGEMENT LEADS TO APOPTOSIS OF HUMAN ISLET CELLS FOLLOWING EXPOSURE TO INTERLEUKIN- $1 \beta$

A.C. Loweth, G.T. Williams, J.H.B. Scarpello, R.F.L. James* and N.G. Morgan, Universities of Keele, Staffs. \& Leicester*, U.K.

Recent work has highlighted the importance of induction of apoptosis to the development of diabetes and has implicated the interaction of Fas ligand with its receptor ( $F$ as) in $\beta$-cell death. Indeed, there is evidence that $F$ as expression is increased on human $\beta$-cells, in response to interleukin-1 $\beta$ (IL-1) treatment. Here, we report that IL-1 alone induces apoptosis in human islets and we confirm, using functional evidence, that IL-1 further sensitises human islets to the induction of apoptosis by a Fas-dependent mechanism. Human islets were cultured for 96 hours in the presence or absence of IL-1, agonistic anti-Fas antibody or both. Islets were dispersed, stained with acridine orange and the \% of apoptotic cells determined by fluorescence microscopy (1000 cells/sample were scored: \% apoptosis (mean \pm SEM): Control: $0.65 \pm 0.06 ;+2 \mathrm{pg} / \mu \mathrm{l} \mathrm{IL-1 \beta \text {: }}$ $1.88 \pm 0.15^{*}$; +anti-Fas: $0.73 \pm 0.11$; + HL-1+anti-Fas: $3.45 \pm 0.53^{* *}$. ${ }^{*}$ p $<0.001$ vs control; ${ }^{* *} p<0.001$ vs IL-1 $\beta$ ). Thus, only after exposure to IL-1 were islets susceptible to anti-Fas-induced apoptosis, suggesting that IL-I may upregulate Fas expression on islets. Western blot analysis confirmed that human islets did not express Fas prior to culture, but that Fas was upregulated in cultured human islets of Langerhans in a time-dependent manner. A striking observation was that, in a total of 6 batches examined, a single, 37kDa band corresponding to Fas ligand was found to be constitutively expressed in normal human islets (Western blotting). Immunohistochemical examination of human pancreas sections revealed that Fas ligand is co-localised with insulin, indicating $\beta$-cell specific expression. Thus, under conditions of cytokine exposure, human islets can express both effector molecules of the Fas/FasL pathway and may be rendered especially susceptible to apoptosis.

\section{0}

HUMAN AND RAT ISLETS ARE PROTECTED AGAINST OXIDATIVE STRESS IN VITRO BY HEAT SHOCK PROTEIN 70 GENE TRANSFER P. Y. Benhamou, C. Moriscot, L. Badet, M. J. Richard, J. Kerr-Conte, F. Pattou and S. Halimi. Endocrinology, CHU, BP 217X, 38043 GRENOBLE, FRANCE.

Susceptibility of pancreatic islets to oxidant stress may compromise islet viability and contribute to primary non function of allo-or xenogenic grafts. We investigated the influence of overexpression of Heat Shock Protein 70 (HSP70) on the viability of rat and human islets.

Islets were transfected with an episomal expression vector containing the human HSP70 cDNA under the control of SV40 promoter, using polycationic liposome DOSPER $\left(1.7 \mu \mathrm{g}\right.$ DNA $/ 10^{5}$ cells, ratio DOSPER/DNA $2 \mu \mathrm{l} / \mu \mathrm{g}$ ). Oxidant stress was induced $48 \mathrm{~h}$ later by xanthine oxidase-hypoxanthine (XO 7.5 to $25 \mathrm{U} / \mathrm{ml}, \mathrm{HX} 0.5 \mathrm{mM}$ ). Islet cell viability was assessed $72 \mathrm{~h}$ after gene transfer by a quantitative colorimetric assay (WST-1 test). Islet function was tested by static incubation assay.

HSP70 gene expression assessed by Western blot was consistently enhanced in transfected islets, comparable to expression achieved by a $90-$ min- $43^{\circ} \mathrm{C}$ heat shock, and could be detected $48 \mathrm{~h}$ post transfer and at least for 10 days. Following XOHX exposure $(25 \mathrm{mU})$, human islet viability was decreased by $21.6 \pm 2.3 \%$. The same stress applied to HSP70transfected islets reduced islet viability by $2.6 \pm 2.6 \%(\mathrm{p}<0.01)$, meaning a protection of $87.8 \pm 17.0 \%$ conferred by HSP70. Similarly, a $64.2 \pm 6.1 \%$ protection was observed when transfected rat islets were exposed to XOHX $(7.5 \mathrm{mU})$. Basal and glucose-stimulated insulin secretion was also significantly improved in HSP70-transfected islets exposed to XOXH stress.

Cationic liposomes are potentially relevant vectors to be used for gene transfer in the islet transplant setting. We suggest that HSP70 gene transfer may be a realistic approach to protect encapsulated islet grafts against the adverse effects of non-specific inflammation. 


\section{OP 36}

\section{Diabetic Embryopathy - Clinical and Experimental Advances}

\section{1}

STIMULATION OF TUMOR NECROSIS FACTOR SECRETION BY HIGH D-GLUCOSE IN UTERINE CELLS IS MEDIATED BY INTERLEUKIN-1. S. Pampfer, S. Cordi, C. Dutrieux, I. Vanderheyden and R. De Hertogh. OBST 5330 Unit, University of Louvain Medical School, Brussels, Belgium.

Recent observations have shown that the synthesis of TNF- $\alpha$ is up-regulated in the uterus of the diabetic pregnant rat at the time of implantation and that TNF$\alpha$ inhibits the rates of cell proliferation and differentiation in implanting embryos. The aim of the present study was to determine whether glucose directly stimulates TNF- $\alpha$ secretion by uterine cells. In a first set of experiments, primary cultures of mouse uterine cells were incubated in either 8 $\mathrm{mM}$ or $28 \mathrm{mM}$ of D-glucose and TNF- $\alpha$ was assayed in the culture supernatants. High D-glucose was found to increased TNF- $\alpha$ release $(P \leq 0.01)$. There was no effect when a hyperosmolar control medium containing L-glucose was used. In a second set of experiments, confluent cultures of the mouse uterine epithelial cell line WEG-1 were treated with either $8 \mathrm{mM}$ or $28 \mathrm{mM}$ of D-glucose. High D-glucose had no effect on TNF- $\alpha$ secretion by WEG-1 cells. When WEG-1 cells were exposed to IL-1 $\beta$, however, a dose-dependent increase in TNF- $\alpha$ secretion was detected in the culture supernatants $(P \leq 0.05$ with the lowest effective $I L-1 \beta$ concentration of $250 \mathrm{pg} / \mathrm{ml}$ ). The impact of $\mathrm{LL}-1 \beta$ on WEG-1 cells was neutralized by adding a molar excess of IL-1B receptor antagonist. The stimulatory effect of D-glucose on TNF- $\alpha$ secretion by primary cultures of uterine cells was also blocked by the $I L-1 \beta$ receptor antagonist. Immunocytochemical analysis of the primary uterine cell cultures revealed that $13 \%$ of the cells were contaminating macrophages. Because $28 \mathrm{mM}$ of D glucose was found to stimulate $1 \mathrm{~L}-1 \beta$ secretion in macrophages of the mouse cell line RAW 264.7 when compared with $8 \mathrm{mM}(\mathrm{P} \leq 0.01)$, it is speculated that the effect of diabetes on TNF- $\alpha$ synthesis in the uterus is mediated via the release of $I L-1 \beta$ by macrophages in response to hyperglycemia. Disruptions in the uterine cytokine network implicated in the regulation of the implantation process may contribute to the peri-implantation embryopathy associated with maternal diabetes.

\section{3}

DECREASED EXPRESSION OF CYCLOOXYGENASE AND PGE2 LEVELS IN RAT EMBRYOS EXPOSED TO A DIABETIC ENVIRONMENT

P. Wentzel, N. Welsh, and U. J. Eriksson, University of Uppsala, Uppsala, Sweden.

The cellular mechanisms of the diabetic embryopathy are not completely understood. Previous experimental studies have suggested that diabetes-induced disturbance of arachidonic acid and prostaglandin (PG) metabolism is associated with the embryonic dysmorphogenesis. The aim of the present work was to determine whether hyperglycemia in vitro or maternal diabetes in vivo affects the expression of the genes coding for cyclooxygenase (COX), which is the rate-limiting enzyme of the PG biosynthesis, thereby altering the production of $\mathrm{PGE}_{2}$, a prostaglandin important for the closure process of the neural tube. To estimate $\mathrm{COX} 1+2$ gene expression and $\mathrm{PGE}_{2}$ concentration, rat embryos were harvested after $24-48 \mathrm{~h}$ of embryo culture in low $(10 \mathrm{mmol} / \mathrm{l} ; 10 \mathrm{G})$ or high glucose concentration $(30 \mathrm{mmol} / \mathrm{l} ; 30 \mathrm{G})$, or on days 10 or 11 of normal $(\mathrm{N})$ or diabetic (D) rat pregnancy, and subjected to semi-quantitative RT-PCR, or $\mathrm{PGE}_{2}$ Enzyme Immuno Assay. We found that both high glucose in vitro and maternal diabetes in vivo caused severe embryonic dysmorphogenesis, and diminished the embryonic and yolk sac gene expression of COX 2 (10G vs. 30G \& N vs. $\mathrm{D}$, day $10,0.05<\mathrm{p}<0.10, \chi^{2}$-statistics), whereas $\operatorname{COX} 1$ expression was not affected in these embryos. The $\mathrm{PGE}_{2}$ concentration in vivo of the $\mathrm{MD}$ embryos was decreased ( $\mathrm{N}$ vs. $\mathrm{D}$, day $10, \mathrm{p}<0.01$, ANOVA). In vitro, however, there was no significant difference in $\mathrm{PGE}_{2}$ concentrations between the $\mathrm{N}$ and $\mathrm{D}$ embryos.

In conclusion, a diabetic environment disturbs the gene expression of embryonic $\operatorname{COX} 2$, which affects the concentration of the developmentally important prostaglandin $\mathrm{E}_{2}$.

\section{2}

HYPERGLYCEMIA CAUSES OXIDATIVE STRESS, INOSITOL DEPLETION AND MALDEVELOPMENT IN THE EMBRYO

U. J. Eriksson and P. Wentzel, Uppsala University, Uppsala, Sweden.

Glucose-induced teratogenesis is related to oxidative stress and hampered prostaglandin biosynthesis. Previously, addition of the antioxidant $\mathrm{N}$-acetylcysteine (NAC) and prostaglandin $\mathrm{E}_{2}$ (PGE2) was shown to diminish glucose-induced embryonic maldevelopment in vitro, in which also a decreased inositol metabolism was implicated. The aim was to investigate putative interrelationships between these different teratological pathways. We exposed embryos in vitro to $30 \mathrm{mM}$ glucose (30G), and to $500 \mu \mathrm{M}$ or $750 \mu \mathrm{M}$ of scyllo-inositol (500SI and $750 \mathrm{SI}$ ), a competitive inositol inhibitor, with the intent to cause embry. onic dysmorphogenesis. We found that $30 \mathrm{G}, 500 \mathrm{SI}$ and $750 \mathrm{SI} \mathrm{em}$ bryos had fewer somites $(16.3 \pm 1.1,21.5 \pm 1.2$ and $19.5 \pm 1.0)$ shorter crown-rump length (CR: $2.8 \pm 0.2 \mathrm{~mm}, 3.3 \pm 0.1 \mathrm{~mm}$ and $3.2 \pm 0.1 \mathrm{~mm}$ ) and higher malformation score (MS; 9.4, 6.4 and 6.9) than control embryos cultured in $10 \mathrm{mM}$ glucose $(10 \mathrm{G})$ (somites: $28.7 \pm 0.3$, CR: $3.4 \pm 0.2 \mathrm{~mm}$, MS: $0.1, \mathrm{p}<0.05$ vs. $30 \mathrm{G}, 500 \mathrm{SI}$ and 750SI, ANOVA and $\chi^{2}$-statistics). Adding $1600 \mu \mathrm{M}$ inositol to the $30 \mathrm{G}$ or $750 \mathrm{SI}$ culture medium partly corrected embryo development (somites: $23.6-23.9$, CR: $3.3-3.5 \mathrm{~mm}$, MS: $3.7-4.2$, p < 0.05 vs. $30 \mathrm{G}$ and $750 \mathrm{SI}$ ), and completely normalized the 500SI embryo development (somites: $28.6 \pm 0.7$, CR: $3.6 \pm 0.1 \mathrm{~mm}$, MS: 0.6). Furthermore, addition of $0.5 \mathrm{mM}$ NAC largely corrected embryogenesis (somites: $26.8 \pm 0.7, \mathrm{CR}: 3.5 \pm 0.1 \mathrm{~mm}$, MS: 1.8 ), whereas addition of $280 \mathrm{nM}$ PGE2 failed to diminish the 500SI-disturbed embryonic maldevelopment

We conclude that high glucose concentration suppresses the embryonic metabolism of inositol and prostaglandins. The inositol depletion is associated with oxidative stress, but seems to be parallel with the inhibition of prostaglandin metabolism, thereby increasing the number of compounds with anti-teratogenic potential in diabetic pregnancy.

\section{4}

OXIDATIVE STRESS MEDIATED LIPID AND DNA ALTERATIONS $\mathbb{N}$ DIABETES. PREVENTION BY VITAMIN E

B. Bonet ${ }^{1,2}$, M. Viana', OI. Aruoma ${ }^{3,4}$, A. Jenner ${ }^{4}$. B. Halliwell ${ }^{4}$ and E. Herrera' ${ }^{1}$ Universidad San Pablo CEU (Madrid-Spain); ${ }^{2}$ Fundación Hospital Alcorcón (Madrid-Spain), ${ }^{3}$ OICA International (Saint Lucia-West Indies), ${ }^{4}$ Pharmacology Group, King's College London (London-UK)

Objectives: l-To study the presence of altereted DNA bases and lipid aldehydes in liver and cmbryos of pregnant diabetic rats and 2- To determine the effects of vitamin $E$ administration upon these parameters

Methods: Diabetes was induced prior to pregnancy by the administration of streptozotocin. Two groups of diabetic rats were performed, one without any supplementation (D) and another treated during pregnancy with vitamin $\mathrm{E}$ (100mg/day) (D+E). A control group was also performed (C). The animals were sacrificed on day 11,5 and the embryo morphology studied. Alterations in the DNA bases (including Fapy-adenine, 5-OH-cytosine, o-OH-guanine), vitamin $\mathrm{E}$ and lipid aldehydes were determined, respectively by gas chromatography/ mass spectronetry; HPLC and by determining the thiobarbituric reacting substances (TBARS).

Results: As expected the percentage of malformations in D rats were $44 \%$, higher than the values observed in $C(7 \%)$ and $D+E(12 \%)$ animals. $D$ rats showed a higher concentration of TBARS in liver, and Fapy-adenine, both in liver and embryos, also a trend toward higher levels of DNA bases altcrations than in the $C$ and the $D+E$ was observed. No differences were observed in the above parameters between the $D+E$ and $C$ groups.

Conclusions: Diabetes during pregnancy is associated with an increased concentration of TBARS in liver, and altered DNA bases, both in liver and embryos. These alterations could be responsible for the diabetic teratogenic effects. The administration of vitamin $\mathrm{E}$ to diabetic rats decreases the lipid and DNA alterations in liver and embryos to levels found in the $\mathrm{C}$ animals 
COMBINED ANTI-TERATOGENIC TREATMENT WITH VITAMIN E AND VITAMIN C IN DIABETIC RAT PREGNANCY

J. Cederberg and U. J. Eriksson, Department of Medical Cell Biology, Uppsala University, Uppsala, Sweden

We have previously shown that the increased risk of embryonic dysmorphogenesis in diabetes can be diminished in vivo by supplementing either $2 \%$ vitamin $\mathrm{E}$ or $4 \%$ vitamin $\mathrm{C}(\mathrm{w} / \mathrm{w})$ to pregnant diabetic rats. The aim of this study was to investigate whether a combination of vitamin $E$ and $C$ would give a protective effect at a lower dose due to synergism between the two antioxidants. Female Sprague-Dawley rats were made manifestly diabetic (MD) with $i . v$. streptozotocin, and nondiabetic females served as controls $(\mathrm{N})$. The MD rats received standard food or food supplemented with either $2 \%$ vitamin $E+4 \%$ vita$\min \mathrm{C}$, or $0.5 \%$ vitamin $\mathrm{E}+1 \%$ vitamin C. At day 20 of pregnancy the offspring were morphologically examined. The MD fetuses showed more cranial malformations (micrognathia) and resorptions ( $19 \%$ and $30 \%$ ) than the $\mathrm{N}$ offspring $(0 \%$ and $4 \%, \mathrm{p}<0.05$, $\chi^{2}$-statistics). The malformations and resorptions decreased in the MD $2+4$ group ( $6 \%$ and $11 \%$ ) compared to the MD group $(0.05<$ $p<0.1$ and $p<0.05$, respectively), whereas the malformations and resorptions of the MD $0.5+1$ group ( $12 \%$ and $20 \%$ ) did not differ from the MD group. The somatic and liver weight of the MD fetuses $(2.5 \pm 0.2 \mathrm{~g}$ and $145 \pm 13 \mathrm{mg}$, ANOVA) were decreased (N: $3.5 \pm$ $0.1 \mathrm{~g}$ and $246 \pm 25 \mathrm{mg}, \mathrm{p}<0.001$ vs. MD) but increased marginally in the antioxidant supplemented MD groups $(2.6-2.7 \mathrm{~g}$ and 182 $186 \mathrm{mg})$. The MD maternal weights were decreased $(269 \pm 15 \mathrm{~g}$ vs. $\mathrm{N}: 385 \pm 7 \mathrm{~g}, \mathrm{p}<0.001$ ) and increased to subnormal values in the vitamin-treated groups (304-313 g, $\mathrm{p}<0.05$ vs. MD).

We conclude that antioxidant treatment is beneficial for offspring and mother in diabetic pregnancy, but the combined dietary supplementation of vitamin $E$ and $C$ does not seem to offer any synergistic advantages.

\section{PREPREGNANCY CLINIC ATTENDANCE: RELATIONSHIP TO CONGENITAL ABNORMALITIES AND HBA1C.}

DWM Pearson, DG Lang and HWS Sutherland, Combined Obstetric Diabetic Clinic, Aberdeen Maternity Hospital, Aberdeen Royal Hospitals NHS Trust, Aberdeen, Scotland, UK, AB25 2ZN

From a geographic area 325 consecutive births were studied in 194 women with established IDDM. Information about contraception and pregnancy planning was widely available and a joint prepregnancy clinic (PPC) held weekly. One hundred and twenty one (37.2\%) pregnancies were preceded by PPC attendance and in this group one major malformation ( $0.8 \%$ ) occurred in a mother who had been advised to defer pregnancy till control improved. In the non attendees at the pre-pregnancy clinic (oPPC) eight (3.9\%) major congenital abnormalities were diagnosed from birth to 1 year of life. In all women who had an infant with a major congenital abnormality, first trimester $\mathrm{HbAlc}$ was either significantly raised or unavailable due to delayed attendance or care. The method of HbAlc analysis changed during the course of the study. In a sub-group of 104 pregnancies (40 PPC, 64 oPPC) HbAlc levels were companed at 5 time periods during pregnancy. Mean maternal HbAlc levels were lower in the PPC group than in the oPPC group at 0-8 weeks $(7.2 \pm 1.6 \%-v$ $8.5 \pm 1.9 \%)$ and 8.16 weeks $(6.4 \pm 1.6 \% \cdot v-7.5 \pm 1.4 \%)$ but identical at $17-24$ weeks $(6.1 \pm 0.9 \%-v \cdot 6.4 \pm 1.3 \%), 25-34(6.4 \pm 1.0 \% \cdot v$ $5.6 \pm 1.2 \%)$ and $35-40$ weeks $(6.4 \pm 1.0 \%-v-6.4 \pm 1.0 \%)$. Attenders for PPC were older $(p=0.0001)$ and more often non-smokers $(p=0.002)$. No significant differences in parity, pregnancy number, pre-pregnancy $B M I$ or age at diagnosis were found. Women with complicated diabetes (White class $F+R$ ) often did not attend for PPC. Conclusion: Attendance at a pre-pregnancy clinic is associated with fewer congenital malformations and better glycaemia during early pregnancy.

\section{7}

\section{OXYDATIVE STRESS AS CAUSAL FACTOR OF} COMPLICATIONS IN DLABETIC PREGNANCY. E.Wender-Ożegowska, J.Koźlik, R.Biczysko, Karol Marcinkowski University School of Medical Sciencies, Poznań,Poland

Maternal diabetes and the consequent hyperglycemia are associated with high frequency of fetal congenital malformations, spontaneous abortions and intrauterine death. Experimental studies have revealed, that hyperglycemia initiates glycosylation of proteins, resulting in decrease of activity of antioxydative enzymes, as well as enhanced production of free radicals, which have been shown to generate disturbaces of embryonic development. The aim of our study was to investigate, whether maternal diabetes is capable of inducing alterations in the parameters of oxidative stress and if changes in oxidative substrates concentration in maternal blood correlate with embryonic development. 51 patients with pregestational diabetes were chosen for the study, 15 of them have lost their pregnancy. In 9 instances the pregnancy was planned Alt patients were subjected to insulin therapy. Glycemia was estimated by daily self control and by measurement of $\mathrm{HbA}_{\mathrm{IC}}$ concentration. Oxidative stress was evaluated by estimation of: superoxide dysmutase (SOD), catalase and peroxidase activity; malondialdehyde (MDA) concentrations in erythrocytes and in maternal blood serum. Before pregnancy the mean glycemia level in the analyzed group was $119,3 \mathrm{mg} / \mathrm{dl}$, and $\mathrm{HbA} / \mathrm{C}$ was $9,35 \%$, and during whole pregnancy it was maintained at a level of $119,9 \mathrm{mg} / \mathrm{dl}$, and $7,85 \%$. The activity of all antioxidative enzymes was lover before, than during pregnancy, and so was concentration of MDA. The investigated oxidative stress substrates (ROS) as well as glycemia level and $\mathrm{HbA} 1 \mathrm{C}$ did not show any significant differences between pregnancies with and without vascular complications. The correlation of ROS with the degree of glycemia control has shown, that SOD activity decreased with increasing glycemia, but MDA concentration increased with increasing glycemia. Patients with spontaneous abortions, malformations and intauterine deaths had significantly higher levels of glycemia and $\mathrm{HbA}_{1 \mathrm{C}}$, concentrations as well as significantly decreased SOD activity.

Oxidative stress may be assumed as a causal factor of unfavourable outcome of diabetic pregnancy

\section{8}

Prospective Analysis of Neurological Development of Children of Mothers with Diabetes Type I and Gestational Diabetes

G. Ircha', M. Zawodniak-Szałapska', K. Cypryk ${ }^{2}$ and J. Wilczyniski'; 'Dept. of Neurology, ${ }^{2}$ Diabetes Care Unit, ${ }^{3}$ Dept. of Fetal-Maternal Medicine, Research Institute Polish Mother's Memorial Hospital, Łódź, Poland

Aim of the study: The purpose of our research work was early diagnosis of neurological damages and neurodevelopment disorders in children of mothers with diabetes type I and gestational diabetes (GDM). The influence of severity of diabetes and metabolic disorders during pregnancy and perinatal complications in newborns on the neurological state of infants from birth to 18 months was evaluated. Material and methods: The prospective neurodevelopment analysis included 35 children of mothers with diabetes type 1 and 122 children of GDM mothers. The children underwent neurological examination in the $3^{\text {rd }}, 6^{\text {th }}, 9^{\text {th }}, 12^{\text {th }}$, and $18^{\text {th }}$ month of life. Presence of symptoms of neurological damages, motoric development and posture control, coordination of vision and movements, social interactions and speech were evaluated. Results: In the group of the children of mothers with diabetes type 1 four infants $(11,4 \%)$ had serious neurological disorders (including 1 developmental anomaly), 2 infants had cerebral palsy, 1 infant had psychomotoric developmental retardation, and 2 infants had slight speech developmental retardation. In the group of the children of GDM mothers only 1 infant had a light form of cerebral palsy. The dependence of frequency and gravity of neurological disorders in children on diabetes metabolic control, serious hypoglycaemia during pregnancy, and diabetes duration and complication was observed $(p<0.05)$. The dependence of frequency and gravity of neurodevelopment disorders on fetopathy, hypoglycaemia, prematurity, and hypoxemic and ischemic conditions in newborns was observed $(p<0.05)$. Conclusion: 1.Neurological anomalies and neurodevelopment disorders were statistically more frequent in the children of mothers with diabetes type 1 than in the children of GDM mothers. 


\section{OP 37}

\section{New Therapies for Type 2 Diabetes}

\section{9}

METABOLIC AND THERMOGENIC EFFECTS OF ACARBOSE IN NIDDM PATIENTS IN SECONDARY FAILURE

B. Brooks, L. Molyneaux, R. Zilkens, K. Willey, G. Ross and D. K. Yue

Diabetes Centre, Royal Prince Alfred Hospital, Camperdown, Department of

Medicine, University of Sydney, NSW, 2006.

Acarbose lowers post-prandial blood glucose (BG) levels by delaying the digestion and absorption of complex polysaccharides and sucrose. In this study, we evaluated the effects of acarbose on: (i) glycaemic control in NIDDM patients in secondary failure (ii) energy expenditure (EE), diet induced thermogenesis (DIT) and respiratory quotient (RQ), using indirect calorimetry. A total of 12 NIDDM patients (M/F: 8/4; Age: $56 \pm 9$ yrs; Duration: $10.1 \pm 4.6 \mathrm{yrs;}$ BMI 29.6 $+2.7 \mathrm{~kg} / \mathrm{m}^{2} ; \mathrm{HbA} 1 \mathrm{c}: 8.8 \pm 0.9 \%$ ) completed 8 weeks treatment with acarbose (100 mg tds). Glycaemic control, EE and DIT were assessed at baseline and week 8. Resting metabolic rate (RMR) was measured for 45 minutes after which patients ate a standard meal ( 440 calories with $60 \mathrm{~g}$ CHO). EE, DIT and RQ were then measured for the next 4 hours. Blood was collected every 15 minutes to evaluate BG and insulin (INS) area under the curve (AUC). At week 8, acarbose was taken with the test meal. After 8 weeks treatment with acarbose, HbA1c was lower compared to baseline $\left(8.8 \pm 0.9 \%\right.$ vs $8.0^{*} \pm 0.9 \% ; p<0.03$, Paired t test). Acarbose acutely lowered post-prandial BG and INS AUC by $18 \%$ and $11 \%$ respectively. The acute changes in BG AUC induced by a test dose of acarbose $100 \mathrm{mg}$ varied amongst individual patients, and correlated with the change in $\mathrm{HbA1c}$ over the 2 month study period $(r=0.87, p<0.01)$. At week 8 , $R Q$ was lower at 30 minutes compared to baseline $(0.86 \pm 0.04$ vs $0.83 \pm 0.05$, $P<0.02$, Paired $t$ test). There was a significant effect of Acarbose on $E E$ and DIT for the first 120 minutes post meal $\left(F_{392} 3.4 ; p<0.05, F_{268} 6.3 ; p<0.01\right.$, respectively, Repeated Measures ANOVA). In conclusion, acarbose changes the pattern of post meal thermogenesis and improves glycaemic control in NIDDM patients in secondary failure. A test dose of acarbose can identify individual's responsiveness to this agent.

\section{1}

THE SAFETY OF ANTI-OBESITY TREATMENT WITH THE NOVEL LIPASE-INHIBITOR, ORLISTAT (XENICAL TM): AN OVERVIEW

\section{W. Canovatchel, Roche Pharmaceuticals, Nutley, New Jersey, USA.}

Orlistat represents a new class of anti-obesity drugs, a highly selective lipaseinhibitor which acts exclusively within the intestinal tract. When taken in combination with a low-fat, moderately hypocaloric diet for 1 year orlistat resulted in significantly greater weight loss than placebo $(p<0.05)$ significantly greater reduction in waist circumference $(p<0.0001)$ and substantial improvements in serum lipid profiles and glucose tolerance status. These benefits were sustained during the second year of treatment, with orlistat-treated patients maintaining the significantly greater weight loss $(p<0.0001)$ and reduced waistline circumference $(p=0.014)$ as well as improved lipid profiles despite a $200-300 \mathrm{kcal} /$ day increase in the diet after 1 year. The most common adverse events were gastrointestinal [GI], reported by $79.8 \%$ of patients treated with orlistat $120 \mathrm{mg}$ tid in year 1 (placebo $=56.8 \%$ ) and by $41.1 \%$ of orlistat-treated patients in year 2 (placebo $=35.1 \%$ ). Oily spotting (26.6\%), flatus with discharge $(23.9 \%)$ and faecal urgency $(22.1 \%)$ were the most frequently reported GI adverse events, which were generally mild and transient. In general the first occurrence of these events was within 3 months of starting therapy. Half of the GI episodes lasted for less than 1 week. There were no clinically relevant changes in haematology, blood chemistry or urinalysis parameters. There were slight decreases in vitamin $\mathrm{D}$ (25-OH-D) but no loss of bone density or alteration of calcium metabolism (assessed by PTH) There were no decreases in essential fatty acid levels and orlistat had no detrimental effect on vitamin A levels. The vitamin E/LDL-cholesterol ratio increased during treatment with both orlistat and placebo and vitamin $\mathrm{K}$ levels (assessed by prothrombin time) remained within the normal range during 2 years of treatment. Because of the lack of systemic uptake, orlistat has a particularly benign safety profile. It is a unique feature of orlistat treatment that, by learning to reduce their fat intake, patients can exercise control over the incidence of the GI adverse events
230

THIS ABSTRACT HAS BEEN WITHDRAWN BY THE AUTHOR.
232

LONG-TERM USE OF REPAGLINIDE VERSUS GLYBURIDE IN TYPE 2 DIABETES.

P. MÜLLER and P. STRANGE, Princeton, NJ, for the REPAGLINIDE STUDY GROUP

The purpose of the study was to compare the long-term (1-year) use of repaglinide (REP) and glyburide $(G)$ in patients with type 2 diabetes. The trial was a prospective, multicenter, double-blind, randomized, parallelgroup assessment of the efficacy and safety of these oral hypoglycemic agents (OHAs). A total of 576 patients were randomized to receive monotherapy with REP ( $n=383$ ) or $\mathrm{G}(\mathrm{n}=193)$. Of these patients, $87 \%$ had previously been treated with OHAs; $13 \%$ were OHA-naïve. During a dose-adjustment period (weeks 1 to 8 ) patients were treated with preprandial doses of REP ( 0.5 to $4 \mathrm{mg}$ with 3 meals). Doses of G were from 2.5 to $15 \mathrm{mg}$. Doses of both study drugs were increased toward a target fasting plasma glucose (FPG) range of 4.4 to $7.8 \mathrm{mmol} / \mathrm{L}$ after which the final dose was maintained for 12 months. REP provided glycemic control similar to that of $\mathrm{G}$. For patients previously treated with OHAs, glycemic control was maintained for 14 months $(0.002$ change from baseline). In patients naive to oral hypoglycemic therapy, a decrease in the proportion of total $\mathrm{HbA}_{1 \mathrm{c}}$ from 0.094 to 0.076 was observed within the first 3 months $(0.018$ change from baseline). The improvement in $\mathrm{HbA}_{\mathrm{lc}}$ was maintained for the rest of the study (change from baseline: 0.009 ). In both groups, the most frequent adverse events possibly related to study drugs were: headache, dizziness, tremor, and increased appetite After 14 months of treatment, body weight decreased by $-0.3 \mathrm{~kg}$ in previously treated patients and increased $2.4 \mathrm{~kg}$ in naive patients. The overall safety profile including changes in proinsulin and lipid levels was similar with both agents. Conclusion: the efficacy and safety of REP is similar to those of $\mathrm{G}$ 


\section{3}

EFFECTS OF PRAMLINTIDE THERAPY: A I-YEAR STUDY IN INSULINREQUIRING TYPE 2 DIABETES

R. Ratner, C. Levetan, S. Schoenfeld, S. Jeffcoate, and O. Kolterman for the Pramlintide Type 2 Study Group. Washington, DC; San Diego, CA, USA

In type 2 diabetes, native amylin levels fall over time, with frank deficiency occurring when patients require insulin. We undertook a prospective, placebocontrolled dose-ranging study of pramlintide in insulin-treated type 2 diabetes. We randomized 539 subjects to receive placebo or pramlintide $30 \mu \mathrm{g}, 75 \mu \mathrm{g}$, or $150 \mu \mathrm{g}$ TID and followed them for 52 weeks. Subjects were obese (mean BMI=30.7), with a mean duration of diabetes of 12 years, and a mean daily insulin requirement of $60 \mathrm{U}$. $\mathrm{HbA}_{\mathrm{lc}}$ concentrations fell to lower levels, and were better maintained over time, in subjects receiving higher doses of pramlintide. Mean relative changes in $\mathrm{HbA}_{18}$ for the intent-to-treat population were:

\begin{tabular}{|c|c|c|c|c|}
\hline & $\begin{array}{c}\text { Placebo } \\
n=132\end{array}$ & $\begin{array}{l}30 \mu \mathrm{g} \\
n=116\end{array}$ & $\begin{array}{c}75 \mu \mathrm{g} \\
\mathrm{n}=133\end{array}$ & $\begin{array}{l}150 \mu \mathrm{g} \\
\mathrm{n}=126\end{array}$ \\
\hline $\mathrm{HbA}_{\mathrm{Ic}}(\%)$ at baseline & 9.15 & 9.00 & 9.27 & 9.23 \\
\hline Relative $\Delta$ at 13 wks & $-5.4 \%$ & $-7,3 \%$ & $-9.3 \%$ & $-10.1 \%$ \\
\hline Relative $\Delta$ at 26 wks & $-3.9 \%$ & $-5.6 \%$ & $-8.0 \%$ & $-8.2 \%$ \\
\hline Relative $\Delta$ at 52 wks & $-2.1 \%$ & $-3.7 \%$ & $-5.0 \%$ & $-6.3 \%$ \\
\hline
\end{tabular}

Based on DCCT findings, this $>5 \%$ relative reduction in $\mathrm{HbA}_{10}$ at 52 weeks in the 75 and $150 \mu \mathrm{g}$ groups would result in a $>20 \%$ reduction in the risk of complications. Differences in $\mathrm{HbA}_{\mathrm{tc}}$ were amplified in those subjects in whom insulin doses were not increased over time. Pramlintide-treated subjects required fewer upward insulin adjustments than those receiving placebo. No differences in frequency or severity of hypoglycemia were seen. With improved glycemic control, pramlintide resulted in a significant weight loss ( $-2.7 \mathrm{~kg}$ for $150 \mu \mathrm{g}$ group; $-1.6 \mathrm{~kg}$ for $75 \mu \mathrm{g}$ group) for all doses compared to placebo. Transient nausea was the most common side effect noted, with tachyphylaxis within 2-4 weeks. We conclude that pramlintide at 75 and $150 \mu \mathrm{g}$ TID doses with insulin improved glycemic control compared to insulin alone, without augmented insulin doses, weight gain, or added hypoglycemic events.

\section{5}

IMPROVEMENT IN INSULIN ACTION AND INCREASE IN FAT OXIDATION

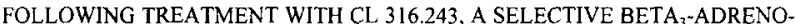
CEPTOR AGONIST IN HUMANS

C. Weyer, P.A. Tataranni, S. Snitker, E. Danforth Jr. and E. Ravussin, CDNS/ NIDDK/ NIH, 4212 N $16^{\text {th }}$ Street, Phoenix, AZ, 85016, USA.

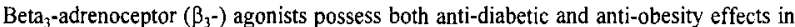
rodent models of obesity and may also have therapeutic potentials in humans. The aim of this randomised, double-blind, placebo-controlled study was to assess the effects of 8 weeks of treatment with $1500 \mathrm{mg} /$ day of CL 316.243 , a highly selective $\beta_{3}$-agonist, on insulin action and energy metabolism in 14 healthy, lean, male subjects [age 22.5 $\pm 3.3 \mathrm{yrs}$, body fat $15 \pm 5 \%$ (mean $\pm \mathrm{SD}$ ), drug/placebo $10 / 4$ ]. Insulin-stimulated glucose uptake $(\mathrm{M})$ and non-oxidative glucose disposal (NOGD, indirect calorimetry) were determined during a 100 min euglycemic glucose clamp (plasma insulin $\sim 60 \mu \mathrm{U} / \mathrm{ml}$ ) before and after 4 and 8 weeks of treatment. 24-hour energy expenditure (24-EE), 24hour respiratory quotient $(24-\mathrm{RQ})$ and the oxidation rates of fat and carbohydrate were measured before and after 8 weeks in a respiratory chamber. Body weight and $\%$ body fat (DEXA) were also determined. After 4 weeks of treatment, CL 316.243 increased $\mathrm{M}(+45 \% \mathrm{p}<0.01)$ and NOGD $(+82 \% \mathrm{p}<0.01)$, but none of these effects persisted for 8 weeks (Table), 24-EE, body weight and \% body fat did not change after 8 weeks, but CL 316.243 markedly lowered $24-\mathrm{RQ}(-7 \pm 2 \%$, Table), indicating a $23 \pm 6 \%$ increase in fat oxidation $(\mathrm{p}<0.01)$ and a $17 \pm 4 \%$ decrease in carbohydrate oxidation $(p=0.05)$. None of these parameters changed in the placebo group.

\begin{tabular}{llll} 
& baseline & 4 weeks & 8 weeks \\
\hline M (mg/kg EMBS/min) & $5.6 \pm 2.0$ & $8.1 \pm 2.3^{* *}$ & $6.1 \pm 1.8$ \\
NOGD (mg/kg EMBS/min) & $3.2 \pm 1.8$ & $5.8 \pm 2.1^{* *}$ & $4.0 \pm 1.4$ \\
24-EE (kcal/day) & $2052 \pm 120$ & n.a. & $2066 \pm 62$ \\
24-RQ & $.855 \pm .017$ & n.a. & $.831 \pm .028 * *$ \\
body weight $(\mathrm{kg})$ & $73.3 \pm 9.0$ & $73.2 \pm 8.8$ & $73.3 \pm 8.9$ \\
\hline
\end{tabular}
$\begin{array}{lccc}\text { body weight }(\mathrm{kg}) & 73.3 \pm 9.0 & 73.2 \pm 8.8 & 73.3 \pm 8.9 \\ * *=p<0.01 \text { vs. baseline, EMBS (estimated metabolic body size })=\mathrm{FFM}+17 \mathrm{~kg}\end{array}$ We conclude that CL 316.243 improves insulin action in humans, but this effect seems to be only transient. At the reported dose, the compound has no effect on energy expenditure or body composition, but it stimulates fat oxidation, which may favour the reduction of fat stores if maintained over time.
CONTINUOUS SUBCUTANEOUS INFUSION OF GLP-1 LOWERS BLOOD GLUCOSE AND REDUCES APPETTTE IN NIDDM PATIENTS

M. Toft-Nielsen ${ }^{1,2}$, S. Madsbad' and J.J. Holst'. ${ }^{1}$ Dept. of Endocrinology, Hvidovre Hospital and ${ }^{2}$ Dept. of Medical Physiology,Panum Institute,Copenhagen, Denmark

The gut hormone GLP.1 has insulintropic and anorectic effects during iv infusion and has been proposed as a new treatment for NIDDM and obesity. The effect of a single se injection is short lasting due to rapid degradation. We, therefore, infused GLP-1(1.2 or $2.4 \mathrm{pmol} / \mathrm{kg} / \mathrm{min})$ or saline subcutaneously for 48 $h$ in randomised order in 11 patients with NIDDM to evaluate the effect on appetite during fixed energy intake, on plasma glucose (PG), insulin, glucagon, postprandial lipidemia, blood pressure(BP), heart rate (HR) and basal metabolic rate (BMR). The high rate infusion resulted in elevations of the plasma concentrations of intact GLP-1 similar to those observed after iv infusion of 1.2 $\mathrm{pmol} / \mathrm{kg} / \mathrm{min}$, previously shown to effectively lower blood glucose in NIDDM patients. Fasting PG (day 2) decreased dose dependently from $13.2 \pm 1.3$ (saline) to $11.3 \pm 1.0 \mathrm{mmol} / \mathrm{(GLP}-1), p=0.001$, during the high rate infusion, and from $12.2 \pm 2.7$ (saline) to $11.8 \pm 2.9 \mathrm{mmol} / \mathrm{h}$ (GLP-1), NS, during the low rate infusion. Correspondingly, $24 \mathrm{~h}$ mean $\mathrm{PG}$ decreased from $14.4 \pm 1.5$ to $12.1 \pm 1.2$ $\mathrm{mmol} / \mathrm{l}, \mathrm{p}=0.005$, (high rate infusion), and from $14.5 \pm 2.9$ to $13.5 \pm 2.8 \mathrm{mmol} /$, NS (low rate infusion). Fasting insulin and C-peptide levels were significantly higher during the high dose GLP-1 administration, whereas glucagon levels were unchanged. Neither triglycerides nor free fatty acids were affected. The GLP-1 administration decreased hunger $(p<0.0001)$, increased satiety $(p=0.001)$, increased fullness $(p=0.02)$, and decreased prospective food intake $(p=0.0004)$. No side effects during GLP-1 infusion were recorded except for a short lasting cutaneous reaction. BMR and HR did not change significantly during GLP-1 administration, but systolic and diastolic BP was slightly lower during the GLP-1 infusion. We conclude that a $48 \mathrm{~h}$ continuous subcutaneous infusion of GLP-1 in NIDDM patients 1) lowers fasting as well as meal-related PG, 2) reduces appetite, 3) has no gastrointestinal side effects, and 4) has a small positive effect on BP.

\section{6}

DO PATIENTS WTTH DIABETES TAKE THEIR ORAL HYPOGLYCAEMIC TABLETS?

GM Brennan. DIR Boyle, AD McMahon, TM MacDonald, RT Jung, RW Newton and AD Morris for the DARTS/MEMO Collaboration, University of Dundee Scotland.

There are no direct data on adherence to therapy in NIDDM. Using the DARTS database (April 1993 to September 1994) we compared the known prescribed sulphonylurea (SU) and metformin (MF) dose ( $\mathrm{mg} /$ day) with tablets supplied. MEMO contains complete data on prescriptions encashed at all Tayside pharmacies. The medically recommended dose and cumulative volume of prescriptions supplied were used to calculate the days of total drug coverage per annum, expressed as the adherence index (AI). Relationships between AI, glycaemic control ( $\mathrm{HbAlc}$ ), age, and duration of diabetes were investigated in all patients taking MF or SU alone. We studied 200 patients, $120 \mathrm{SU}$ and $80 \mathrm{MF}$ treated. There were no differences in age 65 $\pm 11 \mathrm{SU}$ (mean \pm S.D.), $60 \pm 10 \mathrm{MF}$; diabetes duration $4 \pm 5 \mathrm{SU}, 2 \pm 3$ years $\mathrm{MF}$, or $\mathrm{HbA} 1 \mathrm{c} 7.3 \pm 1.4 \% \mathrm{SU}, 7.2 \pm 1.7 \mathrm{MF}$ between groups. 126 patients $(63 \%)$ encashed less tablets than their prescribed dose $(58 \% \mathrm{SU}, 71 \%$ $\mathrm{MF}$ ). The mean AI was 345 days (range 55-891) and 331 days (range 33$662)$ for the SU and MF groups respectively. There was no difference in the AI between the SU and MF groups. These data suggest that almost two-thirds of NIDDM patients are non-adherent regardless of the type of prescribed oral hypoglycaemic therapy. 


\section{OP 38}

\section{Animal Models of Type 1 Diabetes}

\section{7}

THE LEW.1AR1/Ztm-iddm RAT - A NEW IDDM RAT MODEL S. Lenzen*, M. Tiedge", S. Lortz", M. Elsner*, A. Jöms ${ }^{\varsigma}$, G. Klöppel', D. Wedekind $^{\ddagger}$, C. Prokop ${ }^{\ddagger}$, H.J. Hedrich ${ }^{\ddagger}$ "Institutes of Clinical Biochemistry, ${ }^{\ddagger}$ Laboratory Animal Science, and ${ }^{\S}$ Anatomy I, Hannover Medical School, Hannover, and Institute of Pathology, University of Kiel, Kiel, Germany We provide here a first description of a new congenic IDDM rat model (LEW.1AR1/Ztm-iddm) which arose spontaneously last year in an inbred Lewis MHC congenic rat colony in our institution. Diabetes was characterised by weight loss, polydipsia, polyuria, glycosuria, ketonuria, hyperglycemia and hypoinsulinemia. The diabetes incidence was $20 \%$ in this IDDM rat colony with no sex preference. The mean age at diabetes manifestation (blood glucose $21.4 \pm 1.3 \mathrm{mM}$ ) was $61 \pm 2$ days. An analysis of the lymphocyte subsets in peripheral blood of diabetic rats revealed a normal proportion of $T$ lymphocytes as shown by monoclonal antibodies directed against TcR $\alpha \beta$ and $R T \sigma^{a}$. Only at a late stage of the disease a significant reduction of $\mathrm{T}$ lymphocytes was observed in severely diabetic animals. In mildly diabetic rats (blood glucose $<20 \mathrm{mM}$ ) the number of beta cells as well as insulin immunostaining was already reduced in the majority of the islets. All islets were heavily infiltrated with B and T lymphocytes, macrophages and NK cells. Beta cells in areas of insulitis showed characteristic signs of apoptosis in electron microscopy. Quantitative analysis by TUNEL assay revealed 5-12\% apoptotic beta cells. In severely diabetic rats (blood glucose $>20 \mathrm{mM}$ ) most islets were devoid of beta cells but still contained alpha cells and some delta cells. Other organs were not affected. Taken together, this new IDDM rat model has characteristics closer to human IDDM than the established BB rat and the NOD mouse models. In the future this new IDDM rat may be a valuable model to study the genetics of the underlying mechanisms leading to autoimmune diabetes with particular emphasis upon the mutated gene.

\section{9}

IN VIVO SENSIBLIZED LYMPHOCYTES CAUSE ALTERATION OF ANTIGEN EXPRESSION ON B-CELLS IN VITRO

B. Kuttler, H. Wanka and H. J. Hahn; Institute of Pathophysiology, University of Greifswald, Germany

A coculture of lymphocytes with allogeneic islets (MLIC) for $96 \mathrm{~h}$ leads to reduction of B-cells and to an allospecific induction of MHC class II antigens on B-cells. We investigated if peripheral blood lymphocytes (PBL) obtained from allogeneic islet grafted rats (= sensibilization in vivo) cause similar alterations on donor-specific islet cells in vitro. Diabetic BB/OK rats (RT.1") were grafted with 2000 islets (ITx) isolated from LEW.1A rats (RT. $1^{\mathbb{Q}}$ ). Redevelopment of hyperglycaemia (rejection) was observed after $20 \pm 3$ days. $2 \times 10^{5}$ PBL prepared before ITx, after $7 \mathrm{~d}$ of normoglycaemia and after rejection of islets were cocultured with 50 donor-specific islets for $24 \mathrm{~h}$ at $37^{\circ} \mathrm{C}$. For control islets were cultured without PBL. After culture islets were separated from PBL using nylon gauze, dispersed into single cells and used for determination of antigen expression. MHC class I (OX18) and class II (OX6) antigens and an intercellular adhesion molecule (ICAM-1) were detected on B-cells (K14D10) by two-color flow cytometry. After a culture of LEW. IA rat islets for $24 \mathrm{~h}$ we measured $80.9 \pm 2.0 \%$ B-cells $(n=16) .81 .3 \pm 2.7 \%$ of them were $O X 18^{+}$, only $0.9 \pm 0.3 \%$ were $0 \mathrm{X}^{+}$and $19.8 \pm 1.5 \%$ were ICAM- $1^{+}$. PBL obtained before ITx had no effect on OX18 ${ }^{+} B$-cells, induced OX6 on B-cells in 9 of 15 cases (67.3\%) and enhanced ICAM-1 ${ }^{+} B$-cells to $42.7 \pm 3.7 \%$. In contrast, $86 \%$ of the PBL suspensions obtained from normoglycaemic rats at day 7 reduced $O X 18^{+} B$-cells to $40.9 \pm 4.9 \%(p<0.01)$ and induced $0 X 6$ on $B$-cells. These marked effects could never be observed in a 24h-MLIC with lymphocytes from non-grafted rats or after rejection. However, if the culture time was prolonged to $96 \mathrm{~h}$ lymphocytes from nongrafted rats were able to induce MHC class II on B-cells whereas a reduction of OX18 ${ }^{+} B$-cells was not observed.

In summary, the $24 \mathrm{~h}-\mathrm{MLIC}$ is capable to distinguish between effects of sensibilized and non-sensibilized lymphocytes on donor-specific target cells. Reduction of MHC class $I^{+} B$-cells were caused by acutely sensibilized PBL only. The results underline the presence and functional activity of donor-specific lymphocytes in the peripheral blood of islet-grafted BB/OK rats which can be detected by the MLIC. Possibly, this method is usefull for early diagnosis of islet graft destruction.

\section{8}

NOVEL DIABETES PREDISPOSING AND PROTECTING GENES IN THE SPONTANEOUSLY DIABETIC BB/OK RAT

I. Klöting, S. Schmidt and P.Kovacs, Department of Laboratory Animal Science at the Institute of Pathophysiology, University of Greifswald, D17495 Karlsburg, Germany

By several crossing studies it has been demonstrated that the MHC class-II genes of the RTl" haplotype, Iddml, and the lymphopenia, Iddm2, are essential for diabetes development in the BB rat. Using diabetic BB/OK and diabetes-resistant DA rats it has been additionally shown that a third diabetogenic non-MHC gene, Iddm3, mapped on chromosome 18 cosegregates with spontaneous diabetes in the $\mathrm{BB} / \mathrm{OK}$ rat subline. Because mapping results need not be consistent among different crosses, we genetically analysed a new cross population using diabetic BB/OK and diabetes-resistant SHR/Mol rats to evaluate the existence of Iddm3 and to search for additional diabetogenic non-MHC genes using 73 microsatellite markers covering about $63 \%$ of the rat genome. The genetic analysis of $I d d m 1$ and $I d d m 2$ homozygous [(BB/OK x SHR)Fl x BB/OK] first backcross hybrids $(\mathrm{BC} 1)$ confirmed the action of $I d d m 3$ on chromosome 18 and identified 2 new nonMHC loci predisposing as well as protecting diabetes development. The predisposing non-MHC locus, Iddm4, was found near Ighe/D6Mgh2 on chromosome 6 and the protective locus, Iddm 5 r(esistance), was detected around $I g / 2 / \mathrm{Tnt}$ on chromosome 1 . The homologous region of the $I d d m 4$ locus lies on human chromosome 14q32 near the diabetes susceptibility locus IDDMII and Iddm5r on chromosome 11p15 near the IDDM2 locus, known to have some IDDM protective alleles in humans. From these novel findings it is concluded that the diabetogenic phenotype of the BB/OK rat subline is the result of the interaction of predisposing and protecting diabetes genes.

\section{0}

DYNAMICS OF B-CELL DESTRUCTION IN THE NOD MOUSE DIABETES TRANSFER MODEL

M. Füchtenbusch ${ }^{1,2}$ E. Larger ${ }^{2}$ M.F. Richard ${ }^{2}, K$. Thebault ${ }^{2}$, and C. Boitard ${ }^{2}$ ${ }^{1}$ Diabetes Research Institute and 3rd Medical Dept., Krankenhaus MünchenSchwabing, Munich, Germany, ${ }^{2}$ INSERM U342, Hopital Saint-Vincent-de-Paul, Paris, France

It is widely held that the autoimmune destruction of the B-celis during the prediabetic phase of type 1 diabetes is a chronic, lineary process. The aim of this study was to determine whether the activity of the autoimmune stimulation increases decreases or remains stable in the course of this process. As an indirect measure of the activity of the autoimmune stimulation we determined the individual diabetes transfer time after i.v. injection of splenocytes from prediabetic NOD mice of different ages into NOD-SCID mice and correlated this transfer time with the diabetes incidence in the NOD donor mice and their pancreatic insulin content. Three groups $(n=8)$ of NOD mice, aged 5,11 , and 17 weeks (wk) underwent splenectomy and hemipancreatectomy. Then, from each NOD mouse, $10 \times 10^{6}$ splenocytes, collected aseptically in HBSS, were injected into groups of four 6 wk old NOD-SCID mice, respectively. Insulin was extracted from the resected hemipancreas with ammonium hydroxide over $24 \mathrm{~h}$ at $4^{\circ} \mathrm{C}$, and the insulin content was determined by a RIA. 1) diabetes manifestation in the NOD-SCIDs after a mean time of 126 days after transfer of splenocytes from 5 wk old NODs, after 68 days (from 11 wk old NODs), and after a mean time of 43 days (transfer from 17 wk old NODs, 5 vs. 11 wk: $p<0.02,11$ vs. 17 wk: $p<0.001$, linear regression). 2) In the 17 wk old NOD mice, time to diabetes was positively correlated with the diabetes transfer time $(p<0.0001)$ : the later the diabetes manifestation, the later the occurence of the disease in the NOD-SCIDs. 3) Positive correlation of the individual insulin content of the $17 \mathrm{wk}$ old NODs and the diabetes transfer time in the recipient mice $(p<0.0001)$. 4) However, in contrast, no such correlations for these parameters were seen for the 5 and 11 wk ols NOD mice (time to diabetes: 11 $w k, p=0.14,5 w k, p=0.75$; insulin content $11 w k, p=0.81,5 w k, p=0.14$ ). Conclusions: 1) The activity of the autoimmune stimulation seems to increase in the course of $B$-cell destruction. This could be due to epitop spreading and due to a progredient skewing towards a TH1-response. 2) In the late prediabetic phase, close to the manifestation, the immune response seems to be programmed to $B$-cell destruction; it is only at this time, that the pancreatic insulin content predicts the impending manisfestation of the disease. 


\section{1}

iNOS-/- MICE HAVE A REDUCED SENSITIVITY TO IMMUNE MEDIATED $\beta$-CELL DAMAGE: IN VITRO AND IN VIVO STUDIES.

M. Flodström ${ }_{2}$ D.L. Eizirik $\#$, I S. Sandler \# \#pepartment of Medical Cell Biology, Uppsala University, Uppsala Sweden and Tiabetes Research Center, Vrije Universiteit Brussel, Brussels, Belgium.

The role of nitric oxide (NO) for $\beta$-cell destruction in type 1 diabetes remains unclear. The present study evaluate the sensitivity of a mouse genetically deficient in the inducible nitric oxide synthase, iNOS (iNOS- $/$-, background C57BL/6Jx129SvEv), to diabetes induced by multiple low dose streptozotocin (STZ). Furthermore, the effects of in vitro exposure of pancreatic islets to IL-1 $\beta$ were studied. Male control C57BL/ 6 and iNOS- $/$ mice were treated for 5 consecutive days with IP injections of STZ (40 $\mathrm{mg} / \mathrm{kg} \mathrm{BW}$ ) or vehicle (citrate buffer). Untreated C57BL/6 mice showed no increase in blood glucose (BG) levels during a 21 days period, whereas STZ treated C57BL/6 mice gradually developed hyperglycaemia with $12 / 16$ animals having $B G$ levels $>11.1$ $\mathrm{mM}$ on day 21 (MBG of $7.4 \pm 0.5$ and $15.6 \pm 1.6 \mathrm{mM}$ on day 1 and 21 , $\mathrm{n}=16$ ). STZ-treated iNOS-/- mice had no or a minor increase in BG during the observation period with $4 / 17$ mice having a BG level $>11.1$ $\mathrm{mM}$ on day 21 (MBG, $6.9 \pm 0.2$ and $9.8 \pm 0.6 \mathrm{mM}$ day 1 and 21 $\mathrm{p}<0.01$ vs. control C57BL/6 mice day $21, \chi^{2}$-test). Vehicle treated iNOS-/- mice $(n=13)$ kept a normal BG level throughout the period (7.3 $\pm 0.3 \mathrm{mM}$, day 21). No significant decrease in body weight could be observed in any of the groups. A $24 \mathrm{~h}$ exposure period to IL-13 (25 $\mathrm{U} / \mathrm{ml})$ in vitro resulted in an increase in nitrite formation (5-6-fold) and a decrease in glucose induced insulin release and (pro)insulin biosynthesis by islets isolated from C57BL/6 mice, as compared to control islets. Islets from iNOS-/- mice showed neither an increase in nitrite synthesis, nor an impairment in cellular functions after exposure to IL-1 $\beta$. In conclusion, the present findings suggest that NO formation contributes to $\beta$-cell damage both following in vivo treatment with multiple low doses of STZ and following in vitro exposure to IL$1 \beta$.

243

IMPAIRMENT OF MUCOSAL IMMUNE SYSTEM IN THE SMALL INTESTINE OF NOD MICE

D. P. Funda ${ }^{1,2}$, A. Kaas ${ }^{1}$, M-L. Hartoft-Nielsen', T. Bock ${ }^{1}$, K. U. Hartmann ${ }^{3}$, H Tlaskalová-Hogenová ${ }^{2}$, and $\mathrm{K}$. Buschard ${ }^{1}$. 'Bartholin Instituttet, Kommunehospitalet, Copenhagen, Denmark, 'Department of Immunology and Gnotobiology, Institute of Microbiology, Czech Acaderny of Sciences, Prague, Czech Republic, Institute für Experimentelle Immunologie, Philipps-Universităt, Marburg, Germany

Mucosal surfaces represent the major contact area with outer environment and mucosal immune response is known to differ in many respects from the systemic one. Mucosa-associated lymphocytes were reported to form about one half of the early islet-infiltrating mononuclear cells. In addition, $T-\gamma \delta$ lymphocytes were shown to play an important role in suppression of the autoimmune reaction in NOD mice. The aim of this study was to examine lymphocyte subsets within the gut mucosa of NOD mice in comparison with BALB/cJ mice. Using immunohistochemistry, proximal and distal jejunum as well as ileum of 6-week-old NOD and BALB/cJ female mice were stained for $\mathrm{CD}^{+}, \mathrm{CD}^{+}, \mathrm{CD}^{+}, \mathrm{CD} 8 \alpha^{+}, \mathrm{TCR} \gamma \delta^{+}, \mathrm{IgA}^{+}$, and $\mathrm{IgM}^{+}$ cells. By applying several counting methods for each of the markers and/or compartments (lamina propria, epithelium), significantly lower numbers of $\mathrm{CD} 3$ $(88.4 \pm 7.4$ vs. $210.1 \pm 19.1 ; p<0.001), \mathrm{CD4}^{+}(8.4 \pm 0.3$ vs. $14.3 \pm 2.2 ; \mathrm{p}<0.05)$ $\mathrm{CD5}^{+}(29.1 \pm 1.1$ vs. $114.7 \pm 19.3 ; \mathrm{p}<0.001), \mathrm{CD} 8 \alpha^{+}(81.1 \pm 13.5$ vs. $186.5 \pm 17.0$ $\mathrm{p}<0.01)$, and $\mathrm{T}-\gamma \delta^{+}$cells $(34.5 \pm 7.6$ vs. $69.1 \pm 7.9 ; \mathrm{p}<0.001)$ as well as IgA producing cells $(27.1 \pm 1.9$ vs. $43.8 \pm 1.1 ; p<0.001)$ but not IgM cells $(2.6 \pm 0.1$ vs $4.4 \pm 0.7, \mathrm{~ns}$ ) were observed in NOD mice compared to $\mathrm{BALB} / \mathrm{cJ}$ mice. In addition, a significantly lower ratio of intraepithelial to lamina propria $\mathrm{CD} 3^{+}$cells was found in NOD mice compared to BALB/cJ mice $(4.48 \pm 0.79$ vs. $7.9 \pm 1.11, p=0.037)$. No remarkable differences, except for IgA ${ }^{+}$cells, were observed among proximal and distal jejunum, and ileum within each group. Thus, our results show significantly lower number of $T$ and in a lesser extent IgA-producing $B$ cells in the gut of NOD mice in comparison to BALB/cJ mice. The difference is most evident within the intraepithelial $\mathrm{CD}^{+}$cells (i.e. mainly TCR $\gamma \delta^{+}, \mathrm{CD} 8 \alpha{ }^{\dagger}$ ), that may be of importance in induction of mucosal immune response. The impaired mucosal immune system in NOD mice may contribute to autoimunity, e.g. by failing to develop proper immune response to outer environmental antigens.

\section{2}

TISSUE SPECIFICITY OF INTERFERON- $\gamma$ EXPRESSION AND THE GENETIC BACKGROUND CAN AFFECT DEVELOPMENT OF DIABETES

T.Toyonaga, E.Araki, A.Shiragami, H.Motoshima, K.Kaneko, Y.Anami, T.Sasahara, N.Miyamura, H.Kishikawa and M.Shichiri, Department of Metabolic Medicine, Kumamoto University School of Medicine, 1-1-1 Honjo, Kumamoto, 860-8556, JAPAN

The interferon- $\gamma$ (IFN- $\gamma$ ) plays an important roles in the development of insulin dependent diabetes mellitus (IDDM). To analyze the influence of the tissue specific expression of IFN- $\gamma$ and genetic background on the development of diabetes, we microinjected the murine IFN $-\gamma$ transgenes into the pronucleus of fertilized eggs of C57BL/6 mice and established two lines of the transgenic mice. To examine tissue specificity of IFN- $\gamma$ expression, RT-PCR was performed using mRNAs from various tissues (pancreas, liver, spleen, stomach, small intestine, lung, kidney and peripheral blood lymphocyte). One transgenic mouse line (No.5) expressed IFN- $\gamma$ in the liver and the mean concentration of IFN- $\gamma$ in the serum was $3533 \mathrm{pg} / \mathrm{ml}$ at 7 weeks of age. However the No.5 line did not develop diabetes. The other transgenic mouse line (No.44) expressed IFN- $\gamma$ mainly in the pancreas and the mean concentration of IFN- $\gamma$ in the serum was below the detectable level $(125 \mathrm{pg} / \mathrm{ml})$. The No.44 line developed diabetes and the histology of the pancreas showed insulitis. This development of diabetes in No.44 line was inhibited by intraperitoneal administrations of anti-IFN- $\gamma$ antibody. To understand genetic background on the development of diabetes in No.44 line, this line was mated with NOD mice and produced F1(NODxNo44) mice. To our surprise these F1 mice did not develop diabetes at all. These results suggest that both tissue specificity of IFN- $\gamma$ expression and genetic background can affect the development of diabetes in the IFN- $\gamma$ transgenic mice.

\section{4}

1,25-DIHYDROXYVITAMIN D3 INDUCES A Th2 IMMUNE RESPONSE IN NON-OBESE DIABETIC MICE IMMUNIZED WITH GAD65 (524-543),

C. Mathieu, L. Overbergh, K.M. Casteels, O. Rutgeerts and M. Waer. Laboratory for Experimental Medicine and Endocrinology and the Laboratory for Experimental Transplantation, Gasthuisberg, Catholic University of Leuven, 3000 Leuven, Belgium.

Aim : The activated form of vitamin $\mathrm{D}, 1,25(\mathrm{OH})_{2} \mathrm{D}_{3}$, prevents type 1 diabetes in the non-obese diabetic (NOD) mouse and induces an immune shift towards Th2 cytokines, locally in the pancreatic islets as well as in transplanted islets. Here we investigate whether the immune shift induced by $1,25(\mathrm{OH})_{2} \mathrm{D}_{3}$ is a generalised phenomenon or limited to autoantigen-related immune responses. Methods : In vitro :T-lymphocyles from 8 week old control and $1,25(\mathrm{OH})_{2} \mathrm{D}_{3}$ treated NOD mice were isolated from popliteal lymph nodes 10 days after in vivo stimulation with GAD (524-543) or ovalbumin (ova) in the footpath. T-cell proliferation and cytokine production before and after peptide rechallenge in vitro was analysed (ELISA of culture supernatant). In vivo : Control and $\mathrm{I}, 25(\mathrm{OH})_{2} \mathrm{D}_{3}$ treated NOD-mice were immunized in the footpath with GAD or ova. Cytokine profiles were analysed in the draining popliteal lymph nodes by quantitative RT-PCR at different time points after primary as well as after booster injections. Results : Immunization with GAD induced a spontaneous T lymphocyte proliferation in control as well as treated mice. In vitro rechallenge did not result in differences in proliferation between both groups, but a marked shift in cytokine profile was noted : IL4 production was significantly increased, while IFN $\gamma$ levels were decreased in the $1,25(\mathrm{OH})_{2} \mathrm{D}_{3}$ treated group. In vivo, analysis of cytokine levels in draining lymph nodes after $\mathrm{GAD}$ immunization and booster injection showed again a significant increase in Th2 cytokines, especially IL4, IL5 and IL13, and a significant decrease in the Thl cytokine IFNy in $1,25(\mathrm{OH})_{2} \mathrm{D}_{3}$ treated mice. This immune shift towards $\mathrm{Th} 2$ was not seen in the ovalbumin immunized mice, where a rise in IL4 was associated with an even rise in IFN $\gamma$. Conclusion : From these studies we can conclude that $1,25(\mathrm{OH})_{2} \mathrm{D}_{3}$ induces an immune shift from Th1 towards Th2 in peripheral lymphocytes upon stimulation with autoantigen, but not with non-autoantigens. 
OP 39

\section{Leptin in Obesity and Pregnancy}

\section{5}

EXPRESSION OF THE DIFFERENT LEPTIN RECEPTOR ISOFORMS IN THE BRAIN OF DIABETIC AND OBESE HUMANS

B. Burguera, L. Jin, ME Couce, J. Lamsam, MD Jensen and RV Lloyd. Endocrine Unit and Department of Pathology. Mayo Clinic, Rochester, MN 55905. USA Leptin exerts important effects on the regulation of food intake by interacting with a receptor that is alternatively spliced, in the brain. The long form (OB-Rb) has a long intracellular domain which is essential for signal transduction. The specific aim of this study was to further investigate the role that the brain may play in the pathogenesis of diabetes and obesity in humans. For this purpose, we studied the expression of the OB-R mRNA in the brain of obese $(n=5)$, lean $(n=5)$ and diabetic subjects $(\mathrm{n}=4)$, by means of in situ hybridization (ISH), Northern blot (NB) and RTPCR analysis. We used two alternative probes; one that recognizes all known splice variants (common form) and a second one that just recognizes the $\mathrm{OB}-\mathrm{Rb}$. Several brain regions including, hypothalamus, cerebellum, neocortex, entorrhinal cortex amygdala and rostral medulla, were evaluated. ISH studies, using a non-isotopic detection method and appropriate controls, including the use of sense probes, revealed that both common and $\mathrm{OB}-\mathrm{Rb} \mathrm{mRNA}$ are widely distributed in the human brain. The specific ISH signal was, with both probes, detected exclusively in the cytoplasm of the cell body, dendrites and proximal axonal regions of the neurons from the hypothalamic nuclei, Purkinge cells and dentate nuclei of the cerebellum, inferior olivary and cranial nerves nuclei in the medulla, amygdala and neurons from both the neocortex and entorrhinal cortex. The ISH signal obtained in the choroid plexus and ependyma was lower than that in neurons. No significant differences were identified neither among regions nor among the three groups studied. These results, matched those previousiy obtained (Neuroendocrinology 66:145, 1997) where the distribution of the OB-R protein in the human brain was first described. NB and RT-PCR analysis indicated that the OB- $\mathrm{Rb}$ is expressed at a relatively high level in the hypothalamus and cerebellum with lower expression in the choroid plexus. The identity of the PCR products were confirmed by sequencing analysis. Control experiments with the omission of the reverse transcriptase allowed to exclude genomic DNA amplification. Our results suggest that the original hypothesis, based on animal studies, that the $O B$ $\mathrm{Rb}$ was only present in the hypothalamus needs to be reconsidered. The $\mathrm{OB}-\mathrm{Rb}$ isoform seems to be widely expressed in the human brain. Obesity and diabetes seem not to be associated with a down-regulation of the OB-Rb in the brain.

\section{7}

IMPACT OF OBESITY AND LEPTIN TREATMENT ON ADIPOCYTE GENE EXPRESSION IN PSAMMOMYS OBESUS A. Sanigorski, D. Cameron-Smith, P. Lewandowski and G.R. Collier Metabolic Research Unit, School of Nutrition and Public Health, Deakin University, Geelong, Australia.

In this study we have investigated key enzymes involved in lipid metabolism in fat tissues isolated from lean and obese Psammomys obesus. Psammomys obesus (the Israeli sand rat) is a polygenic model of obesity and NIDDM, which naturally develops a range of metabolic and physical characteristics associated with the metabolic syndrome. Psammomys obesus were separated into 2 groups. One group of animals were lean, normoglycemic, normoinsulinemic and normoleptinemic whereas the second group were obese, hyperinsulinemic and hyperleptinemic. Animals in both groups were given intraperitoneal injections three times per day of $15 \mathrm{mg} / \mathrm{kg}$ of body weight of leptin or saline for 7 days. We examined fatty acid metabolism in adipocytes from suprascapular and perirenal fat depots by measuring the mRNA levels of lipoprotein lipase (LPL), hormone sensitive-lipase (HSL), uncoupling proteins 1 and 2 (UCP-1 and UCP-2) and peroxisome proliferatoractivated receptor (PPAR $/ 2$ ) using semi-quantitative RT-PCR, normalised to $\beta$-actin mRNA levels.

Our results showed that obese animals had significantly elevated gene expression levels of LPL, HSL, UCP-2 and PPAR $\gamma 2$ consistent with the 2-3 fold increase in body fat mass in comparison to lean animals. Leptin treatment failed to have any effect on body fat mass or gene expression in obese animals. However, in lean animals, leptin treatment resulted in an $82 \%$ reduction in body fat mass which was accompanied by a tendency for a reduction in LPL, HSL, UCP-2 and a significant decrease in PPAR $\gamma$.

In conclusion, increased fat mass is accompanied by increases in major genes associated with lipid metabolism which may be directly or indirectly regulated by increased PPAR 2 activity. Interestingly, in leptin responsive animals leptin treatment significantly reduced PPAR $\gamma$ expression which may contribute to the reduced fat mass observed in lean animals.

\section{6}

CELL BIOLOGICAL CHARACTERIZATION OF A TRUNCATED HUMAN LEPTIN (FRAMESHIFT 133)

H. Rau, J.P. Whitehead, B.J. Reaves, S. O'Rahilly. Departments of Medicine and Clinical Biochemistry, University of Cambridge, Addenbrooke's Hospital, Cambridge, UK.

Recently, we reported a frame-shift mutation in the human leptin (ob) gene associated with low serum leptin and extreme obesity in 2 homozygous individuals. Preliminary data suggested a defect in the secretion of this truncated mutant leptin. In the present investigation we have performed transient transfection studies in $\mathrm{CHO}$ and $\operatorname{COS} 7$ cells to characterize the biological properties of the mutant leptin. Consistent with our previous observations, only immunoreactive wt leptin was secreted. In pulse-chase experiments, intracellular wt leptin levels decreased upon its secretion into the medium. In contrast, whilst immunoreactive mutant leptin disappeared from cell lysates with similar kinetics to wt leptin $\left(t_{1 / 2} 45 \mathrm{~min}\right)$ it was not detected in the medium. Inhibition of the proteasome, using the inhibitor clasto-lactacystine $\beta$-lactone, led to a significant increase in the intracellular levels of mutant leptin, indicating a crucial role for the proteasome in the degradation pathway. Although intracellular immunoprecipitated wt and mutant leptin levels were comparable, analysis of total cell lysates revealed a 7 -fold increase in total intracellular mutant leptin compared to wt. Size exclusion gel filtration indicated that intracellular mutant leptin accumulated in an aggregated form, presumably as a result of misfolding in the endoplasmic reticulum. Thus, we have shown that the mutant leptin accumulates intracellularly as a consequence of misfolding/aggregation and is subsequently degraded by the proteasome.

\section{8}

PROGRAMMING OF LEPTIN METABOLISM IN EARLY PROTEIN RESTRICTED RATS

C.J. Petry, L. James, S.E. Ozanne, C.L. Wang \& C.N. Hales. Clinical Biochemistry Dept., Univ. of Cambridge, Cambridge, U.K. CB2 2QR

Maternal and early protein restriction in rats has been used to investigate mechanisms behind the finding that restricted fetal growth may increase the risk of developing type 2 diabetes. A number of metabolic changes have thus far been detected in such rats. The present study was initiated to test the hypothesis that leptin metabolism is altered by early protein restriction. Female rats were fed a $20 \%$ protein ('control') or an isocaloric $8 \%$ protein ('LP') diet throughout pregnancy and lactation Female offspring were weaned onto their respective maternal diets and from 70 days of age fed either chow or a cafeteria-style diet. At one year of age cafeteria-fed rats showed excessive weight gain $(\mathrm{p}<0.0001)$ and independently, LP rats were lighter than controls $(\mathrm{p}<0.0001)$ Fasting plasma leptin concentrations were: control-chow $(n=12) 7.5+$ 0.5 , control-cafeteria $(n=14) 46.9 \pm 3.8$, LP-chow $(n=14) 6.9 \pm 1.3$, LP-cafeteria $(n=15) 26.0 \pm 4.1 \mathrm{ng} / \mathrm{ml}$ (effect of early diet, $\mathrm{p}=0.001$ effect of adult diet, $p<0.0001$; interaction $p=0.002$ ). Relative to body weights plasma leptins were still raised in cafeteria-fed rats $(\mathrm{p}<0.0001)$ but this rise was significantly greater in control than in LP rats (interaction between early and adult dietary groups, $\mathrm{p}=0.041$ ): controlchow 18.2 \pm 1.2 , control-cafeteria $61.2 \pm 5.9$, LP-chow $20.7 \pm 3.3$, LP-cafeteria $45.8 \pm 6.1 \mathrm{pg} / \mathrm{ml} / \mathrm{g}$ body weight. This significant interaction, which occurs independently of dietary-induced changes in body weights, suggests that early protein restriction leads to either altered leptin metabolism in the rat adipocyte or possibly a reduction in cafeteria-induced leptin resistance. 
249

EVIDENCE FOR A PHYSIOLOGICAL ROLE OF LEPTIN IN HUMAN PREGNANCY

A. Festa, N. Shnawa, G. Schernthaner and S. M. Haffner. University of Texas Health Science Center, San Antonio, Texas, USA and Department of Medicine I, Rudolfstiftung Hospital, Vienna, Austria.

There is increasing evidence suggesting that leptin is not only a major regulator of adipose tissue metabolism and energy homeostasis, but might also be involved in the regulation of the neuroendocrine and the reproductive system. Recently, it has been shown that leptin is synthesized in and secreted from the human placenta. The aim of our study was to elucidate the role of leptin in human pregnancy by measuring serum leptin levels in a large number of consecutive pregnant women with norma $(n=173)$ and impaired $(n=67)$ glucose tolerance in relation to $B M I$ and various metabolic variables, including specific insulin and proinsulin during a $2 \mathrm{~h}$ OGTT (20-31 weeks of pregnancy). In 53 women serum leptin was measured during pregnancy and post partum (mean: 10.7 months). During pregnancy serum leptin was significantly related to body weight $(r=0.39)$, BMl ( $r=0.44)$, fasting immunoreactive insulin $(r=0.31)$, specific insulin $(r=0.40)$ and proinsulin $(r=0.28$ ) (all $p$-values $=0.0001$ ) but not to age, blood glucose levels and the increment of insulin relative to the increment of glucose in $60 \mathrm{~min}$, as a measure of insulin secretory capacity. Leptin was significantly higher during pregnancy compared to post partum (mean \pm SE: $24.2 \pm 1.4$ vs. $20.0 \pm 1.6 \mathrm{ng} / \mathrm{ml}, \mathrm{p}=0.001$ ). The difference remained significant after adjustment for change in BMI and change in fasting insulin $(\mathrm{p}=0.042)$. In multiple regression analysis $\mathrm{BMI}$ and fasting insulin oredicted $20 \%$ of the variability in leptin levels during pregnancy, whereas in the same women the same variables explained more than twice of leptinvariability post partum $(44 \%)$. In women with gestational diabetes leptin levels were lower compared to women with normal glucose tolerance after adjusting for $\mathrm{BMl}$ and fasting insulin (26.37 vs $16.06 \mathrm{ng} / \mathrm{ml}, \mathrm{p}<0.006$ ) Our data add to the evidence that leptin might be added to the catalogue of placenta derived hormones playing a physiological role during human pregnancy.

\section{0}

FREE LEPTIN, BOUND LEPTIN AND SOLUBLE LEPTIN RECEPTOR CONCENTRATIONS IN SERUM OF HEALTHY WOMEN DURING PREGNANCY

A. Widjaja, R. Hofmann, C. Husemann, R. Horn, A von zur Mühlen and G. Brabant. Dept. of Clinical Endocrinology, Hannover Medical School, Germany Leptin binding proteins have been identified in human serum, however the physiological significance of these binding proteins remain unknown. It has been shown that a relevant fraction of these binding proteins consist of the soluble leptin receptor which is also produced by placental tissue. We have developed new radioimmunoassays to detect free leptin, bound leptin and soluble leptin receptor levels in serum. Thus, the aim of the study was to evaluate the course of leptin and leptin receptor levels during pregnancy in 22 healthy women (mean (SD) age: 30.7 (5.6) yrs). BMI, free leptin, bound leptin, soluble leptin receptor, NEFA, glucose and insulin were determined after an overnight fast at each trimester of gestation. Throughout I. to III. trimester BMI (mean \pm SD) increased (I: $22.9 \pm 3.2$, II: $24.7 \pm 3.0$, III: $26.2 \pm 2.9 \mathrm{~kg} / \mathrm{m}^{2}, \mathrm{p}<0.0001$ ). Free senum leptin levels increased from the I. $(122 \pm 82 \mathrm{pmol} / /)$ to the II. trimester $(181 \pm 89 \mathrm{pmol} / 1 ; \mathrm{p}<0.0001)$ and remained stable thereafter (III: $182 \pm 102 \mathrm{pmol} /$; II.-III. n.s.). Insulin increased from the I $(56 \pm 19 \mu \mathrm{U} / \mathrm{l})$ to the III. trimester $(86 \pm 68 \mu \mathrm{U} / \mathrm{l} ; \mathrm{p}<0.05)$. Bound leptin increased from the II. $(0.43 \pm 0.20 \mathrm{nmol} / \mathrm{l})$ to the III. trimester $(0.57 \pm 0.28 \mathrm{nmol} / 1 ; \mathrm{p}<0.001)$. The increase of soluble leptin receptor levels was not significant between the I. $(3.68 \pm 1.65 \mathrm{nmol} / \mathrm{l})$ and the III. trimester $(4.08 \pm 1.29 \mathrm{nmol} / \mathrm{l})$. Multiple regression analysis revealed that despite progression of pregnancy BMI remained an important predictor for free serum leptin levels (I: $R^{2}=0.54 ; \mathrm{II}$ and III: $\mathrm{R}^{2}=0.35 ; \mathrm{p}<0.0001$ in all). In conclusion: 1) BMI is an important predictor of free serum leptin levels despite the progression of pregnancy, 2) free leptin levels plateau after the II. trimester. This may be due to an increased binding of leptin to a secreted form of the leptin receptor in the III. trimester as reflected by the significant increase of bound leptin whereas leptin receptor levels remained unchanged. Thus, leptin resistance at late pregnancy, induced by a shift in free to bound leptin may allow increased metabolic efficiency in preparation for birth and nursing.

\section{1}

FREE LEPTIN, BOUND LEPTIN AND SOLUBLE LEPTIN RECEPTOR IN NORMAL AND DIABETIC PREGNANCY K. Lewandowski, D. Dunlop, R. Horn, C. O'Callaghan, G. Medley, P. O'Hare and G. Brabant

Department of Biological Sciences, The University of Warwick, Gibbet Hill Road, Coventry CV4 7AL, United Kingdom and Department of Endocrinology, Medizinische Hochschule, Carl Neuberg Strasse 1, Hannover, Germany (R.H., G.B.)

So far there are no data on alterations of free and bound leptin levels during diabetic pregnancy and the role of the soluble leptin receptor in human subjects remains to be explored. We measured free leptin, bound leptin and soluble leptin receptor levels by radioimmunoassays in 20 normal and 19 IDDM subjects at 20 and 30 weeks of gestation and post-partum, and analysed the data using hierarchical statistical models. Results: Total leptin levels rise from 20 to 30 weeks of gestation $(688+/-58$ (mean+/SEM in pmol/1) to $785+162, p=0.009$ ). There is a significant post-partum fall to $445+1-47 \mathrm{pmol} / \mathrm{p}<0.001$. This rise is caused by the rise in the bound leptin levels as there is no significant change in free leptin levels between 20 and 30 weeks $(\mathrm{p}=0.17)$ There is a significant post-partum fall in free leptin levels $(p<0.001)$. Insulin requirements rise in the 3rd trimester, but despite this there was no significant difference in free or bound leptin levels between the normal and diabetic subjects at any stage (free leptin: $223+/-35$ and $266+1-24 ; 237+1-45$ and $223+/-27 ; 109+/-16$ and $104+/-24, \mathrm{p}=0.34$; bound leptin: $410+/-73$ and $428+/-54 ; 501+/ .78$ and $562+/-71$; $330+/-47$ and $271+/-46, p=0.84$ for normals and diabetics at 20,30 weeks and postpartum respectively). Diabetic subjects however had significantly higher soluble leptin receptor levels at all stages $(p<<0.001)$, that rose further in the 3rd trimester from $3742+/-268$ (mean $+/$-SEM in pmol/1) to $4134+/-239$, while in the normal group there was a fall from $3149+/-169$ to $2712+/-123, p=0.05$. There is a linear relationship between the soluble leptin receptor levels and the body mass index present in the diabetic group only.

Conclusions: There is no significant difference in free or bound leptin levels between the normal and IDDM subjects either in pregnancy or post-partum, but female IDDM subjects have significantly higher soluble leptin receptor levels. We speculate that high soluble leptin receptor levels might be implicated in the development of leptin resistance in this group.

\section{2}

RELATIONSHIPS BETWEEN CORD LEPTIN, C PEPTIDE, GROWTH HORMONE \& IGF1 LEVELS IN CONTROL, \& DIABETIC PREGNANCIES E Koukkou ${ }^{1}$ R Edwards ${ }^{2} M$ Wheeler', P Jenkins ${ }^{2}$, LB Taylor ${ }^{2}, A$ Grossman ${ }^{3}$ and C Lowy ${ }^{1} \quad{ }^{1}$ Dept Endocrinology \& Diabetes, UMDS, St Thomas' Hospital, London SE1 7EH, ${ }^{2}$ Netria and ${ }^{3}$ Dept of Endocrinology, St Bartholomew's Hospital, London EC1A 7BE

Although plasma leptin levels have been shown to reflect fat mass in new-borns this association \& relationship with other growth promoting hormones have not been studied in new-borns of diabetic mothers who are prone to an increased fat mass. The aim was to determine cord leptin levels in control, gestational diabetes (GDM) \& established diabetes (EDM) pregnancies and relate these to $\mathrm{C}$ peptide, IGF 1, Growth hormone (GH) \& glucose levels. Maternal and cord blood was taken at delivery from 13 control, 10 GDM and 15 EDM women. Cord plasma glucose levels of controls were $3.1+0.2 \mathrm{SEM}$ mmol/1 compared with $3.1+0.3$ \& $4.4 \pm 0.8 \mathrm{mmol} / /$ in the GDM and EDM respectively. As expected cord C peptide levels were significantly higher in the babies who subsequently developed hypoglycaemia $4.14 \pm 0.6 \mathrm{ng} / \mathrm{ml}$ vs $2.53 \pm 0.5 \mathrm{ng} / \mathrm{ml} p<0.05$ but unexpectively GH levels were also higher in the subsequently hypoglycaemic babies $110+22.9 \mathrm{mu} / 1$ vs $41.8 \pm 5.3 \mathrm{mu} / 1 \mathrm{p}<0.01$. Cord leptin levels were significantly higher in the EDM babies compared with controls $10.6 \pm 6.5 \mathrm{ng} / \mathrm{ml}$ vs $27.0 \pm 5.2 \mathrm{ng} / \mathrm{ml}$ $(\mathrm{p}<0,05)$. There was no difference between the sexes in any of the groups. Cord Leptin/birth weight ratio, a marker of adiposity, correlated with cord $\mathbf{C}$ peptide $(r=0.44 p<0.01)$ and cord GH $(r=0.55 p<0.005)$ but not with cord IGFl but cord IGF1 did correlate with birth weight $(r=0.4 p<0.007)$ the latter probably reflecting lean body mass. The correlation of cord leptin/birth weight ratio with cord C peptide and GH levels could be explained as follows; the quantity of glucose delivered to the fetus determines fetal insulin secretion, the latter regulates fat synthesis and leptin secretion. Leptin is known to inhibit hypothalamic NPY secretion and decreased levels of the latter have been shown to stimulate GH secretion. 
OP 40

\section{Development and Regeneration of $\beta$-Cells}

253

MOLECULAR CLONING OF RAT PAX 4: IDENTIFICATION OF FOUR ISOFORMS IN RAT INSULINOMA CELLS.

K.Sakurai, Y.Tokuyama, K.Yagui, Y.Saito, and A.Kanatsuka Second Department of Internal Medicine, Chiba University School of Medicine, Chiba, Japan

The Pax proteins constitute a family of developmental regulators which are characterized by the presence of the paired domains (PD), a conserved DNA binding motif and are involved in pattern formation during embryogenesis. Pax4 and Pax6 are involved in the differentiation of pancreatic islets. Molecular cloning of rat Pax4 cDNA from rat insulinoma cell line, RINm5F, library by PCR based cloning strategy revealed 4 isoforms: Pax $4 a$, Pax $4 b$, Pax4c and Pax4d. Pax4a is composed of two conserved motif, PD and homeodomain (HD), linker region of $P D$ and HD, and Cterminal region. Pax4b is shorter than Pax $4 a$, because of the deletion of 42 amino acids spanning the first $\alpha$-helix part in HD. Pax $4 c$ is shorter than Pax4a, because of the replacement of the novel nucleotides sequence, resulting in the loss of the third $\alpha$ helix of HD and C-terminal region of Pax4a. Pax4d is truncated form of Pax $4 \mathrm{c}$ by deletion of $\mathbf{4 2}$ amino acids in the linker region. Analysis of tissue distribution using RNA blotting and RT-PCR showed specific expression of Pax4 mRNA in pancreatic islets and RIN cells. RT-PCR confirmed that the mRNAs of 4 isoforms were expressed in RIN cells. In the case of transcription factors genes, alternative splicing gives rise to protein isoforms with distinct or even opposing transcriptional activities. These Pax4 variants may regulate the transcriptional activity of Pax 4 during the development of pancreatic islets.

\section{4}

MOLECULAR CLONING AND GHARACTERISATION OF PAX4: A PAIRED-BOX CONTAINING TRANSCRIPTION FACTOR IN THE PANCREATIC $\beta$-CELL.

S.C. Campbell, H. Cragg, K.I.J. Shennan and K. Docherty. Department of Molecular and Cell Biology, Institute of Medical Sciences, University of Aberdeen, Aberdeen, AB25 2ZD, U.K

The mammalian pancreas emerges as an outpocketing from the embryonic gut at an early stage of development. The role of transcription factors in controlling the ability of pluripotent pancreatic stem cells to give rise to the endocrine and exocrine tissues is becoming better understood. For example, early patterns of expression of the transcription factor PDX1 have identified this homeodomain protein as an essential requirement for the insulin producing $\beta$-cells However, the PAX gene family, which contain a shared sequence motif, the paired box, have also been shown to be important. PAX6 is required for normal islet development while PAX4 has been shown to be essential for differentiation of the pancreatic $\beta$-cells. In the present study we report the cloning of a full-length PAX4 cDNA from a rat $\beta$-cell cDNA library. The PAX4 cDNA comprises 1047 base pairs with a single open reading frame encoding 349 amino acids. Transcription and translation of this gene in vitro generates a single protein of $38 \mathrm{kDa}$. Western blot analysis with a paired-box antibody confirms the presence of a paired-box domain. Molecular cloning of PAX4 will allow the first characterisation of the binding activities of this critical transcription factor and lead to an increased understanding of the roles of the paired-box family in the expression of insulin and other $\beta$-cell genes, such as IAPP.

\section{5}

EXPRESSION OF THE TYROSINE PHOSPHATASE-LIKE PROTEIN IA-2 DURING ISLET DEVELOPMENT IN THE NEONATALRAT

C. Roberts ${ }^{1}$, A. Clark 2 , E. Bonifacio 3 and M.R. Christie 1 Department of Medicine, King's College School of Medicine, London, U.K., Diabetes Research Laboratories, Oxford U.K. and San Raffaele Institute, Milan, Italy.

Islets of Langerhans originate from stem cells within pancreatic ducts both in the foetus and post-natally. During early neonatal life there is an expansion of pancreatic endocrine cell mass through cell growth and neogenesis and a maturation of the insulin secretory response to nutrients. Two tyrosine phosphatase-like proteins that are autoantigens in Type 1 diabetes, IA-2 and IA-2 $\beta$, have been shown to be early markers of pancreatic development in rodents or implicated in the regulation of cell growth. In the present study, immunocytochemical techniques were used to study IA-2 expression in the rat pancreas during the first 10 days of life. IA-2 expression was visualised with a monoclonal antibody, 76F, that recognises epitopes in the juxtamembrane domain of the molecule and which

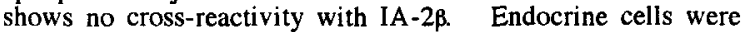
localised using antibodies to insulin and glucagon and pancreatic ducts with antibodies to cytokeratin-20 and CFTR. IA-2 staining on endocrine islet cells showed a progressive increase in intensity from very weak levels at 1 day of age to strong staining at 10 days. Subpopulations of duct cells, particularly those adjacent to islets, also showed strong staining in the 3 and 10 day pancreas. In the adult, IA-2 expression was restricted to the endocrine islet cells. The results demonstrate an increase in islet IA-2 protein expression during the period of functional maturation of islets and identify IA-2 as a potential marker of endocrine stem cells within pancreatic ducts.

\section{6}

ROLE OF $\beta 1$ INTEGRINS IN SPREADING AND FUNCTION OF RAT ISLET $\beta$.CELLS

D. Bosco, P. A. Halban and D. G. Rouiller. Laboratoires de Recherche Louis Jeantet, Centre Médical Universitaire, CH-1211 Geneva 4, Switzerland.

Cell-matrix interactions are implicated in islet structure and function, but their molecular basis is largely unknown. Primary islet cells express $\beta 1$ integrins, with a large proportion of $\alpha 3 \beta 1$, the specific ligand of laminin-5. By immunofluorescence, we now show that $\alpha 6 \beta 1$ integrin is also expressed at the surface of $\beta$-cells (rat $\beta$ cells sorted by FACS and cryosections of rat pancreas). Expression was increased in $\beta$-cells maintained for 2 days with secretagogues (22.2 mM glucose; $0.5 \mathrm{mM}$ IBMX; $1 \mu \mathrm{M}$ glucagon), vs control ( $5.6 \mathrm{mM}$ glucose alone). To study the importance of cell-matrix interactions in insulin secretion from individual cells, the reverse hemolytic plaque assay was used on $\beta$-cells attached for $3 \mathrm{~h}$ on glass slides coated or not with $804 \mathrm{G}$ matrix, which contains high levels of laminin-5. After a 45-min stimulation with $16.7 \mathrm{mM}$ glucose, $86 \%$ of $\beta$-cells secreted insulin when attached on $804 \mathrm{G}$ matrix, compared to $63 \%$ in absence of matrix. Also, the mean plaque area, reflecting the insulin secreted per cell, was increased in cells plated on the matrix ( $3803 \pm 322$ vs $1424 \pm 137 \mu \mathrm{m}^{2}$ in the control). Interestingly, this effect was higher in spreading cells as compared to those remaining round on $804 \mathrm{G}$ matrix. To investigate the role of $\beta 1$ integrins in cell spreading, $\beta$-cells were plated on Petri dishes coated or not with $804 \mathrm{G}$ matrix. After 2 days, spreading of $\beta$-cells was only observed on $804 \mathrm{G}$ matrix and was glucose dependent with $2.7 \pm 1.8,32 \pm 10$ and 67 $\pm 6.5 \%$ of $\beta$-cells spreading at $5.6,11.1$ and $22.2 \mathrm{mM}$ glucose, respectively. In all cases, spreading was prevented by an anti- $\beta$ 1 antibody. In summary, these studies show that 1) $\alpha 6 \beta 1$ is present on the surface of $\beta$-cells and increased by secretagogues, 2) spreading requires an extracellular matrix, increases with secretagogues, and is associated with increased insulin secretion, 3) $\beta 1$ integrins are responsible, at least in part, for $\beta$-cell spreading on $804 \mathrm{G}$ matrix. These results suggest that matrix interactions mediated by $\beta 1$-integrins play a crucial role in the regulation of islet function. 
REG AND CYTOCHERATIN 20 EXPRESSION DURING DUCTAL CELL DIFFERENTIATION AND PROLIFERATION IN AUTOIMMUNE DIABETES

E. Anastasi, E. Ponte, R. Gradini, P. Sale, H. Okamoto, C.Tiberti, A. Bulotta, F. Dotta and U. Di Mario. Endocrinology and Experimental medicine University of Rome "La Sapienza" Rome-Italy/ Department of Biochemistry, Tohoku University School of Medicine, Sendai Japan.

To evaluate a potential islet regeneration in an animal model of autoimmune diabetes, the low-dose streptozotocin mouse (Id-STZ), ductal $\beta$ cell differentiation, by the expression of Reg and cytocheratin 20 (CK20) protein, and proliferation, by bromodeoxyuridine (BrdU) incorporation, were investigated. Diabetes was induced in male C57Bl6/J mice with ld-STZ ( $40 \mathrm{mg} / \mathrm{Kg} \mathrm{b} / \mathrm{w}$ for five consecutive days). The animals were killed at two intervals: at day 10 and 23 from the beginning of the STZ treatment. Five animals were used per investigated time point and each group was evaluated for blood glucose leveis, insulitis, Reg and CK 20 pancreatic expression together with staining for insulin using immunochemical techniques and confocal microscopy. All mice treated with Id-STZ were hyperglycemic and histologica investigations showed a mild or severe insulitis both at day 10 and 23. At day 10 histological analysis revealed a strong Reg and CK20 expression with pancreatic ducts in ld-STZ treated mice but not in control mice. Reg and CK20 immunoreactive cells revealed co-staining with the insulin antiserum. On the contrary, at day 23 sections obtained from pancreata of Id-STZ treated mice reacted weekly with anti-Reg and anti-CK20 antibody. Cell proliferation analyzed by incorporation of $\mathrm{BrdU}$ was observed only in pancreatic sections obtained from mice killed at day 10. In conclusion, this study represents the first direct demonstration of ductal $\beta$ cell differentiation and proliferation in an autoimmune model of diabetes: the Id-STZ. Moreover Reg and CK20 are here demonstrated as useful markers for the evaluation of ductal cell differentiation and proliferation. Preventive or therapeutic strategies of the regeneration of $\beta$ cell in order to enhance the mechanisms involved in $\beta$ cells growth and differentiation can be tested in this model.

HYPERGLYCEMIA INCREASES LACTATE DEHYDROGENASE MRNA LEVELS IN ISLETS FROM 90\% PANCREATECTOMIZED RATS

J.C. Jonas, A. Sharma, H. Ilkova, G. Patanè, S. Bonner-Weir and G.C. Weir. Islet Transplantation and Cell Biology, Joslin Diabetes Center, One Joslin Place, Boston, MA 02215, U.S.A.

Insulin secretion and islet gene expression are markedly impaired in animal models of type 2 diabetes, probably due to altered expression of key islet transcription factors (TF) and loss of $\beta$-cell differentiation induced by the diabetic state. To test the role of hyperglycemia in this process, Sprague-Dawley rats were submitted to $\sim 85-95 \%$ pancreatectomy (Px) or sham surgery. This resulted in Px rats with low $(\mathrm{LH}<5.5 \mathrm{mmol} / \mathrm{l})$, mild (MH) or high $(\mathrm{HH}<8.3 \mathrm{mmol} / 1)$ hyperglycemia from week 2 to 4 . The mRNA levels of key TFs and other genes in total RNA from isolated islets were compared in Px and sham by semiquantitative RT-PCR. PDX-1 mRNA level decreased with increasing glycemia. A similar reduction was observed for the hepatocyte nuclear factors $1,4 \alpha$ and $3 \beta$ and several islet genes, but Beta2 and insulin mRNA levels decreased only in HH. In contrast, lactate dehydrogenase (LDH) mRNA level, that is low in normal islets, gradually increased with glycemia (2, 3 and 5 times sham levels in LH, MH and HH). LDH served as a marker to study the time course and reversibility of changes in islet gene expression after $90 \%$ Px. LDH mRNA level was 136 and $259 \%$ of sham at 1 and 2 weeks. Normoglycemia was restored for the next 2 weeks with phlorizin $(0.4$ $\mathrm{g} / \mathrm{kg}$.day) with virtual normalization of islet $\mathrm{LDH}$ mRNA to $127 \%$ of sham at 4 weeks. Because phlorizin is known to correct hyperglycemia without changing insulinemia, its ability to reverse altered gene expression strongly suggests that glucotoxicity rather than lipotoxicity plays a major role in this process. Our results also show that increased LDH gene expression is a new sensitive marker of islet dysfunction.
ROLE OF BETACELLULIN IN THE DIFFERENTIATION OF PANCREATIC ISLET CELLS

M.-A. Huotari, P. Miettinen, J. Palgi, J. Ustinov, T. Koivisto, E. Lehtonen and T. Otonkoski.

Haartman Institute, University of Helsinki

Epidermal growth factor receptor (EGFR, ErbB1) is expressed throughout the developing pancreas. In mice deficient in EGF-R, development of islets is delayed. We have now studied the expression of other erbB tyrosine kinases (erbB2 and 4) and the effect of their ligands, especially betacellulin (BTC), on the development of the endocrine pancreas of normal and EGFR $(-/)$ mice. Embryonic day 12 (E12) pancreases were incubated in serum-free medium with the factor to be tested for 5 days. At the onset of culture, only glucagon-positive islet cells were present, whereas all 4 endocrine cell types developed in vitro. BTC expression was detectable in the rudiments with RT-PCR. Exogenously added rBTC dramatically affected the development of islet cells decreasing the proportion of glucacon cells $(7.5 \pm 1.6 \%$ of all endocrine cells vs. $54.4 \pm 6.0 \%$ in controls, $\mathrm{p}<0.001$ ) and increasing the proportion of insulin expressing cells $(64.8 \pm 3.5 \%$ vs. $30.1 \pm 4.6$, $\mathrm{p}<0.001)$. Proportion of somatostatin $(17.3 \pm 3.6 \%)$ and PP $(6.9 \pm 2.1$ $\%)$ cells did not change. In the EGFR $(--)$ pancreases betacellulin did not have such an effect, suggesting that EGFF is essential for the action of BTC. Immunoreactive ErbB2/neu protein was found throughout the acinar tissue in the developing pancreas, and erbB-4 was expressed in the ductal epithelium. In the adult mouse pancreas, erbB4 immunoreactivity was only found in the glucagon-producing $\alpha$ cells. These findings suggest that BTC, a member of the EGF-family, may play a role in the differentiation of the B-cells. The ductal localization of erbB4, a putative BTC receptor, is in agreement with a role in islet cell differentiation.

\section{0}

B ALANCE OF ISLET CELL BIRTH AND DEATH OF FETAL AND NEONATAL RATS IS ALTERED BY A LOW PROTEIN DIET THROUGH MECHANISMS WHICH INCLUDE CELL CYCLE KINETICS

B. Reusens, D. J Hill. J. Petrik, C. Remacle and J.J.Hoet. Laboratory of Cellular Biology, Catholic University of Louvain, Louvain-la-Neuve. Belgium; and the Lawson Research Institute, London, Ontario, Canada.

A reduced pancreatic $B$ cell mass at birth and a lower insulin secretion in later life are characteristics of offspring from rats chronically fed a low protein isocalorific diet (LP), A programmed reduction in B cells mass by apoptosis occurs in the neonatal rat, and is balanced by islet cell replication and neogenesis. The apoptosis i associated with the removal of a locally-expressed survival factor, insulin-like growth factor-II (IGF-II). This study investigated the impact of a LP diet on islet cell ontogeny. Pregnant Wistar rats were fed either LP $(8 \%)$ or normal $(20 \%)$ chow. Bromo-dcoxyuridine (BrdU) was administered $1 \mathrm{~h}$ before sacrifice. Pancreata were removed from 19.5 days fetal life to postnatal (PN) day 21. Rats given $20 \%$ protein diet had a low rate of islet cell apoptosis detected by TUNEL method, in fetal and neonatal life $(1-2 \%)$ and a transient increase to $8 \%$ at day 14 PN. Rats receiving LP diet demonstrated a 2-3-fold greater rate of islet cell apoptosis predominantly B cells, at every age studied, and the neonatal peak was advanced by 1-2 days. The islets of the $20 \%$ protein-fed group exhibited a low incidence of proliferating cell nuclear antigen (PCNA) staining of 2-3\%, but this was $50-100 \%$ greater at each age in LP animals. Paradoxically, the incidence of BrdU staining was reduced at each age. Since PCNA detects cells within all stages of the cell cycle, but BrdU only $S$ phase, this suggests a lengthened cell cycle in islet cells from LP animals. In the LP group, the presence of immuno-reactive IGF-II within islet cells, and the abundance of IGF-II mRNA in whole pancreas, was reduced compared to controls. At 21 days PN the insulin content of pancreata from LP animals was significantly reduced compared to controls. The findings support a role for IGF-II in B cell developmental apoptosis, and a possible mechanism involving altered cell cycle kinetics for the $B$ cell defici which results from low protein diet in early life. 
OP 41

\section{Hypoglycaemia}

\section{1}

INTENSIVE TREATMENT MAINTAINS NORMAL GLUCAGON RESPONSE TO HYPOGLYCEMIA IN SHORT-TERM IDDM.

S. Pampanelli, M. Lepore, C. Fanelli, P. Del Sindaco, C. Lalli, M. Ciofetta, G. Calabrese, P. Brunetti and G. B. Bolli*, DIMISEM Perugia, Italy

In DDM, glucagon responses to hypoglycemia (hypo) are normal at onset, but thereafter are rapidly lost within few years. To establish if intensive insulin therapy (IIT, multiple daily insulin injections aiming at near-normoglycemia) since early IDDM onset, contributes to maintaining normal glucagon responses to hypo, two groups (GI, GII) of IDDM patients and 10 nondiabetics $(N)$ were studied with the stepped hyperinsulinemic-hypo clamp (plasma glucose, $\mathrm{PG}$, decreased from 90 to $42 \mathrm{mg} / \mathrm{dl}$ over $360 \mathrm{~min})$. GI $(\mathrm{n}=10)$ and GII $(\mathrm{n}=8)$ had similar IDDM duration ( $14 \pm 5$ vs $16 \pm 4$ months) ( $\mathrm{p}=\mathrm{NS}$ ), but GI was on IIT since IDDM onset ( $\mathrm{HbAlc} 5.8 \pm 0.3 \%$ ), whereas GII was treated subintensively ( $\mathrm{HbAlc}$ $7.4 \pm 0.4 \%$ ) ( $\mathrm{p}<0.05$ ). During hypo, glucagon responses in GI (PG threshold of response $64 \pm 2 \mathrm{mg} / \mathrm{dl}, \Delta$-peak $71 \pm 11 \mathrm{pg} / \mathrm{ml}$ ) were not different from $\mathrm{N}$ (PG threshold $63 \pm 2 \mathrm{mg} / \mathrm{dl}, \Delta$-peak $73 \pm 8 \mathrm{pg} / \mathrm{ml}$ ) ( $\mathrm{p}=\mathrm{NS}$ ). In contrast, in GII glucagon responses occurred at lower PG $(55 \pm 4 \mathrm{mg} / \mathrm{dl})$ and were blunted $(\Delta$-peak $18 \pm 9 \mathrm{pg} / \mathrm{ml})(\mathrm{p}<0.05$ vs $\mathrm{N})$. Basal and glucagonstimulated C-peptide (C-Pep) was greater in GI $(0.27 \pm 0.04$ and $0.54 \pm 0.07$ $\mathrm{nmol} / \mathrm{l})$ than in GII $(0.15 \pm 0.03$ and $0.21 \pm 0.05 \mathrm{nmol} / \mathrm{l})(\mathrm{p}<0.05)$. In GI, but not GII and neither in N, basal C-Pep and C-Pep suppression during hypo correlated with glucagon responses to hypo $(\mathrm{r}=0.57$ and $0.72, \mathrm{p}<0.05)$. When GII was restudied after 3 month IIT (HbAlc 6.0 $0.3 \%$ ), neither CPep nor glucagon responses to hypo improved. Conclusions. Because IIT since early onset of IDDM is critical to maintain normal glucagon responses to hypo, it is important that ITT is initiated as early as possible in IDDM.

\section{3}

RENAL GLUCOSE PRODUCTION COMPENSATES FOR THE LIVER DURING THE ANHEPATIC PHASE OF LIVER TRANSPLANTATION. S.E. Joseph, N. Heaton, D. Potter, A. Pernet, M. Umpleby ${ }^{1}$ and S.A. Amiel. King's College School of Medicine \& Dentistry \& UMDS', London, UK.

The renal contribution to endogenous glucose production (EGP) is controversial in humans. We measured EGP in the absence of the liver, during the anhepatic phase $(\mathrm{AH})$ of liver transplantation in 3 subjects (age $51.3 \pm 11.7$ years). Stable labelling of plasma glucose (PG) was achieved over 2 hours prior to AH by primed continous infusion of $6,6 \mathrm{D}_{2}$ glucose $(1.7 \mathrm{mg} / \mathrm{min})$. PG was maintained at 5.95 $\pm 0.85 \mathrm{mM}$ with labelled $5 \%$ dextrose $(0.98 \pm 0.45 \mathrm{mg} / \mathrm{kg} / \mathrm{min})$ during 1-hr AH. During AH, EGP fell from $3.83 \pm 1.3$ to $0.38 \pm$ $0.17 \mathrm{mg} / \mathrm{kg} / \mathrm{min}$ ( $10 \%$ baseline) but recovered at $\sim 30 \mathrm{~min}$, reaching $3.24 \pm 2.1 \mathrm{mg} / \mathrm{kg} / \mathrm{min}$ by $60 \mathrm{~min}(84 \%$ baseline $)$. Adrenaline, lactate, NEFA and glycerol rose $(0.79 \pm 0.74$ to $3.65 \pm 2.1 \mathrm{nM}$; $\mathrm{p}=0.005 ; 1.88 \pm 0.43$ to $3.46 \pm 1.32 \mathrm{mM}, \mathrm{p}=0.02 ; 565 \pm 333.5$ to $745 \pm 250 \mu \mathrm{M}, \mathrm{p}=0.01 ; 61.5 \pm 13.4$ to $137.8 \pm 130 \mu \mathrm{M}, \mathrm{p}=0.003$ ). As the kidney is the only source of EGP during AH, this shows that renal glucose production increases during $\mathrm{AH}$ associated with a stress response and increased substrate availability. We conclude that the human kidney is a major player in glucose homeostasis and can provide an EGP equivalent to that of the liver. The kidneys may play an important role in recovery from insulin induced hypoglycaemia and in liver failure.

\section{2}

GLIBENCLAMIDE DECREASES GLUCAGON RELEASE DURING MILD HYPOGLYCAEMIA

E.W.M.T. ter Braak, A.M.M.F. Appelman, D.W. Erkelens and T.W. van Haeften, Department of Internal Medicine, Utrecht University Hospital, The Netherlands

Normally, hypoglycaemia leads to glucagon release from the A-cell, which is a first line defense. It has been suggested that glucagon release is also regulated by the B-cell or via the autonomous nervous system, or both. The aim of this study was to investigate the effect of the sulfonylurea glibenclamide on glucagon release during insulin-induced hypoglycaemia. Eight healthy subjects $(4 \mathrm{M} / 4 \mathrm{~F}$, mean $\pm \mathrm{SD}$ age: $47 \pm 6$ years) were investigated with a stepwise hypoglycaemic clamp on two occasions. Glibenclamide ( $10 \mathrm{mg}$ orally) or placebo was administered before the hypoglycaemic clamp in a double blind randomized cross-over fashion. A continuous insulin infusion $(2 \mathrm{mU} / \mathrm{kg} / \mathrm{min})$ was given. Plasma glucose levels were kept constant at the fasting level for 60 min and subsequently allowed to fall gradually to $3.8,3.2$ and $2.6 \mathrm{mmol} / 1$ respectively during $20 \mathrm{~min}$ each: they were then kept constant at these levels for $20 \mathrm{~min}$, before the next hypoglycaemia level was aimed for. At the end of the clamp (glucose $=2.6 \mathrm{mmol} / \mathrm{l}$ ) plasma glucagon, catecholamines, cortisol and growth hormone were significantly increased compared to baseline values (all $p<0.05$ ). At glucose $=3.2 \mathrm{mmol} / \mathrm{l}$ glucagon rose significantly more at the placebo day than at the glibenclamide day (mean $\pm S D$ increase: $8.4 \pm 5.4 \mathrm{vs}$ $1.0 \pm 4.0 \mathrm{pmol} / \mathrm{l}, \mathrm{p}<0.02$ ), while no significant differences were seen for cortisol, growth hormone and catecholamines. At glucose $=3.2 \mathrm{mmol} / \mathrm{l} \mathrm{C}$ peptide was less suppressed with glibenclamide than with placebo (mean $\pm S D$ decrease : $0.14+0.08$ vs $0.30 \pm 0.14 \mathrm{nmol} / \mathrm{l}, \mathrm{p}<0.03$ ), indicative for $B$-cell stimulation with glibenclamide. We conclude that glibenclamide decreases glucagon secretion at mild hypoglycaemia in healthy volunteers, presumably by an inhibitory effect of the B-cell on the pancreatic A-cell. Use of glibenclamide may jeopardize glucose counterregulation.

\section{4}

HYPOGLYCEMIA, GLUCOPRIVATION AND THE DEVELOPMENT OF HYPOGLYCEMIA-ASSOCIATED AUTONOMIC FAILURE

S.D. Bouman, J.E. Bruggink, A.J.W. Scheurink, J.H. Strubbe and A.B. Steffens; Dept. of Animal Physiology, Univ. of Groningen, P.O. Box 14, Haren, The Netherlands

Hypoglycemia-Associated Autonomic Failure (HAAF), an experimental model for hypoglycemia unawareness, is characterized by attenuation of the counterregulatory autonomic and hormonal responses to hypoglycemia. It is generally induced by multiple hypoglycemic episodes caused by consecutive infusions of insulin. The aim of the present study was to investigate whether HAAF is only caused by hypoglycemia or by a reduction in cellular glucose availability in general. Therefore it was studied whether HAAF could also be evoked by consecutive administrations of 2-deoxyglucose (2-DG), a drug that causes hyperglycemia together with intracellular glucoprivation and counterregulatory autonomic and hormonal responses similar to those in insulin-induced hypoglycemia. To this end, two groups of rats were subjected to a setup similar to the classical HAAF experiment (which consists of an insulin administration after two insulin administrations on the preceding day): a control group in which insulin was administered after two consecutive infusions of $0.9 \% \mathrm{NaCl}$ on the preceding day; and an experimental group in which insulin was administered after two consecutive administrations of 2-DG on the preceding day. Insulin dosage was $125 \mathrm{mU} \cdot \mathrm{min}^{-1} \cdot \mathrm{kg}^{-1}$ for 90 minutes, 2-DG dosage was $200 \mathrm{mg} \cdot \mathrm{kg}^{-1}$. The studies were performed in permanently cannulated rats. Blood samples were frequently withdrawn to measure the changes in blood glucose and plasma catecholamine levels. Insulin-induced hypoglycemia (controls) significantly increased adrenaline levels (peak value $217 \pm 69$ (average \pm SEM); $p<0.05$ ) Consecutive administrations of 2-DG on the day preceding the insulin-induced hypoglycemia significantly reduced this adrenaline response to hypoglycemia $(217 \pm 69$ vs $22 \pm 8 \mathrm{ng} / \mathrm{ml} ; \mathrm{p}<0.05)$. The data reveal that reduced glucose availability in general, instead of hypoglycemia per se, may be a crucial factor in the development of HAAF. 
265

INSUFFICIENT ADAPTATION OF HYPOGLYCEMIC THRESHOLD FOR COGNITIVE IMPAIRMENT IN TIGHTLY CONTROLLED DIABETES

M. Taverna, J. M'Bemba, A. Sola, A. Chevalier, G.Slama and J.L. Selam, U. 341 INSERM, Dept of Diabetology, Hotel-Dieu Hospital, Paris, France.

Fixity or adaptation (occuring at lower glycemic levels, as symptoms and hormones) of hypoglycemic threshold for cognitive impairment in tightlycontrolled type 1 diabetic patients is an important and still unsolved question. We studied 19 type 1 patients, aged $37 \pm$ SD $7 \mathrm{yr}$, with diabetes duration $15 \pm 11 \mathrm{yr}$, and various degrees of diabetes control ( $\mathrm{HbA} 1 \mathrm{c}$ range $5.7-9.7 \%$ ) including 8 patients with hypoglycemia unawareness, as assessed by Cox questionnaire. Hypoglycemic thresholds for hormones, symptoms, awareness and cognitive impairment using 4-choice reaction time (4 RT) were measured repeatedly during a $3 \mathrm{~h}$ stepped 5.0 to $2.2 \mathrm{mM}$ hypoglycemic hyperinsulinemic clamp. The nadir hypoglycemic level was also recorded as an indicator of effective counterregulation. Using multivariate analysis, we have found that awareness $(p<0.01)$ autonomic and neuroglycopenic symptoms $(p=0.01$ and $<0.01)$, nadir glycemia $(p<$ 0.01) but not cognitive impairment thresholds were related to HbA1c levels, independently of age or duration of diabetes. Tightly - controlled subjects ( $\mathrm{HbA1c}<8 \%, n=12$ ) adapted all above thresholds though glycemic decrement of cognitive impairment thresholds were modest and not significant, thus placing it at the higher levels in the hierarchy of counterregulatory thresholds : $4 \mathrm{RT}$ accuracy and speed : $3.0 \mathrm{mM}$ and 2.6 $\mathrm{mM}$, epinephrine $2.8 \mathrm{mM}$, neuroglycopenic and autonomic symptoms 2.5 and $2.2 \mathrm{mM}$, awareness $2.3 \mathrm{mM}$ in tightly controlled patients vs $3.5,2.8$ $3.4,3.3,3.1$ and $3.1 \mathrm{mM}$ in other subjects. The tightly-controlled patients also had a lower hypoglycemic nadir and higher unawareness score indicative of higher risk of severe hypoglycemia. We conclude that 1) cognitive impairment threshold is less influenced by the degree of glycemic control than other counterregulatory thresholds, and that this insufficient adaptation may contribute to higher risk of severe hypoglycemia in tightlycontrolled patients, 2) contrary to other reports, accuracy deteriorates earlier than speed in our cognitive tests, thus aggravating further the severe hypoglycemia risk.

\section{7}

EEG-MAPPING: DECREASE OF VIGILANCE IN TYPE 1 DIABETIC PATIENTS WITH HISTORY OF REPEATED SEVERE HYPOGLYCAEMIA Kinga Howorka, J. Pumprla, P. Anderer', B. Saletu' and A. Schabmann ${ }^{2}$ Research Group Functional Rehabilitation and Group Education, Institute of Biomedical Engineering, 'Dept. of Psychiatry, ' 2 Dept. of Applied Psychology, University of Vienna, k.howorka@bmtp.akh-wien.ac.at

Aims: (1) Cross-sectional study of EEG mapping in type 1 diabetic patients with and without previous repeated severe hypoglycaemia, (2) comparison with a control group of non-diabetic persons. Patients and Methods: A vigilance-controlled EEG mapping (10-20 system, significance probability maps) was performed in a relatively euglycaemic state (blood glucose 4.4-11 $\mathrm{mmol} / \mathrm{l})$. EEG data of a group of 14 IDDM-patients without a history of severe hypoglycaemia (age $36 \pm 10$, diabetes duration $16 \pm 9$ years, $H_{b A_{1 c}} 7.5+1.3 \%$ ) were compared with those of 13 IDDM-patients with a history of repeated severe hypoglycaemia (age $36 \pm 11$, diabetes duration $18 \pm 11$ years, $H b A_{c c}$ $7.7+1.1 \%)$, and with those of non-diabetic age- and gender-matched controls Results: Patients with history of severe hypoglycaemia demonstrated a reduction in absolute spectral power in beta band and shifting-to-left of centroid frequencies of theta and beta bands when compared to the nondiabetic control group (up to $\mathrm{p} \leq 0.01$ ). Patients without a history of severe hypoglycaemia showed only a borderline reduction of absolute power in delta band when compared to control group. The most remarkable differences between groups with and without history of severe hypoglycaemia were demonstrated in centroid frequencies in delta and theta bands $(1.3-7.5 \mathrm{~Hz})$ due to the deceleration in patients after repeated hypoglycaemia, $p<0.01$. Conclusions: Patients with history of repeated severe hypoglycaemia demonstrated in non-hypoglycaemic state significantly reduced vigilance when compared to the group without history of severe hypoglycaemia and to the control group, as well. These EEG patterns in hypoglycaemia patients are similar to those seen in pathologic ageing.

\section{6}

REDUCED BETA ADRENERGIC SENSITIVITY AFTER HYPOGLYCEMIA IN TYPE 1 DIABETIC SUBJECTS

A. Fritsche*, M Stumvol1*, S. Sieslack*, M. Grüb*, H.U. Häring*, R.M. Schmülling*, J.E. Gerich ${ }^{\circ}{ }^{*}$ Med. Klinik IV, Universität Tübingen, Germany; ${ }^{\circ}$ University of Rochester, USA

Reduced peripheral tissue sensitivity to catecholamines is suggested to be one of the mechanisms contributing to hypoglycemia unawareness. We have previously shown that in normal subjects antecedent hypoglycemia does not impair but increases sensitivity to catecholamines. In the present study we assessed beta adrenergic sensitivity in 10 type 1 diabetic subjects with no history of hypoglycemia unawareness ( $29 \pm 5$ years old, diabetes duration 13 \pm 8 years, $\mathrm{HbAlc} 7.3 \pm 0.9 \%$ ) using an isoproterenol test after an euglycemic hyperinsulinemic clamp and after a hypoglycemic (blood glucose 3 mmol/l) hyperinsulinemic clamp. Beta adrenergic sensitivity was expressed as the dose of isoproterenol which increased the heart rate by $25 \mathrm{bpm}$ (IC25). During hypoglycemia diabetic subjects had an impaired plasma epinephrine response compared to the normal subjects studied with the same protocol ( $16.7 \pm 5.0$ vs. $40.1 \pm 6.7 \mathrm{ng} / \mathrm{dl}, \mathrm{p}=0.02)$. In diabetic subjects, IC 25 was greater after hypoglycemia than after euglycemia $(1.00 \pm 0.26$ vs. $0.65 \pm$ $0.14, p=0.04$ ) indicating a decrease in beta adrenergic sensitivity. In contrast, controls showed a lower IC25 after hypoglycemia than after euglycemia $(0.83 \pm 0.22$ vs. $1.13 \pm 0.21, p=0.02)$ indicating an increase in beta adrenergic sensitivity. In the control experiments after euglycemia, type 1 diabetic patients seem to have a lower IC25 compared to controls $(0.65 \pm$ 0.14 vs. $1.13 \pm 0.21, \mathrm{p}=0.07$ ) which reflects a compensatorily increased beta adrenergic sensitivity under basal conditions in diabetic subjects with already decreased catecholamine response to hypoglycemia. We conclude that in diabetic subjects a single episode of hypoglycemia reduces beta adrenergic sensitivity indicating that maladaptation of tissue sensitivity to catecholamines contributes to the development of hypoglycemia unawareness.

\section{8}

PREVENTION OF NOCTURNAL HYPOGLYCEMIA IN INTENSIVE THERAPY OF IDDM.

C. Fanelli, S. Pampanelli, M. Lepore, F. Porcellati, L. Bartocci, P. Brunetti and G. B. Bolli*,DIMISEM Perugia, Italy

Hypoglycemia (hypo), especially at night, is a major obstacle for near-normoglycemia in intensive insulin therapy (IIT) of IDDM. To test the hypothesis that the split of evening insulin dose is a key step to preventing nocturnal hypo during IIT, 18 C-peptide negative IDDM patients were randomized to two, 4-month period treatment, with either a mixture of human regular (Hum-R) and NPH before dinner (MLX), or Hum-R at dinner and NPH at 23:30 h (SPLIT) (open, crossover design). A snack at $23: 30 \mathrm{~h}$ was suggested whenever capillary blood glucose (BG) was $<7.0 \mathrm{mmol} / 1$ with $\mathrm{MIX}$ or $6.0 \mathrm{mmol} / \mathrm{l}$ with SPLIT. With both treatments, Hum- $R$ was given at breakfast and lunch. BG was determined prior to each insulin injection every day, and at 23:30 h and 03:00 h every other day. Frequency of nocturnal hypo ( $B G<4.0 \mathrm{mmol} / 1)(14 \pm 3$ vs $3 \pm 2$ episodes/patient-month), fasting $\mathrm{BG}(8.9 \pm 0.8$ vs $7.7 \pm 0.6 \mathrm{mmol} / \mathrm{l})$, variability of day-to-day fasting BG (coefficent of variation $39 \pm 5.2 \mathrm{vs}$ $27 \pm 3.5 \%)$, HbAlc $(7.61 \pm 0.16$ vs $7.05 \pm 0.12 \%)$ were all greater with MIX vs SPLIT $(p<0.05)$. Frequency of daytime hypo was no different with the two treatments. During in-hospital overnight BG monitoring, hypo developed between $00: 30-04: 00 \mathrm{~h}$ in 15 out the 18 patients on MLX, but only in 3 patients on SPLIT. When patients were studied with the stepped hypo clamp, responses of adrenaline, symptoms, and cognitive dysfunction during experimental hypo, were greater in SPLIT than MIX. Conclusions : the split of evening insulin dose is a key approach in IIT of DDM to reduce :1)frequency of nocturnal hypo, 2) fasting $B G, 3$ )variability of $B G$, $4) \% \mathrm{HbA}(\mathrm{c}, 5)$ risk for hypo unawareness and impaired counterregulation to hypo. 


\section{Epidemiology of Type 2 Diabetes}

\section{9}

\section{HIGH PREVALENCE OF FAMILIES WITH BOTH TYPE 1 AND TYPE 2 DIABETES}

H. Li, E. Lindholm, L. Groop, T. Tuomi and the Botnia Research Group, Diabetes and Endocrine Research Laboratory, Dept. of Endocrinology, Lund University, Sweden and Helsinki, Finland

We have previously shown that a family history of type 1 diabetes influences insulin secretion in type 2 diabetic patients. The aim of the present study was to examine the prevalence of familial clustering of both type 1 and type 2 (MIN, mixed IDDM and NIDDM). Among our type 2 diabetes family collections in Finland (the Botnia Study) and Southern Sweden, we selected families with $\geq 2$ diabetic patients. The prevalence of MIN families among the multiplex diabetes families was $14 \%(110 / 788)$ in Finland and $24.5 \%(65 / 265)$ in Southern Sweden. When combined, $22 \%$ (750/3400) of patients with type 2 diabetes revealed a family history of type 1 diabetes. In addition, we investigated the family history of type 2 diabetes in families ascertained through a patient with type 1 diabetes in the Diabetes 2000 Registry in Southern Sweden. 18.4\% (96/523) of patients with type 1 diabetes reported a family history of type 2 diabetes. Patients with type 2 diabetes $(n=270)$ in MIN families differed from patients $(n=382)$ in multiplex type 2 diabetes families with respect to younger age at onset $(54.3 \pm 13.8$ vs. $58.4 \pm 11.3 \mathrm{yr}, \mathrm{p}=0.0001)$, higher prevalence of GADab ( $17.8 \%$ vs. $11.4 \%, \mathrm{p}=0.032)$, and lower fS-C-peptide concentration $(0.57 \pm 0.52$ vs. $0.64 \pm 0.39 \mathrm{nmol} / \mathrm{l}, \mathrm{p}=0.044$, corrected for BMI and duration). MIN patients were more often assigned to insulin therapy than patients with type 2 diabetes $(41.6 \%$ vs. $29.8 \%, p=0.004)$. In conclusion, type 1 and type 2 diabetes occur in about $20 \%$ of multiplex diabetes families. The data suggest that MIN patients represent a large subgroup in the spectrum of diabetes and that these patients have a distinct phenotype.

\section{0}

DIABETES IN FAMILIES STUDY: THE INSULIN RESISTANCE SYNDROME SEGREGATES INTO 5 UNDERLYING FACTORS IN NON-DIABETIC SIBLINGS OF TYPE 2 DIABETIC PATIENTS

R.J.Morris, J.C.Levy, B.A.Barrow, I.J.Ross and R.C.Turner Diabetes Research Laboratories, Nuffield Department of Clinical Medicine Oxford University, Radcliffe Infirmary, Oxford UK

The Diabetes in Families Study is a population-based coliection of sibships of 347 type 2 diabetic patients sampled from GP practices in Oxfordshire and Northamptonshire. 585 non-diabetic siblings with FPG $<10 \mathrm{mmol}^{-1}$ and not on lipid lowering therapy, $44 \%$ male, mean (SD) age 61 (11) y, median (interquartile range) BMI $27.5(24.7,30.8) \mathrm{kg}^{-2} \mathrm{~m}^{-2}$ and FPG $5.5(5.2$, 6.0) $\mathrm{mmol} . \mathrm{I}^{-1}$, were studied to identify factors relating to the insulin resistance syndrome. Principal factor analysis with promax rotation was used considering the variables age, $B M I$, waist circumference, systolic and diastolic blood pressure, haemoglobin $A_{1 c}\left(H_{b A} A_{1 c}\right)$, HOMA derived beta cell function $(\% \beta)$ and insulin sensitivity $(\% S), H D L, L D L$ and VLDL cholesterol and triglycerides. 5 factors were identified: 1) Dvslipidaemia with large $(>0.5)$ positive loadings for VLDL cholesterol $(0.78)$ and triglycerides $(0.81)$ and a moderate $(>0.3)$ negative loading for $\mathrm{HDL}$ cholesterol $(-0.50)$, 2) Obesity with large positive loadings for BMI $(0.79)$ and waist $(0.79)$ and moderate loadings for $\% S(-0.36)$ and $\left.\mathrm{HbA}_{1 \mathrm{c}}(0.34), 3\right)$ Hypertension with large positive loadings for systolic and diastolic blood pressure $(0.71$ and 0.74 , respectively) 4) Aoeing with large positive loadings for age (0.61) and $\mathrm{HbA}_{10}(0.51)$ and a moderate positive loading for LDL cholesterol $(0.39), 5$ ) Insulin resistance with compensatory insulin secretion with large loadings for $\% S(-0.54)$ and $\% \beta(0.75)$. The 5 factors explained $54 \%$ of the variance. Factors for dyslipidaemia, obesity and insulin resistance were moderately intercorrelated $(r=0.35$ to 0.46 ), as were those for hypertension and ageing $(r=0.31)$, but intercorrelations between the 2 groups were weak $(r<0.25)$. In conclusion, 5 underlying factors relating to the insulin resistance syndrome were identified in non-diabetic siblings of type 2 diabetic patients suggesting that the syndrome may comprise several distinct components.

\section{1}

IN NORMOGLYCAEMIC SIBLINGS OF TYPE 2 DIABETIC PATIENTS, OBESITY ACCOUNTS FOR DECREASED INSULIN SENSITIVITY BUT NOT FOR HYPERTENSTON.

Levy J.C., Morris R.J., Barrow B.A., Ross I.J., Turner R.C.

Diabetes Research Laboratories, Radcliffe Infirmary, Oxford UK

In the Diabetes in Families Study, a population-based collection of sibships of 347 type 2 diabetic patients, we have shown that non-diabetic siblings, compared with non-diabetic controls randomly selected from the general population, were more hyperglycaemic, less insulin sensitive and had greater central obesity, hypertension and dyslipidaemia. To determine whether metabolic syndrome variables segregate independently from hyperglycaemia, 98 nomoglycaemic siblings (NGS) were compared with 62 normoglycaemic controls (NGC), each selected for fasting plasma glucose (FPG) $\leq 5.0$ mmol. $I^{-1}$. In groups matching for sex $(33 \%$ vs $34 \%$ male, NGS vs. NGC, respectively, NS) and age (58 vs 58 yrs, NS), FPG was slightly lower in NGS (4.8 vs. $4.9 \mathrm{mmol}^{-1}, \mathrm{p}=0.04$ ). Adjusting for sex, age and FPG, NGS had greater BMI (25.3 vs $23.7 \mathrm{~kg} \cdot \mathrm{m}^{-2}, p=0.01$ ), waist circumference ( 90 vs $85 \mathrm{~cm}, p=0.006$ ), systolic $B P$ (133 vs $123 \mathrm{~mm} \mathrm{Hg}$ $p=0.003$ ), plasma triglycerides (1.2 vs $1.0 \mathrm{mmol}^{-1}, p=0.002$ ), lower HOMA insulin sensitivity (84 vs $96 \% \mathrm{~S}, \mathrm{p}=0.02$ ) and compensatory increase in beta-cell function (117 vs $106 \% \beta, p=0.02$ ). Adjusting for $\mathrm{BMI}$ and waist circumference accounted for differences in insulin sensitivity and beta-cell function, but the difference in systolic blood pressure persisted (131 vs 123 $\mathrm{mm} \mathrm{Hg}, \mathrm{p}=0.04$ ). In conclusion, in normoglycaemic siblings of type 2 diabetic patients, insulin resistance was accounted for by obesity and waist circumference, but systolic hypertension was not, suggesting independent segregation of some metabolic syndrome variables.

\section{2}

ABDOMINAL OBESITY IS ASSOCIATED WITH IMPAIRED NEFA SUPPRESSION IN NORMAL GLUCOSE TOLERANT RELATIVES TO NIDDM SUENECTS

C.Saloranta, C.Forsblom, A.Hyrkkö, M.Lehtovirta and L.Groop. Department of Internal Medicine, University of Helsinki, Finland, and Department of Endocrinology, University of Lund, Sweden.

To study the relation between abdominal obesity and NEFA metabolism at different degrees of glucose intolerance, 47 healthy control subjects (CON), 77 normal glucose tolerant first-degree relatives to NIDDM subjects (NGT), 77 relatives with impaired gluose tolerance (IGT), and 90 subjects with NIDDM (DM) underwent an $75 \mathrm{~g}$ oral glucose tolerance test with measurements of plasma glucose and serum insulin at $0,30,60$ and $120 \mathrm{~min}$, and serum NEFA levels at 0 and $120 \mathrm{~min}$. Each study group was further divided into two groups with $(A O+, W H R>0.90)$ and without $(A O-, W H R<0.90)$ abdominal obesity. These subgroups were matched for age and BMI (CON AO-: $60 \pm 2 \mathrm{yr}, 25.8 \pm 0.4 \mathrm{~kg} / \mathrm{m}^{2}$, CON AO+: $59 \pm 2 \mathrm{yr}, 26.0 \pm 0.4$ $\mathrm{kg} / \mathrm{m}^{2}$; NGT AO-: $47 \pm 2 \mathrm{yr}, 25.2 \mathrm{~kg} / \mathrm{m}^{2}$, NGT AO+: $52 \pm 4 \mathrm{yr}, 25.5 \pm 0.5 \mathrm{~kg} / \mathrm{m}^{2}$; IGT AO-: $57 \pm 2 \mathrm{yr}, 27.8 \pm 0.3 \mathrm{~kg} / \mathrm{m}^{2}$, IGT AO+: $58 \pm 2 \mathrm{yr}, 28.3 \pm 0.3 \mathrm{~kg} / \mathrm{m}^{2} ; \mathrm{DM}$ AO-: $60 \pm 2 \mathrm{yr}, 29.6 \pm 0.7 \mathrm{~kg} / \mathrm{m}^{2}$, DM AO $+60 \pm 1 \mathrm{yr}, 30.3 \pm 0.7 \mathrm{~kg} / \mathrm{m}^{2}$ ). In NGT $\mathrm{AO}+$, NEFA levels were less suppressed at 120 min than in NGT AO- $(171 \pm 13 \mathrm{vs}$ $125 \pm 10 \mu \mathrm{mol} / 1, \mathrm{p}<0.002$ ), and the area under the curve for plasma glucose was higher (NGT AO+ vs NGT AO-; $905 \pm 27$ vs $820 \pm 23 \mathrm{mmol} / \mathrm{p}, \mathrm{p}<0.02$ ). In subjects without family history of NIDDM (CON), suppression of NEFA did not differ between $\mathrm{AO}+$ an $\mathrm{AO}-(144 \pm 13$ vs $132 \pm 17 \mu \mathrm{mol} / \mathrm{l})$. In subjects with IGT or NIDDM, suppression of NEFA was impaired compared to healthy controls (IGT and DM vs CON: $174 \pm 12$ and $242 \pm 21$ vs $144 \pm 13 \mu \mathrm{mol} / \mathrm{l}, \mathrm{p}<0.05$ and $\mathrm{p}<0.001$, respectively) regardless of WHR. Insulin levels were not different between $\mathrm{AO}+$ and $\mathrm{AO}-$ in any subject category. In summary, in normal glucose tolerant relatives to NIDDM patients, abdominal obesity is associated with impaired suppression of NEFA and elevated plasma glucose levels during OGTT. In IGT and NIDDM subjects, NEFA suppression is impaired and unrelated to abdominal obesity. The results indicate, that the association between abdominal obesity and impaired NEFA and glucose metabolism is confined to families with a history of NIDDM 


\section{3}

RELATION OF PROINSULIN AND INSULIN TO INSULIN SENSITIVITY (S $S_{1}$ AND ACUTE INSULIN RESPONSE (AIR) IN SUBJECTS WITH NIDDM: THE IRAS STUDY

L. Mykkänen, D.J. Zaccaro, C.N. Hales and S.M. Haffner, San Antonio, TX, Winston-Salem, NC, Cambridge, UK.

Plasma proinsulin levels are increased relative to insulin leveis in NIDDM patients. This may be due to insulin resistance or a defect in proinsulin processing or insulin secretion. We investigated the association of fasting intact proinsulin (PI), fasting insulin (I), and the proinsulin-to-insulin ratio (PI/I) with $\mathrm{S}_{1}$, estimated by a frequently sampled intravenous glucose tolerance test and the minimal model, and with AIR in 479 subjects with NIDDM in the Insulin Resistance Atherosclerosis Study (IRAS). Levels of I (pmol/l) and PI (pmol//) by quartiles of $S_{1}$ and AIR are shown below (adjusted for age, gender and ethnicity):

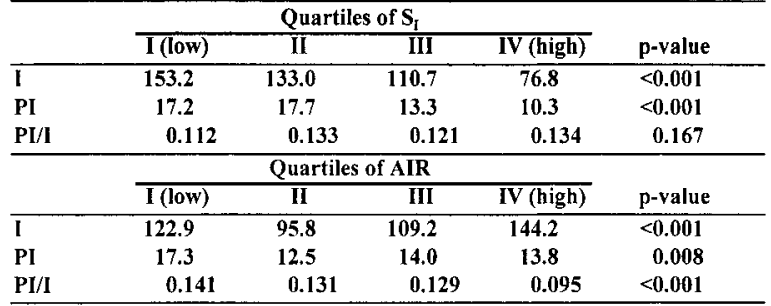

These associations were similar after further adjustment for body mass index. Thus, insulin resistance (low $S_{\text {) }}$ ) is not related to increased PI/I ratio in subjects with NIDDM. In contrast, low AIR is associated with increased PI/I in NIDDM in the IRAS population. These results suggest that increased $\mathrm{Pl} / \mathrm{I}$ is a marker of a defect in proinsulin processing or insulin secretion.

\section{4}

SPLIT PROINSULIN AS A RISK FACTOR FOR TYPE 2 DIABETES B. Zethelius, H. Lithell, C.N. Hales and C. Berne. Dept. of Geriatrics and Dept. of Internal Medicine, Uppsala University, Uppsala, Sweden and Dept. of Clinical Biochemistry, Addenbrooke's Hospital, Cambridge, UK

The aim of this study was to identify predictors of type 2 diabetes, with special reference to insulin-like molecules in 50-year old men with normal intravenous glucose tolerance reinvestigated after 7-14 years and after 20 years. Specific insulin, proinsulin and 32-33 split proinsulin were measured by the double antibody RIA technique in plasma samples frozen in liquid nitrogene. Results were compared to known baseline risk factors for type 2 diabetes, adjusted for age and timespan between the investigations. Risk factors were glucose and insulin concentrations 60 min after an intravenous glucose load, fasting plasma insulin, insulin index (the average of the insulin values at 4 and 6 min divided by fasting insulin concentrations), BMI, office blood pressure and antihypertensive drug treatment. We have not tested all risk factors together due to too few cases of diabetes in the subset with complete data of all risk factors.

Fasting 32-33 split proinsulin alone had a standardized odds ratio (OR) of 3.15 with a $95 \%$ confidence interval (CI) of $1.79-5.56$ at the 7-14 year follow up and was independent of the other determinants, tested one by one, except for the $60 \mathrm{~min}$ insulin concentration after intravenous glucose, (adjusted $\mathrm{OR}=1.98, \mathrm{Cl}=0.84-4.69$ ). At the 20 year follow up fasting 32-33 split proinsulin alone had an $\mathrm{OR}=1.53, \mathrm{CI}=1.09-2.16 \mathrm{but}$ was not independent of the other determinants, tested one by one. We conclude that in normoglycaemic men increased 32-33 split proinsulin predicts the development of type 2 diabetes 7-14 years later, but not 20 years later. The effect is however influenced by the late insulin response, as an indicator of insulin resistance.

\section{5}

THE IMPACT OF GENETIC AND ENVIRONMENTAL FACTORS ON THE INSULIN RESISTANCE SYNDROME AMONG MALE AND FEMALE TWINS. P. Poulsen, A. Vaag, K. Kyvik and H. Beck-Nielsen. Diabetes Research Centre, Odense University Hospital and Genetic Epidemiological Research Unit, Odense University, Denmark.

An strong association has been demonstrated between glucose intolerance ( $2 \mathrm{~h}$ OGTT plasma glucose $\geq 7.8 \mathrm{mM}$ ), hyperinsulinaemia (fasting plasma insulin $>60$ $\mathrm{pM}$ ), abdominal obesity (WHR male $>0.95$; female $>0.85$ ), hypertension (blood pressure $(\mathrm{BP})>160 / 95 \mathrm{mmHg}$ ) and dyslipidaemia (triglycerides $>1.7 \mathrm{mM}$, HDL cholesterol male $<0.9 \mathrm{mM}$; female $<1.1 \mathrm{mM}$ ), which often is referred to as the "insulin resistance syndrome (IRS)". In order to study the relative importance of genetic and environmental factors responsible for these components we examined 303 twin pairs (125 monozygotic (MZ), 178 dizygotic (DZ); 125 male, 178 female) between 55 and 74 years of age. Seventeen twins (1.7\%) fulfilled the criteria of IRS. The concordance rate was higher, though not significantly higher, among $M Z$ twin compared to DZ twins (MZ: $0.29 ; \mathrm{DZ}: 0$ ). The heritability estimates for BMI $(0.88)$ indicated a major genetic component in the development of obesity. The heritability for WHR $(0.06)$, however, was low, indicating a major influence of environmental factors on the distribution of obesity. Glucose tolerance $(0.34)$, insulin resistance (0.28) and plasma triglycerides $(0.40)$ seem to have a major environmental etiological factor. However, a genetic influence on the level of systolic $(0.78)$ and diastolic (0.66) blood pressure and HDL cholesterol $(0.70)$ is indicated due to the relatively high heritability estimates. We observed differences in heritability among male and female twins for BMI (male: 0.54, female: 1.00), fasting glucose (male: 0.16 , female: 0.88 ), systolic BP (male: 1.00 , female: 0.56 ), diastolic BP (male: 0.06 , female: 0.82 ) and triglycerides (male: 0.72 , female: 0.06 ). In conclusion the present study confirms the notion of an multifactorial etiology of the insulin resistance syndrome and its components including both genetic and non-genetic factors. Furthermore, the differences in heritability between male and female twins propose an influence of gender on several of the components of the insulin resistance syndrome.

\section{6}

HIGH BODY IRON STORES PREDICT NON-INSULIN DEPENDENT DIABETES MELLITUS IN MIDDLE-AGED MEN J.T. Salonen, T.-P. Tuomainen, K. Nyyssönen, H.-M. Lakka and K. Punnonen, Research Institute of Public Health and Department of Clinical Chemistry, University of Kuopio, Kuopio, Finland

NIDDM is a common complication of iron overload diseases such as haemochromatosis, and its development is related to iron excess. There are, however, no previous studies in humans of the association of body iron stores with the incidence of NIDDM in healthy populations. We tested the hypothesis that iron accumulation in the body predicts the development of NIDDM in a prospective 4-year follow-up study of 985 randomly sampled nondiabetic ( $\mathrm{fB}$-glucose $<6.0 \mathrm{mmol} / \mathrm{L}$ and no treatment for diabetes) men aged 42-60 from East Finland, an area with high NIDDM incidence. We estimated body iron stores by the ratio of levels of transferrin receptor (TfR) and ferritin in frozen serum samples drawn at baseline examinations for 41 men who later developed diabetes (fB-gluc $\geq 6.7 \mathrm{mmol} / \mathrm{L}$ or $2 \mathrm{~h}$ - $\mathrm{fB}$-gluc $\geq 10.0 \mathrm{mmol} / \mathrm{L}$ or treatment) during the 4-year follow-up and for 82 controls who were matched for age, four measurements of obesity, baseline glucose and insulin and eight other strongest predictors of NIDDM. In a logistic model adjusting for baseline serum triglycerides and glycosylated proteins, men with high iron stores (tfR/ferritin $<94 \mu \mathrm{g} / \mu \mathrm{g}$ ) had a 2.5 fold risk ( $95 \%$ confidence interval for odds ratio, 1.1 to $6.0, \mathrm{p}=0.039$ ) of NIDDM, compared with men with lower iron stores. This is the first study showing an association between body iron stores and the incidence of diabetes. Our data provide support to the theory that elevated body iron stores contribute to the development of NIDDM and warrant further cohort studies and iron depletion trials. 
OP 43

\section{Genetics of Type 1 Diabetes}

\section{7}

IDENTIFICATION OF GLUCOSE INDUCED BETA-CELL GENES USING SUPPRESSION SUBTRACTIVE HYBRIDIZATION

T. Østerbye, K. Buschard, and K. Josefsen. Bartholin Instituttet, Kommunehospitalet, 1399 Copenhagen K, Denmark.

The aim of the study was to identify beta-cell genes involved in the adaption to a high glucose environment. The functional state of the beta cell may play a role in the development of insulin-dependent diabetes, and actively insulin-secreting beta cells express elevated levels of antigens. In the present study we have used Suppression Subtractive Hybridization (SSH) for generating a beta cell library based on a subtraction of resting beta cell mRNA from mRNA isolated from actively secreting beta cells. The subtracted library was differentially screened in a dot blot assay with two cDNA probes synthesized from mRNA isolated from active and resting beta cells, respectively. 24 clones were picked for further characterization. Sequencing showed that 16 clones contained cDNA fragments encoding known proteins, while 8 clones contained cDNA fragments representing genes encoding novel proteins. Four genes were examined by Northern blotting which confirmed enhanced transcription levels during glucose stimulation. The genes selected were a VH-1 like protein tyrosine phosphatase (PTPase), aldolase and two unknown genes. Quantification of the signals obtained by Northern blotting showed induction rates of 5 and 8 fold of the two unknown genes, respectively, whereas the PTPase was induced 7 fold and aldolase 10 fold following 24 hours of glucose stimulation. Thus, the method used in the present study has proven to be fast and efficient; several genes in the beta cell are upregulated by glucose at the mRNA level. These genes include a tyrosine phosphatase and aldolase.

\section{9}

HLA-DMA AND HLA-DMB ALLELES IN GERMAN PATIENTS WITH TYPE 1 DIABETES MELLITUS

T. Siegmund, H. Donner, J. Braun, KH Usadel and K. Badenhoop, Medizinische Klinik I, Schwerpunkt Endokrinologie, Zentrum der Innerem Medizin, Klinikum der JW Goethe-Universität Frankfurt/Main, Germany The HLA-DMA and HLA-DMB genes are located in the HLA-D region between DQ and DP. Four variants of the DMA (DMA*0101-0104) and five of the DMB (DMB*0101-0105) have so far been identified. HLA-DM molecules are required in the process of peptide loading to MHC class II antigens, both regulating the dissociation of class II associated in variant chain peptides (CLIP) and the subsequent binding of exogenous peptides. In order to investigate the immunogenetic heterogeneity within the HLA-D susceptibility region we analysed the distribution of DMA alleles in 99 patients with type 1 diabetes mellitus and 90 healthy controls, of DMB alleles in 88 patients and 79 healthy controls. Patients and controls were all from central Germany. The PCR amplified products were purified and separated on a $10 \%$ polyacryamide gel electrophoresis. Among the recognized four DMA alleles DMA*0102 was significantly less frequent $(11 \%$ vs. $28 \%, p<0.02)$ in patients with type 1 diabetes mellitus. DMB $* 0101$ $\left(70 \%\right.$ vs. $\left.98 \%, p<1,5 \times 10^{-5}\right)$ was also reduced in frequency compared to controls. Comparing patients and controls positive for the type 1 diabetes high risk markers we found a significant association between DMA*0102 and $\mathrm{DQA} * 0501$ ( $9 \%$ vs. $31 \%, \mathrm{p}<0.05)$, as well as $\mathrm{DMB} * 0101$ and DQA*0501 (64\% vs. $100 \%, p<0.04)$. In conclusion HLA DMA*0102 and $\mathrm{DMB} * 0101$ contribute to genetic protection to type 1 diabetes mellitus in individuals with high risk DQA markers in the german population. These data add to the immunogenetic heterogeneity within the strongest predisposing gene region and may help to identify relevant peptide epitopes for class II binding.

\section{8}

LNKAGE ANALYSIS OF IDDM11 IN TYPE 1 DIABETES DANISH MULTIPLEX FAMILIES

F. Pociot, Z.M. Larsen, J. Johannesen, O.P. Kristiansen, J. Nerup, DSGD and DIEGG. Steno Diabetes Center, Gentofte, Denmark.

Genetic susceptibility to insulin-dependent dirhetes mellitus (IDDM) is polygenic and caused by both HLA and non-HLA locl. IDDM11 located on chromosome 14q24.3-q31 showed highly significant evidence for linkage in a mixed population from UK and US. IDDM11 appeared to act primarily in families that show less evidence of susceptibility through the major genetic determinants in the HLA region. However, no replication studies in other populations have been reported. We have analysed this locus in a homogeneous Danish IDDM family material. In total, 251 IDDM families (1041 individuals) with 410 IDDM-affected offspring were genotyped for the microsatellite marker D14S67. Linkage was evaluated in 146 sib-pair families (169 sib pairs) by the SIBPAL software. No evidence of linkage was found ( $p=0.18$ ), although increased sharing was observed (0.525). The total data set was also analysed by the transmission disequilibrium test (TDT). Transmissions (T) and nontransmissions (NT) to diabetic and non-diabetic offspring of most common alleles is shown in the table:

\begin{tabular}{ccccccc}
\hline D14S67 & \multicolumn{2}{c}{ IDDM } & \multicolumn{4}{c}{ non-1DDM } \\
Allele & $T(\%)$ & NT & $\mathbf{P}_{\text {mi }} \leq 0.05$ & $T(\%)$ & NT & $P_{\text {mir }} \leq 0.05$ \\
\hline 7 & $91(52,9)$ & 81 & - & $62(52,5)$ & 56 & - \\
9 & $93(51,7)$ & 87 & - & $49(55,7)$ & 39 & - \\
10 & $120(57,7)$ & 88 & 0.027 & $61(54.5)$ & 51 & - \\
11 & $76(47,2)$ & 85 & - & $43(47,8)$ & 47 & - \\
\hline
\end{tabular}

Thus, alleele 10 showed increased transmission with $57 \%$ T. Stratifying for HLA-DR high (DR3,4) and low (non-DR3,4) risk genotypes, increased T of allele 10 to $67 \%$ in the high-risk group ( $p=0.0017$; corrected by the number of comparisons, $p_{c}=0.009$ ). In contrast to the previous report our data demonstrates that IDDM11 primarily acts in tamilies with major HLA genetic risk determinants. However, we were not able to replicate the original observation of linkage of the IDDM11 locus to disease in a large Danish IDDM family data set without stratifying for HLA-region conferred risk.

\section{0}

THE HLA-E LOCUS IS ASSOCIATED WITH THE SUSCEPTIBILITY TO AND AGE AT ONSET OF TYPE 1 DIABETES MELLITUS

A.G. Demaine, A.D. Hodgkinson and B.A. Millward. Molecular Medicine Research Group, Postgraduate Medical School, University of Plymouth, UK.

There is increasing evidence to suggest that the HLA class I region plays an important role in determining the susceptibility to, and age of onset of type 1 diabetes. Studies have suggested that the region telomeric of HLA-B may harbor a diabetes susceptibility gene. The gene coding for HLA-E is located approximately 650 kilobase telomeric of HLA-B. HLA-E has recently been shown to be one of the cell surface receptors that are involved in natural killer cell lysis. A number of allelic variants of HLA-E have also been identified. The aims of this study was to investigate the frequency of HLA-E alleles in the DNA of 136 randomly selected patients with type 1 diabetes and 78 normal healthy controls. Exon 2 of the HLA-E gene was amplified using the polymerase chain reaction. The products were electrophoresed, transferred to nylon membranes and hybridised to HLA-E aliele specific DIG-labelled oligonucleotides. Four HLA-E alleles, designated $0101,0102,0103$ and 0104 were detected in both the patients and controls. Nine of the 10 possible genotypes were detected. There was a highly significant increase in the frequency of the HLA-E*0101/0103 genotype in the patients compared to controls $\left(\chi_{2}=15.8, p<0.0001, P c=0.001\right)$. When the patients where separated according to age at onset, the HLA-E*0101 genotypes was found in $45.2 \%$ of individuals developing the disease between the age of $10-20$ years compared to only $15.9 \%$ before 10 years of age $(\chi 2=10.9$, $\mathrm{p}<0.001, \mathrm{Pc}=0.01$ ) and was accompanied by an increase in the HLAE*0101/0103 genotype in the latter group. In conclusion, these results suggest that the HLA class I region may be involved in determining the age at onset of type 1 diabetes. 


\section{Risk Factors of the Diabetic Foot}

281

\section{CHRONIC DIABETIC COMPLICATIONS ASSOCIATED WITH FOOT ULCERATION}

L.A.Koeva and H.A.Bohchelian, Clinic of Endocrinology, Medical University, Varna, Bulgaria

The aim of the study was to investigate the relationship between diabetic vascular complications, osteopenia and diabetic foot ulceration. A casecontrol study was carried out. 202 patients with I and II type of diabetes (mean age - 46,1 1 6,7 years, BMI - $24,2 \pm 1,2 \mathrm{~kg} / \mathrm{m} 2$, sex ratio- M/F - 92/ 110) were examined. They were subdivided into two groups matched for age, gender and BMI: group A (cases) - 50 patients with present and former foot ulceration; group B (controls) - 152 - without foot ulceration. Blood glucose level, HbAlc, microalbuminuria, total cholesterol, LDL and HDL - cholesterol, serum calcium and phosphorus, microalbuminuria, transcutaneous oxygen tension, vibration perception threshold, Doppler ankle/brachial pressure index were investigated. Osteodensitometry at the distal and ultradistal radius, electromyography, isotopic angiography, bone scintigraphy, ophthalmoscopy were carried out. The degree of diabetic control and complications were assessed. The estimated frequency of chronic diabetic complications (group A vs group B) was as follows: diabetic retinopathy - $69 \%$ vs $41 \%$ (p<0,001); diabetic polyneuropathy - 64 $\%$ vs $42 \%$ ( $<<0,001$ ), microalbuminuria - 62 vs $40 \%$ ( $<<0,001$ ), peripheral macroangiopathy - 58 vs $23 \%$ ( $<<0,001)$, osteopenia - 38 vs $22 \%(p<0,01)$, osteoporosis - 24 vs $12 \%$ ( $p<0,001)$. Cases with poor glycaemic control were $47 \%$ (group A) vs $19 \%$ (group B) (p<0,001). These data confirm that poor glycaemic control, diabetic retinopathy, nephropathy, polyneuropathy, peripheral macroangiopathy and osteopenia are associated with foot ulceration. Early diagnosis of these chronic diabetic complications and osteoporosis is necessary for prevention of diabetic foot complications.

\section{3}

THE INFLUENCE OF DIFFERENT RISK FACTORS ON THE DIABETIC FOOT E.U.Komeliagina* I.V.Gourieva, A.S.Ametov. Diabetic Foot Centre, International Diabetes Program, Institute of Working Ability and Rehabilitation, Moscow, Russia.

The aim of this study was to analyze the influence of different risk factors on the diabetic foot development. 254 IDDM and NIDDM patients took part in this study. Range of age was 16-86 years, diabetes duration 0-30 years. 104 patients had diabetic foot ulcers: 28 were neuroischemic, 76 were pure neuropathic. The influence of such factors as age, sex, type and duration of diabetes, previous ulcers and amputations, severity of complications (retinopathy, nephropathy, periferal and autonomic neuropathy), ankle/brachial index $<0.8$, hypertension, presence of foot deformities and mycosis were analysed. The NDS was used to diagnose and to guantify periferal neuropathy, ADS was used to estimate autonomic neuropathy. FDS (Foot Deformity Score) was used to quantify foot deformities. The results obtained showed that every form of diabetic foot had their own risk factors. The main risk factor for neuropathic foot was presence of neuropathy. Odds ratio of neuropathy itself (NDS $>5$ and/or ADS $\geq 2$ ) was not high $O R=1.1$ (CI 95\% 0.52-2.97). The OR of neuropathy combined with the foot deformities (FDS $>0)$ was $2.4(\mathrm{Cl} 95 \% 1.13$ 6.75 ). In the combination of neuropathy, foot deformities and mycosis (interdigital and/or nailes) the OR was 3.1 (CI $95 \% 1.02-9.14)$. When the patient had severe nephropathy combined with neuropathy the OR was 8.1 (Cl 95\% 3.91-17.02). The OR of previous toes amputations was 16.1 (CI 95\% 5.2-31.07). The highest risk of foot ulceration had the patients wih neuropathy and proliferative stage of retinopathy : OR was 32.1 (CI 95\% 10.01-68.21). There was no patients with the foot deformities, mycosis, severe nephropathy, proliferative stage of retinopathy and without neuropathy. The ischemic ulcers developed due to the vascular insufficiency. Risk of vascular desease was related to NIDDM, age of patients ( $O R$ of the patients older then 60 years old was 2.4 (CI 95\% 1.12-4.75). Conclusion. In terms of our results we conclude that such risk factors as foot deformities, mycosis, severe nephropathy, proliferative stage of retinopathy play their role on the diabetic foot development only under the presence of neuropathy. So each diabetic patient must be neuropathy screened. We recommend to categorise neuropathic foot risk patients regarding $O R$ of combinations of different risk factors. We suggest revealing neuropathic and ischemic diabetic foot risk factors separetely because of the difference in the preventive measures.

\section{2}

THE RISK OF ULCERATION IN DIABETIC PATIENTS WITH HIGH FOOT PRESSURES AND NEUROPATHY

RG Frykberg, LA Lavery, H Pham, LB Harkless and A Veves. Deaconess Joslin Foot Center. Harvard Medical School, Boston, MA.

High plantar foot pressures in association with peripheral neuropathy have been ascertained as important risk factors for ulceration in the diabetic foot. We have studied a group of 251 diabetic patients of Caucasian $(n=120)$, Black $(n=35)$, and Hispanic $(n=96)$ racial origins with an overall age of $58.5 \pm 12.5$ years (range $20-83$ ). There was an equal distribution of males and females across the entire study population. All patients underwent a complete medical history and lower extremity evaluation for neuropathy and foot pressures. Neuropathic parameters were dichotomized $(0 / 1)$ into two high risk variables: patients with a Vibration Perception Threshold $\geq 25$ were categorized as HiVPT $(n=132)$ and those with Semmes-Weinstein Monofilament tests $\geq 5.07$ were classified as HiSWF $(n=190)$. The mean dynamic plantar foot pressures of three footsteps were measured using the F-Scan Mat System with patients walking without shoes. Patients were divided to a high pressure group (Pmax6, $\geq 6 \mathrm{~kg} \cdot \mathrm{cm}^{-2}, \mathrm{n}=96$ ) and a normal pressure group. An interaction term (HiRisk) was also generated combining those patients with both high pressures and HiVPT $(n=67)$. A total of 99 patients had a current or prior history of ulceration at baseline. Univariate logistic regression of ulceration on Pmax6 yielded an Odds Ratio (OR) of 3.9 $(p=0.000)$. For HiVPT the OR=11.7 $(p=0.000)$, for HiSWF the $O R=9.6$ $(p=0.000)$, and for HiRisk the $O R=7.4 \quad(p=0.000)$. Controlling for age, diabetes duration, sex, and race (all $p<0.05$ ), multivariate logistic regression yielded the following significant predictors of ulceration: Pmax6 (OR=2.1, $p=0.002)$, HiVPT $(O R=4.4, p=0.000)$, HiSWF $(O R=4.1$, $p=0.000$ ). The interaction term was not significant in this model. In a model controlling the same confounders and this variable alone, however, the HiRisk OR=4.1, $p=0.000$. We conclude that both high foot pressures and neuropathy independently predict ulceration in a diverse diabetic population, with the latter having the greater magnitude of effect.

\section{4}

RISK FACTORS OF FOOT ULCERATION IN A LARGE DIABETIC POPULATION: TWO YEAR PROSPECTIVE FOLLOW-UP H Pham, LA Lavery, C Harvey, BI Rosenblum, RG Frykberg, LB Harkless, A Veves. Boston, MA, San Francisco, CA, San Antonio, TX

Diabetic foot ulceration is a preventable condition provided that a successful screening system and preventable care that are applicable in different centers with different practices exist. We present the first two years results of a large prospective clinical trial which examines the risk factors for the development of foot ulceration in 249 patients (125 males, 49 with type $1 \mathrm{DM}$ ) in three separate diabetic foot centers. Mean age was $58 \pm 12$ years (mean $\pm \mathrm{sd}$ ), 118 were White 36 Black and 94 Hispanic, mean DM duration $14 \pm 11$ years. On clinical examination, the Neuropathy Symptom Score was $4 \pm 4$, the Neuropathy Disability Score (NDS) $10 \pm 8$, Vibration Perception Threshold (VPT) $29 \pm 17$ Volts and the Semmes-Weinstein Monofilaments (SWF) $5.42 \pm 1.43$. The first metatarsal joint mobility was $73 \pm 24^{\circ}$ degrees and the subtalar joint (SJM) $24 \pm$ 9. The mean foot pressure was $5.7 \pm 3.0 \mathrm{~kg} . \mathrm{cm}^{-2}$ while peripheral vascular disease was present in 79 patients. For the statistical analysis each lower extremity was considered as a separate entity. Foot ulceration developed in 86 feet $(17 \%)$ during the study period. The NDS at baseline was abnormal in all patients who developed foot ulceration. Univariate logistic regression analysis showed a significantly higher risk for foot ulceration in those with NDS $>5$, (OR 12.8, p <0.001) VPT $\geq 25$ Volts (OR 11.0, p <0.001). SWF $\geq 5.07$ (OR 8.9, $\mathrm{p}<0.01$ ) foot pressures $>6.00{\mathrm{~kg} . \mathrm{cm}^{-2}}^{-2}$ (OR $1.4, \mathrm{p}<0.05$ ), and history of foot ulceration (OR 2.9, $\mathrm{p}<0.001$ ) while lower risk was observed in patients who had high SJM (OR 0.95, p <0.001), were black (OR 0.6, p <0.001) and women (OR $0.36, p<0.001$ ). A multivariate analysis which included all above factors showed statistical significance only for $\operatorname{NDS}(3.8, p<0.001)$, VPT (OR $3.7, p<0.001)$ and history of foot ulceration (OR 1.61, $p<0.001$ ). We conclude that clinical examination and measurement of VPT are the two more robust tests that in conjunction with a history of foot ulceration can predict future foot ulceration in three different centers and a racially mixed population. Other tests such as SWF and foot pressure measurements are also helpful but less robust, probably because of racial differences in the tested population and testing preferences among examiners, and should be used as auxiliary methods. 
OP 45

\section{Quality of Life}

\section{5}

PARENTS' PSYCHOLOGICAL HEALTH AND FAMILY FUNCTIONING IN RELATION TO CHILDREN'S METABOLIC CONTROL AND DIABETES ADAPTATION

B. Lernmark, B. Persson, L.D. Fisher and P-A. Rydelius, Department of Woman and Child Health, Karolinska Institute, Stockholm, Sweden; Department of Biostatistics, School of Public Health, University of Washington, Seattle, USA

In a study of psychological adaptation to type 1 diabetes in 62 children, one parent for each child was assessed for psychological health (Symptoms Checklist) and family functioning (Family Relations scale). The aim was to investigate (1) if the parent variables were important to the child's metabolic control (mean $\mathrm{HbAlc}$ last year) and adaptation to diabetes (questionnaire of adaptation), and (2) if the parent variables were related to the physician's assessment of the child's coping with diabetes (rated on a 5-point scale from "no problems" to "significant problems" in coping). Data were analyzed with stepwise multiple regressions. With metabolic control as dependent variable, the only significant parent variable was "chaotic family functioning." explaining $11 \%$ of the variance $(p=0.006)$. With each subscale of the adaptation index as dependent variable, age of the child, depression and anxiety in the parent were significant to the child's "attitude and behavior to lesting," (29\% of the variance; $p<0.001$ ). The child's age and "family enmeshment" were significant to the child's "attitude to diabetes" $(21 \%$; $p<0.001)$. Psychiatric symptoms in the parent were significant for the child's "feelings about diabetes" and "impact on daily life of diabetes" $(15 \% ; \mathrm{p}=0.005$ and $13 \% ; \mathrm{p}=0.01)$. A regression with the physician's rating as dependent variable and parent variables as potential predictors, showed that psychiatric symptoms in the parent were significant for the child's coping (28\%: $\mathrm{p}<0.001$ ). The result after adding the child's HbAlc as an independent variable (the only child variable significant for the physicians' ratings), showed that metabolic control, family functioning (isolation) and parent's psychiatric symptoms (depression, paranoid ideation, psychoticism) were signficant, explaining 56\% the variance of the physicians' assessment $(\mathrm{p}<0.001)$. Conclusions: The child's metabolic control was only related to chaotic family functioning, while the child's diabetes adaptation mainly was correlated to parental psychological health. The physician's assessment of the child's coping with diabetes was related to variables in the parent and the family, to metabolic control but no other variables in the child.

\section{7}

WELL-BEING AND DIABETES TREATMENT SATISFACTION IN TYPE 2 DIABETIC PATIENTS TREATED WITH INSULIN OR SULPHONYLUREA H. El Ghomari and M.R. Ababou. Endocrinology and Metabolic Diseases, University Hospital Ibnou Rochd, Casablanca, Morocco

Objective of the study was to assess the possible relationship between glycaemic control in patients with type 2 diabetes and their psychological well-being and treatment satisfaction. Methods: Patients $(n=91)$ with unacceptable glycaemic control (fasting blood glucose $>140 \mathrm{mg} / \mathrm{dl}, \mathrm{HbA}_{1 \mathrm{c}}>$ $25 \%$ above normal range) were included. The study had an open, parallel group design with a 4 week run-in period with optimisation of the SU treatment. Patients $(n=77)$ with unacceptable glycaemic control at the end of the run-in period were randomised to a 12 week treatment period with insulin or SU. Two patient questionnaires were used: Well-being (22 items) and diabetes treatment satisfaction ( 8 items). Arabic versions of the questionnaires were used for 73 of the 75 patients completing the study. Results: There is a significant correlation between glycaemic control and both well-being $(r=-0.5315, p=0.000)$ and treatment satisfaction $(r=-0.7267$, $p=0.000$ ). The increased well-being is mainly influenced by the increasing energy levels and less by reduced depression and anxiety levels. During the study the well-being score increased significantly $(p=0.002)$ more in the insulin group (mean score 34.7 (week 4) to 44.7 (week 16)) than in the SU group (mean score 34.6 (week 4) to 40.0 (week 16)). This agrees with the small but better improvement in glycaemic control in the patients starting insulin $\left(\mathrm{HbA}_{1 \mathrm{c}}\right.$ week 4: $10.2 \%$; week 16: $9.1 \%$ ) as compared with the patients continuing with SU $\left(\mathrm{HbA}_{1 \mathrm{c}}\right.$ week $4: 10.6 \%$; week 16: 10.5\%) $(\mathrm{p}=0.036)$ Conclusions: Improving glycaemic control will improve the patient's wellbeing and treatment satisfaction. In this study improving glycaemic control was more successfully done, although to a small extent, when starting insulin as compared with continued optimised SU.

\section{6}

FURTHER DEVELOPMENT OF A QUALITY-OF-LIFE MEASURE FOR IDDM PATIENTS

U. Bott and S. Ebrahim. Clinic of Metabolic Diseases and Nutrition, HeinrichHeine-University Düsseldorf, Germany

Aim of the study was the completion of a validated diabetes-specific quality-oflife scale (DSQOLS) to assess the impact of genetically engineered insulin analogues (IA) on quality of life and treatment satisfaction in subsequent trials. The original DSQOLS was validated in a population based study with 684 adult IDDM patients and comprised 7 subscales (items, Chronbach's $\alpha$ ): preferenceweighted treatment satisfaction $(20,0.78)$, social relations $(11,0.88)$, leisure time flexibility $(6,0.83)$, physical complaints $(8,0.84)$, worries about future $(5,0.84)$, diet restrictions $(5,0.71)$ and daily hassles $(4,0.70)$. Expansion of the instrument was based on semi-structured interviews with 30 patients including different questions regarding daily treatment demands (e.g. diet restrictions, anticipation of insulin action, protection against hypoglycaemia, stability of blood glucose, fear of side-effects of IA). Patients' considerations created 54 new items which were validated in a pilot sample comprising 134 IDDM patients $(51 \%$ women, age $36 \pm$ 11 years, diabetes duration $15 \pm 11$ years; mean \pm SD). 6 of the newly developed items enhanced the diet and 4 items the daily hassles subscale. Factor analysis (varimax rotation) of the 44 items remaining revealed 5 components (items, Chronbach's $\alpha$ ): fear of hypoglycemia $(11,0.92)$, blood glucose fluctuations (11, $0.91)$ burdens of hypoglycemic events $(7,0.87)$, fear of IA $(3,0.80)$, self-efficacy regarding adaptation of insulin dosage $(3,0.56)$. Correlations with validated generic quality of life and treatment satisfaction scales indicated high convergent validity except for self-efficacy and fear of IA. HbAlc showed the strongest correlations ( $\mathrm{p} \leq 0.001)$ with preference-weighted treatment satisfaction $(-.33)$, physical complaints $(-.39)$ and blood glucose fluctuations (-.38). Patients with at least one severe hypoglycaemia (preceding year) achieved poorer scores on the subscales leisure time flexibility $(\mathrm{p}<0.05)$, fear of hypoglycaemia and burdens of hypoglycaemic events $(p<0.001)$. CSII revealed better scores regarding leisure time flexibility, self-efficacy $(\mathrm{p}<0.05)$, diet restrictions, daily hassles $(\mathrm{p}<0.001)$ and burdens of hypoglycaemic events $(p<0.005)$. Keeping strict injection-mealintervals was associated with more fear of hypoglycaemia $(p<0.05)$. Patients using insulin lispro were less fearful regarding potential risks of IA.

The DSQOLS is a valid diabetes-specific quality-of-life measure for IDDM and has sufficient sensitivity to distinguish between different treatment regimen.

\section{8}

FOUR GROUPS OF IDDM PATIENTS: PERSONALITY, PSYCHOPATHOLOGY, OBJECT RELATIONS AND QUALITY OF LIFE.

S. Clerici l, G. Negri l, A Fossati l, C. Maffei l, V. Di Carlo ${ }^{3}$, E. La Rocca ${ }^{2}$ G. Pozza ${ }^{2}$ and A Secchi ${ }^{2}-{ }_{1}$ Medical Psychology and Psychotherapy Unit Neuropshychic Department, ${ }^{2}$ Internal Medicine I Department, ${ }^{3}$ Surgical II Department H San Raffaele Scientific Institute, Milan, Italy

The aim of the study was to evaluate personality, psychopathological aspects and quality of life of four groups of IDDM patients. A sample of I43 IDDM patients was recruited: 33 IDDM (uncomplicated), 44 waiting-list kidney or kidney-pancreas transplantation, 15 kidney transplanted, 51 kidney-pancreas transplanted. In order to evaluate personality, psychopathology and quality of life, the following self-administered questionnaires were used: Personality Diagnostic Questionnaire (PDQ-4+), Bell Object Relations and Reality testing Inventory (BORRTI), Symptoms Check List-90 (SCL-90), Diabetes Quality of Life (DQOL). All tests are well known and validated tools to address these topics. Analysis of variance has shown that there are no significative differences among four groups in PDQ-4+ and BORRTI (Bonferroni test $p<.05$ ). Nevertheless significative differences hare resulted between waiting-list group and kiney-pancreas transplanted group in SCL-90 in "Depression" and "Others" subscales (respectively 13.273 and $8.039, F=3.900$, d.f. $=3 / 139$, $p<05$ ). Significative differences have been found among waiting-list patients versus non complicated diabetes and kidney-pancreas transplanted patients in DQOL questionnaire's subscales: "Satisfaction" (respectively 2.632 versus 3.276 and $3.275, \mathrm{~F}=7.344$, d.f. $=3 / 101, \mathrm{p}<.05$ ) and "Impact" ( 3.666 versus 4.232 and $4.178, F=6.908, \mathrm{~d} . \mathrm{f} .=3 / 111, \mathrm{p}<.05)$. On the whole, results suggest that in IDDM patent's population there are no serious personality disorders or psychiatric pathology. According to literature, kidney-pancreas transplanted patients improve their quality of life expecially as regarded to dialyzed patients. 
OP 46

\section{PKC-Activation and Vascular Function}

\section{9}

IN SITU PROTEIN KINASE C ACTIVITY IN CULTURED FIBROBLASTS FROM TYPE 1 DIABETIC PATIENTS WITH NEPHROPATHY M. Vedovato, E. Iori, C. Marescotti, A. Tiengo, S. Del Prato and R Trevisan. Department of Clinical and Experimental Medicine, Padua, Italy

Protein kinase $\mathrm{C}$ (PKC) plays a key role in the regulation of cellular growth and induction of PKC pathway by hyperglycemia has been associated with many of the cellular changes observed in the long-term complications of diabetes. This study tested the hypothesis that fibroblasts from type 1 diabetic patients who developed nephropathy (DN) have an intrinsic increased PKC activity compared with cells of those without DN. PKC activity was therefore determined in serially passaged, subconfluent, quiescent skin fibroblasts cultured in either normal (5 mM) or high ( $20 \mathrm{mM}$ ) glucose concentrations from 12 type 1 diabetic patients with DN, 12 without DN matched for age diabetes duration and body mass index and 9 healthy subjects. PKC activity was determined by in situ PKC assay that used a highily specific peptide PKC substrate (VRKRTLRRL) in digitoninpermeabilized fibroblasts. In normal glucose in situ PKC activity was significantly higher in patients with $\mathrm{DN}$ both in quiescent state (mean + S.D : 15401 $\pm 1471 \gamma^{33} \mathrm{P}$ cpm.mg protein ${ }^{-1} \cdot \min ^{-1} ; \mathrm{p}<0.01$ ) and after phorbol esters (TPA 1uM) stimulation $(32801+1971, p<0.01$ ) than in those without DN (13273 \pm 1179 and $19277+2281$, respectively) and normal controls (12984 \pm 962 and $19527 \pm 2171$, respectively). Incubation in high glucose concentrations caused an increase in PKC activity both in quiescent state $\left(18951 \pm 1216 \gamma^{3{ }^{33}} \mathrm{P} \mathrm{cpm} \cdot \mathrm{mg}\right.$ protein $\left.{ }^{-1} \cdot \mathrm{min}^{-1}, \mathrm{p}<0.001\right)$ and after TPA stimulation (38458-1840; $p<0.01$ ) in type 1 diabetic patients with $D N$ only, with no significant change in the other two groups. In conclusion, in normal glucose concentration cultured fibroblasts from type 1 diabetic patients with DN have higher PKC activity as compared to those without DN and normal controls. This difference was magnified following incubation of cells in high glucose concentrations. The persistence of enhanced PKC activity in serially passaged cells suggests the presence of an intrinsic abnormality in cell function, which may contribute to the increased DN risk secondary to chronic hyperglycemia.

\begin{abstract}
290
ROLE OF OXIDATIVE STRESS AND PROTEIN KINASE C ACTIVATION IN THE INCREASED RETINAL ENDOTHELIAL CELL MONOLAYER PERMEABILITY INDUCED BY THE DIABETIC MILIEU

F.Pricci', G.Leto', G.Romeo', S.Cordone', E.Albanese', P.Sale', L.Amadio', S.Catalano' ${ }^{1}$, P.Barsotti ${ }^{1}$, L.Pala ${ }^{2}$, C.M. Rotella ${ }^{2}$, U.Di Mario' and G. Pugliese ${ }^{1}$ Universities of ${ }^{1}$ Rome "La Sapienza" and ${ }^{2}$ Florence, Italy

In experimental diabetes, vascular permeability was shown to be increased in tissues targets of long-term diabetic complications, including the retina. We previously showed an increased leakage of differently-sized proteins induced by high glucose or advanced glycation endproducts (AGE)-modified BSA in bovine retinal endothelial cell (BREC) monolayers grown on permeable membranes inserted in culture dishes. This study was aimed at investigating the biochemical mechanisms underlying the increased permeability induced by the diabetic milieu in this in vitro system, with particular reference to the role of the oxidative stress and protein kinase $\mathrm{C}(\mathrm{PKC})$ activation occurring in response to hyperglycemia. To accomplish this objective, ${ }^{125}$-albumin leakage over $5 \mathrm{~h}$ was measured in BREC grown to confluence in high glucose (HG, $30 \mathrm{mM}$ ) vs. normal glucose (NG, 10 $\mathrm{mM}$ ) or on BSA-AGE vs. native BSA. The superoxide-generating system xantine oxidase (XO, $500 \mathrm{U})$ + hypoxantine $(\mathrm{HX}, 10 \mathrm{mM})$, for $15 \mathrm{~min}$ before, or the PKC activator phorbol 12-myristate 13-acetate (PMA, $100 \mathrm{nM}$ ), during permeability assessment, were added to BREC cultured in NG. The antioxidant superoxide dismutase (SOD $150 \mathrm{U} / \mathrm{ml}$ ), the PKC inhibitor staurosporine (St, $10 \mathrm{nM}$ ), both during permeability assessment, or the PKC downmodulator PMA $(100 \mathrm{~nm}$, for 24 hours before) were added to BREC under all the experimental conditions. Albumin leakage at $5 \mathrm{~h}$ was increased by both $\mathrm{XO} / \mathrm{HX}(7.7 \pm 1.3 \%)$ and PMA $(8.0 \pm 1.2 \%)$, vs. control cells $(5.4 \pm 0.7, p<0.001)$, with the maximal increment observed at $1(+97 \%)$ and $3(+86 \%) h$, respectively. BREC grown in HG $(8.3 \pm 1.6 \%$ vs. $5.2 \pm 1.1 \%$ in NG, $p<0.001)$ or on BSA-AGE $(8.0 \pm 1.3$ vs. $4.9 \pm 0.9$ on $B S A, p<0.001)$ showed similar increases, which were virtually prevented by the addition of SOD, St or PMA (for $24 \mathrm{~h}$ ). These data suggest that the increased monolayer permeability induced by HG or BSA-AGE is mediated by increased free radical generation and PKC activation, possibly resulting in cytoskeletal changes and opening of intercellular gaps.
\end{abstract}

\section{1}

ACTIVATION OF PKC $\alpha, \beta_{1}$ and $\beta_{2}$ BUT NOT $\delta$, BY ACUTE HYPERGLYCEMIA IN NON-DIABETIC SUBJECTS IS DUE TO INDIRECT EFFECTS OF GLUCOSE AND NOT REPRODUCED BY ELEVATED GLUCOSE IN VITRO G. Bartling, R. Assert, V. Pirags*, A. Bumbure*, H. Schatz and A. Pfeiffer, Medizinische Universitatsklinik Bergmannsheil, D-44789 Bochum and *Latvian Medical Academy, Dept. of Endocrinology, LV-1050 Riga

Protein kinase $\mathrm{C}(\mathrm{PKC})$ is activated by hyperglycemia in endothelia and several tissues of experimental animals. The enzyme probably participates in the pathogenesis of diabetic ocular complications. We have previously shown an activation of PKC $\beta_{2}$ in thrombocytes of diabetic patients by acute hyperglycemia in vivo. We now investigated whether a $2 \mathrm{~h}$ hyperglycemic clamp activates PKC isoenzymes acutely in healthy volunteers and whether this can be reproduced in vitro. Methods: 6 volunteers were infused with $20 \%$ glucose to a steady state level of $15 \mathrm{mmol} / 1$, somatostatin and insulin to achieve $40 \mathrm{mU} / \mathrm{ml}$. Blood was withdrawn before, at 60 and 120 $\min$. Differential centrifugation yielded pure thrombocytes which were analysed by Western blot relative to a recombinant shortened PKC $\beta_{1}$ standard which was generated in bacteria. Quantification was done with a soft laser densitometer. Results: Acute hyperglycemia of $15 \pm 1 \mathrm{mmol} / \mathrm{l}$ for $120 \mathrm{~min}$ caused a translocation of PKC $\beta_{1}$, $\beta_{2}$ and PKC $\alpha$ to the membranous fraction with increases to 153,302 and $225 \%$ of basal levels ( $n=6, p<0,05)$ for all enzymes and reductions in cytosol to 59,45 and $54 \%$, respectively PKC $\delta$ was not altered. Incubation of the thrombocytes with 16.6 $\mathrm{mmol} / \mathrm{glucose}$ with or without insulin compared to mannitol did not cause activation of PKC in the presence of $\mathrm{Ca}^{2+}$ and $\mathrm{PGI}_{2}$. The phorbol ester TPA caused massive translocation to $700 \%$ of control. Conclusion: Moderate acute hyperglycemia of 15 $\mathrm{mmol} / /$ in vivo clearly activated $\mathrm{PKC} \alpha, \beta_{1}$ and $\beta_{2}$ in human thrombocytes over 120 min in normal controls. Glucose did not translocate PKC $\alpha$ or $\beta$ from normal controls in vitro indicating that generation of diacylglycerol is not operative in thrombocytes. The activation of PKC therefore must be achieved by different mechanisms such as by hormones or metabolic changes occurring in vivo. Similar changes may be relevant in patients with diabetes were we observed a strong activation of PKC by nonglucose related stimuli in vitro.

\section{2}

EFFECT OF HIGH GLUCOSE CONDITIONS ON SUBCELLULAR LOCALIZATION OF PROTEIN KINASE C IN HUMAN ENDOTHELIAL CELLS M.M.E. van Ginneken, W. Engels, P.J.M.R. Lemmens, G. Swennen and B.H.R. Wolffenbuttel. Dept. of Endocrinology, Maastricht University, Maastricht, The Netherlands.

Animal studies indicate that activation of specific PKC subtypes (particularly $\beta_{1}$ ) plays a role in cellular deterioration in diabetes mellitus. Although PKC translocation and activation have been shown biochemically, it is still unknown, to which sites PKC subtypes translocate within the endothelial cell under diabetic conditions. Therefore, it is unclear to which intracellular signalling processes PKC subtypes are related. The aim of the study was to determine the subcellular localization of PKC $\alpha, \beta$ and $\beta_{\|}$, using immunocytochemistry in human endothelial cells (EC-RF24) cultured to confluency under conditions of normal and high $(25 \mathrm{mM})$ glucose and consequently stimulated with PMA, thrombin or glucose $(25 \mathrm{mM})$. Additionally, intracellular calcium levels were determined by fluorescent imaging microscopic techniques. Perinuclear staining of PKC $\alpha$ and $\beta_{\|}$in cells cultured under conditions of normal glucose increases upon stimulation with high glucose, whereas PKC $\beta_{1}$ translocates to the plasma membrane. Nuclear staining of PKC $\beta_{1}$ and $\beta_{11}$ remains unchanged under these conditions. Cultivation of the cells under high glucose conditions, reduced PKC $\alpha$ and $\beta$ staining, whereas PKC $\beta_{\mid 1}$ staining was unchanged. Translocation of PKC subtypes induced by PMA was comparable with high glucose stimulation, whereas thrombin stimulation not only resulted in a preferential translocation of PKC $\alpha$ to the nucleus, but also increased intracellular calcium levels. This study suggests that in human endothelial cells already short-term exposition to elevated glucose concentrations can lead to translocation of various PKC subtypes without influencing calcium levels, probably resulting in an altered PKC-mediated signalling. 
OP 47

\section{Patterns of Insulin Secretion from $\beta$-Cells}

\section{3}

\section{INSULIN GRANULE EXOCYTOSIS OCCURS BETWEEN $\beta$-CELLS IN MOUSE ISOLATED ISLETS}

D.L.A. MacArthur, ' J.F.Morris and ${ }^{1,2}$ A. Clark, ${ }^{1}$ Department of Human Anatomy, ${ }^{2}$ Diabetes Research Laboratories, Oxford, UK.

Insulin granule release is considered to occur largely towards the capillary border of the $\beta$-cell and islet hormone secretion is modulated by paracrine interactions between islet endocrine cells. To quantify insulin granule release in relation to islet cells and capillaries, exocytosis of secretory granules was visualised by the use of tannic acid (TA) which mordants (fixes) released protein granules. Mouse isolated islets were incubated in Hepes buffered Krebs containing $0.2 \% \mathrm{TA}, 20 \mathrm{mM}$ glucose and $0.1 \mathrm{mM} \mathrm{IBMX}$ at $37^{\circ} \mathrm{C}$ in $\mathrm{O}_{2}$ for 30 mins ( $n=3$ expts). Islets were fixed in $2.5 \%$ glutaraldehyde and processed for electron microscopy (em) for quantitative morphometry. Supernatants were collected for insulin measurement by RIA. The proportion of active cells in islet em sections (one or more exocytosis /cell profile) was $19.6+0.23 \%$ (mean \pm SEM) of total cells examined $(n=448)$; insulin was released as single granules, glucagon as multiple exocytoses. Insulin exocytosis was localised mostly between $\beta$-cells ( $81.3 \pm 3.9 \%$ active cells), but also towards capillary remnant $(11.7+.3 .5 \%)$ or at both locations in $7 \pm 2.9 \%$. The observed quantity of insulin granule release was compared with RIA-measured insulin secretion. Predicted granule release was calculated from reduction of RIA measured insulin secretion as a result of granule capture by TA ( $0.063 \mathrm{pmol} / \mathrm{islet})$, measured values of $\beta$-cell and islet areas, thickness of tissue sections and the estimated molecular content of insulin granules $\left(2 \times 10^{5} \mathrm{mols} /\right.$ granule). Predicted insulin granule exocytosis visualised in an em section under these experimental conditions was 5 granules/active cell which corresponds to 2.4 exocytoses/active cell determined experimentally.

Conclusion: Insulin granule exocytosis in isolated islets occurs at intercellular not perivascular borders and from $<20 \%$ of $\beta$-cells under maximal stimulation

\section{5}

PULSATILE RELEASE OF INSULIN FROM ISOLATED NOD MOUSE ISLETS

J.M. Lin, J. Sternesjö, S. Sandler and P. Bergsten, Department of Medical Cell Biology, Uppsala university, Uppsala, Sweden.

Plasma insulin of normal subjects is oscillatory, reflecting coordinated pulsatile release from the pancreatic $\beta$-cells. Loss of the regular plasma insulin oscillatory pattern has been observed in non-diabetic ICA positive first degree relatives to type 1 diabetic patients. The non-obese diabetic (NOD) mouse spontaneously develops type 1 diabetes, which strongly resembles the human disease. To investigate how the disturbances of the regular plasma insulin oscillations relate to the secretory activity of the islets of Langerhans, islets were isolated from non-diabetic female NOD mice of different ages. The diabetes incidence among the females is $72 \%$ by 30 weeks of age. The control group comprised 5 weeks old mice i.e. before pancreatic insulitis is observed. Insulin release was pulsatile $(0.2 \pm$ 0.02 oscillations $/ \mathrm{min}$ ) in the presence of $3 \mathrm{mM}$ glucose with a secretory rate of $21 \pm 3 \mathrm{pmol}^{*} \mathrm{~g}^{-1} \mathrm{~s}^{-1}$. When the glucose concentration was increased to $11 \mathrm{mM}$, the secretory rate rose 8 -fold by modulation of the amplitude of the insulin pulses leaving their frequency unaffected. Islets from 13 weeks old mice had varying degrees of peri-insular infiltration. Basal insulin release was pulsatile with a rate and frequency similar to that recorded from 5 weeks old islets. Although $11 \mathrm{mM}$ glucose caused amplitude modulation of insulin pulses also in these islets, the rise in secretory rate was only 2-3 fold. Similar results were obtained when islets from 25 weeks old mice, most of which had marked insulitis, were perifused in the presence of 3 and $11 \mathrm{mM}$ glucose. In the NOD-mouse model for type 1 diabetes, islets released insulin in pulses despite mononuclear cell infiltration. However, glucose-induced increase of the amplitude of insulin pulses was substantially reduced, which may be related to reduction in glucose metabolism. The mononuclear cells may be responsible for this reduction, since normalization of insulin release was observed after culturing the infiltrated islets for 7 days, which depletes the islet mononuclear cell content and normalizes glucose metabolism.

\section{4}

INHIBITION OF INTRAPANCREATIC GANGLIA CAUSES SUSTAINED AND NON-OSCILLATORY INSULIN RELEASE FROM THE PERFUSED PANCREAS

T. Sundsten, H. Ortsäter and P. Bergsten, Department of Medical Cell Biology, Uppsala university, Uppsala, Sweden.

Insulin release from the isolated pancreas is pulsatile, which is decisive for the appearance of plasma insulin oscillations. In order to obtain the pulsatile release from the pancreas, coordination of the secretory activities between the islets is required. The intrapancreatic ganglia have been proposed to play a part in this coordination. To test this hypothesis, pancreata from male Sprague-Dawley rats were perfused in the absence or presence of tetrodotoxin (TTX), a blocker of neuronal function Pancreata were perfused with a Krebs-Ringer bicarbonate buffer containing $11 \mathrm{mM}$ glucose. Fractions of perfusate were collected every $30 \mathrm{sec}$ and analyzed for insulin with ELISA technique. After $30 \mathrm{~min}$ perfusion insulin release was $7.2 \pm 2.2 \mathrm{pmol}^{*} \mathrm{~min}^{-1}{ }^{*}$ pancreas ${ }^{-1}$. When the perfusion was performed in the presence of $5 \mu \mathrm{M}$ TTX insulin release was $29 \pm 9.4 \mathrm{pmol}^{*}$ min $^{-1 *}$ pancreas ${ }^{-1}$. Insulin release in the absence as well as in the presence of TTX was analyzed by Fourier transformation. Power spectra showed a dominant frequency $(0.21 \pm$ $0.03 \mathrm{osc} / \mathrm{min}$ ) in the insulin release data obtained in the absence of TTX No such dominant frequency component was found in the data obtained in the presence of TTX. To investigate possible effects of TTX on the pulsatile release from the isolated islet, individual islets were perifused in the absence or presence of $5 \mu \mathrm{M}$ TTX at $11 \mathrm{mM}$ glucose. Whereas TTX increased the amplitude of the insulin pulses, their frequency was essentially not affected. We propose that the loss of regular secretory activity from the perfused pancreas in the presence of TTX is caused by impaired coordination between the islets due to inhibition of the intrapancreatic ganglia.

\section{6}

GLUCOSE AND PALMITATE ENHANCE PROINSULIN/NSSULIN RELEASE FROM HUMAN ISLETS THROUGH AN INSULIN DEPLETION EFFECT.

A Björklund, V Grilt Dept Endocrinology, Karolinska Hospital, Stockholm, Sweden Relative hypersecretion of proinsulin is a feature of type 2 diabetes. We investigated whether this feature can be replicated in vitro by long term exposure to elevated glucose and/or fatty acids. Human islets (obtained from Beta Cell Transplant Unit, Brussels) were exposed for $48 \mathrm{~h}$ to to 27 or to $5.5 \mathrm{mM}$ glucose. The ratio of intraislet proinsulin-like molecules (PI) to insulin (I) was 3.7-fold increased after culture at 27 vs $5.5 \mathrm{mM}$ glucose, due to preferential decrease of $\mathrm{I}$. The $\mathrm{PI} / \mathrm{I}$ ratio of secretion was 3.6 -fold increased during $60 \mathrm{~min}$ final incubations at a basal $(3.3 \mathrm{mM})$ glucose concentration. Diazoxide was added to culture media to investigate the importance of overstimulation. In cultures at $27 \mathrm{mM}$ glucose co-culture with diazoxide normalized the intraislet $\mathrm{PI} / \mathrm{I}$ ratio and the $\mathrm{PI} / \mathrm{I}$ ratio of secretion in postdiazoxide incubations at $3.3 \mathrm{mM}$ glucose $(p<0.001$, see table). None of these effects were seen when diazoxide was co-cultured with $5.5 \mathrm{mM}$ glucose

Culture conditions $(48 \mathrm{~h}) \quad$ Islet $\mathrm{PI} / \mathrm{I}$ ratio $(\%)$ Secreted $\mathrm{PI} / \mathrm{I}$ ratio $(\%)$

$27 \mathrm{mM}$ glucose $\quad 7.4 \pm 0.6 \quad 13.9 \pm 0.9$

$27 \mathrm{mM}$ glucose $+325 \mu \mathrm{M}$ diazoxide $1.7 \pm 0.1 * \quad 1.8 \pm 0.1 *$

Culture with $0.2 \mathrm{mM}$ palmitate together with $27 \mathrm{mM}$ glucose decreased islet contents of 1 by $49 \%$. In final incubations at $3.3 \mathrm{mM}$ glucose (following culture with palmitate and $27 \mathrm{mM}$ glucose) the per cent $\mathrm{PI}$ to 1 of secretion was increased to $21.2 \pm 3.8$ versus $13.9 \pm 0.9 \%, \mathrm{p}<0.05$ after culture with $27 \mathrm{mM}$ glucose alone. Culture with palmitate at $5.5 \mathrm{mM}$ glucose, decreased islet content of both PI and 1 and significantly increased the $\mathrm{PI} / \mathrm{ratio}$ of secretion during final incubations after culture, $p<0.05$. Conclusions: Long term exposure of human islets to elevated glucose in vitro increases PI/I. This abnormality is aggravated by fatty acids. The effects, which appear clinically relevant, are likely secondary to depletion of mature insulin granules. 


\section{Alterations in Glucose Metabolism}

\section{7}

TRANSGENIC MICE OVEREXPRESSING HUMAN APOLIPOPROTEIN C1 ARE HYPERLIPIDEMIC AND SHOW AN INCREASE IN INSULIN-MEDIATED GLUCOSE METABOLISM. S.J. Koopmans, M.C. Jong, I. Que, V.E.H. Dahlmans, H.D. Petronilia, J.K Radder, M.H. Hofker and L.M. Havekes. Dept. Endocrinology and Dept. Human Genetics, LUMC and TNO-PG, Leiden, The Netherlands.

Excess of apolipoprotein $\mathrm{C} 1$ (apoC1) on the VLDL particle impairs hepatic uptake of plasma VLDL and leads to hyperlipidemia. ApoC1 mice not only show elevated plasma cholesterol and triglyceride but also elevated free fatty acid (ffa) levels versus wild-type mice $(7.0 \pm 1.2$ vs $1.6 \pm 0.1 ; 9.1 \pm 2.3$ vs $0.4 \pm 0.1$ and $3.0 \pm 0.3 \mathrm{vs} 0.9 \pm 0.1 \mathrm{mmo} / \mathrm{l}$, respectively). The Randle cycle predicts a negative interaction between (insulin-mediated) glucose and $\mathrm{ffa}$ metabolism. To examine whether insulin-mediated glucose metabolism is influenced by primary hyperlipidemia in apoC1 mice, we have performed hyperinsulinemic $(6 \mathrm{mU} / \mathrm{kg} \cdot \mathrm{min})$, euglycemic $(7 \mathrm{mmo} / /)$ clamps with $3-3 \mathrm{H}$ glucose or 9,10-3H-palmitic acid infusions. Fasting (6h) plasma glucose levels were lower in apoc1 vs wild-type mice ( $6.9 \pm 0.8$ vs $9.5 \pm 0.6 \mathrm{mmol} / \mathrm{l}, \mathrm{p}<0.05)$. Insulin-mediated whole body glucose uptake, glycolysis (generation of $3 \mathrm{H}_{2} \mathrm{O}$ ) and glucose storage were increased in apoC1 vs wild-type mice (339+28 vs $200 \pm 11 ; 183 \pm 39$ vs $128 \pm 17$ and $156 \pm 44$ vs $72 \pm 17 \mu \mathrm{mol} / \mathrm{kg} . \mathrm{min}, p<0.05$, respectively). Insulin-mediated whole body ffa uptake, ffa oxidation (generation of $3 \mathrm{H}_{2} \mathrm{O}$ ) and ffa storage were decreased in apoC 1 vs wild-type mice ( $15 \pm 3$ vs $33 \pm 6 ; 3 \pm 2$ vs $11 \pm 4$ and $12 \pm 2$ vs $22 \pm 4 \mu \mathrm{mol} / \mathrm{kg}$. $\mathrm{min}, p<0.05)$ in the face of elevated plasma ffa levels $(1.3 \pm 0.3 \mathrm{vs} 0.5 \pm 0.1 \mathrm{mmol} / \mathrm{p}, \mathrm{p}<0.01)$, respectively. Summary: excess apoC1 on the VLDL particle leads to increased whole body insulin-mediated glucose metabolism and decreased insulin-mediated ffa metabolism in an environment of high plasma ffa concentrations. Conclusion: reasoning in line with the concept of the Randle cycle, this study indicates that apoCt on the VLDL particle is involved in the regulation of tissue free fatty acid uptake and metabolism and, as a consequence, apoC1 influences insulin-mediated glucose metabolism.

\section{8}

EXPRESSION OF GLUCOKINASE IN SKELETAL MUSCLE OF TRANSGENIC MICE DECREASES DIABETIC HYPERGLYCEMIA P. J. Otaegui, T. Ferre, A. Pujol, R. Jimenez, E. Riu and F. Bosch. Department of Biochemistry and Molecular Biology, School of Veterinary Medecine, Universitat Autonoma de Barcelona, 08193Bellaterra, Spain

Skeletal muscle has been postulated to be the most importan tissue involved in the disposal of glucose following oral ingestion. However, part of the limitation in glucose utilization during situations associated with increased glucose transport is likely to be due to feedback inhibition of the hexokinase-II (HK-II) by its product, glucose 6phosphate. In contrast to HK-II, the liver enzyme glucokinase (GK) has a high $\mathrm{Km}$ for glucose and its activity is not inhibited allosterically by glucose 6 phosphate. To determine whether GK expression in skeletal muscle might lead to increased glucose uptake, transgenic mice expressing a myosin-light chain (MCL) promoter/GK chimeric gene were obtained. Fed transgenic mice showed levels of GK mRNA in the skeletal muscle about 4 -fold those found in the liver. However, similar levels of GK protein were noted in muscle and liver of these mice, when analyzed by Western blot. GK activity was detected in the muscle of transgenic mice which have also an increase in the intracellular concentration of glucose 6-phosphate (Con 578 $\pm 58 ; \quad T g 2 \quad 1723 \pm 63 \mathrm{nmol} / \mathrm{g}$ muscle, $\mathrm{p}<0.05$ ) and glycogen (Con $2.8 \pm 0.1 ; \mathrm{Tg} 13.8 \pm 0.2 ; \mathrm{Tg} 2 \quad 4.9 \pm 0.1 \mathrm{mg} / \mathrm{g}$ muscle) These changes in muscle glucose metabolism led to a reduction in blood glucose levels (Con $86 \pm 2 \mathrm{mg} / \mathrm{dl}$; Tg1 72 \pm 2 ; Tg2 $71 \pm 2$; $\mathrm{p}<0.05$ ) and an increase in serum lactate concentration (Con: $10.3 \pm 2.1 \mathrm{mM} ; \quad \mathrm{Tg} 1 \quad 17.7 \pm 1.3 \mathrm{mM} ; \quad \operatorname{Tg} 2 \quad 15.8 \pm 1.2 \mathrm{mM} ; \quad p<0.05)$. Transgenic mice also showed lower levels of blood glucose after an intraperitoneal glucose tolerance test, indicating that GK expression caused an increase in blood glucose disposal by the muscle. Moreover, lower levels of blood glucose were noted in transgenic mice treated with streptozotocin than in control mice treated with streptozotocin. All these results indicated that expression of $\mathrm{GK}$ in muscle increased glucose uptake and utilization, and suggest that engineering skeletal muscle to express the liver enzyme might be an useful approach to counteract diabetic hyperglycemia

\section{9}

PHOTOAFFINITY LABELING OF THE MICROSOMAL GLUCOSE-6PHOSPHATE TRANSPORTER DEFICIENT IN GSD TYPE $1 \mathrm{~b}$

W. Kramer, H.-J. Burger, H. Hemmerle, W.J. Arion and A. Herling Hoechst Marion Roussel Deutschland GmbH, D-65926 Frankfurt, Germany

The terminal step of hepatic glucose production is catalyzed by the glucose-6-phosphatase system, composed of a phosphohydrolase with its active site to the lumen of the endoplasmic reticulum, and transporter proteins for glucose-6-phosphate (T1) and phosphate (T2). Deficiencies of the glucose-6-phosphatase system are the genetic basis for glycogen storage disease type 1 (GSD1). In order to identify the microsomal glucose-6-phosphate transporter T1 deficient in GSD type 1b patients, we have performed photoaffinity labeling of human and rat liver microsomes with photoreactive chlorogenic acid analogues. These chlorogenic acid derived inhibitors specifically inhibit T1 in the nM-range without affecting T2 or the phosphohydrolase. Photoaffinity labeling of rat liver microsomes led to the labeling of polypeptides of $M_{t} 70,57,50,33$, and $31 \mathrm{kDa}$ and of $\mathrm{M}, 70,55,33$, and $31 \mathrm{kDa}$ in human liver microsomes. Treatment of microsomes with $0.3 \%$ Chaps retained the high affinity binding of T1 inhibitors; photoaffinity labeling of such stripped microsomes resulted in the identification of polypeptides of $M_{r} 55,33$, and $31 \mathrm{kDa}$ in human liver microsomes and of 50,33 , and $31 \mathrm{kDa}$ in rat liver microsomes. Photoaffinity labeling of particulate material obtained from a healthy human liver revealed the labeling of polypeptides of $M_{r} 55,33$, and $31 \mathrm{kDa}$ whereas in a biobsy probe of a patient with diagnosed GSD type $1 \mathrm{~b}$ (which was a generous gift by E. Van Schaftingen) the 55 and $33 \mathrm{kDa}$ labeled proteins, were missing. Therefore, proteins of $M_{r} 55 \mathrm{kDa}$ in human liver and $50 \mathrm{kDa}$ in rat liver represent most likely the glucose-6-phosphate transporter of the endoplasmic reticulum.

\section{0}

THIAZOLIDINEDIONE-STTMULATED SKELETAL MUSCLE GLUCOSE UTILIZATION MIGHT BE MEDIATED THROUGH THE NO/CGMP CASCADE B. Leighton and M.E. Young. University of Oxford, U.K.

Thiazolidinediones (TZDs) are a relatively new class of compound, which appears to possess insulin sensitising properties. Recently, TZDs have been found to have acute effects on skeletal muscle glucose utilisation in vitro. The purpose of this study was to investigate the possible mechanism(s) by which TZDs acutely effect skeletal muscle glucose metabolism. Isolated rat soleus muscle preparations were incubated (1.5 hrs) with various concentrations of the TZD BRL49653. BRL 49653 $(10 \mu \mathrm{M})$ increased the rates of both net lactate release (control, $9.02 \pm$ 0.79 , versus BRL49653, $17.35 \pm 0.88 \mu \mathrm{mol} / \mathrm{h} / \mathrm{g}$ wet wt.; $\mathrm{P}<0.001)$ and ${ }^{14} \mathrm{C}$-labelled lactate release (control, $5.70 \pm 0.86$, versus BRL49653, $10.98 \pm 1.10 \mu \mathrm{mol} / \mathrm{h} / \mathrm{g}$ wet wt.; $P<0.001$ ), and decreased rates of glycogen synthesis (control, $2.40 \pm 0.25$, versus BRL49653, $1.69 \pm 0.21 \mu \mathrm{mol} / \mathrm{h} / \mathrm{g}$ wet wt.; $\mathrm{P}<0.05$ ). The rate of ${ }^{14} \mathrm{C}$-labelled lactate release reflects the rate of glucose transport by skeletal muscle in vitro. As these acute effects of BRL 49653 on skeletal muscle glucose utilisation are similar to those of nitric oxide (NO), we investigated whether the BRL49653 effects were via stimulation of the NO/cGMP signalling cascade. L-NAME ( $2 \mathrm{mM})$, a competitive

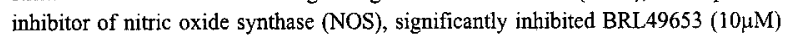
stimulated rates of ${ }^{14} \mathrm{C}$-labelled lactate release (BRL49653, 9.27 \pm 0.96 , versus BRL49653 plus L-NAME, $7.25 \pm 0.47 \mu \mathrm{mol} / \mathrm{h} / \mathrm{g}$ wet wt.; $\mathrm{P}<0.05$ ). The guanylate

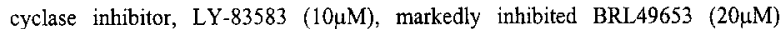
stimulated rates of ${ }^{14} \mathrm{C}$-labelled lactate release (BRL49653, $21.25 \pm 0.92$, versus BRL49653 plus LY-83583, $5.09 \pm 0.44 \mu \mathrm{mol} / \mathrm{h} / \mathrm{g}$ wet $\mathrm{wt}$.; $\mathrm{P}<0.001$ ). In addition,

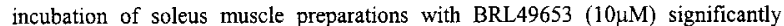
increased soleus muscle cGMP contents (control, $3.99 \pm 0.27$, versus BRL49653, $5.65 \pm 0.33 \mathrm{pmol} / \mathrm{g}$ wet wt: $\mathrm{P}<0.01$ ). These results strongly suggest that the $\mathrm{NO} / \mathrm{cGMP}$ signalling cascade plays a tole in the acute stimulation of skeletal muscle glucose utilisation by TZDs. Whether this cascade plays a role in the sensitisation of skeletal muscle to insulin by chronic TZD treatment requires elucidation. 


\section{Genetics of Type 1 Diabetes}

\section{1}

MUTATIONS IN HEPATOCYTE NUCLEAR FACTOR- $1 \alpha$ IN CAUCASIANS ORIGINALLY CLASSIFIED AS IDDM

L Ambye, AM Møller, F Pociot, J Nerup, T Hansen, O Pedersen.

Steno Diabetes Center and Hagedorn Research Institute, Copenhagen, Denmark.

Mutations in the hepatocyte nuclear factor-1 $\alpha$ (HNF-1 $\alpha$ ) gene is the cause of the type 3 form of maturity-onset diabetes of the young (MODY3), which is characterised by a severe impairment of insulin secretion and an early onset of the disease. Also at onset of diabetes some MODY patients show similar symptoms as IDDM patients. The objective of this study was to estimate the prevalence of MODY3 patients misclassified as IDDM patients. The coding region as well as the minimal promoter of the HNF-1 $\alpha$ gene were examined by single-strand conformational polymorphism scanning followed by direct sequencing in 44 unrelated IDDM patients chosen among the about $10 \%$ of IDDM patients that did not carry any highrisk HLA-genotypes i.e. DR3 and DR4. Three IDDM patients (7\%), negative for GAD65 and IA-2 antibodies, were identified to carry mutations in the HNF-1 $\alpha$ gene. Two patients carried a missense mutation (Cys241Gly) in the region coding for the DNA binding domain and one patient carried a frameshift mutation (P291 fsdelA) which will generate a truncated protein of 341 amino acids. The mutations were all identified in heterozygous form, segregated with diabetes in the nuclear family, and were not identified in 100 unrelated, healthy subjects. Furthermore, family history in two of the three families showed diabetes in four generations, consisting with an autosomal dominant inheritance. Therefore we conclude, that diabetes in about $7 \%$ of Danish IDDM patients without a high-risk HLAgenotype is caused by mutations in the HNF- $1 \alpha$ gene and that approximately $1 \%$ of all IDDM patients actually may have a form of diabetes that is caused by mutations in the HNF-1 $\alpha$ gene. These patients should be reclassified as MODY3 patients.

\section{3}

IS LAMDA ${ }_{s}\left(\lambda_{s}\right)$ A GOOD WAY OF ASSESSING THE CONTRIBUTION OF HLA TO THE OVERALL GENETICS OF IDDM?

E. Tuomilehto-Wolf and J. Tuomilehto. National Public Health Institute, Helsinki, Finland

$\lambda_{s}$ was originally proposed by $N$. Risch in 1987 as a measurement of the genetic contribution of HLA in IDDM. $\lambda_{s}$ was 15 in IDDM based on the lifetime recurrence rate in siblings (6\%) divided by the population frequency $(0.4 \%)$ and the contribution of HLA was $32 \%$ based on the number of affected siblings sharing $0 \mathrm{HLA}$ alleles. As these calculations were the rationale for whole genome searches for non-HLA genes for IDDM the aim of the present study was to find out how $\lambda_{s}$ holds up in a truly populationbased family study of IDDM in Finland (DiMe Study). Of the 184 siblings who shared 0 HLA haplotypes (HLA-A,C,B,DRBI,DQA1,DQBI alleles were defined by serology and molecular methods) with the diabetic proband 7 developed IDDM (3.8\%). One was proven to be a half-sibling, 3 were HLA recombinants ( 2 between $A$ and C, 1 between $B$ and DR) and 3 had parents who possessed 3 to 4 high risk IDDM associated HLA haplotypes. This showed that affected siblings sharing 0 alleles are a heterogeneous group including genetic abnormalities. In addition, the lifetime recurrence risk in siblings is about the same in high and low incidence populations $(6-10 \%)$ while the incidence of DDM varies 40 -fold (from 1 to $40 / 100$ $000 /$ year). The previous $\lambda_{5}$ calculations are based on highly biased studies of families selected for affected sibling pairs which only represent $10-15 \%$ of all IDDM families. In conclusion, $\lambda_{s}$ is not a good way of looking at the genetic contribution of HLA in IDDM as it also ignores the complexity of HLA and the graded susceptibility found for different IDDM associated HLA haplotypes.

\section{2}

ALANINE AT POSITION 74 OF THE DRB CHAIN IN HLA DRB1*04 ALLELES CONFERS SUSCEPTIBILITY TO IDDM K. Badenhoop, H. Donner, T. Siegmund, J. Braun, J. Herwig and K.H. Usadel, Center of Internal Medicine, Medical Clinic 1, and Dept. of Pediatrics, University Hospital Frankfurt am Main, FRG.

Class II genes of the human major histocompatibility complex (MHC) are the primary determinants of susceptibility to type 1 diabetes (IDDM), mainly conferred by the HLA DR4-DO8 haplotype. As these genes are tightly linked, it has been difficult to identify HLA DR or DO alleles as the primary risk markers in IDDM. We therefore investigated the HLA DRB1 *04 subtype distribution in 249 DR4 + individuals $(162$ IDDM patients and 87 controls) in relation to their DOA 1 DOB1 alleles in order to evaluate their their contribution to disease susceptibility. In addition we analysed 89 families with an IDDM proband with respect to the transmission of the HLA DRB $1 * 04$ subtypes to the patients, comprising 130 DR4 + of the 356 investigated haplotypes. HLA DRB1, DOA 1 and DQB1 alleles were assigned by sequence specific primer (SSP) analysis. HLA DRB $1{ }^{*} 0401$ was significantly more frequent in DR4 + patients with IDDM $(66 \%)$ than in controls $(50 \%, p<0.05)$, also in DOB $1 * 0302+$ patients $(68 \%$ vs. $48 \%, p<0.03)$ and was more often transmitted to affected probands in families $\left(p<6 \times 10^{-4}\right)$, HLA DRB1*0402 was found in $8 \%$ of transmitted haplotypes $11 \%$ of nontransmitted, $p<0.02)$, whereas $D R B 1{ }^{*} 0403$ was less frequent in patients $(1 \%$ vs. $13 \%, p<0.02)$ and among transmitted haplotypes, confirming its protective potential. An alanine at position 74 of the DRB chain was found in $49 \%$ of all DRB1*04 transmitted alleles in patients but only in $19 \%$ of those non-transmitted $\left(p<8 \times 10^{-6}\right)$. In conclusion, the Ala74 DRß residue, amongst others, contributes to HLA linked susceptibility in IDDM.

\section{4}

IDENTIFICATION OF 3 NOVEL CD4 GENE PROMOTER POLYMORPHISMS: LINKAGE ANALYSIS IN DANISH IDDM MULTIPLEX FAMILIES

O.P. Kristiansen, F. Pociot, J. Johannesen, A.E. Karlsen, J. Nerup, T. Mandrup-Poulsen, DSGD and DIEGG. Steno Diabetes Center, DK-2820 Gentotte, Denmark.

Recently, evidence for linkage between IDDM and the A4 (113bp) allele of a CTTTTrepeat in the $\mathrm{CD} 4$ gene promoter was reported. The aims of the present study were to screen the CD4 promoter for novel polymorphisms and to investigate for linkage between novel polymorphisms and IDDM in the Danish population. Methods and material: SSCP screening of the CD4 promotor (position $45 \rightarrow 1523 \mathrm{bp}$; M86525) was performed in 20 IDDM and 10 healthy subjects. The allelic variants in the SSCP polymorphic sites were identified by cycle sequencing. Linkage was investigated by the transmission disequilibrium test (TDT) in 148 sibpair and 105 parent-offspring IDDM tamilies. Transmission to 424 affected and 249 non-affected offspring was observed. Polymorphism screening. Three novel polymorphisms were identified: 1) a $T \rightarrow C$ substitution in pos. $474 b p, 2)$ a $G \rightarrow C$ substitution in pos. $1003 \mathrm{bp}$, and 3) a $C \rightarrow G$ substitution in pos. 1343bp. PCR based ('Bsil RFLP, ${ }^{2}$ Mspl RFLP and 3 a mutagenically separated (MS)) assays for the polymorphisms were established. Linkage analysis: The G-allele of the Bsil (pos. 474bp) polymorphism was in complete linkage disequilibrium with allele $A B$ (88bp) of the CD4 (CTTT)-repeat, known not to be associated with IDDM. Both the Mspl (pos. 1003bp) and the BsaXI (pos. 1343bp) alleles showed random transmission to the IDDM offspring, but a non-significant tendency to skewed transmission was observed for both polymorphisms: $82(55 \%)$ G- and $66(45 \%)$ C-alleles (prot $=0.19)$ and $163(55 \%)$ C. and $136(45 \%)$ G-alleles ( haplotype was established. Transmission of the 3 identified haplotypes G-C, C-G and G$G$ were $55 \%\left(167 / 305 ; p_{\text {idt }}=0.1\right), 43 \%\left(65 / 151 ; p_{\text {rdd }}=0.09\right)$ and $48 \%\left(115 / 238 ; p_{\text {id }}=0.6\right)$. In conclusion, we have identified 3 novel polymorphisms in the CD4 gene promoter. None of the found polymorphisms or the resulting Mspl-BsaXI haplotype were significantly linked to IDDM in the Danish population. Thus, it is less likely that any of the novel poly. morphisms confer susceptibility to IDDM development, although the region is linked (CD4 (CTTTT)-repeat) to IDDM. 
305

PHENOTYPE OF PARENTS INFLUENCES THE NUMBER, THE SEX RATIO, AND THE DIABETICS:NONDIABETICS DISTRIBUTION OF THEIR OFFSPRING

S. Schmidt, I. *Rjasanowski, P. Kovacs and I. Klöting, Institute of Pathophysiology, University of Greifswald and * Clinic of Karisburg, Centre for Diabetes and Metabolic Diseases, D-17495 Karlsburg, Germany

The aim of our study was to analyse families with type ldiabetes (d) and their offspring with regard to the influence of phenotype of parents (father $x$ mother; $d x$ nd, nd $x d, d x d$ ) on the number, the sex ratio and the diabetics : nondiabetics distribution of their offspring which were older than 20 years by December 1997. Fron the registry of the Clinic of Diabetes, Garz, and the formerly Central Institute of Diabetes "Gerhardt Katsch“, Karlsburg, we selected 170 families in which the father $(n=44, d x n d)$, the mother $(n=101$, $n d x d)$ or both father and mother $(n=25, d$ $x$ d) were diagnosed as type 1 diabetics. To estimate the diabetes frequency in families of diabetic parents, families with and without diabetic offspring were included. Data were evaluated using the statistical analysis system SPSS, significant differences of mean values were checked by ANOVA analysis, and distributions by $\chi^{2}$ test. Logistic regression was used to evaluate the influence of year and age of diagnosis, number of offspring per family, age of mothers at delivery on diabetes occurrence in the offspring. More than $50 \%$ of diabetic mothers delivered one child only (nd $x d=55 \%$ and $d x d=64 \%$ ). Families with diabetic parents ( $x d$ ) had at the most two children and the sex ratio was significantly disturbed in these families, in which more females than males (25:9) were born. There were no significant differences in the age at onset of offspring from the different families, but diabetes was diagnosed earlier in offspring than in their parents. The diabetes frequency of offspring amounted about $30 \%$ (diabetic father, d $\mathrm{xd}$ ), $14 \%$ (diabetic mother, nd $\mathrm{x}$ d), and $50 \%$ (diabetic father and mother, $\mathrm{dx} \mathrm{d}$ ). Logistic regression analysis clearly documented that the year of diagnosis significantly influenced the different diabetes frequencies between the parental phenotypes demonstrating the importance of the year of diagnosis and of the phenotype of parents which have to be considered in family studies.

\section{6}

MAPPING OF IDDM 12 IN SOUTHERN EUROPEAN POPULATIONS

L. Nisticò, L. Prichard, R. Buzzetti, A. De Cinti, G. Di Genova, R. Tosi, M. SerranoRios, M.T. Martinez, J. Todd, P. Pozzilli. Istitute II Clinica Medica, University of Rome "La Sapienza"

Type 1 diabetes is a polygenic disease. One locus (named IDDM12) has been mapped, by linkage and association studies, on chromosome $2 q 33$ in the CTLA$4 / C D 28$ gene region. In order to refine the localisation of IDDM12 we developed a physical map of CTLA-4/CD28 region that cover an approximate distance of 250 kilobases $(\mathrm{kb})$. Eight new microsatellite markers, besides CTLA-4 microsatellite, were physically linked to the CTLA-4 contig and therelative order have been estabilished for most of them. Genotyping is currently ongoing on a collection of 350 Italian diabetic families. Transmission Disequilibrium Test (TDT) was performed on the most frequentalleles of each marker: none of them, except allele 6 at CTLA-4 $(p=0.014$, families typed $=187)$, showed a significantly distorted transmission. In order to identify the putative ancestral haplotype (hp) carrying the susceptibility allele, we determined, at each marker typed in more than 90 families (5 out of 8 , here named $A$ to $E$ ), the allele in linkage disequilibrium $(L D)$ with allele 6 at the CTLA-4 locus. LD pattern between $A$ and $B$ and between CTLA-4 and D were ambiguous and the corresponding hps are not shown. Subsequently, TDT analysis was performed on two-point hps. So far, four hps, involving four markers encompassing $140 \mathrm{~kb}$, show distortion from random transmission at a similar level of significance. It is expected that after expansion of the map and of the family panel, a peak of genetic association will be defined leading to a more precise localisation of IDDM12.

Markers Hp Freq. T NT P \%

$\begin{array}{llllllll}\text { A-C } & 5-6 & 4.7 & 11 & 3 & 0.034 & 78.6\end{array}$

$\begin{array}{lllllll}\text { B-C } & 5-6 & 26.9 & 33 & 17 & 0.024 & 66\end{array}$

$\begin{array}{lllllll}\text { B-CTLA-4 } & 5-6 & 16.1 & 23 & 10 & 0.024 & 69.7\end{array}$

$\begin{array}{lllllll}\text { C-CTLA-4 } & 6-6 & 14.7 & 22 & 10 & 0.034 & 68.7\end{array}$

$\begin{array}{lllllll}D-E & 6-3 & 8.8 & 10 & 10 & \text { NS } & 50\end{array}$

$\mathrm{Hp}$ : numbers are allele in LD at the corresponding marker pair. Freq.: hp frequency calculated on parents (total identified hp varied from 250 to 316 ) $T$ and NT: Transmissions and Non Transmissions to probands only. \%T percentage

of $T(T / T+N T)$.

\section{7}

POLYMORPHISMS OF INTERFERON-Y GENE ARE ASSOCIATED WITH TYPE I DIABETES

M.M. Jahromi, B.A. Millward and A.G. Demaine. Molecular Medicine Research Group, Postgraduate Medical School, University of Plymouth, UK.

It is well known that type 1 diabeles is caused by an immune-mediated destruction of pancreatic islet $\beta$-cells. Recent studies have shown that genes outside of the HLA region are involved in determining susceptibility to type I diabetes. It is now known that polymorphisms in the coding and non-coding regions of the genes encoding cytokines may contribute to the susceptibility to autoimmune diseases as well as influencing the immune response to foreign antigens. The aim of this study was to investigate a microsatellite CA repeat polymorphism in the first intron of the interferon- $\gamma$ (IFNG) gene in 88 patients with type 1 diabetes and 29 normal controls. The IFNG microsatel lite region was amplified using the polymerase chain reaction with specific oligonucletides primers (the anti-sense was tabelled with 32P-ATP) and the products separated using polyacrylamide gel electrophoresis. The gel was dried and autoradiography performed. Eight alleles were detected and corresponded to 10-17 CA repeats and designated $\mathrm{CA}(10)$ to $\mathrm{CA}(17)$ respectively. The most common allele in both groups of subjects was CA(12). However, there was a highly significant increase in the frequency of this allele in the patients compared to the normal controls (58.5\% vs $27.6 \%$ respectively, $\chi 2=16.7, p<0.00005, P c=0.0005$ ). Interestingly, this allele was more common in those patients who developed the disease between $10-20$ years of age $(69.6 \%)$ than those who developed diabeles before 10 years or after 20 years of age ( $56.5 \%$ and $55.0 \%$ respectively). In conclusion, these preliminary results suggest that the IFNG locus may confer susceptibility to type 1 diabetes. This may be due to an association of these polymorphisms with secretion of interferon- $\gamma$ by immune mediated cells.

\section{8}

ANALYSIS OF INTERFERON Y GENE POLYMORPHISM IN SPANISH TYPE 1 DLABETIC PATIENTS

L. Gallart, E. Mato, M.A. Ottíz A. de Leiva, M. Puig Domingo. Hospital de Sant Pau, Barcelona and the Spanish Diabetes Genome Study Group.

Interferon $y$, as other cytokines, is thought to play an important role in the autoimmune destruction of $\beta$ cells during development of type 1 diabetes (DM1). An association of a polymorphism in the interferon $\%$ gene has been found in several populations. We studied 66 sporadic DM1 cases, 91 controls and 33 muitiplex DM1 families (132 individuals) from sparish population. A CA dinucleotide repeat at the first intron was analysed by PCR (Awata et al.) followed by SSCP (single strand conformational polymorphism) and nonradioactive hybridzation. Allalic and genotypic distributions were analysed by using $x^{2}$ and TDT (1.1 version) tests. Eight different alleles, which have different allelic distributions between sporadic cases and controls ( $\mathrm{p}=0.01$ ), were found. Alleles 5 and 7 were detected only in DM1 patients, but without differences in the genotypic frequency $(p=0.09)$. No evidences for overall transmission disequilibrism using either allele-wise $(p=0.639)$ or genotype wise analysis $(\mathrm{p}=0.639)$ were detected. These results indicate a modest contribution of this interferon $\%$ polymorphism in the pathogenesis of DM1 in spanish population, as the low significance of the case-control study tailed to be replicated by the TDT (supported by SAF $97 / 0251$ ). 
LINKAGE ANALYSIS OF IDDM 13 IN SPANISH TYPE 1 DIABETIC MULTIPLEX FAMILIES

E. Mato', Z.M. Larsen', O.P. Kristiansen', J.Johannesen ${ }^{2}$, L. Gallart', M. Puig-Domingo ${ }^{1}$, A. de Leiva' ${ }^{1}$ J. Nerup ${ }^{2}$, F. Pociot ${ }^{2}$ and SDGSG.

Hospital Sant Pau, Barcelona, Spain ${ }^{1}$ and Steno Diabetes Center, Gentofte, Denmark $^{2}$

Several loci assigned to the long arm of chromosome 2 have been proposed to participate in the polygenic nature of susceptibility to type 1 diabetes (DM1). Recently, a locus at 2q34 (IDDM 13) has been reported to show linkage to DM1 in British, Australian and Danish populations. The aim of our work was to study if linkage between IDDM 13 (D2S164) and DM1 is present in a Spanish diabetes cohort composed by 39 multiplex families with a total of 176 individuals. Genotyping of the D2S164 microsatellite was performed by PCR followed by PAGE, blotting and visualization using a streptavidine-HRP ECL reaction. Statistical analysis was performed using the extended transmission disequilibrium test (version 1.1) for multi-allele marker loci. Evidence for allele-wise $\left(x^{2}=26.51,14 \mathrm{df}, \mathrm{p}=0.022\right)$ and genotype-wise $\left(\mathrm{x}^{2}=\right.$ $41.17,27 \mathrm{df}, \mathrm{p}=0.040$ ) transmission disequilibrium was found. Distorted transmission of alleles $278(\mathrm{p}=0.039)$ and $288(\mathrm{p}=0.001)$ was observed. In conclusion, we found linkage of IDDM 13 to DM1 in a Spanish multiplex family cohort, replicating previous findings, mostly in Danish population. (Supported by SAF 97/0251)

\section{1}

GENETIC SUSCEPTIBILITY (HLA DQA1 AND DQB1) AND AGE AT THE ONSET OF TYPE 1 DIABETES

R. Arcari, G.Bruno, M. Borra, A. Lezo, F. Cerutti' M. Berrino G. Pagano and Piedmont Study Group for Diabetes Epidemiology. Dept. of Internal Medicine; ${ }^{1}$ Dept. of Pediatrics, University of Turin, Italy

Whereas the contribution of HLA DQAl and DQBI alleles to the susceptibility to type 1 diabetes in children has been previously assessed, few studies have been conducted in young adults. In this study, we analysed HLA DQA1 and DQB I alleles in 174 patients with type 1 (n. 85 aged $0-14$ yrs and 89 aged 15-29 yrs at the onset of the disease), randomly selected from the local population-based registry; 174 healthy controls paired with cases for place of birth of parents, randomly selected among bone marrow donors. The frequencies of alleles DQA 1*0301 and DQA1*0501 were, respectively, $29.4 \%$ and $38.8 \%$ in the age group $0-14$ yrs, and $27.5 \%$ and $39.3 \%$ in the age group 15-29 ( $p=n s)$. The frequencies of alleles DQB I*0201 and DQBI*0302 were, respectively, $37.1 \%$ and $27.1 \%$ in the age group $0-14 \mathrm{yrs}$, and $44.4 \%$ and $20.8 \%$ in the age group $15-29$ $(p=n . s)$. The frequencies of the high risk haplotypes DR3/DQA 1*0501-DQB1*0201 and DR4/DQA1*0301-DQB 1*0302 were $32.3 \%$ and $27.1 \%(0-14$ yrs $)$ vs. $34.8 \%$ and $20.8 \%(15-29$ yrs) ( $p=n s$ ). Four diabetogenic heterodimers were found in $38.8 \%$ $(0-14$ yrs $)$ vs $35.9 \% \quad(15-29$ yrs $) \quad(p=n s)$, whereas no heterodimers were found in $3.5 \%$ vs. $13.5 \%(p=0.04)$. A four times increased risk in children than in young adults with four diabetogenic heterodimers was evident $(O R=204.6$ vs $O R=49.6)$. This study suggests that the weight of genetic susceptibility conferred by HLA DQA -DQBI on type 1 risk could be different by age at onset of the disease.
ESTIMATION OF RECURRENCE RISK CONDITIONED BY HLAHAPLOTYPE IDENTITY IN IDDM SIB-PAIRS

T.L.Kouraeva ${ }^{1}$, A.S.Sergeyev', V.V.Nosikov ${ }^{3}$, Y.Y.Kondratiev ${ }^{1,3}$, V.A.Peterkova ${ }^{1}$, and I.I.Dedov', 'Endocrinology Research Centre, ${ }^{2}$ Research Centre for Medical Genetics, and "National Research Centre "GosNII Genetika", Moscow, Russia Previously, in clinical and population based study we have established the relative risk (RR) of IDDM for alleles and genotypes of HLA-DQA1 and DQB1 genes, as well as for their susceptible and protective heterodimers. Recently, we studied IDDM risk distribution in families having both concordant and discordant sib-pairs. HLA-DR antigens and/or highly polymorphic HLA-DQA1/Bl gene alleles were used as genetic markers in the family study. According to HLA-haplotype identity by descent (IBD) scores $(2 ; 1 ; 0)$, all sibships distributed as follows:

\begin{tabular}{lcccc}
\hline & IBD $=2$ & IBD $=1$ & IBD $=0$ & Total \\
\hline Concordant sib-pairs (n) & 18 & 7 & 1 & 26 \\
Discordant sib-pairs (n) & 4 & 10 & 6 & 20 \\
IDDM recurrence risk* (\%) & 18.1 & 3.32 & 0.81 & $\ldots$ \\
\hline
\end{tabular}

* General IDDM recurrence risk value found regardless HLA-haplotype identity status was accepted as $6 \%$;

Thus, IDDM recurrence risk value estimated using IBD for non-affected siblings HLA-identical to affected ones, was the maximum and 3-fold higher than that obtained without IBD/HLA testing (18.1\% vs. 6\%). Recurrence risk value for nonaffected and HLA-non-identical siblings, despite being minimal was, however, 4-fold higher than cumulative (up to $40 \mathrm{yr}$.) RR value found previously in general population $(0.81 \%$ vs. $0.2 \%)$. In conclusion, IBD method used marker haplotypes of HLA-DR/DQ loci is highly informative tool in the study of genetic susceptibility to IDDM. It might provide a genetic basis for high risk group(s) formation in IDDM prediction and prevention trials.

\section{2}

THE ROLE OF DPBI-ENCODED AMINO ACIDS IN SUSCEPTIBILITY TO TYPE I DIABETES IN THREE RACES.

C.L.Perry, C.H.Mijovic, D.Jenkins and A.H.Barnett. Department of Medicine, Birmingham University, Birmingham, England.

The HLA class II genes DR and DQ have been associated with determining susceptibilty to Type I diabetes. Alleles of a third HLA class II gene, DPBI have also been implicated as putative susceptibility determinants by some studies but not by others. A recent paper has indicated that analysis of individual HLA-DR and DQ-encoded amino acids is more relevant to predicting disease susceptibility than considering the identity of the alleles present. The aim of this study was to analyse polymorphic DPBI-encoded amino acids in Chinese, Japanese and Afro-Caribbean Type I diabetic and control subjects, to determine whether these amino acids are associated with susceptibility to the disease. The sites analysed were positions $35,36,55$ and 56 which may influence the spectrum of peptides bound to the DP molecule. The frequency of the presence of Tyrosine at position 35 was significantly increased in the Japanese patients compared to controls (14/84 alleles (16.7\%) vs $1 / 124$ alleles $\left.(0.8 \%) p_{c}=3.78 \times 10^{-4}\right)$. This was not replicated in the other races where the presence of Tyrosine was more common in the control populations (allele frequency in diabetic patients vs control subjects: Chinese $8 / 98(8.2 \%)$ vs $22 / 160$ (13.8\%); Afro-Caribbean $37 / 84(44.1 \%)$ vs $119 / 238$ $(50.0 \%)$ ). No associations between DPB1-encoded amino acids at the other sites analysed and the disease were identified in any race. This lack of consistent association indicates there is no independent role for the DPBIencoded amino acids analysed in determining susceptibility to Type I diabetes. Cross-ethnic studies continue to be useful in distinguishing primary MHCencoded susceptibility determinants from those merely associated with the disease due to linkage disequilibrium. 


\section{3}

\section{LACK OF ASSOCIATION OF IL-1 GENE CLUSTER POLYMORPHISMS IN POLISH CHILDREN WITH TYPE 1 DIABETES}

W. Młynarski, H.W.Witas, R. Sychowski, A. Młynarska and J. Bodalski, Molecular Biology Unit, $2^{\text {nd }}$ Clinic of Children Diseases, Medical University of Lodz, Poland,

Proinflammatory cytokines which are produced by macrophages such as $\mathrm{IL}-1$, play an important role in destruction of $\beta$ cells and are involved in pathogenesis of type 1 diabetes (IDDM). Besides, IL-1 gene cluster on chromosome $2 \mathrm{q}$ is suggested to be a locus for IDDM susceptibility. Thus, it is reasonably to analyse recently reported polymorphisms of IL-1B and IL-1RN genes in patients with type 1 diabetes. 98 patients suffering from type 1 diabetes and 108 healthy controls were examined The substitution at position $-1903 \mathrm{~T} \rightarrow \mathrm{C}$ (Alu I) within promotor region $+5810 \mathrm{G} \rightarrow \mathrm{A}$ (Ita I) within intron 4 and $+5887 \mathrm{C} \rightarrow \mathrm{T}$ (Taq I) within exon 5 of $I L-1 B$ gene as well as $T \rightarrow C$ substitution at +8006 within exon 2 (Msp I) and $\mathrm{T} \rightarrow \mathrm{C}$ transition at +11100 within exon 4 (Ita I) in IL-1RN gene were analyzed by RFLP-PCR technique. Additionally, the polymorphism of tandem repeats within intron 2 of $I L-1 R N$ gene was identified as the size of PCR products. The corrected $\mathrm{Chi}^{2}$ statistical analysis of examined polymorphisms did not show significant differences between IDDM group and healthy controls. The only difference occurred between examined groups referred to genotype TC in promotor of $I L-1 B$ (Alu I). 55 diabetic patients $(56 \%)$ vs. 44 of controls (40.7\%) exhibited TC genotype $(p=0.0387)$. Observed difference seems to be accidentally significant with no influence on IDDM etiopathogenesis. Although we have not found any association between polymorphic sites of IL-1B and $I L-1 R N$ and type 1 diabetes in Polish children, it seems reasonable to examine IL-1 gene cluster which is possibly involved in APC-T cell interaction.

\section{5}

THE DELIMITATION OF 5' AND 3' BOUNDARIES OF IDDM2 LOCUS IN POLISH POPULATION.

M.Krokowski, P.Machejko, K.Wyka, W.Andrzejewski, J.Bodalski, Institute of Paediatrics, University School of Medicine, Lodz, Poland

Insulin gene region on chromosome 1 lq15.5 contains IDDM2 locus associated with genetic predisposition to insulin dependent diabetes mellitus. Because of strong linkage disequilibrium there has not been possible up till now neither to indicate which of several polymorphism in this region is IDDM2 locus itself nor to precisely delimite the disease associated region. In the present study we analysed five polymorphisms located on both sides of the insulin gene to discriminate the disease associated polymorphisms and therefore to limit the region which should contain IDDM2 locus. We analysed the allelic and genotypic distribution of five following polymorphisms in the insulin gene region: $-4217 \mathrm{Pstl},-2221 \mathrm{Mspl},-23 \mathrm{Hphl},+805$ Drall! and $+3580 \mathrm{Mspl}$ in a group of IDDM patients and healthy individuals. Studied group consisted of 100 IDDM patients from Central Poland. The control group consisted of 100 healthy unrelated blood donors. Genotyping of all studied polymorphisms was done using the PCR-RFLP method. The statistical analysis was done by Fisher Exact test. The significant $p$ value was set to 0.05 . Odds ratios were calculated according to Woolf formule. We found that three of studied polymorphisms were associated with insulin dependent diabetes mellitus. This polymorphisms include: $-2221 \mathrm{Mspl}(\mathrm{p}=0,0001 \mathrm{OR}=3,57)-23 \mathrm{HphI}(\mathrm{p}<0,0001$ $O R=4,03 ;)$ and +805 Dralll $(p<0,0001 O R=4,27)$ The most 5 ' located polymorhism -4217 Pstl showed no association with the disease $(p=0,08)$ allowing to limit the $5^{\prime}$ boundary of disease associated region. The most 3' located polymorhism +3580 Mspl also showed no association with IDDM $(p=1,0)$ so we were able to set the $3^{\prime}$ boundary. We present our results as a map of the INS region and-odds ratios.

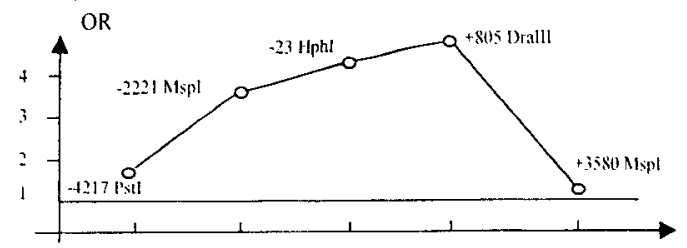

\section{4}

MUTATION SCANNING OF AN IDDM CANDIDATE GENE, GALECTIN-3.

Z.M. Larsen, J. Johannesen, O.P. Kristiansen, J. Nerup, F. Pociot, DSGD and DIEGG. Steno Diabetes Center, Gentofte, Denmark.

IDDM (Insulin-dependent diabetes mellitus) is a polygenic disorder. Combining information from a pathogenic model (Copenhagen Model), genome screening and a new 2-dimensional (2D) gel electrophoresis approach which identifies beta-cell proteins putatively involved in destruction and defence, lead to identification of new IDDM susceptibility genes. Galectin-3 is one of the proteins/genes identified by this approach. Our preliminary results suggested evidence of linkage to IDDM of the galectin-3 gene region as demonstrated by a distorted transmission of D14S980 microsatellite alleles in an extended TDT $(p<0.05)$. To further explore this, in the present study we screened the coding region of galectin-3 for mutation by singlestranded conformation polymorphism (SSCP). An $A \rightarrow T$ point mutation was identified in codon 207, which lead to a Glutamine $\rightarrow$ Histidine substitution (Gin207His). The ATT polymorphism was analysed by PCR-RFLP in 76 healthy controls and 76 IDDM index cases from multiplex families in a case control study. No differences in neither genotypic nor allelic frequencies were found between IDDM cases and controls $(p>0.5)$. The polymorphism was analysed also by the transmission disequilibrium test (TDT) in 88 type 1 diabetes Danish sibpair tamilies. Only 57 fully informative transmissions, from heterozygous parents to affected offspring were revealed. These preliminary results did not show any statistically significant transmission disequilibrium: 33 ' $\mathrm{A}$ ' passed and 24 ' $\mathrm{T}$ ' passed to affected offspring ( $\mathrm{p}=0.23$ ). In summary, galectin-3 is a potential candidate gene for IDDM and some evidence of linkage to the region has been demonstrated. However, the identified GIn207His mutation did not show association/linkage to IDDM in the present data set and is therefore not likely to be the etiological mutation of the region.

\section{6}

HIGHLY PREDICTIVE HLA GENES FOR IDDM AMONG YEMENITE JEWS: MARKERS FOR A RAPIDLY ADVANCING AT-RISK GROUP P. Vardi, S . Israel, E. Sprecher, O. Pinhas-Hamiel, O.J. Kwon, S. Assa, Z. Josefsberg, R. Ofan, H. Ben-Zaken, C. Brautbar, and N. Weintrob. Institute for Endocrinology and Diabetes, SCMCl, and FMRC, Petah Tikva, and Tet-Aviv University, Tel Aviv;Tissue Typing Unit, Hadassah Hospital, Jerusalem, Israel.

Yemenite youth have the highest incidence of IDDM in Israel. Our study was designed to determine whether genetic differences within the HLA complex can explain the higher risk of IDDM in this subgroup. Forty-four IDDM patients (DM) and 76 healthy controls (C) of Jewish Yemenite origin underwent HLA sequence speciflc oligotyping for DRB1, DQA1 and DQB1 genes, and the odds ratio for the different alleles and genotypes was determined. Results showed an exceptionally high odds ratio (OR=220) for the DRB $1^{\star} 03011, \mathrm{DQA1}{ }^{\star} 05, \mathrm{DQB1}{ }^{*} 02 / \mathrm{DRB} 1^{\star} 0402$, $D Q A 1 * 03, D Q B 1 * 0302$ genotype [DM= 26/44, $C=0 / 76, p<0.0001]$, and a high IDDM resistance for the DRB1*0701,DQA1*0201, DQB1*O2 haplotype [DM=0/44, $C=31 / 76, O R=0.02, p<0.0001]$. Conclusion: Molecular class II genotyping shows that Yemenite Jews carry both highly susceptible and highly protective class II HLA genes. The extraordinarily high and low odds ratios shown for both IDDM susceptible and IDDM protective genotypes suggest that such genotyping can be used as the main tool in prediction and primary prevention of IDDM in very young subjects in this community. 
PHENOTYPIC DIFFERENCES BETWEEN DIABETES-PRONE BB RAT SUBLINES COSEGREGATE WITH LOCI ON CHROMOSOMES X AND 10

P. Kovacs and I. Klöting, Department of Laboratory Animal Science at the Institute of Pathophysiology, University of Greifswald, D-17495 Karlsburg, Germany

The spontaneously diabetic BB rat is a well-established animal model in diabetes research. Since that discovery in 1974 many breeding colonies of BB rats have subsequently been established including ourselves. These sublines differ not only in frequency, age at onset and severe of the disease but also in several immunological characteristics most probably caused by genetic differences. Despite that, there are no studies until now investigating the genetic basis of phenotypic differences between BB rat sublines. That prompted us to use diabetic rats of our well-characterized $B B / O K$ subline and of commercially available diabetic $\mathrm{BB} / \mathrm{Wor} / \mathrm{Mol}$ rats $(\mathrm{BB} / \mathrm{Mol})$ for crossing studies in order to get an answer which polymorphic loci between both sublines may be responsible for the obvious differences in diabetes frequency and age at onset of diabetes between BB/OK(50\%, $132 \pm 29$ days) BB/Mol rats (ca. $100 \%$, ca. 80 days). Therefore, diabetic $\mathrm{BB} / \mathrm{OK}$ and $\mathrm{BB} / \mathrm{Mol}$ rats were reciprocally crossed. Fl females of both crosses were backcrossed onto diabetic $\mathrm{BB} / \mathrm{Mol}$ rats resulting $94 \mathrm{BCl}$ hybrids which were analyzed for 30 polymorphic microsatellite markers on 14 chromosomes. For the first time it is shown that a diabetes protective locus on chromosome $\mathrm{X}$ and a gene around the DIOMit 9 locus on chromosome 10 can explain the low frequency (ca. $50 \%$ ) and the late age at onset of diabetes (ca. 130 days) in the BB/OK rat subline, respectively.
MMMUNOHSTOCHEMISTRY OF PANCREAS AND ITS MITOCHONDRIAL DNA REARRANGEMENISIN ADIABETIOSWTTHAMITOCHONDRIAL3243 MUTATTON Nobuaki Kuzruya', Kajuro Komeda², Mitsuhko Noda ${ }^{3}$, Yasunoi Kanazawa', and Masatoshi Kikudhi', Institute for Adult Diseases, Asahi Life Foundation', Animal Reseanch Laboratory, Tokyo Metical Collezeze, and Omiya Metical Center, Jichi Medical Schod ${ }^{3}$

To analyze the immunohistochemical and molecular characteristics of the pancreas tissue in diabetes with a mitochondrial (mt) 3243 mutation, we have stained islets using antibodies to insulin , ghucagon, and somatastatin, and examined mt DNA mutation and rearrangements using PCR, Southern hybridization, primer shift PCR, and sequencing of the amplifed molecules in tissues obtained at autopsy from a patient aged 38 years old with insulin-requiring diabetes, hearing bss and cardiomyopathy. His mother and two brothers showed a mt 3243 mutation, and one brother was implicated to have cartiomyopathy also. The islets were atrophic, but devoid of inflammatory cels. The istet contour was smooth suggesting non-inflammatory nature of the lesion. The number of beta-cells were decreased in comparison with those of alfa- and detta-cels. The 3243 mutation was detectedby PCR in all the tissues exammed Large deletions (about $7 \mathrm{~kb}$ ) were detected using primer shift PCR in genomic DNAs extracted from pancreas and heart. Upon sequencing, one of the multiple deletions in pancreas tissue was the same as reported in CPEO or KSS. The size of deletions were slightly diffenent in each part of the pancreas, but map in a potentially single stranded region during $\mathrm{H}$ strand replication. Directly repeated sequences were present at the boundaries of the deletions. On the contrary, several deletions were found in the cardiac tissue and the position of one dektion was same as reported in mtcardionyopathy. Other tissues such as muscle and liver contained a little amount of debtions. Southern blotting analysis confirmed the presenoe of multiple deletions in both pancreas and heart These results suggest that characteristic mt DNA rearrangements might present in tissues relevant to clinical course in patients with a 3243 mutation, and might be responsible to some extent for the pathogenesis of mt diabetes.

\section{PS 2}

\section{Epidemiology of Type 1 Diabetes}

\section{9}

\section{THE INCIDENCE OF DIABETES MELLITUS IN THE AGE GROUP 15-34 YEARS IN SWEDEN IS DECREASING} L. Nyström ', H. Amqvist ${ }^{2}$, G. Blohmé ${ }^{3}$, J. Bolinder', J. Eriksson ${ }^{5}$, B. Littorin ${ }^{6}$, G Sundkvist ${ }^{6}$, L. Wibell', J. Östman

Department of Epidemiology and Public Health, Umeå University' and Department of Internal Medicine, University Hospital, Linköping ${ }^{2}, G_{0 ̈ t e b o r g}{ }^{3}$, Stockholm ${ }^{4}$, Umea $^{5}$, Malmö $^{5}$ and Uppsala ${ }^{7}$

Since January 1, 1983 all departments of internal medicine, endocrinology, paediatrics and primary health care units in Sweden reports all incident cases of diabetes mellitus in the age group 15-34 years on a standardized form to the Diabetes Incidence Study in Sweden (DISS). Until December 31, 19965553 cases, 3450 men and 2103 women, have been reported corresponding to a yearly incidence of 20.6 and $13.2 / 100000$ for males and females respectively. The incidence in the 5-year age groups 15-19, 20-24, 25-29 and 30-34 years is similar, $20.6,19.1,20.8$, and 22.4 for males and 13.6, 13.2, 13.0, and 12.9 for females. Three out of four $(74.3 \%)$ cases have by the reporting physician been classified as type 1 diabetes, $16.1 \%$ as type 2 diabetes, $1.6 \%$ as secondary diabetes and $8.0 \%$ as not classifiable at onset. From 1983 to 1996 the incidence has decreased with more than $40 \%$, for males from 27.2 in 1983 to 15.5 in 1996 and for females from 15.1 to 8.4. The reduction has been most pronounced between 1983 and 1986 and between 1994 and 1996. If we compare the number of incident cases 1983-93 with $1994-96$ the reduction is $27 \%, 26 \%$ in males and $28 \%$ in females. There is a tendency that the reduction is more pronounced for cases with type 2 diabetes (36\%) and for cases $25-34$ years at diagnosis (33\%). A validity study performed 1983-1987 showed a high level of ascertainment ( $83 \%$ ), in particular for type 1 diabetes $(88 \%)$. Ongoing validity studies does not indicate any tendency towards a higher degree of underreporting during 1994-96. Preliminary figures for 1997 indicates that the reduction in the incidence continues.

\section{0}

THE EFFECT OF PARENTAL AGE AT THE CONCEPTION ON THE AGE-AT. DIAGNOSIS OF IDDM IN THE OFFSPRING

J. Tuomilehto, T. Podar, M. Karvonen, E. Tuomilehto-Wolf, L. Hentonen and P. Onkamo. National Public Health Institute, Helsinki, Finland; University of Tartu, Tartu, Estonia

The purpose of the study was to assess the association between the age-at-diagnosis of IDDM in children diagnosed under 15 years of age and the age of their nondiabetic parents at the conception. Finnish data obtained from the population-based IDDM registry for 1987-1996 were used. The number of eligible childhood onset IDDM cases was 2892. The age-at-diagnosis of IDDM was analyzed by tertiles of age (father's age at conception - cutpoints 28.4, 32.5 years; mother's age at conception - cutpoints 27.6. 31.4 years; offspring's age-at-diagnosis - cutpoints 6.2, 10.5 years) and with linear models. Chi-square tests and the multiple regression analysis were performed using the package SAS. Both paternal and maternal age at conception influenced the age-at-diagnosis of IDDM in offspring. The effect of father's age at conception was more pronounced (Chi-square $=32.7, \mathrm{df}=4, \mathrm{p}<0.001$ ) than mother's age at conception (Chi-square $=18.6, \mathrm{df}=4, \mathrm{p}<0.001$ ). On average, a ten-year increase in parental age at the time of conception was associated with a one year earlier diagnosis of IDDM in the offspring. Paternal age influenced the age-atdiagnosis in both the male and female offspring, while the maternal age had a statistically significant effect only on the male offspring. In the univariate regression analysis, the mean age of parents was also a significant predictor of the age-atdiagnosis of IDDM $(b=-0.091, p<0.0001)$, but not the paternal-maternal age difference $(b=-0.038, p=0.08)$. In the multiple regression analysis the significan predictors of the age-at-diagnosis in the offspring were paternal age at conception $(b=-0.093 p<0.0001)$ and the number of children in the family $(b=0.120, p<0.0001)$. Maternal age lost its significance after paternal age was included in the model. In conclusion, high parental age is associated with the younger age-at-diagnosis of IDDM in non-familial cases of IDDM. In Finland the age of parents at conception is increasing in general and it may have contributed to the increase in younger onset of IDDM. 


\section{1}

REGIONAL VARIATION IN THE INCIDENCE OF CHILDHOOD DIABETES IN NSW AUSTRALIA

NJ Howard, ME Craig, M Silink, and A Chan. Endocrinology Institute, New Childrens Hospital, Sydney, Australia.

Geographic variation in childhood diabetes incidence within countries has been attributed to varying genetic populations and to variations in local environment. In a young country, without long established regional populations, environmental differences may be more relevant to variations in disease incidence.

Aim: To compare the regional incidence of IDDM in children aged 0-14 years (population 1286000 ) in the state of NSW over a period of rising incidence 199296.

Methods: Standardised incidence rates of IDDM for NSW and regional incidence rates were calculated using the NSW Children's Diabetes Register and the Australian Bureau of Statistics 1991 and 1996 population census data. The relative change in IDDM incidence over time was calculated from logarithms of incidence using linear regression, where the regression co-efficient is the change per year expressed as a percentage. Confidence intervals were calculated assuming a Poisson distribution, Chi-squared analysis and the Cochrane-Armitage trend test (StatXact statistical software) were used to compare regional incidences.

Results: The 5-year age standardised incidence was 19.1 per 100000 (95\% CIs 16.9-21.8) for the entire state of NSW with an average rise in boys of $2.9 \%$ and girls of $3.5 \%$ per year. The incidence in children across the 12 statistical subdivisions of NSW ranged from 12.8 (95\% Cls $0.32-71.5)$ to 25.2 per 100000 (95\% CIs 17.0 36.0 ) for the whole group and 2.4 (95\% CIs 0.1-13.1) to 30.7 per 100000 (95\% CIs 0.8 to 171 ) for age 0.4 years. There was a significant variation in incidence by region for the whole group $\left(X^{2}=175,11 \mathrm{df}, \mathrm{p}=0.005\right)$ and $\left(\mathrm{X}^{2}=125.5, \mathrm{p}=0.0005\right)$. Standardised incidence ratios ranged from $67 \%$ to $131 \%$ for $0-14$ years and $20 \%$ to $253 \%$ for $0-4$ years. When the 12 regions were ranked by population density (total population per square $\mathrm{km})$, a significant trend in incidence was found $(p=0.005)$ with a positive correlation between incidence and population density in the $0-4$ year olds but not for the whole population.

Conclusion: At a time of rising incidence of IDDM in children in NSW there is a regional variation in incidence and a relationship between incidence and population density in $0-4$ year olds.

\section{2}

THE ETHNIC FACTOR IN CHILDREN WITH INSULIN - DEPENDENT DIABETES MELLITUS IN ROMANIA

V.Serban for the ONROCAD-Study Group. Departmert of Diabetology, Timisoara - Romania

Nationwide epidemiological investigation performed in children $10-16$ years) in the period of 1992-1997 pointed out an annual incidence of IDDM between 3.57-3.76/100,000 and a cumulated incidence of $0.28 \%$, a fact that placed Romania among the countries with the lowest frequency of that disease. This finding justified the undertaking of a descriptive epidemiological study in order to correlate the incidence with some parameters: ethnic group, territorial distribution, diabetes heredity, breast-feeding period and multi-annual epidemic dynamics of various viral infections. The multi-variant analysis only rendered a significant support for the differential frequency of IDDM in different ethric groups in Romania. Thus, the most endangered ethnic group was that of the Hungarian, characterized by an annual incidence of $7.80 / 100,000$ and a cumulated incidence of $0.55 \%$, similar to the parameters reported in Hungary. We firther specified that the annual incidence for the other ethnic groups ranked at 3.29/100,000 for Romanians, 3.40/100,000 for Gypsies and 4.10/100,000 for Germans, the cumulated incidence being of $0.24 \%$ for Romanians, $0.28 \%$ for Gypsies and $0.26 \%$ for Germans. The sigurficant statistic difference $(\mathrm{p}<0.001)$ motivated the second part of this study, the correlative malvsis of the groups constituted on ethnic criteria with the abovementioned parameters. The epidemiological peculiarities conid not be attributed to the breast-feeding period, geographic factors or the infections diseases. in conclusion, the factor pertaining to diabetes heredity remained further to be discussed, as it seemed to confer an epidemiological individuality to the people belonging to the same ethnic group, not depending on the gengraphic territory or the country to live in. The difference regarding the frequency of IDDM in Gernman children living in Romania versus those in Germany could be explained by the fact that all of the children belonging to the German minority in Romania come from mixed martiages

\section{3}

TEMPORAL TRENDS IN INCIDENCE OF CHILDHOOD ONSET TYPE 1 DIABETES IN DEVON AND CORNWALL, ENGLAND, 1975 - 1996

H.X. Zhao, E. Stenhouse, C. Soper, P. Hughes, E. Sanderson, H. Baumer, A.G Demaine and B.A. Millward. Molocular Medicine Research Group, Plymouth Postgraduate Medical School, University of Plymouth, Plymouth, UK

The aim of the study was to determine the temporal trends in incidence of Type 1 diabetes in children aged 0-14 years old in the far Southwest of England (Cornwall and part of Devon counties) from 1975 to 1996. The incidence data in this study were obtained from two sources. The primary source was from diabetologists, Diabetes Specialist Nurses and hospital medical records and the secondary source from General Practitioners. 480 newly diagnosed cases were ascertained between $01 / 01 / 1975$ and $31 / 12 / 1996$. The overall completeness of ascertainment during the 22 -year study period was $95.6 \%$ (95\% CI: $92.8-98.6 \%$ ) by the capture-recapture method. The annual incidence rates were calculated per 100,000 population and the age adjustment of the rates was done using 5-year intervals with the proportions $1 / 3,1 / 3$ and $1 / 3$ respectively as the standard population as recommended by the DERI Study Group. The increase trends were estimated by fitting the linear regression with the annual incidence data. Between 1975 and 1996, the average annual crude incidence rate was $14.7 / 100,000 /$ year. The standardised incidence rate was $14.5 / 100,000 /$ year. The ascertainment corrected incidence rate was $15.4 / 100,000 /$ year. The significant incidence increase was observed during the study period (the slope coefficient was $0.40, p$ $=0.0025$ ). The regression-based average increase in incidence was $2.7 \%$ per year. The increase in incidence was more obvious in girls than that in boys (3.1 vs. $2.3 \%$ per year). The incidence increased in the three age group $(0-4,5-9$ and $10-14$ yrs) on average by $7.7,2.0$ and $2.0 \%$ per year, respectively. We conclude that the incidence rate of childhood diabetes increased steadily between 1975 and 1996 in this suddy population; children aged 0-4 years showed a significantly faster increase in incidence than the older children, and girls had a faster rate of increase than boys.

\section{4}

INCIDENCE OF INSULIN-DEPENDENT (TYPE 1) DIABETES MELLITUS OVER SIX CONSECUTIVE YEARS AMONG 15-39-YEAR AGED LITHUANIAN POPULATION

R. Ostrauskas, R. Žalinkevicius, A. Norkus and the Lithuanian Epidemiology Diabetes Study Group*.

Institute of Endocrinology, Kaunas Medical Academy, Kaunas, Lithuania

Objective - To document the incidence of insulin-dependent diabetes mellitus in Lithuanian 15-39 years of age population from 1991 to 1996.

Research design and methods-A specifically developed contact system with all endocrinologists/diabetologists and general practitioners involved in the diabetes care covering $100 \%$ of the Lithuanian population aged 15-39, was the initial data source. Annual reports from regional endocrinologist's /diabetologists, statistical note-marks of diabetic patients who visited Medical Units, death certificates and patients' lists from Diabetes Societies remained as secondary independent sources for case ascertainment.

Results - The totals of 649 new cases (420 males and 229 females) of insulindependent diabetes mellitus were recorded among the population 15-39-year of age during the period 1 January 1991 - 31 December 1996. The cumulative incidence density per year was 7.69/100,000 (95\% Poisson distribution confidence interval 7.12-8.31) and was slightly higher among males $(9.86 / 100,000,95 \% \mathrm{Cl} 8.96-10.85)$ than among females $(5,43 / 100,000,95 \% \mathrm{CI}$ 4.81-6.23), $\mathrm{p}<0.0001$. Age standardized overall incidence rates for males and females were 9.89 and 5.49 , respectively. Male/female ratio was 1.80 . Results of the linear regression models showed that the incidence density of insulindependent diabetes mellitus in 15-39-year age group had the tendency to increase.

Conclusions - The results suggest that the incidence data of insulin-dependent diabetes mellitus in Lithuania is appropriate to those in Poland and lower than in other countries of Baltic Sea region. The data contributes to the knowledge of the incidence of insulin-dependent diabetes mellitus in Eastern Baltic countries, an area which until now has been lacking epidemiological data on diabetes among 15-39-year aged population. 


\section{5}

INCIDENCE OF CHILDHOOD ONSET DIABETES MELLITUS TYPE 1 IN CENTRAL POLAND IN 1983-1997

A. Szadkowska, E. Czerniawska, 8. Mianowska, J. Bodalski, Institute of Paediatrics, Medical University of Lodz, Lodz, Poland

The aim of this study was to establish the incidence of diabetes mellitus type 1 in children in the population of four provinces of Central Poland in the years 1983-1997. We estimated the population 532000 children in the age of $0-14$ years. Onset of diabetes mellitus type 1 was considered to be the date of first insulin injection. The data were collected from diabetic outpatients clinics and hospitals. Ascertainment is estimated to be ove $95 \%$. Incidence rates were expressed as the annual number of newly diagnosed diabetic cases per 100000 age-adjusted population. The dependence of the incidence on sex, age, seasonality was analysed. Because of annual differences we compared the mid-period incidence rate from five years periods. A total of 574 patients were registered for a period of 15 years. In 1983-1997 the incidence rate in Central Poland was found to be 6.61. An increase in incidence rate from 5.01 in 1983-87 to 9.17 in 1992-97 was observed. The incidence rate was statistically higher in urban children (urban - $7.59 \mathrm{v}$. rural -4.83 ). There was no significant difference between incidence rate of boys and girls (6.16 v. 7.08 ). Marked seasonal variation of incidence was identified with peak incidence in winter. Central Poland is a region with low incidence of childhood diabetes mellitus type 1. However in the years 1983-1997 we observed the increase trend in the incidence of diabetes mellitus type 1 among children in this part of Poland.

\section{6}

IS THE PUBLIC HEALTH IMPACT OF TYPE 1 DIABETES IN CHILDREN AND ADOLESCENTS UNDERESTIMATED IN GERMANY?

J. Rosenbauer, A. Icks, and G. Giani. Department of Biometrics and Epidemiology, Diabetes Research Institute at Heinrich-Heine-University Düsseldorf, Germany Objective: Currently no nationwide data on incidence and prevalence of Type 1 diabetes in children and adolescents under 20 years are available in Germany. Aim of our analyses was a model-based estimation of nationwide diabetes incidence and prevalence rates using previously reported age-specific nationwide and regional incidence data. Methods: Based on nationwide incidence data in children under 5 years (1993-95), regional age-specific incidence data in children and adolescents under 20 years (age-groups: $0-4,5-9,10-14,14-19$ years) of the Duesseldorf are (1993-95), the federal state of Baden-Württemberg (1987-93), and the former GDR (1988), nationwide age-specific incidence rates were estimated using a Poisson regression model. Assuming no time-dependency, from these incidence rates rough estimates of age-specific prevalence rate were derived by applying age-specific prevalence-incidence-ratios of the former diabetes register (1988). Furthermore, age-standardized incidence and prevalence rates as well as the cumulative incidence were estimated for the age-group 0-19 years (standard population 1996: $17,674,008)$. Results: The nationwide age-specific incidence rates $\left[10^{-5 *}\right.$ pyrs $^{-1}$ $\left(\mathrm{CI}_{95 \%}\right)$ for the age-groups $0-4,5-9,10-14$, and $15-19$ years were estimated as 6.1 (7.6-8.6), $15.3(13.1-17.9), 18.5(17.9-21.5)$, and 25.1 (19.7-32.1), respectively. The corresponding prevalence estimates $\left[10^{-5} * \mathrm{pyrs}^{-1}\right]\left(\mathrm{Cl}_{950}\right)$ were $20.0(18.8-213), 84$ (72.1-98.0), 148.1 (127.4-172.1), and 296.7 (232.2-379.0), respectively. After direct standardization for age, an overall incidence rate of $17.1(12.9-15.5)\left[10^{-5 *}\right.$ pyrs $^{-1}$ and an overall prevalence rate of $140.2(134.3-146.1)\left[10^{-5} *\right.$ pyrs $\left.^{-1}\right]$ was estimated Thus, in Germany there are 3,002 ( $\left.\mathrm{Cl}_{95 \%}: 2,679-3,325\right)$ newly diagnosed diabetic children and adolescents under 20 years per year. The total estimated number of diabetic children and adolescents amounts to $24,743\left(\mathrm{Cl}_{95 \%}: 23,696-25,788\right)$. The estimated cumulative incidence rate of 335.0 (299.2-370.8) implies that one out of 299 ( Cl $\left._{95 \%}: 270-334\right)$ newborns will develop Type 1 diabetes before the age of 20 years. Conclusions:. Our analyses results in about twofold higher estimates of incidence and prevalence of Type 1 diabetes in children and adolescents under 20 years than previously reported from the diabetes register of the former GDR. Thus, the public health impact of Type 1 diabetes in children and adolescents is considerably underestimated in Germany if applying data of the former GDR diabetes register.

\section{7}

MORTALITY DURING UP TO 25 YEARS OF IDDM IN THE ERFURT DISTRICT

U.J.W. Schaver, 2. Medical Clinic, Erfurt Hospital, Erfurt, Germany

The aim of the study was to find out the frequency of death and its associated factors in IDDM within a geographically defined population. The centralized diabetes care system in the former GDR had been the basis for the almost complete registration of diabetic patients. The original charts of all 1132 patients with IDDM onset below age 40 in the Erfurt district between 1966 and 1988 were reviewed up to the end of 1990 . Cumulative mortality rate after 25 years of IDDM was $21.0+0.04 \%$ for all patients and $5.0+0.02 \%$ for patients with IDDM onset below age 20. Death occured after $12+6$ years of diabetes at an age of $39+10$ years. In comparison $(p<0.05)$ to patients with similar age and duration of IDDM at the end of follow-up $(n=620)$ patients who died $(n=61)$ showed a significantly higher BMI (24.4 vs $23.1 \mathrm{~kg} / \mathrm{m}^{2}$ ), were more often obese (21 vs $11 \%$ ) and had higher systolic (133 vs $128 \mathrm{~mm} \mathrm{Hg}$ ) and diastolic blood pressure ( 85 vs $81 \mathrm{~mm} \mathrm{Hg}$ ) at IDDM onset. During the course of IDDM blood pressure remained higher as it did blood glucose (11.3 vs $10.3 \mathrm{mmol} / \mathrm{l}$ ) despite similar insulin doses during the first 10. years of diabetes. Patients who died suffered more often from myocardial infarction ( 10 vs $1 \%$ ), stroke ( 7 vs $1 \%$ ) as well as claudication ( 20 vs $5 \%$ ) and developed more frequently hypertension (46 vs $24 \%$ ), chronic renal failure (18 vs $3 \%)$, end-stage renal failure ( 3.3 vs $0.3 \%$ ) and proliferative retinopathy (10 vs $3 \%)$ during the follow-up period. This cluster resembles the metabolic syndrome known from NIDDM. A subgroup of IDDM may be at especially high risk for long-term complications and early death due
to a concomitantly existing insulin resistance syndrome.

\section{8}

DIABETIC KETOACIDOSIS: A POPULATION-BASED STUDY (1991-1996) *AM Molino, MP de Miguel, JP Marañes, A Charro and Món Sonez. Hospitai Universitaro de San Carlos. *Centro de Salud Maqueda. Madrid. Spain. BACKGROUND: Diabetic ketoacidosis (DKA) is a serious acute complication of the disease. The incidence is unknown in our country, and it has been related to the diabetologic educational level. We had tried to final out such an incidence, as well as the clinical presentation, the managentent and the mortality of the DKA in a health care area in Madrid in order to establish future strategies for improting the diabetes care of our patients. METHOOS: 245 cases of DKA (250 1 ICD- 2 CM) were retrospectively collected from the Hospital data files, which is the referencr hospital for people living in the heath area. Therefore, we used these people for reckoning the incidence figures. RESULTS: $58,8 \%$ of the patients were type 1,55,5\% femae, age -mean (SD)- $46,9(26,2)$ $Y_{s}$ and duration of diatetes 12,3(8,9) $Y_{S}$. DKA incidence was 6.54100 .090 inhabitantsyear (1.34 debuts). There was an increase from 3.7908 .91 between 1991 and 1996 (5.18 to 23.33 in older than 65 Ys). Ondy the $51.8 \%$ of the patients had beens included in a diabetes educational progran (19.4\% in older than 65 years, $p<0.0001)$. Classical symptoms were present in $92,2 \%$ a median of $17 \mathrm{ds}$ prior to admission. The most common precipitating factors were infection (40.4\%), onissionicror in the treatment (24.1\%), and unknown (15.5\%). $18.8 \%$ were new diabetes cases The percentage of nixed cases (osmolality >320 mOsml) increased from 42,9\% in 1991 to 70.5 in $1996,76.7 \%$ in older than 65 years versus $44.8 \%$ in younger patients, $p<0.004)$. The average length of stay in the Hospital was $15(15.3)$ days. $25 \%$ of the patients were admitted to the intensive care unit (ICU). Age, time spent in the ICU and comorbility were independently correlated to the average stay - $\mathrm{p}<0.05$. ICU adnission was correlated to age and $\mathrm{pH}(\mathrm{p}<0.01)$. Mortally was $5.71 \%$ (from $3,57 \%$ in 1992 to $9,84 \%$ in 1996 ), $50 \%$ in the first 16 hours. In $78,6 \%$ of patients the metabolic controf was not reached. The main causes of death were corobrovascular disease (21.4\%), sepsis (21.4\%), and ischemic Gardiopathy (14.3\%). Age, infection, comortility, ICU adnission, and a low level of consciousness at presentation were independenlity correlated to death $(p<0.05)$. CONCLUSiON: Dering the stury-period, DKA incidence in people older than 65 years of age has increased, thus ressiting in an increased percentage of mixed cases. Diabetological education was lower in suchl patients, and their mears length of stay and mortality were highor. 
TARGET HEIGHT AND THE RISK OF IDDM

E Hyppönen, SM Virtanen, M Knip, HK Ákerblom, Childhood Diabetes in Finland -Study Group. Tampere School of Public Health and Medical School, University of Tampere, Children's Hospital, University of Helsinki, Finland.

Children who develop IDDM have been found to be taller than control children before the onset of the disease. The purpose of the present study was to evaluate, whether the differences in the relative height between case and control children observed in present series could be explained by differences in their target height.

All diabetic children < 15 years of age diagnosed from Sept. 1986 to April 1989 were invited to participate in a Finnish nationwide case-control study. Each child was matched by date of birth and sex to a randomly selected population-based control child. Growth data were obtained from well-baby clinics and school health care units for 380 matched case-control pairs, and height of both parents was available for 243 case-control pairs for whom the target height could be calculated.

There were no differences in the relative heights of fathers of diabetic and control children $(-0.09$ SDS vs. -0.15 SDS, $p=0.51)$. Mothers of case girls were talle than those of control girls $(-0.07$ SDS vs. -0.35 SDS, $p=0.02)$, whereas no difference was observed between mothers of case and control boys $(-0.02$ SDS vs. -0.23 SDS, $\mathrm{p}=0.41$ ). The target height did not differ between cases and controls (0.14 SDS vs. 0.09 SDS, $p=0.54$ for boys; 0.11 SDS vs. -0.02 SDS, $p=0.09$ for girls, respectively). Target height was not associated with the risk of IDDM in the conditional logistic regression analysis: odds ratio 0-1 SDS vs. $<0$ SDS and > SDS vs. $<0$ SDS $1.22(95 \% \mathrm{Cl}=0.8-2.0)$ and $2.08(95 \% \mathrm{CI}=0.7-6.6)$ for boys and $1.50(95 \% \mathrm{Cl}=0.9-3.5)$ and $1.03(95 \% \mathrm{CI}=0.4-3.0)$ for girls, respectively The relative height 4 to 1 years before the diagnosis of IDDM remained associated with the risk of IDDM after adjustment for the target height: $>1$ SDS vs. $<1$ SDS crude $\mathrm{OR}=1.67(95 \% \mathrm{CI}=1.1-2.6)$, adjusted $\mathrm{OR}=1.54(95 \% \mathrm{CI}=1.0-2.5)$

These observations indicate that differences in height between cases and controls cannot be explained by the target height. However, information on the growth velocity of parents was not available, so it is not possible to rule out genetically determined rapid growth as a factor associated with the development of IDDM.
330

TRANSIENT AND PERMANENT NEONATAL DIABETES ARE GENETICALLY DIFFERENT DISEASES ?

R. Hermann, A.P. Laine*, L. Tokarska\#, H. Daiatkowak\#, C. Johansson§̧, J. Ilonen* and G. Soltész. Department of Paediatrics, University Medical School of Pécs, Pécs, Hungary, *Department of Virology, University of Turku, Turku, Finland, \#Polish-American Children's Hospital, Krakow, Poland, §Ryhov Hospital, Jönköping, Sweden

Recently, we studied 6 patients with neonatal diabetes, four of which had permanent and two had transient neonatal diabetes. The aim was to investigate the possible genetic heterogeneity of neonatal diabetes. HLA DQ typing revealed that none of these newborns carried IDDM susceptibility genotype. Paternal uniparental isodisomy of chromosome 6 was found by HLA typing in patient "A" with transient neonatal diabetes. Seven different microsatellites covering chromosome 6 have been investigated in all families: D6S334, D6S286, D6S310, D6S308, D6S292, D6S311, D6S403. A fluorescence based DNA fragment analysis was used. The presence of paternal uniparental isodisomy of chromosome 6 affecting the whole chromosome was confirmed in patient " $\mathrm{A}$ ". Paternal isodisomy or partial duplication of paternal chromosome 6 sequences was found neither in permanent neonatal diabetes cases nor in our second case (patient " $\mathrm{B}$ ") with transient neonatal diabetes. Transient neonatal diabetes was associated with macroglossia in Patient " $\mathrm{A}$ ". Transient neonatal diabetes and granulocytopenia was found in Patient " $B$ ". Her brother also have had early onset diabetes and agranulocytosis. Here we propose that transient and permanent neonatal diabetes are genetically different diseases and a gene on chromosome 6 is responsible for the development of transient neonatal diabetes. It has probably a critical role in beta cell maturation/insulin secretion and may also influence foetal nutrition and intra-uterine growth. To explain the lack of paternal uniparental isodisomy of chromosome 6 in patient " $B$ " we hypothesise that the pathogenesis of diabetes and its association with granulocytopenia may be due to a rare genetic anomaly and patient " $B$ " may not represent the classical transient neonatal diabetes.

\section{1}

\section{CLINICO-BIOCHEMICAL CHARACTERISTICS OF THE YOUNG ONSET DIABETIC} PATIENTS IN BANGLADESH

S. Parvin'1, Z. Hassan', J.M.A. Hannan'1, R. Zinnat', NS Chowdhury', A.K. Azad Khan'2 and L. Ali1. 'Research Division, Dept. of Cell \& Molecular Biology, BIRDEM, 2Department of Gastroenterology, BIRDEM, Dhaka, Bangladesh.

The classification of young onset diabetes in a number of tropical countries is still confusing due to the presence of a substantial number of patients with clinical presentation corresponding
to neither Type 1 or Type 2 class of the disease. As a part of an ongoingl attempt to to neither Type 1 or Type 2 class of the disease. As a part of an ongoingl attempt to
characterize the clinico-biochemical features of these patients their secretory capacity was characterize the clinico-biochemical features of these patients their secretory capacity was
explored in this study by measuring the fasting and post-glucose ( 2 hrs after $75 \mathrm{~g}$ oral load) explored in this study by measuring the fasting and post-glucose ( $2 \mathrm{hrs}$ after $75 \mathrm{~g}$ oral load)
levels of C-peptide in the serum of 205 young (under 30 years of age) subjects (Age in yrs levels of $C$-peptide in the serum of 205 young (under 30 years of age) subjects (Age in yrs
$M \pm S D, 23.6 \pm 4.6$. BMI 19.9 of age $(23.8 \pm 4.4)$ and BMI $(19.1 \pm 2.9)$ - matched non-diabetic subjects, without family history of diabetes and without any clinical signs of malnutrition were also studied. The lowest age of the 13 years. Obese patients were only in $9 \%$ cases). In contrast (94\%) patients were underweight by the traditional cut-poin $(\mathrm{BMl}=19)$ and $17 \%$ of total patients had BMI in the range of only $12-15$. However, $6 \%$ of nondiabetic subjects also had BMI 12-15 and they did not develop any glucose intolerance. The fasting levels of glucose in $41 \%$ of the patients was remarkably higher (range, 16.04 $34.19 \mathrm{mmol} / \mathrm{l}$ ) than the usual cut-point of $16 \mathrm{mmol} / \mathrm{l}$ above which ketosis is thought to be developed without intervention. However, none of them showed any clinical evidence of ketosis. Both fasting and post-glucose C-peptide was significantly lower in the diabetic group (C-peptide, pmolll, $M \pm S D$ fasting: $0.49 \pm 0.25$ in Nondiabetic vs $0.42 \pm 0.34$ in diabetic $p<0.02$ post-glucose: $1.63 \pm 0.91$ in Nondiabetic vs $0.95 \pm 0.89$ in diabetic $p<0.001$ ). C-peptide-glucose ratios in the two states revealed that insulin secretory capacity in the face of rising glucose levels is almost negligible in the diabetic group (C-peptide:GI, $M \pm S D$; Control: $0.14 \pm 0.06$ in fasting vs $0.35 \pm 0.20$ in post-glucose states, a $150 \%$ increase in mean value Diabetic: $0.04 \pm 0.05$ in fasting vs $0.05 \pm 0.06$ in post-glucose states, only $25 \%$ increase in the mean value). In the diabetic group there was a high dispersion in the fasting as well as postglucose C-peptide with $27 \%$ values below 0.16 pmolli (usual demarcation point between IDDM and NIDOM) and $17 \%$ values above $0.76 \mathrm{pmol} / \mathrm{h}(>M+1 \mathrm{SD})$. The results suggest the following a) childhood diabetes (<16 yrs) is relatively rare in Bangladesh; b) Low BMI in adult life per se does not lead to diabetes mellitus; c) The insulin secretory capacity in many of these young diabetic subjects is extremely low and overall values are highly heterogenic which may signify the presence of etiopathogenetically different groups of diabetic subjects in the young population.

\section{2}

POPULATION BASED DATA SHOW MATERNAL THYROID DISEASE CONFERS A RISK FOR CHILDHOOD IDDM R Parslow, PA McKinney, K Gurney, R Williams, and HJ Bodansky Paediatric Epidemiology Group, Centre for Health Services Research, University of Leeds, UK Autoimmune conditions including thyroid disease are known from case reports and hospital series to be clinically associated with IDDM. A population-based case control study in Yorkshire aiming to identify risk factors has determined whether there is an excess of autoimmune diseases and conditions marking immune dysfunction in the parents and sibs of children with IDDM. Mothers of 220 children aged 0-14 years diagnosed with IDDM and 433 age sex matched controls were interviewed and details obtained on the following - thyroid disease, rheumatoid arthritis, pernicious anaemia colitis/chronic bowel conditions, hay fever/allergic rhinitis, leukaemia and cancer, coeliac disease, myasthenia gravis, Addisons disease and multiple sclerosis. Conditional logistic regression analysis of matched sets showed a significant excess of maternal thyroid disease ( 20 cases, 12 controls odds ratio (OR) 3.3,95\% CI 1.6-6.8) and IDDM ( 6 cases, 0 controls). None of the mothers had co-incident IDDM and thyroid disease. There was no increased risk for any other conditions. Information was available for fewer fathers ( 136 cases, 243 controls) where there was a non-significant excess of thyroid disease ( 3 cases, 1 control) but a significant risk for IDDM (OR 2.75,95\% CI 1.14-6.61). For siblings no associations were present apart from IDDM (OR 8, 95\% CI 2.328.3). These results suggest a pattern of risk for maternal thyroid disease and IDDM in offspring which is independent of the raised risk for children of IDDM mothers 
AUTOIMMUNE THYROID DISEASE IN TYPE 1 DIABETIC PATIENTS: 5 YEARS FOLLOW-UP. PRELIMINARY RESULTS

G.Cuatrecasas, A.Lucas, I.Salinas, M.L.Granada, A.Sanmartí. Endocrinology department. Hospital Germans Trias i Pujol. Badalona, Spain.

Aim: Few longitudinally studies analyzing thyroid autoimmunity in type 1 diabetic patients have been performed. They basically focus on japanese, swedish and hispanic pre-pubertal children. The aim of this study was to evaluate thyroid function and thyroid antibodies (ATIR), prospectivelly (1993-1997) in a non-selected group of type 1 diabetic patients (p). Patients and methods: We studied 70 type 1 diabetes p, 27 men and 43 women with an average age of 27 years (range 15-55). The mean time of evolution of its diabetes was 12 years (range 5-28). We registered familial history of type 1 and 2 diabetes, thyroid pathology and other autoimmune diseases ( 3 generations), the positivity of microsomal $(\mathrm{TPO}>50 \mathrm{U} / \mathrm{ml})$ and tyroglobulin $(\mathrm{Tg}>150$ $\mathrm{U} / \mathrm{ml}$ ) antibodies (ELISA) and the values of TSH (IRMA) and free $\mathrm{T}_{4}$. These values were evaluated initially and yearly, from 1993 to 1997 . Results: $13,8 \%$ of our patients had familial history of diabetes, $16,9 \%$ history of thyroid diseases and 3,1\% of other autoimmune diseases ( 2 cases of pernicious anemia). $7,7 \%$ had goiter and $30,9 \%$ ATIR+ at least on one occasion, $30,8 \%$ exclusively TPO and $27,7 \% \mathrm{Tg}$. At the beginning of the study, $4 \mathrm{p}(5,7 \%)$ had goiter, $10(14,2 \%)$ ATIR+ and $3(4,3 \%)$ subclinical hypothyroidism (SH). Among the $4 \mathrm{p}$ with goiter at the beginning, 2 had ATIR+ and developed in one case, SH and on the other case a Hashimoto thyroiditis (HT). One of the 2 patients with goiter but ATIR- at the beginning, showed further positivisation of its ATIR and developed a SH. Among the $10 \mathrm{p}$ with ATIR+, 8 showed persistent ATIR+ thereafter, 2 developped goiter, $2 \mathrm{SH}$ and 3 a HT. Among the 3 patients with SH at the beginning, one had goiter and showed ATIR+ thereafter, another did not have goiter nor ATIR and the third showed persistent ATIR+ and developed a HT. Globally 3 patients developed a HT. We also report 1 case of Graves disease concomitantly with the onset of its type 1 diabetes. This means a 5,7\% prevalence of clinical autoimmune thyroid disease. The $3 \mathrm{p}$ who developed HT had ATIR+ at the beginning of the study, only one had goiter and none SH. Conclusions: $36,9 \%$ of our type 1 diabetes patients had ATIR + during the study and $5,7 \%$ developed clinical autoimmune thyroid disease. Antithyroid antibodies seem to predict the onset of autoimmune thyroid disease. ATIR+ do not depend on the diabetes evolution time.

\section{PS 3}

\section{Prediction and Prevention of Type 1 Diabetes}

\section{4}

VARYING RATES OF PROGRESSION TO IDDM IN FIRST DEGREE RELATIVES: GENETIC AND AUTOIMMUNE MARKERS

I.M. Libman, M. Pietropaolo, R.E. LaPorte, and D.J. Becker. University of Pittsburgh, Pittsburgh, PA

Studies have shown that first degree relatives with high risk HLA-DQ heterodimers and presence of autoimmunity to $\beta$ cell antigens are at increased risk of developing diabetes. However, the time of progression to diabetes from the first known evidence of autoimmunity, an important factor in the design of intervention studies, seems to vary and may depend on the presence of several risk factors. We evaluated genetic (presence of HLA-DQ heterodimers) and autoimmune (ICA GAD65 aa and/or IA-2 aa) determinants prior to diagnosis of diabetes in 68 converters to IDDM from a cohort of 5512 first degree relatives who have been followed for 17 years and who presented signs of $\beta$-cell autoimmunity. The 68 converters were classified into three groups: group I (rapid progressors) who developed IDDM in $<5$ years after evidence of $\beta$ cell autoimmunity ( $n=31$ ), group II (slow progressors) who developed IDDM in $\geq 5$ years after evidence of autoimmunity $(n=20)$, and group III (LADA) who started insulin treatment 1 to 8 years after diagnosis of diabetes $(n=17)$. The mean age at start of insulin treatment was $20.1 \pm 12.9$ in group I, 28.5 \pm 15.2 in group II and 42.8 \pm 13.4 in group III Characteristics of the groups were as follows:

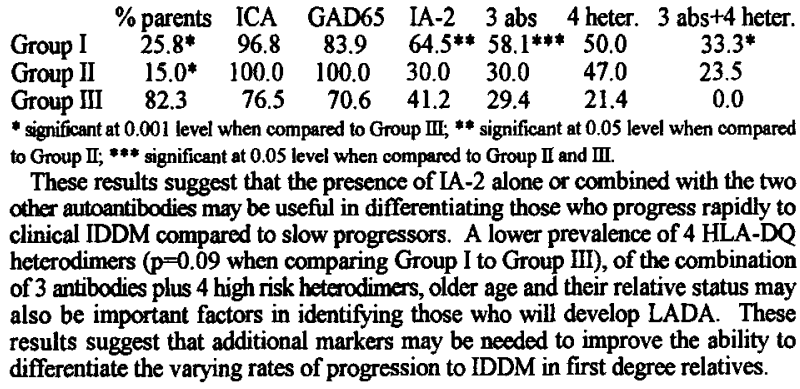

\section{5}

TARGET POPULATION FOR PREVENTION TRIALS OF BETA-CELL AUTOIMMUNITY IN EARLY CHILDHOOD

M. Rewers, G.S. Eisenbarth, C. Elsey, J. Norris, H.A. Erlich, T. Bugawan and G. Klingensmith University of Colorado, Denver, and Roche Molecular Systems, Alameda, USA

Secondary interventions, applied after development of autoimmunity, appear to delay but not prevent the onset of IDDM. To define a high risk group for prevention of autoimmunity, we followed for up to 4 yrs a population-based cohort of U.S. infants: 308 siblings or offspring of IDDM patients and 900 general population newborns (181 with the high IDDM risk HLA-DR3/4,DQB1*0302 genotype). The cumulative incidence of autoimmunity (antibodies to insulin, $G A D_{65}, \mid A-2$ on at least two consecutive visits) by the age of $4 \mathrm{yr}$ and the relative risk (RR) were estimated in SAS Lifetest and Lifereg (with left, interval, and right censoring):

\begin{tabular}{|l|c|c|l|}
\hline Group & $\begin{array}{l}\text { Population } \\
\text { Frequency }\end{array}$ & $\begin{array}{l}\text { Cumulative } \\
\text { Incid.(95\%Cl) }\end{array}$ & $\begin{array}{l}\text { RR } \\
(95 \% \mathrm{Cl})\end{array}$ \\
\hline General population, HLA & & & \\
-not DR3/4,DQB1*0302 & $97.70 \%$ & $1.5(0.2-2.9) \%$ & 1.0 reference \\
-DR3/4,DQB1*0302 & $2.30 \%$ & $3.1(0-7.9) \%$ & $1.4(0.3-2.0)$ \\
A.l siblings/offspring & $\sim 1.00 \%$ & $10.6(3.1-18) \%$ & $6.9(2.7-18)$ \\
-DR3/4,DQB1*0302 & $\sim 0.12 \%$ & $37.9(13-6) \%$ & $36.6(13-101)$ \\
-DR3/401,2,5,DQB1*0302 & $\sim 0.08 \%$ & $41.1(9.6-73) \%$ & $51.4(17-158)$ \\
\hline
\end{tabular}

The analyses were adjusted for race/ethnicity and gender, which were not independent predictors of autoimmunity. Young relatives of IDDM persons with the HLA-DR3/"0401,"0402, or "0405; DQB1"0302 genotype should be targeted for primary prevention trials. The intervention needs to be administered before the age of 9 months, since $30 \%$ of these high risk infants already had autoantibodies at that age. To detect an intervention effect of $50 \%$, a 4 -yr trial with $10 \%$ loss to follow-up and $5 \%$ noncompliance would require 240 participants at an expected autoimmunity risk of $40 \%$ by the age of 4 . Ten times as many newborn relatives $(-2,400)$ would need to be HLA-DR,DQ screened. 


\section{6}

LONG TERM FOLLOW-UP OF FAMILY MEMBERS WITH ICA $\geq 20$ JDF UNITS: WILL THEY ALL DEVELOP DIABETES?

P.J. Bingley, S.G. Gardner, A.J.K. Williams, K. Lawrence, G.F. Bottazzo and E.A.M. Gale. University of Bristol, and St Bartholomew's and the Royal London School of Medicine, UK

Relatives with high titres of islet cell antibodies (ICA) have a $35-40 \%$ risk of IDDM within five years, but their longer term prognosis is unknown. To determine long term risk and factors modulating progression to diabetes, we examined all family members (total 146 , median age $28.7 \mathrm{yr}$, range 2.1-58.6) with ICA $\geq 20$ JDF units from 1,386 families recruited to UK family studies since 1978 . Both the first and the most recent eligible samples were tested for antibodies to insulin, glutamate decarboxylase and IA-2, and HLA-DQ genotype was determined. Non-diabetics had. a $35 \mathrm{~g}$ intravenous glucose tolerance test. 16 developed IDDM within 5 years (rapid progressors), and 45 were non-diabetic at 5 years. Of these 10 went on to diabetes (slow progressors, median follow-up $7.4 \mathrm{yr}$ range 5.7-18.1 yr), while 35 remain non-diabetic (non-progressors, follow-up 6.8 $\mathrm{yr}, 5-17.4 \mathrm{yr}$ ). The overall cumulative risk of diabetes was $19 \%$ within $5 \mathrm{yr}, 35 \%$ within $10 \mathrm{yr}$ and $50 \%$ within $15 \mathrm{yr}$. Rapid and slow progressors were of similar age at entry (18.8 (1.6-45.5 yr) vs $12.3(3.1-42 \mathrm{yr}) \mathrm{p}>0.05)$, but non-progressors were older $(30.5 \mathrm{yr}, 4.5-59, \mathrm{p}=0.01)$. Antibodies to GAD and IA-2 were present in $79 \%$ and $50 \%$ of rapid progressors vs $100 \%$ and $50 \%$ of slow progressors, and $93 \%$ of rapid and $100 \%$ of slow progressors had least one additional islet autoantibody marker. In the non-progressor group 14\% lost ICA ( $<4$ JDF units in the lastest sample). Of those with persistent ICA, $54 \%$ had multiple autoantibodies and $60 \%$ had first phase insulin secretion ( $\Sigma 1+3 \mathrm{~min}$ insulin) below $100 \mathrm{mU} / \mathrm{L}$ (10th centile). We conclude that the risk of diabetes in family members with ICA $\geq 20$ JDF units does not diminish over time, that multiple antibodies predict slow as well as rapid progression to diabetes, and that long term survivors with confirmed, persistent ICA have multiple islet autoantibodies and low first phase insulin secretion. This implies on-going $\beta$ cell damage, and suggests that most individuals will eventually develop autoimmune diabetes.

\section{8}

FLUCTUATION OF AUTOANTIBODY COMBINATIONS OVER TIME IMPLICATIONS FOR RISK ASSESSMENT IN FIRST DEGREE RELATIVES. S.G.Gardner, P.J. Bingley, C.A. Rogers, A.J.K. Williams and E.A.M. Gale, University of Bristol, U,K

Little is known about the stability of islet autoantibody combinations over time. We examined this within a population-based prospective family study by serial testing of islet cell antibodies (ICA) and antibodies to GAD, IA-2 and insulin (IAA) in (1) 267 "antibody positive" relatives with $\geq 1$ marker above the 97.5 th centile of a schoolchild population (median age of relatives at entry $31.8 \mathrm{yrs}$, median follow-up $2.7 \mathrm{yr}$ ), (2) 345 "antibody negative" relatives with initially normal levels retested after $\geq 4$ years (median age $35 \mathrm{yrs}$ ) and (3) 19 family members (age at entry 12.4) who developed IDDM after $4.2(1.7-7.0)$ yr follow-up, in whom the last sample was collected a median of 0.4 years before diagnosis. Of 48 family members with $\geq 2$ markers at entry 13 developed diabetes; 12 retained $\geq 2$ markers up to the time of diagnosis while the other retained high levels of GAD antibodies alone. Of 35 who remain nondiabetic, 23 retained $\geq 2$ markers, 9 retained one marker and 3 lost both markers. Of 219 family members who had a single marker at entry, 3 developed diabetes. Two of these developed $\geq 2$ markers in subsequent samples, and one retained one antibody. Of 216 who remain non-diabetic, only $76(36 \%)$ were antibody-negative in all followup samples, while $27(13 \%)$ showed $\geq 2$ markers in at least one later sample, and 65 (30\%) showed a single marker over a median of 1.6 years of follow-up. A further 33 (16\%) showed fluctuation of levels above and below the 97.5th centile and follow-up sample were unavailable for 18 . Random effects models were fitted using Bayesian analysis with Gibbs sampling in order to assess changes in level of IAA, and GAD and IA-2 antibodies, and none was found to have changed significantly during followup in either those progressing to diabetes or in the "antibody positive" group. After median 4.8 years, 5 initially antibody-negative individuals $(1.5 \%)$ had $\geq 2$ markers and one of these had developed IDDM: $31(9 \%)$ had elevated levels of $\geq 1$ marke (ICA $19(5 \%)$, IA-2 antibodies $2(0.6 \%)$, GAD antibodies $11(3 \%)$, IAA $6(1.7 \%)$ ) Antibody combinations are generally stable, and $\geq 2$ markers above the 97.5 th centile in a single sample offers a sensitive screening test. This study has also shown that multiple antibodies can appear for the first time in those who initially screen negative and that repeat testing is justified in family members with a single marker.

\section{7}

PROLACTIN DELA YED HYPERGLYCEMIA IN MICE INJECTED WITH MULTIPLE LOW DOSES OF STREPTOZOTOCIN

M. Holstad and S. Sandler, Department of Medical Cell Biology, Uppsala University, Uppsala, Sweden.

Prolactin has been suggested to play an important role in the regulation of the immune system because it stimulates lymphocyte proliferation and macrophage function. The stimulatory effect of prolactin on immunity may result from antagonism of glucocorticoid effects. Glucocorticoids are reported to block macrophage IL-1 and TNF- $\alpha$ release, to inhibit the inflammatory actions of IL- 1 and to decrease IFN- $\gamma$ and IL- 2 release by lymphocytes. A severe inflammation often results in elevated levels of circulating glucocorticoids and ACTH, which in turn, reduces the secretion of prolactin. Due to these properties prolactin might influence the development of autoimmune diabetes. The aim of the present study was to examine the effect of prolactin on the development of hyperglycemia and pancreatic insulitis in mice treated with multiple intraperitoneal injections of streptozotocin $(40 \mathrm{mg} / \mathrm{kg}$ body-weight for five consecutive days). It was found that one daily subcutaneous injection of prolactin ( $4 \mathrm{mg} / \mathrm{kg}$ body weight) for 21 days delayed the development of hyperglycemia following the streptozotocin injections. The mice treated with multiple low dose injections of streptozotocin became gradually hyperglycemic, and concomitant treatment with prolactin significantly reduced the elevation in blood glucose levels on day 14 $(p=0.05)$ and $17 \quad(p=0.015)$. Morphological examinations of the pancreas on day 21 of mice receiving STZ injections revealed a marked insulitis in the control group and moderate insulitis in the prolactin treated animals. Prolactin administration alone did not change the serum glucose concentration compared to the correspondingly saline treated group of animals. This study indicate that prolactin may affect hyperglycemia in the early phase of autoimmune diabetes.

\section{9}

DNA VACCINATION WITH PLASMIDS CONTAINING GAD65 AND/OR INTERLEUKIN-4 CDNA: NO EFFECT ON THE INCIDENCE OF DIABETES IN NOD MICE. U. Roll, $R$. O'Rourke, and S. Baekkeskov. UCSF, San Francisco, USA

DNA vaccination involves the delivery of specific cDNA in mammalian expression plasmids by intramuscular, intradermal, or subcutaneous injection. Many studies have shown that DNA vaccines effectively generate strong immunity to proteins encoded by plasmid DNA; few studies have examined the role of DNA vaccination in generating tolerance in autoimmune models, however. The aim of this study is to investigate the potential for DNA vaccination to tolerize NOD mice using murine glutamic acid decarboxylase (mGAD65). In order to promote the differentiation of mGAD65-specific $T$ cells towards a Th2 phenotype, which has been associated with protection from diabetes in the NOD mouse, and to prevent the development of diabetes, CDNA encoding murine interleukin-4 (mlL4) was coinjected. NOD mice ( $\mathrm{n}=15$ per group) received 100ug of plasmid DNA i.m. once per week for four weeks beginning at 3-4 weeks of age. Experimental ams included no injection, plasmids containing full length mGAD65 cDNA alone, mIL4 CDNA alone, or mGAD65 CDNA + mlL4 cDNA. A plasmid containing murine beta galactosidase cDNA was used as a negative control. Blood glucose was monitored weekly to determine the incidence of diabetes. Serum was drawn at weeks 10, 14, 18, 22, 26, and antibody responses to GAD65 were measured by immunoprecipitation using in vitro translated radiolabeled GAD65. The incidence of diabetes at week 30 was: no treatment: $67 \%$; beta-galactosidase: $80 \%$; GAD65: $79 \%$; IL4: $100 \%$; GAD65 + IL4: $100 \%$. No differences were significant, except between GAD65 alone and GAD65 + IL4 ( $p=0.0197$ ). GAD65 antibody responses were seen in mice receiving $M G A D 65$ cDNA with or without IL4 CDNA; no GAD65 antibody responses were seen in IL4 cDNA alone animals or in control mice. This study shows that DNA vaccination using cDNA encoding GAD65 and/or IL 4 does not prevent the development of diabetes in NOD mice. There is some suggestion that IL4 CDNA, when administered as an adjuvant reagent with GAD65, increases the incidence of diabetes relative to GAD65 alone. 
REGIONAL CONTROL OF LYMPHOCYTE HOMING IN AUTOIMMUNE DIABETES

C. Ploix, C. Thivolet, INSERM U 449 Lyon France

Type 1 diabetes results from the lack of tolerance to beta-cell antigens and the expansion of autoreactive $T$ cells. During adoptive transfer experiments using two strains of NOD mice congenic at the Thy-1 locus we have demonstrated that diabetogenic $T$ cells accumulate 3 days after injection in pancreatic lymph nodes (PLN) before infiltrating islets 7days later. We have reported that peripheral tolerance can be achieved by oral administration of insulin conjugated to cholera toxin B-subunit (CTB) in prediabetic mice. Feeding 8week-old NOD mice with $10 \mu \mathrm{g}$ of CTB-insulin induced splenic regulatory $\mathrm{T}$ cells and prevented spontaneous and transferred autoimmune diabetes (PNAS, 1997). Homing of splenic T cells induced in CTB-insulin or CTB fed Thy-1,1+ mice was analysed after injection into Thy-1,2+ recipients mixed or not with Thy-1,2+ diabetogenic $T$ cells. Adoptive co-transfer experiments demonstrated a selective recruitment of Thy-1,1 donor cells originated from CTB-insulin fed mice in the PLN in comparison with control animals $(38 \pm 4 \%$ vs $19 \pm 8 \%, p<0,03)$. This accumulation persisted in absence of diabetogenic $T$ cells with respectively $52 \pm 14 \%$ vs $21 \pm 7 \% \quad(p<0,04)$. Preliminary RT-PCR data showed a concomitant increase in IL-4 mRNA expression that relied on the co-injection of diabetogenic $T$ cells. In addition, PLN cells in vitro stimulated secreted higher IL-4 amounts one week after a single oral dose of CTB-insulin but similar levels of IFN $\gamma$ than CTB fed mice. These results suggest that prevention of autoimmune diabetes following oral CTB-insulin adminis-tration involve the selective migration and retention of protective $T$ cells into lymphoïd tissues draining the site of organ injury.

\section{2}

PREDICTION OF INSULIN TREATMENT IN LATENT AUTOIMMUNE DIABETES IN ADULTS

C. Törn, M.Landin-Olsson, H.Arnqvist, G.Blohmé, Å.Lernmark, F.Lithner, B.Littorin, L.Nyström, B.Scherstén, G.Sundkvist, L. Wibell, J.Östman. Dept of medicine, Lund University Hospital, Lund and regional centres for the Diabetes Incidence Study in Sweden (DISS), Sweden.

In 1992-93 consecutive blood samples were collected from all patients with newly diagnosed diabetes aged $15-34$ years of whom $183 / 782$ were classified as Type 2 or unclassified. Ninety-three patients $(51 \%)$ had ICA, GAD65-antibodies or ICA512. These patients are referred to as LADA. ICA were analyzed using IF, while GAD65- antibodies, ICA512 and C-peptide were analyzed with RIA.The aim was to test the hypothesis that autoantibodies at onset predict a need for insulin within 3 years. Results. LADA: At diagnosis 58/93 (62\%) received insulin therapy and after three years $83 / 90(92 \%)$ were insulin treated. The positive predictive value for insulin treatment after three years in patients positive for all three antibodies was $100 \%$, ICA alone $89 \%$, GAD65-antibodies $93 \%$ and ICA512 89\%. Autoantibody negatives: $24 / 90(27 \%)$ were insulin treated from diagnosis. After three years $38 / 78(49 \%)$ received insulin. LADA and Type 1 patients had the same levels of autoantibodies. C-peptide levels were higher in LADA compared to Type $1,0.37$ versus 0.26 $\mathrm{nmol} / \mathrm{l},(\mathrm{p}<0.001)$. LADA had higher BMI than Type $1,23.6$ versus $21.0,(p<0.001)$. Autoantibody negatives compared to LADA had higher C-peptide median 0.76 and $0.37 \mathrm{nmol} / /(\mathrm{p}<0.001)$ and higher BMI median 29.6 and $23.6(\mathrm{p}<0.001)$. C-peptide decreased in autoantibody positive Type $1(\mathrm{p}<0.001)$, but not in LADA or autoantibody negative patients.

Conclusion: Positivity for one or several autoantibodies predict a future need for insulin in this group of patients.
SENSITIVITY OF ANTI-GAD AND ANTIIA-2 MEASURED BY ELISA, AND ICA, FOR TYPE 1 DIABETES IN NEWLY-DIAGNOSED CHILDREN AND ADOLESCENTS JA WILLIS, RS SCOTT and BA DARLOW Lipid and Diabetes Research Group, Christchurch Hospital, Christchurch, New Zealand

Antibodies directed against $B$-cell antigens are a feature of type 1 diabetes. Measurable in the serum years before clinical symptoms of type 1 diabetes are manifest these molecules are predictive markers of progression to total ß-cell destruction. Islet cell antibodies (ICA), directed against multiple cytoplasmic antigens and antibodies directed against glutamic acid decarboxylase (anti-GAD) and tyrosine phosphatase like protein (antil|A-2), show high prevalence in type 1 diabetes. This study compared the sensitivity of ICA, anti-GAD and antiIA-2 for type 1 diabetes in 114 children and adolescents consecutively diagnosed between January 1989 and September 1996 in Canterbury (NZ). MeantSD of the cases was 9+5 years (range 1-19 years) and 48 cases were girls. Mean $\pm \mathrm{SD}$ blood glucose concentration at presentation was $28 \pm 11$ (range $8.6-67.0 \mathrm{mM}$ ). For those cases where ketones were determined, $95 \%$ of presentations were ketotic. In virtually all cases insulin therapy commenced within 24 hours of diagnosis. Blood samples were taken as soon as possible after diagnosis, typically wittin one week of diagnosis. ICA were measured by indirect immunofluorescence using human pancreas tissue. Anti-GAD and anti-1A-2 were determined by separate ELISA assays using biotinylated antigen on streptavidin-coated microtitre plates from Boehringer Mannheim. Of the 114 cases studied, $110(96 \%)$ individuals were positive for at least one antibody, $103(90 \%)$ individuals were ICA positive. Seven $(6 \%)$ cases were positive for anti-GAD and/or anti-IA-2 in the absence of ICA. ICA, anti-GAD and anti-IA-2 were positive in $65(57 \%)$ of cases, with $14(12 \%)$ cases positive for ICA and anti-GAD and 20 $(18 \%)$ cases positive for ICA and anti-IA-2. That is, anti-GAD and/or anti-IA-2 were positive in $99(96 \%)$ of the 103 ICA positive cases. Anti-GAD and anti-AA-2 determined in combination by these assays therefore show the same sensitivity for type 1 diabetes as ICA. These ELISAS from Boehringer Mannheim (Diaplets $(R)$ anti-GAD/commercial product; Diaplets $(R)$ anti-A2/premarketing lot), based on recombinant B-cell antigens, are easily performed and standardised, and do not require scarce human pancreas tissue. Anti-GAD and anti-IA-2 determination by ELISA provides a valuabie alternative to ICA lesting for identification of individuals at risk for type 1 diabetes. In the context of adult-onset diabeles, these measurements could be used to identify the $15-20 \%$ of such cases who have latent type 1 diabetes and will progress to insulin deficiency.

\section{3}

GAD65 AUTOANTIBODIES IN THE GENERAL POPULATION ARE RELATED TO ABNORMAL OGTT.

O. Rolandsson ', E.Hägg', M. Nilsson', G. Jansson ${ }^{2}$, G. Hallmans' and A. Lernmark ${ }^{3}$. Umeå University, Umeå, ${ }^{2}$ Stenbergska Pimary Health Care Centre, Lycksele, Sweden and ${ }^{3}$ University of Washington, Seattle, WA, USA.

The aim was to test the hypothesis that GAD65 autoantibodies in the general population are related to abnomal oral glucose tolerance test (OGTT). A total of 2293 individuals without diabetes at 30-60 years of age who participates in the Västerbotten County Health Project were subjected to WHO standard OGTT a entry into the study. GAD65 and IA-2 autoantibodies were detcrmined in duplicates by radioligand binding assays. The intrassay $\mathrm{CV}$ was $8 \%$ and $13 \%$, respectively. In a total of $17(0.7 \%)$ individuals with diabetic OGTT, the GAD65 but not LA-2 autoantibody levels were significantly higher than individuals with normal OGTT $(p=0.03)$. In $30-40$ year old women, the frequency of individuals with GAD65 autoantibody levels exceeding the $95^{\text {th }}$ percentile was higher among those with impaired or diabetic OGTT compared to those with a nomal test $(\mathrm{p}=0.002$; Fischer's exact test). There was an increased frequency of individuals with IA-2 autoantibodies exceeding the $95^{\text {th }}$ percentile in 50-60 year old women with impaired or diabetic OGTT compared to those with a normal test $(\mathrm{p}=0.03)$. These relationships were not observed in men. As expected there were relations between BML, age and impaired and diabetic OGTT in both women and men. When it was tested whether GAD65 autoantibudy levels were related to BMI among the subjects with normal OGTT ( $\mathrm{n}=1983$ ), it was found that both 50 (mean BMI 28,$6 ; \mathrm{p}=0.025$ ) and 60 (mean BMI $30.8 ; p=0.00 \mathrm{~J}$ ) year old women with GAD65 auloantibodies exceeding the $99^{\text {th }}$ and $95^{\text {th }}$ percentiles, respectively had an increased BMI compare to GAD65 autoantibody negative 50 (mean BMI 25.3) and 60 (mean BMI 26,5) year olds. It is concluded that GAD65 autoantibodies are associated with impaired or diabetic glucose tolerance in the adult population. GAD65 autoantibody levels in adult women with nonnal OGTT may indicate a possible relation between islet autoimmunity and beta-cell function abnormalities with insulin resistance and obesity. 
344

HIGH AND STANDARD DOSE OF NICOTINAMIDE IN RECENT ONSET INSULIN DEPENDENT DIABETES (THE IMDIAB 6 STUDY)

N. Visalli, G. Ghirlanda, P. Pozzilli on behalf of the IMDIAB Study Group Istitute II Clinica Medica, University of Rome "La Sapienza" and Clinica Medica Università Cattolica; Rome, Italy.

Nicotinamide (NA) administered at the time of clinical onset of IDDM has been shown to protect residual beta cell function. The aim of our multicenter randomized study was to evaluate the effect of two different concentrations of NA on the integrated metabolic parameters during the first year of diagnosis of IDDM. Seventy-four patients have been included in this study ( 39 males and 35 females) with duration of the disease less than 4 weeks, mean age 13.3 years (range 5 to 35 years). Thirty-eight patients ( $25<15$ years and $13>15$ years of age) have been treated for 12 months with $25 \mathrm{mg} / \mathrm{kg}$ daily NA; 36 patients $(28<15$ years and $8>15$ years of age) have been treated for 12 months with $50 \mathrm{mg} / \mathrm{kg}$ NA. All patients have received intensive insulin therapy, 3 or 4 injections daily. Glycated haemoglobin, insulin requirement, and basal C-peptide have been measured at diagnosis and at 3 month intervals during the first year of the disease. Patients results were analyzed taking into account the age at diagnosis. Patients above 15 years of age showed $C$ peptide values significantly higher compared to children throughout the period of observation: 3 months; $1.22 \mathrm{ng} / \mathrm{ml}$ vs $0.71 \mathrm{ng} / \mathrm{ml}(P=.003) ; 6$ months; 1.03 $\mathrm{ng} / \mathrm{ml}$ vs $0.67 \mathrm{ng} / \mathrm{ml}(\mathrm{P}=0.012) ; 9$ months; $1.01 \mathrm{ng} / \mathrm{ml}$ vs $0.58 \mathrm{ng} / \mathrm{m}$ $(P=0.02)$. Children at one year follow-up showed a significant increase in insulin requirement $(P=001)$ compared to adult patients. When analysed all together (children and adults) patients treated with different doses of NA did not show significant differences in HbA1 levels or in insulin requirement Clinical remission was observed in two patients in each treatment group.

In conclusion, $25 \mathrm{mg} / \mathrm{kg}$ of NA are similar to the highest dose of $50 \mathrm{mg} / \mathrm{kg}$ NA in protecting residual beta cell function in recent onset IDDM.

\section{5}

THE SARDINIAN NEWBORN IDDM STUDY: DATA OF THE FIRST THREE YEARS OF FOLLOW UP.

G. Guaita, I. Pelligra, A.M. Setteneri, P. Pitzalis, 'M. Songini, E. Cossu, *M Shattock, *M. Locatelli, R. Cirillo, *G.F. Bottazzo and the IDDM Sardinian Study Groups. Diabetologic Centre, Department of Medical Sciences, University of Cagliari, Italy; ${ }^{\circ}$ Centre for Metabolic Diseases \& Atherosclerosis, San Michele Hospital, Cagliari, Italy; *Department of Immunology, St Bartholomew's \& The Royal London School of Medicine \& Dentistry, London, UK

In order to study the natural history of IDDM in reference to the first appearance of islet-related autoantibodies (I-r-AA), a large cohort of newborn has been recruited in Sardinia, where the incidence of IDDM, together with Finland, is the highest in the world. The main aim of the study is to recruit 30,000 newborn children and follow them for a period of 5 years, while its overall objective is to investigate the environmental, genetic and immunological factors which have led, and continue to lead, to this unusually high incidence of the disease in the Island. We have tested 15,509 cord blood samples, randomly collected in 18 Centres of the 4 Sardinian provinces. The prevalence of ICA was $2.3 \%$ for titres $\geq 5$ JDF-u, $1.7 \%$ for titres between 6 and 20 JDF $u$ and $0.6 \%$ for titres $\geq 20 \mathrm{JDF}-\mathrm{u}$. The prevalence of ICA $\geq 5$ JDF-u in follow up samples from 2,959 children bled at year 1 , from 1,148 at year 2 and from 388 at year 3 was $0.17 \%, 0.78 \%$ and $1.80 \%$ respectively At the 1 st and 2 nd year of follow up, GADA were detected in $0.6 \%$ and $1.5 \%$ of children tested $(2,125$ and 399 , respectively), while IA-2icA were detected in $0.1 \%$ and $1.3 \%$ of the samples tested $(2,117$ and 399 , respectively) Altogether, 26 children included in the follow up were positives for one or more Ir-AA. From the whole cohort, 11 children became diabetic (age 15 to 33 months) of the 9 cord blood tested, only one had ICA, 3 were positive for at least 2 I-r-AA in a sample taken before diagnosis, while one was negative, but became positive for all three I-r-AA at the time of diagnosis. Two children had 2 or 3 I-r-AA at the time of diagnosis, but no sample was available before it. For the remaining 5 children, no blood sample was available neither before or at the time of diagnosis. In summary, these data confirm the trend of an high prevalence of IDDM and immunological susceptibility to the disease in very young children in Sardinia.
346

KARLSBURG IDDM RISK STUDY - COMPARISON OF AUTOANTIBODY PATTERN IN A GENERAL SCHOOLCHILDREN POPULATION WITH NEWLY DIAGNOSED TYP-I-DIABETIC PATIENTS

M. Strebelow, M. Schlosser, B. Ziegler, I. Rjasanowski* and M. Ziegler; Institute of Pathophysiology, University of Greifswald; *Centre of Diabetes and Metabolic Disorders Karlsburg; Germany

Autoantibodies (AAbs) to B-cell antigens precede the development of clinical dia betes. In this study the AAbs against glutamic acid decarboxylase (GADA), tyrosine phosphatase (IA2A), insulin (IAA) and cytoplasmic islet cell antibodies (ICA) were compared between 7,829 schoolchildren $(11 \pm 3$ yrs. $)$ of the Karlsburg IDDM risk study and 109 newly diagnosed IDDM patients $(13 \pm 5$ yrs.). Using the 99 . percentile for GADA and IAA, the 99.5. percentile for IA2A and 20 JDF units for ICA as cutoffs $88 \%(96 / 109)$ of IDDM patients and $2.4 \%(199 / 7,829)$ of schoolchildren were positive for at least one AAb. $14.6 \%(16 / 109)$ and $2 \%(164 / 7,829)$ were positive for only $1 \mathrm{AAb}$ and $73.4 \%(83 / 109)$ and $0.4 \%(34 / 7,829)$ were positive for $\geq 2 \mathrm{AAbs}$ respectively. We found no significant difference in the titres of GADA, IA2A and ICA between IDDM patients and AAb positive schoolchildren. The titres of GADA IA2A and ICA were significantly increased with multiple AAb positivity in both groups. In schoolchildren the frequency of AAbs as well as AAb titres were significantly increased with increasing ICA titres, whereas in IDDM patients only the frequency of additional IA2A and their titres were increased. $98.8 \%(79 / 80)$ of IDDM patients positive for $\geq 2 \mathrm{AAbs}$ have GADA and/or IA2A, however $10 \%(8 / 80)$ were ICA negative. $100 \%(34 / 34)$ of schoolchildren positive for $\geq 2 \mathrm{AAbs}$ could be identified by GADA and/or IA2A, whereas $26.4 \%(9 / 34)$ were ICA negative. Furthermore, multiple AAb positivity in schoolchildren was significantly associated with the genetic IDDM marker HLA-DQB $1{ }^{*} 0302$ and/or * $0201.82 .4 \%(28 / 34)$ of the children positive for $\geq 2 \mathrm{AAbs}$ were positive for at least one of these alleles but not any child was positive for the protective allele ${ }^{*} 0602$. 16 out of these 34 children were investigated by i.v. glucose tolerance tests. $44 \%(7 / 16)$ had impaired glucose tolerance and/or diminished first phase insulin secretion. During the short follow-up period 3 of these children have progressed to IDDM. Our data indicate that the highest diagnostic sensitivity and specificity to identify subjects at risk for IDDM in a general population can be achieved by combined screening for GADA and IA2A.

\section{7}

KARISBURG IDDM RISK STUDY IN SCHOOLCHILDREN - MULTIPLE AUTOANTIBODY POSITIVITY IS STRONGLY ASSOCIATED WITH THE GENETIC RISK ALLELES OF THE HLA-DQB 1 LOCUS

M. Schlosser', R. Wassmuth ${ }^{2}$, M. Strebelow, B. Ziegler and M. Ziegler Institute of Pathophysiology and 'Surgery Hospital, Universitiy of Greifswald, ${ }^{2}$ Institute of Clinical Immunology, University of Erlangen-Nürnberg, Germany

Islet cell autoantibodies are detectable in more than $90 \%$ of IDDM patients at onset of the desease, but also in prediabetic individuals. The genetic predisposition to the disease is strongly positive associated with the occurrence of the HLA-DQB1 alleles ${ }^{*} 0302$ and $*^{*} 0201$ whereas dominant protection is conveyed by the DQBI*0602 allele. In our study 12,558 schoolchildren were screened for autoantibodies (AAb) against glutamate decarboxylase (GADA), protein tyrosinphosphatase (IA2A), insulin (IAA) and islet cell cytoplasmic AAb (ICA). In 199 probands, positive for at least one $A A b$ in re-examination, HLADQB1 alleles were defined by DNA-genotyping. $30.1 \%$ of them were positive for GADA only and $16.1 \%$ for IAA only $(99$. percentile), $19.1 \%$ for IA2A only $(>99.5$ percentile) and $17.6 \%$ for ICA $\geq 20$ JDFU only. These probands have beside associated alleles a high frequency of the protective and other alleles. $18.3 \%(11 / 60)$ of probands positive for GADA only, $13.1 \%(5 / 38)$ for IA2A only, $18.8 \%(6 / 32)$ for IAA only and $25.7 \%(9 / 35)$ for ICA only carried DQB $1 * 0602$ Moreover, a strong titre dependency of the ratio of associated/protective alleles was observed for all AAb specificities. Schoolchildren positive for two biochemically defined $A A B$ only showed at least one of the associated alleles but not the protective allele, i.e. $75 \%$ positive for GADA/IA2A ( $n=4), 100 \%$ positive for GADA/IAA $(n=3)$ and $50 \%$ positive for IA2A/IAA $(n=2)$. All schoolchildren positive for three AAb specificities, GADA/IA2A/ICA $(\mathrm{n}=10)$ and GADA/IAA/ICA $(n=2)$ and positive for all four AAb, GADA/IA2A/IAA/ICA $(n=5)$, carried one of the associated DQB1 alleles. Thus multiple AAb positivity is strongly associated with the presence of IDDM associated risk alleles in the general population. Probands positive for only one AAb and for the protective allele have a lower risk underlining the importance of combined $A A b$ screening and HLA-DQB1 genotyping to assess the risk to develop IDDM to select patients for future intervention trials. 
348

PREVALENCE OF DIABETES-ASSOCIATED AUTOANTIBODIES IN SLIM ELDER PATIENTS WITH DIABETES MELLITUS

A. Pfützner, T. Forst, T. Kunt, M. Löbig, N. Abdoullahnia, R. Maki, M. Engelbach, and J. Beyer, Dept. of Endocrinology, University Hospital Mainz, Germany

Diabetes-associated autoantibodies have been descriebd to be markers for an autoimmune onset of Diabetes mellitus at any age. They can be used to diagnose type 1 diabetes in in elder patients clinically presenting with a pathological oral glucose tolerance test. The goal of this study was to identify the prevalence of Diabetes associated autoantibodies in slim adult patients presenting with recent onset of diabetes. Inclusion criteria were age $>30$ years, BMI below $27 \mathrm{~kg} / \mathrm{m}^{2}$, and $<1$ year of duration of diabetes. The serum of 140 patients was included into the study $(80$ male, 60 female, age( $\pm S T D): 47.2 \pm 11.5 \mathrm{yrs}$;; BMl: $23.1 \pm 2.6 \mathrm{~kg} / \mathrm{m}^{2}$; duration of diabetes $3.3 \pm 4.2$ months). Islet cell antibodies were determined with immunfluorescence test (Euroimmun), while antibodies to GAD and IA2 were measured with radioimmunoassays (both Medipan). The prevalence was $51 / 140(36.4 \%)$ for anti-GAD, $43 / 140(30.7 \%)$ for ICA and $24 / 140(17 \%)$ for anti-IA2 in this group of patients. All three antibodies were seen in $21 / 140$ patients $(15 \%)$, while just one single type of antibodies was seen in $3 / 140$ for ICA $(2.1 \%), 11 / 140$ for antiGAD $(7.9 \%)$, and $3 / 140$ for anti-IA2 $(2.1 \%)$ respectively. Either one of the antibodies was seen in $57 / 140$ patients $(40,7 \%)$. The oldest patients presenting with LADA were 83 years old. One had all three antibodies, while one only showed prevalence of anti-GAD. Additional measurement of ICA and anti-IA2 next to anti-GAD increased the sensitivity for the detection of LADA by $12 \%$. We therefore recommend to include measurement of ICA and IA2 into the routine screening methods for LADA.
349

Incomplete Freund's adjuvant protects NOD mice from Type 1 diabetes. P.E.Beales, R.Liddi, G.Matta, ${ }^{*}$ GP Webb and P.Pozzilli. Dept of Diabetes \& Metabolism, St Bartholomew's Hospital, London ECIA 7BE and *University of East London, London E12 4LZ UK.

As the study of diabetes moves towards the possibility of preventive therapy with immunomodulating drugs the role of adjuvants needs to be addressed Immunomodulating drugs usually require some time to exert an effect and as such usually benefit from being held in suspension rather than dispersed immediately into tissue following injection Oil-based adjuvants such as incomplete Freund's adjuvant (iFA) are often used for this purpose. iFA has hitherto mainly been thought of as "immunologically inert" as, unlike Complete FA, it has no significant ruptured cell membrane content designed in itself to provoke an immune response.

This study looked at diabetes incidence in 24 NOD mice injected intradermally (id) at 8 and 12 weeks of age witl a $1: 1$ mixture of emulsified iFA and saline. 24 litter matched controls were given id injections of saline alone at the same time points. At the conclusion of the study at 32 weeks, splenocytes were collected from all animals and tested against specific antigens thought to be involved in diabetes, namely $\beta$-casein and Glut-? they were also stimulated with $C$ on- $A$, a non specific mitogen. Splenocytes from iFA treated animals showed non-specific immunosuppression with Con-A $(p<0.01)$ whereas the response to $\beta$-casein and Glut -2 was raised in iFA treated animals with respect to controls. Diabetes incidence in control mice was $38 \%$ whereas the iFA treated group had a significantly lower incidence of $13 \%$ ( $p<0.05$ - Chi squared). We suggest that the raised response to specific antigens produced t-cell clones of the non-damaging Th-2 type rather than a damaging Th- 1 infiltration and that increased Th-2 insulitis resulted in a lower incidence of diabetes in the iFA treated group. In conclusion, iFA alone can reduce diabetes incidence in the NOD mouse model of Type 1 diabetes

\section{0}

TIME-COURSE OF ISLET CELLS ANTIBODIES (ICA) DURING EARLY CHILDHOOD IN OFFSPRING OF IDDM PARENTS

C. Lévy-Marchal and the DICA Study Group INSERM U457, Paris, France Prediction of IDDM in first degree relatives is based on the high risk for the development of the disease over 5 yr. associated with ICA determination in these subjects. Calculation of the predictive values infers the persistence of ICA between the test and IDDM diagnosis in the same individuals. If intervention trials prove beneficial screening for high-risk children should take place as early as possible. The aim of this study was to monitor the time-course of ICA in a cohort of young offspring of IDDM parents. A group of 265 children born to 142 IDDM mothers and 127 IDDM fathers were recruited at the age of $3.5 \pm 1.9$ yr. They were prospectively followed for $3 \mathrm{yr}$. At inclusion $12(4.5 \%)$ tested positive for ICA. Annual results for seroconversions to negativity (SC-) and positivity $(\mathrm{SC}+)$ are given below:

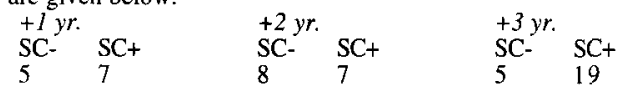

Altogether $27(10 \%)$ children tested positive for ICA after 3yr. follow-up, among whom 10 were $\geq 20 J D F u$. The proportion of ICA positive- and negative-children were significantly different at inclusion $(4,5 \%)$ and after 3 yr. of follow-up (10\%) ( $<<0.01$; Chi2 paired-test). Titers of ICA in JDFu in positive-children did not significantly vary between inclusion and follow-up (27 vs. $19 \mathrm{JDFu}$; $n$ s by Mann-Whitney test).

An additional group of 21 children born to 16 IDDM mothers and 5 diabetic fathers were followed over the same time from birth. Results for ICA testing in cord blood showed 5 positive results among whom 4 from ICA-positive mothers. ICA testing at follow-up of these newborns showed

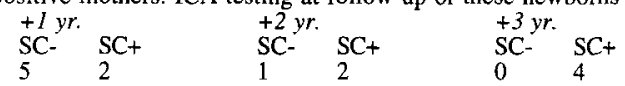

In conclusion our data indicate 1)- that in infants mother-transmitted ICA show a clearance $<1$ yr. as already reported, 2)- high rates of ICA-positive seroconversion $(8.5 \%$ and $24 \%$ in children and newborns, respectively) over 3yr. follow-up in comparison to inclusion. This variability of ICA testing makes difficult screening for high risk children very early in life in young offspring of IDDM parents. 


\section{Environmental Factors}

\section{1}

APPEARANCE OF DIABETES-ASSOCIATED ANTIBODIES IN OFFSPRING OF PARENTS WITH TYPE 1 DIABETES IS INDEPENDENT FROM ENVIRONMENTAL FACTORS

M. Hummel, M. Schenker, A.G. Ziegler and the BABY-DIAB Study Group. Diabetes Research Institute, Munich, Germany.

To examine the influence of perinatal determinants and early environmental events on the appearance of islet autoantibodies we prospectively followed offspring of parents with type 1 diabetes from birth (BABY-DIAB Study). Today, 389 offspring of mothers with type 1 diabetes and 140 children of fathers with type 1 diabetes were followed until at least the third year of life (Min 2.0; Max 8.1 years). Fifty-six children (10.6\%) have one or more islet autoantibody (IAA, GADA, IA2A, or ICA) and 9 $(1.7 \%)$ developed IDDM. Offspring of healthy mothers (and type 1 diabetic fathers) have a significant longer total breast feeding duration compared to offspring of mothers with type 1 diabetes (23 versus 19 weeks, $p<0.05$ ). However, we find no difference in the duration of total and exclusive breast feeding between antibody positive $(a b+)$ compared to antibody negative (ab-) offspring. Furthermore, children of healthy mothers (and type 1 diabetic fathers) have a significant longer duration of gestation ( 39.3 versus 38.4 weeks, $p<0.001$ ) and the diabetic mothers are younger than the diabetic fathers at birth (29 versus 32 years, $p<0.001$ ). However, again neither the gestational duration nor the age of the diabetic parent differ between the $a b+$ and ab-children. Prenatal determinants as age of diabetes onset of the diabetic parent, smoking behaviour of the parents during pregnancy, and frequency of relatives with type 1 and type 2 diabetes do not influence the development of antibodies. Postnatal factors as vaccinations, viral diseases (measles, rubella, mumps), gender, weight, and height do not differ between $a b+$ and ab- children. In summary, we have no evidence that any of the described factors influences the development of islet autoantibodies during the first years of life.

\section{2}

SEASONALITY OF BIRTH IN PATIENTS WITH CHILDHOOD IDDM THE EURODIAB STUDY.

PA McKinney, P Rothwell, E Schober, C Ionescu-Tirgoviste, A Neu, P Pozzilli and $\mathrm{S}$ Gutnikov on behalf of the EURODIAB study group. Paediatric Epidemiology Group, Centre for Health Services Research, University of Leeds, UK.

A significant difference in the monthly pattern of birth in children with IDDM compared to births in the general population has been observed in the UK. A follow-up study using data from 16 registers of the EURODIAB collaborative network investigated whether this phenomenon was present in other populations. The following registers contributed data: UK-Oxford, UK-Leicester, UK-Northern Ireland, Austria, Denmark, Italy-Lazio, ItalySardinia, Lithuania, Luxembourg, Germany-Baden Wuerttemberg, Romania, Spain, Poland, Sweden, Slovakia and Czech Republic. Each area was analysed individually and grouped according to geographical areas of high, medium and low risk. Data on 10,426 births, occurring between 1974 and 1994, of children diagnosed with IDDM between 1989 and 1995 were analysed. For each region the observed numbers of cases each month were compared to those expected based on the regional or national birth rate. Statistical significance of the deviation of the observed to the expected numbers of cases was calculated using the test for seasonality of events designed by Walter and Elwood. The results showed no overall significant seasonality either for high, medium or low risk areas or any individual country apart from Leicester, UK. These findings from a large number of births fail to demonstrate any relationship between seasonality of birth and the onset of IDDM in those under 15 years. Across Europe, environmental factors which vary by season of the year do not appear to have any influence around the time of birth on subsequent development of childhood IDDM. However, this does not preclude seasonal exposures operating in any one particular region.

\section{3}

NUTRITIONAL FACTORS AND RISK OF TYPE 1 DIABETES Sa. Muntoni, P. Cocco $0^{\star}$, G. Aru ${ }^{\star}$, F. Cucca ${ }^{\star \star}$ and S. Muntoni. Centre for Metabolic Diseases and Atherosclerosis, The ME.DI.CO. Association, Cagliari; ${ }^{*}$ Inst. of Occupational Medicine, Cagliari University; ${ }^{* *}$ Dept. of Paediatrics, Cagliari University; Cagliari, Italy.

Several studies have reported an increase in risk of type I diabetes (IDDM) associated with some nutrients. To test this hypothesis, we explored possible correlations between nutritional factors and IDDM incidence with an ecological analysis. Incidence rates of childhood ( $<15 \mathrm{yr})$ IDDM in the late $80 \mathrm{~s}$ in 43 world countries and total and specific per capita mean caloric intake were derived from official sources. Univariate linear regression between IDDM and socio-economic, geographic and dietary indicators was calculated. Variables correlated with IDDM were selected to fit a multiple regression model with IDDM incidence as the outcome. Meat $(\mathrm{r}=0.464, \mathrm{p}<0.01)$ and milk $(\mathrm{r}=0.649, \mathrm{p}<0.001)$ correlated directly, and cereals $(r=-0.544, p<0.01)$ inversely with IDDM. Per capita gross domestic product and average temperature were associated with IDDM incidence in the univariate analysis, but not in the multivariate analysis when dietary variables fitted the model. Moreover, in 11 European countries where IDDM incidence increased over the last $25 \mathrm{yr}$, the variation in caloric intake over the same period correlated with IDDM (total calories $r=0.468$; meat: $r=0.487$; cereal: $r=-0.546$ ), although the correlation coefficients were not significant due to the small number of countries These results suggest that the rising incidence of IDDM since the end of World War II in several countries and among populations migrated to high-incidence/high-income countries could be related, at least in part, by a diet richer in calories and animal protein and/or poorer in cereals early in life in genetically susceptible individuals.

\section{4}

COW'S MILK CONSUMPTION ASSOCIATED WITH PROGRESSION TO CLINICAL IDDM IN SIBLINGS OF CHILDREN WITH DIABETES SM Virtanen, E Hyppönen, E Läärä, L Räsänen, A Aro, M Knip, HK Akerblon, the Childhood Diabetes in Finiand Study Group. School of Public Health and Medical School, University of Tampere; Department of Mathematical Sciences and Department of Pediatrics, University of Oulu; Division of Nutrition and the Children's Hosoital, University of Helsirki; Department of Nutrition, National Fublic Health Institute, Helsinki, Finland.

We followed-up siblings of children with IDCM to find out whether cow's milk consumption during infancy or later in childhood is related to progression to clinical IDDM. A cohort of 725 initially unaffected $0-25$-year-old siblings of 801 index children with IDDM diagnosed in 1986-1989, participated in the nationwide study (82\% of those invited). The siblings were followed for IDDM starting at the diagrosis of DDV in the index child until october 37, 1995. Of all siblings $4.6 \%$ (33/725) developed IDIM during the follow-up time. The age at introduction of suppiementary milk feeding was not significantiy related to the development of IDIM in the siblings. When adjusted for age, sex and matemal age and education, high milk consumption in childhood ( $>3$ glasses daily) was associated with more frequent progression to clinical IDDM than low consumption ( $<3$ glasses daily) (adjusted hazard ratio 3.11, 95\% confidence interval 1.1-9.3, p=0.04). When adjusted also for infant feeding patterns, scmewhat weaker association was observed between high milk consumption and progression to clinical IDIM (adjusted hazard ratio 2.75 , 95\% confidence interval $0.9-8.4, p=0.07$ ). To conclude, this is the first study to suggest that high consumption of $\mathrm{Cow}^{\top} \mathrm{s}$ milk during childhood may be associated with progression to clinical IIIM among siblings of children with diabetes. 
COW'S MLLK CONSUMPTION ENHANCES STREPTOZOTOCIN SENSIBILITY IN WISTAR RATS.

A.Gaisina, T. Kurbanov, Sh. Rohimovo, V.Poltorack', N.Gorbenco', A.Glodkjach and O.Nanova'. Instifute of Physiology, Baku, Azerballan,

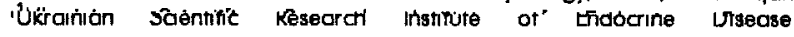
Pharmocotheropy, Kharkov, Ukraine.

Early introduction of cow's milk (CM) into the infant diet is suggested to predispose for IDOM development. The alm of this study was to evaluate the impoct of $\mathrm{CM}$ consumption on streptozotodin (SZ)- sensibility and oxldative status in Wistar rats. Newborn anlmals were glven CM per os for the first 21 doys of life. Controls group (C) received saline alone. To characterize the sensibility to STZ in rats the i. p. GT (2 g glucose $/ \mathrm{kg}$ ) was performed at 3 wesk of age fa doy before and doy after single inlection of non-diabetogenic dose of $5 T \cdot 40 \mathrm{mg} / \mathrm{kg} \mathrm{l}$. p.). No sigrificant differences in bosal blood glucose levels and glucose tolerance were observed between $C M$-treated and $C$ before $S T Z$-Inlection. A low-dose of STZ induced basol hyperglycemic $6.95 \pm 0.30$ vs $5.42 \pm 0.22 \mathrm{mmol} / \mathrm{h}$, p 0.02 ) and increased integral glycemia over glucase load in CMt-treated rata $\{42.45 \pm 4.80$ vs $27.43 \pm 1.60 \mathrm{mmol} / 1, \mathrm{p}<0.01)$, but did not change these parameters in control group. Oxidative status of experimental onimals has been estimated by spectrophotometric determination of diene conlugates (DC) and matonlc dialdehyde (MDA) in liver homogenate. We reveoled that $C M$ introduction for 21 doys promoted oxidative stress In rats Increosing $D C$ level $[141.1 \pm 10.5$ vs $89.6 \pm 3.4 \mathrm{nmol} / \mathrm{mg}$ protein, $p<0.02)$ and MDA content $[59.0 \pm 4.3$ vo $43.5 \pm 3.9 \mathrm{nmol} / \mathrm{mg}$ protein, $p<0.05$ ) in comparison with C. Administration of STZ enhanced oxidative porameters both in $C$ onimals and CM-treated group, but DC content wos higher in CMttreated rols $(215.5 \pm 19.5$ vs $158.9 \pm 14.9 \mathrm{nmol} / \mathrm{mg}$ protein, $p<0.02$ ) compared to $C$. We conduded that early $\mathrm{CM}$ consumption enhanced sensibility to STZ and oxldative stress in rats.
GESTATIONAL SOCIAL STRESS INCREASES GLUCOSE INTOLERANCE IN RATS SECOND GENERATION MALE OFFSPRING

V.Poltorack, N.Krasova, A.Gladkih. Ukrainian Scientific Research Institute of Endocrine Diseases Pharmacotherapy, Kharkov, Ukraine.

The aim of the study was to evaluate the vertical transmission of gestational social stress (GSS) - induced glucose tolerance (GT) disturbances in the first generation female offspring $\left(F_{1}\right)$ to the second generation male offspring $\left(\mathrm{F}_{2}\right)$ and to characterize the sensibility to streptozotocin (STZ) in the above. For GSS creation rats were transferred daily from one rat's association to another within $2^{\text {nd }}-8^{\text {th }}$ day of pregnancy (Group 1). The decrease in GT was shown in their female offspring $\left(\mathrm{F}_{1}\right)$ before mating: integral glycemia over i.p. GTT $(3 \mathrm{~g}$ glucose $/ \mathrm{kg} ; 0,30,60$ and $120 \mathrm{~min}$ ) was $30.9 \pm 3.2 \mathrm{vs} 20.8 \pm 2.6 \mathrm{mmol} / \mathrm{l}$ in controls (Group 2) offspring $(\mathrm{p}<0.05)$. The same GTT was performed in the male $F_{2}(n=16)$ at 45 and 90 days of age. Then Group 1 and Group 2 $F_{2}$ were subjected to a single $S T Z$-injection $(45 \mathrm{mg} / \mathrm{kg}$, i.p.). In 5 days basal glycemia, catalase activity (CA), reduced glutathione (GSH) levels in liver and plasma diene conjugates (DC) contents were determined. It was revealed significant decrease in GT of Group $1 \mathrm{~F}_{2}$ at 90 days of age (integral glycemia over GTT was $36.5 \pm 2.8 \mathrm{vs} 26.9 \pm 2.7 \mathrm{mmol} / \mathrm{l}$ in Group $2 \mathrm{~F}_{2}, \mathrm{p}<0.05$ ). After $\mathrm{STZ}$-treatment basal glycemia formed $16.1 \pm 0.2 \mathrm{vs}$ $9.6 \pm 1.4 \mathrm{mmol} / \mathrm{l}$ in Groups 1 and $2 F_{2}$, compared to $4.2 \pm 0.3 \mathrm{mmol} / \mathrm{l}$ in intact controls (IC) $(n=7, p<0.001)$. Liver $C A$ was increased and GSH levels were decreased similarly in both STZ-treated Groups $F_{2}$ in comparison with IC $F_{2}$ (respectively, $p<0.01, p<0.05$ ). Thus, alterations in antioxidant defense system reactivity revealed after STZ-injection were not depended on GSS availability in ancestors. In conclusion, gestational social stress induced glucose tolerance impairment and enhancement of sensitivity to STZ-hyperglycemic impact in male $F_{2}$ at the sex-maturity period

\section{PS 5}

\section{Clinical Immunology}

\section{7}

CLINICAL AND BIOLOGICAL DIFFERENCES BETWEEN AUTOANTIBODY. POSITIVE AND -NEGATIVE ADULTHOOD-ONSET DIABETES

I. Weets ${ }^{1}$, V. Siraux ${ }^{2}$, J-C. Daubresse ${ }^{3}$, G. Krzentowski ${ }^{2}$, F. Féry ${ }^{4}$, BJ Van der Auwera', DG. Pipeleers', FK. Gorus', and the Belgian Diabetes Registry. 'Diabetes Research Center, VUB, Brussels; Hôpitaux Civils de ${ }^{2}$ Jumet et de ${ }^{3}$ Charleroi; ${ }^{4} \mathrm{HU}$ Erasme, Brussels, Belgium.

Diabetes is heterogeneous in terms of pathogenesis, clinical presentation and biological markers. Disease classification and treatment might benefit from more objective criteria. We therefore measured autoantibodies against islet cells (ICA), insulin (IAA), GAD (GADA) and IA-2 (IA-2-A) in 496 recent-onset diabetic patients aged between 20 and 40 yrs and sampled during the first week after diagnosis, and analyzed clinical and biological findings after stratification for absence or presence of antibodies. Compared to the antibody-negative group $(n=168 ; 34 \%)$, patients with at least one type of antibodies $(n=328 ; 66 \%)$ were significantly younger (median age: 29 vs. 33 yrs; $p<0.001$ ) but presented a similar increase in male/female ratio $(1.8$ vs. $2.0 ; p>0.05)$. Antibody-positivity was associated with a higher reported frequency of polyuria, polydipsia or weight loss ( 97 vs. $82 \%$ in antibody-negative patients; $p<0.001$ ), a lower BMI (21 vs. $\left.25 \mathrm{~kg} / \mathrm{m}^{2} ; p<0.001\right)$ and more frequent insulin treatment ( 88 vs. $51 \% ; p<0.001)$. Median levels of glucose $(359$ vs $305 \mathrm{mg} / \mathrm{dL} ; \quad P<0.001)$ and $\mathrm{HbA} 1 \mathrm{c}(214$ vs. $194 \%$ of mean of local reference range; $p<0.05)$ were higher, but median $C$-peptide levels were lower (41 vs. $83 \%$ of mean of local reference range). Antibody. positive patients showed a higher prevalence of ketonuria (75 vs. $53 \% ; p<0.001)$ and of genotypes predisposing to type 1 diabetes (HLA DQA1*-DQB1* 0301-0302/0501-0201: $22 \%$ vs. $7 \%, \quad p<0.001$; 5'INS I/I: $73 \%$ vs. $53 \% ; \quad p<0.001$ ), but carried less often protective genotypes (no 0301-0302 nor 0501-0201: $15 \%$ vs. $52 \%$; $\mathrm{p}<0.001$ ). In conclusion, autoantibodies occur in most patients with onset between 20 and $40 \mathrm{yrs}$ in association with a more severe phenotype. Metabolic and immunological benefits from early insulin therapy should be assessed during follow-up studies.

\section{8}

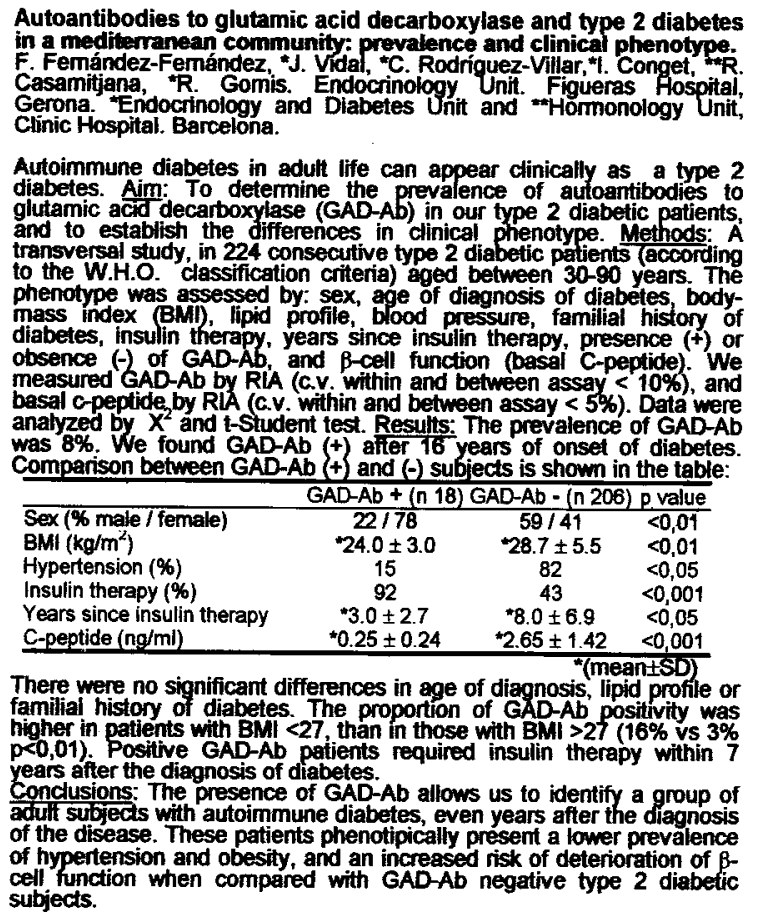


PREVALENCE AND CORRELATION OF ISLET AUTOANTIBODIES (ICA, IA-2, GAD-65) AND THYROID ANTIBODIES IN FIRSTDEGREE RELATIVES OF IDDM PATIENTS

E.Hatziagelaki, C.Jaeger, R.Petzoldt*, J.Seissler** W.A.Scherbaum** and R.G.Bretzel.Third Medical Department and Policlinic, JustusLiebig University, Giessen. "Diabetes Clinic, Bad-Oyenhausen. ${ }^{* *}$ Diabetes Research Institute, University of Duesseldorf, Germany.

Type I diabetes mellitus is often associated with thyroid autoimmunity. Data on the prevalence of thyroid antibodies (TG and TPO), adrenal $(A D-A b)$ and gastric parietal cell antibodies (GPC-Ab), in first-degree relatives of IDDM patients have not been systematically obtained to study their correlation with islet autoantibodies (ICA, IA-2, GAD-65) We determined ICA, IA-2, GAD-65, thyroid-Ab, AD-Ab and GPC-Ab in 842 first-degree relatives (mean age $14.6 \pm 8.7 \mathrm{yrs}$ ) of IDDM patients and inter-relationships of these antibodies were analysed in respect of antibody positivity. Of the 842 first-degree relatives, ICA were found positive in 90 cases $(10.7 \%$ ), anti-GAD in $84(9.9 \%)$, IA-2 in $57(6.8 \%)$ and thyroid-Abs in 28 cases $(3.3 \%)$, respectively. A statistically significant correlation was observed between ICA and thyroid antibodies $(p<0.05)$. A significant association was also seen between IA-2 and thyroid-Abs: higher levels of IA-2 Ab were observed in subjects with TPO-Ab positivity in comparison to those tested negative for (TPO)-Abs $(p<0.01)$. No significant correlation was observed between GAD-65 and thyroid-Abs. There was also no significant assosiation of any antibody (ICA/ IA-2/ GAD-65) with AD-Ab and GPC$A b$. The results of the present study, that prevalence of thyroid-Abs is associated with ICA and IA-2 antibody positivity may predict susceptibility to thyroid disease not only in IDDM patients but also in their relatives who are at risk for IDDM.
FLUCTUATIONS OF ISLET CELL ANTIBODIES AFTER CLINICAL ONSET $\sigma$ F DIABETES DIFFER ACCORDING TO AGE AND TO ANTIGEN-SPECIFICITY

FK. Gorus' ${ }^{1}$ K. Decochez', I. Weets', G. Somers ${ }^{2}$, B. Keymeulen ${ }^{1,2}$, L. Van Gaal', J-L. Coolens', and the Belgian Diabetes Registry. 'Diabetes Research Center and ${ }^{2}$ Dept of Diabetology, VUB, Brussels; ${ }^{3}$ Dept of Endocrinology, UIA, Antwerp; ${ }^{4}$ Dept of Internal Medicine, Salvatorziekenhuis, Hasselt, Belgium.

Antibodies against GAD (GADA) and against IA-2 (IA-2-A) contribute to a major part of islet cell cytoplasmic antibodies (ICA). Their monitoring during the first years of clinical diabetes may provide relevant information for disease classification, treatment and outcome. We determined ICA, IA-2-A and GADA during the first 2 years of clinical diabetes in insulin-treated patients under age 40 yrs $(n=194)$. Patients aged $0-19$ yrs $(n=103 ; 54 \%)$ had a higher prevalence of ICA (86 vs $66 \% ; \quad p<0.001)$ and IA-2-A 67 vs $47 \%$; $p<0.005$ ), but not of GADA, (77 vs $76 \%$;NS) than patients aged $20-39$ yrs $(n=103 ; 54 \%)$. Among the initially positive patients, antibody persistence was noted in $77 \%$ for ICA in $87 \%$ for $\mid A-2-A$ and in $91 \%$ for GADA. Median antibody levels decreased already during the first year after onset for ICA (both age groups) and IA-2-A (0-19 yrs alone) $(p<0.001)$ but only in the second year for GADA (0-39 yrs). Fourty $\%$ of IA-2-A-positive patients (20-39 yrs) and $45 \%$ of GADA-positive subjects $(0-39 \mathrm{yrs})$ reached maximal levels after clinical onset. Likewise, 23 initially negative patients seroconverted later for ICA $(n=7)$, IA-2-A $(n=8)$ or GADA $(n=11)$. Most patients $(n=14)$ were older than 20 yrs and two of them were completely antibody-negative at onset. Twelve patients $(6 \%)$ were persistently negative for ICA, IA-2-A and GADA (8/12 older than $20 \mathrm{yrs}$ ) but still on insulin after two years $(0.27$ to $0.74 \mathrm{lU} / \mathrm{kg})$ and showed a significant rise in insulin antibody levels during treatment. In conclusion, persistance or increase of autoantibodies after clinical onset is more pronounced after age 20 yrs and, in younger patients, for GADA than for IA-2-A. Determination of autoantibodies at onset underestimates the number of cases with autoimmune diabetes.

\section{1}

T CELL RESPONSE TO GAD IN STIFF-MAN SYNDROME, AUTOIMMUNE CEREBELLAR ATAXIA AND TYPE-1 DIABETES: DIFFERENT CYTOKINE PATTERNS?

${ }^{1} \mathrm{M}$. Costa. ${ }^{1} \mathrm{C}$. Roura-Mir, ${ }^{1} \mathrm{M}$. Martí, ${ }^{3} \mathrm{~A}$. Sáiz, ${ }^{3} \mathrm{~F}$. Graus, ${ }^{4} \mathrm{M}$. F. Castanyer, ${ }^{2} \mathrm{~A}$. Sanmartí. 'R. Pujol-Borrell and 'D. Jaraquemada. 'Immunology and 'Endocrinology Units, Hospital Germans Trias i Pujol, Badalona. Neurology Department, Hospital Clínic and Endocrinology Department. Hospital de Bellvitge, Barcelona, Spain.

Glutamic acid decarboxilase (GAD) catalyzes the synthesis of the major inhibitory neurotransmitter gamma aminobutyric acid (GABA). GAD expression is mostly restricted to the central and peripheral nervous system and to the endocrine cells of the pancreas islets of Langerhans. GAD autoantibodies are found in the serum of more than $80 \%$ of newly diagnosed type 1 diabetes patients. High titre, mostly oligoclonal, GAD autoantibodies are also present in the serum and cerebrospinal fluid of patients with central nervous system disorder, stiff-man syndrome. In addition. a recently described putatively autoimmune cerebelar ataxia is also associated to the presence of high-titre GAD-autoantibodies. Both neurological diseases are associated with type I diabetes. AIM: to study the $T$ cell response to GAD in three autoimmune diseases with GAD antibodies. type 1 diabetes, stiff-man syndrome (SMS) and autoimmune cerebelar ataxia (ACA). METHODS: samples from 3 SMS. 3 ACA, 10 newly diagnosed type 1 diabetes patients and 8 diabetic patients with high titre of anti-GAD antibodies were tested for anti-GAD cellular reactivity in $\mathrm{T}$ cell proliferation and cytokine production assays. Control grups were healthy age-matched donors. RESULTS: low but abovecontrol proliferation was found in all patient grups, although $2 / 3 \mathrm{SMS}$ patients and $2 / 8$ high titre GAD-ab diabetes patients showed high reactivity to puritied GAD obtained from recombinant baculovirus infected cells. However, GAD-specific cell lines could be obtained from all patient grups, indicating that GAD-specific $T$ cells were present bu non-proliferative. To characterize the $T$ cell population/s reacting to GAD in these samples. GAD-specific cell lines from each grup were fully stimulated with PMA and ionomicine and the cytoplasmic cytokine production was analysed by flow cytometry. Preliminary results show a heterogeneous $T$ cell cytokine pattern in all samples, with some differences: a population of activated $T$ cells producing IL- + or simultaneousily IFN- $\gamma$ and IL-4 was found in diabetes patients, whereas both SMS and ACA cell lines showed an additional population of cells producing IFN- $\gamma$ only. The percentage of IFN$\gamma$ positive cells was larger in the ACA than in SMS cell lines. The specific population which gets stimulated after culture with purified GAD is now been studied. The results so far indicate that there is heterogeneity in the cellular response to GAD in these patients.

\section{2}

DISTINCT IMMUNE REACTIVITY TO GAD-65 IN STIFF MAN SYNDROME AND TYPE I DIABETES MI Hawa, T Lohmann, RDG Leslie and M Londei

Dept of Diabetes and Metabolism, St Bartholomews Hospital London UK, Dept of Internal Medicine III, University of Leipzig, Germany and Kennedy Institute of Rheumatology, London, UK.

Stiff man Syndrome (SMS) is a very rare disease associated with autoantibodies to glutamic acid decarboxylase 65 (GAD65), a major antigen in insulin dependent diabetes (IDDM). We sought to identify differences in the cellular and humoral immune response to GAD in these two diseases. We therefore compared responses in 8 SMS patients, 15 IDDM patients and 10 controls. Peripheral blood T cells of SMS patients recognised different immunodominant epitopes of GAD 65 from both IDDM patients and healthy controls since: 1) region 81-171 was recognised by $\mathrm{T}$ cells of 4/8 SMS, 0/15 IDDM $(\mathrm{p}<0.01)$ and $1 / 10$ control subjects; 2 ) region 473-555 was recognised by $8 / 15 \mathrm{IDDM}, 0 / 8 \mathrm{SMS}(<0.01)$, and $0 / 10$ control $(p<0.03)$ subjects ; 3 ) region $161-243$ was recognised by $5 / 10$ controls but none of the SMS patients $(\mathrm{p}<0.05)$. Antibodies to GAD were detected in all 6 SMS patients and 8 IDDM patients. All the GAD isotypes were detected in one or more SMS patients but not in IDDM patients ; e.g. GAD IgM isotypes were detected in 1/8 IDDM but $4 / 6$ SMS patients $(p=0.015)$. The majority of patients in both groups had GAD IgG1 antiobodies, consistent with GAD antigenspecific Thl effector cell mechanisms operating in both diseases with a more heterogeneous response in SMS. These observations identify differences between cellular and humoral responses to GAD65 in SMS and IDDM suggesting that the immune response may be disease-related and not an epiphenomenon. 
CELLULAR IMMUNITY TO DIFFERENT COW'S MILK PROTEINS IN IDDM

M.G. Cavallo, L. Monetini, F. Tiberi, F. Barone, L, Valente, P. Pozzilli. Istitute II Clinica Medica, University of Rome "La Sapienza" and Università Campus BioMedico, Rome, Italy

Immune response to cow's milk in IDDM patients is directed against different protein components including BSA, beta casein, beta lactoglobulin and, as we have observed recently, also to alpha casein.

In this study we evaluated cellular immune reactivity to all the above proteins in individual IDDM patients to determine whether responses to different bovine milk proteins are associated in the same individuals. 32 consecutive patients with recent onset IDDM have been tested for T cell reactivity to BSA, beta casein, beta lactoglobulin and alpha casein using a 5 days proliferation assay. Proliferative responses were expressed as stimulation index (SI and positivity defined as 2SD above mean SI of healthy controls.) $34 \%$ of the patients (11/32) reacted to beta casein; $12 \%$ $(4 / 32)$ to beta lactoglobulin; $42 \%(14 / 32)$ to alpha casein; $9 \%(3 / 31)$ to BSA When we evaluated combination of proliferative responses, we found that $43 \%$ of patients did not show reactivity to any of these proteins, $18 \%$ reacted to one single protein, $31 \%$ reacted to 2 proteins and $6 \%$ to three proteins. Response to all 4 proteins was never observed. The associations found most frequently were alpha/beta casein (7 patients) and alpha casein/beta lactoglobulin (4 patients)

In conclusion, we show that the majority of recent onset IDDM patients show cell mediated immune reactivity to at least one protein component in cow's milk; casein is the major component involved in this reactivity to bovine milk; which often occur independently for the different protein components.
ACTIVATION OF CD8 ${ }^{+} T$ CELLS IN TYPE 1 DIABETIC PATIENTS WITH INCREASED BLOOD PRESSURE AND BORDERLINE ALBUMINURIA

B. Hehmke', D. Michaelis' and K.-D. Kohnert". ${ }^{3}$ Institute of Diabetes "Gerhardt Katsch" e.V., Karlsburg; "Institute of Pathophysiology, University of Greifswald and ${ }^{2}$ Center for Diabetes and Metabolic Diseases, Karisburg, Germany

Increased levels of activated $\mathrm{T}$ cells and $\mathrm{T}$ cell subset alterations are present at onset of type 1 diabetes. Whether these abnormalities persist and are related to the development of diabetic complications has not vet been examined. In a crosssectional study including 34 patients with type 1 diabetes (disease duration 5-20 years) we therefore analysed the expression of the HLA-DR activation antigen in $\mathrm{CD}^{+}$and $\mathrm{CD}^{+} \mathrm{T}$ cell subsets using 3-color flow cytometry and investigated the relationship between $T$ cell activation and albumin excretion rate (AER). The patients were classified into 3 groups: normo-group (NG; AER < $10 \mu \mathrm{g} / \mathrm{min}$; $\mathrm{n}=19$ ), borderline-group (BG; AER 10-20 $\mu \mathrm{g} / \mathrm{min} ; \mathrm{n}=10$ ) and micro-group (MG; AER $>20 \mu \mathrm{g} / \mathrm{min} ; \mathrm{n}=5$ ). Systolic blood pressure values (24h profile) in BG- and MG patients $(131.4 \pm 5.2$ and $134.6 \pm 4.8 \mathrm{mmHg}$ ) were significantly $(\mathrm{p}<0.01)$ higher than those in NG patients $(119.6 \pm 8.0 \mathrm{mmHg})$. Compared to normal control group subjects $(\mathrm{n}=10), 24$ (71\%) diabetic patients had elevated levels of activated $\left(C D 3^{+}{ }^{+}\right.$LA-DR $\left.{ }^{+}\right)$T lymphocytes. The percentage of total activated $T$ cells did not differ between the patient groups and was unrelated to blood pressure or AER. However, the composition of the activated T cell fraction was significantly different between the patient and control groups. Predominant activation of $\mathrm{CD8}^{+} \mathrm{T}$ lymphocytes $\left(\mathrm{CD}^{+} \mathrm{HLA}-\mathrm{DR}^{+}>60 \%\right.$ of total $\mathrm{CD}^{+} \mathrm{HLA}-$ $\mathrm{DR}^{+} \mathrm{T}$ cells) was significantly more frequent in $\mathrm{BG}$ patients $(6 / 10,60 \%)$ than in those of MG $(1 / 5,20 \%)$, NG $(1 / 19,5 \%)$ and control $(0 / 10,0 \%)$ groups. The proportion of $\mathrm{CD}^{+}$cells in the fraction of activated T lymphocytes correlated with the AER in NG- and BG patients $(\mathrm{r}=0.412, \mathrm{p}=0.036)$. It is concluded that i) $\mathrm{T}$ cell activation largely persists in long-standing type 1 diabetes and ii) persistent predominant activation of $\mathrm{CD} 8^{+} \mathrm{T}$ cells appears to be associated with the development of diabetic complications.

\section{5}

SEARCH FOR RETROVIRAL SEQUENCES IN HUMAN TYPE I DIABETES

M. Juan, M. Vives-Pi, P. Caro, M. Sospedra, M. Costa, R. Gomis*, R. PujolBorrell. Unitat d'Immunologia. Hospital Germans Trias i Pujol. Universital Autònoma de Barcelona. 08916 Badalona, Spain. "Dept. Endocrinologia Nutrició. Hospital Clínic i Provincial, 08036 Barcelona, Spain.

Autoimmune response to pancreatic $\beta$-cells in type I diabetes is due to complex interactions between genetic and environmental factors of which viral infection remains one of the most attractive candidate to be the trigger. Recent findings suggest that new retroviral sequences of the virus IDDMK $_{12} 22$ are involved on type I diabetes. The aim of this study was to assess the prevalence of this virus in the diabetic and pre-diabetic population in our own geographical area. To that end, sera from recent onset type I diabetic patients (ICA+, $n=12$ and ICA-, $n=8$ ), first degree relatives (ICA+, $n=27$ and ICA-, $n=13)$ and healthy controls $(n=15)$ were collected and total viral RNA was extracted. A semiquantitative and competitive RT-PCR technique for the detection of viral RNA was developed using an internal competitor and specific primers. This competitor (of a higher m.w. than the product), works as a control inside the amplification. It was constructed by PCR from a $\beta$-actin sequence, using hybrid oligonucleotides (IDDMK//3-actin). Specific IDDMK ${ }_{12} 22$ primers were those defined in the original description of the retrovirus for CDNA amplification. Possible contamination by genomic DNA was also assessed by direct PCR of all the samples; positive samples were retreated with DNAse. Quantification of the amplification products was carried out by comparing densitometric values of serial dilutions of the internal competitor with that of the problem sample. Preliminary results point to a correlation between the presence of viral RNA and disease while viral and ICA titters bore an inverse correlation: Only an inverse correlation seems to be present between the level of viremia and ICA titter. Work is in progress to expand the number of patients tested to obtain statistically significant results.

\section{6}

NO ASSOCIATION BETWEEN IDDMK 1,22 , A NOVEL. ISOLATED RETROVIRUS, AND INSULIN-DEPENDENT DIABETES MELLITUS

E Jaeckel ${ }^{*}+, S$ Heringlake* ${ }^{*}, D$ Bergerx, G Brabantx, G Hunsmannt and MP Manns*; *Department of Gastroenterology \& Hepatology, xDepartment of Cinical Endocrinology, Medizinische Hochschule Hannover; +Department of Virology, Deutsches Primatenzentrum Gottingen

Abstract. In the past endogenous retroviral sequences have been isolated from patients suffering from different kinds of autoimmune diseases. Recently, a full length retroviral genome, termed IDDMK 1,222 , was isolated from patients with new onset IDDM. This genome contains a MHC II dependent superantigen within its envelope gene. The viral sequence was found in 10 patients with new onset IDDM, but not in age-matched controls. The aim of the study was to search for the presence of this viral genome by nested RT.PCR in a cohort of 6 patients with new onset IDDM and 6 controls of the same age. We found all samples to be positive without any differences between patients and controls. The same results were obtained with supernatants of activated peripheral blood mononuclear cells. We performed isopycnic ultracentrifugation in sucrose density gradients on all samples and were unable to detect viral particles of the new virus in any of our samples. However, positive signals were obtained with all pellet fractions. RNase, DNase treatment and nested PCRs without reverse transcription showed that the positive signals were probably derived from intracellular RNA and DNA. In summary, no correlation between a positive nested PCR signal for IDDMK 1,22 and diabetes mellitus was found indicating that the new sequence represents just another member of the human endogenous retrovirus (HERV) family with lack of an exogenous counterpart. This work was supported by BMBF grant 01GB9407/2. 
367

IDENTIFICATION OF TYPE 1 DIABETIC PATIENTS WITH INSULITIS AT DIAGNOSIS BY 99mTC-IL2 SCINTIGRAPHY

A. Signore, M. Chianelli, N. Visalli, R. Barone, A. Annovazzi, G Di Leve, M.G. Parisella, P. Pozzilli for the IMDIAB study group*.

Nu.M.E.D. Group, Medicina Nucleare, II Clinica Medica, Università "La Sapienza" Roma, Italy.

Our aim was to investigate the presence and the severity of insulitis in IDDM patients at diagnosis and its relationship with disease remission. We studied 25 newly diagnosed IDDM patients by $99 \mathrm{mTC}-\mathrm{LL} 2$ scintigraphy a non invasive technique for the detection of organ infiltration by activated lymphocytes. Tomographic sections of the abdomen were obtained $1 \mathrm{hr}$ after the injection of $2 \mathrm{mCl} 99 \mathrm{mTC}-\mathrm{IL} 2$ and pancreatic radioactivity was calculated as pancreas to background (bone) ratio $(P / B)$. In all patients metabolic status ( $C$ peptide, HibA 1C, blood glucose, insulin requirement) was monitored every 3 months throughout the study. Results showed that $33 \%$ of patients had a significant pancreatic accumulation of $99 \mathrm{mTC}-\mathrm{LL} 2$ at time of diagnosis. C peptide values 9 months after diagnosis were significantly higher in patients with pancreatic accumulation of $99 \mathrm{mTC}$-IL2 compared to patients with a negative IL2 scintigraphy at diagnosis (1.3 vs 0.63 $\mathrm{ng} / \mathrm{ml} ; \mathrm{p}=0.004)$. These results suggest that a residual mass of insulinproducing beta cells and a pancreatic beta-cell inflammation are present in a subgroup of patients that can be identified by $99 \mathrm{mTC}-1 \mathrm{~L} 2$ scintigraphy; these patients have better metabolic control at 1 year. This tectnique, therefore, may be used for the selection of patients to be treated with adjuvant immunotherapies in an attempt to preserve the residual beta-cell mass and improve long-term metabolic control.

"P Pozzilli, N Visalli, MG Baroni, R Buzzetti, L Nisticò, E Fioriti, C Mesturino, A Signore, MG Cavallo, L Lucentini, MC Matteoli. A Crinò, C Teodonio, R Amoretti, F Paci, M Cervoni, L Pisano, C Suraci, MG Pennatina. B Boscherini, S Stoduto, MT Fonte, F Batelli, G Multari, MA Suppa, GC De Mattia, M Cassone Faldetta, O Laurenti, ML Manca Bitti, G Marietti, D Pitocco, F Ferrazzoli. O Bizzarri, AV Greco, G Ghirlanda

\section{9}

\section{ISLET CELL AUTOANTIBODIES IN CORD BLOOD FROM CHILDREN WHO LATER DEVELOPED IDDM} B. Lindberg ${ }^{1}$, S-A Ivarsson ${ }^{1}, M$. Landin-Olsson ${ }^{4}, G$. Sundkvist ${ }^{2}, L$. Svanberg ${ }^{3}$, and $\AA$. Lernmark ${ }^{5}$ From the Departments of 'Paediatrics, ${ }^{2}$ Endocrinology, ${ }^{3}$ Obstetrics and Gynaecology, Malmö University Hospital, ${ }^{4}$ Department of Medicine, Lund, Univ. of Lund, Sweden and the ${ }^{5}$ Department of Medicine, Univ. of Washington, Seattle, WA, USA.

Islet cell autoantibodies are early markers for IDDM. Our aim was to investigate whether islet cell autoantibodies are present at birth in children who later developed IDDM. Cord blood sera from 85 children who developed IDDM between 10 months and 14.9 years of age were analyzed for GAD65Ab, ICA512Ab and IAA (IA) by quantitative radioligand binding assays and ICA by indirect immunofluorescence. Cord blood sera from 320 randomiy selected matched children were controls. The children who developed IDDM had an increased frequency of cord blood islet cell autoantibodies compared to controls: GAD65Ab was detected in $8 \%(7 / 85)$ patients and $2 \%$ $(5 / 320)$ controls $(p=0.005)$; ICA512Ab in $7 \%(5 / 77)$ patients and $1.4 \%(4 / 288)$ controls $(p=0.02)$; insulin antibodies (IA) in $5 \%(4 / 83)$ patients and $0.3 \%(1 / 320)$ controls $(p=0.007)$; and ICA in $9 \%(8 / 85)$ patients compared to $0.6 \%(2 / 320)$ controls $(\mathrm{D}=0.0001)$. Taken together, $21 \%(18 / 85)$ patients had one or more islet cell autoantibody compared to $4 \%(12 / 320)$ controls $(p=0.0001)$. While none of the control children had more than one antibody, $7 \%(6 / 85)$ children who later developed IDDM were double positive $(p=0.0001)$. Maternal autoantibodies did not explain these results. Only $5 \%(4 / 85)$ of the children who later developed diabetes had a mother with the disease. The increased frequency of cord blood islet cell autoantibodies indicates strongly that the IDDM process is initiated already in utero.

\section{8}

LOW INSULIN SECRETION IN DIABETIC CHILDREN WHOSE LYMPHOCYTES EXPRESS INOS MRNA

M. G. E. Karlsson", M Flodströmb and J. Ludvigsson"

${ }^{2}$ Div of Pediatrics, Faculty of Health Sciences, Linköping University, Linköping, Sweden ${ }^{b}$ Dept Medical Cell Biology, Uppsala University. Uppsaia, Sweden

Background: IDDM is characterized by inflammation in the islets of Langerhans, which destroy the insulin-secreting $\beta$-cells. Inducible Nitric Oxide Synthase (iNOS), produced by the $\beta$-cells or by macrophages, has been associated with cytokinemediated $\beta$-cell dysfunction and destruction.

Aim: To investigate if iNOS can be expressed in lymphocytes (PBMC) and whether this expression is related to the immune process in IDDM.

Patients and Methods: Spontaneous expression of iNOS, IFN- $\gamma$ and IL-4 mRNA measured by RT-PCR was compared with the expression after stimulation with either $\mathrm{GAD}_{65}$ (a.s. 247-279) or ABBOS (pre-BSA a.s. 152-169) in lymphocytes from 15 children with IDDM and from 10 healthy controls (DR3,4). Nitrite was analysed by spectrophotometry, C-peptide by RIA and Insulin Antibodies (IA) with a radioligand assay.

Results: Spontaneous iNOS mRNA expression could be seen in two patients, at onset of IDDM, and in five healthy donors. Two other patients responded with iNOS mRNA after stimulation with either GAD or ABBOS, and two further patients responded to ABBOS. Patients with expression of iNOS mRNA, either spontaneous or after stimulation with GAD or ABBOS, had very low C-peptide at onset of IDDM (p<0.01). Patients with spontaneous expression for iNOS mRNA and/or GADstimulated response had also higher amount of insulin antibodies at onset of IDDM $(p=0.01)$, but not the two patients who only responded to ABBOS. Patients, as well as healthy controls, with spontaneous expression of iNOS mRNA produced more nitrite $(\mathrm{p}<0.05)$. Increased nitrite after stimulation with $\mathrm{ABBOS}$ was related to IL-4 mRNA in both patients and controls $(p<0.05)$ whereas no association was observed between iNOS and IFN- $\gamma$ or IL-4 mRNA either spontaneously or after stimulation with GAD or ABBOS

Conclusion: These results demonstrate that lymphocytes can produce iNOS in both diabetic children and healthy controls. Low insulin secretion related to iNOS expression in lymphocytes may indicate that iNOS is involved in $\beta$-cell destruction.

\section{0}

ELEVATED SERUM IMMUNOREACTIVE LEVELS AND BIOACTIVITY OF TUMOR NECROSIS FACTOR- $\alpha$ IN NIDDM AND OBESITY G.Winkler', F.Salamon ${ }^{1}$, D.Salamon' ${ }^{2}$, G.Speer ${ }^{2}$, G.Harmos ${ }^{1}$, O.Szekeres ${ }^{1}$ M.Kovács ${ }^{2}$ and K.Cseh"; St.John's ${ }^{1}$, Károlyi Hospital ${ }^{3}$ and Semmelweis University School of Medicine', Budapest, Hungary

The aim of our recent study is the comparison of the immunoreactive and biologically active levels of tumor necrosis factor (TNF)- $\alpha$ in patients with insulin resistance. Serum TNF- $\alpha$ and basal C-peptide (Cp) concentrations have been measured in a follow up study of twelve month ( 4 measurements per patients) in 59 patients with NIDDM (Group 1, male/female 32/27, age: X \pm SE $62 \pm 3$ years, BMI: $\left.32.2 \pm 3.1 \mathrm{~kg} / \mathrm{m}^{2}\right)$, 28 patients with android type obesity and normal glucose tolerance (Group 2, 10/18, $49 \pm 4$ years, $39.5 \pm 3.0 \mathrm{~kg} / \mathrm{m}^{2}$ ) and 35 healthy lean controls (Group 3, 19/16, $59 \pm 5$ years, $25.4 \pm 2.4 \mathrm{~kg} / \mathrm{m}^{2}$ ). Immunoreactive TNF- $\alpha$ levels have been determined by ELISA (Sigma). L929 bioassay has been used for the determination of TNF- $\alpha$ bioactivity. Recombinant human TNF- $\alpha$ (Sigma) bas been applied as a standard and monoclonal mouse antiTNF- $\alpha$ immunoglobulins (Bochringer Mannheim) were used for neutralization of the TNF- $\alpha$ bioactivity in the samples. $\mathrm{Cp}$ concentrations have been detected by RIA (Serono). Significantly higher $(p<0.01)$ immunoreactive TNF $-\alpha(X \pm S E$ $10.51 \pm 0.84 \mathrm{pg} / \mathrm{ml})$ and $\mathrm{Cp}$ levels $(4.71 \pm 0.69 \mathrm{ng} / \mathrm{ml})$ have been found in Group 1 , and Group 2 (TNF- $\alpha: 8.86 \pm 0.42, \mathrm{Cp:} 2.81 \pm 0.29$ ) as compared to Group 3 (TNF- $\alpha: 6.32 \pm 0.26, \mathrm{Cp}: 1.46 \pm 0.25$ ). TNF- $\alpha$, bioactivity has also been found to be significantly higher $(p<0.01)$ in Group $1(3.60 \pm 0.40 \mathrm{U} / \mathrm{ml})$ and $2(3.12 \pm$ $0.48)$ as compared to Group $3(0.88 \pm 0.32)$. Both, immunoreactive concentrations and TNF- $\alpha$ bioactivity correlated with the $\mathrm{Cp}$ levels $(\mathrm{r}=0.56)$ and BMI $(0.60)$ in Group 1 , and $2(0.54,0.58)$. The biological activity comparing to the immunoreactive TNF-a levels in Group 1 and 2 seems to be 2.5 times higher than in controls. The results raise the possibility of the presence of a TNF- $a$ variant in these patients. 
CIRCULATING CYTOTOXIC TCl CELLS IN NEWLY MANFESTED TYPE 1 DIABETES.

A. Gessl, G. Beilhack, E. Schober, and W. Waldhäusl. Department of Medicine III, Division of Endocrinology and Metabolism, University of Vienna, Austria.

Recently, we could trace the long known increased HLA-DR expression of circulating $\mathrm{T}$-cells in newly manifested type 1 diabetics to predominant activation of $\mathrm{CD} 45 \mathrm{RA}^{+} \mathrm{CD} 8^{+}$cells. In the present report the phenotype and function of these activated "naive" $\mathrm{CD} 8^{+}$cells was analyzed in detail by four-color flow cytometry vs. healthy subjects. Thereby it was shown that all HLA-DR ${ }^{+} \mathrm{CD}^{2} 5 \mathrm{RA}^{+} \mathrm{CD} 8^{+}$cells strongly expressed adhesion molecules CD11a (integrin $\alpha_{L}$ chain), CD18 (integrin $\beta_{2}$ chain), CD29 ( $\beta_{1}$ chain), CD49d ( $\alpha_{4}$ chain of VLA) and CD54 (ICAM), whereas CD62L (L selectin), the lymph node homing receptor, and the $\beta$, integrin chain, which is specific for gut-specific homing receptors, as well as CD11b (integrin $\alpha_{M}$ chain), that marks non-cytotoxic lymphocytes were absent or only weakly expressed. Although the memory cell marker CD45R0 was undetectable in activated $\mathrm{CD} 5 \mathrm{RA}^{+}$cells the observed adhesion molecules clearly marks them as memory/effector cells. Expression of activation markers CD69, CD38, CD25 (IL-2R $\alpha$ chain) was low, but all cells expressed large amounts of IL2R $\beta$-chains (CD122). A similar population was present in healthy individuals, but their proportion was lower compared to diabetic subjects $(-45 \%)$. Furthermore, cytoplasmic single cell cytokine staining revealed high amounts vs. healthy subjects of IFN- $\gamma$ but not IL-4, which clearly marks these cells as $T_{c}$ I cells. In summary, our study shows activated cytotoxic $T_{c} 1$ cells circulating in the peripheral blood of newly manifested type 1 diabetic patients.
PREVALENCE OF DIABETIC AND COELIAC AUTOANTIBODIES IN FIRST DEGREE RELATIVES OF NON-COELIAC IDDM SUBJECTS

E.Buratti', M.Pocecco', T.Not', A.Città', R. Zoratti' ${ }^{2}$ A. Petrucco ${ }^{2}$, A. Leopaldi' ', F. Cian² D.lafusco ${ }^{3}$, A. Ventura ${ }^{1}$, L. Cattin ${ }^{2}$.

Department of Pediatrics' IRCSS "Burlo Garofolo" and department of Clinical Medicine ${ }^{2}$ University of Trieste; department of Pediatrics II University of Naples $^{3}$

Autoimmune diseases are known to be clustered not only in the same individual, but also in different members of the same family. Recent data support the hypothesis that autoimmune mechanisms might be operative in the pathogenesis of $\mathrm{CD}$ and this prompted as to screen for CD the FDR of IDDM subjects. Methods. The study population comprised 252 IDDM patients, 562 FDR and 2000 bealthy blood donors. The subjects' sera were screened for IgA-endomysium antibody (AEA) by IFA using the human umbilical cord vein (HUCV) tissue as antigenic substrate. Duodenal biopsy was performed in all subjects tested positive for AEA. The FDR sera were also screened for the related IDDM autoantibodies: anti-glutamic acid decarboxylase and anti-IA2. Results. The results are summerized in the table. $19(7.5 \%)$ of IDDM patients have CD. $4(9.5 \%)$ of the FDR of coeliac diabetics have CD. 11 (2\%) of the FDR of AEA-negative diabetics have CD. 6 $(0.3 \%)$ of the 2000 blood donors have CD. $2(4.7 \%)$ of the FDR of cocliac diabetics had positive GAD antibodies. $19(3.6 \%)$ of the FDR of AEA-negative diabetics had positive GAD antibodies and 10 (1.9\%) of the FDR of the same group had IA2 positive antibodies.

\begin{tabular}{|c|c|c|c|c|c|c|c|}
\hline & $\overline{N^{0}}$ & $\begin{array}{c}\text { median } \\
\text { age }\end{array}$ & $\overline{\mathrm{AEA}+}$ & $\begin{array}{c}\text { fiat jejunial } \\
\text { mucosa }\end{array}$ & GAD & IA2 & $\overline{A \mid A}$ \\
\hline coeliac diabetics & 19 & 26 & 19 & 19 & n.d. & n.d. & n.d. \\
\hline fathers/mothers & 22 & 49 & 2 & 2 & 1 & - & 1 \\
\hline brothers/sisters & 13 & 19 & 1 & 1 & - & - & - \\
\hline sons/daughters & 7 & 10 & 1 & 1 & 1 & - & - \\
\hline diabetics. & 233 & 31 & - & $=$ & n.d. & n.d. & n.d. \\
\hline fathers/mothers & 200 & 53 & 4 & 4 & $3 \sqrt{5}$ & 20 & $3 / 2$ \\
\hline brothers/sisters & 159 & 28 & 3 & 3 & $6 / 1$ & $1 / 2$ & $0 / 2$ \\
\hline sons/daughters & 92 & 12 & 4 & 4 & $2 / 2$ & $3 / 2$ & $1 / 2$ \\
\hline blood donors & 2000 & 35 & $\overline{6}$ & 6 & n.d. & n.d. & n.d. \\
\hline
\end{tabular}

Conclusions. Our data demonstrate an increased prevalence of CD in FDR of IDDM subjects with negative AEA test. The prevalence of AEA in FDR of diabetics with negative AEA test is slightly lower than the antibodies related to IDDM autoantibodies.

\section{3}

CELLULAR AND HUMORAL AUTOIMMUNITY AGAINST COW'S MILK PROTEINS IN TYPE 1 DIABETES

E. Sarugeri, N. Dozio, F. Meschi, M.R. Pastore, E. Bosi and E. Bonifacio. Istituto Scientifico San Raffaele, Milan, Italy.

Early exposure to cow's milk has been suggested to be a risk factor for type 1 diabetes and give rise to increased immunity to cow's milk proteins in patients. The aim of our study was to evaluate humoral and cellular autoimmunity against such proteins in patients with recent onset type 1 diabetes and control subjects. Subjects were tested for: a) $T$ cell reactivity against bovine $\alpha$ casein, $\beta$ casein, $\beta$ lactoglobulin and BSA, by a proliferation test performed on fresh peripheral blood mononuclear cells in the presence of each antigen $(20$ and $200 \mu \mathrm{g} / \mathrm{ml})$; b) antibodies against $\alpha$ casein, $\beta$ casein and $\beta$ lactoglobulin, using ELISA and, for $\beta$ casein, also competition RBA. T cell responses against $\alpha$ casein, $\beta$ casein, $\beta$ lactoglobulin and BSA were observed in both patients (range SI: $0.2-22.8, n=23$ ) and control subjects (range SI: $0.1-18.2, n=22$ ), with no differences between groups. Higher levels of $\beta$ casein antibodies were detected in patients by RBA $(p=0.005)$, but only 11 out of 59 patients had levels higher than the 99 th centile of 52 control subjects, and no differences were observed between patients and control subjects using ELISA. Higher levels of IgG $\alpha$ casein antibodies by ELISA were seen in patients $(p=0.015)$. No other differences were detected. No relationship was found between cellular and humoral autoimmunity against individual antigens and no differences were observed with respect to HLA DR status. In conclusion, while increased humoral immunity against $\alpha-$ and $\beta$ casein may be present in a minority of patients with Type 1 diabetes, the data do not show a major alteration in the immune response to cow's milk proteins and therefore do not support a major influence by cow's milk in the pathogenesis of Type 1 diabetes.

\section{4}

INTERLEUKIN 2/ IL-2 RECEPTOR SYSTEM AS A RISK MARKER OF TYPE 1 DIABETES MELLITUS DEVELOPMENT.

A.Kretowski, J.Mysliwiec, M.Szelachowska, C.Brzozowski and I.Kinalska, Department of Endocrinology and Department Medical School Białystok, Poland

Interleukin-2 a Thl lymphocyte-derived cytokine is actually considered to play an important role in the etiopathogenesis of insulin-dependent diabetes mellitus. In the previous studies an increased, decreased and unchanged interleukin 2 levels in patients with recent onset of IDDM were found. These differences could be a result of different metabolic status or/and a different stage of autoimmune process. The aim of our study was to estimate in vitro secretion of $\mathrm{IL}-2$ and $\mathrm{CD} 25$ antigen expression by the peripheral blood lymphocytes $\mathrm{T}$ in subjects at the pre-clinical stage of IDDM, but still without metabolic disturbances. In 27 first degree relatives of IDDM patients with antibodies against different pancreatic islet cells antigens (ICA, GADA, IAA, IA-2) CD25 antigen expression on peripheral blood lymphocytes $T$ was measured by flow cytometry and IL-2 concentration in supernatants of 48- and 72-hours cultures of peripheral whole blood with $10 \mathrm{ug} / \mathrm{ml}$ PHA was estimated by ELISA. The control group was comprised of 34 age and sexmatched healthy volunteers. In the studied high risk IDDM subjects the decreased CD25 expression in peripheral CD4+ lymphocytes $\mathrm{T}$ and a negative correlation between the percentage of CD25+ cells and ICA titres was observed. No differences in IL-2 levels in supernatants of $48 \mathrm{~h}$ and $72 \mathrm{~h}$ blood cultures was found in subjects with single antibody (ICA+) in comparison to healthy controls. A significant increase of IL-2 secretion in $72 \mathrm{~h}$ of PHA stimulation was shown in first degree relatives of IDDM patients with a combination of 3 or more anti-pancreatic.B-cells antibodies. The present study suggests the involvement of interleukin-2 in the pathogenesis of IDDM. The estimation of CD25 antigen expression in the peripheral blood lymphocytes could be an additional immune marker of identification of subjects in prediabetes. 
TRANSFER OF TYPE 1 DIABETES BY BONE MARROW TRANSPLANTATION

D. Iafusco, F. Locatelli*, M.T. Di Tullio, R. Lorini**, F. Casale, P. Indolfi, O. Stoppoloni, G. Ursomanno, C. De Sangro, C. Betterle*** and F. Prisco Department of Paediatrics - Second University of Naples - Italy -Department of Paediatrics - University of Pavia - Italy **Paediatric Clinic - University of Genova - Italy

*** Institute of "Semeiotica Medica" - University of Padova - Italy

Francesco D.S. was diagnosed with Acute Hybrid Lymphoblastic Leukaemia with chromosomic transiocation $t(4 ; 11)$ at the age of 6 months. After chemiotherapy, during the second remission, he was transplanted with bone marrow from his HLAidentical 9 yrs old sister affected by insulin-dependent diabetes since the age of 4 6/12. After tranplantation the patient was treated with Cyclosporin A ( $3 \mathrm{mg} / \mathrm{Kg} /$ die i.v. for 21 days and $7 \mathrm{mg} / \mathrm{Kg} /$ die per os for the following 2 months). He developed intestinal and skin graft versus host disease and cytomegalovirus infection. Chromosomal analyses verified the full chimerism of the recipient. Nineteen months after the transplant he presented type 1 diabetes. When diabetes was diagnosed, the sister presented high-titre islet cell antibodies (ICA, >80 JDFU), which were negative at the moment of bone marrow transplantation to the brother In the transpianted child ICA were found repeatedly negative before transplantation and every 3 months. Fifteen days before the onset of diabetes they were found positive (40 UJDF) together with $\mathrm{GAD}(6,4 \mathrm{U} / \mathrm{ml})$. Diabetes manifested itself with post-prandial hyperglycemia requiring insulin administration before the meals This report suggests transfer of insulin dependent diabetes by bone marrow lymphoid cells. Of course, other mechanisms could theoretically be invoked, such as unknown environmental factors together with the same HLA predisposition in the two siblings.

On the other hand, transmission after bone marrow transplantation of autoimmune diseases ( diabetes, myasthenia gravis, thyroiditis ) has already been described in humans as well as in experimental animal models (non-obese-diabetic mice and BB rats).

\section{7}

DISTURBED IMMUNOREGULATION DURING HYPOGLYCEMIA IN DIABETES MELLITUS TYPE I

I.Koop, P.Preuß, C.Loeffler, S.Will, S.Schreiber, A. Geißler and *K. Ehlenz. Depts. Internal Medicine, Univ. Hospitals *Marburg and Charité, Berlin, FRG

Hypoglycemia is a major threat to the human organism. Defence mechanisms include activation of the sympathetic nervous system and secretion of contrainsulinemic hormones. Our aim was to study the cellular and humoral immune response during hypoglycemia in patients with type 1-diabetes Methods: Six subjects with type 1-diabetes (age 26-46y., duration of diabetes: $7-17 y$.) and 11 healthy controls (22-25y.) were studied by a stepwise hyperinsulinemic hypoglycemic clamp. At the beginning and the end of the clamp the leucocyte count, differential leucocyte count and plasma concentrations of TNF- $\alpha$, IL-1B, IL-6, IL-10, cortisol and epinephrine were determined. Results: Patients with type 1-diabetes revealed no changes in the cellular and humoral immune response during hypoglycemia. As expected, the increase of contrainsulinemic hormones was diminished. In contrast, healthy volunteers showed a significant immune response during hypoglycemia with a rise of peripheral leucocytes, a relative shift to neutrophils and a significant rise of the contrainflammatory cytokine IL-10 (see table). Plasma levels of

\begin{tabular}{|c|c|c|c|c|}
\hline & \multicolumn{2}{|c|}{ type 1-Diabetes } & \multicolumn{2}{|c|}{ lealthy controls } \\
\hline serum glucose & $S m M$ & $2.3 m M$ & $5 m M$ & $2.3 m M$ \\
\hline Leucocytes, GPT/1 & $6.1+0.4$ & $6.4+0.9 \mathrm{n} . \mathrm{s}$ & $6.6+0.3$ & $13.7+0.9 \$$ \\
\hline IL- $10, \mathrm{pg} / \mathrm{ml}$ & $8.1 \pm 0.2$ & $8.9+0.7 \mathrm{n}, \mathrm{s}$. & $<7.8$ (n.d.) & $15.5+2.7^{* *}$ \\
\hline Epinephrine, $\mathrm{nM}$ & $0.25+0.05$ & $1.39 \pm 0.47^{*}$ & $0.23 \pm 0.01$ & $6.70 \pm 0.57 \S$ \\
\hline Cortisol. nM & $318+40$ & $517 \pm 103$ n.s. & $343+54$ & $946 \pm 64 *$ \\
\hline
\end{tabular}
(nean \pm SEM; *:p<0.05; **: p<0.01; $\$:$ p<0.001; n.d.: not detectable)

TNF- $\alpha$, IL- $1 \beta$ and IL- 6 were not altered. Discussion: In contrast to healthy volunteers, the cellular and humoral immune response is impaired in patients with type 1-diabetes. Whether the missing increase of leucocytes and the contrainflammatory cytokine IL-10 is the consequence of reduced stress hormones or reflects a disease-associated phenomenon has to be elucidated.

\section{6}

AUTOIMMUNE CHARACTERISTICS OF CHILDHOOD- AND ADULT-ONSET IDDM

E. Sabbah, K. Savola, T. Ebeling, P. Kulmala, P. Vähäsalo, P. Salmela and M. Knip. Departments of Pediatrics and Internal Medicine, University of Oulu, Oulu, Finland

About one third of patients with IDDM manifest their disease after the age of 20 years. To explore whether there are any differences in the autoimmune features of IDDM presenting in childhood and that diagnosed later, we analyzed all individuals presenting with clinical signs of IDDM over a time period of 7.5 years in a university hospital in Northem Finland with a primary catchment population of about 300,000 for islet cell antibodies (ICA), insulin autoantibodies (IAA) and antibodies to glutamic acid decarboxylase (GADA) and the IA-2 molecule (IA-2A). Two hundred seventeen children and adolescents $(84.4 \%)$ tested positive for ICA at diagnosis, while only 44 adult patients $(38.6 \% ; p<0.001)$ had detectable ICA. The young patients had also higher ICA levels than the adult ones (median $34 v s .0$ JDF units; $p<0.001$ ). Both the frequency and levels of IAA were reduced in the adult patients $(15.8 \% v s$. $54.1 \%$, and median $37.5 v s .74 \mathrm{nU} / \mathrm{ml} ; \mathbf{p}<0.001$ for both). The young patients tested positive for GADA more often $(69.1 \%$ vs. $44.7 \%)$ and had higher antibody levels (median 12 vs. $4 \mathrm{RU} ; \mathrm{p}=0.006$ ) than the adults. The same held true for IA-2A ( 80.2 vs. $41.2 \%$; $p<0.001$, and median 49.2 vs. $0.2 \mathrm{RU} ; \mathrm{p}<0.001$ ). The proportion of patients with multiple autoantibodies $\geq 3$ antibodies) was decreased among the adult patients $(29.8 v$ s. $70.4 \%$; $<<0.001)$, while $40.4 \%$ of them tested negative for all four autoantibodies, this proportion being only $4.3 \%$ among the children and adolescents $(p<0.001)$. Females had higher GADA levels than males among the adult patients (21.2 vs. $2.4 \mathrm{RU} ; \mathrm{p}=0.02$ ). These observations show that childhood-onset IDDM is associated with more frequent and stronger humoral autoimmune responses against beta-cell antigens than adult-onset disease. This suggests that an aggressive autoimmune process is characteristic of a rapid progression to clinical IDDM, as seen in children with newly diagnosed disease.

\section{8}

ISLET CELL ANTIBODY LEVELS AT ONSET OF TYPE I DIABETES: PREDICTIVE VALUE FOR THE DURATION OF CLINICAL REMISSION

A.Jotić, M.Zamaklar, N.M.Lalić. "M.Bukelica, K.Lalić, N. Rajković, Lj. Lukić and P.B.Djordjević. Institute for Endocrinology and "Allergology Clinic, Belgrade. Yugoslavia

The predictive value of islet cell antibody (ICA) levels at onset of Type 1 diabetes regarding the clinical course and the residual beta cell function has not been elucidated. Therefore, the aim of this study was to compare (a) duration of clinical remission (CR) (optimal metabolic control without insulin lasting $>30$ days) and (b) insulin secretion capacity between two groups of patients with recent-onset Type 1 diabetes showing lower ICA titer (40 Juvenile Diabetes Foundation (JDF) units) (group $\mathrm{A}, \mathrm{N}=16$ ) or higher ICA titer ( $80 \mathrm{JDF}$ U) (group B, N=15) at onset of the disease. The ICAs were detected by indirect immunofluorescence on human pancreas cryostate sections. The insulin secretion capacity was evaluated by determining $\mathrm{C}$ peptide levels (RIA) before and after $1 \mathrm{mg}$ iv glucagon stimulation $(0 / 6 \mathrm{~min})$ We found that duration of CR was significantly longer in group A (266 \pm 37 days) than in group $\mathbf{B}(77 \pm 17$ days, $p<0.01)$. In addition, we found that the insulin secretion capacity at onset of Type 1 diabetes was significantly lower in group A $(0.23 \pm 0.05 / 0.45 \pm 0.09 \mathrm{mmol} / \mathrm{l})$ than in group B $(0.41 \pm$ $0.06 / 0.77 \pm 0.08 \mathrm{nmol} / \mathrm{L}, \mathrm{p}<0.05$ ). However, in the state of $\mathrm{CR}$, we found that the secretion capacity was similar in both groups (A: $0.45 \pm 0.06 / 0.79 \pm 0.09$; B: $0.48 \pm 0.09 / 0.81 \pm 0.09 \mathrm{nmol} / 1, \mathrm{p}=\mathrm{NS})$, while the increase in the capacity $(\Delta$ 1) was higher in group A ( $\Delta \mathrm{I} ; A: 0.22 \pm 0.04 / 0.34 \pm 0.03$ vs $B: 0.07 \pm 0.02 /$ $0.04 \pm 0.01 \mathrm{nmol} / 1 . \mathrm{p}<0.05$ ). Our results have demonstrated that recent-onset Type 1 diabetes patients with lower ICA titer, accompanied with lower insulin secretion capacity at onset of the disease, exhibit longer $C R$ with higher increase in residual insulin secretion capacity during CR compared to the patients with higher ICA titer. The results imply that the ICA levels at the onset of disease might have an important predictive clinical value regarding the duration of CR and the relevant increase in insulin secretion capacity in the recent-onset Type 1 diabetes patients. 
Possible role of autoimmunity in the pathogenesis of diabetes mellitus in thalassemia major.

Monge L., Pinach S., ${ }^{*}$ Caramellino L., ${ }^{*}$ Degani G., ${ }^{\circ}$ Bertero M.T., ${ }^{\circ}$ De Leo A.M., §Dall'Omo A., Carta Q.

UOA Diabetologia, *UOA Ematologia, §UOA Immunologia dei Trapianti - AO S.Giovanni Battista della città di Torino; ${ }^{\circ}$ Div. Universitaria Immunologia e Allergologia - Osp.Mauriziano Umberto $1^{\circ}$, Torino - Italy. Introduction: diabetes mellitus (DM) is one of the principal endocrine complications of beta-thalassemia major. Despite chelation therapy, frequent transfusions lead to the chronic accumulation of iron in tissues that induces beta-cell dysfunction in the pancreas and insulin resistance in the liver. The appearance of impaired glucose tolerance is often gradua but, in some cases, the onset of DM is acute with ketosis and severe insulin deficiency. Aims: to evaluate the role of autoimmunity in the pathogenesis of DM associated with thalassemia. Materials and methods: a cross-sectional study of a cohort of 53 individuals ( 3 IF and $22 \mathrm{M}$, age $32.3 \pm 7.4 \mathrm{yrs})$ with beta-thalassemia. Twelve $(22.6 \%)$ had diabetes and were tested for the presence of islet-cell antibodies (ICA) by indirect immunofluorescence (IIF) of human pancreas (positive if $>5 u$ JDF), for serum anti-nuclear antibodies (ANA) by IIF and for serum Cpeptide by RIA. Results: nine individuals (16.98\%) were ICA-positive in the total beta-thalassemic population. Among the twelve diabetic patients five $(41.6 \%)$ were ICA-positive. Of these, three had C-peptide values $<0.4$ $\mathrm{ng} / \mathrm{ml}$. Among non-diabetics, four individuals (9.5\%) were ICA-positive and four $(9.5 \%)$ presented diffuse fluorescent staining of extra-insular tissue. One of the latter was also ANA-positive. Discussion: in our study, some of the cases of DM associated with thalassemia have the clinical and immunological characteristics of type I DM. Furthermore, immunological markers typical of type I DM are more frequent than in the general population. Based on this data, we hypothesize that beta-thalassemia major may represent a disease that is genetically associated with type 1 DM or beta-cell damage caused by iron deposition may not only modify insulin secretion but uncover islet antigens that can trigger an autoimmune response.
CHARACTERISATION OF AUTOREACTIVE EPITOPES OF IA-2 AND IA $2 \beta$ IN TYPE 1 DIABETES

H. Steinbrenner, M. Schott, W.A. Scherbaum and J. Seissler; Diabetes Research Institute Duesseldorf, Germany

Two tyrosine phosphatase-like proteins, IA-2/ICA 512 and IA-2 $\beta$ /phogrin, have been described as major autoantigens in type 1 diabetes. Autoantibodies are directed against the cytoplasmic domains of the proteins (aa 603-979 in human IA-2 and aa $345-718$ in mouse (A-2 $\beta$ ), which possess $74 \%$ sequence similarity. To map humora epitopes, different protein fragments of human IA-2 and mouse IA-2 $\beta$ were generated by PCR amplification of cDNA's followed by in vitro transcription/translation. Using an immunoprecipitation assay we analysed 50 IA-2-antibody (IA-2-A) positive sera from patients with newly-diagnosed type 1 diabetes. The following autoantibody reactivities were found

\begin{tabular}{|l|c|c|c|c|c|}
\hline Human IA-2 [aa] & $605-676$ & $605-772$ & $771-979$ & $699-874$ & $771-930$ \\
\hline Positive sera & $58 \%$ & $95 \%$ & $76 \%$ & $34 \%$ & $65 \%$ \\
\hline $\begin{array}{l}\text { Mouse IA-2 } \beta \text { [aa] } \\
\text { (IA-2 homology) }\end{array}$ & $\begin{array}{c}345-718 \\
(=\text { IA2 } 603-979)\end{array}$ & $\begin{array}{c}345-624 \\
(=\text { IA2 } 603-885)\end{array}$ & $\begin{array}{c}623-718 \\
(=\text { IA2 884-979) }\end{array}$ \\
\hline Positive sera & $68 \%$ & $2 \%$ & $58 \%$ \\
\hline
\end{tabular}

Out of 50 IA-2-A positive sera $34(68 \%)$ had IA-2 $\beta-A$. Blocking studies with IA-2 indicated that all but one (33 of 34) IA-2 $\beta$-A positive sera recognize epitopes that are shared with IA-2. To further characterize these epitopes we investigated the humoral reactivity to peptides of 12 aa residues ( 3 aa overlapping), spanning the regions aa 605-648 and aa 900-979 of human IA-2. Peptide reactivity, however could not be detected in any serum. Our data indicate that IA-2-A may be directed to conformational epitopes located within the $\mathrm{N}$-terminal (aa 605-676) and the middle (aa 771-930) domain of IA-2ic.
DIABETES AUTOIMMUNE MARKERS IN INFANTS BORN TO MOTHERS WITH IDDM

T.G.Gogishvili, R.B.Kurashvili, N.G.Asatiani, M.G.Dundua, L.R.Tsutskiridze, E.L.Shelestova and *A.J.Elphick. Georgian Diabetes Center, Tbilisi, Georgia; *Novo Nordisk A/S, Copenhagen, Denmark.

The researeh was the first attempt to detect autoantibodies to pancreatic $\beta$-cells ever made in Georgia. The main aim of the study was to investigate whether IDDM associated antibodies (ICA, GADA) are produced in prenatal period, and to compare their levels depending on the birth weight (BW) of the newborns. In total 38 women were supervised in the Diabetes in Pregnancy Program, resulting from the WHO/DF twinning between the Israel Diabetes Association and Georgian Diabetes Federation. All the women were introduced to preconception carc, extending from 3 months. After this period 14 of them (mean age $26 \pm 5$ years; disease duration $11 \pm 4$ years) became pregnant and got specialized care during pregnancy and labour. Umbilical vien blood samples were obtained from the newborns to deteet the ICA and GADA levels (ELISA kits, BIOMERICA). Macrosomia was register in 2 cases (14.3\%; BW 4.106-4.200 g), hypotrophia in another 2 infants $(14.3 \% ; B W$ 1.400-2.000 g), and remaining 10 were normalweight $(71.4 \%, B W 2.900-3.000 \mathrm{~g})$. In macrosomic infants one was GADA positive (Optical Density /OD/ - 1.120 \pm 0.32 ), the other had intermediate GADA levels (OD - 1.038 ) and ICA positive sample (OD (0.075). In low-BW ibfants one was GADA positive (OD - 1.203), while no ICA was determined. In normalwelght gronp one infant was ICA positive (OD - 6.81) with intermediate GADA levels (OD - 1.027), two had high GADA levels (OD $1.163 \pm 0.21$ ), and three were ICA positive (OD - 0.53). Thus, onr observations indicate that signs of $\beta$-cell autoimmanity may be introduced already during fetal life and $\mathrm{BW}$ does not play the main role in the development of autoimmunity in infants born to diabetic mothers. The results indicate that it is worth-while to continue present study in a larger population, and the results may have far reaching effects on the diabetes care in Georgia.
IMMUNOLOGICAL AND GENETIC HETEROGENEITY OF DIABETES IN A COHORT OF LEAN NEWLY-DIAGNOSED PATIENTS AGED 30-54 YRS.

A. De Salvia. G. Bruno, R. Arcari, M. Borra, N. Grosso. A. Lezo Q.Carta. M. Trovati, M. Veglio. G. Pagano and Piedmont Study Group for Diabetes Epidemiology, Dept. of Internal Medicine. University of Turin. Italy

In lean diabetic patients the presentation of the discase does not allow to distinguish between type 1 and type 2 diabetes. We have recruited a population-based cohort of 130 lean (body mass index $<25 \mathrm{~kg} / \mathrm{m}^{2}$ ) newly diagnosed patients aged $30-54$ yrs to estimate the frequencies of adult-onset type 1. latent autoimmune and type 2 diabetes. ICA, antiGAD, fasting and glucagon-stimulated Cpeptide, and HLA DQA1-DQB1 susceptibility genotypes were measured. ICA and antiGAD positivities were found, respectively. in $45(34.6 \%)$ and $29(22.3 \%)$ patients, with $59(45.4 \%)$ having either ICA or antiGAD and $15(11.5 \%)$ having both antibodies. With respect to antiGAD negative, patients antiGAD positive had lower levels of age, fasting and stimulated C-peptide values. and higher frequencies of HLA DQAI-DQB1 susceptibility genotypes and insulin-treatment. Similar results were found in ICA positive vs. ICA negative patients. Patients with positivities to both ICA and antiGAD had the lowest stimulated C-peptide values. Frequencies of adult-onset type 1 (stimulated C-peptide $<0.60$ $\mathrm{nmol} / \mathrm{l}$ ). latent autoimmune diabetes (stimulated C-peptide $\geq 0.60$ $\mathrm{nmol} / \mathrm{l}$ and at least one marker of autoimmunity) and type 2 (stimulated C-peptide $\geq 0.60 \mathrm{nmol} / 1$ but no markers) were respectively $16.1 \%(95 \%$ CI $0.4-31.8), 331(95 \%$ CI $19.4-46.8)$ and $50.8 \%(95 \%$ CI $38.8-62.8)$. Patients with latent autoimmune diabetes had intermediate values of insulin secretory impairment and frequency of HLA DQAI-DQBI susceptibilty genotypes. In conclusion. in lean young and middle-aged patients the prevalence of autoimmune insulitis is high, suggesting a slowly evolving form of type 1 diabetes. 
383

DETECTION OF ANTIPHOSPHOLIPID (aPL) AND ANTITHIROID (aAT) ANTIBODIES IN PATIENTS WITH DIABETES MELLITUS (DM) RELATED WITH VASCULAR ALTERATIONS.

J.Martí; R.Pérez;I,Navarro;R.Ferrer;J.Martinez;M.A.Ruiz;E.Ferrer Hospital Francesc de Borja. Gandía

Aim of the study: The aim of our study was confirm the likely association between the natural prevalence of the appearance of $\mathrm{APL}$ in patients with DM and their possible association with micro-mactoangiophatic disorders. At the same time aAT were determined in the same group in order to establish their hypothetical association with the above quoted patients. Patients and methods: 180 diabetic patients (80 from type I and 100 from type II) random selected, 98 males, 82 women; average age 47,6 \pm 18.5 and average of evolution years of disease: $9,3 \pm 7,5$. The following antibodies were determined: Anticardiolipin (ACA) IgG and IgM,

antiphosphatidilserine $\operatorname{IgG}$ and $\operatorname{IgM}$; lupus anticoagulant (LA) and antithiroid. Coagulation parameters were also determined. All the patients were further analysed in order to detect current or previous microangiophatic (nephopathy, retinopathy) or macroangiophatic ( coronariopathy, brain artheriopathy...) disorders.

Results: 71 patients (39\%) presented positive aPL (37\% in type I and $40 \%$ in type II). Resulting that $46(64,7 \%)$ were ACA positive, $13(18,3 \%) \mathrm{AL}$ positive and $12(17 \%)$ both. No difference about vascular alterations was found between the aPL positive group and the aPL negative group ; neither in the total group ( $35 \%$ vs. $41 \%$ ) nor in the DM type I group $(38,7 \%$ vs. $22,4 \%$ ). In the DM type Il group, Fewer vascular disorders were found in aPL positive patients $(32,5 \%$ vs. $56.4 \% ; p<0,05)$. The coagulation parameters were absolutely normal in all the patients. In 22 patients $(12,2 \%)$ aAT were found $(16,2 \%$ in DM type $I$ and $9 \%$ in DM type II). Conclusions: The aPL prevalence is high in our diabetic patients and it's 3.2 times greater than the aAT prevalence. In our group, the aPL prevalence cannot be considered as a determining factor in the appearance of vascular alterations.

\section{4}

IMMUNOLOGICAL EVALUATION OF RECENT-ONSET TYPE 1 DIABETES CORRELATION WITH $\beta$-CELL FUNCTION AND METABOLIC CONTROL C.Dell'Anna, J.Vidal, G.Sesmilo, M.Fernández, C.Rodríguez-Villar R.Casamitjana, R.Gomis and I.Conget. Endocrinology and Diabetes Unit, Hormonology Unit. Hospital Clínic i Universitari, Barcelona, Spain.

Antibodies against the pancreas are recognized as markers of autoimmune $\beta$-cell damage in type 1 diabetes mellitus. The presence of those antibodies and its heterogeneity at diagnosis, has recently been correlated to residual pancreatic function and glycaemic control. The aim of our study was to analyze the relationship between autoantibodies, metabolic control and $\beta$ cell function in recent onset type 1 diabetes. Subjects and methods: Fifty-one type 1 diabetic patients ( 17 male, 34 female, mean age $22.8 \pm 5.7 \mathrm{yr}$ ) were studied at the onset of the disease and again 6 months later. We measured GAD, IA2, IAA (glutamic decarboxylase, tyrosine phosphatase, and insulin) autoantibodies. Pancreatic $\beta$-cell function was assessed by basal pro-insulin, fasting and glucagon-stimulated cpeptide levels. Glycosylated haemoglobin and insulin requirements were also evaluated. Results: GAD positivity was $70 \%$ at the onset of the disease and $61 \%$ after 6 months, for $1 \mathrm{~A} 2$ positivity was $43 \%$ and $41 \%$, and for AAI it was $23 \%$ and $67 \%$, respectively. Both GAD and IA2 at the onset correlated well with values at 6 months ( $\mathrm{r}=0.99$ and $0.90, \mathrm{p}=0.000$ respectively). No significant correlation between autoantibodies (GAD, IA2 alone or combined) and glycosylated haemoglobin or pancreatic function parameters, such as fasting pro-insulin and those parameters derived from glucagon test (fasting and stimulated c-peptide, $\delta$ c-peptide and AUC c-peptide) was found. At 6 months evaluation, parameters of $\beta$ cell function correlate neither with glycosylated haemoglobin nor with insulin requirements. Conclusions: In summary, our findings do not support the possible relationship of autoantibodies with residual $\beta$ cell function and metabolic control in patients with recent onset type 1 diabetes.

\section{5}

LONG-TERM VS SHORT-TERM REMISSION IN TYPE 1 DIABETES: ANALYSIS OF $\mathrm{CD}^{+} \mathrm{T}$ CEL L SUBSETS AND ISLET CELL ANTIBODIES N.M.Lalić.M.L.Lukić,D.Kosec,A.Jotić,M.Zamaklar,K.Lalić,N.Rajković,L.Lukić and P.B.Djordjević, Institute for Endocrinology, Institute for Microbiology and Immunology and Center for Immunological Research, Belgrade, Yugoslavia

It has been previously suggested that changes both in $\mathrm{CD}^{+} \mathrm{T}$ lymphocyte subsets and islet cell antibody (ICA) levels might correlate with the clinical course of the disease in patients with recent-onset Type 1 diabetes. Therefore, this study was aimed to compare changes in $\mathrm{CD}^{+} \mathrm{T}$ cell subsets and ICA levels in peripheral blood between the two groups of the patients showing a long-term ( $>180$ days) clinical remission (CR) (group $A, N=24$, duration of CR $324^{+} /-62$ days) or a short-term ( $<180$ days) CR (group B, $N=26$, duration of $\mathrm{CR} 127^{+} / 32$ days). The CR was defined as optimal metabolic control without insulin lasting $>30$ days. The percentage of memory cell ( $\mathrm{CD} 45 \mathrm{R} 0^{-}$) and naive $\left(\mathrm{CD}^{2} \mathrm{RA} \mathrm{A}^{\mathrm{i}}\right.$ ) $\mathrm{CD}^{+} \mathrm{T}$ cell subsets was analysed by using two-color immuno-fluorescence staining and flowcytometry, while ICA levels were determined by indirect immunofluorescence on human pancreatic tissue sections and expressed in Juvenile Diabetes Foundation (JDF) units. In each patient, the analysis was done sequentially (a) in initial insulin-requiring state (IRS I); (b) at day 30 of CR (CR I); (b) at day 60 of CR (CR II): (c) in relapse of IRS after CR (IRS II). We found that percentage of $\mathrm{CD} 4 \mathrm{CD} 45 \mathrm{R} 0^{\circ} \mathrm{T}$ cells was lower in group $A$ than in group B, both in CR I and CR II $(27.6 / 2.0$ vs $31.9^{+} /-2.1 \% ; 28.4^{+} / 2.0$ vs $32.7^{+} /-2.2 \%$, respectively, $\left.p<0.05\right)$, while simultaneously $\mathrm{CD} 4{ }^{+} \mathrm{CD} 45 \mathrm{RA}^{+} \mathrm{T}$ cells did not differ between the groups. The percentage of both $\mathrm{CD4}^{+} \mathrm{T}$ cell subsets did not differ between the two groups in IRS I and IRS II. Moreover. ICA levels did not differ between group $A$ and group $B$ in $C R I$ and $C R$ II. In IRS I, the ICA levels were lower in group A than in group B (mean ICA titer: 20 vs 60JDFU) but the ICA levels did not differ between the groups in IRS II. Our results signify that duration of CR correlates only with the changes in $\mathrm{CD} 4{ }^{\prime} \mathrm{CD} 45 \mathrm{R} 0^{+} \mathrm{T}$ cells and not in CD4 CD45RA ${ }^{+} T$ cells or ICAs. The results imply that duration of $C R$ could be prolonged by preventing an increase in the $\mathrm{CD} 45 \mathrm{R} 0^{+}$(memory cell) subset of $\mathrm{CD}^{+}$T lymphocytes. 


\section{Experimental Immunology}

\section{6}

\section{DIFFERENTIATION OF IMMUNE REACTIVITY TO GAD 65 PEPTIDES BY TRANSGENIC MOUSE MODELS}

T. Lohmann', R. Boyton ${ }^{2}$, R.D.G. Leslie ${ }^{3}$, D. Altmann ${ }^{2}$, and M. Londei ${ }^{4}$ ${ }^{1}$ Dept. of Internal Med. III, University of Leipzig, Germany; ${ }^{2} \mathrm{Hammer}-$ smith Hosp., 'St. Bartholomew's Hosp., "Kennedy Inst of Rheumatology, London, UK.

IDDM is believed to result from $\mathrm{T}$ cell mediated immune destruction of pancreatic beta cells and GAD 65 has been implicated as important self antigen. We have identified some immunodominant epitopes of GAD 65 in IDDM amongst them GAD 505-519 and 521-535 but only the latter epitope revealed diabetes specifity in identical twin studies. To dissect further T cell immunity to these GAD 65 epitopes, we studied T cell responses in $\mathrm{C} 57 / \mathrm{B} 6$ mice carrying an human transgene for the diabetes associated HLA allele DQ*0302. The mice were immunized with GAD peptides 505-519 and 521-535 in both hind footpads. After 10 days, $T$ cell proliferation and cytokine production were measured in popliteal draining lymph nodes. We observed a significant proliferative response to both peptides, 505-519 and 521-535. T cell responses to both epitopes were restricted at least partially by the $\mathrm{DQ}^{*} 0302$ transgene despite a weak binding affinity of both peptides to this HLA allele. Long term $T$ cell lines against both peptides elicited a high IFNg and IL-10 production. After immunisation of diabetes-prone NOD and diabetesprotected NOD.I-E mice using the same protocol, peptide 505-519 induced comparable $T$ cell proliferation and cytokine production in NOD and NOD. I-E mice whereas peptide 521-535 elicited T cell proliferation and $\mathrm{IL}-10$ production only in NOD mice ( $p<0.05$ to NOD. I-E by Student's $t$ test). In conclusion, GAD peptide 52l-535 selectively induces a $T$ cell response in diabetes susceptible humans and mice. This peptide may be an important epitope of GAD 65 in IDDM and tolerance induction to it a possible therapeutic target.

\section{8}

MUTATIONS IN THE SIGNAL SEQUENCE OF GLYCEROPHOSPHATE DEHYDROGENASE CHANGE ITS INTRACELLULAR DESTINATTON M.E.Fabregat, E.F.Usac, C.Franco , T.Gallart*, C.Enrich and R. Gomis. Endocrinology and Diabetes Department. ${ }^{*}$ Immunology Department 4 Cell Biology Department. IDIBAPS. Hospital Clínic. Villarroel 170, 08036 Barcelona. Spain Islet cell antibodies, a hallmark of type 1 diabetes are directed to a number of autoantigens. We had previously found antibodies against a mitochondrial enzyme FAD-linked glycerophosphate dehydrogenase ( $\mathrm{mGDH}$ ) in type 1 patients. It plays a key role in the recognition of D-glucose metabolism as a stimulus for insulin release from beta cells. Aim: To investigate if some mutations in the signal sequence of mGDH could change its destination to another intracellular compartment involved in antigenic presentation. Methods: We changed, by sitedirected mutagenesis, five aminoacids of $\mathrm{mGDH}$ cDNA signal sequence. We obtained two different constructs. One of them had aminoacids 5 and 8 changed whereas the other construct had aminoacids 31,32 and 33 changed. Mutated and wild type constructs were subcloned, as fusion proteins, into an eukaryotic expression vector (pEGFP-N1) that contained the sequence of a green fluorescent protein (mGDH5i8-EGFP, mGDH313233-EGFP and mGDHwt-EGFP respectively). $\operatorname{COS}-7$ cells were liposome-mediated transiently transfected by these constructs. The location of these mGDH fusion proteins was observed with a confocal scanning laser microscope. Colocalization was analysed incubating transfected cells with an antimitochondrial antibody using as a second antibody a goat anti-mouse Cy3. Results: mGDHwt-EGFP and mGDH5i8-EGFP fusion proteins colocalized with an antimitochondrial antibody. mGDH313233-EGFP fusion protein was mostly located in a cytoplasm like pattem and it disappeared in previously permeabilized cells. Conclusion: Substitution of aminoacids at positions 31,32 and 33 at the signal sequence of the $\mathrm{mGDH}$ are sufficient to misstarget the protein out of the mitochondria. The pattern observed showed a cytoplasmatic distribution. There, it could be associated with class 1 MHC molecules, being transported to the surface membrane and expressed as an antigenic determinant.

\section{7}

BINDING OF GAD65 PEPTIDES TO HLA-DR3/4 CELLS AND CELLULAR IMMUNITY OF NEW TYPE 1 DIABETIC PATIENTS. E. Rharbaoui A. Mayer, M. Bouanani, C. Granier, C.Thivolet, F. Rharbaoui, A. Mayer, M. Bouanani,
A.M. Madec, Montpellier, Lyon, FRANCE.

Autoantibodies and autoreactives T lymphocytes directed against GAD65 have been identified in the circulation before and at the onset of type $\uparrow$ diabetes. Using 12 mer overlapping synthetic peptides spanning the entire human GAD65 sequence, we studied the proliferative responses of PBMC in 26 new-onset
type 1 diabetic subjects. Out of them, $15(58 \%)$ had stimulation index (SI) above the mean +3 SD of controls (SI $\geq 3$ ). PBMC proliferation was also observed in $6 / 16(37.5 \%)$ non-diabetic healthy subjects matched for age, sex. PBMC proliferation against healthy subjects matched for age, sex. PBMC proliferation against significantly associated with type 1 diabetes, $13 / 26(50 \%)$ vs $0 / 16$ in control sujects $(p<0.001)$. In the 21/26 HLA typed type diabetic patients, no correlation could be made between MHC specificity and T cell epitopes but patients with the same HLA-DR or HLADQ alleles showed partially identical T-cell reactivity. We analyzed by flow cytometry the direct binding of biotinylated GAD 65 peptides on EBV-B cell line (DPC) producing monoclonal GAD 65-antibody obtained from a diabetic patient. These cells carried the HLA haplotype strongly associated with type 1 diabetes DRB1*0301/0401- DQB1*0201/0302. Only 11/93 peptides were able to bind specifically to DPC cells as compared to the binding to class II-deficient RJ 2.2 .5 cells. $3 / 4$ of the peptides showing the highest binding (mean fluorescence between 82.4 and 105.3 ) belong to the C-terminal GAD65 region (367-378;373-384;445-456;499-510), in correlation with PBMC proliferation results.

\section{9}

EPITHELIAL CELLS IN ENDOCRINE AUTOIMMUNITY. ARE THEY CAPABLE OF PRESEITING AUTOLOGOUS PEPTIDES?

t. Muntasail. L. Serradeli. II. Cattiltamo and D. Juraquemada. Linitat d'Immunotogia. Lniversitar Auronoma de Bircelona. 08193 Bellaterna. Spain.

Endocrine evithetiai ceils in organ-specific autoimmune diseases such as type I Diabetes or Grates disease are the target of the autommune attack and express class I molecules. It has been postulated that endocrine epithelial cetls could act as APC and inav be resconsible ior the induction and/or maintenance of the in situ autoimmune $\mathrm{CDH}^{+} \mathrm{T}$ zil response. AIM: To study the capaciry of endocrine epithelial cells to process and present iarget auroantigens to $T$ ceils und how class It associated molecules Ii and DMI can arfect the pepride pool and stability of class II molecules. METHODS in vitro: :anstection of rat insulinoma cell line. RINmsF. with HLA-DR $t^{*}(0)+01$. I and HL t-DY cDN ts. Sludy of transport and structural stability of DR molecules and reptides dssociuted in the differenc transtectants. Simidar studies with in rito :utoimmune thyroid epithelial (ells (TFC). RESLLTS: ceil expressing DR atone or DR+Ii fij nor liorm SDS-statle complexes. cells expressing DR+Ii had most DR inclecules wicupied by CLIP and class II expressed by DR+Ii+DM transtectants showed eqular routung and reptide louding patterns. In addition. DR+DM ceils expressed stable necules und al more efticient transport out of the ER than in $\mathrm{DR}^{+}$cells. i.e.. in the ence of Ii. DM exehange peptides and may ha involved in class II exit from the $t$ : Eptheitat ceils are therefore able to express stable tiass II complexes and the starti.

"f these molecules depend on HLA-DM expression. Results were confirmed in in an

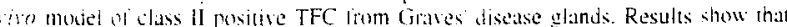
TFC comstitutively express also $1 \mathrm{f}$ and D.M and atiter IFN-y stimulation. The level of

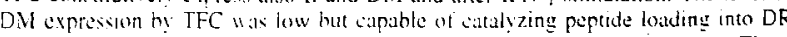
molecules and expressines stable DR-pentide complexes on the IFC surtiace. These colecules auld theretore present peptides to ilutoreacuve T cells. Searching for tisus pecific pentades associated to DR. we characterized the naturally processed peptides
Gnend 16 DRt in the translectants. A lot of peptides were associated with DR

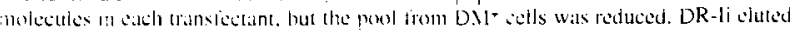

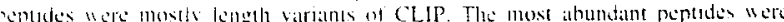

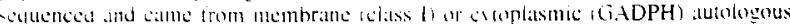

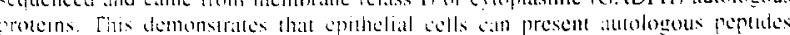

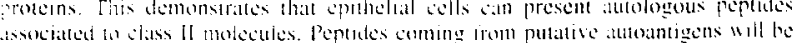

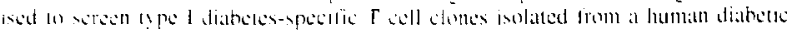

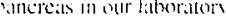


390

HOMOLOGY MODELLING OF I-A ${ }^{7}$ VERIFIES EXISTENCE OF TWO PEPTIDE BINDING MOTIFS (pH 5 AND pH 7) EXPLAINS SEVERAL PROPERTIES OF THIS MHC II ALLELE FROM NOD MICE

G.K. Papadopoulos, Labor. of Inmunol. Dept. of Internal Medicine, Univ. of loannina Medical School, GR-451 10 Ioannina GREECE.

The aim of this study was to discover via homology modelling the most stable energy conformation for several peptides that are autoantigenic and immunogenic in the NOD mice, and thus bound to $\mathrm{I}-\mathrm{A}^{\mathrm{g}}$. Modeling was based on the crystal structure of HLA-DR1. Peptides were aligned in the groove by using two motifs reported in the literature, and obtained at two different experimental conditions: $\mathrm{pH} 5.0$, and $\mathrm{pH}$ 7.0. Energy minimisation via the program Discover, went for 1000 cycles each of the steepest and conjugate gradient methods. The minimised structures obtained violated no physicochemical principles when examined in detail. Minimised energy of a peptide-I-A $\mathrm{A}^{7}$ complex was always higher, thus less stable $(17-81 \mathrm{kcal} / \mathrm{mol})$ when the $\mathrm{pH}$ of the complex was discordant with the $\mathrm{pH}$ corresponding to the peptide register, compared to minimisations with concordance between $\mathrm{pH}$ value and peptide register. We attribute this remarkable $\mathrm{pH}$-dependent motif change to the unique combination of $\beta 9 \mathrm{His}, \beta 56 \mathrm{His}$ and $\beta 57 \mathrm{Ser}$, where the first two residues are charged at endosomal $\mathrm{pH}$, but uncharged at extracellular $\mathrm{pH}$. Thus immunogenic peptides from NOD mice must contain within their native stretch two nonamer non-overlapping sequences, one satisfying the $\mathrm{pH} 5.0$ and the other the $\mathrm{pH} 7.0$ motif. All such known peptides satisfy this criterion. As the majority of peptides generated in the endosome and bound to $\mathrm{I}-\mathrm{A}^{\mathrm{g}^{7}}$ there, cannot be expected to fulfill the neutral $\mathrm{pH}$ motif as well, the corresponding $\mathrm{I}-\mathrm{A}^{87}$-peptide complexes will disso-ciate at the cell surface. This is consistent with the reported innstability of I-A $\mathrm{A}^{7}$ molecules at the cell surface in vivo. Our results provide the structural basis for a unifying hypothesis as to the origin of the $\mathrm{pH} 5.0$ and $\mathrm{pH} 7.0$ motifs, and the instability of $\mathrm{I}-\mathrm{A}^{87}$ in vivo. The also offer a means to test this hypothesis by binding of nonamers to $1-\mathrm{As}^{7}$ either in the endosomal or neutral $\mathrm{pH}$ register.

\section{2}

T-CELL REACTIVITY TO A DOMINANT EPITOPE ON THE TYROSINE PHOSPHATASE LIKE PROTEIN IA-2 IN INSULIN DEPENDENT DIABETES MELLITUS

N. C. Schioot (1), C. J. Hawkes (2),E. J. Stevens (2), S. J. M. Willemen (1) M.R.Christie (2) and B.O.Roep(1) Dept. Immunohematology \& Bloodbank University Hospital Leiden. NL (1); Dept. Medicine and Immunology, King's Callege School of Medicine, London, GB (2)

IDDM is an autoimmune disease in which the insulin- producing pancreatic beta cells are selectively destroyed by a process involving autoreactiveT-cells. Antibodies to the islet antigen IA-2ic appear in $60-70 \%$ of IDDMpatients. The aim of this study was to characterize T-cell responses to IA-2ic. We generated IA-2ic specific T-cell lines from three newly diagnosed IDDM patients. The epitopes were mapped by testing overlapping peptides spanning the entire sequence of human IA-2ic to evaluate whether any IA-2ic epitopes are recognized dominantly. All three T-cell lines specifically reacted to two IA-2 peptides, overlapping by amino acids aa 841-850, and spanning aa 831-860. This suggested that this region of IA-2ic is immunogenic. Then, the relationship between primary T-cell responses to the intact IA-2ic molecule and the two IA-2 peptides that stimulated the $T$ cell lines was investigated. The majority of IDDM patients (14/19) and healthy siblings (14/21) recognized IA-2ic. T cell responses to aa $831-860$ were detected in $9 / 19$ of the IDDM patients and in 15/21 of the healthy sibtings resulting in co-recognition of IA-2ic and aa 831-860 in $60 \%$ of IA-2ic responding subjects (8/14 IDDM patients, $9 / 14$ healthy siblings). Interestingly, a significantly higher proportion of healthy siblings than IDDM patients responded to the lA-2 peptides in the absence of a response to the IA-2 protein. These results indicate that T-cell epitopes defined on basis of IA-2 specific $T$ cell lines are immunogenic in primary assays and can help to further elucidate $T$ cell autoreactivity in IDDM.

\section{1}

NES2Y: A NEW HUMAN ENDOCRINE ISLET CELL LINE

M. Vives, RFL. James*, M. Costa, R. Pujol-Borrell. Unitat d'Immunologia. Hospital Germans Trias i Pujol. Universitat Autònoma de Barcelona. 08916 Badalona, Spain. "Dept. of Surgery. The University of Leicester. Leicester Royal Infirmary. Leicester LE2 7LX, U.K.

The limited access to human pancreatic islets is an important obstacie for the in vitro experiments in type I diabetes mellitus research. Recently, a new insular human cell line -NES2Y- has been generated from insulinsecreting cells obtained after partial pancreatectomy of a patient with persistent hyperinsulinemic hypoglycaemia of infancy (PHHI). The main aim of this work was to characterize the NES2Y cell line in terms of antigenic content and immunophenotype. Cells cultured as monolayer in RPMl 1640 and $10 \%$ vol/vol FCS, were stained by indirect immunofluorescence technique (IFL) for membrane and cytoplasmic antigens and analyzed by flowcytometry. After trypsinization, cells were membrane or cytoplasmic stained -after fixation and permeabilizationusing specific antibodies and appropriate conjugates; groups of 5000 cells were acquired and analyzed by flowcytometry. Results demonstrated that NES2Y cells are strongly positive for insulin (95\%), Cpeptide $(99 \%)$, Glutamic Acid Decarboxilase $(97 \%)$, somatostatin $(75 \%)$ and glucagon $(21 \%)$. The expression of the molecules involved in endogenous presentation pathway, HLA Class I, Transporter Associated with Antigen Processing-1 (TAP1), and proteasome subunits Lowmolecular-mass polypeptides 2 and 7 (LMP2 and LMP7) was found to be constitutive and it could be increased by IFN-gamma treatment. HLA Class II molecules were not membrane expressed in basal conditions but its expression could be induced by cytokines. The cell line NES2Y may provide a useful target for use into immunological as well as functional aspects of Type I diabetes.

\section{3}

MONOCLONAL ANTIBODIES AGAINST TYROSINE PHOSPHATASE RECOGNIZE IDDM-ASSOCIATED IA2 EPITOPES AT THE N-TERMINUS B. Ziegler; J. Seissler*, M. Schlosser, M. Strebelow, N. Morgenthaler** and M. Ziegler; Institute of Pathophysiology Karlsburg, University of Greifswald, *Diabetes Research Institute, University of Düsseldorf, **BRAHMS Diagnostica, GmbH, Berlin, Germany

Autoantibodies (AAbs) against the cytoplasmic domain of LA2 (IA2ic) are an important marker of $\beta$-cell destruction in IDDM. The AAbs are directed against at least 4 distinct epitope regions on IA2 measured with constructs representing the juxtamembrane (JM) and the tyrosine phosphatase (PTP) domains of IA2. The aim of our study was to evaluate whether monoclonal IA2 antibodies (mc-IA2-Abs) generated after immunization of a non-diabetes susceptible Balb/c mouse with human recombinant IA2ic recognize IA2 epitopes targeted by IDDM-associated AAbs. These mc-IA2-Abs are potential suitable to characterize the submolecular specificity of IDDM-associated AAbs to LA2. Mc-IA2-Abs (all of IgG class) were derived by immortalization of the splenocytes by fusion with the myeloma cell line SP2/0. After screening by a radiobinding assay using 125I-LA2ic and cloning, the supernatants were re-examined with $35 \mathrm{~S}$-methionine labelled LA2 deletion mutants by immunoprecipitation. $15 / 16(96 \%)$ mc-LA2-Abs were reactive with the JM region (amino acids aa 605-772), and only 1 mc-lA2-Ab binds to the PTP construct (aa 771979). Only the 125I-IA2 binding of those mc-IA2-Abs reactive with the $\mathrm{M}$ construct was significantly diminished by $66 \%(33 / 55)$ LA2-AAb positive IDDM sera. All mcIA2-Abs failed to bind IA2B. Furthermore, the ICA binding pattern of the mc-LA2Abs were examined on cryosections of human and mouse pancreas. Most of mc-LA2Abs were also detectable on mouse islets indicating that LA2 is an important target of the non-restricted ICA which predict progression to IDDM. This binding differs from that of mc-GAD-Abs which did not stain mouse pancreas. The results show that the JM IA2 region targeted by IDDM-associated IA2-AAbs is also immunogenic in $\mathrm{Balb} / \mathrm{c}$ mice. Further experiments are necessary to generate mc-LA2-Abs recognizing epitopes localized at the C-terminus known to be a prominent binding side for IDDM-associated IA2-AAbs. 


\section{4}

V $\lambda$ SECONDARY REARRANGEMENT IN A HUMAN MATURE B CELL PRODUCING IgM $\lambda$ ANTI-ISLET AUTOANTIBODY

C. Benito, T. Gallart*, J. Fernández-Alvarez, M. Blay*, and R. Gomis.

Endocrinology and Diabetes Department.Immunology Department*.IDIBAPS.Hospital Clínic.Villarroel 170, 08036 Barcelona Spain

Secondary rearrangements (SR) in rearranged V(D)J genes, ("receptor editing"), was believed to occur only in immature $B$ cells. However, recent data in the murine system have shown that SR can also occur in activated mature $B$ cells. In contrast, few data are available about SR in human mature $B$ cells. Here we provide evidence of a SR in the $\mathrm{V} \lambda$ region of a human $E B V$-immortalized mature $B$ cell clone (MB91-EBV) producing IgM anti-islet antibody (ICA), derived from a Type I diabetic patient at the onset of the disease. MB91-EBV was selected and cloned on the basis of its ability to produce IgM $\lambda$ with ICA activity. Moreover, in order to prevent the frequent instability of Ig secretion from EBV clones, a heterohybridoma (HY-MB91) derived from MB91-EBV was also obtained. Analysis of Ig VH and VL genes using DNA and CDNA of both MB91-EBV and HY-MB91, demonstrated a unique VH rearrangement: $V_{H 5-51}-D_{n 1}-J_{H S b}$. In contrast, two $V \lambda$ rearrangements were found: one using the $V_{\lambda_{1}} 1-4$ (DPL11) gene segment linked to $\mathrm{J}_{2}-\mathrm{C}_{3}\left(\mathrm{Ke}^{-} \mathrm{Oz}^{+}\right)$, and the other using $\mathrm{V}_{\lambda}$ 4-1 gene segment linked to the same $\mathrm{J}_{2}-\mathrm{C}_{3}\left(\mathrm{Ke}^{-} \mathrm{Oz}^{+}\right)$. Given that the $V_{\lambda}$ 4-1 gene segment is more distant than $V_{\lambda} 1-4$ from the $J \lambda-C \lambda$ locus, data suggest that $V_{2} 1-4$ was replaced by $V_{\lambda} 4-1$ in the $V_{\lambda} l$ $4-\mathrm{J} \lambda_{2}-\mathrm{C} \lambda_{3}$ primary rearrangement. This $\mathrm{SR}$ abolished ICA activity.

\section{6}

HIGH GLUCOSE CONCENTRATIONS INCREASE THE LPS-STIMULATED PRODUCTION OF TUMOR NECROSIS FATOR- $\alpha$ IN VITRO

M.G. Netea', N. Hancu' and l Baciu'. 'Diabetes Center and Clinic and

'Department of Physiology. Cluj-Napoca University of Medicine and Pharmacy, Romania.

The proinflammatory cytokines tumor necrosis factor- $\alpha$ (TNF) and interleukin- $1 \beta$ (IL-1) play an important role in the pathogenesis of insulin-dependent diabetes mellitus (IDDM). It has been recently shown that glucose can induce the synthesis of TNF and IL-6 in human monocytes. The aim of the present study was to investigate the effect of glucose on unstimulated and lipopolysaccharide (LPS)induced TNF and IL-1 production by human peripheral blood mononuclear cells (PBMC). PBMC from healthy volunteers were preincubated in medium with various glucose concentrations. Mannitol was used in the control samples to achieve similar osmolarity. After $24 \mathrm{~h}$, the cells were washed and incubated for an additional $24 \mathrm{~h}$ with or without $I \mathrm{ng} / \mathrm{mL}$ LPS. TNF and IL- 1 were measured by specific ELISA kits. No effect of glucose on spontaneous release of TNF or IL-1 could be observed. The LPS-stimulated production of TNF was increased from 600 $+47 \mathrm{pg} / \mathrm{mL}$ (glucose concentration $100 \mathrm{mg} / \mathrm{dL}$ ), to $663 \pm 46 \mathrm{pg} / \mathrm{mL}$ (glucose 200 $\mathrm{mg} / \mathrm{dL}$ ), $762 \pm 123 \mathrm{pg} / \mathrm{mL}$ (glucose $400 \mathrm{mg} / \mathrm{dL}$ ) and $930 \pm 163 \mathrm{pg} / \mathrm{mL}$ (glucose 700 $\mathrm{mg} / \mathrm{dL}$ ) (overall Kruskal-Wallis $\mathrm{p}=0.0205$ ). In contrast. no effect of glucose preincubation on LPS-induced IL-1 synthesis was found. In conclusion, high glucose concentrations can increase the stimulated TNF production capacity, with possible important consequences. On the one hand a higher TNF response induced by acute hyperglycemia in the first days of the clinical manifestation of IDDM may further contribute to isiet cell damage. On the other hand. it has been shown that TNF is an important mediator of insulin resistance, and amplification of its synthesis by hyperglicenia may induce a positive feed-back with serious deleterious effects

\section{5}

CHANGES IN FCYRIIIA SIGNALLING
NONENZYMATIC GLUCOSILATION OF IgG

M.Hirt, D.Cheta, I.Popescu, A.Sulica, M.Manciulea. Center of Immunology and "N. Paulescu" Institute, Bucharest, Romania

Our previously reported data have demonstrated that human immunoglobulin $\mathrm{G}$ in monomeric form ( $\mathrm{mlgG}$ ), has an important regulatory role on the human natural cytotoxicity, by occupancy of FcyRIIIA present on the natural killer (NK) cells. We have shown that stimulation of NK cells via FcyRIIIA by mlgG resulted in activation of three protein tyrosine kinases, i.e., LCK, LYN and SYK and no ligand induced activation was observed for FYN, SRC or YES, src-related protein tyrosine kinases which are expressed in NK cells. Increased activity of phosphatidylinositol 3-kinase (PI 3-k) have been determined following the interaction between the $\mathrm{Fc}$ receptor and the specific ligand. Previously we have demonstrated qualitative modifications of some functions of mlgG molecules (cytophilic property, fluorescence property), induced by nonenzymatic glucosilation. To extend these observations we examined the effects induced by the excess of glucose on the mentioned signalling aspects, following triggering the FcyRIIIA on normal human NK cells by glucosilated (in vivo or in vitro) mlgG molecules. Upon triggering FcyRIIIA with glucosilated mlgG an increased activation of LYN, LCK and PI 3-kinase in comparison with those induced by normal $\mathrm{mlgG}$ was determined. Contrary, the very strong activity of SYK induced by ligation of $\mathrm{mlgG}$, was abotished upon interaction between glucosilated $\mathrm{mlgG}$ and Fcy RIIIA. This results suggest that nonenzymatic glucosilation of mlgG induced changes in intracellular signalling patterns which can affect the functions of the cell.

\section{7}

DOUBLE NEGATIVE NKT CELLS MAY BE INVOLVED IN THE TH1 OR TH2 PREDOMINANCE IN AUTOIMMUNE T CELL INFIL TRATES C. Roura-Mir, M. Catálfamo, M. Martí, R. Pujol-Borrell and D. Jaraquemada, Unitat d'Immunologia, Hospital Germans Trias i Pujol, Universitat Autònoma de Barcelona, Badalona, Spain

A TCR homogeneous cell subset of double negative (DN) T cells (CD3+CD4-CD8-), NKT cells, are presumably involved in the development of type 1 diabetes, where they'd be responsible of the regulation of Th1 responses by $\mathrm{CD} 4 \mathrm{~T}$ cells. Th1 responses are functionally predominant in type 1 diabetes as well as other organspecific autoimmune diseases such as Hashimoto's thyroiditis (HT) Others, such as Graves' disease (GD), where stimulating antibodies induce thyroid hiperfunction, are Th2 mediated. The population responsible for the initiation and maintenance of the Th1 or Th2 responses is not known but NKT cells as well as NK and $\gamma \delta$ cells are capable of directing such responses by producing IFN- $\gamma$ or $\mathbb{L}-4$ upon stimulation. The possible role of NKT cells in thyroid autoimmunity and IDDM has been analysed. DN cells were found in relatively large numbers in T cell infiltrates from GD and HT thyroids and a DN cell line was isolated from the $T$ cell infiltrate of a diabetic pancreas. Most high IL4 producing $T$ cells in the thyroid infiltrates were $D N$ a proportion of which expressed the NKT-associated marker CD161 (NKRP1). In addition, expression of V $\beta 11$ and $V \alpha 24$, the TCR associated with human NKT cells was also analysed, showing that the frequency of $\mathrm{V} \beta 11$ and $\mathrm{V}_{\alpha 2} 24$ bearing cells was high compared to the expression found in normal PBL or in total infiltrates. These cells produced $\amalg-4$ and IFN- $\gamma$ after in vitro stimulation. DN cell lines were cytotoxic against epithelial cells but also recognized CDla to dtransfected CR1 cell lines, as determined by cytotoxicity and cytokine production. Our data so far suggest that these double negative $T$ cells may be NKT cells and that this population may have an important role in the induction and/or maintenance of autoreactive $\mathrm{T}$ cell responses. 
398

CELLULAR AUTOIMMUNITY TO PHOGRIN AND IA2 IN THE NOD MOUSE

K. Kelemen, D. Wegmann, C. Wasmeier and J. C. Hutton. Barbara

Davis Center for Childhood Diabetes, Denver, CO, USA.

The structurally-related protein tyrosine phosphatases IA-2 and phogrin are major targets of humoral autoimmunity in type 1 diabetes in Man however their involvement in the disease in experimental disease models (NOD mouse and BB rat) is disputed. In this study we have evaluated cell-mediated autoimmunity to these molecules in the NOD mouse and their role in the pathogenesis and progression of disease. Phogrin and IA-2 were generated as soluble glutathion-S-transferase (GST) fusion products and purified after Factor Xa cleavage. Immunization of NOD mice with purified phogrin resulted in a vigorous $\mathrm{T}$ cell proliferative response which was antigen specific and cross reactive on islet cells. Limiting dilution yielded $12 \mathrm{~T}$ cell clones which reacted to phogrin. 6 of these reacted with crude granule preparation of rat insulinoma tissue consistent with the documented localization of the antigen. All of the phogrin specific $\mathrm{T}$ cell clones were CD4 positive by FACS analysis and were TH1-like as defined by their cytokine production profile as analysed by ELISA. Reactive T-cell lines were also been generated from islet infiltrates from unprimed pre-diabetic NOD mice. These results demonstrate that cellular autoreactivity to phogrin is a feature of the NOD model, that reactive cells respond to the native antigen at levels prevailing in islet tissue and that cellular autoimmunity probably arises in the islet during the course of the spontaneous diasease. Because of the close structural relationship of these molecules, further delineation of $\mathrm{T}$ cell epitopes shared between IA2 and phogrin will facilitate analysis of the role determinant spreading in the progression of type 1 diabetes.

\section{9}

PRESENCE OF SULPHATIDE AND SULPHATED LACTOSYLCERAMIDE IN HUMAN FETAL PANCREATA

A. Kaas, B. Formby, P. Fredman, and K. Buschard. Sansum Medical Research Foundation, Laboratory of immunology, Santa Barbara, USA; Department of Neurochemestry, University of Göteborg, Göteborg, Sweden; and Bartholin Instituttet, Kommunehospitalet, Copenhagen, Denmark.

The aim of the study was to examine for sulphatide and related glycolipids in fetal pancreata. Sulphatide is present in adult islets and anti-sulphatide antibodies are seen in patients with type 1 diabetes. Seventeen pancreata of fetal age from 16 to 24 week were investigated using thin-layer chromatography and a sulphatide-specific mAb Sulph I The tissue was collected independently of the present legally approved study. The concentration of sulphatide was $17.6 \pm 2.0 \mathrm{pmol} / \mathrm{mg}$ wet weight, ranging from 11 in the youngest pancreata to 33 in the 24-weekold pancreas, significantly increasing with age $(p=0.002)$. The corresponding value in adult pancreas was 95 . In contrast, there was no correlation between the age and the sulphatide concentration when this was related to the amount of protein or phosphor, all together indicating that the number of sulphatide-containing cells increases with age but not the quantity of sulphatide per cell. In the fetal pancreata the structure-related sulphatated lactosylceramide was found at a concentration of $3.4 \pm 0.3$ $\mathrm{pmol} / \mathrm{mg}$ wet weight, positively correlated with age $(\mathrm{p}=0.02)$. In contrast to sulphatide, sulphated lactosylceramide was not detected in adult pancreata. Thus, the diabetes autoantigen sulphatide is seen in pancreas early in fetal life and concentrations increase with age. Moreover, an additional related antigen, sulphated lactosylceramide, is expressed during fetal life but is not found in adults.

\section{0}

\section{LINOMIDE DOES NOT PREVENT AUTOIMMUNE} THYREOIDITIS IN NOD MICE.

M.-L. Hartoft-Nielsen, A. Kaas, Å.K. Rasmussen, U. Feldt-Rasmussen, and K. Buschard. University Hospital, Dept. of Endocrinology, Bartholin Instituttet, Kommunehospitalet, Copenhagen, Denmark.

IDDM and autoimmune thyroiditis (AIT) share common pathogenetic mechanisms. Linomide (LS-2616) is a potent immunomodulator and has been reported to prevent IDDM and insulitis in NOD mice and to reduce the incidence of some other autoimmune diseases. The mechanisms of action seem to involve antigen expression by down regulation of macrophage activity and to antagonize the activation of Th1 cells during cellular immune response. In order to investigate the effect of Linomide on the incidence of AIT in female NOD mice we administered Linomide in drinking water to NOD mice from $5^{\text {th }}$ to $17^{\text {th }}$ week of age. The mice were breed in our stables and did not develop diabetes during the study period. Linomide was continuosly present at a concentration of $0.5 \mathrm{mg} / \mathrm{ml}$ in the drinking water of the mice. Consequently each mice had an intake of 100 $\mathrm{mg}$ Linomide per $\mathrm{kg}$ per day. The mice were sacrificed at the end of week 17. The incidence of thyroiditis was evaluated on paraffin HE-stained sections and graduated on a scale from 0 to 4 . We found that $37 \%$ of 38 mice treated with Linomide developed thyroiditis compared to $48 \%$ of 21 controls. Among the mice who developed thyroditis there were no differece in the degree of thyroditis. Thus, there may be a tendency for a beneficial effect of Linomide on AIT but this was not significant $\left(\mathrm{p}=0.42 \mathrm{Chi}^{2}\right.$-test). Linomide has been reported to reduce the incidence of diabetes and insulites i NOD mice and the differently lack of effect in AIT is interesting. Based on our results in NOD mice Linomide can not be recommended for human studies regarding AIT.

\section{1}

PROTECTIVE ROLE OF CELLULAR IMMUNITY ON THE PATHOGENESIS OF NIDDM INDUCED BY VIRUS INFECTION T. Utsugi T. Kanda, 1. Kobayashi, T. Uchiyama, Y. Ohyama, H. Ito, A. Tsuchida, S. Tomono, S. Kawazu, and R.Nagai, Maebashi, Japan IDDM is sometimes induced by virus infection into the pancreatic beta cells in both humans and animals. Recently, We have found that not only IDDM, but NIDDM is also induced by the NDK25 variant of Encephalomyocarditis (EMC) virus in mice, and reported that mature lymphocytes can act as protection against viral infection by using Rag 2 knockout

(Rag 2KO) mice which lack of both $T$ cells and $B$ cells. To investigate which lymphocyte subset play a protective role on the pathogenesis of NIDDM in this model, CD4+T cell knockout mice (CD4KO), CD8+T cell knockout mice (CD8KO), CD4+ and $\mathrm{CD} 8+\mathrm{T}$ cell double knockout mice (CD4KO+CD8KO), and Rag 2KO were infected with the EMC-NDK25 variants. Wild type of $129 / \mathrm{SV}$ mice and C57BL/6 mice that are originaliy diabetes resistant were used as control. OGTT was performed before and 1 weck after infection. Blood glucose levels of Rag 2KO during OGTT at 1 week after infection were significantly higher than those of CD4KO, $\mathrm{CD} 8 \mathrm{KO}, \mathrm{CD} 4 \mathrm{KO}+\mathrm{CD} 8 \mathrm{KO}$, and WT and the insulin content of pancreas in Rag 2KO was significantly lower than those in the other group of mice ( $20 \pm 3$ vs $31 \pm 1$ to $93 \pm 31 \mu \mathrm{g} / \mathrm{g}$ of pancreas, $\mathrm{p}<0.01$ ). Histological study showed mild destruction of pancreatic islets only in Rag $2 \mathrm{KO}$, whereas pancreatic islets were apparently normal in the other group. In conclusion, cellular immunity, essentially B cells, play a protective role on the mechanism of NIDDM induced by virus infection, and the cytolytic activity of virus is not affected by the absence of cellular immunity, suggesting that the destruction of pancreatic beta cells by virus depends on their "affinity" without regard to host cellular immunity. 


\section{Genetics of Type 2 Diabetes}

\section{2}

REFINEMENT TO 6.1-CM OF GENETIC LOCALISATION OF ALSTRÖM SYNDROME ON CHROMOSOME 2p12-13.

F. Macari, C. Lautier, F. Dadoun, A. Girardet, A. Dutour, E. Renard, P. Bouvagnet, M. Claustres, Ch. Oliver and F. Grigorescu. IURC, Montpellier, France.

Alström syndrome is a rare autosomal recessive disorder characterized by retinal degeneration, deafness, hyperlipidemia and non-insulin dependent diabetes mellitus (NIDDM). While the disease-related gene remains unknown, studies of the genetic isolate of French Acadians provisionally located the Alström syndrome on Chr. 2p12-13 within a 14.9-cM interval. To attest this finding and refine the candidate region, we investigated by linkage analysis a consanguineous family of North African origin, in which 3 out of 7 siblings displayed all major features of Alström syndrome, including NIDDM with severe insulin resistance and acanthosis nigricans. Genotyping was performed on an ABI373A DNA automatic sequencer. Five microsatellite markers confirmed the involvement of 14.9-cM interval. To further confirm homogeneity and refine the candidate region, 20 additional markers were investigated. Haplotype analysis and allele segregation revealed that affected children shared the same haplotype and were homozygous for the eight most centromeric markers (D2S291 to D2S2114), over a 6.1cM interval. Significative multipoint lod scores $\left(Z_{\max }=3.95\right)$ were obtained between markers D2S2110-145 and D2S286. Two clusters of known genes are present in this region, the most attractive candidate being the hexokinase II gene. However, except several known polymorphisms, no mutations were detected in the coding region of this gene. In conclusion we confirm the location of Alström syndrome on Chr. 2p12-13 and reduce the genetic interval to $6.1-\mathrm{cM}$ which would considerably reduce the number of Alström candidate genes.

\section{4}

DIABETES-ASSOCIATED DELETION IN MITOCHONDRIAL DNA IN A PATIENT WTTH PEARSON'S PANCREAS-BONE MARROW SYNDROME J.M.W. van den Ouweland, G.J. Bruining', and J.A. Maassen. Department of Molecular Cell Biology, Leiden University Medical Center, Leiden \& ' Department of Paediatrics, Sophia Children Hospital/Academic Hospital Rotterdam, Erasmus University, Rotterdam, The Netherlands

We have recently diagnosed a patient with a variant of Pearson's pancreas-bone marrow syndrome displaying diabetes mellitus, anemia, severe tubulopathy, but no dysfunction of the exocrine pancreas. DNA analysis by Southern blot hybridization showed a large $8 \mathrm{~kb}$ heteroplasmic (i.e. coexistence of wild-type and mutant mitochondrial DNA (mtDNA) molecules) deletion in mtDNA. Heteroplasmy levels ranged from $30 \%$ in cultured fibroblasts to approximately $95 \%$ in liver and kidney biopsies. In leukocyte DNA from her mother and younger sister we were unable to detect the mtDNA deletion, even by PCR, indicating its sporadic occurrence. Direct sequencing of the breakpoint region revealed that the deletion extended 8034 bp from nt 7934 in the cytochrome c oxidase II gene to nt 15968 in the tRNA proline gene, and was flanked by an imperfect 11 bp repeat sequence. We applied mitochondriamediated transformation utilizing $\rho^{0}$ cells as recipients and mitochondria from our patient with Pearson syndrome as donor. Clonal cell lines harboring $0 \%$ to up to $87 \%$ deleted $\mathrm{mtDNA}$ were obtained and studied for mitochondrial functionality. Cells containing $<80 \%$ deleted mtDNA appeared phenotypically normal, whereas cells containing $>80 \%$ of deleted mtDNA resulted in inhibition of overall mitochondrial protein synthesis, as well as reduction of cellular respiration and cytochrome $c$ activity.

We conclude from these results that deletion in mtDNA deletion can act as a diabetogenic factor. Furthermore, the mtDNA deletion is functionally recessive with a steep threshold for expression of the pathogenic phenotype.

\section{3}

THE IMPACT OF A G/A NUCLEOTIDE VARIANT IN THE $\beta$-CELL GLUCOKINASE GENE PROMOTER ON THE $\beta$-CELL FUNCTION IN DANISH CAUCASIANS

Søren A. Urhammer, Torben Hansen, Jesper O. Clausen, Hans Eiberg, Oluf Pedersen. Steno Diabetes Center and Hagedorn Research Institute, Gentofte, Center of Preventive Medicine, Glostrup University Hospital, Copenhagen, Department of Medical Genetics, University of Copenhagen, Denmark

Recently it was shown that a frequently occurring g/a nucleotide substitution at position -30 in the $\beta$-cell specific glucokinase promoter is associated with reduced pancreatic $\beta$-cell function as estimated during an oral glucose tolerance test (OGTT) in Japanese-American subjects. We evaluated the impact of this variant in three different Danish populations. The presence of the variant was determined by PCRRFLP. In a group of 240 unrelated middle-aged glucose tolerant subjects we assessed the $\beta$-cell function as the ratio of the areas under the insulin and $C$-peptide curves, respectively, to that of the glucose curve during the first $30 \mathrm{~min}$ of an OGTT ( $\triangle$ ins $(0$ $30 \mathrm{~min}) / \Delta \mathrm{glu}(0-30 \mathrm{~min})$ and $\Delta \mathrm{Cpep}(0-30 \mathrm{~min}) / \Delta \mathrm{glu}(0-30 \mathrm{~min}))$ with respect to genotype status of the $\mathrm{g} / \mathrm{a}$ variant. Also these ratios adjusted for basal insulin and C-peptide levels were evaluated. No differences in these variables were found between carriers and noncarriers of the variant. The impact of the variant was also examined in a population based sample of 380 young (18-32 years) healthy subjects in which a frequently sampled intravenous glucose tolerance test (IVGTT) was performed. No relationship between the g/a variant and alterations in the acute insulin and C-peptide responses (incremental areas under the curves during the first $0-8 \mathrm{~min}$ ) were detected. Finally the g/a variant was examined in a family study consisting of 230 glucose tolerant offspring of NIDDM patients from 63 families which all underwent a $3 \mathrm{hr}$ frequently sampled OGTT and an IVGTT. The variables (the $\Delta$ ins $(0-30 \mathrm{~min}) / \Delta \mathrm{glu}(0-$ $30 \mathrm{~min})$, the $\Delta C \operatorname{cep}(0-30 \mathrm{~min}) / \Delta \mathrm{gl}(0-30 \mathrm{~min})$, the fasting-insulin-adjusted ratios during the OGTT and the acute insulin and $C$-peptide responses to intravenous glucose) were analyzed by computing the difference in the variables within each sib. pair as the value in the sibling carrying the $\mathrm{a} / \mathrm{g}$ variant subtracted by the value in the sibling without the variant. None of the mean differences were statistically different from zero. We conclude that the g/a nucleotide substitution of the GCK gene promoter has no impact on the pancreatic $\beta$-cell function in glucose tolerant Caucasian subjects of Danish origin

\section{5}

THE STUDY OF NON-INSULIN-DEPENDENT DIABETES MELLITUS GENETIC DETERMINATION

S. Shtandel, ${ }^{1}$ S.Finoghenova and ${ }^{2}$ L.Atramentova. Ukrainian Research Institute of Endocrine Diseases of Pharmacotherapy, Kharkov, Ukraine; 'Institute of Rheumatology, Moscow, Russia; ${ }^{2}$ Kharkov State University, Kharkov, Ukraine

The aim of the study was to assay the genetic determination of noninsulin-dependent diabetes mellitus (NIDDM) and its clinical forms persistent non-insulin dependence (PNID) and secondary insulin dependence (SID). 275 probands and their $1^{\text {st }}$ and $2^{\text {nd }}$ degree relatives (4357 subjects) were entrolled. D.Falconer's, Ch.Smith's and T.Reich's models were tested. The hereditability coefficient "in narrow sense" (average mean $80.0 \%$ ) obtained within the framework of the of the multifactorial threshold D.Falconer's model confirmed once more importance role of genetic factors in the appearance of the disease. The estimations of genetic additional component $\left(G_{A}\right)$, obtained on the date of the $1^{\text {st }}$ and $2^{\text {nd }}$ degree relatives and parents and siblings were differed from each other $(68.7 \pm 4.8 \%$ vs $80.1 \pm 5.7 \%$, NS). This phenomenon and constant presence of genetic epistatic component $\left(\mathrm{G}_{\mathrm{AA}}\right)$ in the versions of decompose of phenotypic variance in NIDDM indicate presence of nonlinear genetic factors in the determination of disease. The study of genetic heterogeneity within the framework of the Ch.Smith's model failed to reveal any genetic independence of NIDDM forms $\left(r_{g}=0.97\right)$. T.Reich's model demonstrated the most influence of the environmental components of phenotypic variance in SID as compared to that in PIND ( $25 \%$ vs $8 \%$ ). It has been shown, that the NIDDM distribution in the population and families may well be described by means of a variant polygene model with essential influence of the major gene. 
406

INSULIN VNTR AND HLA DRB1/DQB1 LOCI AFFECT AGE OF PRESENTATION OF AUTOIMMUNE DIABETES IN ADULTS

V.A. Horton', I.M. Stratton', R. J. Owen', S.T.Bennett', J. A. Todd', R.R. Holman' and R.C. Tumer', 'Diabetes Research Laboratories, 'Wellcome Trust Centre for Human Genetics, Oxford University for the UK Prospective Diabetes Study Group,Oxford, UK.

Glutamic acid decarboxylase antibodies (GADA) and islet cell antibodies (ICA) are present in $12 \%$ of Type 2 diabetes-diagnosed patients. In early adult presentation, patients with antibodies are non-obese but older patients are obese and less often require insulin therapy. Genetic variations may explain the heterogeneity. Allelic variation at both the HLA and insulin VNTR loci account for $40 \%$ of genetic risk in juvenile-onset Type 1 diabetic British Caucasians. We typed 255 antibody positive patients tor the predisposing HLA DR3, DR4, and DQB1 alleles by PCR-artificial RFLP and 306 antibody positive patients by PCR-RFLP at the insulin gene $-23 \mathrm{Hphl}$ site which is in strong linkage disequilibrium with the insulin VNTR. The presence of class III alleles protects against Type 1 diabetes. For analysis, patients were grouped into decades by age at diagnosis (years): $25-34,35-44,45-54,55-$ 65. The high risk DR3/DR4 genotype was present in $38 \%$ of patients with onset at $25-34$ years vs. $14 \%$ in those with onset at $55-65$ years. The nonDR3/nonDR4 genotype increased in frequency as age at diagnosis increased, from $14 \%$ to $34 \%\left(\chi^{2}\right.$ trend test: $\left.p=0.006\right)$. The frequency of the VNTR class III alleles ( $B$ genotypes) for protection against Type 1 diabetes increased from $13 \%$ at $25-34$ years to $40 \%$ at $55-65$ years. $\chi^{2}$ trend test: $p=0.016$ ) compared to $51 \%$ in healthy controls and $27 \%$ in type 1 insulin dependent diabetic patients diagnosed under 17 years of age. The younger age of onset is associated with a greater genetic load for diabetes, and conversely older age of onset with less genetic load.

\section{7}

THE PREVALENCE OF MODY3 AMONG DANISH MODY FAMILIES AND IDENTIFICATION OF 8 NOVEL MODY3 MUTATIONS IN HEPATOCYTE NUCLEAR FACTOR-10 L.T.Dalgaard, L.Ambye, T.Hansen, A.M.Møller, L.Hansen, M.Fridberg, S.A.Urhammer, O.Pedersen. Steno Diabetes Center, Gentofte, Denmark

Maturity onset diabetes of the young (MODY) is a subtype of non-insulin dependent diabetes mellitus (NIDDM), which is characterised by an autosomal dominant mode of inheritance, early age of onset and an impaired insulin secretion. Although MODY is a monogenic form of NIDDM the genetics of this disease is heterogeneous. Mutations in at least 5 different genes have been shown to cause MODY: Hepatocyte Nuclear Factor(HNF)-4 $\alpha$ (MODY1), glucokinase (MODY2), Hepatocyte Nuclear Factor (HNF)-1 $\alpha$ (MODY3), Insulin Promoter Factor (IPF)-1 (MODY4) and Hepatocyte Nuclear Factor(HNF)-1B (MODY5).

The aim of the present study was to estimate the nature and frequency of mutations in the HNF-1 $\alpha$ gene in Danish MODY families. We performed mutational analysis on probands from 14 unrelated MODY families. In all cases there was a family history of diabetes with autosomal dominant inheritance and at least one patient with onset before the age of 25 years. The 10 exons including intron-exon boundaries and the minimal promoter region was amplified by PCR and sequenced directly using an ABI 373 automatic sequencer. We detected 8 new mutations: 1 frameshift (His291 fsdelA), 1 nonsense (Glu234Amber), 5 missense mutations (Arg159Gln, Arg229Pro, Arg271Trp, Pro289Arg, Asn476Ser) and a 7 basepair insertion of the 5'UTR region. In 6 subjects no mutation was detected.

In a previous Danish study 9 MODY families were investigated and 5 MODY3/HNF-1 $\alpha$ mutations were identified (T.Hansen et al. Diabetes 46: 726 $730,1997)$. Thus, the previous study taken together with our present findings indicates that the prevalence of MODY 3 among Danish MODY patients is around $60 \%$. The families in which no MODY3 mutation was identified will undergo linkage analysis to either verify or exclude linkage to known MODY loci.

\section{8}

\section{APOLIPOPROTEIN E POLYMORPHISM IN INDIGENOUS AUSTRALIAN} SUBJECTS WITH NIDDM: A MAJOR CONTRIBUTOR TO DYSLIPIDAEMIA AND CARDIOVASCULAR RISK J.A.G. Shaw ${ }^{1}$ J. Tait ${ }^{2}$. P.K. Lovelock ${ }^{1,3}$ J. B. Kesting ${ }^{1}$. M. Marczak ${ }^{4}$ J. Cardinal ${ }^{1}$ J.R. Berkholz ${ }^{1}$ D. Purdie ${ }^{3}$ and J.T.E. Shaw ${ }^{1}$ Dept of Diabetes \& Endocrinology ${ }^{1}$ and Dept of Chemical Pathology ${ }^{2}$, Princess Alexandra Hospital Brisbane, Centre for Molecular and Cellular Biology, University of Queensland ${ }^{3}$, Redlands Health Service Centre ${ }^{4}$, Dept of Social \& Preventive Medicine, University of Old ${ }^{3}$, Australia

Apolipoprotein (apo) E plays a central role in the metabolism of cholesterol and triglycerides, and is a 299 amino acid protein with three major isoforms: apoE2, apoE3 and apoE4. The biosynthesis of these isoforms is coded by three independent codominant alleles at a single gene locus on chromosome 19q. Because of different receptor binding affinities of the apoE isoforms, the apoE polymorphisms have an impact on plasma lipoprotein metabolism and cardiovascular disease. Allelic frequencies vary among different populations, and may contribute to geographic differences in cardiovascular morbidity and mortality. The indigenous Austraiian population has a high prevalence of cardiovascular disease. Central obesity and NIDDM are common, and the clustering of these conditions with hypertension, hyperinsulinaemia and dyslipidaemia is likely to account for much of the excess cardiovascular risk. It is possible, however, that other specific genetic factors contribute to the adverse lipid status and high cardiovascular death rate of this population. To test this hypothesis, apoE genotypes and plasma lipids were determined for 155 indigenous Australian subjects and compared with those for 113 Caucasian control subjects. The apoE allelic frequencies observed in the indigenous Australian subjects differed from those of the Caucasian subjects (apoE2 0.08 vs 0.06 , apoE3 0.63 vs 0.78 , apoE4 0.28 vs 0.16 ). The frequency of the apoE4 allele was significantly higher in the indigenous Australian subjects $(p<0.001)$ and is amongst the highest reported for any population. By comparison with the homozygous apoE $3 / 3$ indigenous subjects $(n=60)$, indigenous subjects with the apoE4 allele $(n=73)$ tended to have higher triglyceride levels, and had significantly lower HDL-cholesterol levels $(\mathrm{p}=0.01)$. There was no significant difference in total cholesterol or LDL-cholesterol level between the two groups. The high frequency of the apoE4 allele in the indigenous Australian population and its association with adverse lipid status indicate that apoE allelic frequency is one of the cluster of factors contributing to the high cardiovascular morbidity and mortality of this population.

\section{9}

GENTIC DISSECTION OF THE INSULIN RECEPTOR IN 82 DANISH CAUCASIAN NIDDM FAMILIES

L. Hansen, T. Hansen, S. Urhammer, S. Jessing, S. G. Larsen, O. Pedersen. Steno Diabetes Center and Hagedorn Research Institute, Gentofte, Copenhagen, Denmark.

Previous studies of the insulin receptor gene have been confined to either whole gene scannings (exon 1-22) in selected syndromes of insulin resistance: Type $\mathrm{A}$ insulin resistance syndome, polycystic ovary syndrome (PCOS), Acanthosis Nigricans, Leprechaunism and the Rabson-Mendelhall syndrome or merely scanning of the intracellular tyrosine kinase domain (exons 17-22) in Caucasian NIDDM populations. We here report the first whole gene scanning of the insulin receptor gene in Caucasian NIDDM families. The coding sequence of the 22 exons, including the signal peptide, was analysed by PCR-SSCP-heteroduplex scanning in 29 segments and performed on genomic DNA from one diabetic proband from each of the 82 families that were included in the study. In all we found 4 different missense mutations of which the V985M in exon 17 is known to be functionally insignificant, whereas 3 others were novel. In exon 12 we found a R796S change and in exon 22 we found a R1234H and a D $1296 \mathrm{~N}$ replacement. The R796S and the D1296N mutations were detected in the same diabetic subject and is probably on the same allele since both mutations were transmitted to all the offspring of the proband. The $\mathrm{R} 1243 \mathrm{H}$ was also only found in one individual. Unfortunately the sizes of these families were too small to confirm segregation of the variants with NIDDM, so expression studies for the estimation of in vivo and in vitro functioning of the receptor variants have been initiated. If these receptor variants are shown to be functionally significant, insulin receptor variants may represent the so far most important (about $2 \%$ ) identified single genetic contribution to the "common form" of late onset NIDDM among Caucasians. 


\section{0}

FUNCTIONAL EFFECTS OF MUTATIONS AFFECTING GLYCEROPHOSPHATE DEHYDROGENASE IN TYPE 2 DM

E.Femández-Usac, M.Gudayol, J.Vidal, C.Franco, A.Novials, R.Gomis, Endocrinology and Diabetes Unit. IDIBAPS. Hospital Clínic de Barcelona. Spain.

Screening for mutations in the FAD-linked glycerolphosphate dehydrogenase ( $m-G D H)$ has yielded positive results in some individuals with type $2 \mathrm{DM}$. In vitro assessment of the functional effects of such mutations is lacking. The aim of the present study was to evaluate the effect of m-GDH FAD-binding and calcium-binding domain site-directed mutagenesis on enzymatic activity. In vitro site-directed mutagenesis protocol was followed to reproduce the mutations affecting both m-GDH domains found in a single type 2 diabetic patient Mutated m-GDH cDNA was transfected into Cos-7 cells, an eukariotic cell line. Mutated m-GDH mRNA was microinjected in Xenopus oocytes. m-GDH enzymatic activity was measured using a radioisotopic assay. $\mathrm{Cos}_{-7} 7$ cells m-GDH protein content was assessed by Western blot analysis. Differences between groups were assessed by the Mann-Whitney $U$ test and within groups by the Kruskal-Wallis $H$ test. Cos -7 cells transfection $(n=5)$ with the wild-type form of $\mathrm{m}-\mathrm{GDH} \mathrm{cDNA}$ resulted in a $276 \pm 81 \%$ (mean \pm SD) increase in enzymatic activity compared to control cells $(p<0.01) . \mathrm{m}$ GDH enzymatic activity following transfection of either the FAD-binding $(42.9 \pm 24.2 \% ; n=4)$ or calcium-binding $(52.1 \pm 25.8 \% ; n=5)$ domain mutated $\mathrm{m}-\mathrm{GDH} \mathrm{cDNA}$ was significantly lower than that measured in Cos-7 cells transfected with the wild-type form $(\mathbf{p}<0.01)$. Xenopus oocytes microinjected with FAD-binding domain mutated mRNA displayed on average an m-GDH enzymatic activity of $5.8 \%$ of that measured in those microinjected with the wild-type form. In summary, Cos-7 cells are suitable for the assessment of in vitro m-GDH enzymatic activity studies. The present results suggest that both the FAD-binding domain and calcium-binding domain of the $\mathrm{m}-\mathrm{GDH}$ should be considered for screening of putative $\mathrm{m}-\mathrm{GDH}$ mutations in type 2 diabetic patients.

\section{1}

COMMON AMINO ACID SUBSTITUTIONS IN IRS-4 ARE NOT ASSOCLATED WITH RANDOM NIDDM OR INSULIN RESISTANCE.

S. K. Frederiksen', K. Almind', M. G. Ahlgren', S. Urhammer', T. Hansen', J. O. Clausen $^{2}$ and O. Pedersen' ${ }^{1}$ 'Steno Diabetes Center and Hagedorn Research Institute, Copenhagen, Denmark, ${ }^{2}$ Center of Preventive Medicine, Glostrup University Hospital, Copenhagen, Denmark.

The family of insulin receptor substrates (IRS1-4) is defined by proteins with an overall similar structure. IRS-1 and IRS-2 have been shown to play key roles in cellular transmission of the action of insulin, IGF-1 and various cytokines. We have previously identified amino acid polymorphisms in the human IRS-1 and IRS-2 proteins. Given the documented importance of IRS-1 and 2 in insulin signaling and the implications of the disruption of these genes for the pathogenesis of insulin resistance and diabetes, we found that the most recent member of the IRS family, IRS-4, was a relevant candidate to examine for genetic variability which might be associated with subsets of diabetes or insulin resistance. The gene encoding IRS-4 was analysed by the SSCP technique in 83 Danish Caucasians with NIDDM. Four amino acid polymorphisms were identified: Arg411Gly, Gly584Cys, His879Asp and Lys883Thr. In an association study of 324 NIDDM patients and 267 normal glucose tolerant control subjects the polymorphism at codon 411 was found with allelic frequencies of $3.9 \%$ and $5.6 \%$, respectively, and the variant at codon 879 with frequencies of $19.2 \%$ and $18.0 \%$, respectively. Each carrier of the codon 411 variant was also carrier of the variant at codon 879 . The variants at codon 584 and 883 were each only found in one NIDDM patient. The frequencies of the variants at codon 411 and 879 were also determined in 380 young healthy subjects $(4.6 \%$ and $18.1 \%$, respectively). The insulin sensitivity index as estimated by Bergman's minimal model of the young healthy subjects carrying either polymorphism was indistinguishable from the carriers of wildtype IRS-4. Moreover, no males were heterozygous for the IRS-4 polymorphisms indicating that the gene is located on the $\mathrm{X}$-chromosome

In conclusion, amino acid polymorphisns in human IRS-4 are common in Caucasians but do not associate with random NIDDM or with insulin resistance in young healthy subjects.

\section{2}

Identification of a Missense Mutation (P33T) in the Insulin Promoter Factor1 (IPF-1) Gene in an Italian Patient with Early-onset Type 2 Diabetes C. Gragnoli*, T. Lindner*, F. Chiaromonte ${ }^{\circ}$, L. Colasurdo ${ }^{\circ}$, T. D' Antonio $0^{\circ}, F$. Gragnoli, G. Gragnolis, Manetti E*, Signorini AM *, and $G$. Marozzi *Department of Medicine, The University of Chicago, ${ }^{\circ}$ Santo Spirito Hospital, Rome, aUniversity of Studies of Siena, Siena

Maturity-onset diabetes of the young (MODY) is a monogenic form of diabetes mellitus, characterized by an autosomal dominant inheritance and onset usually before 25 years of age. Mutations in five different genes have been associated with MODY: hepatocyte nuclear factor-4a (HNF-4a)/MODY1, glucokinase/MODY2, HNF-1 $\alpha / \mathrm{MODY} 3$, IPF-1/MODY4 and HNF -1B/MODY5 We have been screering a group of 44 unrelated patients, with early-onset Type 2 diabetes who have a positive family history (at least two generations of affected) and onset before 39 years, for mutations in the known MODY genes. The coding region and flanking introns of the IPF-1 gene were amplified using PCR and the products were sequenced directly using an ABI PRISM 377 DNA Sequencer. In one of the 44 patients, there was a $\mathrm{C} \rightarrow \mathrm{T}$ substitution in codon 33 (CCT $\rightarrow \mathrm{ACT}$ ) of exon 1 resulting in a missense mutation, $\mathrm{Pro} \rightarrow \mathrm{Thr}$ ( $\mathrm{P} 33 \mathrm{~T})$. The identified patient has an age of onset of 30 years, a BMI of 33.3 , is treated with insulin and has retinopathy. Her identical twin has an age of onset of 46 years, a BMI of 32.4, is treated with both hypoglicemic oral agents and insulin, and has nephropathy and atherosclerosis. Both patients have carpal tunnel syndrome. To the best of our knowledge, this is only the second mutation identified in the IPF-I gene in subjects with diabetes. These studies are providing a better understanding of the etiology of early-onset Type 2 diabetes in Italians.

\section{3}

\section{IMPAIRMENT OF INSULIN SECRETION IN IMPAIRED GLUCOSE} TOLERANCE

T.W. van Haeften, W. Pimenta, A. Mitrakou, M. Korytkowski, T. Jenssen, H. Yki-Jarvinen, and J.E. Gerich. Dept Internal Medicine Utrecht University, Dept Endocrinology Pittsburgh University, Dept Endocrinology Helsinki University Dept Endocrinology University of Rochester (NY, USA), Dept Internal Medicine University Athens.

Since insulin levels after an oral glucose tolerance test are elevated in subjects with impaired glucose tolerance (IGT), it is often assumed that they have insulin resistance without impaired insulin secretion. We assessed insulin secretion in 98 subjects with IGT ( 59 with a first-degree relative with type 2 diabetes and 39 without, mean \pm sem age $49.3 \pm 1.1$ years, BodyMass Index (BMI) $28.0 \pm 0.5$ $\mathrm{kg} / \mathrm{M}^{2}$, waist/hip ratio (WHR) $0.88 \pm 0.01$ ) and in 185 subjects with normal glucose tolerance (NGT, 100 with a type 2 diabetes relative and 85 without (controls), age $42.9 \pm 0.8$ years, BMI $26.1 \pm 0.3 \mathrm{~kg} / \mathrm{M}^{2}$, WHR $0.82 \pm 0.01$ ) with hyperglycemic clamps (glucose $10 \mathrm{mmol} / \mathrm{L}, 180 \mathrm{~min}$ ). NGT with a type 2 relative had a lower first phase (Geometric mean $(95 \% \mathrm{CI})$ sum plasma insulin 0-10min: 647 (562-745) vs. $796(718-883) \mathrm{pmol} / \mathrm{L}$ ) and second phase secretion (average plasma insulin 140-180min: 266 (239-297) vs. 301 (272-334) pmol/L) than NGT controls (both $\mathrm{p}<0.05$ ). IGT with a type 2 relative and IGT subjects without a type 2 relative had a lower first (Geometric Mean (95\% CI) 480 (378-609) and 449 (359-562) pmol/L), and second phase (199 (160-248) and 209 (176-249) $\mathrm{pmol} / \mathrm{L}$ ) than NGT subjects (all $\mathrm{p}<0.001$ ). Insulin sensitivity index (ISI $=$ glucose infusion rate expressed per $\mathrm{kg}$ LeanBody Mass (kgLBM) divided by average plasma insulin) did not differ between NGT relatives and controls $(0.242 \pm 0.012$ vs. $0.228 \pm 0.011 \mathrm{umol} / \mathrm{kgLBM} / \mathrm{min} / \mathrm{pmol} / \mathrm{L}$ ). Taking age, gender, BMI and WHR as covariates, ISI were decreased both in IGT subjects without a relative and in IGT relatives $(0.179 \pm 0.019$ and $0.217 \pm 0.016 \mathrm{umol} / \mathrm{kgLBM} / \mathrm{min} / \mathrm{pmol} / \mathrm{L}$, respectively, both $\mathrm{p}<0.001$ ) as compared to their NGT counterparts. Conclusion: Insulin secretion is diminished in normo-glucose tolerant first-degree relatives of type 2 diabetes subjects without a change in insulin action. In IGT subjects, both insulin secretion and insulin action are decreased regardless of family history of diabetes. 


\section{4}

MUTATIONS IN THE HNF1 $\alpha$ PROMOTER IN MODY3 AFFECT HNF1 $\alpha$ EXPRESSION AT TRANSCRIPTIONAL LEVEL.

F. Godart, C. Bellanné-Chantelot*, P. Boutin. J.C. Chevre, S. Clauin* ${ }^{*}$, A. Abderrahmani, P. Froguel and B. Bailleul. CNRS ER-10-Lille Institute of Biology (France), ${ }^{*}$ Jean Dausset Foundation-CEPHB (Paris, France).

Mutations in the HNF1 $\alpha$ gene are responsible for MODY3, the most common form of Maturity Onset Diabetes of the Young. If most mutations have been found in the coding region, so far, one variation was recently described in the HNF4 binding site of the HNFl $\alpha$ promoter. Here, we report the identification of two other mutations; a $\mathrm{T}$ to $\mathrm{C}$ substitution at position +8 according to the initiation start identified in an isolated patient with early age of onset, and a $G$ deletion at position +107 which cosegrates with the diabetes through three generations in a MODY family. No other mutations in the HNFl $\alpha$ and Glucokinase coding regions were found in these patients. Functional studies with luciferase reporter constructs containing a $235 \mathrm{bp} \mathrm{HNF1} \alpha$ minimum promoter and transient transfections in HepG2 show that the +8 mutation exhibit a $30 \%$ decrease of promoter activity versus the wild type promoter. According to sequence analysis, this substitution is likely to disrupt the binding of a CCAAT binding protein. Works are in progress to analyse the mutation effects in a $\beta$-pancreatic cell line, and to identify the nuclear factors involved in this process and to characterise the loss of binding activity. These results suggest that monoallelic deregulation of HNF1 $\alpha$ transcription may yield a $\beta$-pancreatic defect leading to MODY 3 phenotype.

\section{6}

MUTATIONS IN THE CALCIUM BINDING-DOMAIN OF THE FAD-LINKED MITOCHONDRIAL GLYCEROLPHOSPHATE DEHYDROGENASE IN TYPE 2 DIABETIC PATIENTS

J.Vidal, M.Gudayol, E. Fernández-Usac, C.Franco, C. Rodríguez-Villar, R.Gomis, A.Novials. Endocrinology and Diabetes Unit. IDIBAPS. Hospital Clínic de Barcelona. Spain.

FAD-linked glycerolphosphate dehydrogenase (m-GDH), the limiting enzyme of the glycerolphosphate shuttle, has been considered a candidate gene for type $2 \mathrm{DM}$. We have recently reported mutations affecting the amino acid sequence of the calcium-binding domain of $\mathrm{m}-\mathrm{GDH}$ in a patient with type $2 \mathrm{DM}$ and his glucose intolerant sister. We aimed to screen for mutations in the $\mathrm{m}$-GDH calcium-binding domain in type 2 diabetic patients and to evaluate the effect of those mutations on insulin secretion in vivo and on m-GDH enzymatic activity in Xenopus oocytes overexpressing the constructed mutant form of the protein Screening for the previously described mutations was performed using Nla III and $T a q I$ restriction enzymes in 115 type 2 diabetics and 90 healthy controls. New mutations were screened using SSCP in a subset of 34 type 2 diabetics and 64 healthy controls. Insulin secretion in vivo was assessed throughout an IVGTT in the glucose intolerant woman positive for the mutations. Overexpressed m-GDH enzymatic activity in Xenopus oocytes was measured using a radioisotopic assay. Any subject was positive for $\mathrm{Nla}$ III digestion. Taq $I$ digestion positive patients were equally frequent in both study groups (type 2 DM: $12.8 \%$, control: $7.8 \%$ ). Direct sequencing of samples from type $2 \mathrm{DM}-\mathrm{Taq}$ I digestion positive patients did not reveal any other mutation. SSCP analysis was unremarkable. First phase insulin release (FPIR) from the IVGTT in the glucose intolerant women (IGTw) was under the fifth percentile of healthy agematched controls (FPIR: IGTw $40.2 \mathrm{mU} / \mathrm{l}$, control $5^{\text {th }}$ percentile $70.8 \mathrm{mU} / \mathrm{l}$ ). In vitro enzymatic activity of the calcium-site directed mutant form of m-GDH in Xenopus oocytes presented a $89.2 \%$ reduction compared to that measured in oocytes overexpressing the wild-type form of the enzyme. In summary, m-GDH calcium-binding domain mutations are not a common finding in type 2 DM. However, functional studies suggest that in same cases their existence may contribute to $\beta$ cell dysfunction.

\section{5}

GFNETIC AND METABOLIC CHARACTERISATION OF MODY IN SPAIN

A Costa, P Boutin, J Vidal, JC Chèvre, C Dell'Anna, R Casamitjana, J Ferrer, P Froguel, G Velho, R Gomis and I Conget. Endocrinology and Diabetes Unit, Hormonology Unit, Hospital Clínic i Universitari, Barcelona, Spain. CNRS EP10, Lille, France. INSERM U342, Paris, France.

Four MODY genes have been identified up to now: HNF-4a/MODY1 (chromosome 20q), glucokinase/MODY2 (chr. 7p), HNF-1a/MODY3 (chr. 12q) and IPF1/MODY4 (chr. 13). We have examined the prevalence of these MODY subtypes in a panel of $10 \mathrm{MODY}$ families of spanish ancestry, and characterised insulin secretion and insulin sensitivity in affected individuals. Mutations were searched by SSCP and/or by direct sequencing. An OGTT was performed in 22 affected family members. Beta-cell function $(\% \mathrm{~B})$ and insulin sensitivity (\%S) were computed from basal insulin and glucose values using the HOMA method. Two families were identified as MODY2 and five as MODY3 whereas no mutations were found in three families (MODYx). MODY2, MODY3 and MODYx subjects were comparable in terms of age, sex, BMI and duration of the disease. Fasting glucose was significantly higher in MODYx subjects than in the other two groups. The area under the curve (AUC) of glucose during the OGTT in MODYx subjects was 22\% higher than in MODY2 and $13 \%$ higher than in MODY3 subjects $(p<0.05)$. AUC of insulin was significantly higher in MODY2 $(7470 \pm 2144 \mathrm{mU} \cdot \mathrm{min})$ than in MODY3 $(1967 \pm 814 \mathrm{mU} \cdot \mathrm{min})$ and MODYx $(1549 \pm 475 \mathrm{mU} \cdot \mathrm{min})$ subjects $(p<0.05)$. Log $B$ was significantly lower in MODYx $(1.27 \pm 0.29)$ than in MODY3 $(1.67 \pm 0.30)$ and MODY2 subjects $(1.80 \pm 0.20),(\mathbf{p}<0.05)$, but no differences between groups were found in terms of $\% \mathrm{~S}$. In conclusion, $50 \%$ of the Spanish MODY families of our panel are MODY 3 and $20 \%$ are MODY2, while $30 \%$ of the families could not be allocated to any of the defects described up to now. MODYx subjects seem to present a more severe form of diabetes, with lower insulin secretion and higher hyperglycaemia, than MODY2 and MODY3 subjects, OGTT being extremely useful in order to further detect such a differences.

\section{7}

LIPOPROTEIN LIPASE POLYMORPHISM ASSOCIATED WITH INCREASED PLASMA TRIGL YCERIDES IN HEALTHY SUBJECTS

M. Klannemark ${ }^{1}$, L. Suurinkeroinen ${ }^{2}$, M. Orho', L. Groop ${ }^{1}$ and M.-R. Taskinen ${ }^{2}$ 'Dept. of Endocrinology, UMAS, Lund University, Malmö, Sweden and ${ }^{2}$ Dept. of Medicine, Helsinki University Central Hospital, Helsinki, Finland.

The lipoprotein lipase (LPL) Asn291Ser polymorphism has previously been associated with familial combined hyperlipidemia and increased risk of ischemic heart disease. The aim of this study was to investigate if the Asn291Ser polymorphism was increased in 197 unrelated Finnish NIDDM-patients (94M/104F, age $64 \pm 12$ years, $\mathrm{HbA}_{1 \mathrm{c}} 7.3 \pm 1.5 \%$ and plasma triglycerides $\left.(\mathrm{Tg}) 2.1 \pm 1.1 \mathrm{mmol} / \mathrm{l}\right)$ and 199 unrelated healthy Finnish 1st degree relatives of NIDDM-patients (NGT-rel; $113 \mathrm{M} / 86 \mathrm{~F}$, age $57 \pm 14$ years, $\mathrm{HbA}_{\mathrm{lc}} 5.4 \pm 0.4 \%$ and $\left.\mathrm{Tg} 1.6 \pm 0.9 \mathrm{mmol} / \mathrm{l}\right)$ vs. 217 unrelated healthy Finnish controls $\left(103 \mathrm{M} / 114 \mathrm{~F}\right.$, age $56 \pm 10$ years, $\mathrm{HbA}_{1 \mathrm{c}} 5.5 \pm 0.4 \%$ and $\operatorname{Tg} 1.3 \pm 0.7 \mathrm{mmol} / \mathrm{l})$, all patricipating in the Botnia study. Asn291Ser was genotyped with allele-specific PCR and agarose electrophoresis. The frequency of the Asn/Ser genotype was similar between the three groups $(6.0 \%$ in controls, $9.5 \%$ in NGT-rel and 6.6\% in NIDDM). However, when subdivided by $\mathrm{Tg}$ (HiTg $>1.7 \mathrm{mmol} / 1$, NorTg $<1.7 \mathrm{mmol} /$ ), the frequency of Asn/Ser was increased in controls with $\mathrm{HiTg}\left(13.0 \%, \chi^{2}=6.3,1\right.$ d.f., $\left.\mathrm{p}<0.05\right)$ and NGT-rel with $\mathrm{HiTg}(20.4 \%$, $\chi^{2}=15.8,1$ d.f., $\left.\mathrm{p}<0.0001\right)$ vs. controls with NorTg $(3.7 \%)$. Additionally, the frequency of the Asn/Ser genotype was increased in NGT-rel with HiTg vs. NGT-rel with NorTg ( $20.4 \%$ vs. $5.5 \%, \chi^{2}=10.0,1$ d.f., $\left.p<0.005\right)$. Totally, 32 controls and NGT-rel carried the Ser allele and had significantly increased $\mathrm{Tg}(1.91 \pm 0.86$ vs. $1.39 \pm 0.82, \mathrm{p}<0.005$ ), decreased plasma HDL cholesterol (HDL; $1.24 \pm 0.25$ vs $1.42 \pm 0.35, \mathrm{p}<0.0005$ ) and decreased plasma HDL2 cholesterol (HDL2; $0.42 \pm 0.22$ vs. $0.55 \pm 0.28, p<0.005)$ compared to Asn homozygotes. These differences were particularly marked in 14 female carriers, whereas no significant differences could be seen in 18 males. Furthermore, these differences could not be seen between carriers and non-carriers with NIDDM. In conclusion, the frequency of the LPL Asn/Ser genotype is increased in healthy individuals with $T g>1.7 \mathrm{mmol} / \mathrm{l}$ and associates with an altered lipid profile (increased Tg, decreased HDL and HDL2), an effect that is particularly prominent in female carriers. Thus, the Asn/Ser genotype may generate an increased risk for dyslipidemia and predispose to early cardiovascular complications in NIDDM. 


\section{8}

RENAL PROXIMAL TUBULAR DEFECTS ASSOCIATED TO THE MODY3 PHENOTYPE.

G. Velho, F. Benqué-Blanchet, M. Vaxillaire, M. Pontoglio, P. Froguel, G. Friedlander, D. Prié. INSERM U342, Paris; Service d'Explorations Fonctionnelles, Hôpital Bichat, Paris: CNRS EP10, Institut Pasteur, Lille; CNRS URA1644, Institut Pasteur, Paris, France.

Mutations in the gene encoding the transcription factor HNF-1a (hepatocyte nuclear factor 1 alpha) have been found in $25-50 \%$ of the MODY families. MODY3/HNF-1a is expressed in the pancreas and the liver, but also in the kidney and other organs. As severe renal tubular defects have been observed in HNF-1a knock-out mice, we have evaluated renal tubular function in MODY 3 subjects. Six patients from three families with three different mutations were studied. We have determined the thresholds of renal resorption of glucose (TM-glucose) and phosphate (TM-PO4), and the rate of renal excretion of aminoacids. The TM-glucose was decreased in all subjects $\left(<9 \mathrm{mM} / 1.73 \mathrm{~m}^{2}\right.$ body surface; usual values: $12-14$ $\left.\mathrm{mM} / 1.73 \mathrm{~m}^{2}\right)$. TM-PO4 was decreased $(<0.60 \mathrm{mM}$; usual values: $0.75-1.45 \mathrm{mM}$ ) only in 2 unrelated patients, who also presented hypophosphatemia $(<0.8 \mathrm{mM}$; usual values: $0.85-1.40 \mathrm{mM})$, with normal plasma parathyroid hormone values. Three patients out of 4 tested showed a moderate increase in the renal excretion of glycine (194-322 $\mathrm{mmol} / \mathrm{mmol}$ of creatinine; usual values: $43-173$ ) and alanine (89-112 $\mathrm{mmol} / \mathrm{mmol}$ of creatinine; usual values: $16-68)$. No patient presented with renal proximal tubular acidosis. Unlike the knock-out mice, the patients did not present phenylcetonuria. In conclusion, the phenotype associated with HNF-1 a mutations in humans is complex and heterogeneous. Defects in the renal resorption of glucose, phosphate and aminoacids may be observed, associated to the pancreatic beta cell defect.

GENETIC ANALYSIS OF AN INDIGENOUS AUSTRALIAN PEDIGREE WITH NIDDM

P.K. Lovelock ${ }^{1,2}$ M Kishimoto-Hashiramoto ${ }^{1}$ I B. Kesting ${ }^{1}$, M Marczak $^{3}$. D.J. Cavallucci ${ }^{1}$. J. Cardinal ${ }^{1}$, J.R Berkholz ${ }^{1}$, and I.T. E. Shaw ${ }^{1}$ Dept of Diabetes \& Endocrinology, Princess Alexandra Hospital Brisbane', Centre for Molecular and Cellular Biology, University of $\mathrm{Qld}^{2}$ and Redlands Health Service Centre ${ }^{3}$, Australia. Non-insulin dependent diabetes mellitus (NDDM) is a major public health problem for Australia. The prevalence of NIDDM among Australians of European descent is 3.4\%, however prevalence rates more than six times higher have been reported for indigenous Australian communities. The average age of onset of NIDDM is lower in this population, with the maximal prevalence at age 40 years. NIDDM is most common in westernised indigenous communities, and correlates with a high prevalence of obesity, hyperinsulinaemia and hypertriglyceridaemia. The epidemiological and pathophysiological characteristics of NDDM in the indigenous Australian population have led to the hypothesis of the "thrifty genotype", however to date the nature of the genetic predisposition is unknown. Three loci contribute to diabetes susceptibility in different European pedigrees with Maturity-Onset Diabetes of the Young (MODY), however the role of these loci in indigenous Australian diabetes is not known. The objective of the study is to identify novel mutations which result in diabetes susceptibility through the application of exclusion mapping with polymorphic markers. This paper describes a single 7 generation pedigree of indigenous Australian descent comprising 246 living subjects from 3 generations, of whom 64 have NIDDM. The mean age of the diabetic subjects in the pedigree is $53 \pm 12$ years $( \pm 1$ SD). 29 are on dietary therapy alone, 33 are on oral hypoglycaemic agents and 2 are on insulin treatment. The mean BMI of the diabetic subjects is $32 \pm 7 \mathrm{~kg} / \mathrm{m}^{2}$ and 16 have clinical evidence of atherosclerotic vascular disease. In order to characterise the genetic abnormality in the pedigree we have commenced linkage analyses with the three loci previously defined as causing dominantly-inherited diabetes. There is no evidence for linkage of NIDDM with the microsatellite markers D12S807 (near the MODY3 locus, chromosome 12q), GCK1 and GCK3 (glucokinase locus, chromosome 7p) or with OL3, D20S527 and D20S112 (near the MODYI locus, chromosome 20q). If these results are confirmed with additional typing we will proceed to a genome-wide scan to identify novel gene(s) contributing to NIDDM susceptibility in this pedigree.
419

SCREENING OF SUR 1 AND KIR6.2 PROMOTERS FOR MUTATIONS IN DIFFERENT TYPES OF TYPE 2 DIABETES

A.L. Gloyn, Y Hashim and R.C. Turner. Diabetes Research Laboratories, Oxford University, Oxford, UK.

The $\beta$-cell ATP regulated K-channel (K-ATP) consists of 2 subunits, the sulphonylurea receptor (SUR 1) and the inwardly rectifying K-channel (Kir6.2) Mutations in the nuclear binding fold regions (NBFs) of SUR 1 and in Kir6.2 have been found to cause persistent hyperinsulinaemic hypoglycaemia of infancy. Population association studies have shown polymorphisms in SUR 1 at exon 18 (T761T) and intron 16 splice acceptor site -3 tagGCC to -3 cagGCC were significantly more prevalent in patients with type 2 diabetes than normal control subjects, suggesting that these polymorphisms could be in linkage disequilibrium with a pathogenic mutation. Mutations in the promoter region of either gene could result in abnormal expression and regulation of K-ATP channel with decreased insulin secretion. We have screened both promoter sequences and the remaining 20 exons of SUR 1 , by single stranded conformational polymorphism (SSCP) analysis in $\beta$-cell deficient subtypes of white caucasian patients with type 2 diabetes who are islet cell antibody (ICA) and glutamic acid decarboxylase antibody (GADA) negative, and in normoglycaemic controls: (i) 20 non-obese (BMI $<27 \mathrm{~kg} \cdot \mathrm{m}^{-2}$ ) who presented at age $<50 \mathrm{yrs}$ with fasting plasma glucose (FPG) $<8 \mathrm{mmol} / \mathrm{l}$ (i.e. similar to glucokinase deficient, MODY 2 patients) (ii) 20 non-obese subjects who presented with FPG $>12$ mmol/l who have marked $\beta$-cell deficiency and a family history of diabetes (iii) 20 type 2 diabetic subjects with both exon 18 and intron 16 mutations (iv) 20 tandomly selected patients with type 2 diabetes (v) 20 normoglycaemic controls. A silent variant in exon 12 of SUR 1 was not associated with diabetes. No polymorphisms were detected in the Kir6.2 promoter. A 3 base insertion/deletion was detected in SUR 1 promoter and is now being evaluated in larger numbers and different ethnic groups, (including the possibility of linkage disequilibrium with the $16 / 18$ putative haplotype), to determine if abnormal expression of SUR 1 could contribute to type 2 diabetes.
421

\section{IS GENETIC DENSITY A KEY DETERMINANT OF AGE-AT-ONSET IN TYPE 2 DIABETES?}

M. Sargın, A Şengül, S. Salman, K. Karşıdağ, E. Özer, F. Salman, Y. Yılmaz, 1. Satman, N. Dinççağ and M.T. Yılmaz. Diabetes Division, Istanbul Faculty of Medicine, and Institute for Experimental Medical Research, Istanbul University, Istanbul, Turkey.

This study is designed to investigate the concept of genetic density and its effect on onset of clinical type 2 diabetes and to define the characteristics of genetic transition in relation to degree of relation to degree of relativity.

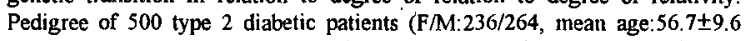
years) who were consecutively admitted to the Outpatient Clinic of Diabetes Unit between 1996 and 1997 were examined based on interrogation for obesity and/or diabetes among $1 \mathrm{st}$, 2nd and $3 \mathrm{rd}$ degree relatives. The study covered 10,017 individuals. Of the 500 type 2 diabetic patients $81.8 \%$ had at least one diabetic relative. Considering all degree relatives, the mean age at onset of diabetes for those who had no diabetic relative was $58.3 \pm 10.4$ years: while diabetes in patients who had one diabetic relative appeared earlier at $53.2 \pm 8.5$ years. Similarly, we noticed that the age-at-onset in patients with two diabetic relatives was $49.0 \pm 6.5$ years, the same parameter in patients with three diabetic relatives was $45.8 \pm 6.4$ years, in those with four or five diabetic relatives was $41.6 \pm 10.3$ years, and finally having more than five diabetic relatives led to youngest-onset diabetes at $38.7 \pm 6.9$ years $(p<0.001$ ). The same trend was observed for increased number of diabetic closer-degree diabetic relatives. No difference was noticed between two sexes. Our findings have suggested that most of the distinct clinical diabetes entities might indeed be younger-onset diabetes forms that were simply negatively correlated to genetic density. 
INCREASED FREQUENCY OF THE PPAR-GAMMA POLYMORPHISM (P12A) IN PATIENTS WITH NIDDM.

W.A. Mann, D. Evans, J. de Heer, D. Wendt, D. Berg, and U. Beisiegel. Medical Clinic, University Hospital Eppendorf, Hamburg, Germany

Peroxisome proliferator activating receptors (PPAR) are important regulators of several genes involved in glucose and lipid metabolism. Activation of PPAR gamma by thiazolidinediones results in amelioration of glucose homeostasis by increasing insulin sensitivity. Thus PPAR gamma may play a role in the manifestation of NIDDM. Recently a polymorphism in the PPAR gamma gene has been described (Pro $12 \mathrm{Ala}$ ). We investigated the frequency of this polymorphism in patients with NIDDM $(n=40)$, with and without accompanying hypertriglyceridemia (HTG). For comparison patients $(\mathbf{n}=92)$ without NIDDM with or without HTG were analysed. The following table shows the number of patients analysed until now, body mass index (BMI) in $\mathrm{kg} / \mathrm{m}^{2}$, plasma triglycerides (TG) in $\mathrm{mg} / \mathrm{dl}$, and the frequency of the $\mathrm{P} 12 \mathrm{~A}$ polymorphism and A-carriers (in \%)

$\begin{array}{lllllllr} & \text { n } & \text { BMI } & \text { TG } & \text { PP } & \text { PA } & \text { AA } & \text { A-car. } \\ \text { + NIDDM + HTG } & 20 & 30 & \mathbf{8 7 4} & 11 & 7 & \mathbf{2} & \mathbf{4 5} \\ \text { + NIDDM - HTG } & 20 & 26 & 176 & 14 & 6 & 0 & 30 \\ & & & & & & & \\ \text { - NIDDM + HTG } & 30 & 28 & \mathbf{4 7 6} & 23 & 7 & 0 & 23 \\ \text { - NIDDM - HTG } & 62 & 24 & 152 & 49 & 12 & 1 & 21\end{array}$

Thus the frequency of the A allele was higher in patients with NIDDM than in patients without NIDDM $(p=0.04)$ Though the highest $A$ allele frequency was patients without NIDDM $(p=0.04)$ Though the highest $A$ allele frequency differ in non diabetics with or without HTG. Our results suggest a role for variation in thePPAR-gamma gene in the pathogenesis of NIDDM.

\section{4}

REDUCING MISCLASSIFICATION BIAS IN THE HOMA INDICES OF INSULIN RESISTANCE AND SECRETION.

A.B. Jenkins', K. Samaras ${ }^{2}$, D.G. Carey ${ }^{3}$ and L.V. Campbell ${ }^{2}$ University of Wollongong', Garvan Institute ${ }^{2}$, NSW and Princess Alexandra Hospital ${ }^{3}$, QLD Australia.

Homeostasis model assessment (HOMA) provides indices of insulin secretion (B) and insulin resistance (B) from fasting glucose and insulin levels. When we applied this model to data from twins, a significant heritability of fasting plasma glucose $(F P G)\left(h^{2}=0.75, p<0.01\right)$ in these data was not accounted for by any heritability of the HOMA indices $\left(B ; h^{2}=0.28, p\right.$ $>0.3 ; \mathrm{R}: \mathrm{h}^{2}=-0.08, p>0.5$ ). This result is consistent with a misclassification between effects due to insulin secretion and resistance in the HOMA indices. We report here evidence of misclassification in the HOMA indices and describe a minor modification to the model which corrects it. Direct measures of insulin resistance (euglycemic ctamp) and secretion ( $N$ glucose measures of insulin resistance (euglycemic damp) and secretion (N glucose
bolus) were obtained in 43 non-diabetic subjects ( $M / F 8 / 33$, Age $39 \pm 11$ (SD) $y r$, BMI $25.6 \pm 4.2 \mathrm{~kg} / \mathrm{m}^{2}$ ). Heritability was estimated by statistical modelling of genetic and environmental influences in data from 214 non-diabetic female subjects (107 twin pairs, Age $52 \pm 14$, BMI 25.244.2). The HOMA model was reformulated, independently of the direct measures data in terms of relationships between $L n$-transformed fasting plasma insulin ( $L(F P I)), F P G$ and insulin sensitivity. The modified indices were obtained from $\beta^{\prime}=(\operatorname{Ln}(\mathrm{FPl})$ c)/FPG and $\mathrm{R}^{\prime}=(\operatorname{Ln}(\mathrm{FPI})-\mathrm{c})^{*} \mathrm{FPG}$ where $\mathrm{c}$ is a constant (-4.64). Indices from both models correlated with the direct measures similariy $(r=0.63$ (R), 0.49 $\left(R^{\prime}\right), 0.45(B), 0.39\left(B^{\prime}\right)$, all $\left.p<0.01\right)$. Directly measured insulin resistance and secretion were not significantly correlated $(r=0.13, p=0.21)$. However, $B$ and $R$ were strongly related $(r=0.78, p<0.0001 \vee 0.13)$ demonstrating substantial misclassification. The relationship between $B^{\prime}$ and $R^{\prime}(r=0.13)$ was not different to that between the two direct measures. Significant heritability of $B^{\prime}:\left(h^{2}=0.68, p<0.01\right)$; and $R^{\prime}\left(h^{2}=0.59, p<0.05\right)$ was found in the twin data. The inaccuracy in the HOMA indices caused by misclassification may lead to erroneous conclusions concerning the roles of insulin resistance and insulin secretion in pathology. The modification we propose significantly reduces the misclassification and exposes separate components of insulin resistance and insulin secretion in the heritability of FPG. We conclude that this modification to the method is necessary for its reliable use.
SCREENING FOR MUTATIONS IN THE GLUCOKINASE (MODY2/GCK) AND HEPATOCYTE NUCLEAR FACTOR $1-\alpha$ (MODY3/HNF1- $\alpha$ ) GENES IN SOUTH-ITALIAN FAMILIES.

C. Bellanné-Chantelot', D. lafusco², H. Blanché', M.R. Puca', S. Clauin', V. Morel', I. Le Gall', G. Thomas' and F. Prisco ${ }^{2}$. 'Fondation Jean DaussetCEPH, Paris, France; ${ }^{2}$ Department of Pediatrics-Second University of Naples, Naples, Italy.

The MODY2/GCK and MODY3/HNF-1 $\alpha$ genes are implicated in maturityonset diabetes of the young (MODY, a form of non-insulino-dependent diabetes mellitus (NIDDM) characterized by an early age of onset and by autosomal dominant inheritance. Here, we report the prevalence of these two MODY genes in 18 unrelated pedigrees recruited in south-Italy and explored after the fortuitous diagnosis of a fasting hyperglycemia occuring in a family history of diabetes. We identified 11 novel mutations in the MODY2/GCK gene: 7 missense mutations (L30P, Q38P, T1491, I225M M235T, E265K, D278E), 2 splice site mutations (K15 +1G->T, L288 -1G->A) and 2 deletions (K104del4, A460del22). In MODY3/HNF-1 $\alpha$ gene, we detected two mutations, one is a novel missense mutation T354M and the other (P291insC) is localized in the polyC tract in exon 4 described as a mutational hot spot. The segregation of these mutations with the diabetes were checked in all families. Surprisingly, three of these mutations correspond to de novo mutations (L3OP and T149I in MODY2/GCK; $\mathrm{P} 291 \mathrm{fsinsC}$ in MODY3/HNF-1 $\alpha$ ). Five patients presenting also a profile of mild hyperglycemia were negative for the MODY2 and MODY3 genes. The observed frequencies in these south-Italian families of $61 \%$ and $11 \%$ for MODY2/GCK and MODY3/ HNF-1 $\alpha$ genes respectively, are quite different from those reported in the litterature for caucasian families. And except one proband who is MODY 3 and who has a more severe form of diabetes, clinical and biological data could not discriminate these families. This emphasizes the interest of the molecular study of MODY as the prevalence of diabetes complications differs between the MODY2 and MODY 3 diabetes. Thus the follow up of young patients presenting a hyperglycemia could be better suited.

\section{5}

DEMONSTRATION OF THE A3243G MITOCHONDRIAL MUTATION IN FAMILIES WITH MATERNALLY INHERITED DIABETES AND DEAFNESS C. Thorns, A. Widjaja", N. Boeck, C. Skamira" and H. Zühlke. Hannover Medical School, Dept's of Biochemistry and "Clin. Endocrinol., D-30625 Hannover, Germany.

The mitochondrial(mt) DNA codes for polypeptides of the oxidative phosphorylation pathway, rRNAs and tRNAs. A point mutation of the mt tRNA A3243G has been observed in some diabetic patients with maternally inherited diabetes. We here decribe two german families with this mutation in some cases suffering from maternally inherited diabetes and deafness (MIDD). We screened 58 diabetics with a possible maternal mode of inheritance. 41 patients (70\%) were treated with insulin, 23 of these patients $(56 \%)$ were initially treated with oral antidiabetic drugs (OADD) and required insulin years later. 17 patients $(30 \%)$ were treated with OADD and diet restrictions. The BMI ranged from $19 \mathrm{~kg} / \mathrm{m}^{2}$ to $41 \mathrm{~kg} / \mathrm{m}^{2}$ (mean $27.9 \mathrm{~kg} / \mathrm{m}^{2}$ ). They developed diabetes mellitus between the age of $12 \mathrm{y}$ to $78 \mathrm{y}$ (mean $42.2 \mathrm{y}$ ). Oral mucosa was obtained for DNA isolation in 21 patients, in the remaining 37 patients EDTA-blood was used. Mucosa cells were lysed with $\mathrm{NaOH}(50 \mathrm{mM})$ and neutralized with Trisbuffer $\left(1 \mathrm{M}, \mathrm{p}_{1} 8.0\right)$, DNA was isolated by phenol-chloroform extraction. For DNA isolation from peripheral blood we isolated white blood cells. A $500 \mathrm{bp}$ fragment encompassing the mutation was amplified using PCR: sense primer (2295-3012): 5'ggatcaggacatcccgat3', antisense primer (3494-3476): 5'ggtttaggggctcttgg3', denaturation for $45 \mathrm{~s}$ at $92^{\circ} \mathrm{C}$, annealing for $40 \mathrm{~s}$ at $59^{\circ} \mathrm{C}$, extension for $45 \mathrm{~s}$ at $72^{\circ} \mathrm{C}, 32$ cycles. Since the mutation creates a restriction site for BSP 120I, the fragment was digested with BSP $120 \mathrm{I}$ followed by electrophoresis on a $1.8 \%$ agarose gel. Using this method with not yet published primers we obtained 2 oligonucleotides of comparable size increasing the sensitivity of our method 2 times (detection of $1 \%$ mutated mtDNA). Hearing acuity was examined with pure tone audiometry. The mutation was found in oral mucosa cells of 7 patients, but not in blood cells. As known from the literature, the mode of inheritance was maternal, all diabetic patients carrying the mutation show hearing loss = MIDD, but age of onset (in our study 36-45y) and BMI $\left(>26 \mathrm{~kg} / \mathrm{m}^{2}\right.$ ) differ from other data. MIDD may be combined with neuronal disorders. 
426

FUNCTIONAL ANALYSIS OF NATURALLY OCCURING VARIANTS IN THE HEPATOCYTE NUCLEAR FACTOR- $4 \alpha$ GENE

AM Møller', L Suaud ${ }^{2}$, SA Urhammer', T Hansen', B Laine ${ }^{2}$, O Pedersen $^{2}$

'Steno Diabetes Center and Hagedorn Research Institute, Copenhagen, Denmark, ${ }^{2}$ U459 INSERM, Lille, France.

The hepatocyte nuclear factor- $4 \alpha$ (HNF- $4 \alpha$ ) protein is a transcription factor belong ing to the steroid/thyroid hormone receptor superfamily and regulates many essential genes involved in liver, kidney and pancreatic islets differentiation and metabolism. Mutations in the gene encoding the HNF-4 $\alpha$ protein are the cause of a form of maturity onset diabetes of the young (MODY1), a subtype of non-insulin-dependent diabetes mellitus (NIDDM). We have previously identified two genetic variants in the HNF- $4 \alpha$ gene in a search for genetic defects causing diabetes in the Danish lateonset NIDDM patients. One of these variants (Val255Met) was exclusively identified in NIDDM patients $(0,8 \%, \mathrm{~N}=477)$, while the other variant (Thr130lle) was associated with NIDDM in the Danish population $(4,7 \%(95 \% \mathrm{Cl}: 3,4-6,0)$ vs. $1,9 \%(0,7-$ $3,1), \mathrm{p}=0,008)$ but not in the closely related Swedish population $(5,4 \%(2,7-8,1)$ vs. $5,1 \%(3,9-6,3))$. In order to examine whether these genetic variants have any impact on the development of NIDDM, the transactivation activity of the two mutants and the wildtype HNF-4 $\alpha$ proteins were examined in transient transfection assays in C33 cells. The DNA binding capability of the mutants and the wildtype HNF- $4 \alpha$ proteins were examined as well. There was no difference in the transactivation activity of the mutants and the wildtype HNF- $4 \alpha$ proteins when examined in cotransfection with luciferase reporter gene constructs carrying the HNF- $4 \alpha$ responsive element of either the HNF-l $\alpha$ (normalised activities (mean $+/$ - SD): wt: $100 \%$ vs. Thr 1301 le: $89,3 \%$ $(80,2-98,4)$ / Val255Met: $86,3 \%(76,8-95,8))$ or the ApoCII promoter (wt: $100 \%$ vs. Thr130lle: $97,3 \%(91,4-103,2)$ / Val255Met: $92,7 \%(83,9-101,5))$. Also the DNA binding activity of the mutants and the wildtype HNF-4 $\alpha$ proteins were similar when examined on the HNF- $4 \alpha$ binding site of the HNF- $1 \alpha$ promoter. Therefore, using the above mentioned assays, no difference between the Thr130Ile, the Val255Met and the wildtype HNF- $4 \alpha$ protein could be identified.
427

ADRENERGIC RECEPTOR AND UNCOUPLING PROTEIN GENE VARIANTS AND SUSCEPTIBILITY TO NIDDM AND RELATED CONDITIONS

P.J.Saker, S.J.Huxtable, E.Kousta, S.Robinson, N.Gharani, G.Conway, S.Franks, C.Warden, G.A.Hitman, D.G.Johnston and M.I.McCarthy. Imperial College School of Medicine; St Bartholomew's and Royal London School of Medicine; Middlesex Hospital London, UK; University of California, Davis, USA.

Defective thermogenesis is a feature of women with gestational diabetes (GDM) and polycystic ovary syndrome (PCOS): both conditions are associated with later NIDDM. Variation in genes regulating this process (B2AR, B3AR, UCP1, UCP2, UCP3) may influence susceptibility to NIDDM, obesity and related clinical phenotypes: the aim of this study was to extend the inventory of variation in these and other relevant candidates and to correlate genomic and phenotypic variation. The entire coding sequence of $B 3 A R$ and UCP1 and exons 3-8 of UCP2, plus immediate 5 ' sequence of B3AR and UCP1 have been screened for mutations in a set of 103 women of mixed ethnic origin with either (a) previous GDM and/or (b) PCOS, the latter selected for extreme BMI and WHR. Apart from the previously-reported B3AR Trp64Arg and UCP1 -3826 variants, we have identified the following non-silent variants. In B3AR, a novel $g \rightarrow$ t polymorphism (in a single Bengali GDM subject) 14bp $3^{\prime}$ of exon 1 with potential consequences for mRNA splicing; in UCP1, a Met229Leu variant (present at $\sim 7 \%$ in all groups with no differences in allele frequency); in UCP2, (i) an Ala55Val variant (exon 4: variant frequency $\sim 40 \%$, no differences in allele $P=0.44$, or genotype frequencies, $P=0.67$, between groups on case-control analysis), and (ii) a $45 \mathrm{bp}$ duplication in the $3^{\prime}$ UTR (variant frequency $\sim 20 \%, P=0.36$ and $P=0.57$, respectively). Within each (GDM, PCOS, control) cohort, we found no association between genotype at these UCP2 loci and weight, BMI, or fasting plasma glucose (all $P>0.05$ ). The phenotypic relevance of these variants was further assessed by TDT in (a) 80 South indian (SI) NIDDM parent-offspring trios and (b) 61 European PCOS trios. These analyses also fail to support a positive role for any of the variants described (eg -3826 variant in $S \mid, P=0.61$, Trp64Arg in PCOS, $P=0.21$; exon 6 duplication in $P C O S, P=0.77$ ). Within the limits of the phenotypes and portions of the genes thus far examined, genomic variation appears to show no correlation with variation in these diabetes- and obesity-related phenotypes.

\section{8}

STUDIES OF THE VARIABILITY IN THE GENES ENCODING THE INSULIN PROMOTER FACTOR-1 AND ITS UPSTREAM ACTIVATOR NEUROD/BETA2 IN DANISH CAUCASIAN NIDDM FAMILIES.

T. Hansen, L. Hansen, S. Urhammer, H. Eiberg, O. Pedersen. Steno Diabetes Center and Hagedorn Research Institute, Gentofte. Dept of Biochemistry and Genetics, University of Copenhagen, Denmark. The recent development of transgenic mouse models carrying null alleles of either the pancreatic homeobox gene, IPF-1, or the endodermal factor NeuroD/Beta2 has shown that animals homozygous for the IPF-1 null alleles have pancreatic agenesis and animals homozygous for the NeuroD/Beta2 null alleles have disorganized islets and failure of proper $\beta$-cell maturation. Both types of transgenic mice dye from diabetes postnatally. In man, a frameshift deletion in exon 1 of the IPF-1 gene has been linked to maturity-onset diabetes of the young (MODY4) in one family. Consequently, we have tested the hypothesis that structural changes in these genes crucial to the morphogenesis of either the whole pancreas (IPF-1) or the islets (NeuroD/Beta2) could be responsible for the onset of diabetes in subsets of NIDDM families of Danish ancestry. By PCR-SSCPheteroduplex analysis we scanned the IPF-1 gene in 5 segments and the NeuroD/Beta2 gene in 6 segments for mutations in one diabetic proband from each of 82 Danish NIDDM families. We found a guanine nucleotide insertion in the 5'-UTR at position - 109 from the translation initiation codon in the IPF-1 gene and in the NeuroD/Beta2 gene we found a frequent A45T and a less frequent $\mathrm{P} 197 \mathrm{H}$ amino acid substitution. The allelic frequency of the A45T polymorphism was 0.36 in 154 NIDDM patients and 0.37 in 210 age matched control subjects whereas the $\mathrm{P} 197 \mathrm{H}$ was equally frequent in each group $(0.03)$ We conclude that mutations in the coding region of IPF-1 and NeuroD/Beta2 are unlikely to contribute to the genetics of NIDDM in the Danish Caucasian population. Further studies are needed to establish the potential role of the NeuroD/Beta2 amino acid variants on insulin secretion in the carriers.

\section{9}

THE ISLET AMYLOID POLYPEPTIDE GENE SER20GLY MUTATION AND GLUCAGON RECEPTOR GENE GLY4OSER MUTATION IN CHINESE

K.S.Xiang,T.S. Zheng,H.J.Lu,D.Q.Sun,J.X.Lu and J.Li. Shanghai 200233, China

It has been reported that the Ser20Gly mutation of the islet amyloid polypeptide (IAPP) gene was associated with type 2 diabetes in Japanese, and the Gly40Ser mutation of the glucagon receptor (GCGR) gene was associated with type 2 diabetes in French and British Caucasians as well as a subset of type 2 diabetes with positive family history in German Caucasians.Since geographic restricted distribution of these mutations in ethnic groups was suggested,The aim of this study is to ascertain the presence of these mutations and its relation to type 2 diabetes in Chinese. 825 Chinese subjects were genotyped for the Ser20Gly mutation of IAPP gene with PCR-RFLP MspI digestion. The mutation was observed in $17 / 609(2.79 \%)$ subjects with type 2 diabetes, and only in $1 / 216$ $(0.46 \%)$ subjects with non-diabetics $(\mathrm{P}<0.05)$. The mutation detected by PCRRFLP was confirmed to be the $A$ to $G$ point mutation in the nucleotide 582 of IAPP gene cDNA encoding the amino acid codon 20 (AGC to GGC). All the mutation carriers detected were heteozygotes. Comparisons of clinical features between mutation carriers and non -carriers in type 2 diabetic patients showed that higher fasting insulin levels 70.44(26.88 184.98)pmol/L vs. 42.18(6.06 293.88) $\mathrm{pmol} / \mathrm{L}(\mathrm{P}=0.004)$, and more patients with diabetic family history $(58.8 \% \mathrm{vs} .21 .4 \%)$ $(\mathrm{P}=0.001)$ were observed in mutation carriers. 276 subjects with type 2 diabetes were genotyped for the Gly40Ser of GCGR gene with PCR-RFLP BstE II digestion. Gly40Ser mutation was not detected in this group. In conclusion,there are significant frequency differences of the IAPP-Ser20Gly and GCGR-Gly40Ser mutations among ethnic groups. Only the former is present in Chinese and is associated with type 2 diabetes.

* Log 10 transformed during statistical analysis and expressed as geometric means with $95 \%$ confidential interval. 
430

AN ASSOCIATION BETWEEN UCP2 AND BODY MASS INDEX IN A SOUTH INDIAN POPULATION

${ }^{\text {ap.G. Cassell, }}{ }^{\mathrm{M}} \mathrm{M}$. Neverova, ${ }^{\text {aS }}$. Janmohammed, ${ }^{\text {a N.C. Uwakwe, }}$ "A. Qureshi, ${ }^{a}$ P.G. Kopelman, ${ }^{b} \mathrm{~A}$. Ramachandran, ${ }^{b} \mathrm{C}$. Snehalatha, ${ }^{\mathrm{C}} \mathrm{D}$. Ricquier, ${ }^{\mathrm{d}} \mathrm{C}$.H. Warden and ${ }^{\mathrm{a}} \mathrm{G} . \mathrm{A}$. Hitman. ${ }^{\mathrm{a}}$ Medical Unit, St. Bartholomew's and The Royal London School of Medicine, London, U.K., 'Diabetes Research Centre, Chennai, India,

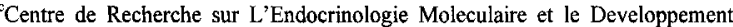
France and "Rowe Institute in Genetics, U.C.L.A., United States of America.

The uncoupling proteins (UCP) play an important role in energy expenditure and therefore are candidate genes for the aetiology of both obesity and Type 2 diabetes. Recently, linkage has been found between markers close to uncoupling protein 2 (UCP2) and resting metabolic rate. The purpose of our studies was to study a newly described UCP2 variant in exon 8 in obesity and Type 2 diabetes. Exon 8 of UCP2 was sequenced and a 45 base pair insertion was identified which is a duplication of the preceding 45 base pairs. DNA was prepared from 449 South Indian subjects from a cross-sectional survey and 71 South Indian families consisting of parents and a child with Type 2 diabetes. DNA was separated by agarose gel electrophoresis after amplification of exon 8. Three genotypes were identified II (homozygous for insertion), ID and DD (homozygous wild). No association or linkage was found beiween Type 2 diabetes and UCP2 variant in the South Indian families using the transmission disequilibrium test. In the parents of the families a positive association was found between BMI and the UPC2 variant ( $p<0.001)$, mean BMI 32.7 for II $(n=5), 25.2$ for ID $(n=47)$ and 24.9 for $\mathrm{DD}$ ( $\mathrm{n}=91$ ). To verify this positive observation we then screened the 449 South Indian samples. The positive association with BMI was replicated in females only, $(\mathrm{p}=0.019)$, mean BMI 26.4 for II $(n=9), 22.1$ for ID $(n=62), 23.2$ for DD $(n=147)$; furthermore, no association was found with Type 2 diabetes $(\mathrm{p}=0.83)$. In conclusion, the UCP2 exon 8 variant appears to be associated with increased BMI but not with Type 2 diabetes in a South Indian ethnic population.

\section{2}

HEPATOCYTE NUCLEAR FACTOR-4 $\alpha$ (MODY1) GENE MUTATIONS IN LATE-ONSET TYPE 2 DIABETICS IN THE UK.

D.E. Shepherd, Y Hashim and R.C. Turner. Diabetes Research Laboratories, Oxford University, Oxford, UK

Mutations in the hepatocyte nuclear factor 4 alpha $(\mathrm{HNF}-4 \alpha)$ gene have been shown to cause autosomal dominant early-onset diabetes in a number of MODY families. We have investigated the role that mutations in HNF- $4 \alpha$ may play in type 2 diabetes in white caucasians. We report an analysis of the MODYl gene in 3 subgroups of non-obese, islet cell antibody (ICA) negative and glutamic acid decarboxylase antibody (GADA) negative UK white caucasian diabetic subjects (i) severe $\beta$ cell deficient with fasting plasma glucose (FPG) $>12 \mathrm{mmol} / \mathrm{L}(\mathrm{n}=68$ ) (ii) glucokinase-like diabetes with mild hyperglycemia FPG $<8 \mathrm{mmol} / \mathrm{L}$ ( $\mathrm{n}=27$ ) and (iii) randomly chosen type 2 diabetic subjects $(n=100)$. The 11 exons and flanking introns of HNF-4 $\alpha$ were screened by Single Strand Conformational Polymorphism (SSCP) analysis and RFLP for known and novel polymorphisms with the variants being sequenced. Two missense mutations have been found, the previously identified $\mathrm{T} / 1130$ and a novel E269D mutation. The $T / 130(\mathrm{Thr} \rightarrow \mathrm{Ile}$ ) has an allele frequency of $6 \%$ (glucokinase subgroup) and $2 \%$ (severe $\beta$-cell subgroup). This polymorphism was identified by Yamagata et al, who found it on the same allele as the amber mutation Q268X in the $\mathrm{R}-\mathrm{W}$ pedigree and at a frequency of $5 \%$ in non diabetic white subjects. The missense mutation E269D is a result of a $\mathrm{G} \rightarrow \mathrm{C}$ nucleotide substitution at codon 269 , Glu(GAG) $\rightarrow$ Asp (GAC) and has been found in a single glucokinase-like patient. This conservative amino acid substitution is unlikely to be a major cause of late onset diabetes. Two novel polymorphisms have also been found in exon 2, one silent and the other in the intron near the intron/exon junction. HNF- $4 \alpha$ mutations are less prevalent than HNF-1 $\alpha$ (MODY3) mutations.

\section{1}

SCREENING OF MATERNAL DIABETIC PATIENTS FOR MITOCHONDRIAL MUTATIONS IN ALL 22 TRNA GENES K. Tsukuda1, T. Asano', Y. Oka ${ }^{2}$ and Y. Yazaki ${ }^{1}:{ }^{1}$ Third Department of Internal Medicine, University of Tokyo, ${ }^{2}$ Third department of Internal Medicine, Yamaguchi University Though diabetogenic mitochondrial DNA defects were reported around the tRNAleu gene so far, mutations in other tRNA genes were reported in neuromuscular disorders. Therefore, mutations around tRNA genes other than IRNAleu gene may play a role in the development of NIDDM and it is thought to be worthwhile to screen maternal diabetic patients for mutations in these regions. For this purpose, we sequenced around all 22 tRNA genes using DNA samples from peripheral blood leukocytes of 30 diabetic patients with a history of strong maternal inheritance. Detected gene mutations were further investigated by comparing the frequencies between $\mathbf{2 4 0}$ maternal diabetic patients (age; $46.0 \pm 14.5$ ) and 240 non-diabetic controls (age; $41.7 \pm 9.3$ ) Dot-blot hybridization method using $32 \mathrm{P}$-labeled sequencespecific oligonucleotides as probes was employed in this screening. Twenty-eight mitochondrial gene defects were detected up to now. Among these, $C$ to $T$ transition at position $456 \mathrm{bp}, \mathrm{G}$ to $\mathrm{A}$ transition at position $5773 \mathrm{bp}$ and $\mathrm{A}$ to $\mathrm{G}$ transition at position $15951 \mathrm{bp}$ were found in $5 / 240$ diabetic patients but $0 / 240$ controls, $3 / 240$ diabetic patients but $0 / 240$ controls and $6 / 240$ diabetic patients but $0 / 240$ controls $(p<0.05)$, respectively. For these mutations may be potentially diabetogenic, we are now investigating clinical phenotypes of theses diabetic patients and screening more diabetic patients and non-diabetic controls for these mutations.

433

HETEROPLASMY OF THE MITOCHONDRIAL A3243G MUTATION AND THE PHENOTYPE OF MATERNALLY INHERITED DIABETES AND DEAFNESS.

E.H.R van Essen, L.M. 't Hart, J.JJansen, J.M.W, van den Ouweiand, J.A. Maassen and H.H.P.J. Lemkes. Depts. of Endocrinology and Molecular Cell Biology, L.U.M.C., C4-R, Albinusdreef 2, 2333 ZA Leiden, The Netherlands.

Maternally Inherited Diabetes and Deafness (MIDD) is a diabetic subtype associated with an A to $G$ mutation in the mitochondrial tRNA (Leu, UUR) gene at basepair 3243. The clinical phenotype of MIDD shows a considerable variation mimicking either type 1 or type 2 diabetes, with similar frequencies of occurrence. A factor that may contribute to the pathogenesis of mitochondrial diabetes is the degree of heteroplasmy of the mitochondrial $\mathrm{A} 3243 \mathrm{G}$ mutation Heteroplasmy is defined as the percentage mutated mitochondrial DNA present in tissues. The aim of this study was to determine the relation between the level of heteroplasmy and the clinical phenotype of MIDD. Heteroplasmy was measured in leukocyte DNA and mouth mucosa cell DNA of 63 individuals that tested positive for the mutation, using the polymerase chain reaction (PCR) with 32P-labelled desoxynucleotides (detection-threshold: 1\% heteroplasmy). Diabetic patients were subdivided into type 2-like and type 1-like. Initiation of insulin therapy within 3 months after diagnosis of diabetes was regarded as a type 1 diabetic phenotype. Of the tested individuals 11 ( 3 male (M), 8 female (F); mean age of onset \pm sd: $30 \pm 7.1$ years) showed a type 1like phenotype, $8(2 \mathrm{M}, 6 \mathrm{~F} ; 40.8 \pm 16.1$ years) a type 2 phenotype, $12(4 \mathrm{M}, 8 \mathrm{~F}$; mean age at diagnosis \pm sd: $39.5+16.3$ years) were diagnosed as Impaired Glucose Tolerant (IGT) and 32 $(15 \mathrm{M}, 17 \mathrm{~F} ; 34.3 \pm 13.5$ years) as Normal Glucose Tolerant (NGT). A significant correlation $(\mathrm{r}=0.9, \mathrm{p}<0.05)$ was observed between heteroplasmy levels in oral mucosa and leukocytes. Heteroplasmy levels ( $\% \pm s d$ ) in type 1-like diabetic patients (oral mucosa: $45 \pm 27.1$, leukocytes: $25.0 \pm 17.5$ ) did not differ significantly from levels in type 2-like diabetic patients (oral mucosa: $34.4 \pm 17.8$, leukocytes: $21.8 \pm 11.7$ ), subjects with IGT (oral mucosa: $37.8 \pm 19.8$, leukocytes: $22.2 \pm 13.9$ ) and subjects with NGT (oral mucosa: 27.1 \pm 22.6 , leukocytes: $17.0 \pm[6.0$ ). There was no correlation between the age of onset of diabetes and the level of heteroplasmy. We conclude that heteroplasmy levels of the investigated tissues are not indicative of the clinical phenotype of the mitochondrial A3243G mutation. 


\section{4}

NO ASSOCIATION BETWEEN HUMAN 32 -ADRENORECEPTOR GENE POLYMORPHISM AND OBESITY OR TYPE 2 DIABETES

J.L. Hedenbro' ${ }^{1}$, M. Carlsson ${ }^{2}$, M. Orho ${ }^{2}$ and L.C. Groop ${ }^{2}$. Departments of Surgery ${ }^{1}$ and Endocrinology ${ }^{2}$, Lund ${ }^{1}$ and Malmö ${ }^{2}$ University Hospitals, Lund University, Sweden.

32-adrenoreceptors (32-ARs) play a central role in the regulation of lipolysis in human adipose tissue. Recently the Gln27Glu polymorphism in the B2-AR gene was reported to be associated with obesity in women. In order to replicate this finding and explore the role of the B2-AR gene in obesity-related NIDDM we studied the Gln27Glu polymorphism in four groups of unrelated Swedish subjects: 1) morbidly obese nondiabetic patients scheduled for bariatric surgery (OBE: $\mathrm{n}=68$, males $=16$, fem $=52$, age $41 \pm 11$ years, BMI $42 \pm 5 \mathrm{~kg} / \mathrm{m}^{2}$ ), 2) obese NIDDM patients (ObeDM: $n=79$, males $=38$, fem $=41$ age $58 \pm 11$ years, BMI $34 \pm 4 \mathrm{~kg} / \mathrm{m}^{2}$ ), 3) lean NIDDM patients (LeanDM: $n=83$, males $=53$, fem $=30$, age $62 \pm 10$ years, BMT $24 \pm 2 \mathrm{~kg} / \mathrm{m}^{2}$ ) and 4) non-diabetic, lean controls (CON: $\mathrm{n}=60$, males $=35$, fern=25, age $62 \pm 11$ years, BMI $26 \pm 4 \mathrm{~kg} / \mathrm{m}^{2}$ ). The $\beta 2$-AR Gln27Glu polymorphism was genotyped using PCR and restriction enzyme digestion followed by agarose gel electrophoresis. Genotype ferquencies are given below, $\mathbf{n}(\%)$ :

$\begin{array}{ccccc}\text { GENOTYPE: } & \text { OBE } & \text { ObeDM } & \text { LeanDM } & \text { CON } \\ \text { B2-AR GIn27Gln } & 17(25) & 23(29) & 31(37) & 20(33) \\ \text { B2-AR GIn27Glu } & 38(56) & 48(61) & 35(42) & 27(45)\end{array}$

B2-AR Glu27Glu $13(19) \quad 8(10) \quad 17(20) \quad 13(22)$

The genotype frequencies of the $\beta 2$-AR Gln27Glu did not significantly differ for either OBE, ObeDM or LeanDM groups when compared with controls $\left(\chi^{2}\right.$-test, 2 d.f., $p=0.45, p=0.09$ and $p=0.88$, respectively). Neither did we observe any significant differences between the clinical variables (BMI, WHR, serum lipids and blood pressure) between the different genotype carriers within the groups. The results were similar when analysed separately for males and females. In conclusion, we foind no association of the B2-AR Gln27Glu polymorphism with obesity or NIDDM in Swedish patients, challenging a role for the $82-\mathrm{AR}$ Glu/Glu genotype in the development of these conditions.

\section{6}

Search for a sixth Maturity-Onset Diabetes of the Young causing-gene: Studies with Twelve Candidate Genes in nine MODY families.

J-C. Chèvre, P. Boutin, E.H. Hani, M.Vaxillaire, G. Velho, N. Vionnet and P. Froguel. Institut de Biologie de Lille, CNRS-EP10, Institut Pasteur de Lille, 1 rue Calmette, BP 245, 59019 Lille cedex, France.

Maturity-onset diabetes of the young (MODY) is a heterogenous monogenic form of non-insulin-dependant diabetes mellitus with a primary defect in insulin secretion. We have identified 9 MODY families in which diabetes is not the result of mutations in any of the five known MODY genes: the glucokinase/MODY2, hepatocyte nuclear factor-1 alpha/MODY3, hepatocyte nuclear factor-4 alpha/MODY1, insulin promoter factor 1MODY4, hepatocyte nuclear factor-1 beta/MODY5 genes. Moreover, no evidence for linkage of those loci with MODY was found. This cohort of families will be useful for identifying other diabetes-susceptibility genes. Twelve other candidate genes encoding for transcription factors implicated in pancreatic development and function have been tested for linkage with MODY in these families using polymorphic markers. These included caudal-type homeo box traanseription factor 3, dimerization cofactor of HNFla, Islet-Brain 1 protein, hepatocyte nuclear factor-3 alpha, CCAAT/Enhancer-Binding Protein-epsilon, hepatocyte nuclear factor-3 gamma, CCAAT/Enhancer-Binding Proteinbeta, Beta-2/neurogenic differentiation factor 1 , paired box homeotic factor 4 , hepatocyte nuclear factor-4 gamma, hepatocyte nuclear factor-3 beta, and hepatocyte nuclear factor6. None of these loci showed evidence for linkage with MODY. Linkage analysis or mutation screening of Islet-1 transcription factor, Nkx2.2 and Nkx6.1 factors genes are in progress.

\section{5}

THE B-CELL GLUCOKINASE GENE PROMOTER G-A VARIANT IS ASSOCIATED WITH HIGHER FASTING GLUCOSE LEVELS

M. Zhang, N.M.S. Wat, E.D Janus*, S.S.M.Chung** and K.S.L.Lam. Department of Medicine, *Clinical Biochemistry Unit and **Institute of Molecular Biology, University of Hong Kong, Hong Kong, China.

The G-A variant at position -30 of the beta-cell glucokinase gene promoter has been reported to be associated with reduced $B$-cell function in middle-aged Japanese-American men. To investigate the relationship between this genetic variant and glucose metabolism in Southern Chinese, we studied 333 unrelated Southern Chinese (aged 47.0 \pm 11.7 years, mean $\pm S D$; range 27-74 years) who were nondiabetic at baseline, according to a $75-\mathrm{g}$ oral glucose tolerance test and WHO diagnostic criteria: 205 had NGT (normal glucose tolerance) and 128 had IGT (impaired glucose tolerance). Repeat testing at 2 years showed that $1 \%$ and $5 \%$ of the NGT and IGT subjects respectively had developed diabetes $(p<0.0001)$. The $G$ to $A$ substitution at position -30 of the glucokinase promoter, resulting in the loss of a Bsi HKAI cut site, was detected after Bsi HKAI digestion of polymerase chain reaction-amplified genomic DNA. Allele frequency of the A subjects $(P=N S)$. Fasting plasma glucose levels were significantly higher in subjects with the $A$ allele $(n=103 ; G / A: A / A=96: 7)$, when compared to the $G / G$ subjects $(n=230)$, at baseline $(5.32 \pm 0.53$ versus $5.15 \pm 0.51 \mathrm{mmol} / \mathrm{L}$ for $\mathrm{G} / \mathrm{G}$ $\mathrm{p}<0.005)$ and at the 2 -year follow up $(5.23 \pm 0.85$ versus $5.01 \pm 0.54 \mathrm{mmol} / \mathrm{L}$ $p<0.005$ ). Higher fasting glucose levels were also found in IGT subjects with the A variant $(5.58+0.53$ versus $5.35+0.51 \mathrm{mmol} / \mathrm{L}$ for $\mathrm{G} / \mathrm{G} ; \mathrm{P}=0.02)$ at baseline and at 2 years $(5.62 \pm 1.10$ versus $5.22 \pm 0.66 \mathrm{mmol} / \mathrm{L}$ for $\mathrm{G} / \mathrm{G} ; \mathrm{p}=0.01)$. In subjects with NGT at baseline, there was no significant difference in fasting plasma glucose levels between different glucokinase genotypes. We conclude that the -30 beta-cell glucokinase gene promoter variant is associated with higher fasting plasma glucose levels, and probably contributes to the development of impaired glucose tolerance in Southern Chinese.

\section{7}

Maternally Inherited Diabetes and Deafness (MIDD): a Multicenter Study P.J. GUILLAUSSEAU, P. MASSIN, B. VIALETTES, H. GIN, J. TIMSIT, A. GRIMALDI, E. KALOUSTIAN, J. CAHEN, P. CHEDIN, E. BERTIN, B. GUERCI, B. POROKHOV, M. VIRALLY-MONOD, D. TIELMANS, and M. PAQUES. Paris, Marseille, Bordeaux, Nancy, Nantes, Reims, France.

Maternally inherited diabetes and deafness (MIDD) is a matrilinearly inherited syndrome consisting of deafness, macular pattern dystrophy, and neuromuscular disorders, which is caused by a mitochondrial DNA mutation, usually $A \rightarrow G$ m RNAt Leu ${ }^{\text {UUR }} 3243$ mutation. We report on a series of 26 cases, 11 men and 15 women, of mean age $48.9 \pm 10$ years $(m \pm S D)$. Two of these patients had impaired glucose tolerance, and 24 diabetes, of which 14 were treated with insulin and 10 not. Insulin therapy was initiated a mean $8.5 \pm 8.9$ years after discovery of the diabetes in 11 cases. The body mass index was less than 27 in all cases but one (95\%). Diabetes was discovered at age $36.8 \pm 8.9$ years (range $20-58$ years). There was no history of ketoacidosis. A familial history of diabetes was evidenced in 23 cases (88\%), with maternal inheritance in 20 cases (77\%). Deafness was evidenced in 25 cases (96\%) and neuromuscular disorders in 14 cases $(61 \%)$. Macular pattern dystrophy was observed in 23 cases $(88.5 \%)$. This was characterized by subretinal deposits in the macular area with, in advanced forms, chorioretinal atrophy. All cases had normal electroretinograms, thus excluding retinitis pigmentosa. The distinctive combination of diabetes, deafness, and/or macular pattern dystrophy should lead to screening for a mitochondrial DNA mutation. 


\section{8}

AMINO ACID VARIANTS OF THE VITAMIN D-BINDING PROTEIN ARE NOT ASSOCIATED WITH TYPE 2 DIABETES IN CAUCASIANS.

T. Klupa ${ }^{1}$, M.T. Malecki ${ }^{1,2}$, M.B. Davidson ${ }^{2}$, J. Sieradzka ${ }^{1}$, J. Frey', J. Sieradzki, and A.S. Krolewski ${ }^{2}$. Dept. Metabolic Diseases, Jagellonian University, Cracow, Poland ${ }^{1}$ and Dept. Genetics and Epidemiology, Joslin Diabetes Center, Boston, MA, USA ${ }^{2}$

Vitamin D-binding protein (DBP) has been reported to be associated with type 2 diabetes mellitus in several populations. There are two known polymorphisms in DBP that result in amino acid variants: at codons 416 GAT $\rightarrow$ GAG $($ Asp $\rightarrow$ Glu $)$ and 420 ACG $\rightarrow$ AAG $(T h r \rightarrow$ Lys). These changes generate Haelll and Styl restriction sites, respectively. The aim of our study was to examine the association of the alleles and genotypes at these codons with type 2 diabetes in Caucasians. The study group consisted of 215 unrelated patients with type 2 diabetes, age $>45$ at diagnosis, and 166 healthy controls, matched for sex and age. Exon 11 was amplified by polymerase chain reaction, and alleles were determined by digestion. Group differences in the distributions of variants at both sites were tested by $\chi^{2}$. At codon 416 , Asp/Glu frequencies were $43 \% / 57 \%$ in the cases and $42 \% / 58 \%$ in the controls ( $\mathrm{p}=0.91$ ), while at codon 420 . Thr/lys frequencies were $70 \% / 30 \%$ and $72 \% / 28 \%$, respectively $(p=0.63)$. The frequencies of the genotypes are presented in the table:

\begin{tabular}{|l|l|l|l|l|}
\hline Codon 416 & Asp/Asp & Asp/Glu & Glu/Glu & $\mathrm{p}=0.4$ \\
\hline Cases & $37(17.2 \%)$ & $110(51.1 \%)$ & $68(31.6 \%)$ & \\
\hline Controls & $30(18.0 \%)$ & $80(48.1 \%)$ & $55(33.1 \%)$ & \\
\hline Codon 420 & Thr/Thr & Thr/Lys & Lys/Lys & $\mathrm{p}=0.8$ \\
\hline Cases & $104(48.3 \%)$ & $94(43.7 \%)$ & $17(7.9 \%)$ & \\
\cline { 1 - 3 } Controls & $86(51.8 \%)$ & $68(40.9 \%)$ & $12(7.2 \%)$ & \\
\cline { 1 - 2 } & &
\end{tabular}

In conclusion, our results do not confirm the association of DBP variants with type 2 diabetes diagnosed after age 45 in Caucasians.

\section{0}

LOW SERUM HDL CHOLESTEROL LEVEL WITHOUT HYPERTRIGLYCERIDAEMIA IN NORMOGLYCAEMIC NONDIABETIC OFFSPRING OF PARENTS WITH CONJUGAL TYPE 2 DIABETES.

T.Kasperska-Czyżyk, K.Jaskólskaładosz, K.Stępień and R.Nowaczyk, Central Clinical Hospital, Warsaw, Poland. In 56 adult normoglycaemic non-diabetic children of parents with conjugal type 2 diabetes (group A) and in 68 control subjects matched for age, sex and body mass (group B) the OGTT $(75 \mathrm{~g})$ was carried out, including measurements of serum insulin (IRI) and C-peptide (CP). In fasting state also the blood lipid profile was determined. In group $A$ serum IRI values were higher than in group $B$ (AUC $1.04 \pm 0.05$ (SEM) vs $0.92 \pm 0.05 \mathrm{nmol} \cdot l^{-1} \cdot h^{-1}, p=0.031$ ). This difference was less evident as regards serum $C P$, resulting in lower serum $C P: \mid R I$ ratio in group $A$. In this group significantly lower than in group $B$ values of serum HDL cholesterol $(1.20 .1$ vs $1.5 \pm 0.1 \mathrm{mmol} / \mathrm{h}$, $p=0.0001)$ and of apoAl (157 \pm 3.9 vs $182 \pm 6.5 \mathrm{mg} / \mathrm{dl}$, $p=0.0005$ ) were found, while in both groups the serum levels of triglycerides, total cholesterol and $\mathrm{apoB}$ were within normal range and didn't differ significantly. We conclude, that the low level of serum HDL cholesterol in non-diabetic offspring of parents with conjugal type 2 diabetes is due to insulin resistance and subsequent increase in the activity of enzymes which control HDL metabolism (e.g. CETP, hepatic lipase).

\section{9}

CANDIDATE GENES IN SOUTH AFRICAN INDIANS WITH TYPE 2 DIABETES

F.J Pirie, D.F York*, A.A Motala, A Amod. M.A.K Omar, J.C. Chevre**, P Froguel|** Departments of Medicine and Molecular Virology*. University of Natal, Durban, South Africa, Institut Pasteur de Lille, France**

South African Indians have a high prevalence of type 2 diabetes with a strong familial tendency. In an attempt to understand the contribution of selected candidate genes to the pathogenesis of type 2 diabetes, the insulin and mitochondrial tRNA ${ }^{\text {let }}$ genes were evaluated in 181 subjects of whom 104 were diabetic, 69 normal glucose tolerant (NGT) and 8 had impaired glucose tolerance (IGT) according to the 1985 WHO criteria. in a subset of 18 individuals, incorporating 7 probands with early onset type 2 diabetes, the giucokinase (GK) and hepatocyte nuclear factor $1 \alpha$ (HNF $1 \alpha$ ) genes were evaluated. Polymerase chain reaction (PCR) - single stranded conformation polymorphism (SSCP) of the insulin gene identified a $G$ to $A$ point mutation at position -329 of the promoter region in a diabetic and a $C$ to $G$ mutation at position -56 in a NGT subject. No SSCP variation was seen in exons 1 and 2 but a variant SSCP pattern was seen in exon 3 . The variant pattern represented a Pst 1 polymorphism in the 3 ' untranslated region of exon 3 . No mutations were found at position 3243 of the mitochondrial tRNA $A^{104}$ gene. The GK gene was normal in the subgroup of individuals with early onset type 2 diabetes but six variant SSCP patterns were found in the HNF $1 \alpha$ gene, located in the promoter and exons $5,6,7,8$ and 9 . The sequence variations responsible for these patterns are currently being determined. In conclusion, the insulin and mitochondrial tRNA ${ }^{\text {leu }}$ genes do not contribute substantially to the pathogenesis of type 2 diabetes in the SA Indian population and the early onset variant of the condition, in a small number of subjects, is characterized by mutations in the HNF $1 \alpha$ gene but not in the GK gene.

\section{1}

ASSOCIATION OF THE SULPHONYLUREA RECEPTOR 1 GENE VARIANTS WITH GESTATIONAL DIABETES MELLITUS AND NIDDM J. Rissanen', A. Markkanen', P. Kärkkäinen', J. Pihlajamäki', S. Heikkinen', P. Kekäläinen ${ }^{1}$ L. Mykkänen ' ${ }^{2}$, J. Kuusisto', P. Karhapää', L. Niskanen ${ }^{1}$ and M. Laakso' 'Department of Medicine, University of Kuopio, 70211 Kuopio, Finland ${ }^{2}$ Department of Medicine, Division of Clinical Epidemiology, the University of Texas, San Antonio, USA

Both gestational diabetes mellitus (GDM) and non-insulin-dependent diabetes mellitus (NIDDM) are characterised by beta cell dysfunction. Because the sulphonylurea receptor 1 (SUR1) mediates insulin secretion in beta cells, we screened the two highly conserved nucleotide binding folds of the SUR1 gene by single-strand conformation polymorphism analysis in 42 patients with GDM and 40 patients with NIDDM from Finland. We identified one amino acid change (S1370A), 4 silent substitutions (R1274R, L830L, T761T and K650K) and 3 intronic variants (agtggc $\rightarrow$ agcggc, ggtgct $\rightarrow$ tgtgct and tagGCC $\rightarrow$ cagGCC). The S1370A, R1274R, L830L, T761T variants and the previously unreported ggtgct $\rightarrow$ tgtgct of intron 21 and the tagGCC $\rightarrow$ cagGCC of exon 24 splice acceptor site were screened in an additional sample of 82 normoglycemic control subjects. An AGG allele of the R1274R polymorphism was more common in patients with GDM $(0.87)$ or NIDDM $(0.87)$ than in control subjects $(0.52)(P<0.001)$. Furthermore, the tagGCC allele of exon 24 splice acceptor site was more common in patients with NIDDM $(0.60)$ than in control subjects $(0.45)(P=0.02)$. We further investigated the association of the S1370A, R1274R, T761T, ggtgct $\rightarrow$ tgtgct and tagGCC $\rightarrow$ cagGCC variants with insulin secretion in a separate sample of 295 subjects with normal glucose tolerance and 38 subjects with impaired glucose tolerance. None of these variants showed any effect on first-phase insulin secretion, fasting, 1-h and 2-h insulin levels. In conclusion, the variants of the SUR1 gene are unlikely to predispose to GDM or NIDDM in Finnish subjects. 


\section{2}

PHOSPHATIDYLINOSITOL 3-KINASE AS A CANDIDATE GENE FOR NON-INSULIN-DEPENDENT DIABETES MELLITUS

M. Kossila, ${ }^{1,2}$, M. Sinkovic ${ }^{2}$, M. Laakso ${ }^{2}$, S. Ylä-Herttuala, ${ }^{1,2},{ }^{1} A . I$. Virtanen Institute and ${ }^{2}$ Department of Medicine, University of Kuopio, Finland

Phosphatidylinositol 3-kinase (P|3K) activity is required for the insulin-stimulated glucose transport. Non-insulin-dependent diabetes mellitus (NIDDM) is in large part caused by insulin resistance which is characterized by decreased glucose transport, glucose uptake, and utilization. Our aim is to study the catalytic subunit $p 110 \beta$ of PI3K as a candidate gene for NIDDM. The first step is to clone $p 110 \beta$ gene from the human genomic library. Four different fragments of $p 110 \beta$ cDNA were used as probes in the screening of the library. Probes were prepared by RT-PCR using human skeletal muscle total RNA as a template. The exon-intron structure of the gene is determined by comparing the genomic sequence to the CDNA sequence. Eighteen positive phage clones were obtained from the library. Seventeen exons of the $p 110 \beta$ gene have so far been localized from genomic clones. They represent $78 \%$ of the cDNA. Fourteen exons have already been screened for variants in NIDDM patients using singlestrand conformation polymorfism (SSCP) analysis but no gene defects have been found. All exons will be localized and screened for variants and these will be expressed, if found, in E. coli. p110 $\mathrm{CDNA}$ will also be cloned into adenovirus and the effects of PI3K activity on glucose transport will be studied in vitro and in vivo using cell culture and diabetic animal models. In conclusion, we have localized 17 exons of the $p 110 \beta$ gene. 14 exons have been screened so far but no variants have been identified. As soon as all exons are localized and screened for variants we can conclude whether or not the $\mathrm{p} 110 \mathrm{\beta}$ gene plays a role in the etiology of NIDDM.

\section{4}

HEPATOCYTE NUCLEAR FACTOR 1-ALPHA (MODY 3) GENE MUTATIONS IN TYPE 2 DIABETICS OF DIFFERENT ETHNICITY

${ }^{1}$ Y. Hashim, 'D.E. Shepherd, ${ }^{2}$ J. Li, ${ }^{2}$ J. Chan and R.C. 'Turner. Diabetes Research Laboratories, Oxford University, Oxford, UK.

Department of Medicine and Therapeutics, The Chinese University of Hong Kong, Hong Hong.

HNFla mutations, cause dominantly inherited Maturity onset Diabetes of the Young Type 3 (Mody 3), that classically presents at age less than 25 yrs. It can ocassionally present as Type 2 diabetes in adults, when the phenotype has been reported as predominantly non-obese with marked beta-cell deficiency causing insulin requiring diabetes. The aim was to determine how frequently HNF $1 \alpha$ mutations are observed in type 2 diabetics of different racial origins, white Causcasians, AfroCarribean, Asian-Indian and Chinese and the nature of associated phenotype We examined 3 subgroups of non-obese ICA- and GADA-negative diabetic white Caucasian subjects, (i) severe beta cell deficient (FPG $=<12 \mathrm{mmol} / \mathrm{l})(\mathrm{n}=68)$ (ii) mild hyperglycaemia (FPG $=<8 \mathrm{mmol} / \mathrm{l})(\mathrm{n}=27)$; (iii) gestational diabetics $(\mathrm{n}=38)$; (iv) randomly selected diabetic subjects $(n=75)$. We examined 25 randomly chosen diabetics and 25 normoglycaemic control subjects from the remaining three racial groups, in whom diabetes present at younger age of onset. We screened for mutations with sequencing and SSCP with mutations verified by RFLP-based assays. Two promoter mutations were found in the mildly hyperglycaemic groups, one being a heterozygous $2 \mathrm{bp}$ deletion in a putative $\mathrm{C} / \mathrm{EBP}$ binding site and a missense mutation in exon 4 (G301A) and exon 7 (C492T) in diabetic patients who had a similar, mild phenotype. One classical Type 2 diabetic had an exon 7 (C498A) missense mutation. Mody 3 mutations were not found in supposedly high-risk, markedly hyperglycaemic patients but more often in patients with glucokinase-like phenotype $(p=0.0019)$. Four novel, non-conservative missense mutations were identified in Afro-caribbean and Chinese diabetics that alter the structure and size of the amino acid. This prevalence does not account fro the early age of onset of diabetes in the ethnic groups. HNF1 $\alpha$ muations appear to occur at low prevalence in several ethnic groups.
443

Clinical and Immunogenetic parameters in families with high incidence of NIDDM. A.DePascale',M.Batistini', S.Barocci ${ }^{2}$, V.Chell', E.Gazzerro',P.Melga', V.Miotti, G.Tosi ${ }^{4}$ and R.Accolla.5. 'Dip. endocrino-metabolico Univ. di Genova, ${ }^{2}$ st. di Immunologia Univ. di Genova, ${ }^{3}$ Osp.Civ.di Udine, ${ }^{4}$ Centro di Biotecnologie Avanzate, Genova, ${ }^{5} D i p$. di Scienze Cliniche e Biologiche, Univ. di Pavia a Varese. Background Many different etiopathogenic factors are involved in the natural history of typell diabetes (NDDM).Among them genetic inffuences are of primary importance, but contrary to diabetes type I (IDDM) the role of specific genes like II class HLA antigens is unclear. Methods We studied 10 families with high incidence of NIDDM (at least two brothers affected). In some families there were cases of both, NIDDM and IDDM.In all cases the diagnosis had already been defined.In the 10 families we selected 47 subjects ( 18 males, 29 females) with BMk $<30$. Fasting and after meal plasma glucose and CDep, HBA1C, ICA, anti-GAD antibodies, autoantibodies to several tissue antigens were measured. We assessed the frequency of the HLA-DQB1, DQA1, DRB1 alleles and carried out the molecular typing of their specificities in 30 NIDDM and 52 healthy control subjects with PCR and Southern biot analysis. Results in the selected populations, 8 males (7 NIDDM, 1 IDDM) and 22 females (21 NIDDM and 1 IDDM) were affected by diabetes. As far the diabetic patients, we observed 1)Male group: age $52.5 \pm 4.6$; BMI $26.1+0.5$; fasting plasma glucose $192+18.6 \mathrm{mg} / \mathrm{dl} ; \mathrm{HbA}_{1 \mathrm{c}} 5.7+0.4 \%$. 2) Female group: age $63.3 \pm 2.8$; BMI $26.2 \pm 0.3$; fasting plasma glucose $196 \pm 17.7 \mathrm{mg} / \mathrm{dl} ; \mathrm{HbA}_{16} 6.0 \pm 0.3 \%$.Data are expressed as Mean \pm Standard Error. The incidence of antibodies to tissues antigens was not significantly different between the affected subjects and the healthy control. No one was positive for antibodies anti-GAD. There were only 3 cases positive for ICA (2 affected females, 1 healthy male). As far as the DQB1 locus only the 0501 group of alleles showed a stastically significant association with protection from disease $(p<0.025)$. None of the other DQB1 alleles was associated to either susceptibility or resistance. As far as the alleles at the DQA1 locus only a borderfine statistically significant association with resistance was found for A1 group of alleles. Contrary to IDDM there was no correlation between "susceptiblen $\alpha-\beta$ DQ heterodimers and the NIDDM status.in $70 \%$ of the families the study of the vertical transmission revealed maternal heredity. This observation suggest that maternal genes, possibly linked to mithocondrial DNA, are involved in the genetic susceptibility to the disease.

\section{5}

ALTERATION OF HSP72i GENE EXPRESSTON AND FUNCTTON INNIDDM Hegectuis E., Kurucz I, Morva A, Vaag A.," Eriksson K-F., * Lehtovirta M, * Huang $X .,{ }^{*}$ Groop L*, Koranyi L BIOREX R\&D, Balatonfured, Hungary, and University of Lund, Wallenberg Laboratory, Malmö, Sweden*

Diabetic complications are associated with altered structure and function of membranes and intracellular proteins. Stress proteins, especially the HSP-family, play a crucial role in folding, refolding and intracellular trafficking of newly synthesized and residential polypeptides therefore, they could be involved in the pathomechanism of diabetic complications. To address this question their cellular level and function in diabetic patients were investigated. Peripheral mononuclear cells were isolated from the blood of 44 NIDDM patients and 28 matched controls, and heat induced $\left(43^{\circ} \mathrm{C}\right.$ for

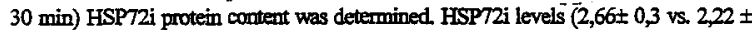
0,4 dens.unit, $\mathrm{p}=0,015$ ) were decreased in cells of NDDM patients. HSP72 levels correlated with the daily albumin excretion $\left(r^{2}=0,254, p<0,05\right)$.

The level of the expression of HSP72i gene in skeletal muscle biopsy samples was also measured by a highly sensitive quantitative RI-PCR Glucose disposal was determined by byperinsulinemic, nomogitycemic clamps in 27 NIDDM patients, in 25 controls and in 12 monozygotic twins, discordant for NDDM. The levels of HSP72i mRNA in diabetic muscle were lower than in control biopsies $1,92 \pm 0,45 \mathrm{vs} .288 \pm 0,36 \mathrm{fmol} /$ $\mathrm{p}<0,00001$ ). The expression of the HSP72i gene was also significantly decreased in both twins compared to controis, with lower levels in the NIDDM $(1,71 \pm 0,1 \mathrm{fmol} / \mathrm{h})$ than in their nondiabetic controls $(1,96 \pm 0,1 \mathrm{fmol} / 1)$. HSP72i mRNA leveis correlated with -giucose disposal rate $(M)(r=0,42, p<0,001)$, -oxidative $(r=0,34, p<0,05)$, and non-oxidative $(r=0,63, p<0,00002)$ ghucose utilization. Conclusions: Our data here indicate that the development of diabetic complications in NIDDM could be related to decreased chaperone expression, as 1/ HSP-72 gene expression markedly decreased and $2 /$ HSP72i function maybe also inpaired in diabetes, what correlates with albuminuria. 3/ The decreased HSP72i expression is partially of genetic origin and 4. the diabetic state further rectuces the expression of HSP-72. 
PARAOXONASE POLYMORPHISM MET-LEU 54 IS ASSOCIATED WITH FATAL MYOCARDIAL INFARCTION IN TYPE 2 DIABETIC PATIENTS

C.J.Groves' , V.A. Horton' ${ }^{1}$ A. Ritchie', R.J. Owen', I. R. Stratton', F.R.Green', R.R. Holman', and R.C. Turner', 'Diabetes Research Labs, ${ }^{2}$ Nuffield Department of Surgery for the U.K. Prospective Diabetes Study, Oxford, UK.

Coronary heart disease (CHD) is the dominant cause of early mortality in Type 2 diabetes mellitus. Oxidation of low-density lipoproteins (LDL) contributes to the development of cardiovascular disease. Paraoxonase, an HDL-associated enzyme, provides protection against the oxidation of LDL and several studies have implicated polymorphisms as risk factors for heart disease. Two polymorphisms of paraoxonase Gln-Arg 191 and Met-Leu 54 were evaluated by PCR-RFLP in 169 Type 2 diabetic patients with cardiovascular disease (angina $n=56$, non-fatal myocardial infarction (MI) $n=95$ and fatal $M I n=18$ ) and 169 control diabetic subjects without evidence of heart disease. Cases and controls were matched for gender, duration of diabetes, age at diagnosis and for fasting plasma glucose, blood pressure. $L D L$, and HDL after 3 months diet therapy. The proportion of patients with LL in control, angina, nonfatal $\mathrm{Ml}$ and fatal Ml was $41 \%, 39 \%, 45 \%$, and $78 \%$, $\chi^{2}$ for trend, $p<0.04$. For fatal MI, the association of $\mathrm{LL}$ compared with controls was $p<0.006$. The addition of data from the 191 poymorphism only slightly increases the association. In conclusion, there was a trend for association of the paraoxonase 54 polymorphism with $\mathrm{Ml}$, with the major effect being for fatal MI. This would be in keeping with decreased activity of paraoxonase, increasing the liquidity and decreasing the stability of atheromatous plaques.

\section{PS 8}

\section{Epidemiology of Type 2 Diabetes}

447

\section{WEIGHT GAIN AND THE RISK OF INSULIN RESISTANCE SYNDROME}

S. A. Everson, D. E. Goldberg, S. P. Heimrich, I. A. Lakka, J. W. Lynch, G. A. Kaplan and J. T. Salonen. Department of Epidemiology, School of Public Health, University of Michigan, Ann Arbor, Michigan, U.S.A. and Research Institute of Public Health, University of Kuopio, Kuopio, Finland

Obesity and increased body weight have been associated independently with hyperinsulinemia, dyslipidemia and hypertension. However, there are few if any studies of the relationship between weight gain from early adulthood to middle age and the development of this cluster of cardiovascular risk factors, known as insulin resistance syndrome. Therefore, we investigated the association between weight gain from the age of 20 years to middle age ( 53 years on the average) and the odds of having developed insulin resistance syndrome, defined as simultaneous existence of hyperinsulinemia (fasting serum insulin of $\geq 11.7 \mathrm{mU} / \mathrm{l}$ ), hypertension (systolic blood pressure of $\geq 160 \mathrm{mmHg}$ or diastolic blood pressure of $\geq 95 \mathrm{mmHg}$ or treatment for hypertension) and dyslipidemia (serum HDL cholesterol of $<1.0 \mathrm{mmo} / \mathrm{l}$ or senum triglycerides of $\geq 2.3 \mathrm{mmol} / \mathrm{l}$ ), by middle age in a population-based sample of 2272 men aged 42-60 years from eastern Finland. For each $5 \%$ increase in body weight, there was a neariy $19 \%$ greater risk of having developed insulin resistance syndrome, adjusting for age and height. Men who had an increase in body weight of $10-19 \%, 20-29 \%$ and $\geq 30 \%$ had a $3.0-$, 4.7- and 10.6-fold risk of having developed insulin resistance syndrome, respectively, by middle age, compared to men who had a weight increase of less than $10 \%$. Adjustment for age, height, physical activity, smoking, education and parental history of diabetes did not alter these findings. These findings show that weight gain from early adulthood to middle age has a strong, graded and independent association with the risk of having developed insulin resistance syndrome by middle-age in men in eastem Finland. This study adds to a large body of literature identifying the deleterious effects of weight gain from early adulthood to middle age

\section{8}

STUDY ON GTT CRITERIA OF ADA BASED ON COMPLICATIONS C. Ito Hiroshima A-Bomb Casualty Council Health Management Center, 3-8-6, Senda-machi, Naka-ku Hiroshima 730-0052, Japan

Purpose: GTT standard proposed by ADA and need of GTT for diagnosis of diabetes mellitus (DM) were studied based on retinopathy (RD) and CHD mortality. Methods: Subjects were 21,958 GTT examinees between 1965 and 1997. Correlation of GTT 2-hr PG to FPG was investigated and prevalence and incidence of RD were analyzed by FPG and 2-br PG. Results: 1) Regression equation of 2-br $P G(x)$ and FPG $(y)$ for those $<60$ years $(n=7,454)$ was $y=56.2+0.342 x \quad(r=0.865)$, and 2 -hr PG of 200 was calculated to be $125 \mathrm{mg} / \mathrm{dl}$ of FPG. 2) According to WHO standard, normal was $53.3 \%$, IGT was $25.5 \%$, and DM was $21.2 \%$, but by ADA standard they were $52.9 \%, 24.2 \%$, and $22.9 \%$, respectively. Cases evaluated to be DM by FPG of $126-139 \mathrm{mg} / \mathrm{dl}$ only were $1.7 \%$. 3) Prevalence of RD by deciles with FPG $\leqq 87$ and $88-92$ was $0.4 \%, 0.7 \%$ for $93-96,1.0 \%$ for $97-99$ and $100-103$, $1.6 \%$ for $104-108,1.7 \%$ for $109-115,1.6 \%$ for $116-125,3.1 \%$ for $126-145$, and $6.4 \%$ for $\geqq 146 \mathrm{mg} / \mathrm{dl}$. Groups $\geqq 126$ showed a significantly higher rate than groups $\leqq 125 \mathrm{mg} / \mathrm{dl}$. Also by 2 -hr PG, in groups with $198-235 \mathrm{mg} / \mathrm{d}$ l the rate was $1.8 \%, 236-300$ was $3.4 \%$ and $\geqq 301$ was $5.7 \%$. Groups $\geqq 198 \mathrm{mg} / \mathrm{dl}$ showed a significantly higher rate than groups $\leq 197.4$ ) Incidence of RD was 1.5 in groups with FPG $\leqq 89,3.0$ for $90-99,2.8$ for $100-109,3.1$ for $110-125,6.9$ for 126-139, and $13.9 / 1000 \mathrm{PY}$ for $140-199 \mathrm{mg} / \mathrm{dl}$. In groups $\geqq 200 \mathrm{mg} / \mathrm{dl}$ the rate was 27.2/1000 PY, showing significant elevation from $126 \mathrm{mg} / \mathrm{dl}$ group. Incidence of RD was 3.0 for FPG of $126-139$ and 2 -hr $P G<200 \mathrm{mg} / \mathrm{dl}$ and at 2 -hr $\mathrm{PG} \geqq 200$ $\mathrm{mg} / \mathrm{dl}$ it was $10.2 / 1000 \mathrm{PY}$ and at FPG of $110-125 \mathrm{mg} / \mathrm{dl}$ it was 2.6 and $5.2 / 1000$ PY, respectively. 2 -hr PG $\geqq 200 \mathrm{mg} / \mathrm{dl}$ was more strongly correlated to incidence of RD than FPG. 5) CHD mortality rate of groups with FPG $\geqq 126$ was significantly higher than groups $\leqq 114 \mathrm{mg} / \mathrm{dl}$. Conclusion: From regression equation, FPG for 2 -hr PG of 200 was $125 \mathrm{mg} / \mathrm{dl}$. Prevalence and incidence of RD elevate from FPG of $126 \mathrm{mg} / \mathrm{dl}$, but as $2-\mathrm{hr} P G \geqq 200 \mathrm{mg} / \mathrm{dl}$ has a greater relationship to RD, it is considered that GTT is important for diaguosis of DM. 
449

PROBLEMS IN PERFORMING SYSTEMATIC REVIEWS IN DIABETES V.M. Montori, S.F. Dinneen and A.J. Farmer. Division of Endocrinology, Mayo Clinic, Rochester, MN, USA and Health Services Research Unit, University of Oxford, UK.

Systematic reviews (SRs) of the literature and meta-analyses of randomized controlled trials (RCTs) are recognized as valid methods for answering clinical questions. The Cochrane Diabetes Group is undertaking SRs in the field of diabetes. In the course of performing a Cochrane SR of dietary marine oil supplementation in Type 2 diabetes we identified 19 RCTs eligible for inclusion. The following hurdles to SR and/or meta-analysis were encountered: (1) explicit diagnostic criteria and a precise classification of diabetes were frequently lacking ( $85 \%$ of RCTs); (2) both crossover ( $42 \%$ ) and parallel group (58\%) designs were used but no consensus exists on how to combine data from these different types of trials; (3) data from the end of the first phase/beginning of the washout period and/or data from the end of the washout/beginning of the second phase were never reported; (4) in the 15 RCTs which reported glycosylated hemoglobin (GH) as an outcome 11 used $\mathrm{HbAlc}(73 \%), 3$ used $\mathrm{HbA} 1(20 \%)$ and 1 used total $\mathrm{GH}(6 \%)$. A reference range for this assay was reported in only 9 RCTs $(60 \%)$ making standardization of this important outcome measure difficult; (5) three of 7 crossover trials which reported $\mathrm{GH}$ as an outcome had a duration of less than 8 weeks raising concerns about a carryover effect on a variable which represents an integrated measure of glycemic control over 12-16 weeks. The quality of a SR depends largely on the quality of its primary studies (RCTs). Our data would call for better collaboration between trialists in diabetes to standardize the outcomes used in RCTs. Such a collaboration would also provide easier access to individual patient data where needed. Journal editors should be more aware of the importance of uniform reporting of RCT data in order to facilitate SR and meta-analyses.

\section{0}

GETTING AND SURVIVING PROSTATE CANCER: ARE MEN WITH DIABETES FORTUNATE?

J.C. Will, F.Vinicor and E.E. Calle; Centers for Disease Control and Prevention and the American Cancer Society, Atlanta, USA

Two recent prospective incidence studies suggest that men with diabetes may be protected from developing prostate cancer. However, several earlier studies are conflicting, probably due to small sample sizes and failure to adjust for important covariates. With hundreds of thousands of male respondents, the 1959-1972 Cancer Prevention Study provided a unique opportunity to explore whether men with diabetes $(n=3,996)$ were more likely to develop prostate cancer during a 13-year follow-up period than were men without diabetes ( $n$ $=209,311)$. Cox proportional hazards analysis with adjustment for known important prostate cancer risk factors yielded no association for diabetes and prostate cancer incidence (incidence density ratio $[\mathrm{IDR}]=1.17,95 \%$ confidence interval $[\mathrm{CI}] 0.85-1.61)$. However, after a diagnosis of prostate cancer, men with diabetes were more likely to die from prostate cancer (IDR $=1.62$, CI 1.02-2.56). Possible reasons for a higher case-fatality rate among men with diabetes include: 1) delayed diagnosis, 2) more "aggressive" prostatic cancer, and 3) restricted use of the most effective prostate cancer treatments. Public health researchers and practitioners should investigate and ensure that all persons with diabetes receive timely and appropriate "non-diabetes" prevention services, including prostate cancer screening in men.

\section{1}

IS INSULIN SENSITIVITY, INSULIN OR BODY FAT AT THE CENTER OF SYNDROME X? THE INSULIN RESISTANCE ATHEROSCLEROSIS STUDY R.B. D'Agostino Jr, R. Hamman, M. Rewers, P. Savage, G. Howard, M. Saad, J. Selby and S.M. Haffner, Winston-Salem, NC, USA

It has been shown that cardiovascular disease (CVD) risk factors (RF), such as dyslipidemia (DYS), hypertension (HYP), and impaired glucose tolerance (IGT), are associated with insulin sensitivity (SI), fasting insulin (FI), 2 hour insulin (2HI), BMI, and waist circumference (W) (i.e. Syndrome X). We sought to establish which index of Syndrome X (SI, FI, 2HI, BMI, W) was most highly associated with an increased prevalence of the three RF (DYS, HYP, IGT). Analyses were performed on 910 nondiabetic persons from the Insulin Resistance Atherosclerosis Study (IRAS). An insulin boosted 12 sample intravenous glucose tolerance test with minimal model analysis was used to estimate SI. Five ordinal logistic regression models estimated the association between the number of CVD $\mathrm{RF}$ (outcome) and the variables: SI, FI, 2HI, BMI or W. Each of these indices of Syndrome $\mathrm{X}$ was significantly related to increased prevalence of the three RF; however, the association was significantly stronger in the model with SI than the other 4 indices $(\mathrm{p}<0.001$ ). Odds ratios for predicting CVD RF for each index were compared and the most striking relationship was for SI. The lowest quartile of SI (most insulin resistant) was associated with a 14.3 fold higher likelihood of multiple CVD RF (see figure). In a multivariable model SI, $2 \mathrm{HI}$, and W were all associated with more CVD RF indicating that each may be contributing independently to aspects of Syndrome X. We conclude that insulin sensitivity (SI) may be the strongest mediator underlying the increasing prevalence of these CVD $\mathrm{RF}$ in Syndrome X.

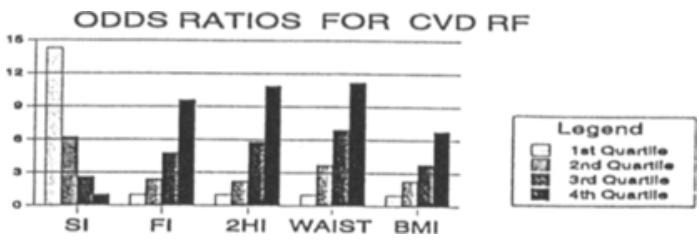

\section{2}

CAN FASTING GLUCOSE EVER BE ADEQUATE TO SCREEN FOR DIABETES?

MP de Courten, JE Shaw, AM Hodge and PZ Zimmet, Melbourne, Australia.

The American Diabetes Association report on diabetes classification suggests a lowering of the fasting plasma glucose (FPG) threshold and the creation of a new category of impaired fasting glucose (IFG), to bring the fasting cut-offs into line with the $2 \mathrm{~h}$ cut-offs, and abolish the need for an oral glucose tolerance test (OGTT). We tested the validity of this by examining OGTT data from 7 southern hemisphere populations. The analysis was restricted to subjects with no previous diagnosis of diabetes $(\mathrm{n}=15246)$. The prevalence of each of the following was determined for each population (subdivided by ethnic group and urbanisation): diabetes using FPG alone (DMF $\mathrm{FPG} \geq 7.0 \mathrm{mmol} / 1)$; diabetes using FPG and $2 \mathrm{hPG}(\mathrm{DM} 2-\mathrm{FPG} \geq 7.0$ or $2 \mathrm{hPG} \geq 11.1 \mathrm{mmol} / 1$ ); IFG (FPG 6.1-7.0mmol/1); IGT (2hPG 7.8$11.1 \mathrm{mmol} / \mathrm{l})$. For females, the prevalence of IGT ranged from 56 $480 \%$ (median 160 ) of the IFG prevalence, and DM2 prevalence was $100-367 \%$ (median 157) that of DMF. For males, IGT prevalence was 31-226\% (median 90) IFG prevalence, and DM2 prevalence was 103$220 \%$ (median 131) DMF prevalence. IFG was less common than IGT in 19 sub-populations, and more common in 12 . The varying relationship between $2 \mathrm{hPG}$ and FPG underlies these differences in prevalence. We used regression analysis to determine the percent of total variation of the FPG-2hPG increment that was explained in each population by age, ethnicity, sex, obesity, lipids, FPG and fasting insulin. This value ranged from $7-42 \%$. Thus, whilst prevalences are significantly and variably affected by using only FPG, we were not able to determine the main factors causing this variability, and suggest that FPG alone is an inadequate substitute for the OGTT for population screening for diabetes. 
453

THE 1997-ADA-AND 1985-WHO CRITERIA: DIABETES PREVALENCE, CARDIOVASCULAR RISK PROFILE AND MORTALTITY. THE HOORN

\section{STUDY.}

J.M. Dekker, F. de Vegt, C.D.A. Stehouwer, G. Nijpels, L.M. Bouter, R.J. Heine. Institute for Research in Extramural Medicine, Vrije Universiteit Amsterdam, The Netherlands

The new American Diabetes Association criteria are based on fasting plasma giucose levels to avoid the burdensome oral glucose tolerance test. We compared the 1997-ADA-criteria with the 1985-WHO-criteria with respect to the prevalence of diabetes, the cardiovascular risk profile and subsequent mortality in the Hoorn Study. In this population-based survey of 2378 men and women aged $50-75$ years, without known diabetes, fasting and 2-hour plasma glucose values and cardiovascular risk factors were determined. Subjects were categorised according to both the 1997-ADA-criteria and the 1985-WHOcriteria. The vital status of all subjects was checked in the municipal registrations, after 6-7 years of follow-up. Age- and sex adjusted relative risks comparing categories of glucose tolerance were estimated by Cox proportional hazards model.The overall prevalence of diabetes was the same for both sets of criteria. However, of the 118 newly diagnosed diabetic subjects (NDM) according to the $1985-$ WHO-criteria, 45 subjects ( $38.1 \%$ ) were not classified as diabetic when using the 1997-ADA-criteria. Similarly, 47 (39.2\%) of $120 \mathrm{NDM}$ subjects according to the 1997-ADA-criteria were classified as IGT or normal when using the 1985-WHO-criteria. Subjects who were diagnosed as diabetic by one of the two sets of criteria only had an adverse cardiovascular risk profile compared to subjects with normal glucose tolerance (NGT). During the followup (6.4 \pm 1.2 years) 224 subjects died. The hazard ratio for NDM compared to NGT differed slightly: $1.9(95 \% \mathrm{Cl}: 1.2-3.0)$ when using the $1985-$ WHO-criteria and 1.7 (95\% Cl: $1.0-2.7)$ when using the 1997-ADA-criteria. In this study both criteria diagnosed a similar number of diabetic subjects, but a large part of the subjects shifted from glucose tolerance categories. With either set of criteria, a number of diabetic subjects with an adverse cardiovascular risk profile will be missed. The hazard ratio for total mortality were similar using either criteria.

\section{4}

ADA VS WHO DIAGNOSTIC CRITERIA FOR DIABETES: IDENTIFICATION OF CLASSES WITH A DIFFERENT PATTERN OF RISK FACTORS.

M. P. Garancini and G. Gallus. Epidemiology Unit, S. Raffaele Institute and University of Milan, Milan, Italy

The opportunity for European countries to adopt the new ADA diabetes diagnostic criteria should be evaluated on the basis of European population data. Our aim was to compare the different classes of subjects identified by both WHO and ADA criteria in order to highlight possible differences in terms of cardiovascular risk factors. The study is based on the data collected through the Cremona population study. Standardised oral glucose tolerance test results as well as further laboratory and clinical data were available. We confined our evaluation to subjects without known diabetes and aged 40 years or more $(\mathrm{n}=1935)$. These subjects were classified as follows: WHO (fasting and/or 2nd h glucose)

\begin{tabular}{ccccc} 
ADA (fasting glucose only) & diabetes & IGT & normal & total \\
\hline diabetes & 23 & 7 & 6 & 36 \\
IFG & 8 & 27 & 68 & 103 \\
normal & 19 & 119 & 1658 & 1796 \\
\hline Total & 50 & 153 & 1732 &
\end{tabular}

Diabetic subjects identified by ADA only $(n=13)$ were younger, fatter and with higher fasting insulin levels than those identified by WHO only $(\mathbf{n}=27)$ (age: $58 \pm$ 13 vs $67 \pm 2, p=0.03$; BMI: $29.4 \pm 1.2$ vs $25.8 \pm 0.8, p=0.02$; insulin: $20 \pm 3$ vs $16 \pm 1$, $\mathrm{p}=0.13$ ). IFG subjects ( $\mathrm{ADA}$ only, $\mathrm{n}=75$ ) were younger $(60 \pm 1$ vs $64 \pm 1$, $\mathrm{p}=0.005)$, fatter $(28.3 \pm 06$ vs $27.1 \pm 04, \mathrm{p}=0.06)$ with higher triglycerides $(138 \pm 6$ vs $120 \pm 3, p=0.04)$ and higher insulin ( $18 \pm 1$ vs $15 \pm 1, p=0.04)$ than IGT subjects (WHO only, $\mathrm{n}=126)$. Analogously, ADA normal subjects $(\mathrm{n}=138)$ were significantly elder than the WHO ones $(n=74)$ and with a significantly healthier cardiovascular risk factors pattern. These differences were confirmed even after controlling for age and gender. In conclusion, despite the ADA criteria are based on lower basal cut-off levels, they identify less abnormal subjects but with higher cardiovascular risk fatcors levels, with respect to the previous diagnostic criteria.

\section{5}

HYPOTHESIS: MALADAPTATION TO CHRONIC EXCESS DIETARY PORTAL VEIN FATTY ACID FLUX, RATHER THAN GENES DETERMINE ETHNIC DIFFERENCES IN GLUCOSE TOLERANCE. Kennedy Cruickshank, Simon Anderson. Manchester University, UK. Excess type II diabetes across most ethnic groups includes African. Caribbean (AfC) and Indian sub-continental if not yet European origin peoples in Britain. We examined the possible role of plasma fatty acids (FA) and serum lipid composition in ethnic differences in glucose tolerance (GT) $75 \mathrm{~g}$ oral GT test results were compared in carefully-taken population samples (77\% response) of $100 \mathrm{AfCs}(53$ women (w)), 113 Gujratis [Guj.] (55w) and 118 Europeans (60w) using 1985 WHO criteria, excluding known diabetes. Among $2 \mathrm{hr}$ normoglycaemics (ng), age/sex adjusted fasting nonesterified (NE) FA were considerably lower in $\mathrm{AfC}(\mathrm{n}=70)$ at 0.42 (mean, $95 \% \mathrm{CI} 0.36-0.48)$ despite obesity than in Guj., $0.58(0.51-0.65)$, or Europeans, $0.58(0.52-0.64), \mathrm{mmol} / \mathrm{l}, \quad(\mathrm{F}=8.2, \quad \mathrm{p}=0.004)$. NEFA were significantly $26-52 \%$ higher in, with no ethnic difference between, those glucose intolerant (GI). Proportions of serum linoleic acid $(18: 2 n-6)$ were lower in AfC (27.4, 26-29, \%) and Europeans $(24,23-26, \%)$ than the 35.3, $34-36.6, \%$ in vegetarian Guj. whose likely fish-derived docosohexanoate $(22: 6 n-3)$ at $1.2,0.8-1.5, \%$ was half or less than AfC $(2.7,2.3-3, \%)$ or Europeans $(2.4,2-2.8, \%)-p<0.001$, in both ng and GI. With BMI and fasting insulin, NEFA were independently associated with $2 \mathrm{hr}$ glucose accounting for much of the ethnic difference. We suggest inadequate adaptation to longterm exposure to excess dietary fatty acids, probably of particular chain length, in context of chronic positive energy balance, determines risk of diabetes, accounting for more of the ethnic difference than does putative genetic variation.

\section{6}

AUTOANTIBODIES TO GLUTAMIC ACID DECARBOXYLASE IN DIABETIC PATIENTS FROM A MULTI-ETHNIC URBAN COMMUNITY T.M.E. Davis' ${ }^{1}$, P. Zimmet ${ }^{2}$, W.A. Davis', D. Bruce ${ }^{1}$ and L. Mackay. ${ }^{3}$ 1. University of Department of Medicine, Fremantle Hospital, Australia, 2. International Diabetes Institute, Melhourne, Australia 3. Department of Biochemistry and Molecular Biology, Monash University, Clayton, Australia. Antibodies to glutamic acid decarboxylase (GADA) are found in most patients with type 1 diabetes. GADA are also a marker of latent autoimmune diabetes of adults (LADA) which is often diagnosed as type 2 diabetes. Small-scale studies of selected samples have suggested ethnic differences in GADA prevalence. To further investigate GADA positivity in diabetic patients from a large, urban, multi-ethnic community, we studied 1381 patients representing $61 \%$ of all those identified in a catchment area of 120,097 people. Anglo-Celts formed $62 \%$ of the sample, Southern Europeans $18 \%$, other Europeans $8 \%$ and Asians 3\%. Amongst Anglo-Celts, GADA positivity was found in $46.0 \%$ of patients with clinically diagnosed type 1 and in $4.2 \%$ of type 2 patients, while the prevalence in Southem Europeans was $22.2 \%$ and $1.7 \%$ respectively. In the full cohort, GADA positive type 2 diabetic patients were younger, more recently diagnosed, were less obese, had a greater $\mathrm{Hb}_{\mathrm{lc}}$, and were more likely to be on insulin than GADA negative subjects $(P<0.05)$. In both type 1 and 2 diabetes, there was a strong independent inverse association between GADA and fasting serum triglycerides $(P<0.001)$. The prevalence of GADA positivity in Anglo-Celt patients was low compared to that in previous studies, regardless of diabetes type. This could reflect the community-based nature and older age structure of the present sample. However, we found even fewer Southem European GADA positive patients with type 1 diabetes and LADA than in the Anglo-Celt group. Although GADA positive patients had usual phenotypic and metabolic features of type 1 diabetes or LADA, the inverse association with serum triglycerides was unexpected and may relate to greater insulin sensitivity compared to GADA negative patients. These data suggest a significant variation by latitude in GADA positivity in diabetic patients of European ethnicity which could have clinical implications. 
RESIDUAL BETA-CELL FUNCTION IN NON-INSULIN DEPENDENT (NIDDM) TYPE 2 DIABETES MELLITUS: CLINICAL AND THERAPEUTICAL CORRELATIONS

A. Sabbatini, E. Borrello, MG. Russo, E. Sciullo, A. Maldonato and F. Fallucca

Cattedra di Diabetologia, Ist. II Clinica Medica, Università "La Sapienza", Roma

It is well known that in insulin dependent type 1 diabetes mellitus the residual beta-cell secretion (RIS) reduces oneself progressively from the time of diagnosis. A greater reduction of RIS has been found associated with a higher prevalence of acute and chronic diabetic complications. The evolution of RIS in the non-insulin dependent type 2 diabeles is extremely longer over the time and, likely, for these reasons the knowledge on the role of RIS in the evolution of type 2 diabetes and its complications are few. For this purpose we evaluated the residual beta-cell function in the last 1000 NIDDM patients of our Diabetic Unit. They were grouped according to four ranges fasting values of $C$-peptide (CPR) plasma values 1) $<0.6$; 2) $0.6-1.2$; 3) $1.2-2.4$; 4$)>2.4 \mathrm{pmol} / \mathrm{l}$. Lower CPR values $(<0.6$ $\mathrm{pmol} / \mathrm{l})$ were found associated with a longer diabetes duration, a higher prevalence of retinopathy (background and proliferant), cataract and neuropathy. On the contrary greatest CPR values were found associated with higher plasma cholesterol, tryglycerides, hypertension and myocardial infarction. These differences were observed in the four groups although they do not differ for age and metabolic control ( $\mathrm{HbA1c}$ and mean monthly blood glucose profile). This study, performed in a large population of NIDDM patients, suggests that a reduced insulin secretion is associated other than a longer duration of diabetes, with a greater prevalence of microvascular complications, whereas the patients with higher insulin levels meet a greater prevalence of atherosclerosis, hypertension and macrovascular complications.

\section{9}

INFLUENCE OF GENDER AND AGE ON THE PREVALENCE OF TYPE 2 DIABETES IN THREE FRENCH REGIONS : THE MONICA STUDY 95-97.

P. Gourdy ${ }^{1}$ J.B. Ruidavets ${ }^{2}$, D. Arveiler ${ }^{3}$, Ph. Amouyel ${ }^{4}$, P. Ducimetière and J.P. Tauber'. Service de diabétologie, CHU Rangueil, Toulouse. ${ }^{2}$ MONICA Toulouse. ${ }^{3}$ MONICA Strasbourg. ${ }^{4}$ MONICA Lille. ${ }^{5}$ INSERM 258, Paris.

The ADA proposed in July 1997 to reduce the fasting plasma glucose (FPG) threshold for the diagnosis of diabetes mellitus (DM) from 7.8 to $7.0 \mathrm{mmol} / \mathrm{l}$. Using successively WHO 85 and ADA 97 criteria, we analyse the prevalence of type 2 DM and mild fasting hyperglycaemia (MFH) in a random sample of 1778 men and 1730 women, aged 35-64 years, having participated in the french MONICA population study, conducted between 1995 and 1997 in Bas-Rhin (BR, East), Haute-Garonne (HG, South-West) and Lille (L, North). A stratification by sex and age was performed in each region. Type $2 \mathrm{DM}$ is defined by a self-reported history of diabetes, or an oral hypoglycemic treatment, or FPG level. MFH is only defined by FPG (WHO 85: 6.1-7.7 mmol/l, ADA 97: 6.1-6.9 mmol/l). 31 insulin-treated subjects $(0.9 \%)$ are excluded of the following analysis

\begin{tabular}{|c|c|c|c|}
\hline 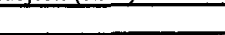 & Men & Women & Total \\
\hline $\begin{array}{l}\text { Type } 2 \text { DM WHO } 85 \\
\text { MFH WHO } 85\end{array}$ & $\begin{array}{c}6.1 \% \\
14.2 \% \\
\end{array}$ & $\begin{array}{l}4.2 \% \\
6.5 \% \\
\end{array}$ & $\begin{array}{r}5.2 \% \\
10.4 \%\end{array}$ \\
\hline $\begin{array}{l}\text { Type } 2 \text { DM ADA } 97 \\
\text { MFH ADA } 97\end{array}$ & $\begin{array}{l}8.3 \% \\
12.0 \%\end{array}$ & $\begin{array}{l}5.4 \% \\
5.3 \%\end{array}$ & $\begin{array}{l}6.9 \% \\
8.7 \% \\
\end{array}$ \\
\hline
\end{tabular}

The large predominance of male subjects observed in Type $2 \mathrm{DM}$ and MFH groups appears significant in each region. According to WHO criteria the prevalence of type $2 \mathrm{DM}$ and MFH are respectively $2.3 \%$ and $6.1 \%$ in $35-44$ years old subjects, $4.0 \%$ and $11.3 \%$ in $45-54,9.3 \%$ and $14.1 \%$ in $55-64(p<0.001)$. The prevalence of Type 2 DM is lower in HG $(4.0 \%)$ than in BR $(5.7 \%)$ and $L(5.8 \%)(\mathrm{p}<0.001)$. By contrast the prevalence of MFH is higher in HG $(14.6 \%)$ than in BR $(8.1 \%)$ and $\mathrm{L}(8.5 \%)$ $(p<0.001)$. These results emphasize the influence of gender, age and geographical location on the prevalence of Type $2 \mathrm{DM}$ and MFH in middle-aged population.
USE OF NEURAL NETWORKS IN THE SCREENING FOR DIABETES IN OBESE PATIENTS.

G. Bardini, E. Mannucci, A. Ognibene*, A. Ciucci, G. Messeri*, and C.M. Rotella. Section of Metabolic Diseases and Diabetology, Dept. of Clinical Pathophysiology, University of Florence, and *Laboratory of Endocrinology, Careggi Hospital, Florence. Viale Pieraccini 6, I-50134 Firenze, Italy.

The American Diabetes Association (ADA) has recently proposed to use Fasting Plasma Glucose (FPG), rather than OGTT, for the screening of diabetes, but FPG could retain a insufficient sensitivity for screening in obese patients. This study is aimed at the evaluation of Neural Networks (NN) in the screening for diabetes in obese patients. NN are a computer algorithm which reproduce the structure of biological nervous systems, allowing the prediction of an unknown output (e.g., diagnosis) from known baseline data, using the experience built on a previous sample of cases with known output. A series of 407 (331 F, $76 \mathrm{M}$ ) obese $\left(\mathrm{BMI}>30 \mathrm{~kg} / \mathrm{m}^{2}\right)$ patients, aged $(\mathrm{m} \pm \mathrm{sd}) 45.0 \pm 14.5$ years and $\mathrm{BMI}$ $37.1 \pm 6.7 \mathrm{~kg} / \mathrm{m}^{2}$ were studied with FPG and OGTT. A random selection of 200 patients was used for training of NN, while the remaining 207 were used to verify NN's ability to predict OGTT-derived diagnoses from baseline clinical and laboratory parameters. Of the 207 patients, 65 were affected by diabetes, 40 by IGT, and 15 by IFG (ADA criteria). Had FPG been used as a screening method with a threshold of 126 $\mathrm{mg} / \mathrm{dl}$, it would have retained a sensitivity of $66.2 \%$. NN identified as "probably diabetic" 97 patients; false positives (19 IGT, 10 IFG), and false negatives were 5 (sensitivity $93.2 \%$, specificity $73.9 \%$ ). In conclusion, providing a few simple baseline clinical and laboratory parameters, NN are able to identify those individuals who are at higher risk of diabetes, and who should therefore undergo OGTT.

\section{0}

RELATIONSHIPS BETWEEN LOW BIRTH WEIGHT AND GLUCOSE TOLERANCE AND INSULIN SENSITIVITY IN A COHORT OF 20 YR OLD SOUTH AFRICANS

NS Levitt, EV Lambert, D Woods, and CN Hales. University of Cape Town South Africa, University of Cambridge, England, UK

An association between low birth weight and both glucose intolerance and insulin resistance has been observed, however, there is little data available from groups undergoing epidemiological transition. We examined these relationships in 20 yr-old offspring of historically disadvantaged primigravid women of mixed ancestry in Cape Town. Subjects ( $N=111$ ) whose birth weights (BW) were either <10th centile ( $S G A, n=59$ ) or between the 25th-75th centile for gestational age (AGA $n=52)$ were studied. $B W(2.35 \pm 0.22$ vs $3.05 \pm 0.20 \mathrm{~kg})$, ponderal index $(2.39 \pm 0.19$ vs $2.60 \pm$ $0.21)$ and placental weight $(483 \pm 68$ vs $551 \pm 69 \mathrm{~g})$ were different in $S G A$ and $A G A$ groups, $(\vec{P}<$ $0.001)$. Maternal weight $(51.4 \pm \overline{9} .1$ vs $61.0 \pm 8.7 \mathrm{~kg})$ and $B M l(21.5 \pm 3.8$ vs $25.0 \pm 3.2)$ were lower in SGA than AGA group ( $<<0.0005)$, however, both groups were equally short in stature $(1.53+0.08$ v $1.55+0.06 \mathrm{~m})$. Anthropometry and oral glucose tolerance were measured in the cohort. WHO criteria were used to diagnose diabetes and impaired glucose tolerance. Insulin and proinsulin data were $\log$ transformed, the $\log (\Delta 30-0$ [insuliny/30min (glucose) ) was calculated $(R A T I O)$ and data adjusted for weight and gender. Current weight $(P=0.001), B M I$ $(P=0.007)$, waist $(P=0.02)$ and head circumference $(P=0.01)$ were all higher in the $A G A$ group.

\begin{tabular}{llcc} 
& & SGA & AGA \\
\hline Glucose: & Fasting (mM) & $4.9 \pm 0.5^{*}$ & $4.7 \pm 0.5$ \\
& $120 \mathrm{~min}(\mathrm{mM})$ & $5.7 \pm 1.6$ & $5.4 \pm 1.1$ \\
Insulin & Fasting (pM) & $45.7 \pm 4.0$ & $38.7 \pm 4.2$ \\
& $120 \mathrm{~min}(\mathrm{pM})$ & $393.3 \pm 47.0$ & $309.8 \pm 51.6$ \\
RATIO & $2.50 \pm 0.04$ & $2.50 \pm 0.05$ \\
Proinsulin (pM) & 4.0 & 4.0 \\
Split proinsulin (pM) $§$ & 7.0 & 7.0 \\
IGT and NIDDM (\%) & $11.9^{* *}$ & 0 \\
\hline
\end{tabular}

( $P=0.056$ and ${ }^{* *} P<0.03$ and $\S=$ geometric mean)

There were no apparent differences in insulin secretion or sensitivity to explain the association between low birth weight and glucose intolerance in this disadvantaged group. These data suggest a complex interplay between intergenerational effects and in utero events. Further work is required to elucidate the underlying mechanisms. (Sponsored: Nestle' Foundation) 


\section{1}

IHAIC PREDICTS CARIJIOVASCULAR MOR'TALITY IN THE GENERAL POPULATION: THE HOORN STUDY

I: de Vegt. J.M. Dekker. C.D.A. Stehouwer. G. Nijpels. L.M. Bouter. and R.J lleine. Institute for Rescarch in Extramural Medicinc. Vrije Universiteit Amsterdam, the Netherlands.

Glycacmic level has been slown to he associated with cardiovaseular (CVD) mortality in dialvetic subiects. We studied whether this association is also present in the generial population in a poputation-based colort study of 2378 subjects withou known diabeles. aged 50 - 7.5 years. Mean follow-up was $6.4 \pm 1.1$ years. Causes of death were extracted from the medical records and classified according to ICD-9. In 185 of 199 deceised the causes of dealh could be retrieved: for 98 subjects the cause of death was cardievascular (ICD-9 codes 390-459 or 798 (sudden death) Relative risks (RR) for cardiovascular mortality were estimated by Cox proportional hazards models for quintiles of HbA lowest $B \mathrm{BA} \mathrm{A}_{1 \mathrm{r}}$-quintile. subjects in the highest quintite were significantly older (63.6 versus 59.7 years). more often hypertensive (38.0 versus $26.4 \%)$. more often cigarette smokers $(45.0 \%$ versus $20.9 \%$ ) and had relatively more abdominal fat (waist-to-hip ratio $(1.92$ versus 0.87 ). The lipid profile was also more atderse (cholesterol 6.87 versus $6.46 \mathrm{mmol} / \mathrm{l}$ and HDL-cholesterol 1.22 versus 1.4 mmol/l). The ayd-and sex-adjusted RR for CVD mortality was $3.43(95 \%$ Cl 1.44 8.18 for the highest relative to the lowest HbA adjustment for hypertension. waist-to-hip ratio, cigarette smoking, total- and IIDLcholesterol fevel. the RR remained statistically significant: $2.43(95 \%$ CI 1.01 5.87). When 118 subjects with plasma glucose values in the diabetic range were cxcluded, the age- and sex- adjusted RR for cardiovascular mortality was 2.91 (9.5 ") (I 1.19-7.10) for the highest relative to the lowest $\mathrm{HbA}_{\mathrm{k}}$-quintile After additional adjustment for $C V D$ risk factors, the $R R$ was no longer significant ( $R R=$ 2.11). 95\% (I (1.85-5.18). probably due to small mumbers of CVD-cases. These results indicate that $\left[H b \Lambda_{k}\right.$ is an independent cardiovascular risk indicator in sulpects witherut dialhetes.
463

\author{
WAIST-TO-HEIGHT RATIO IS AN IMPORTANT \\ PREDICTOR OF DIABETES AND HYPERTENSION \\ M. A. Sayeed', M. G. Kibriya' ${ }^{1}$, P. A. Khanam', A Banu², \\ and A. K. Azad Khan'. Dhaka, Bangladesh
}

1- BIRDEM, Dhaka; 2- INFS, University of Dhaka, Bangladesh

Waist-to-hip ratio (WHR), body mass index (BMl: $w t$ in $\mathrm{kg} / \mathrm{ht}$ in $\mathrm{m}^{2}$ ), skinfold thickness and waist-to-thigh ratio are used to relate diabetes and cardiovascular risk. Short stature also relates these disease events. However, there was no report on anthropometric measure waist-to-height ratio (WHTR), relating adult height with the central obesity. We investigated 2361 (M/F: 1479/882) randomly selected subjects of age $\geq 20 \mathrm{yr}$ for height, weight, waist and hip circumference, blood pressure (BP) and blood glucose. WhO diagnostic criteria were used for non-insulin-dependent diabetes mellitus (NIDDM). Systolic (sHTN) and diastolic (dHTN) hypertension were defined as systolic (SBP) and diastolic (DBP) blood pressure $\geq 140$ and $\geq$ $90 \mathrm{mmHg}$ respectively. Age- and sex-adjusted comparison between subjects with and without disease events (NIDDM vs non-NIDDM, sHTN vs non-sHTN and dHTN vs non-dHTN) showed that the mean \pm SD values of WHR $(p<0.01)$ and WHTR $(p<0.01)$, in either sex, were significantly and consistently higher among those with disease events than those without. The values for $\mathrm{BMl}$, though inconsistent, were also higher in the former. Pearson's correlation coefficients also showed that SBP, DBP and $2 \mathrm{~h}$ post-load blood glucose (2hBG) had significant association with WHR ( $<<0.001$ for all) and WHTR ( $p<0.001$ for all); whereas for $\mathrm{BMl}$, these were inconsistent $\mathrm{Chi}$ sq trend tests for the prevalence of disease events in the lowest through highest quintiles of BMI, WHR and WHTR showed that the highest prevalence of NIDDM, sHTN and dHTN were observed in the highest quintile of WHTR compared with that of BMI and WHR. In multiple regression analysis taking $2 \mathrm{hBG}$, SBP and DBP as the dependent variables in each individual model, adjusted for sex, area and social class, when WHR and WHTR were entered WHTR was proved to be more important predictor for SBP and DBP than WHR; whereas for $2 \mathrm{hBG}$, both WHR and WHTR were equally important. Thus, this study revealed that the waist-to-height ratio is as important as WHR for diabetes and even more important predictor for hypertension, at least, in the developing communities. Moreover this anthropometric measure is simple and yields least error
462

HYPERHOMOCYSTEINAEMIA INCREASES RISK OF 5-YEAR MORTALITY, ESPECIALLY IN DIABETES: THE HOORN STUDY E.K. Hoogeveen ${ }^{1}$, P.J. Kostense ${ }^{1}$, R.J. Heine ${ }^{1,2}$, F. de Vegt ${ }^{1}$, C. Jakobs ${ }^{3}$, J.M Dekker' $^{1}$, G. Nijpels ${ }^{1}$, L.M. Bouter ${ }^{1}$ and C.D.A. Stehouwer ${ }^{1,2} .{ }^{2}$ Institute for Research in Extramural Medicine, ${ }^{2}$ Department of Internal Medicine and ${ }^{3}$ Clinical Chemistry, Vrije Universiteit Amsterdam, The Netherlands. Hyperhomocysteinaemia is an important risk factor for cardiovascular disease and mortality. The aim of the present study was to investigate the predictive value of hyperhomocysteinaemia for mortality in relation to diabetes. We used a case-cohort approach. During 5-year follow-up of a 50-75 year old general Caucasian population-based cohort $(n=2484)$ there were 171 cases of mortality. An age-, sex- and glucose tolerance-stratified random sample $(n=715)$ served as source of controls. Glucose tolerance was assessed by means of two oral glucose tolerance tests, except in subjects with diabetes mellitus who were treated with insulin and/or hypoglycaemic agents. Diabetes was present in 184 subjects. Information on death was obtained by the municipal population registry office. Hyperhomocysteinaemia was defined as serum total homocysteine $>14 \mu \mathrm{mol} / \mathrm{l}$. After stratification by diabetes, we performed a logistic regression analysis to calculate 5year mortality risks. After adjustment for age, sex, hypertension, current smoking, hypercholesterolaemia and serum albumin (a biochemical marker of health), the odds ratio $(95 \% \mathrm{CI})$ for 5 -year mortality for hyperhomocysteinaemia was $1.34(0.87-2.06)$ in nondiabetic and $2.51(1.07-5.91)$ in diabetic subjects. We conclude that hyperhomocysteinaemia is a stronger (1.9-fold) risk factor for mortality in diabetic than in non-diabetic subjects, independent of classical risk factors.

\section{4}

6-YEAR CHANGES IN GLUCOSE TOLERANCE, ASSESSED BY DUPLICATE ORAL GLUCOSE TOLERANCE TESTS. THE HOORN STUDY. G. Nijpels, M. Oudega, A. Jager, JM Dekker, C.D.A. Stehouwer, L.M. Bouter, R.J. Heine. Institute for Research in Extramural Medicine, Vrije Universiteit Amsterdam, The Netherlands

Little is known about the natural history of glucose tolerance in the general population. In the Hoorn Study, we assessed the conversion rates during 6 year follow-up. In 1990, 2484 men and women aged 50-75 years, completed an oral glucose tolerance test (OGTT). An age, sex, and glucose tolerance stratified random sample of 631 subjects had a second OGTT. Subjects were classified according to the WHO-85 criteria, based on the mean fasting and 2 hour glucose values of the two OGTTs. Subjects treated with oral medication or insulin did not perform an OGTT and are denoted as DM-med. In 19961997 all subjects were invited for a follow-up medical examination, including two OGGTs. A total of 407 subjects participated, with a mean follow-up duration of 6,4 years. Of the remaining 224,76 had deceased, 39 had moved, and 109 did not participate. For 3 subjects data on use of medication, and for 2 fasting glucose values were missing. The Table shows changes between glucose tolerance categories.

Table: Glucose tolerance according to the means of fasting and 2-hour glucose levels of duplicate OGTT in 1990-1991 and in 1996-1997. IGT DM DM-med Total

\begin{tabular}{llllll}
$1990-1991$ & & & & & \\
\hline Normal & 157 & 30 & 5 & - & 192 \\
IGT & 31 & 41 & 26 & 12 & 110 \\
DM & 18 & 4 & 19 & 25 & 66 \\
DM-med & - & - & - & 34 & 34 \\
\hline Total & 206 & 75 & 50 & 71 & 402
\end{tabular}

The results show that a large proportion of subjects who were classified as IGT or DM at baseline returned to normal glucose tolerance in a period of 6 years It is not yet clear whether this is due to life style changes or to normal biological variation. However, these results indicate that abnormal glucose tolerance may not be a stable condition. The clinical relevance of this unstability remains to be established. 


\section{5}

THE PREVALENCE BUT NOT THE INCIDENCE OF DIABETES IS INCREASING

B Berger 1, G Stenström 2, and G Sundkvist 3. Depts. of Medicine, Skövde Central Hospital ${ }^{1}$ and Kungälv Hospital 2, and Dept. of Endocrinology, Malmö University Hospital, Malmö ${ }^{3}$, Sweden.

An increased prevalence of diabetes may be due to an increased incidence and/or a prolonged survival. The aim of this study was to clarify these issues using the Skaraborg Diabetes Registry (SDR). Since 1991, SDR prospectively follows the incidence, prevalence, and mortality of diabetes in the entire population (280 000 inhabitants) of a Swedish county. According to SDR, and as expected, the age related incidence of diabetes increased with advancing age (from 22 per 100000 per year for those $0-4$ year old to 1340 per 100000 per year for those $80-84$ year old). The overall incidence rate was, however, constant during the years $(343 \pm 54$ per 100000 per year). Nevertheless, the prevalence of diabetes significantly increased during the observation period (from $2.65 \% 1992$ to $3.45 \% 1997$; $0.16 \%$ per year; $\mathrm{P}=$ 0.03 ). The survival was clearly dependent on the age at diagnosis of diabetes. While 40 patients have died since 1991, only 5 out of 638 patients diagnosed before the age of 15 years had survived to the age of 65 years: On the other hand, patients diagnosed after the age of 60 years seemed to have a survival similar to the background population. Conclusion: The prevalence but not the incidence rate of diabetes is increasing. Although there is a high mortality in patients with an early diagnosis of diabetes, most likely a prolonged survival explains the increasing prevalence of diabetes in the county of Skaraborg.

\section{6}

OBESITY INCREASES THE INCIDENCE OF HYPERINSULINEMIA: A FOUR-YEAR FOLLOW-UP STUDY IN MIDDLE-AGED MEN

H.-M. Lakka, T.A. Lakka and J.T. Salonen. Research Institute of Public Health, University of Kuopio, Kuopio, Finland

Hyperinsulinemia has consistently been associated with obesity in cross-sectional studies. However, on the basis of prospective studies, the time order of the relationship is unclear and the association seems to be population specific. Therefore, we investigated the association of obesity with the incidence of hyperinsulinemia and vice versa in a population-based four-year follow-up study of middle-aged men from eastern Finland. Out of 978 men who had no diabetes, $879 \mathrm{men}$ were defined normoinsulinemic at baseline (nine lowest deciles of fasting serum insulin). Eightyseven men developed hyperinsulinemia (fasting serum insulin in the highest decile, $\geq$ $12.2 \mathrm{mU} / 1)$ during the follow-up. In logistic regression model adjusting for other risk factors, the odds ratios (ORs) for incident hyperinsulinemia in men with body mass index (BMI) of 24.2-25.9, 26.0-27.9 and $\geq 28.0 \mathrm{~kg} / \mathrm{m}^{2}$ (three highest quartiles) were $1.20(95 \% \mathrm{Cl} 0.31-4.58), 3.21(1.03-9.99, \mathrm{p}=0.045)$ and $12.96(4.48-37.50, \mathrm{p}<0.001)$ respectively, compared to men with $\mathrm{BMI}$ of $<24.2$ (lowest quartile). After further adjustment for waist-to-hip circumference ratio (WHR), the respective ORs were 1.04 $(0.69-4.11), 2.71(0.83-10.25, \mathrm{p}=0.098)$ and $10.19(3.18-32.60, \mathrm{p}<0.001)$. The ORs for incident hyperinsulinemia in men who gained weight $0-2.3,2.4-4.6$ and $\geq 4.7 \mathrm{~kg}$ (three highest quartiles) were $1.40(0.53-3.72), 2.86(1.25-6.54, p=0.013)$ and 5.92 (2.76-12.73, $\mathrm{p}<0.001$ ), compared to men who lost their weight (lowest quartile) adjusting for other risk factors including baseline weight. The ORs for incident hyperinsulinemia in men with WHR of 0.89-0.92, 0.93-0.96 and $\geq 0.97$ (three highes quartiles) were $1.13(0.38-3.35), 2.76(1.07-7.14, \mathrm{p}=0.036)$ and $5.53(2.23-13.71$, $p<0.001$ ), compared to men with WHR of $<0.89$ (lowest quartile). This association disappeared after further adjustment for BMI. Baseline hyperinsulinemia was not associated with weight gain during the follow-up. These data in nondiabetic men suggest that obesity and weight gain are independent risk factors for hyperinsulinemia whereas hyperinsulinemia does not predict weight gain.

\section{7}

HDL SUBFRACTIONS AND INCIDENCE OF TYPE 2 DIABETES: OPPOSITE EFFECTS IN MEN AND WOMEN.

A Fagot-Campagna, W Knowler, $\vee$ Narayan, J Saaddine, M Goldschmid, G Beckles, E Gregg and B Howard. DDT, CDC, Atlanta GA. NIDDK, NIH, Phoenix AZ. Medlantic Research Institute, Washington DC, USA.

The relationship between HDL subfractions (measured by ultracentrifugation, HDL $2 b$ being the largest and lightest) and type 2 diabetes incidence was assessed among Pima Indians (mean age 42 years). During a mean follow-up of 10 years, 25 of 50 men and 54 of 123 women developed diabetes. In proportional hazards models controlled for age, body mass index, $2 \mathrm{hr}$ glucose and systolic blood pressure at baseline, high levels of total HDL, HDL2a and HDL 3 but not HDL2b were protective of diabetes in women, whereas a high level of HDL3 was predictive in men (table).

\begin{tabular}{lcccccc} 
& \multicolumn{2}{c}{ Men (n=50) } & \multicolumn{3}{c}{ Women $(\mathrm{n}=123)$} \\
\hline & $\mathrm{GM}^{*}$ & \multicolumn{2}{c}{ HRR $^{* *}$} & $\mathrm{GM}^{*}$ & \multicolumn{2}{c}{ HRR $^{* *}$} \\
\hline HDL total & 1.14 & $3.1[0.91-11]$ & 1.17 & 0.29 & {$[0.13-0.63]$} \\
HDL2b & 0.11 & 1.2 & {$[0.25-6.2]$} & 0.15 & 0.48 & {$[0.20-1.2]$} \\
HDL2a & 0.20 & 2.6 & {$[0.64-10]$} & 0.25 & 0.29 & {$[0.14-0.59]$} \\
HDL3 & 0.81 & 3.8 & {$[1.2-13]$} & 0.74 & 0.48 & {$[0.25-0.90]$} \\
\hline
\end{tabular}

* Geometric means, except for HDL 3 (mean), in mmol/l. **Hazard rate ratios and 95

$\%$ confidence intervals comparing the 90 th with the 10 th percentile.

In models controlled for fasting insulin, a risk factor for diabetes in women only, high levels of HDL and HDL2a were slightly weaker protectors but a high level of HDL3 was a stronger protector of diabetes in women. Results were unchanged in men. In models controlled for alcohol consumption, a risk factor for diabetes in men only, results were unchanged in women, but a high level of HDL 3 was not a significant risk factor in men ( $\mathrm{HRR}=2.9 ; 95 \% \mathrm{CI}[0.69-12])$. These findings suggest that:1) high HDL 3 level in men may be predictive of diabetes because it is influenced by alcohol consumption. 2) high levels of HDL, HDL2a and HDL3 in women remained protective of diabetes after accounting for alcohol consumption and estimated insulin resistance. The sex differences in the effects of HDL subfractions may be due to sex hormones.

\section{8}

THE CAUSE OF DEATH IN JAPANESE NIDDM PATIENTS A HOSPITAL-BASED STUDY 1976-1995

T. Nakagami* ${ }^{*}$, R Kawahara ${ }^{*}$, T. Sato"2, N. Iwasaki" ${ }^{*}$, and Y. Iwamoto" "Diabetes Center, "Department of Hygiene and Public Health, Tokyo Women's Medical College Tokyo Japan

To determine the characteristics of the cause of death in Japanese diabetics, we examined the principal causes of death in 1256 NIDDM patients from 1976-1995. Standardized proportional mortality ratios (SPMRs) were used to compare the number of dead patients with death occurring concurrently among the general population in Japan. The most frequent cause of death was vascular disease $(34.4 \%$ ), including coronary heart disease (CHD)(16.3\%), cerebrovascular disease (CVD) $(13.3 \%)$ and diabetic nephropathy $(\mathrm{DN})(4.8 \%)$, the next frequent was malignant neoplasms $(22.3 \%)$, and then other heart disease $(14.3 \%)$. The ratio of CHD increased from $10.9 \%$ in the 1970 's to $19.0 \%$ in the 1990 's, that of CVD decreased from $14.4 \%$ in the 1970 's to $12.1 \%$ in the $1990^{\prime} \mathrm{s}$, and that of DN decreased from $6.5 \%$ in the 1970 's to $2.9 \%$ in the 1990 's Compared with the number of deaths among the general population in the same period, NIDDM patients died more frequently from diabetes, CHD, other heart disease and infection ( $\mathrm{SPMR}=3.60,2.65,2.35,1.37$ ), but less frequently from $\mathrm{CVD}$ and malignant neoplasms (SPMR $=0.41,0.62$ ). The SPMRs of CHD increased from 1.60 in the 1970's to 3.38 in the 1990's.

In conclusion, the number of death from CHD among Japanese NIDDM patients increased gradually during the 20 -year observation period. 
TYPE II DIABETES IS ASSOCIATED WITH COGNITIVE IMPAIRMENT AND COGNITIVE DECLINE AMONG OLDER WOMEN

E.W. Gregg; K. Yaffe; J. A. Cauley; G. Beckles and K.M.V. Narayan. Centers for Disease Control and Prevention; Atlanta, GA; University of California, San Francisco; CA, University of Pittsburgh, PA, USA

We examined the relationship of diabetes to cognitive impairment and cognitive decline among 7425 Caucasian women aged $\geq 65$ years participating in the Study of Osteoporotic Fractures. Self-reported diabetes and 3 tests of cognitive function (Digit Symbol, Trails B, and modified Mini-mental State Exam (MMSE)) were administered at a baseline visit and again after 4 to 6 years. Cognitive function was measured by mean test scores; Clinically relevant cognitive impairment and cognitive decline were defined according to the worst $10^{\text {th }}$ percentile baseline and change scores, respectively. Compared to women without diabetes, those with diabetes $(n=495,7 \%)$ had significantly increased odds of baseline cognitive impairment on Digit Symbol and Trails B but not MMSE (see Table), adjusted for age, education, visual impairment, estrogen use, and stroke. Women with diabetes had significantly worse change scores on all 3 tests $(p<0.05)$ and increased odds of cognitive decline on Digit Symbol and Trails B, adjusted for potential confounders.

\begin{tabular}{|l|lc|cc|}
\hline & \multicolumn{2}{|l|}{ Odds: baseline cognitive impairment } & \multicolumn{2}{c|}{ Odds: Cognitive Decline } \\
\hline Test & \multicolumn{2}{|c|}{ Diabetes Status } & \multicolumn{2}{c|}{ Diabetes Status } \\
\hline & No & Yes & No & Yes \\
\hline Digit Symbol & 1.0 & $2.13(1.65,2.74)$ & 1.0 & $1.52(1.12,2.08)$ \\
\hline Trails B & 1.0 & $1.56(1.20,2.03)$ & 1.0 & $1.94(1.34,2.66)$ \\
\hline MMSE & 1.0 & $1.09(0.89,1.33)$ & 1.0 & $1.03(0.74,1.43)$ \\
\hline
\end{tabular}

Disease duration was also related to cognitive decline ( $\mathrm{p}$ trend $=0.01$ ). Odds ratios of decline on any test for women with diabetes $<5$ years, 5 to 14 , and $\geq 15$ years were $1.36(0.95-1.95), 1.48(1.05-2.07)$, and $1.53(1.01-2.33)$, respectively, compared to non-diabetic women. These findings suggest diabetes is associated with an accelerated cognitive decline among older women.

\section{SCREENING FOR DIABETES WITH THE NEW DIAGNOSTIC CRITERIA AND PROPOSED SCREENING METHODS.}

A. De Bellis, E. Mannucci, C.M. Rotella, M. Velussi*, and the DIAINF Study Group. Section of Metabolis Diseases and Diabetology, Dept. of Clinical Pathophysiology, University of Florence; *Section of Diabetology, Civil Hospital, Monfalcone; and DIAINF Study Group, Florence, Italy. Via della Mattonaia 17, I- 50137 Firenze, Italy.

This study is aimed at the evaluation of the effect of the application of the diagnostic criteria and screening methods proposed by the American Diabetes Association (ADA) in groups of patients at high risk for diabetes. Using the DIAINF computerised network, which contains clinical data of over 300,000 patients from more than 100 Outpatient Clinics all over Italy, high-risk individuals who had undergone a OGTT for the screening of diabetes between 1994 and 1997 in one of 12 Diabetes Care Units were identified. The sample was composed of 5,720 patients $(2,167 \mathrm{M} ; 3,553 \mathrm{~F})$, with a mean $( \pm \mathrm{sd})$ age of $47.9 \pm 16.5$ years and BMI $28.2 \pm 4.8 \mathrm{~kg} / \mathrm{m}^{2}$, who had undergone OGTT for obesity, previous gestational diabetes, transient hyperglycaemia, or other highrisk conditions. Of those, $25.7 \%$ were affected by diabetes, 15.1 by IGT, and 5.5 by IFG (ADA criteria). Fasting Plasma Glucose (FPG) and 2-hr glucose during OGTT ( $2 \mathrm{hrPG}$ ) showed a good correlation ( $\mathrm{r}=0.56$; $\mathrm{r}=0.72$ in those aged $>70$ years). However, of those with $\mathrm{FPG}<126$ $\mathrm{mg} / \mathrm{dl}, 9.2 \%$ had $2 \mathrm{hrPG}>200 \mathrm{mg} / \mathrm{dl}$ and were therefore classified as diabetic; among patients with FPG between 110 and $126 \mathrm{mg} / \mathrm{dl}$, those with diabetes were $30.2 \% ; 4.9 \%$ of subjects with $\mathrm{FPG}<100 \mathrm{mg} / \mathrm{dl}$ were also diabetic. In conclusion, FPG could be insufficient for the screening of diabetes in high-risk subjects, particularly in those $<70$ years old. The OGTT should be retained as a useful tool for the screening of diabetes in selected individuals.

\section{1}

TYPE II DIABETES AND IMPAIRED GLUCOSE TOLERANCE IN A POPULATION STUDY: 10 YEARS OF FOLLOW UP.

J.A.Vazquez , J.Bayo, V.Elorza, K.Latorre and S.Gaztambide. Department of Endocrinology. Hospital de Cruces. Bilbao. SPAIN.

The aim of the study was to establish the incidence of type II diabetes mellitus (DM) and impaired glucose tolerance ( IGT ) in a population followed up for a 10year period. It is a prospective study in a randomized selected population older than 30 years, stratified by age and sex, conducted in 1985 . The selected population was invited to participate again in 1995. An oral glucose tolerance test ( OGTT) was performed following the OMS criteria. Height, weight and blood pressure were registred.OGTT was performed in $862(87.2 \%$ of the selected people ) in 1985 and in $538(77 \%$ of the selected people ) in 1995 ( 47 were deceased $-6 \%, 8 \%$ and $25.8 \%$ belonged to the normal, IGT and DM gorup respectively - , 86 moved to other cities and 196 refused to collaborate). In 1995, 330 out of 438 people ( $76 \%$ ) with a normal glucose tolerance test in 1985 persisted with a normal test, 85 (19\%) had an IGT and 23 (5.2\%) had DM. Twenty eight out of 58 with IGT in 1985 normalized the test $(48 \%), 19(32.7 \%)$ persisted with IGT and $11(18.9 \%)$ had DM. That means a cummulative annual incidence of $1.9 \%$. Thirty one people were diagnosed of DM by OGTT in 1985,8 of them $(25.8 \%$ ) were dead and 3 of them refused the test in $1995 ; 17$ out of the other 20 ( $85 \%$ ) persisted with DM, 2 (10\%) had IGT and one had normal glucose tolerance. BMI of the group with normal glucose tolerance in 1985 increased $4.9 \%$ ( $4 \%, 6.8 \%$ and $10.8 \%$ respectively for those with normal, IGT and DM in 1995 ). BMI of the group with IGT in 1995 increased $5 \%(3.8 \%, 4.5 \%$ and $8.8 \%$ respectively for those with normal, IGT and DM in 1995). In summary: 1) Mortality was higher in the diabetic group ; 2) The cummulative incidence of DM ( $1.9 \%$ ) from the IGT group were lower than expected ; 3) Incidence of IGT and DM have increased between 1985 and 1995 from $10.4 \%$ and $3.8 \%$ to $19 \%$ and $5.2 \%$ respectively, associated to an increment in weight and age.

\section{2}

INCIDENCE OF TYPE 2 DIABETES MELLITUS AND ITS RISK FACTORS IN SOUTH AFRICAN INDIANS : A 10-YEAR FOLLOW-UP STUDY A.A Motala, F.J Pirie, M.A.K Omar, A Amod and E Gouws* Diabetes Unit, Department of Medicine. University of Natal and Medical Research Council*, Durban, South Africa.

Globally, several cross-sectional epidemiology studies have examined the prevalence of type 2 [non-insulin dependent diabetes mellitus (NIDDM)] in different populations but there are few longitudinal population studies. A prospective community study was undertaken to determine the incidence of type 2 diabetes mellitus and the risk factors associated with its developments in a cohort of South African Indians who had been studied 10 years previously (yr 0/baseline). In the baseline survey, 2479 subjects (> $15 \mathrm{yr}$ ) were studied: using WHO criteria for glucose tolerance based on $75 \mathrm{~g}$ oral glucose tolerance tests (OGTT), the crude prevalence of diabetes mellitus (Diabetes) was $9.8 \%$ and of impaired glucose tolerance (IGT) $5.8 \%$ (age and sex adjusted prevalence, $13 \%$ and $6.9 \%$, respectively). At the $10-y$ r followup study, 563 of the subjects who could be traced had repeat OGTT: of these, $91(16.2 \%)$ were classified as Diabetes and $41(7.3 \%)$ as IGT. Of the subjects who did not have diabetes at baseline $(n=517), 49(9.5 \%)$ progressed to diabetes (PTD) and $40(7.7 \%)$ had IGT; the crude incidence of diabetes was $0.95 \%$ per annum $(9.5 / 1000$ person years). Examination of risk factors predictive of subsequent progression to NIDDM (PTO) was undertaken by analysis of baseline (yr 0 ) age, gender, anthropometric and metabolic characteristics in the 517 subjects. In the multivariate analysis the significant predictive risk factors for diabetes development included obesity (p 0.01 OR $4.6,95 \% \mathrm{Cl} 1.4-14.7$ ) body mass index (BMI) ( 0.006 . OR 1.1,

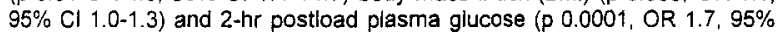
$\mathrm{Cl} 1.4-2.1)$. This long-term study has shown that in South African Indians there is an increased incidence of type 2 diabetes and that significant risk factors for its development include BMI, obesity prevalence and 2-hr plasma glucose. 
473

IMPACT OF THE NEW DIAGNOSTIC CRITERIA FOR DIABETES ON THE NUMBER OF AFFECTED SUBJECTS

H. Pardini, V.C. Pardini and G. Velho. Endocrinology Research Center (CEPEN), Belo Horizonte, Brazil; INSERM U342, Paris, France.

New diagnostic criteria for diabetes mellitus (DM) have been proposed by the American Diabetes Association (ADA), essentially a decrease from 7.8 to $7.0 \mathrm{mM}$ of the fasting plasma glucose (FPG) cutpoint defining DM. We evaluated the impact of the new criteria on the glycemic status of a group of 8517 Brazilian subjects (aged $35 \pm 15$ years, $m \pm S D ; 76 \%$ of women) without previously known diabetes who underwent an oral glucose tolerance test (OGTT) for diagnostic purposes. The frequencies of DM, impaired glucose tolerance (IGT) and normal glucose tolerance (NGT) according to the WHO/1980 criteria were $6.15 \%, 11.68 \%$ et $82.17 \%$, respectively. The new glycemic status would be: $6.61 \%$ DM $11.31 \%$ IGT, $0,61 \%$ impaired fasting glucose (IFG), and $81.47 \%$ NGT. In the 2728 subjects older than 40 years ( $53 \pm 20$ years), the glycemic status by the WHO/1980 criteria was: $16.24 \%$ DM, $22.80 \%$ IGT and $60.96 \%$ NGT. The new glycemic status would be: $17.12 \%$ DM, $22.03 \%$ IGT, $1.36 \%$ IFG and $59.49 \%$ NGT. The ADA does not recommend the routine clinical use of OGTT as a tool for diabetes diagnosis. However, only $20.61 \%$ of the subjects with DM ascertained by the WHO/1980 criteria had FPG $>7.8$ $\mathrm{mM}$, and only $41.56 \%$ of the subjects ascertained by the ADA/1997 criteria had FPG $>7.0 \mathrm{mM}$. In conclusion, the new diagnostic criteria of diabetes lead to an increase of $0.70 \%$ in the number of "hyperglycemic" subjects $(0.12 \%$ to $1.74 \%$ in different classes of age), with an increase of $0.46 \%(0.08 \%$ à $0.88 \%)$ in the number of diabetic subjects in this population without previously known diabetes. Although FPG sensitivity doubles with the new criteria, it remains a poor discriminator of diabetes defined as OGTT 2-hour plasma glucose $>11.1 \mathrm{mM}$

\section{5}

\section{SCREENING FOR NON-INSULIN-DEPENDENT DIABETES IN LUXEMBURG.}

G. Michel, A. Giuliani, M. Keipes, S. Steil and D. Hansen, Direction de la Santé and Association Luxembourgeoise du Diabète, Luxemburg.

In April 1997, the Health Authorities of Luxemburg and the national patient association (ALD) started a national screening campaign for non-insulin dependent diabetes, as a part of the Saint-Vincent Declaration program. During 6 months, persons ( $\geq 40$ years old) and with one of the following risk factors for NIDDM (symptoms of diabetes, obesity, positive family history for diabetes, at least one macrosomial child, dislipidemia and hypertension) were invited by a publicity campaign through media to visit their doctor for a screening of diabetes. All the general practitioners and internists $(n=350)$ were informed about this action. They were invited to fill out a questionnaire for each person. 57 physicians showed a positive interest. Results: The forms of 109 persons (68 female) screened were sent back. Of these 109 persons, $16(15 \%)$ went to their doctor for symptoms of diabetes, $86(79 \%)$ for obesity, $45(41 \%)$ for a positive family history, $66(60 \%)$ for hypertension or dislipidemia and 14 women for a macrosomial child. According to the usual criteria, 29 of $109(27 \%)$ had NIDDM and $39(36 \%)$ had IGT. Of the 29 people with NIDDM, $24(83 \%)$ were obese, 10 had a positive family history for diabetes, 19 had hypertension or dislipidemia (66\%). Mean glucose leve of the whole group was $7.44 \mathrm{mmol} / \mathrm{l}(11.36 \pm 4.93 \mathrm{mmol} / \mathrm{l}$ for diabetics) and cholesterol level was $6.02 \mathrm{mmol} / /$ and mean triglyceride level was 1.90 $\mathrm{mmo} / \mathrm{l}(2.77 \pm 1.96 \mathrm{mmol} / \mathrm{l}$ for diabetics$)$. None of the diabetics had a complication, but 4 persons with IGT had a vascular complication (foot ulcer stroke, heart failure). Applying the new criteria proposed by the ADA, 37 persons of 109 would have had NIDDM. Conclusions : 1/ In this screened population, $27 \%$ had NIDDM, whereas the overall prevalence of diabetes is $2 \%$ and obesity, hypertension and dislipidemia were mostly found as risk factors. 2/ Considering the poor response rate, the perception of diabetes in the general population should be improved.
474

IMPACT OF THE AMERICAN DIABETES ASSOCIATION-1997 DIAGNOSIS CRITERIA ON HIGH-RISK SPANISH POPULATION.

B. Costa, J. Franch, A. Donado, J. Morató, F. Martín, J. Daniel and IGT Research Group. Catalan Institute of Health, Tarragona-Barcelona, SPAIN.

To research into the impact of the new ADA-97 classification and diagnosis criteria on the high-risk Spanish population a multicentric survey was carried out in primary health care. Individuals aged $>40$ years with any one of these 4 major risk factors: obesity, a first degree relative with $\mathrm{DM}$, oral hiperglycaemic drugs and previous abnormality of glucose tolerance were included and diagnosed starting from FPG test and OGTT, according to the OMS-85 rules. A total of 970 individuals were evaluated, 453 males (46.7\%), mean age 58 year-old and $\mathrm{BMI}=30.5 \mathrm{~kg} \cdot \mathrm{m}^{-2}$. OMS-85 diagnoses were: 376 $(38.8 \%)$ normal $[95 \% \mathrm{Cl}=35-41 \%] ; 200(20.6 \%)$ IGT [18-23\%] and 394 $(40.6 \%)$ DM [ $37-43 \%$ ]. In 265 cases (27\%) OGTT was not required and 178 (18.4\%) individuals [16-21\%] fitted to IFG interval (6.1-6.9 mM). After OGTT $65(36.5 \%)$ were displaced to the IGT group and $54(30.3 \%)$ to the DM group. Furthermore, $379(39 \%)$ showed FPG levels $>=7.0 \mathrm{mM}$. A total of $114(11.8 \%)$ of them [10-13\%] were included in the new direct diagnostic interval of DM $(7.0-7.7 \mathrm{mM})$. OGTT concordance were observed in 49 cases (43\%). However, $34(29.8 \%)$ subjects evidenced ITG $(3,5 \%)$ whereas 31 (27.2\%) presented a normal oral tolerance $(3.2 \%)$ after OGTT. ADA-97 diagnoses were: $286(29.5 \%)$ normal tolerance [26-32\%]; $59(6.1 \%)$ IFG [4.6$7.7 \%] ; 166(17.1 \%)$ IGT [15-19\%] and $459(47.3 \%)$ DM [44-50\%]. The application of the new criteria meant a $6.7 \%$ increase of DM and a $3.5 \%$ reduction of IGT. IFG initially concerned nearly $18.4 \%$ but only $1 / 3$ remained the same, as the rest $(2 / 3)$ associated impaired oral tolerance. Thus, there is clear evidence that although the new interval of direct diagnostic of DM would have saved a $12 \%$ OGTT, a $3.2 \%$ of individuals with normal oral tolerance would have been diagnosed of diabetes.

\section{6}

MEASURES OF FETAL GROWTH AND GLUCOSE METABOLISM AMONG 20 TO 68-YEAR-OLD FINNISH SUBJECTS

K. Ylönen', M. Pekkala', L. Groop ${ }^{2}$, S.M. Virtanen ${ }^{3}$, and the Botnia Research Group. Division of Nutrition, Univ of Helsinki, Finland'; Dept of Endocrinology, Lund University, Malmö, Sweden ${ }^{2}$; School of Public Health, Unit of Epidemiology, Univ of Tampere, Finland ${ }^{3}$.

Relationships between measures of fetal growth and glucose metabolism were studied among Finnish subjects with non-insulin-dependent diabetes mellitus (NIDDM), their relatives, and spouses without family history of diabetes, born between $1925-72$. Birth data were found in archives for 295 subjects ( $71 \%$ of those invited). Birth variables used were birth weight, birth length, birth weight to birth length ratio, ponderal index (birth weight divided by birth length ${ }^{3}$ ), head circumference, and head circumference to birth length ratio. The age of men was 48 \pm 11 years, and that of women $46 \pm 12$ years. Subjects participated in a standard oral glucose tolerance test with measurements of glucose and insulin concentrations. According to the WHO criteria, 81 men and 111 women had normal glucose tolerance (NGT), 21 men and 43 women impaired glucose tolerance (IGT), and 22 men and 16 women NIDDM. There were no differences in the birth variables between subjects with NGT, IGT and NIDDM. In linear regression models, adjusting for age, body mass index, waist to hip ratio, length of education, physical activity, smoking, and in men also for alcohol, a low ponderal index, reflecting thinness at birth, was associated with high serum fasting glucose $(p=0.03)$ in men. In women, head circumference was inversely associated with serum fasting $(\mathrm{p}=0.02)$ and $2-\mathrm{h}$ insulin $(p=0.02)$, and incremental insulin area $(p=0.007)$. However, a high head circumference to birth length ratio, proposed to reflect an asymmetrical pattern of development, was also mversely associated with serum 2 -h insulin $(p=0.006)$ and incremental insulin area $(\mathrm{p}=0.04)$. The relationships were independent of duration of gestation. The observations support the idea that impaired fetal growth, whatever the causes are, should be taken into account when searching for factors predisposing to disorders of glucose metabolism. 
477

PREVALENCE OF DIABETES MELLTTUS AND OF IMPAIRED GLUCOSE TOLERANCE IN A CANARIAN POPULATION: CLASSIC AND NEW CRITERIA P. Pablos, F. Rodríguez*, J. Martínez, P. Murado, , A. Macías, V. Sánchez, I. García and C. Santana. Endocrinology and *Preventive Dpts; H. del Pino, Las Palmas, Spain

In order to establish the prevalence of diabetes mellitus (DM) and impaired glucose tolerance (IGT) in our population, we have performed a study in which 585 people have been randomly selected among the population over 30 years old in the council of Santa María de Guia (northern Gran Canaria island). A standard OGTT (75 gr of glucose) was performed in the fasting state to all subjects, excepting those whose fasting glycemia was $>7.8 \mathrm{mM} / \mathrm{L}$. According to the classic W.H.O criteria for fasting glycemia, 365 subjects $(62 \%)$ had normal glucose tolerance, 104 (18\%) had impaired glucose tolerance (IGT) and $116(20 \%)$ had DM. Of these. only $60 \%$ were aware of their diagnosis. The highest prevalence of DM was found in women over 60 years old ( $36 \% ; 68 \%$ of them aware). and the highest prevalence of IGT was found in women between 50 and 60 years (34\%). When applying the new A.D.A criteria for the diagnosis of DM (i.e., those subjects with confirmed fasting glucose $>7 \mathrm{mM} / \mathrm{L}$ instead of $7.8 \mathrm{mM} / \mathrm{L}$ should be classified as having DM, without the need for an OGTT), 6 more subjects had DM (1.02\%): 2 previously classified as normal $(0.34 \%$ of the total, $0.55 \%$ of the normal subiects); and 4 previously classified as having IGT $(0.68 \%$ of the total, $3.84 \%$ of those with IGT). Besides, 2 subjects $(0.34 \%$ of the total) were classified as having DM by OGTT, though their fasting glycemia was normal, and without the OGTT would have been considered normal by both criteria. We conclude that both the prevalence of DM and IGT are high in our population (especially postmenopausal women), and that a large proportion of the diabetic subjects is unaware of their condition. The successive modes of IGT and DM confirm that in our population DM is preceded by IGT. Applying the new fasting criteria for the diagnosis of DM will slightly increase the number of subjects classified as having DM, especially among those previously classified as having IGT.

\section{9}

PREDICTORS OF A LETHAL OUTCOME OF MYOCARDIAL INFARCTIONS IN PATIENTS WITH TYPE 2-DIABETES MELLITUS M.M. Heimesaat, A. Klamann, P. Sarfert, W.H. Schmiegel, and M.A. Nauck. Medizinische Universitätsklinik, Knappschaftskrankenhaus, Bochum-Langendreer, Germany.

After a myocardial infarction, survival rates of Type 2-diabetic patients are distinctly lower in comparison with metabolically healthy subjects. The aim of the present study was to elucidate factors correlated with in-hospital mortality after myocardial infarctions in Type 2-diabetic patients. Therefore, patients with myocardial infarctions treated in our intensive care unit between 01.01 .91 and 30.06 .97 were studied retrospectively. The study groups of surviving and deceased patients with Type 2 diabetes mellitus were compared by univariate analyses (continuous variables: $t$-test; categoric variables: chi-square-test) and by multiple regression analysis (significance: $p<0.05)$. Of 601 patients with myocardial infarctions, $244(40.6 \%$ ) were Type 2 diabetic patients of which $170(69.7 \%)$ survived and $74(30.3 \%)$ died. Deceased diabetic patients were significantly older than diabetic survivors $(\mathrm{p}=0.037)$, had had more often previous infarctions ( $47 \mathrm{vs.} 28 \%$; $\mathrm{p}=0.0076$ ), more often complained about angina pectoris $(\mathrm{p}=0.013)$, and more frequently had been treated with nitrates $(\mathrm{p}=0.023)$ and digitalis $(\mathrm{p}=0.044)$, respectively. Regarding laboratory data, deceased diabetic patients had significantly higher potassium $(\mathrm{p}=0.0006)$, creatinine $(\mathrm{p}<0.0001)$ and $\mathrm{CK}_{M B}(\mathrm{p}=0.008 \mathrm{l})$ levels as well as lower total cholesterol $(\mathrm{p}=0.0006)$ and LDLcholesterol $(p=0.028)$ levels. There were no significant differences in BMI, smoking, duration of diabetes, infarct localization, treatment with thrombolytic agents, sulfonylureas, biguanides, insulin, $\beta$-blockers, molsidomin, calcium antagonists, $\mathrm{ACE}$ inhibitors, and diuretics, as well as $\mathrm{HDL}$ cholesterol, $\mathrm{HbA}_{\mathrm{tc}}$ and totat $\mathrm{CK}$ levels. In the multivariate analysis, however, only previous myocardial infarctions were positively associated with in-hospital mortality $\left(p^{-0}-0.041\right)$. In the light of these findings, previous myocardial infarctions are an important predictor of a lethal outcome of myocardial infarctions in Type 2-diabetic patients.
478

PREVALENCE OF DIABETES MELLITUS (DM) AND IMPAIRED GLUCOSE INTOLERANCE (IGT) IN OBESE WOMEN.

LMB Araújo, M Cardeal, M Britto, J Pousada and T Cruz. Hospital Universitário Prof Edgard Santos, Bahia Federal University, Salvador Bahia, Brazil.

The frequence of DM in obese individuals has been referred as higher than in non obese. We investigated the prevalence of DM and IGT in obese women adjusted for age (age $\leq 40$ and $>40$ years) and degree of obesity $\left(B M I \leq 40 \mathrm{~kg} / \mathrm{m}^{2}\right.$ or $\left.>40 \mathrm{~kg} / \mathrm{m}^{2}\right)$. Three hundred thirty six obese women from an out patient unit of obesity were studied. None of them had previous diagnosis of DM. The mean age $(+D P$ ) was $35.1+9.2$ years (ranged from 15 to 63 years) and mean BMI ( \pm DP) was $40.6 \pm 6.9 \mathrm{~kg} / \mathrm{m}^{2}$ (ranged from 30.3 to $71 \mathrm{~kg} / \mathrm{m} 2$ ). They were submitted to an oral glucose tolerance test $(75 \mathrm{~g})$ and plasma glucose was determined by glucose-oxidase method. The criteria for DM was fasting glucose $>126 \mathrm{mg} \%$ or glucose level some time after glucose administration $>200 \mathrm{mg} \%$ and IGT was fasting glucose $\leq 140$ $\mathrm{mg} \%$ and $2 \mathrm{~h}$ after glucose $>140$ and $<200 \mathrm{mg} \%$. The Epi info, v. 6.0, software was aplied for statistical analysis. Crude prevalence of DM was $10.4 \%$ and IGT was $9,7 \%$ and stratified analysis showed:

\begin{tabular}{|c|c|c|c|c|}
\hline & & Prevalence ratio & $\begin{array}{l}\text { Confidence } \\
\text { interval ICI } 95 \%\end{array}$ & \\
\hline $\begin{array}{l}\text { Diabetes Mellitus (DM) } \\
n=31\end{array}$ & $\begin{array}{l}\text { BMI } \\
\text { Age }\end{array}$ & $\begin{array}{l}1.71 \\
2.18\end{array}$ & $\begin{array}{l}{[0.91-3.23]} \\
{[1.17-4.06]}\end{array}$ & $\begin{array}{l}0,09 \\
0,01\end{array}$ \\
\hline $\begin{array}{l}\text { Impaired glucose } \\
\text { Intolerance (IGT) } n=30\end{array}$ & $\begin{array}{l}\text { BMI } \\
\text { Age }\end{array}$ & $\begin{array}{l}3.70 \\
0.92\end{array}$ & $\begin{array}{c}{[1.70-8.0]} \\
{[0.44-1.94]}\end{array}$ & $\begin{array}{l}0,0003 \\
0,8\end{array}$ \\
\hline
\end{tabular}

We conclude that in obese women the association between obesity and DM was higher in older individuals and not related to the degree of obesity. In contrast, the association between obesity and IGT was higher in heavier individuals and it was independent of age.

\section{0}

DIABETES AND RISK FOR PERIPHERAL ARTERIAL OCCLUSIVE DISEASE

A. Planas, A. Clará, A. Gasol, A. Moner, C. Contreras, R. Hernández, R. Concustell, M.A. Ortiz, J.M. Pou. ABS Pubilla Casas and Hospital de Sant Pau, Barcelona, Spain.

It was estimated the risk for peripheral arterial occlusive disease (PAOD) in patients with type 2 diabetes and its relation to metabolic control, and in glucose tolerance impairment. A cross-sectional study was performed in a representative sample from general population of 1.054 men aged 55 to 74 years. Diabetes, impairment glucose tolerance (IGT) (OMS criteria) and other cardiovascular risk factors were analyzed in all subjects. PAOD was screened by rest ankle/braquial pressure index (ABPI) and it was considered pathological when ABPI was $<0.9$. Prevalence data was: diabetes: $19.1 \%$, IGT: $6.1 \%$. PAOD was $13.1 \%$ in general population and $24.8 \%$ in diabetics. Odd ratio (OR) was adjusted for risk factors associated to PAOD (diabetes, IGT, age, tobacco, hypertension, waist-tohip ratio $>0.95$, tryglicerides, cholesterol and HDL-c ): 2.4 for diabetes (C195\% 1.4-4.1) and only overcome by tobacco exposure and age over 70 years (OR:38.9 and 3.5 respectivelly). IGT was only associated to PAOD when microalbuminuria was present $(p<0,001)$. PAOD was associated to diabetes control (Glycated hemoglobin. HbAlc) and to microalbuminuria but, no relationship was found to diabetes duration and insulin therapy. Adjusted OR for PAOD was: 3.2 (CI95\% 1.2-8.8) for $\mathrm{HbAlc}>8 \%$ and 4.1 (CI95\% 1.7-10.3) for microalbuminuria. HbAlc $>7 \%$ was still associated to PAOD (OR 2.4, CI95\% 1-5.8). Therefore diabetes was the third risk factor associated to PAOD in adult-old men. The HbAlc $>7 \%$ and microalbuminuria were also risk factors in diabetic men. IGT was only PAOD risk factor when it is associated to microalbuminuria. 
OBESITY RELATED RISK FOR HYPERTENSION AND CORONARY HEART DISEASE IN NIDDM SUBJECTS

A Banu ${ }^{\dagger}$, M. A. Sayeed ${ }^{2}$, M. A. Malek ${ }^{1}$, M. G. Kibriya2 2 P. A. Khanam² and A. K. Azad Khan?. 1- INFS, University of Dhaka, 2- BIRDEM, Dhaka, Bangladesh To assess the prevalence of hypertension and coronary heart disease (CHD) in a diabetic population of Bangladesh from a total of 3,583 newly detected NIDDM subjects, 693 ( $M=295$ $F=398$ ) were selected randomly for investigation. The subjects were $30-60$ years old. The diagnosis of CHD was based on cardiogram or exercise tolerance test or both when equivocal. Compared with non-CHD group the subjects with $\mathrm{CHD}$ had significantly higher age $(\mathbf{p}<0.001)$, higher waist-to-hip ratio (WHR, $p<0.01$ ), higher systolic and diastolic blood pressure (SBP and DBP, $p<0.001$ ) and higher fasting plasma glucose (FPG, $p<0.05$ ). There was no difference of CHD prevalence between men and women. Compared with the male subjects the female had significantly higher body mass index (BMI, $\left.\mathrm{kg} / \mathrm{ht} \mathrm{m}^{2}, \mathrm{p}<0.001\right)$, higher SBP $(p=0.001)$ and total plasma cholesterol $(C H O L ~ p<0.001)$; whereas, the male had significantly higher $W H R(p=0.001)$ and DBP $(p=0.051)$. Adjusting for age, sex and obesity glycemia showed no association with WHR, SBP, DBP and CHOL; whereas, it showed significant negative correlation with $B M l(p<0.001)$. In contrast, $B M \mid$ showed significan positive correlation with SBP $(p<0.001)$ and DBP ( $<0.01)$. The CHD prevalence showed significant association with increasing age $(p<0.0001)$, increasing $B M I(p<0.05)$, higher waist girth $(p<0.05$, higher WHR $(p<0.01)$. Increased level of triglycerides $(T G)$ and decreased level of high-density-lipoprotein (HDL) were also significantly related to $\mathrm{CHD}$. Taking BMl, WHR SBP, DBP, FPG, 2-hPG, chol and family history of diabetes or hypertension as the independent risk variables and $\mathrm{CHD}$ as a dependent one, further analysis by stepwise logistio regression showed that increased age, high WHR, high DBP, increased TG and decreased $\mathrm{HDL}$ were the risks for $\mathrm{CHD}$. Very strong risk for $\mathrm{CHD}$ was chol/HDL ratio. Using differen models of logistic regression, it appears that the highest level of risk for $\mathrm{CHD}$ were chol/HDL ratio, high $\mathrm{DBP}$, increasing age, increased TG, and decreased $\mathrm{HDL}$, and moderate risk with high WHR. Advanced age, higher BMI and lower HDL-chol were the independent risks for both SHTN and dHTN. High chol/HDL ratio was the most important and significant biochemical risk factors for all these disease events in the newly detected diabetic population of Bangladesh.
MORTALITY IN INSULIN TREATED DIABETES MELLITUS 1993-1996. NT Raymond, JL Botha, AC Burden, R Gregory, PG McNally, M Davies, PGF Swift and $\mathrm{H}$ Blackledge. Department of Epidemiology and Public Health, University of Leicester. Diabetes Departments, Leicester General Hospital, Leicester Royal Infimary. Leicester Children's Hospital, Leicester, UK.

Estimating diabetes associated mortality is complicated by the inadequacy of official published data; diabetes is not always the underlying cause of death, and may not feature on the death certificate at all. The aim of this study was to determine excess mortality in the population aged 15 years and older with insulin treated diabetes and to examine causes of death. Using a population based register of people with diabetes and Office of National Statistics (ONS) mortality data, record linkage using AutoMatch software was undertaken to determine mortality observed during the years 1993 to 1996 inclusive. After computerised matching records were reviewed to maximise the accuracy of linkage. Standardised mortality ratios (SMR) and 95\% confidence intervals (95\% ci) were estimated using confidence interval analysis software. The England and Wales population and death rates for 1995 were used as the standard. The population with insulin treated diabetes numbered 5358 aged 15 years and older in mid 1995. A total of 706 deaths were identified distributed evenly over the 4 years; 167 in 1993, 173 in 1994,190 in 1995 and 176 in 1996. There were 321 (46\%) females and $385(55 \%)$ males. The median age at death was 71 years, range 19 to 95 years. The overall SMRs ( $95 \%$ ci) for the 4 years were significantly raised for females, 173 (155 to 194) and for males, 158 (142 to 174). Ischaemic heart disease, ICD9 codes $410-414$ accounted for 281 (40\%) deaths, and the SMRs for females 340 (284 to 403) and males 223 (188 to 261 ) were significantly raised. $43(6 \%)$ deaths were due to cerebrovascular disease, ICD9 codes 430-438 with SMRs 116 (76 to 170) for females and 83 (48 to 133 ) for males. The males SMR for all neoplasms was significantly lowered 74 (55 to 99). Despite ongoing improvements in the management of diabetes, significant excess mortality in both males and females with insulin treated diabetes was observed in this study.

\section{3}

TYPE II (NON-INSULIN-DEPENDENT) ARE SHORTER THAN TYPE I (INSULIN-DEPENDENT) DIABETIC PATIENTS

R.E.J. Ryder, R.J. Ryder, N. Kong, F.L. Game, S.L. Jones and K.G. Taylor. City Hospital, Dudley Rd., Birmingham, U.K.

Studies have suggested that smaller babies at birth are more likely to develop Type II diabetes in adult life. No such relationship exists for Type I diabetes. We aimed to investigate the hypothesis that as a result of smaller babies becoming shorter adults, Type II patients are shorter than Type l's. 1402 consecutive diabetic adults (145 Type 1, 85 males; 1256 Type 2, 672 males) having height and weight measured at a hospital, diabetic annual review clinic were studied. The mean +/- SEM height of the Type I patients $(167.97+/-$ $0.81 \mathrm{~cm}$ ) was $4 \mathrm{~cm}$ greater than that of Type II's $(164.00+1-0.27$ $\left.\mathrm{cm} ; \mathrm{p}=1.6 \times 10^{-6}\right)$. By contrast the Type l's $(74.87+/-1.13 \mathrm{~kg})$ weighed, on average, $4.5 \mathrm{~kg}$ less than Type II's $(79.35+/-0.44 \mathrm{~kg}$; $\left.p=1.1 \times 10^{-4}\right)$. The height and weight data combined to give a 2.86 $\mathrm{kg} / \mathrm{m}^{2}$ difference in mean body mass index between Type I $(26.60$ $\left.+/-0.39 \mathrm{~kg} / \mathrm{m}^{2}\right)$ and Type II $\left(29.46+/-0.15 \mathrm{~kg} / \mathrm{m}^{2} ; \mathrm{p}=5.3 \times 10^{-12}\right)$ patients. The differences were maintained when the sexes were analysed separately. Type I $\vee$ Type II height - males: $173.64+/-0.77$ $v 170.08+/-0.28 \mathrm{~cm}, p=8 \times 10^{-7}$; females: $159.95+/-0.89 \vee 157.0$ $+1.0 .29 \mathrm{~cm}, p=7.7 \times 10^{-4}$. These data suggest that Type 2 diabetic patients are not only more obese than Type I, but also are shorter men, on average, about $3.5 \mathrm{~cm}$ shorter, and women about $3 \mathrm{~cm}$ shorter, than their Type / counterparts. This observation does not seem to have been made before.

\section{4}

EPIDEMIOLOGICAL DATA OF THE OVERWEIGHT POPULATION OF DEBRECEN, ESPECIALLY THE OTHER CARDIOVASCULAR RISK FACTORS E. Katona, P. Polgár, D. Páll, Z. Jenei, Zs. Karányi and Gy. Kakuk. I ${ }^{\text {tt }}$ Department of Medicine, University Medical School of Debrecen, Debrecen, HUNGARY.

The aim of the study was to gain data about the frequency of obesity, and about further cardiovascular risk factors of overweight individuals. We surveyed the cardiovascular risk factors of 20000 voluntary inhabitants of Debrecen $(7567$ males, 12322 females), between the age of 30-65. Their average body mass index (BMI) was $23.4 \mathrm{~kg} / \mathrm{m}^{2}$. Only $63.2 \%$ proved to have nornal body weight (group A: BMI<27 $\mathrm{kg} / \mathrm{m}^{2}$ ). Overweight was measured in $30.6 \%$ (group B: $27 \mathrm{~kg} / \mathrm{m}^{2}<\mathrm{BMK}<33 \mathrm{~kg} / \mathrm{m}^{2}$ ) and obesity in $6.2 \%$ (group C: $\mathrm{BMI}>33 \mathrm{~kg} / \mathrm{m}^{2}$ ). In the frequency and degree of overweight there was no significant difference between males and females. Women after menopause (4760) showed a higher number of overweight: group B: $35.5 \%$, group C: $8.3 \%(\mathrm{p}<0.001)$. There were more overweight persons among those with lower education: overweight $37.4 \%$ and obesity $9.2 \%$ ( $p<0.001$ ). Among those performing physical activity on a weekly basis $22.9 \%$ showed overweight and $3 \%$ obesity, as opposed to those not: $32.1 \%$, and $6.8 \%(p<0.001)$. The average BMI of the currently smokers was lower ( $p<0.001$ ), group $B: 24.6 \%$ and group $C: 4.1 \%$ Former smokers had a higher number of overweight (35.1\%) and obesity $8.8 \%$ $(p<0.001) .36 .4 \%$ of persons consuming fat food regularly had overweight and $8.8 \%$ had obesity $(p<0.001)$. There was a clear correlation between blood pressure and the frequency of high BMI: $8.9 \%$ of normal BMI, $20.5 \%$ of group B and $33.3 \%$ of obesity had recognized hypertension ( $p<0.001$ ). The percentage ratio of the frequency of obesity has increased parallel with aging. In case of normal BMI $5.3 \%$, overweigh $8.9 \%$ and obesity $9 \%$ of persons had slightly elevated blood glucose level in the previous history. High blood glucose level was noticed in $2.2 \%, 4.2 \%$ and $4.9 \%$. Family history of heart attack in group A-B-C in relevance to brother-sister $3.3 \%$ $4.8 \%-5.7 \%(p<0.001)$, to mother $5.6 \%-6 \%-7.1 \%(p<0.001)$. The positive family history of fathers' heart attack did not differ in the three groups. Diabetes mellitus was present in group A-B-C: $1.5 \%-3.9 \%-7.4 \%$ ( $p<0.001$ ). Overweight persons have more other cardiovascular risk factors (e.g. diabetes mellitus), thus we have taken care of these individuals, and provided them with life-style and diet advice. At least their follow-up seems to be necessary, which we are carrying on currently. 
Diabetes Complications in Asia.

The DCDCP (Diabcare - Asia) Study Group

Diabetes complications such as retinopathy, blindness, nephropathy, neuropathy, amputation, cerebrovascular disease and coronary heart disease are resulting in increasing disability and mortality. While several reports for studies of diabetes complications were documented in western countries, relatively few studies examining diabetes complications in Asia have been carried out. In the first phase of the Diabetes Care Data Collection Project (DCDCP. 1997), we aimed to provide an overview of the complication status in the Asian diabetes population. A total of about 26.500 patients, with mean age of $58.3 \pm 12.0$ years old were recruited from 154 participating diabetes centres in 6 participating countries (China. Indonesia, Malaysia, Philippines, Thailand and Vietnam). They were assessed for known risk factors associated with occurrence of diabetes complications, eye. feet and severe late complications using well documented medical criteria and tests. Satisfactory compliance was achicved in majority of the patients in terms of body weight. blood pressure, smoking and alcohol intake. However, control of serum lipids levels was poor in about $20-30 \%$ of the patients $-30 \%$ had serum triglycerides $>2.2 \mathrm{mmol} / \mathrm{l}$, $20 \%$ had total cholesterol $>6.5 \mathrm{mmol} / \mathrm{l}$ and $18 \%$ had $\mathrm{HDL}<0.9 \mathrm{mmol} / \mathrm{l}$. Screening for micro- or macroalbumuria was very rare $(7 \%)$ compared to proteinuria monitoring (45\%). Similarly, screening for eye (photocoagulation. cataract, retinopathy and advanced eye diseases) and feet (foot pulse, healed ulcer, acute ulcer, neuropathy, amputation and angioplasty) complications were relatively infrequent (about $30 \%$ ). The average prevalence of cataract, retinopathy. neuropathy, myocardiac infarction and cerebral stroke was 44,33,39,5 and 6 percent respectively. Hence the incidence of patients with cataract and neuropathy was relatively high in the 6 countries studied. In conclusion, our data show that regular screening for microalbuminuria, eye and feet complications was not performed or at least not recorded. As early detection of eye and feet complications as well as renal impairment can provide direction for preventive strategies, the DCDCP study call for vigorous efforts aimed at improving awareness among health professionals of the potential of reducing major long-term diabetes complications in Asia
PREDICTORS OF INSULIN SENSITIVITY IN NON-INSULIN-DEPENDENT DIABETES.

M. Alberiche, C. Targher, R.C. Bonadonna, M. Muggeo and E. Bonora. Division of Endocrinology and Metabolic Diseases, University of Verona, Verona, Jtaly.

Reduced insulin sensitivity is an hallmark of non-insulin-dependent diabetes (NIDDM). However, insulin resistance is associated with several clinical conditions which are often encountered in subjects with NIDDM overweight, central fat distribution, dyslipidemia, hypertension and microalbuminuria. Also hyperglycemia and cigarette smoking adversely affect insulin sensitivity. Aim of the present study was i) to identify the independent predictors of insulin sensitivity in NIDDM, and ii) to establish whether isolated NIDDM is a condition of insulin resistance. We examined 45 subjects with non-insulin-treated NIDDM ( 37 men, 8 women; age $44-67$ yrs; BMI 20-29) undergoing a 4-h euglycemic hyperinsulinemic clamp (20 $\left.\mathrm{mU} / \mathrm{m}^{2} \cdot \mathrm{min}\right)$ combined with $\left[{ }^{3} \mathrm{H}\right]-3$-glucose infusion. Total glucose disposal (TGD) during insulin clamp was independently and negatively associated with hypertension (multiple regression standardized coefficient -0.34 $p<0.01)$, glycated haemoglobin $(-0.30, p<0.01)$, plasma triglycerides $(-0.25$, $p<0.05)$ and smoking $(-0.22, p<0.05)$. The overall variance of TGD explained by these 4 variables was $69.4 \%$. Smoking NIDDM subjects with overweight (BMI 25-29), central fat distribution, hypertension ( $>160 / 95)$, high triglycerides $\left(>200 \mathrm{mg} / \mathrm{dl}\right.$ ) and poor metabolic control $\left(\mathrm{HbA}_{\mathrm{c}} \geq 7 \%\right)$ had TCD significantly lower than non smoking, lean (BMI <25) NIDDM subjects, with normal blood pressure and triglycerides, and good metabolic control ( $16 \pm 2$ vs $31 \pm 4 \mathrm{mg} / \mathrm{min} \cdot \mathrm{kg}$ lean body mass, $\mathrm{p}<0.01$ ). Nevertheless, the latter still had insulin resistance when compared to sex-and age-matched nondiabetic subjects in good health ( $31 \pm 4$ vs $54 \pm 5 \mathrm{mg} / \mathrm{min} \cdot \mathrm{kg}$ lean body mass, $\mathrm{p}<0.01)$. We conclude that hypertension, hypertriglyceridemia, smoking and poor metabolic control are strong predictors of insulin resistance in NIDDM. However, reduced insulin sensitivity can be found also when NIDDM is isolated and well controlled.

\section{PS 9}

\section{Prediction and Prevention of Type 2 Diabetes}

\section{7}

URINARY MYO-/CHIRO-INOSITOL RATIO IS INCREASED IN NONDIABETIC FIRST DEGREE RELATTIVES OF TYPE 2 DIABETES PATIENTS

B. Greuer. H.G. Wahl, R. Werner, A. Volk. E. Maerker, K. Rett, R.M. Schmülling and H.U. Häring. Medizinische Universitätsklinik Abt. IV. Tübingen. Germany

Chiro- and myoinositol are components of inositolglycans and phosphatidylinositolglycans, which may be involved in insulin-sensitive phospholipid signalling pathways, and they have been proposed to exhibit various insulin-mimetic activities in different cell types. It has been shown, that the urinary excretion pattern of inositol isomers is altered for type 2 diabetes patients. Different studies have shown lower chiroinositol and higher myoinositol urinary excretion in diabctic patients compared to normal subjects. Urinary chiroinositol excretion has also been claimed to be an index marker for insulin sensitivity. We therefore examined the urinary excretion pattern of inositol isomers of type 2 diabetes patients, non-diabetic first degree relatives and controls. Quantification of inositol isomers was done by Gas Chromatography Mass Spectrometry. Insulin sensitivity was determined as metabolic clearance rate (MCR) by euglycemic-hyperinsulinemic glucose clamp. For this first part of the study data from 30 type 2 diabetes patients (age $50.9 \pm 9.2$ years. BMI $30.2 \pm 2.6 \mathrm{~kg} / \mathrm{m}^{2}$ ). 31 offsprings (age $34.2 \pm 1.5$ years, BMI $26.0 \pm 0.8$ $\mathrm{kg} / \mathrm{m}^{2}$ ) and 25 controls (age $29.8 \pm 1.8$ years, BMI $22.4 \pm 0.9 \mathrm{~kg} / \mathrm{m}^{2}$ ) was analyzed. In the type 2 diabetes group there was a lower chiroinositol and higher myoinositol excretion than in the control group. The first degree relatives showed the same alterations only in a more moderate way. As a more sensitive parameter, independent from the urine collection period and the patient's compliance we used the myo/chiroinositol ratio:

\begin{tabular}{|c|c|c|c|}
\hline Mean \pm SEM & $\begin{array}{c}\text { Myoinositol } \\
{[\mathrm{mg} / \mathrm{L}]}\end{array}$ & $\begin{array}{c}\text { Chiroinositol } \\
{[\mathrm{mg} / \mathrm{L}]}\end{array}$ & Myo/chiro \\
\hline Control $(\mathrm{n}=25)$ & $67.7 \pm 11.7$ & $11.2 \pm 3.5$ & $\mathbf{2 0 . 0 \pm 7 . 3}$ \\
\hline Offspring $(\mathrm{n}=31)$ & $78.4 \pm 10.7$ & $3.4 \pm 1.3$ & $\mathbf{7 8 . 1} \pm \mathbf{1 1 . 3}$ \\
\hline type 2 diabetes $(\mathrm{n}=30)$ & $127.5 \pm 22.0$ & $1.9 \pm 0.6$ & $\mathbf{1 9 3 . 9 \pm \mathbf { 3 7 . 2 }}$ \\
\hline
\end{tabular}

Type 2 diabetes is associated with increased urinary myo/chiroinositol ratio and this abnormality seems to be present in offsprings before the manifestation of diabetcs.

\section{8}

FOLLOW-UP OF WOMEN WITH GESTATIONAL DIABETES. INCIDENCE AND FACTORS ASSOCIATED WITH LATER DEVELOPMENT OF ABNORMAL GLUCOSE TOLERANCE. L. Herranz; M. T. Garcia-Ingelmo; P. Martin-Vaquero; C. Grande*; M. Jañez**; L. F. Pallardo. Dept. of Endocrinology, Biochemistry* and Gynaecology**. Hospital Universitario La Paz. Madrid. Spain.

Women with gestational diabetes $(G D)$ have an increased risk for later development of diabetes mellitus. The aim of the study was to determine the 6 year cumulative incidence of abnormal glucose tolerance(AGT)(diabetes mellitus and glucose intolerance), to identify factors which may predict future development of AGT and to evaluate the relationship of AGT to other components of the metabolic syndrome. METHODS: 276 women diagnosed as having GD between 1991-95 were prospectively followed with annual oral glucose $(75 \mathrm{~g}$.) tolerance tests (OGTT) and assessment of variables related to the metabolic syndrome. The actuarial method was used to determine the cumulative incidence of AGT. Differences in survival experience for predictive factors were determined by log-rank test. The relation of the area under the curve for OGTT at the end of the study with components of the metabolic syndrome was analysed using correlation coefficients. RESULTS: The 6-year cumulative incidence of AGT was $59.1 \%$ (39.6\% for diabetes mellitus). Predictive factors for AGT were: prepregnancy body mass index, fasting glucose during pregnancy and insulin treatment during pregnancy $(p<0.001)$; gestational age at diagnosis of $C D$, postprandial glucose and $\mathrm{HBA} 1 \mathrm{c}$ during pregnancy and waist-to-hip ratio after pregnancy $(p<0.01)$; and number of abnormal values in the 3-hour OGTT (100 g.) during pregnancy $(p<0.05)$. The degree of glucose tolerance (area under the curve for OGTT) at the end of the study was significantly related to: waist circumference $(r=0.441)$, waist-to-hip ratio $(r=0.378)$, body mass index ( $r$ $=0.353)$, systolic $(r=0.270)$ and diastolic blood pressure $(r=0.258) \quad(p<0.001)$; and triglyceride levels $(r=0.201 ; p<0.01)$. CONCLUSIONS: $G D$ is associated with a considerably increased risk of developing AGT later in life. The severity of glucose intolerance during pregnancy, prepregnancy obesity and abdominal fat distribution predict the occurrence of AGT. AGT in women with previous $G D$ is related to other components of the metabolic syndrome, therefore GD should be considered as an early indicator of the metabolic syndrome. 
489

OBESITY TREATMENT WITH ORLISTAT (XENICAL ${ }^{\text {B }}$ ) HELPS TO PREVENT DETERIORATION IN GLUCOSE TOLERANCE

J.P.H. Wilding, University of Liverpool, UK; B. Stolshek, Roche Pharmaceuticals, Nutley, USA.

Orlistat is a novel anti-obesity drug which acts within the lumen of the bowel to inhibit the hydrolysis of dietary fats. Data from 5 phase III studies were pooled to analyse the effect of orlistat on diabetic status. A total of 1629 obese patients were recruited to the studies and, following 4-week lead-in periods with placebo treatment and a mildly hypocaloric diet $(600 \mathrm{kcal}$ deficit, $30 \%$ calories as fat), subjects were stratified according to initial diet-induced weight loss and randomised to treatment with orlistat $120 \mathrm{mg}$ tid or placebo. After 52 weeks the diet was adjusted such that patients losing $\geq 3 \mathrm{~kg}$ had their diet increased by $200-300 \mathrm{kcal} /$ day. Mean percent weight loss in the orlistat group was $9.2 \%$ vs $5.8 \%$ in the placebo group ( $<<0.05$ ). The shift from baseline diabetic status for patients with normal glucose tolerance $(<7.8 \mathrm{mmol} / \mathrm{L}[<140$ $\mathrm{mg} / \mathrm{dL}] 2 \mathrm{hrs}$ post challenge), impaired tolerance $(\geq 7.8 \mathrm{mmol} / \mathrm{L}$ but $<11$. $\mathrm{mmol} / \mathrm{L}[200 \mathrm{mg} / \mathrm{dL}]$ at $2 \mathrm{hrs})$ or diabetic glucose tolerance $(\geq 11.1 \mathrm{mmol} / \mathrm{L}$ at $2 \mathrm{hrs}$ ) was determined at 52 and 104 weeks. At 52 weeks, $25.0 \%$ of diabetic patients had attained 'normal' status (vs placebo 15.4\%;) and at 104 weeks $18.5 \%$ remained 'normal' (placebo $11.1 \%$ ). A further $43.8 \%$ improved to 'impaired' status at 52 weeks (placebo $15.4 \%$ ) and $43.8 \%$ at 104 weeks (placebo $11.1 \%$ ). There was a significant difference between orlistat and placebo for 'diabetic' patients at 52 weeks $\left(x^{2} p=0.019\right)$ and nearly so at 104 weeks ( $\mathrm{p}=0.053$ ). Of the 'impaired' patients at baseline, $72.2 \%$ improved to 'normal' at 52 weeks (placebo $45.8 \%, p=0.006$ ) and $71.7 \%$ at 104 weeks (vs $47.5 \%)$; differences that were significant at 52 weeks $(\mathrm{p}=0.006)$ and almost so at 104 weeks $(p=0.054)$. There was no significant difference in the proportion remaining 'normal' at 52 weeks $(92.9 \%$ vs $91.4 \% ; \mathrm{p}=0.677)$, but at 104 weeks the difference was significant $(93.8 \%$ vs $86.1 \%$; $p=0.005$ ). Orlistat treatment of obesity may delay the onset of type 2 diabetes in high-risk obese patients.

CLUSTERING OF HYPERTENSION, MICROALBUMINURIA, INSULIN RESISTANCE AND HYPERLIPIDEMIA IN NIDDM FAMILIES.

AE Pontiroli, LD Monti, A Pizzini and PM Piatti, H San Raffaele, Milano, Italy Insulin resistance (IR), arterial hypertension $(\mathrm{AH})$, hyperlipidemia [high triglycerides (TG) and low-HDL (cholesterol)], and microalbuminuria $(\mu \mathrm{A})$ are found in first degree relatives (FDR) of NIDDM patients. Our aim was to check the hypothesis of a family cluster of these abnormalities. In the study SPIDER (an italian acronyme for regional study for prevention of diabetes), we evaluated 86 families that included: 1) at least one NIDDM patient (in 10 families with more than one NIDDM patient, the proband is the patient with the longest duration of NIDDM, the others are considered as FDR); brothers/sisters, FDR; 3 ) spouses without family history of NIDDM, with similar eating and physical hab. its. 103 NIDDM patients, 130 FDR and 60 spouses were studied. FDR and spouses were similar as to age, body weight, diet and physical activity, and frequency of $\mathrm{AH}$. IR was assessed by the HOMA model [fasting glucose (mmol) $x$ true insulin $(\mu \mathrm{U} / \mathrm{ml}) / 22.5]$. At OGTT (75 g), 24 FDR were classified NIDDM, 31 impaired glucose tolerant (IGT) and 75 normal glucose tolerant (NGT), 47 spouses NGT, 13 spouses IGT ( $p<.01$ vs FDR). AH was present in $17 / 34$ families in which the proband had $\mathrm{AH}$ as opposed to 11/48 families in which the proband was negative $(\chi 2=5.34, \mathrm{p}<.05)$; low-HDL was present in $23 / 41$ families in which the proband had low-HDL, as opposed to $10 / 41$ families with a negative proband $(\chi 2=7.30, p<.01) ; 8 / 23$ families with a positive proband for $\mu \mathrm{A}$ had $\mu \mathrm{A}$, as opposed to $1 / 45$ families with a negative proband $(\chi 2=11.36, p<.001) ; 49 / 99$ FDR of probands showing IR had IR, as opposed to $9 / 38$ FDR of probands without IR $\left(\chi_{2}=6.47, p<.02\right)$. High TG and waist/hip $(\mathrm{W} / \mathrm{H})$ ratio did not show family cluster. $\mathrm{IR}$, low-HDL, and high $\mathrm{W} / \mathrm{H}$ ratio were more frequent in FDR than in spouses, while only FDR belonging to families positive for $\mathrm{AH}$ or $\mu \mathrm{A}$ showed a greater frequency of $\mathrm{AH}$ or $\mu \mathrm{A}$ than spouses. Clusters disappeared when FDR with NGT were excluded. These data indicate that $\mathrm{AH}, \mu \mathrm{A}, \mathrm{IR}$ and low HDL-cholesterol cluster in NIDDM families.

\section{0}

THE PREVALENCE OF IMPAIRED GLUCOSE TOLERANCE IN WOMEN AGED 50-59 YEARS IN RELATION TO MEDICAL RISK INDICATORS.

J. Lidfeldt, C-D. Agardh, L. Lindholm, C. Nerbrand, G. Samsioe and B. Scherstén. Department of Community Healih Sciences, Lund University, Lund, Sweden. The aim of the study was to investigate the prevalence of impaired glucose tolerance (IGT) in a geographically defined population of middle-aged women in relation to medical and psychosocial risk indicators. To that end, all $50-59$ year old women living in the Lund area in Southern Sweden were invited to a medical and psychosocial examination in a two-step screening procedure. The population comprises 10870 subjects and the results of the 3000 first examined are presented. The report includes an analysis of questionnaires and laboratory screening of biological variables. Women with $(n=1227)$ one or more pathological variables at the primary screening were referred to a diagnostic follow-up $75 \mathrm{~g}$ oral glucose tolerance tes! (OGT). Data on numbers and means $\pm S D$ are presented and Wilcoxon's rank sum test as well as chisquared test were performed in order to evaluate any relationship between blood glucose levels and origin, family history of diabetes, hypertension or cardiovascular diseases and different biological variables. The results show, that out of the 3000 examined women, $569(18.9 \%)$ were overweight (BMI $\left.\geq 28 \mathrm{~kg} / \mathrm{m}^{2}\right)$ and $342(11.4 \%)$ obese $\left(\mathrm{BM} \mid \geq 30 \mathrm{~kg} / \mathrm{m}^{2}\right)$. The random capillary whole blood glucose was $\geq 8.0 \mathrm{mmol} / \mathrm{L}$ in $234(7.8 \%)$ and $\geq 10.0 \mathrm{mmol} / \mathrm{L}$ in $50(1.7 \%)$ subjects. A fasting $0 \mathrm{GTT}$ was performed in 1227 subjects with a positive screening, out of whom $202(6.7 \%)$ had IGT and 39 $(1.3 \%)$ had diabetes. There was an association between the $2 \mathrm{hr}$ glucose value during the OGT and $B M Y(p=0.0001)$, systolic blood pressure $(p=0.0001)$ and smoking $(p=0.0016)$, irrespective of ethnic origin (multiple regression analysis). However, there was no association between the $2 \mathrm{hr}$ glucose value and family history of diabetes. In conclusion, this study shows a high prevalence of IGT in middle-aged women, and that the glucose intolerance is associated with factors, like BMI and smoking, which if eliminated, may prevent the development of IGT to diabetes mellitus. Furthermore, these factors seem to be more important than a family history of diabetes, indicating that life style factors contribute more to the increasing prevalence of diabetes.

\section{2}

ORLISTAT (XENICAL ${ }^{\circledR}$ ) REDUCES CARDIOVASCULAR DISEASE RISK FACTORS IN OBESE PATIENTS WITH TYPE 2 DIABETES

P. Hollander, Baylor University Medical Centre, Dallas, Texas, USA., C.Lucas and K. R. Segal, Roche Laboratories, Nutley, NJ., USA.

Type 2 diabetes mellitus and obesity are linked frequently with hypertension and hyperlipidaemia as the key components of insulin resistance syndrome, a condition which predisposes to major cardiovascular risk. To determine the independent effect of weight loss and orlistat (a lipase-inhibitor which blocks about $30 \%$ of dietary fat absorption) on risk factors, 321 obese diabetic patients treated with sulphonylureas, were randomised to a mildly hypocaloric diet plus orlistat (OrI)120 mg tid or placebo (Pla) in a 1-year, double-blind, multicenter study. Weight loss at 1 year was significantly greater in the Orl vs Pla group $(6.2 \%$ vs $4.3 \% ; p<0.05)$. Changes in risk factors were calculated for 3 categories: weight loss $<5 \%, 5-10 \%$ or $>10 \%$ initial body weight. Total cholesterol was reduced in Orl patients $(-0.31,-0.38$ and $-0.71 \mathrm{mmoll}$, respectively) and in Pla was elevated over baseline $(+0.01,+0.31$ and +0.18 $\mathrm{mmol} / \mathrm{L}$, respectively; $\mathrm{p}<0.05$ ), indicating an independent beneficial effect of Orl treatment. $\mathrm{HbAlc}$ showed progressive decrease across the weight categories from -0.10 to $-2.29 \%$ in both Orl and $\mathrm{Pla}(\mathrm{p}<0.05)$ with no additive drug effect. Likewise, systolic blood pressure decreased progressively by $-2,-3$ and $-15 \mathrm{mmHg}$ across the weight loss categories in both Orl and $\mathrm{Pla}(\mathrm{p}<0.05)$. Gastrointestinal adverse events such as fatty/oily stools, oily spotting and faecal urgency were mild and transient , and only $10 \%$ of Orl patients had more than 2 episodes of GI AEs. The drop-out rate for ORL was only $15 \%$ compared to PLA $28 \%$. The results of the study show that ORL is an effective adjunct to diet, has an independent beneficial effect on lipid profile, leads to improved glycemia and blood pressure as related to weight loss and is well tolerated in obese patients with type 2 diabetes. 
493

\section{TREATING IMPAIRED GLUCOSE TOLERANCE.} LONG-TERM RESULTS

A.B. Hreidarsson, I.Arnardottir and T. Helgason. Diabetic Clinic, National University Hospital, Reykjavík, Iceland.

This study describes our results to prevent or postpone further deterioration of glucose utilization in patients with IGT. 180 non-pregnant individuals mean age, 56.2 yrs treated for IGT at our clinic were identified. Of these 28 followed for $<9$ months were excluded. 89 (51 males, 38 females) were obese or overweight ( $>10 \%$ above Kemsley standard) and treated with weight reducing diet. Mean follow-up 7.7 yrs. Mean weight at initial visit was $85.4 \pm 1.2$ (SEM kg) , mean lowest $74.3 \pm 1.2 \mathrm{~kg}$ and at last visit $79.7 \pm 1.4 \mathrm{~kg}$. For males this was $88.9 \pm 1.2 \mathrm{~kg}, 77.2 \pm 1.2 \mathrm{~kg}$ and $82.4 \pm 1.4 \mathrm{~kg}$ and for females $78.6 \pm 2.0 \mathrm{~kg}$, $70.3 \pm 2.1 \mathrm{~kg}$ and $76.0 \pm 2.6 \mathrm{~kg}$. Weight reduction from initial to last visit was significant for the whole group - for males $7.3 \%(p<0.001)$ for females $3.3 \%$ $(\mathrm{p}=0.02) .29(33 \%)$ had achieved normal weight, losing on average $10.4 \mathrm{~kg}, 24$ (27\%) had lost some weight, $8.7 \mathrm{~kg}$, but $35(40 \%)$ had failed to lose weight or gained weight. Of those who had lost weight $2(4 \%)$ progressed to diabetes compared to $4(11.5 \%)$ diet failures. Glucose tolerance normalized in $59 \%$ of those who achieved normal weight, in $38 \%$ with some weight loss and in $18 \%$ of diet failures $(p<0.001$ ). 63 ( 32 males, 31 females) were of normal weight. 49 were treated with a small dose of sulphonylurea and 14 were not. All were advised to keep their weight steady and avoid concentrated carbohydrates. Mean follow-up 6.7 years. Glucose tolerance normalized in $37 \%$ of the cases treated with sulphonylurea and in $25 \%$ of those on diet only. For individuals treated with sulphonylurea glucose tolerance improved significantly $(p<0.05)$. Conclusion: Long-term weight reduction was achieved in $60 \%$ of obese individuals with IGT. Weight reduction improved glucose tolerance significantly. Males had more success with weight reduction than females. Also, long- term treatment with sulphonylurea had a beneficial effect on giucose utilization in normal weight subjects with IGT.

\section{4}

THE STOP-NIDDM TRIAL. Study to prevent type 2 diabetes.

J.-L. Chiasson ${ }^{1}$, R. Gomis ${ }^{2}$, M. Hanefeld ${ }^{3}$, R.G. Josse ${ }^{4}$, A. Karasik ${ }^{5}$, M. Laakso ${ }^{6}$ and The STOP-NIDDM Trial Research Group. ${ }^{1}$ Montreal (Canada), ${ }^{2}$ Barcelone (Spain), ${ }^{3}$ Dresden (Germany), ${ }^{4}$ Toronto (Canada), ${ }^{5}$ Jerusalem (Israel), ${ }^{6}$ Kuopio (Finland).

The STOP-NIDDM Trial is an international study on the efficacy of an $\alpha$-glucosidase inhibitor, acarbose, to prevent or delay the development of type 2 diabetes mellitus in an IGT population. Subjects with IGT according to the WHO criteria and a fasting plasma glucose $\geq 5.6 \mathrm{mmol} / \mathrm{L}$ have been randomised in a double-blind fashion to acarbose $100 \mathrm{mg}$ TID or placebo for a mean follow-up of 4 years. Sample size calculation was done using a 2-tailed $\alpha$ of 0.05 and a $1-\beta$ of $90 \%$ assuming: 1) a conversion rate of $7 \%$ per year; 2) a $36 \%$ risk reduction; 3) a $10 \%$ drop-out rate. It was calculated that at least 600 subjects needed to be randomised per treatment group. The primary outcome is the development of type 2 diabetes according to the new criteria using a $75 \mathrm{~g}$ OGTT. The secondary outcomes are changes in blood pressure, lipid profile, insulin sensitivity, cardiovascular events and anthropometric profile. Screening was completed on February 28, 1998. A total of 1418 subjects have been enrolled. The mean age is $53.6 \pm 8.4$ years with a mean BMI $\geq 27 \mathrm{~kg} / \mathrm{m}^{2}$ in $73.3 \%$ of the subjects. A family history of diabetes was known in $85.1 \%$ of the subjects and $36.2 \%$ had known hypertension and $35 \%$ dyslipidemia. Of the women participating, $23.1 \%$ had a history of gestational diabetes. The subjects have now been followed for a mean of 1 year. The drop-out rate is $8.5 \%$ mostly for gastrointestinal side effects. Seventy-seven subjects $(5.8 \%)$ have converted to diabetes. In conclusion, The STOP-NIDDM Trial has completed recruiting, is now in its second year follow-up, and should provide us with a definite answer as whether acarbose can prevent or delay the progression of IGT to type 2 diabetes mellitus by the year 2001

\section{5}

PERFORMANCE OF A TYPE 2 DIABETES SCREENING QUESTIONNAIRE: COMPARING WHO-85 AND ADA-97 CRITERIA

A.M.W. Spijkerman, J.B. Ruige, J.M. Dekker, G. Nijpels, C.D.A. Stehouwer, L.M. Bouter and R.J. Heine. Vrije Universiteit, EMGO Institute, van der Boechorststrat 7, 1081BT Amsterdam. the Netherlands.

Type 2 diabetes screening questionnaires have been developed to identify subjects with possibly type 2 diabetes, in whom the diagnosis is to be confirmed later using an oral glucose tolerance test (OGTT) in keeping with the WHO-85 criteria. It is not yet known how such screening instruments perform when using the fasting criteria established by the ADA in 1997. We evaluated the performance of the Symptom Risk Questionnaire (SR-Q), previously validated by J.B. Ruige (Diabetes Care 1997; 20(4): 491-496), using both the WHO-85 and the ADA-97 criteria. Sensitivity, specificity and positive predictive values (with $95 \% \mathrm{CIs}$ ) were calculated for various cutoff values of the questionnaire. A random population sample of 786 Caucasian subjects, aged 45-74 years and not known to have diabetes, completed the SR-Q. Subjects with a fasting whole blood glucose $>4.9 \mathrm{mmol} / \mathrm{l}$ underwent an oral glucose tolerance test (OGTT). 32 Subjects were diagnosed as having type 2 diabetes using the WHO-85 diagnostic criteria. Applying the ADA-97 criteria, 40 diabetic subjects were identified; 22 subjects were classified as having type 2 diabetes according to both criteria. The cutoff value of $>6$ yielded the best test characteristics of the SR-Q (high specificity with comparatively high sensitivity) for both criteria. Sensitivity was $63 \%(46-81)$, specificity was $69 \%(65-72)$ and the positive predictive value was $7.9 \%$ (4.5-11.4) when the WHO-85 criteria were applied. Making use of the ADA97 criteria the SR-Q (at cutoff value $>6$ ) had a sensitivity of $69 \%(55-84)$, a specificity of $70 \%(66-73)$ and a positive predictive value of $11.2 \%(7.2-15.6)$. Overall performance of the SR-Q showed considerable resemblance between the WHO-85 and the ADA-97 criteria. This correspondence was also shown by the ROC curves that were fairly close to cach other, although the ROC curve of the ADA-9? was a bit more favourable. We conclude that the SR-Q has a similar accuracy for identifying persons with an elevated risk of having diabetes, diagnosed by either the WHO- 85 or the ADA-97 criteria

\section{6}

\section{FRUCTOSAMINE IN EARLY STAGES OF DIABETES MELLITUS}

E. Volkova, E. Gasparian and V. Slobodskoy. Department of Endocrinology, Medical Academy of Postgraduate studies, St. Petersburg, Russia.

Glycosylated proteins are usually used for the determination of the state of metabolism in diabetic patients. However, the role of glycosylated proteins for the prognosis or diagnosis of early stages of DM still remains disputable.We have investigated fasting serum fructosamine level in 25 control healthy subjects with normal weight, without a family history of $\mathrm{DM}$, and in 82 subjects with normal glucose tolerance test (GTT) as well as with various degrees of impaired glucose tolerance ( IGT) with a family history. In all subjects we have estimated the level of IRI and C-peptide - fasting and in response to glucose. The changes of insulin secretion typical for the diabetic state were found in all offsprings of both IDDM and of both NIDDM parents. We assigned 25 offsprings of both IDDM and of both NIDDM parents with normal GTT as subjects with " prediabetes". It was found that the level of fructosamine depended on the degree of disturbances of carbohydrate metabolism, enhancing with progressive deterioration of glucose tolerance. Even patients with «prediabetes» showed significantly higher fructosamine level compared with the findings in healthy subjects $(2.25 \mathrm{nmol} / \mathrm{l})$. These data suggest that fructosamine level can be used as a marker for diagnosis of "prediabetes» in subjects with fructosamine level of $2,5-2,9 \mathrm{nmol} / \mathrm{l}$. IGT can be predicted in subjects with fructosamine level of more than $3,0 \mathrm{nmol} / \mathrm{l}$. 
INCREASING CARDIOVASCULAR CO-MORBWITY AMONG DIABETIC SUBJECTS.

C.A. Baan, J.J.Barendregt and L. Bonneux, Department of Public Health, Erasmus University, PO-Box 1738, 3000 DR Rotterdam, the Netherlands

In the past decades a marked improvement in cardiovascular disease (CVD) survival has been shown. Since diabetes is associated with CVD, an improved CVD survival will have an effect on the diabetes prevalence and comorbidity. We quantitatively examine the impact of these changes.

We used a multi-state life table including three disease processes: coronary heart disease, cerebrovasculair disease and diabetes, which are modeled as continuous time markov processes. The changes in CHD mortality and survival between 1980-83 and 1990-93 in the Netherlands are used to estimate the impact on diabetes prevalence of the decline in mortality selection. Relative risks for total and cardiovascular mortality and survival for diabetics were based on community and population studies.

Preliminary results show that the improved CVD survival between 1980-1983 and 1990-1993 resulted in an age related increase of diabetes prevalence with a maximum of about $10 \%$ (when prevalence is expressed in numbers) and $2 \%$ (rates) for men at high ages. The impact on diabetes prevalence among women is much smaller. The comorbidity of CVD disease and diabetes shows age-related increases that reach 50 to $70 \%$ at higher ages (both sexes). A notable difference between men and women is that the latter also show a considerable increase for ages under 65, while for the former the increase in that age group is more limited. The changes in cardiovascular mortality and survival are unlikely to be a major cause of increasing diabetes prevalence, but they are likely to cause a sizable increase in the comorbidity of cardiovascular disease and diabetes.
BIRTHWEIGHT AND ANALYTIC DATA IN BLOOD OF UMBILICAL CORD: A POSSIBLE ASSOCIATION WITH NIDDM IN ADULT LIFE. A. Becerra, J.M. Arroyo, D. de Luis, G. Piédrola, J.E. Campillo and M.D. Torres. Dept. of Endocrinology, Ramón y Cajal Hospital, Madrid, and Fac. Med., UEX, Badajoz. Spain.

According to the hypothesis of "thrifty phenotype" a low birthweight and a reduced growth in fetal life is associated to the appearance of non-insulin-dependent diabetes (NIDDM), as well as of other clinical correlates of the insulin resistance syndrome (IRS) in adult life. The aim of this study was to investigate the association between anthropometric parameters of the mothers and their newborn children and analytic data in blood of umbilical cord. We performed a cross-sectional study of 96 non-diabetic mothers, aged $28.2 \pm 4.4$ years (range 16-40), and their 96 newborn children. Birthweight (BW) was $3151.9 \pm 370.8 \mathrm{~g}$ (range 2054-3840). We analyzed in mothers: gestational age (GA) and prepregnancy body mass index (BMI); in newborn babies: weight, height, $\mathrm{BMI}$, cephalic (CP) and thoracic (TP) perimeters, and in blood of umbilical cord: glucose, total colesterol (TC), HDL-colesterol and triglycerides (TG) levels. We did not find significant differences between parameters studied in 9/96 babies with low BW $(\leq 2500)$ and those of normal BW $(>2500)$. However, GA was correlated (Pearson's correlation coefficient) with BW $(r=0.28, p=0.005)$ and with TG $(r=0.32$, $p=0.001)$; BW was correlated with TC $(r=-0.28, p=0.006)$, with $C P$ $(r=0.23, p=0.02)$ and with TP $(r=0.21, p=0.04) ;$ and $T G$ was correlated with $\mathrm{CP}(r=0.23, p=0.02)$ and with TP $(r=0.21, p=0.04)$. In conclusion, the association between anthropometric parameters and some analytic correlates of the IRS in blood of umbilical cord suggests that not only the BW but also the gestational age could have some influence on the IRS in adult life.

\section{PS 10}

\section{$\beta$-Cell Development, Replication and Insulin Gene Expression}

\section{9}

\author{
ROLE OF PDX-1 THREONINE PHOSPHORYLATION IN \\ GLUCOSE RESPONSIBLE INSULIN GENE EXPRESSION \\ N. Furukawa, T. Shirotani, H. Kishikawa, K. Kaneko, E. Araki, and \\ M. Shichiri, Department of Metabolic Medicine, Kumamoto \\ University School of Medicine, Kumamoto, Japan
}

To elucidate the mechanism of the glucose responsive expression of human insulin gene, we analyzed the role of pancreatic and duodenal homeobox gene-1 (PDX-1) using a beta cell line MIN6 cells. Previously we reported that PDX-1 bound to A3 element of human insulin gene in a glucose dependent manner. In this study, we evaluated the quantitative and qualitative changes of PDX-1 induced by high glucose. Western blot analysis employing anti-PDX-1 antibody revealed that the amount of PDX-1 in nucleus of MIN6 was not affected by glucose. However, the binding activity of PDX-1 significantly reduced by potato acid phosphatase treatment, suggesting that DNA binding activity of PDX-1 was affected by its phosphorylation status. Western blot analysis using anti-phosphopeptide antibodies demonstrated that phosphorylation of threonine, but not tyrosine or serine, in PDX-1 was increased with high glucosc. We further tried to identify the protein kinase which phosphorylate PDX-1. In in vitro experiment, PDX-1 was phosphorylated by protein kinase $\mathrm{C}$ (PKC) but not by cyclic-AMP dependent protein kinase (PKA) or mitogen-activated protein kinase (MAPK). In conclusion, it was suggested that high glucose increased phosphorylation of some threonine residues of PDX-1 through PKC was followed by the increase of the DNA binding activity of PDX -1 to A3 element, resulting finally in the increase of insulin gene expression.

\section{0}

PDX-1 TURNS FROM AN ACTIVATOR TO A REPRESSOR OF INSULIN PROMOTER IN A DOSE DEPENDENT MANNER IN RIN-38 \& HIT-T15 CELLS. S. Ferber, Y. Cohen, A. Karasik and R. Seijffers. Endocrinology Institute, Sheba Medical Ctr. Tel-Hashomer. Israel.

The aim of the present study was to analyze whether the functional deterioration process which occurs in RIN-38 cells with increase in passage, is reflected in and can be explained by alteration in $\beta$-cell specific transcription factors. We demonstrate that decrease in insulin production in RIN-38 cells that occurs with time in culture is associated with $50+3 \%$ (passage 30, p. 30 ) and $75+2 \%$ (p. 40 ) decrease in basal rat insulin promoter (RIP) activity compared to the same cells at passage 20 (p. 20), and with loss of glucose regulation of this activity (p. 40). This was associated with decrease in the insulin gene transactivators, BETA2 and E47 protein levels, and with paradoxically increased PDX-1 levels $(67 \pm 4 \%$ increase in $p$. 40 vs. p. 20). That increased PDX-1 expression is related to the fall in insulin expression was clearly established by our finding that overexpression of the transcription factor using recombinant adenovirus technology, suppressed insulin promoter activity in a dose dependent fashion in both RIN-38 and HITT15 cells to $10-20 \%$ of its maximal activity, without altering the endogenous BETA2 and E47 nuclear proteins levels (as established by western blot analysis). Interestingly, treating HTT-T15 cells, as opposed to RIN-38 cells, by 1 and 2 multiplicity of infection (moi) of recombinant adenovirus carrying PDX-1 expression, in spite of inducing comparable PDX-1 protein levels in both cell lines, increased insulin promoter activity in the first cells by $45 \pm 3 \%$, but 10 and 20 moi suppressed RIP activity in HIT-T15 cells as well.

We conclude that that PDX-1 dosage is a critical factor affecting insulin promoter activity. Moreover, the balance among the relative quantities of cooperating insulin gene transactivators, may play a crucial role in insulin gene level of expression and may be differently perturbed in different cell lines and states of islet cell dysfunction. The mechanism by which PDX-1 turns from a transactivator of the insulin promoter to its repressor in a dose dependent manner, and the physiological relevance of the phenomenon, will be discussed. 
501

ISLET CELL TRANSCRIPTION FACTOR EXPRESSION IN AR42J CELLS.

J. Palgi, E. Stumpf and T. Otonkoski

Haartman Institute, University of Helsinki

The clonal pancreatic acinar AR42J cells have been reported to differentiate towards an endocrine phenotype by treatment with growth factors, such as Activin A, betacellulin and HGF. In our hands, growth factor treatment of the clone AR42J-B13 resulted in growth inhibition and morphological differentiation towards a neuroendocrine phenotype, but no insulin gene transcripts could be detected. We have investigated the expression levels of various transcription factors in this model by Northern analysis. A high expression level of the islet-associated transcription factors pdx-1, isl-1, Nkx6.1, BETA2, IA-1, Pax4 and Pax6 was found in the control insulinoma cell lines INS-1 and RINm5F. In AR42J cells, pdx-1 BETA2 and IA-1 mRNAs were readily detectable. Pax4 and Pax6 transcripts were found at a very low level, but Pax4 expression was upregulated by Activin A plus HGF. Nkx6.1 and isl-1 were not transcribed in AR42J cells even under growth factor treatment. In addition to these factors known to be involved in islet differentiation, we also investigated the expression of the zinc-finger factors GATA-4 and GATA-6. Both of these were highly expressed in all of the cell lines, and GATA- 6 mRNA level increased markedly during differentiation induced by Activin A plus HGF. Based on these results, the transcription factors $\mathrm{Nk} \times 6.1$ and isl-1 could be critical for the induction of insulin expression in AR42J cells. Furthermore, GATA-4 and GATA-6 may also be involved in pancreatic $\beta$-cells differentiation.

\section{3}

HYPOXIA INCREASES INSULIN MRNA LEVELS BY STIMULATING BINDING OF A PROTEIN TO A HYPOXIA ELEMENT IN RAT INSULIN mRNA

L. Tillmar, C. Westling, C. Carlsson and N. Welsh. Department of Medical Cell Biology, Uppsaia University, Uppsala, Sweden

The 3'-untranslated region of rodent insulin mRNA contains a sequence highly homologous to the previously characterized hypoxia-inducible protein binding site of tyrosine hydroxylase mRNA. Therefore, the aim of this study was to investigate the putative role of hypoxia in the control of insulin gene expression. $16 \mathrm{~h}$ of hypoxia $\left(10\right.$ or $\left.5 \% \mathrm{O}_{2}\right)$ induced a dosedependent increase in insulin mRNA levels. This effect was not additive to the glucose-induced increase in insulin mRNA. Homogenates from isolated rat islets were used for gel shift analysis using a $25 \mathrm{bp}{ }^{32} \mathrm{P}$-labelled RNA oligo with sequence 5'-UCCACCACUCCCCGCCCACCCCUCU, (3'-end of rat insulin I mRNA). Specific binding to this oligonucleotide was observed and the binding was stimulated in vitro by reducing agents and spermine. Cross-linking experiments revealed that the molecular weight of the hypoxia element binding protein was $50 \mathrm{kDa}$. A one hour exposure to $5 \% \mathrm{O}_{2}$ increased binding activity in the absence of reducing agents. Glucose did not affect binding activity when added for $1 \mathrm{~h}$. However, a $3 \mathrm{~h}$ exposure to glucose moderately increased binding activity. The sequence of the wild type hypoxia element or two mutants were introduced into a $\mathrm{pCR}^{\mathrm{TM}} 3-\mathrm{CAT}$ vector downstream of the coding sequence of the CAT reporter gene. The constructs were transfected into rat islet cells and reporter gene mRNA levels were determined using RT-PCR. It was found that a substitution of a cytidine to an adenosine in the core binding site resulted in a decreased mRNA level, whereas a mutation outside the core binding site had little effect. It is concluded that hypoxia increases insulin mRNA contents via stabilization of the messenger. We also speculate that glucose stimulated insulin gene expression may involve hypoxia-activated mechanisms.
TUNGSTATE PROMOTES BETA CELL REPLICATION AND NEOGENESIS IN NEONATAL STREPTOZOTOCIN RATS

J.Fernández-Alvarez, B.Nadal, A.Truc and R.Gomis. Endocrinology and Diabetes Department. IDIBAPS/Hospital Clínic. Villarroel 170, 08036 Barcelona, Spain In an animal model of NIDDM, the neonatally streptozotocin-injected diabetic rats (nSTZ), administration of tungstate ( $\mathrm{Tg}$ ) normalise glycemia with concomitant increase in insulin-secretion and insulin content. Moreover, tungstate treatment increase the $\beta$-cell mass in the pancreas. Aim: To investigate whether this increase in 3-cell mass is accompanied by an augmentation in $\beta$-cellular proliferation . Methods: The animals treated with $\mathrm{Tg}$ were given a solution of $2 \mathrm{mg} / \mathrm{ml}$ of sodium $\mathrm{Tg}$ in distilled water during 1 month. The insulin blood levels was measured by RIA method. Morphometric studies were made using indirect immunofluorescence and stained with anti-insulin antibodies. The number of beta -cell was determinate by image analysis. Beta-cell replication rate was estimated from "in vive" incorporation of 5-bromo-2'-deoxyuridina (BrdU) in $\beta$-cell and double immunofluorescence with anti-BrdU and anti-insulin antibodies. The neogenesis was determined by double immunofluorescence with anti-cytokeratine 20 and anti-insulin and anti-glucagon antibodies. Results: $\mathrm{Tg}$ normalised glycemia in treated diabetic treated animals $(4.8 \pm 0.1 \mathrm{mmol} / 1$ v.s. $8.3 \pm 1.2 \mathrm{mmol} /$, $\mathrm{p}<0.05$ ) and this normalisation in glycemia was correlated with and increase in blood insulin levels $(18.8 \pm 6.7 \mu \mathrm{U} / \mathrm{ml}$ v.s. $60.0 \pm 5.1 \mu \mathrm{U} / \mathrm{m} ; \quad p<0.005)$. Concomitantly a recovery in the number of $\beta$-cell in the pancreas was observed $(0.77 \pm 0.07 \%$ v.S. $1.67 \pm 0.04 \% ; p<0.001)$. Studies of cellular replication showed an increase in the number of beta-cell inside islets $(2.01 \pm 0.01 \%$ v.s. $1.26 \pm 0.01 \%$; $\mathrm{p}<0.001)$ and outside islets $(0.69 \pm 0.01 \%$ v.s. $0.55 \pm 0.01 ; \mathrm{p}<0.05)$. In treated diabetic animals this increase in beta-cell proliferation was correlated with an increase in the number and presence of positive cells for cytokeratine 20 . Conclusion: The normalisation of metabolic status, in tungstate treated nSTZ animals, could be associated to an increase in cellular proliferation mechanisms described in pancreatic islets, beta cell replication and neogenesis (precursor cells)

\section{4}

GLUCOSE AND GLP-1 SYNERGIZE TO INDUCE IMMEDIATE-EARLY RESPONSE GENES IN THE PANCREATIC $B$-CELL LINES INS-1 AND HC9.

S. Susini', E. Roche ${ }^{2}$, J. Buteau', W. Schlege!' and M. Prentki ${ }^{2}$

'University of Geneva, Switzerland and 'University of Montreal, Canada

To link glucose signaling to its long term pleiotropic effects in pancreatic $\beta$-cells, we have investigated whether glucose regulates immediate-early response genes (IEGs) coding for transcription factors implicated in cell proliferation and differentiation Glucose causes a coordinated transcriptional activation of the IEGs c-fos, c-jun, junB, $z i f-268$ and $n u r-77$ in the pancreatic $\beta$-cell line INS-1. This activation is entirely dependent on the presence of the cell permeant cAMP analog chlorophenylthio-cAMP which has only a modest effect by itself. The accumulation of c-fos, junB and nur-77 mRNA occurs at physiological concentrations of glucose ( 3 to $11 \mathrm{mM}$ ), requires a $1-2$ hour time period and is mimicked by other nutrient stimuli including mannose, leucine plus glutamine, and pyruvate. Glucose is synergistic with the glucoincretin peptides GLP-1 and PACAP-38, whereas these neurohormonal agents have no effect at low $(3 \mathrm{mM})$ glucose. Mechanistically, the synergy between glucose and the glucoincretins is not based on cAMP alone as glucose does not further increase intracellular cAMP in response to GLP-1 and PACAP-38. A role for $\mathrm{Ca}^{2+}$ signaling is inferred as the L-type $\mathrm{Ca}^{2+}$ channel blocker nifedipine markedly reduces the induction of $c$-fos and nur-77 by glucose and GLP-1. The induction of IEGs by glucose and chlorophenylthio-cAMP or GLP-1 and the inhibitory effect of nifedipine are also observed in the cell line $\beta \mathrm{HC}$. Finally, both glucose and GLP.1 markedly stimulates DNA synthesis as indicated by measurements of the incorporation of $\left[{ }^{3} \mathrm{H}\right]$-thymidine in INS-1 cells. The results indicate that GLP-1 and PACAP- 38 act as competence factors for the action of glucose on $\mathrm{c}-f \circ s, j u n B$ and nur-77. It is suggested that the synergistic effect of glucose and glucoincretins on the studied IEGs and protooncogenes plays a role in the adaptive processes of the $\beta$-cell to hyperglycemia and the control of B-cell growth. 


\section{5}

Mechanisms underlying the short-term control of insulin gene transcription

I.B. Leibiger, B. Leibiger, T. Moede, P.O. Berggren.

The Rolf Luft Center for Diabetes Research, Dept. Molecular Medicine. Karolinska Institute, S-17l 76 Stockholm, Sweden

The maintenance of glucose homeostasis by insulin requires, in addition to the stimulus-secretion coupling, a highly dynamic control of insulin biosynthesis. Since pancreatic $\beta$-cells are exposed to elevated glucose levels for minutes rather than hours we hypothesised the existence of a short-term transcriptional control. By performing RNA analysis and on-line monitoring of insulin gene expression we were able to provide evidence that insulin gene transcription is affected by glucose within minutes and thus is as dynamically regulated as translation and secretion. Using this strategy and employing inhibitors of protein kinases we found that insulin secreted by the pancreatic $\beta$-cell serves as a major stimulator of the transcription of its own gene via the PI-3 kinase/p70s6k and CaM kinase pathways. Exposure of pancreatic islets, islet cells and HIT-T15 cells to $16.7 \mathrm{mM}$ glucose for only 15 min resulted in a 2-4. fold elevation in (prepro)insulin mRNA levels and led to an enhanced insulin promoter-driven GFP expression in single insulin-producing cells. A similar effect was obtained when $\beta$-cells were stimulated for $5 \mathrm{~min}$ with either 50 $\mathrm{mM} \mathrm{KCl}, 1 \mu \mathrm{M}$ tolbutamide or with physiological concentrations of insulin. Blocking insulin secretion with nifedipine or insulin signalling via receptor tyrosine kinases with HNMPA-(AM $)_{3}$ abolished up-regulation of insulin gene transcription in response to stimulation with either secretagogues or exogenous insulin. Whereas treatment with inhibitors of PKA, PKC, CaM kinases, p70s6k or Pl-3 kinase abolished the glucose-stimulated insulin gene transcription, only inhibition of PI-3 kinase, p70s6k and CaM kinases affected the insulin-stimulated insulin gene transcription. In both cases treatment with MAP kinase inhibitors PD98059 or SB203580 had no effect.

\section{6}

PALMITATE AND OLEATE INDUCE THE IMMEDIATE-EARLY RESPONSE GENES C-FOS AND NUR-77 IN THE PANCREATIC B-CELL LINE INS-1

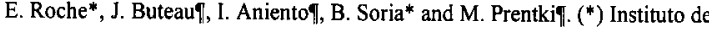
Bioingeniería, Universidad Miguel Hernández, Elche, Alicante (Spain). (\$) Molecular Nutrition Unit, University of Montreal (Canada).

Long term exposure of $B$-celis to fatty acids impairs the secretory response to glucose and may cause cell growth or apoptosis depending on the experimental condition. The mechanism whereby these nutrients exert these effects is not known. These alterations are likely related to changes in the expression level of genes which must be identified. We have investigated the effect of palmitate and oleate on the expression of immediate early-response genes (IEGs) coding for transcription factors implicaled in cell growth, differentiation and apoptosis. Two IEGs have been studied. First, the proto-oncogene $\mathrm{c}$-fos which associates with JUN proteins to form the so-called AP-1 transcription factor, a main regulator of many target genes. Second, nur-77, a member of the hormone nuclear receptor superfamily, whose expression increases during lymphocyte apoptosis. Palmitate and oleate cause a pronounced accumulation of $\mathrm{c}-f \circ s$ and nur-77 transcripts in INS cells. Nuclear run-on transcription assays supported a transcriptional mechanism. The activation displays a bell-shape kinetics with a maximal induction at $\mathrm{lh}$. The effect of the fatty acids is dose dependent between 0.1 and $0.4 \mathrm{mM}$. Myristate is also effective, whereas polyunsaturated, nonmetabolizable and short chain fatty acids barely change the expresion of $\mathrm{c}-\mathrm{fos}$ and nur-77. The C-kinase transduction system is apparently implicated in the action of fatty acids since the effect of palmitate and oleate is almost abolished in protein kinase $\mathrm{C}$-down-regulated cells. Finally, palmitate and oleate increase AP$\mathrm{l}$ activity and the incorporation of $\left[{ }^{3} \mathrm{H}\right]$-thymidine into INS cells. The results indicate that fatty acids induce in B-(INS)cells master genes implicated in the control of cell proliferation and apoptosis. These observations possibly have implications for the understanding of obesity associated diabetes mellitus and the link between fatty acids and tumorigenesis.

\section{7}

SPECIES SPECIFIC TRANSCRIPTION PATTERN AND ACTIVITY OF $\mathrm{Na} / \mathrm{Ca}$ EXCHANGE ISOFORMS IN PANCREATIC $\beta$ CELLS F. Van Eylen ${ }^{1}$, V. Duquesne ${ }^{2}, A$. Bollen $^{2}$ and A. Herchuelz ${ }^{1}$ 'Laboratory of Pharmacology and ${ }^{2}$ Laboratory of Applied Genetics, Brussels University School of Medicine, Bât. GE, 808 route de Lennik, B-1070, Brussels, Belgium.

In the rat pancreatic $\beta$ cell, $\mathrm{Na} / \mathrm{Ca}$ exchange, displays a quite high capacity. The cell is equipped with 2 alternatively spliced NCXI isoforms, $\mathrm{NaCa} 3$ and $\mathrm{NaCa} 7$. The aim of the study was (i) to examine the possible functional differences between these 2 isoforms compared to the heart isoform $\mathrm{NaCa}$ and (ii) to measure $\mathrm{Na} / \mathrm{Ca}$ exchange activity and transcription pattern of NCXI isoforms in pancreatic $\beta$ cells from rat, mouse, hamster and guinea pig. $\mathrm{NaCa}, \mathrm{NaCa} 3$ and $\mathrm{NaCa} 7$ were cloned from human heart or insulinoma, and expressed in HEK293 and $\mathrm{CHO}$ cells. In both expression systems and at equal level of expression, the cell transfected with $\mathrm{NaCal}$ displayed a $50 \%$ lower $\mathrm{Na} / \mathrm{Ca}$ exchange activity than $\mathrm{NaCa} 3$ - and $\mathrm{NaCa} 7$-transfected cells. Different levels of expression of $\mathrm{NaCa}_{3}$ and $\mathrm{NaCa} 7$ were found in the 4 rodent species. Three additional isoforms were found, $\mathrm{NaCa} 9$ and $\mathrm{NaCa} 33$ in guinea pig and $\mathrm{NaCa} 2$ in hamster and mouse, again in different proportions. In addition, $\mathrm{Na} / \mathrm{Ca}$ exchange activity was about $50 \%$ lower in the mouse, the hamster and the guinea pig than in the rat. Our data demonstrate the existence of a difference in intrinsic activity between NCXI isoforms. It also shows the existence of a differential and species specific transcription pattern of $\mathrm{NaCa}$ exchange isoforms in the $\beta$ cell that may explain in part the interspecies difference in $\mathrm{Na} / \mathrm{Ca}$ exchange activity.

\section{8}

MOLECULAR CHARACTERIZATION OF THE $70 \mathrm{kDa}$ POLYPEPTIDE OF THE $\mathrm{Na} / \mathrm{Ca}$ EXCHANGER

R. SABA, A. Van Praet, and A. HERCHUELZ. Laboratory of Pharmacology, Brussels University School of Medicine, Brussels, Belgium.

In pancreatic $\beta$-cells, $\mathrm{Na} / \mathrm{Ca}$ exchange plays an important role in cytosolic $\mathrm{Ca}^{2+}$ concentration regulation and insulin release. The transcription pattern and potential activity of $\mathrm{Na} / \mathrm{Ca}$ exchanger in $\beta$-cells present similarities to the cardiac one. The exchanger is associated with a 160,120 , and $70 \mathrm{kDa}$ polypeptide which nature is poorly understood. We have purified and characterized the $\mathrm{Na} / \mathrm{Ca}$ exchanger from bovine cardiac sarcolemmal vesicles (slv) using a combination of alkaline extraction, DEAE-Sepharose and WGA-affinity chromatography. The final WGAchromatography eluted fraction, enriched in the exchanger, was reconstituted into asolectin liposomes (10:1 w/w lipid:protein ratio) which showed $\mathrm{Na} / \mathrm{Ca}$ exchange activity. Under nonreducing conditions, SDS-PAGE showed a single $70 \mathrm{kDa}$ polypeptide that was further characterized by immunoblots using different purified antibodies raised against specific epitopes in the amino acid sequence of cloned bovine cardiac exchanger : "SWant", polyclonal, raised against the purified exchanger protein; " $\mathrm{NH}_{2}$ ", polyclonal, amino acid sequence (aa) 1-21; "NCX1", polyclonal, aa sequence 393-406; "Exon $\mathrm{F}$ ", polyclonal, aa sequence 622-644. Immunoblots done under reducing gel conditions using "SWant", " $\mathrm{NH}_{2}$ ", and "NCX1" showed 3 bands migrating at 160,120 , and $70 \mathrm{kDa}$ for slv preparations, while "Exon F" reacted only with the 160 and $120 \mathrm{kDa}$ ones. Under none reducing conditions, immunoblots carried out using purified reconstituted $\mathrm{Na} / \mathrm{Ca}$ exchanger showed a single band at $70 \mathrm{kDa}$ that reacted with "SWant", "NH2", and "NCX1"

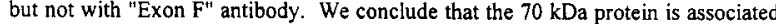
with $\mathrm{Na} / \mathrm{Ca}$ exchange activity, that it has the same $\mathrm{NH}_{2}$-terminal sequence as the cloned bovine cardiac exchanger, and that its amino acid sequence is reduced by at least $35 \%$ from its $\mathrm{COOH}$-terminal portion, compared to that of the cloned exchanger. Accordingly, our preparation may represent a good model for further study of $\mathrm{Na} / \mathrm{Ca}$ exchange activity regulation in different cell types. 


\section{9}

\section{BIOENGINEERING OF GLUCOSE-RESPONSIVE INSULIN- SECRETING SURROGATE CELLS}

M. Elsner, M. Tiedge, N.H. McClenaghan and S. Lenzen, Institute of Clinical Biochemistry, Hannover Medical School, D-30623 Hannover, Germany

Physiological glucose responsiveness is the crucial requirement of a surrogate pancreatic $B$ cell for insulin replacement therapy in insulindependent diabetes. A novel insulin-secreting cell with physiological glucose responsiveness was generated from an early passage glucose unresponsive RINm5F cell clone through overexpression of glucokinase. This surrogate cell had a physiological glucokinase/hexokinase ratio and showed molecular and metabolic characteristics comparable to those of the normal pancreatic $\beta$ cell. Glucokinase overexpressing RINm5F cells exhibited lower basal insulin release and significant responsiveness to millimolar glucose concentrations, with a 2.6 fold increase over basal of insulin release at 5 and $10 \mathrm{mM}$ glucose. Implantation of glucoseresponsive glucokinase overexpressing surrogate cells abolished the diabetic metabolic state of rats made diabetic through injection of streptozotocin. The expression level of the overexpressed glucokinase gene remained stable after implantation as well as the gene expression level of GLUT2 and insulin. In contrast to nontransfected RINm5F control cells, RINm5F-GK cells induced a gradual progressive blood glucose normalisation over a period of 14 days, resulting in blood glucose values in the normal range. This study demonstrates the usefulness of bioengineered glucose-responsive insulin-producing surrogate cells for insulin replacement therapy in IDDM.
510

CHARACTERISATION OF AN ENGINEERED INSULIN-SECRETING
HEPATOCYTE CELL LINE

H.M. Docherty, E.M. Davies*, A.W. Hart, C J Bailey*, K Docherty, and K.I. J Shennan. Department of Molecular and Cell Biology, University of Aberdeen, Institute of Medical sciences, Aberdeen, and *Department of Pharmaceutical Sciences, Aston University, Birmingham UK

The aim of this study was to engineer insulin secreting liver cells for use in the treatment of IDDM and NIDDM. Co-transfection of a human hepatoma cell line, HepG2, with a mutant insulin gene construct, Ins 4 , and the human furin gene, gave rise to a stable cell line, HepG2ins4F.159, which secreted a high level (up to $0.05 \mu \mathrm{g} / 10^{6}$ cells $/ 24$ hours) of mature, biologically active human insulin. The Ins 4 gene construct encodes a mutant proinsulin in which both cleavage sites (at the B chain / C peptide junction and the $C$ peptide / $A$ chain junction) have been engineered to consensus furin cleavage sites. The constitutive release of insulin by HepG2ins4F. 159 cells was not altered by changes of the glucose concentration in the medium, within and above the normal physiological range. However, insulin release was increased by $20 \mathrm{mM}$ arginine (by $22 \%$ ), $1 \mathrm{mg} / \mathrm{ml}$ forskolin (by $52 \%$ ) and $5 \mathrm{mM}$ IBMX (by $32 \%$ ). Intraperitoneal implantation of $1.5 \times 10^{7} \mathrm{HepG}$ 2ins4F. 159 cells into insulin-dependent streptozotocin-diabetic nude mice did not eliminate the dependency of the mice on exogenous insulin injections. However, after 28 days, when exogenous insulin was withheld for 18 hours, plasma human insulin concentrations were in the range 1 $10 \mathrm{ng} / \mathrm{ml}$. Autopsy of mice implanted with HepG2ins4F. 159 cells revealed many vascularised aggregations of cells attached to the diaphragm and mesenteries. These results demonstrate the feasibility of engineering non-neuroendocrine cells to secrete high levels of biologically active human insulin, capable of generating approximately normal circulating insulin concentrations when implanted into streptozotocin-diabetic nude mice.

\section{1}

ENHANCED FUNCTION OF ELECTROFUSION-DERIVED SURROGATE B-CELLS IMPLANTED IN DIABETIC NUDE MICE

Y.H.A. Abdel-Wahaba, E.L. Daviesb,C.J. Baileyb and P.R. Flatta. aSchool of Biomedical Sciences, University of Ulster,Coleraine, UK. bSchool of Life and Health Sciences, Aston University, Birmingham, UK.

The present study aimed to evaluate the insulinotropic activity of electrofusion-derived BRIN-BD11 cells following implantation in streptozotocin-diabetic nude $(\mathrm{nu} / \mathrm{nu}$ ) mice (glycaemia $>24 \mathrm{mmol} / \mathrm{l}$ ). Intraperitoneal implantation of $5 \times 10^{6}$ BRIN-BD11 cells reduced glycaemia so that insulin injections were ceased by $5-16$ days $(<17$ $\mathrm{mmol} / \mathrm{l})$ and normoglycaemia $(<9 \mathrm{mmol} / \mathrm{l})$ was achieved by $7-20$ days. Acute incubation of BRIN-BD11 cells reestablished in culture $9-20$ days after tumour removal, revealed that $16.7 \mathrm{mmol} / \mathrm{l}$ glucose-stimulated insulin release was enhanced by $121 \%(p<0.001)$ compared to nonimplanted (control) cells $\left(5.1 \pm 0.7\right.$ and $5.1 \pm 0.5 \mathrm{vs} 2.3 \pm 0.2 \mathrm{ng} / 10^{6}$ cells $/ 20 \mathrm{~min}, \mathrm{n}=6$ ). Responses to $10^{-9} \mathrm{~mol} / 1 \mathrm{GLP}-1,10^{-8} \mathrm{~mol} / 1 \mathrm{CCK}-8$ or 10 $\mathrm{mmol} / \mathrm{l} \mathrm{L}$-alanine (at $5.6 \mathrm{mmol} / \mathrm{l}$ glucose) were similarly increased by $31-53 \%, 32-43 \%$ and $37-68 \%(\mathrm{p}<0.05-0.01, \mathrm{n}=6)$. Insulin content of preimplanted cells was increased ( $p<0.001 ; 321.4 \pm 31.2$ vs $129.3 \pm 11.84$ $\mathrm{ng} / 10^{6}$ cells, $\left.n=6\right)$, but basal insulin release $(5.6 \mathrm{mmol} / \mathrm{l}$ glucose $)$ was not changed $\left(0.8-0.9 \mathrm{ng} / 10^{6} \mathrm{cells} / 20 \mathrm{~min}\right)$ by any of the experimental manipulations. After 40 days in culture, insulin content declined to $88.6 \pm$ $4.2 \mathrm{ng} / 10^{6}$ cells, $\mathrm{p}<0.01, \mathrm{n}=6$ ) and the secretory responses to $16.7 \mathrm{mmol} / \mathrm{l}$ glucose, $10^{-9} \mathrm{~mol} / 1 \mathrm{GLP}-1,10^{-8} \mathrm{~mol} / 1 \mathrm{CCK}-8$ or $10 \mathrm{mM}$ L-alanine were decreased by $70 \%, 47-54 \%, 42-47 \%$ and $69-73 \%$, respectively $(p<0.05-$ $0.01)$ to achieve values not significantly different from non-implanted Bcells. The significant functional enhancement of electrofusion-derived surrogate B-cells following implantation and restoration of normoglycaemia indicates that in vivo environment can greatly assist Bcell engineering in approaches leading to gene therapy of diabetes.

\section{2}

DETERMINANTS OF THE EFFECT OF LONG-TERM GROWTH HORMONE INCUBATION ON INSULIN SECRETION FROM HUMAN FETAL ISLETS P.B.Djordjević, N.M.Lalić, A.Jotić, I.Paunović, N.Raketić, S.Petković, M.Zamaklar, K. Lalić. Z. Dragojlović, N. Rajković, Lj. Lukić and I. Marković. Institute for Endocrinology, Belgrade. Yugoslavia

We have previously shown that long-term ( 15 day) incubation with growth hormone $(\mathrm{GH})$ has a beneficial effect of preventing the decline of insulin secretion from hunan fetal islets during cultivation in vitro. Therefore, the aim of this study was to evaluate the following determinants of the effect of 15 day incubation with $\mathrm{GH}$ (Genotropin. Kabi Pharmacia, $1000 \mu \mathrm{g} /$ ) on insulin secretion from islets: (a) gestational age of the donor fetuses $(16,18,20,22$ and $24 \mathrm{wk}$ ), (b) type of procedure for obtaining fetal tissue (spontaneous vs prostaglandin-induced abortion); (c) glucose concentration in the culture media $(5,11,16$ or $22 \mathrm{mmol} / \mathrm{)})$. The islets were isolated by collagenase digestion and cultured in vitro $\left(10 \%\right.$ felal calf serum, $37^{\circ} \mathrm{C}$ $5 \% \mathrm{CO} 2$ ). The insulin secretion capacity was evaluated by determining insulin levels in culture media after $I$ hr incubation sequentially with $1.67 \mathrm{mmol} / \mathrm{l}$ glucose and $16.7 \mathrm{mmol} / \mathrm{l}$ glucose $+5 \mathrm{mmol} / \mathrm{l}$ theophyllin and expressed as the percentage of the increase in insulin levels after the high-glucose+theophyllin stimulation. We found first, that the insulin response remained stable after incubation with $\mathrm{GH}$ when the islets originated from 16,18 and $20 \mathrm{wk}$ old fetuses (days 1 vs $15, \mathrm{p}=\mathrm{NS}$ ), while it declined in the islets from 22 and 24 wk old fetuses (days I vs $15 ; 22 \mathrm{wk}: 736.5+/$ $61.6 \%$ vs $478.9+/-39.4 \%, \mathrm{p}<0.05: 24 \mathrm{wk}: 731.4+/-53.4 \%$ vs $422.5+/-47.3 \%, \mathrm{p}<0.05$ ) In the second part of the study, we detected that insulin secretion remained unchanged during the 15 day incubation period $(p=N S$ ) in the islets from spontaneous abortion while the secretion declined in those from prostaglandin-induced abortion (gestational age $16-20 \mathrm{wk}$ ) (days I vs $15: 739.2+1-67.3 \%$ vs $498+/-42.1 \%$; $\mathrm{p}<0.05$ ). Finally, we detected similar insulin secretion of the islets (gestational age $16-20 \mathrm{wk}$. spontaneous abortion) incubated in 5 and $11 \mathrm{mmol} / \mathrm{l}$ glucose (days 1 is $15, p=N S$ ). while it decreased in isicts incubated with $22 \mathrm{mmol} /$ (days I vs $15: 732.3+/-58.4 \%$ vs $187.8+/-21.3 \%, \mathrm{p}<0.05$ ). Our results have demonstrated that the beneficial effect of the long-term incubation with GH was strongly dependent on gestational age of the isolated islets. type of procedure for obtaining fetal tissue and ambiental glucosc concentration which might be of clinical relevance during preparation for hunan fetal islet transplantation in Type I diabetes patients. 


\section{3}

TOWARDS SYNGENEIC GENE THERAPY FOR DIABETES: INSULIN EXPRESSION IN ADULT RAT FIBROBLASTS J.A.M. Shaw ${ }^{a}$, M.I. Delday ${ }^{b}$, A. Hart ${ }^{a}$, H.M. Docherty ${ }^{a}$, C.A. Maltin ${ }^{b}$ and $\mathrm{K}$. Docherty ${ }^{\mathrm{a}}{ }^{\mathrm{a}}$ Department of Molecular and Cell Biology, Institute of Medical Sciences, University of Aberdeen, and ${ }^{\mathrm{b}}$ Rowett Research Institute, Aberdeen, U.K.

Transfection of fibroblasts derived from a diabetic individual with an insulin gene construct and subsequent reimplantation is a novel approach to diabetic gene therapy circumventing immune rejection. The objectives of this study were to establish adult rat dermal fibroblasts in primary culture, determine optimal conditions for transfection and assess insulin secretory dynamics in transfected cells. Dermal fibroblasts were derived from $150 \mathrm{~g}$ Rowett-Hooded-Lister rats. Cells were transfected with a wild type insulin gene construct (pIREShppI1) and a mutant construct (pIRES-hppI $4^{3 \text { UTR }}$ ) in which the PC2 and PC 3 cleavage sites were altered by site-directed mutagenesis to enable proinsulin to insulin processing by furin. Transfection was assessed by radio-immuno-assay (RIA) and immunofluorescence. Transient transfection with Lipofectamine resulted in secretion of (pro)insulin into the medium peaking 24 hours after transfection (pIRES-hppI1 4.0ng ml $\mathrm{m}^{-1}$; pIRES-hppI4 $4^{3^{3} \mathrm{UTR}} 0.23 \mathrm{ng} \mathrm{m}^{-1}$ ) and continuing for 7 days. Insulin-specific RIA demonstrated $22 \%$ proinsulin to insulin processing with pIRES-hppIl vs $90 \%$ processing with pIRES-hppI $4^{3}{ }^{3}{ }^{R}$. In conclusion, constitutive proinsulin secretion has been attained following transfection of adult rat primary dermal fibroblasts with insulin gene constructs, transduction with a mutant construct resulting in significant proinsulin to insulin processing.

\section{5}

PHARMACOLOGICAL CHARACTERIZATION OF A CELL LINE STABLY EXPRESSING THE HUMAN PANCREATIC $\beta$-CELL K KTP COMPLEX

M. Dabrowski ${ }^{1}$, P. Wahl ${ }^{1}$, J. Fuhlendorff ${ }^{2}$ and W.E. Holmes ${ }^{3}$. Dept. Of ${ }^{1}$ Molecular Pharmacology, 2 Diabetes Biology, ${ }^{3}$ Molecular Biology, Novo Nordisk A/S, Novo Nordisk Park, DK-2760 Maaloev, Denmark.

HEK293 cells have been stably transfected with the human SUR1 receptor and the human inward rectifying $\mathrm{K}^{+}$-channel Kir6.2. These subunits constitute the ATP sensitive $\mathrm{K}^{+}$-channel complex ( $\mathrm{K}_{\mathrm{ATP}}$ ) of the human pancreatic $\beta$-cell. We have investigated the ATP sensitive $\mathrm{K}^{+}$-currents in this cell line to determine if the expressed $\mathrm{K}_{\mathrm{ATP}}$-channel can be used as a model for the human $\beta$-cell $\mathrm{K}_{\mathrm{ATP}}$. All experiments were performed at $23^{\circ} \mathrm{C}$ using the whole cell patch-clamp technique. The minimal receptor density estimated from whole cell currents obtained in cells dialyzed with $0.3 \mathrm{mM}$ $\mathrm{MgATP}$ and $0.3 \mathrm{mM} \mathrm{MgADP}$ is $387 \pm 39$ channels $/ \mathrm{pF}$. Tolbutamide was found to block $83 \pm 4 \%$ of the whole cell $\mathrm{K}_{\mathrm{ATP}}$-current: $\mathrm{IC}_{50}=4.7 \pm 0.2$ $\mu \mathrm{M}$ in cells dialyzed with $0.3 \mathrm{mM} \mathrm{MgADP}$ and $0.3 \mathrm{MgATP}$. The new oral antidiabetic compound repaglinide (NovoNorm ${ }^{\mathrm{TM}}$ ) is a very potent $\beta$-cell $\mathrm{K}_{\mathrm{ATP}}$-channel blocker. In cells dialyzed with $0.3 \mathrm{mM} \mathrm{MgATP}, 30 \mathrm{nM}$ repaglinide blocked $95.5 \pm 1.4 \%$ of the whole cell $\mathrm{K}^{+}$-current. There is a significant difference in the timecourse of $\mathrm{K}_{\mathrm{ATP}}$-channel inhibition produced by $100 \mu \mathrm{M}$ tolbutamide and $30 \mathrm{nM}$ repaglinide (both maximal inhibition concentrations). Tolbutamide fully inhibits the current in $\sim 30 \mathrm{~s}$, whereas full effect of repaglinide is obtained after 3-4 minutes. Diazoxide activated the $\mathrm{K}_{\mathrm{ATP}}$-current: $\mathrm{EC}_{50}=33 \pm 3 \mu \mathrm{M}$ in cells dialyzed with $5 \mathrm{mM}$ $\mathrm{MgATP}$ and $0.3 \mathrm{mM} \mathrm{MgADP}$. Other $\mathrm{K}_{\mathrm{ATP}}$-channel openers tested were minoxidil and pinacidil, which activates the $\mathrm{K}^{+}$-current with lower affinity and efficacy than diazoxide. The pharmacological properties tested suggests

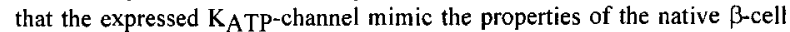
$\mathrm{K}_{\mathrm{ATP} \text {-channel. }}$

\section{4}

MIN6 PSEUDOISLETS: DEVELOPMENT OF AN EXPERIMENTAL MODEL FOR ISLETS OF LANGERHANS

A.C. Hauge-Evans, S.J. Persaud and P.M. Jones; Cellular and Molecular Endocrinology Group, Biomedical Sciences Division, King's College London, London W8 7AH.

Immortalised pancreatic $\beta$-cell lines are widely used as research models in the study of insulin secretion. Here we report that MIN6 3 -cells, which normally grow as a monolayer, formed islet-like structures (pseudoislets) when grown on gelatincoated tissue culture plastic. After 7-9 days in culture the pseudoislets reached a size and morphology similar to isolated primary rat islets maintained in culture. Western blotting studies using antisera against the gap junction protein connexin 43 and the cell adhesion molecule E-CAD ( 80 and $120 \mathrm{kDa}$ isoforms) showed that both molecules were expressed in pseudoislets and in MIN6 monolayer cells. The $80 \mathrm{kDa}$ isoform of E-CAD was expressed to a similar extent in the two forms of MIN6 cells, whereas pseudoislets contained more of the $120 \mathrm{kDa}$ isoform than did monolayer cells. The insulin content of pseudoislets did not differ significantly from the content of monolayer cells (pseudoislets: $9.5 \pm 0.8 \mathrm{ng} / \mu \mathrm{g}$ protein, $\mathrm{n}=6$, monolayers: $9.3 \pm 0.7 \mathrm{ng} / \mu \mathrm{g}$ protein, $\mathrm{n}=6, \mathrm{P}>0.2$ ) and was approximately $15 \%$ of the insulin content found in primary rat islets $(66.5 \pm 19.9 \mathrm{ng} / \mu \mathrm{g}, \mathrm{n}=7$ ). Pseudoislets contained $741.6 \pm 37.0 \mathrm{ng}$ protein/islet while primary rat islets were found to contain $594 \pm 28.5 \mathrm{ng}$ protein/islet. Both nutrient and non-nutrient secretagogues stimulated insulin secretion from pseudoislets. The secretion was measured after 1 hour incubation with nutrients $(2 \mathrm{mM}$ glucose: $143 \pm 52.5$ $\mathrm{pg} /$ islet/h; $20 \mathrm{mM}$ glucose: $352.5 \pm 58.1 ; 10 \mathrm{mM}$ glyceraldehyde: $1347 \pm 204.9$; mean \pm SEM, $n=9)$ and PMA, an activator of protein kinase $C(20 \mathrm{mM}$ glucose + $500 \mathrm{nM}$ PMA: $832.5 \pm 137.3 \mathrm{pg} / \mathrm{islet} / \mathrm{h}$ ). Since the pseudoislets can be handled like primary rodent islets and can be made available in virtually limitless quantities, these results suggest that they may serve as an alternative research model replacing the use of primary rat islets in certain experiments

\section{6}

THE SRC-FAMILY PROTEIN BSK INHIBITS CELL REPLICATION IN INSULIN PRODUCING CELLS.

C. Annerén and M. Welsh. Depart. of Med. Cellbiology, Uppsala University, Box 571, Biomedicum, 57123 Uppsala, SWEDEN

Bsk is a tyrosine kinase of the Src-family expressed in the islets of Langerhans. It has recently been identified and cloned by us from the mouse $\beta$ TC-1 cell line and might have importance for different cellular processes such as growth, differentiation and survival. Two tyrosine residues in the C-terminal tail (tyr-497 and tyr-504) are putatively homologous to tyr-527 in Src, a negative regulator of Srcactivity which renders Src continiously active when mutated. We have shown that NIH3T3 cells expressing double mutated (Y497F/504F) Bsk display a decreased growth rate and have a larger fraction of cells in G1-phase compared to control cells. The aim of the study was to investigate cell growth in RINm5F cells expressing wild type or mutated Bsk as well as studying the contents of different cell cycle proteins in NIH3T3 cells expressing double mutated Bsk. The proliferation of RINm5F cells was determined by cell countings for five days. It was shown that RINm5F cells expressing Y504F-mutated and Y497/504F-mutated Bsk display a significantly decreased growth rate compared to the control cells $(p<0.0005$, ANOVA). Expression levels of different cell cycle proteins (Rb2, p27/Kip1, cdk2, cdk4, cyklin D3 and E2F1) were determined by immunoblotting. It was found that $\mathrm{Rb} 2$ expression is higher in NIH3T3 cells expressing double-mutated Bsk/Iyk compared with the control cells. High p27/Kip1-levels accompanied with low cdk2 levels compared to the control cells was seen in one of the three double-mutant Bskexpressing cell lines. We have also studied levels of expressions of cdk4, cyklin D3 and E2F1 and detected no differences between the Bsk transfected clones and the controls. Preliminary results suggest an increase in p27/Kipl-expression in Y504F-mutated as well as Y497/504F-mutated Bsk/Tyk transfected RINm5F cells compared to Y Thes control cells. These results (cell counting accompanied with replication in insulin producing cells. 


\section{7}

AGING AND CHANGES IN BETA CELL REPLICATION, SLZE AND MASS IN LEWIS RATS.

V. Nacher, M. Biarnés, M. Raurell, M. Pérez-Maraver, J. Soler and E. Montanya. Laboratory of Diabetes and Endocrine Unit. CSUB-H. Bellvitge. Barcelona.

The replicative rate of beta cells decreases with age but it is not known whether it is eventually reduced to zero and how it contributes to the dynamics of beta cell mass. The aim of the study was to determine the evolution of beta cell replication and mass throughout the postweaning life of Lewis rats. Six groups of 6 Lewis rats were studied $1,3,7,10,15$ and 20 months after birth. Pancreas sections were double stained with immunoperoxidase for 5-bromo-2 deoxyuridine (BrdU), and for beta cells or for the endocrine non-beta cells of the islets. Replication was expressed as percentage of beta cell nuclei positive for BrdU; individual beta cell area was determined by planimetry and beta cell mass by point counting morphometry. Differences among groups were determined by ANOVA and Fisher PLSD method. All animals remained normoglycemic until the end of the study. Beta cell replication was progressively reduced in the first months of life (month 1: $1.27 \pm 0.09 \%$, month $3: 0.24 \pm 0.04 \%$, month $7: 0.12 \pm 0.02 \%, \mathrm{p}<0.01$ ), but remained stable thereafter (month 10: $0.14 \pm 0.02 \%$, month 15: $0.10 \pm 0.03 \%$, month 20 : $0.13+0.03 \%$ ). Individual beta cell area increased initially (month $1: 122 \pm 4 \mathrm{um}^{2}$,

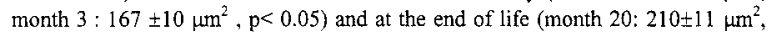
$p<0.05$ vs month 3). Beta cell mass increased progressively: month $1: 2.25 \pm 0.52$ $\mathrm{mg}$; month 3: $5.92 \pm 0.88 \mathrm{mg}$; month 7: $10.22 \pm 1.04 \mathrm{mg}$; month 10: $10.97 \pm 0.34$ $\mathrm{mg}$; month 15: $13.61 \pm 3.21 \mathrm{mg}$; month $20: 15.48 \pm 2.32 \mathrm{mg}(\mathrm{p}<0.01)$. In contrast, exocrine and endocrine non-beta cell mass did not change significantly after month 7. The beta cell mass/body weight ratio remained stable throughout the entire life; in contrast, non-beta cell mass/body weight ration was reduced after month 7. In summary, beta cell replication was progressively reduced in the first 7 months of life, but remained constant thereafter. Both hyperplasia and hypertrophy contributed to increase beta cell mass in the first months, whereas hypertrophy played a major role in late periods of life. The specific and constant adaptation of beta cell mass to body weight may be essential to maintain normoglycemia. Limitations in beta cell replication could disrupt the adaptative capacity of beta cell mass, and contribute to the development of diabetes.

\section{PS 11}

\section{Signal Transduction in $\beta$-Cells}

\section{8}

\section{DUAL EFFECTS OF DIADENOSINE POLYPHOSPHATES ON INS-1 CELLS}

E. J. Verspohl and B. Johannwille. Institute of Pharmaceutical Chemistry, Dep. of Pharmacology, University of Münster, Hittorfstr. 58-62, 48149 Münster, Germany

Recently a role of diadenosine polyphosphates as 2 nd messengers was suggested for insulin secreting cells. In this study we investigated whether receptors for these compounds exist in insulin secreting cells (INS-1) and how these extracellular compounds contribute to insulin release. Binding of $\left[{ }^{3} \mathrm{H}\right] \mathrm{Ap}_{4} \mathrm{~A}$ to INS-1 cell homogenates was time dependent and saturable (one-half maximal after $4 \mathrm{~min}$, maximal at $30 \mathrm{~min} ., 12 \%$ bound of total radioactivity). The rank order of diadenosine polyphosphates displacing $\left[{ }^{3} \mathrm{H}\right] \mathrm{A} \mathrm{p}_{4} \mathrm{~A}$ from binding sites was $A p_{4} \mathrm{~A}=$ $\mathrm{Ap}_{5} \mathrm{~A}>\mathrm{Ap}_{3} \mathrm{~A}=\mathrm{Ap}_{6} \mathrm{~A}$. Adenosine, ATP, UTP, $\alpha, \beta$-meATP, $\beta, \gamma$-meATP, ADP- $\beta S$, 2-MeSATP, and PPADS also displaced $\left[{ }^{3} \mathrm{H}\right] \mathrm{Ap}_{4} \mathrm{~A}$ from binding sites whereas suramin, a compound not structurally related to $\mathrm{Ap}_{4} \mathrm{~A}$, had no displacement activity. Insulin release (RIA) was inhibited dose dependently (max. by $45 \%$ ) by either of the four diadenosine polyphosphates $\left(A p_{0} A, n=3-6\right)$ in the presence of $8.3 \mathrm{mM}$ glucose during a $90 \mathrm{~min}$. (long term) incubation. However, a stimulation of glucase-induced insulin release was observed during perifusion experiments (short term incubation). Due to these contradictory results the stability of $\left[{ }^{3} \mathrm{H}\right] \mathrm{Ap} \mathrm{p}_{4} \mathrm{~A}$ was tested. $\left[{ }^{3} \mathrm{H}\right] \mathrm{Ap}_{4} \mathrm{~A}$ was degraded in the extracellular medium to ATP, ADP, AMP, inosine and mainly adenosine ( $80 \%$ after $90 \mathrm{~min}$ ) with a half-life of about $20 \mathrm{~min}$. During incubation of INS- 1 cells with $\left[{ }^{3} \mathrm{H}\right] \mathrm{Ap}_{4} \mathrm{~A}$ an uptake of radiolabeled compounds occurred. The uptake was inhibited by $\mathrm{NaCN}$ and concanavalin $\mathrm{A}$ indicating a specific uptake mechanism. However, only small amounts of $\left[{ }^{3} \mathrm{H}\right] \mathrm{Ap} \mathrm{p}_{4} \mathrm{~A}$ can be detected in INS-1 cells whereas major amounts consist of $\left[{ }^{3} \mathrm{H}\right] \mathrm{ATP}$. This data indicates that diadenosine polyphosphates increase insulin release via binding sites; quickly emerging extracellular degradation products such as adenosine inhibit insulin release. The binding sites involved may be diadenosine polyphosphate receptors albeit others, such as $P_{1}$ or $P_{2}$ receptors cannot be ruled out.

\section{9}

DIADENOSINE TETRAPHOSPHATE STIMULATES INSULIN AND GLUCAGON SECRETION IN THE PERFUSED RAT PANCREAS.

R.A. Silvestre, J. Rodríguez-Gallardo, P. García, E. Gutiérrez and J. Marco. Clínica Puerta de Hierro, and Dept. Physiology, Universidad Autónoma de Madrid, Madrid, Spain.

Intracellular diadenosine polyphosphates are considered signal molecules that alert the cell to metabolic stress conditions. It has been recently shown that, in the B-cell, glucose induces an increase in the intracellular leveis of these compounds which are effective inhibitors of the ATPregulated $\mathrm{K}^{+}$channels. We have studied the effect of diadenosine tetraphosphate (AP4A) on insulin and glucagon secretion by the perfused rat pancreas. Perfusate consisted of Krebs-Henseleit buffer supplemented with albumin $(0.5 \%)$, dextran T-70 (4\%) and glucose $(5.5$

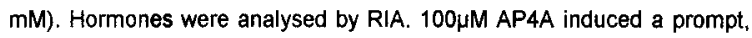
short-lived insulin response (peak at $2 \mathrm{~min}: 18.8 \pm 3.7$, Mean $\pm S E M, n g / m i n$ vs. basal value: $3.7 \pm 0.5 \mathrm{ng} / \mathrm{min} ; \mathrm{p}<0.01$ ). This stimulatory effect of AP4A was abolished $(p<0.01)$ by known inhibitors of insulin release such as somatostatin $(10 \mathrm{nM})$ and diazoxide $(300 \mu \mathrm{M})$. AP4A, at a lower concentration $(15 \mu \mathrm{M})$, similar to that found in glucose-stimulated $B$ cells, evoked a two-fold increase in insulin release (peak at 2 min: $9.8 \pm 1.6$ $\mathrm{ng} / \mathrm{min}$ vs. basal value: $4.2 \pm 0.4 \mathrm{ng} / \mathrm{min} ; \mathrm{p}<0.05)$. In pancreases perfused with a constant glucose concentration, addition of $100 \mu \mathrm{M}$ AP4A to the perfusate was followed by a rapid increase in glucagon release (peak at 3-min: $440 \pm 86 \mathrm{pg} / \mathrm{min}$ vs. basal value: $99 \pm 36 \mathrm{pg} / \mathrm{min} ; \mathrm{p}<0.025$ ). This glucagon response to AP4A was abolished by $10 \mathrm{nM}$ somatostatin $(p<0.01)$. The stimulatory effect of AP4A on insulin and glucagon release favours the concept of this adenosine polyphosphate as being implicated in the triggering mechanism of both $\mathrm{A}$ - and $\mathrm{B}$-cell secretion. 


\section{0}

INTRACELLULAR DIADENOSINE POLYPHOSPHATES: A NOVEL SECOND MESSENGER IN THE STIMULUS-SECRETION COUPLING

Bernat Soria', Jesús Pintor ${ }^{\dagger}$ Juan M. Rovira", Cristina Ripoll, M. Teresa MirasPortugal $^{\dagger}$ and Franz Martin ${ }^{\ddagger}$. Departments of Physiology', Science and Technology $y^{\ddagger}$ and Institute of Bioengineering", Campus de San Juan, Miguel Hernandez University, Alicante. Department of Biochemistry ${ }^{\dagger}$ School of Veterinary, Complutense University, Madrid, Spain.

In pancreatic $\beta$-cells, stimulatory glucose concentrations increase cytosolic diadenosine polyphosphates leveis $\left(\left[\mathrm{Ap}_{\mathrm{n}} \mathrm{A}\right]_{\mathrm{j}}\right)$ to values sufficient to block ATPsensitive $\mathrm{K}^{+}\left(\mathrm{K}_{\mathrm{ATP}}\right)$ channels. To study the metabolic pathways by which pancreatic $\beta$-cells synthesize $\operatorname{Ap}_{\mathrm{n}} \mathrm{A}$ and the mechanism through which $\operatorname{Ap}_{\mathrm{n}} \mathrm{A}$ inhibit $K_{A T P}$ channels, high performance liquid chromatography and patch-clamp techniques were used. Ap A show a glucose- and time-dependent cytosolic concentration increase, parallel to changes observed in adenine nucleotides, though 30- to 50-fold higher. Other fuel secretagogues, leucine and 2ketoisocaproate, rise $\left[\mathrm{Ap}_{\mathrm{n}} \mathrm{A}\right]_{\mathrm{i}}$ to the same level as $22 \mathrm{mM}$ glucose. Blockade of glycolysis or Krebs cycle decreases glucose-induced $\left[A p_{n} A\right]_{i}$. No significant increase in cytosolic $\mathrm{Ap}_{\mathrm{n}} \mathrm{A}$ levels are induced by non-nutrient secretagogues or non-metabolizable nutrient secretagogues. Inorganic pyrophosphatase inhibition with sodium fluoride blocks $22 \mathrm{mM}$ glucose-induced $\left[A p_{n} A\right]_{i} . A p_{n} A$ inhibition of $K_{A T P}$ channel resembles that induced by ATP in efficacy, but shows clear functional differences with it. $\mathrm{Ap}_{4} \mathrm{~A}$ does not restore channel activity after rundown. Besides, both compounds do not compete with each other for the same site. We conclude that nutrient metabolization through pyrophosphatase activation is necessary to induce $\mathrm{Ap}_{\mathrm{n}} \mathrm{A}$ synthesis, which, in turn, constitutes a new, ATPindependent, metabolic regulation of $\mathrm{K}_{\mathrm{ATP}}$ channel activity.

\section{1}

CHARACTERISATION AND FUNCTIONAL ROLE OF CYCLIC AMP PHOSPHODIESTERASES IN CLONAL $\beta$-CELLS

P.R. Flatta, Y.H.A. Abdel-Wahaba, M. Ahmadb, N.J. Pyneb, and B.L. Furmanb. aSchool of Biomedical Sciences, University of Ulster, Coleraine, UK.

bDept. of Physiology \& Pharmacology, University of Strathcylde, Glasgow, UK.

Effects of type selective inhibitors of cyclic AMP phosphodiesterase (CAMP-PDE) were examined using a glucose-responsive clonal $\beta$-cell line (BRIN-BD11) generated by electrofusion. Org $9935(0.02-5 \mu \mathrm{mol} / \mathrm{l})$ a potent and selective PDE3 inhibitor, inhibited cAMP-PDE activity by a maximum of $39 \pm 2 \%$ (control $495 \mathrm{pmol} / \mathrm{min} / \mathrm{ml}$ ) in the pellet but not in the supernatant $\left(\mathrm{IC}_{50} 0.2 \mu \mathrm{mol} / \mathrm{l}\right)$. A high concentration of zaprinast (50 $\mu \mathrm{mol} / \mathrm{l}$ ) inhibited CAMP-PDE by $29 \%$ ( $\mathrm{IC}_{50} 4 \mu \mathrm{mol} / \mathrm{l}$ ), suggesting the presence of PDE1 ( $\mathrm{Ca}^{2+-}$-calmodulin activated). Rolipram (0.05-10 $\mu \mathrm{mol} / \mathrm{l})$, a PDE4 inhibitor, decreased CAMP-PDE activity concentrationdependently in the soluble fraction, with maximum inhibition of around $30 \%$. In acute incubations with stimulatory $16.7 \mathrm{mmol} / 1$ glucose $(1.6 \pm 0.1$ compared with $0.7 \pm 0.1 \mathrm{ng} / 10^{6}$ cells $/ 20 \mathrm{~min}$ for $1.1 \mathrm{mmol} / \mathrm{l}$ glucose; $\mathrm{P}<0.001 ; \mathrm{n}=8), 1-50 \mu \mathrm{mol} / 1$ Org 9935 and another PDE3-selective inhibitor, SK\&F 94836, induced $52-66 \%$ and $40-95 \%$ dose-dependent increases of insulin release $(\mathrm{p}<0.01)$. Rolipram $(1-50 \mu \mathrm{mol} / 1)$ similarly increased insulin secretion by $52-60 \%(\mathrm{p}<0.01)$ but PDE1 inhibition with zaprinast (1-50 $\mu \mathrm{mol} / 1)$ was without effect. At $5.6 \mathrm{mmol} / 1$ glucose, $10^{-9}$ mol/l GLP-1 and $1 \mu \mathrm{mol} / \mathrm{l}$ forskolin induced 2.1 fold increases $(\mathrm{p}<0.001)$ of insulin secretion $\left(1.1 \pm 0.1 \mathrm{ng} / 10^{6}\right.$ cells $/ 20 \mathrm{~min}$ at $5.6 \mathrm{mmol} / 1$ glucose) The stimulatory actions of both agents were enhanced 1.3-2.6 fold by 1-10 $\mu \mathrm{mol} / 1$ Org 9935 or SK\&F 94836 and $1.7-2.2$ fold by rolipram ( $\mathrm{p}<0.01$ 0.001 ). These data demonstrate functional roles of PDE3 and PDE4 in insulin secretion from clonal pancreatic $\beta$-cells and highlight these enzymes as possible targets for new antidiabetic drugs.

\section{2}

RT-PCR IDENTIFICATION OF GaOLF AND CALCIUMREGULATED ADENYLYL CYCLASES IN RAT BETA CELLS ISOLATED BY FLOW CYTOMETRY.

S. Frayon, M. Pessah, C. Boissard, D. Mercan, W.J. Malaisse and J.M. Garel. INSERM U.482, Hôpital Saint-Antoine, Paris, France and Lab. Exp. Med., Brussels Free University, Brussels, Belgium.

Molecular diversity of heterotrimeric $\mathrm{G}$ proteins and adenylyl cyclases for islet cells were already reported, but quantitative expression for such molecules in B cells and non-B cells are lacking. It was shown previously that Goolf which share $88 \%$ homology with Gas is expressed in the endocrine pancreas. Islet $B$ and non-B cells were separated by flow cytometry of dispersed cells from adult female rats labelled with the calcium-sensitive fluorochrome fluo- 3 . The expression of Gaolf and adenylyl cyclases (AC) of types II, III, V and VI was evaluated by reverse-transcriptase polymerase chain reaction (RT-PCR) using specific rat primers and compared to a GAPDH control probe. Southern blot of amplicons generated with specific primers of Gaolf revealed the presence of a specific 540 bp band only in B cells. AC II, $A C$ III, $A C V$ and $A C$ VI were expressed both in $B$ and non-B cells as shown by RT-PCR amplicons. However, AC III was clearly more abundant in non- $B$ cells than in $B$ cells. Moreover, in $B$ cells, the expression of $A C$ VI was higher than that of $A C V$; whereas similar levels of $A C V$ and $A C$ VI were found in non- $B$ cells. We unequivocally demonstrate the presence of mRNA for Gaolf in B cells. Since AC III is known to be stimulated by the calcium-calmodulin complex and that $A C \mathrm{~V}$ and $A C \mathrm{VI}$ are known to be inhibited by calcium, the expression of these adenylyl cyclases in B cells, where cellular ionized calcium concentrations are of importance in the control of insulin release, may have physiological relevance.

\section{3}

EXPRESSION OF FUNCTIONAL ADENYLYLCYCLASE-COUPLED G-PROTEINS IN MOUSE FETUS

H.H. Phan, I. Godin*, K. Regnauld, S. Emami, A. Astésano, C. Boissard and G. Rosselin. INSERM U 482, Paris, FRANCE and "Institut d'Embryologie Cellulaire et Moléculaire UPR 9064, 94736 Nogent-sur-Marne, FRANCE We have previously demonstrated by immunological SDSPAGE analysis the presence of G-protein subunit colf in different pancreatic $\beta$-cell types using antibodies directed against different sequences of this molecule. To determine the possible operational capacity of the adenylylcyclase (AC)-coupled $\mathrm{G}$ proteins signaling system in the early developing pancreas we studied the presence and activator properties of the $G_{\alpha S}$ and $G$ aolf which transduce the signal to activator properties of the Gas and Gaolf which transduce the signal to
the AC, in the fetal endocrine pancreas. The embryonic 14.5-day (E14.5) Balb $C$ mouse pancreas was chosen as a model to explore the presence of AC-coupled $G$ proteins. At this stage, as shown by immunohistochemistry, the glucagon cells were prevalent upon the insulin cells, the Gos was clearly present, and the Gaolf scarcely detectable. We prepared a subcellular fraction of ten E-14.5 pancreases $(120,000 \times \mathrm{g}$ for $60 \mathrm{~min})$. Fifteen $\mu \mathrm{g}$ of subcellular fraction proteins were ADP-ribosylated in presence of activated cholera toxin and I ${ }^{32}$ PINAD. We obtained using SDS-PAGE analysis and autoradiography a radioactive band in the range of $45 \mathrm{kDa}$, related to the functional labeling of the $A C$-coupled $G$ proteins. The presence of Gaolf was thereafter detected after $\left[{ }^{2} \mathrm{P}\right]$ decay as an unique $45-\mathrm{kDa}$ band, using the antibody K-19 (against Goolf; Gramsh) and an ECL kit (Amersham). Since only those $G$ proteins which are in the trimeric form are able to be ADP ribosylated, it can be concluded that functional $G$ proteins are present as soon as in E-14.5 mouse pancreas and could be coupled to the AC. 


\section{4}

EXPRESSION OF GQOLF AND TYPES II AND III OF ADENYLYL CYCLASES IN THE RAT PANCREAS DURING DEVELOPMENT

M. Pessah, N. Ferrand, S. Frayon, T. Demongeot, J. Marais, G. Rosselin and J.-M. Garel. INSERM U.482, Hôpital Saint-Antoine, 75571 ParisCedex 12, France.

Originally Golf was described as an heterotrimeric $G$ protein specifically expressed in the olfactory neuroepithelium and coupled to type III of adenylyl cyclase (AC). Subsequently, it appeared that other tissues may express Goolf. In our laboratory, immunocytochemical and Western-blot analyses have already revealed the presence of Goolf in the $B$ cells of pancreatic islets. Since changes in the expression of Goolf for islet cells were not reported, we have analysed mRNA and protein expressions in rat pancreases before and after birth (fetuses at 15, 18 and 21.5 days of gestation, and rats at $0,3,14,21,30$ days after birth). Olfactory bulbs were used as a tissue control for mRNA and protein expressions. By Northern hybridization, low levels of Gaolf mRNA were detected in fetuses at 21.5 days of gestation when compared with the high levels observed in olfactory bulbs. Large changes in Goolf mRNA occurred during development for olfactory bulbs with maximal values at 14 days; two majors transcripts were observed after short exposure, and two additional minor mRNA after a long exposure. In the pancreas during

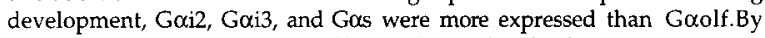
RT-PCR, the expression of Goolf was observed in fetal pancreases at 15 days of gestation. Western-blot using the K19 antibody from Santa-Cruz also detected the protein in pancreases of 21.5-day-old fetuses, and this expression was observed until weaning. mRNA for AC II and AC III were identified by RT-PCR as early as 15 days in fetal pancreases. In summary, mRNAs for Goolf were expressed early in the rat fetus, but at a low level. Since in adults Goolf is present in B islet cells, it may be possible that such expression occurred in the same cell type during development. Moreover, the presence of this $\mathrm{G}$ protein isotype is associated, at each stage studied, with the expression of AC II and AC III.

\section{6}

CELLULAR SPECIFICITY OF ADENYIYL CYCLASE COUPLED SICNALINC SYSTEM IN PANCREATIC A \& B CELLS K. Regnauld, A. Astesano, C. Cespach, C. Rosselin and S. Ennami. INSERM K. Regnauld, A. Astesano, C. Cespach, C. Rosse
U-482 St-Antoine Hospitai 75012 Paris, France.

Recent data suggest the involvement of genetic and functional alterations of several signaling pathways coupled to the AC signaling system in diabetes. The characteristic of this system was thercfore analyzed by immunocytochenistry and Western blot in adult as well as in pancreatic primordium of fetal mice at 14 days of gestation (FM14). Our data were compared: 1 ) to mouse pancreatic $A$ and $B$ celi lines $\alpha$ TC -1 and $\beta T C-3$, and 2) to the cellular distribution of various convertases $(\mathrm{PC})$ innplicated in the maturation of pancreatic hormoness. In adult mousc pancreatic sections, as expected, PC1/PC3 were detected only in B cetls. PC2 was predominantly expressed in the periphery of islets while PC5/6 expression was widely expread in all islet $c$ ells. There was a clor-cut difficence in the distribution spread in all the poristribution of signaling nolecules within islet cells, with predominant expression of 1CLP-1-R, Coolf, and Calcium-dependent ACll isoform in B cells. High
expression of PACAP-R and ACll isotorm was observed in A cells. VIP-I-R expression of PACAP-R and ACIl isotorm was observed in $A$ cells.
expression was scattered in both $A$ and $B$ cells whereas $C$ as was further distributed in isiet and acinar cells. In FM174, PACAP-R, Cox and ACII proteins were accumulated, while the mouse adult $B$ cell markers PC1/PC3, TGLP-1-R, VIP-R, Coolf and ACIII were barely detectable, indicating the predominance of a glucagon-like cell phenotype at this stage of mouse development. Due to the cellular heterogeneity of pancriatic mousc cell lines the specificity of the signaling system was not completely comparable with that of normal mouse. The prisence of ICLP-1-R at least in its immature form and of VIP-R was detected in both colls lines. AC isoforms I II III, IV V VI wire dowe we me

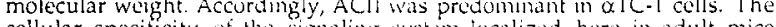
accounts for the differences observed in the cyclic-AMP-dependent stimulation of insulin or glucagon relcase by $B$ and $A$ cells. Furthermore the functional role of AC in the FM14 pancreatic endocrine cells is also suggested, since heterutrimeric $C$ proteins are functional at this point. The precise action of Coolf on insulin sicertion is currently under investigation in our laboratory, after transfection of the corresponding cDNA wild type and activated forms in BTC-3 cells and using the Goolt knockout mouse.

\section{5}

BETA CELL SPECIFICITY OF THE EFFECTS OF GLUCOSE ON ADENINE NUCLEOTIDES

P. Detimary, S. Dejonghe, Z. Ling, D. Pipeleers, F. Schuit and J.C. Henquin. Unité d'Endocrinologie et Métabolisme UCL, and Diabetes Research Center VUB, Brussels, Belgium.

The increase in the ATP/ADP ratio that glucose causes in mouse islets is compatible with a role of adenine nucleotides as second messengers in $\beta$ cells, e.g. as regulators of ATP-sensitive $\mathrm{K}^{+}$channels. The present study examined whether this effect is specific for $\beta$ cells and whether it also occurs in other species. Adenine nucleotides were measured by a luminometric method in dissociated or purified rat islet cells, after incubation at glucose concentrations between 1 and $10 \mathrm{mmol} / \mathrm{l}$. In dissociated cells, glucose produced concentration-dependent increase in ATP and decrease in ADP levels, which resulted in an increase in the ATP/ADP ratio from 4 to 12 . In purified $\beta$ cells, the ratio increased from 3 to 15. In non- $\beta$ cells, the basal ATP/ADP ratio was higher (6) and increased only to 10 . This small change might be explained by contaminant $\beta$ cells $(-10 \%)$. Thus, in purified $\alpha$ cells, the ATP/ADP ratio remained stable around 8 from 1 to $10 \mathrm{mmol} / /$ glucose. On the other hand, glucose oxidation increased in both $\beta$ cells (8-fold) and non- $\beta$ cells (4-fold). In human islet cells, $10 \mathrm{mmol} / /$ glucose almost doubled the ATP/ADP ratio. However, the true change is underestimated because the preparations contain $30-45 \%$ duct cells in which glucose did not affect adenine nucleotides. In conclusion, glucose stimulation increases the ATP/ADP ratio in $\beta$ cells and not in $\alpha$ cells. This specificity and the similarity of the changes occurring in three species reinforce the suggestion that the ATP/ADP ratio serves as a second messenger in glucose-induced insulin secretion.

\section{7}

INVOLVEMENT OF ENDOGENOUS GLUTAMATE IN THE FIRST PHASE OF GLUCOSE-INDUCED INSULIN SECRETION

G. Bertrand, M. Ravier, R. Puech and J. Bockaert. CNRS UPR9023; Montpellier. France.

We have previously shown in vitro and in vivo that exogenous glutamate stimulates insulin release via $\alpha$-amino-3-hydroxy-5methyl-isoxazole-4-propionic acid (AMPA) receptors. Here we explore a role of endogenous glutamate in the glucose-induced insulin secretion in perfused rat pancreas. The low first phase $(5 \mathrm{~min})$ of insulin response to the glucose increase from 4.2 to $8.3 \mathrm{mmol} / \mathrm{l}$ was potentiated when glutamate $(400 \mu \mathrm{mol} / \mathrm{l})$ was added simultaneously with the rise of glucose $(+290 \%, p<0.001)$. In the same experiments, after a 15 min pretreatment with glutamate, no glutamate potentiation was observed. However, a blocker of the AMPA receptor desensitization, cyclothiazide $(10 \mu \mathrm{mol} / \mathrm{l})$, ineffective per se, suppressed the inhibitory effect of the pretreatment with glutamate $(p<0.001)$, indicating a desensitization of AMPA receptors by glutamate. We used this desensitization property of glutamate and the specific antagonist of AMPA receptors (LY303070) to demonstrate that the high first phase of insulin response to the glucose increase from 4.2 to $16.7 \mathrm{mmol} / \mathrm{l}$ included an AMPA receptor component (inhibition of 31 and $28 \%$, respectively; $p<0.01$ ). This was also demonstrated by a potentiating effect of cyclothiazide on the first phase of insulin response to the glucose rise $(+45 \%, p<0.001)$ and its blockade by LY 303070 . The results show that pancreatic AMPA receptors mediate desensitizing insulin responses and suggest that endogenous glutamate could be an amplifier of the first phase of glucose-induced insulin secretion. 
INHIBITION OF PROTEIN-TYROSINE PHOSPHATASES RESTORES INSULIN RESPONSE TO GLUCOSE IN DIABETIC GK RATS

C-G. Östenson, C. Rondinone, S. Gogg, A-C. Sandberg Nordqvist, U. Smith, and S. Efendic, Dept of Molecular Medicine, Karolinska Hospital, Stockholm, and Dept of Internal Medicine, Sahlgrenska Hospital, Göteborg, Sweden The role of protein-tyrosine phosphatases (PTPs) in the B-cell stimulussecretion coupling for glucose has been studied in islets of GK rats, a genetic model of type 2 diabetes, and control Wistar (W) rats. For that purpose, a stable and potent inhibitor of PTPs, bisperoxopicolinato-oxovanadate,

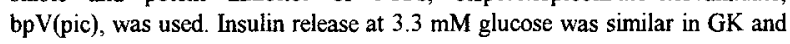
W islets, and stimulated by $16.7 \mathrm{mM}$ glucose to $7.4 \pm 0.9$ and $24.4 \pm 3.7$ $\mu \mathrm{U} /$ islet $/ \mathrm{h}$, respectively $(\mathrm{p}<0.001)$. At $3.3 \mathrm{mM}$ glucose, addition of $10^{-5}-10^{-3}$ $\mathrm{M}$ bpV(pic) augmented insulin release dose-dependently in both GK and W islets, reaching the rates of $28.4 \pm 4.2$ and $18.6 \pm 2.6 \mu \mathrm{U} /$ islet/h, respectively, at $10^{-3} \mathrm{M}$ bpV(pic). At $16.7 \mathrm{mM}$ glucose, $10^{-3} \mathrm{M}$ bpV(pic) induced a similar insulin release in GK and $W$ islets $(20.9 \pm 3.2$ and $29.2 \pm 5.4 \mu \mathrm{U} /$ islet $/ \mathrm{h}, \mathrm{n.s}$.). To study effects of PTP inhibition on B-cell exocytosis, islets were depolarized by $30 \mathrm{mM} \mathrm{KCl}$ in the presence of $0.25 \mathrm{mM}$ diazoxide to keep $\mathrm{K}_{\mathrm{ATP}}$-channels open. In depolarized islets, bpV(pic) $\left(10^{-4}-10^{-3} \mathrm{M}\right)$ enhanced insulin secretion at $3.3 \mathrm{mM}$ glucose about 4-fold in GK rat islets, but only about 2-fold in W rat islets. $\mathrm{BpV}$ (pic) did not affect glucose utilization, measured as production of ${ }^{3} \mathrm{H}_{2} \mathrm{O}$ from $5-{ }^{3} \mathrm{H}$-glucose, in $\mathrm{GK}$ or $\mathrm{W}$ rat islets. Immunoprecipitation of islet homogenates exposed to $\mathrm{bpV}$ (pic) revealed a massive increase in tyrosine phosphorylated proteins. In situ hybridization, using probes to 11 cloned rat PTPs, showed enhanced expression of the PTP NE-3 in liver and islets of the GK rat. In conclusion, impaired glucose-induced insulin release in the GK rat is restored by bpV(pic). This action is not mediated by affecting islet glucose metabolism, but is likely to involve tyrosine phosphorylation of exocytotic proteins. Increased expression of PTP NE-3 may be of pathogenetic significance in this context.
ASSESSMENT OF PANCREATIC B-CELL FUNCTION IN THE GK RAT MODEL OF NIDDM DURING THE PREDIABETIC PERIOD M.-H. GIROIX, M.-N. GANGNERAU, J. MOVASSAT, D. BAILBÉ, B. PORTHA Lab. Physiopathology of Nutrition, CNRS ESA 7059, University PARIS 7, France In the GK rat model of NIDDM, a reduced B-cell mass is considered as a primary feature in the pathological sequence leading to overt diabetes. We have now quantified the insulin secretion in vivo and in vitro during the prediabetic period which spans from birth to 5 weeks. During the 7-21 dayafter birth period, the GK neonates exhibited normal or only moderately elevated basal plasma glucose despite low basal plasma insulin. The rise of their plasma insulin in response to hyperglycemia (IPGTT; $1 \mathrm{~g}$ glucose $/ \mathrm{kg}$ body weight) was very modest ( $\Delta \mathrm{I}$ at 14 days: $1 \pm 0.01 \mathrm{ng} / \mathrm{ml} . \mathrm{min})$ at variance with that of the Wistar $(\mathrm{W})$ control neonates $(\Delta \mathrm{I}$ at 14 days: $10 \pm 2$ $\mathrm{ng} / \mathrm{ml} . \mathrm{min})$. Insulin release by the neonatal islets was studied in vitro over 90 min batch-type incubation. During the 7-21 day-period, GK rat islets released significantly less $(\mathrm{p}<0.001)$ insulin at basal $(2.8 \mathrm{mM})$ glucose concentration, when compared to age-related $\mathrm{W}$ rat islets. Contrasting with the in vivo pattern, the islets isolated from GK neonates amplify their insulin release in response to $16 \mathrm{mM}$ glucose, $10 \mathrm{mM}$ leucine associated or not to $10 \mathrm{mM}$ glutamine, or the combination of $2 \mathrm{mM} \mathrm{Ba}^{2+}$ and $1.4 \mathrm{mM}$ theophylline in the absence of extracellular $\mathrm{Ca}^{2+}$, to the same extent as the age-related $W$ neonatal islets. When tested at 35 days (1 week after weaning) GK rats exhibited severe basal hyperglycemia together with a 2-fold decreased plasma insulin/glucose ratio. However, their isolated islets were still able to amplify their insulin release in response to glucose to the same extent that $\mathrm{W}$ rat islets. The insulin response of GK rat islets to leucine was significantly decreased at this age. In conclusion, our in vivo and in vitro results suggest that in the GK rat, the lack of pancreatic B-cell reactivity to glucose as seen in vivo during the post-natal period 1) is a very early event in the prediabetes sequence, 2) is not due to an intrinsic B-cell dysfunction, 3 ) is unrelated to hyperglycemia.

\section{0}

Carbachol restores insulin release in spontaneously diabetic GK (GotoKakizaki) rat islets by a mechanism largely involving rapid hydrolysis of diacylglycerol to arachidonic acid

Amel Guenifi, Samy M. Abdel-Halim, Michael R. Jirousek* and Suad Efendić Department of Molecular Medicine, Karolinska Institute, Stockholm. Sweden. - Lilly Research Laboratories, Indianapolis, USA

The spontaneously diabetic GK (Goto-Kakizaki) rat presents a markedly impaired glucose-induced insulin release. The current study reports on insulin responses to the phospholipase-C (PLC) stimulator carbachol $(50 \mu \mathrm{M})$ in normal and diabetic GK rats. In the isolated perfused pancreas, carbachol induced exaggerated responses at $3.3 \mathrm{mM}$ glucosel [ $10668 \pm 2355$ in GK vs $2158 \pm 445 \mu \mathrm{U} / \mathrm{min}$ in controls, $p<0.02$ ] and fully normalised response at 16.7 $\mathrm{mM}$ glucose. Similarly strong insulinotropic effects of carbachol were obtained in isolated islets. Exaggerated carbachol responses in GK pancreata were also obtained under conditions in which the $\mathrm{K}_{\text {ATP }}$ channels were inactivated by diazoxide and membranes depolarized by high $\mathrm{KCl}$, indicating that signals directly coupled to exocytosis of insulin are mediating the carbacol effects in endocrine pancreas. Selective metabolic inhibitors of PLC (10 $\mu \mathrm{M}$ U73122), PKC (300 nM LY-333531 or $20 \mu \mathrm{M} L Y-315864)$ and PLA 2 (50 $\mu \mathrm{M} \mathrm{ACA})$ did not affect carbachol effects in normal or GK islets. The impact of rapid hydrolysis of DAG to arachidonic acid was assessed using RHC-80267, a specific diacylglycerol (DAG) lipase inhibitor. In normal islets, $35 \mu \mathrm{M} \mathrm{RHC}$ significantly inhibited carbachol-induced insulin release at $3.3 \mathrm{mM}(p<0.02)$ or $16.7 \mathrm{mM}$ glucose $(p<0.001)$. In $\mathrm{GK}$ islets, RHC had no effect on the already impaired gluocse-induced insulin release but significantly decreased the effects of carbachol at $3.3 \mathrm{mM}$ glucose $(26.6 \pm 3.9 \mathrm{vs} 15.8 \pm 1.5 \mu \mathrm{U} /$ isiet $p<0.02)$ or $16.7 \mathrm{mM}$ glucose $(115.3 \pm 7.2 \mathrm{vs} 33.8 \pm 5.7 \mu \mathrm{U} / \mathrm{islet}, p<0.001)$ Hence, the restorative effects of carbachol on impaired insulin release in GK islets is coupled to exocytosis and dependent on a pathway largely involving rapid hydrolysis of DAG to arachidonic acid.

\section{1}

Glucose enhances adenylyl cyclase responses in normal but not diabetic GK rat islets

Samy M. Abdel-Halim, Amel Guenifi, and Suad Efendić

Department of Molecular Medicine, Karolinska Institute, Stockholm, Sweden

In diabetic GK rat islets, forskolin restores the impaired glucose-induced insulin release at $16.7 \mathrm{mM}$ glucose through enhanced cAMP generation. The current study aims to determine the role of prevatent glucose concentration on adenylyl cyclase (AC) responses to forskolin stimulation. For that purpose, insulin release and cAMP generation in response to $5 \mu \mathrm{M}$ forskolin with 3.3 or $16.7 \mathrm{mM}$ glucose were studied in islets cultured for $48 \mathrm{hr}$ in 5.5 or $11 \mathrm{mM}$ glucose. Insulin release was impaired in GK rat islets, irrespective of culture condition, in response to $3.3 \mathrm{mM}$ and $16.7 \mathrm{mM}$ glucose and was fully restored by forskolin through exaggerated insulin responses. Forskolin elicited different CAMP responses depending on glucose concentration of the culture medium. In normal islets cultured at $11 \mathrm{mM}$ glucose, forskolin increased cAMP levels 6 -fold at $3.3 \mathrm{mM}$ glucose $[24.0 \pm 1.6$ vs $147.9 \pm 13.8$ fmol/islet, $p<0.0001]$ and 5 -fold at $16.7 \mathrm{mM}$ glucose $[18.1 \pm 0.8 \mathrm{vs} 84.9 \pm 9.8$ $p<0.0001]$. In contrast, normal islets cultured at $5.5 \mathrm{mM}$ glucose, forskolin increased cAMP levels only 2 -fold at $3.3 \mathrm{mM}$ glucose $[30.8 \pm 3.0 \mathrm{vs} 60 \pm 15$ fmol/islet] or $16.7 \mathrm{mM}$ glucose [27.3 \pm 3.1 vs $56.8 \pm 9.9$ fmol/islet, $p<0.01]$. Forskolin, however, elicited a consistently exaggerated approximately 8 -fold increase in cAMP generation in GK islets $(p<0.0001)$ irrespective of glucose concentration in culture medium. In conclusion, culturing islets at hyperglycaemic level ( $11 \mathrm{mM}$ glucose) primes and markedly enhances cAMP generation in normal islets in response to forskotin. This enhancing effect of glucose is lost in GK islets. The latter finding can be ascribed to our recent observation of $A C$-Ill enhancement due to mutational changes in $A C$ III gene promoter in the GK rat islets. 
532

EFFECTS OF GLP-1 AND MIDAGLIZOLE AT EU-AND HYPERGLYCEMIA IN A MODIFIED IV GLUCOSE TOLERANCE TEST IN ANESTHETIZED RATS A. Raap. J. Schloos, B. Steckel-Hamann and H.-J. Mest

Dept. of Pharmacology, Beiersdorf-Lilly GmbH, 20253 Hamburg, Germany

We compared glucagon-like peptide 1 (GLP-1) and the imidazoline midaglizole on their effects on insulin and glucose at eu - and hyperglycemia in anesthetized rats. The test agents were administered to fasted male wistar rats $(300-350 \mathrm{~g})$ using an iv loading dose followed by a 30 min infusion. An iv glucose challenge followed $15 \mathrm{~min}$ after infusion start. This allows to examine the effects of the compounds under both basal and elevated blood glucose levels. The whole experiment was performed under pentobarbitone anesthesia. Blood samples were drawn via the carotid artery before, during the infusion at $5,10,15,20,25$ and $30 \mathrm{~min}$ as well as after infusion at 35,45 and $55 \mathrm{~min}$ to determine plasma insulin and plasma glucose. Statistically significant increases of insulin prior to iv glucose were observed with both compounds at doses of 1.3 and $11.3 \mu \mathrm{g} / \mathrm{kg}$ GLP-1 and $5.6-28.9 \mathrm{mg} / \mathrm{kg}$ midaglizole, respectively. However, GLP-1 exhibited a transient increase of insulin which peaked at 10 min and decreased again at 15 min shortly before iv glucose as demonstrated previousiy by other groups.

Tab.: Dose dependent effects on insulin and glucose after an iv glucose challenge

\begin{tabular}{|c|c|c|c|c|c|c|c|c|c|}
\hline \multicolumn{4}{|c|}{ Glucagon-like Peptide 1} & \multicolumn{6}{|c|}{ Midaglizole } \\
\hline$\mu \mathrm{g} / \mathrm{kg}$ & Insulin* & $\Delta \%$ & Glucose $\mathrm{e}^{* *}$ & $\Delta \%$ & $\mathrm{mg} / \mathrm{kg}$ & Insulin** & $\Delta \%$ & Glucose** & $\Delta \%$ \\
\hline 0.4 & $49.2 \pm 10.3$ & +38 & not tested & & 1.2 & $77.6 \pm 14.6$ & +4 & $3401 \pm 276$ & +5 \\
\hline Veh. & $35.7 \pm 2.8$ & & & & Veh. & $74.4 \pm 14$ & & $3250 \pm 364$ & \\
\hline 0.8 & $41.7 \pm 5.6 \mathrm{a}$ & +60 & $2883 \pm 388$ & -8 & 5.6 & $143 \pm 19 a$ & +66 & $3410 \pm 300$ & -13 \\
\hline Veh. & $26.0 \pm 3.7$ & & $3130 \pm 198$ & & Veh & $86 \pm 8.2$ & & $3936 \pm 425$ & \\
\hline 1.3 & $69.6 \pm 7.0 \mathrm{a}$ & +116 & $1242 \pm 219 a$ & -60 & 10.6 & $86.7 \pm 11.2 \mathrm{a}$ & +69 & $2563 \pm 233 a$ & -18 \\
\hline Veh. & $32.2 \pm 4.3$ & & $3126 \pm 351$ & & Veh & $51.2 \pm 7.4$ & & $3118 \pm 287$ & \\
\hline 11.3 & $76.7 \pm 12.1$ a & +136 & $1572 \pm 326 a$ & -52 & 28.9 & $104 \pm 13.8 \mathrm{a}$ & +89 & $1719 \pm 205 a$ & -34 \\
\hline Veh. & $32.5 \pm 5.0$ & & $3307 \pm 341$ & & Veh & $55.3 \pm 6.3$ & & $2607 \pm 305$ & \\
\hline
\end{tabular}

"AUC from peak post iv glucose up to the end of drug infusion ( $30 \mathrm{~min}$ ), doses ( $30 \mathrm{~min}$ infus

In conclusion, in this modified iv glucose tolerance test the effects of GLP-1 and midaglizole can be differentiated at basal as well as elevated glucose.

\section{3}

PKC $\alpha$ AND $\delta$ MEDIATE PHORBOL ESTER BUT NOT GLUCOSE STIMULATED INSULIN SECRETION FROM RAT PANCREATIC ISLETS L. Carpenter, E. Kerr, and T.J. Biden. Garvan Institute ofMedical Research, 384 Victoria St, Darlinghurst, Sydney, Australia 2010.

Protein Kinase C (PKC) has been suggested to play a crucial role in insulin secretion from islets in response to various stimuli but its involvement in glucose stimulated secretion remains controversial. The aim of this project was to determine the specific role of PKC isozymes $\alpha$ and $\delta$ in glucose and phorbol ester stimulated insulin secretion from rat pancreatic islets using adenovirus technology. PKC $\alpha$ and $\delta$ wild type (WT) and kinase dead (KD) mutants were prepared using standard molecular biological techniques and recombined with human adenovirus (Ad5) to generate recombinant Ad5 PKC constructs. Adenovirus was shown to give infection efficiencies exceeding $50 \%$ in whole islets, as determined by immuno-histochemistry and FACS analysis of over-expressed PKC $\delta$. Insulin secretion was measured 3 days post-infection in response to $2.8 \mathrm{mM}$ or $16.8 \mathrm{mM}$ glucose or $500 \mathrm{nM} 12-\mathrm{O}$ Tetradecanoylphorbol 13-acetate (TPA). Insulin secretion (measured by radio-immuno assay) from islets infected with control adenovirus ( $\mathrm{Ad} 5 \mathrm{mx} 17$ ) was shown to be stimulated in response to glucose and TPA and comparable to the uninfected control islets. Whereas islets infected with Ad5 PKC $\alpha$ and $\delta$ WT constructs were shown to have a glucose response similar to the $\mathrm{m} \times 17$ control, the TPA response which was significantly elevated (see Table below). Conversely the Ad5 PKC $\alpha$ and $\delta$ KD mutants did not significantly decrease glucose stimulated secretion, but inhibited the TPA response by 46 and $22 \%$ respectively. These results show that activation of PKC $\alpha$ and $\delta$ are necessary for phorbol ester, but not glucose-stimulated insulin secretion. Insulin secretion $\mathrm{ng} / \mathrm{ml}^{-1} /$ islet $^{-1}$

\begin{tabular}{cccc} 
Ad5 construct & Glucose 2.8mM & Glucose 16.8mM & $500 \mathrm{nM}$ TPA \\
\hline mx 17 & $0.33 \pm 0.04$ & $0.96 \pm 0.06$ & $1.01 \pm 0.06$ \\
PKC $\alpha W T$ & $0.53 \pm 0.07^{*}$ & $1.1 \pm 0.08$ & $1.47 \pm 0.09^{*}$ \\
PKC $\alpha \mathrm{KD}$ & $0.31 \pm 0.04$ & $0.82 \pm 0.06$ & $0.57 \pm 0.05^{*}$ \\
PKC $\delta W T$ & $0.48 \pm 0.08$ & $0.91 \pm 0.11$ & $0.97 \pm 0.10$ \\
PKC $\delta \mathrm{KD}$ & $0.49 \pm 0.07$ & $1.17 \pm 0.08$ & $0.79 \pm 0.06^{*}$ \\
\hline
\end{tabular}

$*=\mathrm{P}<0.05$ vs $\mathrm{m} \times 17$ control. $\mathrm{n}=10-18$

\section{4}

PHOSPHOLIPASE A $\mathrm{A}_{2}$-MEDIATEd ISLET ACTIONS OF CHOLECYSTOKININ ARE CALCIUMINDEPENDENT AND INTERACT WITH PROTEIN KinASE A AND Digl YCERIDE LIPASE E. Simonsson, S. Karlsson and B. Ahrén. Dept. Med., Malmö, Lund Univ., Sweden

Cholecystokinin (CCK) induces insulin secretion through activation of phospholipase $\mathrm{C}$ (PLC) and, as we recently have shown, phospholipase $\mathrm{A}_{2}$ (PLA $\mathrm{P}_{2}$. In this study we characterised CCK-induced PLA, activation in rat islets with regard to $\mathrm{Ca}^{2+}$-independency and interplay with protein kinase $A(P K A)$ and diglyceride lipase. We found that CCK (CCK-8; $100 \mathrm{nM} ; 5.6 \mathrm{mM}$ glucose) induces lysophosphatidylcholine accumulation from $\left[{ }^{3} \mathrm{H}\right]$ palmitate-prelabelled islets (by $170 \pm 39 \% ; \mathrm{p}=0.003$ ), and arachidonic acid (AA) efflux from $\left[{ }^{3} \mathrm{H}\right] \mathrm{AA}$-prelabelled islets $(190 \pm 13 \% ; \mathrm{p}<0.001)$ which both are methods reflecting PLA $\mathrm{A}_{2}$ activity, and that PLA, inhibition by pamylcinnamoylantranilic acid (ACA; $50 \mu \mathrm{M})$ reduces CCK-induced AA efflux $(52 \pm 11 \% ; p=0.001)$ and insulin secretion $(67 \pm 16 \% ; p<0.001)$. Also after removal of extracellular $\mathrm{Ca}^{2+}, \mathrm{CCK}$ increased $\mathrm{AA}$ efflux $(48 \pm 14 \% ; \mathrm{p}=0.006)$ and insulin secretion $(105 \pm 46 \% ; \mathrm{p}=0.025)$. In contrast, carbachol $(100 \mu \mathrm{M})$-stimulated AA efflux and insulin secretion were absent when $\mathrm{Ca}^{2+}$ was omitted. Accordingly, in a $\mathrm{Ca}^{2+}$-deficient environment, ACA reduced CCK-induced AA efflux $(55 \pm 20 \% ; \mathrm{p}=0.020)$ to the same level as in the presence of $\mathrm{Ca}^{2+}$. Since other islet forms of $\mathrm{PLA}_{2}$ require $\mathrm{Ca}^{2+}$ for their activation, the action of $A C A$ under these conditions reflects inhibition of $\mathrm{Ca}^{2+}$.

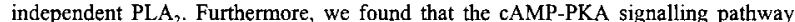
interacts with $\mathrm{CCK}$-induced $\mathrm{PLA}_{2}$ stimulation, since adenylate cyclase activation by forskolin $(1 \mu \mathrm{M})$ reduced CCK-mediated AA efflux $(41 \pm 19 \% ; \mathrm{p}=0.030)$. Moreover we found that the diglyceride lipase pathway contributes to $\mathrm{CCK}$-stimulated $\mathrm{AA}$ formation, since diglyceride lipase inhibition by RHC 80267 (1,6-biscyclohexylideneaminooxycarbonylamino-hexane; $35 \mu \mathrm{M})$ diminished $\mathrm{CCK}$-induced AA efflux to the same extent as ACA, both in the presence $(51 \pm 17 \% ; \mathrm{p}=0.014)$ and in the absence of $\mathrm{Ca}^{2+}(58 \pm 25 \% ; \mathrm{p}=0.041)$. When combining $\mathrm{RHC} 80267$ with ACA, the effects of CCK were almost abolished $(90 \pm 18 \% ; p=0.001$, and $90 \pm 21 \% ; p=0.002$, respectively). In conclusion, this study shows that $\mathrm{CCK}$ activates $\mathrm{Ca}^{2+}$-independent PLA $\mathrm{A}_{2}$ in islets, and that the CCK-mediated PLA $A_{2}$ action interacts with PKA and diglyceride lipase.

\section{5}

INOSITOL HEXAKISPHOSPHATE STIMULATES CALCINEURIN-MEDIATED ENDOCYTOSIS AND PKA-DEPENDENT EXOCYTOSIS IN MOUSE B-CELLS

M. Hoy and J. Gromada. Dept. Islet Cell Physiology, Novo Nordisk A/S, Symbion, Fruebjergvej 3, DK-2100 Copenhagen, Denmark.

Inositol polyphosphates are important for the control of fundamental cellular processes. Here we have explored the effects of inositol hexakisphosphate $\left(\mathrm{InsP}_{6}\right)$ on $\mathrm{Ca}^{2+}$-induced endocytosis and exocytosis in single mouse pancreatic Bcells using the standard whole-cell configuration of the patch-clamp technique and high-resolution measurements of cell capacitance. The rate of increases in cell capacitance, elicited by intracellular dialysis with a $\mathrm{Ca}^{2+}$-EGTA buffer with a free intracellular $\mathrm{Ca}^{2+}$ concentration ([ $\left.\mathrm{Ca}^{2+}\right]_{i}$ ) of $0.8 \mu \mathrm{M}$, amounted to $20 \pm 4 \mathrm{fF} / \mathrm{s}$. Inclusion of $\operatorname{Ins}_{6}(100 \mu \mathrm{M})$ in the pipette solution inhibited the increase in cell capacitance by $60 \%$. This effect of Ins $P_{6}$ was concentration-dependent $\left(\mathrm{K}_{i} 13 \mu \mathrm{M}\right)$ and mimicked by inositol tetra- and penta-kisphosphates but not inositol trisphosphates. In experiments where increases in cell capacitance were elicited by $500 \mathrm{~ms}$ voltage-clamp depolarisations from -70 to $0 \mathrm{mV}$, Ins $\mathrm{P}_{6}$ inhibited exocytosis to the same extent without affecting the whole-cell $\mathrm{Ca}^{2+}$-current. The inhibitory action of $\operatorname{InsP}_{6}$ is likely to reflect the simultaneous operation of two opposite directed processes. In the presence of low and non-exocytotic $\left[\mathrm{Ca}^{2+}\right]_{i}(30$ $\mathrm{nM}$ ), InsP $\mathrm{P}_{6}$ stimulated endocytosis via activation of the protein phosphatase calcineurin (PP2B). Following inhibition of endocytosis by cyclosporin $\mathrm{A}$ or deltamethrin, Ins $\mathrm{P}_{6}$ potentiated $\left[\mathrm{Ca}^{2}\right]_{\mathrm{i}}(0.8 \mu \mathrm{M})$-evoked exocytosis 2-fold This stimulatory action of $\operatorname{Ins}_{6}$ was antagonised by the protein kinase $A$ (PKA) inhibitor Rp-cAMPS but was not affected by protein kinase $C$ inhibition with staurosporine Inhibition of $\mathrm{PP} 1, \mathrm{PP} 2 \mathrm{~A}$ and $\mathrm{PP} 3$ protein phosphateses by okadaic acid was without effect on InsP $\mathrm{P}_{6}$-induced inhibition of exocytosis. These data suggest that Ins $\mathrm{P}_{6}$ exerts a dual action on insulin secretion by simultaneous stimulation of calcineurin-dependent endocytosis and PKA-mediated exocytosis. 


\section{6}

P38 MITOGEN-ACTIVATED PROTEIN KINASE IS NOT REQUIRED FOR INSULIN SECRETION FROM ISLETS OF LANGERHANS.

C.J. Burns, S.L. Howell, P.M. Jones and S.J. Persaud. Cellular and Molecular Endocrinology, King's College London, Campden Hill Road, London, U.K.

The activation of members of the mitogen-activated protein kinase (MAPK) family of enzymes is known to be important in the transduction of extracellular signals into cellular responses. Here we have investigated the possible role of the p38 isoform of MAPK in the regulation of insulin secretion. Western blotting studies using a specific antibody demonstrated that pancreatic $\beta$-cell lines (MIN6, RINm5F $\beta T C 3$ and $\beta T C 6$ ) and isolated rat islets of Langerhans expressed P38 MAPK. Enzyme activity was assessed by $\left[{ }^{32} \mathrm{P}\right]$ incorporation into a substrate peptide of mitogen-activated protein kinase-activated protein kinase 2 (a downstream target of p38 MAPK). p38 MAPK was activated in islets by glucose $(20 \mathrm{mM}$ glucose: $150 \pm 11 \%$ basal activity, $n=4, P<0.01$ ) and in islets and MIN6 $\beta$-cells by cellular stress and by phosphatase inhibitors $[1 \mathrm{mM}$ arsenite: $149 \pm 12 \%$ basal activity (MIN6), $190 \pm 2 \%$ (islets); $0.5 \mathrm{M}$ sorbitol: $142 \pm 6 \%$ (MIN6), $254 \pm 1 \%$ (islets); $100 \mu \mathrm{M}$ pervanadate: $212 \pm 16 \%$ (MIN6), $268 \pm 6 \%$ (islets); $2 \mu \mathrm{M}$ okadaic acid: $131 \pm 5 \%$ (MIN6), $n=4, P<0.005$ in all treatments]. Neither cellular stress nor phosphatase inhibitors significantly stimulated insulin secretion from islets $(P>0.5, n=7)$. In addition, pre-exposure of islets to a range of concentrations $(2.5-80 \mu \mathrm{M})$ of SB203580 (SB), an inhibitor of p38 MAPK, had no significant effect on glucose(G)-induced insulin secretion

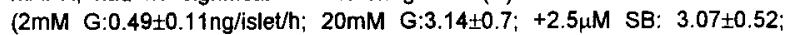
$+80 \mu \mathrm{M}$ SB: $2.82 \pm 0.52 ; n=8-9, P>0.5$ by ANOVA), nor did $\mathrm{SB}(40 \mu \mathrm{M})$ have a significant effect on PMA- or forskolin-potentiated insulin secretion from islets (20mM G: $2.76 \pm 0.21 \mathrm{ng} / \mathrm{islet} / \mathrm{h} ;+500 \mathrm{nM}$ PMA: $7.29 \pm 0.92 ;+500 \mathrm{nM}$ PMA+SB: $7.09 \pm 0.68, \quad P>0.8 ;+10 \mu \mathrm{M}$ forskolin: $6 \pm 0.47 ;+10 \mu \mathrm{M}$ forskolin $+S B: 6.1 \pm 0.55, P>0.8, n=7-8$ ). These results suggest that $\beta$-cells express p38 MAPK but pharmacological activation of this kinase is not sufficient to stimulate insulin secretion and signalling through this pathway is not essential for insulin secretion in response to glucose, nor to activators of protein kinases $A$ and $C$.

\section{7}

L-ARGININE STIMULATION OF GLUCOSE-INDUCED INSULIN SECRETTON THROUGH MEMBRANE DEPOLARIZATION AND INDEPENDENT OF NITRIC OXIDE.

P. Thams and K. Capito. Department of Medical Biochemistry \& Genetics, Biochemistry Laboratory A, University of Copenhagen, Denmark.

The mechanism of L-arginine stimulation of glucose-induced insulin secretion from mouse pancreatic islets was studied. At $16.7 \mathrm{mmol} / \mathrm{L}$ glucose, $\mathrm{L}$-arginine $(10$ $\mathrm{mmol} / \mathrm{L}$ ) potentiated both phase 1 and 2 of glucose-induced insulin secretion. This potentiation of glucose-induced insulin secretion was mimicked by the membrane depolarizing agent tetraethylammonium (TEA, $20 \mathrm{mmol} / \mathrm{L}$ ), which at $16.7 \mathrm{mmol} / \mathrm{L}$ glucose obliterated L-arginine $(10 \mathrm{mmol} / \mathrm{L})$ modulation of insulin secretion. Thus during phase 2 , L-arginine $(10 \mathrm{mmol} / \mathrm{L})$ stimulated glucose $(16.7 \mathrm{mmol} / \mathrm{L})$-induced insulin secretion from $3.44 \pm 0.98(5)$ to $7.00 \pm 1.32(5) \mathrm{ng} / \mathrm{min} / 100$ islets $(\mathrm{P}<0.001)$ in the absence and from $6.61 \pm 1.10(5)$ to $7.51 \pm 1.14(5) \mathrm{ng} / \mathrm{min} / 100$ islets $(P>0.05)$ in the presence of TEA $(20 \mathrm{mmol} / \mathrm{L})$. At $3.3 \mathrm{mmol} / \mathrm{L}$ glucose, $\mathrm{L}$-arginine $(10 \mathrm{mmol} / \mathrm{L})$ failed to stimulate insulin secretion. In accordance with membrane depolarization by the intracellular accumulation of $\mathrm{L}$-arginine, however, $\mathrm{L}$-arginine $\left(10 \mathrm{mmol} / \mathrm{L}\right.$ ) stimulation of insulin secretion was induced by the $\mathrm{K}^{+}$channel inhibitor TEA ( $20 \mathrm{mmol} / \mathrm{L}$ ), which may potentiate membrane depolarization by $\mathrm{L}$ arginine. Furthermore, $\mathrm{L}$-arginine $(10 \mathrm{mmol} / \mathrm{L})$ stimulation of insulin secretion was induced by forskolin $(10 \mu \mathrm{mol} / \mathrm{L})$ or tetradecanoylphorbol 13-acetate $(0.16$ $\mu \mathrm{mol} / \mathrm{L}$ ), which by activation of protein kinase $\mathrm{A}$ and $\mathrm{C}$, respectively may sensitize the exocytotic machinery to $\mathrm{L}$-arginine-induced $\mathrm{Ca}^{2+}$ influx. $\mathrm{L}$-arginine stimulation of glucose-induced insulin secretion was mimicked by the constitutive nitric oxide synthase inhibitor $\mathrm{N}^{\mathrm{G}}$-nitro-L-arginine methyl ester $(10 \mathrm{mmol} / \mathrm{L})$, altogether suggesting that L-arginine-derived nitric oxide neither inhibits nor stimulates insulin secretion in L-arginine-induced insulin secretion. In conclusion, it is suggested that L-arginine potentiation of glucose-induced insulin secretion occurs independent of nitric oxide, but is mediated by membrane depolarization, which stimulates insulin secretion through protein kinase $\mathrm{A}$ and $\mathrm{C}$-sensitive mechanisms.

\section{PS 12 \\ Ion Channels and Exocytosis}

\section{8}

SUR1A2 AND SUR1B, TWO NOVEL FORMS OF THE RAT SULFONYLUREA RECEPTOR.

P. Blache, K. Peyrollier, L. Gros and D. Bataille.Institut National de la Santé et de la Recherche Médicale INSERM U376, CHU Arnaud-de-villeneuve 34295 Montpellier Cedex, France.

ATP-sensitive potassium $\left(K_{\text {ATP }}\right)$ channels play an important role in various cellular responses by linking the cell metabolic activity to its membrane potential and are an important step in controlling insulin secretion or excitability of various muscle ceils. The $K_{\text {arp }}$ channel of $\beta$-cells is constituted of the sulfonylurea-binding protein, SUR1, and the inwardly rectifying $\mathrm{K}^{+}$ channel subunit Kir6.2. Several allelic variants of the human SUR1 have been described and some of them were found to be associated with noninsulin-dependent diabetes mellitus. By RT-PCR analysis of CDNAs to SUR1 gene transcripts from RINm5F insulin-secreting cell line, two cDNAs were obtained which differed by their length. The two novel isoforms, which differ by their size, display significant differences in both the nucleotide and the aminoacid sequences with the originally cloned rat SUR1, all within the nucleotide-binding fold 1 domain. The short isoform shows a 114-nucleotide (38-aminoacids) deletion in a region which codes for two potential transmembrane-spanning helices, leading to a protein with a different membrane topology. These fidings lead us to propose a new terminology for rat SUR molecules: we suggest to call "SUR1A1" the original SUR1 gene transcript, "SUR1A2" the novel isoform and the shortest form SUR1B. RTPCR was used to determine tissue distribution of both SUR1A1 and SUR1A2 and SUR1B MRNAs, respectively. SUR1A1 and SUR1A2 were expressed in pancreas, hypothalamus, medulla oblongata, heart, adrenal and spleen, whereas SUR1B was ubiquitously distributed. In addition, SUR1A1, SUR1A2 and SUR1B are expressed in the rat insulin-secreting cell line RINm5F, in the mouse glucose-responsive insulin-secreting cell line MIN 6 and in the rat somatostatin-secreting cell line RIN T3.

\section{9}

\author{
HUMAN $\alpha$ ENDOSULFINE, A POSSIBLE REGULATOR OF \\ SULPHONYLUREA-SENSITIVE $K_{\text {ATP }}$ CHANNEL \\ L. Héron*, A. Virsolvy* , K. Peyrollier*, F. M. Gribblet, A. Le Cam*, F \\ M. Aschcroftt and D. Bataille*. ${ }^{*}$ INSERM U376, Montpellier, France. \\ tUniversity Laboratory of Physiology, Oxford, United Kingdom.
}

Sulphonylureas, commonly used in the management of non-insulindependent diabetes mellitus (NIDDM), exert their therapeutic action primarily by inhibiting ATP-sensitive potassium (K $\mathrm{K}_{\mathrm{ATP}}$ ) channels in the plasma membrane of pancreatic B-cells and thereby stimulating insulin release. A key question concerns the existence of an endogenous regulator for the KATP channel which is able to mimic the effects of sulphonylureas. We describe here the biological properties of human $\alpha$ endosulfine, a $13 \mathrm{kDa}$ peptide which is a putative candidate for such a role. $\alpha$ endosulfine is expressed in a wide range of tissues including muscle, brain and endocrine tissues. After molecular cloning of the cDNA encoding human $\alpha$ endosulfine, we carried out the expression of the human recombinant protein to determine its biological features. The recombinant $\alpha$ endosulfine displaces binding of the sulphonylurea ${ }^{3} \mathrm{H}$-glibenclamide to $B$-cell membranes in a dosedependent manner with an ED50 of $1 \mu \mathrm{M}$. $\alpha$ endosulfine inhibits cloned $\mathrm{K}_{\mathrm{ATP}}$ channels and stimulates insulin secretion from $B$-cells in culture, in the same range of concentration. We propose that $a$ endosulfine may act as an endogenous regulator of B-cell $K_{A T P}$ channel, which has a key role in the control of insulin release and, more generally, which couples cell metabolism to electrical activity. Finally, the availability of the human $\alpha$ endosulfine cDNA sequence will enable to evaluate the role of this peptide in some pathological states, such as non-insulin-dependent diabetes. 
540

Activation of a $\mathrm{Ca}^{2+}$-activated $\mathrm{K}^{+}$-conductance terminates the burst of action potentials in insulin-secreting pancreatic $\mathrm{B}$-cells

Sven Göpel*, Patrik Rorsman,, Department of Physiology and Neuroscience, Lund University, Sölvegatan 19, S-223 62 Lund (*presenting author)

The pancreatic B-cell generates a characteristic pattern of electrical activity when exposed to glucose and other stimulators of insulin secretion. This electrical activity consists of oscillations in membrane potential between depolarized plateaus (lasting 5-10 s) on which $\mathrm{Ca}^{2+}$-dependent action potentials are superimposed, and repolarized electrically silent intervals. The underlying mechanism(s) remain(s) unestablished. Dispersed islet cells maintained in tissue culture exhibit an atypical electrical activity thus precluding the experimental investigation of the processes involved. We have applied patch-clamp recordings to freshly isolated intact pancreatic islets. A large fraction $(30-40 \%)$ of the superficial cells generated the characteristic pattern of B-cell electrical activity in the presence of glucose. Once the identity of the cells had thus been established, the glucose concentration was lowered to $5 \mathrm{mM}$ to suppress spontaneous electrical activity, the amplifier switched into the voltage-clamp mode and trains of voltage-clamp pulses applied to simulate electrical activity. During the train, an outward current developed reaching an amplitude of typically $20-30 \mathrm{pA}$ (measured at $-40 \mathrm{mV}$ ). Upon cessation of stimulation, the current gradually decayed towards the baseline. The time required for complete deactivation varied between 5 and $15 \mathrm{~s}$ in different cells. The current was carried by $\mathrm{K}^{+}$and the reversal potential changed $\approx 40 \mathrm{mV}$ for a 4.2 -fold increase in the external $\mathrm{K}^{+}$-concentration. The $\mathrm{K}^{+}$ channel blockers apamin $(1 \mu \mathrm{M})$, charybdotoxin $(50 \mathrm{nM})$ and tolbutanide $(0.1 \mathrm{mM})$ were all ineffective but the current was inhibited by nifedipine $(10 \mu \mathrm{M})$. Thapsigargin ( $1 \mu \mathrm{M}$; an inhibitor of the intracellular $\mathrm{Ca}^{2+}$-ATPase) reduced the amplitude and slowed the deactivation of the current. The latter effects were associated with the appearance of uninterrupted firing of action potentials. We propose that the termination of the burst of action potentials in the B-cell results from the activation of $\mathrm{Ca}^{2+}$-activated $\mathrm{K}^{+}$-channels. The current depends on influx of external $\mathrm{Ca}^{2+}$ which feeds forward into mobilization of $\mathrm{Ca}^{2+}$ from intracellular stores culminating in the activation of $\mathrm{K}^{+}$-channels. The latter finding combined with the "negative pharmacology" (lack of effects of the $\mathrm{K}^{+}$-channel inhibitors) raise the possibility that the current is identical to that the $\mathrm{IP}_{3}$-dependent low-conductance $(<1 \mathrm{pS}) \mathrm{Ca}^{2+}$. activated $\mathrm{K}^{+}$-current we have previously described in dispersed cultured $\mathrm{B}$-cells.

\section{1}

EFFECT OF ANTIDIABETIC AGENT, NATEGLINIDE, ON $K_{\text {ATP }}$ CHANNEL IN $\beta$-CELLS: COMPARISON TO GLYBURIDE AND REPAGLINIDE.

S. Hu and S. Wang; Research Department, Novartis Pharmaceuticals Corporation, Summit, NJ 07901, USA

Nateglinide (NAT, also known as A-4166) is an insulinotrophic agent which is chemically and pharmacologically distinct from glyburide (GLY) and repaglinide (REP). Although each agent is known to stimulate insulin release via the signaling cascade initiated by closure of $K_{\text {ATP }}$ channels, the pharmacological actions of NAT are more rapid in onset, shorter in duration, and more sensitive to ambient glucose levels. The aim of the present study was to elucidate the mechanisms underlying the different mode of insulinstimulating actions of these agents through profiling the $K_{\text {ATP }}$ channelblocking activities of NAT, GLY and REP in rat pancreatic $\beta$-cells using the patch-clamp technique in whole-cell configuration. Approximately $85 \%$ of the cells examined were responsive to glucose: the amplitude of the wholecell $K_{A T \rho}$ current decreased e-fold with every $3.7 \mathrm{mM}$ increase of extracellular glucose. NAT, GLY and REP each produced a concentrationdependent inhibition of diazoxide-induced activation of $K_{\text {ATP }}$ current in the presence of $5 \mathrm{mM}$ glucose, with $I_{50} S$ of $7.4 \mu \mathrm{M}, 16.6 \mathrm{nM}$ and $5.0 \mathrm{nM}$, respectively. At elevated glucose $(16 \mathrm{mM})$ the $I_{50}$ of NAT was reduced by 3-fold (to $2.5 \mu \mathrm{M}, \mathrm{p}=0.05$ ) whereas the $\mathrm{IC}_{50} \mathrm{~s}$ of $\mathrm{GLY}(18.9 \mathrm{nM})$ and REP $(7.4$ $n M)$ were slightly increased (NS). Thus, glucose sensitized the $K_{A T P}$ channels in $\beta$-cells to NAT while the effects of GLY and REP were glucoseinsensitive. The time courses of the effects of the agents on $K_{\text {ATp }}$ current were also compared. When tested at $2 x$ the $I C_{50}$ the time to half-maximal inhibition was similar for NAT and GLY $(4.1 \pm 0.3$ and $4.2 \pm 0.5 \mathrm{~min}$ respectively) but longer for REP $(12.2 \pm 2.4 \mathrm{~min})$. The recovery from maximal inhibition was considerably more rapid following removal of NAT $\left(t_{12}=34.8 \pm 3.8 \mathrm{~min}\right)$ than of GLY and REP $(68.1 \pm 4.0$ and $175.0 \pm 1.0 \mathrm{~min}$, respectively, $p<0.001$ ). These findings provide a mechanistic explanation for observations that nateglinide (but not the other agents) sensitizes $\beta$-cells to glucose and has a much shorter duration of action. The more rapid onse of insulin-stimulation observed with NAT than GLY in vivo likely reflects pharmacokinetic differences.

\section{2}

Stevioside stimulates insulin secretion and upregulates insulin II mRNA in pancreatic $\beta$-cells independent of CAMP and $K_{\text {ATP }}$-channel activity. P. B. Jeppesen, S. Gregersen, C. R. Poulsen ${ }^{1}$ and K. Hermansen, Dept. of Endocrinology and Metabolism, Aarhus University Hospital, Aarhus and 'Islet Cell Physiology, Novo Nordisk A/S, Copenhagen, Denmark

The diterpene glucoside stevioside has, as a constituent of the plant Stevia Rebaudiana Bertoni, been used for many years in the treatment of non-insulin dependent diabetes in South America. Recently, we showed that stevioside is a potent, glucose-dependent insulinotropic substance in vitro. The underlying mechanisms of action are, however presently unknown. The aims of the study were to explore whether stevioside acts directly on the $\beta$-cell to release insulin and if so - to study changes in the insulin II $m R N A$ levels. In addition, the effect of stevioside on ATP-sensitive $\mathrm{K}^{+}$-channel activity and cytoplasmic cAMP levels were examined. We found that stevioside potentiated glucose-(16.7 mM)-stimulated insulin release during $60 \mathrm{~min}$ incubations of INS- 1 cells at all concentrations studied $\left(10^{-3}\right.$ to $\left.10^{-9} \mathrm{M} ; \mathrm{p}<0.05\right)$. This was associated with a approximately $20 \%$ increase in the insulin II mRNA content of the mouse islets after $24 \mathrm{~h}$ of stimulation with $16.7 \mathrm{mM}$ glucose in the presence of $1 \mathrm{mM}$ stevioside $(p<0.05)$. In addition to the acute action on $\beta$-cell, stevioside also has a more long-term impact on the mouse islet. It is well known that closure of $\mathbf{K}_{\mathrm{ATP}}$-channels plays an important role for $\beta$-cell depolarization and stimulation of $\mathrm{Ca}^{2+}$-dependent insulin secretion. To examine the possibility that stevioside induces insulin secretion by closure of $K_{\text {ATP- }}$ channels we studied changes in whole-cell $\mathrm{K}_{\mathrm{ATP}}$-currents in normal mouse $\beta$-cells using the standard whole-cell patch-clamp technique. At $5 \mathrm{mM}$ glucose the $\mathrm{K}_{\mathrm{ATP}}$-channel activity was unaltered by $1 \mathrm{mM}$ stevioside. Cyclic AMP is an important second messager in the $\beta$-cell. Stevioside $(0,1 \mathrm{mM})$ did not affect cAMP levels in freshly isolated mouse islet, exposed for $20 \mathrm{~min}$ to this glucoside, whereas ferskolin produced a $400 \%$ stimulation. In conclusion, stevioside has the capability to acutely stimulate insulin secretion from the $\beta$-cells. It also has actions that could reflect a stimulatory longterm effect on insulin secretion. Notably, and in contrast to the well known sulfonylureas, stevioside does not interact with the $\mathrm{K}_{\mathrm{ATP}}$-channels. Stevioside, or analogues here of, may prove a role as a potential new drug for use in non-insulin dependent diabetes mellitus.

\section{3}

A4166, BUT NOT REPAGLINIDE, STIMULATE CA ${ }^{2+}$-EVOKED, $\mathrm{K}_{\mathrm{ATP}}$-CHANNEL INDEPENDENT, SECRETION IN RAT PANCREATIC $\alpha$-AND $\beta$-CELLS

K. Bokvist, M. Høy, C. R. Poulsen, K. Buschard* and J. Gromada. Islet Cell Physiology, Novo Nordisk $\mathrm{A} / \mathrm{S}$ and ${ }^{*}$ Bartholin Institutet, Kommunehospitalet, Copenhagen, Denmark.

The aim of this study was to investigate the cellular mechanisms targeted by the novel compounds repaglinide and A4166 in the stimulussecretion coupling of pancreatic $\alpha$ - and $\beta$-cells. We have used the patchclamp technique to record membrane potentials, ionic currents and exocytosis (measured as changes in membrane capacitance) from single rat $\alpha$ - and $\beta$-cells. The $\alpha$-cell generates action potentials at low glucose levels, a condition associated with glucagon release. This electrical activity is inhibited by high glucose concentrations which is the opposite to what is observed in the $\beta$-cell. Both cell types contained ATPregulated $\mathrm{K}^{+}$channels $\left(\mathrm{K}_{\mathrm{ATP}}\right)$ sensitive to the ATP/ADP ratio. Repaglinide and $\mathrm{A} 4166$ blocked these $\mathrm{K}_{\mathrm{ATP}}$ channels with $\mathrm{IC}_{50}$-values of $22 \pm 14 \mathrm{nM}$ and $410 \pm 220 \mathrm{nM}$ in the $\alpha$-cell and $8 \pm 2 \mathrm{nM}$ and $200 \pm 70 \mathrm{nM}$ in the $\beta$-cell. Both compounds were able to initiate electrical activity in $\alpha$-or $\beta$-cells under otherwise non-stimulatory conditions. Exocytosis was measured in cells infused with the test substance and a fixed (low) cytosolic calcium level $(170 \mathrm{nM})$ in order to study the direct effects of these compounds on the exocytotic machinery. A4166 and the well known sulphonylurea, tolbutamide, enhanced $\mathrm{Ca}^{2+}$-induced exocytosis by $260-450 \%$ in both cell types. Interestingly, repaglinide has no such effects on exocytosis in either cell type. Tolbutamide potentiated $\mathrm{Ca}^{2+}$. induced exocytosis even in the presence of repaglinide. Our data suggest that (1) both rat $\alpha$ - and $\beta$-cells contain $\mathrm{K}_{\mathrm{ATP}}$ channels, (2) $\alpha$-cells exhibit a glucose dependence which is the opposite to that in the $\beta$-cell and ( 3 ) A4166 and sulphonylureas enhance exocytosis in both cell types by binding to an intracellular site not recognised by repaglinide. 
544

HOW DOES GLUCOSE INCREASE INSULIN SECRETION BY THE K+-ATP CHANNEL-INDEPENDENT PATHWAY?

Y. Sato, M. Nenquin and J.C. Henquin. Unité d'Endocrinologie et Métabolisme, University of Louvain, Brussels, Belgium.

By closing K+-ATP channels, glucose promotes depolarization-dependent $\mathrm{Ca}^{2+}$ entry and $\mathrm{Ca}^{2+}$ rise in $\mathrm{\beta}$ cells. $\mathrm{Ca}^{2+-d e p e n d e n t ~ e x o c y t o s i s ~ o f ~ i n s u l i n ~}$ granules is then potentiated by a K+-ATP channel-independent action of glucose. The underlying mechanisms are still unclear. They were studied by the method of islet incubation in the presence of diazoxide to open K+-ATP channels, and $30 \mathrm{mmol} / / \mathrm{K}+$ to restore $\mathrm{Ca}^{2+}$ entry. Long-chain acyl CoAs, which are potentially important mediators of the glucose effect, can be increased in $\beta$ cells by incubation with fatty acids. In low glucose $(3 \mathrm{mmol} / \mathrm{l})$ palmitate, stearate, and oleate did not influence $\mathrm{K}^{+}$-induced insulin secretion even in the presence of bromopalmitate, an inhibitor of fatty acid oxidation. Bromopalmitate alone had no effect, but it decreased the potentiation that the fatty acids produced in high glucose $(20 \mathrm{mmol} / \mathrm{l})$. The possible role of phospholipase $\mathrm{A}_{2}\left(\mathrm{PLA}_{2}\right)$ was then evaluated. Exogenous arachidonic acid (AA) did not mimic or augment glucose effects on insulin secretion in high $\mathrm{K}^{+}$. Inhibitors of PLA $A_{2}$ antagonized the effect of glucose, but their action was not reversed by $A A$ and was associated with a fall in islet ATP. In contrast to acetylcholine, glucose did not induce AA release from high $\mathrm{K}^{+}$stimulated islets. Inhibitors of PI-3 kinase were also found not to impair the action of glucose. In contrast, formycin $A$, an adenosine analogue that is converted to formycin A-triphosphate in islets, increased $\mathrm{K}$-induced insulin secretion in 0 and $3 \mathrm{mmol} / \mathrm{l}$ glucose. In conclusion, long-chain acyl CoAs, PLA, and PI-3 kinase do not seem to be implicated in the $\mathrm{K}^{+}$-ATP channel-independent action of glucose. Further support is provided for a role of adenine nucleotides.

\section{6}

PRESENCE OF THE CALCIUM-SENSING RECEPTOR IN ISLET B-CELLS J. Rasschaert, Y. Deng and W.J. Malaisse. Laboratory of Experimenta Medicine, Brussels Free University, Brussels, Belgium.

In 1993, an extracellular calcium-sensing receptor (CaSR) was cloned from bovine parathyroid tissue. The presence of the receptor was further documented in several tissues involved or not in the control of calcium homeostasis such as kidney and thyroidal C-cells or brain, antral gastrin cells and lens epithelial cells. In parathyroid, activation of the CaSR by increasing extracellular calcium concentration leads to inhibition of parathyroid hormone secretion through a phospholipase C-dependent increase in intracellular calcium. In pancreatic islets, it is well established that a rise in intracellular calcium plays a key role in initiating the process of insulin secretion. In the present study, therefore, we examined whether the calcium-sensing receptor is expressed in pancreatic islet cells. A specific fragment of the CaSR gene was amplified by PCR in islets from normal and GK rats, purified B-cells and two B-cell lines. The PCR products were subcloned and sequenced. A specific $\mathrm{CDNA}$ probe was labelled by the digoxigenin method and used to determine the expression of the CaSR by Northem hybridization. Our results showed that, after PCR amplification, a specific fragment of the CaSR was obtained in kidney (used as positive control), pancreatic islets, purified B-cells, RINm5F and BRIN-BD11 cells. The sequence of the CaSR fragment obtained from purified B-cells was comparable to that reported for the rat kidney CaSR. We also identified specific transcripts of the CaSR gene by Northern blot of total RNA from kidney, islets and RINm5F cells. Our results clearly demonstrate that pancreatic B-cells are a site of calcium-sensing receptor gene expression. Further studies are now in progress to examine whether calcium, in addition to serving its role as a key intracellular second messenger for insulin secretion, could also act as an extracellular first messenger through its $G$ protein-coupled cell surface receptor.

\section{5}

METABOLIC AND K+-ATP CHANNEL-INDEPENDENT SECRETORY ACTIONS OF GLUCOSE AND 2-KETO ACIDS IN CLONAL $\beta$-CELLS

N.H. McClenaghan and P.R. Flatt. School of Biomedical Sciences, University of Ulster, Coleraine, BT52 1SA, N. Ireland, U.K.

Effects of glucose and the 2-keto acids, 2-ketoisocaproate (KIC), 2-ketoiso3-methylvalerate (KMV) and 3-phenylpyruvate (PP) were examined in the clonal glucose-responsive BRIN-BD11 cell line. In acute $20 \mathrm{~min}$ incubations $(n=6), 10 \mathrm{mmol} / 1$ of KIC, KMV and PP initiated 1.5-1.7-fold insulinsecretory responses $(\mathrm{p}<0.01)$ at non-stimulatory $(1.1 \mathrm{mmol} / 1)$ glucose $(2.26 \pm$ $0.02 \mathrm{ng} / 10^{6}$ cells $/ 20 \mathrm{~min}$, mean $\pm S E M$ ). Increasing the glucose concentration to $16.7 \mathrm{mmol} / 1$ evoked a 1.7 -fold insulin-secretory response $(3.74 \pm 0.33$ $\left.\mathrm{ng} / 10^{6} \mathrm{cells} / 20 \mathrm{~min}, \mathrm{p}<0.001\right)$ and potentiated $(1.7-2.2$-fold $)$ the effects of 10 $\mathrm{mmol} / 1$ of each $2-$ keto acid tested $(\mathrm{p}<0.001)$. The responses to glucose, $\mathrm{KIC}$ KMV or PP were significantly reduced by $5 \mathrm{mmol} / 1$ of sodium azide (38-61\%; $\mathrm{p}<0.001), 5 \mathrm{mmol} / \mathrm{l}$ sodium cyanide $(45-72 \%, \mathrm{p}<0.001), 10 \mathrm{mmol} / 12$-cyano-3 hydroxycinnamate $(40-63 \%, p<0.001), 10 \mathrm{mmol} / 12$-cyano-4 hydroxycinnamate $(52-67 \%, p<0.001)$ or $15 \mathrm{mmol} / 1$ mannoheptulose $(53-67 \%$, $p<0.001$ ), emphasizing the critical role of mitochondrial metabolism in their actions. Under depolarizing conditions $(25 \mathrm{mmol} / 1 \mathrm{KCl} ; 14.38 \pm 0.78$ $\mathrm{ng} / 10^{6}$ cells $/ 20 \mathrm{~min}$ ), $16.7 \mathrm{mmol} / 1$ glucose induced a 1.2 -fold increase $(\mathrm{p}<0.05)$ in insulin release. Addition of $10 \mathrm{mmol} / 1$ of KIC, KMV or PP induced a further 1.2-fold increase in insulin output $(\mathrm{p}<0.05)$ over that observed in the absence of 2-keto acid $\left(16.70 \pm 0.51 \mathrm{ng} / 10^{6} \mathrm{cells} / 20 \mathrm{~min}\right)$. Voltage-dependent $\mathrm{Ca}^{2+-}$-channel blockade with $20 \mu \mathrm{mol} / \mathrm{l}$ verapamil, or removal of extracellular $\mathrm{Ca}^{2}+$ abolished both glucose- and 2-keto acidinduced insulin release, clearly establishing the central role of intracellular $\mathrm{Ca}^{2+}$ in these secretory effects. Collectively, these results indicate that $\mathrm{KIC}, \mathrm{KMV}$ and $\mathrm{PP}$, like glucose, can initiate insulin secretion at least partially independently of $\mathrm{K}+$-ATP channel activity, both through their mitochondrial metabolism and regulation of $\mathrm{Ca}^{2}+$ influx.

\section{7}

DFFERENTIAL PATTERN OF GLUCOSE-INDUCED ELECTRICAL ACTIVITY AND INTRACELULAR CALCIUM RISES IN RAT AND MOUSE ISLETS C.M. Antunes', A.P. Salgado', L.M. Rosário ${ }^{1,2}$ and R.M. Santos ${ }^{1,2}{ }^{1}$ Centre for Neuroscience of Coimbra; ${ }^{2}$ Dept. of Biochemistry, Fac. of Sciences and Technology, University of Coimbra, Portugal

Isolated rat islets have been extensively used as an important source of biological material for biochemical and insulin secretion studies. However, in contrast to mouse, microdissected rat islets have not been found amenable to the intracellular recording of electrical activity, a key event in insulin response coupling. We have now undertaken a comparative study of glucose-induced electrical activity (recorded via high-resistance microelectrodes) and rises in cytosolic free calcium concentration ( $\left[\mathrm{Ca}^{2+}\right]$, recorded via fura-2 microfluorometry) using single collagenase-isolated islets from normal Wistar rats and albino mice. Average resting membrane potential in $2 \mathrm{mM}$ glucose was -50 and $-60 \mathrm{mV}$ in rat and mouse $\beta$-cells, respectively. Challenging rat islets with $11 \mathrm{mM}$ glucose evoked rapid $\approx 1$ min.) depolarizations to a plateau at $\approx-30 \mathrm{mV}$ which were accompanied by the firing of low amplitude $(\approx 4 \mathrm{mV})$ action potentials. In contrast, mouse $\beta$-cells displayed a typical bursting pattern of electrical activity consisting of alternating hyperpolarized (silent) and depolarized phases at $\approx-40 \mathrm{mV}$ with superimposed action potentials (amplitude $\approx 10 \mathrm{mV}$ ). A step-wise rise in glucose concentration from 2 to $22 \mathrm{mM}$ induced a dose-dependent depolarization of the rat $\beta$-cell membrane $\left(E C_{50}=6.1 \mathrm{mM}\right)$ and a dose-dependent increase in fractional active phase duration in mouse $\beta$-cells $\left(\mathrm{EC}_{50}=11.0 \mathrm{mM}\right)$, with no detectable changes in burst plateau potential. Consistent with the electrophysiological recordings, raising glucose concentration from 2 to $11 \mathrm{mM}$ evoked pronounced $\left[\mathrm{Ca}^{2+}\right]_{i}$ rises in rat islets. Fast $\left[\mathrm{Ca}^{2+}\right]_{i}$ transients were occasionally observed throughout the ascending phase of these responses. Moreover, in contrast to mouse islets, a step-wise rise in glucose concentration from 2 to $22 \mathrm{mM}$ evoked a dose-dependent increase in $\left[\mathrm{Ca}^{2+}\right]$ $\left(\mathrm{EC}_{50}=7.5 \mathrm{mM}\right)$ with no detectable oscillations at the steady state. This study provides evidence for important inter-species differences in the pattern of glucoseinduced electrical activity and $\left[\mathrm{Ca}^{2+}\right]$, responses.

This work was supported by FCT. 


\section{8}

GLIBENCLAMIDE IMPAIRMENT OF $\beta$ CELL FUNCTION INVOLVES ALTERATIONS OF CYTOPLASMIC CA ${ }^{2+}$ REGULATION.

M. Anello, P. Gilon and J.C. Henquin. Unité d'Endocrinologie et Métabolisme, University of Louvain, Brussels, Belgium.

Chronic treatment with glibenclamide alters the $\beta$ cell response to various insulin secretagogues. The possible mechanisms have been studied with islets cultured for $18 \mathrm{~h}$ in the presence of $10 \mathrm{mmol} / \mathrm{l}$ glucose, with (test) or without (control) a therapeutical concentration of glibenclamide $(10 \mathrm{nmol} / \mathrm{l})$. Acute responses of the islets were then determined in the absence of glibenclamide. Insulin release from test islets was unaffected by sulphonylureas or diazoxide because the drugs failed to increase and decrease $\left[\mathrm{Ca}^{2+}\right]_{i}$ that was already elevated in the absence of any stimulus. The responsiveness to glucose was preserved but modified in several respects. As compared with controls, insulin release by test islets was increased in the absence of glucose, and mainly stimulated between 0-10 instead of $7-20 \mathrm{mmol} / 1$ glucose. The maximum response was halved, but this difference disappeared after correction for the decrease in insulin content. The first phase of glucose-induced insulin secretion was abrogated because of a paradoxical decrease of the high basal $\left[\mathrm{Ca}^{2+}\right]_{i}$ in $\beta$ cells. The second phase was preserved but occurred with little rise of $\left[\mathrm{Ca}^{2+}\right]_{i}$. These abnormalities did not result from alterations of glucose metabolism (NADPH fluorescence). In islets cultured with tolbutamide, glucose-induced $\left[\mathrm{Ca}^{2+}\right]_{\mathrm{i}}$ and insulin changes were normal, except for a lower maximal secretion rate due to the fall in insulin content. These islets, however, behaved like those cultured with glibenclamide if tolbutamide remained present during the acute functional tests. In conclusion, treatment with a low glibenclamide concentration perturbs $\beta$ cell function by permanently blocking $\mathrm{K}^{+}$-ATP channels and raising $\left[\mathrm{Ca}^{2+}\right]_{\mathrm{i}}$. Glucose stimulation of insulin secretion occurs at lower concentrations, is delayed and is largely mediated by a modulation of $\mathrm{Ca}^{2+}$ action on exocytosis.

\section{0}

ENGINEERED PEPTIDES FROM THE H3 DOMAIN OF SYNTAXIN INHIBIT INSULIN RELEASE IN INTACT MOUSE PANCREATIC $\beta$ CELLS

F. Martin", E. Salinas*, J. Vazquez", B. Soria* and J. A. Reig- Departments of "Sciences and Technology, Neurochemistry, Physiology" and Institute of Bioengineering, University of Miguel Hernandez, Campus de San Juan, 03550 Alicante, "Center of Molecular Biology, School of Sciences, University Autonoma of Madrid, Cantoblanco, 28049 Madrid, Spain.

Recent studies show that syntaxin- 1 is implicated in the calcium-dependent secretory pathway in pancreatic endocrine $\beta$-cells. Moreover the functional role of two $23^{\circ}$ segments (Syn A and SynB) of the $\mathrm{H} 3$ domain of syntaxin-1 have been demonstrated In the present study, we have stablished in intact $\beta$-cells a minimal functional active zone of 17 residues of the $\mathrm{H} 3$ domain of syntaxin- 1 , as the very effective uncoupling element of Syn A segment. $13^{\text {er }}$ peptides (Syn-1 to Syn-6) from peptides Syn A, Syn I and one additional peptide, with the same amino acid composition than Syn I but in random sequence to be used as controls (Syn $I_{0}$ ) were synthesized by t-boc chemistries using an authomated solid-phase peptide synthesizer. Peptides were purified by RP-HPLC. The second portion of $13^{\text {th }}$ peptides Syn $I$ and Syn $I_{\varepsilon}$ were subjected to myristoylation. After deprotection of the amino terminus of the peptides, myristic acid was activated "in situ" with BOP. Functional experiments were carried out in digitonin-permeabilized and intact cells incubated for 10 and $30 \mathrm{~min}$ respectively, in the presence of the different synthetic peptides. Insulin was determined by RIA. Basal or stimulated secretion were measured in media containing $5 \mathrm{mmol} / \mathrm{EGTA}, 10 \mu \mathrm{mmol} / 1 \mathrm{Ca}^{2+}$ or $22 \mathrm{mmol} / 1$ glucose. $200 \mu \mathrm{mol} / 1$ of peptides $\mathrm{Syn}$ 2 , Syn-3 and Syn-4 inhibited $\mathrm{Ca}^{2+}$-dependent insulin release $(p<0.0001, \mathrm{n}=6)$ in permeabilized ceils. No additive effects were observed when Syn-2 to Syn-4 were incubated together $(n=4)$. myr-Syn I provoked a dose-dependent inhibition of 22 mmoll glucose-induced insulin release $\left(\mathrm{IC}_{50} \sim 23 \mathrm{mmol} / \mathrm{l}\right)$ in intact cells $(n=4)$. myrSyn $I_{c}$ had not inhibitory effect at $300 \mu \mathrm{mmol} /(n=4)$. These data demonstrated for the first time, that nutrient-induced secretory process can be uncoupled in intact $\beta$ cells by using specific peptides and support previous studies concerning the direct role of specific regions of syntaxin- 1 in the insulin exocytotic process.
549

AMINO ACID TRANSFORMATION OF OSCILLATORY $\mathrm{Ca}^{2+}$ SIGNALS IN MOUSE PANCREATIC B-CELLS AND INTACT ISLETS

M. Ahmed', L. Ali', E. Grapengiesser'2 and B. Hellman'2. 'BIRDEM, Dhaka, Bangladesh and 2Department of Medical Cell Biology, University of Uppsala, Uppsala, Sweden

Glucose-induced increase of cytoplasmic $\mathrm{Ca}^{2+}$ in pancreatic B-cells is usually manifested as slow oscillations from the basal level. The significance of this rhythmicity for maintaining normal 8-cell function with periodic variations of circulating insulin made it of interest to investigate how the oscillatory $\mathrm{Ca}^{2+}$ signal was affected by various amino acids. Cytoplasmic $\mathrm{Ca}^{2+}$ was measured in individual B-cells and islets from ob/ob mice using dual wavelength microfluorometry and the indicator fura-2. The individual B-cells were very sensitive to alanine, glycine and arginine. When added at concentrations as low as $0.1 \mathrm{mM}$ and $0.5 \mathrm{mM}$, each of these amino acids transformed the oscillations into sustained elevation of cytoplasmic $\mathrm{Ca}^{2+}$ in 30 and $60 \%$ respectively of the $\mathrm{B}$ cells. Stimulation of the entry of $\mathrm{Ca}^{2+}$, obtained either by raising the extracellular concentration or by prolonging the open state of the voltage-dependent $\mathrm{Ca}^{2+}$ channels with BAY K 8644 , resulted in reappearance of the rhythmic activity. Oscillatory $\mathrm{Ca}^{2+}$ signals in intact islets were more resistant to transformation by amino acids than those of individual B-celis. Even at $10 \mathrm{mM}$ of glycine, alanine or arginine sustained elevation was observed in only about $55 \%$ of the islets. It is suggested that signals from the adjacent cells make it possible for B-cells situated in islets to overcome a suppression of the oscillatory activity otherwise seen in the presence of amino acids.

\section{1}

COUPLING OF THE $\alpha$-LATROTOXIN RECEPTOR LATROPHILIN TO INSULIN EXOCY'TOSIS IN CLONAL $\beta$-CELLS

J. Lang', .C. B. Wollheim ', M. Pescatori',

R. A. Fisher, A. Grasso ${ }^{4}$ and Y. Ushkaryov ${ }^{5}$

${ }^{\mathrm{I} D i v . ~ d e ~ B i o c h i m i e ~ C l i n i q u e, ~ D e ́ p . ~ d e ~ M e ́ d e c i n e ~ I n t e r n e, ~ C M U, ~ G e n e ̀ v e, ~}$ Switzerland; ${ }^{2}$ Università di Roma, Roma, Italy, ${ }^{3}$ Dep. of Pharmacology, University of Iowa, Iowa City, USA, ${ }^{4}$ Istituto di Biologia Cellulare, CNR, Roma, Italy; and ${ }^{5}$ Dep. of Biochemistry, Imperial College, London, UK

The black-widow spider venom component $\alpha$-latrotoxin $(\alpha-\mathrm{LTX})$ induces massive exocytosis in neurons and evokes exocytotic insulin release in primary $\beta$-celis as well as in the clonal cell lines MIN6 and INS-1, but not in HIT-T15 or RINm5F cells. The effect of $\alpha$-LTX in insulin secreting cells is mediated by latrophilin (LPH), a class II G-protein coupled receptor.

We have investigated the coupling of latrophilin to exocytosis in HIT-T15 cells. Transient transfection of full-length cDNA coding for LPH induced dose dependent insulin release with an $\mathrm{EC}_{50}$ of $0.5 \mathrm{nM}$ for its agonist $\alpha$-LTX. LPH was targeted to discrete areas of the plasma membrane as evidenced by confocal immunocytochemistry. The agonist dependent insulin release is of vesicular origin as it was blocked by co-expression of the light chains of the botulinum neurotoxins $\mathrm{Cl}$ or $\mathrm{E}$ known to cleave syntaxin and SNAP-25, respectively. Coupling of LPH to insulin release was specifically reproduced in streptolysin-O permeabilized cells by a receptor-mimetic peptide corresponding to an intracellular loop of LPH. Therefore it involves most likely heterotrimeric G-proteins. However, the effect of $\alpha$-LTX was insensitive to pertussis toxin. Moreover, the effect of $\alpha$-LTX on insulin release was not altered by concomitant overexpression of the Regulators of $\underline{G}$-protein Signalling RGS3 and RGS3-t, which impeded stimulation of hormone secretion by carbachol and PACAP.

We conclude that latrophilin is capable of mediating the effects of $\alpha$-LTX on insulin exocytosis and induces vesicular release of insulin by coupling to a heterotrimeric $G$-protein distinct from $G_{i o}, G \alpha_{s}$ or $\mathrm{G} \alpha_{q}$. 
CYSTEINE-STRING PROTEINS REGULATE INSULIN EXOCYTOSIS: DEFINITION OF FUNCTIONAL DOMAINS

H. Zhang, W. L. Kelly*, L. H. Chamberlain ${ }^{*}$,

R. Bourgoyne ${ }^{*}$, C.B. Wollheim and J. Lang

Div. of Clinical Biochemistry and * Dep. of Medical Biochemistry, CMU, U. of Geneva, Switzerland; "Physiological Laboratory, U. of Liverpool, U.K

Cysteine-string proteins (Csps) are synaptic vesicle proteins which have been implicated in neurotransmitter release in Drosophila and may interact with presynaptic $\mathrm{Ca}^{2+}$-channels. All $\mathrm{Csps}$ studied to date contain at least four domains: an N-terminal J-domain which shares homology with the DnaJ family of proteins and which directs interactions with the Hsc70 chaperone, an adjacent highly conserved linker region, the defining cysteine rich domain, and a variable $\mathrm{C}$-terminus.

In endocrine insulin-secreting pancreatic $\beta$-cells we found Csps on secretory granules and synaptic-like microvesicles. Vesicle membrane attachment was independent of the palmitoylation of the cysteine-rich "string" region but required the first 82 amino acids, which comprise the J-domain. Csps are necessary for insulin exocytosis as transient reduction of the amount of endogenous Csps by antisense cDNA inhibited $\mathrm{Ca}^{2+}$-induced insulin release Mutational analysis revealed marked differences between the action of Csps in insulin exocytosis and the ability of purified Csps to stimulate Hsc70 ATPase Point mutations within the highly conserved HPD motif of the J-domain abolished activation of $\mathrm{Hsc} 70$, also underscoring the general role of this motif in mammalian J-domain containing proteins. However, these same mutations altered regulation of exocytosis only after additional truncation of the Cterminus as found in the Csp splice variant Csp2. Furthermore, the strikingly conserved linker region adjacent to the J-domain was found to be an importan domain for Csp function in exocytosis, but not for the activation of $\mathrm{Hsc} 70$ ATPase. Since the effects of Csps on exocytosis were still observed in streptolysin-O permeabilised cells, Csps also regulate secretion independent of the modulation of ion fluxes.

These observations establish the requirement for Csps in exocytosis and define functional domains necessary for this process.

\section{4}

$\mathrm{Ca}^{2+}$ INDUCED LOSS OF $\mathrm{Ca}^{2+} /$ CALMODULIN-DEPENDENT PROTEIN KINASE II ACTIVITY IN PERMEABILIZED RAT ISLETS OF LANGERHANS T.E. Harris, S.J. Persaud and P.M. Jones. Cellular and Molecular Endocrinology, King's College London, Campden Hill Road, London, U.K. An elevation in cytosolic $\mathrm{Ca}^{2+}$ is a pivotal step in the stimulation of insulin secretion from pancreatic $\beta$-cells, an event thought to be mediated via the activation of the $\mathrm{Ca}^{2+} /$ calmodulin-dependent protein kinase II (CaMK II). We have shown previously that $\mathrm{Ca}^{2+}$-induced insulin secretion from electrically permeabilized islets is transient and that $\mathrm{Ca}^{2 *}$-induced phosphorylation of endogenous islet proteins is greatly reduced when permeabilized islets are exposed to $10 \mu \mathrm{M} \mathrm{Ca}^{2+}$ for up to $30 \mathrm{~min}$. We have now used $\mathrm{Ca}^{2+}$-treated permeabilized islets to investigate whether [i] immunoreactive CaMK II protein is decreased and [ii] whether $\mathrm{Ca}^{2+} /$ calmodulin-induced CaMK II enzyme activity is reduced. Electrically permeabilized rat islets were incubated in zero $(0 \mu \mathrm{M})$ or high $(10 \mu \mathrm{M}) \mathrm{Ca}^{2+}$ buffer for up to $30 \mathrm{~min}$ and extracts were prepared for immunoblotting and enzyme assays. CaMK II immunoreactivity was detected as a protein of $58 \mathrm{kDa}$ in extracts of normal rat islets. Electrical permeabilisation of rat islets did not reduce CaMK II immunoreactivity. Incubation of permeabilized islets in $10 \mu \mathrm{M} \mathrm{Ca}{ }^{2+}$ for up to $\mathbf{3 0 m i n}$ did not cause any reduction in this $\mathbf{5 8} \mathrm{kDa}$ protein compared to zero $\mathrm{Ca}^{2+}$ controls as determined by densitometric scanning $(5 \mathrm{~min}, 85 \%$ control: $15 \mathrm{~min}, 106 \%$; $30 \mathrm{~min}, 113 \%$ ). However, when $\mathrm{Ca}^{2+} /$ calmodulin-induced CaMK II enzyme activity was measured in similar extracts, a large reduction was observed in extracts of islets incubated in high $\mathrm{Ca}^{2+}$ buffer $(1 \mathrm{~min}$, 93.6 $\pm 4.6 \mathrm{fmol} / \mathrm{islet} / \mathrm{min} ; 5 \mathrm{~min}, 81.4 \pm 2 ; 15 \mathrm{~min}, 31.8 \pm 15.5 ; 30 \mathrm{~min}, 28.7 \pm 1.5$; $p<0.001$ by ANOVA, mean $\pm S E M, n=4)$. In contrast, there was no decrease in activity in extracts of permeabilized islets incubated in zero $\mathrm{Ca}^{2+}$ buffer for the same periods of time $(1 \mathrm{~min}, 118.6 \pm 12.4 \mathrm{fmol} / \mathrm{is} / \mathrm{et} / \mathrm{min} ; 30 \mathrm{~min}$, $115.5 \pm 10.8 ; p>0.8$ by ANOVA, mean $\pm S E M, n=4)$. These results suggest that the desensitization of $\mathrm{Ca}^{2+}$-induced insulin secretion from permeabilized islets reflects a loss of CaMK II activity rather than CaMK II protein.
GRANULE MOVEMENT AND EXOCYTOSIS IN $\beta$ HC9 CELLS Samira Daniel, Mitsuhiko Noda and Geoffrey W.G. Sharp. Dept. of Pharmacology, Cornell University, Ithaca, NY 14853-6401

Exocytosis in the pancreatic $\beta$-cell involves a coordinated sequence of granule movement to the plasma membrane, i.e., translocation, docking, priming and fusion, that are highly conserved from yeast to higher mammals. The SNAREs synaptotagmin, syntaxin, SNAP-25 and synaptobrevin are present in the particulate fraction of the $\beta \mathrm{HC} 9$ cell-line as expected from their location in granules and the plasma membrane. We have identified the SNARE complex associated with docked granules by co-immunoprecipitation of the granule SNARE synaptobrevin by an antibody against the plasma membrane protein syntaxin. We find that stimulation of insulin release by glucose, $\mathrm{KCl}$ or mastoparan results in the disassembly of the complex due to exocytosis. The $\alpha_{2}$-adrenergic agonist, norepinephrine, which inhibits exocytosis, prevents this disassembly of the core complex. Yohimbine, a competitive inhibitor of norepinephrine, can, in turn, block the inhibitory effect of norepinephrine on exocytosis. Importantly, in association with perifusion studies, we can determine the size of the readily releasable pool of granules and the rate at which granules from the reserve pool are mobilised to the plasma membrane under different physiological conditions. Thus, the first phase of glucosestimulated insulin secretion $(\sim 5-10 \mathrm{~min})$ is associated with the discharge of the docked pool. During the second phase (10-60 min), the pool of docked granules is restored so that the rate of translocation of granules from the reserve pool to the docked pool exceeds the rate of granule release. Therefore, glucose stimulates both the discharge and re-filling of the docked granule population in the $\beta \mathrm{HC} 9$ cell.

\section{5}

DIFFERENTIAL LOCALISATION AND PHOSPHORYLATION OF MYOSIN IIA AND IIB IN Ca ${ }^{2+}$-DEPENDENT INSULIN SECRETION

J.R.Wilson*, R.I.Ludowyke and T.J.Biden. Garvan Institute of Medical Research, Sydney, AUSTRALIA

Activation of protein kinases by nutrient stimuli plays an important role in $\mathrm{Ca}^{2+}$-dependent insulin secretion. Substrates for these kinases and their roles in the latter stages of insulin secretion are poorly defined. We have recently shown that $\mathrm{Ca}^{2+}$-dependent insulin secretion from both rat pancreatic islets and clonal RINmSF cells is associated with increased phosphorylation of myosin II heavy chain (MHC) on serine (ser) and threonine (thr) residues ( 2 fold and 6 fold respectively). The aim of the current study was to further define the role of MHC by examining the subcellular localisation and differential phosphorylation of the myosin IIA and IIB isozymes during $\mathrm{Ca}^{2+}$-dependent insulin secretion. In fractionation experiments RINm5F cell lysates were separated into triton soluble (cytosolic and membrane) and triton insoluble (cytoskeletal associated) fractions and proteins were separated by $7.5 \%$ SDS-PAGE. Myosin and thr/ser phosphorylation were detected by immunoblotting. Using isoform specific antibodies we found that myosin IIA and IIB displayed subtle differences in subcellular localisation. Myosin IIA appears to be predominantly in the triton soluble fraction $(90 \pm 2$ vs $10 \pm 2 \%)$ whereas myosin IIB is more evenly distributed between the triton soluble and triton insoluble fractions $(64 \pm 4.6 \mathrm{vs}$ $36 \pm 4.6 \%$ ). This correlated well with the findings that myosin IIA appears to undergo a greater increase in thr phosphorylation than myosin IIB in response to agents increasing the cytosolic free $\mathrm{Ca}^{2+}$ concentration and that the majority of phosphorylated myosin is found in the triton soluble fraction. Preliminary confocal microscopy provides further evidence indicating differences in isoform subcellular localisations and also suggests that these locations may change upon stimulation of the cell. The results suggest that MHC IIA and IIB exert different cellular functions, and that there is a myosin subpool (probably MHC IIA) that is regulated by thr phosphorylation. As myosin has been shown to be involved in a number of cellular functions including exocytotic processes, these findings might suggest an important and specific role of myosin in the mediation of $\mathrm{Ca}^{2+}$-dependent insulin secretion. 
556

MULTIPLE PLASMA MEMBRANE $\mathrm{Ca}^{2+}$-ATPase ISOFORMS EXPRESSED IN RAT PANCREATIC $\beta$ CELLS.

A. Kamagate, F. Van Eylen and A. Herchuelz. Laboratory of Pharmacology, Brussels University School of Medicine, Brussels, Belgium.

The plasma membrane $\mathrm{Ca}^{2+}-\mathrm{ATPase}$ (PMCA) is thought to be important for $\mathrm{Ca}^{2+}$ homeostasis in the pancreatic $\beta$-cell. Up to now, the isoforms present in the $\beta$-cell have been studied at only 1 of the 3 alternative splicing sites (site $C$ ). Alternative splicing options of transcripts of the four currently known rat PMCA (rPMCA) genes were analyzed at sites $A, B$, and $C$ in insulin producing cells by RT-PCR using primers flanking the alternative splicing sites $A$ and $B$ + C. Data obtained indicate that $\beta$-cells, RINm5F, and islets of Langerhans express at site $A$ PMCA 1x, 2y, 2w, 3z, $4 x$ whereas islets of Langerhans contained PMCA $4 z$ in addition. Two B-site splicing products were observed for $\mathrm{PPCA} 1$ in islets of Langerhans, generating 2 transcripts $1 \mathrm{~h}$ and $1 \mathrm{k}$, the latter lacking the exon coding for the 10th transmembrane domain. For both sites $\mathrm{B}$ and $\mathrm{C}$, we identified PMCA lhb, $1 \mathrm{fb}$, 2hb, 3ha, 3hc, $4 \mathrm{hb}$ in $\beta$-cells, RINm5F and islets of Langerhans, in addition to PMCA4ha expressed by the latter. We suggest the existence of rPMCA1xhb, rPMCA2yhb, rPMCA2whb, rPMCA32ha, rPMCA3zhc and rPMCA4xhb in rat pancreatic $\beta$-cells, RNm5F cells and islets, plus 2 additional isoforms (rPMCA 1xkb, rPMCA4zha) in islets of Langerhans. Our data show that there are 2 different sites of alternative splicing operating in all four PMCA genes: sites $\mathrm{B}$ and $\mathrm{C}$ for rPMCAI, and sites $A$ and $C$ for rPMCA2, rPMCA 3 and rPMCA4. Hence, $\mathrm{Ca}^{2+}$-ATPase isoforms expressed in rat pancreatic $\beta$-cells have multiple splice sites, corresponding to protein domains having regulatory role : regulation by acidic phospholipids at site $\mathrm{A}$, regulation by calmodulin and protein kinases at site $\mathrm{C}$ and no $B$ spliced out isoform that may be inactive. This provides a full range of $\mathrm{Ca}^{2+}-\mathrm{ATPases}$ for cytosolic free $\mathrm{Ca}^{2+}$ regulation in the $\beta$-cell.

\section{8}

BOTH $\alpha$-D-GLUCOSE AND $\beta$-L-GLUCOSE PENTAACETATES POTENTIATES ELECTRICAL ACTIVITY AND CYTOSOLIC Ca** IN MOUSE PANCREATIC ISLETS

A.B. Ropero, R. Pomares, J.V. Sánchez-Andrés, A. Nadal, B. Soria and W.J. Malaisse*.

Department of Physiology, Institute of Bioengineering, School of Medicine, Miguel Hernández University. Aptdo. 18, E-03550 San Juan de Alicante, Spain. * Laboratory of Experimental Medicine, Brussels Free University. 808 Route de Lennik, B-1070 Brussels, Belgium.

Electrical activity of $\beta$-cells and cytosolic $\mathrm{Ca}^{2+}$ concentration $\left(\left[\mathrm{Ca}^{2+}\right] \mathrm{i}\right)$ were monitored in mouse pancreatic islets exposed to the pentaacetate esters of $\alpha-D$-glucose, $\beta-D$-galactose and $\beta$-L-glucose, all tested at $1.7 \mathrm{mM}$ concentration. The electrical activity of $\beta$-cells was recorded from microdissected islets of Langerhans, and $\left[\mathrm{Ca}^{2+}\right]_{\mathrm{i}}$ was monitored using Indo-1 fluorescence from islets of Langerhans, both obtained from albino mice. In the presence of $5 \mathrm{mM}$ D-glucose, $\alpha$-D-glucose pentaacetate induced electrical activity $(n=6)$ and increased $\left[\mathrm{Ca}^{2+}\right]_{i}(\mathrm{n}=4)$ whilst $\beta$-D-galactose pentaacetate failed to do so ( $n=4$ and 6 ). The electrical and cationic response to the $\mathrm{D}$-glucose ester occurred with a delay between 5 and $10 \mathrm{~min}$, the ester-induced increase in $\left[\mathrm{Ca}^{2+}\right]_{i}$ being suppressed in the absence of extracellular $\mathrm{Ca}^{2+}(n=2)$. As a rule, $\beta-L$ glucose pentaacetate also failed to evoke biophysical responses in the islets exposed to $5 \mathrm{mM} D$ glucose. However, in the presence of $10 \mathrm{mM}$ L-leucine the L-glucose ester induced electrical activity $(\mathrm{n}=3)$. In the presence of the amino-acid, $\alpha$-Dglucose pentaacetate produced an increase in the electrical activity in 5 of 6 cells tested. In these conditions, when $\beta$-D-galactose pentaacetate was tested, not only did not potentiate L-leucine-elicited electrical activity but it abolished L-leucine effect. These findings, which parallel the insulinotropic action of selected hexose pentaacetates suggest a dual mode of action linked to both the metabolism of their carbohydrate moiety and a direct effect of the ester itself upon a yet unidentified receptor system.

\section{7}

ACETYLCHOLINE STIMULATES EXOCYTOSIS BY ACCELERATION OF GRANULA MOBILISATION IN MOUSE PANCREATIC B-CELLS J. Gromada, M. Høy, E. Renström", K. Bokvist, L. Eliasson", S. Göbel", P Rorsman" Novo Nordisk, Copenhagen, Denmark and "Lund University, Sweden.

Capacitance measurements of exocytosis were used in combination with digital $\mathrm{Ca}^{2+}$ imaging to investigate how acetylcholine (ACh) stimulates $\mathrm{Ca}^{2+}$-induced insulin secretion in single mouse pancreatic B-cells. ACh increased exocytotic responses elicited by voltage-clamp depolarisations 2.3 -fold. This effect was mediated by activation of muscarinic receptors and dependent on elevation of the cytoplasmic $\mathrm{Ca}^{2+}$-concentration $\left(\left[\mathrm{Ca}^{2+}\right]_{i}\right)$, by mobilisation of $\mathrm{Ca}^{2+}$ from thapsigargin-sensitive intracellular stores, localised to the secretory pole of the Bcell. Interplay between intracellular $\mathrm{Ca}^{2+}$-stores and exocytosis is facilitated by the organisation of the B-cell and electron microscopy revealed that endoplasmic reticulum and the secretory granules frequently juxtapose. The ACh-induced elevation of $\left[\mathrm{Ca}^{2}\right]_{\mathrm{i}}$ accelerated the refilling of the readily releasable pool of granules and its size increased 4-fold in the presence of the neurotransmitter. $\mathrm{ACh}$ also interfered with buffering of $\left[\mathrm{Ca}^{2+}\right]_{\mathrm{i}}$ following depolarisation-induced $\mathrm{Ca}^{2+}$. entry and the duration of the associated transients increased 5-fold. Thus, $\mathrm{Ca}^{2+}$ following a burst of action potentials was present at concentrations sufficient to stimulate pool refilling $\left(\mathrm{K}_{\mathrm{d}}=0.2 \mu \mathrm{M}\right)$ for much longer periods in the presence than in the absence of the neurotransmitter. The stimulation of granule mobilisation reflected activation of CaM-kinase II and myosin light chain kinase suggesting that $\mathrm{Ca}^{2+}$ and $\mathrm{ACh}$ control the exocytotic capacity of the B-cell by regulating the interactions between the cytoskeleton and the secretory granules.

\section{9}

ELECTRICAL ACTIVITY AND CYTOPLASMIC CA CA $^{2+}$
OSCILLATIONS IN GLUCOSE-STIMULATED $\beta$-CELLS

S. Dryselius, E. Grapengiesser, B. Hellman and E. Gylfe. Department of Medical Cell Biology, Uppsala University, Uppsala, Sweden.

The role of voltage-dependent $\mathrm{Ca}^{2+}$ entry for glucose generation of slow oscillations of the cytoplasmic $\mathrm{Ca}^{2+}$ concentration $\left(\left[\mathrm{Ca}^{2+}\right]_{i}\right)$ was evaluated in individual mouse pancreatic $\beta$-cells. Like depolarization with $\mathrm{K}^{+}$, a rise of the glucose concentration resulted in an enhanced influx of $\mathrm{Mn}^{2+}$, which was inhibited by nifedipine. This antagonist of $\mathrm{L}$-type $\mathrm{Ca}^{2+}$ channels also blocked the slow oscillations of $\left[\mathrm{Ca}^{2+}\right]_{i}$ induced by glucose. Parallel measurements of $\left[\mathrm{Ca}^{2+}\right]_{i}$ and electrical activity revealed that the slow oscillations occur in perfect synchrony with bursts of action potentials, the elevation of $\left[\mathrm{Ca}^{2+}\right]_{i}$ being proportional to the frequency of the action potentials. A similar relationship was obtained when $\mathrm{Ca}^{2+}$ was replaced with $\mathrm{Sr}^{2+}$, which was removed from the cytoplasm at only $40 \%$ of the rate found for $\mathrm{Ca}^{2+}$. In some experiments the slow $\left[\mathrm{Ca}^{2+}\right]_{i}$ oscillations were superimposed with pronounced spikes temporarily arresting the action potentials. It is concluded that the glucose-induced slow oscillations of $\left[\mathrm{Ca}^{2+}\right]_{i}$ are caused by periodic depolarization with an influx of $\mathrm{Ca}^{2+}$ through L-type channels. $\mathrm{Ca}^{2+}$ spiking, due to intracellular mobilization, may be important for chopping the slow oscillations of $\left[\mathrm{Ca}^{2+}\right]_{i}$ into shorter ones characterizing $\beta$-cells situated in pancreatic islets. 
THE INFLUENCE OF CELL NUMBER ON $\mathrm{CA}^{2+}$ OSCILLATIONS IN CLUSTERS OF $\beta$ CELLS

F.C. Jonkers, P. Gllon and J.C. Henquin. Unité d'Endocrtnologie et Métabolisme, University of Louvain, Brussels, Belgium.

During continuous stimulation with glucose, intact islets display regular oscillations of cytosolic $\left[\mathrm{Ca}^{2+}\right]_{1}$, whereas the oscillations are inconsistent and varlable in single $\beta$ cells. Here, we tested the hypothesis that a minimum number of $\beta$ cells must be coupled for appearance of regular oscillations. Normal mouse islets were dispersed into single cells and clusters of different stzes, and cultured for 1-4 days. $\left[\mathrm{Ca}^{2+}\right]_{1}$ was then measured by the fura-2 technique during continuous stimulation with $15 \mathrm{mmol} / 1$ glucose. One third of single cells were unresponsive. In responsive cells, $\left[\mathrm{Ca}^{2+}\right]_{1}$ oscillations had a mean frequency of $0.16 / \mathrm{min}$, and were variable in amplitude and duration. $90 \%$ of cell doublets showed oscillations of greater regularity but still low frequency $(0.20 / \mathrm{min})$. In small (3-5 cells) or large (up to 50 cells) clusters, all cells showed $\left[\mathrm{Ca}^{2+}\right]_{1}$ responses, usually characterized by regular oscillations. However, the frequency plateaued at $-0.25 / \mathrm{min}$. Mean $\left[\mathrm{Ca}^{2+}\right]_{\mathbf{i}}$ did not increase with the cluster size. Extending the culture from 1 to 4 days did not affect the characteristics of the response in single cells, but caused appearance of sustained rises in $\left[\mathrm{Ca}^{2+}\right]_{1}$ in $20 \%$ of the clusters. In cell doublets, both regular and irregular oscillations of $\left[\mathrm{Ca}^{2+}\right]_{1}$ were synchronous in the two cells. In larger clusters, both adjacent and non-adjacent cells displayed synchronous $\left[\mathrm{Ca}^{2+}\right]_{\mathrm{f}}$ changes. In no cluster could an irregular or sustained elevation of $\left[\mathrm{Ca}^{2+}\right]_{\text {, be }}$ ascribed to asynchronous responses in different cells. In conclusion, the heterogeneity of glucose-induced $\left[\mathrm{Ca}^{2+}\right]_{1}$ changes in single $\beta$ cells disappears when the cells form small clusters. However, an increase in cluster size hardly affects the frequency of the oscillations, which is similar to that of the slow oscillations present in intact islets.

\section{2}

PARTIAL INHIBITION OF THE Na/K PUMP UNMASKS $\mathrm{Na}^{+}$ OSCILLATIONS IN GLUCOSE-STIMULATED PANCREATIC $\beta$-CELLS E. Grapengiesser. Department of Medical Cell Biology, Uppsala University, Sweden.

The importance of the $\mathrm{Na} / \mathrm{K}$ pump for the cytoplasmic concentration of $\mathrm{Na}^{+}\left(\left[\mathrm{Na}^{+}\right]_{i}\right)$ in glucose-stimulated mouse $\beta$-cells was analyzed using dual-wavelength microfluorometry and the indicator SBFI. Under conditions known to induce large-amplitude oscillations of cytoplasmic $\mathrm{Ca}^{2+}(11 \mathrm{mmol} / 1$ glucose $)\left[\mathrm{Na}^{+}\right]_{i}$ usually remained low and stable at 10-14 mmol/l. Partial suppression of the $\mathrm{Na} / \mathrm{K}$ pump with $50 \mu \mathrm{mol} / \mathrm{l}$ ouabain resulted in oscillations of $\left[\mathrm{Na}^{+}\right]_{\mathrm{i}}$ in $65 \%$ of the cells (frequency $0.13 \pm 0.01 \mathrm{~min}^{-1}$; amplitudes $4.4 \pm 0.3 \mathrm{mmol} / \mathrm{l}$ ). The oscillations were unaffected by the presence of tetrodotoxin but disappeared when the medium was depleted of $\mathrm{Ca}^{2+}$ or supplemented with methoxyverapamil. The analysis of the ouabain effect was facilitated by replacing extracellular $\mathrm{Ca}^{2+}$ with 5 $\mathrm{mmol} / 1 \mathrm{Sr}^{2+}$. In the $\mathrm{Sr}^{2+}$-containing medium oscillations of $\left[\mathrm{Na}^{+}\right]_{\mathrm{i}}$ were seen in $>70 \%$ of the $\beta$-cells exposed to $11 \mathrm{mmol} / \mathrm{l}$ glucose. Ouabain $(50 \mu \mathrm{mol} / \mathrm{l})$ modified these oscillations by increasing their amplitudes almost threefold and reducing the frequency from once every $3 \mathrm{~min}$ to once every $10 \mathrm{~min}$. A relationship between oscillations of cytoplasmic $\mathrm{Sr}^{2+}$ and $\mathrm{Na}^{+}$was apparent both from observations of similar frequencies and for the modifications obtained with ouabain. It is concluded that the glucose-induced oscillations of cytoplasmic $\mathrm{Ca}^{2+}$ result in a rhythmic entry of $\mathrm{Na}^{+}$usually balanced by the $\mathrm{Na} / \mathrm{K}$ pump.
PROTEIN KINASE A AS DETERMINANT FOR SLOW AND FAST $\mathrm{Ca}^{2+}$ OSCILLATIONS IN B-CELLS AND PANCREATIC ISLETS

E. Gylfe and Y.J. Liu. Dept. of Medical Cell Biology, Uppsala University, Sweden

Pancreatic $\beta$-cell and islets of Langerhans commonly respond to $11 \mathrm{mM}$ glucose with slow oscillations (0.3-0.9 $\left.\mathrm{min}^{-1}\right)$ of the cytoplasmic $\mathrm{Ca}^{2+}$ concentration $\left(\left[\mathrm{Ca}^{2+}\right]_{\mathrm{i}}\right)$. In islets a regular fast pattern $\left(2-7 \mathrm{~min}^{-1}\right)$ is often superimposed on the slow oscillations. Agents increasing cAMP promote the fast islet pattern and also induce less regular fast $\left[\mathrm{Ca}^{2+}\right]_{\mathrm{i}}$ oscillations and pronounced $\left[\mathrm{Ca}^{2+}\right]_{i}$ spiking in the individual $\beta$-cells. We have now tested the effect of the protein kinase $A$ inhibitor $R_{P-}$ cAMPs on oscillatory $\mathrm{Ca}^{2+}$ signalling. $\mathrm{R}_{\mathrm{P}}$-cAMPs was found to inhibit the fast oscillations in islets transforming the mixed oscillatory pattern into a slow one. However, at high concentrations Rp-cAMPs also suppressed the slow oscillations. Since such an effect may be due loss of cell coordination after inhibition of gap-junctional coupling we tested the effects of $R_{P}$-cAMPs on individual $\beta$-cells. It was found that the individual $\beta$-cells sometimes reacted to high concentrations of $\mathrm{R}_{\mathrm{P}}-\mathrm{cAMPs}$ with attenuation of the slow oscillations. The data indicate that the disappearance of the fast oscillatory signalling in islets is due to inhibition of intracellular $\mathrm{Ca}^{2+}$ release after desensitization of inositol 1,4,5-trisphosphate receptors. Further studies will have to clarify if the attenuation of the slow oscillations in individual cells represents an effect on the voltage-dependent $\mathrm{Ca}^{2+}$ channels per se or on determinants of the membrane potential.

\section{3}

ROLE OF VOLTAGE-DEPENDENT $\mathrm{Na}^{+}$CHANNELS FOR RHYTHMIC $\mathrm{Ca}^{2+}$ SIGNALLING IN GLUCOSE-STIMULATED PANCREATIC $\beta$-CELLS.

M. Eberhardson, E. Grapengiesser. Dept. of Medical Cell Biology, Uppsala University, Sweden

The role of voltage-dependent $\mathrm{Na}^{+}$channels for glucose induction of rhythmic $\mathrm{Ca}^{2+}$ signalling was studied in mouse pancreatic $\beta$-cells using dual wavelength fluorometry and the indicator fura-2. A rise of glucose from 3 to $11 \mathrm{mmol} / 1$ induced slow oscillations $\left(0.2-0.5 \mathrm{~min}^{-1}\right)$ of the cytoplasmic $\mathrm{Ca}^{2+}$ concentration $\left(\left[\mathrm{Ca}^{2+}\right]_{i}\right)$, which persisted when adding the $\mathrm{Na}^{+}$channel blocker tetrodotoxin. However, in the presence of the $\mathrm{Na}^{+}$channel agonist veratridine (1-10 $\left.\mu \mathrm{mol} / 1\right)$, the glucose-induced oscillations were replaced by repetitive and pronounced transients of $\left[\mathrm{Ca}^{2+}\right]_{\mathrm{i}}\left(1.7-3.5 \mathrm{~min}^{-1}\right)$ arising from the basal level. This effect of veratridine was reversed by tetrodotoxin. The $\left[\mathrm{Ca}^{2+}\right]_{i}$ transients obtained in the presence of veratridine were amplified by increasing extracellular $\mathrm{Ca}^{2+}$ and disappeared in $\mathrm{Ca}^{2+}$-deficient medium or when blocking the voltage-dependent $\mathrm{Ca}^{2+}$ channels by methoxyverapamil. The intracellular $\mathrm{Ca}^{2+}$-ATPase inhibitor thapsigargin caused a slight amplification of the transients. When the $\beta$-cells were depolarized with $100 \mu \mathrm{mol} / 1$ tolbutamide or $10 \mathrm{mmol} / 1$ ketoisocaproate, veratridine induced transients of $\left[\mathrm{Ca}^{2+}\right]_{\mathrm{i}}$ similar to those obtained in glucosestimulated cells but failed to do so in $\beta$-cells depolarized with 100 $\mu \mathrm{mol} / 1$ quinine or $30 \mathrm{mmol} / \mathrm{l} \mathrm{K}^{+}$. The results indicate that the $\mathrm{Ca}^{2+}$ oscillations normally observed in glucose-stimulated $\beta$-cells occur without involvement of voltage-dependent $\mathrm{Na}^{+}$channels. However, the activation of such channels might represent a useful approach in the development of drugs promoting insulin secretion. 
564

\section{REGULATION OF PANCREATIC $B$ CELL GLUCOKINASE ACTIVITY BY A GLUCOSE-SENSITIVE PROTEIN FACTOR}

M. Tiedge, M. Elsner and S. Lenzen, Institute of Clinical Biochemistry, Hannover Medical School, D-30623 Hannover, Germany

Regulation of glucose-induced insulin secretion is crucially dependent upon glucokinase function in pancreatic $\beta$ cells. It was the aim of this study (1) to determine if the stimulation of glucokinase (GK) in rat pancreatic islets is regulated by glucose or by $B$ cell metabolism and (2) if glucose regulates intracellular binding and activity of GK in insulin-secreting cells. Islets were incubated for $24 \mathrm{~h}$ with glucose, $\alpha$-ketoisocaproic acid (KIC) or mannoheptulose $(\mathrm{MH})$ and thereafter characterised for GK mRNA, protein and enzyme activity. Glucose $(10 \mathrm{mM})$ as well as $\mathrm{KIC}(10 \mathrm{mM})$ induced $\mathrm{GK}$ mRNA but not GK protein expression. In contrast only glucose was effective to induce GK enzyme activity. MH decreased intrinsic glucokinase activity below the activity level observed at $2 \mathrm{mM}$ glucose. Thus in B cells glucokinase mRNA is regulated by metabolism whereas GK enzyme activity was exclusively regulated by glucose. Permeabilization experiments on bioengineered GK overexpressing RINm5F insulinoma cells were performed to characterise intracellular GK binding and activity states. A diffusible GK fraction with high specific activity after permeabilization with digitonin could be distinguished from a matrix-bound fraction after extraction with Triton X-100. The low GK activity of the matrix-bound fraction was due to a hitherto unknown protein factor which was inactivated through proteolytic digestion or heat inactivation. Glucose $(10 \mathrm{mM})$ induced a significant long-term increase of the diffusible high activity GK fraction Our results provide evidence that a protein factor regulates the intracellular activity state of GK in insulin-secreting cells in dependence upon the nutrient state, which may function as a regulatory protein in the $B$ cell.
565

Secreted insulin stimulates glucokinase gene transcription in pancreatic $\beta$-cells

B. Leibiger, T. Moede, I.B. Leibiger, P.O. Berggren.

The Rolf Luft Center for Diabetes Research, Dept. Molecular Medicine, Karolinska Institute, S-171 76 Stockholm, Sweden

Control of the pancreatic $\beta$-cell transcription unit of the glucokinase gene $(\beta \mathrm{GK})$ has been suggested to be regulated by similar mechanisms as the insulin gene. Recently we were able to show that insulin, secreted in response to glucose stimulation, up-regulates the transcription of its own gene via the PI-3 kinase/p70s6 kinase and CaM kinase pathways. The aim of the present study was to evaluate whether insulin, secreted in response to glucose stimulation, activates $\beta G K$ transcription and if so whether the same signalling pathways as involved in insulin gene transcriptional control are involved. By employing nuclear run-off analysis we were able to show that stimulation of insulinproducing HIT-T15 cells with $5 \mathrm{mU}$ insulin/ml for $5 \mathrm{~min}$ led to an 4 -fold increase in $\beta \mathrm{GK}$ transcription initiation $30 \mathrm{~min}$ following start of stimulation. On line monitoring of $\beta G K$ promoter-driven GFP expression showed enhanced expression following stimulation with either $16.7 \mathrm{mM}$ glucose for 15 min or $5 \mathrm{mU}$ insulin/ml at substimulatory glucose concentrations in transfected primary $\beta$-cells or HIT cells. This elevation was abolished when insulin secretion was prevented with $\mathrm{L}$-type $\mathrm{Ca}^{2+}$ channel blockers and when blocking insulin signalling with the receptor tyrosine kinase inhibitor HNMPA-(AM) and was more pronounced following overexpression of insulin receptors. Combining insulin stimulation with the protein kinase inhibitor treatment showed that $\beta \mathrm{GK}$ promoter driven transcription was sensitive to CaM kinase inhibitors but not to inhibitors of PI-3 kinase or p70s6k, the latter kinases being involved in the control of insulin gene transcription. Therefore, our data show that $\beta G K$ transcriptional control does not employ signalling pathways identical to those of insulin gene transcription.

\section{6}

EFFECT OF D-GLUCOSE UPON D-FRUCTOSE METABOLISM IN RAT PANCREATIC ISLETS

O. Scruel, A. Sener and W.J. Malaise. Laboratory of Experimental Medicine, Brussels Free University, Brussels, Belgium

It was recently observed that D-glucose confers to glucokinase positive cooperativity towards D-fructose. This could conceivably result in an enhancing action of the aldohexose upon the ketohexose phosphorylation in pancreatic islet B-cells. The major aim of the present study was to investigate the latter hypothesis. The influence of D-glucose $(10 \mathrm{mmol} / \mathrm{l})$ upon the metabolism of $\mathrm{D}$-fructose (also $10 \mathrm{mmo} / \mathrm{l}$ ) was examined in rat pancreatic islets. The aldohexose decreased D-[5- $\left.{ }^{3} \mathrm{H}\right]$ fructose utilization by $14.2 \pm 3.7$ $\%$. It doubled, however, the oxidation of $\mathrm{D}-\left[\mathrm{U}-{ }^{14} \mathrm{C}\right] \mathrm{g}$ lucose, indicating that the aldohexose stimulates preferentially the oxidative, as distinct from anaerobic, modality of glycolysis. The glucose-induced increment in D-[U $\left.{ }^{14} \mathrm{C}\right]$ fructose oxidation was grossly proportional to the concentration of the aldohexose up to $10 \mathrm{mmol} / \mathrm{l}$, and progressively declined at higher concentrations of the latter sugar. D-glucose also augmented the oxidation of D-[2- $\left.{ }^{14} \mathrm{C}\right]$ fructose, $\mathrm{D}-[3,4-$ $\left.{ }^{14} \mathrm{C}\right]$ fructose and $\mathrm{D}-\left[6-{ }^{14} \mathrm{C}\right] \mathrm{glucose}$ to respectively $131.3 \pm 7.8 \%, 175.3 \pm 4.7$ $\%$ and $132.5 \pm 6.9 \%$ of control value, but failed to affect the oxidation of D$\left[1{ }^{14} \mathrm{C}\right]$ fructose which averaged $98.1 \pm 4.4 \%$ of its control value. Thus, $\mathrm{D}-$ glucose decreased the fractional contribution of the pentose shunt to the generation of $\mathrm{CO}_{2}$ and $\mathrm{D}$-glyceraldehyde 3-phosphate from the ketohexose. As judged from the paired ratio between ${ }^{14} \mathrm{CO}_{2}$ output and $\mathrm{D}-\left[5{ }^{3} \mathrm{H}\right]$ glucose utilization, the aldohexose slightly increased the inflow into the Krebs cycle of dicarboxylic metabolites derived from endogenous nutrients relative to that of fructose-derived acetyl-CoA. At the same concentrations of the two sugars, no significant effect of D-fructose upon $\mathrm{D}-\left[5{ }^{3} \mathrm{H}\right]$ glucose utilization or D-[U$\left.{ }^{14} \mathrm{C}\right]$ glucose oxidation was detected. It is proposed that the glucose-induced remodeling of D-fruetose metabolism may coordinate and optimize the B-cell secretory response to the two hexoses, e.g. following food intake.

\section{7}

OVER-EXPRESSION OF LACTATE DEHYDROGENASE A INHIBITS GLUCOSE-INDUCED INSULIN SECRETION IN MIN6 $\beta$-CELLS.

C. Zhao and G.A. Rutter. Department of Biochemistry, University Medical School, Bristol BS8 1TD, U.K.

Islet $\beta$-cells express very low levels of $L$-lactate dehydrogenase activity. In order to determine whether this is important for glucose-stimulated insulin secretion we have prepared MIN6 cell lines stably overexpressing lactate dehydrogenase A (LDHA) under cytomegalovirus immediate-early gene promoter control. cDNA encoding LDHA, subcloned into the mammalian expression vector pCDNA3, was introduced into MIN6 $\beta$-cells (passage \#18) by lipopolyamine-mediated tranfection. Stable clones were selected after 3 weeks, and expanded in the presence of $500 \mu \mathrm{g} \cdot \mathrm{m}^{-1} \mathrm{G} 418$. In mock-transfected cells, incorporating only the empty pCDNA3 backbone, LDH activity was $14.2 \pm 7.3$ nmol.min.mg.protein ${ }^{-1}(n=4$ separate cultures, mean \pm S.E.M). In these cells, elevated glucose concentrations ( 30 vs $3 \mathrm{mM}$ ) stimulated secretion by $10.4 \pm 1.6$-fold. By contrast, in two clones expressing LDH activity at $100 \pm 52.0$, and $91 \pm 37$ nmol.min.mg protein ${ }^{-1}$, insulin secretion was stimulated by glucose by 2.7 \pm 0.2 , and $2.1 \pm 0.4$-fold ( $n=6$ separate cultures), respectively. By contrast, the extent of stimulation of insulin secretion by high $\mathrm{K}^{+}(56 \mathrm{mM})$ was not significantly different in each cell line whereas the effect of 10 $\mathrm{mM}$ leucine was slightly enhanced in LDHA-expressing cell lines. Each cell line displayed closely similar activities of hexokinase, glucokinase, and malate dehydrogenase. Low levels of LDH activity would therefore appear important to ensure correct sensing of extracellular glucose by islet $\beta$-cells, possibly by ensuring high flux of glucose carbon towards mitochondrial oxidation. Changes in LDH activity may therefore contribute to loss of $\beta$-cell glucose responsiveness in some forms of noninsulin dependent diabetes mellitus. 
THE IMPACT OF CIS AND TRANS FATTY AICDS ON INSULIN RELEASE AND FATTY ACID OXIDATION IN INS-1 CELLS

K.K Alstrup, B.Brock and K.Hermansen, Department of Endocrinology and Metabolism, Aarhus University Hospital, Tage-Hansen gade 2, DK-8000 Aarhus C, Denmark Both in vitro and in vivo studies in animals have revealed that elevated levels of free fatty acids (FFA) induce impaired $\beta$-cell functions corresponding to the abnormalities being present in NIDDM. In addition to the level of fatty acids also the chain length and degree of unsaturation of importance. However, it is not known how the spatial configuration (cis/trans form) of fatty acids influences $\beta$-cell function. The aim of the present study was to elucidate the influence of the trans and cis forms of $\mathrm{Cl}$ 8:1 fatty acids on insulin release and fatty acid oxidation in the glucose sensitive $\beta$-cell line INS-1. INS-1 cells were cultured for 3 days in a modified RPMI medium containing different amounts of cis and trans fatty acids. After culture, insulin secretion during one hour at high glucose (glucose stimulated insulin secretion (GSIS)) and at low glucose (basal insulin secretion) as well as fatty acid oxidation were determined. Insulin was measured by RIA; fatty acid oxidation was estimated as production of ${ }^{14} \mathrm{CO}_{2}$ from $\left[\mathrm{U}^{14} \mathrm{C}\right]$ Palmitic acid during 2 hours. ANOVA followed by Newman-Keuls test was used for comparison. INS-1 cells cultured for 3 days in increasing concentrations $(0.05-0.4 \mathrm{mM})$ of fatty acids bound to $0.5 \% \mathrm{BSA}$ in the presence of $5.0 \mathrm{mM}$ glucose showed an increased basal insulin secretion for all tested fatty acids. However, $0.2-$ $0.4 \mathrm{mM}$ cis vaccenic acid (cis C18:1 $\Delta 11$ ) caused a higher basal insulin secretion compared to cells cultured with trans vaccenic acid (trans $C$ 18:1 $\Delta 11)(\mathrm{p}<0.05)$. When oleic acid (cis C 18:1 $\Delta 9$ ) and elaidic acid (trans C 18:1 $\Delta 9$ ) was tested a similar tendency could not be demonstrated. GSIS in cells cultured in increasing concentrations of oleic acid $(0.05-0.4$ $\mathrm{mM}$ ) was not altered. In contrast, GSIS was stimulated dose dependently with increasing concentrations of elaidic acid $(0.05-0.4 \mathrm{mM})$. Neither cis nor trans vaccenic acid did alter GSIS, Fatty acid oxidation in the presence of $3.3,8.3$ and $16.7 \mathrm{mM}$ glucose was increased for all fatty acids tested $(\mathrm{p}<0.05)$. In cells cultured with $0.4 \mathrm{mM}$ elaidic acid the fatty acid oxidation at 8.3 and $16.7 \mathrm{mM}$ glucose was significantly higher than in the presence of 0.4 $\mathrm{mM}$ oleic acid. Also $0.4 \mathrm{mM}$ trans vaccenic acid tended to increase the fatty acid oxidation compared to cis vaccenic acid at all glucose concentrations tested. In conclusion: We have demonstrated, that a differential effect on insulin release from Ins-1 cells after long term exposure to isomeric cis and trans fatty acids. The differential effect can not be described to changes in fatty acid oxidation
ALTERED LIPID RATHER THAN GLUCOSE METABOLISM CONTRIBUTES TO ELEVATED BASAL INSULIN SECRETION IN B(INS) CELLS CHRONICALLY EXPOSED TO FATTY ACIDS

F. Assimacopoulos-Jeannet', L. Segall ${ }^{2}$, E. Roche ${ }^{2}$, N. Lameloise ${ }^{1}$, B. Corkey ${ }^{3}$ and M. Prentki ${ }^{2}$

${ }^{1}$ University of Geneva, Switzerland, ${ }^{2}$ University of Montreal, Canada and ${ }^{3}$ Boston University, USA

Long term exposure of pancreatic islets or B(INS)-cells to free fatty acids (FFA) causes high basal secretion and impairs glucose induced insulin release. To bette understand the mechanism(s) whereby FFA cause these effects we sought to determine whether changes in glucose and/or lipid metabolism by FFA may explain these two alterations of insulin secretion. B(INS)-cells were exposed for 3 days to $0.4 \mathrm{mM}$ oleate bound to $0.5 \%$ BSA and were subsequently incubated for $30 \mathrm{~min}$ at low $(5 \mathrm{mM})$ or high $(20 \mathrm{mM})$ glucose, afterwhich various metabolites and parameters of cell activation were measured. With respect to glucose metabolism, glucose usage (assessed with ${ }^{3}[\mathrm{H}]$ glucose), glycogen, glucose-6-phosphate and citrate contents and the activity of pyruvate dehydrogenase were unaffected by oleate. Nonetheless, the fatty acid caused a modest $(25 \%)$ reduction in glucose oxidation at both glucose concentrations. With respect to fatty acid metabolism, oleate caused a marked deposition of triglycerides, increased fatty acid oxidation by twofold and reduced the basal concentration of malonyl-CoA (the physiological regulator of fat oxidation). $\mathrm{O}_{2}$ determinations and fluorimetric measurements of NAD(P)H indicated that a 3 day exposure of INS cells to oleate resulted in elevated basal respiration and redox state and a dampening of the glucose response for both respiration and $\mathrm{NAD}(\mathrm{P}) \mathrm{H}$. In addition the maximum rate of respiration of $\mathrm{NNS}$ cells, assessed in the presence of FCCP, was markedly increased. The data demonstrate that FFA alter the energy metabolism of $B(\mathrm{INS})$-cells. We conclude that the main response of INS cells to long term exposure to FFA is to increase the mitochondrial capacity to oxidize fatty acids, an event which may contribute to cause high basal insulin secretion via increased reducing equivalents and ATP production. By contrast, glucose metabolism remains largely unaffected by fatty acids and consequently changes in glucose metabolism cannot explain the altered secretory properties of cells chronically exposed to fatty acids.

\section{0}

FATTY ACID-REGULATION OF THE EXPRESSION OF UNCOUPLING PROTEN-2 IN INSULIN-PRODUCING CELLS. N. Lameloise', O. Boss', W-F. Pralong ${ }^{2}$, M. Prentki ${ }^{3}$, J-P. Giacobino' and F. Assimacopoulos-Jeannet ${ }^{1}$.

${ }^{1}$ University of Geneva, ${ }^{2}$ Modex Thérapeutiques Incl, Lausanne, Switzerland, ${ }^{3}$ University of Montreal, Canada.

Uncoupling protein-2 (UCP2) is expressed in a wide variety of tissues in rodents and humans. Its expression in yeast has been shown to partly uncouple respiration from ATP synthesis. In endothelial cells, the protein has been reported to decrease the production of free radicals. An increase in the cytosolic ATP/ADP ratio is one of the signals by which glucose stimulates insulin secretion. The presence of UCP2 in pancreatic $\beta$-cells could, by uncoupling the mitochondria, decrease ATP production in response to glucose and thereby decrease glucose-stimulated insulin secretion without any change in glucose metabolism. The expression of the protein was therefore studied in rat islets and in the $\beta$-cell line INS-1E. In INS-IE cells, UCP2 mRNA is increased 2 fold after $16 \mathrm{~h}$ exposure to 0.4 $\mathrm{mM}$ palmitate or oleate but remains unchanged by high glucose or leptin. In contrast, agents which increase cAMP, like forskolin, decrease UCP2 mRNA by $50 \%$. In INS-1E cells, analysis of the mitochondrial potential, using flow cytometry, shows a $12 \%$ decrease after long term (48h) but not short term exposure to fatty acids. Measurements of UCP2 by Western blotting show equal amounts of UCP2 in INS-IE cells and in isolated rat islets, where it is mostly expressed in the $\beta$-cell.

Altogether, the data indicate that the protein is present and its expression regulated by fatty acids in insulin-producing cells. The precise role of UCP2 in the mitochondria of $\beta$-cells (uncoupling, free radical generation, others) remains to be determined.

\section{1}

TEMPORAL RELATIONSHIPS IN THE INITIATION OF NUTRIENTINDUCED INSULIN RELEASE IN MOUSE PANCREATIC B-CELLS

G. Larsson-Nyrén and J. Sehlin. Department of Histology and Cell Biology, Umeå University, Umeå, Sweden.

Glucose-induced insulin secretion in isolated islets is characterized by a silent lag-period of about 1-2 min before the first-phase of insulin release. The mechanisms behind this lag-period are still unclear. The aim of the present study was to investigate the underlying mechanisms by studying the effects on lag-period of glucose concentration and nutrient secretagogues bypassing glycolysis, i.e. L-leucine and 2-

ketoisocaproate (KIC). We were using islet perifusion with high timeresolution - the perifusate was fractionated at $15 \mathrm{~s}$ intervals. Increase in glucose concentration from 3 to $7 \mathrm{mM}$ glucose elicited a significant stimulation of insulin secretion above baseline after $135 \mathrm{~s}(\mathrm{P}<0.05$ for the 135-150 s period). An increase in glucose concentration from 3 to 20 $\mathrm{mM}$ shortened the lag-time to $60 \mathrm{~s}$ ( $\mathrm{P}<0.005$ for the $60-75 \mathrm{~s}$ period). With a glucose concentration of $7 \mathrm{mM}$ during the preperifusion and a subsequent stimulation of the $B$-cells at $20 \mathrm{mM}$ glucose, a further reduction in the lag-time to $30 \mathrm{~s}$ ( $\mathrm{P}<0.02$ for the $30-45 \mathrm{~s}$ period) was observed.We also investigated the effects of the amino acid L-leucine and its deamination product, 2-ketoisocaproate (KIC) on the early insulin release dynamics. These nutrient stimuli of insulin secretion bypass the metabolic flux through the glycolytic pathway. Onset of the secretory response from $17 \mathrm{mML}$-leucine was elicited after $30 \mathrm{~s}$ $(P<0.005$ for the $30-45 \mathrm{~s}$ period). An even shorter lag-period was observed for $17 \mathrm{mM} \mathrm{KIC}$, i.e. $15 \mathrm{~s}$ ( $\mathrm{p}<0.005$ for the $15-30 \mathrm{~s}$ period). The results suggest that the lag-period observed when abruptly stimulating resting $B$-cells with a maximal glucose challenge may be partly due to the glycolysis. As it has previously been shown that B-cell ATP levels are initially lowered at such stimulation, it is suggested that the lag-period is due to the balance between ATP consumption and production initially counteracting an elevation of the $B$-cell ATP level. 


\section{2}

TIME RELATIONSHIP BETWEEN RISES IN REDUCED PYRIDINE NUCLEOTIDES AND INSULIN RELEASE

P. Lindström. Department of Histology and Cell Biology, Umeå University, Sweden

Abnormalities in first phase insulin release is an early indicator of faulty islet function in both Type I and Type II diabetes. To evaluate possible coupling of events for the initiation of insulin release, $I$ have dissected temporal relationships of components of rat pancreatic islet stimulus-secretion coupling. Isolated rat pancreatic islets were perifused under a microscope equipped for microfluorometry. Insulin release, NAD(P)H/NAD(P) ratios, and cytoplasmic calcium were studied in parallel. The rise in insulin release started later in experiments with mannose $(60-75 \mathrm{~s}, \mathrm{p}<0.05 \mathrm{Mann}$ Whitney $u$ test) when compared with glucose. Insulin release started earlier in overnight cultured islets stimulated with $20 \mathrm{mmo} / /$ glucose $(60 \mathrm{~s}, \mathrm{P}<0.05)$ or 3 $\mathrm{mmol} / \mathrm{l}$ glucose $+17 \mathrm{mmol} / \mathrm{l}$ mannose (75-90 s, $\mathrm{P}<0.05$ ) when compared with freshly prepared islets. The protein kinase C activator $100 \mathrm{nmol} / \mathrm{l}$ phorbol-12-myristate-13acetate shortened the lag time $(60-75 \mathrm{~s}, \mathrm{P}<0.01)$. There was no difference between 12 20 , or $30 \mathrm{mmol} / \mathrm{glucose}$ with regard to timing of onset of insulin release or rise in $\mathrm{NAD}(\mathrm{P}) \mathrm{H}$ fluorescence. The timing of rise in $\mathrm{NAD}(\mathrm{P}) \mathrm{H}$ was the same in experiments with mannose when compared with equimolar concentrations of glucose but the maximum effect of mannose was lower than that of glucose at all concentrations tested (up to $30 \mathrm{mmol} /$ ). After 4 days' culture the rise in $\mathrm{NAD}(\mathrm{P}) \mathrm{H}$ fluorescence started slightly earlier $(15 \mathrm{~s}, \mathrm{P}<0.01)$ then in freshly prepared islets. Increasing mitochondrial metabolism by $20 \mathrm{mmol} / /$ ketoisocaproate induced an earlier rise in $\mathrm{NAD}(\mathrm{P}) \mathrm{H}$ fluorescence and insulin secretion when compared with stimulatory glucose. The time of rise in cytoplasmic calcium was the same for glucose and mannose. Conclusion: It is probably not the time of onset of increased hexose metabolic flux or differences in the initial amount of glycolytic signal(s) for exocytosis that govern the timing of onset of exocytosis. Instead, mitochondrial metabolism (probably ATP production), degree of protein phosphorylation at late steps in the exocytotic process, and kinetic properties of the rate of generating cell messages may be important.

\section{3}

THEORETICAL STUDY OF THE ROLE OF Na/Ca EXCHANGE IN THE REGULATION OF THE $\beta$-CELL ELECTRICAL ACTIVITY.

D. Gall ${ }^{1}$, I. Susa ${ }^{2}$ A. Herchuelz ${ }^{1}$ and K. Bokvist ${ }^{3}$ ' Lab. de Pharmacodynamie, ${ }^{2}$ Service de Chimie Physique, Université Libre de Bruxelles, Brussels, Belgium and ${ }^{3}$ Islet Cell Physiology, Novo Nordisk A/S, Copenhagen, Denmark.

Glucose-stimulated insulin secretion from pancreatic $\beta$ cells is associated with the generation of electrical activity which consists in groups of action potentials superimposed on a plateau potential (bursts) interceded by silent more repolarized intervals. The burst duration is influenced by glucose concentration but the mechanisms involved remain the subject of much debate. The role of a Na/Ca exchange current in the regulation of the electrical activity of the mouse pancreatic $\beta$ cell was investigated by combining the patchclamp technique and mathematical modeling. We have recorded electrical activity from freshly isolated islets in the presence of 11 $\mathrm{mM}$ glucose before, during and after reducing extracellular $\mathrm{Na}$ concentration from $140 \mathrm{mM}$ to $30 \mathrm{mM}$. This partial removal of extracellular $\mathrm{Na}^{+}$results in a transient shortening of the plateau phase (during which action potentials are generated) and a slight (5 $\mathrm{mV}$ ) hyperpolarization. Moreover, we have extended existing mathematical models of the pancreatic $\beta$ cell to include the $\mathrm{Na} / \mathrm{Ca}$ exchange current. Our numerical simulations of $\beta$-cell electrical activity reproduce the observed effects of $\mathrm{Na}^{+}$-removal. Furthermore, the model predicts that the activity of the exchanger is influenced by glucose-induced changes in the cytoplasmic $\mathrm{Na}^{+}-$ concentration and that this could contribute to the effects of the secretagogue on $\beta$-cell electrical activity. We have also extended the model to a cluster of heterogeneous cells with gap-junctional coupling. Our results show that the exchanger plays a substantial role in the regulation of cytosolic free $\mathrm{Ca}^{2+}$ inside the islet.

\section{5}

HIERARCHY OF THE SIGNALS DRIVING PULSATILE INSULIN SECRETION

M.A. Ravier, P. Gilon and J.C. Henquin. Unité d'Endocrinologie et Métabolisme, University of Louvain, Brussels, Belgium.

Three types of signals occurring in $\beta$ cells have been proposed to trigger the oscillations of insulin secretion induced by glucose: oscillations of metabolism, cytoplasmic $\left[\mathrm{Ca}^{2+}\right]_{i}$ and protein kinase activity. This study was an attempt to establish the hierarchy between these three mechanisms. We followed the standard procedure of repetitive and sustained depolarizations of mouse islets with high $[\mathrm{K}]_{0}$ in the presence of diazoxide. In a medium containing $10 \mathrm{mM}$ glucose, repetitive increases of $[\mathrm{K}]_{0}$ induced rises in $\left[\mathrm{Ca}^{2+}\right]_{\mathrm{i}}$ and pulses of insulin secretion. Continuous stimulation of PKA by dibutyryl cAMP (or forskolin) or PKC by a phorbol ester (PMA) did not dissociate the $\left[\mathrm{Ca}^{2+}\right]_{i}$ and insulin responses from the $[\mathrm{K}]_{0}$ rises. However, the amplitude of the insulin pulses was consistently increased, while that of the $\mathrm{Ca}^{2+}$ pulses was either increased (PKA activation) or decreased (PKC activation). Receptor-mediated (acetylcholine + GLP-1) activation of both kinases increased the secretory response without altering the pacing imposed by the $\left[\mathrm{Ca}^{2+}\right]_{i}$ oscillations. When $\left[\mathrm{Ca}^{2+}\right]_{i}$ was steadily elevated by a sustained rise of $[\mathrm{K}]_{0}$, insulin secretion was stable. Under these conditions, oscillations of metabolism imposed by periodic variations in the glucose concentration resulted in damped oscillations of secretion, much smaller than those occurring when $\left[\mathrm{Ca}^{2+}\right]_{i}$ fluctuates and metabolism is stable. These results indicate that oscillations of $\left[\mathrm{Ca}^{2+}\right]_{i}$ exert a greater control on the oscillations of insulin secretion than do $\mathrm{Ca}^{2+}$-independent variations of protein kinase activity or of other metabolic signals. That oscillations of metabolism entrain $\left[\mathrm{Ca}^{2+}\right]_{i}$ oscillations remains possible. 


\section{6}

\section{PULSATLE INSULIN RELEASE FROM METABOLICALLY INHIBITED ISOLATED MOUSE ISLETS}

J. Westerlund. J.M. Lin, E. Mörtberg and P. Bergsten, Department of Medical Cell Biology, Uppsala university, Uppsala, Sweden.

Insulin release from islets is pulsatile, which is decisive for the appearance of plasma insulin oscillations. Type 2 diabetics have a deranged plasma insulin pattern. Although the cause of this alteration is not known disturbances in the $\beta$-cell metabolism are implicated in the pathogenesis of the disease. We have studied how the dynamics of insulin release are affected by metabolic inhibition. Insulin release from individual islets was measured with a sensitive ELISA in the presence of antimycin A (AA) or dinitrophenol (DNP). When $10 \mu \mathrm{M}$ AA was added to the perifusion medium insulin release decreased from $226 \pm 70$ to $6 \pm$ $1 \mathrm{pmol}^{*} \mathrm{~g}^{-1 *} \mathrm{~s}^{-1}$ within $1 \mathrm{~min}$ in the presence of $11 \mathrm{mM}$ glucose. The persisting secretory activity was oscillatory without change in frequency $(0.24 \pm 0.03 \mathrm{osc} / \mathrm{min})$ and was not further affected by prolonged exposure ( $30 \mathrm{~min}$ ) to AA. When metabolism was lowered by reducing the glucose concentration to $3 \mathrm{mM}$ glucose or omitting the sugar, insulin release was $2 \pm 1 \mathrm{pmol}^{*} \mathrm{~g}^{-1}{ }^{*} \mathrm{~s}^{-1}$ and oscillatory $(0.27 \pm 0.03 \mathrm{osc} / \mathrm{min})$, similar to what was found in the presence of $11 \mathrm{mM}$ glucose and $10 \mu \mathrm{M}$ AA. Insulin secretion in the presence of $3 \mathrm{mM}$ glucose or in the absence of glucose was not further reduced when 10 or $100 \mu \mathrm{M}$ AA or $250 \mu \mathrm{M}$ DNP was added to the perifusion medium. On the contrary, after prolonged ( $30 \mathrm{~min}$ ) exposure of the islets to the inhibitors, a gradual rise in secretion was observed, possibly related to increase in cytoplasmic $\mathrm{Ca}^{2+}$ concentration. The amplitude of glucose-stimulated pulses of insulin release was effectively reduced by metabolic inhibition without affecting the frequency of the insulin pulses. The remaining secretory activity, comparable to that obtained at low glucose concentrations, may represent a minimal secretory rate of the islet which cannot be reduced by metabolic inhibition. The deranged plasma insulin pattern in Type 2 diabetics may in part be due to reduction in insulin pulse amplitude from the islets.

\section{7}

ALLELIC VARIATION AT THE INS VNTR LOCUS IS ASSOCIATED WITH CHANGES IN THE PATTERN OF PULSATILE INSULIN RELEASE S. Almed ${ }^{1}$, S.T. Benmett ${ }^{2}$, S.J. Huxtable ${ }^{2}$, J.A. Todd ${ }^{2}$, D.R. Matthews ${ }^{1}$ and S.C.L. Gough ${ }^{3}$ ${ }^{1}$ Radcliffe infirmary, Oxford: ${ }^{2}$ University of Oxford and The Wellcome Trust Centre for Human Genetics, Oxford: ${ }^{3}$ University of Birmingham, Birmingham Heartlands Hospital. Allelic variation at the insulin gene (INS) VNTR (variable number of tandem repeats) locus on chromosome $11 \mathrm{p} 15.5$ is associated with type 1 diabetes, polycystic ovary syndrome and perhaps type 2 diabetes and hyperinsulinaemia. The INS VNTR has been shown to regulate INS expression although its effect on insulin secretion remains unclear. The aim of this study was to determine whether allelic variation at the INS VNTR locus is associated with differences in insulin secretion in healthy adult subjects. Thirty-one individuals with a normal OGTT underwent an intravenous glucose tolerance test with one-minute sampling, seventeen homozygote for the shorter class I VNTR allele and fourteen for the longer class III allele (matched for age and sex). Because of reported differences in class I allele transmission in type 1 diabetic families class 1 homozygotes had specific length class 1 alleles, sized accurately by fluorescence-based PCR and electrophoresis detection using ABI (373A) DNA sequencer. No significant differences were found in total insulin secretion $(p=0.30)$, first-phase insulin response $(p=0.68)$ or in beta cell function (HOMA) $(p=0.75)$ between the two groups. There were however differences in the pattern of basal pulsatile insulin secretion. Homozygotes for class I alleles had two dominant oscillatory cycles one with 9-minute oscillations, and the other with cycles of 13-minute periodicity. The class III homozygotes showed a single dominant peak corresponding to oscillations with a 14- minute periodicity. In addition, the peak after-load plasna glucose concentration was higher in those with class I alleles ( 15.29 vs $13.01 \mathrm{mmol} / \mathrm{l}, \mathrm{p}=0.03$ ). It is possible that basal insutin pulsatility affects glucose handling. Although major differences in the overall amount and rate of insulin secretion were similar in the two groups, there were differences in the pattern of secretion. The significance of this needs further evaluation.

\section{8}

DESENSITIZATION OF MITOCHONDRIAL $\mathrm{Ca}^{2+}$ AND INSULIN SECRETION IN THE $\beta$-CELL WITH PRESERVED ATP RESPONSE. P. Maechler, E.D. Kennedy, H. Wang, and C.B. Wollheim. Div. de Biochimie Clinique, University Medical Centre, Geneva, Switzerland.

The role of the mitochondria in the desensitization of insulin secretion to repeated stimuli was investigated. In rat pancreatic islet cells, insulin secretion stimulated by the mitochondrial substrate methyl-succinate was associated with a rise in mitochondrial $\left[\mathrm{Ca}^{2+}\right]$ $\left(\left[\mathrm{Ca}^{2+}\right]_{m}\right)$. Both secretion and $\left[\mathrm{Ca}^{2+}\right]_{m}$ were markedly blunted during a second pulse of the stimulus. In the $\beta$-cell line INS-1 similar results were observed when a $5 \mathrm{~min}$ interval separated two $5 \mathrm{~min}$ pulses. In contrast, ATP generation monitored in INS-1 cells expressing cytosolic luciferase rose to the same extent during both exposures to methylsuccinate. Succinate activates the electron transport chain at complex II, as does $\alpha$-glycerophosphate. As a consequence, the mitochondrial membrane hyperpolarizes, promoting ATP synthesis and $\mathrm{Ca}^{2+}$ influx into the mitochondria through the $\mathrm{Ca}^{2+}$ uniporter. Desensitization was further studied in Staphylococcus $\alpha$-toxin permeabilized INS-1 cells. increasing extramitochondrial $\left[\mathrm{Ca}^{2+}\right.$ ] from $100 \mathrm{nmol} / \mathrm{L}$ to $500 \mathrm{nmol} / \mathrm{L}$ enhanced succinate oxidation 4 fold. At $500 \mathrm{nmol} / \mathrm{L} \mathrm{Ca}{ }^{2+}, 1 \mathrm{mmol} / \mathrm{L}$ succinate caused a blunted $\left[\mathrm{Ca}^{2+}\right]_{\mathrm{m}}$ rise upon the second, compared to the first, stimulation. These effects were mimicked by $\alpha$ glycerophosphate and there was cross desensitization between the two compounds. The rise in $\left[\mathrm{Ca}^{2+}\right]$ was not attenuated when succinate was added at $500 \mathrm{nmol} / \mathrm{L} \mathrm{Ca}^{2+}$ following its application at $100 \mathrm{nmol} / \mathrm{L} \mathrm{Ca}^{2+}$, a condition in which no $\left[\mathrm{Ca}^{2+}\right]_{\mathrm{m}}$ increase occurs. This suggests that the uniporter itself, rather than the respiratory chain, is desensitized since the mitochondrial membrane was always hyperpolarized by succinate. Furthermore, succinate hyperpolarized the mitochondrial membrane both during first and second stimulation. These results emphasize the key role of the mitochondria not only in the stimulation of insulin secretion, but also in its desensitization.

\section{9}

ALTERATIONS OF CYTOPLASMIC CA ${ }^{2+}$ REGULATION IN MOUSE ISLETS UNRESPONSIVE TO GLP-1

P. Gilon, D. Flamez, A. Van Breuseghem, L. Schrocchi, D.J. Drucker, J.C. Henquin and F. Schuit. Unité d'Endocrinologie et Métabolisme UCL and Diabetes Research Centre VUB, Brussels, Belgium, and Banting and Best Diabetes Centre, Toronto, Canada.

It has been suggested that regular exposure to GLP-1 is important for $\beta$ cells to maintain normal responsiveness to glucose. Mice with a null mutation for the GLP-1 receptor (GLP-1 R -/-) display abnormalities of $\beta$ cell function in vivo and in vitro. Because of the critical role of $\mathrm{Ca}^{2+}$ in the regulation of insulin secretion, we compared the changes in cytoplasmic $\left.\mathrm{Ca}^{2+}\left(\mathrm{Ca}^{2+}\right]_{\mathrm{i}}\right)$ in islets isolated from control and (GLP-1 R /-) mice. The islets were cultured for 2 days before being loaded with fura-PE3 for $\left[\mathrm{Ca}^{2+}\right]_{i}$ measurement by microspectrofluorimetry. Increasing the glucose concentration from 3 to 15 mmol/ produced a biphasic rise in $\left[\mathrm{Ca}^{2+}\right]_{\text {, }}$, with oscillations during the second phase in control islets. In (GLP-1 $\mathrm{R}-/$-) islets, the rise in $\left[\mathrm{Ca}^{2+}\right]_{\mathrm{i}}$ was larger and without oscillations. Whereas most control islets did not show $\left[\mathrm{Ca}^{2+}\right]_{\mathrm{i}}$ changes, (GLP-1 R - ) islets usually displayed $\left[\mathrm{Ca}^{2+}\right]_{\mathrm{i}}$ oscillations in response to $7 \mathrm{mmol} / /$ glucose. This greater sensitivity of (GLP-1 R $-/$ ) islets to glucose cannot be ascribed to changes in $\mathrm{K}^{+}$-ATP or $\mathrm{Ca}^{2+}$ channels because tolbutamide and high $\mathrm{K}^{+}$did not produce larger $\left[\mathrm{Ca}^{2+}\right]_{i}$ rises in (GLP-1 R -/) islets than in controls. In contrast to GLP-1, that was ineffective in (GLP-1 R \%-) islets, other cAMP-raising agents (forskolin, GIP) similarly increased $\left[\mathrm{Ca}^{2+}\right]_{i}$ in control and (GLP-1 $\mathrm{R} \%$ ) islets. Image analysis revealed that the glucose-induced $\left[\mathrm{Ca}^{2+}\right]_{i}$ changes were less well synchronized between different regions in the (GLP-1 R -/) than in control islets. In conclusion, disruption of the GLP-1 receptor appears to increase the ability of $\beta$ cells to raise $\left[\mathrm{Ca}^{2+}\right]_{i}$ but decreases the synchrony of these changes within the islet. These abnormalities may contribute to alterations of insulin secretion. 
580

FUNCTIONAL HETEROGENEITY OF PANCREATIC ISLETS. T. Aizawa, T. Kaneko, M. Komatsu, H. Yajima, M. Nagai, K.

Yamauchi, and K. Hashizume. Shinshu University, Matsumoto, Japan. Insulin secretion by freshly isolated rat single islets was systematically analyzed at $37^{\circ} \mathrm{C}$ in $\mathrm{KRB}$ buffer. Islet size was individually measured with a microscope and small, medium and large were defined as the diameter being $<100 \mu \mathrm{m}, \geq 100$ and $<150 \mu \mathrm{m}$, and $\geq 150 \mu \mathrm{m}$, respectively. In perifusion, 32 islets (14 small, 10 medium, and 8 large) were singly placed into a mini-column, and perifused with a flow rate of $180 \mu \mathrm{l} / \mathrm{min}$ : glucose concentration was raised from 3 to $16.7 \mathrm{mM}$ and effluent collected at $1-5 \mathrm{~min}$ intervals for $30 \mathrm{~min}$. In incubation experiments, 60 islets ( 23 small, 22 medium, 15 large) were singly incubated in $0.22 \mathrm{ml}$ buffer, and sequentially exposed to $3,8.5,14.2$, and $20 \mathrm{mM}$ glucose for $30 \mathrm{~min}$ respectively: buffer was aspirated at the end of each $30 \mathrm{~min}-$ incubation for insulin RIA. In perifusion, $16.7 \mathrm{mM}$ glucose elicited a biphasic insulin release in all of large and in 9 medium islets. Whereas, it produced a gradually increasing 'monophasic' insulin release without the initial peak in 11 out of 14 small islets. Nevertheless, the release by the small islets was dependent on $\mathrm{Ca}^{2+}$ influx through the L-type voltagedependent $\mathrm{Ca}^{2+}$ channels because it was obliterated by nifedipine. In incubation, i) all islets but 3 small ones showed a concentration-dependent response to glucose, ii) the larger the islet, the lower the $\mathrm{EC}_{50}$, and iii) both basal and the maximum insulin output correlated well with the islet insulin content. Islet size and insulin content were positively cotrelated as well. In conclusion, 1) glucose-induced first phase insulin release consists of secretion predominantly from the larger islets, 2) high glucose elicits a concentration-dependent, not all or none, insulin release from cach islet, and 3) the larger the islets, the more insulin content and output. Change in islet subpopulation may well be an etiologic factor for physiological and pathophysiological alteration in insulin secretion.

\section{1}

IN VIVO EFFECTS OF HYPERGLYCAEMIA ON PANCREATIC D CELL MASS AND PLASMA SOMATOSTATIN CONCENTRATION IN RATS C. Magnan, V. Parent, J. Philippe*, and A. Ktorza. Université Paris 7 , CNRS ESA 7059, Paris, France, ${ }^{*}$ Centre Médical Universitaire, Genève, Suisse

Glucose not only stimulates insulin secretion and synthesis but also increases the pancreatic B cell mass. Glucose is also a potent stimulator of somatostatin release by the pancreatic D cell. However its effect on the $D$ cell mass is poorly documented. We investigated the effect of a $48 \mathrm{~h}$ glucose infusion in unrestrained normal rats on pancreatic $D$ cell mass and plasma somatostatin concentration. Plasma glucose was maintained at about $25 \mathrm{mM}$ throughout the infusion. This resulted in a fivefold increase in plasma insulin concentration. These rats were named HG-HI rats. Control rats were infused with saline (C rats). Using morphometric investigations, we observed a dramatic increase in D cell mass in HG-HI rats compared to controls (C: $14 \pm 4 \mu \mathrm{g}, \mathrm{n}=4$; HG-HI: $66 \pm 7 \mu \mathrm{g}, \mathrm{n}=4$, $\mathrm{p}<0.001)$. Plasma somatostatin levels were significantly increased during the first $6 \mathrm{~h}$ of glucose infusion (from $26 \pm 1$ to $37 \pm 3 \mathrm{pg} / \mathrm{ml}, \mathrm{n}=8, \mathrm{p}<0.01$ ) but dropped thereafter to basal values until $48 \mathrm{~h}$. Because this situation could be related to an interplay between hyperglycaemia and hyperinsulinaemia, $48 \mathrm{~h}$ euglycaemic / hyperinsulinaemic clamps were performed in unrestrained rats by simultaneous infusion of glucose and insulin which induced a rise in the plasma insulin level similar to that observed in HG-HI, whereas glycaemia remained similar to those of HG$\mathrm{HI}$ rats (EG-HI rats). In EG-HI rats, D cell mass was unchanged compared to controls (C: $14 \pm 4 \mu \mathrm{g}, \mathrm{n}=4$ : EG-HI rats: $13 \pm 3 \mu \mathrm{g}, \mathrm{n}=4$, NS). Plasma somatostatin was not modified by insulin under euglycaemic conditions. In conclusion, a $48 \mathrm{~h}$ increase in plasma glucose but not in plasma insulin concentration has a potent trophic effect on pancreatic D cell mass. This effect is paralleled by an increase in $D$ cell function in the first $6 \mathrm{~h}$ of glucose infusion, which can be thereafter counterbalanced by the inhibitory effect of insulin. These inhibitory effects seems require high plasma glucose concentration as reported by Gerber and coworkers (Endocrinology, 1981, 109).

\section{2}

Oscillatory insulin secretion after i.v. glucose stimulus in controls and newly diagnosed type 2 diabetic patients

H.J. Lüddeke, R. Renner and K.D. Hepp Krankenhaus München Bogenhausen, Diabeteszentrum Iil.Med.Abt 81925 München Englschalkingerstr. 77, Gemany

Basal oscillatory insulin secretion is well documented whereas little is known about the pulsatile characteristics of insulin secretion after i.v. glucose stimulation. Patients : 6 newly diagnosed male type 2 diabetic patients, 6 male controls. Age $55 \pm 9(41 \pm 7)$, BMI $27,1 \pm 1,3(24,8 \pm 1,0) \mathrm{HbA1c} 7,1 \pm 0,8$ $(5,1 \pm 0,3)$. Methods : modified IVGTT ( $0,3 \mathrm{~g}$ glucose $/ \mathrm{kg}$ i.v. in $30 \mathrm{sec}$. Insulin and glucose at $-30,-10,-5$, then simultaneously in periods of 1 minute from 0 to $30 \mathrm{~min}, 40,50,60,90,120,150,180 \mathrm{~min}$. Insulin with double antibody Elisa, proinsulin free (Abbot), Glucose with GOD-method (Beckman analyzer). Results : Individual and mean secretion profiles in diabetic patients and controls demonstrate an oscillatory time course. During a 30 min period 3 ground waves (including the first insulin peak) can be detected. The first peak in controls and diabetics was measured after an average time period of 4 (4-5) min after injection. Area under the curve of this peak for the diabetic patients is about $40 \%$ of the controls. The maximum of the second peak in controls is at $10(9-11) \mathrm{min}$, in patients at 14 (14-15) $\mathrm{min}$. The third peak reaches its maximum in controls at $22(21-24)$ min and varies between 20-24 min in patients. In the course of these secretion peaks the oscillatory amplitude decreases in the controls and increases in patients. Conclusion Poststimulatory insulin secretion ist pulsatile in controls and even more pronounced so in patients. The time period between the first two peaks is shorter, the period between the second and third peak nearly equals the oscillation period in the basal state.Apparently the maximum secretion in type 2 diabetic patients is reached by means of cycles with increasing amplitudes.

\section{3}

Induction or Rapid Pursatile Insulan Secretion in Healthy Humans by Periodic GLUCOSE INFUSION.

N. Pørksen, C. Jurl, T. Grøfte, M. Holi.ingdal,J. Sturis,J. D.Veldhuis and O. SCHMITZ". Arhus and Copenhagen, Denmark, and Charlottesville, VA, USA.

The release of insulin is coordinated as common secretory bursts. This pattern is quantitatively dominant as at least $75 \%$ of overall insulin release and is important for optimal insulin action on liver, muscle and adipose tissues. An intrapancreatic (neuronal) pacemaker has been hypothesized as coordinating mechanism, since the pattern is preserved in the isolated perfused pancreas. In contrast, ultraradian pulses may be entrained by oscillatory glucose infusion. The possible influence of glucose concentration changes to induce insulin secretory bursts is not known. Therefore the purpose of the present study was to examine if pulsatile insulin secretion may be induced by small changes in the circulating glucose concentrations. To examine this, we studied 13 healthy subject following overnight fast, by minutely blood collection from a peripheral vein for analysis of insulin and glucose concentration time series. 6 subjects were studied with glucose infusion of $6 \mathrm{mg} / \mathrm{kg} / \mathrm{min}$ for 1 minute every 10 minutes from 10 to 90 minutes. In a matched control group (n=7) no glucose was infused. The concentration time series were analyzed for significant periodicities and timely correlation by autocorrelation and cross correlation analysis. Insulin concentration time series were deconvolved for analysis of secretory patterns allowing estimates of mean puise interval, mass amplitude, duration and for basal (time-invariant) secretion. We found that infusion of glucose at $6 \mathrm{mg} / \mathrm{kg} / \mathrm{min}$ caused oscilations in the glucose concentrations of $\sim 5 \%(\sim 0.25 \pm 0.05 \mathrm{mM})$, resulting in oscillations of $\sim 100 \pm 15$ $\%$ in insulin concentrations with similar periodicities at a lag time of 2 minutes Deconvolution analysis revealed $(0.01<p<0.00001)$ higher secretory burst mass $(47$ $\pm 10 \mathrm{vs} 16 \pm 3 \mathrm{pmol} / \mathrm{L} / \mathrm{pulse}$ ) and interval $(9.8 \pm 0.3 \mathrm{vs} 6.1 \pm 0.5 \mathrm{~min} / \mathrm{pulse}$ ) with less variability in interval (CV $8 \pm 2$ vs $29 \pm 3 \%$ ) and very high degree of coordination of release into common bursts at glucose induction. Breakthrough pulsatile release between induced pulses was not observed. We conclude that the in vivo pulsatile insulin reiease pattern may be induced by discrete episodic glucose infusion, resulting in small glucose changes and large insulin oscillations. We believe that this novel method may be important in assessing beta cell sensitivity to glucose at disease, predisease and conditions of pathological metaholism. 
DISORDERLY INSULIN RELEASE PROCESSING IN NIDDM ASSESSED BY APPROXIMATE ENTROPY

O.Schmitz, N. Porksen, C. Juhl, J.D. Veldhuis, P.C. Butler, and S.M. Pincus. Aarhus, Denmark; Charkttesville, VA, USA; Edinburgh, Scotland; and Guilford, CT, USA In vivo insulin release is characterized by a pulsatile secretory pattern, reflected as quantifiable oscillations of peripheral insulin concentrations. Individual $\beta$-cell secretion is likely resulting from serial metabolic events including cyclic glycolysis, depolarization, influx of calcium and exocytosis. NIDDM is characterized by impaired insulin secretion including disturbed pulsatile insulin release. The latter may signify defect steps involved in the cyclic release pattern. Approximate Entropy (ApEn), a scale independent method for quantification of data regularity, has proven useful in detection of pathophysiological pituitary, adrenal and islet hormonal release patterns, assessed as increased disorderliness (increased ApEn values). ApEn measures logarithmic likelihood that runs of pattems reproduce on next incremental comparison. For insulin increased disorderliness evidence defects in the cyclic release proces or dyscoordinate release from islet population. To assess if insulin release is disorderly in NIDDM we studied 14 NIDDM patients (D) and 10 healthy control (C) subjects (fasting plasma glucose $8.7 \pm 0.5$ vs $5.2 \pm 0.1 \mathrm{mM}$ ). Following an ovemight fast blood (1 cc) was collected minutely for 75 minutes, and serum analyzed for insulin in triplicate by ELISA (intraassay $\mathrm{CV}<3 \%$, and interassay $\mathrm{CV}<5 \%$ ). Insulin concentration time series were analyzed by spectral analysis, and for regularity by ApEn. Insulin concentrations tended to be higher in D $(42 \pm 5 \mathrm{VS} 34 \pm 6 \mathrm{Pm})$. Spectral analvsis density was lower in $\mathrm{D}(0.15 \pm 0.03$ vs $0.26 \pm 0.04, \mathrm{p}<0.05)$. Furthermore data regularity was significantly reduced in $\mathrm{D}$ (ApEn $0.80 \pm 0.01$ is $0.75 \pm 0.008$ $p=0.006)$ and $A p E n$ values correlated to fasting glucose $(p=0.02, r=0.61)$. Increasing glucose concentration in $\mathrm{C}$ by glucose infusion did not affect $A \mathrm{pEn}$ values, indicating that the detected disorderliness in $\mathrm{D}$ was not due to acute hyperglycemia. We conclude that the insulin release processing in NIDDM is characterized by significant disorderliness. This may reflect impairment of steps involved in the cyclic $\beta$-cell metabolic processess or dyscoordination.
PS 13

\section{Modulation of Insulin Secretion}

\section{5}

INSULINOTROPIC ACTION OF THE POLYACETATE ESTER OF NONNUTRIENT MONOSACCHARIDES

W.J. Malaisse, A. Laghmich, L.E. Flores and M.M. Kadiata. Laboratory of Experimental Medicine, Brussels Free University, Brussels, Belgium

The pentaacetate ester of $B$-L-glucose was recently reported to stimulate insulin release, albeit to a lesser extent than $\alpha$ - or $\beta$-D-glucose pentaacetate. It was now observed that other polyacetate esters of non-nutrient monosaccharides also display positive insulinotropic action. For instance, the anomers of 2-deoxy-D-glucose tetraacetate, whilst inhibiting insulin release evoked by D-glucose $(8.3$ and $16.7 \mathrm{mmol} / \mathrm{l})$ when tested at a $10.0 \mathrm{mmol} / \mathrm{l}$ concentration, augment insulin output from islets exposed to D-glucose (8.3 $\mathrm{mmol} / \mathrm{l})$, L-leucine $(10.0 \mathrm{mmol} / \mathrm{l})$ or succinic acid dimethyl ester (SAD; 10.0 $\mathrm{mmol} / \mathrm{l}$ ) when tested at concentrations in the 0.2 to $1.7 \mathrm{mmol} / \mathrm{l}$ range. Both the negative and positive insulinotropic effects of 2-deoxy-D-glucose tetraacetate are more pronounced in the case of the $\alpha$ - than $\beta$-anomer. Likewise, D-mannoheptulose hexaacetate $(1.7 \mathrm{mmol} / \mathrm{l})$, which inhibits both the metabolism and secretory response to D-glucose, enhances insulin release from islets exposed to SAD $(10.0 \mathrm{mmol} / \mathrm{l})$. Under suitable experimental conditions, L-glucose pentaacetate, 2-deoxy-D-glucose tetrancetate and Dmannoheptulose hexaacetate (all $1.7 \mathrm{mmol} / \mathrm{l}$ ) also augments insulin secretion in islets from diabetic Goto-Kakizaki rats. Relative to the paired reference value recorded in the absence of these or other monosaccharide esters, their enhancing action upon insulin release is often more marked in the diabetic rats than in control animals. For instance, such is the case for the increases in insulin output evoked by $\alpha$-D-glucose pentaacetate in the presence of $\mathrm{L}$ leucine, 2-deoxy-D-glucose tetraacetate in the presence of D-glucose, and either B-D-glucose pentaacetate or D-mannoheptulose hexaacetate in the presence of SAD. The anomeric preference may be perturbed, however, in the diabetic rats. These findings raise the idea that selected esters of non-nutrient carbohydrates could be used as insulinotropic tools in NIDDM.

\section{6}

\section{STIMULATION OF INSULIN SECRETION BY SEMICYCLIC AMIDINES}

S. Ullich*, S. Hartmann ${ }^{5}$ and A.W. Frahm ${ }^{\text {s }}$ Instiute of Physiology (*) and Department of Pharmacy ${ }^{5}$ ), Albert-Ludwigs-University, Hermann-Herder-Str. $7\left(^{*}\right)$ and 9 ( $),$ D - 79104 Freiburg, Germany.

Semicyclic amidines are known to stimulate insulin secretion. The aim of the present study was to investigate whether $(R, R)-/(S, S)$-enantiomers of these amidines exert distinct effects on insulin secreting cells. Therefore, two series of enantiomerically pure (97-99 \%) cis-2-substituted (Isopropyl-, Phenyl-, Cyclohexyl-, Benzyl-, Cyclopentyl- and Cyclohexylmethyl-) $\mathrm{N}$ cyclopentaneamidines have been synthesized. At the highest concentration tested $(100 \mu \mathrm{mol} / \mathrm{l})$ the amidines had no toxic effects on insulin-secreting INS- 1 cells. All 24 compounds elevated insulin release between $44 \%$ and $376 \%$ at $10 \mu \mathrm{mol} / 1$, whereby effectiveness increased with increasing steric hindrance of the $\alpha$-carbon atom attached to the lactamidine function Surprisingly, the most hydrophobic 2-cis-cyclohexylmethyl-substituted semicyclic amidine, which stimulated insulin release $3.5 \pm 0.3$-fold $(n=$ 12) at $10 \mu \mathrm{mol} / \mathrm{l}$, abolished secretion at higher concentrations $(100 \mu \mathrm{mol} / \mathrm{l})$. Enantiomer discrimination (between $20 \%$ and $60 \%$ ) was observed in some cases such as 2-cis-cyclohexyl- and -cyclopentyl-substituted compounds. To investigate the underlying mechanism effects on membrane voltage, membrane conductance and $\left[\mathrm{Ca}^{2+}\right]_{j}$ have been studied. Semicyclic amidines depolarized INS- 1 cells and generated action potentials. This was accompanied by a decrease of membrane conductance from $20.7 \pm 3.7 \mathrm{nS}$ to $4.9 \pm 1.4 \mathrm{nS}(\mathrm{n}=7)$. At the same time, $\left[\mathrm{Ca}^{2+}\right]_{\mathrm{i}}$ and $\left[\mathrm{Ca}^{2+}\right]_{\text {; }}$-oscillations increased. At high concentrations, where inhibition of secretion has been observed, $\left[\mathrm{Ca}^{2+}\right]_{\mathrm{i}}$ still rose upon application of the amidine. This indicates an additional inhibitory pathway downstream to the elevation of $\left[\mathrm{Ca}^{2+}\right]_{i}$. In conclusion, semicyclic amidines stimulate insulin secretion by depolarisation-induced increases of $\left[\mathrm{Ca}^{2+}\right]_{i}$. 
Stimulation of insulin release by a new compound S-22068. Evidence for a novel imidazoline binding site in $B$ cells L. Le Brigand ${ }^{1}$, A. Virsolvy 1 , D. Manechez ${ }^{2}$, J. . Godfroid ${ }^{3}$, B. Guardiola-Lemaitre ${ }^{2}$, F. M. Gribble ${ }^{4}$ F. M. Ashcroft ${ }^{4}$ and D. Bataille ${ }^{1} .{ }^{1}$ Inserm U376, Montpellier, 2I.R.I.S, Courbevoie, ${ }^{3}$ Laboratoire de Pharmacologie Moléculaire, Paris, France, 4University Laboratory of Physiology, Oxford, United Kingdom.

We investigated the mechanism of action of S-22068, a new drug with an imidazoline structure displaying interesting features for treatment of type II diabetes. Its effect on insulin release and on calcium fluxes in the MIN6 B cell line was compared to that of other secretagogues such as glucose, sulfonylureas or classical imidazolines. We show that S-22068 stimulates insulin release via an increase in the intracellular calcium concentration. S-22068-stimulated insulin release was totally suppressed by inhibitors of voltage-dependent calcium channels and by activators of ATP-dependent potassium (K $\mathrm{K}_{\mathrm{ATP}}$ ) channels. This mechanism of action resembles that of sulfonylureas, although S-22068 did not interact with the sulfonylurea receptor. Using patch-clamp experiments on Xenopus laevis oocytes injected with cloned Kir6.2, we observed that S-22068 acts directly on this K ATP channel subunit, similarly to other imidazolines. On the other hand, S-22068 stimulated insulin release even at non-stimulatory glucose concentrations, in contrast to efaroxan, an imidazoline whose activity is strictly dependent upon glucose stimulation. This discrepancy suggests that the new drug acts through a novel type of binding site, a hypothesis confirmed by the observation that efaroxan, RX821002 and phentolamine, three imidazoline compounds which stimulate insulin release, inhibited the specific binding of $3 \mathrm{H}-\mathrm{R} \times 821002$ to the low affinity imidazoline binding site, whereas S-22068 did not. In conclusion, S-22068 stimulates insulin release via its interaction with the Kir6.2 subunit of $\mathrm{K}_{\mathrm{ATP}}$ channels through a novel binding site and might be the basis for a new pharmacological approach to the management of type II diabetes.

\section{9}

THE IMIDAZOLINE RX871024 INDUCES PROSTGLANDIN SYNTHESIS AND INHIBITS INSULIN SECRETION FROM FRESHLY ISOLATED RAT ISLETS

M. Mourtada, S.A.Smith* and N.G. Morgan. Department of Biological
Sciences, Keele University, Staffs, UK and "Department of Vascular Sciences, Keele University, Staffs, UK and *Department of Vascular
Biology, SmithKline Beecham Pharmaceuticals, Harlow, Essex, UK.

Imidazoline derivatives have attracted attention as possible insulin secretagogues for use in type II diabetes and recently one such compound, RX871024, was reported to stimulate insulin secretion from cultured islets. We have studied the effects of this agent in freshly isolated and cultured rat islets and have observed that it exerts opposite effects on insulin secretion under these two conditions. In islets cultured for $18 \mathrm{~h}$ in RPMI-1640, RX871024 induced a concentration-dependent increase in insulin secretion induced by $20 \mathrm{mM}$ glucose $\left(\mathrm{EC}_{50} \sim 30 \mu \mathrm{M}\right)$ whereas, in fresh islets, it caused a marked inhibition of glucose-induced insulin secretion (20mM glucose: $6.0 \pm 0.38 \mathrm{ng} / \mathrm{islet} / \mathrm{h} ; 20 \mathrm{mM}$ glucose +

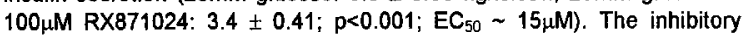
response to RX871024 was not altered by co-incubation with $\alpha_{2^{-}}$ antagonists confirming that it was not mediated by any $\alpha_{2}$-agonist activity of the compound. By contrast, the inhibitory effect of RX871024 was prevented by inclusion of either indomethacin or flurbiprofen to inhibit islet cyclo-oxygenase activity (20mM glucose: $4.7 \pm 0.34 \mathrm{ng} / \mathrm{islet} / \mathrm{h}$; $20 \mathrm{mM}$ glucose $+100 \mu \mathrm{M}$ RX871024:2.4 $\pm 0.39 ; 20 \mathrm{mM}$ glucose +

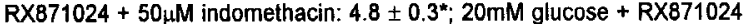
$+50 \mu \mathrm{M}$ flurbiprofen: $4.4 \pm 0.22^{*} ;{ }^{*} p<0.001$ relative to RX871024). In support of this, inhibition of insulin secretion by RX871024 correlated with an increase in islet prostaglandin $E_{2}$ production (20mM glucose: $47.3 \pm$

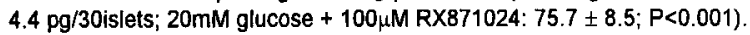
Overall, these results suggest that RX871024 can induce an increase in islet prostaglandin production which then causes inhibition of glucoseinduced insulin release. These findings cast doubt on the therapeutic potential of RX871024 as an insulin secretagogue.
588

SENSITIZATION OF ISLETS TO GLUCOSE - A NEW ACTION FOR IMIDAZOLINE INSULIN SECRETAGOGUES

S.L.F. Chan, D. Manechez* and N.G. Morgan. Cellular Pharmacology Group, Keele University, U.K. and ${ }^{*}$ Institut de Recherches Internationales Servier, Courbevoie, France.

Prior short term exposure of pancreatic $\beta$-cells to a variety of compounds, including high glucose, increases their insulin secretory response to subsequent re-stimulation: a phenomenom known as "priming" or "sensitization". In this study, we have investigated whether imidazoline compounds, a recently-characterised class of insulin secretagogues, are also able to prime the insulin secretory response of rat islets to a glucose challenge. Efaroxan $(100 \mu \mathrm{M})$ caused a marked monophasic and reversible increase in insulin release in islets perifused at $5 \mathrm{mM}$ glucose (mean secretion rate over exposure period, $1.39 \pm 0.08 \mathrm{pg} / \mathrm{islet} / \mathrm{min}$ versus control, $0.43 \pm 0.01$; $p<0.001$ ). When the glucose concentration was subsequently increased to $10 \mathrm{mM}$, these efaroxan-pretreated islets responded with a significantly enhanced rate of insulin release $(1.43 \pm 0.06 \mathrm{pg} /$ islet/ $\mathrm{min}$ versus $0.89 \pm 0.04$; $\mathrm{p}<0.001$ ). By contrast, $100 \mu \mathrm{M}$ idazoxan (an imidazoline which does not potentiate insulin release), was not able to sensitize islets to glucose. The priming response was not, however, unique to efaroxan since both antazoline and a newly-synthesized imidazoline, S22068, also sensitized rat islets to glucose. Thus, S22068 $(100 \mu \mathrm{M})$ caused a significant increase of the secretion rate at $5 \mathrm{mM}$ glucose $(0.98 \pm 0.11 \mathrm{pg} /$ islet/ min versus $0.42 \pm 0.02 ; \mathrm{p}<0.001)$, which was readily reversible upon removal of the drug. A subsequent increase in glucose concentration (from $5 \mathrm{mM}$ to $10 \mathrm{mM}$ ) was accompanied by a marked enhancement of secretion from these S22068-pretreated islets $(1.39 \pm 0.07 \mathrm{pg} /$ islet/ $\min$ versus $0.74 \pm 0.03 ; \mathrm{p}<0.001)$. These results provide the first evidence that imidazoline insulin secretagogues can sensitize islets to glucose and support the proposal that this new class of drugs may be useful adjuncts for use in the management of type II diabetes.
590

\section{4-HYDROXY-2-NONENAL INHIBITS GLUCOSE-INDUCED} INSULIN SECRETION

I. Miwa, S. Taniguchi, and N. Ichimura, Meijo University, Nagoya, Japan

Oxidative stress is elevated in diabetes mellitus, resulting in the acceleration of lipid peroxidation. The effect of 4-hydroxy2-nonenal (HNE), one of the major aldehydic products of lipid peroxidation, on the ability of isolated rat pancreatic islets to secrete insulin was examined in this study. HNE concentrationand time-dependently inhibited insulin secretion induced by glucose: insulin secretion was inhibited by $50 \%$ when measured after incubation of islets with $100 \mu \mathrm{mol} / \mathrm{L} \mathrm{HNE}$ for $1 \mathrm{~h}$. HNE at $100 \mu \mathrm{mol} / \mathrm{L}$, however, did not inhibit insulin secretion induced by leucine, but rather stimulated it. Insulin secretion induced by glyceraldehyde was inhibited by only $15 \%$ by $100 \mu \mathrm{mol} / \mathrm{L}$ HNE. On the other hand, insulin secretion induced by glyceraldehyde + glucose was inhibited by $60 \%$ by $100 \mu \mathrm{mol} / \mathrm{L}$ HNE. Effect of HNE on lactate production and some enzyme activities in islets revealed that the glycolytic pathway, the mitochondrial catabolism of pyruvate, the glycerol phosphate shuttle, and the pyruvate malate shuttle are not the site of action of HNE. We conclude that HNE, at concentrations likely to occur in tissues, preferentially inhibits glucose-induced insulin secretion, although the target of the aldehyde is not yet known 


\section{1}

INHIBITION OF INSULIN SECRETION FROM PANCREATIC $\beta$-CELLS BY BUTANEDIONE MONOXIME

G.-D. Li and R.H. Luo. National University Medical Institutes, National University of Singapore, Singapore

The effect of 2,3-butanedione monoxime (BDM), a chemical phosphatase that interferes with actin-myosin interaction via inhibiting myosin kinases, on insulin secretion was investigated in transformed $\beta$-cells (HIT-T15). Pre-treatment of cells with BDM for 30 min inhibited insulin secretion from intact cells both at resting (glucose-free medium) and upon stimulation by secretagogues in a dosedependent manner. Insulin release induced by $\mathrm{KCl}(40 \mathrm{mM})$, which depolarizes membrane potential resulting in opening voltage-sensitive $\mathrm{Ca}^{2+}$ channels, was reduced maximally by $74 \%$ over $30 \mathrm{~min}$ by $50 \mathrm{mM}$ BDM, with an $\mathrm{IC}_{30}$ of $\sim 20$ $\mathrm{mM}$. No BDM effect occurred below $3 \mathrm{mM}$. Resting insulin secretion was diminished by $48 \%$ with $50 \mathrm{mM}$ BDM. Activation protein kinase A (PKA) by raising cyclic AMP with forskolin did not relief the inhibitory effect of BDM on insulin secretion, since it also decreased insulin release due to glucose $(10 \mathrm{mM})$ plus forskolin $(1 \mu \mathrm{M})$, with a similar dose-response pattern to that seen in high $\mathrm{K}^{+}$stimulation. Insulin secretion during both acute phase $(0-15 \mathrm{~min})$ and late phase (16-35 $\mathrm{min}$ ) of stimulation was affected, albeit a tendency of more potent inhibition on the later phase. In contrast to intact cells, $30 \mathrm{mM}$ BDM failed to alter either basal insulin release (at $0.1 \mu \mathrm{M} \mathrm{Ca}^{2+}$ ) or exocytotic secretion triggered by $\mathrm{Ca}^{2+}(2-20 \mu \mathrm{M})$ from digitonin-permeabilized cells over 8-min stimulation. Therefore, the effect of BDM on cytosolic free $\mathrm{Ca}^{2+}$ concentrations $\left(\left[\mathrm{Ca}^{2+}\right]_{i}\right)$ was also examined. $\left[\mathrm{Ca}^{2+}\right]$, rises evoked by $10 \mathrm{mM}$ glucose or $40 \mathrm{mM} \mathrm{K}{ }^{+}$were inhibited by BDM higher than $10 \mathrm{mM}$ and the dose-response relationship was similar to its inhibition of insulin secretion in intact cells. This BDM effect was observed either after pre-exposure to the compound or when it was added during elevation of $\left[\mathrm{Ca}^{2+}\right]$. These results suggest that BDM inhibits insulin release from $\beta$-cells mainly via blocking voltage-operated $\mathrm{Ca}^{2+}$ conductance, probably by modulating non-PKA-mediated phosphorylation status of the $\mathrm{Ca}^{2+}$ channels.

\section{3}

MINIGLUCAGON (GLUCAGON 19-29) INHIBITS, IN THE PM RANGE, INSULIN RELEASE THROUGH A CA ${ }^{2+}$-DEPENDENT PATHWAY

S. Dalle, P. Blache, F. Bergeron, D. Le-Nguyen and D. Bataille, INSERM U 376, CHU Arnaud-de-Villeneuve, 34295 Montpellier, France

Miniglucagon, the C-terminal (19-29) fragment of glucagon, is processed from the mother-molecule and modulates its action. The islets A-cells contain miniglucagon ( $3-5 \%$ of the glucagon content). We addressed the possibility of a control of insulin release by miniglucagon which, accordingly, might act as a local regulator of the islet physiology. Using the MIN-6 cells which respond to glucose in the physiological range as a cellular model, we show that miniglucagon, at concentrations ranging from 0.01 to $1000 \mathrm{pM}$, dose-dependently inhibited insulin release whatever the secretagogue used for stimulation (glucose, glucagon, tGLP-1 or sulfonylurea) with half-maximal inhibition close to $1 \mathrm{pM}$. At $1 \mathrm{nM}$, miniglucagon almost completely reversed the secretagogue action. While miniglucagon did not introduce any change in the basal, glucagon or tGLP1-stimulated cyclic AMP levels, calcium influx was reduced in parallel with inhibition of insulin release. When opening voltage-sensitive calcium channels using Bay $\mathrm{K}-8644$ or $\mathrm{KCl}$ as a depolarising agent, miniglucagon blocked insulin release and calcium uptake in the same picomolar range, strongly suggesting that the miniglucagon action involves closure of that channels. The miniglucagon action was totally abolished after pre-treatment of the cells with pertussis toxin (PTX). It is concluded that miniglucagon is a good candidate for being a novel local regulator of islet physiology. Its mode of action is independent from cyclic AMP and appears to imply the closure of the beta cell voltage-sensitive calcium channels via a PTX-sensitive G-Protein.
592

EXPRESSION OF MULTIPLE SOMATOSTATIN RECEPTOR SUBTYPES IN ZUCKER RAT ISLETS AND FUNCTIONAL TYPING OF THE SOMATOSTATIN INDUCED INHIBITION OF INSULIN SECRETION

A.L. Pallett', V. Emilsson', D. Coy ${ }^{2}$, J. Taylor ${ }^{3}$, C-W Woon ${ }^{3}$ and M.A Cawthorne ${ }^{1}$. Clore Laboratory, University of Buckingham, UK (1) University of Tulane, New Orleans, Lo, USA (2) and Biomeasure Inc., Milford, Ma, USA (3)

Somatostatin receptor (SSTR) sub-types are characterised by different analogue specificities and distinct but often overlapping specificity of tissue distribution. In the present study we have examined the expression of the 5 SSTR in rat islets and determined which receptor sub-type mediates inhibition of insulin secretion. We find mRNA expression of all five receptor sub-types occurs in isolated pancreatic islets of lean Zucker rats but in age-matched obese fa/fa rats, receptor expression is reduced. The mean fold reduction is SSTR-1, 2.6; SSTR-2, 2.3; SSTR-3, 2.2; SSTR-4, 3.0 and SSTR-5, 2.0-fold. A series of analogues designed to have selectivity for particular sub-types, particularly SSTR-5 and SSTR2 , have been synthesised and their sub-type selectivity evaluated using ligand binding and microphysiometer studies in $\mathrm{CHO}$ cells expressing the individual cloned rodent and human SSTR sub-types. Inhibition of glucose-stimulated insulin secretion was determined using isolated pancreatic islets from Wistar rats. Analogues of somatostatin that are selective for the type 5 SSTR such as BIM 23268, BIM 23295 and BIM 23052 are potent inhibitors of insulin secretion, whereas SSTR-2 agonists were essentially inactive. BIM 23268 inhibits glucose-stimulated insulin secretion with an EC50 of $0.43 \mathrm{nM}$. BIM 23268 is also selective for the human SSTR-5 receptor. These studies suggest that BIM 23268 is a potential inhibitor of inappropriate insulin secretion in man.

\section{4}

GLUCAGON RECEPTORS ON HUMAN ISLET-CELLS CONTRIBUTE TO GLUCOSE COMPETENCE OF INSULIN RELEASE

P. Huypens, Z. Ling, D. Pipeleers and F. Schuit. Diabetes Research Center, Vrije Universiteit Brussel, Brussels, Belgium.

Glucose-induced insulin release from pancreatic $\beta$-cells is controlled by a synergistic interaction of signals derived from glucose metabolism and cAMP which is produced after receptor stimulation by peptides of the secretin family. The role of glucagon-like peptide-1 (7-36)amide and glucose-dependent insulinotropic polypeptide as gut hormones amplifying glucose-induced insulin release has been well documented in rodents and in man. However the participation of pancreatic glucagon in this regulating process is still controversial. The present study has investigated expression and activity of glucagon receptors in human islet cell preparations, containing at least 45 percent insulin-positive $B$-cells. Analysis of human islet cell RNA revealed the presence of glucagon receptor mRNA with a partial ( $689 \mathrm{bp}$ ) sequence that is identical to the previously published human liver glucagon receptor mRNA. Incubation of human islet cells with $1 \mu \mathrm{M}$ of the glucagon-receptor specific antagonist des-His ${ }^{1}$-[Glu $\left.{ }^{9}\right]$-glucagon-amide abrogated $55 \pm 7$ $\%$ of the glucose-induced insulin release from these cells (mean $\pm \mathrm{SEM} ; \mathrm{n}=5 ; \mathrm{P}<0.05$ ). This antagonist also suppressed the insulin secretory response to glucagon, glucagon-like peptide-1 (736)amide and glucose-dependent insulinotropic polypeptide by approximately 55\%. In conclusion, these data document the presence of glucagon receptors in human islets and illustrate their functional role in $\beta$-cells. They are consistent with the occurrence of type II diabetes in individuals with mutations in the glucagon receptor gene. 
THE INSULINOTROPIC EFFECT OF ENDOTHELIN-1 IS MEDIATED INDIRECTLY VIA THE ISLET $\alpha$-CELLS: EVIDENCE FROM LIGAND BINDING AND SECRETORY STUDIES. Gregersen $^{1}$, S., Brock ${ }^{1}$, B., Kofod ${ }^{2}$, H., and Hermansen ${ }^{1}, K$. 'Dept. of Endocrinology and Metabolism', Aarhus University Hospital, ${ }^{2}$ Novo Nordisk, Bagsvaerd, Denmark.

The endothelium derived peptide endothelin-1 (ET-1) is present in elevated circulating concentrations in diabetes and hypertension. The peptide has potent insulinotropic actions in vivo and in vitro and these effects of ET-1 were originally thought to be direct $\beta$-cell effects. We have, however, not been able to a show direct insulinotropic action of ET-1 nor evidence for ET-receptors on B-cells. Instead our recent data suggests that the islet $\alpha$-cells may play an important role for the ET-1-induced insulin release. To further explore this possibility we have performed ligand-binding studies on $\alpha$-TC1.9 cells. ${ }^{125}$ I-ET- 1 was used as tracer and BQ123 $\left(\right.$ ET $_{\mathrm{A}}$-receptor antagonist) and $B Q 788\left(E_{B}\right.$-receptor antagonist) for displacement studies. Interestingly, a specific binding of ${ }^{123}$ I-ET-1 was found on the $\alpha$-cells ( $k_{d}$ approximately $10 \mathrm{nM}$ ). The binding of ${ }^{123}$ I-ET-1 could be displaced by BQ123 whereas BQ788 was unable to displace. Thus, the $\mathbf{E T}_{\mathrm{A}}$-receptor subtype is present on the $\alpha$-cells. Subsequently, we wanted to see if this receptor is functional and carried out incubation experiments on $\alpha$-TC1.9 cells with and without ET-1 (100 $n M)$. We found a slight, but significant $(p<0.05)$ increase in the ET1-induced glucagon secretion. In conclusion: specific $\mathbf{E T}_{\mathbf{A}}$ receptors exist on the $\alpha$-cells and ET-1 stimulation leads to glucagon secretion. We suggest that the $\alpha$-cells plays a crucial role for the insulinotropic action of ET-1. The intraislet action of ET-1 therefore seems to be of a unique paracrine nature.
Involvement of adrenergic receptors in the regulation of insulin release in transgenic mice with overexpressed B-cell glucocorticoid receptors

Zong-Chao Ling, Behrous Davani, Claes-Göran Östenson, Sam Okret, Suad Efendić, and Akhtar Khan

Dept. of Molecular Medicine, Karolinska Hospital and Dept. of Medical Nutrition, Huddinge Hospital, Karolinska Institute, Stockholm, Sweden.

Transgenic mice (TG) with overexpressed glucocorticoid receptors in the beta cell demonstrate the inhibition of glucose-induced insulin release both in vivo and in isolated islets. Islet glucose-6-phosphatase activity and glucose cycling (glucose $\longrightarrow$ glucose-6-P) was also increased in these islets. In the present work we studied the effect of $\alpha-2$ adrenergic blocker benextramine $(10 \mu \mathrm{M})$ on glucose-induced insulin release as well as the activity of glucose-6-phosphatase in islets from TG and normal mice. Glucose-6-phosphatase activty was determined in sonicated islets by measuring the hydrolysis of $\left[C_{-}{ }^{14}\right]$ glucose-6-P to $\left[C-{ }^{14}\right]$ glucose. As expected islets from TG mice secreted less insulin (UU/isleth) than control islets at $16.7 \mathrm{mM}$ glucose [120.6 \pm 7.9 vs $166.6 \pm 9.6 ; p<0.001,(n=12)]$ Benextramin completely normalized insulin release $(n=5)$ in TG mice islets (167.7 \pm 9.9 vs $113.1 \pm 3.6$ in untreated islets) but did not influence insulin release in control islets. In parallel, benextramine significantly decreased glucose-6-phosphatase activity (pmol/islet/h) $(n=5)$ in TG mice islets (6.10 \pm 0.5 vs $3.3 \pm 0.2 ; p<0.01)$ and had no effect on control islets $(2.9 \pm 0.2$ vs $2.6 \pm 0.3$. These findings suggest that glucocorticoid mediated inhibition of insulin release involves $\alpha$-adrenergic receptors which in turn augments glucose-6-phosphatase activity and decreases insulin release.
597

EFFECT OF PROLONGED EXPOSURE TO HIGH GLUCOSE ON THE RELEASE OF INSULIN AND PROINSULIN BY HUMAN B CELLS K. Hostens, Z. Ling. D. Pipeleers and C. Van Schravendijk. Diabetes Research Center, Vrije Universiteit Brussel, Laarbeeklaan 103, B-1090 Brussels, Belgium

Diabetes is often associated with a discordance in circulating levels of proinsulin and insulin, which is responsible for an increased proinsulin over insulin ratio. The present study investigates whether such discordance can occur as a result of prolonged exposure to elevated glucose concentrations. Human islets preparations $(n=6)$ were cultured for 10 to 13 days at either 6 (control) or $20 \mathrm{mmo} /$ liter (high) glucose. High glucose culture led to a 5-fold lower cellular insulin content ( $p<0.05$ versus control) but did not affect the proinsulin content so that the cellular ratio in proinsulin over insulin was markedly increased ( 10 percent versus 2.6 in control). Glucose-induced insulin release from high glucose cultured cells was, in absolute terms, 2 -fold lower than in control preparations; when corrected for their lower cellular insulin content, these cells exhibited the same secretory activity as control cells, confirming that lower amounts of released insulin in type 2 diabetes do not necessarily reflect an impaired glucose regulation of release. Furthermore, proinsulin release from high glucose cultured cells was significantly elevated both in relative and in absolute terms, a finding which also correlates with in vivo observations in type 2 diabetes. The underlying mechanisms for this increased proinsulin release have not yet been fully identified. The observed changes in proinsulin and insulin release result in 7- and 11fold $(p<0.05)$ higher proinsulin over insulin ratios in the medium collected after, respectively, 5 and $20 \mathrm{mmol} /$ iter glucose pulses. It is concluded that prolonged exposure of human $B$ cells to high glucose levels leads to a lower glucose-induced insulin release and higher proinsulin release. This secretory pattern is caused by the sustained state of activation in the majority of $B$ cells, and includes a depletion in the cellular content of insulin but not proinsulin.

\section{8}

RATE OF CHANGE OF GLUCOSE CONCENTRATION HAS LITTLE IMPACT ON HUMAN INSUL!N SECRETION RATES

J. Sturis, C. Juhl, T. Grøtte, O. Schmitz and N. Pørksen, Scientific Computing, Novo Nordisk A/S, Bagsværd, Denmark, and Medical Department M, Aarhus University Hospital, Aarhus, Denmark.

The secretion of insulin is dependent on many variables and presumably primarily on concentrations of circulating glucose. A physiologically relevant control mechanism would involve detection of changes in the glucose concentration to adjust for rapidly increasing or decreasing glucose concentrations. A rate-sensor (for rapidly increasing glucose concentration) has been reported in the isolated perfused rat pancreas. We aimed to assess the role of increasing and decreasing glucose concentrations on overall insulin release rate. Six healthy non-obese volunteers were studied on three separate occasions after a 10-hr overnight fast, after which insulin, C-peptide and glucose concentrations were measured every $5 \mathrm{~min}: 1$ ) following $60 \mathrm{~min}$ of glucose intusion $(10 \mathrm{mg} / \mathrm{kg} / \mathrm{min}$ ) the infusion was stopped and samples collected until glucose was normalized, 2) following $60 \mathrm{~min}$ at basal conditions, the glucose infusion rate was stepwise increased every $40 \mathrm{~min}$ to 2,4 , and $8 \mathrm{mg} / \mathrm{kg} / \mathrm{min}$ and 3) following $60 \mathrm{~min}$ of glucose infusion at $8 \mathrm{mg} / \mathrm{kg} / \mathrm{min}$, this infusion was stepwise decreased to 4,2 , and 0 $\mathrm{mg} / \mathrm{kg} / \mathrm{min}$. Insulin secretion rates were calculated from C-peptide concentrations by deconvolution analysis using standard kinetic parameters. A linear regression model with glucose concentration and delta glucose (defined as the change in glucose concentration between successive sampling points) as the independent variables and with insulin secretion rate as the dependent variable was used on all the data pooled. $A$ good model fit was observed $(r=0.75)$, and not surprisingly, the calculated insulin secretion rates were highly influenced by the prevailing glucose concentrations $(p<0.0001)$, whereas the rate of change in the glucose concentration did not contribute significantiy $(p=0.17)$. Our study suggests that in healthy, non-obese humans, insulin release is predominantly influenced by overall glucose concentration, whereas we did not find a significant impact of the glucose concentration change. This finding may be dependent on the relatively slow changes in glucose concentration in our study. 
Mechanism and Location of the Distal Inhibitory Effect of Norepinephrine and Somatostatin on Insulin Secretion. Geoffrey W.G. Sharp, Samira Daniel, Mitsuhiko Noda, Linming Shen, and Susanne G. Straub. Department of Pharmacology, Comell University, Ithaca, NY, USA.

In the $\beta H C-9$ and HIT cell lines, we have shown that norepinephrine and somatostatin inhibit insulin secretion at a "distal" site in stimulussecretion coupling. This was demonstrated in two ways. First, by the inhibition of $\mathrm{Ca}^{2+}$-stimulated insulin secretion in $\beta \mathrm{HC}-9$ cells permeabilized with $\alpha$-toxin, so that the intracellular compartment is clamped and unaffected by inhibitor action on ion channels. Secondly, by depolarization of HIT cells and simultaneous $\mathrm{Ca}^{2+}$ and capacitance measurements under patch clamp conditions. To determine the location of the site at which this distal inhibition occurs, we identified the docked granule pool in the BHC-9 cell. This was done by immunoprecipitation of the core complex of docked granules. We co-immunoprecipitated the granule SNARE protein synaptobrevin by antibodies against syntaxin, and co-immunoprecipitated the plasma membrane SNARE protein syntaxin by antibodies against synaptobrevin. We then showed that the core complex was associated with the $\alpha$-subunits of $\mathrm{Gi}$ and Go, the heterotrimeric G-protein mediators of inhibitor action. Subsequently, we measured the core complex associated with the docked granule pool after stimulation of the cells with glucose or $\mathrm{KCl}$, in the presence or absence of norepinephrine. Stimulation of release (exocytosis) by glucose or $\mathrm{KCl}$ resulted in discharge of the docked granules. In the presence of norepinephrine, insulin secretion was blocked and neither glucose nor $\mathrm{KCl}$ discharged the docked granules. Thus, the site of the distal inhibitory action is at a point after granule docking and prior to the exocytotic fusion step in stimulus-secretion coupling. In additional studies, we tested the hypothesis that protein phosphatase 2B (calcineurin) is a mediator of the distal inhibitory effect. Inhibition of insulin release was unaffected by the protein phosphatase $2 \mathrm{~B}$ antagonists cyclosporin A or deltamethrin. Thus, calcineurin appears not to be involved in the mechanisms of inhibition of insulin release.

\section{PS 14}

\section{Cytokines and $\beta$-Cell Degeneration}

\section{1}

INSULIN AND GLUCAGON RELEASE OF ISLETS PREPARED FROM A TYPE 1 DIABETIC PATIENT

P. Marchetti, R. Lupi, S. Del Guerra, F. Dotta, C. Lencioni, E. Anastasi, L. Marselli, M. Carmellini, R. Navalesi. Dip. Endocrinologia e Metabolismo, Pisa, Italy

In Type 1 diabetes (DM) insulin release (IR) is reduced or absent, and glucagon secretion (GS) in response to glucose and/or aminoacids is altered. A direct in-vitro characterization of the function of islets (Isls) from DM patients has never been performed. We report on IR and GS of Isls isolated from a 14 yr-old DM girl (HLADR3 positive; ICA, anti-GAD and anti-IA2 positive) who died accidentally shortly after diagnosis. Insulin content (IC) of freshly prepared DM Isis ranged 2.1 to 4.9 $\mathrm{ng} /$ islet (about $25 \%$ of control Isls, Ctrl). IR during static incubation ( $\mu \mathrm{U} / \mathrm{islet} / 45$ min, mean \pm d) from DM Isls was $0.8 \pm 0.1$ at $3.3 \mathrm{mM}$ glucose $(3.3 \mathrm{G}$ ) and increased significantly at $3.3 \mathrm{G}$ plus $20 \mathrm{mM}$ arginine (Arg, $1.5 \pm 0.6), 16.7 \mathrm{mM}$ glucose $(16.7 \mathrm{G}$ $1.6 \pm 0.7)$, and $16.7 \mathrm{G}$ plus $1 \mathrm{mM}$ IBMX $(2.0 \pm 0.2)$. These figures were consistently lower than the respective values from Ctrl. Perifusion studies showed that the reduction occurred with both the $1 \mathrm{st}$ and 2 nd phase IR. When normalized for IC, the differences between DM Isls and Ctrl remained significant at $16.7 \mathrm{G}(1.6 \pm 0.3 \mathrm{vs}$ $2.6 \pm 0.6 \%)$ and $16.7 \mathrm{G}$ plus IBMX $(1.9 \pm 0.2$ vs $3.0 \pm 0.2 \%)$. After 3 weeks of culture, IC of both DM Isls and Ctrl decreased of 50-60\%. IR was still lower from DM Isls when expressed as $\mu U /$ islet $/ 45 \mathrm{~min}$, but not when normalized for IC (DM Isls vs Ctrl: $3.8 \pm 2.4$ vs $3.0+0.7 \%$ at $3.3 \mathrm{G}, 9.7 \pm 3.4$ vs $6.5 \pm 2.2 \%$ at $3.3 \mathrm{G}$ plus $\mathrm{Arg}$, and $7.8 \pm 1.2$ vs $6.5 \pm 0.5 \%$ at $16.7 \mathrm{G}$. Following exposure for $24 \mathrm{~h}$ to either 50 $\mathrm{U} / \mathrm{ml} \mathrm{IL}-1$ beta, or a combination of IL-1 beta, gamma interferon $(1,000 \mathrm{U} / \mathrm{ml})$ and TNF alpha $(1,000 \mathrm{U} / \mathrm{ml}), \mathrm{IR}$ changes from the cultured TyDM islets were more severe than from non-diabetic islets, including no significant increase of IR at $16.7 \mathrm{G}$ vs $3.3 \mathrm{G}$ with the single cytokine and paradoxical reduction of IR at $16.7 \mathrm{G}$ with the cytokine mixture. GR (pg/ml) from DM Isls during static incubation was similar at $3.3 \mathrm{G}(20.3 \pm 1.1)$ as $16.7 \mathrm{G}(20.9 \pm 1.8)$, and the defect of suppression by high glucose (suppression that was normally observed with Ctrl), did not change upon culture. The addition of $20 \mathrm{mM}$ Arg to $3.3 \mathrm{G}$ caused a similar, significant increase of GR from both DM Isls $(24.4 \pm 1.9)$ and Ctrl $(27.6 \pm 1.8)$. Thus, IR from the Isls of this DM patient showed defects that were mainly quantitative; however, the improvements of IR after a period of culture suggests that some of the alterations were due at least in part to the native islet environment. GR from DM Isls showed loss of suppression by high glucose (that remained unchanged upon culture), and a maintained sensitivity to arginine.
Abnormal islet glucose Sensing Induced ey Chronic Microimfusion of NOREPINEPHRINE AND SEROTONIN IN VENTROMEDIAL HYPOTHALAMUS OF HAMSTER.

Y. Liang,S.Q..Luo, H.sheng, and A.H.Cincotta. Ergoscience, Boston, USA

Important roles for hypothalamic neuroendocrine regulation of metabolism in the pathogenesis of diabetes are becoming increasingly evident. For example, the ventromedial hypothalamus ( $\mathrm{VMH}$ ) plays an important role in the regulation of insulin release. Furthermore, a significant increase in VMH monoamine (norepinephrine and serotonin) level has been observed in type II diabetic animal models. The aim of this study was to investigate whether chronic infusion of norepinephrine $(\mathrm{N}, 0.6 \mu \mathrm{mol} / \mathrm{day})$ and serotonin ( $\mathrm{S}, 60 \mathrm{nmol} /$ day) into the VMH could disrupt normal islet glucose (G) sensing. Hamsters receiving a 5 -week infusion of $\mathrm{N} / \mathrm{S}$ became insulin resistant and $G$ intolerant compared with controls receiving vehicle (V). Using isolated islets and static incubation, we found that the dose-dependent curve of $G$ stimulated insulin release was markedly shifted to the left in islets from the $\mathrm{N} / \mathrm{S}$ group compared with islets from $\mathrm{V}$ group. There was a 4-fold increase in insulin release at $5 \mathrm{mM} \mathrm{G}(19.9 \pm 3.7$ vs. $5.1 \pm 1.0 \mathrm{fmol} / \mathrm{ng} \mathrm{DNA} / \mathrm{h}$, respectively, $\mathrm{P}<0.01)$ and 1.7 -fold increase at $7.5 \mathrm{mM} \mathrm{G}(42.7 \pm 5.6$ vs. $25.8 \pm 5.6 \mathrm{fmol} / \mathrm{ng}$ DNA/h, respectively, $P<0.05)$. At higher $G$ concentrations $(10-20 \mathrm{mM})$, no significant difference in insulin release was observed between $N / S$ and $V$ groups. Islet perifusion with $15 \mathrm{mM} \mathrm{G}$ showed similar kinetics of bi-phasic insulin release between N/S and V groups. Microinfusion of $\mathrm{N}$ or $\mathrm{S}$ alone had no such significant effect on islet glucose sensing. Neither islet cAMP content (basal or after $15 \mathrm{~min}$ exposure to 5 or $15 \mathrm{mM} \mathrm{G}$ ) nor islet tissue PKC- $\alpha$ and PKC- $\varepsilon$ contents (tested by immunoblotting) differed between $\mathrm{N} / \mathrm{S}$ and $\mathrm{V}$ groups. However, islet insulin-release responses to neurotransmitters $(1 \mu \mathrm{M}$ norepinephrine or $10 \mu \mathrm{M}$ acetylcholine) added to the incubation buffer were lost in the N/S group compared with that in the V group. Our results suggest that abnormal islet glucose sensing induced by N/S microinfusion into the VMH may be partly the consequence of changes in autonomic nervous system control of the endocrine pancreas.

\section{2}

GROWTH FACTOR EFFECTS ON FAS-MEDIATED APOPTOSIS OF CYTOKINE-TREATED NEONATAL RAT ISLETS OF LANGERHANS M.Harrison., IC. Green. Biochemistry Laboratory, University of Sussex, Department of Pharmacy, University of Brighton, E. Sussex, U.K

We have previously shown that cytokines induce cell death in islets by apoptotic and non-apoptotic processes. We have found that exposure to cytokines induces expression of the apoptotic cell death mediating receptor, Fas. The aim of this study was to determine if pre-treatment of neonatal rat islets with the growth factors, IGF, TGFB, or insulin would protect them from cytokine induced, Fas-mediated, apoptosis. Neonatal rat islets were obtained through collagenase digestion of pancreata and pretreated, groups of 200 islets were treated with either TGFß $(3.3 \mathrm{x}$ $\left.10^{-1} \mathrm{M}\right)$, IGF $\left(10^{-8} \mathrm{M}\right)$ or insulin $(25 \mathrm{ng} / \mathrm{ml})$ for $24 \mathrm{~h}$. Islets were then treated with the combined cytokines, IL-1B (50pM), TNF $\alpha$ (50pM) and IFN $\gamma\left(5 \mathrm{Uml}^{-1}\right)$ for a further 24 hours and made into single cells. These single cells were incubated $\left(37^{\circ} \mathrm{C}\right)$ with or without a cytolytic anti-Fas antibody and cell counts were carried out at timed intervals to determine Fas-mediated cell loss. Even without the addition of the cytolytic anti-Fas antibody, cytokine treated islets had $32.7 \% \pm 2.6$ less cells than control islets. One hour after trypsinisation, the cytokine treated cells were reduced in number by $50 \% \pm 10.6$ vs. $25 \% \pm 5.6$ for control islet cells. Growth factor pre-treatment resulted in improved cell survival-IGF had $13 \%$ more cells, TGFB $21 \%$ and insulin $10.3 \%$ and after $60 \mathrm{~min}$, IGF $19.8 \%$, TGFB $18.5 \%$, insulin $9.6 \%$ (although the insulin effect was not significant). No change in this reversal of cell loss was observed for cells incubated with the cytolytic anti-Fas antibody. We conclude that growth factors do protect against cytokine-induced cell death but not via the Fas-mediated programmed cell death pathway. 


\section{3}

PROMOTER POLYMORPHISMS IN THE INOS GENE AND STRAIN-DEPENDENT DIFFERENCE IN SENSITIVITY OF ISLETS OF LANGERHANS TO IL-1 $\beta$

J. Johannesen, F. Pociot, A.E. Karlsen, T. Mandrup-Poulsen, J. Nerup. Steno Diabetes Center, Gentofte, Denmark

Nitric oxide (NO) may be a necessary but not sufficient mediator of cytokine mediated selective $\beta$-cell destruction. Previously, we have described a NO correlated difference of $\mathrm{LL}-1 \beta$ sensitivity in vivo and in vitro of islets from two rat strains, Brown Norway (BN) being more resistant than Wistar Kyoto (WK). The aim of the study was to i) examine the time-response of IL-13 effects on isolated islets from both strains and ii) sequence the iNOS promoter region of these rat strains for polymorphisms. Methods: 150 islets from 5-7 day-old rats of both strains were incubated in $300 \mu \mathrm{l}$ RPMI $1640+0.5 \%$ HS with $0-150 \mathrm{pglL}-1 \beta / \mathrm{ml}$ (dose-response) for 2 48 hours (time-response). The incubation media were examined for insulin and nitrite accumulation. Semi-quantitative iNOS mRNA analysis was performed and the iNOS promoter region from both strains cloned and sequenced. Results: Dose-response experiments (24 hours $\mathrm{IL}-1 \beta$ exposure) showed that insulin and nitrite responses were dose and strain dependent (all $\mathrm{p}<0.02,2$ way ANOVA). At $15 \mathrm{pgl \textrm {L }}-1 \mathrm{~B} / \mathrm{ml}$ higher nitrite accumulation was seen for WK vs BN $\langle 370 \pm 68,1$ vs $284 \pm 102 \%$ of ctr., $p<0.05$ ). Time-response experiments ( $15 \mathrm{pglL}$ $1 \mathrm{~B} / \mathrm{m}$ ): Insulin and nitrite responses correlated significantly with time and strain (all $p<0.03,2$ way ANOVA). After $12 \mathrm{hrs}$ of IL-1 $1 \beta$ incubation nitrite was only released from WK islets $(p<0.02)$. The INOS mRNA content, expressed relative to a house-keeping mRNA ( $\beta$ glycoronidase), was determined after 4,12 and 24 hours of IL-1 $\beta$ incubation with a peak value at 12 hrs for both strains. NNOS mRNA response significantly correlated to time and strain ( $p=0.0002$ and 0.01 , resp., 2 way ANOVA). Polymorphism screening: 2042 bp of the promoter region and exon 1 were cloned from $B N$ and WK. In the $5^{\prime}$ end of the cloned promoter region a deletion of $10 \mathrm{bp}(\mathrm{WK})$ and $\mathrm{a}$ ' $\mathrm{T}$ ' $(\mathrm{BN}) \rightarrow{ }^{\prime} \mathrm{C}$ ' (WK) substitution were identified in the same GTrepeat structure. Finally, a 'A' (BN) $\rightarrow$ 'G' (WK) polymorphism was seen in exon 1 (the 5 'UTR of the iNOS gene). Conclusion: BN and WK respond with different sensitivities to IL-1 $\beta$ in-vitro by dose-response and time-response and in vivo. The differences, especially in the $5^{\prime}$ end of the iNOS promoter, may have functional implications.

\section{4}

REGULATION OF RAT AND HUMAN ISLET NITRIC OXIDE SYNTHASE BY GLUCAGON AND EFFECTS ON INSULIN SECRETION

V. D. Belin, J. G. Mabley, R. F. L. Jamesa, S. M. Swiffa, H. Clayton a and I. C. Green. Biochemistry Department, University of Sussex and Pharmacy Department, University of Brighton, Brighton U.K. aDepartment of Surgery, University of Leicester, Leicester, U.K. Cytokines induce nitric oxide synthase (iNOS) in rat and human islets of Langerhans and in the insulin secreting cell line RIN5F. The subsequent nitric oxide formation inhibits insulin secretion. We have previously found that glucagon $(0.1,1 \mu \mathrm{mol} / /)$ decreases iNOS activity and expression induced by $1 \mathrm{~L}-1 \mathrm{~B}(10 \mathrm{pmol} / \mathrm{l})$ in RIN5F cells. The aim of this study was to investigate the effect of glucagon and cyclic AMP (forskolin) on cytokine induction of nitric oxide synthase and cytokine action, in rat and human islets. The insulin secretory response was assessed by radioimmunoassay and iNOS expression was determined by Wesfern blotting. IL-1 B (100pmol/l) decreased glucose-induced insulin secretion of rat islets, from 2.0 to $0.4 \mathrm{ng}$ insulin/islet $(p<0.001)$. Glucagon $(0.01,0.1,1 \mu \mathrm{mol} / l)$ partially reversed interleukin-1B's inhibitory effect from 0.4 to $1.1,1.2$ and $0.9 \mathrm{ng}$ insulin/islet respectively $(p<0.05)$. Treatment of human islets with a combination of cytokines (IL-1B 100pmol/l, TNF $100 \mathrm{pmol} / \mathrm{l}$, IFN $\gamma 500 \mathrm{U} / \mathrm{ml}$ ), for $48 \mathrm{~h}$, stimulated glucose-induced insulin secretion from 6.1 to $17.3 \mathrm{ng}$ insulin/islet $(p<0.001)$. Pretreatment with glucagon $(1 \mu \mathrm{mol} / \mathrm{l})$, for $1 \mathrm{~h}$, partially reversed the cytokine stimulatory effect on human islets insulin secretion, from 17.3 to $10.4 \mathrm{ng}$ insulin/islet $(p<0.01)$. Glucagon $(1 \mu \mathrm{mol} / /)$ or forskolin $(10 \mu \mathrm{mol} / 1)$ decreased iNOS expression induced by cytokines in rat and human islets as shown by Western blotting. In conclusion, we demonstrated that glucagon regulates cytokine induction of nitric oxide synthase and decreases cytokine effects on insulin secretion in rat and human islets of Langerhans.
605

REGULATION OF THE INDUCIBLE FORM OF NITRIC OXIDE SYNTHASE PROMOTER IN INSULIN-PRODUCING CELL S

M.I. Darville and D.L. Eizirik, Diabetes Research Center, Vrije Universiteit Brussel Laarbeeklaan 103, B-1090 Brussels, Belgium.

Cytokines may contribute to beta-cell damage in type 1 diabetes mellitus. The radical nitric oxide, generated by the inducible form of nitric oxide synthase (iNOS), is a potential mediator of cytokine-induced beta-cell dysfunction. In rat pancreatic islets and insulin-producing cell lines interleukin-1B (IL-1B) induces expression of iNOS mRNA and increases NO production, an effect potentiated by interferon- $\gamma$ (IFN- $\gamma$ ). In human islets both IL- $1 B$ and IFN- $\gamma$ are required for iNOS expression. We have previously shown that both the transcription factors nuclear factor- $\mathrm{KB}(\mathrm{NF}-\mathrm{kB})$ and interferon regulatory factor-1 (IRF-1) are activated by cytokines in rodent and human islets, but there are no direct information on the iNOS promoter regulation in insulinproducing cells. We presently investigated the iNOS transcriptional regulation by cytokines in both rat insulin-producing RINm $5 \mathrm{~F}$ cells and in primary FACS-purified rat beta-cells. For this purpose, the $1.5-\mathrm{kb}$ rat $\mathrm{iNOS}$ promoter region and $5^{\prime}$ deletants of it were cloned upstream of the luciferase reporter gene and the activity regulation by cytokines of these constructs was studied in transient transfection experiments. We observed that the region extending up to $-1002 \mathrm{bp}$, and containing a distal and a proximal NF-кB binding sites, a $\gamma$-interferon activated site (GAS) and two adjacen IFN-stimulated response elements (ISRE), is required for a 12 -fold IL-13 $(30 \mathrm{U} / \mathrm{ml}$ induction $(\mathrm{P}<0.01$ vs control; $n=6)$ and a 4 -fold potentiation by IFN- $\gamma(1000 \mathrm{U} / \mathrm{m})$ $(\mathrm{P}<0.01$ vs IL-1B; $\mathrm{n}=6)$ of promoter activity in RINm5F cells. Site-mutation analysis showed that both the distal and proximal NF- $\mathrm{KB}$ sites and GAS are necessary for IL$1 B$-induced iNOS expression ( $\mathrm{P}<0.01$ vs wild-type promoter; $\mathrm{n}=6$ ). The distal NF- $\kappa \mathrm{B}$ site, GAS and ISRE are necessary for IFN- $\gamma$ potentiation. In primary beta-cells, the promoter region extending up to -1002 bp was also required for a 10 -fold IL-1B induction ( $\mathrm{P}<0.01$ vs control; $n=4$ ) and site-mutation analysis confirmed a role for both the distal and proximal NF- $\kappa B$ sites $(P<0.05$ vs wild-type promoter; $n=3)$. In these cells, IFN- $\gamma$ neither increased IL-1B-induced iNOS promoter activity nor iNOS mRNA expression as analyzed by RT-PCR, and site-mutation in the GAS and ISRE did not prevent IL- $1 B+/$ - IFN- $\gamma$-induced iNOS promoter activity. The present data indicate that in tumoral RINm5F cells both NF- $\mathrm{KB}$ sites, GAS and IRF-1-binding site are important for iNOS expression. On the other hand, in primary rat beta-cells NF$\kappa B$ is the main responsible for iNOS expression.

\section{6}

INVOLVEMENT OF PI-3 KINASE IN IL-1ß INDUCED

ACTIVATION OF NFKB IN $\beta$-CELLS

A.K. Andersson and S. Sandler, Department of Medical Cell Biology, Uppsala University, Uppsala, Sweden.

Exposure of rodent pancreatic islets to IL-1 $\beta$ suppresses $\beta$-cell function Activation of the transcription factor NFxB, expression of the gene for inducible nitric oxide synthase activation and subsequent nitric oxide (NO) formation is suggested to mediate $\mathrm{IL}-1 \beta$ induced $\beta$-cell damage. The aim of this study was to elucidate the role of phosphatidylinositol 3 kinase (PI-3 kinase) in $\mathrm{IL}-1 \beta$ mediated nuclear translocation of $\mathrm{NF} \kappa \mathrm{B}$ in $\beta$-cells. Interaction of PI-3 kinase with the type $1 \mathrm{LL}-1$ receptor might be an early event in $\mathrm{L}$ - $1 \beta$ stimulated $\beta$-cells. Wortmannin, a fungal metabolite, has been shown to be a potent irreversible inhibitor of PI-3 kinase without affecting the upstream signaling events. In the present study we wanted to test if blocking of early IL-1 receptor signaling events by wortmannin affects $N F \kappa B$ translocation in $\beta$-cells. For this purpose RINm5F cells were pretreated with $500 \mathrm{nM}$ or $100 \mathrm{nM}$ wortmannin for $45 \mathrm{~min}$ and then exposed to $\mathrm{L}-1 \beta(25 \mathrm{U} / \mathrm{ml})$ from 20 min up to $6 \mathrm{~h}$. Nuclear protein exctracts were prepared and tested for $N F \times B$ content by binding to a $\left[\alpha-{ }^{32} \mathrm{P}\right] \mathrm{dATP}$ labeled oligonucleotide probe representing the NFKB consensus sequence and then separated on a polyacrylamide gel (electrophoretic mobility gel shift assay). Nuclear extracts from untreated cells and cells treated with $I L-1 \beta$ or wortmannin alone were used as controls. Wortmannin at both concentrations inhibited NFkB translocation effectively after $20 \mathrm{~min}$ exposure to $\mathrm{IL}-1 \beta$. Since wortmannin is unstable in aquous solutions the inhibitory effect decreased with time. Preliminary results from messurements of nitrite accumulation in medium from isolated normal rat islets exposed for IL $1 \beta$ show that wortmannin may also counteract NO formation. In conclusion our findings suggest that PI- 3 kinase is involved in IL- $1 \beta$ mediated translocation of $\mathrm{NF}_{\mathrm{K}} \mathrm{B}$ in $\beta$-cells. 


\section{7}

BLOCKING OF THE PI3-KINASE PATHWAY INDUCES ISLET CELL APOPTOSIS IN SERUM-FREE CONDITIONS, WHEREAS MAP. KINASE INHIBITION HAS NO SUCH EFFECT.

S.Rasilainen, J.Ustinov and T. Otonkoski. Haartman Institute, University of Helsinki

We investigated the significance of PI3-kinase and MAP-kinase pathways in the differentiation and apoptosis of fetal porcine pancreatic cells. The cells were incubated with specific PI3-kinase and/or MAPkinase inhibitors, LY294002 (LY) and PD098059 (PD), respectively, in serum-free medium and in the presence of $10 \%$ human serum. Samples were collected at various time points for measurement of DNA and insulin content and at 2 weeks cells were fixed on cytocentrifuge slides for the measurement of cell-specific apoptosis using TUNEL combined with insulin immunocytochemistry, In serum-free conditions, LY progressively decreased the DNA content (and cell number) and simultaneously increased the insulin content per DNA up to 8-fold. The loss of cells was apparently due to an increased rate of apoptosis (in beta cells: contr. $0 \%, \mathrm{LY} 3.7 \%$, in other cells: contr. $0.7 \%, \mathrm{LY} 7.1 \%$ ) $P D$ did not induce apoptosis and neither changed the insulin content. However, the increased apoptosis induced by LY was blocked by PD. In the presence of serum, LY increased the insulin content less effectively and no apoptotic cells were present. Nicotinamide appeared to potentiate both the apoptotic and differentiation-inducing effects of LY. PD had a negative effect on the insulin content in serumcontaining medium. In conclusion, inhibition of the PI3-kinase pathway in serum-free conditions induces both differentiation and apoptosis of fetal porcine B-cells, whereas MAP-kinase inhibition has an opposite effect. Serum contains factors protecting the cells against apoptosis.

\section{8}

CO-INDUCTION OF ARGINASE AND NITRIC OXIDE SYNTHASE IN THE INSULIN-SECRETING CELL LINE RIN5F P. Stickings, A.J. Bone and J.M. Cunningham. Department of Pharmacy, University of Brighton, Brighton, East Sussex, U.K.

The aim of this study was to determine the effect of cytokine treatment on the activities of arginase and nitric oxide synthase (NOS) in the insulin-secreting cell line RIN5F. In pancreatic beta cells nitric oxide is a mediator of pro-inflammatory cytokine-induced cytotoxicity. The rate of NOS-catalysed generation of NO is determined by both the availability of the substrate arginine and the activity of other arginine-utilizing enzymes such as arginase. Cells were cultured \pm cytokines (interleukin $1 \mathrm{~B} 100 \mathrm{U} / \mathrm{ml}$ plus interferon gamma $6 \mathrm{U} / \mathrm{ml}$ ) for $18 \mathrm{~h}$. Cytosols were incubated with U-14C-L-arginine $(20 \mu \mathrm{M})$ for 1 h at $37^{\circ} \mathrm{C} \pm$ an inhibitor of arginase (valine, $50 \mathrm{mM})$ or NOS (N-methyl-arginine, $1 \mathrm{mM}$ ). Samples were applied to Dowex cation-exchange columns and water-eluted radioactivity was quantified to determine formation of the arginase product urea and the NOS product citrulline. Cytokine treatment increased synthesis of citrulline (control $1.44 \pm 0.05 \mathrm{pmol} / 10^{6}$ cells seeded $/ \mathrm{h}$, cytokine-treated $\left.23.88 \pm 0.76 \mathrm{pmol} / 10^{6} \mathrm{cells} / \mathrm{h}\right)(\mathrm{P}<0.01)$ and generation of medium nitrite (control $<0.4 \mathrm{nmol} / 10^{6} \mathrm{cells} / 18 \mathrm{~h}$, cytokinetreated $10.68 \pm 1.78 \mathrm{nmol} / 10^{6}$ cells $/ 18 \mathrm{~h}$ ). This was accompanied by a significant increase in urea formation (control $21.34 \pm 6.16 \mathrm{pmol} / 10^{6}$ cells $/ \mathrm{h}$, cytokine-treated $59.15 \pm 4.69 \mathrm{pmol} / 10^{6}$ cells $\left./ \mathrm{h}\right)(\mathrm{P}<0.01)(\mathrm{n}=3$ experiments). Additional preliminary data suggest that the increase in arginase-catalysed urea formation is due primarily to the effects of interleukin $1 B$ rather than interferon gamma. These results provide evidence for expression and induction of arginase in RIN5F cells. Agents which selectively induce beta-cell arginase may offer a means to control cytokine-stimulated, NO-mediated beta-cell cytotoxicity.

\section{9}

\section{PROTEASOME INHIBITORS PROTECT ISLETS} AGAINST IL-1B INDUCED SUPPRESSION

J. Sternesjö and S. Sandler, Department of Medical Cell Biology, Uppsala University, Uppsala, Sweden.

The aim of this study was to examine the role of the proteasome in IL- $1 B$ induced suppression of rat islets. The proteasome inhibitor MG115 (N-carbobenzoxyl-leu-leu-norvalinal) and the two calpain inhibitors allMET (N-acetyl-leu-leu-methional) and norLEU $(\mathrm{N}$ acetyl-leu-leu-norleucinal) were used for this purpose. Rat islets were cultured in medium RPMI $1640+10 \%$ fetal calf serum and exposed for $48 \mathrm{~h}$ to $25 \mathrm{U} / \mathrm{ml} \mathrm{\Pi L}-1 \mathrm{~B}$ with the inhibitors $(1,10$ and 100 $\mu \mathrm{M})$ added $30 \mathrm{~min}$ before IL-1B. The islet glucose metabolism, as measured by the glucose oxidation rate (pmol/10 islets $\times 90 \mathrm{~min}$ ), was $380 \pm 57$ in the control group and $115 \pm 17(\mathrm{p}<0.001)$ in the IL$1 \beta$ treated group. Pretreatment with MG115 $(10 \mu \mathrm{M})$ and norLEU $(100 \mu \mathrm{M})$ resulted in an oxidation rate of $253 \pm 38$ and $262 \pm 23$ ( $<<0.001$ compared to IL-1B), thus counteracting IL-1B induced suppression. The calpain inhibitor allMET did not have this effect. Nitric oxide formation, as measured by nitrite production (pmol/islet $x h)$, was $0.23 \pm 0.07$ in control islets and $0.59 \pm 0.05(\mathrm{p}<0.001)$ in the $\mathbb{L}-1 B$ treated group. IL-1B induced nitrite formation was blocked by MG115 $(10 \mu \mathrm{M})$ and norLEU $(100 \mu \mathrm{M})$ while allMET was without effect. To further elucidate the effects of MG115 a time study was performed. When adding the inhibitor $30 \mathrm{~min}$ before and $1 \mathrm{~h}$ after $\mathrm{LL}-1 \beta$ islets were protected, while addition 3 and $6 \mathrm{~h}$ after the cytokine did not influence IL-1B induced suppression of glucose oxidation. This was paralleled in nitrite production, where a nadir was reached in the group with the inhibitor added after $1 \mathrm{~h}$. After this time point, nitrite production started to rise and adding the inhibitor 6 $\mathrm{h}$ after the cytokine resulted in nitrite levels comparable to IL-1B alone. We conclude that these data indicate that the proteasome has a role in IL-1B induced suppression of islet function.

\section{0}

CELLULAR SPECIFICITY OF IL-1B INDUCED MITOGEN ACTIVATED PROTEIN KINASE ACTIVITY IN ISLETS OF LANGERHANS.

N. Aa. Andersen", D. Pavlovic", D. Eiziriks and T. Mandrup-Poulsen". "Steno Diabetes Center, Gentofte, Denmark; ${ }^{s}$ Diabetes Research Center, Vrije Universiteit, Brussel, Belgium.

IL- $1 B$ has been demonstrated to activate mitogen activated protein kinases (MAPKs) (ERK1/2 and p38) in isolated islets of Langerhans from neonatal rats. The aim with this study was to investigate the cellular specificity of cytokine induced MAPK activity. Isolated islets from rats were dispersed and sorted by FACS based on autoflourescence. The sorted cells were left to reaggregate into pseudo-islets. The two cell populations consisting of purified beta cells and nonbeta cells were incubated for $20 \mathrm{~min}$ with or without $60 \mathrm{U} / \mathrm{ml}$ rhIL-1ß, $1000 \mathrm{U} / \mathrm{ml}$ rrIFNy or both after which the cells were lysed. The lysates were investigated for the phosphorylation of the substrates Elk-1, ATF-2 and HSP25 by ${ }^{32} \mathrm{P}$ incorporation in the substrate proteins. The transcription factors Elk-1 and ATF-2 are mainly phosphorylated by the MAPKs ERK1/2, and by JNK, ERK $1 / 2$ and $p 38$ respectively, wheras HSP25 is phosphorylated by p38 via a downstream kinase MAPKAPK-2. The phosphorylated proteins were separated by SDS-PAGE and visualized by autoradiography. IFN $\gamma$ alone did not increase phosphorylation of the substrates in either beta or non-beta cell populations. $\mathrm{IL}-1 \mathrm{~B}$ alone increased the phosphorylation of all three substrates in both cell populations. The phosphorylation of Elk-1 was increased about 10 -fold in both cell populations. The phosphorylation of ATF-2 was increased about 6 -fold in non-beta cells and 12-fold in beta cell populations $(\mathrm{P}<0.05)$. HSP-25 phosphorylation was increased 3 -fold in the non-beta cells and 16 -fold in the beta cells $(\mathrm{P}<0.05)$. IFN $\gamma$ failed to potentiate IL-1 1 induced phosphorylation in either cell population. These data suggest that $\mathrm{IL}-\mathrm{IB}$ induced p38 activity is much more pronounced in beta than non-beta cells, suggesting that attenuated signalling by p38 MAPK may contribute to the lack of cytokine induced iNOS expression in non-beta cells 


\section{1}

ALTERATIONS OF INSULIN RESPONSE TO GLUCOSE AND ARGININE BY NITRIC OXIDE SYNTHASE BLOCKADE: INVOLVEMENT OF CYTOCHROME C REDUCTASE ACTIVITY

A. Lajoix ${ }^{1}$, M. Royel, V. Bourginel, T. Chardès 1 , S. Roux ${ }^{1}$, D. HillaireBuys $^{2}$, P. Masiello ${ }^{3}$, G. Ribes ${ }^{1}$, R. Gross ${ }^{1}$. 1 UMR 9921 du CNRS et ${ }^{2}$ UPRES EA 1677, Univ. Montpellier I, France, ${ }^{3}$ Ist. Patol. Gen. Univ. Pisa, Italy.

We could previously show that decreased NO production accounts only partly or not for the alterations of respectively glucose and arginine insulinotropic effects induced by Nitric Oxide Synthase (NOSc) blockade with L-N $\omega$-Nitro-L-Arginine Methyl Ester (L-NAME). Since, NOSc, in addition to NO production is also able to exert cytochrome $\mathrm{c}$ reductase activity, we investigated whether changes in the latter could be involved in the effect of L-NAME, an inhibitor of NO production, devoid of effect on cytochrome $c$ reductase activity. Therefore, we studied in the isolated perfused rat pancreas, the effects of L-NAME $(5 \mathrm{mmol} / \mathrm{l})$ on glucose (11 $\mathrm{mmol} / 1)$ and arginine $(5 \mathrm{mmol} / \mathrm{l})$ induced insulin secretion in the presence or absence of miconazole, a selective inhibitor of NOSc cytochrome $c$ reductase activity. Miconazole $(10 \mu \mathrm{mol} / \mathrm{l})$ was ineffective on insulin secretion induced by either $\mathrm{KCl}(5 \mathrm{mmol} / \mathrm{l})$ or tolbutamide $(100 \mathrm{mg} / \mathrm{l})$ in the presence of $5 \mathrm{mM}$ glucose and only slightly reduced mean integrated insulin response to a 20 min increase in glucose concentration to $11 \mathrm{mmol} / 1$ (respectively $178.6 \pm 13.9$ and $154.6 \pm 15.9 \mathrm{ng} \times 20 \mathrm{~min}$ in the absence and the presence of miconazole). However, the strong increase of glucose effect due to NOSc blockade with LNAME was drastically reduced from $796.8 \pm 50.0$ to $177.0 \pm 10.6 \mathrm{ng} \times 20 \mathrm{~min}$ $(p<0.001)$ in the presence of miconazole $(10 \mu \mathrm{mol} / \mathrm{l})$. Likewise, miconazole was able to brought back the strong potentiation of arginine effect by LNAME from $954.9 \pm 35.1 \mathrm{ng} \times 20 \mathrm{~min}$ to $269.0 \pm 15.8 \mathrm{ng} \times 20 \mathrm{~min}(\mathrm{p}<$ 0.001 ). Our data show that L-NAME induced alterations of glucose and arginine insulinotropic effects result mainly, from an increased cytochrome $\mathrm{c}$ reductase activity of NOSc.

\section{2}

\author{
INTERLEUKIN-1 $\beta$ STIMULATES JUN N-TERMINAL AND P38 \\ KINASE ACTIVITIES IN INSULIN SECRETING $\beta$-CELLS. \\ C.D. Major and B. A. Wolf. University of Pennsylvania, Philadelphia, PA.
}

IL-I $\beta$ has been implicated as an immunological effector molecule that inhibits insulin biosynthesis and secretion, oxidative metabolism, and mediates $\beta$-cell death. Mitogen-activated protein kinase (MAPK) cascades have been reported to mediate cytokine signaling in a variety of cell types. To test the hypothesis that MAPKs mediate IL- $1 \beta$ signaling in $\beta$-cells, the effect of IL- $1 \beta$ on two cytokine-activated members of the MAPK family, the Jun N-terminal kinase (JNK) and p38, was assessed using the insulin-secreting $\beta-\mathrm{TC} 3$ and $\mathrm{RINm} 5 \mathrm{~F}$ cell lines. $\beta \mathrm{TC} 3$ and RINm5F cells were cultured in complete RPMI-1640 media supplemented with $10 \%$ fetal bovine serum. Expression of ERK, JNK, and p38 MAPKs was confirmed by immunoblotting in $\beta$-cell lines as well as in isolated rat islets. To determine whether IL-1 $\beta$ induced MAPK activation, $\beta$-cells were stimulated with $100 \mathrm{U} / \mathrm{ml}$ of IL- $1 \beta$ or vehicle for 0-20 minutes. Cells were lysed and MAPKs were immunoprecipitated using antibodies selective for $\mathrm{JNK} 1$ or $\mathrm{p} 38$. Antibody complexes were subjected to an in vitro kinase reaction in the presence of $\left[\gamma^{32} \mathrm{P}\right]$ ATP using $\mathrm{c}-\mathrm{jun}$ or ATF-2 as substrates for JNK1 and $\mathrm{p} 38$, respectively. Reaction products were separated by SDS-PAGE and MAPK activation measured as ${ }^{32} \mathrm{p}$ incorporation into phosphorylated substrates via autoradiography. Results indicate that after 20 minutes, IL-I $\beta$ caused an increase in JNK activity in both $\beta T C 3$ and RINm5F cells compared to controls $(10.5 \pm 2.6$ fold, and $9.2 \pm 3.1$ fold respectively, $n=5, p<0.05$ ). Similarly, IL-1 $\beta$ increased p38 activity over controls ( $\beta$ TC3: $4.5 \pm 1.1$ fold, RINm5F: $22.1 \pm 0.9$ fold, $n=2$ ). The effect of $I L-1 \beta$ was time-dependent for both MAPKs. Activity was observed within $5 \mathrm{~min}$, peaked at $10 \mathrm{~min}$, and returned to basal levels within 2 hours. Further, the activity of both JNK and p38 was selective for $\mathrm{IL}-1 \beta$ as $\mathrm{TNF}-\alpha(1000 \mathrm{U} / \mathrm{ml})$ and IFN- $\gamma(500 \mathrm{U} / \mathrm{ml})$ were without effect. In summary, IL-1 $\beta$ selectively activates the stress-activated protein kinases JNK and p38 in $\beta$-cells in a time-dependent manner. These results support the hypothesis that NNK and $p 38$ mediate IL- $1 \beta$ induced $\beta$-cells death.

\section{3}

DYNAMIC CHANGES IN PROTEIN EXPRESSION IN SYNGENEIC ISLET TRANSPLANTS DURING IDDM DEVELOPMENT IN DP-BB RATS

T. Sparre, U. B. Christensen, P. Mose Larsen*, S. J. Fey*, A. E. Karlsen, F. Pociot, C. Gottredsen\#, B. Richter\#, T. Mandrup-Poulsen and J. Nerup. Steno Diabetes Center, Gentofie, Den mark, ${ }^{*}$ Center for Proteome Analysis, Odense, Denmark, \# Novo Nordisk, Bagsværd, Denmark Interleukin-1 13 induces impaired $B$-cell function and destruction in vitro, and has been proposed to be involved in the pathogenesis of IDDM. IL-1B induced B-cell impairment is dependent upon protein synthesis. We have previously demonstrated that transplantation of 200 syngeneic neonatal islets to 30 days old DP-BB rats do not change incidence or time of onset of diabetes. The pattern of cellular infiltrate seen in transplants mirror the pattern seen in islets in situ. By high resolution 2-dimensional gel electrophoresis it is possible to separate approximately 2200 proteins according to molecular weight and pl. IL-1B has been shown to induce statistically significant changes in expression level of 82 proteins in DP-BB rat islets in vitro. Aim: To study if the $82 \mathrm{LL}-1 \mathrm{~B}$ induced changes in islet protein expression levels in vitro can be re-identified in syngeneically transplanted DP-BB rat islets during spontaneously developing IDDM in vivo. Methods: 200 neonatal DP-BB rat islets were transplanted under the kidney capsule of 30 days old DP-BB rats and excised at day 7, 12, 23 or 37 after transplantation or at onset of diabetes. The transplants, IL-1B stimulated DP-BB islets and normal islets were labelled with [ ${ }^{35} \mathrm{~S}$ )-methionine and prepared for two dimensional gel electrophoresis. The fluorographs of the gels were analysed on a Biolmage computer programme. In situ hybridization for IL- 1 B mRNA was performed on transplants from day 7 . Expression levels were compared with non stimulated islets. Results: Of the 82 proteins found to change expression levels in DP-BB islets stimulated with IL-1B 81 proteins could be re-identified in the transplants. The expression levels of 65 of the 82 proteins were significantly changed in transplants compared to normal islets $(p<0.01)$. Changes in expression of 28 proteins were consistent during all prediabetic time points. 3 proteins were only changed at onset of diabetes. Presence of IL-1B in transplants from day 7 prior to cellular infiltrate was demonstrable by mRNA in situ hybridization. Conclusion: Changes in protein expres sion induced by IL-1B in vitro are also seen during IDDM development in DP-BB syngeneic transplants in vivo. The presence of IL-1B early on in syngeneic transplants and identical changes in expression levels of islet proteins in vitro and in vivo are compatible with the hy pothesis that $I L-1 B$ is involved in the pathogenesis of IDDM in the DP-BB rat.

\section{4}

HSP 70 EXPRESSION IN ISLETS OF DIABETES-PRONE BB RATS DURING RECOVERY FROM EXPOSURE TO INTERLEUKIN-1BETA

G. Wachlin, B. Kuttler, D. Schröder, I. Klöting and S. Schmidt, Institute of Pathophysiology of the University of Greifswald, Karlsburg, Germany

Interleukin-lbeta $(\mathrm{IL}-1 \beta)$ has been demonstrated to be involved in the destruction of pancreatic islet beta-cells. It was the aim of the study to investigate the IL- $1 \beta$ induced stress response of pancreatic islets from diabetesprone $\mathrm{BB}$ rats and the ability of such islets to recover from injury. Isolated pancreatic islets were precultured for $3 \mathrm{~d}$ and than exposed to $\mathrm{LL}-1 \beta(10 \mathrm{U} / \mathrm{ml})$ for $24 \mathrm{~h}$ or remained untreated (control). Groups of cytokine-exposed and control islets were then used for functional tests or were cultured without $I L-1 \beta$ for further $6 \mathrm{~d}$ (recovery). Immediately after cytokine treatment insulin content, glucose-stimulated insulin release and total protein biosynthesis were significantly diminished compared to control islets while no membrane alterations were detectable in cytokine-pretreated islets as measured by spontaneous ${ }^{51} \mathrm{Cr}$-release. Surface antigen expression was measured on single islet cells by FACS analysis using OX 18 (MHC class I), OX 6 (MHC class II), ICAM-1 (adhesions molecule) and K14D10 (beta-cell reactive). The addition of $\Pi L-1 \beta$ to the culture medium caused a reduction of beta-cell number $(p<0,01)$ and an increase of ICAM- $1^{+}$beta-cells $(p<0,001)$ while the expression of MHC class I and class II was hardly diminished. Islet proteins were separated by SDSPAGE followed by immunoblotting. Synthesis of heat shock protein 70 (HSP 70) was detectable up to $4 \mathrm{~d}$ during recovery of islets after exposure to $\mathrm{IL}-1 \beta$. Though $6 \mathrm{~d}$ after cytokine-treatment insulin storage was comparable with controls and islets were able to respond on high glucose chalłange with increased insulin release, but there was still a significant difference $(p<0,001)$ to control islets. Our results suggest that in response to $\mathrm{IL}-1 \beta$ protective mechanisms including expression of HSP 70 are activated for up to $4 \mathrm{~d}$ in pancreatic islet beta-cells of BB rats, however, the essential beta-cell function of glucose responsiveness remained impaired even after $6 \mathrm{~d}$ of culture without $\mathrm{IL}-1 \beta$. 


\section{5}

CYTOKINE-INDUCED ACTIVATION OF THE TRANSCRIPTION FACTORS STAT1 AND NF-KB IN INS-1 CELLS

N. Sekine, T. Ishikawa, T. Okazaki, M. Hayashi and T. Fujita. Fourth Department of Internal Medicine, University of Tokyo, Tokyo, Japan. Interferon- $\gamma$ (IFN- $\gamma$ ), in combination with tumor necrosis factor- $\alpha$ $(\mathrm{TNF}-\alpha)$, exerts deleterious effects in pancreatic $\beta$-cells, possibly through the induction of nitric oxide synthase (iNOS). The present study was conducted to investigate signalling mechanisms mediating the effects of these cytokines using a differentiated insulin-secreting cell line, INS-1, with special reference to the activation of cytokine-inducible transcription factors STAT1 and NF- $\mathrm{kB}$. Proliferation of INS- 1 cells was affected neither by $100 \mathrm{U} / \mathrm{ml}$ IFN- $\gamma$ nor by $50 \mathrm{ng} / \mathrm{ml} \mathrm{TNF}-\alpha$, whereas the combination of the two cytokines elicited severe cytotoxicity. Similarly, expression of iNOS mRNA was induced by IFN- $\gamma$ plus TNF- $\alpha$, but not by either cytokine alone. At variance with previous reports, IFN- $\gamma$ failed to phosphorylate JAK2 tyrosine kinase in INS-1 cells, but tyrosine phosphorylated STAT1. Electrophoretic mobility shift assay was performed to examine DNA-binding activities of ST AT 1 and NF-KB in INS-1 cells exposed to the cytokines. IFN- $\gamma$ stimulated DNA-binding of nuclear proteins to the IFN- $\gamma$-activated sequence in the promoter of the iNOS gene as well as that of the interferon regulatory factor-1 (IRF-1) gene. TNF- $\alpha$ did not affect DNA binding of STAT1, but stimulated NF-KB binding, which was further increased by the addition of IFN- $\gamma$. In conclusion, IFN- $\gamma$ activates ST AT 1 via a mechanism that appears to be JAK2-independent, and potentiates TNF- $\alpha$-induced NF- $\mathrm{kB}$ activation in INS-1 cells. Since the promoter of the iNOS gene contains binding sites of ST AT 1, IRF-1 and NF-KB, activation of these factors by IFN- $\gamma$ may contribute to the synergistic induction of iNOS, and eventually to $\beta$-cell destruction, by the combination of IFN- $\gamma$ and TNF $-\alpha$.

\section{7}

DNA ADDUCT FORMATION AND INDUCTION OF THE DNA REPAIR PROTEIN AP ENDONUCLEASE IN RAT AND HUMAN PANCREATIC ISLETS FOLLOWING EXPOSURE TO GENOTOXIC AGENTS C.A. Delaney ${ }^{1,2.3 .}$ N. Springer ${ }^{2}$, A. Claus' ${ }^{2}$, G.E. Kisby' ${ }^{2}$ and D.L. Eizirik ${ }^{1,4}$. 'Dept of Medical Cell Biology, Uppsala University, Sweden, ${ }^{2}$ Center for Research on Occupational and Environmental Toxicology, Oregon, USA, ${ }^{3}$ Cancer Research Unit, University of Newcastle upon Tyne, UK, "'Diabetes Research Center, Vrije Universiteit Brussel, Belgium.

Exposure to environmental genotoxins may contribute to beta cell dysfunction and destruction in diabetes mellitus. The damaging effects of these agents may be due at least in part to the formation of DNA adducts and the reduced activity of the DNA repair protein apurinic/apyrimidinic endonuclease (APE). In this study, we further explored the mechanisms by which streptozotocin (STZ), nitric oxide (NO), peroxynitrite (ONOO) and methylazoxymethanol acetate (MAMOAc) lead to beta cell damage in human and rat pancreatic islets. By using immuno slot blotting together with antibodies directed against lesions within the DNA, we measured the levels of 2 major alkylation products, i.e $\mathrm{O}^{6}$ methylguanine ( $\left.\mathrm{O}^{6} \mathrm{MeGua}\right)$ and $\mathrm{N}^{7}$ methylguanine $\left(\mathrm{N}^{7} \mathrm{MeGua}\right)$ adducts. APE activity was assessed in parallel by a novel method based upon the repair of a depurinated plasmid. There were no detectable DNA adducts in control rat islets and in islets exposed to NO or ONOO. However, there was an increased amount of DNA adducts (fmol/ug DNA) in rat islet cells following $12 \mathrm{~h}$ exposure to STZ ( $1 \mathrm{mM} ; 21303 \pm 7783$ for $\mathrm{O}^{6} \mathrm{MeGua}$ and 15394 $\left.\pm 6569 \mathrm{~N}^{7} \mathrm{MeGua}\right)$ or MAMOAc $\left(0.05 \mathrm{mM} ; 5736 \pm 3737 \mathrm{O}^{6} \mathrm{MeGua}\right.$ and $5963 \pm$ $2127 \mathrm{~N}^{7} \mathrm{MeGua}$ ) $(\mathrm{n}=3)$. In rat islets APE activity was significantly reduced $(\mathrm{P}<0.05$ vs controls; $\mathbf{n}=4-5)$ following $12 \mathrm{~h}$ exposure to STZ $(1 \mathrm{mM})$, NO $(0.5 \mathrm{mM})$, ONOO $(0.2 \mathrm{mM})$ and MAMOAc $(0.05 \mathrm{mM})$. In human islets exposure to STZ $(6 \mathrm{mM})$ for $12 \mathrm{~h}$ also decreased APE activity $(\mathrm{P}<0.05$ vs controls; $\mathrm{n}=3$ ). In conclusion, the alkylating agents STZ and MAMOAc may contribute to beta cell death by both inducing DNA adducts and reducing activity of the DNA repair protein APE. On the other hand, the radical NO reduces APE activity without inducing detectable $\mathrm{O}^{6} \mathrm{MeGua}$ and $\mathrm{N}^{7} \mathrm{MeGua}$ DNA adducts, suggesting that these two events may be dissociated following radical-induced damage.

\section{6}

MECHANISMS FOR IMPAIRED TNF-a AND IL-1b RELEASE IN MACROPHAGES FROM DIABETIC (DB/DB) MICE

S.N.Zykova, M.Berdal, R.Seljelid and T.G.Jenssen. Institute of Clinical Medicine, University of Tromse, Norway

Impaired function of immune cells may be one of the possible causes of the susceptibility to infection and delayed wound healing in diabetes mellitus. This can be due to either a genetic defect related to diabetes, or, metabolic factors, or both. Macrophages have a crucial tole for the immune defense as well as the production of extracellular matrix. The aim of the present study was to compare the cytokine release from cultured peritoneal macrophages in a murine NIDDM model (strain $\mathrm{C} 57 \mathrm{BL} / \mathrm{Ks}-\mathrm{db} / \mathrm{db}$ ) with macrophages from heterozygous phenotypically normal mice of the same strain. The number of animals in each group was 10-12. All results are presented as mean \pm s.e.m.. Cultured diabetic macrophages differed from control cells morphologically: they showed relative inability to stretch when attached to the plastic surface and had a tendency to cluster. Cytokine release from LPS-stimulated macrophages cultured in medium with $5 \mathrm{mmol} / \mathrm{l}$ glucose were lower in diabetic mice compared to controls (TNF-a: $719.05 \pm 60.18$ vs. $2466.50 \pm 70.23 \mathrm{pg} / \mathrm{ml}$, IL-1b: $323.62 \pm 8.69$ vs. $553.65 \pm 20.19 \mathrm{pg} / \mathrm{ml}, \mathrm{p}<0.05$ for both). Elevation of glucose up to $10 \mathrm{mmol} / \mathrm{l}$ inhibited LPS-stimulated TNF-a $(328.58 \pm 42.65$ vs. $978.18 \pm 25.33 \mathrm{pg} / \mathrm{ml})$ and IL- $1 \mathrm{~b}(205.37 \pm 9.53$ vs. $307.56 \pm 10.64 \mathrm{pg} / \mathrm{ml}$ ) release, which was still significantly lower in cells from diabetic animals compared to lean controls $(p<0.05)$. Elevation of glucose up to $20 \mathrm{mmol} / 1$ did not cause further inhibition of either TNF-a $(290 \pm 50.11$ vs. $898 \pm 34 \mathrm{pg} / \mathrm{ml})$ or IL-1b $(196.91 \pm 15.95$ vs. $271.01 \pm 32.51$ $\mathrm{pg} / \mathrm{ml}$ ) release in diabetic and control cells. We conclude that incubation of diabetic macrophages in medium with normal glucose concentration does not restore diminished TNF-a and IL-1b release. Glucose in elevated concentrations also inhibits cytokine release from both diabetic and normal macrophages in vitro. IL-1b and TNF-a release is impaired in diabetic mice both due to elevated glucose concentrations and an intrinsic cellular defect.

\section{8}

PEROXYNITRITE CAUSES STIMULATION OF INSULIN SECRETION AND DNA DAMAGE IN HIT-T15 CELLS.

S. Thomas ${ }^{1,3}$, S.J.Dunmore ${ }^{3}$, and I.C.Green ${ }^{1,2},{ }^{1}$ Biochemistry $^{2}$ department, University of Sussex, UK, ${ }^{2}$ Pharmacy department, University of Brighton, UK and ${ }^{3}$ Health Sciences, University of Wolverhampton, UK.

In autoimmune diabetes beta-cells may be exposed to free radicals exogenously, from activated immune cells, and endogenously via cytokine induction. Nitric oxide and superoxide from either source can react with each other to form peroxynitrite $\left(\mathrm{ONOO}^{-}\right)$. This study examines the acute effects of peroxynitrite $(0-1.0 \mathrm{mmo} / \mathrm{h})$, made from the ozonation of azide, on insulin secretion and DNA damage in the ozonation of azide, on insulin secretion and DNA damage in
insulin-containing HIT-T15 cells. To test for effects on insulin secretion HIT-T15 cells were challenged with glucose \pm peroxynitrite for $1 \mathrm{~h}$. To assess the DNA-damaging effects of peroxynitrite the Comet assay, in which DNA damage can be determined in single cells, was used. Chemically synthesised peroxynitrite, when applied exogenously, dose-dependently enhanced glucose-induced insulin secretion from $1.7 \pm 0.2 \mathrm{ng}$ insulin per $10^{5}$ cells to $3.2 \pm 0.4 \mathrm{ng}$ at 0.5 $\mathrm{mmol} / \mathrm{l}$ peroxynitrite $[5$ experiments; $\mathrm{P}<0.01]$. Glutathione $(1 \mathrm{mmol} / \mathrm{l})$, a known scavenger of peroxynitrite, reversed the potentiating effect of peroxynitrite $(0.5 \mathrm{mmol} / \mathrm{l})$ on insulin secretion to $1.9 \pm 0.3 \mathrm{ng}[5$ experiments]. Peroxynitrite $(0.25 \mathrm{mmol} / \mathrm{l})$ caused extensive DNA damage in HIT-T15 after a 15 min treatment period. This DNA damage was not uniform but arose from the point of application of peroxynitrite to the slide and was not repairable after $48 \mathrm{~h}$. Inactivated peroxynitrite had no effect on insulin secretion or DNA damage. We conclude that peroxynitrite enhances insulin secretion but causes DNA damage and that the latter effect may contribute to beta-cel dysfunction 
619

ENHANCING EFFECT OF PERTUSSIS TOXIN ON APOPTOSIS INDUCED BY S-NITROSOGLUTATHIONE OR INTERLEUKIN-1 $\beta$ IN RINm5F CELLS.

J. Elliott and N.G. Morgan. Department of Biological Sciences, Keele University, Staffs, UK.

It has been demonstrated previously that sodium fluoride can induce apoptosis in RINm5F cells and that pretreatment with pertussis toxin (Ptx) which prevents the activation of members of the Gi/o family of Gproteins, results in an enhancement of this response. In the present study, the effect of Ptx pretreatment on apoptosis induced by a nitric oxide donor [S-nitrosoglutathione (GSNO)], or by interleukin-1 $\beta$ (IL-1 $\beta$; which upregulates inducible nitric oxide synthase leading to increased NO production) was investigated. RINm5F cells were treated with $400 \mu \mathrm{M}$ GSNO in the absence or presence of $0.2 \mu \mathrm{g} / \mathrm{ml}$ Ptx for 24 hours or with $200 \mathrm{pg} / \mathrm{ml} \mathrm{IL-1 \beta}$ in the absence or presence of $0.2 \mu \mathrm{g} / \mathrm{ml} \mathrm{Ptx}$ for 48 hours. The extent of cell death was quantified with trypan blue and apoptosis was confirmed by acridine orange staining. Ptx did not directly induce cell death but pretreatment of RIN cells with $\mathrm{Ptx}$ resulted in a significant enhancement of GSNO induced cell death (Control: $4927+1-378$ dead cells/ml; Ptx: 4904+/-489; GSNO: 16862+/-3521; GSNO plus Ptx: 35212+/-3148; $\quad \mathrm{p}<0.01$ vs GSNO alone). Ptx pretreatment also significantly enhanced IL-1 $\beta$ induced cell death (Control: $3346+/-402$; Ptx: $3741+/-522 ; \quad I L-1 \beta: 6120+/-650 ; \quad I L-1 \beta$ plus Ptx: 12084+/-733; $p<0.001$ vs $\mathrm{IL}-1 \beta$ alon $\theta$ ). In the case of $\mathrm{IL}-1 \beta$, the enhancing effect of $\mathrm{Ptx}$ was evident at all concentrations of the cytokine between 100 and $400 \mathrm{pg} / \mathrm{ml}$. These results provide the first evidence that one or more Ptx sensitive G-proteins is involved in a protective mechanism which reduces the pancreatic beta cell sensitivity to apoptosis induced by chemical NO donors or IL-1 $\beta$.

\section{0}

ALLOXAN EFFECTS ON HUMAN AND MOUSE ISLET INSULIN SECRETORY PATTERN DURING GLUCOSE PERIFUSION

B. Tyrberg and L.A.H. Borg. Department of Medical Cell Biology, Uppsala University, Uppsala, Sweden.

Alloxan is a selective B-cell cytotoxic substance. Experiments in vitro have however, indicated that the sensitivity of human islets to alloxan differs from that of rodent islets. The present study is a discriminative characterization of the insulin secretory pattern of human and mouse islets after exposure to alloxan in vitro. Approximately equal volumes of human (5 organ donors, $\beta$-cell Transplant, Brussels) and mouse (NMRI) islets were perifused in small chambers under a constant flow of $\mathrm{J} \mathrm{ml} / \mathrm{min}$ at $37^{\circ} \mathrm{C}$. During the perifusions, an initial stabilization phase of $20 \mathrm{~min}$ without glucose was followed by $5 \mathrm{~min}$ with or without addition of $2 \mathrm{mM}$ alloxan, $5 \mathrm{~min}$ with $2.8 \mathrm{mM}$ glucose, $40 \mathrm{~min}$ with $28 \mathrm{mM}$ glucose and thereafter $20 \mathrm{~min}$ with $2.8 \mathrm{mM}$ glucose. Medium was continuously sampled for insulin measurements (RIA). Both the human and mouse islets showed a biphasic secretory pattern in control experiments. The human islets were not affected in any of the secretory phases by alloxan exposure ( $96-120 \%$ insulin secreted vs. control, $\mathrm{n}=10$ ). However, mouse islets increased their secretion dramatically when exposed to alloxan $(329 \pm 70 \%$ vs, control, $n=10, p<0.001)$, but were not able to respond to the following glucose stimulus (first phase: $22 \pm 5 \%$ vs, control, $\mathrm{p}<0.01$; second phase: $28 \pm 6 \%$ vs. control, $p<0.001)$ and, finally, increased their secretion after withdrawal of the glucose stimulus $(301 \pm 85 \%$ vs. control, $\mathrm{p}<0.05$ ). The present study demonstrates that human islets have a biphasic glucose stimulated insulin secretory pattern in vitro, which is not affected by alloxan in any phase. On the contrary, mouse islets loose their biphasic response pattern after alloxan exposure. Furthermore, the initial stimulation of insulin secretion during alloxan exposure of mouse islets, probably caused by rapid redox potential alterations, is not observed during exposure of human islets to alloxan.

\section{1}

\section{PROTECTION OF RINm5F CELLS AGAINST NO TOXICITY} THROUGH OVEREXPRESSION OF ANTIOXIDANT ENZYMES

S. Lortz, M. Tiedge, and S. Lenzen, Institute of Clinical Biochemistry, Hannover Medical School, D-30623 Hannover, Germany

The generation of NO is crucial for beta cell destruction in autoimmune diabetes. It was the aim of this study to investigate the synergistic action of reactive oxygen species in the toxicity of chemically-generated NO. We therefore exposed bioengineered $\mathrm{RINm} 5 \mathrm{~F}$ insulin-secreting cells overexpressing different patterns of the antioxidant enzymes catalase (CAT), glutathione peroxidase (GPX) and superoxide dismutase (SOD) to the NO releasing compounds sodium nitroprusside (SNP), S-nitroso-Nacetyl-D, L-penicillamine (SNAP) and 3-morpholinosydnonimine (SIN-1). The resistance of these cells against the NO donors was quantified by the MTT assay. Overexpression of antioxidant enzymes provided significant protection against toxicity of all NO donors with an individual specificity related to their chemical properties. In particular the GPX overexpressing cells showed a significantly higher resistance against SNP and SNAPinduced toxicity, whereas CAT overexpressing cells provided protection against SIN-1. SOD overexpression protected against SNAP and SNP with lower efficacy than GPX overexpression but was more effective against SIN-1 toxicity. The synergistic toxic effect of NO and reactive oxygen species could be demonstrated through a combined exposure of RINm5F cells towards $\mathrm{H}_{2} \mathrm{O}_{2}$ or hypoxanthine/xanthine oxidase and SNAP or SIN-1. The moderate toxic effect of NO donors at sublytic concentrations was increased by sublytic concentrations of free oxygen radical donors in an overadditive manner. Our results indicate that in particular the overexpression of GPX and SOD with efficient transfer vectors may be a therapeutic concept to prevent $\mathrm{NO}$ radical-mediated toxicity in autoimmune diabetes.

\section{2}

DNA DAMAGE AND INSULIN SECRETION OF RAT AND HUMAN ISLETS EXPOSED TO FREE RADICAL AND CYTOKINE TREATMENTS V. Hadjivassiliou, R.F.L. James*, S.M. Swift*, H. Clayton*, M.H.L. Green and I.C. Green Biochemistry Department, University of Sussex and Pharmacy Department, University of Brighton, East Sussex, UK "Department of Surgery, University of Leicester, Leicester, UK

The aim of this study was to compare rat and human islet responses to damaging treatments. Effects on nucleor DNA integrity and insulin secretory responsiveness were determined. Islets were exposed for $48 \mathrm{~h}$ to nitric oxide (S-nitrosoglutathione $500 \mu \mathrm{mol} / \mathrm{l}$ ), peroxynitrite $(1 \mathrm{mmol} / \mathrm{l})$, applied in glucose and serum free medium, or combination of cytokines (IL-1 $13100 \mathrm{pmol} / \mathrm{I}$, TNF $\alpha$ $\alpha 100 \mathrm{pmol} / \mathrm{I}$ and rat IFN $-\gamma 2 \mathrm{U} / \mathrm{ml}$ or human IFN- $\gamma 500 \mathrm{U} / \mathrm{ml}$ ). There was significant DNA damage in human islets as indicated by an increase in nucleus plus comet length $(\mu \mathrm{m})$ from control $37.5 \pm 1.8(\mathrm{~N}=13)$ to $54.2 \pm 1.7(\mathrm{~N}=5)$ $p<0.001$ for nitric oxide, $55.0 \pm 6.8(\mathrm{~N}=4) \mathrm{p}<0.01$ for peroxynitrite and $53.1 \pm 5.3(\mathrm{~N}=5) \mathrm{p}<0.01$ for cytokine treatments. Rat islets showed extensive and very similar DNA strand breakage induced by all treatments. Rat islet insulin secretory responsiveness to glucose $(20 \mathrm{mmol} / 1)$, expressed as a $\%$ of islet insulin content $(3.5 \%, \mathrm{~N}=5$ expts) was inhibited following nitric oxide or cytokine treatments (to $2.2 \%, \mathrm{~N}=3$ or $1.0 \%, \mathrm{~N}=3 \mathrm{p}<0.05$ respectively). Human islet insulin secretion was maintained following nitric oxide treatment (from $5.5 \%$, $\mathrm{N}=8$ to $5.6 \%, \mathrm{~N}=7$ ), but was increased after peroxynitrite or cytokine treatments $(13.2 \%, N=6 p<0.01)$. The results are consistent with cytokine effects in both species being free radical mediated. We conclude that rat and human insulin secretion is differentially affected by $48 \mathrm{~h}$ cytokine treatments. DNA in islets of both species is equally susceptible to damage by free radical and cytokine treatments. 


\section{3}

EFFECTS OF TROGLITAZONE ON INTERLEUKIN 1B-INDUCED B CELL CYTOTOXICITY

A. Dunger, S. Berg and U. Fischer*, Gerhardt Katsch Institute of Diabetes Karlsburg, * Inselklinik Heringsdorf, Germany

Interleukin $1 B$ (IL1)-induced $B$ cell cytotoxicity which is partly mediated by nitric oxide (NO) is suggested to be implicated in the autoimmune destruction of IDDM The insulin sensitizing compound troglitazone (TGZ) has been shown to influence islet NO production. Therefore, this study aimed at elucidating (1) the efficacy of TGZ in reducing cytokine-induced NO generation and (2) whether TGZ-mediated reduction of NO generation may prevent $B$ cell dysfunction and DNA damage. Rat islets were treated for $20 \mathrm{~h}$ with human IL $1(10 \mathrm{U} / \mathrm{ml})$ or TGZ $(10 \mu \mathrm{mol} / \mathrm{l})$ alone or in combination. Also, islets were exposed in arginine-free medium to IL1 or TGZ in the presence of $\mathrm{N}$-nitro-L-arginine methylester (NAME, $1 \mathrm{mmol} / \mathrm{l}$ ), a competitive inhibitor of NO synthase. Control islets remained untreated or were cultured in the presence of DMSO the solvent for TGZ. Results: TGZ and NAME prevented IL1induced islet NO production (measured as nitrite accumulation in the culture medium) (IL1: $6.80 \pm$ SEM0.46, TGZ+IL1: $3.39 \pm 0.50$, NAME+IL1: $2.40 \pm 0.12$ $\mathrm{pmol} /$ islet $\mathrm{p}<0.01)$. ILI reduced islet insulin release by $50 \%(\mathrm{p}<0.01)$ while TGZ on its own had a stimulatory effect on insulin output compared to control islets, and completely counteracted the $\mathrm{ILl}$-induced inhibition. NAME+ILl-treated islets released as much insulin as control islets did. However, post-culture glucose stimulation of insulin secretion in the absence of either compound revealed a significant inhibition of $B$ cell function both in IL1- and in TGZ+IL1-treated islets, while the response of NAME+ILl-pretreated islets was comparable to that of control islets. TGZ did not alter islet cell viability (trypan blue, ethidium bromide/acridine orange-technique), but prevented ILI-induced viability loss. The abundance of cytoplasmic histone-associated DNA fragments (HADF) was assessed by means of a sandwich ELISA was increased by $50 \%$ in ILI-treated islets as compared to controls $(\mathrm{p}<0.01)$. HADF were not influenced by NAME TGZ or by DMSO alone. TGZ completely prevented the IL1-mediated increase in HADF which was also significantly reduced but not completely normalized by NAME. In conclusion, TGZ reduces the NO generation of islets which in turn may prevent their structural and functional damage. TGZ does, however, not protect the islets from long-lasting functional impairment. The observed effect of TGZ may partly be explained by its competition for a common binding site with ILl.

\section{5}

OVEREXPRESSION OF MHC CLASS 1 AND RAPID LOSS OF IA2/ICA-512 AND INSULIN INDUCED BY MULTIPLE LOW-DOSE STREPTOZOTOCIN

Z.C.LI, L.J.Zhao, F.A.Karlsson and S.Sandler, Departments of Medicine and Medical Cell Biology, Uppsala University, Uppsala, Sweden

The expression of $\mathrm{MHC}$ class $1, \mid \mathrm{A} 2 / \mathrm{CCA}-512$ protein tyrosine phosphatase and insulin, all key molecules in human autoimmune diabetes, were examined in the multiple low-dose streptozotocin (MD-STZ) mouse, an experimental model of type 1 diabetes. C57BL/Ks mice were injected i.p. day $1-5$ with $40 \mathrm{mg} / \mathrm{kg} \mathrm{STZ}$ daily. Blood glucose was determined and pancreata were sampled for morphometric analysis on days $3,10,14,21$ and 28 . Immunofluorescence staining was performed for MHC class 1 and IA2/ICA 512 on cryostat sections. Insulitis was observed on sections stained with hematoxylin-eosin. |A2/ICA-512 density was determined by confocal microscopy. MD-STZ treatment caused hyperglycaemia from day 7 and onwards in parallel with a fall in the number of insulincontaining cells. A rapid reduction of IA2/ICA-512 was detectable at day 3 , and reached the lowest level on day 7 (mean of optical density $124 \pm 12$ day $0 ; 80 \pm 13$ at day $3 ; .41 \pm 6$ day $7 ; 43 \pm 7$ day $10 ; 35 \pm 2$ day $14 ; 51 \pm 8$ day $21 ; 39 \pm 4$ day 28 ). MHC class 1 staining was very weak in control pancreatic islets. In contrast, the MD-STZ model displayed strong signals day 7-28 with maximum on day 10. An intiltration of lymphocytes was observed with the highest insulitis score on day 14. Thus, the recruitment of lymphocytes seems to follow upon an overexpression of MHC 1 molecules brought about by the streptozotocin treatment. The rapid reductions of the $1 \mathrm{~A} 2 / 1 \mathrm{CA}$ 512 and insulin prior to maximal MHC class 1 expression indicates that the major fraction of the lymphocytes are recruited into the islet area by non-antigen specific mechanisms.

\section{4}

PIOGLITAZONE PREVENTS MICE FROM MULTIPLE LOW-DOSE STREPTOZOTOCIN-INDUCED INSULITIS AND DIABETES

H. Ando, T. Takamura, Y. Nagai, H. Yamashita and K. Kobayashi. First Department of Internal Medicine, School of Medicine, Kanazawa University, Ishikawa, Japan

Macrophage infiltration into pancreatic islets is thought to be an initial event inducing insulitis in the development of type I diabetes. Activated macrophages produce not only nitric oxide (NO) by itself, but also cytokines to induce NO synthase (iNOS) in $\beta$ cells to degenerate $\beta$ cells. An insulin sensitizing agent, thiazolidinedione is a direct ligand for peroxisome proliferator-activated receptor (PPAR)- $\gamma$, recently reported to inhibit macrophage activation including cytokine production and iNOS expression. To test a hypothesis that PPAR- $\gamma$ activation by thiazolidinediones could block insulitis development in the course of type I diabetes by inhibiting macrophage activation, we evaluate the effect of pioglitazone, a thiazolidinedione compound, on the development of multiple low-dose streptozotocin (MD-STZ)-induced autoimmune diabetes in mice. CD-1 mice $(171 \pm 8 \mathrm{mg} / \mathrm{dl})$ intraperitoneally injected with 5 daily subdiabetogenic doses of STZ $(40 \mathrm{mg} / \mathrm{kg}$ body wt) developed mononuclear cell infiltration in and around islets followed by hyperglycemia $2-4$ weeks after STZ-injection $(479 \pm 46 \mathrm{mg} / \mathrm{dl}$ at Day 28 , $p<0.01$ vs control). Oral administration of pioglitazone $(0.01 \%$ food admixture) from 7 days before STZ treatment prevented CD-1 mice from development of diabetes induced by MD-STZ $(254 \pm 49 \mathrm{mg} / \mathrm{dl}$ at day 28 , $p<0.02)$. Histologically, pioglitazone blocked the infiltration of mononuclear cells into islets in MD-STZ mice. Our results lighten the novel therapeutic aspect of thiazolidinedione to prevent autoimmune disease including type I diabetes. Mechanisms underlying the preventive effects of pioglitazone will be discussed.

\section{6}

DIAZOXIDE MODIFIES STREPTOZOTOCIN INDUCED BETA-CELL DAMAGE M. Kullin, Z.C. Li, E. Björk, S. Sandler and F.A. Karlsson, Departments of Medicine and Medical Cell Biology, Uppsala University, Uppsala, Sweden

The beta-cell sensitivity to damage is likely to be influenced by their functional activity. We have investigated a putative protective effect of diazoxide, an inhibitor of insulin release acting at the level of the ATP dependent potassium channel, on beta-cell damage induced by streptozotocin (STZ). Islets were isolated from male Sprague-Dawley rats and precultured for 3 days in medium RPMI $1640+10 \%$ fetal calf serum, and then incubated for 30 minutes with diazoxide and subjected to $0.5 \mathrm{mM}$ or $1 \mathrm{mM} \mathrm{STZ}$ treatment for another 30 minutes in the presence of diazoxide. Insulin secretory capacity, insulin content and islet morphology were studied directly or after 24 hours of recovery in culture. STZ treatment alone reduced insulin secretion and insulin content to $54 \%(p=0.001)$ and $56 \%(p=0.0007)$ respectively, after 24 hours compared to control islets. Islets pretreated with $0.5 \mathrm{mM}$ diazoxide had partially preserved secretory capacity and insulin contents and differed from the STZ group $(p=0.0083$ and $p=0.012)$. STZ reduced the number of insulin positive cells, whereas their shape and number were partially preserved in the diazoxide treated islets. These results demonstrate a protective effect in vitro of diazoxide on islets exposed to STZ, which may be related to the activity of the ATP dependent potassium channel in the beta-cell. 


\section{7}

INTERLEUKIN-9 (IL-9) REDUCES T CELL-MEDIATED INSULITIS IN AUTOIMMUNE PRONE NONOBESE DIABETIC (NOD) MICE.

M-C. Many, I.M. Colin, D. Au, J-F Denef, and ${ }^{+}$J-C Renauld. Histology and ${ }^{+}$Experimental Units, Catholic University of Louvain, and Ludwig Institute for Cancer Research, Brussels Belgium.

The understanding of IDDM has been greatly facilitated by the availability of animal models such as NOD mice that develop insulitis at 4-6 weeks (w) of age, leading to overt diabetes in animals of this strain after $30 \mathrm{w}$. Increasing evidence suggests at least in part that autoimmune insulitis observed in NOD results from an excessive activation of Th1 cells, and/or a defective control by Th2 cells. In the present study, we analyze whether treatment of NOD mice with IL-9 $(1 \mu \mathrm{g} /$ day $)$, a Th2 cytokine, could prevent or at least reduce the progression of insulitis.

Four different experimental groups of 9 weeks old NOD mice $(N=5)$ were used : (1) animals received a single injection of IL-9 and were sacrificed 6 days later; (2) animals received 1 injection/day for 6 days and were sacrificed the next day; (3) animals received the same treatment as in (2) but were sacrificed 8 weeks later and (4) animals received 6 daily injections +3 weekly injections until sacrifice. For each group, age-paired untreated animals were used as controls. After sacrifice, the pancreas was removed, and processed for a morphological quantification on 5- $\mu \mathrm{m}$ thick serial sections. The percentage of islets showing signs of insulitis was then quantified.

In control animals of groups 1 and 2, the percentage of Langherhans islets being infiltrated by inflammatory cells was $42.7 \pm 2.5 \%$ (mean \pm SEM). It was not changed in treated animals of group 1 and was reduced to $12 \pm 0.7$ $\%(p<0.05)$ in group 2 . At 18 weeks of age (groups 3 and 4$)$, nearly all islets $(93.2 \pm 6.8 \%)$ were infiltrated in control animals. However, in treated animals, the percentage was reduced to $47.9 \pm 6.3 \%(p<0.05$ ) in group 3 and to $20.9 \pm 3.8 \%(p<0.05)$ in group 4

In conclusion, our data show that IL-9 significantly reduces the degree of insulitis in NOD mice and suggest therefore that this Th2 cytokine could prevent or delay the onset of the diabetes.

\section{8}

KINASE INHIBITOR EFFECTS ON SPONTANEOUS AND CYTOKINE. INDUCED APOPTOSIS IN HIT-T15 CELLS AND NEONATAL RAT ISLETS

N.E. John, V.D. Belin, M.J. Harrison, N. Aabenhus Andersena, T. Mandrup-Poulsen ${ }^{a}$ and I.C. Green. Biochemistry Lab., Univ. of Sussex and Pharmacy Dept., Univ. of Brighton, UK; aSteno Diabetes Center, Gentofte, Denmark

Mitogen-activated and stress-activated protein kinase (MAPK/SAPK) activation has been observed in neonatal rat islets following exposure to IL-1B. The aim of this study was to investigate how specific inhibitors of MAPK/SAPK pathways affect spontaneous or cytokineinduced apoptosis. HIT-T15 cells $\left(2 \times 10^{5}\right)$ and groups of 150 islets from 8 day old rats were treated in $300 \mu$ RPMI for up to $48 \mathrm{~h}$ with PD98059 (10 and $100 \mu \mathrm{mol} / \mathrm{l})$, a MEK inhibitor, or SB203580 (10 and $50 \mu \mathrm{mol} / \mathrm{l}$, a p38 inhibitor. Apoptosis and lysis were determined using acridine orange cytochemistry and Hoechst 33342/propidium iodide staining. Combined cytokines (IL-1B, TNF $\alpha$ and IFN $\gamma$ ) increased apoptosis in HIT-T15 cells from $0.45 \%$ to $2.56 \%(p<0.005)$. Apoptosis was also increased in response to $48 \mathrm{~h}$ treatment with $\mathrm{SB}(10 \mu \mathrm{mol} / 1)$ and PD (100 $\mu \mathrm{mol} / \mathrm{l})$ [control, 0.45\%; SB, 1.8\%; PD $3.4 \mathrm{p}<0.01$ ]. Levels of cytokine-induced apoptosis remained high when either of the kinase inhibitors was added. Similarly, when islets were exposed to a combination of $\mathrm{SB}(10 \mu \mathrm{mol} / \mathrm{l})$ and $\mathrm{PD}(10 \mu \mathrm{mol} / \mathrm{l})$, an increase in apoptosis was seen [control, $0.46 \% ; \mathrm{PD}+5 \mathrm{~B}, 1.35 \%, \mathrm{p}<0.05$ ] and these levels of apoptosis remained high in the presence of IL- IB. We conclude that inhibition of MAPK/SAPK pathways using specific inhibitors promotes spontaneous apoptosis in both HIT-T15 cells and neonatal rat islets, although the magnitude of this effect is greater in HIT-T15 cells. These inhibitors were incapable of lowering cytokineinduced apoptosis in either HIT-T15 cells or neonatal rat islets.

\section{9}

\section{TOXIN-BASED SELECTION OF RIN CELLS WITH IMPROVED} REGULATION OF INSULIN SECRETION

$\mathrm{K}$. Bloch and P.Vardi. The Institute of Endocrinology and Diabetes, SCMCI, Petah Tikva \& Felsenstein Medical Research Center, Tel-Aviv University, Israel

We previously demonstrated that repeated exposure of RNm cells to a high dose of streptozotocin (STZ) induces not only selection of STZ-resistant but also of a insulin-enriched cell subpopulation. The aim of this study was to select a toxin resistant cell subpopulation by alloxan (AL) treatment and to compare regulation of insulin secretion from these cells to those selected by STZ and to untreated cells. The selection of resistant cells was performed by repeated exposure of RINm cells to a high dose of STZ ( $10 \mathrm{mM})$ or AL ( 20 $\mathrm{mM})$. Cell resistance was evaluated by the MTT method and by ${ }^{3} \mathrm{H}$ thymidine incorporation. Established resistant cell strains and naive RNm cells at the same passage were analyzed by determination of intracellular insulin content insulin concentration in the culture medium and sensitivity to glucose and cAMP-dependent secretagogues (IBMX and forskolin). Our data indicate that both STZ and AL select cell subpopulations with higher resistance to both toxins, probably due to a lower expression of GLUT-2 as indicated by 3-O-methyl-glucose competitive assay. Intracellular insulin contents and insulin concentrations in the culture medium (11 mM glucose) were 2-fold higher for AL selected and 5- and 3-fold higher for STZ selected cells when compared to naive $R I N m$ cells, respectively $(p<0,001)$. Incubation of cells with increasing glucose concentrations $(0,3$ and $15 \mathrm{mM}$ ) showed some insulin response in resistant strains, but had no effect in naive RINm cells. Adenylate cyclase activator (forskolin, $1 \mu \mathrm{M}$ ) and phosphodiesterase inhibitor (IBMX, 0.1 mM) potentiated insulin secretion approximately 2 -fold for AL selected and 4-fold for STZ selected cells at $5 \mathrm{mM}$ glucose ( $p<0.001$ ), but had very little or no effect on insulin secretion in naive RINm cells. In conclusion: our results suggest that diabetogenic toxins (STZ and AL) can be used in order to select toxin-resistant strains of RIN cells with improved regulation of insulin secretion.

\section{0}

POLYAMINES MAY INDUCE PROLIFERATION AND APOPTOSIS OF ISLET CELLS IN A DOSE DEPENDENT MANNER

Ch. Laue, A. Neumeyer, J. Schrezenmeir. Institute of Physiology and Biochemistry of Nutrition, Federal Research Center, Kiel, Germany

NIDDM is associated with reduced insulin sensitivity and hyperinsulinemia which is later followed by a decline in insulin secretion. The pathogenesis is based on a genetic disposition, but particularly nutritional factors also play a crucial role. Spermidine, a polyamine which is present in a number of foods from animal sources, is known to be involved in several processes of cell proliferation and differentiation. Therefore neonatal rat islets were cultured for 14 days in RPMI containing different concentrations $\left(10^{-12}\right.$ to $\left.10^{-3} \mathrm{Mol}\right)$ of Spermidine. Proliferation was determined by a BrdU-cell-ELISA. BrdUincorporation significantly $(p<0.01)$ increased from $95 \pm 5 \%$ proliferation/islet (mean $\pm S E M, n=80$ ) with $10^{-12}$ to $130 \pm 9 \%$ with $10^{-5} \mathrm{Mol}$ spermidine. This effect was combined with a marked increase of the basal (at $5,5 \mathrm{mM}$ glucose) and glucose stimulated (at $16,6 \mathrm{mM}$ glucose) insulin secretion (basal insulin: $45 \pm 4$ at $10^{-12}$ Mol spermidine and $69 \pm 14 \mu \mathrm{U} / 24 \mathrm{~h} / \mathrm{s} / \mathrm{s}$ et at $10^{-6}$ Mol, stimulated insulin: $73 \pm 7$ at $10^{-12} \mathrm{Mol}$ and $113 \pm 14 \mu \mathrm{U} / 24 \mathrm{~h} /$ islet at $10^{-6} \mathrm{Mol}$ (mean $\pm S E M$, $n=40, p<0.03$ ). However with higher spermidine doses, proliferation decreased significantly to $48 \pm 9 \%\left(10^{-3} \mathrm{Mol} \mathrm{p}<0.01\right)$. This rebound was associated with an increase in apoptosis as assessed by an ELISA for the determination of cytoplasmatic histone-associated DNA fragments (monoand oligonucleosomes). Concentrations of spermidine above $10^{-5}$ entanced apoptosis significantly to $218 \pm 17 \%\left(10^{-4} \mathrm{Mol}\right)$ e.g. $341 \pm 38 \%\left(10^{-3} \mathrm{Mol}\right)$ (mean $\pm S E M, n=48, p<0.01$ e.g. 0.001 ). Insulin secretion reflected this phenomenon by a lacking stimulatory response to glucose with $10^{-4}$ and $10^{-3}$ Mol spermidine. In conclusion factors inducing islet cell proliferation induce higher insulin secretion which may be due to an increased islet cellularity and may explain hyperinsulinemia in the early stage of NIDDM. The induction of apoptosis by the same factors at higher doses may explain the secondary failure of islets at later stages of the disease. Polyamines may act according to this dual key model as indicated by these results. 


\section{1}

\section{DEMONSTRATION OF B-CELL APOPTOSIS IN AN ACCELERATED MODEL OF AUTOIMMUNE DIABETES}

P. Augstein, L. A. Stephens*, J. Allison*, A. G. Elefanty*, M.

Institute of Diabetes „Gerhardt Katsch" Karlsburg e.V., Germany; The Walter and Eliza Hall Institute for Medical Research, Australia*

In the spontaneously-diabetic non-obese diabetic (NOD) mouse it has been difficult to demonstrate $B$-cell apoptosis in vivo due to the long period over which B-cell destruction occurs. In this study, NOD.scid mice were crossed with transgenic mice expressing the co-stimulatory molecule B7-1 (CD80) on their $B$ cells (RIP-B7-1 NOD.scid mice). The adoptive transfer of splenic $T$ cells from diabetic NOD mice into RIP-B7-1 NOD.scid recipients ( $\mathrm{n}=16)$ resulted in an accelerated onset of diabetes (mean \pm SD: $15 \pm 1.7$ days), which occurred in all recipient mice within a very narrow time frame (12-16 days). In comparison, diabetes onset in the non-transgenic NOD.scid littermates $(n=9)$ occurred significantly later (mean $\pm S D: 29 \pm 6.2$ days; $p<0.001$ ), and the time at which individual mice developed diabetes was far less predictable. Insulitis and the percentage of islets positive for apoptosis as detected by the TUNEL-technique were significantly greater in B7-1 NOD.scid mice. Confocal microscopy after double labelling of TUNEL ${ }^{+}$cells with both B7-1 and insulin confirmed that the cells undergoing apoptosis were $B$ cells. This study in an accelerated model of diabetes provides clear evidence in vivo that $B$ cells in the autoimmune lesion undergo death by apoptosis.
GENERATION OF AN ASSAY TO STUDY ISLET DESTRUCTION IN THE NON OBESE DIABETIC (NOD) MOUSE MODEL.

Mette Ejrnæs, Laura Bowie, Anne Cooke, and Birgitte Michelsen. Hagedorn Research Institute, Nicls Steensens Vej 6,2820 Gentofte, Denmark

Insulin dependent diabetes mellitus (IDDM) results from the autoimmune mediated process in which the insulin-producing pancreatic $\beta$ cells are being destroyed. This process involves $\mathrm{CD} 4^{+}$and $\mathrm{CD} 8^{+}$lymphocytes homing to and infiltrating the islets. Eventually, the $\mathrm{CD} 8^{+} \mathrm{T}$ cells are believed to be the cells responsible for the final $\beta$ cell destruction and seems to be associated with a response towards an islet cell autoantigen. Several islet specific autoantigens have been discovered. One of these being glutamic acid decarboxylase (GAD) against which autoreactive T cells and autoantibodies have been discovered, both in man and in an animal model of IDDM, the nonobese diabetic mouse (NOD). Aim: In order to test the role of islet autoantigens in the islet destructive phase of diabetes we have generated a cytotoxicity assay whereby $\mathrm{T}$ cell mediated islet destruction can be measured in vitro. Method: Islet single cells are labelled with triaminopentaacetate (TDA). Upon effector cell mediated lysis of the target cell TDA is released to the medium. In an europium solution released TDA will form a complex which becomes fluorescent when pulsed with excitation light. Results: We have shown that a GAD specific $\mathrm{CD} 8^{+}$clone can lyse NOD islet single cells presenting GAD67 peptide at $40-45 \%$ and BALB/c islet single cells at $23-27 \%$ for effector:target ratios $3: 1$ and $6: 1$, respectively. Islet single cells from C57BL/6 mice presenting GAD67 peptide were lysed at 3-4\%. Incubating islets from the three mouse strains without peptide and with the GAD67 specific $\mathrm{CD} 8^{+}$ clone, lysis percentages of 15 and $17 \%$ were observed from NOD islets compared to $\mathrm{BALB} / \mathrm{c}$ and C57BL/6 islets which were lysed close to zero. This could reflect two things: First, a relatively higher spontaneous GAD67 expression on NOD islets compared to $\mathrm{BALB} / \mathrm{c}$ and $\mathrm{C} 57 \mathrm{BL} / 6$. Secondly the NOD islets being more sensitive to effector cells and cytokines produced by these. We are now in position to compare the $\beta$ cell toxicity of various immune effector cells and cytokines using islet cells as targets.

\section{3}

THE PANCREATIC ISLET BLOOD FLOW IN PREDIABETIC FEMALE NOD MICE IS INCREASED

S. Sandler, L. Jansson and P.-O. Carlsson. Department of Medical Cell Biology, Uppsala University, Uppsala, Sweden. Presently we tested the hypothesis that changes in pancreatic islet blood flow correlate with the difference in diabetes incidence between male and female non-obese diabetic (NOD) mice. Blood flows were determined by a microsphere technique. In animals aged $10 \quad(P<0.05)$ and 14 wks $(P<0.001)$, the islet blood perfusion was threefold higher in female NOD mice, both when compared with age-matched male NOD mice and age- and sex-matched control ICR mice. At 5 wks of age islet blood flow was similar in all groups. Administration of a bolus dose of aminoguanidine (AG; a blocker of inducible nitric oxide synthase) to 10 wks old animals selectively and markedly decreased islet blood flow in female NOD mice $(P<0.01)$, whereas islet blood flow in ICR mice and in male NOD mice remained unaffected. AG neither affected mean arterial blood pressure nor whole pancreatic blood flow in any of the groups. These combined findings suggest that the diabetes-prone female NOD mice have an increased islet blood flow, which is mediated by an excessive production of nitric oxide formed by inducible nitric oxide synthase. The islet blood hyperperfusion may augment homing to the pancreatic islets of inflammatory cells and soluble factors involved in $\beta$-cell destruction during the development of Type 1 diabetes in this animal model. The observed gender difference in the blood flow response could therefore, at least partially, explain why female NOD mice are more prone to develop hyperglycemia than the males.

\section{4}

INFECTION OF ISOLATED HUMAN PANCREATIC ISLETS WITH VARIOUS STRAINS OF COXSACKIE VIRUS B4: EFFECTS ON THE INSULIN RELEASE AND ISLET MORPHOLOGY

G. Frisk, K. Jansson, D. L. Eizirik, H. Diderholm, and C. Hellerström Dept of Women and Child Health, Dept of Clinical Virology, Dept of Medical Cell Biology, Uppsala, Sweden, and Dept of Metabolism and Endocrinology, Vrije Universiteit Brussel, Brussels, Belgium

The aim of the present study was to characterize the interactions between different strains of Coxsackie virus B4 (CBV-4) and human islet cells. For this purpose isolated human pancreatic islets were maintained in tissue culture (medium RPMI 1640; $5.5 \mathrm{mM}$ glucose) and infected with the following strains of CBV-4: VD 2921 , V89 4557, V345 and JVB $\left(10^{3}-10^{4} \mathrm{TCID}_{50^{\prime}} /\right.$ well $)$ followed by an additional $48 \mathrm{~h}$ culture period. Infected and uninfected (control) islets were then cultured for $24 \mathrm{~h}$ in medium containing either 5.5 or $16.5 \mathrm{mM}$ glucose followed by a final $24 \mathrm{~h}$ culture period at $5.5 \mathrm{mM}$ glucose. Virus replication and changes in islet morphology were assessed after $1,24,48,72$ and $96 \mathrm{~h}$ in culture. VD 2921 , V89 4557 and JVB replicuted well, whereas the remaining strain, V345 had a slower replication in the human islet cells. All strains, except VD 2921, caused islet cells lysis within the $96 \mathrm{~h}$ period. The morphology of the islets infected with VD 292 I did not differ from that of the control islets. of the islets infected with VD 2921 did not differ from that of the control islets.
Control islets showed a strong $(9.6 \pm 1$. 9-fold; $n=28)$ insulin response to high glucose, whereas islets exposed to V89 4557 and JVB responded significantly less $(2.5 \pm 0.6$-fold: $n=7 ; P<0.01$ and $1.4 \pm 0$.9-fold $n=5 ; P<0.01$ ). The insulin release in response to glucose of the VD 2921 and V 345 infected islets was lower than that of the controls (7.5 \pm 2 .9-fold; $n=7 ; P>0.05$ and $5.4 \pm 3.4$-fold; $n=5$ ) although the difference was not significant $(P>0.05)$. The results suggest that some strains of CBV-4 replicate well in human pancrealic islets in vitro although this may not always be associaled with lysis of the islet cells. The present observations support the view that certain strains of CBV- 4 are present observations support the view that certain strains of CBV-4 are
cytotropic to human islet cells including the protoype strain of CBV-4 (JVB) which was previously considered not to be diabetogenic in mice. Lasting CBV-4 infection without islet cell lysis may predispose to progressive autoimmune islet cell damage and diabetes. (Supported by grants from the European Community, the Juvenile Diabetes Foundation International, The National Institutes of Health and the Swedish Medical Research Council). 


\section{Islet and Pancreas Transplantation}

\section{5}

\section{TARGETTED EXPRESSION OF GREEN FLUORESCENT PROTEIN AS THE BASIS FOR SORTING HUMAN $\beta$-CELLS.}

P.A. Halban, K. Meyer, Larry G. Moss ${ }^{2}$, Lina Moitoso de Vargas², J Oberholzer', P. Morel ${ }^{1}$ and J.-C. Irminger. Jeantet Research Laboratories and 'Department of Surgery, University of Geneva, Switzerland and ${ }^{2}$ Tufts University School of Medicine/ New England Medical Center, Boston, USA.

Since routine methods for sorting rat islet cells based on autofluorescence and cell size are not readily applicable to human cells, we have developed a new technique for sorting $\beta$-cells based upon targetted expression of green fluorescent protein (GFP). Islet cells are infected with recombinant (replication deficient) adenovirus expressing GFP under the control of the insulin promoter (Adeno.R|P-GFP). Whereas both $\beta$ - and non- $\beta$-cells are infected by the virus, only $\beta$-cells should express GFP, allowing for their sorting by fluorescence activated flow cytometry (FACS). To confirm this, human islets were obtained by collagenase digestion and dispersed into single cells by treatment with enzyme-free dissociation buffer. Cells were incubated with Adeno.RIP.GFP at $10^{8}$ ptu (plaque forming units) $/ \mathrm{ml}$ for $1 \mathrm{~h}$ After 2 days of culture to allow for maximal expression of GFP, cells were fixed for immunofluorescence with preserved GFP fluorescence, incubated with either anti-insulin or anti-glucagon serum followed by rhodamine labelled second antibody and examined by fluorescence microscopy. Of the total population, $54 \%$ and $14 \%$ were positive for insulin or glucagon respectively. Of the insulin-containing cells, $93 \%$ were also GFP-positive whereas none of the glucagon-positive cells co-expressed GFP. All of the GFP-positive cells were also found to express insulin. By FACS, infection with Adeno.RIP-GFP led to appearance of a new population of cells with fluorescence 10-50 times higher than controls, allowing for efficient sorting based on this parameter alone. In conclusion, this method allows for highly selective expression of GFP in human $\beta$-cells which can in turn be used as the basis for sorting human $\beta$-cells from non- $\beta$-cells by FACS.

\section{7}

LONG TERM TREATMENT WITH DIAZOXIDE IMPROVES B-CELL FUNCTIONS OF ISLETS TRANSPLANTED TO A DIABETIC ENVIRONMENT S.Hiramatsu and V.Grill Department of Molecular Medicine, Endocrine and Diabetes Unit, Karolinska Hospital, Stockholm, Sweden and Department of Internal Medicine. Section of Endocrinology, University of Trondheim, Trondheim. Norway We tested whether long term stimulation by hyperglycemia impairs B-cell functions to a major part by chronically excessive stimulation. To this end effects of diazoxide treatment were investigated. Islets from inbred Wistar-F rats were transplanted to recipients with streptozocine-induced diabetes. Recipients received grafts of 200 and 20 islets under left kidney capsules and were then treated with diazoxide $(0.2 \%$ in food) for 8 weeks. Average blood glucose levels of treated and control rats were $31.6 \pm 1.3$ and $34.0 \pm 0.9 \mathrm{mM}$ respectively (n.s.). Levels were higher in treated rats one week after transplantation but not later. After 8 weeks, graft bearing kidneys were perfused. Insulin responses to arginine were higher in diazoxide treated rats, as were contents of insulin and insulin mRNA (table).

\begin{tabular}{ccccc}
\hline \multicolumn{3}{c}{$\begin{array}{c}\text { insulin secretion (fmol/min) } \\
27.8 \mathrm{mM} \text { glucose } \\
10 \mathrm{mM} \text { arginine }\end{array}$} & $\begin{array}{r}\text { insulin content } \\
(\mathrm{ng} / \mathrm{islet})\end{array}$ & $\begin{array}{r}\text { mRNA level } \\
(\mathrm{pg})\end{array}$ \\
\hline control & $0.7 \pm 0.5$ & $26.8 \pm 13.5$ & $5.9 \pm 0.7$ & $79.8 \pm 14.7$ \\
treated & $8.0 \pm 6.5$ & $135.1 \pm 38.2^{*}$ & $23.4 \pm 3.4^{* *}$ & $145.1 \pm 30.2$ \\
\hline
\end{tabular}

In a second series of experiments, diazoxide was given for 2 weeks after transplantation, followed by 6 weeks without treatment. There was no effect on insulin secretion but a tendency for increased insulin contents $(6.6 \pm 1.1 \mathrm{vs} .9 .1 \pm 1.3$, $\mathrm{p}=0.14)$ and a significant increase in mRNA $(89.2 \pm 18.3$ vs. $134.8 \pm 6.0, \mathrm{p}=0.04)$. Long term treatment with diazoxide improves B-cell functions of islets transplanted into hyperglycemic conditions. Results indicate that excessive stimulation of B-cell is a major cause of impairment both early and late after transplantation.

\section{6}

EFFECT OF TEMPERATURE CULTURE ON HLA DR mRNA

EXPRESSION IN HUMAN PANCREATIC ISLET PREPARATIONS.

Desailloud R, Pattou F, Kerr-Conte J, Gmyr V, Proye C, Lefebvre J.

Department of Endocrinology, Endocrine Surgery and Cell Culture. University

Hospital of Lille, France.

In rodents, culture pretreatment especially at low temperature appears beneficial 10 islet allograft survival presumably due to the depletion of antigen presenting celis or reduction of MHC class II antigens. Even though islets are of ten cultured belore clinical allotransplantation, effects of culture on the expression of MHC II antigens in human istets remain unknown. The aim of the study was to examine the effects of culture on the expression of HLA DR mRNA in human islet preparations. Human pancreatic glands $(n=7)$ were obtaincd from adult heartbeating organ donors. After isolation and purification following the standard semi-automated technique, isless preparations were cultured for seven days either at $27^{\circ} \mathrm{C}$ or at $37^{\circ} \mathrm{C}$. We used mcdium containing either $10 \%$ fetal calf serum (FCS) or 2\% UltruserG (US) a scrum substitute. Influence of culture on HLA DR mRNA expression (chain $\beta$ 5'region) was cvalualed using semi-quantitative RT-PCR. HLA DR mRNA cxpression was indexed to actin's. Antigen expression at the surface level was assessed with immunocytochenistry. Before culture, index of $\mathrm{HLA}$ DR mRNA expression was $0.49 \pm 0.12$ (mean \pm sem). After culture at $27^{\circ} \mathrm{C}$, inder was $(0.50 \pm 0.13$ with medium containing FCS and $0.46 \pm 0.13$ with medium containing serum substitute $\left(27^{\circ} \mathrm{C}\right.$ culture vs before culture $\mathrm{p}=(0.3)$. In contrast, after culture at $37^{\circ} \mathrm{C}$, index was $0.84 \pm 0.09^{*}$ with medium containing FCS and $0,76 \pm 0,09^{*}$ with modium containing scrum substitute $\left(*: 37^{\circ} \mathrm{C}\right.$ culture is basal $\left.p<0,01\right)$. Immunocytochemical study of cultured islets locillised HLA DR antigens mostly on nonendocrine contaminating tissuc. Enhancement of HLA DR mRNA cxpression was concomittint to a phenotypic switch from acinar toward ductal cells. HLA DR mRNA expression is enhanced during culture at $37^{\circ} \mathrm{C}$ and not modificd with cuiture at $27^{\circ} \mathrm{C}$. Our results question the polential bencfit of culture before human islets transplantation. When culture is requisite, temperature of $27^{\circ} \mathrm{C}$. should be prelerred.
638

EFFECT OF ANTIFIBROTIC COMPOUND HOE 077 ON THE FIBROTIC OVERGROWTH AROUND BARIUM ALGINATE MICROCAPSULES

W. Zhang', Ch. Laue', A. Hyder ${ }^{1}$, G. Kloppel ${ }^{2}$, M.Bickel $^{3}$ and J. Schrezenmeir ${ }^{1}$ ${ }^{\prime}$ Institute of Physiology and Biochemistry, Federal Research Center, Kiel; ${ }^{2}$ Institute of Pathology, University of Kiel; ${ }^{3}$ Hoechst Marion Roussel Deutschland, Frankfurt, Germany.

Microencapsulation of pancreatic islets has been proposed as an alternative method to prevent graft rejection in allo- and xenotransplantation without immunosuppression. One of the most important factors affecting long-term viability of encapsulated islets is the cellular adherence and the pericapsular fibrotic infiltration (PCI). The antifibrotic compound HOE 077 was found to be effective in the inhibition of hepatic fibrogenesis in the rat. The goal of this study is to investigate the effect of HOE 077 on the fibrotic overgrowth around the microcapsules. Porcine islet cells were encapsulated in barium alginate beads, and implanted beneath the liver capsules of male normoglycaemic Balb/c mice. HOE 077 was given orally, dissolved in the drinking water in a concentration of $1.5 \mathrm{mg} / \mathrm{ml}(\mathrm{n}=10)$; the controls received only water $(n=10)$. After four weeks, the microcapsules were retrieved for histological examination. The severity of reaction to the capsules was determined by measuring the thickness of the pericapsular fibrotic overgrowth. The viability (\%) of the porcine islet cells was assessed with a score quantifying the grade of damage. Within the observation period, the alginate capsules of the control group were surrounded with cell-rich, growing fibrotic tissue. The thickness of the PCI was $62.12 \pm 3.84 \mu \mathrm{m}$ and the viability was $15.16 \pm 2.32 \%$ (mean \pm SEM). In contrast, only a slight fibrotic reaction can be detected in the treated group; the thickness of the PCI was $41.44 \pm 2.45 \mu \mathrm{m}$ and the viability was $23.08 \pm 2.45 \%$. Compared to the group without HOE 077 the use of this drug resulted in a thinner pericapsular fibrotic layer $(p<0.0001)$ and higher viability of the encapsulated islets $(p<0.05)$. Our data suggest that administration of the antifibrotic compound HOE 077 could be a new approach for the prevention of the PCI that impedes graft survival. 
639

PROTECTIVE EFFECT OF 15-DEOXYSPERGUALIN ON TRANSPLANTED ISLETS

$\mathrm{C}-\mathrm{H}$. Kuo, J-H. Juang and B.R-S. Hsu. Division of Endocrinology and Metabolism, Chang Gung Memorial Hospital, Taipei, Taiwan, R.O.C.

Nonspecific inflammation is an important factor in primary graft failure. In the present study, we examined the protective effect of 15-deoxyspergualin (DSG), a macrophage immunomodulatory agent, on the transplanted islets. An insufficient number of islets (150 islets) were syngeneically transplanted under kidney capsule of streptozotocin-diabetic C57BL/6 mice. Twelve recipients received daily intraperitoneal injection of DSG, $0.625 \mathrm{mg} / \mathrm{kg}$, for 4 weeks after transplantation. Twenty-one recipients without DSG treatment were used as control. At 4 weeks, the recipients' blood glucose (BG), body weight (BW), 30 min $B G$ during intraperitoneal glucose tolerance test ( $30 \mathrm{~min} B G)$ and glycosylated hemoglobin $\left(\mathrm{HbA}_{1} \mathrm{C}\right)$, as well as the insulin content (IC) of the graft are shown in Table.

\begin{tabular}{lcc}
\hline & DSG & control \\
\hline $\mathrm{BG}(\mathrm{mg} / \mathrm{dl})$ & $225 \pm 37^{*}$ & $327 \pm 25$ \\
$\mathrm{BW}(\mathrm{g})$ & $21.8 \pm 0.5$ & $22.3 \pm 0.5$ \\
$30 \mathrm{~min} \mathrm{BG}(\mathrm{mg} / \mathrm{dl})$ & $282 \pm 19^{\star}$ & $378 \pm 23$ \\
$\mathrm{HbA} \mathrm{A}_{1 \mathrm{c}}(\%)$ & $5.1 \pm 0.4^{\star}$ & $7.6 \pm 0.4$ \\
$\mathrm{IC}(\mu \mathrm{g})$ & $3.41 \pm 1.13^{*}$ & $0.60 \pm 0.22$ \\
\hline${ }^{*} \mathrm{P}<0.05$ vs. control &
\end{tabular}

Furthermore, there was more B-cells mass in DSG group. In conclusion, DSG can protect the islet graft from nonspecific, macrophage-mediated injury.
LOCAL TH1 CYTOKINE MRNA EXPRESSION AFTER ALLOGENEIC ISLET TRANSPLANTATION IS INFLUENCED BY PRETRANSPLANT ISLET CULTURE.

A. El-Ouaghlidi, H. Jahr, G. Pfeiffer, and R. G. Bretzel. Third Medical Department, University of Giessen, Germany.

The aim of this study was to characterize the inflammatory and immunological response to allogeneic transplanted rat islets by measuring cytokine mRNA expression locally at the transplantation site. For this purpose we transplanted freshly prepared or cultured (1 day $37^{\circ} \mathrm{C}$ in TCM-199 medium) Lewis rat islets under the kidney capsula of Wistar Furth rats. The graft was retrieved three days post transplantation by nephrectomy, total RNA was extracted, and cytokine expression was evaluated using semiquantitative RT-PCR and indexed to a housekeeping gene ( $\beta$-actin) expression. The macrophage derived proinflammatory cytokines $\mathrm{IL}-1 \beta$ and TNF $\alpha$ as well as the Th1 cytokines IFNy and IL-2, and the Th2 cytokines IL-4, IL-10, and TGF $\beta 1$ were measured. The expression of proinflammatory/Th1 cytokine transcripts was enhanced in islet grafts from one day cultured vs. freshly prepared islets (IL-2: $0.029 \pm 0.03$ vs $0.000 \pm 0.000$ IFN $\gamma: 0.091 \pm 0.016$ vs $0.026 \pm 0.008$, TNF $\alpha: 1.733 \pm 0.724$ vs 0.742 \pm 0.309 ). No significant difference was found for Th2 cytokine and IL$1 \beta$ mRNAs (IL-4: $0.940 \pm 0.281$ vs $1.217 \pm 0.356$, IL-10: $0.272 \pm$ 0.022 vs $0.354 \pm 0.177$, TGF $\beta 1: 0.000 \pm 0.000$ vs $0.000 \pm 0.000$, IL1 $\beta: 0.885 \pm 0.381$ vs $0.685 \pm 0.033$ ) for precultured vs. fresh islets, respectively. These results show, that the early engraftment of islets will be determined by the balance of the magnitude of the inflammatory attack (higher after transplantation of cultivated islets) and the susceptibility of islets to this attack (higher in freshly prepared or $22^{\circ} \mathrm{C}$ cultivated islets).

\section{1}

LYMPHOKINE RELEASE FROM LYMPHOMONOCYTES OR LYMPHOCYTES IN RESPONSE TO FRESH OR CULTURED HUMAN AND BOVINE ISLETS.

L. Marselli, C. Tellini, R. Giannarelli, C. Lencioni, M. Ferdeghini, S. Del Guerra, R. Lupi, F. Scatena, S. Guida, R. Navalesi, and P. Marchetti. Dipartimento di Endocrinologia e Metabolismo, Sezione Metabolismo, University of Pisa, Italy

The present study evaluated the release of several lymphokines (LK) from human lymphomononuclear cells (LMC) or lymphocytes (LC) in response to allo- or xenoislets, and the role of islet culture. Human LMC were prepared from healthy volunteers. LMC aliquots were cultured at $37^{\circ} \mathrm{C}$ on plastic for $60 \mathrm{~min}$, to allow the monocyte-macrophages to adhere, and the floating cells (LC) were then harvested. Purified human (HI) or bovine (BI) islets (fresh or 3-4 week cultured) were co-cultured for 3 days with $1 \times 10^{6} / \mathrm{ml}$ of either LMC or LC. Then, $\gamma$-interferon (IFN, U/ml) interleukin-2 (IL-2, U/ml), -6 (IL-6, pg/ml) and $-10(\mathrm{IL}-10, \mathrm{pg} / \mathrm{ml})$, and $\alpha$-tumor necrosis factor (TNF, pg/ml) were measured. Compared to LMC without islets (LMCCtrl), IFN, IL-2 and IL-6 release was significantly and similarly higher with fresh $\mathrm{HI}$ (respectively $1.17 \pm 0.07,1.17 \pm 0.12$ and $3848 \pm 1537$ ) or $\mathrm{BI}$ (respectively $1.66 \pm 0.59$, $1.45 \pm 0.20$ and $9567 \pm 2270$ ). IL-10 production was higher with fresh BI (39.32 \pm 4.13 ) than $\mathrm{HI}(13.47 \pm 5.20)$, and TNF release did not increase significantly neither with allo(169.15 23.47) nor xeno-islets $(120.0 \pm 18.21)$ compared to LMC-Ctrl $(114.18 \pm 18.6)$. Culturing the islets significantly decreased the release of IFN and IL-2 with both HI and BI and the release of IL-10 with BI, did not affect IL-6 levels, and caused an increase of TNF production with both $\mathrm{HI}(458 \pm 65)$ and $\mathrm{BI}(406 \pm 121)$. After removal of the monocyte-macrophage cells, the production of IFN and IL-2 was similar as from LMC, with no difference between fresh $\mathrm{HI}$ (respectively $1.76 \pm 0.30$ and $1.56 \pm 0.20$ ) and $\mathrm{BI}$ (respectively $2.02 \pm 0.36$ and $1.67 \pm 0.27$ ). The release of IL-6, IL- 10 and TNF in response to fresh $\mathrm{HI}$ was also similar from LC (respectively $4958 \pm 2969,11.21 \pm$ 6.36 , and $143 \pm 32$ ) as from LMC. However, in response to $\mathrm{Bl}$ the release of these latter cytokines from LC (respectively $910 \pm 474,5.94 \pm 1.37$, and $71.83 \pm 11.04$ ) was significantly lower than from LMC. Culturing the islets caused a significant decrease of IFN, IL-2 and IL-6 production from LC challenged with BI. These results show that $\mathrm{HI}$ and $\mathrm{BI}$ cause a similar increase of the release of IFN and IL-2 from human LMC, whereas BI have a more marked effect on IL-10 production. Removal of monocytemacrophage cells causes a marked reduction of IL-6, IL-10 and TNF production in response to xeno-islets. Culturing the islets alters the pattern of $L K$ release, with possible positive effects (reduction of IFN, IL-2 and IL-6), but also with potentially negative changes (increase of TNF).

\section{2}

DETECTION OF PORCINE RETROVIRUS-RELATED SEQUENCES AND REVERSE TRANSCRTTASE ACTIVITY TO ASSESS THE RETROVIRAL RISK DURING XENOTRANSPLANTATION

L. Martignat, B. Clémenceau, A. Jestin, P. Saï. Immuno-Endocrinology, University/INRA, ENVN, Atlanpole, BP 40706, 44307 Nantes cedex 03. France.

One difficulty to be solved before xenotransplantation of porcine islets during type I diabetes is the risk of transmitting infectious agents, particularly retroviruses. We have developed two sensitive complementary PCR-derived detection tests to assess this risk. The first is designed to detect a novel porcine retrovirus-like sequence related to a recently described human endogenous retrovirus (HERV-L). Primers for the species-specific detection of this porcine endogenous sequence were designed and tested. The second test is a productenhanced reverse transcriptase assay (PERT assay) optimized for the detection of porcine reverse transcriptase activity. As the use of specific pathogen free (SPF) porcine tissues could minimize the risk of transmitting conventional infectious agents, we used the latter test to detect reverse transcriptase activity in the culture supernatants from certain SPF pig cells. Additionally, PCR primers for detection of a different type $\mathrm{C}$ porcine retrovinus, able to infect some human cells in vitro, have been reported by another group. Using these tests, we developed an experimental in vitro infection assay of human immortalized lymphocytes with porcine retroviruses. This model is useful to study the risk of transmitting porcine retroviruses to human celis in the immunogenetic and immunosupression context of pig islet xenotransplantation during diabetes. 
643

IN VITRO XENORECOGNITION OF ADULT PIG PANCREATIC ISLET CELLS BY SPLENOCYTES FROM NON-OBESE DIABETIC OR NON-DIABETESPRONE MICE

A.S. Rivereau, S. You, S. Lalain, E. Gouin, P. Sai. Immuno-Endocrinology, University/INRA, ENVN, Atlanpole, BP 40706, 44307 Nantes cedex 03, France.

In vitro studies were conducted in the NOD mouse to investigate the mechanisms possibly involved in cell-mediated rejection of pig islet xenografts in the NOD mouse Mouse splenocytes responded in vitro to pig islet cells (PIC) since primary proliferations were detected in non-diabetes-prone Balb/c $(\mathrm{p}<0.04)$ or NOD $(\mathrm{p}<0.001)$ mice. Moreover, NOD mice displayed a higher ( $p<0.003$ ) splenocyte response to PIC [SI: $5.8 \pm 0.7$ ] than did Baib/c mice (SI: $2.3 \pm 0.3$ ), whereas responses to pig stimulator splenocytes were similar in both strains. The proliferation of NOD splenocytes to PIC was lower $(\mathrm{p}<0.0001$ ) than allogeneic response to Balb/c islet cells, but similar to syngeneic proliferation to NOD islet cells. In both NOD and Balb/c mice, splenocyte proliferation to PIC was abolished $(\mathrm{p}<0.01)$ when $\mathrm{CD}_{4}{ }^{+}$cells were blocked with antibodies, whereas the blocking of $\mathrm{CD}_{\mathrm{s}}{ }^{+}$cells gave a non-significant effect. The main T-splenocyte subsets involved were restricted to mouse MHC Class II as they did not proliferate in the presence of MoAbs directed at I-A molecules. NOD and Balb/c splenocyte proliferation to PIC was abolished after removal of plastic-adherent APC, indicating that the major activation pathway was indirect. Purified $\mathrm{CD}_{4}^{+}$or $\mathrm{CD}_{8}^{-}$cells alone did not proliferate in response to PIC but recovered a proliferative ability when mixed with APC. $\mathrm{CD}_{4}$ cells, alone or in the presence of APC, did not respond to PIC. Both Th1 and Th2 splenocytes were involved in response to PIC since IFNy and IL-4 productions increased significantly ( 300 -fold and 11 -fold, respectively, $0<0.02$ for both), whereas the increase in IL-10 was only 1.5 -fold. The IFNy/IL 4 and IFNy/IL 10 ratios stimulated by PIC were not different with NOD and Balb/c splenocytes. In conclusion, mouse cell-mediated reaction against adult PIC mainly involves Class IIrestricted $\mathrm{CD}_{4}^{+} \mathrm{T}$ lymphocytes of $\mathrm{Th} 1$ and $\mathrm{Th} 2$ subtypes, with indirect pathway for the recognition. Although of low intensity, this cell-mediated reaction constitues an obstacle to pig islet engraftment in the mouse, though not necessarily more insurmountable than alloreactivity. The peculiarity of NOD mouse splenocytes, in terms of proliferation against pig islets, suggests that the study of islet xenograf rejection should take the immunogenetic context of diabetes into account.

\section{4}

SPLEEN CELLS OF NON-OBESE DIABETIC MICE FED WITH PIG SPLENOCYTES DISPLAY MODIFIED PROLIFERATION AND REDUCED AGGRESSIVENESS IN VITRO AGAINST PIG ISLET CELLS

S. You, E. Gouin, P. Sai. Immuno-Endocrinology, University/INRA, ENVN, Atlanpole, BP 40706, 44307 Nantes cedex 03, France.

A new possibility for modifying xenogeneic reaction to pig islet cells, which involves pre-feedings with pig spleen cells, was investigated in the NOD mouse. Compared to controls, mice fed pig spleen cells displayed much higher splenocyte proliferation in response to pig spleen and islet ceils ( $\mathrm{p}<0.0001$ ). This enhanced proliferation was specific for the species providing the fed cells. Positive relationships $(p<0.01)$ were found between increased splenocyte proliferation in response to pig cells and the number of cells per feeding or the number of daily feedings. Concomitantly, while coincubation with splenocytes from control mice led to inhibition of both basal and stimulated insulin releases from pig islet cells $(p<0.001$ ), this aggressiveness was abolished $(p<0.001)$ after coculture with splenocytes from mice fed with pig spleen cells. The proliferative responses of splenocytes from fed or control mice to pig islet or spleen cells were abolished after removal of plastic-adherent cells, indicating that the major indirect pathway of $\mathrm{T}$-cell activation was unchanged by pig spleen cell feeding. The main T-splenocyte subsets involved were restricted to MHC Class II as they did not proliferate in the presence of MoAbs directed at I-A molecules. In fed or control mice, the blocking of CD4+ T cells with MoAbs led to abolition of proliferation ( $p<0.002$ ), while the blocking of $\mathrm{CD}_{8}{ }^{+}$led to a less marked effect. However, an increase in the blocking effect of anti-CD8 MoAbs was noted in mice fed pig cells ( $p$ 0.02 ). In control mice, the main splenocyte subset involved during proliferation in response to pig islet cells was Th1, since IFNy increased significantly $(p<0.01)$ while IL-10 increased only slightly. The main change observed in mice fed pig cells was a marked increase in basal IL-10 production ( $<<0.01$ ) and basal IL-10/IFNy ratio $(p<0,001)$. It scems likely that feeding with pig spleen cells shifted the Th1/Th2 balance towards a dominance of Th2 Class II-restricted CD4+ T-cells, which may have been conducive to activating CD8+ suppressor $T$ cells. In any event, oral administration of pig cells modified xenogeneic cellular response, which may have implications for xenografts of pig islets. In a more general sense, physiological feeding of cells from xenogeneic species appear to have effects on the immune system.

\section{5}

PERIFUSION ANALYSIS OF INSULIN SECRETION FROM SPECIFIC PATHOGEN-FREE LARGE.WHITE PIG ISLETS SHOWS SATISFACTORY FUNCTIONAL CHARACTERISTICS FOR XENOGRAFTS IN HUMANS

E Gouin*t, A-S Rivereau*, V Duvivier', S Darquy', E Larher*, S You*, A Jestin ${ }^{8}, G$ Reach', P Sai*. * Immuno-Endocrinology, University/NRA/ENVN, Nantes; 'Zoopole and 'Molecular Biology, Ploufragan; 'TNSERM U341, Paris; France Though the pig appears to be the islet donor of choice for grafts, there may be a risk of transmission of infectious agents to humans. In this contexi, we adopted a strategy of islet isolation from pigs raised and killed in specific pathogen-free (SPF) conditions. The present study investigated the function of SPF pig islets to determine whether they react qualitatively and quantitatively to nutriments, hormones and neuromediators with which they would be confronted in man. B cells from 20 Large-White SPF pigs were functionally intact after 7 days in culture. Insulin stimulation indexes (SI) of 3.1 $\pm 0.2,2.2 \pm 0.1$, and $4.4 \pm 0.3$ were found respectively for $30 \mathrm{mmol} / \mathrm{l} \mathrm{K}, 100 \mu \mathrm{mol} / \mathrm{l}$ tolbutamide and $10 \mathrm{mmol} / / \mathrm{l}$ theophylline. Basal insulin secretion $(72.2 \pm 7.6 \mu \mathrm{U} / \mathrm{min})$ had already increased significantly $(p<0.001)$ with $5.5 \mathrm{mmol} / 1$ glucose $(184.2 \pm 25.5$ $\mu \mathrm{U} / \mathrm{min}$, SI: $2.5 \pm 0.6$ ), indicating that the threshold stimulatory concentration was comparable to that of human islets. Insulin secretion increased in a glucose dosedependent manner ( $p<0.001$ ) with $11.0 \mathrm{mmol} / \mathrm{l}$ and $22.0 \mathrm{mmol} / \mathrm{l}$ glucose. The phenomenon of "glucose memory" was apparent in these pig islets. Arginine stimulated $(p<0,001)$ insulin secretion dose-dependently with $5 \mathrm{mmol} / \mathrm{l}$ and 10 mmol/l. The ketone body B-hydroxybutyrate $(10 \mathrm{mmol} / \mathrm{l})$ also induced insulin secretion. Insulin release was stimulated by $4 \mu \mathrm{mol} / 1$ gastric inhibitory peptide, revealing sensitivity to the hormonal enteroinsular axis, and by $2 \mu \mathrm{mol} / 1$ glucagon Parasympathetic cholinergic influence was studied using $500 \mu \mathrm{mol} / / \mathrm{l}$ carbamylcholinc, which increased insulin secretion. The influence of orthosympathetic control and of stress situations was also studied. As in human islet response, epinephrine and the $\alpha 2$ agonist clonidine $(50 \mu \mathrm{mol} / 1)$ inhibited insulin secretion. Finally pre-culture of islets may be beneficial for graft outcome, provided that no deterioration in islet function occurs. A prolonged 21-day culture of pig islets showed no decrease in insulin response to glucose, arginine and $\mathrm{K}^{+}$, even with an unaltered threshold stimulatory glucose concentration. Thus, Large-White SPF pigs and the application of our isolation procedure provided islets with characteristics reproducibly compatible with potential utilisation for regulation of glycaemia under physiologic and physiopathologic situations in humans.

\section{6}

AUTOANTIBODIES IN ISLET GRAFTED IDDM PATIENTS: PREVALENCE AND CORRELATION WITH GRAFT FUNCTION

C. Jaeger, M.D. Brendel, M. Eckhard, E. Hatziagelaki, B.J. Hering and R.G. Bretzel. Third Medical Department, University of Giessen, Germany

For islet cell antibodies (ICA) and GAD 65 antibodies we have shown that both types of autoantibodies can persist or occur after clinical islet transplantation despite sustained immunosuppression. The purpose of the present study was to add the new antibody specificity LA-2 to this antibody panel and to investigate the autoantibody prevalences in relation to the islet graft function in 23 islet transplanted IDDM patients. Intraportal infusion of allogeneic islets was performed in $23 \mathrm{C}$-peptide negative IDDM patients and complete islet graft failure was defined as reappearance of permanent Cpeptide negativity $(<0.2 \mathrm{ng} / \mathrm{ml})$ in the serum. ICA, GAD 65 antibodies and IA-2 antibodies were monitored prior to islet transplantation and regularly afterwards. IA-2 antibodies were found positive in 4 out of $23(17 \%)$ patients, GAD 65 antibodies were positive in 11 out of $23(47 \%)$ and islet cell antibodies were positive in 5 out of $23(21 \%)$ individuals. Interestingly, none of the patients was exclusively positive only for IA-2 antibodies. Looking at all three antibodies together we observed generation of autoantibodies either pre-and/or posttransplantation in 11 out of 23 islet transplanted IDDM patients. Most important, we revealed a significant (Kaplan Meier estimate, $\mathrm{p}<0.05$ ) difference in the individual transplant survival times of those individuals presenting with one or more autoantibodies compared to the group of autoantibody negative IDDM recipients of an intrahepatic islet allograft. Taken together, IA-2 antibodies did not identify additional individuals being negative for GAD 65 antibodies or ICA. Furthermore, the results of the present study suggest recurrent autoimmunity directed to transplanted B-cells to be a relevant mechanism in islet graft failure despite standard immunosuppressive therapy. The detection of autoantibodies may help to identify individuals at high risk for recurrent autoimmunity. 
COUNTERREGULATION AFTER PANCREAS TRANSPLANTATTON

C.Dieterle ${ }^{1}$, R.Scheuer ${ }^{1}$, J.Mojto ${ }^{1}$, A.Weigel ${ }^{1}$, W.D.Illner ${ }^{2}$ and W.Land ${ }^{2}$ and R.Landgraf ${ }^{1}$. ${ }^{1}$ Department of Internal Medicine, Klinikum Innenstadt, Munich. ${ }^{2}$ Division of Transplant Surgery, Klinikum Grosshadern, Munich Abnormalities in secretion of counterregulatory hormones, partially as a result of autonomic neuropathy, is a common phenomenon in long lasting diabetes. Aim of this study was to find out whether pancreas transplantation (PTX) is able to normalize or ameliorate counterregulation. Insulin-induced hypoglycaemia test was performed in 61 type 1 diabetics after succesful pancreas grafting. 14 patients received a duct-occluded segmental pancreas graft (SPTX), 47 a bladder-drained whole pancreas (WPTX). Eight healthy volunteers served as a control group (CG). Before and after intravenous injection of $0.075 \mathrm{IU} / \mathrm{kg}$ insulin, blood was collected for measurements of glucose, C-peptide, glucagon, growth hormone, prolactin, epinephrine, norepinephrine and cortisol. Basal glucose levels were significantly higher in CG. The fall in blood glucose was slower in PTX with a nadir after $30 \mathrm{~min}$ in comparison to $20 \mathrm{~min}$ in CG. C-peptide secretion was suppressed in both groups, however C-peptide levels were before and after insulin injection significantly higher in PTX. Secretion of catecholamines, glucagon, cortisol and growth hormone was stimulated in PTX and CG. However, the peak value of each counterregulatory hormone was significantly higher in CG. Glucagon levels, basal and stimulated, were significantly higher in graft recipients with WPTX. Other differences in secretion of counterregulatory hormones between SPTX and WPTX were not found. Pancreas graft recipients, with a normal glucose tolerance in an OGTT performed at the same time, displayed significant higher peak values for cortisol, epinephrine and norepinephrine. Despite of successful PTX disturbances in counterregulation were still found. The size of the transplanted pancreas as well as the grade of normalization of glucose metabolism influence the secretion of counterregulatory hormones. Prospective studies will investigate the longterm effect of PTX on autonomic neuropathy and counterregulation.

\section{PS 16 \\ Amylin}

\section{8}

GLIPIZIDE ADMINISTRATION LEADS TO AMYLOIDOSIS IN A CAT MODEL OF TYPE 2 DIABETES

M. Hoenig ${ }^{1}$ and T.D. O'Brien ${ }^{2}{ }^{1}$ Department of Pharmacology, University of Georgia College of Veterinary Medicine, Athens, GA. ${ }^{2}$ Department of Veterinary Diagnostic Medicine, University of Minnesota, St. Paul, MN.

The objective was to evaluate the effect of insulin and glipizide treatment on islet amyloid formation in cats with type 2 diabetes.

Eight castrated male purpose-bred Domestic Shorthair cats were used in this study. After a partial pancreatectomy which was followed by a 4 week recovery period, diabetes was induced with daily injections of growth hormone and oral administration of dexamethasone. All cats were stable diabetics after 4 months following this induction regimen indicated by glycosylated hemoglobin $(\mathrm{gHb})$ levels of $3 \%$, fasting glucose concentrations of $362 \mathrm{mg} / \mathrm{dl}$, and $\mathrm{k}$-values of 0.45 (mean) which were significantly different from $1.5 \%, 88$ $\mathrm{mg} / \mathrm{dl}$, and 1.22 , respectively, at entry of study ( $<<0.0001$ for all three). Four of the cats were then treated with insulin (Humulin $N$ ) and 4 were treated with $5 \mathrm{mg}$ glipizide three times daily. Glucose was measured weekly at insulin peak action time. In addition, intravenous glucose tolerance tests were performed before and in 4 week intervals during the treatment period with measurements of $\mathrm{gHb}$, insulin, glucose, and IAPP. The cats were euthanatized after 18 month of diabetes management. All cats on glipizide treatment had significantly higher amylin concentrations than the insulin treated cats $(p<0.001)$ and all 4 glipizide treated cats had islet amyloid deposits with scores of $1+$ (minimal; $n=1), 2+($ mild; $n=1)$ and $3+($ moderate; $n=2)$, while only one of the insulin treated cats had a $1+$ amyloid score.

This study shows a. that the diabetic cat is an excellent model for type 2 diabetes, $b$. that glipizide increases IAPP secretion from the islets of cats with induced diabetes mellitus, and c. that glipizide therapy is associated with greater deposition of IAPP-derived amyloid in the pancreatic islets compared to insulin therapy.

\section{9}

EFFECTS OF ISLET AMYLOID POLYPEPTIDE ON IGF-IINDUCED GLUCOSE DISPOSAL IN SKELETAL MUSCLE Dimitriadis $^{1}$, E. Crowne ${ }^{1}, A$. Clark ${ }^{2}$ and D. B. Dunger ${ }^{1}$. Department of Paediatrics ${ }^{1}$ and Diabetes Research Laboratory ${ }^{2}$, Oxford UK. Islet amyloid polypeptide (IAPP) reduces rates of insulin-induced glucose disposal in skeletal muscle but the mechanisms are unclear. Insulin-like growth factor I (IGF-I) and insulin regulate glucose disposal in skeletal muscle acting through different receptors. To determine if the effects of IGF-I are modulated by IAPP, glucose uptake and disposal was examined in rat soleus muscle strips. Rates of glucose transport (GT, 3-Omethyl[ $\left[{ }^{3} \mathrm{H}\right]$ glucose), glycogen synthesis (GS, [ $\left.\mathrm{U}^{14} \mathrm{C}\right]$-glucose) and glycolysis (GL, lactate formation) were examined in the presence of IGF-l $(0.04,0.4$ and $4 \mathrm{nM})$ or insulin $(0.07,0.7$ and $7 \mathrm{nM})$ and IAPP (10nM). IAPP decreased IGF-I-stimulated GT $\left(3.4 \pm 0.1^{*}\right.$, $4.1+0.1^{*}$ and $4.5+0.2^{\star}$ vs $4.2+0.1,5.0+0.3$ and $4.8+0.2 \mu \mathrm{mol} / \mathrm{h} / \mathrm{g}$, $\left.{ }^{*} p<0.01\right)$ and $G S^{-}\left(0.9 \pm 0.1^{+}, 1.1 \pm 0.1^{+}\right.$and $2.1 \pm 0.1^{+}$vs $2.4 \pm 0.2$, $3.6 \pm 0.2$ and $5.8 \pm 0.7 \mu \mathrm{mol} / \mathrm{h} / \mathrm{g},{ }^{\circ}<0.001$ ) but did not decrease $\mathrm{GL}(10.7 \pm 0.7,11.9 \pm 1.1$ and $12.5 \pm 1.1$ vs $10.4 \pm 0.5,11.9 \pm 0.6$ and $12.01 \pm 0.8 \mu \mathrm{mol} / \mathrm{h} / \mathrm{g}$ ). IAPP also decreased insulin-stimulated GT $\left(3.9 \pm 0.2,4.3 \pm 0.2^{*}\right.$ and $6.0 \pm 0.3$ vs $3.9 \pm 0.1,5.0 \pm 0.3$ and $6.5 \pm 0.3$, $" p<0.05)$ and GS $\left(1.0 \pm 0.2^{+}, 1.9 \pm 0.3^{+}\right.$and $3.6 \pm 0.2^{+}$vs $2.0 \pm 0.3$, $4.3+0.5$ and $7.6 \pm 0.6 \mu \mathrm{mol} / \mathrm{h} / \mathrm{g},{ }^{+} \mathrm{p}<0.01$ ), while $\mathrm{GL}$ was not affected. Conclusions: 1. IAPP reduces both IGF-I and insulininduced glucose metabolism in skeletal muscle and its effects are selective: IAPP decreases the sensitivity of GT and GS to IGF-I or insulin but does not affect GL. 2. IAPP actions are likely to be mediated by post-receptor or other mechanisms since IGF-I and insulin act via different receptors in skeletal muscle. 
650

EVIDENCE FOR MODULATION OF POSTPRANDIAL GLUCAGON SECRETION BY ENDOGENOUS AMYLIN IN MAN

B. Ludvik, K. Thomaseth, M. Clodi, J. Nolan, R. Prager, G. Pacini, and J. Olefsky. San Diego, USA, Vienna, Austria, and Padova, Italy

Amylin is cosecreted with insulin and is assumed to modulate gastric emtpying and post-prandial glucagon secretion. To directly evaluate the secretion of glucagon, amylin and insulin in humans, we performed hepatic vein catheterization experiments (HVC) in 6 lean, healthy controls (C, $45 \pm 4$ yrs., BMI: $26.5 \pm 0.9 \mathrm{~kg} / \mathrm{m}^{2}$ ), 6 obese, insulin resistant subjects $\left(O, 45 \pm 2\right.$ yrs., BMI: $\left.35.3 \pm 1.2 \mathrm{~kg} / \mathrm{m}^{2}\right)$ and 6 patients with NIDDM (D, $51 \pm 5 \mathrm{yrs}$., BMl: $30.8 \pm 1.1 \mathrm{~kg} / \mathrm{m}^{2}$ ). Splanchnic blood flow was assessed by infusion of indocyanine green dye. Following glucose administration ( $75 \mathrm{gms}$ ) the respective peptides and glucose were measured every $15 \mathrm{~min}$ over a period of $240 \mathrm{~min}$. A circulatory model ws used to calculate mass balance through the liver. Assuming a $25 \%$ hepatic extraction of glucagon, the total amount of portally released glucagon was $21 \pm 2 \mu \mathrm{g} / \mathrm{L} \cdot 4 \mathrm{~h}$ in $\mathrm{C}, 27 . \pm 5$ in $\mathrm{O}$ and $28 \pm 5$ in D (ns by ANOVA). Compared to the fasting profile, glucagon secretion was unchanged in $\mathrm{C}$ (variation: $-3.5 \pm 14 \%$, reduced in $\mathrm{O}(-25 \pm 12 \%)$, increased in $\mathrm{D}(36 \pm 21 \%, \mathrm{p}=0.0051)$. During OGTT insulin increased by $55 \pm 5 \mathrm{nmol} / \mathrm{L} \cdot 4 \mathrm{~h}$ in $\mathrm{C}, 134+31$ in $\mathrm{O}, 16 \pm 6$ in $\mathrm{D}(\mathrm{p}=0.001)$. Amylin was increased by $0.61 \pm 0.12 \mathrm{nmol} / \mathrm{L} \cdot 4 \mathrm{~h}$ in $\mathrm{C}, 1.07 \pm 0.29 \mathrm{in} \mathrm{O}$, and only by $0.06 \pm 0.09$ in $\mathrm{D}(\mathrm{p}<0.01)$ depicting the relative hyposecretion in $\mathrm{D}$. Glucagon secretion correlated inversly with amylin increase $(r=-0.6$, $p<0.01)$, however not with insulin increase $(r=-0.2, p=0.4)$. In conclusion, we directly demonstrated postprandial hyposecretion of amylin and hypersecretion of glucagon in patients with NIDDM. The inverse correlation between amylin and glucagon secretion supports the contention that amylin might act as a modulator of postprandial glucagon secretion.

\section{1}

Amylin is SECRETEd in a Pulsatile Pattern in Healthy Humans.

C. Juhl, N. Pørksen, J. Veldhuis, S. Pincus and O. Schmitz, Aarhus, Denmark, Charlottesville, VA . and Guilford, CT.

Amylin is stored in the beta-cell granules and co-secreted with insulin in response to nutrient stimuli. Anylin circulates in a non-glycosylated and different glycosylated forms. The aim of the present study was to elucidate the pattern of stimulated amylin secretion with respect to heigh frequency pulses. Seven healthy subjects (3 male, 4 temale, age $27.8 \pm 5.1$, BMI $21.5 \pm 2.5 \mathrm{~kg} / \mathrm{m}^{2}$ ) with no family history of diabetes mellitus were infused with glucose at a constant rate $(2.0 \mathrm{mg} / \mathrm{kg} / \mathrm{min}$.) for $180 \mathrm{~min}$. From time 90 to $180 \mathrm{~min}$. blood was collected every minute and analyzed in triplicate for non glycosylated amylin, total amylin and insulin. All time series were detrended by subtraction of 7-point moving average and no smoothing was performed prior to mathematical analysis. Mean plasma concentration of amylin, amylin like peptide, insulin and glucose were $2.77 \pm 1.21 \mathrm{pM}, 7.60 \pm 1.73 \mathrm{pM}$ $50.4 \pm 17.5 \mathrm{pM}$ and $105.4 \pm 5.9 \mathrm{mg} / \mathrm{dl}$ respectively. A method of random shuffle spectral analysis was developed, and spectral density peak values were tested for being significantly different from random shuffle values. 90 minute time series of amylin, amylin like polypeptide and insulin were analyzed for periodicity and regularity. By spectral analysis $6 / 7,6 / 7$ and $5 / 7$ showed significant spectral density peaks. Autocorrelation analysis revealed significant pulsatility in $5 / 7,7 / 7$ and $4 / 7$ respectively. The dominant frequencies of oscillations were determined by spectral analysis of pooled data from all seven subjects to be 4.7, 4.2 and 5.6 minutes respectively. This was in good accordance with frequencies obtained by autocorrelation analysis performed on pooled data. Regularity of oscillations was evaluated by the recently introduced scale- and model-independent statistic approximate entropy ( $\mathrm{ApEn}$ ). ApEn was found to be significantly different from random $A p E n$ in $6 / 7$ subjects indicating a high degree of regularity. In conclusion circulating amylin concentrations equivalent to other hormones show oscillations resembling a regular pulsatile secretory pattern of non-glycosylated as well as glycosylated anylin.

\section{2}

TRANSGENIC CORRECTION OF INSULIN RELEASE AND GLUCOSE CLEARANCE DEFECTS IN ISLET AMYLOID POLYPEPTIDE NULL MICE.

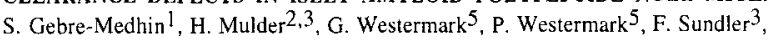
B. Ahrén ${ }^{5}$ and C. Betsholtz ${ }^{1}$. Departments of ${ }^{1}$ Medical Biochemistry, Göteborg University; ${ }^{2}$ Cell and Molecular Biology, ${ }^{3}$ Physiology and Neuroscience, ${ }^{4}$ Medicine at Malmö University Hospital, Lund University; ${ }^{5}$ Pathology, Linköping University; Sweden.

The proposed inhibitory action of islet amyloid polypeptide (IAPP or amylin) on insulin release and blood glucose clearance has gained support by the finding that IAPP null mutant $\left(\mathrm{IAPP}^{-/ \%}\right.$ ) male mice display potentiated insulin release and improved glucose tolerance in oral and iv glucose tolerance tests (GTT). The aim of the present investigation was to study the glucoregulatory impact of a rat insulin-1 promoter-human IAPP transgene (rip-hIAPP) when present in IAPP- ${ }^{-}$or wild-type $\left(\mathrm{IAPP}^{+/+}\right)$genetic backgrounds. Male mice with the genotypes IAPP $/ \%$, IAPP $/++$ IAPP $/-$ rip-hIAPP or IAPP ${ }^{+/+}$rip-hIAPP were subjected to ivGTT with measurements of circulating insulin and blood glucose. IAPP-/-rip-hIAPP mice and $\mathrm{IAPP}^{+/+}$controls displayed nearly identical insulin responses. The insulin response in $1 \mathrm{APP}^{+/+}$or IAPP $/$rip-hIAPP mice was significantly lower than in mice with the $1 \mathrm{APP}^{-/-}$genotype alone (at 5 minutes after glucose infusion: $82.3 \pm 269$ or $1073 \pm 261$ vs $1941 \pm 692 \mathrm{pmol} / /$, respectively, $P<0.05)$. Blood glucose levels in IAPP $+/+$ controls and IAPP $/ /$ rip-hIAPP were comparable. However, glucose levels in IAPP $/++$ or IAPP $/ /$ rip-hIAPP mice were significantly higher than in mice with the IAPP $\%$ genotype alone (at 30 minutes after glucose infusion: $13,9 \pm 0,7$ or $14,3 \pm 1,1$ vs $10,7 \pm 1,1 \mathrm{mmol} / \mathrm{l}$, respectively, $\mathbf{P}<0.05$ ). Finally, although blood glucose levels did not differ between IAPP $+/+$ rip-hIAPP mice and IAPP $+/+$ controls. IAPP ${ }^{+/+}$rip-hIAPP mice displayed significantly lower second phase insulin responses than $\mathrm{AAPP}^{+/ t}$ controls (at 20 minutes after glucose infusion: $470 \pm 30$ vs $786 \pm 120 \mathrm{pmol} / /$, respectively, $\mathrm{P}<0.05$ ). These results provide a causal link hetween the IAPP- $/$ genolype and phenotype, and imply that endogenous IAPP acts to inhibit glucose-stimulated insulin release and blood glucose clearance in the mouse.

\section{3}

THE HUMAN AMYLIN ANALOGUE PRAMLINTIDE SUPPRESSED GLUCAGON SECRETION IN PATIENTS WITH TYPE 2 DIABETES M.S. Fineman, K. Organ, and O.G. Kolterman. CA, USA

People with diabetes have an exaggerated glucagon response to the ingestion of protein. This glucagon response can contribute to hyperglycemia by increasing hepatic glucose output via increased glycogenolysis and gluconeogenesis. The synthetic human amylin analogue, pramlintide, has been shown to suppresses plasma glucagon in amylin deficient people with type 1 diabetes during hyperglycemia but not during hypoglycemia. Patients with type 2 diabetes have a blunted and delayed amylin response to nutrients and pramlintide therapy may suppress the increase in glucagon observed in those patients. To test this hypothesis, we measured plasma glucagon in patients with type 2 diabetes who were treated with an infusion of pramlintide. The study utilized a randomized, placebo controlled, single-blind, 2-visit crossover design in which 12 patients on insulin therapy (INS) and 12 patients controlled by diet or oral agents but not insulin $(\mathrm{D} / \mathrm{O})$ received a 4 hour intravenous infusion of pramlintide at $100 \mu \mathrm{g} / \mathrm{hr}$ (PRAM) or placebo (PBO). Patients started the infusion at $\mathrm{T}=0$ and received their normal morning therapy (insulin or oral agents) at $\mathrm{T}=30 \mathrm{mins}$. At $\mathrm{T}=60 \mathrm{mins}$, patients were given a $7 \mathrm{kcal} / \mathrm{kg}$ body weight standardized liquid meal challenge (Sustacal(B)) Plasma glucagon concentrations were measured by RIA (Linco Research Inc.) and plasma pramlintide concentrations were measured by IEMA. The mean maximal plasma pramlintide concentration was $592 \pm 25 \mathrm{pM}$. Plasma glucagon concentrations 30 minutes post Sustacal $\circledast$ increased significantly $(\mathrm{P}<0.02)$ during $\mathrm{PBO}$ infusion compared to PRAM infusion. For INS glucagon increased $12.6 \pm 3.3 \mathrm{pg} / \mathrm{ml}$ on PBO compared to $1.9 \pm 1.5 \mathrm{pg} / \mathrm{ml}$ on PRAM. For D/O plasma glucagon increased $15.2 \pm 3.2 \mathrm{pg} / \mathrm{ml}$ on PBO compared to $4.1 \pm 2.3 \mathrm{pg} / \mathrm{ml}$ on PRAM. Total glucagon secretion from Sustacal $\mathbb{B}$ administration to the end of the infusion ( $60-240 \mathrm{mins}$ ) was also significantly reduced in both groups when on PRAM compared to PBO. The mean \pm SEM plasma glucagon $\mathrm{AUC}_{60.240}$ on $\mathrm{PBO}$ was $9200 \pm 580 \mathrm{pg}^{*} \mathrm{~min} / \mathrm{ml}$ compared to $8096 \pm 581 \mathrm{pg}^{*} \mathrm{~min} / \mathrm{ml}$ on PRAM $(\mathrm{P}=0.005)$ for INS. For D/O, AUC 60.240 was $8483 \pm 487 \mathrm{pg}^{*} \mathrm{~min} / \mathrm{ml}$ on PBO compared to $7450 \pm 429 \mathrm{pg} * \mathrm{~min} / \mathrm{ml}$ on PRAM $(\mathrm{P}=0.01)$ We conclude that the administration of the human amylin analogue, pramlintide, significantly reduced the elevated postprandial plasma glucagon concentrations observed in patients with Type 2 diabetes. This mechanism may contribute to the lowering of the postprandial plasma glucose concentrationsthat has been reported in previous studies. 


\section{4}

CONCENTRATION-RESPONSE FOR GLUCAGONOSTATIC EFFECT OF PRAMLINTIDE IN RATS

K. Beaumont, B. Gedulin, C. Jodka, R. Lawler and A. Young. Amylin Pharmaceuticals, Inc., 9373 Towne Centre Dr., San Diego, CA 92121 , USA

Pancreatic glucagon secretion is inhibited by insulin and by the 37 amino acid peptide hormone, amylin, which is also secreted from pancreatic $B$-cells. Insulin and amylin are both deficient in type 1 and insulin-requiring type 2 diabetic individuals, and their absence may be implicated in the hyperglucagonemia and exaggerated glucagon response to amino acids often seen in those conditions. In the present study we examined the effect of pramlintide, a synthetic human amylin analogue, on amino acid-stimulated glucagon secretion. Changes in arginine-stimulated glucagon secretion were measured in anesthetized male Harlan Sprague Dawley rats infused intravenously with pramlintide $(0,0.1,1$, or $10 \mu \mathrm{g} / \mathrm{h} ; \mathrm{n}=7,7,6,7$ respectively) from $30 \mathrm{~min}$ before until $120 \mathrm{~min}$ after arginine $(2 \mathrm{mmol}$ infused over $10 \mathrm{~min}$ ). To equalize the effects of glucose and insulin upon glucagon secretion, plasma glucose was clamped at $5.8 \mathrm{mM}$ following a primed/ continuous insulin infusion $(12 \mathrm{mU}+5 \mathrm{mU} / \mathrm{kg} / \mathrm{min})$, beginning $60 \mathrm{~min}$ before the arginine infusion. Pramlintide infusion inhibited the plasma glucagon response to arginine (measured as the $60 \mathrm{~min}$ increment in areaunder-the-curve above the pre-arginine baseline) by a maximum of $56 \pm 5.3 \%$ ( $P<0.01$ vs saline controls). Analysis of glucagon response as a function of calculated steady-state piasma pramlintide concentration revealed an EC of $30.4 \mathrm{pM} \pm 0.38 \mathrm{log}$ units. These data indicate a glucagonostatic potency of pramlintide that could prove beneficial for therapy with this agent in type 1 and insulin-requiring type 2 diabetic patients.

\section{5}

CYTOTOXICITY OF HUMAN ISLET AMYLOID POLYPEPTIDE TO $\beta$-TC CELLS IS MEDIATED VIA APOPTOSIS AND NECROSIS

'C.E. Higham, 'M.Lewis, 'J.F. Morris and ${ }^{12}$ and A. Clark. 'Department of Human Anatomy and 'Diabetes Research Laboratories, Oxford UK

Islet amyloidosis is accompanied by progressive reduction of $\beta$-cells in type 2 diabetes. Amyloid fibrils are toxic to endocrine and neuronal cells in vitro by an unknown mechanism, possibly related to the conformation of the amyloidogenic peptide. To investigate toxicity of islet amyloid fibrils, the structural properties and toxic effects of synthetic hIAPP obtained from three different commercial sources was determined. Peptide purity was determined using HPLC and mass spectrometry. $B-\mathrm{TC}$ cells and mouse islets were cultured in media containing synthetic hIAPP $(12 \mu \mathrm{M})$ for up to 48 hours. Cell viability and mode of death was examined by fluorescence and electron microscopy. The vital dyes calcein-AM (detects intact cytoplasm) and propidium iodide, (PI) (binds to DNA when membrane integrity is compromised) were used together with annexin $\mathrm{V}$ (binds phosphatidylserine on its exposure during apoptosis). Terminal UTP nick end labelling (TUNEL),

fluorescently labels DNA strand breaks identified apoptosis. $\beta T C$ cells, but not islets, were susceptible to hIAPP fibrils in a time dependent manner. hIAPP (Bachem), incubated with cells for $24 \mathrm{~h}$, induced $40 \%$ cell death (PI+ve), $12 \%$ annexin $\mathrm{V}+\mathrm{ve}$ cells and $25 \%$ TUNEL + ve cells $(n=3, p<0.01$ for all labels). hIAPP from 3 commercial sources caused different degrees of cytotoxicity over $24 \mathrm{~h}$; PI+ve cells, $12 \%$, Advanced Biotechnology, $<5 \%$, Peninsula. IAPP from the 3 sources were pure as determined by mass spectrometry and HPLC; Bachem IAPP by HPLC contained a dimerised form.

Conclusion; hIAPP fibrils are toxic in a time dependent manner to $\beta$ cells but not islets; both apoptosis and necrosis are involved in this toxic process

\section{6}

INSULIN AND POLYIONIC SULPHONATES MODIFY HUMAN ISLET AMYLOID POLYPEPTIDE FIBRIL AGGREGATION IN VITRO

'D.S. Powell, ${ }^{1,2}$ E.T.A.S. Jaikaran, ${ }^{2}$ B. Laube, ${ }^{2}$ D.L.A. MacArthur, ${ }^{3}$ P.E Fraser, ${ }^{4}$ W.A. Szarek, and ${ }^{1,2}$ A. Clark. ${ }^{1}$ Diabetes Research Laboratories, ${ }^{2}$ Dpt. of Human Anatomy, Oxford UK, ${ }^{3} \mathrm{CNDR}$, University of Toronto, Canada, ${ }^{4} \mathrm{Dpt}$ of Chemistry, Queens University, Kingston, Canada

Human islet amyloid polypeptide (hIAPP) forms amyloid fibrils in type 2 diabetes by unknown mechanisms. Islet amyloidosis is associated with deterioration of insulin secretion. To identify mechanisms of fibril aggregation and potential therapeutic inhibitors of amyloid formation, biological and biochemical systems have been developed; techniques included an ELISA assay for immunodetection of non-aggregated hiAPP, cultured islets isolated from hIAPP transgenic mice and electron microscopy (em). Fibril formation

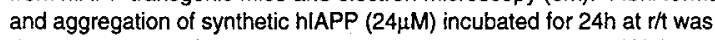
determined by ELISA assay of fibril supernatants. After 24h, hIAPP in the supernatant was reduced by $75.7 \pm 2.7 \%\left(\triangle \mathrm{hlAPP}_{24}\right)$ indicating timedependent formation of precipitated fibrils. $\triangle$ hIAPP ${ }_{24}$ was inhibited by $61.6 \pm$ $7.2 \%$ at $\mathrm{pH} 3.0$ and reduced by polywinyl sulphonate (PVS, $0.4 \mathrm{mM}$ ) and insulin $(2.4 \mu \mathrm{M})$ but not by heparin sulphate $(40 \mathrm{mM})$ calcium $(5 \mathrm{mM})$, proinsulin $(2.4 \mu \mathrm{M})$ or somatostatin $(2.4 \mu \mathrm{M})$. Ultrastructure of synthetic hIAPP fibrils (em) was modified by insulin which was shown to be bound to the fibrils by immuno-em. islets isolated from hlAPP transgenic mice were cultured in RPMI 1640 , pius $16 \mathrm{mM}$ glucose.PVS $(10 \mu \mathrm{M})$ for 6 days. Islet amyloid formed in hIAPP mouse islets was determined by quantitative morphometry of em images and was not changed by PVS.

Conclusion; Interaction of IAPP and insulin occuring in secretory granules could stabilise both peptides in vivo. PVS-related compounds may provide data for design of therapeutic inhibitors of amyloid formation in diabetes.

\section{7}

SYNTHETIC HUMAN PRO-ISLET AMYLOID POLYPEPTIDE FORMS AMYLOID-LIKE FIBRILS IN VITRO.

${ }^{1}$ R.L. Hull, ${ }^{2}$ E.T.A.S. Jaikaran, ${ }^{3}$ L.C. Serpell, ${ }^{3}$ P.E. Fraser, ${ }^{2}$ A. Clark and ${ }^{1} M$. Landon.' School of Biomedical Sciences, University of Nottingham, UK; ' ${ }^{2}$ Diabetes Research Laboratories and Department of Human Anatomy, University of Oxford, UK; ${ }^{3}$ CRND, University of Toronto, Canada.

Human islet amyloid polypeptide (IAPP, amylin) is derived from a 67 amino acid precursor, proLAPP, by post-translational proteolysis in the secretory granules of islet $\beta$-cells and is the principal component of islet amyloid found in the pancreatic islets of over $90 \%$ of type 2 (non-insulin dependent) diabetic subjects. The factors influencing fibrillogenesis of human IAPP are unknown but could be related to abnormal processing of prolAPP. To determine the fibrillogenic properties of this precursor peptide, synthetic human prolAPP was produced by solid state synthesis and the intra-molecular disulphide bond formed following purification. The secondary structure of proIAPP in water was determined by circular dichroism to be predominantly random coil; addition of heparin at a $20: 1 \mathrm{w} / \mathrm{w}$ ratio with prolAPP produced an immediate shift toward $\beta$-structure. Amyloid-like fibrils were detected in preparations of proIAPP $(12-120 \mu \mathrm{M})$ by electron microscopy: fibril formation was both concentration and time dependent (1 hour- 10 days). Heparin $(20: 1 \mathrm{w} / \mathrm{w})$ accelerated fibril formation and influenced fibril morphology. Immunogold labelling of synthetic fibrils using antibodies raised against different epitopes of human prolAPP demonstrated immunoreactivity and confirmed that the fibrils were formed from intact prolAPP.

Conclusions: Synthetic prolAPP forms amyloid-like fibrils in vitro. Abnormal proteolysis of proIAPP in type 2 diabetes could result in the production of intracellular or extracellular proLAPP fibrils contributing to islet amyloid deposition. 
658

ELEVATED PROINSULIN BUT NOT ISLET AMYLOID POLYPEPTIDE ACCOMPANIES DECLINING $\beta$-CELL FUNCTION IN TYPE 2 DIABETES C.M. McNamara, A. Clark and R.C. Turner. Diabetes Research Laboratories, Oxford, UK

Islet amyloid formation could result from raised secretion of islet amyloid polypeptide (IAPP) and contribute to declining $\beta$-cell function leading to insulin therapy in type 2 diabetes. Raised proinsulin (PI) concentrations have been detected in subjects before onset of diabetes. The aims of this study were to determine if plasma IAPP and PI concentrationsare elevated in patients with glucose intolerance or with diabetes. Onehour infusion of glucose test $\left(180 \mathrm{mg} / \mathrm{m}^{2} / \mathrm{min}\right)$ was used in 19 non-diabetic subjects, 58 non-diabetic 1st-degree relatives of type 2 diabetics and 39 type 2 diabetic subjects; blood samples were collected at time zero and at 1-hour. The nondiabetic and control subjects (ND) were stratified in three groups based on fasting glucose; Normal (N); fpg $<5.5 \mathrm{mmol} / \mathrm{n}, \mathrm{n}=52$, Intermediate glucose $(\mathrm{IG})$; fpg $>5.5<6.1 \mathrm{mmol} /$, $\mathrm{n}=14$, Impaired Fasting Glucose (IFG); fpg $>6.1 \mathrm{mmol} / \mathrm{h}, \mathrm{n}=11$. There was no significant differences in gender or BMI in the ND groups but the IFG subjects were significantly older (N,48y; IG,46y, IFG,59y, p<0.05). The diabetics (D) were stratified according to treatment with insulin treated group being older than diet or oral treatment $(\mathrm{p}<0.05)$. C-peptide (CP), IAPP and PI concentrations were increased in IFG group compared to $\mathrm{N}(\mathrm{p}<0.05)$. PV/CP\% and IAPP/CP\% were not different in $\mathrm{ND}$ groups, indicating proportional changes with increased fpg. In the diet and oral treated D fasting CP and IAPP were similar to those in IFG but were lower ( $\mathrm{p}<0.0001)$ in insulin treated group. However, fasting PI was elevated in all groups of diabetics ( $p<0.001$ ) and PI/CP\% was elevated in insulin treated group $(p<0.001)$. Conclusions: IAPP and PI secretion rises significantly and in proportion to $\mathrm{CP}$ with deteriorating glucose tolerance in nondiabetic subjects; there was no evidence of disproportionately increased IAPP production at any stage. Disproportionately increased PI occurred in diabetic subjects in contrast to a decline in CP and IAPP

\section{9}

ROSIGLITAZONE REDUCES OVEREXPRESSION OF INSULIN AND AMYLIN mRNA IN HYPERTROPHIED PANCREATIC ISLETS

S.A. Smith, D. Boam ${ }^{1}$, M.A. Cawthome ${ }^{2}$, J. Sidaway ${ }^{1}$, M. Newman, M. Wilkinson, S. Dunmore ${ }^{2}$ and C.A. Lister. SmithKline Beecham Pharmaceuticals, Harlow, UK, ${ }^{1}$ University of Manchester, UK and

2 University of Buckingham, UK.

Insulin resistance and hyperinsulinemia in the Zucker fatty rat are accompanied by pancreatic islet hypertrophy and increased $\beta$-cell insulin content. Oral administration of rosiglitazone (RSG; BRL-49653), a potent PPAR $y$ agonist insulin sensitiser, normalises impaired glucose tolerance and reduces hyperinsulinemia in the Zucker rat. Here, we determined islet hormone and mRNA expression profiles in male Zucker lean and fatty rats (9-11 weeks old), treated with RSG ( $3 \mathrm{umol} / \mathrm{kg} / \mathrm{day}$, orally) for 21 days. Plasma insulin and amylin concentrations of control fatty rats were 20- and 26-fold greater than those of lean rats. RSG significantly reduced ( $<<0.001)$ plasma insulin by $56 \%$ and amylin by $70 \%$ in fatty rats but not in lean rats. Pancreatic content of insulin and amylin (acid-ethanol extraction and radioimmunoassay), was increased in control fatty rats versus leans (3-fold and 2-fold, respectively), but unlike the reduction in plasma concentrations, RSG had no effect on fatty rat islet insulin or amylin content. Insulin and amylin mRNA levels (northern blot analysis) were elevated 4- to 5-fold in control obese rats versus leans, and RSG significantly reduced $(\mathrm{p}<0.001)$ expression of both mRNA's by about $50 \%$. RSG had no effect on insulin or amylin gene expression in lean rats. Hypersecretion of insulin from isolated perfused pancreata, observed under both basal $(2.8 \mathrm{mM}$ glucose $)$ and glucosestimulated $(16.7 \mathrm{mM})$ conditions was unaffected by 21 day treatment of fatty rats with RSG. These results demonstrate that RSG selectively reduces insulin (and amylin) gene expression in Zucker fatty rats, without affecting islet hormone content. These changes likely result from a reduced requirement for insulin gene transcription as a secondary consequence of cnhanced peripheral insulin sensitivity and suggests that RSG may have a therapeutically relevant $\beta$-cell sparing or protective effect.

\section{0}

ROSIGLITAZONE INCREASES PANCREATIC ISLET AREA, NUMBER AND INSULIN CONTENT, BUT NOT INSULIN GENE EXPRESSION

C.A. Lister, D. Boam ${ }^{1}$, D. Bretherton-Watt ${ }^{1}$, M. A. Cawthorne ${ }^{2}$, G. Moore, S. Loughborough, J. Warrack, M. Wilkinson and S.A. Smith. SmithKline Beecham Pharmaceuticals, Harlow, UK, IUniversity of Manchester, UK and ${ }^{2}$ University of Buckingham, UK

Treatment of C57BI/KsJ $d b / d b$ diabetic mice with the potent PPAR $\gamma$ agonist insulin sensitiser, rosiglitazone (RSG; BRL-49653), normalises hyperglycemia and pancreatic $\beta$-cell insulin content. We have determined the temporal relationship between changes in blood glucose and pancreatic insulin of $d b / d b$ mice during a 10-day treatment with RSG (30 umol/kg of diet). Blood glucose was lower than pre-dose values $(19.5 \pm 4.7 \mathrm{mM}$; mean $\pm S D, n=5)$ after 3 days treatment $(13.4 \pm 3.3 \mathrm{mM}, \mathrm{n}=5, \mathrm{p}<0.05)$, and was normalised after 6 days. Pancreatic insulin content was increased after 6 , but not 3 days therapy. Quantitative image analysis of pancreatic sections immunostained for insulin revealed that islet area was increased by 50 $65 \%(p<0.05)$ after 6 or 10 days treatment. In contrast, islet density (islets/mm 2 pancreas) was increased 2 -fold after 3 days and was characterised by the appearance of many small clusters of cells intensely stained for insulin, suggesting enhanced islet neogenesis. The effects of RSG on islet hormone and mRNA expression profiles after correction of hyperglycemia were also determined. Islet insulin of control $d b / d b$ mice was reduced by $70 \%$ relative to non-diabetic lean littermates whereas amylin was unchanged. RSG increased insulin content 3.2-fold and amylin 2.2-fold, but had no effect on glucagon. Insulin and amylin mRNAs were significantly lower in control $d b / d b$ mice than in leans, but unlike the increases in hormone content, RSG had no effect on insulin or amylin gene expression. RSG-induced expansion of islet area, density and hormone content are probably consequences of reduced secretory pressure on the $\beta$ cell following normalisation of glycemia. This suggests that RSG may have $\beta$-cell sparing or protective actions. 


\section{LADA}

\section{1}

$\beta$-CELL FUNCTION IN NONDIABETIC OFFSPRING OF PATIENTS WITH LATE ONSET AUTOIMMUNE DIABETES IN ADULTS (LADA) Authors: I. Vauhkonen ', L. Niskanen', J. Karjalainen' ${ }^{2}$, T. Tuomi', M. Uusitupa' and M. Laakso'; Departments of Medicine, Kuopio University Hospital, Kuopio, Finland; Department of Pediatrics', University of Oulu, Oulu, Finland; Diabetes and Endocrine Research ${ }^{3}$, Malmö University Hospital, Malmö, Sweden There are no studies that have evaluated first phase and maximal insulin secretion capacity and insulin sensitivity in the offspring of non-insulin dependent diabetic probands (NIDDM) with ICA and/or GAD positivity. Therefore, we evaluated insulin secretion capacity with oral and intravenous glucose tolerance tests and hyperglycemic clamp, and the rates of whole body glucose uptake with the euglycemic clamp in 36 offspring of NIDDM patients who were ICA- and/or GADpositive during the 10-year follow-up from the diagnosis (Fh-LADA-group) and 19 healthy control subjects without a family history of diabetes mellitus. The acute insulin response during the first $10 \mathrm{~min}$ of an intravenous glucose tolerance test was lower in the Fh-LADA-group than in the control group (incremental plasma insulin area under the curve: $2097 \pm 206$ and $3428 \pm 512 \mathrm{pmol} / / \mathrm{min}$ for the Fh-LADAgroup and for the control group, respectively; $P<0.01$ ). Maximal insulin secretion capacity was lower in the Fh-LADA-group $(2097 \pm 206 \mathrm{pmol} / \mathrm{l} \cdot \mathrm{min})$ than in the control group ( $3428 \pm 512 \mathrm{pmol} / 1 \cdot \mathrm{min} ; P<0.01)$. The rates of whole body glucose uptake were similar between the groups $(86.2 \pm 6.2$ and $83.7 \pm 4.3 \mu \mathrm{mol} / \mathrm{LBM} / \mathrm{min}$ for the Fh-LADA-group and for the control group, respectvely) and no differences in oxidative and nonoxidative glucose disposal were found. We conclude that the first phase and maximal insulin secretion capacity are reduced in the middle-aged nondiabetic offspring of patients with LADA. Genetic defect(s) leading to impaired insulin secretion in NIDDM is likely to be involved in the pathogenesis of LADA

\section{3}

LADA IS CHARACTERIZED BY DECREASED INSULIN SECRETION BUT SIMILAR DEGREE OF INSULIN RESISTANCE AS NIDDM

ÅL Carłsson, L Groop, G Sundkvist, T Tuomi, Dept. Endocrinology, Malmö University Hospital, Sweden and the Botnia Research Group, Finland.

GADab+ patients diagnosed with NIDDM (LADA) are shown to develop insulin deficiency during the course of the disease, but it is not known whether this is primary (present at diagnosis) or develops as a consequence of chronic hyperglycemia or represents an adaption to a high insulin senstivity. To address these questions we compared 11 newly diagnosed LADA patients with 11 GADab- NIDDM patients matched for sex, age $(55 \pm 11$ vs. $57 \pm 9 \mathrm{yr})$, duration $(1.7 \pm 1.2$ vs. $3.2 \pm 1.2 \mathrm{yr})$, and BMI $\left(27.3 \pm 4.2 \mathrm{vs}, 26.2 \pm 2.7 \mathrm{~kg} / \mathrm{m}^{2}\right)$. The groups had similar fB-glucose $(9.6 \pm 4.3$ vs. $8.6 \pm 2.2 \mathrm{mmol} / \mathrm{L})$ and $\mathrm{HbAl}_{\mathrm{c}}(7.7 \pm 2.3$ vs. $6.3 \pm 1.0 \%)$ levels. The insulin response 10 intravenous arginine $(5 \mathrm{~g})$ was studied at three $\mathrm{B}$-glucose levels (fasting 14 and $28 \mathrm{mmol} / \mathrm{L}$ ). The insulin response to increasing glucose (AIRgluc) and arginine-glucose (AIRarg) concentrations were significantly lower in LADA compared with GADab- NIDDM at all glucose levels (both $\mathrm{p} \leq 0.04$ ).
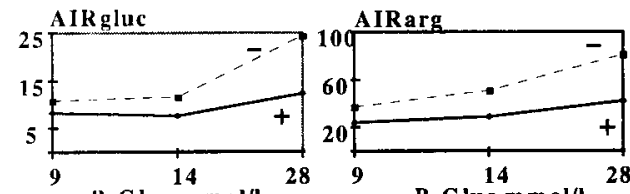

Glucuse disposal rate measured during a euglycemic hyperinsulinemic clamp was similar in 13 LADA patients and 13 matched GADab- NIDDM patients $(3.1 \pm 2.2 \mathrm{vs}$ $3.2 \pm 3.0 \mathrm{mg} / \mathrm{kg} / \mathrm{min}$ ).

Conclusions: LADA is characterized by decreased beta-cell capacity and marked insulin resistance early in the course of the disease. The finding that LADA is metabolically different from NIDDM points at a different etiopathogenic back ground for the disease.

\section{2}

LESS EVIDENCE OF THE METABOLIC SYNDROME IN LADA THAN IN COMMON NIDDM.

B.Isomaa, M.Henricsson, T. Tuomi, L. Sarelin, L. Groop and the Botnia Research Group. Jakobstad Hospital (Finland), Helsingborg Hospital (Sweden), University of Lund (Sweden)

LADA (latent autoimmune diabetes in adults) is defined as GAD ab positive diabetes with age at onset after 35 years. The aim was to compare the presence of chronic diabetic complications between 63 (M/F 30/33) LADA patients (age $70.3 \pm 10.9$, duration $14.0 \pm 7.1$ and $\mathrm{HbA}_{1 \mathrm{c}} 8.4 \pm$ $1.4 \%$ ) and 63 ( M/F 30/33) GAD-ab negative NIDDM patients (age 68.9 \pm 10.5 , duration $12.1 \pm 6.5$ and $\mathrm{HbA}_{1 \mathrm{c}} 8.2 \pm 1.4 \%$ ) participating in the Botnia Study. Moderate and severe retinopathy (Wisconsin scale) was seen in $15 \%$ and $10 \%$ of LADA and in $16 \%$ and $5 \%$ of NIDDM (NS). Clinical neuropathy was seen in $30 \%$ of LADA vs $27 \%$ of NIDDM (NS).and microalbuminuria (>20 $\mathrm{\mu g} / \mathrm{min}$ ) in $27 \%$ vs $24 \%$ (NS). The prevalence of cardiovascular disease did not differ between LADA and NIDDM ( $55 \%$ vs. $59 \%$ ). However, LADA patients had lower prevalence of hypertension ( 55 $\%$ vs. $75 \% ; \mathrm{p}=0.03$ ), lower BMI ( $27.5 \pm 4.9$ vs. $29.6 \pm 5.3 ; \mathrm{p}=0,02$ ), lower WH ratio $(0.92 \pm 0.08$ vs. $0.96 \pm 0.08 ; p=0.006)$, higher HDL2-cholesterol ( $0.49 \pm 0.26$ vs $0.36 \pm 0.13 \mathrm{mmol} / / \mathrm{p}=0.003$ ) vs $\mathrm{NIDDM}$. In a logistic regression analysis performed in the LADA patients $\mathrm{HbA}_{\mathrm{tc}}(\mathrm{RR} 2.0 ; \mathrm{p}=$ $0.02)$ and duration $(\mathrm{RR} 1.2 ; \mathrm{p}=0.01)$ predicted retinopathy, age $(\mathrm{RR} 1.2$; $\mathrm{p}=0.004)$ predicted neuropathy whereas $\mathrm{HbA}_{1 \mathrm{l}}(\mathrm{RR} 1.7 ; \mathrm{p} 0.02)$ predicted coronary heart disease (CHD). In conclusion, LADA can be distinguished from common NIDDM by less features of the metabolic syndrome. In patients without other risk factors for cardiovascular disease poor glycemic control is associated with an increased risk of CHD.

\section{4}

PREVALENCE AND CHARACTERISTICS OF LATENT AUTOIMMUNE DLABETES IN A CUBAN TYPE 2 DIABETES COHORT

E Cabrera-Rode ${ }^{1}$, O Diaz-Horta ${ }^{1}$, A Rendón ${ }^{1}$, G Molina ${ }^{1}$, LR Suarez ${ }^{1}$, P Perich ${ }^{1}$, M

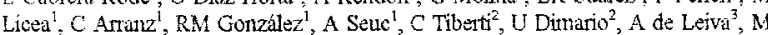
Puig-Domingo ${ }^{3}$

Instituto de Endoctinologia, La Habana. Cuba'; University of Rome "La Sapienza". Italy ${ }^{2}$; Hospital de Sant Pau, Barcelona, Spain ${ }^{3}$

Identification of late onset type 1 diabetes also called latent autoimmune diabetes of the adult (LADA) may have therapentic implications either becalse of the necessity of early insulin treatment and for the potential of immune intervention trials in this subgroup. LADA has been reported to be as prevalent as $20 \%$ annong individuals initially diagnosed as type 2 diabetics. The aim of our work was to evaluate the prevalence of I,ADA in a cohort of 1005 type 2 cuban diabetics (age 56.4111) by means of $1 C A, G A D$ and IA2 abs detection. ICA were found in $2.8 \%(2 \% / 1005)$ of type 2 patients in contrast to $85 \%$ of a group of typel diabetes $(85 / 100)$ and $0.5 \%$ of control population (1/200). GADab were found in $89 \%$ (26/29) of LADA, in $72 \%$ $(72 / 100)$ of typel and in $0.5 \%$ of controls $(1 / 200)$, while IA2ab were present in $20 \%$ (6/29) of LADA, $65 \%(65 / 100)$ of typel and 0 in controls. LADA group distinguished for having no family history of typel diabetes, a lower family history of type 2 diabetes, a lower weight (BMI $24 \pm 3.9$ vs $27.3 \pm 5.5, \mathrm{p}=0.04$ ), earlier diabetes diagnosis and duration of disease ( $5.8 \pm 8.5$ vs $9.6 \pm 8.9$ yr, $\mathrm{p}=0.018)$ and were younger $(52.7 \pm 11$ vs $57.3 \pm 10 \mathrm{yr}, \mathrm{p}=0.012)$, in comparison to $\mathrm{ICA}$ negative type 2 group. Among LADA, 50\% showed a low basal C-peptide and were treated with insulin. We conclude that LADA shows a low prevalence among this large cohort of cuban type 2 diabetic patients; the immunologic and clinic characteristics of these patients cleatly suggest a slow progressing form of autommune B-cell destruction with therapeutic implications. 
PS 18

\section{Insulin Action: Signal Transduction and Insulin Resistance}

\section{5}

\section{CELLULAR PROCESSING AND ACTIVITIES OF INSULIN AND} INSULIN ANALOGUES

W.C. Duckworth, J. Fawcett, R. G. Bennett, and F. G. Hamel, VA Medical Center and University of Nebraska Medical Center, Omaha, Nebraska USA

Insulin analogues may have properties which do not correlate with accepted mechanisms of insulin actions, e.g. receptor binding. Thus studies of the properties of various analogues may provide new insights into insulin action with both basic and clinical implications. We have compared selected insulin analogues (lys $\mathrm{s}^{\mathrm{B} 28}$, pro ${ }^{\mathrm{B} 29}$-insulin (-

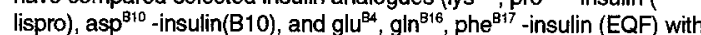
insulin in terms of receptor binding, cellular processing, and effects on various cellular pathways affected by insulin. Binding and processing of insulin and lispro were equivalent in hepatocytes whereas binding of EQF was decreased and B10 increased. Cellular processing of B10 was slowed relative to insulin. The cellular half life of insulin and lispro was 25 min whereas B10 was $75 \mathrm{~min}$. Biological effects of analogues on DNA synthesis (thymidine incorporation) were not significantly different for insulin $\left(\mathrm{EC}_{50}=5.8 \mathrm{nM}\right)$, lispro $(1.8 \mathrm{nM})$ and EQF $(0.86 \mathrm{nM})$ but was for $B 100.64 \mathrm{nM}(\mathrm{p}<0.01)$. Maximal effects of $B 10$ were also greater than insulin showing a correlation between mitogenesis and cellular processing. Effects on protein degradation in liver and muscle were also examined. Insulin inhibited protein degradation in liver by $29 \%$ with an $\mathrm{EC}_{50}=37 \mathrm{nM}$. B10 was not different but lispro was significantly different $(4.5 \mathrm{nM})$. In myocytes insulin $\left(\mathrm{EC}_{50}=2.5 \mathrm{nM}\right)$, lispro $(6.3 \mathrm{nM}) \times \mathrm{EQF}(15 \mathrm{nM})$ inhibited protein degradation equally with $\mathrm{B} 10(66 \mathrm{nM})$ having increased effects. These results show that insulin analogues have variable insulin like effects depending on the activity and the tissue studied. Differences in cellular processing may help explain the different activities.

\section{6}

PATHOGENIC ROLE OF MUTATIONS IN INSULIN AND IGFRECEPTORS AND IRS-1 GENES IN INSULIN RESISTANCE.

C. Lautier, F. Macari, S. Ait El Mkadem, E. Renard, D. Rokiki, J. Bringer, C Jaffiol, R. Smith and F. Grigorescu. IURC, Montpellier, France and Joslin Diabetes Center, Boston, USA.

To understand the role of gene defects in insulin action in the pathogenesis of insulin resistance we investigated the prevalence of mutations in receptors for insulin (IR) and IGF-1, as well as the IRS-1 substrate in patients with various degrees of insulin resistance and acanthosis nigricans (AN). Sequencing of PCR products was performed on an ABI373A automatic DNA sequencer. In the IR gene Cys524 $\rightarrow$ Stop[OPAL] and Arg 1092 $\rightarrow$ Trp mutations were found in a leprechaun patient. Homozygous Tyr6 $66 \rightarrow$ Cys and compound heterozygote Asp59 $\rightarrow$ Gly and Leu62 $\rightarrow$ Pro were found in 2 Type A patients. No IR mutations were found in patients $(n=17)$ with polycystic ovary (PCO) and AN displaying less severe insulin resistance (basal insulin $28.8 \pm 7 \mu \mathrm{U} / \mathrm{ml}$ ) and obesity $(\mathrm{BMI}=33.8 \pm 1.5)$. Despite alterations in IGF-1 binding and/or receptor autophosphorylation in some severe insulin resistant patients, no mutations were found in the IGF-1 receptor gene, except for 8 silent polymorphisms. Investigation of IRS-1 revealed one new mutation His $834 \rightarrow$ Tyr. The Gly972 $\rightarrow$ Arg variant of IRS 1 was found either in association with IR mutations in some Type A patients and relative carriers or as a unique genetic alteration in $35.7 \%$ of patients with PCO and AN, including one homozygous carrier. The Gly972 $\rightarrow$ Arg variant was found in only $5 \%$ of PCO patients $(n=35)$ without insulin resistance (basal insulin $=7.65 \pm 0.4$ $\mu \mathrm{U} / \mathrm{ml}$ ), who presented mixed lean and obese phenotypes. We conclude that the severity of insulin resistance correlates with cumulative gene defects in insulin action, which may act in an epistatic manner in the pathogenesis of insulin resistance.

\section{7}

DIFFERENTIAL ROLE OF IRS-1 AND IRS-2 IN L6 MUSCLE CELLS EXPRESSING THE ARG ${ }_{1152}>$ GLN INSULIN RECEPTOR. C. Miele, M. Caruso, P. Formisano, V. Calleja, R. Auricchio, F. Andreozzi, F. Oriente, E. Van Obberghen and F. Beguinot. DBPCM \& CEOS - CNR, Federico II University of Naples, Italy and INSERM U145, Nice, France.

We have analyzed insulin signal in L6 skeletal muscle cells expressing the constitutively active $A \operatorname{Arg}_{1152}>\mathrm{Gln}$ insulin receptor $\left(\mathrm{IR}_{1152}\right.$ ), which transduces proliferative but not metabolic insulin responses. Basal tyrosine phosphorylation of IRS-1 in IR $_{1152}$ expressing cells (Mut) was only slightly increased. Exposure to insulin increased IRS-1 phosphorylation by 10-fold in both the Mut and the wild-type cells (Wt) Grb-2 association to IRS-1, MAP kinase activity and thymidine incorporation were similarly induced by insulin in Mut and Wt cells. In contrast to IRS-1, IRS-2 was constitutively phosphorylated on tyrosine in the Mut cells and its phosphorylation was not further stimulated upon insulin addition. Grb-2 and PI 3-kinase association to this substrate and glycogen synthase activity were maximal in Mut cells under basal conditions and showed no change in response to insulin. Pre-treatment with the PI 3-kinase inhibitor Wortmannin inhibited both the constitutive activity of glycogen synthase in Mutcells and its insulin-stimulated activity in the $\mathrm{Wt}$, while having no effect on thymidine incorporation by the two cell lines. At variance with the $W t$ receptor, the $\mathrm{IR}_{1152}$ mutant did not phosphorylate Shc or stimulate its association to Grb-2 in L6 cells, either in the absence or in the presence of insulin. Thus, MAP kinase activation and mitogenic signalling reflect IRS-1 phosphorylation in L6 cells expressing the constitutively active $\mathrm{IR}_{1152}$ mutant. In contrast, metabolic signals from $I_{I I 52}$ receptors appear to be mediated by IRS-2, which is constitutively phosphorylated in mutant expressing cells. These data suggest an important role of IRS-2 in insulin regulation of glucose storage in skeletal muscle, at least in part, mediated via PI 3-kinase.

\section{8}

CYCLIC NUCLEOTIDE PHOSPHODIESTERASE 3 INHIBITORS DETERMINATION OF PDE3A OR 3B ISOFORM-SPECIFICITY T Shirakawa ${ }^{1},{ }^{*}$ T Nagaoka ${ }^{*}{ }^{*}, 2$ VC Manganiello ${ }^{3}, \mathrm{G} \mathrm{Gao}{ }^{3}, \mathrm{~K}$ Kobayashi ${ }^{2}, \mathrm{Y}$ Fujita-Yamaguchi', 'Dept of Molecular Biology, BRI, City of Hope, Duarte CA, USA, ${ }^{2}$ First Dept of Internal Medicine, Kanazawa Univ Medical School, Kanazawa, Japan, ${ }^{3}$ NHLBI. NIH, Bethesda MD, USA

Cyclic nucleotide phosphodiesterase 3 (PDE3), one of seven related PDE gene families, catalyzes hydrolysis of both CAMP and cGMP (1). The availability of family-specific inhibitors has facilitated understanding physiological functions of individual PDE families. Inhibition of PDE3, for example, has been shown to result in stimulation of cardiac contractility, inhibition of platelet aggregation, relaxation of vascular and airway smooth muscle, inhibition of the antilipolytic action of insulin in adjpocytes, and stimulation of insulin secretion in pancreatic $b$ cells. Molecular cloning revealed the presence of two PDE3 isoforms, 3A and 3B. Since our tissue specific expression studies demonstrated differential regulation of PDE3A and $3 \mathrm{~B}$ in rodent models of diabetes (2), we examined isoform-specificity of existing PDE3 inhibitors. Human PDE3A and 3B were expressed in Sf9 cells using recombinant baculovirus. Crude homogenates of those $\mathrm{S}$ 99 cells expressed either PDE3A or 3B as confirmed by immunoblotting with isoform-specific antibodies. PDE3A or 3B activity was measured at a substrate concentration of $0.05 \mathrm{mM}$ cAMP in the absence or presence of differing concentrations of PDE3 inhibitors including cGMP, milrinone, cilostamide, trequinsin, and three structurally-related "KF" compounds, from which IC50s were determined. The results indicated that PDE3 inhibilors thus far examined hardly distinguish PDE3A from 3B except that PDE3A was x 2.5 more sensitive to inhibition by cGMP than PDE3B. This study suggested similar inhibition spectra for two PDE3 isoforms by readily-available PDE3 inhibitors, which obliges further screening to identify PDE3 isoform specific inhibitors. (1) Degerman et al. J. Biol. Chem. 272(1997) 6823 (2) Nagaoka et al. Diabetes (1998) in press. 


\section{9}

Differential Regulation of Hormone Sensitive Lipase in Macrophages and Adipocytes : Potential Implications for Atherosclerosis

L.O'Rourke and P.R. Shepherd.

Dept. of Biochemistry, University College London,UK.

The mechanisms by which NIDDM dramatically increases atherosclerotic risk remains porly understood. Accumulation of cholesterol esters in macrophage fcam cells is an imporant early event in atherosclerosis. The neutral cholesterol esterase. controlling cholesterol ester breakdown in macrophages, is identical to hormonesensitive lipase (HSL) in adipocytes. In adipocytes insul in decreases HSL activity suggesting insulin may also regulate cholesterol ester homeostasis in macrophages. Therefore we are investigating the regulation of HSL in macrophages. We find that components of the signalling pathwav by which insulin decreases HSL activity in adipocytes are also in macrophages, these include the $\alpha$ $\beta$ and $\delta$ isoforms of classla PI 3-kinases and PDE3B. The $\alpha$ and $\beta$ isolorms are recruited by insulin into tyrosine phosphorylated complexes. Western blots for HSL show two bands at 80 and $85 \mathrm{kD}$ in both $3 \mathrm{~T} 3 \mathrm{~L}-1$ adipocytes and $J 774$ ? macrophages. Chronic exposure to insulin results in an incrcase in the level of both the 80 and $85 \mathrm{kD}$ proteins in adipocytes whereas the levels of HSL do not change in macrophages. HSL activity was immunoprecipitated from both cell types and macrophages contained $62.5 \%$ of the activity measured in adipocytes. Insulin acutely decreases HSL activity in macrophages and adipocytes. In adipocytes this inhibition persists but in macrophages it is only observed in the first 10 minutes following insulin stimulation. We find no evidence that agents that raise cAMP levels stimulate HSL activity in macrophages, which contrasts with the situation in adipocytes. These results suggest that the HSL protein is identical in adipocytes and macrophages but that there are differences in the regulation by insulin. Studies are ongoing to determine the effects of insulin on HSL in macrophages and whether disruption of insulin signalling pathways could block changcs in HSL activity thus contributing to cholesterol ester accumulation in these cells.

\section{1}

IN VIVO INSULIN INCREASES THE Ka OF GLUCOSE 6PHOSPHATE FOR GLYCOGEN SYNTHASE IN CALORIERESTRICTED MONKEYS.

HK Ortmeyer, L Huang, J Lamer and BC Hansen. Univ of Maryland, Baltimore, MD and Univ of Virginia, Charlottesville, VA USA

In skeletal muscle (sm) of normal subjects, the concentration of glucose 6-phosphate (G6P) where the activity of glycogen synthase (GS) is half maximal (Ka) is decreased by in vivo insulin, and the fractional activity (fv) of GS is increased without change in GS maximal activity (Vmax). We have previously shown that chronic calorie restriction (CR) resulted in the prevention of obesity and type 2 diabetes, and that in a subgroup of CR monkeys, insulin during a euglycemic hyperinsulinemic clamp caused an unexpected decrease in sm GSfv. These same monkeys had the lowest whole-body glucose disposal rate (M), the greatest increase in sm G6P content and the greatest increase in sm glycogen phosphorylase activity during a clamp compared to the $\mathrm{CR}$ monkeys with normal insulin action. To determine whether this highly unusual decrease in GSfv with insulin was due to increased phosphorylation (increased $\mathrm{Ka}$ ), we measured the activity of sm GS at 9 different G6P concentrations before and during a clamp in $6 \mathrm{CR}$ monkeys. Ka increased $(n=4)$ and $V \max$ decreased $(n=5)$ during the clamp. Basal $\mathrm{Ka}$ was inversely related to basal GSfy ( $\mathrm{r}=-0.94$, $\mathrm{p}<0.002$ ). $\mathrm{Ka}$ and sm G6P content were positively related under insulin-stimulated conditions $(r=0.93, p<0.005)$. The change in $\mathrm{Ka}$ (insulin-stimulated minus basal) was inversely related to $M(r=-0.94$, $\mathrm{p}<0.002)$ and positively related to the change in sm G6P content $(r=0.93, p<0.005)$. We conclude that moderate $C R$ results in an increase in phosphorylation of GS and a decrease in Vmax of GS in the presence of insulin during a euglycemic hyperinsulinemic clamp indicating a reversal of nomal insulin action at skeletal muscle.

\section{0}

INSULIN REGULATES THE EXPRESION OF DNA REPAIR ENZYMES R. Perfetti. Division of Endocrinology and Metabolism, Cedars-Sinai Medical Center and University of California Los Angeles, Los Angeles, CA 90048.

Diabetes is characterized by premature cellular aging. Accumulation of products of oxidative damage into DNA has been proven deleterious to cells, contributing to the development of the microangiopathic complications of the disease. Herein, we demonstrated that insulin regulates the expression of genes involved in the nuclear excision repair pathway, a fundamental mechanism to remove DNA lesions, preserve DNA integrity, aild ultimately prevent cell death.

We evaluated the effect of receptor-linked tyrosine kinase activation in the expression of the XPD gene. XPD has intrinsic helicase activity and plays a dual role in both transcription and nucleotide repair. Chinese hamster ovary (CHO) cells transfected with the human insulin receptor (CHO/HIRc) showed a 3-fold increase in XPD mRNA levels when compared to control CHO/neo cells $(p<0.01)$ However, cells overexpressing a mutant insulin receptor known to have impaired kinase-related signaling (CHO/YF3) had XPD mRNA levels similar to control $\mathrm{CHO}$ /neo cells. The addition of insulin to serum-starved cells led to an increase in XPD mRNA levels in both $\mathrm{CHO}$ /neo and $\mathrm{CHO} / \mathrm{HIRc}$ cells. Half-maximum insulin stimulation was obtained with $12.5 \mathrm{ng} / \mathrm{ml}$ of insulin in the culture medium. Peak insulin-stimulated XPD expression occurred in $2-4 \mathrm{~h}(>40 \%)$ in $\mathrm{CHO} /$ neo cells and in $1-2 \mathrm{~h}$ in $\mathrm{CHO} / \mathrm{HIRc}(>220 \%)$. Insulin acted primarily, but not exclusively, at the transcription level of XPD. Moreover, inhibition of protein synthesis by cycloexamide induced a marked decrease of XPD mRNA levels in insulin treated cells, suggesting that the turnover rates of the messenger RNA coding for XPD vary according to the state of insulin receptor activation. We demonstrated an increased resistance to UV-induced DNA damage in cells transfected with normal insulin receptors but not in cells expressing a kinase-deficient insulin receptor, indicating that stimulation of the insulin receptor phosphorylation was responsible for the effect observed. Finally, internucleosomal DNA fragmentation, a marker of preapoptosis, was significantly inhibited in cells transfected with the human insulin receptor compared to control cell lines, and the addition of insulin to the culture medium further protected against DNA fragmentation. These data suggest the increase in XPD expression by activation of membrane associated tyrosine kinase is likely to play an important role in the cellular response leading to repair of damaged DNA.

\section{2}

OBESITY INFLUENCES INSULIN ACTION MORE THAN TYPE 2 DIABETES MELLITUS

G.Šindelka, J.Škrha, and J.Hilgertová. Dept.of Internal Medicine 3, Faculty of Medicine 1, Charles University, Prague, Czech Republic

Insulin resistance was found as in Type 2 diabetes mellitus as in obese non-diabetic subjects. The aim of this study was to compare the insulin action in monstrose obesity with and without diabetes and in Type 2 diabetic patients with different degree of obesity. Total number of 36 Type 2 diabetic patients was subdivided according to body mass index (BMI) into those with monstrose obesity (MODM, $\mathrm{BMI}>40, \mathrm{n}=6$ ), with medium obesity (MEODM, BMI 31-40, $\mathrm{n}=16$ ), with mild overweight (MIODM, BMI 26-30, $\mathrm{n}=9$ ) and without obesity (NODM, BMI 21$26, n=5$ ). A group of monstrose obese non-diabetic persons (MONDM, BMI $>40$ $\mathrm{n}=5$ ) and 12 non-obese healthy persons were used as the controls. Patients in all groups were of comparable age and of diabetes control $\left(\mathrm{HbA}_{\mathrm{Ic}}\right.$ was $\left.7.1 \pm 0.5 \%\right)$. Isoglycemic hyperinsulinemic clamps on Biostator were performed with the insulin infusion rate $1 \mathrm{mU} \cdot \mathrm{kg}^{-1} \mathrm{~min}^{-1}$ during $120 \mathrm{~min}$. Basal glucose concentration $\left(\mathrm{G}_{\mathrm{o}}\right.$ mmol. $\left.I^{-1}\right)$, glucose disposal rate $\left(\mathrm{M}, \mu \mathrm{mol} \cdot \mathrm{kg}^{-1} \cdot \mathrm{min}^{-1}\right)$, insulin sensitivity index $\left(\mathrm{M} / \mathrm{l}, \mu \mathrm{mol} . \mathrm{kg}^{-1} \cdot \mathrm{min}^{-1}\right.$ per $\left.\mathrm{mU} \cdot \mathrm{I}^{-1} \times 100\right)$ and metabolic clearance rate of glucose $\left(\mathrm{MCR}_{\mathrm{G}} \mathrm{ml}^{\mathrm{kg}} \mathrm{kg}^{-1} \cdot \mathrm{min}^{-1}\right)$ were calculated in all subjects. Results are means $\pm \mathrm{SEM}$

\begin{tabular}{|c|c|c|c|c|c|c|}
\hline & MONDM & MODM & MEODM & MIODM & NODM & C \\
\hline BMI & $53.1 \pm 3.8$ & $48.0 \pm 2.9$ & $33.4 \pm 2.9$ & $28.6 \pm 0.8$ & $24.9 \pm 0.7$ & $23.9 \pm 0.8$ \\
\hline $\mathrm{G}_{0}$ & $5.1 \pm 0.5$ & $9.5^{a} \pm 2.0$ & $10.3^{z} \pm 1.2$ & $8.1^{2} \pm 1.4$ & $8.1^{\mathrm{a}} \pm 0.9$ & $4.1 \pm 0.3$ \\
\hline$M$ & $16.7^{6} \pm 2.6$ & $22.5^{8} \pm 4.4$ & $25.6^{6} \pm 1.9$ & $34.7 \pm 4.3$ & $36.8 \pm 4.3$ & $37.5 \pm 3.0$ \\
\hline $\mathrm{M} / \mathrm{I}$ & $8.2^{\mathrm{a}} \pm 1.4$ & $12.9^{\mathrm{a}} \pm 3.0$ & $21.6^{8} \pm 1.8$ & $29.6^{6} \pm 2.6$ & $47.5 \pm 9.5$ & $48.9 \pm 5.0$ \\
\hline $\mathrm{MCR}_{\mathrm{g}}$ & $2.9^{a} \pm 0.5$ & $2.5^{a} \pm 0.5$ & $3.0^{\mathrm{a}} \pm 0.4$ & $5.3^{\mathrm{b}} \pm 0.6$ & $5.4^{6} \pm 1.0$ & $8.6 \pm 1.1$ \\
\hline
\end{tabular}

Statistical significance as compared to healthy subjects: "p<0.001, $\mathrm{p}<0.05$.

No difference was observed in calculated variables between non-obese Type 2 diabetic patients and healthy controls except metabolic clearance rate of glucose An inverse relationship was found between BMI and insulin sensitivity index $\mathrm{M} / \mathrm{A}$ in a total cohort of 53 persons $(r=-0.68, p<0.001)$. Insulin receptor characteristics on erythrocytes showed slightly decreased receptor number in obese Type 2 diabetic patients $(p<0.05)$ as compared with non-obese controls. We conclude that $B M I$ is the strongest predictor of insulin sensitivity which may influence insulin action mainly on postreceptor level whereas well controlled diabetes has a small effect. 
673

INTERACTIONS OF HEMIPANCREATECTOMY AND SYSTEMIC DIVERSION OF PANCREATIC VEINS IN INSULIN SENSITIVITY

J. Radziuk, P. Barron, J. Davies, and S. Pye, Ottawa Civic Hospital, Ottawa, Canada

Chronic peripheral insulin entry decreases systemic insulin sensitivity. Both peripheral entry and decreased $\beta$ cell mass can contribute to a decrease in glucose tolerance. The interaction of these two factors on peripheral insulin sensitivity was therefore studied in four groups of dogs: (i) D - pancreatic venous drainage diverted from the portal vein to the inferior vena cava $(\mathrm{n}=8)$ (ii) $\mathrm{S}$ - sham operation with reanastomosis of veins to the portal vein $(\mathrm{n}=8)$ (iii) $\mathrm{H}$ - hemipancreatectomy with resection of the tail of the pancreas $(\mathrm{n}=7)$ (iv) HD - both resection and diversion $(n=8)$. Following recovery from surgery, each animal underwent a hyperinsulinemic euglycemic clamp during an insulin infusion at $0.8 \mathrm{mU} / \mathrm{kg}-\mathrm{min}$. A concurrent infusion of $\left[6-{ }^{3} \mathrm{H}\right]$ glucose was used to measure the metabolic clearance of glucose $\left(\mathrm{MCR}_{\mathrm{g}}\right)$, and insulin sensitivity $\left(S_{l}\right)$ was quantitated as the ratio of $\mathrm{MCR}_{\mathrm{g}}$ to circulating insulin. The results demonstrated a decrease (mean \pm sem) in $S_{1}$ from $0.38 \pm 0.04$ to $0.20 \pm 0.03(p<0.02)$ for $S$ and $D$ respectively and a parallel decrease from $0.39 \pm 0.03$ to $0.23 \pm 0.04(p<0.05)$ for $H$ and HD. Similar patterns were present under basal conditions. The decrease in $S_{1}$ in $D$ was primarily due to a fall in $\mathrm{MCR}_{\mathrm{g}}$ (from $10.0 \pm 0.3$ to $6.7 \pm 0.3 \mathrm{ml} / \mathrm{kg}$-min, $\mathrm{p}<0.05$ ). In $\mathrm{HD}$ the decrease in $S_{1}$ resulted from a fall in MCR of insulin and therefore an increase in insulin concentrations $(26 \pm 1$ vs $40 \pm 4 \mu \mathrm{U} / \mathrm{ml}$ for $\mathrm{H}$ and $\mathrm{HD}$ resp, $p<0.02$ ). Since the secretory capacity of the resected pancreas is reduced, these data suggest that hyperinsulinemia is not the only cause of the insulin resistance which results from the diversion of pancreatic venous drainage to the periphery, although it is likely to aggravate it.

\section{5}

EFFECTS OF EXERCISE TRAINING AND ACE INHIBITION ON INSULIN ACTION IN INSULIN-RESISTANT RAT MUSCLE.

E. J. Henriksen, M. S. Steen, K. R. Foianini, E. B. Youngblood, and T.

R. Kinnick. University of Arizona, Tucson, AZ 85721-0093 U.S.A.

The purpose of this investigation was to determine the interactions of endurance exercise training and chronic angiotensin-converting enzyme (ACE) inhibition (trandolapril, T) on glucose tolerance, insulin action on muscle glucose transport activity, and lipidemia in the insulin-resistant, dyslipidemic obese ( $\mathrm{f} a / \mathrm{fa}$ ) Zucker rat. Four groups of obese animals were studied: sedentary controls, T-treated $(1 \mathrm{mg} / \mathrm{kg}$ body wt/day by gavage for 6 weeks), exercise-trained (treadmill running at $24-30 \mathrm{~m} / \mathrm{min}, 8 \%$ grade, $90 \mathrm{~min} /$ day for 6 weeks), or combined T-treated and exercise-trained. Exercise training, alone or in combination with $\mathrm{T}$, significantly $(p<0.05)$ increased peak oxygen uptake by $31-34 \%$. Similar decreases in fasting plasma insulin $(34 \%)$ and free fatty acids (31\%) were achieved with exercise training alone or in combination with $\mathrm{T}$ treatment. Compared to control, exercise training or $\mathrm{T}$ treatment individually caused smaller areas under the curve (AUC) for the glucose (12-14\%) and insulin (28-33\%) responses to an oral glucose tolerance test. The largest decreases in the glucose AUC (40\%) and insulin AUC (53\%) were observed in the combined treatment group. Similarly, whereas exercise training alone or T treatment alone improved insulin-stimulated $(2 \mathrm{mU} / \mathrm{ml})$ glucose transport in epitrochlearis $(26-34 \%)$ or soleus $(39-41 \%)$ muscles, the greatest improvements in insulin action in these muscles $167 \%$ and $107 \%$, respectively) were demonstrated in the combined treatment group. Collectively, these results indicate that endurance exercise training and chronic $\mathrm{T}$ treatment in combination improve oral glucose tolerance and insulin-stimulated glucose transport in insulin-resistant skeletal muscle to a greater extent than either intervention individually.
674

CHRONIC BRADYKININ TREATMENT ENHANCES INSULIN ACTION IN THE INSULIN-RESISTANT OBESE ZUCKER RAT.

E. B. Youngblood, S. Jacob, D. L. Fogt, D. B. Dal Ponte, G. J. Dietze, and E. J. Henriksen. The University of Arizona, Tucson, AZ 857210093 U.S.A.; and Max-Grundig-Klinik, 77815 Bühlerhöhe, Germany.

Bradykinin (BK) may mediate the beneficial effect of angiotensin converting enzyme inhibitors on insulin-stimulated glucose transport in insulin-resistant skeletal muscle. Therefore, the effects of chronic BK treatment of insulin-resistant obese Zucker rats on glucose tolerance and muscle glucose transport stimulated by insulin or contractions were investigated. BK was administered subcutaneously (twice daily at $\mathbf{4 0}$ $\mu \mathrm{g} / \mathrm{kg}$ body weight) for 14 days. Compared to a saline-treated obese group, the BK-treated obese animals had significantly $(p<0.05)$ lower fasting plasma levels of insulin (20\%) and free fatty acids (26\%), while plasma glucose was not different. During an oral glucose tolerance test, the glucose and insulin responses (areas under the curve, AUC) were $21 \%$ and $29 \%$ lower, respectively, in the BK-treated obese group. The glucose-insulin index, the product of the glucose and insulin AUCs and an indirect index of peripheral insulin action, was $52 \%$ lower in the BK-treated obese group compared to the obese control group. Moreover, glucose transport activity (assessed using $1 \mathrm{mM} \mathrm{2-}$ deoxyglucose uptake) in the isolated epitrochlearis muscle stimulated by a maximally-effective dose of insulin $(2 \mathrm{mU} / \mathrm{ml})$ was $52 \%$ greater in the BK-treated obese group. Contraction-stimulated (10 electricallystimulated tetani) glucose transport activity was also enhanced by $35 \%$ as a result of the BK treatment. In conclusion, these findings indicate that in the severely insulin-resistant obese Zucker rat, chronic in vivo treatment with BK can significantly improve whole-body glucose tolerance, possibly as a result of the enhanced insulin-stimulated skeletal muscle glucose transport activity observed in these animals. 
PS 19

\section{Insulin Action: Cardiovascular Effects}

\section{6}

INSULIN-INDUCED VASODILATION: NITRIC OXIDE OR ADENOSINE ? C.H.A. de Haan, A.J.H.M. Houben, P.W. de Leeuw, B.H.R. Wolffenbuttel, P. Smits", N.C. Schaper. Dept. of Internal Medicine, University Hospital Maastricht and "University Hospital Nijmegen.

The mechanism of insulin-induced vasodilation is still unclear. We studied whether release of nitric oxide (NO) and adenosine ( $\mathrm{ADN}$ ) is involved and if their release depends upon stimulation of glucose metabolism. Methods: 21 healthy men, age 22 (19-23) yrs (median + interq. ranges), BMI $22.8(20.5-23.7) \mathrm{kg} / \mathrm{m}^{2}$, were studied. Insulin $(7 \mathrm{mU} / \mathrm{min})$, with or without concomitan glucose $5 \%$ (regional glucose $15 \mathrm{mmol} / \mathrm{l}$ ), was infused into the bractial artery on two occasions (INS and INS/GLYC). Forearm blood flow (FBF) was measured using plethysmography. To determine the role of NO, in $12 \mathrm{men}$ L-NMMA $1-2-8 \mu \mathrm{mol} / \mathrm{min}$ was infused i.a. before insulin and after $225 \mathrm{~min}$. To study the role of $\mathrm{ADN}$, in 9 men draflazine (DRAF, adenosine uptake blocker) 100-250-500-1000 ng/min/dl forearm was infused after $180 \mathrm{~min}$ insulin infusion. In a control experiment only draflazine was infused. Flow data are expressed as index (infused/contralateral arm). Results: Mean regional insulin levels were $90 \mathrm{mU} / \mathrm{l}$ on all days, systemic glucose levels did not change. INS: FBF index increased by $44 \%(30-77)$ at $t=180$ (Wilcoxon, $\mathrm{p}<0.01$ vs $\mathrm{t}=0$ ). NS/GLYC: $\mathrm{FBF}$ index rose by $75 \%(35-117)$ at $\mathrm{t}=180(\mathrm{p}<0.02$ vs $\mathrm{t}=0)$, indicating more vasodilation than with INS $(\mathrm{p}=0.05)$. During INS and INS/GLYC both L-NMMA-induced vasoconstriction as well as DRAF-induced vasodilation increased relatively to baseline. L-NMMA EC 30 (conc. inducing $30 \%$ constriction) decreased from 83 to $26 \mathrm{nmol} / \mathrm{ml}$ during $\mathrm{NS}$, and from 105 to $25 \mathrm{nmol} / \mathrm{ml}$ during INS/GLYC (both $\mathrm{p}<0.03$ ), without any differences between INS and INS/GLYC, DRAF: EC30 on the control day was $74 \mathrm{ng} / \mathrm{ml}, 45 \mathrm{ng} / \mathrm{ml}$ during INS and $20 \mathrm{ng} / \mathrm{ml}$ during INS/GLYC (INS and INS/GLYC both $\mathrm{p}<0.05$ vs. control day), with no differences between INS and INS/GLYC. Conclusions: Concomitant hyperglycemia during hyperinsulinemia augments insulin-induced vasodilation. The decrease in EC30 of DRAF and L-NMMA during INS indicates that insulin stimulates the release of $N O$ and $\mathrm{ADN}$ but no further stimulation was found during INS/GLYC, although the vasodilation was markedly augmented. These data indicate that, apart from a glucose independent stimulation of both NO and $A D N$, insulin also induces vasodilation through a glucose dependent mechanism.

\section{8}

THE ROLE OF ADENOSINE IN THE VASODILATOR RESPONSE TO INSULIN

E.J.Zandbergen, G.Vervoort, C.J.J.Tack, N.C.Schaper*, J.A.Lutterman and P Sinits. Depts. of Medicine and Pharmacology, University Hospital Nijmegen and "Maastricht, The Netherlands.

Hyperinsulinemia induces vasodilation in human skeletal muscle. This vasodilation is impaired in insulin-resistant conditions. The mechanism mediating the vasodilation has not yet been completely clarified. Based on animal data, we hypothesised that stimulation of the adenosine receptor in vascular smooth muscle cells is involved in insulin-induced vasodilation. A hyperinsulinemic euglycemic clamp was performed in 24 healthy subjects to investigate the vasodilator response to insulin in the forearm. The subjects were randomly assigned to three groups. In group 1, placebo was infused into the left brachial artery (experimental arm). Draflazine (an adenosine uptake blocker, $250 \mathrm{ng} / \mathrm{min} / \mathrm{dl}$ ) and theofylline (an adenosine receptor antagonist, 50 $\mathrm{mg} / \mathrm{min} / \mathrm{dl}$ ) were infused in group 2 and 3. Forearm blood flow (FBF) was measured by plethysmography. The FBF-ratio (FBF experimental arm/FBF control arm) was used to test our hypothesis. Data are expressed as means \pm SE. Statistical analysis was performed by ANOVA. Hyperinsulinemia induced vasodilation; $F B F$ in the control arm increased from $1.8 \pm 0.2$ to $2.3 \pm 0.3$ $\mathrm{ml} / \mathrm{min} / \mathrm{dl}(\mathrm{p}<0.001)$. In the placebo group FBF-ratio decreased by $18.2 \pm 9.4 \%$ $(\mathrm{p}<0.01)$. The change in FBF-ratio in the draflazine-group was significantly less prominent than in the placebo group $(-1.6 \pm 13.1$ vs $18.2 \pm 9.4 \%, \mathrm{p}=0.01)$. The decrease in FBF-ratio was more pronounced in the theophylline group $(30.9 \pm 5.2$ vs $18.2 \pm 9.4 \%, p<0.01)$. These results demonstrate that the insulininduced increase in blood flow is augmented during adenosine uptake blockade by infusion of draflazine, whereas this was diminished during adenosine receptor antagonism by theophylline. These results are in agreement with our hypothesis that insulin-induced vasodilation is, at least partly, mediated by the release of adenosine

\section{7}

SYSTEMIC, RENAL AND OCULAR HEMODYNAMIC EFFECTS OF LARGININE AND INSULIN

L. Schmetterer, S. Dallinger, O. Findl, R. Hitsch and M. Wolzt. Department of Clinical Pharmacology, Institute of Medical Physics, Department of Ophthalmology, University of Vienna, Währinger Gürtel 18-20, A-1090 Vienna, Austria

$\mathrm{L}$-arginine, the precursor of nitric oxide, induces systemic hypotension when intravenously infused in healthy subjects. The mechanism behind this vasodilator effect has not yet been completely elucidated. There is, however, evidence that the vasodilator action of L-arginine is at least partially mediated by endogenous insulin. The aim of the present study was to investigate the hemodynamic effect of intravenous $\mathrm{L}$-arginine $(1 \mathrm{~g} / \mathrm{min}$ over $30 \mathrm{~min})$ during a high dose insulin clamp $(6 \mathrm{mU} / \mathrm{kg} / \mathrm{min})$ or during placebo infusion. The study was performed in a randomised three-way cross over design in 12 healthy male subjects using D-arginine as a control substance (1 $\mathrm{g} / \mathrm{min}$ over $30 \mathrm{~min}$ ). Mean arterial pressure was measured non-invasively. Renal plasma flow and pulsatile choroidal blood flow were assessed by PAH clearance technique and laser interferometric measurement of fundus pulsation amplitude, respectively. Whereas D-arginine did not influence any parameter under study, L-arginine significantly decreased mean arterial pressure $(-8 \%, \mathrm{p}<0.006)$ and increased renal plasma flow $(+8 \%, \mathrm{p}<$ $0.001)$ and fundus pulsation amplitude $(+10 \%, \mathrm{p}<0.001)$. Insulin alone did not affect mean arterial pressure, but significantly increased renal plasma flow $(+16 \%, \mathrm{p}<0.001)$ and fundus pulsation amplitude $(+8 \%, \mathrm{p}<0.001)$. The effect of co-administration of insulin and L-arginine on renal plasma flow $(+34 \%)$ and fundus pulsation amplitude $(+28 \%)$ was markedly higher than the sum of the effects exerted by the two drugs alone. This study supports the concept that the stereospecific vasodilatory effect of L-arginine is at least partially independent of endogenous insulin release.

\section{9}

Contribution of vascular endothelial cells to the regulation of transcapillary insulin-transport

T.C. Wascher, F. Brunner*. Dept. Internal Medicine and *Inst. of Pharmacology and Toxicology, University of Graz, Austria

Capillary endothelial cells are believed to limit the transport of insulin from the vascular to the interstitial space, thus limiting hormonal action at target sites. This study examined the contribution of the endothelium to the regulation of transcapillary insulin transport (TIT) in rat hearts in vitro.

Hearts were perfused with a protein-free buffer which results in increased generation of interstitial fluid that was collected at the surface of ventricles. Insulin $(0.1-1 \mathrm{U} / \mathrm{l})$ was added to the perfusate, and its transfer kinetics to and clearance from the interstitium were analysed from insulin measurements in transudate of hearts with intact or collagenase-disrupted endothelium. In endothelium-intact hearts $(n=5-8)$, steady-state insulin in transudate was $30 \pm 2 \%, 53 \pm 1$ $\%, 103 \pm 6 \%$ and $97 \pm 4 \%$ of perfusate concentrations at $0.1,0.2,0.5$, and $1 \mathrm{U}$ insulin $/$, respectively. The corresponding apparent rate constants for transport, $\mathrm{k}_{\mathrm{in}}$, increased from 0.11 to $\sim 0.27 / \mathrm{min}$, indicating a non-saturable transport. In endothelium-disrupted hearts $(\mathrm{n}=3-5), \mathrm{ki}_{\mathrm{n}}$ values were $\sim 2$-fold higher, and the same concentration ratios were observed, suggesting a contribution of reabsorption processes back into the vascular space in the generation of the gradient. Finally, inhibition of endothelial nitric oxide (NO) formation by $\mathrm{N}^{G}$-nitro-L-arginine $(200 \mu \mathrm{mol} / \mathrm{l})$ affected neither $\mathrm{k}_{\text {in }}$ nor the extent of TIT.

We conclude that (i) insulin is transported by a bi-directional convective transport rather than by a receptor-mediated mechanism; (ii) capillary endothelial cells affect TIT by slowing the transfer to the interstitium and (iii) endothelium-derived NO is without effect on TIT in this model. 
680

INSULIN'S EFFECTS ON SKELETAL MUSCLE MEAN FLOW, FLOW HETEROGENEITY AND MUSCLE BLOOD VOLUME IN PATIENTS WITH ESSENTIAL HYPERTENSION

H. Laine, J. Knuuti, U. Ruotsalainen, T. Utriainen, V. Oikonen, M. Raitakari, P. Vicini, C. Cobelli, P. Nuutila and H. Yki-Järvinen. Turku and Helsinki, Finiand; Padova, Italy.

To determine whether the integrity of insulin's effects on muscle blood flow and blood volume are altered in essential hypertension we studied 11 newly-diagnosed untreated mildly hypertensive men (age $35 \pm 1$ years, BMI $25.2 \pm 0.4 \mathrm{~kg} / \mathrm{m}^{2}$, blood pressure $141 \pm 4 / 96 \pm 2 \mathrm{mmHg}$ ) and 11 matched normotensive men. Positron emission tomography (PET) combined with $\left[{ }^{15} \mathrm{O}\right] \mathrm{H}_{2} \mathrm{O},\left[{ }^{15} \mathrm{O}\right] \mathrm{CO}$ was employed for direct measurement of skeletal muscle blood flow and volume. Whole body glucose uptake was measured using euglycemic clamp technique $(5 \mathrm{mU} / \mathrm{kg} / \mathrm{min})$. In the basal state both mean skeletal blood flow $(1.7 \pm 0.2$ vs $2.7 \pm 0.4 \mathrm{ml} / 0.1$ $\mathrm{kg} \cdot \mathrm{min}$, hypertensive vs normotensive, $p<0.05$ ) and blood volume $(3.0 \pm 0.2$ vs $3.5 \pm 0.2 \mathrm{ml} / 0.1 \mathrm{~kg}, p<0.05)$ were significantly lower in the hypertensive than the normal subjects. During insulin stimulation mean

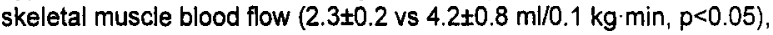
the flow response $(p<0.05)$, volume $(3.1 \pm 0.2$ vs $4.0 \pm 0.2 \mathrm{~m} / 0.1 \mathrm{~kg}$ muscle, $p<0.05)$ and the volume response $(p<0.05)$ as well as the whole body glucose uplake $(41 \pm 4$ vs $59 \pm 4 \mu \mathrm{mol} / \mathrm{kg} \cdot \mathrm{min}, \mathrm{p}<0.005)$ were significantly lower in the hypertensive patients. True flow heterogeneity, as judged from the coefficients of variation (relative dispersion) was comparable between the groups basally and during hyperinsulinemia, while insulin stimulation increased absolute dispersion of blood flow significantly more in the normal than the hypertensive subjects $(p<0.05)$. We conclude that mean flow, its absolute dispersion and blood volume exhibit insulin resistance in patients with essential hypertension.

\section{1}

INSULIN INCREASES LEFT VENTRICULAR EJECTION FRACTION AND MAXIMAL WORKLOAD DURING EXERCISE IN HEALTY MEN F. C. Sasso, T. Salvatore, D. Cozzolino, G. Sessa, D. Capobianco*, L. Mansi*, S. Gentile, M. Persico, S. Turco and R. Torella. Unit of Internal Medicine *Unit of Nuclear Medicine Second University of Naples, Italy.

The insulin is known as a vasoactive peptide that is able to influence the behaviour of the cardiovascular system. We have investigated the role of $\beta$ cell hormone on left ventricular function at rest and during a physical stress in healthy men. The effects of normal saline (test $\mathrm{A}$ ) and insulin-glucose (insulin $=1.7 \mathrm{mU} \cdot \mathrm{kg}^{-1} \cdot \mathrm{min}^{-1} ;$ glucose $=6 \mathrm{mg} \cdot \mathrm{kg}^{-1} \cdot \mathrm{min}^{-1}$ ) (test B) infusion on left ventricular ejection fraction (LEVF), left ventricular end-diastolic volume (LVEDV), and mean blood pressure (MBP) at rest and during dynamic exercise were examined by radionuclide ventriculography. The workload, performed by bicycle ergometer, was increased by steps of 25 watts until maximal exercise. Nine healthy men (age 28.4 $\pm 3.4 \mathrm{yrs}, \mathrm{M} \pm \mathrm{SD}$, BMI $23.1 \pm$ $1.3 \mathrm{~kg} / \mathrm{m}^{2}$ ) were enrolled. Coronary artery disease and others cardiovascular diseases as well as diabetes were preliminary excluded. Only subjects without risk factors for coronary artery disease were selected. Statistical analysis was made by using one-way analysis of variance (ANOVA). When differences were significant by ANOVA test, Student's $t$ test for paired data was performed. Rest- and stress-LVEF were significantly higher during test $B$ than test A $(70.6 \pm 1.9$ vs $66.3 \pm 2.3 \%$; $<<0.05$, and $80.7 \pm 2.2$ vs $70.2 \pm 2.1 \%$; $\mathrm{p}<0.001)$. At rest and in dynamic conditions no significant differences there were both in LVEDV and in MBP between test A and test B. During exercise a higher maximal workload (max systolic blood pressure $\times \max$ heart rate) was achieved in test B than test $A(27,650 \pm 1,250$ vs $29,570 \pm 1,630 ; p<$ 0.05 ). In conclusion it may be speculated that in healthy men: 1) insulin directly influences left ventricular contractility, as indicated by increased LEVF without variations in pre- and post-load; 2) insulin increases maximal workload during exercise

\section{2}

ACUTE EFFECT OF INSULIN ON THE COAGULATION AND FIBRINOLYSIS PATHWAY IN PATIENTS WITH NIDDM

K. Takanashi, T. Inukai, I. Kurihara and Y. Takemura, Department of Medicine, Koshigaya Hospital, Dokkyo University School of Medicine, Koshigaya 343-0845, Japan

It has been accepted that chronic hyperglycemia leads to accelerate the coagulation pathway and simultaneously to suppress the fibrinolysis pathway, however the role of insulin on the pathogenesis remains to be unclarified. We therefore tried to solve the problem in patients with non-insulin-dependent diabetes mellitus (NIDDM), using the euglycemic insulin clamp (GC) method by an artificial endocrine pancreas. Studies were conducted in 20 NIDDM patients (12 males and 8 females, $57.6 \pm 10.1 \mathrm{yrs}$ ). We adopted the GC-method suggested by DeFronz et al. and considered glucose infusion rate (GIR) as the insulin resistance. Blood was taken in each patient before and after the GCmethod, and then various plasma factors associated with the coagulation and fibrinolysis pathway were immediately measured. Body mass index and serum IRI showed significant positive correlations with GIR in NIDDM patients $(\mathrm{P}<0.05)$. A marked decrease in blood glucose (the end point: $100 \mathrm{mg} / \mathrm{dl}$ ) and a rapid increase in serum IRI were observed after the GC-method. Plasma levels of plasminogen activator inhibitor 1 (PAI-1) and thrombomodulin (TM) decreased significantly after the GC-method, respectively (PAI-1; before: $10.3 \pm 5.2 \mathrm{ng} / \mathrm{ml}$, after $6.5 \pm$ $2.5 \mathrm{ng} / \mathrm{ml}, \mathrm{P}<0.05$, TM; before: $22.0 \pm 4.8 \mathrm{ng} / \mathrm{ml}$, after $19.7 \pm 4.5 \mathrm{ng} /$ $\mathrm{ml}, \mathrm{P}<0.001)$. Other plasma factors including PT, fibrinogen, plasmin, TAT, t-PA and protein $\mathrm{C}$ did not exhibit significant alterations. Conclusions: We demonstrated remakable decreases in PAI- 1 and TM associated with thrombosis after the GC-method in NIDDM patients, which provides an evidence that insulin itself plays an important role in those alterations.

\section{3}

EFFECT OF INSULIN ON BLOOD PRESSURE IN ESSENTIAL HYPERTENSION

T. Heise, L. Heinemann. M. Stoffels, U. Schaden, and P. T. Sawicki, Clinic of Metabolic Diseases and Nutrition, Heinrich-Heine-University Dusseldorf, Germany

There is a close link between insulin resistance, hyperinsulinemia and essentia hypertension. The aim of this study was to investigate the effect of two weeks of insulin administration on blood pressure in patients with essential hypertension. In a prospective. randomized, double-blind, cross-over study 23 hypertensive, non-diabetic patients ( 19 male, age $40 \pm 1$ ) years (mean \pm SD), BMI $31 \neq 5 \mathrm{~kg} / \mathrm{m}^{2}$ ) without antihypertensive medication, injected either $\mathrm{NPH}$-insulin $(0.3 \mathrm{IE} / \mathrm{kg}$ body weighidday) or placebo subcutaneously for a period of two weeks, separated by a wash-out period of 14 days. Body weight, 24-hour blood pressure and office blood pressure values were recorded at the beginning and at the end of cach treatment phase. Concomitantly, serum insulin, C-peptide, fructosamine, and insulin sensitivity were determined.

Following insulin administration. serum insulin concentrations and the insu$\mathrm{lin} / \mathrm{C}$-peptide-ratio were significantly higher when compared to placebo (insulin: $168 \pm 178$ vs. $131 \pm 122 \mathrm{pmol} / \mathrm{l}$, insulin/C-peptide ratio: $16 \pm 7$ vs. $11 \pm 4 \mathrm{pmol} / \mathrm{nmol}$, $\mathrm{p}<0.05)$. whereas fructosamine concentrations were lower $(239 \pm 20$ vs. $245 \pm 18$ $\mu m o l / l, p<0.05$ ). Mean office blood pressure values decreased during insulin administration from $130.7 \pm 12.7$ to $128.3 \pm 12.4 \mathrm{~mm} \mathrm{Hg}$ and remained unchanged in the placebo group: $131.8 \pm 13.3$ and $131.9 \pm 13.1 \mathrm{~mm} \mathrm{Hg}, \mathrm{p}=0.02$ for insulin vs. placebo. Insulin decreased mean 24 -hour blood pressure values by $-4.7 \pm 9.0 \mathrm{~mm}$ $\mathrm{Hg}$. while placebo had no influence $(+0.2 \pm 6.3 \mathrm{~mm} \mathrm{Hg}, p=0.03)$. There were no significant differences in weight. BMI and insulin sensitivity within or between insulin and placebo. These data argue against the postulated pressor action of insulin in essential hypertension. In obese, insulin resistant, hypertensive patients subcutaneous insulin administration exerts a small blood pressure lowering effect. 
DIMINISHED WAVE REFLECTION IN THE AORTA:

A NOVEL PHYSIOLOGICAL ACTION OF INSULIN

J. Westerbacka, I. Wilkinson, T. Utriainen, S. Vehkavaara, J. Cockcroft and H. YkiJärvinen. Helsinki, Finland; Cardiff and Edinburgh, U.K.

We determined whether insulin has direct effects on large arteries in vivo. Ten normal men were studied on two occasions, once during a $360 \mathrm{~min}$ infusion of saline $(O)$, and once during maintenance of normoglycemic hyperinsulinemia (stepwise insulin infusions of 1,2 and $5 \mathrm{mU} / \mathrm{kg} \cdot \mathrm{min}$ each for $120 \mathrm{~min}$ for a total of $360 \mathrm{~min}, \bullet$ ). Central aortic pressure waves were synthetized from those recorded in the periphery using applanation tonometry and a reverse transfer function every 30 minutes. This allowed determination of the augmentation index (the pressure difference between the first and second systolic peaks expressed as a percentage of pulse pressure), which is known to be regulated by nitric oxide as

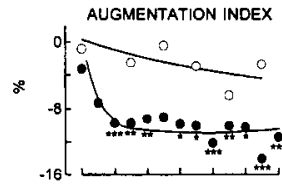

PERIPHERAL RESISTANCE

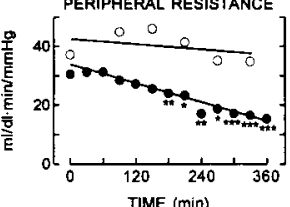

TIME (min) During the first $120 \mathrm{~min}$, insulin changed neither heart rate, systolic or diastolic blood pressure, peripheral (forearm) blood flow or vascular resistance. Augmentation ( $\mathrm{mmHg}$ ) as well as the augmentation index decreased, however, significantly by insulin but not saline within $60 \mathrm{~min}$ (Figure). These changes were further accentuated by insulin over the entire study period. These data provide the first evidence of a direct physiological effect of insulin on large arteries. The decrease in augmentation reflects either an increase in large vessel compliance or vasodilatation of large arteries. Resistance to this action of insulin could explain the association of insulin resistance and hypertension.

PS 20

\section{Hormonal Action (Other)}

\section{6}

\section{EXENDIN-4 IS MORE POTENT THAN GLP-1 IN LOWERING PLASMA GLUCOSE IN DIABETIC MICE.}

A.A. Young, S.P. Bhavsar, R LaChappell and J.J. Watkins. Amylin Pharmaceuticals Inc, 9373 Towne Centre Drive, San Diego, CA, 92121, USA

Exendin-4, a 39 amino acid peptide from Gila monster (Heloderma suspectum) venom with $52 \%$ sequence similarity to glucagon-like peptide-1 (GLP-1), is reported to bind and activate GLP-1 receptors. In this study, we compared the effects of exendin-4 and GLP-1, on plasma glucose concentrations in diabetic C57BL/6 J-m+/+ Leprob (db/db) mice. Animals were deprived of food for 2 hours, injected intraperitoneally with either saline, exendin- $4(0.001,0.01,0.1,1$ and $10 \mu \mathrm{g} /$ mouse) or GLP-1 $(0.1,1,10,100$ and $1000 \mu \mathrm{g} /$ mouse). Plasma glucose was measured just before and 1 hour after injections. Exendin-4 injections dose-dependently reduced plasma glucose by up to $39 \%$ from $505 \pm 18$ to $307 \pm 11 \mathrm{mg} / \mathrm{dL}(P<0.0001)$ with an $\mathrm{ED}_{50}$ of $0.011 \mu \mathrm{g} /$ mouse $\pm 0.12 \mathrm{log}$ units. In separate kinetic studies, this $0.011 \mu \mathrm{g}$ intraperitoneal dose of exendin- 4 was calculated to result in a plasma concentration of $10 \mathrm{pM}$ in $\mathrm{db} / \mathrm{db}$ mice 1 hour after injection. In the same diabetic model, GLP-1 injections dose dependently reduced glucose by a similar amount ( $37 \%$, from $505 \pm 18$ to $318 \pm 16$ $\mathrm{mg} / \mathrm{dL} ; P<0.0001)$ but $\mathrm{GLP}-1$ was 880 -fold less potent $\left(\mathrm{ED}_{50} 9.74\right.$ $\mu \mathrm{g} /$ mouse $\pm 0.33 \log ; P<0.0001$ vs. Exendin $\left.E D_{50}\right)$. In summary, intraperitoneal injections of exendin-4 or GLP-1 each lowered plasma glucose in diabetic $d b / d b$ mice, but exendin-4 was $\sim 3$ orders of magnitude more potent than GLP-1.
EFFECTS OF GLYCOMETABOLIC CONTROL ON HYPERTENSION IN KIDNEY AND PANCREAS TRANSPLANTED PATIENTS

E. La Rocca, I. Baragetti, A. Caumo, P. Fiorina, V. Di Carlo, G. Pozza and A. Secchi. Scientific Institute San Raffaele Milan Italy

Pathophysiology of hypertension in diabetes is not clearly defined. In particular, the effect of insulin on vascular tone, and the role of hyperglycaemia on endothelial function is under discussion in literature. To investigate the relationship among hypertension, glucose $(\mathrm{mg} / \mathrm{dl})$ and free insulin (F IRI $\mathrm{uU} / \mathrm{ml}$ ), a cohort of 67 IDDM hypertensive patients with ESRF were studied. 2 months after kidney and pancreas transplantation 24 of them were hypertensive (group $\mathrm{H}$ ) and 23 normotensive (group N). During the same period, glucose levels and F IRI secretion was studied by the 24 hours metabolic profile, OGTT and IVGTT. The Index of insulin secretion ( $\triangle \mathrm{AUC}$ ) and insulin sensitivity (SIGalvin) were evaluated on IVGTT, according Galvin mathematical model . During the 24 hours metabolic profile, postprandial glucose levels were higher in $\mathrm{H}$ than $\mathrm{N}$ group (H vs $\mathrm{N}$ glucose $\mathrm{mg} / \mathrm{dl} 13 \mathrm{pm}: 173 \mathrm{vs} 143 \mathrm{p}=.03 ; 14 \mathrm{pm:} 172$ vs $130 \mathrm{p}=.008 ; 17 \mathrm{pm}: 131$ vs $113 \mathrm{p}=.03 ; 21$ pm 127 vs $104 \mathrm{p}=.038$ ), while no differences were observed in terms of F IRI levels. After OGTT, a delayed F IRI secretion and higher glucose levels were observed in group $\mathrm{H}$ than in group $\mathrm{N}$ (H vs $\mathrm{N}$ glucose : $30 \mathrm{~min}$ : 139 vs $120 \mathrm{p}=.02 ; 60 \mathrm{~min}: 156$ vs $128 \mathrm{p}=$ $.02 ; 90 \mathrm{~min}: 156$ vs $123 \mathrm{p}=.008 ; 120 ; 149$ vs $127 \mathrm{p}=.09$ ). IVGTT analysis showed a first phase of insulin secretion higher in $\mathrm{N}$ than in $\mathrm{H}$ group (H vs $\mathrm{N} \triangle A U C$ 0-10 min: $437.15 \mathrm{vs} 655.02 \mathrm{p}=.02$ ). No statistical differences were observed in terms of insulin sensivity (H vs $\mathrm{N}-\mathrm{F}$ IRI ml/uU- SIGalvin: 1 . 297E-05 vs 1.43E-05). In conclusion the association between hypertension, lower blood glucose levels and higher insulin secretion in kidney and pancreas transplanted patients, suggests a key role of glucose and insulin on haemodinamic of peripheral vascular system and on hypertension pathophysiology.

\section{7}

ACTIVATION OF ENDOTHELIAL NITRIC OXIDE SYNTHASE (eNOS) BY C-PEPTIDE

T. Kunt', T. Forst', E.Closs' ${ }^{2}$, T. Wallerath ${ }^{2}$, U. Foerstermann ${ }^{2}$, R. Lehmann ', A. Pfuetzner', O. Harzer', M. Engelbach' and J. Beyer', Dept. of Endocrinology ${ }^{1}$ and Inst. of Pharmacology ${ }^{2}$, University of Mainz, Germany

Recent studies have demonstrated that C-peptide reveals biological functions like increased glucose uptake in muscle cells, improvement of renal and nerval function and particularly of capillary blood flow. The aim of this study was to characterize the effect of C-peptide on endothelial nitric oxide synthase (eNOS). The measurement of eNOSactivation was performed by means of a reporter cell assay (increase of cGMP production in RLF-6 cells) using the supernatant of Cpeptide $(6.6 \mathrm{nM})$ treated bovine aortic endothelial cells (BAEC), human umbilical vein endothelial cells (HUVEC) and the endothelial cell line EaHy. Calcium influx was shown by Fluo3/FuraRedtechnique. Stimulation of BAEC with $6.6 \mathrm{nM} \mathrm{C}$-peptide increased the cGMP production to $186.3+/-11.2 \%(\mathrm{p}<0.01)$ after 2 minutes and to 120.4 + $-14.7 \%$ (n.s.) after 30 minutes versus untreated cells. HUVEC and EaHy failed to activate eNOS. Production of cGMP in BAEC and activation of eNOS respectively were able to be suppressed completely by inhibition with nitro-arginin and were also not detectable in calcium-free media (108.3+/-18.6\% after 2 and $111.4+i-6.0 \%$ after 30 minutes; all n.s.). Therefore, intracellular calcium transients were measured showing a significant increase of Fluo3/FuraRed-ratio of $28.3+/-4.3 \%(\mathrm{p}<0.001)$. The presented data show that C-peptide is able to increase calcium influx with subsequent activation of eNOS in aortic endothelial cells. This may explain the vasodilating effect of $\mathrm{C}$-peptide of recent in vivo studies. 
688

\section{ENHANCEMENT OF INSULIN RECEPTOR ACTIVITY BY C-PEPTIDE}

AAF Sima, PR Scrinivas, S Kommaraju, S Verma, ${ }^{*} J$ Wahren, G Grunberger; Wayne State University, Detroit, MI U.S.A.,

*Karolinska Institute, Stockholm, Sweden

There is no known biological function attributed to C-peptide apart from facilitating folding of proinsulin chains. Cleavage of proinsulin results in the release of equimolar amounts of insulin and C-peptide. The kidney is the main target of C-peptide uptake, where it is excreted into urine Previous studies however demonstrate that short term infusion of $\mathrm{C}$-peptide improve glucose utilization and reduce glomerular hyperfilteration in type 1 diabetes. The molecular mechanisms or targets involved in mediating these actions of C-peptide have not been described. In the present study, we examined whether $\mathrm{C}$-peptide could exert its action via the insulin receptor. We demonstrate that C-peptide $(1.0 \mathrm{nM})$ enhances the insulin-stimulated tyrosine kinase activity of partially purified human insulin receptors by almost 1.5 fold. A parallel increase was observed in the autophosphorylation activity of the insulin receptor at the same concentrations. When rat 1 fibroblasts expressing the human insulin receptor (HIRcB) were incubated with C-peptide for a period of $30 \mathrm{~min}$ prior to insulin stimulation, a marked increase in tyrosine phosphorylation of the $\beta$-subunit of the insulin receptor was observed confirming our results on isolated receptors. C-peptide stimulated aminoacid uptake and revealed a 2-fold increase in uptake of $\left[{ }^{14} \mathrm{C}\right]$-aminoisobutyric acid in $\mathrm{HIRcB}$ cells. Binding studies revealed that $\mathrm{C}$-peptide does not compete with insulin for binding on the insulin receptor $\alpha$-chain at concentrations that enhance insulin-induced tyrosine kinase activity. These results demonstrate for the first time that C-peptide may actually possess a biological function in enhancing insulin signaling. Further investigations of the biological role of C-peptide could lead to therapeutic applications in diabetes.

\section{0}

\section{IESTOSTERONE REPLACEMENT, INSULIN SENSITIVITY AND SERUM LEPTIN} LEVELS IN HYPOGONADIC AND IN TYPE 2 DIABETIC PATIENTS I. Sinay, O. Levalle, M. Gurfinkiel, A. Nagelberg, and P. Arlas - Centro de Endocrinología, Hospltal Francés, la Rloja 951, B. Aires, Argentina Aims: to study the effect of testosterone (To) replacement on insulin sensitivity (IS) and serum leptin (Le) levels in patients with hypogonadism (HG) and in type 2 diabetic subjects (D2) with normal/low circulating To levels. Subjects: eight patients with HG laged $27.9 \pm 4.9$ y.. BMi $24.6 \pm 1.5 \mathrm{~kg} / \mathrm{m}^{2}$, basal To levels $1.1 \pm 0.4 \mathrm{ng} / \mathrm{ml}$ means \pm SEM), 7 patients with D2 $\left(64.3 \pm 2.1\right.$ y., BMl $27.3 \pm 1.7 \mathrm{~kg} / \mathrm{m}^{2}$, basal To levels $3.5 \pm 0.5 \mathrm{ng} / \mathrm{ml}$ in good metabolic controll, and 8 healthy controls (CON) [38.5 \pm 9.1 y.. BM! $24.8 \pm 1.2 \mathrm{~kg} / \mathrm{m}^{2}$, basal To $5.2 \pm 0.5 \mathrm{ng} / \mathrm{ml}$ ) Methods: we measured weight, height, waist/hip ratio, blood pressure. serum lipids, fructosamine, total To, fasting insulin and glucose levels. as well as Le concentrations. IS was evaluated by means of the short i.v. insulin tolerance test, and expressed by the $\mathrm{Km}_{\mathrm{m}} \mathrm{value}$. After initia testing, patients received 3 To enanthate injections (Testoviron Depot $8,250 \mathrm{mg}$ i.m. every 10 days). Evaluations were repeated 5 days after the last injection. Statistics: Wilcoxon's test for paired data, or one-way ANOVA followed by Tukey's test. Results: D2 patients showed clearly elevated plasma glucose and fructosamine levels, and a lower IS, as compared to $H G$ and CON subjects $\left(K_{\Pi}: 2.3 \pm 0.4,4.4 \pm 0.3\right.$ and $5.3 \pm 0.2$, respectively; $p<0.05$. After replacement, serum to levels rose markedly in D2 and $H G$ patients to $9.0 \pm 0.8$ and $10.0 \pm 1.1 \mathrm{ng} / \mathrm{ml} 10<0.01$ vs. basal); in D2 patients, IS increased (Km: $3.1 \pm 0.4 ; p<0.02$ vs. basal), fructosamine levels decreased ( $<<0.05$ vs. basall, and Le concentrations remained unchanged. To treatment did not alter IS in HG patients, and lowered Le levels $(7.8 \pm 1.4$ vs. $4.9 \pm 1.3 \mathrm{ng} / \mathrm{ml} ; \mathrm{p}<0.01)$. The remaining physical and biochemical parameters showed no modifications. Conclusions: the induced increase in To levels led to a significant amelioration of is and metabolic control in D2 patients. leaving Le concentrations unchanged. Conversely, in $H G$ subjects hormonal replacement did not modify $I S$, and diminished serum Le levels. Thus, changes in IS and le concentrations observed after intensive To replacement in our patients seem not to be correlated.
689

IS IGFBP-1 OF IMPORTANCE TO THE BLOOD-GLUCOSE REGULATION IN PUBERTAL DIABETIC BOYS?

I.Zachrisson, K. Brismar, G.Dahlquist and M.Wallensteen Department of Paediatrics, Danderyd Hospital, Stockholm, Sweden.

We have recently shown that blood glucose variability is correlated to growth rate in pubertal diabetic children. To further study whether growth factors have any impact on blood glucose levels, we analysed the relations between B-Glucose, insulin, GH, IGF-I and IGFBP-1 in diabetic boys during and after puberty. Method: We have, in 5 diabetic boys in Tanner stage 3 and 6 in Tanner 5 , during 24 hours, continuously collected

blood samples every $30 \mathrm{~min}$. Each sample was analysed for B-Glucose, S-Insulin, SGH, S-IGF-I and S-IGFBP-1. Results: We found a positive correlation between BGlucose and IGFBP-1 and a negative correlation between B-Glucose and insulin in the same time point. No correlation was found between B-Glucose and GH. (Table below)

\begin{tabular}{r|c|c|c|c|c|c|} 
& \multicolumn{2}{c}{$\log$ IGFBP-1 } & \multicolumn{2}{c|}{ Insulin } & \multicolumn{2}{c|}{$\log \mathbf{G H}$} \\
\cline { 2 - 7 } Tanner 3 & $\mathbf{r}$ & $\mathbf{p}$ & $\mathbf{r}$ & $\mathbf{p}$ & $\mathbf{r}$ & $\mathbf{p}$ \\
\cline { 2 - 7 } Tanner 5 & 0,52 & 0,004 & $-0,52$ & 0,004 & $-0,13$ & 0,49 \\
In a multiple regression analysis we have standardised for the effect of insulin, & 0,74 & $<0,001$ & $-0,52$ & 0,007 & 0,08 & 0,68
\end{tabular}

In a multiple regression analysis we have standardised for the effect of insulin, demonstrating an independent effect of IGFBP-I on the B-Glucose level (Table below, B-Glucose is the dependent variable).

\begin{tabular}{cc|c|c|c} 
& \multicolumn{2}{c|}{ Tanner 3 } & \multicolumn{2}{c}{ Tanner 5 } \\
\cline { 2 - 5 } Total model & $\mathbf{R}^{\mathbf{2}}=\mathbf{0 , 2 5}$ & $\mathbf{p}<\mathbf{0 , 0 1}$ & $\mathbf{R}^{\mathbf{2}} \mathbf{0 , 6 3}$ & $\mathbf{p}<\mathbf{0 , 0 1}$ \\
& $\mathbf{r}$ & $\mathbf{p}$ & $\mathbf{r}$ & $\mathbf{p}$ \\
log IGFBP-1 & 0,29 & 0,30 & 0,64 & $<0,001$ \\
Insulin & $-0,29$ & 0,31 & $-0,29$ & 0,04 \\
logGH & \multicolumn{2}{c}{ No significant effect } & \multicolumn{2}{|c}{ No significant effect }
\end{tabular}

Conclusions: The B-Glucose level in diabetic boys co-variates with IGFBP-1 independently of the insulin level. Insulin suppresses IGFBP-1, which is an important regulator of free IGF-I. In animal studies IGF-I has been found to activate, to a significant extent, the glucose transport in muscles, via the same intracellular pathway as insulin. Thus, the level of IGFBP-1 seems to be a factor of importance for the regulation of the B-Glucose level in pubertal boys with diabetes.

\section{1}

EFFECTS OF OBESITY ON REGULATION OF P450 AROMATASE ACTIVITY BY INSULIN AND GLUCOCORTICOID IN HUMAN ADIPOSE TISSUE. PG McTernan, A Anwar, M Eggo, AH Banett, PM Stewart, and S Kumar. Dept of Medicine, University of Birmingham, Birmingham, England.

P450 aromatase activity in adipose tissue is important for oestrogen production which may have a paracrine role in promoting pre-adipocyte proliferation and thus contribute to adiposity. Our aim was to investigate the hormonal of aromatase in mature human adipocytes (Ad) and pre-adipocyte cells. P450 aromatase activity was assessed in adipocytes from abdominal subcutaneous (sc) fat tissue isolated from obese (BMI $>25)$ and non-obese (BMI<25) females undergoing elective surgery. Ad were cultured in serum-free medium for 3 days with either cortisol $\left(10^{-6} \mathrm{M}\right)$ or insulin $(500 \mathrm{nM})$ or a conbination of both. Aromatase activity was expressed as $\mathrm{fmol} / \mathrm{mg} / \mathrm{h}$ mean $+/$. SEM. In non-obese control subjects sc $A d(n=4)$ basal activity $(217+/-50$ fmol $/ \mathrm{mg} / \mathrm{h}$ ) was stimulated by cortisol (F) $(491+/-78 \mathrm{fmol} ; \mathrm{p}<0.05)$ and $\mathrm{F}+\mathrm{insulin}$ $(2187+/-371 \mathrm{fmol} ; \mathrm{p}<0.0005)$ but not insulin alone (pN.S). Aromatase activity was higher in Ad treated with $\mathrm{F}+$ insulin compared to $\mathrm{F}$ or insulin alone $(\mathrm{p}<0.05)$. In obese sc $\mathrm{Ad}(\mathrm{n}=5)$ basal activity $(293+/-25 \mathrm{fmol} / \mathrm{mg} / \mathrm{h})$ was stimulated by $\mathrm{F}(55 \mathrm{G}+/$. $70 \mathrm{fmol} / \mathrm{mg} / \mathrm{h} ; \mathrm{p}<0.005)$ and $\mathrm{F}+$ insulin $(711+/-64 \mathrm{fmol} / \mathrm{mg} / \mathrm{h} ; \mathrm{p}<0.0005)$, but not insulin alone $(366+/-86 \mathrm{fmol} / \mathrm{mg} / \mathrm{h} ; \mathrm{p}=\mathrm{N}$.S). In obese sc Ad no significant differences in aromatase activity were demonstrated when $F$ +insulin was compared to $F(p=N . S)$ however comparison of $\mathrm{F}+$ insulin with insulin alone showed significance $(\mathrm{p}<0.05)$ Comparison of treatments between obese and non-obese Ad indicated that $\mathrm{F}+$ insulin stimulated aromatase activity higher in non-obese than obese females. Pre-adipocyte cell data showed a similar trend. In conclusion: (1) aromatase from non-obese individuals is regulated by insulin acting synergistically with cortisol; (2) Ad from non-obese individuals treated with insulin+cortisol demonstrate greater sensitivity in aromatase activity compared to obese individuals; (3) aromatase activity is hormonally regulated, however as adiposity increases there is loss of synergy between insulin+cortisol which may have ramifications for the insulin resistant state. 
EFFECTS OF LONG TERM GH-TREATMENT ON INSULIN SENSITIVITY, GLUCOSE EFFECTIVENESS, AND $\beta$-CELL SENSITIVITY IN PUBERTAL AND PREPUBERTAL GH-DEFICIENT PATIENTS.

L.Guazzarotti ${ }^{1}$, G.Toffolo ${ }^{2}$, C.Sgattoni ${ }^{1}$, C.Cobelli ${ }^{2}$ and E.Bartolotta

'Divisione di Pediatria, Ospedale S.Lucia, Recanati (MC), 2Dipartimento di Elettronica ed Informatica, Universita di Padova, Italy

An increase of Growth Hormone (GH) within the physiologic range can cause insulin resistance in man. To evaluate the effect of a long term $\mathrm{GH}$ treatment on glucose metabolism in pubertal (P) and prepubertal (PP) state, we studied 7P and 3PP subjects with partial GH-deficiency (5Fand5M,age $=12 \pm 3.3$, Mean \pm $\mathrm{SD})$, treated with $\mathrm{GH}$ for a minimum of 2 to a maximum of 9 years $(5.6 \pm 2.4)(\mathrm{GH}$ dose $=0.66 \pm 0.06 \mathrm{U} / \mathrm{Kg} / \mathrm{w}$ ). Four $\mathrm{P}$ and $3 \mathrm{PP}$ normal subjects (4Fand $3 \mathrm{M}$, age $=11.2 \pm$ $1.2 y r s)$ without metabolic and hormonal disorders, were used as controls.All patients had a normal glucose tolerance after an OGTT and a normal body weight. Insulin sensitivity SI $\left(\mathrm{min}^{-1} \mu \mathrm{U}-1 \mathrm{ml}^{1} \mathrm{10}^{4}\right)$ and glucose effectiveness SG $\left(\mathrm{min}^{-1} \times 10^{2}\right)$ was evaluated from a frequently sampled iv glucose tolerance test (dose $=0.3 \mathrm{gr} / \mathrm{Kg} 50 \%$ dextrose) by using the minimal model method.An index of $\beta$-cell sensitivity, AIR ( $\mu \mathrm{U} \mathrm{ml-1} \mathrm{min),} \mathrm{was} \mathrm{calculated} \mathrm{as} \mathrm{the} \mathrm{incremental} \mathrm{area}$ under the insulin curve between 0 and $10 \mathrm{~min}$ after glucose injection. In the group of PP subjects SI was significantly lower in patients than in controls (6.2 \pm $1.3 v s 18.1 \pm 7.5 ; P<0.05)$ while $S G$ was significantly higher $(2.8 \pm 0.2 v s 2.2 \pm$ $0.3 ; P<0.04$ ). In the group of $P$ subjects $S I$ and $S G$ did not show a significant

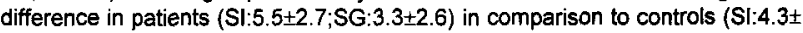
$1.7 ; \mathrm{SG}: 2.1 \pm 0.1)$. AIR was significantly higher in PP patients in comparison to controls $(560 \pm 83$ vs $235 \pm 30: P<0.003)$, but it was similar in the group of $P$ subjects ( $681 \pm 133 \mathrm{vs} 563 \pm 182$ ). Also basal plasma insulin was significantly higher in PP patients in comparison to controls $(6.0 \pm 1.0 v 52.7 \pm 0.6 \mu$ $\mathrm{U} / \mathrm{ml} ; \mathrm{P}<0.007)$, but it was similar in the group of $\mathrm{P}$ subjects $(8.4 \pm 4.0 \mathrm{vs} 7.5 \pm$ 3.4).In conclusion: a) $\mathrm{GH}$ treatment decreases $S I$ and increases $\beta$-cell sensitivity in PP subjects, but these effects are not evident in P subjects; b)the differences between $P$ and PP subjects does not seem to be due to the GH treatment duration; c)our results suggest that the decrease of SI and increase of $\beta$-cell sensitivity, linked to puberty, probably mask the GH treatment diabetogenic effects in $\mathbf{P}$ patients.

\section{4}

NORMOGLICAEMIC ACROMEGALY AS A MODEL OF EARLY INSULIN RESISTANCE

D.Piniewska, Z.Lewandowski, W.Bonicki. and A.Czyżyk. The Medical University of Warsaw, Poland

Hypothesis: Can acromegaly with normal glucose tolerance (NGT) simulate an early stage of effects of insulin resistance? The answer to this question is important for prospective studies in the development of NIDDM. The aim of the study was to refer the hormonal state of people with acquired insulin resistance during NGT stage (acromegalics, group $A, n=16$ ) to healthy humans (group $\mathrm{H}, \mathrm{n}=43$ ) and to humans with impaired glucose tolerance (group HIGT, $n=12$ ). We estimated fasting serum insulin (INS), C-peptide (CP), growth hormone (GH), insulin-like growth factor-l (IGF-l), prolactin $(P L)$, plasma glucagon (GG) concentrations, and the area under the glycaemic curve (AUC) in OGTT. The Spearman correlation coefficient and ANOVA were used in the statistical analysis. Using the previously presented index $=(\mathrm{CP} / \mathrm{GG}) /(\mathrm{INS} / \mathrm{CP})$ based on interrelations among fasting molar hormone concentrations, we distinguished, in A group, people with higher blood glucose levels $(\mathrm{Bgl})$ of OGTT $(\mathrm{p}<0.05)$ still remaining within normal limits according to the WHO criteria. The particular Bgl of OGTT in A group with index<518 were $5.35,8.46,8.71,7.18,5.26(\mathrm{mmol} / \mathrm{l})$ but with index 2518 were $4.52,7.41,6.58,5.54,4.84$. The similarity beteen groups $A$ and $H$ was expressed through the significant correlation between AUC and particular Bgl of OGTT, whereas the analogy between $A$ and HIGT by lack of correlations between fasting PL or IGF-I concentrations and the particular Bgl of OGTT. Such significant, but negative correlations were, however, observed in group $\mathrm{H}$. Conclusion: The metabolism in acromegaly with NGT appears to rest between that of a healthy subjects and humans with IGT. We propose acromegaly with NGT be employed as a model in prospective studies of initial effects of exposure to insulin resistance due to the easier determining the time period of insulin resistance in such subjects.
CHANGES IN EXPRESSION OF G $\alpha$ ISOTYPES AND ADENYLYL CYCLASES ISOFORMS AFTER DEXAMETHASONE TREAT-MENT IN RINm5F BETA CELLS

N. Ferrand, M. Pessah, J. Marais, S. Frayon and J.-M. Garel. INSERM U.482, Hôpital Saint-Antoine, 75571 Paris-Cedex 12,France

Others have already shown that dexamethasone decrease insulin secretion in the RINm5F beta pancreatic cell line and that transfection of the glucocorticoid receptor was associated with a reduction in insulin release. Since it is known that inhibition of insulin secretion by galanin in RIN m5F involved the Gi2 heterotrimeric protein, we have investigated the effects of dexamethasone on the expression of different $G \alpha$ isotypes and adenylyl cyclase isoforms (AC). RINm5F cells were treated for 1 to 5 days with doses of dexamethasone ranging from $0.1 \mathrm{nM}$ to $1 \mu \mathrm{M}$. Decreases in Goi2 mRNA were observed under $10 \mathrm{nM}$ to $1 \mu \mathrm{M}$ dexamethasone for 3 days; no effect was seen at $0.1 \mathrm{nM}$. Treatment with $1 \mu \mathrm{M}$ dexamethasone during 3 days elicited marked morphological changes leading to cell detachment. Under these conditions, the mRNA level of Goi2 evaluated by northem hybridization was sharply reduced. Goi3 mRNA was decreased, however, a similar reduction was observed for the control cyclophilin mRNA probe. When compared to the control probe, the mRNA level for $G \alpha$ s was increased. Western blotting of Gai2 revealed no change in the amount of protein after dexamethasone treatment. This finding may suggest a rapid fall in the half-life of Goi2 mRNA without change in the turn-over of the protein under dexamethasone treatment. RT-PCR determination of the amount of AC II and AC III showed no change after glucocorticoid treatment, however, amounts of proteins detected by Western-blot were decreased. Thus, a translationnal effect of dexamethasone on AC II and AC III synthesis could not be excluded. In summary, the inhibition of insulin secretion induced by dexamethasone could be partly due to a decrease in the expression of ACII and AC III.

\section{5}

THE BENEFICIAL EFFECT OF ESTROGEN IS WEAKENED BY DIABETES: A STUDY ON AORTA FROM DIABETIC RATS

C. Bolego, A. Cignarella, V.Zancan, C. Pinna, R. Zanardo and L.Puglisi Institute of Pharmacological Sciences, Via Balzaretti, 9, 20129 Milan, Italy

Estrogens are known to exert a protective effect against cardiovascular disease. However, women with diabetes have three times the cardiovascular risk as compared with age-matched non-diabetic women. Our previous study on aortic rings of ovariectomized (OVX) female rats treated with 17-B-estradiol $\left(E_{2}\right)$ demonstrated that the beneficial effect of estrogen may be related to an enhanced basal release of nitric oxide (NO) from endothelial cells. In the present study, in order to understand why estrogen protection is abolished in diabetes, we evaluated vascular responses in $\mathrm{OVX}$, streptozotocin-diabetic female rats receiving or not $E_{2}$ replacement. Concentration-response curves to norepinephrine (NE) showed attenuation of the contractile response in diabetic, with respect to non-diabetic preparations, which was further impaired in diabetic, $E_{2}$ deprived rats (ANOVA, $p<0.001$ ). The basal release of NO, as evaluated by responses to superoxide dismutase and $\mathrm{N}^{\mathrm{G}}$-methyl-L-arginine (L-NMA) in NE precontracted aortic rings, was found to be impaired in diabetic tissues, no further effect being induced by $E_{2}$ deprivation (L-NMA: $E_{\max }$ $183.5 \pm 8.7$ vs $149.8 \pm 6.7, p<0.05$ ). The endothelium-dependent relaxation produced by carbachol did not change between the three groups, whereas the relaxation produced by histamine was enhanced by diabetes, with no further effect elicited by $E_{2}$ deprivation (ANOVA, $p<0.001$ ). Finally, the relaxation induced by sodium nitroprusside, an endothelium-independent relaxant agent, was comparable between groups. These results suggest that the lack of protective effect of estrogen in diabetes may be mainly ascribed to the failure of estrogen to affect the basal release of NO, which is impaired in the aorta of diabetic rats. This finding may have implications for the understanding of the interplay between estrogen protection and diabetes in women. 
PS 21

\section{Hormone Receptors}

\section{6}

MAPPING OF THE SECOND RECEPTOR BINDING DOMAIN ON THE INSULIN MOLECULE BY ALANINE SCANNING MUTAGENESIS

P. De Meyts, C. Kristensen, P. Holst, L. Larsø, T. Kjeldsen, L. Schäffer, A. S. Andersen and R. M. Shymko, Hagedorn Research Institute and Diabetes Research, Novo Nordisk, 2820 Gentofte and 2880 Bagsværd, Denmark.

Alanine scanning mutagenesis (i.e. replacement of key amino acids by alanine) in either the ligand or the receptor has proved to be a powerful tool in investigating the structure-activity relationships in ligand-receptor interactions. Recent work has suggested that insulin binding to its receptor (IR) involves two distinct binding surfaces, one made of residues that partially overlap with the insulin surface involved in dimerization, and a second one that comprises residues (including Leu A13 and Leu B17) involved in insulin hexamerization. A study of 19 new insulin analogues with alanine substitutions on both surfaces of the insulin molecule using a low affinity purified, soluble, extracellular IR domain (sIR) has confirmed the importance of residues on the dimer forming surface of insulin (A2, A3, A19, B6, B8, B23, B24) for IR binding, but did not provide conclusive evidence regarding the putative second binding surface also involved in hexamer formation. We are now studying the potency of these analogues for insulin binding to the intact IR in the membrane of IM-9 lymphocytes or cells overexpressing the IR cDNA, as well as their potency in stimulating lipogenesis in isolated rat adipocytes and thymidine incorporation in cells overexpressing the IR. The direct interaction of the insulin analogues with the purified extracellular domain (SIR) has also been studied using surface plasmon resonance on the BIAcore instrument (BIAcore $A B$, Uppsala), where the sIR behaves in a manner more similar to the intact IR. These assays have confirmed a role of residues in the hexamer forming surface of insulin in high affinity binding and biological potency. For example, mutation of Leu at $\mathrm{A} 13$ and B17 to Ala results in a decrease in lipogenic potency correlated with a decrease in the rate of association of the analogues to the sIR on the BIAcore. The combination of these assays should allow a complete mapping of the residues involved in insulin -IR interaction

\section{7}

WITHDRAWN

\section{8}

HYBRID INSULINANSULIN-LIKE GROWIH FACTOR-I RECEPTORS ARE NOT INCREASED IN SKE ETAL MUSCLEOFOBESEANDNIDDMPATIENTS D.Spampinato ${ }^{\circ}$ G.Pandini ${ }^{\circ}$, S.Graci ${ }^{\circ}$, A.Iuppa*, F.Fullone*, V.Trischitta $\$$, R.Vigneri ${ }^{\circ}$ and L.Frittitta ${ }^{\circ}$. Instit Int Med and *Depart of Surgery, Univ of Catania; §Div End, Scient Instit CSS S. G. Rotondo; Italy.

Hybrid receptors (HR), constituted by one $\alpha-\beta$ insulin receptor (IR) moiety and one $\alpha-\beta$ insulin-like growth factor type-I receptor (IGF-I-R) moiety, have been reported to be increased in skeletal muscle of NIDDM patients. These findings suggest a possible role of HR in insulin resistance by sequestrating typical IR for a different ligand. In those studies, HR have been measured by an indirect assay based on ligand binding. Aim: To directly quantify the alteration of HR content in insulin resistant subjects. Methods: We first developed a specific (less than 5\% cross reactivity with IR and IGF-I-R), sensitive (125 pg the lowest HR amount measurable) and reproducible (intra and inter-assay variation coefficient $<10 \%$ ) ELISA for HR. HR were first immunocaptured by a bound solid phase anti-IR antibody and then specifically quantified by a biotynilated anti- IGF-I-R antibody. HR content was measured in skeletal muscle specimens from 9 obese subjects (Ob, BMI $>27 \mathrm{Kg} / \mathrm{m}^{2}$ ), 6 untreated non-obese NIDDM patients (fasting plasma glucose $8.7 \pm 1.3 \mathrm{nM} / \mathrm{l}$, BMI $28 \pm 2.9$, mean \pm S.E.M.) and 9 age and sex matched controls (C, BMI 24.7 \pm 0.8 ). Results: Muscle HR content was similar in $\mathrm{Ob}, \mathrm{NIDDM}$ and $\mathrm{C}(4.96 \pm 0.34,5.35 \pm 0.9,7.17 \pm 0.6 \mathrm{ng} / \mathrm{mg}$ protein, respectively, $\mathrm{p}=\mathrm{n} . \mathrm{s}$.). Moreover, when all 24 specimens were considered together, HR content tended to be negatively correlated with basal insulin levels, a marker of insulin resistance $(r=-0.40, p=0.056)$. Conclusion: These data indicate that HR content increase in skeletal muscle is not a consistent feature in obese and NIDDM patients and, therefore, it is not a relevant factor in determining insulin resistance in these patients.

\section{9}

THE EXSPRESSION AND ROLE OF THE INSULIN-LIKE GROWTH FACTOR I RECEPTOR IN MALIGNANT HEMANGIOPERICYTOMAS T.Čabrijan', Z.P.Pavelić, V.Zjačić1, V.Goldoni', Ž.Čabrijan², V.Altabas', S.Kaštelan' and K.Pavelićs. 'Department of Internal Medicine, Clinical Hospital "Sestre Milosrdnice", 2Department of Internal Medicine, Clinical Hospital "Dubrava", "BDivision of Molecular Medicine, Ruđer Bošković Institute, HR-10000 Zagreb, Croatia.

Hemangiopericytomas (HPC) are rare soft tissue tumors originating from contractile pericapillary cells-pericytes, very often associated with severe hypoglycemia. The aim of the study was to analyse the insulin-like growth factors (IGFs) in 19 tumors collected from our human tumor bank network. Seven of them were associated with severe hypoglycemia. Six of these were retroperitoneal and one was located in the leg. To address this issue we were using tissue specimens by routine histology and immunohistochemistry, RNA extraction, reverse transcriptase-polymerase chain reaction (PCR), IGF I binding and cultivation of HPC cells. 11 of 19 tumors were positive for IGF II mRNA $(57,9 \%)$. Almost $90 \%$ of HPC-s expressed IGF I receptor mRNA (17 out of 19). All of $19 \mathrm{HPC}$-s examined were IGF IR positive with regard to specific receptor binding. The potential role of IGF IR as a growth promoting factor in malignant HPC was studied using antisense oligonucleotides and monoclonal antibody $\alpha$ IR3 that specifically inhibit IGF IR synthesis or activity. Ten $\mu$ M IGF IR antisense oligonucleotides significantly inhibited the growth of HPC cells in culture by $\sim 50 \%$. Monoclonal antibody against IGF IR ( $\alpha$ IR3) also significantly inhibited HPC proliferation. Taken together, our results clearly show that IGF IR is expressed in almost all hemangiopericytomas examined and that inhibition of receptor activity can impede tumor proliferation in vitro. The data suggest that IGF IR may play an important role in the genesis and progression of malignant hemangiopericytomas. 


\section{0}

THE ADRENERGIC REGULATION OF LIPOLYSIS AND BLOOD FLOW IN HUMAN SKELETAL MUSCLE IN VIVO

E. Hagström-Toft, S. Enoksson', E. Moberg, J. Bolinder and P. Arner. Department of Medicine and the Research Center, 'Department of Vascular Surgery, Huddinge Hospital, Karolinska Institute, S-141 86 Huddinge, Sweden.

A marked lipolytic activity in human skeletal muscle, which can be stimulated in vivo by catecholamines, has recently been demonstrated but little is known about its regulation. Therefore, adrenergic regulation of lipolysis and blood flow was investigated in healthy, normal weigh subjects in vivo, using microdialysis of the gastrocnemius muscle. First, the muscle was locally perfused with beta-adrenoceptor blocking agents during a hypoglycaemic, hyperinsulinaemic clamp, which induces a lipolytic response (increased glycerol levels) in skeletal muscle tissue, due to an increase in circulating catecholamines. Perfusion with nonselective (propranolol) and beta ${ }_{2}$-selective (ICI 118,551) blocking agents, respectively, counteracted the hypoglycaemia-induced lipolysis $(\mathrm{p}<0.01$ for both drugs), whereas perfusion with metoprolol (beta - -blocker) did not affect the glycerol response. Secondly, selective beta-adrenoceptor agonists were perfused in situ into skeletal muscle during resting conditions. Beta $a_{2}$-adrenoceptor stimulation with terbutaline induced a concentration-dependent increase in skeletal muscle glycerol levels and in tissue blood flow (ethanol clearance), whereas perfusion with beta,

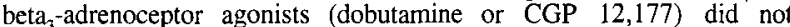
influence the glycerol concentration or blood flow. Local perfusion with the alpha,-adrenoceptor blocker yohimbine did not affect adrenalineinduced increase in dialysate glycerol levels but attenuated adrenalinemediated vasoconstriction $(\mathrm{p}<0.01)$. In conclusion, in skeletal muscle tissue only the beta ${ }_{2}$-subtype is of importance among beta-adrenoceptors for regulation of lipolysis and blood flow. This is in contrast to adipose tissue, where beta - and beta ${ }_{3}$-adrenoceptors are also involved. Alpha adrenoceptors seem to be of importance mainly for blood flow regulation.

\section{1}

FUNCTIONAL ACTIVITIES OF THE MURINE AND RAT PROXIMAL PROMOTER REGIONS OF THE GLUCAGON RECEPTOR GENE IN A RAT MEDULLARY THYROID CARCINOMA CELL LINE

A. Geiger, G. Salazar and A. Kervran. INSERM U376. CHU A. de Villeneuve. Rue Doyen G. Giraud. 34295 Montpellier Cedex 5. France.

The rat hepatoma (FAO, FTO2B) and the insulinoma (RINm5F, RINT3) cell lines, which derive from tissues expressing the glucagon receptor, have lost their sensitivity to glucagon. The CA-77 cell line, derived from a rat medullary carcinoma of the thyroïd, expresses this receptor and has been used to determine the transcriptional activities of murine and rat upstream regions of the glucagon receptor gene. Using RT-PCR, glucagon receptor mRNA was observed in CA-77 cells and was undetectable in hepatoma and insulinoma cells. Primer extension assay indicated that a major transcription start site (TSS) was located 174 base pairs upstream from the ATG in the rat gene. Transient transfection in CA-77 cells of different length constructs (spanning region -799 bp to +16 bp from TSS) of the rat 5 -flanking region in front of luciferase gene indicated that a sequence extending 35 bp upstream from TSS was sufficient for maximal trancription activity. Using mouse proximal promoter constructs $(-571$ bp to -1 bp from TSS), the maximal activity was reached with a region extending $63 \mathrm{bp}$ upstream from TSS. A sequence of $20 \mathrm{bp}$, in the mouse proximal promoter region, was able to increase in a copy-dependent manner the activity of the heterologous minimal thymidine kinase promoter. In conclusion, these data point out a dramatic decrease in glucagon receptor mRNA expression in rat hepatoma and insulinoma cells. In CA-77 cells, transfection of a limited region of the proximal murine and rat glucagon receptor promoters were sufficient for maximal expression of the reporter gene constructs. In the murine promoter, a 20 bp sequence might be the target of transcription factors that regulate positively the glucagon receptor gene.

\section{2}

HIGH GLUCOSE MODULATES NUCLEOTIDE RECEPTOR-ACTIVATED RESPONSES IN HUMAN SKIN FIBROBLASTS

A. Solini, P. Chiozzi* R. Fellin and F. Di Virgilio*, Departments of Internal Medicine II and General Pathology*, University of Ferrara, Ferrara, Italy

The physiological role of purinergic receptors (PR) of the P2Y and P2X subtypes is still unknown, although their involvement in many cellular function (proliferation, cytokine secretion, insulin secretion, cell death) has been proposed. They are expressed and have been characterized in neuronal cells and tumor lines, while little information is available about their presence in human primary cells. Aim of this study was a) to characterize PR in human skin fibroblasts (HF), that share several features with smooth and striated muscle cells and are directly involved in chronic degenerative diseases such as atherosclerosis, and b) to explore the potential role of different glucose concentrations (GluC) in modulating their activity. In $\mathrm{HF}$ from forearm skin biopsies grown at 5.5 and 22 mM GluC, we evaluated: a) immunoreactivity by Western blot with a specific anti P2X7 antiserum; b) stimulation of plasma membrane depolarization by benzoyl-benzoyl-ATP (BzATP); and c) activation of $\mathrm{Ca}^{2+}$ influx by intracellular trapping of Fura2-AM. We found that HF express an atypical P2X7 receptor, since stimulation with ATP or BzATP caused a slow but relevant uptake of lucifer yellow and no significant cytotoxicity (LDH release $<3 \%$ in 6 hours) BzATP also induced a striking increase in cytoplasmic microvesicle formation that did not appear to be due to increased pinocytosis and was fully reversible upon nucleotide removal. The pharmacological sequence for plasma membrane depolarization was BzATP $>$ CTP $>$ ATP $=$ ATPY $S=2$ MeATP $(100>52>>11$ $=12=12 \%$ ). Oxidised-ATP and, to lesser extent PPADS, were inhibitors Depolarization triggered by CTP, in contrast to that caused by BzATP, was fully inhibited by removal of extracellular $\mathrm{Ca}^{2+}$. Cells also expressed a yet to be characterized G-protein-coupled P2Y receptor, as shown by the fast release of stored $\mathrm{Ca}^{2+}$. The pharmacological sequence for $\mathrm{Ca}^{2+}$ release was: ATP $\geq \mathrm{CTP}$ $>>$ BzATP $=$ GTP $=$ UTP $=$ ATPY $S(278 \geq 240 \gg 160=163=170=152 \mathrm{nM})$ ADP, 2MeS-ATP and $\alpha \beta \mathrm{Me}-\mathrm{ATP}$ were inactive. $\mathrm{HF}$ at $22 \mathrm{mM}$ Glu, even in absence of changes in protein expression, showed a striking enhancement of all nucleotide-dependent changes (ATP depolarization: $+50 \%$ respect to $5.5 \mathrm{mM}$ glucose; $\mathrm{Ca}^{2+}$ release: $419 \mathrm{nM}$ ). We suggest that $\mathrm{HF}$ express a P2Y receptor and an atypical P2X7 ATP-gated channel and that high glucose modulates functional responses mediated by $P R$ 


\section{Glucose Transport}

\section{3}

INSULIN AND IGF-I REGLLATION OF GLUCOSE METABOLISM IN ADULT SKELETAL MUSCLE CELLS EXPRESSING EPITOPE-TAGGED GLUT4 A.B. Bentz, R.M. Shymko, P. De Meyts and C. Reynet, Hagedorn Research Institute, Gentofte, Denmark

Insulin is a major physiological stimulus of glucose transport in skeletal muscle, and acts mainly by translocating GLUT4 glucose transporters from intracellular vesicles to the plasma membrane. Insulin-like growth factor type I (IGF-I) appears also to be important in several aspects of muscle cell metabolism. We examined the acute effect of insulin and IGF-I on glucose uptake, GLUT4 translocation and glycogen synthesis in $\mathrm{C} 2 \mathrm{C} 12$ myoblasts stably expressing GLUT4 tagged with a myc-epitope in the first exofacial loop of the transporter. We measured in parallel the rate of 2deoxyglucose (2-DG) uptake and cell surface GLUT4myc content (by myc-antibody binding) in intact cells upon $20 \mathrm{~min}$ incubation with increasing concentrations of insulin or IGF-I. Both insulin and IGF-I stimulated 2-DG uptake and cell surface GLUT4myc content in a dose-dependent manner with a direct correlation between the increase in 2-DG uptake and in GLUT4 translocation $\left(\mathrm{R}^{2}=0.94\right.$ and 0.92 upon insulin and IGF-I stimuli, respectively). In both assays, maximal stimulation with insulin and IGF-1 were 2- and 2.2-fold above basal respectively, but IGF-I was approximately 10-times more potent than insulin in increasing 2-DG uptake $\left(\mathrm{ED}_{50}-2\right.$ $\mathrm{nM}$ for insulin, $\mathrm{ED}_{50} \sim 0.2 \mathrm{nM}$ for IGF-I) and GLUT4 translocation $\left(\mathrm{ED}_{50} \sim 7 \mathrm{nM}\right.$ for insulin, $\mathrm{ED}_{\mathrm{s}_{0}} \sim \mathrm{nM}$ for IGF-I). We then assayed the rate of glycogen synthesis after 1 hour incubation with insulin or IGF-I. Both ligands stimulated glycogen synthesis in a dose-dependent manner with similar maximum effects (1.9-fold and 2-fold above basal respectively, basal value $=59.3+6.6 \mathrm{pmol}$ glucose $/ \mathrm{min} / \mathrm{mg}$ protein), and interestingly insulin and IGF-I seemed equally potent in increasing glycogen synthesis $\left(\mathrm{ED}_{50} \sim 0.2 \mathrm{nM}\right)$. Furthermore, we compared the binding of insulin, highaffinity insulin analog X92, IGF-I and $\operatorname{des}(1-3)$ IGF-I on cell monolayers at equilibrium. Competitive binding data, analyzed by fitting to a one-site binding model, showed the presence of 30 to 40 -times more IGF-I than insulin receptors at the cell surface. In conclusion, IGF-I appears not only to stimulate metabolic signaling through the insulin receptor, as usually believed, but clearly in these cells through its own receptor, although both ligands may also stimulate their heterologous receptors and possibly insulin/IGF-I hybrids receptors

705

INVOLVEMENT OF BRADYKININ IN EXERCISE INDUCED INCREASE OF GLUCOSE UPTAKE IN RAT SKELETAL MUSCLE T. Taguchi, M. Uehara, K. Kaneko, T. Yano, E. Araki, H. Kishikawa, and M. Shichiri, Department of Metabolic Medicine, Kumamoto University School of Medicine, Kumamoto, Japan

We have previously demonstrated that bradykinin (BK) could potentiate insulin induced glucose uptake in dog skeletal muscle and rat L6 myocytes. In this study, we evaluated whether BK was involved in exercise induced glucose uptake in rat skeletal muscle. Saline or HOE140 (bradykinin B2 antagonist; $500 \mu \mathrm{g} / \mathrm{kg} /$ day) was subcutaneously infused in male Wister rats for 5 days using osmotic minipump. Three groups of rats were prepared as follows : 1) Sedentary with saline injection (S), 2) Exercise with saline injection (E; swimming for $1 \mathrm{~h}$ ), and 3) Exercise with HOE-140 injection (E-H). Before and after exercise, their blood glucose levels were measured. Blood glucose levels decreased significantly after exercise in $E$ group (from $84 \pm 3$ to $78 \pm 2 \mathrm{mg} / \mathrm{dl} ; \mathrm{p}<0.05$ ), while increased significantly from $85 \pm 2$ to $107 \pm 13 \mathrm{mg} / \mathrm{dl}$ in E-H group $(\mathrm{p}<0.05)$. Furthermore, to estimate glucose metabolism in vitro, 2-deoxyglucose uptake and GLUT4 translocation were evaluated using isolated skeletal muscle from these rats. E group and E-H group showed significant $(\mathrm{p}<0.05)$ increase in 2-deoxyglucose uptake in purified plasma membrane as compared with S group $(190 \%, 171 \%$, and $100 \%$ in E, E$\mathrm{H}$, and $\mathrm{S}$ group, respectively). Although there was no significant difference in the total GLUT4 contents of the muscle homogenate among three groups, GLUT4 translocation in plasma membrane was significantly $(\mathrm{p}<0.05)$ higher in E group (194\%) than in S group and E-H group (100\% and $152 \%$, respectively). In conclusion, BK may be involved in the modulation of the exercise induced GLUT4 translocation in rat skeletal muscle, resulting in the decrease of blood glucose during exercise.

\section{4}

RELATIONSHIP BETWEEN GLUCOSE TRANSPORTER 4 (GLUT4) AND FIBER TYPE IN HUMAN MUSCLE.

M.Gaster*, P.Poulsen", A.Handberg', H.D. Schrøder* and H. Beck-Nielsen". Dept Of Pathology*, Dept. Of Medical Endocrinology", Odense University Hospital, Odense. Dept. Of Medical Physiology', University of Copenhagen, Copenhagen, Denmark.

Insulin stimulated glucose uptake is positively correlated with the percentage of slow-oxidative fibres in skeletal muscle in man, suggesting that GLUT4 is expressed more abundantly in oxidative than in glucolytic muscle fibres. Our aim was to investigate the distribution pattern of GLUT4 in slow vs. fast fibres in sections of skeletal muscle biopsies from healthy fasting volunteers and after a $2 \mathrm{~h}$. hyperinsulinemic, euglycemic clamp $(40 \mathrm{mU} / \mathrm{m} 2 / \mathrm{min}$.). GLUT4 immunoreactivity was visualised by an immunoperoxidase reaction and the fibre types by an immunophosphatase reaction (slow $=$ WB-MHCs, fast $=$ my 32) and analysed by stereology. Only GLUT4 immunoreactivity sites associated with the plasma membrane were counted. The fiber type area in each field was estimated by pointcount. The expression of GLUT4 in the two fiber types was estimated by dividing the number of GLUT4-immunoreactive sites in each fiber type by its area. $(n=4$ 8 biopsier). We found that GLUT4 expression in slow fibres obtained in the basal condition was $3.01(2.83-3.23)$ compared to $2.33(2.01-2.92)$ in fast fibres (mean, range) ( $p<0.025$, students t-test). After insulin stimulation GLUT4 expression were $3.43(3.00-3.80)$ vs. $2.16(1.91-2.28)$ in slow vs fast fibres $(p<0.02)$. Insulin stimulation did not significantly change GLUT4 expression in slow nor in fast fibres. Thus, by measuring GLUT4 immunoreactivity in individual fibres of human skeletal muscle, we were able to demonstrate that GLUT4 is more abundantly expressed in slow compared to fast fibres. These results could partially explain the variations found in insulin stimulated glucose uptake among healthy subjects by variations in fiber type composition.

\section{6}

GLUCOSE UPTAKE IN ADIPOCYTES CAN BE STIMULATED BY $\mathrm{Ca}^{++}$-RELEASE AND BY NITROGEN OXIDE

A.R. Sørensen, S. Rasmussen, G.M. Danielsen, and P. Kurtzhals, Novo Nordisk A/S, Novo Alle, 2880 Bagsvaerd, Denmark Glucose uptake in both muscle and fat is stimulated by insulin In muscle, both contraction and nitrogen oxide (NO) also stimulate glucose transport by distinct pathways which are independent of both insulin and PI3 kinase. To examine whether similar pathways exist in fat, we have here studied the effect of chemical stimulation mimicking the contraction- and NO-stimulation in muscle on 2-deoxyglucose (2-DG) uptake in primary adipocytes from mice. Sodium nitroprusside (SNP) served as NO donor, and N-(6-aminohexyl)-5-chloro-1naphtalenesulfonamide (W7) as $\mathrm{Ca}^{++}$-releasing agent. The inhibitors dantrolene (DAN) and $N^{6}$-monomethyl-L-arginine (LNMMA) for $\mathrm{Ca}^{++}$-release and NO synthase, respectively, were included in the study. These inhibitors did not alone change the glucose uptake. SNP $(10 \mathrm{mM})$ and W7 $(100 \mu \mathrm{M})$ increased 2-DG uptake $2.3 \pm 0.3$ fold and $1.6 \pm 0.1$ foid, respectively. The maximal stimulation with insulin was $6.6 \pm 1.3$ fold. DAN and $L$ NMMA had no effect on SNP and W7 stimulated 2-DG uptake. In contrast, the $\mathrm{PI} 3$ kinase inhibitor LY294002 reduced the stimulation of 2-DG uptake by both insulin, SNP and W7 in a concentration dependent manner. We conclude that glucose uptake in adipocytes can be stimulated by $\mathrm{Ca}^{+*}$-release and by NO as in muscle, though to a smaller degree, and that in adipocytes the pathways through which these stimuli increase glucose uptake are PI3-kinase dependent. 


\section{7}

RESPECTIVE MOLECULAR EFFECTS OF INSULIN AND METFORMIN ON GLUCOSE

UPTAKE USING THE XENOPUS OOCYTE MODEL AS EXPRESSION SYSTEM.

D. Detaille ${ }^{1}$, P. Devos ${ }^{1}$ and N. Wiernsperger ${ }^{2}$. ${ }^{1}$ University of Namur, Belgium. ${ }^{2}$ Lipha, Lyon, France

The goal of this study was to better define, in molecular terms, the role of metformin on glucose transport and transporters since a failure at this level characterizes diabetes in the target cells of insulin. The experimental approach consisted in injecting Xenopus oocytes with the in vitro synthesized RNA encoding the muscle transporter GLUT4. The uptake of 2-deoxy-D-glucose by both uninjected and injected oocytes were then carried out under conditions of initial velocity to allow for the determination of kinetic constants. Studies achieved with uninjected cells clearly indicate that $10 \mu \mathrm{M}$ metformin, when combined with $2 \mu \mathrm{M}$ insulin, has no effect on the apparent $\mathrm{Km}$ for hexose ( $2.4 \mathrm{vs} 2.1 \mathrm{mmol} / \mathrm{h}$ ) but elicites a further increase in the $V \max \left(63.5\right.$ vs $45.4 \mathrm{pmol}$ oocyte $\mathrm{e}^{-1} \mathrm{~min}^{-1}$ ). On the other hand, oocytes expressing the protein GLUT4 show a markedly enhanced hexose transport activity. Kinetic analysis however reveals that the response to insulin was similar to that observed for the endogenous oocyte carrier. Indeed, oocytes transfected with GLUT4 and then exposed to insulin show only a twofold increase in their capacity to take up 2-deoxyD-glucose $\left(107.1 \pm 23.4\right.$ vs $\left.56.1 \pm 13.9 \mathrm{pmol}_{\text {oocyte }}-1 \mathrm{~min}^{-1}, \mathrm{n}=4\right)$. Moreover, in the presence of metformin, we mainly observed a decrease in the $\mathrm{Km}$ for hexose transport ( 3.3 vs $2.4 \mathrm{mmol} / /$ ), the Vmax being weakly affected. These findings are consistent with the notion that, in transformed oocytes, insulin contributes to the recruitment of new transporters to the oocyte cell surface whereas the drug induces the functional activation of the protein GLUT4. This hypothesis has been partly explored by measuring the transport activity after a prior treatment of oocytes with parathyroid hormone (PTH), a substance known to impair the intrinsic activity of GLUT4 in adipocytes. Interestingly, we observe that $100 \mathrm{ng} / \mathrm{ml}$ PTH eliminates the full stimulatory action of metformin on glucose uptake whereas the insulin effect is only partially prevented. The whole data strongly support the idea that metformin improves the glucose homeostasis by altering profoundly the intrinsic properties of pre-existing or newly synthesized glucose curriers.
708

INCREASED GLUCOSE UPTAKE IN SOLEUS MUSCLE OF HYPOINSULINEMIC EUGLYCEMIC MICE

M Ruiz ${ }^{2}$, F Martin ${ }^{2}$ and B Soria ${ }^{1}$. Departments of Physiology ${ }^{1}$ and Science and Tecnology ${ }^{2}$ and Institute of Bioengineery ${ }^{1,2}$, Campus of San Juan, Universidad Miguel Hernández, 03550 San Juan, Alicante, Spain.

The main aim of the study was to investigate the effects mechanisms of adaptation to a reduction of about $50 \%$ in serum insulin levels with maintenance of euglycemia in an animal model. We studied 8-12 week old male Swisss mice in which a $60^{\circ}$ pancreatectomy causes a decrease of serum insulin of about $50 \%$ from controls with maintenance of euglycemia. We studied 3 groups: Px: $60 \%$ pancreatectomized mice; Ps: "sham" pancreatectomized mice, and $\mathrm{CtI}$ : control mice. 15 days after pancreatectomy the soleus muscles were surgically removed for studies. Studies of glucose uptake were made with the method of dilution of ${ }^{3} \mathrm{H}-2-\mathrm{DOG}$, by incubating the muscles with insulin $0 \mathrm{nmol} / /$ (basal) and $1.25 \mathrm{nmol} /$ (insulin estimulated). For calculation of GLUT-4 Km and Vmax we used GLUT-4 enriched muscle membrane vesicles obtained after muscle digestion with collagenase and $\mathrm{KCl}$. GLUT-4 was measured by Western blot using a monoclonal antibody. Basal glucose uptake was not significantly different in Ctl $(13.87 \pm 1.11 \mathrm{nmol} / \mathrm{g} ; \mathrm{n}=7)$; Ps $(12.91 \pm 1.85 \mathrm{nmol} / \mathrm{g}$. $\mathrm{n}=4)$, and $\mathrm{Px}(10.36 \pm 1.49 \mathrm{nmol} / \mathrm{g} ; \mathrm{n}=6)(\mathrm{p}>0.05)$. Insulin estimulated glucose uptake was not significantly different in $\mathrm{Ctl}(57.81 \pm 6.54 \mathrm{nmol} / \mathrm{g} ; \mathrm{n}=7)$ and $\mathrm{Ps}(79.25$ $\pm 19.46 \mathrm{nmol} / \mathrm{g} ; n=4)(p>0.05)$ but it was significantly greater in $P x(152.89 \pm 15.46$ $\mathrm{nmol} / \mathrm{g} ; \mathrm{n}=4)$ than $\mathrm{Ctl}(\mathrm{p}<0.0001)$ and Ps $(\mathrm{p}<0.01)$. GLUT-4 Km was not significantly different in $\mathrm{Ctl}(16.5 \pm 0.5 \mathrm{mM} ; \mathrm{n}=3)$ and $\mathrm{Px}(18.4 \pm 2.1 \mathrm{mM} ; \mathrm{n}=5)$ GLUT -4 Vmax was significantly different in $P x(0.242 \pm 0.017 \mathrm{pmol} / \mathrm{ug} / 300 \mathrm{~s} ; \mathrm{n}=4)$ from $\mathrm{Ctl}(0.041 \pm 0.002 \mathrm{pmol} / \mathrm{ug} / 300 \mathrm{~s} ; \mathrm{n}=4)(\mathrm{p}<0.001)$. Basal GLUT-4 was not different in CtI group $(0.8 \pm 0.1 \mathrm{OD}, n=4)$ and $\mathrm{Px}(0.9 \pm 0.07 \mathrm{OD} ; \mathrm{n}=4)(\mathrm{p}>0.05)$ Insulin (1.25 nmol/l) increased GLUT-4 in both Ctl ( $1.72 \pm 0.09 \mathrm{OD}, \mathrm{n}=4)$ and $\mathrm{Px}$ $(1.84 \pm 0.12 \mathrm{OD} ; \mathrm{n}=4)(\mathrm{p}<0.05)$. We conclude that in this animal model there is an adaptation to hypoinsulinemia consisting of a doubling of insulin estimulated glucose uptake that is, at least in part, dependent on a change in GLUT-4 Vmax but not in the total number of GLUT-4 recruited to the plasma membrane

\section{PS 23}

\section{Gastro-Entero-Pancreatic Factors}

\section{9}

\section{INTRAPORTAL GLP-1 ADMINISTRATION AUGMENTS THE INSULIN RESPONSE TO PORTAL GLUCOSE VIA NON-MUSCARINIC NERVES B. Balkan and X. Li. Novartis Pharmaceuticals Corp., Summit, NJ} The incretin glucagon-like peptide 1 7-36 amide (GLP-1) is an important factor in prandial glucose homeostasis. Besides the prominent augmentation of insulin release to glucose, several tissues have been suggested as direct targets. The liver is the first organ to be encountered by endogenously released GLP-1 and absorbed nutrients. Hepatocytes have been reported to increase glycogen synthesis upon stimulation with GLP-1. We therefore studied whether GLP-1 in the portal vein (PV) would alter the disposition of a PV glucose load. PV GLP-1 has been shown to increase the firing rate of hepatic vagal afferent nerves. We therefore also determined whether any effects of PV GLP-1 could be prevented by nervous blockade. Studies were performed in conscious, chronically catheterized, overnight fasted rats. PV GLP-1 (10pmol/kg, n=9) augmented the integrated insulin response (area under the curve, AUC in $\mu \mathrm{U} / \mathrm{ml}^{*} 10 \mathrm{~min}$ ) to an PV glucose bolus $(500 \mathrm{mg} / \mathrm{kg})$ by $47 \%(2108 \pm 243 \mathrm{vs} 1162 \pm 119, p<0.01)$. This effect was reversed by pre-treatment with the ganglionic blocker chlorisondamine $\mathrm{HCl}(3 \mathrm{mg} / \mathrm{kg}, i N$, AUC: $1298 \pm 142)$, but was not affected by peripheral muscarinic blockade $(0.5 \mathrm{mg} / \mathrm{kg}$ methyl-atropine, $i v, n=7$, AUC: 2039 \pm 316 ). PV injection of GLP-1 markedly improved the glucose

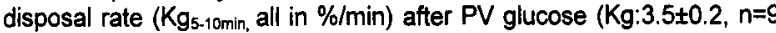
vs $2.7 \pm 0.3$ in controls, $n=7, p<0.05$ ). Ganglionic blockade again reversed the effects of the GLP-1 injection (Kg:2.8 $\pm 0.3, n=5)$. It is concluded that PV GLP-1 greatly affects handling of a PV glucose load This effect appears to be mediated by augmented insulin release However, the data suggest that $B$-cell stimulation is evoked via a neura reflex that may be triggered in the liver and is non-muscarinic in nature. Since absorbed nutrients and GLP-1 first appear in the portal system this mechanism may constitute a major pathway of GLP-1 action during meals.

\section{0}

GLP-1 INCREASES SECRETORY BURST MASS OF PULSATLE INSULIN SECRETION IN PATIENTS WITH IMPAIRED GLUCOSE TOLERANCE AND TYPE 2 DIABETES R. Ritzel ${ }^{1}$, M. Schulte ${ }^{1}$, M. Nauck ${ }^{2}$, W. März ${ }^{2}$, N. Pørksen ${ }^{3}$, W. Schmiegel ${ }^{1}$ and M.A. Nauck', 'Med. Univ,-Klinik, Knappschafts-KH, Bochum, Germany, ${ }^{2}$ Clinical Chemistry, Freiburg, Germany, ${ }^{3}$ Dep. of Endocrinology and Metabolism, Aarhus, Denmark

The gut hormone GLP-1 increased secretory burst mass and amplitude of pulsatile insulin secretion in healthy volunteers, whereas burst frequency was not affected. The influence of GLP-1 on parameters of pulsatile insulin secretion in patients with impaired glucose tolerance (IGT) and Type 2 diabetes has not yet been studied. Therefore eight Type 2 diabetic patients ( $64 \pm 9$ years, BMI $28.9 \pm 7.2$ $\left.\mathrm{kg} / \mathrm{m}^{2}, \mathrm{HbA}_{1 \mathrm{c}} 7.9 \pm 1.4 \%\right)$ and eight patients with impaired glucose tolerance $(63 \pm$ 10 years, $\left.\mathrm{BMl} 31.7 \pm 6.4 \mathrm{~kg} / \mathrm{m}^{2}, \mathrm{HbA}_{1 \mathrm{c}} 5.7 \pm 0.5\right)$ were studied in the fasting state after / during i.v. GLP-I (Saxon Biochemicals, Hannover, FRG, $1.2 \mathrm{pmol}^{-1} \mathrm{~kg}^{-1} \mathrm{~min}^{-1}$, started at 10:00 p.m. in the evening before) or placebo and compared to eight healthy volunteers $\left(62 \pm 7\right.$ years, BMI $\left.27.7 \pm 4.8 \mathrm{~kg} / \mathrm{m}^{2}, \mathrm{HbA}_{\mathrm{lc}} 5.4 \pm 0.5\right)$. Blood was sampled continuously over $60 \mathrm{~min}$ (roller-pump) in l-minute fractions for the measurement of plasma glucose and insulin. The detection and quantification of pulsatile insulin secretion was performed by deconvolution analysis. Statistics: ANOVA, t-Tests (significance: $\mathrm{p}<0.05$ ). GLP-1 normalised plasma glucose concentrations $(6.4 \pm 2.1 \mathrm{mmol} / \mathrm{l}$ vs. placebo $9.8 \pm 4.1 \mathrm{mmol} / 1)$ in Type 2 diabetic patients. GLP-1 significantly increased insulin burst mass (Type 2 diabetes: placebo $26.3 \pm 4.7$, GLP-1 $44.2 \pm 6.8$ pmoll $^{-1}$ pulse ${ }^{-1}, p=0.007$; IGT: placebo $31.0 \pm 5.6$, GLP-1 $45.1 \pm 8.3$ pmoll $^{-1}$ pulse ${ }^{-1}, \mathrm{p}=0.019$ ) and burst amplitude (Type 2 diabetes: placebo $11.1 \pm 1.8, \mathrm{GLP}-117.6 \pm 2.7 \mathrm{pmoll}^{-1} \mathrm{~min}^{-1}, \mathrm{p}=0.006$; IGT: placebo $13.3 \pm$ 2.1, GLP-1 $18.4 \pm 3.2 \mathrm{pmol}^{1-1} \mathrm{I}^{-1} \mathrm{~min}^{-1}, \mathrm{p}=0.02$ ). Insulin secretory burst frequency was not affected by GLP-1 (Type 2 diabetes: placebo $9.5 \pm 0.9$, GLP-1 $10.9 \pm 0.8$ pulses/hour, $\mathrm{p}=0.15$; IGT: placebo $10.4 \pm 0.8$, GLP-1 $10.1 \pm 0.6$ pulses/hour, $\mathrm{p}=$ 0.76 ). In conclusion, intravenous GLP-1 normalises plasma glucose in Type 2 diabetic patients and increases oscillations of basal insulin secretion by selective amplification of insulin secretory burst mass. 
711

LONG-LASTING ANTIDIABETIC EFFECT OF A DIPEPTIDYL PEPTIDASE IV-RESISTANT ANALOGUE OF GLP-1.

B. Thorens, W. Dolci and R Burcelin. Institut of Pharmacology and Toxicology, Lausanne, Switzerland

Glucagon like peptide one (GLP-1) stimulates insulin secretion in a glucose dependent manner. Its insulinotropic activity is preserved in non-insulin-dependent diabetic patients and allows a complete correction of diabetic hyperglycemia. The therapeutic use of this peptide is however limited by its short half-life due to rapid in vivo degradation by dipeptidylpeptidase IV (DPPIV). To overcome this draw back we report that replacing alanine at position 8 of the peptide by glycine made the peptide (GLP-1Gly8) resistant to proteolysis by DPPIV as monitored by HPLC and a biological assay. This change slightly decreased the affinity of the peptide for its receptor (IC50 $0.4 \pm 0.1$ and $1.4 \pm 0.6 \mathrm{nM}$ for GLP-1 and GLP-1-Gly8, respectively) but did not change the efficiency to stimulate accumulation of intracellular CAMP (EC50 $0.25 \pm 0.5$ and $0.36 \pm 0.06 \mathrm{nM}$ for GLP-1 and GLP-1-Gly8, respectively). To determine the in vivo effects of GLP-1-Gly8, we generated glucose intolerant $\mathrm{C} 57 \mathrm{Bl} / 6 \mathrm{~J}$ mice by feeding them a high-fat, sugar-free, diet. An acute intraperitoneal injection of GLP-1-Gly8 could efficiently normalize glucose tolerance and fasting hyperglycemia even when injected up to 4 hours before initiation of glucose tolerance tests whereas the effect of GLP-1 was lost even when injected 10 minutes before glucose challenge. The effect of GLP-1-Gly8 correlated with a persistant increase in insulin and decrease in glucagon levels. In conclusion, GLP-1Gly8 has very significantly improved therapeutic capabilities as compared to that of the GLP-1. GLP-1-Gly8 represents a more promissing peptide than GLP-1 for the treatment of NIDDM since it could be used at much lower doses and with a more flexible schedule of administration.

\section{3}

AMINOPEPTIDASE RESISTANT GLYCATED GLUCAGON-LIKE PEPTIDE1[7-36]AMIDE SHOWS POTENT INSULINOTROPIC ACTION IN VIVO

M. Mooney, C.M.N. Kelly, P.R. Flatt and F.P.M. O'Harte. School of Biomedical Sciences, University of Ulster, Coletaine, N. Ireland, UK.

The insulinotropic action of the gut hormone glucagon-like peptide-1(7-36)amide (tGLP-1) is well established. This study examined the degradation of tGI.P-1 and glycated tGLP- 1 in plasma and effects on insulin release and glucose homeostasis in vivo. Amino-terminally glycated tGLP-1 (identified by mass spectrometry $M_{r}$ 3463.8) was prepared under hyperglycaemic reducing conditions and purified by reversed-phase HPLC. tGLP-1 or glycated tGLP-1 $(10 \mu \mathrm{g})$ were incubated for various times $\left(0-4 \mathrm{~h}\right.$ at $\left.37^{\circ} \mathrm{C}\right)$ with human plasma $(20 \mu \mathrm{l})$ in $50 \mathrm{mmol} / \mathrm{l}$ tricthanolamine $/ \mathrm{HCl}$ buffer ( $\mathrm{pH} \mathrm{7.8,} \mathrm{final} \mathrm{volume} 0.5 \mathrm{ml}$ ) and reaction mixtures separated on HPLC. After $4 \mathrm{~h}$ incubation $54 \%$ of tGLP-1 remained intact and the remainder was converted to GLP-1(9-36)amide. Breakdown of tGLP-1 was substantially reduced ( $85 \%$ intact) by diprotin A ( $5 \mathrm{mU})$ an inhibitor of dipeptidyl-peptidase IV. In contrast, glycated tGLP1 was completely resistant to in vitro degradation in plasma up to $4 \mathrm{~h}$. Acutc effects were tested in vivo in Wistar rats $(250-300 \mathrm{~g}, \mathrm{n}=6)$ following i.p. injection of each peptide $(40 \mu \mathrm{g} / \mathrm{kg})$ with glucose $(18 \mathrm{mmol} / \mathrm{kg})$. Glucose administration alone (control) resulted in peak plasma concentrations of (mean \pm SEM) $21.4 \pm 1.4 \mathrm{mmol} / \mathrm{l}$ for glucose and $16.3 \pm 1.7 \mathrm{ng} / \mathrm{ml}$ for insulin after $15 \mathrm{~min}$. However, following tGLP1 and glycated tGLP-1 peak glucose concentrations $(15 \mathrm{~min})$ were $14.2 \pm 1.5$ and $15.1 \pm 0.6 \mathrm{mmol} / 1$ and insulin concentrations $26.0 \pm 1.8$ and $24.8 \pm 2.9 \mathrm{ng} / \mathrm{ml}$, respectively. The area under the curve (AUC) for glucose from 0 to $90 \mathrm{~min}$ following tGLP-1 $(358.1 \pm 36.5 \mathrm{mmol} / \mathrm{h} / \mathrm{h})$ or glycated tGLP-1 $(271.2 \pm 27.1)$ were significantly lower $(p<0.01)$ than after glucose alone $(565.3 \pm 43.4)$. Significantly higher AUC for plasma insulin concentrations were recorded for both tGLP-1 $(666.3 \pm 27.9 \mathrm{ng} / \mathrm{ml} / \mathrm{h})$ and glycated tGLP- 1 (643.1 \pm 24.3$)$ compared to controls $(p<0.001,337.0 \pm 19.3$ ). No significant differences were found between the AUC for tGLP- 1 and glycated tGL.P-1. In conclusion N-terminally glycated tGLP-1 is resistant to aminopeptidase degradation and retains its insulinotropic and antihyperglycaemic activities.

\section{2}

GLYCATED GASTRIC INHIBITORY POLYPEPTIDE EXHIBITS AMINOPEPTIDASE RESISTANCE AND ENHANCED INSULINOTROPIC ACTIVITY.

F.P.M. O'Harte, M. Mooney and P.R. Flatt. School of Biomedical Sciences, University of Ulster, Coleraine, N. Ireland, UK

Gastric inhibitory polypeptide (GIP) is an important element of the enteroinsular axis which enhances postprandial glucose-stimulated insulin release. This study examined the degradation of GIP and glycated GIP in plasma and effects upon insulin release and glucose homeostasis in rats. Monoglycated GIP (identified by mass spectrometry $M_{T}$ 5147.2) was prepared under hyperglycaemic conditions in the presence of sodium cyanoborohydride and purified by RP-HPLC. GIP or glycated GIP $(10 \mu \mathrm{g})$ were incubated for various times $\left(0.4 \mathrm{~h}\right.$ at $\left.37^{\circ} \mathrm{C}\right)$ with human plasma $(20 \mu \mathrm{l})$ in $50 \mathrm{mmol} / \mathrm{l}$ tricthanolamine $/ \mathrm{HCl}$ buffer $(\mathrm{pH} 7.8$, final volume $0.5 \mathrm{ml})$. The reaction was terminated by acidification and the products scparated by HPLC. After $4 \mathrm{~h}$ incubation $71 \%$ of GIP and $99 \%$ of glycated GIP remained intact. The breakdown to GIP(3-42) was almost completcly abolished in the presence of diprotin A ( $5 \mathrm{mU})$ an inhibitor of dipeptidyl-peptidase IV (DPP-IV). Acute effects of GIP and glycated GIP were tested in Wistar rats $(250-300 \mathrm{~g}, \mathrm{n}=6)$ following combined i.p. injection of peptide ( 50 $\mu \mathrm{g} / \mathrm{kg}$ ) with glucose $(18 \mathrm{mmol} / \mathrm{kg}$ ). Glucose administration alone (control) resulted in peak plasma concentrations of (mean \pm SEM) $21.4 \pm 1.4 \mathrm{mmol} / \mathrm{l}$ for glucose and $16.3 \pm 1.7 \mathrm{ng} / \mathrm{ml}$ for insulin after $15 \mathrm{~min}$. However, following GIP and glycated GIP these plasma parameters rose to $13.9 \pm 0.9$ and $10.7 \pm 0.6 \mathrm{mmol} / \mathrm{l}$ for glucose and $22.9 \pm 3.0$ and $21.0 \pm 1.5 \mathrm{ng} / \mathrm{ml}$ for insulin. respectively. The area under the curvo (AUC) for gluciose from 0 to $90 \mathrm{~min}$ following i.p. GIP $(311.2 \pm 25.2 \mathrm{mmol} / / \mathrm{h})$ was significantly lower $(p<0.001)$ than for glucose controls $(565.3 \pm 43.4)$. Furthermore the AUC for glucose after i.p. glycated GIP $(210.7 \pm 31,1)$ was lower compared to AUC for controls $(p<0.001)$ and GIP $(p<0.05)$. Significantly higher AUC for plasma insulin concentrations were recorded for both GIP $(479.7 \pm 22.9 \mathrm{ng} / \mathrm{ml} / \mathrm{h})$ and glycated GIP $(503.3 \pm 29.7)$ compared to controls $(p<0.01,337.0 \pm 19.3)$. In conclusion Nterminally glycated GIP is resistant to degradation by plasma DPP-IV activity and has cnhanced insulinotropic and antihyperglycaemic actions in vivo.
714

LY315902: ENHANCING THE ACTIVITY AND TIME ACTION OF GLUCAGON-LIKE PEPTIDE-1 (GLP-1) IN VIVO

S.R. Myers, J.E. Baker, C.L. Broderick, M.D. Clephane, V.K. Williams, and R.K Workman. Lilly Reasearch Laboratories, Indianapolis, IN, USA. While clinical studies have demonstrated efficacy of the intestinal hormone GLP-1 in patients with type 2 diabetes, its rapid inactivation by dipeptidylpeptidase IV and its rapid plasma clearance limit the use of native GLP-I as a pharmaceutical entity. LY315902 (IP7,R26,K34(C8) GLP-1), a protease-protected, fatty-acid-acylated analog of GLP-1, was designed to overcome these defects. To examine LY315902 in vivo, 3-hour euglycemic and byperglycemic $(8.3 \mathrm{mmol} / \mathrm{l})$ clamp studies were conducted in chronically cannulated, overnight-fasted dogs. Sixty minutes after initiation of the clamp, a subcutaneous bolus ( $3 \mathrm{nmol} / \mathrm{kg}$ ) of GLP-1(7-37)OH or LY315902 was administered, and the activity (insulin change area under the curve; ICAUC) and time action of the peptides were compared. Combining both fatty acid acylation of GLP-1 with protease protection (LY315902) more than doubled the activity and time action of the compound without compromising the glucose dependence of the peptide (see table). These data: 1 ) suggest that protecting GLP-1 from protease inactivation and decreasing its plasma clearance can lead to a morc pharmaceutically relevant form of GLP-1 and 2) support the development of LY315902 as a potential treatment for type 2 diabetes.

\begin{tabular}{|c|c|c|c|}
\hline & $n$ & ICAUC $(\mathrm{nmol} / 1 \bullet \min )$ & Time Action (min) \\
\hline \multicolumn{4}{|c|}{ Euglycemic clamp } \\
\hline Vehicle & 5 & $-0.2 \pm 1.2$ & $0 \pm 0$ \\
\hline GLP-1 & 5 & $1.0 \pm 1.4$ & $2 \pm 1$ \\
\hline LY315902 & 5 & $0.9 \pm 1.9$ & $0 \pm 0$ \\
\hline \multicolumn{4}{|c|}{ Hyperglycemic clamp } \\
\hline Vehicle & 9 & $4.0 \pm 2.4$ & $5 \pm 5$ \\
\hline GLP-I & 10 & $12.8 \pm 2.8 *$ & $24 \pm 7$ \\
\hline LY315902 & 8 & $25.1 \pm 3.8 * \dagger$ & $76 \pm 14 * 1$ \\
\hline
\end{tabular}


715

IMPAIRED POSTPRANDIAL RELEASE OF GLUCAGON-LIKE PEPTIDE1 IN PATIENTS WITH TYPE 2 DIABETES

J. Lindqvist, J. Pigon, J.J. Holst and S. Efendic. Dept. of Endocrinology and Diabetology, Karolinska Hospital, Stockholm, Sweden

GLP-1 is released from intestinal endocrine L-cells in response to orally administered mutrients, especially carbohydrates and fat. The aim of the present study was to characterise the effect of a standardised $621 \mathrm{kcal}$ mixed meal on the release of glucose, insulin, C-peptide, glucagon, glucose-dependent insulinreleasing polypeptide (GIP) and GLP-1 in terms of total Area under Curve (AUC) during $3 \mathrm{~h}$ after the meal. In the study participated 20 well-matched normalweight (BMI 20-26) subjects; 7 healthy control subjects, and 13 patients with type 2 diabetes of whom 6 patients had an acceptable metabolic control on diet treatmen alone and 7 patients experienced secondary failure on oral sulphonylurea (SU) Highly specific radioimmunoassays, determining only biologically active hormone, were used for the investigation. Logarithmically transformed data were compared using Dunnett's test for multiple comparison of means. As expected, postprandial glucose levels were significantly elevated in both diabetes groups during the entire investigation. Insulin levels proved lower in the SU failure group as compared to controls only during the first 90 minutes of the investigation $(13,993$ vs. 32,001 , $p<0.01$ ), as did $C$-peptide levels in both diabetes groups (102 and 127 vs. 202 $\mathrm{p}<0.01$ and $\mathrm{p}<0.05$, respectively). Glucagon, however slightly elevated in the diabetes groups (total area 7,390 and 6,184 vs. 4,699), and GIP (total area 9,28 and 9,873 vs. 10,392 ) were insignificantly different from controls. The GLP-1 response during the last 90 minutes and the total 180 minutes was markedly decreased and delayed in the SU failure group (area 90-180 minutes $960 \mathrm{vs} .1,511$ total area 1,856 vs. $2,826, p<0.05$ ), while the diet-treated group displayed an intermediary release of GLP-1. This alteration in GLP-1 response may play a role in the pathogenesis of glucose intolerance, and specifically the decreased insulin and enhanced glucagon secretion characterising this disease

\section{7}

INITIATION OF PANCREATIC BETA CELL PROLIFERATION IN YOUNG MICE

A. Edvell and P. Lindström. Department of Histology and Cell

Biology, Umeå University, Sweden

Compensatory beta cell proliferation in response to increased metabolic demand has been observed in several animal models. The aim of this study was to identify factors related to increased food intake and increased insulin release, that can initiate such compensatory growth

To do this, we have studied islet cell BrdU- and ${ }^{3} \mathrm{H}$-thymidine-labeling index in young obese-hyperglycemic mice (Umea ob/ob) and their lean littermates. Double staining with insulin antibodies show that islet cell proliferation is largely confined to beta cells. We find that ob/ob mice show an elevated beta cell proliferation rate at 20 days of age, and that the rise in blood glucose starts at day 22.20 days old lean normoglycemic mice were used to study factors that can initiate beta cell proliferation. Islet cell labeling index was enhanced from $1.6 \pm 0.17 \%(n=18)$ to $3.3 \pm 0.4 \%(n=13, P<0.01)$ when $40 \mathrm{nmol} / \mathrm{kg}$ GLP-1 was injected subcutaneously for two days, and to $3.1 \pm 0.5 \%$ $(\mathrm{n}=12, \mathrm{P}<0.02)$ when $5.4 \mathrm{nmol} / \mathrm{kg}$ human C-peptide was injected. L5 -Hydroxytryptophan, $375 \mu \mathrm{mol} / \mathrm{kg}$ inhibited the proliferation. After culture islet cell proliferation rate was the same in lean and obese mouse islets also when cultured with $10 \%$ serum from the obese littermates. When cultured in RPMI medium containing $27.6 \mu \mathrm{mol} / /$ C-peptide an enhanced islet cell labeling index was observed $(3.2 \pm 0.6$ $\%$ vs $1.4 \pm 0.3 \%$ for controls, $n=8, P<0.02$ ). We conclude that beta cell proliferation in response to metabolic demand can be triggered by substances involved in the physiological regulation of insulin release other than glucose, such as GLP-1. C-peptide may function as a mediator of such responses.
716

GLUCAGON-LIKE PEPTIDE-1 STIMULATES INSULIN SECRETION AND GLUCOSE EFFECTIVENESS IN MICE.

G Pacini and B Ahrén, LADSEB-CNR, Padova, Italy and Dept Medicine, Lund University, Malmö, Sweden

Glucagon-like peptide-1 (GLP-1) has been proposed as a new treatment modality for diabetes due to its potent insulinotropic activity. Whether GLP-1 in addition affects glucose uptake is still subject of debate. We therefore evaluated its effect on glucose disposal in normal mice. Non-fasted female NMRI mice were injected iv with glucose $(1 \mathrm{~g} / \mathrm{kg}$ ) with or without synthetic human GLP-1 at doses ranging from $10 \mathrm{pmol} / \mathrm{kg}$ to $100 \mathrm{nmol} / \mathrm{kg}$. Seven blood samples $(75 \mu 1)$ were taken within $50 \mathrm{~min}$, and glucose and insulin data were analysed with the minimal model technique. GLP-1 markedly and dose-dependently increased insulin secretion with a maximal effect obtained at 30 $\mathrm{nmol} / \mathrm{kg}\left(\mathrm{AUC}_{\text {insulin }} 188 \pm 29(\mathrm{n}=26)\right.$ vs. $51 \pm 6 \mathrm{nmol} / \mathrm{lm}$ in in controls $(\mathrm{n}=34)$, $\mathrm{p}<0.001$ ). Concomitantly, the iv glucose tolerance index was markedly increased by GLP-1 $\left(\mathrm{K}_{G} ; 5.2 \pm 0.3\right.$ vs $\left.2.8 \pm 0.2 \% / \mathrm{min}, \mathrm{p}<0.001\right)$. Also glucose effectiveness $\left(\mathrm{S}_{\mathrm{G}}\right)$ was increased by the peptide $(0.053 \pm 0.006$ vs. $0.036 \pm 0.003 \mathrm{~min}^{-1}, \mathrm{p}<0.012$ ), due to increased glucose effectiveness at zero insulin (GEZI; $0.048 \pm 0.006$ vs. $0.015 \pm 0.004 \mathrm{~min}^{-1}, \mathrm{p}<0.001$ ), whereas basal insulin effectiveness was reduced by GLP- $1(0.005 \pm 0.001$ vs. $0.021 \pm 0.003$ $\left.\min ^{-1}, \mathrm{p}<0.001\right)$. In contrast, the insulin sensitivity index $\left(\mathrm{S}_{\mathrm{I}}\right)$ was reduced by GLP-1 $\left(0.14 \pm 0.02\right.$ vs. $\left.0.58 \pm 0.0810^{-4} \mathrm{~min}^{-1} / \mathrm{pmol} / 1, \mathrm{p}<0.001\right)$. The GLP-1 receptor antagonist, exendin ${ }_{9,39}$, inhibited the actions of GLP-1. We conclude that GLP-1 is a potent insulinotropic agent which stimulates glucose elimination and glucose effectiveness, but inhibits both dynamic and basal insulin sensitivity in mice. The marked potentiation of insulin secretion and the exaggeration of glucose elimination verify that GLP-1 might be useful in the treatment of diabetes. The inhibition of insulin sensitivity might counteract its antidiabetogenic action, but may also be of advantage to protect against hypoglycemia when GLP-1 is given at high doses.

\section{8}

GASTROINTESTINAL EFFECTS OF PRAMLINTIDE

M. Denaro, B. Gedulin, C. Jodka and A. Young. Amylin Pharmaceuticals, Inc., 9373 Towne Centre, San Diego, CA, 92121, USA

Pramlintide is a synthetic analogue of human amylin, a peptide hormone cosecreted with insulin from pancreatic -cells in response to nutrient stimuli. Amylin regulates gastric acid secretion, gastric emptying, exocrine pancreatic secretion and postprandial glucagon secretion. To investigate the effect of pramlintide on gastrointestinal function, we examined its effects on pentagastrin-stimulated acid secretion and on cholecystokinin octapeptide (CCK-8) stimulated secretion of lipase and amylase. To study acid secretion, rats chronically implanted with gastric cannulae were injected s.c. with $125 \mu \mathrm{g} / \mathrm{kg}$ pentagastrin and $\mathbf{4 0} \mathrm{min}$ later with s.c. saline or with pramlintide $(0.1$, $1,10 \mu \mathrm{g} / \mathrm{rat} ; n=9,7,3,6$ ). Gastric contents obtained by flushing the cannulae every $10 \mathrm{~min}$ were titrated to measure acid production and the aggregate acid secreted over the next 60 min measured. Pramlintide suppressed gastric acid secretion by up to $93.8 \pm 1.4 \%(P<0.001)$ with an $E D_{50}$ of $0.0061 \mu \mathrm{g} \pm 0.43 \log$ units (at least as potent as amylin, for which this is a physiological effect). To study exocrine pancreatic secretion, halothane-anesthetized Sprague Dawley rats were cannulated via common bile and pancreatic ducts so that bile flow drained into the duodenum while exocrine pancreatic secretion was collected. Pancreatic juice was collected every $15 \mathrm{~min}$ from $t=-30$ to $t=+90 \mathrm{~min}$ after subcutaneous injection of $1 \mu \mathrm{g}$ CCK-8. Total secreted lipase and amylase activity was measured every $15 \mathrm{~min}$. Pramlintide injected s.c. five min before CCK-8 injection at doses $0.01,0.03,0.1$ and $0.3 \mu \mathrm{g}(n=2,5,5,14)$ in comparison with saline injections $(n=13)$ suppressed CCK-8 stimulated pancreatic amylase activity respectively by $38 \%, 34 \%, 42 \%$ and $25 \%(P<0.05$ $0.01,0.01$ and 0.01 , respectively) and lipase activity by $55 \%, 49 \%, 51 \%$ and $34 \%,(P<0.01,0.02,0.01$ and 0.006 , respectively) without affecting basal enzyme secretion. These doses are calculated from separate kinetic studies to result in plasma pramlintide concentrations ranging from $\sim 5$ to $\sim 30 \mathrm{pM}$. Thus, in addition to its reported effects to slow gastric emptying and inhibit postprandial glucagon secretion, pramlintide also inhibits, with similar potency, pentagastrin-stimulated gastric acid secretion and CCK-stimulated secretion of lipase and amylase from the exocrine pancreas in the rat. We propose that through this spectrum of effects, pramlintide may coordinately regulate the rate of nutrient appearance in plasma. 
MODY3 MUTATIONS AFFECT TRANSCRIPTIONAL ACTIVITY OF HEPATOCYTE NUCLEAR FACTOR I ALPHA (HNF1 $\alpha$ ).

M. Vaxillaire ${ }^{1,2}$, A. Abderrahmani ${ }^{1}$, P. Boutin ${ }^{1}$, B. Bailleul ${ }^{1}$, M. Yaniv ${ }^{2}$, P. Froguel $^{1}$ and M. Pontoglio ${ }^{2}{ }^{1}$ Institut de Biologie de Lille, CNRS EP10, Institut Pasteur, Lille and ${ }^{2}$ Unité des Virus Oncogènes, CNRS URA 1644, Institut Pasteur, Paris, France.

Hepatocyte nuclear factor 1 alpha $(H N F 1 \alpha$ ) is an atypical dimeric homeodomain-containing protein that is expressed in liver, intestine, stomach, kidney and pancreas. Mutations in the HNF1 $\alpha$ gene are associated with an autosomal dominant form of non-insulin-dependent diabetes mellitus called maturity-onset diabetes of the young (MODY3). More than 60 different mutations have been so far identified spanning the functional domains of HNF1 $\alpha$, many of which involve highly conserved amino acid residues among vertebrates. The present work aimed to investigate the molecular mechanisms by which MODY 3 mutations could affect HNFl $\alpha$ function. For this purpose, we generated eleven mutants resulting from amino acid substitutions or protein truncation, and characterized their transcriptional activation capacity, nuclear localization, protein stability and DNA binding. We show that all these mutations decrease or abolish transcriptional activity of HNFl $\alpha$ in transient transfection assays. MODY3 mutants can be classified into three different groups: decreased transactivation (Class I), loss of transactivation (Class II) and loss of transactivation with a dominant negative effect on the wild type protein (Class III). The functional defects are caused either by decreased protein stability, defective DNA binding or impaired intrinsic transactivation. These data suggest that MODY 3 could be elicited by two different mechanisms: loss of function mutation resulting in haploinsufficiency, and formation of a dominant negative protein. Moreover, our analysis sheds new light on the structure of a homeoprotein playing a key role in pancreatic $\beta$ cell function.
Chronic Exposure of HIT-T15 Cells to Supraphysiologic glucose Concentrations Decreases Activity of the Hepatocyte Nuclear Factor one Response Element.

A. Abderrahmani, M. Vaxillaire*, C. Rouault*, M. Pontoglio*, G. Reach*, M. Yaniv*, P. Froguel, B. Bailleul and V. Poitout*, Lille and Paris *, France.

Binding activity of the HNF-1 (Hepatocyte Nuclear Factor one) is decreased in hepatocytes from diabetic rats, and is recovered upon normalization of blood glucose. This study was designed to investigate the effects of prolonged exposure of insulin-secreting cells to elevated glucose concentration on HNF-1 activity. HIT-T15 cells were chronically cultured in 0.8 or $11.1 \mathrm{mM}$ glucose for 35 passages. Electromobility shift assays failed to demonstrate any differences in the binding activity of nuclear proteins extracted from both conditions to a nucleotide probe containing an HNF-1 binding site. Late passage HIT-T15 cells were transfected with chloramphenicol acetyl transferase reporter genes controled either by the 5 -regulatory regien of the human insulin gene (INSCAT), or by a multimerized HNF-1 response element (B28CAT). As expected, relative expression of INSCAT / RSVLUC was 20-fold lower in cells chronically cultured in high glucose $(5.1 \pm 0.4$ vs. 100 $\pm 15, n=3, p<0.01$ ). In addition, relative expression of B28CAT / RSVLUC was 10-fold lower in cells chronically cultured in high glucose than in cells chronically cultured in low glucose $(11 \pm 8$ vs. $100 \pm 37, n=3, p<0.05)$. We conclude that chronic exposure of insulin-secreting HITT15 cells to elevated glucose concentrations markedly decreases the activity of the HNF-1 response element, with no change in the binding activity of cognate factors.

\section{PS 24 \\ Insulin Resistance: Tissue and Cellular Level}

\section{1}

RESISTANCE OF GLUCONEOGENESIS TO SHORT-TERM REGULATION BY INSULIN IN CULTURED OBESE ( $f a / f a$ ) RAT HEPATOCYTES.

J.J. Carrillo, M.C.G. Lechuga, A. Esteban-Gamboa and J.E. Felíu. Departamento de Bioquímica, Facultad de Medicina, Universidad Autónoma de Madrid. Spain.

We have previously reported that the impaired short-term regulation of liver glucose production by insulin in the obese Zucker ( $f a / f a$ ) rat is due, at least in part, to the increase in the hepatic content of fructose 2,6bisphosphate (F26P) and to changes in key regulatory enzyme activities of the glycolytic/gluconeogenic pathway in response to the chronic hyperinsulinemia present in these animats. The aim of the present study was to investigate the short-term modulation of gluconeogenesis (GNG) by insulin in primary cultures of obese $(f a / f a)$ and lean $(F a /-1$ rat hepatocytes, maintained in the absence of insulin. Cells were cultured for 24 hours in $\mathrm{M}-199$ with $10 \mathrm{mM}$ glucose, T3 $(1 \mu \mathrm{mol} / \mathrm{l})$ and dexamethasone $(1 \mu \mathrm{mol} / \mathrm{l})$. Glucokinase, 6-phosphofructo 1 -kinase pyruvate kinase and 6-phosphofructo 2-kinase $a$ (PFK2a) activities, as well as the concentration of F26P, were significantly higher in obese than in lean rat hepatocytes, while the phosphoenolpyruvate carboxykinase activity was lower. In good agreement with these findings, GNG (lactate/pyruvate, $10 / 1 \mathrm{mmol} / \mathrm{l}$ ) was decreased in obese rat hepatocytes $(11.1 \pm 1.8$ vs $26.2 \pm 1.5 \mathrm{nmol}$ glucose $/ \mathrm{mg}$ protein $x$ $30 \mathrm{~min}$, respectively, for obese and lean rat cells). In cultured lean rat hepatocytes, insulin caused a dose-dependent inhibition of GNG, together with a significant increase in both PFK2a activity and F26P concentration (maximal effects: $25 \%, 23 \%$ and $20 \%$ vs the basal values, at $10 \mathrm{nmol} / 1$ insulin, respectively). In contrast, in obese rat hepatocytes, insulin did not significantly modify any of these metabolic parameters. In conciusion, our results show that primary cultures of obese $\{\mathrm{fa} / \mathrm{fa}\rangle$ rat hepatocytes maintain -for at least 24 hours- the glycolytic/gluconeogenic phenotype characteristic of the liver of adult obese $(\mathrm{fa} / \mathrm{fa}\rangle$ rats, together with a marked resistance of GNG to shortterm modulation by insulin.

\section{2}

PHYSIOLOGICAL GROWTH HORMONE REPLACEMENT CAUSES LIVER INSULIN RESISTANCE ASSOCIATED WITH LIPOLYSIS

S. D. Mittelman and R. N. Bergman, University of Southern California, Los Angeles, CA, United States

It is known that elevation of growth hormone levels can cause insulin resistance in vivo. Since we have previously shown that the majority of insulin action to suppress endogenous glucose production (EGP) is mediated via insulin suppression of lipolysis, the present study was performed to test A) whether physiologic growth hormone $(\mathrm{GH})$ levels can cause liver insulin resistance, and $B$ ) whether these changes may be due to GH stimulation of lipolysis. The effects of GH on EGP and lipolysis were examined under both basal and hyperinsulinemic conditions. Euglycemic clamps were performed on conscious dogs $(n=6)$, with somatostatin (1 $\mu \mathrm{g} / \mathrm{kg} / \mathrm{min})$. Insulin $(0.3 \mathrm{mU} / \mathrm{kg} / \mathrm{min})$ and glucagon $(1.0 \mathrm{ng} / \mathrm{kg} / \mathrm{min})$ were replaced portally throughout the basal period $(-150$ to $0 \mathrm{~min}$ ). Canine growth hormone was infused at either a "replacement" dose (hGH, 15 $\mathrm{ng} / \mathrm{kg} / \mathrm{min}$ ), or saline was given as a control. At $\mathrm{t}=0$, the portal insulin infusion rate was increased to $1.5 \mathrm{mU} / \mathrm{kg} / \mathrm{min}$. Both FFA and EGP were

\begin{tabular}{|c|c|c|c|}
\hline \multirow{2}{*}{ Basal } & $\begin{array}{c}\text { EGP } \\
(\mathrm{mg} / \mathrm{kg} / \mathrm{min})\end{array}$ & $\begin{array}{l}\text { FFA } \\
(\mathrm{mM})\end{array}$ & \multirow{7}{*}{$\begin{array}{l}\text { at each insulin dose (see } \\
\text { Table). This study shows } \\
\text { that physiological levels } \\
\text { of growth hormone can } \\
\text { cause insulin resistance of } \\
\text { both the liver and the } \\
\text { adipocyte. These resuits } \\
\text { are consistent with the } \\
\text { hypothesis that insulin } \\
\text { acts indirectly to suppress } \\
\text { EGP via suppression of }\end{array}$} \\
\hline & & & \\
\hline \multirow{2}{*}{$\begin{array}{c}\text { With GH } \\
\text { No GH } \\
\text { Increment } \\
\text { due to GH } \\
\text { Hyperinsuline }\end{array}$} & $\begin{array}{l}2.4 \pm 0.3 \\
1.9 \pm 0.2\end{array}$ & $\begin{array}{l}0.65 \pm 0.11 \\
0.43 \pm 0.07\end{array}$ & \\
\hline & $\operatorname{mia}^{0.4 \pm 0.2}$ & 0.2 & \\
\hline With GH & $1.0 \pm 0.5$ & 0.1 & \\
\hline No GH & $0.5 \pm C$ & 0.04 & \\
\hline $\begin{array}{l}\text { necrer } \\
\text { due to }\end{array}$ & $0.5 \pm 0.4$ & $0.11 \pm 0.04$ & \\
\hline
\end{tabular}


ACUTE EFFECTS OF SPECIFIC FATTY ACIDS ON INSULIN-MEDIATED GLUCOSE METABOLISM IN ISOLATED SOLEUS MUSCLE.

A.L. Thompson, M.Y.C. Lim-Fraser, E.W. Kraegen and G.J. Cooney Garvan Institute of Medical Research, Darlinghurst, NSW Australia 2010

Skeletal muscle insulin resistance is associated with increased triglyceride storage and increased intramuscular long chain acyl CoAs, but the mechanism by which lipids influence insulin action is unclear. To investigate any direct interaction between specific lipid species and insulin action in skeletal muscle. the effects of fatty acids on insulin-mediated glucose metabolism were determined in vitro. Soleus muscle strips from Wistar rats were preincubated, for up to 6 hours, at $37^{\circ} \mathrm{C}$ in the presence of $5.5 \mathrm{mM}$ glucose and fatty acid. Control muscles were preincubated in the absence of fatty acid for the same length of time. After preincubation, muscles were transferred to $3 \mathrm{ml}$ of similar medium (without fatty acid) that also contained radioactive tracers and insulin (10 (basal) or 300 (stimulated) $\mu \mathrm{U} / \mathrm{ml}$ ) to assess glucose uptake (GU) and glycogen synthesis (GS). Insulinstimulated GS was decreased to $51 \%$ of control $(p=0.005)$ in a time-

\begin{tabular}{cccccc|cccc} 
& Insulin & \multicolumn{3}{c|}{ Time with $2 \mathrm{mM}$ Palmitate $(\mathrm{h})$} & \multicolumn{4}{|c}{ Palmitate concentration (mM) } \\
& $(\mu \mathrm{UU} / \mathrm{ml})$ & 0 & 2 & 4 & 6 & 0 & 0.5 & 1 & 2 \\
\hline GU & 300 & 3.76 & 3.23 & 2.9 & 2.51 & 2.82 & 2.94 & 2.72 & 2.89 \\
& & \pm 0.40 & \pm 0.41 & \pm 0.25 & \pm 0.11 & \pm 0.27 & \pm 0.29 & \pm 0.31 & \pm 0.34 \\
GS & 300 & 1.39 & 0.81 & 0.69 & 0.71 & 1.62 & 1.43 & 1.16 & 1.00 \\
& & \pm 0.23 & \pm 0.15 & \pm 0.08 & \pm 0.07 & \pm 0.16 & \pm 0.20 & \pm 0.09 & \pm 0.08
\end{tabular}

Mean \pm SEM expressed as $\mu$ mol/h/g wet wt; $\mathrm{n}=6-9$

dependent manner. There was a small decrease in basal $(p=0.048)$ and insulin-stimulated $(\mathrm{p}=0.049)$ GU. Preincubation for $4 \mathrm{~h}$ with $0-2 \mathrm{mM}$ palmitate decreased insulin-stimulated GS dose-dependently $(\mathrm{p}<0.02)$ Oleate $(\mathrm{ImM})$ also decreased insulin-stimulated GS (control $1.88 \pm 014$ oleate $1.24 \pm 0.13 ; p<0.02$ ) whilst $1 \mathrm{mM}$ linoleate decreased GS (control 1.88 \pm 0.14 ; linoleate $0.91 \pm 0.07 ; \mathrm{p}<0.001$ ) and $\mathrm{GU}$ (control $3.60 \pm 0.14$ linoleate $2.67 \pm 0.35 ; \mathrm{p}<0.01$ ). The results indicate that fatty acids may affect glucose transport/phosphorylation and glycogen synthesis differentially. The effects of different fatty acids support a hypothesis that specific lipid metabolites may contribute to the induction of insulin resistance in muscle.

\section{5}

TROGLITAZONE DIRECTLY AND ACUTELY INHIBITS PALMITATE OXIDATION IN RAT SKELETAL MUSCLE.

C.Fümsinn, S.Neschen, B.Brunmair, M.Roden, P.Nowotny and W.WaldhăusI Dept.Med.III, Div.Endocrinol.Metab., University of Vienna, Austria.

Troglitazone is a potent insulin sensitizer when orally administered for several weeks. We have previously shown that troglitazone acutely stimulates skeletal muscle glucose metabolism in vitro in a non-insulinlike catabolic fashion ( $B r$ J Pharmacol 122, 1367-1374, 1997). We now investigated the effects of short-term $(90 \mathrm{~min}$ ) troglitazone exposure on palmitate oxidation by isolated rat soleus muscle. The drug exerted a very distinct inhibitory effect on insulin stimulated $(30 \mathrm{nmol} / /)$ palmitate oxidation with the maximal effect reached at $20 \mu \mathrm{mol} / \mathrm{h}$ troglitazone (relative change vs. intraindividual control: $5 \mu \mathrm{mol} / 1,-17 \pm 5 \%, p<0.01$; $10 \mu \mathrm{mol} / \mathrm{l}-14 \pm 5 \%, p<0.025 ; 20 \mu \mathrm{mol} / \mathrm{s},-52 \pm 2 \%, \mathrm{p}<0.0001 ; 40 \mu \mathrm{mol} / /$, $-44 \pm 4 \%, p<0.001 ; 80 \mu \mathrm{mol} / 1,+7 \pm 11 \%$, ns). The effect was obviously insulin-independent, because similar results were obtained in the absence of the hormone $(5 \mu \mathrm{mol} / \mathrm{l},-13 \pm 7 \%$, ns; $10 \mu \mathrm{mol} / \mathrm{l},-15 \pm 4 \%$, $\mathrm{p}<0.02 ; 20 \mu \mathrm{mol} / \mathrm{n},-36 \pm 5 \%, \quad \mathrm{p}<0.0001 ; 40 \mu \mathrm{mol} /,-20 \pm 7 \%, \quad p<0.05 ;$ $80 \mu \mathrm{mol} / /, \quad-3 \pm 5 \%$, ns). Inhibition of palmitate oxidation was accompanied by unchanged rates of glucose oxidation (relative change vs. intraindividual control at $20 \mu \mathrm{mol} / \mathrm{l}$ troglitazone: basal conditions: $-7 \pm 8 \%$, ns; insulin-stimulated conditions: $-17 \pm 10 \%, n s$ ), increased rates of lactate release (basal conditions: $+53 \pm 7 \%, p<0.0001$; insulinstimulated conditions: $+46 \pm 5, p<0.0001$ ), and decreased rates of glycogen synthesis (basal conditions: $-11 \pm 9 \%$, ns; insulin-stimulated conditions: $-30 \pm 6 \%, p<0.001$ ). In conclusion, acute troglitazone exposure markedly inhibits palmitate oxidation as accompanied by increased anaerobic glycolysis in isolated skeletal muscle. It remains to be elucidated in what way acute and direct inhibition of falty acid oxidation contributes to troglitazone's insulin sensitizing and antidiabetic action in vivo.
THE THIAZOLIDINEDIONE BM13.1258 INSULIN-INDEPENDENTLY INCREASES MUSCLE GLUCOSE OXIDATION IN OBESE RATS.

S.Neschen, B.Brunmair, M.Roden, W.Waldhäusl, and C.Fürnsinn Dept.Med.III, Div.Endocrinol.Metab., University of Vienna, Austria. BM13.1258 is " new thiazolidinedione compound with distinct antidiabetic efficacy. In the present study, we investigated the effects of BM13.1258 on skelatal muscle glucose metabolism. Soleus muscles prepared from insulin-resistant obese Zucker rats (fa/fa) treated or not with BM13.1258 for ten days (2 mg/d) were incubated in the absence or presence of $10 \mathrm{nmol} / \mathrm{insulin}$. BM13.1258 induced distinct insulinsensitization as reflected by an increase in insulin-stimulated glycogen synthesis (glucose incorporation into glycogen, $\mu \mathrm{mol} / \mathrm{g} / \mathrm{h}$ : control, $3.02 \pm$ 0.18 vs. BM $13.1258,4.26 \pm 0.28 ; p<0.002)$, but unchanged basal rates of glycogen synthesis (control, $1.82 \pm 0.22$ vs. BM13.1258, 1.76 \pm 0.16 ; $\mathrm{ns})$, what represents a more than doubled insulin-induced increment over basal (control, $+1.19 \pm 0.28$ vs. BM13.1258, $+2.50 \pm 0.20 ; p<0.002$ ). Insulin sensitization was accompanied by marked increases in the rates of glucose oxidation, which were of similar extent in the absence of insulin $\left(\mathrm{CO}_{2}\right.$ produced from nmol glucose/g/h: control, $421 \pm 27 \mathrm{vs}$. $B M 13.1258,806 \pm 90 ; p<0.001)$ and in the presence of insulin (control, $513 \pm 52$ vs. BM13.1258, 938 $\pm 58 ; p<0.0001$ ). Glucose transport showed a BM13.1258-dependent increase in basal rates (cpm ${ }^{3} \mathrm{H}-2$-deoxyglucose transport/mg/h: control, $254 \pm 14$ vs. BM13.1258, $313 \pm 12$; $p<0.01)$ and a more pronounced increase under insulin-stimulation (control, $501 \pm 24$ vs. BM13.1258, 655 $\pm 33 ; p<0.002$ ) hence reflecting the sum of increased substrate requirements associated with glycolytic and glycogenic flux. In conclusion, BM13.1258 affects muscle glucose metabolism not only by insulin sensitization, but also by eliciting a distinct increase in glucose oxidation, which revealed not to depend on the presence of insulin.

\section{6}

MUSCLE TRIGLYCERIDE AND GLYCOGEN CONCENTRATIONS IN MONOZYGOTIC AND DIZYGOTIC TWINS

M.Lehtovirta', E.Laurila ${ }^{\mathrm{I}}$, J.Kaprio"l and L.Groop". Dpts of Medicine' and of Public Health" ${ }^{\mathrm{W}}$, University of Helsinki, Finland and Dpt of Endocrinology", University of Lund, Malmo, Sweden.

Increased intramuscular triglyceride $(\mathrm{mTg})$ concentrations have been reported in insulin-resistant NIDDM subjects. It is, however, not known whether this represents a primary, genetic, feature or develops secondary to increased fat mass. To address this question we correlated mTG with measures of energy metabolism and body composition and estimated intra-twinpair correlation coefficients in 22 monozygotic

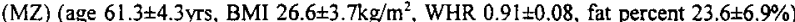
and 20 dizygotic (DZ) $\left(63.5 \pm 2.3 y \mathrm{rs}, 26.5 \pm 3.0 \mathrm{~kg} / \mathrm{m}^{2}, \quad 0.93 \pm 0.11,24.6 \pm 6.2 \%\right)$ nondiabetic twins, who participated in 3 studies each: 1) $75 \mathrm{~g}$ OGTT, 2) euglycemic hyperinsulinemic $\left(45 \mathrm{mU} / \mathrm{m}^{2}\right)$ clamp in combination with indirect calorimetry and 3) basal biopsy from the vastus lateralis muscle for the study of mTG and glycogen (G) concentrations. Results: There was no difference in mTG $(50.6 \pm 21.6$ vs $53.6 \pm 30.0$ $\mathrm{mmol} / \mathrm{kg}$ dry weight) or $G(360 \pm 101$ vs $327 \pm 90 \mathrm{mmol} / \mathrm{kg}$ dry weight) between $\mathrm{MZ}$ and DZ twins. mTG correlated with body fat mass $(r=0.44 ; p=0.0007)$, basal FFA $(r=0.39$; $\mathrm{p}=0.003)$ and glucose area during OGTT $(\mathrm{r}=0.37 ; \mathrm{p}=0.005)$. $\mathrm{G}$ correlated with fasting glucose $(r=0.44 ; 0.0008), F F M(r=0.32 ; 0.015)$, fasting insulin $(r=0.32 ; p=0.016)$ and body weight $(r=0.26 ; p=0.05)$. No associations were observed between $m T G$ or $G$ and insulin sensitivity or metabolic rate.

The intraclass correlation coefficients: $\quad M Z \quad P \quad D Z \quad P$ $\begin{array}{lllll}\text { The intraclass correlation coefficients: } & M Z & p & D Z & p \\ \text { Weight } & 0.68 & <0.0001 & 0.43 & 0.023\end{array}$

Fat percentage

Glucose uptake

$\begin{array}{rrrr}0.78 & 0.000 & 0.32 & 0.078 \\ 0.006 & -0.09 & 0.64\end{array}$ mTG

$\begin{array}{lllll}0 & 0.16 & 0.10 & 0.05 & 0.43\end{array}$

Conclusion: Both genetic (intrapair correlation in $\mathrm{MZ}$ twins) and environmental (correlation with fat mass) factors seem to contribute to $\mathrm{mTG}$ in man. Elevated $\mathrm{mTO}$ cannot explain skeletal muscle insulin resistance in these subjects. 
EICOSAPENTAENOIC ACID AMELIORATES INSULIN RESISTANCE, BUT INCREASES MUSCLE TRIGLYCERIDE, IN OLETF RATS

M. Kusunoki', T. Hara', T. Nakamura', H. Ogawa', F. Sakakaibara', M. Yazima', K. Asai', K. Usui', A. Iriyama', K. Yamanouchi', S. Kakumu', and L. Storlien ${ }^{3}$. ${ }^{1}$ First Department of Internal Medicine, Aichi Medical University, Japan; 'Laboratory of Biomedical Control, Hokkaido University, Japan and ${ }^{3}$ Metabolic Research Centre, University of Wollongong, Australia

Fish oils containing omega- 3 polyunsaturated fatty acids ( $n-3$ PUFAs) have been shown to be beneficial in rodents models of insulin resistance. The aim was to investigate the effects of eicosapentaenoic acid (EPA, 20:5 n-3) on insulin action in the obese and insulin resistant Otsuka Long Evans Tokushima Fatty (OLETF) rat. Eleven month old male OLETF rats were divided into 3 groups ( $n=6$ for all measures) fed either lab Chow or Chow supplemented with sufficient EPA to provide either 0.3 or 1.0 grams $/ \mathrm{kg}$ body weight/day. At the end of 10 weeks feeding hyperinsulinemic, euglycemic clamps were used to assess insulin action and soleus muscle was freeze-clamped for measurement of triglyceride (TG) and glycogen levels. EPA improved insulin action in a dose-dependent manner. glucose infusion rate to maintain euglycemia was: Chow $14.7 \pm 3.9 \mu \mathrm{mol} \cdot \mathrm{min}^{-1} \cdot \mathrm{kg}^{-1} ; 0.3 \mathrm{EPA}$ group 19.5 \pm 3.8 , and 1.0 EPA group 32.1 \pm 5.5 , p<0.01 vs. the other groups. Weight gain did not differ between groups. Circulating TG was decreased by EPA (Chow $2.7 \pm 0.2 \mathrm{mM} ; 0.3 \mathrm{EPA}$ group $2.4 \pm 0.2 ; 1.0 \mathrm{EPA}$ group $1.5 \pm 0.1, \mathrm{p}<0.01$ vs. the other groups). In contrast, muscle TG increased in the EPA-treated groups (Chow $29.9 \pm 3.4 \mathrm{mg} / \mathrm{g}$ wet weight; $38.7 \pm 6.5$ in the $0.3 \mathrm{EPA}$ group; and $42.0 \pm 2.5$ in the 1.0 EPA group, $\mathrm{p}<0.05$ vs the Chow group).Muscle glycogen did not differ between groups. In summary, EPA, a long-chain n-3 PUFA, improved insulin action in the aged OLETF rat even though it increased muscle TG levels. The positive effects of EPA may then relate either to increased incorporation of PUFAs into muscle membranes or to effects solely at the liver. In conclusion, dietary EPA has a beneficial effect on insulin action in the OLETF rat but the mechanism is not decreased muscle TG levels.
MUSCLE GLYCOGEN REGULATION IN THE EVOLUTION OF HUMAN OBESITY TO DIABETES

A. Golay, R. Munger, F. Assimacopoulos, E. Bobbioni, E. Jéquier and J.P. Felber. University Hospital Geneva and Institute of Physiology, Lausanne, Switzerland.

The purpose of the work was to study changes in muscle glycogen regulation in the evolution of obesity towards diabetes. A group of 33 obese patients was subdivided into 3 groups according to their degree of glucose intolerance: 12 with normal glucose tolerance (NGT), 7 with impaired glucose tolerance (IGT) and 14 with diabetes (D). They were compared to 11 nonobese subjects $(\mathrm{N})$. They were submitted to a euglycemic, hyperinsulinemic clamp associated with indirect calorimetry. Muscle needle biopsies were taken before and at the end of the 2-hour clamp for measurements of glycogen synthase (GS) and phosphorylase (GP) activity and glycogen concentration [gly]. This study confirms the gradual increase in plasma NEFA and decrease in glucose oxidation and storage in the evolution of obesity towards diabetes. In NGT, a negative correlation between [gly] and GS activity is observed $(r=0.837 ; p=0.003)$ and a positive correlation between [gly] and GP activity. In IGT, the negative correlation between [gly] and GS does not exist anymore as well as in the diabetic group; whereas the positive relationship between [gly] and GP is still significant $(r=0.917 ; p=0.001)$. In the diabetic group, the glycogen regulation does not exist anymore with no correlation between GS and GP. The endogenous regulation of glycogen concentration between [gly] and both GS and GP activity is progressively lost. The loss of this regulation is confirmed by the diminution of the sensitivity of GS to G-6-P and could be one explanation for the evolution from obesity towards diabetes.

\section{9}

\section{RAPID OSCILLATIONS IN OMENTAL LIPOLYSIS}

L. Getty, M. Dea, S. D. Mittelman, M. Ader, R. N. Bergman, University of Southern California, Los Angeles, CA, USA.

It has been shown that in the basal state, plasma insulin oscillates with a period of 8-14 min. Recently, we have shown that free fatty acids (FFA) also oscillate rapidly in plasma. This study was performed to further investigate the presence of rapid FFA oscillations and their association with insulin. Blood samples were drawn from $24 \mathrm{~h}$-fasted, conscious dogs at 1 min intervals for 1 hour $(n=4)$. Samples for measurement of FFA, glycerol, and insulin were taken simultaneously from the carotid artery and portal vein. The oscillatory frequency of each metabolite was determined by spectral analysis. Both arterial and portal insulin showed a rapid oscillation with a mean period of $9 \mathrm{~min}(5.5-11 \mathrm{~min})$. Arterial FFA and glycerol showed a rapid oscillation with a mean period of $5.8 \mathrm{~min}$ (5.0-6.2 $\mathrm{min})$. In all dogs, the FFA and glycerol concentrations were significantly higher in the portal vein than the carotid artery (FFA: $0.70 \pm .01 \mathrm{mM}$ arterial vs. $0.76 \pm .01 \mathrm{mM}$ portal, $\mathrm{p}<0.001$ for each dog; glycerol: $1.37 \pm .26 \mathrm{mg} / \mathrm{dl}$ arterial vs. $1.78 \pm .36 \mathrm{mg} / \mathrm{dl}$ portal, $\mathrm{p}<0.001$ for each $\operatorname{dog}$ ). Calculation of the portal-arterial difference for either FFA or glycerol allows for a direct measurement of omental lipolysis. The portalarterial gradient for both FFA and glycerol showed an oscillatory profile, suggesting that there is pulsatile lipolysis from the omentum. Spectral analysis showed that this pulsatile release is at a mean frequency of 5.5 $\min$ (4.4-8 $\mathrm{min}$ for both FFA and glycerol). It is possible that the pulsatile release of FFA from the omentum is driven by the rapid arterial insulin oscillation. However, for each dog the arterial insulin frequency did not correspond with the omental FFA or glycerol frequency. It is also possible that the oscillatory release of FFA from the omentum is controlled by a neural signal.

\section{0}

DIRECT MEASUREMENT OF THE LUMPED CONSTANT FOR 1 $\left[{ }^{14} \mathrm{C}\right]$-2-DEOXY-GLUCOSE IN VIVO IN HUMAN SKELETAL MUSCLE $T$. Utriainen, S. Lovisatti, S. Mäkimattila, A. Bertoldo, $R$. Bonadonna, S. Weintraub, R. DeFronzo, C. Cobelli and H. YkiJärvinen. University of Helsinki, Finland; Universities of Padova and Verona, Italy; University of Texas HSC at San Antonio, USA.

The lumped constant (LC) is used to convert clearance rates of 2 deoxy-D-glucose to those of glucose. There are currently no data to validate the widely used assumption of an LC of 1.0 for human skeletal muscle in positron emission tomography -studies. We determined the $L C$ for $1-{ }^{14} \mathrm{Cl}$-2-deoxy-glucose (2-DG) in 18 normal male subjects (age $29 \pm 2 \mathrm{yrs}$, BMI $24.8 \pm 0.8 \mathrm{~kg} / \mathrm{m}^{2}$ ) under fasting, physiological $(1 \mathrm{mU} / \mathrm{kg} \cdot \mathrm{min}$ insulin infusion for $180 \mathrm{~min}$ ) and supraphysiological (5 $\mathrm{mU} / \mathrm{kg} \cdot \mathrm{min}$ ) hyperinsulinemic conditions. Normoglycemia was maintained using the euglycemic clamp technique. The $L C$ was directly measured using a new triple tracer technique. $3-\left[{ }^{3} \mathrm{H}\right]$ glucose, $2-\mathrm{DG}$ and ${ }^{12} \mathrm{C}$-mannitol were injected as an intrabrachial artery bolus at $165 \mathrm{~min}$. The concentration of ${ }^{3} \mathrm{H}$ glucose and ${ }^{14} \mathrm{C}$-DG (dpm/ml plasma) and of ${ }^{12} \mathrm{C}$-mannitol $(\mu \mathrm{mol} / \mathrm{l}$, by gas chromatography mass spectroscopy) were determined in 50 blood samples withdrawn from the ipsilateral deep forearm vein over 15 minutes after the bolus injection. The clearance rate (clr) of each tracer was determined from the area under the washout curve divided by the dose. The LC was calculated from the equation (2-DG $-\mathrm{G}_{\mathrm{cr} r}-\mathrm{Man}_{\mathrm{ctr}}$ ) / ( $\left.{ }^{3} \mathrm{H}-\mathrm{Glu}_{\mathrm{clr}}-\mathrm{Man}_{\mathrm{crl}}\right)$. The LC averaged $1.26 \pm 0.08$ (range 1.06-1.43), $1.15 \pm 0.05 \quad(0.99-1.39$ ) and $1.18 \pm 0.05(0.97-1.37)$ under fasting conditions and during the $?$ and $5 \mathrm{mU} / \mathrm{kg} \cdot \mathrm{min}$ insulin infusions (NS between the different insulin concentrations, mean LC 1.2, $p<0.01$ vs 1.0 . In conclusion, the LC for 2-DG in human skeletal muscle is constant over a wide range of insulin concentrations and averages 1.2. 


\section{1}

NO REGIONAL DIFFERENCES IN THE SENSITIVITY OF THE SUBCUTANEOUS ADIPOSE TISSUE TO THE ANTI-LIPOLYTIC EFFECT OF INSULIN IN OBESE, DIABETIC AND NON-DIABETIC WOMEN.

J. R. Katz', C. Weyer', K. Rave', S. W. Coppack' and J. Yudkin'. 'Center for Diabetes and Cardiovascular Risk, Whittington Hospital, UCLMS, Archway Road, London N19 3UA, UK and ${ }^{2}$ Dept Metabolic Diseases and Nutrition, University of Duesseldorf, Moorenstr. 5, 40225 Duesseldorf, Germany

Regional differences in subcutaneous adipose tissue (SAT) lipolysis may play a role in the disposition to predominately abdominal (abd) or femoro-gluteal (fem) fat accumulation. Local differences between both SAT sites were found in their sensitivity to the lipolytic effect of exercise. In the present study we aimed to investigate whether the sensitivity of SAT to the anti-lipolytic effect of insulin is a) different between the abd and fem region and b) related to whole body insulin action on glucose disposal. Eleven obese women [age $48 \pm 9 \mathrm{yrs}$, BMI $42.5 \pm 9.8 \mathrm{~kg} / \mathrm{m}^{2}$ (mean \pm SD), diabetic/nondiabetic 4/7] underwent a 2-step-euglycemic glucose clamp [90 min baseline, 120 min step I (insulin infusion rate (IIR): $0.25 \mathrm{mU} / \mathrm{kg} / \mathrm{min}$ ) and $120 \mathrm{~min}$ step 2 (IIR $0.50 \mathrm{mU} / \mathrm{kg} / \mathrm{min})]$. Interstitial glycerol concentrations ('lipolysis index') were measured at baseline (B-GLY) and at the end of step 1 (C1-GLY) and step 2 (C2GLY) of the clamp at abd and fem SAT sites by microdialysis (CMA/ $60,0.3 \mu \mathrm{l} / \mathrm{min})$. Regional SAT blood flow was determined by ${ }^{133} \mathrm{Xe}$ wash-out. Stimulated glucose uptake $[\mathrm{M},(\mathrm{mg} / \mathrm{kgFFM} / \mathrm{min})]$ was measured during the last $30 \mathrm{~min}$ of the clamp. BGLY was similar in both SAT regions $[322 \pm 79$ (abd) vs. $370 \pm 119$ (fem) $\mu \mathrm{mol} / /$, n.s. $]$. During the clamp, Cl-GLY was suppressed by $22 \%$ (abd, n.s.) and $27 \%$ (fem, n.s.) and $\mathrm{C} 2$-GLY was suppressed by $41 \%$ (abd, $\mathrm{p}<0.05$ ) and $43 \%(\mathrm{fem}, \mathrm{p}<0.05$ ) as compared to B-GLY. Abd and fem suppression of glycerol (SUP-GLY) were closely related $(r=0.89, p<0.001)$ without regional differences. $M$ was positively correlated with SUP-GLY [ $\mathrm{r}=0.75$ (abd), $\mathrm{r}=0.78$ (fem), $\mathrm{p}<0.01]$, but not with B-GLY. There was no relationship between either B-GLY or SUP-GLY and the waist-to-hip ratio Diabetic subjects showed a lower $M[2.7 \pm 0.6$ vs. $4.4 \pm 1.3, p<0.05]$ and a trend towards a lower SUP-GLY ( $31 \pm 15$ vs. $49 \pm 14 \%, p=0.09$ ) as compared to non-diabetic subjects. These findings suggest that a) there are no regional differences in the anti-lipolytic effect of insulin between the abdominal and the femorogluteal SAT site in obese women and b) the sensitivity of the SAT to the anti-lipolytic effect of insulin is related to whole body insulin action on glucose disposal.
732

ARACHIDONIC ACID AND BRL-49653 ENHANCE GLUCOSE UPTAKE IN 3T3 L1 ADIPOCYTES

C.Baker, J.B.Prins, C.L.Loizou and S.O'Rahilly. Departments of Clinical Biochemistry and Medicine, Cambridge University, Addenbrookes Hospital, Hills Road, Cambridge CB2 2QR

Dietary fatty acids have been shown to modulate insulin sensitivity, although the mechanism whereby this occurs is unknown. Thiazolidinediones are potent insulin sensitising agents which act via the nuclear hormone receptor PPAR $\gamma$. The endogenous ligand for this receptor is thought to be a member of the prostanoid family. We hypothesised that fatty acid composition may modulate insulin sensitivity by influencing production of endogenous activators of PPAR $\gamma$. 3T3 $\mathrm{Ll}$ adipocytes were incubated with various fatty acids or the thiazolidinedione BRL- 49653 for $48 \mathrm{~h}$. Of the ten fatty acids studied, arachidonic acid showed the most marked enhancement of basal and insulin-stimulated glucose uptake (3.8 fold and 1.9 fold respectively compared with vehicle control; all $\mathrm{p}<0.001$ ). A similar effect was observed for BRL-49653 ( 2.5 fold and 1.6 fold respectively, all $\mathrm{p}<0.001$ ). In a time course experiment arachidonic acid enhanced basal and insulin-stimulated glucose uptake after $1 \mathrm{~h}$ of supplementation whereas BRL-49653 had no effect up to $48 \mathrm{~h}$. Thus arachidonic acid, a known precursor of intracellular prostanoids, has effects on basal and insulin-stimulated glucose uptake in $3 T 3 \mathrm{~L} 1$ adipocytes which closely resemble those induced by thiazolidinediones. However, the time course of action of these two compounds differ which suggests possible differences in their precise modes of action.

\section{PS 25}

\section{Insulin Resistance: Intracellular}

\section{3}

PHYSIOLOGICAL CHARACTERIZATION AND MUTATIONAL ANALYSIS OF THE INSULIN RECEPTOR GENE IN A PATIENT WITH SEVERELY INSULIN RESISTANT DIABETES MELLITUS.

H. Vestergaard, L. Hansen, S. Jessing, S. G. Larsen, O. Pedersen. Steno Diabetes Center and Hagedorn Research Institute, Gentofie. Dept of Endocrinology, Herlev University Hospital, Copenhagen, Denmark.

A 14 year old girl with 3 years of known diabetes was referred to Steno Diabetes Center. She presented with acanthosis nigricans, fasting hyperglycemia $(14.4 \mathrm{mmol} / \mathrm{l})$, fasting hyperinsulinemia (3982 pmol/1, ref. Interval: $5-69 \mathrm{pmo} / /)$, and elevated $\mathrm{HbA}_{1 \mathrm{c}}(14.4 \%$, ref. Interval: $4.1-6.4 \%)$ and non-esterified fatty acids $(1.22 \mathrm{mmol} / \mathrm{h}$ vs. 0.56 $\pm 0.15 \mathrm{mmol} / \mathrm{l}$ in 6 healthy non-diabetic women). During hyperinsulinemic and euglycemic clamp conditions her total glucose disposal rate was one-fifth of the total glucose disposal rate compared to 6 non-diabetic women $\left(85 \mathrm{vs.} 436 \pm 31 \mathrm{mg} / \mathrm{m}^{2} / \mathrm{min}\right)$ and one-third compared to 6 diabetic women ( 85 vs. $278 \pm 14$ ). Because of the severe insulin resistance and acanthosis nigricans which occasionally is associated with structural changes in the insulin receptor, we studied the insulin receptor gene for mutations. The coding sequence of the 22 exons, including the signal peptide, was analyzed by PCR-SSCPheteroduplex scanning in 29 segments, and in exon 2 we found a previously unreported T184I amino acid substitution. This mutation was inherited from her mother who is slightly insulin resistant (fasting serum insulin, $200 \mathrm{pmol} / \mathrm{lvs} .39 \pm 20 \mathrm{pmol} / \mathrm{l}$ in 80 non-diabetic women) but otherwise normal. We conclude that the novel T184I variant of the insulin receptor confers insulin resistance and if not directly pathognomonic it may well be an important factor in the pathogenesis of acanthosis nigricans.

\section{4}

NOVEL MUTANT ALLELES OF THE INSULIN RECEPTOR GENE. S. Riqué, N. Potau, D. Mauricio, S. Ferragut, V. Marcos, L. Ibáñez, C. Nogués and A. Carrascosa. Hospital Universitari Materno-Infantil Vall d'Hebron. Barcelona. Spain.

Insulin resistance of genetic syndromes has been well characterized as having mutations in the insulin receptor gene. We have studied the structure of insulin receptor gene in four patients with extreme insulin resistance. A 27 year old boy with Rabson-Mendenhall syndrome (RM), and three girls with Type A insulin resistance. Two of them were sisters ( $\mathrm{Al}$ and $\mathrm{A} 2$ ) with different phenotype, and the other girl (A3) had acanthosis nigricans, polycystic ovary pattern, high androgen levels and fasting hyperinsulinemia $(350 \mu \mathrm{U} / \mathrm{ml})$. A $75 \mathrm{~g}$ oral glucose test was normal. The boy was diabetic and treated with high insulin doses, and the girls had hyperinsulinemia with normal fasting glucose levels. Amplification of the 22 exons by PCR showed no structural aberrations. The single strand conformation polymorphism (SSCP) revealed abnormal patterns in some exons. Automatic sequencing of the insulin receptor gene demonstrated that the insulin receptor gene of patient $\mathrm{RM}$ presented a missense mutation in the maternal allele substituting Lys (AAA) for Asn (AAC) at codon 15 in exon 2 and a nonsense mutation at codon 1000 changing an Arg (CGA) into a Stop (TGA) in the paternal allele. Patient Al presented a missense mutation substituting Leu (TTG) for Val (GTG) in heterozygous state at codon 140 in exon 2 and a mutation in heterozygous state in the acceptor splice site of intron 21 which changes the consensus sequence AG into AA. This second mutation was present in the insulin receptor gene of her sister (A2). Patient A3 presented a missense mutation substituting Val (GTG) for Ala (GCG) in heterozygous state at codon 1028 in exon 17. The relationship between genotype and phenotype in our patients seems to be coincident, but patient $\mathrm{A} 3 \mathrm{had}$ an heterozygous mutation with a severe phenotype. To our knowledge $\mathrm{Leu}^{140}, \mathrm{Val}^{1028}$ and the mutation in the acceptor splice site of the intron 21 have not been previously described. We can conclude that the severe insulin resistance state of these patients is due to these mutations in the insulin receptor gene 
METFORMIN POTENTIATES PHOSPHATIDYLINOSITOL-3' KINASE BY IRS-2 RECRUTMENT IN RAT HEPATIC TISSUE.

F. Grigorescu, P. Poucheret, O. Bouix, C. Lautier, E. Renard, F. Macari, J. Bringer, G. Cros, N. Wiensperger and J.J. Serrano. IURC and Pharmacology Dept., Montpellier and Lipha International, Lyon, France.

In order to understand the molecular mechanism of action of metformin, a biguanide commonly used in treatment of NIDDM, we have investigated its in vivo effects on insulin signaling in rat hepatic tissue. Intraportal bolus injection of insulin (1.5 IU) was performed in Wistar rats at fast, 90 min after oral administration of 4-400 $\mathrm{mg} / \mathrm{kg}$ metformin. Hepatic tissue was excised at 1,5 and $10 \mathrm{~min}$, solubilized and submitted to immunoprecipitation with antibodies against insulin receptor, IRS-1 and 2 and p85 $\alpha$ subunit of PtdIns $3^{\prime}$ kinase. Proteins were resolved in SDS-PAGE and submitted to immunoblotting. PtdIns $3^{\prime}$ kinase activity was measured by thin layer chromatography. Tyrosine phosphorylation of the insulin receptor revealed by antiphosphotyrosine antibodies was maximun at $1 \mathrm{~min}$ after insulin stimulation and slowly decreased by 5 and 10 min. Metformin at maximal dose stimulated by 2 fold the phosphorylation and the association of the insulin receptor with IRS-2 at 5 and $10 \mathrm{~min}$ but not those of IRS-1. Similarly, metformin stimulated by 3 fold the PtdIns 3 'kinase activity in immunoprecipitates of IRS-2 but not in IRS-1 precipitates. These data, combined with our previous results indicating the potentiation in vitro of insulin receptor kinase after both acute and chronic treatment of metformin, suggest that this drug may act in the early steps of insulin transduction, preferentially implicating IRS-2 substrate in rat hepatic tissue. Specific action of metformin in hepatic tissues may give a new insight in mechanisms involved in therapeutic correction of transduction defects associated with insulin resistance.

MOLECULAR ALTERATION OF THE INSULIN SIGNAL TRANSDUCTION PATHWAY IN IMMORTALIZED LYMPHOBLASTS FROM PATIENTS WITH ESSENTIAL HYPERTENSION J. Niggemann, M. Koenen and J. Eckel, Molecular Cardiology, Diabetes Research Institute, Düsseldorf, Germany

The association of essential hypertension and insulin resistance is well esta blished, however, the molecular basis of this interaction has remained completely unknown. Epstein-Barr virus immortalized lymphoblasts from patients with essential hypertension have now been used to study the components of the insulin signal transduction pathway in order to identify primary defects free of systemically-induced alterations. Cell lines obtained from four normotensive (NT) and four hypertensive (HT) patients were kept in a continuous culture in the presence of $10 \% \mathrm{FCS}$ and were transferred to a serum-free buffer before stimulation with insulin $\left(10^{-7} \mathrm{M}, 10 \mathrm{~min}\right)$. Immunoprecipitation (IP) of the insulin receptor (IR) indicated an enhanced autophosphorylation of the IR betasubunit ( 2 fold) in HT patients with an unaltered protein expression of the IR. Propagation of insulin signaling was then analyzed at the level of IRS-1 and IRS-2. Stimulation with insulin produced a marked increase in the tyrosine phosphorylation of IRS-1 in cells from NT patients. This effect was reduced by $49 \pm 13 \%(n=4)$ in HT patients. This was paralleled by a profound reduction in the protein expression of IRS- 1 in HT cells $(89 \pm 8 \%$ in IRS-1 IP and $62 \pm 17 \%$ in cell lysates). Protein expression of IRS-2 was also reduced, but to a lesser extent (about $60 \%$ in IP and $\mathbf{4 2 \%}$ in cell lysates). Downstream insulin signaling was then analyzed at the level of PI 3-kinase. In control cells insulin produced a four fold increase in p85-associated PI 3-kinase activity with no difference between NT and HT cells. Further, expression of the p85 adapter subunit was not different between the two groups. In conclusion, our data show a profound alteration of the insulin signaling cascade at the level of IRS protein expression in cells from HT patients. This defect is compensated by an increase in the IR tyrosine kinase activity and enables an unaltered downstream signaling to PI 3-kinase. We conclude that the downregulation of IRS-1 and -2 must be geneticaily fixed and may render HT cells more susceptible to exogenous factors finally leading to the manifestation of insulin resistance in these patients. (Supported by DFG SFB 351, C2 and EU COST B5)
DIVERGENT EFFECTS OF ALTERED GLYCEMIA ON EARLY AND INTERMEDIATE SIGNALING EVENTS IN GOTO-KAKIZAKI (GK) RATS

Y. Kawano, X.M. Song, A. Krook, S. Efendic, R.A. Roth, H. Wallberg-Henriksson and J.R. Zierath. Stockholm, Sweden and Stanford, CA, USA.

Hyperglycemia may directly contribute to the development of insulin resistance in NIDDM through alterations in insulin signaling in peripheral tissues. To explore the role of hyperglycemia on insulin signaling in skeletal muscle, GK diabetic rats were treated with phlorizin (PHL) or vehicle (VHL) for 4 wks. Thereafter, key components of the insulin-signaling cascade were assessed in glycolytic (EDL) and oxidative (soleus) skeletal muscle. Normalization of blood glucose in the GK diabetic rat by PHL-treatment improved, but did not fully restore glucose tolerance $(\mathrm{p}<0.05$ vs VHL-treated-GK rats). PHL-treatment increased maximal insulinstimulated glucose transport in soleus by $38 \%(\mathrm{p}<0.05$; vs VHL-treated) and fully restored insulin sensitivity in EDL muscle. Insulin-stimulated IRS-1 tyrosine phosphorylation was reduced by $43 \%(\mathrm{p}<0.01)$ in $\mathrm{GK}$ soleus compared to controls, with no apparent defect noted in EDL. Similarly, IRS-1-associated PI-3 kinase activity was reduced by $51 \%(p<0.01)$ in GK soleus compared to controls, with no in EDL. Despite reduced PI 3-kinase activity in GK soleus, protein expression of the p85 subunit of PI3-kinase was not altered. Defects in IRS-1 tyrosine phosphorylation and IRS- 1 associated PI-3 kinase activity in soleus muscle were not improved in PHL-treated GK rats. Activity of the serine/threonine kinase Akt, a major target of PI 3-kinase generated signals, was also assessed. Insulin-stimulated Akt activity was reduced by $65 \%$ in GK soleus ( $p<0.01$; vs non-diabetic), whereas, insulin was without effect on Akt in GK EDL muscle. Restoration of glycemia with PHL-treatment completely normalized Akt activity in both soleus and EDL muscle to non-diabetic levels. In conclusion, improved glucose tolerance in diabetic GK rat by PHL-treatment fully restores the downstream response of PI 3-kinase, including Akt activity and glucose transport, despite a persistent defect in early insulin signaling elements. Thus, in diabetic skeletal muscle, distal rather than proximal steps to PI 3-kinase appear to be sensitive to changes in glycemia
738

CIRCULATING SOLUBLE TNF- $\alpha$ RECEPTOR 2 LEVELS ARE ASSOCIATED WITH INSULIN RESISTANCE

JM Fernández-Real, M. Broch ${ }^{\star}$, J. Vendrell ${ }^{\star}$, C. Gutiérrez ${ }^{*}$, R. Casamitjanat, C

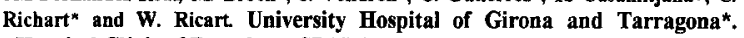
+Hospital Clínic of Barcelona. SPAIN

Recent studies have shown that the tumor necrosis factor (TNF) system is implicated in the insulin resistance of human obesity. The purpose of this study was to explore whether activation of the TNF system, as measured by the levels of circulating tumor necrosis factor receptors 1 and 2 (sTNFRI and STNFR2) and TNF- $\alpha$, is associated with insulin resistance. Nineteen men (mean age $35.4 \pm 2$; BMI 27.8 \pm 1.2 , range 22.2-35.7) and 17 women (age 33.9 \pm 1.4 ; BMI 28.4 \pm 0.8 , range 19-37.9)] were studied. STNFR2 levels correlated with BMI $(r=0.50$ $\mathrm{p}=0.002$ ), fat-free mass $(r=0.61, p<0.0001)$ and waist-to-hip ratio (WHR) $(r=0.39, p=0.02)$, basal glucose levels $(r=0.45, p=0.007)$ and with area under the curve for glucose (AUCglucose) after OGTT $(\mathrm{r}=0.42, \mathrm{p}=0.013)$. sTNFR2 also correlated negatively with insulin sensitivity $\left(\mathrm{S}_{\mathrm{l}}\right)$, evaluated using the frequently sampled intravenous glucose tolerance test with minimal model analysis ( $r=$ $0.38, \mathrm{p}=0.02$ ), and with $\mathrm{S}_{\mathrm{l}}$ derived from the oral glucose tolerance test $(\mathrm{OGTT})(\mathrm{r}=-0.56, \mathrm{p}=0.001)$. Plasma sTNFR1 and TNF- $\alpha$ levels were not associated with any of these variables. As WHR influenced both SI and STNFR2 levels, we constructed a Multiple Linear Regression to predict $S_{\mathrm{t}}$, with WHR and sTNFR2 as independent variables. In this model, both WHR $(p=0.0078)$ and sTNFR2 levels $(\mathrm{p}=0.025)$ contributed to $47 \%$ of the variance in $\mathrm{S}_{\text {. }}$. However, after controlling for fat-free mass, all the correlations between $S_{\mathrm{L}} \mathrm{S}_{\mathrm{L}}$ from the OGTT and sTNFR2 levels disappeared, indicating that fat-free mass was significantly influencing these associations. In summary, plasma sTNFR2 levels, but not sTNFR 1, were proportional to body mass index, waist-to-hip ratio, fat-free mass (a well-known confounder in the evaluation of insulin sensitivity), basal and postload glucose levels, and insulin resistance. These findings support TNF- $\alpha$ as a system regulating insulin action in human obesity. 
CO-TRANSFECTION OF INSULIN RECEPTOR AND PKCB2 IN HEK 293 CELLS: EFFECT OF SERINE TO ALANINE EXCHANGES IN THE INSULIN RECEPTOR

V. Strack, A.M. Hennige, *B. Bossenmaier, M. Kellerer and H.U. Hăring Universităt Tübingen, Innere Medizin IV, Otfried-Müller-Str. 10, 72076 Tübingen, Germany. *Bochringer Mannheim GmbH, Abt. Molekularbiologie, 68305 Mannheim, Germany.

We have previously shown that co-transfection of insulin receptor (HIR) wildtype and protein kinase $C$ (PKC) $\beta 1$ and $\beta 2$ inhibits insulin stimulated autophosphorylation of HIR after stimulation with TPA. The underlying mechanism how PKC inhibits HIR auto-phosphorylation is unclear, one potential mechanism involves phosphorylation of serine residues in the insulin receptor B-subunit. In order to identify potential regulatory domains we exchange serine to alanine at 16 candidate positions. To determine whether one of these serine residues is essential for the inhibitory effect of PKC $\beta$ on insulin receptor signalling we co-transfected HIR mutants with PKC 32 in HEK 293 cells. Cells were stimulated with TPA (10 M, $30 \mathrm{~min}$ ) and insulin $\left(10^{-7} \mathrm{M}, 5 \mathrm{~min}\right)$. HIR autophosphorylation was determined by pTyr antibodies, expression of HIR mutants and PKC $\beta$ was monitored by the respective antobodies. We found that all HIR mutants could be inhibited by TPA stimulated PKC 2 , however there were quantitative differences in the inhibitory effect. The inhibition was less pronounced in HIR-994, HIIR-1023/25 and HIR exchanges in the C-terminus (HIR-1258, HIR-1293/94, HIR-1308/09). As described earlier, serine to alanine exchanges in these domains lead to increased HIR autophosphorylation. It is therefore difficult to decide whether these domains are indeed specifically required for the inhibitory effect of PKC $\beta 2$ on HIR or whether the reduced ability of PKC $\beta 2$ to inhibit these HIR constructs reflects the activity state of these receptor mutants.
EXPRESSION OF PC-1 IN INSECT CELLS AND CHARACTERIZATION OF ITS ANTI-INSULIN RECEPTOR KINASE ACTIVITY.

S. Tokita. M. Ito. Y. Takeuchi, and M. Ihara. Tsukuba Research Institute. Banyu Plannaceutical Co., Ltd.. Ibaraki, Japan.

PC-1 is a membrane glycoprotein expressed in a form of homodimer. PC-1 has its enzymatic activity as an ccto-phosphodiesterase(PDE)/pyrophosplatase(PPase). It has recently been suggested that, in insulin resistant subjects. expression of PC-1 is elevated in association with a decrease in the activity of insulin receptor tyrosine kinasc (IR-TK), suggesting a close linkage between PC-1 expression and insulin resistance. The purpose of this study is to investigate the anti-[R-TK activily of PC. 1 and to elucidate its inhibitory mechanism. Methods: Baculovirally expressed human PC-1(rhPC-1) was partially purified by lectin-affinity chromatography. The hydrolylic activity of rhPC-1 was measured using n-NPP (p-nitrophenylphenyl phosplonate) as a substrate. IR-TK. purified from IM9 cells, was estimated for insulin $(0.1-10 \mu \mathrm{M})$-stimulated autophosphorylation in the presence of rhPC-1. Results: (1)Homodimeric rhPC-1 was indeed expressed in the insect cells, as estimated al Mr $220 \mathrm{KDa}$ by an immunoblot analysis. The hydrolytic activity of rhPC-1 was comparable to that of native PC-1 (Km for $\mathrm{p}-\mathrm{NPP}$ : rhPC- $1 / \mathrm{nPC}-1=$ $240 / 210 \mu \mathrm{M}) .(2)$ The rhPC-1 inlibited IR-TK activity in a dose dependent manner $(\mathrm{EC} 5()=\sim 80 \mathrm{U} / \mathrm{ml})$ and exerted little effect on human EGF receptor TK activity. (3) J-()91,122 and orthovanadale were found to inhibit the hydrolytic activity of the rhPC- $1(\mathrm{IC} 50=0.2-10 \mu \mathrm{M})$. Some of them also suppressed the rhPC-1 inhibition of IR-TK activity (IC50 $=0.5-10 \mu \mathrm{M})$, whereas the others did not show the significant effects (IC50> -100 $\mu \mathrm{M}$ ).(4) The rhPC-1 inhibited IR-TK activity more potently $(>100)$ folds) than did bovine intestine PDE-I, despitt of the revealed considerable homology at active sites of the two diesterases. Conclusions: These results suggest that anti-IR activity of PC-1 is not simply explained hy ATP depletion through the hydrolytic activity of PC-1. Chemicals we discovered may be useful to investigate the mechanism of $\mathrm{PC}-1$ action over IR.
741

CIRCULATING TUMOR NECROSIS FACTOR $\alpha$ (TNF $\alpha$ ) CONCENTRATIONS ARE ELEVATED IN TYPE 2 DIABETES AND RELATE TO THE DEGREE OF GLYCEMIA

C Tsigos', F. Giardina', I Kyrou', P. Tsiotra, ${ }^{1}$ A. Mitrakou ${ }^{2}$ and S. Raptis ${ }^{2}$. 'Hellenic National Diabetes Center and ${ }^{2}$ 2nd Department of Internal Medicine, Research Institute and Diabetes Center, University of Athens, Evangelismos Hospital, Athens, Greece.

The expression of the cytokine tumor necrosis factor $\alpha$ (TNF $\alpha$ ) is elevated in obesity in both adipose and muscle tissue and is thought to play a critical role as mediator of peripheral insulin resistance. To examine whether circulating $T N F \alpha$ levels are elevated in type 2 diabetes and whether they relate to glycemia level, we compared the plasma levels of TNF $\alpha$ and of the other main endocrine cytokine, interleukin-6 (IL-6), (both measured by Elisa) in 12 type 2 male diabetic patients (treated with diet only) and 11 non diabetic controls, who were matched for age and body mass index (BMI) (49 \pm 2 vs $47 \pm 3$ years and 29.1 \pm 1.2 vs $31.6 \pm 1.9$, respectively) and gave no history of recent or active infection. Glucose and insulin levels were measured at 0,1 and 2 hours after a $75 \mathrm{gr}$ oral glucose load. No differences were observed in plasma IL-6 levels between the two groups. In contrast, TNF $\alpha$ levels were significantly elevated in the diabetic patients compared to the control subjects $(5.48 \pm 0.72$ vs. $3,41 \pm$ $0.57 \mathrm{pg} / \mathrm{ml}, p<0.02$ ) and correlated significantly with basal and 2 hour post-OGTT glucose levels $(r=0.80, p<0.01)$. TNF $\alpha$ levels did not correlate, however, to BMl or weight-to-hip ratio (WHR). In conclusion, circulating $T N F \alpha$ levels are elevated in type 2 diabetes mellitus, independently of the presence of obesity, and appear to relate the degree of both fasting and post-prandial glycemia.

\section{2}

CHRONIC HYPERINSULINAEMIA DOES NOT REGULATE PC-1 GENE EXPRESSION AND PROTEIN CONTENT.

B. Costanzo ${ }^{\circ}$, P. Sbraccia*, M. D'Adamo*, G. Tamburrano*, V. Trischitta $\$$ and L. Frittitta ${ }^{\circ} .{ }^{\circ}$ Instit Int Med, Univ of Catania; $*$ Div End 3 Univ "La

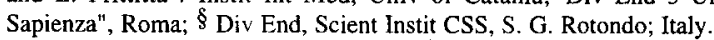

Glycoprotein PC-1, a plasma membrane ectoenzyme and an inhibitor of insulin receptor tyrosine-kinase, is increased in skeletal muscle and adipose tissue of healthy, insulin resistant, hyperinsulinaemic subjects. It is not known whether PC-1 content increase is secondary to hyperinsulinaemia. Aim: To verify whether hyperinsulinemia may modulate PC-1 gene expression and protein content. Methods: PC-1 gene expression (by competitive PCR) and PC-1 protein content (by ELISA) was measured in skeletal muscle of 8 patients with Insulinoma (I), a natural model of chronic hyperinsulinaemia which is not secondary to insulin resistance. Data were compared to those obtained in 8 control subjects (C) matched for sex $(4 \mathrm{M} / 4 \mathrm{~F}$ vs $4 \mathrm{M} / 4 \mathrm{~F})$, age ( $40 \pm 6.8$ yrs vs $47.7 \pm 4.3)$, BMI $(27.7 \pm 1.3$ $\mathrm{kg} / \mathrm{m}^{2}$ vs $25.5 \pm 1.4$ ) and whole body insulin sensitivity as assessed by euglycaemic clamp $(M=6.1 \pm 0.7 \mathrm{mg} / \mathrm{kg} \cdot \mathrm{min}$ vs $5.8 \pm 0.7)$. Results: First, the $\mathrm{PC}-1$ protein content was significantly correlated to the $\mathrm{PC}-1$ gene expression $(r=0.8, p<0.05)$ indicating that no difference in $P C-1$ transcript processing and post translational events occurs in the presence of hyperinsulinaemia. Second, both PC-1 content $(23.1 \pm 2.9 \mathrm{vs} 27.1 \pm 5 \mathrm{ng} / \mathrm{mg}$ protein) and gene expression ( $728 \pm 77$ vs $804 \pm 258$ cDNA copy/40ng RNA) were similar in muscle tissue specimens from I and C. Moreover, no correlation was observed between fasting plasma insulin concentration and PC-1 protein content. Conclusions: These data indicate that chronic hyperinsulinaemia does not regulate PC-1 gene expression and protein content in human skeletal muscie and suggest that the increased PC-1 protein content reported in skeletal muscle of insulin resistant subjects is not secondary to hyperinsulinaemia. 


\section{3}

LONG-TERM MODULATION BY INSULIN OF KEY REGULATORY ENZYMES OF GLUCOSE METABOLISM IN CULTURED OBESE (fa/fa) RAT HEPATOCYTES.

A. Esteban-Gamboa, M.C.G. Lechuga, J.J. Carrillo and J.E. Felíu. Departamento de Bioquímica, Facultad de Medicina, Universidad Autónoma de Madrid. Spain.

In obese (fa/fa) Zucker rats, glucose metabolism in liver and peripheral tissues is resistant to short-term modulation by insulin. Nevertheless, the rise in the key glycolytic enzyme activities in the liver, together with the reduction of the key gluconeogenic enzymes, suggest a possible long-term adaptation of the glycolytic/gluconeogenic pathway to the chronic hyperinsulinemia present in these animals. The aim of the present study was to investigate the long-term modulation by insulin of glucokinase (GK), pyruvate kinase (PK) and phosphoenolpyruvate carboxykinase (PEPCK) activities, as well as the expression of GK and PEPCK mRNAs, in primary cultures of hepatocytes from obese $(\mathrm{fa} / \mathrm{fa})$ and lean $(F /-)$ rats. Hepatocytes were isolated from 48-hour fasted animals and cultured for 24 hours in $\mathrm{M}-199$ in the presence of $10 \mathrm{mM}$ glucose, T3 $(1 \mu \mathrm{mol} / \mathrm{/})$ and dexamethasone $(1 \mu \mathrm{mol} / \mathrm{l})$. Insulin $10.1,1$ and $10 \mathrm{nmol} / \mathrm{l}$ ) or saline were added 4 hours after plating. In cultured lean rat hepatocytes, insulin caused a statistically significant and dosedependent increase in GK and PK activities, as well as a diminution of PEPCK activity $133 \%, 20 \%$ and $42 \%$, respectively, vs the corresponding saline values, at $1 \mathrm{nmol} / \mathrm{l}$ insulint. In good agreement with these findings, insulin raised the expression of GK mRNA and reduced that of PEPCK mRNA $1100 \%$ and $35 \%$ vs the saline values, respectively, at $10 \mathrm{nmol} / \mathrm{l}$ insulin). In contrast, in cultured obese rat hepatocytes, insulin did not modify GK or PK activities, or alter GK mRNA levels; however, this hormone caused a significant decrease in both the activity and mRNA expression of PEPCK. Our results indicate that primary cultures of obese $(f a / f a)$ rat hepatocytes present resistance to the long-term positive modulation of GK by insulin, while the negative control of PEPCK by this hormone is maintained.

\section{4}

A NEW ISOPRENYL SYNTHASE, WHICH IS OVER-EXPRESSED IN OBESITY AND INDUCED BY ADIPOGENESIS, SYNTHESIZES THE PRENYL MOIETY USED IN G-PROTEIN PRENYLATION

D. Vicent, E. Maratos-Flier, and C.R. Kahn. Joslin Diabetes Center, Boston, MA 02215, USA.

In order to identify molecules that contribute to insulin resistance we have studied gene expression in muscle of the ob/ob mouse, a genetic model of obesity and extreme insulin resistance by using the mRNA differential display method. One of the over-expressed mRNAs encoded a 300 amino acids protein that was identified as the mouse geranylgeranyl pyrophosphate synthase (GGPP synthase) by its homology with proteins cloned from yeast and fungus. Two mRNAs of 4.3 and $1.7 \mathrm{~kb}$ were detected in Northern blot analysis. Western blot analysis of tissue homogenates using specific antipeptide antibodies revealed a single band of $34.8 \mathrm{kD}$ which expression level in different tissues correlates with the $4.3 \mathrm{~kb}$ mRNA. The mRNA expression was increased in skeletal muscle, liver and fat of the ob/ob mouse between 5- and 20 -fold by Northern blot analysis. Western blots also showed a 2 -fold over-expression of the protein in muscle and fat, but not in liver where the dominant isoform is encoded by the $1.7 \mathrm{~kb}$ mRNA. Differentiation of 3T3L1 fibroblasts into adipocytes induced GGPP synthase expression more than 20-fold. Using the immunoprecipitated protein, we found that mammalian GGPP synthase not only synthesizes GGPP but also its metabolic precursor, farnesyl pyrophosphate (FPP). FPP and GGPP are used for protein isoprenylation in animal cells. This modification is required for the function of Ras related small GTPases and trimeric $\mathbf{G}$ proteins involved in G proteincoupled receptors signaling. These results suggest that the expression of GGPP synthase, a key regulator of protein isoprenylation, is regulated in multiple tissues in obesity and adipogenesis. Altered regulation of Gproteins isoprenylation might be a major factor determining the ability of the cells in responding insulin stimulation in obesity.

\section{PS 26}

\section{Insulin Resistance: Cardiovascular}

\section{5}

\section{INSULIN-MEDIATED VASODILATION AND GLUCOSE UPTAKE ARE FUNCTIONALLY RELATED IN MAN.}

\section{S.J. Cleland, S. Ueda, J.R. Petrie, H.L. Elliott and J.M.C. Connell.} Department of Medicine \& Therapeutics, University of Glasgow, Glasgow, UK.

There is a positive relationship between insulin sensitivity and basal endothelial nitric oxide production in healthy volunteers; furthermore, insulin-mediated vasodilation has been shown to be endotheliumdependent. We hypothesised, therefore, that insulin-mediated vasodilation and insulin sensitivity should also be directly related. Eighteen healthy, normotensive male volunteers (age 26 \pm 5.4 years) attended on two occasions for measurement of whole-body insulin sensitivity using the hyperinsulinaemic euglycaemic clamp, and forearm vasodilation in response to an intra-arterial infusion of insulin by bilateral venous occlusion plethysmography. Insulin-mediated glucose uptake (M) for the group (mean \pm S.D.) was $10.0 \pm 2.2 \mathrm{mg} \mathrm{kg}^{-1}$ $\mathrm{min}^{-1}$, while the percentage change in forearm blood flow ratio (\%FBFR) for the group (median, interquartile range) was $28.2 \%$ (13.6, 48.6). In univariate analysis, $M$ was significantly correlated with \%FBFR $\left(r_{s}=0.60, p<0.05\right)$, but not with BMI $\left(r_{s}=-0.42\right)$, age $(r=-$ $0.39)$ or MAP $(r=0.13)$. In multiple regression analysis, \%FBFR remained a significant independent predictor of $M\left(R^{2}(a d j)=0.48, t=\right.$ $3.23, p<0.01$ ) in a model involving BMI, age and blood pressure. From these data, we conclude that (1) insulin-mediated vasodilation may be an important determinant of insulin-mediated glucose uptake, and (2) glucose uptake modulates the vascular response to insulin, possibly at an endothelial level.

\section{6}

INSULIN RESISTANCE AND INDICES OF ENDOTHELIAL FUNCTION : THE BARILLA STUDY

I. Zavaroni, , L. Monti, P. Massironi, A. Zuccarelli, P. Gasparini, A. Barilli, Valsecchi G, Phan VC, Pontiroli AE and PM. Piatti .Institute of Medical Clinic Parma University and Scientific Institute H.San Raffaele, Milano University

Altered endothelial function documented by low levels of nitric oxide (NO) and high levels of endothelin-1 (ET-1) has been found in diabetic in hypertensive and in patients with CHD disease. Since all these conditions are associated with insulin resistance it has been hypothesized that abnormalities of endothelial function are present in insulin resistant subjects. To test this hypothesis 421 healthy subjects, factory workers, have been examined and divided into 4 quartiles on the basis of their insulin area after OGTT, as index of insulin resistance. Subjects with diabetes, hypertension and CHD disease have been excluded. The comparison (mean $\pm S$.E.) has been done between the subjects in the 4th quartile, the most insulin resistant and in the 1st quartile, the most insulin sensitive, matched for age, BMI and gender. Fasting nitric oxide, endothelin-1 and selectin $E$ levels have been measured and compared in the two groups. Results :the insulin resistant subjects (quartile 4; $n=49$; age $52 \pm 1$ years, BMI $26.7 \pm 0.3 \mathrm{Kg} / \mathrm{m}^{2}$ ) compared to the insulin sensitive subjects (quartile $1 ; n=49$; age $52 \pm 0.9$ years; BMI $26.3 \pm 0.3 \mathrm{Kg} / \mathrm{m}^{2}$ ), show significantly higher levels of plasma insulin, plasma glucose, triglycerides, lower levels of HDL cholesterol, higher blood pressure. The indices of endothelial function were: NO $24.9 \pm 1.7$ vs $18.1 \pm 1.0$ $\mathrm{mmol} / \mathrm{l}$ (p<0.001); ET-1 6.1 \pm 0.6 vs $7.0 \pm 0.4 \mathrm{ng} / \mathrm{ml}$ (NS); Selectin E $61.2 \pm 5.0 \mathrm{vs} 52.1 \pm 3.9 \mathrm{ng} / \mathrm{ml}$ (NS) in the 4 th and $1 \mathrm{st}$ quartile respectively. A significant correlation was found between plasma NO levels and fasting insulin $(r=0.34 ; p<0.001), 1 \mathrm{~h}$ insulin $(r=0.32 ; p<0.01), 2 \mathrm{~h}$ insulin $(r=0.22 ; p<0.05)$, triglyceride $(r=0.45 ; p<0.001)$, uric acid $(\mathrm{r}=0.22 ; \mathrm{p}<0.05)$. The multiple regression analysis shows that NO is independently related to triglycerides and insulin area .Conclusion: the results indicate that plasma NO metabolites are higher, not lower in hyperinsulinemic, insulin resistant subjects. This unexpected relationship in individuals at increased risk for CHD requires further evaluation. 
VASCULAR INSULIN RESISTANCE IN SMALL ARTERIES OF ZUCKER OBESE RATS AND AGEING ZUCKER LEAN RATS.

T.B.Bödvarsdóttir, H.C.M. Boonen, B. Voss and, R.D. Carr. Department of Diabetes Pharmacology, Novo Nordisk A/S, Bagsvaerd, Denmark.

It has been suggested that one of the factors contributing to peripheral insulin resistance in diabetes, is an observed change in haemodynamic responses to insulin. Insulin-induced vasodilatation may contribute to increasing nutritive blood flow and hence glucose disposal from the blood. Resistance arterial function may thus contribute significantly to peripheral insulin resistance. We tested whether age, penipheral insulin resistance, or both, affect insulin-induced changes in small artery reactivity. Femoral (FrA) and mesenteric (MrA) resistance sized arteries were isolated from 12 and 30 week (WK) old insulin resistant Zucker obese (Ob) and insulin-sensitive lean $(\mathrm{Ln})$ rats. The vessels were mounted in a wire myograph at their individual optimal lumen diameter for recording of isometric force development. Vessels were incubated for one hour in the presence or absence of $0.1 \mathrm{mU} / \mathrm{ml}$ insulin (INS), after which cumulative concentration- response curves were constructed for phenylephrine (PHE) and serotonin (SHT). In Ln rats, INS, as compared to control, significantly reduced maximal force development in FrA from 12WK rats ( $\mathrm{E}_{\mathrm{MAX}}$ in $\mathrm{mN} / \mathrm{mm}$ ) to PHE by $36 \%(3.9 \pm 0.2$ vs $2.5 \pm 0.6)$ and $5 \mathrm{HT}$ by $38 \%$ $(3.9 \pm 0.4 \mathrm{vs} 2.4 \pm 0.5)(\mathrm{p}<0.05)$. Furthermore, In Ln rats, INS tended to increase $\mathrm{E}_{\mathrm{MAX}}$ in 30 WK rats to PHE by $19 \%(3.3 \pm 0.5$ vs $3.9 \pm 0.4)$ and to $5 \mathrm{HT}$ by $18 \%$ ( $3.5 \pm 0.5$ vs 4.1 \pm 0.4 ) (NS), respectively. In Ob rats, INS, as compared to control, did not change $E_{\text {MAX }}$ to PHE ( $3.0 \pm 0.5$ vs $\left.2.6 \pm 0.4\right)$ and $5 H T$ T (2.2 \pm 0.6 vs $\left.2.7 \pm 0.4\right)$ in FrA from $12 \mathrm{WK}$ rats and $30 \mathrm{WK}$ rats $(4.7 \pm 0.6$ vs $4.4 \pm 0.7)$ and $(5.1 \pm 0.6$ vs $4.7 \pm 0.8)$, respectively. Sensitivities to PHE and SHT were unaffected by INS. In MrA of both $\mathrm{Ln}$ and $\mathrm{Ob}, \mathrm{E}_{\mathrm{MAx}}$ and sensitivities to PHE and 5HT were unaffected by INS. These results indicate that insulin i) reduces small artery reactivity in vitro, depending on the anatomical location of the artery, ii) this effect appears age dependent and iii) it is impaired in an animal model of peripheral insulin resistance. The significance of the regionality and lack of vascular insulin responsiveness in the development/maintenance of peripheral insulin resistance has to be further established.
POST-EXERCISE $\beta$-ADRENERGIC STIMULATION OF ADIPOSE TISSUE LIPO LYSIS AND BLOOD FLOW IS UNALTERED IN OBESE INSULIN RESISTANT MEN. E. Børsheim", P. Lönnroth, S. Knardahl", and P.A. Jansson. "The Norwegian University of Sport and Physical Education, National Institute of Occupational Health, Oslo, Norway, and The Lundberg Laboratory for Diabetes Research, Sahlgrenska University Hospital, Gothenburg, Sweden.

The aim of this study was to determine if an exercise bout increases the lipolytic response to $B$-adrenoceptor stimulation, and to characterize adipose tissue blood flow (ATBF) post-exercise. Eight lean (L; BMl:23.6 $22.1 \mathrm{~kg} \cdot \mathrm{m}^{-2}$ (Mean $\pm S D$ ); plasma insulin: $5.6 \pm 1.5 \mathrm{mU} \cdot \cdot^{-1}$; heart rate: $49 \pm 6$ beats $\cdot \min ^{-1}$ ) and eight obese men (O; BMl:29.0 $\pm 1.9 \mathrm{~kg} \cdot \mathrm{m}^{-2}, p<0.001 \mathrm{vs.} \mathrm{L} ;$ insulin: $11.5 \pm 4.6 \mathrm{mU} \cdot \mathrm{l}^{-1}, \mathrm{p}=0.004 \mathrm{vs}$. L; heart rate: $65 \pm 11$ beats $\cdot \mathrm{min}^{-1}, \mathrm{p}=0.004 \mathrm{vs}, \mathrm{L}$ ) were investigated in the postabsorptive state with abdominal subcutaneous microdialysis and ${ }^{133} \mathrm{Xe}$ clearance. A stepwise in situ infusion of the non-selective B-adrenoceptor agonist isoprenaline $\left(10^{-8}, 10^{-7}\right.$ and $\left.10^{-6} \mathrm{~mol} \cdot \cdot^{-1}\right)$ was given two hours before and two hours after exercise (90 min at $50 \%$ of maximal $\mathrm{O}_{2}$ uptake). No difference in lipolysis was found neither between groups nor between response to pre- and post-exercise isoprenaline infusion. However, when the vasodilating agent hydralazine $\left(\left.0.125 \mathrm{~g} \cdot\right|^{-1}\right)$ was infused together with isoprenaline to control blood flow, there was a reduced maximum lipolytic response in both groups post-exercise: (L:251 $\pm 42 \mu \mathrm{mol} \cdot \mathrm{H}^{-1}$ (Mean \pm SE); $\left.0: 288 \pm\left. 77 \mu \mathrm{mol} \cdot\right|^{-1}\right)$ vs. pre-exercise $\left(L: 352 \pm\left. 62 \mu \mathrm{mol} \cdot\right|^{-1}, p=0.045\right.$ vs. post; $0: 380 \pm 94 \mu \mathrm{mol} \cdot F^{-1}, p=0.021$ vs. post). Pre-exercise ATBF was similar in the

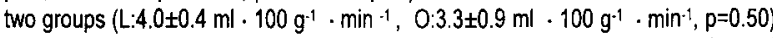
and doubled during $3.5 \mathrm{~h}$ post-exercise. However, the increase in ATBF was delayed in the obese subjects $(\mathrm{p}=0.032$ ).

In conclusion, the results indicate that adipose tissue lipolysis responsiveness to $B$. stimulation is not altered post-exercise. Lipolytic response is not altered in obese subjects despite significantly higher insulin levels, indicating that the antilipolytic effect of insulin is reduced. In the recovery period, ATBF is delayed in insulin resistant obese vs. lean subjects.
749

FETAL GROWTH IS ASSOCIATED WITH GLUCOSE UPTAKE AND BLOOD PRESSURE BUT NOT WITH PLASMA LIPIDS

L. Byberg, P. McKeigue ${ }^{2}$ and H. Lithell. Department of Public Health and Caring Sciences / Geriatrics Unit, Uppsala University, Uppsala, Sweden, and "Epidemiology Unit, London School of Hygiene \& Tropical Medicine, London, UK

Reduced size at birth has been related to disease outcome in adult life. The aim of this study was to investigate the strength of potential relations between components of the insulin resistance syndrome (IRS) and birthweight. In 1970-73, a metabolic investigation of all 50-year-old men living in Uppsala, Sweden, included 1268 men with recorded birthweights. A follow-up investigation was performed at age 70 $(n=737)$. IRS at age 50 was defined if [hypertension] and [diabetes or elevated levels of fasting glucose or insulin] and [elevated levels of serum triglycerides or reduced HDL cholesterol] were present simultaneously. Height, weight and BMI were positively related to birthweight. At age 50, systolic blood pressure, fasting insulin and glucose, and truncal fat (subscapular to triceps skinfold ratio) were inversely related to birthweight. At age 70, insulin sensitivity increased and PAI-1 activity decreased with increasing birthwejght, regardless of BMI (slope $(95 \%$ confidence interval) for $1 \mathrm{SD}$ increase in the variable and $1 \mathrm{~kg}$ increase in birthweight: $.18(.06$ to .31$)$ and $-.25(-.40$ to -10 ), respectively). There were no significant relationships between birthweight and serum triglycerides (slope at age $50:-.06(-.14$ to .03$)$ ) or $\mathrm{HDL}$ cholesterol (slope at age 50: $.01(-.11$ to .12)) measured at either investigation. The prevalence of IRS at age 50 was higher with lower birthweight, a trend strengthened by restricting the analysis to term births ( $\geq 38$ weeks gestation). In the lowest birthweight group $(<3.25$ $\mathrm{kg}$ ) $19.1 \%$ men had IRS and in the highest $(\geq 4.25 \mathrm{~kg}$ ) $6.2 \%$ had IRS ( $\mathrm{p}$ for trend: 0.008 ). A $1 \mathrm{~kg}$ decrease in birthweight was related to an increased risk of having the IRS at age 50 of 2.97 . We conclude, on the basis of these two cross-sectional investigations, that birthweight is inversely related only to some of the components of the IRS in men, namely blood pressure, glucose, insulin, insulin resistance, and PAI-1 levels, and not to serum triglycerides and HDL cholesterol. Thus, the suggested "small-baby syndrome" is not equivalent to the IRS.

\section{0}

SKELETAL MUSCLE CAPILLARY PERMEABILITY OF INSULIN IS INDEPENDENT OF BLOOD FLOW.

S. Gudbjörnsdóttir, J. Wahren* and P. Lönnroth. Lundberg Laboratory for Diabetes Research, Sahlgrenska University Hospital, and *Dep. of Clinical Physiology, Karolinska Hospital, Sweden.

Interstitial concentrations of insulin and glucose in skeletal muscle are markedly lower than in plasma, suggesting that capillary delivery of insulin to interstitial fluid is rate limiting for the insulin effect. It has been suggested that insulin delivery to muscle interstitial fluid is mediated both by passive diffusion and active transendothelial receptor-mediated transport and that plasma flow rate regulates insulin transcapillary transport. To evaluate this, permeability surface area product (PS) for insulin and glucose were measured using the forearm model, arterial and deep venous catheterization, intramuscular microdialysis and a two-step euglycemic hyperinsulinemic insulin clamp in ten healthy young males (age $26.5 \pm 1.3$, BMI $23 \pm 2.8$, (mean \pm SE)). Insulin infusion rate Insulin infusion rate P-value

$120 \mathrm{mU} / \mathrm{m}$

Plasma insulin mU/1 $230 \pm 68$

Plasma glucose $\mathrm{mmol} / \mathrm{l} \quad 6.02 \pm 0$.

Glucose infusion rate $\mathrm{mg} / \mathrm{kg} / \mathrm{min} 11.2 \pm 0.5$

Interstitial/arterial insulin ratio $0.36 \pm 0.1$

Interstitial/arterial glucose ratio $\quad 0.45 \pm 0.04$

Blood flow $\mathrm{ml} / \mathrm{min} / 100 \mathrm{ml} \quad 4.4 \pm 0.7$

PS insulin $\mathrm{ml} / \mathrm{min} / 100 \mathrm{mg} \quad 0.6 \pm 0.2$

PS glucose $\mathrm{ml} / \mathrm{min} / 100 \mathrm{~g} \quad 2.2 \pm 0.8$

$573 \pm 58$

$6.1 \pm 0.2$

$13.1 \pm 0.8$

$0.48 \pm 0.1$

$0.45 \pm 0.04$

$6.2 \pm 0.8$

$0.4 \pm 0.1$

$4.5 \pm 1.6$

P-value

ns $<0.0001$

ns $<0$. ns ns described for inulin and considerably lower than for glucose. Furthermore, the interstitial arterial concentration ratio for insulin did not change at increased plasma insulin levels. PS for insulin and glucose did not increase significantly with insulin-induced increase in blood flow. The results suggest that insulin-induced increase of plasma flow is not accompanied by significantly augmented capillary recruitment and that non-saturable diffusion is the most important transcapillary route for insulin. 
751

FLOW-MEDIATED IMPAIRMENT IN THE ABILITY OF EXERCISE TO STIMULATE GLUCOSE UPTAKE IN OBESITY AS MEASURED USING $\left[{ }^{15} \mathrm{O}\right]-\mathrm{H}_{2} \mathrm{O},\left[{ }^{15} \mathrm{O}\right]-\mathrm{O}_{2}\left[{ }^{18} \mathrm{~F}\right]-F D G$ AND PET

Pirjo Nuutila, Kirsti Larmola,Vesa Oikonen, Pauliina Peltoniemi, Teemu Takala, Jukka Kemppainen, Hanna Laine, Ulla Ruotsalainen, Juhani Knuuti, Hannele Yki-Järvinen. Departments of Medicine, University of Turku and Helsinki, and Turku PET Centre, Finland.

We compared the ability of insulin and exercise to stimulate muscle blood flow and glucose uptake in 8 obese subjects (BMI $36 \pm 2 \mathrm{~kg} / \mathrm{m}^{2}$ ) and 10 nonobese age matched subjects (BMI $22 \pm 1 \mathrm{~kg} / \mathrm{m}^{2}$ ) during euglycemic hyperinsulinemic conditions (serum insulin $70 \mathrm{mU} / \mathrm{L}$ ) and isometric exercise in one leg (rectus femoris muscle). Muscle blood flow, oxygen consumption and glucose uptake were measured simultaneously in both femoral regions using $\left[{ }^{15} \mathrm{O}\right]$-labeled water, $\left.\left.{ }^{15} \mathrm{O}\right]-\mathrm{O}_{2},{ }^{18} \mathrm{~F}\right]-\mathrm{FDG}$ and positron emission tomography (PET). Muscle oxygen consumption was 15 -fold higher in the exercising muscle compared to the resting contralateral muscle and similar in both groups during exercise ( 30 $\mathrm{mL} / \mathrm{kg}$ muscle $\cdot \mathrm{min}$ ). The obese subjects exhibited resistance to both insulin stimulation of glucose uptake in the resting leg muscles ( $26 \pm 8$ vs $43 \pm 5 \mu \mathrm{mol} / \mathrm{kg}$ muscle $\mathrm{min}, p<0.05$, for obese vs. nonobese) and to exercise stimulated glucose uptake $(100 \pm 18$ vs $149 \pm 15 \mu \mathrm{mol} / \mathrm{kg}$ muscle $\min , p<0.05$, respectively). The latter defect could be attributed to impaired ability of exercise to stimulate blood flow in the obese $(13 \pm 9$ vs $22 \pm 3 \mathrm{~mL} / \mathrm{kg}$ muscle $\mathrm{min}, \mathrm{p}<0.01$, for obese vs. nonobese). We conclude that obese subjects exhibit insulin resistance of glucose uptake at rest and a defect in the ability of exercise to stimulate both blood flow and glucose uptake. The defect in exercise induced increase in blood flow could impair the ability of physical training to improve glucose homeostasis in the obese.
752

ANTI-PLATELET AGENT, CILOSTAZOL, IMPROVES METABOLIC DERANGEMENT OF GLUCOSE IN OBESE NIDDM MODEL RATS

I. Nakahara. Y. Shiba, M. Matsuhisa, T. Tomita. M. Lida, M. Ikeda, M, Kubota. Y. Yamasaki, and M. Hori., First Department of Medicine, Osaka University School of Medicine, Osaka, Japan

The aim of this study was to examine the relationship between muscular blood flow (MBF) and insulin sensitivity (IS) in an obese NIDDM model, Otsuka Long-Evans Tokushima Fatty (OL) rats, and whether an anti-platelet agent, Cilostazol (CILO, Otsuka Pharmaceutical. Japan), which has a vasodilator action, affect the metabolic derangenent of glucose in these rats. [Methods] Ten $\mathrm{OL}$ rats were divided into 2 groups. a group of CILO-treated from 24- to 32-week-age (CILO (+), 26.1 \pm 0.6 $\mathrm{mg} / \mathrm{day})$ and a group of the diabetic control (CILO (-)). Those 2 groups of $\mathrm{OL}$ and the normal control, Long-Evans Tokushima Otsuka (LE) rats were studied ( $n=5$ in each study group). Hind-limb MBF was assessed by using a laser-Doppler flowmeter at 24- and 32-wcek-age. and IS was assessed as the metabolic clearance rate of glucose using a euglycemic hyperinsulinemic clamp at 32-week-age in each group. [Results] At 24-wcek-age, MBF in OL $(5.6 \pm 1.4 \mathrm{ml} / \mathrm{min} / 100 \mathrm{~g}$, mean \pm $\mathrm{SD})$ was significantly lower than $\mathrm{LE}(6.5 \pm 0.6 \mathrm{ml} / \mathrm{min} / \mathrm{l00} \mathrm{g})$. At 32-week-age, MBF in CILO $(+)$ was significantly increased to a similar level $(7.4 \pm 2.0$ $\mathrm{ml} / \mathrm{min} / 100 \mathrm{~g})$ with $\mathrm{LE}(6.7 \pm 0.7 \mathrm{ml} / \mathrm{min} / 100 \mathrm{~g})$, and was significantly higher than CILO (-) $(5.2 \pm 0.8 \mathrm{ml} / \mathrm{min} / 100 \mathrm{~g})$. At 32 -week-age, fasting plasma glucose level in CILO $(+)(6.7 \pm 0.5 \mathrm{mmol} / \mathrm{l})$ was comparable with $\mathrm{LE}(6.8 \pm 0.5 \mathrm{mmol} / \mathrm{l})$ and was significantly lower than CILO (-) $(8.3 \pm 0.7 \mathrm{mmol} / \mathrm{l})$. IS in CILO (+) $(21.6 \pm 3.0$ $\mathrm{m} / \mathrm{kg} / \mathrm{min})$ was significantly higher than CILO (-) $(14.6 \pm 4.8 \mathrm{ml} / \mathrm{kg} / \mathrm{min})$, but was significantly lower than LE $(36.9 \pm 4.5 \mathrm{~m} / \mathrm{kg} / \mathrm{min})$

These findings suggest that the decrement of MBF contributes to the development of insulin resistance. and the amelioration of 'this defect by vasodilative therapy may improve metabolic derangement of glucose in obese diabetic rats.

\section{3}

Impairment of glucose tolerance in survivors of myocardial infarction (MI) and in their first degree relatives (FDR)

H-D Klimm, S Jacob, S Schlageter, D Schmitt-Köppler, M Weber, S Scherer, A Volk, K Rett, W Renn, $H$ Keller, G Weismann, H-J Augustin, W Mär and H-U Häring, MI-Kid Centres, Univ. of Heidelberg, Mannheim, Freiburg and Tübingen, Germany

Type 2 Diabetes (DM) and impaired glucose tolerance (iGT) are important risk factors for coronary heart disease (CHD). However, only few studies specifically looked at the presence of disorders of GT in MI-patients. Furthermore, as children of these patients are at higher cardiovascular (cv) risk, early detection and intervention seems important. The aim of the study is to explore the cv risk factors in MI and in their asymptomatic offspring (FDR). So far we examined $50 \mathrm{Ml}$ patients, an age-and body-mass-index (=BMI)-matched control group [C, i.e. 50 subjects $(63,5 \mathrm{vs} 63,2 \mathrm{yrs}$, and $26,8 \mathrm{vs} 26,7 \mathrm{~kg} / \mathrm{m} 2)$ ] with no history of $\mathrm{CHD}$ or DM and 78 FDR. All, except for the known diabetics, underdetermination of insulin. According to the $2 \mathrm{hr}$ plasma glucose post OGTT (WHO), they were subdivided in 3 groups, $N:<7,8 \mathrm{mM} / \mathrm{L}$, $\mathrm{GGT}:<11,1 \mathrm{mM} / \mathrm{L}$ and $\mathrm{DM}:>11,1 \mathrm{mM} / \mathrm{L}$. In $\mathrm{M} \mid$ $38 \%$ were DM and $26 \%$ were iGT, while in C $17 \%$ were found to have an impairment \begin{tabular}{l|l|l|l|l|l} 
Age & BMI & N & TGT & DM \\
\hline
\end{tabular} \begin{tabular}{l|l|l|l|l|l|l} 
MI & $63,5 y$ & 26,8 & $36 \%$ & $26 \%$ & $38 \%$ \\
\hline
\end{tabular} \begin{tabular}{l|l|l|l|l|lll}
\hline FDR & $36,7 y$ & 25,6 & $75 \%$ & $13 \%$ & $12 \%$ & $28 \%$ of $C$ and $45 \%$ of FDR of those with N
\end{tabular} This study finds a high prevalence of iGT and DM in MI. As usually this was assessed only by a history of the disease and not by an OGTT, all MI with an unknown DM or IGT were not detected. Thus, the relevance of DM and iGT in MI might have been underestimated. Moreover, the FDR showed a high frequency of IGT or DM indicating that this young, asymptomatic group is at higher metabolic risk. Furthermore in all groups, a substantial proportion of $N$ had hyperinsulinemia. Thus an impairment of GT is not only very frequent in $\mathrm{MI}$, it is already present to a considerable degree in their asymptomatic FDR. These findings are currently substantiated by a larger sample size.

\section{4}

INSULIN DEPENDENT AND INDEPENDENT MYOCARDIAL GLUCOSE UPTAKE IN DIABETIC DOGS

A.I.Khomazjuk, A.P. Nescheret, I.V Gonchar and N.V. Okhrimenko. Institute of Endocrinology \& Metabolism, Vyshgorodska 69, Kyiv, Ukraine.

There is an ongoing controversy regarding the existence of insulin (I) resistance to heart muscle in diabetes. The aim of this study was to elucidate the influence of I on myocardial glucose $(G)$ uptake. Experiments were performed on 12 dogs with overt untreated diabetes (D; fasting G $15.4 \pm 1.2$ $\mathrm{mmol} / \mathrm{l}$ after 6 weeks of $75 \mathrm{mg} / \mathrm{kg}$ alloxan injection, i.v.) and 12 control dogs (C; fasting G $4.0 \pm 1.2 \mathrm{mmol} / \mathrm{l})$. Catheterization and extracorporal programmed autoperfusion of left coronary artery, catheterization of heart and continuous drainage of coronary sinus were used under chloralose anaesthesia. Myocardial G, lactate, pyruvate, NEFA, glycerol and $\mathrm{O}_{2}$ uptake (MU) were determined by calculation of coronary arteriovenous difference (AVD) at constant perfusion flow and controlled cardiohaemodynamic. There were no significant difference in MU D and C (AVD 0.42+0.05 and $0.40 \pm 0.04 \mathrm{mmol} / \mathrm{l}$, resp.) but extraction ratio (ER) was diminished in D ( 0.03 and 0.10 , resp.) At the nadir of hypoglycaemia (G $2.6+0.3 \mathrm{mmol} / \mathrm{l})$ on $30^{\text {th }}$ min of $I$ injection $(1,0 \mathrm{IU} / \mathrm{kg}$, i.v.) AVD and ER of $\mathrm{G}$ were $0.42 \pm 0.04 \mathrm{mmol} / \mathrm{l}$ and 0.16 , resp. in C but in $D$ arterial $G$ decreased only to $11.3 \pm 1.8 \mathrm{mmol} / \mathrm{l}$, AVD and ER increased to $0.72 \pm 0.08 \mathrm{mmol} / 1$ and 0.06 , resp. On the $60^{\text {th }} \mathrm{min}$ after I injection D was still hyperglycaemic $(9.41 \pm 1.3 \mathrm{mmol} / \mathrm{l}), \mathrm{AVD}$ and ER were $0.60 \pm 0.11 \mathrm{mmol} / 1$ and 0.06 , resp. We have recently demonstrated that MU of lactate, pyruvate and NEFA during I induced hypoglicaemia were attenuated due to adrenergic activation of glycolysis and lypolysis. But interaction of substrates, insulin and counterregulatory hormones in myocardial metabolism in vivo needs in further investigation. We conclude from our data that in basal state in $D$ there is no deficiency in $G$ to heart muscle due to hyperglicaemia but I stimulated MU of $\mathrm{G}$ is attenuated and its time course is slowed. 


\section{Insulin Resistance: Whole Body}

\section{5}

\section{CHANGES IN MUSCLE MORPHOLOGY IN SUBJECTS WITH IMPAIRED GLUCOSE TOLERANCE AND TYPE II DIABETES}

Anu Hedman and Hans Lithell, Dpt. of Public Health and Caring Sciences/Geriatrics Unit, Uppsala University, Uppsala, Sweden.

Skeletal muscle accounts for up to $85 \%$ of the glucose that is metabolized following a glucose load. Peripheral insulin sensitivity has been associated with the alterations in the fiber type distribution and capillary supply in the muscle. In a cohort of 70year-old men in Uppsala, a muscle biopsy from vastus lateralis muscle was performed in order to investigate the differences in muscle morphology between the groups with different degree of insulin sensitivity. The different types of muscle fibers were detected by myofibrillar ATPase method and capillaries (per $\mathrm{mm}^{2}$ ) were visualized with Amylase-PAS method. Insulin sensitivity was investigated by hyperinsulinemic euglycemic clamp procedure. Comparison of 64 IGT subjects, 71 subjects with NIDDM and 177 healthy untreated controls was made. Compared to controls, the subjects with IGT and NIDDM had a higher number of glycolytic type IIB fibers and a decreased number of oxidative type I fibers, which was statistically significant even independent of obesity in the NIDDM group. The subjects with NIDDM, but not with IGT, had a significantly lower capillary density compared to healthy controls.

\begin{tabular}{llll} 
& Controls & IGT & NIDDM \\
\hline \% of type I fibers & 51.3 & $47.7^{*}$ & $45.1^{* *}$ \\
\% of type IIB fibers & 18.7 & $21.8^{*}$ & $24.3^{* *}$ \\
Capillary density in $\mathrm{mm}^{2}$ & 324 & 317 & $302^{*}$ \\
M/I & 6.06 & $4.22^{* * *}$ & $3.22^{* * *}$ \\
\hline
\end{tabular}

$\mathrm{P}<0.0001$ compared to contro

Insulin sensitivity index $(\mathrm{M} / \mathrm{I})$ was positively correlated to type I fibers $(\mathrm{r}=0.27$. $0.49 ; \mathrm{P}<0.05)$ and negatively correlated with type IIB fibers $(\mathrm{r}=-0.32--0.51 ; \mathrm{P}<$ $0.01)$ in all groups even after the adjusting for obesity $(r=-0.36 ; P<0.05)$, which strongly contributes to the increase in type IIB fibers. Thus, insulin resistance was accompanied by decreased number of insulin-sensitive type I fibers, increased number of type IIB fibers and capillary rarefaction in the skeletal muscle in the patients with impaired insulin sensitivity. This was more pronounced in patients with NIDDM than in subjects with IGT.

\section{7}

EVIDENCE OF DAY-NIGHT CHANGES IN INSULIN SENSITIVITY IN TYPE 2 DIABETES MELLITUS

G. Perriello, W. Pimenta, S. Pampanelli, F. Porcellati, M. Lepore, P. Lucidi, M.C. Cordoni, P. Brunetti and G. B. Bolli*, DIMISEM Perugia, Italy

Day-time changes in insulin sensitivity in patients with type 2 diabetes mellitus have long been suspeted. Previous studies failed to demostrate diurnal variation in insulin sensitivity and their effects on glycemic control. To assess the clinical relevance and mechanisms of daynight variability in glucose metabolism, we studied 7 diet-treated type 2 diabetics $(5 \mathrm{M}, 2 \mathrm{~F}$; Age $56 \pm 1$; BMI $27.6 \pm 0.9)$ during a 3-hour isoglycemic-hyperinsulinemic ( $10 \mathrm{mU} / \mathrm{m}^{2} / \mathrm{min}$ ) clamp using a combination of isotopic $\left(6,6-{ }^{2} \mathrm{H}_{2}\right.$-glucose) and balance (forearm) techniques. All patients were studied on two occasions, a week apart, between 0500 and $0800 \mathrm{~h}$ (Study AM) and between 1700 and $2000 \mathrm{~h}$ (Study PM), after the same interval of fasting ( 9 hours). Before starting experiments, plasma glucose was greater in the morning $(6.6 \pm 0.3 \mathrm{mmol} /)$ than in the afternoon $(5.1 \pm$ $0.2 \mathrm{mmol} / \mathrm{l},(\mathrm{p}<0.05)$. During steady-state $(150-180 \mathrm{~min})$ plasma glucose $(5.2+0.2$ and $5.1 \pm 0.2 \mathrm{mmol} / \mathrm{l}, \mathrm{AM}$ and $\mathrm{PM} ; \mathrm{p}=\mathrm{NS})$ and insulin $(202 \pm 21$ and $198 \pm 18 \mathrm{pmol} / \mathrm{AM}$ and $\mathrm{PM}, \mathrm{p}=\mathrm{NS}$ ) levels of clamp experiments, the amount of exogenous glucose needed to maintain euglycemia resulted lower in study AM than in PM $(2.68 \pm 1.11$ vs $6.87 \pm 0.81 \mu \mathrm{mol} / \mathrm{kg} / \mathrm{min}$, $\mathrm{p}<0.05$ ). Systemic glucose production was higher in study AM than in $P M$ $(6.47 \pm 0.53$ vs $1.98 \pm 0.32 \mu \mathrm{mol} / \mathrm{kg} / \mathrm{min}, \mathrm{p}<0.05)$, whereas neither systemic $(9.15+0.51$ and $8.85+0.49 \mu \mathrm{mol} / \mathrm{kg} / \mathrm{min})$ nor muscle glucose utilisation $(162 \pm 16$ and $160 \pm 11 \mu \mathrm{mol} / \mathrm{min}, \mathrm{p}=\mathrm{NS})$ were different. In conclusion, during physiological hyperinsulinemia Type 2 diabetic patients exhibit important changes in day-night insulin sensitivity, due to variations in systemic glucose production.

\section{6}

INCREASED MUSCLE TRIGLYCERIDES CONTENT IN YOUNG, NON-OBESE FIRST DEGREE RELATIVES OF NIDDM PARENTS: A ${ }^{1}$ H-NMR ASSESSMENT. G. Perseghin, P. Scifo, F. De Cobelli, A Vanzulli, A. Del Maschio, G. Pozza and L. Luzi. Istituto Scientifico H San Raffaele Milan, Italy

Insulin resistance is the best predictor for the development of NIDDM and it has been suggested that intramuscular triglycerides store is a pathogenetic factor for its development. To test this hypothesis 14 young, non-obese offspring of NIDDM parents $\left(7 \mathrm{M} / 7 \mathrm{~F}\right.$, age $=30 \pm 1 \mathrm{ys}, \mathrm{BMI}=22 \pm \mathrm{l} \mathrm{kg} / \mathrm{m}^{2}$, Ideal Body Weight $=104 \pm 3 \%$, WHratio $=0.85 \pm 0.03$ ) and 14 normal healthy subjects matched for age, gender, antropomorphic parameters, physical activity and life habits, underwent localized proton nuclear magnetic resonance spectroscopy ('H-NMR) of the soleus (fiber type l, slow twich, insulin sensitive) and of the tibialis anterioris (fiber type II, fast twitch, insulin resistant). ${ }^{1} \mathrm{H}-\mathrm{NMR}$ has been recently validated for the assessment of intramyocellular lipids. Measurements of intramyocellular lipids in Arbitrary Units (AU) were obtained using a phantom of triglycerides solution as external standard. Offspring of NIDDM parents were characterized for slightly increased fasting plasma insulin $(9.8 \pm 1.6 \mathrm{vs} 7.7 \pm 0.9 \mu \mathrm{U} / \mathrm{ml} ; \mathrm{p}=0.05)$, glucose $(93 \pm 4 \mathrm{vs} 84 \pm 3 \mathrm{mg} / \mathrm{dl} ; \mathrm{p}=0.0 \mathrm{l})$ and triglycerides ( $115 \pm 28$ vs $80 \pm 9 \mathrm{mg} / \mathrm{dl} ; \mathrm{p}=\mathrm{NS}$ ). Intramyocellular triglycerides content in the soleus muscle was increased in offspring of NIDDM parents ( $1047 \pm 40$ vs $636 \pm 53$ $\mathrm{AU}: \mathrm{p}<0.00 \mathrm{l})$ but not in the tibialis anterioris $(493 \pm 52 \mathrm{vs} 395 \pm 52 \mathrm{AU} ; \mathrm{p}=0.15)$. A subgroup of 10 subjects ( 5 offspring and 5 normals) underwent a euglycemic ( 90 $\mathrm{mg} / \mathrm{dl}$ )-hyperinsulinemic $(85 \mu \mathrm{U} / \mathrm{ml})$ clamp to assess insulin sensitivity and simple regression analysis between the $\mathrm{M}$-value and the intramyocellular triglycerides in the soleus showed a signilicant relationship (adjusted $R^{2}=0.25 ; p=0.01$ ). In summary olfspring of NIDDM parents showed increased intramyocelluar triglycerides concentration in the most insulin sensitive muscle fibers. This finding suggests that intramyoceliular accumulation of triglycerides mny play a primary role in the development of insulin resistance and that might be considered a valuable index of risk to develop diabetes in the future. Further studies need to be perfornted to assess the qualitutive composition in free fatty acids of the intramyocellular triglycerides.

\section{8}

INSULIN DEFICIENCY OR RESISTANCE AS THE INITIATING FACTOR OF GLUCOSE INTOLERANCE IN JAPANESE MigRANTS?

S.R.G. Ferreira, S.G.A. Gimeno, L.J. Franco, M. Iunes and JBDSG. Federal University of São Paulo. Rua Botucatu, 740 - 04023-062 - São Paulo, SP, Brazil

Hyperglycemia results from an unbalance between insulin secretion and peripheral sensitivity in subjects with type 2 diabetes (DM). Although Japanese migrants present high prevalence of DM, its classic phenotype including obesity, is not seen among them. The aim of this study was to evaluate if insulin deficiency or resistance is the underlying mechanism initiating glucose intolerance in Japanese migrants living in Brazil. Estimates of $\beta$-cell function and insulin resistance were obtained by the homeostasis model assessment (HOMA), according to the mathematical formulae described by Matthews (1985). The values of fasting glucose and insulin were derived from an oral glucose tolerance test, used to classify the subjects according to WHO criteria. In the population sample $(n=530), 349$ subjects had normal (NGT) and 90 impaired glucose tolerance (IGT), and the 91 diabetics were excluded from the analysis. HOMA estimates of $\beta$-cell function and resistance were compared among 3 groups of subjects: NGT, with and without family history of DM, and IGT. Their mean ages and male/female ratios were similar. Higher body mass index, mean blood pressure, fasting and 2-hr insulin and proinsulin levels and lower HDLcholesterol were found in the IGT group as compared to the others. While HOMA estimates of $\beta$-cell function did not differ among the groups (medians, $25.0,30.9$ and 44.6 , for NGT with and without family history, and IGT, respectively), HOMA estimates of resistance were higher in the IGT group (median, 1.06) as compared to the NTG groups (medians, 0.51 and 0.60 , with and without family history) $(\mathrm{p}<0.001)$. Our data favor the hypothesis that insulin resistance rather than insulin deficiency may represent the underlying mechanism of glucose metabolism disturbance in Japanese-Brazilians. 
LOW-DOSE ANGIOTENSIN CONVERTING ENZYME INHIBITOR THERAPY DOES NOT IMPROVE INSULN SENSITIVITY IN NORMOTENSIVE TYPE 2-DIABETIC PATIENTS

H. Tillil, C. von Boxberg, F. Buschkotte, J. Köbberling, Dept. of Medicine, Ferdinand Sauerbruch-Klinikum Elberfeld, Klinikum Wuppertal, Teaching Hospital of the Heinrich Heine University of Düsseldorf

Short term captopril therapy has been described to improve glucose metabolism by increasing insulin sensitivity in hypertensive type 2-diabetic patients. Aim of the study: It should be investigated whether this effect can also be observed in normotensive type 2-diabetic patients independently of a reduction in blood pressure. Patients and methods: Insulin sensitivity was measured by the euglycemic hyperinsulinemic glucose clamp technique in 20 normotensive type 2 -diabetic patients without insulin treatment $(4 / 16$ $\mathrm{F} / \mathrm{M}$, age $55.2 \pm 6.5$ years, BMI $27.5 \pm 4.4 \mathrm{~kg} / \mathrm{m}^{2}$ ) before and after a 4 -week randomized placebo controlled doubleblind therapy with ramipril $2.5 \mathrm{mg} /$ day (mean+SD). Results: After a 4-week therapy mean 24-h blood pressure did not change significantly in the ramipril (systolic: $129 \pm 11 \mathrm{~mm} \mathrm{Hg}$ before vs $127 \pm 9 \mathrm{~mm} \mathrm{Hg}$ after therapy, $\mathrm{p}=0.58$; diastolic: $73.5 \pm 5$ vs $76 \pm 3 \mathrm{~mm} \mathrm{Hg}$ $p=0.22$ ) nor in the placebo group (systolic: $135 \pm 12$ vs $135 \pm 10 \mathrm{~mm} \mathrm{Hg}$, $\mathrm{p}=0.92$; diastolic: $80 \pm 5$ vs $78 \pm 6 \mathrm{~mm} \mathrm{Hg}, \mathrm{p}=0.23$ ). There was no significant change of insulin sensitivity (M-value) in the ramipril nor in the placebo group (ramipril: $3.9 \pm 2.4$ vs $3.7 \pm 2.1 \mathrm{mg} / \mathrm{kg} \cdot \mathrm{min}, \mathrm{p}=0.61$; placebo: $4.0 \pm 2.3$ vs $4.3+2.6 \mathrm{mg} / \mathrm{kg} \cdot \min , \mathrm{p}=0.10)$. $\mathrm{HbA}_{1 \mathrm{c}}$ did not change significantly in the ramipril group $(7.6 \pm 1.0$ vs $7.5 \pm 1.1 \%, p=0.37)$ nor in the placebo group $(7.7 \pm 1.3$ vs $7.4 \pm 1.6 \%, p=0.28)$. Summary and conclusion: A 4-week therapy with a low dose of ramipril had no effect on the blood pressure and did not improve insulin sensitivity in normotensive type 2-diabetic patients. The effect of ramipril therapy on insulin sensitivity needs to be investigated in hypertensive type 2-diabetic patients.
FIVE-YEAR PROGRESSION OF THE METABOLIC SYNDROME IN RELATIVES OF TYPE 2 DIABETIC PATIENTS.

S.M.A.Bennett, P.A.Shearing, A.Littlefield, M.D.Brown, L.A.Ashworth, M.W.Stewart and M.Walker. Department of Medicine, University of Newcastle, Newcastle upon Tyne, UK

AIMS: to examine the progression over five years of the Metabolic Syndrome in relatives of type 2 diabetic patients. BACKGROUND: relatives of type 2 diabetic patients are at increased risk of cardiovascular disease and diabetes. We have previously reported that firstdegree relatives have more features of the Metabolic Syndrome than control subjects with no family history of diabetes. METHODS: 97 relatives ( 42 males, 55 females), age (mean $\pm S D$ ) $45.0 \pm 9.5$ years, underwent a $75 \mathrm{~g}$ OGTT, assessment of fasting lipids and insulin, and anthropometric measurements at baseline and after five years. Changes were compared by paired t-tests. RESULTS: using baseline data we identified two groups of relatives: those with no features $(n=30)$, and those with three or more features $(n=16)$ of the Metabolic Syndrome (obesity, hypertension, dyslipidaemia, abnormal glucose tolerance and hyperinsulinaemia). The relatives with no features showed significant increases (means [SD differences]) over five years in BMI $\left(23.9,25.5[2.4] \mathrm{kgm}^{-2}, p=0.001\right)$, triglycerides $(0.84,1.05$ [0.41] $\left.\mathrm{mmoll}^{-1}, p=0.008\right)$ and insulin resistance [HOMA IR] $(0.87,1.64$ $[0.97], p<0.001)$. There was no further progression in those with three or more features, although they showed decreased HOMA $\beta$-cell function. CONCLUSION: relatives of type 2 diabetic patients who do not have the Metabolic Syndrome show a progressive development of an adverse cardiovascular risk profile, whilst those in whom the Metabolic Syndrome is established show little further increase in their risk over five years.

\section{1}

Normal Glucose Induced Suppression of Production But Impaired Stimulation of Disposal in Type 2 Diabetes: Evidence for a Concentration-Dependent Defect in Uptake.

Michael F. Nielsen, Rita Basu, Steven Wise, Andrea Caumo, Claudio Cobelli and Robert A Rizza.

Rochester, MN; Milan, Italy; Aarhus, Denmark

To determine whether people with type 2 diabetes are resistant to the effects of glucose, nine diabetic and ten nondiabetic subjects were studied on three occasions Endogenous hormone secretion was inhibited with somatostatin and insulin concentrations were kept constant at individually determined "basal" insulin concentrations (defined as that necessary to maintain glucose at $\sim 5 \mathrm{mM}$ ) from 0 to 180 minutes then increased to $\sim 200 \mathrm{pmol} / \mathrm{L}$ from 181 to 360 minutes (high insulin infusion). Glucose was clamped at either 95,130 or $165 \mathrm{mg} / \mathrm{dl}$ on each occasion. In the presence of basal insulin concentrations, a progressive increase in glucose concentration from 95 to 130 to $165 \mathrm{mg} / \mathrm{dl}$ was accompanied by a comparable and progressive decrease $(p<0.01)$ in EGP (measured by $\left[6{ }^{3} \mathrm{H}\right]$ glucose), total glucose output (measured by $\left[2-{ }^{3} \mathrm{H}\right]$ glucose) and incorporation of ${ }^{14} \mathrm{CO}_{2}$ into glucose (a qualitative index of gluconeogenesis) indicating normal hepatic (and probably also renal) response to glucose. In the nondiabetic subjects, an increase in glucose concentration from 95 to 130 to $165 \mathrm{mg} / \mathrm{dl}$ resulted in a progressive increase in glucose disappearance during both the low $(p<0.01)$ and high $(p<0.01)$ insulin concentrations. In contrast, in the diabetic subjects, whereas an increase in glucose from 95 to $130 \mathrm{mg} / \mathrm{dl}$ resulted in an increase in glucose disappearance during both the low ( $p<0.01$ ) and high ( $p<0.01$ ) insulin concentration, a further increase in glucose concentration to $165 \mathrm{mg} / \mathrm{dl}$ had no further effect on glucose disappearance at either low or high insulin concentration $(p>0,38)$. We therefore conclude that whereas glucose induced stimulation of its own uptake is abnormal in type 2 diabetes, glucose induced suppression of endogenous glucose production and output is not. The abnormality in uptake occurs in the presence of both low and high insulin concentrations and is evident at glucose concentrations above but not below $130 \mathrm{mg} / \mathrm{d}$ implying a defect in a glucose responsive step.

\section{2}

INSULIN ACTION AND SECRETION IN OFFSPRING OF PATIENTS WITH TYPE 2 DIABETES.

A. Volk, W. Renn, E. Maerker, B. Dahl, M. Haap, H.U. Häring and K. Rett

Medizinische Klinik, Abtl. IV, Universität Tübingen

Offspring of patients with type 2 diabetes (FDR) are at increased risk for type 2 diabetes (DM2). It is a matter of controversy, whether insulin action or secretion -or both- are disturbed in FDR. In 154 young (mean 33 ys, range 18-50ys), glucose tolerant FDR we directly assess both insulin sensitivity [glucose metabolic clearance rate (MCR)] and insulin secretion. 154 age-, sex- and weight-matched subjects without known family history of DM2 serve as controls (CL). MCR is determined by euglycaemic-hyperinsulinaemic glucose-clamp. Insulin secretion and clearance during an oral glucose load (oGTT:40g glucose $/ \mathrm{m}^{2}$ ) are estimated by deconvolution techniques applied to stimulated serum Cpeptide using the kinetics published by Polonsky et al.. MCR is scattered across a wide range in both FDR and CL (1.9-16.5 ml/ $/ \mathrm{kg} / \mathrm{min})$. Average MCR in FDR is $10 \%$ lower $(\mathrm{p}<0,05)$. Prevalence of insulin resistance (IR) is $44 \%$ in FDR vs. $26 \%$ in CL. According to MCR $44 \%$ of the FDR are classified as insulin resistant (IRFDR) and $34 \%$ as insulin sensitive (ISFDR). MCR and insulin clearance are correlated in FDR $(r=0,4 ; p<0,05)$. Secretion curve is significantly elevated in IRFDR compared to ISFDR and total insulin response (AUC) is higher in IRFDR (72 \pm 8 vs. $\left.29 \pm 2 \mathrm{nmol} / \mathrm{l}^{*} 120 \mathrm{~min} ; \mathrm{p}<0,01\right)$. In IRFDR secretion progressively rises until $60 \mathrm{~min}$ whereas in ISFDR secretion peaks at $30 \mathrm{~min}$. When secretion is expressed as fold increase above basal, early phase $(0-30 \mathrm{~min})$ is significantly lower in IRFDR $(5,3 \pm 0,4$ vs. $7,3 \pm 0,5 ; p<0,01)$ whereas late phase secretion (60-120min) of ISFDR and IRFDR are identical. Absolute hyperinsulinaemia but delayed and reduced relative secretion in IRFDR is dependent on the presence of overweight (ow;BMI $>25 \mathrm{~kg} / \mathrm{m}^{2}$ ). Secretory response of lean (L) and ow ISFDR and L IRFDR are similar and significantly different from ow IRFDR. Finally, degree and prevalence of IR is higher in FDR than CL. IR in FDR is associated with reduced insulin clearance. In presence of ow IR is characterized by delayed and reduced relative early phase secretion and absolute hyperinsulinaemia. 


\section{3}

INADEQUACY OF HOMA TO DETECT BETA CELL DYSFUNCTION IN INDIVIDUALS WITH IMPAIRED GLUCOSE TOLERANCE

A.Mitrakou, D.Platanisiotis, X Orfanos, F. Giardina and S.A.Raptis

$2^{\mathrm{ND}}$ Dept of Internal Medicine Research Institute and Diabetes Center Athens University Evangelismos Hospital

Homeostasis Model Assessment (HOMA) is a widely used method to assess beta cell function and insulin sensitivity Abnormal beta cell function and insulin resistance have been determined to play important role in the deterioration of individuals from normal glucose tolerance to impaired glucose Therefore to determine whether HOMA could be used as a sensitive method to assess alterations in beta cell function and insulin sensitivity in people with impaired glucose tolerance, we compared HOMA derived values for beta cell function and insulin sensitivity in 200 individuals with impaired glucose tolerance (IGT)and 463 individuals with normal glucose tolerance classified using the WHO criteria. The increment in the $30 \mathrm{~min}$ plasma insulin concentration (30min $\mathrm{Pl}$ ) was used as an independent measurement of beta cell function because it has been shown to predict development of IGT and /or diabetes. We found that HOMA derived values of insulin resistance were greater in IGTs than NGTs $(2.16 \pm 0.1$ vs $2.94 \pm 0.2, p<0.001)$. However estimates of beta cell function using the HOMA was quite similar in IGTs and NGTs $(129 \pm 8.7$ vs $137 \pm 7.5 p=0.5)$. In contrast the 30 min PI was significantiy reduced in IGTs compared to NGTs ( $293 \pm 9$ vs $226 \pm 10 \mathrm{pmol} / \mathrm{L}, \mathrm{p}<0.001)$ Furthermore there was no correlation between beta cell function as assessed by HOMA and the $30 \mathrm{~min} \mathrm{PI}(r=0.095, p=0.032$ ) We therefore conclude that while HOMA may detect changes in insulin sensitivity between individuals with IGT and NGT, it is inadequate to detect differences in beta cell function

\section{4}

CORONARY ARTERY DISEASE RISKS PREDICTED BY INSULIN RESISTANCE, LIPIDS AND HYPERTENSION IN NONDIABETIC INDIVIDUALS

WHH Sheu, CY Jeng, MS Young, YA Ding and Y-T Chen Taichung and Taipei, Taiwan, ROC

Although insulin resistance, plasma lipids concentrations and hypertension are frequently associated with coronary artery disease (CAD), their relative contributions and predictive power were still unclear particularly in those of nondiabetic subjects. We examined these risk factors in 96 nondiabetic patients with angiographically documented CAD and 96 age, sex, BMImatched controls. Patients with CAD had significantly higher fasting cholesterol (C); LDL-C and triglyceride while lower HDL-C concentrations as compared with controls $(\mathrm{p}<0.02-0.001)$. In response to a $75 \mathrm{~g}$ ora glucose tolerant test, glucose (area $31.6+0.8$ vs $21.0+0.3 \mathrm{mmol} / . \mathrm{h}, \mathrm{p}<0.001$ ) and insulin $(1866 \pm 126$ vs $1272 \pm 78 \mathrm{pmol} / \mathrm{l} . \mathrm{h}, \mathrm{p}<0.01)$ responses were also higher in patients with CAD when compared to those of controls. Degree of insulin resistance as measured by insulin suppression test were higher in patients with CAD (steady state plasma glucose (SSPG) values $12.2+0.4$ vs $8.1+0.4 \mathrm{mmol} / \mathrm{l}, \mathrm{p}<0.001$ ) than nonCAD. If one considers HDL-C $<0.9$ $\mathrm{mmol} / \mathrm{h}, \mathrm{LDL}-\mathrm{C}>4.1 \mathrm{mmol} / \mathrm{l}$, triglyceride $>2.3 \mathrm{mmol} / \mathrm{l}, \mathrm{SSPG}>10.5 \mathrm{mmol} / 1$ and history of hypertension as separate risk factors for $\mathrm{CAD}$, individuals displaying 3 or more risk factors were found 10 times more frequently in patients with CAD than in controls. By logistic regression analysis, SSPG values was the strongest risk followed by lower HDL-C, triglyceride, LDL$\mathrm{C}$ and hypertension, all of which account for $35.7 \%$ of CAD in these subjects. In conclusion, 1), nondiabetic CAD patients were glucose intolerant, hyperinsulinemic and insulin resistant 2) insulin resistance, plasma lipids values and hypertension together accounted, for one third of the development of CAD while insulin resistance was the strongest predictor in nondiabetic individuals.

\section{5}

PC-1 CIRCUlates IN hUMAN PLASMa: RELATIONSHIP WITH INSULIN RESISTANCE AND ASSOCIATED ABNORMALITIES L. Frittitta*. A. Natali ${ }^{\circ}$, R. Baratta*, S. Graci*, ID. Goldfine\&, R. Vigneri*, E Ferrannini ${ }^{\circ}$ and V. Trischitta*^. ${ }^{*}$ Instit Int Med, Univ of Catania; ${ }^{\circ}$ Instit Clin Physiol, CNR Pisa; ^Div End, Scient Instit CSS, SG Rotondo; Italy. §Diab Res Lab, Univ of CA, S Francisco; USA.

Membrane glycoprotein PC-1 is overexpressed in both skeletal muscle and subcutaneous adipose tissue of healthy non diabetic subjects with a reduced whole body insulin sensitivity and it is associated to a diminished insulin receptor tyrosinekinase activity. PC-1, therefore, may play an important role in the development of insulin resistance (IR) and related diseases. The aim of the present study was to verify whether soluble PC-1 is present in human plasma and whether circulating PC-1, as muscle PC-1 content, is related to the subject insulin sensitivity. Fifty normotensive subjects, 25 insulin sensitive (S) with a $M$ value at euglycaemic clamp=6.9 \pm 0.3 $\mathrm{mg} / \mathrm{kg} \cdot \mathrm{min}(\mathrm{mean} \pm \mathrm{SE}$ ) and 25 insulin resistant $(\mathrm{R}), \mathrm{M}$ value $=4.3 \pm 0.2$ and 20 hypertensive subjects $(\mathrm{Ht})$ with a mean blood pressure $(\mathrm{MBP})>107 \mathrm{mmHg}$ and $\mathrm{M}$ value $=4.9 \pm 0.4$ were studied. BMI was significantly $(p<0.05)$ higher in $R(28.4 \pm 1.1)$ and $\mathrm{Ht}(27.2 \pm 0.7)$ than in $S(25.1 \pm 0.7)$. At Western blot, by immunoprecipitation and blotting with different anti-PC-1 antibodies, 3 bands were identified in human plasma at 130, 90 and $80 \mathrm{KDa}$. Plasma PC-1 content (measured by a specific ELISA) ranged $2.82 \mathrm{ng} / \mathrm{ml}$ and was significantly higher in $\mathrm{S}$ than in $\mathrm{R}$ and $\mathrm{Ht}(36.7 \pm 4.5 \mathrm{vs}$ $22.7 \pm 3.0$ vs $18.3 \pm 4.3$ respectively, $p<0.005$ by 1 way ANOVA (esi). When all 70 subjects were considered altogether, $\mathrm{PC}$ - 1 content was significantly correlated with the waisthip ratio $(r=-0.46, p<0.0001)$ MBP $(r=-0.32, p<0.01)$, and HDL/total cholesterol ratio $(r=0.24, p<0.05)$. Since these data were different from expected ones we also measured PC-1 content in the skeletal muscle of additional 20 subjects. Data indicated an inverse correlation between plasma and muscle $\mathrm{PC}-1$ content $(\mathrm{n}=20, \mathrm{r}=$ $0.5, \mathrm{p}<0.05$ ). In conclusion: 1) soluble PC-1 is present in human plasma; 2) PC-1 concentration in plasma is inversely related to skeletal muscle PC-1 content; 3) a low plasma PC- 1 content is associated with reduced insulin sensitivity, abdominal fat accumulation, high blood pressure and dyslipidaemia; 4) these findings may sugges the use of plasma PC-1 measurement as a possible marker in prospective studies aimed to understand the role of PC-1 in the development of insulin resistance and related diseases.

\section{6}

BROMOCRIPTINE PLUS METFORMIN AMELIORATES SEVERE GLUCOSE INTOLORANCE AND INSULIN RESISTANCE INDUCED BY A HIGH FAT DIET IN HAMSTERS.

A H. CINCOTTA ${ }^{* 1,2}$, P. SCISLOWSKI J. JOSLIN ${ }^{2}$, R. PREVELIGE

S. PHANEUF ${ }^{2}$, and S. R. CINCOTTA ${ }^{2}$, Charlestown, MA

Polypharmacia for the treatment of diabetes is founded on the principle that two compounds which reduce hyperglycemia by independent mechanisms may be combined to produce additive effects on glycemic control. Bromocriptine (BC) and metformin (MET) may be two such compounds; bromocriptine altering central regulation of metabolism and metformin reducing hepatic glucose production. The present study investigated the additive effects of bromocriptine and metformin on reducing body weight, glucose intolerance, and hyperinsulinemia in hamsters fed a high fat diet. Male Syrian hamsters, held on 14-hr daily photoperiods, were fed a high energy ( $4.4 \mathrm{kcal} / \mathrm{g}) 49 \%$ fat, 35\% carbohydrate, $15 \%$ protein (by energy contribution), vitamin supplemented diet for 3 weeks. Animals (ave. $\mathrm{BW}=190 \mathrm{~g}$ ) were then divided into 4 groups and treated with either bromocriptine $(4 \mathrm{mg} / \mathrm{kg}$ ) at light onset, metformin $(200 \mathrm{mg} / \mathrm{kg}$ ) at light offset, both BC plus MET, or vehicle at both times of day for 3 weeks while held on the high fat diet. Glucose tolerance tests (GTT) (3g glucose $/ \mathrm{kg}$ ) were performed $24-\mathrm{hr}$ following the termination of treatment. A six week treatment with the high fat diet produced severe glucose intolerance (glucose $=270 \mathrm{mg} / \mathrm{dl}$ and insulin $=498 \mu \mathrm{U} / \mathrm{ml}$ at $120 \mathrm{~min}$ after glucose administration). Neither BC nor MET affected the GTT area under the glucose curve while $\mathrm{BC}$ plus MET reduced it by $36 \%(\mathrm{P}<0.05)$. BC and $\mathrm{BC}$ plus MET but not MET alone reduced the GTT area under the insulin curve (by $67 \%$ relative to control; $\mathrm{P}<0.01$ ). Furthermore, $\mathrm{BC}$ but not MET reduced basal hyperinsulinemia $(60 \%$, from $279 \pm 69$ to $112 \pm 16 \mu \mathrm{U} / \mathrm{ml} ; \mathrm{P}<0.05)$ which was further reduced to $65 \pm 11 \mu \mathrm{U} / \mathrm{ml}$ by $\mathrm{BC}$ plus MET treatment $(\mathrm{P}<0.01$ relative to control and $\mathrm{BC}$ groups). BC and MET reduced body weight by $12 \mathrm{~g}$ and $11 \mathrm{~g}$, respectively, but $\mathrm{BC}$ plus MET reduced body weight by $26 \mathrm{~g}(\mathrm{P}<0.01$ relative to control and $\mathrm{P}<0.05$ relative to $\mathrm{BC}$ and MET groups). These results indicate that $\mathrm{BC}$ and MET can be combined to produce additive and robust improvements in the obese-insulin resistant condition in animals maintained on a high fat diet. Further studies indicate that this interactive drug effect on carbohydrate metabolism is associated with an increased capacity for skeletal muscle fatty acid oxidation as assessed by palmitate oxidation in vitro. 


\section{7}

EXENDIN-4 DOSE-DEPENDENTLY DECREASES HEMOGLOBIN $A_{1 C}$ AND IMPROVES INSULIN SENSITIVITY IN DIABETIC FATTY ZUCKER RATS

B. Gedulin, C. Jodka, J. Hoyt and A. Young. Amylin Pharmaceuticals, Inc., 9373 Towne Centre Dr., San Diego, CA 92121, USA

Exendin-4, a 39 amino acid peptide from the venom of the Gila Monster (Heloderma suspectum) with some structural similarity to glucagon-like peptide-1 (GLP- $t$ ), acutely decreases plasma glucose in diabetic $d b / d b$ mice and diabetic thesus monkeys (Macaca mulata). Here we examined the peptide's potential antidiabetic properties in Diabetic Fatty Zucker (DFZ) rats in 2 separate studies. In the first, DFZ rats were injected twice daily with either saline $(n=6)$ or $100 \mu g$ exendin-4 $(n=6)$. Change in Hemoglobin $A_{1}$ $\left(\Delta \mathrm{HbA}_{1 c}\right)$ over 5 weeks was greater in exendin-treated than in saline-treated rats $(-2.85 \pm 0.17$ units; $P<0.0001$ vs pretreatment; $P<0.01$ vs saline controls $1.26 \pm 0.33$ units at 5 weeks). $\Delta \mathrm{HbA}_{1 \mathrm{c}}$ in lean rats treated with exendin or saline was $-0.21 \pm 0.07$ and $-0.67 \pm 0.06$ units, respectively, a lesser effect than in hyperglycemic animals. Insulin sensitivity, measured as glucose infusion rate per measured insulin concentration $(\mathrm{M} / \mathrm{l})$ in a hyperinsulinemic euglycemic clamp 22 hours after the last exendin/saline injection, was increased $107.8 \pm 12.8 \%(P<0.01)$. In a second dose-response study, DFZ rats ( $n=3-5 /$ group) were injected twice daily with saline or exendin-4 in doses of $0.1,1,10$ or $100 \mathrm{ug}$ for 5 weeks. In contrast to the first study, saline-treated DFZ rats increased their $\mathrm{HbA}_{t c}$ with time $\left(\Delta \mathrm{HbA}_{1 \mathrm{c}}=1.30 \pm 0.17\right.$ units; $P<0.001$ vs pretreatment) but exendin-4 dose-dependently retarded this rise $\left(E D_{50}\right.$ $0.28 \mu \mathrm{g} \pm 0.33 \mathrm{log}$ units; $P<0.01$ ANOVA). Exendin-4 treatment dosedependently increased glucose infusion rate measured during 60-180 min of the clamps from $8.6 \pm 0.45$ (control) to $12.61 \pm 0.96 \mathrm{mg} / \mathrm{kg} / \mathrm{min}$ in the high dose group $(P<0.005)\left(E_{50} 3.5 \mu \mathrm{g} \pm 0.26 \mathrm{log}\right)$. In summary, 5 weeks treatment of Diabetic Fatty Zucker rats with exendin- 4 was associated with a reduction in $\mathrm{HbA}_{\mathrm{lc}}$ and an improvement in insulin sensitivity.
768

VENTROMEDIAL HYPOTHALAMIC LESIONS IN OBESE, INSULINRESISTANT RODENTS ATTENUATES SYMPTOMS OF DIABETES.

K. G. Bina, J. Li, and A. H. Cincotta, ErgoScience Corp., 100 First Ave., Charlestown, MA 02129, USA.

In lean rodents, damage to the ventromedial hypothalamus ( $\mathrm{VMH})$ results in hyperphagia, insulin-resistance (IR) and subsequent obesity suggesting that the activity in VMH is required to maintain the lean/insulinsensitive state. However, increased noradrenergic levels and activity within the VMH is also associated with obesity and IR. We used two rodent obeseIR models, namely the genetically obese $a b / o b$ mouse and the seasonally obese Syrian hamster, to test the hypothesis that the VMH is also required to maintain the obese/IR condition. Therefore, VMH lesions of obese-IR animals may improve their condition. We performed radiofrequency lesions of the VMH in female $o b / o b$ mice (BW 30g; $\mathrm{n}=20$ ) and obese male hamsters (BW 190g; $n=5)$. Sham operated mice $(n=28)$ and hamsters $(n=16)$ served as controls. Lesion sites were verified at the end of the study. Unlike VMH lesions in lean rodents, by two weeks VMH-lesioned $a b / a b$ mice showed a significant decrease $(\mathrm{p}<0.001)$ in food consumption and body weight compared to sham controls. Elevated blood glucose and FFA levels also decreased by $28 \%(\mathrm{p}<0.0001)$ and $29 \%(\mathrm{p}<0.0001)$ respectively in lesioned mice compared to sham controls. Syrian hamsters also showed a significant reduction in food consumption and body weight by three weeks after VMH lesions. In addition, the intravenous glucose tolerance test in lesioned hamsters revealed significantly lower glucose $(15 \%)$ and insulin (46\%) levels compared to controls. FFA levels were also reduced by $32 \%$ following VMH lesions. Thus, VMH lesion in genetically and seasonally obese-IR rodents alleviate symptoms of diabetes and obesity. Our results taken together with lesion studies in lean rodents suggest that the VMH can support both the lean/insulin-sensitive or obese/insulin resistant states as a function of its internal organization.

\section{9}

CHRONIC INFUSION OF SEROTONIN INTO THE VENTROMEDIAL HYPOTHALAMUS INDUCES GLUCOSE INTOLERANCE IN HAMSTERS S. Luo, Y. Liang, J. Joslin, S. Hodge, J. Luo and A.H. Cincotta. Ergo Sci. Corp., Charlestown, MA 02129, USA

Alterations of brain serotonergic activities have been described in both diabetic patients and animal models of diabetes. We have shown increased serotonergic activity in the VMH of glucose intolerant hamsters. Since the VMH is known to have a major neural regulatory role in peripheral metabolic activities, we examined whether chronic increases in the local concentration of serotonin within the VMH play a role in the pathogenesis of diabetes. Vehicle, or different doses of serotonin $(6,12$ or $60 \mathrm{nmol} /$ day $)$ were infused continuously into the right VMH of male Syrian hamsters through osmotic minipumps placed subcutaneously. Glucose tolerance tests (GTT)(1.5 g glucose $/ \mathrm{kg}$ bw, iv) were conducted after 5 weeks of infusion. Serotonin infusion dose dependently induced glucose intolerance $(F=3.713, p<0.03)$. Serotonin at $60 \mathrm{nmol} /$ day produced a substantial increase of the GTT glucose total area under the curve by $35 \%(\mathrm{p}<0.05)$ and $33 \%(\mathrm{p}<0.05)$, compared to vehicle and serotonin at $6 \mathrm{nmol} /$ day respectively. Food intake measured at the fourth week of infusion and body weight gain were not significantly different among groups, although there was a trend towards serotonin infused hamsters gaining more weight. Pancreatic endocrine function was also examined using isolated islets and static incubation technique. Compared with islets from vehicle treated animals, islets from hamsters receiving 60 $\mathrm{nmol} /$ day serotonin infusion showed a 2 -fold increase of insulin release at 5 $\mathrm{mM}$ glucose $(2.8 \pm 0.5$ vs. $5.1 \pm 0.4 \mathrm{fmol} / \mu \mathrm{g}$ DNA $/ \mathrm{h}, \mathrm{P}<0.01)$ and a dramatic decrease at $15 \mathrm{mM}$ glucose $(33.5 \pm 1.8 \mathrm{vs} .21 .6 \pm 3.0 \mathrm{fmol} / \mu \mathrm{g} \mathrm{DNA} / \mathrm{h}, \mathrm{P}<0.01)$. Contrariwise, glucose-induced insulin release in islets from hamster receiving 6 or $12 \mathrm{nmol} /$ day serotonin showed no significant difference compared with that from vehicle group. The present findings that sustained increases in VMH serotonin alters pancreatic endocrine function and induces glucose intolerance strongly suggest that altered VMH serotonergic activity can contribute to the pathogenesis of diabetes.

\section{0}

\section{THE ROLE OF INSULN SECRETION AND RESISTANCE IN THE PATHOGENESIS OF TYPE 2 DIABETES IN CHINESE}

\author{
W.P. Jia, K.S. Xiang, J.X. Lu,Y.M. Zheng and J.L. Tang, Dept. of \\ Endocrinology and Metablism, Shanghai Sixth People Hospital, \\ Shanghai 200233,P.R.China
}

To investigate the impact of insulin secretion and insulin resistance on the development of glucose intolerance in Chinese. Given 75-gram standard oral glucose tolerance challenge, 467 Chinese subjects who had been in Shanghai for more than 10 years ( 279 nonobese, 188 obese) were divided into three groups: normal glucose tolerance (NGT group), and impaired glucose tolerance/impaired fasting glucose (IGT/IFG group), as well as type 2 diabetes (DM group) according to American Diabetes Association (ADA) criteria which was issued in 1997. Homeostasis model assessment (HOMA) was applied to assess the status of insulin secretion and insulin resistance, while insulinogenic index (the ratio of the increment of insulin to that of plasma glucose $30 \mathrm{~min}$ after a glucose load, $\left.\Delta I_{0} / G_{i 0}\right)$ was applied to assess the early phase insulin secretion. Adjusted with age and body mass index (BMI),our data indicated that HOMA insulin resistance index (HOMAIR) increased significantly not only in IGT/IFG group compared with NGT group (in nonobese subjects: $2.96_{ \pm} 1.62$ vs $1.89 \pm 1.36, \mathrm{P}=0.001$ and in obese subjects: $4.16 \pm 0.34$ vs $2.79 \pm 0.16, \mathrm{P}=0.005$, respectively) but also in $\mathrm{DM}$ group compared with IGT/IFG group (in nonobese subjects: $3.82+0.26$ vs

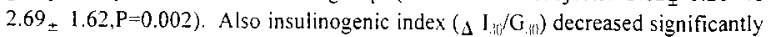
in DM group compared with IGT/IFG grcup (in obese subjects: $12.15,8.16 \mathrm{vs}$ $46.16 \neq 7.15, \mathrm{P}=0.05$ ). Whether obese or not , there was no significant change of $\Delta \mathrm{I}_{30} / \mathrm{G}_{30}$ between IGT/IFG group and NGT group. Our conclusion comes to that both insulin secretion and insulin resistance may contribute to the pathogenesis of type 2 diabetes in Chinese, and insulin resistance may play a more important role. 
DIAZOXIDE IMPROVES INSULIN SENSITIVITY IN ZUCKER OBESE RATS R.D. Carr, S. Gronemann, B. Hansen, K.F. Press, J. Sturis and C.L. Brand Diabetes Pharmacology I, Novo Nordisk A/S, DK-2880 Bagsvaerd, Denmark. The glucose lowering and insulin sensitising effects of chronic diazoxide (DZ $50 \mathrm{mg} / \mathrm{kg}$ bid for 7-9 wks) were investigated in male Zucker Obese (ZO) rats (9-10 weeks old at study start). Rats were allocated, with matched fasting plasma glucose (FPG), to receive either $D Z$ or vehicle (C). Lean male litter mates (ZL) received C. Rats were not dosed 2-5 days prior to tests. All tests were conducted in $18 \mathrm{hr}$ fasting rats. Pre-instumented, conscious rats underwent euglycaemic, hyperinsulinaemic $\left(5 \mathrm{mU} \cdot \mathrm{kg}^{-1} \cdot \mathrm{min}^{-1}\right)$ clamp during which hepatic glucose production (HGP) and glucose disappearance $\left(R_{d}\right)$ rates were measured using the isotope dilution method. Results are mean $\pm S E M ; n=4-10$; ANOVA or t-test: " $p<0.05$ vs $Z L-C ;{ }^{* *} p<0.05$ vs ZO-C; ND: not determined

GIR: glucose infusion rate. ZL-C $\quad$ ZO-C $\quad$ ZO-DZ

Pre-treatment variables$$
\text { ZL-C }
$$
body weight $(\mathrm{g})$ plasma glucose (mM)

$230 \pm 73$

$5.0 \pm 0.1$

$341 \pm 5^{*}$

$339 \pm 7^{*}$ plasma insulin (pM)

$16 \pm 7$

$7 \pm 0.3^{*}$

Post-treatment variables body weight ( $\mathrm{g}$ ) plasma glucose (mM)

\section{$313 \pm 8$} plasma insulin (pM)

$$
5.4 \pm 0.2
$$

$499 \pm 16^{*}$

$563 \pm 77^{*}$

Clamp variables plasma glucose (mM) plasma insulin (pM) GIR $\left(\mathrm{mg}^{\prime} \mathrm{kg}^{-1} \cdot \mathrm{min}^{-1}\right)$ HGP (\% inhibition) $\mathrm{R}_{\mathrm{d}}$ (\% stimulation) $52 \pm 17$

$6.8 \pm 0.4^{*}$

$480 \pm 20^{*}$ $5.4 \pm 0.2^{* *}$ $487 \pm 59^{*}$

$\begin{array}{lll}\text { ND } & 6.6 \pm 0.2 & 6.9 \pm 0.2 \\ \text { ND } & 1348 \pm 206 & 1371 \pm 146 \\ \text { ND } & 7.2 \pm 0.8 & 12.7 \pm 0.6^{* *} \\ \text { ND } & -46 \pm 8 & -78 \pm 5^{* *} \\ \text { ND } & 64 \pm 10 & 1166^{* *}\end{array}$
In conclusion, DZ reduced FPG presumably by improving peripheral and hepatic insulin sensitivity in the $Z O$ rat.
GLUCOSE EFFECTIVENESS COMPENSATES FOR INSULIN RESISTANCE AND INADEQUATE INSULIN SECRETION IN OLD RATS. M. Ader, J.M. Richey, and R.N. Bergman, University of Southern California, Los Angeles, CA.

Aging is characterized by glucose intolerance associated with insulin resistance. Although insulin secretion may partially compensate for resistance, the role of glucose effectiveness (GE) in aging is less clear. We calculated GE and insulin sensitivity $\left(S_{1}\right)$ in young (6 mos) and old $(28$ mos) rats. $S$, was assessed from hyperinsulinemic $(1 \mathrm{mU} / \mathrm{min}$ per $\mathrm{kg}$ ) euglycemic clamps ( $\mathrm{n}=2$ at each age), as [glucose infusion/(sinsulin $\times$ glucose)]. GE and its components of glucose-mediated stimulation of glucose uptake and suppression of glucose production were estimated by previously validated two compartment modeling of the combined injection of cold and labeled glucose $(0.3 \mathrm{~g} / \mathrm{kg}, 20 \mu \mathrm{Ci} / \mathrm{kg})$ during somatostatin ( $n=5$ and $n=3$ for young and old rats). Old rats were insulin resistant $\left(1.78\right.$ vs $3.68 \times 10^{-4} \mathrm{~d} / / \mathrm{min} \mathrm{kg}$ per $\left.\mu \mathrm{U} / \mathrm{ml}\right)$ and exhibited a compensatory doubling of fasting insulin (21 \pm 8 vs $10 \pm 0.4 \mu \mathrm{U} / \mathrm{ml} ; p<0.05$ ). Similarly, glucose effectiveness was increased in old rats, also compensating for insulin resistance. GE was $0.089 \pm 0.034 \mathrm{~min}^{-1}$ in old rats, nearly 2 -fold higher than in young rats $\left(0.047 \pm 0.008 \mathrm{~min}^{-1} ; p<0.05\right)$. This increase was largely due to a $>200 \%$ increase in the production component of $\mathrm{GE}$ $\left(0.042 \pm 0.02\right.$ vs $0.014 \pm 0.007 \mathrm{~min}^{-1}$ for old and young rats). These data suggest that glucose effectiveness may compensate for impaired insulin action in the aging rat model when insulin secretion is inadequate. Understanding the mechanisms by which glucose effectiveness is increased in resistant states is critical to elucidating the pathogenesis of impaired glucose tolerance.

\section{3}

DELAYED OCCURRENCE OF INSULIN RESISTANCE IN A NEW EXPERIMENTAL MODEL OF NIDDM

M. Taouis ${ }^{1}$, C. Broca ${ }^{2}$ P. Masiello ${ }^{3}$ M. Roye ${ }^{2}$, M. Manteghetti ${ }^{2}$ M. Derouet', R. Gross ${ }^{2}$ and G. Ribes ${ }^{2}$ ' ' SRA, INRA, Nouzilly. ${ }^{2}$ UMR 9921 CNRS and UPRES 1677, Montpellier I France. ${ }^{3}$ Ist. Patol. Generale, Pisa, Italia.

We have previously reported that adult rats treated with streptozotocin (STZ) $(65 \mathrm{mg} / \mathrm{kg}$. IV) plus nicotinamide at the dose of $230 \mathrm{mg} / \mathrm{kg}$ (NA 230 ) show a relative deficiency in glucose induced insulin secretion due to a decrease in pancreatic insulin stores reaching about $40 \%$ of normal ones. The aim of the present study was to explore whether peripheral insulin resistance could also develop in this new experimental NIDDM model. For this purpose, in Wistar rats STZ + NA. 230 administered, glucose-induced insulin response and insulin binding to peripheral tissues were simultaneously evaluated at day 7, 15, 30,60 after diabetes induction. Insulin response to glucose was studied in the isolated rat pancreas by increasing glucose concentration ( 5 to $11 \mathrm{mmol} / \mathrm{l}$ ) and ${ }^{[25} \mathrm{I}$ porcine insulin binding was tested using rat crude liver membranes. Our results revealed that glucose-induced insulin response was significantly reduced as early as day 7 after diabetes induction. Areas under the curve for glucose stimulation $/ 20 \mathrm{~min}(\mathrm{AUC} / 20 \mathrm{~min})$ were $227 \pm 13 \mathrm{ng}$ versus $696 \pm 53 \mathrm{ng}$ in normal animals $(\mathrm{p}<0.01)$. Similar reductions were observed at day 15,30 , 60 (AUC/20 min: $229 \pm 18,182 \pm 30$ and $170 \pm 23 \mathrm{ng}$, respectively; $\mathrm{p}<$ 0.01 ). Insulin binding to liver membrane progressively decreased from day 15 to reach a significant difference at day $60(-25 \%, \mathrm{p}<0.05)$ with respect to controls. Thus in this new experimental model of NIDDM induced by NA + STZ and characterized by a defective insulin responsiveness to glucose, a secondary insulin resistance develops, both effects mimicking at least in part, the major features of human NIDDM.

\section{4}

AMARANTH OIL AMELIORATES DEXAMETHASONEINDUCED GLUCOSE INTOLERANCE IN AGING RATS

M.Gorshunska, N.Gorbenko, O.Ivanova and 'T.Gopeziy. Ukrainian Scientific Research Institute of Endocrine Diseases Pharmacotherapy, Kharkov, Ukraine. 'Agricultural University, Kharkov, Ukraine Amaranth oil (AO) possesses free radicals scavenger activity and it is widely used as anti-inflammatory agent. The imbalance between pro- and antioxidants in diabetes and possible link of oxidative stress and insulin resistance provide justification for antioxidant treatment, especially in old patients. To elucidate $\mathrm{AO}$ antidiabetic potential its ability to correct dexamethasone (D)- induced alterations of glycaemic pattern in aging rats was investigated. Male Wistar rats (18-mo-old) were injected D $(0,125 \mathrm{mg} / \mathrm{kg} /$ die s.c. for 13 days). Control rats (C) were given vehicle alone. One group of D-treated rats received AO $(5 \mathrm{ml} / \mathrm{kg} /$ die per os) starting 4 days after first $D$-injection. At the end of the study fasted rats were subjected to a glucose tolerance test (GTT, $2 \mathrm{~g} / \mathrm{kg}$ i.p.). Dtreatment induced a significant rise in basal plasma insulin $(\mathrm{p}<0.02)$ and glucose levels $(7.0 \pm 0.9 \mathrm{mmol} / \mathrm{l}$ vs $4.6 \pm 0.3 \mathrm{mmol} / 1, \mathrm{p}<0.02)$ compared to C. Moreover, glucose tolerance was impaired after D-administration (integral glycaemia over GTT was $67.0 \pm 8.9 \mathrm{mmol} / \mathrm{l}$ vs $29.6 \pm 0.8 \mathrm{mmol} / \mathrm{l}$, $\mathrm{p}<0.01$ ) in comparison with non-treated controls. Administration of AO prevented development of D-induced hyperglycaemia $(4.5 \pm 0.3 \mathrm{mmol} / \mathrm{l}$ vs $7.0 \pm 0.3 \mathrm{mmol} / \mathrm{p}, \mathrm{p}<0.02$ ) and improved glucose tolerance reducing integral glycaemia over GTT 1.5 fold $(p<0.02)$ in comparison with $D$ treated rats. The basal and glucose induced plasma insulin levels were significantly decreased $(p<0.05)$ after $A O$ treatment. The results allow the conclusion that $A O$ is able to prevent $\mathrm{D}$-induced hyperglycaemia and improved glucose tolerance in aging rats. We suggest the use of $A O$ in diet may have potential therapeutic benefit in the NIDDM treatment. 
ONR YRAR GROWTE BORMONB TRRAMARANY HTHECT ON INSULINSENSITIVITY STATUS MND GRNG EXPRESSION Y Xhalfallah*123, H Vidall, M Laville ${ }^{1} 2, \mathrm{~N}^{2}$ Vegal, Vallier ${ }^{1}$, JP Rioul2, G Sassolas ${ }^{3}$. ${ }^{1}$ INSERM U449, ${ }^{2}$ Human Nutrition Research Center and ${ }^{3}$ Nuclear Medecine Center, Faculty RTH Laennec, rue G Paradin, 69372 Lyon Cedex 08, France.

Growth hormone (GH) may control metabolic pathways by acting on the activity of key enzymes and/or by changing their expression levels. The aim of our study was to characterize the in vivo effect of $\mathrm{GH}$ on insulin sensitivity and mRNA levels of key genes involved in metabolic pathways and insulin action. Six secondary GH deficient adults were studied before and one year afte GH substitution dose-treatment. Insulin sensitivity was determined before and during $\mathrm{GH}$ treatment using a 3 hour euglycemic hyperinsulinic clamp $(12 \mathrm{pmol} / \mathrm{kg} / \mathrm{min})$. Muscle and abdominal subcutaneous adipose tissue biopsies were taken before and at the end of the clamps. Levels of insulin receptor, PI-3 kinase (PI3K), glut4, glycogene synthase, PFK-1 and LPL MRNA were determined by RT competitive-PCR. The results showed that, in spite of significant IGFl increase (158 \pm 17 vs $77 \pm 9 \mathrm{ng} / \mathrm{ml}$ ) endogenous glucose production $(10.9 \pm 0.6$ vs $11.3 \pm 0.4$ $\mu \mathrm{mol} / \mathrm{kg} / \mathrm{min}$ ) and insulin stimulated glucose disposal ( $40 \pm 3$ vs $47 \pm 3 \mu \mathrm{mol} / \mathrm{kg} / \mathrm{min})$ were not significantly modified by $G H$ treatment. Treatment increased significantly LPL mRNA in muscle $(70 \pm 7$ vs $38 \pm 2$ amoles/ $/ \mathrm{g}$ total RNA, $p<0,05)$ and adipose tissue $(4860 \pm 880 \mathrm{vg}$ $2490 \pm 260$ amoles/ $\mu g$ total RNA, $p<0.051$, but did not change the basal levels of other mRNAs. Before $\mathrm{GH}$ insulin was not able to modify the levels of the measured mRNAs while after treatment, it induced a significant increase in muscle glut 4 gene expression $(\Delta=30$ vs 7 amoles $/ \mu$ g total RNA, $p<0.05)$.

\section{PS 28}

\section{Insulin Sensitivity: Methods}

\section{6}

BAYESIAN IDENTIFICATION OF A NEW TWO-COMPARTMENT MINIMAL MODEL CORRECTS $\mathrm{S}_{\mathrm{G}}$ OVERESTIMATION AND SI UNDERESTIMATION. A. Caumo and C. Cobelli. San Raffaele Scientific Institute, Milano, and Department of Electronics and Informatics, University of Padova, Padova, Italy.

The minimal model (MM) indices of glucose effectiveness, $S_{G}$, and insulin sensitivity, $\mathrm{S}_{\mathrm{I}}$, are widely used to characterize glucose tolerance. Recent reports, however, indicate that $S_{G}$ is overestimated and $S_{I}$ underestimated. The use of a single- instead of a two-compartment description of glucose kinetics has been indicated as a major source of error. Aim of this study was to develop a new approach to $S_{G}$ and $S_{I}$ estimation based on the bayesian identification of a two-compartment minimal model (2CMM). A standard IVGTT $(0.3 \mathrm{~g} / \mathrm{kg} \mathrm{BW})$ was performed in 6 normal dogs and 14 normal humans. Glucose and insulin data were interpreted with both MM and 2CMM. 2CMM had the same structure of MM (irreversible glucose loss and insulin action in the accessible pool) except for the presence of a second nonaccessible pool. It was identified in each study by using a physiological constraint on the exchange rate parameters between the glucose pools and a bayesian parameter estimation technique. Since $M M$ and $2 \mathrm{CMM}$ fractional indices $\mathrm{SG}_{\mathrm{G}}$ and $\mathrm{SI}$ refer to different volumes, they were multiplied by their respective accessible pool volumes, $\mathrm{V}$ and $\mathrm{V}_{1}$, where the former is the $\mathrm{MM}$ volume of glucose distribution and the latter is the accessible pool volume of 2CMM. Results (Meant SEM) are reported below.

\begin{tabular}{|c|c|c|c|c|}
\hline & $\begin{array}{c}\mathrm{S}_{\mathrm{G}} \mathrm{V} \\
(\mathrm{d} \mathrm{l} / \mathrm{kg} \min )\end{array}$ & $\begin{array}{c}\mathrm{S}_{\mathrm{I}} \mathrm{V} \\
\cdot\left(10^{-4} \mathrm{dl} / \mathrm{kg} \mathrm{min}\right. \\
\text { per } \mu \mathrm{U} / \mathrm{ml})\end{array}$ & $\begin{array}{r}{\left[\mathrm{S}_{\mathrm{G}} \mathrm{V}_{1}\right]_{2 \mathrm{c}}} \\
(\mathrm{dl} / \mathrm{kg} \mathrm{min})\end{array}$ & $\begin{array}{c}{\left[\mathrm{S}_{\mathrm{I}} \mathrm{V}_{1}\right]_{2 \mathrm{c}}} \\
\left(10^{-4} \mathrm{~d} \mathrm{l} / \mathrm{kg} \text { min }\right. \\
\text { per } \mu \mathrm{U} / \mathrm{ml})\end{array}$ \\
\hline $\begin{array}{l}\text { Dogs } \\
(\mathrm{n}=6)\end{array}$ & $0.099 \pm 0.014$ & $8.6 \pm 2.0$ & $0.054 \pm 0.016^{*}$ & $16.3 \pm 2.8 *$ \\
\hline $\begin{array}{l}\text { Humans } \\
(n=14)\end{array}$ & $0.046 \pm 0.005$ & $8.0 \pm 2.5$ & $\begin{array}{r}0.030 \pm 0.003^{*} \\
*^{*} p<0.05\end{array}$ & $\begin{array}{l}10.6 \pm 2.4^{*} \\
M M\end{array}$ \\
\hline
\end{tabular}

In summary, 2CMM yields an index of glucose effectiveness which is lower than that provided by $\mathrm{MM}(-45 \%$ in dogs and $-35 \%$ and humans) and an index of insulin sensitivity which is higher than that provided by MM ( $+90 \%$ in dogs and $+33 \%$ in humans). We conclude that the bayesian identification of $2 \mathrm{CMM}$ corrects the $\mathrm{MM}$ overestimation of glucose effectiveness and underestimation of insulin sensitivity.

\section{7}

SIZE OF THE INTRAVENOUS GLUCOSE LOAD AND MINIMAL MODEL-DERIVED $S_{1}$ AND $S_{a}$ : APPLICATION TO OBESITY

M.R. Letiexhe, A.J. Scheen and P.J. Lefebvre P.J. Division of Diabetes, Nutrition \& Metabolic Disorders, CHU Sart Tilman, Liège, Belgium.

Intravenous glucose tolerance test (IVGTT) has been used to assess insulin secretion and action in obesity. As the glucose load is usually adjusted to body weight (BW), obese subjects receive a much higher glucose dose than lean controls. In order to avoid a possible bias, minimal model-derived indices of insulin sensitivity $S_{1}$ and glucose effectiveness $S_{0}$, coefficient of glucose assimilation $\left(\mathrm{K}_{\mathrm{o}}\right)$, and insulin secretion rate (ISR, estimated by deconvolution of plasma C-peptide levels) were calculated during IVGTTs : 1) using $0.3 \mathrm{~g}$ glucose $/ \mathrm{kg} \mathrm{BW}$ in 10 obese non-diabetic subjects $(5 \mathrm{~F} / 5 \mathrm{M}$; age: $24.5 \pm 2.0$ years; BW: $114.3 \pm 3.5 \mathrm{~kg}$; BMI: $39.5 \pm 1.6 \mathrm{~kg} / \mathrm{m}^{2}$; average glucose load of $34.3+1.1 \mathrm{~g}$ ); and 2) using 0.3 and $0.5 \mathrm{~g}$ glucose $/ \mathrm{kg} \mathrm{BW}$ (at 2 week-intervals) in 10 lean controls (5F/5M; age: $24.1 \pm 0.7$ years; BW: $64.8 \pm 2.0 \mathrm{~kg}$; BMI: $21.6 \pm 0.4 \mathrm{~kg} / \mathrm{m}^{2}$; average glucose load of $19.4 \pm 0.6 \mathrm{~g}$ and $32.4 \pm 1.0 \mathrm{~g}$ respectively). ISR/180 min was higher in obese subjects $(81.3 \pm 10.6 \mathrm{nmol})$, whatever the glucose dose used in controls $(29.8 \pm 3.1 \mathrm{nmol}$ with $0.3 \mathrm{~g} / \mathrm{kg}$, $\mathrm{p}<0.0002$, and $40.1 \pm 3.6 \mathrm{nmol}$ with $0.5 \mathrm{~g} / \mathrm{kg}, \mathrm{p}<0.002)$. $\mathrm{K}_{\mathrm{o}}$ was significantly lower in obese subjects $\left(1.36 \pm 0.17 \mathrm{~min}^{-1}\right)$ when compared to that of controls after $0.5 \mathrm{~g} / \mathrm{kg}\left(1.94 \pm 0.14 \mathrm{~min}^{-1}, \mathrm{p}<0.02\right)$ but not after $0.3 \mathrm{~g}$ glucose $/ \mathrm{kg}\left(1.65 \pm 0.16 \mathrm{~min}^{-1}, \mathrm{NS}\right) . \mathrm{S}_{\mathrm{I}}\left(10^{-5} \mathrm{~min}^{-1} . \mathrm{pmol}^{-1} . \mathrm{L}\right)$ was significantly decreased in obese subjects $(4.01 \pm 0.96)$, whatever the glucose load used in controls $\left(p<0.0025\right.$ and $p<0.0015$ respectively); however, $S_{1}$ of controls was slightly lower after $0.5 \mathrm{~g}$ glucose $/ \mathrm{kg}(9.61 \pm 1.26)$ than after $0.3 \mathrm{~g} / \mathrm{kg}$ $(14.28 \pm 2.55, p<0.05) . S_{\mathrm{C}}$ was similar in obese and lean subjects, whatever the glucose load. In conclusion, even if the size of the glucose load may slightly affect the results, severely obese non-diabetic subjects showed decreased $S_{1}$, higher ISR and similar $S_{a}$ indices as compared to those of lean controls, whatever the intravenous glucose load injected in the latter. 
ESTIMATE OF INSULIN SENSITIVITY WITH THE HOMEOSTASIS MODEL ASSESSMENT. VALIDATION STUDIES AGAINST THE GLUCOSE CLAMP

G. Targher, M. Alberiche, M. Zenere, F. Saggiani, R. Bonadonna, M. Muggeo and $E$. Bonora. Division of Endocrinology and Metabolic Diseases, University of Verona, Italy.

Several investigators used the homeostasis model assessment (HOMA) as a surrogate measure of in vivo insulin sensitivity in man. This method, however, has not been sufficiently validated against the glucose clamp, which is unanimously regarded as the reference technique for the measurement of insulin sensitivity. In the present study we have compared the measurement of insulin sensitivity obtained by a 4 hour euglycemic $(\sim 5$ $\mathrm{mmol} / \mathrm{l})$ hyperinsulinemic $(\sim 300 \mathrm{pmol} / \mathrm{l})$ clamp with that estimated by the HOMA in 115 subjects with various degrees of glucose tolerance and insulin sensitivity. We have found a strong correlation between clamp-measured (total glucose disposal, TGD) and HOMA-estimated insulin sensitivity ( $r=-$ $0.820, p<0.001$ ), without any substantial difference in men $(r=-0.800)$ and women $(r=-0.796)$ as well as in younger $(r=-0.811)$ and older $(r=-0.897)$, in nonobese $(r=-0.817)$ and obese $(r=-0.786)$ in nondiabetic $(r=-0.754)$ and diabetic $(r=-0.695)$, and in normotensive $(r=-0.786)$ and hypertensive $(r=-$ 0.762 ) subjects. After stratification of subjects into quartiles of HOMA values and TGD values, the coefficient of agreement in the categorization of individuals for insulin sensitivity was good (weight $\mathrm{k}=0.65$ ).

We conclude that the HOMA is a reliable method to estimate in vivo insulin sensitivity in man. Since the HOMA is simple, straightforward and cheap, it can be used in large scale or epidemiological studies in which only a single fasting blood sample is available.

\section{PS 29}

\section{Carbohydrate Metabolism}

\section{9}

THE EFFECTS OF EXERCISE ON GLUCOSE METABOLISM IN SKELETAL MUSCLE CAN BE REPRODUCED BY NITRIC OXIDE

M.E. Young and B. Leighton. University of Oxford, U.K.

It is hypothesised that nitric oxide (NO) and cGMP are mediators of contraction-stimulated glucose utilization in skeletal muscle. Immediately after erercise or contraction, the rates of glucose transport and metabolism remain elevated in skeletal muscle, thus enabling glycogen repletion. The aim of this study was to investigate the role of $\mathrm{NO} / \mathrm{cGMP}$ in glycogen re-synthesis. Isolated rat soleus muscle preparations were incubated for 30 minutes with the NO donor sodium nitroprusside (SNP; 15mM), washed (incubation for 15 minutes without SNP), followed by incubation (without SNP) for various times in fresh medium. Prior NO exposure increased rates of both lactate release (control, $6.17 \pm 0.77$, versus SNP, $9.04 \pm 0.68$ $\mu \mathrm{mol} / \mathrm{h} / \mathrm{g}$ wet wt.; $\mathbf{P}<0.01$ ) and glycogen synthesis (control, $0.89 \pm 0.12$, versus SNP, $2.20 \pm 0.31 \mu \mathrm{mol} / \mathrm{h} / \mathrm{g}$ wet wt.; $\mathrm{P}<0.001$ ), even 80 minutes after the wash stage. In addition, cGMP levels remained elevated within skeletal muscles pre-exposed to NO when compared to control muscles (5-fold higher, even 50 minutes post-SNP exposure; $\mathrm{P}<0.001$ ). Nitric oxide synthase (NOS) activity is higher in muscles primarily composed of type II fibres. The fibre type composition, in order of decreasing type II fibres, in various skeletal muscies is white gastrocnemius (84\%) > $\operatorname{EDL}(56 \%)>$ red gastrocnemius $(8 \%)>$ soleus $(0 \%)$. We found the descending order of sensitivity of soluble guanylate cyclase (sGC) to activation by $10 \mu \mathrm{M}$ SNP (a submaximal concentration) in skeletal muscle extracts (shown as percentage of maximal sGC activity) to be white gastrocnemius $(69 \pm 3 \%)>\operatorname{EDL}(23 \pm 3 \%)>$ red gastrocnemius $(9 \pm 1 \%)>$ soleus $(3 \pm 1 \%)$. In addition, prior incubation of muscle preparations with SNP $(15 \mathrm{mM})$ causes a marked increase in the sensitivity of $5 G C$ to NO (2-fold increase; $P<0.01$ ). We propose that the sustained elevated cGMP contents in skeletal muscles previously incubated with SNP are caused by the increased sensitivity of sGC to endogenously produced NO. We also hypothesise that sustained elevated cGMP levels are responsible for the increased rates of glucose transport and glycogen synthesis observed in skeletal muscle after exercise or contraction.

\section{0}

INCREASED GLYCOGEN FROM GLUCONEOGENESIS WITH PROLONGED FASTING IN HUMANS REFED WITH GLUCOSE. A. Lteif and W. F. Schwenk, Department of Pediatrics and Adolescent Medicine, Rochester, MN

We have previously shown in fasted dogs refed with glucose that fasting significantly increases intrahepatic UDP-glucose flux (an estimation of the rate of glycogen synthesis) and the amount of UDP-glucose formed via the indirect pathway. To assess the importance of the indirect pathway after different periods of fasting in humans and to determine whether fasting followed by refeeding also affects the rate of glycogen synthesis, five normal volunteers received a six hour infusion of glucose at $20 \mu$ $\mathrm{mol} / \mathrm{kg} / \mathrm{min}$, along with [3-3H] glucose (to assess direct hepatic uptake of glucose), [U-14C]lactate (to label UDP-glucose via the indirect pathway) and $[1-13 \mathrm{C}]$ galactose (to estimate UDP-glucose flux), after fasting overnight or for 2.5 days. With two additional days of fasting, UDPglucose flux during refeeding was not significantly increased $(9.24 \pm 1.35$ $\mathrm{v} / \mathrm{s} 10.69 \pm 1.22 \mu \mathrm{mol} / \mathrm{kg} / \mathrm{min})$. UDP-glucose formed via direct uptake of glucose was similar on the 2 study days $(600 \pm 0.8 \mathrm{v} / \mathrm{s} 5.04 \pm 1.19 \mu$ $\mathrm{mol} / \mathrm{kg} / \mathrm{min}$ ), while the amount of UDP-glucose formed via the indirect pathway was increased $(3.24 \pm 0.73 \mathrm{v} / \mathrm{s} 5.65 \pm 0.62 \mu \mathrm{mol} / \mathrm{kg} / \mathrm{min}, \mathrm{p}<0.05)$. Similarly, endogenous glucose production was higher after 2.5 days of fasting $(3.37 \pm 0.56 \mathrm{v} / \mathrm{s} 4.67 \pm 0.44 \mu \mathrm{mol} / \mathrm{kg} / \mathrm{min}, \mathrm{p}<0.05)$. These changes reflecting increased gluconeogenesis occurred despite higher insulin levels after 2.5 days of fasting $(130.22 \pm 18.24 \mathrm{v} / \mathrm{s} 252.57 \pm 55.7 \mathrm{pmol} / \mathrm{l}$, $\mathrm{p}<0.05$ ). We conclude that with increased fasting in humans, gluconeogenesis is an increasingly important pathway not. only for subsequent glycogen repletion but also for persistent endogenous glucose production. 


\section{1}

SUBCELLULAR LOCALISATION OF KEY ENZYMES OF GLYCOGEN SYNTHESIS. A NOVEL CONTROL MECHANISM OF GLYCOGEN METABOLISM.

J.C. Ferrer, S. Baqué, E. Cid, R.R. Gomis and J.J. Guinovart. Departament de Bioquimica i Biologia Molecular. Universitat de Barcelona, 08028 Barcelona. Spain

The accepted model of glycogen synthesis involves three different steps: initiation by glycogenin, a self-glucosylating enzyme, the elongation step catalysed by glycogen synthase, and the ramification of the polysaccharide by the branching enzyme. Glycogen synthase, whose activity is highly regulated by covalent modification as well as by allosteric effectors, is believed to be the key enzyme in the control of the overall process. Glycogen synthesis, the main pathway of non-oxidative glucose metabolism, is active in most mammalian tissues. However, this polysaccharide accumulates to a largest extent in the liver and the skeletal muscle, and these tissues are therefore extremely important in maintaining blood glucose homeostasis. Our studies show that glycogen synthase changes its localisation in the cell in response to glucose. The liver isoform is uniformly distributed throughout the cytosol of the hepatic cell in the absence of glucose and moves towards the periphery in response to glucose and insulin Alternatively, the form of glycogen synthase found in muscle is localised to the nucleus and exits this cellular compartment when glucose is present. We have also shown that the form of glycogenin prevalent in muscle binds to actin microfilaments through a common actin-binding motif, which is present in its primary sequence but is absent in the sequence of the glycogenin isoform characteristic of liver. These facts delineate differences between the hepatic and the muscular metabolism of glycogen. The interaction of these enzymes with different subcellular stnuctures and their movement in response to glucose may represent a novel regulatory mechanism of glycogen metabolism. This model opens up a new area of study: from the molecular mechanisms controlling the intracellular distribution of these enzymes to the alterations that they may suffer in diabetes.
782

GLYCOGEN STORAGE IN L6 CELLS ARE INCREASED BY OVEREXPRESSION OF GLYCOGENIN

B.F. Hansen', P. Jensen' and F. Barbetti'. 'Diabetes Biology, Novo

Nordisk AVS, DK-2880 Denmark and ${ }^{2}$ Mol Pat Diabetes Unit, HS Raffaele Scientific Institute, Milan, 20132, Italy.

Glycogenin is the protein core which upon the glycogen molecule is built. Hypothetically, the maximal attainable glycogen concentration in cells are limited by the expression of glycogenin, since no free (non-glucosylated) glycogenin is found in cells. In order to test this idea we transfected L6 myoblasts with glycogenin or empty vector as control. Three stable clones were isolated, two overexpressing glycogenin and one expressing the control vector. Maximal glycogen storage capacity was evaluated by exposing cells to high insulin $(100 \mathrm{nM})$ and glucose $(25 \mathrm{mM})$ concentrations for $5 \mathrm{~h}$. Glycogen concentration in cells was measured by the hexokinase method after acid hydrolysis. The glycogen concentration increased rapidly $(\sim 3$ fold) during the first $2 \mathrm{~h}$ in both cells overexpressing glycogenin and the controf line; after $2 \mathrm{~h}$ no further increase was seen. Notably, the glycogen concentrations were found to be significantly (ANOVA, $p<0.001$ ) higher overall during the $5 \mathrm{~h}$ in cells overexpressing glycogenin compared to control cells. Glycogen synthesis was also measured as incorporation of ${ }^{14} \mathrm{C}$-glucose into glycogen during the $5 \mathrm{~h}$. The two first hours of incubation glycogen synthesis was identical, however from $2 \mathrm{~h}$ and onwards a significant effect of overexpressing glycogenin was seen (ANOVA, $\mathrm{p}<0.001$ ). Since glycogen synthesis continues to increase after $2 \mathrm{~h}$ incubation, despite constant total glycogen concentrations, recycling of glucose in the glycogen molecule must take place. The same phenomenon was observed in parental (non-transfected) cells. In conclusion, glycogenin overexpression in a skeletal muscle-like cell line is able to augment the total glycogen concentration and glycogenin might therefore play a role in determining maximal glycogen storage capacity.
783

ETHNIC DIFFERENCES IN GLUCOSE AND LIPID METABOLISM IN OBESE SOUTH AFRICAN WOMEN. $C$ Punyadeera, NJ Crowther, M-T van der Merwe*, AR Immelman and IP Gray. Department of Chemical Pathology, South African Institute for Medical Research and *Department of Medicine, Faculty of Health Sciences, University of the Witwatersrand, York Road, Parktown 2193, Johannesburg.

Studies in South Africa have shown that black obese women have higher serum FFA levels and are more insulin resistant (IR) than their white counterparts. The aim of our study was to determine whether the higher IR observed in the black group was due to the inhibitory effect of elevated FFA levels on glucose uptake and metabolism. Glucose metabolism was measured in 8 black and 8 white obese females using measurement of ${ }^{13} \mathrm{CO}$, levels in breath following the ingestion of a $75 \mathrm{~g}$ glucose load containing $0.5 \mathrm{~g} 1-{ }^{13} \mathrm{C}$-labelled glucose. Breath and blood samples were taken over a 7 hour period during which C-peptide and glucose levels were similar but proinsulin $(1.9 \pm 0.6 \mathrm{pM}$ vs $4.6 \pm 0.9$; $6.1 \pm 1.3$ vs $11.8 \pm 1.9 ; 11.9 \pm 1.9$ vs $24.9 \pm 5.6 ; 6.8 \pm 0.7$ vs $17.0 \pm 4.6$ at $0,30,120$ and 180 mins; $p<0.05$ for all) and ${ }^{13} \mathrm{CO}_{2}$ breath levels $(4.7 \pm 0.7 \%$ vs $7.7 \pm 1.1 ; 19.1 \pm 1.4$ vs $25.1 \pm 2.4$ at 60 and 150 mins respectively; $p<0.05$ for both) were lower and insulin higher $(621 \pm 144$ vs $288 \pm 40 \mathrm{pM}$ at 120 mins; $\mathrm{p}<0.05)$ in the black subjects. FFA levels were higher in the black subjects at 30 and 360 mins $(677 \pm 80 \mu \mathrm{M}$ vs $202 \pm 78 ; 696 \pm 39$ vs $490 \pm 48$ respectively; $\mathrm{p}<0.005$ for both). We conclude that lower glucose metabolism in the black obese subjects is a result of higher FFA levels which may in turn decrease hepatic extraction of insulin. Furthermore, conversion of proinsulin to insulin is increased in the black subjects possibly as a result of a B-cell attempt to maintain euglycaemia in the face of high insulin resistance.

\section{4}

GLUCOSE AND LACTATE LOWERING BY DICHLOROACETATE IN DIABETIC RATS IS CORRELATED WITH TISSUE PYRUVATE DEHYDROGENASE ACTIVITY J. Gao, X. Liu, E. Kaplan and W. Mann. Diabetes Pharmacology, Novartis Pharmaceuticals Corp., 556 Morris Ave., Summit, NJ 07901, USA

The pyruvate dehydrogenase (PDH) complex catalyzes the first irreversible reaction in the mitochondrial oxidation of glucose. PDH activity is decreased in tissues of diabetic humans and diabetic animals. The purpose of the study was to determine if an activation of tissue PDH is correlated with an improvement of glycemia and lactataemia in diabetic animals. Male Sprague-Dawley rats were rendered diabetic with dexamethasone ( $2.5 \mathrm{mg}$, s.c.). Plasma glucose and lactate concentrations were matched for the groups ( $\mathrm{n}=6$ /group), which were then orally treated with dosing vehicle (control) or dichloroacetate (DCA, a known PDH activator) at 250,500 and $1,000 \mu \mathrm{mol} / \mathrm{kg} / \mathrm{day}$ for 4 days. On day $5(24 \mathrm{~h}$ after the last treatment) a blood sample was collected and the tibialis anterior muscle (TA), liver and heart were taken for the measurement of PDH activity. Plasma glucose and lactate levels and tissue PDH activities after the treatment are shown in the table below (* indicating $\mathrm{P}<0.05$ vs. the control group):

Group Glucose Lactate PDH (mU/mg protein)

$\begin{array}{lccccr} & (\mathrm{mg} / \mathrm{dl}) & (\mathrm{mM}) & \text { TA } & \text { Liver } & \text { Heart } \\ \text { Control } & 388 \pm 24 & 5.4 \pm 0.2 & 0.1 \pm 0.0 & 0.1 \pm 0.0 & 1.5 \pm 0.6\end{array}$

$\begin{array}{llllll}\text { DCA250 } & 438 \pm 22 & 4.9 \pm 0.2 & 0.2 \pm 0.0 & 0.1 \pm 0.0 & 0.6 \pm 0.2\end{array}$

$\begin{array}{llllll}\text { DCA500 } & 294 \pm 46 & 3.3 \pm 0.3^{*} & 0.3 \pm 0.0^{*} & 0.2 \pm 0.0 & 4.0 \pm 1.4^{*}\end{array}$

$\begin{array}{llllll}\text { DCA } 1,000 & 244 \pm 45^{*} & 3.3 \pm 0.2^{*} & 1.5 \pm 0.3^{*} & 1.1 \pm 0.2^{*} & 12.1 \pm 2.8^{*}\end{array}$

There is a highly adverse correlation between the tissue PDH activity and plasma glucose and lactate levels after the treatment for TA $(\mathrm{r}=-0.68, \mathrm{P}<0.001 ; \mathrm{r}=-0.50$, $\mathrm{P}<0.01)$, liver $(\mathrm{r}=-0.65, \mathrm{P}<0.001 ; \mathrm{r}=-0.38, \mathrm{P}=0.07)$ and heart $(\mathrm{r}=-0.79, \mathrm{P}<0.001 ; \mathrm{r}=-$ $0.54, \mathrm{P}<0.01$ ). In conclusion, (1) glucose and lactate lowering and $\mathrm{PDH}$ activation by DCA are dose-dependent; and (2) tissue PDH activity is adversely correlated with plasma glucose and lactate levels. Thus, PDH activation may have caused the improvement of glycemia and lactataemia in these diabetic animals. 
SPLANCHNIC GLUCOSE UPTAKE (SGU) FOLLOWING DIFFERENT AMOUNTS OF ORAL GLUOSE IN MAN

A. Kautzky-Willer, Th. Aigmüller, W. Waldhäusl, A. Hofer, and B. Ludvik, Vienna, Austria

The magnitude of SGU in human subjects following different amounts of orally administered glucose has not yet been evaluated lacking a method to repeatedly measure SGU. We have recently developed and validated a non-invasive technique (OG-CLAMP) to measure glucose disposal rate (GDR), SGU and time required for glucose absorption. This method combines an euglycemic, hyperinsulinemic clamp $\left(120 \mathrm{mU} / \mathrm{m}^{2} / \mathrm{min}\right)$ and an oral glucose load (OGL) during steady-state of glucose disposal. To study the effect of different OGLs on the extent of SGU we perfomed three OGCLAMPs on different days in 7 healthy male subjects (age $=24 \pm 2$ yrs, $\mathrm{BMI}=23.4 \pm 1.3 \mathrm{~kg} / \mathrm{m}^{2}$ ) employing OGLs with 25,75 , and $100 \mathrm{gms}$, respectively. Mean GDR was $11.3 \pm 0.7 \mathrm{mg} / \mathrm{kg} / \mathrm{min}$. SGA was $2.3 \pm 1.4 \%, 13.4 \pm 3.1 \%$, and $23 \pm 3.5 \%$ following 25,75 , and $100 \mathrm{gms}$ glucose. SGU was significantly lower following the 25 gms compared with the $75(p<0.05)$ and $100 \mathrm{gms} \operatorname{OGL}(\mathrm{p}<0.01,75$ vs. $100 \mathrm{gms}$ $\mathrm{p}<0.05$ ). The time required for absorption of the OGL was $70 \pm 2.4$ min for $25 \mathrm{gms}, 131 \pm 5.4 \mathrm{~min}$ for $75 \mathrm{gms}(\mathrm{p}<0.001 \mathrm{vs} .25 \mathrm{gms})$, and $151 \pm 7.8 \mathrm{~min}$ for $100 \mathrm{gms}$ ( $\mathrm{p}<0.01$ vs. $75, \mathrm{p}<0.001$ vs. $25 \mathrm{gms}$ ). In conclusion, increasing the amount of orally administered glucose enhances SGU and the time required for resorption of an OGL in healthy humans. These results support the importance of the portal arterial glucose gradient as a modulator of the magnitude of SGA following oral glucose administration.

\section{PS 30}

\section{Carbohydrate Metabolism: Hepatic Glucose Production}

787

EFFECTS OF AGE, GENDER AND BODY WEIGHT ON RENAL AND HEPATIC GLUCOSE RELEASE IN POSTABSORPTIVE HUMANS

C. Meyer, M. Stumvoll, J. Dostou, M. Mitrakou and J. Gerich, University of Rochester, Rochester, NY, USA

In postabsorptive humans, liver and kidney account for about $75 \%$ and $25 \%$ of overall glucose release (OGR), respectively. However, it is well established that aging reduces renal mass and renal function. One might therefore anticipate decreased renal glucose release (RGR) with aging. To test this hypothesis, we assessed the effect of age (as well as gender and body weight) on RGR and hepatic glucose release (HGR) in 58 normal postabsorptive humans ( $32 \mathrm{male}$ 26 female) ranging from $20-65$ years of age and from $44-116 \mathrm{~kg}$ body weight. RGR was determined using the standard combination of isotopic (6-3H glucose) and organ balance (renal vein catheterization) techniques, and HGR was calculated as the difference between OGR and RGR. RGR and HGR were significantly lower in women $(140 \pm 11$ and $593 \pm 28 \mu \mathrm{mol} / \mathrm{min}$, respectively) than in men $(220 \pm 14$ and $685 \pm 21 \mu \mathrm{mol} / \mathrm{min}, \mathrm{p}<0.001$ and 0.012 respectively); RGR, but not HGR, was also significantly lower in the oldest (136 $\pm 23 \mu \mathrm{mol} / \mathrm{min})$ than in the youngest quartile $(201 \pm 9 \mu \mathrm{mol} / \mathrm{min}, \mathrm{p}=0.016)$. However, analysis of covariance indicated that gender did not affect either RGR or HGR whereas body weight was an independent determinant of both RGR ( $r=$ $0.690, p<0.001)$ and HGR $(r=0.886, p<0.001)$, and age was an independent determinant of RGR $(r=-0.274, p=0.039)$ but not of HGR $(r=-0.163, p=$ 0.224 ). Thus, an increase in body weight from $50 \mathrm{~kg}$ to $100 \mathrm{~kg}$ increased RGR $150 \%$ and HGR $\sim 100 \%$. In contrast, an increase in age from 20 to 60 years decreased RGR by $\sim 50 \%$ but decreased HGR only by $\sim 12 \%$. Age and body weight accounted for $83 \%$ of the variation in RGR and $97 \%$ of the variation in HGR. We conclude that body weight is the most important determinant of both renal glucose release and hepatic glucose release and that with aging bepatic glucose release becomes relatively more important than renal glucose release for glucose homeostasis.

\section{INSULIN-LIKE EFFECTS OF TUNGSTATE IN CULTURED HUMAN} MUSCLE FIBRES.

S. Baqué and J.J. Guinovart. Departament de Bioquimica i Biologia Molecular. Universitat de Barcelona, 08028 Barcelona, Spain.

The insulin-like effects of sodium tungstate in two diabetic animal models have recently been described on our laboratory. We have shown that this compound, when administered orally, is able to normalise glycaemia as well as the hepatic metabolic parameters of treated animals. However, the action of tungstate in muscle tissue has not been studied. Muscle is the major site of insulin-stimulated glucose disposal, either through the accumulation of glycogen, or through the glycolytic pathway, contributing to the maintenance of blood glucose homeostasis. In order to elucidate the effects of tungstate in muscle tissue, an in vitro model consisting of primary cultured muscle from human biopsies has been established. Differentiated muscle fibres were incubated in the presence of $100 \mathrm{nM}$ insulin, sodium tungstate (ranging from 0.01 to $1 \mathrm{mM}$ ), or the combination of both, and different metabolic parameters were analysed. Insulin elicited a more than two-fold increase in glucose 6-phosphate levels, and a $60 \%$ increase in glycogen deposition. Although $0.1 \mathrm{mM}$ sodium tungstate raised glucose 6-phosphate levels in the muscle fibres to the same extent as insulin, it was unable to increase total glycogen content. Tungstate and insulin showed a synergistic effect on the deposition of glycogen, which correlated with the activation state of glycogen synthase. The glycolytic flux was also stimulated by $0.1 \mathrm{mM}$ tungstate as assessed by lactate production measurements. The hexokinase and pyruvate kinase activities were not modified by incubation of the fibres with insulin and/or tungstate, but a significant increase was observed in the 6-phosphofructo 1-kinase activity. Furthermore, tungstate largely potentiated the increase in 6-phosphofructo 1-kinase produced by insulin. Our results show that in cultured human muscle, tungstate exerts insulin-like actions and acts synergistically with insulin stimulating the two main pathways of glucose disposal, glycogen synthesis and glycolysis.

\section{8}

SPLANCHNIC AND RENAL GLUCOSE PRODUCTION IN THE POSTABSORPTIVE STATE IN HEALTHY HUMANS

K Ekberg, BR Landau, A Wajngot, V Chandramouli, S Efendic, H Brunengraber, and J Wahren. Depts of Clinical Physiology and Endocrinology, Karolinska Hospital Stockholm, Sweden and Depts of Medicine and Nutrition, Case Western Reserve Univ., Cleveland, Ohio The aim of the study was to evaluate simultaneously A-V net exchange of gitucose across kidney and liver and the uptake of glucose to the splanchnic and renal tissues. Seventeen healthy subjects, age $27 \pm 1 \mathrm{yrs}$, BMI $22.6 \pm 0.6 \mathrm{~kg} / \mathrm{m}^{2}$, were studied in the postabsorptive state by arterial, hepatic and renal venous catheterization and given a primed constant infusion of either $\left(6{ }^{3} \mathrm{H}\right)$ glucose $(\mathrm{n}=10 ; 20 \mu \mathrm{Ci}, 10 \mu \mathrm{Ci} / \mathrm{h}$ for $3 \mathrm{~h})$ or $\left(\mathrm{U}^{1{ }^{13}} \mathrm{C}_{6}\right)$ glucose $(\mathrm{n}=7 ; 30 \mu \mathrm{mol} / \mathrm{kg}, 0.5 \mu \mathrm{mol} / \mathrm{kg} / \mathrm{min}$ for $5 \mathrm{~h})$ Net exchange was determined from regional blood flow and arterial and regional venous glucose concentrations. Uptake of glucose to the splanchnic and renal tissues were measured from the ${ }^{3} \mathrm{H}$-activity or ${ }^{15} \mathrm{C}$-enrichment $(\mathrm{M}+6)$ in arterial and regional venous plasma, regional blood flow and arterial and regional venous glucose concentrations. Results:

\begin{tabular}{lccc}
\hline Glucose & $\left(6-{ }^{3} \mathrm{H}\right)$ glucose & $\left(\mathrm{U}-{ }^{13} \mathrm{C}_{6}\right)$ glucose & \\
\hline Ra & $11.4 \pm 0.7$ & $13.6 \pm 0.6$ & $\mu \mathrm{mol} / \mathrm{kg} / \mathrm{min}$ \\
Spl uptake & $1.9 \pm 0.2$ & $2.3 \pm 0.5$ & $\mu \mathrm{mol} / \mathrm{kg} / \mathrm{min}$ \\
Spl Net Exch. & $-9.6 \pm 1.4$ & $-9.9 \pm 1.0$ & $\mu \mathrm{mol} / \mathrm{kg} / \mathrm{min}$ \\
Renal uptake & $-0.5 \pm 0.3$ & $0.8 \pm 0.4$ & $\mu \mathrm{mol} / \mathrm{kg} / \mathrm{min}$ \\
Renal Net Exch. & $-0.3 \pm 0.4$ & $0.2 \pm 0.2$ & $\mu \mathrm{mol} / \mathrm{kg} / \mathrm{min}$
\end{tabular}

Net splanchnic exchange of glucose and splanchnic glucose uptake together accounted for $90-100 \%$ of glucose Ra. Neither a significant renal net exchange nor a renal uptake of glucose were found. Calculated from the ${ }^{13} \mathrm{C}$-enrichments in arterial and hepatic and renal venous plasma the renal contribution to whole body glucose production was $5 \pm 2 \%(p<0,05)$. It is concluded that the liver is the predominant site of glucose production in the postabsorptive state and that the renal contribution to whole body glucose turnover is small, if any. 
789

INHIBITION OF HEPATIC GLUCOSE PRODUCTION BY INTERFERENCE WITH THE HEPATIC GLUCOSE-6-PHOSPHATASE SYSTEM

A.W. Herling, H.-J. Burger, H. Hemmerle, G. Schubert, and D. Schwab HOECHST MARION ROUSSEL Deutschland GmbH, Frankfurt, Germany

Reduction of hepatic glucose production (HGP) is a significant approach for developing drugs for treatment of Type II diabetes mellitus (NIDDM). Glucose-6-Phosphatase (G6Pase) catalyzes the terminal step of HGP. G6P translocase (T1) facilitates access of G6P to the catalytic site and exerts a significant control on G6P hydrolysis. Synthetic derivatives of chlorogenic acid $(\mathrm{CHL})$ are potent inhibitors of T1. Two new $\mathrm{CHL}$ derivatives, $\mathrm{S} 3483$ and S 4048, inhibited G6P hydrolysis in intact, but not in disrupted rat liver microsomes with $\mathrm{IC}_{50}$ values of 100 and $2 \mathrm{nmol} / \mathrm{l}$, respectively. We investigated the effect of these compounds on fructose-stimulated gluconeogenesis in isolated perfused livers of starved rats, and on glycogenolysis using livers from fed rats for perfusion. Both compounds concentration-dependently inhibited HGP in the same concentration range for both experimental conditions. in starved rats, euglycemia is maintained by gluconeogenesis. 2 hrs infusion with S $3483(60 \mathrm{mg} / \mathrm{kg} / \mathrm{h}$ i.v. $)$, reduced HGP from $7.6 \mathrm{mg} / \mathrm{kg} / \mathrm{min}$ to 2.8 $\mathrm{mg} / \mathrm{kg} / \mathrm{min}$. Both compounds caused dose dependent hypoglycemia likely due to the reduction of gluconeogenesis driven HGP in starved rats. In fed rats, glycogenolysis was induced by i.v. injection of glucagon resulting in a transient hyperglycemia. Infusion of CHL derivatives abolished this hyperglycemic peak. Inhibition of either gluconeogenesis (starved rats) or glycogenolysis (fed rats + glucagon) was accompanied by dose dependent increases of hepatic glycogen and G6P as determined at the end of the experiments, after 5 hours of infusion. In conclusion, the compound induced effects on HGP, blood glucose, and metabolite concentrations demonstrate that the hepatic G6Pase system is the locus of pharmacological interference of $\mathrm{CHL}$ derivatives. Supplementary studies will determine if $\mathrm{CHL}$ derivatives will become useful to reduce HGP and lower blood glucose in NIDDM.

\section{0}

GLUCOSE PER SE CONTROL ON ENDOGENOUS GLUCOSE PRODUCTION AND CLEARANCE RATE ALSO ORIGINATES FROM A PLASMA-DLLAYED SIGNAL.

M. Simeoni. A. Caumo. A. Basu. R. A. Rizai and C. Cohelli. Padova and Milano. llaty: Rochester. MN. ISA

Glucose efled per se on endugenous glucome production (ECP) and plasthit clearance rale (PCR) platys an impontant role in glucose tolerance. However, tittle is known about the timing of glucose control on EGP and PCR. To clarify this issue we investigated the dynamic re]ationship belween the time courses of glucose, EC.P. and PCR when glucose exhibited a prandial-like protile with insulin maintained at the basal level with at somalustatin infusion. Eight nondiabetic subjects wero studied. Exugenous glucuse was administered in such a way to mimic glucose plistma profile after a meal. $\Lambda$ glucose tricer ( $\left[6-{ }^{3} \mathrm{H} / g /\right.$ lucose) was added to exogenous glucose (Hot-GinO). thus allowing us to accurately assess EGP. Wo lound that. whereas glucose peaked al 90 min and was back to bisal at $300 \mathrm{~mm}$ EGP reateded its natir later. al 195 min. and was lan below its hasal level by the end at the study. Kinetic nurdelling of glucose and ECP profiles revealed that ECil is controlled by both plasua and phasma-delayed gleose signals. The presence of a glucose-inceliated inhibition of PCR wats demonstrated by comparing the measured profile of $\left[6-{ }^{3} \mathrm{H}\right] \mathrm{glucose}$ concentation with that predicted by Rildziuk's twoconpartment model af glucose kinetics assuming a consiant PCR (i.c. $k_{(j)}=$ constint). The model well described the tracer data during the first $30 \mathrm{~min}$,

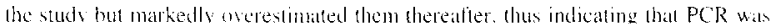
nol constant but was progressively inhibited, The time course of PCR inhihition wils calculated from the sime model by allowing for a time-varying fractional disappearance ratc. kn (1). Model andilysis of the time course of $\mathrm{k}_{01}(t) \mathrm{vs}$, glueose concentration revealed that PCR is inhibited not directly by glucose in plasma hu by glucose in a remote comparment. In conclusion, glucose exerts its elfects on EGP and PCR via bodl plasmia and plasma-deliyed signals. We speculate that these results should help to sim ta out the individual effects of vlucose and insulin on top and PCR when glucose and insulin vary together. e.g. during a meal.

\section{1}

INHIBTION OF HEPATIC GLUCOSE PRODUCTION BY LIPOIC ACID IS ASSOCIATED WITH INCREASED ACYL-CARNITINE PRODUCTION M. Khamaisi", A. Gutman ${ }^{\#}$, A. Rudich*, R. Potashnik*, HJ.Tritschler ${ }^{\wedge}$, and N. Bashan ${ }^{*}{ }^{*}$ Clinical Biochemistry, Ben-Gurion University, Beer-Sheva, "Hadassah Medical Center, Jerusalem, Israel, and ^ASTA Medica, Frankfurt, Germany.

Lipoic acid (LA) is a unique antioxidant which increases peripheral glucose utilization in diabetic patients. This study was conducted to investigate whether inhibition of hepatic glucose production (HGP) could be an additional mechanism for LA's hypoglycemic action. Intravenous injection of $100 \mathrm{mg} / \mathrm{kg} \mathrm{LA}$ to fasting non diabetic or streptozotocin-induced diabetic rats caused a rapid reduction in blood glucose. with no effect on circulating insulin levels. In vivo conversion of fructose to glucose was not inhibited by LA, whereas gluconeogenesis flux from alanine was completely prevented, as assessed by loading tests. A reduction in acetyl CoA concentration ( $80 \pm 10 \%$ in non diabetic and $60 \pm 8 \%$ reduction in diabetic rats) was observed following LA, suggesting reduced in-vivo activity of pyruvate carboxylase (PC). To investigate the mechanism for reduced acetyl CoA, FFA, ketone bodies, carnitine and acyl carnitine levels were determined. Plasma FFA and bound carnitine levels were increased following LA by -2 fold in both non diabetic and diabetic rats. In accordance, free carnitine levels were reduced following LA treatment $(13 \pm 0.2$ to $7 \pm 0.3$ in non diabetic, $12 \pm 0.4$ to $9.6 \pm 0.4$ in diabetic, $P<0.05)$, with no elevation in plasma ketone bodies. Mass spectroscopy reviled that increased plasma levels of C16, C18, C18:1 and C18:2 -carnitines, but not lipoyl-carnitine could account for the increase in bound carnitine levels, in both non diabetic and diabetic rats. While direct sequestration of carnitine by LA was excluded, these results suggests an inhibition of mitochondrial long chain fatty acid $\beta$ oxidation, which in turn result in reduced liver acetyl $\mathrm{COA}$ and hence $\mathrm{PC}$ activity. These provide mechanism for inhibition of liver gluconeogenesis by acute, high dosage of LA.

\section{2}

\section{LIVER GLUCOKINASE GENE TRANSFER INTO RAT SKELETAL MUSCLE IN VIVO: METABOLIC IMPACT AND CONTROL OF GLUCOSE HOMEOSTASIS}

J.C. Jiménez-Chillarón, S. Chico, S. Arias and A.M. Gómez-Foix Department of Biochemistry and Molecular Biology. University of Barcelona, 08028. Spain.

Glucose utilization in skeletal muscle is limited by transport and/or glucose phosphorylation. To evaluate the role of glucose phosphorylation in limiting its rate of utilization, we have delivered to skeletal muscle the rat liver glucokinase, wich in contrast to hexokinase II is not inhibited by glucose 6-phosphate. Previous experiments have showed that expression of glucokinase, by means of recombinant adenovirus-mediated gene transfer (AdCMV-LGK), in cultured human fibers, leads to the acute stimulation of glucose uptake and consumption by cells (unpublished results, Baqué et al.). We have tested the potential utility of this genetic engineering for enhancing muscle glucose disposal in vivo. Delivery of transgenes was accomplished by intramuscular injection (i.m.) to newborn Wistar rats (2-3 days) in the right hind leg with either control adenovirus (AdCMV-lacZ) or AdCMV-LGK viruses. AdCMV-lacZ served as a negative control for metabolic effects due to viral protein expression, and was also used to evaluate the proportion of muscle fibers expressing the transgene. The expression of glucokinase was detected by western blot and by determination of glucose phosphorylating activity at high glucose concentration. Immunoblotting of total cell homogenates from muscle tissue showed glucokinase expression in muscles injected with AdCMV-LGK, 15 days to 2 months after adenovirus manipulation. The glucokinase expression varied over a wide rarige as may be expected from differences in the efficiency of delivery. The presence of glucokinase was correlated with an increase in the muscle glucose phosphorylating capacity. To evaluate the changes in glucose homeostasis, one month injected animals were subjected to an intraperitoncal (i.p.) glucose tolerance test. Rats expressing glucokinase cleared glucose faster than controls although the effect was only manifested during the raise in glucose. Fed and fasted basal blood glucose was also decreased in glucokinase expressing animals. Therefore, glucokinase delivery to muscle may contribute to increase muscle glucose disposal and improve glycemic control and it may represent a potential therapeutic strategy for diabetes. 
REGULATION OF GLYCOGENESIS BY PORTAL-ARTERIAL GLUCOSE GRADIENTS IN PERFUSED RAT LIVER

O.Mokuda, and Y.Sakamoto

Teikyo University, Ichihara, Japan

To study effects of portal(P)-arterial(A)

glucose gradients on the hepatic glycogenesis,

the liver of fasted rats was perfused. $35 \mathrm{ml}$ of

$\mathrm{KRB}$ buf'er with $2 \mathrm{mM}$ glucose, $3 \mathrm{mM}$ lactate, 20

$\mu \mathrm{g} / 1$ insulin, and $(1-14 \mathrm{C})-\mathrm{g}$ lucose or $(U-14 \mathrm{C})-$

lactate was recirculated at flow rates of $14 \mathrm{ml}$

$/ \mathrm{min}$ via $\mathrm{P}$ route and $7 \mathrm{ml} / \mathrm{min}$ via A route.

Glucose was infused at a rate of $27.75 \mu \mathrm{mol} / \mathrm{min}$

into the $P$ route ( $P$-experiment) or the $A$ route

(A-experiment), resulting the $\mathrm{P}-\mathrm{A}$ glucose

gradients of +1.98 or $-3.96 \mathrm{mM}$. Incorporation

of $14 \mathrm{C}$ from $14 \mathrm{C}-\mathrm{glucose}$ into glycogen was

higher in the $\mathrm{P}$-experiment than in the $\mathrm{A}$ -

experiment $(0.25 \pm 0.02$ vs $0.18 \pm 0.02 \%$ for 20

min, $P<0.01$ ), and not influenced by insulin.

Incorporation of $14 \mathrm{C}$ from $14 \mathrm{C}-1$ actate into

glycogen was not different between the $\mathrm{P}-$ and $A$-experiments in the absence of insulin. This activity was more strongly stimulated by

insulin in the $\mathrm{P}$-experiment than in the $\mathrm{A}$ -

experiment $(0.49 \pm 0.03$ vs $0.40 \pm 0.03 \%$ for 20

min, $P<0.05)$. It is suggested that the $P-A$

glucose difference has an important role in the

regulation of hepatic glycogen synthesis from exogenous glucose and gluconeogenesis.

\section{PS 31}

\section{Protein Metabolism}

794

COMBINED INSULIN RESISTANCE OF GLUCOSE AND PROTEIN METABOLISM IN ACROMEGALY.

A. Battezzati, M. Losa, S. Benedini, L. Piceni Sereni, A. Fattorini, P. Mortini and L. Luzi. Istituto Scientifico H. San Raffaele, Milano, Italy.

Growth hormone (GH) counteracts insulin action on glucose metabolism frequently causing glucose intolerance and diabetes in acromegalic patients (ACRO). However, both hormones promote protein accretion, and it is unclear whether insulin resistance in acromegaly also involves protein metabolism. Since insulin suppresses proteolysis in vivo, we studied whether ACRO impairs the antiproteolytic effect of insulin. Seven non-diabetic ACRO (GH 5.7-81.1 ng/ml, IGF $1286-1107 \mathrm{ng} / \mathrm{ml}$ ) and 6 healthy controls (CON, GH 0.6-1.2 $\mathrm{ng} / \mathrm{ml}$ ) matched for sex, age and body weight underwent an infusion of $\left[6,6-{ }^{2} \mathrm{H}_{2}\right]$ glucose and [1${ }^{13} \mathrm{C}$ leucine during an euglycemic insulin clamp (1 $\left.\mathrm{mU} / \mathrm{kg} / \mathrm{min}\right)$ to assess insulin sensitivity of glucose and endogenous leucine flux (ELF), an index of proteolysis. Postabsorptively, ACRO and CON had a comparable p- glucose $(96.5 \pm 1.4$ vs $91.5 \pm 2.5 \mathrm{mg} / \mathrm{dl})$, glucose flux $(2.60 \pm 0.11$ vs $2.19 \pm 0.19 \mathrm{mg} / \mathrm{kg} / \mathrm{min})$, p-leucine

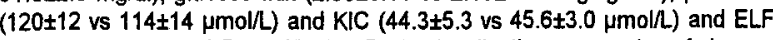
$(105.2 \pm 2.7$ vs $1075 \pm 1.5 \mu \mathrm{mol} / \mathrm{kg} / \mathrm{h})$. During insulin, the suppression of glucose production was impaired in ACRO (0.45 \pm 0.10 vs $0.01 \pm 0.01 \mathrm{mg} / \mathrm{kg} / \mathrm{min}, p<0.05$ ), and the stimulation of glucose uptake was markedly reduced $(2.69 \pm 0.07$ vs $5.93 \pm 0.30 \mathrm{mg} / \mathrm{kg} / \mathrm{min}, \mathrm{p}<0.01$ ). In addition, the insulin suppression of $p$-leucine $(-23.5 \pm 3.7 \%$ vs $-41.3 \pm 4.0 \%, p<0.05)$ and $\mathrm{KIC}(-21.9 \pm 7.4 \%$ vs $-50.1 \pm 5.3 \% \mathrm{p}<0.05)$ was defective. Finally, insulin suppression of ELF $(-8.9 \pm 1.6 \%$ vs $-19.3 \pm 1.4 \%$ $p<0.01$ ) was impaired. Pooling all subjects, GH and ELF suppression were inversely related $(p<0.05)$. Two ACRO were studied again 6 months after succesful adenectomy and showed a near normalization in insulin sensitivity of glucose fluxes and a modest improvement in insulin sensitivity of proteolysis. In summary, the insulin stimulation of glucose uptake and the suppression of glucose production and proteolysis are all impaired in non-diabetic ACRO. In conclusion, ACRO are severely insulin resistant for both glucose and protein metabolism, indicating a generalized defect in insulin signaling. A reduced insulin anabolic effect may in part reduce the stimulation of protein accretion by $\mathrm{GH}$ in acromegalic patients.

\section{5}

MUSCLE PROTEIN ANABOLISM DURING MEAL ABSORPTION IS IMPAIRED IN THE ELDERLY DUE TO INSULIN RESISTANCE AT THE PROTEN METABOLISM LEVEL.

E.Volpi, B. Mittendorfer and R.R.Wolfe. University of Texas Medical Branch, Shriners Hospital, 815 Market St., Galveston, TX 77550, USA.

The reduction of muscle mass that takes place with aging is possibly due to an inappropriate response of muscle protein anabolism to the meal. However, we have recently shown that muscle protein anabolism is stimulated by exogenous amino acids alone in the elderly as in the young. Thus, we hypothesized that if the loss of muscle in the elderly is due to an altered response to meal this could be due to an impairment in the response to the physiologic hyperinsulinemia occurring during meal intake. We measured leg muscle protein kinetics by the means of femoral arterio-venous catheterization and stable isotope ( $\mathrm{L}$-[ring- ${ }^{2} \mathrm{H}_{5}$ ]phenylalanine) infusion in 5 healthy young $\left(30 \pm 3\right.$, BMI $\left.25 \pm 1 \mathrm{~kg} / \mathrm{m}^{2}\right)$ and 4 healthy elderly $(72 \pm 1 \mathrm{yrs}$, BMI $26 \pm 1 \mathrm{~kg} / \mathrm{m}^{2}$ ) volunteers, with normal glucose tolerance, in the post-absorptive state (PA) and during the administration of a 3 hour amino acid-glucose meal (AG) given in small boluses every 10 minutes in order to maintain the plasma amino acid concentrations at steady state. Meal intake induced a significant increase in arterial phenylalanine delivery to the leg (young: $236 \pm 37$ vs $663 \pm 132$; elderly: $248 \pm 54$ vs 474 $\pm 118 ; \mathrm{nmol} / \mathrm{min}^{-1} \cdot 100 \mathrm{ml} \mathrm{leg}^{-1}$; PA vs AG, $\mathrm{p}<0.01$ ) with no differences between the groups. However, the meal-induced increase in phenylalanine net balance (young: $-31 \pm 9$ vs $71 \pm 16$; elderly: $-18 \pm 4$ vs $35 \pm 5$; $\mathrm{nmol} / \mathrm{min}^{-1} \cdot 100 \mathrm{ml} \mathrm{leg}{ }^{-1}$; PA vs AG, $p<0.001$ ) and rate of disappearance (young $35 \pm 12$ vs $115 \pm 22$; elderly $41 \pm 6$ vs $72 \pm 16 \mathrm{nmol} / \mathrm{min}^{-1} \cdot 100 \mathrm{ml} \mathrm{leg}{ }^{-1} ; \mathrm{PA}$ vs $\left.\mathrm{AG}, \mathrm{p}<0.001\right)$ across the leg were blunted in the elderly (time by group interaction: $p<0,05$ ). During the meal leg blood flow increased in the young whereas it decreased in the elderly (young: $3.54 \pm 0.60 \mathrm{vs}$ $4.31 \pm 0.79$; elderly: $3.82 \pm 0.75$ vs $3.22 \pm 0.63 ; P A$ vs $A G$, time by group interaction: $\mathrm{p}<0.05$ ). Since in the elderly the response of muscle protein anabolism to amino acids alone is normal, we conclude that the observed impairment of muscle protein anabolism during amino acids plus glucose is likely due to alterations in insulin sensitivity at the muscle protein metabolism level. 
Title: Insulin deprivation inhibits mucosal protein synthesis in type I diabetes mellitus. Michael R. Charlton MD and K. Sreekumaran Nair MD. Endocrine Research Unit, Mayo Clinic and Foundation, Rochester, MN. USA.

Background: Although insulin deficiency is associated with net protein catabolism in patients with type I diabetes mellitus, whole body protein synthesis increases during insulin deficiency. Regional protein turnover studies have demonstrated that the increase in whole body protein synthesis during insulin deprivation in patients with type I diabetes mellitus occurs in the splanchnic region while mixed muscle protein and myosin heavy chain synthesis is unchanged. We hypothesized that an increased rate of protein synthesis occurs in mucosal proteins in patients with type I diabetes mellitus during insulin deficiency. Methods: We determined gut mucosal protein synthesis in six patients with type I diabetes mellitus during insulin deprivation using a primed continuous infusions of $\mathrm{L}-\left[1-{ }^{13} \mathrm{C}\right]$ leucine. Results were compared with six patients with type I diabetes mellitus during insulin treatment, and six non-diabetic healthy controls. Mucosal tissue samples were obtained endoscopically. Results: The fractional synthesis rates (FSR) of mixed mucosal proteins in the three groups are shown below:

\begin{tabular}{lccc}
\hline Non-diabetic controls & $\begin{array}{c}\text { Type I DM-insulin } \\
\text { dependent }\end{array}$ & $\begin{array}{c}\text { Type I DM- } \\
\text { insulin treated }\end{array}$ \\
\hline Free insulin (pmol) & $32 \pm 3$ & $86 \pm 13$ & $6 \pm 1$ \\
FSR $(\% / h)$ & $2.5 \pm 0.1$ & $1.7 \pm 0.2+$ & $2.4 \pm 0.2$ \\
\hline Free insulin levels were measured in pmol/L. FSR $=$ fractional synthesis rate of
\end{tabular}

Free insulin levels were measured in pmol/L. FSR $=$ fractional synthesis rate of mixed mucosal proteins. $t$ indicates that the FSR of mixed mucosal proteins was significantly lower in insulin deprived type I diabetic subjects than in either nondiabetic healthy controls $(P=0.04)$ or type I diabetic subjects during insulin treatment $(P=0.01)$. Conclusions: Insulin deficiency is associated with decreased fractional synthesis of mucosal proteins in subjects with type $\mathbf{I}$ diabetes mellitus. Insulin treatment reverses this effect. The increase in splanchnic protein synthesis that occurs during insulin deficiency must occur at a site other than mucosal proteins, such as among the hepatically synthesized plasma proteins.

\section{PS 32 \\ Lipids I}

\section{7}

n-3 FATTY ACIDS INCREASE FATTY ACID OXIDATION IN DIETARY INDUCED INSULIN RESISTANCE AND HYPERTRIGLYCERIDEMIA

E. Sebokovaa, E. Bergenea,b, L. Madsenc, J. Ukropeca, B. Liskaa, E. Dyroeyc, A. C. Rustanb, I. Klimesa and R. K. Bergec, alnstitute of Experimental Endocrinology, Slovak Academy of Sciences, Slovak Republic, bSchool of Pharmacy, University of Oslo, Norway, eDepartment of Clinical Biology, Division of Biochemistry, University of Bergen, Norway

Hypertriglyceridemia and high levels of plasma free fatty acids (FFA) are associated with atherosclerosis, type 2 diabetes (NIDDM) and insulin resistance (RR). We have previously shown that treatment of rats with $n-3$ polyunsaturated fatty acids ( $n-3$ PUFA) causes a reduction in the plasma levels of these lipids and an improvement of insulin action. This study was undertaken to assess the role of fatty acid oxidation in liver, heart and skeletal muscle for lowering of plasma lipids, and thus for improvement of IR. Male albino rats (Wistar) were fed for 3 weeks either basal (BD) or high (63 cal\%) sucrose (HS) diets containing ca. $20 \mathrm{cal} \%$ saturated fat. The fat in the HS diet was replaced with fish oil-derived $\mathrm{n}^{-3}$ fatty acids (Triomar, Pronova, Norway) (FO), the average intake of n-3 PUFA being $3.4 \mathrm{~g} / \mathrm{kg} / \mathrm{day}$. Fatty acid oxidation was assessed by measuring the enzyme activities of carnitine palmitoyltransferase (CPT) I and II in post-nuclear fractions from freeze clamped tissues. All data are presented as mean $\pm \mathrm{SEM}, \mathrm{p}<0.05$ unless otherwise stated. Feeding the animals HS diet led to a 2 -fold increase in plasma postprandial triglyceride (Tg) [BD: $2.4 \pm 0.2 ; \mathrm{HS}: 5.1 \pm 0.3$ mmol. $\left.\mathrm{I}^{-1}\right]$ levels, whereas fasted plasma FFA [BD: 0.65 0.03 , HS: $0.56 \pm 0.02$ mmol. $\left.\mathrm{I}^{-1}\right]$ and ketone bodies ( $\beta$ hydroxybutyrate) [BD: $11.4 \pm 1.7$, HS: $10.4 \pm 0.8 \mathrm{mg} / \mathrm{dl}]$ remained unchanged. Supplementation with $\mathrm{n}-3$ PUFA to the HS diet led to a 5 -fold decrease in postprandial $\mathrm{Tg}\left[\mathrm{HS}+\mathrm{FO}: 0.95 \pm 0.09 \mathrm{mmol} \mathrm{1}^{1 /}\right]$ and a 2 -fold decrease in fasted FFA [HS+FO: $0.29 \pm 0.02 \mathrm{mmol} . \mathrm{r}^{-1}$ ]. While the fasted level of ketone bodies remained unchanged [HS+FO: $9.7 \pm 1.4 \mathrm{mg} / \mathrm{dll}$, the ratio of ketone bodies to FFA was increased 1.8-fold [HS: 18.6, HS+FO: 33.2]. Hepatic CPT I activity was slightly increased

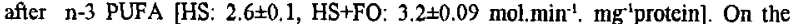
other hand, the CPT II activity was induced more than 3 -fold in comparison to the

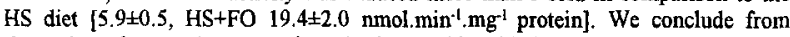
these data that an increased hepatic fatty acid oxidation may contribute to the decrease in plasma lipids after treatment with n-3 PUFA in dietary induced hypertriglyceridemia and insulin resistance.

\section{8}

A RE-APPRAISAL OF THE ROLES OF MUSCLE AND ADIPOSE TISSUE FOR GLYCEROL AND LACTATE RELEASE IN MAN J. Bolinder, E. Hagström-Toft, D. Kerckhoffs, E. Moberg and P. Arner. Dept of Medicine, Huddinge Hospital, Karolinska Institute, S-141 86 Huddinge, Sweden.

Glycerol production (lipolysis index) and lactate release were quantitatively assessed in muscle and adipose tissue in vivo, using microdialysis measurements of interstitial glycerol and lactate concentrations in combination with determination of local blood flow ( ${ }^{133} \mathrm{Xe}$ clearance technique) in the two tissues (gastrocnemius muscle and abdominal subcutaneous adipose tissue). The experiments were carried out in 12 healthy, normal-weight subjects in the fasting state and during $240 \mathrm{~min}$ following a $75 \mathrm{~g}$ oral glucose load. At baseline, blood flow was similar in adipose tissue and muscle; following glucose ingestion adipose tissue blood flow was almost doubled whereas muscle blood flow remained unchanged. In the fasting state, the estimated glycerol and lactate production rates were 4 times higher and $50 \%$ lower, respecively, in adipose tissue than in muscle $(p<0.01)$. After glucose ingestion glycerol production in adipose tissue was transiently reduced by about $30 \%(\mathrm{p}<0.02)$; in muscle a similar but more protracted decrease in glycerol release was registered $(\mathrm{p}<0.01)$. However, glycerol release remained 3-4 times higher in adipose tissue than in muscle after the oral glucose load. I

$n$ response to glucose ingestion, the release of lactate was increased 2fold in adipose tissue, whereas in muscle the corresponding increase was only $25 \%$. It is concluded that adipose tissue is the main source of glycerol, in the fasting state as well as after glucose ingestion. A significant proportion of the glycerol release, however, apparently originates from muscle. In the basal state the production of lactate is higher in muscle than in adipose tissue, whereas after carbohydrate intake the increase in lactate release predominantly occurs in adipose tissue. 


\section{9}

EFFECT OF HYPERGLYCAEMIA AND HYPERTRIGLYCERIDAEMIA ON LOW-DENSITY LIPOPROTEIN OXIDISABILITY IN HEALTHY MEN.

T. Pelikánová, L. Kazdová, P. Wohl, A. Žák and V. Nováková. Institute for Clinical and Experimental Medicine and 1st Medical Faculty of Charles University, Prague, Czech Republic

increased oxidative modification of low-density lipoproteins (LDL) has been implicated as independent risk factor for atherosclerosis. LDL oxidation in vitro is stimulated by glucose itself. The aim of our study was to evaluate the effect of acutely-induced hyperglycaemia and hypertriglyceridaemia on LDL oxidisability in 13 healthy subjects. The susceptibility of $\mathrm{LDL}$ to $\mathrm{Cu}^{2+}$ induced oxidative modification, and plasma concentrations of $\alpha-(\alpha T)$ and $B+\gamma-$ tocopherol $(\beta+\gamma T)$ were under following conditions lasting 4 hours: a) hyperglycaemic clamp (12 mmol//:HG), b) hyperglycaemic clamp with infusion of somatostatin in order to decrease the insulin production (HGS) c) infusion of Intralipid ( $0.15 \mathrm{~g}$ of fat. $\left.\mathrm{kg}^{-1} \cdot \mathrm{h}^{-1}, 1\right)$, and d) a time-controlled study with saline infusion $(\mathrm{C})$. Conjugated diene production did not change during HG and HGS compared to C ( HG: $564 \pm 75$ vs $560 \pm 76 \mathrm{mmol} / \mathrm{mg}$ of LDL protein; HGS: $564 \pm 70$ vs $582 \pm 72 \mathrm{mmol} / \mathrm{mg}$ of LDL protein; C: $443 \pm 85 \mathrm{vs}$ $450 \pm 70 \mathrm{mmol} / \mathrm{mg}$ of LDL protein), while it significantly decreased during I $(589 \pm 100$ vs $524 \pm 87 \mathrm{mmol} / \mathrm{mg}$ of LDL protein; $p<0.05$ ). No significant changes in the lag time of diene production (HG: $74.8 \pm 20.0$ vs $76.8 \pm 26.7$ $\mathrm{min}$; HGS: $66.9 \pm 9.4$ vs $69.2 \pm 15.7 \mathrm{~min}, \mathrm{l}: 69.0 \pm 22.3$ vs $86.8 \pm 18.3 \mathrm{~min}$ C: $71.15 \pm 15.0$ vs $78.5 \pm 20.1 \mathrm{~min}$ ), in thiobarbituric acid reactive substances concentration and in oxidative modification of protein moiety measured by the emission fluorescence spectra were found. Decrease in diene production during Intralipid infusion was accompanied by increase in $\alpha \mathrm{T}(22.2 \pm 3.4 \mathrm{vs}$ $24.0 \pm 3.5 \mathrm{umol} / \mathrm{l} ; \mathrm{p}<0.001)$ and $\beta+\gamma \mathrm{T}(2.3 \pm 1.4$ vs $16.9 \pm 7.6 \mathrm{umol} / \mathrm{l} ; \mathrm{p}<$ $0.001)$. The results suggest that 4 hour in vivo induced hyperglycaemia is not able to increase the LDL oxidisability. Infusion of Intralipid even decreases LDL susceptibility to oxidative modification probably due to high tocopherol content in lipid emulsion. The acute in vivo experiments do not exclude the opposite long-term effects. (Supported by IGA MZ CR, No. 4903-3)

\section{1}

RELATIONSHIP OF INSULIN SENSITIVITY WITH ABDOMINAI FAT AND MUSCLE LONG CHAIN ACYL COA's (LCACs) IN MEN

D.J. Chisholm, A.M. Poynten, B.A. Ellis, S.M. Furler, A.J. Lowy, E.W. Kraegen, L.V. Campbell and G.J. Cooney. Garvan Institute of Medical Research, St Vincent's Hospital, Sydney, NSW, Australia

We and others have demonstrated a strong inverse relationship between central abdominal fat and insulin sensitivity in humans and between muscle triglyceride content and insulin sensitivity in animals. Therefore a large abdominal fat depot may provide an excess systemic supply of fatty acids which accumulate in muscle and generate insulin resistance. We propose that muscle LCAC content is a better indicator of lipid availability than triglyceride in human muscle biopsies. Estimation of triglyceride content from muscle biopsies may be confounded by very small amounts of contaminating fat; however LCAC content (by HPLC) of adipose tissue is $20 \%$ that of muscle. We have therefore determined LCAC content of vastus lateralis muscle, as an indicator of muscle fatty acid availability, in 9 males (age 57-75, BMI 25-38) undergoing knee surgery. Results were correlated with insulin sensitivity by euglycemic clamp (insulin level approximately $70 \mathrm{mU} / \mathrm{L}$ ), serum triglycerides and abdominal fat (by dual $x$-ray absorptiometry). Muscle LCAC ranged from 3.25 to $5.25 \mathrm{nmol} / \mathrm{g}$ tissue, abdominal fat ranged from 0.9 to $3.2 \mathrm{~kg}$ and insulin sensitivity (glucose infusion rate) from 1 to $10.5 \mathrm{mg} / \mathrm{min} / \mathrm{kg}$ fat free mass. Insulin sensitivity (glucose infusion rate/fat free mass) correlated negatively with abdominal fat $(\mathrm{Kg}), \mathrm{r}^{2}=0.49, \mathrm{p}<.03$ and with total muscle LCAC's, $r^{2}=0.46 p<.05$, but the relationship between abdominal fat and LCAC's did not reach statistical significance. Serum triglycerides did not show a significant relationship to either insulin sensitivity or abdominal fat. This data supports the importance of muscle fatty acid availability, as well as abdominal fat, in the generation of insulin resistance in man.
800

PROTEIN RESTRICTION ALTERS THE REGULATION OF TRIACYLGLYCEROL TURNOVER BY INSULIN DURING PREGNANCY

M.J. Holness and M.C. Sugden, Department of Biochemistry, Faculty of Basic Medical Sciences, St. Bartholomew's and the Royal London School of Medicine and Dentistry, Queen Mary and Westifield College, London, U.K.

Moderate protein restriction throughout gestation, which leads to foetal growth retardation, is associated with impaired suppression of plasma triacylglycerol (TAG) levels by hyperinsulinemia. The study investigated the molecular basis for this impaired action of insulin through examining the impact of moderate protein restriction (providing an isocaloric $8 \%$ vs. $20 \%$ protein diet) during pregnancy on the liporegulatory actions of insulin to suppress hepatic TAG secretion (estimated using Triton WR 1339) and enhance TAG utilisation. Protein restriction did not affect maternal energy intake or body weight gain, but liver weight at day 19 was reduced. Postabsorptive plasma TAG concentrations were 1.9 fold higher in the protein-restricted (PR) group. Rates of TAG accumulation after Triton WR 1339 administration were 1.6 fold higher $(P<0.0 .5)$ in the PR group $(4.53 \pm 0.36$ $\mu \mathrm{moles} / \mathrm{m} / \mathrm{h}$ ) than in the control (C) group (2.92 $\pm 0.33 \mu \mathrm{moles} / \mathrm{m} / \mathrm{h})$. Increased postabsorptive TAG production as a consequence of mild protein restriction during pregnancy may therefore underlie post-absorptive hypetriglyceridemia. Plasma TAG concentrations declined in response to $2 \mathrm{~h}$ of insulin infusion $(4.2 \mathrm{mU} / \mathrm{kg} / \mathrm{min})$, with an overall decline of $33 \%(\mathrm{P}<0.0 .5)$ in the $\mathrm{C}$ group and $48 \%(\mathrm{P}<0.0 .5)$ in the $\mathrm{PR}$ group. Although TAG concentrations after $1 \mathrm{~h}$ of insulin infusion remained 1.7 fold higher $(P<0.05)$ in the $P R$ group, the continued infusion of insulin for a further hour attenuated the differences between the groups. Hyperinsulinemia suppressed TAG secretion by $42 \%(P<0.05)$ and $51 \%(P<0.01)$ respectively in the $C$ and $P R$ groups. Nevertheless, TAG secretion rates during steady-state hyperinsulinemia remained 1.3 fold higher in the PR group. Since TAG concentrations decline at a greater rate, while at the same time, TAG secretion is enhanced, the resulis demonstrate more rapid TAG utilisation during hyperinsulinaemia as a consequence of protein restriction. Higher rates of TAG turnover during hyperinsulinemia may be viewed as part of a homeostatic strategy to reduce the maternal requirement for gluconeogenesis from available amino acids, optimising the fetal protein supply.

\section{2}

RELATIONSHIP BETWEEN HYPERTRIGLYCERIDEMIA AND INSULIN SENSITIVITY: AN EUGLYCEMIC CLAMP STUDY

A. Patzak, S. Fischer, B. Kindel, C. Kirch, K. Fücker, M. Hanefeld. Institute and Policlinics of Clinical Metabolic Research, Medical Faculty, Technical University Dresden, Germany.

Although disturbances of glucose metabolism are frequently associated with elevated levels of trigiycerides, it has been difficult to define the order in which these derangements appear and how they relate to each other. Therefore we investigated insulin sensitivity in 21 male hypertriglyceridemic patients without diabetes mellitus (no lipid lowering therapy for at least 6 weeks) in comparison to 18 male healthy subjects (HS) by means of euglycemic hyperinsulinemic clamp technique according to DeFronzo. In a $75 \mathrm{~g}$ oral glucose tolerance test 14 of the hypertriglyceridemic patients showed a normal (HTG) and 7 an impaired glucose tolerance (HTG-IGT). The following parameters significantly differed between the groups (HTGIGT vs. HTG vs. HS): age ( $49.0 \pm 10.7$ vs. $40.5 \pm 9.7$ vs. $25.7 \pm 1.8$ years), body mass index $\left(30.1 \pm 3.6\right.$ vs. $25.9 \pm 3.3$ vs. $\left.22.6 \pm 1.8 \mathrm{~kg} / \mathrm{m}^{2}\right)$. Fasting glucose was significantly higher in the HTG-IGT ( $5.4 \pm 0.4 \mathrm{vs.} 4.5 \pm 0.5 \mathrm{vs}$. $4.6 \pm 0.4 \mathrm{mmol} / \mathrm{l})$. The following parameters significantly differed between the HTG-IGT and the HTG, respectively, and the HS: triglycerides (3.2 士 $1.0 v s .4 .8 \pm 3.1$ and $0.8 \pm 0.4 \mathrm{mmol} / \mathrm{l})$, fasting insulin $(123.2 \pm 80.6 \mathrm{vs}$. $182.4 \pm 98.5$ and $35.8 \pm 18.1 \mathrm{pmol} / /)$, insulin sensitivity $(2.5 \pm 1.7 \mathrm{vs} .4 .3 \pm$ 2.0 and $14.1 \pm 4.1 \mathrm{mg} \times \mathrm{kg}$ body weight ${ }^{-1} \times \min ^{-1} \times$ insulin ${ }^{-1}$ ). Triglyceride levels correlate with insulin senitivity independently of age $(p=0.004)$. The hypertriglyceridemic patients with normal glucose tolerance (HTG) nearly were of normal weight and showed the highest triglyceride and fasting insulin levels, normal fasting glucose, and decreased insulin sensitivity. Our data strongly suggest that hypertriglyceridemia per se is associated with a reduced insulin sensitivity that further deteriorates in overweight hypertriglyceridemic patients with an impaired glucose tolerance. 
INTRAPERITONEAL INSULIN AND SUBCAPSULAR HEPATIC STEATOSIS IN DIABETIC PATIENTS ON CAPD

J. Lahtela, P. Nevalainen, T. Kallio, J. Mustonen and A. Pasternack. Medical School, University of Tampere, Tampere, Finland.

Continuous ambulatory peritoneal dialysis (CAPD) is a common replacement therapy of end-stage renal disease in diabetic patients. Intraperitoneal insulin (IP) administration, considered theoretically the most physiologic insulin replacement modality, is readily available. This therapy results in equal or better glycemic control and improved insulin sensitivity than the use of subcutaneous (SC) insulin but may induce lower HDL-cholesterol and higher LDL-cholesterol levels. This longitudinal study was done to evaluate the effects of IP insulin on peritoneal membrane permeability (peritoneal equilibration test, PET), serum lipids and glycemic control. In a cross-sectional manner an ultrasound of the liver was done. The data on insulin treatment, doses, dialysis, episodes of peritonitis, serum albumin and liver enzymes were recorded. Pearson correlation coefficient was used to test covariation. A total of 13 patients with type I diabetes participated, 5 women and 8 men. The mean age was $42.9 \pm 7.3$ (SD) years, the duration of diabetes $27.8 \pm 6.6$ years and the duration of CAPD 21.5 \pm 18.4 months. Four patients used SC insulin throughout and 9 were on IP insulin. Seven patients with IP insulin showed subcapsular liver steatosis $(6.4-12.0 \mathrm{~mm})$. In 4 patients with SC insulin no liver steatosis was seen. The thickness of the subcapsular steatosis correlated with three measures of PET: 10-minute $\left(r^{2}=0.79\right.$, $\mathrm{p}=0.008)$ and 2-hour $\left(\mathrm{r}^{2}=0.63, \mathrm{p}=0.032\right)$ dialysate creatinine and 2-hour dialysate glucose $\left(\mathrm{r}^{2}=0.79, \mathrm{p}=0.007\right)$. In patients using IP insulin plasma HDL -cholesterol correlated with PET results: 2 -hour $\left(\mathrm{r}^{2}=0.66, \mathrm{p}=0.008\right)$ and 4-hour $\left(r^{2}=0.48, p=0.049\right)$ dialysate creatinine. Thus we confirmed that high IP concentrations of glucose together with insulin induce subcapsular liver steatosis. Direct correlation of PET to the degree of hepatic steatosis suggests the role of insulin in the increased permeability of peritoneal membranes. Decrease in plasma HDL-cholesterol may be, at least partly, the result of increased peritoneal permeability and leakage to the peritoneal fluid during IP insulin treatment.

\section{PS 33 \\ Lipoproteins}

\section{4}

EVALUATION OF PARINARIC ACID AS A PROBE TO MONITOR LDL IN VITRO OXIDATION IN TYPE 2 DIABETES

T. Teerlink, E.E. Musch, S.J.L. Bakker, R.J. Heine and P.G. Scheffer. Departments of Clinical Chemistry and Endocrinology. Research Institute for Endocrinology, Reproduction and Metabolism, Academic Hospital Vrije Universiteit, Amsterdam, The Netherlands.

We have used the fluorescent, oxidation-sensitive probe parinaric acid (PnA) to determine the resistance of LDL against copper-induced oxidation in 48 Type 2 diabetic patients (HbA1c $6.2 \pm 1.0 \%$ ). The main aim of the study was to compare an early stage of LDL oxidation as measured by $\mathrm{PnA}$ oxidation with a later stage of the oxidation process characterized by formation of conjugated dienes (CD), with respect to several determinants of LDL oxidation. After its incorporation into LDL, PnA oxidation was measured by monitoring fluorescence decay after addition of copper $\mathrm{CD}$ formation was measured by monitoring absorbance at $234 \mathrm{~nm}$. PnA-lagtimes correlated significantly with $C D$-lagtimes $(\mathrm{r}=0.82, \mathrm{P}<0.001)$, but were considerably shorter (20.7 +4.3 vs $49.6 \pm 6.6 \mathrm{~min}, \mathrm{P}<0.001)$. PnA-lagtimes and CD-lagtimes were both significantly associated with $L D L$ size: $r=0.30, P=0.038$ and $r=0.33$, $\mathrm{P}=0.022$, respectively. With respect to LDL lipid composition, PnA-lagtimes and CD-lagtimes correlated significantly with free cholesterol content $(r=0.43, P=0.003$ and $\mathrm{r}=0.31, \mathrm{P}=0.033$, respectively) and with triglyceride content $(\mathrm{r}=0.36, \mathrm{P}=0.012$ and $r=0.40, P=0.005$, respectively). Regarding the antioxidant content of LDL, no association with vitamin $\mathrm{E}$ was observed, whereas only PnA-lagtime was associated with the coenzyme $\mathrm{Q} 10$ content $(\mathrm{r}=0.30, \mathrm{P}=0.036)$. CD-lagtime, but not PnAlagtime, showed a significant negative association with plasma lipid peroxides measured as malondialdehyde $(r=-0.41, P=0.004)$. In conclusion, both methods are related with $\mathrm{LDL}$ size and composition. The two methods monitor different stages of the oxidation process, as reflected by the difference in average lagtimes observed. PnA oxidation is particularly suitable to study relations with LDL antioxidants that are consumed early in the oxidation process, whereas $\mathrm{CD}$ formation reflects the formation of lipid peroxides.

\section{5}

THE DENSE LDL PHENOTYPE AND NIDDM. ASSOCIATION WITH LIPOPROTEIN LEVELS AND VISCERAL OBESITY.

L. Van Gaal*, M. Wauters*, I. Mertens*, F. Peiffer*, I. De Leeuw* and J.-P. Després**. *Dept. of Endocrinology, University Hospital Antwerp, Belgium and **Lipid Research Center, CHUL Research Center, Québec, Canada.

The objective of this study was to investigate the potential relationship between visceral obesity and the small dense LDL phenotype in type 2 diabetics. Visceral fat (VAT), measured by CTscan at level L4-L5, dense LDL particles and lipid parameters were evaluated in 105 NIDDM subjects ( 69 men and 36 women; mean age $59 \pm 10$ years and mean BMI $27.8 \pm 4.2$ ), not treated with insulin or hypolipidemic agents. The oxidizability of the non-HDL lipid fraction was evaluated by measuring TBARS. LDL peak particle size was estimated by nondenaturating 2 $16 \%$ gradient gel electrophoresis. The LDL particle score, reflecting the proportion of small dense LDL particles, correlated strongest with triglycerides ( $r=0.73$ $p<0.001)$, and negatively with HDL cholesterol $(r=0.59 ; p<0.001)$. While LDL cholesterol levels showed no association at all with the small dense LDL particle score, ApoB showed a good correlation $(r=0.39 ; p<0.001)$ with it, and the ratio of ApoB/LDL even better $(r=0.59 ; p<0.001)$. TBARS formation after 3 hours correlated significantly with LDL particle score $(r=0.51 ; \mathrm{p}<0.001)$. Comparing lowest $(<22.4)$ and highest $(>23.0)$ tertiles for LDL particle score, significant $(p<0.001)$ differences were found for all these parameters. Metabolic control of diabetes, measured as $\mathrm{HbAl} \mathrm{c}$, was associated with the $\mathrm{LDL}$ particle score $(\mathrm{r}=0.26$; $\mathrm{p}=0.004) . \mathrm{HbAlc}$ was significantly lower in the lowest $(7.8 \pm 1.9)$ compared to the highest $(8.8 \pm 1.6 ; \mathrm{p}=0.03)$ tertile of particle score, as well as fasting glucose and glucose $2 \mathrm{~h}$ after a glucose load. VAT showed a significant correlation with the LDL particle score $(r=0.29 ; \mathrm{p}=0.005)$. A clear difference was also seen for VAT between lowest and highest tertiles $(\mathrm{p}<0.001)$ of particle score. However, using multiple regression analysis, VAT was no independent determinant of the small dense phenotype since triglycerides and HDL were the most important parameters (explaining up to $60 \%$ of the variance, $p<0.001$ ). For men and women separately, similar results were found. We conclude from these results that the high triglycerides - low HDL cholesterol profile, frequently found in NIDDM with visceral fat accumulation, is a strong determinant of the very atherogenic small dense LDL phenotype. 
DIENE PRODUCTION KINETICS DURING IN VITRO LDL OXIDATION ARE ASSOCIATED WITH INSULIN SENSITIVITY IN TYPE 2 DIABETES S.J.L. Bakker, P.G. Scheffer, E.E. Musch, T. Teerlink, R.J. Heine. Research Institute for Endocrinology, Reproduction and Metabolism, Academic Hospital Vrije Universiteit, Amsterdam, The Netherlands.

Insulin resistance is alleged to play an important role in the excessive atherosclerosis in type 2 diabetes mellitus. The key phenomenon in the development of atherosclerosis is thought to be subendothelial oxidation of low density lipoprotein (LDL) particles. The aim of this study was to evaluate the association between measured insulin sensitivity and in vitro LDL oxidizability in subjects with type 2 diabetes. Patients and methods: 12 males and 14 females, treated with diet or oral hypoglycaemic tablets, age $61 \pm 7.8$ years, BMI $31.0 \pm 6.3$ $\mathrm{kg} / \mathrm{m}^{2}, \mathrm{HbAlc} 6.1 \pm 1.2 \%$ (range $4.1-8.3 \%$ ) were studied. Insulin sensitivity was measured as insulin-mediated glucose disposal (M-value) during an euglycaemic hyperinsulinaemic clamp. In vitro susceptibility to oxidation of LDL was evaluated following the kinetics of conjugated diene formation in the presence of $\mathrm{Cu}^{+4}$ ions, resulting in a time to start of oxidation (lag-time), maximal speed of diene production (max-rate), and the quantitiy of dienes produced (diene-prod). Contents of anti-oxidants such as vitamin $E$ (vit-E), co-enzyme Q10 and B-carotene (BC) were measured in isolated LDL particles. Results: The M-value correlated significantly with max-rate $(\mathrm{r}=0.57, \mathrm{P}=0.002)$ and diene-prod $(\mathrm{r}=0.51, \mathrm{P}=0.008)$. The M-value also correlated with vit-E ( $r=0.61, P=0.001)$ and $B C(r=0.50$, $\mathrm{P}=0.009$ ). There was no significant correlation of the $\mathrm{M}$-value with lag-time. Conclusion: Insulin sensitivity in subjects with type 2 diabetes mellitus is associated with kinetics of diene production during in vitro copper-induced LDL oxidation, and these kinetics are associated with the vitamine $E$ and B-carotene content of the LDL particles. Although most studies on LDL oxidizability only include the lag-time, it seems prudent also to include the kinetics of diene production after this lag-phase in future studies.

\section{8}

METABOLIC BASIS OF LOW HIGH DENSITY LIPOPROTEIN IN SUBJECTS WITH IMPAIRED GLUCOSE TOLERANCE

J. Pietzsch, U. Julius, S. Nitzsche, and M. Hanefeld. Institute \& Policlinic of Clinical Metabolic Research, Technical University Dresden, Germany

The in vivo kinetics of HDL apolipoproteins A-I and A-II (apoA-I, apoA-II) were studied in six subjects with impaired glucose tolerance (IGT) and six controls with normal glucose tolerance (NGT) using a stable isotope approach. During a 12 -h primed, constant infusion of $\mathrm{L}-\left[{ }^{13} \mathrm{C}_{6}\right]$-phenylalanine tracer enrichment was determined in HDL apoA-I and apoA-II. Rates of protein synthesis and catabolism were estimated using one-compartmental models. Triglycerides were higher in IGT $(1.33 \pm 0.21$ vs $0.84 \pm 0.27 \mathrm{mmol} / \mathrm{L}$, $\mathrm{p}<0.05)$, but in the normal range. HDL cholesterol and apoA-I levels were significantly lower in IGT $(1.07 \pm 0.15$ vs $1.36 \pm 0.14 \mathrm{mmol} / \mathrm{L}, \mathrm{p}<0.05$; $0.94 \pm 0.10$ vs $1.34 \pm 0.07 \mathrm{~g} / \mathrm{L}, \mathrm{p}<0.01)$. Cholesteryl ester transfer protein (CETP) activity was significantly higher in IGT $(34.1 \pm 5.5$ vs $25.4 \pm 5.3$ $\mathrm{nmol} / \mathrm{ml} \times \mathrm{h}^{-1}, \mathrm{p}<0.05$ ). The mean fractional catabolic rate (FCR) of HDL apoA-I was significantly higher in IGT $\left(0.34 \pm 0.05 v s 0.26 \pm 0.03 \mathrm{~d}^{-1}, \mathrm{p}<0.05\right)$ while the HDL apoA-I production rate (PR) as well as the PR and FCR of HDL apoA-II showed no differences between the two groups. There were significant correlations between HDL apoA-I FCR and the following parameters: HDL apoA-I $(r=-.902, p=0.000)$, plasma triglycerides $(r=.743$, $\mathrm{p}<0.01)$, HDL triglycerides $(r=.696, p<0.01)$, insulin $(r=.765, p<0.01)$, proinsulin $(r=.797, p<0.01)$, and CETP activity $(r=.646, p<0.05)$. These data support the hypothesis that the decrease in HDL cholesterol and apoA-I levels in IGT is exclusively the result of an enhanced apoA-I catabolism. Increased CETP activity appears to be the most important modulator of HDL catabolism in these patients. Our results seem to be an early metabolic finding under insulin resistant conditions even when other lipid parameters, especially plasma triglycerides, still appear to be not or only weakly affected.
IS LDL SUBFRACTION PROFILE MODIFIED IN NIDDM BY DIFFERENT DIABETES TREATMENT AT SIMILAR LEVEL OF GLUCOSE CONTROL?

L. Patti, G. Romano, F. Innelli, L. Di Marino, G. Annuzzi, G. Riccardi, and A.A Rivellese. Department of Clinical and Experimental Medicine, Federico II University Medical School, Naples, Italy.

LDL subfraction profile, particularly the prevalence of small dense LDL is considered an important cardiovascular risk factor. Since an increase in small dense LDL has been reported in NIDDM patients, the aim of this study was to evaluate the effect of subcutaneous insulin (INS) and sulphonylurea (SUL) therapy, at similar levels of blood glucose control (BGC), on this very important lipid parameter. Nine normolipidemic NIDDM men (56 \pm 3 yrs, BMI $\left.26.5 \pm 0.9 \mathrm{Kg} / \mathrm{m}^{2}\right)$ (M \pm SEM) after a 3week wash-out period, were randomly assigned to INS or SUL for 2 months and then shifted to the other treatment. Both treatments were changed only during the first month and then kept constant when a good BGC was reached. At the end of the two treatments, BCG, plasma lipids, LDL subfraction profile (LDL1 $d=1.025-1032$ LDL2 $\mathrm{d}=1.032-1.040, \mathrm{LDL} 3 \mathrm{~d}=1.040-1.060 \mathrm{~g} / \mathrm{ml}$, isolated by density gradient ultracentrifugation), plasma post heparin lipoprotein and hepatic lipase (LPL, HL) activities were evaluated. Although $\mathrm{BGC}$ was similar at the end of both periods ( $\mathrm{HbA}_{1} \mathrm{c} 7.4 \pm 0.3$ vs $7.0 \pm 0.2 \%$ ) (INS vs SUL), INS compared to SUL therapy reduced significantly plasma TG $(0.9 \pm 0.1$ vs $1.1 \pm 0.1 \mathrm{mmol} /, \mathrm{p}<0.05)$ and, despite no change in LDL concentration, it induced: 1) a significant increase in LDL1 both for absolute $(44.5 \pm 3.6$ vs $36.5 \pm 3.7 \mathrm{mg} / \mathrm{dl}, \mathrm{p}<0.02)$ and relative lipid amount $(25.3 \pm 2.3$ vs $20.7 \pm 2.1 \%, \mathrm{p}<0.03) ; 2$ ) no change in LDL2 neither in absolute ( $80.4 \pm 9.4$ vs $76.4 \pm 11.3$ $\mathrm{mg} / \mathrm{dl}$ ) nor relative amount $(43.5 \pm 2.0 \mathrm{vs} 41.1 \pm 2.7 \%)$;) a decrease in LDL 3 for both absolute $(59.0 \pm 9.8$ vs $76.1 \pm 16.8 \mathrm{mg} / \mathrm{dl}$, ns) and relative amount $(31.2+3.0 \mathrm{vs}$ $38.3 \pm 3.8 \%, \mathrm{p}<0.03)$.; 4$)$ a significant decrease in HL $(247.2 \pm 22.3$ vs $263.5 \pm 22.6$ $\mathrm{mU} / \mathrm{ml}, \mathrm{p}<0.05)$. The $\%$ decrease in $\mathrm{HL}$ after INS was inversely related to the $\%$ increase in LDL1 $(\mathrm{r}=-0.79, \mathrm{p}<0.02)$ and directly to \% decrease in LDL3 ( $\mathrm{r}=0.69$, $\mathrm{p}<0.05$ ). In conclusion, INS therapy vs sulphonylureas has a positive, antiatherogenic effect on LDL subfractions in NIDDM patients, inducing an increase in larger, less dense LDL particles. This change in LDL distribution profile is related to a decrease in HL activity.

\section{9}

TROGLITAZONE REDUCES THE RATE OF VDL SYNTHESIS AND POOL SIZE IN PATIENTS WITH NIDDM.

Robinson A.C.J.1, Gray R.G., Bannister P.A., Gallagher J.J., Robinson S., Johnston D.G. Venkatesan $\mathrm{S}$.

Unit of Metabolic Medicine, St. Mary's Hospital Medical School, Norfolk Place, Paddington, London W2 1PG and 'Bishop Auckland Hospital, Cockton Hill Road, Bishop Auckland, County Durham, DL14 6AD.

Troglitazone (TGZ) reduces plasma triglyceride levels by up to $30 \%$ in patients with NIDDM. To investigate this effect further we have conducted a placebo-controlled double-blind study in 24 diet treated diabetic subjects randomised to receive TGZ $600 \mathrm{mg}$ daily, TGZ $200 \mathrm{mg}$ daily or placebo for 8 weeks. Secretion of very low density lipoprotein apolipoprotein B100 (VLDL apoB) was measured directly using a primed, constant intravenous infusion of $1-\left[{ }^{3} \mathrm{C}\right]-$-leucine, monitoring isotopic enrichment by gas chromatography-mass spectrometry. There were no significant changes in plasma triglyceride, total cholesterol or $\mathrm{HDL}$ cholesterol in the individual treatment groups, although patients did not have severe hypertriglyceridaemia before treatment. Analysis of all subjects who received TGZ revealed a $22 \%$ reduction in plasma triglyceride when compared to baseline, geometric mean 161 $\mathrm{mg} / \mathrm{dl}\{$ range 79 to $327 \mathrm{mg} / \mathrm{dl}\}$ vs. $124 \mathrm{mg} / \mathrm{dl}$ (61) to $252 \mathrm{mg} / \mathrm{dl}\} \mathrm{p}=0.018 ; 1.8\{0.9-$ $3.7 \mathrm{mmol} / \mathrm{i}$ vs $\uparrow .4\{0.7-2.8 \mathrm{mmol} / \mathrm{l}\}$. TGZ $600 \mathrm{mg}$ once daly reduced VLDL apoB pool size by $33 \%$ compared to baseline, (2.45 mg/kg $\{1.06$ to $5.63 \mathrm{mg} / \mathrm{dl}$ vs. $1.65 \mathrm{mg} / \mathrm{kg}$ $\{0.62$ to $4.42 \mathrm{mg} / \mathrm{dl}\} \mathrm{p}=0.014$ ) and reduced the absolute synthetic rate of $\mathrm{VDL}$ apoB by $58 \%(0.94 \mathrm{mg} / \mathrm{kg} / \mathrm{hr}\{0.41$ to $2.15 \mathrm{mg} / \mathrm{kg} / \mathrm{hr}\}$ vs. $0.40 \mathrm{mg} / \mathrm{kg} / \mathrm{hr}\{0.14$ to 1.13 $\mathrm{mg} / \mathrm{kg} / \mathrm{hr}\}, \mathrm{p}=0.020$ ). No significant changes were seen in the placebo or TGZ 200 $\mathrm{mg}$ od. groups. We conclude that TGZ (6COmg daily) markedly reduces the absolute secretion rate of VLDL particles. We suggest that this is the mechanism of the drug's triglyceride lowering effect. 
EFFECTS OF QUANTITATIVE TRAIT LOCI FOR LIPID PHENOTYPES IN THE RAT ARE INFLUENCED BY AGE.

J. van den Brandt, P. Kovács and I. Klöting, Department of Laboratory Animal Science at the Institute of Pathophysiology University of Greifswald, D-17495 Karlsburg, Germany

The analysis of genetic factors involved in lipid regulation might shed light on genetic mechanisms underlying features of the coronary heart disease, hypertension, non-insulin-dependent diabetes and obesity which are major causes of ill health in industrial societies. Our previous study on the backcross hybrids derived from a cross of the spontaneously hypertensive rat (SHR/Mol) and the spontaneously diabetic $\mathrm{BB} / \mathrm{OK}$ rat demonstrated the existence of quantitative trait loci (QTL) affecting lipid phenotypes on chromosome 4 and suggestive linkage of lipid phenotypes with markers on chromosome 1. Since the previous study was performed with backcross hybrids at 12 wøeks of age, and it is known that lipid phenotypes can show age-related differences, in this study the effect of QTLs (chromosome I and 4) on serum triglycerides and cholesterol was analysed at 20,28 and 32 weeks of age of backcross hybrids. The results of this study showed that the effect of QTL on chromosome 4 (between Il-6 and D4Mit 9 ) for serum triglycerides was maximal at 20 weeks of age, but disappeared at 32 weeks of age. On the other hand the effect of QTL on serum total cholesterol on chromosome 4 (Npy-Spr) was maximal at 32 weeks of age. In contrast to the first study (12 weeks), the longitudinal investigation showed significant linkage of DIMit14 marker with lipid phenotypes on chromosome 1. Our Findings indicate the necessity of considering the important role of age in QTL analysis of phenotypes showing temporal changes.

\section{PS 34}

\section{Free Fatty Acids}

\section{1}

PLASMA FATTY ACID UTILIZATION AND FATTY ACID BINDING PROTEIN CONTENT ARE DIMINISHED IN SKELETAL MUSCLE OF TYPE 2 DIABETIC SUBJECTS

E.E.Blaak, A.J.M. Wagenmakers, J.F.C. Glatz, B.H.R. Wolffenbuttel, G.J. Kemerink, C.J.M. Langenberg, G.A.K. Heidendal, and W.H.M. Saris. Dept Human Biology, NUTRIM, Maastricht University, The Netherlands.

Impairments in muscle fatty acid (NEFA) metabolism were studied in patients with type 2 diabetes. 8 obese patients with mild type 2 diabetes $(50.6 \pm 2.4 \mathrm{y}$, body fat: $33.8 \pm 2.1$, HbA1C: $6.4 \pm 0.3$ ) and 8 non-obese controls (CON: $51.8 \pm 2.4$ y, body fat: $16.8 \pm 1.9 \%$ ) were studied using the forearm balance technique and indirect calorimetry during infusion of the stable isotope tracer $\mathrm{U}-{ }^{13} \mathrm{C}$-palmitate after an overnight fast and during infusion of the nonselective $B$-agonist isoprenaline (ISO, $20 \mathrm{ng} / \mathrm{kg} \cdot \mathrm{LBM}^{-1} \cdot \mathrm{min}^{-1}$ ). Addtionally, markers of skeletal muscle oxidative, glycolytic, and fatty acid transport capacity were determined in biopsies from the vastus lateralis muscle. Both during fasting as well as ISO-infusion, muscle NEFA uptake (CON vs type 2; fast: $449 \pm 69$ vs $258 \pm 42$, and ISO: $715 \pm 129$ vs $398 \pm 70 \mathrm{nmol}^{-100 \mathrm{ml} \text { tissue }}$ 1 . $\left.\mathrm{min}^{-1}, \mathrm{P}<0.05\right)$, and release were lower in type 2 diabetes as compared to CON. Also, muscle plasma NEFA oxidation during ISO-infusion was blunted in type 2 diabetes (CON vs type 2; ISO: $446 \pm 274$ vs $16 \pm 70 \mathrm{nmol} \cdot 100 \mathrm{ml}$ tissue $\left.{ }^{-1} \cdot \mathrm{min}^{-1}, \mathrm{P}<0.05\right)$. The diminished skeletal muscle fat utilization in type 2 diabetes was accompanied by a lowered cytosolic content of fatty acid binding protein (CON vs type 2; $1.19 \pm 0.20$ vs $0.50 \pm 0.12 \mathrm{mg} / \mathrm{g}$ total protein, $P<0.01$ ), a lowered activity of 3-hydroxyacyl-COA dehydrogenase (CON vs type 2: $58 \pm 9$ vs $29 \pm 7 \mathrm{U} / \mathrm{g}$ total protein, $\mathrm{P}<0.05$ ) and a tendency towards a lowered citrate synthase activity (CON vs type 2: $105 \pm 19$ vs $63 \pm 11 \mathrm{U} / \mathrm{g}$ total protein). in summary, there is a pronounced reduction in skeletal muscle NEFA uptake and oxidation of type 2 diabetic subjects both during overnight fasted and ISO-stimulated conditions. The diminished fat utilization was accompanied by a lowered cytosolic FABP content and a lowered oxidative capacity, indicating that a lowered cytosolic transport capacity is involved in disturbances in fat utilization.

\section{2}

MEASUREMENT OF NON-ESTERIFIED FATTY ACIDS IN INTERSTITIAL FLUID OF SUBCUTANEOUS ADIPOSE AND MUSCLE TISSUE

'A. Wutte, ${ }^{1} G$. Sendlhofer, ${ }^{1 .}{ }^{2}$ M. Ellmerer, ${ }^{2}$ L. Schaupp, 'G.A. Brunner and ${ }^{1} T . R$, Pieber. 'Department of Internal Medicine, Diabetes and Metabolism, University Graz, ${ }^{2}$ Institute of Electro- and Biomedical Engineering, Department of Biophysics, University of Technology Graz, Austria

Non-esterified fatty acids (NEFA) play a potential role in the pathogenesis of insulit resistance and type 2 diabetes. Aim of this strdy was to develop a method for direct access to extracellular fluid of the subcutaneous adipose and muscle tissue. Method: The novel method of open flow microperfusion is based on a macroscopic perforated double lumen catheter perfused by a sterile Krebs Ringer solution. The macroscopic perforations of the outer lumen of the catheter enable a free exchange of molecules independent of their size between the interstitial fluid of the surrounding tissue and the perfusate solution. The partially equilibrated fluid was transported to the collecting vial by a high precision roller pump at a flow rate of 1.0 $\mu \mathrm{l} / \mathrm{min}$. In basic evaluation studies, zero flow rate protocols showed a flow dependent recovery of NEFA, albumin, urea, and glucose. We measured albumin and NEFA in 5 healthy male volunteers [age (mean \pm SD $23 \pm 2$ years, BMI $22,5 \pm 1.2$ $\mathrm{m}^{2} / \mathrm{kg}$ ] after overnight fast. Interstitial fluid was sampled in intervals of $30 \mathrm{~min}$, corresponding plasma samples were obtained. NEFA were measured using NEFA-C (WAKO Chemicals, Germany). Results: In the fasting state NEFA concentration in the sampled fluid of muscle was (mean \pm SEM) $0.33 \pm 0.128 \mathrm{mmol} / \mathrm{l}$ and significantly higher compared to subcutaneous adipose tissue $(0.10 \pm 0.01 \mathrm{mmol} / \mathrm{l}, \mathrm{p}=0,008)$. NEFA levels in the corresponding plasma samples were $0.44 \pm 0.098 \mathrm{mmol} / 1$. Discussion: Open flow microperfusion allows direct measurement of NEFA concentrations in subcutaneous adipose and muscle tissue. In the fasting state NEFA levels in muscle are considerably higher compared to adipose tissue. This novel technique will provide valuable insight into human lipid and carbohydrate metabolism 
813

CARDIAC AND FEMORAL MUSCLE FFA UPTAKE IN PATIENTS WITH IGT OR NIDDM AS STUDIED WITH 14(R, S)-[18F]FLUORO-6-THIA HEPTADECANOIC ACID AND PET

AK Turpeinen, TO Takala, P Nuutila, M Luotolahti, M Haaparanta, J Bergman, H Hămäläinen, MIJ Uusitupa and J Knuuti; Turku PET Centre, University of Turku, and Departments of Medicine and Clinical Nutrition, University of Kuopio, Finland

Impaired glucose tolerance (IGT) and non-insulin-dependent diabetes mellitus (NIDDM) are associated with multiple abnormalities in plasma and cellular lipid metabolism, which may play a fundamental role in the pathophysiology of these diseases. To investigate free fatty acid (FFA) metabolism in the heart and skeletal muscle, we studied 8 men either with IGT or mild NIDDM (glucose-intolerant group; age $49+1$ years, body mass index $29+1 \mathrm{~kg} / \mathrm{m}^{2}$ ) and 8 healthy men (control group; $48 \pm 1$ years, $25 \pm 1 \mathrm{~kg} / \mathrm{m}^{2}$, mean $\pm \mathrm{SEM}$ ) Cardiac and femoral muscle FFA uptake rates were measured in the fasting state with positron emission tomography (PET) and $14(R, S)-{ }^{18} F$ ffluoro-6-thiaheptadecanoic acid $\left(\left[{ }^{18} \mathrm{~F}\right] \mathrm{FTHA}\right)$, a fatty acid tracer trapped into the cell after undergoing initial steps of beta-oxidation. Serum glucose and insulin concentrations were higher in the glucose-intolerant group during the PET study, but serum FFA levels were comparable in the two groups. No differences were observed in myocardial FTHA uptake between the glucose-intolerant and control groups $(5.6+1.0$ vs. $5.2+1.0 \mu \mathrm{mol} / 100 \mathrm{~g} / \mathrm{min}$, glucose-intolerant vs. control, $p=N S)$. Interestingly, femoral muscle FTHA uptake $(0.31+0.01$ vs. $0.41 \pm 0.01 \mu \mathrm{mol} / 100 \mathrm{~g} / \mathrm{min}, \mathrm{p}=0.02$ ) was lower in the glucose-intolerant group. In conclusion, in the fasting state, subjects with IGT or NIDDM showed a normal myocardial but reduced femoral muscle FFA uptake as compared to control subjects. These data argue against the hypothesis that increased oxidation of serum FFA, via the competition of glucose and FFA as fuel sources, is the primary cause for impaired peripheral glucose utilization and insulin resistance commonly observed in IGT and NIDDM.

\section{4}

INITIAL EFFECTS OF FREE FATTY ACIDS ON GLUCOSE-TRANSPORT / PHOSPHORYLATION IN SKELETAL MUSCLE IN MAN

M. Krssak, H. Stingl, S. Gruber, A. Hofer, W. Waldhäusl, and M. Roden, Vienna, Austria

In isolated rat muscle, free fatty acids (FFA) induce insulin resistance by decreasing glucose oxidation with subsequent intracellular accumulation of glucose- 6 phosphate (Glu-6-P). Recently, evidence has been provided for an additional inhibition by FFA of glucose uptake. To test this hypothesis we studied 6 healthy men ( $25 \pm 1 \mathrm{yrs} ; 22.1 \pm 1.3 \mathrm{~kg} / \mathrm{m}^{2}$ ) during euglycemic-hyperinsulinemic clamp tests in the presence of (i) elevated (HIGH; $1.48 \pm 0.18 \mathrm{mM}, \mathrm{p}<0.00001$ vs $\mathrm{CON}$ ), (ii) fasting (FAST; $0.40 \pm 0.05 \mathrm{mM}, \mathrm{p}<0.01$ vs CON) FFA concentrations induced by iv infusion of Intralipid $(20 \%, 1.5 \mathrm{ml} / \mathrm{min})+/$-heparin $[0.2 \mathrm{IU} /(\mathrm{kg} \mathrm{min})]$, and (iii) suppressed FFA levels (CON: $<0.01 \mathrm{mM}$ ). Concentrations of Glu-6-P, anorganic phosphate $\left(\mathrm{P}_{\mathrm{i}}\right)$, and phospho-creatine $(\mathrm{PCr})$ in calf muscle were measured noninvasively every $3.2 \mathrm{~min}$ for $180 \mathrm{~min}$ by using ${ }^{31} \mathrm{P}$-NMR spectroscopy. Whole-body glucose uptake increased similarly in all studies until $140 \mathrm{~min}$, then it dropped only in the presence of high FFA levels [160-180 min; HIGH: $7.3 \pm 0.6$ $\mathrm{mg} /(\mathrm{kg} \min )$ vs CON: $9.4 \pm 0.7 \mathrm{mg} /(\mathrm{kg} m \mathrm{~m}), \mathrm{p}<0.001 ;$ FAST: $7.9 \pm 0.6 \mathrm{mg} /(\mathrm{kgmin})$, ns]. The insulin-induced increase of intramuscular Glu-6-P was blunted during lipid infusion from $60 \mathrm{~min}$ ( $\Delta \mathrm{Glu}-6-\mathrm{P}$; HIGH: $0.091 \pm 0.002 \mathrm{mM}, \mathrm{p}=0.033$, FAST: $0.145 \pm 0.006 \mathrm{mM}$, ns vs CON: $0.135 \pm 0.002 \mathrm{mM})$ until $180 \mathrm{~min}(0.090 \pm 0.002 \mathrm{mM}$ $p=0.013,0.106 \pm 0.002 \mathrm{mM}, p=0.032$ vs CON: $0.157 \pm 0.002 \mathrm{mM}$ ). Muscular $P_{i}$ increased in all groups until $68 \mathrm{~min}\left(\triangle \mathrm{P}_{\mathrm{i}} ; \mathrm{HIGH}: 0.36 \pm 0.02 \mathrm{mM}\right.$, FAST: $0.39 \pm 0.05$ $\mathrm{mM}, \operatorname{CON}: 0.34 \pm 0.06 \mathrm{mM})$, but then slowly declined until $180 \mathrm{~min}\left(\Delta \mathrm{P}_{\mathrm{i}}\right.$ $0.12 \pm 0.05 \mathrm{mM}, 0.19 \pm 0.05 \mathrm{mM}$, and $0.3 \mathrm{l} \pm 0.06 \mathrm{mM}$ ). PCr concentrations continuously decreased in all groups $(\triangle \mathrm{PCr} ; 160-180 \mathrm{~min} ;-0.59 \pm 0.01 \mathrm{mM},-0.65 \pm 0.10$ $\mathrm{mM},-0.54 \pm 0.14 \mathrm{mM}$ ). Conclusion: the early decline of muscle Glu-6-P concentrations indicates that FFA primarily cause a defect of glucose transport/phosphorylation in man.

\section{5}

THE USE OF BROMOPALMITATE AS A TRACER TO STUDY FATTY ACID UPTAKE IN INDIVIDUAL TISSUES OF CONSCIOUS RATS

S. Furler, N. Oakes*, G. Cooney, M. Lim-Fraser, B. Ljung* and E. Kraegen Garvan Institute of Medical Research, Sydney, Australia *Astra-Hässle, Mölndal, Sweden

Because bromopalmitate $(\mathrm{Br} P)$ is not oxidised in the mitochondria, it has been proposed as a suitable analog for the study of tissue free fatty acid (FFA) uptake. We administered $\mathrm{BrP}$ to chronically-cannulated overnightfasted conscious male Wistar rats $(-300 \mathrm{~g})$ to examine the effect of acute administration of a $B$-oxidation blocker (etomoxir: $15 \mu \mathrm{mol} / \mathrm{kg}$ ). A single purified isomer of $\left[9,10-{ }^{3} \mathrm{H}\right]$-bromopalmitate $\left({ }^{3} \mathrm{H}-\mathrm{BrP}\right)$ and $\left[1{ }^{14} \mathrm{C}\right]-$ palmitate $\left({ }^{14} \mathrm{C}-\mathrm{P}\right)$ were infused into the jugular vein for $4 \mathrm{~min}$. After a further $12 \mathrm{~min}$ rats were killed and tissues excised. Labelled FFAs were extracted from carotid-artery plasma samples. Whole-body clearance of tracer was estimated from tracer plasma dynamics. Tissue clearance rates were calculated as tissue tracer content/integrated plasma tracer concentration.

\begin{tabular}{|l|c|c|}
\hline Plasma FFA(mM) & $0.53 \pm 0.04$ & $1.07 \pm 0.13^{*}$ \\
${ }^{3} \mathrm{H}-\mathrm{BrP}$ Clearance $\left(\mu 1 . \mathrm{min}^{-1} \mathrm{~g}^{-1}\right)$ & & \\
Whole-body & $48 \pm 1$ & $14 \pm 2^{*}$ \\
Heart & $225 \pm 24$ & $35 \pm 6^{*}$ \\
Diaphragm & $66 \pm 5$ & $23 \pm 3^{*}$ \\
Epididymal fat & $12 \pm 1$ & $12 \pm 2$ \\
\hline
\end{tabular}

In analyses including additional rats (total $n=20$ ), with a range of nutritional states, whole-body clearance of ${ }^{3} \mathrm{H}-\mathrm{BrP}$ and ${ }^{14} \mathrm{C}-\mathrm{P}$ correlated well $(R=0.63, P=0.003)$. In etomoxir-treated rats $(n=9)$ similar correlations (all $P<0.05)$ existed for clearance estimates in heart $(R=0.82)$, diaphragm $(R=0.72)$ and fat $(R=0.68)$. Together, these results confirm, in conscious animals, the functionality of ${ }^{3} \mathrm{H}-\mathrm{BrP}$ to trace FFA uptake. The 4 -fold reduction in muscle ${ }^{3} \mathrm{H}-\mathrm{BrP}$ clearance induced by etomoxir suggests that early steps of FFA uptake are closely regulated by its rate of oxidation.

\section{6}

\section{ASSESSMENT OF TISSUE-SPECIFIC LIPID METABOLISM IN VIVO USING} A NOVEL FATTY ACID TRACER TECHNIQUE

N. Oakes, A. Kjellstedt, T. Clementz, S. Furler*, E. Kraegen*, M. ÖlwegårdHalvarsson, A. Jenkins ${ }^{\star *}$ and B. Ljung. Astra-Hässle, Mölndal, Sweden; *Garvan Inst. Med. Research and * University of Wollongong, NSW, Austratia.

Following resolution of $\left[9,10-{ }^{3} \mathrm{H}\right]-(\mathrm{R}, \mathrm{S})$-2-bromopalmitate, one of the purified isomers $\left({ }^{3} \mathrm{H}-\mathrm{BrP}\right)$ was used as a partially metabolisable analogue to trace free fatty acid (FFA) transport and initial metabolism, but not $\beta$-oxidation. In most tissues, the resulting ${ }^{3} \mathrm{H}$-products are effectively trapped. In contrast, $\left[\mathrm{U}-{ }^{14} \mathrm{C}\right]$ palmitate $\left({ }^{4} \mathrm{C}-\mathrm{P}\right)$, following transport and initial metabolism, is either stored or undergoes oxidation. We define indices of total FFA utilisation (FAU), and incorporation into storage products (FAS), based on tissue ${ }^{3} \mathrm{H}$ and ${ }^{14} \mathrm{C}$ activities respectively, $16 \mathrm{~min}$ following the start of a $4 \mathrm{~min}$ intravenous infusion of $\mathrm{a}^{3} \mathrm{H}$ $\mathrm{BrP}$ and ${ }^{14} \mathrm{C}-\mathrm{P}$ mixture. Experiments were performed in anaesthetised $350 \mathrm{~g}$ male Wistar rats. Unilateral hindlimb muscle contraction was induced by sciatic nerve stimulation. The relationship between ${ }^{3} \mathrm{H}-\mathrm{BrP}$ and ${ }^{14} \mathrm{C}-\mathrm{P}$ uptake was studied by comparing a control group (CON) to a group given etomoxir (ETX), a $\beta$-oxidation inhibitor. Plasma clearance rate of ${ }^{3} \mathrm{H}-\mathrm{BrP}$ was lower (by $39 \%$ ) than ${ }^{14} \mathrm{C}$-P. Correspondingly, slower ${ }^{3} \mathrm{H}-\mathrm{BrP}$ metabolism was indicated in all tissues examined: FAU (as \% FAS) were in ETX group; skeletal muscle (SM) $37 \%$, white adipose tissue (WAT) $38 \%$. Thus, FAU could be used as a relative, but not as an absolute measure of fatty acid utilisation rate. Of the noncontracting hindlimb SMs, FAU and FAS were higher in red than white muscles. In the CON group, sciatic nerve stimulation induced substantial increases in FAU (but not FAS) in red gastrocnemius (by $111 \%$ ), white gastrocnemius (by $241 \%$ ) and EDL (by $285 \%$ ), compared to the contralateral non-stimulated leg $(P<0.01)$. The contraction-induced increases in FAU were abolished by etomoxir. Taken together our findings show that ${ }^{3} \mathrm{H}-\mathrm{BrP}$ metabolism (tissue ${ }^{3} \mathrm{H}$ retention) traces local total fatty acid utilisation. In conclusion, our method employing the simultaneous use of ${ }^{3} \mathrm{H}-\mathrm{BrP}$ and ${ }^{14} \mathrm{C}-\mathrm{P}$ tracers provides a new useful tool for in vivo studies of tissue-specific free fatty acid transport, utilisation and metabolic fate (oxidative vs non-oxidative). 


\section{7}

MALONYL COA ACUTELY REGULATES FATTY ACID OXIDATION IN RAT MUSCLE IN VIVO.

D. Dean, D. Chien, A. Saha, D. Vavvas, J.P. Flatt, and N. Ruderman. Boston and Worcester, MA.

Malonyl $\mathrm{CoA}$ acutely regulates fatty acid oxidation in liver by inhibiting carnitine palmitoyltransferase I (CPTI). Whether malonyl CoA plays a similar role in skeletal muscle is less clear. While in vitro experiments suggest a inverse relationship between malonyl $\mathrm{CoA}$ and fatty acid oxidation, the concentrations of malonyl $\mathrm{CoA}$ found in whole muscle $(1-5 \mu \mathrm{M})$ appear to be sufficiently high to totally inhibit the muscle isoform of CPTI $(\mathrm{Ki}=0.03 \mu \mathrm{M})$. The aim of this study was to examine this conundrum in rats fasted for 48 hours and allowed ad libitum refeeding for various durations up to 24 hours. The amount of $\mathrm{O}_{2}$ consumed and $\mathrm{CO}_{2}$ produced in individual rats at various times allowed for in vivo respiratory quotient (RQ) analysis. Muscle and liver malonyl CoA and LCFA-carnitine levels were measured using standard biochemical procedures. Other parameters were measured using commercially available kits or standard biochemical techniques. Refeeding produced the expected increases in plasma insulin ( 5 -fold; $\mathrm{p}<0.05$ ) and decreases in plasma FFA $(-70 \% ; \mathbf{p}<0.05)$ within 3 hours. RQ values were 0.82 at baseline and increased to $0.93,1.0,1.05$, and 1.08 after $1,3,12$, and 18 hours of refeeding, respectively, indicating inhibition of fat oxidation. In soleus and gastrocnemius muscles, the increases in RQ at each time point correlated closely $\left(\mathrm{r}^{2}=0.94 ; \mathrm{p}<0.05\right)$ with the progressive $(50-250 \%)$ increases in malonyl CoA. LCFAcarnitine was also diminished suggesting a concurrent inhibition of CPT1. Hepatic changes were similar, although in contrast to muscle, malonyl $\mathrm{CoA}$ levels peaked at 3 hours and then decreased. Additionally, refeeding had a moderate effect on muscle acetyl CoA carboxylase (ACC) activity, whereas hepatic ACC activity was increased. The results strongly suggest refeeding following a 48 hour fast leads to changes in malonyl COA in muscle that occur rapidly, inhibit CPT1 and thus play a major role in in the inhibition of fatty acid oxidation. They also suggest that, malony] $\mathrm{COA}$ levels in liver and muscle are regulated by different mechanisms.

\section{9}

ALTERNATIONS IN LIPID METABOLISM OF $o b / o b$ MICE TREATED WITH DOPAMINERGIC AGONISTS.

P.W.D. Scislowski, Y. Zhang, R. Prevelige, S.Phaneuf and A.H. Cincotta Ergoscience Corp. Charlestown, MA, U.S.A.

We have previously shown that a two week treatment of $o b / o b$ mice with dopamine receptor agonists bromocriptine (BC) and SKF 38393 (SKF) normalized hyperglycemia and hyperlipidemia. The aim of present study was to identify the sequence of metabolic events ameliorating diabetes/obese conditions in $a b / a b$ mice. Female $o b / o b$ mice $36 \pm 3 \mathrm{~g}$ were injected i.p. at IHALO (hours after light onset) with vehicle (control group) or $\mathrm{BC} / \mathrm{SKF}$ both $10 \mathrm{mg} / \mathrm{kg} \mathrm{Bw}$ (treated group). Animals were sacrificed at 4 HALO after 1,3 and 7 days of treatment. One day of treatment resulted in a decrease of plasma FFA from $0.81 \pm 0.06$ to $0.6 \pm 0.04 \mathrm{mM}$ and TG from $194 \pm 30$ to $155 \pm 16 \mathrm{mg} / \mathrm{dl}, \mathrm{p}<0.05$ and a modest decrease by $(50 \mathrm{mg} / \mathrm{dl})$ blood glucose. Isoprenaline $\left(10^{-7} \mathrm{M}\right)$ stimualted lipolysis by $50 \%$ in adipocytes isolated from control mice vs. $25 \%$ in treated group. Although oxidation of $1-\mathrm{C}^{14}$ palmitic acid by heart and liver homogenates was not significantly different between two groups, the heart oxidation of palimtate in the presence of $\alpha$-glycerophosphate( $\alpha$-GP) of treated mice was $12 \%$ higher $(\mathrm{p}<0.01)$ than in control group indicating a decrease of TG formation. In the liver the decrease of esterification of $\alpha$-GP was associated with the reduction in plasma level of B-hydroxybutyrate $105 \pm 5$ vs $172 \pm 20 \mu \mathrm{M}$ $(\mathrm{p}<0.05)$ indicating a lower hepatic FFA flux. Three days treatment resulted in significant reduction of adipocyte size $0.89 \pm 0.07 \mathrm{vs.} 1.15 \pm 0.04 \mu \mathrm{g} \mathrm{lipid/cell} \mathrm{and}$ diminution of adipose lipoprotein lipase activity $35 \pm 4 \mathrm{vs.} 64 \pm 7 \mu \mathrm{mol}$ fatty acid/h/1 $10^{6}$. After 7 days of treatment blood glucose was reduced by $260 \mathrm{mg} / \mathrm{dl}(\mathrm{p}<0.01)$, plasma TG level was further reduced to $130 \pm 8 \mathrm{mg} / \mathrm{dl}$, while FFA concentration did not differ from the value after 1 day of treatment. The rate of basal lipolysis was similar to value from day 1 of treatment:0.72 \pm 0.08 pmoles glycerol $/ / 10^{6}$ cells, however isoprenaline $\left(10^{-7} \mathrm{M}\right)$ failed to stimulate lipolysis in adipocytes from treated mice. Taken together our results revealed that the amelioration of hyperglycemia and hyperlipidemia in $o b / o b$ mice treated with $\mathrm{BC} / \mathrm{SKF}$ is achieved by densensitization of lipolysis to adrenergic stimuli and decrease of TG formation in liver and heart.

\section{8}

DYSREGULATION OF INSULIN'S ANTI-LIPOLYTIC ACTION AS A CONSEQUENCE OF PROTEIN RESTRICTION DURING PREGNANCY

M.C. Sugden, M.J. Holness and L.G.D. Fryer Department of Biochemistry, Faculty of Basic Medical Sciences, St. Bartholomew's \& the Royal London School of Medicine \& Dentistry, Queen Mary \& Westield College, London, U.K. Impaired suppression of plasma non-esterified fatty acid (NEFA) concentrations during hyperinsulinemia is revealed when pregnant rats are maintained on a protein-restricted diet. We examined whether this is due to dysregulation of adpocyte lipolysis by insulin or counter-regulatory hormones. Lipolysis (assessed from glycerol release) was examined in mesenteric and parametrial adipocytes prepared from late (19-20 day) pregnant rats. Moderate protein restriction (provision of an isocaloric diet containing $8 \%$ vs. $20 \%$ protein) throughout pregnancy did not influence basal rates of lipolysis. Protein restriction modified the lipolytic response to noradrenaline in a depot-specific manner: although the pattern or magnitude of the lipolytic response to noradrenaline was not modified with mesenteric adipocytes, maximal rates of noradrenaline-stimulated glycerol release from parametrial adipocytes were significantly higher (by $40 \% ; P<0.05$ ) in cells prepared from proteinrestricted (PR) rats compared with control $(C)$ rats. In the $\mathrm{C}$ group, half-maximal antilipolytic effects of insulin were observed at $\approx 8$ and $\approx 10 \mu \mathrm{U} / \mathrm{ml}$ insulin with parametrial and mesenteric adipocytes. Protein restriction resulted in $\approx 2$ fold increases in apparent IC50 values for inhibition of lipolysis (half-maximal effects of insulin at $=17 \mu \mathrm{U} / \mathrm{ml}$ and $\approx 21$ $\mu \mathrm{U} / \mathrm{ml}$ with parametrial and mesenteric adipocytes), with a negligible anti-lipolytic action of insulin at $<15 \mu \mathrm{U} / \mathrm{ml}$. Insulin's anti-lipolytic action at a physiologically high concentration $(75 \mu \mathrm{U} / \mathrm{ml})$ was unimpaired. The study demonstrates a significant enhancement of noradrenaline-stimulated lipolysis at low insulin concentrations by moderate protein restriction during pregnancy. The results are consistent with increased post-absorptive FA production as a consequence of a persistent effect of mild protein restriction to suppress the adipocyte's anti-lipolytic response to insulin. By the provision of alternative energy substrates, amino acids that might otherwise be used as precursors for glucose production via gluconeogenesis are "spared" for use by the fetus. In addition, glycerol, produced as a result of adipose-tissue lipid breakdown, can be used as a gluconeogenic precursor. 


\section{Obesity}

\section{0}

WAIST/HIP RATIO IN TYPE 1 (INSULINDEPENDENT) AND TYPE 2 (NON-INSULINDEPENDENT) DIABETIC SUBJECTS

R.Lichiardopol and C.Dumitrescu, "N.Malaxa" Hospital, Bucharest, Romania.

As part of the insulin resistance syndrome, abdominal fat accumulation is frequently associated with type 2 diabetes. We do not know a similar association in type 1 diabetic subjects. Objective:to evaluate the relationship of central fat distribution with age, BMI, blood pressure and fasting serum lipids in type 1 and type 2 diabetic subjects. 134 diabetic subjects, 61 type 1 (27 males, 34 femeles) and 73 type 2 (33 males, 40 females) were investigated. Type 2 subjects had greater mean (t SD) values (analysis of variance) for waist/hip (WHR) ratio $10.984 \pm$ 0.07 vs $0.839 \pm 0.06 ; p<0.001)$, BMI $(29.6 \pm 4.7$ vs 23.614.1; $p<0.001)$, age $(54.1 \pm 8.7$ vs $43.0 \pm 15.3$ ys; $p<0.001)$, serum triglycerides $(2.34 \pm 0.67$ vs $1.77 \pm 0.50 \mathrm{mmol} / \mathrm{L} ; \mathrm{p}<0.001)$ sistolic $(152.03+23.03$ vs $125.78 \pm 21.60 \mathrm{~mm} \mathrm{Hg:} p<0.001)$ and diastolic $(84.12 \pm 13.65$ vs $72.67 \pm 10.19 \mathrm{~mm} \mathrm{Hg} ; \mathrm{p}<0.001)$ blood pressure and lower mean HDL-cholesterol levels $(1.38 \pm 0.26$ vs $1.60 \pm 0.27 \mathrm{mmol} / \mathrm{L} ; \mathrm{p}<0.001)$. Insulin sensitivity, assessed as glucose disappearance rate (KITT), after iv bolus insulin injection $(0.1 \mathrm{U} / \mathrm{Kg})$ was correlated $w$ only in type 2 diabetic subjects $(x=-0.57 ; n=20 ; p<0.01)$. Moreover, it was no correlation between WHR and mean daily insulin dose $(\mathrm{U} / \mathrm{Kg})$ in type 1 diabetic subjects. WHR was correlated with serum triglycerides levels $(r=0.47 ; n=55 ; p<0.001)$, sistolic and tiastolic blood pressure ( $r=0.32 ; n=73 ; 0<0.01)$ in tipe 2 diabetic subjects, but only with age $(r=0.40 ; n=61 ; p<0.01)$ in type 1 diabetic subjects. Our data suggests that abdominal fat accumulation is produced by different mechanisms, being associated with aging in type 1 , and with insulin resistance syndrome in type 2 diabetic subjects.

\section{2}

LIPID ABNORMALITIES NN TYPE 2 DIABETIC SUBJECTS: EFFECT OF OBESITY AND BODY FAT DISTRIBUTION

M.Boemi, F.Romagnoli and P.Fumelli. Diabetology Unit, I.N.R.C.A., Ancona (Italy)

Aim of the present study was to evaluate the frequency of abnomalities of ipid profile in relation to body fat excess and to central obesity. A cohort of 553 (287 women and 266 men, mean age $66.0 \pm 8.5$ years) consecutive type 2 diabetic outpatients were studied. Only subjects with a known duration of diabetes longer than 2 years were enrolled. Criteria for exclusion were: active lipid-lowering therapy, hypothyroidism, sexus hormones replacement treatment, and renal failure. Patients were grouped according to BMI ( 24 for wornen and 25 for men = normal, $>24 / 25<30$, overweight, $>30$ obese), to WHR $>0.85$ central obesity) and to WAIST cincumference $(<80$ or 94 nomal, $>80<88$ or $>94<102$ mild, $>88$ or $>102 \mathrm{~cm}$ severe visceral adiposity in women and men respectively). Measures were repeated at 2 and 4 months after baseline. The same timing was used to determine total, $L D L, H O L$ cholesterol and tygliceride concentration and HbA1c. Hypercholesterolemia (HCh) was defined as cholesterol concentration $>200$

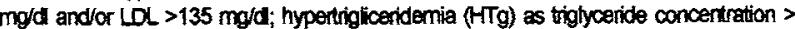
$200 \mathrm{mg} / \mathrm{dl}$ and low HDL( $(\mathrm{Hal})$ as $\mathrm{HDL}<35 \mathrm{in}$ men or $<42 \mathrm{mgdi}$ in women. The mean of the 3 deteminations was used. Diet was not modified during the study. HCh frequency was simitar in nomal, overweight and obese subjects $(46.5 \%, 46.9 \%$ and $44.6 \%$ respectively); frequencies of $\mathrm{HTg}$ and $\mathrm{LHdl}$ were higher but not significantly increased in overweight and obese patients compared to nomal ones (28.7\% and $32.4 \%$ vs $20.9 \%$ $24.0 \%$ and $23.9 \%$ vs $14.0 \%$ ). Central obesity was associated with not significantly higher frequency of $\mathrm{HTg}$ and $\mathrm{LHdl}$ as evaluated both by WHR ( $30.4 \%$ and $23.5 \mathrm{vs} 19.4 \%$ and $15.3 \%$ in nomal WHR individuals). Among subjects with severe visceral adiposity the frequency of LHAll was slightly increased compared to normal and mild subgroups ( $25.1 \%$ vs $19.6 \%$ and $18.5 \%$ ). As concems to $\mathrm{HbA1c} \%$ values, all the cited subgroups were omogeneous. There was a weak association between obesity and viscera adiposity and hyper-dyslipidemia in our sample. The increased cardiovascular risk in obese type 2 diabetic patients is probably due to quallative rather than quantitative lipoprotein modification.

\section{1}

FAT DISTRIBUTION AND METABOLIC CONTROL IN PATIENTS WITH TYPE 2 DIABETES IN ITALY.

E. Pierazzuoli, S. Turco*, E. Mannucci, A. Marsilii**, and the DIAINF Study Group. Sect. Diabetology, Endocrinology, Univ. of Florence; *Internal Medicine, Federico II Univ. of Naples; **Sect. Diabetology, Gerontology, Univ. of Florence; DIAINF Study Group, Florence. Via della Mattonaia 17, 50137 Firenze, Italy.

Aim of this study is the assessment of body fat distribution in type 2 diabetic patients in Italy and its relationship with metabolic control. The data were collected using the DIAINF computerised network, which contains clinical data of over 300,000 patients. Waist-Hip Ratio (WHR) was measured in a consecutive series of $6,647(3,664 \mathrm{~F}, 2,983 \mathrm{M})$ patients with type 2 diabetes, aged $(\mathrm{m} \pm \mathrm{sd}$ ) $65.2 \pm 16.1$ years, with $\mathrm{BMI}$ $29.4 \pm 5.1 \mathrm{~kg} / \mathrm{m}^{2}$, and $\mathrm{HbA}_{1} \mathrm{c} 7.2 \pm 1.9 \%$, from 30 Diabetes Care Units all over the country. In females, WHR was $0.87 \pm 0.08(0.91 \pm 0.08$ in the North; $0.89 \pm 0.08$ in the Centre; $0.87 \pm 0.09$ in the South; $0.84 \pm 0.07$ in the Islands; $p<0.001)$. In males, WHR was $0.95 \pm 0.06(0.98 \pm 0.07$ in the North, $0.95 \pm 0.07$ in the Centre, $0.93 \pm 0.09$ in the South, and $0.95 \pm 0.06$ in the Islands; $\mathrm{p}<0.001$ ). At multivariate analysis, $\mathrm{HbA}_{1} \mathrm{c}$ showed a significant inverse correlation with BMI (adj. $\mathrm{r}=0.27$ ), both in males (adj. $r=0.27$ ) and in females (adj.r=0.28). After adjustment for BMI, sex, age, and duration of diabetes, no significant correlation of WHR with $\mathrm{HbA}_{1} \mathrm{c}$ was observed (adj.r=0.04). In conclusion, the android-type fat distribution appears to be a common feature of patients with type 2 diabetes in Italy, particularly in the North of the country. Central distribution of fat mass cannot be considered a predictor of metabolic control, after adjustment for other potentially confounding variables.

\section{3}

HYPERGLYCAEMIC SIBLNGS OF TYPE 2 DIABETIC PATIENTS HAVE GREATER CENTRAL OBESITY THAN THEIA NORMOGLYCAEMIC SIBLINGS BUT SIMILAR PHYSICAL ACTIVITYINDICES

OM Herlihy, BA Barrow, JC Levy, NJ Wareham ${ }^{1}$ and RC Turner. Diabetes Research Laboratories, Radcliffe Infirmary, Oxford, UK and 'Department of Community Medicine, University of Cambridge, UK Familiality of obesity and insulin resistance have been demonstrated in siblings of type 2 diabetic patients. The factors that determine glycaemia between siblings within familes have not been determined. Obesity and physical inactivity are potential candidates.

Ten age and gender matched sibling pairs, discordant for fasting plasma glucose (FPG); i.e. one sibling with impaired fasting glucose (IFG: FPG $>6.0 \mathrm{mmol} /$ ) and one with normal fasting glucose (NFG: FPG $<6.0 \mathrm{mmo} / /$ ): $4 \mathrm{M}: 6 \mathrm{~F}$, age mean \pm SD $55.6 \pm 10$ and $54.5 \pm 8.4 \mathrm{yrs}$ (IFG and NFG respectively), were studied to evaluate anthropometry, HOMA derived insulin resistance (\%S) and beta-cell function $(\% \beta)$, and physical activity index (PAI): the ratio of total energy expenditure (EE) to basal metabolic rate, using 5 day continuous heart rate monitoring, with individual calibration of the relationship between EE and heart rate. IFG vs. NFG sibs had similar BMI $\left(27.7+4.0\right.$ vs $\left.25.8+4.1 \mathrm{kgm}^{-2}, p=0.18\right)$, greater waist circumference ( $96 \pm 14$ vs $87 \pm 7 \mathrm{~cm}, p=0.002)$, reduced \%S (55 (38-81) vs $78(53-115), p<0.005)$, similar $\% \beta(83 \pm 25$ vs $96 \pm 22, p=0.17)$. $\mathrm{PAl}$ did nol differ between the groups (geometric mean (1SD range): 1.43 $(1.24-1.65)$ vs $1.52(1.20-1.94)$, respectively, $p=0.56)$ in the IFG and NFG groups. No differences were detected in blood pressure or lipid profiles. We conclude that differences in glucose tolerance between siblings of type 2 diabetic subjects were associated with differences in central adiposity and insulin resistance, but not in physical activity. 


\section{4}

RAPID WEIGHT GAIN AND INSULIN RESISTANCE IN SEVEN-YEAR-OLD SOUTH AFRICAN CHILDREN

N.J. Crowther, N. Cameron*, J. Trusler and I.P. Gray. Department of Chemical Pathology, South African Institute for Medical Research and *Department of Anatomical Sciences, Faculty of Health Sciences, University of the Witwatersrand, York Road, Parktown 2193, Johannesburg.

A number of studies have demonstrated that glucose tolerance falls with decreasing birth weight and that people with low birth weight and high body mass index (BMI) as adults are those at greatest risk of developing non-insulin-dependent diabetes mellitus (NIDDM). The aim of our study was to investigate the relationship between glucose tolerance and birth weight in a population of 7-year-old black South Africans for whom longitudinal anthropometric data were available. Oral glucose tolerance tests (OGTTs) were performed on 152 subjects and significant inverse correlations were found between birth weight and the total amount of insulin secreted during the first 30 minutes ( $r$ $=-0.19, p=0.04)$ and last 90 minutes $(r=-0.19, p=0.04)$ of the OGTT and also between birth weight and the 30 minute glucose levels $(r=-0.20, p=0.02)$. Children who had high weight velocities between birth and 7 had high levels of adiposity as measured by skinfold thickness and BMI. There was also a significant positive correlation between weight velocity and insulin resistance $(r=0.18, p$ $=0.04$ ) as measured using homeostasis model assessment (HOMA). Thus, low birth weight in conjunction with rapid childhood gains in both weight and subcutaneous fat, produces poor glucose tolerance in 7-year-old children and may make these individuals susceptible to the development of NIDDM later in life.

\section{5}

GLUCOCORTICOIDS STIMULATE PAI-1 GENE EXPRESSION AND PRODUCTION IN HUMAN VISCERAL ADIPOSE TISSUE

C.M. Halleux, B.A. Reul, P.J. Declerck and S.M. Brichard. Unité d'Endocrinologie et Métabolisme UCL Brussels and Farmaceutische Biologie en Fytofarmacologie KUL Leuven, Belgium.

Enhanced production of type-1 plasminogen activator inhibitor (PAI-1) by visceral fat could partly be responsible for the development of cardiovascular diseases in central obesity. The mechanisms underlying the overproduction of PAI-1 are still unclear. The direct contribution of hormones has never been addressed in human visceral adipose tissue. Because omental, but not subcutaneous, fat may generate active cortisol, we particularly focused on the effects of glucocorticoids. We also examined the role of cAMP. Visceral adipose tissue was obtained from patients undergoing elective abdominal surgery (age : $48 \pm 3 \mathrm{yr}, \mathrm{BMI}: 30 \pm 2 \mathrm{~kg} / \mathrm{m}^{2}$; mean \pm SEM), and explants were cultured in MEM. Dexamethasone added for 24 $h$ to the medium increased the secretion of PAI-1 by adipose tissue in a concentration-dependent manner with a threshold at $10 \mathrm{nM}$, a half-stimulation at $30 \mathrm{nM}$ and a maximal effect at $1 \mu \mathrm{M} .1 \mu \mathrm{M}$ dexamethasone increased PAI-1 production from $980 \pm 160$ (basal) to $1870 \pm 280 \mathrm{ng} / \mathrm{\mu g}$ tissue DNA after $24 \mathrm{~h}$ ( $\mathrm{n}=$ $8, p<0.01$ ). This was preceded by a 2 -fold rise in PAI-1 mRNA levels between 4 $8 \mathrm{~h}$ of culture, an effect which was specific as glyceraldehyde-3-phosphate dehydrogenase gene expression was unaffected by the hormone. Besides the positive control by glucocorticoids, PAI-1 gene expression was negatively regulated by cAMP. Thus, the addition of CAMP for $24 \mathrm{~h}$ to the medium decreased PAI-1 mRNA abundance by $85 \%$ under basal conditions and $45 \%$ in the presence of dexamethasone. In conclusion, we provide evidence for a reciprocal regulation of PAI-1 by dexamethasone (positive effector) and cAMP (negative effector) in cultured human visceral adipose tissue. This regulation operates at the pretranslational level. The stimulation by glucocorticoids could contribute to the enhanced production of PAI-1 levels associated with central obesity and, thereby, be a link between accumulation of visceral fat and cardiovascular diseases.

\section{6}

INCREASED ADIPOSE MASS IN TRANSGENIC MICE OVEREXPRESSING THE P-ENOLPYRUVATE CARBOXYKINASE GENE

S. Franckhauser-Vogel, A. Pujol, B. Su, J. Visa, A. Arbós, A. Casellas and F. Bosch, Dept. Biochemistry and Mol. Biology, Fac. Veterinaria, Univ. Autònoma Barcelona, 08193-Bellaterra, Spain

In the western world there has been a marked increase in the prevalence of obesity in recent decades. It is now well established that obesity is a risk factor for type 2 diabetes. In our laboratory we aim to study the mechanisms that lead to type 2 diabetes, particularly the relationship with obesity. Several animal models of obesity and diabetes are available that show alterations in the expression of genes for transcription factors, hormones or receptors. Nevertheless, to study the role of alterations in key steps in the metabolic pathways leading to triglyceride formation, we have developed transgenic mice overexpressing the P-enoipyruvate carboxykinase (PEPCK) gene in adipose tissue. PEPCK supplies glycerol for triglyceride synthesis. This pathway, termed glvceroneogenesis, is required for re-esterification of free fatty acids. We have obtained five lines of transgenic mice that overexpress PEPCK under the control of the aP2 promoter (aP2/PEPCK chimeric gene). Analysis by Northern blot has shown that transgenic animals expressed higher levels (about 2- to 5-fold) of PEPCK mRNA than control mice. PEPCK activity was also increased (about 2-fold) specifically in adipose tissue of transgenic mice. Preliminary results indicated that 3-month-old transgenic mice increased lipid accumulation. Histological studies showed hypertrophia and hyperplasia of adipocytes. Thus, this animal model may provide new insights into the mechanisms leading to the development of obesity and type 2 diabetes. 


\section{PS 36}

\section{Treatment of Obesity}

\section{7}

THE EFFECT OF ESTROGEN THERAPY (ET) ON CENTRAL FAT, LIPIDS AND VASCULAR DISTENSIBILITY IN TYPE 2 DIABETES K. Samaras', C.S. Hayward ${ }^{2}$ D. Sullivan ${ }^{3}$, R. Kelly ${ }^{2}$ and L.V Campbell ${ }^{1}$.

1 Garvan Institute of Medical Research; 2 St Vincent's Hospital; 3 Royal Prince Alfred Hospital, Sydney, Australia.

The major increase in heart disease in women with Type 2 diabetes is predominantly attributed to disturbed lipid and glucose metabolism. central obesity and vascular dysfunction. While ET in non-diabetic women reduces heart disease risk by $50 \%$, whether such cardioprotection occurs in women with diabetes is unclear. The effects of ET on lipid metabolism, glycaemic control, body composition and vascular parameters in postmenopausal females with Type 2 diabetes were examined ( $\mathrm{n}=14$, mean $\pm \mathrm{SD}$ age $58 \pm 6$ yrs, BMI $29.5 \pm 4.8 \mathrm{~kg} / \mathrm{m}^{2}$ ). Subjects randomly received conjugated equine estrogen $(0.625 \mathrm{mg}$ daily) or no treatment for 6 months, then crossed over. Fasting glucose, tota and HDL cholesterol, triglycerides, LDL size, NEFA, HbAlC and SHBG, resting energy expenditure, total and central abdominal fat (by dual energy $\mathrm{x}$-ray absorptiometry), $24 \mathrm{hr}$ blood pressure (BP) and vascular distensibility (by applanation tonometry) were measured. ET resulted in significant reductions in HbAlC $(-0.3 \pm 0.2$ vs $0.6 \pm 0.4 \%$, $\mathrm{p}=0.04)$, total cholesterol $(-0.63 \pm 0.12$ vs $0.17 \pm 0.19 \mathrm{mmol} / \mathrm{L}, \mathrm{p}=0.001)$ and central abdominal fat $(-175 \pm 51$ vs $-24 \pm 56 \mathrm{~g}, \mathrm{p}=0.05)$, compared to no treatment. There were increases in SHBG $(42.1 \pm 5.6$ vs $-10.3 \pm 5$.1 $\mathrm{nmol} / \mathrm{L}, \mathrm{p}=0.0001)$ and energy expenditure $(33 \pm 23 \mathrm{vs}-38 \pm 23 \mathrm{~kJ} /$ day, $\mathrm{p}=0.04)$ and a greater decrease in LDL size with ET $(-0.32+0.22 \mathrm{~nm}$ vs $-0.16 \pm 0.22$, NS). Total body fat, glucose, triglycerides, NEFAs, 24 hour BP control and vascular distensibility were unchanged. We conclude that postmenopausal ET in overweight females with Type 2 diabetes improves major predictors of heart disease. Whether these translate to diminished morbidity and mortality as observed in nondiabetic women awaits further study.

\section{9}

GLUCAGON-LIKE PEPTIDE 1 INCREASES SATIETY AND REDUCES FOOD INTAKE IN OBESE PEOPLE.

E. Năslund, B. Barkeling, M.K.Gutniak, J.J. Holst S. Rössner and P.M. Hellström. Division of Surgery, Danderyd Hospital, Karolinska Institute, Obesity Unit, Huddinge University Hospital, Multidisciplinary Pain Center Kronan, Karolinska Institute, Dept. of Medical Physiology, PANUM Institute, Copenhagen, Denmark, Dept. of Hepatology and Gastroenterology, Karolinska Hospital, Stockholm, Sweden,

The present study was designed to investigate the effect of a short acting ( 4 hours) and long acting ( 8 hours) infusion of $0.75 \mathrm{pmol} / \mathrm{kg} / \mathrm{min}$ Glucagon-Like Peptide 1 (GLP-1) in 14 morbidly obese patients (BMI $40.55 \pm 5.2$ ) on gastric emptying, food intake and hunger. In the first experiment series, intravenous infusion of GLP-1 started at the beginning of breakfast and continued during the following 4 hours. Oral paracetamo $(1.5 \mathrm{~g})$ was given at the start of meals as a marker for gastric emptying. In the second series of experiments, the infusion was continued for 8 hours. Lunch and dinner were served after $\mathbf{4}$ and $\mathbf{8}$ hours respectively. Hunger, satiety and food choice were assessed with VAS and food choice questionnaires before and after the meals. GLP-1 reduced postprandial glucose concentrations but no subject recorded hypoglycemia. Insulin and glucagon concentrations were unchanged. However, the insulinogenic indices were increased. Gastric emptying was significantly retarded after breakfast and lunch, $p<0.001$. Food intake at breakfast was not changed, but when GLP-1 infusion was prolonged, a reduction of a daily energy intake by $24 \%(206 \pm 61 \mathrm{Kcal}, \mathrm{p}<0.008)$ was observed. Eating rate was significantly lower at lunch and dinner. In both experiments GLP-1 reduced feelings of hunger, desire to eat and prospective consumption after a meal. In conclusion, infusion of GLP-1 in massively obese patients resulted in decreased hunger and increased fullness postprandially. Food intake was decreased and gastric emptying delayed suggesting an influence of GLP-I on signals from the stomach.

\section{8}

\section{CENTRAL AND PERIPHERAL ADMINISTRATION OF EXENDIN-4 REDUCES FOOD INTAKE IN RATS.}

S.P. Bhavsar, J.J. Watkins and A.A. Young. Amylin Pharmaceuticals Inc, 9373 Towne Centre Drive, San Diego, CA, 92121, USA

Exendin-4, a 39 amino acid peptide from Gila monster (Heloderma suspectum) venom with $52 \%$ sequence similarity to glucagon-like peptide- 1 (GLP-1), is reported to bind and activate GLP-1 receptors. In rats, intracerebroventricular (but not intraperitoneal) GLP-1 is reported to inhibit food intake. We investigated the effect of intraperitoneal (IP) or intracerebroventricular (ICV) administration of exendin-4 on food intake in Sprague Dawley Rats (weight $300-350 \mathrm{~g}$ ) in which lateral ventricular guide cannulae had been implanted. At least ten days after implantation, rats were injected just before the onset of the dark cycle with $0-10 \mu \mathrm{g}$ of exendin-4 via ICV route $(2 \mu \mathrm{l} /$ dose, $N \geq 4$ rats/dose) or with $0-100 \mu \mathrm{g}$ of exendin- 4 via IP route ( $n \geq 8$ rats/dose). Similar experiments were performed using GLP-1. Food intake was measured by the difference in food weight 2 hours after presenting it to the rats. Exendin-4, given ICV, inhibited food intake by up to $94 \%$ (at $3 \mu \mathrm{g}$ and $10 \mu \mathrm{g}$ doses) with an $\mathrm{ED}_{50}$ of $0.16 \mu \mathrm{g} \pm 0.08 \mathrm{log}$. GLP-1, injected ICV was at least 72-fold less potent than ICV exendin-4 (apparent $\mathrm{ED}_{50} \uparrow 1.6 \mu \mathrm{g} \pm 0.06 \mathrm{log}$ ) and inhibited food intake by a maximum of $50 \%$ with a 10ug ICV dose. IP exendin-4 inhibited food intake by a similar magnitude as ICV administration, but was 68 -fold less potent via this route $\left(E_{50} 11 \mu \mathrm{g} \pm\right.$ $0.58 \mathrm{log}$ ). In contrast to the actions of exendin-4, GLP-1 injected IP had no effect on food intake at any dose up to $1000 \mu \mathrm{g} / \mathrm{rat}$. In summary, the greater potency on inhibition of food intake with ICV vs IP routes of exendin-4 administration is consistent with a central site for this action. The enhanced potency of ICV and IP exendin-4 over GLP-1 for inhibition of food intake indicates a possible utility of exendin-4 in appetite control.

\section{0}

POSITIVE EFFECTS ON BODY WEIGHT RESULTING FROM PRAMLINTIDE THERAPY IN TYPE 1 AND TYPE 2 DIABETES O. Kolterman, F. Whitehouse, R. Ratner, J. Rosenstock, S. Schoenfeld, S. Jeffcoate. San Diego CA; Detroit MI; Washington DC; and Dallas TX, USA

In diabetes, there is a deficiency of both insulin and amylin resulting from loss of $\beta$-cell function. The deficiency is absolute in type 1 ; postprandial responses are deficient in type 2 diabetes. The effects of SQ insulin and pramlintide (PRAM), a synthetic analog of human amylin, on body weight were evaluated in two randomized, double-blind, placebo-controlled trials, one each in type 1 and 2 . Type 1 subjects received insulin with 30 or $60 \mu \mathrm{g}$ PRAM or placebo QID for 52 weeks. PRAM-treated subjects exhibited significant improvements in $\mathrm{HbA}_{\mathrm{t}}(-0.39 \%$ vs $-0.09 \%$ for placebo, $\mathrm{p}<0.004$ ) with no increase in the frequency or severity of hypoglycemic events. With improvements in $\mathrm{HbA}_{\mathrm{tc}}$, in patients with weight recorded at all visits $(\mathrm{n}=317)$, significant decrease in body weight in PRAM-treated subjects $(-1.3 \mathrm{~kg}$ vs placebo, $\mathrm{p}=0.01)$ was observed at 52 weeks. By baseline BMI, weight was maintained $(+0.1 \mathrm{~kg})$ among lean subjects $(\mathrm{BMI}<27 ; \mathrm{n}=110)$ and substantial weight loss $(-1.8 \mathrm{~kg}$ from baseline) observed in PRAM-treated subjects BMI $\geq 27$ $(n=53)$. This contrasts with DCCT, where after 1 year, subjects in both groups had weight gain $\left(+2.4 \mathrm{~kg}\right.$ for conventional therapy, mean $\Delta \mathrm{HbA}_{1 \mathrm{c}}+0.2 \%$; $+5.1 \mathrm{~kg}$ for intensive therapy, mean $\Delta \mathrm{HbA}_{\mathrm{lc}}-2.0 \%$ ). Type 2 subjects received insulin with 30 , 75 , or $150 \mu \mathrm{g}$ PRAM or placebo TID for 52 weeks. For those with values at each visit, $\mathrm{HbA}_{\mathrm{lc}}$ was reduced $(-0.30 \%,-0.47 \%,-0.64 \%$ for $30,75,150 \mu \mathrm{g}$, respectively vs $-0.20 \%$ for placebo, NS) at 52 weeks with no increase in the frequency or severity of hypoglycemic events. In patients with weight recorded at all visits $(n=345)$, differences from baseline at week 52 for PRAM-treated subjects were $-1.6,-1.4,-2.7$ $\mathrm{kg}$ for $30,75,150 \mu \mathrm{g}$, respectively, vs placebo (all doses $\mathrm{p}<0.05$ ). Both $\mathrm{BMI}<27$ and $\geq 27$ showed significant weight loss. In both studies, the initiation of PRAM therapy was associated with mild, transient nausea that dissipated by 4 weeks but significant weight loss occurred even in those patients that did not report Gl symptoms. Thus, compared with placebo, PRAM was found to have beneficial effects on body weight while improving $\mathrm{HbA}_{1 c}$ values in both type 1 and type 2 diabetes. 


\section{1}

ROSIGLITAZONE ENHANCEMENT OF UNCOUPLING PROTEIN-1 EXPRESSION IN BROWN ADIPOCYTES IS BLOCKED BY WY 14643

T.Teruel and S.A.Smith. SmithKline Beecham Pharmaceuticals, Harlow, Essex, $U K$.

Rosiglitazone (RSG, BRL-49653), a PPAR $\gamma$ (peroxisomal proliferator activated receptor) agonist insulin sensitiser, promotes differentiation of rodent brown fat pre-adipocytes and increases expression of UCP-1 (uncoupling protein-1). UCP-1 is expressed only in brown fat and mediates the unique thermogenic activity of this tissue by uncoupling substrate oxidation from ATP generation. Brown adipocytes express both $\alpha$ and $\gamma$ isoforms of PPAR and the presence of a putative PPAR response element in the 5'-flanking region of UCP-1 suggests that PPARs may control transcription of this gene. The involvement of PPAR subtypes in regulation of UCP-1 expression was assessed directly by measuring UCP1 mRNA levels in cultured fetal rat brown adipocytes treated with the PPAR $\gamma$-selective agonist, rosiglitazone, or the PPAR $\alpha$-selective agonist hypolipidemic agent, Wy 14643. Brown adipocytes were prepared from 20 day old rat fetuses by collagenase digestion. Isolated adipocytes were cultured in Eagle's Minimal Essential Medium supplemented with $10 \%$ Fetal Calf Serum for $4 \mathrm{hr}$, followed by incubation in serum-free medium for $20 \mathrm{hr}$. Cells were then exposed to compounds for up to $48 \mathrm{hr}$ before quantitation of UCP-1 mRNA. RSG $(10 \mathrm{nM}-10 \mathrm{uM})$ increased UCP-1 expression in a concentration- $\left(\mathrm{EC}_{56}=30 \mathrm{nM}, \max\right.$ increase 4-fold) and time-dependent manner $\left(\mathrm{t}_{1 / 2}=12 \mathrm{hr}\right)$. Increases in UCP $-1 \mathrm{mRNA}$ were accompanied by parallel increases in UCP-1 protein, determined by western blot analysis. In contrast. Wy 14643 alone (10-100 uM) had no significant effect on UCP-1 expression but blocked the RSG-stimulated UCP-1 expression. This study is the first to demonstrate that the transcriptional activity of PPAR $\gamma$ can be negatively modulated by PPAR $\alpha$ ligands. These data suggest that the expression of UCP-1 and hence thermogenic capacity of brown adipose tissue can be directly regulated by RSG, which may impact on whole body energy balance.

\section{PS 37}

\section{Animal Models of Obesity}

\section{3}

DYSREGULATION OF NEUROPEPTIDES NOT RESPONSIBLE FOR THE DEVELOPMENT OF OBESITY IN PSAMMOMYS OBESUS. G. Morton, P. Zimmet, ${ }^{1}$ and G.R. Collier, Metabolic Research Unit, Deakin University, Geelong 3216, Australia; ${ }^{1}$ International Diabetes Institute, Melbourne 3162, Australia.

Leptin is an important regulator of energy balance and thought to act on the hypothalamus to reduce food intake and increase energy expenditure. Although leptin's exact mode of action is unknown, there are several possible mediators including neuropeptide Y (NPY), melanin concentrating hormone (MCH), pro-opiomelanocortin (POMC) and the melanocortin-4 receptor (MC4-R). Central administration of NPY or MCH causes hyperphagia, however subsequent studies have demonstrated that NPY knockout mice have normal food intake and body weight, suggesting NPY may not be essential for certain feeding responses. The melanocortin system featuring POMC and the MC4-R is also implicated in energy homeostasis, with agonists of the MC4-R reducing food intake and disruption of the MC4-R resulting in obesity. To examine these neuropeptides, we have utilized Psammomys obesus, a polygenic animal model of obesity which naturally develops a range of metabolic and physical characteristics. Psammomys obesus were categorized into three distinct groups, Group A : lean, normoinsulinemic, normoglycemic and normoleptinemic; Group B : obese, hyperinsulinemic, normoglycemic and hyperleptinemic and Group C : obese, hyperinsulinemic, hyperglycemic and hyperleptinemic. The aim of this study was to investigate differences in hypothalamic NPY, MCH, POMC, MC4-R mRNA gene expression by comparative RT-PCR in the three groups of Psammomys obesus.

\begin{tabular}{|c|c|c|c|c|c|}
\hline Group & $\begin{array}{c}\text { Body weight } \\
(\mathrm{g})\end{array}$ & $\begin{array}{c}\text { NPY/ } \\
\text { B-ACTIN }\end{array}$ & $\begin{array}{c}\text { MCH/ } \\
\text { B-ACTIN }\end{array}$ & $\begin{array}{c}\text { POMC / } \\
\text { B-ACTIN }\end{array}$ & $\begin{array}{c}\text { MC4-R/ } \\
\text { B-ACTIN }\end{array}$ \\
\hline $\mathrm{A}(\mathrm{n}=10)$ & $188 \pm 3$ & $1.85 \pm 0.13$ & $1.12 \pm 0.08$ & $0.85 \pm 0.08$ & $1.12 \pm 0.07$ \\
\hline $\mathrm{B}(\mathrm{n}=10)$ & $226 \pm 8$ & $1.89 \pm 0.28$ & $0.90 \pm 0.11$ & $0.91 \pm 0.04$ & $1.13 \pm 0.02$ \\
\hline $\mathrm{C}(\mathrm{n}=10)$ & $242 \pm 3$ & $1.96 \pm 0.22$ & $0.99 \pm 0.12$ & $1.00 \pm 0.09$ & $1.21 \pm 0.13$ \\
\hline
\end{tabular}

There was no difference in gene expression in any of the hypothalamic neuropeptides examined. In conclusion, dysregulation of NPY, MCH, POMC and MC4-R gene expression is unlikely to be involved in the development of obesity in Psammomys obesus.

\section{2}

SIBUTRAMINE INDUCES WEIGHT LOSS AND IMPROVES GLYCEMIC CONTROL IN OBESE PATIENTS WITH TYPE 2 DIABETES MELLITUS K. Fujioka', S.P. Weinstein', E. Rowe ${ }^{2}$, and P. Raskin ${ }^{3}$; 'Scripps Clinic, San Diego, CA; ${ }^{2}$ Knoll Pharmaceutical Co, Mt. Olive, NJ; ${ }^{3}$ UTSW Med Ctr, Dallas, TX. Sibutramine (Sib; MERIDIA ${ }^{\oplus} ;$ Reductil), a novel serotonin and norepineplirine reuptake inhibitor, was recenty approved in the US for managing obesity. This 24-wk, randomized, double-blind, placebo (Pcb)-controlled, parallel-group study evaluated the effect of $\mathrm{Sib}(20 \mathrm{mg} / \mathrm{d})$ on weight loss and glycemic control in obese patients with type 2 diabetes uncontrolled by diet or oral antidiabetic therapy. After a 5-wk nun-in period (all received Pcb), patients were randomized to Sib or Pcb; Sib patients started with $5 \mathrm{mg}$ and were titrated up by $5 \mathrm{mg}$ every 2 wk through wk 6 . Sib patients received $20 \mathrm{mg}$ in wk 6-24. Analyses for completers and categorical data were performed using the Kruskal-Wallis test ( ${ }^{*}$ indicates $P<.05 \mathrm{vs} \mathrm{Pcb}$ ). Of 175 patients $(93 \mathrm{M}, 82 \mathrm{~F}$ ) randomized ( $89 \mathrm{Sib}, 86 \mathrm{Pcb}$; mean characteristics: age, 54.2 yrs; weight, $99 \mathrm{~kg}$; BMI, $34 \mathrm{~kg} / \mathrm{m}^{2}$; fasting plasma glucose, $182 \mathrm{mg} / \mathrm{dL} ; \mathrm{HbA}_{1}$ $8.34 \%), 60 \mathrm{Sib}(67 \%)$ and $6 \mathrm{I} \mathrm{Pcb}(71 \%)$ patients completed the study. For 24-wk completers, mean actual and percent changes from baseline weight for Sib and Pcb patients, respectively, were $-4.3 \mathrm{~kg}^{*}$ and $-4.5 \% *$, and $-0.3 \mathrm{~kg}$ and $-0.4 \%$. The proportion of patients achieving $\geq 5 \%$ and $\geq 10 \%$ weight loss in Sib and Pcb groups, respectively, were $33 \%^{*}$ and $8.3 \%^{*}$, and $0 \%$ and $0 \%$. Mean changes in BMI were $-1.5 \mathrm{~kg} / \mathrm{m}^{2 *}$ for Sib vs $-0.1 \mathrm{~kg} / \mathrm{m}^{2}$ for Pcb. Sib produced larger mean reductions in waist circumference than Pcb $(-3.4 \mathrm{~cm}$ vs $-2.0 \mathrm{~cm})$. Weight loss $(\geq 5 \%)$ with Sib was associated with improvements in glycemic control; mean changes from baseline in $\mathrm{HbA}_{1 c}$ and fasting plasma glucose ( $\mathrm{mg} / \mathrm{dL}$ ) for $\mathrm{Sib}(25 \%$ weight responders) vs all Pcb patients, respectively, were -0.28 and $-8.2 *$ vs +0.25 and +15.8 . Sib was well tolerated. The type, incidence, and severity of reported adverse events were comparable between Sib and Pcb. Quality of life assessments showed significant* inprovements with $\mathrm{Sib}$ in general health, social functioning, and bodily pain scales. Conclusion: Sibutramine at $20 \mathrm{mg} / \mathrm{d}$ is safe and produces significant weight loss associated with improved glycemic control in obese patients with type 2 diabetes.

\section{4}

OVERFEEDING, LEPTIN AND THE DEVELOPMENT OF NIDDM AND OBESITY IN PSAMMOMYS OBESUS.

A. de Silva, M. Coughlan, P. Zimmet' and G.R. Collier. Metabolic Research Unit, School of Nutrition and Public Health, Deakin University, Geelong, Australia 3216, ${ }^{1}$ International Diabetes Institute, Melbourne 3162, Australia.

Psammomys obesus (Israeli sand rat) is a polygenic model of obesity and NIDDM which naturally develops a spectrum of metabolic characteristics when fed a relatively energy-dense diet of laboratory chow. Sucrose-feeding is an established method of promoting obesity in laboratory rodents and the aim of the present study was to examine the interaction between leptin, the leptin receptor $\mathrm{Ob}-\mathrm{Rb}$, and overfeeding in Psammomys obesus. Animals were categorized into three distinct groups, group A: lean, normoinsulinemic, and normoglycemic, group B: obese hyperinsulinemic, nornoglycemic and hyperleptinemic and group $\mathrm{C}$ : obese hyperinsulinemic, hyperglycemic and hyperleptinemic. Treated animals were allowed ad lib. access to a $5 \%$ sucrose solution. Comparative RT-PCR was utilised to examine changes in the level of $\mathrm{Ob}-\mathrm{Rb}$ gene expression in the liver, hypothalamus, and suprascapular fat, and $\mathrm{Ob}$ gene expression in suprascapular fat. Sucrose feeding increased the rate of body weight gain in all groups, however only the increase in groups $\mathrm{A}$ and $\mathrm{B}$ was significant ( $\mathrm{p}=0.003$ and $\mathrm{p}=0.01$, respectively). The percent body fat also tended to be higher in the sucrose-fed animals, although only group $\mathrm{A}$ animals showed a significant difference $(p=0,04)$. Group B animals developed hyperglycemic $(p<0.05)$. Interestingly, sucrose-feeding resulted in a significantly lower ratio of leptin-to-percent body fat when compared to control animals $(\mathrm{p}=0.006$ ). Hypothalamic $\mathrm{Ob}-\mathrm{Rb}$ gene expression was increased in sucrose-fed $\mathrm{A}$ and $\mathrm{C}$ animals only $(p=0.02)$, while there was no difference in the level of $\mathrm{Ob}-\mathrm{Rb}$ mRNA expression in either the liver or adipose tissue of sucrose-fed animals. In conclusion, sucrose feeding resulted in increased energy intake and increased body weight. This change in body weight was accompanied by decreased leptin levels and increased hypothalamic leptin receptor gene expression. These results suggest that sucrose feeding may have a direct effect on circulating leptin concentrations, resulting in an increased energy consumption, thereby allowing animals to overeat. 
835

HEPATIC LIPOTOXICITY IS ASSOCIATED WITH THE DEVELOPMENT OF NIDDM IN PSAMMOMYS OBESUS P. Lewandowski, D. Cameron-Smith, A. Sanigorski and G.R. Collier. Metabolic Research Unit, School of Nutrition \& Public Health, Deakin University, Geelong, Australia.

Excessive cytosolic lipid accumulation is thought to have a toxic effect and has been associated with the development of NIDDM. In this study we examined the underlying mechanism which may lead to excessive hepatic fat accumulation in a polygenic model of obesity and diabetes. Psammomys obesus (the Israeli sand rat) naturally develops a range of metabolic and physical characteristics associated with obesity and NIDDM. Animals were classified and into three groups, group A (normoglycemicnormoinsulinemic), group B (normoglycemic-hyperinsulinemic) and group C (hyperglycemic-hyperinsulinemic). Animals were studied in the fed state and following a $24 \mathrm{hr}$ fast. Hepatic CPT enzyme activity was measured in isolated mitochondrial extracts and mRNA level of key regulatory enzymes of fat metabolism were expressed as a ratio to $\beta$-actin mRNA, following semi-quantitative Rt-PCR. The development of hyperglycemia in the diabetic $\mathrm{C}$ animals was associated with excessive liver TG accumulation, an increase in mRNA levels of cytosolic fatty acid binding protein (L-FABP), acetyl-CoA carboxylase (ACC) and glycerol phosphate acyltransferase (GPAT). The expression of lipoprotein lipase (LPL) and uncoupling protein 2 (UCP-2) was decreased, whereas apolipoprotein B100 (Apo-B) mRNA and CPT activity was unchanged. Fasting corrected the hyperinsulinemia present in group $B \& C$, reduced the hyperglycemia previously found in group C, but did not alter liver TG levels. In fasted animals group $C$ had increased L-FABP and GPAT expression. LPL mRNA and CPT activity were decreased in fasted group $C$ animals, while ACC, UCP-2 and Apo-B expression was not changed. These findings suggest with the onset of NIDDM less fat is entering the hepatocyte however endogenous lipid production increases, the hepatocyte does not increase uncoupled or $\beta$ oxidation of this cytosolic lipid nor does it increase VLDL synthesis. Thus cytosolic lipid continues to accumulate within the hepatocyte where it may have toxic effects via increasing levels of long chain fatty acyl-CoA esters leading to impaired insulin sensitivity or altered hepatic glucose production.

\section{6}

INSULIN SECRETORY DEFECTS IN DIABETES-PRONE AND -RESISTANT LINES OF PSAMMOMYS OBESUS: EFFECT OF NUTRITIONAL STATE. R. Nesher, D.J. Gross, E. Cerasi, N. Kaiser. Dept. of Endocrinology and Metabolism, Hebrew University - Hadassah Medical Center, Jerusalem, IL

Insulin secretory response to glucose was assessed in islets of Psammomys obesus, a model of Type $2 \mathrm{DM}$, exhibiting predisposition for nutrition-evoked diabetes, obesity and insulin resistance associated with reduced pancreatic insulin stores. Islets of diabetes-resistant (DR) Psammomys exhibited normal

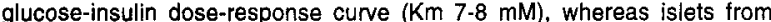
diabetes-prone (DP) Psammomys, either in the pre-diabetic state [low energy (LE) diet] or with overt diabetes [high energy (HE) diet] exhibited a shift to the left (Km 4-5 mM). HE-induced hyperglycemia lead to $90 \%$ depletion in islet insulin stores in DP but not in DR Psammomys: $18 \mathrm{hr}$ fast resulted in 50\% reduction of plasma glucose and 4.5 fold increase in stored insulin. Islets of DR Psammomys displayed strong insulin response to glucose comparable to rat islets while hyperglycemic DP Psammomys showed reduced early insulin response, and delayed and attenuated late insulin response to glucose. Regardless of diet and diabetic predisposition, Psammomys islets exhibited a significant delay in insulin release 'turn-off' following cessation of the glucose stimulus. No significant differences were observed between islets of DP and DR Psammomys in their sensitivity to calcium-free medium (+EGTA), diazoxide $(100 \mu \mathrm{M})$, or epinephrine $(10 \mu \mathrm{M})$. To assess the mechanism underlying the differential response to glucose, islet hexokinase and glucokinase activities were assayed. Islets of DP Psammomys on HE diet exhibited 4.5 fold increase in their capacity to phosphorylate glucose as compared to islets of the DR line, resulting from an increase in Vmax of both enzymes, while the $\mathrm{Km}$ values remained unaltered. DP Psammomys maintained on LE diet exhibited 2 fold increase in capacity to phosphorylate glucose. Conclusion: The increased capacity to phosphorylate glucose in the DP Psammomys, is affected by both diet and the genetic makeup; it may be responsible for the left shift of the glucose-insulin dose-response curve and contribute to the prevailing hyperinsulinemia and accelerated pancreatic insulin depletion observed in this animal model of Type $2 \mathrm{DM}$.

\section{7}

Prolonged diet-induced hyperglycemia in Psammomys obesus leads to $\beta$-cell apoptosis and irreversibility of the diabetic syndrome E. Ziv, R. Ben-Sasson, H.Bar-On and E. Shafrir Diabetes Unit \& Dept. of Biochemistry, HU-Hadassah Medical Center, Jerusalem, Israel The desert rodent Psammomys progresses into full fledged diabetes on high energy diet from stage $\mathbf{A}$ of normoglycemia and normoinsulinemia trough stages B (hyperisulinemia), C (hyperglycemiathyperinsulinemia) and D (peak hyperglycemia, loss of plasma and $\beta$-cell insulin). The continuous glucose elevation is driven by remarkable insulin resistance until $\beta$-cell collapse. However, stage $\mathbf{C}$ fully reverts to $\mathbf{A}$ by placing the animals on a low energy diet for $2 \mathrm{wk}$ (glucose $14.1 \rightarrow 4.5$ $\mathrm{mmol} / \mathrm{l}$, insulin $268 \rightarrow 45 \mathrm{mu} / \mathrm{l}, \mathrm{n}=10$ ). At stage $D$ no reversal was possible (glucose $24.0 \rightarrow 13.7 \mathrm{mmol} / \mathrm{l}$, insulin $22 \rightarrow 28 \mathrm{mu} / 1, \mathrm{n}=6$ ). To study this lack of reversal we stained the islets of Psammomys at stages A-D for insulin with biotylinated peroxidase-linked anti-insulin and for apoptosis by terminal deoxynucleotidyl transferase biotin-dUTP nick end labeling (TUNEL), visualising the DNA cleavage sites with 3aminoethylcarbazole. Psammomys at stage A, had ample, insulin laden, apoptosis negative $\beta$-cells. At stage $\mathbf{B}$ hypertrophy and hyperplasia of $\beta$-cells was seen $(160 \%$ of $A$ by morphometry), with partial insulin depletion, due to secretion overstimulation, but without apoptosis. At stage $\mathbf{C}, \beta$-cell mass in islets decreased to that of $\mathbf{A}$, insulin content was reduced, but the TUNEL staining was still almost negative. At stage $\mathbf{D}$, $\beta$-cells became virtually devoid of insulin, their mass only $50 \%$ that of A, showing a strong TUNEL reaction. These findings indicate that the apoptotic nuclear fragmentation coincides with the inability to recover from the hyperglycemia-induced secretory overtaxation, causing an irreversible damage to the insulin secretion apparatus. 
PS 38

\section{Obesity and TNF $\alpha$}

838

EFFECTS OF STORAGE, ANTICOAGULANTS AND FREEZETHAW CYCLES ON STABILITY OF IL-6, TNF- $\alpha$ AND LEPTIN

FLOWER CL, AHUJA RH, YUDKIN JS, COPPACK SW, MOHAMEDALI V, UCLMS, LONDON, UK

Levels of $\Pi L-6, T N F-\alpha$ and leptin are raised in obesity, cardiovascular disease and NIDDM, but results are conflicting. Discrepancies may be due to effects of storage, anticoagulants, or previous freeze-thaw cycles of samples. These parameters were investigated in 20 non-diabetic subjects (sex 10M:10F; age median \{IQR $25\{22-34\} \mathrm{yrs}$; BMI 22 \{21-27\} $\mathrm{kg} \cdot \mathrm{m}^{-2}$ ). Methods: (a) blood samples were allowed to stand for $1,2,4$ and 6 hours at RT prior to separation and freezing at $-70^{\circ} \mathrm{C}$; (b) blood samples were separated and the plasma allowed to stand at $+4^{\circ} \mathrm{C}$ for $1,2,4$ and 6 hours prior to freezing; (c) blood was taken into tubes with lithium heparin, sodium citrate, EDTA or no anticoagulant and immediately separated and frozen; (d) plasma samples were repeatedly frozen and thawed for up to 6 cycles. Results: (a) TNF- $\alpha$ levels increased significantly after 6 hours (up to $12 \%$ ) compared to baseline ( $=$ separated and stored within 30 minutes of collection); $\mathrm{L}-6$ levels decreased by $8 \%$ within 1 hour $(\mathrm{p}=0.03$ ) and by $15 \%$ after $2(p=0.004), 4$ and 6 hours $(p<0.001)$. Leptin levels did not change; (b) while there appeared to be a decrease in levels of TNF- $\alpha$, IL- 6 and leptin, none were significantly altered for up to 6 hours; (c) leptin levels were not affected by the anticoagulant, whereas TNF- $\alpha$ and IL- 6 were stable in EDTA and senum but less so with lithium heparin and sodium citrate; (d) leptin was stable for up to 3 freeze-thaw cycles, being reduced after this but both $I L-6$ and TNF- $\alpha$ decreased significantly after one cycle. Conclusions: The method of storage had opposing effects on IL- 6 and TNF- $\alpha$. Leptin remained unchanged. Anticoagulants affected TNF- $\alpha$ and IL- 6 levels but not those of leptin, as did the number of freezethaw cycles. Opposing effects of storage on levels of cytokines could have major implications to study outcome.

\section{9}

TUMOR NECROSIS FACTOR $\alpha$ AND ITS RECEPTOR IN IDENTICAL TWINS DISCORDANT FOR OBESITY

T. Rönnemaa and K. Pulkki. Departments of Medicine and Clinical Chemistry, University of Turku, Finland

Tumor necrosis factor $\alpha$ is expressed in adipose tissue and may be involved in the pathophysiology of insulin resistance. Information about serum levels of TNF- $\alpha$ and TNF- $\alpha$-receptors in human obesity are limited. Therefore, we studied the effects of genetic background and obesity on circulating TNF- $\alpha$ and soluble TNF $\alpha$ receptor concentrations in man by examining their serum levels in 23 identical twin pairs ( 9 male, 14 female) who had on average 18 $\mathrm{kg}$ intra-pair difference in body weight. Mean \pm SD fasting serum TNF- $\alpha$ concentration was $44.1 \pm 34.4 \mathrm{ng} / \mathrm{l}$ in obese and $34.2 \pm 21.7$ $\mathrm{ng} / \mathrm{l}$ in lean cotwins $(\mathrm{p}=0.051)$. The respective soluble TNF- $\alpha-$ receptor concentrations were $1989 \pm 335 \mathrm{ng} / \mathrm{l}$ and $1840 \pm 305 \mathrm{ng} / 1 \mathrm{p}$ $=0.004)$. The intra-pair difference in TNF- $\alpha$-receptor level was independent of body fat distribution determined as visceral fat area by MRI. The intra-pair difference in soluble TNF- $\alpha$-receptor concentration correlated positively with the difference in percent body fat $(\mathrm{r}=0.57, \mathrm{p}=0.004)$. The variation in TNF $\alpha$ level between the pairs was 1.35 -fold higher than the variation within the pairs. The variation in soluble TNF- $\alpha$-receptor level between the pairs was 1.86 -fold higher than the variation within the pairs. Our results show that serum soluble TNF- $\alpha$-receptor concentration is associated with obesity independent of genetic factors but genetic factors also contribute markedly to its circulating levels. The results are compatible with findings that the expression of TNF- $\alpha$ is increased in obesity.

\section{0}

ADIPOSE TISSUE TNF- $\alpha$ EXPRESSION IS INCREASED IN OBESE MALES AND ITS CORRELATION WITH BMI IS GENDER SPECIFIC H.A. Koistinen', J.P. Bastard ${ }^{2}$, P. Ebeling', E. Dusserre, ${ }^{3}$, B. Hainque ${ }^{2}$, P. Vallier $^{3}$, S. Perche ${ }^{3}$, M. Laville, V.A. Koivistot, and $\mathrm{H}$. Vidal ${ }^{2}$. ${ }^{1}$ Helsinki University Central Hospital, Helsinki, Finland; ${ }^{2}$ Hôpital la Salpêtrière, Paris, and ${ }^{3}$ INSERM U449, Lyon, France.

The expression of tumor necrosis factor (TNF)- $\alpha$ in adipose tissue is increased in obesity. Whether this is equal in both genders is not known. We examined the expression of subcutancous adipose tissue TNF- $\alpha$ expression (competitive RT-PCR assay) in 80 nondiabetic subjects with both genders and a wide range of obesity (mean BMI $33.4 \pm 1.1 \mathrm{~kg} / \mathrm{m}^{2}$, (mean $\pm \mathrm{SE}$ ), range 17.8 to $62 \mathrm{~kg} / \mathrm{m}^{2}$ ). The expression of TNF- $\alpha$ was 2.2 -fold higher in 30 obese males (BMI $\left.35.7 \pm 1.8 \mathrm{~kg} / \mathrm{m}^{2}\right)$ than in 8 lean men (BMI $\left.23.1 \pm 0.4 \mathrm{~kg} / \mathrm{m}^{2}\right)(0.40 \pm 0.06 \mathrm{vs}$ $0.18 \pm 0.02 \mathrm{amol}$ TNF$-\alpha$ mRNA $/ \mu \mathrm{g}$ total RNA, $P=0.051$ ). However, the TNF- $\alpha$ expression was not different in 32 obese females (BMI $37.5 \pm 1.4 \mathrm{~kg} / \mathrm{m}^{2}$ ) than in 10 lean females (BMI $\left.21.9 \pm 0.6 \mathrm{~kg} / \mathrm{m}^{2}\right)(0.33 \pm 0.03$ vs $0.27 \pm 0.04$ amol TNF$\alpha$ mRNA/ $\mu$ g total RNA, $P=0.391$ ). The expression of TNF- $\alpha$ in all subjects correlated positively with BMI $(r=0.41, P<0.001)$. This was due to the correlation in males $(r=0.65, P<0.001)$, whereas this association was not observed in females $(\mathrm{r}=0.042$, NS). A $240 \mathrm{~min}$ euglycemic hyperinsulinemia (120-150 mU/l) did not change the expression of TNF- $\alpha$ in a subgroup of 7 lean males (BMI $23.4 \pm 0.4 \mathrm{~kg} / \mathrm{m}^{2}$, glucose disposal rate [M] $64.7 \pm 2.7$ $\mu \mathrm{mol} / \mathrm{kg} / \mathrm{min}$ ), or in 10 age-matched obese insulin resistant males (BMI $30.5 \pm 1.0 \mathrm{~kg} / \mathrm{m}^{2}, \mathrm{M} 36.2 \pm 2.8 \mu \mathrm{mol} / \mathrm{kg} / \mathrm{min}$ ). There was no correlation between $M$ and TNF- $\alpha$ expresssion in these subgroups. In conclusion, subcutaneous adipose tissue TNF- $\alpha$ mRNA expression: 1) is increased in obese males, 2) correlates with obesity in males but not in females, 3 ) is not regulated by acute hyperinsulinemia. Thus, while adipose tissue TNF- $\alpha$ expression is increased in obese males, there is a gender difference in its regulation particularly in extreme obesity. 
PS 39

\section{Leptin}

\section{1}

OBESITY AND LEPTIN LEVELS DISTRIBUTION IN TWO ABORIGINAL POPULATIONS FROM CHILE: MAPUCHE AND AYMARA NATIVES.

F. Pérez.Bravo, JL. Santos, C. Albala, M. Calvillán, D. Montalvo, G. Larenas, E. Carrasco. Molecular Biology and Epidemiology Units. INTA; Diabetes Unit. San Juan de Dios Hospital. University of Chile. University of Tarapacá (Arica, Chile) and University La Frontera (Temuco, Chile). (Supported by FONDECYT 1960395 and Bristol Myers/Squibb/Mead/Jonhson Unrestricted Grant)

Leptin may play an important role in the regulation of body weight by influencing energy intake and expenditure. Several studies have suggested that there are both gender and ethnic differences in serum leptin levels. Population based studies are useful in examining the importance of leptin in human obesity and type 2 diabetes. We examined the relationship of leptin regarding to obesity in two native populations with very low prevalence of type 2 diabetes. METHODS: Leptin levels were determined by RIA and correlated with BMI, sex and ethnic background; 208 mapuche natives from Temuco (63 males and 145 females, mean age $46.5 \pm 15.5$ years) and 164 aymara natives from Arica (64 males and 100 females, mean age 46.4 \pm 18 . lyears ) were included in this study. RESULTS: Both populations have a very low prevalence of type 2 diabetes $1.65(0.45-4.17)$ in mapuche and $1.23(0.26-3.56)$ in aymara natives. Obesity status (BMI>27.5 $\mathrm{k} / \mathrm{m}^{2}$ ) was more prevalent between mapuche natives ( $48 \%$ ) than aymara natives ( $31 \%$ ) (BMI $27.6 \pm 4.4$ vs $26.4 \pm 4.8$; $\mathrm{p}<0.05$ ). T he leptin levels were increased in the mapuche groups compared with aymara natives $(14.8 \pm 19.1 \mathrm{ng} / \mathrm{ml}$ vs $10.2 \pm 13.8 \mathrm{ng} / \mathrm{ml} ; \mathrm{p}<0.01) \quad$ Leptin concentrations in obese subjects were significantly increased in both ethnic groups in relation to lean status: $21.4 \pm 20.3 \mathrm{ng} / \mathrm{ml}$ and $19.8 \pm 19.3 \mathrm{ng} / \mathrm{ml}$ (NS) (mapuches and aymaras respectively) vs $9.1 \pm 12.8 \mathrm{ng} / \mathrm{ml}$ and $5.8 \pm 7.1 \mathrm{ng} / \mathrm{ml}$ (NS) in lean status (mapuches and aymaras respectively). When we compared the leptin distribution by sex, similar values were found between the mapuche and aymara men $(5.1+9.3 \mathrm{ng} / \mathrm{ml} \mathrm{vs} 4.2+5.9 \mathrm{ng} / \mathrm{ml}$; NS). In contrast, in the women group, we found higher levels of leptin with a slight differences by ethnic background between mapuche and aymara natives $(19.1 \pm 21.7 \mathrm{ng} / \mathrm{ml}$ vs $14.2 \pm 15.9$ $\mathrm{ng} / \mathrm{ml} ; \mathrm{p}<0.05)$ CONCLUSIONS:Higher leptin concentrations are associated with obesity in both ethnic groups. However the leptin levels in the aymara natives were significantly decreased compared to mapuche subjects

\section{3}

SERUM LEPTIN, INSULIN RESISTANCE AND BLOOD PRESSURE IN ADOLESCENTS AND MIDDLE-AGED MEN

H. Hirose, I. Saito, H. Kawabe, M. Tsujioka H. Maruyama, and T. Saruta Health Center and internal Medicine, Keio University School of Medicine. 35 Shinanomachi, Shinjuku-ku, Tokyo160-8582, Japan

The obese gene product leptin, secreted from adipocytes, was discovered to serve as a satiety factor and to play an important role in regulating body weight. Serum leptin level reportedly increase with the degree of obesity. There is, however, dispute as to leptin-induced insulin resistance. The present study was designed to clarify the relationship between blood pressure and $8 \mathrm{MI}$, plasma glucose, serum insulin and leptin levels in 290 Japanese male adolescents (15-16 years old) and 167 middle-aged men. In adolescents, BMl in hypertensive group was higher than normotensives $(22.4 \pm 3.6$ vs $20.2 \pm 2.0, \mathrm{P}<0.01)$, as well as leptin $(3.8 \pm 2.0$ vs $2.5 \pm 1.3 \mathrm{ng} / \mathrm{ml}, \mathrm{P}<0.05)$ and insulin resistance by homeostasis model assessment $(1.35 \pm 0.41$ vs $1.0 \pm 0.48, P<0.05)$. Simple linea correlation revealed that mean blood pressure (MBP) correlated with $B M]$ $(r=0.24), \log$ [leptin] $(r=0.14)$, insulin (log, $r=0.16)$ and insulin resistance $(\log , r=0.17)(P<0.02$ for all). After adjustment for age and BMI, MBP correlated with neither leptin $(r=0.01)$ nor insulin $(r=0.10)$. In middle-aged men, BMl in hypertensive group was higher than the normals $(24.0 \pm 2.5 \mathrm{vs}$ $22.7 \pm 2 . P<0.05)$ as well as leptin $(3.7 \pm 1.9$ vs $2.9 \pm 1.5 \mathrm{ng} / \mathrm{ml}, P<0.05)$. Simple linear correlation revealed that MBP correlated with $B M \mid(r=0.27)$, leptin $(r=0.22)$ and insulin resistance $(r=0.18)(P<0.03$ for all). After adjustment for age and BMI, MBP correlated with neither leptin $(r=0.10)$ nor insulin resistance $(r=0.14)$. These results indicate that serum leptin levels are associated with blood pressure in both adolescents and middleaged men. However, these associations were considered not to be mediated through insulin resistance, but through obesity itself.

\section{2}

PLASMA LEPTIN CORRELATES WITH INSULIN RESISTANCE IN DYSLIPIDEMIC HYPERTENSIVE PATIENTS

K.H. Malminiemi, O.I. Malminiemi and J.T. Lahtela

Tampere University Hospital, РОВ 2000, Tampere, Finland

The anorexigenic and thermogenic hormone, leptin, may have independent clinical value in determination of metabolic syndrome. The associations between fasting plasma leptin and the variables related to insulin sensitivity were evaluated. An oral glucose tolerance test IOGTT, glucose dose $1.2 \mathrm{~g} / \mathrm{kg}$ ) was performed 3 times at 6 -month intervals with 187 non-diabetic, dyslipidemic (LDL $>4 \mathrm{mM}$ or HDL-ratio $<0.2$ ) patients on anti-hypertensive monotherapy. Insulin sensitivity index (ISI) was approximated using a formula validated with euglycemic clamp test. 105 men and 64 women with the mean age of 50 years (range $32-65$ ) and body mass index (BMI) of $28.4 \mathrm{~kg} / \mathrm{m}^{2}$ completed the study. All measured data were included in linear correlation and regression analyses $(n=543$ ).

All insulin variables in OGTT were highly significantly (p $<10^{-6}$ ) associated with fasting plasma leptin. The linear correlation coefficients between $|S|$ and leptin were $r=-0.648$ in men and $r=-0.604$ in women. Parallelly, insulin secretory response in OGTT, area under the curve (AUC $0.2 \mathrm{~h}$. correlated also with leptin: $r=0.543$ in men and $r=0.531$ in women. Glucose variables in OGTT correlated only weakly with leptin; incremental AUC: $r=0.272$ in men and $r=0.126$ in women. From the fasting serum lipids and lipoproteins, only triglycerides $(p<0.01)$ and apoB $(\mathrm{p}<0.05)$ showed statistically significant association with leptin in both genders. The linear correlation coefficients between BMI and leptin were 0.546 in men and 0.627 in women. Although the average body weight decreased only $0.8 \mathrm{~kg}$ during the one-year follow-up, its change was the most predictive indicator for the change of fasting plasma leptin. Leptin did not associate statistically significantly with serum cholesterol, fasting glucose levels, age, apoE phenotype, fibrinogen, urate, smoking. ethanol consumption or treated blood pressure.

In conclusion, fasting plasma leptin is associated with insulin sensitivity and hyperinsulinemia in dyslipidemic hypertension.

\section{4}

EFFECTS OF ACUTE OR PROLONGED EXPOSURE TO LEPTIN ON ISOLATED HUMAN ISLET FUNCTION.

R. Lupi, S. Del Guerra, M. Maffei, A. Bertacca, L. Marselli, C. Lencioni, C. Tellini, M. Camellini, F. Mosca, R. Navalesi, P. Marchetti. Dipartimento di Endocrinologia e Metabolismo, Sezione Metabolismo, via Paradisa 2, Ospedale Cisanello, Pisa, Italy.

To investigate whether leptin has a direct effect on insulin secretion (IS, hereby expressed as $\mu \mathrm{U} / \mathrm{ml}$, mean $\pm S D$ of at least 8 replicates of any given experimental condition) from human islets (HI), we prepared HI from four pancreata by collagenase digestion and denstity gradient purification, and exposed them to varying concentrations of recombinant human leptin (HL). In a first set of experiments, HI were acutely challenged for $45 \mathrm{~min}$ with $3.3(3.3 \mathrm{G})$ or $16.7(16.7 \mathrm{G}) \mathrm{mmol} / \mathrm{glucose}$ without or with the addition of $1,5,20,50$ or $500 \mathrm{ng} / \mathrm{ml} \mathrm{HL}$. The effect on IS of 20 and $500 \mathrm{ng} / \mathrm{ml}$ leptin was evaluated also at $3.3 \mathrm{G}$ plus $20 \mathrm{mmol} / 1$ arginine $(\arg )$. In a second set of experiments, HI were cultured for $24 \mathrm{~h}$ at $5.5 \mathrm{mmol} / \mathrm{glucose}$, without or with 20 or $500 \mathrm{ng} / \mathrm{ml} \mathrm{HL}$ in the medium. Then, the islets were challenged with either 3.3G, 3.3G plus arg, or 16.7G. In the HL acute experiments, IS from control islets was $11.8 \pm 4.2$ at $3.3 \mathrm{G}, 36.8 \pm 12.8$ at $3.3 \mathrm{G}$ plus arg $(\mathrm{p}<0.01$ vs $3.3 \mathrm{G})$ and $23.0 \pm 7.4$ at $16.7 \mathrm{G}(\mathrm{p}<0.01$ vs $3.3 \mathrm{G})$. No acute effect of 1 to $50 \mathrm{ng} / \mathrm{ml} \mathrm{HL}$ on IS was observed. In the presence of $500 \mathrm{ng} / \mathrm{ml} \mathrm{HL}$ a significant $(\mathrm{p}<0.01)$ decrease of IS at $3.3 \mathrm{G}(6.7$ $\pm 2.2)$ and $16.7 \mathrm{G}(7.5 \pm 1.6)$, but not at $3.3 \mathrm{G}$ plus arg $(27.9 \pm 10.2)$ was found. After $24 \mathrm{~h}$ exposure to $\mathrm{HL}$, a significant $(\mathrm{p}<0.01)$ reduction of glucose stimulated IS from HI was observed: IS at $3.3 \mathrm{G}$ was $5.4 \pm 12$ and $2.9 \pm 0.9$ from the islets pre-cultured with 20 or $500 \mathrm{ng} / \mathrm{ml} \mathrm{HL}$, respectively, and the release of insulin at $16.7 \mathrm{G}$ was $8.2 \pm$ 3.3 and $5.6 \pm 2.0$ from HI previously cultured with the low or high HL concentration, respectively. However, IS in response to $3.3 \mathrm{G}$ plus arg was again preserved from the islets exposed either to 20 (IS: $31.8 \pm 9.1$ ) or 500 (IS: $49.8 \pm 13.8$ ) $\mathrm{ng} / \mathrm{ml} \mathrm{HL}$. These secretory results were confirmed when IS was expressed as percent of islet insulin content. The present study shows that an acute inhibition of IS from HI by HL occurred only at pharmacological concentration of $\mathrm{HL}$ and that a prolonged exposure of $\mathrm{HI}$ to both physiological and pharmacological HL levels caused a significant reduction of glucose, but not arginine stimulated insulin release. 


\section{5}

THE EFFECTS OF REPEATED COLD-WATER EXPOSURE ON PLASMA LEPTIN CONCENTRATION IN HUMANS

A. Zeyl, J.M. Regan, M.J. Patterson, N.A.S Taylor, A.B. Jenkins, Department of Biomedical Science, University of Wollongong, NSW Australia 2522 Leptin secreted from adipocytes appears to act as a signal feeding back energy store status to mechanisms regulating energy expenditure and appetite. However adipose tissue has functions other than energy storage, in particular subcutaneous fat has a thermal insulating role. We are therefore iinvestigating a potential role for general and local body temperatures in the regulation of circulating leptin and implications this may have for the regulation of total and regional adiposity. This study investigated the effects of repeated cold exposure on circulating leptin levels. Seven lean male subjects (BWt $73.1 \pm 2.5(\mathrm{sem}) \mathrm{kg}, \mathrm{BMI}=23.7 \pm 0.2 \mathrm{~kg} / \mathrm{m}^{2}$ ) underwent 15 days cold acclimation consisting of daily chest-deep immersion (12-1.30pm) in $18^{\circ} \mathrm{C}$ water. On days 1,8 and 15 blood samples were taken before (to, after stabilisation in $20^{\circ} \mathrm{C}$ air) and after 60 minutes of immersion and body composition was estimated from total body water (TBW) determined by ${ }^{3} \mathrm{H}_{2} \mathrm{O}$ dilution. Body core temperature (Tc, 3 sites) and skin temperature (Tsk, 8 sites) were monitored continuousiy before and during immersion. Serum leptin was assayed by homologous RIA. Data were analysed by ANCOVA. The acclimation led to a significant increase in serum leptin $(20 \pm 9 \%, p<0.05)$ which is unlikely to be accounted for by the calculated $5 \%$ (NS) increase in body fat. Tc was not significantly affected by acclimation, but Tsk showed a pattern of increase similar to that of leptin, risng from $24.8 \pm 0.6^{\circ} \mathrm{C}(\mathrm{t0}$, day 1) to $27.2 \pm 0.5$ ( ( to, day $15, p<0.05$ ). We conclude that cold acclimation increases senum leptin in a way more closely related to skin temperatures than to body core temperature. Our pilot studies in other subjects demonstrate substantial variations in the temperature of subcutaneous fat $\left(29-34^{\circ} \mathrm{C}\right)$ at an ambient temperature of $23^{\circ} \mathrm{C}$. We suggest that these low temperatures of insulating subcutaneous fat may reduce leptin secretion and hence make insulating fat less visible to central mechanisms regulating appetite and energy expenditure. Interindividual variations in thermal adaptions could therefore contribute to inter-individual variations in adiposity.

\section{7}

EFFECT OF CATECHOLAMINES ON LEPTIN SECRETION

M. Stumvoll, A. Fritsche, E. Metzinger, O. Tschritter, M. Kellerer, H.U. Häring, Med. Klinik IV, Universität Tübingen, Germany.

While insulin is an important stimulator of leptin secretion, in vitro data suggest that catecholamines inhibit leptin secretion. In the present studies we have examined the effect of endogenous catecholamines during 3 hours hypoglycemic hyperinsulinemic clamp in $10(4 \mathrm{~F})$ normal subjects and $10(3 \mathrm{~F})$ type 1 diabetic subjects on leptin secretion. Furthermore, we examined the effect of exogenous catecholamines (4 hours of isoproterenol infusion 30 $\mathrm{ng} / \mathrm{kg} / \min$ in $6(3 \mathrm{~F})$ normal subjects) on leptin secretion whereby free fatty acids (FFA) were suppressed by acipimox. Euglycemic hyperinsulinemic control experiments showed an increase in serum leptin in type 1 diabetic patients $(6.2 \pm 1.4$ to $7.8 \pm 1.8 \mathrm{ng} / \mathrm{dl}, \mathrm{p}<0.05)$ as well as in normal subjects $(6.1 \pm$ 0.9 to $7.2 \pm 1.1 \mathrm{ng} / \mathrm{dl}, \mathrm{p}<0.05$ ). During hypoglycemic hyperinsulinemic clamp epinephrine increased from $4.8 \pm 0.7$ to $40.1 \pm 6.7 \mathrm{ng} / \mathrm{dl}$ in control and from $7.1 \pm 2.6$ to $16.7 \pm 5.0 \mathrm{ng} / \mathrm{dl}$ in diabetic subjects. In type 1 diabetic subjects, leptin increased from $5.6 \pm 1.1$ to $7.6 \pm 1.7 \mathrm{ng} / \mathrm{dl}(\mathrm{p}<0.05)$ but remained unaltered in normal subjects $(5.5 \pm 0.7$ to $5.7 \pm 0.9 \mathrm{ng} / \mathrm{dl})$. Moreover, the increase in serum leptin was negatively correlated with the increase in plasma epinephrine $(r=0.5, p=0.02)$ indicating a suppressive effect on leptin secretion. During hypoglycemic hyperinsulinemic clamp, plasma FFA levels in normal subjects $(0.45 \pm 0.04 \mathrm{mM})$ were double that in type 1 diabetic subjects $(0.25 \pm 0.05$, $\mathrm{p}=0.008$ ). The increase in leptin was also correlated with the decrease in plasma FFA levels $(\mathrm{r}=0.68, \mathrm{p}=0.001)$. During isoproterenol infusion with suppressed FFAs $(0.09 \pm 0.02 \mathrm{mM})$ leptin levels did not change (baseline: $5.0 \pm$ $1.2,120 \mathrm{~min}: 4.4 \pm 1.1,240 \mathrm{~min}: 5.1 \pm 1.5 \mathrm{ng} / \mathrm{dl}$ ). We conclude that under hyperinsulinemic conditions in humans increased plasma epinephrine seems to suppress leptin secretion. Since exogenous elevation of catecholamines with simuitaneously suppressed FFAs had no short-term effect on leptin secretion, the effect of catecholamines on leptin secretion may be mediated by FFAs.

\section{6}

EVIDENCE FOR LEPTIN REGULATION OF FOOD INTAKE IN HUMANS H. Larsson, S. Elmståhl*, G. Berglund and B. Ahrén, Dept. of Medicine and *Dept. of Community Medicine, Lund University, Malmö, Sweden

Obesity is an important contributor to the metabolic syndrome and type 2 diabetes, and in obese humans, plasma leptin levels are high. Leptin is known to inhibit food intake in mice. It is not known, however, whether leptin regulates food intake and thus affects the development of obesity in humans. Therefore, our aim was to study whether serum leptin levels are related to habitual dietary intake in 64 healthy women with normal glucose tolerance. Dietary habits were assessed with a modified diet history method. Body fat content was measured using bioelectrical impedance. In the 64 women aged (mean \pm SD) $58.6 \pm 0.4 \mathrm{yr}$., serum leptin was $19.3 \pm 12.7 \mathrm{ng} / \mathrm{ml}$, BMI $25.0 \pm 3.5 \mathrm{~kg} / \mathrm{m}^{2}$, body fat content $31.6 \pm 4.3 \%$, fasting glucose $4.6 \pm 0.5 \mathrm{mM}$ and fasting insulin $56 \pm 21 \mathrm{pM}$. Leptin levels were negatively correlated to total energy intake $(r=-0.34, p=0.006)$, carbohydrate intake $(r=-0.36, p=0.004)$ and total $(r=-0.27$, $p=0.034)$ as well as saturated fat intake $(r=-0.31, p=0.014)$. Leptin was correlated to the absolute but not to the percentual intake of these nutrients. When normalized for body fat content, the correlations remained significant. Comparing the quartiles with the lowest (LL, $7.0 \pm 1.7 \mathrm{ng} / \mathrm{ml}, \mathrm{n}=16$ ) and the highest (HL, $38.2 \pm 8.0 \mathrm{ng} / \mathrm{ml}, \mathrm{n}=16$ ) leptin levels, the HL group had a higher body weight, BMI and body fat content but similar waist to hip ratio compared to the LL group. Despite the higher body weight, the HL group had a lower energy intake $(1838 \pm 424 \mathrm{vs}$. $2311 \pm 669 \mathrm{kcal} /$ day, $\mathrm{p}=0.023$ ), lower carbohydrate ( $199 \pm 46$ vs. $259 \pm 77 \mathrm{~g} /$ day, $\mathrm{p}=0.013$ ) and saturated fat intake ( $30 \pm 11$ vs. $42 \pm 17 \mathrm{~g} / \mathrm{day}, \mathrm{p}=0.031$ ) than the LL group. In contrast, the differ ences in total fat (HL 76 \pm 26 vs. LL $96 \pm 35 \mathrm{~g} /$ day, $\mathrm{p}=0.082$ ) and protein intake $(74 \pm 15$ vs. $85 \pm 19 \mathrm{~g} /$ day, $\mathrm{p}=0.068$ ) were not significant. There were no differences between the LL and HL groups in percentual intake of the different nutrients. To summarize, we found that serum leptin is negatively associated with the absolute dietary intake of energy, carbohydrate and fat, but not to the percentual amount of the nutrients. Thus, the study shows that leptin is related to the quantity rather than to the quality of habitual food intake in humans.

\section{8}

PLASMA LEPTIN AND TUMOR NECROSIS FACTOR ALPHA IN TYPE I DIABETES MELLITUS M.Lechleitner, T. Koch, W. Sturm, C. Götsch, H.Hoppichler, J.R. Patsch Internal Medicine, University of Innsbruck, Austria

The complex interactions between leptin, the gene product of the obesity gene, and insulin make it difficult to evaluate the influence of leptin alone on metabolic control parameters and cardiovascular risk factors. Because type I diabetes is defined by the absence of endogenous insulin secretion we have determined the correlation of leptin, and tumor necrosis factor alpha, to BMl, blood pressure, duration of diabetes, glycated hemoglobin (HbA1c), plasma lipid levels and flowmediated dilatation (FMD) in 44 patients with type I diabetes. Plasma leptin was measured by a radioimmunoassay kit, Pearson's correlations were calculated to determine relations between variables. Mean plasma leptin levels were $3.1 \pm 1.9$ $\mathrm{ng} / \mathrm{mL}$ in men and $11,8 \pm 9.7 \mathrm{ng} / \mathrm{mL}$ in women $(p<0.005)$. Leptin levels showed a strong positive correlation to $\mathrm{BMI}$ in all of our normal-weight diabetics $(0.75 ; p<0.003)$. A strong positive correlation between leptin and insulin was present in diabetic women $(0.73 ; p<0.003)$, but not in men. In diabetic men leptin correlated with blood pressure and triglyceride values. TNFalpha showed a significant positve correlation to leptin, insulin and BMI in women, but not in men. Plasma leptin levels thus revealed in diabetic patients, in accordance to the results in non-diabetic persons, a strong correlation to BMI and were significantly higher in women than in men. A significant correlation of leptin to triglycerides and blood pressure, parameters of insulin resistance, was only found in men. 


\section{9}

FREE AND SPECIFICALLY BOUND LEPTIN AND INSULIN SECRETION IN IMPAIRED GLUCOSE TOLERANCE (IGT)

W. Leonhardt, R. Horn*, G. Brabant*, M. Breidert, Th. Temelkova-Kurktschiew, K. Fücker and M. Hanefeld, Institute and Policlinic of Clinical Metabolic Research, Technical University Dresden, *Department of Clinical Endocrinology, Medizinische Hochschule Hannover, Germany

Only very limited data are available concerning the interaction between leptin and insulin in impaired glucose tolerance (IGT). This relation may be further influenced by the fact that a part of circulating leptin is bound to a specific binding protein. Therefore, we studied free and bound leptin in 136 patients $(77$ males, 59 females) with IGT, in connection with the course of glucose, insulin, proinsulin and C-peptide during an oral glucose tolerance test (oGTT). A newly developed radioimmunoassay for free and bound leptin was used in which concentration and percent of free leptin showed similar relationships. Leptin concentrations increased with body mass index (BMI) and were higher in females than in males. In three tertiles of leptin (16-58,60-160, and $169-932$ pmol/l) mean fasting insulin levels were 65,93 , and $100 \mathrm{pmol} / \mathrm{l}$. The increase was significant also after correction for BMI and gender. Insulin concentrations and responses during the oGTT increased with the leptin level, too. The relationship was also significant in multiple regression analysis with BMI and gender as covariates. Similar relationships to the leptin level were observed also for the concentrations of $\mathrm{HbA} 1 \mathrm{C}$ and $\mathrm{C}$ peptide but not of glucose and proinsulin. Our data strongly suggest that there is a regulatory relationship between leptin and insulin secretion in impaired glucose tolerance.

\section{0}

LEPTIN ATTENUATES DECREASES OF GLUCOSE, INSULIN, AND GLUCAGON DURING FASTING IN MICE VIA SYMPATHETIC NERVES $B$ Ahrén and PJ Havel, Dept Medicine, Lund University, Malmö, Sweden and Dept Nutrition, University of California, Dovis, Ca, USA

Decreases of circulating leptin might contribute to the neuroendocrine response to starvation, since leptin inhibits the increase of glucocorticoids and the reduction of thyroid and reproductive hormones which accompany starvation. We examined whether decreases of leptin might also contribute to changes of glucose metabolism accompanying fasting in normal mice. Recombinant human or mouse leptin ( $300 \mu \mathrm{g} /$ mouse) or saline was administered ip prior to and after 12 $h$ of fasting in female NMRI mice. Blood samples were taken before and after $24 \mathrm{~h}$ of fasting. Fasting in saline-treated mice $(\mathrm{n}=24)$ reduced body weight from $27.3 \pm 0.9$ to $25.1 \pm 0.6 \mathrm{~g}(\Delta=-2.2 \pm 0.4 \mathrm{~g})$, plasma glucose from $8.0 \pm 0.3$ to $4.3 \pm 0.3 \mathrm{mmol} / 1(\Delta=-3.6 \pm 0.4 \mathrm{mmol} / \mathrm{l})$, plasma insulin from $214 \pm 39$ to $76 \pm 16$ $\mathrm{pmol} / \mathrm{l}(\Delta=-137 \pm 35 \mathrm{pmol} / \mathrm{)})$, plasma glucagon from $63 \pm 7$ to $31 \pm 4 \mathrm{pg} / \mathrm{ml}(\Delta=$ $-32 \pm 7 \mathrm{pg} / \mathrm{ml})$ and plasma leptin from to $4.1 \pm 0.3$ to $2.0 \pm 0.2 \mathrm{ng} / \mathrm{ml}(\Delta=-2.1 \pm 0.2$ $\mathrm{ng} / \mathrm{ml}$; all $\mathrm{p}<0.001)$. Treatment with human leptin $(\mathrm{n}=24)$ during the fasting did not affect the reduction in body weight $(\Delta=-2.3 \pm 0.3 \mathrm{~g})$ but attenuated the reduction in plasma glucose (by $33 \% ; \Delta=-2.2 \pm 0.3 \mathrm{mmol} / \mathrm{l}, \mathrm{p}=0.038$ vs. saline) and plasma insulin (by 74\%; $\Delta=-36 \pm 12 \mathrm{pmol} /, \mathrm{p}=0.010$ ), and abolished the reduction in plasma glucagon $(\Delta=-1 \pm 7 \mathrm{pg} / \mathrm{ml}, \mathrm{p}<0.001)$. The same attenuation of the reduction in glucose (by $25 \%, \mathrm{p}=0.036$ ), insulin (by $56 \%, \mathrm{p}=0.023$ ) and glucagon (by $82 \%, \mathrm{p}=0.018$ ) was seen after administration of mouse leptin $(n=12)$. Leptin administration did not attenuate fasting-induced reductions of circulating glucose, insulin or glucagon in mice after chemical sympathectomy with 6-hydroxydopamine $(40 \mathrm{mg} / \mathrm{kg}$ iv $48 \mathrm{~h}$ prior to fasting; $\mathrm{n}=12$ in both groups). Therefore, leptin attenuates the reductions of circulating glucose, insulin and glucagon during $24 \mathrm{~h}$ fasting in mice by a mechanism requiring intact sympathetic innervation. Hence, leptin seems to contribute to the adaptive response in glucose metabolism to fasting and starvation.

\section{1}

LEPTIN-STIMULATED JANUS KINASE-2 (JAK-2) PHOSPHORYLATES IRS-2 IN HEK 293 CELLS. M. Kellerer, 'J. Mushack, 'E. Seffer, ${ }^{+} A$. Ullich, "H.U. Häring

"Eberhard-Karls-Universität, Tübingen, ${ }^{+}$Max-PlanckInsitut für Biochemie, Martinsried

We have previously shown that leptin stimulation of $\mathrm{C}_{2} \mathrm{C}_{12}$ myotubes induces PI3-kinase activation. This appears to involve JAK-2- and IRS-2-dependent pathways. In the present study we used transient transfection in HEK 293 cells with JAK-2, insulin receptor (HIR), insulin receptor substrate-1 (IRS-1) and IRS-2 as well as the tyrosine phosphatase PTP1C to analyze further the interaction of insulinand leptin-dependent signaling pathways. Leptin $(10 \mathrm{ng} / \mathrm{ml})$ stimulates the autophosphorylation of JAK-2 significantly $(p<0.001, n=4)$. This effect is suppressed when cells are cotransfected with HIR or PTP1C. Cotransfection of JAK2 and IRS-2 revealed a strong leptin-dependent phosphorylation of IRS-2 at $10 \mathrm{ng} / \mathrm{ml}$ while IRS-1 showed only minor phosphorylation at $10 \mathrm{ng} / \mathrm{ml}$ leptin. The data suggest that 1) HIR-dependent signals seem to antagonize leptin effects on JAK-2 phosphorylation. 2) PTPIC suppresses JAK-2dependent phosphorylation. 3) Leptin-stimulated JAK-2 phosphorylates preferentially IRS-2, not IRS-1 at low leptin concentrations in HEK 293 cells.

\section{2}

EFFECTS OF METFORMIN AND VANADIUM ON LEPTIN SECRETION FROM CULTURED RAT ADIPOCYTES.

Peter J. Havel, Wendy M. Mueller, Kimber L. Stanhope, and Francine Gregoire, Dept. of Nutrition, Univ. of California, Davis, CA, U.S.A.

Leptin is involved in the regulation of energy balance. We have reported that glucose utilization regulates leptin expression and secretion from isolated rat adipocytes. Since the antidiabetic drugs, vanadium and metformin act to increase glucose uptake by peripheral tissues, we examined leptin secretion, glucose uptake, and lactate production from primary cultures of isolated rat adipocytes incubated for 96 hours with metformin or vanadium. Adipocytes $(100 \mu \mathrm{l}$ packed cells per well) were anchored in a defined matrix of basement membrane components (Matrigel) with media containing 5.0 mM glucose. Metformin $(n=6-19$ per dose) at concentrations from 0.25 to $1 \mathrm{mM}$ increased glucose uptake in the presence of $0.16 \mathrm{nM}$ insulin by $14 \pm 7 \%(\mathrm{p}<0.05)$ to $70 \pm 15 \%$ $(p<0.0001)$ over insulin alone. Concentrations higher than $5.0 \mathrm{mM}$ inhibited glucose uptake. Metformin from 0.5 to $5.0 \mathrm{mM}$ increased lactate production by $65 \pm 31 \%(p<0.05)$ to $155 \pm 45 \%(p<0.0025)$. At concentrations from 1.0 to $100 \mathrm{mM}$, metformin inhibited leptin secretion by $38 \pm 7 \%$ to $86 \pm 4 \%$ (all $p<0.0001$ ). From $0-5.0 \mathrm{mM}$, leptin secretion was inversely related to the percent of glucose released as lactate $(r=-0.64 ; p<0.0001)$. Vanadium at concentrations from $5-20 \mu \mathrm{M}(n=$ $6-14)$ increased glucose uptake by $23 \pm 6 \%(p<0.0025)$ to $44 \pm 12 \%$ $(p<0.01)$ and increased lactate production from $18 \pm 8 \%(p<0.05)$ to 63 $\pm 21 \%(p<0.02)$. Leptin secretion was inhibited by $38 \pm 6 \%$ to $67 \pm 8 \%$ (all $p<0.0001$ ). Similar to metformin, leptin secretion was inversely related to the conversion of gilucose to lactate $(r=-0.65 ; p<0.0001)$. We conclude that both metformin and vanadate increase glucose uptake and inhibit leptin secretion for cultured adipocytes. The inhibition of leptin secretion is related to an increase in the conversion of glucose to lactate. 
853

LEPTIN INHIBITS POTENTIATION OF INSULIN SECRETION IN MOUSE ISLETS WITHOUT ALTERING CYTOSOLIC Ca ${ }^{2+}$ LEVELS V. Poitout, C. Rouault, J-C. Marie, M. Guerre-Millo, and G. Reach INSERM U341, Hôtel-Dieu, CNRS ESA 7059, Université Paris 7, and INSERM U465, Institut Biomédical des Cordeliers, Paris, France.

Different effects of leptin on insulin secretion have been reported in several studies. We have recently demonstrated that physiological concentrations of leptin had no effect on glucose-induced insulin secretion (GSIS) in normal rodent islets. In contrast, leptin was shown to inhibit GSIS potentiated by IBMX, a phosphodiesterase inhibitor known to increase intracellular cAMP levels. This study was designed to further characterize the mechanisms by which physiological concentrations of leptin could modulate insulin secretion in normal mouse islets. Isolated islets were incubated for $60 \mathrm{~min}$ in the presence of either 2.8 or $16.7 \mathrm{mM}$ glucose and various secretagogues, with or without $10 \mathrm{ng} / \mathrm{ml}$ recombinant murin leptin. The results indicated that : 1) In normal CD mouse islets, leptin had no effect on GSIS ( $n=6, N S)$, but inhibited IBMX-potentiated GSIS by $31 \pm 4 \%(413 \pm 86$ vs. $519 \pm 79 \mu \mathrm{U} / \mathrm{islet}$ $\mathrm{n}=7, \mathrm{p}<0.05)$, and carbachol-potentiated GSIS by $25 \pm 8 \%(340 \pm 79$ vs. $427 \pm 68, n=9, p<0.05$ ). 2 ) In contrast to the above results, leptin had no effect on either IBMX- or carbachol-potentiated GSIS in islets from $d b^{P A S} / d b^{P A S}$ mice, bearing a mutation affecting all isoforms of the leptin receptor. Further, $C D 1$ mouse islets were loaded with the fura-2 probe and analysed for their concentrations of cytosolic free calcium $\left[\mathrm{Ca}^{2+}\right]_{i}$ by microfluorometry during perifusion studies. The islets were perifused with a buffer containing $2.8 \mathrm{mM}$ glucose (basal) and then with $16.7 \mathrm{mM}$ glucose + 0.1 $\mathrm{mM}$ IBMX. This resulted in a sharp increase of $\left[\mathrm{Ca}^{2+}\right]_{i}$ over basal levels which reached a plateau. Thereafter, the addition

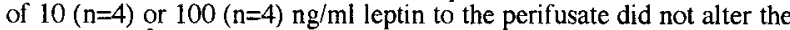
initiated $\left[\mathrm{Ca}^{2+}\right]_{i}$ response. These results indicate that, in normal mouse islets, physiological concentrations of leptin can inhibit potentiation of insulin secretion at a site distal to increased calcium levels, through a direct effect mediated by the leptin receptor.

\section{5}

In Zucker Diabetic Fatty rats plasma leptin correlates with plasma insulin rather than with body weight

S.W.J. Janssen ${ }^{1,2}$, G.J.M. Martens', I. van der Heijden ${ }^{1,2}$, H.A. Ross ${ }^{2}$, C.G.J. Sweep ${ }^{3}$, and A.R.M.M. Hermus ${ }^{2}$. 'Dept. of Animal Physiology, Faculty of Science; ${ }^{2}$ Dept. of Endocrinology and ${ }^{3}$ Dept. of Chemical Endocrinology, Faculty of Medical Sciences, University of Nijmegen, The Netherlands.

The obese $(o b)$ gene product leptin is secreted from adipose tissue and acts in the hypothalamus to regulate body energy stores. In vitro experiments have shown that insulin increases both leptin mRNA expression and leptin secretion, while in vivo the insulin effect has been shown only on the mRNA level. Here, we have investigated the relationship between plasma insulin and leptin in vivo. In Zucker Diabetic Fatty (ZDF) rats, aa animal model for NIDDM, body weight, plasma insulin, and plasma leptin were measured longitudinally from 10 to 25 weeks of age. In ZDF control rats, body weight and plasma levels of leptin and insulin increased gradually to $\sim 350 \mathrm{~g}, \sim 5.5 \mathrm{ng} / \mathrm{ml}$ and $-1.5 \mathrm{ng} / \mathrm{ml}$, respectively, at week 25 . In the ZDF control rats, plasma leptin levels and body weight were significantly correlated (males $\mathrm{r}=0.91$; females $\mathrm{r}=0.90$, both $\mathrm{p}<0.0 \mathrm{l}$ ) Body weights of male ZDF rats did not significantly change with age, whereas body weights of female ZDF rats increased significantly during the whole period. In 10-week old male ZDF rats, plasma insulin levels were $19.7 \pm 2.1 \mathrm{ng} / \mathrm{ml}$ and decreased to $1.6 \pm 0.2$ $\mathrm{ng} / \mathrm{ml}$ at week $25(\mathrm{p}<0.05)$. Plasma leptin levels were $19.4 \pm 0.6 \mathrm{ng} / \mathrm{ml}$ at 10 weeks and decreased to $4.6 \pm 0.4 \mathrm{ng} / \mathrm{ml}$ at 25 weeks $(\mathrm{p}<0.05$ ). In 10 -week old female ZDF rats, plasma insulin levels were $11.3 \pm 1.8 \mathrm{ng} / \mathrm{ml}$ and increased to $24.0 \pm 4.4 \mathrm{ng} / \mathrm{ml}$ at 13 weeks $(\mathrm{p}<0.01)$ and subsequently decreased to $4.3 \pm 1.1 \mathrm{ng} / \mathrm{ml}$ at 25 weeks $(\mathrm{p}<0.05)$. Plasma leptin levels of 10 -week old $Z D F$ female rats were $34.5 \pm 1.9 \mathrm{ng} / \mathrm{ml}$ and increased to $44.9 \pm 3.6 \mathrm{ng} / \mathrm{ml}$ at 13 weeks $(p<0.01$ ). Thereafter, these levels decreased to $22.9 \pm 1.0$ $\mathrm{ng} / \mathrm{ml}$ at 25 weeks $(\mathrm{p}<0.05$ ). In both male and fernale ZDF rats, significant correlations were found between plasma leptin and insulin (males $r=0.98$; females $r=0.81$, both $\mathrm{p}<0.01)$ but not between plasma leptin and body weight. Our results indicate that in ZDF rats plasma leptin correlates with plasma insulin rather than with body weight, suggesting an important role for insulin in the modulation of leptin secretion.

\section{4}

LEPTIN ALTERS IAPP SECRETION IN MOUSE PANCREATIC ISLETS

E. Karisson, M. Stridsberg and S. Sandler, Depts. of Medical Cell Biology and Medical Science, Uppsala University, Uppsala, Sweden

Leptin receptors are expressed in pancreatic $\beta$-cells. However, leptin's role in islet hormone secretion is essentially unknown. In the present study we aimed to elucidate leptin's effect on isolated pancreatic NMRI mouse islets, by examining islet amyloid polypeptide (IAPP) and insulin secretion in acute experiments and after $48 \mathrm{~h}$ exposure to leptin (1-100 nM). For the latter purpose islets were cultured in medium RPMI $1640+10 \%$ fetal calf serum. Leptin's effects on islet cell replication, islet glucose metabolism and islet hormone content was also examined. Glucose-stimulated IAPP and insulin secretions were measured at different glucose concentrations in the presence or absence of leptin. Leptin reduced IAPP secretion both acutely (103 \pm 12 versus $129 \pm 13 \mathrm{fmol} / 10$ islets $\times \mathrm{h}$ ) at $10 \mathrm{nM}$ leptin and after $48 \mathrm{~h}$ exposure of $10 \mathrm{nM}$ and $100 \mathrm{nM}$ leptin $(50 \pm 8$ and $48 \pm 7$ versus $69 \pm$ $11 \mathrm{fmol} / 10$ islets $x h$ ), whereas only minor effects were found on insulin release i.e. an inhibition in islets cultured with $1 \mathrm{nM}$ leptin $(6.2$ \pm 1.4 versus $8.2 \pm 2.0 \mathrm{pmol} / 10$ islets $\times \mathrm{h}$ ), $\mathrm{P}<0.05$, Student's paired $t$ test. Islet glucose oxidation rate was enhanced to $135 \%$ of the control by $1 \mathrm{nM}$ leptin, but decreased to $55 \%$ at higher concentrations of leptin in acute experiments, $\mathrm{P}<0.05$, Student's paired $t$-test. In contrast, glucose metabolism was not affected in long-term experiments. Moreover, leptin did not influence islet (pro)insulin synthesis or cell replication rate. In conclusion, we show that leptin affects islet IAPP release more than insulin release. Furthermore, the regulation of hormone secretion appears to be dissociated from glucose metabolism, an effect previously described in islets after exposure to certain cytokines.

\section{6}

FAILURE OF LEPTIN TO DIRECTLY AFFECT GLUCOSE METABOLISM OF RAT SKELETAL MUSCLE IN VITRO. B.Brunmair, R.Furtmüller, M.Roden, M.Nemecz, W.Waldhäusi, C.Fürnsinn Dept.Med.III, Div.Endocrinol.Metab., University of Vienna, Austria. Studies on different isolated tissues provided evidence that leptin may directly modulate cellular glucose handling. The present study was performed to elucidate leptin's action on basal and insulin-stimulated glucose metabolism of native muscle tissue, which under physiological circumstances is the quantitatively most important target tissue of insulin. Isolated rat soleus muscle strips were incubated for $t \mathrm{~h}$ in the absence or presence of leptin $(0$, 1,10 , or $100 \mathrm{nmol} /$ ) under basal or insulin-stimulated conditions. No effects of leptin were found on the rates of ${ }^{3} \mathrm{H}$-2-deoxy-glucose transport (basal: control, $314 \pm 14 ; 1 \mathrm{nmol} / /$ leptin, $320 \pm 17 ; 10 \mathrm{nmol} /$ leptin, $314 \pm 13 ; 100 \mathrm{nmol} / /$ leptin, $322 \pm 16$; insulin-stimulated: control, $690 \pm 33 ; 1 \mathrm{nmo} / /$ leptin, $691 \pm 29 ; 10 \mathrm{nmol} /$ leptin, 665 26; $100 \mathrm{nmol} / \mathrm{l}$ leptin, $664 \pm 27 ; \mathrm{cpm} / \mathrm{mg} / \mathrm{h} ; \mathrm{n} . \mathrm{s}$. vs. respective control) and on net glucose incorporation into glycogen (basal: control, $1.75 \pm 0.18 ; 1 \mathrm{nmol} / /$ leptin, $2.01 \pm 0.13 ; 10 \mathrm{nmol} / /$ leptin, $1.92 \pm 0.11 ; 100 \mathrm{nmol} / \mathrm{h}$ leptin, 1.81 \pm 0.13 ; insulin-stimulated: control, $5.98 \pm 0.40 ; 1 \mathrm{nmol} / \mathrm{l}$ leptin, $5.93 \pm 0.30 ; 10 \mathrm{nmol} / /$ leptin, $5.46 \pm 0.25 ; 100 \mathrm{nmol} / \mathrm{l}$ leptin, $5.85 \pm 0.30 ; \mu \mathrm{mol} / \mathrm{g} / \mathrm{h}$; n.s. vs. respective control). Likewise, prolonged leptin treatment of muscle specimen for $6 \mathrm{~h}$ failed to affect ${ }^{3} \mathrm{H}$-2-deoxy-glucose transport (basal: control, $437 \pm 21 ; 50 \mathrm{nmol} / \mathrm{l}$ leptin, $421 \pm 17$; insulin-stimulated: control, $61 \pm \pm 47 ; 50$ $\mathrm{nmol} / /$ leptin, $570 \pm 46 ; \mathrm{cpm} / \mathrm{mg} / \mathrm{h}$; n.s. vs. respective control) and on net glucose incorporation into glycogen (basal: control, $0.48 \pm 0.02 ; 50 \mathrm{nmol} / \mathrm{l}$ leptin, $0.44 \pm 0.03$; insulin-stimulated: control, $3.05 \pm 0.48 ; 50 \mathrm{nmo} / /$ leptin, $3.52 \pm 0.37 ; \mu \mathrm{mol} / \mathrm{g} / \mathrm{h}_{i} \mathrm{n.s}$. vs. respective control). In parallel, leptin' did neither modulate muscle glycogen content nor the rates of aerobic and anaerobic glycolysis. Further experiments revealed that the inability of leptin to directly affect muscle glucose handling prevailed independent of muscle fiber type (parallel experiments in epitrochlearis muscle) as well as of ambient insulin concentration $(0-30 \mathrm{nmol} / \mathrm{f})$. Thus, our findings fail to support speculations on a physiological role of direct insulin-mimetic or insulin-desensitizing leptin effects on skeletal muscle. 
PS 40

\section{Clinical Diabetes}

\section{7}

CORRELATION BETWEEN RANDOM PLASMA GLUCOSE (RPG) AND HBAIC, IMPLICATIONS FOR FOLLOW-UP AND DIAGNOSIS FWF Hanna, BG Issa, ETK Obuobie and JR Peters, University Hospital of Wales, Cardiff, U.K.

Introduction: Incidence of micro- and macrovascular complications of DM have been shown to rise sharply with $\mathrm{HbAlc}$ exceeds $7 \% \quad$. HbAlc is the measurement of choice for monitoring DM and implementing changes in therapy. HbAlc $>7 \%$ was also found to imply the need for pharmacological intervention, arguing for its use as a diagnostic test for treatment-requiring DM . On the other hand RPG $>11.1 \mathrm{mmol} / \mathrm{L}$ is diagnostic of DM. Aim \& Methods: To investigate the correlation coefficient $(\mathrm{r})$ of $\mathrm{HbAlC}$ to RPG in a diabetic clinic [1777 Follow-up (FU) and 285 new (N) referrals, from January 1995 onwards]. The following correlations were made, a) an overall correlation between RPG and $\mathrm{HbAlc}, \mathrm{b}$ ) RPG and $\mathrm{HbAlc}$ above and below $7 \%, \mathrm{c}) \mathrm{HbAlc}$ and $\mathrm{RPG}$ above and below $11.1 \mathrm{mmol} / \mathrm{L}$. Results:

$\begin{array}{lcccccc} & \text { No. } & \begin{array}{c}\mathbf{r} \\ \text { Overall }\end{array} & \begin{array}{c}\mathbf{r} \\ \text { HbAlc } \\ <7 \%\end{array} & \begin{array}{c}\mathbf{r} \\ \text { HbAlc }\end{array} & \begin{array}{c}\mathbf{r} \\ \text { RPG }\end{array} & \begin{array}{c}\mathbf{r} \\ \mathrm{RPG}\end{array} \\ & & & <\% & <11.1 & \geq 11.1 \\ \text { Type 1 DM, FU } & 557 & 0.12 & 0.01 & 0.11 & 0.06 & 0.2 \\ \text { Type 2 DM, FU } & 1220 & 0.12 & 0.04 & 0.07 & 0.2 & 0.01 \\ \text { Type 1 DM, N } & 82 & 0.06 & 0.68 & -0.04 & 0.09 & 0.12 \\ \text { Type 2 DM, N } & 203 & 0.27 & 0.21 & 0.17 & 0.23 & 0.09\end{array}$

None of these values reached a statistical significance. Discussion:

These results indicate a poor correlation between HbAlc and RPG. RPG is a poor indicator of glycaemic control, merely reflecting instantaneous glycaemic state. Its routine use in the diabetic outpatient clinic should be abandoned. The poor correlation of RPG $>11.1$ and $\mathrm{HbAlc}$ argues against the suggestion that the latter is a good diagnostic test for treatment-requiring diabetes

\section{8}

ESTIMATION OF GLYCOLATED HEMOGLOBIN FROM BLOOD GLUCOSE MEASUREMENT DATA

M. Mäkipääa1, P. Ebeling ${ }^{2}, P$. Heinonen ${ }^{3}$ and E. Oja ${ }^{1}{ }^{1}$ Helsinki University of Technology, Espoo; ${ }^{2}$ Helsinki University Hospital, Helsinki; ${ }^{3}$ Nokia Mobile Phones, Espoo, Finland

It is generally difficult to assess the $\mathrm{HbA}_{1 \mathrm{c}}$ level based on the average blood glucose level for a population of patients. The goal of the study is to develop methods for patient specific estimation of the $\mathrm{HbA}_{\mathrm{lc}}$ level based on the self-monitoring blood glucose data. 21 IDDM patients participated in an open study for nine months. The mean age $( \pm \mathrm{SD})$ was $35.6 \pm 7.0$ years, diabetes duration $14.5 \pm 7.1$ years and BMI $23.5 \pm 2.3 \mathrm{~kg} / \mathrm{m}^{2}$. Blood glucose values were collected on average 3.2 times a day $(\min 1.4$, max 5.6) using a blood glucose meter. HbA $1 \mathrm{lc}$ levels were determined in a central laboratory approximately once a month using a HPLC method, for a total of 190 measurements. A parametric density estimate was fitted using a maximum-likelihood procedure to blood glucose measurements in a certain range of days before each $\mathrm{HbA}_{1 \mathrm{c}}$ measurement. The parameters were then used to find the patient specific dependency between the blood glucose data and $\mathrm{HbA}_{1 \mathrm{c}}$ level. The estimate for $\mathrm{HbA}_{\mathrm{ic}}$ was found as trimmed mean of estimates given by models with different ranges of days $(40,50, . ., 90)$. Leave-one-out type cross-validation was used to provide a realistic assessment of the estimation accuracy. The model was found to give a significantly better estimate of the $\mathrm{HbA}_{1 \mathrm{c}}$ level in terms of absolute estimation error than was given by using only the other $\mathrm{HbA}$ ic values from the patient, either the previous $\mathrm{HbA}_{1 \mathrm{c}}$ measurement, the mean of previous measurements or a linear trend fitted to the measurements (for each, $\mathrm{P}<0.001$ ). Also, the estimate was found more accurate than was the 60-day average of the blood glucose values $(\mathrm{P}<0.001)$. The 60 -day average was found best for this group. The correlation between the blood glucose average and $\mathrm{HbA}$. level using the average, 0.61 (95\% CI 0.51-0.69) was improved to 0.89 $(0.85-0.92)$ using the model. Median absolute estimation error was 0.23 $\mathrm{HbA}_{1 \mathrm{c}} \%$. In conclusion, the utilization of a patient specific blood glucose based models for HbAlc estimation gives an increased estimation accuracy and promises to make possible new tools for determining the patient's level of control from self-monitoring data in the future.

\section{9}

In type I diabetic patients with a short duration of disease injection of insulin and meal intake does not influence muscle tissue oxygen tensions. D. Luf, C. Maisch, T. Luithle, E. Stopper, V. Hofmann-Krück, *N. Benda $4^{\text {ih }}$ Department of Internal Medicine and "Department of Medical Biometry, University of Tübingen, Tübingen, Germany

Background: In type I diabetic patients, both insulin injection and meal intake have been shown to increase muscular blood flow. Whether this leads to changes of substrate concentration amd consequently metabolic changes is unknown. Substrate concentrations measured directly within the muscular tissue may have more impact on metabolism than microcirculatory changes We, therefore, polarographically evaluated muscle tissue oxygen tensions before and after both the injection of insulin and the intake of breakfast in type I diabetic patients and normal control subjects.

Methods: Ten type I diabetic patients (mean age $30 \pm 8$ yrs, diabetes duration $4.1+2.5 \mathrm{yrs}, \mathrm{HbAlc} 7.6 \pm 1.3 \%$, normal results of standard autonomic cardiovascular reflex testing), treated with an intensified conventional insulin therapy, and 13 healthy control subjects with comparable age, sex distribution, weight, and physical fitness were investigated. Oxygen tension histograms each containing 200 single measurements, were sampled in the right anterior tibial muscle before and 70-80 minutes after the injection of NPH-insulin $(14.5 \pm 5.0 \mathrm{IE})$ and normal insulin $(11.3+5.3 \mathrm{IE})$, i.e. $50 \mathrm{~min}$. after the intake of breakfast ( $48 \mathrm{~g}$ carbohydrate, $16 \mathrm{~g}$ fat, $8 \mathrm{~g}$ protein).

Results: In diabetic patients, blood glucose concentrations increased significantly from $163 \pm 73$ to $199 \pm 61 \mathrm{mg} / \mathrm{dl}$ (in normals from $79 \pm 5$ to $95 \pm 19 \mathrm{mg} / \mathrm{dl}$ ), insulin concentrations from $125 \pm 92$ to $263 \pm 137 \mathrm{pmol} / \mathrm{l}$ (in normals from $80 \pm 32$ to $184 \pm 115 \mathrm{pmol} / \mathrm{l}$ ), heart rate increased significantly in both groups, blood pressures did not change. Muscular $\mathrm{pO} 2$ before insulin injection was $27.2 \pm 7.9 \mathrm{mmHg}$ in diabetics and $26.7 \pm 8.1 \mathrm{mmHg}$ (n.s.) in normals. In both groups, there was no significant change of oxygen tensions after insulin injections and breakfast: $28.3 \pm 10.4$ vs. $28.6 \pm 4.9$ (d.m. vs normals, n.s.).

Conclusion: Neither in type I diabetic patients nor in normal controls, physiological increases of both insulin and blood glucose concentrations induced increases of muscular oxygen tensions. Reported changes of microcirculation may have no metabolic impact in daily practice.

\section{0}

BODY COMPOSITION DURING THE FIRST YEAR AFTER DIAGNOSIS AND TREATMENT OF INSULIN DEPENDENT DIABETES MELLITUS. AM Rosenfalck, T Almdal, J Hilsted and S Madsbad. Hvidovre University Hospital, Copenhagen, Denmark.

Insulin deficiency is associated with a negative nitrogen balance, protein catabolism and loss of muscle mass. Thus insulin dependent diabetes mellitus (IDDM) is a potential catabolic disease with weight loss as a classical symptom at onset. The dimensions and the composition of the weight loss has only partly been described. The aim of the present study was to describe body composition in patients with newly onset insulin dependent diabetes mellitus from onset and during the first year after initiation of insulin therapy.

Body composition and total body water were estimated in $10\left(80^{\circ}\right.$ and $\left.2 \%\right)$ newly onset IDDM patients, age 31,5 $\pm 3,2$ (SD) years, by means of a DXA whole body scan and ${ }^{3} \mathrm{H}_{2} \mathrm{O}$ at onset and after $1,3,6$ and 12 months. The reported weight loss was $6,3 \pm 2,5$ $\mathrm{kg}$, range (1,5-10,0 kg), which was in agreement with a body weight $6,2 \mathrm{~kg}$ lower than expected as compared to a reference population (Metropolitan). BMI was 20,8 $\pm 1,6(19,2-23,4) \mathrm{kg} / \mathrm{m}^{2}$. The weight loss represented a significant reduced fat mass $25 \%$ or $-0,9 \mathrm{SD}$ lower than an age and sex matched reference population, whereas the lean body soft tissue mass was within normal range, only reduced corresponding to an initial reduction in the total body water volume. The regional fat distribution showed a uniform reduction in fat mass. During the first year HbAlc gradually decreased from $11,8 \pm 1.8 \%$ to $7.9 \pm 1,5 \%(\mathrm{p}<0.002)$, while the insulin dose increased from $0,36 \pm 0,2$ til $0,44 \pm 0,2 \mathrm{IU} / \mathrm{kg}$ (NS). Body weight increased $4,3 \mathrm{~kg}(\mathrm{p}<004)$, however still around $2 \mathrm{~kg}$ below normal weight. The fat mass increased from 12,0 4,2 to $13,6 \pm 4,1 \mathrm{~kg}$ (p<0.05) and the lean mass from $51,3 \pm 9,1$ to $53,8 \pm 8,6 \mathrm{~kg}$ $(\mathbf{p}<0.006$ ). Total body water increased from $41,6 \pm 5,91$ to $42,8 \pm 6,41$ (NS) during the first month and remained thereafter stable.

In Conclusion: Patients with newly onset IDDM are characterized by a $10 \%$ weigh reduction primarily as a reduction of the fat mass whereas the lean body soft tissue mass surprisingly seems to be normal. 
861

MICROVASCULAR VASODILATORY RESERVE IN NON-DIABETIC OFFSPRING OF TWO NIDDM PARENTS

B.C. Lee, J.M. Humphreys, A.C. Shore, A.T. Hattersley and J.E. Tooke. Department of Vascular Medicine, Postgraduate Medical School, University of Exeter, Exeter, UK.

The microvascular vasodilatory response to local heating is decreased in subjects with fasting hyperglycaemia who are at risk of developing NIDDM. Such microvascular abnormalities may be involved in the pathogenesis of diabetic microangiopathy. It is currently unclear whether impaired microvascular vasodilatory reserve is a consequence of insulin resistance and/or its associates in the prediabetic state or is a primary abnormality that precedes the emergence of insulin resistance. This study aimed to investigate microvascular vasodilatory reserve in non-diabetic offspring of two NIDDM parents. These individuals have previously been shown to have metabolic abnormalities and have a high risk of NIDDM. Using laser Doppler fluximetry we assessed maximum microvascular hyperaemic response to local heating of the skin in 21 non-obese, glucose-tolerant offspring of two NDDM parents (10 M, age 40.5 [19-54] yrs, median and range), and 21 age and sex matched controls. All had fasting blood glucose $<5.5 \mathrm{mmol} / \mathrm{l}$ and normal OGTT. Maximum hyperaemic response did not differ between the two groups, (1.53 [0.84-2.55] V vs $1.56[0.86-2.19] \mathrm{V}$ controls). Baseline fasting insulin level was $67.9[10.0-103.7] \mathrm{pmol} / /$ in offspring and 40.5 [10.0-93.9] pmol/l in control subjects respectively. The area under glucose curve during OGTT in the offspring was significantly higher compared to controls $(13.6$ [10.8-17.4] mmol/l h vs $12.0[9.1-15.1] \mathrm{mmol} / \mathrm{h}, p=0.001 \mathrm{Mann}$-Whitney). These data suggest that microvascular hyperaemia appears normal in glucose-tolerant, normoglycaemic offspring of two NIDDM parents even in the presence of relative hyperinsulinaemia and reduced glucose clearance. These findings provide no support for the concept that a defect in microvascular vasodilatory reserve precedes the development of diabetes or its metabolic precursors although specific endothelial dependent mechanisms remain to be determined.

\section{2}

A new family with autosomal dominant hyperinsulinism: intersubject variation in insulin secretion

K. Hoogenberg', C. Rouwé ${ }^{2}$, R.P.F. Dullaart' ${ }^{1}$, Departments of Endocrinology $y^{1}$ and Pedriatic Endocrinology $y^{2}$, University Hospital Groningen, The Netherlands

Familial autosomal dominant hyperinsulinism has been reported in only a few families thus far. Hyperinsulinemic (fasting plasma insulin $25 \mathrm{IU} / \mathrm{l}, \mathrm{C}$ peptide $0.374 \mathrm{nmol} / \mathrm{l}$ ) hypogycemia (fasting blood glucose (BG) $2.1 \mathrm{mmol} / \mathrm{l}$ ) was diagnosed in a 4 yrs old boy evaluated for convulsions. He was then successfully treated with diazoxide $(9 \mathrm{mg} / \mathrm{kg} /$ day $)$. The mother and grandmother had occasional convulsions and the deceased great-grandfather suffered from attacks of inappropriate behaviour that were relieved by eating. In the mother, BG was $1.6 \mathrm{mmol} / \mathrm{l}$ after a $40 \mathrm{~h}$ fast with an insulin level of 28 IU// and a C-peptide level of $0.424 \mathrm{nmol} / \mathrm{l}$. The grandmother had a BG of 1.8 $\mathrm{mmol} / \mathrm{l}$ after a $62 \mathrm{~h}$ fast with an insulin level of only $3 \mathrm{IU} / \mathrm{l}$ and a $\mathrm{C}$-peptide level of $0.165 \mathrm{nmol} / 1$. A hyperglycemic clamp (BG clamped at 8 and 32 $\mathrm{mmol} / \mathrm{l}$, both for $2 \mathrm{~h}$ ) demonstrated a 2.75 -fold elevated insulin level (491 IU// at the end of the clamp) compared to controls (mean (SD) $180 \pm 45 \mathrm{IU} / \mathrm{l}$ ) with a preserved first phase insulin secretion. In the grandmother, plasma insulin at the end of the clamp (233 $\mathrm{JU} / \mathrm{l})$ was not different from controls. A $C$-peptide suppression test revealed a moderately elevated C-peptide level $(0.212 \mathrm{nmol} / \mathrm{l}$, ref. $<0.080 \mathrm{nmol} / \mathrm{l})$ and a blunted counterregulatory hormonal response in the grandmother. Pedigree analysis did not show consanguinity and suggested that 4 other children of the deceased great-grandfather and 2 aunts of the propositus also had hypoglycemia. It is concluded that this family has autosomal dominant hyperinsulinism. Excessive insulin secretion appears to vary considerably among cases. Impaired counterregulatory response, consequently to repeated hypoglycemia, may contribute to hypoglycemia when insulin secretion is only slightly increased. The genetic defect in this family is currently under study.

\section{3}

FASTING AND $I N$ VIVO HUMAN INSULIN RELEASE PROCESSES.

T. Grøfte, N. Pørksen, C. Juhl, J. D. Veldhuis, S. M. Pincus, and O. Schmitz. Aarhus, Denmark; Charlottesville, VA, 22908, USA; Guilford, CT, 06437 Insulin is released as secretory bursts superimposed on basal release. Overall contribution of secretory bursts is recently quantitated as at least $75 \%$, and main regulation of insulin secretion is through perturbation of mass and frequency of these secretory bursts. The mode of delivery of insulin into the circulation seems important for insulin action, and therefore physiological conditions that alter the pattern of insulin relase may affect insulin action through this mechanism. In order to assess the mechanisms by which fasting changes the mass, frequency, amplitude and overall contribution of pulsatile insulin secretion as well as to investigate the orderliness and insulin glucose interplay at fasting we used a recently validated deconvolution model and the novel Approximate Entropy and Cross Approximate Entropy to examine the above during 10 and 58 hours of fasting in 7 healthy subjects. The subjects were studied for 40 minutes before $(0-40)$ and 40 minutes during (70-110) a glucose infusion $(2.5 \mathrm{mg} / \mathrm{kg} / \mathrm{m})$. We found that the pulsatile insulin release pattern was preserved, and that at fasting, overall insulin release is adusted to needs by reduced mass $(10.1 \pm 1.7$ vs $16.0 \pm 3.2, \mathrm{p}<0.05)$, but similar frequency ( $6.3 \pm 0.4$ vs $6.1 \pm 0.4 \mathrm{~min} /$ pulse) of the bursts. In both states glucose infusion caused increased $(p<0.05)$ mass $(100-200 \%)$ and frequency $(\sim 20 \%)$. The orderliness of the insulin release process (likely reflecting the tightness of the cyclic metabolic processess that drive oscillatory insulin release) was improved at long term fasting ( $0.98 \pm 0.07$ vs $1.17 \pm 0.03$, ApEn arbitrary units, $p<0.01$ ). Furthermore, the orderliness of insulin to glucose changes may reflect the ability of insulin to control glucose concentrations and hence reflect insulin sensitivity. This insulin-glucose interplay evaluated by croos Approximate Entropy was significantly impaired at the matched glucose in the fasting (insulin resistant) state, suggesting a novel application of this mathematical approach to evaluate physiology and pathophysiology.

\section{4}

SEASONAL VARIATION OF HbA1c IN IDDM CHILDREN. A POPULATION STUDY 1994-1996.

Nordfeldt S, Ludvigsson J, Div of Pediatrics, Dept of Health and Environment, University Hospital, Linköping, Sweden Seasonal variation of $\mathrm{HbAlc}$ has been demonstrated in conventionally treated IDDM subjects. Such variation may have both clinical and scientific implications. Does multiple insulin therapy with active self-control prevent seasonal variation of HbAlc?

Patients and Methods: A geographic population of 120 IDDM patients aged 3.0-18.9 years (median 15.0), with age at onset 0.515.6 years (median 6.6) and duration 2.0-16.6 years (median 5.3) registered their HbAlc at visits every 2-4 months during 1994-96, altogether 918 determinations during 277 patient years.

Results: HbAlc decreased in springtime and increased in autumn.

\begin{tabular}{|lccc|}
\hline & no. of samples & HbAle mean \pm SD & range \\
Feb-April & 228 & $7.5 \pm 1.2$ & $4.7-11.9$ \\
May-Aug & 255 & $7.3 \pm 1.1$ & $4.6-11.1$ \\
Oct-Dec & 262 & $7.6 \pm 1.2$ & $4.7-12.3$ \\
\hline
\end{tabular}

Differences were significant between Feb-April and May-Aug $(\mathrm{p}=0.020)$ as well as between May-Aug and Oct-Dec $(\mathrm{p}=0.002)$ using Mann-Whitney U-test. Lowest values were seen in May and August months, and the highest in March

Conclusion: A seasonal variation of HbAlc can be detected on a population level also in intensively treated IDDM children. This should be considered in short-term studies of metabolic control. The tendency to decreasing HbAlc in spring season, when young subjects increase outdoor physical activity, needs attention with regard to increased risk for severe hypoglycemia. 


\section{5}

ORDERLINESS OF INSULIN SECRETION IS ENHANCED IN NON INSULIN DIABETES MELLITUS (NIDDM) FOLLOWING OVERNIGHT BETA CELL REST.

L.L. Kjems ${ }^{1}$, T. Laedtke', N. Pørksen ${ }^{3}$, O. Schmitz ${ }^{3}$, J.D. Veldhuis ${ }^{4}$ and P.C. Butler' University of Edinburgh, Scotland ${ }^{\prime}$; University of Aarhus, Denmark ${ }^{3}$ and University of Virginia Charlottesville', USA

Insulin secretion is abnormal in patients with NIDDM. One of the earliest measurable abnormalities of insulin secretion is increased disorderliness of insulin concentration profiles with time. The orderliness of hormone concentrations in plasma depend in part on intact feedback mechanisms between hormone secretion and action. Since both insulin secretion and insulin action are abnormal in patients with NIDDM, it is not surprising that a highly specific and sensitive statistical measure of orderliness, approximate entropy (ApEn), detects abnormal and highly disordered insulin concentration profiles during modest hyperglycemia in first degree relatives of patients with NIDDM. One possible cause of disordered insulin secretion in NIDDM is chronically increased stimulation of $\beta$ cells. To address this, we measured orderliness of insulin secretion in NIDDM before and after $\beta$ cell rest and in matched controls before and after $\beta$ cell stimulation. 11 NIDDM patients were studied, once after overnight hyperglycemia $(8 \mathrm{mmol} / \mathrm{l})$ achicved by a glucose and $0.9 \% \mathrm{NaCl}$ infusion (SAL) and on a second occasion after concurrent overnight hyperglycemia and $\beta$ cell rest achieved with somatostatin (SMS) and hormone replacement which were discontinued shortly before study $(7: 00 \mathrm{am})$. Blood was then sampled at 1 minutes intervals for 60 minutes for insulin assay while plasma glucose was clamped at 8 mmol/l during both SAL and SMS. 6 controls were similarly studied, once at $4 \mathrm{mmol} / \mathrm{l}$ and on a second occasion at $8 \mathrm{mmol} / \mathrm{l}$. The resulting insulin concentration profiles were subject to analysis for approximate entropy. Results: Disorderliness decreased in NIDDM patients after overnight $\beta$ cell rest (ApEn $1.42 \pm 0.01$ vs $1.377 \pm 0.04, \mathrm{p}<0.05$ ). In contrast, disorderliness increased in control subjects during $\beta$ cell stimulation by hyperglycaemia $(8 \mathrm{mmol} / \mathrm{l})$, with the consequence that stimulated $\beta$ cells in control subjects secreted insulin with a comparable degree of disorderliness to that observed in patients with NIDDM (8 mmol/l) prior to $\beta$ cell rest $(1.42 \pm 0.01$ vs $1.42 \pm 0.01$, NS) Conclusions: We conclude that the increased disorderliness of insulin secretion in patients with NIDDM is, at least in part, a function of the chronic stimulation of $\beta$ cells.

\section{7}

B-CELL REST IN TYPE 2 DIABETES - EFFECTS ON INSULIN-SECRETION AFTER DIAZOXIDE AND INSULIN

M. Guldstrand, U. Adamson, P.-E. Lins and V. Grill*, Division of Internal Medicin Danderyd Hospital and Department of Endocrinology and Diabetology, *Karolinska Hospital, Stockholm, Sweden

In type 2 diabetes the first-phase glucose-induced insulin secretion is low while the second-phase usually is exaggerated.The latter is probably explained by a potentiating effect by chronic hyperglycemia. Our aim of this study was to inhibit insulin secretion to test the hypothesis if pancreatic rest could influence the insulin secretory capacity. Eight patients (4 men), age 53 (48-59) years, BMI 33.2 $\mathrm{kg} / \mathrm{m}^{2}(28.2-41.9)$, HbAlc $8.9 \%(8.2-9.5$, ref. $<5.2 \%)$ with a diabetes duration of 13.2 (8-23) years were included in an open randomised cross-over study. Oral antidiabetic therapy was discontinued temporarily two days before study start. B-cell rest was induced in two ways 1) by long-time ( 90 hours) euglycemic clamp alone and 2) by additional diazoxide treatment $100 \mathrm{mg} \mathrm{x} 3$ for 3 days. Insulin response was studied day 1 and 5 after intravenous arginine at start (pulse 1) and after glucosinfusion at $\min 90$ (pulse 2) raising the blood glucose about 10 $\mathrm{mmol} / \mathrm{h}$. Baseline c-peptide levels were reduced after insulin alone and after insulin+diazoxide $(1.00 \pm 0.11, \mathrm{vs} 0.48 \pm 0.27 \mathrm{nmol} /, \mathrm{p}<0.001$ and $0.92 \pm 0.24$, vs $0.57 \pm 0.28 \mathrm{nmol} / \mathrm{l}, \mathrm{p}<0,009$, respectively; mean $\pm \mathrm{SD})$. Baseline glucagon levels were also reduced $(187 \pm 88$, vs $128 \pm 24 \mathrm{nmol} / \mathrm{h}, \mathrm{p}<0.04$ and $155 \pm 63$, vs $113 \pm 68$ nmol/l, $\mathrm{p}<0.02$, respectively; meant $\mathrm{SD}$ ). Insulin release after arginine (pulsel) was reduced after insulin $(-56 \% ; p=0.02)$ as well as after insulin $+(-47 \% ; p=0.10)$. First-phase insulin (pulse 2) was slightly decreased after insulin $(-8 \% ; p=0,99)$ but increased $(+44 \% \mathrm{p}=0.04)$ after insulin+diazoxide. There were improvements in insulin release after insulin+diazoxide from day 1 pulse 1 to pulse 2 day $5(+40 \%$ $\mathrm{p}=0.03)$, and after insulin alone $(+25 \% \mathrm{p}=0.10)$. The second-phase insulin secretion was increased after insulininfusion but after insulin+diazoxide there was a tendency to reduction. We conclude that after diazoxide given in addition to insulin therapy first-phase insulin secretion capacity appears to be improved. This pilot study encourages further and extended trials exploring long term effects after $\beta$-cell rest on metabolic control in type 2 diabetes.

\section{6}

QUANTITATIVE INDICES OF BETA-CELL FUNCTION DURING GRADED GLUCOSE INFUSION

G. Toffolo, ${ }^{\wedge}$ M. K. Cavaghan, ^K.S. Polonsky and C. Cobelli. Department of Electronics and Informatics, University of Padova, Italy and ${ }^{\wedge}$ Department of Medicine, The University of Chicago, Chicago, Illinois, USA.

Availability of quantitative indices of insulin secretion in an individual is important to monitor $\beta$-cell function over time and to compare different groups with impaired glucose tolerance. They are currently evaluated from the Cpeptide minimal model from i.v. glucose tolerance test (TVGTT) data, i.e during a gross glucose perturbation. However, the response of $\beta$-cells to a more physiological slowly increasing/decreasing stimulus may uncover novel features of $\beta$-cell function. Here, plasma $\mathrm{C}$-peptide and glucose concentrations from a low-dose graded glucose infusion protocol (seven 40min periods: 0-4-8-16-8-4-0 $\mathrm{mg} \mathrm{kg} \mathrm{min}^{-1}$ ) in 7 normal subjects (age $=27 \pm 4 \mathrm{yr}$, body weight $=68 \pm 10 \mathrm{~kg}$ ) were analysed. A model of insulin secretion assuming a linear control of glucose, used in conjunction with a two compartment model of C-peptide kinetics, failed to describe the data. A model also including a control of the rate at which glucose increases performed satisfactorily. The model provided pancreatic insulin secretion (ISR) in each individual, and three indices of pancreatic sensitivity to glucose: $\Phi_{s}, \Phi_{b}$ and $\Phi_{b}$ which quantify respectively the control exerted on ISR by the above-basal glucose level (static control), by the rate at which glucose increases (dynamic control), and by basal glucose. The insulin sensitivity index $S$, was also estimated from glucose and insulin data of this protocol by using the glucose minimal model. Indices were (MeantSD): $\Phi_{\mathrm{s}}=17.5 \pm 4.9\left(10^{9} \mathrm{~min}^{-1}\right) ; \Phi_{\mathrm{d}}$ $=319 \pm 220\left(10^{9}\right) ; \Phi_{b}=4.6 \pm 1.3\left(10^{9} \mathrm{~min}^{-1}\right) ; \mathrm{S}=5.3 \pm 4.3\left(10^{4} \mathrm{~min}^{-1} /\left(\mu \mathrm{Uml}^{-1}\right)\right)$.

In conclusion, a novel approach to quantify $\beta$-cell function has been proposed. Since insulin sensitivity can be estimated from the same protocol, it also allows an easy and straightforward normalization of $\beta$-cell function indices to insulin sensitivity. This normalization appears mandatory in assessing $\beta$-cell function in pathophysiological and drug therapy studies.

\section{8}

PREVALENCE OF HEPATITIS C VIRUS ANTIBODIES IN PATIENTS WITH DIABETES MELLITUS: RELATION TO NOSOCOMIAL TRANSMISSION

JM. PETIT, JB. BOUR, S. AHO, A. CASTANEDA, S. RUDONI, C DAMATTE, G. VAILLANT, B. VERGES, JM. BRUN. Hopita universitaire, Dijon, France.

The association of diabetes mellitus (DM) and hepatitis $\mathrm{C}(\mathrm{HCV})$ has been recently described. In several reports, prevalence of DM in patients with $\mathrm{HCV}$ was around $20 \%$ and significantly higher compared with other types of chronic liver disease. One study found a higher prevalence of HCV $(11.5 \%)$ in diabetic patients in comparison with blood donors. Moreover, outbreaks of hepatitis virus attribuable to finger stick devices for capillary blood sampling have been reported The possibility of patient-to-patient $\mathrm{HCV}$ transmission during hospital admissions in diabetic units must be evaluated. The objective of this study is to evaluate the prevalence of HCV infection in diabetic population and to investigate the influence of nosocomial transmission. We investigate hepatitis $\mathrm{C}$ virus markers in 248 patients with diabetes mellitus in comparison with 14100 blood donors. HCV was detected in 8 diabetic patients $(3.2 \%)$. A higher prevalence of $\mathrm{HCV}$ infection was observed in diabetic patients in comparison with blood donors $(3.2 \% \mathrm{vs} 0.04 \%, p<0.01$ ). Most of anti-HCV positive patients ( 7 of 8 , or $87.5 \%$ ) presented a main risk factor of $\mathrm{HCV}$ transmission with a previous blood transfusion (3), a previous major surgical procedure (3) or a professional transmission (1). In this study none of the patients had an intravenous drug addiction. Previous hospital admissions in diabetologia departement, or used of finger stick devices for capillary blood sampling was not associated with a increased prevalence of HCV infection. In conclusion: We find a increased prevalence of HCV antibodies in patients with diabetes mellitus. A screening of $\mathrm{HCV}$ antibodies in diabetic population with prior blood transfusion or previous major surgical procedure could be recommended. This study was unable to demonstrate a responsability of the use of finger stick devices for capillary blood sampling for the higher prevalence of $\mathrm{HCV}$ infection in diabetic population. 


\section{9}

ISLET CELL AND AUTOANTIBODIES PREVALENCE AND CHANGES IN RESPONSE TO INTERFERON $\alpha$ IN PATIENTS WITH CHRONIC VIRAL HEPATITIS.

S. Piquer ', C. Hernandez ${ }^{2}$, J. Enríquez ${ }^{1}$, A. Ross', J.Genescá ${ }^{2}$, E. Bonifacio ${ }^{3}$, R. Simó ${ }^{2}$, M. Puig-Domingo ${ }^{1}$

1. Hospital de Sant Pau. Barcelona (Spain); 2. Hospital Vall d'Hebrón.

Barcelona (Spain); 3. Istituto Scientifico San Raffaele. Milan (Italy)

We have previously reported a higher than expected prevalence of virus $C$ chronic hepatitis (HVC) infection in diabetic patients than in blood donors matched by recognized risk factor to acquire HVC infection $(15 \%$ vs $2.5 \%$ control population). Furthermore other autors have demonstrated a high prevalence of diabetes mellitus among HVC. Also type I diabetes has been reported to develop in patients treated with interferon $\alpha$. The aim of our work was to explore the possible involvement of autoimmune $\beta$ cell damage in a large cohort of HVC. We studied the prevalence of islet cell autoantibodies in 231 non diabetic HVC and 273 sex and age matched controls. GADab were positive in 4/231 in HVC patients and one control while IA2ab were negative in all of them. Forty-six HVC patients were treated for 24 weeks with interferon and islet autoantibodies measured at the end and 6 months after the treatment; either GAD and IA2 abs remained negative at both time points in all patients while thyroid abs became positive in $5 / 46(9.5 \%)$. Additionally, in a group of unselected HVC diabetic patients (mean age at diagnosis $45 \pm 5$ ) only 1/26 showed low titre islet cell abs. We conclude that islet cell autoimmunity is not associated to HVC, nor to interferon alfa treatment in this large group of patients. It is suggested that the excess of prevalence of diabetes mellitus among HVC patients is probably related to an impaired insulin action/secretion associated to a chronic infection by virus $\mathrm{C}$ hepatitis.
870

POST-TRANSPLANTATION DIABETES IN KIDNEY TRANSPLANT RECIPIENTS

Bastos M, Baptista C, Rodrigues D, Ruas L, Alves R, Freitas L, Bastos C, Carvalheiro M, Mota A, Furtado L and Ruas M Departments of Endocrinology and Diabetes, Urology and Transplantation. Nephrology and Dialysis. University Hospital. Coimbra. PORTUGAL

Post-transplantation diabetes is known since Starzl 1964. Steroids, ciclosporine and genetic are the main causes. Aim: to evaluate the prevalence of post-transplantation diabetes in transplanted kidney recipients and their evolution. Patients and Methods: 725 kidney transplants (1980-Oct1997). Excluded 32 diabetics. Studied 693 patients. Diagnosis (OMS/ADA): two fasting plasma glucose (FPG) $\geq 140 \mathrm{mg} / \mathrm{dl}$ or an $2 \mathrm{H}$ OGTT glucose $>200 \mathrm{mg} / \mathrm{dl}$ or requiring insulin treatment. All patients received a cadaver kidney (Tx), triple immunossupression: azathioprine (AZA)+cyclosporine (CyA)+steroids (ST); Glucagon Test (1mg/IV); Statistic mean+2SD, Actuarial survival Wilcoxon. Results:68 post-transplant diabetic patients

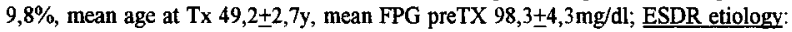
unknown $41,2 \%$, Glomenulonephritis $17,6 \%$, Policystic D. 16,2\%,Pyelonephritis C $13,2 \%$, Nephrosclerosis $8,8 \%$, others $3 \%$. Diabetes in the family in $33,8 \%$. Mean time of dialysis $47,7 \pm 11,9$ months $(\mathrm{m})$, mean time $\mathrm{Tx}$ to diagnosis $14,1 \pm 6,3 \mathrm{~m}$. At diagnosis: FPG 224,5 $\pm 31,3 \mathrm{mg} / \mathrm{dl}$; IMC 28,4+1,6; serum creatinine $1,5 \pm 0,2 \mathrm{mg} / \mathrm{dl}$; Total steroids dose $7,2 \pm 2,3 \mathrm{gr}$; Total AZA dose $22,5 \pm 10,9 \mathrm{gr}$; CyA dose 4,8 $\pm 0,6 \mathrm{mg} / \mathrm{kg} /$ day; Glucagon Test: PepC 0, 3,3+0,7 $\mathrm{ng} / \mathrm{ml}$ at $6,5,2 \pm 1 \mathrm{ng} / \mathrm{ml}$. Treatment: $72,1 \%$ started insulin and $100 \%$ diet and blood glucose monitoring. Now 56 patients are followed with functioning graft (mortality $11,8 \%$, graft lost $17,6 \%$ ). On insulin $57,1 \%$, diet and blood glucose monitoring $100 \%$ with a HbAlc $7+0,4 \%$; mean serum creatinine $1,4 \pm 0,2 \mathrm{mg} / \mathrm{dl}$; Steroid dose $6,7 \pm 0,9 \mathrm{mg} / \mathrm{d}$; Cya $3,1 \pm 12,3$ $\mathrm{mg} / \mathrm{kh} / \mathrm{d}$; mean diabetes duration $43,1+12,3 \mathrm{~m}$. Complications: background retinophathy 3, vascular 13, infection 19 , neoplasia 5 , rejection 25 , urological 14 Actuarial patient survival a) Non diab.: $1 Y 94,75 \%$, 5thY $86,46 \%$ and $10^{\text {th }} \mathrm{Y} 76,15 \%$ b) Post-t. diab.: 1stY 95,49\%, 5thY 93,27\%, 10thY68,3\% ( $p=0,183)$. Actuarial graft survival a) Non diab. 15 tY $90,39 \%$, 5thY $76,2 \%$ and $10^{\text {th }}$ y $60,34 \%$ b) Post-t. Diab. 1stY $94,3 \%$, 5thY $85,2 \%$ and 10 thY $62,26 \%(p=0,193)$. Conclusions: prevalence of post-transplantation diabetes was $9,8 \%$ in our Center. Good results needs a multidisciplinary team

\section{1}

CLINICAL AND GENETIC FINDINGS IN THE WOLFRAM (DIDMOAD) SYNDROME.

D. Cucinotta and F. De Luca on behalf of the Italian Study Group on the Wolfram Syndrome, University of Messina Medical School,Messina,Italy

The Wolfram Syndrome (WS) or DIDMOAD is a genetic disorder in which diabetes mellitus (DM) is associated with diabetes insipidus (DI), optic atrophy (OA), deafness (D) and, less frequently, with urinary tract abnormalities, gonadal atrophy and neurological findings. It has been suggested that mitochondrial DNA alterations can be involved in the aetiology of this syndrome, but data are controversial. We abstracted and reviewed the medical records from 18 patients (11 females,mean age 19.8 \pm 7 years) with at least 2 of the 4 most salient WS clinical features,recruited from 10 Italian clinics. Mitochondrial DNA from the patients' blood was examined for the 3243 tRNA-Leu point mutation and the 7.6 and $8.5 \mathrm{~Kb}$ deletions, which have been previously reported in some WS patients. An insulin-requiring DM was the first-appearing clinical manifestation of the disease in all the patients but one, with a mean age at diagnosis of $13.5 \pm 4.7$ years. It was also the most frequent one $(100 \%)$, followed by OA $(89 \%)$, D $(83 \%)$, DI $(55 \%)$ and urinary tract dilatation $(50 \%)$. Other less common clinical features were primary hypogonadism $(11 \%)$, megaloblastic anemia (5\%) and epilepsy (5\%). The 4 cardinal features of WS were present at the same time in 9 patients $(50 \%$ ), while in other 5 patients $(28 \%) 3$ major components of WS were observed. The mitochondrial DNA 3243 tRNA-Leu mutation and 7.6 or $8.5 \mathrm{~Kb}$ deletions were not detected in the blood of any patient. These data demonstrate that 1) WS has a variable clinical expressivity, with a constant feature represented by DM; 2) due to their frequency,urinary tract abnormalities should be included among the main clinical findings of the syndrome and 3) mitochondrial DNA alterations do not seem primarily involved in the aetiology of the disease. 


\section{Devices}

\section{2}

A THREE-MONTH, MULTICENTER CLINICAL TRIAL OF THERAPY WITH INHALED HUMAN INSULIN IN TYPE 2 DIABETES MELLITUS W.T. Cefalu, R.A . Gelfand, and I.A. Kourides for the Inhaled Insulin Phase II Study Group. Winston-Salem NC, Groton CT, and San Carlos CA, USA. A new dry powder insulin (I) formulation and aerosol delivery system allow reproducible dosing of rapid-acting I by inhalation. Therapeutic quantities of $I$ are delivered to the systemic circulation with 1-2 inhalations per dose. To compare the efficacy and safety of an inhaled I (INH) with a conventional injection regimen (SC), 51 patients with type 2 diabetes from 10 study sites were randomly assigned, after a 1-mo run-in, to $\mathbb{N H}$ or $\mathrm{SC}$ treatment for $3 \mathrm{mo}$. INH patients received pre-meal INH plus bedtime injected Ultralente I; SC patients continued their pre-study I ( $2-3$ injections daily). Baseline $\mathrm{HbA}_{\mathrm{ic}}$ was $8.7 \pm 1.4 \%$ in INH and $7.8 \pm 0.9 \%$ in SC. QID home monitoring was reviewed weekly for all patients and I adjusted to target pre-meal glucose of $100-160 \mathrm{mg} / \mathrm{dl}$. Glycemic control, evaluated by the 3-mo decrement in $\mathrm{HbA}_{1 \mathrm{c}}$, was the same in INH $(-0.7 \pm 0.7 \%)$ and SC $(-0.7 \pm 0.7 \%)$ patients. Weight gain in the 3-mo study was less for INH $(-0.4 \pm 3.0 \mathrm{~kg})$ than for $\mathrm{SC}(1.1 \pm 1.8 \mathrm{~kg})$ $(p=0.04)$. Mild-moderate, but not severe, hypoglycemia occurred in both groups ( 0.8 episodes per subject-month in INH and 1.0 in SC). Results of a patient satisfaction questionnaire significantly favored $\mathrm{NNH}$, and $92 \%$ of patients treated with $\mathbb{I N H}$ chose to continue in a 1-yr extension of $\mathbb{I N H}$ treatment. Pulmonary function tests (spirometry, lung volumes, and diffusion capacity) were unchanged after 3 mo of INH therapy. In conclusion, I treatment with INH was well tolerated with little hypoglycemia and no weight gain, well liked by patients, and as efficacious as $\mathrm{SC} I$ in managing type 2 diabetes .

\section{3}

ADD-ON THERAPY WITH INHALED HUMAN INSULIN IN TYPE 2 DIABETIC PATIENTS FAILING ORAL AGENTS: PRELIMINARY RESULTS OF A MULTICENTER TRIAL. *S Berger, MH Davidson, I Kourides, WH Landschulz and RA Gelfand for the INHALED INSULIN PHASE II STUDY GROUP, Chicago IL, Groton CT, San Cartos CA.

A new dry powder aerosol delivery system has been developed that allows reproducible inhalation dosing of regular human insulin (I). To examine whether this short-acting I delivery system can offer therapeutic benefit in a no-injection regimen, we investigated whether type 2 diabetics failing oral agent (OA) therapy can improve glycemic control by adding pre-meal inhaled insulin (INH) to their usual $\mathrm{OA}$. Eligibility required $\mathrm{HbA}_{1 \mathrm{c}}>8.0 \%$ despite therapeutic doses of a sulfonlurea (e.g., $\geq 5 \mathrm{mg} / \mathrm{d}$ glyburide) and/or metformin $(\geq 1.5 \mathrm{~g} / \mathrm{d})$. After a 1-mo run-in, 60 subjects from 10 sites were randomized to a 3 mo treatment period of either continued usual $O A$ alone or usual $O A$ plus INH, 1-2 puffs pre-prandially TID. INH doses were titrated based on QID glucose testing. Interim $\mathrm{HbA}_{1 \mathrm{c}}$ results are shown for the first 22 patients to complete 8 wks of randomized therapy:

\begin{tabular}{|c|c|c|c|c|c|c|}
\hline Therapy & & Baseline & Wk 8 & $\Delta$ Wk 8 & Wk12 & $\Delta$ Wk12 \\
\hline INH & Mean (\%) & 10.2 & 8.3 & -2.0 & 7.2 & -2.6 \\
\cline { 2 - 7 } plus OA & SEM [N] & $0.4[10]$ & $0.3[10]$ & $0.4[10]$ & $0.3[5]$ & $0.8[5]$ \\
\hline \multirow{2}{*}{$\begin{array}{c}\text { OA } \\
\text { Alone }\end{array}$} & Mean (\%) & 10.1 & 9.6 & -0.4 & 9.8 & +0.2 \\
\cline { 2 - 7 } & SEM [N] & $0.5[12]$ & $0.4[12]$ & $0.3[12]$ & $0.5[10]$ & $0.2[10]$ \\
\hline
\end{tabular}

Starting with comparable, suboptimal baseline glycemic control, patients on $\mathrm{OA}$ alone showed little change in $\mathrm{HbA}{ }_{1 \mathrm{c}}$ after 8-12 wk, while those receiving INH add-on therapy showed a marked $(-2 \%)$ improvement in $\mathrm{HbA}_{1 c}(p<.05)$. INH has been very well tolerated. There have been no episodes of severe hypoglycemia. These early results suggest that in patients with type 2 diabetes failing oral agents, adjunctive therapy with INH markedly improves glycemic control with low risk of hypoglycemia. Data from the complete study will be presented.

\section{4}

PRLIMINARY STUDY OF ORAL POLYLACTIDE MICROCAPSULATED INSULIN IN VITRO AND IN VIVO.

Ma Xue Yi, Pan Guo Min, Lu Ze et al. Beijing304 hospital of PLA; Institute of chemistry, the Chinese Academy of Science, Beijing, China.

Abstract:[Aim]:Oral insulin(ins) administration is the ideal delivery way for all ins-injected DM patients. But it will be quickly denatured and degraded in gut. We prepared polylacted(PLA) to microencapsulate the regular ins(RI) to prevent above destroyed procedure.[Method]:PLA was synthesized from $L$ lactid by ring-opening polymerization. Pork RI was used for microencapsulation.30 STZ-DM rats were gavagely fed by oral capsulated RI of doses of 33u,50u, 60u, 80u respectively. Their BGs were measured at 15,30 min and per hour until 10h after gavage. Uncapsulated RI was fed in 5 DMrats as control.[Results]:The average capsulated rates for $R I$ were $82 \%, 93 \%$ and $44 \%$ when average diameter of microcapsulated ins were $1.2-2 \mu \mathrm{m}, 2-5 \mu$ m and 10-20 $\boldsymbol{\mu} \mathrm{m}$ respectively. In vitro microcapsulated RI comulative release were $38 \%, 58 \%, 65 \%$ and $74 \%$ respectively in $2,6,8,10 \mathrm{~h}$. The average $\mathrm{BG}$ were reduced by $28 \pm 7.7 \%, 36 \pm 17 \%, 54 \pm 14 \%$ and $78 \pm 21.2 \%$ in 30 DM-rats with dose of $30 \mathrm{u}, 50 \mathrm{u}, 60 \mathrm{u}$ and $80 \mathrm{u}$. The peak time of capsulated ins releasing was at $6-7 \mathrm{~h}$ in vitro,but in vive it was at $7-8 \mathrm{~h}$. [Discussion]:PLA microcapsulated ins sustained release ins in vitro and in vivo. The effects for BG decrease were as high as $\mathbf{7 8} \pm \mathbf{2 1 . 2 \%}$ during 8 h.PLA microcapsulated ins may prevent ins from denature and degrade by enzyme in gut. It may be a hopeful oral ins preparation.

\section{5}

BETTER LONG TERM GLYCEMIC CONTROL IVITH CONTINUOUS SUBCUTANEOUS INSULIN INFUSION.

$D$. Bell and F. Ovalle. The University of Alabama at Birmingham, Alabama. USA

Intensive insulin therapy with achievement of near normoglycemia has been proven to decrease microvascular complications in type 1 diabetes. So far there have been no long term studies comparing long term glycemic control by means of insulin pump therapy (CSII) with multiple daily injections (MDI) in routine clinical practice. Te performed a retrospective analysis on all active patients $(n=90)$ on CSII of one of the authors. The primary end point of the study was to compare the average glycemic control (as documented by frequent measurements of glycated Hgb), during the three year period prior to initiation of CSII vs the average glycemic control during the 3 year period following the first year of CSII. We included all patients for whom we had data on for at least one year of each of those periods (pre and post CSII) and only those patients who had been on a MDI prior to CSII $(n=58)$. A1C's for the year of initiation of CSII were not included. The mean $\mathrm{Hgb} \mathrm{Alc}$ for the 3 year period prior to CSII (on MDI) was $8.4 \%(+/-0.1 \mathrm{SEM})$ vs a mean $\mathrm{Hgb}$ A1c of $7.7(+/-0.1$ SEM) for the 3 year period following the first year of CSII. This $0.7 \%$ improve ment in $A 1 \mathrm{C}$ is statistically significant $(\mathrm{p}=0.001)$ We conclude that intensive insulin therapy with CSII improves glycemic control even in patients that are already utilizing MDI. 
876

ARE INSULIN KIINETICS REPRODUCIBLF, AFTER LONG-TERM INTRAPERITONEAL INSULIN TREATMENT IN PATIENTS WITH TYPE I DIABETES V. I.ASSMANN-VAGUF, C. AI.ESSIS, Ph. VAGUE and P. BELICAR, University Timone Hospital - Marseille - FRANCE

Insulin bioavailability after subcutaneous injection is not very reproducible and this accounts at least partly for difficulties in tight-control diabetes. In order to assess reproducibility of IP infused insulin kinetics, we studied on two occasions 11 patients (age: $46.3 \pm 9$ years $(\mathrm{m} \pm \mathrm{SD}$ ) ), with type 1 diabetes (mean duration: $21 \pm 6$ years), treated by an implantable programmable pump (Minimed MIP 2001) infusing 21 PH Hoeclist* insulin intraperitoneally. Insulin kinetics were studied by measuring frec plasma insulin levels every 30 minutes (after the meals) or 60 minutes, on two separated days, with an interval varying from 6 to 60 months. Insulin doses ( basal rate and boluses ) were similar on the two days. Results were compared using Student's test and ANOVA for paired values. Nocturnal and fasting fnee insulin levels were not different (mean \pm sem: $8 \pm 0.9$ versus $7.3 \pm 0.5 \mathrm{mU} / \mathrm{L}$ ). After the bolus, time to peak and return to basal level were not significantly different. Conversely, magnitude of the peak was significantly lower on the second day of testing (mean \pm sem : $16 \pm 5.4$ versus $29.5 \pm 8 \mathrm{mU} / \mathrm{L}$ ), as were post-bolus AUC (mean \pm sem: 20 versis $30 \mathrm{mU} / \mathrm{L}^{-1}$ ) and total AUC (mean \pm sem : 58.2 versus 84.3 $\mathrm{mU} / \mathrm{L}^{-1}$ ). Intrapatient coefficient of variation was $32.4 \%$ for the peak, and $25.6 \%$ for post-bolus AUC. Many factors could explain these differences : increased insulin antibody levels, position of the catheter tip, underdelivery of insulin duc to backflow or peritoneal tissue overgrowth. None of them was the only responsible. To eliminate the possible rolc of insulin antibodies and causes of underdelivery, we tested twice 5 newly implantert patients at one month interval.The plasma free insulin peak and post-bolus AUC were not significantly different during the 2 procedures ( respectively $36.4 \pm 7$ vs $28 \pm 8 \mathrm{mU} / \mathrm{L}$ and $35 \pm 14$ vs $30.5 \pm 13 \mathrm{mUhL}^{-1}\left(\mathrm{l}^{\text {rst }} \mathrm{vs} 2^{\text {nd }}\right.$ day of testing) ). Intrapatient coefficient of variation was $19.6 \%$ for the peak and 26 $\%$ for post-bolus AUC. So in diabetic patients treated by IP infused insulin with implantable pumps, insulin kinetics are reproducible. But the magnitude of the peak following the bolus can be altered on a long-term basis. Specific factors related to the technique as the anti-insulin antibody levels and insulin underdelivery by the pump could account for this result.

\section{8}

MULTIPLE DAILY INJECTION VS. INSULIN PUMP: A CRITICAL ANALYSIS IN IDDM BY MEANS OF CONTINUOUS TISSUE GLUCOSE MONTORING

M.I. Salgado, F. Stemberg, U. Hoss, R. Gessler and R Fussgänger. Institute of Diabetes Technology at the University of Ulm, Ulm Germany

Intensified insulin therapy (IIT) may be performed either by multiple daily injection (MDI) regime or by means of an insulin pump (continuous subcutaneous insulin infusion/CSID). Different studies show the benefits of the latter when compared to MDI. However, no study has been yet based on complete glucose profiles to evaluate the metabolic state of both therapy modalities. We designed a study, in order to establish whether if there are any differences between the glucose metabolic state of type 1 diabetic patients undergoing MDI and CSII by means of continuous tissue glucose monitoring (CTGM). We performed a 24h CTGM in 10 $\mathrm{MDI}$ and $10 \mathrm{CSII}$ patients. No statistical difference was observed between the studied groups regarding age, diabetes duration, $\mathrm{BMI}$ and $\mathrm{HbA}_{1 \mathrm{c}}$. The CTGM was carried out with a self developed glucosensor based on the enzymatic-amperometric glucose measurement combined with the microdialysis technique. Patients were asked to perform their usual treatment during CTGM. Additionally, capillary glucose values were obtained as reference. The following results based on the minutely glucose profile provided by the CTGM were obtained for MDI and CSII, respectively: glucose range: $352-29 \mathrm{mg} / \mathrm{dl}$ and $387-57 \mathrm{mg} / \mathrm{dl}$; mean tissue glucose: $127 \pm 22 \mathrm{mg} / \mathrm{dl}$, and $154 \pm 29 \mathrm{mg} / \mathrm{dl}$; mean amplitude of glucose excursion $206 \pm 77 \mathrm{mg} / \mathrm{dl}$ and $248 \pm 66 \mathrm{mg} / \mathrm{dl}$. The hypoglycemia frequency (glucose $<70 \mathrm{mg} / \mathrm{dl}$ ) was 1.1 events/patient/day and 0.9 events/patient/day while a dawn phenomenon was observed in every second patient/day in both MDI and CSIl patients. Our study showed that the MDI group presented a better metabolic state than the CSII group when evaluated by CTGM. These results contradict the actual literature, and could be explained by the "passive" attitude adopted by the CSII patients regarding the insulin pump efficacy. Further studies should be performed to confirm the presented hypothesis.

\section{7}

COMPARISON OF INSULIN LISPRO AND REGULAR INSULIN IN CONTINUOUS INSULIN INFUSION PUMP THERAPY

J.H. Holcombe, S. Zalani, H. Hoen and C. Harris. Eli Lilly and Company, Indianapolis, IN, USA

Insulin lispro (Humalog() (LP) is a phosphate-buffered insulin analog with quicker onset and shorter duration than regular human insulin (HI). These properties suggest that LP may be useful in treating diabetes patients who use insulin pumps. In this open-label, randomized, cross-over study, we compared glucose control between LP and buffered regular insulin (HII)(Velosulin BR@) in 58 patients (ages 14-58 yrs) receiving CSI (MiniMed). After a 2 week lead-in period of treatment with HI, patients were randomized to either continue $\mathrm{HI}$ or to switch to $L P$ for 12 weeks. At that point, patients switched to the alternate insulin for another 12 weeks. Bolus and basal insulin doses were adjusted to optimize control for each patient. Bolus insulin doses were given directly before meals. Test meals (Sustacal $\mathbb{B}$ $360 \mathrm{cal}$ ) were done at 0,12 , and 24 weeks, during which fasting and postprandial ( $\mathrm{PP})$ glucose values were measured. Glucose levels $(\mathrm{mmol} / \mathrm{L}$, mean + sd) at baseline and end of each treatment period are shown.

\begin{tabular}{|l|c|c|c|c|}
\hline & Baseline & Lispro & Regular & $\mathbf{P}^{*}$ \\
\hline $\mathrm{FBG}$ & $8.0 \pm 3.0$ & $8.7 \pm 4.5$ & $\mathbf{8 . 0 + 4 . 0}$ & 0.299 \\
\hline $1 \mathrm{hr}$ PP & $13.2 \pm 3.8$ & $11.1 \pm 4.3^{\mathrm{g}}$ & $13.2 \pm 4.7$ & 0.012 \\
\hline $\mathrm{hr}$ PP & $12.1 \pm 4.2$ & $9.6 \pm 4.1^{\mathrm{b}}$ & $12.5 \pm 4.6$ & 0.001 \\
\hline $\mathrm{HbAl} l_{c} \%$ & $7.74 \pm .97$ & $7.41 \pm .97^{\mathrm{b}}$ & $7.65+.85$ & 0.004 \\
\hline
\end{tabular}

${ }^{*}$ Lispro vs Regular; ${ }^{a} \mathrm{p}=0.012$ vs baseline, ${ }^{b} \mathrm{p}<0.001$ vs baseline

The number of patients reporting severe hypoglycemia ( $\mathrm{SH}$ ) was similar between treatments $(L P=4 ; H I=3)$, for a total $S H$ rate of $24 / 100$ patient years. The rate of tubing occlusion did not differ between the treatments. A questionnaire at study end revealed that $82 \%$ of patients preferred LP, $11 \%$ preferred $\mathrm{HI}$, and $8 \%$ had no preference. We conclude that insulin lispro is safe and effective in CSII therapy, and that insulin lispro significantly lowers $\mathrm{HbAl}_{\mathrm{c}}$ and 1- and 2-hr PP glucose levels compared with regular insulin.

\section{9}

Definition of insulin requirement in type 1 diabetes by continuous subcutaneous infusion of insulin (Insulin Requirement Assessment - IRA method): follow-up after twelve months.

Maghenzani G, Dani F, Grassi G, Monge L, Petraroli G, Pinach S, Benaduce E, Carta Q. - U.O.A. Diabetologia - Azienda Ospedaliera S. Giovanni Battista della città di Torino; Italy.

The insulin requirement of out-patients receiving intensive insulin therapy must be checked regularly. For this purpose, we have devised a method for assessing insulin requirement by means of insulin treatment with continuous subcutaneous infusion. Twenty-one individuals with type I diabetes $(12 \mathrm{~F}$ and $9 \mathrm{M}, \mathrm{C}$-peptide-negative, aged $42 \pm 17$ yrs, duration of diabetes $18 \pm 8$ yrs, BMI $24 \pm 3$, on four daily insulin injections with bedtime intermediate insulin) were subjected to the IRA method while hospitalized in our Division, after which they were followed as out-patients for a year to establish (i) the long-term efficacy in improving glycemic control and (ii) its usefulness in re-setting insulin treatment.

With respect to pre-hospitalization values (HbAlc $9.17 \pm 1.3 \%$ ), plasma glucose control had improved significantly at the two-month stage $(8.5 \pm 1 \%, \mathrm{p}<.001)$ and was maintained after six $(8.39 \pm 1.2 \%, p<.0000)$ and twelve months $(8.5 \pm 1.1 \%, p<.0004)$. The $24 \mathrm{~h}$ insulin requirements $(0.60 \pm 0.1 \mathrm{U} / \mathrm{Kg})$, which had decreased significantly at the time of discharge, was found to increase in the following twelve months but remained lower than pre-admission values $(0.66 \pm 0.13$ vs $0.61 \pm 0.15 \mathrm{U} / \mathrm{Kg} \mathrm{p}<.025)$. Adjustment of the insulin doses according to the daily eating habits led to a slight decrease in the doses prescribed at discharge for breakfast and lunch while the dinner dose progressively increased $(0.180 \pm 0.05 \mathrm{U} / \mathrm{Kg}$ vs $0.191 \pm 0.05 \mathrm{U} / \mathrm{Kg} \mathrm{p}<.0008)$. The substantial decrease in the intermediate bedtime dose was confirmed after twelve months; the requirement for intermediate insulin proved to be $21.6 \%$ of the total insulin dose. The BMI was sinificantly increased at twelve months $(24.13 \pm 2.7$ vs $25.14 \pm 2.8$, p<.0004). During the follow-up period, no patient suffered major episodes of hypoglycemia or ketosis.

These data confirm the validity of the IRA method for the long-term maintenance of glycemic control, despite the decrease in insulin dose, thanks to the re-assessment of the insulin dose and the relationship between regular and intermediate insulin. Our data highlight a decidedly lower bedtime dose of intermediate insulin than that utilized in intonsive treatment schedules. 
RELIABILITY OF INTERSTITIAL GLUCOSE MEASUREMENTS BY SUBCUTANEOUS GLUCOSE SENSORS IN HUMANS

K. Rebrin, G.M. Steil*, W.P. Van Antwerp and J.J. Mastrototaro

MiniMed Inc, Sylmar, CA, *Joslin Diabetes Center, Boston, MA, USA

The accuracy of continuous glucose measurements using subcutaneous (sc) glucose sensors depends on the in vivo calibration of the sensor. For sensors to achieve wide spread use, the calibration must be performed by patients using home blood glucose meters. In the present study, conducted in a hote setting, blood and sensor glucose data were analyzed from 11 diabetic patients on intensive insulin therapy. The sensor was placed sc in the abdominal area for between 72 and 96 hours. The patients calibrated the sensor daily and blood glucose was measured with their glucose meter and YSI at least hourly during daytime. Sensor glucose values were stored every 5 min. Agreement between the glucose sensor (GS) and blood glucose (GB) was assessed as percent difference ( $\% \mathrm{D}=100[\mathrm{~GB}-\mathrm{GS}] / \mathrm{GB})$. The mean $\% \mathrm{D}$ based on patient calibration was less than $2 \%(1.7 \pm 4.2 \%$; not different from $0, p>.05$ ) indicating that the sensor accurately reflects average blood glucose. Further, the Standard Deviation (STD) of the \%D was calculated for each subject, sensor and blood values were within $26.3 \pm 2.6 \%$. Note, in addition to errors in the sensor glucose signal, the STD measure includes a variance component due to the blood meter (estimated to be up to $\pm 15 \%$ ) as well as an unknown error term due to differences between blood and sc glucose dynamics. Finally, to determine if calibrations as performed by the patient could be improved upon, theoretical calibrations of the sensor data based on the best fit linear regression of all the data over a given day were calculated retrospectively. The mean $(-5.3 \pm .8 \%)$ and STD $(25.4 \pm 2.0 \%)$ of the $\% \mathrm{D}$ for these theoretical calibrations were not significantly different from those obtained by the patients ( $p>05$ for both). Thus, the subcutaneous glucose sensor operated with daily patient derived calibrations accurately reflects blood glucose values continuously up to four days and provides much more information about glucose dynamics than occasional glucose monitoring.
DOES EXPOSURE AT 3,000 m AFFECT PERFORMANCE OF GLUCOSE METERS?

O.Pecchio*, C.Rossi, M.Migliardi, M.Deandrea, A.Grassi, D. Fonzo and M.Veglio. Italian Alpine Club Medical Commission*, Dpt of Endocrinology, Ospedale. Mauriziano, Torino, Italy

Self blood glucose (BG) monitoring is mandatory for insulin-dependent diabetic patients who perform exercise at high altitudes where $B G$ meter (BGM) could be not reliable because of changes in temperature, humidity, barometric and partial $\mathrm{O} 2$ pressure. The present study aimed at evaluating the in field performance of 2 reflectance meters at $3000 \mathrm{~m}$ altitude. METHODS: the Lifescan One Touch II (Ortho Diagnostics) (OT) and the Glucometer Elite II (Bayer) (GE) reflectance meters were tested at sea level (SL) and at $3000 \mathrm{~m}$ altitude (AL) on 6 type 1 diabetic patients. BG was simultaneously assessed on a total of 30 capillary samples both at $\mathrm{AL}$ and at SL with OT and GE. A venous blood sample was simultaneously drawn from the contralateral antecubital vein in a sodium fluoride tube, centrifuged and stored at $-20^{\circ}$ : BG was assayed with a glucose oxidase (GO) method within 3 days. This last assessment was taken as reference method. The blood samples were drawn from 7 a.m. till 1 p.m. in fasting, resting, exercise and post-prandial conditions in order to test reliability at different BG levels. Measurement linearity was tested using Pearson's correlation coefficient; the mean of the differences between BGM and GO results represented the bias between the methods while the accuracy was expressed as percent error (PE). RESULTS: Altitude: GE vs GO: $r=$ 0.981 , bias $-4.9 \pm 21.6 \mathrm{mg} / \mathrm{dl}$, PE $8.1 \%$; OT vs GO: $\mathrm{r}=0.985$, bias $13.3 \pm$ $22.7 \mathrm{mg} / \mathrm{dl}$, PE $6.4 \%$. Sea Level: : GE vs GO: $\mathrm{r}=0.960$, bias $5.2 \pm 26.5$ $\mathrm{mg} / \mathrm{dl}$, PE $4.1 \%$; OT vs GO: $\mathrm{r}=0.946$, bias $5.6 \pm 30.1 \mathrm{mg} / \mathrm{dl}$, PE $0.5 \%$ CONCLUSIONS: BGM performance is similar and good at sea level. At a moderate high altitude, similar to that usually attained for winter skiing or summer walking a trend to underestimate BG for GE and to overestimate for OT was observed. However the bias is not clinically meaningful with both BGMs.

\section{2}

BLOOD GLUCOSE MONITORING AND METABOLIC CONTROL IN TYPE 1 DIABETES

AD Morris, DIR Boyle, SA Greene, TM MacDonald and RW Newton for the DARTS/MEMO Collaboration.

University of Dundee, Scotland.

The intensive treated cohort of the DCCT performed self-monitoring of blood glucose at least four times daily but the relationship between blood glucose monitoring and metabolic control is controversial. Using the DARTS/MEMO database we evaluated (i) the extent of home glucose monitoring in the community; and (ii) the relationship between glucose monitoring use and metabolic control. We used all prescriptions (1993 to 1994) obtained from all pharmacy outlets to evaluate the relationships between glucose monitoring equipment obtained (number of strips), age, duration of diabetes, $\mathrm{BMI}$ and glycaemic control ( $\mathrm{HbAlc})$ in an unselected patient group similar to the DCCT cohort. One bundred and eleven patients aged $17 \pm 5$ (mean \pm S.D; range 10-25 years) years, diabetes duration $7.6 \pm 5.5$ years, $\mathrm{HbAlc} 8.8 \pm 1.9 \%$ were studied. Fiftyeight $(52 \%)$ obtained no strips at all over the study period. In the remainder, the mean number of strips obtained was $278 \pm 243$ per annum (range 50-850). There was a significant inverse relationship between strips obtained and $\mathrm{HbAlc}$ which remained when adjusted for all other covariates $(p=0.035$ ). In summary; (i) the majority of young patients with IDDM perform no home glucose monitoring; (ii) the majority of those who do encash prescriptions obtain insufficient strips to perform one glucose measurement per day; and, (iii) no patient encashed sufficient to perform four tests per day, and (iv) there is an association between strips obtained and metabolic control. This must be regarded as a crucial issue in assessing any intensive monitoring regimen.

\section{3}

COMPARATIVE STUDY OF SMALL SAMPLE VOLUME EFFECTS WITH BLOOD GLUCOSE METERS FOR SELF MONITORINO

H.-J. Verlohren, T. Ziegler and Th. Nitsche, local diabetes specialist Leipzig

Reliable systems for blood glucose self monitoring are essential for diabetics to achieve normoglycemic metabolic levels. During ambulant or stationary instruction of blood glucose measurement the patients have to be informed about the relevant safety parameters, especially the glucose value deviations due to blood volume errors. The necessary blood volume (BV) is indicated as the nominal volume or admissible volume by the manufacturer of the meter. The most frequent and misguiding volume error is the use of small sample volumes leading to drastic underestimations of the actual glucose levels.

The blood glucose test systems Accutrend alpha [Aa] / Accutrend Sensor [AS] (Boehringer, Mannheim), Glucometer Elite 2000 [E2] / Glucometer DEX [DE] (Bayer), One Touch II [OT] / Gluco Touch [GT] (Lifescan), and Precision QID [QI] / Precision G [PG] (MediSense) were compared. Different sample volumes of $1.5,2,3.5,5,7,9,15,25 \mu$ l with sodium fluoride stabilised venous blood in the concentration range $8.7-11.3 \mathrm{mmol}$ glucose $/ 1$ were examined in 10-fold replicates. The sample volumes were applied to the different glucose meters with a microliter pipette.

All the evaluated systems delivered reproducible results (CVs of $1.4-4.2 \%$ ) when applying the nominal volume. When small sample volumes were applied incorrect readings occured - particularly too low results or error readings. The range of the sample volumes used was -10 to $-90 \%$ less than those recommended, and this varied according to the type of instrument. Only QI, PG and GT did not deliver false blood glucose results. When a small sample volume was used in the case of $\mathrm{QI}$ and PG the measurement did not start, due to the specially electrode setup. In the case of GT an error message was produced. The evident risks of underestimation of glucose levels by patients due to small sample errors should be taken into account by all manufactures. 
884

Rapid Blood Glucose Meters for Newborn Babies

F. Wehrhoff, H.H. Günter, B. Schneider, F. Dresssler and F. Degenhardt Oststadtkrankenhaus Hannover, Podbielskistr.380, D-30659Hannover

Measuring the blood glucose in newborn babies is an important diagnostic means of monitoring the neonatal metabolism. Since severe hypoglycaemic episodes can lead to neurological damage, blood glucose determinations in the newborn must be reliable, particularly in the region of 1.1-4.4 mmol/l.

Eight rapid blood-glucose instruments were compared (One Touch basic, One Touch II: Lifescan - Johnson \& Johnson; Precision Q.I.D.: Abbott; Glucometer Elite: Bayer Diagnostics; Accutrend Sensor, Reflolux S, Glucotrend: Boehringer Mannheim; and Hemocue-B-Glucose: Mallinckrodt Medical) in respect of their suitability for blood glucose control in newborn babies. To determine the validity situation, comparative measurements were carried out on 390 samples of capillary blood from 55 newbom babies of mothers with gestational diabetes, using the eight rapid-test meters and the glucose dehydrogenase laboratory method (COBAS MIRA S, Hoffmann-La Roche). The coefficients of determination were between 45 and $88 \%$ and the standard error of the methods was between 0.4 and $0.8 \mathrm{mmol} / 1$. The number of measurements outside the range of $0.8 \mathrm{mmol} / 1$ was between 5.1 and $35 \%$. The coefficients of variation for inseries precision were between 6.3 and $23.4 \%$ and the standard deviations were $0.2-0.6 \mathrm{mmol} / \mathrm{l}$. The measured sensitivities and specificities at $2.2 \mathrm{mmol} / \mathrm{l}$, the limiting value for neonatal hypoglycaemia, were respectively between 44 and $98 \%$ and between 85 and $100 \%$. The mean deviation of the methods was between 3.5 and $23.8 \%$. None of the instruments examined satisfied in full the strict measurement accuracy requirements, but there is no doubt about the need for a rapid-test meter to supplement laboratory determinations if optimal monitoring of the newborn is to be ensured. Glucotrend came closest to fulfilling the requirements, giving the closest approximation to the laboratory result. Glucotrend also has the advantage of needing a very small sample volume $(3 \mu \mathrm{l})$ and of the possibility of coating the test strips separately with blood.
885

NEW APPROACHES TO INSULIN PEN DEVELOPMENT ENSURE BENEFITS TO PATIENTS AND HEALTH CARE PROFESSIONALS J.A. Llewelyn, J.K. Malone, J.M. Martin and J. Mailhot, Erl Wood Manor, UK The aim of this study was to assess whether a new approach to insulin pen development would result in benefits to patients and healthcare professionals (HCP). The approach included a number of unique process steps to ensure that important product features were incorporated into the final design of a new 3.0 $\mathrm{mL}$ reusable pen. The acceptability and functionality of the pen was tested in a clinical trial. The 5 to 7 week study in 25 centres in Australia, Canada and the Netherlands, involved 289 type $1(45 \%)$ and type $2(55 \%)$ patients with diabetes. All patients had been administering insulin for a minimum of 3 months using either syringes (25\%), Novopen 3 devices $(73 \%)$ or both $(2 \%)$. Mean patient ag was 45 yrs (range: 13-74 yrs) and mean duration of diabetes was 8.4 yrs (range: 0.3-52.0 yrs). Acceptability of the new device (HumaPen) was assessed by a questionnaire developed using input from a consultative panel of patients and HCPs. At study entry $89 \%$ of device users (DU) and $81 \%$ of syringe users (SU) were satisfied with their current method of insulin administration. At study completion HumaPen features rated most highly were ease of correcting the insulin dose (95\% DU, $97 \% \mathrm{SU})$, reading the dose $(97 \% \mathrm{DU}, 95 \% \mathrm{SU})$, ease of learning ( $91 \% \mathrm{DU}, 95 \% \mathrm{SU})$, ease of changing cartridges ( $81 \% \mathrm{DU}, 87 \% \mathrm{SU})$, and ease of priming ( $73 \%$ DU, $89 \%$ SU). Previous DU thought that HumaPen was easier to correct the dose ( $85 \%)$, read the dose number $(75 \%)$, and change cartridges $(58 \%$ ) compared to Novopen 3 . The Novopen 3 was ranked as a better size by $50 \%$ of DU, however, HumaPen was considered to have a better weight $(62 \%)$ and be more comfortable to hold (51\%). HCPs $(n=38)$ ranked the following features of HumaPen as important when recommending it to patients: can dial back easily without wasting insulin (79\%), easy to read numbers in dose window $(71 \%)$ and easy to learn $(52 \%)$. Although most patients entered the study "satisfied" with their existing device, more than half ( $53 \%$ ) chose to stay on HumaPen after experiencing its features and benefits. This study supports the hypothesis that a development process focused from the onset on tangible patient and $\mathrm{HCP}$ benefits can produce a preferred insulin pen device.

\section{6}

B-D 31 GAUGE $5 \mathrm{~mm}$ PEN NEEDLE IS SAFE AND EFFECTIVE IN CHILD̉REN J. L. Parkes', J. McGonigle', B. Ginsberg', M. Tan'. R. Jamal ${ }^{2}$, 'Becton Dickinson and Co., Franklin Lakes, New Jersey, USA., ${ }^{2}$ Nutri-fit Canada

Shorter, thinner pen needles are being develced for less traumatic insulin injections. To evaluate efficacy, the new Becton Dickinson (B-D) 31 gauge $5 \mathrm{~mm}$ pen needle, compatible with all B-D and Novo insulin pens, was tested in 55 patients, 5-16 years old. The study commenced with a 3-week period with all subjects using the B-D 30 gauge $8 \mathrm{~mm}$ needle followed by two, 3-week random crossover segments using these needles or the B-D 31 gauge $5 \mathrm{~mm}$ investigational needles. Subjects injected regular, NPH, or premixed insulin with their own B-D or Novo insulin pen. Patients "pinched-up" for those injections using $8 \mathrm{~mm}$ needles and did not "pinch-up" when using the $5 \mathrm{~mm}$ needles. Glucose control was evaluated via fructosamine measurements and daily self-monitoring of blood glucose (SMBG) There was no significant difference in fructosamine values from baseline for the $5 \mathrm{~mm}$ vs $8 \mathrm{~mm}$ needles $($ mean $=369 \mu \mathrm{M}, 366 \mu \mathrm{M}$, respectively: $\mathrm{p}=0.43$ ). Painwise $95 \%$ confidence intervals for percentage of SMBG values falling within, above and below target values showed no significant differences. There was no significant difference in number of unexplained hypoglycemic or hyperglycemic episodes during use of the 5 vs $8 \mathrm{~mm}$ needles and no difference in irritation at the injection site. A significantly greater number of subjects reported leakage from the injection site when using the $5 \mathrm{~mm}$ needle $(85 \%)$ than when using the $8 \mathrm{~mm}$ needle $(67 \% ; \mathrm{p}=$ $0.03)$, however blood glucose control was not affected. There was no significant difference in plunger depression pressure between the two needles $(p=0.06)$ or in the perceived pain of injection (rated on a 1-5 scale) between the two needle types ( $\mathrm{p}$-value $=0.53$ ). Fifty-two percent $(52 \%)$ of the children preferred using the $5 \mathrm{~mm}$ needle, $35 \%$ preferred using the $8 \mathrm{~mm}$, and $13 \%$ indicated no preference. The B-D 31 gauge $5 \mathrm{~mm}$ pen needle has been shown to be a medically equivalent alternative for children with diabetes, especially for those fearful of injections, and may be a better choice for those who do not "pinch-up".

\section{7}

PAIN PERCEPTION AND BLEEDING IN DIABETIC CHILDREN WHEN USING DIFFERENT SIZES OF INJECTION NEEDLES

R. Hanas ${ }^{1}$, L. Lytzen ${ }^{2}$, I Franzén ${ }^{3}$, E. Johansson ${ }^{1}$, AS. Karttunen ${ }^{1}$ and J. Ludvigsson ${ }^{3}$. Department of Pediatrics, Uddevalla' and Linköping ${ }^{3}$, Sweden and Novo-Nordisk $\mathrm{A} / \mathrm{S}^{2}$, Denmark.

In this double-blind, randomized, controlled study the aim was to compare pain and bleeding when using different needles. 40 children and adolescents with IDDM aged 8-20 years (mean 15.9) with a diabetes duration of $0-16$ years (mean 6.8) participated. The test products were B-D MicroFine $+\mathrm{G} 29(12.7 \times 0.33 \mathrm{~mm})$ and NovoFine G30 $(8 \times 0.30 \mathrm{~mm})$. NovoFine $\mathrm{G} 28(12 \times 0.36 \mathrm{~mm})$ and a placebo (without needle) were used as reference. The patients received 6 injections ( 1 in thigh and 1 in abdomen of each needle type) with test media and 2 dummy injections at 2 separate study visits. Pain was recorded on a $10 \mathrm{~cm}$ visual analogue scale (VAS) with faces. We found no statistically significant difference in pain perception between the needles but all were clearly separated from placebo (ANOVA $\mathrm{p}=0.0001)$. Pain perception, VAS score $\mathrm{cm}($ mean $\pm \mathrm{SD})$ :

\begin{tabular}{lllll}
\multicolumn{1}{r}{ Needle } & G28 & G29 & G30 & Placebo \\
\hline Abdomen & $2.7 \pm 2.1$ & $2.6 \pm 1.8$ & $2.8 \pm 1.7$ & $0.3 \pm 0.6$ \\
Thigh & $2.3 \pm 1.7$ & $2.1 \pm 1.8$ & $2.1 \pm 1.6$ & $0.4 \pm 0.6$
\end{tabular}

Abdominal injections were more painful than thigh injections (ANOVA $\mathrm{p}=0.0099$ ). Bleedings were less common with the G29 needle than with the G28 needle (Friedman $\mathrm{p}=0.028$ ). The frequency of intracutaneous injections was $1.2-5.0 \%$ (Friedman $p=n$.s.). Leakage of insulin was found in 9$18 \%$ of abdominal and $19-29 \%$ of thigh injections (Friedman $\mathrm{p}=\mathrm{n} . \mathrm{s}$.). The VAS pain score when taking a blood glucose test was $1.5 \pm 1.7$. In conclusion we found low pain perceptions with all 3 needles with no difference between the needle types. 


\section{Pharmacological Agents}

\section{8}

EFFECT OF SODIUM TUNGSTATE ON INSULIN AND GLUCAGON SECRETION. STUDY IN THE PERFUSED RAT PANCREAS.

J. Rodríguez-Gallardo, E. Gutiérrez, P. Garcia, R.A. Silvestre and J. Marco. Clínica Puerta de Hierro, and Dept. Physiology, Universidad Autónoma de Madrid, Madrid, Spain

It has recently been reported that, in diabetic rats, oral treatment with tungstate salts normalises glycaemia and hepatic glucose metabolism. We have investigated the effect of sodium tungstate on insulin and glucagon secretion in the perfused pancreas isolated from normal fed rats. Perfusate consisted of Krebs-Henseleit buffer supplemented with albumin $(0.5 \%)$, dextran T-70 ( $4 \%)$ and glucose $(5.5 \mathrm{mM})$. Hormones were analysed by RIA Tungstate infusion (5 $\mathrm{mM}$ ) markedly stimulated insulin secretion $(F 15,45=11.3 ; p<0.01)$. This insulin response was short-lived and resembled the typical first phase of glucose-induced insulin release. Direct infusion of tungstate into the pancreas does not seem to be toxic to the B-cell since it did not alter the insulin response to a subsequent glucose stimulus (incremental area: $29 \pm 11$, Mean \pm SEM, $\mathrm{ng} / 10 \mathrm{~min}$ vs. $29 \pm 6.8 \mathrm{ng} / 10 \mathrm{~min}$ in control experiments). Infusion of $5 \mathrm{mM}$ tungstate did not significantly modify glucagon release $(F 10,30=1.9 ; N S)$. Oral tungstate treatment (50 $\mathrm{mg} /$ day for eight days) did not significantly modify food or water intake (food: $19.9 \pm 0.9$ vs. $20.7 \pm 0.4 \mathrm{~g} /$ day in control experiments; water: $26.8 \pm 0.7$ vs. $27.9 \pm 0.5 \mathrm{ml} /$ day); body weight gain was reduced (values at day 8 of treatment: $195 \pm 6 \mathrm{~g}$ vs. $217 \pm 4 \mathrm{~g}$ in control rats; $p<0.01$ ). Pancreases obtained from tungstate treated rats showed a greater insulin response to an increase in perfusate glucose levels (from 5.5 to $9 \mathrm{mM}$ ) than controls (incremental areas: $60 \pm 6 \mathrm{ng} / 5 \mathrm{~min}$, and $20 \pm 6 \mathrm{ng} / 5 \mathrm{~min}$, respectively: $p<0.01$ ). It is concluded that the reduction of glycaemia caused by sodium tungstate may be, at least in part, due to its insulinotropic effect.

\section{0}

INFLUENCE OF ACARBOSE ON POSTPRANDIAL INSULIN REQUIREMENTS IN PATIENTS WITH TYPE 1 DIABETES L. JUNTTI-BERGGREN, a J. PIGON, a J.J. HOLST, b S. EFENDIC a aKarolinska Hospital, Stockholm, Sweden and ${ }^{b}$ University of Copenhagen, Denmark

The primary objective of this double-blind, placebo-controlled, randomized, cross-over study was to investigate of the influence of acarbose on insulin requirementsin type 1 diabetic patients following a standard meal. The secondary objective was to investigate the effect of acarbose on postprandial triglycerides and gastrointestinal peptides. The effect of acarbose on oxidative glucose metabolism was also assessed, using indirect calorimetry. Ten patients with type 1 diabetes were evaluated on two occasions using an artificial pancreas (Biostator ${ }^{R}$ ). After normalisation of blood glucose, patients received a standardised meal, together with acarbose $(100 \mathrm{mg})$ or placebo. The insulin required for maintenance of normoglycaemia was assessed over 180 minutes; blood samples were taken every $15-30$ minutes. A double-blind, randomized, cross-over design ensured that all patients received both acarbose and placebo (separated by $10 \pm 3$ days). The primary efficacy parameter was Isoglycaemic Meal-related Insulin Requirement (IMIR). Acarbose induced a statistically significant beneficial decrease in mean IMIR, compared with placebo (acarbose: $5171.7+$ (SD) $2282.6 \mathrm{mU}$ vs. placebo: $8074.5 \pm$ (SD) $3045.4 \mathrm{mU}$; $\mathrm{p}=0.003$ ). For GIP, there was a statistically significant reduction using acarbose compared with placebo in terms of both AUC $(p=0.006)$ and $C_{\max }(p=0.022)$, but not for $t_{\max }(p>0.05)$. There were no statistically significant differences between acarbose and placebo with regard to GLP-1, postprandial triglycerides, carbohydrate oxidation, or fat oxidation (all $p>0.05$ ). These results indicate that acarbose reduces postprandial insulin requirement in patients with type 1 diabetes.

\section{9}

$\beta_{3}$-ADRENERGIC AGONISTS STIMULATE SKELETAL MUSCLE GLUCOSE UPTAKE IN ASSOCIATION TO AN INCREASE IN GLUTI GENE EXPRESSION. L. Forga, F.i. Milagro and J.A. Martínez. Dpto. Fisiología y Nutrición, Universidad de Navarra, y Sección de Endocrinología, Hospital de Navarra, 31008 Pamplona, SPAIN. $\beta_{3}$-adrenergic receptors are involved in the regulation of glucose homeostasis. Thus, some $\beta_{3}$-adrenergic agonists have shown a hypoglycemic effect owing to an increase in peripheral tissue glucose uptake; however, the mechanisms involved are yet unclear. In this context, Trecadrine ${ }^{*}$, a molecule with affinity for $\beta_{3}$-adrenoceptors, produced a potent effect in an alloxan-diabetes model in rats after 4 days of administration (1.0 $\mathrm{mg} / \mathrm{kg}$.day), where hyperglycemia decreased from $289 \pm 39$ to $149 \pm 32 \mathrm{mg} / \mathrm{dl}(\mathrm{p}<0.05)$ with no modifications in plasma insulin levels. The aim of this study is to assess the effects of Trecadine on in vitro glucose uptake in skeletal muscle (gastrocnemius, soleus and extensor digitorum longus from both the two hindlimb legs of Wistar rats). Each muscle was incubated at $37^{\circ} \mathrm{C}$ in Krebs-Henseleit buffer, $\mathrm{pH} 7.4$, with $0.1 \%$ bovine seroalbumin and $1 \mathrm{mM} 2$-deoxy-D-glucose. From each rat, muscles frorn one leg were incubated with Trecadrine ${ }^{\infty} 10^{-4} \mathrm{M}$, and those from the other leg without it. After 30 minutes of incubation with $0.03 \mu \mathrm{Ci} / \mathrm{ml}$ of $\left[{ }^{14} \mathrm{Cl} 2\right.$-deoxy-D-ghucose and 0.03 $\mu \mathrm{Ci} / \mathrm{ml}$ of $\left[{ }^{3} \mathrm{H}\right]$ mannitol, radioactivity inside the cells was measured in a liquid scintillation counter. Trecadrine ${ }^{\infty}$ significantly enhanced $\left[{ }^{14} \mathrm{C} 2\right.$-deoxy-D-glucose uptake in these muscles from $8.1 \pm 1.4$ to $11.2 \pm 2.1 \mu \mathrm{mol} / \mathrm{ml}$ of intracellular waterxmin $(\mathrm{p}<0.05)$. On the other hand, facilitative glucose carrier GLUT4 and GLUTI mRNA was quantified by RT-PCR in gastrocnemius after Trecadrine ${ }^{\infty}(1.0 \mathrm{mg} / \mathrm{kg}$.day) was orally administered for 4 days to alloxan-diabetic Wistar rats. GLUTl gene expression significantly increased in Trecadine -treated rats as compared to that of GLUT4. Thus, the ratio GLUT4:GLUT1 mRNA in non-treated rats was $0.70 \pm 0.07$, but it decreased to $0.49 \pm 0.05$ in Trecadine ${ }^{\infty}$-treated rats $(\mathrm{p}<0.05)$. As a conclusion, Trecadime ${ }^{\star}$ appears to enhance ghcose uptake in skeletal muscle through a mechanism that is non-insulindependent. A stimulation of GLUTl gene expression instead of that of GLUT4 occurs, suggesting that an enhancement in cell membrane GLUT1 is involved in the $\beta_{3}$. adrenergic agonist-induced glucose uptake.

\section{1}

NATEGLINIDE (A-4166) CONTROLS GLYCEMIA IN DIET-TREATED TYPE 2 DIABETIC PATIENTS

J.J.M Deijns, K.P Bouter. Bosch Medicentre, Den Bosch, NL; M. Hanefeld, University Dresden, D; Ch. Gultard, Novartis Pharma, Basel, $\mathrm{CH}$.

Main objective: to evaluate the effects on glycemic control of 4 dose levels of nateglinide and placebo $(\mathrm{pl})$ administered before main meals. Method: doubleblind, randomized, 12-week parallel group study with a Sustacal@ challenge (liquid meal, $250 \mathrm{kcal}$ ). Subjects: 289 patients were randomized. The mean age was $56.4 \pm 10.2$ years; female/male ratio $33 / 67$ and $93 \%$ were Caucasian. The mean BMI was $28.5 \pm 3.4 \mathrm{~kg} / \mathrm{m}^{2}$. Results: At baseline (BL) there were no clinically relevant differences between the 5 groups. Mean $H b A_{1 c}$, FPG, fructosamine, total insulin $\mathrm{AUC}_{0-1 \mathrm{n}}$ and total glucose $\mathrm{AUC}_{0-4 \mathrm{n}}$ at $\mathrm{BL}$ and changes from $B L$ at week 12 or with the last value carried forward were:

\begin{tabular}{|c|c|c|c|c|c|c|}
\hline & BL & pl & $30 \mathrm{mg}$ & $60 \mathrm{mg}$ & $120 \mathrm{mg}$ & $180 \mathrm{mg}$ \\
\hline $\mathrm{HbA}_{1 \mathrm{c}}(\%)$ & 8.4 & +0.07 & -0.20 & $-0.38^{*}$ & $-0.55^{\star \star \star \star}$ & $-0.56^{* * *}$ \\
\hline FPG $(\mathrm{mmol} / \mathrm{L})$ & 10.1 & +0.22 & -0.42 & $-0.46^{\star}$ & $-0.92^{* *}$ & $-0.56^{*}$ \\
\hline Fructosamine $(\mu \mathrm{mol} / \mathrm{L}$ & 352 & +6.6 & -1.8 & $-18.1^{\text {tkt }}$ & $-23.7^{\star \star \star \star}$ & $-27.6^{\text {*nth }}$ \\
\hline $\mathrm{AUC}_{0-1 \mathrm{~h}}$ insulin (hr.mU/L) & 105 & +6.0 & +19.0 & $+35.5 . * \pm$ & $+49.0^{\star \star \star \star}$ & $+47.0^{*+14}$ \\
\hline$A \cup C_{0-h h}$ glucose (hr.mmol/L) & 48.5 & +3.2 & $-3.6^{* \pm}$ & $-8.0^{\hbar \pm \hbar+}$ & $-8.5^{\hbar \star \hbar}$ & $-8.1^{* \hbar \star}$ \\
\hline
\end{tabular}

All doses of nateglinide were well tolerated; no treatment emergent adverse events were observed vs pl, except an increase of signs and symptoms suggestive of hypoglycemia $(\mathrm{H})$. $\mathrm{H}$ was confirmed by a home blood glucose $\leq 2.8 \mathrm{mmol} / \mathrm{L}$ in $4(1.8 \%)$ subjects. All episodes of $\mathrm{H}$ were of mild nature, except one considered as moderate and another as severe. No subject discontinued because of $\mathrm{H}$. No clinically relevant hematological or biochemical laboratory changes were observed. Conclusions: nateglinide improved glycemic control in a dose-dependent manner in the tested dose-range by reducing fasting and post-prandial hyperglycemia through improved prandial insulin secretion. All tested doses were well tolerated. 


\section{2}

EFFECTS OF TROGLITAZONE ON FATTY LIVER AND HEPATIC INSULIN CLEARANCE IN PATIENTS WITH NIDDM

S. Katoh ${ }^{2}$, J. Yokoyama ${ }^{1}$, N. Tajima ${ }^{1}$, Y. Ikeda ${ }^{1}$ and K. Ikeda ${ }^{2}$

1 Jikei Univ. Sch. of Med., Tokyo, ${ }^{2}$ Kawaguchi Municipal Medical Center, Saitama, Japan

We examined effects of troglitazone on fatty liver, hepatic insulin clearance and fat distribution in 22 patients with NIDDM. Patients were divided into troglitazone-treated (T) group $(n=9)$ or control (C) group $(\mathrm{n}=13)$ and followed for 24 weeks. Fatty liver was evaluated by mean hounsfield unit of liver $(L m H U)$. Visceral fat area $(\mathrm{V})$ and subcutaneous fat area (S) were measured by the abdominal $\mathrm{CT}$. The hepatic insulin clearance was calculated from serum $\mathrm{C}$-peptide/insulin ratio (CPR/IRI) in fasting state. In C group, the body mass index after the treatment significantly decreased compared with that before the treatment, but did not significantly change in $T$ group. In $T$ group, $\mathrm{LmHU}(51.5 \pm 8.3$ ) and $C P R / R I \quad(0.28 \pm 0.07)$ after the treatment significantly $(p<0.01)$ increased compared with $\mathrm{LmHU}(36.4 \pm 12.4)$ and $\mathrm{CPR} / \mathrm{IRI}(0.19 \pm 0.05)$ before the treatment. In $T$ group, $\mathrm{HbA} 1 \mathrm{c}$, fasting IRI and glucose after the treatment significantly decreased compared with those before the treatment. In $\mathrm{C}$ group, $\mathrm{LmHU}$, $C P R /|R I, H b A| c$, fasting $|R|$ and glucose did not significantly change. $V, S$ and $V / S$ ratio did not significantly change in bath group. In conclusion, fatty liver may. contribute to hyperinsulinemia and troglitazone treatment improves fatty liver, hepatic insulin clearance and hyperinsulinemia.

\section{4}

EFFICACY AND SAFETY OF ACARBOSE IN PATIENTS WITH TYPE 2 DIABETES INADEQUATELY CONTROLLED WITH INSULIN THERAPY

T. Taylor, J. Magner, A. Krol, and D. E. Kelley West Haven, CT and Pittsburgh, PA The efficacy, safety and tolerability of acarbose as compared to placebo was examined in patients with type 2 diabetes inadequately controlled with diet and insulin in a multicenter, double-blind, placebo-controlled comparison study. A total of 195 patients were randomized in the study, which consisted of a 2-week screening period followed by a 24-week treatment period. Acarbose was forced fitrated from $25 \mathrm{mg}$ tid to $50 \mathrm{mg}$ tid after 4 weeks, and then titrated to $100 \mathrm{mg}$ tid after 12 weeks based on efficacy. The daily dose of insulin was to be held constant throughout the study. The primary criterion of efficacy was the change from baseline in $\mathrm{HbA}_{1 \mathrm{C}}$ at endpoint. Secondary efficacy criteria inciuded changes from baseline in fasting and postprandial glucose and triglyceride levels. Safety was assessed by evaluating vital signs, laboratory values and adverse events. Seventy-three placebo and 72 acarbosetreated patients were valid for efficacy analysis. At endpoint, placebo patients had a mean increase in $\mathrm{HbA}_{16}$ of $0.11 \%$ while acarbose patients had a mean decrease in $\mathrm{HbA}_{10}$ of $0.58 \%$, for a statistically significant placebo-subtracted mean decrease of $0.69 \%(p=0.0001)$. Acarbose-treated patients had significantly lower postprandial plasma glucose values at 60 $(p=0.0178), 90(p=0.0004)$ and 120 ( $p=0.0001)$ minutes compared to placebo patients. There was a trend to lower fasting glucose values in the acarbose-treated group. Acarbose significantly reduced serum triglyceride levels $90(p=0.005)$ and $120(p=0.0133)$ minutes after a meal compared to placebo. For patients valid for safety analysis, six patients $(6 \%)$ taking placebo and 19 patients (20\%) taking acarbose discontinued from the study prematurely due to adverse events. The most common adverse events in the acarbose group were gastrointestinal. There was no significant difference in hypoglycemic events between groups, and no hepatic transaminase elevations greater than 1.8 times the upper limit of normal. This study demonstrates that acarbose is safe and effective in improving glycemic control in patients with type 2 diabetes who are inadequately controlled with insulin therapy.

\section{3}

LONG-TERM OBSERVATION OF REMISSION IN SECONDARY FAILURE OF ORAL DRUGS IN NON-INSULIN DEPENDENT DIABETIC PATIENTS.

J.Ruxer, J.Loba, W.Torzecka and J.Drzewoski. Metabolic Diseases and Gastroenterology Department, Medical University of Łódż, Łódź, Poland.

Secondary failure of oral drugs (SFOD) has not had, up to date, any widely accepted management schemes. It is well established that the strict glycaemic control with insulin in non-insulin-dependent diabetic (NIDDM) patients restores sensitivity to sulfonylureas. However, there are few long-term studies of SFOD remission duration following insulin therapy. The aim of this study was to evaluate the effectiveness of short-term intensive insulin therapy with intravenous pump (IVII) as a method of sulfonylureas sensitivity restoration. The material consists of 35 NIDDM patients with real SFOD, aged $\leq 65 \mathrm{yrs}$, treated with glibenclamide $(15 \mathrm{mg} / \mathrm{d})$ and metformin $(1500$ $\mathrm{mg} / \mathrm{d}$ ). Patients included into the study were subject to IVII for 3 to 5 days, until near normoglycaemia was achieved. Subsequently, all patients returned to previous oral therapy. The metabolic control of each patient was checked every six months. The results of the study are shown in the table

\begin{tabular}{|c|c|cc|}
\hline $\begin{array}{c}\text { Cumulative } \\
\text { observation time } \\
\text { [months] }\end{array}$ & $\begin{array}{c}\text { No of patients } \\
\text { under } \\
\text { observation }\end{array}$ & Patients with SFOD-remission & [\%] \\
\hline 6 & 35 & 16 & 45.7 \\
\hline 12 & 35 & 12 & 34.3 \\
\hline 24 & 33 & 7 & 21.2 \\
\hline 36 & 32 & 5 & 15.6 \\
\hline 48 & 31 & 4 & 12.9 \\
\hline
\end{tabular}

The longest period of SFOD remission observed in one patient lasted 71 months. Conclusion: 1 . IVIl seems to be an effective method of oral drugs sensitivity restoration. 2. Although the number of patients with SFOD remission decreased in time, it was possible to maintain remission over 48 months in some cases.

\section{5}

EFFICACY AND SAFETY OF ACARBOSE IN PATIENTS WITH TYPE 2 DIABETES INADEQUATELY CONTROLLED WITH METFORMIN

J. Magner, T. Taylor, A. Krol and J. Rosenstock W. Haven, CT and Dallas, TX The efficacy, safety and tolerability of acarbose as compared to placebo was examined in patients with type 2 diabetes inadequately controlled with diet and metformin in a multicenter, double-blind, placebo-controlled comparison study. A total of 168 patients were randomized in the study, which consisted of a 1-week screening period followed by a 6 -week, single-blind placebo pretreatment period and a 24-week double-blind treatment period. Acarbose was forced fitrated from $25 \mathrm{mg}$ tid to $50 \mathrm{mg}$ tid after 4 weeks. and then titrated to $100 \mathrm{mg}$ tid after 12 weeks based on efficacy. A stable dose ( $2000 \mathrm{mg} / \mathrm{day}$ or $2500 \mathrm{mg} / \mathrm{day}$ ) of metformin was to be maintained. The primary criterion of eficacy was the change from baseline in $\mathrm{HbA}_{\mathrm{Ic}}$ at endpoint. Secondary efficacy criteria included changes from baseline in fasting and postprandial plasma glucose, serum insulin and triglyceride levels. Seventy-four patients in each group were valid for efficacy analysis. An analysis of covariance model was used to adjust mean $\mathrm{HbA}_{1 \mathrm{c}}$ values to a common baseline of $8.33 \%$. At endpoint, placebo patients had a mean increase in $\mathrm{HbA}_{1 \mathrm{c}}$ of $0.08 \%$ while acarbose patients had a mean decrease in $\mathrm{HbA}_{1 \mathrm{c}}$ of $0.57 \%$, for a significant placebo-subtracted mean decrease of $0.65 \%(p=0.0001)$. Placebo patients had an increase of $1.8 \mathrm{mg} / \mathrm{dL}$ in mean fasting glucose while acarbose patients had a decrease of $12.7 \mathrm{mg} / \mathrm{dL}(\mathrm{p}=0.0213$ ). Acarbose patients had significantly lower postprandial glucose values at $60(p=0.0001), 90(p=0.0001)$ and $120(p=0.0001)$ minutes compared to placebo patients. Acarbose lowered fasting and postorandial serum insulin levels and postprandial triglyceride levels. For patients valid for safety analysis, three patients $(4 \%)$ taking placebo and 10 patients $(12 \%)$ taking acarbose discontinued from the study prematurely due to adverse events. The most common adverse events in the acarbose group were gastrointestinal. Acarbose did not significantly increase hepatic transaminases when added to metformin therapy. This study demonstrates the safety and efficacy of acarbose in improving glycemic control in patients with type 2 diabetes who are inadequately controlled with metformin therapy. 


\section{6}

AY-4166 INCREASES THE SENSLTIVITY OF INSUTIN SBSCRETION TO GLUCOSE IN ISOLATLED RAT PANCREAS

Y. Sakamoto, S. Morimoto and O. Mokuda

Teikyo University school of Nedicine, Ichihara Japan

To study the effect of AY-4166(AY), a new antidiabetic agent on the glucose-st imulated insul in secretion in isolatd perfused rat pancreas. This drug is a D-phenylalanine derivative that is a non-sulfonylurea oral hypoglycemic agent. Stabilization perfusion with $5 \mathrm{mM}$ glucose was carried aut for $10 \mathrm{~min}$, and then insulin secretion was stimulated by $5,7.5,10$ and $15 \mathrm{mug}$ glucose in the various concentration of $\mathrm{AY}(0,3,10$ and $30 \mu \mathrm{M})$. In the presence of $5 \mathrm{mM}$ glucose, $\mathrm{AY}$ did not affect insulin secretion at concentrations below 10\%M and significantly increased at 30,M (IRI release was $83,87,177$ and $241 \mu \mathrm{U}$ curing $20 \mathrm{~min}$ perfusion with $0,3,10$ and 30, $\mathrm{MAY}$, respectivery). In the dependency of insulin secretion on the glucose concentration at 5, 7.5, 10 and 15 m glucose, AY $(3, M)$ did not affect the basal insul in secretion at $5 \mathrm{mMglucose}$, and no stimulating effect of the crug was found on the maximally glucose-stimulated insulin secretion. However, we could find out a most significant potency in the dose-response curve, i.e., the doseresponse curve of the insulin secretion to glucose was shifted to the left side parallel by AY. These results suggest that $\mathrm{AY}$ is not a stimulator releasing insul in independently of glucose concentrations, but a potentiator of insulin.AY is a unigue oral antidiabetic agent that increases insulin release in the state of hyperglycemia, but does not stimulate insulin secretion at euglycemic level in an optimal dose.

\section{8}

TRIGLYCERIDE-LOWERING ACTION OF CILOSTAZOL IN RELATION TO ITS

EFFECT ON INSULIN RESISTANCE Third Department of Internal Medicine, Hirosaki University School of Medicine, 5 Zaifu-cho, Hirosaki, 036-8562 Japan

Cilostazol (CSZ), a potent phophodiesterase (PDE) III inhibitor, has a triglyceride' (TG)-lowering act 10n. As CSZ potentiates blood supply and could improve insul in resistance, its effect on plasma $T G$ levels in relation to its effect on insul in resistance were investigated in consecutive 15 subjects ( 9 men and 6 women, mean age of $55 \mathrm{yr}$ mean BMI of $27 \mathrm{~kg} / \mathrm{m}^{2}$ ) with hypertrigi yceridemia ( $(\mathrm{G})>150 \mathrm{mg} / \mathrm{d}$ l) and hyper insul inemia (I, plasma IRI level $2 \mathrm{hr}$ after oral glucose load $(O G)>50 \mu \mathrm{U} / \mathrm{ml}$ ) but without fasting hyperglycemia (FPG<140 mg/dl). Eugl ycemic clamp (EC), intravenous hyperglycemia ( $\mathrm{FG}<140 \mathrm{mg}$ /dI). Eugl ycemic clamp (EC), intravenous fat tolerance test (IVFII) and OG were performed on separate days before and following administration of daily $200 \mathrm{mg}$ of CSZ for 8 weeks. Following CSZ treatment $T G$ and $I_{2}$ levels declined significantly (TG:228 $\pm 73 \rightarrow 148 \pm 53 \mathrm{mg} / \mathrm{dl}$, mean $\pm \mathrm{SD} ; \mathrm{I}_{2} 96 \pm 64$ $\overrightarrow{5} 54+25 \mu \mathrm{U} / \mathrm{ml}, \mathrm{p}<0.01)$, whi Te BMI and plasma glucose levels at $2 \mathrm{hr}$ after $\mathrm{OG}$ showed no significant change. Glucose infusion rate (GIR) in EC revealed significant improvement $(3.9 \pm 1.6 \rightarrow 4.9 \pm 2.1$ $\mathrm{mg} / \mathrm{kg} / \mathrm{min}, \mathrm{p}<0.01)$. In addition, there was a negative corre 7 ation between the depress in TG and the increase in GIR $(r=-0.61, p<0.01)$ The decreased IRI levels during OG was attributable not only to improvement in insul in resistance but to increased insul in clearance, as the insul in clearance during EC increased signifcantly $\left.497 \pm 75 \rightarrow 562 \pm 94 \mathrm{ml} / \mathrm{m}^{2} / \mathrm{min}, 0<0.02\right)$. In addition ant $i$ ipol ytic action of insul in, evaluated by FFA decay dur ing EC, was not impaired, despite the possible effect of the PDE IIIinhibitory action of CSZ to accelerate lipolysis. The exponential decay of the incremental TG level in the IVFTT improved $(0.7 \pm$ $0.3 \rightarrow 0.9 \pm 0.5 \%$ min, $p<0.1)$. However, we failed to demonstrate any correlation between the decrease in TG level and the increase in TG clearance after CSZ administration. In conclusion, CSZ simultaneously lowered TG levels and insul in resistance, In addition TG clearance improved however, the lack of significant correlation with the decrease in TG level may indicate that correlation with the decrease in TG level may indicate that mechanisms other than TG clearance, such as decreased hepatic TG
production would be involved in the TG-lowering action of CSZ.

\section{7}

PHENSUCCINAL ATTENUATES THE DEVELOPMENT OF MULTIPLE LOW-DOSE STREPTOZOTOCIN-INDUCED DIABETES

N. Gorbenko, V. Poltorack, A. Gladkih and O. Ivanova. Ukrainian Scientific Research Institute of Endocrine Diseases Pharmacotherapy, Kharkov, Ukraine

The low-toxic succinate derivative phensuccinal $(\mathrm{Ph})$ is a novel antioxidant which is currently in clinical trials for the prevention of late diabetic complications. The aim of the study was to determine whether Phtreatment would protect from the diabetogenic effects of multiple lowdose of streptozotocin (STZ). Male C57B16/J mice were given STZ (50 $\mathrm{mg} / \mathrm{kg} / \mathrm{die}$ i.p. for 5 consecutive days). One group of animals was treated with $\mathrm{Ph}(25 \mathrm{mg} / \mathrm{kg}$ per os) for a week prior to the first STZ-injection and for the further two weeks. Control diabetic and intact mice have been included in the study. Plasma samples were analyzed for glucose and insulin at the day 15 from the diabetes induction. Lipid peroxidation intensity was assessed in liver homogenate by determination of malonic dialdehyde (MDA) content. All pancreata were graded for insulitis calculated as percentage of infiltrated islets multiplied by the degree of infiltration (score). At the end of the experiment plasma glucose level was significantly lower in Ph-treated group ( $13.7 \pm 1.0 \mathrm{vs} 30.2 \pm 3.7 \mathrm{mmol} / \mathrm{l}$, $\mathrm{p}<0.01)$ and plasma insulin content was increased by $40 \%(\mathrm{p}<0.05)$ in comparison with non-treated diabetic controls. Moreover, $\mathrm{Ph}$ supplementation suppressed STZ-induced oxidative stress reducing hepatic MDA content by $50 \%(\mathrm{p}<0.01)$ compared to control diabetic group. Histological examination of pancreata revealed that administration of $\mathrm{Ph}$ diminished insulitis scores (35\% vs $77 \%, \mathrm{p}<0.05$ ) in comparison with non-treated diabetic controls. These results suggest that antioxidant $\mathrm{Ph}$ attenuates the development of multiple low-dose STZinduced diabetes in mice, reducing basal hyperglycemia and suppressing lymphocyte infiltration into pancreatic islets

\section{9}

Effect of A-4166 on postprandial hyperglycemia in GK rats Y. Kitahara, M. Akiyoshi, Y. Tsuchiya, S. Fujitani, T. Ikenoue, K. Okazaki and N. Kondo. Central Research Labs. Ajinomoto. Co., Inc. Yokohama, Japan

A-4166 is a new oral hypoglycemic agent that suppresses the postprandial hyperglycemia. To clarify the importance of suppression of the postprandial hyperglycemia in NIDDM, we compared the effect of A-4166 on the glucose and lipid metabolism with that of glibenclamide(GB) in Goto-Kakizaki (GK) rats. $50 \mathrm{mg} / \mathrm{kg}$ of A-4166 or $1 \mathrm{mg} / \mathrm{kg}$ of GB were administered to GK rats by oral gavage before meal twice a day for 18 weeks. A-4166 treatment significantly lowered blood glucose level at $1 \mathrm{hr}$ after meal at week 3 (A-4166, $192.2 \pm 9.1 \mathrm{mg} / \mathrm{dl}$ vs. GB, $244.2 \pm 11.5 \mathrm{mg} / \mathrm{dl} ; \mathrm{p}<0.05$ ) and its effect persisted throughout the experimental period. Oral glucose tolerance test at week 18 showed the lower blood glucose levels at 30min after glucose load in A-4166 treated rats $(A-4166,231.6 \pm 8.4 \mathrm{mg} / \mathrm{dl}$ vs. $G B, 260.2 \pm 7.3 \mathrm{mg} / \mathrm{dl} ; \quad P<0.05)$. Pancreatic insulin contents in A-4166 treated rats after 18-weeks treatment showed a trend to be higher than that in GB-treated rats (A-4166, $124.57 \pm 7.46 \mu \mathrm{g} / \mathrm{g}$ pancreas; GB, $102.26 \pm 7.39 \mu \mathrm{g} / \mathrm{g}$ pancreas). These results of glucose tolerance test and pancreatic insulin contents might contribute to the improvement of insulin secretion in response to glucose. After 18-weeks treatment with A-4166, HbA1C level did not deteriorate (Week $0,5.56 \pm 0.24 \%$ vs. Week $18,6.03 \pm 0.13 \%, \mathrm{p}=0.07$ ), while $\mathrm{HbA} 1 \mathrm{C}$ in GB-treated rats was significantly increased (Week $0,5.46 \pm 0.21 \%$ vs. Week $18,6.21 \pm 0.14 \%$, $\mathrm{p}<0.001$ ). NEFA at week 18 in $\mathrm{A}-4166$ treated rats was also low level compared with that in GB-treated rats $(A-4166,0.31 \pm 0.02 \mathrm{mEq} /$; GB, $0.42 \pm 0.04 \mathrm{mEq} / /)$. These results suggest that strict control of postprandial glucose concentration by A-4166 is a beneficial approach for improving glucose and lipid metabolism in NIDDM. 


\section{0}

ADDITION OF GLIMEPIRIDE SIGNIFICANTLY IMPROVES GLYCAEMIC CONTROL IN TYPE 2 DIABETIC PATIENTS INSUFFICIENTLY CONTROLLED ON METFORMIN G. Charpentier', I. Eugène-Jolchine ${ }^{2}$ and $M$. Kabir'. 'Department of Internal Medicine and Diabetology, Corbeil Hospital and ${ }^{2} \mathrm{HMR}$, Paris, France

Glimepiride is a new long-acting sulfonylurea given once daily. The aim of this multicentre, randomised, double blind and double dummy trial was to compare the efficacy and safety of glimepiride (G) given in combination with metformin (M) against either drug given as monotherapy in type 2 diabetic patients not well controlled on $M$ alone. Patients under $M$ alone $(850 \mathrm{mg}$ t.i.d.) for 4 weeks at least were randomised in three parallel groups to either $M, G$ or $G+M$. G was titrated through a 12-week period (initial dose: $1 \mathrm{mg}$ q.d., maximum dose : $6 \mathrm{mg}$ q.d.) followed by an 8 -week maintenance phase. The $\mathrm{M}$ dose $(850 \mathrm{mg}$ t.i.d.) was kept constant throughout the study. The primary endpoint was $\mathrm{HbAlc}$ at 20 weeks. A total of 372 patients (mean \pm SD : age $56 \pm 8$ years, diabetes duration $8 \pm 6$ years, BMI $29.4 \pm 4.1 \mathrm{~kg} / \mathrm{m}^{2}$ ) with baseline values of $6.5 \pm 1.1 \%$ for $\mathrm{HbAlc}$ (norma range $3.7-4.4 \%$ ) and $190 \pm 30 \mathrm{mg} / \mathrm{dl}$ for fasting blood glucose (FBG) were treated $\mathrm{G}+\mathrm{M}$ significantly ( $\mathrm{p}<0.001)$ improved glycaemic control as compared to both monotherapy groups $[\mathrm{HbAlc}(\%)$ change : $+0.07 \pm 1.20(\mathrm{M}),+0.27 \pm 1.10(\mathrm{G})$, $-0.74 \pm 0.96(\mathrm{G}+\mathrm{M}) ;$ FBG $(\mathrm{mg} / \mathrm{dl})$ change : $+15 \pm 64(\mathrm{M}),+13 \pm 56(\mathrm{G})$ $-32 \pm 39(\mathrm{G}+\mathrm{M})]$. Postprandial blood glucose was also significantly $(\mathrm{p}<0.001)$ lower with $\mathrm{G}+\mathrm{M}$ Ichange : $+0.19 \pm 1.06(\mathrm{M})-0.02 \pm 0.91(\mathrm{G}),-0.45 \pm 0.7$ $(\mathrm{G}+\mathrm{M})]$. The frequency of symtomatic hypoglycaemia was similar in monotherapy (M: $12 \%, G: 13 \%)$ and higher for the combination ( $\mathrm{G}+\mathrm{M}: 22 \%)$. Severe episodes were reported in three patients in each of $\mathrm{G}$ and $\mathrm{G}+\mathrm{M}$ groups. The percentage of patients with adverse events was $52.0 \%(\mathrm{M}), 46.0 \%(\mathrm{G})$ and $49.7 \%(\mathrm{G}+\mathrm{M})$, with a slightly higher incidence of gastrointestinal events in the $\mathrm{M}$ group ( $\mathrm{M}: 13.3 \%, \mathrm{G}: 6.7 \%, \mathrm{G}+\mathrm{M}: 9.5 \%$ ). In conclusion, addition of glimepiride to metformin in type 2 patients insufficiently controlled with metformin alone provided a significant improvement in glycaemic control and was wel tolerated.

\section{1}

Extreme metformin-induced lactic acidosis successfully treated with bicarbonate haemodialysis

ARNO J. SOOMERS, CEES J TACK. Medical Center Alkmaar, The Netherlands and Clinical Neuroscience Branch, NIH, Bethesda, USA

Metformin, a representative of the class of biguanide oral hypoglycaemic agents is in use since 1957 but was only recently (in 1995) introduced in the US. As a result, there may be a tendency to a more frequent use in general, and in elderly populations in particular. Its rare but potentially lethal side-effect lactic acidosis, occurs especially in patients with known risk factors like renal failure. We recently treated a 75 year old female with a history of type 2 diabetes treated with glibenclamide ( $5 \mathrm{mg}$ tid) and metformin $(850 \mathrm{mg}$ tid). Two months before admission, serum creatinin level was $91 \mu \mathrm{mol} / \mathrm{L}$. Immediately after admission for abdominal pain, leucocytosis, renal failure (serum creatinin $713 \mu \mathrm{mol} / \mathrm{L}$ ) and lactic acidosis (serum lactate level $18 \mathrm{mmol} / \mathrm{L}$, normal <2), she underwent emergency laparotomy that was negative. Post- operatively, a few hours after admission, circulatory shock developed and she was diagnosed as metformin-associated lactic acidosis, and treated with vasopressive drugs and acute intermittent bicarbonate haemodialysis. Metformin level before the first dialysis was $61.3 \mathrm{mg} / \mathrm{L}$, afterwards $23.4 \mathrm{mg} / \mathrm{L}$ (Merck/Lipha, France; therapeutic zone $<5 \mathrm{mg} / \mathrm{L}$ ). The extreme acidosis (minimal arterial $\mathrm{pH} 6.73$ !) normalised during bicarbonate dialysis, blood pressure recovered and after two days she was extubated successfully. She refused further renal replacement therapy and died after 5 more days.

This case illustrates that severe metformin-associated lactic acidosis can occur in older patients with previously "normal" creatinin levels, as plasma levels may not be a good indicator of renal function. Furthermore, even extreme metformininduced lactic acidosis can be successfully treated with acute bicarbonate haemodialysis.
902

NATEGLINIDE (A-4166), A NEW INSULINOTROPHIC AGENT, CONTROLS PRANDIAL HYPERGLYCEMIA IN TYPE 2 DIABETIC PATIENTS K.P Bouter, J.J.M Deijns. Bosch Medicentre, Den Bosch, NL; M. Hanefeld, University Dresden, D; Ch. Guitard, Novartis Pharma, Basel, $\mathrm{CH}$. Main objective: to evaluate the prandial effects of 4 dose levels of nateglinide and placebo (pl) administered before main meals. Method: double-blind, randomized, 12-week, parallel group study. A Sustacal( $\mathbb{B}$ challenge (liquid meal, $250 \mathrm{kcal}$ ) was performed at week 0,4 and 12 . Blood samples were obtained at baseline (BL), $15 \mathrm{~min}, 30 \mathrm{~min}, 1 \mathrm{hr}, 2 \mathrm{hr}$ and $4 \mathrm{hr}$ after the challenge. Subjects: 243 subjects were tested at week 12. Rosults: The mean glucose and insulin levels at selected time points, and the area under the curve for 0-4 hours $\left(\mathrm{AUC}_{0,-4 \mathrm{~h}}\right.$ ) adjusted for insulin/glucose at baseline were

\begin{tabular}{|c|c|c|c|c|c|}
\hline & $\mathrm{PI}$ & $30 \mathrm{mg}$ & $60 \mathrm{mg}$ & $120 \mathrm{mg}$ & $180 \mathrm{mg}$ \\
\hline Insulin $\mathrm{BL}(\mu \mathrm{U} / \mathrm{ml})$ & 17.3 & 15.8 & 18.3 & 22.5 & 15.5 \\
\hline Insulin $15 \mathrm{~min}(\mu \mathrm{U} / \mathrm{ml})$ & 28.1 & 34.1 & 43.3 & 46.6 & 32.4 \\
\hline Insulin $1 \mathrm{~h}(\mu \mathrm{U} / \mathrm{ml})$ & 39.1 & 53.5 & 56.2 & 64.9 & 58.3 \\
\hline Insulin $4 \mathrm{~h}(\mu \mathrm{U} / \mathrm{ml})$ & 16.7 & 17.3 & 18.7 & 18.7 & 20.2 \\
\hline $\mathrm{AUC}_{0-4 \mathrm{~h}}$ insulin (hr.mU/L) & +6.2 & +16.8 & +25.5 & $+32.6^{*}$ & $+39.5^{\star \star}$ \\
\hline Glucose BL $(\mathrm{mmol} / \mathrm{L})$ & 10.4 & 10.2 & 9.6 & 9.8 & 9.9 \\
\hline Glucose $1 \mathrm{~h}(\mathrm{mmol} / \mathrm{L})$ & 14.9 & 13.5 & 12.4 & 12.7 & 13.1 \\
\hline Glucose $2 \mathrm{~h}(\mathrm{mmol} / \mathrm{L})$ & 12.7 & 11.1 & 9.9 & 9.8 & 10.4 \\
\hline Glucose $4 \mathrm{~h}(\mathrm{mmo} / \mathrm{L})$ & 9.4 & 8.5 & 7.6 & 7.0 & 7.4 \\
\hline AUC $_{0-4 h}$ glucose (hr.mmol/l) & +0.6 & $-2.7^{*}$ & $-6.4^{* * *}$ & $-5.0^{\star k \star}$ & $-6.4^{\star \star \star \star}$ \\
\hline
\end{tabular}

Changes vs placebo (ANOVA model): " $p<0.05$, ${ }^{* *} p<0.01,{ }^{* * *} p<0.001$

$A$ rapid, dose-dependent increase in insulin secretion was observed $15 \mathrm{~min}$ post-challenge with a return to baseline values $4 \mathrm{hr}$ post-challenge.This rapid prandial increase of insulin induced a significant reduction of post-prandial glucose excursions in a dose-dependent manner. Conclusions: nateglinide exerts its blood glucose lowering effect by restoring early prandial insulin secretion and diminishing meal-related glucose fluctuations.

\section{3}

Contraindications To Metformin Therapy Are Generally Disregarded D. Nahrwold, E. - H. Egberts and A. Holstein. Medizinische Klinik I, Klinikum Lippe - Detmold, Detmold, Germany Metformin is well established in the therapy of NIDDM due to its anihyperglycaemic effect and its positive influence on insulin resistance. Nevertheless, the application of the substance is limited due to the known contraindications ( $\mathrm{Cl}$ ), especially renal impairment and tissue hypoxia. In a cross section analysis, we evaluated, if metformin therapy in practice was critically proceeded and sufficiently monitored. 221 consecutive NIDDM patients ( 111 female, 110 male; age $66 \pm 11,5$ yrs; BMI 29 $\pm 5,6 \mathrm{~kg} / \mathrm{m}^{2} ; \mathrm{HbA} 1 \mathrm{C} 9,3 \pm 5,6 \%$; daily dosage $1200 \pm 540 \mathrm{mg}$ ) pre treated with metformin were examined on $\mathrm{Cl}$ of biguanide administration. All patients were hospitalized between January 1995 and December 1997 in a general district hospital due to acute medical problem or for improvement of diabetic treatment. A medical history, a complete physical examination, ECG and an elaborate laboratory profile were done and $30 \%$ underwent coronary angiography. This examination revealed the definite $\mathrm{Cl}$ of renal impairment in $21 \%$ of all patients (creatinine $>1,2 \mathrm{mg} / \mathrm{dl}$ or creatinine clearance $<60 \mathrm{ml} / \mathrm{min}$ ) $; 24 \%$ had cardiac failure (NYHA II IV $\left.{ }^{\circ}\right) .23 \%$ showed coronary heart disease with the immediate need of intervention ( Iysis, PTCA, stent or ACVB ). $6 \%$ had respiratory and $2 \%$ hepatic insufficiency; arterial occlusion IV $\circ$, chronic alcohol abuse and pregnancy were present in another $6 \%$. During the therapy with metformin, $14 \%$ of all diabetics developed cerebral ischaemia, in $6 \%$ an acute malignant tumor was diagnosed. Moreover in only $22 \%$ of all patients no $\mathrm{Cl}$ were present. $52 \%$ of the diabetics exceeded the age of 65 . The $\mathrm{Cl}$ of biguanides were generally neglected in the presented cohort; furthermore co - morbidity and old age of NIDDM patients were regarded insufficiently. Conclusions: Many patients were at risk for obtaining a lactic acidosis due to metformin therapy. Further education for physicians is needed for the treatment of patients with NIDDM. 


\section{4}

INFLUENCE OF ACARBOSE AND GLIBENCLAMIDE ON INSULIN SENSITIVITY IN TYPE 2 DIABETES: RESULTS OF A CLAMP-STUDY S. Fischer, M. Hanefeld, H. Rietzsch, A. Patzak, U. Schwanebeck, J. Wildbrett, K. Fücker. Medical Faculty of the Technical University Dresden, Germany. Insulin resistance is the dominant metabolic defect in type 2 diabetes. The aim of this double blind study was to compare the effect of acarbose and glibenclamide on glucose disposal rate during euglycaemic hyperinsulinaemic clamp. 77 type 2 diabetic patients (mean age 58,7 years, mean BMI $27,3 \mathrm{~kg} / \mathrm{m}^{2}$ ), insufficiently treated with diet alone, were randomized to 3 different treatment regimen for 16 weeks: $3 \times 100 \mathrm{mg}$ acarbose $(n=25)$ or $3 \times 1 \mathrm{mg}$ glibenclamide $(n=27)$ or $3 \times 1$ placebo $(n=25)$. Measurements of fasting plasma glucose, plasma glucose 1 hour after breakfast, $\mathrm{HbA}_{1 \mathrm{C}}$, fasting insulin and euglycaemic hyperinsulinemic clamp investigation according to De Fronzo were performed before and after the 16 weekly treatment period under metabolic ward conditions. After therapy BMI was significantly diminished in the acarbose and placebo group, but significantly increased in the glibenclamide group. Fasting plasma glucose was reduced after acarbose $(10,6 \mathrm{vs} .9,7 \mathrm{mmol} / \mathrm{l}, \mathrm{n.s}$.) and glibenclamide $(11,6$ vs. $8,2, p<0,05)$ and non significantly enhanced after placebo $(11,1$ vs. 11,7), plasma glucose postprandial decreased after acarbose $(13,8$ vs. $11,4, \mathrm{p}<0,05)$ and glibenclamide $(14,6$ vs. $11,4, \mathrm{p}<0,05)$ and was unchanged after placebo $(13,8$ vs. 13,7$) \mathrm{HbA}_{1 \mathrm{C}}$ remained unchanged in the acarbose group, decreased significantly after glibenlamide and rose significantly after placebo. No significant changes were observed in fasting insulin in the 3 treatment groups. Neither acarbose nor glibenclamide influenced significantly insulin sensitivity (Mc). Insulin sensitivity raised non significantly in the acarbose and smaller in the placebo group, whereas there was no change after glibenclamide (acarbose: $\mathrm{Mc}$ before treatment $2.3 \mathrm{mg} \cdot(\mathrm{kg} \text { body weight } \mathrm{min} \cdot \mathrm{insulin})^{-1}$ vs after treatment 3.2; placebo: Mc before 2.6 vs after 3.0 ; glibenclamide: $\mathrm{Mc}$ before 2.2 vs after 2.1 ). Our results show, that glibenclamide improves glycemic control more than acarbose, but improvement of glycemic control by glibenclamide was not associated with increase of insulin sensitivity. Obviously improved postprandial glucose control after acarbose led to increased insulin sensitivity.

\section{5}

TROGLITAZONE AS LONG TERM THERAPY IS SUPERIOR TO GLIBENCLAMIDE

M.Gliese ${ }^{1}$, J.Saltevo ${ }^{2}$, J.Schulze ${ }^{3}$, S.Kench ${ }^{4}$ and L.Frith ${ }^{4}$. 'Glostrup, Denmark, ${ }^{2} J y v a s k y l a$, Finland, ${ }^{3}$ Dresden, Germany, ${ }^{4}$ Glaxo Wellcome Research \& Development, UK

Troglitazone(T.) targets insulin resistance improving the diabetic state in terms of glycaemic control and multiple cardiovascular risk markers. In this multicentre study the efficacy, safety and tolerability of T. $100 \mathrm{mg}, 200 \mathrm{mg}$ and $600 \mathrm{mg}$ in type 2 diabetics, was compared with that of optimally titrated glibenclamide (Glib) 785 patients with $\mathrm{FCBG}<12 \mathrm{mmol} / \mathrm{L}$ were randomised to one of the above treatment arms for one year. Analysis was performed on log transformed data using analysis of covariance allowing for effects due to baseline, country and treatment. Results are presented as adjusted geometric mean at 12 months.

$\begin{array}{lllll} & \text { T100mg } & \text { T200mg } & \text { T600mg } & \text { Glib } \\ \text { HbA }(\%) & 7.7^{\mathrm{b}} & 7.7^{\mathrm{b}} & 7.2 & 7.3 \\ \text { FSG (mmol/L) } & 10.5^{\mathrm{b}} & 9.9 & 8.8^{\mathrm{a}} & 9.5 \\ \text { Specific insulin( }(\mu / \mathrm{U} / \mathrm{mL}) & 8.9^{\mathrm{a}} & 8.3^{\mathrm{a}} & 7.3^{\mathrm{a}} & 11.2 \\ \text { HDL-C (mmo/L) } & 1.00 & 1.00 & 1.07^{\mathrm{a}} & 0.99 \\ \text { Triglycerides (mmol/L) } & 2.04 & 1.98 & 1.71^{\mathrm{a}} & 1.93\end{array}$

The table below shows analysis of responders. $\mathrm{HbA}_{\mathrm{lc}}$ responders were classified as patient with $\mathrm{a} \geq 1 \%$ decrease from baseline and FSG responders with $\mathrm{a} \geq 1.67 \mathrm{mmol} / \mathrm{L}$ decrease from baseline.

$\begin{array}{lllll} & \text { T100mg } & \text { T200mg } & \text { T600mg } & \text { Glib } \\ \text { HbAtc (\%) } & 48(25 \%)^{\mathrm{b}} & 40(21 \%)^{\mathrm{b}} & 73(39 \%) & 69(36 \%) \\ \text { FSG (mmo/L) } & 47(25 \%) & 59(31 \%) & 93(51 \%)^{*} & 65(33 \%)\end{array}$

(a $p<0.05$ and superior to $G i b, b p<0.05$ and superior to troglitazone)

T. $600 \mathrm{mg}$ is as effective as optimally titrated Glib. in reducing $\mathrm{HbA}_{\mathrm{lc}}$ and more effective in reducing and maintaining levels of FSG over 12 months of treatment. T. also shows improvements in important cardiovascular risk markers compared to Glib. Overall incidence of adverse events during treatment with T. were similar to Glib. Hypoglycaemic events were higher in the Glib group ( $7 \%$ vs $1 \%$ ). Three to six percent of patients on $\mathrm{T}$. had ALT $>3 \times$ ULN compared with $2 \%$ on Glib, which all resolved. Conclusion: T. $600 \mathrm{mg}$ significantly improves and maintains glycaemic control and reduces cardiovascular risk markers (HDL-C and TGs).

\section{7}

THE IN VIVO EFFECT OF THE NEW ORAL HYPOGLYCEMIC AGENT (AY-4166) ON GLUCOSE TURNOVER IN THE HIGH FED DIET. INDUCED OR IN HEREDITARY INSULIN RESISTANCE OF RATS I. Klimeš, B. Lišḱa, D. Gašperíková, J. Ukropec, E. Bergene, E. Šeböková, Inst. Exp. Endocrinology, Slovak Acad. Sci., Bratislava, Slovak Republic AY-4166, a phenylalanine derivative (i.e. N-[(trans-4-iso-propylcyclohexyl)-carbonyl]-D-phenylalanine) is a hypoglycemic agent, which has been shown to improve blood glucose levels in some models of type 2 diabetes and insulin resistance. This is mainly due to the rapid and short term lasting stimulation of insulin release. A possible extrapancreatic action of AY -4166 has not yet been investigated. Therefore, insulin action (euglycemic hyperinsulinemic $/ 6.4 \mathrm{mU} \cdot \mathrm{kg}^{-1} \cdot \mathrm{min}^{-1}$ / clamp plus ${ }^{3} \mathrm{H}$-2-deoxyglucose $/ 50$ hyperinsulinemic $/ 6.4 \mathrm{mU} . \mathrm{kg}$. min $/$ clamp plus $\mathrm{H}$-2-deoxyglucose 150 $\mathrm{mCi} /$ tracer administration) was studied after 3 weeks on either standard
laboratory animal (BD) diet or high $(70 \mathrm{cal} \%)$ fat (HF) diet in normal control (C) or in hereditary insulin resistant (hHTg) rats which were given a single dose of AY-4166 (10 mg per kg BW, i.v.) 60 min after clamp commencement. HF feeding $(F)$ reduced the glucose infusion rate (GIR) required to maintain eyglycemia to about $50 \%$ of $C$ (C-BD: $28.4 \pm 5.8$ vs C. HFF: $15.2 \pm 0.4 \mathrm{mg} . \mathrm{kg}^{-1} \cdot \mathrm{min}^{-1} ; \mathrm{p}<0.00 \mathrm{l}$ ). In hHTg rats, HFF did not further $\left.\mathrm{HFF}: 15.2 \pm 0.4 \mathrm{mg} \cdot \mathrm{kg} \cdot \mathrm{min}^{2} ; \mathrm{p}<0.001\right)$. In hHTg rats, HFF did not further
pronounce the pre-existing decrease (hHTg-BD: $20.2 \pm 0.4)$ of GIR of $\mathrm{hHTg}$ animals fed BD. AY -4166 changed GIR neither in $\mathrm{C}$, nor in the $\mathrm{hHTg}$ group. Estimated glucose disposal (Rd) (C-BD:32.3 \pm 1.9 vs C-HF: $25.5 \pm 1.9 \mathrm{mg} . \mathrm{kg}$ min $\left.^{-1}, \mathrm{p}<0.001\right)$ and glucose metabolic index $\left(\mathrm{Rg}^{\prime}\right.$, from accumulation of phosphorylated deoxyglucose) in skeletal muscles (e $Q$ femoris: C. $\mathrm{BD}: 25.6 \pm 1.5$ vs C-HF: $12.3 \pm 1.1 \mathrm{mmol} / 100 \mathrm{~g} \cdot \mathrm{min} / \mathrm{p}<0.001$ ) were reduced by $\mathrm{BD}: 25.6 \pm 1.5$ vs $\mathrm{C}-\mathrm{HF}: 12.3 \pm 1.1 \mathrm{mmo} / 100 \mathrm{~g} \cdot \mathrm{min}, \mathrm{p}<0.001)$ were reduced by
$\mathrm{HFF}$ in $\mathrm{C}$ rats and did not restore by concomitant AY -4166 bolus. Nevertheless, a single dose of AY-4166 to HF fed hHTG rats brought back their Rd (hHTg-HF:23.5 \pm 1.3 vs hHTg-HF plus AY-4166: $31.0 \pm 3.5 \mathrm{p}<0.03$ ) and $\mathrm{Rg}^{\prime}$ (e.g. Soleus m.: hHTg-HF: $29.2 \pm 3.2$ vs hHTg-HF plus AY-4166: $41.3 \pm 4.0$ to values of the control group on BD. In summary, a) a single bolus administration of AY-4166 to control or to the insulin resistant $\mathrm{hHTg}$ rats, fed either a control or the HF diets, did not abolish the reduction of GIR fed either a control or the HF diets, did not abolish the reduction of GIR
required to maintain euglycemia during hyperinsulinemic clamps; b) required to maintain euglycemia during hyperinsulinemic clamps; b)
nevertheless, AY-4166 caused a significant increase of the estimated plasma glucose disposal (Rd) and skeletal muscle glucose metabolic index (Rg') of hHTG rats fed the HF diet; c) we suggest that A-4166 may have an extrapancreatic action but this needs be proven using a long-term administration plan of A-4166. 


\section{8}

LONG-TERM EFFICACY AND SAFETY OF REPAGLINIDE IN COMPARISON WITH GLIBENCLAMIDE IN SUBJECTS WITH TYPE 2 DIABETES

B.H.R. Wolffenbuttel and R. Landgraf, on behalf of the Dutch and German Repaglinide Study Group, Dept. of Endocrinology, Univ Hosp Maastricht, The Netherlands, and Diabetes Centre, Univ of Munich, Germany Repaglinide (Repa) is a newly-developed oral antihyperglycaemic agent $(\mathrm{OHA})$, which exerts its effect by stimulating insulin secretion. We compared the efficacy and safety of this drug with Glibenclamide (Glib) in a 1-year, multicentre, randomized double-blind study. A total of 424 subjects, age $61 \pm 9$ yrs, duration of diabetes 8 (range $0.5-35$ ) yrs, 154 women, 270 men, body mass index $28.3 \pm 3.5 \mathrm{~kg} / \mathrm{m}^{2}, \mathrm{HbA}_{3 \mathrm{c}} 7.1 \pm 1.4 \%$, and fasting plasma glucose (FPG) $10.8 \pm 3.1 \mathrm{mM}$ participated. The majority of them was previously treated with sulfonylurea, alone or in combination with metformin. The patients were randomized to a $2: 1$ ratio of Repa $(0.5-4 \mathrm{mg}$ tid) and Glib (1.75-10.5 mg daily) treatment, and after a 6-8 week titration period they were followed for 1 year. The trial was completed by 320 subjects, $211(74 \%)$ in the Repa and $109(78 \%)$ in the Glib group. $\mathrm{HbA}_{1 \mathrm{c}}$ initially decreased in both groups, and then increased during the second half year of the maintenance period, to a similar extent in Repa $(0.58 \%$ vs. at screening) and Glib-treated $(0.45 \%)$ subjects. In OHA-naive subjects, a sustained improvement of metabolic control couto be observed with both drugs, which was slightly better with Glib $\left(\triangle \mathrm{HbA}_{1 \mathrm{c}}\right.$ $-2.4 \%$ vs. $-1.0 \%, p<0.05)$. The same trends were seen with FPG. Serum lipids did not change. $15 \%$ of Repa-treated and $13 \%$ of Glib-treated subjects withdrew from the study due to adverse events, mostly hyperglycaemia. No difference in adverse events between both drugs were reported. The incidence of hypoglycaemias was also not different between both treatments. We conclude that Repaglinide is a safe and effective oral blood-glucose lowering agent. Its rapid onset of action and hepatic clearance allows meal-related administration, also in subjects with impaired kidney function

\section{9}

REPAGLINIDE: A DOUBLE-BLIND, RANDOMIZED, DOSERESPONSE STUDY.

T. G. HEDBERG, W-C. HUANG, NovoNordisk, 100 Overlook Center, Princeton, NJ 08540

This randomized, placebo-controlled, double-blind, crossover study with $a 7 x$ 7 Latin square design monitored 24-hour serum glucose concentrations to assess the pharmacokinetics and dose response relationships of repaglinide (REP) in patients with type 2 diabetes. After a 12-day washout period 26 patients received each of the following treatments for 5 consecutive days: placebo, or REP at dosages of either $0.125 \mathrm{mg}, 0.25 \mathrm{mg}, 0.5 \mathrm{mg}, 1.0 \mathrm{mg}, 2.0$ $\mathrm{mg}$, and $4.0 \mathrm{mg}$ preprandially ( 3 meals/day). There was no washoul between treatments. On day 5 of each treatment a 30-point glucose and REP sampling was performed over 24 hours. Dose-response relationships were observed for all doses of REP. At increasing REP doses. glucose levels decreased. Over the 24 hour period studied, AUC serum glucose was significantly lower than placebo for all REP doses; and serum levels showed dose-proportionality Mean slopes for all REP dose profiles were negative in the elimination phase indicating no accumulation. Overall, dose appears to correlate positively with both serum drug levels and glycemic control.

\begin{tabular}{|c|c|c|c|c|c|c|}
\hline \multicolumn{7}{|c|}{ Serum Glucose - Mean $\mathrm{AUC}_{0-24}$ (mg/dL.hr) (\% difference from placebo) } \\
\hline Placebo & $0.125 \mathrm{mg}$ & $0.25 \mathrm{mg}$ & $0.5 \mathrm{mg}$ & $1.0 \mathrm{mg}$ & $2.0 \mathrm{mg}$ & $4.0 \mathrm{mg}$ \\
\hline 6131.61 & $\begin{array}{l}5590.89^{*} \\
(-9 \%)\end{array}$ & $\begin{array}{l}5340.96^{*} \\
(-13 \%)\end{array}$ & $\begin{array}{l}5020.37^{*} \\
(-18 \%)\end{array}$ & $\begin{array}{l}4874.93^{*} \\
(-20 \%)\end{array}$ & $\begin{array}{l}4842.49^{\star} \\
(-21 \%)\end{array}$ & $\begin{array}{l}4711.51^{\star} \\
(-23 \%)\end{array}$ \\
\hline \multicolumn{7}{|c|}{ Serum Repaglinide - Mean of Log Transformed Pharmacokinetic Variables } \\
\hline & $0.125 \mathrm{mg}$ & $0.25 \mathrm{mg}$ & $0.5 \mathrm{mg}$ & $1.0 \mathrm{mg}$ & $2.0 \mathrm{mg}$ & $4.0 \mathrm{mg}$ \\
\hline$A \cup C_{0.20}$ & 3.22 & 3.96 & 4.43 & 5.33 & 6.16 & 6.89 \\
\hline $\mathrm{C}_{\max 020}$ & 2.17 & 2.95 & 3.35 & 4.22 & 4.87 & 5.55 \\
\hline
\end{tabular}

* $\mathrm{p} \leq 0.05$ compared with placebo

\section{0}

A RANDOMIZED, PLACEBO-CONTROLLED, DOUBLE-BLIND, FIXED-DOSE STUDY OF REPAGLINIDE.

W. W. CHEATHAM and P. STRANGE, Princeton, NJ for the REPAGLINIDE STUDY GROUP

The safety and efficacy of repaglinide (REP) at 2 dose levels vs placebo was assessed in a multicenter, randomized, double-blind study. Eligible patients with type 2 diabetes were randomized to 1 of 3 groups: REP I-mg $(n=140)$. REP 4-mg $(n=146)$, or placebo $(n=75)$. Study medications were administered preprandially (with 3 meals) for 24 weeks. Both REP doses were well tolerated. Adverse events (AEs) possibly or probably related to study drug were reported for $24 \%$ of placebo patients and $21 \%$ of all REP patients. The most frequent AE was dizziness, occurring in $4 \%$ of placebo patients, and in $1 \%$ and $5 \%$ of the 1- and 4-mg REP patients, respectively. Hypoglycemic episodes increased with increasing doses of REP: $11 \%$ in the placebo group, $27 \%$ in the REP I-mg group, and $36 \%$ in the REP $4-\mathrm{mg}$ group. Most events were mild; none were severe. This frequency of hypoglycemic episodes is expected in a fixed-dose clinical trial, and is normally lower in clinical practice. For OHA-naïve patients, mean decreases in the proportion of total $\mathrm{HbA}_{\mathrm{ic}}$ for both REP groups from baseline (1-mg: 0.093; 4-mg: 0.092) to end of treatment (1-mg:0.076; 4mg: 0.074) were observed (change from baseline: 0.013 and 0.019 ); vs an increase from 0.085 to 0.092 for the placebo group (change from baseline: 0.01 ). For previously treated patients, mean decreases in the proportion of total $\mathrm{HbA}_{1 c}$ from 0.087 and 0.085 for REP 1 - and 4-mg, respectively, to 0.082 were noted (change from baseline: 0.004 and 0.002 ): vs an increase from 0.087 to 0.1 for the placebo group (change from baseline: 0.014$)$ REP was safe and effective in reducing $\mathrm{Hb}_{\mathrm{ic}}$ vs placebo by the end of the study

\section{1}

REPAGLINIDE PHARMACOKINETICS IN PATIENTS WITH RENAL IMPAIRMENT VERSUS HEALTHY VOLUNTEERS

J. L. RUCKLE' ${ }^{1}$ and V. HATORP ${ }^{2}$, Tacoma, WA, ${ }^{2}$ Bagsvaerd, DK

This study compared the pharmacokinetics of single and multiply dosed repaglinide (REP) between healthy subjects and those with various degrees of renal impairment. Subjects (aged 18-64 years) were stratified by mild, moderate, and severe renal impaiment (determined by creatinine clearance for 2 consecutive 24 -hour periods). On day 1 , non-hemodialysis and healthy subjects received a single 2-mg dose of REP. On day 2 they began preprandial multiple dosing ( 3 meals/day) for 5 days, and received a final $2-\mathrm{mg}$ dose on day 7 . Hemodialysis subjects received a single 2 -mg dose on 2 occasions separated by a washou period of 7 to 14 days. AUC, $C_{\max }, T_{\max }$, and $T_{4}$ were obtained after the single dose regimen (day 1) for all subject groups (24- and 48-hr) and (2) after the multipie-dose regimen (day 7) for non-hemodialysis and heaithy subjects. A significant increase $(p \leq 0,05)$ appcared from day 1 to day 7 in AUC between healthy and mild to moderate renally impaired subjects $(+120.8 \%)$. This was more pronounced in severely impaired subjects $(+199.7 \%)$. Baseline AUC was similar for all renally impaired subjects. After multiple doses, $C_{\text {max }}$ was essentially unchanged for healthy subjects but increased for both the renally impaired and henodialysis subjects. Overall, $\mathrm{T}_{\mathrm{w}_{\mathrm{m}}}$ and $\mathrm{T}_{\max }$ were similar between subject groups despite multiple dosing. REP binding ranged from $95.1 \%$ to $97.5 \%$ for all treatment groups. Hypoglycemia, dizziness, tremor, and sweating were the most frequent adverse events. Hypoglycemic frequency did not differ between healthy and renally impaired subjects; and no hemodialysis subjects experienced liypoglycemia. REP dose adjustment does not appear necessary for renally impaired subjects, but initial doses should be increased gradually 


\section{2}

a Comparison of the Pharmacokinetics of Repaglinide in Healthy Si BJECTS WITH THAT IN SUBJECTS WITH CHRONIC LIVER DISEASE V.Hatorp' ${ }^{\mathbf{1}}$ and G. Haug-Pihale ${ }^{2}$. ${ }^{1}$ Novo Nordisk A/S, Bagsvaerd, DK; ${ }^{2}$ APEX, Munich, Germany

Repaglinide (REP) is a prandial glucose regulator (PGR) with a unique physiological insulin release profile. This single centre, single-dose, open-label study, compared the pharmacokinetics of REP in healthy subjects $(n=12,53.2 \pm$ 5.6 years) with those in subjects $(\mathrm{n}=12,52.9 \pm 7.3$ years) with chronic liver disease (CLD). CLD was defined as a Child Pugh Grade of $\mathrm{B}$ or $\mathrm{C}$ and a caffeine clearance below $0.8 \mathrm{ml} / \mathrm{min} \cdot \mathrm{kg}$. Following a $10 \mathrm{~h}$ overnight fast, subjects received a single REP $4 \mathrm{mg}$ dose. From individual 20 -point, $48 \mathrm{~h}$, concentrationtime profiles obtained prior to dosing until the morning of day 3 , pharmacokinetic parameters were calculated. Subjects with CLD had significantly higher and more prolonged serum levels of both total and unbound REP. AUC, $P<0.01$; Cmax $P<0.01$ compared with the healthy subjects. For CLD subjects AUC was correlated $(\mathrm{r}=-0.7)$ with caffeine clearance $(\mathrm{P}=0.017) . \mathrm{T}_{1 / 2}$ was in the range 0.6 to $14.7 \mathrm{~h}$ for the CLD group. Protein binding of REP was similar for both subject groups (97\%). There were no serious adverse events or withdrawals during the trial. A total of 36 and 29 adverse events were reported by 12 CLD and 11 healthy subjects, respectively. In both groups, 24 hypoglycaemic events were reported by 0 subjects. In conclusion, higher and more prolonged REP serum concentrations in CLD subjects did not result in an increased incidence of hypoglycaemic episodes compared to healthy subjects.

\begin{tabular}{|c|c|c|c|c|}
\cline { 2 - 5 } \multicolumn{1}{c|}{} & $\begin{array}{c}\mathbf{A U C} \\
\left(\mathbf{n g} / \mathbf{m} \mathbf{l}^{*} \mathbf{h}\right)\end{array}$ & $\mathbf{C}_{\max }(\mathbf{n g} / \mathbf{m l})$ & MRT $(\mathbf{h})$ & $\mathbf{t}_{\max }(\mathrm{h})$ \\
\hline $\begin{array}{c}\text { CLD group } \\
\text { (mean, SD) }\end{array}$ & $368.9,233.4$ & $105.4,31.6$ & $5.9,4.4$ & $0.8,0.5$ \\
\hline $\begin{array}{c}\text { Healthy group } \\
\text { (mean, SD) }\end{array}$ & $91.6,67.0$ & $46.7,24.3$ & $1.2,0.4$ & $0.8,0.5$ \\
\hline
\end{tabular}

\section{4}

REPAGLINIDE COMPARED TO GLIPIZIDE IN THE TREATMENT OF TYPE 2 DIABETIC PATIENTS

A. Dejgaard', S. Madsbad ${ }^{2}$, B. Kilhovd ${ }^{3}$, 1. Lager ${ }^{4}$ and P. Mustajoki $i^{5}$. Novo Nordisk AS, Bagsværd, DK; ${ }^{2}$ Hvidovre Hosital, Copenhagen; ${ }^{3}$ Aker Sykehus, Oslo, $\mathrm{N}$; ${ }^{4}$ Centralsjukhuset, Kristianstad, S; ${ }^{5}$ Peijaksen Sairaala, Vantaa, SF

Repaglinide (rep) is a prandial glucose regulator (PGR), the first of a new class of oral antidiabectic agents (carbamoylmethylbenzoic acid derivative (CMBA))

It is metabolised in the liver and has a short T-1/2 of 1 hour. Accordingly it is administered at main meals like insulin is administered as bolus in Type 1 diabetic patients. In a randomised, multicenter, double-blind parallel-group design 250 tablet- or diet treated Type 2 patients were treated for 12 months with either Repaglinide $0,5-4 \mathrm{mg}$ at each meal or Glipizide 5-15 mg daily (B.D.). There was no statistical difference between the two groups at entry as regards age, sex,

weight, Body Mass Index, HBA1c, fasting blood glucose or C-peptide. In an intention to treat analysis Repaglinide showed superiority over Glipizide in mean change of $\operatorname{HBA} 1 \mathrm{C}(0,6 \%, \mathrm{p}<0.05)$ and fasting blood glucose $(0,9 \mathrm{mmol}, p<0,05)$ during the 12 months. Furthermore patients treated with Glipizide had a higher (non-significant) frequency of hypoglycaemic reaction. No changes were seen in lipid metabolism in either treatment group during the trial.

It can be concluded that Repaglinide with its novel dose regimen is an efficacious and safe treatment of Type-2 diabetic patients.
913

REPAGLINIDE CAN BE GIVEN IN A FLEXIBLE PREPRANDIAL DOSING REGIMEN IN PATIENTS WITH TYPE 2 DIABETES. T.C. Marbury', V. Hatorp', P. Damsbo'and P. G. Muller'. ' ${ }^{2}$ OCRC Orlando, Florida, USA; ${ }^{2}$ Novo Nordisk A/S, Bagsvaerd, DK; ${ }^{3}$ Novo Nordisk Inc., Princeton New Jersey, USA

Repaglinide (REP) is a novel fast-acting prandial glucose regulator (PGR) with a short duration of action. This in-patient 28 day study was conducted to compare the efficacy of $1 \mathrm{mg}$ repaglinide (therapeutic dose range: $0.5-4.0 \mathrm{mg}$ ), given preprandially, in maintaining glycaemic control in type 2 diabetic patients who either miss or have an extra meal (mixed regimen), with those who have three regular meals (fixed regimen) a day. After a 1 week "in-patient diet" stabilisation period, 25 patients (18 men and 7 women) were randomised to either a fixed regimen of three meals/day or one of two mixed regimens consisting of repeating patterns of two, three or four meals/day over a 20 -day period. On the 21 st day, every patient received the 3 meals/day regimen. Mean fructosamine concentrations within the two meal regimens decreased significantly ( 3.10 to 2.68 $\mathrm{mmol} / \mathrm{l}$ - fixed-meal; 3.37 to $2.85 \mathrm{mmol} / \mathrm{l}$ - mixed-meal; $\mathrm{P}<0.05$ ), but there was no significant difference between the regimens. Fasting blood glucose levels and serum glucose profiles during the study, were not significantly altered after stabilisation by the number of meals consumed. Based on 37-point blood glucose profiles, AUC over 24 hours was not significantly different between both meal regimens. When lunch was omitted, blood glucose levels remained stable until the next meal. Repaglinide was well tolerated and no hypoglycaemic events were reported. In conclusion, preprandial (meal time) treatment with repaglinide is effective in maintaining glycaemic control irrespective of the number of meals consumed in a day

\section{5}

EFFECTS OF TUNGSTATE ADMINISTRATION TO ZUCKER DIABETIC FATTY RATS

A. Barberà, M.C. Muñoz and J..J. Guinovart. Departament de Bioquimica i Biologia Molecular. Universitat de Barcelona, 08028 Barcelona, Spain

Sodium tungstate is an insulin-like agent whose effects have recently been characterised in two experimental models of diabetes, STZ-diabetic rats and nSTZ-diabetic rats. Here we study the effects of the oral administration of tungstate to Zucker Diabetic Fatty rats (ZDF), an animal model of NIDDM. In these animals, hyperglycaemia appears when they are seven weeks old, and the blood glucose concentration reaches $25 \mathrm{mM}$ at the $11^{\text {th }}$ week. The ZDF rats show insulin resistance associated with a decrease in GLUT4 transporters in the skeletal muscle. Healthy (ZLC) and diabetic (ZDF) rats were treated orally with a $2 \mathrm{mg} / \mathrm{ml}$ sodium tungstate solution for two months. When this compound was administered to 13-week-old diabetic rats, a $30 \%$ decrease in blood glucose concentration and a lower level of triglyceridemia were observed. However, tungstate treatment did not restore the lower levels of glucokinase activity or glucose 6-phosphate found in the diabetic animals. When younger animals, 7.5 weeks old (at the beginning of hyperglycaemia) or 4.5 weeks old (normoglycaemic), were treated with tungstate, the appearance of hyperglycaemia was delayed. Moreover, after one month of treatment the animais had a glycemia about $11 \mathrm{mM}$, significantly lower than the values found in the untreated diabetic animals $(25 \mathrm{mM})$. When the hepatic metabolism was analysed in treated ZDF rats, glucokinase and pyruvate kinase activities increased after the treatment. Furthermore, glucose 6-phosphate values were quite similar to those found in control animals. The administration of tungstate to pre-diabetic animals stimulated hepatic glucose utilisation by increasing in the activity of the glycolytic enzymes, thus leading to a significant decrease in hyperglycaemia. 
916

GLICLAZIDE STIMULATES GLUCOSE TRANSPORT IN SKELETAL MUSCLE THROUGH PI 3-KINASE ACTIVATION.

E. Rodríguez, N. Pulido, S. Terol, R. Romero, B. Casanova, F. J. Arrieta and A. Rovira. Fundación Jiménez Díaz (U. A. M.). Madrid. Spain.

We have previously shown that gliclazide directly stimulates glucose uptake in perfused rat hindquarters. The aim of this work was to determine the possible mechanism of action of gliclazide on glucose transport in soleus muscle. Paired experiments were performed with the two soleus of each rar clamped and isolated from Wistar rats $(180-200 \mathrm{~g}$ ) and incubated in KHB buffer, $\mathrm{pH} 7.4$. ${ }^{3} \mathrm{H}$-deoxi-glucose uptake was measured in basal conditions and after addition of gliclazide $300 \mu \mathrm{g} / \mathrm{ml}$ (70 $\mathrm{min}$ of incubation). Gliclazide produced a significant increase on glucose transport over the basal value $(3.84 \pm 0.4$ vs $2.7 \pm 0.3 \mathrm{nmol} / \mathrm{g} . \mathrm{min}$ mean $\pm S E M, n=11, p<0.05$ ). To know whether the stimulatory effect of gliclazide is produced through the PI-3 kinase activity, we measured the glucose transport in soleus muscle incubated in the absence or the presence of gliclazide or insulin $10^{-9} \mathrm{M}$ with $1 \mu \mathrm{M}$ wortmannin (a potent inhibitor of PI 3-kinase activity). Wortmannin blocked the stimulatory effect on basal glucose uptake of both gliclazide ( $93 \%$ of basal) and insulin (109\% of basal). In a second series of assays, clamped muscles were incubated with gliclazide or insulin in the absence or presence of wortmannin. The stimulatory effect of gliclazide on glucose transport was inhibited by wortmannin $(3.8 \pm 0.3$ vs $2.43 \pm 0.5, p<0.05)$, and wortmannin also inhibited the insulin-stimulated glucose transport $(6.9 \pm 0.5 \mathrm{vs}$ $3.3 \pm 0.6, p<0.05$ ). In conclusion, the acute effect of gliclazide on glucose transport seems to be produced through the activation of PI 3-kinase enzyme.

\section{8}

INHIBTION OF RAT DISACCHARIDASE BY AN ANTIHYPERGLYCEMIC PLANT EXTRACT O. Faruque', R.A. Bethe', J.M.A. Hannan', B. Rokeya', L. Ali', N. Nahar', M. Mosihuzzaman' and A.K. Azad Khan'. 'Dept. of Phamacology, BIRDEM; 2Dept of Chemistry, University of Dhaka, Dhaka, Bangladesh

Delaying of carbohydrate digestion and absorption is a recognized therapeutic approach in the management of diabetes mellitus. Although few drugs relying on this approach are already available improved agents in this group with better potency and less side effects are still required. Extracts of few of the plants have previously been shown to flatten the glucose tolerance curves both in nondiabetic and NIDDM model rats. In the present study hot water extract (ME02) of one of those plants were investigated for its mechanism of action by exploring the effect of the extract on disaccharidase activity and on serum fructosamine and insulin levels of nondiabetic and NIDDM model rats. Two different doses of the extract, 0.5 $\mathrm{g} / \mathrm{kg}(\mathrm{E} 0.5)$ and $1.0 \mathrm{~g} / \mathrm{kg}(\mathrm{E} 1.0)$, were fed daily to the rats for 4 weeks. The control rats (EO) were fed only with water. The last feeding was given after 20 hours fasting of the rats which were then sacrificed. The small intestine between the part just below the duodenum and the part just above the cecum were cut longitudinally, rinsed with ice cold saline and homogenized. The homogenate $(20 \mu \mathrm{ll})$ was incubated with $40 \mathrm{mmol} / \mathrm{h}$ sucrose at $370 \mathrm{C}$ for 1 hour and glucose, converted from sucrose by disaccharidase, was measured by glucoseoxidase method. The protein in the homogenate was determined by using a sensitive spectrophotometric kit (Bio-Rad, USA) and disaccharidase activity was calculated by the concentration of glucose converted from sucrose as $\mu \mathrm{mol}$ glucose $/ \mathrm{mg}$ protein/h. Serum fructosamine was measured by using spectrophotometric method (Boehringer Mannheim, Germany) and insulin by microelisa technique (Crystal Chemical, USA). Serum fructosamine levels were decreased both in normal and NIDOM rats in a dose-dependent manner (S Fructosamine, mmolh, $\mathrm{M} \pm \mathrm{SD}$, Nondiabetic: $86.6+35.8,80.1 \pm 5,77.8+4.8$ and NIDDM: $91.1+7.2,71.9+6.35,62.4+10.7$ in $E 0, E 0.5 \& E 1.0$ Groups respectively). Difference in fructosamine levels, by ANOVA (Bonferroni at $5 \%$ level of significance), was significant between EO-E1.0 in nondiabetic, and EO and EO-E0.5 and EO-E1.0 in NIDDM rats. The levels of fructosamine were paralleled by the levels of enzyme activity ( $\mu \mathrm{mol}$ glucose/mg protein/h, Nondiabetc; $2.3 \pm 0.1,1.8 \pm 0.2,1,6 \pm 0.1$ and NIDDM: $2.8 \pm 0.3,1.9 \pm 0.2,1.7 \pm 0.4$ in EO, E0.5\& E1.0 Groups respectively). The enzyme inhibitory activity in both dosage groups were significantly higher than the control group $(p<0.05)$ in normal as well as NIDDM rats. The insulin levels remained unchanged in all the groups. The result suggest that extract ME02 has no stimulating action on B cells and its anthyperglycemic effect is related to the inhibition of disaccharidase activity in the gut.

\section{7}

EFFECTS OF METFORMIN ON FUNCTIONAL CAPILLARY DENSITY IN DIABETIC HAMSTERS

E. Bouskela, F.Z.G.A. Cyrino and N. Wiernsperger ${ }^{1}$. Laboratório de Pesquisas em Microcirculação, State University of Rio de Janeiro, RJ, Brazil and 'Diabetic Microangiopathy Research Unit, Lipha/Inserm, Villeurbanne, France

The aims of our study were to determine, using the hamster cheek pouch preparation, the effects of metformin (N-N'-dimethylbiguanide) on functional capillary density [(FCD), number of red-cell-perfused capillaries per observation field] in (1) control conditions (before ischemia) and (2) during reperfusion after $90 \mathrm{~min}$ ischemia [(I/R) obtained with a cuff placed around the neck of the everted pouch] in moderately (glycemia between 160 and $240 \mathrm{mg} / \mathrm{dl}$ ) and severely (glycemia $>240 \mathrm{mg} / \mathrm{dl}$ ) diabetic hamsters. Diabetes was induced by 2 (moderate) or 3 (severe) i.p. injections of streptozotocin $(50 \mathrm{mg} / \mathrm{kg} /$ day $)$ given in 2 or 3 days, respectively. The animals were divided in 4 groups: moderately (placebo and metformin-treated) and severely (placebo and metformin-treated) diabetic. The animals were treated for 30 days after the onset of diabetes with either vehicle or $20 \mathrm{mg} / \mathrm{kg} /$ day metformin, once a day. The cheek pouch was placed under an intravital microscope coupled to a closed circuit TV system to determine FCD. FCD in moderately and severely diabetic hamsters (1) in control conditions tented to be higher in metformin-treated animals (moderately - placebo: $89.7 \pm 3.7$ and metformin: $114.0 \pm 3.5$ and severely - placebo: $80.5 \pm 2.7$ and metformin: $90.8 \pm 2.4$ ) and (2) at the onset of reperfusion was significantly higher in metformintreated groups (moderately - placebo: $54.7 \pm 3.4$ and metformin: $96.5 \pm 2.9$ and severely - placebo: $56.8 \pm 4.1$ and metformin: $73.7 \pm 4.8$. Our results show that metformin is able to improve FCD which is an important determinant of tissue oxygenation.

\section{9}

RETARDATION OF INTESTINAL. CARBOHYDRATE DIGESTION AND ABSORPTION BY AN ANTIHYPERGLYCEMIC PLANT MATERIAL EXTRACT

B. Rokeya', JMA Hannan', S. Haque', L. Ali', N. Nahar', M. Mosihuzzaman'2 and A.K. Azad Khan', 'BIRDEM, 'Dept of Chemistry, University of Dhaka, Dhaka, Bangladesh

A hot water extract (M001) from a tropical plant material has previously been shown to fiatten the postprandial rise of blood glucose in both IDDM and NIDDM model rats. In the present study the effects of the extract on carbohydrate digestion and absorption and on serum insulin levels in normal and NIDDM model rats were investigated both in acute and 4-week chronic studies with different dosages $(0.5 \mathrm{~g} / \mathrm{kg}$ and $1 \mathrm{~g} / \mathrm{kg}$ ). Carbohydrate digestion was evaluated by measuring the amount of sucrose remaining in 6 different parts of the gastrointestinal tract after feeding of sucrose. Sucrose solution ( $2.5 \mathrm{~g} / \mathrm{kg}$ body weight) was fed to $20 \mathrm{hrs}$ fasted rats with or without extract ( $500 \mathrm{mg} / \mathrm{kg}$ body weight). Insulin level (by microelisa technique) was estimated at different timings (fasting, 30, 60,120 and $240 \mathrm{~min}$ ) after sucrose load. The GITs of rats, sacrificed at $30,60,120$ and $240 \mathrm{~min}$, were removed and washed with ice-cold normal saline. The wash-out fluid, acidified by $\mathrm{H}_{2} \mathrm{SO}_{4}$ and neutralized by $\mathrm{NaOH}$, was analyzed for its glucose content (by enzymatic-colorimetric methods) liberated from sucrose and the remaining sucrose in the gut was estimated by back calculation of glucose. In the NIDDM Control Group (CG) sucrose loading led to a rapid increase of blood glucose (mmoli, $M \pm S D$, fasting $4.45+0.65,30 \mathrm{~min} 17.17+1.50,60 \mathrm{~min} 16.79+1.82,120 \mathrm{~min} 9.10+0.75$ and $240 \mathrm{~min} 4.21+0.52$ ) whereas in the Extract Group (EG) the blood glucose response was considerably flattened (fasting $5.31 \pm 0.39,30 \mathrm{~min} 12.26 \pm 1.39,60 \mathrm{~min} 10.40 \pm 0.93,120 \mathrm{~min} 7.72+0.58$ and $240 \mathrm{~min}$ $6.29+0.26)$. The change in the blood glucose were inversely related by the residual gastrointestinal sucrose content of the 2 groups of rats (Table).

\begin{tabular}{|l|l|l|l|l|l|l|l|l|}
\hline \multirow{2}{*}{$\begin{array}{l}\text { Gut } \\
\text { Segment }\end{array}$} & \multicolumn{2}{|c|}{$30 \mathrm{~min}$} & \multicolumn{2}{c|}{$60 \mathrm{~min}$} & \multicolumn{2}{c|}{$120 \mathrm{~min}$} & \multicolumn{2}{c|}{$240 \mathrm{~min}$} \\
\cline { 2 - 9 } & $\mathrm{CG}$ & $\mathrm{EG}$ & $\mathrm{CG}$ & $\mathrm{EG}$ & $\mathrm{CG}$ & $\mathrm{EG}$ & $\mathrm{CG}$ & $\mathrm{EG}$ \\
\hline Stomach & $37 \pm 3$ & $120 \pm 7$ & $8 \pm 1$ & $24 \pm 3$ & $3 \pm .6$ & $6 \pm 1$ & $0.6 \pm .1$ & $2.0 \pm .3$ \\
\hline SIU $20 \mathrm{~cm}$ & $14 \pm 3$ & $9 \pm 3$ & $2.0 \pm .4$ & $4.1 \pm .6$ & $1.1 \pm .3$ & $1.9 \pm .3$ & $0.6 \pm .1$ & $1.3 \pm .1$ \\
\hline Middle & $6 \pm 2$ & $7 \pm 2$ & $0.7 \pm .1$ & $3.2 \pm .4$ & $1.2 \pm .3$ & $2.5 \pm .6$ & $0.6 \pm .2$ & $2.9 \pm .6$ \\
\hline SIl $20 \mathrm{~cm}$ & $2 \pm 1$ & $1 \pm .3$ & $0.6 \pm .1$ & $1.8 \pm .3$ & $0.4 \pm .1$ & $2.1 \pm .3$ & $0.7 \pm .1$ & $3.3 \pm .2$ \\
\hline Cecum & $1 \pm .5$ & $2 \pm 1$ & $0.8 \pm .1$ & $1.4 \pm .1$ & $0.6 \pm 1$ & $1.9 \pm .2$ & $0.3 \pm .1$ & $2.9 \pm .2$ \\
\hline $\mathrm{LI}$ & $1 \pm .4$ & $1 \pm .6$ & $0.3 \pm .1$ & $0.8 \pm .2$ & $0.5 \pm 1$ & $0.9 \pm .2$ & $0.2 \pm .1$ & $0.6 \pm .1$ \\
\hline
\end{tabular}

SIU: Small intestine upper, SIL: Small intestine lower, LI: Large intestine, Mean \pm SD, result in mg

The serum insulin levels were unchanged both in acute and chronic studies. The results indicate that the reported antihyperglycemic activity of the extract is related to the delaying of carbohydrate digestion and absorption from the gut. 
920

\section{EFFECT OF METFORMIN ON GLUCOSE METABOLISM} IN TYPE 2 DIABETES MELLITUS

H.G. Wahl, D. Overkamp, M. Stumvoll, A. Fritsche, H.U. Häring and R.M.

Schmülling. Medizinische Universitätsklinik Abt. IV, Tübingen, Germany Metformin has been shown in many studies to lower plasma glucose levels in patients with type 2 diabetes mellitus. The aim of our study was to investigate the effect of metformin on glucose metabolism in type 2 diabetes patients by stable isotope methodology. U-13C glucose $(7 \mathrm{mg} / \mathrm{kg}$ ) was used to asses total glucose oxidation in addition to endogenous glucose production. $3-13 \mathrm{C}$ glucose $(300 \mathrm{mg})$ was used in a second experiment to asses more specific the effects of metformin on the pyruvate dehydrogenase complex involved in the oxidative decarboxylation of pyruvate to Acetyl-CoA. Glucose oxidation was determined by indirec calorimetry $\left(\mathrm{VCO}_{2}\right)$ and mass spectrometry $\left({ }^{13} \mathrm{CO}_{2}\right)$. Lactate kinetics were calculated by analysis of $\mathrm{U}-13 \mathrm{C}$ lactate derived from $\mathrm{U}-13 \mathrm{C}$ glucose. Patients were admitted to the clinic the night before the first experiment and stayed for three days until the end of the second tracer experiment. After 10 weeks of metformin treatment this experimental setup was repeated. The initial dose of $850 \mathrm{mg}$ of metformin was increased every second week up to a final dose of $2550 \mathrm{mg}$ unless the patient had a fasting plasma glucose (FPG) below $120 \mathrm{mg} / \mathrm{dL}$, which did not happen in this first six patients (age $42 \pm 7$ years, duration of diabetes $5 \pm 1$ years). The effect of metformin on melabolic profile is shown in the following table (Mean \pm SEM, Chol=cholesterol. TG=triglyceride, FFA=free fatty acids).

\begin{tabular}{|c|c|c|c|c|c|c|c|c|}
\hline & $\begin{array}{c}\mathrm{HbAlc} \\
{[\%][]}\end{array}$ & $\begin{array}{c}\text { FPG } \\
\text { [mg/dLI }\end{array}$ & $\begin{array}{l}\text { Insulin } \\
{[\mathrm{pmol} / \mathrm{L}]}\end{array}$ & $\begin{array}{l}\text { Lactate } \\
\text { [munothl }\end{array}$ & $\begin{array}{l}\text { BMI } \\
{\left[\mathrm{kg} / \mathrm{m}^{2}\right]}\end{array}$ & $\begin{array}{l}\text { Chol } \\
\text { ImgdLI }\end{array}$ & $\begin{array}{c}\mathrm{TG} \\
\text { [mg d d }]\end{array}$ & $\begin{array}{c}\text { FFA } \\
{[\mathrm{mmol} / \mathrm{L}]}\end{array}$ \\
\hline & & $175 \pm 11$ & $187 \pm 4$ & $1.3 \pm$ & $40 \pm 4$ & $216 \pm 9$ & $254+52$ & $1.3 \pm 0.2$ \\
\hline & & & 18 & $1.7 \pm 0$. & $39 \pm$ & $181 \pm 6$ & $201 \pm 36$ & \\
\hline
\end{tabular}

Getformin reduces endogenous glucose production significantly from $23 \pm 0.1$ to $2.0 \pm 0.1 \mathrm{mg} / \mathrm{kg} / \mathrm{min}$ with no change in glucose clearance of $1.3 \pm 0.1 \mathrm{ml} / \mathrm{kg} / \mathrm{min}$ Based on the U-13C lactate kinetics there seems to exist a group of patients with accelerated generation of lactate from U-13C glucose. There was an increase in glucose oxidation based on 3-13C glucose as tracer in contrast to no significant change of $\mathrm{U}-13 \mathrm{C}$ glucose oxidation which points to the pynuvate dehydrogenase complex as one possible site of metformin action.

\section{2}

ROSIGLITAZONE (BRL49653) MONOTHERAPY HAS SIGNIFICANT GLUCOSE EFFECT IN TYPE 2 DIABETIC PATIENTS

H.E. Lebovitz, J Patel, J Dole and R Parwardhan. New York NY, Collegeville $P A$ Rosiglitazone (RSG) is the most potent member of the thiazolidinedione class in clinical development. In a multi-centre, placebo-controlled trial, 493 patients with a fasting glucose between 140 and $300 \mathrm{mg} / \mathrm{dL}$, were randomly assigned to treatment with placebo, RSG $4 \mathrm{mg}$ or RSG $8 \mathrm{mg}$ per day administered as a twice daily regimen for $26 \mathrm{wks}$, following a $4 \mathrm{wk}$ placebo run-in period. The ITT efficacy results are summarized below

\begin{tabular}{|c|c|c|c|}
\hline \multirow{5}{*}{$\begin{array}{l}\mathrm{HbAlc}(\%) \text {-baseline } \\
\text { mean } \Delta \text { from baseline (SD) } \\
\text { comparison with placebo\# } \\
95 \% \mathrm{CI}\end{array}$} & Placebo $(n=158)$ & RSG 4mg (n=166) & RSG8mg (n=169 \\
\hline & 9.04 & $9.02^{\circ}-1-x$ & \\
\hline & $0.92(1.21)$ & $-0.28(1.27)$ & $-0.56(1.38)$ \\
\hline & & & \\
\hline & - & $(-1.52,-0.89)$ & $(-1.85,-1.22)$ \\
\hline FPG (mg/dL) - baseline & 228.8 & 226.9 & 219.7 \\
\hline mean $\Delta$ from baseline (SD) & $18.9(64.5)$ & $.38 .4(52.4)$ & $.54 .0(51.4)$ \\
\hline comparison with placebo" & $\cdot-$ & $-57.7^{\mathrm{tat}}$ & $-76^{* *}$ \\
\hline $95 \% \mathrm{Cl}$ & - & $(-70.9,-44.6)$ & $(-89.2,-62.9)$ \\
\hline (\# adjusted mean diffe & $p<0.0$ & & \\
\hline
\end{tabular}

These results demonstrate that RSG had a clinically and highly statistically significant glucose lowering effect compared to placebo and baseline. The change from baseline in $\mathrm{HbAlc}$ in the subset of patients who had previously failed to be controlled on dietary therapy alone demonstrated a greater change from baseline in the RSG treated groups:

HbA Ic (\%) (diet failure subset)

baseline 45 8.54 mean $\Delta$ from baseline (SD) 0.47 (1.14)

$$
44
$$$$
.83(0.93
$$$$
8.51
$$$$
-0.91(1.04)
$$

Similar proportions of patients in each treatment group reported adverse experiences. There were no serious adverse experiences related to the liver or hypoglycemis. In conclusion, rosiglitazone monotherapy has been demonstrated to be well tolerated and effective in lowering FPG and HbAlc in Type 2 diabetic patients.

\section{1}

LONG-TERM TREATMENT WITH SULFONYLUREAS ENHANCES THE EXHAUSTION OF NNSULIN SECRETION IN NIDDM PATIENTS

K.Ueda, K.Tatsumi, T.Nakagawa, Y.Shimajiri, E.Matsumoto, T.Sanke, and K.Nanjo. The First Department of Medicine, Wakayama University of Medical Science, Wakayama, Japan

The aim of this study was to investigate whether long-term treatment with Sulfonylureas (SU) may enhance the exhaustion of insulin secretion of NIDDM patients. The capacity of insulin secretion was evaluated using glucagon test ( $\triangle C P R 5^{5}$ ) in 13 NIDDM patients treated with SU (SU group) and 6 NIDDM patients treated with insulin (INS group) during 5 to 10 years-follow-up. At the baseline, $\triangle \mathrm{CPR} 5^{\prime}$, age, DM duration and body mass index were matched between two groups. $\triangle \mathrm{CPR} 5^{3}$ of SU group significantly decreased in time-dependent manner as compared with INS group [d $\triangle C P R 5$ ' $(\mathrm{ng} / \mathrm{ml} / \mathrm{yr}):-0.112 \pm 0.022$ vs $-0.019 \pm 0.018, \mathrm{p}<0.05$ (mean $\pm \mathrm{SE}$ )] . In SU group, $\triangle \mathrm{CPR} 5^{\prime}$ of poorly controlled (HbAlc $\geqq$ $8.5 \%$ ) patients or patients with family history of NIDDM showed relatively marked decreasing tendency. In particular, $\triangle C P R 5^{\circ}$ of poorly controlled patients with family history of NIDDM was obviously less than that of other patients ( $\mathrm{d} \triangle \mathrm{CPRS} \mathrm{s}^{\prime}:-0.202 \pm 0.074 \mathrm{vs}-0.072 \pm 0.041$ ). These data suggest that long-term treatment with $\mathrm{SU}$ enhances the exhaustion of insulin secretion in poorly controlled NIDDM patients with family history of NIDDM

\section{3}

\section{ACARBOSE COMBINED WITH METFORMIN IMPROVES THE GLYCEMIC CONTROL IN NIDDM PATIENTS WITH OVERWEIGHT}

S. Halimi, M.A. Le Berre and V. Grangé. CHU Grenoble, 38043 Grenoble, France.

Acarbose, the first alpha-glucosidase inhibitor, is known to improve glycemic control in patients with non-insulin dependant diabetes mellitus (NIDDM) managed with diet alone. However, it is controversial whether this drug is effective when combined in patients treated with biguanide. Method: the objective of this study was to test the efficacy of acarbose in combination with metformin. This double blind randomized study consisted in adding acarbose or placebo in patients previously treated with metformin, for at least 2 months, for a treatment period of 6 months. During the first 2 months, $50 \mathrm{mg}$ of acarbose or placebo where given three times per day respectively. After two months, a non forced titration to $100 \mathrm{mg}$ three times per day was possible. Patients: 129 patients completed the study (acarbose:59, placebo:70) and were selected for the intention to treat analysis. There was no statistically significant difference between the two groups concerning the demographic characteristics. Patients had a diabetes history (median) 7 years and 8 months for the acarbose group and 5 years and 6 months for the placebo group $(p<0.05$ ). The mean BMI was respectively 30.13 $3.46 \mathrm{~kg} / \mathrm{m}^{2}$ (acarbose) and $29.4 \pm 3.89 \mathrm{~kg} / \mathrm{m}^{2}$ (placebo). Results: In the analysis of covariance,the baseline value was included as covariate. This analysis showed a significant difference between the treatments groups for $\mathrm{HbAlc}(\mathrm{p}<0.01)$, and for fasting serum glucose $(p<0,01)$.

$\begin{array}{rrrr}\text { HbAlc } & \text { HbAlc } & \begin{array}{r}\text { Fasting } \\ \text { glycemia }\end{array} & \begin{array}{r}\text { Fasting } \\ \text { glycemia } \\ \text { placebo }\end{array} \\ \text { acarbose } & \text { Placebo } & \text { acarbose } & \end{array}$

Baseline $8.58 \pm 1.13 \quad 8.54 \pm 1.12 \quad 10.57 \pm 3.06 \quad 10.48 \pm 2.64$ End point $7.88 \pm 1.50 \quad 8.77 \pm 1.53 \quad 9.58 \pm 3.11 \quad 11.73 \pm 3.72$

Conclusion: in NIDDM patients with overweight not controlled by metformin alone, combination with acarbose is effective in improving glycemic control. 


\section{4}

ROSIGLITAZONE THERAPEUTIC INDEX: SEPARATION OF EFFICACY AND HAEMODILUTION

L.Pickavance, P.S. Widdowson, R.E. Buckingham* and J.P.H. Wilding. Dept. of Medicine, Liverpool University, U.K. \& SmithKline Beecham Pharmaceuticals*, Harlow, U.K.

In rodent models of insulin resistance and in Type 2 diabetic human subjects, thiazolidinedione (TZD) insulin-sensitising agents improve glucose metabolism but cause haemodilution. To date, the therapeutic ratio of TZDs for glucose lowering and haemodilution has not been determined. Rosiglitazone (RSG), the only aminopyridyl glitazone and a 2nd generation PPAR $\gamma$ activator, is the most potent member of the class and we have determined a therapeutic ratio for this agent in dietary obese, insulin-resistant rats. Thirty-six male Wistar rats $(\sim 200 \mathrm{~g})$ were fed a highly palatable, high-fathigh-sugar diet for 8 weeks to induce mild obesity ( 313 世 $6 \mathrm{~g}$ vs $293 \pm 7 \mathrm{~g}$ in age-matched chow-fed controls; $\mathrm{p}<0.05$ ). Obese rats (6/group) then received daily treatment for $\mathbf{2 1}$ days by gavage with control vehicle or RSG $(0.3,1,3,10$ or $30 \mathrm{mg} / \mathrm{kg}$ body weight $)$. At the end of treatment, efficacy of RSG was assessed by incorporating values of fasting plasma glucose and insulin into the Homeostasis Model Assessment equation. Dose-dependent improvements in insulin sensitivity were observed, with a threshold of $1 \mathrm{mg} / \mathrm{kg}$ ( $4.63 \pm 0.50$ vs $6.23 \pm 0.76$ in obese controls; $\mathrm{p}<0.05$ ). Two further indices of drug action, fasting plasma free fatty acids (FFAs) and epididymal fat pad mass, were also significantly altered by the drug, though at a lower threshold dose. Thus at $0.3 \mathrm{mg} / \mathrm{kg}$, RSG evoked a fall in FFAs $(0.42 \pm 0.06$ vs $0.67 \pm 0.08 \mathrm{mM}$ in controls; $\mathrm{p}<0.01)$ and an increase in epididymal fat pad mass $(16.25 \pm 1.53$ vs $12.56 \pm 1.40 \mathrm{~g}$ in controls; $\mathrm{p}<0.05)$. By contrast, the threshold dose of RSG for eliciting haemodilution (packed cell volume: $45.5 \pm 0.5$ vs $47.2 \pm 0.3 \%$ in controls; $p<0.05$ ) and concomitant cardiac hypertrophy $(2.19 \pm 0.06$ vs $1.93 \pm 0.06 \mathrm{~g} ; \mathrm{p}<0.001)$ was $10 \mathrm{mg} / \mathrm{kg}$. These results indicate that for RSG, using insulin sensitivity as the index of efficacy, the therapentic index in the dietary obese male $W$ iscar rat, is $>3$ and $\leq 10$. In conclusion, our data demonstrate that RSG's therapeutic activity is separable from its effects on haemodilution.

\section{5}

RESULTS OF 1-YEAR STUDY WITH PRAMLINTIDE THERAPY IN TYPE 1 DIABETES: EFFECT ON METABOLIC CONTROL AND SAFETY PROFILE

J. Rosenstock, F. Whitehouse, S. Schoenfeld, S. Jeffcoate, and O. Kolterman for the Pramlintide Type 1 Study Group. Dallas TX; Detroit MI; San Diego CA, USA

In type 1 diabetes, achieving and maintaining target metabolic control remains a major challenge, especially if postprandial hyperglycemia, weight gain, and the potential for hypoglycemic events are to be avoided. Pramlintide is a synthetic analog of the human $\beta$-cell hormone amylin, which is deficient in type 1 diabetes. The aim of this 52-week, multicenter, double-blind, placebo-controlled study was to evaluate the effects of pramlintide therapy on metabolic control in people with type 1 diabetes. 477 subjects were treated subcutaneously with insulin and either pramlintide $(30 \mathrm{or} 60 \mu \mathrm{g}$ ) or placebo QID. Mean age was $\sim 40 \mathrm{yrs}$, mean duration of pramlintide $(30 \mathrm{or} 60 \mu \mathrm{g})$ or placebo QID. Mean age was $\sim 40$ yrs, mean duration of
diabetes was $\sim 17$ yrs and mean baseline $\mathrm{HbA}_{1 \mathrm{c}}$ was $\sim 8.8 \%$. Effects of pramlintide on $\mathrm{HbA}_{\mathrm{lc}}$ (change from baseline at 52 weeks) are shown in the table for all subjects completing the study and for those with baseline $\mathrm{HbA}_{\mathrm{lc}} \geq 8$ who did not vary insulin dose more than $20 \%$ during the study.

\begin{tabular}{|c|c|c|}
\hline & Pramlintide 30 or $60 \mu \mathrm{g}$ & Placebo \\
\hline $\begin{array}{l}\text { Patients with } \mathrm{HbA}_{\mathrm{lc}} \text { at all visits } \\
\text { (including baseline }<8 \% \text { ) }\end{array}$ & $\begin{array}{r}-0.39 \% * \\
(\mathrm{n}=171)\end{array}$ & $\begin{array}{l}-0.09 \% \\
(\mathrm{n}=161) \\
\end{array}$ \\
\hline $\begin{array}{l}\text { Baseline } \mathrm{HbA}_{1 \mathrm{c}} \geq 8 \% \text { and insulin } \\
\text { variability } \leq 20 \%\end{array}$ & $\begin{array}{c}-0.83 \% \text { (NS) } \\
(n=40)\end{array}$ & $\begin{array}{c}-0.16 \% \\
(\mathrm{n}=33) \\
\end{array}$ \\
\hline
\end{tabular}
$(n=40)$

* $p<0.004$; statistically significant difference from placebo

Improvements in glucose control were achieved without an increase in the frequency of severe hypoglycemic events. With this improved control, subjects treated with pramlintide achieved an average reduction in body weight of $1.3 \mathrm{~kg}$ compared with placebo $(\mathrm{p}=0.01)$ and an average increase in serum HDL/LDL cholesterol ratio of $8.6 \%$ compared with placebo $(\mathrm{p}=0.0008)$. The most common drug-related side effect was mild nausea, which generally dissipated during the initial 4 weeks of therapy. In conclusion, compared with insulin alone, the addition of pramlintide in type 1 diabetes resulted in metabolic benefits including significant improvements in glucose control, body weight, and HDL/LDL ratio without an increase in the incidence of severe hypoglycemic events.

\section{6}

4-HYDROXYISOLEUCINE IMPROVES GLUCOSE TOLERANCE IN NORMAL AND NIDDM ANIMALS

C. Broca ${ }^{1}$, R. Gross ${ }^{1}$, P. Petit ${ }^{1}$, Y. Sauvaire ${ }^{2}$, M. Manteghetti ${ }^{1}$, P. Masiello ${ }^{3}$. R. Gomis ${ }^{4}$ and G. Ribes ${ }^{1}{ }^{1}$ Lab. Pharmacol. Fac. Med. (UMR 9921 CNRS and UPRES 1677). ${ }^{2}$ Lab. Rech. Subst. Nat. Veget. (UPRES 1677) Montpellier France. ${ }^{3}$ Istit. Patol. Gen. Pisa Italia. ${ }^{4}$ Diabetes Unit. Hosp. Clin. Barcelona Spain.

We have previously shown that 4-hydroxyisoleucine (4-OH-Ile) extracted from Trigonella foenum graecum $L$ seeds is a novel amino acid potentiator of insulin secretion, both in rat and human isolated islets. The present study was designed to investigate whether 4-OH-Ile could have antidiabetic properties in vivo. For this purpose, intravenous (IVGTT) or oral (OGTT) glucose tolerance tests were performed in normal rats, normal dogs and NIDDM rats made diabetic by injections of nicotinamide $(230 \mathrm{mg} / \mathrm{kg}$ i.p. $) 15$ min before streptozotocin $\left(65 \mathrm{mg} / \mathrm{kg}\right.$ i.v.). Our results show that: $\left.1^{\circ}\right)$ during IVGTT in anesthetized fed normal rats, $4-\mathrm{OH}-\mathrm{Ile}(18 \mathrm{mg} / \mathrm{kg}$ i.v. $)$ enhanced glucose induced insulin secretion $(+125 \%)$ and thus improved glucose tolerance: area under the curve for $30 \mathrm{~min}$ (AUC/30 min) was $5421 \pm 125 \mathrm{mg}$ versus $6459 \pm 67 \mathrm{mg}$ in controls, $p<0.01 .2^{\circ}$ ) a similar effect was obtained with 4-OH-Ile during OGTT in conscious normal dogs (AUC/45min: $5027 \pm 107 \mathrm{mg}$ versus $6290 \pm 140 \mathrm{mg}$ in controls, $\mathrm{p}<0.01) .3^{\circ}$ ) in NIDDM rats, an acute administration of $4-\mathrm{OH}-\mathrm{ll}(50 \mathrm{mg} / \mathrm{kg}$ i.v.) restored glucose induced insulin response (AUC/30min: $116 \pm 14 \mathrm{ng}$ versus $87 \pm 7 \mathrm{ng}$ in NIDDM control rats, $\mathrm{p}<0.05) .4^{\circ}$ ) in NIDDM rats a daily administration of $4-\mathrm{OH}-\mathrm{Ile}(50 \mathrm{mg} / \mathrm{kg}$ i.p.) for 6 days provoked a significant increase in glucose induced insulin response but also a significant effect on glucose tolerance (AUC/30min: $6569 \pm 101 \mathrm{mg}$ versus $7841 \pm 72 \mathrm{mg}$ in NIDDM controls, $\mathrm{p}<0.05$ ). In conclusion 4-hydroxyisoleucine clearly improves in vivo the insulin response to glucose in normal and NIDDM animals. This new insulinotropic agent may be considered with potential interest for the treatment of NIDDM. 


\section{Hypoglycaemia}

\section{7}

SUPPRESSION OF ADRENERGIC ACTIVITY DOES NOT PREVENT HYPOGLYCEMIA-ASSOCIATED COGNITIVE DYSFUNCTION

D.J. Becker, C.M. Ryan, and C. Suprasongsin. Children's Hospital of Pittsburgh, 3705 Fifth Ave, Pittsburgh PA 15213, USA

Previous work has demonstrated a relationship between increased levels of epinephrine during experimental hypoglycemia and cognitive dysfunction. To assess the effect of hypoglycemia independent of epinephrine, we used adrenergic blockade to prevent the $\alpha$ and $\beta$ adrenergic actions of epinephrine and norepinephrine stimulated by hypoglycemia. Specifically, propanolol ( $\beta$ adrenergic blocker) was infused at a dose of $1.4 \mu \mathrm{g} / \mathrm{kg} / \mathrm{min}$ after a loading bolus of $140 \mu \mathrm{g} / \mathrm{kg} / \mathrm{min} \times 2 \mathrm{~min}$, and phentolamine ( $\alpha$ adrenergic blocker) was infused at a dose of $7.1 \mu \mathrm{g} / \mathrm{kg} / \mathrm{min}$ after a loading dose of

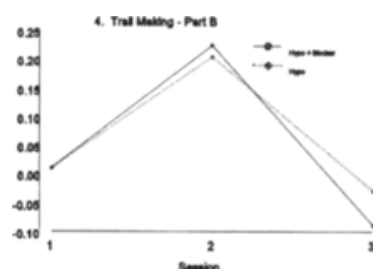
$71 \mu \mathrm{g} / \mathrm{kg} / \mathrm{min} \times 2 \mathrm{~min}$ in normal saline. Twenty-three diabetic subjects (mean age $=17.6 \mathrm{yrs}$ ) participated in two hypoglycemic clamps: one standard clamp in which mild hypoglycemia ( 3.3 $\mathrm{mmol} / \mathrm{L}$ ) was maintained for 45 minutes prior to cognitive assessment, and one with $\alpha$ and $\beta$ adrenergic blockade. Sustained attention was measured with the Digit Vigilance Test, mental flexibility was assessed with Trail Making B, and decision-making efficiency was assessed with the computerized Letter Rotation Test. We found that adrenergic blockade did not prevent the occurrence of hypoglycemia-associated cognitive deterioration on any measure, nor did it have any effect on the degree of induced anxiety, as indexed by the State Anxiety Inventory. We conclude that the adrenergic hormonal changes occurring during hypoglycemia are markers of CNS autonomic changes, but do not have any major direct effects on factors influencing cognitive function in diabetics.

\section{8}

GENDER DIFFERENCES IN COUNTERREGULATION DURING HYPOGLYCAEMIA IN TYPE I DIABETIC PATIENTS

A. MARAN, C. CREPALDI, E. IORI, C. MARESCOTTI, A. AVOGARO, S.

DEL PRATO and A. TIENGO. Department of Clinical and Experimental Medicine, University of Padova, Italy.

It has been previously demonstrated that during insulin-induced hypoglycaemia countrerregulatory responses are brisker in males than females non-diabetic subjects. To test the hypothesis that these differences are maintained in diabetic individuals, we have studied 14 Type I diabetic patients ( $7 \mathrm{M}$ and $7 \mathrm{~F}$, age $37 \pm 2$ vs $33 \pm 3 \mathrm{yrs}$, diabetes duration $14 \pm 1$ vs $13 \pm 1 \mathrm{yrs}$, HbAlc $8.7 \pm 0.2$ vs $8.6 \pm 0.2 \%$, all $p^{-}$N.S.) without loss of awareness of hypoglycaemia during a stepwise hypoglycemic clamp study (blood glucose maintained at euglycaemia for 40 minutes then reduced stepwise to $3.6,2.8$, $2.4 \mathrm{mmol} / \mathrm{l}$; insulin infusion $1.5 \mathrm{mU} / \mathrm{kg} / \mathrm{min}$ ). Plasma counterregulatory hormones, symptomatic responses and cognitive function (4-choice reaction time test) were measured at euglycemia and throughout the hypoglycemic clamp. Adrenaline response was significantly lower in females (peak $1.6 \pm 0.7$ vs $0.74 \pm 0.3 \mathrm{pmol} / \mathrm{ml}, \mathrm{p}=0.02)$ starting at lower blood glucose $(3.5 \pm 0.2$ vs $2.7 \pm 0.2 \mathrm{mmol} / \mathrm{l}, \mathrm{p}=0.01$ ). Autonomic symptoms (i.e. sweating, warmness, anxiety, shakiness) started at a similar glucoses in both sexes $(2.8 \pm 0.1$ vs $2.6 \pm 0.1 \mathrm{mmol} / \mathrm{l}, \mathrm{p}=0.2$ ), while neuroglycopenic symptoms (i.e. confusion, dizziness, lack of concentration, blurred vision) were experienced in females at lower blood glucose $(3.1 \pm 0.1$ vs $2.3 \pm 0.1 \mathrm{mmol} / \mathrm{l}, \mathrm{p}=0.001)$. Cognitive dysfunction occurred earlier in males than females (loss of accuracy: $3.1 \pm 0.2$ vs $2.4 \pm 0.2 \mathrm{mmol} / \mathrm{l}, \mathrm{p}=0.02$, speed deterioration: $3.2 \pm 0.2$ vs $2.6 \pm 0.2 \mathrm{mmol} / \mathrm{l}$, $p=0.02$ ). In conclusion, in Type I diabetic patients without hystory of hypoglycaemia unawareness the glucose threshold for counterregulatory responses and cognitive dysfunction are higher in females than in males. This must be taken into account whenever the counterregulatory responses to insulin-induced hypoglycemia are investigated or interpreted, not only in normal subjects but also in IDDM patients.

\section{9}

DECREASE IN THE NEUROENDOCRINE RESPONSE TO HYPOGLYCEMIA DURING THE FIRST YEAR OF TYPE 1 DIABETES IN MAN

J.Hilsted and M.B.Damholt, Dept of Endocrinology, Hvidovre Hospital, Denmark.

The aim of the study was to describe peripheral somatic and autonomic nerve function in diabetic patients during the first year after the diagnosis of type 1 diabetes. Methods : All patients with newly diagnosed type 1 diabetes submitted to our department during two years were invited to the study. The patients were evaluated within 1 month and 3,6 and 12 months after the diagnosis. At each visit the evaluation comprised history; clinical examination;blood tests; electrophysiology, biothesiometry, tests of heart rate variability and insulininduced hypoglycemia (method : á modum Bolli and White) during which blood for hormone analysis was drawn. Results: Eight of eleven patients accepted to participate. Five patients completed the study. Median $\mathrm{Hb}_{\mathrm{AlC}}$ was $12 \%(10.0,12.8)\left(25 \%\right.$ and $75 \%$ fractiles) at baseline. After one year median $\mathrm{HB}_{\mathrm{A} 1}$ had decreased to $7.9 \%(7.3,8.8)$. In one case hypoglycemia could not be achieved at baseline. Results of the 3 - and the 12 months evaluation are therefore compared. The maximal p-epinephrine concentration during hypoglycemia decreased significantly at 12 months $(p<0.05)$. Further insignificant decrease in the maximal $\mathrm{p}$-norepinephrine concentration $(\mathrm{p}=0.14)$ and in the maximal incremental $\mathrm{p}-\mathrm{PP}$ concentration during hypoglycemia $(p=0.08)$ was found. The latter has been suggested to be a marker of vagal function. There was no significant differences in the lowest blood glucose concentration achieved $(p>0.14)$. No significant changes in somatic peripheral nerve function, orthostatic tests or heart rate variability tests could be detected. Conclusion: Our data suggest that a decrease in the neuroendocrine response to hypoglycemia, which may predispose to hypoglycemia associated autonomic dysfunction, is detectable as early as within the first year after diagnosis of type 1 diabetes. The present results support the hypothesis that hypoglycemia associated autonomic dysfunction is not attributable to classical autonomic neuropathy.

\section{0}

\section{THEOPHYLLINE AND GLUCOSE RECOVERY AFTER} HYPOGLYCAEMIA IN PATIENTS WITH TYPE 1 DIABETES

A. Hvidberg Hellebek, A. Rosenfalck, N.J. Christensen and J. Hilsted Departments of Internal Medicine and Endocrinology, Hvidovre Hospital and Herlev Hospital, Copenhagen, Denmark.

We tested the hypothesis that long term administration of theophylline augments glucose recovery after insulin induced hypoglycaemia. Eleven healthy subjects (mean(95\%confidence interval) BMI 23 (22-24), age $28(26-31)$ y) and eight insulin dependent diabetes patients (BMI 24 (21-26) age 31 (26-35) y. duration of diabetes $3(1-5)$ y hemoglobin A1c $8.2(6.8-9.6))$ were made hypoglycaemic by $60 \mathrm{~min}$ insulin infusion $\left(40 \mathrm{mU} / \mathrm{m}^{2}\right)$ after two weeks' oral therapy with Euphyllin Retard (theophylline). The dose of Theophylline (350 $\mathrm{mg}$ bid) was adjusted to maintain serum levels of $55-85$ microM. Plasma glucose nadir was 254 (2.31-2 77) mmol/l after Euphyllin Retard and $2.27(2.05-2.48) \mathrm{mmol} / \mathrm{l}$ after placebo (mean difference $0.26(0.05-0.58) \mathrm{mmol} / \mathrm{l}, \quad \mathrm{p}=0.0949$ ) for healthy control subjects and $2.56(2.07-3.04) \mathrm{mmo} / / \mathrm{l}$ and $2.19(1.37-2.65)$ mmol/l (mean difference $0.38(0.12-0.63) \mathrm{mmol} / \mathrm{l}, \mathrm{p}=0.0112$ ), respectively, for diabetic patients. We found an increase in glucose area under the curve after theophylline treatment for healthy control subjects $(p=0.0292)$ and for diabetic patients $(p=0.0241)$. There were no concomitant significant increases in plasma c-AMP $(p=0.60$ and $p=0.32$, respectively), or in glucose production rate rate $(p=0.16$ and $p=0.71$, respectively). In conclusion the study demonstrated a small significant increase in glucose recovery. The mechanism is uncertain since plasma c-AMP and glucose production rate did not increase. Whether the increase in glucose recovery is large enough to be of biological significance in the clinical prevention of insulin induced hypoglycaemia remains to be settled. 


\section{1}

GENDER DIFFERENCE IN THE COUNTER-REGULATORY RESPONSE TO CENTRAL NEUROGLYCOPENIA IN MICE

S. Karlsson A. Scheurink, A. Steffens and B. Ahrén, Dept Medicine, Lund University, Malmö, Sweden, Dept Animal Pysiol, Groningen, Netherlands The counter-regulatory response to neuroglycopenia involves autonomic nervous activation and changes in islet hormone secretion. We studied whether the acute antonomic and islet hormone responses to neuroglycopenia exhibit gender differences. The glucose analogue, 2deoxy-D-glucose (2DG; $50-500 \mathrm{mg} / \mathrm{kg}$ ), which induces central neuroglycopenia, or saline (controls) was injected iv. to non-fasted mice. Blood was sampled after $10 \mathrm{~min}$ and analysed for its content of insulin, glucagon, glucose, adrenaline or noradrenaline. Basal plasma levels of glucose $(10.3 \pm 0.3$ vs. $8.7 \pm 0.5 \mathrm{mmol} / \mathrm{l} ; \mathrm{P}<0.05)$ and insulin $(372 \pm 72$ vs. $168 \pm 18 \mathrm{pmol} / \mathrm{l} ; \mathrm{P}<0.05)$ were higher in male $(\mathrm{M})$ vs. in female (F) mice. Basal glucagon levels were lower in $\mathrm{M}(74 \pm 10 \mathrm{pg} / \mathrm{ml})$ versus in $\mathrm{F}(126 \pm 13 \mathrm{pg} / \mathrm{ml} ; \mathrm{P}<0.01, \mathrm{n}=10) .2 \mathrm{DG}(500 \mathrm{mg} / \mathrm{kg})$ increased plasma glucagon levels by $818 \pm 106 \mathrm{pg} / \mathrm{ml}$ in $\mathrm{F}$ vs. by $323 \pm 49 \mathrm{pg} / \mathrm{ml}$ in $\mathrm{M}(\mathrm{P}<0.01)$. Plasma insulin, glucose and noradrenaline levels after 2DG did not differ between genders. 2DG $(500 \mathrm{mg} / \mathrm{kg})$ increased plasma adrenaline levels to the same extent in both genders (by $5.5 \pm 0.6 \mathrm{ng} / \mathrm{ml}$ in $\mathrm{F}$ vs. by $4.8 \pm 1 \mathrm{ng} / \mathrm{ml}$ in $\mathrm{M}$; n.s.) indicating similar degreee of autonomic activation by $2-\mathrm{DG}$ in $\mathrm{M}$ and $\mathrm{F}$. The secretory glucagon response ( $2 \mathrm{~min}$ ) to iv. carbachol (muscarinic agonst; $30 \mu \mathrm{g} / \mathrm{kg}$ ) was $451 \pm 84 \mathrm{pg} / \mathrm{ml}$ in $\mathrm{F}$ vs. $91 \pm 21 \mathrm{pg} / \mathrm{ml}$ in $\mathrm{M}(\mathrm{P}<0.001 ; \mathrm{n}=16)$. Similarly, clonidine ( $\alpha_{2}$-adrenergic agonist; $13 \mu \mathrm{g} / \mathrm{kg}$ ) increased glucagon levels by $152 \pm 17 \mathrm{pg} / \mathrm{ml}$ in F vs. $43 \pm 13 \mathrm{pg} / \mathrm{ml}$ in $\mathrm{M}(\mathrm{P}<0.001 ; \mathrm{n}=18)$. It is concluded that, whereas the degreee of autonomic activation to central neuroglycopenia by 2DG does not differ between genders, the glucagon response is lower in $M$ due to a lower sensitivity of the glucagon producing cells to cholinergic and adrenergic activation.

\section{2}

\section{SEVERE HYPOGLYCAEMIA IN AN UNSELECTED} POPULATION WITH TYPE 1 DIABETES

M.van de Laak, E.ter Braak, A.Appelman, R.Stolk and W.Erkelens, Department of Internal Medicine and Julius Center for Patient Oriented Research, Utrecht University Hospital, The Netherlands The price to pay for good glycaemic control is an increase in severe hypoglycaemia(SH). Many efforts are being made in this field, including both basic research and intervention strategies. The aim of this study was to explore the magnitude of the problem, investigating the incidence of $\mathrm{SH}$, potential predictors for $\mathrm{SH}$ and the burden of the problem for individual patients in an unselected population, after implementation of the results of the DCCT. 195/211(92\%) consecutive out-patients filled out a questionnaire on the spot. Additional data were obtained from their files. Subjects who reported at least $1 \mathrm{SH}$ (assistance required) over the past 12 months were identified as cases $(40,5 \%)$; the others were employed as controls. Nearly half of the cases had at least $1 \mathrm{SH}$ complicated by a coma or insult(CSH). The incidence of $\mathrm{SH}$ and of $\mathrm{CSH}$ respectively had been at least 1,5 and 0,4 episodes per pat/yr. The majority of cases and controls was on intensive insulin treatment $(84 \%$ vs $81 \%$, ns). They had similar age (mean \pm SD: $41+14)$, sex (M/F:45/55), HbAlc $(7.8 \pm 1.2 \%)$, insulin dose $(0.74 \pm 0.24$ $\mathrm{U} / \mathrm{kg} / \mathrm{day}$ ). Duration of DM was $22+11$ years for cases vs $19+13$ for controls $(\mathrm{p}=0.08)$. No significant differences were detected regarding excercising habits, alcohol consumption, smoking, caffeine use, frequency of SMBG, blood glucose targets or co-medication. SH was significantly related to retinopathy (Odds Ratio(95\% CI)): $2.3(1.3-4.1)$ and nefropathy:2.3(1.1-5.0). Fear of hypoglycaemia (HFS worry scale) was much higher in cases (scores: $17 \pm 9$ vs $12 \pm 9, p<0.001)$. We conclude that in this well controlled unselected population SH is a very common complication that causes serious distress. Subjects with microvascular complications seem to be more at risk.

\section{3}

DOES INSULIN LISPRO PRESERVE THE PHYSIOLOGICAL DEFENCES TO HYPOGLYCAEMIA DURING INTENSIVE INSULIN THERAPY?

S.R.Heller, S.A.Amiel, I.A.Macdonald and R.B.Tattersall, Northern General Hospital, Sheffield, Kings College Hospital, London, Queen's Medical Centre, Nottingham, UK

We and others have shown that lispro in a basal/bolus regimen during intensive insulin therapy (IIT) produces equally good glycaemic control but reduces the frequency of hypoglycaemia. We tested the hypothesis that reduced hypoglycaemic events could result in improved physiological and symptomatic defences to hypoglycaemia in tightly controlled patients using insulin lispro when compared to the use of conventional insulins. Ten patients with IDDM ( 4 female, mean age $33 \pm 3 y$, duration of diabetes $12 \pm 2 y$ ) underwent IIT in an open, randomised cross-over study, with a 2 month run-in and 4 month treatment periods using either lispro (lp) or human soluble insulin (hs) before meals and human NPH at night. Total reported hypoglycaemic episodes (lp vs hs, 123 vs $128, \mathrm{P}=\mathrm{ns})$ and $\mathrm{HbA}_{10}(6.1 \pm 0.2$ vs $6.6 \pm 0.3 \%, \mathrm{P}=0.08)$ were similar at the end of 4 months. At the end of each 4 month period, we measured symptomatic, counterregulatory and cognitive responses (4-choice reaction time) and glycaemic thresholds during hypoglycaemia induced with a slow fall hyperinsulinaemic clamp (plateaus of $5,4.5,3.5$ and $2.5 \mathrm{mmol} / \mathrm{l}$ ). We found similar overall responses of adrenaline (AUC, $105 \pm 19$ vs $96 \pm 29$ nmol. min. $\mathrm{I}^{-1}, \mathrm{P}=0.6$ ), cortisol

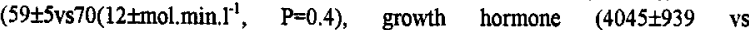
$4027 \pm 1571$ liu.min. $\mathrm{I}^{\mathrm{t}}, \mathrm{P}=0.8$ ) and total symptom score $4170 \pm 316$ vs $4006 \pm 270$, $\mathrm{P}=0.5)$. Glycaemic thresholds for rises in adrenaline $(3.1 \pm 0.2$ vs $3.1 \pm 0.2 \mathrm{mmol} / 1$, $\mathrm{P}=1)$, cortisol $(2.2 \pm 0.1$ vs $2.2 \pm 0.1 \mathrm{mmol} / \mathrm{P}=0.6)$, growth hormone ( $3.3 \pm 0.15$ vs $2.9 \pm 0.2 \mathrm{mmol} /, \mathrm{P}=0.053)$, symptoms $(3.2 \pm 0.2$ vs $3.3 \pm 0.1 \mathrm{mmo} / /, \mathrm{P}=0.8)$ and impaired cognitive function $(3.0 \pm 0.2$ vs $3.0 \pm 0.2 \mathrm{mmol} /, \mathrm{P}=1)$ were also similar We conclude that intensive treatment with insulin lispro results in equivalent physiological defences to hypoglycaemia as those obtained using human soluble insulin, perhaps because in these patients, counterregulatory and symptomatic responses were not impaired on soluble insulin.

\section{4}

Does the brain become tolerant to sustained caffeine use? Implications for treatment hypoglycaemia unawareness

J.M Watson, M. J. Weiss, M.J. Lunt, D. A. Cavan and D. Kerr, Royal

Bournemouth Hospital, Bournemouth, UK.

Caffeine augments the symptoms and signs of hypoglycaemia and is a potential therapy for hypoglycaemia unawareness. The aim of this study was to determine whether individuals become tolerant to the acute effects of caffeine ingestion, 12 healthy volunteers ( 6 women, aged $25-36$ years, all left-hemisphere dominant) consumed, using a double-blind, randomised controlled design either $200 \mathrm{mg}$ of caffeine (C-replete) twice daily or placebo (C-naive) for one week, including the morning of a study. Following baseline measurements, the perception of and physiological responses to acute caffeine ingestion (200mg) were examined over the subsequent $120 \mathrm{~min}$. Blood glucose was not allowed to fall below $4.0 \mathrm{mmol} / \mathrm{l}$

\begin{tabular}{|c|c|c|c|}
\hline \multirow{2}{*}{\multicolumn{4}{|c|}{$\begin{array}{c}\text { Base-mean } \pm \mathrm{SE} \\
\text { Middle cerebral artery velocity }(\mathrm{cm} / \mathrm{s})\end{array}$}} \\
\hline & & & \\
\hline C-replete & $61.4 \pm 2.7$ & $55.7 \pm 2.6 * *$ & $-5.7(-8.0,-3.4)$ \\
\hline C-naïve & $64.9 \pm 3.0$ & $55.6 \pm 2.5 * *$ & $-9.3(-11.4,-7.1) \#$ \\
\hline \multicolumn{4}{|c|}{ Systolic Blood pressure (mmHg) } \\
\hline C-replete & $116.5 \pm 4.7$ & $124.2 \pm 6.1 * *$ & $7.6(4.1,11.1)$ \\
\hline C-naive & $113.5 \pm 3.5$ & $126.8 \pm 5.8 * *$ & $12.0(5.0,18.7)$ \\
\hline \multicolumn{4}{|c|}{ Diastolic Blood pressure (mmHg) } \\
\hline C-replete & $64.9 \pm 2.4$ & $68.9 \pm 3.0 * *$ & $4.1(2.3,5.8)$ \\
\hline C-naïve & $63.2 \pm 1.36$ & $71.2 \pm 2.7 *$ & $8.2(4.9,11.5) \#$ \\
\hline \multicolumn{4}{|c|}{ 4-Choice Reaction Time (sec) } \\
\hline C-replete & $0.51 \pm 0.01$ & $0.49 \pm 0.01^{*}$ & $0.02(0.01,0.04)$ \\
\hline C-naïve & $0.51 \pm 0.01$ & $0.49 \pm 0.01 *$ & $0.02(0.00,0.03)$ \\
\hline
\end{tabular}

$\#=p<0.01 \mathrm{C}$-replete vs. C-naïve: ${ }^{*}=p<0.05,{ }^{* *}=p<0.005$ Base vs. Max change

Baseline feelings of tension and pleasure were significantly worse in the caffeine replete state $(p<0.05$ ). However, following caffeine ingestion energetic aspects of mood improved only when the subject was caffeine-naive $(p<0.5)$. Overall these results suggest that people do not become completely tolerant central effects of caffeine. If these effects are identical during hypoglycaemia, caffeine could prove to be a useful agent in improving hypoglycaemia awareness, albeit with pressor effects. 
PS 44

\section{Insulin Therapy I}

\section{5}

PREMIXED FORMULATIONS OF INSULIN LISPRO: ACTIVITY PROFILES IN TYPE 1 DIABETIC PATIENTS

L. HEINEMANN, K. RAVE, C. WEYER, L. PAUL, U. GUDAT*, P, ROACH*, J. WOODWORTH", T. HEISE; Department of Metabolic Dis., Heinrich-Heine-University, Düsseldorf, Germany; "Eli Lilly, USA

NPL-insulin, a novel protamine-retarded formulation of insulin lispro (LP), allows formulation of stable insulin mixtures. In an open randomised crossover study we investigated the blood glucose lowering effect of two different mixtures: Mid Mixture (MM; LP/NPL 1:1) and Low Mixture (LM; 1:3) in 6 type 1 diabetic patients (age $36 \pm 5$ years, BMI $24.4 \pm 2.0 \mathrm{~kg} / \mathrm{m}^{2}$, diabetes duration $15 \pm 3$ years (mean $\pm S D$ )). During a baseline period of $3 \mathrm{~h}$ blood glucose was adjusted to $5.0 \mathrm{mmol} / \mathrm{l}$ by means of an i.v. insulin infusion. One hour after s.c. injection of $0.3 \mathrm{U} / \mathrm{kg}$ of one of the two preparations by means of a syringe in the abdominal region the i.v. insulin infusion was stopped. The experiments ended either after $1140 \mathrm{~min}$ or when blood glucose increased above $11.0 \mathrm{mmol} / \mathrm{l}$. With this experimental set-up glucose infusion rates (GIR) necessary to keep glycaemia at $5.0 \mathrm{mmol} / /$ reflect changes in insulin action over time, whereas duration of insulin action is indicated by an increase in glycaemia. The maximal metabolic activity $\left(\mathrm{GIR}_{\max }\right)$ is higher with a greater $\mathrm{LP}$ content $(\mathrm{MM}>\mathrm{LM}$; $5.8 \pm 1.2$ vs. $3.8 \pm 1.8 \mathrm{mg} / \mathrm{kg} / \mathrm{min} ; \mathrm{p}=0.017$ ). The time to maximal metabolic activity $\left(\mathrm{t}_{\max }\right)$ was comparable (113 \pm 8 vs. $\left.123 \pm 20 \mathrm{~min}, \mathrm{NS}\right)$. MM induced a higher maximal activity $\left(A \cup C_{0.360}\right.$ min $)$ within the first $6 \mathrm{~h}$ after injection $(1.34 \pm 0.22$ vs. $0.91 \pm 0.34$ $\mathrm{g} / \mathrm{kg} / 360 \mathrm{~min}, \mathrm{p}=0.025$ ). Blood glucose increased from the glucose clamp target level of $5.0 \mathrm{mmol} / 1$ to values $>7.0 \mathrm{mmol} / \mathrm{l}$ more rapidly with $\mathrm{MM}$ (lower content of NPLinsulin) than with $\mathrm{LM}(865 \pm 127$ vs. $988 \pm 134 \mathrm{~min} ; \mathrm{p}=0.031)$. Glycaemia increased further to values $>11.0 \mathrm{mmol} / \mathrm{l}$ in 4 patients with $\mathrm{MM}$, but in one patient only with LM. In conclusion, this study shows, that the two formulations studied have a maximal pharmacodynamic effect $2 \mathrm{~h}$ after injection and allow blood glucose control for up to $15 \mathrm{~h}$. With higher LP content maximal metabolic effect rises whereas duration of action decreases. These time-action profiles of MM and LM obtained in type 1 diabetic patients are in accordance with published data from healthy volunteers.

\section{7}

PHARMACOKINETICS OF INSULIN LISPRO MIDMIXTURE COMPARED TO HUMULIN 50/50

F. Holleman, J.W. Vermeijden, J.R. Woodworth*, E.M. Kuck, P. Roach* and J.B.L. Hoekstra. Diakonessenhuis, Utrecht, The Netherlands and *Eli Lilly, Indianapolis, USA.

The pharmacokinetics of Humulin $50 / 50(\mathrm{H})$ and insulin lispro MidMixture (MM), a 50/50 mixture of insulin lispro and NPL, were studied in 9 IDDM patients participating in a randomized $2 * 3$ month crossover trial, at two study days before and after the crossover visit. The patients had individually standardized meals at $08.00,11.00$ 12.00 , and $15.30 \mathrm{hrs}$; physical exercise was identical and limited on both study days; Insulin dose was individually standardized but $H$ was injected 30 minutes before breakfast, and MM was injected immediately before breakfast.

Patients had an age of $31.2 \pm 8.1$ years, a duration of diabetes of $11.2 \pm 8.1$ years, $\mathrm{HbA} 1 \mathrm{C}$ of $7.3 \pm 1.4 \%$ and $\mathrm{BMI}$ of $24.7 \pm 2.4 \mathrm{~kg} / \mathrm{m}^{2}$. Pharmacokinetic data were as follows ( $p$-values from paired T-tests):

\begin{tabular}{|l|l|l|l|}
\hline & Lispro MM & Humulin 50/50 & p-value \\
\hline Cmax (ng/l) & $4021 \pm 2020$ & $2378 \pm 1178$ & 0.002 \\
\hline Time to Cmax (min.) & $52 \pm 8$ & $137 \pm 59$ & 0.001 \\
\hline AUC 0-630 min. & $711 \pm 247$ & $600 \pm 251$ & 0.007 \\
\hline AUC 0-240 min. & $506 \pm 196$ & $382 \pm 180$ & 0.004 \\
\hline AUC 240-630 min. & $205 \pm 89$ & $218 \pm 113$ & ns \\
\hline
\end{tabular}

Initial glucose was $10.8+4.1 \mathrm{mmol} / \mathrm{for} \mathrm{MM}$ and $10.3 \pm 3.9 \mathrm{mmol} / \mathrm{l}$ for $\mathrm{H}$. Glucose values reached a nadir of $6.2 \pm 3.6 \mathrm{mmol} / \mathrm{lat} 210 \pm 37$ $\mathrm{min}$. after breakfast with MM and a nadir of $5.3 \pm 2.5 \mathrm{mmol} / \mathrm{l}$ at $203 \pm$ 25 min. after breakfast with $\mathrm{H}$. While the rapid onset of action of insulin lispro is retained in the MidMixture, this did not result in improved postprandial glycemia in this study setting.

\section{6}

ANTIOXIDANT DEFENSES ARE REDUCED DURING MEAL IN INSULIN-REQUIRING TYPE-2 DIABETICS THE EFFECT OF INSULIN LISPRO

C. Taboga, N. Bortolotti, L. Tonutti, S. Lizzio, A Russo, D. Santoro, A. Ceriello. University of Udine and Udine General Hospital, Eli Lilly Italia, Italy

The mechanism by which hyperglycaemia causes or impacts chronic complications of diabetes mellitus is unknown. One potential link is the hyperglycaemic contribution to the generation of free radicals, which may alter antioxidant defenses.Total plasma radical-trapping parameter (TRAP) evaluates plasma antioxidant capacity due to known and unknown antioxidants present in the plasma as well as their mutual cooperation. In this study two standard meals (372 $\mathrm{Kcal}, 49 \%$ as carbohydrates, mainly as complex carbohydrates, $40 \%$ as fat, and $11 \%$ as protein) were administered to 17 insulinrequiring type-2 diabetic patients. Glycaemia was mantained between 7.2 and $8.3 \mathrm{mmol} / \mathrm{l}$ before the start of each study. The meal started $30 \mathrm{~min}$ after regular insulin [1] $(0.15 \mathrm{U} / \mathrm{Kg})$ or $10 \mathrm{~min}$ after insulin Lispro [L] $(0.15 \mathrm{U} / \mathrm{Kg})$ administration. Glycaemia and TRAP were measured from 0 to $120 \mathrm{~min}$. Basal glycaemia was not different at the start of the two tests (L:9.3 $\pm 0.7, \mathrm{I}: 9.5 \pm 0.5 \mathrm{mmol} /$; $\mathrm{M} \pm \mathrm{SE}$ ), while the increase of post-prandial glycaemia was more relevant during $I$ administration than during $L(p<0.001)$. TRAP was significantly reduced during meals ( $p<0.02$ vs basal). The $\%$ of TRAP vs basal was significantly lower during I than $L$ at 90 and $120 \mathrm{~min}(90 \mathrm{~min}: 103.2 \pm 3.3$ vs $92.1 \pm 1.6 \%, \mathrm{p}<0.02 ; 120 \mathrm{~min}$ : $100.4 \pm 3.2$ vs $94.7 \pm 1.6 \%, p<0.05$ ). This finding suggests that during meal free radicals are produced and that this effect is convincingly linked to the level of post-prandial hyperglycaemia. $L$ treatment may significantly contribute to preserve TRAP from mealinduced reduction in diabetes.

\section{8}

\section{LISPRO INSULIN FOR TREATING IDDM-PATIENTS}

\section{THE LISPRO IN HUNGARY STUDY}

G. Jermendy ${ }^{\star}$, G. Tamás, G. Winkler and the Lispro In Hungary Study Group. *Bajcsy-Zsilinszky Hospital, Budapest, Hungary. In order to assess the safety and the efficacy of lispro insulin 123 IDDMpatients $(63$ men, 60 women; age: $34.9 \pm 9.3$ years; duration of diabetes $11.7 \pm 8.2$ years; duration of intensive conservative insulin therapy $4.8 \pm 4.0$ years; $x+S D)$ were investigated in an open, 16 week-long, multicenter $(25$ centres) study in Hungary. Safety of the treatment with lispro insulin was evaluated by registration of adverse events and numbers of hypoglycaemic episodes (blood glucose $<2.5 \mathrm{mmol} / \mathrm{l}$ ) while efficacy was assessed by measuring $\mathrm{HbA}_{1 \mathrm{c}}$ values and blood glucose home monitoring. Following the recruitment period, IDDM-patients were treated for 4 weeks with human unmodified regular insulin given 30 min before meals ( 3 times daily) and basal insulin twice daily. Subsequently, patients were switched to lispro insulin given immediately before meals with unchanged basal insulin supplementation for 12 weeks. Only one, obviously unrelated adverse event was reported during lispro treatment-period. The number of hypoglycaemic episodes did not differ significantly $(\mathrm{p}=0.61)$ during human unmodified regular insulin period ( 170 episodes in 4 weeks $=0.34$ episode/week/patient) compared to that of lispro insulin period (565 episodes in 12 wccks $=0.38$ episode/week/patient). The postprandial ( $90 \mathrm{~min}$ after breakfast) blood glucose values were significantly $(\mathrm{p}=0.0026)$ lower $(8.1 \pm 2.4 \mathrm{mmol} / \mathrm{l})$ during lispro treatment compared to those during human unmodified regular insulin therapy $(8.8 \pm 2.2 \mathrm{mmol} / \mathrm{l})$. The $\mathrm{HbA}_{1 \mathrm{c}}$ values were significantly $(\mathrm{p}=0.031)$ lower at the end $(7.59 \pm 1.38 \%)$ than at the beginning $(7.77 \pm 1.49 \%)$ of treatment with lispro insulin. According to the patients' questionnaires, more flexible lifestyle was the most common declared benefit related to the use of lispro insulin. Thus, the safety as well as the efficacy of lispro insulin treatment in IDDM-patients were confirmed in Hungary 
EFFECT OF LISPRO vs HUMAN REGULAR INSULIN ON LONG. TERM BLOOD GLUCOSE CONTROL IN IDDM: IMPORTANCE OF TIME-INTERVAL BETWEEN INSULIN INJECTION AND MEAL.

E. Torlone, P. Del Sindaco, C. Lalli, M. Ciofetta, P. Brunetti and G. B. Bolli*, DIMISEM Perugia, Italy

In previous studies in which Lispro (+variable NPH) was given as s.c. injections at meals, HbAlc decreased by $\sim 0.3 \%$ vs human regular insulin (Hum-R) injected $10.40 \mathrm{~min}$ prior to meal. However, in reality many IDDM patients inject Hum-R strictly at meal-time. To establish the effect of Lispro on HbAlc in IDDM patients who are used to inject Hum-R strictly at mealtime, 28 IDDM patients were divided into two groups (GI, $\mathrm{n}=14$, GII, $\mathrm{n}=14$ ). Each group was studied for 6 months (two periods of 3 months each, open, crossover design). GI patients were randomized to either Lispro(+variable NPH) or Hum-R injected strictly at mealtime. GII patients were randomized to Hum-R given either at mealtime or 10-40 min prior to meals. Bedtime NPH was continued in both groups. In GI, total insulin daily dose was similar with the two treatments, but $\sim 27 \%$ more NPH and $-25 \%$ less short-acting insulin was needed in the Lispro vs Hum$R$ treatment. Hypoglycemia (capillary blood glucose $<4.0 \mathrm{mmol} /$ ) was less frequent with Lispro vs Hum-R $(5.1 \pm 0.6$ vs $8.9 \pm 0.9$ episodes/patientmonth). HbAlc was $6.23 \pm 0.19$ with Lispro and $6.89 \pm 0.13 \%$ with Hum-R $(p<0.05)$. In GII, hypoglycemia was more frequent with Hum-R at mealtime than $10-40 \mathrm{~min}$ before meals $(8.5 \pm 0.8$ vs $4.7 \pm 0.5$ episodes/patient-month) and HbAlc greater $(6.86 \pm 0.11$ vs $6.51 \pm 0.10 \%)$ $(p<0.05)$. Conclusions. The classic rule of eating the meal $10-40 \mathrm{~min}$ after injection of Hum-R itself decreases HbAlc by $0.25 \pm 0.04 \%$. Use of Lispro is especially beneficial to \% HbAlc and frequency of hypoglycemia of patients who commonly inject Hum-R and immediately eat.

941

LISPRO INSULIN COMPARED WITH REGULAR INSULIN IN CIRRHOTIC PATIENTS WITH TYPE 2 DLABETES MELLITUS

S. Gentile, F. C. Sasso, S. Turco*. Department of Geriatrics, $2^{\text {nd }}$ University of Naples, and * Departments of Clinical and Experimental Medicine, Federico $2^{\text {nd }}$ University of Naples, Italy

The treatment protocol of patients with type 2 diabetes (DM2) and liver cirrhosis (LC) is based on the administration of regular insulin before meals. However, frequent after-meal hyperglycemic spikes and late hypoglycemic episodes (HY) are frequent in such patients. Aim: to compare the effect of short-acting insulin lispro (Humalog ${ }^{\oplus}$, Eli Lilly Italia) (HMO) with that of regular insulin (Humulin $R^{3}$ ) (HUI). Patients: 47 subjects with DM2 and well compensed LC, 29F and $18 \mathrm{M}$, age range $45-67 \mathrm{y}$, mean age $58 \pm 6 \mathrm{y}$, diabetes duration $12 \pm 5 \mathrm{y}$, BMI $27.3 \pm 4$, previous insulin treatment al least for 12 months (range 1-4y). Protocol: pts were educated to self-monitor glycemia and then were randomly assigned to two sequential 2-months treatments: a) HUO immediately before each meal, and b) HUI 20 min before each meal; insulin doses $\mathrm{ID}$ (U/day) were adjusted according to the glycemias collected at home every week before and $2 \mathrm{~h}$ after each meal and at 03.00 AM. A quality-of-life questionnaire (Q) was administered after each treatment. The mean number of HY (defined as glycemia $<2.8 \mathrm{mmol} / \mathrm{l}$ with or without symptoms) per patient per treatment period and $\mathrm{HbAlc}(\%, \mathrm{HPLC})$ were recorded. Mean glycemias $( \pm \mathrm{SD}$ ) (before the 3 meals [MBe] and $2 \mathrm{~h}$ after the 3 meals [MAf]) were calculated. Results are reported in Table. We observed in pts under HUO, compared to HUI: 1) a significant reduction of HY; 2) a more stable glycemic level both before and after meals $(p<0.01): 3$ ) a $Q$ score significantly higher. Conclusion: the results encourage the use of short-acting insulin lispro in pts with DM2 and LC, especially those affected with hepatic encefalopathy, in which $\mathrm{HY}$ can be particularly misleading or complicating. \begin{tabular}{lccccccc}
\hline & BWt & ID & ID/kg & HY & MBe & MAf & HbA $_{1 \mathrm{c}}$ \\
HUMULIN R & $78 \pm 7$ & $35 \pm 7$ & $0.46 \pm 6$ & $7 \pm 3$ & $13 ! \pm 24$ & $197 \pm 76$ & $7.3 \pm 1.1$
\end{tabular} $\begin{array}{llllllll}\text { HUMALOG } & 75 \pm 6 & 33 \pm 5 & 0.40 \pm 6 & 2 \pm 3 * & 139 \pm 16 & 133 \pm 34^{*} & 7.0 \pm 0.9\end{array}$ ${ }^{*} p<0.01$ vs Humulin $R$
FAILURE OF RAT AND DOG MODELS TO PREDICT SUBCUTANEOUS ABSORPTION OF INSULIN ASPART IN MAN

Plum, A, Andersen, L and Agerso, H. Novo Nordisk A/S, Målov, DK Insulin aspart (LA) [B28 A sp human insulin] a novel short acting insulin analogue was developed to resemble human insulin (HI) in all biological aspects, but with an earlier onset of action and a shorter duration of effect. $l n$ vivo screening was performed in normal pigs, the preferred animal model for the development of s.c. administered insulin preparations. However, the pharmacokinetics of IA were determined in rats and dogs, as part of the toxicity program. This pharmacokinetic program was designed as single dose studies via the s.c. and i.v. route, and as repeated s.c. studies (over 1 week), with all administrations at 3 dose levels in order to investigate the degree of accumulation. As expected, similar pharmacokinetic parameters following i.v. administration of IA and HI were obtained in rats, dogs, pigs and humans.

However, although differences were predicted in the s.c. pharmacokinetics of IA and $\mathrm{HI}$ in dogs and rats, neither of the two species illustrated the expected faster onset of action or faster s.c. absorption of IA, although the pharmacokinetics did show a faster elimination of IA after i.v, administration compared to s.c. administration. This predicted difference could only be shown in pigs and later in man. The main explanation seems to be the structural differences of the subcutis, which in dogs and rats is of a more loose structure, consisting of less lipid than in pig and man. This difference results in generally faster kinetics after s.c. absorption, making it difficult to differentiate between compounds such IA and $\mathrm{HI}$ in rats or dogs, where the main difference seen is the reduction of dissociation time as part of the rate-limiting s.c. absorption

Conclusion: only the pig model predicts s.c. absorption of insulin aspart in man.

\begin{tabular}{|c|c|c|c|c|c|c|c|c|}
\hline \multirow[t]{2}{*}{$\begin{array}{l}\text { Species/ } \\
\operatorname{dose}(U / k g)\end{array}$} & \multicolumn{2}{|c|}{$\begin{array}{l}\operatorname{man} \\
(0.10)\end{array}$} & \multicolumn{2}{|c|}{$\begin{array}{l}\text { pig } \\
(0.125)\end{array}$} & \multicolumn{2}{|l|}{$\begin{array}{l}\text { dog } \\
(1.0)\end{array}$} & \multicolumn{2}{|l|}{\begin{tabular}{|l|} 
rat \\
$(6.0)$ \\
\end{tabular}} \\
\hline & $\overline{\mathbf{I A}}$ & HI & IA & HI & IA & HI & IA & $\mathrm{HI}$ \\
\hline$t_{1 / 2}(\min )$ & 76 & 122 & 77 & 121 & 67 & 57 & 22 & 23 \\
\hline $\mathbf{C}_{\max }(\mathrm{pM})^{*}$ & 246 & 102 & 204 & 122 & 3146 & 2871 & $1.7 \times 10^{3}$ & $1.8 \times 10^{3}$ \\
\hline$t_{\operatorname{lnax}}(\min )$ & 52 & 145 & 73 & 99 & 46 & 60 & 15 & 15 \\
\hline $\begin{array}{l}\mathrm{CL}^{* *} \\
(\mathrm{l} \cdot \mathrm{min} / \mathrm{kg})\end{array}$ & $=$ & - & 0.019 & 0.021 & 0.055 & 0.048 & 0.044 & 0.058 \\
\hline
\end{tabular}

Doses for man and pig were comparable with respect to effect, but cannot be dircctly

* Calculated based on intravenous data.

\section{2}

THE CHEMICAL STABILITY OF INSULIN LISPRO PROTAMINE SUSPENSION AND INSULIN LISPRO MIXTURES

K. S. Looney, M. R. DeFelippis, J. D. Hofer, and B. H. Frank. Eli Lilly and Company, Lilly Research Laboratories, Indianapolis, USA

The rapid acting monomeric insulin analog, insulin lispro, has been cocrystallized with the basic protein protamine to form an intermediate acting suspension formulation, insulin lispro protamine suspension (NPL). NPL is formulated with soluble insulin lispro in ratios of 25:75 (Insulin Lispro Low Mixture, LM) and 50:50 (Insulin Lispro Mid Mixture, MM) to provide mixtures with the benefits of the rapid timeaction of insulin lispro combined with the basal activity of NPL. The aim of the present study was to assess the chemical stability of NPL, $\mathrm{LM}$ and $\mathrm{MM}$ at $5^{\circ} \mathrm{C}$ for 24 months. Insulin lispro potency as determined by reversed phase HPLC decreased $1.4 \pm 0.2 \mathrm{U} / \mathrm{mL}$ for NPL, $\mathrm{LM}$ and $\mathrm{MM}$ formulations, and the immediately available insulin lispro decreased $3.6 \pm 0.3 \mathrm{U} / \mathrm{mL}$ for $\mathrm{LM}$ and $2.0 \pm 0.7 \mathrm{U} / \mathrm{mL}$ for $\mathrm{MM}$. High molecular weight proteins as determined by the proposed $\mathrm{Ph}$. Eur. HPLC method increased $0.79 \pm 0.14 \%, 0.87 \pm 0.06 \%$ and $0.67 \pm 0.03 \%$ for NPL, LM and MM, respectively. The related substances as determined by reversed phase HPLC increased $0.87 \pm 0.06 \%$. The purity was also evaluated by a complementary reversed phase HPLC method that determined the aspartyl and isoaspartyl B3 desamidos, showing an increase of $0.10 \pm 0.01 \%, 0.13 \pm 0.03 \%$ and $0.19 \pm 0.02 \%$ for the aspartyl B3 desamido and $0.08 \pm 0.01 \%, 0.11 \pm 0.02 \%$ and $0.29 \pm 0.02 \%$ for the isoaspartyl B3 desamido for NPL, LM and MM, respectively. The supernatant insulin lispro in NPL, A21-desamido insulin lispro, mcresol, phenol and $\mathrm{pH}$ also were evaluated during the stability testing and did not exhibit significant change from the initial values. NPL, LM and MM preparations demonstrate excellent chemical stability for 24 months at $5^{\circ} \mathrm{C}$. 


\section{3}

IMPROVED POSTPRANDIAL GLYCEMIA DURING TREATMENT WITH A LISPRO/NTERMEDIATE-ACTING INSULIN MIXTURE, MIX25

P. Roach, M.E. Trautmann, J.H. Anderson, and the Mix25 Study Group, Indianapolis, Indiana

Mix 25 is an insulin mixture containing $25 \%$ lispro and $75 \%$ NPL, a sustained-release lispro-protamine formulation. Mix 25 was compared to human $30 / 70$ (30\% regular insulin, $70 \% \mathrm{NPH}$ ) in 87 individuals with type 2 diabetes in a 6-month randomized crossover study. Mix 25 was injected 6 minutes before and 30/70 was injected 25 minutes before breakfast and dinner (mean reported injection times). Self-monitored blood glucose (BG) data collected at 2 and 3 months were combined to provide meanon-therapy results. Mix 25 resulted in smaller mean postprandial BG excursions ( $2 \mathrm{~h}$ postprandial minus preprandial BG) after breakfast and dinner while providing similar glycemic control at other time points.

\begin{tabular}{|l|c|c|c|}
\hline \multicolumn{1}{|c|}{ (mean data) } & LM & $30-70$ & p-value \\
\hline Fasting BG & 8.68 & 8.56 & NS \\
2h ppBG & 9.03 & 9.91 & .011 .005 \\
2h Excursion & 0.47 & 1.35 & .002 \\
\hline Pre-lunch BG & 6.94 & 6.90 & NS \\
2h ppBG & 10.12 & 9.69 & NS \\
2h Excursion & 3.27 & 2.83 & NS \\
\hline Pre-dinner BG & 9.50 & 9.44 & NS \\
2h pp BG & 9.61 & 10.21 & NS \\
2h Excursion & 0.11 & 0.77 & $<.001$ \\
\hline Bedtime BG & 9.06 & 9.61 & $\mathrm{NS}$ \\
\hline 3 AM BG & 7.98 & 7.77 & $\mathrm{NS}$ \\
\hline
\end{tabular}

Mean insulin doses were similar for both treatments at both 2 and 3 months (AM: 0.36-0.37 U/kg; PM: 0.26-0.28 U/kg), as were 30-day hypoglycemia rates. $\mathrm{HbA}_{1} \mathrm{c}$ levels were not different at endpoint (Mix 25: 7.82\%; 3070: 8.05\%, $\mathrm{p}=.41$ ). Mix 25 provided the convenience of immediate pre-meal injection, similar overall glycemic control, and better postprandial BG control compared to human $30 / 70$.

\section{4}

COMPARATION OF INTENSIVE THERAPHY WITH MIXED LISPRO/NPH VERSUS REGULAR/NPH INSULIN IN TYPE 2 DM. J.A. Piniés, E. Ugarte, J. Alonso, R. Bilbao, and I. Goicolea. Servicio de Endocrinologia del Hospital de Cruces. Baracaldo.Vizcaya. Espáía.

Introduction. Clinical trial experience with intensified insulin regimenes in type 2 diabetes is limited. Implantable insulin pupm therapy and multiple daily insulin injections have been shown to be secure and effective in controlling type 2 diabetes. Objetives. The present study was designed to determined whether multiple daily insulin injections with Lispro injected in combination with NPH insulin improve glucose control and offers any specific advantages over Regular insulin in combination with NPH insulin in type 2 diabetic patients. Material and methos: Sixty five type 2 diabetic patients, most of them in conventional insulin theraphy. without severe cronic complications attending the outpatient's clinic of Cruces Hospital were randomized divided in two groups. Group $1(n=33)$ was treated with 3 daily insulin injections with Lispro in combination whit NPH insulin and group 2 (n=32) was treated with Regular insulin injected in combination with NPH insulin before the 3 main meals during 7 months. Initially, no differences in sex, age, years of diabetes evolution, BMI, dose of insulin, cronic complications, glycemic control lipid profile and $\mathrm{HbAlc}$ between the groups were found. $\mathrm{C}$ - peptide response after glucagon was higher in group 2 versus group $1(p=0,027)(10$ of 33 patients in group 1 had positive GAD antibodies and 7 of these had $C$ - Peptide after glucagon $<0,19$ nmol/l versus 3 of 32 positive GAD antibodies in group 2 with C-peptide after glucagon $>0,67 \mathrm{nmol} / 1)$. Results. Initial $\mathrm{HbAlc}$ was 8,2 and $7,8 \%$ in group 1 and 2 and improved during the study ( $\mathrm{p}<0,005$ and $\mathrm{p}<0,051$ respectively). Results at the end of the study are expresed in the next table:

\begin{tabular}{|c|c|c|c|c|c|c|}
\hline & $\begin{array}{l}\text { Instilin } \\
\text { (UI/Kg) }\end{array}$ & $\begin{array}{l}\text { Weight } \\
\text { gain }(\mathrm{Kg})\end{array}$ & $\begin{array}{l}\text { HbAlc } \\
\text { (\%) }\end{array}$ & $\Delta$ HDLe & $\begin{array}{l}\text { TA } \\
\text { reducction }\end{array}$ & $\begin{array}{l}\text { Severe } \\
\text { bypogly }\end{array}$ \\
\hline Group 1 & 0,95 & $2 \pm 4$ & 6,7 & yes & yes & 1 \\
\hline \multirow[t]{2}{*}{ Group 2} & 0,92 & $1,8 \pm 3$ & 6.7 & no & yes & 5 \\
\hline & ns & ns & ns & is & is & ns \\
\hline
\end{tabular}

In the last month of treatment, HbAlc continued decreasing significantly only in the group 1. There were no differences in mild hypoglycemias between the 2 groups. Conclusions. 1. Intensive insulin theraphy with mixed Lispro/NPH and regular/NPN is effective and secure in controlling type 2 diabetes improving $\mathrm{HbAlc}$. 2. No significant advantages were found betwenn the two regimenes of intensive insulin theraphy in this study.

\section{5}

THE COMBINATION OF LISPRO INSULIN WITH NPH IMPROVES OVERALL BLOOD GLUCOSE CONTROL IN IDDM PATIENTS.

G. Annuzzi, L. Benzi, C. Coscelli, S. Del Prato, D. Fedele, A. Galluzzo, R Giorgino and D. Santoro. (Naples, Pisa, Parma, Padova, Palermo, Bari, Florence) Italy.

While insulin Lispro has been reported to improve post-prandial blood glucose (PPBG), less consistent effects on overall blood glucose control in IDDM patients as measured by glycated haemoglobin levels have been shown. This might be due to Lispro short time action profile that would be not long enough to cover till nex meal, especially when dietary habits are characterized by long time intervals between meals. Therefore, the aim of this study was to define the most appropriate strategy for Lispro therapy. Eightysix IDDM patients (age 32 \pm 7 yrs, BMI 23 \pm 3 $\mathrm{kg} / \mathrm{m}^{2}$, diabetes duration $12 \pm 7 \mathrm{yrs}, \mathrm{M} \pm \mathrm{SD}$ ) were studied in a multicenter randomized comparative (vs Regular) crossover (3 month) study in which intermediate acting insulin (NPH), in addition to the dinner or bed-time injection, was also added if necessary at breakfast and lunch. The number of daily injections (inj) was kept constant during the study ( $43 \%$ and $57 \%$ of patients received 3 and 4 inj/day, respectively). Total daily insulin dose before the study ( $41 \pm 11 \mathrm{IU}$ ) did not change during the two study periods. At either breakfast and lunch NPH was more often associated to Lispro than to Regular insulin $(41 \%$ vs $17 \%$, respectively, $\mathrm{p}<0.001$ ). On average the Lispro/NPH ratio was $70 / 30$ at breakfast and lunch, and $40 / 60$ at dinner. Fasting and pre-prandial blood glucose were similar, while PPBC improved after Lispro compared to Regular insulin (breakfast: $149 \pm 43$ vs $167 \pm 49$ $\mathrm{mg} / \mathrm{dl}$; lunch: $150 \pm 48$ vs $163 \pm 48 \mathrm{mg} / \mathrm{dl}$, dinner: $145 \pm 49$ vs $167 \pm 44 \mathrm{mg} / \mathrm{dl}$, ANOVA: $\mathrm{p}=0.003$ ). HbAlc was also significantly improved after Lispro: $8.1 \pm 0.9$ vs $8.3 \pm 0.8 \%, p=0.046$. Rate of hypoglycemia was instead not different between the two treatments. In conclusion, Lispro improves overall blood glucose control in IDDM patients without increasing insulin dose, daily insulin injections and incidence of hypoglycemic episodes. This can be achieved by an optimal combination of insulin Lispro and intermediate acting insulin (NPH) when the time intervals between meals are too long.

\section{6}

EFFECTS OF MULTIPLE INJECTION THERAPY WITH LISPRO HIGH MIX INSULIN ON THE CATECHOLAMINE RESPONSE TO HYPOGLYCAEMIA

M.M.J. Janssen, N. Masurel, R.P.L.M. Hoogma*, M. Seubert, W. Devillé, C. PoppSnijders, R.J.Heine. Research Institute for Endocrinology, Reproduction and Metabolism. Vrije Universiteit. P.O. Box 7057, 1007 MB Amsterdam. *Dep.of Internal Medicine, Groene Hart Hospital, Gouda, The Netherlands.

Several studies have demonstrated the importance of optimal basal insulin replacement during treatment with lispro insulin in insulin-dependent diabetes mellitus (IDDM) patients. Optimal basal insulin replacement and patient convenience is offered by basal bolus therapy with a premixture of $75 \%$ insulin lispro and $25 \%$ neutral protamine lispro (NPL) before meals (lispro high mix) and NPL at bedtime. We tested the effect of this regimen on counterregulatory responses to hypoglycaemia. The study had an open, randomized, parallel design. We studied $19 \mathrm{IDDM}$ patients (15 men, 4 women, age (average) 30.4 (SD: 6.4) years, diabetes duration $12.8(7.2)$ years, $\mathrm{HbAlc} 7.2(0.6) \%$ ). During the 8-10 week control period patients used regular insulin before meals and NPH at bedtime. After this period patients were randomized to regular insulin before meals and NPH at bedtime or lispro high mix before meals and NPL at bedtime for 12 to 14 weeks. At the end of each period a hypoglycaemic clamp was performed during which blood glucose was lowered from $4 \mathrm{mmol} / \mathrm{L}$ to $3.5,3.0$ and $2.5 \mathrm{mmol} / \mathrm{L}$ over 300 minutes by means of constant intravenous infusion of regular insulin and a variable infusion of a glucose $20 \%$ solution. Differences in magnitude and threshold of catecholamine responses between regular and lispro high mix treatment were tested in a multiple linear regression analysis corrected for baseline values and for HbAlc at the start of the final clamp. Magnitude of response was defined as the incremental area under the curve (AUC), and threshold as the time at which a sustained increase above the average plus two standard deviations of hormone concentrations at $4 \mathrm{mmol} / \mathrm{L}$ was reached. No statistically significant differences between lispro high mix and regular were detected for AUC noradrenalin, threshold noradrenalin or threshold adrenalin. The AUC adrenalin was significantly lower for lispro high mix than for regular: 31.7 (2.5-171.8) versus 36.9 (13-453.6) nmol/L.min, median (range), $\mathrm{p}=0.005$. The clinical relevance of this finding remains to be established. 


\section{7}

\section{TREATMENT OF DIABETIC CIRRHOTIC PATIENTS WITH} LISPRO ANALOGUE.

D. Bruttomesso, M. Zanon, P. Angeli, C. Girardi, E. Iori, M.C. Marescotti, D. Santoro, A. Tiengo , S. Del Prato . University of Padova, italy.

Liver disease associated with diabetes ( $L D+D M)$ is characterized by severe insulin resistance accounting for large doses of exogenous insulin though satisfactory metabolic control is seldom achieved. Since liver underinsulinization may account for poor control, we tested whether the quick rise in plasma insulin attainable with s.c.Lispro injection vs regular insulin (HR) may provide an advantage. $14 \mathrm{LD}+\mathrm{DM}(4 \mathrm{~F} / 10 \mathrm{M} ; 57.4 \pm 8.3 \mathrm{yrs}$ old $20.0 \pm 4.0 \mathrm{~kg} / \mathrm{m}^{2} ; 51 \pm 3 \mathrm{U} /$ day; Child A/B class: AST $=47 \pm 9 \mathrm{UI}$; ALT $=58 \pm 16$ UI; Albumin $=43 \pm 1 \mathrm{gr} / \mathrm{l} ; \mathrm{PT}=75 \pm 5 \%$ ) were recruited. A 3 or more injection/day regimen (HR at meals plus evening Humulin I) was ensured in all patients during a 4 wk run-in. Patients were then randomly maintained in the same treatment or switched to Lispro. After 2 mos, treatments were crossed-over and patients followed up for 2 more mos. At the beginning and end of each arm, patients received a standard meal preceded by s.c. Lispro ($5 \mathrm{~min}=11.4 \pm 1.0 \mathrm{U})$ or $\mathrm{HR}(-30 \mathrm{~min}=11.6 \pm 1.2 \mathrm{U})$. Daily insulin did not change ( $52 \pm 4$ vs $51 \pm 3 \mathrm{U} /$ die), but pre-meal plasma glucose was lower after $\mathrm{HR}$ (13.8 \pm 0.9 vs. $11.4 \pm 0.9 \mathrm{mmol} / 1)$. With Lispro, plasma insulin reached a higher $(+3.1 \pm 0.4$ vs $2.2 \pm 0.3 \mathrm{ng} / \mathrm{ml})$ and earlier peak $(1.1 \pm 0.1 \mathrm{vs} 1.9 \pm 0.1 \mathrm{hr}$; both $\mathrm{p}<0.05)$ but similar AUC $(12.4 \pm 1.3$ vs $11.7 \pm 1.2 \mathrm{ng}-\mathrm{hr} / \mathrm{ml})$. Plasma glucose excursion $(+4.7 \pm 1.4$ vs $3.7 \pm 1.1 \mathrm{mmol} / \mathrm{l})$, $2 \mathrm{hr}$ plasma glucose $(18.3 \pm 1.2 \mathrm{vs}$ $14.3 \pm 0.8)$ and glucose tolerance $(\triangle \mathrm{AUC}=14.8 \pm 3.0$ vs $6.2 \pm 2.3 \mathrm{mmol}-\mathrm{hr} / \mathrm{l})$ were improved $(p<0.05)$. This improvement was associated with less endogenous insulin secretion (AUC C-peptide $=43.3 \pm 14.4$ vs $15.6 \pm 7.8 \mathrm{ng}$ $\mathrm{hr} / \mathrm{ml} ; \mathrm{p}<0.05)$. In conclusion, Lispro in LD+DM patients improves postprandial glucose profile and reduces stimulation of residual $\beta$-cell function. However, a certain degree of pre-meal hyperglycemia is apparent. Therefore, Lispro provides an advantage in $L D+D M$, but adjustments of long-acting insulin administration may be necessary.

\section{8}

A NEW LONG-ACTING INSULIN (HOE901) DEMONSTRATES LESS NOCTURNAL HYPOGLYCAEMIA WHEN COMPARED WITH PROTAMINE INSULIN IN A CLINICAL TRIAL.

D.R. Matthews ${ }^{1}$ and C. Pfeiffer ${ }^{2}$ for the Multicentre HOE901 Research Group, Oxford, UK. ${ }^{1}$ Radcliffe Infirmary, Oxford: ${ }^{2}$ Hoechst AG, Frankfurt

We report the results of a 4 week multicentre, multinational, parallel three-group study of HOE901 which is a novel human insulin analogue manufactured using

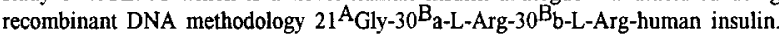
HOE901 is less soluble at physiological pH than native human insulin, and thus precipitates locally in the skin after subcutaneous injection. This delays its absorption and prolongs its duration of action. The trial used one of two formulations of HOE901 or protamine insulin (NPH) as an adjunct to therapy in NIDDM patients who had moderate glycaemic control on oral agents. 204 NIDDM patients were recruited. Inclusion criteria were $\mathrm{HbAlc}>7.0 \%$, age $40-80$ years, BMI 21-35. Patients were currently taking sulphonylurea or sulphonylurea plus metformin or acarbose. After a two week screening phase they were randomised to three treatment groups - two different formulations of HOE901 $(n=64 ; n=72)$ or $\mathrm{NPH}$ insulin $(\mathrm{n}=68)$. Median age was 59 years, median duration of NIDDM 9 years; median BMI 27.4. There were no statistical differences between glycaemic control on either of the HOE901 formulations or NPH insulin. The HbAlc reduced in all groups by $0.8 \%(\mathrm{p}<0.0001)$ from baseline. However, there were significantly less hypoglycaemic episodes on HOE901 (7.3\% HOE901 vs. 19:1\% NPH; $\mathrm{p}<0.037)$ In conclusion, HOE 901 is an effective long-acting insulin which was characterised in this trial against protamine insulin by less overnight symptomatic hypoglycaenia.

\section{9}

THE ABSORPTION OF HOE 901 IN HEALTHY SUBJECTS.

D. Owens, S. Luzio, J. Tinbergen ${ }^{\circ}$ and R. Kurzhals ${ }^{\circ}$. Diabetes Research Unit, Llangdough Hospital, Penarth, UK, and ${ }^{\circ}$ Hoechst Marion Roussel, Frankfurt, Germany.

The absorption characteristics of $\mathrm{I}^{125}$ labelled HOE 901 were studied in 2 studies in healthy subjects. Study A compared the absorption of two formulations of HOE $901(15$ or $80 \mu \mathrm{g} / \mathrm{ml}$ zinc content $)$ and NPH insulin. The insulins were administered as a single dose of $0.15 \mathrm{U} / \mathrm{kg}$ s.c. in the abdominal wall. Study B compared HOE $901(30 \mu \mathrm{g} / \mathrm{ml}$ zinc) following an injection of $0.2 \mathrm{U} / \mathrm{kg}$ into the abdomen, arm or leg. Both studies were randomised, three way cross-over design. Twelve subjects were studied in each study. The absorption rate was determined by external gamma counting at the injection site for 24 hours. Blood samples were taken for plasma glucose, C-peptide, and insulin.

All HOE 901 formulations had similar absorption patterns, while NPH had a faster absorption rate. The mean disappearance time of $25 \%$ radioactivity (T75\%) for both trials is summarised in the following table.

\begin{tabular}{|l|c|c|c|c|c|c|}
\hline \multirow{2}{*}{} & \multicolumn{3}{|c|}{ Study A } & \multicolumn{3}{c|}{ Study B } \\
\cline { 2 - 7 } & $\mathrm{NPH}$ & $\begin{array}{c}\mathrm{HOE} \\
901[15]\end{array}$ & $\begin{array}{c}\mathrm{HOE} \\
901[80]\end{array}$ & $\begin{array}{c}\mathrm{HOE} \\
901[30]\end{array}$ & $\begin{array}{c}\mathrm{HOE} \\
901[30]\end{array}$ & $\begin{array}{c}\mathrm{HOE} \\
901[30]\end{array}$ \\
\hline Inj. site & abdomen & abdomen & abdomen & abdomen & arm & leg \\
\hline T75\%* & $3.21^{* *}$ & 8.75 & 11.00 & 13.20 & 11.90 & 15.30 \\
\hline$*$ hours, $* *$ p $<0.001$ & & & &
\end{tabular}

The exogenous plasma insulin profile (C-peptide corrected immunoreactive insulin) showed a peak for NPH at 3-5 hours, whereas the HOE 901 profile was peakless. HOE 901 showed reduced variation between injection sites, indicating its freedom from injection site-dependency. In view of its prolonged and peakless absorption characteristics HOE 901 may be a suitable basal insulin for diabetic patients requiring insulin.

\section{0}

INSULIN LISPRO AND REGULAR INSULIN: POSTPRANDIAL GLUCOSE AND INSULIN IN GESTATIONAL DIABETES MELLITUS (GDM).

L. Jovanovic, S. Ilic, D. Pettitt, M. Gutierrez, and E.J. Bastyr III. Sansum Medical Research Institute, 2219 Bath street, Santa Barbara, CA.

Elevated postprandial glucose in pregnancy increases risk of macrosomia. Thus the treatment strategy should be designed to lower those levels. It has been shown in nonpregnant diabetic patients that insulin lispro lowers postprandial glucose levels Therefore we designed the study to compare insulin lispro with regular human insulin for the following: plasma glucose, serum insulin and C-peptide concentrations before, and 60,120 , and $180 \mathrm{~min}$ after a standardized meal test.Thirty-five woman with GDM (14-32 weeks of gestation) who failed on diet were randomized to receive either lispro or regular insulin before the meal test. At baseline, a meal test (30kacl/kg; $40 \%$ carbohydrate, $20 \%$ protein, $40 \%$ fat) was given along with a subcutaneous injection of $0.179 \mathrm{U} / \mathrm{kg}$ of either regular insulin (at $-30 \mathrm{~min}$ ) or with lispro insulin (at $-5 \mathrm{~min}$ ).

\begin{tabular}{|c|c|c|c|c|}
\hline Time & Fasting & $60 \mathrm{~min}$ & $120 \mathrm{~min}$ & $180 \mathrm{~min}$ \\
\hline \multicolumn{5}{|c|}{ FLASMA GWOCOSE (mmol) } \\
\hline LSFRO & $4.33 \pm 0.78$ & $5.56 \pm 0.7$ & $494 \pm 122$ & $3.89 \pm 0.94^{*}$ \\
\hline REGLAR & $4.44 \pm 0.56$ & $6.11+0.83$ & $5.50 \pm 122$ & $4.78 \pm 1.23$ \\
\hline \multicolumn{5}{|c|}{ SERUM NSUUN ( $\mathrm{mmd}$ ) } \\
\hline USPRO & $80.64 \pm 35.76$ & $543.66 \pm 144.18^{*}$ & $371.76 \pm 140.40$ & $17880+55.92$ \\
\hline REGULAR & $101.64 \div 59.46$ & $781.80 \pm 188.10$ & $354.30 \pm 131.88$ & $23640 \pm 83.76$ \\
\hline \multicolumn{5}{|c|}{ C-PEPTIDE (pondL) } \\
\hline USPRO & $1284 \pm 9.54$ & $23.1 \pm 4.74^{*}$ & $20070 \pm 11.40$ & $15.48+1260$ \\
\hline REGULAR & $10.44 \pm 5.34$ & $43.80 \pm 11.40$ & $30.42+4.86$ & $26.16 \pm 9.84$ \\
\hline \multicolumn{5}{|c|}{ SERUMLSPROLEVES (pmo/L) } \\
\hline LISFRO & 0 & $15.90 \pm 864$ & $11.70+5.34$ & $6.12+3.18$ \\
\hline
\end{tabular}

$* p<.00$

As shown above, lispro insulin was able to significantly lower glucose level after meal test at the 180 min point. Of interest is that treatment with lispro insulin resulted in a lower $60 \mathrm{~min}$ insulin and C-peptide levels, perhaps reflecting biological response that's not reflected by statistics. In conclusion insulin lispro improves postprandial glucose concetration and thus may be considered as the optimal treatment in GDM 
PLASMA ALBUMIN BINDING RETARDS APPEARANCE OF ACYLATED INSULIN IN INTERSTITIAL FLUID.

M.K. Dea, M. Hamilton-Wessler, M Ader, D.M. Moore, J Markussen * and R.N. Bergman. U. of Southern California School of Medicine, Los Angeles, CA, USA and Insulin Research, Novo Nordisk A/S, DK2880 Bagsvaerd, Denmark.

Lys ${ }^{\mathrm{B} 29}$-tetradecanoyl des(B30) human insulin (NN304) is designed to improve insulin therapy due to protracted action. The main aim of this study was to determine the transendothelial transport (TET) and clearance rates of NN304 in comparison to native insulin. Euglycemic clamps were performed in anesthetized dogs. NN304 was infused at $3.6(n=6), 10.2(n=6)$ or $18(n=6) \mathrm{pmol} / \mathrm{min} / \mathrm{kg}$ for $320 \mathrm{~min}$, then stopped for $340 \mathrm{~min}$. Porcine insulin was infused into controls $(n=4)$ at $10.2 \mathrm{pmol} / \mathrm{min} / \mathrm{kg}$ for $180 \mathrm{~min}$, then stopped for $180 \mathrm{~min}$. Arterial plasma and hindlimb lymph, representative of interstitial fluid (ISF), were sampled, and compartmental models describing the transendothelial transport rates and clearance of NN304 and insulin were developed. These models assume that only the unbound NN304 is available for TET or clearance. 5 parameters were identified for NN304: TET, plasma clearance, ISF clearance, plasma volume, and rate of unbinding from albumin. For insulin, only 4 parameters were identified, as the rate of unbinding from albumin does not apply. None of the parameters differed between the three doses of NN304 ( $p>0.29$ ). Based on the binding constant of the analog for albumin, $1.7 \%$ and $3.3 \%$ of NN304 are unbound in plasma and lymph, respectively. Transendothelial transport rates were 10 fold higher for NN304 vs. controls $(p=0.020)$. Fractional clearance of the unbound NN304 pool was 4.0 fold $(p=0.008)$ and 2.6 fold $(p<0.001)$ higher for plasma and interstitial fluid, respectively. Despite the faster transport rates for NN304, the effective transport rates are slower for NN304 because albumin sequesters more than $96.7 \%$ of the NN304 making it unavailable for transport across the endothelium or for clearance. Thus, this study concludes that the extended action of NN304 is due to reduced clearance secondary to albumin binding The delayed onset of glucose uptake is due to slower appearance in interstitial fluid, also secondary to albumin binding.

\section{3}

LISPRO MIX25 REDUCES POSTRANDIAL GLUCOSE COMPARED TO HUMAN INSULIN 30/70 AND NPH IN NIDDM PATIENTS

V.A. Koivisto, J.A. Tuominen, and P. Ebeling. Helsinki University Hospital, Helsinki, Finland

Mixtures of short- and long-acting insulins are increasingly being used, particularly for the treatment of NIDDM patients. Lispro Mix25 represents a new formulation of insulin lispro (25\%) and insulin lispro protamine suspension (NPL 75\%). We compared the premeal use of Mix25, human insulin 30/70 (Mixtard) and NPH (Protaphan) in 22 insulin-treated NDDM patients $\left(12 \mathrm{M} / 10 \mathrm{~F}\right.$, age $62 \pm 1 \mathrm{yrs}$ (mean $\pm \mathrm{SE}$ ), BMI $30 \pm 1 \mathrm{~kg} / \mathrm{m}^{2}$, duration of diabetes $15 \pm 2$ yrs and of insulin therapy $6 \pm 1$ yrs, insulin dose $65 \pm 6 \mathrm{U} / \mathrm{d}$, $\mathrm{HbA}_{1 \mathrm{c}} 7.9 \pm 0.2 \%$, C-peptide $0.58 \pm 0.06 \mathrm{mmol} / \mathrm{l}$ ). Each patient was studied 3 times at 1 week intervals in a double blind, randomized fashion. After an overnight $(10-12 \mathrm{~h})$ fast patients received $36 \pm 4 \mathrm{U}$ of test insulin S.C. in the abdominal wall. Ten minutes after insulin injection they ingested a breakfast meal $(500 \mathrm{kcal}, 60 \%$ carbohydrate, $20 \%$ fat, $20 \%$ protein), identical in all studies. Blood samples were taken before and at 10-30 min intervals for 240 min after the breakfast meal. Fasting serum glucose levels were similar before the Mix 25, 30/70 and NPH studies $(9.9 \pm 0.4,9.5 \pm 0.3$ and $9.6 \pm 0.3 \mathrm{mmol} / \mathrm{l}$, respectively). Ten healthy individuals $(6 \mathrm{M} / 4 \mathrm{~F}$, age $56 \pm 1 \mathrm{yrs}$, BMI $28 \pm 1$ $\mathrm{kg} / \mathrm{m}^{2}$ ) were studied using a similar breakfast meal. After Mix25 administration in NIDDM patients, the area under serum glucose curve was larger than in healthy subjects, but $36 \%$ smaller than after $30 / 70(\mathrm{p}=0.008)$, and $56 \%$ smaller than after NPH $(p=0.003)$. The difference in glucose response emerged at $30 \mathrm{~min}$ and was consistent thereafter. The peak rise in serum glucose was also smaller $(\mathrm{p}<0.01)$ after Mix $25(4.2 \pm 0.4 \mathrm{mmol} / \mathrm{l})$ than after $30 / 70(5.2 \pm 0.3 \mathrm{mmol} / \mathrm{l})$ or NPH $(6.3 \pm 0.2 \mathrm{mmol} / \mathrm{)})$

In conclusion: after the injection of Mix25 the rise in postprandial serum glucose in NIDDM patients: 1) is less than after human 30/70 or NPH insulins, and 2) is closer to a physiologic postprandial glucose response than with the other two insulins.
ELEVATION IN FREE FATTY ACIDS INFLUENCES ALBUMIN-BINDING BUT NOT METABOLIC EFFECTS OF FATTY ACID ACYLATED INSULIN, NN304. M. Hamilton-Wessler, J. Markussen*, and R.N. Bergman. Dept. of Physiology\&Biophysics, U. of Southern California School of Medicine, Los Angeles, CA, USA and "insulin Research, Novo Nordisk A/S, Bagsvaerd, Denmark.

Binding of the myristic acid acylated insulin, NN304, to albumin at fatty acid binding sites results in prolongation of NN304 action. This study examined the influence of elevated free fatty acids (FFA) on NN304 action. Liposyn II (L) was infused i.v. at three doses (L1-3: $0,0.5$ or $1.5 \mathrm{ml} / \mathrm{min}$ ) throughout euglycemic clamps (somatostatin, and $0.6 \mathrm{mU} / \mathrm{min} / \mathrm{kg}$ i.v. human insulin (HI) or NN304 infusion for $320 \mathrm{~min}$ in normal dogs; $\mathrm{N}=6$, with 6 exps/animal). Basal FFA was $0.68 \pm 0.15 \mathrm{mM}$, and levels for $L 1-3$ were $0.22 \pm 0.02,0.83 \pm 0.04$ and $2.95 \pm 0.19 \mathrm{mM}$, respectively. Plasma steady-state(SS) HI was $212 \pm 40 \mathrm{pM}$ ( $P>0.50$ across doses). SS total NN304 (albumin-bound plus free) declined from $2750 \pm 98$ at $L 1$ to $2351 \pm 200$ and $1774 \pm 128 \mathrm{pM}$ at $L 2$ and $L 3(P<0.05)$, reflecting an increase in NN304 clearance of $20 \pm 7$ and $58 \pm 10 \%$, due to displacement from albumin by elevated FFA. Yet, effects on SS giucose production (EGP) and utilization (Rd) were similar between $\mathrm{HI}$ and NN304 (P>0.10). SS similar between $\mathrm{Hl}$ and $\mathrm{NN304}(\mathrm{P}>0.10)$. SS
EGP (Fig.A) fell from basal at $\mathrm{L} 1$ and rose significantly at $L 2$ and $L 3(P<0.01$ vs. $L 1)$. SS Rd (Fig.B) increased from basal at L1, and fell slightly at $L 2$ and $L 3 \quad(P<0.05$ vs. $L 1)$. Conclusions: 1) These findings are consistent with competition between FFA and NN304 for binding sites on albumin when FFA are markedly elevated; 2) Acute elevations in FFA have similar effects on native insulin and NN304 action to increase EGP and reduce Rd, counteracting potentially negative outcomes of displacement of NN304 from albumin.

\section{4}

TOPICAL TREATMENT WITH MILD OXIDANTS FACILITATES TRANS-DERMAL DELIVERY OF INSULIN

U. Wormser and A. Sintov, The Institutes for Applied Research, E.D Bergmann Campus, Ben-Gurion University of the Negev, Beer-Sheva, Israel Parenteral administration of insulin is associated with serious clinical problems including inappropriate maintenance of blood levels of insulin and glucose, resulting in a variety of complications. The objective of the presen study was to develop an effective system for transdermal delivery of insulin. The following assay systems were emploved. The abdominal skin of anesthetized rats was treated with povidone-iodine ointment $(10 \%)$ for 3 hours. After washing, regular human insulin solution (Humulin R $50 \mathrm{IU} / 0.5$ $\mathrm{ml}$ in a non-leaky open cylinder or in a patch) was applied. At selected time intervals, tail bleeding was carried out for glucose determination. Franz diffusion cells were used for in vitro penetration studies of insulin. Our findings showed a time-dependent reduction of plasma glucose levels $(80 \%$ decrease after 4 hours) with povidone iodine pretreatment whereas no hypoglycemic effect was obtained without such pretreatment. Other oxidants such as silver protein and potassium permanganate had similar effect to that of povidone iodine. In glucose tolerance tests, when subcutaneous injection of D-glucose ( $4 \mathrm{M}, 0.8 \mathrm{ml})$ was carried out 30 minutes after insulin patch application, a significant suppression of plasma glucose elevation was observed. Interestingly, in the in vitro system insulin penetrated the abdominal skin of rat, hairless mouse and human without an oxidant pretreatment while the permeated hormone retained its biological activity. However, in the skin flap system of the living animal the penetrated insulin was unable to cause hypoglycemic effect when injected into fasted animals. It is concluded that insulin is inactivated during the skin permeation process in the living animal and that a topical pretreatment by mild oxidants may inhibit these inactivating mechanism(s). This system nuay be employed as a new approach for transdermal delivery of insulin. 


\section{5}

INSULIN PRODUCTION BY ENGINEERED MUSCLE CELLS

L. Gros, E. Riu, L. Montoliu, M. Ontiveros, L. Lebrigand and F. Bosch. Department of Biochemistry and Molecular Biology, School of Veterinary Medecine, Universitat Autonoma de Barcelona, 08193-Bellaterra, Spain.

Type 1 diabetic patients depend dramatically on insulin replacement therapy, which involves the administration of intermediate- or long-acting insulin, together with short-acting insulin to mimic physiological insulin profiles. However, the delayed-action preparations available are not generally able to produce smooth background levels of insulin, resulting either in hyperglycemia or hypoglycemia. Muscle cells were tested for long-term delivery of active human insulin as an approach to achieve a constant basal level of insulin. Thus $\mathrm{C}_{2} \mathrm{C}_{12}$ mouse myoblast cells were stably transfected with a chimeric gene obtained by linking the myosin-light chain 1 (MLC1) promoter to the human proinsulin gene, containing genetically engineered furin endoprotease cleavage sites (MLC1/Insm). When differentiated, $\mathrm{C}_{2} \mathrm{C}_{12}$ Insm myotube cells expressed high levels of insulin mRNA and protein, while no insulin was detected in myoblast cells. HPLC fractionation of culture medium and cell extracts from differentiated $\mathrm{C}_{2} \mathrm{C}_{12}$ Insm cells revealed that about $90 \%$ of the proinsulin was processed to mature insulin. In addition, these cells released significant levels (about $100 \mu \mathrm{U} / 10^{6} \mathrm{cells} / \mathrm{h}$ ) of mature insulin to the medium. The hormone was biologically active since an increase (about 2.5 -fold) in glucose uptake by the differentiated $\mathrm{C}_{2} \mathrm{C}_{12}$ Insm cells was observed. Furthermore, insulin produced by $\mathrm{C}_{2} \mathrm{C}_{12}$ Insm cells was able to block the expression of the endogenous $\mathrm{P}$ enolpyruvate carbox ykinase (PEPCK) gene in FTO-2B rat hepatoma cells. Thus, our results indicated that genetically engineered differentiated $\mathrm{C}_{2} \mathrm{C}_{12}$ Insm cells synthesized, processed and secreted active insulin, and they were also able to remove glucose very efficiently. This suggests that the use of engineered myotube cells continuously secreting a defined level of insulin might be a useful approach to improve the efficacy of insulin injection treatment.

\section{PS 45}

\section{Insulin Therapy II}

\section{6}

\section{REDUCTION OF THE RISK OF INTRAMUSCULAR INSULIN INJECTION WITH THE 8 MM LENGTH NEEDLES IN THIN DIABETIC CHILDREN \\ N. Tubiana-Rufi, N. Belarbi*, L. Du Pasquier, M. Polak, G. Licha-Munz, C. Delcroix, M. Hassan* and P. Czernichow. \\ Departments of Pediatric Diabetology and * Radiology. Hôpital Robert Debré, Paris. France}

The technique of insulin injection varies among different centers or countries. Direct methods of evaluation of the intramuscular (IM) vs subcutaneous (SC) location of the insulin deposit, according to the injection technique, in well defined populations, are needed. We have shown previously, by the mean of a non invasive method (ultrasonography : US), that the frequency of IM insulin injections with the $12.7 \mathrm{~mm}$ needles was high in diabetic children, even with a skinfold, and inversely related to their body mass index (BMI). Objective : to assess whether the $8 \mathrm{~mm}$ length needles are able to reduce the frequency of IM assest whether the $8 \mathrm{~mm}$ length needles are able to reduce the frequency of $1 \mathrm{M}$ $\mathrm{mm}$ length needles on the occurrence of IM injections, localized by US, in children whose BMI was s60th percentile for age and sex. Each patient was his/her own control : 2 standardized insulin injections $(12.7 \mathrm{vs} 8 \mathrm{~mm}$, in a randomized order) were performed by a nurse into a two-finger pinch. Two consecutive studies were performed in 2 groups of 25 children, on 2 injection sites : arms and thighs. Results : in the 50 children (mean age $=11.5 \pm 3.5 \mathrm{yrs}$, mean $\mathrm{BMI}=38 \pm 18$ th percentile), the IM injection frequency was $86 \%$ with the $12,7 \mathrm{~mm}$ needles and $38 \%$ with the $8 \mathrm{~mm}$. The Mac Nemar and Prescott tests showed a significant reduction of the IM frequency with the $8 \mathrm{~mm}$ needles in arms $(p<0.01)$ and thighs $(p<0.01)$. The efficiency of the $8 \mathrm{~mm}(\mathrm{IM} / 12.7 \mathrm{~mm}$ and $\mathrm{SC} / 8 \mathrm{~mm}$ ) was found for half of the children whose injection was performed in the arms and for the $2 / 3$ rd of the children who injected in the thighs. The distance measured by US from the skin surface to muscular fascia (DSM), with a skinfold, was significantly higher in the group where the $8 \mathrm{~mm}$ were efficient $(n=24)$ than in those where they were not efficent $(n=19)$ : respectively, $9.8 \pm 2.2$ vs $6.8 \pm 2.1 \mathrm{~mm}, p<0,0001$. The efficiency of the $8 \mathrm{~mm}$ was not significantly related to age, sex, percentile of BMI, injection device or site. For a DSM threshold at $8 \mathrm{~mm}$ at the injection site, the sensibility and specificity of the method to predict the efficiency of the $8 \mathrm{~mm}$ were calculated at $79 \%$. In conclusion : the $8 \mathrm{~mm}$ length needles allowed to significantly, but not totally, reduce the risk of intramuscular insulin injection in thin diabetic children.

\section{7}

REGULAR INSULIN IN THE TREATMENT OF OBESE TYPE 2 DIABETICS

Ch. Koenen, D. Weise, T. Witton, P. de Faber, C. Hasslacher St. Joseph Hospital ,Heidelberg, Germany

The most widely used insulin treatment in type 2 diabetics is the injection of an intermediate-acting insulin once or twice per day, sometimes in combination with oral agents. In obese type 2 diabetics this is often combined with a weight gain, an increase in insulin resistance, and episodes of hypoglycemia. The goal of this study was to determine whether these disadvantages could be avoided by using regular insulin without deteriorating metabolic control. Twenty-one obese type 2 diabetics $(B M \mid>26)$ were treated for one year with injections of regular insulin prior to every meal. After a year, body weight, $\mathrm{HbA} 1 \mathrm{c}$, triglycerides, and cholesterol were measured. This data was compared to a group of 36 type 2 diabetics that were treated with conventional insulin therapy over the same time. Both groups were comparable in age, body weight, diabetes duration, and gender. Both groups underwent the same patient education program prior to initiation of the treatment. The patients treated with regular insulin gained, on average, less weight $(1.2 \mathrm{~kg}$ vs. $4.0 \mathrm{~kg})$ and had no episodes of hypoglycemia. The conventional insulin therapy group reported a total of three episodes of severe hypoglycemia. In both groups metabolic control was improved ( $\mathrm{HbA} 1 \mathrm{c}-1.4 \%$ and $-1.6 \%$ ). Additionally, cholesterol (-64 $\mathrm{mg} \%$ and $-56 \mathrm{mg} \%$ ) and triglycerides (-19 $\mathrm{mg} \%$ and $-21 \mathrm{mg} \%$ ) levels were reduced. Using regular insulin prior to every meal did improve metabolic control without a significant weight gain. Quality of life could be improved by reducing weight gain and episodes of hypoglycemia. 
SUSTAINED IMPROVEMENT OF GLYCAEMIC CONTROL BY INSULIN TREATMENT IN PATIENTS WITH TYPE 2 DIABETES

T. Lindström, Department of Endocrinology and Diabetes, The University Hospital, S-581 85 Linköping, Sweden

Objective. To evaluate the long-term efficacy of insulin treatment of patients with Type 2 diabetes and secondary failure to oral hypoglycaemic agents. Research Design and Methods. We have previously reported results on glycemic control and other metabolic variables in 21 patients after insulin treatment for a median of 27 months (T. Lindström et al. Diabetes Care 17; 719-21, 1994). The patients had initially taken part in 2 short-term trials but then received usual care. Six of these patients have died by cardiovascular diseases. The remaining 15 patients were reevaluated after a median of 110 (range 92-131) months of insulin treatment.

\begin{tabular}{llllll}
\hline & $\begin{array}{l}\text { Before } \\
\text { insulin }\end{array}$ & & 27 months & $\begin{array}{l}110 \\
\text { months }\end{array}$ & $\begin{array}{l}\text { prob. } 0-110 \\
\text { months }\end{array}$ \\
\hline $\mathrm{HbAlc} \mathrm{( \% )}$ & $8.9 \pm 0.2$ & $<0.0001$ & $6.8 \pm 0.4$ & $7.3 \pm 0.3$ & $<0.001$ \\
fasting blood glucose & $11.9 \pm 0.8$ & $<0.05$ & $7.4 \pm 0.8$ & $7.9 \pm 1.1$ & $<0.01$ \\
glucose after breakfast & $17.2 \pm 1.0$ & $<0.001$ & $11.6 \pm 0.9$ & $10.5 \pm 0.9$ & $<0.0001$ \\
body weight & $71.2 \pm 3.2$ & $<0.0001$ & $77.9 \pm 3.2$ & $78.8 \pm 3.2$ & $<0.0001$ \\
Body mass index & $24.4 \pm 0.8$ & & $26.7 \pm 0.8$ & & \\
insulin dose (4 weeks) & $51.3 \pm 5.2$ & & $45.5 \pm 7.2$ & $79.5 \pm 10$ & $<0.001$ \\
fasting insulin & $6.1 \pm 0.8$ & $<0.01$ & $11.7 \pm 1.6$ & $12.3 \pm 2.0$ & $<0.01$ \\
insu!in after breakfast & $16.4 \pm 2.0$ & $<0.001$ & $42.7 \pm 4.5$ & $48.0 \pm 7.1$ & $<0.0001$
\end{tabular}

Plasma total cholesteroi concentration was $5.33+0.38 \mathrm{mmol} / \mathrm{l}$ during treatment with oral agents and $5.64 \pm 0.28$ at the 110 months evaluation (ns); total triglycerides 1.99 \pm 0.45 and $1.56 \pm 0.25 \mathrm{mmol} / \mathrm{l}$ respectively (ns). There were non-significant decreases of plasma VLDL cholesterol and triglycerides and a non-significant rise of plasma HDL cholesterol concentration at the 110 months evaluation. Conclusion. Insulin treatment gives long-term improvement of glycaemic control and might therefore improve the prognosis of patients with Type 2 diabetes and normal or moderate overweight when failing on oral agents. In spite of weight gain there is no adverse effect on plasma lipoprotein concentrations.
IS NORMOGLYCEMIA ACHIEVED BY INTENSIFIED INSULIN THERAPY? 6-DAY CONTINUOUS TISSUE GLUCOSE MONTTORING IN IDDM

F. Sternberg, M.I. Salgado, U. Hoss, R. Gessler and R. Fussgänger. Institute of Diabetes Technology at the University of UIm, Ulm, Germany

Normoglycemia is a primary goal in diabetes care. Intensified insulin therapy (IIT) delays the onset and slows the progression of diabetic late complications while decreasing the $\mathrm{HbA}_{1 \mathrm{c}}$. However, does IIT actually lead to normoglycemia? We developed a glucosensor which on-line and continuously monitors the subcutaneous glucose. The system is based on the enzymatic-amperometric glucose measurement in combination with the microdialysis technique. We designed a study, in order to evaluate the ability of IIT to achieve normoglycemia in 6 type I diabetic patients (2W:4M, age: $42 \pm 9$ years, BMI: $23.8 \pm 2.3 \mathrm{~kg} / \mathrm{m}^{2}, \mathrm{HbA}_{1 \mathrm{c}}: 6.5 \pm 0.7 \%$, diabetes duration: $22 \pm 17$ years) by means of 3 series of $48 \mathrm{~h}$ continuous tissue glucose monitoring (CTGM) in each patient. During CTGM, patients remained on the ward, went to work or spent their normal life. They were asked to control their capillary glucose at least 5 times/day. We did not interfere with the patients' therapy decisions, which remained always unchanged. By means of the CTGM we calculated the fasting glucose, mean glucose, mean amplitude of glucose excursion (MAGE), hypoglycemia frequency (glucose $<70 \mathrm{mg} / \mathrm{dl}$ ) and dawn phenomenon. Altogether we obtained 6 days of CTGM in each of our patients. $70 \%$ of the fasting glucose values were hyperglycemic, while $11 \%$ were hypoglycemic and $19 \%$ normoglycemic. Mean glucose was $120 \pm 30 \mathrm{mg} / \mathrm{dl}$ (mean range: $294 \pm 88-38 \pm 13 \mathrm{mg} / \mathrm{dl}$ ). Glucose fluctuated between 440 and $24 \mathrm{mg} / \mathrm{dl}$ and MAGE was $256 \pm 95 \mathrm{mg} / \mathrm{dl}$. The hypoglycemia frequency was of 2.3 events/patient/day, while a dawn phenomenon was present in $56 \%$ of the cases. The sensor signal correlated significantly with the capillary glucose. Despite the acceptable $\mathrm{Hb} \mathrm{A}_{1 \mathrm{c}}$ values, only one patient presented relatively good glucose values resembling near normoglycemia. In the other patients, IIT by far did not achieve normoglycemia. Even though the mean glucose values were close to normoglycemia, these by no way represent the daily glucose profile. A glucosecontrolled insulin delivery system may achieve normoglycemia.

\section{0}

COMBINED TREATMENT WITH METFORMIN AFTER UNSUCCESSFUL INSULIN THERAPY IN OBESE TYPE 2 DIABETIC PATIENTS

S. Tenés, F.J. Ampudia-Blasco, J.T. Real, M. Civera, R. Carmena. Diabetes Reference Unit, Endocrinology Dep., Clinic University Hospital Valencia, Spain. Some obese type 2 diabetic patients had difficulties to reach treatment objectives although elevated insulin doses are employed. The purpose of this study was to evaluate the effects of metformin addition $(1700 \mathrm{mg} /$ day) to a previous unsatisfactory insulin treatment. Twenty-eight obese type 2 diabetic patients selected from the outpatient clinic (21 women (75\%), age $58.1 \pm 8.8$ y (mean \pm SD), BMI $33.8 \pm 4.7 \mathrm{~kg} / \mathrm{m}^{2}$, diabetes duration $15.2 \pm 8.5 \mathrm{y}$, insulin treatment since $6.4 \pm 6.2$ $y$, with $0.81 \pm 0.34 \mathrm{U} / \mathrm{kg} /$ day) were allocated to two treatment modalities: $(A, n=17)$ insulin bedtime+metformin, with reduction of insulin doses and $(B, n=11)$ insulin b.i.d+metformin, without reduction of insulin doses. Fasting blood glucose (FBG), $\mathrm{HbA}_{1 \mathrm{c}}$, insulin dose and weight were collected from the initial and the most recent visit. Results from the whole cohort of patients after $4.6 \pm 1.8$ months (2-9) were:

\begin{tabular}{|c|c|c|c|c|c|}
\hline & $\begin{array}{l}\text { FBG } \\
(\mathrm{mg} / \mathrm{dl})\end{array}$ & $\begin{array}{l}\mathbf{H b A _ { 1 c }} \\
(\%)\end{array}$ & $\begin{array}{l}\text { insulin dose } \\
\text { (U/kg/d) }\end{array}$ & $\begin{array}{l}\text { weight } \\
\text { (kg) }\end{array}$ & $\begin{array}{l}\text { BMI } \\
\left(\mathbf{k g} / \mathrm{m}^{2}\right)\end{array}$ \\
\hline pre-metformin & $228 \pm 69$ & $9.1 \pm 1.9$ & $0.81 \pm 0.34$ & $84.5 \pm 11.2$ & $33.8 \pm 4.7$ \\
\hline post-metformin & $188 \pm 81$ & $8.1 \pm 1.4$ & $0.62 \pm 0.36$ & $83.1 \pm 11.1$ & $33.3 \pm 4.7$ \\
\hline p value & 0.009 & 0.0009 & 0.003 & 0.31 & 0.34 \\
\hline
\end{tabular}

The comparison of both groups (A vs. B) showed a major weight reduction in group A $(-2.91$ vs. $+0.79 \mathrm{~kg}, \mathrm{p}=0.004)$ but a greater diminution of $\mathrm{HbA}_{\mathrm{lc}}$ in group $B$ $(-0.51$ vs. $-1.87 \%, p=0.05)$. Reduction of insulin doses had a positive correlation with the magnitude of weight decrease $(r=0.43, p=0.02)$ and a negative correlation with the reduction of $\mathrm{HbA}_{1 \mathrm{c}}(\mathrm{r}=-0.44, \mathrm{p}=0.01)$. We conclude that the addition of metformin to previous insulin treatment improves glycaemic control and lowers insulin dose. Combined treatment with metformin and insulin prevents weight gain and may induce significant weight loss when previous insulin doses are reduced

\section{1}

METABOLIC EFFECTS OF I.M. VS. S.C. INJECTION OF SOLUBLE INSULIN AND INSULIN LISPRO IN HEALTHY SUBJECTS

J. Hermberger, T. Heise, K. Rave, R. Bender, S. Hirschberger*, L. Heinemann; Department of Metabolic Diseases and Nutrition, HeinrichHeine-University, Düsseldorf, Germany; *Novo Nordisk, Germany The aim of the study was to compare the glucodynamic and pharmacokinetic effects elicited by subcutaneous (s.c.) and intramuscular (i.m.) injection of soluble insulin (SI) and insulin lispro (LI). Twelve healthy subjects $(27 \pm 2$ years; BMI $23 \pm 2 \mathrm{~kg} / \mathrm{m}^{2}$; mean \pm SD) participated in this euglycaemic glucose clamp study (blood glucose $5.0 \mathrm{mmol} / /$; basal i.v. insulin infusion $0.3 \mathrm{mU} / \mathrm{kg} / \mathrm{min}$ ). On four different study days SI (Actrapid HM, NOVO NORDISK) and $\mathrm{L} !$ (Insulin

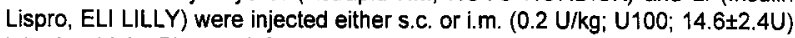
into the thigh. Glucose infusion rates (GIR) and serum insulin concentrations (IN) were determined for $\mathbf{4 2 0}$ min after injection. Parameters calculated are shown in the table. In conclusion, the clinically known, more rapid effect of i.m. applied $\mathrm{SI}$ in comparison to s.c. injected SI was quantified in this study for the first time. The time action profile of i.m. administered SI was between s.c. injected SI and s.c. and i.m. applied $\mathrm{LI}$. The later exhibited a similar metabolic profile irrespective whether injected into subcutis or muscle.

\begin{tabular}{|c|c|c|c|c|}
\hline & SI s.c. & Slim. & LI s.c. & Ll i.m. \\
\hline$G \mid R_{\max (m g / k g / m i n)}$ & $7.8 \pm 2.3^{\text {\#S }}$ & $9.7 \pm 2.3$ & $11.1 \pm 2.3$ & $10.4 \pm 2.7$ \\
\hline GIR early $t_{50 \%(\min )}$ & $50 \pm 14$ & $56 \pm 13$ 触 & $44 \pm 15$ & $43 \pm 10$ \\
\hline$G I R t_{\max (\min )}$ & $179 \pm 34$ * * & $138 \pm 29$ & $116 \pm 26$ & $120 \pm 28$ \\
\hline GIR AUC ${ }_{0-120 \mathrm{~min}\left(g / \mathrm{kg}^{+} 120 \mathrm{~min}\right)}$ & $0.50 \pm 0.15^{* 35}$ & $0.60 \pm 0.18^{108}$ & $0.81 \pm 0.26$ & $0.78 \pm 0.25$ \\
\hline GIR AUC ${ }_{0-420 \mathrm{~min}\left(g / \mathrm{kg}^{*} 420 \mathrm{~min}\right)}$ & $2.55 \pm 0.70$ & $2.54 \pm 0.74$ & $2.81 \pm 0.70$ & $2.78 \pm 0.89$ \\
\hline$N t_{\max (\min )}$ & $156 \pm 52$ * * * & $127 \pm 47^{\top}$ & $78 \pm 24$ & $91 \pm 29$ \\
\hline$\left[N \mathrm{C}_{\max (\mathrm{pmol})}\right.$ & $182 \pm 51$ *\# & $244 \pm 52$ & $229 \pm 65$ & $236 \pm 42$ \\
\hline $\mathbb{N}^{\mathrm{N}} \mathrm{AUC} \mathrm{C}_{0-120 \mathrm{~min}}\left(\mathrm{nmol} \mathrm{n}^{*} 120 \mathrm{~min}\right)$ & $13.2 \pm 3.4^{\text {* * }}$ & $19.1 \pm 6.0$ & $20.6 \pm 5.4$ & $20.9 \pm 5.5$ \\
\hline
\end{tabular}

" significant differences of s.c. vs. i.m. SI, " s.C. SI vs. s.C. LI, "i.m. SI vs. S.C.

$\mathrm{LI}^{5}$ s.c. SI vs. i.m. LI, and i.m. SI vs. i.m. LI. MeantSD. 
962

IMPROVEMENT OF B-CELL FUNCTION BY INTENSIVE INSULIN TREATMENT IS RAPIDLY INDUCED AND RAPIDLY LOST

L Karvestedt, $G$ Andersson, S Efendic, P Clauson and V Grill, Clinic of Geriatrics Sabbatsbergs Hospital and Dept. of Endocrinology Karolinska Hospital, Stockholm

Intensive insulin treatment (IT) may improve insulin secretion in NDDM. Whether duration of IT is important for induction and persistence of improvement is unclear. Therefore we measured parameters of B-cell function in NIDDM patients ( $\mathrm{HbAlC}>7 \%$ and previously on SU tablets) at different time points before, during and after a 9 weeks of IT, consisting of 4 daily insulin injections. IT was followed by combination therapy (CT) consisting of SU + bedtime insulin. A decrease in proinsulin/insulin ratio $(\mathrm{P} / \mathrm{T})$ and an increase in glucagon-stimulated $\mathrm{C}$ peptide was noted 3 days after initiating IT. These effects remained stable (P/I) or slightly improved (C-peptide) during 9 weeks of IT but were lost $(\mathrm{P} / \mathrm{I})$ or attenuated ( $C$-peptide) 3 days after IT cessation (Table). Attenuated C-peptide did not differ from a control group, $n=10$, which, in parallel to the IT group, started and then continuously upheld $\mathrm{CT}$.

Fasting blood Proinsulin/Insulin Glucagonstimulated

$\begin{array}{llll} & \text { glucos mM } & \text { ratio } & \text { C-peptid, nmol/1 } \\ \text { Before IT } & 12,4 \pm 0,9 & 0,43 \pm 0,09 & 0,77 \pm 0,19 \\ \text { 3 days after initiation } & 11,5 \pm 0,6 & 0,29 \pm 0,05^{*} & 1,28 \pm 0,19^{*} \\ \text { 9 weeks after initiation } & 10,5 \pm 0,5 & 0,27 \pm 0,05 & 1,20 \pm 0,26^{*} \\ 3 \text { days post IT } & 9,5 \pm 0,6 * & 0,40 \pm 0,09 & 1,17 \pm 0,18^{*}\end{array}$

Values are Mean $\pm S E M, n=10, * p<0,05$ or less

Nine weeks of IT reduced $\mathrm{HbAlC}$ more than CT $(-2,0 \% \pm 0,5$ vs $-1,4 \% \pm 0,2)$. This effect was completely lost 8 weeks after switching IT to CT. Contrariwise, an extra gain in BMI $\left(0,9 \mathrm{~kg} / \mathrm{m}^{2} \pm 0,2 \mathrm{vs} 0,5 \mathrm{~kg} / \mathrm{m}^{2} \pm 0,1\right.$ in the continously CT group) persisted 8 weeks after switching IT to CT. In conclusion: 1)IT for 9 weeks reduces $\mathrm{HbAlc}$ more effectively than $\mathrm{CT}, 2$ ) extra weight gain during IT persist after cessation of IT, 3) improvement of B-cell function by IT is mostly gained initially, 4) a lasting effect is not produced.

\section{4}

INTENSIVE OR CONVENTIONAL INSULIN THERAPY IN TYPE 2 DIABETES A. Kademann, R. Schiel, U.A. Müller, University of Jena Medical School, Department of internal Medicine II, Jena, Germany

Up to the present there is controvery about intensive or conventional insulin therapy in type 2 diabetic patients. In a cross-sectional study $90 \%(n=117)$ of all insulin treated type 2 diabetic patients aged 16 to 60 years and living in a large city $(100,247$ inhabitants) were examined. Of the total of 117 patients, 40 (34\%) had an intensive (ICT, $\geq 2$ injections of normal and $\geq 1$ injection of NPH/mixed-insulin/d, $\geq 1$ linsulin-dose adjustment/week, $\geq 2$ blood-glucose selftests/d) and 77 patients $(66 \%)$ a conventional insulin therapy (CIT). Patients with ICT had a higher insulin dosage/d $(0.71 \pm 0.32$ vs $0.47 \pm 0.21 . \mathrm{U} . \mathrm{kg}$ body $w t / d, p<0.001)$, were younger $(50.5 \pm 6.7$ vs $54.0 \pm 5.9$ years, $p=0.004)$ and had $a$. tendency to a better HbA1c $(8.7 \pm 2.2$ vs $9.2 \pm 2.0 \%, p=0.23$, HPLC, Diamat $($, normal range $4.4-5.9 \%)$. There was a negative correlation between $\mathrm{HbA} 1 \mathrm{c}$ and the frequency of blood-glucose self-tests/week $(r=-0.23, p=0.019)$ and the number of insulin-dose adjustments/week $(r=-0.33, p<0.001)$. There were no differences between the groups of patients with ICT and CIT as regards bodymass index $\left(29.7 \pm 4.9\right.$ vs $\left.28.0 \pm 4.5 \mathrm{~kg} / \mathrm{m}^{2}, p=0.06\right)$, diabetes duration since diagnosis $(12.3 \pm 6.9$ vs $12.2 \pm 7.5$ years, $p=0.96$ ), duration of insulin therapy $(4.2 \pm 3.5$ vs $4.5 \pm 4.8$ years, $p=0.67)$, incidence of acute complications (hypoglycaemia, comata), prevalence of diabetes long-term complications and educational level. Quality of life was assessed using a standardized questionnaire according to Bradley et al. There were also no differences between the groups: ICT $48.5 \pm 8.9$ vs CIT $51.1 \pm 11.4, p=0.25) .2 .7 \pm 1.9$ years prior the cross-sectional study in 20 of the 40 patients with ICT, successive attending our clinic, an intervention was started: As lack of good quality of diabetes control and/or need for more flexibility these patients changed from CIT to ICT: The change lead to an improvement of $\mathrm{HbA1C}$ from $9.7 \pm 1.9 \%$ to $8.8 \pm 2.6 \%(\mathrm{p}=0.21)$. Up to date, in eiderly patients with type 2 diabetes an ICT is indicated in "problem"-patients with lack of good quality of diabetes control under CIT and/or need for more flexibility. In other patients, there is no evidence for benefit of ICT over CIT.

\section{3}

HIGHER INSULIN REQUIREMENT IN GIRLS COMPARED TO BOYS: REMISSION PHASE IN 878 PEDIATRIC PATIENTS WITH IDDM

R.W. Holl, W. Hecker, M. Grabert, B. Bartus, E. Heinze, A. Thon and M. Holder. Department of Pediatrics, University of U/m and Children's Hospital, Stuttgart, Germany

The remission phase following diagnosis and initial treatment of diabetes is characterized by good metabolic control despite low insulin requirement. Partial remission is defined by an insulin requirement of less than $0.5 \mathrm{U}$ per kg of body weight. Using a computer documentation system specifically designed for diabetes mellitus, patients from 2 adjacent diabetes centers wer evaluated. Mean daily dose of insulin was related to duration of diabetes, age at diagnosis and gender. A total of 878 pediatric patients with type-1 diabetes were evaluated ( 439 boys, 439 girls, mean age at diagnosis $8.1 \pm 0.1$ years). During the first 3 months after diagnosis, the average insulin dose was $0.49=$ $0.01 \mathrm{U} / \mathrm{kg}, 54 \%$ of patients fulfilled the criterion of partial remission. After 1 year of diabetes, the insulin dose increased to $0.65 \pm 0.01 \mathrm{U} / \mathrm{kg}(27 \%$ in remission), and after 2 years the respective numbers were $0.78 \pm 0.01 \mathrm{U} / \mathrm{kg}$ ( $10.4 \%$ of patients in remission). After 3 years, only $5 \%$ of patients still fulfilled the criterion of partial remission. Insulin requirement during the first years of diabetes was not different between children with a prepubertal ( $<11$ years, $n=633$ ) or pubertal $(n=245)$ onset of diabetes. However, 36 patients with a diabetes onset beyond 15 years of age tended to require less insulin compared to children with a younger age at onset. When boys and girls were compared, a striking difference was present: The insulin requirement was significantly higher in girls compared to boys during the first 4 years of diabetes: After 1 year, $32 \%$ of boys, but only $23 \%$ of girls were in partial remission ( $\mathbf{0}<0.0005$ ). After 2 years, the respective numbers were $12.2 \%$ for boys and $8.3 \%$ for girls $(p<0.001$ ). This gender difference was present both in children with a prepubertal and in children with a pubertal onset of diabetes. In conclusion, partial remission as defined by an insulin requirement of less than $0.5 \mathrm{U} / \mathrm{kg}$ can be present for up to 3 years. Remission is significantly more pronounced and lasts longer in boys compared to girls. As this effect is present in both prepubertal and puberta children, sex steroids cannot be the major cause of this phenomenon.

\section{5}

CORRECTION OF INCIDENTAL HYPERGLYCEMIA IN HOSPITALIZED DIABETIC PATIENTS

J.B.L. Hoekstra, F. Holleman, E.M. Kuck, I van der Tweel and D.W Erkelens. Diakonessenhuis, Utrecht, The Netherlands.

An insulin dosing nomogram for rapid and safe correction of incidental hyperglycemia in hospitalized patients was studied. Participants were screened for glucose levels $\geq 14 \mathrm{mmol} / \mathrm{l}$ at $9 \mathrm{p.m}$. on five consecutive days. Aiming for a glucose of $6 \mathrm{mmo} / /$, the insulin dose necessary to correct hyperglycemia was calculated as follows: (rounded glucose value - 6) $B M I{ }^{*} 0.04 I U$. Patients were randomized to double-blind correction with regular insulin (R) or insulin lispro (L). Up to three corrections per patient were allowed. Glucose samples were taken at $t=0,120,180$ and 240 minutes. Adequate glycemia was defined as glucose between 3 and 10 $\mathrm{mmol} / \mathrm{l}$. Several co-variables known to influence glucose concentrations were registered.

Sixty-four corrections were performed in 37 NIDDM patients ( 9 men) with median age of 74 years (range, 40-91), duration of diabetes of 9 years $(0$ 40 ) and BMI of $25.9 \pm 3.7 \mathrm{~kg} / \mathrm{m}^{2}$. For $L, 16$ of 29 corrections resulted in adequate glycemia after $120 \mathrm{~min}$ and 19 of 29 after 240 minutes; 3 corrections resulted in hypoglycemia. For $R$, these figures were 7 of 35,23 of 35 and 2 . Thus, only 42 of 64 corrections resulted in adequate glycemia. The difference between $L$ and $R$ at $120 \mathrm{~min}$. was significant (Pearson $\chi^{2}, p$ $=0.008$ ). Average glucose fell from $15.8 \pm 2.9$ to $7.6 \pm 3.8 \mathrm{mmol} / \mathrm{l}$ Unfortunately, no correlation existed between the administered dose and the fall in glucose. A multiple regression analysis (which used only the first complete data set of each patient) revealed that the presence of fever was associated with a decreased fall, and physical activity was associated with an increased fall in glucose concentration. The use of $L$ was associated with an increased fall in glucose concentration at $t=120$ minutes. Insulin lispro resulted in a more rapid correction that makes early reintervention possible. However, due to the heterogeneity of the population, it is not possible to use this nomogram to correct incidental hyperglycemia. 
Glimepiride and Insulin Multicenter Study in NIDDM (GIM Study): hypoglycaemic events and perceived barriers to good glycaemic control. M.H.A. Stehouwer', J.A.E. Lumeij ${ }^{2}$, H.J. Ader ${ }^{3}$, F.J. Snoek ${ }^{2}$ and R.J. Heine ${ }^{1}$ 'Research Institute for Endocrinology, Reproduction and Metabolism, 2Department of Medical Psychology and ${ }^{3}$ Department of Biostatistics and Epidemiology. Vrije Universiteit, Amsterdam, The Netherlands In this multicenter study we compared the efficacy and incidence rate of hypoglycaemic episodes between 3 treatment regimens in 243 obese type 2 diabetic patients. aged 39-75 year, with secondary failure to sulfonylurea and metformin. During the run-in phase patients were treated with glimepiride and metformin. After 3 months patients with $\mathrm{HbAIc}>7.0 \%$ were randomized to: A) glimepiride-NPH insulin at bedtime, B) NPH insulin twice daily and C) 30/70 mixture of shortacting and NPH insulin twice daily. Therapeutic aim was HbAlc levels $\leq 6.5 \%$. During 9 months of treatment number and severity of hypoglycaenic episodes, $\mathrm{HbAlc}$ and weight change were assessed. Mean $\mathrm{HbAlc}$ achieved at 9 months was significantly higher in group $\mathrm{A}: 8.9 \%$ versus $8.3 \%$ and $8.4 \%$ in groups $\mathrm{B}$ and $\mathrm{C}(\mathrm{P}<0.05)$. No serious hypoglycaemic events (coma,seizure) were observed. The number of mild hypoglycaemic events in group A was significantly lower than in group B and C: 54 versus 107 and 102 (odds-ratio $2.12 ; 95 \% \mathrm{CI} 1.49-3.02$ ). Mean weight gain was comparable in all 3 groups: $3.9,5.1$ and $4.7 \mathrm{~kg}$, respectively (NS). As the target $\mathrm{HbAlc}$ of $6.5 \%$ was achieved in only a minority of patients a questionnaire was developed with 17 statements concerning possible causes, of which the perceived importance was indicated by the 24 diabetologists on a scale from 1 to 10 . Of the insulin therapy-related statements severe co-morbidity and the patients fear of hypoglycaemia were ranked the highest: 108.4 and 106.6 on a scale of 0 to 240 . Significant correlations at $\mathrm{P}=0.01$ level were found between statements with presumed adverse effects of a high insulin dose ('Insulin is atherogenic') and patient related statements as for example 'Lack of motivation' and 'Patient refuses insulin therapy'. In conclusion, in the glimepiride+NPH insulin treated group the incidence rate of hypoglycaemic episodes was significantly lower but at a higher HbAlc level. Good glycaemic control was achieved only in the minority of patients. The main barriers to achieve target glycaenia as perceived by the treating physicians were risks associated with insulin therapy and tight glycaemic control.
TREATMENT OF THE DAWN PHENOMENON IN IDDM ADOLESCENTS WITH AMORPHOUS ZINC INSULIN (SEMILENTE) AT BEDTIME. L. Barkai' ${ }^{1}$ A. Soós ${ }^{1}$ and L. Madácsy ${ }^{2}$, II. Dept. of Paediatrics, Imre Haynal Univ. of Health Sciences, Miskolc ${ }^{1}$ and I. Dept. of Paediatrics, Semmelweis Univ., Budapest ${ }^{2}$, Hungary.

Early morning hyperglyeaemia without nocturnal hypoglycaemia (dawn phenomenon) is a common finding in adolescents with insulin-dependent diabetes mellitus (IDDM) and contributes to the deterioration of metabolic control during puberty. The aim of this study was to examine whether Semilente, an amorphous zinc insulin with kinetics different from NPH insulin, is better suited to alleviate the dawn phenomenon in adolescent patients. This

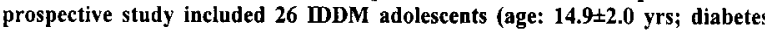
duration: $7.8 \pm 3.6 \mathrm{yrs}$ ) treated with multiple insulin therapy. All patients suffered from profound dawn phenomenon which could not be satisfactorily suppressed by a bedtime injection of NPH insulin. Bedtime insulin was switched to Semilente insulin and patients were followed up for $15.9 \pm 10.1$ months. Change in metabolic control (fasting and mean daily blood glucose of 1 month and 3-monthly measured $\mathrm{HbA}_{\mathrm{Ic}}$ of 1 year before and after switch, respectively), insulin requirement, BMI and rate of hypoglycaemia was assessed to estimate the effect of therapeutic modification. As a result of change in therapy, fasting Imedian (95\% CI): 13.05 (12.35-14.50) vs 9.20 (8.07-9.92) mmol/; $p<0.0001]$ and mean daily blood glucose $[10.70$ (10.23-11.45) vs 9.20 $(8.50-9.56) \mathrm{mmol} / \mathrm{p} ; \mathrm{p}<0.0001]$ values and $\mathrm{HbA}_{\mathrm{tc}}$ levels $[10.30$ (9.06-10.79) vs $8.80(8.11-9.20) \% ; p=0.0024]$ decreased significantly, meanwhile a diminution in bedtime $[0.32(0.28-0.41)$ vs $0.25(0.20-0.34) \mathrm{U} / \mathrm{kg} / \mathrm{day} ; \mathrm{p}=0.0078]$ and total daily insulin requirement $[1.14(1.00-1.32)$ vs $1.02 \quad(0.98-1.11) \mathrm{U} / \mathrm{kg} / \mathrm{day}$; $\mathrm{p}=0.0117]$ was observed. Neither the rate of hypoglycaemia nor the BMI changed significantly in response to change in tberapy. It is concluded that Semilente insulin given at bedtime is effective to suppress dawn phenomenon and to improve long term metabolic control in adolescents with WDM.

\section{PS 46}

\section{Diabetes Education - Health Care Delivery}

968

SUCCESSFUL INTRODUCTION OF AN ANNUAL HEALTH CHECK FOR PEOPLE WITH DIABETES TO DETECT DIABETIC COMPLICATIONS M. Grüßer*, P. Hartmann ${ }^{*}, K$. Hoffstadt", M. Spraul** and V. Jorgens ${ }^{* *}$ *Central Research Institute for Ambulatory Health Care in Germany, Cologne, "Volkswagen Health Insurance, Wolfsburg, Germany, "*Heinrich-HeineUniversity of Düsseldorf, Department for Metabolic Diseases

There should be an annual health check for all people with diabetes to ensure early detection of diabetic complications in primary health care. The quality of documentation of these examinations in primary care is known to be very poor. Therefore in a model project an annual check for diabetic complications was introduced into ambulatory care in a city of 128.000 inhabitants where 50 $\%$ of the population were insured by one compulsary health insurance. The project began in November 1997 and will continue for 3 years. Primary care physicians are remunerated for the documentation of screening for diabetic complications, focusing on a detailed examination of patients' feet. Ophthalmologists are remunerated for the documentation of screening for diabetic eye disease. One copy of the results is handed out to the patients. Complete documentation is the prerequisite for remuneration of the physicians ( $£ 24$ for general practitioners, $£ 7$ for ophthaimologists). Within the first 6 weeks of the project $65 \%$ of the primary care physicians and $100 \%$ of the ophthalmologists had participated in the project. 666 patients were examined by primary care physicians and 503 by ophthalmologists. First results: age $64 \pm 12$ years, $54 \%$ female, diabetes diagnosed since $9 \pm 8$ years, insulin treated $33 \%$ of patients since $7 \pm 8$ years. Plantar ulcera were diagnosed in $3 \%$; the number of amputations was 14 (2 due to accidents). In $17 \%$ the examination with a Semmes-Weinstein-Monofilament (10 g) was pathologic. $38 \%$ of the patients had plantar hyperkeratosis. In patients having started insulin therapy below the age of 40 years $(n=49)$ mean $\mathrm{HbA} 1 \mathrm{c}$ was $8.1 \pm 2.3 \%$, in these probably type 1 diabetic patients the Monofilament test was pathologic in $10 \%$. The results will permit the provision of shared care programmes based upon the actual requirements of the patients (e. g. referal for specialised foot care for high risk patients).

\section{9}

DIABETES NIGHT CLINIC (DNC) MEDICAL QUALITY APPROVAL PROCEDURE ACCORDING TO ASD CRITERIA

B. Lacner', K. Bergmann', H. Förster', H.P. Filz' ${ }^{2}$, H. Burkhard ${ }^{3}$ and G. Giani ${ }^{3}$ 'Bethesda Hospital Essen, ${ }^{2}$ Luisen Hospital Lindenfels, ${ }^{3}$ Diabetes Research Institute, Dep. Biometrie and Epidemiology, University Dussseldorf - Germany

Problem: Equal opportunity and the improvement of quality of live (aims of the St. Vincent Declaration), will be made possible below DNC conditions through retention of the social and professional integrity during a structured training after the guidelines of the German Diabetes Society (DDG). Method: Examined were the data of those type I diabetics which were structural trained 1/94-12/96 in the DNC. $96 \%$ of the patients were capable of performing work. The ASD criteria were determined at the training date as well as 12-15 months later: $\mathrm{HbA}_{10}$ number of hypoglycemias (with external aid), frequency of the ketoacidoses (with hospital reception), number of days in hospital - referring to the last 12 months each. The 2-times Wilcoxon-sign-ranktest for the patient individual differences between the event frequencies was carried out. Stay in hospital was subjected besides to the McNemar symmetry test. Results: Follow-up examination: 64 of 75 type I diabetics could be examined (85\%). Average

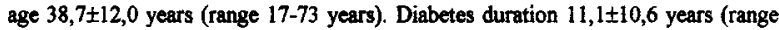
0,5-45 years). The patients registered in the drop-out-analysis were significantly younger. Age: 28,1 17,6 years (range 16-38 years), $p<0,05$; Diabetes duration 7,0 $\pm 5,8$ years (range 0,5-18 years). One year atter the training the $\mathrm{HbA}_{1 \mathrm{c}}$ (normal: $4,4-6,4 \%$ ) dropped of $8,2 \pm 1,8 \%$ (range $5,5-13,6 \%$ ) to $6,9 \pm 1,1 \%$ (range $5,0-10,0 \%$ ) $=$ average reduction of $1,3 \pm 1,8 \%$ ( $t$-test $p<0,05 \%$ ). The number of the hypoglycemias dropped to 0,30 from 1,03 per patient year and appeared significantly itself in a reduced manner $(p=0,02)$. The incidence of the ketoacidoses found itself in a not significant decreased manner $(p=0,13)$ with reduction from 0,06 to 0,00 per patient year. The number of the hospital days per patient year did not sink significantly to 3,83 $(p=0,50)$ from 4,95. The McNemar symmetry test did not show significantly $(p=0,44)$ to itself. 16 patients had been treated in-patient before the DNC, 11 patients had been treated in-patient after the DNC training. Conclusion: The quality of the metabolism adjustment according to ASD criteria in the DNC is identically with results achieved by a hospital stay. The model is farther of an important economical importance by lowering the primary and secondary costs. 
970

ATTENTION TO INJECTION TECHNIQUE IS ASSOCIATED WITH A LOWER FREQUENCY OF LIPOHYPERTROPHY IN INSULIN TREATED TYPE 2 DIABETIC PATIENTS

B. B. Nielsen, L. Museaus and P. Gæde, Steno Diabetes Center, Gentofte, Copenhagen, Denmark.

Aim: To evaluate the effect of intensified patient education and care on the frequency of lipohypertrophy in insulin treated type 2 diabetic patients. Methods: 160 type 2 diabetic patients with microalbuminuria were randomized to a group receiving stepwise intensified, multifactorial treatment of several risk factors for the outcome of type 2 diabetes over a 4 yr period $(n=80)$ and a group receiving standard treatment. The insulin treated patients in the intensive group received intensified education and care with frequent training of injection technique and palpation of injection sites with visits every 8 week while the control group received standard education and care in a routine outpatient clinic. Lipohypertrophy was defined as a palpable subcutanous swelling larger than $0,5 \times 0,5 \mathrm{~cm}$ in one or more injection sites not used for 24h. Results: 40 patients $\left(29 \delta^{\circ}\right)$ in the intensive group and 31 patients $\left(15 \delta^{3}\right)$ in the control group were treated with insulin. $\mathrm{HbA}_{1 \mathrm{c}}$ at the start of insulin treatment was the same in both groups (mean (SO) $(9,6(1,1)$ in the intensive group vs $10,5(1,1) \%$, while the known duration of diabetes was longer in the control group $(11,8(5,9)$ vs. $14,7(6,6)$ yr, $p=0,05$ (Mann-Whitney)). $8(20 \%)$ patients in the intensive group and $17(55 \%)$ patients in the control group developed lipohypertrophy, $p=0,01$ (logistic regression)). In multiple logistic regression with group allocation, sex, age, known duration of diabetes, $\mathrm{HbA}_{1}$. at start of insulin treatment, duration of insulin treatment present insulin dose, number of daily injections, needle length and circulating levels of insulin antibodies as covariates the only significant variable was group allocation. Conclusion: Intensified education and care are associated with a smaller proportion of patients with lipohypertrophy in insulin treated type 2 diabetic patients with microalbuminuria as compared to standard education and care

\section{2}

THERAPEUTIC GROUP EDUCATION FOR THE FOLLOW-UP OF PATIENTS WITH NON INSULIN.DEPENDENT DIABETES.

M Trento, P Passera, M Tomalino, F Pagnozzi, P Vaccari, M Bajardi, GM Molinatti and M Porta. Department of Internal Medicine, University of Turin. Torino, Italy.

Aims: The clinical effectiveness of therapeutic group education, compared to individual consultations with a specialist, as the main form of follow-up for patients with non insulin treated non insulin-dependent diabetes was tested in the course of a randomized controlled clinical trial. Design: Fifty-five patients were enrolled in a structured course of group education sessions held every 3months, whereas 57 controls continued to be seen by a doctor in the clinic with the same time intervals. Results: After 1 year the patients receiving group education had improved their general knowledge of diabetes, assessed by a validated questionnaire (mean scores, 19.4 vs $15.3, \mathrm{p}<0.001$ ) and their score answering a questionnaire designed to explore their choice of conduct in situations of potential risk for people with diabetes $(147 \pm 2.7$ SD vs. $112 \pm$ $3.0, \mathrm{p}<0.001)$. They had also reduced their body weight $(77.5$, range $57-117$, vs 76.0 , range $53-109, p<0.005)$ and body mass index $(29.7 \pm 4.4$ vs $29.0 \pm$ $4.3, p<0.005$ ), whereas no significant changes had occurred in the control group. In the test patients, the difference between levels of glycated haemogobin $\left(\mathrm{HbA}_{\mathrm{lc}}\right)$ before $(7.21 \pm 1.34)$ and at the end $(7.12 \pm 1.29)$ of the trial year showed a trend to improved metabolic control in the patients who had received group education compared to the controls $(\mathrm{p}<0.05)$ and correlated negatively with the final score of the conducts questionnaire $(r=-31$, $\mathrm{p}<0.005$ ). Among these patients, the decrease in $\mathrm{HbA}_{\mathrm{Ic}}$ correlated with its levels at the beginning of the study $(\mathrm{r}=-0.44, \mathrm{p}<0.005)$. No changes in quality of life were observed using a validated modification of the DQOL questionnaire. Conclusions: Although the long-term outcome needs further evaluation, these results suggest that, in busy clinics, structured group education may be a valid alternative to formal consultations for the follow-up of patients with non insulin treated, non insulin-dependent diabetes.

\section{1}

LEARNING GOOD EATING HABITS PLAYING COMPUTER GAMES AT SCHOOL : A 2000 CHILDREN EXPERIMENTATION

M.C. Turnin ${ }^{1}$, O. Couvaras ${ }^{2}$, B. Jouret ${ }^{3}$, M.T. Tauber ${ }^{3}$, C. Bolzonella-Pène ${ }^{1}$, O. Bourgeois ${ }^{1}$, D. Fabre ${ }^{1}$, A. Rouzaud ${ }^{3}$ and J.P. Tauber ${ }^{1}$ 1. Service de Diabétologie-Nutrition, 2. Service d'Endocrinologie Pédiatrique, 3. Service de Gastro-Entérologie-Nutrition Pédiatrique, CHU-Toulouse, France.

Computer games for nutritional education, developed by nutritionists and pediatricians, were evaluated in 1997 with 2000 primary school children with the support of the Ministry of Education. Two groups of children were constituted by randomization of 16 schools located in a french South-West town. Both groups (games group and control group) were given nutritional teaching by their teachers. In addition, the children in the "games" group played computer games in pairs, two hours a week for five weeks. This was a case-control study comparing the results of the two groups by dietetic knowledge tests and dietary records after the completion of nutritional education. The mean age of the children was 9 years (7-12 years). BMI values were $>90$ th percentile for $23.7 \%$ of children and $>97$ th percentile for $11.1 \%$. Dietary knowledge tests results are better in the games group $(\mathrm{p}<0.0001)$. The children in the games group had a significantly better balanced diet for a caloric intake of about 1900 kilocalories : more carbohydrate $46.4 \pm$ $0.2 \%$ vs $45.7 \pm 0.2 \%, p<0.05)$, less fat $(37.1 \pm 0.1 \%$ vs $37.6 \pm 0.2 \%, p<$ $0.05)$, less protein $(16.5 \pm 0.1 \%$ vs $16.7 \pm 0.1 \%, p<0.05)$, less saccharose $(11.5 \pm 0.1 \%$ vs $12.2 \pm 0.2 \%, p<0.001)$, more calcium $(p<0.001)$ and more fiber $(p<0.01)$. The games group had a better snack at 10 a.m., a less copious lunch and a less important picking $(\mathrm{p}<0.000 \mathrm{l})$. The "games" group children developed a greater interest concerning diet. They discussed more about diet a home. In conclusion, it is possible for children to learn good eating habits by playing computer games !

Study supported by a Clinical Research Hospital Program from the French Ministry of Health.

\section{3}

THE IMPACT OF CONTINUOUS EDUCATION ON LIFE STYLE IN TYPE 2 DIABETIC PATIENTS WITH MICROALBUMINURIA Results from a 4 year intervention study

M. Beck, P. Gæde, J. Obel, S. Kohlwes, B. Nielsen, P. Vedel and O. Pedersen, Steno Diabetes Center, Copenhagen, Denmark.

Aim: To assess the effect of continuous education in life style (diet, exercise and smoking) as part of an intensified multifactorial intervention over a 4 year period in type 2 diabetic patients with microalbuminuria. Protocol: 160 patients, age 45-65 yrs were randomly assigned either to an intensive group focusing on change of habits as well as polypharmacological treatment or to a control group receiving conventional treatment. The overall educational approach in the intensive group was the empowerment model using the patients psychosocial skills to bring changes in their personal behaviour and the transtheoretical stages of a change model based on integrating the stages and processes of behaviour alterations. Diet intervention focused on dietary fat and carbohydrate. Total diet intake was estimated by dietary history interviews and calculation from food tables. Exercise and smoking habits were evaluated by interviews. Patients in the intensive group were offered smoking cessation courses and received nicotine substitution for free. $R e$ sults after 4 yrs: Diet (energy composition): $34 \%$ vs. $38 \%$ fat (intensive vs. control) $(p=0.001)$ and $42 \%$ vs. $39 \%$ carbohydrate $(p=0.003)$. Smoking habits: 6 patients in the intensive group $(p=0.01)$ and 5 patients in the control group $(p=0.02$ ) maintained smoking cessation after 4 years $(p=n s$ between groups). The exercise time pr week increased within the intensive group (mean (SD)) (163 (23) to 215 (23) minutes, $p=0.04$ ), however compared to the change in the control group (174 (24) to $202(24)$ ) minutes, $p=0.3$ ) this change was not statistically significant. Conclusion: Continuous education of type 2 diabetic patients over a $4 \mathrm{yr}$ period was associated with life style changes considered to reduce the risk of macrovascular complications. 


\section{4}

PROGNOSTIC FACTORS OF OUTCOME IN HYPEROSMOLAR NONKETOTIC DIABETIC COMA

S.Balić, N.Vučić, V.Pilaš and A.Bilić

Division of Endocrinology and Intensive Care Unit,Dept. of Internal medicine. GH Sveti Duh, Zagreb, Croatia Hyperosmolar nonketotic diabetic coma (HNDC) is an acute complication of predominantly NIDDM. It is associated with high mortality rate ranging from $30-50 \%$. The aim of this study was to assess the prognostic value of invasive hemodynamic variables on the outcome. Thirty one patient with HNDC underwent right heart catheterization with the purpose of pressure-guided fluid replacement. The catheterization was performed to avoid frequent complications of fluid overload or insufficient fluid repletion, both of which occur if this condition is managed empirically or by central venous pressure. The decision to insert catheter was made if patient was hemodynamically unstable. The hemodynamic data were collected upon catheterization and 24 hours later. The overall mortality rate at the seventh day upon admission was $35 \%$. The receiver-operating characteristic curve was constructed for each hemodynamic finding regarding outcome of disease. Logrank analysis was performed for each cut-off point to see whether it may separate significantly survivors from fatalities. The best prognostic indicator was the ratio of left ventricular stroke work index and pulmonary capillary wedge pressure after 24 hours of catheter insertion. Its value $>4$ was associated with the survival rate of $84 \%$, while only $22 \%$ patients with this index $<4$ survived $(p<0.0001)$. This index is considered as one of the best parameters of myocardial function in the critically ill patients. Our data allow conclusion that myocardial dysfunction has a significant role in determining the outcome of disease.

\section{5}

BASIC CARE OF DIABETIC PATIENTS THROUGH SELF-SUSTAINING APPROACH: A BANGLADESH EXPERIENCE

I. Ishrat', F. Mahtab', B. Majumder'2 and L. Ali2, 'Diabetic Association of Bangladesh, 2BIRDEM, Dhaka, Bangladesh

Care of diabetic patients requires substantial and continuous flow of resources posing great challenge to health care services all over the world. This is a special problem for a developing country like Bangladesh due to detection of more and more of the existing patients and also due to a rapid increase in the number of patients resulting from industrialization and urbanization. Diabetic Association of Bangladesh has created a unique example by providing basic health care to a large number of diabetic patients (around 250000 registered patients in all of its projects) free of cost. However, the capital cost and about $30 \%$ of the recurrent cost of such care have, so far, been supported by the public fund (government, charity, community participation etc). This dependence on extra-organizationalfund may create some uncertainty regarding the sustainability and financial viability of its projects especially in the context of the rapid increase in the number of patients. From mid-1996 DAB initiated a special project - National Diagnostic Network (NDN) which aims to provide basic health care to diabetic patients with totally selfgenerated resources. With 7 Out-patient Centers in various areas of Dhaka and a Central Laboratory the Network provides basic health care and advanced diagnostic facilities to both diabetic and non-diabetic patients. The income generated by selling services to the non-diabetic patients and also to the diabetic patients for extra-diabetic problems is diverted to the free basic health care of diabetic patients. The present study has investigated the financial performance of the project during the last 1.5 years in the context of its major objectives. The financia transactions of all NDN components, from the very beginning, were analyzed and the incomeexpenditures were classified in different heads. The data shows that the total capital cost for NDN, up to the present time, is $1.14 \mathrm{~m}$ US $\$$ (Medical equipment 0.98 , electrical $\&$ other supporting equipment 0.08 , furniture 0.06 , initial running capital 0.02 ). The Network has so far registered 4261 patients and they have been provided with basic health care services including health and nutrition education. The total number of patient visits is 79195 . For both non-diabetic and diabetic patients the actual cash outflow is $0.77 \mathrm{~m}$ USS in contrast to the total inflow of 0.40 $m$ US\$ which shows a recovery rate of $52 \%$. The cost of the free care $60 \%$ of the usual setting rate) has found to be $0.289 \mathrm{~m}$ US $\$$ and if it is added, the recovery rate becomes about $91 \%$. The information shows that with a modest investment it is possible to create and organization even in a developing country for providing quality health care to diabetic patients through a selfsustained approach.

\section{6}

DUTCH DIABETES CARE: LESSONS FOR THE 21TH CENTURY

Prof.Dr G.E.H.M.Rutten, General Practitioner, Professor of Diabetology in General Practice. Department of Family Medicine, University of Utrecht, Netherlands.

National guidelines on Type 2 Diabetes care were published by the Dutch College of General Practitioners in 1989. In the autumn of 1998 the updated guidelines will be published. These incorporate both the experiences with the implementation of the former guidelines and the newest insights on 'evidence based' diabetes management. This study aims at formulating an implementation-strategy of the updated guidelines. For this purpose, the results of an implementation study among 73 GP's and 998 patients in three regions in the Netherlands will be discussed. The level of implementation was assessed by scrutinizing all patients' files with regard to seven guidelines. An 'implementation-score' 0-7 was assessed per GP. Factors that explain the implementation were gathered during a semi-structured interview with each GP. A postal questionnaire (to 599 patients, response rate $84 \%$ ) was used to measure patient's reasons for non-compliance. The recorded performance during at least one of two years was $58 \%$ for three monthly blood glucose measurement, $84 \%$ for annually blood pressure measurement, $49 \%$ for anpually creatinin measurement, $13 \%$ for foot examination and $37 \%$ for funduscopy. The mean implementation score was 1.9 A multiple regression analysis revealed that taking part in a diabetes service system, recent postgraduate diabetes education, a broad conception of the GP's task and a busy surgery were the most important factors explaining the 'implementation-score'. If the GPs' were asked about the reasons for non-adherence to the guidelines, it became clear that medical arguments and the compliance of patients were most often mentioned. About $70 \%$ of the patients reported problems in keeping their diet. Other diabetes related activities were troublesome for $10-20 \%$ of the patients. From the patient's perspective, lack of feedback and social support were most frequently mentioned as an explanation for non-compliance. The implementation of the new set of guidelines on diabetes type 2 should not only focus on knowledge-improvement of the general practitioners, but also on their attitudes towards practice-organisation and patient education.

\section{7}

Current Management of Diabetes in Asia The DCDCP (Diabcare - Asia) Study Group

The Diabetes Care Data Collection Project (DCDCP, 1997), a collaboration between Novo Nordisk and national diabetes associations of 6 participating countries (China Indonesia, Malaysia, Philippines, Thailand and Vietnam), was one of the largest studies conducted in Asia. It was targeted to provide an overview of diabetes management and late complication status in Asia and a assessment tool for local interventions so as to improve diabetes care standards in each of the participating country. In this study, 154 participating diabetes centres, selected by the DCDCP local committee in each participating country to represent the various regions within the country and the country itself, were involved. Data from a cohort of about 26,500 patients with more than 12 months of diabetes management were collected. Of all the patients recruited, $95 \%$ were diagnosed as type 2 diabetes with a mean age $( \pm S D)$ of $58.3 \pm 12.0$ years and mean duration of diabetes of $8.2 \pm 6.7$ years. The mean BMI was $24.4 \pm 5.5 \mathrm{~kg} / \mathrm{m}^{2}$ and $64 \%$ of the patients had BMI $<25 \mathrm{~kg} / \mathrm{m}^{2}$. The use of glycated haemoglobin $\left(\mathrm{HbAl}_{\mathrm{c}}\right)$ as assessment of metabolic control was underutilised (19\% of all patients) compared to fasting plasma glucose (FPG) assessment $(91 \%)$ Although the accessibility to $\mathrm{HbAl}_{\mathrm{c}}$ measurement varied considerably among the 6 countries, it was lacking in $80 \%$ of all patients. Of all the respondents, only $18 \%$ had HbAlc within the normal range ( $\mathrm{HbAlc}<6.5 \%$ ) while $61 \%$ showed poor glycemic control (HbAlc $>7.5 \%)$. The mean HbAlc level for type 1 diabetes $(9.2 \pm 2.7 \%)$ was higher than the type 2 diabetes $(8.5 \pm 2.4 \%)$. In addition, FPG measurements also corroborates that majority $(60 \%)$ of the patients had poor glycemic control (FPG $>7.8 \mathrm{mmol} / \mathrm{l})$. Mean FPG level for type 1 diabetes $(10.3 \pm 5.1 \mathrm{mmol} / \mathrm{l})$ was also higher than the type 2 diabetes $(9.4 \pm 3.8 \mathrm{mmol} / \mathrm{l})$. Self-monitoring data indicates that the proportion of patients practising blood or urine glucose monitoring was very low, which could due to only a small proportion of patients being educated on selfmonitoring intervention. Majority (82\%) of the patients were treated with oral hypoglycemic agents (OHAs), $17 \%$ with insulin and $7 \%$ with insulin and OHA combination therapy. In conclusion, the result presented indicates that the majority of the patients involved in this study bad poor glycemic control and calls for a need in improving the glycemic status of the patients as safely as possible. 
978

ASSESSMENT OF TREATMENT SATISFACTION AND UNDERSTANDING OF DIABETES IN DIABETES CARE IN POLAND.

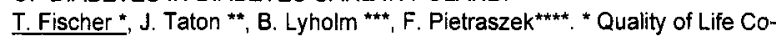
ordinator, Novo Nordisk. "Medical Teams. Department of Internal Medicine and Diabetology, Warsaw Medical School, Poland. ***Statistical Teams, Dis-

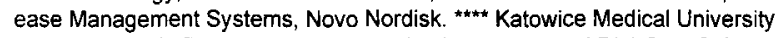
Diabetes care in Poland, was monitored using an adapted DiabCare BIS. 2 validated questions from the WHO DTSQ were used to assess treatment satisfaction and understanding of diabetes. 4077 diabetes patients were included in the national population based study. $45 \%$ were type I while $55 \%$ were type II. $48 \%$ of the patients were recruited from tertiary care, $35 \%$ from secondary care, and $17 \%$ from primary care. $55 \%$ of the patients were female while $45 \%$ were male. The level of treatment satisfaction was analysed using a stepwise linear regression model. HbA1C $(p<0.0001)$, diabetes type $(p<0.01)$, and age $(p<0.01)$ related negatively to satisfaction levels, while the use of pen $(p<0.0001)$, education in selfmonitoring $(p<0.0001)$, and duration of diabetes $(p<0.0001)$ seemed to affect treatment satisfaction positively. Level of care, gender, hypoglyceamic events and education on healthy eating did not contribute significantly $(\rho>0.05)$ to the regression model. It was further found that higher levels of $H b A 1 c$ related to lower understanding of diabetes $(p<0.0001)$. Understanding varied significantly between levels of care: Patients in tertiary care rated higher understanding $(p<0.005)$ than patients in secondary care, who again rated their understanding higher than patients in primary care. Use of pen ( $p<0.0001)$, degree of selfmonitoring $(p<0.0001)$, and frequency of $h y-$ perglycaemic events $(p<0.05)$ related positively. Diabetes type, hypoglycaemic events, gender, duration, age and the provided education did not enter the model. In conclusion, these results suggest that treatment satisfaction and understanding of diabetes improve along improved metabolic control (lower $\mathrm{HbA1c}$. Moreover, understanding of diabetes is related to level of care, leaving room for improvement primarily in primary and secondary care.

\section{9}

DIABETES CARE IN GENERAL PRACTICE. MAIN RESULTS FROM A NATIONAL INTERVENTION STUDY.

N. de Fine Olivarius' A.H. Andreasen ${ }^{1.2}$, H. Beck-Nielsen ${ }^{3}$ M. Horder ${ }^{4}$ and P.A Pedersen'. 'Central Research Unit of General Practice. 'Department of Biostatistics. Copenhagen University. ${ }^{3}$ Medical Dpt. M and ${ }^{1} \mathrm{Clinical}$ Chemical Dpt. Odense University Hospital. Denmark.

Our aim has been to examine in a randomized controlled trial whetler a broadly defined patient-centred intervention in order to optimize quality of diabetes carc supplied to non-insulin-dependent diabetic patients results in reduced morbidity and mortality. The study is based mainly on optimized utilization of existing resources with the general practitioner as co-ordinator of diabetes care for each individual diabetic patient. In 1989-91 487 general practitioners included al newly diagnosed diabetic patients aged 240 years on their practice list. 649/614 patients were included in the intervention/control group. Interventions: Regular follow-up and screening for diabetic complications and proposing and pursuing one of three categories of quality of diabetes care with well-defined goals for each individual patient for blood glucose. HbAlc, diastolic blood pressure (DBP). total cholesterols (CHOL), fasting triglvcerides (TRIG) and body weigh (BW). Only standard therapies are cmployed. These efforts are supported by cducational initiatives as e.g. folders and annual seminars for the gencral practitioncrs and reports on individual patients at least once a vear. A major follow-up cximination has been performed in both groups in 1995-97: $\mathrm{HbAlc}=8.5 / 9.0 \%$ (normal range: $5.4-7.4 \%) . p=0.0001$ (medians. intervention/control group. Wilcoxon test): DBP $=80 / 8+\operatorname{mmHg} . \quad p=0.17 ; T R I G=1.77 / 1.89 \mathrm{mmol} / 1 ; \mathrm{p}=0.15 . \mathrm{CHOL}=6.0 \% 1.1$ munol/l. $\mathrm{p}=0.15 ; \quad \mathrm{BW}=80.0 / 80.7 \mathrm{kgs} . \quad \mathrm{p}=0.41$ : urinary albumin $/ \mathrm{creatininc}$ ratio $=1.48 / 1.69 \mathrm{mg} / \mathrm{mm} \mathrm{mol}, \mathrm{p}=0.61$ : overall mortality $=22.5^{5} / 23.9 \%, \mathrm{p}=0.54, \log -$ rank test. Data are currently further scrutinized. We conclude that the interventional model seems fit for implementing into the clinical setting in gencral practice
980

LATE COMPLICATIONS MEASURED IN THE NATIONAL DIABETES QUALITY ASSESSMENT PROGRAMME IN POLAND

B. Lrholm * J. Taton **: * Statistical teams. Disease Management Systems, Novo Nordisk. ** Medical teams. Department of Internal Medicine and Diabetology, Warsaw Medical School, Poland.

As an integrate part of the national network for monitoring diabetes care in Poland, we assessed the prevalence of late complications and risk factors using an adapted version of the DiabCare BIS. The programme has now been working for 2 years and the first preliminary longitudinal comparisons can be made. In this work we compared the St Vincent targets among 1817 IDDM patients from 1997 to the 1271 IDDM patients included in the 1996 study. $\mathrm{Pa}$ tients in 1997 were distributed between 3 levels of care, while all patients in 1996 were recruited only from tertiary care. In the assessment programme 1996 we reported a prevalence of blindness to be $3.2 \%$ while the 1997 figures has increased slightly to $3.3 \%$. The measured prevalence of myocardial infarction increased from $1.5 \%$ in 1996 to $2.1 \%$ in 1997 while prevalence of cerebral stroke increased from $0.3 \%$ to $0.9 \%$ in 1997 . The prevalence of ESRD increased slightly from $0.6 \%$ to $1.0 \%$. Amputations above ankle was stable at $0.7 \%$ while the prevalence of amputations below ankle changed from $0.1 \%$ in 1996 to $0.6 \%$ in 1997. Improvements in the data collection strategy in 1997 enables us to analyse differences in prevalence rates between tertiary, secondary and primary care. The figures are listed as prevalence rates in (tertiary/secondary/primary care ). In 1997 we found the following distribution of the prevalence of blindness $(2.4 / 4.5 / 5.7)$, stroke $(0.7 / 1.1 / 2.9)$, myocardial infarction (1.5/2.5/12.1), ESRD $(0.5 / 2.1 / 0.0)$, amputations above ankle $(0.6 / 0.9 / 0.0)$, amputations below ankle $(0.6 / 0.4 / 2.9)$. We conclude that the prevalence of late complications within tertiary care is stable, while prevalence rates between levels of care is very heterogeneous. The challenges in the Polish diabetes care structure will be to adapt structure and develop expertise so that it suits different groups of patients.

\section{1}

GROWTH IN MEDICAL CARE COSTS AFTER DIAGNOSIS OF TYPE 2 DLABETES

H.S. Glauber, J.B. Brown, G.A. Nichols, and A.W. Bakst; Kaiser Permanente Northwest, Portland OR, SmithKline Beecham Pharmaceuticals, Collegeville PA It is widely assumed that improved diabetes care will decrease future medical care expenditures. However, cost growth in diabetes has never been described. We identified--and matched to non-DMs--all members of Kaiser Permanente Northwest Region, a nonprofit HMO, who were diagnosed with Type 2 diabetes (T2DM) in 1988 through 1995. We calculated total (per person) medical care costs and the excess costs caused by T2DM, in constant 1993 dollars for 8 years following diagnosis.

Per Person Medical Care Costs by Year Since Diagnosis of T2DM

\begin{tabular}{|c|c|c|c|c|c|}
\hline $\begin{array}{c}\text { \# of } \\
\text { Cases }\end{array}$ & $\begin{array}{c}\text { Post-DX } \\
\text { Year }\end{array}$ & $\begin{array}{c}\text { Total } \\
\text { Costs }\end{array}$ & $\begin{array}{c}\text { T2DM- } \\
\text { Caused } \\
\text { Costs }\end{array}$ & $\begin{array}{c}\text { Ratio of DM- } \\
\text { Caused Costs } \\
\text { to Total Costs }\end{array}$ & $\begin{array}{c}\text { \% Change in } \\
\text { T2DM-Caused } \\
\text { Costs }\end{array}$ \\
\hline 8685 & 1 & $\$ 4007$ & $\$ 2504$ & 0.6 & --- \\
\hline 6743 & 2 & 3862 & 2292 & 0.59 & $-8.5 \%$ \\
\hline 5097 & 3 & 3911 & 2264 & 0.58 & $-1.2 \%$ \\
\hline 3738 & 4 & 4082 & 2319 & 0.57 & $+2.4 \%$ \\
\hline 2703 & 5 & 4329 & 2248 & 0.52 & $-3.1 \%$ \\
\hline 1833 & 6 & 4259 & 2319 & 0.55 & $+3.2 \%$ \\
\hline 1076 & 7 & 4364 & 2566 & 0.59 & $+10.7 \%$ \\
\hline 502 & 8 & 4984 & 3072 & 0.62 & $+19.7 \%$ \\
\hline
\end{tabular}

The incremental costs of T2DM accounted for $52.63 \%$ of total per-person medical costs, and average excess cost of $\$ 2448$. Despite available therapy, or perhaps because of it, annual excess costs were substantial from the moment of diagnosis, although they dropped slightly as a percent of total costs. After year five, incremental costs began to accelerate. The acceleration of diabetes-related costs coincided in this population with failure to maintain good gtycemic control on sulphonylurea treatment, initiation of insulin, and progression of diabetic complications 
982

COSTS OF CARDIOVASCULAR AND RENAL COMPLICATIONS IN TYPE 2 DIABETES BY STAGE OF DISEASE

J.B. Brown, K.L. Pedula, H.S. Glauber, and A.W. Bakst. Kaiser Permanente Northwest, Portland OR; SmithKline Beecham Pharmaceuticals, Collegeville PA

The costs caused by the complications in patients with Type 2 diabetes (T2DM) have never been directly estimated by stage of progression. We identified all members of Kaiser Permanente Northwest Region, a nonprofit HMO, who were diagnosed with T2DM from 1988 through 1995. Using electronic data, we identified and staged their cardiovascular (CVD) and renal complications. CVD stages ranged from preventive drug therapy to events requiring hospitalization (cardiovascular, cerebrovascular or peripheral vascular). Renal stages ranged from microalbuminuria through advanced renal impairment (chronically elevated serum creatinine, impaired creatinine clearance or diagnosis of chronic renal disease) to end-stage renal disease (dialysis or transplantation). We then used ordinary least squares to regress the total cost per person with T2DM on age, sex, and stage of complications.

Annual Added Medical Care Costs of Complications of Type 2 Diabetes By Stage

\begin{tabular}{lccc}
\hline \multicolumn{1}{c}{ Complication } & Prevalence & $\begin{array}{c}\text { Added } \\
\text { Cost } \\
\text { Per Stage }\end{array}$ & $\begin{array}{c}\text { Cumulative } \\
\text { Cost } \\
\text { By Stage }\end{array}$ \\
\hline $\begin{array}{l}\text { No CVD or Renal Disease (intercept) } \\
\text { Cardiovascular Disease }\end{array}$ & $23 \%$ & $\$ 2,020$ & $\$ 2,020$ \\
$\quad$ 1. BP/lipid drugs only & $7 \%$ & $\$ 1,362$ & $\$ 3,382$ \\
2. Other CVD drugs or cardiologist visit & $39 \%$ & $\$ 1,033$ & $\$ 3,053$ \\
3. Advanced CVD events & $29 \%$ & $\$ 7,346$ & $\$ 9,366$ \\
Renal Disease & $12 \%$ & $\$ 1,357$ & $\$ 3,377$ \\
1. Tested, abnormal result & $10 \%$ & $\$ 3,990$ & $\$ 6,010$ \\
2. Advanced renal impairment & $1 \%$ & $\$ 15,685$ & $\$ 17,705$ \\
\hline 3. Chronic dialysis or transplantation & & & \\
\hline
\end{tabular}

Conclusion: Renal and cardiovascular costs rise dramatically as these complications worsen, eventually greatly exceeding the costs associated with complication free Type 2 patients.

\section{3}

DIADOQ: AN ELECTRONIC MEDICAL RECORD SYSTEM - EVALUATION BY DIABETES SPECIALISTS IN PRACTICES

G. Köhler ${ }^{1}$, T. Koschinsky', B. Haastert', W. Moser' ${ }^{2}$, T. Diedrich', G. Muller ${ }^{3}$, R. Engelbrecht ${ }^{2}$, F.A. Gries' and the DIADOQ-Group. 'Diabetes Forschungsinstitut an der Heinrich-Heine-Universität Düsseldorf, ${ }^{2}$ GSF - Forschungszentrum für Umwelt und Gesundheit Neuherberg, ${ }^{3}$ Institut für Medizinische Informatik und Biometrie Dresden

The DIADOQ project aims at improving the quality of care for diabetic patients by providing decision support within diagnosis, therapy planning, and monitoring of diabetes. A problem-oriented medical record system for diabetes specialists in outpatient clinics, based on structured data entry has been developed. The data set is based on EURODIABETA, DiabCare and the Diabetes Passport. Two knowledge bases are integrated. - To assess the acceptance in general practice the system was demonstrated to 33 diabetes practioners and the concept of computer-assisted diabetes management was introduced by a standardized evaluation procedure. This included biographical data, involvement in diabetes care, routine handling of computers, attitude towards electronic medical records, and more specific groups of questions with regard to DIADOQ e.g.: attitude towards its application in the clinical setting, satisfaction with the content of the system, user friendliness, expected effects on quality of care, perceived usefulness and a total assessment scale. The rating was positive for all groups of questions: the satisfaction with the content of the system was positive in $100 \%$, user friendliness in $79 \%$, expected improvement of quality of care in $94 \%$, perceived usefulness in $71 \%$ and a positive total assessment scale in $89 \% .91 \%$ of the practioners predicted that the medical staff of their practice would work with the system. $78 \%$ were interested in a computer-based medical record, $61 \%$ would test DIADOQ, while $59 \%$ would implement the system at its present state for routine work. The most frequent arguments in support were expected improvement of quality of medical records and diabetes care. Frequent arguments to refuse routine implementation were complexity, waste of time and missing integration into the particular docters office systems. The effect on quality of care was estimated most positively by practices of 1000-1500 patients, perceived usefullness was greatest in practices with $\leq 30 \%$ diabetic subjects. The results of this pilot study suggest that DIADOQ is quite well accepted by diabetes practioners, and it should be integrated into existing docters office systems for routine work.

\section{4}

DIABCARE Q-NET: IMPLEMENTATION RESULTS

D. Westphal, K. Piwernetz, M.R. Gallego, F. Storms, M. Massi-Benedetti, R. Landgraf, A. de Leiva, L. Kleinebreil, K. Stæhr-Johansen, M. Porta, S. Skeie, M. Fahlen and E. Hardt-Stremayr; DiABCARE Office, Munich, Germany and DIABCARE Consortium.

The Quality Development Cycle of DIABCARE Q-Net is now implemented in pilot regions within Europe: France, Portugal, Spain, Netherlands, Germany, Italy, Norway, Austria and Sweden. Initiatives are started in U.K. and Greece. The implementation is based on the data collection using the DIABCARE Diabetes Data Set, which contains those data items necessary to monitor the quality of care of an individual diabetes patient. More than 50,000 DIABCARE Diabetes Data Sets are collected all over Europe so far: $>20,000$ from 150 hospitals and 70 diabetologists in France, $>12,000$ from 258 General Practitioners (GP's) and 4 hospitals in Portugal, 3,000 from diabetologists and other specialists in Netherlands, 15,000 from 80 centres ( $80 \%$ hospitals, GP's, Diabetes Centres) in Germany, 1,500 from 16 centres in Spain, $>1,500$ from 49 Diabetes Centres in Italy, 150 from 1 hospital and 5 GP's in Norway. Depending on the situation in each country different tools for data collection are used, e.g. the software program DIABCARE Data for Windows in Spain and Netherlands or the DIABCARE Fax System in Italy and Germany (Bavaria). Other tools are the paper version of the BIS, the EPI-INFO program from WHO or data export from other software programs.

This presentation will give an overview of the state-of-the-art situation of the implementation process. Alt countries are now in the process of setting up national networks by linking regional servers or clients.

International benchmarking using a European network and server are also implemented and in use. The benefit of this network must result in a continuous quality development system for diabetes care across Europe. This target will be achieved by strengthening the national implementation processes under their responsibilities and individual solutions. The umbrella of these national implementations is the St. Vincent Declaration. The European network was developed in the project DIABCARE Quality Network in Europe from the TELEMATICS APPLICATION Programme (1994-1998), European Commission DG XIII. 
PS 47

\section{Psychology}

\section{5}

WELL-BEING AND SYMPTOMS IN RELATION TO INITIATION OF INSULIN THERAPY IN NIDDM.

JJJ de Sonnaville, FJ Snoek, LP Colly, W Devillé, D Wijkel, RJ Heine. Research Centre Primary/Secondary Health Care, Amsterdam Thrombosis Service and Laboratory for General Practitioners, Institute for Endocrinology, Reproduction and Metabolism, Vrije Universiteit, Amsterdam, The Netherlands.

A descriptive, prospective 2-years cohort study was performed to determine the influence of insulin therapy on well-being and treatment satisfaction in NIDDM patients. The study population consisted of 272 NIDDM patients of Dutch origin, $>40$ years of age, with known diabetes duration $>3$ months, and treated with diet and/or oral hypoglycaemic agents, participiting a regional shared care project. Dependent variables in the logistic regression analysis were scores on the Type II Diabetes Symptom Checklist, the Profile of Mood States, and questions regarding general well-being and treatment satisfaction. Potential determinants under study were age, sex, known diabetes duration, insulin dose and duration of insulin therapy, comorbidity, baseline and change of metabolic parameters and cardiovascular risk factors. Of 157 patients $(58 \%)$ a baseline and 2 years questionaire was available. During 2-years of follow-up, 39 of them (24.8\%) were treated with insulin. Initiation of insulin therapy was significantly associated with improved glycaemic control (mean HbAlc $8.2 \pm$ sd 1.4 to $7.4 \pm 0.9 \%, p=0.001$ ) and weight gain (BMI $27.1 \pm 3.9$ to $28.6 \pm 4.3 \mathrm{~kg} / \mathrm{m}^{2}, \mathrm{p}=0.000$ ). Of all symptom and well-being scores only feelings of emotional fatigue worsened significantly, but modestly $(0.4$ to 1.7 on a scale of 0.0 (best) -10.0 (worst), $p=0.02$ ). Although diabetes management with insulin was experienced as more demanding $(p=0.04)$, treatment satisfaction score was not adversely influenced $(2.5$ to $1.9, \mathrm{p}=0.39)$. High insulin doses were significantly and independently associated high symptom scores (total score, hypoglycemic score), and with low mood (displeasure score, anger, tension, emotional fatigue) and perceived state of health. In conclusion, initiation of insulin therapy in NIDDM improves glycaemic control effectively, has little influence on physical and psychological wellbeing dimensions, and does not affect treatment satisfaction.

\section{7}

\section{FACTOR STRUCTURE AND FACTOR STABILITY OF THE (BRADLEY) WELL-BEING QUESTIONNAIRE.}

F. Pouwer ${ }^{1}$, F.J. Snoek ${ }^{1}$, H.J. Adèr ${ }^{2}$, H.M. van der Ploeg ${ }^{1}$ and R.J. Heine ${ }^{3}$ Department of Medical Psychology ${ }^{2}$ Department of Biostatistics and Epidemiology ${ }^{3}$ Department of Endocrinology. Institute for Endocrinology, Reproduction and Metabolism, Vrije Universiteit, Amsterdam, the Netherlands.

Background: The literature suggests that psychological problems such as depression and anxiety are underdiagnosed by non-psychiatric practitioners from $50 \%$ to $70 \%$ of the time. In St Vincent Declaration Action Programme for Diabetes it is advised to monitor the psychological well-being of diabetic patients by means of Bradley's Well-being Questionnaire (W-BQ). The detection-rate of patients with low psychological well-being can probably be improved by the systematic use of this instrument in diabetes care. However, research on the psychometric properties of the W-BQ is still scarce and results are inconsistent. Aim: To investigate the factor structure and the factor stability of the Dutch adaptation of the W-BQ. Methods: Confirmatory factor analyses were used to test the fit of two factor solutions described by the designer of the scale. A random sample of members of the Dutch Diabetic Organisation $(n=765)$ filled out the $W-B Q$. This sample was randomly divided into group $A(n=385)$ and $B(n=380)$. Results: Confirmatory factor analyses in group A showed that a three factor solution with 12 items provided the best fit to the data. The stability of this three factor model was tested in group B in subsamples of IDDM $(n=196)$ and NIDDM patients $(n=184)$. A stable three factor solution emerged. Conclusion: The best description of the latent structure of the W-BQ can be given by three reliable factors, measuring Positive Wellbeing ( 4 items), Negative Well-being (4 items) and Energy (4 items).

\section{6}

USE AND METABOLIC EFFECTS OF TRICYCLIC ANTIDEPRESSANT DRUGS IN DIABETIC PATIENTS. W.A. Davis and T.M.E. Davis, University Department of Medicine, Fremantle Hospital, Fremantle, Australia.

Tricyclic antidepressant drugs (TCA) may worsen glucose tolerance. Although there are few data on the epidemiology and treatment of depressive illness in diabetes. patients with chronic disease have an increased risk of depression that may itself influence glycaemia. The aim of the present study was to investigate the prevalence of TCA treatment in diabetic patients and the impact of this group of drugs on metabolic control. 1426 subjects (mean age 62 years; $48.5 \%$ males; $91 \%$ with type 2 diabetes) were recruited to the Fremantle Diabetes Study (FDS; a community-based prospective study of care, control and complications) between 1993 and 1996 representing $63 \%$ of all diabetic patients identified in a population base of 120,097 A comprehensive FDS database has details of anmual clinical and biochemical assessments. Each of those taking TCA at recruitment was identified and matched with two others not taking antidepressant medication in a retrospective case-contro design. Matching variables were age, gender, diabetes type and treatment, and body mass index. Out of the total FDS cohort, 91 patients $(6.4 \%)$ were taking antidepressant therapy. Of these, 75 patients $(82 \%)$ were on TCA. Compared with the 150 controls, the odds of those on TCA having a fasting plasma glucose $\geq 8.0$ $\mathrm{mmol} / \mathrm{L}$ was $0.85(95 \%$ confidence interval $(\mathrm{Cl}) 0.49$ to 1.49$)$ and for a $\mathrm{HbA}_{\mathrm{tc}}$ $8.0 \%$ was 0.82 ( $95 \%$ CI 0.47 to 1.45 ). Serum lipid profiles were also similar in the two groups $(P>0.1) .88 \%$ of those on TCA claimed to monitor their blood glucose compared to $82 \%$ of controls $(P=0.3)$. There were no significant differences in the frequency of general practitioner, specialist or clinic visits in relation to diabetes between the two groups $(P>0.2)$. These data show that one in 16 diabetic patients in a community-based sample of diabetic patients were treated for depression, the majority taking TCA. The use of self-monitoring and supervised care by those on TCA was similar to those of matched control subjects, and there were no differences in glycaemic control. TCA remain a common form of treatment for depression in diabetes and do not appear to have significant adverse metabolic effects.

\section{8}

IMPACT OF THE SWITCH FROM REGULAR HUMAN INSULIN TO INSULIN LISPRO ON THE QUALITY OF LIFE - THE GERMAN QOL STUDY P. Herschbach, U. Lindner, M. Trautmann, G. Duran, and A. Pfützner, München, Bad Homburg, and Mainz, Germany

Improvements in the overall glycemic control may also positively influence the quality of life of diabetic patients, in particular if practical therapeutic changes lead to regimens that are more convenient for the patient. In comparison to treatment with regular human insulin $(\mathrm{HI})$, the time action profile of the rapid acting insulin analog lispro (LP) allows patients to omit the injection to meal interval and to skip snacking. In order to investigate the QOL impact of a switch from HI to LP in intensive insulin treatment, a prospektive, open-labeled, uncontrolled study was performed using the QSD-R and the DTSQ questionnaires to assess changes in quality of life and treatment satisfaction. In the QSD-R, eight major topics of daily diabetic life are reflected in $\mathbf{4 5}$ items: leisure time, depression, hypoglycemia, diet, sickness, work, partnership, and patient-doctor relation. 2216 patients (1011 female, 1205 male, mean age (tSTD): $41.3 \pm 13.3$ years, 1793 type 1 patients, 423 type 2 patients, duration of diabetes: $13.1 \pm 10.0$ years) at 254 study sites participated into the trial. The questionnaires were answered at baseline and at endpoint after 3 months of treatment. Other observation parameters were hemoglobin $\mathrm{A} 1 \mathrm{c}$, body weight and adverse events. The questionnaires were analyzed calculating overall ranking scores for DTSC and QSD and subscores for the major topics of QSD.

The switch from $\mathrm{HI}$ to LP lead to significant improvements in $\mathrm{HbAtc}$ $(7.81 \pm 0.3 \%$ to $7.66 \pm 0.3 \%, p<0.001)$, increase in treatment satisfaction (DTSQ-score: $26.4 \pm 6.3$ to $29.9 \pm 6.0, p<0.001$ ) and decrease in the stress due to diabetes (overall QSD score: $1.06 \pm 0.04$ to $0.88 \pm 0.04$ ). Significan reductions were also seen in each of the subscores of QSD with major improvements concerning leisure time, diet, and hypoglycemia.

Due to the study design, it is not possible to estimate the impact of LP per se on the outcome results of the study. However, it can be concluded that the overall circumstances of a switch from $\mathrm{HI}$ to insulin lispro may result in a general improvement of the quality of life of diabetic patients. 


\section{9}

VALIDATION OF A DIABETES QUALITY-OF-LIFE MEASURE FOR NIDDM PATIENTS

K. Lohr, U. Bott and S. Ebrahim. Clinic of Metabolic Diseases and Nutrition, Heinrich-Heine-University Düsseldorf, Germany

Quality-of-life scales are usually applied to different types of diabetes, although IDDM and NIDDM patients suffer from different diabetes related restrictions and burdens. Aim of the study was the validation of a new questionnaire, specifically designed to measure quality-of-life and treatment satisfaction in NIDDM patients. Item development based on semi-structured interviews with 28 NIDDM patients, which revealed 66 items: treatment goals ( 9 items), treatment satisfaction (9), physical complaints (7), fear of hypoglycaemia (10), worries about future/late complications (6), social restrictions (8), daily hassles (7), diet restrictions (10). Validation was performed in a pilot sample of 92 NIDDM patients $>50$ years old: $48 \%$ women, age $68 \pm 8$ years, diabetes duration $10 \pm 10$ years, insulin therapy $40 \%$, oral agents $51 \%$; mean \pm SD). The product of patients' ratings of preferred treatment goals and treatment satisfaction provided a preference-weighted treatment satisfaction score (PWTSS). The remaining subscales were subjected to factor analysis which guided the elimination of 5 items to improve homogeneity (items, Cronbach's $\alpha)$ : physical complaints $(6,0.89)$, fear of hypoglycaemia $(10$, $0.94)$, worries about future/late complications $(6,0.88)$, social restrictions $(5$, $0.84)$ daily hassles $(7,0.89)$, diet restrictions $(9,0.91)$. All subscales and the PWTSS were significantly correlated with the physiscal health and mental health scales of a validated generic measure (SF-12). With increasing age diabetesspecific burdens regarding physical complaints (.23), worries about future (.32), diet restrictions (.29) and social restrictions (.26) decreased. A longer diabetes duration was associated with more physical complaints $(-26)$ and worries about future $(-.22)$. Patients with a higher body mass index perceived stronger diet restrictions $(-.27)$. Insulin-treated patients had poorer quality-of-life scores on any subscale. After adjustment for age, diabetes duration, $\mathrm{HbAlc}$ and co-morbidity in regression analyses 3 subscales (physical complaints, fear of hypoglycaemia, daily hassles) revealed still significant differences $(p<0.05)$. Patients with signs of peripheral neuropathy achieved poorer scores on the physical complaints $(\mathrm{p}<$ $0.01)$ and worries about future subscales $(p<0.05)$

The questionnaire is a reliable and valid measure of diabetes quality-of-life in NIDDM and appears particularly useful to compare different treatment regimen.

\section{PS 48}

\section{Clinical Pregnancy}

\section{0}

\section{POOR GLYCAEMIC CONTROL IN EARLY PREGNANCY IN TYPE 1 DIABETES IS NOT ASSOCIATED WITH FETAL MACROSOMIA}

R.C. Templea, V.J. Aldridge", P.J. Heyburn", M.J. Sampson", R.H. Greenwood", K.P. Stanley', M.B. Kelly" "Bertram Diabetes Centre and 'Department of Obstetrics, Norfolk and Norwich Health Care NHS Trust, Norwich, UK

It is believed that good glycaemic control in pregnancy in Type 1 diabetes reduces the risk of congenital malformation and macrosomia. Recent studies suggest that poor control in early rather than late pregnancy has a greater influence on birthweight. The aim of this study was to investigate the relationship between hyperglycaemia, as measured by HbAlc and fructosamine, and birthweight in pregestational Type 1 diabetics. All pregestational Type 1 diabetics who delivered between 1990 and 1995 were included in the study. HbA1c (normal range $<5.8$ ) was measured monthly during pregnancy, and from 1991, fructosamine (normal range $<285 \mu \mathrm{mol} / \mathrm{L}$ ) was measured fortnightly. Macrosomia was defined as birthweight above the 90th centile. We report the results of 120 live births. $47(39 \%)$ of infants were macrosomic. There was no significant difference in glycaemic control between macrosomic pregnancies and non-macrosomic pregnancies at booking (HbA1c $6.5 \pm 1.5$ vs $6.3 \pm 1.7 \%$ NS, fructosamine $337 \pm 59$ vs $322 \pm 70$ umol/L, NS), at 12 weeks (HbA1c $5.8 \pm 0.9$ vs $5.7 \pm 1.5 \%$ NS, fructosamine $297 \pm 41$ vs $287 \pm 56 \mu \mathrm{mol} / \mathrm{L}$ NS), at 24 weeks (HbA1c $5.2 \pm 1.0$ vs $5.2 \pm 1.3 \%$ NS, fructosamine $263 \pm 26$ vs $254 \pm 42 \mu \mathrm{mol} / \mathrm{L} \mathrm{NS}$ ) and at 34 weeks (HbA1c $5.4 \pm 0.9$ vs $5.2 \pm 1.3 \%$ NS, fructosamine $228 \pm 26 \mathrm{vs} 233 \pm 38 \mu \mathrm{mol} / \mathrm{L} \mathrm{NS}$ ). 37 (79\%) of macrosomic babies and $32(44 \%)$ of non-macrosomic babies were delivered by Caesarean section. In conclusion, poor glycaemic control, as measured by HbA1c or fructosamine, is not a predictor for macrosomia in Type 1 diabetes.

\section{1}

PREGESTATIONAL OVERWEIGHT AND OUTCOME OF PREGNANCY M. Mancone, R. Fresa. F. Carlino. G. Russo, M. Torelta, S. Carbone and S. Sgambato, Dept. of Gerontology and Metabolic diseases, II ${ }^{\text {nd }}$ University of Naples Obesity is one of the most important risk factor for gestational diabetes mellitus (GDM). We followed 138 pregnant woman screened for GDM up to delivery to evaluate weather is a relationship between obesity and perinatal or maternal complications. We studied 4 groups: 1) 36 normal weight non diabetic patients (mean

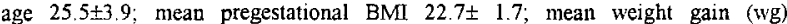
$13.06 \pm 6.05$ at 37 weeks of gestation): II) 34 overweight non diabetic patients (mean age 26.6 \pm 4.8 ; mean pregestational BMI $26.64 \pm 4.0$; mean wg $14.0 \pm 6.3$ at 37 weeks); III) 25 normal weight GDM patients (mean age 25.16 \pm 5.43 ; mean pregestational $\mathrm{BM}$ 24.02 \pm 1.2 ; mean wg $12.0 \pm 1.8$ at 37 weeks; mean time of diagnosis of GDM 23.92 \pm 6.2 weeks); IV) 43 overweight GDM patients (mean age $29.43 \pm 6.97$; mean pregestational BMI 29.84 \pm 3.86 ; mean wg 12.15 \pm 5.4 at 37 weeks; mean time of diagnosis of GDM 25.2 \pm 6.54 ). We considered a pregestational BMT $>25$ as abnormal and we used Carpenter and Coustan criteria for screening and diagnosis of GDM. Student's and $\chi^{2}$ test are used for statistic analysis. Statistic analysis showed significant statistic difference of perinatal and maternal complication rate between II and III group and no significant difference of number of LGA or macrosomia in the same groups. In conclusion, our data show that obesity alone can influence both intrauterine fetal growth and birth weight but not perinatal or maternal complications. *I vs III, $\mathrm{p}<0.01$; ${ }^{\circ} \mathrm{II}$ vs III, $\mathrm{p}<0.05$; $L I$ vs III, $\mathrm{p}<0.001$; ${ }^{\wedge}$ II vs III, $\mathrm{p}<0.005:$ §II vs IV, $\mathrm{p}<0.001$

\begin{tabular}{l|c|c|c|c} 
& $\mathrm{I}$ & $\mathrm{II}$ & III & IV \\
\hline Time of delivery & $39.8 \pm 1.2$ & $39.2 \pm 1.7$ & $39.7 \pm 0.8$ & $38.7 \pm 1.5$ \\
\hline Birthweight $(\mathrm{kg})$ & $3.418 \pm 0.506$ & $3.497 \pm 0.534$ & $3.381 \pm 0.289$ & $3.573 \pm 0.435$ \\
\hline LGA & $2.7 \%(1 / 36)$ & $20.5 \%(7 / 34)$ & $16 \%(4 / 25)$ & $18.6 \%(8 / 43)$ \\
\hline Macrosomia & $8.3 \%(3 / 36)$ & $11.7 \%(4 / 34)$ & 0 & $11.6 \%(5 / 43)$ \\
\hline Perinatal compl & $13.8 \%(5 / 36)^{*}$ & $17.6 \%(6 / 34)^{\circ}$ & $40 \%(10 / 25)$ & $27.9 \%(12 / 43)$ \\
\hline Maternal compl. & $2.7 \%(1 / 36)^{*}$ & $2.9 \%(1 / 34)^{\wedge}$ & $28 \%(7 / 25) \perp$ & $32.5 \%(14 / 43) \S$
\end{tabular}


992

PROGNOSTIC VALUES OF BLOOD FLOW VELOCITY IN PREGNANCY COMPLICATED BY DIABETES.

J.Wilczyński, P.Hincz, K.Szaflik and P.Pawłowicz; Research Institue Polish

Mother's Memorial Hospital, Lódź, Poland

The aim of our study was evaluation of blood flow velocity used in assessment of fetal welfare. Material and methods: We included 110 pregnant women with type 1 and 2 diabetes, hospitalized and followed-up in PMMH in 1992-1997. All the women were classified according to White. Good metabolic control (mean blood glucose level $<100 \mathrm{mg} / \mathrm{dl}$ ) was achieved in 37 patients- group I and remaining 73 patients with poorly controlled diabetes constitute group II. We assessed selected blood flow velocity parameters in the umbilical artery, the middle cerebral artery and the fetal descending aorta. The software of the sonograph (Acuson $128 \mathrm{XP} / 10$ ) allowed an evaluation of the systolic/diastolic ratio (S/D), the resistance index (RI) and the pulsatility index (PI). All the newborns were estimated according to the 1 and 5 minute Apgar score compared with umbilical blood $\mathrm{pH}$ values. The statistical analysis assessed the following parameters of the applied biophysical methods: accuracy, sensitivity, specificity, the positive and the negative predictive values. Results: In the group I in comparison with the 5th minute Apgar score the highest sensitivity was established for RI in fetal descending aorta $(100 \%)$ with specificity $32,1 \%$ and positive and negative predictive value consecutively $32,1 \%$ and $100 \%$. The highest specificity had RI in the middle cerebral artery - $88,9 \%$, with sensitivity, positive and negative predictive values consecutively $17,9 \%, 83,3 \%$ and $25,8 \%$. S/D in the middle cerebral artery and $\mathrm{S} / \mathrm{D}$ and RI in fetal descending aorta had the highest accuracy $(48,6 \%)$ among the assessed parameters. In the group II the highest sensitivity was also set up for $\mathrm{RI}$ in fetal descending aorta $(76,5 \%)$, but the specificity for all evaluated parameters did not exceed $50 \%$ (range $25,6-38,5 \%$ ). Conclusions: 1 .The highest sensitivity in both groups was found for RI in fetal descending aorta; 2.Estimation of blood flow velocity is valuable method for assessment of fetal well-being in pregnancy complicated by diabetes type 1 and 2 .

\section{4}

MATERNAL BIRTH WEIGHT AND INSULIN RESISTANCE IN NON-DIABETIC MOTHERS: A FURTHER CONFIRMATION FOR THE THRIFTY PHENOTYPE HYPOTHESIS? G. Seghieri, S. Sani, M.C. Breschi, R. Anichini, and L. Alviggi. Dpt. of Internal Medicine, Hospital of Viareggio, and Diabetes Unit, Spedali Riuniti, Pistoia, Italy. According to the 'thrify phenotype' hypothesis a low birth weight is associated to appearance of non-insulin-dependent diabetes mellitus, as well as of other clinical correlates of the insulin resistance syndrome during the adult life. Whether the correlates of insulin sensitivity in a physiological insulin resistance status such as pregnancy, as well as the neonatal weight can be in some way predicted by the maternal birth weight is the question raised by this study. Both glucose and insulin plasma levels were evaluated basely, at $60 \mathrm{~min}$ and at $120 \mathrm{~min}$ after an oral 100 g-glucose load in a group of 190 non-diabetic pregnant women, tested between the $24^{\text {th }}$ and the $28^{\text {th }}$ gestational week. Offspring's neonatal weight was, on average, significantly higher in the group of the highest quartile of maternal birth weight even after adjusting for gestational age, neonatal sex, maternal height or maternal caloric intake $(p=0.001)$. While no significant differences were observed in maternal glucose area under the curve across the quartiles of maternal birth weight, insulin area as well as the ratio (insulin area/glucose area) were inversely related to maternal birth weight, both of them being significantly higher in the first quartile of maternal birth weight than in the fourth, also after adjusting for pregestational body mass index, body weight increase during pregnancy, parity and time of oral test $\left(44.1 \pm 1.68(\mathrm{SD})\right.$ vs $30.9 \pm 3.7 \mathrm{nmol} \mathrm{r}^{-1} 2 \mathrm{~h}$ and respectively $54 \pm 1.7$ vs $36.8 \pm 1.8 \mathrm{nmol} / \mathrm{mol} ; \mathrm{p}<0.05$ for both). In conclusion these results demonstrate that a higher maternal birth weight predicts the delivery of fatter babies, being, moreover, associated with a lower insulin response to a standardised oral load of glucose. All this could suggest that 'physiologic' insulin resistance during pregnancy is higher in mothers with a low birth weight, providing further evidence to support the thrifly phenotype hypothesis.
993

PROGNOSTIC VALUE OF BIOPHYSICAL METHODS IN PREGNANCY COMPLICATED BY DIABETES.

P.Hincz, J.Wilczyński, P.Pawłowicz and K.Szaflik; Research Institute Polish

Mother's Memorial Hospital, Lódź, Poland

The aim of our study was evaluation of biophysical methods used in assessment of fetal welfare. Material and methods: Our study included 110 pregnant women with type 1 and 2 diabetes, hospitalized and followed-up in PMMH in 1992-1997. Biophysical monitoring of fetal well-being included fetal movement count (FMC) non-stress test (NST), biophysical profile of fetus (BPF), amniotic fluid index (AFI) and blood flow velocimetry in the umbilical artery and fetal vessels (middle cerebral artery and descending aorta). All the newborns were estimated according to the $1^{\text {st }}$ and $5^{\text {th }}$ minute Apgar score $(<7$ and 8-10). Results: The comparison of NST with the Apgar score revealed the highest predictive values of the test: positive $83,3 \%$ and negative $74,3 \%$ with sensitivity and specificity consecutively $61,2 \%$ and $90,2 \%$. FMC had also high positive and negative predictive values: $69,2 \%$ and $69,0 \%$ consecutively with sensitivity and specificity $55,1 \%$ and $80,3 \%$. Analysis of BPF revealed also its high positive predictive value $-80,0 \%$ (negative $72,0 \%$ ) with sensitivity and specificity $57,1 \%$ and $88,5 \%$. Diagnostic accuracy of these tests were above $70 \%$ (range $70,1-77,3 \%$ ). In blood flow velocimetry parameters the highest sensitivity was established for RI in fetal descending aorta (100\%) with specificity $32,1 \%$ and positive and negative predictive value consecutively $32,1 \%$ and $100 \%$ The highest specificity had RI in the middle cerebral artery - 88,9\%, with sensitivity, positive and negative predictive values consecutively $17,9 \%, 83,3 \%$ and $25,8 \% . \mathrm{S} / \mathrm{D}$ in the middle cerebral artery and $\mathrm{S} / \mathrm{D}$ and $\mathrm{RI}$ in fetal descending aorta had the highest accuracy $(48,6 \%)$ among the assessed parameters. Conclusions: 1.The highest positive predictive value for fetal well-being was set up for non-stress test (NST), biophysical profile (BPF) and fetal movement count (FMC); 2.Analysis of blood flow velocimetry parameters revealed the highest sensitivity for resistance index (RI) in fetal descending aorta.

\section{5}

Predicting the risk of macrosomia (LGA) in pregnancies of type 1 diabetic women: usefulness of a precise method $\mathrm{HbA}_{\mathrm{lc}}$ in $3^{\text {rd }}$ trimester R. Linkeschova, R. Kimmerle, W. Klockenbusch*, and M. Berger. Dep. of Metabolic Diseases and Nutrition, Dep. of Gynecology*, Heinrich-Heine University, Düsseldorf

The level of hyperglycemia that accelerates fetal growth can be subtle and difficult to assess based on ambulatory blood glucose profiles. The usefulness

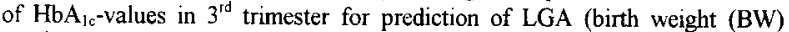
$>90^{\text {th }}$ percentile) in type I diabetic pregnancies has been questioned. Methods: All singelton pregnancies of type 1 diabetic women cared for at our diabetes clinic from 1987-95 (170 newborns, perinatal mortality 0 ) excluding White $F$ and $>1$ pregnancy per woman were analysed for associations of maternal $3^{\text {rd }}$ trim. $\mathrm{HbA}_{\mathrm{ic}}$ (HPLC, normal 4.2-6.1\%, normal pregnant 3.7-5.4\%) with LGA yes $/$ no and birth weight ratio ( $=$ multiple of median reference $\mathrm{BW}$ ) adjusting for prepregnancy weight and weight gain. Results: 29 LGA (BW 4373 (314) g) and 100 non-LGA infants (BW $3202(626) \mathrm{g}$ ) fulfilled the inclusion criteria. Matcrnal age (29 (4) vs. 28 (5) vrs). duration of diabetes (13 (6) vs. 13 (8) yrs), primiparity ( 62 vs. $71 \%$ ) and gestational wk at delivery (39 (2) vs. 39 (1)) were comparable between the two groups. Maternal $\mathrm{HbA}_{10}$ in $3^{\text {rd }}$ trim and prepregnancy weight was higher in LGA than in non-LGA-infants $(6.0(0.8)$ vs. $5.5(0.7) \%, \mathrm{p}=0.001: 66.4(9.5)$ vs. $62.8(8.1) \mathrm{kg}) ; \mathrm{p}=0.04$. The odds of LGA increased with $\mathrm{HbA}_{1 \mathrm{c}}$ in $3^{\text {rd }}$ trim. $(2.2(95 \% \mathrm{CI} 1.2-4.0), \mathrm{p}=0.01$;.multiple regression). LGA-risk was normalized $(=10 \%)$ at $\mathrm{HbA}_{1 \mathrm{c}}$ of $4.6(95 \% \mathrm{CI} 3.6$ $5.6) \%$. High $\mathrm{HbA}_{10}(\geq 5.6 \%)$ poorly predicted LGA (predictive value $36(95$ $\% \mathrm{CI} 24-51) \%$ ). However. low $\mathrm{HbA}_{\mathrm{Ic}}(\leq 5.5 \%)$ had a high negative predictive value for LGA $(88$ ( $95 \%$ Cl $75-94) \%$ ). The BW-ratio of the whole group $(n=129)$ was significantly associated with $\mathrm{HbA}_{\mathrm{lc}}$ in $3^{\text {rd }}$ trim. $(\mathrm{p}=0.004)$ and with prepregnancy weight $(p=0.002)$. Conclusion: To achieve normal rates of LGA in infants of type 1 diabetic mothers $\mathrm{HbA}_{\mathrm{Ic}}$ in $3^{\text {rd }}$ trim. should be within I SD of the normal non-pregnant mean. $\mathrm{HbA}_{1 \mathrm{c}}$-values within this range measured by a precise method can be useful in negatively predicting LGA. 


\section{6}

GESTATIONAL DIABETES IS ASSOCIATED WITH INCREASED RISK OF ADVERSE MATERNAL-FETAL OUTCOMES

D.M. Jensen, B. Sørensen, J.G. Westergaard and H. Beck-Nielsen, Odense University Hospital, Denmark

Aim: To investigate the influence of Gestational Diabetes Mellitus (GDM) on maternal-fetal outcome. Study design: 143 GDM pregnancies and 143 controls with similar risk profiles but normal $75 \mathrm{~g}$ oral glucose tolerance test (OGTT) were investigated retrospectively. The two groups were matched on the basis of age, parity and pre-pregnancy body mass index (BMI). Data was collected from obstetric records and birth records. Results: Baseline variables: The GDM group had a significantly higher frequency of the following risk factors: glucosuria ( $32,2 \%$ vs $13,3 \%), \mathrm{GDM}$ in previous pregnancy $(16,1 \%$ vs $2,2 \%$ ), family history of diabetes mellitus ( $48,3 \%$ vs $34,2 \%$ ) and non-caucasian ethnic origin $(15,4 \%$ vs $1,4 \%)$. No significant difference was found regarding smoking and previous delivery of a macrosomic or stillborn child. Maternal outcomes: the frequency of pregnancy-induced hypertension $(24,5 \%$ vs. $12,6 \%)$ and cesarean delivery $(32,9 \%$ vs $21,0 \%)$ was significantly higher in the GDM group than in the control group. Fetal outcomes: children of GDM mothers had a significantly higher incidence of macrosomia (birth weight $4500 \mathrm{~g}$ ) and neonatal hypoglycemía compared to controls (20\% vs $9 \%$ and $10,5 \%$ vs $0 \%$ resp.). The minimum blood glucose value measured in the newborn during the first 48 hours was significantly lower in the GDM group $(2,57 \pm 0,89$ vs. $3,64 \pm 0,91 \mathrm{mmol} / \mathrm{l})$. There was no difference between the groups in apgar scores, length and birth weight, but when the latter was corrected for gestational age, the GDM babies were significantly heavier $(3744,65 \pm 646.98$ vs $3593,65+577,97 \mathrm{~g}$ ). Conclusion: Pregnancies complicated by GDM, despite intensive treatment, carry an increased risk of pregnancy-induced hypertension, infant macrosomia and neonatal hypoglycemia compared to matched control pregnancies with an otherwise similar risk profile.

\section{7}

The outcome of diabetic pregnancy in a centralised system of diabetcs care A Hodeanu, R Hera, D. Hudita and C. Ionescu-Tirgoviste, Clinic of Diabetes and Clinic of Obstetrics and Gynecology, I. Cantacuzino Hospital, Bucharest, Romania

This study is a retrospective analysis of births amongst diabetic women over a four year period - 1994-1998 who were registered in the Obstetrics Clinic of the 1 . Cantacuzino Hospital catering to almost all pregnant diabetics in Bucharest where 45000 diabetics exist for a population of 2.3 million. In this period, there were a total of 9775 births $(2290$ - 1994; 2496 - 1995; 2542 1996 and $2447-1997)$ of which there were $67(0.6 \%)$ births in patients with diabetes mellitus. Of these, 58 cases $(86.5 \%)$ were type 1 diabetics, 2 cases (2.98\%) type 2 diabetics and 7 cases (10.4\%) were gestational diabetics. The age of these patients were between $20-25$ yrs. in 26 cases $(38.8 \%), 26-35 \mathrm{yrs}$. in 23 cases $(34.3 \%)$ and greater than 35 yrs. in 10 cases $(14.9 \%)$. With the exception of the cases of gestational diabetes, the duration of diabetcs varied between 3 months and 20 yrs. The average value of $\mathrm{HbAlc}$ was $8.4 \pm 0.9 \%$. Three patients presented with more than background retinopathy and one case was associated with nephropathy. The average gestational age at birth was 3-6 wecks ranging between $29-41$ weeks. Caesarean birth occurred in 48 cases $(71.6 \%)$ and 2 cases Bracht method of assisted delivery (2.98\%). Spontaneous births occurred in only 17 cases $(25.3 \%)$. Birth weights varied between $1000 \mathrm{~g}$ and $4400 \mathrm{~g}$ of which: $<2500 \mathrm{~g}$ were 12 cases $(17.9 \%$ ), between $2500-4000 \mathrm{~g}$ 44 cases $(65.6 \%)$ and $>4000 \mathrm{~g}$ in 4 cases $(13.8 \%)$. Sex distribution was $42 \mathrm{~F}$ $(62.6 \%)$ and $25 \mathrm{M}(37.3 \%)$. Complications of pregnancy were: 3 precclanpsia, 2 cases of abruptio placentae, and 1 cases of placenta previa. Fetal complications were: 9 still births $(13.4 \%), 4$ premature $(10.4 \%), 3$ acute hydramnious $-4.4 \%$ and 1 fetal malformations $(1.4 \%)$. Conclusions: 1 . the incidence of diabetic pregnancy of $38 / 100000 / y r$. is one of the lowest in Europe; 2. deaths and malformations comprised $14.8 \%$ (10 cases); 3 . spontaneous births comprised only $1.4 \%$ of cases. 4 a a special programme directed at eliminating or reducing these negative outcomes is urgently required.

\section{8}

GESTATIONAL DIABETES PROGRAMME: SIMILAR OBSTETRIC AND NEONATAL RESULTS TO THE GENERAL PREGNANT POPULATION. E. Baliestar, E. Pizarro,A.Palaudàries,C.Monfort *,J.Solé* *and E.Genover.* Diabetes \& Endocrinology Unit. Diabetic Education*. Obstetric Unit * * Hospital del Consorci Sanitari de Mataró (CSM), Barcelona.Catalonia.Spain The Gestational Diabetes Mellitus (GDM) Community Programme, comprises 11 municipalities ( 190.000 inhabitants) attended by Primary and Women's Care Centers with our hospital (CSM) as the reference center. Aims: to determine the incidence of GDM and by ethnia (native-Caucasian, Moroccan and Central African) and to evaluate clinical, obstetric, neonatal and postpartum glucose tolerance testing (PPGTT) results during 1994-97. Method: four years of universal screening with $50 \mathrm{~g}$ oral glucose at the beginning of gestation and/or between 24-30 weeks.GDM being diagnosed by NDDG and ADA criteria.Diet was isocaloric and home glucose monitoring (HGM):1 fasting and 3 postprandial-1hour (4-readings/day) was required. Optimum control: fasting $<95 \mathrm{mg} / \mathrm{dl}$ and postprandial $<130 \mathrm{mg} / \mathrm{dl}$. If HGM were higher 2 o more times in a week, insulin treatment was initiated. PPGTT with 75g (WHO criteria) were administered. Results: of the 3.757 pregnant, 323 had GDM with a global incidence of $8.60 \%$.By ethnic group: $9.07 \%$ for native-Caucasian, $7.39 \%$ for Moroccans and $1.60 \%$ for Central Africans. The average age of the GDM was $31.36 \pm 4.58$ years, without diferences by ethnic groups. In non-GDM was $28.68 \pm 4.83$ years with a significant lower age for Moroccan and Centro African.Insulin was necessary in $27.2 \%$ of GDM mostly in multiple doses.Ponderal increment during gestation was similar for GDM with and without insulin $(9.36 \mathrm{Kg}$ vs. $9.02 \mathrm{Kg}$ ). No statistically significant differences were found between non-GDM and GDM for : weeks gestation at delivery ( $39.16 \pm 1.55$ wks vs $39.11 \pm 1.42$ wks), delivery mode, birth weigh $(3230 \mathrm{~g}$ vs. $3248 \mathrm{~g}$ ), percentage of macrosomia $>3999 \mathrm{~g}$ ( $5.11 \%$ vs. $6.23 \%$ ), 5 -minutes Apgar score $\leq 6$ ( $3.49 \%$ vs. $3.26 \%$ ) and mortality $(5.79 \%$ vs. $3.11 \%$ ). Only neonatal hipoglycemia $<40 \mathrm{mg} / \mathrm{dl}$ at 2 hours in GDM was higher than in non-GDM ( $8.8 \%$ vs. $4.5 \%$ ). PPGTT results : $5.26 \%$ had impaired glucose tolerance and $1.05 \%$,diabetes melitus.Conclusion: this programme of defection and treatment attain similars results in gestational diabetes women to compare as general pregnant population.The programme achieve the target of St Vincent Declaration about pregnancies outcomes.

\section{9}

THE CONTRIBUTION OF MATERNAL GLUCOSE AND INSULIN LEVELS ON BIRTH WEIGHT IN NORMAL PREGNANCY

O. Giampietro, P. Bay*, F. Piazza and E. Matteucci. Clinica Medica II, Clinica Ostetrica*, Pisa, Italy.

The relationship of fetal growth and maternal glucose tolerance has long been sought. We studied 80 healthy pregnant women (age $28 \pm 5$ years) both at $24 \pm 2$ and $33 \pm 1$ weeks of gestation as for glucose tolerance by performing both 50-g 2-hour glucose challenge test, GCT, and 100-g 3hour oral glucose tolerance test, OGTT (areas under glycemic and insulinemic curves, AUGC and AUIC, were geometrically calculated). No pregnancy was complicated by gestational diabetes or hypertension. The mean gestational age of the infants was $39 \pm 2$ weeks. By dividing pregnant women according to neonatal weight (after excluding four cases with birth weight $<2.5 \mathrm{~kg}$ and lower gestational age, $36 \pm 1 \mathrm{wk}$ ), 65 of them delivered normal-weight babies (NGA, $3.3+0.3 \mathrm{~kg}, 39 \pm \mathrm{l}$ wk) while 11 large babies $>3.9 \mathrm{~kg}$ (LGA, $4.6 \pm 1 \mathrm{~kg}, 39 \pm 1 \mathrm{wk}$ ). LGA mothers were older than NGA ( $31 \pm 5$ vs $28 \pm 4 \mathrm{yr}, \mathrm{p}<0.05)$; in addition, they had higher prepregnancy BMI $\left(25 \pm 5 \mathrm{vs} 22 \pm 3 \mathrm{~kg} / \mathrm{m}^{2}, \mathrm{p}<0.05\right)$, AUIC at 2 nd trimester GCT $(3.4 \pm 1.5$ vs $2.5 \pm 1.3 \mathrm{mU} \mathrm{min} / \mathrm{ml}, \mathrm{p}<0.05)$ and fasting glycemia at 3 rd trimester $(85 \pm 8$ vs $79 \pm 8 \mathrm{mg} / \mathrm{dl}, \mathrm{p}<0.05)$. The presence of a single abnormal glycemia (1-h GCT value $>130 \mathrm{mg} / \mathrm{dl}$ or OGTT criteria of Carpenter and Coustan exceeded) divided pregnant women into 19 who had at least one abnormal value ( $\mathrm{HG})$ and 61 who did no

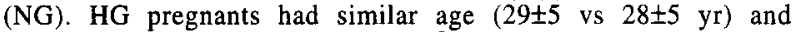
prepregnancy BMI ( $23 \pm 3$ vs $\left.23 \pm 4 \mathrm{k} / \mathrm{m}^{2}\right)$ than NG, but higher neonatal $(3.8 \pm 1.2$ ys $3.3 \pm 0.6 \mathrm{~kg}, \mathrm{p}<0.05$ ), higher AUGCs during GCT and OGTT at 2nd $(\mathrm{p}<0.01)$ and 3rd $(\mathrm{p}<0.001)$ trimesters, yet similar AUICs. Neonatal weight was positively correlated (multiple R $0.42, p<0.01$ ) with maternal age and fasting insulinemia at 2 nd trimester. We conclude that 1) birth weight pregressively increases, just in the normal range with increasing maternal glycemia, even if maternal tolerance remains normal; 2) the risk of out-of-range birth weights significantly increases with increasing maternal age, basal BMI and post-load insulinemia. 
1000

MATERNAL LIPID METABOLISM AND FETAL MACROSOMIA

M Jolly, R Amin, V Anyaoku, J Soares, K Murphy, T.G Teoh, D Johnston and S Robinson. Section of Endocrinology \& Metabolic Medicine and, Imperial College School of Medicine, St Mary's Hospital, London.

Maternal obesity, even in the absence of hyperglycaemia, is a major risk factor for fetal macrosomia. We therefore aimed to test the hypothesis that abnormal maternal fatty acid metabolism associated with insulin insensitivity in non-diabetic mothers was associated with fetal macrosomia. Twelve obese pregnant women were compared with 7 lean pregnant women and 9 non-pregnant controls. Insulin sensitivity and NEFA suppression were assessed with the frequent-sampling intravenous glucose tolerance test. Specific Insulin was used for modelling purposes. Maternal body mass index was highest in the obese pregnant women ( $n-p$ $25.0 \pm 4.9$, obese-preg $34.5 \pm 5.3$, lean-preg $25.1 \pm 2.1 \mathrm{~kg} / \mathrm{m}^{2}$ ). Fasting plasma glucose was significantly higher in the obese pregnant women (n-p $4.9 \pm 0.5$, obese-preg $5.2 \pm 1.0$, lean-preg $4.4 \pm 0.8 \mathrm{mmol} / \mathrm{h}, \mathrm{p}<0.05$ ) although glucose tolerance was not significantly different in this group (n-p $2.1 \pm 0.7$, obese-preg $1.7 \pm 0.8$, lean-preg $2.0 \pm 0.7 \%$, NS). Insulin sensitivity was decreased in pregnancy and nonsignificantly further decreased in the obese pregnant women (n-p 18.5 \pm 17.1 , obesepreg $3.0 \pm 1.7$, lean-preg $4.0 \pm 3.810^{-4} \mathrm{~min}^{-1}$ per $\mathrm{pM} . \mathrm{l}^{-1}, \mathrm{p}<0.01, \mathrm{n}-\mathrm{p} \mathrm{v}$ either pregnan

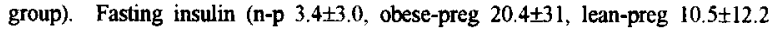

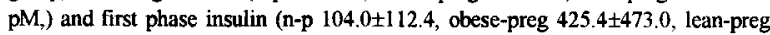
$271.8 \pm 216.0 \mathrm{pM}$,) were increased in pregnancy, but not significantly further increased in obese pregnant women. Data on 6 macrosomic and 11 non-macrosomic infants are available. NEFA nadir was higher in women who had a macrosomic compared to a non-macrosomic fetus (mac $119 \pm 102 \mathrm{v}$ non-mac $100 \pm 56, \mathrm{p}<0.01$ ) as was LDL-cholesterol (mac 3.4 \pm 0.7 v non-mac $2.5 \pm 1.1, p<0.05$ ). In conclusion insulin resistance is increased in normal pregnancy although not significantly further increased in obese compared to lean pregnant women. Lipid and glucose metabolism may play a role in fetal macrosomia for non-diabetic mothers

\section{1}

IS THERE RELATION BETWEEN TRIGLYCERIDE LEVELS AND MACROSOMIA IN GESTATIONAL DLABETES ?

P Fontaine $e^{1.3}$, A Vambergue $^{1,3}$, O Verier-Mine ${ }^{3}$, Lefebvre $^{3}$, M Cordonnier ${ }^{3}, \mathrm{MC}$ Nuttens ${ }^{2,3},{ }^{1}$ Service d'endocrinologie, ${ }^{2}$ Département d'Information Médicale, CHRU, 59037 Lille Cedex, France, ${ }^{3}$ DIAGEST group.

Intensive treatment of gestational diabetes (GD) often normalizes plasma glucose levels. Does it also improve triglyceride (TG) levels ? Does TG levels influence rate of macrosomia? In a multicentric prospective study, we diagnosed 221 GD (Carpenter and Coustan criteria). Fasting and post-prandial $\mathrm{TG}$ were measured in $197 \mathrm{GD}$ at each diabetologic visit. All had intensive dietetic counselling and selfmonitored blood glucose 4 times a day. Insulin was added if weekly venou glycemia was fasting $\geq 5.3$ and/or 2 hours post-prandial $\geq 6.6 \mathrm{mmol} / 1$. «Goodcontroly group ( $\mathrm{n}=125)$ achieved mean venous glycemia fasting $\leq 4.9$ and 2 hours post- prandial $\leq 6.6 \mathrm{mmol} / \mathrm{l}$. «Nol-good-control " group $(\mathrm{n}=72)$ did not. " Goodcontrol " vs « not-good-control * were younger (29.1 (5.6) vs 32.3 (5.9) years $\mathrm{p}<0.001$ ) and leaner before pregnancy (prepregnancy BMI : $24.7(4.9)$ vs 27.9 (5.8) ; p $<0.001$ ). Insulinotherapy was given to $36.0 \%$ of « good-control » and $64.3 \%$ of « not-good control ». During treatment, the means of fasting and postprandial TG levels werc lower in " good-control " Fasting $2.19(1$ 52) vs 247 (1.65) mmol/l, $\mathrm{p}<0.05$; post prandial :2.33 (1.52) vs 2.63 (1.64) mmol/l, $\mathrm{p}<0.05$ ) Mean fasting and mean post-prandial TG were correlated with mean fasting and mean post-prandial glycemia. These differences and correlations persisted after adjustment on prepregnancy BMI and gestational age. There were no association between pregnancy hypertension and TG. Large for gestational age infants (LGA) were less frequent in " good-control » $(16,1 \%$ vs $34,7 \% ;$ p $<0.01)$. After adjustment on 35 years old, prepregnancy BMI, parity, and educational level. LGA was still less frequent among " good-control 》 (OR=0.41;95\% CI $[0.28$ $0.601)$. Fasting and post-prandial TG levels did not influence the rate of LGA in " good-control " and «not-good-control». The normalization of plasma glucose level may contribute to lower fasting and post-prandial TG levels. This decreasc does not secm to influence the rate of LGA.

\section{2}

MATERNAL HYPERGLYCEMIA IS NOT THE ONLY CAUSE OF MACROSOMIA LESSONS LEARNED FROM A COHORT OF 2028 GESTATIONS

W Ricart, C Bach, JM Fernández-Real, J Biarnes, J Vidal, D Cabrero and J Sabria. Hospital Universitario Doctor Josep Trueta. Girona. SPAIN Maternal hyperglycemia has been implicated as the major cause of neonatal macrosomia. Wo examined the predictive value of maternal age (MA), ethnic group ( $E G$ ), maternal pregestational body mass index (BMI), maternal weight increase, serum glucose after a $50 \mathrm{~g}$ glucose overload test (SG50), glucose tolerance status -normal (85\%), glucose intolerance $(3.9 \%)$ or gestational diabetes (11\%)-, and gestational duration (GD) on birth weight (BW) and LGA in 2028 consecutive gestations. HbA1 was evaluated in the gestational diabetic subgroup. BW was expressesd as a \% of the 50 th percentile adjusted by sex and gestational age obtained from a 12000 births. LGA was defined as BW above 9oth percentile for sex and gestational age. In a subgroup of 298 patients with chromosomal risk, amniotic fluid glucose (AFG) and insulin (AFI), and maternal serum glucose (MSG) and insulin (MSI) concentrations at 14-16 weeks of gestation were measured. BW was classified in quartiles. In the univariate analysis a difference of MA $(p=0.02), G D(p=0.007)$, SG50 $(p=0.03)$, EG $(p=0.0000)$ and for $B M l(p=0.0000)$ between the higher and lower $B W$ quartil was demostrated. A slight difference for glucose tolerance status $(p=0,08)$ and AFG $(p=0.06)$ was found. In a multivariate analysis only BMI and SG50 were predictive for $B W$. In the analysis of the gestational diabetic subgroup BMI and slightly $\mathrm{HbA1}(\mathrm{p}=0.08)$ had a predictive value. No differences in the prevalence of $L G A$ between non-gestational diabetic and gestational diabetic subgroups were found. Differences in BMI $(p=0.00001)$ and EG $(p=0.001)$ were demonstrated in the analysis between normal $B W$ and LGA groups. The multivariate analysis showed that only BMI, both in all the cohort and in the gestational diabetic subgroup, were predicitve for macrosomia. In conclusion, maternal glucose metabolism, but specially the pregravid overweight, independent from hyperglycemia, are associated with increased birthweight in our population.

\section{3}

PRE-PREGNANCY MICROALBUMINURIA PREDICTS PREECLAMPSIA IN INSULIN DEPENDENT DIABETES. $P$ Ekbom, E Stage, $K$ Norgård, A Svenningsen, $P$ Clausen, U Feldt-Rasmussen, B Feldt-Rasmussen, L Nielsen, L Molsted-Pedersen, P Damm and ER Mathiesen. Obstetrisk Clinic and dept. of Medical Endocrinology and Nephrology, Steno Diabetes Center and Rigshospitalet, Copenhagen, Denmark. Our aim was to determine whether presence of microalbuminuria prior to pregnancy can predict development of preeclampsia in women with insulin dependent diabetes mellitus (IDDM). A prospective cohort study including all pregnant Caucasian women with IDDM admitted to our obstetric clinic prior to 17 weeks of gestation with a living foetus, from January 1996 to May 1997 was performed. Women with diabetic nephropathy (urinary albumin excretion> 300 $\mathrm{mg} / 24 \mathrm{~h}, \mathrm{n}=5)$ or a history of hypertension $(\mathrm{n}=1)$ were excluded. Values of $24 \mathrm{~h}$ urinary albumin excretion (UAE), blood pressure and haemoglobin $A_{1 c}$ within 2 years prior to pregnancy were obtained from local records and presence of microalbuminuria was defined as UAE $30-300 \mathrm{mg} / 24 \mathrm{~h}$ (geometric mean of $2-4$ consecutive urine samples). During pregnancy UAE, office blood pressure and $24 \mathrm{~h}$ blood pressure were recorded. Preeclampsia was defined as office blood pressure $>140 / 90 \mathrm{mmHg}$ and UAE $>300 \mathrm{mg} / 24 \mathrm{~h}$. Seventy-four consecutive pregnancies resulted in 68 deliveries later than 24 weeks of gestation. Mean age of the 68 women was 30 (SD 5) years, duration of diabetes 13(9) years, prepregnancy UAE 11 (range $1-265) \mathrm{mg} / 24 \mathrm{~h}(\mathrm{n}=64,94 \%)$, blood pressure $118 / 75$ (11/7) $\mathrm{mmHg}$ and $\mathrm{HbA}_{1 \mathrm{c}} 7,9(1,3) \%$. Six $(60 \%)$ out of 10 women with prepregnancy microalbuminuria developed preeclampsia compared to $2(4 \%)$ out of 54 women with normal UAE ( $p<0,001)$. Using multiple logisitic regression analysis pre-pregnancy microalbuminuria was the best predictor for development of preeclampsia The relative risk of developing preeclampsia in women with pre-excisting microalbuminuria was 16 (95\% confidence interval 4-69) compared to women without microalbuminuria. Conclusion: Pre-existing microalbuminuria predicts preeclampsia in women with IDDM. 


\section{4}

NON SUPPRESSIBILITY OF GLUCAGON IN LATE PREGNANCY AND IN PREVIOUS GESTATIONAL DIABETIC WOMEN

C. Beis, E. Anastasiou, M. Alevizaki, S.I. Grigorakis, G. Philippou and A. Souvatzoglou. 1st Endocrine Section and Diabetes Centre, ALEXANDRA University Hospital, 11528 Athens.

Absence of glucagon suppressibility after OGTT has been reported in subjects with IGT and NIDDM. We investigated this possible abnormality in previous gestational diabetic women (ex-GDM) with normal OGTT. Furthermore we studied fasting $\left(0^{\prime}\right)$ and post glucose glucagon levels in normal and GDM pregnancy, as available data is somewhat conflicting. We examined, 2-4 months postpartum, 17 ex-GDM women with normal OGTT (WHO criteria) age $38 \pm 5$ years and BMI $27.2 \pm 5.2$ and 17 controls matched for age and $\mathrm{BMl}$. In addition we investigated $20 \mathrm{GDM}$ women (NDDG criteria) aged $32.2 \pm 5.1$ years with pre-pregnancy BMI $26.7 \pm 4.2$, and 26 normal pregnant women (Npregn) matched for age and BMl, in the $28^{\text {th }} \cdot 32^{\text {nd }}$ pregnancy weeks. Glucose, insulin and glucagon levels were measured during a $75 \mathrm{~g}$ or $100 \mathrm{~g}$ OGTT respectively. Glucagon (gluc) levels were suppressed at $90^{\prime}$ and $120^{\prime}$ in controls; percent difference $(\Delta \%)$ of glucagon at 90 and 120 minutes minus glucagon $0^{\prime}$ was as follows: $\Delta$ gluc $90^{\circ}=-10 \pm-$ $15 \%, p=0.02, \Delta$ gluc $120 '=-10 \pm-11 \%, p=0.001$, one sample t test. In ex-GDM women glucagon levels were not suppressed, but were even increased: $\Delta$ gluc $^{\prime} 0^{\prime}=14 \pm 13 \%, \quad p<0.061$ and $\Delta$ gluc $^{\prime} 0^{\prime}=10 \pm 14, \quad p=0.007$. During pregnancy glucagon levels at $O^{\prime}$ and at $180^{\prime}$ were significantly higher in GDM compared to Npregn: $62.3 \pm 11.5$ vs $53.1 \pm 11.8 \mathrm{pmo} / \mathrm{L}, \mathrm{p}<0.005$ and $63.7 \pm 16.1$ vs $53.4 \pm 16.9, p<0.005$ respectively. There was no glucagon suppression at any time during OGTT in GDM or in Npregn. We sugges that: (a). Absence of the suppressibility of glucagon in ex-GDM women with normal OGTT might be involved in the natural history towards glucose intolerance. (b). No suppression of glucagon in normal late pregnancy as well as in pregnancy complicated by GDM may be due to "physiological" insulin resistance during this period.

\section{6}

INTENSIVE INSULIN TREATMENT OF PREGNANT DIABETIC WOMEN SUPPORTED BY TELEMATIC SYSTEM. RESULTS FROM 2 YEARS STUDY

\section{J.Wójcicki, P.Ładyżyński, J.Krzymień*, E.Jóźwicka*, J.Blachowicz,} E.Janczewska**, K.Czajkowski** and W. Karnafel*. Inst.Bioc.\& Biom.Eng PAS, Clin.Gastr. \& Met.Dis. MA*, I \& II Clin.Obst. \& Gynaec. MA**,Warsaw, Poland In order to assess effectiveness of the telematic system on glycaemic control of the pregnant diabetic women two randomized groups of patients (15 persons each), selected according to established protocol of the study, will be evaluated. One with routine clinical control every 3 weeks and other with telematic transinission of the data allowing on daily examination of the patient state and if necessary intervention by the physician. Both groups are trained using the same educational program during 3 days of hospitalization. Designed and developed system consists: central clinical control system with originally designed software system "Diapret" allowing for visualization of the long-term metabolic control and patient's teletransmission modules with Bayer Glucometer $\mathrm{M}+$, modem and dial-up or cellular phone. Till now 17 patients ( 9 in control group and 8 in study group) with average for each patient $143.9 \pm 6$ days of monitoring in control and $160.3 \pm 6.5$ days in study group have been evaluated. Both groups were very similar at the start point of the study regarding to glycaemic control (MBG=8.6 $\pm 2.2 \mathrm{vs8.5} \pm 1.3$ $\mathrm{mmol} / \mathrm{l})$ duration of the diabetes $(7.7 \pm 5.7$ vs $10.6 \pm 3.2$ years) end level of intelligence ( $103 \pm 11$ vs $103 \pm 13$ pts). Obtained results indicated that an application of the telematic system leads especially to: (a) significantly higher stability of the blood glucose data represented by much lower variability of MBG (c.v.= $5.8 \% \mathrm{vs} 14.4 \%, \mathrm{p}<0.01$ ), J-index (c.v. $=15.8 \%$ vs $31.2 \%, \mathrm{p}<0.05$ ), (b) significant difference between glycaemia courses during Iast 10 weeks of the study due to better glycaemic control in the study group $(\triangle \mathrm{MBG}=0.6 \pm 0.5 \mathrm{mmol} / \mathrm{l}, \Delta \mathrm{J}=6.4 \pm 4.8$, $\mathrm{p}<0.001$ ). It seems that an application of the developed and studied system, which allows on daily examination of the metabolic control of the patient leads to significant improvement of the intensive insulin treatment. Final conclusion will be made after termination of the project which is scheduled on the end of this year.

\section{5}

THE BIRTH OF A DIABETES IN PREGNANCY CENTER IN GEORGIA

N.Asatiani, A.Elphick*, R.Kurashvili, M.Natsvlishvili, T.Chanturia**, 2. Tbilisi, Georgia, ${ }^{* *}$ Dept. of Obstetrics and Gynecology, Rabin Medical Center, Tel-Aviv, Israel; *Novo Nordisk A/S, Copenhagen, Denmark.

Georgian Diabetes in Pregnancy Program was initiated in cooperation with the Rabin Medical Center and the Israel Diabetes Asseciation (IDA), resulting from an IDF twinning partnership between the IDA and Georgian Diabetes Federation. The aim of this study is to compare pregnancy outcome before and after implementation of the Program. Participants were sixtheen women with IDDM. Fourteen of them had one or more prior pregnancies complicated by Diabetes Mellitus (1991-1995). None had received qualified medical care, and 13 of the 38 pregnancies $(34.2 \%)$ ended in neonatal deaths, $10(26 \%)$ in spontaneous abortions and $10(26.3 \%)$ in induced abortions. Only in 5 cases $(13.1 \%)$ overweight infants were born. Patients' mean age was $27 \pm 6$ years, and diabetes duration $10 \pm 6$ years. Under the Program, that commenced in October 1996, patients reported 3 month before conception for pregnancy care. By the end of this period HbAlc levels dropped to $7.6 \pm 0.5 \%(P<0.001)$ and insulin doses decreased to $0.5 \pm 0.06 \mathrm{IU} / \mathrm{kg}(\mathrm{P}<0.01)$. After conception, the women were supervised by a multidisciplinary team. At the end of the third trimester, the mean HbAlc level was $7.2 \pm 0.2 \%(\mathrm{P}<0.05)$ and insulin requirement, compared to the first trimester, increased to $0.9 \pm 0.08 \mathrm{IU} / \mathrm{kg}(\mathrm{P}<0.001)$. There were no severe maternal complications throughout pregnancy. All 16 women gave birth to liveborm offsprings between the 36 th and 39 th weeks of gestation (6 cesarean sections, 10 vaginal deliveries). Fetal birth weight ranged from 2.3 to $4.1 \mathrm{~kg}$ These preliminary results indicate that our Program has significantly reduced the high pre-program levels of neonatal mortality, and morbidity, as well as the incidence of matermal complications. We hope to continue patient education, and intensive pre- and postnatal care in wider groups of women with Diabetes Mellitus, thus providing an equal opportunity for motherhood to all women.

\section{7}

QUALITY OF CARE PROVIDED TO GESTATIONAL DIABETIC WOMEN: A BASIC INFORMATION SHEET FOR DIABETES AND PREGNANCY Gy. Tamás, Á. Gy. Tabák, P. Turbucz, T. Hidvégi, I.Földesi and Zs. Kerényi, Depts. of Med., Budapest, Györ, Szentes, Hungary

Quality assurance is of utmost importance in all levels of health care. As part of an on-going continuous quality improvement project, we evaluated diagnostic measures and outcomes of care for 134 gestational diabetic women (mean age: 28.9+6.1[+SD; range:16-43]yrs; GDM: n=117; Gestational-Impaired Glucose Tolerance; G-IGT [75g oGT'T; WHO]:n=17; 5 twins) by level of service delivery using a newly developed standardized Basic Information Sheet for Diabetes and Pregnancy (BIS-DP; WHOIDF/Euro). BIS-DP contained process (documentation [history, diagnosis, booking], monitoring, intervention) and outcome (intermediate: metabolic parameters, risk factors, progress of complications; true: perinatal morbidity, mortality, congenital inalformation [CM], stillbirth, materna] hypertension, discharge) indicators and data on reclassification. Diabetes centres (C) were responsible for the care of 47 patients. Diabetes units (U) cared for 117 women. No significant differences were observed between $C$ and $U$ with respect to mean maternal age, time of booking, birth weight. perinatal morbidity and mortality. $\mathrm{CM}(\mathrm{n}=4)$ were found in $\mathrm{U}$ group only. No data in $U$, however, on booking/third trimester HbA1c (C: $6.5 \%$ [4.89.5; range - 4.9\% [3.4-6.8]; performed in 12 and 24/47 cases), microalbuminuria (C: 7/47 investigated) and hypertension (10 cases of chronic/pregnancy induced in $\mathbf{C}$ ) were marked. $\mathbf{C}$ women had a higher section rate ( 33 vs $6 \%$; $\mathrm{P}<0.001) ; 7 / 47$ used self monitoring compared to none in U. Fifteen/47 C women (IDDM: 2; IGT: 2; healthy: 11) and 6/117 U cases (NIDDM: 2; IGT:1; healthy: 3) were reclassified shortly after delivery ( $C$ vs $U$ P $<0.001$ ). In conclusion, BIS-DP as a tool in quality improvement strategies proved a wide gap between recommended international standards for GDM care and the care actually received even at tertiary level (C). Standardized, structured data sets may have the potential to influence organizational factors in health care delivery and to improve care of GDM women. 
PS 49

\section{Experimental Pregnancy}

1008

IMPAIRED ENDOTHELIUM-DEPENDENT RELAXATION IN PREGNANT OFFSPRING OF DIABETIC RATS

R.T. Gerber, K.Holemans ${ }^{1}$, F.A. Van Assche ${ }^{1}$ and L.Poston. Fetal Health Research Group, Departments of Obstetrics and Gynaecology, St Thomas' Hospital, London SE1 7EH and ${ }^{1}$ Katholieke Universiteit Leuven, 3000 Leuven, Belgium.

Offspring of streptozotocin-induced diabetic rats $(O D)$ are insulin resistant in adult life and develop hyperglycaemia and insulin resistance in pregnancy (Holemans K et al. J Endocrinol 199;

131:387-393). Gestational diabetes in women is associated with an increased incidence of vascular disorders, particularly pre-eclampsia. The aim of this study was to assess vascular function in pregnant $O D$ rats (POD). Using a myograph relaxation to acetylcholine (ACh) was assessed in small mesenteric arteries from pregnant control and POD. Arteries were preconstricted with $5 \mu \mathrm{M}$ noradrenaline \pm indomethacin $\left(10^{-5} \mathrm{M}\right)$. Max relaxation to $\mathrm{ACh}$ was significantly impaired in arteries from POD (\% of noradrenaline induced tone; POD: $94.30 \% \pm 1.34, \mathrm{n}$ $=10$ vs pregnant control: $98.43 \% \pm 0.60, n=9 ; p<0.01$ ).

Indomethacin abolished any differences in $\mathrm{ACh}$ relaxation, suggesting a defect in prostacyclin synthesis or increased constrictor prostanoid production in POD. Relaxation to sodium nitroprusside was not different between the groups. Fetuses ( $20 \mathrm{~d}$ gestation) from POD were significantly heavier than those of pregnant controls (POD: $2.08 \pm$ $0.04 \mathrm{~g}, \mathrm{n}=79$ vs pregnant control: $1.86 \pm 0.02 \mathrm{~g}, \mathrm{n}=56 ; \mathrm{p}<0.01$ ). In conclusion pregnant offspring of diabetic (POD) have a reduction in endothelium-dependent relaxation. These observations are comparable to human gestational diabetes which is associated with endothelial dysfunction and macrosomia. Pregnant offspring of diabetic rats may be a good model for human gestational diabetes.

\section{0}

\section{LOW-PROTEIN DIET DURING GESTATION AND LACTATION} DID NOT MODULATE INSULIN RESISTANCE IN SHR

\section{K. TANIGAWA, T. MIURA and E. ISHIHARA Department of}

Clinical Nutrition, Suzuka University of Medical Science, Mie, Japan

The effect of a low-protein diet (LPD) during gestation and lactation on insulin resistance and hypertension was examined in spontaneously hypertensive rats (SHR). Offspring born to LPD-fed rats ( $8 \%$ protein) had significantly lower body weights than the controls. Their weight failed to catch up to control levels until 12-weeks of age, despite feeding with the control diet after weaning. Blood pressure at 12weeks old did not differ depending on whether the rats were fed with or without LPD. Euglycemic insulin clamp $(6 \mathrm{mU} / \mathrm{kg} / \mathrm{min})$ was also performed, but there was no difference in glucose infusion rate between the two groups. An intravenous glucose tolerance test $(0.5$ $\mathrm{g} / \mathrm{kg}$ bw) revealed that glucose tolerance was unimpaired in the offspring of rats fed the LPD, but their plasma insulin levels were significantly $(p<0.05)$ lower at 5 and $15 \mathrm{~min}$ after a glucose load. Although the protein content of the pancreas was lower $(p<0.05)$ in the rats fed the $L P D$, pancreatic insulin stores did not differ between the two groups. Therefore, growth retardation due to LPD did not modulate insulin resistance or blood pressure in SHR, but insulin secretory activity may be influenced by LPD during gestation and lactation.

\section{9}

EFFECT OF SOCIAL STRESS AND GESTATIONAL INSULIN DEFICIENCY ON GLUCOSE TOLERANCE IN RATS OFFSPRING N.Krasova, V.Poltorak, A.Gladkih, L.Sergienko, G.Brizgalova. Ukrainian Institute of Endocrine Diseases Pharmacotherapy, Kharkov, Ukraine. The aim of the study was to investigate the impact of maternal social stress (MSS) against the background of gestational insulin deficiency (GID) on glucose tolerance in male and female Wistar rats offspring (O) For MSS creation rats were transferred daily from one rats association to another within $2^{\text {nd }}-8^{\text {th }}$ day of pregnancy, GID was rendered by STZ injection $(45 \mathrm{mg} / \mathrm{kg}$, i.p.) on the second day of pregnancy. The maternal cohort consisted of 50 pregnant rats exposed MSS, GID, MSS+GID and controls. An i.p. GTT ( 3 g glucose $/ \mathrm{kg})$ was performed in $\mathrm{O}(\mathrm{n}=32)$ at 45 days of age Disturbances in glycemic pattern during GTT were revealed in MSS male $\mathrm{O}$ : plasma glucose levels did not return at $120 \mathrm{~min}$ to basal value (respectively, $7.6 \pm 0.4$ and $4.9 \pm 0.6 \mathrm{mmol} / \mathrm{l}, \mathrm{p}<0.01$, vs $5.3 \pm 0.3$ and $4.9 \pm 0.3 \mathrm{mmol} / \mathrm{l}, \mathrm{NS}$, in controls). GID impaired glucose tolerance in $\mathrm{O}$ independently from gender: integral glycemia levels over GTT were $31.3 \pm 2.0$ and $31.2 \pm 2.2 \mathrm{mmol} / \mathrm{l}$, respectively, in male and female $O$ in comparison with $25.4 \pm 1.1$ and $24.2 \pm 1.2 \mathrm{mmol} / 1$ in controls $(\mathrm{p}<0.05)$. MSS+GID also induced glucose intolerance in both gender $\mathrm{O}$ : glycemia didn't return to basal value at $120 \mathrm{~min}$ after glucose load and formed $9.3 \pm 2.1$ vs $4.7 \pm 0.6 \mathrm{mmol} / 1(\mathrm{p}<0.05)$ in male and $7.3 \pm 0.8$ vs $4.4 \pm 0.2 \mathrm{mmol} / \mathrm{l}(\mathrm{p}<0.05)$ in female compared to $5.2 \pm 0.5$ vs $5.2 \pm 0.4$ $\mathrm{mmol} / \mathrm{l}$ (NS) for control female. MSS enhanced GID-induced glucose tolerance disturbances in male $\mathrm{O}$ : integral glycemia was $40.7 \pm 2.4$ vs $31.3 \pm 2.0 \mathrm{mmol} / \mathrm{l}(\mathrm{p}<0.02)$, respectively, in MSS+GID and GID 0 in comparison with $25.4 \pm 1.5 \mathrm{mmol} / 1(\mathrm{p}<0.001)$ in MSS offspring. Thus maternal social stress strengthened gestational insulin deficiency-induced glucose intolerance in rats male offspring at puberty and did not change it in female offspring

\section{1}

TISSULAR LIPOPROTEIN LIPASE ACTIVITY AND MRNA EXPRESSION IN STREPTOZOTOCIN DIABETIC PREGNANT RATS

E. Herrera, L. Blanco, and A. Martín-Hidalgo, Universidad San Pablo-CEU and Departamento Investigación, Hospital Ramón y Cajal, Madrid, Spain

Hypertriglyceridemia is a common feature both in normal pregnancy and in diabetes, and usually becomes exagerated when both conditions coincide. Since a decreased clearance of circulating triglyceride-rich lipoproteins caused by decreased adipose lipoprotein lipase (LPL) activity could contribute to such change, the present work was addressed to study both LPL activity and $m R N A$ expression in different tissues of virgin $(V)$ and 20 day pregnant rats (P) made diabetic (D) with $40 \mathrm{mg}$ i.v. streptozotocin/ $/ \mathrm{kg}$ body weight given before mating. Control rats (C) receiving the buffer were studied in parallel. PC rats had higher plasma triglyceride (TG) levels than VC, and although $D$ enhanced TG in both groups, values in PD remained higher than in VD. Plasma insulin levels were higher in PC than in VC, whereas the decrease caused by $D$ was greater in $P$ than in $V$, disappearing the differences between both groups. Adipose tissue LPL activity and mRNA expression were lower in $P C$ than in VC, and the effect of $D$ decreasing both variables was greater in $\mathrm{V}$ than in $\mathrm{P}$ rats. Mammary gland LPL activity and mRNA expression were higher in $\mathrm{PC}$ than in $\mathrm{VC}$, but the effect of $\mathrm{D}$ decreasing both variables was greater in P than in V. LPL activity and mRNA expression in red fiber skeletal muscle were slightly lower in PC and VC, and D hardly enhanced these variables in P rats. Parallel changes in LPL activity and mRNA indicate that long term control by insulin of LPL activity is tissue specific and is made at the transcriptional level, and it is suggested that the different responsiveness detected among the tissues to either $P$ or $D$ depends on their respective insulin sensitivity in late pregnancy, which also affects plasma TG levels. 
PLASMA PANCREASTATIN-LIKE AND CATECHOLAMINE LEVELS IN GESTATIONAL DIABETES

JA Lobón Hernández, V Sanchez-Margalet*, González Jiménez A Fernández Soto ML, Goberna R* and Escobar-Jiménez F. Department of Endocrinology, Univ. Hospital , Granada, *Depart of Clinical Biochemistry, Investigation Unit, Univ. Hospital Virgen Macarena, Seville, Spain.

Pancreastatin (PST) effects raised the hypothesis of these peptide being a general autocrine, paracrine and endocrine inhibitor. Since we had prevously demonstrated the counterregulatory effect of PST on insulin action, and other study had show tha PST levels seem to increased in the third trimester of pregnancy: whether PST may be involved in the pathophysiology of Gestational Diabetes Mellitus (GDM)?. The AIM of the present study was to evaluate plasma levels of glucose and hormones involved in glucose metabolism in lean subjects with GDM compared with those in lean subjects with normal pregnancy. PATIENTS AND METHODS: 23 non-obese (BMI $<27 \mathrm{~kg} / \mathrm{m} 2)$ pregnant subjects were employed for the study. 12 subjects had previously been diagnosed of GDM and 11 were control pregnant subjects. Selection criteria for GDM were those recommended by National Diabetes Data Group. Fasting blood samples were obtanined at late pregnancy ( 301 weeks). Plasma glucose, insulin, glucagon, PST, epinephrine (E) and norepinephrine (EN) were measured. Values are means SEM. Student's t test was used for comparisons, with differencies bieng considered significant at $\mathrm{P}<0.05$. RESULTS: Gestational diabetic subjects had significantly higher insulin levels than control pregnant subjects (18 1 versus $151 \mathrm{mU} / \mathrm{ml}$ ), whereas glucose and glucagon levels where comparable in both groups. However increased catecholamines levels ( $E$ and NE) were found in the GDM. We also found increased PST levels in these patients, compared with control pregnant group (46 2 versus $302 \mathrm{~mol} / \mathrm{l}$ ). Actually, PST levels positively correlated witn both $\mathrm{E}(\mathrm{r}=0.34)$ and $\mathrm{EN}(\mathrm{r}=0.80)$ levels. CONCLUSIONS Catecholamine and PST levels were found elevaed in GDM. These counterregulatory hormones may play a role in the insulin resistance syndrome of GDM
FASTING TRIGLYCERIDE LEVEL MODULATES INSULIN RESPONSE IN GESTATIONAL DIABETES

F.J. Ampudia-Blasco, J.F. Ascaso, A. Rodrigo, R. Carmena. Diabetes Reference Unit, Endocrinology Dep., Clinic University Hospital Valencia, Spain.

Basal hypertriglyceridaemia, which is frequently found in normal and pathologic pregnancies, has been associated with insulin resistance in other conditions. The aim of this study was the evaluation of insulin response in gestational diabetes in relation to fasting triglyceride (TG) level. A cohort of 89 patients who had an oral glucose tolerance test (OGTT, $100 \mathrm{~g}$ glucose) and a complete lipid profile in the $26^{\text {th }}$ to $36^{\text {th }}$ week of gestation were selected. Patients were classified according to the level of TG in 2 groups: group 1 with $\mathrm{TG}<200 \mathrm{mg} / \mathrm{dl}(\mathrm{n}=44$, mean $\pm \mathrm{SD}, 159 \pm 27$ $\mathrm{mg} / \mathrm{dl})$ and group 2 with $\mathrm{TG} \geq 200 \mathrm{mg} / \mathrm{dl}(\mathrm{n}=45,292 \pm 101 \mathrm{mg} / \mathrm{dl})$. Insulin secretion was evaluated by means of the insulin and C-peptide profiles during OGTT. Both groups (group 1 vs. 2) were comparable in age (31.9 \pm 4.2 vs. $32.6 \pm 4.9$ y), BM before pregnancy ( $25.7 \pm 4.6$ vs. $\left.25.1 \pm 3.5 \mathrm{~kg} / \mathrm{m}^{2}\right)$, weight gain $(8.3 \pm 4.9$ vs. $8.6 \pm 3.7$ $\mathrm{kg}$ ) previous pregnancies $(1.22 \pm 1.55 \mathrm{vs}, 1.24 \pm 1.49)$ and mean $\mathrm{HbA}_{1 \mathrm{c}}(4.5 \pm 0.3 \mathrm{vs}$ $4.4 \pm 0.4 \%$ ). Samples were obtained at $31.9 \pm 1.9$ (group 1) and $32.7 \pm 2.2$ weeks (group 2, NS). As well as TG, total cholesterol levels were also increased in group 2 (group 1 vs. $2,216 \pm 35$ vs. $255 \pm 44 \mathrm{mg} / \mathrm{dl}, \mathrm{p}<0.05$ ), but HDL ( $69 \pm 16$ vs. $64 \pm 13$ $\mathrm{mg} / \mathrm{dl})$ and LDL cholesterol fractions ( $124 \pm 30 \mathrm{vs} .135 \pm 42 \mathrm{mg} / \mathrm{dl})$ were similar. The basal-, peak- and area under the curve (AUC) values from insulin and C-peptide profiles were:

\begin{tabular}{|c|c|c|c|}
\hline & $T G<200 \mathrm{mg} / \mathrm{dl}$ & TG $\geq 200 \mathrm{mg} / \mathrm{dl}$ & p value \\
\hline basal insulin $(\mu \mathrm{g} / \mathrm{ml})$ & $16.0 \pm 6.5$ & $17.5 \pm 9.2$ & 0.4 \\
\hline ulin $(\mu \mathrm{g} / \mathrm{ml})$ & $191.4 \pm 115.4$ & $258.0 \pm 147.1$ & 0.02 \\
\hline AUC-insulin $\left(\mu \mathrm{g} / \mathrm{ml}{ }^{\star} 180 \mathrm{~min}\right)$ & $22189 \pm 12$ & $29807 \pm 16914$ & 0.02 \\
\hline e (ng/ml) & $2.15 \pm 1.26$ & $2.69 \pm 1.42$ & 0.08 \\
\hline $\mathrm{de}(\mathrm{ng} / \mathrm{ml})$ & $13.83 \pm 5.39$ & $20.01 \pm 13.57$ & 0.01 \\
\hline AUC C-peptide (ng/ml * $180 \mathrm{~min}$ ) & $1758 \pm 635$ & $2420 \pm 1236$ & 0.004 \\
\hline
\end{tabular}

In conclusion, fasting hypertriglyceridaemia was related with an increased insulin response in pregnant women with gestational diabetes. Hyperinsulinism with increased TG may indicate indirectly a greater level of peripheral insulin resistance in these subgroup of patients with gestational diabetes.

\section{4}

GESTATIONAL GLUCOSE INTOLERANCE AND MATERNAL SOCIAL STRESS DEPRESS SEXUAL BEHAVIOUR IN MALE OFFSPRING G.Brizgalova, L.Sergienko, V.Poltorack, T.Bondarenko and N.Karpenko. Ukranian Research Institute of Endocrine Diseases Pharmacotherapy, Kharkov, Ukraine

The aim of the study was to evaluate the combined influence of gestational glucose intolerance (GGl) and social stress (SS) during early pregnancy on sexual behaviour in male offspring. In Wistar rats GGI was induced by a single dose of streptozotocin $(45 \mathrm{mg} / \mathrm{kg}$ i.p.) on day 2 of pregnancy, then rats were exposed SS by change of rat's association until the 9th day of pregnancy. In 3-month-old offspring the sexual behaviour was examined. Frequency and latency of mountings, intromissions, ejaculations and postejaculation refractory period (PRP) were recorded during $15 \mathrm{~min}$ test. Behavioural studies have shown that $\mathrm{GGI}$ increased latency of mountings and intromissions by 2.3 and 3.0 fold respectively ( $p<0.001$ vs controls), suggesting a low activity of sexual motivation in offspring. Only tendency to decrease of ejaculation frequency was noted. SS depressed copulative phase in offspring decreasing the ejaculation frequency ( $p<0.05$ vs controls) and lengthened PRP (389 $2+15.4 \mathrm{~s}$ vs $3042+28.3 \mathrm{~s}$ in controls, $p<0.05$ ). GGl combined with SS increased the latency of mountings, intromissions and ejaculations by $2.3,2.6$ and 1.4 fold respectively $(p<0.001$ vs controls). PRP was significantly lengthened $(420.6 \pm 20.1 \mathrm{~s}$ vs $304.2+28.2 \mathrm{~s}$ in controls, $\mathrm{p}<0.01$ ). As a result only $50 \%$ offspring had the 2nd ejaculation within test. We conclude that both GGI and SS disturb sexual behaviour suppressing different phases of sexual cycle. GGI and SS supplement the effects of each other when combined, weakening both sexual motivation and copulative activity in male offspring. 
PS 50

\section{Exercise}

1015

STIMULATION OF RAT MUSCLE GLUCOSE UPTAKE BY 5AMINOIMIDAZOLE-4-CARBOXAMIDE RIBOSIDE.

R. Bergeron, R.R. Russell III, L.H. Young, J.M. Ren, A. Lee, and G.I. Shulman Yale University School of Medicine, Depatment of Internal Medicine, $333 \mathrm{Ceda}$ Stret. P.OBox 208020, New Haven, CT 06520-8020, U.S.A

AMPectivated protein kinæse (AMPK) is stimulated by execise and can incease glucose uptake in the perfised rat musde preparation The objective of the present study was to determine the role of AMPK in skeletal musde glucose transport in vivo. Awake Sprague-Dawley rats were studied under basa fasting insulin concentrations (somatostatin $1.0 \mu \mathrm{g}^{\circ} \mathrm{kg}^{-1} \bullet \mathrm{min}^{-1}$; and basal insulin repbocment: 0.1 $\mathrm{mU} \bullet \mathrm{kg}^{-1} \cdot \mathrm{min}^{-1}$ ). Animals were either infised with sal ine or 5 -aminoimidazole-4 carboxamide riboside (AICAR: $40 \mathrm{mg} \mathrm{kg}^{-1}$ bolus and $7.5 \mathrm{mg} \bullet \mathrm{kg}^{-1} \cdot \mathrm{min}^{-1}$ constant infision), an analogue of AMP known to activate AMPK Gluose tramsport rates were calaulated from $2-[1,2-\mathrm{H}]$-deoxygluoose-6-phosphate uptake. To courteract the hypoglyœmic effoct of AICAR, exogenous gluœse was infised at a rate of $49.7 \pm 3.2$ $\mu \mathrm{mol} \bullet \mathrm{kg}^{-1} \cdot \mathrm{min}^{-1}$ (meantsem) which mairtained a plasma glucse concentration of 5 $\mathrm{mM}$. The basal plasma lactate concentration progressively increased from $0.64 \pm 0.03$ to $6.2240 .35 \mathrm{mM}(P<0.001)$ folbwing $75 \mathrm{~min}$ of AICAR infision whereas it remained stable in the saline group. The glucose-6-phosphate conœentrations were significanty elcuated $(P<0.005)$ in both lateral $(L G)$ and medial gastrocnemis $(M G)$ of AICAR infused animals compared to animals receiving saline (respectivdy; $1.03 \pm 0.23$ vs $0.15 \pm 0.02$; and $1.59 \pm 0.35$ vs $0.32 \pm 0.08 \mu \mathrm{mol}^{\circ} \mathrm{g}^{-1}$ ). AICAR induced more than two fold inceases in musde gluosse uptake in the soleus and both the LG and MG compared to saline infised animals (respectively $18.5 \pm 2.4$ vs $7.8 \pm 2.3$ : $\mathrm{P}=0.006 ; 10.2+1.2$ vs $4.8 \pm 0.5: \mathrm{P}=0.0006$; and $12.9 \pm 2.6$ vs $5.6 \pm 0.9: \mathrm{P}=0.03$ $\left.\mu \mathrm{mol} \bullet \mathrm{kg}^{-1} \bullet \mathrm{min}^{-1}\right)$. CONCLUSION: These data demonstrate that the activation of the AMPK pathway increases skeletal muscle glucose uptake and suggest that this pathway may be important in the execise-induced incease in glucose transport

\section{6}

EFFECTS OF EXERCISE ON METABOLIC AND MITOGENIC SIGNALING PATHWAYS IN HUMAN SKELETAL MUSCLE.

M. Björnholm ${ }^{\S}$, U. Widegren ${ }^{*}$ X.J. Jiang ${ }^{\S}$, A. Krook ${ }^{\S}, M$. Tally ${ }^{\#}$, R.A. Roth ${ }^{\dagger}$ J. Henriksson", H. Wallberg-Henriksson ${ }^{\S}$, and J.R. Zierath". ${ }^{\S}$ Dept. of Clinical Physiology \& "Molecular Medicine, Karolinska Hospital, "Dept. of Physiology and Pharmacology, Karolinska Institute, Stockholm, Sweden and the ${ }^{\dagger}$ Dept. of Molecular Pharmacology, Stanford Univ. School of Med., Stanford, CA, USA.

The molecular signaling mechanisms by which muscle contractions leads to changes in glucose metabolism and gene expression remain largely undefined. We assessed whether exercise activates MAP kinase proteins (ERK1/2, SEK1 and p38 MAP kinase), as well as Akt and PYK2 in skeletal muscle from healthy volunteers obtained during and following one-leg cycle ergometry at $\sim 70 \% \mathrm{VO}_{2} \max$. Insulin levels decreased from $98.6 \pm 15$ to $35.9 \pm 4.2 \mathrm{pM}$ following $60 \mathrm{~min}$ of exercise. IGF-1 levels increased slightly from $245 \pm 17$ to $270 \pm 18 \mu \mathrm{g} / 1$ following $60 \mathrm{~min}$ of exercise Exercise led to a $31 \pm 8$-fold increase in ERK $1 / 2$ phosphorylation, with a maximal effect noted after $30 \mathrm{~min}$ of exercise. Upon recovery, ERK1/2 phosphorylation rapidly decreased to resting levels. Exercise increased phosphorylation of SEKI by $2.5 \pm 0.4$-fold and p38 MAP kinase by $2.2 \pm 0.7$-fold. In contrast to ERK $1 / 2$ and SEK1, p38 MAP kinase phosphorylation was increased in non-exercised muscle upon cessation of exercise. Repeated biopsy sampling had no effect on ERK1/2, SEK1 and p38 MAP kinase phosphorylation. Interestingly, phosphorylation of the transcription factor CREB was increased in non-exercised muscle upon cessation of exercise Exercise did not increase Akt kinase activity or increase tyrosine phosphorylation of PYK2. However insulin led to a 5-fold increase in Akt activity in isolated human skeletal muscle. Furthermore, hyperosmosis led to a 2.5 -fold increase in tyrosine phosphorylation of PYK2 in rat soleus muscle. In conclusion, our data do not support a role of Akt or PYK2 in exercise/contraction induced signaling in human skeleta muscle. Importantly, we show exercise has divergent effects on parallel MAP kinase pathways. The extent to which these different pathways are activated by physical exercise may influence transcriptional events in skeletal muscle.

\section{7}

SELECTIVE EFFECT OF PRIOR EXERCISE IN ENHANCING INSULIN ACTION IN DIFFERENT MUSCLES AND LIVER

S. Pye, L. Matchett, and J. Radziuk, Ottawa Civic Hospital, Ottawa, Canada

Exercise acutely increases sensitivity to insulin in some but not all studies. To examine the basis for this effect, 4 groups $(n=6$ each) of untrained Sprague Dawley rats $(200-230 \mathrm{~g})$ underwent (i) a $4 \mathrm{hr}$ swim followed by a $3 \mathrm{~h}$ recovery, SR (ii) a $4 \mathrm{~h}$ swim followed by a $3 \mathrm{hr}$ insulin infusion (29pmol/kg-min) SI, (iii) $4 \mathrm{~h}$ rest $+3 \mathrm{~h}$ "recovery" (RR) and (iv) $4 \mathrm{~h}$ rest $+3 \mathrm{~h}$ insulin infusion (RI). Glucose was clamped at basal during insulin infusions and $\left[6-{ }^{3} \mathrm{H}\right] \mathrm{glucose}$ was infused throughout the study. Plasma levels of tracer and glucose were measured during the $3 \mathrm{~h}$ recovery or insulin infusion period. After the final blood sample, the rats were sacrificed and the soleus (Sol), white (WG) and red (RG) gastrocnemii, and liver (L) were freeze-clamped. Total and labelled glycogen were determined in all samples. New synthesis (by direct pathways) was calculated from the $\left[6-{ }^{3} \mathrm{H}\right] \mathrm{glucose}$ content of the glycogen divided by the mean specific activity of glucose during the recovery ( \pm insulin) period. Total glycogen rose or remained unchanged (relative to RR) in all muscles except in SR where it decreased. New glycogen synthesis was minimal in RR $(0.9 \pm 0.1,0.4 \pm 0.1,0.1 \pm 0.1 \mathrm{mg} / \mathrm{kg}$ and $0.15 \pm 0.05$ in Sol, RG, WG and L) and changed to $0.9 \pm 0.3,1.7 \pm 0.5,1.0 \pm 0.3$ and $0.4 \pm 0.1 \mathrm{mg} / \mathrm{kg}$ for Sol, $R G$, WG and $L$ in $S R$ and to $3.5 \pm 0.5,3.8 \pm 0.6,0.9 \pm 0.2$ and $0.8 \pm 0.3 \mathrm{mg} / \mathrm{kg}$ in $\mathrm{RI}$. When insulin infusion followed exercise (SI), new glycogen synthesis was additive (SR+RI), $4.7 \pm 0.5 \mathrm{mg} / \mathrm{kg}$ for Sol and supra-additive for the remaining muscle groups and liver: $11.9 \pm 1.5,5.3 \pm 1.3$ and $2.8 \pm 0.7 \mathrm{mg} / \mathrm{kg}$ for $R G$, WG and $L(p<0.05)$. These data indicate that new glycogen synthesis is enhanced synergistically by insulin and prior exercise but only in those muscle groups (RG, WG) where exercise alone increases glycogen synthesis and in liver (SI). Thus the differential effect of exercise on insulin action in various tissues could decrease the systemic detectability of the effect.

\section{8}

EXERCISE-INDUCED INCREASE IN PLASMA LACTATE DOES NOT SUBSTITUTE FOR BRAIN GLUCOSE DURING HYPOGLYCEMIA.

P. Bottini, C. Lalli, A. Baccarelli, S. Pampanelli, M. Lepore, M. Ciofetta, P. Del Sindaco, F. Santeusanio and G.B. Bolli*, DIMISEM Perugia, Italy

To establish if increased plasma lactate (Lact) during exercise (Ex) substitutes for glucose as brain fuel during hypoglycemia (Hypo), 7 nondiabetics (N) and 6 IDDM patients (D) were studied. Adrenaline (A), noradrenaline (NA), autonomic (AS) and neuroglycopenic (NS) symptoms were measured during Ex (Study 1, S1), or Hypo (Study 2, S2), or Ex+Hypo (Study $3, \mathrm{~S} 3$ ). Steady state Ex (cycloergometer, $80 \mathrm{~min}$ at 110 watts) was performed in $\mathrm{S} 1$ and $\mathrm{S} 3$, whereas in $\mathrm{S} 2$ subjects remained seated. Insulin $(2 \mathrm{mU} / \mathrm{Kg} / \mathrm{min})+$ variable glucose were infused i.v. from 20 to $80 \mathrm{~min}$ to induce Hypo in S2 and S3 (plasma glucose $2.8 \mathrm{mmol} / 1$ between $60-80 \mathrm{~min}$ ) or to maintain euglycemia (plasma glucose $5 \mathrm{mmol} / \mathrm{l}$ ) in $\mathrm{S} 1$.

\begin{tabular}{|c|c|c|c|c|c|c|c|}
\hline & & \multicolumn{2}{|c|}{ Ex (S1) } & \multicolumn{2}{|c|}{ Hypo (S2) } & \multicolumn{2}{|c|}{ Ex+Hypo (S3) } \\
\hline & $(\min )$ & 0 & $60-80$ & 0 & $60-80$ & () & $60-80)$ \\
\hline $\bar{N}$ & Lact (mM) & $1.3 \pm 0.1$ & $2.9 \pm 0.1^{*}$ & $1.2 \pm 0.1$ & $1.5 \pm 0.1$ & $1.1 \pm 0.1$ & $3.3 \pm 0.2 *$ \\
\hline & $A(n M)$ & $0.3 \pm 0.1$ & $0.6 \pm 0.1^{*}$ & $0.2 \pm 0.1$ & $2.6 \pm 0.4^{*}$ & $0.3 \pm 0.1$ & $6.9 \pm 0.6 *$ \\
\hline & NA (nM) & $1.7 \pm 0.2$ & $5.5 \pm 0.5^{*}$ & $1.7 \pm 0.2$ & $2.2 \pm 0.2^{*}$ & $2.3 \pm 0.2$ & $7.3 \pm 0.4^{*}$ \\
\hline & AS score & $1.6 \pm 0.8$ & $5.2 \pm 1 * a$ & $1 \pm 0.3$ & $5.5 \pm 1$ * & $1.5 \pm 0.4$ & $8 \pm 0.7^{*}$ \\
\hline & NS score & $0.2 \pm 0.1$ & $3 \pm 0.1^{*}$ & $0.4 \pm 0.2$ & $4.9 \pm 1.2^{*}$ & $0.6 \pm 0.2$ & $7 \pm 0.9^{*}$ \\
\hline D & Lact (mM) & $1.7 \pm 0.3$ & $3.8 \pm 0.5^{*}$ & $1.1 \pm 0.1$ & $1.5 \pm 0.2^{*}$ & $1.5 \pm 0.4$ & $3.6 \pm 0.3^{*}$ \\
\hline & $\bar{A}(\mathrm{nM})$ & $0.3 \pm 0.1$ & $0.8 \pm 0.1^{*}$ & $0.3 \pm 0.1$ & $1.9 \pm 0.4^{*} \dagger$ & $0.3 \pm 0.1$ & $3.3 \pm 0.7^{*} \dagger$ \\
\hline & $\mathrm{NA}(\mathrm{nM})$ & $1.9 \pm 0.3$ & $5.2 \pm 0.3^{*}$ & $1.7 \pm 0.2$ & $2.2 \pm 0.1^{*}$ & $2 \pm 0.3$ & $6.5 \pm 0.5^{*}$ \\
\hline & AS score & $1.5 \pm 0.2$ & $6.9 \pm 0.4^{*}$ & $0.3 \pm 0.1$ & $5.6 \pm 2.3^{*}$ & $0.8 \pm(0.1$ & $7.7 \pm 0.9^{*}$ \\
\hline & NS score & $1.8 \pm 0.1$ & $5.4 \pm(1) .4^{*}$ & $0.4 \pm 0.1$ & $6 \pm 1.5^{*}$ & $0.5 \pm 0.1$ & $7 \pm 1.3^{* *}$ \\
\hline
\end{tabular}

${ }^{*} \mathrm{p}<0.05$ vs $0 ;$ tp $<0.05$ vs $\mathrm{N} ; \mathbf{9 p}<0.05$ vs $\mathrm{S} 3$

Thus, Ex-induced increase in Lact does not substitute for glucose as brain fuel during hypo. In IDDM, the physiological Ex+Hypo induced potentiation of A and symptoms observed in non-diabetics, is reduced independently of plasma lactate. 
DAILY PROFILES OF INSULIN DOSE EQUIVALENTS OF EXERCISE (IDE) IN DIABETIC CHILDREN ON BASAL-BOLUS REGIMES

U. Fischer, C. Ramirez, G. Weigt, H. Boltz, and E. Salzsieder; Kinder Reha-Zentrum Putbus, Inselklinik Heringsdorf and Gerhardt Katsch Institute of Diabetes Karlsburg. Germany

Hypoglycaemia in otherwise healthy type-1 diabetic patients is frequently caused by difficulties in adapting s.c. insulin doses (scID) to exercise activities. In hypoglycaemia prevention of adult patients, we have previously validated a nomogram relating IDE of a work load to the steady state heart rate (SSHR). This study was designed to relate under well-controlled conditions the $24 \mathrm{~h} \mathrm{HR}$ profile to IDE of varying daily activities. 30 children of either sex (9-16 years old diabetes duration 1-11 years, $\mathrm{HbA}_{1 \mathrm{c}} 5.9-11.2 \%$ ), intensively treated according to the basal (NPH, Semilente ${ }^{\mathrm{R}}$ ) bolus (regular insulin) principle, were studied during a summer camp: (a) bicycle ergometer tests were performed to identify the individual SSHR vs. work load (25-125 W) function after breakfast, lunch or supper; (b) HR (Polar Vantage) and physical activities were monitored over $24 \mathrm{~h}$ and were related to blood glucose, scID, and meals. The 8-grade physical activity score comprised sleeping, sedentary activities/meals, house keeping/shopping, walking, hiking/swimming, outdoor sports, biking/ jogging/ dancing, and romping/football; (c) Using the results of test (a), HR profiles were modelled in terms of work load $(W)$ at 15 min intervals, which - using the mentioned nomogram - were transformed into IDE profiles. Results: Above grade-2 activities, and except transient states such as rising or hygiene, there were clear-cut individual IDE profiles which could be related to blood glucose profiles under consideration of scID. On the average, the daily sum of IDE amounted to $8-20 \%$ of applied doses of regular insulin. The results did not depend neither on daytime nor on $\mathrm{HbA}_{1 .}$. But they were significantly related to the individual training conditions as characterised by means of work-related increase in SSHR and lactate concentration during test (a) in that IDE varying between 0.004 and $0.1 \mathrm{IU}$ of regular insulin per $\mathrm{W} \cdot \mathrm{h}$, was higher in well-trained patients $(\mathrm{p}<0.01)$. - These findings facilitate individwal recommendations on the reduction of SCID before planned exercise activities.
NO EFFECT OF LONG-TERM PHYSICAL EXERCISE ON THE GLYCEMIC CONTROL IN TYPE 1 DIABETES PATIENTS; A CROSS-SECTIONAL STUDY

P.C. Ligtenberg", M.C.A. Blans", I. van der Tweef, J.B.L. Hoekstra , and D.W. Erkelens". "Dept. of Internal Medicine, University Hospital Utrecht, P.O.Box 85500,3508 GA Utrecht; "Centre for Biostatistics, Utrecht University; 'Dept. of Internal Medicine, Diakonessen Hospital, Utrecht, The Netherlands

Physical activity was shown to be inversily related to mortality risk in type 1 diabetes patients. It is not clear whether the reduced death rate was due to an ameliorated glycemic control or occurred from reduction of other risk factors. In the present study, the relation between levels of physical activity and glycemic control was evaluated in a cross-sectional observation. Medical and demographic data besides blood samples for glycated hemoglobin and lipid profile were collected in consecutive type 1 diabetes patients between 18 and 45 years of age without late complications. A self-reported questionnaire was used to determine the degree of physical activity. Additonal information on insulin therapy, and the self measurement of blood glucose levels (SMBG) was obtained. Correlation coefficients and analysis of variance were used for statistical analyses. Three hundred and sixty three type 1 diabetes patients were screened. Data of 221 patients were analyzed. No correlation was observed between the different levels of physical activity and glycemic contro or lipid profile. Females showed a significantly higher total physical activity index than males $(p=0.004)$, mostly due to the leisure-time activity index. More active patients used a lower amount of insulin $(r=-0.20, p=0.002)$ than less active patients. SMBG at least once a day was associated with a significantly lower glyHb. HDL- cholesterol was inversily correlated with the total number units of insulin per day (males: $r=-0.27, p=0.003$, females: $r=$ $-0.21, p=0.04$ ).

Glycemic control and lipid profile were not found to be associated with longterm physical exercise in type 1 diabetes patients. However, a higher physical activity level was related to a lower insulin dosage, which may be of benefit. Exercise apparently did not negatively affect long-term glycemic control, so it can be safely advised to people with type 1 diabetes on the condition that metabolic balance is maintained.

\section{1}

THE EFFECT OF EXERCISE TRAINING ON MUSCLE LIPID METABOLISM IN A RAT MODEL OF TYPE 2 DIABETES

M.Straczkowski, I.Kowalska, I.Kinalska and J.Górski* Department of Endocrinology and "Department of Physiology, Medical School, Białystok, Poland

The aim of the present study was to estimate the influence of exercise training on glucose tolerance and muscle lipid metabolism in a rat model of type 2 diabetes. Four groups of rats were examined: I - control group vehicle at 2 days of age and standard diet; II - low dose of streptozotocin (STZ - $45 \mathrm{mg} / \mathrm{kg}$ ) at 2 days of age and standard diet; III - vehicle and high fat diet (HFD - 59\% calories as fat) from 8 to 11 weeks of age; IV - STZ + HFD - model of type 2 diabetes. An intravenous glucose tolerance test with the determination of basal and post load insulin was performed before the final experiments. In all groups the glycogen and triglycerides (TG) contents were measured in white and red part of the gastrocnemius muscle and in the liver in basal conditions and after 4 weeks of exercise training on a treadmill. HFD rats developed hyperinsulinemia and STZ/HFD hyperglycemia. Both groups which received STZ showed impaired first phase insulin secretion. Exercise training did not improve glucose tolerance in the STZ/HFD and insulin response in the HFD group. Muscle and liver TG content was markedly higher in the studied groups in comparison to control. We observed a significant positive correlation between the red gastrocnemius TG content and fasting insulin and insulin/glucose index in the control and HFD group. Exercise training increased TG content in the control group in all examined tissues, did not affect tissue TG in the HFD group and decreased liver TG in the STZ and STZ/HFD groups. After exercise training there were still markedly higher white and red gastrocnemius TG contents in HFD and STZ/HFD groups vs control. We conclude that exercise training did not completely reverse metabolic disturbances in that model of type 2 diabetes.

\section{2}

EFFECTS OF 2-WEEK PROGRAM OF PHYSICAL ACTIVITY ON INSULIN RESISTANCE IN OBESE INSIULIN-INDEPENDENT DIABETICS M. Čizmić, R. Dragojević and S. Živanjé. Clinic of Endocrinology and Institute for Medical Researches MMA, Belgrade, Yugoslavia

This paper discusses influence of the programmed physical activity (PA) on the insulin resistance $(\mathrm{M} / \mathrm{I})$ and biochemical indicators of atherogenesis in obese NIDDM. Twelve subjects $42-52$ years old, with BMI $26-30 \mathrm{~kg} / \mathrm{m}^{2}$ and the history of NIDDM $>1$ year, were divided at rancom into 2 groups. During 2 weeks, 5 subjects (group C) were on $1600 \mathrm{kcal}$ diet. In the group $E(n=7)$, with the same diet, there was applied PA program (35-min sessions of walking on treadmill, intensity of $60-65 \%\left(\mathrm{VO}_{2}\right)$ max, frequency $\left.5 / w k\right)$. At the beginning and at the end of the 2-week period, the following was measured in both groups: $\left(\mathrm{VO}_{2}\right)$ max from progressive test of loading on ergocycle, $\mathrm{M} / \mathrm{I}$ by the method of hyperinsulin euglycemic clamp and biochemical parameters. In the group $E$, together with increase of $\left(\mathrm{VO}_{2}\right) \max \{31.10 \pm 5.45$ vs $26.79 \pm 4.48$ $\mathrm{ml} / \mathrm{kg} / \mathrm{min} ; \mathrm{p}<0.001)$, there was achieved a significant decrease of: insulin resistance $(M / 1=2.34 \pm 0.7$ vs $1.08 \pm 0.46 \mathrm{mg} / \mathrm{kg} / \mathrm{min} / \mathrm{mU} ; 0<0.001$ ). PAl-1 activity $(3.40 \pm 0.72$ vs $5.09 \pm 1.74 \mathrm{U} / \mathrm{ml} ; \mathrm{p}<0.05)$, coagulation $X$-factor, TG, $L D L, A p O B$, glycemia on an empty stomach and HbA1C ( $p<0.05)$. No differ ences were found in $\mathrm{BMl}\left(28.10 \pm 1.65 \mathrm{vs} 28.68 \pm 1.65 \mathrm{~kg} / \mathrm{m}^{2}\right)$, VII and VIII faCtors of coagulation, total and $\mathrm{HDL}$ cholesterol and ApoA1. In the group $\mathrm{C}$, no differences were found in $\left(\mathrm{VO}_{2}\right) \max (26.65 \pm 5.58$ vs $25.15 \pm 4.55$ $\mathrm{ml} / \mathrm{kg} / \mathrm{min}), \mathrm{BMl} 27.11 \pm 2.73$ vs $\left.27.16 \pm 3.01 \mathrm{~kg} / \mathrm{m}^{2}\right), \mathrm{M} / \mathrm{l}(1.03 \pm 0.64$ vs $0.93 \pm 0.25 \mathrm{mg} / \mathrm{kg} / \mathrm{min} / \mathrm{mU}$ ), or in other variables. The research has shown that the increase of $\left(\mathrm{VO}_{2}\right)$ max achieved in 2-week program, has effects on decrease of $\mathrm{M} / \mathrm{I}$ and suppression of atherogenesis in obese N:DDM. 
MECHANISM OF EXERCISED INDUCED HYPOGLYCEMIA DURING SULFONYLUREA TREATMENT IN TYPE 2 DIABETIC PATIENTS

Jens Jørn Larsen, Flemming Dela, Sten Madsbad and Henrik Galbo. The Copenhagen Muscle Research Centre, National University Hospital, and Department of Medical Physiology, University of Copenhagen, Denmark

The primary rationale for sulfonylurea treatment in type 2 diabetic patients is related to the major pharmacological effect of sulfonylureas to stimulate insulin secretion and thereby lower plasma glucose concentrations. Acute exercise of moderate intensity also decreases plasma glucose concentrations in type 2 diabetic patients and the risk might exist that the glucose lowering effect of both exercise and sulfonylurea in the fasting patient could increase the probability of hypoglycaemic incidents. We therefore studied 8 postabsorptive type 2 diabetic patients after oral administration of sulfonylurea ( $7 \mathrm{mg}$ Daonil, glibenclamide) (day S); during $60 \mathrm{~min}$ of ergometer bicycle exercise at $55 \pm 3 \%$ of $\mathrm{VO}_{2}-\max$ (day $\mathrm{E}$ ) and during a combination of these conditions (day $\mathrm{S}+\mathrm{E}$ ). During exercise on both day $\mathrm{E}$ and day $\mathrm{S}+\mathrm{E}$ a comparable rate of decrease in insulin concentration was observed throughout the exercise period $(-0.22 \pm 0.10 \mathrm{pmol} / \mathrm{min}$ versus $-0.28 \pm 0.052 \mathrm{pmol} / \mathrm{min}$, day $E$ and day $S+E(p>0.05))$. However, throughout the exercise period absolute concentrations of insulin were lower on day $E$ compared to day $S+E(47.38 \pm 5.45$ and $34.5 \pm 4.71, \mathrm{pmol} / \mathrm{l}(\mathrm{p}<0.05)$ due to oral administration of sulfonylurea during protocol S+E. Glucose concentrations were comparable between all three days of investigation just before start of exercise. However, the decreases of plasma glucose concentrations during exercise was on day $\mathrm{S}+\mathrm{E}$ higher when compared to both day $\mathrm{S}$ and day $E(-0.0348 \pm 0.0093$ versus $-0.0215 \pm 0.0045$ and $-0.0160 \pm 0.0022$ $\mathrm{mmol} / / \mathrm{min}, \mathrm{S}+\mathrm{E}$ versus $\mathrm{E}$ and $\mathrm{S}$, respectively $(\mathrm{p}<0.05)$. Consequently, glucose reached a lower nadir at the end of exercise than during both protocol $\mathrm{S}$ and $\mathrm{E}$ (6.72 $\pm 1.12 \mathrm{mmol} / 1$ versus $8.06 \pm 0.86 \mathrm{mmol} / 1$ and $7.63 \pm 0.96 \mathrm{mmol} /$, respectively, $p<0.05$ ). Hepatic glucose output (HGO) was decreased on day S+E when compared to day $\mathrm{E}$ $(2.3 \pm 0.3$ versus $3.1 \pm 0.3 \mathrm{mg} / \mathrm{min} / \mathrm{kg}$ ). In conclusion, the hypoglycemic action of orally administered glibenclamide is enhanced by exercise in type 2 diabetic patients due to increased absolute concentrations of insulin resulting in an enhanced drop in plasma glucose. We therefore suggest that type 2 diabetic patients should be advised to reduce the dose of this drug before endurance exercise.

\section{PS 51}

\section{Nutrition and Diet Therapy}

\section{4}

\section{GLYCAEMIC EQUIVALENTS: A NEW BASIS FOR FOOD EXCHANGE IN DIABETES}

J.C. Brand Miller, S. Colagiuri, V. Swan, M. Colagiuri and P. Petocz

Human Nutrition Unit, Department of Biochemistry, University of Sydney 2006 and Prince of Wales Hospital, Randwick, NSW, Australia

The current exchange system based on 10 or $15 \mathrm{~g}$ carbohydrate portions, assumes that the same amount of carbohydrate will produce a similar glycemic response irrespective of the food. A scientifically based exchange system must consider both the quantity and type (glycaemic index) of carbohydrate. Using the published GI and carbohydrate contents of foods, we calculated the amount of any particular food that would be the theoretical 'glycaemic equivalent' (GE) of one slice of white bread. One GE was equivalent to $16 \mathrm{~g}$ low amylose rice (raw weight), 33 $\mathrm{g}$ spaghetti (raw weight), $15 \mathrm{~g}$ comflakes (with $100 \mathrm{~mL}$ milk), $155 \mathrm{~g}$ fruit yogurt, $85 \mathrm{~g}$ for banana, $220 \mathrm{~mL}$ for orange juice, $130 \mathrm{~g}$ for baked beans and $14 \mathrm{~g}$ for jelly beans. Our aim was to confirm that the theoretical GEs of 20 common foods were equivalent in practice. In addition, the 'dose-response' effect of $1,2,3,4$ or 6 GEs in one meal was also determined in a sub-set of foods. Groups of healthy $(\mathrm{n}=10)$ and NIDDM ( $n=8)$ subjects were studied after an overnight fast. As predicted, the observed mean \pm SEM areas under the plasma glucose curve (AUC) were similar ( $p>0.05$ ), differing by less than $\pm 20 \%$. Insulin responses were more variable with significant differences among the foods $(\mathrm{P}<0.05)$. The dose-response relationship between number of GEs eaten in one meal and the AUC for glucose and insulin was highly significant $(p=0.000)$. Thus the GE approach accurately predicts the same degree of postprandial glycaemia although not necessarily equivalent insulin demand. The end product of these studies will be a validated exchange list of quantities of foods which are equivalent in their glycaemic impact that may ultimately lead to improvements in long term glycaemic control.

\section{5}

MECHANISMS OF THE HYPOGLYCEMIC EFFECT OF WATER SOLUBLE FIBER IN NON INSULIN DEPENDENT DIABETIC PATIENTS

R. Giacco, G.Clemente, M. Mancini*, A. D’Avanzo*, G.Mansueto ${ }^{\circ}$, F.Brighenti^, G. Lasorella, AM. Rivieccio ${ }^{\circ}$, A.A. Rivellese ${ }^{\circ}$ and G. Riccardi ${ }^{\circ}$. I.S.A. CNR, Avellino; ${ }^{\circ}$ Dep. of Clinical and Experimental Medicine, - "Federico II" University, Naples; *S.G.Moscati Hospital -Avellino; ^DISTAM, Milan University, Italy.

The hypoglycemic effect of water soluble fiber has been attributed to: 1) viscosity; 2) colonic fermentation; 3 ) physical structure of food. To evaluate the role of each of these mechanisms, 10 NID diabetic patients with a mean age of $54 \pm 7$ years (M $\pm S D$ ), BMI $26 \pm 4 \mathrm{~kg} / \mathrm{m}^{2}$, diabetes duration $5 \pm 4$ years and in therapy with diet or diet plus glibenclamide, participated in the study. The patients consumed, in random order and at 1 week interval, 4 isoenergetic diets (CHO 55\%, fat 30\%, protein 15\%) different only in water-soluble fiber content: a) control diet (fiber $12 \mathrm{~g}$ ); b) control diet $+15 \mathrm{~g}$ of guar (fiber $27 \mathrm{~g}$ ), which is viscous; c) control diet $+15 \mathrm{~g}$ of hydrolyzed guar (fiber $27 \mathrm{~g}$ ), which is not viscous and induces colonic fermentation and d) naturally fiberrich diet (fiber $28 \mathrm{~g}$ ), which is rich in viscous fiber, ferments and reduces the accessibility of starch to amylolitic enzymes. For each diet we measured for 24-hour plasma glucose, insulin, triglyceride, gastric emptying time and colonic fermentation (Breath $\mathrm{H}_{2}$ ). The guar rich diet compared to the control diet did not increase colonic fermentation but significantly delayed gastric emptying ( $15.8 \pm 6.6$ vs $29.5 \pm 11.3 \mathrm{~m} 1 / \mathrm{h}$; $\mathrm{p}<0.002$ ); conversely the hydrolyzed guar diet compared to the control diet did not delay gastric emptying but increased significantly Breath $\mathrm{H}_{2}(+52 \% ; \mathrm{p}<0.005)$. The naturally fiber-rich diet delayed gastric emptying $(14.6 \pm 7.8$ vs $29.5 \pm 11.3 \mathrm{ml} / \mathrm{h}$; $\mathrm{p}<0.05)$ and increased breath $\mathrm{H}_{2}$ by about $51 \%(\mathrm{p}<0.005)$. Of the three test diets only the naturally fiber-rich one decreased postprandial plasma glucose $(-63 \%, p<0.03)$, insulin $(-31 \% ; \mathrm{p}<0.01)$ and triglyceride incremental areas $(-66 \% ; \mathrm{p}<0.04)$ in comparison with the control diet. In conclusion: 1) only the diet made up of naturally fiber-rich foods improves plasma glucose and lipid metabolism; 2) neither delayed gastric emptying nor colonic fermentation represent the main hypoglycemic mechanisms of water soluble fiber; conversely physical structure of food might play, at least in acute conditions, a more important role. 
Influence of a B-glucan enriched bedtime snack on nocturnal blood glucose levels in diabetic children

Schober E, Rami B, Zidek Th.

Department of Pediatrics, University of Vienna, Austria

Nocturnal hypoglycaemia is a frequent problem for diabetic children and an important factor limiting attempts to achieve normoglycaemia. To reduce or avoid nocturnal hypoglycaemia in children with diabetes in many countries a bedtime snack is included in the regular dietary prescriptions.

The aim of our study was to compare the glycaemic effect of a bedtime snack $B$ glucan enriched (Nestle) with the glycaemic effect of a conventional equicaloric bedtime snack in 38 normally active diabetic children during 12 nights. During the study period 1823 BG values could be evaluated. $11.1 \%$ of all measurements $(\mathrm{n}=203)$ were below $3.3 \mathrm{mmol} / \mathrm{l}, 7.0 \%(\mathrm{n}=128)$ below 2.8 $\mathrm{mmol} / \mathrm{l}$ and $3.1 \%(\mathrm{n}=56)$ below $2.2 \mathrm{mmol} / \mathrm{l}$. No severe hypoglycaemia occurred during the observation period. There was no significant difference in the frequency of hypoglycaemic values between boys and girls and no difference in the prevalence of low BG levels between children with a HbAlc below and above $8 \%$. For the differences in BG levels between 18.00 and 22.00 and 22.00 and 02.00 a significant influence of the type of bedtime snack could be observed. $(\mathrm{p}<0.05)$. During the nights with conventional snacks blood glucose increased from $7.8 \pm 4.4 \mathrm{mmol} / /$ to $9.4 \pm 5.3 \mathrm{mmol} / \mathrm{l}$ and subsequently fell to 6.0 $\pm 3.6 \mathrm{mmol} / \mathrm{l}$ while in the nights with B-glucan enriched snacks blood glucose increase was only from $8.4 \pm 4.9 \mathrm{mmol} / \mathrm{l}$ to $8.6 \pm 5.2 \mathrm{mmol} / \mathrm{l}$ between 18.00 and 22.00 falling to $6.4 \pm 4.2 \mathrm{mmol} / \mathrm{l}$ until 02.00 . However the mean $B G$ values and the frequency of hypoglycaemic BG values (arbitrarily defined as BG $<3.3$ mmol/l) did not differ significantly between the two types of bedtime snacks. Our investigation showed that low BG levels during the night are very common and that a fiber enriched bedtime snack reduced the postabsortive $B G$ fluctuation but did not reduce the frequency of nocturnal hypoglycaemia. Other therapeutic strategies are needed to minimize the risk of nocturnal hypoglycaemia in diabetic children.
W-3 POLYUNSATURATEd FATTY ACIDS INDUCED ALTERATIONS IN PLATELET AND THE ERYTHROCYTE METABOLISM IN NIDDM PATIENTS A.ASerhivenko, A.M.Uibanovich M.E.Kordaba, V.O.Serhivenko, I.V.Gorbachevska, T.Y.Djus and L.M.Serhiyenko. Department of Endocrinology, Lviv Medical University, Lviv, Ukraine

Dietary supplementation with fish oil, a source of highly polyunsaluraled $w-3$ fatty acids has been proposed as an antithrombotic and atherosclerotic therapy. High dictary intake of sicosapentzenoic acid (EPA) and docosahexaenoic acid (DHA) is of special interest in the possibility of influencing the metabolism of lipids and synthesis of eicosanoids. The aim of this study was to assess the long term effect of EPA and DHA on the activities of protein-kinase $C$ (PK-C), $\mathrm{Na}^{+}, \mathrm{K}$-ATPase, $\mathrm{Ca}^{2+}, \mathrm{Mg}^{2+}$ - ATPase, levels of phosplolipids and fatty acids in the membranes of erythrocytes and the levels of the ${ }^{125}\left[-6\right.$-ketoprostaglandin $F_{l \alpha}\left(6+k e t o P G F_{1 \alpha}\right)$ and ${ }^{125}$ I-thromloxane $\mathrm{B}_{2}\left(\mathrm{TXB}_{2}\right)$ in the blood plasma, activities of superoxide dismutase (SOD). gluthation peroxidase in the RBCs'. lipid peroxidation of RBCs', concentration of conjugated dienes (CD), total plasnua antioxidative capacity (TPAC) in NIDDM patients. It has been discovered that the progress of diabetic micro-and macrovessel disorders is accompanied by a decrease of the activities of PK-C. Na, $\mathrm{K}^{+}$- ATPase and $\mathrm{Ca}^{2+} . \mathrm{Mg}^{2+}$-ATPase in the RBC's menubranes, SOD, gluthation peroxidase in the RBC's and TPAC. There is a considerable increase in the TXB, level, erythrocyte lipid peroxidation. level of $\mathrm{CD}$ and a decrease in senum 6-keto-PGF $F_{1 \alpha,}$ phospholipids and potyunsaturated fatty acids in the RBCs' membranes. We have carried out an analysis of fish oil effects on the above indices in NIDDM patients. 53 patients ( $58 \pm 6$ years, $26 \mathrm{~m} / 27 \mathrm{f}$ ) were allocated into two treatment groups. The $1 \mathrm{st}$ group (11=27) was receiving capsules of fish oil every day (1,75 g EPA $1,75 \mathrm{~g} \mathrm{DHA}$ and $0,1 \% \alpha$-10copherol acetate) and the 2nd group ( $\mathrm{n}=26$ ) was receiving placeto capsules of olive oil. All patients were on the same die. After 2 months of treatment there was a decrease in TXB. level $(p<0,001)$, level of $\mathrm{CD}(\mathrm{p}<0,01)$ with simultanoous increases in EPA level, EPA/arachidonic acid ratio, activities of PK-C $\left(22,49 \pm 3,13\right.$ pmol ${ }^{32} \mathrm{P} / \mathrm{mg}$ protein per $\left.1 \mathrm{~min}, \mathrm{p}<0.001\right) . \mathrm{Na}^{+} . \mathrm{K}^{+}$-ATPase $(\mathrm{p}<0.001), \mathrm{Ca}^{2+}, \mathrm{Mg}^{2+}$-ATPase and gfuilation peroxidase $(\mathrm{p}<0,001)$ and the concentration of 6-ketoPGF ${ }_{1 \alpha}$ in the firs grom. Therefore it seems that a $3,5 \mathrm{~g}$ fish oil treatment during following 2 months result the tendency of normalizing the state of prostacyclin $\mathrm{I}_{2}$-thromboxane A. system activeness of membrane-bound enzymes. some indexes of the oxidative stress. In conclusion, EPA and DHA at muderate doses may exer antithrombic effects and may be used for prophylaxis and treatment of diabetic angiopathies.

\section{8}

\section{LONG-TERM IMPACT OF VITAMIN E ON GLYCAEMIC PATTERN AND LIPID PEROXIDATION IN DIABETIC RABBITS}

O.Ivanova, V. Poltorack, N. Gorbenko and A. Gladkih. Ukrainian Scientific Research Institute of Endocrine Diseases Pharmacotherapy. Kharkov, Ukraine

There is increasing evidence that diabetes is associated with oxidative damages resulting from disturbed equilibrium between pro- and antioxidations. The aim of the study was to determine effect of long-term supplementation with vitamin $E(E)$ on metabolic control and oxidative status in rabbits with absolute insulin insufficiency. Male chinchilla rabbits were made diabetic by i.v. injection of dithizone $(35 \mathrm{mg} / \mathrm{kg} \mathrm{b.w}$.). Control rabbits $(\mathrm{C})$ were given vehicle alone. In a week after diabetes induction animals were randomized into two group: one group was untreated to act as diabetic control (D) and the other group received $\mathrm{E}$ $(50 \mathrm{mg} / \mathrm{kg} / \mathrm{die})$ in the diet for 6 months. At the end of the study fasted rabbits were subjected to a glucose tolerance test (GTT, $2 \mathrm{~g}$ glucose $/ \mathrm{kg}$ per os). Diabetes was associated with basal hyperglycaemia, impaired glucose tolerance and reduced basal plasma insulin levels. Administration of $\mathrm{E}$ decreased basal hyperglycaemia ( $8.8 \pm 0.3$ vs $\mathrm{D}: 18.5 \pm 2.3, \mathrm{p}<0.05, \mathrm{C}: 4.2 \pm 0.3 \mathrm{mmol} / \mathrm{l})$, improved glucose tolerance (integral glycaemia over GTT was $58.6 \pm 2.7$ vs D: $111.3 \pm 6.3, p<0.02$, C: $24.9 \pm 0.8 \mathrm{mmol} / \mathrm{l}$ ) and increased basal plasma insulin by $32 \%$ in comparison with non-treated diabetic control. Supplementation with $\mathrm{E}$ suppressed lipid peroxidation reducing malonic dialdehyde. MDA contents $(0.10 \pm 0.01$ vs $D: 0.24 \pm 0.02, \mathrm{p}<0.02, C: 0.18 \pm 0.02 \mu \mathrm{mol} / \mathrm{mg}$ protein) compared to both intact and diabetic controls. These results suggest that long-term supplementation with $\mathrm{E}$ can improve metabolic parameters including suppression of lipid peroxidation intensity in rabbits with dithizone-induced diabetes. Thus, the use of vitamin $\mathrm{E}$ in diet may have potential therapeutic benefit in the diabetes treatment

\section{9}

EFFECT OF AGING ON B-CELL MASS AND GLUCOSE TOLERANCE IN ADULT RATS SUBMITTED TO PERINATAL MALNUTRITION

B. BREANT, A. GAROFANO and P. CZERNICHOW, INSERM U457, Hospital Robert Debré, Paris, F.

We have recently shown that maternal food restriction during late pregnancy in rats decreased B-cell mass in the offspring at birth Prolonged maternal undernutrition until weaning (day 21) leads to $66 \%$ reduction in $\beta$-cell mass and number at that age, although $B$-cell proliferation remains normal. The consequences of malnutrition on $B$ cell status and glucose tolerance at adulthood were studied. Beta-cell fraction (insulin-positive) and proliferative capacity were determined by quantitative morphometry both in the head and in the tail of pancreas after in vivo incorporation of BrdU, in 3 month- and 12 month-old male animals, fed ad libitum from weaning. Glucose tolerance was studied by means of OGTT at both ages. At 3 months of age, previously malnourished $(R)$ animals demonstrated $35 \%$ decreased $B$-cell fraction, with a $50 \%$ decrease in the head of the pancreas whereby $B$-cell proliferation was similar to that of controls. In the tail part, B-cell fraction was only slightly impaired but B-cell proliferation was increased by $37 \%(0.63 \pm 0.07 \%)$, as compared to controls $(0.46 \pm 0.06 \%$ $\mathrm{p} \leq 0.01$ ), however insufficient to fully restore $B$-cell mass. While total $B$ cell mass continued to increase until 12 months of age in control animals $(9.0 \pm 1.8 \mathrm{mg})$ it stopped in $\mathrm{R}$ animals $(4.5 \pm 0.7 \mathrm{mg}, \mathrm{p}<0.001)$. Moreover, aging led to a loss of insulin response to oral glucose in $\mathbf{R}$ animals, $80 \%$ of which developed glucose intolerance at 12 months of age. In conclusion, perinatal undernutrition strongly and durably impairs $\beta$-cell development. Subsequent renutrition is followed by a period of increased $B$-cell proliferation, however insufficient to fully restore $B$-cell mass. In such animals with decreased $B$-cell mass the increased insulin demand of aging leads to $B$-cell exhaustion and precipitates the animals in glucose intolerance. 
1030

\section{MODIFIED INSULIN SECRETION IN RESPONSE TO NEUROTRANSMITTERS IN ISLETS FROM MALNOURISHED RATS T. Leon-Quinto ${ }^{1}$, A. Nadal ${ }^{1}$, B. Portha ${ }^{2}$ and B. Soria}

'Department of Physiology and Institute of Bioengineering. Miguel Hernández University. 03550 San Juan(Alicante). Spain

${ }^{2}$ Lab. Physiopathologie de la Nutrition CNRS ESA 7059, Université Paris 7, Paris, France

We have recently reported that rats submitted to protein-energy malnutrition ( $65 \%$ energy and $5 \%$ protein) for 4 weeks after weaning, exhibited decreased basal plasma insulin and glucose levels as well as increased plasma levels of epinephrine and norepinephrine. Moreover, the plasma catecholamine increase is likely attributable to an increase in sympathetic nervous activity since we have demostrated that malnourished rats presented a simpathetic hyperactivity and weak parasympathetic activity. Our study was designed to investigate the effects of this chronic anomalies observed in vivo on insulin secretion. Insulin secretory responses were studied in isolated islets by static incubation. Islets from malnourished rats presented a desensitization to glucose and acetylcholine $(\mathrm{ACh})$. In order to determine wheter this lack of response to $\mathrm{ACh}$ was correlated with a lack of increment in intracellular free calcium, we measured the intracellular free calcium with indo-1 fluorescence in the presence of carbachol. However, we have not found any differences between islets from control and malnourished rats in the fluorescence ratio $(\Delta R)$, being $\Delta R=0.83 \pm$ 0.10 and $0.95 \pm 0.06$ (arbitrary units), respectively.

On the other hand, islets from malnourished rats were hypersensitive to norepinephrine, a characteristic observed also in islets from STZ rats. Thus, in the presence of stimulatory glucose concentrations, the decrease in insulin secretion was significantly larger that in control islets. This hypersensitization to norepinephrine was corroborated by using simultaneously norepinephrine and yohimbine, since the reactivation of the insulin secretory response was larger in islets from malnourished rats.

Our findings suggest an impaired neural control of insulin release in islets from malnourished rats

\section{PS 52}

\section{Gastrointestinal Autonomic Neuropathy}

\section{1}

HYPERINSULINAEMIA MODIFIES GASTROINTESTINAL MOTOR REFLEXES

Th. Lingenfelser, W. Renn, R. Kadow, W. M. Sun, T. Hinrichs, H. G Wahl, J. Dent and V. Gross. Department of Gastroenterology, Klinikum Amberg, Germany, and Gastrointestinal Medicine, Royal Adelaide Hospital, South Australia.

In healthy subjects physiological hyperglycaemia $(\sim 10 \mathrm{mM})$ modifies gastrointestinal motor function. Subsequent hyperinsulinaemia due to hyperglycaemia may mediate this phenomenon. We investigated the effect of physiological hyperinsulinaemia on antropyloroduodenal motility in 10 healthy volunteers. Blood samples were taken during normoinsulinaemia $(50 \mathrm{pM})$ and mild hyperinsulinaemia $(300 \mathrm{pM})$ in randomized order and cross-over fashion, i.e. hyperinsulinaemia-normoinsulinaemia $(\mathrm{H} / \mathrm{N})$ vs. normoinsulinaemiahyperinsulinaemia $(\mathrm{N} / \mathrm{H})$. Antropyloroduodenal motor responses were evaluated after repetitive proximal duodenal distension with a non-compliant ultrathin bag during phase I activity. Intrabag volumes increased by $4 \mathrm{~mL}$ at each distension step (from 12 to $48 \mathrm{~mL}$ ) of $2.5 \mathrm{~min}$ duration (10 min intervals). A manometric sleeve-sidehole assembly recorded number and amplitude of antral, pyloric and duodenal contractions, tonic and phasic activity of isolated pyloric pressure waves (IPPW), and intraduodenal pressure. Proximal duodenal distension induced IPPW and duodenal pressure waves, and raised basal pyloric and intraduodenal pressure. In both studies, hyperinsulinaemia increased these motor responses at each distension step (e.g. at $48 \mathrm{~mL}$ ): number of IPPW $(1.9 \pm 0.6 / 2.0 \pm 0.8, \mathrm{~N} / \mathrm{H}$ vs. $2.5 \pm 0.8 / 2.0 \pm 0.6, \mathrm{H} / \mathrm{N}, \mathrm{p}<0.05)$, amplitude of IPPW $(32.5 \pm 0.8 / 77.0 \pm 6.9, \mathrm{~N} / \mathrm{H}$ vs. $58.5 \pm 1.1 / 38.8 \pm 2.5, \mathrm{H} / \mathrm{N}, \mathrm{p}<0.05)$, basal pyloric pressure $(7.3 \pm 1.1 / 9.0 \pm 1.3, \mathrm{~N} / \mathrm{H}$ vs. $8.2 \pm 1.7 / 7.1 \pm 1.2, \mathrm{H} / \mathrm{N}, \mathrm{p}<0.05)$, and intraduodenal pressure $(8.3 \pm 1.1 / 12.6 \pm 1.5, \mathrm{~N} / \mathrm{H}$ vs. $12.9 \pm 1.2 / 11.2 \pm 1.3, \mathrm{H} / \mathrm{N}$ $\mathrm{p}<0.05)$. Insulin per se has a major impact on gastrointestinal motor reflexes after duodenal distension in healthy subjects.

\section{2}

CUTANEOUS ELECTROGASTROGRAPHY IN EVALUATION OF TYPE 1 DIABETES MELLITUS GASTRIC NEUROPATHY. H. Mayaudon, B. Cariou, B. Ceccaldi, O. Dupuy, B. Bauduceau, O. Farret and C. Molinié. Hôpital d'Instruction des Armées Bégin, 69 avenue de Paris. 94160 Saint-Mandé. France.

The aim of this study was to assess gastric neuropathy in type 1 diabetes mellitus and its relationships with cardiac autonomic neuropathy. Methods: 54 type 1 diabetes mellitus asymptomatic patients, aged $43 \pm 12$ years, and 15 healthy subjects participated in the study. None received medical treatment apart from insulin Cutaneous electrogastrography (EGG) was recorded for 4 hours before, during and 4 hours after the ingestion of a standard meal. The electrogastrography dominant frequency was divided into three bands: bradygastria $(<2$ cycles per minute $\mathrm{cpm})$, normal $(2-4 \mathrm{cpm})$ and tachygastria $(4-10 \mathrm{cpm})$. The assessment of diabetic autonomic neuropathy was based on the five Ewing tests, time domain indexes (SDNN/5 mn, RMSSD, pNN50) and frequency domain indexes (total power, high-frequency power, lowfrequency power LFP) which were analyzed from 24 hours continuous ECG record. Results: Tachygastria is significantly more common during the entire period of recording ( $38 \pm 5$ vs $23 \pm 11,8 \%, 0<0,001$ ), before $(37 \pm 6$ vs $26,5 \pm 8,9 \%, p<0,001)$, during $(41 \pm 7,8$ vs $23 \pm 10,5 \%, p<0,001)$ and after the meal ( 37 vs $29 \%, p<0,01$ ) in diabetic patients than in controls. The percentage of dominant electrical frequency in normal range is significantly lower in diabetic patients than in controls $(49 \pm 6$ vs $63,3 \pm 11,1 \%, \mathrm{p}<0,001)$. Tachygastria is correlated with duration of diabetes $(r=0,234, p<0,05)$ but not with glycemic control. Abnormalities in gastric myoelectrical activity are not correlated with Ewing tests, time domain and frequency domain indexes. Conclusions: EGG is a relevant technique for the detection of gastric dysrythmias which are present in diabetic patients without gastrointestinall symptoms. There is no association between cardiac autonomic neuropathy and gastric myoelectrical activitiy. 
AUTONOMIC NEUROPATHY AND HELICOBACTER PYLORI IN DYSPEPTIC PATIENTS WITH TYPE 2 DIABETES MELLITUS

S. Turco, B. Oliviero, F.C. Sasso, R. Torella and S. Gentile. $1^{\text {st }}$ and $2^{\text {nd }}$ University of Naples, School of Medicine, Naples, Italy

A high prevalence of upper gastrointestinal (GI) symptoms has been reported in patients with diabetes mellitus (DM). Autonomic neuropathy (AN) and Helicobacter pylori (Hp) infection bay be considered as risk factors for dyspepsia (DY) in pts with type 2 DM (DM2). Nevertheless, a systhematic evaluation of these parameters has been never performed in DM2 pts. Aim: to evaluate the frequency of $\mathrm{Hp}$ infection in a large series of DM2 pts with DY in comparison with N-DM pts, and to correlate the endoscopic diagnoses with both $\mathrm{AN}$ and $\mathrm{Hp}$ infection. Patients: all patients and control subjects were selected after administration of a questionnaire on upper gastrointestinal symptoms (pts that have previously undergone to a gastroscopy and a gastrectomy or taking drugs active on the upper GI tract or antibiotics were not included): they were 164 consecutive DM2 pts, $86 \mathrm{M}$ and $78 \mathrm{~F}$, with mean age 52 $18 \mathrm{SD}$ (range 45-61) diabetes duration $14.6 \pm 8 \mathrm{y}, \mathrm{HbA} 1 \mathrm{c} 8.3 \pm 1.4 \%$ (HPLC), BMI $29.7 \pm 1.5 \mathrm{~kg} / \mathrm{m}^{2} ; 164$ N-DM controls were comparable for socioeconomic status and matched for sex age, body weight \pm 5 . Methods: all pts underwent: I) an upper endoscpy (EGDS), 2) multiple biopsies of antral mucosa addressed to histological diagnosis and to the search of $\mathrm{Hp}, 3$ ) a complete set of 5 cardiovascular tests (as previously described Diab Res Clin Invest 1996, 31:87). Results: $20.1 \%$ and $29.3 \%$ of DM2 and N-DM pts had peptic ulcer, respectively (p n.s.); chronic gastritis was found in $35.4 \%$ and $50 \%(\mathrm{p}<0.01)$, and DY without uicer and gastritis in $44.5 \%$ and $20.7 \%(\mathrm{p}<0.01)$ The prevalence of AN was higher in DM2 pts than in N-DM $(74.4 \%$ vs $50 \%$ respectively; $\mathrm{p}<0.01$ ) for all the endocsopic diagnoses. The prevalence of AN was higher in the Hp positive DM2 groups than in the negative $(83.6 \%$ vs $11.9 \%$; $p<0.901$ ). Conclusion: data clearly demonstrate: 1) a significant higher frequency of Hp infection in DM2 patients than in non diabetic subjects, and 2) a significant association of $\mathrm{Hp}$ infection with the atonomic neuropathy. Motility alterations duc to AN could explain the more frequent infection in diabetics, even though a generical predisposition to infections may be non excluded. Therefore. DM2 pts with DY and AN are under high risk for Hp infection.

\section{5}

PROGNOSIS OF DIABETIC GASTROPARESIS

M-F Kong, M Horowitz, KL Jones, J Wishart, and P Harding

Department of Medicine, Royal Adelaide Hospital, Adelaide, South Australia, 5000

The application of scintigraphic techniques has established that gastric emptying is delayed in about $50 \%$ of outpatients with longstanding diabetes mellitus. This observation challenges the assumption that diabetic gastroparesis is associated with a poor prognosis. Between 1984 and 1989, 86 outpatients with diabetes (66 type 1,20 type 2,40 male, 46 female) underwent assessments of solid and liquid gastric emptying (scintigraphy) diabetic complications (retinopathy, nephropathy), autonomic nerve function (cardiovascular reflex tests) and glycaemic control (glycated haemoglobin). Of these 86 patients solid emptying (\% retention at $100 \mathrm{~min}$ ) was delayed in $57 \%$ and liquid emptying $\left(\mathrm{T}_{50}\right)$ in $28 \%$. At follow-up in 1998, 61 patients were known to be alive, 19 had died (mainly from cardiovascular or renal disease) and 6 were lost to follow-up. Results are shown in the table (mean values):

\begin{tabular}{lccc}
\multicolumn{2}{l}{ Results are shown in the table (mean values): } & & \\
& $\begin{array}{c}\text { Alive } \\
(61)\end{array}$ & $\begin{array}{c}\text { Deceased } \\
(19)\end{array}$ & $p$ \\
\hline Age at baseline (years) & 46 & 52 & NS \\
Solid retention at 100 min (\%) & 55 & 58 & NS \\
Liquid 50\% emptying time (min) & 30 & 36 & NS \\
Autonomic nerve dysfunction (score) & 1.97 & 3.0 & 0.047 \\
Retinopathy (score) & 0.84 & 1.42 & 0.013 \\
Glycated haemoglobin (\%) & 9.4 & 9.9 & NS \\
Serum creatinine (mmol/) & 0.10 & 0.15 & NS \\
\hline
\end{tabular}

In the group who had died there was an increased prevalence of autonomic neuropathy and retinopathy, but no difference in gastric emptying. Thus, in this large cohort of patients there is no evidence that delayed gastric emptying, per se, is associated with a poor prognosis.
RELATIONSHIP BETWEEN GASTRIC EMPTYING AND $H$ PYLORI IN INSULIN DEPENDENT DIABETIC PATIENTS DA. de Luis, D Boixeda, J M Cordero, C Caballero, A Becerra. and $\mathrm{H}$ de la Calle, $\mathrm{H}^{\mathrm{a}}$ Ramón y Cajal, Madrid, Spain.

Alterations in gastric emptying (GE) were observed in diabetic patients, due to different etiologies such as autonomic neuropathy. Helicobacter pylori $(\mathrm{Hp})$ infection can alter the GE in non-diabetic population. The aim of this study is to determine a possible relationship between $\mathrm{Hp}$ infection and gastric emptying in insulindependent diabetes mellitus patients (IDDM). Thirteen IDDM patients with gastritis associated to $\mathrm{Hp}$ infection were studied. A scintigraphy (DTPA- ${ }^{90} \mathrm{TC}$ ) study of the stomach was made after of a standard meal. Gastric inflammatory markers such as gastrin pepsinogen $\mathrm{I}$, and pepsinogen $I I$ levels were measured. After the initial study, a $\mathrm{Hp}$-eradicating treatment was made and after three months the same studies were performed. After eradicating treatment, no gastritis findings were oberved in 5 patients (group I), persistent gastritis findings were observed in 8 patients (group II). In group I the half time of GE was $(32.1+10.6 \mathrm{~min} . v \mathrm{~s}$ $58.3 \pm 16 \mathrm{~min} ; \mathrm{p}<0.05$ ), before and after treatment, respectively. No differences in GE were observed in group II $(30 \pm 9.8 \mathrm{~min}$. vs. $37.8+21.5 \mathrm{~min}$; $\mathrm{n.s})$. The levels of gastrin $(62+9 \mathrm{pg} / \mathrm{ml}$ vs $56.9 \pm 16.4 \mathrm{pg} / \mathrm{ml} ; \mathrm{ns})$, pepsinogen I $(74 \pm 38.9 \mathrm{ng} / \mathrm{ml}$ vs $51.2 \pm 25.9$ $\mathrm{ng} / \mathrm{ml} ; \mathrm{p}<0.05)$ and pepsinogen II $(10.9 \pm 4.8 \mathrm{ng} / \mathrm{ml}$ vs $5.4 \pm 2.6$ $\mathrm{ng} / \mathrm{ml} ; \mathrm{p}<0.005$ ) in the group I decreased after treatment, the ratio $\mathrm{ppgI/H}$ increased. No changes were found in group II. There was a correlation between half time emptyig and gastrin levels $(r=0,5 ; p<0,05)$. The delay of $G E$ after eradication of $\mathrm{Hp}$-gastritis in IDDM patients could be related with the improvement in the inflammatory markers secondary to the gastritis. Hp infection could be another patologic factor in the alterations of gastric emptying present in IDDM.

\section{6}

LONG-TERM EFFECT OF CISAPRIDE ON GASTRIC EMPTYING AND GLUCOSE CONTROL IN DIABETICS WITH GASTROPARESIS R.A. Honegger', W. Schwizer ${ }^{2}$, R. Lehmann 1 , P. Kunz ${ }^{2}$, C. Feinle ${ }^{2}$, M. Fried ${ }^{2}$ and G.A. Spinas'. Divisions of 'Endocrinology and Diabetes and ${ }^{2}$ Gastroenterology, University Hospital Zurich, Switzerland

Impaired gastric emptying is a major reason for brittle diabetes. Short-term application of cisapride effectively improves gastric emptying. However, its efficacy if administered on a long-term basis and in particular its effect on glucose control has not yet been shown. The present study investigates the effect of cisapride on gastric emptying and glucose control during long-term administration. Eight insulin-treated patients with diabetes (mean diabetes duration 24.6 years) and severe autonomic neuropathy with delayed gastric emptying, as determined by radioopaque marker emptying, were randomly treated in a double-blind, cross-over design with cisapride $20 \mathrm{mg}$ b.i.d and placebo, for 3 months each. Glycemic control was assessed by daily measurements of 4 - 7 capillary glucose levels, and $\mathrm{HbA1c}$ was determined every 3 months. Gastric emptying of a mixed solid standard meal was measured by scintigraphy at the beginning of the study, after 3 and 6 months, respectively. Chronic administration of cisapride resulted in a significant improvement of gastric emptying of solids $\left(t_{1 / 2}=104 \mathrm{~min}(95 \% \mathrm{Cl} 77-158)\right.$ in the cisapride vs $126 \mathrm{~min}(95 \% \mathrm{Cl} 92-199)$ in the placebo group (n.s.), being significant in 2 out of 8 patients. Glycemic control (mean $\pm S D$; $n=6660$ measurements) was not different on cisapride $9.2 \pm 1.6 \mathrm{mmol} / \mathrm{l}$ as compared to placebo $8.9 \pm 1.7 \mathrm{mmol} / \mathrm{n}$ (n.s). During both treatment periods there was a reduction in $\mathrm{HbA} 1 \mathrm{c}(8.0 \% \pm 1.3$ to $7.8 \% \pm 1.1$ on cisapride vs $8.0 \% \pm 1.1$ to $7.6 \% \pm 1.0$ on placebo; n.s.). Long-term administration of cisapride has no effect on gastric emptying in the majority of patients with severe gastroparesis and, does not improve overall glycemic control. In these patients tachyphylaxis to chronic cisapride treatment may be a possible explanation for the loss of efficacy, in contrast to the well established benefit of short-term use. 
1037

HYPERINSULINEMIA DECREASES ERYTHROMYCIN-INDUCED GALLBLADDER EMPTYING BUT NOT GASTRIC MOTILITY IN NORMAL SUBJECTS.

L. Scionti, E. Distrutti*, C. Marino, R. Bosso*, C. Vermigli, E. Puxeddu, P. Bottini, S. Fiorucci* and F. Santeusanio. Dipartimento di Medicina Interna e Scienze Endocrine e Metaboliche and *Istituto di Gastroenterologia ed Endoscopia Digestiva, University of Perugia, Italy To study whether hyperinsulinemia (HI) affects erythromycin-induced gallbladder emptying and gastric motility, 5 normal subjects underwent a 3-hour euglycemic hyperinsulinemic $(\sim 100 \mu \mathrm{U} / \mathrm{mI})$ clamp. Erythromycin $(1 \mathrm{mg} / \mathrm{kg})$ was infused after 2 hours of $\mathrm{HI}$ and gallbladder volume (GV) and gastric motility, expressed as motility index (MI), were measured every 10 minutes over the last hour of HI. GV and MI were measured by sonography using a $3.5 \mathrm{MHz}$ convex transducers according to the methods of Dodds and Bolondi, respectively. In a separate day, E was infused after 2 hours of saline infusion (SAL). Results are expressed as mean+SEM and are shown in the table:

\begin{tabular}{lccccccc}
\hline min & 0 & 10 & 20 & 30 & 40 & 50 & 60 \\
\hline Gallbladder volume (\% of basal) & & & & \\
SAL & 100 & $68 \pm 4^{*}$ & $68 \pm 1^{*}$ & $86 \pm 9$ & $84 \pm 6$ & $87 \pm 9$ & $80 \pm 13$ \\
HI & 100 & $86 \pm 7$ & $95 \pm 8$ & $96 \pm 11$ & $106 \pm 13$ & $112 \pm 6$ & $106 \pm 10$ \\
\hline Gastric & Motility Index $\left(x 10^{-1}\right)$ & & & & - \\
SAL & $0.3 \pm 0$ & $26 \pm 4 \#$ & $10 \pm 1 \#$ & $4 \pm 2 \#$ & $0.6 \pm 0$ & $2 \pm 1$ & $2 \pm 2$ \\
HI & $0.4 \pm 0$ & $23 \pm 5 \#$ & $11 \pm 2 \#$ & $5 \pm 2 \#$ & $2 \pm 2$ & $0.9 \pm 0$ & $0.8 \pm 0$ \\
\hline
\end{tabular}

${ }^{*} \mathrm{p}<0.05$ vs time $0 ; \# \mathrm{p}<0.001$ vs time 0 .

Two-way ANOVA showed that the time course of GV after $\mathrm{E}$ was significantly $(\mathrm{p}<0.0001)$ different between SAL and HI, whereas no differences were found in gastric MI. In conclusion, HI reduces the erythromycin-induced gallbladder emptying in normal subjects without affecting gastric motility. The inhibitory effect of $\mathrm{HI}$ could play a role in the pathogenesis of gallstones in type 2 hyperinsulinemic diabetic patients.
1038

SHORT-TERM HYPERGLYCEMIA IMPAIRS THE MEALINDUCED GALLBLADDER EMPTYING IN NORMAL SUBJECTS.

A. Baccarelli, P. Bottini, E. Distrutti*, C. Marino, C. Vermigli, E. Puxeddu, R. Bosso*, S. Fiorucci* and L. Scionti. Dipartimento di Medicina Interna e Scienze Endocrine e Metaboliche and *Istituto di Gastroenterologia ed Endoscopia Digestiva, University of Perugia, Italy

To evaluate whether hyperglycemia can influence the meal-induced gallbladder emptying, 6 normal subjects ate a mixed meal during a 5 hour hyperglycemic clamp. The mixed meal $(500 \mathrm{Cal}, 40 \%$ carbohydrates, $20 \%$ lipids, $40 \%$ proteins) was eaten after 2 hours of hyperglycemia (HYPER) and gallbladder volume (GV) and gastric motility, expressed as motility index (MI), were checked every 15 minutes over the last 3 hours of HYPER. GV and MI were measured by sonography using a $3.5 \mathrm{MHz}$ convex transducers according to the methods of Dodds and Bolondi, respectively. In a separate day, meal was eaten during saline infusion (SAL). Results are expressed as meantSEM. Mean plasma glucose was $208.1 \pm 4.5 \mathrm{mg} / \mathrm{dl}$ during HYPER and $84.0 \pm 1.7 \mathrm{mg} / \mathrm{dl}$ during SAL. GV decreased after meal both during HYPER and SAL, but the reduction was faster $(78.8 \pm 7.1 \%$ of basal value vs $100.8 \pm 11.7 \%$ after $15 \mathrm{~min}$ ) and more marked $(52.1 \pm 7.1 \%$ vs $70.0 \pm 12.4 \%$ ) during SAL than HYPER. Twoway ANOVA showed that the curves were significantly $(\mathrm{p}<0.0001$ ) different. Meal increased gastric MI both during SAL and HYPER, but it was lower during HYPER than SAL at all the time points. The curves were significantly $(p<0.0002)$ different by two-way ANOVA. In conclusion, the meal-induced gallbladder emptying is reduced by hyperglycemia in normal subjects. The inhibitory effect of HYPER could play a role in the pathogenesis of gallstones in diabetic patients.

\section{0}

PANCREATIC POLYPEPTIDE RESPONSE TO FOOD IS NOT SUITABLE FOR EVALUATION OF VAGAL FUNCTION IN DIABETIC PATIENTS

M.B.Damholt and J.Hilsted, Dept. of Endocrinology, Hvidovre Hospital, Denmark.

The first phase secretion of pancreatic polypeptide (PP) in response to food ingestion is mediated by n.vagus and is compromised in patients with diabetic autonomic nerve dysfunction. Decreased PP response to a meal has also been demonstrated in IDDM patients without signs of peripheral nerve damage. The aim of this study was to evaluate plasma PP (p-PP) response to a standardised meal in IDDM patients without signs of neuropathy and in healthy subjects. Further we aimed to investigate if PP secretion capacity could be evaluated as the response to an L-arginine infusion, which in vitro has been shown to stimulate PP secretion in the isolated pancreas. Methods: Based on standard heart rate variability tests and clinical examination all persons were healthy and without signs of peripheral nerve dysfunction. Experiments were performed after an overnight fast. Eighteen healthy subjects (age $25 \pm 2$ years (mean \pm SD) ) and 11 IDDM patients (age $28 \pm 5$ years) with short term diabetes $(3.4$ \pm 2.9 years) participated in a standard meal test. On two occasions L-arginine was infused intravenously $(0.5 \mathrm{~g} / \mathrm{kg}$ body weight $/ 45 \mathrm{~min})$ with or without injection of 1 mg. atropine iv. at start of infusion. Ten healthy persons (age $25 \pm 2$ years) and 6 IDDM patients (age $25 \pm 5$ years, diabetes duration $5.3 \pm 2.8$ years) took part in the infusion studies. Results: $p-P P$ response to a meal was significant in both groups ( $p<$ 0.0001 , ANOVA, log. data), but no difference in first phase peak p-PP between the groups could be found ( $p=0.6$, Mann-Withney U-test). However 3 healthy persons and 1 IDDM patient were practically without p-PP response to food ingestion. No pPP response could be detected during L-arginine infusion in either experiment. Conclusion: L-arginine does not stimulate the PP-cell in vivo and then there is no known method to estimate if the pancreatic PP-secretion capacity is intact in the individual patient as basis for evaluation of the vagal-mediated response. Some healthy persons do not respond with PP secretion to meal ingestion, thus it is not a suitable parameter for evaluation of vagal function in the individual patient. 
PS 53

\section{Neuropathy}

1041

\section{SURAL NERVE PERINEURIAL ABNORMALITIES RELATE TO NEUROPATHIC SEVERITY IN DIABETIC PATIENTS.}

M.Ghani, R.Malik, D.Walker, S. Hayat, S.Tesfaye, A.J.M Boulton and J.D.Ward. Department of Medicine, Manchester Royal Infirmary, Diabetes Unit, Royal Hallamshire Hospital, UK

The perineurium is a highly important structural and functional barrier which regulates peripheral nerve function. We have performed detailed electronmicroscopic studies on perineurial abnormalities in sural nerve biopsies from 35 diabetic patients without $(\mathrm{NN})(\mathrm{n}=10)$, with mild $(\mathrm{MN})$ $(n=11)$ and severe (SN) $(n=14)$ neuropathy and 10 control subjects $(C)$. Perineurial sheath thickness (PST) $(\mu \mathrm{m})$ and the Intraperineurial space $(\mathrm{IS})(\mu \mathrm{m})$ did not differ significantly between $\mathrm{C}(15.2 \pm 1.7,1.3 \pm 0.2)$, $\mathrm{NN}-(16.4+1.9,1.3 \pm 0.1), \mathrm{MN}-(15.9+1.5,1.1 \pm 0.1)$ and $\mathrm{SN}-(17.7 \pm 1.2$, $1.2 \pm 0.1)$ respectively. Perineurial cell thickness $(\mathrm{PCT})(\mu \mathrm{m})$ was significantly increased in $\mathrm{NN}(0.64 \pm 0.1, \mathrm{P}<0.002)$ but did not differ in MN $(0.43 \pm 0.1)$ or $\mathrm{SN}(0.47 \pm 0.1)$ compared with controls $(0.46 \pm 0.03)$. Perineurial lamellar no.(PLN) was increased in diabetic patients (NN-7.4 \pm $0.5, \mathrm{P}=0.5, \mathrm{MN}-8.1 \pm 0.2, \mathrm{P}<0.05, \mathrm{SN}-8.0 \pm 0.2, \mathrm{P}<0.03$ ) compared to $\mathrm{C}$ $(7.0 \pm 0.4)$. Perineurial cell basement membrane thickness (PCBMT) $(\mu \mathrm{m})$ was significantly increased in diabetic patients $(\mathrm{NN}-0.23 \pm 0.02, \mathrm{P}=0.20$, MN-0.25+0.01, $\mathbf{P}<0.0005, \quad \mathrm{SN}-0.29+0.01, \mathrm{P}<0.0001)$ compared with controls $(0.19 \pm 0.01)$. Peroneal motor nerve conduction velocity and vibration perception threshold correlated with PCT $(r=0.4, p<0.01, r=-$ $0.49, \mathrm{P}<0.01), \mathrm{PLN}(\mathrm{r}=-0.35, \mathrm{P}<0.05, \mathrm{r}=0.40, \mathrm{P}<0.03)$ and PCBMT $(r-$ $0.45, \mathrm{P}<0.01, \mathrm{r}=0.4, \mathrm{P}<0.02)$ respectively but not with either TPT or IS. Thermal discrimination threshold correlated with TPT $(\mathrm{r}=0.55, \mathrm{P}<0.001)$, IPS $(r=0.41, P<0.01)$, PLN $(r=0.36, P<0.05)$ and PCBMT $(r=0.55$, $P<0.0001)$. Myelinated fibre density correlated with PCBMT ( $r=-0.47$, $\mathrm{P}<0.01$ ) but not TPT, IPS, PCT or PLN. Perineurial abnormalities may play a role in the pathogenesis of human diabetic neuropathy as they relate to neuropathic severity.

\section{2}

PERIPHERAL NERVE STRUCTURAL ABNORMALITIES DO NOT EXPLAIN FUNCTIONAL DEFECITS IN THE STREPTOZOTOCIN DIABETIC RAT.

D. Walker, R.Malik, A. Carrington, S.A.Cannan, D. Sawicki, A.J.M Boulton, and J. Sredy. Department of Medicine, Manchester Royal Infirmary, UK, The Institute for Diabetes Discovery, USA.

The streptozotocin diabetic rat is the most commonly employed animal model of diabetic neuropathy characterised by a reduction in nerve conduction velocity and blood flow. Whether or not stnuctural abnormalities underlie such abnormalities is unclear. 10 control animals and 10 diabetic animals were studied. Motor nerve conduction velocity $\mathrm{ms}^{-1}$ was significantly reduced in diabetic animals (D) $41.3+2.3$ compared to controls (C) $46.2+4.5$ $(p<0.0001)$. Tibial nerve glucose $(p<0.001)$, sorbitol $(p<0.001)$ and fructose $(p<0.001)$ levels were elevated and myoinositol $(p<0.001)$ was reduced in D v C. Detailed morphometric assessment of the Tibial nerve was carried out to determine underlying abnormalities of the myelinated nerve fibres and endoneurial capillaries. No significant difference was observed for fascicular area (D-0.20 \pm 0.08 v C $-0.19 \pm 0.07$, ns), myelinated fibre density (D-10312 3705 v C-10058 \pm 3571 , ns), regenerative cluster density (D-81.6 \pm 34.7 v C$71.4+26, \mathrm{~ns})$, myelinated fibre (D-38.9 + 2.4 v C-38.6 +6.2, ns) and axon (D-17.8 \pm 1.5 $\mathrm{C}-17.9 \pm 3.1, \mathrm{~ns}$ ) areas. No significant difference was observed in endoneurial capillary density (D-41.7 $\pm 8.9 \times$ C $-39.1 \pm 11.1)$ basement membrane area (D-16.7 +2.4 v C-16.8 $\pm 1.1, p=0.81$ ) or endothelial cell profile no. (D-5.1 +0.3 v C-5.3 $+0.7, p=0.81$ ). However paradoxically, endothelial cell area was reduced in $\vec{D}(21.7 \pm 2.6)$ compared to $C(24.8 \pm$ $2.6), p=0.07$ and luminal area was significantly increased in $D(35.4 \pm 5.1)$ compared to $\mathrm{C}(25.0 \pm 7.7), \mathrm{p}<0.03$. In conclusion the reduction in nerve conduction velocity in the streptozotocin diabetic rat cannot be attributed to a loss of myelinated fibres or axonal atrophy. Furthermore, the increase in luminal area and reduction in endothelial cell size without endothelial cell hyperplasia or basement membrane thickening fails to provide a structural basis for the reduction in nerve blood flow and infact argues against a structural basis for nerve blood flow reduction.

\section{3}

THE EARLY CHANGES OF CEREBRAL MORPHOLOGY AND LEARNING AND MEMORY FUNCTION IN STZ-DM RATS

J. S. Hu, X. Y. Ma, S. L. Sheng, et al.

Endocrinology Department, 304 hospital,PLA, Beijing, China.

Abstract: [Aim]:There is high incidence of depression and dementia in longtermed DM patients. Our study is to investigate the early impact of hyperglycemia on CNS. (Method]: 40 male Wistar rats(BW 150 200g) were divided into two groups, group one: STZ-diabetic rats $(n=20)$, group two: normal control rats $(n=20)$. At the 1st, 2 nd and 3rd month of $D M$ seperately, water maze, brain ulfrastructure and immunohistochemistry of NF, NGF, NT-3 were studied. [Results]: We found that the swimming time of water maze has significant difference between the two groups as early as 1 month after diabetes( $(40.001)$. Examination of transmission electron microscopy showed that there were scattered degenerative neurons in the rat hippocampal CA1 region, temporal cortex, and septal area after one month of diabetes, but no micrangium change was found. After diabetes for 3 months, the neuron degeneration became more significant and apparent, and the degenerative neurons were wrapped by microglial cells. In the hippocampal CA1 region nearons decrease of NT-3, NGF-erpressing were apparent at one month after diabetes, and decrease of NF-expressing neurons and axons were also significant at three month after diabetes in comparing with the controlled rats. [Conclusion]; This results suggested that hyperglycemia may cause neurodegeneration of the brain in very early stage, as well as behavior abnormal and the neuron damage became mpre and more apparent along with the time of diabetes. Being lack of micrangium damages under electro micrrocope, it was suggested that the metubolistic factors were the main cause for the brain morphological damage.

\section{4}

ANTIOXIDATIVE DEFENSE IN DIABETIC PERIPHERAL NERVE: ROLE FOR ALDOSE REDUCTASE AND SORBITOL DEHYDROGENASE I.G. Obrosova, H.-J. Lang and D.A. Greene, University of Michigan, Ann Arbor, MI, USA and HMR-HoechstAG, Frankfurt, Germany

The study was aimed at evaluating the role for two enzymes of the sorbitol pathway aldose reductase(AR) and sorbitol dehydrogenase (SDH) in diabetesinduced changes of markers of oxidative stress (total 4-hydroxyalkenal and MDA levels) and antioxidative defense (GSH levels and superoxide dismutase (SOD), catalase (Cat), glutathione peroxidase (GSHPx), glutathione transferase (GSHTrans), glutathione reductase (GSSGRed) and total quinone reductase (TQRed) activities) in peripheral nerve. 3-w STZ-diabetic rats(D) were treated with/without SDI (SDI-157(HMR), I00 mg/kg/d, in the drinking water) while 6-w $\mathrm{D}$ were treated with/ without ARI (sorbinil, $65 \mathrm{mg} / \mathrm{kg} / \mathrm{d}$, in the diet for $2 \mathrm{w}$ after 4 w of untreated diabetes). 4-HA+ MDA levels (measured with kits from Oxis Int) were increased in both $3-\mathrm{w}$ and $6-\mathrm{w} \mathrm{D}(0.136 \pm 0.048$ and $0.174 \pm 0.040 \mu \mathrm{mol} / \mathrm{g}$ vs $0.077 \pm 0.028$ and $0.108 \pm 0.034$ in controls $(C)$ ), and this increase was even higher in 3-w D+SDI $(0.179 \pm 0.061)$ and was corrected in 6-w D+ARI $(0.080 \pm 0.026)$. GSH levels (assayed spectrofluorometrically with O-phthaldialdehyde) were decreased in $3 \mathrm{w}-\mathrm{D}(0.87 \pm 0.11 \mathrm{vs} 1.15 \pm 0.018 \mu \mathrm{mol} / \mathrm{g}$ in $\mathrm{C}, \mathrm{p}<0.002)$ and this decrease progressed in D+SDI $(0.66 \pm 0.16, p<0.01$ vs D) while GSH depletion in 6w D was corrected by ARI. SOD, Cat, GSHPx were similar in C and 3-w D, but were increased in D+SDI. SOD and Cat were lower in 6-w D vs C, and SOD but not $C$ at was partially corrected by ARI. TQRed was lower in 3-w D vs C, and was further decreased by SDI, while TQRed deficit in 6-w D was partially corrected by ARI. Sorbitol(S) and fructose(F) levels in D+SDI were $7.34 \pm 1.77$ and $1.73 \pm 0.78$ $\mu \mathrm{mol} / \mathrm{g}$ (vs $1.65 \pm 0.36$ and $6.66 \pm 2.10$ in $\mathrm{D}$, and $0.15 \pm 0.03$ and $1.22 \pm 0.15$ in C), while both $S$ and $F$ levels in D+ARI were lower than in C. In conclusion, impairment of antioxidative defense in peripheral nerve progresses with the duration of diabetes. Increased AR is an important (but not the only) mechanism of diabetesinduced oxidative stress while SDH has a protective role and its inhibition exacerbates oxidative damage. 


\section{5}

ENDONEURIAL CYTOSOLIC AND MITOCHONDRIAL REDUCTIVE STRESS INTERACTIONS BETWEEN ELEVATED GLUCOSE LEVELS AND HYPOXIA Y. Ido, J.R. Nyengaard, E. Ostrow, and J.R. Williamson; Washington University, St. Louis, MO, USA

The objective of these studies was to assess effects of hyperglycemia and hypoxia on (sciatic nerve) endoneurial glucose metabolism and on free cytosolic $\mathrm{c}_{(\bullet)}$ and mitochondrial ${ }_{(m)} \mathrm{NAD}^{+} / \mathrm{NADH}$ (decreased ratios indicate reductive stress). Endoneuria from normal rats, some fed tolrestat, $0.2 \mathrm{mmol} / \mathrm{kg}$ bwt/day for 7 days), were incubated $2 \mathrm{~h}$ in 5 or $30 \mathrm{mM}$ glucose (glu) in media at $5 \%$ (low) or $95 \%$ (hi) $\mathrm{O}_{2}+$ $5 \% \mathrm{CO}_{2} . \mathrm{NAD}^{+} / \mathrm{NADH}_{\mathrm{c}}$ and $\mathrm{NAD}^{+} / \mathrm{NADH}_{\mathrm{w}}$ were estimated by the redox metabolite indicator method based on substrate levels of lactate dehydrogenase and glutamate dehydrogenase $\left(+\mathrm{NH}_{4}\right)$, respectively. These metabolites, endoneurial glucose 6 phosphate (G6P) and fructose 6-phosphate (F6P), and medium lactate were measured enzymatically. G6P at hi- $\mathrm{O}_{2}$ was $108 \pm 8(\mathrm{X} \pm \mathrm{SD}) \mathrm{pmol} / \mu \mathrm{gDNA}$ in $5 \mathrm{glu}$ and increased $55 \%(\mathrm{p}<0.005)$ in $30 \mathrm{glu}$; low $-\mathrm{O}_{2}$ decreased $\mathrm{G} 6 \mathrm{P} 34 \%(\mathrm{p}<0.005)$ in 5 and $30 \mathrm{glu}$. F6P at hi- $\mathrm{O}_{2}$ was $24 \pm 6 \mathrm{pmol} / \mu \mathrm{gDNA}$ in $5 \mathrm{glu}$ and increased $33 \%(\mathrm{p}=0.021)$ in $30 \mathrm{glu}$; low$\mathrm{O}_{2}$ decreased F6P $33 \%$ (p<0.05) in 5 and $30 \mathrm{glu}$. $\mathrm{NAD}^{+} / \mathrm{NADH}_{\mathrm{c}}$ at hi- $\mathrm{O}_{2}$ was $847 \pm 87$ in $5 \mathrm{glu}$ and decreased $36 \%(\mathrm{p}<0.002)$ in $30 \mathrm{glu}$ (due to a 2.3 fold increase in endoneurial lactate $(\mathrm{p}<0.001)$ vs a $48 \%$ increase $(\mathrm{p}=0.014)$ in pyruvate). Low- $\mathrm{O}_{2}$ decreased $\mathrm{NAD}^{+} / \mathrm{NADH}_{\text {c }}$ to $136 \pm 18$ in $5 \mathrm{glu}$ and to $108 \pm 20$ in $30 \mathrm{glu}(\mathrm{p}<0.0001$ for both 5 and $30 \mathrm{glu}$ ); these decreases were due to 6.5 and 5.3 fold increases in endoneurial lactate $(\mathrm{p}<0.0001)$ with no change in pyruvate. $\mathrm{NAD}^{+} / \mathrm{NADH}_{\mathrm{m}}$ at hi- $\mathrm{O}_{2}$ was $49 \pm 16$ in $5 \mathrm{glu}$ and fell $43 \% \quad(\mathrm{p}=0.082)$ in $30 \mathrm{glu}$; low- $\mathrm{O}_{2}$ decreased $\mathrm{NAD}^{+} / \mathrm{NADH}_{\mathrm{m}} 25 \%(\mathrm{p}<0.01)$ in 5 and $30 \mathrm{glu}$. Lactate production at hi- $\mathrm{O}_{2}$ was $20 \pm 4$ $\mathrm{nmol} / \mu \mathrm{gDNA}$ in $5 \mathrm{glu}$ and increased $68 \%(\mathrm{p}<0.001)$ in $30 \mathrm{glu}$; low $-\mathrm{O}_{2}$ increased lactate production 10 fold in 5glu and 7.4 fold in 30glu ( $<<0.0001$ for 5 and $30 \mathrm{glu}$ ). Tolrestat prevented the decrease in $\mathrm{NAD}^{+} / \mathrm{NADH}_{\mathrm{c}}$ at $30 \mathrm{glu}$ but did not affect lactate production or $\mathrm{NAD}^{+} / \mathrm{NADH}_{\mathrm{m}}$. We suggest that these additive effects of $30 \mathrm{glu}$ and low $\mathrm{O}_{2}$ on $\mathrm{NAD}^{+} / \mathrm{NADH}_{\mathrm{c}}$ may exacerbate diabetic neuropathy via effects on substrate levels of dehydrogenase enzymes implicated in diabetic complications.

\section{7}

POLYOL PATHWAY INHIBITORS DOSE-DEPENDENTLY PRESERVE NERVE FUNCTION IN DIABETIC RATS P. Oates, T. Schelhorn, M. Miller, E. Hammerlund, C. Ellery, D. Beebe and J. Hakkinen. Pfizer Inc, Groton, CT, USA

The purpose of this study was to compare the dose-dependent protective effects of an aldose reductase inhibitor (ARI) vs. a sorbitol dehydrogenase (SDH) inhibitor (SDI) on tibial motor nerve conduction velocity (NCV) in diabetic rats. Rats made diabetic with $60 \mathrm{mg} / \mathrm{kg}$, i.p., streptozocin were given ARI zopolrestat (Z) (Alond ${ }^{\mathrm{TM}}$ ) (Study 1) or SDI CP-166,572 (Study 2) for 4 weeks in chow or drinking water respectively. Each study had 5 groups. Study 1: nondiabetic (N1) diabetics untreated (Db1) or treated with $Z$ at $\sim 10,25$ or $50 \mathrm{mg} / \mathrm{kg} / \mathrm{day}$ (groups Z10, Z25, Z50); Study 2: nondiabetic (N2), diabetic untreated (Db2), or treated with SDI at $\sim 50,100$ or $250 \mathrm{mg} / \mathrm{kg} /$ day (SD50 SD100, SD250). Standard electrophysiological and biochemical methods were used. ARI or SDI did not affect body weight, plasma glucose, glycated hemoglobin, or serum B-hydroxybutyrate. In Study $1 \mathrm{NCV}$ values $(\hat{\mathrm{x}} \pm \mathrm{SD}(\mathrm{n}), \mathrm{m} / \mathrm{s})$ fell from $\mathrm{N}=61.5 \pm 3.6$ (9) to Dbl $=52.1 \pm 2.3 \quad(13) \quad(\mathrm{p}<0.05)$; the ARI-treated groups had dosedependent NCV protection: $Z 10=54.0 \pm 3.8(15), Z 25=56.2 \pm 4.6$ (14), $\mathrm{Z} 100=60.1 \pm 5.5(13)(\mathrm{Z} 100 \mathrm{NS}$ vs. $\mathrm{N} 1, \mathrm{p}<0.05$ vs. Db1). Similar NCV changes were seen in Study 2: $N 2=61.4 \pm 2.9(10)$ vs $\mathrm{Db} 2=49.4 \pm 4.3(9)(\mathrm{p}<0.05)$; the SDI-treated groups also showed dosedependent NCV protection: SD50 $=53.5 \pm 2.7(9)$, SD $100=55.3 \pm 6.4(8)$ $\mathrm{SD} 250=57.8 \pm 5.0(9)$. Nerve fructose levels, elevated in $\mathrm{Db} 1$ and $\mathrm{Db} 2$ groups, were strongly suppressed by ARI or SDI, and correlated $(\mathrm{p}<0.05)$ with NCV values in the SDI-treated group. These results indicate that in diabetic rats inhibition of elevated metabolic flux through either polyol pathway step prevents tibial motor NCV loss, and they suggest that metabolism through SDH plays a key pathogenic role.

\section{6}

OXIDATIVE STRESS, AND NA/ K ATPASE ACTIVITY IN DIABETIC RATS: ALDOSE REDUCTASE INHIBITOR WITHDRAWAL STUDIES

M. Basso', D. Banas', K-D Lai ${ }^{1}$, TC Hohman', NE Cameron ${ }^{2}$ and MA Cotter ${ }^{2}$. 'Wyeth-Ayerst, Princeton, NJ, USA and 'Biomedical Sciences, Aberdeen University, Scotland UK

Aldose reductase inhibitor (ARI) treatment corrects nerve conduction velocity (NCV) deficits in diabetic rats. Treatment benefits have been linked to ARI effects on ouabain sensitive $\mathrm{Na} / \mathrm{K}$ ATPase activity (ATPase) as well as increased resistance to oxidative stress and improved endoneurial blood flow (EBF). To distinguish between these mechanisms, sciatic NCV, EBF, reduced glutathione levels (RG) and ATPase were measured in diabetic rats treated for 12-14 days with an ARI (WAY-121,509,10 mg/kg) and following a 5-8 day washout period. NCV and EBF reduced $17.4 \%$ and $48.6 \%$, respectively ( $p<0.001$ ) by 8 weeks of diabetes, were restored by ARI-treatment. Within 3-4 days of ARI withdrawal both $\mathrm{NCV}$ and EBF dropped to diabetic levels ( $\mathrm{p}<0.001)$. In contrast nerve sorbitol and fructose levels (11.2-fold and 5.4-fold elevated by diabetes) were reduced to $12 \pm 2 \%( \pm 1$ SEM) and $25 \pm 3 \%$ of nondiabetic levels, respectively $(\mathrm{p}<0.001)$ with ARI treatment and remained below nondiabetic levels 5 days after drug withdrawal. ATPase was $41 \pm 5 \%$ reduced by diabetes $(\mathrm{p}<0.001)$, and partially restored $(60 \pm 8 \%$; $\mathrm{p}<0.001)$ by ARI treatment. With ARI withdrawal, the ATPase decline was delayed compared to $\mathrm{NCV}$, taking 8 days to reach the diabetic level. The best correlation with nerve function was nerve RG which was $30 \pm 2 \%$ decreased by diabetes $(\mathrm{p}<0.001)$ and $63 \pm 2 \%(\mathrm{p}<0.01)$ corrected by ARI treatment. On ARI withdrawal, RG declined to the diabetic level within 2-4 days. Lack of concordance between $\mathrm{NCV}$, polyol and ATPase suggest that decreased blood flow and impaired antioxidant protection make the most important contributions to neuropathic changes in diabetic rats.

\section{8}

\section{THE EFFECTS OF THYROTROPIN RELEASING HORMONE IN EXPERIMENTAL DIABETIC NEUROPATHY}

H. Gul, M. Ozata*, O. Yildiz, Z. Odabasi**, G. Deniz, A. Isimer, and I.C. Ozdemir*. Dept. Of Pharmacology, *Endocrinology and Metabolism, and **Neurology, GATA School of Medicine, 06018 Etlik, Ankara, TURKEY

Thyrotropin releasing hormone (TRH) is a well-known effective drug in experimental and clinical spinal injury. But the effects of TRH in diabetic neuropathy is not known. The aim of this study was to investigate the electrophysiologic effects of TRH in the streptozotocin induced diabetic rats. We used three group of rats : (1) Non-diabetic control (NC, $n=10)$, (2) Diabetic control (DC, $n=8$ ), (3) Diabetic-TRH (DTRH, $n=9$ ). TRH treatment and electrophysiological measurements were conducted one month after the induction of diabetes. TRH was given intraperitoneally in a dose of $600 \mu \mathrm{g}(3 \mathrm{ml})$ to each rat of DTRH group and $3 \mathrm{ml}$ serum saline were given to the two other groups. Nerve conduction velocity (NCV), measured in caudal nerve, and $\mathrm{N}_{2}$ latency of somatosensory evoked potentials (SEP) and were measured 75 minute after injection of TRH or serum saline. SEP latencies were $27.58 \pm 0.45,32.08 \pm 0.82,28.90 \pm 0.55 \mathrm{~ms}$ and $\mathrm{NCV}$ values were $28.10 \pm 0.80,23.80 \pm 0.42,27.90 \pm 0.68 \mathrm{~m} / \mathrm{s}$ in NC, DC, DTRH groups respectively. SEP latency was lenghtened in DC group and shortened in DTRH group slightly but not significantly $(\mathrm{P}>0.05)$. NCV was significantly reduced in DC group and TRH improved NCV in DTRH group significantly $(\mathrm{P}<0.05)$. Our findings suggest that TRH is effective on peripheral neuropathy in experimental STZ-induced diabetes as revealed by NCV measurements. 
EFFECT OF Y-128 ON NEUROPATHY IS NOT DEPEND ON ITS POWERFUL THROMBOXANE $\mathrm{A}_{2}$ INHIBITION.

Y. Hayashi, T. Morishita, H. Satoh and N. Goto. Iruma-shi, Saitama 358, Japan, Yoshitomi Pharmaceutical Industries, LTD.

Y-128 has an inhibitory action of thromboxane(TX) synthase. To clarify the efficacy of Y-128 against diabetic(DM) neuropathy, we studied the effect of Y-128 on motor nerve conduction velocity (MNCV) and nerve regeneration(NREG) rate in STZ(60-65 $\mathrm{mg} / \mathrm{kg}$ i.v.)-induced DM rats. 1)MNCV study. Two weeks after the STZ treatment, $Y \cdot 128(0.03-3 \mathrm{mg} / \mathrm{kg}, \mathrm{n}=9 \cdot 10)$ or $O K Y \cdot 046(5-50 \mathrm{mg} / \mathrm{kg}$ b.i.d., $n=10$ ), other TX synthase inhibitor was administered orally for 4 weeks. At the end of treatment, caudal MNCV in DM rats declined significantly (DM:39.6 $\pm 0.2 \mathrm{~m} / \mathrm{s}$ vs. normal: $43.1 \pm 0.3 \mathrm{~m} / \mathrm{s}, \mathrm{P}<0.01$ ). Y-128 dose-dependently prevented the MNCV defect at $0.1 \mathrm{mg} / \mathrm{kg}$ and above. At $3 \mathrm{mg} / \mathrm{kg}$ dosing, $71 \%$ prevention was observed $(\mathrm{P}<0.01)$. On the other hand, OKY.046 caused no preventive effect(DM: $38.3 \pm 0.3$ $\mathrm{m} / \mathrm{s}$ vs. OKY $04650 \mathrm{mg} / \mathrm{kg}$ b.i.d.: $38.5 \pm 0.4 \mathrm{~m} / \mathrm{s}, \mathrm{P}=\mathrm{NS}$ ) while sciatic endoneurial $\mathrm{TXB}_{2}$ content was decreased to the same level of that of Y-128. Y-128, but not OKY-046 prevented the nerve myo-inositol reduction. 2)NREG study. Five weeks after the STZ treatment, the left sciatic nerve was crushed twice for $20 \mathrm{sec}$. Then, Y-128(3 mg/kg, $\mathrm{n}=18)$ or $\mathrm{OKY}-046(50 \mathrm{mg} / \mathrm{kg}$ b.i.d., $\mathrm{n}=17)$ was administered orally for 11 days. In DM rats, NREG distance evaluated by pinch test was decreased significantly(DM: $26.5 \pm 1.5 \mathrm{~mm}$ vs. normal: $37.6 \pm 0.7 \mathrm{~mm}$,

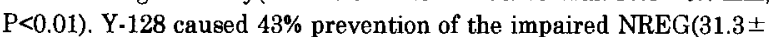
$0.8 \mathrm{~mm}, \mathrm{P}<0.05$ ). In contrast, $\mathrm{OKY}-046$ did not prevent it significantly $(29.0 \pm 1.3 \mathrm{~mm}, \mathrm{P}=\mathrm{NS})$. Thus, $\mathrm{Y}-128$ is effective against experimental DM neuropathy. But the mechanism(s) is not considered to depend on its TX synthase inhibition.
NEUROVASCULAR RESPONSES AND SUBSTANCE P IN IDDM PATIENTS T. Forst, T. Kunt, A. Pfützner, T. Magin, S. Schmidt, M. Engelbach and J. Beyer. University Hospital Mainz; Dept. Endocrinology and Metabolism; Mainz; Germany In IDDM patients neurovascular function is known to be impaired. Substance P binding sites have been demonstrated on endothelial cells and an alterated neuronal secretion of substance P might be implicated in disturbances of neurovascular control. The aim of the present study was to compare neurovascular function and plasma substance $\mathbf{P}$ levels in IDDM patients. Therefore, the microvascular response to thermal injury and the iontophoresis of $1 \%$ acetylcholine was investigated in 23 IDDM patients (age: $39.2 \pm 2.4$ years; duration of diabetes: $12.2 \pm 2.4$ years;

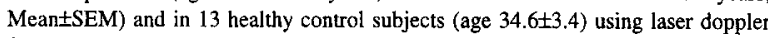
fluxometry (LDF, MBF 3D, Moor Instruments, Devon, UK). All subjects were free from peripheral macrovascular disease or foot ulceration and without any vasoactive drugs. Small nerve fibre function was assessed by the investigation of cold, heat and pain perception thresholds (Path Tester, PHYWE, Göttingen, Germany). Substance P plasma levels were measured by a competitive ELISA using purified plasma samples (C-18 reverse phase cartridges). Thirteen of the IDDM patients showed pathological results in small nerve fibre evaluation. The group of diabetic patients with small nerve fibre injury showed a decreased microvascular response following mild thermal injury compared to the diabetic group without neuropathy and the healthy control group ( $35.2 \pm 6.7$ vs. $69.7 \pm 10.4 \mathrm{AU}, \mathrm{p}<0.01$; vs. $102.2 \pm 34.9 \mathrm{AU}$, $\mathrm{p}<0.05$; respectively). In addition, a reduced microvascular response to acetylcholine was observed in the neuropathic diabetic group compared to the diabetic group without neuropathy and the control group (4.011.1 vs. $21.9 \pm 9.5 \mathrm{AU}$, $\mathrm{p}<0.05 ;$ vs. 17.5 $\pm 4.2 \mathrm{AU}, \mathrm{p}<0.001$; respectively). In diabetic patients plasma substance $P$ levels were significantly decreased compared to the control group $(10.4 \pm 0.5$ vs. $13.9 \pm 0.8 \mathrm{pg} / \mathrm{ml}, \mathrm{p}<0.001)$. No difference in plasma substance $P$ levels was found between diabetic patients with or without neuropathy $(10.2 \pm 0.6 \mathrm{vs}$. $10.5 \pm 0.7 \mathrm{pg} / \mathrm{ml}$ ). No association was found between substance $P$ and the microvascular response to thermal injury $(r=0.01, p=0.48)$ or stimulation with acetylcholine $(r=0.23, p=0.10)$. Our study confirm an impaired microvascular function in diabetic patients with small fibre neuropathy. Reduced plasma substance $P$ levels in diabetic patients are not associated to small nerve fibre function or neurovascular control.

\section{1}

DISCRIMINATIVE VALUES OF CLINICAL AND NEUROPHYSIOLOGCAL PARAMETERS IN CARPAL TUNNEL SYNDROME IN DIABETICS M. Reljanović, A Barada, R. Bilić, J. Kovjanić and Ž. Metelko. Vuk Vrhovac Institute, Dugi dol 4a, Zagreb; Orthopedic Clinic, Zagreb, Croatia

The aim of the study was to determine the discriminative values of different clinical and neurophysiological tests in diabetics with typical symptoms of carpal tunnel syndrome CTS (Group 1) compared with diabetics without those symptoms but with clinically present diabetic polyneuropathy (DPN, Group 2). Group 1 consisted of 141 diabetics with mean age $53.2( \pm 10.0)$, diabetes duration 13.5 years $( \pm 8.9)$, while 29 diabetics with mean age $52.4( \pm 9.8)$, diabetes duration 9.4 years $( \pm 7.5)$ were in Group 2 . Three standard clinical tests (Tinel's sign, Phalen's wrist flexion test and arm compression test) were shown to be sensitive up to $95 \%$, but insufficiently specific in distinguishing CTS and DPN (average $35 \%$ specificity). Median nerve motor conduction velocity, amplitude, distal latency, sensory conduction velocity in hand and amplitude as well as vibration perception threshold differentiated the two groups significantly (all $\mathrm{p}<0.01$ ), all demonstrating worse values in Group 1. Warm and cold perception threshold in hand as well as sympathetic skin response were not statistically different in the two groups. Ulnar nerve neurophysiological parameters were shown to be slightly better in Group 1. To determine parameters which best differentiate the two groups, median/ulnar nerve quotient was calculated for all neurophysiological parameters. Median/ulnar nerve quotient of sensory conduction velocity in hand was shown to have the best discriminative value (Group $1=0.73 \pm 0.14$; Group $2=0.97 \pm 0.11$, $p<0.0001$ ). Neurophysiolocal parameters have demonstrated better discriminative values in differentiating diabetics with CTS and DPN than clinical tests.

\section{2}

THE ROLE OF SENSORY SYMPTOMS IN THE ASSESSMENT OF DIABETIC POLYNEUROPATHY

G.D. Valk', P.A. Grootenhuis', J.Th.M. van Eijk', L.M. Bouter' and F.W. Bertelsmann'. 'EMGO-Institute, 'Department of Neurology, Vrije Universiteit Amsterdam

Recently, we have established the existence of two dimensions of sensory symptoms of diabetic polyneuropathy, pain and sensory alteration (e.g. complaints of numbness and paraesthesiae). To determine the role of sensory symptoms in assessing and following the course of diabetic polyneuropathy, we assessed the construct validity and reproducibility of the measurement of symptom severity of neuropathic pain and sensory alteration. In addition, we assessed the smallest detectable difference (SDD) on group level, to investigate the potential of detecting changes in clinical status (responsiveness). Seventy-eight patients with stable diabetic polyneuropathy were investigated on three separate occasions with a test-retest interval of 17 and 52 weeks, mean age 49.5 yrs. (range 27-65), 43 male and 35 female, mean diabetes duration 21.3 yrs. (range 1-46). Sensory symptoms were quantified using a previously validated questionnaire. Small nerve fibre function was measured on the basis of temperature discrimination thresholds for warmth and cold (TDT warmath and TDT cold, respectively), and large nerve fibre function was measured by testing sensory and motor nerve conduction velocities (SNCV and MNCV, respectively) and vibration perception thresholds (VPT). Neuropathic pain was only significantly associated with TDTold, and with the MNCV of the tibial nerve (correlation coefficients $(r) 0.26, p=0.04$ and 0.29 , $p=0.03$, respectively). Sensory alteration was associated with almost all nerve function tests except the SNCV and MNCV of the ulnar nerve ( $\mathrm{r}$ ranging from 0.26-0.53, $\mathrm{p}$ value from $0.04-0.0001$ ). The measurement of both dimensions of sensory symptom severity proved to be sufficiently reproducible (test-retest correlation $0.85-0.89$ ). The SDD (standardised to the corresponding mean of test and retest. group size $=100$ ) of the measurement of sensory alteration and neuropathic pain were almost the same $(9 \%$ and $12 \%$, respectively). In conclusion, sensory alteration appears to be useful in the assessment of diabetic polyneuropathy in daily clinical practice. In addition, the standardised measurement of symptom severity is reproducible and responsive and can, therefore, be useful for monitoring the course of polyneuropathy in clinical trials . 
1053

'NUMBNESS OF THE FEET' IS A POOR INDICATOR FOR POLYNEUROPATHY IN TYPE 2 DIABETIC PATIENTS

L.V. Franse', G.D. Valk' J.H. Dekket, R.J. Heind.2 and J.Th.M. van Eijk ! EMGO-

Institute, ${ }^{2}$ Department of Internal Medicine, Vrije Universiteit Amsterdam

Early diagnosis of diabetic polyneuropathy can decrease patient morbidity by allowing for therapeutic interventions, including patient education and regular foot surveillance Despite guidelines recommending yearly foot examination by general practitioners in patients with diabetes, chart reviews reveal that foot assessment for neuropathy is only seldomly performed. Patient history might be a quick and simple manner to identify patients with diabetic polyneuropathy in daily clinical practice. The aim of our study was to investigate if measurement of the severity of neuropathic symptoms can be used for this purpose. Type 2 diabetic patients $(n=578)$ from 20 general practices in the Netherlands, were examined. All patients underwent a Clinical Neurological Examination (CNE: pinprick sense, light touch sense (cotton wool), ankle jerk and vibration sense ( $128 \mathrm{~Hz}$ tuning fork)) and filled in a questionnaire listing neuropathic sensory complaints. Lineair regression analysis was used to estimate the association of the severity of neuropathic sensory complaints with the outcome of the CNE. Two dimensions of complaints existed: Pain and sensory alteration (numbness and parasthesiae). In contrast to pain, complaints of sensory alteration was associated with the $\mathrm{CNE}$ score. Of the dimension of sensory alteration the question about 'numbness of the feet' appeared to be the only item significantly related to $\mathrm{CNE}$ score $(\beta=0,35$ $p<0,01)$. Covariates age $(B=0,34 p<0,001)$, diabetes duration $(B=0,02 p<0,55)$ and $\mathrm{HbAlc}(\beta=0,07 p=0,05)$ did not confound this relation. However, with a sensitivity of only $32 \%$ and a specificity of $81 \%$, the positive and negative predictive values of this one question are very low. It can thus be concluded that the patient history is a poor indicator for diabetic polyneuropathy. Therefore, a clininal neurological examination has to become a routine part of the yearly diabetes consult.

\section{5}

PERIPHERAL NERVE REFRACTORY PERIOD IN DIABETES.

A.L. Carrington and S.A. Cannan. Institute for Diabetes Discovery, Branford, CT, USA.

Nerve function is often assessed in clinical and experimental diabetes as the conduction velocity of both motor and sensory nerve fibres. However, there are a number of problems associated with its measurement and the interpretation of the results obtained. The refractory period (RP) of such nerve fibres is also impaired in diabetic patients. Therefore, the aim of this study was to assess RP in vivo and in vitro, in order to determine if this impairment holds true for rats with short term diabetes. Two studies were carried out using control and streptozotocin-induced diabetic rats of four weeks duration. In the first study, the RP profile of the compound sensory nerve action potential (CSNAP) of the Saphenous nerve was measured in vivo, under isoflurane anesthesia. Paired stimuli (100uS, 20V) were used to determine the amplitude of a test CSNAP as a percentage of its maximum value, over an interstimulus interval (ISI) range of 2 to $0.2 \mathrm{~ms}$. In the second experiment, the RP profile was measured in the sciatic nerve (mixed) in vitro, using suction electrodes and bicarbonate buffered saline. A similar stimulation protocol was used as above, except that the amplitude of the test CNAP was expressed as a percentage of the conditioning CNAP over the interstimulus range of 4 to $0.4 \mathrm{~ms}$. In both studies diabetic rats were hyperglycaemic and had a lower body weight than controls. The RP profiles were expressed as the mean ISI required to produce a $50 \%$ decrease in the test CNAP. This ISI was estimated by linear interpolation between the responses that were immediately above and below $50 \%$. There were no significant differences found between controls and diabetics respectively (Study 1 : $0.60 \pm 0.04\{9\}$ v $0.67 \pm 0.3\{10\} \mathrm{ms}, p=0.14$; Study $2: 0.67 \pm 0.03\{6\}$ v 0.62 $\pm 0.02\{7\} \mathrm{ms}, \mathrm{p}=0.21$, [Mean $\pm \mathrm{SE}\{\mathrm{n}\}$, two-sample $t$-test]). These studies indicate that there may not be a clinically relevant difference in refractory period, which represents a measurement of a specific neuronal membrane function, between diabetic rats and controls at this early stage of diabetes.

\section{4}

THERMAL THRESHOLD TESTING-A QUANTITATIVE METHOD TO DETECT EARLY CHANGES IN SMALL NERVE FIBRES FUNCTION IN DIABETES

A. Korzon-Burakowska, J. Allawi, S. Burakowski and M. Przeżdziak. Department of Diabetology, Medical University, Gdańsk; Unit for Metabolic Medicine, Guy's Hospital, London.

The aim of the study was to determine whether testing thermal thresholds for cold and hot can detect early impairment in small fibre function in diabetic patients with no clinical evidence of neuropathy. Using the"Triple $T$ " Thermal Threshold Tester (Medilec ,UK) we have recorded thermal thresholds on the dorsum of the foot in diabetic patients without clinical evidence of neuropathy (group I, $n=54,29$ males, BMI 29.1 \pm 3.1 ), diabetic subjects with clinical neuropathy (group II, $n=48$, 29 males, BMI $27.1 \pm 3.3$ ) and in the group of 24 healthy volunteers (group III, 13 males, BMI 24.5 \pm 3.3 ). Mean age of subjects in all groups was $49.1 \pm 12.6$ years with no statistically significant difference between the groups. Diabetes control in group Il was worse than in group I (mean $\mathrm{HbAlc} 9.2 \pm 1.9 \%$ and $8.3 \pm 1.6 \%$ respectively, $\mathrm{p}=0.01$ ). Mean diabetes duration in group I was $106,4 \pm 84.5$ and in group II $141.3 \pm 104.9$ months $(p=0.06$ ). There was no statistically significant difference in thermal thresholds between men and women in each group. The mean thermal thresholds in groups I, II and III were: for hot $2.35^{\circ} \mathrm{C} ; 7,27^{\circ} \mathrm{C} ; 0.58^{\circ} \mathrm{C}$ and for cold $1.07^{\circ} \mathrm{C} ; 5,86^{\circ} \mathrm{C} ; 0.36^{\circ} \mathrm{C}$ respectively. There was statistically significant difference in hot and cold thermal thresholds between groups I and II $(\mathrm{p}<0.00001$ for hot and cold) and between groups I and III ( $p<0.001$ for hot, $p<0.01$ for cold) Conclusions: Diabetic patients with no clinical neuropathy have significantly impaired thermal thesholds as compared to the controls. This suggests that therma threshold testing may be a valuable method for detecting early changes in small fibres function which can not be found on clinical examination.

\section{6}

FACTOR ANALYSIS OF THERMAL AND VIBRATION SENSORY THRESHOLDS IN YOUNG IDDM PATIENTS.

Ofra Kalter-Leibovici ${ }^{1,2}$. Gill Yosipovich ${ }^{3}$, Uri Gabbay ${ }^{1}$, Moshe Karp ${ }^{2}$

1-Gertner Institute for Epidemiology \& Health Policy Research, Sheba Medical Center Tel-Hashomer: 2-Institute for Endocrinology \& Diabetes. Sclnneider Children's Mcdical Center of Israel, Petach-Tikva; 3-Department of Dermatology. Rabin Medical Center. Beilinson Campus. Petach-Tikva, Israel.

Objectives: a.) Identify factors that represent relationships among sets of interrelated thermal and vibration threshold variables: b.) Look for risk markers that are significantly and independently related to these factors.

Subjects: 148 patients ( $47.3 \%$ males), with young-onset IDDM. Median duration of diabetes: 11.4 years (range: $0.25-35.5$ ); median current age: 22.3 years (range:

13.8-44.3). Location: An outpatient hospital diabetes clinic. Methods: Thermal (cold. warmth and heat-related pain) and vibration sensory thresholds were evaluated over the thenar and methatarsal area. Data on patients' current age and age at onset of diabetes. mean cumulative glycosylated hemoglobin values since 1978 , tobacco consumption. presence of hypothyroidism, blood lipid profile. blood pressure. current height. weight and $\mathrm{BMI}$ values were collected.

Results: Factor analysis of the thresholds variables produced 3 factors: factor-1 which correlates with thenar cold. warmth and vibration sensation thresholds; factor-2 which correlates with methatarsal cold. warmth and vibration sensation thresholds; and factor-3 which correlates with thenar and methatarsal heat-related pain thresholds. More than $75 \%$ of the total variance is attributable to these 3 factors. In a multivariate analysis, the following risk markers were significantly and independently associated with factor-1: mean glvcosylated hemoglobin during the last year of follow-up and total-cholesterol values: factor-2: mean glycosylated hemoglobin values in the first vears of follow-up, total-cholesterol values and current age; factor-3: the presence of hypothyroidism.

Conclusions: Our data point at three distinct subsets of sensory thresholds in IDDM patients, with possible different underlying mechanisms. Glycemic control and total-cholesterol levels were significantly and independently associated with cold. warmth and vibration thresholds in the upper and lower limbs. Age was further significantly and independently related to cold. warmth and vibration thresholds in the lower limbs. In contrast, hypothyroidism was the only risk marker found to be significantly and independently related to heat-associated pain thresholds. 
THE IMPACT OF PERIPHERAL SENSORY NEUROPATHY ON BONE DENSITY IN PATIENTS WITH IDDM

M. Rix, H. Andreassen, P. Eskildsen. Dept.Int. Med., Roskilde County Hospital Koge, DK-4600 Koge, Denmark.

Introduction: IDDM is associated with reduced bone mineral density (BMD). The pathogenesis of the diabetic bone disease is, however, not fully understood, but the microangiopathy seen in many of these patients may be of importance. The aim of the present study was to investigate how peripheral sensory neuropathy (PSN), a part of the microangiopathic complex, affects BMD locally in the extremities and in the axial skeleton of patients with IDDM. Patients: Groun 1: 21 males with IDDM and severe PSN, mean age (range) 57 (50-66) years, weight: 78 (64-104) kg, HbAlc 8.2 (6.3-10.4)\% and with a duration of diabetes of 28 (9-59) years. Groun 2 $21 \mathrm{IDDM}$ patients with mild/absent PSN matched to patients of Group 1 regarding sex, age, weight, and duration of diabetes. Group 3: 21 sex-, weight- and age matched normal control subjects. Methods: BMD was measured by DEXA in the spine, femur and distal forearm and expressed as z-scores (values normalized to age and sex matched mean, Hologic reference population) and by Quantitative Ultrasound in the calcaneus expressed as Broadband Ultrasound Attenuation (BUA $\mathrm{dB} / \mathrm{MHz}$ ). Vibration perception threshold (VPT) in the feet was determined by biothesiometry. Severe PSN was defined as VPT $>36$ Volts; mild/absent PSN as a VPT <25Volts. Results: In Group 1 BMD z-scores were reduced in the spine ($1.01 \pm 0.34)$, the femur $(-0.94 \pm 0.25)$ and the forearm $(-1.10 \pm 0.36), p<0.01$. In Group 2 BMD was also reduced in the spine $(-0.60 \pm 0.26)$, the femur $(-0.55 \pm 0.25)$ and the forearm $(-1.05 \pm 0.36), p<0.05$. Group 3 had normal BMD: $-0.23 \pm 0.25$, $0.10 \pm 0.21,-0.07 \pm 0.25$ in the spine, femur and forearm respectively. Group 1 had significantly lower BMD than Group 3 in the femur $(p<0.05)$. BUA of the calcaneus was significantly lower in Group $1(108 \pm 3)$ than in Group $2(115 \pm 2)$ and Group 3 (115 \pm 2$)$, (p<0.05); but no difference was found between Group 2 and Group 3. Conclusions: 1) IDDM is associated with reduced BMD. 2) IDDM patients with severe PSN have reduced bone density of the calcaneus compared to IDDM patients without PSN and normal control subjects. 3) PSN in itself or as a part of the microangiopathic complex is associated with reduced bone density locally in the foot as well as in the skeleton in general in patients with IDDM
Is impaired glucose tolerance associated with autonomic and peripheral neuropathy?

Daniela Brugger, Hans-J. Lüddeke, Rolf Renner and K. Dietrich Hepp Krankenhaus München Bogenhausen, Diabeteszentrum, Englschalkingerstr. 77, 81925 München

Controversial data exist about the occurence of autonomic and peripheral neuropathy in patients with impaired glucose tolerance (IGT). Patients: After screening of 337 relatives of patients with type 2 diabetes (part of the STOP NIDDM Study) 42 subjects with impaired glucose tolerance (repeated testing) were identified and matched with 20 controls with normal glucose tolerance for age, sex and BMI. Age (controls in parentheses): $57 \pm 8$ (54 \pm 6 ), BMI $28.9 \pm 4$ (28.4 \pm 3$),$ WHR $0.94 \pm 0.07(0.95 \pm 0.1)$, glucose $0 \mathrm{~min} 109 \pm 8(93 \pm 6) \mathrm{mg} / \mathrm{dl}, 120$ min $167 \pm 15(115 \pm 10) \mathrm{mg} / \mathrm{dl}$. Methods: Autonomic nerve function tested by the -ProSciCard"-Analyzer during rest and deep respiration. Peripheral nerve function by sensory perception threshold for heat and cold. Results (only significant differences):

\begin{tabular}{l|lll}
\hline & IGT & Controls & $P$ \\
\hline Coefficient of heart rate variation at rest & $3.4 \% \pm 1.2$ & $4.6 \% \pm 1.6$ & 0.03 \\
Spectral analysis frequency $0.05-0.15 \mathrm{~Hz}$ & $0.4 \pm 0.4$ & $0.9 \pm 1.0$ & 0.002 \\
Frequency $0.15-0.5 \mathrm{~Hz}$ & $0.4 \pm 0.4$ & $0.8 \pm 0.9$ & 0.04 \\
Coefficient of variation (deep breathing & $7.6 \% \pm 3.9$ & $9.9 \% \pm 3.6$ & 0.03 \\
Perception threshold for heat & $12.2^{\circ} \mathrm{C} \pm 1.1$ & $11.2^{\circ} \mathrm{C} \pm 1.1$ & 0.004 \\
Perception threshold for cold & $5.5^{\circ} \mathrm{C} \pm 1.2$ & $4.8^{\circ} \mathrm{C} \pm 0.4$ & 0.005 \\
\hline
\end{tabular}

No significant differences: beats/min. during rest and deep respiration, lowfrequency band $(0.01-0.05 \mathrm{~Hz})$ and QTc-interval. Conciusion: Atthough no patient showed clinical symptoms, a significant deviation in autonomic and peripheral nerve function was found in subjects with impaired glucose tolerance.

\section{9}

NEUROQOL - THE FIRST SPECIFIC QUALITY OF LIFE SCALE FOR DIABETIC NEUROPATHY

L Vileikyte, C Bundy, J Shaw, B Tomenson, T Walsh and AJM Boulton, Manchester Royal Infirmary, Manchester UK.

Although diabetic neuropathy may result in severe morbidity and consequent effects on quality of life (QOL), to date, no specific QOL measures have been developed. Our aim was to develop a theoretically based, clinically relevant neuropathy-specific $Q O L$ instrument. An initial pool of 50 items, including a neuropathy-specific symptom checklist and psychosocial domains, was derived from an expert panel and semi-structured interviews with focus groups comprising 62 diabetic patients ( 15 controls and 47 with variable degrees of neuropathy). The initial self-report scale was then piloted on 153 diabetic patients with and without neuropathy (mean age 54.3 years; $46.4 \%$ males), on two occasions, 5-21 days (mean 11 days) apart in order to test psychometric properties. The sensitivity and specificity in the identification of those with neuropathy was, for the symptom checklist $79.2 \%$ and $89.3 \%$ respectively, and $72.6 \%$ and $82.1 \%$ for the psychosocial domains. Factor analysis: a) of symptom checklist revealed significance for 3 factors (pain; diffuse sensorimotor symptoms; negative symptoms/ impotence) accounting for $65.8 \%$ of variance; b) of psychosocial domains revealed significance for 3 factors (psychological, social and mixed psycho-social) accounting for $65.0 \%$ of variance. This $\mathrm{QOL}$ measure has a high degree of internal consistency (Cronbach's alpha 0.89 for physical, 0.94 for psychosocial domains), and excellent test-retest reliability $(\mathbf{r}=0.90)$. Correlations between relevant SF-36 subscores and our newly derived subscales (construct validity) were highly significant, varying from 0.36 to 0.75 . We conclude that a neuropathy-specific QOL scale has now been devcloped and validated. 


\section{Diabetic Foot}

1060

REPRODUCIBILITY OF SCREENING TESTS FOR DETECTION OF DIABETIC NEUROPATHY.

Goicolea I, Villar G, Izuzkiza AJ, Vicente MA, Vázquez JA.

Department of Endocrinology. Hospital de Cruces. Vizcaya. Spain.

Diabetic neuropathy (DN) is a major long term complication of diabetes, but it lacks a reliable diagnostic test. This study evaluates the reproducibility of several procedures used in the diagnosis of DN. A total of 112 type 2 diabetic patients (56\% women), with a mean age of $60 \pm 7 \mathrm{yr}$. and a mean diabetic duration of $13 \pm 8 \mathrm{yr}$. were asessed for DN using the following procedures: 1) Clinical asessment of symptoms; 2) SemmesWeinstein ${ }^{\mathrm{TM}}$ (SW) monofilament examination, 3) Achiles reflex, 4) Vibration sense threshold at the base of the hallux tested with a tunning fork (TF) and a biotensiometer (Bio). Six months later, all patients were re-examined by the same investigator. Patients affected with other known causes of neuropathy were excluded. Pearson correlation coefficient and Cohen's kappa were used for asessment of reproducibility of quantitative (Bio) and qualitative test, respectively. Results.

\begin{tabular}{|c|c|c|c|c|c|}
\hline & Kappa & $\mathbf{R}$ & S.E. & $95 \%$ C. I. & $p$ \\
\hline DN symptoms & 0.46 & & 0.065 & & $<0.001$ \\
\hline SW filament & 0.99 & & 0.07 & & $<0.001$ \\
\hline TF right toe & 0.43 & & 0.059 & & $<0.001$ \\
\hline TF left toe & 0,57 & & 0.06 & & $<0.001$ \\
\hline Right achilles reflex & 0,68 & & 0.07 & & $<0.001$ \\
\hline Left achiles reflex & 0.87 & & 0.07 & & $<0.001$ \\
\hline Bio $>15$ volts & 0.65 & & 0.70 & & $<0.001$ \\
\hline Bio (Quantitative) & & 0,77 & & $0.69-0.84$ & $<0.01$ \\
\hline
\end{tabular}

Conclusion: All diagnostic tools evaluated for DN are fairly reproducible although the SW monofilament shows the highest values. The choice of one or other test will depend on other characteristics, such as sensitivity, specificity, simplicity and cost-effectiveness.

\section{1}

DETECTION OF THE INSENSATE FOOT WITH THE SEMMES WEINSTEIN MONOFILAMENT : POSSIBLE SOURCES OF ERROR

M. McGill, L. Fan, R. Spencer, L. Molyneaux and DK Yue. Diabetes Centre, Royal Prince Alfred Hospital, Camperdown and Department of Medicine, University of Sydney, NSW, Australia

The $5.07 / 10 \mathrm{gm}$ monofilament (MF) is recommended as a simple device to identify the insensate foot. This study aimed to evaluate the accuracy of MF and optimum sites for testing. 2 batches of MF $(n=14, n=50)$ were calibrated using a Mettler Balance. Results demonstrated buckling force of neither batch is $10 \mathrm{gm}$, and differs from the theoretical value by up to $6 \mathrm{gm}(6.8 \mathrm{gm}$, range 4.1 $10.3 \mathrm{CV} 29 \% ; 7.2 \mathrm{gm}$, range 7.1-7.9, CV $4.9 \%$. Biological significance of these differences was tested in 117 subjects using 5 and $15 \mathrm{gm}$ MF on 5 sites; 1 -great toe, 2-dorsum between 1st and 2nd metatarsals, 3-plantar aspect of 1st metatarsal, 4-plantar aspect of 5th metatarsal, 5-plantar aspect of arch. Using $5 \mathrm{gm}$ MF as the standard, testing with 15gm MF showed:-

\begin{tabular}{|l|l|l|l|l|l|}
\hline$\% n=117$ & Site 1 & Site 2 & Site 3 & Site 4 & Site 5 \\
\hline Sensitivity & 87.5 & 62.5 & 58.3 & 71.4 & 47.0 \\
\hline Specificity & 100 & 97.8 & 95.7 & 100 & 100 \\
\hline
\end{tabular}

The problem of inaccurate buckling force of MF can be overcome by The problem of inaccurate buckling force of MF can be overcome by
application of Euler's Buckling Theory (Force $\propto$ Young's modulus $\times \pi d^{4} / 64$ length of filament ${ }^{2}$ ), where buckling force can be changed by altering the length of the filament. Using Biothesiometer $>40$ as the standard, testing of 132 subjects with a calibrated $10 \mathrm{gm}$ MFat the 5 sites showed:-

\begin{tabular}{l|l|l|l|l|l}
$\% \mathrm{n}=132$ & Site 1 & Site 2 & Site 3 & Site 4 \\
\hline
\end{tabular}

\begin{tabular}{|l|l|l|l|l|}
\hline Sensitivity & 43.8 & 68.1 & 70.8 & 72.9 \\
\hline Speciticity & 96.4 & 89.3 & 91.7 & 90.5 \\
\hline
\end{tabular}
If insensate foot is defined as one of 5 sites cannot feel MF: sensitivity $83 \%$, specificity $77 \%$, if one or both of Sites 3 or 4 cannot feel MF: sensitivity $80 \%$, specificity $86 \%$. In conclusion, these results demonstrate that i) each batch of MF must be calibrated as incorrect buckling force affects accuracy of clinical and epidemiological studies ii) the site(s) selected in testing sensation is dependent on time available for testing and degree of sensitivity and specificity required. In general, a single site is not sufficiently sensitive but a combination of Site 3 or 4 represents a reasonable compromise.

\section{2}

BIO-IMPEDANCE TO ASSESS HYDROSTATIC SYSTOLIC TOE PRESSURE IN THE DIABETIC FOOT.

B. Beyenal-ögmen, E. Chantelau. Diabetes-Fußambulanz Heinrich-Heine-Universität Düsseldorf, Germany Hydrostatic measurement of systolic toe blood pressure (HSTBP) is reliable in feet with calcified arteries, when sphygmomanometry fails. The leg being lifted above heart level, weaning of the big toe's arterial pulsations indicates HSTBP.Bioimpedance was assessed to monitor the weaning of arterial pulsations(impedance sphygmography ISG; Medis GmbH, Ilmenau, Germany). Methods: In a pilot study in 30 healthy volunteers, a tourniquet was placed above the ankle; ultrasound ( $8 \mathrm{mHz})$ and ISG were used simultaneous$I_{y}$ to detect arterial pulsations in the big toe, in the sitting and supine position after opening the tourniquet. In a clinical study, HSTBP was assessed by big toe ISG in 48 diabetic legs with PIVD subjected to arteriography before vascular surgery. Results: In the pilot study,ultrasound and ISG signals occurred at nearly identical systolic pressure levels (difference 2.4 (SEM 0.6) $\mathrm{mm} \mathrm{Hg}, r=0.99, p=0.001)$. In the clinical study, HSTBP $\leq 50 \mathrm{~mm} \mathrm{Hg}$ was associated with multiple occlusions ( 3 legs with $\leq 1,9$ legs with 2 , and 12 legs with $\geq 2$ arteries occluded); by contrast, HSTBP > $50 \mathrm{~mm}$ Hg indicated single occlusions (14 legs with $\leq 1,8$ legs with 2 , and 2 legs with $\geq 2$ occluded arteries; $x^{2}$ contingency $\left.p<0.05\right)$. In conclusion, assessment of big toe arterial pulsation by ISG was reliable. HSTBP $<50 \mathrm{~mm}$ Hg indicates severe PIVD ( $\geq 2$ arterial occlusions).
1063

BONE TURNOVER MARKERS IN ACUTE CHARCOT NEUROARTHROPATHY

${ }^{2} \mathrm{PL}$ Selby, ${ }^{1} \mathrm{~EB}$ Jude, ${ }^{1} \mathrm{~J}$ Burgess, ${ }^{2} \mathrm{~S}$ Page, ${ }^{3} \mathrm{ME}$ Edmonds, ${ }^{3} \mathrm{~A}$ Foster, ${ }^{1}$ EB Mawer, 'JE Adams and ${ }^{1} \mathrm{AJM}$ Boulton. ' Department of Medicine and Radiology, Manchester Royal Infirmary, ${ }^{2}$ Queens Mcdical Centre, Nottingham, ${ }^{3}$ Kings College Hospital, London, UK.

Few previous studies have reported on potential pathogenetic mechanisms in the Charcot foot because of the relative rarity of this condition. In a three-centre study we have now investigated 25 patients with acute Charcot neuroarthropathy (Criteria for diagnosis: severe peripheral neuropathy, temperature difference $>2^{\circ}$ between feet, palpable pulses, charactcristic radiological changes and positive bisphosphonate bone scan), and compared with them with 14 -age matched neuropathic controls.

Measurements included plasma osteocalcin, urinary hydroxyproline, urinary DPD crosslinks and plasma bone specific alkaline phosphatase (BSAP). Results showed significant elevation of BSAP in patients with charcot neuroarthropathy $(19.2 \pm 1.7$ vs $12.2 \pm 0.8 \mathrm{lU} / \mathrm{l}$; $p=0.0002)$. No significant changes were seen between the other biochemical parameters. The sensitivity and specificity of BSAP for the diagnosis of charcot neuroarthropathy were 0.27 and 1.00 , respectively. We conclude that the acute phase of Charcot neuroarthropathy is characterised by increased bone turnover and therefore elevation of BSAP may be an additional useful marker for the diagnosis of acute Charcot foot 
1064

POWER SPECTRAL ANALYSIS OF HEART RATE VARIATIONS IMPROVES ASSESSMENT OF AUTONOMIC NEUROPATHY IN PATIENTS WITH CHARCOT NEUROARTHROPATHY

A.Jirkovská, P.Bouček, V.Woskovâ, J.Hosová, J.Skibová and J.Pumprla a. Institute for Clinical and Experimental Medicine, Prague, Czech Republic and ${ }^{x}$ Institute for Biomedical Technique und Physisc, Vienna, Austria

Conclusive diagnosis of Charcot foot may be difficult mainly in the early stage. Assesment of autonomic neuropathy (AN) can help in the diagnostic procedure. The aim of the study was to compare short-term power spectral analysis and Ewing's battery (R-R interval analysis during deep breathing, standing-lying and Valsalva manoeuvre) of cardiovascular autonomic function tests in patients with Charcot neuroarthropathy $(\mathrm{CN})$. Both investigations were performed on a computer-aided system for the examination of heart rate variability (Varia Pulse TF 3, Sima Media, Olomouc, CZ). A group of 18 patients ( 10 with Type 1 and 8 persons with Type 2 diabetes with disease duration of $15 \pm 7$ years) with early stage $\mathbf{C N}$ did not differ in mean age ( $52 \pm 14$ and $53 \pm 8$ years) from 30 sex-matched non-diabetic control group. Four patients could not be assessed by complete battery of Ewing's tests (because of non-compliance or contraindications) and one patient by spectral analysis. Results: All patients with $\mathrm{CN}$ assessed by Ewing's battery had AN taken as abnormality in two of three tests, the mean values of these tests differ significantly from those of the control group with the exception of standing-lying 30:15 rations, the best sensitivity (by $95 \%$ specificity) was shown for the I-E difference by deep breathing, $R_{R_{\max }} / R_{R_{\min }}$ and Brake Index during standing-lying and Valsalva Ratio, the worst sensitivity was found with orthostatic blood pressure fall. The patients with $\mathrm{CN}$ differred significantly from the control group also in all parameters of spectral analysis with the exception of the low frequency (LF) to high frequency (HF) ratio, the best sensitivity by $95 \%$ specificity had the parameter PSD low in successive lying position (PSD Lo 3) and the sum of total power in standing and successive lying positions (TP 2+3). Using the 5th percentil of PSD Lo 3 and TP $2+3$ in the control group as the cut point for $\mathrm{AN}, 94 \%$ and $82 \%$ respectively of patients with $\mathrm{CN}$ had AN by spectral analysis. We conclude that spectral analysis may help considerably in diagnosing early stage Charcot foot because it can well identify AN, offers a shorter examination time and is better tolerated by patients compared to classical Ewing's test battery.

\section{5}

THE DIAGNOSIS OF PEDAL OSTEOMYELITIS IN DIABETIC PATIENTS.

T.Kearney $y^{2}$, K. Pointin ${ }^{b}$, D. Cunningham ${ }^{b}$, W. Gedroyc ${ }^{b}$, S. Robinson ${ }^{a}$, R. Elkeles ${ }^{a}$ Department of Metabolic Medicine $e^{a}$, Institute of Radiology ${ }^{b}$, St. Mary's Hospital, Paddington, London.

Osteomyelitis in patients is a source of major morbidity, necessitating prolonged hospitalisation. There is no single, reliable, diagnostic tool; plain films are inaccurate, bone scans fail to differentiate Charcot's arthropathy from infection, and the role of human pooled immunoglobulin (Hig) scans remain unclear Magnetic resonance imaging (MRI) may prove to be sensitive and useful in localising infection. We therefore aimed to compare these four diagnostic modalities. Thirteen diabetic patients with possible pedal osteomyelitis were recruited. Nine were found to have osteomyelitis based on clinical, microbiological, and surgical fincings. All were scanned with all four modalities and the results compared. MRI scans correctly identified all positive cases, with two false positive cases and excelled in localising infection, allowing for surgical planning. Plain scans and Hig scans were less sensitive $(67 \%, 89 \%$ respectively), were difficult to interpret and were poor in localising infection. Bone scans showed a sensitivity of $89 \%$ with a negative predictive value of $100 \%$, but failed to differentiate Charcot's arthropathy from osteomyelitis. In conclusion, our study suggests that $\mathrm{X}$-rays and Hig scans are not diagnostically helpful; bone scans are a useful screening tool, but when positive an MRI scan should be sought to confirm the diagnosis in diabetic patients.

\section{6}

HIGH RESOLUTION ULTRASOUND FOR THE DIAGNOSIS OF OSTEOMYELITTS IN THE DIABETIC FOOT SYNDROME M.D. Enderle, S. Coerper, H.P. Schweizer, A.F.Kopp, C. Meisner, H.D. Becker, H.U. Haring, and D. Luft. University of Tubingen, Tubingen, Germany.

Background: Osteomyelitis of the foot is a major cause for morbidity and mortality in diabetic patients and its detection is a critical issue in the treatment of the diabetic foot. Objective: To assess the impact of ultrasound in the diagnosis of osteitis / osteomyelitis in the diabetic foot syndrome. Research design and methods: We prospectively investigated 19 consecutive diabetic patients $(2 \mathrm{f}, 17 \mathrm{~m}$, age $60.7 \pm 9.8$ y, BMI $27.0 \pm 3.8 \mathrm{~kg} / \mathrm{m}^{2}$, diabetes duration $11.0 \pm 8.2$ y, HbAlc $8.1 \pm 1.1 \%$, creactive protein $3.9 \pm 4.3 \mathrm{mg} / \mathrm{dl}$ ) with clinical suspicion of bone infection of the foot (classification of Wagner $\geq 2$ ). A high resolution ultrasound system (Esaote / Biosound, Munich) with a linear array transducer up to $13.0 \mathrm{MHz}$ and an axial resolution of $0.12 \mathrm{~mm}$ was used. The sonographic results were compared with both plain film radiography (PFR) and magnetic resonance imaging (MRI). Histopathology after metatarsal resection served as the gold standard.

Results: In 14 out of 19 patients histopathology confirmed osteitis / osteomyelitis $(74 \%)$. Ultrasound showed a sensitivity of $78.6 \%(95 \% \mathrm{CI}=49.2-95.3 \%)$ : PFR $69.2 \%(38.3-90.0 \%)$; MRI: $100 \%(75.3-100 \%)$. The specificity of ultrasound was $80.0 \%$ (28.4 - 99.5\%); PFR: $80.0 \%$ (28.4 - 99.5\%); MRI: $75 \%(19.4-99.4$ $\%)$. The positive predictive value was $91.7 \%(61.5-99.8 \%)$; PFR: $90.0 \%(55.5$ $99.7 \%)$; MRI: $92.9 \%(66.1-99.8 \%)$. The negative predictive value was $57.1 \%$ (18.4 - 90.1\%); PFR: $50.0 \%(15.7$ - 84.3\%); MRI: $100 \%(29.2$ - $100 \%)$

Conclusions: High resolution ultrasound is a useful tool for the diagnosis of osteomyelitis / osteitis and compares well to other methods in diabetic patients with clinical suspicion for this complication.

\section{7}

RADIOIMMUNE IMAGING OF DIABETIC FOOT INFECTIONPLANAR AND SINGLE PHOTON EMISSION TOMOGRAPHY H.A.Bohchelian, A.D.Klisarova and L.A.Koeva, Clinic of Endocrinology, Department of Radiology, Medical University, Varna, Bulgaria The aim of the study was to evaluate the clinical efficacy of immunoscintigraphy in planar and single photon emission computer tomographic (SPECT) imaging in diabetic foot infection. Thirty patients with I and II type diabetes with foot ulceration and possible infection (mean age - 54,6 I 7,2 years, BMI- $24,3 \pm 1,4 \mathrm{~kg} / \mathrm{m}^{2}$, sex ratio - M/F - 16/14) were included in the study. All patients underwent a clinical examination, electromyography, radiography, immunoscintigraphy, investigation of blood glucose level, HbA1c, Doppler ankle/arm pressure index, bacteriological cultures. Immunoscintigraphy with $740 \mathrm{MBq}$ monoclonal antigranulocyte antibody (MAb) BW 250/183 conjugated with technetium (Tc) - 99m was carried out in order to prove or exclude an inflammatory foot process. Final diagnosis was verified by bacteriological cultures and a long-term follow -up.The following results were obtained-osteomyelitis was present in 5 cases, cellulitis-in 22 patients, non-infected neuropathic foot ulcers - in 3 cases. SPECT study was applied at a second stage in all cases when planar immunoscintigraphy was insufficient to distinguish soft-tissue inflammation from osteomyelitis. The analysis of diagnostic validity in detection and precise localization of foot infection by the applied method revealed diagnostic accuracy - $92 \%$. SPECT technique provided more positive scan findings and higher accuracy than planar imaging. Antigranulocyte immunoscintigraphy with $99 \mathrm{~m}$-Tc-labelled monoclonal antigranulocyte antibody is an efficient method for early diagnosis and adequate therapeutic decision in diabetic foot infection. SPECT imaging is superior to planar scanning for precise localization and differentiation between osteomyelitis and cellulitis. 
1068

THE ROLE OF NITRIC OXIDE SYNTHASE AND ARGINASE IN DIABETIC FOOT ULCERS

EB Jude, AJM Boulton and I Appleton". Department of Medicine/ Diabetes, Manchester Royal Infirmary and The School of Biological Sciences", The University of Manchester, Manchester, U.K.

Diabetic foot ulcers (DFUs) are a major clinical concern with increased morbidity and mortality which may, in severe cases lead to amputation. L-arginine is metabolised by one of two pathways; nitric oxide synthase (NOS) and arginase. The metabolism of L-arginine can result in tissue destruction if via NOS or matrix deposition if by arginase. In this study we have investigated the activity, protein levels (by Western blot analysis) and cellular distribution (using immunocytochemistry) of these two enzymes in skin adjacent to foot ulcers (DFU), diabctic skin (DS) and normal skin (NS), with a minimum of $n=10$ per group. The results demonstrated significantly increased $(p<0.001)$ levels of both total and

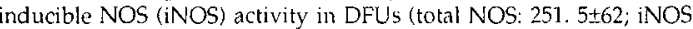
$323 \pm 66 \mathrm{pmol} L$-citrulline/mg protein) compared to DS $(132.5 \pm 31 ; 123 \pm 23$ ) and NS $(172 \pm 76 ; 143 \pm 48)$. Inducible NOS was the major isoform with the macrophage being the predominant cellularsource. Arginase activity was significantly increased $(p<0.01)$ in DFUs $(1.4 \pm 0.45 \mathrm{~kg}$ urea/ $\mathrm{mg}$ protein) in comparison to DS (0.22 \pm 0.07$)$ and NS $(0.16 \pm 0.05)$. The protein levels corroborated with the activity data, with the fibroblast being the major source. The spatial and cellular distribution of the two enzyme systems was distinct. This therefore leads us to speculate that the raised levels of NOS in DFUs may contribute to the impaired healing of DFUs. Furthermore, the increased levels of arginase could account for the characteristic callus formation in DFU.
1069

SERIAL MEASUREMENTS OF FOOT ULCER MICROVASCULAR OXYGEN SATURATION LEVELS PREDICT HEALING

N.D. Harris, S.M. Rajbhandari, S. Tesfaye S and J.D. Ward

Diabetes Research Unit and Department of Medical Physics, Royal Hallamshire Hospital, Sheffield, UK

Adequate oxygenation is a prerequisite for tissue repair and healing of diabetic foot ulcers. However, little is known about the changes in oxygenation of foot ulcers during this process. We measured the microvascular oxygen saturation of foot ulcers in a group of diabetic patients, to investigate the characteristics of ulcers which heal without any intervention. Microvascular oxygen saturation was measured within the ulcer, its margin and the identical site on the contra-lateral (control) limb using a microlightguide spectrophotometer. We studied 13 patients with diabetic foot ulcers (20 ulcer sites). Surgical intervention was planned in 5 patients ( 8 ulcers sites) which did not show any signs of healing due to underlying ischaemia or osteomyelitis. In 12 ulcer sites (mean area 2.6 $\mathrm{cm}^{2}$ ) the measurements were repeated at $5( \pm 2)$ weeks when the area had reduced to between $30 \%$ and $70 \%$ of the original size (mean area $1.3 \mathrm{~cm}^{2}$ ). In these patients, there was a significant reduction in oxygen saturation (initial $60 \%$ vs $47 \%, \mathrm{p}<0.01$ ) within the ulcers, with a trend for its reduction at the margin ( $49 \%$ vs $44 \%$; $p=0.09$ ). There were no significant changes in control site $(43 \%$ Vs $40 \%)$. There were no significant changes in the oxygen saturation of the ulcers that did not heal $(44 \%$ vs $41 \%, n=7)$ at $5( \pm 2)$ weeks and their size remained unchanged (mean $1.2 \mathrm{~cm}^{2}$ vs $1.4 \mathrm{~cm}^{2}$ ). In conclusion, there is normally a reduction in the microvascular oxygen saturation of foot ulcers as they heal. Serial measurements of oxygen saturation may be useful for the early identification of ulcers that require surgical intervention.
1070

FACTORS AFFECTING PRIMARY HEALING IN DIABETIC FOOT LESIONS

Ch. Manes, Th. Mesimeris, Th. Melekos, E. Voskopoulos, E. Papadeli and N. Papazoglou. Diabetes Unit and Unit of HBO Treatment $-1^{\text {st }}$ General Hospital "St. Paul". Thessaloniki - GREECE

Peripheral Vascular Disease (PVD), neuropathy (DN) and the resulting tissue hypoxia (TH) are considered as the major contributing factors in the aetiopathogenesis of foot ulceration (FU). To investigate the role of the above factors in the healing process in diabetic foot lesions a prospective study was undertaken. 44 diabetic patients (28 males, 6 type 1) were studied. Mean age and diabetes duration were $61,32 \pm 9.1$ and $13,13 \pm 8,29$ yrs respectively. Presence and severity of DN was assessed by a) clinical examination using score techniques and b) quantitative sensory testing (estimation of Vibration Perception Threshold -VPT using the biothesiometer and Pressure Perception by the Semmes-Weinstein monofilaments). PVD was diagnosed clinically and confirmed by measuring the ankle pressure index (API). Foot lesions and tissue oxygenation were evaluated according to the Wayner's grading system and to the measurements of $\mathrm{TCPO}_{2}$ in normal and hyperbaric conditions (2,4 ATA) respectively. After a two month period of treatment the relative risk (RR) for amputation was calculated in patients with severe ischaemia neuropathy and reduced $\mathrm{TCPO}_{2}$. Results: 1) Ulcers: a) grade I $\mathrm{n}=\mathbf{2}$, grade $2 n=25$, grade $3 n=8$, grade $4 n=9$. b) neuropathic $n=17$, neuroischaemic $n=23$ and ischaemic $n=4$, c) group A: 28 were healed $(63,6 \%)$, group $B: 10 \quad(22,7 \%)$ improved and group $\mathrm{C}: 6(13,6 \%)$ failed. 2$)$ In univariate analysis no differences were established between group A and B reganding the presence or severity of DN, $\mathrm{PVD}, \mathrm{FU}$ and $\mathrm{TH}$. Values of API and $\mathrm{TCPO}$ (in 2,4 ATA) were lower in group C than in patients of group $A_{1}$ (groups $A+B=$ group $\left.A_{1}\right)(0,87 \pm 0,07$ vs $0,55 \pm 0,36$ and $419,77 \pm 223,08$ vs $133,33 \pm 126,79 \mathrm{~mm} \mathrm{Hg}$ respectively, $p<0,05$ in both cases). DN and severe foot lesions were more prevalent in group $C$ than in group $A_{1}(p<0,05)$. 3) Logistic regression analysis showed that only severity of DN and $\mathrm{TcPO}_{2}$ (in 2,4 ATA) were independent predictors of wound healing $(\mathrm{p}<0,05)$. 4) $\mathrm{RR}$ for amputation (group C) was 6,02 for low TcPO2 (in 2,4 ATA) $(\leq 200 \mathrm{~mm} \mathrm{Hg}), 3,17$ for critical PVD and 2,92 for severe DN. Conclusion: Tissue oxygenation and severity of DN are critical determinants of diabetic ulcer healing.

\section{1}

RUPTURE OF THE PLANTAR FASCIA IN THE DIABETIC FOOT LEADS TO TOE DORSIFLEXION DEFORMITY R. Taylor, G.D. Stainsby and D.L. Richardson. Royal Victoria Infirmary, Newcastle upon Tyne, U.K.

Excess pressure under metatarsal heads leads to ulceration, although the genesis of the predisposing foot abnormality has been unclear. The typical at risk foot exhibits dorsiflexed toes. Normally, toe position is maintained by the plantar fascia which straightens and plantar-flexes the toes during weight bearing. We hypothesised that the fascia may commonly rupture in longstanding diabetes and therefore carried out MRI scans of the fascia (foot dorsiflexed) in 12 consecutive patients with dorsiflexed toes. The patients ( 4 Type 1,2 insulin treated Type 2 and 6 non-insulin dependent) had a mean $\pm S D$ age $58 \pm 3.5 \mathrm{y}$, duration of diabetes $16.1 \pm 2.2 \mathrm{y}, \mathrm{HbAlc} 8.9 \pm 0.5 \%$, serum creatinine $90.2 \pm 0.5 \mathrm{uM}$, urinary albumin/creatinine ratio $8.8 \pm 0.1$ and 9 had microvascular complications of diabetes. All 12 patients exhibited a concertina-ed and discontinuous plantar fascia indicating rupture The appearance was observed in none of 12 matched diabetic subjects with straight toes. The existence of an acute rupture syndrome therefore may be postulated, and we have observed this in a Type 1 diabetic colleague who presented to us directly, rupture with oedema being seen on MRI scan. Glycosylation of connective tissue decreases compliance and is likely to increase chance of rupture. These observations offer a dramatic new insight into the pathophysiology of the diabetic foot and carry major implications for design of footware. 
BETA-1,3-D POLYGLUCOSE AND GROWTH FACTORS IMPROVE WOUND HEALING IN DIARETES MELLITUS M.Berdal, R.Seljelid and T.G.Jenssen, University of Tromse, Tromse, Norway Healing of diabetic wounds is delayed in diabetes mellitus, partly by way of altered cellular mechanisms in the healing wound. During the earty phase of the healing process the macrophages infiltrate the wound and take part in the inflammatory response by producing cytokines like Tumor Necrosis Factor- $\alpha$ (TNF- $a$ ) and growth factors like Platelet-Derived Growth Factor (PDGF) and Insulin-like Growth Factor-1 (IGF-1).

Monocyte-macrophage dysfunctions have been described in diabetes mellitus. Beta-1,3-D polygheose (BDP) is known to improve macrophage chemotaxis and secretory activity. The present study was undertaken to see if $\beta-1,3-D$ polyglucose or PDGF +IGF-1 would improve wound healing. Cutaneous wounds were established on the back of either diabetic (C57B1-db/db)- or non-diabetic (C57Bl-db/+)-mice. Six groups were studied: $1 \mathrm{db} / \mathrm{db}$-mice with placebo wound treatment ( $\mathrm{n}=24$, average blood glucose (BG) $26,0+1,3 \mathrm{mmo} / \mathrm{h}, \mathrm{p}<0,05), 2 \mathrm{db} / \mathrm{db}$ mice treated locally with BDP, 1 dose $(\mathrm{n}=12, \mathrm{BG} 26,4 \pm 1,7 \mathrm{mmol} / \mathrm{)}), 3 \mathrm{db} / \mathrm{db}-\mathrm{mice}$ treated locally with BDP, 5 doses $(\mathrm{n}=13$, BG $26,2 \pm 1,1 \mathrm{mmol} / \mathrm{)}), 4 \mathrm{db} / \mathrm{db}$-mice treated locally with BDP, 10 doses $(n=12$, BG $25,8 \pm 2,05 \mathrm{db} / \mathrm{db}$-mice treated locally with PDGF + IGF-1 (n=12, BG 26,3+2,2 mmol/h) and 6 non-diabetic control mice without treatment $(n=15, B G-1 \pm 0,3 \mathrm{mmol} /)^{-}$. Biopsies from placebo-treated diabetic wounds showed decreased infiltration of macrophages. The LPS-stimulated release of TNF- $\alpha$ from isolated peritoneal macrophages was only $1 / 8$ of that from non-diabetic mice. The percentage reduction in wound area after 10 days for group $1-6$ was: $3 \pm 2,2 \pm 2,5,17 \pm 3,11 \pm 3,16 \pm 5$ and $69 \pm 3 \%$ (mean \pm SEM, $p<0,05$ ). The corresponding results after 15 days were: $28 \pm 3,28 \pm 4$, $49+3,53+4,50+11$ and $95+1 \%(p<0,05)$.

Conclusion: Macrophage function as judged by TNF- $\alpha$ release, is impaired in diabetes. Topic applications of $\beta-1,3-D$ polyglucose or PDGF + IGF-1 in diabetic mice improve wound healing significantly despite serious hyperglycemia.
ELECTRICAL STIMULATION AS AN ADJUNCT TO HEAL DIABETIC FOOT ULCERS

E.J.G. Peters, D.G. Armstrong and L.A. Lavery. University of Texas Health Science Center at San Antonio, Texas, U.S.A.

The purpose of this study was to evaluate the effectiveness of electrical stimulation as an adjunct to diabetic wound care. The study is designed as a twelve-week randomized, double blind, placebo-controlled trial consisting of two groups of 20 diabetic patients with neuropathic foot ulcers. No significant differences between the groups were present concerning Hbalc levels, age, sex and neuropathy. Inclusion criteria included: transcutaneous oxygen $>30 \mathrm{mmHg}$, grade 1 or II ulcer older than 4 weeks. Exclusion criteria were: cardiac conductivity abnormalities, osteomyelitis and history of malignancy. The treatment group received nocturnal electrical stimulation from an electrical stimulation device, delivered trough a dacron-mesh silver nylon stocking. Following instruction as to its proper use, a dose of 50 volts (DC) at 80 pulses per second was delivered every night for an 8 hour period. The placebo group used identically functioning inactivate electrical stimulation units. All patients used a removable cast to offload the foot. Furthermore, both groups received traditional wound care consisting of weekly debridements. For evaluation of wound closure, weekly acetate tracings and photographs were made Microvascular assessment consisted of transcutaneous oxygen pressure measurements. Outcomes included: success of wound closure, hospitalization, presence or absence of infection and proximal amputation and compliance. $65 \%$ of the patients healed in the group treated with stimulation and $35 \%$ healed with placebo $\left(\chi^{2}=3.60, p=0.058\right)$. When patients within the treatment group were stratified by compliance (compliant defined as electrical stimulation use $>20$ hours a week), a significant trend was identified between compliant patients ( $71 \%$ healed), non-compliant patients $(50 \%$ healed) and patients that used placebo $(35 \%$ healed $)\left(\chi^{2}\right.$ for trend $\left.=4.35, p<0.04\right)$. These data suggest that electrical simulation may enhance wound healing when used in conjunction with appropriate offloading and local wound care. Therefore, it is likely to contribute to the earlier return to functional activity and increased patient satisfaction.

\section{4}

\section{THE EFFICACY OF INJECTED LIQUID SILICONE IN THE REDUCTION OF FOOT PRESSURES AND CALLUS FORMATION IN THE DIABETIC FOOT.}

C.H.M. van Schie ${ }^{1}$, A. Whalley', L. Vileikyte', T. Wignall', S. Hollis ${ }^{2}$ and A.J.M. Boulton'. 'Department of Medicine, Manchester Royal Infirmary, UK and ${ }^{2}$ Medical Statistics Unit, Lancaster University, Lancaster, UK.

Case studies have suggested the therapeutic use of silicone injections in the neuropathic or painful foot and this was tested in a randomised double-blind trial. Twenty-nine diabetic neuropathic patients without peripheral vascular disease were randomised to active treatment consisting of 6 injections of 0.2 ml liquid silicone in the plantar surface of the foot or to equal volume of saline (control). There were no significant differences in age and neuropathy status (confirmed by quantitative sensory testing) between the two groups. All injections were done under the metatarsal heads at sites with callus or high pressures. Barefoot plantar pressures (pedobarography) and subcutaneous thickness under the metatarsal heads (planscan ultrasound device) were measured at baseline, 3, 6 and 12 months after first injection. Throughout the study patients were seen by the same podiatrist for all podiatry treatment. Patients who received active treatment had significantly increased subcutaneous thickness at injection sites compared to the control group (31.8 vs $0.7 \%)(p<0.0001)$ and corresponding significantly decreased plantar pressures $(-20.6$ vs $+0.5 \%)(p<0.05)$ at 3 months. Similar figures were seen at 6 and 12 months. The results confirm the efficacy of silicone injections under metatarsal heads to increase subcutaneous thickness and reduce plantar pressures thereby reducing the risk of foot ulceration.

\section{5}

EVALUATION OF THE EFFECT OF PERCUTANEOUS TRANSLUMINAL ANGIOPLASTY IN PATIENTS WITH THE DIABETIC FOOT

V.Wosková, A.Jirkovská, J.Peregrin and J.Skibová. Institute for Clinical and Experimental Medicine, Department of Diabetes, Prague, Czech Republic.

The aim of this study was to evaluate the effectiveness of percutaneous transluminal angioplasty (PTA) in patients with the diabetic foot treated at a diabetic foot clinic. The study was conducted to evaluate 54 diabetic patients (17 and 37 with Type-1 and 2 diabetes, respectively, 33 males, 21 females, with a mean age of $59 \pm 12$ years and mean diabetes duration of $18 \pm 9$ years) undergoing technically successful primary PTA in 1995-1997. The patients were followed up for 11 \pm 7 months (range 1-27 months) after PTA. The criteria assessed included clinical benefit defined as improvement in claudication or resolution of rest pain, healing ulcers or healing minor amputations, ankle brachial index (ABI) changes (in $\mathbf{2 5}$ lower limbs) and transcutaneous oxygen tension (in the last 8 consecutive patients). Of the $\mathbf{5 7}$ evaluated lower limbs, the indications for PTA included non-healing ulcer (Wagner II-III) in $19(33 \%)$, gangrene (Wagner IV) in $19(33 \%)$, claudications in $12(21 \%)$ and rest pain in $7(13 \%)$ lower limbs. Angioplasty was performed separately in $4(7 \%)$ iliac arteries, in $16(28 \%)$ femoral arteries, in $24(42 \%)$ popliteal arteries and its branches. In 13 limbs $(23 \%)$, PTA was performed simultaneously in femoral and crural arteries. In 8 lower limbs, a repeat PTA was involved. Complications of PTA occurred in 4 (7\%) patients (bleeding at puncture site 2 cases, and renal function impairment and congestive heart failure one case each). Results: PTA led to clinical benefit in $38(67 \%)$ limbs: ulcer healed in $11(19 \%)$ cases, minor amputation healed in $12(21 \%)$ cases, claudications improved in $10(18 \%)$ cases, and rest pain resolved in $5(9 \%)$ cases. ABI improved significantly $(0.58 \pm 0.3$ vs $0.75 \pm 0.3 \mathrm{p}<$ 0.001 ). In 7 of 8 examined patients, a rise in transcutaneous oxygen tension was seen after PTA $(24 \mathrm{mmHg}$, range $1-49$ vs $46 \mathrm{mmHg}$, range $3-73$, p < 0.05 ). Conclusion: Our results suggest that PTA is an effective method for the treatment of peripheral arterial disease in diabetic patients. It has little risk of complications, it is repeatable and has a potentially beneficial outcome. 
1076

MICROVASCULAR REACTIVITY IN THE NEUROPATHIC FOOT REMAINS IMPAIRED AFTER SUCCESSFUL REVASCULARIZTION

A Veves, S Arora, P Smakowski, H Pham, FW LoGerfo. Microcirculation Lab, BIDMC, Harvard Medical School, Boston, MA, USA

We have examined the effect of successful large vessel revascularization on the microcirculation of the neuro-ischemic diabetic foot. We have tested 13 diabetic ischemic patients (DI group, 11 Males, age $62 \pm 3$ years, mean $\pm S E$, $D M$ duration $20 \pm 3$ yrs) before and 4-6 weeks after bypass operation and have compared them to 15 diabetic neuropathic (DN, $13 \mathrm{M}$, age $60 \pm 2, \mathrm{DM}$ dur. $22 \pm 3$ ), 7 non-neuropathic patients (D, 5 $M$, age $55 \pm 6$, DM dur. $15 \pm 4$ ) and 12 healthy controls (C, $8 M$, age $58 \pm$ 3 ). We employed single point and scanner laser Doppler imaging to measure the foot skin vasodilation in response to heating to $44^{\circ} \mathrm{C}$ and to iontophoresis of $1 \%$ acetylcholine (Ach, endothelial dependent response) and $1 \%$ sodium nitroprusside (NaNP, endothelial independent). The response to heat increased from $289 \% \pm 90$ pre-operatively (increase over baseline) to $427 \% \pm 61 \quad(p<0.05)$ post-operatively, but still was comparable to the DN group $(318 \% \pm 51)$ and lower than $D(766 \% \pm 220)$ and $C(891 \% \pm 121)$ groups $(p<0.0001)$. The Ach response also improved from $6 \% \pm 4$ pre-operatively to $26 \% \pm 8$ post-operatively ( $p<$ $0.05)$ and was similar to $D N(18 \% \pm 3)$ and $D(38 \% \pm 8)$ groups, but still lower than $C(48 \% \pm 9, p<0.001)$. The NaNP response improved from $10 \% \pm 4$ to $29 \% \pm 9(p<0.05)$ and was similar to DN $(25 \% \pm 9), D(32 \% \pm$ $6)$ and $C(40 \% \pm 5)$. The neurovascular response, which depends on normal function of the $\mathrm{C}$ nociceptive fibers, was similar at baseline $(5 \% \pm$ 9), post-operatively $(14 \% \pm 10)$ and the DN group $(33 \% \pm 21)$ but it was dramatically reduced when compared to $D(110 \% \pm 40)$ and $C(198 \% \pm$ 54) groups $(p<0.001)$. We conclude that impaired vasodilation in the diabetic neuropathic lower extremity leads to functional ischemia which improves but is not fully corrected by successful bypass surgery and therefore, diabetic neuropathic patients may still be at high risk for developing foot ulceration or failure to heal an existing ulcer despite adequate correction of large vessel blood flow.

\section{7}

BENEFITS OF A MULTIDISCIPLINARY APPROACH IN THE MANAGEMENT OF RECURRENT DIABETIC FOOT ULCERATION V.Dargis, O.Pantelejeva, A.Jonushaite, L.Vileikyte* and A.J.M.Boulton*. Rehabilitation Hospital, Kaunas, Lithuania, "Manchester Royal Infirmary, UK

A history of previous ulceration is the strongest risk factor for diabetic foot ulceration and subsequent amputations, and the greatest challenge is to reduce the incidence of recurrent foot problems. In this study we therefore aimed to measure the ability of a multidisciplinary foot clinic to reduce recurrent ulceration in a two year prospective study, 145 purely neuropathic diabetic patients with a history of previous foot ulceration were entered into the study. All patients were screened for neurovascular status at baseline, and received footcare education. The intervention group $(n=56)$ were seen by the multidisciplinary team of chiropodists, nurses and physicians and received regular chiropody at least every 3 months with re-education at each visit and the provision of specialist footwear as required. Control subjects $(n=89)$ were screened in the same manner, received baseline education and were then followed in the local practices on a three monthly basis. There were no significant differences (mean \pm SD) in age (intervention $59.2 \pm 13.4$ vs controls, $58.5 \pm 11.5$ years) or duration of diabetes $(14.0 \pm 7.1$ vs $15.6 \pm 7.8$ years). The ratio of genders was similarly NS. Neuropathic status did not differ between intervention and control groups (vibration perception threshold $31.1 \pm 12$. vs $33.9 \pm 11.2 \mathrm{~V}$, neuropathy disability score $8.1 \pm 1.4$ vs $7.9 \pm 1.7$ ). No patient had clinical evidence of peripheral vascular disease. The intervention group had significantly less recurrent ulcers during the two year period than the controls $(30.4 \%$ vs $58.4 \%$ : $p<0.001)$. Thus, this prospective study has demonstrated the efficacy of the multidisciplinary foot clinic together with the provision of specialist footwear in the long term management of those patients with a history of recurrent ulceration.

\section{8}

DIABETIC LOWER EXTREMTTIES AMPUTATION - RIO DE JANEIRO, BR., $90-96$ E.R.S. SPICHLER; D. SPICHLER; C.S.F. MARTINS; L.J. FRANCO and I. LESSA Health Ministry, Rio de Janeiro, Brazil

The aim is to estimate LEA incidence, its amputation level, survival and fatality rates of diabetic amputces and to evaluate their costs. Amputation register provides information about 34 hospital units. 2,823 amputees were enrolled from 90-96. Capturerecapture method was employed to estimate LEA incidence. Diagnosis were coding as vascular insufficiency-IV, diabetes mellitus-DM, trauma-TR, tumor-TU, gangrene emphysematous-GE, and osteomyelitis-OS. Amputations were categorized as toes, foot, below knee (BK), above knee (AK), and hip. Diabetic amputee death certificates were reviewed for all deaths registered in the state of Rio de Janeiro $(336,182)$. Survival rates were analyzed according to an actuarial table, allowing us to calculate a cumulated fatality rate. Mantel test was calculated to evaluate statistical significances for sex differences. Amputation direct costs were evaluated for 96, (hospital stay, medical and surgical treatments, hospital staff) as well as indirect costs (lost of productivity). Annual estimated incidence rate of global LEA was 13.8/100,000. Diabetic related LEA incidence rate was $180.6 / 100,000$, representing a 100 -fold greater risk of LEA, than those without diabetes, in Rio de Janeiro municipality. Concerning to global LEA, mean age was $58.5 \pm 19.4$ yr. for men and $64.4 \pm 18.0 \mathrm{yr}$. for women. Sex ratio was 1.4:1. Diagnosis demonstrated: TU $28(1 \%)$; GE 50(1.8\%); OS 57(2\%); TR 183(6.5\%); DM 923(32.7\%) and VI 582(56\%). The increase was significant for DM and VI $(p<0.05)$. VI was the major cause of LEA until 94, in contrast to DM, in 95. A significant increase of LEA was observed in the 40-79 yr. group. AK amputation level was higher than BK $(p<0.01)$. VI and DM represented a major cause of reamputations $(p<0.005)$. V represented $50 \%$ of bilateral amputations, while DM, $34.6 \%$. Survival probability from diabetics was $54.9 \%$ for men and $59.4 \%$ for women $(p<0.001)$. Cumulated fatality rate was $25 \%$ for men and $29.6 \%$ for women $(0<0.001)$. Age/sex distribution for DM LEA at the time of death, demonstrated mean age for men $61.4 \pm 16.83 \mathrm{yr}$. and mean age for women $68.3 \pm 11.1 \mathrm{yr}$., $p<0.02$. The costs of amputation averaging $\approx$ US\$ 3,021 per case. The total costs for LEA in the diabetic population have been estimated at US $\$ 10,660,000$ for 96 . To lower LEA incidence, preventive action through education, foot care programs, and early detection of DM must be intensified.

\section{9}

WHAT IS THE EFFECT OF AMPUTATIONS ON THE QUALITY OF LIFE IN DIABETIC PATIENTS?

M.R. Childs, E.J.G. Peters, D.G. Armstrong and L.A. Lavery. University of Texas Health Science Center at San Antonio, Texas, U.S.A.

Persons with diabetes have a high incidence of amputation which, it may be postulated, has a detrimental influence on quality of life. However, we are unaware of any study in the medical literature to explain how amputations affect the daily lifestyle, physically and socially of diabetic patients. The purpose of this study was to evaluate the effect of amputation on the quality of life. We used the Sickness Impact Profile to evaluate functional status. This instrument was developed to provide a measure of perceived health status and is sensitive enough to detect changes or differences that occur over time or between groups. We enrolled 124 diabetic patients, in approximately a 1:2 case to control ratio. Cases were defined as those receiving a lower extremity amputation and controls were defined as those with no history of amputation. Study participants received a physical examination and interview to evaluate exposure variables including demographic data, general medical, surgical, diabetes and diabetes related complication history, lower extremity vascular and neurological assessment. Only the physical dimension ( $33.5 \pm 14.9$ vs. $22.3 \pm 14.7, \mathrm{p}<0.001)$ and the total SIP-score $(27.6 \pm 9.9$ vs. $22.5 \pm 10.3, p=0.013)$ revealed a significant lower value for amputees. The psychosocial dimension score on the other hand, showed no significant difference $(14.9 \pm 8.9$ vs. $15.2 \pm 10.0)$. Factors among amputees that had a significant association with lower SIP scores included longer duration of diabetes $(>10$ years), sensory neuropathy (VPT $>$ $25 \mathrm{~V}$ ), and male sex. ANOVA further suggested that high level amputations had a stronger influence on the physical dimension of the SIP than lower level amputations $(p=0.024)$. Interestingly, bilateral amputees did not have significanlly lower scores on any of the SIP dimensions than people with a unilateral amputation. It can be concluded that amputations in diabetics seem to have a substantial influence on the physical element of life, while the effects on the psychosocial aspects are not substantial. 
PS 55

\section{Retinopathy}

\section{0}

RETINAL CYTOSOLIC AND MITOCHONDRIAL REDUCTIVE STRESS: INTERACTIONS BETWEEN HYPERGLYCEMIA AND HYPOXIA

J.R. Williamson, J.R. Nyengaard, E. Ostrow, and Y. Ido; Washington University, St. Louis, MO, USA

The objective of these studies was to assess effects of hyperglycemia vs hypoxia on retinal glucose metabolism and free cytosolic $_{(\mathrm{c})}$ and mitochondrial $\mathrm{m}_{(\mathrm{m})}$ $\mathrm{NADH} / \mathrm{NAD}^{+}$. Retinas from normal rats, some fed tolrestat $(0.2 \mathrm{mmol} / \mathrm{kg}$ bw/day for 7 days), were incubated for $2 \mathrm{~h}$ in 5 or $30 \mathrm{mM}$ glucose (glu) at $5 \%$ (low) or $95 \%$ (hi) $\mathrm{O}_{2}+5 \% \mathrm{CO}_{2} . \mathrm{NADH} / \mathrm{NAD}_{\mathrm{c}}^{+}$and $\mathrm{NADH} / \mathrm{NAD}_{\mathrm{m}}^{+}$were estimated by the redox metabolite indicator method based on substrate levels of lactate dehydrogenase and glutamate dehydrogenase $+\mathrm{NH}_{4}$. These metabolites, glucose 6-phosphate (G6P), fructose 6-phosphate (F6P), and medium lactate were measured enzymatically. G6P at hi $\mathrm{O}_{2}$ was $2.52 \pm 0.76(\mathrm{X} \pm \mathrm{SD}) \mathrm{pmol} / \mu \mathrm{gDNA}$ in $5 \mathrm{glu}$ and increased 2.7 fold $(\mathrm{p}<0.001)$ in 30glu. Low $\mathrm{O}_{2}$ decreased G6P 27\% (p=ns) in 5 and $48 \%(\mathrm{p}<0.01)$ in 30 glu. F6P was undetected in $5 \mathrm{glu}$ at both $\mathrm{O}_{2} \mathrm{~s}$. F6P in $30 \mathrm{gluc}$ was $1.24 \pm 0.36 \mathrm{pmol} / / \mu \mathrm{gDNA}$ at hi $\mathrm{O}_{2}$ and fell $45 \%(\mathrm{p}<0.01)$ at low $\mathrm{O}_{2}$. NADH/NAD ${ }_{\mathrm{c}}$ at hi $\mathrm{O}_{2}$ was $26 \pm 4 \times 10^{-4}$ in $5 \mathrm{glu}$ and increased $58 \%(\mathrm{p}<0.01)$ in $30 \mathrm{glu}$ due to a 1.9 fold increase $(\mathrm{p}<0.01)$ in retinal pyruvate vs a 3 fold increase $(p<0.001)$ in lactate; at low $\mathrm{O}_{2} \mathrm{NADH} / \mathrm{NAD}^{+}$ increased 11 fold in 5 and 20 fold in $30 \mathrm{glu}$ ( $\mathrm{p}<0.001$ for both) due to $\geq 86 \%$ decreases in pyruvate $(\mathrm{p}<0.001)$ vs $1.5-4.7$ increases $(\mathrm{p} \leq 0.01)$ in lactate at 5 and $30 \mathrm{glu}$. Lactate production at hi $\mathrm{O}_{2}$ was $19.3 \pm 3.7 \mathrm{nmol} / \mu \mathrm{g}$ DNA in $5 \mathrm{glu}$ and increased $54 \%(\mathrm{p}<0.0 \mathrm{I})$ in 30glu; at low $\mathrm{O}_{2}$ it increased $33 \%(\mathrm{p}<0.01)$ in 5 and $56 \%(\mathrm{p}<0.001)$ in $30 \mathrm{glu}$. $\mathrm{NADH} / \mathrm{NAD}_{\mathrm{m}}^{+}$did not differ in 5 or $30 \mathrm{glu}$ at hi $\mathrm{O}_{2}\left(68 \pm 13 \times 10^{-3}\right)$ but increased 2 fold ( $p<0.001$ ) at low $\mathrm{O}_{2}$ in 5 and $30 \mathrm{glu}$. Tolrestat prevented increased $\mathrm{NADH} / \mathrm{NAD}^{+}$at $30 \mathrm{glu}$ but had no effect on lactate production or NADH/NAD ${ }_{m}^{+}$. Conclusions: $30 \mathrm{glu}$ and low $\mathrm{O}_{2}$ increase lactate production via increased glucose phosphorylation and activation of phosphofructokinase, respectively; 30glu and low $\mathrm{O}_{2}$ also increase $\mathrm{NADH} / \mathrm{NAD}^{+}$by different mechanisms. The combined effects of hyperglycemia and hypoxia on $\mathrm{NADH} \mathrm{NAD}_{\mathrm{c}}^{+}$may exacerbate diabetic retinopathy via effects on substrate levels of dehydrogenase enzymes implicated in diabetic complications.

\section{2}

EFFECTS OF MODIFIED LDL ON CULTURED RETINAL CAPILLARY CELLS: MECHANISMS OF TOXICITY AND MITIGATION BY PRE-ENRICHMENT WITH $\alpha$-TOCOPHEROL

I.J. Lyons, W. Li, D.F. Gates, K. Moller, R.L. Klein, M-X Fu, J.W. Baynes, S.R. Thorpe, and A.J. Jenkins. Medical University of $S C$, Charleston, and Univeristy of SC, Columbia, S.Carolina, USA.

Aim: To determine whether pre-enrichment of Low Density Lipoproteins (LDL) with $\alpha$ tocopherol mitigates its adverse effects, following in vitro glycation, oxidation or glycoxidation, fowards cultured bovine retinal capillary endothelial cells (RCEC) and pericytes. Methods: LDL (unsupplemented and supplemented with $\boldsymbol{\alpha}$-tocopherol in plasma, before isolation) from non-diabetic humans was modified in vitro by glycation, minimal oxidation, and glycoxidation. Bovine RCEC and pericytes were exposed to $100 \mathrm{ug} / \mathrm{ml}$ LDL protein for 3 days. Cell viability was determined by cell counting. Cell supernatant levels of plasminogen activator inhibitor-1 (PAI-1) and endothelin-1 (ET-1) were measured by ELISA, and nitrite by spectroscopy. To assess mechanisms of toxicity, retinal cells were exposed to native (N)-LDL, and to LDL modified by glycation and/or minimal oxidation and by marked $\left(\mathrm{Cu}^{2+4}\right.$ oxidation with and without prior glycation, in the presence and absence of catalase, superoxide dismutase (SOD), and protein kinase $C$ (PKC) inhibitors. Results: Relative to N-LDL, modified LDL reduced viability of RCEC by a mean of $41 \%$, and of pericytes by $25 \%$. Pre-enrichment of LDL with $\alpha$-tocopherol abolished the reduction in cell viability. Enrichment with $\alpha$-tocopherol did not reduce the measured extent of LDL modification. LDL supplementation with $\alpha$ tocopherol reduced RCEC PAI-1 (17.1 \pm 4.0 vs $22.3 \pm 4.3 \mathrm{pg} /$ cell, mean $\pm S E M ; p=0.0002)$ and increased nitrite $(2.93+0.05$ vs $1.34+0.04 \mu \mathrm{mol} / \mathrm{l}, \mathrm{p}<0.0001)$ but had no effect on ET-1. These effects of $\alpha$-tocopherol may inhibit clot formation and favor vasodilation, In the second study, mean RCEC viability following exposure to modified LDL was $63 \%$ of N-LOL. SOD and PKC inhibitors improved viability to $84 \%$ and $100 \%$ of $N-L D L$ respectively $(p<0.001)$, but catalase had no significant protective effect $(69 \%$ of $N$-LDL). Conclusion: Increasing the $\alpha$ tocopherol content of plasma lipoproteins could be of value in retarding diabetic retinopathy. Superoxide anion and PKC, but not hydrogen peroxide are involved in the response of retinal capillary cells to modified LDL.

\section{1}

N-METIIYL-D-ASPARTATE RECEPTORS PAR'TICIPATE IN THE RETINAL DAMAGE DUE TO THE HYPOGLYCAEMIA \$. Karadeniz, F. Tekeli, L. Bilgiç, B. Güvener, M. Küçük and H. Koyuncuoğlu. Institute for Experimental Medicine and Istanbul Faculty of Medicine, Istanbul University, Turkey

Excitatory amino acids (EAAs) are implicaled in the development of neuronal cell damage following periods of reversible cerebral ischaemia and/or insulin-induced hypoglycaemic coma. Glutamate, which is one of the major EAAs in the central nervous system and also a major neurotransmitter in the retina, is blamed for the neuronal injury during hypoglycaemia via the $\mathrm{N}$-methyl-D-aspartate (NMDA) subtype glutamate receptor. We investigated histopathological changes due to the insulin induced hypoglycaemia and the efrect of MK-801, a NMDA receptor antagonist, on the retina in the Wistar rats. MK-80I was given $0.3 \mathrm{mg} / \mathrm{kg}$ IV twice on the first day and once on the subsequent three days (daily $501 \mathrm{~kg}$ bolus regular insulin injections through the penis vein). Eyes were enucleated on the last day. The thickness of the ganglion cell- and the nerve fibre layers were significantly increased in the hypoglycaemic group $(n=9)$ compared to the control group $(n=16)$ and also compared to the MK-801 group $(n=7)(11.5 \pm 3.2 \mu \mathrm{m}$ vs $9.7 \pm 2.8 \mu \mathrm{m}, \mathrm{p}<0.05$ and $11.5 \pm 3.2 \mu \mathrm{m}$ vs $8.4 \pm 1.8 \mu \mathrm{m}, p<0.05)$. No significant morphological changes were revealed with hematoxylene-eosin staining on light microscope. As a result, we have induced listopathological changes in the retina following hypoglycaemia. Its prevention with MK-80I shows that NMDA receptors are involved in the retinal insult due to the hypoglycaemia. In the clinic, these findings may be of importance in diabetic patients on insulin treatment who may have sometimes severe hypoglycaemia and in patients with severe reactive hypoglycaemia.

\section{3}

INHIBITION OF CATARACT DEVELOPMENT AND LIPID PEROXIDATION BY $\alpha$-LIPOIC ACID IN SAND RATS. Z. MADAR ${ }^{1}$, D. BORENSHTEIN ${ }^{1}, M$. VERMAN ${ }^{2}$, S. MOKADI ${ }^{2}$, A. POLLACK $^{3}$, and H-J. TRISCHLER ${ }^{4} .{ }^{1}$ Faculty of Agriculture, Rehovot, ISRAEL, ${ }^{2}$ Haifa, IS, ${ }^{3}$ Rehovot, IS and ${ }^{4}$ Frankfurt, Germany

Cataract, one of the major causes of age-dependent visual impairment, is a late complication of diabetes. Reactive oxygen species are implicated in this disease condition. We investigated the effects of $\alpha$-lipoic acid, a potent antioxidant, on cataract development, lens aldose reductase activity (AR), lenticular reduced-glutathione (GSH) content and lipid peroxidation in sand rats (a model for type II diabetes). Sand rats were fed a 'high' calorie diet and treated with or without i.p. injection of $\alpha$-lipoic acid, ( $30 \mathrm{mg} / \mathrm{kg}$ ) for 26 days. Daily plasma glucose profiles and cataract incidence were evaluated. Glucose profiles were significantly reduced after 18 and $24 \mathrm{~d}$ on $\alpha$-lipoic acid compared with those not receiving $\alpha$-lipoic acid. After $18 \mathrm{~d}$, mean cataract grade value was $1.7+0.3$ in animals injected with of $\alpha$-lipoic acid $\nu$ s. $3 \pm 0.4$ in sand rats without treatment $(P<0.05)$. Compared with untreated animals, lens AR activity was significantly lower in sand rats treated with $\alpha$-lipoic acid for $26 \mathrm{~d}(12.8+2.3$ vs. $5.2+1.2 \mu \mathrm{mol} \mathrm{NADPH} / \mathrm{mg}$ protein $/ \mathrm{min}$, respectively $P<0.01$ ). Concomitantly, significantly higher lenticular protein and GSH contents were found in sand rats treated with $\alpha$-lipoic acid Lipid peroxidation, measured as TBARS after $26 \mathrm{~d}$, decreased 2 -fold in liver of treated animals $(P<0.05)$. These results suggest that decreased hyperglycemia, in parallel with lower AR activity and higher content of GSH obtained by $\alpha$-lipoic acid, led to a significant preventive effect on cataract development in diabetic sand rats. This study supports the usefulness of $\alpha$-lipoic acid in the treatment of diabetes. 
1084

IN VIVO EFFECT OF AN ISOPROSTANE, 8-EPI-PGF $2 \alpha$ ON RETINAL CIRCULATION IN DIABETIC AND NON-DIABETIC RATS

N. WIERNSPERGER, E. MICHOUD, M. LECOMTE and M. LAGARDE Diabetic Microangiopathy Research Unit. LIPHA - INSERM U352. INSALyon. 69621 Villeurbanne cedex. France

The in vivo effects of one of the $F_{2}$ isoprostanes, 8-iso-PGF ${ }_{2 \alpha}$, which is elevated in plasma of non insulin-dependent diabetic patients, was characterized on the retinal circulation in non-diabetic and diabetic rats. Retinal hemodynamic responses to isoprostanes were quantitated in vivo in rats using a video fluorescein angiography system. Vascular diameters and retinal mean circulation time were determined before and after $5 \mu \mathrm{l}$ intra-vitreous injection of 8-epi-PGF $2 \alpha\left(10^{-5}\right.$ to $10^{-3} \mathrm{M}$ ), 8-epi-PGF $2 \alpha 10^{-4} \mathrm{M}+\mathrm{SQ}_{29,548} 10^{-3} \mathrm{M}$ (an inhibitor of $\mathrm{TXA}_{2}$ receptor), $9 \beta-\mathrm{PGF}_{2 \alpha} 10^{4} \mathrm{M}$ ) (another $\mathrm{PGF}_{2}$ epimer, distinct from 8-epi-PGF ${ }_{2 \alpha}$ without known biological activity) or the carrier in nondiabetic animals. Diabetic rats received either 8-epi-PGF ${ }_{2 \alpha} 10^{-4} \mathrm{M}$, or the carrier). Compared to control animals, diabetic rats presented in the basal state a venous vasodilation $(p<0.01)$ and a non-significant arterial vasodilation $(p=0.06)$, without modification of retinal mean circulation time. After intravitreous injection of 8-iso- $\mathrm{PGF}_{2 \alpha}$, a significant arterial vasoconstriction was observed in control but not in diabetic animals. This vasoconstriction was concomitant with increased retinal mean circulation time in control but not in diabetic rats. No vasoconstriction was observed after injection of either the carrier, $9 \beta-\mathrm{PGF}_{2 \alpha}$ or 8 -iso- $\mathrm{PGF}_{2 \alpha}+\mathrm{SQ} 29,548$. This is the first direct observation that the isoprostane 8-epi-PGF $2 \alpha$ is a potent vasoconstricting agent in the retina. It occurs at the arterial but not venous level, and is likely mediated through a TXA $\mathrm{A}_{2}$-like receptor. Differences observed in retinal mean circulation time between control and diabetic animals suggest altered adaptative mechanisms toward vasoconstrictor substances (such as isoprostanes) in diabetic rats.

\section{5}

CURATIVE EFFECTS OF BIMOCLOMOL ON VISUAL PATHWAY ABNORMALITIES IN FREELY MOVING DIABETIC RATS

K. Bíró, * $G$. Juhász, J. Pálhalmi, T. Kukorelli

Biorex Res. \& Dev. Co., Veszprém, *Dept. of Comparative Physiology, Eötvös Loránd University, Budapest. Hungary

A method of electrode implantation for simultaneous measuring of electroretinogram (ERG) and visual evoked potential (VEP) in conscious streptozotocin-diabetic (STZ, $45 \mathrm{mg} / \mathrm{kg}$ i.v.) rats was used to study the development of early signs of retinopathy and the time course of recovery by bimociomol. From the 4 th postoperation day experimental sessions were performed weekly on dark-adaptated male $\mathrm{Cr}$. Wistar rats before and after verification of diabetes over 1 month. Curative treatment with 10 $\mathrm{mg} \mathrm{kg}{ }^{-1}$ bimoclomol i.p. was initiated for a further month. 1-month nontreated diabetic and nondiabetic groups were also included Weekly monitoring showed a gradual decrease in each oscillatory potential (OP) magnitude and their sum (OP $1-3$ by $67.9 \%$ ) and the b-wave and VEP P1 subcurve areas ( 48.5 and $58.9 \%$, resp.) by the end of month 1 . Improvements of diabetic deficits by $50.8 \%$ for OP1-3; by $88.3 \%$ for b-wave and by $54.3 \%$ for VEP P1 (for all $p<$ $0.05)$ were achieved after 3 -wk treatment. The b-wave and VEP P1 latency prolongations $(30$ and $23 \%$, resp., $p<0.01$ ) were obvious only after 1-month of diabetes. Bimoclomol shortened the latencies by about $48 \% \quad(p<0.05)$ and $19 \%$, resp. After a subsequent washout period of 1 -wk, responses declined to diabetic level Results may be explained by cytoprotective effect of bimoclomo on retinal glia and/or neurons against diabetes-related ischemic cell damages.

\section{6}

THE EFFECTS OF TAURINE AND VITAMIN E ON NA,K-ATPASE AND ANGIOTENSIN-CONVERTING ENZYME ACTIVITIES IN STREPTOZOTOCIN-INDUCED DIABETIC RATS.

M.A.S. Di Leo ${ }^{1}$, L. Todaro ${ }^{1}$, S.A. Santini ${ }^{2}$, G. Marra' ${ }^{1}$, P. Cotroneo ${ }^{1}$, S. Cercone ${ }^{1}$, P. Magnani ${ }^{1}$, L. Antico ${ }^{3}$, A.V. Greco ${ }^{1}$, and G. Ghirlanda' Institutes of ${ }^{1}$ Intemal Medicine, ${ }^{2}$ Chemical Biochemistry and 3Ophthalmology, Catholic University, Rome, Italy.

To investigate the effects of taurine and vitamin $E$ on the activity of retinal Na,K-ATPase and on the activity of angiotensin-converting enzyme (ACE) in the serum and retina of streptozotocin (STZ)-induced diabetic rats, we sacrificed after 4 months of disease different groups of animals fed with standard diet and taurine- or vitamin E-supplemented diet. As markers of lipid peroxidation, conjugated dienes were measured in rat retinas. Na,K-ATPase and ACE activities were measured by spectrophotometry. No difference in senum activity of ACE was found between nomal and diabetic rats, independently of diet. Retinal ACE activity was comparable in normal rats $(13.6 \pm 4.3 \mathrm{U} / \mathrm{L} / \mathrm{mg}$ protein) and diabetic rats with standard diet $(12.8 \pm 2.5)$, but was significantly reduced in diabetic rats $(p<0.05)$ with taurine- $(9.9 \pm 1.8)$ and vitamin $E$ supplemented diet $(9.5 \pm 3.3$ ). In comparison with nomal rats, retinal $\mathrm{Na}, \mathrm{K}-\mathrm{ATPase}$ activity was significantly decreased $(p<0.05)$ in diabetic rats with standard diet $(60 \%)$ and with vitamin E-supplemented diet $(40 \%)$ but not in those with taurine-supplemented diet. A significant increase of conjugated dienes $(p<0.0001)$ was found in retinas of diabelic rats but not in those of diet-supplemented rats with respect to normal rats. These results suggest that the effects of taurine and vitamin $E$ seem to be related to merely local effects in the retina. Taurine and vitamin $\mathrm{E}$ contribute to delay early oxidant damage in the course of diabetic retinopathy.

\section{7}

PROGRESSION OF RETINOPATHY IN THE DCCT COHORT AFTER 4 YEARS OF FOLLOWUP IN THE EPIDEMIOLOGY OF DLABETES INTERVENTIONS AND COMPLICATIONS (EDIC) STUDY J. LACHIN, P. CLEARY, M. DAVIS, D. NATHAN, S. GENUTH AND THE EDIC RESEARCH GROUP, Rockville, MD, U.S.A.

At the DCCT conclusion in 1993, patients completed a detailed closeout assessment. All were referred to a personal physician and encouraged to maintain as low a blood glucose as safely possible. Of $144 \mathrm{I}$ original DCCT patients, 1396 have agreed to participate in the 10 year EDIC followup. In 1997, EDIC year 4, fundus photographs were scheduled for all patients. Over the 4 years of EDIC, the average $\mathrm{HbAlc}$ in the former DCCT intensive group (INT) was $8.1 \%$ compared to $8.4 \%$ in the former conventional group (CON) $(p<.01)$.

We assessed the relative risk (RR) of retinopathy worse by 3 or more steps from DCCT baseline, or of the prevalence of serious retinopathy, between the original DCCT INT and CON groups at EDIC year 4. Among the 971 patients evaluated at EDIC year 4 , the prevalence of retinopathy at DCCT closeout and after four years of EDIC and the unadjusted reduction in risk (RR) were:

$$
\text { DCCT Closeout EDIC Year 4 Risk Reduction }
$$$$
\begin{array}{lllllll}
\text { CON } \% & \text { INT } \% & \text { CON } \% & \text { INT } \% & \text { RR } & 95 \% \text { C.I. } & \mathrm{P}=
\end{array}
$$

$\begin{array}{lllllllll}\geq 3 \text { step } & 44.4 & 25.3 & 46.7 & 17.6 & 62.3 & 53.3-69.6 & <.0001\end{array}$

$\begin{array}{lrrrrrrr}\text { SNPDR } & 9.3 & 4.2 & 14.4 & 4.2 & 70.9 & 53.1-82.0 & <.0001\end{array}$

$\begin{array}{lllllllll}\text { PDR } & 6.5 & 3.8 & 12.8 & 4.0 & 68.9 & 48.9-81.1 & <.0001\end{array}$

$\begin{array}{lllrllll}\text { HRC } & 2.6 & 1.3 & 8.5 & 3.1 & 63.2 & 34.5-79.3 & .0007\end{array}$

$\begin{array}{llllllll}\text { CSME } & 6.9 & 6.7 & 11.4 & 4.0 & 65.1 & 42.1-78.9 & <.0001\end{array}$

$\geq 3$ step worsening from DCCT baseline; SNPDR, severe non-proliferative

retinopathy; PDR, proliferative diabetic retinopathy; HRC, high risk characteristics and CSME, clinically significant macular edema. These risk reductions persisted after adjustment for primary versus secondary DCCT cohort, years of treatment in DCCT, and duration of IDDM

Conclusion: Four years after the mean of 6.5 years of DCCT therapy, the risk of vision threatening retinopathy is significantly less in the former DCCT INT than the CON treatment group. 
1088

INCIDENCE AND RISK FACTORS FOR RETINOPATHY IN TYPE 1 and TYPE 2 DIABETES

Villar G.,Goicolea I., Vicente M.A., Garcia Y., Vazqquez J.A. Hospital de Cruces.Baracaldo.48903Spain

The aim of this study is to assess the incidence of diabetic retinopathy (DR) and factors responsable for its appearance. A large cohort of 1.362 subjects (389 with type 1 and 973 with type 2 diabetes) who attended the specialized diabetic clinics of our Health Area and had a normal ocular fundus examination were followed for a period of 24 months, evaluating the progression to DR and identifying the risk factors for it. Results: Thirty-one type 1 patients $(8 \%)$ and $198(20,3 \%)$ type 2 patients were lost in the follow-up. The incidence of DR was 7,2 and 8,3 cases $/ 100$ patients-year in type 1 and type 2 subjects, respectively. Distribution of risk factors is shown in the table. Type 1 diabetes Type 2 diabetes

No DR DR $p$ No DR DR $p$ $\begin{array}{lcccccc}\text { UAE } \geq 30 \mathrm{mg} / 24 \text { hours } & \mathbf{8 , 1} & \mathbf{8 , 7} & \mathbf{0 , 0 2} & \mathbf{8 , 1} & \mathbf{8 , 6} & \mathbf{0 , 0 2} \\ & \mathbf{1 0 , 9} \% & 22 \% & \mathbf{0 , 0 4} & 25,9 \% & \mathbf{3 6 , 5 \%} & \mathbf{0 , 0 4}\end{array}$ $\begin{array}{lllllll}\text { Systolic BP (mmHg) } & 116,6 & 119,7 & 0,2 & 143,6 & 147,2 & 0,07\end{array}$ $\begin{array}{lllllll}\text { Diastolic BP (mmHg) } & 69,8 & 73,3 & \mathbf{0 , 0 4} & \mathbf{8 0 , 2} & 79,7 & 0,6\end{array}$ $\begin{array}{lcccccc}\text { Triglyceride (mg/d) } & 185 & 203 & \mathbf{0 , 0 0 7} & 225 & 224 & 0,9\end{array}$ $\begin{array}{lllllll}\text { Smoking habits } & 34 \% & 30,8 \% & 0,6 & 13,2 \% & 14,7 \% & 0,8\end{array}$ $\begin{array}{llllllll}\text { Years of diabetes } & 9,5 & 14,1 & <0,0005 & 11,5 & 12,6 & 0,1\end{array}$ In logistic regression analysis, independent predictors were duration of diabetes $(p=0,03)$ and urinary albumin excretion $\geq 30 \mathrm{mg} / 24$ hours $(\mathrm{p}=0,04)$ in type 1 diabetes and glycated hemoglobin $(p=0.04)$ in type 2 .

Conclusion. The incidence of DR is similar than the results of other works in type 1 diabetes, but unexpectedly high in type 2 , probably because these patients treated in specialized clinics are more complicated and not representative of type 2 diabetic population.

\section{9}

SERUM N-ACETYL-ß-GLUCOSAMINIDASE PRECEDES MICROALBUMINURIA IN DEVELOPMENT OF DIABETIC RETINOPATHY

J.Škrha, J.Hilgertová, and V.Stibor. Dept. of Internal Medicine 3, Dept. of Ophthalmology, Faculty of Medicine 1, Charles University, Prague, Czech Republic

An increased serum $\mathrm{N}$-acetyl- $\mathrm{B}$-glucosaminidase(NAG) activity has been found in patients with diabetic microangiopathy. The aim of this study was to consider if NAG activities may be a prognostic marker of diabetic retinopathy. We evaluated 33 Type ldiabetic patients( 18 men and 15 women,mean age $39 \pm 8$ yrs) with albustix negative urine in twelve-year follow-up study. Total cohort was separated into patients who did not develope diabetic retinopathy (Group A, $\mathrm{n}=10$ ), who developed or worsened diabetic retinopathy during follow-up (Group $B, n=12$ ) and who had persisted diabetic retinopathy (Group $\mathrm{C}, \mathrm{n}=11$ ) since the beginning of observation. The ophthalmological findings were confirmed by fluorescent angiography. Control group consisted of 20 healthy persons of comparable age and body mass index Fasting serum NAG activity and albumin:creatinine ratio evaluated in the firs morning urine were determined yearly. The results at the beginning (I) and twelve years later (II) are expressed as means and 2SD ranges in the Table.

\begin{tabular}{|c|c|c|c|c|}
\hline & Group A & Groun B & Group C & Controls \\
\hline $\begin{array}{l}\text { Albuminuria I } \\
\text { (g/mol creat.) }\end{array}$ & $\begin{array}{c}0.58 \\
(0.23-1.47)\end{array}$ & $\begin{array}{c}0.59 \\
(0.12 \cdot 3.42)\end{array}$ & $\begin{array}{c}1.04^{*} \\
(0.20-5.62)\end{array}$ & $\begin{array}{c}0.45 \\
(0.17-1.14)\end{array}$ \\
\hline II & $\begin{array}{c}0.47 \\
(0.20-1.18)\end{array}$ & $\begin{array}{c}1.0^{*} \\
(0.14-7.1)\end{array}$ & $\begin{array}{c}0.82 * \\
(0.21-3.7)\end{array}$ & \\
\hline $\begin{array}{l}\text { fS-NAG } \\
(\mathrm{U} / \mathrm{l})\end{array}$ & $\begin{array}{c}13.8 \\
(10.0-19.0)\end{array}$ & $\begin{array}{c}18.9^{*} \\
(12.8 \cdot 28.1)\end{array}$ & $\begin{array}{c}18.6^{*} \\
(13.3-25.9)\end{array}$ & $\begin{array}{c}15.3 \\
(10.7-20.1)\end{array}$ \\
\hline II & $\begin{array}{c}14.9 \\
(11.6-19.1)\end{array}$ & $\begin{array}{c}20.1^{*} \\
(13.6+29.7)\end{array}$ & $\begin{array}{r}17.2 * * \\
(9.2-30.2)\end{array}$ & \\
\hline
\end{tabular}

Statistical significance as compared to the control group: *p<0.01**p<0.05 A development of retinopathy (Group B) was accompanied by the increase of albuminuria whereas serum NAG activites were higher already prior to these vascular changes. This contrasts with no changes either of albuminuria or of NAG observed in Group A. Already developed retinopathy (Group C) was associated with higher albuminuria and NAG activities at the beginning and no changes during follow-up. We may conclude that serum NAG activities may be increased prior to manifestation of diabetic retinopathy and earlier than microalbuminuria developes.

\section{0}

TITLE: Screening for Diabetic Retinopathy: Outcome of ophthalmological referral. AUTHORS: R.L. Gibbins, D.R. Owens, J.C. Allen, Diabetes Research Unit, University of Wales College of Medicine, Cardiff. UK.

The Welsh Community Diabetic Retinopathy Study (WCDRS) investigated general practitioners (GPs) ability in detecting sight threatening diabetic retinopathy (STDR) using ophthalmoscopy or $35 \mathrm{~mm}$ retinal photography compared with a reference standard provided by the Royal Postgraduate Medical School, London (RPMS). This report analyses the outcome of subjects referred to ophthalmologists during the first phase of WCDRS. European Field Guide criteria were used to determine need for referral. Data were obtained by examining subject's primary care records. $186(31 \%)$ subjects were eligible for referral. Data were available on $166(89 \%) .143$ $(86 \%)$ of these had STDR according to GPs, RPMS or both. The mean interval between referral and ophthalmological review for these was 11.7 weeks, range $1-36.101(54 \%)$ subjects had no previous record of specialist assessment for STDR. 3 did not attend clinic appointments. Ophthalmologists diagnosis and treatment are shown in the table.

\begin{tabular}{|c|c|c|c|c|c|}
\cline { 3 - 6 } \multicolumn{2}{c|}{} & \multicolumn{4}{|c|}{ Ophthalmologists outcome : n (\%) } \\
\hline Subject category & Seen (n) & No DR & BDR & STDR & Rx Laser \\
\hline GP/RPMS +ve & 39 & $1(3)$ & $9(23)$ & $29(74)$ & $22(56)$ \\
\hline RPMS +ve, GP -ve & 22 & $1(5)$ & $11(50)$ & $10(45)$ & $7(32)$ \\
\hline GP +ve, RPMS -ve & 40 & $20(50)$ & $16(40)$ & $4(10)$ & $1(3)$ \\
\hline TOTAL & 101 & $22(22)$ & $36(36)$ & $43(43)$ & $30(30)$ \\
\hline
\end{tabular}

(BDR = background diabetic retinopathy; +ve and -ve categories are for STDR) $10(50 \%)$ of the 20 subjects in the GP/RPMS +ve and RPMS +ve, GP -ve categories thought initially to have BDR by ophthalmologists developed STDR within 18 months, increasing the prevalence of agreed STDR from $10.4 \%$ to $12.5 \%$. $30(5 \%)$ newly identified subjects with STDR needed immediate laser treatment. These data suggest that the time intervals recommended in the Field Guide for seeing subjects with STDR are not met in UK practice. Agreement between RPMS diagnosed STDR and ophthalmologists rose from $64 \%$ initially to $80 \%$ after 18 months with RPMS being more sensitive. Improved co-ordination between primary screening services and ophthalmologists will be required to ensure effective use of specialist resources in treating diabetic eye disease.

\section{1}

INTERNET-BASED SCREENING FOR DIABETIC RETINOPATHY B.Liesenfeld ${ }^{1}$, M.Lungershausen ${ }^{1}$ H.Bornemann ${ }^{1}$, S.Kluthe ${ }^{2}$, W.Piehlmeier, c.Birkmann ${ }^{2}$, G.Mann ${ }^{2}$, M.Obermaier $^{3}$, I.Ugi ${ }^{3}$, M.Porta ${ }^{4}$, T.Bek, S.Aldington ${ }^{6}$, H.Mayer ${ }^{5}$, K.D.Hepp ${ }^{1}$, E.Kohner'; Diabetes Centre Munich-Bogenhausen, GSF-medis-Institute ${ }^{2}$, Dep. of Ophthalmology of the Technical University of Munich ${ }^{3}$, Dep. of Internal Medicine of the University of Turin ${ }^{4}$, Dep. of Ophthalmology of the University of Aarhus ${ }^{5}$, Retinopathy Grading Centre of the Imperial College London, Dep. of Ophthalmology of the Munich-Harlaching Hospital ${ }^{7}$, Division of Medicine of St. Thomas' Hospital London?

In a clinical trial we investigated the quality of the remote assessment of digital images of the retina obtained in the routine setting of a busy regional centre for diabetes care to detect diabetic lesions. 129 consecutive patients with type 1 or 2 diabetes mellitus without previous laser therapy or history of glaucona were screened using the following approach: two digital images and two conventional colour slides per eye (dilated pupils; image angle 50 degrees; macula and papilla centered, respectively) were captured. The digital images were transmitted to five expert grading centres via the Internet Structured reports were returned via electronic mail from grading centres to guide further treatment. The colour slides were graded by a single expert of one of the grading centres using the same structured report. All study patients were examined by ophthalmologists (not specialised in diabetic retinopathy) of our clinic using slit-lamp biomicroscopy. For the five grading centres sensitivities were $74,85,88,90$ and $90 \%$ for the diagnosis ,any stage of diabetic retinopathy or macula oedema“ (specificities $41,61,65,75$ and $82 \%$ ) obtained on the basis of the digital image with reference to the conventional colour slides (positive predictive values (PPV) 43,51,56,62 and $67 \%$, negative predictive values (NPV) $86,89,91,91$ and $93 \%$ ). The ophthalmologist's examination resulted in a sensitivity of $67 \%$ and a specificity of 92\% (PPV 81\%, NPV 85\%). CONCLUSION: Screening for diabetic retinopathy with digital images by experts resulted in a higher sensitivity but lower specificity compared to ophtalmologists performing slit-lamp biomicroscopy 
1092

Retinopathy in subjects with Increased Fasting Plasma Glucose Levels

Karunakaran S, Addington S, North B, Ryder R, Holman RR for the EDIT study group,

University of Oxford,UK

631 subjects thought to be at increased risk of Type 2 diabetes with an increased fasting plasma glucose (FPG) of $5.5-7.7 \mathrm{mmol} / 1$ inclusive on two consecutive occasions were recruited into the Early Diabetes Intervention Trial (EDIT). Exclusions were known diabetes, active macrovascular disease, impaired renal function uncontrolled hypertension, life threatening illness or drugs that affect glucose metabolism. 596 subjects with mean(SD) FPG $6.0(0.5) \mathrm{mmol} / \mathrm{h}$, age $52(10)$ years, body mass index $28.5(4.5) \mathrm{kgm} 2$ had four-field colour retinal photographs taken and graded using the "ETDRS final" grading scale by 2 independent assesors. Mean values from twin $75 \mathrm{gm}$ oral glucose tolerance tests classified $46 \%$ of subjects as normal glucose tolerance(NGT), $40 \%$ as impaired glucose tolerance(IGT) and $15 \%$ as diabetes mellitus(DM) using WHO criteria. $21.4 \%$ of subjects had retinopathy with $16 \%$ having microaneurysms only and $5.4 \%$ having haemorrhages and / or exudates in addition. $21 \%$ of NGT, $25 \%$ of IGT and $25 \%$ of DM subjects had retinopathy. One IGT subject with an $\mathrm{fpg}$ of $6.3 \mathrm{mmo} / \mathrm{h}$ had proliferative retinopathy. Conclusion:The glycaemic threshold for developing diabetic retinopathy is probably lower than the present WHO or ADA diagnostic FPG levals for diabates.

\section{3}

OCULAR BLOOD FLOW AND GLUCOSE PLASMA LEVELS IN PATIENTS WITH IDDM

S. Dallinger, O. Findl, B. Rami, K. Polak, R. Hitsch, E. Schober, A. Wedrich, M. Wolzt and L. Schmetterer. Department of Clinical Pharmacology, Institute of Medical Physics, Department of Ophthalmology, Department of Pediatry, University of Vienna, Währinger Gürtel 18-20, A-1090 Vienna, Austria

There is evidence that retinal blood flow is altered in patients with diabetes mellitus. However, there is considerable controversy regarding the exact nature of these perfusion abnormalities. This may in part be caused by the different methods used to assess retinal hemodynamics. In addition, many of these studies included patients with different types of diabetes and concomitant diseases. Retinal blood flow may, however, also be influenced by the actual glucose plasma levels. To test this hypothesis we enrolled 57 subjects with IDDM. Inclusion criteria were age at diagnosis $<15$ years and duration of disease between 13 and 18 years. Retinal blood flow was measured with scanning laser Doppler flowmetry (Heidelberg Retina Flowmeter) in the patient's right eyes. Pulsatile ocular blood flow and flow velocities in the ophthalmic artery were assessed with laser interferometric measurement of fundus pulsation and color Doppler imaging, respectively. There was a significant association between glucose plasma levels and retinal blood flow $(r=0.45, p<0.010)$. In contrast, hemodynamic parameters in the choroid and the ophthalmic artery were not associated with glucose plasma levels. Our data indicate that plasma glucose is an important determinant of retinal blood flow in patients with IDDM.

\section{4}

LY33353I SINGLE ESCALATING ORAL DOSE STUDY IN HEALTHY VOLUNTEERS

D. Demolle, J.M. de Suray, F. Vandenhende and C. Onkelinx. Lilly

Development Centre, 11 rue Granbonpré, 1348 Mont-Saint-Guibert, Belgium.

Background and objectives: LY333531 is a specific inhibitor of protein kinase $C \beta$ (PKC $\beta$ ) that is being developed for the treatment of diabetic retinopathy. The objectives of this study, which was a first administration to man, were: a) to assess safety, tolerability and pharmacokinetics (PK) of LY333531 and 338522, its active metabolite, after escalating single oral doses in healthy male volunteers, and b) to compare the PK of LY333531 and 338522 in males and non-fertile females after single oral doses. Methods: The study was subject- and investigator-blind. In the dose escalation phases, groups of 9 subjects participated in a maximum of three sessions. In each session, 6 received a set dose of LY 333531 and 3 received placebo in a randomized order. Doses varied from 0.25 to $256 \mathrm{mg}$.

Results: Sixty subjects participated in the study. LY333531 was very well tolerated and the incidence and type of adverse events were identical in placebo and treated groups. There were no clinically significant changes in ECG, cardiac output, vital signs and laboratory tests compared to baseline. The $C_{\max }$ and $A U C_{(0-24}$ h) of both LY333531 and 338522 increased linearly but less than proportionally with dose. For both components, two phases of elimination were observed (first $t_{1 / 2}$ up to 3.6 and $7.5 \mathrm{~h}$ and second $\mathrm{t}_{1 / 2}$ up to 39.5 and $41.4 \mathrm{~h}$, respectively, for LY $33353 \mathrm{I}$ and 338522 ). The exposure ratio between parent and metabolite was fairly constant among subjects. No sex differences were observed.

Conclusion: LY.333531 is safe and well tolerated up to a single dose of $256 \mathrm{mg}$. 


\section{Treatment of Diabetic Nephropathy}

\section{5}

\section{RAMIPRIL REDUCES ALBUMIN EXCRETION RATE IN NORMOTENSIVE IDDM PATIENTS WITH MICROALBUMINURIA}

J P O'Hare', A Michael ${ }^{2}$ and G Viberti ${ }^{3}$ on behalf of the ATLANTIS study group

'University of Warwick, U.K. ${ }^{2}$ Hoechst Marion Roussel, Denham,

U.K. ${ }^{3}$ Unit for Metabolic Medicine, UMDS, Guy's Hospital,

London, U.K.

The aim was to assess whether low (1.25mg) and/or standard (5mg) doses of ramipril can exert a nephroprotective effect in incipient diabetic nephropathy. The study was performed as a multicentre randomized placebo controlled double blind parallel group trial with a minimum follow-up of two years. Eligible subjects were men and women between 18 and 65 years of age, with a diagnosis of IDDM of a minimum duration of 6 months, and with microalbuminuria as defined by a urinary albumin excretion rate (UAER) of $20-200 \mu \mathrm{g} /$ minute. Subjects were required to be normotensive, defined as having a systolic blood pressure of $<150 \mathrm{mmHg}$ $(<165 \mathrm{mmHg}$ if between 50 and 65 years) and a diastolic blood pressure of $<90 \mathrm{mmHg}$. 140 subjects were enrolled and 98 completed the two year period or longer. UAER was measured using 2 timed overnight urine collections every 3 months. Glomerular filtration rate (GFR) was measured every 6 months using the iohexol method. The change in UAER from baseline to year 2 showed a statistically significant difference between placebo and the two ramipril groups pooled together $(\mathrm{p}=0.013)$. A statistically significant difference was also observed between placebo and ramipril $5 \mathrm{mg}$ at year $2(\mathrm{p}=0.010)$. There was an observable difference between placebo and ramipril $1.25 \mathrm{mg}$ although this did not reach statistical significance $(p=0.079)$, and there was no difference between two ramipril groups $(p=0.41)$. No statistically significant difference was observed between placebo and ramipril $5 \mathrm{mg}$ in GFR at year 2 ( $p=0.11$ ) after adjusting for sex and baseline GFR. The overall conciusions are that normotensive, insulin-dependent diabetic subjects with microalbuminuria benefit from treatment with ramipril, and that such benefit can be demonstrated as early as two years after commencing treatment, by a reduction in urinary albumin excretion rate as compared to baseline. The reduction is associated with a small decrease in mean arterial blood pressure for both doses of ramipril.

\section{7}

TIGHT BLOOD PRESSURE CONTROL RETARDS PROGRESSION OF NEPHROPATHY IN TYPE 2 DIABETICS

S. Jelić, S. Dimković, D. Kandić, N. Kostić, G. Bojković and Z.Čaparević

Clinical Hospital Center »Dr Dragiša Mišović«, Belgrade, Yugoslavia

In type 2 diabetes microalbuminuria is weaker predictor for development of overt nephropathy but important indicator of higher risk for cardiovascular diseases. The authors are presenting results of five-year prospective study including 30 type 2 diabetic patients without persistent proteinuria treated for hypertension either with $25-75 \mathrm{mg}$ of captopril $(\mathrm{n}=17)$ or $5-10 \mathrm{mg}$ of amlodipin daily $(n=13)$. Results of 20 age-, sex-, body mass index and initial albuminuria-matched type 2 diabetic patients non-complaing with the antihypertensive treatment regimen were retrospectively analyzed and compared with the results of the treated subjects. Good metabolic control existed in all three groups of patients throughout the testing-period. Fairly acceptable blood pressure control was achieved with captopril (170/100 vs. $138 / 85 \mathrm{mmHg}$ ) as well as with amlodipin $(170 / 101$ vs. $137 / 87$ ) within the first year of treatment $(\mathrm{p}<0.01)$. In patients without tight blood pressure control urinary albumin excretion (UAE) inclined at the average rate of $14.93 \%$ per year. During five year of investigation 6 of them (30\%) became macroproteinuric. On the contrary, in patients treated with amlodipin annual increment of UAE was at the level of only $3.76 \%$ with only $2(15 \%)$ patients expressing overt nephropathy during five years. Moreover, none of the patients treated with captopril became macroproteinuric while UAE in this group displayed an decrement of $-2.06 \%$ per year. Tight blood pressure control retards deterioration of microalbuminuria and reduces cardiovascular risks in type 2 diabetic patients. Reverse effect of captopril on neuropathy progression could be hypothetically explained by its protective role against free radical injury in endothelial, glomerular and tubular cells.

\section{6}

EFECTSOF QUNAPRILONRENALHAEMODYNAMICS AND ALBUMINEXCRETION RATE IN TYPE 1 DIABETIC PATIENTS. LACK OF CORRELATIONS TO Na-L COUNTERTRANSPORT.

\section{G. Kourtoglou, D. Karamitsos, N. Karatzas, T. Didangelos, S. Bacatselos, A.Vyzantiadis.}

Diabetes Center, B' Prop. Medical Clinic and Lab of Nuclear Medicine, Aristotelian

University, Hippocration Hospital, Thessaloniki, Greece

The aim of our study was to investigate the effects of quinaprit on the renal haemodynamics and the abumin excretion rate(AER) in normotensive type 1 diabetic pts with or without microalbuminuria and to correiate the results with the $\mathrm{Na}-\mathrm{L}$ countertransport activity (CTA) in red cells. Twenty two pts were included with mear age 34,2 years (range 20-54) and mean duration of diabetes 15,6 years (range 7-20). The AER was measured immunoturbitometrically and the renal heamodynamics were measured radioisotopically with ${ }^{51}$ CrEDTA and ${ }^{125}$ hippuran before and 24 h after the administration of $10 \mathrm{mg}$ of quinapril (phase A) and after three months of $10 \mathrm{mg}$ quinapri daily (phase B). The Na-Li CTA was measured according to method of Canessa et al. Results in table.

$\begin{array}{llll}\text { Nomcalbuminunuric pts }(r=11) \\ \text { baseline } & \text { Change\% A } & \text { Change\%B } \\ \text { Mean BP } & 91.7 \pm 4 & -10.8^{*} & -10.9^{*} \\ \text { GFR } & 132+10 & -4.4 & -13.4 \\ \text { ERPF } & 659 \pm 44 & +13.8^{*} & +12.6^{*} \\ \text { FF } & 0.2+0.02 & -17.4^{*} & -25^{*} \\ \text { RVR } & 8.6 \pm 0.7 & -21.2^{*} & -22.4^{* *} \\ \text { AER } & & & \end{array}$

Microabumiruric pts( $n=11)$ baseline Change\% A Change\% $\begin{array}{lll}89.4 \pm 2.3 & -3.7 & -2.7\end{array}$ $\begin{array}{lll}115 \pm 12 & -7.8 & -8.6\end{array}$ $648 \pm 48+0.4 \quad-4.1$ $0.17 \pm 0.01-10.8 \quad-4.6$ $8.9 \pm 0.7 \quad-6.9 \quad-1.1$ $138+22-40^{\text {t* }}-52^{\star *}$

Na-Li CTA 0.2330 .026 $0.246+0.022$

(values in meantSE, " $=0<0.05$, *t $p<0.005$ with Wilcoxon test)

In 9 pts with GFR> $125 \mathrm{~m} / \mathrm{min} / 1.73 \mathrm{~m}^{2}$ a significant fall of GFR was observed in the phase $B(20 \% \mathrm{p}=0.01)$ and a significant rise in ERPF in phase $\mathrm{A}(+12.2 \% \mathrm{p}=0.018)$. The $\mathrm{Na}$-Li CTA was not related to the basic GFR nor to the difference between the baseline GFR value and that observed at the end of the trimester. It is concluded that quinapril in type 1 diabetic pts, with or without incipient nephropathy, reduced microalbuminuria when present, and imoroved the renal heamodynamics (GFR, FF, ERPF and RVR) especially in pts with normal AER and increased GFR. The renal hemodynamic response was not related to Na-LI CTA

\section{8}

DECREASE OF MICROALBUMINURIA DURING SIMVASTATIN THERAPY IS PRECEDED BY A REDUCTION OF URINARY GLYCOSAMINOGLICANS. G.TONOLO, P.BRIZZI, *M.FORMATO, M.F.ANGIUS, M.M.ATZENI, M.G.MELIS, M. CICCARESE, *G.M.CHERCHI, M.MAIOLI. Diabetologia, Istituto di Clinica Medica and* Biochimica Applicata, University of Sassari, Italy

The decrease of LDL cholesterol (LDL-C) induced by simvastatin is associated with a reduction in urinary albumin excretion rate (AER) in hypercholesterolemic normotensive microalbuminuric NIDDM [1]. The aim of this study was to confirm these effects in microalbuminuric hypertensive NIDDM (NIDDM-M+H+). After 2 months wash-out from the previous antihypertensive therapy (0), 16 NIDDM-M+H+ $\left(\mathrm{HbA}_{1} \mathrm{c}<7.5 \%\right)$ were included in the study if diastolic blood pressure (DBP) $>95$ and $<105 \mathrm{mmHg}$, plasma LDL-C $>3.9$ and $<6.5 \mathrm{mmol} / \mathrm{l}$. They received simvastatin $20 \mathrm{mg} / \mathrm{day}$ for 10 months with 3 months washout (WO) at the end. Total and HDL Cholesterol, triglycerides, apolipoprotein $A_{1}$ and $B$, Lipoprotein (a), AER (mg/24h), systolic and diastolic blood pressure, body weight were measured monthly, while $24 \mathrm{~h}$ urinary glycosaminoglicans excretion (GAG, mg/g.Creatinine) and their low-sulphate chondroitin fraction (\%LSC) were measured at time 0,5 and 10 months and after the WO. Preliminary results for the first 9 patients who completed 10 months simvastatin therapy plus additional three months washout from simvastatin are given. Data are mean \pm SEM, or median and (range) for AER. $* \mathrm{p}<0.05, * * \mathrm{p}=0.02,{ }^{* * *} \mathrm{p}<0.01$ vs. time $0 ; \S p<0.05, \S \S p<0.01$ vs. time 10

$\begin{array}{ccccc}\text { Time } & \mathbf{0} & \mathbf{5} & \mathbf{1 0} & \text { wo } \\ \text { DBP } & 94 \pm 3 & 90 \pm 4 & 86 \pm 2 * & 92 \pm 2 \\ \text { LDL-C } & 4.76 \pm 0.34 & 2.85 \pm 0.23^{* * *} & 2.95 \pm 0.21^{* * *} & 4.8 \pm 0.23 \S \S \\ \text { AER } & 98(45-351) & 87(35-329) & 70(27-210)^{*} & 119(48-410) \\ \text { GAG } & 5.3 \pm 2.7 & 3.5 \pm 1.12 & 2.9 \pm 0.95 * * & 4.7 \pm 0.7 \S \\ \text { \%LSC } & 30.3 \pm 11.7 & 22.5 \pm 6.2^{*} & 20.1 \pm 6.7^{*} & 22.8 \pm 3.1\end{array}$

Simvastatin significantly decreased LDL-C and this effect was associated with a significant decrease in AER and DBP preceded by a significant decrease of both total urinary GAG and the \% of LSC. At WO, a significant increase in total GAG together with a not significant increase in DBP and AER was observed. In conclusion our data are consistent with the hypothesis that simvastatin might lower AER in NIDDM$\mathrm{M}+\mathrm{H}+$ via an amelioration of the glomerular basement membrane.

[1] G Tonolo et al Diabetes Care, vol20, number 12, December 1997 
EFFECT OF GLUCOSAMINOGLYCANS IN CHILDREN WITH DIABETIC NEPHROPATHY .

I.I.Mishina, L.N.Scherbacheva, V.A.Peterkova, Endocrinology Research Centre, Moscow, Russia.

It was shown that the loss of glucosaminoglycan (GAG) content by glomerular basement membrane especially negatively- charged heparan sulphate impairs membrane charge-selectivity and causes albuminuria. The aim of study was to evaluate the antiproteinuric effect of GAG (sulodexide) in children with diabetes mellitus. 10 children (age 14,7 $\pm 0,6 \mathrm{yr}$., duration of diabetes $10,1 \pm 0,7 \mathrm{yr}$.) with good metabolic control $\left(\mathrm{HbA}_{\mathrm{I}} \quad 11,6 \pm 0,4 \%\right)$ were administered the low-molecular heparin Sulodexide (Alfa Vasserman-Italy). All patients had persistent microalbuminuria $(88,6 \pm 16,2 \mu \mathrm{g} / \mathrm{min})$. The treatment was continued for 4 weeks (600 Lipoproteinlipase releasing units (LRU)/ day intramuscularly for 2 weeks, and orally 500 LRU - one capsule twice daily for 2 weeks). The albumin excretion rate wich was measured weekly during dosing and 4 weeks after end of the treatment was used as main evaluation parameter. The significant decrease of albumin excretion rate was already reached after first week of treatment $(28$ $\pm 7,5 \mu \mathrm{g} / \mathrm{min}, \mathrm{p}<0,01)$ and was maintained during the treatment period. At end of treatment and 4 weeks after withdrawal of drug the mean values albumin excretion rate were still- lower than before treatment $(43,6 \pm 11,4 \mu \mathrm{g} / \mathrm{min}, \mathrm{p}<0,05$ vs. $44,6 \pm 13,2$ $\mu \mathrm{g} / \mathrm{min}, \mathrm{p}<0,05$ respectively). No side effects were observed throughout the treatment period. In conclusion, sulodexide administration possesses the antiproteinuric effect and might be useful for the therapy of diabetic nephropathy in children.

\section{PS 57}

\section{Clinical Nephropathy}

\section{0}

MICRO AND MACROALBUMINURIA PREVALENCES IN THE EPIDEMIOLOGY OF DIABETES INTERVENTIONS AND COMPLICATIONS (EDIC)

P. Cleary, M. Molitch, and M. Steffes for the EDIC Research Group, Rockville, MD, USA

The Diabetes Control and Complications Trial (DCCT) demonstrated that intensive therapy (INT) reduced the risk for the development of microalbuminuria by $39 \%$ compared to conventional therapy (CON). Whether this reduction represented absolute prevention or a delay in the onset of nephropathy was not determined from the DCCT. EDIC is a prospective multicenter 10 year observational study of the course of complications in the DCCT cohort. This report presents the prevalence of microalbuminuria ( $\geq 21 \mu \mathrm{g} / \mathrm{min}$ or $\geq 28 \mu \mathrm{g} / \mathrm{min}$ ) and clinical albuminuria $(\geq$ $208 \mu \mathrm{g} / \mathrm{min}$ ) and the risk reduction (RR) after four years of EDIC followup for patients originally assigned to INT during the DCCT. During EDIC a timed four-hour urine collection for the measurement of albumin excretion rate (AER, $\mu g / \mathrm{min}$ ) every two years.

$$
\text { EDIC }
$$$$
\text { YEAR: }
$$

\begin{tabular}{|c|c|c|c|}
\hline YEA & $\mathrm{T}$ & & \\
\hline & INT & $\mathrm{CON}$ & $\underline{\mathrm{RR}}$ \\
\hline & 660 & 666 & \\
\hline$\%$ AER $\geq 21$ & 12.4 & 20.4 & $39 \%$ \\
\hline$\% A E R \geq 28$ & 7.4 & $\$ 7.0$ & $56 \%$ \\
\hline$\% A E R \geq 208$ & 1.4 & 3.5 & $60 \%$ \\
\hline $\mathrm{HbA} 1 \mathrm{c} \%$ & 8.00 & 8.4 & \\
\hline & $p$ & & \\
\hline
\end{tabular}

\begin{tabular}{lrr}
\multicolumn{2}{c}{$3-4$} & \\
$\frac{\text { NT }}{640}$ & $\frac{\text { CON }}{648}$ & RR \\
13.3 & 23.6 & $44 \%$ \\
9.1 & 19.3 & $53 \%$ \\
1.9 & 7.4 & $74 \%$ \\
8.2 & 8.4 & \\
p<. 01 &
\end{tabular}

$\underline{95 \% \mathrm{Cl}}$

The EDIC results indicate that patients treated with INT for an average of 6.5 years during the DCCT continue to experience a reduction in risk (39$74 \%$ ) for the development of microalbuminuria and progression to clinical albuminuria up to four years iater.

\section{1}

EARLY SIGNS OF RENAL AND CARDIOVASCULAR INVOLVEMENT IN CHILDREN WITH TYPE 2 DIABETES MELLITUS

A. Körner, M. Dobos, B. Vásárhelyi, A. Szatmári, L. Madácsy and T. Tutassay

$1^{\text {st }}$ Dept. Pediatrics, Semmelweis Medical University, Budapest, Hungary Several data suggest that pre-clinical type 2 diabetes mellitus (DM) is not a benign condition and significant complications are present in adult patients already at the diagnosis. The aim of our study was to investigate the markers of early renal and cardiovascular involvement in our pediatric population with type $2 \mathrm{DM}$. Between 1990 and 1996 altogether 28 children with type $2 \mathrm{DM}$ have been diagnosed at our department. Detailed analysis has been performed in 10 children (aged 15.9; 13.0 18.3 years). Data were compared to those of 8 age and sex matched healthy controls. Type $2 \mathrm{DM}$ was diagnosed by oral ghucose tolerance test according to WHO criteria. All patients exhitbited marked hyperinsutinemia. Ambulatory blood pressure monitoring (ABPM) was carried out by multiple blood pressure measurements with a portable automatic monitor. Sodium-Lithium countretransport (SLCT) Na ${ }^{+}{ }^{+}$ATPase ( NKA) activity have been measured in the erythrocytes. All blood pressure readings were well within the 90 percentile of the age and sex matched normative blood pressure data for children and adolescents. However in half of the diabetic children the diurnal index was less than $10 \%$ ( non dippers). Left ventricular mass was within the normal range in all patients investigated. Red blood cell SLCT activity $(257 \pm 28 \mathrm{~mm} 0 / / \mathrm{RBC} / \mathrm{h})$ and NKA activity $(288 \pm 24 \mathrm{mmol}$ $\mathrm{ATP} / \mathrm{mg}$ prot/h)were indistiguishable from those in healthy controls $(281 \pm 23 \mathrm{mmol} / \mathrm{h}$ $\mathrm{RBC} / \mathrm{h}$ and $290 \pm 27 \mathrm{nmol} \mathrm{ATP} / \mathrm{mg}$ prot/h, respectively). Urinary altumin excretion in 3 diabetic children was already in the microalbuminuric range, however there was no statistical difference in albumin excretion between diabetic children and controls ( mean; range: $13.7 ; 5.5-34.7$ and $10.2 ; 5.6-18.6 \mu \mathrm{g} / \mathrm{min}$. NS)

Conclusion: early signs of renal involvement occur already in young patients with type 2 DM. 
REDUCED RENAL FUNCTION IN DIABETES IN THE ABSENCE OF MICROALBUMINURIA. S. Panagiotopoulos, C. Tsalamandris, T. Smith, T. Allen, and G. Jerums. Endocrinology Unit, Uni Melb, A\&RMC, Heidelberg, Australia.

Reduced renal function in diabetes without microalbuminuria has been previously observed in small numbers of Type I and Type 2 diabetic patients. We conducted a 5 year prospective cohort study in 47 Type 1 and 56 Type 2 diabetic patients with initial albumin excretion rate (AER) $<200 \mu \mathrm{g} / \mathrm{min}$, without clinical evidence of nondiabetic renal disease. Three baseline measurements of creatinine clearance (CC) and AER were compared with 3 measurements taken 5 years later. GFR was estimated using the Cockroft-Gault (CG-GFR) formula and was also measured isotopically (DTPA-GFR) in 51 patients. The patients were classified according to their final CC and AER status: Normal = normal AER $+\mathrm{CC} \geq 75 \mathrm{ml} / \mathrm{min} / 1.73 \mathrm{~m}^{2}$, LowCC $=$ normal AER + CC $\leq 75 \mathrm{ml} / \mathrm{min} / 1.73 \mathrm{~m}^{2}$ and HighAER $=$ high AER $(\geq 20$ $\mu \mathrm{g} / \mathrm{min}$ ) irrespective of $\mathrm{CC}$. Final measures of renal function (table) revealed the LowCC group had CG-GFR and DTPA-GFR lower than the "Normal" group and comparable to the HighAER group. The 5 year change $(\Delta)$ in measures of renal function indicated significant declines in CC and CG-GFR for all 3 groups. The LowCC group (73\% women, $58 \%$ Type 2 diabetes) had significantly lower $24 \mathrm{hr}$ urinary creatinine excretion compared with the other 2 groups. However, they were of comparable age and diabetes duration to the HighAER group. Analysis of covariance showed a strong, independent difference between groups for $\mathrm{CC}$ (partial $F=16.3, p<0.0001$ ) even after adjustment for age (partial $F=5.9, p<0.02$ ), sex, weight, and type of diabetes (all ns). In conclusion, the results suggest that up to $30 \%$ of Type 1 and Type 2 diabetic patients may have reduced renal function without microaltuminuria. Analysis of covariance in the Normal and LowCC groups revealed a significant contribution to LowCC of increased age and low $24 \mathrm{hr}$ urinary creatinine. The relative contributions of a decrease in muscle mass as opposed to an intrarenal disease process remain to be assessed.

opposed to an intrarenal disease process remain to be assessed.
\begin{tabular}{|l|c|c|c|}
\hline mean \pm SEM & Normal $(\mathrm{n}=40)$ & Low CC $(\mathrm{n}=33)$ & HighAER $(\mathrm{n}=30)$ \\
\hline Age (years) & $43.8 \pm 2.7$ & $60.2 \pm 2.9^{*}$ & $61.0 \pm 2.4^{*}$ \\
CC $\left(\mathrm{ml} / \mathrm{min} / 1.73 \mathrm{~m}^{2}\right)$ & $95.0 \pm 1.8$ & $59.2 \pm 2.4^{*}$ & $69.3 \pm 5.0^{*}$ \\
CG-GFR $\left(\mathrm{ml} / \mathrm{min} / 1.73 \mathrm{~m}^{2}\right)$ & $97.7 \pm 3.2$ & $70.7 \pm 4.5^{*}$ & $67.7 \pm 4.9^{*}$ \\
DTPA-GFR $\left(\mathrm{ml} / \mathrm{min} / 1.73 \mathrm{~m}^{2}\right)$ & $127.5 \pm 4.5$ & $83.4 \pm 9.3^{*}$ & $86.7 \pm 7.5^{*}$ \\
Plasma creatinine $(\mu \mathrm{M})$ & $81 \pm 2$ & $97 \pm 5$ & $118 \pm 12^{*}$ \\
Microalbuminuria $(\mu \mathrm{g} / \mathrm{min})$ & $9.9 \times / \div 1.1$ & $8.8 \times /+1.1$ & $114 \times / \div 1.2^{*}$ \\
$\Delta C C\left(\mathrm{ml} / \mathrm{min} / 1.73 \mathrm{~m}^{2}\right)$ & $-10.8 \pm 3.4$ & $-15.3 \pm 2.6$ & $-16.0 \pm 2.6$ \\
$\Delta C \mathrm{CG}-\mathrm{GFR}\left(\mathrm{ml} / \mathrm{min} / 1.73 \mathrm{~m}^{2}\right)$ & $-5.6 \pm 1.6$ & $-9.3 \pm 2.1$ & $-14.4 \pm 2.2^{*}$ \\
\hline
\end{tabular}

$* 0<0.05$ compared to Normal group using Fisher's LSD post hoc test.
URINARY ALBUMIN EXCRETION IN UPPER BODY OBESITY.

M.D. Jensen and S. Nielsen, Endocrine Research Unit, Mayo Clinic, Rochester, MN 55905, USA

Elevated urinary albumin excretion (UAE) has been associated with insulin resistance and is suggested to be elevated in prediabetic individuals. Upper body obesity, especially visceral obesity, predicts insulin resistance and development of type 2 diabetes. Whether UAE clusters with insulin resistance traits in upper body obese subjects was examined in 50 subjects with a wide range of body fat and body fat distribution. All volunteers had normal blood pressure, normal glucose tolerance, and were maintained on a controlled diet for 2 weeks. UAE was assessed from 3 overnight urine collections, body composition by whole body DXA scanning and abdominal CT scanning. Fasting insulin and insulin responses to oral glucose were significantly increased in the obese subjects, who also tended to have more dyslipidemia, slightly, but not significantly greater blood pressure, and significantly more visceral fat than lean subjects. These differences were more apparent in subjects with upper body obesity (waisthip $\geq 0.85$ (women); $\geq 0.93$ (men)). UAE was normal in obese and upper body subjects and not different from that of lean subjects. UAE ranged from 0.3 to 8.3 $\mu \mathrm{g} / \mathrm{min}$ in lean subjects and from 0.2 to $7.2 \mu \mathrm{g} / \mathrm{min}$ in obese subjects Visceral fat accumulation correlated significantly with fasting plasma triglycerides $(r=0.49, \quad \mathrm{p}<0.05 ; \mathrm{r}=0.42, \quad \mathrm{p}<0.05$, women and men, respectively) and insulin $(r=0.56, p<0.01 ; r=0.62, p=0.001$, women and men, respectively) concentrations, but not with UAE. UAE was not significantly correlated with body composition, blood pressure, plasma levels of glucose, lipids, insulin, or insulin under the OGTT curve. Thus obese, even upper body obese subjects, with increased visceral and total body fat, high plasma insulin and triglycerides, and low HDL cholesterol concentrations do not have elevated UAE. This suggests that UAE is not closely associated with these characteristics and implies a later onset of abnormal albuminuria in the course of the insulin resistance syndrome.

\section{4}

EFFECTS OF ACUTE EXPOSURE AT 3,000 $\mathrm{m}$ ON BLOOD PRESSURE AND ALBUMIN EXCRETION IN TYPE 1 DIABETES. M.Veglio, C.Rossi, M.Migliardi, M.Deandrea, A.Grassi, D. Fonzo and O.Pecchio*Italian Alpine Club Medical Commission*, Dpt of Endocrinology, Ospedale.Mauriziano, Torino, Italy

It is known that acute altitude hypoxia (over 4,000 m) increases AER in healthy subjects, while the effect on blood pressure (BP) is debated. This study aimed at verifying the effect of milder hypoxia $(3000 \mathrm{~m})$ in normoalbuminuric insulin dependent diabetics (IDD). Subjects and methods: 6 uncomplicated IDD, 4 males and 2 females, mean age 25.2 yrs (range 18-42) in good glycemic control (HbAlc 6.8 $1.1 \%$ ), were investigated at sea level (Torino, alt.200 m) and after ascent by car and cable car to $2950 \mathrm{~m}$. A $24 \mathrm{~h}$ ambulatory blood pressure monitoring, was followed by an in field exercise test (a $1500 \mathrm{~m}$ walk on a step path with 150 $\mathrm{m}$ gradient) performed at $70 \%$ of the heart rate at $\mathrm{VO}_{2} \max$, as determined by a previous treadmill test performed in normobaric hypoxia $(3,000 \mathrm{~m})$. Blood glucose and lactate were assessed in fasting condition, before, immediately, 5 and $15 \mathrm{~min}$ after the stop. The AER was assessed overnight and on a $3 \mathrm{~h}$ urine collection before and after the beginning of the exercise.. Results: at altitude the night-time diastolic BP was higher than at sea level $(73.1 \pm 2.6$ vs $65.4 \pm 4.1 \mathrm{mmHg} ; \mathrm{p}<.01)$, while the day time systolic BP was lower $(121.3 \pm 10.8$ vs $126.4 \pm 10.2 \mathrm{mmHg}: \mathrm{p}<.01)$; the AER was higher overnight, before exercise, and particularly during exercise $(2.1 \pm 1.8$ vs $0.9 \pm 0.3 \mu \mathrm{g} / \mathrm{min}, \mathrm{p} \mathrm{NS} ; 5.6 \pm 4.2 \mathrm{vs} 1.4 \pm 0.8 \mathrm{\mu g} / \mathrm{min}, \mathrm{p}<.03$ and $15.9 \pm 9.1$ vs $6.3 \pm 3.8 \mathrm{\mu g} / \mathrm{min}, \mathrm{p}<.01$, respectively). The systolic and diastolic BP peak at the end of the exercise increased at altitude $(167.3 \pm 18.7$ vs $150.5 \pm 19.7$ $\mathrm{mmHg}$ and $98.5+20.6$ vs $68.3+9.8 \mathrm{mmHg}$ ). Conclusions : in IDD, AER increases significantly with exercise even at $3000 \mathrm{~m}$, while BP is not constantly higher, suggesting a prevalent effect of moderate hypoxia through an increase in capillary permeability. Diabetic patients, even if uncomplicated, could be at higher risk of hypoxia induced endothelial disfunction.

\section{5}

RENAL VOLUME AND VASCULAR RESISTANCE IN TYPE 2 DIABETIC PATIENTS WITH MICROALBUMINURIA R.Nosadini, A.Saller, P.Fioretro, M.Dalla Vestra, C.Abaterusso, G.C.Bombonato, M.Bolognesi, A.Favaro, G.Crepaldi, A.Gatta and D.Sacerdoti, University of Padua and Sassari, CESI-CNR, Italy.

Nephromegaly often characterizes type I (D1) with microalbuminuria (MA) and may be related to the presence of diabetic microangiopathy. Conflicting results have been reported in patients with type 2 diabetes (D2). The reason for this difference is not clear. Macroangiopathy may be more frequently associated with microangiopathy in D2 than D1. Ischemic nephropathy could explain both the presence of small kidneys and increased intrarenai vascular resistance in D2. Severe interstitial fibrosis and tubular athrophy could also be explained by ischemia. Kidney biopsies in D2 with MA were classified by light microscopy as: CI-normal or near normal renal structure; CII-typical diabetic nephropathology; CIII-atypical patterns of renal injury with more severe tubulo-interstitial and arteriolar than glomerular changes. Our aim was to compare right/left kidney volumes $(\mathrm{KV})$ and pulsatility index (PI) of interlobar arteries measured by eco-color doppler of 11 controls matched for age ( $50 \pm 8 \mathrm{yrs}, \mathrm{M} \pm \mathrm{S}$.D.) with those of $22 \mathrm{D} 2$ with MA who were divided in 3 groups according to the patterns of renal lesions (CI $\mathrm{n}^{\circ} 7, \mathrm{CII} \mathrm{n}^{\circ}$ 8 and $\mathrm{CIII} \mathrm{n}^{\circ}$ 7). Both PI and $\mathrm{KV}$ were higher in $\mathrm{CI}$, $\mathrm{CII}$ and $\mathrm{CIII}$ than in controls (PI: $0.85 \pm 0.08 ; \mathrm{KV}: 226 \pm 41 \mathrm{ml}, \mathrm{p}<0.05$ for both). CI, CII and CIII patients had similar rates of MA and glomerular filtration. PI was higher in CII and CII than in $\mathrm{CI}(\mathrm{p}<0.05$ ) (CI vs CII vs CIII: $1.06 \pm 0.08$ vs $1.68 \pm 0.35$ vs $1.37 \pm 0.27$.). No differences were observed with regard to KV (CI, CII, CIII; $360 \pm 80,350 \pm 48$, $301 \pm 44 ; \mathrm{ml}$, n.s.). In conclusion nephromegaly is a common characteristic of D2 with MA. On the contrary more severe increase in intrarenal vasular resistance was observed only in D2 in whom MA was associated with typical and atypical lesions, irrespective of the severity of glomerular or interstitial injury. If small kidneys and high intrarenal vascular resistance are peculiar features of macroangiopathy, rather than typical diabetic microangiopathy, intrarenal arteriosclerosis alone is unlikely to explain the different patterns of renal injury between $\mathrm{CII}$ and $\mathrm{CIII}$, which could rather reflect a heterogeneous response to diabetic challenge in D2. 


\section{6}

Progression from microalbuminuria to overt nephropathy in NIDDM: The Reciever Operating Characteristic curve analysis.

Yaijma Y,Jin Y,Moriya T,Matoba K. Kitasato Univ.Schl.of Med, Japan There has so far been no long-term follow-up study to evaluate clinical adequacy of the cut-off value of urinary albumin-creatinine ratio(U-ACR) for microalbuminuria(MA) in relation to progression to overt nephropathy(ON) in NiDDM. The aim of this study is to determine the cut-off value of U-ACR for MA and to evaluate its clinical adequacy in relation to the progression from MA to ON by long-term follow-up study in NIDDM. One hundred fifty seven non-proteinuric NIDDM patients(pts), 54 \pm 12 (M $\pm S D$ ) years(yrs) of age and known diabetes duration of $9 \pm 7 \mathrm{yrs}$, were randomly recruited and followed up for 9 years. U-ACR and urinary creatinine were measured bimonthly by immunonepherometry and Jaffe's method, respectively. ON was diagnosed when 3 successive urines showed persistently overt proteinuria. Negative linear correlation was shown between the $\log$ initial U-ACR values and the periods from MA to ON $(r=,-0.53, p<0.002)$. The Receiver Operating Characteristic curve $(\mathrm{ROC})$, based on the initial U-ACR values and the rates of progression from MA to ON during 9 years of follow-up, was analysed, and the cut-off value of U-ACR for MA was determined as $25 \mu \mathrm{g} / \mathrm{mg}$ $(2.8 \mathrm{mg} / \mathrm{mmol})$. Its sensitivity and specificity were $67.7 \%$ and $69.8 \%$, respectively. Based on the cut-off value, 59 of 157 non-proteinuric NIDDM patients were initially diagnosed as microalbuminuric, and $21(35.6 \%)$ of them and $10(10.2 \%)$ of 98 normoalbuminuric subjects progressed to ON during 9 years of follow-up. Compared with Caucasian microalbuminuric NIDDM pts, the progression from MA to ON is suggeted to be more frequent in Japanese NIDDM pts.

\section{7}

DIABETIC NEPHROPATHY AMONG POLYNESIANS

D.Simmons, D.J.Scott, L.Haru, A.Cecil and T.Kenealy. South Auckland Diabetes Project, Middlemore Hospital, Auckland, NZ

Polynesians have high rates of end stage renal failure (ESRF) due to Type 2 diabetes. This study described the proportion of patients with progressive nephropathy in a multi-ethnic cohort first studied in 1991 and followed up in 1996-7. Included were all patients from the diabetes services and those attending randomly selected general practices over a 17 week period. At both times a questionnaire, blood and an overnight urine sample were taken. After excluding 52 Europeans $(E)$ and 35 Polynesians $(P)$ who had died, 53E and 84P who had moved or were uncontactable, $160 / 226(68 \%) E$ and $63 / 93(68 \%) P$ agreed to participate in the repeat survey. By this time, mean ages were $E: 65 \pm 12$ and $P: 61 \pm 8$ years $(p<0.05)$, duration of diabetes was $15 \pm 9$ and $14 \pm 7$ years, blood pressures were $144 \pm 22 / 85 \pm 9$ and $148 \pm 23 / 88 \pm 8$ (ns/p<0.05 respectively) and $\mathrm{HbA1C}$ was $7.2 \pm 1.6 \%$ and $8.1 \pm 2.4 \%$ respectively ( $p<0.001)$. Receiving antihypertensive therapy were $62 \% \mathrm{E}$ and $64 \% \mathrm{P} ; 23 \% \mathrm{E}$ and $32 \% \mathrm{P}$ were receiving insulin therapy. Over the 6 years, the geometric mean urinary albumin/creatinine excretion ratio (UACR) increased from 1.9 to $4.4 \mathrm{mg} / \mathrm{mmol}$ creatinine in $E$ and from 7.3 to $14.8 \mathrm{mg} / \mathrm{mmo}$ creatinine in $P$. The proportion with normal UACR and serum creatinine ( $<2.5$ and $<0.12 \mathrm{mmol} / \mathrm{An}$ in males and $<3.5$ and $<0.10$ in females) dropped from $69 \%$ to $47 \%(E)$ and $44 \%$ to $27 \%(P)$. Progression from normal to microalbuminuria occurred in $39 \% \mathrm{E}$ and $46 \% \mathrm{P}$, onto nephropathy in $33 \% \mathrm{E}$ and $43 \% \mathrm{P}$ and on to ESRF in $0 \% \mathrm{E}$ and $31 \% \mathrm{P}$ ( $p<0.01$ ). The ethnic difference in UACR in 1997 was eliminated in an analysis of covariance after adjusting for age $(p<.05)$, UACR in $1994(p<0.001), \mathrm{HbA1c}(p<0.05)$ and diastolic blood pressure $(p<0.01)$. In conclusion, $P$ have a higher rate of progression from nephropathy to ESRF than $E$, and this is associated with poorer blood pressure and glycaemic control.

\section{8}

\section{COMPARATIVE CHARACTERISTICS OF OVERT DIABETIC} NEPHROPATHY IN TYPE 1 AND TYPE 2 DIABETES.

R.P. Bain, R.E. Ratner, and J-P. Weurth for the ACTION 1 and 2 Study Groups. George Washington University Biostatistics Center, Medlantic Research Institute and Alteon, Inc.

Concurrent clinical trials in overt nephropathy were undertaken in type 1 and type 2 diabetes (ACTION 1 and 2). Eligibility criteria were comparable and focused on the presence of $>500 \mathrm{mg}$ proteinuria $/ 24$ hours. Presence of retinopathy was required, or diabetic nephropathy was proven by renal biopsy. 588 type 2 and 688 type 1 diabetic patients were enrolled in the trial. Type 2 subjects were 18 years older and had a greater ethnic minority contribution ( $32 \%$ vs 9\%) than type 1 subjects ( $\mathrm{p}<0.001$ for both). Known duration of diabetes was 9 years shorter for type 2 diabetics ( $17 \mathrm{vs}$ 26 years, $\mathrm{p}<0.001$ ). Type $2 \mathrm{~s}$ were significantly heavier (BMI 32 vs 25 , $\mathrm{p}<0.001$ ), and more hypertensive (151/81 vs $139 / 82, \mathrm{p}<0.001)$. Despite comparable entry requirements, the type 2 subjects had significantly greater proteinuria ( $4.1 \mathrm{gm} / 24$ hour vs $2.7 \mathrm{gram}, \mathrm{p}<0.001$ ) but lower renal function as measured by both 24 hour creatinine clearance and iothalamate GFR (54 $\mathrm{ml} / \mathrm{min} / 1.73 \mathrm{~m}^{2}$ vs $59, \mathrm{p}<0.001$ and $52 \mathrm{ml} / \mathrm{min} / 1.73$ vs $66, \mathrm{p}<0.001$, respectively). Microvascular disease, as manifested by both retina (ETDRS) score $(p<0.001)$ and retinal events $(p<0.001)$ were more common in type 1 diabetes, whereas macrovascular discase (MI, LVH, LBBB and ST segment depression) was more common in type 2 diabetes ( $\mathrm{p}<0.001$ ). Despite similar entry criteria, patients with type 2 diabetes present with more significant proteinuria and worse renal function, but shorter duration of diabetes. Diabetic nephropathy is more associated with microvascular complications in type 1 and macrovascular disease in type 2 diabetes.

\section{9}

BLOOD PRESSURE SELF MONITORING VERSUS OFFICE MEASUREMENTS AS A PREDICTOR OF PROGRESSION IN DIABETIC NEPHROPATHY

A. Trocha, K. Rave, R. Bender, Peter T. Sawickl; Department of Metabolic Diseases, Heinrich-Heine-University, Düsseldorf, Germany

Office blood pressure measurements may overestimate blood pressure. In a six years follow-up study we assessed the impact of blood pressure as assessed by self-monitoring $\left(\mathrm{BP}_{\mathrm{S}}\right)$ versus office blood pressure measurements $\left(\mathrm{BP}_{\mathrm{O}}\right)$ on the progression of diabetic nephropathy. 77 IDDM patients $(37$ women, $40 \mathrm{men}$ ) with overt nephropathy, and with arterial hypertension were followed for a mean ( $\pm S D$ ) period of $6.2 \pm 2.8$ years. At baseline patient characteristics were: age $37 \pm 8$ years; diabetes duration $23 \pm 6$ years; smokers $31 / 77$; body mass index $24 \pm 3 \mathrm{~kg} / \mathrm{m}^{2}$; serum creatinine $131 \pm 56 \mu \mathrm{mol} / \mathrm{l}$; glomerular filtration rate (GFR) (calculated from Cockroft formular) $73 \pm 30$ $\mathrm{ml} / \mathrm{min}$; $\mathrm{BP} \circ 166 / 94$ and $\mathrm{BP}_{\mathrm{s}} 159 / 94 \mathrm{~mm} \mathrm{Hg}$; proteinuria $2.5 \pm 2.4 \mathrm{~g} / 24 \mathrm{~h}$; and $\mathrm{HbA}_{1 \mathrm{C}} 7.8 \pm 1.7 \%$. All patients received a blood pressure measurement device and an extensive advice in blood pressure measurement. Self monitoring of blood pressure twice a week before taking medication was recommended Progression of nephropathy was calculated individually as mean decline of GFR per year. Mean visit frequency was $2.6 \pm 1.5$ per year. During the following period $\mathrm{BP}_{\mathrm{s}}$ decreased to $138 / 83$ and $\mathrm{BP}_{\mathrm{o}}$ to $154 / 89 \mathrm{~mm} \mathrm{Hg} ; \mathrm{HbA}_{1 \mathrm{c}}$ increased to $8.1 \pm 1.1 \%$; and proteinuria remained unchanged. The decline of GFR was $4.1 \pm 5.6 \mathrm{ml} / \mathrm{min} /$ year. Baseline- and follow-up parameters were included in a stepwise multiple regression analysis with the decline of GFR per year being the dependent variable. Loss of GFR was significantly correlated with mean proteinuria $(p=0.001)$; mean $B P_{s}(p=0.001)$; and baseline $\mathrm{HbA}_{1 \mathrm{c}}(p=0.001)$ When compared to $\mathrm{BP}_{\mathrm{o}}$ the loss of renal function was better predicted by $\mathrm{BP}_{\mathrm{S}}$ $\left(R^{2}=0.52\right.$ vs. 0.42$)$. Likewise, the simple correlation between $B P_{S}$ and $G F R$ decline was higher than with $B P_{0}(r=0.33$ vs. 0.16$)$. We conclude that seif monitoring of blood pressure is a better predictor of progression in diabetic nephropathy when compared to office blood pressure measurements. 
SIMPLIFIED IOHEXOL PLASMA CLEARANCE: A RELIABLE TECHNIQUE FOR GLOMERULAR FILTRATION RATE MEASUREMENT IN DIABETIC PATIENTS.

G. Penno, S. Bandinelli, M. Pilo, M. Nannipieri, L. Rizzo, A. Marsocci, R. Navalesi and L. Pucci. Dept. of Endocrinology and Metabolism, University of Pisa, Italy

Both follow-up of patients with diabetic nephropathy (the clinical practice) as well long term intervention studies (the clinical research setting) require repeated measurements of renal function (GFR). Contrast medium iohexol plasma clearance (bolus injection) shows excellent agreement with tracers clearance throughout a wide range of renal functions. Agreement was assessed in 41 diabetic patients (15 type 1,26 type 2) with a wide range of plasma creatinine $(0.80-6.38 \mathrm{mg} / \mathrm{dl})$. Repeated determinations of the iohexol plasma clearance gave excellent agreement $(r=0.990)$ and a total $\mathrm{CV}$ of $3.5 \pm 3.3 \%$ (range $0.7-10.7 \%)$. The correlation between multiple sample clearance (13-16 samples up to $300-$ $1440 \mathrm{~min}$ in relation to serum creatinine levels) of iohexol (5 milliliters Omnipaque, Nycomed, Oslo) and ${ }^{51} \mathrm{Cr}$-EDTA $(\sim 1 \mu \mathrm{Ci} / \mathrm{kg}$ body weight) was high $(r=0.965)$. To make the test less cumbersome, a seven-sample design was validated by comparison with both the full-sample schedule and the three-sample approach by Brøchner-Mortensen. Validation was performed in 83 diabetic patients (48 type 1,35 type 2). The best estimates of parameters of the biexponential (full- and seven-sample design) and of the monoexponential function (three-sample approach) as well their variability (variance and covariance matrix analysis) were obtained by DIMSUM. This is an expert system for multiexponential model discrimination. Full-sample GFR was $80.3 \pm 43.8 \mathrm{ml} / \mathrm{min}$, sevensample GFR was $79.4 \pm 43.9 \mathrm{ml} / \mathrm{min}(\mathrm{r}=0.990)$, three-sample GFR was $79.8 \pm 45.2 \mathrm{ml} / \mathrm{min}(\mathrm{r}=0.972)$. The mean $\pm \mathrm{SD}$ coefficient of variation of the different approaches were $2.7 \pm 1.4 \%, 4.1 \pm 2.7 \%$ for the full-sample and the seven-sample approaches, respectively, and signifi-cantly higher for the three-sample design $(6.9 \pm 3.4 \%, \mathrm{p}=0.0001)$. Thus, the BrøchnerMortensen method to determine GFR after iohexol single injection does not provide an accurate estimate of parameters for GFR calculation. The simplified approach gives acceptable errors in GFR calculation, allows a good estimate of GFR througthout a wide range of renal function and is advantageous in the clinical management of diabetic patients.

\section{2}

DECREASE IN GLOMERULAR FILTRATION RATE IN PATIENTS WITH TYPE 2 DIABETES IS LINKED TO ATHEROSCLEROSIS.

H.Taniwaki, T.Kawagishi, Y.Nishizawa, E. Ishimura, M. Emoto, Y. Okuno, and H.Morii. Second Department of Internal Medicine, Osaka City University Medical School, 1-5-7, Asahi-machi, Abeno-ku, Osaka, 545 8586, JAPAN

The aim of this study is to assess the effects of atherosclerosis on the glomerular filtration rate (GFR) in NIDDM patients with and without microalbuminuria. A total of 61 Japanese patients with NIDDM were recruited. They ranged in age from 40 to 69 years $(28$ males and 33 females). Each subject was collected a 24-hr urine sample for the quantitative analysis of albumin. Absence of albuminuria was defined as a urinary albumin excretion level of $<30 \mathrm{mg} / 24-\mathrm{h} \quad(\mathrm{n}=36)$, and microalbuminuria as $30-300 \mathrm{mg} / 24-\mathrm{h}(\mathrm{n}=25)$. The GFR was estimated by ${ }^{99 \mathrm{~m} T c}$-DTPA method. As indices of atherosclerosis, we measured the intimal-medial thickness (IMT) and the distensibility of the carotid artery using a high resolution B-mode ultrasonography and an echo-tracking system, and the resistance index (RI) of the renal interlobar arteries by pulsed Doppler sonography. The clinical characteristics of the NIDDM patients with and without microalbuminuria did not differ except for the duration of NIDDM, which was longer in the patients with microalbuminuria. The GFR also did not differ between the patients with and without microalbuminuria. The GFR was significantly correlated with the patient's age $(r=-0.256, p<0.05)$, carotid IMT $(r=-0.326, p<0.05)$, carotid stiffness $\beta(r=-0.449, p<0.001)$, and the renal arterial $R I$ I $r=$ $0.365, \mathrm{p}<0.05$ ). Multiple regression analysis revealed that the factors independently associated with the GFR were carotid IMT, carotid stiffness $\beta$, and renal arterial $R I\left(R^{2}=0.108, p=0.0102 ; R^{2}=0.208, p=0.0003\right.$; $\mathrm{R}^{2}=0.130, \mathrm{p}=0.0043$ ). The decline in GFR in NIDDM patients with early stage nephropathy may be due in part to atherosclerosis.
Glomerular hyperfiltration in the prediction of diabetic nephropathy - A prospective follow-up study.

E-L. Stattin', G. Dahlquist', B Persson ${ }^{2}$ and S. Rudberg ${ }^{2}$. Department of paediatrics, Umeå University Hospital ${ }^{1}$ and Department of Woman and Child Health, Paediatric Unit, Karolinska Institute ${ }^{2}$, Stockholm, Sweden.

We have previously reported that glomerular hyperfiltration after 12 years mean duration of insulin-dependent diabetes mellitus ( IDDM) predicts incipient nephropathy ( IN) 8 years later. The aim of the present study was to determine the predictive value of glomerular filtration rate ( GFR) on diabetic nephropathy in the same cohort after another 8-10 years of follow-up. The cohort consisted initially of 75 patients ( 40 males, 35 females), aged $17.1 \pm 2.5$ ( mean, SD) with a diabetes duration $\geq 8$ years. GFR, albumin excretion rate ( AER), blood pressure and haemoglobin Alc were measured every two years during the first 8 years of follow-up. After 16 to 18 years end point data were collected from medical records. Sixty-one patients, with $29.0 \pm 2.7$ years (mean, SD) duration of IDDM participated to the end point. Nine of 61 patients developed ON ( defined as persistent AER $>200 \mu \mathrm{g} / \mathrm{min}$ ). All but three had an initial GFR exceeding the upper range of the method $\left(\geq 125 \mathrm{ml} / \mathrm{min} / 1.73 \mathrm{~m}^{2}\right)$. Eleven patients developed $\mathrm{IN}$ (defined as persistent AER 20-200 $\mu \mathrm{g} / \mathrm{min}$ ). Seven of them had GFR $\geq 125$ $\mathrm{ml} / \mathrm{min} / 1.73 \mathrm{~m}^{2}$. When analysing initial hyperfiltration as a risk-factor for the development of $\mathrm{IN}$ or ON odds ratio was $=3.93(95 \% \mathrm{CI} ; 1.04-14.85)$. The positive predictive value for $\mathrm{IN}$ and $\mathrm{ON}$ within 29.0 years of duration when having an initial glomerular filtration rate $\geq 125 \mathrm{ml} / \mathrm{min} / 1.73 \mathrm{~m}^{2}$ was $68 \%$. Patients who developed IN or ON had similar entry values of age, age at onset, duration of IDDM, AER, blood pressure and HbAlc (as also HbAlc at 8 years of follow-up) as those remaining normoalbuminuric. Conclusion: Increased glomerular filtration rate is a strong risk-factor for the development of diabetic nephropathy.

\section{3}

THECOURSEOFGLOMERULAR FLTRATIONRATE INALBUMNURIC NONHNSULN DEPENDENT DIABETTC PATENTS WTH ORWTHOUT DABETIC GLOMERLLOPATHYY. P.K. Christensen, M-A. Gall and H-H. Parving, Steno Diabetes Center, Niels Steensens Vej 2, DK-2820 Gentofte, Denmark.

To evaluate the cause of albuminuria a kidney biopsy was pertormed in 34 consecutive NIDDM patients with persistent alburninuria $(>300 \mathrm{mg} / 24 \mathrm{hr}$ ). The biopsy revealed diabetic glomenulosclerosis in 26 (25M/1F) patients (DG-group), age 52+2 (meantSE) years and non-diabetic glomerulopathies in 8 (7W1F) patients (NDG-group), age $54 \pm 3$ years. Patients were followed prospectively for a median of 7.7 (range (1.0-14.2)) years with glomerular fittration rate (GFR) measurements from onset of persistent albuminuria, at least once a year. Avterial blood pressure and alburninuria were measured at least twice a year. Eighty-one percent in the DG-group received anthypertensive teatment vs $75 \%$ in the NDG-group. GFR decreased from $82(24-146)$ to $38(2-116) \mathrm{m} / \mathrm{min} 11.73 \mathrm{~m}^{2}$ ( $p<0.001$ ) with median rate of decline in GFR of $5.6(0.3-21.6) \mathrm{m} / \mathrm{min} / y$ ear (DG-group) and in the NDG-group from $107(89-135)$ to $90(17-119) \mathrm{m} / \mathrm{min} / 1.73 \mathrm{~m}^{2}(\mathrm{p}<0.05)$ with median rate of decline in GFR of $2.5(0.3-7.6) \mathrm{m} / \mathrm{min} / \mathrm{year}, \mathrm{p}<0.05$ between groups. Abuminuria increased from $1.4(0.3-7.2)$ to $2.6(0.1-21.6) g / 24 \mathrm{hr}$ ( $p<0.05$ ) (DG-group) and decreased in the NDG-group from $2.2(0.8-8.7)$ to $0.8(0.2-2.5) g / 24 \mathrm{hr}(\mathrm{p}=0.05)$. Mean arterial blood pressure (MABP) decreased from $118+3$ to $104 \pm 3 \mathrm{~mm} \mathrm{Hg}(p<0.05$ ) (DGgroup) while it remained unchanged in the NDG-group $106 \pm 3 \mathrm{~mm} \mathrm{Hg}$ vs $105 \pm 3 \mathrm{~mm} \mathrm{Hg}$. In the DG-group the rate of decine in GFR correlated with systolic blood pressure $(r=0.62$, $p<0.001), \mathrm{MABP}(r=0.52, p<0.01)$, albuminuria $(r=0.55, p<0.005)$ and GFR at entry $(r=$ $0.45, p<0.05$ ). The same analyses based on mean values during the follow-up period revealed: Systolic blood pressure $(r=0.62, p<0.001), \operatorname{MABP}(r=0.52, p<0.001)$ albuminuria $(r=0.55, p=0.005)$ as progression promolers for rate of decline in GFR. Mean time to doubling of baseline serum creatinine was 99 months (95\% Cl: $80-119$ ) in the DG-group vs 151 months (95\% Cl: $130-172)$ in the NDG-group ( $p=0.055$ ). Our study demonstrated a more rapid decline in GFR, acceterated development of end stage renal disease and a progressive raise in alburninuria in NIDDM patients with diabetic glomerulosclerosis compared to NIDDM patients with non-diabetic glomerulopathies. 
1114

Does a renal artery stenosis must be searched in NIDDM nephropathy? J.P Courrèges, J.Bacha, E. Aboud, L. André

Service of internal medecine - Diabetologia - Vascular diseases

Centre hospitalier - Bd Dr Lacroix - NARBONNE - 11100 - FRANCE

Renal antery stenosis (RAS) is a common etiology (about 15\%) of terminal renal insufficiency (RI), while nephropathy is one of the most usual complication in NIDDM. What are the prevalence and the profile of RAS in NIDDM nephropathy? RAS has been searched (duplex color scan) in a 75 NIDDM consecutive population with nephropathy (creatininemia clearance $\leq 60 \mathrm{ml} / \mathrm{mn}$ and/or proteinuria $\geq 300$ $\mathrm{mg}$ ), $\leq 80$ y old, 26 females $/ 49$ males (sex Ratio : 0,53 ), mean age $: 67,7 \pm 6,9$ y old, diabetes duration : $10,9 \pm 7,1$ y, mean creatininemia : $144 \pm 61 \mu \mathrm{mol} / 1+$ clearance $53 \pm 15 \mathrm{ml} / \mathrm{mn}$. 63 have $\mathrm{RI}$ and 53 proteinuria, $95 \%$ suffer from hypertension. A significant RAS (stenosis $\geq 70 \%$ ) has been diagnosed in 16 patients $(22 \%)$ and confirmed by arteriography $(n=11)$ or angio $R M N(n=5) .12(75 \%)$ have unilateral and $4(25 \%)$ bilateral stenosis, $70 \%$ of renal echography are abnormal. This ischemic nephropathy group (IN) was composed by 5 females/11 males (Sex Ratio: 0,45 ) mean age : $69,3 \pm 6$ y old, BMI : $26,4 \pm 4$, HBAIC : $8,4 \pm 1,2 \%$, cholesterol : $210 \pm$ $20 \mathrm{mg} / \mathrm{l}$, triglycerides : $255 \pm 11 \mathrm{mg} / \mathrm{l}$. Retinopathy prevalence : $31 \%$, mean arterial pression SBP : $148 \pm 15$ DBP : $82 \pm 7 \mathrm{MBP}: 111 \pm 10 \mathrm{mmHg}$, these data are no significant versus non ischemic nephropathy group (NIN/n $=59$ ). Are differents (IN vs NIV) $\mathrm{P}<0,05$ Diabetes duration: $143+5,7$ vs $10 \pm 7$ y smoking : (50 vs $29 \%$ ) and severe macro angiopathy prevalence ( 60 vs $34 \%$ ) : coronary disease : 50 vs $25 \%$ - legs arteritis : 31 vs $10 \%$ (carotid stenosis 6 vs $8 \%$ NS). The study of.renal function (IN vs NIP) shows $(P<0,01)$ significant increasing of creatininemia (173 \pm 65 vs $136 \pm 60 \mu \mathrm{mol} / \mathrm{l})$ decreasing of creatininemia clcarance $(37 \pm 10 \mathrm{vs} 57 \pm 16 \mathrm{ml} / \mathrm{mn})$ while proteinuria is less important ( $530 \pm 310$ vs $1150 \pm 800 \mathrm{mg} / 24 \mathrm{~h}) .10(19 \%)$ in proteinuric group, $16(26 \%)$ in RI have a RAS. RAS high risk is found in patients with creatininemia $\geq 150 \mu \mathrm{mol} / \mathrm{l}(32 \%)$, creatininemia clearance $\leq 30 \mathrm{ml} / \mathrm{mn}(38 \%)$ microalbuminuria + RJ $(36 \%)$.

Conclusion :In NIDDM nephropathy, renal artery stenosis is frequent $(22 \%)$ and must be searched cspecialy in severe or evolutive renal insufficiency (creatinine ? $150 \mu \mathrm{mol} / \mathrm{l}$ ) in males, smokers, with resistant hypertension and/or severc macroangiopathy and duplex color scan seem the better way of RAS screening.

\section{6}

Conventional and color duplex Doppler ultrasound in diabetic nephropathy

V. Božikov, M. Sučić, D. Soldo-Jureša, B. Brkljačić, D. DelićBrkljačić and V. Mrzljak, University Hospital "Dubrava", Avenija izviđača 6, Zagreb, Croatia.

The aim was to compare conventional and color duplex Doppler ultrasonic findings in diabetic nephropathy. In 190 diabetic patients and 85 healthy examinees (controls) conventional ultrasound was used to assess renal length, parenchymal thickness and cortical echogenicity. Doppler resistance indexes (RIs) were measured in intrarenal arteries, as indicators of renal vascular resistance. According to clinical stage, the patients were classified into 3 groups. RIs were correlated with renal functional tests. Renal length and parenchymal thickness in patients with clinically asymptomatic phase of the disease were significantly increased compared to controls. Significant differences were not observed between controls and patients with clinically manifest nephropathy. In advanced renal disease kidneys were significantly smaller. Cortical hyperechogenicity was observed only in very advanced disease. Elevation of RIs accompanied progression of diabetic nephropathy. Ris correlated well with renal function, and pathologic values of $\mathrm{R}$ $(\geq 0.70$ ) were observed in $15 \%$ in the asymptomatic group and in $87 \%$ in the group with advanced nephropathy. Conclusion: R elevation in diabetic nephropathy can be observed in earlier stages of the disease, as compared to changes detectable by conventional ultrasound. Color duplex Doppler ultrasound enhances diagnostic performances of ultrasound in diabetic renal disease.
1115

DOPPLER ULTRASONOGRAPHY DATE PRECEDE ALBUMIN EXCRETION RATE IN DIABETIC PATIENTS?

B.Brkljačić, S.Ljubić, I.P.Renar, Ž.Metelko, A.Barada, G.Roglić

The Vuk Vrhovac University Clinic, Zagreb, Croatia

Diabetic nephropathy is a frequent microvascular complication in diabetic patients. Histopathologic changes can be found primarily in the vascular compartement, which can cause the elevation in renal vascular resistence (RVR), manifested by increased value of Doppler renal resistance index (DRRI). Resistance index is one of the most useful and reliable parameters in the spectral flow velocity waveform analysis. Forty-two patients with NIDDM aged 33-65 years, with diabetes duration of the 1-24 years ( 20 males and 22 females ) were included in the study. DRRI was correlated with the duration of diabetes mellitus, systolic and diastolic blood pressure, albumin excretion rate (AER) and Tamm-Horsfall protein excretion rate (THPER). Significant rank correlation was found between the DRRI and the disease duration $(\mathrm{R}=0.4218, \mathrm{p}=0.005)$, $\mathrm{DRRI}$ and the systolic blood pressure $(R=0.7544, p=0.000)$, DRRI and AER $(R=0.4217, p=0.005)$ and DRRI and THPER $(R=-0.3264, p=0.055)$. There was no significant correlation between DRRJ and diastolic blood pressure $(R=0.2634$, $\mathrm{p}=0.091$ ). The negative correlation between DRRI and THPER can be explained by expected lowerin of THPER with the longer duration of diabetes. These results indicate that kidney Doppler ultrasonography can help to identify ealier stages of diabetic nephropathy. Detection of byperteusion, an early consequence of RVR elevation, could precede detection of AER

\section{7}

99mTe-DIMERCAPTOSUCCINIC ACID UPTAKE IN DIABETIC NEPHROPATHY PATIENTS

M.Rajić, M. Bogićević, S.Ilić, S.Antić, M.Avramović, B.Mitić and V.Stefanović Department of Nuclear Medicine, Clinical Center, Niš, Yugoslavia

Tubular uptake of $99 \mathrm{mTc}$-dimercaptosuccinic acid (99mTc-DMSA) is established as a reliable marker for quantitative estimation of renal function. The aim of this sludy was to evaluate tubular function in diabetic nephropathy patients by studying $99 \mathrm{mTc}$-DMSA biodistribution. Seventeen insulin-dependen overt diabetic nephropathy patients with normal serum creatinine levels (DN I) and 10 diabetic patients with renal fajlure (DN II) were included in the study. Glomerular filtration rate, estimated by $99 \mathrm{mTc}$-diethylenetriaminepentaacetic acid clearance was found $80.5 \pm 27.2 \mathrm{ml} / \mathrm{min}$ in DN I patients, $38.0+4.5 \mathrm{ml} / \mathrm{min}$ in DN II patients and $120.7 \pm 9.9 \mathrm{ml} / \mathrm{min}$ in 12 healthy volunteers. Tubular function was estimated by determining renal uptake of $99 \mathrm{mTc}$-DMSA and whole blood activity 2 and 4 h after injection, as well as 4 h cumulative urinary excretion. All values obtained were expressed as percentage of applied activity:

\begin{tabular}{|c|c|c|c|c|c|}
\hline \multirow[t]{2}{*}{ Group } & \multicolumn{2}{|c|}{ Renal uptake } & \multicolumn{2}{|c|}{ Blood activity } & \multirow{2}{*}{$\begin{array}{l}\text { Urine activity } \\
4 \mathrm{~h}\end{array}$} \\
\hline & $2 \mathrm{~h}$ & $4 \mathrm{~h}$ & $2 \mathrm{~h}$ & $4 h$ & \\
\hline Control & $47.1 \pm 6.3$ & $51.6 \pm 6.9$ & $26.0 \pm 3.1$ & $19.2 \pm 2.9$ & $10.8 \pm 2.4$ \\
\hline DN I & $17.6 \pm 5.6^{n}$ & $20.9 \pm 6.8^{n}$ & $38.9 \pm 9.5^{\circ}$ & $27.3 \pm 7.2^{x}$ & $26.0 \pm 11.3^{6}$ \\
\hline DN II & $7.3 \pm 1.5^{\circ .4}$ & $11.7 \pm 2.1$ & $53.5 \pm 16.5^{\text {ac }}$ & $39.6 \pm 12.3^{\text {xe }}$ & $10.0 \pm 4.6$ \\
\hline
\end{tabular}

Significantly decreased renal uptake associated with a high blood activity and increased urinary loss of $99 \mathrm{mTc}$-DMSA demonstrated tubular dysfunction in overt diabetic nephropathy patients with normal serum creatinine. In patients with resial failure tubular uptake was found to be more lowered. $4 \mathrm{~h} 99 \mathrm{mTc}$ DMSA renal uptake was found reduced in DN I and DN II patients for 59 and $77 \%$, while glomerular filtration rate was decreased only for 33 and $68 \%$, respectively. These results point out to more severe tubular than glomerular dysfunction in studied diabetic nephropathy patients. 
GLOMERULAR AND TUBULAR DISFUNCTION IN TYPE II DIABETIC PATIENTS

L.Sokolić, S.Ljubić, I.P.Renar, Ž.Metelko; The Vuk Vrhovac University Clinic, Zagreb, Croatia

Recent studies have demonstrated that except glomerular several tubular markers relates to renal complication of diabetes. In present study albumin excretion rate (AER) was considered as a marker of glomerular damage, whereas Tamm-Horsfall protein excretion rate (THPER) as a marker for distal tubular function. Glycosylated hemoglobin $(\mathrm{HbAlc})$ level was used as a measure blood ghucose control. Seventy-two patients with NIDDM aged $30-65$ years, with diabetes duration of 1-30 years ( 32 males and 42 females ) were included in the study. Correlatin between AER, THPER, disease duration, systolic and diastolic blood pressure and $\mathrm{HbA} 1 \mathrm{c}$ level have been studied. Significant rank correlation was found between AER and diastolic blood pressure $(R=0.2412, p=0.037)$ and AER and THPER $(R=-3094$, $p=0.0271)$ as well a correlation between THPER and disease duration $(R=-$ $0.4951, p=0.0002$ ). Significant difference in THPER value between patients with less than fifteen years of disease duration and patients with more than fifteen years disease duration was also found $(p<0.0464)$. In conchusion, these results may indicate a lack of relationship between glomerular and distal tubular dysfunction. As we found the former was influenced by diastolic blood pressure and the latter by disease duration. We can noted the importance of blood pressure regulation in glomenular protection. In contrast to this opservation, tubular dysfunction might progress, despite of blood pressure and glycemia regulation, with longer diabetes duration.

\section{0}

NO ASSOCIATION OF SODIUM-LITHIUM COUNTERTRANSPORT WITH MICROALBUMINURIA IN YOUNG BANGLADESHI DIABETIC PATIENTS

L. Ali1, R. Hada2, Z. Hassan', H.U. Rashld, A.B.M.S. Alam³, A.K. Azad Khan', N.S. Chowdhury'. 'Department of Cell \& Molecular Biology, BIRDEM, 2Department of Nephrology, IPGM\&R, 3Department of Chemistry, Dhaka University, Dhaka -1000 , Bangladesh

Raised $\mathrm{Na}+/ \mathrm{Li}+$ counter-transport activity in RBCs is claimed to be an early marker of nephropathy in IDDM patients. However, the role of the transport system in NIDDM patients is still controversial. We have studied a group ( $n=37)$ of newly diagnosed young and normotensive Bangladeshi diabetic patients (Age in years, $M \pm S D 22 \pm 5, B M$ ) 18.61 \pm 4.82 ) to investigate whether their blood glucose, C-peptide (by ELISA) and particularly microalbuminuria (albumin measured by immunoturbidimetry) are related with any abnormality in the $\mathrm{Na}^{+} / \mathrm{Li}^{+}$counter-transport activity (lithium measured by atomic absorption spectrophotometry). Nineteen age and BMl-matched controls (Age: $23+4$, BMI: 19.82 2.82 ), without family history of diabetes and hypertension, were also investigated. All of the patients were clinically NIDDM, but they had extremely variable degree of hyperglycemia and C-peptide levels ffasting - serum glucose, mmoll, $M \pm S D$, 16.14 \pm 5.91 , and $C$-peptide, pmol/, $M \pm S D$, median (range), $0.253(0.028-2.47) 2$ hour postprandial - glucose $28.94+8.02$ and C-peptide $0.379(0.053-4.159)]$. The urinary albumin levels of control and diabetic subjects were significantly different [urinary albumin-creatinine ratio (ACR), $\mathrm{mg}$ of albumin/mmol creatinine, median (range) 0.589 $(0.371-2.41)$ in control vs $1.495(0.232-7.045)$ in diabetic, $p=0.013$ by Mann-Whitney Test]. The $\mathrm{Na}+/ \mathrm{Li}+$ counter-transport activities did not vary between the two groups [mmol of lithium / g protein / hour, median (range), $0.171(0.039-0.623$ ) in controls vs 0.150 $(0.045-0.542)$ in diabetic]. Taking the already established range of normal ACR levels in our population $8(22 \%)$ of the diabetic patients were found to have microalbuminuria. Comparison of the microalbuminuric and nomoalbuminuric groups for $\mathrm{Na}+/ \mathrm{Li}+$ countertransport activity $0.127(0.058-0.542)$ and $0.206(0.045-0.481)$ respectively did not show any statistical difference. Grouping the subjects into low C-peptide $(<16$ pmol/ - the traditional cut-point between IDDM and NIDDM) and high C-peptide did not also reveal any difference between the groups regarding the transport activity. The data suggest that $\mathrm{Na}+\mathrm{Li}+$ counter-transport may not be a marker for diabetic nephropathy in young Bangladeshi patients.
$\mathrm{Na}^{+}-\mathrm{Li}^{+}$COUNTERTRANSPORT KINETICS, ALBUMIN EXCRETION AND ENDOTHELIAL DYSFUNCTION IN TYPE 2 DIABETES

K.D. Kohnert, W. Besch, J. Strese, E. Zander and W. Kerner, University of Greifswald and Clinics for Diabetes and Metabolic Diseases, Karlsburg, Germany

Erythrocyte sodium-lithium countertransport activity (SLC) is elevated in type 1 diabetes with subclinical and clinical nephropathy. In type 2 diabetes, however, the relationships between SLC activity and diabetic nephropathy or a generalized dysfunction of the vascular endothelium are not clear. We therefore examined a cohort of 75 type 2 diabetic patients, mean age $59.9 \pm$ 11.0 , diabetes duration $11.5 \pm 7.1$ (SE) years, and characterized the relationships between SLC, albumin excretion rate (AER), plasma levels of von Willebrand factor ( $\mathrm{VWF}$ ), a marker of endothelial dysfunction, and blood pressure. Patients were classified on the basis of AER into normoalbuminuric (AER $\leq 15 \mu \mathrm{g} / \mathrm{min}$ ) and micro-/macroalbuminuric (AER $>15$ and $>200 \mu \mathrm{g} / \mathrm{min}$ ). SLC was measured as sodium-stimulated lithium efflux from lithium loaded erythrocytes, and the sodium affinity constant $\left(\mathrm{K}_{\mathrm{m}}\right)$ and maximal velocity $\left(\mathrm{V}_{\max }\right)$ were calculated by linear regression analysis using the Eadie-Hofstee method. Compared with normoalbuminuric patients, micro-/macroalbuminuric patients had higher systolic blood pressure (sBP) (median 140 vs. $130 \mathrm{~mm} \mathrm{Hg}, p=0.006$ ), plasma triglyceride (TG) levels (median 3.2 vs. $2.1 \mathrm{mmol} / \mathrm{h}, \mathrm{p}=0.001)$ and fasting plasma glucose $(9.7 \pm 2.9$ vs. $8.3 \pm 2.3, p=0.029$ ). In stepwise multiple regression analysis, the significant determinants of AER were SBP $(r=0.521, p=0.008), K_{n}$ for SLC $(r=0.354, p=0.002)$ and $v W F(r=0.443, p=0.004)$. However, neither SLC activity, $\mathrm{HbAlc}$, fasting plasma glucose, total and $\mathrm{HDL}$ cholesterol nor BM and duration of diabetes did appear as significant determinants. In conclusion, AER in type 2 diabetes is related to hypertension, altered kinetics $\left(\mathrm{K}_{\mathrm{m}}\right)$ of the $\mathrm{Na}^{+}-\mathrm{Li}^{+}$countertransporter, and endothelial dysfunction.

\section{1}

INSULIN METABOLISM AND ACTION IN TYPE 1 DIABETICS WITH MILD NEPHROPATHY

M. Svensson and J.W. Eriksson, Dept.of Medicine, University Hospital, Umeå, Sweden

Renal failure is accompanied by a reduction in insulin degradation and insulin sensitivity. To elucidate insulin metabolism and action we utilized the euglycemic hyperinsulinemic clamp technique (insulin infusion at $56 \mathrm{mU} / \mathrm{m}^{2} / \mathrm{min}$ ) to study 13 patients ( 5 males, 8 females), age $43.8 \pm 2.6$ (mean \pm SEM) years, diabetes duration $33.5 \pm 2.5$ years, $\mathrm{HbAlc} 7.0 \pm 0.4 \%$ and BMI $25.7 \pm 1.0 \mathrm{~kg} / \mathrm{m}^{2}$. Albumin excretion rate (AER) was measured in overnight urine collections and glomerular filtration rate (GFR) determined by ${ }^{51} \mathrm{Cr}$ EDTA clearance. GFR was $92.2 \pm 6.4 \mathrm{ml} / \mathrm{min} / 1.73 \mathrm{~m}^{2}$ (range 52-135) and AER $139 \pm 40 \mu \mathrm{g} / \mathrm{min}$ (range 25-448). Ten patients had micro$(20-200 \mu \mathrm{g} / \mathrm{min}$ ) and three macroalbuminuria ( $>200 \mu \mathrm{g} / \mathrm{min}$ ) and none GFR less than $50 \mathrm{ml} / \mathrm{min} / 1.73 \mathrm{~m}^{2}$. An overnight insulin infusion preceded the glucose clamp to normalize glycemia. Insulin sensitivity, assessed as glucose uptake at steady state (M-value) $6.4 \pm 0.8 \mathrm{mg} / \mathrm{kg} / \mathrm{min}$ and insulin sensitivity index (ISI $=100 \times \mathrm{M} / \mathrm{plasma}$ insulin) $7.29 \pm 0.8100 \mathrm{mg} / \mathrm{kg} / \mathrm{min} /(\mathrm{mU} / \mathrm{l})$ was normal. There was an inverse relationship between $M$ and AER ( $p=0.091$ ) but not between $M$ or ISI and GFR $(\mathrm{p}=0.434, \mathrm{p}=0.632$ ). Insulin clearance (MCR-I) calculated at steady state by dividing insulin infusion rate with the plasma insulin level was $16.7 \pm 1.1 \mathrm{ml} / \mathrm{kg} / \mathrm{min}$ and no relationship to AER $(p=0.558)$ or GFR $(p=0.992)$ was found. BMI was not significantly associated with $\mathrm{M}(\mathrm{p}=0.183)$, ISI $(\mathrm{p}=0.136)$ or MCR-I $(p=0.168)$ probably because all patients were non-obese. CONCLUSION: In type 1 diabetics with mild nephropathy AER may be linked to insulin resistance but not to insulin clearance. GFR alterations within a normal or near-to-normal range does not affect either insulin sensitivity or insulin clearance. 
1122

IS CYSTATIN C A USEFUL PARAMETER IN THE FOLLOW-UP OF DIABETIC NEPHROPATHY?

C. Van Campenhout, K. Van Cotthem, I. De Leeuw, L. Van Gaal and K. Van Acker, Lab. Immunology and Dept. Endocrinology, University Hospital Antwerp, B-2650 EDEGEM, Belgium

The estimation of glomerular filtration rate (GFR) is an essential tool in the follow-up and treatment of diabetic patients especially since optimalisation of metabolic control and antihypertensive drugs are known to stabilize or inhibit the progression to diabetic nephropathy. The classical methods to measure GFR are either sophisticated and difficult to repeat on an ambulatory basis (CR* EDTA, insulin) or are influenced by non-renal determinants (creatinine-clearance). This last method, although used the most in clinical practice, is dependent on dietary protein intake and muscle mass Moreover, serum creatinine measurement is analytically less reliable in the presence of acidosis and deteriorated protein metabolism, often present in decompensated diabetes. In recent years several papers suggest that serum concentrations of cystatin $\mathrm{C}$, a non glycosylated $13 \mathrm{kD}$ protein correlates with GFR and looks to be independent of protein intake and muscle mass. Cystatin $C$ was measured in 46 type 1 diabetic patients with stable metabolic control using a latex enhanced nephelometric method (Behring reagents, BNII nephelometer) and the results were compared with values of serum creatinine, GFR measured with creatinine clearance and the level of microalbuminuria. The median serum concentration of cystatin C was $0.83 \mathrm{mg} / \mathrm{l}$ (range 0.45-1.68) and did not correlate with the level of metabolic control measured with $\mathrm{HbA}_{\mathrm{ic}}(\mathrm{I}=0.006)$. As expected serum creatinine (median: $75.1 \mu \mathrm{mol} / 1$, range $53.0-150.3)$ correlated significantly with cystatin C: Spearman's rank correlation $\mathrm{r}=0.657, \mathrm{p}=0.0001$. A significant negative correlation was found with the $24 \mathrm{H}$ creatinine clearance: $r=-0.420, p=0.006,95 \% \mathrm{Cl}:-0.6399$ to -0.1373 . Serum cystatin $\mathrm{C}$ correlates with the amount of albumin excreted in $24 \mathrm{~h}: \mathrm{r}=0.345, \mathrm{p}=0.021,95 \% \mathrm{Cl}$. 0.0610 to 0.5776 . This significant correlation persists when microalbuminuria is repeatedly in the pathological range $>30 \mathrm{mg} /$ day: $r=0.479, \mathrm{p}=0.037,95 \% \mathrm{Cl}: 0.0457$ to 0.7601 . From this data it can be concluded that the measurement of cystatin $\mathrm{C}$ is an easy, reliable parameter in the follow-up of diabetic patients prone to the development of nephropathy. Further studies are needed to prove the value of the assay in patients submitted to intervention studies.

\section{4}

Microalbuminuria and peripheral arterial disease are independent predictors of all-cause and cardiovascular mortality, especially among hypertensive subjects; Five year follow-up of the Hoorn Study A Jager', PJ Kostense 1,2, HG Run', RJ Heine ${ }^{1,3}$, G Nijpels', JM Dekker', LM Bouter', and CDA Stehouwer,', 'EMGO Institute, 'Department of Epidemiology and Bouter'. and CDA Stehouwer. Microalbuminuria (MA) is a strong predictor of all-cause mortality. It has been proposed that MA reflects generalized atherosclerosis and may thus predict mortality. To investigate this hypothesis, we studied the predictive values of MA and of peripheral arterial disease (PAD), a generally accepted marker of generalized atherosclerosis.

An age-, sex-, and glucose-tolerance stratified sample $(n=631)$ of a populationbased cohort aged $50-75$ years was followed prospectively for 5 years. At baseline, the albumin-to-creatinine ratio (ACR) was measured in an overnight spot urine sample; MA was defined as ACR above $2.0 \mathrm{mg} / \mathrm{mmol}$. PAD was defined as an ankle-brachial presssure index below 0.90 and/or a history of a peripheral arterial bypass or amputation. At follow-up, the vital status was determined from the mortality register of the local municipality. Cardiovascular death (CVD) was defined as codes 390-459 according to the ninth edition of the International Classification of Disease.

After 5 years of follow-up, 58 subjects had died (24 of CVD). Both MA and PAD were associated with a two-fold increase in all-cause mortality, which decreased to about 1.6 after adjusting for age, sex, diabetes mellitus, hypertension, levels of total and HDL-cholesterol, body mass index, smoking habits and preexistent ischemic heart disease. The relative risk (RR) of mortality associated with MA and PAD were more pronounced among hypertensive and diabetic than among normotensive and non-diabetic subjects, respectively. When MA and PAD were both included in the multivariate analysis, the RRs of all-cause and cardiovascular montality were 1.9(0.9-3.9) and 4.8(1.8-12.5) for MA and 1.9(0.9-4.1) and 3.2(1.2-8.9) for PAD.

Both microalbuminuria and peripheral arterial disease are strong independent predictors of cardiovascular and all-cause mortality. This may be more pronounced in the presence of hypertension and/or diabetes mellitus than in their absence. These data argue against the idea that MA affects mortality risk through generalized atherosclerosis.

\section{3}

IS URINARY ACTIVITY OF N-ACETYL-BETA-GLUCOSAMINIDASE (NAG) AN POSSIBLE EARLY MARKER OF DIABETIC NEPHROPATHY?

V. Dimitrijevic-Sreckovic, P. Djordjevic, Lj. Mandic*, V. Djurdjic*, M.Zamaklar. N.Lalic and B.Sreckovic**. Institute for Endocrinology, Diabeles and Metabolic Diseases, Faculty of Medicine and Faculty of Chemistry*, Institute of Cardiology**. Clinical Center, 11000 Belgrade. Yugoslavia.

The aim of the study was to establish NAG as an early marker of diabetic nephropathy prior to microalbuminuria (MA). NAG urinary activity is a sensilive indicator of tubulocyte lesions. The study included 70 Insulin Dependent Diabctes Mellitus (IDDM) patients, clustered according to presence of vascular complications and unsatisfactory metabolic control. Urinary NAG has been determined spetrophotometrically with susbstrate glucopiranozide. The statistical significance of the difference of the urine NAG activity between IDDM patients-the first four groups: newly diagnosed-I (NAG-2.6 IU/L), patients without complications-II (2.1 IU/L), patients with retinopathy-II] $(1.8 \mathrm{IU} / \mathrm{L})$ and patients with retinopathy alld polyneuropathy-IV $(2.3 \mathrm{JU} / \mathrm{L})$ and patients with retinopathy, polyneuropally, nephropathy-V (5.5 IU/L) confirmed increased urine NAG activity in nephropathic diabetics suggesting renal damage. The conclusion was confirmed by the correlation of the urine NAG activity and MA in patients with nephropatly. With the development of the diabetic nephropathy through the stages from the incipient (NAG-4 $66 \mathrm{IU} / \mathrm{L}$ ), manifest nephropathy $(5.05 \mathrm{IU} / \mathrm{L}$ ) up to chronic renal failure $(6.2$ [U/L), the increase of urinary NAG level has been noticed reflecting the progression of renal damage. Activity of urinary NAG was studied in patients without MA and disease lasting over 2 years. Elevated NAG value was recorded in $25 \%$ of patients with disease lasting 2-10 years, $40 \%$ of patients with lasting $11-15$ years and $43 \%$ in patients with history of the disease $>15$ years. The urine NAG activity may serve as an carly marker of diabetic nephropathy prior to MA. In patients not having MA of various duration of the disease $1 / 3$ of patients showed the increased urine NAG; activity prior to $\mathrm{MA}$, which might serve as a possible indicator of the future nephropathy development.

\section{5}

CARDIOVASCULAR MORBIDITY AND EARLY MORTALITY CLUSTERS IN PARENTS OF IDDM PATIENTS WITH DIABETIC NEPHROPATHY

L.Tarnow, J.Fagerud, P.Rossing, F.S.Nielsen, F.Cambien ", and H.H Parving

Steno Diabetes Center, Gentofte, Denmark \& "INSERM SC7, Paris, France

A familial predisposition has been proposed as a determinant of the increased mor. bidity and mortality from cardiovascular disease (CVD) in insulin dependent diabetic (IDDM) patients with diabetic nephropathy. The insertion allele of the insertion(I)/deletion(D) polymorphism in the angiotensin converting enzyme (ACE) gene seems to protect against coronary heart disease in diabetic and non-diabetic subjects. We investigated the parents of 163 IDDM patients with nephropathy and parents of 163 sex- and age-matched normoalbuminuric patients with IDDM. In a Kaplan-Meier survival analysis of eligible parents total parental mortality was sig. nificantly increased in parents of patients with nephropathy $(121 / 244=50 \%)$ as compared to parents of normoalbuminuric patients $(119 / 269=44 \%), p=0.008$, with an increase in cardiovascular deaths $48 / 244=20 \%$ vs $42 / 269=16 \%, p<0.05$. Fur. thermore, the combined morbidity and mortality from CVD in parents of nephropathic patients was greater than that in the parents of normoalbuminuric IODM patients $146(38-54) \%$ vs $36(28.44) \%, p=0.055$. Among both patients with and without nephropathy those who had had a cardiovascular event were more likely to have a family history of CVD (odds ratio, $1.7(95 \% \mathrm{Cl}, 1.0$ to $2.9, \mathrm{p}<0.001)$ than those who had not experienced such an event. Fathers of patients homozygous for the l-allele of the ACEIID polymorphism had significantly less myocardial infarction as compared to fathers of parents with other genotypes, $p=0.03$. In conclusion: Car. diovascular morbidity and early mortality clusters in parents of IDDM patients with diabetic nephropathy. The ACE/ID polymorphism contributes to explain the increased morbidity from CVD. 
THE RELATIONSHIP BETWEEN DIABETIC NEPHROPATHY AND ISCHEMTC CARDIOPATHY: STUDY OF AN OUT-PATIENT TYPE 2 DIABETIC POPULATION A. Cimino, L. Rocca, A. Girelli, U. Valentini. Unita' Operativa Diabetologica, Brescia, Italy. It is a well known fact that nephropathy in type 2 diabetic patients is associated with an increased prevalence of cardiovascular disease. The aim of this study was to identify the patients with diabetic nephropathy in our ambulatory population and evaluate the association with ischemic cardiopathy and the relative risk of its development. 1648 patients (age $<70$ years, 901 males, 747 females, an average age of $59.7+6$ years and a disease duration of $8.2 \pm 3.9$ years) with type 2 diabetes were studiedat our diabetologic centre from the period 1990-1997; all patients on entry had an accurate medical history taken, underwent clinical exams as well as ECGs, fundoscopy, microalbuminuria dosage (al least 3 samples collected in a greater than 3 month period), complete urine analysis, urine cultures and other exams targeted in diagnosing nephropathy and cardiology. All non ischemic cardiopathy (hypertensive, valvular, dilated) and non diabetic nephropathy was excluded; sources of information on cardiovascular events and the diagnosis of ischemic cardiopathy besides the
archives of the centre were the Brescian Civil Hospital (the reference hospital for most of the patients followed) archives for patient admissions. Statistic analysis involved the utilization of $\mathbf{X}^{2}$ tests; a linear model for the evaluation of the averages in the various groups; and logistic regression to assess the relative risk of the development of ischemic cardiopathy. The average period of observation was $3.1+2.3$ years. On entry $1241 \quad(75.3 \%)$ normal albuminuric, $282(17.1 \%)$ microalbuminuric, $103(6.3 \%)$ macroalbuminuric and $22(1.3 \%)$ patients with $C R F$. The prevalence of retinopathy and arterial hypertension was seen to be significantly greater in nephropathic patients with respect to normal albuminuric patients (prevalence of retinopathy in normal albuminuric patients $13.6 \%$, microalbuminuric $25 \%$, macroalbuminuric $35 \%, \mathrm{CRF}$ patients $40 \%$; the prevalence of arterial hypertension in normal albuminuric patients $28 \%$, microalbuminuric $39 \%$, macroalbuminuric $49 \%$ and in CRF patients $40 \%)(\mathrm{p}<0.001)$. At the beginning of follow-up $192(11.6 \%)$ of the 1648 patients had ischemic cardiopathy, this group further subdivided into a large prevalent group $(p<0.001)$ with nephropathy $(10.2 \%$ of normal albuminuric, $15.2 \%$ of microalbuminuric, $14.5 \%$ of with nephropathy $(10.2 \%$ of normal albuminuric, $15.2 \%$ of microalbuminuric, $14.5 \%$ of
macroalbuminuric and $31 \%$ of patients with CRF). In the following period of observation 139 macroalbuminuric and $31 \%$ of patients with $\mathrm{CRF}$ ). In the following period of observation 139 was seen to be significantly greater in patients with a diagnosis of nephropathy $(11.3 \%$ of microalbuminuric, $12.6 \%$ of macroalbuminuric, and $18.2 \%$ of patients with $\mathrm{CRF}$ ) with respect to patients with normal albuminuria $(7.2 \%)(\mathrm{p}<0.001)$. Utilizing a logistic regression test we were able to show a significant association between the development of ischemic cardiopathy and the values of albuminuria at the start of the observation period $(p<0.01)$. The results of this study confirm the existence in type 2 diabetes of an association between nephropathy and ischemic cardiopathy, the presence of albuminuria being a strong predictive factor in the development of the latter
PREVALENCE OF LEFT VENTRICULAR HYPERTROPHY IN TYPE 1 DIABETIC PATIENTS WITH DIABETIC NEPHROPATHY

A. Sato, L. Tarnow, and H-H. Parving, Steno Diabetes Center, Gentofte, DK The excess mortality in diabetic nephropathy is mainly due to end stage renal failure and cardiovascular disease. Left ventricular hypertrophy is an independent risk factor for myocardial ischaemia, coronary heart disease, heart failure, ventricular dysihythmia, and sudden death. Consequently, the aim of our crosssectional study was to evaluate left ventricular structure and function in type 1 diabetic patients with diabetic nephropathy. M-mode and doppler echocardiography was performed in 105 type 1 diabetic patients with nephropathy (61 males, age 44(9) years, albuminuria, median (range); $567(10-8188) \mathrm{mg} / 24 \mathrm{~h}$, serum creatinine $109(53-558) \mu \mathrm{mol} /$, and 140 type 1 diabetic patients with normoalbuminuria ( 79 males, age $47(10)$ years, albuminuria $8(1-30) \mathrm{mg} / 24 \mathrm{~h}$, and serum creatinine $81(55-121) \mu$ moll. The patients with and without nephropathy were comparable with respect to sex, body mass index, duration of diabetes. Arterial blood pressure was slightly higher in patients with nephropathy: $140 / 79(17 / 9) \mathrm{mm} \mathrm{Hg}$ vs $134 / 78(15 / 8) \mathrm{mm} \mathrm{Hg}, p<0.01$, and a lot of these patients received antihypertensive medication, 84 vs $17 \%$, respectively. Left ventricular mass index was elevated in the nephropathic group $100.6(23.9) \mathrm{g} / \mathrm{m}^{2}$ as compared to the normoalbuminuric group $91.4(21.9) \mathrm{g} / \mathrm{m}^{2}, \mathrm{p}=0.002$. Furthermore, the prevalence of left ventricular hypertrophy was higher in patients with nephropathy $23(14-31) \%$ as compared to patients with normoalbuminuria $9(5-14) \%, p=0.003$. A multiple linear regression analysis with backward selection revealed that only systolic blood pressure and male gender were independent risk factors for left ventricular mass index in patients with nephropathy $\left(r^{2}=0.34\right)$. Diastolic function, assessed from transmitral flow velocity ratio $(E / A)$ and isovolumic relaxation time, was significantly reduced in patients with vs without nephropathy: $1.17(0.29)$ vs $1.34(0.32)$, and $81.7(16.5)$ vs $74.6(14.5), p<0.005$. Systolic function was about the same and normal in both groups. Our study suggests that left ventricular hypertrophy and diastolic dysfunction occur rather early in the course of diabetic nephropathy.

\section{8}

NEED TO IMPROVE THE TREATMENT OF CARDIOVASCULAR RISK FACTORS IN TYPE 2 DIABETIC PATIENTS WITH NEPHROPATHY.

MJ Carrera, N. Gómez, T. Micaló, M. Sahún, C. Vinzia and E. Montanya. C.A.P Prat, Comellà, L'Hospitalet, H. Viladecans and CSUB-Hospital Bellvitge. Barcelona

Patients with diabetic nephropathy (DN) have a strong risk for cardiovascular disease (CVD). The aim of the study was to determine the prevalence of $\mathrm{CV}$ risk factors and CVD, and the achievement of recommended therapeutic goals, in a population of type 2 diabetic patients with newly identified DN attending 4 primary health care centers coordinated with a teaching Hospital. Patients in the 35-70 yr age-range were included if they had micro- $(30-300 \mathrm{mg} / 24 \mathrm{~h})$ or macro- $(>300$ $\mathrm{mg} / 24 \mathrm{~h}$ ) albuminuria in at least 2 of 3 consecutive $24 \mathrm{~h}$. urine collections. Patients with creatinine $>150 \mu \mathrm{mol} / \mathrm{l}$ were excluded. Hypertension (HT) was defined as systolic blood pressure (BP) and/or diastolic BP $\geq 140 / 90 \mathrm{mmHg}$, and/or antihypertensive therapy; dyslipidemia as serum cholesterol $\geq 5.2 \mathrm{mM}$ and/or serum triglyceride $\geq 2.3 \mathrm{mM}$, and/or lipid regulator therapy. CVD was considered present if patients had angina or previous myocardial infartion (CHD), peripheral vascular disease (PVD), stroke or transient ischemic attack (CRVD). 108 patients were identified, $67 \%$ men, age $58.9 \pm 7.3$ yr, diabetes duration $14.6 \pm 8.5 \mathrm{yr}, 68 \%$ with microalbuminuria. $\mathrm{CV}$ risk factors: $55 \%$ were current or previous smokers, $76 \%$ had $\mathrm{HT}$ and $81 \%$ dyslipidemia. Control of $\mathrm{CV}$ risk factors: $\mathrm{HbA}_{1 \mathrm{c}}$ was $\leq$ mean $+6 \mathrm{SD}$ in $38 \%$ patients, BP $<140 / 90$ in $45 \%$, and serum cholesterol $<5.2 \mathrm{mM}$ in $32 \%$. Only $35 \%$ of patients on antihypertensive therapy and $28 \%$ of patients on lipid regulator therapy had achieved the therapeutic goals. $39 \%$ patients had CVD : $49 \%$ PVD, $44 \%$ $\mathrm{CHD}$, and $26 \%$ CRVD. Patients with CVD were older $(61.6 \pm 6.1$ vs $57.1 \pm 7.8 \mathrm{yr}, \mathrm{p}<$ 0.05 ), had longer duration of diabetes $(16.6 \pm 8.8 \mathrm{vs} 13.0 \pm 7.8 \mathrm{yr}, \mathrm{p}<0.05)$ and a tendency to higher prevalence of smoking $(\mathrm{p}=0.06)$. Macroalbuminuria was associated with a higher risk for CVD than microalbuminuria (Odds ratio :2.85). In contrast, sex distribution, body mass index, and prevalence of HT and dyslipidemin were similar in patients with and without CVD. Control of CV risk factors was similar in patients with and without CVD. In summary, type 2 diabetic patients with $\mathrm{DN}$ showed a high prevalence of $\mathrm{CV}$ risk factors and $\mathrm{CVD}$. However, the control of $\mathrm{CV}$ risk factors was poor in this high risk population. Detection of DN will lead to more aggressive treatment of $\mathrm{CV}$ risk factors.

\section{9}

REDUCED PREVALENCE OF CARCNNOMAS IN DIABETIC PATIENTS WTTH GLOMERLLOSCLEROSIS

E.D. Schleicher, H.G. Hagedorn and A.G. Nerlich. Dept. of Internal Medicine IV Tübingen, Dept. of ENT-Diseases and Instinute of Pathology, LudwigMaximillians-Universitat;; München, Germany

The role of chronic metabolic diseases, such as diabetes mellitus, on the induction and maintainance of malignant growth is still unclear. A possible interaction between diabetes and carcinoma, however, is suggested by recent findings indicating that transforming growth factor (TGF-B) play a crucial role in the pathogenesis of diabetic complications, particularly glomerulosclerosis and carcinogenesis and/or metastasis. It was our aim to study if diabetic subjects with overt signs of chronic matrix accumulation, e.g. glomerulosclerosis, show different prevalence for carcinomas since this differentiation has not been performed in earlier larger autopsy series. In our study we performed a retrospective statistical analysis on 5000 consecutive, non-selected autopsy cases. Since presence of glomerulosclerosis was used as indicator for chronic TGF- $B$ action all diabetic renal section were histologically evaluated In our study population ( $>10$ years of age) a total incidence of $9.8 \%(n=488)$ diabetic patients and a total of $34 \%$ patients with histopathologically verfied carcinoma $(\mathrm{n}=1699)$ was determined. The tumors covered all common carcinoma types which were not differently distributed in the diabetic and non-diabetic cohort. The age- and sex ratio was matched between diabetic, non-diabetic and carcinoma patients, particularly the diabetic group was not younger than the carcinoma group. Systemic and coronary arteriosclerosis were significantly more frequent in diabetics than in non-diabetics $(p<0.0001)$ although the rate of myocardial failure was unchanged. The rate of carcinomas in the diabetic group with nodular and diffuse glomerulosclerosis was 2.5 - $(p<0.0001)$ and 1.9-fold lower $(\mathrm{p}<0.0027)$. Furthermore, the statistical evaluation showed significantly fewer rates of lymphogenic $(p<0.0001)$; haematogenic $(p<0.0002)$ metastates in the diabetic group than in non-diabetics with no difference with respect to the tumor stage. In conclusion, our retrospective statistical analysis on an unselected series of autopsy cases provides evidence that diabetes mellitus with overt glomerulosclerosis is associated with significantly lower prevalence of carcinomas when compared to individuals without renal microangiopathy. 
PS 58

\section{Genetics of Diabetic Nephropathy}

\section{0}

LACK OF CONTRIBUTION OF LIPID GENE POLYMORPHISMS TO IDDM NEPHROPATHY - THE GENEDIAB STUDY.

S. Hadjadj, Y. Gallois, B. Bouhanick, G. Berrut, G. Guilloteau and M. Marre for the Génétique de la Néphropathie Diabétique (GENEDIAB) Study Group -Angers - France Genetic susceptibilty contributes to the development of diabetic nephropathy (DN). We searched for a contribution of genetic polymorphisms within lipids : LipoProtein Lipase (LPL - mutation Asn 291 Ser), Cholesteryl Ester Transfert Protein (CETP $\mathrm{B} 1 / \mathrm{B} 2$ ) and apolipoprotein E (Apo E - E2/E3/E4), by studying 494 IDDM patients with proliferative retinopathy and various stages of DN. Total Cholesterol (TC), fasting Triglycerides (TG), Apolipoproteins AI and B, and Lp(a) were measured. Genetic polymorphisms were determined by PCR-based detection. LPL, CETP and Apo $\mathrm{E}$ genetic allelic or mutations frequencies $(\chi 2$ for trend) were not different regarding renal complications (table). There was a significant association (ANOVA) between the stages of $D N$ and TC $(F=6.65 ; p=0.0002)$, TG $(F=8.84 ; p<0.0001)$, Apo $B(F=6.03 ; p=0.0007)$ or $L p(a)$ concentrations $(F=4.25 ; p=0.006)$ (Meant SD) (Table)

\begin{tabular}{lllllll} 
Nephropathy & Absent & Incipient & Estabished & Advanced & X2/F & $P$ \\
LPL (+/-) & $0.99 / 0.01$ & $0.99 / 0.01$ & $0.99 / 0.01$ & $1 / 0$ & 1.65 & 0.20 \\
CETP B1/B2 & $0.56 / 0.44$ & $0.63 / 0.37$ & $0.59 / 0.41$ & $0.61 / 0.39$ & 1.19 & 0.28 \\
ApoE2/E3/E4 & $0.05 / 0.85 / 0.1$ & $0.07 / 0.78 / 0.15$ & $0.05 / 0.85 / 0.1$ & $0.05 / 0.85 / 0.1$ & 0.02 & 0.89 \\
CT [mM] & $5.50 \pm 1.19$ & $5.31 \pm 1.19$ & $5.96 \pm 1.65$ & $5.94 \pm 1.55$ & 6.65 & 0.0002 \\
TG [mM] & $1.13 \pm 0.41$ & $1.17 \pm 0.51$ & $1.64 \pm 0.78$ & $1.84 \pm 0.87$ & 8.83 & 0.0001 \\
Apo AI [g/l] & $1.52 \pm 0.39$ & $1.44 \pm 0.26$ & $1.50 \pm 0.37$ & $1.45 \pm 0.31$ & 0.44 & 0.72 \\
Apo B [g/l] & $0.88 \pm 0.16$ & $0.88 \pm 0.19$ & $1.04 \pm 0.30$ & $1.07 \pm 0.26$ & 6.03 & 0.0007 \\
Lp(a) $[\mathrm{g} /]]$ & $0.08 \pm 0.10$ & $0.07 \pm 0.08$ & $0.11 \pm 0.17$ & $0.10 \pm 0.10$ & 4.25 & 0.006 \\
\hline
\end{tabular}

These results support no or marginal effects of a genetic component leading to altered lipid metabolism for the progression of DN.

\section{2}

USE OF EXTREME PHENOTYPES IN ASSOCIATION STUDY OF GENETIC SUSCEPTIBILITY TO DLABETIC NEPHROPATHY IN IDDM PATIENTS Y.Y.Kondratiev ${ }^{1,2}$, M.V.Shestakovaa ${ }^{1}$, M.S.Shamkhalova ${ }^{1}$, L.A.Tchugunova ${ }^{1}$, N.M.Gorashko' ${ }^{2}$, D.A.Chystyakov', O.K.Vikulova', R.I.Tourakoulov', I.I.Dedov', and V.V.Nosikov' ${ }^{2}{ }^{1}$ Endocrinology Research Centre and ${ }^{2}$ National Research Centre "GosNII Genetika", Moscow, Russia

Case-control studies of genetic susceptibility to diabetic nephropathy (DN) often yield conflicting results. It seems to depend on temporal "maturation" of DN phenotype, its heterogeneity, continuous distribution in IDDM population and overlapping in compared groups of patients. To overcome such phenotype uncertainty, we used two extreme clearly defined phenotypes: DN occurrence (DN+, 14 patients with IDDM duration $\leq 15 \mathrm{yr}$. and urinary albumin excretion rate (UAER) $\geq 300 \mathrm{mg} / 24 \mathrm{~h}$ ) and DN absence (DN-, 31 patients with IDDM duration $220 \mathrm{yr}$. and UAER $\leq 200$ $\mathrm{mg} / 24 \mathrm{~h}$ ). Insertion/deletion (I/D) polymorphism in angiotensin I-converting enzyme gene $(A C E)$ and (CA)n microsatellites located nearby aldose reductase $(A L R 2)$ and catalase ( $C A T)$ genes were determined by PCR technique. Relations between ID/ACE genotypes and metabolic DN risk factors were studied by multiple regression analysis, differences in allele/genotype frequencies were analysed by Fisher's exact test. We observed increased frequency of $D$ allele and depletion of $I$ allele in DN+ group compared to $D N$ - group: 75.0 vs. $46.8 \%$ and 25.0 vs. $53.2 \%$, respectively $(\mathrm{P}<0.02), I D / A C E$ genotype frequencies were also different in both groups: $I I-0.0$ vs. $25.8 \%(\mathrm{P}<0.05) ; I D-50.0$ vs. $54.8 \%(\mathrm{NS})$ and $D D-50.0$ vs. $19.4 \%(\mathrm{P}<0.05)$, respectively. Besides $I D / A C E$ polymorphism, blood pressure and UAER but not glycaemic control were found to contribute to rapid DN development. Both IDDM groups did not differ significantly in distribution of alleles of polymorphic markers located nearby $A L R 2$ and $C A T$ genes. In conclusion, use of extreme $\mathrm{DN}+$ and $\mathrm{DN}$ phenotypes allowed us to demonstrate that: 1) genetic susceptibility or resistance to $D N$ might be attributed rather to $I / D$ polymorphism of $A C E$ gene than to polymorphic markers of two other candidate genes; and 2) found genetic marker seemed to rather protect IDDM patients from than render them susceptible to DN.

\section{1}

Genetic factors in the development and progression of renal disease in type 1 diabetic patients.

S. De Cosmo, V. Tassi, M. Margaglione^, S. Thomas^^, GP Piras, R. Trevisan*, P. Cavallo Perin§, S. Bacci, D. Colaizzo^, C. Cisternino, O. Ludovico, G. Di Minno $^{\wedge}, V$. Trischitta and GC Viberti^n

Division of Endocrinology and $\mathrm{N} U$ nit of Thrombosis and Atheroscierosis, CSS San Giovanni Rotondo 'Unit for Metabolic Medicine, Hospital Brotzy, Cagliari, "Chair of Metabolic Medicine, University of Padova, §Chair of Internal Medicine, "Chair of Metabolic Medicine, University of Padova, \$Chair of Internal Medicine, London U.K.

Genetic factors are involved in the development of diabetic nephropathy (DN) in type 1 diabetes. We have examined the relationship between the polymorphism of three candidate genes, Angiotensin Converting Enzyme (ACE): $/$ D Plasminogen Activator inhibitor-1 (PAl-1):4G/5G and Decorin: 179/183/185 and Plasminogen Activator Inhibitor-1 (PAI-1):4G/5G and Decorin: 179/183/185 and
the development and progression of DN. These genes were selected because of the possible involvement of their products in the pathophysiology of diabetic kidney disease.

We compared, in an association study, 130 type 1 diabetic patients with normoalbuminuria [albumin excretion rate (AER) range 2-16 $\mu \mathrm{g} / \mathrm{min}$ ] and duration of disease longer than 15 years (mean \pm SD: $25 \pm 8$ yrs) with 168 type 1 diabetic patients with albuminuria, 58 microalbuminurics and 110 macroalbuminurics (AER $21-6900 \mu \mathrm{g} / \mathrm{min}$ ), with mean \pm SD diabetes duration of $25 \pm 9 \mathrm{yrs}$. Eighty-two patients with macroalbuminuria (AER range 200-6900 $\mu \mathrm{g} / \mathrm{min}$ ) were followed prospectively for an average of $6.9 \mathrm{yrs}$ (range: 2.1-19) with six monthly measurements of creatinine clearance ( $\mathrm{CrCl}$ )(Kockroft formula). Gene polymorphisms were determined by PCR. Patients with micro and macroalbuminuria had similar gene distribution and are presented as a single group. There was no difference in the distribution of polymorphism of the three candidate genes between patients with normoalbuminuria or albuminuria. In the group of 82 patients with macroalbuminuria the average rate of decline of $\mathrm{CrCl}$ was $6.2 \pm 5 \mathrm{ml} / \mathrm{min} / \mathrm{yrs}$. Patients with the genotype ACE II and Decorin 179/183 showed a slower rate of disease progression (II $2.9 \pm 2.8$ vs $D D+\mid D: 6.7 \pm 6$ $\mathrm{ml} / \mathrm{min} / \mathrm{year} \mathrm{p}<0.05 ; 179-183: 3.5 \pm 3$ vs $183-183: 6.5 \pm 5 \mathrm{ml} / \mathrm{min} /$ year $\mathrm{p}<0.05$ respectively). There was not interaction between these two genes polymorphisms and their effect was independent of blood glucose and blood pressure control. The 3 candidate genes examined in this study are not associated with the development of DN but progression of disease appears related to polymorphism of the ACE and Decorin gene.

\section{3}

RELATIONSHIP BETWEEN GLOMERULAR HYPERFILTRATION AND ANGIOTENSIN CONVERTING ENZYME INSERTION/DELETION POLYMORPHISM IN IDDM CHILDREN AND ADOLESCENTS.

B.Bouhanick,Y.Gallois,S.Hadjadj,G.Berrut,JM.Limal,M.Marre ;Angers, France Glomerular Hyperfiltration may predict diabetic nephropathy in IDDM. Some studies suggest that the Angiotensin Converting Enzyme (ACE) D allele is associated with diabetic nephropathy. We studied the relationship between Glomerular Hyperfiltration (high GFR) and ACE Insertion/Deletion (I/D) polymorphism of 76 IDDM children and adolescents (age 10-20 years) without diabetic nephropathy: age $16 \pm 3$ years : $44 \mathrm{M} / 32 \mathrm{~F}$; IDDM duration $7 \pm 4$ years ; age at IDDM onset $9 \pm 4$ years ; HbAlc $9.5 \pm 1.9 \%$. Glomerular Filtration Rate (GFR) was estimated by the plasma disapearance of $0.5 \mu \mathrm{Ci}{ }^{\mathrm{s}} \mathrm{CrEDTA} / \mathrm{kg}$ body weight and corrected by $1.73 \mathrm{~m}^{2}$ body surface (N:99-134 high GFR $\geq 135 \mathrm{ml}$.min '.1.73 $\mathrm{m}^{2}$ ). ACE I/D genotype was determined by a double PCR method, and ACE plasma levels by ELISA. $29(38 \%)$ patients displayed high GFR. An association between high GFR and ACE (I/D) polymorphism was observed $\left(\chi^{2}=7.09\right.$ $\mathrm{p}=0.029$ ).

$\begin{array}{lllll}\text { Genotype } & \text { DD } & \text { ID } & \text { II } & \% \text { allele D } \\ \text { High GFR } & 4 & 20 & 5 & 48.3\end{array}$

Normal GFR 19 - 19 or

This association was due to a reduced proportion of DD genotype among high GFR $\left(\chi^{2}=6.03, p=0.014\right)$ and not due to an excess of II genotype $(\chi 2=0.04$, $\mathrm{p}=0.83$ ). Age, IDDM duration, age at IDDM onset, and $\mathrm{HbAlc}$ were not different between genotypes. Patients with high GFR had low plasma ACE levels : $457 \pm 157 \mu \mathrm{g} / \mathrm{L}$ vs $553 \pm 186 \mu \mathrm{g} / \mathrm{L}(\mathrm{p}=0.027)$. These results suggest a paradoxical association between glomerular hyperfiltration and ACE (I/D) polymorphism caracterized by a defect of the DD genotypes IDDM children and adolescents with high GFR. 
Insertion-deletion (JD) Polymorfism of Angiotensin-Converting-Enzyme Gene, Plasma ACE Activity and Glomerular Filtration Rate in DDM Patients. A. Manto, P. Cotroneo, L. Todaro, MNannipieri, A. Bertacea', S. Bandinellio L. Puccio, D. Pitoceo, G. Ghirlanda* and R Navalesi ${ }^{\circ}$. Dept of Internal Medicine, Catholic University . Rome and Dept of Endocrinology and Metabolism, University of Pisa, Italy.

UD polymorfism of ACE gene is correlated with plasma ACE activity and this activity is increased in diabetic subjects with microvascular complications. The role of ACE gene in the pathogenesis of nephropathy in IDDM patients is controversial. Hyporfiltration is the easly stage of diabetic nephropathy but its role in the development of renal disease is unclear. Wo ovaluated the ACE gene VD polymorfism related to glomerumar filtration rate (GFR) in 81 DDM pationts (mean age $35.6 \pm 10$ yrs, mean disease duration $14 \pm 8$ yrs) and in 13 healthy subjects (mean age $35.6 \pm 10$ yrs). ACB polymorphism was studied by PCR, GFR by injection of 51 Cr-EDTA, plesma ACE activity by spectrophotometry. GFR was higher in $\mathbb{D D M}$ patients vs controls: $140 \pm 26$ $\mathrm{m} / \mathrm{min} / \mathrm{m}^{2}$ vs $113 \pm 17 \mathrm{~m} / / \mathrm{min} / \mathrm{m}^{2}$ (p<0.02). $40 \mathrm{IDDM}$ with GFR higher than $95^{\circ}$ percentage of controls (135 m//min/m ${ }^{2}$ ) were considered hyperfiltering (49\%). Plasma ACE activity was similas in the hyperfillering and normofiltering patients: $27.4 \pm 16$ UI vs $29.6 \pm 11.7$ U, but it was higher vs

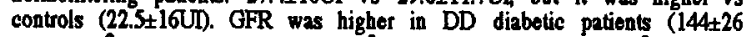
$\left.\mathrm{m} / \mathrm{min} / \mathrm{m}^{2}\right)$ vs $\mathrm{ID}\left(138 \pm 27 \mathrm{~m} / \mathrm{min} / \mathrm{m}^{2}\right)$ and I $\left(136 \pm 25 \mathrm{~m} / \mathrm{min} / \mathrm{m}^{2}\right)$ patients but these differences are not significant; $55 \%$ of hyperfiltering and $39 \%$ of normofiltering patients had DD genotype, $30 \%$ of hyperfiltering and $46 \%$ of normofilitering had $\mathbb{D}$ genotype, $15 \%$ of hyperfiltering and $15 \%$ of normofiltering had II genotype. ACE plesma activity is higher in DD vs D and II patients (36.6 19 UI vs $23.6 \pm 10.2 \mathrm{UI}$ and vs $13.6 \pm 5 \mathrm{UI}, p<0.001$ ). In diabetic patients with DD genotype the albumin excretion rate (AER) is higher than in $\mathbb{D}$ patients $(6.7 \pm 5.7$ vs $3.3 \pm 2.5, p<0.05)$. We hypothesize that ID polymorphism of ACE gene may affect the GFR and AER and contributes with the metabolic control to development of diabetic nephropathy.

\section{6}

ANGIOTENSIN I-CONVERTING ENZYME GENE POLYMORPHISM: WHY IS HETEROZYGOSITY INCREASED IN BOTH IDDM AND NIDDM PATIENTS? I.I.Dedov' ${ }^{1}$, Y.Y.Kondratiev 1,2, L.M.Demurov², N.M.Gorashko², G.G.Mamaeva', L.N.Scherbachova' ${ }^{1}$, T.M.Milenkaya', and V.V.Nosikov', 'Endocrinology Research Centre and ${ }^{2}$ National Research Centre "GosNII Genetika", Moscow, Russia

Using standard PCR technique, we have previously found increased frequency of $D$ (deletion) allele and, respectively, decreased frequency of $I$ (insertion) allele of angiotensin I-converting enzyme ( $A C E)$ gene, along with unexpectedly high $D$ allele homozygosity in healthy subjects from general Moscow population. To assess a contribution of $I D / A C E$ gene polymorphism to genetic susceptibility to diabetic microand macroangiopathy, now we studied distribution of the marker in juvenile onset ( $\leq 15$ yr.) IDDM patients and adult onset $(240 \mathrm{yr}$.) NIDDM patients. All patients were free from advanced vascular complications. Differences in allele/genotype frequencies were analysed by Fisher's exact test and considered significant at $P<0.05$.

$\begin{array}{lccccc}\text { Group/P value } & \text { Healthy (1) } & \text { IDDM (2) } & \text { NIDDM (3) } & \mathrm{P}_{1-2} & \mathrm{P}_{1-3} \\ \mathrm{n} & 165 & 115 & 115 & & \\ \text { Frequency of: } & & & & & \\ I \text { allele } & 0.358 & 0.404 & 0.404 & \mathrm{NS} & \mathrm{NS} \\ D \text { allele } & 0.642 & 0.596 & 0.596 & \mathrm{NS} & \mathrm{NS} \\ \text { II genotype } & 0.200 & 0.113 & 0.174 & <0.05 & \mathrm{NS} \\ I D \text { genotype } & 0.315 & 0.583 & 0.461 & <0.0001 & <0.01 \\ D D \text { genotype } & 0.485 & 0.304 & 0.365 & <0.002 & \mathrm{NS}\end{array}$

Thus, frequency of $I$ allele only tended to increase and $D$ allele to decrease equally in IDDM and NIDDM patients compared to healthy subjects, whereas heterozygous genotype was more frequent in diabetic patients (especially in IDDM) significantly. Recently, angiotensin II (AII) has been found to influence both islet blood flow and insulin secretion, and ACE (and AII) concentration might be partly related to $I D / A C E$ gene polymorphism. In conclusion, observed distribution of $A C E$ genotype frequencies towards increased heterozygosity might be relevant also to IDDM and NIDDM pathogenesis, besides susceptibility/resistance to any angiopathy.
ASSOCIATION OF ACE GEN POLYMORPHISM AND DIABETIC NEPHROPATHY IN TYPE 2 DIABETIC PATIENTS

J.M. Pou, A. Planas*, A. Clara**, A. Gassol**, A de Moner*, C Contreras*, J. Marugat**, F. Vidal**, L. Gallart and M.A. Ortiz.. Dpt. Endocrinology, Hosp S. Pau, **Dept. Vascular Hosp Mar. *Area Básica Hospitalet. Autonomous University of Barcelona.

Several candidate genes have been investigated to elucidate genetic factors responsible for vascular complications. Identification of genetic factors predisposing to chronic vascular complications in diabetes mellitus will permit identification of susceptible individuals for diabetic nephropathy. We investigated whether or not the ACE genotype is associated with diabetic nephropathy in type 2 diabetic patients. It was studied a group of type 2 diabetic patients (188 patients, sex: $M$, age: $59.7 \pm 11.3 \mathrm{y}$, diabetes duration: $11.4 \pm 4.32 \mathrm{y}$ ) was investigated from a cohort of 655 men. Nephropathy was observed in $29 \%$ of patients and peripheral artheriopathy was seen in $33.3 \%$ (clinical and subclinical) ACE gen polymorphism was measured by $P C R$ with specific primers for $D$ and I fragment amplification. DD polymorphism was oberved:

$\begin{array}{lccc} & \text { D/D-ACE (\%) } & \text { OR (C195\%) } & \text { P } \\ \text { Diabetic population } & 20.63 & & \\ \text { Diabetic nephropathy } & 40.7 & 3.87,1.26-12.03 & <0.0067 \\ \text { Peripheral artheriopathy } & 47.87 & 3.50,1.56-11.54 & <0.0044 . \\ \text { Nephropathy+artheriopathty } & 61.4 & \mathbf{8 . 7 , 5 . 4 - 1 1 . 2 3} & =0.0001\end{array}$

These results suggest that ACE D/D polymorphism could . be a potent marker for association between nephropathy and artheriopathy in type 2 diabetic patients.Further studies are required for confirmation of these preliminary results.

\section{7}

ANTIPROTEINURIC EFFECT OF ANGIOTENSIN I-CONVERTING ENZYME IHIBITORS (ACE) IN THE YOUTH WTTH DIABETIC NEPHROPATHY AND ACE GENE POLYMORPHISM

G.I.Sivous', E.P.Kasatkina', L.M.Demurov', E.A.Voichik', Y.Y.Kondratiev ${ }^{2}$, D.A.Chistyakov', I.A.Strokov' and V.V.Nosikov'. 'Paediatric and Adolescence Department of Endocrinology Chair of Russian Academy for Advanced Medical Education, ${ }^{2}$ National Research Centre "GosNII Genetika", Moscow, Russia. Overt diabetic nephropathy (DN) is infrequent in patients with relatively short duration of juvenile-onset insulin-dependent diabetes mellitus (IDDM). Now we studied effect of angiotensin I-converting enzyme (ACE) inhibition on urinary albumin excretion rate (UAER) in $68 \mathrm{IDDM}$ patients with mean age $16.4 \pm 2.5 \mathrm{yr}$, mean IDDM duration $8.0 \pm 4.1 \mathrm{yr}$. with special respect to insertion/deletion (//D) ACE gene polymorphism. All patients received ACE inhibitors (40 patients received enalapril in dosage $5-10 \mu \mathrm{g}$ daily and 26 patients - ramipril in dosage 2,5-5 $\mu \mathrm{g}$ daily) during 3-9 months. UAER and $\mathrm{HbA}_{1}$ were determined by standard assays. ID/ACE gene polymorphism was analysed using polymerase chain reaction technique. Student's t-test and Fisher's exact test were used for data comparison.

According to extent of albuminuria reduction ( $\triangle$ UAER, \%) after ACE inhibitor treatment, all patients were divided in "responders" $(R, \Delta>0 \%)$ and "non-responders" (NR, $\Delta=0) . \mathbf{R}(\mathrm{n}=52)$ and NR $(\mathrm{n}=16)$ groups did not differ significantly in either IDDM duration ( $8.1 \pm 4.4$ vs. $7.9 \pm 3.1$ yr.) or $\mathrm{HbA}_{1}$ level $(11.8 \pm 2.2$ vs. $12.1 \pm 1.7 \%$ ). However, in $\mathrm{R}$ group compared to NR group we observed a tendency for prevalence of I allele to increase ( $34.6 \mathrm{vs}$. $25.0 \%$ ) and D allele to decrease $(65.4$ vs. $75.0 \%)$, as well as for genotype prevalence to redistribute: II 15.4 vs. $12.5 \%$; ID 38.5 vs. $25.0 \%$; DD 46.1 vs. $62.5 \%$, respectively. Conclusion:ID/ACE gene polymorphism might underle part of individual variability in response to ACE inhibition and help to choose more efficient treatment regimen. 


\section{8}

PAI-1 GENE POLYMORPHISM MAY INVOLVED RETINOPATHY AND MICROALBUMINURIA IN CHILDREN WITH TYPE 1 DIABETES

A. Młynarska, H.W. Witas, W. Młynarski, R. Sychowski, A. Szadkowska W. Andrzejewski and J. Bodalski, $2^{\text {nd }}$ Clinic of Children Diseases, Institute of Paediatrics, Medical University of Lodz, Poland

The variation of plasma level the plasminogen activator inhibitor-1 (PAI-1) which is observed to be associated with PAI-1 gene polymorphism, is suggested as factor involved in diabetic microangiopathy. Present studies were performed to elucidate an impact of total PAI-1 and its active fraction as well as PAI-1 gene promotor polymorphism $(4 G / 5 G$ at position -675 ) on retinopathy and microalbuminuria in children with type 1 diabetes (IDDM). $11(12.9 \%)$ of 85 studied IDDM patients (aged 16.5 \pm 3.8 ) after 6.2 \pm 4.2 years of diabetes duration exhibited retinopathy and/or microalbuminuria. Obtained results did not reveal any statistically significant differences in level of plasma total PAI-1 and active PAl-1 in both groups of patients $(33.4 \pm 20.3 \mathrm{ng} / \mathrm{mL}$ $3.46 \pm 7.2 \mathrm{ng} / \mathrm{mL}$ and $41.9 \pm 29.6 ; 4.75 \pm 14.1$, respectively) and in 46 of age matched control subjects $(5.4 \pm 8.3$ and $46.1 \pm 23.9$, respectively). We did not find any influence of patients age, diabetes duration and values of $\mathrm{HbAlc}$ as well as fructosamine concetration on the values of studied parameters. However, the genetic studies using ASA-PCR method confirmed 4G/4G genotype in 5 (45.4\%) patients with microangiopathy (mIDDM) and in 11 (14.9\%) patients without microangiopathy (wmIDDM), also $4 \mathrm{G}$ positive genotypes $(4 \mathrm{G} / 4 \mathrm{G}+4 \mathrm{G} / 5 \mathrm{G})$ in $9(81.8 \%)$ of mIDDM and in $33(44.6 \%)$ of wmIDDM patients; $p=0.044$ and $p=0.047$, respectively (4G aliele frequency 0.636 and 0.297 , respectively for each group; $p=0.004$ ). There was no involvement of PAI-1 gene promotor polymorphism in PAI-1 plasma level in all studied patients. Interestingly, the significantly increased level of total PAI-1 in mIDDM group $(76.6 \pm 21.0)$ in contrast to $27.7 \pm 14.3$ in wmIDDM was found concerning patients with $4 \mathrm{G} / 4 \mathrm{G}$ genotype $(p=0.040$ ). Our observations enable us to suggest the involvement of PAI-1 gene promotor variation in retinopathy and/or microalbuminuria. It is likely that the effect of $4 \mathrm{G} / 5 \mathrm{G}$ polymorphism on diabetic microangiopathy may be mediated through local concentration of PAI1 in retinal and renal circulation rather than its plasma leve

\section{0}

CHARACTERISATION OF THE OSMOTIC RESPONSE ELEMENTS OF THE ALDOSE REDUCTASE GENE IN TYPE 1 DIABETIC PATIENTS

A.E. Heesom ${ }^{\prime}$, A. Graham ${ }^{2}$, B.A. Millward ${ }^{\prime}$ and A.G. Demaine' Molecular Medicine Research Group, Postgraduate Medical School, University of Plynouth, UK ${ }^{1}$, Zeneca Pharmaceuticals, Alderley Park, Cheshire, UK ${ }^{2}$.

There is increasing evidence that genetic as well as metabolic factors play an important role in the susceptibility to diabetic nephropathy (DN) in patients with type 1 diabetes. Aldose reductase (ALR2) is the first and rate-limiting enzyme of the polyol pathway. A highly polymorphic $\mathrm{AC}(\mathrm{n})$ microsatellite locus (5'ALR2) located 2.1 kilobase (kb) upstream of the initiation site is strongly associated with the susceptibility to, and protection from, $D N$ in type 1 diabetes. An increased frequency of the $\mathrm{Z}+2 / \mathrm{Z}+25^{\prime} \mathrm{ALR} 2$ genotype is found in patients without microvascular complications after 20 years of diabetes. In contrast, the frequency of the Z-2/Z-2 genotype is increased in patients with DN. At least 3 osmotic regulatory sequences (ORE's) are located in close proximity to the 5'ALR2 locus. ORE's increase ALR2 gene transcription in cells exposed to hyperosmotic stress. The aim of this study was to determine whether polymorphisms within the ORE's may account for the association of the 5 'ALR2 locus with DN. Therefore, 12 patients with type 1 diabetes were selected, 6 of whom had the 5'ALR2 'protective' genotype without complications $(Z+2 / Z+2)$; the remaining 6 had the DN susceptibility genotype $(Z-2 / Z-2)$. Genomic DNA from these patients was used in the polymerase chain reaction to amplify across the OREs. The products were then purified prior to DNA sequencing. No polymorphisms within the ORE's were found in the DNA of any of the 12 patients. We were unable to confirm the presence of a previously reported functional ORE by extensive sequencing of a cosinid containing at least $4 \mathrm{~kb}$ of the 5 ' flanking end of the ALR2 gene. In conclusion, the association between ALR2 and DN cannot be explained by polymorplisms in the ORE's therefore suggesting that the genetic lesion lies within the coding region of the gene.

\section{9}

Association of a polymorphism in the $\beta_{3}$ adrenergic receptor gene with development of nephropathy in NIDDM subjects.

Grzeszczak W., Saucha W., Zychma M., Żukowska-Szczechowska E. Department and Clinic of Internal Medicine and Diabetology, Zabrze, Poland

Diabetic nephropathy is one of the major long-term complication in both IDDM and NIDDM. In previous investigations it was proved that diabetic nilieu and genetic factors play an important role in the development of diabetic nephropathy. The $\beta_{3}$ adrenergic receptor ( $\beta_{3}$-AR) gene polymorphism (cytosine to thymidine substitution in codon 64 of the gene, resulting in tryptophan to arginine replacement) was previously identified as a risk factor for proliferative retinopathy in NIDDM.

The aim of this study was to investigate the association between the $\beta_{3}$ AR gene polymophism and nephropathy in a large group of NIDDM patients.

The protocol included 591 unrelated NIDDM subjects (398 females and 193 males) randomly selected from a colort of 941 NIDDM patients witi established renal status (according to albumin to creatinine ratio in three consective trine samples). Parients with microalbuminuria $(\mathrm{n}=255)$ and overt proteinuria (n=124) were considered the two groups of cases. Normoalbuminuric patients with known diabetes dimation of at least 10 years were ircluded into a control group $(n=212)$. All patients were Caucasians, attendants of the same diabetic clinic and resided in the same area at the time of the study. DNA was obtained from peripheral blood leukocytes. $\beta_{3}$ AR gene polymorphism was determined by polimerase chain reaction - restriction fragment analysis using Mval endonuclease.

\begin{tabular}{lccc} 
& Nomialbunimuria & Microalbuminuria & Proteinuria \\
\hline $\mathrm{n}$ & 212 & 255 & 124 \\
$\mathrm{Trp} / \mathrm{Trp}$ & $170(80.2 \%)$ & $216(84.7 \%)$ & $98(79.0 \%)$ \\
$\mathrm{Frp} / \mathrm{Ag}$ & $30(18.4 \%)$ & $37(14.5 \%)$ & $24(19.4 \%)$ \\
$\mathrm{Arg} / \mathrm{Arg}$ & $3(1.4 \%)$ & $2(0.8 \%)$ & $2(1.6 \%)$ \\
\hline
\end{tabular}

We observed no significant differences in genotype distributions and allele frequencies between the investigated groups $\left(\chi^{2}\right.$ test). The study revealed that polymorphism of $\beta_{3}$ adrenergic receptor gene is not a major factor contributing to predisposition to diabetic nephropathy in examined population.

\section{1}

PI " POLYMORPHISM OF THE PLATELET GLYCOPROTEIN IIIA IS NOT ASSOCIATED WITH DIABETIC NEPHROPATHY IN NIDDM PATIENTS L. Scaglione, R. Gambino, E. Lillaz, M. Cassader, G. Pagano and P. Cavallo-Perin Dipartimento di Medicina Interna, Università di Torino, Italy

Recently, a strong association between the $\mathrm{Pl}^{\wedge 2}$ allele of the platelet glycoprotein IIIa (GP IIIa) and myocardial infarction has been described. This polymorphism is due to the leucine ${ }^{33} \rightarrow$ proline substitution of the GP IIIa. Diabetic nephropathy is a strong and indipendent risk marker for cardiovascular morbidity and mortality in type 2 diabetic patients: the mechanisms of this association are largely unknown The aim of this study is to evaluate whether the presence of diabetic nephropathy in type 2 diabetic patients is associated with the $\mathrm{Pl}^{A}$ polimorphism. $\mathrm{Pr}^{\wedge}$ genotyping was performed in all type 2 diabetic nephropathic patients attending the outpatient diabetic clinic $(\mathrm{n}=93)$. The $\mathrm{Pl}^{\mathrm{A}}$ genotyping was performed on 89 normoalbuminuric type 2 diabetic patients matched with the cases for sex, and duration of diabetes, and on 98 healthy subjects. Genomic DNA was amplified by PCR, cleaved by enzimatic digestion with $\mathrm{MspI}$.

\begin{tabular}{|c|c|c|c|}
\hline \multirow[b]{2}{*}{ Variable } & \multicolumn{2}{|c|}{ Type 2 diabetic patients } & \multirow{2}{*}{ Healthy subjects } \\
\hline & nephropathic & normoalbuminuric & \\
\hline Genotype & n. $(\%)$ & n. (\%) & n. $(\%)$ \\
\hline 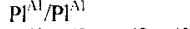 & $76(82)$ & $60(67)$ & $70(7 \mathrm{I})$ \\
\hline $\mathrm{Pl}^{11} /\left.\mathrm{P}\right|^{12}+\mathrm{P}^{12} / \mathrm{Pl}^{1.2}$ & $17+0(18)$ & $27+2(33)$ & $27+1(27)$ \\
\hline \multicolumn{4}{|l|}{ Allelic frequency } \\
\hline $\mathrm{Pl}^{11}$ & 0.91 & 0.87 & 0.85 \\
\hline $\mathrm{Pl}^{12}$ & 0.09 & 0.13 & 0.15 \\
\hline
\end{tabular}

In the whole diabetic group, the $\mathrm{P} !^{\mathrm{A} 2}$ frequency is not significantly different from that of the healthy subjects $\left(0.13\right.$ vs $0.15, \mathrm{p}^{=0.7)}$. The $\mathrm{Pl}^{\mathrm{A}}$ allele frequency is lower in the nephropathic group than in normoalbuminuric group or healthy subjects group. The results of this study do not support the hypotesis that the $\mathrm{Pl}^{\mathrm{i}}$ polymorphism of the platelet GP IIIa may explain the increased incidence of cardiovascular disease in type 2 diabetic nephropathic patients. 


\section{2}

P $]\left.^{A I / P}\right|^{A 2}$ POLYMORPHISM OF PLATELET GPIIIa RECEPTOR AND MICROANGIOPATHY IN TYPE I DIABETIC PATIENTS

L. Pucci, M. Nannipieri, S. Bandinelli, M. Pilo, R. Mangili, R. Navalesi and G. Penno. Dept. of Endocrinology and Metabolism. University of Pisa; Division of Medicine, Istituto Scientifico San Raffaele, Milano, Italy Diabetes clusters renal and cardiovascular complications. Genes that influence factors affecting cardiovascular risk (genes involved in haemodynamics, extracellular matrix, lipids and thrombosis) may be studied in relation to nephropathy. The gene encoding GPIIIa shows polymorphism $\left(\mathrm{P}^{A I} / \mathrm{P}^{A 2}\right)$; the $\left.\mathrm{P}\right|^{A 2}$ allele was associated with cardiovascular events. In 368 type 1 diabetics we evaluated the relation between GPIIIa gene polymorphism and diabetic nephropathy and retinopathy; 76 healthy controls were also studied. A fragment of 247 bp (exon 2) was amplified by PCR (primers: 5'-CTGCAGGAGGTAGAG-AGTCGOCATAG-3' and 5'-CTGCAATCCTCTGGGGACTGACTTG-3'). For the detection of the point mutation, CDGE (Constant Denaturing Gel Electrophoresis) was employed after optimum denaturing conditions setting by perpendicular DGGE (Denaturing Gradient Gel Electrophoresis). Some samples were further analysed by direct sequencing. The allele frequency of $\mathrm{Pl}^{A 2}$ was $11,6 \%$ in IDDM and $8,6 \%$ in controls. In both cohorts genotype distribution was consistent with the frequencies predicted by the Hardy-Weinberg equilibrium (IDDM: $\chi^{2}=5,586 \mathrm{p}=0,061$; controls: $\chi^{2}=0,044 \mathrm{p}=0,833$ ). Al/A1 and A1/A2 genotypes occurred in $77 \%$ and $23 \%$ of IDDM, $83 \%$ and $17 \%$ of controls respectively. Patients were stratified in: a) normoalbuminuric (NA, AER<20 $\mu \mathrm{g} / \mathrm{min}, \mathrm{n} .234,64 \%$ ), microalbuminuric $(\mu \mathrm{A}, \mathrm{AER}=20-200$, n. $78,21 \%)$ and macroalbuminuric patients (MA, AER $>200$; n.56, 15\%); b) patients with no retinopathy (n.124, 34\%), background retinopathy $(\mathrm{n} .120,32 \%)$ and proliferative retinopathy (n.124,34\%). The distribution of GPIIla genotypes was: $177(76 \%)$ Al/AI and $57(24 \%) A l / A 2$ in NA; $62(79 \%) A 1 / A 1$ and $16(21 \%) A l / A 2$ in $\mu \mathrm{A}$; $42(75 \%) A 1 / A I$ and $14(25 \%) A 1 / A 2$ in MA $\left(\chi^{2}=0,16 ; p=0,68\right) ; 96$ $(77 \%) A I / A 1$ and $28(23 \%) A 1 / A 2$ in IDDM with no retinopathy, 92 (77\%)AI/AI and $28(23 \%) A 1 / A 2$ in patients with background retinopathy, $96(77 \%) A 1 / A I$ and $28(23 \%) A I / A 2$ in patients with proliferative retinopathy $(p=0,98)$. In a large cohort of Caucasian patients with type 1 diabetes, no association was observed between the $\mathrm{P}^{A} / / \mathrm{P}^{A 2}$ polymorphism of the GPIIIa and diabetic nephropathy and retinopathy.

\section{3}

MTHFR GENE MUTATION IS NOT ASSOCIATED WITH DIABETIC NEPHROPATHY

M. Odawara, M. Asano, K. Tada, K. Ohkubo, K Yamashita. University of Oxford, University of Tsukuba. Nephropathy is a major complication of diabetes mellitus, which is often related to premature death. Genetic predisposition has been implicated in its development. Previous studies indicate that methylenetetrahydrofolate reductase(MTHFR) genotype is associated with diabetic microangiopathy, which suggest that this mutation may be associated with diabetic nephropathy. To investigate its role in the development of diabetic nephropathy, we analysed 295 unrelated Japanese patients with NIDDM with or without nephropathy for the presence of the C-T mutation in nucleotide position (np) 677 of the MTHFR gene. Age, duration of diabetes, onset age of diabetes, systolic and diastolic blood pressure were matched between the two groups. The frequencies of $\mathrm{C} / \mathrm{C}(\mathrm{Ala} / \mathrm{Ala}), \mathrm{C} / \mathrm{T}(\mathrm{Ala} / \mathrm{Val})$, and $\mathrm{T} / \mathrm{T}(\mathrm{Val} / \mathrm{Val})$ genotypes were $35.0 \%, 47.5$ $\%$, and $17.5 \%$ in patients with overt nephropathy, and $37.3 \%, 45.5 \%$, and $17.3 \%$ in patients with microalbuminuria, while in diabetic patients without nephropathy, the frequencies were 29.0 $\%, 52.4 \%$, and $18.6 \%$. No association of the Val/Val genotype with diabetic nephropathy was observed in the analysed patients with micro- or macro-albuminuria. Unlike previous reports on diabetic microangiopathy, MTHFR genotype is unlikely to play a major role in the pathogenesis of diabetic nephropathy in Japanese.

\section{4}

HLA AND INSULIN (INS) GENES IN CAUCASIANS WITH TYPE 1 DIABETES AND NEPHROPATHY.

TA Chowdhury, PH Dyer, AH Barnett and SC Bain Department of Medicine, University of Birmingham and Birmingham Heartlands Hospital, Birmingham, UK.

Epidemiological observations and the presence of familial clustering suggests that genetic factors are important in the pathogenesis of nephropathy in type 1 diabetes. HLA and INS genes have been previously implicated in susceptibility to nephropathy, although studies have been small poorly controlled. INS has also been implicated in macrovascular disease. The aim of this study was to clarify the role of HLA and INS in type 1 diabetic nephropathy. HLA haplotypes were determined by the phototyping method. INS was typed using the $-23 \mathrm{H}$ ph1 RFLP analysis. 258 patients with type 1 diabetes and nephropathy (presence of retinopathy, hypertension and persistent proteinuria) were compared to 200 recently diagnosed subjects (background diabetic population) and 158 subjects with long duration of diabetes ( $>20$ years) and no nephropathy. All subjects were white Caucasian. No significant difference was observed between the three groups in distribution of HLA-A, B, C, DR, DQA1 and DQB1 alleles. No difference between the three groups in INS allele or genotype frequencies was observed. This is the largest study examining HLA and INS genes in diabetic nephropathy. We conclude that these loci do not play a significant role in susceptibility to nephropathy in Caucasian patients with type 1 diabetes in the UK.

\section{5}

GENETIC AND NON-GENETIC RISK FACTORS FOR PROGRESSION OF DIABETIC NEPHROPATHY IN NORMOTENSIVE TYPE 1 PATIENTS $P$. Jacobsen, $K$. Rossing, L. Tarnow, $P$. Rossing, $C$. Mallet*, $O$. Poirier*, $F$. Cambien ${ }^{*}$, H.-H. Parving. Steno Diabetes Center, Gentofte, Denmark *Institut National de la Santé et de la Recherche Médicale SC7, Paris, France The impact of putative genetic and non-genetic risk factors for progression of diabetic nephropathy was elucidated in 59 normotensive patients with type 1 diabetes. The following progression promoters were determined: insertion/deletion polymorphism in the angiotensin converting enzyme gene, blood pressure, albuminuria, haemoglobin $A_{1 C}$, cholesterol, smoking, retinopathy, height and gender. We studied the natural history by measuring ${ }^{51} \mathrm{Chrom}-\mathrm{EDTA}$ plasma clearance at yearly intervals at least 3 times during (median (range)) $5,5(2,2-18,3)$ years. At baseline the 3 groups (II, $n=11 ; I D$, $n=25$ and $D D, n=23)$ had comparable glomerular filtration rate $\left(\mathrm{ml} / \mathrm{min} / 1,73 \mathrm{~m}^{2}\right)$ (mean (SD)): 103 (16); 99 (19); 113 (22), arterial blood pressure, albuminuria and haemoglobin $A_{1 c}$. During the study period no significant differences were observed in: the rate of decline in glomerular filtration rate $(\mathrm{m} / \mathrm{min} / \mathrm{year})$ (median(range)) $0,9(10,6$ to $-1,9) ; 2,5(12,9$ to $-4,4) ; 1,4(10,8$ to $-1,9)$, blood pressure, albuminuria, haemoglobin $A_{1 C}$ or cholesterol between the 3 groups $(\mathrm{Il} ; \mathrm{D} ; \mathrm{DD})$, respectively.

A multiple linear regression analysis of putative baseline risk factors revealed that albuminuria, short statue and male gender independently predict an enhanced decline in glomerular filtration rate, $R^{2}$ (adjusted) $=0,33 ; p<0,002$. The only independent progression promoter during the follow-up period was albuminuria, $R^{2}$ (adjusted) $=0,37 ; p<0,0001$. Our study revealed a rather slow progression of kidney disease in normotensive type 1 patients with diabetic nephropathy. Albuminuria, short statue and male gender act as progression promoters in such patients. 
PS 59

\section{Pathogenesis of Diabetic Nephropathy}

1146

MODIFIED LOW DENSITY LIPOPROTEINS INDUCE MITOGEN ACTIVATED PROTEIN KINASE ACTIVITY AND TGF- $\beta$ IN MESANGIAL CELLS

A.J. Jenkins, V. Velarde, J. Christopher, R. Mayfield, K.C. Joyce, T.J. Lyons, and A.A. Jaffa. Medical University of South Carolina, Charleston, S.C., U.S.A.

Background: Low-density lipoproteins (LDL) modified by glycation and/o oxidation may contribute to the development of diabetic nephropathy. LDL may affect mesangial cell (MC) proliferation and function, however, the mechanism remain undefined. The Mitogen Activated Protein Kinase (MAPK) pathway and Transforming Growth Factor- $\beta$ (TGF $\beta$ ) mediate changes in cell viability and function. Aim: To examine the effects of normal and modified LDLs on MAPK and TGF- $\beta$ expression in rat MCs. Methods: Experiments were conducted four times. For MAPK studies, normal and modified (mildly oxidized, glycated, glycoxidized, highly oxidized, highly glycoxidized) LDL at various concentrations $(0,10,25,50,75$ and $100 \mu \mathrm{g} / \mathrm{ml})$ were added to quiescent MC for 5 minutes. MAPK activation was measured by western blot using antiphospho-MAPK antibodies. Cells were also exposed to $25 \mathrm{ug} / \mathrm{ml}$ modified LDL in the presence of inhibitors of calmodulin (W7, $75 \mathrm{uM}$ ), protein kinase C (PKC) (bisindolyl malernide, $2 \mathrm{uM}$ ), Stc kinase (PPI, $10 \mathrm{uM}$ ) and MAPK Kinase (PD 98059, $40 \mathrm{uM}$ ). For assessment of TGF$\beta$, quiescent MC were exposed to $100 \mathrm{ug} / \mathrm{ml} \mathrm{LDL}$ for 24 hours. mRNA levels of TGF $\beta$ and the "housekeeping gene" GAPDH were measured by northern blots. Statistical analyses were by ANOVA. Results with $\mathrm{p}<0.05$ were regarded as significant. Results: MC exposure to LDL produced a dose-dependent increase in MAPK activity, with a peak response at $50 \mu \mathrm{g} / \mathrm{ml}$. At lower concentration $(10 \mu \mathrm{g} / \mathrm{ml})$ the modified LDLs were more potent stimulators of MAPK activity, than normal LDL. Pretreatment of MC with inhibitors of calmodulin, PKC, Src kinase and MAPK kinase blocked MAPK activation. Relative to normal LDL, glycated LDL produced a significant 2-fold increase in TGF- $\beta$ mRNA. Conclusion: LDL modified as in diabetes stimulates MAPK and TGF- $\beta$ in cultured MC. MAPK activation involves calmodulin, PKC, Src kinase and MAPK kinase. Changes in these signalling molecules may contribute to the development of diabetic glomenulosclerosis

\section{7}

TRANSGENIC OVERPRODUCTION OF TRANSFORMING GROWTH FACTORB1 INDUCES PROGRESSIVE FUNCTIONAL CHANGES IN THE KIDNEY S. Krag', R.Østerby ${ }^{2}$, C. Nielsen' ${ }^{1}$ and L. Wogensen '. 'Research Laboratory for Biochemical Pathology and ${ }^{2} \mathrm{EM}$ laboratory Aarhus Kommunehospital Denmark.

Many studies have shown an association between transforming growth factor- $\beta 1$ (TGF- $\beta 1$ ) and diabetic nephropathy. Thus, we created a transgenic mouse, with local TGF- $\beta 1$ overproduction targeted to juxtaglomerular cells in the kidney by the Ren-lc promoter. These mice develop progressive glomerulosclerosis with increased extracellular matrix deposition in the mesangial area, at least in part consisting of the basal membrane components collagen IV and laminin. The aim of the present study was to investigate the TGF- $\beta 1$ induced functional changes in our transgenic model. $24 \mathrm{hrs}$ urine collection and blood sampling was performed. We compared transgenic (tg) mice with matched nontransgenic (non-tg) littermates at the age of $1,1 \frac{1}{2}, 2,3,4$ and 5 months. We performed ${ }^{\text {sI }} \mathrm{Cr}$ EDTA clearance on $t g$ and non-tg mice at the age of 3 months. Results are mean \pm SEM. Our results show that the tg mice suffer from polydipsia from the age of 1 month $(p<0.01)$ and polyuria from the age of $11 / 2$ months $(p<0.01)$. At all ages tg mice have an increased plasma BUN compared to non- $t g$ (at 3 montbs tg $13.0 \pm 2,1 \mathrm{mM} \mathrm{n}=6$ vs non-tg $6,9 \pm 0.6$ $\mathrm{mMn}=6)(\mathrm{p}=0.05)$ and increased urinary albumin excretion from the age of 2 months (at 3 months $7,8 \pm 2,8 \mu \mathrm{g} / 24 \mathrm{hrs}, \mathrm{n}=6$ vs. $0,8 \pm 0,25 \mu \mathrm{g} / 24 \mathrm{hrs}, \mathrm{n}=6, \mathrm{p}<0.05$ ), in both cases there is a progresive development with age. GFR was decreased to $3.1 \pm 0.59 \mu 1 / \mathrm{min} / \mathrm{g}$, $\mathrm{n}=7$ in $\operatorname{tg}$ vs non-tg $12.3 \pm 1.18 \mu \mathrm{l} / \mathrm{min} / \mathrm{g} \mathrm{n}=5(\mathrm{p}<0.01)$, in addition the $\mathrm{tg}$ mice had a reduced ${ }^{31} \mathrm{Cr}$-EDTA distribution volume $(17 \pm 0.85 \%$ of bodyweight $\mathrm{n}=7)$ vs. non-tg $(28 \pm 2.49 \%$ bodyweight $n=5)$. Uitrastructurally the 1 month old mice did not differ in glomerular volume, but tg mice had borderline increased basement membrane thickness ( $147 \pm 7.2$ vs $13\lfloor \pm 4.2 \mathrm{~nm}, \mathrm{p}=0.08$ ).

In conclusion, we have shown that localized overexpression of TGF- $\beta 1$ in vivo cause progressive changes in the kidney function. The polyuria may indicate that tubular functional changes occur before glomerular changes because albuminuria and morphological changes is not observed until later stages.

\section{8}

GLUCOSE-INDUCED ACTIVATION OF THE TGF-ß1-PROMOTOR IN MESANGIAL CELLS

Weigert, Cora; Pfeiffer ${ }^{1}$ Andreas; Häring, Hans; Schleicher, Erwin; Medizinische Klinik und Poliklinik IV, Universität Tübingen and ${ }^{1}$ Medizinische Klinik der Universitätsklinik Bergmannsheil, Bochum; Germany

Previous experiments revealed that increased glucose concentration cause a dose-dependent elevation in TGF- $B 1$ production in cultured mesangial cells. The de-novo synthesized TGF-B1 leads to an enhanced synthesis of components of the extracellular matrix e.g. fibronectin and collagen. Therefore, this in-vitro model was used to simulate the in-vivo situation in the glomerulum of diabetic patients i.e. increase in mesangial matrix and glomerular basement membrane thickness. It was the aim of our study to investigate the molecular mechanism of the glucose-induced TGF- $B 1$ production. To clarify, if the TGF- $B 1$ induction occurs at the transciptional level we established an in-vitro transfection model involving a construct of the human TGF- $B 1$ promotor and luciferase as indicator. Briefly, glomerular mesangial cells were transfected with a pGL3-vector containing the CDNA of the TGF- $\beta 1$ promotor $(-453$ to +11$)$ ligated to the cDNA of luciferase. Determination of the luciferase activity reflects the activation of TGF- $\beta 1$ promotor. Hence, using this system, transcription can be measured sensitively. Upon elevation of the glucose concentration to $30 \mathrm{mM}$ the promotor activity was increased 1.6 fold. To simulate the suggested involvement of protein kinase $C$ in the glucose-induced TGF- $\beta 1$ induction we used tumor promoting phorbolester, strong PKC activator, and found a 3.3 fold increase in TGF- $\beta 1$ promotor activity. This increase led to TGF- $\beta 1$ protein production since elevated TFG- $B 1$ levels were found in the respective cell supernatants. In conclusion, our results show that the high glucoseinduced TGF-BI production is caused by an increase in TGF-BI gene transcription. Furthermore, the activation of the TGF-B1 promotor by PKC activtion suggests that the transcription factor AP1 is involved.

\section{9}

\section{SEROTONIN ENHANCES THE PRODUCTION OF TYPE IV} COLLAGEN IN HUMAN MESANGIAL CELLS

T. Sasahara, M. Kasho, S. Kobori*, M. Sakai, Y. Anami, T. Matsumura, and M. Shichiri, Department of Metabolic Medicine, Kumamoto University School of Medicine, Kumamoto, Japan.

* Diabetes Center, Kumamoto National Hospital, Kumamoto, Japan

It has been reported that plasma serotonin (5-HT) level in diabetic subjects was higher than that in normal subjects. In the present study, we investigated the effect of 5-HT on the production of type IV collagen in human mesangial cell (HMC), and further elucidated how could 5-HT enhance type IV collagen sysnthesis. 5-HT stimulated the production of type IV collagen in HMC, which was inhibited by ketanserin and sarpogrelate hydrochloride (5-HT2A receptor antagonists), but not by ondansetron (5-HT3 receptor antagonist). 5-HT increased the bioactivities of both active and total TGF- $\beta$ in mesangial cells. 5-HT enhanced production of type IV collagen was completely inhibited by an anti-TGF- $\beta$ antibody. A PKC inhibitor, calphostin C, inhibited 5-HTinduced increase in type IV collagen secretion, and the activity of membrane PKC was increased by 5-HT. Phorbol ester activated type IV collagen production as well as active and total TGF- $\beta$. Calphostin C completely inhibited 5-HT-enhanced activity of active TGF- $\beta$ but did not inhibit exogenous TGF- $\beta$-induced increase in type IV collagen secretion. Our results suggested that 5-HT-enhanced production of type IV collagen in HMC was mediated by activation of PKC and subsequent increase in active TGF- $\beta$ activity. It is suggested that high plasma concentration of 5HT in diabetic patients may enhance the production of type IV collagen in glomerular mesangial cells, thereby resulting in partly the development of diabetic nephropathy 
EFFECT OF 5-SEROTONIN ${ }_{2 A}$ RECEPTOR ANTAGONIST ON THE DEVELOPMENT OF DIABETIC NEPHROPATHY IN EARLY STAGE S. Kobori, T. Sasahara*, M. Sakai*, T. Matsumura*, M. Kasho*, Y. Hirashima, T. Takahashi and M. Shichiri*, Diabetes Center Kumamoto National Hospital, *Department of Metabolic Medicine, Kumamoto University, Kumamoto, Japan

We have reported in vitro that serotonin (5-HT) enhanced the production of type IV collagen in human mesangial cells and that the production of type IV collagen was inhibited by sarpogrelate hydrochloride, $5-\mathrm{HT}_{2 \mathrm{~A}}$ receptor antagonist. It is considered that expansion of extracellular matricies in mesangial cells may induce the development of diabetic nephropathy. Therefore, we in vivo examined the effect of comparative short-term treatment for 6 months with sarpogrelate hydrochloride in type II DM patients $(n=8)$ with microalbuminuria and without diabetic retinopathy and neuropathy, and diabetic macroangiopathy as compared with the control $(n=8)$ without treatment. There was no significant difference in levels of fasting blood glucose, glycated hemoglobin, and motor and sensory conduction velocities between both groups for 6 months. Urinary albumin excretion rates in treatment-group $(n=8)$ with sarpogrelate hydrochloride was relatively decreased from $35.6 \pm 6.7 \mu \mathrm{g} / \mathrm{min}$ to $24.3 \pm 7.2 \mu \mathrm{g} /$ $\min$ for 6 months, whereas those in non-treatmrnt group $(n=8)$ was slightly not but significantly increased from $34.8 \pm 7.3 \mu \mathrm{g} / \mathrm{min}$ to 38.6 $\pm 6.9 \mu \mathrm{g} / \mathrm{min}$. It is suggested that the developement of diabetic nephropathy in early stage may be inhibited by $5-\mathrm{HT}_{2 \mathrm{~A}}$ receptor antagonist. However, to clarify this mention, long-term study should be performed.

\section{LOCALISATION OF RENAL BRADYKININ RECEPTORS IN EXPERIMENTAL DIABETES}

S. Youssef, M. Jasik, B. Rizkalla, D. Casley, T. Allen and M.E. Cooper. Department of Medicine, University of Melboume, Austin \& Repatriation Medical Centre, Repatriation Campus, West Heidelberg, VIC, Australia 3081.

Previous studies have shown that diabetes is associated with altered plasma and renal tissue kallikrein. This study aims to explore the changes in BK-receptor levels in streptozotocin-induced experimental diabetes using in vitro autoradiography. Male Sprague Dawley rats were randomised into two groups, control (sham injected); and diabetic (streptozotocin $50 \mathrm{mg} / \mathrm{kg} \mathrm{BW}$ ). Tracer was prepared by labelling the BK-B2 specific ligand, HPP-Hoe 140 with ${ }^{125}$ I using chloramine T. Animals were sacrificed at 3 weeks and kidneys were removed and snap frozen in liquid nitrogen. Frozen sections were cut at $15 \mu \mathrm{m}$ and mounted onto gelatin-coated slides. Tissues were incubated in pre-incubation buffer $(170 \mathrm{mM}$ Tris- $\mathrm{HCl} \mathrm{pH} 7.6,0.1 \%$ BSA and $0.05 \%$ bacitracin), then for 3 bours at $4^{\circ} \mathrm{C}$ in incubation buffer (pre-incubation buffer with (racer). The tissues were then washed, dried and placed under X-ray film for two days. Binding was detected in the inner stripe of the outer medulla with moderate binding in the inner medulla in both control and diabetic rats. Binding was inhibited by the presence of $1 \mu \mathrm{M}$ Hoe 140 . No binding was detected in the renal cortex.

$\begin{array}{cccccc} & \text { n } & \begin{array}{c}\text { Plasma Glucose } \\ (\mathrm{mmol} / \mathrm{l})\end{array} & \begin{array}{c}\text { Body Weight } \\ (\mathrm{g})\end{array} & \begin{array}{c}\text { Systolic BP } \\ (\mathrm{mmHg})\end{array} & \begin{array}{c}\text { Medullary } \\ \text { Binding }\left(\mathrm{B} / \mathrm{B}_{0}\right)\end{array} \\ \text { Control } & 5 & 10.5 \pm 0.5 & 348 \pm 11 & 109 \pm 11 & 1.0 \pm 0.06 \\ \text { Diabetic } & 5 & 38.1 \pm 2.6^{*} & 227 \pm 19^{*} & 117 \pm 15 . & 1.46 \pm 0.04^{*} \\ \text { Data are expressed as mean } \pm \mathrm{SEM}^{*}{ }^{*} \text { p }<0.01 \text { vs control. }\end{array}$
Data are expressed as mean $\pm S E M ; * p<0.01$ vs control. Quantitation of X-ray film showed $\sim 50 \%$ increase in [ ${ }^{125}$ I]HPP-Hoe 140 binding in the diabetic kidney, suggesting increased BK-B2 receptors levels. Using emulsion autoradiography, [ ${ }^{125}$ ] HPP-Hoe 140 binding was localised to medullary vasa recta. These results suggest that increased BK activity within the diabetic kidney may be mediated by increased BK receptor levels. Increased BK-B2 receptors may be involved in the early renal haemodynamic alterations observed in experimental diabetes.

\section{2}

CHANGES IN THE GROWTH HORMONE-IGF-I AXIS IN NONOBESE DIABETIC MICE

Y. Segev, D. Landau, R. Eshet, A. Flyvbjerg and M. Phillip. Molecular Endocrinology Lab., Soroka Medical Center, Ben Gurion University of the Negev, Beer Sheva, Israel.

Growth hormone (GH) may have a role in the development of diabetic complications. In non obese diabetic (NOD) mice spontaneous IDDM, renal hypertrophy and glomerular lesions reminiscent of human disease develop. We have previously reported a persistent elevation in renal extractable IGF-I protein in DM-NOD mice up to 30 days from the onset of glycosuria. In the present study we investigated the changes in the GH-IGF-I axis in hyperglycemic NOD-mice. Diabetic female NOD mice and their age and sex-matched controls were sacrificed at 4,14 and 30 days after the onset of glycosuria. Serum IGF-1 levels decreased and serum GH levels increased at 4 weeks of diabetes $(45 \pm 24 \%$ and $182 \pm 32 \%$ of age-matched controls respectively, $p<0.05$ ). Insulin therapy did not prevent the changes in serum IGF-I. GH receptor (GHR) and GH binding protein (GHBP) mRNA levels were decreased in liver but unchanged in kidney tissue of the diabetic animals. Binding assays performed on liver membranes revealed a decreased receptor binding capacity in diabetic mice at one month of diabetes in comparison to controls $(118 \pm 26 \mathrm{Vs}$. $475 \pm 63$ fmol receptor $/ \mu \mathrm{g}$ protein respectively; $\mathrm{p}<0.001$ ). $\mathrm{GH}$ receptor affinity remained unchanged. Insulin therapy did not reverse the decrease in liver GHR message and binding capacity.

In summary, increased serum GH levels are documented in NOD diabetic mice, similarly to the changes described in humans. The decrease in GHR levels and decreased serum IGF-I suggest a state of GH resistance at the hepatic level, whereas the lack of down regulation of GHR message at the kidney level points to a possible increased biological effect for $\mathrm{GH}$ at this target organ for diabetic complications.

\section{3}

THE EFFECT OF GROWTH HORMONE ON THE DEVELOPMENT OF ADVANCED NEPHROPATHY IN EXPERIMENTAL DIABETES MELLITUS D. Landau, E. Israel, A. Flyvbjerg, M. Phillip and Y. Segev. Dept. of Pediatrics, Faculty of Health Sciences, Ben Gurion University of the Negev, Beer Sheva, Israel.

Diabetic nephropathy is the most severe complication of DM. It is characterized by glomerular hyperfiltration and renal hypertrophy, followed by albuminuria and glomerulosclerosis (GS). In the streptozotocin (STZ) model renal hypertrophy and albuminuria occurs, but no subsequent GS or uremia develop, even after a long term follow up. Previous studies demonstrated the involvement of the growth hormone (GH)-insulin-like growth factor- I (IGF-I) system in diabetic nephropathy. Renal IGF-I levels are increased in STZ induced DM. GH serum levels are increased in diabetic patients but are decreased in the STZ diabetic model. Since GH receptors are expressed in renal tissues, we speculated that GH may have a direct effect on the kidneys and enhance the development of diabetic GS. In the present study we investigated whether exogenous GH injected into STZ induced diabetic rats caused changes in renal function and promoted sclerogenic changes. Adult female STZ-induced ( $55 \mathrm{mg} / \mathrm{kg} \mathrm{IP)} \mathrm{DM} \mathrm{rats} \mathrm{(DGH)} \mathrm{and} \mathrm{healthy}$ control rats $(\mathrm{CGH})$ were injected with bovine $\mathrm{GH}(10 \mathrm{mg} / \mathrm{kg} /$ day) for 3 months STZ induced diabetic (D) and control (C) untreated groups were also included in the study. Insulin was not administered and the rats remained hyperglycemic but non ketotic. The rats' weight, glomerular filtration and albuminuria levels were recorded. The kidneys were weighed at sacrifice. The weight of group $\mathrm{C}$ rats increased by $39.8 \pm 5 \%$ compared to $53 \pm 6.3 \%$ in the CGH group. In the D group the rats' weight increased by only $7.3 \pm 5.1 \%$, while in the DGH group the mean weigh increased by $18.2 \pm 3.8 \%(p<0.05$ by ANOVA). The kidney weight to body weight ratio was significantly elevated in both the DGH $(0.8 \pm 0.05 \%)$ and $\mathrm{D}(0.7 \pm 0.02 \%)$ groups in comparison to the $\mathrm{C}(0.42 \pm 0.05 \%)$ and CGH $(0.3 \pm 0.007)$ groups. Creatinine clearance increased only in the $D(143.2 \pm 13.4 \%$ of time 0$)$, but not in the other groups, including DGH. The urinary albumin to creatinine ratio $(\mu \mathrm{g} / \mathrm{mg}$ ) increased to from 60 to 894 in the DGH vs. 279 in the D group during the study period. In summary, $\mathrm{GH}$ has a moderate preventive effect on diabetic induced growth suppression. In contrast, GH treated diabetic rats have a higher degree of hypertrophy associated with less hyperfiltration and more albuminuria. Thus, GH may have a potentially deleterious effect in diabetic nephropathy. 
1154

EXPRESSION AND ACTIVITY OF NITRIC OXIDE SYNTHASE DURING THE ACUTE PHASE IN STREPTOZOTOCIN DIABETIC RATS

S. Keynan, B. Hirshberg, N. Levin-Iaina, H. Dahan, E. Reinhartz ${ }^{1}$, H.Ovadia $^{1}$ and I. Raz. The Departments of Internal Medicine and Neurology ${ }^{1}$, Hadassah University Hospital, P.O. Box 12000 Jerusalem 91120, Israel.

In diabetes mellitus, the kidney responds with hyperfiltration and hypertrophy. The increase in renal size is maximal during the first week of diabetes. There is increasing evidence that nitric oxide (NO) plays an important role in the pathophysiology of renal hyperfiltration and hypertrophy during the early phase of diabetes. The aim of this study was to characterize the effects of experimental diabetes during the first week $(3,5$, and 7 days) on NO synthase expression and activity in the kidney and other organs. We measured NO synthase activity (using the conversion of ${ }^{3}[\mathrm{H}]$-Arginine to ${ }^{3}[\mathrm{H}]$ Citrulline) in the kidney, liver, heart, lung and spleen. Endothelial NO synthase protein expression was evaluated in the kidney and liver by Western blotting. Nitric oxide synthase localization in the kidney was mapped by NADPH-diaphorase staining. In the kidney cortex NO synthase activity decreased already $30 \mathrm{~h}$ after induction of diabetes $982 \pm 301,1709 \pm 445$ (dpm/min/mg protein) respectively, and remained significantly low. However, endothelial NO synthase protein levels were unchanged. The NO synthase activity in the diabetic liver was significantly increased compare to controls after 3 days reaching a maximal value at day $5(10480 \pm 5180,3855 \pm 1476$ $\mathrm{dpm} / \mathrm{min} / \mathrm{mg}$ protein), respectively. Kidney NADPH-diaphorase stain specific to macula densa was significantly lower in the diabetic kidney as compared to controls $(14.0 \pm 6.0,36.13 \pm 7.43, \%)$, respectively. Creatinine clearance was higher in experimental diabetic rats from the third day of diabetes. The levels of $\mathrm{NO}$ stable metabolites $\mathrm{NO}_{2}, \mathrm{NO}_{3}$ ) measured in the urine of diabetic rats were decreased significantly. These results suggest that the decrease in diabetic kidney cortex NO synthase activity during the first week of diabetes may play an important role in the development of renal hypertrophy in the early stage of diabetes.
1155

EFFECTS OF AMINOGUANIDINE ON OXIDATIVE STRESS AND
NITRIC OXIDE RELEASE IN DIABETIC RAT KIDNEYS

D.G.Yavuz,B.Küçükkaya,H.O Ersöz,A.S.Yalçın,K.Emerk and S.Akalın Marmara University Medicine Faculty, Istanbul, Turkey

Reactive oxygen intermediates and increased lipid peroxidation have been implicated in the development of diabetic complications.Aminoguanidine (AG),an inhibitor of advanced glycation has been shown to attenuate the progression of renal disease in experimental models of diabetes. $A G$ also acts as a nitric oxide synthase inhibitor, and the precise mechanisms of this action remains to be elucidated.

The aim of this study was to evaluate the effects of $\mathrm{AG}$ on oxidative stress parameters as well as the release of NO in diabetic rat kidneys.

After induction of diabetes with streptozotocin $(60 \mathrm{mg} / \mathrm{kg}$ i.p) female wistar rats were divided in 2 groups. Group $A(n=9)$ was given $A G$ bicarbonate $(1 \mathrm{~g} / \mathrm{L})$ in drinking water, group $B(n=8)$ was diabetic contol rats given only tap water,group $C$ $(n=8)$ was non-diabetic controls. At the end of the 8 week period,rats were sacrificed and their renal tissue samples were dissected and stored at $-70^{\circ} \mathrm{C}$ until use.

NO release was measured by the $\mathrm{H}_{2} \mathrm{O}_{2}$-induced chemiluminescence method ,lipid peroxidation (MDA=malondialdehyde),protein oxidation ( $\mathrm{PO}$ ), sulphydryl group measurements(total $-\mathrm{SH}$ ) were done by spectrophotometric assays.

Results: total-SH (area under curve) (mmol/g tissue) (mmol carbonyl/ $\mathrm{g}$ tissue)

Group A $461844 \pm 174330 * * \quad 1378,4 \pm 73$ Group B $497703 \pm 89525^{*} \quad 1346.8 \pm 86 \quad 655.8 \pm 7.2$ Group C $\quad 2440310 \pm 489211 \quad 1338.1 \pm 61 \quad 431.5 \pm 8.8$ $2.9 \pm 0.2$ ${ }^{*} \mathrm{p}<0.05 ;{ }^{* *} \mathrm{p}<0.01$ vs control group

Conclusion:We have observed that diabetes decreases $\mathrm{H}_{2} \mathrm{O}_{2}$ induced NO release AG does not increase the NO release but significantly decreases protein oxidation and lipid peroxidation in diabetic rat kidneys.

\section{6}

GAMMA-LINOLENIC ACID (GLA) AND ANTIOXIDANTS REDUCE HYPERCALCIURIA IN DIABETIC PREGNANT RATS.

C.M. Simán, R. Pikgongarm, H.O. Garland, K. Hamilton, J.D. Glazier and C.P. Sibley. School of Biological Science and Department of Child Health, University of Manchester, U.K.

Diabetes causes hypercalciuria which is worsened by pregnancy. Diabetes in the mother may also impair fetal mineralization. Possible causes of these effects are altered expression in the kidney and placenta of the calcium transporting proteins calbindin (CaBP) $9 \mathrm{k}$ and $28 \mathrm{k}$. GLA (an essential fatty acid) and antioxidants have beneficial effects on various diabetes-related disorders. We have studied the effects of GLA, ascorbate (Asc), GLA + Asc, and the new compound ascorbyl-GLA on calcium handling in the kidney and placenta of diabetic pregnant rats.

Rats were treated from weaning with $0.5 \mathrm{~g} / \mathrm{kg} /$ day of GLA or ascorbyl-GLA or $0.3 \mathrm{~g} / \mathrm{kg} / \mathrm{day}$ of Asc in the diet. At 8 weeks of age, rats were made diabetic with $60 \mathrm{mg} / \mathrm{kg}$ of streptozotocin i.p. and mated one week later. At gestational day 20.5 urine was collected over 24 hours in metabolism cages. Urinary calcium concentration was determined by atomic absorbance. Kidneys, placentas and fetuses were collected at day 21.5

Renal calcium excretion was increased in diabetic rats $(1.9 \pm 0.1 \mathrm{mmole} /$ day, $\mathrm{n}=5)$ compared to controls $(0.1 \pm 0.05, \mathrm{n}=5 ; \mathrm{p}<0.01)$ and was reduced with all treatments (Gla 1.1 $\pm 0.2, \mathrm{n}=6$; Asc $1.1 \pm 0.1, \mathrm{n}=4$; Gla + Asc $0.9 \pm 0.2, \mathrm{n}=4$; ascorbyl-Gla $0.8 \pm 0.1, \mathrm{n}=4$; [ $<0.05$ for all, ANOVA]). CaBP $28 \mathrm{k}$ mRNA in kidney was not changed by diabetes or treatment, thus not explaining the hypercalciuria or treatment effect. Placental $\mathrm{CaBP} 9 \mathrm{k}$ mRNA correlated inverscly with the placenta:fetal weight ratio $\left(\Gamma^{2}=0.6, p<0.001\right)$, therefore the previously reported decrease in diabetic placental $\mathrm{CaBP} 9 \mathrm{k}$ expression is likely to be secondary to the diabetes-induced growth retardation rather than a placental malfunction. Treatment did not affect fetal or placental weight or CaBP $9 \mathrm{k}$ expression.

In conclusion, treatment of diabetic rats with GLA and/or antioxidants reduces hypercalciuria without altering expression of renal $\mathrm{CaBP} 28 \mathrm{k}$.

\section{7}

ABNORMAL REGULATION OF INTEGRIN EXPRESSION IN NIDDM: EFFECTS OF HYPERTENSION AND ALBUMINURIA

PA Senior, TH Thomas and SM Marshall. Department of Medicine, Newcastle University. NE2 4HH, UK.

Defects in the insertion of vesicles into the cell membrane may be responsible for the clinical and metabolic abnormalities seen in NIDDM. We used flow cytometry to examine the phorbol myristate actate (PMA) stimulated expression of the $\alpha \mathrm{M}$ integrin (CD11b) on polymorphonuclear leukocytes (PMNL) from NIDDM subjects with hypertension ( $\mathrm{HT}, \mathrm{n}=7$ ), hypertension plus microalbuminuria (HTMA, $\mathrm{n}=6$ ) or neither (NT, n=7) and 7 normal controls (NC). In NC, PMA caused rapid CD11b up-regulation by $5 \mathrm{~min}$. (median (range): $7976(4599-15590)$ sites/cell) which continued to $15 \mathrm{~min}$. (17784 (15711 - 40695) sites/cell) then more slowly until 30 $\min$. (19284 (15247 - 22956) sites/cell). The rate of increase (the slope of sites/cell plotted against time) was similar in NC and HT $(373(150$ - 546) y $452(284$ - 609) sites $/$ cell $/ \mathrm{min}, \mathrm{p}=0.22)$, but higher in NT (756 (498 - 1084) sites $/ \mathrm{cell} / \mathrm{min}, \mathrm{p}=0.004$ $v \mathrm{NC})$ and HTMA (757 (389-1283) sites/cell//min, $p=0.01 \mathrm{v} \mathrm{NC)}$ since CD1 lb expression continued to rise steadily between 15 and $30 \mathrm{~min}$. (NT: 20645 (16847 29418) to 26832 (20351 - 29786), HTMA: 19888 (15530 - 53959) to 27788 (20063 43265) sites/cell). Co-incident with this, a proportion of PMNL were downregulating $\mathrm{CD} 1 \mathrm{lb}$, to become $\mathrm{CD} 1 \mathrm{lb}$ negative. The proportion of $\mathrm{CD} 1 \mathrm{lb}$ positive PMNL at 5 min. was similar in all groups NC $85.5(74.0-97.7) \%$, NT $82.6(58.5$ $87.0) \%$, HT $85.1(74.9-92.2) \%$, HTMA $84.1(83.0-90.1) \%$, but was significantly lower in NC at $15 \mathrm{~min}$. (NC $61.7(46.7-75.5) \%$, NT $80.6(62.3-84.2) \%$, HT 72.8 $(55.6-86.8) \%$, HTMA $81.7(72.0-90.6) \%$ : ANOVA $p=0.01)$ and $30 \mathrm{~min}$. $(\mathrm{NC}$ $49.3(43.2-62.7) \%$, NT $76.9(53.9-79.9) \%$, HT $68.4(46.5-74.9) \%$, HTMA 77.1 $(66.7-88.4) \%$ : ANOVA $p=0.003)$. While CD1 lb expression is enhanced in NT and HTMA, failure of integrin down-regulation was common to all diabetic groups Thus regulation of integrin expression is clearly abnormal in NIDDM and there may be important differences in integrin expression within subgroups of NIDDM. Abnormalities of vesicle insertion may be important in the pathogenesis of NIDDM and its complications. 
PLASMA TISSUE INHIBITOR OF METALLOPROTEINASE-1 IS ELEVATED IN TYPE 1 DIABETICS

D Gordon', P Maxwell ${ }^{2}$ and PM Timms ${ }^{2}$ Departments of Medicine ${ }^{1}$ and Clinical Biochemistry ${ }^{2}$ Stobhill NHS Trust, Glasgow G21 3UW Scotland.

Introduction Increased collagen concentrations in the kidney of diabetic patients is important in the pathogenesis of diabetic renal disease. This increased collagen deposition may arise from increased synthesis and/or a decrease in collagen degradation. Collagen is degraded by a group of enzymes called metalloproteinases the activity of which is inhibited by the antiproteinases called tissue inhibitor metalloproteinases (TIMPs) the best documented of which is TIMP-1. Aim Our aim was to investigate the levels of TIMP-1 in patients with type 1 diabetes. Methods 25 patients with type 1 diabetes had blood taken for TIMP-1 measurement. All these patients were clinically and biochemically screened to exclude hypertension, liver disease and any disorder of connective tissue. Any patients who had had surgery or a significant pathology during the last year were also excluded. Seventeen normal controls satisfying the above inclusion criteria were used for comparisons. Heparinised plasma was used in the ELISA assay for TIMP-1. The TIMP-1 results in the diabetic group were non Gaussian distributed and were compared with the normal control using the Mann Whitney test.

Results The TIMP-1 levels $\mathrm{ng} / \mathrm{ml}$ expressed as $95 \%$ confidence limits were significantly higher in the diabetic patients $(160-916) p<0.001$ in comparison with the controls (61-325). Using Spearman's rank correlation there was no correlation between TIMP-1 concentrations and duration of diabetes, presence of microalbuminuria or $\mathrm{HbA} 1 \mathrm{c}$ levels. Conclusion Our evidence suggests that the increase in collagen deposition found in type 1 diabetic patients may be due to decreased degradation.

\section{0}

AUTOIMMUNITY AGAINST KIDNEY ANTIGENS IN TYPE 1 DIABETIC PATIENTS AND THEIR RELATIVES

P. Migliorini*, B. Marchini*, E. Matteucci and O. Giampietro. Clinica Medica II, Immunologia Clinica*, Pisa, Italy.

Type 1 insulin-dependent diabetes mellitus (IDDM) is an autoimmune disease leading to chronic complications. Diabetic nephropathy shows a familial clustering. Our aim was to investigate the possible contribution of autoimmunity to diabetic nephropathy in families of type 1 diabetic probands. Immunoglobulins $\mathrm{G}$ against renal antigens were detected by immunoblot technique in sera from 67 healthy control subjects (age $38 \pm 11 \mathrm{yr}), 50$ patients with IDDM ( $32 \pm 12 \mathrm{yr}$; disease duration $13 \pm 8 \mathrm{yr}$ 23 uncomplicated, 10 with retinopathy, 13 with microalbuminuria) 53 parents of IDDM probands ( $55 \pm 9 \mathrm{yr}$ ) and 43 siblings ( $31 \pm 11 \mathrm{yr}$ ). Were also measured serum creatinine and urinary excretion rates (UER) of albumin, lysosomal NAG and brush-border AAP. From the biochemical viewpoint, the main characteristic of diabetic patients was the increase in UER of AAP $(p<0.05)$ and NAG $(p<0.01)$ of the microalbuminuric group. Antibodies specific to kidney antigens were detected in $26(38 \%)$ controls, $27(54 \%)$ IDDM patients, $25(47 \%)$ parents and $25(58 \%)$ siblings. Antibodies against multiple renal antigens $(\geq 3)$ were present in $5(7 \%)$ controls, 7 (14\%) IDDM, $6(11 \%)$ parents, $16(37 \%)$ siblings $(p<0.01)$. Antibodies specific to $64-66 \mathrm{kD}$ antigens were detected in 2 (3\%) controls, 5 (10\%) IDDM, $5(9 \%)$ parents and $5(12 \%)$ siblings. Albuminuria resulted higher $(\mathrm{p}<0.05)$ in IDDM probands with multiple as well as anti-64-66 kD antibodies. Siblings with multiple antibodies showed no biochemical abnormalities, while those positive for antibodies specific to 64-66 kD antigens had higher enzymuria (both $\mathrm{AAP}$ and NAG, $\mathrm{p}<0.05$ ). In conclusion, the prevalence of IgG antibodies specific to multiple kidney antigens is higher in siblings of type 1 diabetic patients than in healthy. Multiple antibodies and antibodies specific to 64-66 kD antigens seem associated with signs of glomerulo-tubular dysfunction. To clarify the role of autoantibodies as cause or consequence of renal damage further studies are needed.
ALTERED INTRACELLULAR ANTIOXIDANT ENZYME PRODUCTION MAY ACCOUNT FOR THE GENETIC PREDISPOSITION TO DIABETIC NEPHROPATHY

A. Ceriello, A. Morocutti, F. Mercuri, M. Moro,G. Damante, G.C. Viberti. University of Udine, Udine, Italy, Guy's Hospital, London, UK

It has been suggested that oxidative stress may play an important role in the pathogenesis of diabetic complications. It has been demonstrated that active oxygen species induce antioxidant enzyme expression in some tissues. This effect is considered a defensive mechanism. The influence of a genetic predisposition on diabetic nephropathy development has been hypothesized. In this study the effect of long-term (12-14 weeks) exposure to normal ( $5 \mathrm{mM}$ ) and high $(22 \mathrm{mM})$ glucose concentration on fibroblast antioxidant enzyme gene and activity expression from IDDM patients with (DN) [9] and without nephropathy (D) [6] and non diabetic control subjects (C) [6] has been evaluated. In normal glucose concentration CuZnSOD, MnSOD, Catalase and GPX activity and mRNA expression were not different between the three groups. In high glucose condition CuZnSOD mRNA (DN: $10 \pm 2.1$ vs $3.4 \pm 1.2$, mRNA/GAPDH, p $<0.03, \mathrm{M} \pm \mathrm{SE}$; D: $9.2 \pm 2.9$ vs $4.4 \pm 1.2$, p $<0.05$; C: $13.9 \pm 4.3$ vs $7.3 \pm 2$, $p<0.05$ ) and activity (DN: $0.6 \pm 0.07$ vs $0.4 \pm 0.05, \mathrm{U} / \mathrm{mg}$ protein, $\mathrm{p}<0.001: \mathrm{D}$ : $0.5 \pm 0.05$ vs $0.3 \pm 0.002, p<0.01 ; C: 0.9 \pm 0.1$ vs $0.5 \pm 0.04, p<0.02$ ) increased similarly in all groups ( $\mathrm{p}=\mathrm{NS}$ by ANOVA), Catalase mRNA (DN: $3.2 \pm 0.5 \mathrm{vs}$ $3.4 \pm 0.5, p=N S$; D: $5.6 \pm 1$ vs $3 \pm 0.4, p<0.05 ; C: 5.5 \pm 0.4$ vs $3.1 \pm 0.2, p<0.001$ ) and activity (DN: $0.2 \pm 0.02$ vs $0.2 \pm 0.03, \mathrm{p}=\mathrm{NS} ; \mathrm{D}: 0.4 \pm 0.05$ vs $0.2 \pm 0.02$, $p<0.01: C: 0.5 \pm 0.02$ vs $0.2 \pm 0.02, p<0.001)$ increased only in $D$ and $C(p<0.01$ by ANOVA), GPX mRNA (DN: $2.3 \pm 0.5$ vs $2.2 \pm 0.5$, $\mathrm{p}=\mathrm{NS} ; \mathrm{D}: 2.6 \pm 0.3 \mathrm{vs}$ 1. $5 \pm 0.2, p<0.05 ; C: 4.9 \pm 0.5$ vs $1.9 \pm 0.3, p<0.001$ ) and activity (DN: $0.7 \pm 0.01$ vs $0.7 \pm 0.01, \mathrm{p}=\mathrm{NS} ; \mathrm{D}: 1.4 \pm 0.1$ vs $0.7 \pm 0.03, \mathrm{p}<0.001 ; \mathrm{C}: 1.6 \pm 0.1$ vs $0.8 \pm 0.03, p<0.005)$ increased only in $D$ and $C(p<0.001$ by ANOVA), while MnSOD did not change. These data confirm that high glucose induces antioxidant defenses in cells. However, this defensive mechanism seems to be significantly altered in fibroblasts from DN. A defective intracellular antioxidant enzyme generation during the exposure to hyperglycaemia-generated oxidative stress may account for the genetic predisposition to diabetic nephropathy development.

\section{1}

THE ROLE OF AUTONOMIC NEUROPATHY IN THE NATURAL HISTORY OF DIABETIC RENAL DISEASE P. Stella and T. J. Orchard, Pittsburgh, PA

Previous studies have suggested that much of the excess mortality seen in diabetic subjects with cardiac autonomic neuropathy (CAN) is linked to diabetic renal disease (DN).To investigate this link further we examined whether CAN (defined by an expiration/inspiratin (E/I) ratio $<=1.1$ ) predicted the incidence of DN. Data came from the 10-year follow-up Pittsburgh Epidemiology of Diabetes Complications (EDC) Study in which DN is defined as at least two of three timed urines having an albumin excretion rate (AER) $>200 \mathrm{ug} / \mathrm{min}$, and microalbuminuria as AER 20-200 $\mathrm{ug} / \mathrm{min}$. Subjects are examined every 2 years. During an 8-year follow-up period DN developed in 26 patients (from 371 without DN at baseline), while 22 patients were microalbuminuric at two (or more) exam cycles 2 years apart (from 205 subjects without MA at baseline). Univariate analyses showed poor glycemic control $(p=0.002)$, elevated triglyceride $(p=0.035)$ and fibrinogen $(p=0.032)$ levels, hypertension $(p=0,000)$ and decreased $E / I$ ratio $(p=0.000)$ to be significantly associated with the incidence of DN, while higher age $(\mathrm{p}=0.026)$, increased HbAlc $(\mathrm{p}=0.007)$ and LDL-C $(p=0.057)$ levels, smoking status (ever) $(p=0.019)$ and decreased $E / I$ ratio $(p=0.056)$ predicted incidence of MA. Cox proportional hazard modeling showed elevated $\mathrm{HbAlc}(\mathrm{p}=0.013)$, decreased $E / \mathrm{I}$ ratio $(p=0.0025)$ and hypertension $(p=0.0142)$ to be independent predictors of development of $\mathrm{DN}$, while the incidence of MA was highly predicted only by older age $(p=0.0153)$ and poor glycemic control $(p=0.0006)$. Since CAN was a predictor of DN and has a higher and earlier incidence than does DN, we suggest that it may be either involved in the pathogenesis of DN or at least act as an early marker of those more susceptible to DN 
PS 60

\section{Glycation}

1162

PREPARATION OF MONOCLONAL ANTIBODIES SPECIFIC FOR CML AND NON-CML AGE. H.MIURA, Z.MAKITA*, T.ITOH, M.WAKASA, and T.KOIKE*, Tomakomai and Sapporo*, Japan

Recent findings have indicated that $\mathrm{N}^{\mathbb{E}}$-(carboxymethyl)lysine (CML) is a marker of lipid peroxidation rather than a major source of advanced glycation end-products (AGE), and that there is no evidence of increased oxidation-mediated CML in diabetes, so that non-CML AGE might possibly play an important role in diabetic complications. A method of distinguishing between CML and non-CML AGE is necessary for understanding of $A G E$ formation in vivo.

We prepared AGE-proteins by 12 weeks incubation of BSA, HSA, bovine RNase, lysozyme, and gelatin with $0.5 \mathrm{M}$ glucose, and prepared AGE-peptide by digestion of AGE-HSA with proteinase K. Immunization of mice was done with AGE-BSA and a mixture of AGEpeptide and poylcation poly-L-arginine as an enhancer of uptake of the peptide antigen into antigen-presenting cells (APCs). Then we compared the effects of both antigens. The antigenicity of AGE-BSA was higher, but the specificity of AGE-peptide was greater, so we used both antigens by changing the challenge time. Monoclonal anti-AGE antibodies were prepared from mice immunized with AGE-BSA followed by a mixture of AGE-peptide and poly-L-arginine, or a mixture only. We obtained two types of monoclonal anti-AGE antibodies. One recognized CML-BSA, being CML-specific, and the other did not recognize CML-BSA, being non-CML AGE-specific. Both antibodies showed no immunoreactivity for the carrier protein and their immunoreactivity for AGE was not affected by the reduction of Amadori adducts on the protein

These monoclonal anti-AGE antibodies might be useful for distinguishing between CML and non-CML AGE, and the non-CML AGE-specific monoclonal anti-AGE antibody may contribute to our understanding of the clinical role of $\mathrm{AGE}$ toxicity in diabetic complications.

\section{3}

\section{NON-ENZYMATIC GLYCATION OF PLASMA AND CELLULAR PROTEINS IN A SHORT-TERM STZ-DIABETIC RAT MODEL}

J. Litchfield, J. Letsinger, B. Haigh, D. Guralski, A. Carrington, S. Cannon and J. Sredy Institute for Diabetes Discovery, Branford, CT, USA

The chemical modification of proteins by non-enzymatic glycation and Advanced Glycation Endproduct (AGE) formation are increased with diabetes but their role in metabolic and physiological changes are not well understood. In an effort to describe these reactions better, we identified early changes in tissue proteins after the onset of diabetes by studying the time dependent increase of plasma and cellular protein glycation in a short-term STZ-diabetic rat model. Glycation of plasma proteins were assayed by boronate chromatography (HPLC) while cellular proteins from kidney, liver and nerve were assayed by ELISA and western blot procedures using a monoclonal anti-Amadori antibody and a polyclonal anti-CML antibody. Fasting blood glucose levels elevate $4.5-5.5$-fold following injection of STZ $(40 \mathrm{mg} / \mathrm{kg}$ ). Glycation of plasma proteins increased $3.0 \pm 0.15(n=5)$ fold in 4 days in diabetic animals. After 7 days, protein glycation in liver, kidney and nerve tissues increased $2.5,4.3$ and 1.7 fold respectively, compared to non-diabetic control animals. Western blot analysis further revealed that several cellular proteins become glycated quickly after STZ injection. The advanced glycation endproduct CML was not detected in tissue samples at these early timepoints. Our data show that following STZ injection, blood glucose concentration increases and rapid nonenzymatic glycation of plasma and cellular proteins may lead to further metabolic and physiologic changes associated with glucose toxicity.

\section{4}

BENFOTIAMIN INHIBITS THE FORMATION OF ADVANCED GLYCATION END PRODUCTS IN DIABETIC RATS

H.-P. Hammes ${ }^{1}$, R.G. Bretzel ${ }^{1}$, K. Federlin ${ }^{1}$, S. Horiuchi ${ }^{2}$, T. Niwa ${ }^{3}$, and $H$. Stracke ${ }^{l}{ }^{1}$ III. Med. Dept. Justus-Liebig-University, Giessen, Germany; ${ }^{2}$ Dept. of Biochemistry, Kumamoto, Japan; ${ }^{3}$ Nagoya University, Nagoya, Japan

Advanced glycation end products (AGE) are involved in the pathogenesis of diabetic microvascular complications and several compounds, including thiamin, have been reported to inhibit AGE formation. We tested the effect of benfotiamin, a lipid soluble thiamin prodrug, and thiamin on the formation of a newly described AGE-type reaction product of arginine with 3-deoxyglucosone, imidazolone, and on the glycoxidation product $\mathrm{N}^{\varepsilon}$-(Carboxymethyl-)Lysine (CML) formation in tissues of streptozotocin ( $65 \mathrm{mg} / \mathrm{kg}$ body weight)-diabetic WISTAR rats. Benfotiamin $(100 \mathrm{mg} / \mathrm{kg} \mathrm{x}$ day) and thiamin $(70.18 \mathrm{mg} / \mathrm{kg} \mathrm{x}$ day) were administered orally for 6 months and levels of imidazolone and CML were measured in protein extracts using immuno-blotting and quantitative image analysis. In diabetic animals (DC, $\mathrm{n}=10, \mathrm{HbAl} 11.1 \pm 4.8 \%$ ) CML-levels in neural tissues were elevated 3.5 fold $(553.2 \pm 70.2 \mathrm{kAU})$ compared with non-diabetic animals $(\mathrm{NC}, \mathrm{n}=13, \mathrm{HbA1} 5.1 \pm 0.6 \%$ CML $165.2 \pm 25.4 \mathrm{kAU} ; \mathrm{p}<0.001$ vs NC; Bonferroni Multiple Comparison Test). Imidazolone-type AGE were increased 5.1 fold (NC $118.1 \pm 35.8 \mathrm{kAU}$; DC $604.4 \pm 61.6 \mathrm{kAU} ; \mathrm{p}<0.001)$. Benfotiamin administration in diabetic rats $(\mathrm{D}+\mathrm{B}, \mathrm{n}=11, \mathrm{HbAl} 13.09 \pm 3.57 \%)$ induced a major inhibition of neural imidazolone-type AGE formation $(231.5 \pm 43.6 \mathrm{kAU} ; \mathrm{p}<0.01$ vs $\mathrm{DC})$ and completely prevented diabetesinduced glycoxidation (193.0 $\pm 31.2 \mathrm{kAU} ; \mathrm{p}<0.001 \mathrm{vs} \mathrm{DC})$. Treatment with thiamin did not significantly affect AGE or CML levels. These results suggest that benfotiamin effectively inhibits imidazolone-type AGE and glycoxidation product formation in target tissues of microvascular complications in-vivo. The structural and functional correlates are presently under investigation.

\section{5}

Effects of an Aldose Reductase Inhibitor on Carboxymethyllysine Levels in Aorta from Galactose-fed Dogs and in Erylhrocyles from Diabetic Paticnts Y. Hamadia, Y. Kasuya, J. Nakamura, T. Komori, K. Naruse, S. Chaya, K Kato, S. Horiuchi", N. Koh, N. Hotia. Nagoya University School of Medicine, Nagoya. Japan. "Kumamoto Universily School of Medicine, Kumamoto, Japan

To elarily the involvement of aldose reductase in the formation of advanced glycation end products, we detcrmined the level of carboxymethyllysine (CML) in aorta from galactose-fed dogs as well as in erythrocyles from putients with non-insulin dependent diabetes (NIDDM), and examined the ctfects of epalrestat (Ep). an aldose reductase inhibitor, on the CML levels. Male beagle dogs were fed with $30 \%$ galactose diet including 25 or $50 \mathrm{mg} / \mathrm{day}$ of Ep for 42 months. After victimized, the aorta was removed and homogenized in phosphatc-huffered salinc, and centrifuged to obtain extracts. Fasting blood samples were collected from II healthy voluntecrs and 40 paticnts with NIDDM. Ten of the patients had heen trealed with $150 \mathrm{mg}$ of epalrestat per day for at least 2 months prior to the examination and 30 patients were those frec from the compound. Blood samples were also collected from 8 patients before and after administration of epalrestat for 2 months. The amount of CML in extracts was determined by a competitive enzyme-linked immunosorbent assay using monoclonal antibodies to CML. The CML levels were elevated in aorta from galactose-fed dogs as compared with those fed with control diet $(2.08 \pm 0.39 \mathrm{vs} 1.40 \pm 0.6$ units/g proteins). This increase in CML was prevented by the administration of a high dose of Ep $(1.31 \pm 0.28$ units/g proteins $)$. The erythrocyte CML level was significantly elevated in diabetic patients than in non-diabetic individuals $(50.3 \pm 6.7$ vs $30.1 \pm 5.7$ units/mg proteins, $\mathrm{p}<0.05)$ and was significantly lower in patients who had been treated with epalrestat compared with those free from the compound $(29.2 \pm 4.3$ vs $50.3 \pm 6.7$ units $/ \mathrm{m}$. proteins, $p<0.05)$. The treatment of patients with cpalrestat for 2 months diminished the level of CML $(44.5 \pm 8.8$ v $22.2 \pm 8.8$ units/mg proteins, $p<0.05)$ without changes in the plasma glucose and $\mathrm{HhA}$, levels. We conclude that the aldose reductase plays a tangible role in the production of CML and the polyol pathway is likely to contribute to the glycation reaction. 
ADVANCED GLYCATION ENPRODUCT (AGE) LEVELS IN EYE LENS PROTEINS AND TAIL TENDON COLLAGEN FOLLOWING DIFFERENTIAL ISLET TRANSPLANTATION IN DIABETIC LEWIS RATS

A. Gabriele, S. Morano, S. Morelli, E. Sagratella, P. Castaldo, R. Cipriani, *M Vetri, * V. Caltabiano, * F. Purrello, E. Vecci. U. Di Mario and M. Sensi. Endocrinology, Clinica Medica 2, University of Rome "La Sapienza" and *Clinica Medica, University of Catania, Italy.

Normalizing the metabolic control should correct the abnormal and damaging accumulation of AGEs which occurs, as consequence of diabetic hyperglycemia. over proteins. To verify this hypothesis, islet transplantation was performed in diabetic inbred Lewis rats 15 days (A study), 4 months (B study) and 8 months (C study) after streptozotocin-induced diabetes. Normal and diabetic rats represented the control groups. Transplanted rats metabolic control was evaluated by OGTT. Other metabolic parameters (glycemia, glycosilated hemoglobin and body weight) were determined in all animals at the end of each study $(A=12, B=8$ and $C=12$ months respectively). AGE levels were determined by spectrofluorimetry in eye lens proteins and by ELISA in tail tendon collagen. Metabolic parameters were significantly altered in diabetic rats $(p<0.05-0.001)$ versus normal and transplanted rat groups. which also showed normal OGTTs. AGE levels were significantly increased in diabetic rats $(p<0.05-0.001)$ versus control rats in both tissues and at all times. AGE levels in study $A$ and $B$ transplanted rats were similar to those of normal rats, but were significantly decreased $(p<0.05-0.01)$ when compared to the respective diabetic rats groups. In study $\mathrm{C}$ transplanted rats $\mathrm{AGE}$ values remained significantly higher $(\mathrm{p}<0.05)$ versus normal rats, and lower than the relative diabetic group values, although not significantly. Thus very early or early islet transplantation is capable of preventing or reducing hyperglycemia-associated biochemical protein alterations. If however transplantation is performed later in the course of diabetes, a beneficial effect cannot be achieved. even if euglycemia has been maintained for the whole time following islet transplantation. These results suggest the importance of early preventive therapies against the biochemical effects of diabetic hyperglycenia.

\section{8}

EVALUATION OF THE SERUM ADVANCED GLYCATION ENDPRODUCT MEASUREMENT IN PATIENTS WITH NIDDM T. Inukai, Y. Fujiwara, K. Tayama and Y. Takemura, Department of Medicine, Koshigaya Hospital, Dokkyo University School of Medicine, Koshigaya 343-0845, Japan

Advanced glycation endproduct (AGE) is known to be produced from proteins through Maillard reaction, and plays an important role in the development of atherosclerosis and in the symptom of senility. We therefore investigated the relationship between serum level of AGE (S-AGE) and diabetic pathogenesis in patients with non-insulin-dependent diabetes mellitus (NIDDM). Studies were performed in 90 NIDDM patients ( 47 males and 43 females, $59.7 \pm 9.4$ yrs) and age-matched 24 healthy control subjects. S-AGE was measured by ELISA. Diabetic patients showed a significant increase in S-AGE compared with control subjects (DM: $4.6 \pm 1.1 \mathrm{mU} / \mathrm{ml}$, cont.: $3.5 \pm 0.3$ $\mathrm{mU} / \mathrm{ml}, \mathrm{P}<0.05)$. The frequency of patients with an increase in S-AGE among all diabetic patients was $51 \%(46 / 90)$. Although there was no difference of S-AGE between gender, S-AGE was positively correlated with the duration of $\mathrm{DM}(\mathrm{R}=0.468, \mathrm{P}<0.05)$, but not with body mass index, blood pressure, FPG and serum HbAlc. A significant positive correlation was found between S-AGE and urinary albumin excretion $(\mathrm{R}=0.408, \mathrm{P}<0.05)$. S-AGE in patients with macroalbuminuria was significantly elevated compared with those with normoalbuminuria (macro.: $5.3 \pm 1.4 \mathrm{mU} / \mathrm{ml}$, normo.: $4.3 \pm 0.8 \mathrm{mU} / \mathrm{ml}, \mathrm{P}<0.05$ ). S-AGE in patients with proliferative diabetic retinopathy was significantly higher than in those without retinopathy $(\mathrm{P}<0.05)$. Conclusions: The present data suggest that in NIDDM patients an increment in S-AGE is strongly influenced by the duration of DM, and is closely correlated with the development and/or the progression of diabetic microangiopathy such as nephropathy and retinopathy.

\section{ADVANCED GLYCATION HEMOGLOBIN CORRELATION WITH DIABETIC MICROANGIOPATHY}

Z.Turk, I. Kovačević, B. Benko and Ž. Metelko

Institute Vuk Vinovac, Zagreb, Croatia

Background The consequence of hyperglycemia is the formation and accumulation of advanced glycation endproducts (AGE) on tissue macromolecules. Experimentally, it is well documented that AGEs are implicated in the pathogenesis of late complications of diabetes. However, the investigation of AGE in vivo in diabetic patients was not feasible due to the inaccessibility of various tissues for analysis. The finding that AGEs are also formed on hemoglobin and that Hb-AGEs exists during the lifetime of the red cells, opens a possibility to assess a parameter of advanced glycation. Therefore, we related Hb-AGE as an index of long-term glycation with the duration of diabetes, patients' age and presence of retinopathy and/or nephropathy.

Methods Hb-AGE and HbA1c were measured in 100 patients with a mean duration of diabetes of $7 \mathrm{yrs}$ (range 1-30yrs) and mean age 47yrs (range 18-79 yrs) Thirty-eight patients had clinically established retinopathy and/or nephropathy. $\mathrm{Hb}$ AGE was quantified by the competitive ELISA technique using polyclonal anti-AGERNase-antibodies to detect AGE immunoreactivities of proteins precipitated in red cell hemolysate. Results are expressed as AGE units/mg Hb.

Results The mean level of Hb-AGE did not significantly differ between diabetics with microangiopathy $(9.43 \pm 2.5$, range $4.4-14.5)$ and patients without complications (9.6 +2.0 , range 5.6-13.6). Hb-AGE did not correlate with age ( $r=0.09)$, diabetes duration $(r=0.05)$ or severity of retinopathy/nephropathy. Similarly, there was no correlation of the same parameters in the group of patients without microangiopathy. A week correlation $(r=0.3)$ between $H b A 1 c$ and $H b-A G E$ level was observed in 65 patients $(\mathrm{HbA} 1 \mathrm{c}>8 \%$ ) considered to be in poor glycemia control ( $\mathrm{Hb}-\mathrm{AGE}=9.5+2.3 \mathrm{U} / \mathrm{mg} \mathrm{Hb})$

Interoretation The amount of hemoglobin linked AGEs does not correlate with the presence or absence of retinopathy and/or nephropathy. It seems that $\mathrm{Hb}$-AGE reflects only the metabolic status, both in the subjects with and without complications.

\section{9}

FORMATION OF ADVANCED GLYCATION END PRODUCTS (AGES) MAY PARTICIPATE IN THE DEVELOPMENT OF CORONARY HEART DISEASE IN PATIENTS WITH TYPE 2 DIABETES

Kilhovd B, Berg TJ, Thorsby P, Birkeland KI, Hanssen KF, Aker Diabetes Research Centre and Hormone Laboratory, Aker University Hospital, Oslo, Norway.

Recent epidemiological evidence suggests that hyperglycaemia is associated with diabetic macrovascular disease. One mechanism might be through the formation of AGEs. We therefore measured serum levels of AGEs and the glycoxidation product $\mathrm{N}^{\varepsilon}$-(carboxymethyl)lysine (CML), a specific AGE, in a population of type 2 diabetic subjects with clinical evidence of coronary heart disease (CHD), and compared the results to what was obtained in a population of type 2 diabetic patients without CHD, and in an age-matched, non-diabetic population. Material and methods: Patients $(32 \mathrm{M}, 21 \mathrm{~F})$ aged $59.3 \pm 6.2($ mean $\pm \mathrm{SD})$ years were followed for a median of 5 years in the OCTOPUS study. Levels of lipids, $\mathrm{HbA}_{1}$, AGEs and $\mathrm{CML}$ were measured at inclusion and subjects were classified as having CHD or not on the basis of a full clinical investigation. AGEs and CML were also measured in a group of non-diabetic control subjects $(17 \mathrm{M}, 17 \mathrm{~F})$. Serum levels of AGEs were measured by polyclonal anti-AGE antibodies, and CML-levels by monoclonal anti-CML antibodies in a competitive fluorescence immunoassay. Results: Diabetic patients with CHD had significantly higher mean levels of AGEs $(8.2 \pm 2.0$ vs. $7.0 \pm 1.9 \mathrm{U} / \mathrm{ml} \mathrm{p}=0.03)$ and triglycerides $(2.6 \pm 1.7$ vs. $1.7 \pm 0.9 \mathrm{mmol} / \mathrm{l}, \mathrm{p}<0.05)$ than those without $\mathrm{CHD}$, while the mean levels of CML, total-, LDL, and HDL-cholesterol and HbAlc did not differ between the two groups. Serum levels of AGEs and CML were significantly elevated in diabetic patients compared to controls: AGEs $7.4 \pm 2.0$ vs. $4.1 \pm 1.4 \mathrm{U} / \mathrm{ml}$ and $\mathrm{CML}$ $16.1 \pm 6.9$ vs. $10.5 \pm 6.3 \mathrm{U} / \mathrm{ml}$, both $p<0.0001$. In diabetic patients, levels of $\mathrm{AGES}$ did not correlate to levels of $\mathrm{HbA}_{1 \mathrm{c}}$, triglycerides or cholesterol, but highly significantly to levels of CML $(r=0.61, p<0.0001)$. Conclusions: Serum levels of AGEs are significantly higher in patients with $\mathrm{CHD}$ than in those without. Mean serum levels of AGEs and CML were significantly increased in type 2 diabetic patients compared to non-diabetic controls. 
PS 61

\section{Autonomic Neuropathy}

1170

BAROREFLEX SENSITIVITY IN DIABETIC PATIENTS WITH
POLYNEUROPATHY

POL YNEUROPATHY
Cs. Lengyel, T. Török*, T.T. Várkonyi, P. Légrády, P.
Kempler*** L. Rudas* and J. Lonovics, 1st Dept. of Medicine, *Intensive Care Unit, A. Szent-Györgyi Medical University, Szeged; **1st Dept. of Medicine, Semmelweis Medical University, Budapest, Hungary

In the healthy population, there is a great variation in the baroreflex sensitivity (BRS). The aim of the present study was to analyse the spontaneous BRS in the resting supine position and after standing up in patients with long-standing IDDM with autonomic and sensory neuropathy. Methods: 12 IDDM patients (age: $47.8 \pm 4.6$ years, duration of IDDM: $26.8 \pm 3.9$ years, HbAlc: $9.1 \pm 0.4 \%$, BMI: $26.1 \pm 1.5 \mathrm{~kg} / \mathrm{ms}^{2}$; mean $\pm \mathrm{SE}$ ) and 12 healthy sex, age and weight-matched control subjects were studied. Autonomic neuropathy was assessed by means of five standard cardiovascular reflex tests. The peripheral sensory nerve function was characterized via current perception thresholds (CPTs) measured with the Neurometer. The blood pressure was measured continuously with the Finapres 2300 . The ECG signal was detected continuously by means of a Siemens Sirecust 730 ECG. The signals were fed through an analog-digital converter into a computer and analysed off-line. The BRS was calculated in the supine position and after standing up. Results: The mean autonomic score in the IDDM patients was $5.7 \pm 0.7$ and all had elevated CPTs [peroneal nerve: $2000 \mathrm{~Hz}: 6.6 \pm 0.9$ vs $3.5 \pm 0.2,250 \mathrm{~Hz}: 5.0 \pm 1.1$ vs $1.6 \pm 0.1,5 \mathrm{~Hz}: 3.0 \pm 0.01$ vs $0.7 \pm 0.07$, median nerve: $2000 \mathrm{~Hz}: 4.1 \pm 0.6$ vs $2.7 \pm 0.2,250 \mathrm{~Hz}: 3.3 \pm 1.0$, vs $1.0 \pm 0.1,5 \mathrm{~Hz}: 2.3 \pm 0.1$ vs $0.5 \pm 0.06$ (mA), $p<0.05$; IDDM $v s$ control]. In the IDDM group, the BRS was decreased in the supine position $[3.7 \pm 0.6$ vs $13.3 \pm 3.7(\mathrm{~ms} / \mathrm{mm} \mathrm{Hg})$, $p<0.001]$ and also after standing up $[2.2 \pm 0.6$ vs $5.7 \pm 0.8(\mathrm{~ms} / \mathrm{mm} \mathrm{Hg})$, $p<0.01]$ relative to the control values. In the IDDM group, there was no significant difference between the BRS values measured in the supine and standing positions. In the control group, the BRS was decreased $(p<0.01)$ after standing up in comparison with the resting position. Conclusions: In diabetic polyneuropathy, 1) the BRS was decreased both in the resting supine position and after standing up as compared with the healthy controls, 2) the BRS after standing up was not decreased relative to the supine position. In the IDDM patients with polyneuropathy, a severe impairment of the cardiovascular adaptation mechanism was found.

\section{2}

A MODIFICATION OF THE 30:15 AUTONOMIC FUNCTION TEST. EFFECT OF LEANING FORWARD

N. Tentolouris, A. Linos, E. Stambulis, K. Papageorgiou and N. Katsilambros. 1st Department of Propaedeutic Medicine and Neurologic Department, University of Athens, Greece

The classical 30:15 autonomic function test consists of measurement of the $R R$ cardiac intervals at the 30th and 15th cardiac beats immediately after standing up from the lying position. The present study deals with similar measurements (30:15) after resuming the standing up position following a 2 minute interval during which the examined persons remained in leaning forward position (LF). 105 diabetic persons (DP) (type 1: $n=55$; type 2: $n=50$ ) and 40 controls, matched by age and sex with the $D P$, were examined. They underwent the standardized battery of five Ewing tests for autonomic neuropathy (AN) and also the leaning fonward manoeuvre. AN was diagnosed when 2 of the 5 Ewing fests were abnormal. There were 40 DP without AN (AN-) and 65 with AN (AN+). Sensitivity (\%) (S), specificity (\%) (SP), positive (\%) (PPV) and negative predictive value (\%) (NPV) of the classical 30:15 (C/r) and the LF ratio (LFr) (with the 95\% confidence intervals) were as follows respectively: $67.5(58.5-76.5)$ vs $77.3(69.3-85.3)^{*}$ $71.4(63.4-79.40)$ vs $76.6(68.6-84.6)^{*}, 87.6(81.6-93.6)$ vs $89.2(83.2-95.6)^{*}$, $50.0(41.0-59.0)$ vs $57.5(48.5-66.5)^{*}\left({ }^{*}>0.05\right)$. The coefficients of variation were 6.91 and $7.2 \%$ for the Clr and LFr respectively. The passage from LF to the standing up position caused greater tachycardia than the classical lying to standing test in all subjects and until the 15th beat all DP showed a similar response to heart rate. At the 30th beat, although the heart rate of AN- and controls was restored AN+ continued to have tachycardia. The resulted low LFr clearly indicates parasympathetic damage. So, the cardiac stress after LF manoeuvre seems to unmask an underlying parasympathetic damage to a similar degree as the classical test. Conclusion: The proposed LFr can discriminate between AN- and AN+ DP to a similar degree as the Clr. In addition, it is simple and there is no need for the examined persons to lie down.

\section{1}

INDICES OF SPONTANEQUS BARARECEPTOR REFLEX SENSITIVITYREFERENCE VALUES

M. Weck, J. Tank, RM Baevski, A. Fender, KF Graves, AR Baevski Clinic Bavaria Kreischa,FRG

Objectives: Several tests of baroreceptor reflex sensitivity (BRS) were developed and described in the literature using different approaches to induce blood pressure changes and a variety of mathematical approaches to calculate the index as the quotient of induced heart period changes over the causing change in systolic blood pressure in $\mathrm{ms} / \mathrm{mmHg}$. Available normal values of BRS decrease with age, are higher for rising pressures and range between 5 to $50 \mathrm{~ms} / \mathrm{mmHg}$ or even higher. The goal of our studies was to evaluate different spectral and statistical indices of spontaneous BRS in normal volunteers of different age and sex for routine use in rehabilitaion medicine. Material and Methods: 257 investigations in normal voluntecrs $(f=124$, $m=133$ ) were analysed. The R-peaks of ECG and the systolic and diastolic finger arterial pressure values were detected on a beat-to-beat basis after $10 \mathrm{~min}$. of prerest for $7 \mathrm{~min}$. supine and for $150 \mathrm{~s}$ during deep breathing $(\mathrm{dB}, 6 / \mathrm{min}$.). Power spectral analysis was performed by means of an FFT algorithm. Spectral BRS indices (alpha) were calculated as the quotient of the mean amplitudes in the LF band $(0.1 \mathrm{~Hz})$ of heart period duration and systolic blood pressure. In addition the sequential BRS indices were calculated as the slope of the linear regression lines for spontaneous increases (BRS+) and decreases (BRS-) in systolic blood pressure and the related R$R$ intervals. Results: We did not find significant differences between male and female subjects. Analysis of age groups per decades revealed significant decrease of BRS at the age above fourty years $(\mathrm{P}<0.05)$. The lower 5 th percentiles for two different age groups ( $<40$ years and $\geq 40$ years) were $5 \mathrm{~ms} / \mathrm{mmHg}$. Results are presented as mean \pm SD. Group diffrences were tested with an ANOVA Scheffe F-test $(P<0.05)$. BRS indices calculated by different methods were correlated significantly.

\begin{tabular}{|c|c|c|c|c|c|c|}
\hline BRS & $<20(20)$ & $<30(79)$ & $<40(60)$ & $<50(49)$ & $<60(36)$ & $\geq 60(20)$ \\
\hline$+\mathrm{db}$ & $31 \pm 15$ & $29 \pm 17$ & $23 \pm 12$ & $16 \pm 8$ & $12 \pm 6$ & $9 \pm 5$ \\
\hline+ sup & $28 \pm 13$ & $20 \pm 11$ & $15 \pm 8$ & $9 \pm 4$ & $8 \pm 5$ & $8 \pm 3$ \\
\hline
\end{tabular}

Conclusion: Spontaneous BRS limits of $5 \mathrm{~ms} / \mathrm{mmHg}$ might used to detect baroreflex impairement. To avoid overinterpretation time series must be controled for extrasystolic beats, sufficient systolic pressure rises and significant correlation between systolic blood pressure and R-R intervals.

\section{3}

AUTONOMIC NEUROPATHY IN NIDDM PATIENTS WITH MICROALBUMINLRIA

P.Bouček. T.Pelikánová, A.Jirkovská. B. Vodičková and J.Skibová, Institute for Clinical and Experimental Medicine. Prague. Czech Republic

Microalbuminuria (MAU) is a strong predictor of increased cardiovascular mortality in NIDDM patients. The relation of the presence of MAU to cardiac autonomic function was assessed in patients with NIDDM Autonomic function testing was performed in 16 NIDDM patients with MAU (20-200ug/min), 17 NIDDM without MAU (<20ug/min) and 30 controls of comparable age and sex. Standard tests of heart rate variations (HRV) during quiet $\left(\mathrm{CV}_{\mathrm{R}-\mathrm{s}}\right)$ and deep breathing $(\mathrm{I}-\mathrm{E}, \mathrm{L} / \mathrm{E})$, lying to standing $\left(R-R_{30} / R-R_{15}, R-R_{\max } / R-R_{\min }\right.$. BI). Valsava maneuver (VR) and svstolic blood pressure variation after standing up ( $\triangle \mathrm{SBP}$ ) were used. HRV while standing (1) and lying (2) was also assessed by frequency domain analysis providing quantitative parameters: spectral power (TotPo, LF, HF), spectral power density (PSD-LF.PSD-HF) and spectral power ratio (LF/HF). Examinations were performed using a telemetric computer-aided device (VariaPulse TF3, SimaMedia. Olomouc, Czech Republic) and fast Fourier transform for spectral analysis. Results: Significant ( $(0<0,05)$ differences in results of several tests of autonomic function $\left(C V_{R-R}\right.$. I-E, I/E, R-R $R_{\max } / R-R_{\min }$ BI. VR) and spectral analysis parameters (TotPo 1 and 2, LF 1 and 2. PSD-LF 2. PSD-HF 2) were found between NIDDM patients with MAU and controls. In the NIDDM group without MAU only heart rate reaction to standing and Valsalva indexes differed significantly from the controls. Comparison of the two groups of NIDDM patients demonstrated significant differences in HRV during deep breathing and spectral analysis parameters TotPo, LF. PSD-LF and PSD-HF while lving. Conclusion: The presence of MAU in NIDDM patients is associated with a more extensive impairment of cardiac autonomic function. Autonomic neuropathy may contribute to the increased risk of cardiovascular mortality of NIDDM patients with microalbuminuria. 
INFLUENCE OF CARDIAC AUTONOMIC NEUROPATHY ON URINARY ALBUMIN EXCRETION RATE IN PATIENTS WITH TYPE 1 DIABETES.

B. Bauduceau, H. Mayaudon, O. Dupuy, B. Ceccaldi. B. Cariou,

$X$. Chanudet and $P$. Larroque. Hôpital d'Instruction des Armées Bégin.

69 avenue de Paris. 94160 Saint-Mandé. France.

The aim of this study was to evaluate the influence of diabetic cardiac autonomic neuropathy (CAN) on the development of diabetic nephropathy. Methods: 43 subjects ( $40 \pm 12,2$ years) with type 1 diabetes mellitus were studied. None of the patients were taking cardio-vascular treatment and they were free of nephropathy (urinary albumin excretion UAE $<30$ $\mathrm{mg} / 24 \mathrm{~h}$ ). 24-hour ambulatory blood pressure monitoring (ABPM) and ambulatory electrocardiogram monitoring were performed. CAN evaluation was based on the five Ewing cardiovascular tests, time domain indexes (SDNN/5 mn, RMSSD, pNN50) and frequency domain indexes (total power TP, high-frequency power HFP, low-frequency power LFP). These 43 subjects were divided into two groups according to the median $U A E$ (8mg/24h) : group 1: UAE < $8 \mathrm{mg} / 24 \mathrm{~h}, \mathrm{~N}=24$ : group 2: UAE > 8mg/24h, $N=19$. Résults: Age, sex, body mass index, smoking and serum lipids, duration of diabetes, glycemic control and $24 \mathrm{~h}-\mathrm{ABPM}$ did not differ significantly between the 2 groups. The mean of the five cardiac autonomic function tests was significantly higher in group $2(1,05 \pm 0,83$ vs $0,46 \pm 0,48$ $p<0,01)$. Group 2 had a reduction in total power on $24 \mathrm{~h}$, daytime and nightime periods ( $24 \mathrm{~h}: 2521 \pm 1186$ vs $4221 \pm 2679 \mathrm{~ms}^{2}, \mathrm{p}<0,01$; daytime: $2451 \pm 966$ vs $3708 \pm 2447 \mathrm{~ms}^{2}, \quad p<0,03$; nightime: $3050 \pm 1610$ vs $\left.5227 \pm 3568 \mathrm{~ms}^{2}, p<0,01\right)$. HFP and LFP were aiso significantly lower in group 2 for the three periods. Time domain indexes of group 2 were lower than those of group 1 but the difference was significant only for the vaga indexes. Conclusions: CAN, and more particularly parasympathetic neuropathy, could have a causative role in the development of diabetic nephropathy. An increase in blood pressure variability, resulting from a decrease in heart rate variability, could explain the relationship between CAN and nephropathy.

\section{6}

DIVERSE EFFECTS OF DIABETIC NEUROPATHY AND MICROANGIOPATHY ON CUTANEOUS VASOMOTOR RESPONSES P.Tretjakovs, A.Jurka, I.Taivans and A.Helds. Research Institute of Experimental and Clinical Medicine, Riga, Latvia

Abnormal microvascular control mechanism is a mutual occurrence in diabetic neuropathy and microangiopathy. Aim: to investigate hyperemic responses at sites with different prevalence of arteriovenous shunt vessels and nutritive microvasculature. Subjects: 20 healthy controls (C), 16 diabetics with signs of peripheral autonomic neuropathy $(N)$ and 18 diabetics with microangiopathy (retinopathy and/or nephropathy) without neuropathy (M). The groups were matched for age, sex, BMI, HbAlc, type and duration of diabetes. We recorded cutaneous blood flow changes, induced by $3 \mathrm{~min}$ arterial occlusion (cuff above the knee), local heating $\left(44^{\circ} \mathrm{C}\right)$ and electromagnetic field $(0.9 \pm 0.6 \mathrm{mTelsa}, 80 \pm 34 \mathrm{~Hz}, 60 \mathrm{~min})$, on the pulp (wavelength $780 \mathrm{~nm}$ ) and on the dorsum $(633 \mathrm{~nm}$ ) of the big toe using Laser Doppler flowmetry (Periflux 4001, Perimed). Results showed decrease $(p<0.05)$ in the percentage change $(\Delta \mathrm{Hmax})$ in all the diabetics, compared with controls, independently of test. After arterial occlusion neuropathics had the shortest time (mean \pm SEM s) to hyperemic maximum, while in microangiopathics it was prolonged, especially in the pulp (N 16 163 $\mathrm{s}, \mathrm{M} 62 \pm 19 \mathrm{~s}$ vs $\mathrm{C} 30 \pm 4 \mathrm{~s}, \mathrm{p}<0.05$ ), besides microangiopathics had the lowest $\Delta H_{\max }$ at both sites $(p<0.05)$. During heating neuropatics had the lowest $\Delta$ Hmax on the dorsum $(p<0.05)$, but microangiopathics - on the pulp $(p<0.05)$. During electromagnetic test neuropathics had the lowest $1 \mathrm{~min}$ $\triangle$ Hmax (mean \pm SEM \%) at both sites, but especially on the pulp (N $27 \pm 7 \%, 41 \pm 9 \%$ vs C $56 \pm 11 \%, \quad p<0.05$ ). In conclusion, diabetic neuropathy, in comparison to microangiopathy, has diverse effects on the cutaneous vasomotor responses in both thermoregulatory and nutritive microvasculatures, depending on the hyperemic test.
SKIN MICROCIRCULATION CHANGES IN RESPONSE TO DEEP INSPIRATION AMONG DIABETIC SUBJECTS WITH INDICATIONS OF AUTONOMIC NEUROPATHY I. Kyriazis, P. Tsapogas, A. Hatzilia, I. Ioannidis, G. Avraam, D. Nikolakopoulos, and N. Katsilambros. First Dep. Propaedeutic Medicine, Medical School, University of Athens, Laikon Hospital, Athens, Greece.

In order to evaluate changes of skin blood flow in response to deep inspiration as an additional test indicating the existence of autonomic diabetic neuropathy, we compared the reduction of skin blood flow using Laser Doppler Flowmetry with the classical beat to beat variation test. We examined 18 diabetic subjects (males 13 , females 5 , type $1: 3$, type $2: 15$, age $20-74$ years, BMI $24.6 \pm 3.6$, mean duration of diabetes $9.3 \pm 8.8$ years, blood glucose $10.7 \pm 0.9 \mathrm{mmol} / \mathrm{L}, \quad \mathrm{HbA}_{\mathrm{lc}}=8.4 \pm 2.1 \%$ ). Macroangiopathy was meticulously excluded. Skin microcirculation was recorded on the dorsal surface of left foot between first and second metatarsal during two deep inspirations. Skin temperature ranged between $32-34^{\circ} \mathrm{C}$. Mean percent change of flux (FC) was calculated. ECG recordings were obtained during 5 cycles of inspiration and expiration. The mean ratio of expiratory/ inspiratory $R R$ ( $R R T$ ) (normal $\geq 1.3$, marginal 1.1-1.3, abnormal $<1.1$ ), mean difference of expiratory minus inspiratory $R R$ (RRd), (normal $\geq 0.68 \mathrm{~mm}$ ) and mean difference of heart pulses/min during inspiration minus pulses during expiration (Pd) (classification according to criteria based on age) were calculated. ANOVA was used throughout Results are shown in table.

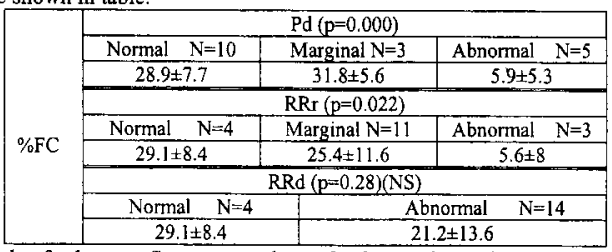

These results further confirm recent data of other authors showing that a poor microcirculation response to deep inspiration may represent an additional tool to the existing cardiovascular tests of autonomic neuropathy.

\section{7}

ABNORMAL BLOOD PRESSURE PROFILE IN DIABETIC PATIENTS WITH SEVERE AUTONOMIC NEUROPATHY: INFLUENCE OF VASOPRESSIN P.T. Monteagudo', H. Gavras', O. Kohimann Jr', A.B. Ribeiro' and M.T. Zanella ${ }^{1}$ Federal University of São Paulo, Brazil; '2University of Boston, U.S.A.

Diabetic patients with advanced autonomic neuropathy (AN) usually have a worse evolution of other complications, associated with large fluctuations of blood pressure (BP) with posture and persistently high BP levels during sleep. It is postulated that vasopressin's (AVP) anti-diuretic action is important for the maintenance of BP in these patients. Objective: to evaluate the effect of vascular $V_{1}$ AVP receptor blockade over BP and over hydroelectrolytic profiles in diabetic (DM) patients with severe AN. Methods: Nine DM aged $41.7 \pm 11.9 \mathrm{y}$, with DM duration of $16.8 \pm 4.6 \mathrm{y}$, body mass index of $22.8 \pm 3.2 \mathrm{~kg} / \mathrm{m}^{2}, \mathrm{HbAlc}$ of $7.7 \pm 2.4 \%$ and an AN score of $8 \pm 2$ (normal $<2$ ), were placed in a hospital for 7 days, on insulin therapy only, under a normossodic, normocalemic diet. On days 4 and 7 , they were submitted to a $24 \mathrm{hr}$ BP monitoring, and blood and urine sampling every $4 \mathrm{hr}$ for sodium (Na), potassium. creatinine, osmolality measurements and excrection fraction(EF) and renal clearance (RCl) calculations. On these days, either placebo or AVP specific $V_{1}$ receptor antagonist $\left(\mathrm{d}\left(\mathrm{CH}_{2}\right)_{5} \mathrm{Tyr}(\mathrm{Me}) \mathrm{AVP}\right.$-AVPi; $\left.0.5 \mathrm{mg}\right)$ were administered IV. at $1 \mathrm{p} . \mathrm{m}$. Statistic: One way ANOVA RM for multiple comparisons; ' $t$ 'test between placebo-AVPi. and Spearman for correlations. Significant if $\mathrm{p}<0.05$.

\begin{tabular}{|c|c|c|c|c|c|c|c|}
\hline \multirow{2}{*}{\multicolumn{2}{|c|}{$\frac{\text { Results: }}{\text { periods }}$}} & $8-12 \mathrm{hr}$ & $12-16 \mathrm{hr}$ & $16-20 \mathrm{br}$ & $20-24 \mathrm{hr}$ & $0-8 \mathrm{hr}$ & $\triangle \mathrm{BP}(\%)$ \\
\hline & & $122 \pm 9$ & $125 \pm 11$ & $134 \pm 14^{8}$ & $139 \pm 14^{*}$ & $130 \pm 16$ & $0.5 \pm 8.7$ \\
\hline & $A V P i$ & $124 \pm 21$ & $119 \pm 19$ & $125 \pm 14$ & $130 \pm 14^{*}$ & $124 \pm 13^{*}$ & $-3.6 \pm 10.2$ \\
\hline $\begin{array}{l}\text { DiastBP } \\
\text { mmHg }\end{array}$ & $\begin{array}{l}\text { plac } \\
A V P i\end{array}$ & $\begin{array}{l}77 \pm 10 \\
78 \pm 15\end{array}$ & $\begin{array}{l}79 \pm 9 \\
75 \pm 15\end{array}$ & $\begin{array}{l}82 \pm 11 \\
79 \pm 12^{5}\end{array}$ & $\begin{array}{l}83 \pm 12^{3} \\
79 \pm 13^{5}\end{array}$ & $\begin{array}{c}77 \pm 12 \\
72 \pm 11^{*}\end{array}$ & $\begin{array}{r}0.4 \pm 12.6 \\
-7.410 .9 *\end{array}$ \\
\hline
\end{tabular}
The $12 \mathrm{hr}$ nocturnal $\mathrm{NaEF}$, diuresis and $\mathrm{RCl}$ were significantly greater than the diurnal ones in the placebo group. NaEF correlated with DBP in AVPi group and with SBP and $\mathrm{RCl}$ in placebo group. Our data show that $\mathrm{AN}$ is related to progressive elevation of $\mathrm{BP}$ in the vigil period and no normal fall of BP during sleep. The blockade of BP elevation with AVPi administration, without a preserved simpathetic nervous system, points to importance of vasopressin on maintenance of BP levels and on an alteration of $24 \mathrm{hr}$ BP profile. In these patients, the vasoconstrictor action of AVP seems to be more evident than its renal action, and electrolytics variation seems to be secundary of $\mathrm{BP}$ changes. 
1178

AUTONOMIC NEUROPATHY AND HYPERTENSION IN TYPE 2 DIABETES MELLITUS: IS THERE RELATIONSHIP?

K. Keresztes, P. Kempler, I. Barna, A Marton, Zs. Hermányi, Zs. Jakab, P. Vargha and R. de Châtel 1st Department of Medicine, Semmelweis University, H-1083 Budapest, Korányi S.u. 2/a, Hungary

The aim of our study was to evaluate a connection between cardiovascular autonomic neuropathy (CAN) and hypertension in non insulin dependent diabetes mellitus (NIDDM). 33 patients with NIDDM were studied (mean age: $58,1 \pm 6,7 \mathrm{ys}$, mean duration of diabetes: $8,8 \pm 5,4 \mathrm{ys}$, males: 8 , females 25). The five standard reflex-tests of cardiovascular autonomic function were applied, twenty-four-hour-long blood pressure monitoring (ABPM) was performed by Meditech ABPM 02 device. Severity of CAN was characterized by the number of abnormal reflex indices on patient

Severity of CAN correlated significantly positively with mean systolic blood pressure values $(p<0,01)$ just as with systolic hypertensive time indices $(p<0,01)$ and systolic hyperbaric impact $(p<0,05)$. Severity of sympathetic CAN was significantly positively related to mean systolic $(p<0,001)$ and diastolic $(p<0,05)$ blood pressure values as well as systolic $(p<0,01)$ and diastolic $(p<0,02)$ hypertensive time indices and systolic and diastolic hyperbaric impact values (both: $\mathrm{p}<0,05$ ). Analysing the relationship between ABPM parameters and the five autonomic tests separately, the increase of diastolic blood pressure during the sustained handgrip test correlated significantly negatively with mean systolic and diastolic blood pressure values (both: $p<0,001)$, systolic $(p<0,01)$ and diastolic $(p<0,001)$ hypertensive time indices as well as systolic $(\mathrm{p}<0,01)$ and diastolic $(\mathrm{p}<0,05)$ hyperbaric impact Mean systolic blood pressure $(p<0,05)$, systolic hypertensive time indices $(p<0,01)$ and systolic hyperbaric impact $(p<0,02)$ correlated also significantly negatively with the Valsalva ratio

Impaired parasympathetic and sympathetic integrity leading to autonomic imbalance seem to be associated with hypertension in NIDDM

\section{0}

SYMPATHETIC CONTROL OF THE CARDIOVASCULAR SYSTEM IN OBESE SUBJECTS. IMPLICATIONS IN THE INVESTIGATIONS OF DIABETICS.

P. Valensi, O. Smagghue, J. Pariès, J. Sibony-Prat, J.R Attali. Laboratory of Nutrition and Metabolic Diseases, Jean Verdier Hospital, Paris-Nord University, Bondy, France.

Data on sympathetic nervous system activity in obese subjects are controversial in the literature. We have studied sympathetic control of the cardiovascular system in non-diabetic obese subjects by performing three kinds of investigations: sustained handgrip test for 5 minutes in 63 obese subjects compared with 35 controls, spectral analysis of systolic blood pressure (BP) variations in the standing position using a Finapres device in 62 obese subjects compared with 38 controls, and peripheral vasoconstrictor responses to sympathetic activation using a laser doppler probe in 57 obese subjects compared with 14 controls. During the handgrip test the increase in systolic BP was slightly lower and the increase in diastolic BP was significantly lower in the obese subjects with cardiac parasympathetic dysfunction (detected by analysing heart rate variations during standardized tests) compared with controls. The spectral analysis of systolic BP variations showed a lower value of the mid-frequency peak (around $0.10 \mathrm{~Hz}$ ) in the obese subjects over 40 years as compared to controls $\left(10.0 \pm 0.6 \mathrm{mmHg} / \mathrm{Hz}^{1 / 2}\right.$ vs 11.9 $\pm 0.8 \mathrm{mmHg} / \mathrm{Hz}{ }^{1 / 2}, \mathrm{p}<0.05$ ). Three tests of sympathetic activation were used to study cutaneous vasoconstrictor response: deep-breathing, sitting-tostanding and Valsalva. The decrease in cutaneous blood flow during the deep breathing test was significantly lower in the obese subjects than in the controls $(43.9 \pm 3.1 \%$ vs $79.1 \pm 23.0 \%, p<0.01)$. These results strongly suggest that sympathetic control of the cardiovascular system is reduced in human obesity and this factor should be taken into account when interpreting the results in overweight diabetic patients.

\section{9}

INFLUENCE OF DIABETIC AUTONOMIC NEUROPATHY ON CEREBRAL VASCULAR REACTIVITY TO HYPERCAPNIA.

C. Tantucci, P. Bottini, C. Fiorani, M.L. Dottorini, L. Scionti, F Santeusanio, L. Provinciali, C.A. Sorbini and G. Casucci, DiMISEM University of Perugia and Clinica di Semeiotica Medica and Clinica di Neuroriabilitazione, University of Ancona, Italy

To investigate the influence of autonomic nervous system on cerebral blood flow changes to hypercapnia 17 insulin-treated male diabetics. matched for age and duration of disease, 8 without autonomic neuropathy (D), 5 with predominantly parasympathetic damage ( $\mathrm{PH}-), 4$ with both parasympathetic and sympathetic damage $(\mathrm{PH}+)$, and 5 male controls of comparable age (C), underwent a $\mathrm{CO}_{2}$ rebreathing test (Read's method). During the test minute ventilation $\left(\mathrm{V}_{E}\right)$, mouth-occlusion pressure $\left(\mathrm{P}_{0.1}\right)$, end-tidal $\mathrm{PCO}_{2}\left(\mathrm{PetCO}_{2}\right)$ were measured together with the mean blood flow velocity in the middle cerebral artery (MCAV) by transcranial Doppler at each occlusion manouvre. The increase of MCAV, expressed as percentage of basal value, i.e. cerebral vascular reactivity (CVR), was calculated at 45 , $50,55,60 \mathrm{mmHg}$ and at peak value of PetCO 2 Data are mean \pm SD. The slope of the linear relationship between $\mathrm{P}_{0.1}$ and $\mathrm{PetCO}_{2}$ was higher in $\mathrm{PH}+$ $\left(0.50 \pm 0.1 \mathrm{~cm} \mathrm{H} 2 \mathrm{O} \cdot \mathrm{mm} \mathrm{Hg}^{1}\right)$ than in $\mathrm{C}(0.43 \pm 0.1), \mathrm{D}(0.42 \pm 0.2)$, and in $\mathrm{PH}-(0.29 \pm 0.1)$. The relationship between MACV and $\mathrm{PetCO}_{2}$ was linear in $\mathrm{C}, \mathrm{D}$, and $\mathrm{PH}-$, the respective slopes amounting to $(2.59 \pm 0.5 ; 2.55 \pm 0.8$ and $1.88 \pm 0.1 \mathrm{~cm} \cdot \mathrm{sec}^{-1} \cdot \mathrm{mmHg}^{-1}$ ). In contrast this relationship was exponential in $\mathrm{PH}+$ due to a progressively greater increase in $\mathrm{MACV}$ at higher level of $\mathrm{PetCO}_{2}$. In fact, while CVR was $33 \pm 13 \%$ in $\mathrm{C}, 23 \pm 16 \%$ in D, $23 \pm 16 \%$ in $\mathrm{PH}-$ and $23 \pm 21 \%$ in $\mathrm{PH}+$ at $\mathrm{PetCO}_{2} 50 \mathrm{mmHg}$, the corresponding values at $\mathrm{PetCO}_{2} 60 \mathrm{mmHg}$ amounted to $68 \pm 14 \%, 63 \pm 35 \%, 54 \pm 27 \%$ and $87 \pm 31 \%$. Our data indicate that: 1) $\mathrm{PH}$ - have a decreased CVR at all levels of $\mathrm{PetCO}_{2}$. 2) $\mathrm{PH}+$ exhibit an enhanced $\mathrm{CVR}$ to higher levels of $\mathrm{PetCO}_{2}$ suggesting a sympathetic inhibitory effect on vasodilating response of cerebral blood vessels; 3) the greater neuromuscular output shown by $\mathrm{PH}+$ in response to $\mathrm{CO}_{2}$ seems not to be related to an impaired $\mathrm{CVR}$.

\section{1}

INFLUENCE OF OBESITY ON VAGAL CONTROL OF HEART RATE VARIATIONS. IMPLICATIONS IN THE ASSESSMENT OF DIABETICS J.R Attali, J. Pariès, J. Sibony-Prat, D. Benidjer, B. Lormeau, P. Valensi. Diabetology, Jean Verdier Hospital, Paris-Nord University, Bondy. France.

Some studies have suggested that overweight may be associated with cardiac autonomic dysfunction. The aim of the present work was to compare autonomic control of heart rate variations in nondiabetic obese subjects and NIDD's and to evaluate its determinants in nondiabetic obese subjects. Three standardized tests : lying-to-standing (LS), deep breathing (DB) and Valsalva (V), were performed in 188 nondiabetic obese subjects with a normal oral glucose tolerance test and 88 NIDD's. According to a linear multiple regression including groups of subjects (obese or NIDD's) and age, each tes correlated negatively with age, but there was no significant difference between obese subjects and NIDD's. In $30 \%$ of the obese subjects, at least one of these tests was abnormal. The determinants of heart rate variations were analysed in 86 obese subjects. Heart rate variations during $L S$ and $D B$ correlated negatively with fasting blood giucose $(p=0.013$ and 0.001 respectively), during DB negatively with waist circumference and waist-to-hip ratio $(\mathrm{p}<0.001$ and 0.0001 respectively) and during $\mathrm{V}$ negatively with insulinemia at fasting and $120 \mathrm{~min}$ after oral glucose intake ( $p=0.03$ for both). In 25 obese women, spectral analysis of heart rate variations during a 5 -min controlled breathing test showed that the high frequency peak, which depends on vagal control, was significantly lower than in a series of 22 age-paired female controls with norma body weight $\left(4.32 \pm 0.66\right.$ beats $/ \mathrm{min} / \mathrm{Hz}^{1 / 2}$ vs $8.18 \pm 0.75$ beats $\left./ \mathrm{min} / \mathrm{Hz}{ }^{1 / 2}, \mathrm{p}<0.0001\right)$ and correlated negatively with age $(p=0.05)$, waist circumference $(p=0.008)$ and waist-to-hip ratio $(p=0.002)$, the correlations with the two latter parameters being significant after adjusting for age. These results suggest that 1) cardiac autonomic dysfunction is frequent in nondiabetic obese subjects, 2) obesity, particularly an excess in android adiposity, exerts a major influence on vagal control of heart rate variations, 3) these findings should be taken into account when assessing cardiac autonomic function in NIDD's. 
1182

AUTONOMIC DYSFUNCTION EVIDENCED BY A SQUATTING TEST IN TYPE 1 DIABETIC PATIENTS : EFFECT OF DISEASE DURATION J.C. Philips, A.J. Scheen, M. Marchand and P.J. Lefebvre. Div. of Diabetes, Nutrition and Metabolic Disorders, CHU Sart Tilman, Liège, Belgium.

In order to study the influence of the duration of type 1 diabetes on the cardiovascular autonomic regulation, three groups of patients with different disease durations (group $1: \leq 10$ years, $4.2 \pm 0.9$ years, $n=19$; group 2 : $11-20$ years, $16.3 \pm 0.7$ years, $n=22$; group $3:>20$ years, $27.0 \pm 0.9$ years, $\mathrm{n}=23$ ) were submitted to a standardized squatting test ( $1 \mathrm{~min}$ standing, $1 \mathrm{~min}$ squatting and $1 \mathrm{~min}$ standing). Heart rate (HR) and mean arterial blood pressure (MAP) were continuously monitored using a Finapres ${ }^{R}$ device (Ohmeda, Louisville, USA). Reference values were obtained in three groups of healthy volunteers matched for sex, age and BMI. Cardiac autonomic neuropathy (CAN : decreased $E / l$ ratio during deep breathing, after adjustment for age) was observed in $7 \%, 32 \%$ and $80 \%$ of diabetic subjects in groups 1,2 and 3, respectively, and correlated with other complications (peripheral neuropathy, retinopathy and nephropathy). During the squatting test, group 3 was characterized by : 1) a reduced bradycardia during squatting and a dampened tachycardia after standing (overall HR rise : $+16 \pm 2 v s+30 \pm 3$ beats/min in controls; $p<0.001) ; 2)$ a similar overall drop in MAP $(-40 \pm 3$ $v s-35 \pm 2 \mathrm{~mm} \mathrm{Hg}, \mathrm{NS})$, but with a significant delay of recovery to baseline values ( $39 \pm 4$ vs $20 \pm 2$ sec; $p<0.0001) ; 3$ ) a marked decrease in baroreflex gain (slope of the regression line relating R-R intervals to MAP changes) $1.6 \pm 0.3$ us $3.9 \pm 0.5 \mathrm{msec} / \mathrm{mm} \mathrm{Hg}$ p $<0.0005 ; 4)$ abnormal R-R SqT ratios defining "SqT vagal" during squatting $(0.92 \pm 0.02$ vs $0.86 \pm 0.02, \mathrm{p}<0.05)$ and "SqT sympathetic" after squatting $(1.08 \pm 0.02$ vs $1.22 \pm 0.03, \mathrm{p}<0.0001)$ All indices of diabetic group 3 were different from those of groups 1 or 2 ( $p<0.01$ or less). In conclusion, long-lasting type 1 diabetes is frequently associated with NAC and cardiovascular autonomic dysfunction can be easily and quantitatively assessed by a squatting test.

\section{4}

REDUCTION OF LOW-FREQUENCY BAND SPECTRAL POWER AND CENTROID FREQUENCY IN ANALYSIS OF HEART RATE VARIABILITY IN DIABETIC PATIENTS WITH HYPOGLYCAEMIA UNAWARENESS

J. Pumprla, Kinga Howorka, P. Anderer', B. Saletu', M. Krieger, A. Schabmann ${ }^{2}$ Research Group Functional Rehabilitation and Group Education, Institute of Biomedical Engineering, 'Dept. of Psychiatry, ${ }^{2}$ Dept. of Applied Psychology, University of Vienna, j.pumprla@bmtp.akh-wien.ac.at

Aim: To compare patterns of spectral analysis of heart rate variability (HRV) in patients without and with hypoglycaemia unawareness in diabetes. Patients and Methods: Short-term spectral analysis of HRV in positions supine-standing-supine (each 300 seconds, low-frequency /LF/ band 0.05 $0.15 \mathrm{~Hz}$, high-frequency $/ \mathrm{HF} /$ band $0.15-0.5 \mathrm{~Hz}$ ) was performed under standardised conditions in two groups of IDDM-patients without and with hypoglycaemia unawareness $(x \pm S D: n=14 / 15$, age $36 \pm 10 / 36 \pm 11$, diabetes duration $16 \pm 9 / 18 \pm 11$ years, $H \mathrm{bA} A_{1 \mathrm{c}} 7.9 \pm 0.4 / 8.0 \pm 0.6 \%$ ), using the evaluation system VariaPulse TF3 ${ }^{\circ}$. Results: When compared to those without unawareness, patients with hypoglycaemia unawareness demonstrated a statistically significant decrease of LF band cumulative spectral power ( $x \pm$ SEM: $\ln \left[\mathrm{ms}^{2}\right]=7.8+0.3$ vs. $7.0 \pm 0.3, p=0.048$ ), LF band cumulative coefficient of component variance $(3.5 \pm 0.4$ vs. $2.5 \pm 0.3, p=0.047)$ and centroid frequency of LF band during standing $(81.8 \pm 6.1 \mathrm{vs} .65 .7 \pm 4.2 \mathrm{mHz}$, $\mathrm{p}=0.036$ ). Conclusions: IDDM-patients with hypoglycaemia unawareness demonstrate lower spectral power and centroid frequency in LF band when compared to those without unawareness. One of possible explanations of this finding could be a slowing of rhythms and/or reduction of heart rate control as depicted in LF band during short-term spectral analysis of HRV. Large-scale study is needed to elucidate this interesting phenomenon.

\section{3}

ASSESSMENT OF DIABETIC AUTONOMIC NEUROPATHY BY SCATTERPLOT METHOD

V.Crasset, Ph.Schoenfeld and S. Degré. Department of Cardiology, Erasme University Hospital, Brussels, Belgium.

Heart rate variability (HRV) has been proved sensitive to detect diabetic autonomic neuropathy but non linear signal analysis by scatterplot method (SP) has never been tested in this setting. We compared scatterplot method to classical HRV parameters in type I diabetic patients. HRV was evaluated on $24 \mathrm{~h}$. Holter in 3 groups of pts matched for age and body mass index: pts with diabetic neuropathy (DIAN): $n=13$, diabetics without neuropathy (DIA): $n=10$ and normal controls $(C): n=33$. Time-domain parameters (HR, SD, pNN50 and rMSSD), frequency-domain (total power, low/high frequency ratio), and SP: length $(L)$ and width $(W)$ of the scatter diagram were measured Results: HR SD pNN50 rmssd Total Low/high L W $\begin{array}{lllllllll}\text { DIAN } & 82.18 & 105.1 & 3.15 & 19.97 & 1844 & 4.17 & 756 & 112\end{array}$ $\begin{array}{lllllllll}\text { DIA } & 77.18 & 129.5 & 11.61 & 35.31 & 3791 & 2.75 & 877 & 177\end{array}$ $\begin{array}{lllllllll}\text { C } & 76.64 & 152.2 & 14.69 & 45.23 & 5093 & 2.25 & 1062 & 222\end{array}$ $p$ value (unpaired Student T-Test)

$\begin{array}{lllllllll}\text { CIDIAN } & 0.02 & 0.001 & 0.0001 & 0.0001 & 0.0001 & \text { NS } & 0.0003 & 0.0001\end{array}$ $\begin{array}{llllllll}\text { DIA/DIAN NS } & \text { NS } & 0.028 & 0.018 & 0.034 & \text { NS } & \text { NS } & 0.01\end{array}$ C/DIA NS NS NS NS NS NS NS NS Our data indicate that width and lenght of SP is reduced in DIAN as classical prameters of HRV. Width seems to point specifically to the cardiac autonomic disturbance linked to diabetes mellitus. The prognosis value of SP remains to be determined in type I diabetic patients.

\section{5}

POWER SPECTRAL ANALYSIS OF ACUTE INSULIN MEDIATED SYMPATHOEXCITATION IN HEALTHY SUBJECTS

B.T. Kinsley. E. Marqusec. C. Broadbridge and R. Freeman. Harvard Medical School, Boston, MA USA

Insulin mediated sympathoexication may play a role in the cardiovascular morbidity that accompanies the hyperinsulinemia in diabetes mellitus. There is no sensitive non-invasive method to quantify such sympathoexcitation. The aim of this study was to identify the changes in cardiovascular sympathetic nervous system activity induced by acute hyperinsulinemia using Power Spectral Analysis (PSA) of variability in heart rate (HR) and blood pressure (BP). Data is presented on 17 healthy subjects (age 28 \pm 2 ). All underwent a supine euglycemic hyperinsulinemic clamp study (12pmol/ $\mathrm{kg} / \mathrm{min})$. PSA of HR and $\mathrm{BP}$, forearm vascular resistance (FVR). HR and BP responses to Valsalva maneuver (VM) and norepinephrine levels were measured at baseline and during euglvcemic hyperinsulinemia. During the clamp procedure insulin levels increased from $90 \pm 6$ to $1620 \pm 156 \mathrm{pmol} / \mathrm{ml}, \mathrm{p}<0.001$. BP $(106 \pm 2 / 65 \pm 2$ vs $109 \pm 2 / 63 \pm 2 \mathrm{mmHg})$ and $\mathrm{HR}(59 \pm 2$ vs $60 \pm 2 \mathrm{bpm})$ did not change. FVR decreased from $59 \pm 9$ to $53 \pm 6, p<0.05$ - suggesting skeletal muscle vasodilatation. With hyperinsulinemia, BP fall in phase 2 of $\mathrm{VM}$ decreased $(111 \pm 5$ vs $102 \pm 6 \mathrm{mmHg}, \mathrm{p}<0.05)$ and the rise in BP over baseline in phase 4 $(22 \pm 3 \mathrm{vs} 33 \pm 5 \mathrm{mmHg}$. $p<0.01)$ increased - suggesting an increase in peripheral resistance. Maximum HR during VM increased ( $95+3$ vs $104 \pm 3 \mathrm{bpm}, p<0.001$ ) - suggesting increased sympathoexcitaition and/or decreased parasympathetic restraint. PSA of low frequency (LF) power of the BP increased (5.5 \pm 1.1 vs $\left.8.1 \pm 1.6 \mathrm{mmHg}^{2} / \mathrm{Hz}, \mathrm{p}<0.008\right)$-a measure of sympathetic vasomotor activityand the ratio of $L F$ to high frequency (HF) heart rate power increased $(0.7 \pm 0.2$ vs. $1.1 \pm 0.3, p=0.02$ )- a measure of relative sympathetic to para-sympathetic balance. Acute hyperinsulinemia is associated with (1) skeletal muscle vasodilatation (2) increased sympathoexcitation as assessed by VM (3) increased sympathetic vasomotor activity and a relative increase in sympathetic to para-sympathetic balance using PSA (t) stable BP and HR. In conclusion PSA provides a sensitive measurement of sympathoexcitation and provides us with a non-invasive means to assess the hrperinsulinemic state. 
1186

CARDIOVASCULAR REFLEX TESTS AND SPECTRAL ANALYSIS OF HEART RATE VARIATION PREDICT MEDIAL CALCINOSIS.

A. Kunz, C.I. Maisch, V. Hofmann-Krück, *J.W. Kaiser, \#C. Meisner, H.-U. Häring, and D. Luft. $4^{\text {th }}$ Dept. of Internal Medicine, *Dept. of Radiology and \#Institute for Medical Information Processing, Eberhard-Karls-University, Tübingen, Germany

Background: Medial arterial calcification (MAC) often confounds the quantification of arterial perfusion pressures of the lower limb in diabetic patients. The correlation of cardiovascular reflex tests with MAC which is thought to be caused by a damage of sympathetic nerves is weak since those tests predominantly represent parasympathetic activity. Therefore, we evaluated wether the low and medium frequency components of the heart rate spectral analysis (SPA) allow any conclusions as to the presence of MAC. Methods: In 53 diabetic patients (10 type-1 and 43 type-2 diabetes mellitus) in our out-patient clinic standard cardiovascular reflex tests (I/E ratio, $\mathrm{L} / \mathrm{S}$ ratio, Valsalva ratio), spectral analysis, and a standardised roentgenogramm of both feet as well were evaluated. Results: In 23 patients who were significantly older and suffered longer from diabetes MAC of the arteries of the feet was detectable. The $\mathrm{E} / \mathrm{t}$ ratio showed the highest sensitivity for the detection of $\mathrm{MAC}(0,36,95 \% \mathrm{CI}: 0,17-0,59)$. Low and medium frequency components of the SPA were less sensitive: 0,09 (CI: 0,01-0,28) and 0,17 (CI $0,05-0,39)$, respectively. The specifity of pathologic results was generally high $(0,82-1,00)$. The positive predictive value of the $I / E$ ratio was 0,62 (CI: $0,31-0,86$ ) and of the medium frequency band 0,80 (CI; $0,28-1,00$ ). The sensitivity of MAC to predict pathologic results of either cardiovascular reflex tests or frequency bands of spectral analysis varied from zero to 0,80 . Conclusions: Pathologic results of heart rate analysis point to concomitant MAC whereas MAC has only limited predictive value for pathologic heart rate variability.

\section{7}

QT-Interval Prolongation in Diabetic Subjects: Effects on Mortality

P. Diem, S.L. Suter, L. Zanchin, A. Teuscher

Bern, Zurich and Langenthal, Switzerland.

It has been suggested that diabetic autonomic neuropathy (DAN) resulting in QT-interval prolongation predisposes to cardiac arrhythmia and sudden death, and as a consequence leads to increased mortality. This hypothesis was tested prospectively in a cohort of 196 diabetics (age: $45.5 \pm 0.4$ years $\mathrm{m} \pm \mathrm{SEM}$; gender: $104 \mathrm{f}, 96 \mathrm{~m}$; duration of diabetes $10.0 \pm 0.5$ years)

QT-intervals were measured on baseline EKGs using a digitizer (Calcomp). QT was corrected for the respective heart rate using Bazett's formula (QTc). Life/death status was assessed after $11.2 \pm 0.2$ years $(\mathrm{m} \pm \mathrm{SEM})$. Survival analysis was performed using the Cox proportional hazards model with survival time as the dependent variable in the regression model. After adjustment for age and duration of diabetes, QTc was a significant predictor of all-cause mortality:

$\begin{array}{llll} & \operatorname{Exp}(\mathrm{Coef}) & 95 \%-\mathrm{CI} & \mathrm{p} \\ \text { All subjects }(\mathrm{n}=196) & 1.010 & 1.001-1.019 & 0.02\end{array}$

When analyzing patients with and without proteinuria at baseline separately, duration of QTc was a significant predictor of all-cause mortality only in patients with proteinuria:

$\begin{array}{llll} & \text { Exp(Coef) } & 95 \% \text {-CI } & \mathrm{p} \\ \text { Proteinuria }(\mathrm{n}=43) & 1.024 & 1.005-1.044 & 0.01\end{array}$

$\begin{array}{llll}\text { No proteinuria }(n=153) & 1.000 & 0.989-1.011 & 0.98\end{array}$ Conclusion: These data indicate that DAN resulting in QT-interval prolongation is associated with increased mortality in patients with diabetes and coexistent proteinuria.

\section{8}

IS THERE A RELATIONSHIP BETWEEN CARDIOVASCULAR, GASTROINTESTINAL AUTONOMIC AND PERIPHERAL SENSORY NERVE FUNCTION IN TYPE 2 DIABETIC PATIENTS ? Zs. Hermanyi, K. Keresztes, A. Marton, and P. Kernpler I.st. Internal Medicine Clinic of the Semmelweis Med.University, Hungary Little is known on the relationship between gastrointestinal (GI) autonomic and peripheral sensory (PS) nerve function in diabetic patients. The connection between GI motility disturbances and glycaemic control is also poorly understood. We examined 52 Type 2 diabetic patients (mean age: 64 ' +6.7 years, mean duration of diabetes: $13.1+7.2$ years, males: 21 , females:29) and 20 healthy subjects as controls. Oesophageal transit time (OTT), using scintigraphy and the five standard tests of cardiovascular (CV) autonomic function were evaluated. PS nerve function was studied by the Neurometer (Neurotron Inc, Baltimore,USA). Neuroselective current perception threshold (CPT) for $2 \mathrm{kHz}$ is an indicator for large fiber function, while lower frequency $5 \mathrm{~Hz}$ CPT correlates with small fiber function. CPT for peroneal nerves (digital branches) were assessed. Thirtyseven patients $(71.1 \%$ ) had prolonged OTT ( $6 \mathrm{sec}$ ). These patients had higher CPT values $(4.97+0.74 \mathrm{~mA}$ vs. $4.24+6.6 \mathrm{~mA}$, p 0.05$)$ reflecting hypaesthesia and higher autonomic score $(3.1+0.5$ vs. $2.6+0.7)$ as a sign of $\mathrm{CV}$ autonomic impairement, compared to diabetic patients with normal OTT $(9.8+1.4$ vs. $7.0+1.2, p$ 0.05). Conclusion: impaired oesophageal motility is associated with poor glycaemic control and PS neuropathy in Type 2 diabetic patients. Morecver, a tendency on a relationship between $\mathrm{CV}$ and $\mathrm{GI}$ autonomic dysfunction was observed.

\section{9}

GUSTATORY SWEATING AND ANTI-SYMPATHETIC GANGLIA ANTIBODIES. C.A. Abbott', J.E. Shaw, R.A. Metcalfe ${ }^{2}$, A.P. Weetman ${ }^{2}$ and A.J.M. Boulton'. Department of Medicine, Manchester Royal Infirmary ${ }^{1}$ and Department of Medicine, University of Sheffield ${ }^{2}$, UK.

Gustatory sweating (GS) is a well-described complication of diabetes, usually associated with autonomic and peripheral neuropathy. It has recently been demonstrated, however, that GS is very common in diabetic nephropathy and resolves in $70 \%$ of patients undergoing renal transplantation, which is at odds with the causal hypothesis of aberrant nerve regrowth. Furthermore, a single case report has demonstrated an association between $\mathrm{GS}$ and serum anti-sympathetic ganglia antibodies (ASGA), suggesting an autoimmune mechanism for GS. We studied this relationship further in diabetic renal patients, including a subgroup who had undergone renal transplantation. 36 subjects were questioned about GS and blood was analysed for ASGA, renal function and $\mathrm{HbA}_{1 \mathrm{c}}$. Tests of autonomic and peripheral nerve function plus GS were performed. 26/36 patients had history of GS, including all 12 transplant patients. 11/12 transplant patients found GS had improved post-transplant, yet 6/12 still had ASGA. 90\% (9/10) of patients with ASGA and 65\% (17/26) of patients without ASGA had GS history ( $n s \chi^{2}$ test). Only $20 \%(3 / 15)$ of patients with ongoing GS and $33 \%(7 / 21)$ of patients without GS had ASGA (ns). Correlation analyses showed no relationships between ASGA levels and GS, neuropathy or renal function, however, measured GS correlated with serum creatinine $(r=0.533, p=0.011)$. The results indicate that there is little or no association between GS and ASGA levels in renal patients, and that the dramatic improvements to GS post-transplant are not accompanied by immunosuppression of ASGA. Furthermore, renal dysfunction appears to be an important determinant of GS. Thus, rather than aberrant nerve regrowth or an autoimmune mechanism, GS may be linked to the reversible biochemical and hormonal changes of nephropathy. 


\section{0}

LONG-TERM EFFECT OF QUINAPRIL ON CARDIOVASCULAR REFLEX TESTS IN PATIENTS WITH DIABETIC AUTONOMIC NEUROPATHY

T. Didangelos, V. Athyros, D. Karamitsos, A. Papageorgiou, G. Kourtoglou, A. Kontopoulos. Divisions of Diabetology and Cardiology, 2nd Prop Clinic of Internal Medicine, Aristotelian University, Hippokration Hospital, Thessaloniki Greece.

The long-term effect of an angiotensin converting enzyme inhibitor (ACE) on diabetic autonomic neuropathy (DAN), using the cardiovascular reflex tests (CRT), has not been studied. 43 consecutive patients (pts) $(19 \mathrm{M}$ and $24 \mathrm{~W}$ ), of median age 52 years were included at the time period that definite DAN was confirmed, as this was established if the values of at leas two of CRT became recently abnormal. The expiration/inspiration (E/I) ratio, standard deviation (SD) and mean circular resultant (MCR) of R-R intervals, the Valsalva index, the $30: 15$ ratio, and the blood pressure response to standing were measured at 3 month intervals for a period of 18 months. Moreover, vibration perception threshold (VPT) was assessed. Pts were randomized to quinapril $(n=21)$ or placebo $(n=22)$. All measured indices, except the Valsalva index, deteriorated in all 22 pts on placebo during the 18 month follow-up. Deterioration became significant at month 15 in most variables. 14 pts developed DAN related symptoms. Quinaprit, by 18 th month, improved significantly the $E / l$ ratio $(1.2 \pm 0.08$ vs $1 \pm 0.06$ ) SD (34 \pm 2.4 vs $25 \pm 2.2)$ and MCR (24 \pm 2.2 vs $14 \pm 1.6)$ of $R-R$ intervals as well as the $30: 15$ index $(1.2 \pm 0.08$ vs $1 \pm 0.07)$ and postural hypotention (13 2.1 vs $21 \pm 2.4 \mathrm{mmHg}$ ). These changes were significant in comparison to baseline $(p<0.05)$ and placebo $(p<0.01)$. Quinapril had no significant effect on the Valsalva index $(1.40 .07 \pm$ vs $1.3 \pm 0.06)$ and VPT $(20 \pm 1.9$ vs $23 \pm 2)$ No patient on quinapril developed DAN related symptoms. The data suggest that quinapril has a long-term beneficial effect on DAN. This effect of an ACE inhibitor might contribute to the improvement of autonomic imbalance, which is implicated in the expression of annoying and/or lifethreatening manifestations of DAN.

\section{1}

LONG-TERM EFFECT OF CONVERTING ENZYME INHIBITION ON CIRCADIAN SYMPATHETIC AND PARASYMPATHETIC ACTIVITY IN PATIENTS WITH DIABETIC AUTONOMIC NEUROPATHY

D. Karamitsos, V. Athyros, T. Didangelos, A. Papageorgiou, H. Boudoulas*, A. Kontopoulos. Divisions of Diabetology and Cardiology, 2nd Prop Clinic of Internal Medicine, Aristotelian University, Hippocration Hospital, Thessaloniki, GREECE. Cardiology Division *, Ohio State University, Columbus, Ohio, USA.

Autonomic nervous system function in patients (pts) with diabetic autonomic neuropathy (DAN), displays an abnormal circadian pattern compared to normal subjects; it probably plays an important role in the onset of acute cardiovascular syndromes, which display a similar pattern of occurrence. The effect of angiotensin converting enzyme (ACE) inhibitors on the circadian pattern of autonomic function in DAN pts has not been studied. Heart rate variability (HRV) frequency domain indices were assessed in 60 pts with DAN at baseline and one year after therapy with quinapril $(n=30)$ or placebo $(n=30)$ on a 24-hour electrocardiogram. Normal subjects $(n=30)$ and pts with diabetes meflitus without DAN $(n=30)$ were used as controls. The baseline circadian variation of fractional normalized power in DAN pts was abolished, with pronounced dominance of low over high frequency normalised power during the whole 24-hour period, but mainly morning and night. After one year of treatment, quinapril increased parasympathetic (P), decreased sympathetic (S) activity and improved sympathovagal (SV) interaction, as manifested by an increase in normalized high frequency power, and a decrease in normalized low frequency power and their ratio in the morning (07.00 a.m. to 15.00 p.m.) and night (23.00 p.m. to $07.00 \mathrm{a} . \mathrm{m}$.) time intervals $(-20 \%$ and $-31 \%$, respectively, $p<0.01$ vs baseline and $p<0.001$ vs placebo). The data suggest that quinapril increased $P$, decreased $S$ activity and improved SV interaction in pts with DAN at hours most adversely manifested (morning and night). Since autonomic function is an important contributor in the onset of acute myocardial infarction, arrhythmias and sudden cardiac death, improvement of autonomic function in DAN pts may prove beneficial.

\section{2}

ERECTILE DYSFUNCTION IN DIABETIC PATIENTS IN ITALY

D. Fedele F. Santeusanio, C. Coscelli, A. Bortolotti, L. Chatenoud, F. Parazzini. The Italian Study Group on Diabetic Neuropathy and Istituto M. Negri, Milan (Italy) Erectile dysfunction (ED) is a common complication in male diabetic patients. Its prevalence and risk factors are not well characterized. The purpose of this study was to investigate a large number of diabetic men in Italy. Men aged 20-69 years with type 1 or type 2 diabetes mellitus, 1-30 years duration, attending follow-up visits on randomly selected days in 178 diabetes centers of the North, Center and South of Italy, were recruited for the study during the period of May-September 1996. A total of 10.157 entered the study. The average number of men at each center was 57 . All subjects were administered a confidential questionnaire by a maximum of two weil trained diabetologists per center. The questionnaire consisted of two parts. In the first anagraphical and clinical data (age, weight, height, marital status, cigarette smoking, alcohol consumption, diabetic treatment, diabetes related complications) were recorded. Patients were also asked about their ability to achieve and maintain an erection sufficient for satisfactory sexual performance. Only if they answered that they were unsatisfied, they were defined to have ED and the interview was completed with the second part of questionnaire in order to define its severity. Incomplete or complete ED was defined when respectively some or all sexual performances were unsatisfactory. Among 10.157 collected questionnaire, 9868 were considered valid. $3534(35,8 \%)$ subjects reported ED $(2483,70,3 \%$, incomplete; $1038,29,4$, complete). The prevalence increased with age $(4,6 \%$ in men aged $20-29 ; 45,5 \%$ in men aged more than 60 years; test for trend, $\mathrm{p}=0,0001$ ). After correction for age, subjects with type 2 diabetes reported less frequently ED than those with type 1 (Odds Ratio,OR, $0,7 \%$, CI $0,6-0,8$ ). ORs for ED were respectively 1,3 and 2,0 for men with diabetes lasting 6-10 and $11-30$ years in comparison with men with diabetes lasting $<5$ years. ORs of ED were respectively 1,7 and 2,3 in men with fair (HbAlc 7,5-9\%) and poor (HbAlc $>9 \%$ ) metabolic control, in comparison with men with good metabolic control ( $\mathrm{HbAlc}<7,5 \%$ ). A history of diabetes related complications (arterial, retinal or renal diseases, and neuropathy) was associated with an increased risk of ED, as well other associated diseases (hypertension, hyperlipidemia, heart disease). Finally in comparison with non smokers, the OR of ED for curtent smokers was $1,5(95 \%$ CI 1,3-1,6) and 1,4 for ex-smokers (CI 1,3-1,6). In conclusion, this large study indicates that even in Italy, the prevalence of ED is very high and that it is well correlated with age, duration of diabetes, metabolic control, the presence of other diabetic complications and smoking habit.

\section{3}

SILDENAFIL (VIAGRA ${ }^{\text {TM}}$ ) IMPROVES INTERCOURSE SUCCESS IN PATIENTS WITH ERECTILE DYSFUNCTION AND DIABETES. M.S. Rendell on behalf of the Sildenafil Study Group, Creighton Diabetes Center, Omaha, Nebraska, USA.

Sildenafil is an orally active and selective inhibitor of cGMP-specific phosphodiesterase 5 in the corpus cavernosum. The effect of sildenafil on intercourse success rates was assessed in a 12-week, double-blind, placebo-controlled, dose-escalation study of 268 men ( $n=136$ sildenafil, $\mathrm{n}=132$ placebo) with erectile dysfunction (ED) and diabetes mellitus. The $50-\mathrm{mg}$ starting dose could be adjusted to $100 \mathrm{mg}$ or $25 \mathrm{mg}$ based on efficacy and tolerability. Subjects completed an event $\log$ of erectile activity each time they engaged in sexual activity, recording whether intercourse was successful. The mean age of the subjects was 57 years (range 27 to $79 \mathrm{yr}$ ), with a mean duration of ED of 5.6 years (range 0.6 to $24 \mathrm{yr}$ ), and mean a duration of diabetes of 12.1 years (range 1.8 to $50.5 \mathrm{yr})$. The majority of men had type II diabetes $(81.3 \%)$. At week 12 , the proportion of men with at least one successful attempt at sexual intercourse was $61 \%$ for the sildenafil group, which was approximately three times greater than that for the placebo group (22\%). For men who had at least one successful intercourse, the proportion of attempts at intercourse that were successful was $74 \%$ for sildenafil versus $51 \%$ for placebo. Sildenafil was well tolerated, with no subject discontinuing treatment due to an adverse event. In conclusion, treatment with sildenafil results in improved intercourse success rates in patients with $\mathrm{ED}$ and diabetes. 


\section{4}

Erythropoietin Responsive Anaemia in Diabetic Autonomic Neuropathy with and without Nephropathy

Watkins PJ Winkler AS, Marsden J and Chaudhuri KR King's Diabetes Centre, King's College Hospital, London SES 9RS, UK

Erythropoietin (EPO) production by the kidney is stimulated chiefly by anaemia and hypoxia and is modulated by the sympathetic nervous system, and in animal models sympathectomy leads to reduced EPO levels. We have examined a group of type I (IDDM) diabetic patients with established symptomatic diabetic autonomic neuropathy (DAN) to determine whether there is evidence of anaemia due to EPO depletion. The study comprised 19 IDDM patients, (mean age $37.5 \pm 9.7$ years (SD) duration of diabetes $21.7 \pm 9.6$ years) all of whom had postural hypotension (systolic BP fall on standing $>30 \mathrm{~mm} \mathrm{Hg}$ ); 17 had diabetic diarthoea, 4 severe gastroparesis, 4 bladder paresis and 8 gustatory sweating. The mean heart rate variation on deep breathing was severely reduced $(3.8 \pm 2.5$ (SD), normal $>12$ beats $/ \mathrm{min}$ ). Mean serum creatinine was $96.1 \mu \mathrm{mol} / /$; none exceeded $120 \mu \mathrm{mol} /$. The DAN patients were anaemic $(\mathrm{Hb} 11.1 \pm 1.6 \mathrm{~g} / \mathrm{dl}$, range $8.1-14.6 \mathrm{~g} / \mathrm{dl})$ compared to matched non neuropathic IDDM controls ( $\mathrm{Hb} 13.8 \pm 0.9 \mathrm{~g} / \mathrm{dl} ; \mathrm{p}<0.001)$. Serum EPO concentration in two groups of anaemic DAN patients (mean $\mathrm{Hb} 9.1 \pm 0.7 \mathrm{~g} / \mathrm{dl}$ and $10.9 \pm 0.6 \mathrm{~g} / \mathrm{dl}$, EPO $11.2 \pm 8.4 \mathrm{mU} / \mathrm{ml}$ and $11.7 \pm 4.3 \mathrm{mU} / \mathrm{ml}$, respectively) was compared to comparably anaemic non-diabetic iron deficient patients (mean $\mathrm{Hb} 8.4 \pm 0.3 \mathrm{~g} / \mathrm{dl}$ and $10.6 \pm 0.7 \mathrm{~g} / \mathrm{dl}$, EPO $96.8 \pm 32.6 \mathrm{mU} / \mathrm{ml}$ and $38.8 \pm 8.3 \mathrm{mU} / \mathrm{ml}$, respectively; $\mathrm{p}<0.00$ compared to DAN patients). EPO in non-anaemic DAN patients and healthy controls was not significantly different $(17.9 \pm 12.2 \mathrm{mU} / \mathrm{ml}$ and $9.6 \pm 10.2 \mathrm{mU} / \mathrm{ml}$, respectively)

Treatment of four anaemic DAN patients (mean $\mathrm{Hb} 10.4$, range $9.9-11.6 \mathrm{~g} / \mathrm{dl}$ ) with EPO (25 IU/kg BW subcutaneously thrice weekly for three months) increased the $\mathrm{Hb}$ in every case to a mean of 13.4 (range $12.4-14 \mathrm{~g} / \mathrm{dl}$ ), with a corresponding fall three months after treatment was withdrawn. EPO responsive normochromic anaemia occurs in some IDDM patients with symptomatic autonomic neuropathy. Neither EPO depletion nor the presence of anaemia were related to the presence of proteinuria, microalbuminuria, or serum creatinine level

\section{PS 62}

\section{Endothelium and Vasomotion}

\section{5}

\section{VASCULAR REACTIVITY IN NON-INSULIN DEPENDENT DIABETIC SUBJECTS: A MEASURE OF ENDOTHELIAL DYSFUNCTION?}

\section{S.E. BALDEWEG, S.W. COPPACK, J.S. YUDKIN, DEPARTMENT OF MEDICINE, UNIVERSITY COLLEGE LONDON, UK}

Insulin induced vasodilation is dependent on endothelially generated nitric oxide (NO). Vascular response to acetylcholine (ACh), but not sodium nitroprusside (SNP), is also dependent on endothelial NO synthesis Vasodilation responses are diminished in insulin resistant states, such as NIDDM, perhaps thereby contributing to the insulin resistance itself. This could reflect endothelial dysfunction or could also result from impaired responsiveness to NO. We examined the relationship between vascular reactivity to insulin, $A C h, S N P$ and insulin sensitivity in 10 subjects with uncomplicated NIDDM (7M:3F, age $58.5+19.5 y r s$, BMI $27.6 \pm 6.9 \mathrm{~kg} / \mathrm{m}^{2}$ ). We used plethysmography to measure forearm vasodilator response to intraarterially administered ACh $(0.15,0.45,1.5,4.5$ and $15 \mu \mathrm{g} / \mathrm{dl} / \mathrm{min}, 5 \mathrm{~min}$ per dose) and SNP ( 1,2 and $4 \mu \mathrm{g} / \mathrm{dl} / \mathrm{min}$ ) as well as insulin-induced vasodilation during a euglycaemic hyperinsulinaemic clamp (insulin at $40 \mathrm{mU} / \mathrm{m}^{2} / \mathrm{min}$, plasma glucose at $6 \mathrm{mmol} / \mathrm{l}$ ) which was also used to evaluate insulin sensitivity All subjects had oral hypoglycaemic medication withdrawn for at least three weeks prior to study. We found no relation of vascular response to either $\mathrm{ACh}$ $(r=-0.23, p=0.73$ at $15 \mu \mathrm{g} / \mathrm{dl} / \mathrm{min})$ or insulin $(r=0.23, \mathrm{p}=0.59$ at $120 \mathrm{~min})$ with insulin sensitivity. We also found no relation between response to SNP and insulin sensitivity $(\mathrm{r}=0.26, \mathrm{p}=0.46$ at $4 \mu \mathrm{g} / \mathrm{dl} / \mathrm{min})$. However, we found a strong relation between vascular response to SNP and BMI $(r=-0.69, p=0.006$ at $2 \mu \mathrm{g} / \mathrm{dl} / \mathrm{min}$ and $r=0.68, p=0.016$ at $4 \mu \mathrm{g} / \mathrm{d} / / \mathrm{min}$ ). There was a significant relationship between $\mathrm{BMI}$ and insulin sensitivity $(r=-0.68 \mathrm{p}=0.032$ at $120 \mathrm{~min})$ but no relationship between $\mathrm{BMT}$ and response to either $\mathrm{ACh}(\mathrm{r}=-0.02, \mathrm{p}=0.93)$ or insulin $(r=-0.29, p=0.30$ ). In our cohort no association was detected between endothelial dysfunction and insulin resistance. Our results may reflect an impaired response to exogenous NO in NIDDM by an alternative mechanism.

\section{6}

ACUTE HYPERGL YCEMIA IMPAIRS RESISTENCE VESSEL FUNCTION IN INSULIN TREATED DIABETICS M. Francesconi* C. Koizar* and T.C. Wascher\#, ${ }^{*}$ Rehabcenter Alland, \#University Clinic Graz, Austria

Impaired postischemic reactive hyperemia, as found in patients with hypertension or hypercholesterolemia, is considered as a marker of impaired resistance vessel function. Acute postprandial hyperlipidemia has been shown to result in vascular dysfunction even in healthy subjects. The impact of postprandial hyperglycemia on resistance vessel reactivity, on the other hand, remains to be established. Aim of the present study was to investigate whether postprandial hyperglycemia influences reactive hyperemia $(\mathrm{RH})$ in insulin treated diabetics. The study was performed in 10 diabetics on insulin therapy after obtaining informed consent ( $5 \mathrm{male} / 5$ female, age $47 \pm 4$ years, HbA1c 7.8 \pm 0.4 ) and 6 age matched healthy controls. $R H$ was measured in the forearm by venous occlusion plethysmography after 5 minutes of ischemia. The measurement was performed in the fasting state and 90 minutes after ingestion of a test meal (cont. $50 \mathrm{~g}$ carbohydrates) without insulin substitution. In diabetics blood glucose increased from $172 \pm 21 \mathrm{mg} / \mathrm{dl}$ to $298 \pm$ $29 \mathrm{mg} / \mathrm{dl} \quad(0<0.001)$ postprandially. This resulted in (1) a significant increase of resting blood flow $(3.38 \pm 0.31$ to $4.48 \pm 0.35$ $\mathrm{ml} / \mathrm{min} / 100 \mathrm{ml}, \mathrm{p}<0.017$ ) and (II) in a reduced peak postischemic flow $(56.62 \pm 7.54$ to $39.58 \pm 4.70 \mathrm{ml} / \mathrm{min} / 100 \mathrm{ml}, p<0.017)$. In healthy controls, a similar effect of the meal on resting blood flow was observed but postischemic flow was unaltered in the postprandial state.

In conclusion, our data provide evidence that acute postprandial hyperglycemia is able to impair resistance vessel function in insulin treated diabetics. 
FOREARM BLOOD FLOW IN TYPE 1 DLABETICS DURING SYSTEMIC HYPERGLYCAEMIA

A.V. Macklin, G.C. Viberti. and 'J. Ritter Unit for Metabolic Medicine, Guy's Hospital, London, UK. 'Department of Clinical Pharmacology, St Thomas' Hospital, London.

An increase in forearm (muscle) blood flow (FBF) is observed in patients with Type 1 diabetes and poor glycaemic control, and is reduced after weeks of improved control by intensified insulin therapy. Acute systemic hyperglycaemia results in an increase in GFR, heart rate, blood pressure \& retinal blood flow within 1 hour. Conversely, local hyperglycaemia for up to 24 hours does not alter FBF in non diabetic subjects. There is no data on the effect of acute systemic hyperglycaemia on FBF in subjects with Type 1 diabetes.

Aim: To investigate the effect of systemic hyperglycaemia, in the absence of hypoinsulinaemia, on forearm blood flow.

Methods: 5 Subjects with Type 1 diabetes (4M/1F) age $30 \pm 4.9$ (mean $\pm S D$ ), without evidence of retinopathy or clinical evidence of macrovascular disease were studied. They had albumin:creatinine ratios <1.2, serum total cholesterol $<4.6 \mathrm{mmol} / \mathrm{l}$, blood pressure $<140 / 90 \mathrm{mmHg}$ and were taking no vasoactive drugs. Median duration of diabetes was 6 years (range 2-10). HbAlc was $7.7 \pm 1.1 \%$ (mean $\pm S D$ ) .

A hyperinsulinaemic glucose clamp was performed using an infusion of $0.5 \mathrm{mU} / \mathrm{kg} / \mathrm{min}$ of insulin \& FBF was measured using strain gauge plethysmography. Systemic glucose was clamped at either 5 (NG) or $12 \mathrm{mM}$ (HG) and FBF measured by strain gauge plethysmography during the last 30 minutes of steady state hyperglycaemia. The order of euglycaemia \& hyperglycaemia was randomly allocated for each patient.

Insulin levels were $348.4 \pm 274.2 \mathrm{pmol} / \mathrm{l}$. There was no change in FBF during acute systemic hyperglycaemia (NG $1.43 \pm 0.68 \mathrm{ml} / 100 \mathrm{ml}$ muscle/min vs HG $1.42 \pm 0.55 \mathrm{ml} / 100 \mathrm{ml}$ muscle $/ \mathrm{min} \mathrm{p}=0.92$ ).

Acute systemic hyperglycaemia, in the absence of hypoinsulinaemia does not alter resistance vessel function as measured by FBF. This suggests that the mediator for the increased FBF seen in poorly controlled Type I diabetics is not hyperglycaemia per se.

\section{9}

INFLUENCE OF METABOLIC CONTROL AND DURATION OF DISEASE ON MICROVASCULAR DYSFINCTION IN DIABETES ASSESSED BY LASER DOPPLER ANEMOMETRY M.F. Meyer and H. Schatz. University Hospital Bergmannsheil, Department of Internal Medicine, Bürkle-de-la-Camp-Platz 1, D-44789 Bochum

Postocclusive reactive hyperaemia is reduced and delayed in type 1 and type 2 diabetes as shown by videophotometric capillaroscopy and laser Doppler fluxmetry. The aim of this study was to examine by means of the new technique of laser Doppler anemometry whether type 1 and type 2 diabetic patients differ in the relation of impaired skin microcirculation to metabolic control and duration of diabetes. 16 patients with type 1 diabetes and 19 patients with type 2 diabetes were investigated and subdivided in patients with good" ( $\mathrm{HbAlc}<7.5 \%)$ and ,bad" ( $\mathrm{HbAl}>7.5 \%$ ) metabolic control and in patients with a duration of diabetes of more than 10 or less than 10 years. Two age- and sex-matched control groups comprising 16 and 19 non-diabetic subjects served as controls. The capillary blood cell velocity (CBV) was measured in the dorsal middle phalangeal area of the fourth finger of the left hand by laser Doppler anemometry. CBV in single capillaries was studied during rest and after 3-min arterial occlusion. Resting CBV was similar in diabetic patients and control subjects. Peak CBV was reduced in type 1 diabetic patients $(0.69 \pm 0.08 \mathrm{~mm} / \mathrm{s}$ vs. $0.96 \pm 0.07 \mathrm{~mm} / \mathrm{s}, \mathrm{p}<0.05)$ independent of diabetes duration and metabolic control, whereas peak CBV in type 2 diabetes was decreased only in patients with bad metabolic control $(0.54 \pm 0.04 \mathrm{~mm} / \mathrm{s}$ vs. $0.70 \pm 0.04 \mathrm{~mm} / \mathrm{s}, \mathrm{p}<0.05)$. Time to peak CBV was markedly prolonged both in type $1(33.8 \pm 4.8 \mathrm{~s}$ vs. $13.6 \pm 1.9 \mathrm{~s}$, $\mathrm{p}<0.001)$ and type 2 diabetic patients $(46.8 \pm 8.5 \mathrm{~s}$ vs. $16.4 \pm 2.2 \mathrm{~s}, \mathrm{p}<0.001)$. With regard to metabolic control time to peak CBV was prolonged in type 2 diabetes only in the group of patients with $\mathrm{HbAlc}>7.5 \%(56.6 \pm 14.8 \mathrm{~s}$ vs. $13.7 \pm 2.7 \mathrm{~s}, \mathrm{p}<0.01)$. Disease duration had no significant influence on time to peak in type 1 and 2 diabetes. The results indicate that in type 2 diabetes actual metabolic control is of a greater importance for the microvascular dysfunction than in type $\mathbf{i}$ diabetes and that there is no evident relation to the duration of disease.
EPTECES OF ACUTE AND CHRONTC FYPEAGLYCAEMIA ON CORONARY REACTIVITY B. Rocsis, I. Pósa, G. Pogátsa, K.Z. Roltai. György Gottsegen National Institute of Cardiology, Budapest, Hungary

Coronary vascular reactions were studied in normal (NG; $5 \mathrm{mnol} / \mathrm{l}$ ) and in high glucose concentration (HG; 25 mol/1) media, as vell as in diabetic (0) vessels, in vitro. Possible involvement of the cyclooxygenase pathway in the alterations induced by hyperglycaenia was investigated. Left anterior descending (CAD) coronary arteries of 15 metabolically healthy and 5 alloxandiabetic $\{560 \mathrm{~mol} / \mathrm{kg}$ alloxan tetrahydrate, $i, v$, Sigma $\}$ young mongrel dogs of both sexes, weighing $19-24 \mathrm{~kg}$ were dissected under pentobarbital anaesthesia (133 $\mu \mathrm{mol} / \mathrm{kg}$ Nembutal,i.v., Sanofi), freed from fat and connective tissues and cut into 3-4 ma rings. Vascular rings were suspended in organ chambers containing $5 \mathrm{ml}$ of $\mathbb{N G}(\mathrm{n}=10)$, IG $(n=5)$ or hyperosmotic (HO; $20 \mathrm{mmol} / \mathrm{l}$ mannitol, $n=5$ ) krebs buffer (pH $7.4,37^{\circ} \mathrm{C}$ ) constantly bubbled with a nixture of 958 , and $58 \mathrm{CO}_{2}$. Changes in isonetric tension were registered by a microdynamoreter ( $\mathrm{F}-50$, Hugo Sachs). Relaxation elicited by acetylcholine (Ach 3 naol/1-10 $\mu \mathrm{mol} / 1$ added in a cunulative manner) - compared to the ef fect of 1 mol/l sodium nitroprusside as 1008 - was studied in LAD rings precontracted with $P G P$. Indothelium dependent relaxation was impaired $(p<0.05)$ in $1 G$ as well as in D coronaries compared to controls, characterized

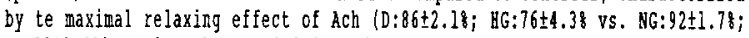

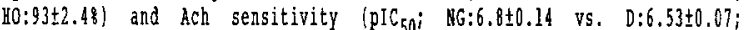
HG:6.59\$0.17; 10:6.46+0.16). Ach sensitivity was decreased (p<0.05) by indomethacin ([NDO;3 $\mathrm{mmol} / \mathrm{l}$ ) in all investigated groups, but the maximal relaxing effect of Ach vas further impaired ( $p(0.05)$ after INDO incubation in the diabetic group only $(0: 75+58)$. These results suggest that both acute and chronic hyperglycaemia may result in impaired endotheliub dependent relaxation in coronary arteries. Impaired relaxation of diabetic coronaries is worsened by the inhibition of the synthesis of vasodilator prostanoids.

\section{0}

MICROVASCULAR FUNCTION AND THE ROLE OF POSTPRANDIAL GLUCOSE EXCURSION.

C. Le Dévéhat, T, Khodabandehlou and M. Vimeux - Unité de Recherches d'Hémorhéologie Clinique, Service de Diabétologie-EndocrinologieNutrition, Centre Hospitalier 58000 NEVERS (France)

Aim: To evaluate direct and immediate microcirculatory consequences of an hyperglycemic spike in 10 insulin dependent diabetic patients (IDDM) and 5 healthy controls, matched for sex, age and body mass index.

Material and methods : The hyperglycemia was induced at a level of 15.6 $\mathrm{mmol} / \mathrm{L}$ for 1 hour using glucose infusion via the GCIS Biostator. Microcirculatory cutaneous blood flow and vasomotion were determined in dorsal big toe at skin temperature by a laser Doppler (LD) fluxmeter (PF4 Perimed). The transcutaneous oxygen tension (TcPO2) was recorded in parallel in dorsal foot heated to $44^{\circ} \mathrm{C}$ by an Oxymonitor (SM 361 Hellige).

Results : Hyperglycemia induced in IDDM's significant increases in LD blood flux and vasomotion $(21.7 \pm 13 \mathrm{PU}$ and $4.8 \pm 0.7 \mathrm{cycles} / \mathrm{min}$ during hyperglycemia vs $12.9 \pm 7.5 \mathrm{PU}$ and $4.2 \pm 1.3$ cycles/min during normoglycemia) which persisted despite of the glycemia normalization. The increase in LD flux was due to that in the concentration of moving blood cells ( $25.4 \pm 14.3 \mathrm{CU}$ during hyperglycemia vs $17.3 \pm 8 \mathrm{CU}$ during normoglycemia) while the velocity of moving blood cells remained unchanged indicating a stasis phenomenon. No significant change was observed in the controls. There was in IDDM's a significant decrease in TcPO2 $(51 \pm 10 \mathrm{mmHg}$ during hyperglycemia vs $54 \pm 9 \mathrm{mmHg}$ during normoglycemia) whilst it increased as a consequence of the hyperglycemic spike in the controls $(66 \pm 9 \mathrm{mmHg}$ during hyperglycemia vs $63 \pm 7.4$ mmHg during normoglycemia).

Conclusion : Hyperglycemic spike at a level frequently observed in IDDM's in the post prandial state induces functional microcirculatory abnormalities such as an hyperperfusion most likely through the nonnutritive microvascular compartments and altered tissue oxygenation. 


\section{1}

IN VIVO NO-SYNTHESIS DEPENDENT ENDOTHELIAL DYSFUNCTION CHARACTERIZES PATIENTS WITH NIDDM S. Vehkavaara, S. Mäkimattila, R. Bergholm, M.-R. Taskinen and $H$. Yki-Järvinen, University of Helsinki, Helsinki, Finland

In vivo vasodilatory responses to intra-arterial infusions of endotheliumdependent (acetylcholine, ACh), NO-dependent (ACh plus L-NMMA, an inhibitor of NO synthesis) and -independent (sodium nitroprusside, SNP) vasoactive agents were determined in a large group of male patients with uncomplicated NIDDM $\left(\mathrm{n}=49\right.$, age $53 \pm 1 \mathrm{yrs}$, BMI $28 \pm 1 \mathrm{~kg} / \mathrm{m}^{2}$, duration $5 \pm 1$ yrs, $\mathrm{HbA}_{\mathrm{ic}} 7.9 \pm 0.2 \%$ ) and 13 matched normal males. The blood flow response to $\mathrm{ACh}$, normalized to the response to SNP was $30 \%$ lower in patients with NIDDM $(0.698 \pm 0.037)$ than in normal subjects $(1.025 \pm 0.18$, $p<0.01)$. Blood flows during combined infusions of ACh and L-NMMA were comparable $(4.9 \pm 0.4 \mathrm{vs} 4.7 \pm 0.8 \mathrm{ml} / \mathrm{dl} \cdot \mathrm{min})$ demonstrating that the impaired response to $\mathrm{ACh}$ was NO-synthesis dependent. NIDDM patients had $88 \%$ increased VLDL-triglycerides $(\mathrm{p}<0.005), 68 \%$ increased fasting insulin levels $(\mathrm{p}<0.02)$ and $15 \%(\mathrm{p}<0.05)$ lower LDL cholesterol than normal subjects. In simple (Spearman) regression analysis within NIDDM patients, endothelial function was not related to age, BMI, blood pressure, $\mathrm{HbA}_{1 \mathrm{c}}$, LDL-cholesterol, VLDL-triglycerides, smoking, blood pressure, albumin excretion or levels of lipid-soluble antioxidants. We conclude that NIDDM patients are characterized by a defect in the NO-synthesis dependent component of endothelium-dependent vasodilatation. This early alteration in vascular function is identical to that known to correlate with LDL-cholesterol in nondiabetic subjects and with $\mathrm{HbA}_{1 \mathrm{c}}$, in IDDM. In NIDDM, however, this defect, thought to precede atherosclerotic vascular disease, cannot be attributed to classic cardiovascular risk factors.

1203

PLASMA NITRITES ARE INCREASED IN WELL METABOLICALLY CONTROLLED TYPE 1 DIABETIC PATIENTS.

B.Wierusz-Wysocka, D.Zozulińska M.Kempa, M.Skowroński and A.Murawska. Poznań Diabetic Center, Poznań, Poland.

Nitric oxide (NO) is a short-lived messenger molecule serving a wide variety of functions in different tissues and is metabolized to $\mathrm{NO}_{2}{ }^{-}$and $\mathrm{NO}_{3}{ }^{-}$in humans. It plays an important role in pathogenesis of type 1 diabetes and it's late complications. The aim of the study was to estimate plasma nitrate anion $\left(\mathrm{NO}_{2}{ }^{-}\right)$concentration in type 1 diabetic patients. The study was performed in 30 well metabolically controlled patients (18 female and 12 male, aged $30.2 \pm 10.6$ years, duration of diabetes $8.4 \pm 6.8$ years, $\mathrm{HbA} 1 \mathrm{c} 6.5 \pm 1.2 \%$ ) and 20 poorly metabolically controlled patients ( 12 female and 8 male, aged $29.8 \pm 9.8$ years, duration of diabetes $8.0 \pm 4.8$ years, $\mathrm{HbAlc}$ $11.2 \pm 1.6 \%$ ). $\mathrm{NO}_{2}^{-}$level was measured with the use of a calorimetric micromethod, where nitrate reductase catalyses the conversion of $\mathrm{NO}_{3}^{-}$to $\mathrm{NO}_{2}^{-}$. The $\mathrm{NO}_{2}^{-}$plasma concentration was significantly higher in well matabolically controlled type 1 diabetic patients in comparison with poorly controlled diabetic patients and healthy subjects $(41.99+4.02$ vs $33.33+2.44$ and $16.68 \pm 2.16 \mu \mathrm{mol} / 1$, respectively, $p<0.05, p<0.0001)$. The nitrite concentrations did not correlate with HbAlc $(r=-0.03, \mathrm{p}>0.05)$. The results support the concept that metabolism of nitrite oxides in diabetes is disturbed independently from the degree of metabolic control.
NO EFFECT OF HYPERGLYCEMIA ON EXCRETION OF NITRIC OXIDE METABOLITES IN HEALTHY MEN AND IDDM PATIENTS - THE ROLE OF VOLUME LOAD

J.Kř̉žová, T.Pelikánová and L.Kazdová. Institute for Clinical and Experimental Medicine, Prague, Czech Republic

Local production of nitric oxide $(\mathrm{NO})$ in the kidney affects renal hemodynamics and sodium metabolism. Last year we found that IDDM patients have a lower response to furosemide administration in terms of elimination of $\mathrm{NO}_{2}^{-} \mathrm{NO}_{3}^{-}\left(\mathrm{NO}_{\mathrm{x}}\right)$ metabolites and show a lower response to hyperglycemia compared to a control group. The aim of the study was to determine whether a role in the pathological response of diabetic patients is not played by differences in the volume load during tests. We measured renal hemodynamics, sodium and $\mathrm{NO}_{\mathrm{x}}$ excretion in two 90-minute periods under a) euglycemic (period I, $5 \mathrm{mmol} / \mathrm{L}$ ) hyperglycemic (period II, $12 \mathrm{mmol} / \mathrm{L}$ ) clamp in a group of 19 IDDM (EHDM) patients without miroalbuminuria, b) euglycemichyperglycemic clamp in 12 healthy controls (EHC) and c) euglycemic-euglycemic conditions in controls (EEC) with a comparable water load. We noted $\mathrm{NO}_{\mathrm{x}}$ excretion in EHDM did not differ between periods I and II ( $352 \pm 56 \mathrm{vs} 323 \pm 39 \mathrm{nmol} / \mathrm{min})$. By contrast, $\mathrm{NO}_{x}$ excretion significantly rose in EHC in period II ( $425 \pm 198$ vs $559 \pm 195$ $\mathrm{nmol} / \mathrm{min} ; \mathrm{p}<0.05)$. However, the rise in $\mathrm{NO}_{\mathrm{x}}$ excretion is comparable in $\mathrm{EHC}$ and EEC $(+32 \%$ vs $+47 \%)$. The glomerular filtration rate, assessed by inulin clearance, and effective renal plasma flow, assessed by clearance of para-amino-hippuric acid, were comparable in IDDM patients and controls and did not vary within periods I and II of all examinations. Sodium excretion fraction $\left(\mathrm{EF}_{\mathrm{Na}}\right)$ declined in period II comparably in EHDM and EHC (EHDM: $1.84 \pm 0.73$ vs $1.67 \pm 0.59 \% ; p<0.05$; EHC $2.6 \pm 0.7$ vs $2.1 \pm 0.4 \% ; p<0.05)$; by contrast, $\mathrm{EF}_{\mathrm{Na}} \mathrm{did}$ not change during EEC $(2.72 \pm 1.2$ vs $2.77 \pm 1.13 \%)$. We conclude hyperglycemia leads to increased sodium reabsorption by a mechanism not mediated by changes in the renal production of NO. The increase in $\mathrm{NO}_{\mathrm{x}}$ metabolites in urine during hyperglycemia is not dependent on blood glucose levels but is related to the volume load which probably also contributes to the changes in NO production regulation we observed in IDDM patients. (Supported by grant No. 4242-3, IGA MZ C $R$ )

\section{4}

DETERMINANTS OF POSTISCHEMIC HYPEREMIA IN PATIENTS WITH DIABETES MELLITUS

C. Koizar*, M. Francesconi* and T.C. Wascher\#, "Rehabcenter Alland, \#University Clinic Graz, Austria

Dysfunction of resistance arteries is thougth to be an early reversible stage in the developement of arteriosclerosis. The influence of metabolic parameters on reactive hyperemia $(\mathrm{RH})$, a useful tool for monitoring resistance vessel function, remains to be established.

Therfore we measured $\mathrm{RH}$ (forearm) by venous-occlusion plethysmography in 100 diabetics (D) and 60 normal controls (C). Statistical comparison was performed using multiple regression analysis.

Over all, no significant differences between $D$ and $C$ where observed by group comparison. In $C$ only gender was significantly related to peak hyperemia $(p<0.011)$ and no other variable contributed to the variation observed. In $D$ on the other hand in addition to their gender $(p<0.007)$ also actual glycemia $(p<0.029)$, duration of diabetes $(p<0.025)$, fasting state $(p<0.028)$, and a history of hypertension $(p<0.030)$ showed significant correlation to peak RH. Further investigation revealed a loss of the correlation between peak reactive hyperemia and actual blood glucose observed in the fasting state $(p<0.001)$ in nonfasting $D$, indicating postprandial alterations of resistence vessel reactivity.

In conclusion, our results suggest, that in $D$, in addition to gender diabetes related variables with a focus on postprandial alterations influence postischemic reactivity of resistence vessels and might contribute to vascular dysfunction in diabetes. 
FUNCTIONAL MICROVASCULAR DYSFUNCTION IN TYPE 1 DIABETIC PATIENTS.

T. KHODABANDEHLOU, C. LE DEVEHAT and M. VIMEUX - Unité de Recherches d'Hémorhéologie Clinique, Service de DiabétologieEndocrinologie-Nutrition, Centre Hospitalier 58000 Nevers (France)

Aim: In a previous study, the Veno-Arteriolar Reflex (VAR) of skin microcirculation was found impaired in diabetics without microangiopathy (Clin Hemorheol and Microcirc 17, 1997, 357-362). In the present investigation, we studied whether such early functional abnormality is related to diabetes duration (DD) and/or the glycated hemoglobin level ( $\mathrm{HbAIC}$ ) in Type 1 diabetics patients. Patients and methods : Patients were 41 Type 1 diabetics without clinical evidence of microvascular complications. They were divided into 3 sub-groups once according to the DD $(<5$ yrs 11 patients ; from 5 yrs to 15 yrs 15 patients; $>15$ yrs 15 patients), and then according to the $\mathrm{HbA} 1 \mathrm{C}$ level $(<7.5 \% 12$ patients ; from $7.5 \%$ to $9.5 \% 17$ patients ; $>9.5 \% 12$ patients). The VAR in dorsal foot was assessed by measuring the laser Doppler blood flux changes (via the Perimed PF4 fluxmeter) during a passive lowering of the foot to $50 \mathrm{~cm}$ below the heart.

Results and discussion : The VAR decreased progressively with increasing DD : $49 \pm 18 \%$ controls $; 42 \pm 10 \%$ diabetics with DD $<5$ Yrs $; 30.5 \pm 18 \%$ diabetics with $5<\mathrm{DD}<15$ Yrs ; $27.6 \pm 24 \%$ diabetics with $\mathrm{DD}>15$ Yrs. The decrease was statistically significant after a mean DD of $11 \pm 3$ yrs (extreme values : $5-15$ yrs) in comparison to either the controls or the patients with DD less than 5 years. This finding supports the hypothesis that a sympathetic nervous dysfunction of skin microvasculature is virtually inevitable after a mean duration as long as 11 Yrs of Type 1 diabetes. The three diabetic groups divided according to the $\mathrm{HbAlC}$ level showed significantly lower value of the VAR than that achieved in the controls ( $49 \pm 18 \%$ controls; $32 \pm 22 \%$ diabetics with $\mathrm{HbAlC}<7.5 \% ; 35 \pm 18 \%$ diabetics with $7.5 \%<\mathrm{HbA} 1 \mathrm{C}<9.5 \% ; 30 \pm 19 \%$ diabetics with $\mathrm{HbA} 1 \mathrm{C}>9.5 \%$ ). In a multivariate analysis taking age, $\mathrm{DD}$ and $\mathrm{HbAIC}$ into account, the VAR correlated significantly and negatively with DD $(p<0.03)$ while it does show no dependency on the HbAlC.

Conclusion : The failure of the VAR may be predicted by the duration of diabetes. The demonstration that the VAR is similarly impaired in the three diabetic groups irrespective of the $\mathrm{HbAlC}$ level does not exclude the possible effect on the VAR of either the level of the circulating glucose or any variation in its concentration.
PRESERVED ENDOTHELIUM-DEPENDENT VASODILATION IN SUBJECTS WITH UNCOMPLICATED TYPE 2 DIABETES.

J.R.Cockcroft, D. J.White, S.R.Page and A.G.Gazis. Departments of Therapeutics and Diabetes, Endocrinology and Nutrition, University Hospital, Nottingham, UK

This study assessed vascular endothelial function in subjects with Type 2 diabetes. Type 2 diabetes increases the risk of cardiovascular discase. Abnormal vascular endothelial function, an early marker for atheroma, has been reported in subjects with Type 2 diabetes. Many of the subjects previously studied have had characteristics affecting endothelial function. We studied 21 patients $(14 \mathrm{~m})$ with uncomplicated Type 2 diabetes and 21 controls $(10 \mathrm{~m})$, none of whom had had a clinical cardiovascular event. Groups were normoalbuminuric ( $\mathrm{ACR} \mathrm{m}<2.5, \mathrm{f}<3.5 \mathrm{mgmmolcreat} \mathrm{i}^{-1}$ ) and matched (mean $\pm 95 \% \mathrm{CI}$ ) for age $(55.4 \pm 4.4$ vs $55.3 \pm 4.6 ; \mathrm{p}=0.97$ diabetes vs control), body mass index $\left(26.0 \pm 1.5\right.$ vs $\left.25.2 \pm 1.3 \mathrm{kgm}^{-2} ; \mathrm{p}=0.38\right)$, total cholesterol $\left(5.2 \pm 0.4\right.$ vs $5.3 \pm 0.7 \mathrm{mmoll}^{-1} ; \mathrm{p}^{-0.75}$ ) and diastolic blood pressure ( $78 \pm 3$ vs $75 \pm 4 \mathrm{mmHg}: \mathrm{p}=0.27) . \mathrm{Hb}_{\mathrm{Alc}}(7.0 \pm 1.5$ vs $4.8 \pm 0.6 \% ; \mathrm{p}<0.01)$ and systolic blood pressure $(145 \pm 14$ vs $129 \pm 15 \mathrm{mmHg}: \mathrm{p}<0.01)$ were higher in the diabetes group. Selection of these subjects minimises the effect of confounding influences on endothelial function, allowing closer examination of the effect of diabetes alone on vascular endothelial dysfunction. Using intra-arterial drug infusion with forearm venous occlusion plethysmography, responses to sodium nitroprusside (an endothelium-independent dilator), acetylcholine and bradykinin (endothelium-dependent) were measured. Area under dose response curves for nitroprusside ( $63.4 \pm 10.9$ vs $50.7 \pm 9.5 ; \mathrm{p}=0.08$ ), acetylcholine $(39.4 \pm 11.8$ vs $31.6 \pm 14.8 ; \mathrm{p}=0.40)$ and bradykinin $(25.6 \pm 7.0 \mathrm{vs}$ 19.1 $\pm 5.7: p=0.14$ ) were not significantly different between diabetes and control groups. In subjects with uncomplicated Type 2 diabetes, endotheliumdependent and independent vasodilation is normal. Supported by the British Heart Foundation

\section{7}

ORAL VITAMIN E DOES NOT IMPROVE VASCULAR ENDOTHELIAL FUNCTION IN SUBJECTS WTTH TYPE 2 DIABETES

A.G.Gazis, D.J.White, S.R.Page and J.R. Cockcroft. Departments of Therapeutics and Diabetes, Endocrinology and Nutrition, University Hospital, Nottingham, UK

This was a randomised. double-blind, placebo-controlled trial of the effects of $1600 i u$ vitamin $E$ daily on vascular endothelial function, an early marker of atherosclerosis, in 48 patients with Type 2 diabetes. The risk of developing cardiovascular disease is increased in subjects with diabetes. In epidemiological and intervention studies in both animals and humans, increased vitamin $\mathrm{E}$ intake is associated with lower cardiovascular risk. All (48) subjects selected for study were normotensive $(<160 / 90 \mathrm{mmHg})$, normoalbuminuric ( $\mathrm{ACR} \mathrm{m}<2.5,<<3.5 \mathrm{mgmmolcreat}^{-1}$ ) and had normal cholesterol $\left(5.13 \pm 0.92 \mathrm{mmoll}^{-1}\right.$, mean $\left.\pm \mathrm{SD}\right)$. After 6 weeks' run-in, forearm venous occlusion plethysmography during intra-arterial infusion of endothelium-independent (nitroprusside) and dependent (acetylcholine, bradykinin) vasodilators was performed. This was repeated following 8 weeks' vitamin $E$ or placebo treatment. During the study, vitamin $E$ levels increased ( $28.2 \pm 6.2 \mu \mathrm{mmoll}^{-1}$ to $66.0 \pm 19.7$ vs $27.3 \pm 9.9$ to $26.1 \pm 8.3$ placebo) and $\mathrm{Hb}_{\mathrm{AlC}}$ was unchanged $(6.4 \pm 1.1 \%$ to $6.4 \pm 1.0$ vs $7.5 \pm 1.4$ to $7.7 \pm 1.6)$. Vitamin $E$ did not affect (ANOVA for repeated measures) area under dose response curves for nitropnusside (51.2 pre-treatment to 43.9 post vitamin $E$ vs 56.5 to 54.7 for placebo: $p=0.33$ ), acetylcholine ( 37.8 to 30.1 vs 26.1 to $25.8 ; \mathrm{p}=0.20$ ) or bradykinin ( 23.9 to 22.5 vs 22.3 to $24.3 ; \mathrm{p}=0.53$ ). 8 weeks' vitamin $\mathrm{E}$ treatment does not improve endothelium-dependent or independent vasodilation in subjects with normotensive, normoalbuminuric Type 2 diabetes. Dose, duration of treatment and target population need to be defined before vitamin $\mathrm{E}$ can be recommended to subjects with Type 2 diabetes. Supported by the British Heart Foundation.

\section{8}

NITRIC OXIDE SYNTHASE INHIBITION IN PATIENTS WITH EARLY IDDM

K. Schmetterer, G. Domer, P. Fasching, M. Wolzt and L. Schmetterer. Department of Clinical Pharmacology, Institute of Medical Physics, Department of Ophthalmology, Department of Internal Medicine III Division of Endocrinology, University of Vienna, Währinger Gürtel 18-20, A-1090 Vienna, Austria

There is evidence from previous animal and human studies that the Larginine/nitric oxide pathway is altered in patients with IDDM. We have previously shown that patients with long-standing IDDM have a reduced systemic and ocular reactivity to intravenous administration of L-NMMA, an inhibitor of nitric oxide synthase. The aim of the present study was to investigate whether this altered reactivity is also observed in IDDM patients with short diabetes duration. 8 patients with IDDM duration of less than eight years and 8 healthy controls were studied and received stepwise increased doses of L-NMMA $(1.5 \mathrm{mg} / \mathrm{kg}, 3 \mathrm{mg} / \mathrm{kg}, 6 \mathrm{mg} / \mathrm{kg})$. Mean arterial pressure was measured non-invasively. Renal plasma flow and pulsatile choroidal blood flow were assessed by PAH clearance technique and laser interferometric measurement of fundus pulsation amplitude, respectively. LNMMA dose-dependently decreased renal plasma flow and fundus pulsation amplitude in both study cohorts. However, no significant differences were observed between the two study groups. At $6 \mathrm{mg} / \mathrm{kg}$ L-NMMA renal plasma flow was decreased by $-22 \%$ in IDDM patients $(\mathrm{p}<0.001)$ and by $-23 \%$ in healthy controls $(\mathrm{p}<0.001)$. The same dose reduced fundus pulsation amplitude by $-24 \%$ in IDDM patients $(\mathrm{p}<0.001)$ and by - $28 \%(\mathrm{p}<0.001)$ in healthy subjects. These data suggest that changes in the vascular responsiveness to inhibition of the L-arginine/nitric oxide pathway in IDDM patients is a secondary functional phenomenon and not observed in patients without long-term diabetes. 
1209

C708/T POLYMORPHISM AT THE ANP GENE IS RELATED TO ANP PLASMA LEVELS AND ENDOTHELIAL DYSFUNCTION IN IDDM PATIENTS.

M. Nannipieri, L. Pucci, G. Penno, S. Bandinelli, A. Clerico, L. Rizzo and R. Navalesi. Dept. of Endocrinology and Metabolism, University of Pisa, Italy.

Recently, we described an association between a new polymorphism $(C 708 / T)$ at the ANP gene and microalbumunuria, but not with overt nephropathy, in IDDM patients. Now, we investigate the putative relationship between this polymorphism and both plasma ANP levels as well widespread microvascular permeability as assessed by transcapillary escape rate of albumin (TERalb). Plasma ANP levels (IRMA method) and TERalb (initial plasma disappearance of 125 I labelled human serum albumin) were measured in 35 normotensive IDDM patients: 19 homozygous for the wild allele (WW), 16 heterozygous (WM). The two groups were well matched for sex, age, BMI, diabetes duration and blood pressure. No differences were found for current fasting plasma glucose (220 \pm 24 vs $201 \pm 28 \mathrm{mg} / \mathrm{dl})$ and $\mathrm{HbAlc}(8.08 \pm 0.7$ vs $8.18 \pm 0.4 \%)$, previous two years mean HbAlc $(7.8 \pm 0.6$ vs $8.2 \pm 0.4 \%)$. ANP levels were higher in WW than in WM $(13.7 \pm 5.9$ vs $8.0 \pm 2.6 \mathrm{pg} / \mathrm{ml}, \mathrm{p}<0.0035)$. Consistently, TERalb was higher in WW than in WM patients $(7.9 \pm 2.2$ vs $5.9 \pm 1.7 \% / \mathrm{h}$, $\mathrm{p}=0.0075$ ). IDDM patients were stratified by AER levels in normoalbuminuric (NA n. 21: WW, 12; WM, 9) and microalbuminuric (mA n. 14: WW, 7; WM 7). A significant difference in TERalb between WW and WM genotypes persisted in NA and $\mathrm{mA}$ patients analysed separately (NA: $7.5 \pm 2.3$ vs $4.9 \pm 1.5 \% / \mathrm{h}, \mathrm{p}=0.013 ; \mathrm{mA}: 9.0 \pm 1.8$ vs $7.2 \pm 0.7 \% / \mathrm{h}, \mathrm{p}=0.049$ ). A stepwise regression including diabetes duration, BMI, sBP and $\mathrm{dBP}$, HbAlc, previous 2-year mean HbAlc, log-transformed AER and ANP genotype, versus TERalb, considered as the dependent variable, showed that $A N P$ genotype and AER were independently associated with TERalb in the whole diabetic cohort (step 1: $\mathrm{r}=-0.45$, F-test 6.06; step 2: multiple $\mathrm{r}=0.59$, F-test 6.26). In conclusion, in normotensive type 1 diabetic patients, the mutated genotype (intron I, C708/T) of the hANP gene is associated with lower plasma ANP levels and reduced transvascular leakage of albumin, an estimate of endothelial function.

\section{1}

INSULIN RESISTANCE AND ENDOTHELIAL DYSFUNCTION IN HYPERTENSIVE, ATHEROSCLEROTIC NON-DIABETIC SUBJECTS. S. Bandinelli, G. Dell'Omo, M. Nannipieri, L. Pucci, A. Marsocci, R. Navalesi, R. Pedrinelli and G. Penno. Dept. of Endocrinology and Metabolism, Dept. of Cardiology, University of Pisa, Italy.

Patients with atherosclerosis and/or hypertension exhibit both endothelial dysfunction and impaired insulin action. However, no direct association between insulin resistence and endothelial dysfunction was demonstrated. To evaluate this relation, the transcapillary escape of albumin (TERalb), influenced by endothelium, extracellular matrix properties and by haemodinamic forces, and insulin resistance assessed by the homeostasis model (HOMA) were measured in 96 non diabetic males: 23 healthy controls (C), 37 essential hypertensives ( $\mathrm{EH}), 23$ atherosclerotic subjects (ATH) and 13 hypertensive-atherosclerotic ones (EH/ATH). The four groups were similar for age, serum creatinine and fibrinogen. BMI was lightly higher in $\mathrm{EH}$ and $\mathrm{EH} / \mathrm{ATH}(\mathrm{p}=0.004)$. Atherosclerotic groups (ATH, EH/ATH) had higher total- and LDL-cholesterol levels ( $<<0.005$ ), ApoB $(p<0.01)$, triglycerides ( $p=0.02$ ) compared with $C$ and $E H$. Systolic and diastolic 24 hour ambulatory $\mathrm{BP}$ were significantly higher $(p=0.0001)$ in $E H$ and $E H / A T H$, and similar in $C$ and ATH. Urinary albumin excretion (logAER) was higher in EH/ATH $(159 \pm 73 \mu \mathrm{g} / \mathrm{min}$ : $\mathrm{M} \pm \mathrm{SE})$, but similar in $\mathrm{C}(8.8 \pm 2.0)$, EH $(12.9 \pm 2.3)$ and ATH $(7.5 \pm 0.8)$ $(p=0.0001)$. Serum albumin, haematocrit, plasma volume and intravascular mass of albumin did not differ in the four groups. Serum albumin and haematocrit were stable in each subject in the TERalb test. TERalb was $6.5 \pm 1.6 \% / \mathrm{h}$ in controls and higher $(\log$ TERalb, $\mathrm{p}=0.0001)$ in EH (9.1 $\pm 2.6, p<0.01)$, ATH $(10.2 \pm 2.6, p<0.001)$ and EH/ATH $(10.4 \pm 3.3$, $p<0.001)$. HOMA IR was $2.0 \pm 0.20$ in controls and higher $(p=0.07)$ in EH $(2.65 \pm 0.22)$, ATH $(2.33 \pm 0.31)$ and EH/ATH $(2.54 \pm 0.32)$. Positive linear regression was found between TERalb and BMI $(r=0.23, p=0.024)$. Stepwise regression showed that (model 1) presence of atherosclerosis $(\mathrm{r}=0.393)$, hypertension (cumulative $\mathrm{r}=0.521)$ and BMI $(\mathrm{r}=0.546)$, and (model 2) atherosclerosis $(\mathrm{r}=0.337)$, hypertension $(\mathrm{r}=0.421)$ and HOMA IR $(r=0.439)$, independently contribute to TERalb variability. These data suggest a relation between widespread endothelial dysfunction and insulin action and provide further evidence for a relation with one of the main features (increased body weight) of the insulin resistance syndrome.
1210

IMPAIRED ENDOTHELIUM DEPENDENT VASODILATATION IN WOMEN WITH PREVIOUS GESTATIONAL DIABETES MELLITUS

E Anastasiou, JP Lekakis, M Alevizaki, C Papamichael, J Megas, SF Stamatelopoulos and A Souvatzoglou. 1st Endocrine Section and Diabetes Centre and Dept Med Therapeutics, ALEXANDRA Hospital, 11528 Athens

The aim of the present study was to assess whether otherwise healthy women with a history of Gestational Diabetes Mellitus (ex-GDM) may have abnormalities in endothelial function at a very early stage, before glucose intolerance occurs. Twenty three women with previous GDM aged $34.7 \pm 4.9$ and 18 healthy women matched for age were examined. A $75 \mathrm{~g}$ OGTT was performed; insulin levels and biochemical parameters were also measured. Using high resolution ultrasound we measured vasodilatory responses of the brachial artery during reactive hyperemia (endothelium dependent vasodilatation) and after nitroglycerin administration, an endothelium independent vasodilator. Flow-mediated dilatation (FMD) was significantly decreased in ex-GDM compared to controls $(1.2+3.1 \%$ vs $10.9+4.5 \%$ respectively, $p<0.001$ ). There was no difference in nitrate-induced dilatation (exGDM $23.8 \pm 7.5 \%$ vs controls $28.8 \pm 9 \%$ ). Ex-GDM had significantly higher BMI $(27.8 \pm 4.6$ vs $24.1 \pm 2.7, p<0.01)$ and basal insulin resistance (HOMA) $(4.0 \pm 1.9$ vs $2.8 \pm 0.9, p<0.05)$ than controls. Multiple regression analysis showed that this decrease in the exGDM group was independent of obesity. Serum uric acid levels were significantly higher in ex-GDM group $(237.9 \pm 35.7$ vs $172.5 \pm 23.8 \mathrm{mmol} / \mathrm{L}, p<0.001)$. Serum uric acid levels correlated inversely with FMD $(r=-0.66, p<0.001)$. There were no differences between the two groups in blood pressure or lipid levels. In conclusion, endothelial dysfunction, which is considered one of the first signs in the development of atherogenesis, is already present in lean as well as in obese women with a history of GDM, even when they appear with normal glucose tolerance.

\section{2}

DISTURBED FLOW-ASSOCIATED BRACHIAL ARTERY DILATION IN GLUCOSE-TOLERANT, INSULIN-RESISTANT FIRST-DEGREE RELATIVES OF SUBJECTS WITH TYPE 2 DIABETES

B. Balletshofer, K. Rittig, M. Enderle, A. Volk, E. Maerker, M. Pfohl, K. Rett and H.U Häring, Medizinische Klinik Abt. IV, Universităt Tübingen, Germany

It is well established, that vascular endothelium plays a central role in the atherogenic process and there is strong evidence of a correlation between endothelial dysfunction and insulin resistance. Measurement of flow-associated dilation of the brachial artery is a reliable test for endothelial dysfunction. We examined 42 first-degree relatives (FDR) of patients with type 2 diabetes (16 $\mathrm{m}, 26 \mathrm{w}$, mean age 33,9 years) with normal oral glucose tolerance test (OGTT) Endothelium-dependent vasodilation and intima-media-thickness (IMT) were measured using high resolution ultrasound (13 MHz, Esaote AU4 idea, Munich, Germany). Brachial artery diameter was assessed under baseline conditions, during reactive hyperemia (with flow increase causing endothelium-dependent dilation) and after sublingual administration of glyceryltrinitrate (as an endothelium-independent dilator). Insulin sensitivity was measured with the euglycaemic hyperinsulinaemic glucose clamp technique (insulin infusion 1 $\left.\mathrm{mU} / \mathrm{kg} \mathrm{min}^{-1}\right) .15$ of the examined FDR were classified as insulin resistant (IR) with a glucose metabolic clearence rate (MCR) $<6 \mathrm{ml}^{*} \mathrm{~kg}^{-1 *} \mathrm{~min}^{-1}, 13$ as insulin sensitive (IS; MCR > 8) and 14 as borderline (MCR 6-8). Flow-associated endothelium-dependent dilation (FAD\%) was 4,6+1,0\% in $\mathbb{R}$ and $10,3+1,4 \%$ in IS ( $p=0,0025$, Wilcoxon Rank Sum Test). There was no difference in age, smoking habits, lipids, $24 \mathrm{~h}$-blood pressure and endothelium-independent dilation capacity. There was a also no difference in $\mathrm{MT}(0,54 \mathrm{~mm}$ in $\mathbb{R}$ and $0,49 \mathrm{~mm}$ in IS).

We conclude, that even in glucose-tolerant normotensive FDR, clamp-derived insulin resistance is associated with endothelial dysfunction. According to our results, testing of endothelial function with ultrasound technique might allow early identification of persons at high-risk for atherosclerosis. 
WAIST-TO-HIP RATIO IS ASSOCIATED WITH A SELECTIVE LOSS OF MICROVASCULAR REACTIVITY IN YOUNG HEALTHY MEN.

R.C. Bonadonna, T. Monauni, B. Brunato, A. Cretti, L. Bertolini, E. Bonora and M. Muggeo. Division of Endocrinology and Metabolic Diseases, Verona, Italy.

Waist-to-hip ratio (W/H) is a predictor of NIDDM, hypertension and atherosclerosis. Endothelial (E) dysfunction preceeds both hypertension and atherosclerosis, and is present in NIDDM. E damage goes through several phases: an early one, in which sensitivity to muscarinic agonists is lost; and a late one, in which sensitivity to kininergic agonists also is lost. These phases are thought to reflect lesions of different classes $(i, o$ and $q$ ) of $\mathrm{G} \alpha$-proteins, coupling muscarinic (M1, M2 and M3) and kininergic (B2) receptor signals to phospholipase C (PLC) and $E$ nitric oxide synthase (ecNOS), with subsequent nitric oxide (NO) synthesis and vasorelaxation. Aim of this study was to assess whether $\mathrm{W} / \mathrm{H}$ is related to $\mathrm{E}$ function, independently of age, blood pressure, circulating lipids and adiposity, utilizing acetylcholine ( $\mathrm{ACH}$ ), an $\mathrm{M}$-receptor agonist, bradykinin (BK), a $\mathrm{B} 2$ receptor agonist, and sodium nitroprusside (SNP), an NO donor. We studied 19 healthy male (age: $23.7 \pm 0.6 \mathrm{yrs}$ ), nonobese (BMI: $24.3 \pm 0.43 \mathrm{~kg} / \mathrm{m}^{2}$; W/H: $0.88 \pm 0.01$ ), non smoking subjects, with normal blood pressure and circulating lipids, by employing the forearm (F) perfusion technique and measuring the $F$ blood flow (FBF: $\mathrm{ml} / \mathrm{min} / \mathrm{kg}$ of $\mathrm{F}$ ) response to the intra-arterial infusion of graded doses of $\mathrm{ACH}(3,9$ and $30 \mu \mathrm{g} / \mathrm{min} / \mathrm{kg}$ of F), BK $(40,120$, and $400 \mathrm{ng} / \mathrm{min} / \mathrm{kg}$ of F), and $\operatorname{SNP}(1,3$, and $10 \mu \mathrm{g} / \mathrm{min} / \mathrm{kg}$ of $\mathrm{F}$ ). Neither BMI, nor blood pressure, nor lipids, nor fasting plasma insulin were correlated to $\mathrm{FBF}$ responses to $\mathrm{ACH}$, BK or SNP (p ranging from 0.20 to 0.90 ). W/H was correlated to the FBF response to ACH $(r=-0.57, p=0.01)$, but not to BK $(r=-0.35, p=0.15)$ or SNP $(r=0.34, p=0.16)$. In multiple regression analysis, the relationship between $\mathrm{W} / \mathrm{H}$ and $\mathrm{FBF}$ response to ACH was independent of BMI, blood pressure, lipids, FBF responses to SNP and $\mathrm{BK}$, and fasting insulin. Thus, high W/H is an independent indicator of selective fall in microvascular reactivity to $\mathrm{ACH}$, which may be due to a reduced stimulation of PLC/ecNOS, possibly secondary to a lesion of Gai- and/or Gao-proteins.

\section{5}

\section{EFFECTS OF FREE FATTY ACIDS AND INSULIN ON ENDOTHELIAL-DEPENDENT VASODILATATION} L Lind, A Fugmann, B Wessby, J Millgård, C Berne, $\mathrm{H}$ Lithell. Depts of Medicine and Geriatrics, University Hospital, S-75185 Uppsala, Sweden.

Fatty acids impair endothelium-dependent vasodilatation (EDV) and insulin has been shown to enhance EDV. In order to clarify how these two metabolic alterations interfere with EDV, 10 healthy volunteers were studied at two occasions. Intralipid and heparin or saline, were infused for 4 hours at a rate which increased the serum free fatty acid concentration from $0.37 \pm 0.19 \mathrm{SD}$ to $2.2 \pm 1.3 \mathrm{mmol} / \mathrm{l}$. During the last 2 hours of the experiment euglycemic hyperinsulinaemia ( $83 \pm 24$ $\mathrm{mU} / 1)$ was added. EDV and endothelial-independent vasodilatation (EIDV) were evaluated in the forearm by local intra-arterial infusion of metacholine or sodium nitroprusside at baseline and after 2 and 4 hours of infusions. Intralipid and heparin infusion decreased EDV from 27.6 48.7 to $21.0 \pm 5.7 \mathrm{ml} / \mathrm{min} / 100 \mathrm{ml}$ tissue $(\mathrm{p}<0.01)$. This effect was reversed by hyperinsulinaemia $(28.9 \pm 6.7 \mathrm{ml} / \mathrm{min} / 100 \mathrm{ml}$ tissue, $p<0.01$ ). Euglycemic hyperinsulinemia alone increased EDV ( from $27.3 \pm 8.4$ to $30.4 \pm 9.5 \mathrm{ml} / \mathrm{min} / 100 \mathrm{ml}$ tissue, $p<0.01$ ), while EIDV was not significantly altered by any of the metabolic interventions. In conclusion, the present study showed that an acute increase of serum free fatty acids decreased EDV, an action that was reversed by elevating plasma insulin.
INFLUENCE OF OVERWEIGHT AND GLYCEMIA ON ACETYLCHOLINEINDUCED SKIN VASODILATION IN OBESE SUBJECTS AND NIDD's.

O. Smagghue, J. Pariès, B. Lormeau, J.R Attali, P. Valensi. Department of Endocrinology-Diabetology-Nutrition, Jean Verdier Hospital, Paris-Nord University, Bondy. France.

Alterations of endothelium-dependent vasodilation have been reported in noninsulin-dependent diabetic patients (NIDD's). Laser-doppler is an accurate tool for investigating microcirculatory vasomotricity. The aim of the present study was to examine the role of overweight and blood glucose levels in these changes. Fiftytwo nondiabetic obese subjects, 18 NIDD's and 23 healthy controls with normal body weight were compared. Cutaneous blood flow (CBF) was measured by laserdoppler (Periflux) on the right index, at baseline and after local iontophoretic administration of acetylcholine. Acetylcholine was delivered by using an anodic current of $0.1 \mathrm{~mA}$ for 30 seconds, $0.2 \mathrm{~mA}$ for 20 seconds and finally $0.2 \mathrm{~mA}$ for 30 seconds. At baseline, the mean values and standard deviations of CBF did not differ significantly between the three groups. After application of the three stimuli, CBF increased in the three groups after a similar lag time. The peak of CBF occurred significantly earlier in obese subjects and NIDD's than in the controls, and CBF also returned to basal values earlier as compared to the controls, but the time lags did not differ significantly between obese subjects and NIDD's. After each of the three stimuli, the peak level of CBF did not differ significantly between the three groups. In the whole series of 96 subjects, both the peak time and the time of recovery of CBF after each stimulus correlated negatively with BMI. The peak time and the time of recovery after the first stimulus also correlated negatively with glycemia 2 hours after $75 \mathrm{~g}$ glucose taken orally in the obese subjects $(p=0.03$ and 0.02 respectively) and post-prandial glycemia in NIDD's ( $\mathrm{p}=0.002$ and 0.07 respectively). In conclusion, in both obese subjects and NIDD's, the acetylcholineinduced cutaneous vasodilative response is more rapid and less sustained than in normal subjects. Overweight and blood glucose levels seem to play a determinan role in this particular kinetic pattern which suggests more a microcirculatory smooth muscle dysfunction than an endothelial alteration. An excess of free radicals might be involved in this disorder.

\section{6}

SHORT-TERM AGGRESSIVE LDL-LOWERING IN ATHEROSCLEROTIC PATIENTS: EFFECTS ON ENDOTHELIAL-MEDIATED VASODILATATION AND CAPILLARY PERMEABILITY

R. Navalesi, G. Penno, G. Dell'Omo, M. Nannipieri, L. Pucci, A. Marsocci, R. Pedrinelli and S. Bandinelli. Dept. of Endocrinology and Metabolism, Dept. of Cardiology, University of Pisa, Italy.

Consistent evidence shows an improvement of arteriolar endothelial function after LDL-cholesterol lowering. Less clear is how early this takes place. It is unknown the effect of treatment with HMG-CoA reductase inhibitors on the function of the capillary bed where most of the endothelial cells lie. High-dose $(40 \mathrm{mg} / \mathrm{die})$ simvastatin was administered for one month in 18 non-diabetic (OGTT-fasting glucose: $97 \pm 12 \mathrm{mg} / \mathrm{dl}$, HbAlc: $5.7 \pm 0.35 \%$ ), non-obese (BMI: $26.1 \pm 1.82 \mathrm{~kg} / \mathrm{m}^{2}$ ) normotensive (24-hour ABPM: $124 \pm 9 / 74 \pm 6 \mathrm{mmHg}$ ) males (age: $58 \pm 9$ years) with atherosclerotic peripheral vascular disease and LDL-cholesterol levels $>130 \mathrm{mg} / \mathrm{dl}(173 \pm 36)$. Main outcomes: 1 . Forearm vasodilatation [percent blood flow (FBF, ml/min/dl, venous plethysmography) changes from baseline] to intra-arterial acetylcholine $(7.5,15,30 \mu \mathrm{g} / \mathrm{min} \times 5 \mathrm{~min}$ each), an endothelial-mediated vasodilator; 2 . Transcapillary albumin escape rate (TERalb, \%/h, i.v. 125I-human albumin, the fraction of the intravascular albumin leaving the vascular bed per hour), a measure of the capillary permeability; 3. Maximum forearm vasodilatation (13-min ischemia peak flow) to account for non specific vasodilation. Simvastatin reduced LDLcholesterol by $45 \pm 11 \%$ (to $102 \pm 26 \mathrm{mg} / \mathrm{dl}, \mathrm{p}<0.0001$ ). No significant changes were observed in the response to $\mathrm{ACH}(+194 \pm 27 \%, 362 \pm 59 \%$, $534 \pm 68 \%$ vs $+215+29 \%, 401 \pm 46 \%, 585 \pm 49 \%$ at $7.5,15$ and $30 \mu \mathrm{g} / \mathrm{min}$ $\mathrm{ACH}$ infusion, respectively). On the contrary, TERalb decreased by $19 \pm 25 \%$ from $10.4 \pm 2.0 \% / \mathrm{h}$ to $8.4 \pm 2.9 \% / \mathrm{h}(\mathrm{p}=0.0096)$. Diastolic $\mathrm{BP}$ decreased from $81.3 \pm 6.2$ to $76.8 \pm 5.8 \mathrm{mmHg},(\mathrm{p}<0.02)$ with no relation with TERalb reduction. Post-ischaemic FBF was stable $(56.4 \pm 11.9$ vs $59.6 \pm 15.1 \mathrm{ml} / \mathrm{min} / \mathrm{dl}$ ). Improvement of arteriolar endothelial function $(\mathrm{ACH})$ resulted still inconsistent after one-month aggressive simvastatin therapy, in spite of a drastic reduction in LDL-cholesterol. The reduced TERalb due to simvastatin over the same period indicates an influence of LDL-cholesterol on capillary permeability, and it suggests that changes in capillary and arteriolar function may follow different time-courses. 
1217

GLYCLAZIDE AS A FREE RADICAL SCAVENGER IN THE ENDOTHELIUM DEPENDENT VASODILATION.

A. Costa e Forti, A.A. Peter, L.R.L. Martin; K.W.P. Gomes, A.P.A. Silva and M.C. Fonteles. Clin. Res. Unit - UFC/UECe/CIDH - Av. José Bastos, 3390 For-Bz, 60934

The anti-oxidant defenses in diabetic patients are found to be generally impaired cellular levels of reduced gluthation (GSH) and serum concentrations of vitamin $\mathrm{C}$, both primary antioxidants, are reduced in diabetics when in poor glvcaemic control. Diabetic vascular tissues are targets to pharmacological derangements especially at the endothelial cells, which can be reversed by superoxide dismutase, an activity that may be correlated to the control of free radicals formation. The aim of this work was to evaluate bradykinin (Bk) endothelial responses, comparing GSH, a natural free radical scavenger, and glyclazide, an antidiabetic drug, in alloxan $(150 \mathrm{mg} / \mathrm{Kg})$ diabetic rabbits. Rabbit kidnevs were isolated and perfused with Krebs-Henseleit solution in a open system at $30^{\circ} \mathrm{C}$, delivering a flow rate of $10 \mathrm{ml} / \mathrm{min}$. Noradrenaline (NOR) was infused at the concentration of $1(\mathrm{M}$ into the renal artery, promoting a great vasoconstriction which reached a steady state response $2-3 \mathrm{~min}$ after the infusion onset. NOR was administrated in two successive cycles of 20 minutes each, with an interval of $10 \mathrm{~min}$ for drug wash out. During this period BK was injected as a bolus, cumulatively into the renal artery in the presence of GSH (1(M) or glyclazide (1(M). The results are expressed as percentage of decrease in the perfusion pressure $((\mathrm{P \%})$, for each dose of $\mathrm{Bk}$

\begin{tabular}{l|l|l|l|l|l}
\hline BK $(\mathrm{M})$ & $4.10^{-8}$ & $8.10^{-8}$ & $1,6.10^{-7}$ & $3,2.10^{-7}$ & $6.4 .10^{-7}$ \\
\hline Control & $4.7 \pm 5.5$ & $5.4 \pm 10.1$ & $0.8 \pm 11$ & $-4.1 \pm 13$ & $-1.4 \pm 1.8$ \\
GSH & $14 \pm 7.9$ & $19.4 \pm 5$ & $19.5 \pm 8.4$ & $17 \pm 11.3$ & $15.2 \pm 14.3$ \\
gly & $16.4 \pm 1.1$ & $18.4 \pm 8.9$ & $21.5 \pm 7.2$ & $21.2 \pm 9$ & $24.4 \pm 8.4$
\end{tabular}

The results show that glyclazide potentiates the BK induced vasodilatation . and acts as GSH possibly as a free radical scavenger effects.

\section{8}

CHANGES IN THE CHOLINERGIC RESPONSE OF ARTERIOLES IN DIABETIC RATS ARE NOT DUE TO HYPERGLYCEMIA PER SE

AA van Lambalgen, JH. Reitsma, C Demirci*, GC van den Bos, CDA Stehouwer, GJ Tangelder. Lab for Physiology, ICaR-VU, Vrije Universiteit, Amsterdam, The Netherlands and *Dept of Biol Istanbul Üniversitesi, Turkey

The role of hyperglycemia for the change in the cholinergic response of arterioles during Diabetes Mellitus (DM) is unclear. Using intravital microscopy, we studied in the spinotrapezius muscle the relation between the

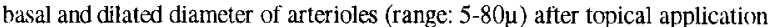
of acetylcholine (ACh: $10^{-3} \mathrm{M}$ ) in 4 groups of rats ( $\mathrm{n}=6$ per group): DM, iv injection of streptozotocin $(70 \mathrm{mg} / \mathrm{kg}) 6$ weeks prior to the experiment; $\mathbf{C 1}$, controls for DM; GL, muscle superfused with glucose (20mM) for $4 \mathrm{hrs}$; $\mathbf{C 2}$, controls for GL. Afterwards $\mathrm{N}^{\mathrm{c}}$-nitro-L-arginine (LNNA; $10^{-4} \mathrm{M}$ ) was used to block the nitric oxide production. Linear regression lines $(\mathbf{y}=\mathbf{a x}+\mathbf{b})$ werc calculated per group between the basal (x) and ACh-dilated (y) diameters of all the arterioles $\geq 20 \mu \mathrm{m}$ (AA: arcade arterioles; $\mathrm{n}=\sim 45 /$ group) and $<20 \mu \mathrm{m}$ (TA: transverse and terminal arterioles; $\mathrm{n}=\sim 40 /$ group). $\mathrm{C} 1$ and $\mathrm{C} 2$ were pooled (C): these groups were comparable. All relationships were significant; $\mathrm{r}$ values: $>60$. For AA the slopes (a) of the lines were $\sim 1$ for all groups, the intercepts (b) were $\sim 25$ in C and GL, but 13 in DM; after LNNA the slone decreased markedly in DM (by $\sim 45 \%$ ), only minor in GL (by $\sim 15 \%$ ), and not in C. So, only in DM, all AA showed less dilatation alter ACh, but increased NO-dependency. For 'TA, in $\mathrm{C}$ the slope was $\sim 1$ and the intercept $\sim 10$, but in GL the slope $(0.8)$ had decreased and the intercept $(-20)$ increased, whilc in DM the opposite was found, slope: $\sim 2$. intercept: zero; after LNNA, in DM the slope decreased by $25 \%$, but in GL and C intercepts decreased by 25 and $50 \%$, respectively. The effect of DM on dilatation of TA after ACh is thus sizedependent: less dilatation in small $(<10 \mu \mathrm{m})$, but more in the bigger TA. The differences between DM and GL, however, show that changes in the cholinergic response in DM cannot be explained by hyperglycemia per se.

\section{9}

EFFECTS OF NEUTRAL ENDOPEPTIDASE 24-11 AND ANGIOTENSIN-CONVERTING ENZYME INHIBITORS ON THE FEMORAL VASCULAR CONDUCTANCES IN STREPTOZOTOCIN-TREATED RATS.

V. ARBIN, N. CLAPERON and J. PEYROUX, Laboratoire de Pharmacologie, Université René Descartes, Paris, France.

In streptozotocin (STZ)-treated rats, resting femoral vascular conductances (FVC) have been shown to be reduced versus control rats. It has been suggested that in anaesthetized normoglycaemic rats, a continuous reiease of bradykinin (BK) from endothelial cells could contribute to vascular tone. In vivo, Angiotensin-converting enzyme (ACE) and Neutral endopeptidase 24-11 (NEP 24-11) appear to be the two main enzymes involved in BK degradation. We have studied the effects of captopril (C $2 \mathrm{mg} \mathrm{kg} \mathrm{kg}^{-1} \mathrm{i}$. v. bolus+1 ${\mathrm{mg} . \mathrm{kg}^{-1}}^{-1} 30 \mathrm{~min}^{-1} \mathrm{i} . \mathrm{v}$. infusion) and retrothiorphan a selective NEP inhibitor (RT, 25 mg.kg-1 i.v. bolus $+12.5 \mathrm{mg.kg}^{-1} .30 \mathrm{~min}^{-1}$ i.v. infusion) alone or in combination on FVC in STZ-treated rats. The haemodynamic responses to inhibitors were assessed by conductance determination (femoral blood flow, $\mathrm{ml} \mathrm{min}^{-1} / \mathrm{mean}$ arterial pressure, $\mathrm{mm} \mathrm{Hg} \times 100$, arbitrary units) using the transit time utrasound technic for continous determination of blood flow in femoral artery. $\mathrm{C}$ or $\mathrm{C}+\mathrm{RT}$ were given alone or after L-NAME (10 mg. $\left.\mathrm{kg}^{-1}\right)$, after the B2 receptor antagonist HOE $140(100$ or $\left.300 \mu \mathrm{g} \cdot \mathrm{kg}^{-\mathrm{I}}\right)$ and after HOE $140\left(300 \mu \mathrm{g} \mathrm{kg}^{-1}\right)+$ the kininase 1 inhibitor $\mathrm{DL}-2$ mercaptomethyl-3-guanidinoethylthiopropanoic acid (MGTA, $10 \mathrm{mg} \cdot \mathrm{kg}^{-1}$ ) in five separate experiments. FVC before and during the different treatments were compared using one way analysis of variance for repeated measures followed by Scheffe test. In control rats, $\mathrm{C}$ alone or $\mathrm{C}+\mathrm{RT}$ increased $\mathrm{FVC}(1,43 \pm 0.16$ and $1,29 \pm 0.16, \mathrm{P}<0.01$ vs resting value $0,75 \pm 0.08$ ) and L-NAME, HOE 100 or $300 \mu \mathrm{g} . \mathrm{kg}^{-1}$ or MGTA + HOE $300 \mu \mathrm{g} . \mathrm{kg}^{-1}$ inhibited these vasodilator effects. In hyperglycaemic rats, FVC were increased only in presence of $C+R T(0,68 \pm 0.13, P<0.01$ vs resting value $0,34 \pm 0.07)$ This vasodilation was blunted in presence of L-NAME or MGTA + HOE $300 \mu \mathrm{g} . \mathrm{kg}^{-1}$ but not in presence of HOE alone. These results suggest that $C+R T$ could unmask a continuous release of BK which increases FVC mainly by nitric oxide $(\mathrm{NO})$ release in the femoral vascular bed of normo- and hyperglycaemic rats. Endogenous BK could induce NO reiease after B2 receptor activation and/or by supplying carboxylic $L$ arginine after its hydrolysis by kininase 
PS 63

\section{Endothelium in Vitro Studies}

\section{0}

TROGLITAZONE INHIBITS CYTOKINE-INDUCED PLASMINOGEN ACTIVATOR INHIBITOR-1 SECRETION FROM ENDOTHELIAL CELLS E. Nohara, M. Yokoyama, T. Takamura, Y, Nagai and K. Kobayashi.. First Department of Internal Medicine, School of Medicine, Kanazawa University, Ishikawa, Japan.

Troglitazone, an insulin sensitizer, has been known to improve glucose intolerance, insulin resistance and dyslipidemia, thereby suggesting that it inhibits the initiation and progression of atherosclerosis. Plasminogen activator inhibitor-1 (PAI-1) plays an important role in the pathogenesis of atherosclerosis, and its level is elevated in patients with insulin resistance. The biosynthesis of PAI- 1 in endothelial cells is stimulated by various inflammatory cytokines such as tumor necrosis factor (TNF)$\alpha$ or interleukin (IL)-1 $1 \beta$, which are released from monocytes / macrophages by oxidative stress. Thus we investigated the direct effects of troglitazone on PAI-1 secretion from human umbilical vein endothelial cells (HUVECs). HUVECs were cultured in the medium containing $0.4 \%$ serum with or without troglitazone $(1$ and $10 \mu \mathrm{M})$ in the presence of TNF- $\alpha(100 \mathrm{ng} / \mathrm{ml})$ or $\mathrm{IL}-1 \beta(10 \mathrm{ng} / \mathrm{ml})$ for $24 \mathrm{~h}$, and the medium contents of PAI-1 were measured by ELISA method (TintElize PAI-1, Biopool, Sweden). TNF- $\alpha$ and IL-1 $\beta$ significantly ( $p<0.02$ vs control) increased PAI- 1 contents to $1.99 \pm 0.16$ and $2.42 \pm 0.42$ fold, respectively. Troglitazone (10 $\mu \mathrm{M})$ significantly inhibited TNF- $\alpha$-induced $(54.3 \pm 0.02 \%, p<0.01)$ and IL- $1 \beta$ induced $(40.8 \pm 0.02 \%, p<0.01)$ PAI-1 secretion, and these inhibitory effects were dose-dependent. Cell viability evaluated by trypan blue exclusion was not influenced by troglitazone. These data suggest that troglitazone may prevent the initiation and progression of atherosclerosis by direct inhibition of PAI-1 secretion from HUVECs. We have recently reported that troglitazone inhibits cytokineinduced monocyte chemoattractant factor (MCP)-1 expression in hurnan mesangial cells. Also in HUVECs, we assume that the inhibitory effects of troglitazone on PAI- 1 levels may be at the level of gene expression. Effects of troglitazone on the PAI-1 mRNA contents in HUVECs will be presented.

\section{2}

THE FORMATION OF EICOSANOIDS IN A HUMAN ENDOTHELIAL CELL LINE UNDER DIABETIC CONDITIONS

W. Engels, P.J.M.R. Lemmens, M.M.E. van Ginneken and B.H.R Wolffenbuttel. Dept. Endocrinology, Maastricht University, Maastricht, The Netherlands.

Microvascular dysfunction in diabetes is characterized by protein kinase $\mathrm{C}$ (PKC)-mediated endothelial cell activation. This will lead to a change in the formation of vasoactive substances like NO and eicosanoids. The aim of this study was to assess in which respect the profile and the amount of eicosanoids is influenced by short- and long-term exposition of a human endothelial cell line EC-RF24 to elevated levels of glucose. Stimulation of control cells, cultured at $5 \mathrm{mM}$ glucose, with thrombin resulted in mobilization of calcium followed by translocation of PKC $\alpha$, and the production of prostacyclin, $\mathrm{PGF}_{2 a}, \mathrm{PGE}_{2}$ and thromboxane $A_{2}$. (TXA). When the cells were cultured for 15 doublings in the presence of $25 \mathrm{mM}$ glucose, thrombin stimulation revealed a shift in the eicosanoid profile from vasodilating (prostacyclin, $P G E_{2}$ ) to vasoconstricting $\left(T X A, P G F_{2 \alpha}\right.$ ). It could be calculated that the prostacyclin/TXA ratio decreased from 5.7 to 1.4 Additionally, endothelial cells grown at $25 \mathrm{mM}$ glucose showed an increased sensitivity towards thrombin when compared to control cells. In contrast, acute elevation of the glucose concentration to $25 \mathrm{mM}$ did not gave a significant change in basal eicosanoid formation nor calcium mobilization, whereas mobilization of PKC $\alpha$ and $\beta$ could be observed. However, activation of PKC by the phorbol ester PMA did not result in a stimulation of eicosanoid formation nor calcium mobilization. These results indicate that endothelial cells under diabetic conditions exhibit increased sensitivity to receptor agonists like thrombin, which is accompanied by changes in intracellular signalling and a change of the eicosanoid profile towards vasoconstrictory compounds, leading to alterations of vascular reactivity in diabetes mellitus.

\section{1}

Activation of human endothelial cells and fibroblasts is regulated by different mechanisms.

X.L. Du, G.Z. Sui, C. Bünting, Th. Koschinsky, P. Rösen, Diabetes Research Institute, Düsseldorf, Germany

Activation of endothelial cells and fibroblasts contribute to the pathogenesis of vascular complications in diabetes. To investigate the underlying mechanisms and the effect of hyperglycaemia, fibroblasts (F) and human umbilical vein endothelial cells (EC) were incubated with glucose (5 to $30 \mathrm{mM}$ ). The generation of reactive oxygen intermediates (ROI) was measured by preloading the cells with dichlorodihydrofluoresceine, activation of NF-кB by EMSA, onitrotyrosylation by Western blot. Incubation of EC and $\mathrm{F}$ with high glucose led to rapid increase in the generation of ROI in both types of cells. In addition, NF- $\mathrm{kB}$ became activated dose dependently. The maximal activation was reached after an incubation of 4 hours. $\mathrm{o}$ nitrotyrosylation was detectable in proteins with a molecular weight in the range of 18 to $182 \mathrm{kDa}$. The generation of $\mathrm{ROI}$ and the activation of NF-KB were inhibited in both types of cells by antioxidants ( $\alpha$ tocopherol $10-100 \mu \mathrm{g} / \mathrm{ml}$, lipoic acid $0.5-10 \mu \mathrm{M}$ ), whereas L-NMMA $(100 \mu \mathrm{M})$, an inhibitor of NO-synthase, was effective only EC, but not in F. PKC activation promoted the formation of ROI and activation of NF-кB only in F, but not in EC. These data suggest that different mechanisms of cell activation are effective in EC and F. In F the activation of PKC by a glucose dependent mechanism plays a decisive role for the generation of $\mathrm{ROI}$ and activation of NF- $\mathrm{KB}$. In contrast, EC seem to me more sensitive to ROI and the activation is depending on the formation of ROI and nitric oxide suggesting that the cytotoxic effects of high glucose are mediated in EC by peroxynitrite.

\section{3}

EFFECTS OF TOLRESTAT ON PROTEIN METABOLISM IN HUMAN ENDOTHELIAL CELLS CULTURED IN HIGH GLUCOSE M. Sternberg, A.-M. Grigorova-Borsos, P. Urios, A. Sanon, G. Mozère, G. Hirbec and R. Guillot. Département de Blochimie, Faculté de Médecine Broussais-Hôtel-Dieu, Université Pierre et Marie Curie, Paris, France.

Thickening of capillary basement membranes, associated with type IV collagen (CIV) accumulation, is characteristic of diabetic microangiopathy. An increase in CIV content of the aortic wall is also observed in diabetic macroangiopathy. In order to analyse the importance of the polyol pathway in the development of CIV metabolism alterations observed in diabetic angiopathy and their prevention by aldose reductase inhibitors, we studied the effects of tolrestat $(T)$ on the high(H) glucose(G)-induced alterations of $\mathrm{CIV}$, type IV collagenase and total protein (P) production by human umbillcal vein endothellal celis (EC). Primary cultures were exposed to 5.5, 16.7 and $27.8 \mathrm{mmol} / \mathrm{G}$, with and without 55 or $110 \mu \mathrm{mol} / \mathrm{T}$, for 3 days after beginning of confluence. We measured the soluble CIV secreted in the culture supernatant ( $\mathrm{S}$ ) and the insoluble CIV accumulated in the extracellular matrix and cell fraction (MCF), by ELISA. ${ }^{14} \mathrm{C}$ C Proline incorporation into the total collagenous and noncollagenous (nc) $P$ secreted in the $S$ was determined. Gelatin zymography of the $S$ showed that EC produce almost exclusively $72 \mathrm{kDa}$ gelatinase degrading CIV. Therefore we determined total gelatinase activity (ga), after dissociation from TIMPs and activation by p-aminophenyimercuric acetate. $H G$ increased $C I V$ amount in the $S(+155 \%$ at $27.8 \mathrm{mmoV} /$, $p<.001)$ and in the MCF $(+240 \%$ at $27.8 \mathrm{mmol} /, \mathrm{p}<.005)$. T reduced these increases significantly $(-54 \%$ and $-44 \%$ respectively at $110 \mu$ mol/ $)$. At $H G$, ga per $\mathrm{ml}$ of $\mathrm{S}$ was reduced and the $72 \mathrm{kDa}$ gelatinase band measured on the zymogram scan was lowered. T lowered ga further at HG (in spite of its corrective effect on CIV accumulation), but also at $5.5 \mathrm{mmol} / \mathrm{G}$. $T$ is not a corrective effect on CIV accumulation, but also at 5.5 mmoln G. T is not a $(+24 \%)$ and $\mathrm{nc}(+30 \%) \mathrm{P}$ in the $\mathrm{S}$. T lowered the synthesis of collagenous $(-21 \%)$ and $n c P(-38 \%)$ at $H G$, but also of $n c P(-22 \%)$ at $5.5 \mathrm{mmol} / \mathrm{l}$. In conclusion, $T$ decreases the synthesis of $n c P$, including $72 k D a$ collagenase, at $5.5 \mathrm{mmol} / \mathrm{l}$ and $\mathrm{HG}$, by a mechanism which remains to be elucidated. The corrective effect of $\mathrm{T}$ on the HG-induced hyperproduction of $\mathrm{CIV}$ and total collagenous $\mathrm{P}$ may be attributed to polyol pathway dysregulation prevention. 
EFFECT OF GLUCOSE ON ADHERENCE OF NEUTROPHILS TO ENDOTHELIAL CELLS

Y.K. Kim, S.M. Son and I.J. Kim

Department of Internal Medicine, School of Medicine, Pusan National University, Pusan, Korea

The aim of this study were to assess the relationship between endothelial dysfunction and neutrophil adhesion induced by high glucose. Rabbit aortic endothelial cells (RAECs) in primary culture were incubated for various times up to 24 hours to evaluate TNF$\alpha$ response and neutrophil adhesion under different glucose
concentrations $(0,5.5,11$ and $22 \mathrm{mM})$. To assess neutrophil adhesion, ${ }^{51} \mathrm{Cr}$ labeled rabbit neutrophils were added to RAECs monolayers at a neutrophil/endothelial cell ratio of 10:1 with different glucose concentration. We also examined ${ }^{3} \mathrm{Cr}$ released from ${ }^{51} \mathrm{Cr}$ labeled endothelial cells and endothelial cell detachment were used as indices of endothelial cell injury with different alucose us indices of cendoneltal cell inution with diferent glucose concentrations. Glucose concentration resembling hyperglycemic condition $(22 \mathrm{mM})$ significantly increased TNF-a release from cultured raboit endothelial cells $(212.5 \pm 39.6 \mathrm{pg} / \mathrm{mL}$ for $5.5 \mathrm{mM}$ vs. $445.5 \pm 67.8 \mathrm{pg} / \mathrm{mL}$ for $22 \mathrm{mM}, \quad p<0.01$ ) Neutrophils were more adhesive to endothelium under high glucose concentration than control $(30.6 \pm 3.7 \%$ vs. $64.5 \pm 6.2 \%, p<0.05)$ RAECs incubated in high glucose resulted in significant increase in ${ }^{5} \mathrm{Cr}$ release when compared with in control( $35.8 \pm 1.3 \%$ vs. $39.2 \pm$ $1.8 \%, p<0.05)$. Endothelial cell detachment was significantly greater when RAECs in high glucose rather than in control(5.9 $1.9 \%$ vs $8.6 \pm 1.8 \%, 0<0.01$ ) This study shows that high olucose timus $8.6 \pm 1.0 \%$, $p<0$. with results suggest that glucose directly causes increased interaction between neutrophil and endothelial cell through a TNF-a dependent mechanism.

\section{INDUCTION OF E-SELECTIN EXPRESSION BY OXIDIZED CHYLOMICRONS}

A. Jagla and J. Schrezenmeir, Federal Research Centre, D-24103 Kiel, Germany

Non-insulin-dependent diabetes mellitus is frequently associated with premature atherosclerosis. Besides other factors, an increased expression of endothelial cell adhesion molecules seems to be a crucial event in early states of atherogenesis/macroangiopathy. Adhesion molecules have shown to be induced by high glucose-levels and by certain lipoproteins, especially oxidized low-densitylipoproteins.

We have previously shown that triglyceride-rich lipoproteins (chylomicrons) as well stimulate the expression of E-selectin and VCAM-1; however, there seem to be differences between different chylomicron preparations. We therefore investigated, whether oxidative modification of these lipoproteins could be a factor determining their stimulatory effects on cells. Chylomicrons $110 \mathrm{mg}$ $\mathrm{Tg} / \mathrm{ml}$ ) were oxidized by addition of $\mathrm{CuSO}_{4}(10$ and $100 \mu \mathrm{M})$ for $6 \mathrm{~h}$ at $37^{\circ} \mathrm{C}$. After dialysis, chylomicrons were diluted with cell culture medium $(1 \mathrm{mgTg} / \mathrm{ml})$ and incubated with human umbilical vein endothelial cells (HUVEC) for $5 \mathrm{~h}$, followed by immunocytometric determination of E-Selectin. E-Selectin expression was stimulated by oxidized chylomicrons to $243 \pm 14 \%(10 \mu \mathrm{M} \mathrm{Cu})$ and to $310 \pm 20 \%$ $(100 \mu \mathrm{M} \mathrm{Cu})(\mathrm{MW} \pm \mathrm{SEM} ; \mathrm{n}=40 ; \mathrm{p}<0,001)$ compared to control $(=100 \%)$.

Several authors showed oxidation products in chylomicrons after ingestion of oxidized fat. Since diabetes is frequently associated with higher levels of chylomicrons in the postprandial state and with decreased antioxidative protectants (e.g. vitamin $E$ and $C$ ), the uptake and incorporation of oxidized fat in chylomicrons could - according to our results - contribute to endothelial activation and thereby promote the development of atherosclerosis.

\section{6}

High glucose increases the formation of nitrotyrosine as marker of oxidative stress in human vein endothelial cels (HUVEC).

G.Z. Sui, X.L. Du, G.Z. Sui, P. Rösen, Diabetes Research Institute, Düsseldorf, Germany

Peroxynitrite is rapidly formed from nitric oxide and superoxide anions. Besides its cytotoxic effects peroxynitrite can lead to onitrotyrosylation of proteins and might, therefore, be used as a stable, long term indicator of oxidatice stress. To investigate the effect of high glucose (HG) on the formation of nitrotyrosine, human umbilical vein endothelial cells were incubated with glucose $(5$ to $40 \mathrm{mM})$ for up to 6 hrs, and the o-nitrotyrosine formation was determined by western-blot using a specific antibody against o.nitrotyrosylated proteins. In the Western blot numerous bands specifically for nitrotyrosin in the range of $18 \mathrm{KDa}$ to $180 \mathrm{KDa}$ were obtained after incubating the cells with the NO-donor sodium nitroprusside (positive control) or HG $(20,30$, $40 \mathrm{mM}$ ) but not if cells were incubated with low glucose $(5 \mathrm{mM})$. The onitrosylation was inhibited by L-nitro-arginine, a specific inhibitor of NO-synthase $(100 \mu \mathrm{M}$, preincubation for 1 hour) and antioxidants. These results can be taken as indication that hyperglycaemia leads to an enhanced formation of peroxinitrite and exerts oxidative stress on endothelial cells. The previously reported increased generation of nitric oxide and superoxide anions in hyperglycaemia are in line with this assumption. Since inhibitors of NO-synthase are able to prevent not only the o-nitrosylation, but also the activation of NF-кB and apoptosis, we assume that peroxinitrite mediates the cyctotoxic effects of HG. Determination of o-nitrotyrosin might be a useful marker of oxidative stress in diabetes.
1227

EFFECTS OF AN INHIBITOR OF PROTEIN KINASE C ON ENDOTHELIAL CELL PROLIFERATION.

F Pomero, A Molinar Min, M La Selva, GM Molinatti and M Porta. Department of Internal Medicine, University of Turin, Italy.

Aims: The Protein Kinase C (PKC) family of enzymes is involved in the regulation of vital cell functions, including signal transduction, DNA synthesis, the turnover of hormone and growth factors receptors and the activity of $\left(\mathrm{Na}^{+}-\mathrm{K}^{+}\right)$-ATPase. Abnormal PKC activity has been suggested to play a role in the pathogenesis of the vascular complications of diabetes. We studied the effects of an inhibitor of PKC isoforms $\beta_{1}$ and $\beta_{2}$ (LY379196, generously provided by Ely Lilly, Indianapolis) on the replication of cultured human umbilical vein endothelial cells (HUVEC) in the presence of physiological $(5.6 \mathrm{mmol} / \mathrm{l})$ and high $(28 \mathrm{mmol} / \mathrm{l})$ concentrations of glucose. Materials and Methods: HUVEC in secondary culture were incubated with physiological or high glucose with or without LY379196 for 7 days. Cell replication was assessed by cell counts in a haemocytometer and by a Cell Proliferation Kit (Boehringer Mannheim, Germany) which measures the activity of mitochondrial dehydrogenase in vital cells. Results: a dose-response curve (range $100 \mathrm{nmol} / \mathrm{l}$ to 10 $\mu \mathrm{mol} / \mathrm{l}$ ) showed approximately $50 \%$ inhibition of cell replication at between 500 $n \mathrm{~mol} / \mathrm{l}$ and $1 \mu \mathrm{mol} / \mathrm{L}$ LY379196. Results are shown as percentages of values in 5.6 $\mathrm{mmol} / \mathrm{l}$ glucose. Means $\pm \mathrm{SD}$ of cell counts were: $89.7 \pm 11.4$ for glucose $28 \mathrm{mmol} /$ $(p=0.003$ vs glucose $5.6 \mathrm{mmol} / /$ ), $65.1 \pm 14.4$ for glucose $5.6+500 \mathrm{nmol} / / \mathrm{LY} 379196$ ( $\mathrm{p}=0.000$ vs gluc. 5.6 ), $61 \pm 11.5$ for glucose $28 \mathrm{mmol} / \mathrm{l}+500 \mathrm{nmol} / \mathrm{l} \mathrm{LY} 379196$ ( $\mathrm{p}=0.001$ vs gluc. 28 ), $52.3 \pm 22.8$ for glucose $5.6 \mathrm{mmol} / \mathrm{l}+1 \mu \mathrm{mol} / \mathrm{l} \mathrm{LY} 379196$ $(\mathrm{p}=0.001$ vs gluc.5.6), $44.9 \pm 11.5$ for glucose $28 \mathrm{mmol} / \mathrm{l}+1 \mu \mathrm{mol} / \mathrm{l} \mathrm{LY} 379196$ ( $\mathrm{p}=0.000$ vs gluc. 28). Cell proliferation (absorbance at $580 \mathrm{~nm}$ ) was: $84.6 \pm 13.2$ for glucose $28 \mathrm{mmol} / \mathrm{l}(\mathrm{p}=0.035$ vs gluc 5.6$), 70.2 \pm 15.7$ for glucose $5.6+500 \mathrm{nmol} / 1$ LY379196 ( $\mathrm{p}=0.006$ vs gluc. 5.6$), 62.2 \pm 18.3$ for glucose $28+500 \mathrm{nmol} / 1 \mathrm{LY} 379196$ ( $p=0.056$ vs gluc. 28), $57.2 \pm 20.8$ for glucose $5.6+1 \mu \mathrm{mol} / \mathrm{LY} 379196(\mathrm{p}=0.004$ vs Gluc. 5.6), $52.1 \pm 15.7$ for glucose $28+1 \mu \mathrm{mol} / 1 \mathrm{LY} 379196$ ( $\mathrm{p}=0.01$ vs gluc. 28 ) Conclusions: The addition of a PKC inhibitor to the culture system reduces the replication of HUVEC to a parallel extent in physiological and high glucose confirming that at least some $\mathrm{PKC}$ isoforms play a crucial role in the regulation of endothelial cell growth and suggesting that high glucose and PKC inhibition may impair endothelial replication through separate mechanisms. 
ELEVATED VASCULAR ENDOTHELIAL GROWTH FACTOR IN TYPE I DIABETIC PATIENTS WITH DIABETIC NEPHROPATHY

P. Hovind, L. Tarnow, P.B. Đstergaard and H.H. Parving. Steno Diabetes Center, Denmark.

The Steno hypothesis suggests that albuminuria reflects widespread vascular damage (proliferative retinopathy and severe macroangiopathy) due to a generalised vascular (endothelial) dysfunction. Vascular endothelial growth factor (VEGF) is a potent cytokine family that induces angiogenesis and markedly increases endothelial permeability. We measured VEGF with an ELISA technique in 199 type I diabetic patients with diabetic nephropathy $(122$ males, age $44 \pm 10$ years, diabetes duration $27 \pm 8$ years, GFR $(\mathrm{ml} \mathrm{x}$ $\left.\min ^{-1} \times 1.73 \mathrm{~m}^{-2}\right) 75$ (10-143), and in 188 long-standing type I diabetic patients with normoalbuminuria 1117 males, age $43 \pm 10$ years, diabetes duration $26 \pm 8$ years. Plasma levels of VEGF were significantly increased in patients with nephropathy as compared to the normoalbuminuric group: median (range): 45.7 (22-410) ngll vs 27.1 (22-355) ngll, respectively, $p<0.0001$. This difference was ascribed to elevated VEGF levels in men with nephropathy: $51.8(22.410)$ vs $22(22-308), p<0.0001$. No differences were found between females with and without nephropathy: 37.8 (22.325) vs $36.6(22.335) \mathrm{ng} / \mathrm{l}$. Plasma VEGF was below the detection limit $(22 \mathrm{ng} / 1)$ in 60 patients with nephropathy and 93 patients with normoalbuminuria, $p<0.0001$. Plasma levels of VEGF tended to be higher in nephropathic patients with proliferative retinopathy $(n-138)$ as compared to simplex retinopathy $(n=61): 55.4$ (22.363) vs $43.2(22.410) \mathrm{ng} / \mathrm{l}, \mathrm{p}=0.10$. A similar trend was demonstrated in the normoalbuminuric patients: lack of retinopathy(n=65): 23.9 (22.353); simplex(n=105): 27.3 (22-308), and proliferative(n-18): 34.9 (22.113) $\mathrm{ng}$ il, NS. The level of VEGF was nearly identical in proteinuric patients with GFR above the median $\left(102 \pm 19 \mathrm{~m} / \mathrm{min} / 1.73 \mathrm{~m}^{2}\right)$ vs patients below the median $\left(46 \pm 18 \mathrm{~m} / \mathrm{min} / 1.73 \mathrm{~m}^{2}\right)$. Our data in men suggest that VEGF is elevated early in the course of diabetic nephropathy, the importance of VEGF for the initiation and progression of diabetic nephropathy remains to be established.
HYPOXIA-INDUCED VASCULAR ENDOTHELIAL GROWTH FACTOR GENE EXPRESSION IS POTENTIATED BY ELEVATED GLUCOSE LEVELS

M.Meier', C.J.O'Callaghan', B.Gallacher', E.Stand ${ }^{2}$ and B.Williams'. ${ }^{1}$ Cardiovascular Research Institute, University of Leicester, UK; ${ }^{2}$ Diabetes Research Institute, Munich, Germany.

Vascular endothelial growth factor (VEGF) is a powerful endothelial cellspecific mitogen which potentially increases vascular permeability. It has been implicated in the pathogenesis of diabetic vasculopathies. Hypoxia induces the expression of VEGF in many tissues and more recently, we have shown that elevated glucose concentrations also increase VEGF expression. The present study thus examined the interaction between hypoxia and glucose in the regulation of VEGF expression after exposure of human vascular smooth muscle cells (VSMC) to control or high glucose levels $(5 \mathrm{mmol} / \mathrm{L}$ or $20 \mathrm{mmol} / \mathrm{L}$ ) for 48 hours prior to exposure to hypoxia (microaerophilic or anaerobic). High extracellular glucose concentrations $(20 \mathrm{mmol} / \mathrm{l})$ increased VEGF mRNA expression (2.4-fold) by human VSMC in a time (maximal 6-12 hours) and glucose concentration dependent (maximal $20 \mathrm{mmo} / \mathrm{L}$ ) manner. Hypoxia had a more potent effect on VEGF mRNA levels (10-fold increase), maximal between 12-24 hours. This effect was hypoxia-dose dependent (anaerobic vs. microaerophilic environment.). Under microaerophilic hypoxia, pre-exposure to high glucose significantly augmented VEGF mRNA expression vs. control glucose + microaerophilic hypoxia (high glucose 4 -fold vs. normal glucose). An altered Redox state is unlikely to be responsible for this interrelationship, as addition of pyruvate $(1-15 \mathrm{mmol} / \mathrm{L})$ or lactate $(1-15 \mathrm{mmol} / \mathrm{L})$ had no effect Superoxide dismutase (SOD) (10-500U) prevented glucose-induced but not hypoxia-induced VEGF expression, thereby suggesting that glucose-induced VEGF expression may be dependent on free-radical production. In conclusion, these results indicate that hypoxia and elevated glucose concentrations can interact to induce VEGF expression by human VSMC. This interaction is evident even in microaerophilic conditions. These observations suggest a mechanism whereby co-existing tissue hypoxia and hyperglycaemia could synergise to induce neovascularisation and other manifestations of endothelial dysfunction in diabetes mellitus.

\section{PS 64}

\section{Markers of Endothelial Damage}

\section{0}

URINARY ENDOTHELIN IN ADOLESCENTS AND YOUNG ADULTS WITH IDOM RELATION TO INDICES OF DIABETIC ANGIOPATHY.

M. Maniati-Christidi, M. Peppa and M. Dracopoulou, C. DacouVoutetakis

Endocrine Unit and Diabetes Center A' Dept. of Pod. Athens Medical School Athens, Helles

Endothelin is a potent vasoconstrictive peptide which might play $a$ roke in diabetic angiopathy in general and in diabetio nephropathy in particular. The aim of this study was to nephropathy in particular. The aim of this study was to
investigate a)alterations of urinary endothelin (UET1) in investigate a)alterations of urinary endothelin (UET1) in
adolescents and young adults with IDDM and $\mathrm{b}$ ) the relation of UET1 to indices and risk factors of diabetic angiopathy. We studied 130 10DM patients, aged 15.2+4.9yrs and diabetes

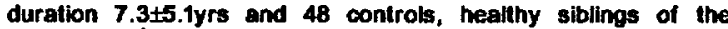
diabetics, of comparable age. The total 24 hour UET1 excretion was higher in diabeties then in controls $(10866 \pm 7270$ and $6598 \pm 3294 \mathrm{p} / 24 \mathrm{~h}$, respecti- vely, $p=0.000$ ). This difference was also noted if male and female diabetics were separately compared to controls. In diabetics, but not in controls, the 24hour UET1 values were greater in males than in females $(p=0.018)$. In IDDM subjects, UET1 showed a linear relationship with age $(p=0.002)$, urinary albumin $(p=0.000)$ serum creatinine $(p=0.001$ ) $p=0.003)$ $(p=0.003)$, HbAlc ( $p=0.041)$ and intimal plus medial thickness of carotid arteries. These data suggest that UET1 is elevaled quite early (in adolescence) in IDDM subjects and it is positively correlated with indices of diabetic micro and macroangiopathy possibly indicating a role of UET1 in the pathogenesis of diabetic angiopathy.

\section{1}

DETERMINATION OF CIRCULATING PLASMA ENDOTHELIN-1 CONCENTRATION IN TYPE 1 DIABETES MELLITUS

B. Sármán, M. Tóth*, K Farkas, A. Somogyi and Zs. Tulassay

$2^{\text {nd }}$ and ${ }^{*} 1^{\mathbf{s}}$ Department of Medicine, Semmelweis University of Medicine, Budapest, Hungary

Diabetes mellitus is well known for its association with macro- and microvascular disease. Besides known coupled risk factors endothelin-1 has been thought to play a role in the development of vascular disease. Endothelial dysfunction found in diabetes mellitus contributes to impaired production and secretion of endothelium derived relaxing factor, nitric oxide and constricting factors, the best known of which is endothelin-1. Damaged release of vasoactive substances and the disturbed permeability of the vascular endothelium in patients with diabetes mellitus can lead to accelerated vascular damage and induce angiopathy. The aim of the authors was to determine how circulating plasma endothelin-1 concentration differ between type 1 diabetic patients and healthy control subjects and to investigate whether endothelin-1 levels may be related to late djabetic complications. 36 non-diabetic heatthy control subjects (C) and 25 patients with type 1 diabetes mellitus (DM) -matched in age (C: $37.39 \pm 10,02$ years, DM:35,8 112,71 years), sex (C : 17 male and 19 female, DM: 11 male and 14 female), and body mass index (C $24,75 \pm 5,00 \mathrm{~kg} / \mathrm{m}^{2}$, DM: $23,67 \pm 3,81 \mathrm{~kg} / \mathrm{m}^{2}$ ) -were studied. Mean duration of diabetes was $10.0 \pm 7.31$ years; mean $\mathrm{HbAl}_{\mathrm{C}}$ value in $\mathrm{C}: 5.11 \pm 0.36 \%$, in DM:7.90 $\pm 2.53 \%$. Endothelin-1 level was measured by self-developed radioimmunoassay technology based on Perinsula antibody. There was no significant difference between endothelin- 1 concentration of type 1 diabetic patients $(2.72 \pm 1.08 \mathrm{ng} /)$ and healthy control subjects $(2.29 \pm 0.80 \mathrm{ng} /), p=0,080$. However in patients who had late diabetic complications $n=10$ (nephropathy, retinopathy, neuropathy, macrovascular lesion), we found that plasma endothelin-1 concentration was signiticantly higher $(3.14 \pm 1.44 \mathrm{ng} /)$ than in the group of healthy controls $(2,29=0.80 \mathrm{ng} /), p<0,05$. These results support the notion that endothelin-1 may play a central role in the development of diabetic angiopathy and therefore contributes to diabetic complications. 
1232

VASOACTIVE FACTORS OF VASCULAR ENDOTHELIUM IN PATIENTS WITH NIDDM WITH RENAL LESIONS

M. Shamkhalova, M. Shestakova, L. Chugunova and I. Dedov. National Endocrinology Center, Moscow, Russia

The role of the endothelium in adjustment of vascular tone and intrarenal haemodymanics is mediated by the interaction of powerful vasoactive factors. The aim of our study is to investigate a role of these factors - endothelin-1 (ET-1), prostacyclin (PGI2) and tromboxane $\mathrm{A} 2$ (TXA2) in the initiation and progression of diabetic renal disease. We studied plasma levels of PGI2, ET-1 and TXA2 in 31 NIDDM patients. 18 of them had no sings of renal involvement, 13 patients showed overt diabetic nephropathy (DN) - AER $>300 \mathrm{mg} /$ day. In the former patients the blood concentration of vasodilating factor PGI2 than of vasoconstricting factor ET-1 and TXA2. In diabetic nephropathy the balance of vasoactive factors shifted to predominance of vasoconstrictors ET-1 and TXA2. Results $(M=S E M)$ :

\begin{tabular}{|lccc|}
\hline Parameters & Controls & AER $<300 \mathrm{mg} /$ day & AFR $>300 \mathrm{mg} /$ day \\
\hline & $\mathrm{n}=8$ & $\mathrm{n}=18$ & $\mathrm{n}=13$ \\
\hline PGI2 $(\mathrm{pg} / \mathrm{ml})$ & $413 \pm 101$ & $350 \pm 87$ & $323 \pm 92$ \\
\hline TXA2(pg/ml) & $364 \pm 95$ & $149 \pm 21^{*}$ & $990 \pm 142^{*}$ \\
\hline ET-1 $(\mathrm{pg} / \mathrm{ml})$ & $1,3 \pm 0,3$ & $1,5 \pm 0,2$ & $2,1 \pm 0,2^{*}$ \\
\hline
\end{tabular}

${ }^{*} p<0,05$ compared to controls and patients with AER $<300 \mathrm{mg} /$ day Such rearrangement of vasoactive factors in higher quantities of vasoconstrictors in diabetes mellitus may initiate or promote progression of DN with resultant spasm of afferent glomerular vessels, reduced glomerular filtration and renal blood flow rates, arterial hypertension and increased thrombogenesis. We can conclude that elevated levels of ET-1 and TXA2 in diabetics and their rise with progression of diabetic nephropathy are likely to act as pathogenetic factors underlying onset and progression of nephropathy.

\section{3}

EFFECT OF A 1-YEAR TREATMENT WITH RAMIPRIL OR ATENOLOL ON ENDOTHELIAL MARKERS IN PATIENTS WITH TYPE-2 DIABETES Eibl N, Krugluger W*, Wagner S*, Schnack Ch, Hopmeier P \& Schernthaner G (1.Med. Department and *Central Laboratory, Rudolfstiftung Hospital, Vienna) Endothelial dysfunction is associated with increased cardiovascular risk in patients with type-2 diabetes and increased urinary albumin excretion rate (UAER). Results of endothelia! markers as endothelin-1 (ET-1), von Willebrand factor (vWF) and thrombomodulin (TM) are conflicting in patients with diabetic late complications. The aim of our study was to compare the effect of blood pressure reduction after one year of treatment by two different antihypertensive drugs, the ACE inhibitor ramipril and the beta blocker atenolol on plasma-ET-1, vWF and thrombomodulin. We investigated 56 patients with type-2 diabetes and increased UAER (Age:63 1, 5yrs; HbAlc: $7,5 \pm 0,3 \%$; UAER: $151 \pm 8 \mathrm{mg} / 24 \mathrm{~h}$ ) that were randomised in 2 groups receiving ramipril $(n=27)$ or atenolol $(n=29)$. All endothelial markers were significantiy increased in diabetic patients compared to 32 controls (ET-1: $1,3 \pm 0,16$ vs $0,75 \pm 0,2 \mathrm{pg} / \mathrm{ml}$, p $<0,04$; vWF: $308 \pm 26$ vs $165 \pm 25 \%, \mathrm{p}<0,001$; TM: $30,1 \pm 1,8$ vs $23,7 \pm 1,3 \mathrm{ng} / \mathrm{ml}, \mathrm{p}<0,02$ ). After 1 year of treatment blood pressure values declined significantly but no significant change of endothelial markers could be demonstrated in either group.

\begin{tabular}{|l|c|c|c|c|}
\hline & Atenolo & $(n=29)$ & Ramipril & $(n=27)$ \\
\cline { 2 - 5 } & OMo & 12 Mo & OMo & 12 Mo \\
\hline RR syst. $(\mathrm{mmHg})$ & $180(170-190)$ & $155^{*}(144-160)$ & $170(160-183)$ & $150^{*}(140-160)$ \\
RR diast $(\mathrm{mmHg})$ & $100(95-104)$ & $85^{*}(80-90)$ & $100(95-105)$ & $90^{*}(83-90)$ \\
ET-1(pg/ml) & $1,3 \pm 0,2$ & $1,2 \pm 0,2$ & $1,2 \pm 0,2$ & $1,3 \pm 0,3$ \\
TM (ng/ml) & $26,9 \pm 1,4$ & $25,8+1,7$ & $32,9 \pm 3,1$ & $30,7 \pm 2,1$ \\
WWF (\%) & $330 \pm 33$ & $321 \pm 34$ & $289 \pm 43$ & $292 \pm 41$ \\
UAER (mg/24h) & $158 \pm 8$ & $166 \pm 7$ & $143 \pm 7$ & $150 \pm 8$ \\
& $* 0<0,001$ & & & \\
\hline
\end{tabular}

The endothelial markers (ET-1, VWF, TM) did not show an intercorrelation and were also not related to UAER, metabolic or lipid parameters. Despite a significant decline of UAER ( $147 \pm 8$ vs $120 \pm 8 \mathrm{mg} / 24 \mathrm{~h}, \mathrm{p}<0,01$ ) in a subgroup of 23 patients there was no significant change in ET-1, vWF or TM levels

Our data indicate that endothelial injury seems to be persistent in patients with type- 2 diabetes and microalbuminuria.

\section{4}

DOES INSULIN INFLUENCE VON WILLEBRAND FACTOR RELEASE IN NORMAL, GLUCOSE INTOLERANT AND DIABETIC SUBJECTS ?

L. Kessler, A. Azimzaded, M L Wiesel, V. Legaludec, P. Wolf, J.P. Cazenave and M. Pinget, Service d'Endocrinologie, * Fondation Transplantation UPRES JE 2601, Hôpitaux Universitaires, Strasbourg, France

Von Willebrand factor ( $\mathrm{vWf}$ ) is a predictive marker of diabetic angiopathy. Its release, independant of glycemic control, could be influenced by the hyperinsulinemia existing in both type I and type II diabetes. The aim of this work was to study the role of insulin on plasmatic vWf release in yivo, in normal and diabetic subjects and in vitro, on cultivated human endothelial cells. Material and Method: prospectively, an oral glucose tolerance test (OGTT) followed by plasmatic measurements of glucose, insulin, C peptide was performed in patients exhibiting diabetes risk factors. $v \mathrm{Wf}$ antigen and plasminogen activator inhibitor (PAI) were measured in plasma at 0,30 and 180 minutes of OGTT. In vitro, human normal endothelial cells isolated from saphen vein were incubated in the presence of thrombine $(10 \mathrm{U} / \mathrm{ml})$ and human insulin $(0.25$ and $250 \mathrm{mIU} / \mathrm{ml})$. After 2 and 6 hours, vWf was measured in the culture medium by ELISA $(n=3)$. Results: 28 patients were enclosed in the study (March 1, 1998): 11 patients (age $=41+11.8$ years, BMI $=$ $29.4 \pm 4.7$ ) have a normal glycemic profil (groupe I) , 7 patients (age $=43.5 \pm 11$ years, $\mathrm{BMI}=31 \pm 4.1$ ) developped glucose intolerance (groupe II) and 10 (age = $55.2 \pm 10.21$ years, $\mathrm{BMI}=33+8.1$ ) proved diabetic (groupeIII). In group I, insulinemia $(\mathrm{t}=0 ; 10.4 \pm 3.8 \mu \mathrm{U} / \mathrm{m})$ increased to $45.5+18.3 \mu \mathrm{U} / \mathrm{ml}$ at 30 minutes and returned to $17.2 \pm 7.3 \mu \mathrm{U} / \mathrm{ml}$ at the end of the test while $\mathrm{vWf}$ levels were normal $(82 \pm 22 \%)$ and the PAI levels were increased $(19.6 \pm 10.6 \mathrm{ng} / \mathrm{ml})$ all along the test. In both groups II and III, $v \mathrm{Wf}$ and PAI were similar to group I while insulinemia was significantly increased to $62 \pm 19.2 \mu \mathrm{U} / \mathrm{ml}$ at 30 minutes in group II and to $39.23 \pm 17.5 \mu \mathrm{U} / \mathrm{mI}$ at 180 minutes in group III $(p<0.05)$. After 2 and 6 hours of incubation, vWf levels expressed as \% of basal secretion were repectively of $76 \%$ and $91 \%$ in presence of insulin at $0.25 \mathrm{mIU} / \mathrm{ml}$ and decreased to $52 \%$ and $69 \%$ for a concentation of 250 $\mathrm{mIU} / \mathrm{ml}$. In contrast, after thrombine stimulation, the $\mathrm{vWf}$ levels were increased by a factor of 3.7 and 5.0. In conclusion: in vitro, high concentation in insulin decreased the $\mathrm{vWf}$ release from human normal endothelial cells. In normal and diabetic subjects, insulin did not stimulate $\mathrm{vWf}$ release. 
PS 65

\section{Adhesion Molecules and Vascular Complications}

\section{5}

\section{PLASMA INTERCELLULAR ADHESION MOLECULE-1 LEVELS} IN PATIENTS WITH TYPE II DIABETES MELLITUS

S. GÜLER*, B. ÇAKIR*, B. DEMIRBAȘ ${ }^{\star}$, U. ÖNDE**, G. GǗRSOY*, R. SERTER* and Y. ARAL*. Ankara State Hospital *Department of Endocrinology and Metabolism and **Department of Microbiology, Ankara, Turkey.

It has previously been reported that plasma soluble intercellular adhesion molecule-1 (sICAM-1) level is increased in patients with diabetes mellitus. Aim of our study was to investigate any relation between plasma sICAM-1 levels and diabetic retinopathy, proteinuria and bypertension in patients with type II diabetes mellitus. Although plasma sICAM-1 level was higher in 68 diabetic patients compared to the healthy control subjects $(n=21)$, this difference was not statistically significant $(391.65 \pm 120.40 \mathrm{ng} / \mathrm{mL}$ vs $348.92 \pm 89.97 \mathrm{ng} / \mathrm{mL}$, p >0.05). Plasma sICAM-1 levels showed positive correlation with fasting plasma glucose $(r=0.25, p=0.04)$, postprandial plasma glucose $(r=0.31, p=$ 0.01) and HbA1C levels $(r=0.26, p=0.04)$. Patients with postprandial plasma glucose levels $251 \mathrm{mg} / \mathrm{dl}$ or over had higher sICAM-1 levels compared to patient with postprandial plasma glucose below $251 \mathrm{mg} / \mathrm{dL}$ (427.71 \pm 103.67 $\mathrm{ng} / \mathrm{mL}$ vs $340.10 \pm 127.58 \mathrm{ng} / \mathrm{mL} ; \mathrm{p}=0.003$ ). sICAM-1 level was significantly higher in diabetic patients with proteinuria than in diabetics without proteinuria (430.25 $\pm 104.33 \mathrm{ng} / \mathrm{mL}$ vs $369.19 \pm 124.51 \mathrm{ng} / \mathrm{mL}, p<0.05$ ). sICAM-1 level was significantly lower in diabetic patients with hypertension than in normotensive diabetics $(358.26 \pm 124.81 \mathrm{ng} / \mathrm{mL}$ vs $416.46 \pm 112.24$ $\mathrm{ng} / \mathrm{mL}, \mathrm{p}<0.05$ ). Mean sICAM-1 level was $405.88 \pm 115.38 \mathrm{ng} / \mathrm{mL}$ in diabetics with nonproliferative retinopathy, $398.62 \neq 93.98 \mathrm{ng} / \mathrm{mL}$ in diabetics with proliferative retinopathy , and $369.44 \pm 149.19 \mathrm{ng} / \mathrm{mL}$ in diabetic patients with no retinopathy; differences between the groups did not reach statistical significance. In conclusion our data suggest us a relation between sICAM-1 and diabetic metabolic control, and sICAM-1 may have a role in the pathogenesis of diabetic microvascular complications.

\section{7}

INTRACELLULAR GSH VALUES INFLUENCE VCAM-1 PLASMA LEVELS IN TYPE 2 DIABETES MELLITUS.

G.De Mattia, *M.C.Bravi, O.Laurenti, *M.Cassone-Faldetta, O.De Luca, A. Arrniento,F.Sperli,C.Ferri. Chair of I Clinica Medica, Fondazione "A.Cesalpino", University "La Sapienza" Rome, *Department of Internal Medicine, University of L'Aquila, Italy.

Elevated plasma soluble vascular adhesion molecule-1 (sVCAM-1) levels have been recently described in type 2 diabetic patients and suggested to be due to increased oxidative stress. According to this, antioxidant agents, namely $\mathrm{N}$-acetylcysteine (NAC) and pyrrolidine dithiocarbamate, reduced VCAM-1 expression by human vascular endothelial cells. Aim of our study was to verify the effects of antioxidant therapy on plasma sVCAM-1 levels in vivo. Therefore, we studied 15 type 2 diabetic patients and 10 healthy controls, excluding subjects affected by inflammatory and neoplastic diseases. In diabetic patients intraerythrocytic GSH and SVCAM-1 were tested at baseline and after chronic administration of NAC $(1200 \mathrm{mg} /$ die for 1 month). Statistical analysis was performed by t-test for paired data and by the linear regression coefficient. With respect to control group, diabetic patients showed elevated sVCAM- $1 \quad(707.9 \pm 52.5$ vs $627.3 \pm 84.6 \mathrm{ng} / \mathrm{ml} p=0.007)$ and reduced GSH levels $(6.0 \pm 0.7$ vs $7.1 \pm 0.9 \mathrm{umol} / \mathrm{gHb}, p=0.002)$. After NAC treatment $\mathrm{sVCAM}-1$ significantly decreased $(707.9 \pm 52.5$ vs $663.1 \pm 73.9 \mathrm{ng} / \mathrm{ml}$, $p=0.01)$ while GSH significantly increased $(6.0 \pm 0.7$ vs $6.3 \pm 0.8$ $\mu \mathrm{mol} / \mathrm{gHb}, p=0.04)$. In diabetic patients baseline sVCAM-1 and GSH levels were negatively correlated. $(r=-0.6, p=0.01)$. In conclusion, NAC treatment increased intraerythrocytic GSH levels and decreased plasma soluble VCAM-1 levels. This finding could be due to reduced oxidative stress, in turn responsible for the activation of oxidativesensitive NF-kB pathway, and the consequent upregulation of VCAMI.

\section{6}

THE EFFECT OF SMOKING ON PLASMA LEVELS OF CICAM-1 IN TYPE 1 DIABETIC PATIENTS

G.Zoppini, G.Targher, L.Bertolini, A.Guerriero, M.Muggeo Division of Metabolic Diseases, University of Verona, Italy

The aim of the study was to evaluate plasma levels of circulating intercellular adhesion molecule-1 (cICAM-1) in 54 type 1 diabetic patients and 20 controls and to study the effect of smoking on this molecule. The two groups were well matched for sex (M/F 30/24 vs $12 / 8)$, age ( $32 \pm 1$ vs $32 \pm 1)$, BMI $(24 \pm 0.5$ vs $23 \pm 0.6 \mathrm{Kg} / \mathrm{mq})$, blood pressure $(128 \pm 2.4 / 81 \pm 1$ vs $123 \pm 2.2 / 80 \pm 1 \mathrm{mmHg})$, plasma lipids (CT $4.7 \pm 0.1$ vs $5.0 \pm 0.1 \mathrm{mmol} / \mathrm{l}$; TG $1.1 \pm 0.1$ vs $1.1 \pm 0.1 \mathrm{mmol} / \mathrm{l}$ ) and for the proportion of smokers ( 33 vs $50 \%$ ). The concentration of cICAM-1 (ELISA) was elevated in type 1 patients compared to control ( $279 \pm 8$ vs $224 \pm 12 \mathrm{ng} / \mathrm{ml}, \mathrm{p}<0.001)$. In both groups smoking showed a significant association with plasma levels of cICAM-1. In diabetic patients the relation between smoking and cICAM-1 was dose-dependent with a significant trend $(p<0.001$ for trend), in detail: nonsmokers $(261 \pm 7 \mathrm{ng} / \mathrm{ml})$, less than 10 cigarettes/day $(294 \pm 20 \mathrm{ng} / \mathrm{ml})$ and more than 10 cigarettes/day $(341 \pm 21 \mathrm{ng} / \mathrm{ml})$. The results of the present study confirm the presence of elevated levels of cICAM-1 in type 1 patients and suggest a harmful effect of smoking. Thus, our study strengthen the importance of quitting smoking in young type 1 diabetic patients.

\section{8}

LEUKOCYTE ROLLING IN DIABETIC RAT SKELETAL MUSCLE. JH Reitsma, FM Jacobs, S IJsselmuiden, MH van Wijhe, C Demirci*, $A A$ van Lambalgen and GJ Tangelder. Lab for Physiology, Research School Icar-VU, Vrije Universiteit Amsterdam, The Netherlands, and *Department of Biology, Istanbul Universitesi, Turkey.

As the functional and mechanical properties of endothelial cells and leukocytes change during the development of diabetes mellitus, a disturbed interaction of leukocytes with the vessel wall may arise. Such an altered interaction may partially explain the (micro)vascular complications associated with diabetes mellitus. To test this hypothesis, we measured leukocyte-vessel wall interactions in the spinotrapezius muscle of both control rats (non treated, $n=7$ ), and diabetic rats ( 6 weeks after STZ injection, $60 \mathrm{mg} / \mathrm{kg}, \mathrm{n}=6$ ). Using intravital microscopy, we were able to ascertain rolling leukocytes flux and velocity in the microcirculation of the rat. Blood samples were collected at 0 and 6 weeks after STZ injection to describe leukocyte population. STZ treatment resulted in elevated glucose levels $(27 \mathrm{mM}$ vs $8 \mathrm{mM}$ in control) and lowered mean arterial pressure (115 vs 135 $\mathrm{mmHg}$. No change in cardiac index and hematocrit was observed. The rolling leukocyte flux (number of rolling leukocytes/min corrected for venular diameter) increased in diabetic animals (146 vs. 59 $\mathrm{p} \leq 0.05)$, the mean rolling velocity $(-50 \mu \mathrm{m} / \mathrm{s})$, however, remained unaltered. Simultaneously, we observed an increase in the absolute number of leukocytes (10.4 vs $\left.5.4 \times 10^{9} / 1\right)$, and a shift in relative mononuclear/polynuclear leukocyte frequency $(73 / 27$ vs $88 / 12 \%)$ for diabetic vs control rats, respectively. In conclusion, the increased rolling leukocyte flux observed in diabetic rat microcirculation affirms the hypothesis of an altered leukocyte-vessel wall interaction. Whether this altered leukocyte flux is due to a shift in leukocyte population remains to be investigated. 
PS 66

\section{Oxydative Stress and Complications}

\section{9}

IN VIVO ANTIOXIDANT STATUS AND BASAL LEVELS OF DNA DAMAGE IN WHITE BLOOD CELLS OF IDDM PATIENTS

C.R. Barnett1, M.P.A. Hannon-Fletcher 1, M.J. O'Kane ${ }^{2}$, K.W. Moles², and Y.A. Barnett1. 'Cancer and Ageing Research Group, University of Ulster Coleraine, N. Ireland, BT52 1SA. ${ }^{2}$ Altnagelvin Hospital, Londonderry, N Ireland, BT47 1SB

Inter-individual differences in the efficacy of defence systems against free radicalinduced damage may contribute to differences in the onset of complications. The current study has assessed in vivo antioxidant status and basal levels of DNA damage in white blood cells from IDDM patients. 25ml of peripheral blood was collected from 23 IDDM patients and 32 age- and sex-matched control subjects. Levels of antioxidants were determined in plasma and red cell concentrate using commercial kits. Lymphocyte, monocyte and neutrophil fractions were separated by density gradients and basal levels of DNA damage were quantitated using the alkaline comet assay. Levels of glutathione peroxidase $(44.3 \pm 1.97$; $37.13 \pm 1.97 \mathrm{U} / \mathrm{gHb}, \mathrm{p}<0.05)$, uric acid $(290.3 \pm 12.05 ; 219.83 \pm 13.92 \mu \mathrm{mol} / 1$, $\mathrm{p}<0.05)$, vitamin $C(4.43 \pm 0.45 ; 2.64 \pm 0.51 \mu \mathrm{g} / \mathrm{ml}, \mathrm{p}<0.01)$ and superoxide dismutase $(304.1 \pm 19.96 ; 1172.06 \pm 57.69 \mathrm{U} / \mathrm{gHb}, \mathrm{p}<0.01)$ were significantly decreased, and catalase was significantly increased $(44.5 \pm 2.65 ; 57.16 \pm 4.80$ $\mathrm{k} / \mathrm{gHb}, \mathrm{p}<0.01$ ), in IDDM patients compared to controls. There was significantly more DNA damage in the neutrophils $(2.3 \pm 0.23 ; 4.75 \pm 0.46$, \%Tail DNA Damage, $p<0.001$ ), but no significant differences in levels of DNA damage in lymphocytes or monocytes, from IDDM patients compared to controls Disturbances in neutrophil function within diabetic patients have been reported elsewhere. In addition such neutrophils are known to be in a chronic low state of activation generating sufficient reactive oxygen species (ROS) to induce DNA damage in vitro. The results of our study suggest that the ROS are actually causing DNA damage within the neutrophils themselves, which may be exacerbated by the observed altered antioxidant status in the IDDM patients. As such, disturbances in neutrophil function observed in diabetic patients may be attributable to the increased levels of DNA damage in these cells.

\section{1}

ADEQUATE ANTIOXIDANT DEFENCE IN TYPE 1 DIABETES? E. Szaleczky, K. Farkas, B. Sármán, I. Kocsis and A. Somogyi 2nd Department of Medicine, Semmelweis University of Medicine, Budapest, Hungary

Diabetes is accompanied by an imbalance between prooxidant and antioxidant factors and this oxidative stress could play a role in the development of diabetic complications. These observations have been made on newly diagnosed of badly controlled diabetics with remarkable hyperglycaemia and all the metabolic changes necessarily accompanying relative insulin deficiency. 40 non-diabetic and 25 type I diabetic subjects matched in age and BMI - were studied in order to evaluate the effect of diabetes controlled by intensive insulin treatment on the antioxidant status. Mean duration of diabetes was $10.6 \pm 7.95$ years; a mean HbAlc value of $\mathbf{7 . 8 9} \pm \mathbf{2 . 5 3 \%}$ implied a good blood glucose control. All the diabetic subjects were on intensive insulin therapy. Serum total sulfhydril group content was measured spectrophotometrically using Ellman reagent, Glutathion Peroxidase activity by the method of Paglia and Valentine (Ransel), Superoxide Dismulase activity was measured in a xanthine/xanthine oxidase system (Ransod); for determination of plasma total antioxidant status Randox TAS kit (Abts, peroxide and $\mathrm{H}_{2} \mathrm{O}_{2}$ ) was used. Of the serum major antioxidants uric acid and albumin levels and total sulfhydril groups content were significantly lower in the diabetic group ( 287 vs. $224 \mu \mathrm{M}, 53$ vs. 50 $\mathrm{g} /$, and $7.39 \mathrm{vs.} 6.66 \mathrm{mmol} / \mathrm{gprotein}, \mathrm{p}<0.001, \mathrm{p}<0.05$, and $\mathrm{p}<0.001$ ). Of the antioxidant enzyme activities only whole blood Glutathione Peroxidase was impaired in patients with diabetes ( $56 \mathrm{vs.} 51 \mathrm{U} / \mathrm{gHb}, \mathrm{p}<0.05$ ), while erythrocyte Superoxide Dismutase and plasma Glutathione Reductase did not differ in the two groups. Similarly Total Antioxidant Status - a measure of overall antioxidant defence in plasma - was not statistically different in controls and diabetics. In a randomly selected cohort of IDDM patients with good indices of glycemic control we could not find signs of a severe imbalance of the antioxidant enzyme system. Type 1 diabetes, if blood glucose is strictly controlled by intensive insulin therapy, is only partly accompanied by changes in the prooxidant - antioxidant balance.

\section{0}

ANTIOXIDATIVE SYSTEMS IN WELL METABOLICALLY CONTROLLED TYPE 1 DIABETIC PATIENTS

A.Majchrzak, M.Kempa, D.Zozulińska and B. WieruszWysocka. Poznań Diabetic Center, Poznań, Poland

Oxidative stress plays an important role in the pathogenesis of chronic complications of diabetes. The aim of the study was to evaluate the antioxidative systems in blood obtained from well metabolically controlled diabetic patients. The study was entered by 30 patients with type 1 diabetes ( 18 female and 12 male, aged $30.2 \pm 10.8$ years with mean duration of disease $8.37 \pm 6.56$ years, $\mathrm{HbAl}$ c $6.8 \pm 1.6 \%) .24$ healthy, sex- and agematched volunteers served as controls. We evaluated the levels of plasma ceruloplasmin (micromethod of $\mathrm{p}$ phenylendiamine oxidation), reduced glutathione in erythrocyte lysate (colorimetric method by Bioxytech S.A) and serum glutathione peroxidase (ELISA method by Bioxytech S.A.). We found significantly higher reduced glutathione level in diabetic patients in comparison with controls $(11.01 \pm 0.78$ vs $3.75 \pm 0.47 \mu \mathrm{mol} / 1, \mathrm{p}<0.001)$. The levels of ceruloplasmin and glutathione peroxidase did not differ significantly from values obtained in healthy subjects $(39.72 \pm 2.73$ vs $41.50 \pm 2.85$ $\mathrm{mg} / \mathrm{dl}$ and $5.51 \pm 0.42$ vs $5.45 \pm 0.16 \mathrm{ng} / \mathrm{ml}$, respectively, $\mathrm{p}>0.05$, $p>0.05$ ). The results obtained in our study suggest that good metabolic control of diabetes might normalize the function of antioxidative systems in type 1 diabetic patients.

\section{2}

EARLY MPAIRMENT OF ANTIOMDANT DERENCES IN TYPE 1 DIAEETIC WOMEN WTHOUT COMPLCATIONS AND IN GOOD METABOLC CONTROL

P. Cotroneo, G. Marra, S.A Santini*, A. Manto, D. Pitocco, L. Todaro, M. and G.Ghirlanda. Dept of Internal Med. Catholic University and Biological Chemistry ${ }^{*}$, Rome, Italy.

Mang apidemiological studies suggest that the protective effect of female sen is loss in presence of diabetes mellitus. Accumulating evidences suggest that oxidative ingury caused by tree radicals contributes to the development of diabetic complications, Recently was reported that IDDM patients shom a decreased antioxidant defences regardless of duration of disease, metabolic control ar the presence of disbetic complications. We determined plasm antioxidant defences, rneasuring the total radical-trapping capacity (TRAP) and mazkers of oxida tive stress such as lipid hydroperoxides (ROOHs) in 3 ? IDDM patients with short duration of disease (mean $6 \pm 3$ yrs; ramge $1-10 \mathrm{yts}$ ), without evidence of clinical diabetic complications and good metabolic control (mean Hb.A1c: 7,4t1.1\%), no assuming othey drugs than insulin, compared with 22 control subjects. Significative differences were found between females and males in diabetic subjects in TRAP and ROOHs levels (TRAF 579.8+95.4 vo $720.3 \pm 111.2 \mathrm{mmol} / 1, \mathrm{~F}=0.0002 \mathrm{~F}$ w. M, ROOH $8.1 \pm 1.7$ s $6.7 \pm 2.1 \mathrm{mmol} / 1, \mathrm{P}=0.03$ F vs. M). Significative irverse relationship was found between TRAF and ROOHs in all disbetic patients $\left(\mathrm{P}<0.0001, \mathrm{R}^{2}=0,685\right)$. No relationships were found between age, HbA1c, lipid profile, uic scid and duration of disease with the parameters of oxidative stress considesed. Our data suggest that type 1 diabetic patients show an early reduced antioxidant capacity ard augnented levels of lipid peyoxides, during the course of disease. This alteyation seems to appear more early in disbetic women, offering a probable pathogenetic explanation of the ligher cardionssculay complications observed in disbetic woman respect to the general popula tion in the epiderriological studies. 
EVIDENCE AGAINST A DIABETES-INDUCED GENERALIZED INCREASE OF OXIDATIVE DAMAGE IN LIVER CYTOSOLIC PROTEINS

M. Portero-Otin, R. Pamplona, D. Riba, C. Ruiz, M. A. Lafarga, M.J. Bellmunt and J. Prat Metabolic Pathophysiology Research Group. Dept de Ciencies Mediques Básiques, Universitat de Lleida, Avda Rovira Roure 44. E-25198 SPAIN.

Despite available experimental evidence suggest an increased oxidative stress in diabetes the protein oxidative damage has not been evaluated in this disease. In this work, we have analysed several markers of protein structural modifications induced by free radicals in liver cytosolic fractions of rats with streptozotocin-induced diabetes. Protein oxidation was assessed using Westem blot, by a chromatographic assay for carbonyl quantification, and by other compositional measurements. In order to relate values of carbonyl and other modifications in terms of protein degradation, the proteolytic capacities of cytosol were evalualed using oxidatively modified proteins. Moreover, proteasome activity was measured using the fuorogenic substrate succinyl-Leu-Leu-Val-tyrosine-4-methylcoumarin-7-amide since proteasome activity has been implied in the tumover of oxidatively damaged proteins. Surprisingly, the Westem-blot for protein carbonyl showed a higher staining in samples from control rats. The chromatographic quantitation confirmed the fact that carbonyl content is not increased in diabetes $(2.85 \pm 1.18$ vs $4.25 \pm 1.69 \mathrm{nmol}$ carbonylymg protein, diabetic vs control; $p=0.1)$. Results did not show significant differences between diabetic and control

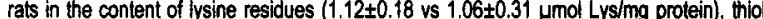
side chains $(52.21 \pm 7.88$ vs $48.12 \pm 7.49 \mathrm{nmol} / \mathrm{mg}$ protein), and side chain amino $(0.86 \pm 0.16$ vs $0.76 \pm 0.1\{\mathrm{mg}$ eq BSA $\mathrm{mg}$ protein). Diabetic cytosol exhibit significantly lower proteolysis over oxidatively modified superoxide dismutase (11\% lower, $p<0.05$ ), while proteolysis over native superoxide dismutase or bovine serum albumin was not significantly different. Further confirmation of decreased cytosolic proteolysis was obtained comparing the digestion of a known proteasome substrate $(165 \pm 19.7 v s \quad 231.7 \pm 36$ AFUs, $\rho<0.005)$ Despite other types of oxidative damage (glyco or lipoxidative modificalions) have not been evaluated in this work, the present data suggest that the steady state levels of oxidative damage of proteins in the soluble fraction of liver homogenates are not increased by the diabetic status.
ORAL VITAMIN E SUPPLEMENTATION AND OXIDATIVE STRESS IN TYPE 1 DIABETIC PATIENTS AND CONTROLS

MJSampson a , A Langrish - Smith a , RHGreenwood a ,PJ Heybum ${ }^{\text {a }}$, RC Temple ${ }^{a}, S$ Astley $S b, C W a r d ~ C a$ and S Southon $S$ b a Bertram Diabetes Research Unit, Bertram Diabetes Centre, Norfolk and Norwich Healthcare NHS Trust, Norwich and ${ }^{\mathfrak{b}}$ Institute of Food Research, Colney, Norwich, UK.

Increased oxidative stress and damage to lipoproteins, endothelial cells and DNA may increase vascular risk in Type 1 diabetes. Antioxidant supplementation might theoretically reduce direct oxidative damage and susceptibility to oxidative damage The aim of this study was to examine the impact of oral vitamin E supplementation on measures of oxidatively induced damage in Type 1 diabetes. In a double - blind, placebo - controlled randomised prospective trial we studied the effect of eight weeks vitamin E (400 iu) supplementation on oxidatively induced DNA single strand breaks (SSB) and copper induced low density lipoprotein (LDL) oxidisability in 41 Type 1 diabetic patients (mean age $39 \pm 1.1$ yrs SE; mean HbA1C $8.7 \pm 0.2 \%$ ) and 36 matched non - diabetic controls. SSB were measured using the COMET assay, directly visualising SSB in lymphocyte DNA. Groups did not differ in LDL oxidisability or SSB rates at baseline. Vitamin $E$ supplementation had no significant effect on lag phase measurements in diabetic patients $(59.8 \pm 2.2$ mins SE baseline vs $59.8 \pm 3.8$ after 8 weeks) or controis ( $56.5 \pm 3.2$ mins baseline ys $69.0 \pm 4.5$ mins after 8 weeks) and had no effect on SSB rates in diabetics or controls, either basally or after treatment with hydrogen peroxide ex vivo. Plasma vitamin $\mathrm{E}$ levels increased significantly in vitamin E supplement groups. Vitamin E supplementation at standard dosages for 8 weeks does not reduce oxidative damage or susceptibility to damage in either Type 1 diabetic patients or controls.
1245

HAEMOSTATC STATUS AND ENDOTHELIAL FUNCTON IN UNCOMPLCATED, WELL CONTROLLED IDDM PATIENTS

G. Marra, A Tordi *, S. Di Gregorio*, P. Cotroneo, A Manto, D. Pitocco, L. Todaro, M. M. Ariaso*, M V. Ciarla*, R Strom* and G.Ghirlanda Dept of Internal Med. Catholic University. and * Dept of Biochemistry, Univ. La Sapienza, Rome, Italy.

Diabetic pationts have an hiepher risk of corriovescular discase. Disturbances of haemostatic system are well-identified risk fectors for coronary heart disease. We evaluated the coagulation gystem in 57 IDDM patients (mean age 31.447.7 yrs, duration of disease $11.5 \pm 6.5 \mathrm{Ym}, \mathrm{HbAlc} 7.4 \pm 1.1 \%$ ) without hypertension, dy slipidemin and vascular disease. We examinated the following permetera fibrinogen antigen (F As), fibrimosen Chus (F C), fibronectin (PNT), $\mathrm{Cl}$ inhibitor antigen and \% (Cl I As), phesminozen (PLO), al macroglobulin (a2 M), D-dimers (D-dim), fibrin monomens (FM), endothetin (ET), C reactive protein (RCR). The results are stown below:

\begin{tabular}{|c|c|c|c|}
\hline & DDM & Controls & P Valuo \\
\hline $\mathrm{n}^{\circ}$ & 57 & 44 & \\
\hline$\overline{F A g}(\mathrm{mg} / \mathrm{dl})$ & $244 \pm 51$ & $267 \pm 55$ & 0.03 \\
\hline$\overline{\mathrm{FC}}(\mathrm{m} / \mathrm{g} / \mathrm{d})$ & $231 \pm 60$ & $238 \pm 60$ & ns \\
\hline FNT $(\mathrm{g} / \mathrm{l})$ & $0.33 \pm 0.06$ & $0.38 \pm 0.06$ & $<0.001$ \\
\hline CI IA $(\mathrm{mg} / \mathrm{dl})$ & $31.4 \pm 4.4$ & $29.0 \pm 3.4$ & 0.003 \\
\hline $\mathrm{Cl} 1 \%$ & $103 \pm 11$. & $102 \pm 11$ & as \\
\hline PLG\% & $92 \pm 17$ & $101+12$ & 0.002 \\
\hline${ }^{2} 2 \mathrm{M}(\mathrm{mg} / \mathrm{dl})$ & $226 \pm 53$ & $191+45$ & 0.001 \\
\hline D-dim. (ng/1) & $305 \pm 187$ & $226 \pm 32$ & 0.04 \\
\hline FM (ng/) & $19.6 \pm 11$ & $8.6 \pm 5.5$ & $<0.001$ \\
\hline PCR $(\mathrm{mg} / \mathrm{dl})$ & $1.6 \pm 1.8$ & $0.7 \pm 0.4$ & 0.005 \\
\hline ET (fmol/ml) & $2.8 \pm 3.6$ & $0.5 \pm 0.02$ & $<0.001$ \\
\hline
\end{tabular}

There is a inverse conrelation between FNT and ET $(p=0.05, \mathrm{R}-0.32)$ and a direct correbtion botwoen ET and D-Dim. In IDOM pationts wo found: 1) an endothetial didunetion ( $\uparrow$ ET and PCR, \ FNT); 2) a prothrombotic attus ( $\uparrow$ FM);3) an increased fibrinolyais ( $\uparrow$-Dim and $\downarrow \mathrm{PLO}$ ); 4) an increase of fibrinolyzis inhibitors ( $\uparrow \mathrm{Cl}$ I $\mathrm{As}$ and $\uparrow \mathrm{a} 2 \mathrm{M}$. These date suggest an earty endothelial stress and an hypercongulability status in uncomplicated, wollcontrolied IDDM patients.

\section{6}

HYPERGLYCAEMIA AFFECTS MITOCHONDRIAL DNA CONTENT, BUT NOT OXIDATION OF DNA AND PROTEINS OF DIABETIC PATIENTS

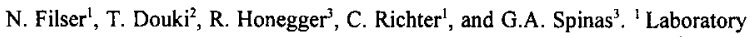
of Biochemistry I, Swiss Federal Institute of Technology, Zurich; ${ }^{2} \mathrm{CEA}$, Grenoble, France; ${ }^{3}$ Department of Endocrinology and Diabetes, University Hospital, Zürich.

Increased production of reactive oxygen species (ROS) and lipid peroxidation may contribute to vascular complications in diabetes. In the present study the effect of chronic hyperglycaemia on mtDNA content, as well as on DNA and protein oxidation was assessed. Twenty male diabetic patients $(10$ with $\mathrm{HbAlc}<8 \%, 10$ with $\mathrm{HbAlc}>8 \%$ were studied. MtDNA content. Nine agematched healthy volunteers served as control subjects. 80HdG and protein oxidation was measured. DNA was extracted from purified lymphocytes from whole blood. MtDNA was linearised, separated on a $0.8 \%$ agarose and Southernblotted gel was hybridised against a human mtDNA probe. The hybridised membrane was analysed with ImageQuant. $80 H d G$ was assayed by HPLC combined with electrochemical detection. Determination of carbonyl content of oxidatively modified proteins was performed using tritiated $\mathrm{NaBH}_{4}$. Results: Patients with $\mathrm{HbAl}>8 \%$ exhibited significantly decreased $m$ tDNA content $(0.83 \pm 0.1 ; n=6)$ as compared to diabetics with $\mathrm{HbAlc}<8 \%(1.57 \pm 0.1 ; n=6)$ and controls $(1.25 \pm 0.21 ; n=4)(p<0.001)$. The levels of $80 \mathrm{HdG}$ were different between the groups: Subjects with $\mathrm{HbAlc}>8 \%$ having $0.0129 \pm 0.0032$, as compared to $0.0156 \pm 0.0015$, and $0.0171 \pm 0.0036 \mathrm{pmol} 80 \mathrm{OHdG} / \mu \mathrm{g} \mathrm{DNA}$ in subjects with $\mathrm{HbAlc}<8 \%$ and controls, respectively. Lymphocytes derived from males with $\mathrm{HbA} 1 \mathrm{c}>8 \%$ exhibit a slightly higher $(1.08 \pm 0.19)$, those patients with $\mathrm{HbAlc}<8 \%$ a lower level $(0.85 \pm 0.12)$ of protein oxidation than that of the control group $(1.0 \pm 0.12 ;$ n.s.). Since well- and poorly-controlled diabetic patients do not differ with regard to oxidative damage to DNA and proteins, we conclude that diabetes-induced oxidative stress is not sufficient to oxidatively damage DNA and proteins of peripheral lymphocytes. On the other hand a loss of mtDNA appears to be a sensitive marker for oxidative stress and possibly contributes to diabetic complications related to hyperglycaemia. 
PLASMA 8-EPI-PGF2 $\alpha$ - AN EFFICIENT NEW MARKER FOR OXIDATIVE STRESS IN PATIENTS WITH IDDM

A. Staudinger, A. Festa, N. Shnawa, G Schernthaner and EJ Menze Dept. of Immunolgy, University of Vienna, Austria and Dept. of Medicine I Rudolfstiftung Hospital, Vienna, Austria.

There is considerable evidence linking oxidative stress to the pathogenesis of diabetes-associated complications. However, interrelationships between markers of oxidative stress are still controversial, and a single, sufficiently stable and thus reliably measurable marker of oxidative stress is still lacking. The aim of our study was therefore to investigate in patients with IDDM ( $\mathrm{n}=72$, mean $\pm \mathrm{SE}$ : age: $34.6 \pm 1.3$ years, $\mathrm{HbA1C}$ : $7.8 \pm 0.1 \%$, diabetes duration: $11.9 \pm 1.2$ years) the interrelationships of direct and indirect markers of oxidative stress, including 8-epi-PGF $2 \alpha$ (8-IP), a recently described specific marker of lipid peroxidation, in comparison to healthy, age-matched control subjects ( $n=30$, age: $32.3 \pm 1.9$ years)

Patients with IDDM had significantly higher levels of 8 -IP $(437 \pm 50$ vs $197 \pm 30 \mathrm{pg} / \mathrm{ml}, \quad p<0.0001$ ), soluble vascular cell adhesion molecule-1 (sVCAM-1: $1223 \pm 73$ vs. $938 \pm 79 \mathrm{ng} / \mathrm{ml}, \quad p<0.02$ ) and antibodies agains oxidised LDL (oLab: $833.2 \pm 54.2$ vs. $616.0 \pm 60.0 \mathrm{mU} / \mathrm{ml}, p<0.03$ ) whereas serum antioxidative capacity (TAS: $1.14 \pm 0.05$ vs. $1.37 \pm 0.08 \mathrm{mmol} / \mathrm{l}, \mathrm{p}<0.02$ was significantly reduced. No significant differences were detected in plasma levels of von Willebrand factor (measured by two different assays) superoxid dismutase (SOD) and endothelin-1. 8-IP was related to oLab $(r=0.34, p<0.005)$ and inversely related to SOD-activity in serum $(r=-0.32$ $p<0.007)$ and erythrocytes $(r=-0.23, p<0.03$ ). Mean HbA1c (determined by multiple measurements over a period of 6-96 months) was correlated to 8 IP $(r=0.27, p<0.03)$. The correlation of metabolic control with 8-IP was more pronounced in patients with poor metabolic control $(\mathrm{HbA} 1 \mathrm{c}>7 \%)(\mathrm{r}=0.43$, $p<0.002$ ). In patients with normoglycemia (blood glucose $<120 \mathrm{mg} / \mathrm{dl}, n=28$ ) a trend towards lower 8-IP levels compared to hyperglycemic patients $(n=44)$ was found $(326 \pm 51$ vs. $501 \pm 72 \mathrm{pg} / \mathrm{ml}, \mathrm{p}=0.05)$. We conclude that 8 epi-PGF2 $\alpha$, a specific marker of lipid peroxidation, is an efficient marker for hyperglycemia-associated oxidative stress in patients with IDDM.

\section{9}

\section{TROGLITAZONE (TRG) DIMINISHES CYTOTOXICITY} INDUCED BY OXIDIZED LOW-DENSITY LIPOPROTEIN(LDL)

Mitsunobu Kawamura, Keiko Ashidate, Shigeru Miyazaki, Tamio Teramoto, ${ }^{*}$ Masatoshi Ishii. Div. of Endocrinol. and Metabo., Dept. of Intern. Med., Tokyo Teishin Hosp. , * Dept. of Med. , Teikyo Univ. School of Med.

Purpose: In the present study, in vitro inhibition of LDL oxidation and the protective effect of TRG on cell cytotoxicity induced by oxidized LDL ( OxLDL) were investigated. Metholds: Susceptibility of LDL to oxidative modification was evaluated by the system using $\mathrm{Cu}^{2+}$ or MeO-AMVN. Cytotoxicity of OxLDL was studied on J 774 macrophages by MTT assay.

Results: (1) The oxidative modification of LDL induced by $\mathrm{Cu}^{2+}$ or MeO-AMVN was inhibited by TRG in dosedependent manner. In vitro inhibition of LDL oxidation by TRG was stronger than vitamin $\mathrm{E}$, but milder than probucol. (2) When the LDL incubated with TRG was dialyzed against PBS , antioxidant effect of TRG on LDL oxidation was kept. (3) TRG diminished cytotoxicity induced by OxLDL on both LDLspecific and tissue-specific antioxidant actions. Its effects were similar to probucol and stronger than vitamin $\mathrm{E}$.

Conclusion: It was suggested that TRG leads to antiatherogenic effect not only by antidiabetic effect but also by LDL-specific and tissue-specific antioxidant actions.
EVALUATION OF GLYCATION, OXIDATION AND COAGULATION PARAMETERS IN TYPE 2 DIABETICS WITH AND WITHOUT PERIPHIERAL VASCULOPATHY

M. Garbeglio,A. Lapolla, S. Proto, P. Carraro*, P. Bonvicini*, G. Giglia,i

Martano, R. Toniato,D. Fedele. Institute of Internal Medicine Chair Metabolic

Disease Padova University. *DPT of Laboratory Medicine Padova (Italy).

We verify the possible relationship between peripheral arteriopathy, a typica expression of diabetic macroangiopathy, and some parameters of early non enzymatic glycation (furosine, $\mathrm{HbA}_{\mathrm{c}}$ ), oxidation (malonil dialdeide: MDA, Total Radical Trapping: TRAP) and of coagulation/fibrinolysis (fibrinopeptide A, PAIantigen. PAI activity). We studied 81 type 2 diabetics without periphera vasculopathy $(\mathrm{L} . \mathrm{W} .>0.80$ ), mean age $63.9+6.3 \mathrm{yrs}$ (Group $\mathrm{A}$ ), and 18 type 2 diabetics with peripheral vasculopathy $(1 . W .<0.80)$ ), mean age $64+4.5 \mathrm{yrs}$, mean duration disease $6.4 \pm 4.5 y$ rs (Group B); as controls 22 healthy subjects matched for sex and age were studied (Group C). Mean ( + SD) values of early glycation parameters were significantly higher in diabetic patients than in controls (mean furosine : Group A $0.44 \pm 0.2 \mathrm{ng} / \mathrm{ml}$, Group $B \quad 0.39 \pm 0.1 \mathrm{ng} / \mathrm{ml}$, Group $C$ $0.26+0.06 \mathrm{ng} / \mathrm{ml}$; A vs. C p $<0.01$, B vs C $<<0.02$. $\mathrm{HbA}_{1 \mathrm{c}}$ : Group A $7.6+1.3 \%$ Group B $8.3+1.7 \%$, Group C $5.6+0.4 \% ; A$ vs. $C$ and $B$ vs. C $p<0.001$ ). MDA values were significantly higher in diabetic subjects than in control ones (Group $A$ $1.01+0.2 \mu \mathrm{mol} / \mathrm{l}$, Group B 1.18+0.4 umol/1, Group C $0.80+0.2 \mu \mathrm{mol} / \mathrm{l}$; A vs. C and $B$ vs. $C$ p $<0001$ ); while significantly lower mean values in diabetic patients than in controls were found for TRAP (Group A 837.5 $\pm 113 \mu \mathrm{mol} /$, Group B $893 \pm 84$ $\mu \mathrm{mol} / \mathrm{l}$, Group $\mathrm{C} 961.9 \pm 16 \mu \mathrm{mol} / \mathrm{l} ; \mathrm{A}$ vs. $\mathrm{C}$ and B vs. C $\mathrm{p}<0.001$ ), and Vit $E$ (Group A $9.26+2.2 \mathrm{mg} / \mathrm{L}$ Group B $8.66+2.1 \mathrm{mg} / 1 \mathrm{l}$ Group $C 11.5+0.8 \mathrm{mg} / \mathrm{l} ; \mathrm{A}$ vs. $\mathrm{C}$ and $\mathrm{B}$ vs $C p<0.001$ ). Among the coagulation/fibrinolysis markers mean values of tPA were significantly higher in diabetic subjects than in control ones (Group $A$ $10.8+3.3 \mathrm{ng} / \mathrm{ml}$, Group B $9.2+4.3 \mathrm{ng} / \mathrm{ml}$, Group C $6.6+3 \mathrm{ng} / \mathrm{ml}$; A vs. C $p<0.001$, B vs $C p<0.05$ ). All these parameters were not significantly different in diabetic patients affected by peripheral vasculopathy with respect to those withou peripheral vasculopathy. However considering all the diabetic subjects the I.W index was negatively related to $\mathrm{HbAlc}(r=-0.3 ; \mathrm{p}<0.05)$ to MDA $(r=-0.28 ; \mathrm{p}<$ $0.005)$ and fibrinogen $(r=-0.23 ; p<0.05)$ suggesting their possible role in macroangiopathy development. These data however need to be confirmed by studying a wide number of diabetics affected by peripheral vasculopathy.

\section{0}

GLYCATION OF L-ARGININE IS CATALYZED BY IRON AND IS RESULTED IN FREE RADICAL PRODUCTION

I.Wittmann, *J.Belágyi, *L.Pótó, Z.Wagner and J.Nagy. 2nd Dept. of Medicine, *Central Research Laboratory, University Medical School of Pécs, Hungary.

The elevated level of advanced glycation endproducts is due to their overproduction (diabetes mellitus), to their increased intake by foods and to their decreased elimination by kidney. The aim of the present study was to investigate the glycation of L-arginine (substrate of nitric oxide synthase enzyme).

We used electron spin resonance (ESR) to detect free radical production during the glycation. We incubated L-arginine with glucose at different temperatures. The electron donor activity of the glucose and $\mathrm{L}$-arginine was measured colorimetrically using ferric iron with ferrozine. Fig. 1. showes pyrazine radical generated at $90 \mathrm{oC}$. Fig.2. demonstrates the catalytic activity of iron and temperature dependence of the glycation. We could not detect free radical signal below $65 \mathrm{oC}$ because of the sensitivity limit of the ESR method. The ferrozine method proved a time dependent reduction of ferric iron by glucose $(n=3$, correlation coefficient: $r=0.98)$ and by $L-$ arginine $(r=0.94)$, but reduction by glucose was 4.7 times faster.
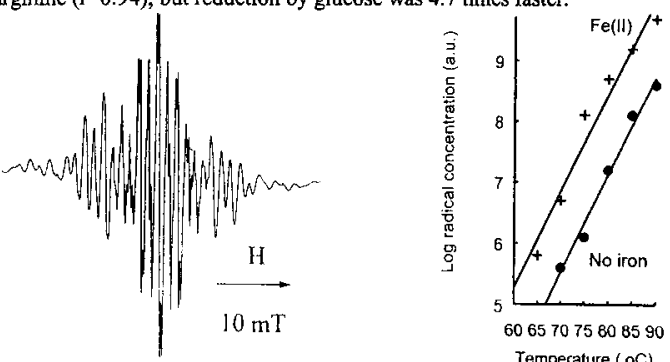

Fig.1. ESR signal of pyrazine radical Fig. 2. Temperature dependence of pyrazine produced by glucose $+\mathrm{L}$-arginine These results demonstrate that glycation of L-arginine produces pyrazine radical, this process is catalyzed by iron, and the electron source can be the glucose. 
EFFECT OF VITAMIN E TREATMENT ON MICROCIRCULATION IN TYPE 1 DIABETIC PATIENTS

J. Vertommen, W. Engelen, B. Manuel y Keenoy and I. De Leeuw, Dept. Endocrinology, Univ. Antwerp, B-2610 Antwerp, Belgium.

Recent data illustrates that increased oxidative stress in diabetic patients can be involved in the pathogenesis of different diabetic long term complications. Results in experimental diabetes suggest a favourable effect of anti-oxidant therapy on the oxidative imbalance, peripheral blood flow and consequently on the onset or the progress of some complications. Therefore 44 IDDM patients (m/f: 28/16) with stable diabetic control and minor complications were treated with dl- $\alpha$-tocopherol (vit $\mathrm{E} ; 3 \times 250 \mathrm{IU} / \mathrm{day}$ ). At start and after 6 months the cutaneous blood flow wa recorded with a Laser-Doppler (Perimed 4001) at skin temp. and at $42^{\circ} \mathrm{C}$ on the ventral surface of the elbow, the extensor surface of the leg, the tip of the third finger and the big toe. Transcutaneous oxygen tension $\left(\mathrm{pO}_{2}\right)$ was determined (Radiometer TINA) at $42^{\circ} \mathrm{C}$ on the forearm and dorsum of the foot. Arterial occlusion $(<200 \mathrm{~mm}$ $\mathrm{Hg}$ ) was applied until $\mathrm{pO}_{2}$ was $<2 \mathrm{~mm} \mathrm{Hg}$. After release of the blood flow the reactive hyperaemic response was followed. Results were the mean of triplicate measurements. Blood viscosity was measured as an important determinant of peripheral blood flow. Plasma vit $E$ increased from 16.7 to $24.1 \mathrm{mg} / \mathrm{l}(\mathrm{p}<0.0007)$ and vitamin $A$ increased simultaneously from 74.4 to $90.6 \mathrm{mg} / 1(\mathrm{p}<0.003) . \mathrm{HbA}_{1 \mathrm{c}}$ tended to decrease $(7.94 \%$ to $7.69 \% ; p=0.06)$. The erythrocyte viscocity decreased from 8.33 to $8.01 \mathrm{mPa} . \mathrm{s}(\mathrm{p}<0.002)$. Baseline $\mathrm{pO}_{2}$ in the arm decreased from 42.2 to 37.1 $\mathrm{mm} \mathrm{Hg}(\mathrm{p}<0.025)$ but remained unchanged in the foot. After occlusion, foot $\mathrm{pO}_{2}$ decreased slower to zero (n.s.) and returned faster to baseline level (149 s to $129 \mathrm{~s}$ $\mathrm{p}<0.02$ ). The same trend was observed at the arm. The blood perfusion of the finger tended to increase, as expressed by the resting L-D value as well as the $42^{\circ} \mathrm{C}$ L-D value but this observations were borderline significant $(p<0.09)$. Other results will be discussed in detail. We conclude that Vit $E$ is well tolerated in diabetics and tends to decrease the $\mathrm{HbA}_{1 \mathrm{c}}$. Although the resting $\mathrm{pO}_{2}$ in the arm is slightly lower the overall tissue oxygenation seems to be ameliorated. This is accompanied by an improvemen in peripheral blood flow as observed by the changes in blood viscosity and L-D signals of the cutaneous blood flow. Further research and finer observation techniques are necessary to determine the long term influence of this therapy on the microcirculation in IDDM.

\section{3}

EFFECT OF HYPERGLYCAEMIA AND HYPERTRIGLYCERIDAEMIA ON EXCRETION OF NITRIC OXIDE METABOLITES IN HEALTHY MEN. R. Śimková, T. Pelikánová, L. Kazdová, R. Komers, J. Malý. Institute for Clinical and Experimental Medicine, Prague, Czech Repubiic.

Nitric oxide (NO) is a relatively stable free radical that contribute to regulation of renal haemodynamics and sodium handling. The aim of our study was to evaluate the effect of acutely-induced hyperglycaemia and hypertriglyceridaemia on renal NO production in 13 healthy subjects. Urinary $\mathrm{NO}_{2}{ }^{-} / \mathrm{NO}_{3}{ }^{-}\left(\mathrm{NO}_{\mathrm{x}}\right)$ levels as a marker of renal production of $\mathrm{NO}$ were assessed under basal conditions and four different examinations lasting 4 hours: a) hyperglycaemic clamp (12 mmol// HG), b) hyperglycaemic clamp with infusion of somatostatin in order to decrease the insulin production (HGS), c) infusion of Intralipid $\left(0.15 \mathrm{~g}\right.$ of fat. $\left.\mathrm{kg}^{-1} \cdot \mathrm{h}^{-1} ; 1\right)$, and d) a timecontrolled euglycaemic isovolumic conditions $(\mathrm{C})$. There were comparable increases in $\mathrm{NO}_{\mathrm{x}}$ excretions during $\mathrm{HGS}(0.06 \pm 0.05 \mathrm{vs} 0.59 \pm 0.57 \mu \mathrm{mol} / \mathrm{min}$; $\mathrm{p}<0.01), \mathrm{HG}(0.40 \pm 0.20$ vs $0.95 \pm 0.8 \mu \mathrm{mol} / \mathrm{min} ; \mathrm{p}<0.01)$ and during $\mathrm{C}$ $(0.08 \pm 0.06$ vs $0.53 \pm 0.40 \mu \mathrm{mol} / \mathrm{min} ; \mathrm{p}<0.01)$. On the other hand the rise in $\mathrm{NO}_{x}$ during Intralipid infusion was significantly higher than in $\mathrm{C}(+0.88$ vs $+0.44 \mu \mathrm{mol} / \mathrm{min} ; \mathrm{p}<0.05$ ). The differences in urinary sodium excretion during examinations were not statistically significant and did not correlate with $\mathrm{NO}_{x}$. We conclude that a) acute hyperglycaemia lasting 4 hours does not affect the renal NO production and the increase in $\mathrm{NO}_{x}$ excretion could be the consequence of the volume expansion, and b) acutely-induced hypertriglyceridaemia is accompanied by the rise in renal NO production. (Supported by IGA MZ CR, No. 4242-3)

\section{INCREASED PEROXYNITRITE PRODUCTION IN DIABETIC} PLATELETS

R.A. Rabini ${ }^{1}$ P. Fumelli, A. Vignini ${ }^{2}$, N. Moretti ${ }^{2}$, M. Tannous ${ }^{3}$, B. Zielinski ${ }^{4}$, B. Mutus ${ }^{3}$ and L. Mazzanti ${ }^{2}-{ }^{1}$ Department of Diabetology INRCA, Ancona (Italy), ${ }^{2}$ Institute of Biochemistry, University of Ancona, Ancona (Italy), ${ }^{3}$ Department of Chemistry and Biochemistry, ${ }^{4}$ Department of Biological Sciences, University of Windsor, Windsor Ontario, Canada

The inducible form of the enzyme nitric oxide synthase ( $i \mathrm{NOS}$ ) originally detected in macrophages and endothelial cells can produce superoxide as well as NO, reacting to yield peroxynitrite (ONOO). The aim of the present work was to investigate $\mathrm{ONOO}^{\circ}$ production in platelets from diabetic patients, as iNOS-dependent peroxynitrite has been recently implicated in the pathophysiology of atherosclerosis. We compared by confocal microscopy platelets from 15 healthy subjects, 14 type 1 and 15 type 2 diabetic patients with respect to L-Arg/NOS-dependent dichlorofluorescein (DCF) formation, which under the conditions employed is an accurate detector of ONOO' production. The platelets were then immunologically probed for $i$ NOS, which has been recently detected in megakaryocytes of subjects with coronary artery disease. Type 1 and Type 2 platelets produced larger levels of peroxynitrite in comparison to normal in the presence of $100 \mu \mathrm{M}$ extracellular L-Arg (pixel intensities $=0.75 \pm 0.49$ in healthy subjects, $2.53 \pm 0.67$ in Type 2 patients, $3.79 \pm 0.77$ in Type 1 patients, $p<0.01$ ). Western blots of intraplatelet proteins indicated that $i$ NOS was absent in control subjects, while $i$ NOS was evident in both Type $1(n=12)$ and Type $2(n=13)$ diabetic patients. The blot densities for Type 1 and Type 2 were $2.63 \pm 0.84$ and $7.3 \pm 5.11$ respectively. These results suggest that diabetes-related factors turn on $i$ NOS production in platelets, which give rise to peroxymitrite, a previously undetected source of platelet damage in diabetes.

\section{4}

SERUM SELENIUM AND ZINC CONCENTRATIONS OF TYPE I DIABETICS UNDER INTENSIVE INSULIN TREATMENT

J. Molnár ${ }^{1}$, P. Pusztai ${ }^{1}$, P. Molnár ${ }^{2}, A$. Somogyi $^{1}$ and $z$. Tulassay ${ }^{1}$, Semmelweis University, Medical School, 2nd Dept. of Medicine, 1088 Budapest, Szentkirályi 46., 2Central Food Research Institute, 1022 Budapest, Herman Otto 15, Hungary

Atherosclerosis is usually accelerated in diabetes. Certain trace elements may play an important role to help to prevent or to slow down this process. Selenium and zinc are essential trace elements with antioxidant properties acting mainly via antioxidant enzymes (glutathione peroxidase, superoxide dismutase). The serum selenium and zinc concentrations of 22 type I diabetic subjects and 40 controls - matched in age and BMI - were determined. All diabetics were on intensive insulin therapy, the mean $\mathrm{HbA1c}$ value was $7,5 \pm 0,4 \%$. Both serum selenium and zinc concentration were measured by atomic absorption spectrometry. The analytical method was verified by analysis of a human reference serum (Seronorm). Glutathione peroxidase activity was determined using the method of Paglia and Valentine (Ransel) and superoxide dismutase activity in a xanthine/xanthine oxidase system (Ransod). Type I diabetics had a slightly higher serum selenium concentration $(86.6 \pm 4,4 \mu \mathrm{g} / \mathrm{l})$ and a significantly higher mean zinc concentration $(0.84 \pm 0.03 \mathrm{mg} / \mathrm{l})$ than the control group $(83,9 \pm 2,4 \mu \mathrm{g} / /$ and $0.76 \pm$ $0.02 \mathrm{mg} / \mathrm{l}$ respectively; $p<0.05)$. Glutathione peroxidase was impaired in patients with diabetes $(56$ vs. $51 \mathrm{U} / \mathrm{gHb}, p<0.05$ ) while erythrocyte superoxide dismutase did not differ in the two groups. The higher serum zinc concentration of diabetics can be partly attributed to the frequent consumption of food with high protein and zinc content, however, insulin therapy may also have contributed to the increase in serum zinc concentration. 


\section{PS 67}

\section{Bone Density}

1255

\section{BONE DENSITY IN TYPE 2 DLABETES IN RELATION WITH THE TYPE OF OBESITY ADRENAL FENCTION}

I. Trznadel-Morawska. J. Sicradzki. B. Katra: Department of Metabolic Diseases of Jagellonian University. Cracow. Poland

In type 2 diabetes associated with obesity bone density is dependent on such protective factors as hyperinsulinism. insulin resistance. insulin-like growth factors. free bone turnover. also obesity. Cortisol $(\mathrm{C})$ accompanying obesity is a resorptive factor. Aim of the study was // assessment of bone density in patients with type 2 diabetes associated with various types of obesity and cortisol level: $2 /$ analysis of the relation between androgen (androgen metabolites) and obesity in type $i$ patients. $4+$ patients with type 2 diabetes were investigate. Bone density was measured by ultrasound (Lunar. USA). The patients were subdivided into groups: I without osteoporosis $(-1 \leqslant T$ score $<1)$. II threatening osteoporosis $(-2.5 \leqslant T s c<-1)$ and III with osteoporosis (Tsc<-2.5). Types of obesity were determined from waist/hip ratio and tissue fat thickness as measured by densilometn using DPX (Lunar USA). Biochemical determinations: cortisol. endogenous insulin. 17-ketosteroids $(17 \mathrm{ks}) \mathrm{HbAlC}$ were performed in the individual T-score groups. In group III the BMI $(29.6+/-\mathrm{SD}=4.68)$ and mean $\mathrm{C}$ level $(21.5+/-9.73 \mathrm{ug} / \mathrm{ml})$ were the highest. In group I and II the level of $17 \mathrm{ks}(37.4+/-10.95$ and $38.2+/-19.71 \mathrm{umol} / \mathrm{h}$ respecticely) was higher than that in group III. The following correlations were significant negative between $S O S$ and $C(r=0.30, p<0.05)$ and positive between SOS and insulin $(r=0.80, p<0.05)$. BUA and $17 \mathrm{ks}(r=0,4 . \mathrm{p}<0.05)$. We conclude that 1 / The lowest density in type 2 diabctes with the highest body weight. central obesity in men and gynoidal obesity in women. elevated $C$ and correlations between cortisol and SOS, and between $17 \mathrm{ks}$ and BUA suggest that in these patients there is an imbalance between the protective and resorptive factors. $2 /$ the lowest level of $17 \mathrm{ks}$ in patients with the lowest bone density and correlation between $17 \mathrm{ks}$ and BUA may indicate decreased anabolic effects in this group. 3/ The relation betwcen bone density and the type of obesity confirms the influence of both protective and resorptive factors on bone density changes in rarious types of obesity

\section{6}

BONE MASS PATTERNS AND THEIR CLINICAL SIGNIFICANCE IN INSULN DEPENDENT DIABETES

D.J.Hadjidakis,B.A.Iconomidou,M.E.Sfakianakis,A.G.Mitrakou,A.E.Raptis S.A.Raptis. $2^{\text {nd }}$ Dpt. Intern. Medicine.Research Institute and Diabetes Center Athens University,Evangelismos Hospital,Athens, Greece

Although bone mass has been reported as diminished in most studies pertaining io type I diabetes,some points remain still uncertain such as anatomic regions,clinical significance and influence of either disease duration or degree of glucose control. The aim of our stucy was the investigation of bone mass behavior in insulin-dependent (IDDs) patients and its comparison (paired matching) to that of healthy persons $(\mathrm{HL})$ with similar anthropometric characteristics from our population.Forty eight IDDs, 28 males (age $28.1 \pm 6.8 \mathrm{yrs}$, mean $\pm 1 \mathrm{SD}$,duration of diabetes $10.8 \pm 5.9$ yrs) and 20 females ( $33.7 \pm 8.7$ yrs, $15.7 \pm 8.2$ yrs respectively) were studied.Bone Mineral Density (BMD) was measured at $\mathrm{L}_{2}-\mathrm{L}_{4}$ vertebrae and proximal femur by Dual Energy X-ray Absorptiometry.BMD of either vertebrae or femoral neck ( $\mathrm{FN}$ ) in females did not differ significantly between IDDs and $\mathrm{HL}$ $(0.99 \pm 0.12$ vs $1.02 \pm 0.1$ and $0.83 \pm 0.13$ vs $0.88 \pm 0.13$, paired $t$-test). In contrast BMD of male IDDs was significantly lower both in vertebrae $(1.01 \pm 0.11$ vs $1.09 \pm 0.15$, $p<0.05)$ and FN $(0.86 \pm 0.13$ vs $0.98 \pm 0.12, p<0.001) .18 \%$ of the male IDDs were in the osteoporotic and $54 \%$ in the osteopenic range according to W.H.O. criteria (osteoporosis T-score $<-2.5$. osteopenia $-2.5<\mathrm{T}<-1$ ). Regarding female IDDs the respective figures were $5 \%$ and $40 \%$.A statistically significant positive correlation was observed between vertebral $B M D$ and $B M I, r=0.39, p<0.05$ and a negative one with $\mathrm{GHbA}_{1 \mathrm{c}}, \mathrm{r}=-0.41 . \mathrm{p}<0.05$ in mates, whereas a positive correlation existed between vertebral $B M D$ and duration of diabetes $(r=0.53, p<0.01)$ as well as $F N$ $\mathrm{BMD}$ and $\mathrm{BMI}(\mathrm{r}=0.38, \mathrm{p}<0.05)$ in females.In conclusion, male IDDs present with lower density of trabecular and mixed bone compared to HL persons while female IDDs do not differ.The risk for clinical osteoporosis is greater in male IDDs.Glucose control seems to affect adversely trabecular bone of males, while obesity influences favorably trabecular bone in males and mixed bone in females.
1257

EFFECT OF HORMONAL REPLACEMENT THERAPY ON CARBOHYDRATE METABOLISM IN WOMEN IN PERIMENOPAUSAL PERIOD

T. Pertyński, K. Cypryk, I. Nadel and E. Kowalska; Research Institute of Polish Mother's Memorial Hospital, Łodź, Poland

Beneficial influence of hormonal replacement therapy (HRT) on calcium, phosphor and lipids metabolism is well known. Effect of HRT on carbohydrate metabolism, especially in women with high risk of diabetes, is still unclear.

Aim of study: Evaluation of 6 months HRT (17 $\beta$-estradiol and norethisteron in continous oral therapy) influence on selected parameters of carbohydrate metabolism in perimenopausal women.

Materials and methods: Study group consists of 52 women at age between 40-67 years (mean $52 \pm 5,8$ ), divided into following groups: A - with normal body mass $\left(B M l<25 \mathrm{~kg} / \mathrm{m}^{2}\right)$, B-overweighted (BMI $\left.-25-30 \mathrm{~kg} / \mathrm{m}^{2}\right)$, C-obese $\left(\mathrm{BMI}>30 \mathrm{~kg} / \mathrm{m}^{2}\right.$ ). Results: Before treatment. There was no differencies in fasting blood glucose levels and AUC-glucose between groups A, B and C. Fasting blood insuline levels were lower in A group than in B group ( $p>0.05)$ and $C$ group $(p<0.05)$; also AUC-insuline

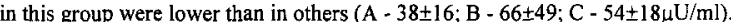

After treatment. In group A - women with normal body mass, significant decrease in fasting blood glucose and AUC glucose was observed ( $p<0.05$ ). In others groups these parameters did not change. After 6 months of HRT treatment higher fasting blood glucose levels were noted in obese and overweighted women than in the group

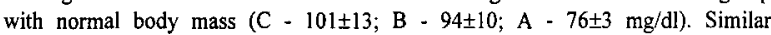
correlation was obserwed in AUC glucose $(\mathrm{p}<0.05)$

After HRT insulin levels and AUC insulin were without significant changes, although in B group there was increase in fasting insulin levels and AUC insulin levels $(p>0.05)$.

Conclusions: Beneficial influence of HRT on carbohydrate metabolism was obserwed in women with normal body mass. Changes in the group of women with $\mathrm{BM}>25 \mathrm{~kg} / \mathrm{m}^{2}$ are unclear. 


\section{PS 68}

\section{Pathogenic Mechanisms of Complications}

\section{8}

HEPARIN ATTENUATES THE PROLIFERATING EFFECTS EXERTED BY INSULIN IN HUMAN RENAL VASCULAR SMOOTH MUSCLE CELLS.

F.Cavalot, I.Russo, G.Anfossi, L.Mattiello, P.Massucco, F.Tassone, P.Perna, A.Giori, D.Fontana (*), and M.Trovati. Diabetes and Urology (*) Units, Dept. of Clinical and Biological Sciences of the University of Turin, San Luigi Hospital, Orbassano (Turin) Italy.

Diabetic patients frequently present endogenous (Type 2) or exogenous (Type 1) hyperinsulinaemia and show reduced vascular heparin-like anionic charges. Insulin increases and heparin inhibits human vascular smooth muscle cell (hVSMC) proliferation. High insulin and low heparin concentrations should play a role in atherogenesis. To evaluate the interrelationships between insulin and heparin in these cells, hVSMC isolated from branches of renal artery were cultured and submitted to proliferation experiments in $10 \%$ serum additioned with: a)insulin (240-1920pmol/l), b)heparin (10-300 U/ml), c) insulintheparin at the same concentrations. After $72 \mathrm{hr}$ incubation, VSMC were trypsinized and counted. Results (cells $\times 5 \times 10^{3}, \mathrm{~m} \pm \mathrm{sem}$ ): 1)insulin dose-dependently stimulated hVSMC proliferation: $29.8 \pm 1.8$ without insulin, $42.5 \pm 2.4$ with insulin $960 \mathrm{pmol} / \mathrm{l}$, $55.8 \pm 1.8$ with insulin $1920 \mathrm{pmol} / 1$ (ANOVA, $\mathrm{p}<0.0001$ ); 2 )heparin dose-dependently inhibited hVSMC proliferation: $29.8 \pm 1.8$ without heparin, $17.3 \pm 1.1$ with heparin $100 \mathrm{U} / \mathrm{ml}, 11.7 \pm 1.1$ with heparin $300 \mathrm{U} / \mathrm{ml}$ (ANOVA, $\mathrm{p}<0.0001$ ); 3) heparin inhibited insulin-induced proliferation (ANOVA, p<0.0001): i.e., with $10 \mathrm{U} / \mathrm{ml}$ heparin, proliferation induced by $960 \mathrm{pmol} / / \mathrm{insulin}$ decreased from $42.5 \pm 2.4$ to $27.7 \pm 0.6$, proliferation induced by $1920 \mathrm{pmol} / /$ insulin decreased from $55.8 \pm 1.8$ a $26.5 \pm 1.2$. Thus, in hVSMC heparin inhibits insulininduced proliferation. Loss of heparin-like anionic charges could therefore increase the insulin-induced proliferating influence in hVSMC, and therefore enhance the atherogenic effect of the hormone.

\section{0}

Increased transcapillary sodiumfluorescein leakage in the skin of normo- and microalbuminuric Type 1 diabetic patients; amelioration by angiotensin-converting enzyme inhibition.

P.H.N. Oomen ${ }^{1}$, K. Hoogenberg' ${ }^{2}$, J. Jager', W.D.Reitsma', R.P.F. Dullaart ${ }^{2}$, A.J. Smit', Departments of General Internal Medicine' ${ }^{1}$ and Endocrinology ${ }^{2}$, University Hospital Groningen, The Netherlands.

Capillary leakage of sodium-fluorescein ( $\mathrm{Na} F$ ) in the skin reflects capillary permeability and may be a marker of diabetes-associated microcirculatory alterations. We evaluated transcapillary $\mathrm{NaF}$ leakage in the skin of 10 matched normoalbuminuric and 10 microalbuminuric Type 1 diabetic patients (diabetes duration $>10$ years) and 10 healthy control subjects. The microalbuminuric patients were restudied after 6 weeks of treatment with angiotensin-converting enzyme (ACE) inhibitor enalapril, $10 \mathrm{mg}$ once daily. All subjects were evaluated at a blood glucose level of $5 \mathrm{mmol} / \mathrm{l}$. Transcapillary leakage of $\mathrm{NaF}$ was assessed by videodensitometry for 20 minutes under standardised conditions. Transcapillary $\mathrm{NaF}$ leakage was strongly increased in the normoalbuminuric Type 1 diabetic patients compared to control subjects $(\mathrm{p}<0.001)$ and was still further increased in the microalbuminuric Type 1 diabetic patients ( $p<0.01$ as compared to the normoalbuminuric patients). Enalapril treatment in microalbuminuric patients reduced NaF leakage significantly $(\mathrm{p}<0.05)$. After treatment, NaF leakage was no longer different from that in the normoalbuminuric patients. This reduction in $\mathrm{NaF}$ leakage was accompanied by a decrease in blood pressure and albuminuria. In conclusion, capillary permeability is clearly elevated in normoalbuminuric Type 1 diabetic patients with long-standing disease, and the excess elevation found in microalbuminuric Type 1 diabetic patients is ameliorated by ACE-inhibition treatment. Measurement of NaF leakage in the skin appears to be a useful tool to document capillary permeability in intervention studies.

\section{EFFECTS OF HEPARIN ON VASCULAR PERMEABILITY IN DIABETIC RATS}

P.S. Oturai. Novo Nordisk Research, Bagsvaerd, Denmark.

Heparin treatment has been shown to have beneficial effects in diabetic nephropathy. Albuminuria reflects general vascular dysfunction, including increased permeability, in diabetes. We aimed to evaluate effects of low molecular weight heparin (LMWH) treatment on vascular permeability in diabetic rats. Male Sprague-Dawley rats with streptozotocin induced diabetes ( $65 \mathrm{mg} / \mathrm{kg}$ i.v.) were randomly allocated to LMWH (6 mg/kg s.c.) or placebo treatment in two sub-studies. Normal rats dosed placebo served as nondiabetic controls. In one study, transcapillary escape rate of albumin (TER) was measured after eight months diabetes. TER $(\% / h)$ was calculated from the disappearance rate of ${ }^{125} \mathrm{I}$-albumin from the blood in anaesthetised rats. In the second study, tissue albumin clearance was measured after six weeks diabetes in awake rats. I.v. injected ${ }^{131} \mid$ - and ${ }^{125}$-albumin were used as markers for cleared and blood contained albumin. Tissue clearance of ${ }^{131} \mathrm{I}-$ albumin ( $\mu \mathrm{g}$ plasma / $\mathrm{g}$ tissue per $\mathrm{min}$ ) was calculated from tracer activities in various tissues and arterial blood samples. Glycaemia $(20-30 \mathrm{mM})$ did not differ among the diabetic groups. TER increased by $24 \%$ in diabetic rats $(p<0.05)$. LMWH did not significantly affect this increase. ${ }^{131}$-albumin clearance was increased by diabetes in eye, skin and skeletal muscle. A tendency towards a lowering effect of LMWH was observed in the eye $(p=0.17)$, but $L M W H$ did not significantly affect increased clearance in any organ.

\begin{tabular}{lccc}
\hline \multicolumn{1}{c}{ Mean $\pm S D$} & Normal & Diabetic & Diabetic+LMWH \\
\hline TER (\%/hour) & $14.1 \pm 3.3$ & $17.5 \pm 3^{*}$ & $15.7 \pm 2.7$ \\
\hline Clearance $(\mu \mathrm{g} / \mathrm{g} \times \mathrm{min})$ & & & \\
Eye & $230 \pm 50$ & $310 \pm 70^{*}$ & $250 \pm 70$ \\
Skin & $230 \pm 30$ & $400 \pm 70^{*}$ & $520 \pm 180$ \\
Muscle & $100 \pm 20$ & $170 \pm 120^{*}$ & $240 \pm 160$ \\
\hline
\end{tabular}

* $P<0.05, * * P<0.01$ vs Normal. Diabetic groups do not differ.

In conclusion, low molecular weight heparin treatment does not affect increased vascular permeability in experimental diabetes.

\section{1}

A NEW METHOD TO NVESTIGATE CAPILLARY PERMEABILITY TO ALBUMIN IN VIVO IN DIABETIC RATS

F. Cohen-Boulakia, P. Valensi, R. Lestrade, D. Mesangeau, H. Boulahdour, C. Hort-Legrand, A. Behar. Laboratory of Nutrition and Metabolic Diseases, Paris-Nord University, Bondy and Biophysics Department, UFR Broussais Hôtel-Dieu, Paris, France.

In diabetic patients, capillary permeability to albumin (CPA) is often increased in a reversible way before the occurrence of microangiopathic complications. Invasive methods have been previously used to investigate CPA in diabetic rats. The aim of the present work was to study the onset of CPA disorders and to follow them in diabetic rats. We have developed a noninvasive isotopic method we had previously described in humans, which consists of injecting intravenously $99 \mathrm{~m}$-Technetium labeled albumin and counting radioactivity externally with a multigate spectrometry before, during and after a 6-min venous compression on the hind legs. After removal of venous compression, the radioactivity curve decreases rapidly and interstitial albumin retention (AR) may be calculated. The analysis of the curve by the Fast Fourier Transform provides an index for lymphatic uptake of interstitial albumin, LF/HF, defined by the ratio of the amplitudes of the low and high frequency peaks. In 17 normal Wistar rats, 60 to 240 days old, $A R=0.46 \pm 0.28 \%$, $\mathrm{LF} / \mathrm{HF}=0.13 \pm 0.04 \%$. In 16 diabetic rats (N5STZ) with blood glucose between 22 and $47 \mathrm{mmol} / 1$, the same test was performed three times, in 100 , 130 and 180 days old.animals The results were successively: $\mathrm{AR}=5.0 \pm$ $2.0 \%, 17.1 \pm 3.4 \%$ and $22.5 \pm 3.2 \%, \mathrm{LF} / \mathrm{HF}=1.5 \pm 0.4 \%, 2.1 \pm 0.4 \%$ and 3.5 $\pm 0.5 \%$. AR and $\mathrm{LF} / \mathrm{HF}$ were always significantly higher in the diabetic rats. These data suggest that 1) CPA is early increased in this model of diabetic rats ; 2) this disorder is associated with a lymphatic dysfunction ; 3) this in vivo noninvasive method may be used to follow the natural history of the functional microcirculatory disorders in diabetic rats and to compare it with microangiopathic lesions and to investigate drug effects. 


\section{2}

HIGH GLUCOSE ATTENUATES INSULIN-STIMULATED CHEMOKINESIS IN NORMAL HUMAN NEUTROPHILS THROUGH PROTEIN KINASE C

J. Sehlin and P.-A. Oldenborg. Department of Histology and Cell Biology, Umeå University, Umeå, Sweden

The increased sensitivity to infections in diabetes has long been associated with changes in neutrophil function. However, while most studies have focused on the involvement of hyperglycemia in the defective neutrophil function, knowledge on the role of insulin in mediating neutrophil dysfunction is scanty. In the present work, insulin (40-160 $\mu \mathrm{U} / \mathrm{ml})$ dosedependently stimulated chemokinesis in normal human neutrophils in the presence of $5 \mathrm{mM}$ D-glucose but this effect of insulin $(160 \mu \mathrm{U} / \mathrm{ml})$ was totally inhibited by increasing the glucose concentration from 5 to $15 \mathrm{mM}$

D-glucose. It was tested whether this effect of glucose is mediated by increase in protein kinase $\mathrm{C}(\mathrm{PKC})$ activiy. Activation of $\mathrm{PKC}$ with phorbol 12-myristate 13-acetate (PMA) at 0-100 nM dose-dependently inhibited neutrophil random locomotion in the absence of insulin. Suboptimal concentrations of PMA (0.1-0.5 nM) inhibited the chemokinetic effect of $160 \mu \mathrm{U} / \mathrm{ml}$ insulin in a dose-dependent way. The specific PKC-inhibitor bisindolylmaleimide did not affect the insulinstimulated chemokinesis at $5 \mathrm{mM}$ glucose but restored the chemokinetic effect of insulin at $15 \mathrm{mM}$ glucose. These results therefore suggest that glucose-induced PKC-activation may mediate the inhibitory effects of high glucose levels on insulin-stimulated chemokinesis in normal human neutrophils.

\section{3}

DIABETES COMPLICATIONS CORRELATE WTH LEVELS OF SKIN COLLAGEN VARIABLES, INDEPENDENT OF HBAIC

$S$. Genuth, V. Monnier, and $O$. Bautista. Case Western Reserve University, Cleveland $\mathrm{OH}$, and George Washington University, Rockville MD, U.S.A.

The effect of intensive treatment (IT) vs, conventional treatment (CT) on skin collagen variables was studied in 216 subjects from the DCCT cohort, 122 IT and 94 $\mathrm{CT}$. Skin biopsies were performed near study end. Furrosine $(\mathrm{F})$, pentosidine $(\mathrm{P})$, carboxymethyllysine (C), relative fluorescence (RF), acid soluble (AS) and pepsin soluble (PS) collagen were measured. Median values for the primary prevention subjects are given in pmole/mg collagen $(F, P, C)$, Relative Units (RF) and \% (AS, PS). $p$ values were determined by Wilcoxon Rank Sum Test.

$\begin{array}{lcccccc} & \text { F } & \text { P } & \text { C } & \text { RF } & \text { PS } & \text { AS } \\ \text { IT } & 605 & 23.8 & 466 & 166 & 7.4 & 0.54 \\ \text { CT } & 903 & 26.5 & 533 & 182 & 5.0 & 0.42\end{array}$

p $\quad 001 \quad 001 \quad 001 \quad 004 \quad 001 \quad 006$

Multiple logistic regression was used to model the prevalence of selected DCCTdefined complication outcomes vs. the full set of collagen variables with $(+)$ and without (-) adjustment for HbAic and separately for each treatment group.

I'T

CT

IT vs CT

HbAlc adjusted

$R^{2}-R^{2} R^{+}$

$\geq 3$ microaneurisms $26 \quad .019 \quad 33$.005

AER> 40mg/day 23 as 6 ns

clinical neuropathy $36 \quad .004 \quad 37 \quad .003$

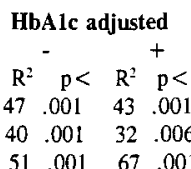

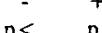

$.014 \quad .007$

.061 ns

ns ns

Conclusions: 1)Biochemical (F,P,C,RF) and physicochemical (PS,AS) abnormalities in skin collagen were significantly less after 6 years of IT compared to $C T$. 2) The prevalence of complications was significantly associated with the degree of skin collagen abnomalities independent of $\mathrm{HbAlc}$. This raises the possibility that skin collagen measurements could be used to supplement $\mathrm{HbAlc}$ in predicting the future risk of diabetic complications.

\section{4}

\section{FACTORS EFFECTING ELEVATED SERUM ADENOSINE} CONCENTRATION IN DIABETIC PATIENTS

B. Kopff ${ }^{1}$, M. Kopff ${ }^{2}$, K. Szosland ${ }^{1}$ and J. Drzewoski ${ }^{1}$

Metabolic Diseases and Gastroenterology Department, Medical University of kodź - Poland, ${ }^{2}$ Department of Biochemistry, Military Medical University of $k$ dó - Poland.

The aim of the study was to determine the level of glycaemia, fructosamine (FA), glycated haemoglobin, serum adenosine concentration and the presence of diabetic late complications. The subjects were a group of 80 diabetic patients (with and without late diabetic complications) treated with insulin. Controls were 40 non-diabetic patients. In blood specimens from all these patients serum adenosine concentration, glycated haemoglobin level and for subgroup of 36 diabetic and 18 controls patients' serum fructosamine (FA) concentration were measured. In diabetic patients the stages of late diabetic complications such as retinopathy and nephropathy were evaluated. The serum adenosine concentration was significantly higher in diabetic than in control group patients $0,251 \pm 0,079$ versus $0,13 \pm 0,048 \mu \mathrm{mol} / \mathrm{l}$. Significantly higher in diabetic group were: glycaemia $(9,72 \pm 1,05 \mathrm{~mol} / \mathrm{h}$ versus $4.52 \pm 0.23)$ and HbA1c $(8,61 \% \pm 0,35$ versus $5,61 \pm 0,21)$. In the diabetic group there was exponential correlation between glycaemia and adenosine concentration ( $p<0.01$; coefficient 0,34028 ) and linear correlation with $\mathrm{HbA1c}$ level was found $(p<0,05$ coefficient $=-0,27332)$. In the diabetic group there was also correlation found between the stage of retinopathy $(p<0,01$; coefficient $=$ 0,3386 ), nephropathy ( $0<0,01$; coefficient $=0,42295$ ) and adenosine serum concentration. No correlation between adenosine and FA level was found in both groups. Also there was not relationship of glycaemia and HBA1C with serum adenosine neither in control group nor in diabetic subgroup with values of glycaemia and $\mathrm{HbA}_{1 \mathrm{c}}$ within range found in controls'.

Conclusion: the obtained results suggest that there may exist a correlation between serum adenosine level and late diabetic complication as a result of changes in high-energy substances metabolism.

\section{5}

ALTERED ADENOSINE-INDUCED RELAXATION OF PENILE CORPORAL TISSUE FROM DIABETIC RATS: POSSIBLE MECHANISMS S. Gür" and B. Öztürk *Univ. of Ankara, Fac. of Pharmacy, Dept. of Pharmacology, Numune Hospital, Dept. of Urology, Ankara, TURKEY.

Diabetes mellitus is associated with an increased incidence of erectile dysfunction. Purine nucleotides, such as adenosine triphosphate (ATP) and adenosine (Ado) produce concentration-dependent relaxant responses in corpus cavemosum tissue. To determine whether or not the relaxant responses to adenosine triphosphate (ATP) and adenosine (Ado) were impaired in diabetic rats, we have firstly performed an in vitro examination of corpus cavemosum tissue obtained from STZ-induced diabetic rats. Sixteen rats were randomly divided into the streptozotocin treated diabetic $(n=8)$ and control $(n=8)$ groups. Diabetic and control animals were maintained for 8 weeks. The mounted smooth muscles were pretreated with guanethidin (5 $\mu \mathrm{mol}$ per liter) and atropin (1 $\mu \mathrm{mol}$ per liter) and contraction was induced in isolated strips of corporal smooth muscle by phenylephrine $\left(10^{-5} \mathrm{M}\right)$ : then relaxation was assessed with administration of two agents: Ado $\left(10^{-8}-10^{-3} \mathrm{M}\right)$ and ATP $\left(10^{-9}-10^{-3} \mathrm{M}\right)$. Ado-induced relaxation was enhanced, as evidenced by a nigher degree of muscle relaxation after the administration of agent in the tissue from diabetic $(D)$ rats than in that from control $(C)$ rats $\left(C \mathrm{pD}_{2}\right.$ : $4.22 \pm 0.23 ; \max .21 .3 \pm 3.52$, versus $\mathrm{D} \mathrm{pD}_{2}: 4.78 \pm 0.15 \quad P<0.05 ; \max .61 .0$ $\pm 4.6, P<0.01$ ). The relaxant effect of ATP appeared to be unaltered in diabetic corpus cavernosum compared to control corpus cavernosum.Furthermore, we have attended to investigate a possible mechanisms of these enhanced relaxant responses to Ado in diabetic preparations. Treatment of corporal muscle with nonspesific $\mathrm{K}^{+}$channel inhibitor tetraethylammonium (TEA) prevented impaired maximum relaxant response to adenosine. These enhanced responses to Ado induced relaxation were not unaffected by inhibitor of nitric oxide synthase by $N^{G}$-nitro Larginine methyl ester ( $L$ - NAME). These results suggest that Ado levels may be diminished in the medium and, so relaxant responses to Ado also increase in diabetic state. Our findings further suggest that adenosineinduced relaxation appears to be involved in enhanced $K^{+}$channel activation or endothelium-dependent hyperpolarizing factor. This impairment may also contribute to the diabetes-induced impotence in men. 
PS 69

\section{Platelets, Coagulation, Rheology}

\section{6}

HYPERSENSITIVITY OF PLATELETS IN DIABETICS AND THEIR ROLE IN THE PROGRESSION OF VASCULAR COMPLICATIONS

A. Sobol, C. Watala, W. Torzecka. Laboratory of Haemostatic Disorders, Department of Laboratory Diagnostics and Department of Gastroenterology and Metabolic Disorders, Medical University of kodż, Poland

The significance of platelets hypersensitivity in the progression of vascular complications in diabetes remains disputable. The aim of the study was to evaluate platelet function and reactivity in diabetics. Subjects: Forty-five diabetic subjects aged 51.8ะ16.8 yrs. (19 IDOM:26 NIDDM) including 29 subjects with retinopathy (7 with proliferative) and 14 patients with nephropathy) were compared with 25 well-matched healthy individuals. Methods: Platelet activation and reactivity was evaluated using flow cytometry, platelet membrane fluidity using ESR spectroscopy and platelet morphoiogy - using a Technicon blood cell counter. Results: Diabetic subjects with retinopathy showed significantly elevated of platelet membrane P-selectin (a marker of platelet activation, $p<0.05$ ) and GPIb $\alpha$ (subunit of von Wiliebrand factor receptor, $p<0.01$ ), increased abundance of fibrinagen receptor (CD41, $p<0.02)$ and increased fraction of platelet microparticles $(p<0.05)$. In addition, patients with proliferative retinopathy showed significantly higher expression of P-selectin $(p<0.05)$ compared to diabetics with non-proliferative retinopathy. In patients with nephropathy the expression of P.-seiectin and fraction of microparticles were higher as referred to patients without nephropathy $(p<0.05)$. Thrombin-induced ex vivo platelet stimulation was significantly depressed in diabetic subjects compared to controls $(p<0.001)$, which might point to depressed platelet reactivity and the increased platelet rupturelconsumption in diabeles Platelet membrane lipid fluidity was reduced in numerous diabetics, atthough on average the changes were not significant. Neither platelet activation and reactivity nor the alterations in platelet membrane fluidity were related to the quality of diabetes metabolic control. Overal;, platelet volume distribution mode, $\mathrm{P}$-selectin expression and platelet microparticles appeared as the parameters which discriminated most significantty control subjects, IDDM and NIDDM patients ( $p<0.001$ or less). Conclusions: The increased P-selectin and platelet microparticles might indicate the enhanced activation of circulating platelets and the intensified release reaction from platelet granules and underlie the depressed reactivity in diabetics. Such a pronounced platelet hypersensitivity might predominantly contribute to the prevalence and progression of diabetic vascular complications.

\section{7}

PLATELET SENSITIVITY TO PROSTACYCLIN IS REDUCED IN OBESITY.

G.Anfossi, I.Russo, P.Massucco, L.Mattiello, F.Cavalot, F.Tassone, P.Perna, A.Giori, and M.Trovati. Diabetes Unit, Dept. of Clinical and Biological Sciences of the University of Turin, San Luigi Gonzaga Hospital, Orbassano (Turin) Italy.

Insulin resistance syndrome shows an increased atherothrombotic risk. In obesity, classical insulin-resistant state, platelets are less sensitive to the anti-aggregating effects of insulin and nitrates, substances acting via cyclic guanosine-monophosphate. To investigate whether platelets from obese patients also present a resistance to the anti-aggregating effect of prostacyclin, an agent acting via cyclic adenosine-monophosphate (cAMP), we studied, after overnight fast, 8 healthy subjects: M/F 4/4, 30.4 \pm 2.5 yrs, BMI $21.8 \pm 0.9$, insulin $(\mu \mathrm{U} / \mathrm{ml}) /$ glucose $(\mathrm{mg} / \mathrm{dl})$ ratio $0.07 \pm 0.01$; ii) 9 obese normotensive patients: M/F 5/4, 33.2 22.9 yrs, BMI 35.4 43.5 , insulin $(\mu \mathrm{U} / \mathrm{ml}) /$ glucose $(\mathrm{mg} / \mathrm{dl})$ ratio $0.23 \pm 0.05$. Platelet aggregation was measured on platelet-rich plasma (PRP) with $2.4 \mu \mathrm{mol} / / \mathrm{ADP}$. To evaluate the sensitivity to prostacyclin, PRP was preincubated for 3 $\mathrm{min}$ with its stable analogue Iloprost $(125-2000 \mathrm{pg} / \mathrm{ml})$ before adding ADP. In PRP incubated for $3 \mathrm{~min}$ with Iloprost, we also measured CAMP (RIA). Iloprost dose-dependently reduced platelet aggregation in both subject groups ( $p=0.0001$ ) at all the doses used; however, concentrations able reduce platelet responses by $50 \%$ (IC-50) were much higher in obese subjects than in controls: $634.9 \pm 62.4 \mathrm{pg} / \mathrm{ml}$ vs $200.0 \pm 30.8 \mathrm{pg} / \mathrm{ml}(\mathrm{p}=0.0001)$. Iloprost dose-dependently increased cAMP in both subject groups $(\mathrm{p}=0.0001)$; obese parients, however, reached cAMP values lower than controls (i.e.: with lloprost 1000 $\mathrm{pg} / \mathrm{ml}, 56.4 \pm 2.4$ vs $116.4 \pm 10.4 \mathrm{pmol} / 10^{9}$ platelets: $\mathrm{p}=0.0001$ ). Thus, platelet sensitivity to prostacyclin is reduced in obesity. Resistance to anti-aggregating agents (nitrates, insulin and prostacyclin) could be a feature of the insulin-resistance syndrome involved in atherogenesis.

\section{8}

ENHANCED ACTIVATION AND CONSUMPTION OF BLOOD PLATELETS MAY RESULT IN THEIR REDUCED REACTIVITY IN PATIENTS WITH TYPE 2 DIABETES MELLITUS Boncler M., Więclawska B., Różalski M., Trojanowski Z.*, Watala C. Laboratory of Haemostatic Disorders, Medical University of todt and ${ }^{*}{ }^{\text {nt }}$ Clinic of Internal Diseases, Military Medical Academy, Lódź, Poland

As a result of platelet hypersensitivity in patients with diabetes, circulating blood platelets undergo more frequent episodes of the release of platelet granule contents. Such an augmented release leads to the accelerated platelet consumption, the formation of platelet size gradient, and contributes to the increased platelet turnover and the reduced platelet survival in diabetic individuals. Our aim was to monitor whether platelet hypersensitvity on diabetes contributes to the altered platelet reactivity and response to activating agents. Platelet reactivity was evaluated by flow cytometric monitoring of the expressions of selected surface membrane antigens and microparticles in platelets (1) following their activation with strong agonists (thrombin. TRAP. microparticles in platelets (1) following their activation with strong agonists (thrombin, TRAP,
ADP) (2) following their incubation in the presence of activating agents $(5 \mathrm{mM}$ EDTAK $)$ ADP), (2) following their incubation in the presence of activating agents (5 mM EDTAK2)
(static modeis) and (3) following $1 \mathrm{hr}$ agitation (dynamic model). The studied type 2 diabetic individuals showed the decreased expression of the subunit $\alpha$ of glycoprotein lb by up to $12.5 \%$ $p<0.01$ ) the reduced expression of $P$-selectin in platelets activated ex vivo with thrombin, ADP or TRAP (by up to $40 \%, 21 \%$ and $36 \%$, resp., $0<0.01$ or less), and following $1 \mathrm{hr}$ agitation (by $23 \%, p<0.02$ ) and the increased number of platelet microparticles ( $p<0.05$ or less). These changes altogether might indicate the enhanced activation of circulating platelets and their depressed reactivity. We further observed higher numbers of platelets of the extreme dimensions: both very small exhausted platelets and very large, young and hypersensitive plateiets were much more frequent in diabetics compared to controls $(p-2<0.01)$ We showed that more distinct bimodality of platelet distribution in diabetic patients resulted from the accelerated thrombopoiesis in diabetic subjects, as we found the elevated fractions of reticulated platelets in diabetic patients (by $78 \%, p<0.02$ ). Overall, we conclude that the reduced platelet reactivity and the altered platelet size distribution of diabetic platelets might result from more frequent episodes of platelet activation in crrculating blood and the augmented piatele rupture/consumption in diabetic patients. These alterations are likely to contribute to the accelerated platelet renewal and the increased thrombopoiesis in diabetic individuals.

\section{9}

HYPERREAGIBILITY OF PKC TO DIFFERENT STIMULI IN PLATELETS OF TYPE 2 DIABETIC PATIENTS A.Bumbure, G.Bartling, H.Schatz, V.Pirags, A.Pfeiffer. Bochum, Germany and Riga, Latvia

Platelet activity is increased in patients with diabetes. We previously demonstrated an increased activation of blood platelet $\mathrm{PKC} \beta$ in vivo in response to acute hyperglycemia. We now aimed to identify underlying mechanisms of this phenomenon by investigating the in vitro responsiveness of platelet $\mathrm{PKC} \beta$ to different stimuli.

Platelets of type 2 diabetes patients $(n=10)$ and controls $(n=6)$ were separated by differential centrifugation and incubated $60 \mathrm{~min}$ in 300 $\mathrm{mg} / \mathrm{dL}$ (16.5 $\mathrm{mM})$ glucose or mannitol solution. $P K C \beta_{\text {। }}$ was determined quantitatively by Western blot using laser densitometry. Platelet separation and incubation were performed in presence of $\mathrm{PGI}_{2}$ to inhibit aggregation

In platelets of diabetic patients an extensive increase of $P K C \beta_{1}$ activity in response to incubation with glucose $(480 \%, \mathrm{p}<0.05)$ as well as to incubation with mannitol $(280 \%, \mathrm{p}<0.05)$ was observed However. similar pattern of platelet $\mathrm{PKC} \beta_{1}$ hyperactivation was observed after 60 min incubation of diabetic platelets in isotonic Krebs-Ringer solution. There was no significant translocation of $P K C \beta$, under these conditions in controls.

In conclusion, we observed in vitro activation of diabetic platelet PKC $\beta$ in response to hyperglycemia, hyperosmolarity as well as to other non specific stimuli. This indicates hyperreagibility of PKC $\beta$ in platelets of diabetic patients most likely related to the increased intracellular calcium 
A RANDOMIZED PLACEBO CONTROLLED TRIAL ON THE EFFECT OF $\alpha$-TOCOPHEROL ON THE PLATELET FUNCTION IN TYPE 1 DIABETES M. Van der Planken, F. Vertessen, W. Engelen, J. Vertommen and I. De Leeuw, Dept. Endocrinology-Hematology, Univ. Hosp. Antwerp, B-2650 Edegem, Belgium

Several studies suggest an important role of hyperfunctional platelets in the pathogenesis of diabetic vasculopathy. Since antioxidants are believed to have some inhibitory effect on the development of this chronic complication this study wants to examine the effect of the daily administration of $750 \mathrm{mg}$ of dl- $\alpha$-tocopherol during a period of 6 months on platelet function versus placebo in a randomized, double blind protocol in 44 type 1 diabetic patients under stable and acceptable metabolic control. Platelet function was studied in platelets rich plasma (PRP) prepared from citrated whole venous blood. PRP platelet aggregation tests were performed with a classic turbidimetric aggregation using a collagen suspension and ADP, as exogenous agonists. Platelet procoagulant activity (PPCA) in PRP samples was studied with a manually performed Stypven clotting time (SCT) and a clotting test (PF3KCT) for measuring platelet factor 3 availability. In the placebo group no significant changes in ADP and collagen aggregation amplitudes could be disclosed when comparing the start values with those obtained after 3 and 6 months. On the contrary ADP aggregation in the vitamin $E$ treated group decreased significantly $(p<0.05)$ from $69.12 \%$ (sd 13.1) at the start to $60.3 \%$ (sd 15.7) after 3 and $64.5 \%$ (sd 14.9) after 6 months. Collagen aggregation amplitudes remained at the same level after 6 month $(80.5 \%$, sd 6.5$)$ as compared to the start $(81.2$, sd 10.8). PPCA measured with both methods did not show significant differences in the placebo and $\alpha$-tocopherol treated groups. Since a pilot study with a new device the "Platelet function anaiyzer" (PFA) has demonstrated a significant $(p<0.005)$ shorter "closing time" (CT) of collagen/epinephrine coated membranes with citrated full blood from diabetic patients as compared to non-diabetic controls, we could compare the CT in 16 vitamin $E$ treated and 14 placebo-treated patients at the end of the study. In the firs group COL/EPI CT's were no longer different from controls $(116.9 \mathrm{sd} 22.9 \mathrm{sec}$ versus $128.8 \mathrm{sd} 32.7 \mathrm{sec}$.) but significantly longer ( $\mathrm{p}<0.025$ ) as compared to placebo patients $(103.8 \mathrm{sd} 23.1 \mathrm{sec}$.). From this data it can be concluded that supplementation with $\alpha$-tocopherol can decrease the platelet hyperreactivity in type 1 diabetic patients.

1272

GENETIC AND ENVIRONMENTAL DETERMINANTS OF PLASMINOGEN ACTIVATOR INHIBITOR-1 ACTIVITY IN CHINESE DIABETIC PATIENTS

K.S.L.Lam, N.M.S.Wat, B.S.H.Lau and E.D.Janus*. Dept. of Medicine and *Clinical Biochemistry Unit, University of Hong Kong, Hong Kong, China

The 4G/5G polymophism in the promotor of the plasminogen activator inhibitor-1 (PAI1) gene was found to be a genetic determinant of plasma PAI-1 activity in Caucasians, but not in Pima Indians in whom, however, an association with diabetic retinopathy was found. To investigate its role in determining plasma PAIl activity and development of diabetic complications, we studied 469 unrelated Chinese subjects: 173 non-diabetic controls, 239 NIDDM and 57 IDDM patients. PAI-1 4G/5G genotype was assessed by allele-specific polymerase chain reaction of genomic DNA. Plasma PAI-1 activity and antigen levels were measured by colorimetric and ELISA assays respectively using commercial kits. No significant difference in PAI-l antigen or activity levels was found among the 3 genotypes the relative frequencies of which were similar among the 3 groups of subjects: $-4 \mathrm{G} / 4 \mathrm{G}: 4 \mathrm{G} / 5 \mathrm{G}: 5 \mathrm{G} / 5 \mathrm{G}=30: 52: 18 \%$. NIDDM patients had higher levels of PAI-1 antigen and activity than controls $(p<0.0001)$ and IDDM patient $(\mathrm{p}<0.0001)$; they also had higher triglyceride $(\mathrm{p}<0.001)$ and lower HDL-cholestero (p<0.001) levels compared to the other 2 groups. In a multiple regression model triglyceride, $\mathrm{BMI}$ and $\mathrm{HDL}$-cholesterol were the only significant independen determinants of PAI-1 activity, contributing $17 \%, 4 \%$ and $2 \%$ respectively to its variance in NIDDM patients. The association between triglyceride and PAI-1 activity or antigen was strongest with the $4 \mathrm{G} / 4 \mathrm{G}$ genotype, being significant for all 3 groups of subjects ( $\mathrm{p}<0.05$ to $\mathrm{p}<0.0001$ ) and weakest with the $5 \mathrm{G} / 5 \mathrm{G}$ genotype, being significant only in NIDDM patients $(\mathrm{p}<0.01)$. Compared to those with $5 \mathrm{G} / 5 \mathrm{G}$, diabetic patients with the $4 \mathrm{G}$ allele bad a higher prevalence of retinopathy $(\mathrm{p}<0.05)$. We conclude that in this Chinese population, the PAI-1 promotor $4 \mathrm{G} / 5 \mathrm{G}$ genotype is not a significant determinant of plasma PAI-1 activity, but may determine increases in PAI-1 expression in response to hypertriglyceridaemia. The $5 \mathrm{G} / 5 \mathrm{G}$ genotype is apparently protective against retinopathy, similar to findings in Pima Indians.
The differential contribution of coagulo-fibrinolytic system to the progress of carotid atherosclerosis in subjects with mild hyperglycemia and diabetics. M.KODAMA, T.WATARAI, K.SAKAMOTO, R.YOSHIOKA, T.TAGAWA, M.KUBOTA, Y.YAMASAKI and M.HORI

First Department of Medicine, Osaka University Medical School, OSAKA, JAPAN Though, high activity of blood coagulation factor VII and high concentration of fibrinogen have been reported in diabetic subjects, whether these abnormalities are primary or secondary to diabetic macroangiopathy remains to be clarified. To clarify whether the coagulo-fibrinolytic abnormalities are observed in diabetics and mild hyperglycemic subjects with and without advanced atherosclerosis, we measured intimal plus medial arterial wall thickness of the carotid artery (IMT) using ultrasound high resolution B-mode imaging and the profiles of the coagulo-fibrinolytic system on 138 diabetic patients without any symptoms of diabetic macroangiopathy (NIDDM;m/f:96/42), 266 mild hyperglycemic subjects (BLDM;m/f:216/50), and 24 nomal subjects (N/C;m/f:13/11). PAI-1 concentraion and activity of blood coagulation factor VII were significantly higher in BLDM with and without advanced carotid atherosclerosis than $\mathrm{N} / \mathrm{C}(141.0 \pm 121.1 \mathrm{ng} / \mathrm{ml}$ vs 53.1 $\pm 46.2 \mathrm{ng} / \mathrm{ml}(\mathrm{N} / \mathrm{C}), 121.8 \pm 33.2 \%$ vs $102.3 \pm 25.5 \%(\mathrm{~N} / \mathrm{C}$ ) respectively, (mean \pm SD) $\mathrm{p}(0.05)$. PAI-1 was significantly higher in BLDM with advanced atlerosclerosis $(159.5 \pm 123.4 \mathrm{ng} / \mathrm{ml})$ than N/C. NIDDM showed higher concentration of fibrinogen $(320.5 \pm 84.4 \mathrm{mg} / \mathrm{dl}$ ws $223.7 \pm 34.0 \mathrm{mg} / \mathrm{dl}(\mathrm{N} / \mathrm{C})$ ) and higher activity of von Willebrand Factor $(134.5 \pm 49.9 \%$ vs $100.3 \pm 38.7 \%(\mathrm{~N} / \mathrm{C})$ than N/C. The high level of fibrinogen concentration was seen in NIDDM withou advanced carotid atherosclerosis $(303.0 \pm 74.2 \mathrm{mg} / \mathrm{dl}$ vis $223.7 \pm 34.0 \mathrm{mg} / \mathrm{dl}(\mathrm{N} / \mathrm{C})$ ) and it was significantly correlated to IMT $(r=0.3)$. These data suggested that the coagulo-fibrinolytic abnormality in BLDM is different from that in NIDDM irrespective of presence of the advanced carotid atherosclerosis. PAI-1 might be a risk factor for carotid atherosclerosis in subjects with mild hyperglycemia, and fibrinogen might be a risk factor for carotid atherosclerosis in diabetics.

\section{3}

PAI-1 LEVRIS ARE DRCREASED BY INSULIN TRRATYENT IN RATIENTS WITH TYPE 2 DIABETES MELLITUS WITE ENDOTHELIAL DYSPUNCTION.

Galajoja P., Balấz D., Martinka R., Šutarík L., Kubisz P., Mokán̆ M. Department of Internal Medicine, National Haemostasis and Thrombosis Centre $^{2}$, University Bospital, Martin, Slovak Republic.

Relation between hyperinsulinemia (BI) and increased PAI-1 levels is well-known in patients with insulin resistance syndrone. According to new multifactorial and multicompartmental model of PAI-1 production, insulin stimulates PAI-1 synthesis in "retabolic" compartment (visceral adipocytes, hepatocytes) and inhibites cytokine-induced endothelial PAI-1 production. Physiological postprandial BI has been accompanied by decreased PAI-1 levels during circadian rhytm. PAI-1 levels are decreased during long-term insulin application. Inhibitive insulin effect on PAI-1 production is not fully understood, but can be realized in endothelial pool. In our study we examined 34 patients with type 2 diabetes mellitus (DM) treated with sulfonylurea (SU) regimens, 24 patients with 2-3 month long-acting insulin (INS) treatnent and 15 age-matched normoinsulinenic healthy controls. We evaluated levels of von Willebrand factor (VWF), thrombomodulin (TM), tissue factor pathway inhibitor (TFPI), platelet factor PF, $\mathrm{TPA}$, PAI-1, C-peptide, triglycerides, HBA1C and BMI. Patients with type 2 DM were divided into subgroups with and without endothelial dyafunction according to WWF level $0.14 \mathrm{IV} / \mathrm{ml}$. There were significant differences of PAT-1 levels among patients without endothelial dysfunction treated by SU (median 48.1, range 14-108 $\mathrm{ng} / \mathrm{ml}$ ) or INS $(27.9$, $2-53 \mathrm{gg} / \mathrm{ml})$ and patients with endothelial dysfunction treated by SU $(80.7$, 43-217 ng/ml) or INS resp. $(16.7,7-34 \mathrm{ng} / \mathrm{ml}$ ) (ANOVA $0<0.001)$. Type $2 \mathrm{DM}$ subgroups were not different in parameters of metabolic compensation and platelet activation. VWF, TM and TPPI levels were elevated in groups with endothelial dysfunction. Insulin treatment was accompanied by decreased PAI-1 leveis especially in patients with endothelial dysfunction. We suggest that long-term insulin application may decreased PAI-1 levels by direct inhibitive action on endothelial PAI-1 compartnent. This hypothesis requires futher research. 
Arterial Wall and Liver of Diabetic Rats exhibit Increased Synthesis of Plasminogen Activator Inhibitor-1 (PAI-1).

A. PANDOLFI, A.GIACCARI*, R. POLISHCHUCK** L. MORVIDUCCI*, E. VITACOLONNA, F. CAPANI and A. CONSOLI. Università d'Anunzio,Chieti; * Universita Cattolica, Roma and " Consorzio Mario Negri Sud. S. Maria Imbaro, ITALY

Increased PAI-1 plasma content and activity have been described in NIDDM. Liver is among the main tissues responsible for PAI-1 synthesis and release into the circulation, liver PAl-1 levels, however, have not been investigated in the diabetic animal. Furthermore, hyperglycemia increases PAI-1 synthesis in arterial wall cells in culture, suggesting impairement of local fibrinolysis in diabetes. However, little is known about PAI-1 localization in arterial wall cells of diabetic animals. Therefore, aim of this study was to measure by immunohystochemistry PAl-1 levels in livers and arterial walls of diabetic and control rats. To this end, diabetes was induced in 10 Sprague Dowley rats by a $90 \%$ pancreatectomy. Five weeks later, we studied PAl-1 antigen localization (immunohistochemical staining) in the liver $(n=7$, weight $224 \pm 16 \mathrm{~g}$. blood glucose $306 \pm 12 \mathrm{mg} / \mathrm{dll}$ ) and in the aorta wall ( $\mathrm{n}=10$, weight $235 \pm 14 \mathrm{~g}$, blood glucose $301 \pm 9 \mathrm{mg} / \mathrm{dl}$ ) of the diabetic animals and of 14 control rats (weight $227 \pm 6 \mathrm{~g}$, blood glucose $117 \pm 4 \mathrm{mg} / \mathrm{dl}$ ). As compared to liver tissue from control animals, in livers from diabetic rats analysis by Laser Scanning Confocal Microscopy revealed increased fluorescence for PAl-1 antigen ( $754 \pm 25$ vs $299 \pm 14$ arbitrary fluorescence units, $p<0.0001)$. The same analysis revealed increased fluorescence for PAI-1 antigen also in the aorta wall of the diabetic animals ( $339 \pm 18$ vs $274 \pm 9$ arbitrary fluorescence units, $p<0.005$ ). This data provide the first direct evidence that diabetes can induce increased PAl-1 synthesis in the liver and in the arterial wall. This suggests that in diabetes the liver can be an important source for increased plasma PAI-1 and local fibrinolysis can be affected by increased PAI-1 levels in the arterial wall.

\section{6}

\section{INCREASED THROMBO-EMBOLIC RISK IN PERIOPERATIVE PERIOD IN DIABETIC PATIENTS}

J.Gizło, *M.Zozulińska, A.Tokarz, \#D.Zozulińska, J.Sowier and "K.Zawilska. Department of Surgery, "Dept.of Haematology, \#Dept.of Internal Medicine and Diabetology, Karol Marcinkowski University of Medical Sciences

Thrombo-embolic complications create a great problem in the perioperative period. The aim of the study was to evaluate selected parameters of hemostasis in diabetic patients in the perioperative period. The study was entered by a group of 17 diabetic patients hospitalized due to abdominal surgery performed in general anaesthesia ( 12 female, 5 male, aged $60.2 \pm 13.9$ years, mean diabetes duration $12.3 \pm 10.7$ years). Patients presented good metabolic control of diabetes ( $(\mathrm{bA} 1 \mathrm{c} 7.2+1.4 \%$ ). All patients were given standard antithrombotic prevention with Calciparin (Sanof-Winthrop) $2 \times 5000 \mathrm{IU} / 24$ hours. Before and 1,3 and 5 days after the surgery we assessed the following parameters: plasma concentration of fibrinogen, peptide $F_{1+2}$, thrombin-antithrombin complex (TAT), complex of tissue plasminogen activator and it's inhibitor (tPA-PAI), platelet factor 4 (PF4) and the activity of antithrombin III (AT III) and protein $C$. In comparison with healthy subjects we observed significantly higher values of PF4 and TAT before and after the surgery. We did not observe statistically significant differences in the other parameters.

The results are shown in a table:

\begin{tabular}{|c|c|c|c|c|c|}
\hline parameter & $\begin{array}{l}\text { Healthy } \\
\text { subjects }\end{array}$ & $\begin{array}{l}\text { before } \\
\text { surgery }\end{array}$ & $\begin{array}{c}\text { 1st day after } \\
\text { surgery }\end{array}$ & $\begin{array}{c}\text { 3rd day after } \\
\text { surgery }\end{array}$ & $\begin{array}{c}\text { 5th day after } \\
\text { surgery }\end{array}$ \\
\hline$F g(g /)$ & $3.6 \pm 0.4$ & $4.2 \pm 0.2$ & $3.7 \pm 0.2$ & $4.2 \pm 0.3$ & $4.6 \pm 0.3$ \\
\hline$F 1+2(\mu g /)$ & $5.5 \pm 3,5$ & $11 . \overline{7} \pm 1.1$ & $12.1 \pm 1.1$ & $12 . \overline{0} \pm 1.3$ & $11 . \overline{1} \pm 1.2$ \\
\hline TAT $(\mu \mathrm{g} /)$ & $2.4 \pm 1.5$ & $31.7 \pm 10.8^{*}$ & $44.4 \pm 10.3^{*}$ & $42.7 \pm 7.6^{*}$ & $64.7 \pm 5.3^{*}$ \\
\hline ATII $(\%)$ & $110 \pm 30$ & $128 . \overline{0}+13.9$ & $128 . \overline{3} \pm 16.2$ & $126 . \overline{0} \pm 18.2$ & $140.6 \pm 13.4$ \\
\hline Protein C (\%) & $105 \pm 35$ & $100.3 \pm 3.6$ & $100.5 \pm 6.9$ & $87.1 \pm 5.7$ & $103.6 \pm 5.6$ \\
\hline tPA-PAl $(\mu \mathrm{g} /)$ & $6 \pm 1,5$ & $4.7 \pm \overline{0} .6$ & $8.5 \pm \overline{1} .4$ & $5.4 \pm 0.8$ & $6.0 \pm 0.9$ \\
\hline PF4 $(\mu g /)$ & $4.5+1.4$ & $82.7 \pm 6.6^{*}$ & $72.4 \pm 10.7^{*}$ & $73.9 \pm 8.9^{*}$ & $82.9 \pm 9.5^{*}$ \\
\hline
\end{tabular}

* $\mathrm{p}<0.05$ in comparison with healthy subjects

The results obtained suggest the presence of hypercoagulability indicators despite the standard prevention with the use of unfractionated heparins in the perioperative period in diabetic patients.
DO ELEVATED PLASMA PROTHROMBIN LEVELS CORRESPOND TO GENETIC VARIATION IN THE 3'-UNTRANSLATED REGION OF THE PROTHROMBIN GENE IN TYPE 2 DIABETIC PATIENTS?

Różalski M., Boncler M., Pietrucha T.* Watała C

Laboratory of Haemostatic Disorders and *Department of Biochemistry, Medical University of Lódź, Lódź, Poland

Recently, occasional reports pointed to the possibte correlation between the genetic polymorphism $20210 \mathrm{G} / \mathrm{A}$ in $3^{\prime}$-untranslated region in prothrombin gene and the elevated prothrombin level in blood plasma particularly in the cases of familial thrombophilia. The coexistence of various impairments resulting in the haemostatic imbalance in diabetes mellitus implies that the increased plasma prothrombin levels could underlie the prothrombotic state often found in diabetic subjects. Moreover, as far as diabetes itself constitutes an additional independent risk factor in the development of vascular complications, and the elevated leveis of prothrombin fragment $F 1+2$, the marker of thrombin generation, have been reported in diabetes, it raises a possibility of the occurrence of the prothrombin gene polymorphism in diabetic patients more frequently. Our aim was to verify the hypothesis whether the eievated plasma prothrombin levels in some patients might correspond to the genetic variation in the 3 '-untransiated region of the prothrombin gene in patients with type 2 diabetes mellitus. We revealed that the averaged plasma level of prothrombin fragment $F 1+2$ in an overall group of 128 type 2 diabetic patients was not significantly different compared to controls ( $2.93 \pm 2.40 \mathrm{nM}$ vs. $2.21 \pm 1.83 \mathrm{nM}$ ); in selected patients ( $16 \%)$, however, the level of $\mathrm{F} 1+2$ was vastly increased $(7.70 \pm 1.83 \mathrm{nM})$, although these patients showed no evident

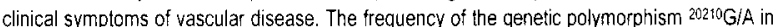
3 -untranslated region in prothrombin gene in the examined group of diabetic subjects $(1.3 \%)$ was not significantly higher compared to the group of control individuals and did not correspond to the increased $F 1+2$ plasma levels: all the diabetic patients with the markedly increased levels of $F 1+2$ were wild type homozygotes $20210 \mathrm{G} / \mathrm{G}$. Overall, we conclude that the genetic polymorphism $20210 \mathrm{G} / \mathrm{A}$ in $3^{\prime}$-untranslated region in prothrombin gene is not evidently associated with the increased plasma thrombin generation in the examined group of diabetic patients. Other, yet undefined, compounding factors might underlie the accelerated thrombogenesis occurring in some patients with diabetes mellitus

\section{7}

PROCOAGULANT PROFILE OF HEMOSTATIC PARAMETERS IN PATIENTS WTTH TYPE 2 DIABETES AND VISCERAL OBESITY Kozek E., Katra B., Sieradzki J., Department of Metabolic Diseases, Jagiellonian University, Kraków, Poland

Introduction. Abnormalities of coagulation and fibrynolysis may be a risk factor of cardiovascular diseases in diabetic patients with obesity. The aim of the study was to evaluate hypercoagulation parameters in patients with type 2 diabetes mellitus depending on the type of obesity taking into account gender, metabolic control and blood lipids. Material and Methods. The study population consisted of 45 patients with type 2 diabetes - 22 women after menopause (mean age $62.63 \mathrm{SD} 8.7 \mathrm{yrs}$ ) and 23 men (mean age 61.08 SD 7.99 yrs). Dual - energy X-ray absorptiometry (DEXA) was used to analyze regional distribution of fat to calculate the visceral waist region (VW) to gynoid hip region (GH) ratio and the visceral fat to total fat (VF/TF) ratio. Fibrinogen concentration, $C$ protein (pC), factor VIl (fVII), antithrombin III (ATIII) and alpha 2 antiplasmin (alpha 2AP) activity were determined with the Chromo Time System (Behringwerke). Results. The VW/GH ratio was significantly higher in men than in women (mean $1.13 \mathrm{SD} 0.21$ vs mean $0.86 \mathrm{SD} 0.19, \mathrm{p}<0.001$ ), similar to the VF/TF ratio (mean 10.7 SD $1.67 \%$ vs mean $7.75 \mathrm{SD} 1.18 \%, \mathrm{p}<0.001$ ). In men with the VF/TF ratio $>10 \%$ as compared to those with the ratio $<10 \%$ the activity of $\mathrm{C}$ protein was significantly lower (mean 94.52 SD $17.73 \%$ vs mean 117.51 SD $23.64 \%$, p<0.05); ATIII activity was also significantly lower (mean 103.66 SD $8.69 \%$ vs 114.99 SD $10.41 \%, p<0.01$ ). A negative correlation was found between the VF/TF ratio and $C$ protein activity $(\mathrm{r}=-0.30, \mathrm{p}<0.05)$ and between the $\mathrm{VW} / \mathrm{GH}$ ratio and alpha $2 \mathrm{AP}$ activity $(\mathrm{r}=-0.38$, $p<0.05$ ). Step-wise multiple regression analysis revealed that the VF/TF ratio is an independent factor influencing fVI activity apart from triglyceride concentration, whereas the VW/GH ratio affects independently alpha $2 \mathrm{AP}$ activity. Conclusion. In men with type 2 diabetes mellitus and visceral obesity the lower activity of coagulation inhibitors such as C protein and ATIII indicates hypercoagulation. In men and women after menopause with type 2 diabetes visceral obesity is an independent risk factor of increased fVI activity with the procoagulative properties. 


\section{8}

HIGH THROMBOMODULIN IN YOUNG PEOPLE WITH TYPE-1 DIABETES: EVIDENCE OF ENDOTHELIAL INJURY IN CHILDHOOD DIARETES ?

T.A. Elhadd, M. McLaren, R.W. Newton, S.A. Greene and JJF Belch University Department of Medicine \& Child Health, Ninewells Hospital \& Medical School, Dundee DD1 9SY, UK

Diabetes mellitus is associated with high morbidity and mortality from vascular disease. Endothelial dysfunction is now established to be the early manifestation of diabetic vascular disease. Thrombomodulin (TM) is a surface glycoprotein of endothelial cells which acts as a cofactor in the thrombin-catalysed activation of protein $\mathrm{C}$. It has been suggested that TM may be a more sensitive marker for endothelial damage than von Willibrand Factor (VWF) since TM appears only to be released by endothelial cell injury whereas vWF can be released by other mechanisms. It has been shown that plasma levels of TM are increased in adults patients with early diabetic nephropathy. We have measured plasma levels of vWF and TM by ELISA method in 52 young diabetic subjects, age (medianrange) of 15 (9-22) years, diabetes duration 6.6 (1-20) years, and HbAlc 8.7 $\%(5.4-11.7)$. and in 29 age and sex matched normal controls. None of the diabetic patients had clinical evidence of vascular disease, and all had urinary microalbumin in the normal range. Plasma levels of TM are significantly increased in the diabetic group compared to the control subjects-6! (4l-72) $\mathrm{ng} / \mathrm{ml}$ and $45(26-56) \mathrm{ng} / \mathrm{ml}$ respectively $\mathrm{p}<0.0046$, Mann Whitney. There were no significant differences in the levels of vWF. Our results suggest that in the juvenile diabetic subjects there is evidence of endothelial cell damage in the absence of clinical vascular disease. It also suggests that TM may be a more sensitive marker for endothelial cell damage than $\mathrm{vWF}$.

\section{9}

PHYSICAL EXERCISE NORMALIZES THROMBOMODULIN LEVELS IN TYPE 1 AND TYPE 2 DIABETIC PATIENTS

M.Rigla ${ }^{1}$, J.Mateo ${ }^{2}$, A.Caixàs ${ }^{1}$, J.Fontcuberta ${ }^{2}$, A. de Leiva ${ }^{1}$, A.Pérez Endocrinology ${ }^{1}$ and Hematology ${ }^{2}$ Dpts. H. Sant Pau. UAB. Barcelona

The aim of the study was to evaluate the influence of physical exercise on various soluble markers of endothelial damage, in a group of type 1 ( 7 male, 7 female, age $25 \pm 6$ years, BMI $24 \pm 3 \mathrm{~kg} / \mathrm{m}^{2}, \mathrm{HbA}_{\mathrm{lc}} 7.3 \pm 1 \%$, diabetes duration $6 \pm 5$ years) and type 2 ( 9 male, 4 female, age $54 \pm 6$ years, BMI $26 \pm 3 \mathrm{~kg} / \mathrm{m}^{2}, \mathrm{HbA}_{1 \mathrm{c}} 7.4 \pm 1 \%$, diabetes duration $9 \pm 5$ years) diabetic patients (DM) free of chronic complications. We evaluated the effect of a 3 month physical training program (3-5 days/week, $1852 \pm 387$ $\mathrm{Kcal} /$ week) on Thrombomodulin (TM), von Willebrand Factor, Plasminogen Activator Inhibitor 1 and tissue-type Plasminogen Activator, measured by standard ELISA kits and Tissue Factor Pathway Inhibitor (TFPI), analysed according to Sandset et al. After the studied period BMI and $\mathrm{HbA}_{1 \mathrm{c}}$ did not change for both groups while $\mathrm{VO}_{2}$ max increased significantly $(\mathrm{p}<0.05)$ in the type I DM group. Among the pre-exercise coagulation parameters studied, only the TM plasmatic levels were significantly higher in type 1 and type $2 \mathrm{DM}$ than in their respective matched control groups $(79.3 \pm 105$ vs $28.5 \pm 10 \mu \mathrm{g} / 1, \mathrm{p}<0.005$ and $50.0 \pm 16$ vs $31.1 \pm 8 \mu \mathrm{g} / \mathrm{l}, \mathrm{p}<0.05)$. After the exercise program TM levels decreased $(p<0.05)$ in both experimental subsets and became similar to those observed in their respective control groups $(58.1 \pm 88$ vs $28,5 \pm 10 \mu \mathrm{g} / \mathrm{l}$; ns, for the type 1 and $38.2 \pm 11 \mathrm{vs} 31.1 \pm 8 \mu \mathrm{g} / \mathrm{l}$, ns; for the type $2 \mathrm{DM}$ )

In conclusion, physical exercise reduces to normal values TM levels in type 1 and type 2 diabetic patients without long term complications.

\section{0}

POSTPRANDIAL COAGULATION ACTIVATION: THE EFFECT OF A SINGLE DOSE OF GLIBENCLAMIDE.

I.Ioannidis, C.Tsoukala, C.Panayotopoulou, E.Maglara, Y.Anastasopoulou, T.Mandalaki, and N.Katsilambros. First Dpt Propaedeutic Medicine, Athens University Medical School, Laiko Hospital, GR 11527 Athens Greece.

Previous data indicate that postprandial (pp) hyperglycaemia in diabetic subjects, even those who are considered to be in good metabolic control, activates coagulation. The aim of this study was to investigate the effect of the administration of a single dose of glibenclamide on the $p p$ activation of the coagulation as compared to placebo. We designed a crossover randomised study. In the morning after an overnight fast, each diabetic subject ( $n=16$, age $50-68$ yr.) consummed a standard meal $(600 \mathrm{Kcal}$, Carbohydrates $40 \%$ Lipids $50 \%$ Proteins $10 \%$ ) preceeded by one tablet of glibenclamide (G, $5 \mathrm{mg}$ ) or placebo (pl). The two tests were performed randomly, with an interval of 7 days. Blood samples were collected at baseline as well as 2 and 4 hours after the meal to measure blood glucose, insulin, c-peptide and fibrinogen, d-dimers, F1+2 (prothrombin fragments 1+2) and TAT (thrombin-antithrombin complex) As expected pp levels of glucose were lower and of insulin and c-peptide were higher after $G$. Main findings were; a) significant $p p$ increase of fibrinogen, ddimers, F1+2 and TAT, (ANOVA, $p<0,001, p<0,05, p<0,01$ and $p<0,01$ respectively) b) pp levels of the above parameters are significantly lower after $G$ as compared to pl (Table, paired t-test)

\begin{tabular}{|c|c|c|c|c|c|}
\hline & & & Baseline & 2 hours & 4 hours \\
\hline \multirow[t]{2}{*}{ Fibrinogen } & \multirow[t]{2}{*}{$\mathrm{mg} \%$} & $\overline{\mathrm{pl}}$ & $220 \pm 47$ & $281 \pm 43^{\text {t* }}$ & $272 \pm 54^{*}$ \\
\hline & & $G$ & $207 \pm 37$ & $239 \pm 46^{\star \star}$ & $265 \pm 48^{*}$ \\
\hline \multirow[t]{2}{*}{ D-Dimers } & \multirow[t]{2}{*}{$\mathrm{ng} / \mathrm{ml}$} & pl & $233 \pm 121$ & $268 \pm 147^{\star}$ & $380 \pm 262^{* / 1}$ \\
\hline & & $G$ & $223 \pm 191$ & $240 \pm 158^{*}$ & $275 \pm 228^{\star \star \star}$ \\
\hline \multirow[t]{2}{*}{$F 1+2$} & \multirow[t]{2}{*}{$\overline{\mathrm{nM}}$} & pl & $1,3 \pm 0,8$ & $4,0 \pm 3,5^{\star}$ & $3,3 \pm 0,5^{\star}$ \\
\hline & & G & $1,8 \pm 1,7$ & $2,5 \pm 2,3^{*}$ & $2,1 \pm 2,6^{*}$ \\
\hline \multirow[t]{2}{*}{ TAT } & \multirow[t]{2}{*}{$\mu g / /$} & $\mathrm{pl}$ & $11,3 \pm 13,0$ & $40,5 \pm 40,0$ & $54 \pm 44^{*}$ \\
\hline & & G & $13,6 \pm 21,0$ & $40,5 \pm 35.0$ & 4 \\
\hline
\end{tabular}

Placebo vs. Glibenclamide ${ }^{*} p<0.05{ }^{* *} p<0.01$

Thus, the $\mathrm{pp}$ activation of coagulation is significantly reduced after -acute a least- $G$ administration.

\section{1}

MOLECULAR PARAMETERS OF THE ACTIVATION OF THE COAGULATION IN CHLDREN WITH DIABETES MELLITUS T. 1 J.Teisseyre, E.Piontek and H.Lukasiewicz. The Children's Memorial Health Institute, Warsaw, Poland.

Diabetes mellitus is associated with high prevalence of vascular disease and is considered a thrombosis prone state. Numerous studies have shown that coagulation disorders occur in the course of adult diabetes t.l, resulting a prethrombotic state. Little is known about haemostasis in diabetic children without any signs of long term complications. Sensitive and specific methods are available to detect a procoagulant imbalance in haemostasis. The aim of the study was to evaluate some molecular parameters of the prethrombotic state: thrombin-antythrombin complexes (TAT), prothrombin fragments $1+2$ $(\mathrm{F} 1+2)$ and $\mathrm{D}$-dimers in diabetic children, compared to duration of disease and glycometabolic control (glucose, $\mathrm{HbAlc}$, fructosamine levels). All the subjects had examined: platelets count, APTT, INR, clotting time after recalcination and fibrinogen level, too. We investigated 72 diabetic children (mean age $11,76 \pm 5,5$ years), duration of disease $0-17,4$ years (mean 3,51 $\pm 3,86$ ). Control group consisted of 19 healthy children (mean age $9,6 \pm 4$, lyears). Compared to healthy children, diabetic patients displayed significantly higher mean levels of TAT complexes $(\mathrm{p}=0,0002)$. Statistically significant positive correlation were found with the TAT and glucose levels $(r=0,224 \mathrm{p}=0,03)$ and negative correlation with TAT and duration of diabetes $(r=0,241 \mathrm{p}=0,04)$. In the group of diabetic children we found more elevated levels of F1+2 $(34,72 \%)$, than in controls $(10,53 \%)$-Fisher's exact test $\mathrm{p}=0,04$. Positive correlation was found between $F 1+2$ levels and: HbAlc concentrations $(r=0,275 p=0,019)$ as well as glucose levels $(r=0,275 p=0,019)$. D-dimers tevels were normal in both investigated groups. These results demonstrate slight impairment of the haemostatic balance in diabetic children that is possible prethrombotic state represents an important factor in pathogenesis of microangiopathy. 
CIRCULATING FACTOR XIII (FXIII) LEVELS AND FXIII CODON 34 GENOTYPE IN TYPE 2 DIABETES MELLITUS M.W. Mansfield, H.P. Kohler, R.A.S. Ariëns and P.J. Grant Unit of Molecular Vascular Medicine, University of Leeds, UK.

Genotype at a polymorphism coding for coagulation factor XIII (FXIII) A-subunit has been related to myocardial infarction. To investigate the relationships of FXIII codon 34 genotype and measures of circulating FXIII in type 2 diabetes (DM), FXIII fibrin cross-linking activity was determined using a cadaverine incorporation assay and antigen levels of the catalytic FXIII A-subunit and carrier FXIII B-subunit were measured by ELISA in 173 type 2 DM patients ( 96 males). FXIII codon 34 genotype was determined by PCR and SSCP analysis. Levels of FXIII activity were similar in males and females but levels of both subunit $\mathrm{A}$ (mean $\pm \mathrm{SD}) 122 \pm 28 \vee 114 \pm 29 \%(p=0.05)$ and B-subunit $134 \pm 26$ v $119 \pm 25 \%(p<0.0005)$ were higher in female than male patients respectively. Levels of FXIII B-subunit showed a consistent correlation with markers of insulin resistance; BMI, fasting insulin, PAI1 , triglyceride, factor VII:C $(r>0.16, p<0.05)$ and hypertension. FXIII activity (mean $\pm \mathrm{SD}$ ) increased with number of $\mathrm{T}$ alleles at the codon 34 polymorphism; G/G $80 \pm 20 \%, \mathrm{G} / \mathrm{T} 126 \pm 20 \%$ and T/T $146 \pm 21 \%$ $(p<0.0005)$. In contrast $\mathrm{T} / \mathrm{T}$ subjects had lower levels of both the A-and B-subunits than other subjects: A-subunit antigen G/G $118 \pm 28 \%$, G/T $121 \pm 28 \%$ and $\mathrm{T} / \mathrm{T} 93 \pm 25 \%(p=0.004)$ and $\mathrm{B}$-subunit antigen $\mathrm{G} / \mathrm{G}$ $123 \pm 24 \%$, G/T $132 \pm 29 \%$ and T/T $107 \pm 18 \%(p=0.002)$. These results indicate that the FXIII A-subunit codon 34 polymorphism has functional significance and that FXIII may play a role in the development of the vascular complications of diabetes.
FIBRINOGEN IS CLOSELY RELATED TO VARIOUS METABOLIC PARAMETERS AND MICROVASCULAR COMPLICATIONS IN CHINESE TYPE II DIABETES C.C.Chow, W.B.Chan, V.T.F.Yeung, J.C.N.Chan, W.Y.So and C.S Cockram. Department of Medicine \& Therapeutics, Prince of Wales Hospital, Chinese University of Hong Kong, Hong Kong, China. Fibrinogen has been shown to be associated with microalbuminuria in both type I and type II diabetes. However, its relationship with other metabolic parameters and complications had not been well established in type $\Pi$ diabetes. In this cross-sectional study, we examined 141 consecutive type II diabetic patients newly referred to our tertiary diabetes clinic serving a population exceeding 1 million from September to November 1996. Detail demographic data, risk factors and complications were documented by a computerized structural approach modified from DiaCare Database. We found that fibrinogen was correlated with age $(r=0.31, p<0.001)$, duration of diabetes $(r=0.26$, $p=0.002)$, HDL cholesterol $(r=-0.17, p=0.050)$ and waist hip ratio $(\mathrm{r}=0.28, \mathrm{p}=0.001)$. On multivariate analysis, waist hip ratio remained significant $(B=6.45, p<0.007)$. Furthermore, fibrinogen was correlated with cataract $(p=0.005)$, peripheral neuropathy $(p=0.007)$ and microalbuminuria $(r=0.30, p<0.001)$. On multivariate analysis, fibrinogen remained strongly correlated with microalbuminuria $(B=0.403, p=0.001)$. In addition, association of various risk factors with complications, similar to other large-scale epidemiology study in type I diabetes, were also demonstrated. We conclude that central obesity is a key determinant of fibrinogen in keeping with the pathogenesis of metabolic syndrome. Fibrinogen may also play a contributory role to the onset of microvascular complications in type II diabetes.

\section{4}

ACTIVATION OF MONOCYTE TISSUE FACTOR IN POORLY CONTROLLED NIDD's. EFFECT OF PENTOXYFILLINE.

B. Lormeau, D. De Prost, F. Lerendu, P. Wechsler, P. Valensi, A. Mercadier, J.R Attali. Department of Endocrinology-Diabetology-Nutrition, Jean Verdier Hospitał, Bondy. INSERM U 291, Bichat Hospital, Paris. CTS, Avicenne Hospital, Bobigny. Hoechst Laboratory, Paris La Défense. France.

The synthesis and expression of tissue factor (TF) and the adhesion related to the expression of beta 2 integrin and in particular Mac 1 receptors are two monocyte functions which are abnormally activated in diabetes. We have previously shown that pentoxyfilline (PTX) reduces in vitro TF production by normal and diabetic monocytes stimulated by endotoxine and reduces leucocyte expression of Mac 1 receptors and the monocyte adhesion to endothelium. The aim here was 1) to compare the expression of monocyte TF, thrombin-anti thrombin 3 complexes (TAT), factor VII and activated VII factor, plasma FT and thrombomoduline (TM) in 33 poorly-controlled NIDD's (HbAlc $=9.73 \pm 1.49 \%$ ) and 26 controls $; 2$ ) to evaluate the effect of a one-month double blind trial of PTX vs placebo on these parameters. Plasma TF and TAT were increased in NIDD's as compared to controls $(252.7 \pm 13.8$ vs $191.3 \pm 21.4 \mathrm{pg} / \mathrm{ml}, \mathrm{p}=0.01$ and 4.4 $\pm 1.0 \mathrm{vs} 3.3 \pm 0.2 \mathrm{\mu g} / \mathrm{l}, \mathrm{p}=0.09$ ). The monocyte expression of Mac 1 receptors did not differ significantly in the two groups but the monocyte expression of $\mathrm{CD} 25$ receptors was higher in NIDD's ( $66 \pm 22$ vs $0.15 \pm 0.02 \%, p<0.05$ ). TM and activated VII factor did not differ in the two groups. TM correlated with waist to hip ratio in NIDD's $(r=0.39, p<0.05)$. The incubation of normal monocytes with plasma from NIDD's induced a higher FT expression as compared to the effect of plasma from controls ( 362 \pm 20 vs $250 \pm 22 \mathrm{mu} / 10^{6}$ monocytes, $\mathrm{p}<0.05$ ). Pentoxyfilline did not induce any significant effect on these parameters. In conclusion, hypercoagulability in diabetic patients is here supported by the increase of TAT complexes and the activation of the FT pathway and the stimulating effect of plasma from diabetic patients on FT production by normal monocytes. A short-term treatment by pentoxyfilline did not induce a significant effect at variance with in vitro results.

\section{5}

IMPAIRED RBC DEFORMABILITY IN NIDDM CORRELATES WITH ELEVATED INTRACELLULAR FREE CALCIUM-ION J. Fujita, T. Adachi, Y. Yamada, H. Ishida, K. Tsuda and Y. Seino Department of Metabolism and Clinical Nutrition, Kyoto University Faculty of Medicine, Kyoto 606-8507, Japan

OBJECTIVE - To explore the mechanisms underlying the impaired RBC deformability (RBC-df) in diabetic patients, the relationship between $\mathrm{RBC}$ intracellular free calcium-ion concentration $\left(\left[\mathrm{Ca}^{2+}\right] \mathrm{i}\right)$ and $\mathrm{RBC}$-df, and the effects of a $\mathrm{Ca}^{2+}$. channel blocker on $\left[\mathrm{Ca}^{2+}\right] \mathrm{i}$ and $\mathrm{RBC}$-df were evaluated.

METHODS - We examined 48 patients with NIDDM and 24 control subjects. [ $\left.\mathrm{Ca}^{2+}\right] \mathrm{i}$ was determined using fura-2, and $\mathrm{RBC}-\mathrm{df}$ by filtration method expressed as Deformability Index (DI). Erythrocytes were treated with nisoldipine to evaluate the effects of a $\mathrm{Ca}^{2+}$-channel blocker.

RESULTS $-\left[\mathrm{Ca}^{2+}\right] \mathrm{i}$ was significantly higher $(82.7 \pm 2.2$ vs. $77.2 \pm$ $1.8 \mathrm{nmol} / \mathrm{RBC}, \mathrm{p}<0.001)$, and DI was significantly lower $(0.16 \pm$ 0.06 vs. $0.22 \pm 0.03, p<0.01)$ in NIDDM than in controls. There was a significant correlation between $\mathrm{HbA}_{\mathrm{lc}}$ and $\left[\mathrm{Ca}^{2+}\right] \mathrm{i}(\mathrm{r}=0.38$, $\mathrm{p}<0.01)$, between $\mathrm{HbA}_{1 \mathrm{c}}$ and DI $(\mathrm{r}=-0.51, \mathrm{p}<0.01)$, and between $\left[\mathrm{Ca}^{2+}\right] \mathrm{i}$ and DI $(\mathrm{r}=-0.42, \mathrm{p}<0.01)$. Stepwise multiple regression analysis revealed $\mathrm{HbA}_{\mathrm{lc}}$ and $\left[\mathrm{Ca}^{2+}\right] \mathrm{i}$ as independent determinants for the impaired RBC-df. Nisoldipine treatment in vitro significantly decreased $\left[\mathrm{Ca}^{2+}\right] \mathrm{i}$, and significantly improved $\mathrm{RBC}$-df There was a significant correlation between the degree of $\left[\mathrm{Ca}^{2+}\right] \mathrm{i}$ decrease and the degree of $\mathrm{DI}$ increase.

CONCLUSIONS - These data indicate that the impaired RBCdf in NIDDM may at least partly be attributed to the elevated $\left[\mathrm{Ca}^{2+}\right] \mathrm{i}$ and poor glycemic control. In addition, the $\mathrm{Ca}^{2+}$-channel blocker affects favorably both $\left[\mathrm{Ca}^{2+}\right] \mathrm{i}$ and $\mathrm{RBC}-\mathrm{df}$. 
PS 70

\section{Atherosclerosis}

\section{6}

C-TYPE NATRIURETIC PEPTIDE CONCENTRATIONS A NEW MARKER OF ATHEROSCLEROSIS IN NIDDM.

A. Fernandez-Cruz, J. Ibarra R. Fernandez Durango, A. Ripodas, E. Pintor, and R. Patiño. Cardiovascular Preventive Center. Hospital Universitario San Carlos. 28040 Madrid (Spain)

The natriuretic peptide family consists of atrial natriuretic peptide,brain natriuretic peptide and C-type natriuretic peptide (CNP). CNP is thought to be a vascular peptide. Is synthesized and released from culture and endothelial cells. Moreover the binding of CNP to the atrial natriuretic peptide receptor-B, express in smooth muscle cells $(S M C)$, generates cGMP which mediates vasodilation. CNP is also antimitogenic and inhibits the proliferation of vascular SMC. Thus CNP may have an important role in controlling vascular tone and vascular remodelling. We have developed a sensitive and specific RIA for CNP which is able to detect IR-CNP in human plasma.In chronic renal failure IR-CNP is significantly increased in the circulation. Many epidemiological studies have shown that persistent proteinuria in diabetes represents a subgroup with very high risk of cardiovascular disease. The present study was designed to examine the relationship between CNP and atherosclerosis in a group of both NIDDM with (Group $1 \mathrm{n}=25$ ) and without macrovascular disease (Group $2 n=22$ ) and NIDDM patients with absence of foot pulses on examination, without any evidence of macrovascular disease (Group 3 $\mathrm{n}=9$ ). Aged from 44 to 75 years, mean $64 \pm 8$ yrs; mean duration of diabetes $18+8 \mathrm{yrs}$. IR-CNP was found to be significantly elevated in Group $1(2.25 \pm 1.4 \mathrm{pg} / \mathrm{ml}$ when compared with 25 age matched controls $0.58 \pm 0.34 \mathrm{pg} / \mathrm{ml} . \mathrm{p}<0.01)$; and groups 2 and $3(1.2+0.9$ and $13 \pm 1.2$ $\mathrm{pg} / \mathrm{ml}$. respectively $p<0.05$ in both cases).

In the present study, we have shown for the first time the significant association between elevated plasma IR-CNP concentrations in NIDDM patients, and the increase prevalence of macrovascular disease.

\section{7}

LONG-TERM EFFECTS AFTER INTRAVENOUS PGE1 INFUSION IN DIABETIC AND NON DIABETIC PATIENTS

A. Rodriguez-Caballero, F. Pazos*, J.M. Carceller, M.J. Chinchetru* and J.A. Amado. Anaesthesic and ${ }^{*}$ Endocrine Services, Hospital U. Marqués de Valdecilla, 39008-Santander, Spain

People with diabetes have a risk of arteriosclerotic occlusive disease two to five times that of non-diabetic individuals. Platelet aggregation has been shown to be increased in diabetes, which may play a role in the pathogenesis of this vascular disease. Also, it has been demonstrated that PGE1 promote improvement in pain and ischemic ulcers in diabetic and non diabetic patients. The aim of this study was to compare the effects of intravenous PGE1 in diabetic and non diabetic patients with rest pain and/or ischemic ulceration due to arteriosclerotic occlusive disease. 28 patients: 9 diabetic and 19 non diabetic, with peripheral occlusive disease in stage III or IV Leriche-Fontaine were followed from 6 to 12 months after 3 weeks of intravenous infusion of PGE1. The previous arteriography showed that reconstructive surgery or angioplasty were not indicated. Two groups were compared by $\chi^{2}$ test. There were no significant differences between both groups in age, sex distribution, ischemic skin lesions or other risk factors. The follow up examination showed that in $8 / 9$ diabetic and $17 / 19$ non-diabetic patients, rest pain improved, only 2 non-diabetic patients required higher doses of analgesics during the treatment with PGE1. In $7 / 7(100 \%)$ diabetic and $5 / 9(55 \%)$ non diabetic patients, ulcers decreased in size during or some weeks after infusion $(p<0.05) .13$ patients in the non diabetic group and 5 in the diabetic one required extremity amputation by 6-12 months (ns). On the other hand, 1 diabetic and 5 non diabetic patients required vascular reconstructive surgery, and 2 in the non diabetic group required spinal-cordstimulation. Side effects associated with the administration of PGE1 like headache, nausea or vomiting were similar in both groups. This study shows that intravenous PGE1 was more effective in healing diabetic ischemic ulcer which suggests that prostanoid pathways might be implicated in diabetic arteriosclerotic occlusive disease. There were no differences in pain relief and the need of extremity amputation between both groups. Also, we could not find any differences in side effects.

\section{8}

RISK OF NNTERMITENT CLAUDICATION AND LOWER LIMB AMPUTATIONS IN AN URBAN COHORT OF NIDDM PATIENTS

P.Luźniak, A.Czech and J.Tatoń,

Department of Diabetology, Warsaw Medical University, Poland.

Accordingly to the local observation the morbidity due to diabetes type II is $5 \%$, in the same time $50 \%$ of the population of persons who underwent the lower limb amputation is formed by diabetics type II. The risk of amputation for diabetics type II is equal to 20 . Therefore the study in a cohort of 1334 type II diabetics from the demographically defined area under long term care of the Warsaw Central Diabetic Care Station was undertaken. The risk factors were determined in a subgroup with intermittent claudication and in comparative group of diabetics after amputation. In all 1334 participants the following studies were performed: standardized clinical examination, determination of the specific for diabetes mellitus type II characteristics and the quality of therapy. Among patients under study $8.25 \%$ presented intermittent claudication and $1.05 \%$ underwent lower limb amputation. Among 14 cases in 5 amputation was made above the knee, in 4 below the knee and in 5 within the foot. The following risk factor were assessed: age, sex, diabetes duration, obesity, blood pressure, fasting and postprandial glycemia, cholesterol, triglycerides, creatinine in plasma, daily proteinuria and smoking. Univariate analysis indicated, that the significant risk factors for intermittent claudication were: diabetes duration, postprandial glycemia and daily proteinuria. In the group after amputation the significant risk was connected with diabetes duration and daily proteinuria. Differences in other parameters were not statistically significant. The multivariate analysis of all variables has indicated that the significant risk factor for intermittent claudication are male sex, diabetes duration and smoking. It did not apply to diabetics after amputation. Conclusions. The delineation of the risk factors for intermittent claudication offers the chance of selecting the groups of patients for early therapeutic measures aimed at prevention of this syndrome and its sequela.

\section{9}

A TRANSCRANIAL DOPPLER STUDY OF CEREBROVASCULAR REACTIVITY IN DIABETIC PATIENTS.

C. Roberti ${ }^{1}$, P. Gargiulo ${ }^{2}$, G. Mancini ${ }^{1}$, M. Solaro' ${ }^{1}$, B. Romani ${ }^{2}$, T. Battocletti ${ }^{2}$, V. Vaccari ${ }^{2}$ and E. Zanette ${ }^{1}$ Department of Neurological Sciences; ${ }^{2}$ Endocrinology I University "La Sapienza", Rome, Itab

Diabetes mellitus (DM) is associated with vasculopathies such as atherosclerosis, thromboembolism and microvascular disease of kidney, heart and retina. Much speculation surrounds the pathophysiology of these lesions, but no experimental data are available for the cerebrovascular disease, which is often associated with DM Recently, a relationship between DM, hyperinsulism and multiple lacunar infarcts has been demonstrated. Since an impairement of the cerebrovascular reactivity could be an index af cerebral microangiopathy, we evaluated the effects of hyper- and hypocapnia on the middle cerebral arteries of type I and type II diabetic patients, with transcranial Doppler. Thirty-six diabetic patients (18 type I, 8 females, 10 males mean age $37.3 \pm 12.2$ years $S D$, mean duration of disease $13.9 \pm 9.2 ; 18$ type II, 7 females, 11 males, mean age $60.2 \pm 4.1 \mathrm{SD}$, mean duration of disease $10.7 \pm 7.1$, in good metabolic conditions, and 36 healthy subjects comparable for age and sex were included. All patients underwent a color duplex of the extracranial arteries, a transcranial Doppler and a vasoreactivity test. The middle cerebral artery mean velocities were recorded bilaterally and the end-tidal $\mathrm{CO}_{2}$ partial pressure was monitored. The recordings included basal condition, hypercapnia $\left(\mathrm{CO}_{2} 6 \%\right)$ and hyperpnea. The reactivity index for hypercapnia $\left(\mathrm{RICO}_{2}\right)$, reactivity index for hyperpnea (RIHp) and total vasomotor reactivity (VMR) were measured. $\mathrm{RICO}_{2}$ was $3.3 \pm 1.2$ in type I and $3.9 \pm 1.3$ in type II; RIHp was $3.3+0.8$ in type I and $3.5 \pm 0.7$ in type II; VMR was $69.8 \pm 15.1$ in type I and $77.2 \pm 13.8$ in type II. We did not find significant difference of the above indices between the two groups of patients and compares to the control groups (ANOVA). These results may be due to the good metabolic control these diabetic pantients have had in the last years. 
CORRELATES OF LOWER EXTREMITY ARTERIAL DISEASE IN TYPE I DIABETES

The Epidemiology of Diabetes Interventions and Complications: Followup of the DCCT Cohort

T. Orchard, P. Cleary, S. Genuth and D. Nathan for the EDIC Study

Group, Rockville, MD, USA

The DCCT showed a very significant benefit of intensive treatment targeted to normalization of blood sugar in preventing or delaying the microvascular complications of Type 1 diabetes; however, the role of glycemia in causing macrovascular complications is uncertain. An association between mean $\mathrm{HbA} 1 \mathrm{c}$ over an average of 6.5 years of follow-up in the DCCT and a biological marker of atherosclerosis, carotid wall intima medial thickness (IMT) measured in the same subjects at the beginning of EDIC, was not seen. In this report, a marker of peripheral vascular atherosclerosis, a depressed ankle:brachial (blood systolic pressure) ratio is examined using data from the first EDIC examination of 1326 subjects who had a mean age of 35 years and mean duration of diabetes of 14.5 years. Four ratios were calculated, including left and right dorsalis and posterior tibialis. Excluding 4 subjects with ankle pressures suggestive of medial wall calcification (ankle pressure $>75 \mathrm{mmHg}$ above brachial), $10.8 \%$ of women and $8.6 \%$ of men had at least one ankle/brachial ratio $<0.9$. In women, systolic blood pressure was the only significant $(\mathrm{p}=0.002)$ predictor, while in men age $(p=0.001)$ and ever being a smoker $(p=0.009)$ were predictors. HbA1c, diabetes duration and prior-DCCT treatment group were not predictors in either gender. These results are consistent with the carotid IMT findings and suggest that the glycemic link to macrovascular association is more complex than the association with microvascular complications. Further study of the DCCT cohort in EDIC will delineate the risk factors for clinical atherosclerosis as the patients develop more clinical events.
ANGIOTENSIN CONVERTING ENZYME GENE POLYMORPHISM IS ASSOCIATED WITH PERIPHERAL ARTHERIOPATHY IN TYPE 2 DIABETIC PATIENTS

M.A. Ortiz*, A. Planas**, A. Clara**, J- Marugat**, J.M. Pou*. P. Travé F. Vidal. Dept of Endocrinology and Nutrition. Hospital Sta. Creu i S. Pau*. Hospital del Mar*. Autonomous University of Barcelona.

Cardiovascular disease (CVD) is the most frequent cause of mortality in general population and in type 2 diabetic patients. Several genetic factors could play a role in the development of CVD. DD Angiotensin converting enzyme (ACE) gen polymorphism could be associated to diabetic nephropathy and CVD. This study was designed to stimated peripheral artheriopathy in a cohort of men, and its association to the presence of $\mathrm{DD}$ polymorphism ACE gen. It was investigated a representative sample from both general (654 males, age: range 55-75 years) and diabetic (118 males, age: range 55-75 years) population. Peripheral artheriopathy was screening by rest ankle/braquial pressure index (ABPI). ABPI was considered pathological when was lower than 0.9. ACE gen polymorphism was determined by PCR with specific primers for D and I fragment amplification. Frequency of diabetes in the investigated group of subjects was $19.1 \%$. Peripheral artheriopathy (PA) (clinical and subclinical) was detected in $13.1 \%$ in the whole group, and in $24.8 \%$ of the diabetic population.. DD polymorphism was observed.

$\begin{array}{lcccc} & \text { D/D ACE (\%) } & \begin{array}{c}\text { Odd Ratio } \\ \text { (CI95\%) }\end{array} & \text { P } \\ \text { general population WO/PA } & 18.67 & & \\ \text { general population W/PA } & 44.71 & 3.52,1.63-7.61 & =0.0003 \\ \begin{array}{l}\text { Diabetic population WO/PA } \\ \text { diabetic population W/PA }\end{array} & 20.03 & 47.82 & 3.50 .1 .58-11.54 & <0.0044\end{array}$

Therefore DD genotype was associated to peripheral artheriopathy in both diabetic and non diabetic population. DD ACE genotype may be considered a marker of risk for periferal artheriopathy.

\section{2}

ANGIOTENSIN CONVERTING ENZYME(ACE) GENE POLYMORPHISM AND EARLY CAROTID ATHEROSCLEROSIS IN CHINESE NIDDM PATIENTS

N.M.S. Wat S.C.W. Cheung* M.T. Chau* and K.S.L. Lam Department of Medicine and, Diagnostic Radiology*, Queen Mary Hospital, Hong Kong, China

Carotid intima-media thickness (IMT) which is related to clinical cardiovascular diseases affecting other arteries such as the coronaries, has been shown to be increased in NIDDM patients, known to be at higher risk of atherosclerosis. In Caucasian diabetic patients, an association between the ACE-insertion/deletion (I/D) polymorphism of the ACE gene and autopsy findings of aortic atherosclerosis has also been reported. To investigate the relationship between the ACE I/D polymorphism and early atherosclerosis as indicated by increased MT in Chinese we studied $100(\mathrm{M} / \mathrm{F}$ 45/54) unrelated NDDM patients, aged $47.8+16.4$ years (mean+SD). ACE genotyping was performed using polymerase chain reaction. Carotid IMT was measured by a single observer using high-resolution B-mode ultrasonography. Mean IMT measurement was $0.7 \pm 0.2 \mathrm{~mm}$ among the whole group. MT measurement correlated significantly with age, duration of disease, systolic blood pressure and LDL-cholesterol (all $p<0.01$ ), and HbAlc $(p<0.05$ ). Prevalence of plaques (localized increased IMT $\geq 1.2 \mathrm{~mm}$ ) on either or both sides of the carotid arteries increased with increasing age $(8,28,57$ and $70 \%$ respectively by quartile) $(p<0.001)$, and among patients having ischaemic heart disease $(67 \%$ versus $33 \%$ $\mathrm{p}<0.005$ ). The presence of plaques was significantly increased in patients with the $D$ allele $(50 \%$ versus $27 \%$ in $I / I$ homozygous; $p=0.023$ ) There was no significant difference in age, duration of disease, body mass index, blood pressure, fasting blood glucose, HbAIc or lipid profile between the two groups (DD + ID versus $I / /$ ). In conclusion, the ACE I/D polymorphism is associated with early atherosclerosis, as reflected by increased carotid MT, in Chinese NIDDM patients, and may serve as a marker to identify NDDM patients at increased risk of cardiovascular mortality so that early preventive measures can be implemented.

\section{3}

DIABETES AND CAROTID ATHEROSCLEROSIS. PROSPECTIVE RESULTS FROM THE BRUNECK STUDY.

E. Bonora, " S. Kiechl, "J. Willeit, ${ }^{*} F$. Oberhollenzer, ${ }^{*} \mathrm{G}$. Egger, R. Bonadonna and M. Muggeo. Division of Endocrinology and Metabolic Diseases, University of Verona and "Division of Internal Medicine, Hospital of Bruneck, Italy; "Department of Neurology, University of Innsbruck, Austria. It is well established that diabetes increases the risk of main clinical manifestations of cardiovascular disease (myocardial infarction, stroke). On the contrary, the relationships existing between diabetes and the various steps of the atherosclerotic process are poorly understood. In addition diabetic patients often carry multiple metabolic, hacmodynamic and haemocoagulative defects. Thus, the independent contribution of diabetes to the development or the progression of atherosclerotic lesions is not well defined. We have examined prospectively 826 subjects aged $40-79$ years, randomly selected from the general population of Bruneck (northestern Italy). All subjects had an eco-duplex assessment of common and internal carotid arteries (16 regions of interest on both sides) in 1990 and 1995. Sixty eight subjects had IGT and 58 had NIDDM at baseline. In all subjects the following parameters were assessed: BMI, WHR, lipids, apoproteins A1 and B, Lp(a) and its phenotype, fibrinogen, antithrombin III, APC ratio, urate ferritin, blood pressure, smoking, daily alcohol intake, physical activity. All subjects but known diabetic individuals had an OGTT. Logistic regression analysis indicated that in subjects without carotid atherosclerosis in 1990 $(n=501)$, the development of plaques during the follow-up period $(n=126)$ was not independently related to the presence of IGT or NIDDM in 1990. On the contrary, in subjects with carotid atherosclerosis in $1990(n=325)$ the progression to stenosis $>40 \%(\mathrm{n}=92)$ was independently related to both IGT $(\mathrm{OR}=3.1, \mathrm{Cl}=1.3-7.6)$ and NIDDM $(\mathrm{OR}=7.1, \mathrm{Cl}=1.8-28.5)(\mathrm{p}=0.001)$ at baseline These results document that IGT and, to a greater extent, NIDDM are predictors of advanced (carotid stenosis) but not early atherosclerosis, suggesting that both abnormalities of glucose tolerance are independently involved in the progression rather than the initiation of the atherosclerotic process. 
HBAIC IS AN INDEPENDENT DETERMINANT OF CHANGES IN CAROTID ARTERY COMPLIANCE IN IMPAIRED GLUCOSE TOLERANT SUBJECTS R.A.J.M. van Dijk, G. Nijpels*, J.M. Dekker*, L.M. Bouter*, R.J. Heine* and C.D.A. Stehouwer, Department of Intemal Medicine, University Hospital Vrije Universiteit, Institute for Cardiovascular Research Vrije Universiteit and ${ }^{*}$ Institute for Research in Extramural Medicine, Vrije Universiteit, Amsterdam, The Netherlands.

Background Impaired Glucose Tolerance (IGT) is associated with increased risk for macrovascular disease. In this study, we assessed compliance in the carotid artery as an early indicator of macrovascular disease and sought to identify determinants for alterations in compliance and related vessel wall properties. Methods At baseline we selected a group of 113 subjects (mean age 63.7 yrs, 25-75 perc: $57.8-70.0$ yrs, 53 males) with IGT according to WHO criteria from a random population sample. Using an ultrasound system right carotid artery diameter was measured during 10-20 heart beats at two follow-ups (2.3 years, range $0.5-3.5$, and 5.0 years, $4.2-6.4$ ). Simultaneously, blood pressure was measured in the brachial artery. Resuits Carotid artery diameter and B-stiffness showed an increase ( 6.85 to $6.97 \mathrm{~mm}, \mathrm{P}<0.001$ and 12.6 to $13.9, \mathrm{P}<0.001$ respectively). Meanwhile, there was a decrease in circumferential arterial strain (CAS, 5.0 to $4.7, \mathrm{P}=0.001$ ), cross-sectional compliance ( $\left(\mathrm{CC}, 0.46\right.$ to $0.44 \mathrm{~mm}^{2} \mathrm{kPa}^{-1}, \mathrm{P}=0.037$ ) and distensibility coefficient (DC, 12.3 to $11.410^{-3} \mathrm{kPa}^{-1.4}, \mathrm{P}=0.001$ ), all indicating arterial stiffening. Meanwhile, there was no significant change in blood pressure nor in heart rate. In multiple linear regression analysis $(n=96)$ correcting for age, sex, mean arterial pressure and follow-up time, HbAlc-levels proved to be a determining factor for relative changes in CAS $(\beta=-0.237, \mathrm{P}=0.028)$, DC $(B=-0.226, P=0.039)$ and $B$-stiffness $(B=0.246, P=0.023)$. After additional correction for smoking habit, total serum cholesterol level and body mass index, $\mathrm{HbAlc}$ showed to be a determining factor for change in $C C(\beta=-0.232, P=0.043)$ too. An independent relation between $\mathrm{HbAlc}$ and diameter could not be demonstrated. Conclusion We demonstrated a process of carotid artery stiffening and widening. However, the increase in diameter could not compensate for the decrease in DC, as shown by the decrease in CC. Furthermore, $\mathrm{HbAic}$ level proved to be a determinant of arterial function, suggesting a role for hyperglycaemia in the process of deteriorating function of elastic elements in the arterial wall.
PROSPECTIVE EVALUATION OF URINARY N-ACETYL-GLUCOSAMINIDASE WITH RESPECT TO MACROVASCULAR DISEASE IN TYPE 2 DIABETICS R. Weitgasser ${ }^{1}$, F.Schnő̈12, B. Gappmayer', I.Kartnig' and S.Sailer ${ }^{1}$

'2nd Dept. of Medicine, Salzburg General Hospital, Salzburg, Austria

${ }^{2}$ Center for Rehabilitation, Grossgmain, Austria

It is known that diabetic macroangiopathy is the main reason for reduced life expectancy in type 2 diabetic patients. Microalbuminuria has been shown to serve as an early marker for cardiovascular morbidity and mortality besides predicting overt diabetic nephropathy. In a random overview in elderly type 2 diabetic patients we were able to detect an association of urinary $\mathrm{N}$-acetyl-glucosaminidase (NAG) excretion and macrovascular disease which lead to our current study. We prospectively followed 124 patients with type 2 diabetes over 7 years. At baseline age, diabetes duration, sex, blood pressure, total cholesterol, HDL-cholesterol, triglycerides, serum creatinine, urinary albumin excretion, urinary NAG excretion, a history of myocardial infarction, stroke and peripheral arteriovascular disease as well as antidiabetic treatment, concomitant medication and smoking habits were determined. After a mean of $7+/-0.5$ years patients were examined again, and multivariate logistic regression models were calculated. Meanwhile 58 patients died, 35 of these from cardiovascular endpoints which were defined as myocardial infarction and heart failure, stroke and severe peripheral arteriovascular disease (gangrene, amputation). Age, diabetes duration, systolic blood pressure, serum total cholesterol and microalbuminuria were found to be parameters of significant value $(p<0.05)$ in these patients. Analyzing the subgroup of 65 patients still alive we found that urinary NAG excretion was significantly elevated in patients with myocardial infarction and peripheral arteriovascular disease $(8.0+/-2.3 \mathrm{U} / \mathrm{L})$ but not in those with a stroke $(3.5+/-0.7$ $\mathrm{U} / \mathrm{L}$ ), $\mathrm{p}<0.05$. There was also a positive predictive trend for the development of myocardial infarction and peripheral arteriovascular disease with respect to NAG excretion, but this was not statistically significant $(\mathrm{p}=0.07)$. NAG was not different for hypertensive and normotensive subgroups in our study. In conclusion we may assume that urinary NAG could serve as an adjunct marker to urinary albumin excretion in patients with preexisting respectively at risk for the development of severe macrovascular disease.

\section{6}

Fiber intake, serum cholesterol levels and cardiovascular disease in European individuals with type 1 diabetes

M. Toeller ${ }^{1}$, A.E. Buyken ${ }^{1}$, G. Heitkamp ${ }^{1}$, G. de Pergola ${ }^{2}$, F. Giorgino ${ }^{2}$, J. H. Fuller ${ }^{3}$ and the EURODIAB IDDM Complications Study Group

I Diabetes Research Institute, Düsseldorf, Germany; ${ }^{2}$ University of Bari, Italy; ${ }^{3}$ UCL Medical School, London, UK

Cross-sectional analysis of dietary fiber intakes in European type 1 diabetic patients enrolted in the EURODIAB IDDM Complications Study for potential relationships to serum cholesterol levels and the prevalence of cardiovascular disease. Dietary intake was assessed by a standardized 3day dietary record. For analysis of fiber intake (total, soluble and insoluble) and its associations with cardiovascular disease (past history or electrocardiogram abnormalities) complete data were available from 1050 male and 1012 female individuals. Relationships of fiber intakes to serum cholesterol levels (total, HDL and LDL-cholesterol) were examined in 926 men and 881 women with type 1 diabetes. Higher intakes of total fiber $(\mathrm{g} / \mathrm{day})$ were independently associated with significantly higher levels of HDL-cholesterol in male $(p=0.01)$ and female individuals $(p=0.03)$. Fiber intakes of men with type 1 diabetes were also inversely related to ratios of total cholesterol to HDL-cholesterol $(\mathrm{p}=0.0001)$ and levels of $\mathrm{LDL}$ cholesterol $(p=0.0002)$. A protective effect of total fiber intake against cardiovascular disease was observed for female subjects, where a significant trend was maintained after adjustment for potential confounders including energy and saturated fat ( $p=0.03$ vs $p=0.2$ in men). Results were similar in separate analyses with soluble or insoluble fiber. The present study demonstrates that higher fiber intakes are independently related to beneficial alterations of the serum cholesterol pattern in men and to a lower risk for cardiovascular disease in European insulin-dependent women. Beneficial effects can already be observed for fiber amounts within the range commonly consumed by outpatients with type 1 diabetes.

\section{7}

Atherosclerosis in type 2 diabetic and non-diabetic subjects with and without clinical coronary artery disease: The Insulin Resistance Atherosclerosis Study

S.M. Haffner, R. D'Agostino Jr., M.F. Saad, D.H. O'Leary, P.J. Savage, M. Rewers, J. Selby, R.N. Bergman, L. Mykkänen, San Antonio, TX.

Type 2 diabetes (DM) is associated with a two-to four-fold increase in the risk of clinical coronary artery disease (CAD). It has been suggested that diabetic subjects without clinical CAD should be treated as aggressively for cardiovascular risk factors as subjects with prior MI. This would be warranted if DM subjects without clinical CAD would have accelerated CAD similar to that of non-diabetic subjects with clinical CAD. To assess this suggestion, we compared the intima-media wall thickness (IMT) in the common carotid artery (CCA) and internal carotid artery (ICA) in 43 DM subjects with clinical CAD $446 \mathrm{DM}$ subjects without clinical CAD, 47 non-diabetic subjects with clinical $\mathrm{CAD}$ and 975 non-diabetic subjects without clinical $\mathrm{CAD}$ in the Insulin Resistance Atherosclerosis Study (IRAS). All data was adjusted for age, gender, ethnicity and clinic. Both DM and CAD were significantly associated with increased atherosclerosis in the CCA. Likewise, DM was significantly associated with increased atherosclerosis in the ICA; however, CAD was no associated with ICA IMT. As expected, DM subjects with CAD had the greatest IMT while non-DM subjects without CAD had the least atherosclerosis. Subjects with DM but without CAD had slightly greater IMT than non-diabetic subjects with $C A D$ although these differences were not statistically significant. Diabetic subjects even without $C A D$ have extensive atherosclerosis. Our results support the suggestion that DM subjects should be treated as aggressively for cardiovascular risk factor management as subjects with preexisting $C A D$. 
INSULIN PRODUCTION AND INSULIN SENSITIVITY IN THE KLOTHO MUTANT MOUSE, A NOVEL ANIMAL MODELFOR HUMAN AGEING.

Y. Ohyama, T. Utsugi, T. Ohno, T. Uchiyama, H. Ito, S. Tomono, S. Kawazu, M. Kuro-o, Y. Nabeshima, and R. Nagai,

Maebashi and Tokyo, Japan

We have identified a novel gene, termed klotho ( $\mathrm{kl})$, that is involved in the suppression of several ageing phenotypes. A defect of $\mathbf{k l}$ gene expression in mice results in a syndrome that resembles human ageing, including arteriosclerosis, emphysema, osteoporosis, skin atrophy, and infertility. The gene encodes a membrane protein that shares sequence similarity with $\beta$-glucosidase enzymes. To see whether mice homozygous for the $\mathrm{kl}$ mutation $(\mathrm{kl} / \mathrm{kl}$ mice) show abnormal glucose metabolism, OGTT was examined at 6-8 weeks of age. Insulin content of the pancreas was measured by radioimmunoassay. Blood glucose level during OGTT in $\mathrm{kl} / \mathrm{kl}$ mice was significantly lower than that in wild mice. Whereas, insulin content of the pancreas in $\mathrm{kl} / \mathrm{kl}$ mice was significantly lower than that in wild mice. This defective insulin production was supported by the northem blot analysis that showed lower level of mRNA expression of insulin in $\mathrm{kl} / \mathrm{kl}$ mice. To understand low blood glucose with the defective insulin production, insulin tolerance test was performed. The insulin sensitivity in $\mathrm{kl} / \mathrm{kl}$ mice was higher than that in wild mice. In $\mathbf{k l} / \mathbf{k l}$ mice, augmented expression of Glut 4 in skeletal muscles was demonstrated both by northern blot analysis and western blot analysis. We conclude that (1) the klotho gene product is associated with insulin production and insulin sensitivity, and (2) arteriosclerosis in $\mathrm{kl} / \mathrm{kl}$ mice is independent of insulin resistance.

\section{PS 71}

\section{Homocysteine and Cardiovascular Disease}

\section{9}

PLASMA HOMOCYSTEINE AND SERUM FOLATE LEVELS IN DIABETES V.J. MoCam, K.G. Stanton, F.M. vanBodkmer, V. Bucke and S. Vasitaran Department of Endocrinology and Diabetes, Reyal Perth Hospital, Perth WA 6001

Depertment of Biochemistry, Poyal Perth Hospital, Ferth wa 6001

Mild to moderate hyperhancoysteinamia has recently been recognised as a risk factor for corcnary heart disease and mey be associated with deficiencies of vitamin cofactors, perticularly folate. he studied the relationship between plasia horocysteine, senm folate and a metrylenetetrahydrofolate reductase (MIHFR) gene polymonghism in patients with Type 1 and Type 2 diabetes. Sinoe 1973, patients atterding the Poyal Ferth Hospital Diabetic Clinic have participated in a tho yar survey of diabetic complications and risk factors. Since Mey 1996, plasta harocysteine, detemined by a HPC method and MIHFR gene polymorphiom, by PCR Hinf I restriction enzyme digestion have been included in the assessment in addition to the corventional risk factors for coronary hoart disease. Analysis of 410 stbjects revealed an inverse relation between plarme hamocysteine and senm folate $(p<0.001)$. As fand by others, plama horocysteine was higher in those hombygaus for the MTHFR gone mutation but anly if the serum folate was low. Somewhat paradoxically, mutation with ine was higher in those homosygus for the MTHR gere mutation with low senu folate than those with low folate alane. Hncyyosity for the mutation was also associated with a significantly higher red cell folate $(p<0.001)$ probably due to the metabolic block folate metabolism. It is concluded that low serum folate increases plama homocysteine, a potential risk factor for cononary heart disease
1300

SERUM TOTAL HOMOCYSTEINE LEVELS IN NON-INSULIN DEPENDENT DIABETICS AT THE START OF INSULIN THERAPY J. Drzewoski, L. Czupryniak, B. Chwatko*, and E. Bald*. Metabolic Diseases and Gastroenterology Department, Medical University of todz: "Environmental Chemistry Department, University of Łódz, Łódz, Poland.

A high serum total homocysteine ( $\mathrm{tHC}$ ) level has recently been shown to be an independent risk factor for atherosclerosis and cardiovascular disease the conditions specifically frequent in non-insulin dependent diabetes, particularly of poor metabolic control. The aim of the study was to evaluate serum $\mathrm{tHC}$ concentration in orally-treated non-insulin dependent diabetic patients with poor metabolic profile and thus requiring insulin treatment. The study subjects were divided into two groups: I - 14 diabetic patients, in whom therapy with maximum doses of oral agents failed and insulin treatment was going to be initiated (mean age $64.4 \pm 4.2 \mathrm{yrs}, \mathrm{BMI} 26.1 \pm 2.7 \mathrm{~kg} / \mathrm{m}^{2}$, duration of diabetes $9.3 \pm 3.6$ yrs, $\mathrm{HbA}_{1 \mathrm{c}} 8.9 \pm 1.1 \%$ ); II (controls) -8 well-matched, well controlied diabetics treated with oral agents (mean age $65.2 \pm 3.2 \mathrm{yrs}$, BM $26.8 \pm 3.1 \mathrm{~kg} / \mathrm{m}^{2}$, duration of diabetes $9.0 \pm 3.2 \mathrm{yrs}, \mathrm{HbA}_{1 \mathrm{c}} 6.4 \pm 0.7 \%$ ). Subjects with any overt cardiovascular disease were excluded from the study. Fasting serum tHC and insulin concentrations were assessed by high-performance liquid chromatography and radioimmunologic method, respectively. The blood for the assays was drawn immediately prior to the first insulin injection. The mean ( $\pm S D$ ) fasting serum tHC in group I subjects was $21.1 \pm 6.5$ $\mathrm{nmol} / \mathrm{ml}$ compared to $15.6 \pm 2.4 \mathrm{nmol} / \mathrm{ml}$ in group $\|(p<0.001)$. In contrast mean fasting serum insulin level was lower in group I than in group II (13.6 t 5.5 vs $21,3 \pm 7.8 \mu \mathrm{lU} / \mathrm{ml} ; p<0.01)$. In conclusion, non-insulin dependent diabetic patients with poor metabolic control and at the start of insulin treatment had higher fasting serum tHC levels and lower fasting serum insulin concentrations than diabetics sufficiently controlled with oral drugs. 
1301

LACK OF CHANGE OF HOMOCYSTEINE BY THE DECREASE OF PROTEINURIA IN DIABETIC PATIENTS WITH NEPHROPATHY

A. Chico, F. Blanco*, A. Córdoba*, R. Arcelus*, J. Puig, J.M. Pou and A. Pérez. Dpts. of Endocrinology and Biochemistry ${ }^{*}$, Hospital Santa Creu i Sant Pau, UAB Barcelona. Barcelona, Spain.

Homocysteine (Hcy) and albumin excretion rate (AER) are considered good predictors of cardiovascular morbidity and mortality. Recently, we observed that Hcy concentrations are related to AER in patients with diabetes mellitus. The aim of the study was to evaluate the effect of the decrease of proteinuria using enalapril on Hcy concentrations in diabetic patients with nephropathy without renal failure. We studied 15 diabetic patients (10 IDDM and 5 NIDDM; 10 men and 5 women; age $46 \pm 16$ years; diabetes duration $22 \pm 7$ years) with nephropathy without renal failure (creatinine $<120 \mu \mathrm{mol} / \mathrm{L}$ ), before and after 3 months of treatment with enalapril. Fasting and post-methionine load ( $1 \mathrm{mg} / \mathrm{kg}$ ) plasma Hcy concentrations were determined by HPLC. Vitamin B12, red cell folate, glycated haemoglobin, glucose, trygliceride, cholesterol, HDL-c, VLDL-c, LDL-c, lipoprotein(a), microalbuminuria, proteinuria, creatinine and clearance of creatinine were measured by standard methods. Fasting and post-methionine $\mathrm{Hcy}$ concentrations at baseline were $6.4 \pm 1.6 \mu \mathrm{mol} / \mathrm{L}$ and $18 \pm 5 \mu \mathrm{mol} / \mathrm{L}$ respectively. After 3 months of treatment with enalapril, proteinuria decreased from $328 \pm 227 \mathrm{mg} /$ day to $194 \pm 76 \mathrm{mg} /$ day $(\mathrm{P}<0.05)$ and microalbuminuria from $139 \pm 148 \mu \mathrm{g} /$ minute to $65 \pm 49 \mu \mathrm{g} /$ minute $(P=0.05)$. However, fasting and pot-methionine Hcy levels did not change after treatment $(7.3 \pm 1.5$ and $21 \pm 7 \mu \mathrm{mol} / \mathrm{L}$, respectively). No significant changes were observed in the other studied parameters. Fasting Hcy correlated with vitamin B12, red cell folate and proteinuria. Post-methionine Hcy correlated with creatinine and age. In conclusion, our findings suggest that decrease in proteinuria after short-term treatment with enalapril does not modify Hcy levels in diabetic patients with nephropathy.

\section{2}

PLASMA HOMOCYST(E)INE LEVELS IN TYPE 2 DIABETIC NEPHROPATHIC PATIENTS

R. Gambino, L. Scaglione, M. Cassader. E. Siano, N. Alemanno, G. Pagano, and P. Cavallo-Perin

Dipartimento di Medicina Interna, Università di Torino, ltaly

Micro-, and macroalbuminuria are strong and indipendent predictors of cardiovascular morbidity and mortality in type 2 diabetic patients (NIDDM) The effect of micro-, and macroalbuminuria on cardiovascular morbidity and mortality persists even after controlling for the conventional risk factors, such as dyslipidemia and hypertension; the mechanisms underlying this association are not completely understood. Elevated plasma homocyst(c)ine levels represent an indipendent risk factor for premature cardiovascular disease. The aim of this study is to evaluate whether moderatly increased plasma homocyst(e)ine levels may explain the increased risk for cardiovascular disease in micro-, macroalbuminuric (nephropathic) NIDDM. We studied 78 consecutive nephropathic NIDDM attending the outpatient diabetic clinic and 70 normoalbuminuric NIDDM, matched for sex and duration of diabetes. Homocyst(e)ine levels were measured using HPLC with electrochemical detection. We found no significant difference between nephropathic and normoalbuminuric patients regarding age, duration of diabetes, sex diastolic blood pressure and $\mathrm{HbA}_{\mathrm{lc}}$ levels, whereas creatinine and systolic blood pressure levels were significantly higher in the nephropathic group ( $p=0.017$ and $p=0.019$, respectively). Plasma homocyst(e)ine levels were not significantly different both in the nephropathic as well as normoalbuminuric groups.

\begin{tabular}{llll} 
& $\mathrm{n}$ & AER $(\mu \mathrm{g} / \mathrm{min})$ & Homocyst(e)ine $(\mathrm{ng} / \mathrm{ml})$ \\
\hline Normoalbuminuric NIDDM & 70 & $5.7 \pm 5.0$ & $12.3 \pm 6.1$ \\
Nephropathic NIDDM & 78 & $145.2 \pm 179.6$ & $11.4 \pm 4.7$
\end{tabular}

These results suggest that homocyst(e)ine does not play a role in increasing the cardiovascular risk in nephropathic NIDDM. This conclusion deserves a confirmatory evaluation by a prospective study.

\section{3}

\section{FACTORS INFLUENCING PLASMA HOMOCYSTEINE AS DETERMINANT OF CARDIOVASCULAR RISK IN TYPE 2 DIABETES MELLITUS}

A. Passaro, A. Solini, K. D'Elia, PL. Pareschi*, M. Carantoni, R. Fellin Department of Internal Medicine II and Diabetes Division*, University of Ferrara, Italy

Plasma homocysteine (Hcy) has been recognized as cardiovascular risk factor (CVRF) in the general population. Its role as either independent CVRF or its role in influencing others parameters is still matter of debate. We have evaluated the effect of duration of the disease and metabolic control in influencing Hcy levels in a group of type diabetic subjects without renal impairment, determining and its role as independent CVRF in this population. We measured fasting plasma glucose, HbA1c, blood pressure values, and Hcy in 208 type 2 diabetic subjects aging from 41 to 88 years, with a wide range of disease duration (0-41 yrs). Mean fasting plasma glucose was $148 \pm 54 \mathrm{mg} / \mathrm{dl}$ and $\mathrm{HbA} 1 \mathrm{C} 8.3 \pm 2.0 \%$. Mean blood pressure values were $143 \pm 18$ over $87 \pm 8 \mathrm{mmHg}$. In type 2 diabetic subjects we observed a highly significant relationship with both duration of disease $(p=0.000, r=0.68)$ and $\mathrm{HbA1C}(\rho=0.000, r=0.64)$. After adjustment for age, BMl, duration of disease, blood pressure values, levels of $\mathrm{HbA1c}$, vitamine-B12 and folate, mean Hcy level was still significantly higher in type 2 diabetic subjects with $C H D$ versus those without $C H D(12.0 \pm 3.1 \mathrm{mmol} / \mathrm{l}$ is $10.2 \pm 2.8, p=0,03$ ). In a stepwise multiple regression analyses including gender, age, smoking habits, systolic blood pressure, HbA1c, duration of disease, family history for CHD. Hcy level resulted as independent risk factor for $C H D\left(R^{2}=0.33, p=0.02\right)$. In a logistic regression analysis, Hcy resulted to be even predictive of $C H D(p=0.03)$. Our observations show that although in type 2 diabetic patients Hcy is strictly influenced either by duration of the disease and by the degree of metabolic control, it still mantains a role as marker potentially predictive of increased $\mathrm{CV}$ risk. 


\section{Hypertension}

\section{4} PATIENTS

M Bojestig*, H Amqvist*, B E Karlberg*, and J Ludvigsson Department of *Internal Medicine and Paediatric, University Hospital, Linköping, Sweden.

We have performed a prospective, populationbased, long term follow up study of complications in type 1 diabetic patients with onset before the age of 15 between 1961-1980, in all 213 patients. Ninety-two percent was followed from diagnosis to 1991 or death. Blood pressure was measured at least once a year from onset of diabetes to end of follow up. Severe retinopathy was defined as the first date of laser treatment. The patients were divided into different groups according to the individual mean value of diastolic blood pressure 1985-91. The cumulative incidence of hypertension (>140/90 $\mathrm{mmHg}$ ) was $27 \%$ after 25 years duration of diabetes. Hypertension was more common among males then females, $40 \%$ vs. $18 \% \mathrm{p}=0,02$. Thirty-five percent of the patients had neither severe retinopathy nor nephropathy at onset of hypertension. Out of the 31 patients who developed hypertension four-teen (45\%) had nephropathy at onset of hypertension compared to $5(3 \%)$ patients among those 165 patients who were normotensive at end of follow up $p<0.0001$. Forty-five percent $(n=14)$ of the patients had severe retinopathy at onset of hypertension compared to $13 \%(\mathrm{n}=21)$ among the normotensives $p<0.0001$.

Mean value of diastolic Degree of retinopathy at follow up 1991 (fundus photo) blood pressurel 985-91 No Ret Simplex Ret Severe Ret p-value $\begin{array}{lllll}75 \mathrm{mmHg} \text { (numbers) } & 23 & 29 & 5 & \text { vs. } 75<85<0,05\end{array}$

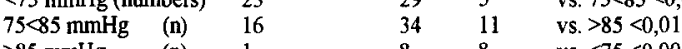

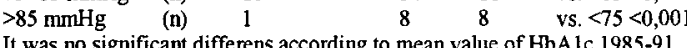
It was no significant differens according to mean value of tbAlc 1985-91 between the groups.

Thus hypertension (>140/90mmHg) is common annong young patients with IDDM, quite often becomes manifest after the onset of nephropathy and/or severe retinopathy. Diastolic blood pressure seems to be associated with the development of retinopathy.

\section{5}

Effects of smoking on 24-h ambulatory blood pressure and autonomic function in normoalbuminuric IDDM patients.

P. L. Poulsen, E. Ebbehøj, K.W. Hansen, and C. E. Mogensen, Medical Dep. M, (Diabetes \& Endocrinology), Aarhus University Hospital, Denmark.

Background: Smoking is an important risk factor for development and progression of diabetic nephropathy. The mechanisms by which smoking increases albuminuria and promotes nephropathy are unknown. Considering the acute pressor effect of smoking and the close association between blood pressure elevation and development of diabetic nephropathy, blood pressure increase might be implicated in the association between smoking and diabetic nephropathy. However, amongst non-diabetics, smokers have repeatedly been found to have lower blood pressure than non-smokers. This is possibly mediated by autonomic adjustment to sustained sympathetic stimulation by nicotine. However, as impaired modulation of sympathovagal activity has been described in diabetes the finding of lower blood pressure in smokers may not necessarily be extrapolated to diabetic patients. In diabetic patients, the effect of smoking on blood pressure and autonomic function remains unclarified. Methods: We examined 24-h ambulatory blood pressure (oscillometric technique) and autonomic function (short-term power spectral analysis as well as conventional tests) in 24 smokers and 24 non-smokers matched for sex, age and diabetes duration All patients were nomoalbuminuric DDM patients. Smoking status was assessed by questionnaire with confirmatory determinations of urinary cotinine

Results: Diabetic smokers had significantly higher 24-h mean arterial blood pressure $(94 \pm 6.7 \mathrm{mmHg}$ compared to diabetic non-smokers $90 \pm 5.8 \mathrm{mmHg}, \mathrm{p}=0.04$ ) including higher diastolic night blood pressure ( $68 \pm 7.3 \mathrm{mmHg}$ vs $64 \pm 5.2 \mathrm{mmHg}, p=0.03)$. Smokers had also significantly higher $24 \mathrm{~h}$ heart rate $(80 \pm 7.2$ compared to $72 \pm 9.2 \mathrm{~b} / \mathrm{min}, \mathrm{p}<0.001)$. In addition, smoking was associated with significantly reduced short-term RR interval variability (supine low frequency component $\left(5.45 \pm 1.29 \mathrm{ln} \mathrm{ms^{2 }}\right.$ in smokers compared to $6.31 \pm 1.11$ in non-smokers, $\mathrm{p}<0.02)$ as well as reduced brake index $(33.5 \pm 14.5$ in smokers vs $42.1 \pm 16.0$ in non-smokers, $p<0.05$ ). Conclusions: Diabetic smokers have significantly higher $24 \mathrm{~h}$ blood pressure compared to diabetic non-smokers. This finding, contrasting the effect of smoking amongst non-diabetics, is possibly mediated by coexisting abnormal postural responses in autonomic cardiac regulation in diabetic smokers. Blood pressure elevation, persisting throughout $24 \mathrm{~h}$, might be operative in the association between smoking and development of diabetic nephropathy.

\section{6}

ARTERIOLAR CHANGES, GLYCAEMIC EXPOSURE, HYPERTENSION AND TYPE 2 DIABETES MELLITUS.

P. Biondi, D. Scorzoni, D. Manservigi ${ }^{\circ}$, P.L. Pareschi',M. Barca, F. Pellicano ${ }^{\circ}$ R.G.Urso, L. Longhini, G.F. Musacci and C. Longhini. Dipartimento Medicina Clinica Sperimentale, Università; ' Servizio Diabetologia Azienda Ospedaliera. Ferrara, Italy. Aim: to evaluate the role of glycaemic exposure and the contribution of associated mild-to-moderate hypertension on the genesis of arteriolar structural changes (ASC) in noninsulin dependent diabetes mellitus subjects (DM) within a wide range of metabolic compensation. Methods: a total of 161 subjects (mean age $61.17 \pm 7.63$ ys) were studied, divided in five groups: 36 with DM in good glycaemic control (DMg); -23 with DM in poor glycaemic control (DMp), -24 with $\mathrm{DMg}$ and hypertension, (DMgHy) - 39 with DMp and hypertension (DMp-Hy), -39 control subjects (CS). The groups were matched for age, duration of diabetes and hypertension. The glycaemic control was evaluated using the average of all glycated haemoglobin values measured during the course of the diabetes $(\mathrm{mHbAl})$; a value $<7.5 \%$ was selected as an expression of good glycaemic control. ASC were assessed by the indirect evaluation of minimal vascular resistance (MVR, PRU) calculated with a strain-gauge plethysmographic study performed at forearm level. Systolic (SBP, $\mathrm{mmHg}$ ), diastolic (DBP, $\mathrm{mmHg}$ ) and mean (MBP, $\mathrm{mmHg}$ ) blood pressure average values of 24 hours were evaluated noninvasively. The statistical analysis was performed by ANOVA and simple linear regression tests. Results: MVR was higher in DMp-Hy $(3.73 \pm 0.61)$ than in DMg-Hy, DMp and DMg (3.27 $\pm 0.47,3.31 \pm 0.63$ and $3 \pm 0.55$ respectively; $p<5)$; in all groups MVR was higher in than CS $(2.58 \pm 0.24, p<.5)$. The correlations between MVR and BP average values and between MVR and mHbAlc were

\begin{tabular}{|c|c|c|c|c|c|}
\hline & \multicolumn{5}{|c|}{ MVR } \\
\hline & $\overline{\text { DMg }}$ & DMp & DMz-Hy & DMp-Hy & $\overline{\mathrm{CS}}$ \\
\hline \begin{tabular}{|l|} 
SBPP \\
\end{tabular} & $\begin{array}{ll}\text { n.s } \\
\end{array}$ & n.s & $1=.45^{\circ}$ & {$\left[=.41^{* *}\right.$} & n.s.s \\
\hline DBP & n.s & $\begin{array}{ll} \\
\end{array}$ & $=4]^{\circ}$ & $r=36^{*}$ & n.s \\
\hline$\overline{\mathrm{MBP}}$ & n.s & n.s. & {$\left[=.43^{*}\right.$} & $\Gamma=4^{*}$ & n.s \\
\hline HbAlc & $\mathrm{r}=.55^{* *}$ & $\mathrm{r}=.49^{\circ \mathrm{*t}}$ & $\Gamma=.43^{*}$ & {$\left[=32^{*}\right.$} & us \\
\hline
\end{tabular}

Conclusion: ASC are related to the degree of glycaemic exposure. The association of hypertension is a worsening factor and its ability to produce damage is highest in poorly compensated patients.

\section{7}

EFFECTS OF NIDDM AND ETHNICITY ON PLASMA RENIN ACTIVITY IN HYPERTENSIVE SUBJECTS.

J Valabhjia, C Konga, C Poulter', SAMCG Thom ${ }^{b}$, MC Feher ${ }^{c}$, RS Elkeles ${ }^{2}$ and S Robinson'. 'Department of Metabolic Medicine and 'Department of Clinical Pharmacology, St Mary's Hospital, London. 'Department of Clinical Pharmacology, Chelsea and Westminster Hospital, London.

The role of the renin-angiotensin-aldosterone axis in hypertension associated with NIDDM is not established. We therefore compared plasma renin activity (PRA) in hypertensive NIDDM subjects to that in hypertensive non-diabetic subjects. PRA was measured in 41 hypertensive NIDDM subjects and 12 hypertensive non-diabetic subjects, using a radioimmunoassay that measures rate of formation of angiotensin I from endogenous substrate. Subjects were not taking $\mathrm{ACE}$ inhibitors or angiotensin II antagonists. PRA was significantly lower in the NIDDM group than in the non-diabetic group $(0.30 \pm 0.35 \mathrm{pmol} / \mathrm{ml} / \mathrm{hr}$ (median \pm interquartile range) vs. $1.15+1.23 \mathrm{pmol} / \mathrm{ml} / \mathrm{hr} ; \mathrm{p}<0.02$ ), implying relative suppression of the renin-angiotensin-aldosterone axis. Age range in the NIDDM group was 47-70 years (median 62) and in the non-diabetic group was 43-70 years (median 52); the NIDDM group had significantly greater body-mass index $\left(28 \pm 3 \mathrm{~kg} / \mathrm{m}^{2}\right.$ vs. $\left.25 \pm 6 \mathrm{~kg} / \mathrm{m}^{2} ; \mathrm{p}<0.05\right)$; the groups were matched for sex. NIDDM subjects were subdivided according to ethnicity; the 13 Afro-Caribbean and 28 non-Afro-Caribbean NIDDM subjects were matched for age, sex and body-mass index. There was no significant difference in PRA between Afro-Caribbean and nonAfro-Caribbean NIDDM subjects $(0.30 \pm 0.40 \mathrm{pmol} / \mathrm{ml} / \mathrm{hr}$ vs. $0.40 \pm 0.40$ $\mathrm{pmol} / \mathrm{ml} / \mathrm{hr}, \mathrm{p}=0.70$ ). Low PRA in hypertensive Afro-Caribbean subjects is recognised, and could explain the poor efficacy of ACE inhibitors in this group. We have found that PRA is reduced in NIDDM to the extent that Afro-Caribbean ethnicity contributes no further reduction. This implies that further studies of ACE inhibitor efficacy in NIDDM are required. 
1308

TREATMENT OF HIGH BLOOD PRESSURE IN TYPE 2 DIABETES WITH MICROALBUMINURIA ON THE BASIS OF THE VITh REPORT OF THE JOINT NATIONAL COMMITTEE.

F. Frigato, M. Velussi, B. Muollo, M. Trevisan, F. Pierulli, M. Sambataro, R. Nosadini. University of Sassari and Padova, CESI-CNR, CAD of Monfalcone, Mestre, Contarina.

The guidelines of the JNC-VIth Report (Arch. Intern. Med. 1997) conclude that ACE inhibitors (ACE) are more beneficial than other agents in patients with Type 1 (DI) and 2 diabetes (D2), but that there is also evidence from prospective, randomized, controlled trials of renoprotective effects of dihydropyridines and nondihydropyridines calcium antagonists (CCBs). Our aim was to evaluate using metanalysis statistica approach the results of 3 randomised double blind, trials carried out from 1989 till 1995 in $234 \mathrm{D} 1$ and D2 with microalbuminuria (MA), serum creatinine $<1.9 \mathrm{mg} / \mathrm{dl}$, reated either by $A C E$ or $C C B s$ associated with alpha/beta blockers $(A / B)$ and thiazides (T) to maintain blood pressure (BP) $<130-135 / 85 \mathrm{mmHg}$. BP was evaluated every 6 months in $97 \mathrm{D} 1$ with $\mathrm{MA}$ (age: $41 \pm 11$, years, $\mathrm{M} \pm \mathrm{SD}$ ) and diabetes duration $20 \pm 7$ years treated either by lisinopril (42) or slow release nifedipine (45) for 3 years. $21 \%$ of D1 with lisinopril and $18 \%$ of D1 with nifedipine received also T: $8 \%$ and $11 \%$ of the 2 groups, respectively, needed a 3 rd drug (A/B); $44 \%$ of D1 with lisinopril and $57 \%$ of D1 with nifedipine (n.s) had BP $<130 / 85 \mathrm{mmHg}$. We also studied $137 \mathrm{D} 2$ with $\mathrm{MA}$, age $58 \pm 7$ years and diabetes duration $7 \pm 4$ years treated either with cilazapril/captopril (62) or with amlodipine/rifedipine slow release (65) for a median period of 38 months (range $9-64$ ) $79 \%$ of D2 with ACE and $82 \%$ of D2 with CCBs needed the association with T. $48 \%$ and $51 \%$ of the 2 groups of D2 respectively needed a 3 rd drug (A/B). $21 \%$ of D2 with $\mathrm{ACE}$ and $26 \%$ of D2 with CCBs had BP values $<130 / 85 \mathrm{mmHg}$. One D1 on nifedipine suddenly died and one D2 on nifedipine had myocardial infarction. No stroke events were observed. These rates of occurrence of cardiovascular complications between ACE and CCBs were not significantly different. Conclusions: $50 \%$ of D1 and $75 \%$ of D2 with MA have high BP according to JNC VIth Report, despite treatment with 2 and even with 3 antihypertensive agents. It is suggested that association rather than altemative treatment with ACE and CCBs could allow a more successful treatment of $B P$ in D1 annd D2 with MA.

\section{9}

ANTTHYPERTENSIVE TREATMENT AND BLOOD PRESSURE PROFILE IN HYPERTENSIVE TYPE 2 DIABETIC PATIENTS WITH MICROALBUMINURIA F. J. Martinez, M. P. Martinez, V. Sánchez, C. Santana, I. Garcia, A. Macias and P.de Pablos. Endocrinology Dpt, Hosp. N. S. del Pino, Las Palmas de Gran Canaria, Spain

In order to study the effect of antihypertensive treatment with an ACE inhibitor (Trandolapril $2 \mathrm{mg}=\mathrm{T}$ ), a calcium channel blocker (Diltiazem $300 \mathrm{mg}=\mathrm{D}$ ), and an angiotensin II antagonist (Losartan $50 \mathrm{mg}=\mathrm{L}$ ) on the daily blood pressure profile and the changes in the nocturnal blood pressure dip in a group of patients with type 2 diabetes mellitus, mild-moderate hypertension and persistent microalbuminuria, 70 patients with the mentioned diagnosis were studied (age $58 \pm 10$ years, 39 women and 31 men); after a 15-day washout period, they were randomly allocated to receive $\mathrm{T}$ (n = 24), $\mathrm{D}(\mathrm{n}=24)$ or $\mathrm{L}(\mathrm{N}=22)$. After 1 month. $12.5 \mathrm{mg}$ of hydrochlorothiazide were added if office blood pressure was $>130 / 85 \mathrm{mmHg}$. At baseline and after 12 weeks the following measurements were performed: body weight; office blood pressure; ambulatory $24 \mathrm{hr}$. blood pressure monitoring (ACP 2200 monitors); plasma glucose, urea, urates, creatinine, sodium, potassium, transaminases (AST, ALT) and lipids (total cholesterol, HDL and LDL fractions, triglycerides); albuminuria (three $24 \mathrm{~h}$ collections), creatinine clearance and insulin sensitivity index (standard insulin suppresion test). All treatments were well tolerated and equally effective for the control of office blood pressure. The percentual values of the nocturnal dip were at baseline $8.7+2.8,8.9+2.9$ and $9.1+3.0$ for $T, D$ and $L$, respectively; after 12 weeks they were $14.8+3.2,10.9+2.8$ and $11.3+3.1$ for $\mathrm{T}, \mathrm{D}$ and $\mathrm{L}(\mathrm{p}=0.034$ for the difference in the $\mathrm{T}$ group; $p>0.05$ for $\mathrm{D}$ and $\mathrm{L}$ ). The changes of the nocturnal blood pressure dip did not correlate significantly with any of the studied clinical or biochemical variables, except for the changes of microalbuminuria, which were $-53 \%$, $-18 \%$ y $-19 \%$ for $T, D$ and $L$; an inverse linear correlation was found, with $r=-0.37$ and $\mathrm{p}<0.01$. In conclusion, $\mathrm{T}, \mathrm{D}$ and $\mathrm{L}$ were effective and well tolerated in the treatment of hypertensive type 2 diabetic patients with persistent microalbuminuria, but only $T$ improved significantly the blood pressure profile, with partial recovery of the nocturnal dip. This effect was associated with a greater reduction in albuminuria.
1310

\section{TRANDOLAPRIL RESTORES CIRCADIAN BLOOD PRESSURE VARIATION IN NORMOALBUMINURIC NORMOTENSIVE DIABETICS} L. Czupryniak, M. Wiśniewska-Jarosińska, E. Małecka-Panas, and J. Drzewoski. Metabolic Diseases and Gastroenterology Department, Medical University of $€$ odź; todź, Poland.

It is well established that circadian blood pressure $(\mathrm{BP})$ variation manifesting itself in at least $10 \%$ BP fall at night is impaired in diabetes mellitus. It is believed that 30 to $50 \%$ of normotensive diabetics fail to show nocturnal BP fall. This condition may lead to myocardial overload and thus contribute to the increased risk of cardiovascular disease present in diabetes. The aim of the study was to evaluate the effect of 14-day treatment with angiotensin converting enzyme inhibitor trandolapril on circadian BP variation in diabetic patients. The study subjects were 12 normoalbuminuric normotensive insulin-dependent diabetics (mean age $32.4 \pm 4.2 \mathrm{yrs}$, BMI $21.1 \pm 2.3 \mathrm{~kg} / \mathrm{m}^{2}$, duration of diabetes $6.2 \pm 2.7$ yrs, $\mathrm{HbA}_{1 \mathrm{c}} 6.9 \pm 1.6 \%$ ), with night-to-day $\mathrm{BP}$ loss less than $10 \%$ in systolic or diastolic pressure ('non-dippers') Day/night BP variation was examined using ABP-Monitor Mobil-O-Graph (I.E.M. Stolberg, D). Recordings were made at 20 min intervals during the day and $30 \mathrm{~min}$ intervals during the night. Trandolapril $2 \mathrm{mg}$ was given once daily, before morning meal. The measurements were performed 1) at baseline; 2) 24 hours after the first drug dose; and 3 ) after 14 days of the treatment. Total mean $(I S D)$ values of systolic $B P$, diastolic $B P$, night fall in systolic and diastolic BP were 1) at baseline: $127.3 \pm 5.4 \mathrm{mmHg} ; 90.4 \pm 4.8 \mathrm{mmHg} ; 3.0 \pm$ $2.8 \% ; 8.3 \pm 6.5 \% ; 2$ ) after the first dose: $114.2 \pm 7.7 \mathrm{mmHg} ; 81.4 \pm 8.3$ $\mathrm{mmHg}(p<0.01$ vs baseline); $4.8 \pm 3.5 \% ; 6.0 \pm 5.1 \%$; and 3 ) after 14 -day treatment: $116.3 \pm 8.9 \mathrm{mmHg} ; 74.4 \pm 10.5 \mathrm{mmHg}$ ( $p<0.01$ vs baseline); 16.7 $\pm 8.1 \% ; 18.1 \pm 11.1 \%$ ( $p<0.001$ vs baseline and after-24-hours values), respectively. In conclusion, within 14 days of trandolapril treatment circadian $\mathrm{BP}$ variation was successfully restored in normoalbuminuric normotensive insulin-dependent diabetic patients.

\section{1}

ANGIOTENSIN CONVERTING ENZYME INHIBITION AND ARTERIAL ENDOTHELIAL FUNCTION IN IDDM

R. McFarlane, R. McCredie, R. Zilkens, L. Molyneaux, D.K. Yue and D.S. Celermajer. Diabetes Centre and Department of Cardiology, Royal Prince Alfred Hospital and Department of Medicine, University of Sydney, Sydney, Australia.

Endothelial dysfunction is a key early event in atherogenesis. It can be studied by a non-invasive high resolution vascular ultrasound technique which measures flow mediated dilatation (FMD) of the brachial artery after a 4.5 minute occlusion of blood flow. Angiotensin converting enzyme (ACE) inhibitors block the formation of the vaso-constrictor angiotensin II and have recently been shown to improve small vessel endothelial function when given intra-arterially to IDDM subjects. We report the results of a randomised double-blind cross over trial investigating whether ACE inhibition improves large vessel endothelial function in IDDM subjects. 20 IDDM subjects (mean age $28 \pm 5 \mathrm{yrs}$;duration $15 \pm 10 \mathrm{yrs}$; M/F:13/7) with FMD below mean of control population at baseline were enrolled. Subjects had no physical evidence of atherosclerosis, had never smoked, had blood pressure $<160 / 90 \mathrm{mmHg}$ and cholesterol $<6.0 \mathrm{mmol} / \mathrm{L}$. Subjects were randomised to either perind opril ( $4 \mathrm{mg}$ ) or hydrene (Triamterene $50 \mathrm{mg}$ with Hydrochlorothiazide $25 \mathrm{mg}$ ) for 12 weeks, followed by a 6 week washout before cross-over to the other treatment. FMD was measured before and at the end of each treatment. Although perindopril lowered blood pressure significantly $(3.5 / 2.9 \mathrm{mmHg}, \mathrm{p}<0.03)$, there was no significant effect of either perindopril or hydrene on FMD (FMD before perindopril $4.6 \pm 2.5 \%$, after $4.1 \pm 3.4 \%$; FMD before hydrene $5.4 \pm 3.6 \%$, after $6.0 \pm 3.3 \% ; F=1.9, p=0.1$ ) Therefore, although ACE inhibition may acutely improve microvascular endothelial function, we were unable to demonstrate a significant benefit of 3 month ACE inhibitor therapy on endothelial function in the large arteries of young adults with IDDM 


\section{2}

MANIDIPINA VERSUS AMLODIPINA IN TYPE \| DIABETIC PATIENTS WITH HYPERTENSION: COMPARISON IN DUBLE BLIND.

M. Oliviero, S. Turco, P. Tammaro, I. Pietropaolo, N. Di Palma Esposito, E Arezzi and A. Celentano, Department of Clinical and Experimental Medicine, University "Federico II" Naples, Italy.

Aim of the study was to value the action of Manidipina (M) in type II diabetic patients with hypertension comparated at Amiodipina (A). 26 type II diabetic patients (age $62.3 \pm 6.8 \mathrm{y}$; duration of diabetes $12.6 \pm 5.9 \mathrm{y}$; BMI $28.3 \pm 5.1 ; \mathrm{HbA}_{1} \mathrm{C} 7.01 \pm 2.85$ ) with hypertension (diastolic blood pressure DBP $>95<115 \mathrm{mmHg}$ ) were selected. After 2 weeks of wash out and 2 weeks of run-in with placebo, the patients were assigned random at treatment with M $10 \mathrm{mg} / \mathrm{die}$ or A $5 \mathrm{mg} / \mathrm{die}$ for 2 weeks;after this time, the patients non normalized (DBP $>90 \mathrm{mmHg}$ ) and non responders (reduction of DBP $<10 \mathrm{mmHg}$ ) increased treatment at cuble dose. The time of the study was 24 weeks. At basal and final time were determined $\mathrm{HbA}_{1} \mathrm{c}$ fasting and post-prandial blood glucose, proteinuria of 24h, ECG, Ambulatory Blood Pressure Monitoring (ABPM) and echocardiographic color-doppler study. No significant difference resulted for metabolic parameter after 24 weks in both treatments. The responders (decreased DBP $>10 \%$ ) were $96 \%$ for both treatments; the normalized were $67 \%$ for $M$ and $68 \%$ for $A$. $M$ and $A$ decreased sistolic blood pressure (SBP) $(p<0.003)$ and DBP $(p<0.001)$ in clinical recordings as well as SBP $(p<0.007)$ and DBP $(p<0.005)$ at $A B P M ;$ moreover both treatments decreased SBP and DBP day-time $(p<0.02)$ and night-time $(p<0.04)$. No significant difference were showed of heart frequence. Hough-to-peak ratio of DBP was $70 \%$ for $M$ and $71 \%$ for $A$. Echocardiographic parameters showed a significant reduction of left ventricular diameter $(p<0.02)$ of telesistolic stress $(p<0.003)$ and of sistemic vascular resistances $(p<0.01)$ in both treatments whereas the Left Ventricular Mass did not change. $M$ versus $A$ determined percentual increased of the ratio stroke volume/pulse pressure $(p<0.05)$. In conclusion $A$ and $M$ have comparable antihypertensive effects in type II diabetic patients without interference on metabolic parameters

\section{3}

COMPARATIVE EFFECTS OF CALCIUM-ANTAGONISTS AND ACEINHIBITORS ALONE OR IN A FIXED COMBINATION ON BLOOD PRESSURF AND ALBUMINURIA IN HYPERTENSIVE TYPE I DIABETIC PATIENTS.

R. Trevisan on behalf of MODHAM (Management of type 2 Diabetes with Hypertension And Microalbuminuria) Study Group. Italy and United Kingdom.

The aim of this study was to compare the effects of a fixed combination of a calciumchannel blocker and ACE-inhibitor (Verapamil + Trandolapril) vs single monotherapy antihypertensive treatment (either Verapamil or Trandolapril) and vs Nifedipine on albumin excretion rate (AER) and insulin sensitivity in hypertensive type II diabetic patients with microalbuminuria. We performed a six-month, single blind randomised, four arm parallel group study ( 4 week placebo followed by a 24 week active treatment period) in 72 hypertensive microalbuminuric type II diabetic patients allocated to Verapamil + Trandolapril $(180+2 \mathrm{mg}$ )(GROUP 1), Trandolapril (2 mg) (GROUP 2), Veraparnil (240 mg)(GROUP 3) and Nifedipine (30 mg)(GROUP 4). AER was determined at baseline, and every 3 months. Insulin sensitivity (assessed by euglycaemic hyperinsulinemic clamp) and $24 \mathrm{~h}$ ambulatory blood pressure (by SpaceLabs 90202) were determined at the beginning and at the end of the study period. Results for mean blood pressure (MBP), AER and insulin sensitivity, expressed as whole body ghucose disposal (M) (mean $\pm S D$, except for AER given as geometric mean and $95 \%$ C. I.) Baseline data:

\begin{tabular}{|c|c|c|c|c|}
\hline & GROUP 1 & GROUP 2 & GROUP 3 & GROUP 4 \\
\hline $\mathrm{MBP}(\mathrm{mmHg})$ & $116.5 \pm 8$ & $115.5 \pm 8$ & $118.1 \pm 7$ & $116.2 \pm 9$ \\
\hline AER $(\mu \mathrm{g} / \mathrm{min})$ & $79(55-103)$ & $65(45-85)$ & $72(47-97)$ & $47(36-58)$ \\
\hline $\mathrm{M}(\mathrm{mg} / \mathrm{kg} / \mathrm{min})$ & $6.9+3.2$ & $6.6 \pm 2.8$ & $6.3 \pm 2.0$ & $6.3 \pm 2.0$ \\
\hline \multicolumn{5}{|c|}{ At the end of the study: } \\
\hline MBP $(\mathrm{mmHg})$ & $101.1 \pm 6$ & $101.0 \pm 7$ & $104.5 \pm 8$ & $98.7 \pm 8$ \\
\hline AER $(\mu \mathrm{g} / \min )$ & $41(23-59)$ & $36(22-51)$ & $62(31-93)$ & $36(20-53)$ \\
\hline $\mathrm{M}(\mathrm{mg} / \mathrm{kg} / \mathrm{min})$ & $5.9 \pm 2.1$ & $6.6 \pm 2.0$ & $6.3 \pm 2.9$ & $5.2 \pm 1.9$ \\
\hline
\end{tabular}

In conclusion, ACE-inhibitors and Calcium channel blockers were equally effective in reducing blood pressure and AER in type II diabetic patients. The antihypertensive therapies used and reduction of blood pressure had no effect on insulin sensitivity

\section{4}

AMBULATORY BLOOD PRESSURE MONITORING DURING PREGNANCY IN TYPE 1 DIABETIC WOMEN

L. Flores, I. Levy, S. Martinez*, R. Gomis and E. Esmatjes. Endocrinology and Diabetes Unit, Obstetrics \& Gynecology Department*, Hospital Clinic de Barcelona, Universitat de Barcelona, Spain.

Increased risk for hipertensive disorders has been described in Type 1 diabetic women during pregnancy. Currently, casual blood pressure (BP) measurement is the most frequently used screening test for hypertension in pregnancy. However, the usefulness of 24-h ambulatory BP monitorization (ABPM) has been recently suggested in non-diabetic pregnant women. The Aim of this study has been to establish the evolution of profiles of ABPM throughout pregnancy in normotensive type 1 diabetic women, and to determine if the BP patterns are able to define a population at risk for developing pregnancy-related hypertensive disorders.

Patients and Method: This is a prospective study in which 24-h ABPM was carried out three times during pregnancy $\left(1^{\text {st }}\right.$ : weeks $7-12 ; 2^{\text {nd }}$ : weeks $20-24$ and $3^{\text {rd }}$ : weeks $30-34)$ in twenty normotensive type 1 diabetic patients Results: Seven women (35\%) developed hypertensive disorders during the $3^{\text {rd }}$ trimester and $13(65 \%)$ remained normotensive. ABPM data was broken down according to presence or absence of hypertension as follows. Value are means \pm DS

\begin{tabular}{|c|c|c|c|c|c|c|}
\hline & & & & & & $30-34$ \\
\hline $\begin{array}{l}\text { Systolic BP } \\
24 \mathrm{H}\end{array}$ & $\begin{array}{r}\text { Norma } \\
78+5.6\end{array}$ & $\begin{array}{c}\text { HTA } \\
114.8+6.7^{*}\end{array}$ & $\begin{array}{r}\text { Nomal } \\
107.6+5.7\end{array}$ & $\begin{array}{c}\text { HTA } \\
118.5+10.8^{*}\end{array}$ & $\begin{array}{r}\text { Norm } \\
113.3+5.0\end{array}$ & $130.3+5.0^{*}$ \\
\hline Day & $110.8 \pm 5.9$ & $117.4 \pm 7.3^{*}$ & $110.9 \pm 6.1$ & $121.0 \pm 12.3^{*}$ & $116.6 \pm 5.7$ & $131.6 \pm 5.8^{*}$ \\
\hline Night & $98.0 \pm 6.7$ & $105.5 \pm 5.5^{*}$ & $96.6 \pm 7.7$ & $110.2 \pm 7.4^{*}$ & $103.7 \pm 7.5$ & $126.0 \pm 7.4^{*}$ \\
\hline Drastolic BP & Normal & HII & Nomita & HTA & $\begin{array}{r}\text { Norm } \\
68744\end{array}$ & माA \\
\hline $24 \mathrm{H}$ & & & $63.5 \pm 4.0$ & $68.7 \pm$ & $68.7 \pm 4.4$ & $78.1 \pm 4.9^{*}$ \\
\hline Da) & $66.3 \pm 4.3$ & $67.7 \pm 3.9$ & $66.6 \pm 4.1$ & $71.0 \pm 5.7^{*}$ & $71.8 \pm 4.2$ & $79.6 \pm 6.1^{*}$ \\
\hline Night & $54.7 \pm 5.9$ & $58.8 \pm 4.1$ & $53.0+5.9$ & $61.4 \pm 2.8^{*}$ & $59.6 \pm 7.1$ & $72.5 \pm 5.5^{*}$ \\
\hline
\end{tabular}
The best threshold value of BP for each measurement at $1^{\text {si }}, 2^{\text {nd }}$ and $3^{\text {to }}$ trimesters of gestation by means of the receiver-operator characteristic curve was established. The night systolic BP at $2^{\text {nd }}$ trimester was the best overall predictor of later hypertensive disorder (cut-off level $105 \mathrm{mmHg}$; sensitivity: $85 \%$, specificity: 92\%) *p $<0.05$ Conclusions: ABPM from patients who will develop hypertensive disorders during pregnancy show different $B P$ patterns that those from normotensive ones all along the three trimesters. The thresholds derived from our data could provide a tool for estimate the risk for developing pregnancy-related hypertensive disorders in type 1 diabetic women.

\section{5}

BLOOD PRESSURE SELF MONITORING IN PATIENTS WITH TYPE 1 DIABETES MELLITUS, NEPHROPATHY AND HYPERTENSION

K. Magnusson, B. Gröbel, L. Heinemann, K. Rave, R. Bender, P.T. Sawicki. Dep. of Metab. Diseases, Heinrich-Heine University Düsseldorf, Germany

In patients with essential hypertension office blood pressure measurements (OBPM) may overestimate blood pressure values. We investigated the difference between OBPM, 24-h ambulatory blood pressure monitoring (ABPM), and blood pressure self monitoring (BPSM) in hypertensive patients with type 1 diabetes mellitus and diabetic nephropathy. In addition, three different BPSM devices were evaluated. Twenty-one hypertensive diabetic patients (age $45 \pm 8$ years, duration of diabetes $32 \pm 12$ years $\left(\right.$ mean \pm SD) ) with micro- or macroproteinuria (proteinuria $>60 \mathrm{mg}^{2} 4 \mathrm{~h}^{-1}$ ) participated in this study. The BPSM values during a period of 2 to 3 months were compared to OBPM done by a physician. Thereafter each patient was asked to perform three BPSM measurements per day for a three week period with three different devices: one sphygmomanometer (SM), and two oscillometric devices, one for the upper arm (OUA) and one for the wrist (OW); all BOSO, Jungingen, Germany). In random order each device was used for seven days. At the beginning and at the end of this threeweek period ABPM (TM 2420, BOSO) was performed. OBPM resulted in higher blood pressure values than BPSM (systolic $153 \pm 23$ vs. $128 \pm 10, p<0.001$; diastolic $86 \pm 9$ vs. $79 \pm 4 \mathrm{~mm} \mathrm{Hg}, \mathrm{p}<0.001$ ). $76 \%$ of systolic OBPM values differed by $>10 \mathrm{~mm}$ $\mathrm{Hg}$ from the BPSM ( $76 \%$ higher, $0 \%$ lower). $62 \%$ of diastolic OBPM values differed by $>5 \mathrm{~mm} \mathrm{Hg}$ ( $52 \%$ higher, $10 \%$ lower). Similar results were obtained when OBPM was compared to ABPM (systolic $153 \pm 23$ vs. $129 \pm 16$, $p<0.001$; diastolic $86 \pm 9$ vs. $80 \pm 7 \mathrm{~mm} \mathrm{Hg}, \mathrm{p}<0.005$ ). The three different devices used for BPSM gave similar BP values when compared to ABPM (SM $127 \pm 13 \mathrm{~mm} \mathrm{Hg}$; OUA $130 \pm 14 \mathrm{~mm} \mathrm{Hg}$; OW $132 \pm 13 \mathrm{~mm} \mathrm{Hg}$; ABPM $129 \pm 80 \mathrm{~mm} \mathrm{Hg} ;$ n.s.). When compared to both upper-armBPSM-devices readings of the oscillometric wrist device resulted in significantly higher diastolic values $(3 \pm 6 \mathrm{~mm} \mathrm{Hg} ; \mathrm{p}<0.05)$. OBPM measurements overestimate blood pressure in hypertensive patients with type 1 diabetes and diabetic nephropathy. For therapy control and for the diagnosis of hypertension in these patients OBPM values are therefore of limited use. BPSM devices for the upper arm are preferable, because oscillometric self monitoring using a wrist device can result in higher diastolic blood pressure values. 
1316

ROLE OF AMBULATORY BLOOD PRESSURE IN DETECTING DIABETIC MICROVASCULAR COMPLICATIONS

"O.DEYNELÍ, "H.ö.ERSÖZ, "D.GOGAS, Y.BUDAK and "S.AKALIN

"Marmara University Endocrinology Department isTANBUL/TÜRKIYE

Elevated serum sialic acid concentration precedes the development of persistant microalbuminuria in diabetic patients. The aim of this study is to evaluate the relation between altered circadian blood pressure pattern and diabetic microvascular complications. We performed 24 hour ambulatory blood pressure monitoring; autonomic neuropathy tests; fundoscopic retinal examinations; $\mathrm{HbAlC}$, lipid profile, serum sialic acid, 24 hour urinary albumin excretion and creatinine clearance measurements in healthy controls $(C)(n=13)$, normoalbuminuric $(N)(n=31)$ and microalbuminuric $(\mathbf{M})(\mathrm{n}=\mathbf{2 0})$ NIDDM patients. Although there was no significant difference between day and night blood pressures and heart rates between groups, the amount of dipping of sistolic blood pressure and MAP in $\mathbf{M}(5.5 \pm 3 \%, 7.1 \pm 4 \%)$ was decreased compared with $\mathrm{C}(10 \pm 2 \% ; 12.3 \pm 2 \%)(\mathrm{p}<0.001)$ and $\mathbf{N}(10.1 \pm 4 \% ; 8.3 \pm 4 \%)$ $(p<0.04)$. Dipping of diastolic blood pressure was decreased in $\mathbf{M}(9.6 \pm 5 \%)(p<0.003)$ compared with $\mathrm{C}(14.9 \pm 3 \%)$. Prevalance of non-dippers was increased in $\mathbf{M}(72 \%)$ compared with $\mathbf{N}(48 \% ; p<0.04)$ and $\mathbf{C}(15 \% ; p<0.001)$. Serum sialic acid concentrations were increased in non-dippers $(6135 \pm 74 \mathrm{mg} / \mathrm{dl})$ compared with dippers $(52.71 \pm 5.6 ; p<0.0009)$ and controls $(51.28 \pm 6.3 ; \mathrm{p}<0.003)$. In $\mathbf{N}$, serum sialic acid concentrations of non-dippers $(56.88 \pm 5.62)$ was increased compared with dippers $(50.2 \pm 2.44, p<0.01)$. Prevalance of autonomic neuropathy was higher in the non-dipper group $(44.5 \%)$ than in the dippers $(10 \%, p<0.02)$ and $\mathrm{C}(0 \% ; 0<0.02)$. In the diabetics there was a positive correlation between serum sialic acid concentration and urinary albumin excretion rate $(r=0.453 ; p<0.004)$, a negative correlation between the amount of blood pressure dipping and serum sialic acid concentrations $(r=-0.61 ; p<0.0001)$. In conclusion, an impaired circadian blood pressure pattern is associated with diabetic microvascular complications, in particular the development of microalbuminuria. Increased serum sialic acid concentrations in normoalbuminuric non-dippers further supports this conclusion.

\section{7}

PREDISPOSITION FOR THE DEVELOPMENT OF DIABETES MELLITUS IN BB RATS IS ASSOCIATED WITH ELEVATED BLOOD PRESSURE

S. Berg. A. Dunger, P. Heinke, U. Fischer* Gerhardt Katsch Institute of Diabetes. Karlsburg, and * Inselklinik, Heringsdorf, Germany

Diabetic nephropathy and hypertension are associated complications in diabetes mellitus. It is not yet elucidated whether elevated blood pressure (BP) results from impaiment of kidney function or is a result of genetic predisposition for the development of diabetes. Therefore, diabetes-prone, nondiabetic $(\mathrm{ndBB}, \mathrm{n}=6)$ and diabetic ( $d B B, n=5$, age at manifestation $20.3 \pm 3.9$ weeks) $B B$ rats and nondiabetesprone LEW.1W rats (LEW, $n=6$ ) were monitored from 14th to 30 th week of age for non-fasting glycaemia (PG). BP and renal function. Diabetic animals were treated with one daily insulin injection. Telemetrically measured systolic (SBP) and diastolic $\mathrm{BP}$, heart rate and motor activity and as well as albumine (AE) and creatinine excretion (CREA) were followed at 4 week-intervals. Results: An elevation in BP was observed in all BB rats before diabetes onset compared to age-matched LEW. An additional increase in SBP occurred with diabetes manifestation. Alerations in kidney function were not detectable before diabetes manifestation as demonstrated by $\mathrm{AE}$ (Table) and CREA (week $14 \mathrm{dBB} 86.1 \pm 20.5 \mathrm{vs}$. LEW $109.7 \pm 4.0 \mu \mathrm{mol} / \mathrm{d}$. NS; week $26 \mathrm{dBB} 157.5 \pm 26.8$ vs. LEW $107.4 \pm 10.5 \mu \mathrm{mol} / \mathrm{d}$, p<0.01). Conclusion: BB rats are prone to hypertension. Alterations in kidney function become after onset of diabetes.

\begin{tabular}{|c|c|c|c|c|c|c|c|c|c|}
\hline age & \multicolumn{3}{|c|}{$\mathrm{PG}(\mathrm{mmol} / \mathrm{l}) \#$} & \multicolumn{3}{|c|}{ SBP (mmHg) \# } & \multicolumn{3}{|c|}{$\mathrm{AE}(\mu \mathrm{g} / \mathrm{d}) \#$} \\
\hline week & ndBB & $\mathrm{dBB}$ & LEW & ndBB & $\mathrm{dBB}$ & LEW & ndBB & $\mathrm{dBB}$ & LEW \\
\hline 14 & $\begin{array}{l}6.1 \pm \\
0.8 \\
\end{array}$ & $\begin{array}{l}6.1 \pm \\
0.4\end{array}$ & \begin{tabular}{|l|}
$5.3 \pm$ \\
0.1 \\
\end{tabular} & $\begin{array}{l}120.4 \pm \\
4.9\end{array}$ & $\begin{array}{l}125.1 \pm \\
2.1^{*}\end{array}$ & $\begin{array}{l}110.7 \pm \\
6.8\end{array}$ & $\begin{array}{l}5.22 \pm \\
3.1\end{array}$ & $\begin{array}{l}6.7 \pm \\
2.5\end{array}$ & $\begin{array}{l}9.6 \pm \\
3.5\end{array}$ \\
\hline 18 & $\begin{array}{l}6.0 \pm \\
0.7 \\
\end{array}$ & $\begin{array}{l}6.1 \pm \\
1.0\end{array}$ & $\begin{array}{l}5.6 \pm \\
1.1 \\
\end{array}$ & $\begin{array}{l}121.9 \pm \\
4.6^{*}\end{array}$ & $\begin{array}{l}126.6 \pm \\
4.3 *\end{array}$ & $\begin{array}{l}108.9 \pm \\
6.7\end{array}$ & $\begin{array}{l}10.5 \pm \\
7.3\end{array}$ & $\begin{array}{l}9.2 \pm \\
0.9\end{array}$ & $\begin{array}{c}13.0 \pm \\
6.8\end{array}$ \\
\hline 22 & $\begin{array}{l}5.8 \pm 0 \\
4^{\circ} \\
\end{array}$ & $\begin{array}{l}11.1 \pm \\
5.7\end{array}$ & $\begin{array}{l}5.1 \pm \\
0.6^{\circ} \\
\end{array}$ & $\begin{array}{l}120.9 \pm \\
4.9 * \circ\end{array}$ & $\begin{array}{l}132.4 \pm \\
5.4^{*} \\
\end{array}$ & $\begin{array}{l}110.8 \pm \\
6.1 \\
\end{array}$ & $\begin{array}{l}12.4 \pm \\
7.4 \\
\end{array}$ & $\begin{array}{l}95.8 \pm \\
60.5 \\
\end{array}$ & \begin{tabular}{|l|}
$16.5 \pm$ \\
12.3 \\
\end{tabular} \\
\hline 26 & $\begin{array}{l}5.5 \pm 0 . \\
6^{\circ} \\
\end{array}$ & $\begin{array}{l}20.5 \pm \\
4.6 \\
\end{array}$ & $\begin{array}{l}5.1 \pm \\
0.6^{\circ} \\
\end{array}$ & $\begin{array}{l}123.4 \pm \\
4.9^{*}\end{array}$ & $\begin{array}{l}130.8 \pm \\
3.5^{*} \\
\end{array}$ & $\begin{array}{l}107.4 \pm \\
9.8 \\
\end{array}$ & $\begin{array}{c}8.4 \pm \\
5.5 \\
\end{array}$ & $\begin{array}{l}75.9 \pm \\
37.4\end{array}$ & $\begin{array}{l}6.7 \pm \\
3.8 \\
\end{array}$ \\
\hline 30 & $\begin{array}{l}6.0 \pm 1 \\
2^{\circ} \\
\end{array}$ & $\begin{array}{l}15.0 \pm \\
5.2\end{array}$ & $\begin{array}{l}5.2 \pm \\
0.7^{\circ} \\
\end{array}$ & $\begin{array}{l}124.4 \pm \\
9.3 *\end{array}$ & $\begin{array}{l}131.4 \pm \\
0.2 * \\
\end{array}$ & $\begin{array}{l}105.7 \pm \\
6.7\end{array}$ & $\begin{array}{r}8.0 \pm \\
4.5^{\circ}\end{array}$ & $\begin{array}{l}99.8 \pm \\
42.5^{*}\end{array}$ & $\begin{array}{l}6.0 \pm \\
6.3 \\
\end{array}$ \\
\hline
\end{tabular}

\# MEAN \pm SD. ${ }^{*}$ p $<0.05$ vs.LEW, ${ }^{\circ} \mathrm{p}<0.05$ vs. dBB

\section{8}

STREPTOZOTOCIN-INDUCED DIABETES IN DAHL SALT SENSITIVE RATS: A MODEL THAT COMBINES DIABETES AND HYPERTENSION F.R.L. Crijns, I.J.M Korstjens, H.A.J. Struijker Boudier* and B.H.R. Wolffenbuttel. Depts. of Endocrinology and Pharmacology*, University Maastricht, The Netherlands.

Although the association of diabetes and hypertension is well-known in human insulin-dependent diabetic subjects, a well described pathophysiological animal model is lacking. We studied the effects of streptozotocin (STZ)-induced diabetes in hypertensive Dahl Salt Sensitive rats (DSS) and normotensive Wistar rats (WR). At the age of 12 weeks, diabetes was induced by injection of STZ (70 mg/kg) in DSS (DSS-D, $\mathrm{n}=5$ ) and WR (WR-D, $\mathrm{n}=11$ ). Non-diabetic control animals received vehicle only (DSS-C, $n=5$ and WR-C, $n=10$ ). Eleven weeks after diabetes induction, mean arterial pressure (MAP) was measured in conscious rats, at baseline and after bolus injections of acetylcholine (ACh, 0.1 $\left.1 \mu \mathrm{g} \cdot \mathrm{kg}^{-1} \cdot \mathrm{min}^{-1}\right)$. After perfusion fixation, several arteries were excised for morphometric analysis. Body weights in both diabetic groups were significantly lower compared to non-diabetic rats, whereas blood glucose and $\mathrm{HbA}_{1 \mathrm{c}}$ levels were markedly elevated. Mean arterial pressure (MAP) was elevated in DSS-C and DSS-D (mean \pm SD: $137 \pm 5$ and $132 \pm 12$ vs. $101 \pm 9$ and $92 \pm 7 \mathrm{mmHg}$; $\mathrm{p}<0.001$ ). ACh decreased MAP dose-dependently but to a lesser extent in both diabetic groups. Thoracic aorta, carotid and superior mesenteric artery of both hypertensive groups show hypertrophy of the wall compared to the normotensive groups. Wall to lumen ratio (W/L) of carotid and superior mesenteric arteries of WR-D is significantly smaller compared to WR-C whereas W/L of both hypertensive groups is comparable. Mesenteric small arteries of both diabctic groups show hypertrophic outward remodeling due to an increased flow to the mesentery in diabetes, which is more pronounced in DSS-D. In conclusion, STZ-induced diabetes in Dahl Salt Sensitive rats is a suitable model for the combination of hypertension and diabetes, is associated with endothelial dysfunction, and leads to hypertrophy of the arterial wall. 


\section{Coronary Heart Disease}

\section{9}

PLASMINOGEN ACTIVATOR INHIBITOR I DAYTIME PATTERN AND INSULINEMIA IN TYPE 2 DIABETES WITH CORONARY HEART DISEASE

K.Lalić, P.B.Djordjević N.M.Lalić, M.Zamaklar, V.Dimitrijević, A.Jotić. M.llić, N.Rajković, Lj.Lukić, Institute for Endocrinology, Belgrade, Yugoslavia

Daytime fluctuation pattem of plasminogen activator inhibitor-1 (PAI-I) levels have been demonstrated in healthy subjects, exhibiting the highest value in the moming $(8: 00 \mathrm{~h})$ and the nadir in the late aftemoon $(18: 00 \mathrm{~h})$ However, the relations between the diumal fluctuation pattem of PAI-1 and plasma insulin levels, known to be important independent risk factors for coronary heart disease (CHD) in Type 2 diabetes patients as well as in nondiabetics, has not yet been clarified. Therefore, the aim of this study was to evaluate simultaneously fasting PAI-l (determined by plasminogen/ chromogenic plasmin substrate assay) and plasma insulin levels (determined by RIA) at 8:00 vs 18:00h in 40 Type 2 diabetes patients with angiographically documented CHD (group A), in 32 Type 2 diabetes patients without CHD (group B), 38 nondiabetic subjects with CHD (group C) and in 20 age-matched healthy controls (group D). In group A and in group C, PAIlevels did not show a significant decrease between 8.00 and $18.00 \mathrm{~h}(\mathrm{~A}: 6.5+1$ 0.6 vs $6.3+/-0.4 \mathrm{U} / \mathrm{ml} ; \mathrm{p}=\mathrm{NS}$; $\mathrm{C}: 4.9+/-0.2$ vs $4.5+/-0.2 \mathrm{U} / \mathrm{ml} ; \mathrm{p}=\mathrm{NS}$ ). In contrast, both in group $\mathrm{B}$ and in group $\mathrm{D}, \mathrm{PAI}-1$ levels decreased during the same interval (B: $4.1+/-0.3$ vs $2.9+/-0.2 \mathrm{U} / \mathrm{ml} ; \mathrm{p}<0.05 \mathrm{D}: 2.7+/-0.2$ vs $0.7+1-$ $0.1 \mathrm{U} / \mathrm{ml} ; p<0.01$ ), while the decrease was significantly more profound in group $D$ than in group $B(27.4+1-10.7 \%$ vs $88.7+1-6.2 \% ; \quad p<0.05)$. Simultaneously, both at 8:00 and 18:00, we found increased fasting plasma insulin levels in group $A(26.8+/-2.7$ and $33.4+/-4.9 \mathrm{mU} /$, respectively) being higher compared to group $\mathrm{B}(15.9+/-2.2$ and $19.5+/-2.8 \mathrm{mU} /$, respectively $\mathrm{p}<0.01)$ and in group $\mathrm{C}(18.9+/-3.1$ and $22.3+/-2.9 \mathrm{mU} /$, respectively) compared to group $\mathbf{D}(9.7+/-0.9$ and $10.3+/-1.1 \mathrm{mU} /$, respectively; $\mathrm{p}<0.01)$ while they did not differ significantly within each group. The results imply that the alterations in the diurnal fluctuation pattem of PAI-1 activity, observed only in patients with $\mathrm{CHD}$, both diabetic and nondiabetic, might be induced by a prolonged daily exposure to elevated plasma insulin levels.

\section{1}

CONGESTIVE HEART FAILURE DESPITE PRESERVED SYSTOLIC FUNCTION AFTER ACUTE MYOCARDIAL INFARCTION IN PATIENTS WITH DIABETES C. Rask-Madsen, B. Brendorp, S.Z. Abildstrom, L. Køber, C. Torp-Pedersen, P. Hildebrandt, and T. Melchior, on behalf of the TRACE Study Group. Gentofte and Frederiksberg University Hospitals, Copenhagen, Denmark.

Background. A putative explanation for the inferior prognosis of diabetic patients after acute myocardial infarction (AMI) is diastolic dysfunction of the left ventricle sometimes known as "diabetic cardiomyopathy". The aim of this study was to study the risk of congestive heart failure (CHF) and mortality among patients with diabetes mellitus (DM) and preserved systolic function of the left ventricle in a large database of consecutive cases of AMI Methods. 6676 patients were screened for the Trandolapril Cardiac Evaluation (TRACE) study. Analyses were based on data from 2179 patients with a WMI $\geq 1.6(\sim$ left ventricular ejection fraction $\geq 50 \%)$. CHF was defined as a history of heart failure or Killip class $\geq 2$ during hospitalization for AMI. A diagnosis of DM was based on history. Left ventricular systolic function was measured by echocardiography on day 2-6 after the AMI and expressed as wall motion index (WMil). Survival status was obtained for all patients with 6 years of follow-up. Results. The frequency of DM was $7 \%$. CHF was present in $46 \%$ of diabetic patients and $33 \%$ of patients without DM. In a linear logistic regression analysis DM was associated with $\mathrm{CHF}$ with an odds ratio of $1.6(95 \% \mathrm{Cl} 1.1-2.3)$. in a Cox regression analysis CHF was associated with mortality with a risk ratio of $2.0(1.2-3.4)$ for patients with DM and $1.6(1.3-1.8)$ for patients without DM. Conclusion. Diabetes is independently associated with an increased risk for CHF among patients with preserved left ventricular systolic function after AMi. These results are in favor of the existence of a clinically relevant diastolic dysfunction after AMI specific for diabetes. CHF in diabetic patients with preserved systolic function is associated with an independent mortality risk similar to that in patients without DM.

\section{0}

THE IMPORTANCE OF ADMISSION PLASMA GLUCOSE IN NON-DIABETIC PATIENTS WITH MYOCARDIAL NFARCTION. A. Norhammar, L. Rydén and K. Malmberg. Department of Cardiology, Karolinska Hospital, Stockholm, Sweden.

Admission plasma glucose (AG) predicts inhospital mortality following acute myocardial infarction (AMI) both in non-diabetic and in patients with diabetes. This have been attributed to stress caused by myocardial dysfunction. The influence of $A G$ on long-term prognosis is however poorly investigated. If an elevated AG reflects a compromised glycometabolic state it may predict an unfavourable long-term outcome and identify a group of patients in possible demand for metabolic intervention. The main aim with this study was to investigate the relationship of $A G$ and long-term outcome following an AMI. Methods: Between 1 Jan.-31 Dec. 1995, a total of 300 patients fulfilled the diagnostic criteria of AMI at our CCU. Their hospital records were collected and retrospectively checked for relevant information during hospital stay and from outpatient visiss until I June 1997. Patients still alive were interviewed.

Results: Of the 264 non- diabetic patients 197 had available AG. The followup time ranged from $1.5-2.5$ years. Mean age was $68 \pm 12$ years. $72 \%$ were male.

\begin{tabular}{|lllll|}
\hline Parameter & $\begin{array}{l}\text { Event } \\
\text { (N) }\end{array}$ & $\begin{array}{c}\text { Yes } \\
(\mathrm{AG} ; \mathrm{mmol} / \mathrm{l})\end{array}$ & $\begin{array}{c}\text { No } \\
(\mathrm{AG} ; \mathrm{mmol} / \mathrm{l})\end{array}$ & $\mathbf{P}$ \\
\hline 1.Death & 60 & $8.9 \pm 3.5$ & $7.8 \pm 2.7$ & 0.015 \\
2.Reinfarction & 12 & $10.7 \pm 5.5$ & $8.0 \pm 2.8$ & 0.003 \\
3.CHF & 20 & $10.1 \pm 4.6$ & $7.9 \pm 2.7$ & 0.002 \\
Combined $(1+2+3)$ & 79 & $9.1 \pm 3.7$ & $7.5 \pm 2.3$ & 0.0005
\end{tabular}

Conclusion: Admission plasma glucose in patient with an AMI was associated with an increased mortality and morbidity during long-term follow up in nondiabetic patients. AG may be a marker of future atherothrombotic disease and not only be related to the degree of acute stress. Future prospective studies should address $c u$ t of levels of $A G$ above which treatment with non-pharmacological or pharmacological methods should be tested

\section{2}

Accelerating impact of diabetes on mortality in the years following an acute myocardial infarction.

T Melchior', L Køber ', CR Madsen², M Seibæk', G Jensen', P Hildebrandt ${ }^{3}$, C Torp-Pedersen ${ }^{2}$ on behalf of the TRACE Study Group. Dept of Medicine B, Division of Cardiology, Rigshospitalet (1), Gentofte University Hospital (2), and Frederiksberg Hospital (3). Denmark.

Diabetes has an adverse effect on mortality in patients with acute myocardial infarction (AMII). The development of risk associated with diabetes during long-term follow-up after AMI has not been studied in detail. Aim: We have studied the time-related changes of risk associated with diabetes during 6 years of follow-up after AMI in patients screened for entry in the TRandolapril Cardiac Evaluation trial. Mothods and Results: During the period 1990-92 6676 patients with AMI were screened in 27 Danish Coronary Care Units. The diagnosis of diabetes was based on history. Survival of all patients was obtained through the Danish Personal Registry. Diabetes was present in $719(11 \%)$ of the patients. Patients with diabetes were slightly older ( 69 years vs. 67 years), comprised of a higher proportion of women $(41 \%$ vs. $31 \%)$ and had a higher prevalence rate of heart failure $(69 \%$ vs. $51 \%)$ than nondiabetic patients $(p<0.001)$. The 5 year mortality rate was higher in patients with diabetes than without $162 \%$ vs. $38 \%, p<0.00011$. The time related influence of diabetes and other risk factors on mortality are shown in the Table:

$$
\begin{array}{llll}
\text { Factor: } 0-30 \mathrm{dav} & 30 \mathrm{dav}-3 \mathrm{yr} & 4-6 \mathrm{yr} \text { after } \mathrm{M}) \\
\mathrm{DM} & 1.03(\mathrm{p}=0.83) & 1.43(\mathrm{p}<0.001) 1.74(p<0.001) \\
\mathrm{CHF} & 2.65(\mathrm{p}<0.001) 1.93(p<0.001) 1.43(p<0.001) \\
\text { Age } & 1.04(p<0.001) 1.05(p<0.001) 1.05(p<0.001)
\end{array}
$$

Conclusion. Diabetes has an adverse independent effect on mortality which increases over time 
1323

FASTING INSULIN PREDICTS MORTALITY FOLLOWING ACUTE MYOCARDIAL INFACTION IN NON-DIABETIC MALES

o. Snorgaard, L. Kaber, J. Carlsen, and C. Torp-Petersen on behalf of the TRACE study group. Frederiksberg University Hospital, Gentofte University Hospital and MEDICON A/S, Copenhagen, Denmark.

The prognostic value of fasting insulin, fasting glucose, glycated haemoglobin (HbAlc) and urinary albumin excretion (UAE) measured at day 2-5 after admission with myocardial infarction was evaluated in a subgroup of 494 nondiabetic caucasian patients screened for participation in the TRAndolapril Cardiac Evaluation (TRACE) study. 151 females and 343 males alive at day 6 after admission (48 subjects with known djabetes mellitus excluded) were followed for 5 years. Age was 67(58-75)yrs [median(quartiles)], fasting insulin 9.4(6.4-13.6) $\mu \mathrm{U} / \mathrm{ml}$, glucose 5.5(5.1-6.0)mmol/, $\mathrm{HbAlc} 4.8(4.4-5.2) \%$ and UAE $13.1(5.7-32.4) \mu \mathrm{g} / \mathrm{min}$. 5-year all-cause mortality was $35 \%$. In univariate Cox analyses, a quartile higher insulin, glucose, HbAlc, or UAE were associated with the following increased relative risks: $R R=1.26(1.09-1.44), p=0.0012$; $R R=1.21(1.06-1.39), p=0.0059 ; R R=1.25(1.09-1.43), p=0.0016$, and $1.29(1.13$ 1.49), $\mathrm{p}=0.0003$, respectively. 5-year mortality in subjects with normal UAE $(61 \%)$, with microalbuminuria $(34 \%)$ and with proteinuria $(5 \%)$ were $34 \%, 41 \%$, and $64 \%$, respectively, $p<0.0001$. In a multivariate Cox proportional hazards regression analysis for 5-year mortality, insulin was the only of these parameters of independent importance $[R R=1.17(1.01-1.35), p=0.034]$ together with age, congestive heart failure and left ventricular function. Excluding 21 subjects with $\mathrm{HbAlc}$ above the normal range (6.1\%) and probable diabetes or impaired glucose tolerance, did not change the results of the analyses. Separate multivariate analyses in the two gender revealed, that insulin concentration was an independent predictor of mortality only in males. Thus, fasting insulin is an independent predictor of 5-year all-cause mortality after myocardial infarction in non-diabetic males.

\section{4}

RISK MARKERS OF LONG-TERM OUTCOME IN PATIENTS WITH DIABETES AND ACUTE MYOCARDIAL INFARCTION. L. Rydén, A.Norhammar, H. Wedel and K. Malmberg. Department of Cardiology, Karolinska Hospital, Stockholm, Sweden.

Introduction and Methods: Despite recent improvements in acute coronary care diabetic patients with acute myocardial infarction (AMI) has a high mortality. This has in particular been linked to female sex. In the randomised prospective study, DIGAMI, insulin-glucose infusion followed by intensive insulin treatment reduced one year mortality by $30 \%$. The present report describes long-term (mean 3.4 years; range 1.6 to 5.6) prognostic factors and effects of concomitant treatment in diabetic patients with AMI by applying uniand multivariate statistics on the DIGAMI cohort $(\mathrm{n}=620)$.

Results: During follow-up there were 102 deaths in the treatment group compared to 138 in the control group $(\mathrm{p}=0.011$ ). Age, previous $\mathrm{CHF}$, diabetes duration, admission blood glucose and HbAlc at admission were independent predictors of mortality among all patients, while previous AMI, angina pectoris, hypertension, smoking or female sex had no predictive value. Administration of betablockers had a striking independent effect on survival, in particular among control patients. Thrombolysis was most efficient in the infusion group. Conclusion: Mortality in diabetic patients with AMI is predicted by age, previous myocardial damage and the severity of the diabetic state, however, not by conventional cardiovascular risk factors or female sex. Betablockers exert striking secondary preventive effect in diabetics with AMI, especially in controls, possibly sharing some mechanisms of action with insulin such as reducing free fatty acids.

\section{5}

ISCHEMIC HEART DISEASE BY THALLIUM SCINTIGRAPHY IN TYPE 2 DIABETES MELLITUS AND CONTROL ASYMPTOMATIC SUBJECTS I. Castells, F. Rius, L. Rubio, M. Fraile, D. Pereferrer, I. Salinas, R. Romero and A Sanmarti. Endocrinology, Nuclear Medicine, Nephrology and Cardiology Service. Hospital "Germans Trias i Pujol". Badalona, Barcelona, Catalonia. Spain Aim: To evaluate the prevalence of silent Ischemic Heart Disease (IHD) among asymptomatic type 2 diabetic (DM2) patients and to identify the factors influencing its development. Subjects and methods: 98 DM2 patients 60 women, age $56.7+/-7$ years, duration of diabetes $12.8+/ .8 .3$ years), recruited from 1994 ; of them, 32 had diabetic nephropathy ( 25 with microalbuminuria and seven with overt proteinuria). The exclusion criteria were: evidence of cardiovascular disease or angina symptoms, anomalous ECG, autonomic neuropathy and other major illnesses. We recorded from all patients: family history, diabetic evolution and complications, hypertension and smoking habit. A general blood analysis was drawn and 3 samples of 24-hour microalbuminuria was collected. Dypiridamole plus exercise thallium myocardial perfusion scintigraphy was performed all DM2 patients and a 20 non-diabetic volunteers, matched for age, sex, smoking habit and colesterol level, as a control group. Results: We found $36(36.7 \%)$ DM2 patients with thallium studies suggesting IHD vs one (1/20: $5 \%$ ) person in the control group ( $p 0.005$ ). Among diabetic subjects, the prevalence of IHD was especially higher in patients with diabetic nephropathy $(17 / 65: 25.7 \%$ in the non-diabetic nephropathy group, $13 / 25: 52 \%$ in the microalbuminuria group and $6 / 7: 85.7 \%$ in the proteinuria group; $p 0.001$ ). Creatinina and microalbuminuria, were significatly higher in patients with IHD (p 0.01 and $\mathrm{p} 0.003$ respectively). A tendency without statistic significance was found, among colesterol, smoking habit and IHD. Conclusion: Silent IHD prevalence among DM2 subjects is higher that in controls. This increased prevalence of IHD was prominent in patients with diabetic nephropathy (higher in microalbuminuric, and maximum in proteinuric). Long-term prognostic value of these findings are not know and follow-up of these patients will be needed.

\section{6}

THE EXERCISE STRESS TEST IS MORE ACCURATE THAN MYOCARDIAL SCINTISCANNING WHEN ASSESSING SILENT MYOCARDIAL ISCHEMIA IN DIABETIC PATENTS,

V. Viviani, P. Valensi, F. Paycha, R.N. Sachs, A. Ramadan, M. Tonton-Moderc, A. Nitenberg, J.R. Attali. Jean Verdier Hospital, Bondy and Louis Mourier Hospital, Colombes, France.

Although the sensitivity $(90 \%)$ and specificity $(85 \%)$ of the thallium myocardial scintigraphy (TMS) is higher than for the exercise stress test (ST) (sensitivity : $65 \%$ specificity : $80 \%$ ) in the general population, the value of TMS in assessing silent myocardial ischemia related to coronary stenoses in diabetic subjects is still controversial. The aim was to evaluate the ST performances and compare them with those for TMS found during exercise, in diabetic patients with more than two cardiovascular risk factors but without cardiac symptoms and with a normal 12-lead ECG. 169 diabetic patients underwent a TMS associated with maximal exercise. A coronary angiography was performed when the test was clinically and/or electrically positive, and/or if a thallium defect was found. The ST was electrically and/or clinically positive in 19 cases, 9 of whem showed TMS defects. The TMS showed defects in 27 other patients with a normal ST. Thus 46 patients had signs of silent myocardial ischemia. A coronary angiography was performed in 32 of them and showed significant stenoses in 14 cases. The sensitivity, specificity and positive predictive value (PPV) for the detection of coronary stenoses were $59 \%, 80 \%$ and $77 \%$ for the ST, and $82 \%$, $13 \%$ and $52 \%$ for the TMS. When all the clinical, electrical and thallium scan criteria during the ST were considered together, the PPV was $53.1 \%$. In addition, the PPV of the TMS, when the ST was negative, was $37 \%$. These results suggest that $1 /$ the sensitivity is higher for TMS than for the ST, but the specificity and PPV are far lower ; $2 /$ when the ST is maximal the scintigraphic image is slightly contributive. Therefore the standard ST should be considered first when assessing silent myocardial ischemia in diabetic patients. If the test is below maximal effort or impossible to carry out, a TMS coupled with dipyridamole is justified. 
EVALUATION OF DIFFERENT THERAPEUTIC METHODS IN NIDDM PATIENTS WITH CHRONIC ISCHEMIC HEART DISEASE

R.B.Kurashvili, N.G.Asatiani, M.G.Dundna, M.E.Natsvlishvill, L.R.Tsutskiridze and L.R.Nikoleishvili. Georgian Diahetes Center, Thillsi, Georgia.

The present study was performed to assess the efficacy of the combined therapy (Insulin/Oral Hypoglycaemic Agents) versus conventional Insulin therapy in NIDDM patients with secondary sulphonylurea failure and Chronic Ischemic Heart Disease (CIHD), In our study 63 patients with NIDDM associated with CIHD were observed (age rang 46 - 69 yrs; diabetes duration12.5 $\pm 4.5 \mathrm{yrs}$; BMI $27.4 \pm 2.1 \mathrm{mg} / \mathrm{m} 2)$. CIHD was diagnosed based on the ECG data and the stresstest results. Specific changes on the ECG proved that 8 patients had Myocardial Infaretion. Patients were devided into three groups: Group 1 (20 patients) sulphonylurea (Gliclazide) treatment was continued in the daytime and NPH Insulin administration at bedtime was started. Group 2 (22 patients) - received Metformin and Gliclazide and bedtime NPH - injections. Group 3 ( 21 patients) - sulphonilurea treatment was discontinued and twice daily Insulin injections started. The baseline characteristics did not differ significantly between the Groups. Repeated examinations were performed 6 month after the initiation of the study. Positive ECG changes were observed in 17 patients $(5$ - Group 1;6 Group 2; 6 - Group 3). No negative ECG changes were registered. HbA1c levels dropped significantly in all the Groups (Gr. 1 - 10.6 $\pm 0.5 \%$ vs $7.6 \pm 0.4 \%$; (P<0.001); Gr. $2-10.4 \pm 0.3 \%$ vs $7.4 \pm 0.3 \%$ (P<0.001); Gr. $3-10.5 \pm 0.6 \%$ vs $8.1 \pm 2.1 \%(P<0.001)$. Following Insulin doses were administered; Gr. 1 $0.32 \pm 0.04 \mathrm{IU} / \mathrm{kg} ;$ Gr. $2-0.26 \pm 0.031 \mathrm{U} / \mathrm{kg} ;$ Gr. $3-0.49 \pm 0.06 \mathrm{IU} / \mathrm{kg}$. In Groups 1 and 2 body weight decreased $(1.9 \pm 0.9 \mathrm{~kg}$, and $2.4 \pm 0.5 \mathrm{~kg}$, respecively), while in Group 3 it increased $(1.2 \pm 0.4 \mathrm{~kg})$. The results achieved indicate that in NIDDM patients with secondary sulphonylurea failure combination of Metformin, Gliclazide and Insulin is more effective than Insulin monotherapy. Besides, combined Insulin/Oral Hypoglycaemic Agents, as well as conventional Insulin therapy did not cause (IHD aggrevation in our NIDDM patients.
CORONARY COLLATERAL CIRCULATION IN DIABETIC PATIENTS WITH CORONARY ARTERY DISEASE

A. Melidonis, S. Heraklianou, A. Kamaratos, D. Damianaki, V. Pisimisis, S. Foussas

Diabetic Unit \& Cardiology Dept of Tzanio General Hospital, Piraeus - Greece

Background : Although it is well established that Diabetes Mellitus (DM) induces more severe Coronary Artery Disease (CAD), it is not known whethes it contributes to the development of coronary collateral circulation in our study we examine the extension and functional capacity of the coronary collateral circulation in diabetic patients with CAD in comparison with non-diabetic patients with CAD.

Methods: The study group consisted of 223 diabetic patients with angiographically established $C A D$ (Group $A$ ) which were compared with 210 nor-diabetic patients with CAD (Goup B). The two groups were comparable according to age ( $60.3 \pm 8$ yrs vs $58.8 \pm 9$ yrs) and the known ntsk factors for CAD. The study of the coronary angiographies was performed by two independent angiographers, which examined the degree and the extension of stenosis and the collateral circulation at the affected coronary vessels. The collateral circulation was categorized using the circulation at the aftected coronary vessels. The collateral circulation was categorized using the Rentrop grading system of 0 to ill in relation to the presence and extension ofthe coilateral filling of coronary
vessels).

Results : We observed that group A had more frequently grade il colateral circulation in Results : We observed that group A had more frequently grade il colderal circulation in comparison with group B (13.2\% vs $8.7 \%$, pro.05), but there were no differences between the two groups in relation to grade O,I, collateral circulation When we stratied the two groups
according to sex and age (age $<55$ yrs and, 55 yrs) the following results emerged: group $A$ males aged i 55 yrs had more frequently grade lil collateral circulation $(20.2 \%$ vs $3.4 \%$ p 0.001$)$ and less frequently complete absence of collaterals (grade 0$)(7.5 \%$ vs $90.8 \%$, pro.01) Grade lil collateral circulation mainly develops at the left anterior descending (LAD) (28\% vs $3.4 \%, p<0.01)$ and the right coronary artery (RCA) $30 \%$ vs $6.9 \%$, prO.OI), where the total occlusions of coronary arteries usually develop (proximal LAD : $9.3 \%$ vs $16 \%$, p 0.01 and proximal RCA : $14.1 \%$ vs $7.1 \%$. p (0.05). Group A males aged ( 55 yrs in comparison with females of the same group had more frequently grade II collateral circulation $(20.2 \%$ vs $2.2 \%$, pro.01). We did not observe any difference in relation to the collaterals between group $A$ and group $B$ males aged i 55 yrs. When we analyzed the risk foctors for $C A D$, we observed that group $A$ males aged $>55 y$ is were less

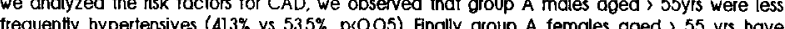
frequentiy hypertensives (45\% vs $53.5 \%$. pro..5). Finally group A females aged i 55 yrs have gore

age group (IIT) vs 4.4\%, pr O.OS). with $C A D$ develop more frequently coronony collotes Conduslon : Diabetic patients with CAD develop more frequently coronary collateral circulation in companison with non-diabetics, especially males ages less than $55 \mathrm{yrs}$. The collateral circulation mainly develops at the LAD and RCA

\section{9}

THE EFFECTS OF ANGIOTENSIN CONVERTING ENZYME GENE POLYMORPHISM ON THE LEFT VENTRICULAR FUNCTION AND MASS IN PATIENTS WITH DIABETES MELLITUS

T.Erbas, L.Tokgozoglu, K.Aytemir, M.Alikasifoglu, M. Kabukcu, S.Kes, E.Tuncbilek and O.Gedik.

Hacettepe Medical School. Departments of Endocrinology. Cardiology and Clinical Genetic, Ankara, Turkey.

The polymorphism of the angiotensin converting enzyme (ACE) has been shown to predispose to various cardiovascular disease. Since the ACE gene has been found to be highly expressed in the endothelial cells and the heart, it would be interesting to see if the ACE genotype contributes to any cardiac abnormalities in diabetic patients. Therefore this study was performed to evaluate the potential relationship between ACE genotypes and the left ventricular function and mass in seventy five diabetic patients (IDDM; $n=23$, mean age $=33 \pm 11$ yr, duration of diabetes $=12 \pm 10 \mathrm{yr}$, NIDDM; $n=52$, mean age $=58 \pm 10 \mathrm{yr}$, duration of diabetes $12 \pm 9 \mathrm{yr}$ ). Seventy-five, age matched healty subjects served as the control group for diastolic function parameters. The genotype analysis was performed by the polymerase chain reaction method. The frequency of the DD allele was $22.7 \%, \mathrm{DI}$ allele $60 \%$, and 11 allele $17.3 \%$. These were compared to data in a healty nondiabetic population where DD allele was $34.3 \%$, DI $55.7 \%$ and $1110 \%$. The DD genotype correlated with microalbuminuria in IDDM $(\mathrm{p}=0.04)$, and presence of myocardial infarction in NIDDM ( $p=0.03$ ). There was no correlation between end diastolic and end systolic volumes, ejection fraction and genotype in either group. Furthermore, diastolic function parameters such as E/A ratio and isovolumic relaxation time also failed to show an association with ACE genotype. The left ventricular mass failed to show an association with genotype in IDDM (LVM=184 $\pm 72 \mathrm{~g}$ ), but was significantly correlated to DD genotype in NIDDM (LVM $=223 \pm 74 \mathrm{~g}$ ) $(p=0.001)$. There was no association between ACE genotype and systolic and diastolic function parameters evaluated by echocardiography. However left ventricular mass was found to be significantly correlated with DD genotype in patients with NIDDM.

\section{0}

EFFECT OF CILAZAPRIL ON MICROALBUMINURIA AND LEFT VENT. RICULAR MASS IN NON-INSULIN DEPENDENT DIABETES NIDDM) M.Spring,A.Raptis,D.Barnes, E.Murphy, J.Chambers, ${ }^{1}$, F.Sorensen ${ }^{2}$, D.Wohler ${ }^{3}$, and G.Viberti. Units for Metabolic Medicine and Cardiology', UMDS, Guys Hospital, London, U.K., IMOR ${ }^{2}$, Lorrach, Germany and Roche Ltd ${ }^{3}$, Basel, Switzerland. Microalbuminuria predicts early cardiovascular disease in NIDDM. Left ventricular hypertrophy is another powerful independent predictor of cardiovascular events. In this randomized, double-blind study we compared the effect of 48 weeks treatment with cilazapril on albumin exeretion rate (AER) and left ventricular mass (LVM) in microalbuminuric (AER 20-200 $\mathrm{gmin}^{-1}$ ) NIDDM patients. The patients who had never previously taken ACE inhibitors, nor had clinical, ECG or echo evidence of ischaemic heart disease were divided into 2 groups, A: cilazapril $5 \mathrm{mg} / \mathrm{day}(\mathrm{n}=45,32 \mathrm{male})$ and B: placebo ( $n=46,32$ male). The groups, $A$ and $B$, were matched for age $60.6+8.4$ vs $60.4+8 \mathrm{yr}$, diabetes duration $8.1+5$ vs $8.9 \pm 6 \mathrm{yr}$ and $\mathrm{HbA}_{1} \mathrm{c} 8.6+1.5$ vs $8.7+1.4 \%$. Blood pressure (BP) was the mean of $\overline{2}$ sitting measurements after $1 \overline{0}$ minutes rest. AER was the median of 3 successive timed overnight urine collections at baseline and the mean of 2 similar collections 12, 24 and 48 weeks after starting treatment. LVM was evaluated at baseline and the end of treatment by M-mode echocardiography and the LVM index (LVMI) calculated as the ratio of LVM and body surface area. Baseline BP (4 weeks after stopping any previous anti-hypertensive treatment) was similar (mean+SD) $137 \pm 22 / 77 \pm 12$ vs $137+19 / 74 \pm 14$ and remained so during treatment $134 \pm 21 / 76 \pm 11$ vs $137 \pm 19 / 77 \pm 11$ for groups $A$ and $B$ respectively. AER was

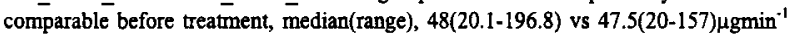
but during treatment decreased by $23 \%$ in group A compared to an increase of $13 \%$ in group $B(p=0.004)$. Although LVMI decreased in group A from $135.3+41.2$ to $132 \pm 33.5 \mathrm{gm}^{-2}$ and increased in group $B$ from $121.8+31.4$ to $127.3+38.2 \mathrm{gm}^{-2}$ the between group difference was not statistically significant. In conclusion, cilazapril reduces AER in microalbuminuric NIDDM patients independent of BP, but LVMI is not affected after 48 weeks treatment. 
1331

DOPPLER ECHOCARDIOGRAPHIC ASSESSMENT OF LEFT VENTRICULAR DIASTOLIC FUNCTION IN DIABETES MELLITUS

\section{Chira and M. Motocu - The $4^{\text {th }}$ Medical Clinic, U.M.F. Cluj-Napoca}

We utilized Doppler echocardiography to characterize left ventricular diastolic function in 42 patients with diabetes mellitus (mean age $42 \pm 11$ years, $75 \%$ male) ho had no symptoms of heart failure and had normal left ventricular systolic function. Data were compared with those in 46 normal control subjects of similar age and gender. Heart rate, systemic blood pressure, and cardiac dimensions (wall thickness, left atrial and left ventricular cavity dimensions) were similar and not significantly different in patients and controls. As a group, patients showed significantly increased deceleration time and decreased rate of decline of flow velocity in early diastole $(p<0.0001$ and $p<0.01$, respectively $)$ when compared to controls. Individual patient analysis showed that $10(24 \%)$ of the 42 patients with diabetes mellitus had 2 or more abnormal Doppler indexes of diastolic function consistent with a pattern of impaired left ventricular relaxation. The most common abnormalities were increased deceleration time (> $224 \mathrm{~ms} ; 9$ patients), ptolonged isovolumic relaxation time (> $103 \mathrm{~ms} ; 8$ patients) and reduced rate of decline of flow velocity in early diastole $(<2.1 \mathrm{~m} / \mathrm{s} ; 5$ patients). In addition, peak early diastolic velocity was reduced $(<43 \mathrm{~cm} / \mathrm{s})$ in 3 patients and early to atrial peak flow velocity ratio was reduces $(<1)$ in 2 patients. Comparison of subgroups of patients with and without abnormal Doppler indexes showed no significant differences with regard to age, gender, heart rate, systemic blood pressure, severity of diabetes mellitus, and cardiac dimensions. This Doppler echocardiographic analysis demonstrates that diastolic abnormalities may be present in patients with diabetes mellitus, even in absence of symptoms of cardiac failure or left ventricular systolic dysfunction. These diastolic abnormalities suggest an intrinsic myocardial abnormality in patiens with dabetes mellitus; however, whether they represent a preclinical phase of myocardial involvement or an intrinsic feature of the primary myocardial disease process in diabetes mellitus remains to be elucidated.

\section{2}

GLUCOSE METABOLISM DISTURBANCES IN MEN REFERRED FOR CORONARY ARTERIOGRAPHY

I.Kowalska, M.Strączkowski, H.Gajewska*, J.Prokop*, W.Kochman*, B.Telejko, I.Kinalska and W.Musiał* Department of Endocrinology and *Department of Cardiology, Medical School, Białystok, Poland

The leading cause of mortality in type 2 diabetic patients is cardiovascular disease. The aim of the present study was the estimation of the prevalence of impaired glucose tolerance (IGT) and type 2 diabetes in men referred for coronary arteriography without previous history of diabetes mellitus. The study was carried out in 264 men (mean age 53.9+9.3, BMI-27.8+3.8). All patients had an oral glucose tolerance test (OGTT). Plasma levels of fasting and post load insulin, HbAlc, total cholesterol, HDL and LDL cholesterol, triglycerides, fibrinogen, PAI, platelets and urinary albumin excretion were measured. Among the patients studied 141 patients $(53 \%)$ had disturbances of glucose metabolism (IGT-29,17\%, $n=77$; type 2 diabetes mellitus - $24,2 \%, n=64$ ). In coronary arteriography significant changes in coronary arteries were observed in 226 patients. We observed a significantly higher prevalence of the three coronary arteries stenosis in patients with type 2 diabetes ( $p .<0,001$ in comparison to the men with normal glucose tolerance and p. $<0,01$ compared to the IGT group). Diabetic patients had markedly higher levels of plasma fibrinogen, PAI, platelets and post load insulin and lower ejection fraction. The significant correlations between post load insulin and platelets ( $p .<0,001 ; r=0,42$ ), fibrinogen $(p,<0,001 ; r=0,40)$ and between PAI and urinary albumin excretion ( $\mathrm{p} .<0,01 ; r=0,32$ ) were also found. We conclude that about half of our patients with coronary artery disease have unrecognized disturbances of glucose metabolism. Early diagnosis and treatment of disturbed glucose tolerance is a key factor in the prevention of coronary artery disease.

\section{3}

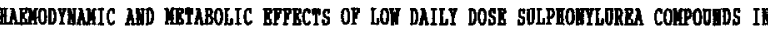
THE COROLARI CIRCOLAFIOR OP DOGS

I. Pósa, R. Mocsis, M. Z. Roltai and G. Pogátsa. György Gottsegen National Institute of Cardiology, Budapest, Hungary

The ain of the study was to compare the haemodynanic and metabolic side-effects of glibenclamide (GB; $n=6)$, glipizide $(G P ; n=6)$ and glimepiride $(G M ; n=6)$ in the coronary circulation of cogs. Yean arterial blood pressure $(B P)$, heart rate $(\mathbb{R}\}$, myocardial contractile force, and the rate of change of nyocardial contraction and relaxation were measured during iv. administration $\left(0.4-2-5-8 \mu \mathrm{mo} \mathrm{I}^{*} \mathrm{~kg}^{-1}\right)$ of the drugs. Arterial (PA; a. carotis) and venous (PV; sinus coronarius) pyruvate concentrations vere determined. Rate-pressure product (HR $\&$ BD) and the arteriovenous pyruvate difference $(\mathrm{PV}-\mathrm{PA})$ vere calculated. $G B$ and $\mathrm{GM}$ proved to be hypotensive (G1: $-218, p<0.001 ; G B:-388, p<0.001$ ), while blood pressure was slightly enhanced by GP (GP: $\$ 98, p<0.001)$. The effect of glipizide vas significantly different from that of $G B(p<0.05)$ and $G N(p<0.01)$. GB reduced the heart rate (6B: $-328, p<0.05$ ), but the other two compounds practically did not alter this paraneter (GK: $-68,0<0.05 ; G P:-28,0<0.01$ ). All the conpounds was found to be negative inotropic (GK: $-528, \mathrm{p}<0.001$; GB: $-498, \mathrm{p}<0.05 ; \mathrm{GP}:-268$, $p<0.001\}$. The rate of change of myocardial contraction was decreased by the drugs (GN: $-458, p<0.05 ; G B:-528, p<0.05 ; G P:-218,0<0.001$ ). Rate-pressure product was decreased by $G B$ and GM (GM: $-218, p<0.001 ; G B:-578, p<0.01)$, however it vas noderately changed by GP $(G P:-4 \%, p<0.001)$. This difference vas significant between $G M$ and $G P(p<0.01)$. Arterial and venous pyruvate concentrations were reduced by all the drugs (Arterial: GB: $-568, p<0.05 ;$ GK: -298 , p<0.05; GP: -128 , $p<0.001$. Venous: $G B:-388, p<0.05 ; G P:-78, p<0.05)$, excepted $G M$, which enhanced the venous concentration (GV: $+268, p<0.05$ ). The arteriovenous pyruvate difference vâs also decreased by the compounds (GB: -108 , $0<0.01$; GM: -88 , p<0.001; GP: -348 , p 0.001$\}$. According to the results, glipizide influences blood pressure and ratepressure product less than the other components do. In the case of the further haemodynamic and metabolic parameters, these drugs exert sinilar cardiovascular side-effects, and they possibly reduce the nyocardial production and/or uptake of pyruvate.

\section{4}

FATTY ACIDS PROTECT THE DIABETIC HEART DURING LOW FLOW ISCHAEMIA: ${ }^{31}$ P NMR STUDY

L.M. King, R.J. Sidell, B.E. Jones, G.K. Radda and K. Clarke. Dept Biochemistry, University of Oxford, South Parks Rd, Oxford OX1 3QU Free fatty acids (FFA) are the major energy substrates of the heart, especially in diabetics. However, elevated fatty acids may add to ischaemic injury, possibly by an accumulation of deleterious metabolic intermediates. Whether the diabetic heart has increased or decreased ischaemic injury is controversial. The conflicting reports may be due to the type of substrate used in the study. The aim of this work was to determine the role of FFA in low flow ischaemia in control and diabetic rat hearts. Wistar rats (250$300 \mathrm{~g}$ ) were injected with streptozotocin (STZ), $50 \mathrm{mg} / \mathrm{kg}$ in citrate buffer pH 4.5, or vehicle. After 7 weeks, hearts were removed from anaesthetised animals for perfusion, with continuous measurement of function via an intraventricular balloon. Myocardial high energy phosphate levels and $\mathrm{pHi}$ were monitored using ${ }^{31} \mathrm{P}$ nuclear magnetic resonance spectroscopy (NMR). Control hearts were perfused with $11 \mathrm{mM}$ glucose and $3 \%$ BSA throughout $32 \mathrm{~min}$ low flow $(0.5 \mathrm{ml} / \mathrm{min})$ ischaemia, and after $30 \mathrm{~min}$ reperfusion had recovered $57 \pm 9 \%$ of their original contractile function. When $1.2 \mathrm{mM}$ palmitate (FFA) was present throughout ischaemia and reperfusion, recovery was $69 \pm 10 \%$ (n.s.d.). In diabetic hearts, recoveries were significantly lower in the absence of FFA ( $26 \pm 10 \%, \mathrm{p}<0.05$ vs. control) than in the presence of FFA $(45 \pm 16 \%)$. ATP and PCr levels were little different between groups during ischaemia and on reperfusion. pHi declined the most during ischaemia in the control glucose only hearts. In conclusion, FFA were protective in low flow ischaemia, and did not result in increased intracellular acidosis. STZ rat hearts showed impaired tolerance to ischaemia in the absence of FFA, but the presence of FFA largely reversed the deleterious effects. These results suggest that FFA are not deleterious during ischaemia, especially in diabetic hearts. 


\section{Lipids II}

\section{5}

NEW DYSLIPEMIC PHENOTYPES IN PATIENTS WITH TYPE 2 DIABETES MELLITUS

AM Wägner, F Calvo\#, A Castellvi*, M Rigla, R Bonet*, J Ordbñez*, and A Pérez. Endocrinology and Nutrition and Biochemistry* Deps. Hospital de Sant Pau. Barcelona. \# H. Clínico. Zaragoza. Spain.

The concentration of LDL cholesterol (c) does not stand for all atherogenic particles. Apolipoprotein $B(A p o B)$ concentrations reflect the total amount of LDL, IDL and VLDL particles, and their increase is considered a cardiovascular risk factor. The aim of this study was to evaluate the prevalence of dyslipemic phenotypes, including those dependent on $\mathrm{ApoB}$, in DM2 patients. One hundred subjects ( 63 men, 37 women, aged $58.3 \pm 11.6$ years, diabetes duration of $9.8 \pm 8.9$ years, $56 \%$ on insulin treatment) were studied. HDLc was determined through a direct method without precipitation, and VLDL and LDLc were calculated using Friedewald's equation, and by ultracentrifugation if triglycerides (TG) $>3.45$ $\mathrm{mmol} / /$. ApoB was measured by an immunoturbidimetric assay. Levels of $\mathrm{LDLC}$ $<3.4 \mathrm{mmol} / \mathrm{l}$ and of $\mathrm{ApoB}<1.0 \mathrm{~g} / \mathrm{l}$ were considered desirable. Nineteen percent of the patients had LDLc $>4.2 \mathrm{mmol} /, 27 \%$ TG $>2.25 \mathrm{mmol} /, 22 \%$, HDLc $<$ $0.9 \mathrm{mmol} / \mathrm{l}$ and $50 \% \mathrm{ApoB}>1.2 \mathrm{~g} / 1$. Their phenotypic distribution is displayed on the table.

\begin{tabular}{|c|c|c|c|c|c|c|}
\hline Ila & Ilb & $\begin{array}{c}\text { hyperApoB } \\
\text { hyperTG }\end{array}$ & $\begin{array}{c}\text { hyperApoB } \\
\text { normoTG }\end{array}$ & $\begin{array}{c}\text { IV } \\
\text { hipoalfalipo- } \\
\text { proteinemia }\end{array}$ & normal \\
\hline 13 & 6 & 11 & 20 & 10 & 8 & 32 \\
\hline
\end{tabular}

Only $45 \%$ and $19 \%$ of the patients had desirable levels of LDLc and ApoB, respectively. In conclusion, given the high prevalence of hyperapoB and the increased cardiovascular risk of these subjects, ApoB should be routinely measured in normolipemic and hypertriglyceridemic patients with DM2

\section{6}

FATTY ACID COMPOSITION OF PHOSPHOLIPIDS AND NEUTRAL LIPIDS FROM HUMAN DIABETIC SMALL ARTERIES AND VEINS BY A NEW TLC METHOD. M. Lecomte, M. Claire, M. Deneuville, and N. Wiernsperger. Diabetic Microangiopathy Research Unit, LIPHA-INSERM U352, INSA-Lyon, 69621 Villeurbanne, France and INSERM U359, 97159 Pointe-à-Pitre, Guadeloupe.

It has been suggested that lipid peroxidation, resulting from oxidative stress, may play a role in the pathogenesis of diabetic complications. Therefore, the aim of this study was to compare polyunsaturated fatty acid composition of small arteries and veins ( $<500 \mu \mathrm{m}$ diameter) obtained from diabetic (D) or non-diabetic (ND) patients undergoing arterio-venous shunt surgery. Small vessels were dissected and stored in liquid nitrogen before analysis by a new TLC method. Briefly, biopsies were applied directly in holes made in the silica gel of the concentration area of a TLC plate. The piate, holding blopsies, was applied onto a steel plate precooled in liquid nitrogen until complete freezing of biopsies before lyophilization under vacuum. The plate was further submitted to $\mathrm{TLC}\left(\mathrm{CHCl}_{3}: \mathrm{CH}_{3} \mathrm{OH}, 80: 8 \mathrm{v} / \mathrm{V}\right)$, phospholipids and neutral lipids were scraped, transmethylated and analyzed by gas chromatography for fatty acid composition. Unexpectedly, similar fatty acid composition was obtained for arteries $(N D=5, D=5)$ and veins $(N D=7, D=3)$ from diabetic or non-diabetic subjects. In phospholipids, major changes consisted of a $20 \%$ decrease of arachidonic acid $(20: 4 n-6)(p=0.07)$, a $40 \%$ decrease of its elongation product $22: 4 n-6(p<0.05)$ and $30 \%$ increase of $18: 2 n-6(p<0.05)$ in diabetic vessels. In neutral lipids, 20:4 n-6 was also diminished by $60 \%(p<0.05)$ in diabetic vessels whereas oleic acid increased by $15 \%(p<0.01)$. This loss of arachidonic acid in small diabetic vessels extend to vascular tissues observations previously made on plasmatic phospholipids and neutral lipids. These results suggest increased peroxide formation or alternatively impaired $\Delta 6$-desaturase forming 20:4 $n-6$, in the vascular wall of small vessels in diabetic patients.

\section{7}

THE ATHEROGENIC LIPID PROFILE (ALP) AND SULFONYLUREA (SU), METFORMIN (MET) AND INSULIN (INS) USE IN DIABETES M.A. Charles, M. Lin, P. Williams, R. Superko and P. Lim, Diabetes Research Center, Tustin, CA and Berkeley Heart Lab, San Mateo, CA., USA The ALP is strongly associated with coronary heart disease. We've reported that $85 \%$ of diabetes (DM) patients have the ALP. This report describes whether diabetes treatment with SU, MET or INS are related to the ALP. 72 consecutively recruited DM patients (Pts) were studied for lipids using the Lipid Research Clinic methods and LDL peak particle diameter (PPD, $\AA$ ) and HDL2 (\%) using polyacrylamide gradient gel electrophoresis. DM Pts using insulin had larger mean LDL PPD, lower triglyceride concentrations and higher HDL-cholesterol and HDL2. In contrast, Pts using SU and MET had smaller mean LDL PPD, higher triglyceride concentrations, and less HDLcholesterol and HDL2.

\begin{tabular}{lrrllrrrrr} 
U=user & SU & \multicolumn{4}{l}{ MET } & \multicolumn{1}{c}{ INS } \\
N=nonuser & $\mathrm{U}$ & $\mathrm{N}$ & \multicolumn{1}{l}{ P value } & \multicolumn{1}{c}{$\mathrm{U}$} & $\mathrm{N}$ & $\mathrm{p}$ & $\mathrm{U}$ & $\mathrm{N}$ & $\mathrm{p}$ \\
LDL PPD $(\AA)$ & 257 & 263 & $<.008$ & 254 & 261 & $<.01$ & 261 & 254 & $<.006$ \\
HDL2(\%) & 33 & 42 & $<.01$ & 32 & 39 & $<.05$ & 43 & 29 & $<.0001$ \\
Triglycerides & 188 & 119 & $<.01$ & 195 & 142 & $<.05$ & 116 & 215 & $<.0001$ \\
LDLc & 105 & 117 & $>.15$ & 109 & 113 & $>.69$ & 113 & 111 & $>.83$ \\
HDLc & 41 & 53 & $<.002$ & 43 & 49 & $<.08$ & 53 & 40 & $<.0001$
\end{tabular}

The HDLc and HDL2 differences between INS and SU U \& N, and the triglyceride differences between INS $U$ \& $N$ remain significant when adjusted for age, BMI, A1C, diabetic complications and cigarette and antilipid and other drug use, except for the MET data which did not remain significant after removal of gemfibrozil users. 8 Pts using gemfibrozil had smaller mean LDL PPD $(p<.03)$ less of their HDL mass as HDL2 $(p<.03)$ and concomitantly higher triglycerides. These associations suggest that some oral agents may not adequately control the ALP compared to DM Pts treated with INS.

\section{8 \\ HIGH LIPOPROTEIN(a) LEVELS IN IDDM AND NIDDM PATIENTS WITH MACROALBUMINURIA.}

Authors: D. Acosta, F. Relimpio, F. Losada, A. Pumar, J.L. Serrera $\left({ }^{*}\right)$, M.A. Mangas and R. Astorga. Center: Servicio de Endocrinologia. $\left.{ }^{*}\right)$ Servicio de Bioquímica Clínica. Hospital Universitario Virgen del Rocío. Sevilla

In order to detect a possible relationship between urinary albumin excretion and lipoprotein(a) levels in both type 1 and type 2 DM, 588 type 1 and 1065 type 2 diabetic patients were consecutively studied and their urinary albumin excretion rate and lipoprotein(a) levels were measured by means of immunonephelometric assays. Lipoprotein(a) levels $(\mathrm{mg} / \mathrm{dl})$ were (median [range]) 17 [196], 16 [183], 23 [468] for type I diabetic patients with normo, micro and macroalbuminuria, respectively (ANOVA, $\mathrm{F}=2.27$, $\mathrm{p}=0.1)$, and 21 [272], 20 [236], 28 [418] for type 2 diabetic patients with normo, micro and macroalbuminuria, respectively (ANOVA. $F=3.38$. $\mathrm{p}<0.05$ ). In the latter case, a post hoc analysis showed statistically significant differences between patients with macro and patients with microalbuminuria, as well as between the formers and those with normoalbuminuria. Current smoking habit was associated with lower lipoprotein(a) levels in type 2 DM (13.5 [418] vs. 22 [272], p <0.01). After considering the effect of age, duration of diabetes and BMI in a multi-way ANOVA with covariates considering jointly both types of DM, a statistically significant association with lipoprotein(a) levels was still observed for the urinary albumin excretion rate category $(F=3.45, p<0.05)$ and the smoking habit $(F=4.35, p<0.05)$. These data prove the existence of an association between macroalbuminuria and high Ip(a) levels mainly in type $2 \mathrm{DM}$, although a similar trend was observed for type $1 \mathrm{DM}$. Likewise, the smoking habit had an independent association with lower lp(a) levels 
1339

LP(a) AND CARDIOVASCULAR DISEASE IN DIABETES MELLITUS: A MULTIVARIATE ANALYSIS IN CAUCASIAN POPULATION

R. Simó, C. Hernández, P. Chacón', L. García ${ }^{2}$, A.Lecube and J. Mesa Diabetes Unit and 'Biochemistry Dept. Hospital General Vall d'Hebron. ${ }^{2}$ Endocrinology Dept. Hospital Mutua de Terrassa. Barcelona. Spain.

Lipoprotein(a) [Lp(a)] is a risk factor for cardiovascular disease (CVD) in non diabetic subjets, but its contribution to CVD in diabetic patients remains a conflicting topic. The aim of the study is to establish whether macroangiopaty is associated with serum $\operatorname{Lp}(\mathrm{a})$ and its phenotypes in diabetic patients. For this purpose we determined serum Lp(a) by ELISA (c.v. inter and intrassay: $5.3 \%$ and $4.6 \%$, respectively) in 191 consecutive diabetic patients (69 IDDM and 122 NIDDM). Lp(a) phenotypes were assessed by SDS-PAGE followed by immunoblotting and grouped by size in small (F,B,S1,S2), big (S3,S4) and null. To assess past and present evidence of CVD, a standardized questionnaire plus ECG were used. Furthermore a careful physical examination were performed. Statistical tests: t-Student [Lp(a) log. transformed], $\chi 2$, logistic regression analysis (dependent variable: presence of CVD; independents variables: $\mathrm{Lp}(\mathrm{a})>20$ $\mathrm{mg} / \mathrm{dl}$, age, BMI, smoking habit, total cholesterol, LDL-C, HDL-C, triglycerides, $\mathrm{HbAlc}$, albumin excretion rate, HTA, duration of diabetes) Diabetic patiens with CVD $(\mathrm{n}=47)$ presented higher but nonsignificant $\mathrm{Lp}$ (a) concentrations that patients without it $(n=144)$ : median $10.5 \mathrm{mg} / \mathrm{dl}$ [Cl 95\%:0.5-40] vs $6 \mathrm{mg} / \mathrm{dl}$ [Cl 95\%:0.5-33.5]. We also observed a trend toward high prevalence of small phenotypes in patients with CVD in comparison with patients without CVD (small $59,4 \%$, big $35.3 \%$ and null $5,4 \%$ vs. small $36.6 \%$, big $50,4 \%$ and null $12.5 \% ; p=0.08$ ). Finally, $L p(a)$ serum concentration $>20 \mathrm{mg} / \mathrm{dl}$ was independently associated to the presence of CVD in the logistic regression analysis $(p=0.01$, odds ratio 6.33 [CI 95\%:4.8-7.8]). We conclude that in diabetic patients serum $\operatorname{Lp}(\mathrm{a})$ concentration $>20 \mathrm{mg} / \mathrm{dl}$ is an independent risk factor for CVD.

\section{1}

A SIGNIFICANT RELATIONSHIP BETWEEN PAI-1 AND LP(a) IS PRESENT IN NIDDM PATIENTS WITHOUT COMPLICATIONS I. Testa', R. Testa ${ }^{2}$, C. Pieri ${ }^{3}$, M. Marra, C. Simolla ${ }^{4}$, S. Manfrinil ${ }^{3}$ and A. R. Bonfigi ${ }^{2}$, ${ }^{1}$ Institute of Intemal Medicine, University of Anoona, ${ }^{2} \mathrm{Center}$ of Biochemistry and ${ }^{3}$ Center of Cytology, Dept. of Gerontological Research, ${ }^{4} \mathrm{Center}$ of Demographic and Statistical Studies, INRCA, Ancona, Italy

Previously, we found a relationship between pasminogen activator inhibitor type(PAI-1) and lipoprotein(a) (Lp(a)) in NIDDM patients. We hypothesized that this relationship could to be due to a compensatory mechanism able to lower the risk of hypofibrinolysis as found in type II diabetes mellitus. The aim of this work was to investigate whether the relationship between PAI-1 and Lp(a) could be associated with the presence of diabetic complications. Other vascular risk factors able to influence the fibrinolytic parameters such as glycemia, obesity, hypertension, dislipidemia and oxidative stress were also taken into account. Sixty-six NIDDM patients without diabetic complications (age 62.7 \pm 11.7 years), 45 NIDDM patients with complications (age 65.3 \pm 9.1 years) as well as 31 control subjects (age $57.3 \pm 12.7$ years) were studied. Plasma concentrations of $\mathrm{Lp}(\mathrm{a}), \mathrm{PAI}-1$ antigen and activity and the main parameters of lipo- and glycometabolic balance were determined. Antioxidant defense was assayed as oxygen radical absorbance capacity of serum $\left(\mathrm{ORAC}_{\mathrm{OH}}\right)$. Statistically significant differences among controls and the two diabetic groups were found for fasting glucose, cholesterol, triglycerides and ORAC $\mathrm{OHb}_{\text {while }}$ no statistically significant differences were evident for PAI- 1 antigen and activity and Lp(a). Regression analysis of Log PAI-1/Lp(a) showed a significant correlation only in diabetic patients without complications $(=-0.57, p<0.001)$. These results show that the presence of a relationship between PAI- 1 and Lp(a) is characteristic of a diabetic population without complications cmphasizing the suggestion that this relationship could be a compensatory mechanism of the fibrinolytic system to limit the risks of hypofibrinolysis. On these basis, a lack or a loss of capacity to balance Lp(a) and PAI1 could potentially contribute to the pathogenesis of the diabetic complications.

\section{0}

LIPOPROTEIN(A) CONCENTRATIONS AND APOLIPOPROTEIN(A) PHENOTYPES IN LONG-LASTING IDDM. RELATIONSHIP TO SURVIVAL? M. Auinger, E. Trenkwalder, C. Feinböck, K. Irsigler, H. Dieplinger and F. Kronenberg. $3^{\text {rd }}$ Med. Dep. and L. Boltzmann Institute, Hospital Lainz Vienna and Institute of Medical Biology and Human Genetics, University of Innsbruck, Austria

High lipoprotein(a) $[\mathrm{Lp}(\mathrm{a})]$ plasma concentrations are a genetically determined risk factor for atherosclerotic complications. In healthy individuals $L p(a)$ concentrations are almost exclusively controlled by the apo(a) gene locus on chromosome $6 \mathrm{q} 2.6-\mathrm{q} 2.7$. More than 30 alleles at this highly polymorphic gene locus determine a size polymorphism of apo(a). There exists an inverse correlation between the size (molecular weight) of apo(a) isoforms and $\mathrm{Lp}(\mathrm{a})$ concentrations. Average $\mathrm{Lp}(\mathrm{a})$ levels are high in individuals with low molecular weight apo(a) isoforms and low in those with high molecular weight isoforms. Patients with IDDM have a markedly increased risk for atherosclerotic complications and are reported to have elevated $L p(a)$ concentrations. We investigated in a large group of 332 patients with IDDM with a disease duration ranging from 1 to 61 years whether the apo(a) size polymorphism is associated with a survivorship effect in these patients. We therefore grouped patients depending on their duration of IDDM in 5-years steps. The frequency of low molecular weight apo(a) isoforms continuously decreased with increasing disease duration from $42 \%$ in patients with a short disease duration up to five years to $18 \%$ in those with a duration of more than 35 years $(p=0.001) . \operatorname{Lp}(\mathrm{a})$ concentrations showed a tendency to lower values in groups with longer duration of IDDM without reaching significance. These data suggest a strong effect of the genetically determined apo(a) size polymorphism on the long-term survival in patients with IDDM. This effect might be caused by an increased rate of fata atherosclerotic complications in patients with low molecular weight apo(a) isoforms

\section{2}

THE RELATIONSHIP BETWEEN BODY MASS INDEX, ABDOMINAL FAT DISTRIBUTION, INSULIN SENSITIVITY AND PLASMA LIPIDS L.K.M. SUMMERS, B.A. FIELDING, V. ILIC and K.N. FRAYN, Oxford Lipid Metabolism Group, Radcliffe Infirmary Oxford OX2 6HE UK

Age-matched type II diabetics, obese and lean non-diabetics were studied to identify relationships between BMI, abdominal fat distribution, insulin sensitivity and plasma lipid concentrations. 6 diet- or tablet-controlled type II diabetics, 5 obese and 6 lean non-diabetics (3 females in each group) underwent magnetic resonance imaging to assess abdominal subcutaneous and visceral fat areas. Fasting blood sampling and euglycaemic-hyperinsulinaemic clamps were performed. BMI, total, subcutaneous and visceral abdominal fat areas differed between the groups $(P<$ $0.05)$, as did glucose concentration $(P<0.01)$ and $\mathrm{HbA}_{1 \mathrm{c}}(P<0.05)$. In lean nondiabetics there were strong relationships between $\mathrm{BMI}$ and glucose concentration $\left(\mathrm{r}_{\mathrm{r}}\right.$ $=0.88, P<0.05)$, insulin sensitivity and total cholesterol concentration $\left(\mathrm{r}_{\mathrm{s}}=-0.83\right.$, $P<0.05)$ and between visceral fat area and fasting insulin concentration $\left(\mathrm{r}_{8}=0.89\right.$, $P<0.05)$. In obese non-diabetics the last was also true $\left(r_{s}=-0.90, P<0.05\right)$, and there were also strong relationships $(P<0.05)$ between total fat area and triglyceride concentration, BMI and HDL-cholesterol concentration and visceral fat area and $\mathrm{HbA}_{\mathrm{ic}}$. In type II diabetics, BMI was related to total cholesterol concentration $\left(\mathrm{r}_{\mathrm{s}}=0.83, P<0.05\right)$. (As they were receiving treatment indices of glycaemic control were not studied). Over all subjects. BMI correlated with fasting insulin concentration $\left(r_{s}=0.51, P<0.05\right)$, while total fat area correlated with both fasting insulin concentrations $\left(r_{\mathrm{s}}=0.62, P<0,01\right)$ and insulin sensitivity $\left(\mathrm{r}_{\mathrm{s}}=-0.54\right.$, $P<0.05)$. Visceral fat area correlated with HDL-cholesterol $\left(\mathrm{r}_{\mathrm{s}}=-0.63, P<0.01\right)$, triglyceride $\left(\mathrm{r}_{\mathrm{s}}=0.59, P<0.05\right)$ and fasting insulin concentrations $\left(\mathrm{r}_{\mathrm{s}}=0.75, P<\right.$ $0.05)$, as well as insulin sensitivity $\left(r_{\mathrm{s}}=-0.56, P<0.05\right)$. Insulin sensitivity correlated with HDL-cholesterol $\left(r_{3}=0.52, P<0.05\right)$ and NEFA concentrations $\left(r_{s}\right.$ $=-(0.49, p<0.05)$. Overall, higher total and visceral fat areas were related to a worsening of atherogenic indices and reduced insulin sensitivity was associated with a worsening of the lipid profile. In type II diabetics, however, neither total nor visceral fat areas or insulin sensitivity were related to an adverse lipid profile, and we conclude that in diabetes other factors predominate in determining cardiovascular risk. 


\section{3}

The effect of diabetic control on post-prandial cholesterol esterification and transfer to atherogenic lipoproteins

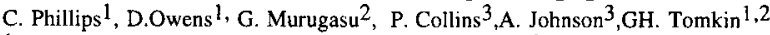
${ }^{1}$ Department of Clinical Medicine, Trinity College, ${ }^{2}$ The Adelaide Hospital, ${ }^{3}$ Department of Biochemistry, Royal College of Surgeons in Ireland, Dublin Ireland Cholesteryl ester transfer protein (CETP) regulates cholesterol transfer between HDL and the apo B-containing lipoproteins particularly in the post-prandial state. Raised CETP levels have been described in diabetes and may be associated with an atherogenic lipoprotein profile. This study examines the effect of improved diabetic control, CETP and lecithin:cholesteryl transfer protein (LCAT) - the enzyme which esterifies of cholesterol prior to its transfer by CETP. Six non-insulin-dependent diabetic (NIDDM) patients (fasting blood glucose $9.6 \pm 1.3 \mathrm{mmol} / 1$ and $\mathrm{HbAlc} 9.8 \pm 5.2 \%$ ) were examined prior to and following good control (blood glucose $6.2 \pm 0.6 \mathrm{mmol} / \mathrm{l}$ and $\mathrm{HbAlc} 4.9 \pm 0.5 \%$ ). A high fat test meal was given and blood samples taken fasting and at 2, 4, 6, 8 and 10 hours post-prandially. Chylomicrons, VLDL, LDL and HDL were isolated by sequential ultracentrifugation and their composition determined. CETP and LCAT activities were determined in the same assay by measuring $\left[{ }^{3} \mathrm{H}\right]$ cholesterol esterification and transfer between the patients' lipoproteins. There was no significant change in fasting plasma triglycerides or HDL cholesterol on good control but plasma LDL cholesterol decreased significantly $(\mathrm{p}<0.05)$. Fasting CETP and LCAT decreased following good control and post-prandial CETP and LCAT decreased significantly $(p<0.0001$, analysis of repeated measures). There was a reduction in post-prandial chylomicron and VLDL cholesterol and an increase in HDL but these were not significant. There was a significant positive correlation between the increase in HDL cholesterol after good control and the reduction in CETP $(\mathrm{p}<0.05)$ and a negative correlation between decrease in LDL cholesterol and decrease in CETP $(r=-0.9, p<0.02)$. This study suggests that improvement in diabetic control was associated with a reduction in transfer to atherogenic apoB-containing lipoproteins due to a reduction in LCAT and CETP.

\section{4}

Post-prandial lipaemia with triglyceride (TG) enrichment of VLDL particles results in increased free radical production and deterioration of endothelial function in non-insulin dependent diabetes (NIDDM)

L.M. Evans', J. Graham², J.S. Davies', R.A. Anderson', G. Ellis', S. Jackson', M.P. Frennaux' ${ }^{1}$. A. Rees'. ' University of Wales College of Medicine, Cardiff, Wales. 'L Liverpool John Moores University, Liverpool, England.

Background: Elevated levels of TG rich lipoproteins in both the fasting and postprandial state may promote the deposition of unstable atherosclerotic plaques, putative mechanisms include enhanced oxidative stress and endothelial dysfunction. NIDDM mechanisms include enhanced oxidative stress and endothelial dysfunction. NIDDM
patients have an excess mortality from vascular disease and exhibit prolonged and patients have an excess mortality from vascular disease and exhibit proionged and studied the relationship between endothelial function, free radical release and the qualitative lipoprotein changes, during post-prandial lipaemia (PPL) in NIDDM Methods: 12 NIDDM patients with no history of vascular disease and moderate glycaemic control (mean HbAlc 7.9\% \pm 1.7 ) were studied, $(7 \mathrm{M}, 5 \mathrm{~F}$ ), mean age $47.8 \pm$ 6.5 years. Subjects were studied following a 12 -hour overnight fast and again 4 hours after the ingestion of a standard fatty meal. Lipoprotein profiles were assessed using a new non-ionic iodinated, density gradient medium iodixanol. Small differences in electrophoretic mobility of VLDL, LDL and HDL enables subfractionation and analysis of lipid content of these subclasses. Oxidative stress was assessed by directly measuring carbon centred free radicals (FR) in venous blood by means of electron paramagnetic resonance spectroscopy. Endothelial function was assessed by measuring flow-mediated, endothelial dependent, dilatation (FMD), of the left brachial measuring flow-mediated, endothelial dependent, dilatation (FMD), of the left brachial artery using a high-resolution ultrasonic vessel wall tracking system. Results: [Mean
土SD] There was significant TG enrichment of VLDL in the post-prandial $\pm S D]$ There was significant TG enrichment of VLDL in the post-prandial phase, with no change in TG content of either HDL or LDL (fasting VLDL TG content $65.1 \pm$ $10 \%, 4$ hour post-prandial VLDL TG content $70.8 \pm 7.6 \%$; $p<0.001$ ). Venous FR (Results expressed in arbitrary units) increased significantly post-prandially $(0.22$ 0.05 vs $3.2 \pm 2.55 ; \mathrm{p}<0.05$ ). This was mirrored by a significant decrease in FMD $(2.57 \% \pm 0.97 \%$ vs $1.33 \pm 1.1 \% ; p<0.05)$. Conclusions: PPL in NIDDM results in significant TG enrichment of VLDL particles which is associated with both increased FR production and deterioration in endothelial function. The prolonged PPL in NIDDM results in continued exposure of the vasular end The prolium TGRL, which may impair endothelial function, either directly, or indirectly via altered FR production.

\section{5}

IMPACT OF A I-FABP GENE POLYMORPHISM ON INSULIN RESISTANCE IN YOUNG MEN WITH POSTPRANDIAL HYPLIR'IRIGL YCERIDAEMIA $S$ Fenselau ${ }^{*}$, J Schrezenmeir' and $L$ Bajer ${ }^{2}$, 'Federal Research Center Institute of Biochemistry and Physiology of Nutrition, Kiel, Germany, NIDDK, National Institutes of Health, Phoenix, Arizona, USA

In nondiabetic Pima indians an alanine to threonine substitution $(A \rightarrow G$ at Codon 54) of the intestinal fatty acid binding protein (I-FABP) was associated with higher fasting insulin concentrations, lower insulin stimulated uptake rates and higher lipid oxidation rates. In a Caco-2 cell model transfected cells expressing the $G$ altele for I-FABP showed a higher LCFA-transport and a higher secretion of triglycerides. We genotyped the I-FABP polymorphism in young male subjects which presented with early signs of a metabolic syndrome (excessive postprandial ( $p p$ ) triglycerides, higher $p p$ FFA, higher glucose and insulin levels, higher intraabdominal fat (High Responders $=H R, n=22$ ) compared to controls (Normal Responders $=N R, n=55$ ) and analysed the data with regard to changes in metabolic parameters characterizing impaired glucose metabolism. The subjects were at mean $25 \pm 4$ years old and had a BMI of $23.7+2.4 \mathrm{~kg} / \mathrm{m}^{2}$. Age and BMl did not differ between $H R$ and NR. We found the frequency of the A-containing and G-containing alleles to be the same in both groups (HR: $A$ allele $=0.73$ and $G$ allele $=0.27$, NR: $A$ allele $=0.75$ and $G$ allele $=0.73$ ). in addition, we could not find differences between $A A$ and $A T / T T$ subjects in fasting triglycerides (AA: $1.14 \pm 0.47 \mathrm{mmol} / \mathrm{t}$ vs AT/TT: $1.09 \pm 0.32$ mmol/l), in fasting glucose (AA: $4.9 \pm 0.5 \mathrm{mmol} / \mathrm{l}$ vs AT/TT: $5.02 \pm 0.56$ ) and in fasting insulin levels (AA:10.7\$6.1 mU/I vs AT/TT: $9.75 \pm 5.5$ $\mathrm{mU} / \mathrm{I})$ neither in the whole group nor divided in HR and NR comparing the different polymorphisms. Based on these data we conclude that the genetic variation in I-FABP does not explain the phenomenon of postprandial triglyceride high response in young male subjects in a caucasion population.

\section{6}

Hormone replacement therapy for non-insulin-dependent diabetes mellitus. The effect of low-dose oestrogen vs oestrogen/progesterone on lipoproteins

Tomkin GH, Collins P, Johnson A, Owens D. Department of Clinical Medicine Trinity College Dublin, The Adelaide Hospital Dublin, Department of Biochemistry, The Royal College of Surgeons in Ireland Dublin, Ireland

Low-dose oestrogen/progesterone (HRT) may increase patient compliance long-term but the cardioprotective effects in diabetes are unknown. This study examined the effect of 6 months treatment with low dose oral oestrogen (1mg 17-b-estradiol) $\left(\mathrm{E}_{2}\right)$ vs low dose combined E2+norethisterone acetate (NETA) (1 mg E $2+0.5 \mathrm{mg}$ NETA). Thirty six post-menopausal non-insulin-dependent diabetic (NIDDM) patients in moderate control (mean $\mathrm{HbAlc} 7.9 \%$ ) and serum oestradiol level $<50 \mathrm{pg} / \mathrm{ml}$ were examined. Serum lipids were measured by enzymatic colorimetric methods and serum Lp(a) by an ELISA assay. Cholesteryl ester transfer protein (CETP) was determined as the capacity of patients plasma to transfer $\left[{ }^{3} \mathrm{H}\right]$-cholesterol from pooled HDL to pooled LDL. Mean serum oestrogen changed from $17 \pm 15 \mathrm{pg} / \mathrm{ml}$ to $120.8 \pm 43 \mathrm{pg} / \mathrm{ml}$ in the $\mathrm{E}_{2}$ group and from $20 \pm 10 \mathrm{pg} / \mathrm{ml}$ to $106.3 \pm 49 \mathrm{pg} / \mathrm{ml}$ for the E2+NETA group $(p<0.0001)$. Patients mean blood glucose and HbAlc did not change during the study. Serum cholesterol fell from $6.8 \pm 1.4$ to $6.2 \pm 0.8 \mathrm{mmol} / 1(\mathrm{p}<0.005)$ and $\mathrm{LDL}$ cholesterol fell from $4.8 \pm 1.0$ to $3.9 \pm 0.7 \mathrm{mmol} / 1(\mathrm{p}<0.0001)$ in the $E_{2}$ group. In the E2+NETA group serum cholesterol fell from $6.1 \pm 1.1$ to $5.3 \pm 1.3 \mathrm{mmol} / 1$ ( $\mathrm{p}<0.005)$ and $\mathrm{LDL}$ from $4.2 \pm 1$ to $3.3 \pm 0.9 \mathrm{mmol} / \mathrm{l}$ $(p<0.005)$. HDL cholesterol did not change significantly in either group nor did fasting triglycerides. $\mathrm{Lp}(\mathrm{a})$ did not change significantly in the E2 group but in the E2+NETA group there was a significant decrease ( $<<0.05$ paired t-test).CETP increased significantly only in the E2+NETA group from $119 \pm 30$ to $136 \pm 31 \mu \mathrm{mol} / \mathrm{ml} / \mathrm{h}$ ( $\mathrm{p}<0.001$ paired ttest). The increase in CETP without change in HDL cholesterol, the reduction in LDL cholesterol and the reduction in $\mathrm{Lp}(\mathrm{a})$ are potentially cardioprotective. Low dose combined HRT may be the treatment of choice for NIDDM due to the improvement in the lipoprotein profile. 
1347

POSTMENOPAUSAL HORMONE REPLACEMENT THERAPY AND LIPID PARAMETERS IN WOMEN WITH TYPE 2 DIABETES

PJ Manning, AR Allum and SD Jones, Dunedin Hospital, Dunedin, NZ Hormone replacement therapy (HRT) has a significant beneficial effect on lipid parameters in non-diabetic postmenopausal women. Little is known, however, of its effect in women with Type 2 diabetes. The aim of this study was to determine the effect of combined continuous HRT on lipid parameters and glycaemic control in these women. The study design was a randomised double-blind, placebo-controlled trial. Subjects were randomised to receive either combined continuous HRT (conjugated equine oestrogen $0.625 \mathrm{mg} /$ day and medroxyprogesterone acetate 2.5 $\mathrm{mg}$ /day) or placebo. Fasting blood samples were drawn at $0,+13$ and +26 weeks, and analysed for lipoprotein profile, $\mathrm{LP}(\mathrm{a})$, fibrinogen, PAI-1, glucose and $H B A_{I C}$. 61 subjects (mean age 64 years) enrolled into the study, 32 in the placebo group and 29 in the HRT group. At baseline there were no significant differences in any variable between the 2 groups. 8 subjects did not complete the study period (1 placebo, 7 HRT). Total cholesterol levels at +26 weeks were $6.76 \pm 1.21 \mathrm{mmol} / 1$ in the placebo group and $5.59 \pm 0.97 \mathrm{mmol} / /$ in the HRT group $(\mathrm{p}<0.001)$. LDL cholesterol was $4.64 \pm 1.12 \mathrm{mmol} / \mathrm{l}$ in the placebo group and $3.52 \pm 0.78$ $\mathrm{mmol} / \mathrm{l}$ in the HRT group $(\mathrm{p}<0.001)$. There were no significant differences in HDL cholesterol, triglyceride, $\mathrm{Lp}(\mathrm{a})$, fibrinogen, PAI- 1 , fasting glucose or $\mathrm{HbA}_{\mathrm{IC}}$. Patient weight increased significantly in the HRT treated group. In conclusion, combined, continuous HRT use in postmenopausal women with Type 2 diabetes results in a significant reduction in total and LDL cholesterol without impairing glycaemic control or elevating triglyceride levels. Tolerability of HRT remains a problem.

\section{8}

LIPID SOLUBLE ANTIOXIDANT VITAMIN STATUS IN IDDM PATIENTS WITH DYSLIPIDAEMIA

A. Somogyi ${ }^{1}$ E. Szaleczky ${ }^{1}$, P. Puszai ${ }^{1}$, K. Farkas ${ }^{2}$ and B. Sámán ${ }^{1}$.

${ }^{\prime} 2^{\text {nd }}$ Department of Medicine, Semmelweis School of Medicine, , ${ }^{2}$ Bajcsy-Zsilinszky Hospital, Budapest, Hungary

Previous studies have shown that metabolic diseases and their co-occurrence can alter the antioxidant defence system including antioxidant vitamins of plasma. In order to assess the antioxidant vitarmin status, 24 insulin-dependent diabetic patients and 33 matched control subjects were examined. Vitamin $\mathrm{E}$ and A concentrations were determined using HPLC method. The patients were divided into hyper/dyslipidaemic (with hypercholesterinaemia hypertrigliceridaemia high $L p(a)$ level, hyper-apoß-lipoproteinaemia atherogen dyslipidaemia, presence of small dens LDL) or normolipidaemic and diabetic or non-diabetio sibgroups. I. Piasma vitamin $A$ and $E$ levels of the hyper/dyslipidaemic group ( $n=37)$ were significantly $(\mathrm{p}<0.05)$ higher than those of nomolipidaemic subjects $(\mathrm{n}=20)(2.20 \pm 0.57 \mathrm{v}$ $1.74 \pm 0.25 \mu \mathrm{mol} /$, and $33.67 \pm 9.82$ is $23.91 \pm 3.82 \mu \mathrm{mol} / \mathrm{x}+\mathrm{SD})$. Calculating vitamin/cholesterol ratios. vitamin E/cholesterol ratio was similar in the two groups, while vitamin $\mathrm{A}$ cholesterol ratio showed a higher value in the normolipidaemic group $(0.42 \pm 0.07 \mathrm{w}$ $0.37 \pm 0.11) .2$. Comparing these subjects as diabetic $(n=24)$ and non-diabetic $(n=33)$ groups no significant difference could be found in vitamin $E$ levels, whereas vitamin A level was considerably lower in the diabetic group ( $1.79+0.41 \mathrm{vs} 2.26+0.52 \mu \mathrm{mol} /$ ). The results were the same considering both vitamin/cholesterol ratios. 3. Plasma vitamin A level of hyper/dyslipidaemic diabetic $(\mathrm{n}=13)$ and normolipidaenic non-diabetic $(\mathrm{n}=10)$ subjects did not differ significantly, while a higher vitamin A/cholesterol ratio was found in the metabolically healthy group $(0.45 \pm 0.07$ us $0.32 \pm 0.11)$. In contrast, plasma vitamin $E$ level in the dyshyperlipidaemic diabetic group was significantly higher $(36.5 \pm 5.2$ vs $25.62 \pm 3.80 \mu \mathrm{mol} /)$ with a disappearing difference in vitamin $\mathrm{E} /$ cholesterol ratio. Plasma vitamin $\mathrm{E}$ concentration does not differ between type 1 diabetic patients and healthy controls even when they suffer from hyperlipidemia. However plasma vitamin A concentration is significantly decreased in diabetic patients regardless of their lipid levels.

\section{9}

VITAMIN E CONCENTRATION IN HIPERLIPIDEMIA AND IN IDDM

P. Pusztai, B. Sármán E Szaleczky, J. Molnár, and A Somogyi

$2^{\text {txd }}$ Department of Medicine, Semmetweis University of Medicine, Budapest, Hungary

Lipid-soluble vitamin $\mathbf{E}$ plays an important protective role against oxidative damage to lipoproteins. The authors have measured vitamin $\mathrm{E}$ concentration in plasma samples and HDL and VLDL-LDL lipoprotein fractions of 40 non-diabetic controls $(C)$ and 25 type I diabetic patients (DM) The groups were adjusted for age (C. $38.0 \pm 10.2$ years; DM: 35. $1+2.7$ years), sex (C: 19 males, 21 females, DM: 11 males, 14 females), and body mass index (C: $24.83 \pm 4.82: \mathrm{DM}: 23.35 \pm 3.81 \mathrm{~kg} / \mathrm{m} 2$ ). Mean $\mathrm{HbAl}_{\mathrm{C}}$ value in $\mathrm{C}: 5.16 \pm 0.4 \%$, in DM: $7.89 \pm 2.53 \%$. The mean duration of diabetes was $10.06 \pm 7.95$ years. Vitamin $\mathrm{E}$ was measured by HPLC. Results 3 . Vitamine E had higher plasma concentration in subjects with hyperlipidemia in the control group (CH); and in the group of diabetic patients $(\mathrm{DMH})$ as compared to those with normolipidemia $(\mathrm{CN} ; \mathrm{DMN})(\mathrm{CH}: 32.96 \pm 4.34 \mathrm{CN}: 25.14 \pm 3.28 \mu \mathrm{M}$, $\mathrm{DMH}: 38.54 \pm 15.75 \mathrm{DMN}: 25.75 \pm 5.50 \mu \mathrm{M})$. 2. When vitamin $\mathrm{E}$ concentrations were corrected for serum cholesterol, the ratio was reversed $(\mathrm{CH} .5 .32+0.86 \mathrm{CN} .5 .92 \pm 0.94 \mu \mathrm{M}$, DMH: $5.82 \pm 1.24 \mathrm{DMN}: 5.93 \pm 1.04 \mu \mathrm{M}) 3$. HDL vitamin $\mathrm{E}$ content did not differ between normo- and hyperlipidemic subjects (CN: $10.5 \pm 2.1 \mathrm{CH}: 9.98 \pm 3.04 \mu \mathrm{M}, \mathrm{DMN}: 10.54 \pm 2.53$ $\mathrm{DMH}: 12.44+3.31 \mu \mathrm{M}$ ). 4. Vitamin $\mathrm{E}$ concentration in VLDL-LDL fraction was higher in hyperlipidemic subjects $(\mathrm{CH}: 22.98 \pm 4.48 \quad \mathrm{CN} 14.64 \pm 3.12 \mu \mathrm{M}, \mathrm{DMH} 26.10 \pm 14.65$ $D M N: 15.21 \pm 4.71 \mu M$ ). In controls: when Vitamin $E$ of the VLDL-LDL fraction was corrected for cholesterol in that lipoprotein fraction, it was higher in nomolipidemic subjects $(\mathrm{CH}: 66+0.98, \mathrm{CN} \cdot 5.47 \pm 1.30 \mu \mathrm{M})$ In diabetic patients: Vitamin $\mathrm{E}$ concentration is significantly higher in VLDL-LDL fraction of hyperlipidemic subjects. This difference disappears when vitamin E concentration is corrected for cholesterol (DMN:5.34 \pm 2.31 DMH $5.46 \pm 1.56 \mu \mathrm{M}$ ). Conclusion: In the plasma of non-diabetic hyperlipidemic subjects, vitanin $E$ concentration is lower that in normolipidenic subjects when it is corrected for the faction's cholesterol content. In HDL and VLDL-LDL fractions of diabetic patients, vitamin $E$ does not differ between normo- and hyperlipidemic subjects when it is corrected for the fractions' cholesterol content, but cholesterol-corrected antioxidant vitamin $\mathrm{E}$ remained low in VLDL-LDL fractions even when the diabetic subject suffers from hyperlipidemia
1350

\section{ANTIOXIDANT VITAMIN A STATUS IN IDDM AND} HYPERLIPIDAEMIA

K. Farkas ${ }^{1}$, B. Sármán ${ }^{2}$, J. Molnár ${ }^{2}$, 1. Kocsis ${ }^{2}$ and A. Somogyi' ${ }^{2}$ 'Bajcsy-Zsilinszky Hospital, ${ }^{2} 2^{\text {nd }}$ Department of Medicine, Semmelweis School of Medicine, Budapest, Hungary.

In order to assess the antioxidant vitamin status, 25 IDDM patients (19 males, 21 females, age $38.0 \pm 10.2$ years; body mass index: $24.83 \pm 4.82 \mathrm{~kg} / \mathrm{m}^{2}, \mathrm{X} \pm \mathrm{SD}$ ) and 40 matched control subjects were examined. Using HPLC method vitamin A concentrations of the plasma, HDL and VLDL-LDL lipid fractions were compared grouping them according to their lipid status (normo- and hyperlipidaemic subgroups). Comparing the hyper- and normolipidaemic control groups, vitamin A concentration of the plasma was significantly $(p<0.05)$ higher in the former group $(2.44 \pm 0.52 v 2.08 \pm 0.43 \mu \mathrm{mol} / \mathrm{l})$, whereas vitamin $A /$ cholesterol ratio was higher in the normolipidaemic group $(0.39+0.10$ vs $049+0.11)$. Vitamin A concentration of the VLDL-LDL fraction of hyperlipidaemic control subjects was significantly higher $(0.26 \pm 0.08$ is $0.18 \pm 0.07 \mu \mathrm{mol} / \mathrm{l})$ with a disappearing difference in vitamin $\mathrm{A} /$ cholesterol ratio of the fractions. Vitamin A concentration and vitamin A/cholesterol ratio of the HDL fraction of hyperlipidaemic controls were found to be higher than those of normolipidaemic subjects $(2.17 \pm 0.48$ vs $1.89 \pm 0.36$ and $1.86 \pm 0.70 v s \quad[.38 \pm 0.57 \mu \mathrm{mol} / \mathrm{l})$. Plasma concentration of vitamin $\mathrm{A}$ in normo- and hyperlipidaemic diabetic groups did not differ considerably ( $1.65 \pm 0.32$ vs $2.09 \pm 0.07$ $\mu \mathrm{mol} / \mathrm{l})$, while vitamin $\mathrm{A} /$ cholesterol ratio was significantly lower in the hyperlipidaemic group $(0.23 \pm 0.10$ vs $0.39 \pm 0.10)$. Vitamin A concentration of the VLDL-LDL fraction of hyperlipidaenic IDDM-patients was significantly higher than that of normolipidaemic patients $(0.29 \pm 0.01 \mathrm{v} 0.14 \pm 0.06 \mu \mathrm{mol} / \mathrm{l})$ again with $a$ disappearing difference in vitamin A/cholesterol ratio of the fractions. Vitamin A concentration and vitamin A/cholesterol ratio of the HDL fraction were not different in the normo-and hyperlipidaemic diabetic groups. In conclusion, hyperlipidaemia in both diabetic and healthy control subjects was associated with a lower plasma vitamin $\mathrm{A} /$ cholesterol ratio suggesting an impaired antioxidant defence system. 
EFFECT OF VITAMIN E SUPPLEMENTATION ON THE PEROXIDABILITY OF RED BLOOD CELL MEMBRANES FROM TYPE I DIABETIC PATIENTS

B. Manuel y Keenoy, H.L. Shen, W. Engelen, J. Vertommen and I. De Leeuw. Dept.Endocrinology, Univ. Antwerp, B-2610 Antwerp, Belgium.

Supplementation with antioxidants has been proposed as a complementary therapy to prevent and/or delay long term complications of diabetes and several clinical studies have shown the beneficial effects of vitamin $E$ supplementation on lipid profiles, lipoprotein peroxidability and protein glycation in diabetics. In this study we concentrate on the effects of vitamin $E$ on cell membrane lipid peroxidation. For this purpose 44 diabetics in good metabolic control and without major complications were randomised into 2 groups : group S received dl- $\alpha$-tocopherol (250 IU 3 times daily) and group $P$ received placebo in a double blind basis. Red blood cell membranes were isolated from each patient at inclusion (visit 1), after 3 months (visit 2) and after 6 months (visit 3) and lipid peroxidability was monitored by the production of TBARS (thiobarbituric reactive substances) and fluorescent products induced by an in vitro incubation with $2 \mathrm{mM}$ tert-butyl hydroperoxide (t-bOOH). At inclusion both goups were comparable as regards patient characteristics and membrane peroxidability. In group $\mathrm{S}$ the increase in serum vitamin $\mathrm{E}$ from a median of $14.8 \mathrm{\mu g} / \mathrm{ml}$ (range $9.7-27.1$ ) in visit 1 to $30.3(14.9-46.6)$ in visit $2(\mathrm{p}<0.001$ ) and $25.2(10.7-39.3)$ in visit $3(\mathrm{p}<0.005)$ was accompanied by a decrease in $\mathrm{t}-\mathrm{bOOH}$ induced TBARS from $2.03 \mathrm{nmol}$ MDA equivalents/mg protein $(0.40-3.26)$ in visit to $1.31(0.26-6.36)$ in visit $2(\mathrm{p}<0.05)$ and $1.29(0.21-5.97)$ in visit 3 . Although TBARS were not related to serum vit $E$ at inclusion, the increase in serum vit $E$ correlated with the decrease in TBARS in visit $3(\mathrm{r}=-0.60 \mathrm{p} 0.02)$. Lagtime of fluorescence formation increased from $20 \mathrm{~min}(9-68)$ in visit 1 to 23 (11-83) in visit 2 to $35(12-54)$ in visit 3 (ns). However, slope of fluorescence: $0.27 \mathrm{FU} / \mathrm{min}(0.09-1.40)$ and membrane thiol content : $150 \mathrm{nmol}$ GSH equiv/mg protein (124-190) as well as tbOOH-induced decrease in thiols: 42 (33-94) and fatty acid composition did not change significantly. In group $\mathbf{P}$ these parameters remained stable. These results show that supplementation with vitamin $\mathrm{E}$ is effective in decreasing $\mathrm{RBC}$ membrane susceptibility to $\mathrm{t}-\mathrm{bOOH}$ induced in vitro peroxidation and that this effect is mediated by the increase in vitamin $\mathrm{E}$ levels rather than by any changes in fatty acid composition or membrane thiol content. The relevance of decreasing membrane peroxidability in the prevention of complications should be further investigated.

\section{PS 75}

\section{Cardiovascular Risk Factors and Mortality}

\section{2}

INCIDENCE OF ACUTE CORONARY HEART DISEASE (CHD) AND STROKE EVENTS IN CHILDHOOD-ONSET IDDM

C.Sarti, J.Tuomilehto, K.Borch-Johnsen, T.Forsen, A.Molarius and A.Reunanen. National Public Health Institute, Helsinki, Finland.

The aim of this prospective analysis was to determine the incidence of CHD, stroke and any cardiovascular disease (CVD) in a populationbased cohort of 5148 young-onset IDDM patients diagnosed with IDDM at the age of 18 years or under. The effect of age at onset of IDDM, attained age, gender, diabetes duration and of the presence of diabetic nephropathy (DN) on the risk of these CVD events was evaluated. A total of $159 \mathrm{CVD}$ events (58 CHD, 57 strokes) were diagnosed during the follow-up of 10-24 years. There were no cases of CVD diagnosed before 12 years' duration of IDDM. In IDDM patients with $\mathrm{DN}$ the cumulative incidence of $\mathrm{CHD}$, stroke and $\mathrm{CVD}$ by the age of 40 years was $13 \%, 20 \%$ and $43 \%$, respectively, compared with $4 \%, 2 \%$ and $7 \%$ in non-DN IDDM patients, similarly in men and women. In DN-IDDM patients compared with non-DN IDDM patients the relative risk of $\mathrm{CHD}$ was 10.3 for $\mathrm{CHD}, 10.9$ for stroke and 10.0 for any CVD. The event rates were similar in men and women by the duration of diabetes and attained age. The risk of CVD increased steadily by $2.3 \% / y e a r$ after the diagnosis of DN. The-at-onset of IDDM did not influence the risk of CVD when the duration of IDDM and DN status were controlled for. $\mathrm{DN}$ is the main determinant of acute CVD events in young-onset IDDM patients. The risk of CHD and stroke in IDDM patients is similar for men and women.

\section{3}

INFLUENCE OF NON-INSULIN DEPENDENT DIABETES MELLITUS ON SHORT-TERM SURVIVAL AFTER STROKE. A PROSPECTIVE STUDY. C. Bambini, G. Seghieri, F. Tomei, A. Rapuano, and A. Valicenti. Dpt. of Internal Medicine, Hospital of Viareggio, Viareggio, Italy.

Diabetic patients have a worse long-term prognosis after stroke than control nondiabetic subjects. Whether presence of diabetes may worsen prognosis early after hospitalisation, and whether mortality is associated to diabetes or to other confounders such as age, sex, hypertension, or dyslipidaemia are the questions raised by this study. All patients who were consecutively admitted to Hospital with diagnosis of both ischemic or haemorragic stroke, confirmed with standardised clinical and CT criteria, during the period 1.1.1996 - 31.12.1996, were admitted to the study. In all cases prognosis (mortality) was then recorded during the period of 12 months after stroke. A group of 211 patients affected with stroke (106 males and 95 females), aged $74 \pm 11$ (SD) years, was admitted to the medical wards of our Hospital, which represents the unique medical resource for this kind of illness from a 100,000 inhabitants' area. During this period 18 deaths among males and 19 deaths among females were observed (one-year-survival rates: $83 \%$ and $80 \%$ respectively). In front of a similar prevalence of non-insulin dependent diabetes mellitus (NIDDM) in both sexes ( $43 \%$ in men and $34 \%$ in women), the one-yearmortality rate was about two-threefold higher in the group of diabetic patients among females $(31.43 \%$ vs $13.33 \%$ ), while similar mortality rates were observed between diabetic and non diabetic subjects in the male group $(17.4 \%$ vs $16.7 \%)$. After Kaplan-Meier life table technique, the mortality incidence was significantly higher in diabetic patients only in the female group $(\mathrm{p}=0.03$; log-rank test). This latter association was further confirmed only in the group of female patients, by Cox regression analysis, using age, blood pressure, hematocrit, smoking habit, serum cholesterol and creatinine as covariates $(p=0.04)$. In conclusion, according to these data, NIDDM is a strong independent predictor of short-term mortality after stroke only in the females. 


\section{4}

CARDIOVASCULAR MORBIDITY AND MORTALITY IN DIABETIC PATIENTS IN THE NORTH OF PORTUGAL

C. Neves, J.L. Medina, J.P. Lima-Reis, F. Lopes and P. Melo. Endocrinology Unit and Statistics Service. S. Joăo Hospital, University of Oporto, Portugal.

The aim of our study was to evaluate the incidence of and mortality from cardiovascular disease (CVD) in diabetic and nondiabetic patients admitted to a central hospital in the North of Portugal between 1989 and 1996. We retrospectively analysed data from all patients aged 25 years or older who satisfied the International Classification of Diseases (9th version) criteria for CVD (ICD9 codes 390 - 459). Results are expressed as means + SD or percentages. Statistical analysis was performed with Student's 1 -test, $\chi^{2}$ test or Fisher exact test. A two-tailed $p$ value $<0.05$ was considered significant. Diabetic patients [42.5\% men (M) and $57.5 \%$ women $(\mathrm{M})]$ were significantly older than nondiabetic patients (48.4\% $M$ and $51.6 \%$ W $(67.8+5.6$ vs 61.8 \pm 7.5 years, $p<0.01$ ). The duration of hospitalization was significantly higher in diabetic patients than in nondiabetic patients $(19.0 \pm 14.7$ vs $14.7 \pm 9.7$ days, $p=0.003$ ). The incidence of fatal and nonfatal cardiovascular events was $18.5 \%(n=4630)$ in diabetic patients and $12.6 \%(n=27989)$ in nondiabetic patients. Diabetic patients had an incidence of cerebrovascular disease $(8.5 \%)$, coronary heart disease $(5.9 \%)$ and peripheral vascular disease $(0.7 \%)$ that was not significantly different from that in nondiabetic patients $(5.3 \%, 4.5 \%, 0.6 \%$, respectively). There were no significant differences in the incidence of cardiac heart failure and cardiogenic shock in diabetic and nondiabetic patients $(3.4 \%$ vs $2.2 \%$ ). There was an excess of cardiovascular deaths among the diabetic patients but this was not significant $(36.7 \%$ vs $25.1 \%)$. The mortality rate after myocardial infarction (MI) was significantly higher in diabetic patients than in nondiabetic patients $(10.3 \%$ vs $3.1 \%, p=0.04)$. In conclusion, the high mortality rate of diabetic patients after CVD, particularly after MI, imply that primary and secondary preventive strategies should become an integral part of their medical care.

\section{6}

PREDICTORS OF 10-YEAR MORTALTY IN PATIENTS WITH NONINSULIN-DEPENDENT DIABETES MELITUS

M.A. Gall', E. Knudsen', P. Hougaard', K. Borch-Johnsen' and H.-H. Parving1. 'Steno Diabetes Center, Gentofte, ${ }^{2}$ Novo Nordisk ASS, Bagsvaerd, Denmark. We prospectively evaluated the impact of micro- and macroalbuminuria and other putative risk factors for mortality in 549 (296 M/253 F) non-insulindependent diabetic (NIDDM) patients. Definitions: normoalbuminuria (urinary albumin excretion rate (UAE) $30 \mathrm{mg} / 24 \mathrm{~h}$ ) microalbuminuria (UAE $30-299$ $\mathrm{mg} / 24 \mathrm{~h}$ ), macroalbuminuria (UAE $\geq 300 \mathrm{mg} / 24 \mathrm{~h}$ ). Mean age at entry was $59 \pm$ $\mathrm{mg} / 24 \mathrm{~h}$ ), macroalbuminuria (UAE $\geq 300 \mathrm{mg} / 24 \mathrm{~h})$. Mean age at entry was $59 \pm$
11 (SD) years. During the 10 -year follow-up period, $34 \%(111 / 323)$ of patients with normoalbuminuria, $59 \%(89 / 151)$ with microalbuminuria, and $69 \%(52 / 75)$ with macroalbuminuria had died, $p<0.001$ (normoalburminuria vs micro- and macroalbuminuria) and $p=0.05$ (microalbuminuria vs macroalbuminuria). 132 patients died from cardiovascular disease (CVD) 18 from uraemia 24 from cancer, 19 from infections, and 59 from other/unknown causes. CVD mortality
rate per 100 person-years of observation was $1.8,4.6$, and 5.1 in patients with normo-, micro-, and macroalbuminuria, respectively, p <0.001 (normoalbuminuria vs micro- and macroalbuminuria) and $\mathrm{p}=\mathrm{NS}$ (microalbuminuria vs macroalbuminuria). Multivariate Cox proportional hazards model, after stepwise exclusion, revealed the following predictors of death from all causes and CVD:

\begin{tabular}{|c|c|c|}
\hline Variable & \multicolumn{2}{|c|}{$\begin{array}{l}\text { Mortality (relative risk (95\% CI)) } \\
\text { Allcause }(\mathrm{N}=252) \mathrm{CVD}(\mathrm{N}=132)\end{array}$} \\
\hline 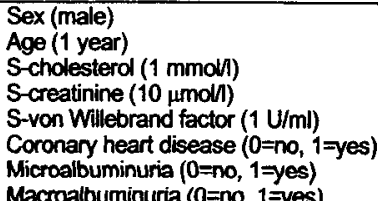 & \begin{tabular}{|l|}
$1.42(1.07-1.90)$ \\
$1.08(1.06-1.10)$ \\
$1.16(1.07-1.25)$ \\
$1.04(1.02-1.07)$ \\
$1.19(1.03-1.37)$ \\
$2.16(1.65-2.82)$ \\
$1.80(1.35-2.40)$ \\
$2.20(1.52-323)$
\end{tabular} & $\begin{array}{c}1.76(1.19-2.59) \\
1.09(1.06-1.11) \\
1.24(1.14-1.35) \\
- \\
- \\
3.08(2.14-4.41) \\
2.75(1.87-4.05) \\
2.78(170-456)\end{array}$ \\
\hline
\end{tabular}

In conclusion: abnormally elevated UAE and elevated s-cholesterol level, both potentially modifiable risk factors, indicate a substantially increased all-cause, mainly CVD, mortality risk in NIDDM patients.

\section{5}

PLASMA FIBRINOGEN LEVELS AND MORTALITY IN NON-INSULINDEPENDENT DIABETES MELLITUS

E. Knudsen, M.A. Gall, P. Rossing, F. S. Nielsen and Hans-Henrik Parving Steno Diabetes Center, Gentofte, Denmark

Elevated levels of $p$-fibrinogen is an independent risk factor for cardiovascular disease (CVD) in the general population As non-insulin-dependent diabetic (NIDDM) patients suffer increased morbidity and mortality from CVD, we performed a prospective study to evaluate the impact of p-fibrinogen level on mortality in 192 Caucasian NIDDM patients <70 years of age, followed for a median of 6.4 (range $0.2-7.1$ ) years. Normoalbuminuria (NOR, urinary albumin excretion (UAE) $<30 \mathrm{mg} / 24 \mathrm{~h}$ )) was present in $53 \mathrm{M} / 44 \mathrm{~F}$, microalbuminuria (MIC, UAE 30-299 mg/24 h) in $31 \mathrm{M} / 23 \mathrm{~F}$, and macroalbuminuria (MAC, UAE $\geq 300 \mathrm{mg} / 24 \mathrm{~h}$ ) in $34 \mathrm{M} / 7 \mathrm{~F}$ patients at baseline. S-creatinine increased with increasing levels of albuminuria: NOR (71 (43-143)) (median (range)), MIC (79 $(48-170)$ ), and MAC $(90(54-539)) \mathrm{mmol} / \mathrm{l}$, respectively ( $p<0.001)$. The level of p-fibrinogen increased with increasing levels of albuminuria: NOR (8.5 (4.5 14.2)), MIC (8.8 (6.1-16.7)), and MAC (10.0 (5.7-16.9)) mmol/I, respectively $(p<0.001)$. Ejght percent $(n=8)$ of NOR patients, $28 \%(n=15)$ of MIC patients and $54 \%(n=22)$ of MAC patients died during follow up: 25 from CVD, 3 from uraemia, 17 from other/unknown causes. Univariate Cox regression analysis revealed age-adjusted p-fibrinogen level to predict all-cause mortality: pfibrinogen (1 mmol/l) $122(1.09-1.38)$ (relative risk $(95 \% \mathrm{Cl})$ ) $(p<0.005)$ but not CVD mortaly: P-fibrinogen (1 mmoll), $1.09(0.92-1.30)$ ) (p=0.31 not CVD (1 proportional hazards model, after stepwise exclusion, revealed the following independent predictors of all-cause mortality

\begin{tabular}{||l|l|l||}
\hline Variable & Relative risk $(95 \% \mathrm{Cl})$ & p-value \\
\hline LogioUAE (factor 10) & $2.05(1.40-3.02)$ & $<0.005$ \\
Coronary heart disease & $2.00(1.05-3.82)$ & $<0.05$ \\
(0=no, 1=yes) & $1.07(1.02-1.13)$ & $<0.005$ \\
Age $(1$ year) & $1.01(1.00-1.01)$ & $<0.005$ \\
S-creatinine $(1$ mmol/l) & & \\
\hline
\end{tabular}

Our study revealed a progressive rise in p-fibrinogen with increasing UAE in NIDDM patients. The importance of $p$-fibrinogen level as an independent risk factor for mortality in NIDDM remains to be established.

\section{7}

INCREASED MORTALITY IN NIDDM PATIENTS ON ANTIHYPERTENSIVE TREATMENT WITH CALCIUM CHANNEL BLOCKERS A. Melander ${ }^{1,2}$, G. Lindberg', J. Olsson', J. Ranstam ${ }^{1}$ and L. Råstam ${ }^{2}$. 'The Swedish Network for Pharmacoepidemiology (NEPI), Malmö University Hospital and ${ }^{2}$ Department of Community Medicine, Lund University, Malmö, Sweden.

The safety of calcium channel blockers, especially in patients with NIDDM, has been questioned. Therefore, we investigated the mortality of NIDDM patients using calcium channel blockers, or beta blockers, or both, as antihypertensive medication. A cohort study was performed on data from NIDDM patients who between 1985 and 1994 were residents of two towns (population $\sim 46,000$ ) in southern Sweden. Patients were followed from the date of their first prescription of either drug in 1985 or later. Patients who had used both types of drugs between 1984 and 1994 were evaluated separately. They were included when the second drug was prescribed. All patients were followed until the end of 1995 or until death. Differences in mortality were calculated using the Proportional Hazard's method. The crude relative mortality risk in users of calcium channel blockers was 2.00 (95\% confidence interval 0.99-4.03) vs. users of beta blockers. After multivariate adjustment for characteristics at inclusion, the relative mortality risk was 2.26 (1.05-4.87). These results suggest an elevated overall mortality risk in NIDDM patients using calcium channel blockers as antihypertensives. The influence from comorbidity on the relation will be evaluated. 


\section{HYPERINSULINAEMIA PREDICTS CARDIOVASCULAR MORBIDITY AND MORTALITY IN NON-DIABETIC MEN}

P Nilsson 1 , B Hedblad ${ }^{1,2}$, K-F Eriksson', and G Berglund ${ }^{1}$. From the ${ }^{2}$ Department of Medicine, and the ${ }^{2}$ Department of Community Medicine, University of Lund, Malmö University Hospital, S-205 02 Malmö, Sweden. Prospective studies have indicated that hyperinsulinaemia is a risk factor for ischaemic heart disease in middle-aged, caucasian males, even corrected for metabolic disturbances. However, no study so far has investigated the role of insulin for the prediction of total mortality, which was the aim of this study. We collected data on 6.074 non-diabetic males (mean age 47 years), with no cardiovascular disease at baseline, as part of a screening investigation between 1974-1984 in Malmö, Sweden. Investigations included a medical history, clinical characteristics (weight, height, blood pressure), and the measurement of fasting serum lipids (cholesterol, triglycerides), blood glucose and serum insulin. Mean follow-up time was 16.5 years for first ischaemic cardiac event and total mortality, based on data derived from local as well as national registers. Mean levels of fasting insulin was 10.5 (SD: 9.3; median 8.0) $\mathrm{mU} / \mathrm{L}$. Subjects were divided into decentiles of fasting insulin levels. A hyperinsulinaemic group, defined as having the highest decentile of insulin (22-140 mU/L), comprised 539 subjects with at total of 75 cardiac events (8.8/1.000 person-years) during the follow-up time. They were compared to the rest of the men, comprising 5.535 subjects (insulin $1-21 \mathrm{mU} / \mathrm{L}$ ) with 422 events (4.7/1.000 person-years) during follow-up. Corresponding figures for deaths were 100 deaths $(11.7 / 1.000$ person-years $)$ in the top decentile group, compared to 630 deaths (7.0/1.000 person-years) in the rest of the men. The differences between groups in unadjusted incidence rates were significant $(p<0.001)$. Fasting insulin concentrations at baseline predicted the subsequent development of ischaemic cardiac events with an odds ratio of $1.32(95 \% \mathrm{Cl}: 1.00-1.73 ; \mathrm{p}<0.05)$, and total mortality OR:1.33 (1.05-1.67; $<0.05)$, even after the adjustment for covariates such as age, hypertension, hyperlipidaemia, body mass index, fasting blood glucose, and smoking habits in Cox proportional hazard models. In conclusion, fasting hyperinsulinaemia is an independent risk factor for ischaemic cardiac events and total mortality, adjusted for the traditional major risk factors, but also for the degree of fasting glycaemia, in non-diabetic men free of cardiovascular disease at baseline.

\section{0}

TYPE 2 DIABETES AND CORONARY ARTERY DISEASE (CAD): CLINICAL, ANGIOGRAPHIC AND MORTALITY DATA

A. Natali, S. Vichi, S. Severi, E. Toschi, A. L'Abbate, E. Ferrannini C.N.R. Coronary Unit, Institute of Clinical Physiology and Department of Internal Medicine, University of Pisa, Pisa, Italy.

To evaluate the impact of diabetes on CAD we reviewed the clinical, angiographic, and follow-up data of all the patients undergoing coronary angiography over the decade 1983-1992 in our Coronary Unit. As compared to non-diabetics ( $n=1,984)$, diabetics $(n=269)$ were: older ( $60 \pm 8$ vs $55 \pm 10 \mathrm{yrs}$ ) and heavier (BMI: $27 \pm 3$ vs $26 \pm 3 \mathrm{~kg} \mathrm{~m}$ $\left.{ }^{2}\right)$, more often female (27 vs $19 \%$ ) or hypertensive (56 vs $38 \%$ ), and less frequently smokers (13 vs $23 \%$ ) ( $\mathrm{p}<0.001$ or less for all). Anginal symptoms and prevalence of previous myocardial infarction (44 vs $38 \%$ ) were similar in the two groups. Abnormal coronaries were more frequently found in diabetics ( $85 v s 67 \%$ ) essentially due to a higher prevalence of 3-vessel disease (30.5 vs 15.1\%, p<0.0001). The sum of all angiographically detectable lumen stenosis (ATS score) was higher in diabetics than in non-diabetics $(352 \pm 232$ vs $211 \pm 201$ units, p< 0.0001 ). After adjusting for age, BMI, total cholesterol, triglycerides, family history of CAD, cigarette consumption, and hypertension, diabetes was associated with +119 ATS units in men and +145 ATS units in women. No association was found between ATS score and disease duration, treatment or in-hospital plasma glucose. After a mean follow-up of 88 months, $18.6 \%$ diabetics and $9.7 \%$ nondiabetics died of a cardiac cause. By Cox analysis, after accounting for other risk factors diabetes was still associated with $22 \%$ greater risk of cardiac death [CI:14-56\%]. When the ATS score was entered into the model, diabetes lost its independent predictive power. We conclude that diabetes is associated with more severe coronary atherosclerosis, which largely explains the shorter life expectancy of diabetic patients referred to a Coronary Unit.
Effects of Alcohol Consumption on Mortality in Diabetic Subjects

B. Müller, A. Teuscher, P. Diem

Bern, Switzerland.

Recent studies in the general population have shown an improvement in mortality with moderate alcohol intake.

To test whether similar protective effects of alcohol can be seen in diabetes mellitus we followed a cohort of 200 diabetics (age $45.5 \pm 0.4$ years $\mathrm{m}+\mathrm{SEM}$; gender: $104 \mathrm{f}, 96 \mathrm{~m}$; duration of diabetes $10.0 \pm 0.5$ years) over $11.2 \pm 0.2$ years. Three patients were iost for follow up. The underlying cause of each death was determined from a copy of the death certificate, autopsy and terminal illness reports, and other medical records. Survival analysis was performed using the Cox proportional hazards model with survival time as the dependent variable in the regression model. After adjustment for age and duration of diabetes, alcohol consumption was associated with slightly (but statistically not significantly) increased all-cause mortality:

$\begin{array}{llll} & \operatorname{Exp}(\mathrm{Coef}) & 95 \%-\mathrm{CI} & \mathrm{p} \\ \text { Age } & 1.068 & 1.013-1.125 & 0.0144 \\ \text { Duration of diabetes } & 1.103 & 1.034-1.177 & 0.0030\end{array}$

Alcohol consumption $\quad 1.013 \quad 0.998-1.028 \quad 0.0930$

After additional adjustment for coronary heart disease, alcoho consumption significantly increased all-cause mortality:

$\operatorname{Exp}$ (Coef) 1.016 (95\%-CI: 1.001-1.031), $\mathrm{p}=0.0411$.

Even in patients with moderate alcohol comsumption $(1-12 \mathrm{~g} / \mathrm{d})$ all-cause mortality risk ratio was significantly increased:

RR 2.073, 95\%-CI: 1.214-3.539, $\mathrm{p}=0.0116$.

Conclusion: In contrast with data obtained in the general (mainly non-diabetic) population, this study shows that the risk of death from all causes is increased in diabetics consuming alcohol.

\section{1}

HYPERTRIGLYCERIDEMIA AS A RISK FACTOR FOR CARDIOVASCULAR DISEASE.

L.D. Monti, S. Allibardi, P.M. Piatti, G Valsecchi, S Costa V.C. Phan, G. Pozza and M. Samaja. Istituto Scientifico H San Raffaele, University of Milan, Milan, Italy

It is well known that hypertriglyceridemia is an independent risk factor for cardiovascular disease but the related pathogenetic mechanisms need further investigation. Aim of the study was to investigate the role of increased triglyceride levels under condition of costant hyperglycemia $(12 \mathrm{mmol} / \mathrm{h})$ and hyperinsulinemia $(200 \mu \mathrm{U} / \mathrm{ml})$ in Langendorff-perfused isolated rat heart. Variable amounts of $20 \%$ Intralipid were added to the perfusion buffer to obtain triglyceride levels of $0(\mathrm{~N}=9)$; $1200(\mathrm{~N}=9) ; 2200(\mathrm{~N}=7) ;$ e $4000 \mathrm{mg} / \mathrm{dl},(\mathrm{N}=6)$. All hearts were stabilized for $20 \mathrm{~min}$ at flow $=15 \mathrm{ml} / \mathrm{min}$ at the different metabolic conditions for baseline measurements. Then, hearts underwent low-flow ischemia for $60 \mathrm{~min}$ by reducing flow to 1.5 $\mathrm{ml} / \mathrm{min}$, followed by $30 \mathrm{~min}$ reperfusion under the same conditions of baseline. At baseline, the heart rate (HR), the myocardial contractile work (LVDPxHR), the end-diastolic pressure (EDP) and the coronary perfusion pressure (CPP) were not influenced by the increased triglyceride levels. In the post-ischemic period, there was

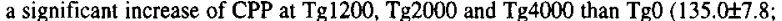
$137.5 \pm 8.6 ; 151.8 \pm 12.3$ vs $98.1 \pm 6.4 \mathrm{mmHg} ; \mathrm{p}<0.005)$. EDP was signifcantly higher at $\mathrm{Tg} 4000$ than $\mathrm{Tg0}(50.3 \pm 10.7$ vs $17.1 \pm 4.3 \mathrm{mmHg} ; \mathrm{p}<0.05)$. The percentages of recovery for LVDPxHR were $82.1 \pm 7.0 \%$ at $\mathrm{Tg} 0,95.1 \pm 6.4 \%$ at $\mathrm{Tg} 1200,70.0 \pm 13.0 \%$ at $\mathrm{Tg} 2200$ and $51.5 \pm 6.3 \%$ at $\mathrm{Tg} 4000$ ( $<<0.02 \mathrm{Tg} 4000$ vs $\mathrm{Tg} 0$ and $\operatorname{Tg} 1200$ ). There was a significant correlation between triglyceride levels and LVDPXHR $(r=0.46, p<0.01)$; between triglyceride levels and post-ischemic EDP $(r=0.54, p<0.002)$; and between triglyceride levels and post-ischemic CPP $(r=0.61$, $\mathrm{p}<0.0003$ ).

These data suggest that hypertriglyceridemia is a severe negative factor for the recovery of myocardial contractile work after ischemia. 
1362

CLASSES OF BMI AND CARDIOVASCULAR RISK FACTORS IN A POPULATION OF PATIENTS WITH TYPE 2 DIABETES

S.Bo, L. Gentile, V. Ghia

Diabetic Clinic, Hospital of Asti, Italy.

All patients with type 2 diabetes, followed at the Diabetic Clinic of Asti, have been evaluated. They are 2113 subjects, that is $1.6 \%$ of reference population. The patients have been divided according to classes of body mass index (BMI); the first tertile correspond to patients with normal body weight $(\mathrm{BMl}<26.1$ $\left.\mathrm{kg} / \mathrm{m}^{2}\right)$; the second to overweight $\left(\mathrm{BMI} \geq 26.1\right.$ and $\left.<30.1 \mathrm{~kg} / \mathrm{m}^{2}\right)$ and the third to obese subjects $\left(\mathrm{BMI} \geq 30.1 \mathrm{~kg} / \mathrm{m}^{2}\right)$. For each patient age, years of diabetes, mean systolic and diastolic blood pressure, mean glycated haemoglobin $\left(\mathrm{HbA}_{1 \mathrm{c}}\right)$, total and HDL-cholesterol, tryglicerides, uric acid, C-peptide, smoking habits, alcohol consumption, exercise during work and leisure have been evaluated. Systolic and diastolic blood pressure, tryglicerides, total cholesterol, uric acid, $\mathrm{HbA}_{\mathrm{Ic}}$ significantly rose with the increase of BMI, while HDL-cholesterol decreased in patients in the highest terzile of BMl. However, smoking and alcohol consumption, age and years of diabetes also significantly fell with the increase of BMI. The frequences of CAD and peripheral artery disease were not significantly different in the three tertiles of BMI. Age, number of cigarettes, years of smoking, duration of diabetes and low HDL-cholesterol values were significant predictors of macroangiopathic complications in a logistic regression model. It is therefore possible that factors which could not or scarcely be modified (age, years of diabetes, HDL-cholesterol) play a relevant role in the pathogenesis of cardiovascular disease in this population of diabetic patients: nevertheless it is mandatory that type 2 diabetic patients quit smoking.

\section{3}

MICROALBUMINURIA PROGRESSION AS AN EARLY RISK MARKER FOR ATHEROSCLEROSIS IN NIDDM.

$X$. Salgado, J. Ibarra A. Fernandez-Cruz, J.M. Pedrajas, R. Fernandez Durango, M. Ruiz Yagüe, E. Pintor, and R. Patiño. Cardiovascular Preventive Center. Hospital Universitario San Carlos, 28040 Madrid (Spain)

The purpose of this prospective study was to investigate in 53 patients ( 21 $\mathrm{F} / 32 \mathrm{M}$; aged from 42 to 70 years, mean $57.6 \mathrm{yrs}$; duration of diabetes $9 \mathrm{yrs}$ with baseline albumin excretion between $20-200 \mu \mathrm{g} / \mathrm{m}$; serum $\mathrm{Cr}<1.2 \mathrm{mg} / \mathrm{dl}$ and absence of any major chronic disease), whether the rate of progression of microalbuminuria (MA) measured as the absolute yearly change in albumin excretion rate $(\triangle \mathrm{AER}$ ), could be a risk marker for macrovascular disease events (MAV), (MI, sudden death, unstable angina/revascularization, congestive heart failure, stroke, amputation). The patients were followed from January 1, 1989 to December 31, 1996. MA was measured by duplicate RIA overnight urine samples twice per year. Progression of MA ( $\triangle A E R$ ) was assessed in each patient, from the slope of the regression line calculated using the median value of at least four single determinations per year. A baseline conventional risk factors were determined in all patients. 4 AER showed marked inter-individuals variations from -23 to $120 \mu \mathrm{g} / \mathrm{m} / \mathrm{yr}$. Twenty two patients had one or more MAV events. Eight patients died. In stepwise Cox multiple regression analysis the only independent factors significantly associated with risk for MAV were: serum HDL cholesterol levels, [RR ( $95 \%$ $\mathrm{Cl})$ ], $0.35(0.16-0.71)$, previous history of MAV, $5.1(2.1-13.4)$ and $\triangle A E R$ $1.01(1.002-1.02), p \leq 0.02$. Twenty three of the patients $(43 \%)$ with stable or regressing MA $(\triangle \mathrm{AER} \leq 0 \mu \mathrm{g} / \mathrm{m} / \mathrm{yr}$ ) had a MAV incidence rate of $3.7 \mathrm{per}$ 100 person $y r$, while those with a rather rapid progression $(14 / 53,27 \%$. $\triangle A E R>20 \mu \mathrm{g} / \mathrm{m} / \mathrm{yr}$ ) had an incidence rate of 11.1 per 100 person yr. (RR $3.48,1.2-9.8,95 \% \mathrm{Cl}, \mathrm{p}=0.018$ ). In spite of the limitations of the study, our data showed that the measurement of the rate of progression of MA may be a more sensitive MAV risk marker than the presence of MA "per se".

\section{4}

\section{GENETIC PREDISPOSITION TO VASCULAR COMPLICATIONS OF} DIABETES.

ML Izmajlowicz, C Groves*, V Horton*, R Owen*, I Stratton*, RC Turner*, FR Green. Nuffield Department of Surgery, John Radcliffe Hospital and *Diabetes Research Laboratories, Radcliffe Infirmary, Oxford, UK.

The aim of the study is to investigate whether there is an association between common genetic polymorphisms and the risk of myocardial infarction (MI) or retinopathy (by retinal photography, Wisconsin score) in non-insulin-dependent diabetes mellitus (NIDDM). Patients are selected from the United Kingdom Prospective Diabetes Study. Approximately 200 cases with each complication and 200 matched controls were genotyped for four polymorphisms. Cases and controls were matched prospectively for gender, age, duration of disease, fasting plasma glucose, blood pressure, with Ml patients matched additionally for smoking, and LDL and HDL cholesterol. Polymorphisms studied, all of which have been found to be associated either with MI itself or atherosclerosis, include the plasminogen activator inhibitor-1 (PAl-1) promoter $4 \mathrm{G} / 5 \mathrm{G}$, the tissue plasminogen activator (tPA) alu insertion/deletion, factor XIIA P/L564 and stromelysin (Sly) promoter 5A/6A. The PAI-1 and Sly polymorphisms were genotyped by PCR with fluorescently labelled primers and resolution of the allelic PCR products each differing by one basepair, on the ABI377. The factor XIIA P/L564 polymorphism was genotyped by PCR using a mutagenized primer and PstI digestion. The PPA alu ins/del polymorphism was genotyped by PCR using primers spanning the insertion followed by size separation. Paired data were analyzed by McNemar's test and retinopathy score using analysis of variance. Data analysis is currently underway for PAI-1, tPA and Sly. In 542 subjects, the factor XIIIA L564 allele was associated with retinopathy ( $\mathrm{p}=0.019$, RR1.6) with a co-dominant effect: median Wisconsin retinopathy scores were 10 10 for the PP564 homozygotes, 2010 for heterozygotes and $31<31$ for the LL564 homozygotes, with a significant test for trend. This polymorphism was not associated with MI. Thus, common genetic polymorphisms may predispose certain individuals to more severe vascular complications of NIDDM.

\section{5}

RISK-FACTORS FOR THE DEVELOPMENT OF MICROVASCULAR COMPLICATIONS IN YOUNG PATIENTS WITH DIABETES.

B.S.Olsen, J.Johannesen, A.K.Sjølie, B.Thorsteinsson, S.Pramming, K.BorchJohnsen, P.Hougaard, H.B.Mortensen and the Danish Study Group of Diabetes in Childhood. Department of Paediatrics, Glostrup Hospital, Copenhagen, Denmark.

The DCCT showed a close relationship between metabolic control and diabetes related complications in adolescents and adults with Type 1 diabetes. Whether the same relation exists in younger children is, however, less evident. A Danish nationwide cohort of children and adolescents with Type 1 diabetes was followed for 9 years on three occasions $(1987,1989,1995)$ with assessment of metabolic control and development of complications in kidneys, eyes and nerves.

The aim of the 1995 follow-up study was to determine risk-factors and prevalence of complications in young Danish patients with diabetes. Furthermore, the significance of the pre-and postpubertal diabetes duration was analyzed in relation to development of microvascular complications.

Clinical information, $\mathrm{HbA}_{\mathrm{tc}}, \mathrm{AER}$, arterial blood-pressure, fundus photos (central reading) and vibration perception threshold (VPT) was obtained from 353 patients ( $50 \%$ of the inception cohort), mean age: $20.7 \pm 3.3$ years and mean diabetes duration: $13.2 \pm 3.2$ years. $\mathrm{HbA}_{1 \mathrm{r}}$ (normal range $4.3-5.8$, mean $5.3 \%$ ) and AER (upper normal limit (95\%): $20 \mu \mathrm{g} / \mathrm{min}$ ) in at least two timed overnight urine collections were analyzed centrally. Average $\mathrm{HbA}_{\mathrm{ic}}$ was $9.7 \pm 1.7 \%($ mean $\pm \mathrm{SD}$ ) Elevated AER ( $>20 \mu \mathrm{g} / \mathrm{min}$ ) was diagnosed in $12.8 \%$ of the patients. Risk-factors for elevated AER (1995) were high AER $(1989)(\mathrm{p}<0.001)$ and high $\mathrm{HbA}_{\mathrm{lc}}(1989)$ $(\mathrm{p}<0.001)$. Retinopathy was present in $58 \%$ and risk-factors were long pre$(\mathrm{p}<0.01)$ and post-pubertal $(\mathrm{p}<0.001)$ diabetes duration and high $\mathrm{HbA}_{1 \mathrm{c}}$ level $(1989)(\mathrm{p}<0.0001)$. Elevated VPT $(>6.5 \mathrm{~V})$ was shown in $60 \%$ and related to male $\operatorname{sex}(p<0.05)$, older age $(p<0.0001)$ and elevated AER $(1989)(p<0.05)$. The present study confirmed the close association between long-term metabolic control and the development of microvascular complications in young diabetic patients. The prepubertal diabetes duration contributes to the development of diabetic retinopathy but to a lesser extent than the post-pubertal duration. There is a major need for the development of better management guidelines and quality assessment programmes for young people with diabetes. 
1366

RELATIONSHIP BETWEEN INCIDENCE OF MACROANGIOPATHY AND PROGRESSION TOWARD

ALBUMINURIA IN NON-INSULIN DEPENDENT DIABETES : A

PROSPECTIVE STUDY. L. Alviggi, G. Seghieri and R. Anichini. Dpt. of Internal Medicine, Hospital of Viareggio, and Diabetes Unit, Spedali Riuniti of Pistoia, Italy.

To evaluate whether presence of albuminuria as well as progression to clinical albuminuria could be related to appearance of macroangiopathy in non-insulin dependent diabetes mellitus (NIDDM), a cohort of 293 NIDDM patients was evaluated at the clinical onset of illness and yearly throughout a period of six years. Every year the incidence was monitored of both increased urinary albumin excretion rates (AER $>20 \mu \mathrm{g} / \mathrm{min}$, assessed as the mean value of six-monthly collected 24-hr urine samples) and of great vessel disease such as cerebrovascular disease (CVD), coronary heart disease (CHD) or peripheral vascular disease (PVD), all of which diagnosed by means of standardised clinical and instrumental criteria. Presence of albuminuria increased by about two/threefold the relative risk for incidence of macroangiopathy (2.38 for CVD, 2.05 for CHD and 2.94 for PVD). Patients were then stratified as Progressors ( $P, n=32)$ whether they had significantly higher AER values $>20 \mu \mathrm{g} / \mathrm{min}$ after 6 years as compared with the basal value, or as NonProgressors (NP, $n=261$ ) whether the six-year AER value was $<20 \mu \mathrm{g} / \mathrm{min}$ as well as non significantly different from the baseline value. Progressors had a poorer metabolic control as compared to the NP group $(7.4 \pm 1.3 \%$ vs $6.6 \pm 1.3 \%, p<0.05)$, also after adjusting for sex, age, body weight, duration, hypertension, plasma creatinine or insulin therapy by means of a multiple logistic analysis, being moreover $\mathrm{HbAlc}$ the unique predictor of progression $(p=0.001)$. Furthermore, in this model, single odd ratios were linearly related to $\mathrm{HbAlc}$ throughout its range. Incidence of macroangiopathy was independently related to progression to albuminuria also after adjusting for sex, age, duration, HbAlc, blood pressure, plasma creatinine, smoking, and insulin therapy by using a Cox regression model $(p=0.0003)$. In summary in NIDDM progression to albuminuria is a potent independent predictor of macroangiopathy, while metabolic control represents the unique significant predictor of progression toward clinical albuminuria. 